\title{
SUPPORTING DOCUMENT FOR THE HISTORICAL TANK CONTENT ESTIMATE FOR
}

\section{BY TANK FARM}

\section{WORK ORDER ER4945}

\author{
Prepared for
}

Westinghouse Hanford Company

$\checkmark$ June 1994

Prepared by

ICF Kaiser Hanford Company

Richland, Washington 


\section{DISCLAIMER}

Portions of this document may be illegible in electronic image products. Images are produced from the best available original document. 


\section{DISTRIBUTION SHEET}

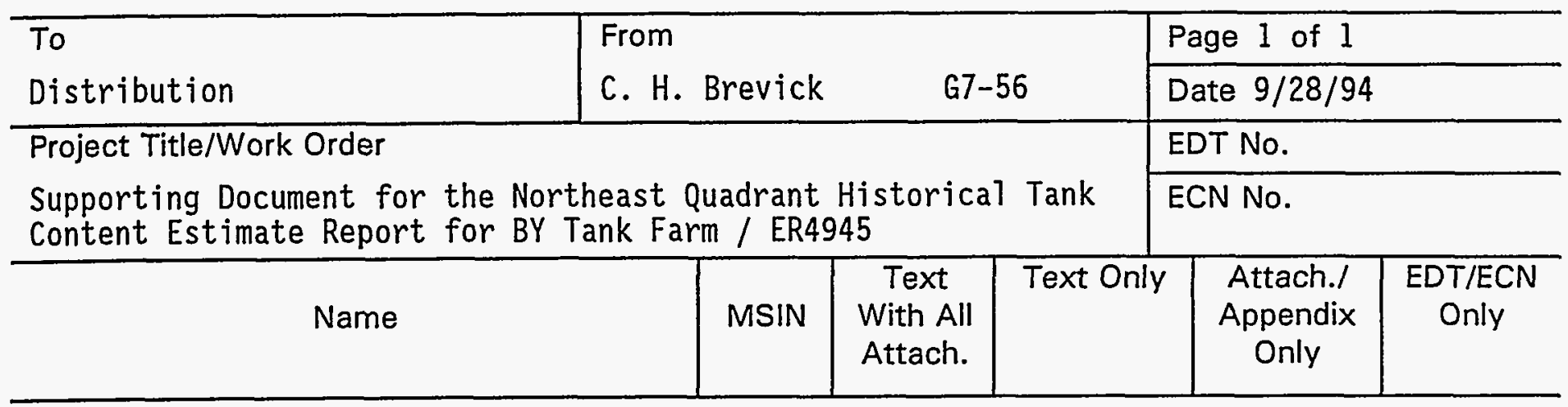

\section{Westinghouse Hanford Company}

T. M. Brown

G. Jansen

P. Sathyanarayana

B. C. Simpson -

TFIC (Tank Farm Information Center)

Central Files

OSTI

$\begin{array}{ll}R 2-12 & X \\ H 6-33 & X \\ R 2-12 & X(2) \\ R 2-12 & X \\ R 1-20 & X \\ L 8-04 & X(2) \\ L 8-07 & X(2)\end{array}$

ICF-Kaiser Hanford Company

C. H. Brevick

L. A. Gaddis

Y. W. Hoffmann

E. D. Johnson

R. L. Newe 11

W. W. Pickett

A. C. Walsh

ICF-KH Publications

ICF-KH Doc Control

$\begin{array}{ll}G 7-56 & X(2) \\ G 7-57 & X \\ S 2-54 & X \\ G 7-57 & X \\ E 6-46 & X \\ G 7-57 & X \\ G 7-57 & X \\ E 6-63 & 0 \\ G 3-11 & X\end{array}$

Department of Energy - Richland Operations

J. M. Clark

$57-54 \quad X$

Los A7 amos National Laboratory

S. F. Agnew

Los Alamos National Laboratory

$x(2)$

MS-J586 - CST-14

P.0. Box 1663

Los Alamos, NM 87545

\section{Los ATamos Technical Associates}

D. S. DeLorenzo

Los Alamos Technical Associates

750 Swift, Suite 4

Richland, WA 99352 


\begin{tabular}{l|l}
\hline $\begin{array}{l}\text { 2. To: (Receiving Organization) } \\
\text { TWRS }\end{array}$ & $\begin{array}{l}\text { 3. From: (Originating Organization) } \\
\text { ICF KH }\end{array}$ \\
\hline 5. Proj./Prog./Dept./Div.: & $\begin{array}{l}\text { 6. Cog. Engr.: } \\
\text { L.A. Gaddis }\end{array}$ \\
ER4945 & \\
8. Originator Remarks: & \\
Supporting Document produced under W.0. ER4945, provides \\
supporting data and in-depth characterization information \\
for NE Quadrant Historica] Tank Content Estimate Report for \\
BY-Tank Farm.
\end{tabular}

11. Receiver Remarks:

Tank Farms

$N / A$

13. Permit/Permit Application No.: $N / A$

14. Required Response Date:

$N / A$

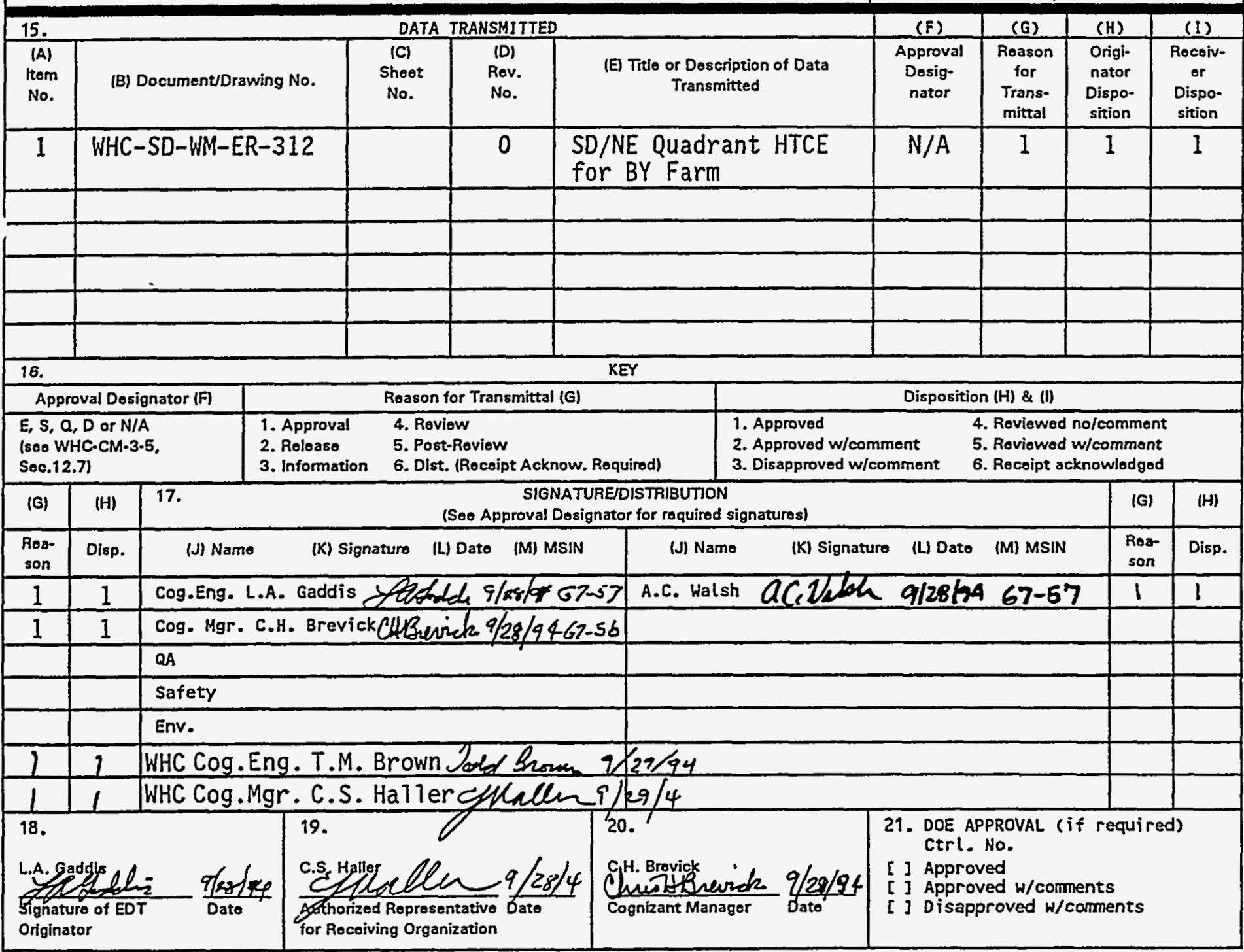


2. Title

Supporting Document for the North East Quadrant Historical Tank Content Estimate Report for BY-Tank Farm

\section{Key Hords}

BY-Tank Farm, Tank Waste Characterization, Historical Tank Content Estimate, Historical Waste Transfer, tank waste level data, tank physical information, drywe11, 1iquid observation well, waste temperature, waste sampling data, analytical data.

\section{APPROVED FOR}

3. Number

WHC-SD-WM-ER-312
4. Rev No.

0
6. Author

Name: C.H. Brevick,

L.A. Gaddis, A.C. Walsh

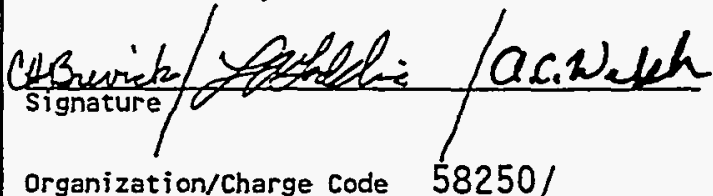

Organization/Charge code 58250/ ER4945/Tank Farm Technical Support

\section{Abstract}

This Supporting Document provides historical in-depth characterization information gathered on BY-Tank Farm, such as historical waste transfer and level data, tank physical information, temperature data, sampling data, and drywell and liquid observation we11 data for Historical Tank Content Estimate Report of the NE Quadrant and the Hanford 200 East Areas.

8. PONoOSE AND USE OF DOCUMENT - This document was prepared for use within the U.S. Department of Energy and its contractors It is to be used onty to perform, direct, or integrate work under U.S. Department of Energy contracts. This docyntht is not approved for public release unt reviewed.

PATENT STATUS - This document since it is transmitted in advance of patent clearance is made aroilable in confidence solely for use in performanee of work under contracts with the U.S. Department of entergy. This document is not towe published nor its contents ptlerwise disseminated or used for purpose-ether than specified above before patent approval for such release or tee has beep secured, upon request, from the Patent Counsel, U.S. Departmentof Energy field Office, Richland, WA.

DISCLAIMER - This report was prepared as an account of work sponsored by an agency of the United States Government. Neither the United States Government nor any agency thereof, nor any of their employees, nor any of their contractors, subcontractors or their employees, makes any warranty, express or implied, or assumes any legal liability or responsibility for the accuracy, completeness, or any third party's use or the results of such use of any information, apparatus, product, or process disclosed, or represents that its use would not infringe privately owned rights. Reference herein to any specific commercial product, process, or service by trade name, trademark, manufacturer, or otherwise, does not necessarily constitute or imply its endorsement, recommendation, or favoring by the United States Government or any agency thereof or its contractors or subcontractors. The views and opinions of authors expressed herein do not necessarily state or reflect those of the United States Government or any agency thereof.

9. Impact Level $N / A$
10. RELEASE STAMP

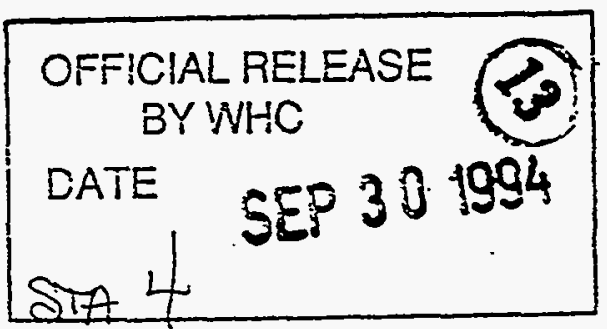




\section{RELEASE AUTHORIZATION}

\begin{tabular}{|ll|}
\hline Document Number: & WHC-SD-WM-ER-312, REV.0 \\
\hline Document Title: & $\begin{array}{l}\text { Supporting Document for the North East Quadrant } \\
\text { Historica1 Tank Content Estimate Report for BY-Tank } \\
\text { Farm }\end{array}$ \\
\hline Release Date: & September 28, 1994 \\
\hline
\end{tabular}

$* * * * * * * * * * * *$

This document was reviewed following the procedures described in WHC-CM-3-4 and is:

APPROVED FOR PUBLIC RELEASE

**************

WHC Information Release Administration Specialist:

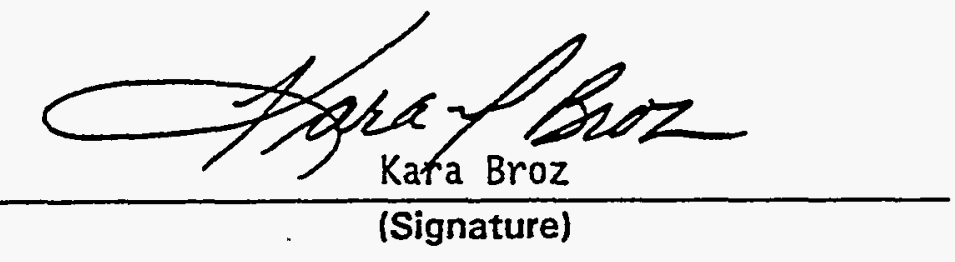

September 28, 1994

(Date) 


\title{
SUPPORTING DOCUMENT FOR THE HISTORICAL TANK CONTENT ESTIMATE FOR
}

\author{
BY TANK FARM
}

WORK ORDER ER4945

Prepared by

ICF Kaiser Hanford Company

Richland, Washington

for

Westinghouse Hanford Company

Trincipar Lead Engineer $\frac{9 / 28 / 94}{\text { Date }} \frac{\text { Toxy Wabh }}{\text { Technical Author }} \frac{9-28-94}{\text { Date }}$

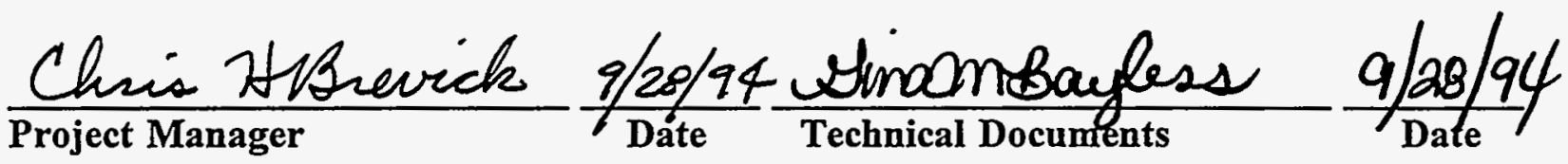

Westinghouse Hanford Company

$\frac{\text { CHlalle }}{\text { Characterization Support }} \frac{9 / 28 / 94}{\text { Date }}$ 
1.0 Introduction . . . . . . . . . . . . . . . . 1

1.1 Purpose . . . . . . . . . . . . . . . . . . . . 1

1.2 Scope . . . . . . . . . . . . . . . . . 1

1.3 Approach . . . . . . . . . . . . . . . . . . 1

1.3.1 Surveillance Techniques for the Hanford

1.3.2 Radioactive Mixed Waste Storage Tanks . . 3 Approach and Development of Supporting Information Contained in the Historical Tank Content Estimate and Supporting Documents . . . . . . . . . . . . 5

1.3.3 Approach and Development of the ProcessBased Historical Tank Content Estimate - 8

1.3.4 Assumptions . . . . . . . . . . . 10

1.4 Historical Information Quality . . . . . . . . . 13

1.5 Background NE Quadrant . . . . . . . . . . . . 16

1.5.1 Waste Generating Processes . . . . . . 17

1.5.2 Waste Management Operations . . . . . . 21

1.5.3 Miscellaneous Waste Sources and

1.6 1.5.4 Timeline . . . . . . . . . . . . . 24

Safety Issues . . . . . . . . . . . . . . . . 27

1.6.1 Watch List Safety Issues . . . . . . 27

1.6.2 Non-Watch List Safety Issues . . . . . 28

2.0 BY Tank Farm . . . . . . . . . . . . . . . . 31

2.0.1 BY Tank Farm History ......... 31

2.0.1.1 BY Tank Farm Waste History . . . . 32

2.0.1.2 BY Tank Farm Temperature History . . 32

2.0.1.3 BY Tank Farm Integrity . . . . . 33

2.0.2 BY Tank Farm Historical Sample Analysis

2.0.3 Data Current status of BY Tank Farm : : : : 34

2.0.3.1 BY Tank Farm In-Tank Photographs . . 34

2.1 Tank 241-BY-101 . . . . . . . . . . . . . . . 35

2.1.1 241-BY-101 Tank History . . . . . . . 35

2.1.1.1 Waste History Tank 241-BY-101: . 35

2.1.1.2 Temperature History 241-BY-101 . . 36

2.1.1.3 Integrity of Tank 241-BY-101... 36

2.1.1.4 Historical Sample Analysis Data for Tank 241-BY-101 . . . . . . . 37

2.1.2 Current Status of Tank 241-BY-101... . 37

$\begin{array}{ll}2.1 .2 .1 & \text { Waste Layer Volume Approximation } \\ & \text { for Tank 241-BY-1 . . } 37\end{array}$

2.1.2.2 In-Tank Photograph 241-BY-101: . 37

2.1 .3 Synopsis Tank 241-BY-101 ....... 38

2.2 Tank 241-BY-102 . . . . . . . . . . . 39

2.2.1 241-BY-102 Tank History ...... . 39

2.2.1.1 Waste History Tank 241-BY-102 . . 39

2.2.1.2 Temperature History 241-BY-102... 40

2.2.1.3 Integrity of Tank 241-BY-102.... 40

2.2.1.4 Historical Sample Analysis Data for 
Tank 241-BY-102 . . . . . . . . . . 41

2.2.2 Current Status of Tank 241-BY-102 . . . . 41

2.2.2.1 Waste Layer Volume Approximation

for Tank 241-BY-102 . . . . . . . . 41

2.2.2.2 In-Tank Photograph 241-BY-102 . . . 41

2.2.3 Synopsis Tank 241-BY-102 . . . . . . . 41

2.3 Tank 241-BY-103 . . . . . . . . . . . . . . . . . . 42

2.3.1 241-BY-103 Tank History . . . . . . . . 42

2.3.1.1 Waste History Tank 241-BY-103 . . . 42

2.3.1.2 Temperature History 24I-BY-103 . . . 43

2.3.1.3 Integrity of Tank 241-BY-103... . 43

2.3.1.4 Historical Sample Analysis Data for

Tank 241-BY-103 . . . . . . . . . . 44

2.3.2 Current Status of Tank 241-BY-103. . . . 44

$\begin{array}{ll}2.3 .2 .1 & \text { Waste Layer Volume Approximation } \\ & \text { for Tank 241-BY-103 . . . . . . . } 44\end{array}$

2.3.2.2 In-Tank Photograph 241-BY-103.. 44

2.4 Tank 241-BY-3opsis Tank 241-BY-103 • . . . . . . . 45

2.4.1 241-BY-104 Tank History . . . . . . . . . . . 46

2.4.1.1 Waste History Tank 241-BY-104 . . . 46

2.4.1.2 Temperature History 241-BY-104. . . 46

2.4.1.3 Integrity of Tank 241-BY-104. . . . 47

2.4.1.4 Historical Sample Analysis Data for

Tank 241-BY-104 .......... . 48

2.4.2 Current Status of Tank 241-BY-104. . . . 48

2.4.2.1 Waste Layer Volume Approximation

for Tank 241-BY-104 . . . . . . . . 48

2.2

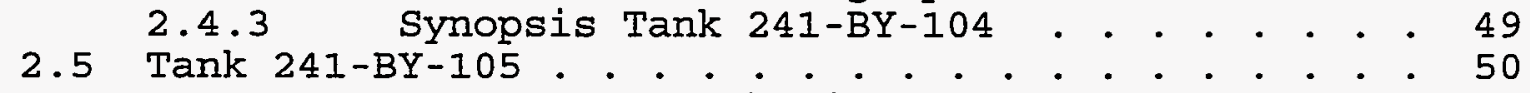

2.5.1 241-BY-105 Tank History . . . . . . . . 50

2.5.1.1 Waste History Tank 241-BY-105 . . . 50

2.5.1.2 Temperature History 241-BY-105 . . . 50

2.5.1.3 Integrity of Tank 241-BY-105 . . . . 51

2.5.1.4 Historical Sample Analysis Data for

Tank 241-BY-105 .......... 52

2.5.2 Current Status of Tank 241-BY-105. . . . 52

2.5.2.1 Waste Layer Volume Approximation

for Tank 241-BY-105 . . . . . . . 52

2.5.2.2 In-Tank Photograph 241-BY-105 . . . 52

2.5.3 Synopsis Tank 241-BY-105 . . . . . . . 53

2.6 Tank 241-BY-106. . . . . . . . . . . . . . . . . . 54

2.6.1 241-BY-106 Tank History . . . . . . . . . 54

2.6.1.1 Waste History Tank 241-BY-106 . . . 54

2.6.1.2 Temperature History 241-BY-106. . . 55

2.6.1.3 Integrity of Tank 241-BY-106. . . . 55

2.6.1.4 Historical Sample Analysis Data for

Tank 241-BY-106 . . . . . . . . 56

2.6.2 Current Status of Tank 241-BY-106... . 56

2.6.2.1 Waste Layer Volume Approximation

for Tank 241-BY-106 . . . . . . . 56

2.6.2.2 In-Tank Photograph 241-BY-106 . . 56

2.6.3 Synopsis Tank 241-BY-106 . . . . . . . . 56 
2.7 Tank 241-BY-107 . . . . . . . . . . . . . . . . . . 57

2.7.1 241-BY-107 Tank History . . . . . . . . . 57

2.7.1.1 Waste History Tank 241-BY-107 . . . 57

2.7.1.2 Temperature History 241-BY-107. . . 58

2.7.1.3 Integrity of Tank 241-BY-107 . . . 58

2.7.1.4 Historical Sample Analysis Data for

Tank 241-BY-107 . . . . . . . . . 59

2.7.2 Current Status of Tank 241-BY-107 . . . . 59

2.7.2.1 Waste Layer Volume Approximation

for Tank 241-BY-107 ....... 60

2.7.2.2 In-Tank Photograph 24I-BY-107 . . . 60

2.7 .3 Synopsis Tank 241-BY-107 . . . . . . . . 60

2.8 Tank 241-BY-108 . . . . . . . . . . . . . . . . . 61

2.8.1 241-BY-108 Tank History . . . . . . . . 61

2.8.1.1 Waste History Tank 241-BY-108 . . . 61

2.8.1.2 Temperature History 241-BY-108 . . . 61

2.8.1.3 Integrity of Tank 241-BY-108 . . . . 62

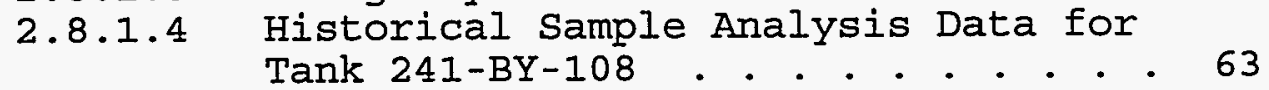

2.8.2 Current Status of Tank 241-BY-108. . . . 63

2.8.2.1 Waste Layer Volume Approximation

for Tank 241-BY-108 . . . . . . 63

2.8.2.2 In-Tank Photograph 241-BY-108 • • 63

2.8 .3 Synopsis Tank 241-BY-108 . . . . . . . . 63

2.9 Tank 241-BY-109. . . . . . . . . . . . . . . . . . 64

2.9.1 241-BY-109 Tank History . . . . . . . . 64

2.9.1.1 Waste History Tank 241-BY-109 . . . 64

2.9.1.2 Temperature History 241-BY-109. . . 65

2.9.1.3 Integrity of Tank 241-BY-109... . 65

2.9.1.4 Historical Sample Analysis Data for

Tank 241-BY-109 .......... . 66

2.9.2 Current Status of Tank 241-BY-109. . . . 66

2.9.2.1 Waste Layer Volume Approximation

for Tank 241-BY-109 ....... . 67

2.9.2.2 In-Tank Photograph 241-BY-109 . . . 67

2.9 .3 Synopsis Tank 241-BY-109 . . . . . . . . 67

2.10 Tank $241-\mathrm{BY}-110$. . . . . . . . . . . . . . . . . . 68

2.10.1 241-BY-110 Tank History . . . . . . . . 68

2.10.1.1 Waste History Tank 241-BY-110 . . . 68

2.10.1.2 Temperature History 241-BY-110 . . . 69

2.10.1.3 Integrity of Tank 241-BY-110 . . . 69

2.10.1.4 Historical Sample Analysis Data for

Tank 241-BY-110 . . . . . . . . 70

2.10.2 Current Status of Tank 241-BY-110 . . . 70

2.10.2.1 Waste Layer Volume Approximation
for Tank 241-BY-110 . . . . . . . . 70

2.10.2.2 In-Tank Photograph 241-BY-110 . . . 70

2.10 .3 Synopsis Tank 241-BY-110 . . . . . . . . 71

2.11 Tank 241-BY-111 . . . . . . . . . . . . . . . . 72

2.11.1 241-BY-111 Tank History . . . . . . . . . 72

2.11.1.1 Waste History Tank 241-BY-111 . . . 72

2.11.1.2 Temperature History 241-BY-III • . . 72

2.11.1.3 Integrity of Tank 241-BY-11I. . . . 73

2.11.1.4 Historical Sample Analysis Data for 
WHC-SD-WM-ER-312, Rev. 0

Tank 241-BY-111 . . . . . . . . . . 73

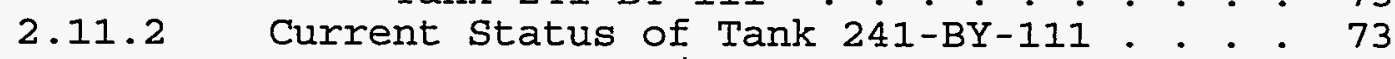

2.11.2.1 Waste Layer Volume Approximation

for Tank 241-BY-111 . . . . . . 74

2.11.2.2 In-Tank Photograph 241-BY-111 . . . 74

2.11 .3 Synopsis Tank 241-BY-111. . . . . . . . 74

2.12.1 241-BY-112 Tank History . . . . . . . . . 75

2.12.1.1 Waste History Tank 241-BY-112 . . . 75

2.12.1.2 Temperature History 241-BY-112 . . . 76

2.12.1.3 Integrity of Tank 241-BY-112 . . . 76

2.12.1.4 Historical Sample Analysis Data for

Tank 241-BY-112 . . . . . . . . . . 76

2.12.2 Current Status of Tank 241-BY-112. . . . 77

2.12.2.1 Waste Layer Volume Approximation

for The Tank 241-BY-112 . . . . . . 77

2.12.2.2 In-Tank Photograph 241-BY-112 . . . 77

2.12 .3 Synopsis Tank 241-BY-112 . . . . . . . . 77 


\section{APPENDICES}

Appendix A. Appendix $\mathrm{B}$. Appendix $\mathrm{C}$. Appendix D. Appendix E. Appendix F. Appendix $G$. Appendix $\mathrm{H}$. Appendix I. Appendix $J$. Appendix $K$.

Glossary

References

Tank Level Histories and Data

Temperature Graphs and Data

Surface Ievel Graphs and Data

Drywell Graphs and Data

Riser Configuration and Tank Cross Section

Sampling Data

Tank Photographs

Unknown Tank Transfers

Tank Layering Comparison and Data 


\section{INFORMATION FEEDBACK CARD}

\section{SUPPORTING DOCUMENT FOR THE HISTORICAL TANK CONTENT ESTIMATE FOR BY TANK FARM \\ ER4945}

\section{COMMENTS AND CONTRIBUTIONS}

The reader is requested to utilize this card to comment on this working document, report any discrepancies, or contribute new information to improve the accuracy and content of the report. please use the space provided below, add additional pages if necessary, and return to the addressee on the reverse side. 
WHC-SD-WM-ER-312, Rev . 0

Mr. Carl s. Haller

Manager, Characterization Support

Westinghouse Hanford Company

P.O. Box 1970

Richland, WA 99352 
WHC-SD-WM-ER-312， Rev. 0

\subsection{Introduction}

\subsection{Purpose}

This document provides historical evaluations of the radioactive mixed wastes stored in the Hanford Site 200-East Area underground single-shell tanks (SSTs). A Historical Tank Content Estimate has been developed by reviewing the process histories, waste transfer data, and available physical and chemical characterization data from various Department of Energy (DOE) and Department of Defense (DOD) contractors.

The historical data will supplement information gathered from in-tank core sampling activities that are currently underway. A tank history review that is accompanied by current characterization data creates a complete and reliable inventory estimate. Additionally, historical review of the tanks may reveal anomalies or unusual contents that are critical to characterization and post characterization activities. Complete and accurate tank waste characterizations are critical first steps for DOE and westinghouse Hanford Company safety programs, waste pretreatment, and waste retrieval activities.

\subsection{Scope}

The scope of this document is Iimited to the SSTs in the BY Tank Farm of the northeast quadrant of the 200 East Area. For summarized characterization data on all SST's in the northeast quadrant, refer to the Historical Tank Content Estimate Report (Brevick 1994). The northeast and southeast quadrants represent an approximate geographical division of the 200-East Area tank farms. The division was made as follows:

NE Quadrant: A, AX, B, BX, BY, C

$S E$ Quadrant: AN, AZ, AP, AW, AY, SY

The following map depicts the 200-East Area.

\subsection{Approach}

This report is a compilation of work performed by ICF Kaiser Hanford Company, Los Alamos National Laboratories, and Westinghouse Hanford Company.

Westinghouse Hanford Company requested that ICF Kaiser Hanford Company review the history of the tanks in each of the four quadrants and incorporate the tank layering models and inventory estimates being developed by Los Alamos National Laboratories into the report. Historical waste transfer and level data, tank physical information, temperature data, sampling data, and drywell and liquid observation well data have been compiled for this report. 

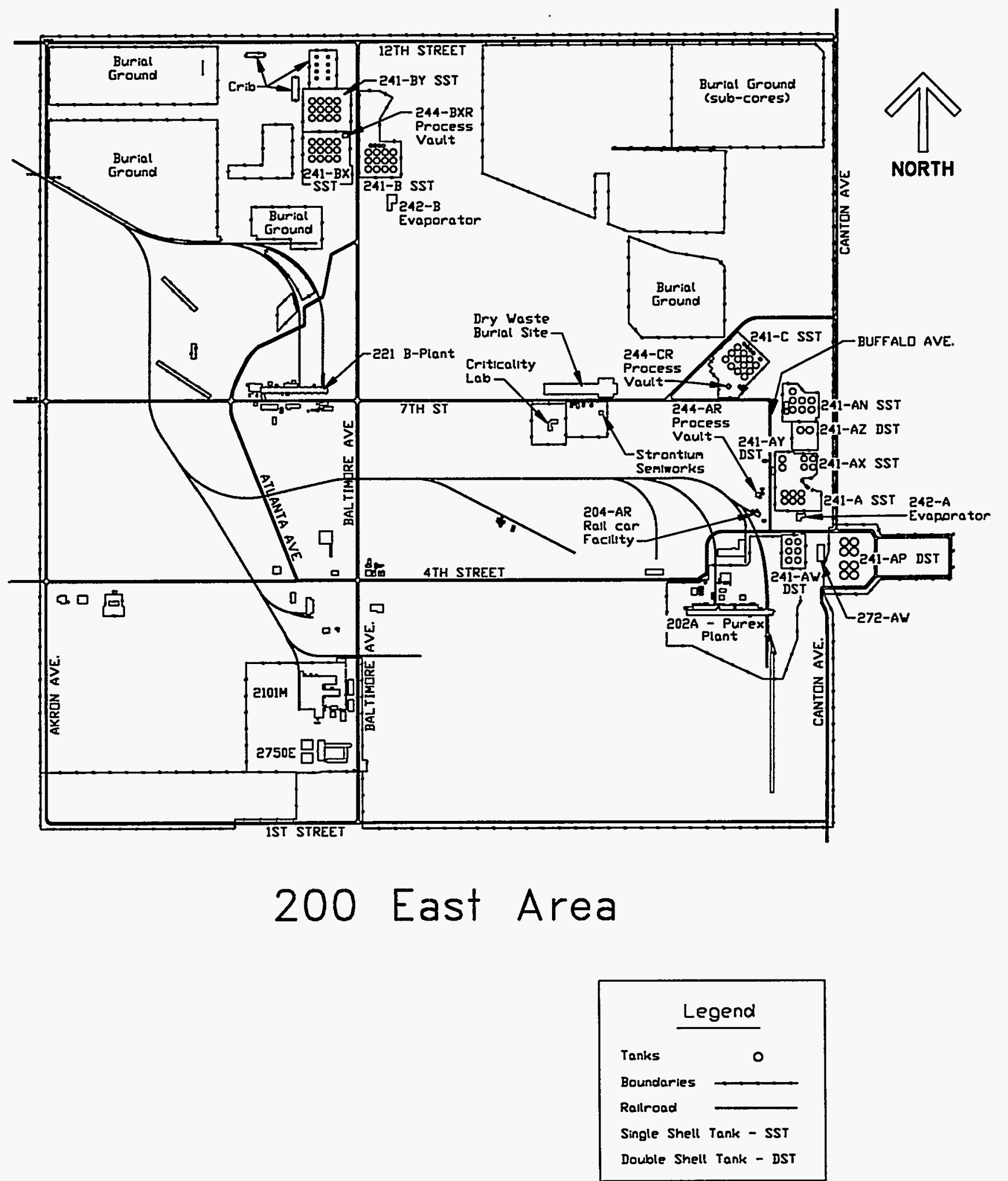
1.3.1 Surveillance Techniques for the Hanford Radioactive Mixed Waste Storage Tanks

Surveillance information is used for tank characterization. Tank integrity (i.e., sound, assumed leaker, assumed re-leaker) is investigated by comparing the changing liquid levels to liquid observation well data and drywell activity. Waste temperatures can be used to correlate the inventories of heat-generating radionuclides $\left({ }^{137} \mathrm{Cs}\right.$ and $\left.{ }^{91} \mathrm{Sr}\right)$ in the tanks. The instrumentation and methods used for tank surveillance are described in the following sections.

\section{In-Tank Surface Levels}

Surface levels within the SSTs are monitored by instruments consisting of calibrated steel tapes with an electrode plummet on the end that will complete a circuit when it contacts a conducting surface (i.e., liquid). The surface level measurements are usually taken with Food Instrument Corporation gauges and manual tapes. The tank monitoring device, manufactured by the Food Instrument Corporation, automatically moves up and down and contacts the waste. The gauge is often set to the intrusion mode by placing the plummet a short distance above the waste. If an intrusion occurs in the tank, the Food Instrument Corporation gauge will record the event when the surface level rises to meet the plummet. The manual tapes are similar to the Food Instrument Corporation gauge, except that manual operation of the tape and manual recording of the readings are required. Some tanks contain both types of level monitoring devices. The measurements are recorded on a computer automated surveillance system (CASS).

\section{- Interstitial Iiquid Level (Iiquid Observation Wells)}

Food Insturment Corporation gauges and manual tapes are limited to surface level measurement; therefore, liquid observation wells in many tanks have been installed specifically to monitor the interstitial liquid level within the SSTs. The interstitial liquid is the liquid trapped within and underneath the solid waste. Fiberglass or TEFZEI-reinforced epoxy-polyester resin liquid observation well tubes (by E.I. du Pont de Numours and Company) are sealed on the bottom and vertically installed approximately 1 in. from the tank bottom. Gamma, neutron, and acoustic probes may be lowered into the liquid observation wells to determine the interstitial liquid levels within the tank. A gamma probe detects the difference between saltcake and saltcake containing liquid, based on the principle that the liquid is the primary source of gamma emissions. A neutron probe distinguishes between the existing dry saltcake, wet saltcake, and liquid phases. Distinct differences exist in the neutron count rates of each material type. An acoustic probe emits a pulse that is reflected off the interface between the outer wall of the liquid observation well and the waste. A fiberglass/liquid interface produces a minimal reflected pulse and a fiberglass/air interface produces a maximum pulse reflection. Wet saltcake will fall between the limits appropriately; therefore, interpretation of the records will determine the liquid level. Each probe scan is fully automatic and 
WHC-SD-WM-ER-312, Rev. 0

successful scans are input to the computer automated surveillance system.

Internal Tank Temperatures (Thermocouples)

Temperatures usually are recorded automatically in the Computer Automated Surveillance System (CASS). Temperatures are monitored with thermocouple trees that are comprised of stainless steel pipe with 4 to 18 thermocouples. Usually, the lowest sensor is located approximately $4 \mathrm{in.} \mathrm{from} \mathrm{the} \mathrm{tank} \mathrm{bottom} \mathrm{and} \mathrm{the} \mathrm{other}$ sensors progress up the tree at 2-ft intervals. More frequent temperature readings are available for a few select tanks due to an upgrade in the surveillance data acquisition system to the tank monitor and control system (TMACS) in 1991.

Generally, a single thermocouple sensor is sent down the Iiquid observation well to take semiannual readings in tanks that do not contain a thermocouple tree. The temperatures are recorded manually in degrees Fahrenheit and are entered into the surveillance analysis computer system (SACS) that is currently using the ORACLE database.

\section{External Leak Detection (Drywells)}

Drywells are located around the circumference of each SST and throughout the tank farms to determine if tank wastes have leaked. The drywells are generally 6-in. diameter carbon steel pipe, between 75 - and 250-ft deep. The wells are termed dry because they are not deep enough to reach ground water. Scan profiles of radiation or moisture in the soil as a function of well depth are obtained with gamma radiation or neutron sensors. Scintillation monitors, Geiger-Mueller instruments, and neutron sensors are used to monitor the drywells. Scintillation monitors are radiation detection instruments based on the principle that Iight pulses are produced in some materials when they are exposed to radiation. Geiger-Mueller instruments detect low-level beta and gamma radiation. Neutron probes are equipped with a neutron source and detector that determine if moisture is present in the soil. The Erequency of and probe used for drywell monitoring are specified in a schedule from the "Waste storage Tank Status and Leak Detection Criteria" (Welty 1989).

Drywell readings are recorded at 1 -ft intervals. The peak readings and the depth of the readings are recorded on a summary sheet. If a drywell indicates a new or unusual increase in activity, a rerun is requested and the drywell is retested. The wells are sealed when they are not being tested.

The drywells described in this report have a 6-digit computer identification number: the first two digits denote the tank farm (i.e., $\mathrm{A}=10, \mathrm{AX}=11, \mathrm{~B}=20, \mathrm{BX}=21, \mathrm{BY}=22$, and $\mathrm{C}=30$ ), the third and fourth are the last two digits of the tank number (i.e., 241-A-105 would be 10-05 and 00 indicates a non-specific tank), and the fifth and sixth describe the clockwise location of the well from due north. For example, the identification number of the first drywell for Tank 241-BY-102 would be 22-02-01. 
Laterals (i.e., horizontal drywells) are positioned under some SSTs to detect radionuclides in the soil which could be indicative of tank leakage. The drywells are monitored by radiation detection probes and occasionally by a temperature probe. Laterals have 4in. Inside diameter steel pipes located 8 to 10 ft below the concrete base of the tank, and each tank has three laterals. Laterals are located only in the A Tank Farm and a portion of the SX Tank Farm.

\section{In-Tank Photography}

In-tank photography is another waste volume determination method used to resolve in-tank measurement anomalies and determine tank integrity. Photographs can help determine sludge and liquid levels, provide a degree of corroboration with other waste management processes, and are a source of information on foreign objects contained in the tanks. The photos can be compared to see if tank wastes with similar histories have similar characteristics.

1.3.2 Approach and Development of Supporting Information Contained in the Historical Tank Content Estimate and Supporting Documents

Extensive information was gathered for each tank. This document contains a comprehensive data set on each tank, that are summarized in the Historical Tank Characterization Estimates.

\section{Tank Level Histories}

The tank level histories are graphical representations of waste levels over the lifetime of a tank. They include information such as $\mathrm{pH}$, in-tank photo dates, waste types, transfer data, and physical data. Information from the mid 1940 s to 1980 was compiled from two Westinghouse Hanford Company documents (Anderson 1990 and Welty 1988). Data from 1977 to the present were obtained from the monthly waste status summary reports. The following Hanford site operating contractors prepared summaries: Atlantic Richfield Hanford Company from 1973 to 1977, Rockwell Hanford Operations from 1977 to 1987, and Westinghouse Hanford Company from 1987 to present. Script files were imported from a spreadsheet to AutoCAD 12 to graph the surface level data. Iiquid observation well data were obtained from the Westinghouse Hanford Company Tank Farm Surveillance group in a spreadsheet format. Additional information was gathered from various documents and comments were added directly to each drawing.

The liquid, solid, and interstitial liquid levels (liquid observation wells data) are shown on the tank level history graphs as volumes and tank elevations. The following formulas were used to relate the volumes to the respective elevation for each tank farm design: 
FORMULAS

$\frac{\text { Total Gallons }}{2750 \frac{\text { Gallons }}{\text { Inch }}}=$ Total Inches

$\frac{\text { Total Gallons }-12,500 \text { Gallons }}{2750 \frac{\text { Gallons }}{\text { Inch }}}+12$ Inches $=$ Total Inches

$\frac{\text { Total Gallons }-12,500 \text { Gallons }}{2750 \frac{\text { Gallons }}{\text { Inch }}}=$ Total Inches

$\frac{\text { Total Gallons }-18,500 \text { Gallons }}{2750 \frac{\text { Gallons }}{\text { Inch }}}+12$ Inches $=$ Total Inches

$\frac{\text { Total Gallons }-590 \text { Gallons }}{196 \frac{\text { Gallons }}{\text { Inch }}}+6$ Inches $=$ Total Inches

\begin{tabular}{|c|l|c|}
\hline Equation No. & Applicable Tank Farms: & Tank Diameter \\
\hline 1 & A, AN, AP, AW, AX, AY, AZ, SY & $75 \mathrm{ft}$ \\
\hline 2 & BY, S, TX, TY & $75 \mathrm{ft}$ \\
\hline 3 & B, BX, C, T, U & $75 \mathrm{ft}$ \\
\hline 4 & SX & $75 \mathrm{ft}$ \\
\hline 5 & B, C, T, U (200 Series) & $20 \mathrm{ft}$ \\
\hline
\end{tabular}


WHC-SD-WM-ER-312, Rev. 0

\section{Temperature Plots}

There are two categories of temperature data, one or few temperature readings per day and thermocouple temperature data. The documentation used for the first category includes Waste status Summary Reports (Roberts, R.E. 2/58 - 10/58, Thress, M.A. 11/58 2/59, Lentz, J.E. 3/59-12/60, and Roberts, R.E. 6/61 - 6/62), RHO$\mathrm{CD}-1172$, and HW-83906. The thermocouple temperature data were obtained from the Westinghouse Hanford Company ORACLE database commonly known as the Surveillance Analysis Computer System (SACS) and various retrieved hand-entered temperature data sheets. Temperature versus time plots were developed for both categories of temperature data. Historical temperature plots cover the time span between January 1957 and January 1973. The common temperature span used for these plots is 350 degrees. There are no tanks that have temperature data outside the sliding 350 degree temperature range.

No thermocouple information has been located prior to 1973 . The thermocouple temperature information was parsed in an EXCEL spreadsheet and graphed to show each individual thermocouple probe. January of 1973 is then the starting date for all thermocouple plots. The temperature range for all tank thermocouple plots in all tank farms was kept to a 120 degree $F$ span, except where the data exceeded this range. In the few tanks where the temperature span exceeds this sliding range the data are shown using the smallest temperature range possible. A consistent time frame and temperature range is essential in comparing temperature information from one tank to another. If documentation was available, thermocouple elevations were included in the plots for tanks currently containing thermocouple trees. Thermocouples are ordered from the bottom to the top of the tank. Therefore, a plot of thermocouple 1 over time would indicate the temperature near the bottom of the tank. In-tank temperature information is available in Appendix D.

\section{Surface Level Plots}

The surface level data from 1991 to 1993 were obtained from the Westinghouse Hanford Company ORACLE database. The information was parsed in an EXCEL spreadsheet and graphed to show the waste level versus time. Current in-tank surface level information is available in Appendix $\mathrm{E}$.

\section{Drywell Plots}

The drywell information was obtained from the manual records of the Westinghouse Hanford Company Tank Farm Surveillance Group. Data from 1990 to the present were input into an EXCEL spreadsheet and graphed to show the peaks in counts/second $(c / s)$ and the peak depths in feet (ft) versus the date on the same chart. Tank drywell information is available in Appendix F.

Tank Profiles and Other Drawings

The drawings included in this report have been compiled from several sources including as-built drawings, the Waste storage Tank Status and Leak Detection Criteria (Welty 1988), and the Riser Configuration Document for Single-Shell waste Tanks (Alstad 1991). 
The risers and penetrations throughout the tank dome are used for many purposes. Some risers were installed during the original tank construction and others were added later.

Through the years, sleeves or smaller risers were installed in many of the larger risers. For the riser configurations in this report, a tank may have a riser number followed by a letter which means that a larger riser was fitted with smallex sleeves. Depending on the intended use, the riser sleeves can be as large as 12 in. or as small as 4 in. For example, a saltwell pump and screen require a 12-in. riser but a thermocouple tree requires only a 4-in. riser. The tank profile drawings are in Appendix $\mathrm{K}$.

\section{Photographs}

The tank farm photos were labeled to indicate the relative position of each tank in the farm and arrows were added to show the cascade series of each tank farm.

The latest in-tank and tank farm photos were obtained from Pacific Northwest Laboratory. In-tank photos have been arranged to show the contents of each tank. The auxiliary equipment within each photo collage was identified if possible and labeled appropriately. The latest in-tank and tank farm photos are available in Appendix I.

\section{Historical Sample Analysis Data}

The sampling data were obtained from the WHC Tank Waste and Retrieval Group. Tables were made for the samples that were taken prior to september 1989. The information was compiled in EXCEL to present the data in as similar a manner as possible. The tables include physical data, chemical analysis data by component, and radiological analysis data by component. Any other data included with the sample references are summarized as text. Tank historical sample analysis data are available in Appendix $\mathrm{H}$.

1.3.3 Approach and Development of the Process-Based Historical Tank Content Estimate

The process-based historical tank content estimates presented in this report were taken from work being performed by Los Alamos National Laboratories. The tank content estimates are the primary source of tank characterization data until the tank characterization reports are completed. The tank characterization reports will incorporate current core sampling and analysis results with historical-based tank content estimates to present the most current evaluation of the tank waste constituents.

Generating historical tank content estimates involves management and evaluation of large quantities of data. Several smaller components of the data management effort make up the system of generating historical tank estimates. The components are described in the following sections. 


\section{Waste Status and Transaction Record Summary Database}

The waste status and transaction record summary database (i.e., transaction summary) is a database of all known waste transfer records. Historically, when waste was pumped from a process plant to tank or tank to tank, a record of the pumped waste volume and type was kept. The transaction summary is the recorded waste level of the solids and supernatant.

The waste status and transaction record summary database is being partitioned into four separate sections (i.e., quadrants). The waste status and transaction record summary database for the northeast quadrant is available in WHC-SD-WM-TI-615 Rev. 0 (Agnew 1994).

\section{Tank Layering Model}

The tank layering model is derived from the waste status and transaction record summary database. The purpose of the tank layering model is to predict the waste types and volumes in each tank. The transaction summary database records the types of waste that enter each tank and the corresponding increase in the solids volume from each waste entry. Each waste type that increases the solids volume in the tank is recorded in the tank layering model. Therefore, the completeness and accuracy of the information in the tank layering model is directly related to the completeness and accuracy of the transaction summary database.

The tank layering model was created to develop historical tank content estimates. The results of the tank layering model are reported in this document. The tank layering model is presented graphically with the inventory estimate described below in Appendix $\mathrm{K}$.

\section{Defined Waste Types}

Early in the development of the historical tank content estimates, a need to compile the chemical compositions of all known waste types into one source document was recognized. This compilation was performed by Los Alamos National Iaboratories. The Hanford defined waste document was developed independently of the waste status and transaction record summaries database and the tank layering model. However, the Hanford defined waste list will be used with the tank layering model to obtain the actual tank waste inventory estimates as described below.

The Hanford defined waste document gives the compositions for all known Hanford waste types that went into the double-shell and single-shell tanks. For more details of the methods used to develop these waste types, refer to the Hanford Defined Wastes Document (Agnew 1994).

\section{Inventory Estimates}

The end result of combining the tank layer model and the Hanford defined waste types is the tank inventory estimate. When the volumes and compositions of each waste type in a particular tank are known from the tank layering model and the Hanford defined 
wastes document, a calculation of the total chemical inventory in the tank is performed. The individual tank results are presented in tabular form in Appendix $\mathrm{K}$.

The inventory estimates developed by Los Alamos National Laboratory are quality checked against existing data from current sample analyses, thermal and physical models, and historical records. The checking constitutes a large portion of the time required to develop the estimates. When estimates are checked against existing data, the models used may be reevaluated. Thus, the process of generating estimates will iterate and the associated errors will be reduced over time as the historical knowledge of the tank waste operations increases.

\section{I.3.4 Assumptions}

The quality of the historical information available for the tanks often requires that assumptions and judgement calls be made on the applicability and validity of the data. Some assumptions were used to interpret the data.

\section{Temperature Data}

The lines joining the data points on the temperature plots were detached to show gaps in data of more than three years.

The single thermocouple tree in some tanks was removed and replaced with two trees. The original single thermocouple tree and tree. 1 data were plotted on the same graph, and the tree 2 data were graphed separately.

No conclusion has been made on the status of thermocouples with less than 5 data points. In general, when there is thermocouple data with less than 5 data points, the data are retained on the temperature data sheets, but have not been plotted. Some possible explanations for the limited data are that the thermocouple failed, it was inoperable when readings were taken, or the operator misrepresented the number of thermocouples and the temperature data in the tank.

The thermocouples used in the trees were installed as purchased. There were no field calibrations before 1991. In 1991, a survey of Ferrocyanide Watch List thermocouple trees was conducted. The thermocouple tree probe temperatures were tested against probes inserted in liquid observation wells or by other methods as specified in Engineering Evaluation of Thermocouples in FeCN Watchlist Tanks, (Bussell 1992). Thermocouples, with equipment that could be repaired outside the tank, were made operable at this time.

It is assumed that the sinusoidal variances in the data can be attributed to seasonal and diurnal trends which are clear after 1991 due to an upgrade in thermocouple surveillance to a computerized data acquisition system, i.e., Tank Monitor and 
Control system (TMACS). Also, in general, the temperatures decrease from the bottom to the top of the tanks.

All the temperature data received by ICF Kaiser Hanford Company was reviewed for suspect data points. Data that obviously deviate from the temperature trend for a any tank are marked as suspect and designated in the comment column as either "suspect data (low)" or "suspect data (high)". Typically, suspect data sharply deviates from the data trend 15 degrees $F$ or more (See historical and thermocouple temperature data in Appendix D Temperature Graphs and Data).

After review of the data, it was determined that some data was recorded in degrees Celsius rather than in degrees Fahrenheit as it was electronically received. The data are converted to Fahrenheit and evaluated against the rest of the data set. Where the converted data are found to be consistent with the temperature trend, they are designated in the comment column as "raw data assumed degree C". Other delineations used to flag suspect data or substantiate the degree Celsius conversions are found from the Tank Waste Information Network system (TWINS) database, property of Battelle - Pacific Northwest Laboratory. Data recorded in degree $C$ or input incorrectly and confirmed with TWINS are delineated in the comments column as: "degree C/TWINS confirm" and. "wrong input/TWINS confirm", respectively (See historical and thermocouple temperature data in Appendix D - Temperature Graphs and Data).

In addition, some of the lines of data were flagged in the comment column by Westinghouse Hanford Company (WHC) before transmission of the data. Some of the WHC comment flags include "cass" or "SET\#2 FROM CASS". It is assumed that "cass", an acronym for Computer Automated Surveillance System, applies to all the data taken on that particular day, and that "SET\#2 FROM CASS" applies to the contiguous data designated under the TREE SET \# column as 2 (See historical and thermocouple temperature data in Appendix D Temperature Graphs and Data).

Sources of temperature data are located in the footnotes of both the temperature plots and data. Some Historical Temperature plots use data from. Waste status summary Reports (Roberts, R.E. 2/58 - 10/58, Thress, M.A. 11/58 - 2/59, Lentz, J.E. 3/59-12/60, and Roberts, R.E. $6 / 61$ - 6/62). If these repórts are used, footnotes on the temperature plots indicate by including the source of data concisely as from "Waste status summary Reports" (See historical temperature plots in Appendix D - Temperature Graphs and Data).

\section{Drywell Data}

The drywell data did not appear to have had a quality review; therefore, the following assumptions were made about the data:

-A data point is considered suspect if an apparent rerun has been performed. 


$$
\text { WHC-SD-WM-ER-312, Rev. } 0
$$

- A data point is considered to be a rerun if the preceding data are erratic and the rerun is performed on the same day if monitored daily, within one or two days if monitored weekly, or within several days if monitored monthly or yearly.

-If suspect data were indeed relevant, the appropriate surveillance organizations would have followed the appropriate procedures to identify any problems.

\section{Sampling Data}

The data obtained from the historical analytical information are understood to be widely variable in type, quality, and scope. The data range from process control data obtained from a grab sample on a few selected analytes to a total characterization suite performed on a core sample. There are no specific quantitative data quality criteria for the information incorporated into the historical tank content estimate. A qualitative assessment of the information sources is done during construction of the historical tank content estimate. The available data are assessed for suitability and consistency in the study and each data source is weighted appropriately for its contribution to the overall estimates.

When the sampling data were compiled, a sample was considered a solids sample if it was greater than $30 \%$ solids. Sludge/slurry samples were included.

\section{Inventory Estimates}

Solid waste formations vary by type and are determined individually by examination of the historical fill records. Further refinement and bounding of the solids formation are provided by conformation to physically attainable systems (e.g., waste volumes contained in the tanks do not exceed the tank capacity and negative void fractions are not possible).

Waste stream compositions were based on process flow sheet information and previous analytical data.

Solids were evenly distributed throughout tank. The solids were assumed to be evenly distributed throughout the slurry during their transfer and their settling rate was assumed to be uniform throughout the tank.

Complete settling of the sludge occurred and the solids settled in a pseudo-pancake formation (i.e., flat layers conforming to the boundaries of the tank) which was a simplifying assumption. Localized mounding and pocketing in various areas of the tank may have occurred due to inlet/outlet phenomena (e.g., cascades and transfers from the floating suction pumps that remove or deposit sludge). This phenomenon would have affected only a small fraction of the tank area and does not impact the overall results of this study. However, spatial variability within physical configuration and process history is acknowledged. No method is currently available that would adequately model this discrepancy. An effort 
is underway to develop this concept and incorporate it into the tank characterization process, but it is incomplete at this time.

Solids from a specifically designated waste type are homogeneous, which is a simplifying assumption. Variation and layering of the sludge due to component concentration differences between batches, precipitate particle size, and particle density were quite likely. The effect of these factors on the distribution of analytes within the solids is unknown; however, the effect was probably small and not detectable with the present sample collection and analysis process.

Solids compaction did not occur and was not accounted for in the study which is a simplifying assumption. Although compaction probably occurred in the history of the tanks because of the formation of additional solids layers on top of each other, the degree of compression was not known and, therefore, was not incorporated.

Density and other physical characteristics of the solids were determined by individual waste type using available information or were based on approximate physical analogues.

The capacity of the tanks was never exceeded during transfers. Tank capacity is dependent on fill status, fill order, tank location in a cascade, and tank type.

Leaking of the tanks was not incorporated during the time of the study but was accounted for at the end.

Cascades, tank transfers, and crib line effluents were normally free of particulate; therefore, the majority of solids were confined to their initial receiver tanks and were not cribbed or transferred extensively from tank to tank. This assumption based on cases of plugged cascade lines when appreciable amounts of solids tried to cascade, and the pumping equipment typically was not designed to transfer of substantial quantities of solids (except when sluicing or specifically transferring solids). Monitors on the crib discharge lines should have indicated significant solids contamination, and the tank farm operating procedures would have prevented solids discharges to the ground.

\subsection{Historical Information Quality}

The quality of historical data compiled for this report varied greatly. Some data came from published documentation. Other data were retrieved electronically from various Westinghouse Hanford Company databases. The quality of data used in the estimates is described in the following section.

\section{Level Histories}

The level history narrative is designed to give a general description of the waste types contained in a specific tank from startup to the present. The history presents physical 
characteristics of the waste such as waste $\mathrm{pH}$ values and the latest maximum temperature of the waste. It also presents information on when the tank was removed from service, if it was stabilized, the method of stabilization, if it ever leaked, and the current stabilization status. It is not designed to be a detailed summary of every waste type and transaction for that tank. The waste types are general and usually are given only for large transactions (i.e., if a waste is not of sufficient volume to significantly impact the total volume of the tank then it was not reported in the narrative). - For a more complete record of transactions and types of wastes involved, see the waste status and transaction record summary (Agnew 1994).

The accuracy of the tank level histories relies on the completeness of the Westinghouse Hanford Company document "A History of the 200 Area Tank Farms" (Anderson 1990); the monthly Westinghouse Hanford Company, Atlantic Richfield Hanford Company, and Rockwell Hanford Operations waste summary reports; and the waste status and transaction record summary (Agnew 1994).

Surface level readings may be affected by several factors: plummet error, flushing water accumulation, and waste surface irregularities. Crystalline wastes (i.e., saltcake) can build up on the end of the plummet gradually and contact the waste which indicates a false surface level increase. Significant level discrepancies occur when the buildup breaks off or when the measuring instrument plummet is flushed to remove the saltcake buildups. Flushing the Food Instrument Corporation Gauge, manual tapes, or any other equipment may cause accumulated wash water to collect under the plummet which indicates a false increase in the overall volume of waste within the tank. Surface level readings often are difficult to obtain from tanks that have a relatively dry saltcake surface. Some tanks have crystalline buildup on in-tank equipment (i.e., pumps, thermocouples, liquid observation wells, and other protruding equipment). As the supernatant is pumped from the tanks, the crystalline structure may remain attached to the equipment and be suspended above the liquid. Therefore, an accurate surface level measurement would be difficult if the breakup of the crystalline structure was inconsistent and a nonuniform waste surface was created. Steel tapes that are bent or warped from operation or those discarded on the waste surface are other sources of altered surface level readings.

Routine measurements of solids and sludges in most SSTs were not reported in the Anderson document until 1965. The solids measurements are taken with a steel donut on a string and operators interpret the sludge level. The interpretation could introduce a wide range of variability in the recorded solids level. Sometimes estimates taken from photographic data and saltwell pumping activities are the only indications of the solids levels.

\section{Iiquid Observation Well Data}

The liquid observation well data were obtained electronically

from the Westinghouse Hanford Company Surveillance Group. Some 
discrepancies exist in the liquid observation well readings for tanks with liquid waste surfaces. The discrepancies were supposed to be addressed in a December 1993 rebaselining of the liquid observation well data. Agreement between the liquid observation well plots and the data obtained from the surveillance group has been verified; however, errors in the data prior to the exchange of information could still exist. The liquid observation well data were understood to be unverified prior to the exchange. Therefore, the data should be reviewed before being used for any design purposes or safety evaluations.

\section{Temperature Readings}

The single-shell tank temperature profiles are provided by electronic data from Westinghouse Hanford Company. Agreement between the thermocouple plots and the data obtained from the surveillance group has been verified; however, errors in the data prior to the exchange of information could still exist. Thermocouples used in the thermocouple trees were purchased and installed according to the American society of Testing and Materials (ASTM) standard. There were no field calibrations before 1991 when a survey of Ferrocyanide watch Iist thermocouple trees was conducted and a few of the thermocouple trees were compared against a thermocouple probe inserted in a liquid observation well.

During a review of the plots, some thermocouples were found to have outlying data points and many of them have only one or two readings. The unusual readings may be the result of the thermocouples functioning on only one occasion, the operator misrepresenting the number of thermocouples in the tank, unnecessary thermocouples readings, or possible thermocouple failure.

Occasionally the operator would read the temperature in degrees celsius instead of degrees fahrenheit and would not indicate this on the data sheets. The thermocouple data were understood to be unverified prior to the exchange. An attempt has been made by ICF Kaiser Hanford to validate and verify the data, however, the data should be checked before being used for any design purposes or safety evaluations.

\section{Surface Level Data}

The surveillance automated computer system data were obtained electronically from the Westinghouse Hanford Company Surveillance Group and were plotted. The data are actual surface levels recorded from the surveillance equipment. If the surveillance equipment in a particular tank riser was removed from service, the readings may show a level change when a new instrument and/or riser is used, especially if the waste surface shows severe heterogeneity.

Agreement between the plots and the data obtained from the surveillance group has been verified; however, errors in the data prior to the exchange of information could still exist. The surveillance automated computer system data were understood to be 
unverified prior to the exchange. Therefore, the data should be reviewed before being used for any design purposes or safety evaluations.

\section{Drywell Data}

The drywell information is received on data sheets for each drywell. If the readings are questionable, Westinghouse Hanford Company often will request a rerun of the drywell scan. Because the reruns are not indicated on the data sheets, it can be difficult to determine if the data sheet has been superseded. In some wells, several scans occurred on the same day with no explanation; therefore, the scan with the accepted results is not identifiable.

\section{Riser Configuration}

The riser configuration was determined from the WHC Riser Configuration Document for Single-Shell Waste Tanks (Alstad 1991). The information was current as of June 1991; therefore, any changes made after that date would not be included.

\section{Photographs}

The photo collages were made from the latest available in-tank photographs. In some tanks, photos have not been taken since the early 1970s. Some tanks had fogging problems in the vapor space which prevented use of the latest photos. Sometimes a review of the older photos was useful to determine the waste changes. Collages made from older photos may not be indicative of current tank contents, especially if the tank has been pumped since the last photo date.

Historical Sample Analysis Data

The historical sample results only refer to sample and analysis information obtained before August 1989. The historical sample and analysis results have not been validated by the characterization program. When validation occurs, they will be reissued in a later revision of the Historical Tank Content Estimate report and supporting documentation. The sample data often were retrieved from memos that indicated the results of the sample analysis. The analytical methods, holding times, and quality control information are unavailable. The location at which the sample was taken was not provided which leads to integration of some uncertainty into the sampling results. The analytical scope performed in many cases was quite narrow which limited the available data set. However, the data set is the only available historical chemical characterization information.

\subsection{Background NE Quadrant}

The SSTs located on the Hanford Site near Richland, Washington were built between 1943 and 1964 to provide interim storage for high-level nuclear wastes. Processes such as plutonium separation from spent nuclear fuel and uranium metal recovery generated the millions of gallons of mixed radioactive hazardous waste solutions stored within these tanks. 
Several waste tanks are on watch lists. The definition of a watch list tank is explained in a later section of this report. Wastes stored in the $\mathrm{NE}$ quadrant were generated mostly from Plutonium Uranium Extraction (PUREX) and a Bismuth Phosphate Plant (B Plant); however, some tanks in the quadrant received wastes from the other onsite separation processes. The separation processes went through many changes as new technologies became available. Liquid waste volumes were reduced by evaporators, in-tank solidification units, or chemical precipitation of radionuclides. The resulting supernatant was disposed of in the ground. Vaults were used for temporary storage or treatment of waste and they were the intermediate storage point for tank waste or waste awaiting further processing. A special rail car facility in the NE quadrant was used to unload onsite waste that was distributed to an evaporator, tanks, or processing facilities. The facilities were sources of the waste that exists in the SSTs of the NE quadrant.

\subsubsection{Waste Generating Processes}

Although not all of the processes listed below contributed waste directly to tanks in the $\mathrm{NE}$ quadrant, the waste they generated could have been indirectly transferred to the quadrant through tank to tank transfers. The plants and processes that generated waste now contained in the SSTs and the DSTs are presented in chronological order.

\section{T Plant}

T Plant was the first-full scale separations plant at Hanford. It was constructed in 1944 and was used as a separation facility for irradiated production reactor fuel until 1956. The bismuth phosphate separation process used at $T$ Plant was identical to the one used at B Plant. Since 1957, T Plant has been used as a decontamination and repair facility. The facility was modified in 1978 for storage of pressurized water reactor (PWR) core II fuel assemblies. T Plant provided facilities for decontamination, decomposition, repair, testing, shipping, and burial of contaminated tools and equipment from onsite and offsite locations.

The following wastes were sent to SSTs: waste solution from equipment decontamination efforts at $T$ Plant, decontamination waste (DW), metal waste (MW) from the bismuth phosphate process, firstcycle (IC) decontamination waste from the bismuth phosphate process, and second-cycle (2C) decontamination waste from the bismuth phosphate process.

\section{B Plant}

B Plant was constructed between August 1943 and February 1945 . The bismuth phosphate process ran until 1952. One of the first plants built along with $\mathrm{T}$ Plant, $\mathrm{B}$ Plant was designed to separate plutonium from uranium and the bulk of fission products in irradiated fuel via the bismuth phosphate separation process. In 1968, B Plant became a waste fractionation plant and was retrofitted to remove cesium 137 and strontium 90 from PUREX acid 
waste, high-level supernatant liquids, and sludge from self-boiling liquid wastes.

B Plant contained 40 processing cells that served several functions (e.g. equipment storage, radionuclide purification, liquid waste concentration, and solvent extraction). Cells 5, 23, 24 and 25 were used as liquid waste concentrators for the B Plant processing. Cell 23 of B Plant was used as a waste concentrator from July 28, 1967 to February 2, 1968. Because B Plant was being upgraded to a waste. fractionation plant, the Cell 23 concentrator was running specifically to concentrate intermediate level waste in the single-shell tanks.

In 1974, the Waste Encapsulation and Storage Facility (WESF) was added to $B$ plant to encapsulate strontium and cesium that was recovered from the $B$ Plant process. The cesium capsules were leased commercially for irradiation of food, medical supplies, and lumber. The strontium and cesium capsules are stored in water basins for future retrieval. Currently most of B Plant is going through closure except for the plant sections that are needed to support the WESF mission. The closures result from a 1991 evaluation of the future B Plant mission.

The following B Plant waste streams were sent to SSTs: B Plant high-level waste (B), B Plant flush (BFSH), low-level waste from the waste fractionation plant (BL), complex concentrate (CC) or (CCPL), complexed waste (CPLX), fission products waste (FP), metal waste (MW), REDOX ion exchange waste processed at B Plant (RIX), ion exchange (IX), strontium sludge (SRS), cell 5 tank 6 waste (56), first-cycle waste (1C), and second-cycle waste (2C).

\section{Bismuth Phosphate Process}

The $B$ and $T$ Plants used a bismuth phosphate extraction to remove plutonium from uranium fuel elements. The extraction waste that resulted was a metal waste that still contained $90 \%$ of the fission products and $99 \%$ of the original uranium. The metal waste was sent to specific tank cascades in the 200-East and 200-West Areas. First-cycle decontamination waste (1C), which contained approximately $10 \%$ of the fission products, was also stored in other underground waste tanks in the 200 Areas.

\section{Plutonium Finishing Plant}

The Plutonium Finishing Plant (PFP or $Z$ Plant), previously called Plutonium Recovery and Finishing Operations, began operation in late 1949 to process plutonium and prepare plutonium products. PFP operations included plutonium handling, plutonium reclamation, plutonium conversion, and decontamination and decommissioning.

Waste from this plant contained minor amounts of fission products and low concentrations of plutonium and other transuranic elements, but was high in metallic nitrates. Initially, the waste was discharged via cribs to soil columns, which absorbed the transuranic elements and retained them close to the point of 
WHC-SD-WM-ER-312, Rev. 0

discharge. Later waste from $\mathrm{Z}$ plant was stored along with other waste in underground tanks.

The following waste was sent to tanks: waste from the Plutonium Reclamation Facility and the remote mechanical $C$ line (Z).

Uranium Oxide Plant

The 224-U Building was completed in 1944 as part of the $U$ Plant complex. - The building was converted to a $\mathrm{UO}_{3}$ plant in 1951. The 224-UA building was constructed in 1957 with six installed calciners. The $\mathrm{UO}_{3}$ plant was able to handle the uranyl nitrate hexahydrate (UNH) stream from REDOX, $U$ Plant, and PUREX. The $\mathrm{UO}_{3}$ plant shut down in 1972 and restarted in 1984 with the feed lines from REDOX and the $U$ Plant canyon disconnected. Since 1984, there have been 17 campaigns at the plant that averaged 8 days each. The plant can calcine UNH much faster than the PUREX plant can produce it. Final deactivation was ordered for the plants in 1992. In April 1993, the $\mathrm{UO}_{3}$ plant resumed operations to convert 200,000 gal of remaining UNH to uranium oxide powder. A final deactivation plan for the facility was prepared in the summer of 1993 and will be carried out in the next few years.

In the basic $\mathrm{UO}_{3}$ process, calcining consisted of concentrating and heating liquid UNH until it was converted to a stable orangeyellow powder. The nitric acid in the UNH solution was also recovered in the same process. The $\mathrm{UO}_{3}$ powder was the base material needed to manufacture uranium hexaflouride ( $\left.\mathrm{UF}_{6}\right)$ which is the primary feed material for gaseous diffusion plants in the United states.

\section{REDOX}

The Reduction and oxidation extraction (REDOX) plant was built between May 1950 and August 1951 and operated until July 1967. Uranium and plutonium were extracted from solid uranium metal slugs that were irradiated in the Hanford reactors or piles into a methyl isobutyl ketone (hexone) solvent by a continuous solvent extraction process. The REDOX process was the first at Hanford to recover both plutonium and uranium.

The waste stream which was slightly acidic contained fission products and large quantities of aluminum nitrate that were used to promote the extraction of plutonium and uranium. The waste was then neutralized and stored in SSTs.

The following wastes were sent to SSTs: REDOX process waste resulting from the dissolution of aluminum and zircaloy fuel element cladding, REDOX coating waste (CWR), REDOX high-level waste (R), and the supernatant liquid portion of waste generated by the REDOX process, REDOX supernatant (RSN). RSN is found above the sludge in the underground storage tanks. 
$U$ Plant (221-U) was built as one of three original bismuth phosphate process facilities although it was not used for that purpose. U Plant was modified extensively and used for the uranium recovery process. U Plant operated from 1952 to 1958. The main canyon at $U$ Plant currently stores failed equipment.

Uranium in waste from the $\mathrm{BiPO}_{4}$ process initially was stored in the SSTs. Later it was mined by sluicing, dissolved in nitric acid, and processed through a solvent extraction process. For every gallon of metal waste originally stored, the uranium extraction process yielded approximately 1.4 gal of UR waste. The waste volume increase made development of a technology that would reduce the volume of the stored waste a priority. This need resulted in the development/implementation of the ferrocyanide scavenging process.

The waste sent to the SSTs from U Plant was called tributyl phosphate (TBP). In reality it contained very little TBP; therefore, to eliminate confusion, it will be referred to as uranium recovery (UR) waste in this report.

\section{Strontium Semiworks}

The strontium or hot semiworks processing facility (i.e., C Plant) was built in 1949 as a hot pilot plant for the REDOX process, but was later converted to a pilot plant for the PUREX process. In 1960, the plant was reactivated and used later as a process demonstration for the conversion of $B$ Plant to a waste fractionation facility and was used to recover strontium 90, promethium 147, and cesium 144 from PUREX waste. The building and building site have been decontaminated and decommissioned.

The strontium semiworks waste sent to SSTs was known as hot semiworks (HS), strontium semiworks (SSW), and fission products waste (FP).

\section{PUREX}

The Plutonium Uranium Extraction (PUREX) plant (i.e., A Plant) was an advanced solvent extraction process that used tributyl phosphate in a paraffin hydrocarbon solvent to recover uranium and plutonium from nitric acid solutions of irradiated uranium. PUREX was built between April 1953 and April 1955, it operated until 1972 when it was closed for 11 years. Two thorium campaigns were conducted in the PUREX plant between 1966 and 1971. The irradiated waste was similar to the PUREX waste but it contained thorium and uranium 233 rather than uranium and plutonium which was extracted from typical PUREX waste. It began operating again in November 1983 and was shut down in 1988 after a safety violation. The PUREX Plant was shut down several times between 1988 and 1990 for various reasons. In October 1990, the PUREX Plant was put in standby mode and was eventually identified for closure in December 1992 by Secretary of Energy James Watkins. 
The following PUREX waste streams were sent to sSTs: cladding waste (CW) or (CWP), organic solvent wash waste (OWW), neutralized PUREX plant acid waste (P), low-level waste from PUREX (PL), PUREX sludge supernatant liquid (PSS), organic wash waste using sodium carbonate (CARB), cesium feed (CF), and concentrated neutralized high-level waste (1WW) .

\subsubsection{Waste Management Operations}

This section describes the different waste concentrating methods used in the 200 Areas. Evaporating, concentrating, and scavenging are all methods used to reduce liquid volumes or precipitate solids out of the supernatant solutions. The operations are presented in chronological order.

\section{2-T Evaporator}

The 242-T Evaporator was built in the early 1950s to reclaim nonboiling waste storage capacity in existing tanks. The evaporator was shut down in the summer of 1955 and modified for tributyl phosphate scavenging, although scavenging was never performed in this evaporator. The 242-T Evaporator restarted in 1965 and operated until 1976. Tank 241-TX-118 was the feed tank for the evaporator.

The following wastes were sent to tanks from the $242 \mathrm{~T}$ Evaporator: Slurry product from the evaporator bottoms (EB), evaporator feed (EF), and terminal liquor (TL).

\section{2-B Evaporator}

The 242-B Evaporator was built south of the B Tank Farm and started operating in December 1951. It received feed waste until November 1954 and was shut down in september 1955. The evaporator was never reactivated. Tanks $241-B-105$ and -106 were used as the bottoms and feed tank respectively. The evaporator operated at atmospheric pressure.

\section{Ferrocyanide scavenging}

Ferrocyanide scavenging was developed to reduce waste volumes. The ferrocyanide flow sheet was first tested in U Plant in October 1953. In-farm scavenging was completed in March 1956 and December 1957 for the 200-West and 200-East Areas respectively. The ferrocyanide scavenging program was designed to remove fission products such as ${ }^{137} \mathrm{Cs}$ (half life: $28.6 \mathrm{Yr}$ ) and ${ }^{90} \mathrm{Sr}$ (half life: $30.2 \mathrm{Yr}$ ) from the UR and $1 \mathrm{C}$ wastes as a precipitate. Because the ${ }^{90} \mathrm{Sr}$ was essentially insoluble in the neutralized UR waste, the strontium compounds would precipitate as phosphates, sulfates, or hydrated oxides when the acidic waste was neutralized. However, common cesium compounds are quite soluble even at alkaline pHs. Development of the nickel ferrocyanide scavenging process provided a relatively simple process to precipitate ${ }^{137} \mathrm{Cs}$ and enhance the precipitation of ${ }^{90} \mathrm{Sr}$. The precipitating chemicals were added in stainless steel process vessels with agitation (i.e. U Plant, T Plant, or the $C R$ vault) and the waste slurry was routed to specific SSTs for settling. After the ${ }^{137} \mathrm{Cs}$ and ${ }^{90} \mathrm{Sr}$ precipitates settled, the 


$$
\text { WHC-SD-WM-ER-312， Rev. } 0
$$

clarified supernatant was sampled and disposed of in cribs. After the ${ }^{137} \mathrm{Cs}$ and ${ }^{90} \mathrm{Sr}$ precipitates settled, the clarified supernatant was sampled and disposed of in cribs.

\section{In-Tank Solidification}

The primary function of the in-tank solidification systems was to concentrate the nonboiling waste directly inside of specially designed tanks that would produced a saltcake slurry. Each in-tank solidification system included a heat exchanger for water evaporation and a-series. of bottoms tanks. The first in-tank solidification unit (ITS \#1) operated with an airlift circulator through Tank 241-BY-102 and the second in-tank solidification unit (ITS \#2) operated with a similar circulator through Tank 241-BY112. Tank 241-BY-112 contained a 4,000 kW electric immersion heater. Tank 241-BY-101 contained an in-tank solidification prototype that was used only for demonstration purposes. In-tank solidification units 1 and 2 began operating in 1965 and 1968, respectively. In 1971 in-tank solidification unit 1 became the cooler for in-tank solidification unit 2. Both units were shut down in 1974 .

\section{REDOX Concentrator}

The REDOX concentrator was used for volume reduction of dilute 200-West Area tank farm wastes by removing water. The concentrator received tank farm wastes from July 28, 1967 until June 30, 1972. Cell $D$ in the REDOX process was the concentration and neutralization section of the plant.

\section{2 -S Evaporator/Crystallizer}

The 242-S Evaporator/Crystallizer began operating in November 1973 and shut down in 1981. It was designed as a reduced pressure (i.e., partial vacuum) evaporator/crystallizer. Aqueous salt wastes were converted in the evaporator to salt crystals for storage in underground tanks in the $S$ and SX Tank Farms.

The following waste types were sent to SSTs from the 242-S Evaporator: non-complexed waste concentrated until the solution was nearly saturated with sodium aluminate known as double-shell slurry feed (DSSF), slurry product from the evaporator bottoms (EB), evaporator feed (EF), Hanford defense residual Iiquor (HDRL), partial neutralized feed waste (PNF), and terminal liquor (TL).

\section{2-A Evaporator/Crystallizer}

Construction of the 242-A Evaporator/Crystallizer was completed in 1976. The evaporator began operation in March 1977 with a design life of 10 years. In 1987, design changes were developed to extend the facilities operating life by 10 years. The evaporator was shut down in April 1989 because of regulatory issues and restarted in May 1994 after extensive modifications.

The following evaporated wastes were sent to SSTs: evaporator bottoms from B Plant low-level waste feed (BLEB), non-complexed waste concentrated in evaporators known as double-shell slurry feed 
WHC-SD-WM-ER-312, Rev. 0

(DSSF), evaporator feed (EF) or (EVAP), evaporator bottoms (EB), and terminal liquor (TL).

1.5.3 Miscellaneous Waste Sources and Equipment

There are various other sources of waste on the Hanford site, as well as other material added to the tanks. Some wastes are from the 300 Area, 100 Area production reactors, various laboratories, and catch tanks. Unique contents added to SSTs included laboratory wastes, diatomaceous earth, Portland cement, shroud tubes, ceramic balls, experimental fuel elements, and relatively small amounts of enriched uranium, plutonium, cobalt, and natural uranium.

The following wastes contribute to SST waste: diatomaceous earth (DE), Hanford defense residual liquor (HDRL), Hanford laboratory operations ( $\mathrm{HLO})$, filtered Hanford water $\left(\mathrm{H}_{2} \mathrm{O}\right)$, phosphate decontamination waste from $\mathrm{N}$ reactor $(\mathrm{N})$, and non-complexed waste (NCPL).

\section{Critical Mass Lab}

The critical mass lab was used to develop data on the factors that are necessary to make a criticality or self-sustained nuclear reaction occur. Because plutonium is the key radioactive element on the Hanford Site, the research was based on plutonium criticality safety in various fuel cycles. This facility was located next to the strontium semiworks and ran from the early 1950s to the early 1980s. The plutonium used in the lab was reprocessed in PUREX.

244-AR, -BXR, and-CR Process Vaults

There are three process vaults in the NE quadrant: the AR Vault, the BXR Vault, and the CR Vault. The vaults were composed of several process vessels or tanks used to prepare the waste for treatment or storage. Specific wastes from tanks can be pumped temporarily to the vaults and the wastes can be sent directly to desired tanks or processing facilities later.

The AR Vault was designed and constructed between 1964 and 1968 and is located next to the A and AX Tank Farms. The AR Vault has been in standby mode since 1978 .

The 244-BXR Vault is located south of the 241-BX Tank Farm and was constructed between 1950 and 1951. The BXR Vault began operating in 1952 and became inactive in 1956. The waste in the vault was difficult to handle, so the vault was high-pressure steam jetted in 1976. The vault was interim isolated after 1976 and interim stabilized in March 1985. Tank BXR-004 was removed in 1963 .

The CR Vault was constructed in 1952 and is located next to the C Tank Farm. Saltwell waste from the C Tank Farm is interim stored in the $C R$ Vault. The 244-BXR and-CR Vaults were constructed to aid in the recovery of uranium from bismuth 
WHC-SD-WM-ER-312, Rev. 0

phosphate metal waste. The $C R$ Vault received waste from the Strontium Semiworks Plant en route to the 241-C Tank Farm.

204-AR and 204-S Railroad Car Facilities

The 204-AR rail car unloading facility was built in 1981 and replaced the 204-S rail car unloading facility. The facilities were built for unloading radioactive liquid waste tank cars.

Iiquids or slurries from the $1314-\mathrm{N}$ Building in the 100 Area and the 340 . Building in the 300 Area are unloaded at the 204-AR unloading facility.

\subsubsection{Timeline}

The following timeline represents the times and spans of different events that occurred during the lifetime of major Hanford plants in the 200-East and 200-West Areas. The plants were the main contributors of waste currently contained in SSTs. The following list of abbreviations and corresponding meanings are used on the timeline:

PUREX: Plutonium uranium extraction process

$\mathrm{UO}_{3}$ : Uranium trioxide

PFP : $\quad$ Plutonium finishing plant

RG-RB : Rubber glove - remote button line

RMA: Remote mechanical "A" Iine

RSE : $\quad$ Recuplex solvent extraction

RMC: Remote mechanical "C" line

PRF : $\quad$ Plutonium reclamation facility

PCB : Polychlorinated biphynels

WESF : Waste encapsulation and storage facility

ITS: $\quad$ In-tank solidification (units 1 and 2)

PWR: $\quad$ Pressurized water reactor

REDOX: Reduction oxidation process 


\section{PROCESSES PRODUCING WASTE CONTAINED IN 200 AREA TANK FARMS}

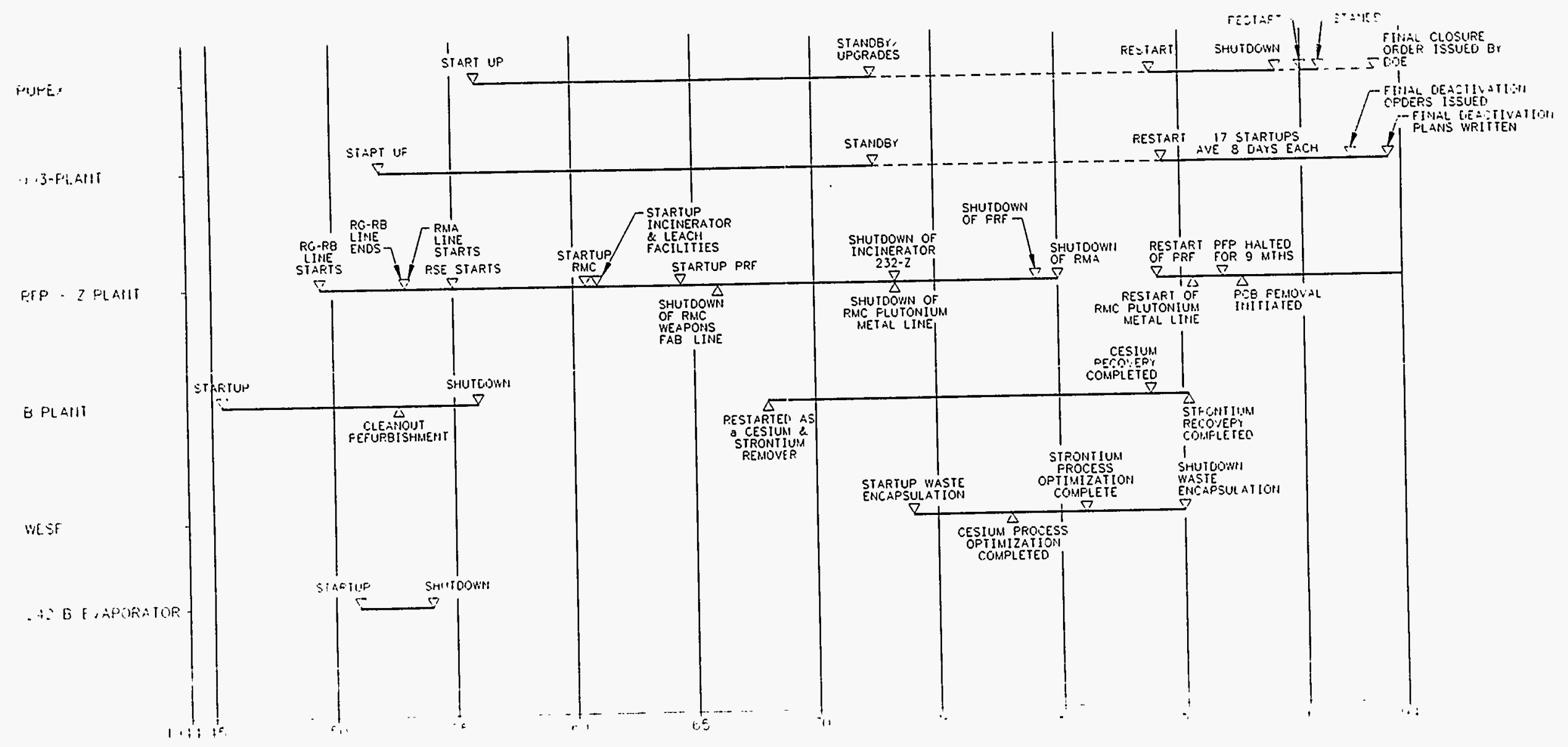




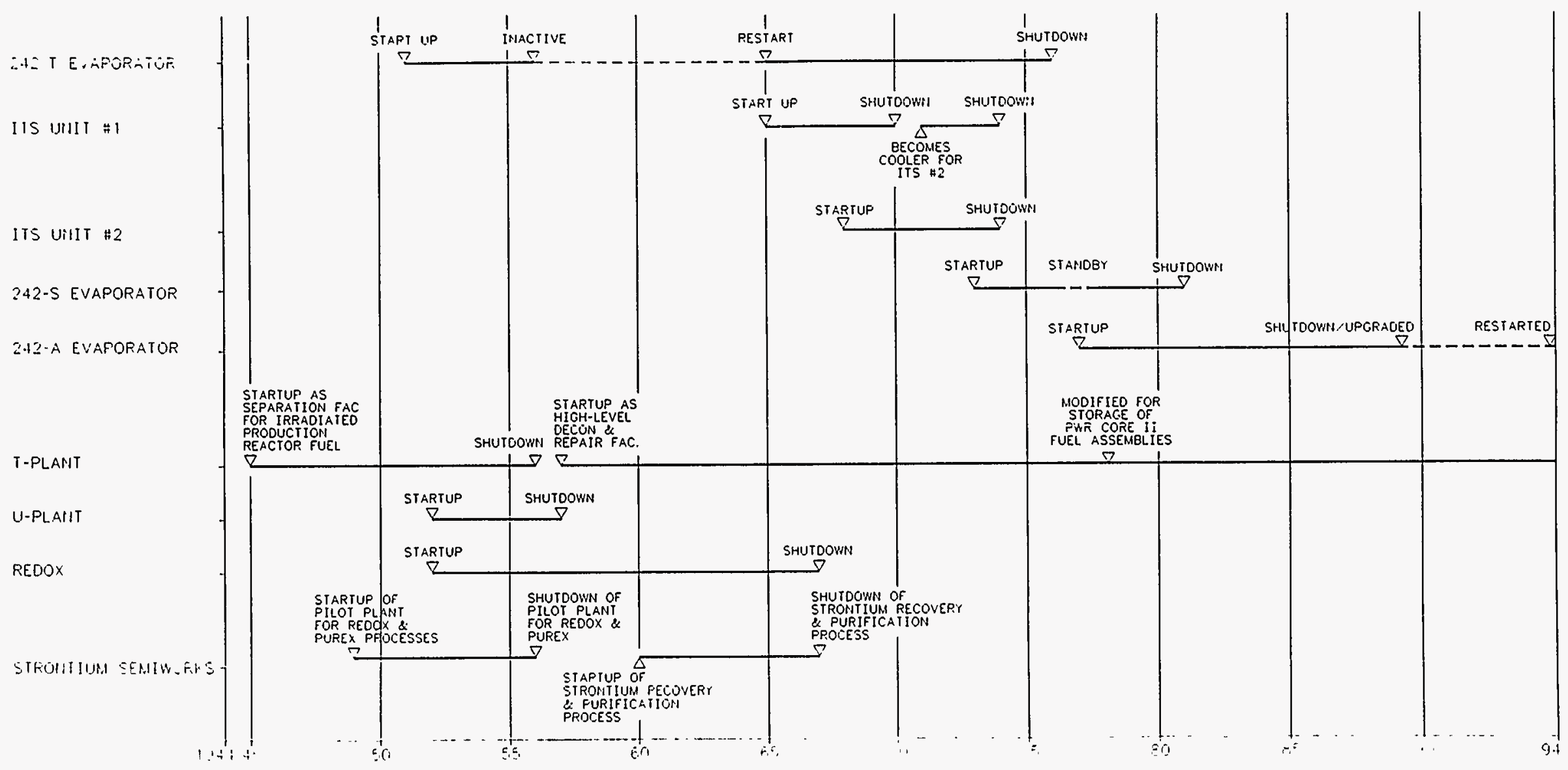


WHC-SD-WM-ER-312, Rev. 0

\subsection{Safety Issues}

The safety issues that effect the tanks can be divided into two groups: watch list and non-watch list. The watch lists are listings of the tanks believed to pose potential safety hazards to the environment and the public. Non-watch list issues are of concern because they may impact the environment.

\subsubsection{Watch List Safety Issues}

Issues in these tanks were identified as "issues/situations that contain most necessary conditions that could lead to worker (onsite) or offsite radiation exposure through an uncontrolled release of fission products" under Public Law 101-510, Section 3137, of the National Defense Authorization Act of Fiscal Year 1991 (i.e., the Wyden Amendment). As of October 1993, 45 SST's and 6 double-shell tanks are on a watch list. In the $\mathrm{NE}$ quadrant, 21 of the 66 SSTs are on a watch list. The four watch list designations described in this report are: ferrocyanide, hydrogen/flammable gas, organic salts, and high-heat load. The following sections provide a general description and criteria of the different watch lists. For a more complete explanation, refer to the Hanford Site Tank Farm Facilities Interim Safety Basis (WHC 1993).

\section{Ferrocyanide}

A tank containing or believed to contain greater than 1,000 gram moles of ferrocyanide (dry basis) is on the Ferrocyanide watch List.

\section{Hydrogen and Flammable Gas}

Most tanks were placed on the Hydrogen/Flammable Gas Watch Iist because of the potential to contain flammable gases rather than the verified presence of hazardous concentrations. Hydrogen/Flammable Gas Watch List tanks have been identified as unreviewed safety questions due to the concern of a flammable gas burn resulting in a radiological release.

\section{- Organic Salts}

Tanks containing or believed to contain more than the equivalent of $10 \%$ by weight of sodium acetate or 3 wt: total organic carbon (TOC) on a dry basis received a watch list designation as in the Tank Farm Surveillance and Waste Status Summary Report (Hanlon 1993).

\section{High-Heat Loads}

Tanks with a heat load greater than $40,000 \mathrm{Btu} / \mathrm{hr}$ are designated as high-heat load tanks. Tank 241-C-106 is the only high-heat load tank on the High-Heat Load Watch Iist.

A tank that does not appear on a watch list but has a heat load less than 40,000 Btu/hr is considered a low-heat load tank by default. 
The following table lists the tanks in the NE quadrant that appeared on a watch list as of October 1993. A current list is published monthly in the Tank Farm Surveillance and Waste status Summary Report (Hanlon 1993).

NE Quadrant Watch List Tanks

\begin{tabular}{||l|l|l|l||}
\hline Tank & Category & Tank & Category \\
\hline A-101 & Hydrogen & BY-108 & Ferrocyanide \\
\hline AX-101 & Hydrogen & BY-110 & Ferrocyanide \\
\hline AX-103 & Hydrogen & BY-111 & Ferrocyanide \\
\hline B-103 & Organic Salts & BY-112 & Ferrocyanide \\
\hline BX-102 & Ferrocyanide & C-103 & Organic Salts \\
\hline BX-106 & Ferrocyanide & C-106 & High-Heat Load \\
\hline BY-103 & Ferrocyanide & C-108 & Ferrocyanide \\
\hline BY-104 & Ferrocyanide & C-109 & Ferrocyanide \\
\hline BY-105 & Ferrocyanide & C-111 & Ferrocyanide \\
\hline BY -106 & Ferrocyanide & C-112 & Ferrocyanide \\
\hline BY -107 & Ferrocyanide & & \\
\hline
\end{tabular}

1.6.2 Non-Watch Iist Safety Issues

Tank leaks are a safety hazard because of the potential chemical and radioactive liquid releases to the ground. Corrosion is the main cause of tank leaks and stabilization, integrity, and intrusion prevention are discussed in the corrosion section of this report. Criticality, tank bumps, and toxic vapor releases are other safety issues that do not require a watch list designation and/or continual monitoring under the Wyden Amendment.

\section{Corrosion}

Corrosion is believed to be caused by localized or general reduction in SST mild carbon liner thickness. Localized liner thickness reduction is caused by three types of corrosion: pitting corrosion, stress corrosion cracking, and crevice cracking. General liner thickness reduction may be caused by uniform corrosion.

The SSTs were removed from service on or before November 21, 1980 and no longer accept wastes. When the SSTs are deactivated, an effort is made to minimize potential leaks into the earth by reducing the liquid waste contained in the SSTs. Primary stabilization is the first deactivation process of removing the supernatant or free liquid above the solid wastes within the SSTs. Supernatant is removed by turbine or jet pumps in a saltwell system, low-volume submersible pumps, or in situ drying. In 
saltwell systems, the liquid is pumped from the waste through a pipe screen in a manner similar to ground water pumping. Tanks with less than 50,000 gal of drainable interstitial liquid and less than 5,000 gal of supernatant liquid are known as interim stabilized tanks. Liquid waste is sent to the evaporator/crystallizer concentrating facilities and the concentrated waste is deposited into double-shell tanks (DSTs).

Eventually, SSTs will be interim stabilized and prepared for intrusion prevention. (formerly known as interim isolation). Stabilization efforts will be completed prior to final closure of the SSTs. Partial isolation of the tanks includes sealing the piping and risers that are not required for pumping or other stabilization methods. Intrusion prevention is the total physical and administrative effort involved in sealing inactive storage tank accesses against liquid additions while still allowing for longterm surveillance.

There are 67 assumed/confirmed leaking SSTs in the 200 East and West Areas with 32 in the NE quadrant. The remaining 34 tanks in the $\mathrm{NE}$ quadrant are sound. There are 51 interim stabilized tanks in the NE quadrant and 15 noninterim stabilized tanks. Intrusion prevention is in place for 46 SSTs in the NE quadrant, and 20 tanks are at the partial interim isolation stage.

Criticality

Criticality is an inadvertent self-sustained nuclear chain reaction. Studies have concluded that a nuclear criticality accident in the tank farms is probably not an imminent risk. However, definitive knowledge of the fissile material inventory and distribution within the tanks is lacking. Therefore, criticality remains a safety issue. The key criticality radionuclide on the Hanford site is plutonium. A fissile material criticality prevention specification of $125 \mathrm{~kg}$ has been placed on the tanks. An unusual occurrence report involving nuclear criticality was filed in June 1991 for Tank 241-C-104. An inventory of $56 \mathrm{~kg}$ was calculated later which indicated a low probability for criticality. Criticality is considered an unreviewed safety question but is not affected by the Wyden Amendment according to the Hanford Site Tank Farm Facilities Interim Safety Basis (WHC 1993).

- Tank Bumps

Tank bumps have been an issue at Hanford since 1953 with the last bump occurring in 1968. The A Tank Farm is known to have had tank bumps. Tank bumps occur when heat from the hotter solid waste in the lower portion of the tank is suddenly transferred to a near boiling supernatant. Mixing can be achieved by "roll over" or sudden restart of airlift circulators which results in rapid fluid vaporization and a sudden internal tank pressurization that causes a bump. The three documented bumps that have occurred were due to airlift circulator failure followed by rapid startup of the airlift circulators which causes rapid mixing. Two key factors have been identified to control bumping: liquid and solid temperatures must be maintained as far away from the local boiling point as possible 
and airlift circulators and ventilation systems must be operated as designed. A tank bump is extremely unlikely if airlift circulators and vent flow rates are maintained at 500 to $1,000 \mathrm{ft}^{3} / \mathrm{min}$ per tank and $50 \mathrm{ft}^{3} / \mathrm{min}$ to the airlift circulators, according to the Hanford Site Tank Farm Facilities Interim Safety Basis (WHC 1993).

\section{- Toxic Vapor Releases}

The safety concern of toxic vapor releases have been analyzed recently at Hanford. Odors have been released from the C Tank Farm in the NE quadrant and from Tank 241-SY-101. Tank 241-C-105 has experienced releases that have resulted in worker exposure. If toxic vapors are determined to exist in a tank, three safety recommendations should be followed: determine a release rate, determine offsite and onsite exposure concentrations, and determine if the release is a spray effect. The entire issue of toxic gas releases at the tank farms has just begun to be understood, Hanford Site Tank Farm Facilities Interim Safety Basis (WHC 1993). 


\subsubsection{BY Tank Farm History}

The BY Tank Farm is located in the north-central end of the 200-East exclusion area, east of Baltimore Avenue. The BY Tank Farm was constructed for the operating contractor, General Electric Company, during 1948 and 1949. Because the BY Tank Farm is a modified B Tank Farm design, the tanks are considered "second generation." - The BY Tank Farm contains twelve 100 series; 758,000gal; 75-ft diameter single-shell tanks. The riser pattern is modified to gain more uniform access to the tank interior. The tanks were designed for a maximum fluid temperature of $220^{\circ} \mathrm{F}$. The BY Tank Farm initially operated to back up the BX Tank Farm. In the BY Tank Farm, tanks are connected in a three-tank cascading series. The BY tanks are connected to the end tanks of the BX Tank Farm. The cascade overflow height is approximately 272 in. from the tank bottom.

The BY tanks contain non-complexed waste. Nine tanks are on the Ferrocyanide Watch List and 5 are confirmed or assumed leaking tanks. The 1993 color aerial photograph of the BY Tank Farm shows the tank orientation and a north arrow. The arrows between the tanks represent the cascade overflow lines and the flow direction.

The tanks were built under project $\mathrm{C}-271$ and are covered by specification HW-3783 "Additional Waste Storage Facilities 200-East Area." The tanks are approximately $75 \mathrm{ft}$ in diameter, with an operating depth of $23 \mathrm{ft}$, and are covered with approximately 8 ft of overburden.

Various coatings and sealants were used to prevent leaks and intrusions. The tank liner is protected from the outside with a 3-ply membrane waterproofing between the steel liner and the encompassing concrete. The outer steel liner surfaces are covered with a 3-ply asphalt waterproofing, a second layer of 3/4-in. Gunite (sprayed concrete), and a third layer of 2-in. by 2-in. welded reinforcing fabric. The concrete wall was poured in three even lifts after steel reinforcement was installed. The inside of the steel liner was sandblasted and one coat of Du Lux Seachrome Primer was applied. Lead flashing was installed along the top of the steel liner to protect the waterproofing membrane. Upon completion of the tank domes, the tanks were covered externally with a 3-ply asphaltic waterproof membrane and Gunite reinforced with wire fabric. The inside of the tank was given a final primer coat. The concrete dome ceiling is protected with three coats of a hardening solution. Exterior surfaces of dome risers and encasing pipes of overflow and tie lines were cleaned thoroughly and given one coat of coal-tar primer followed by one hot coat of coal-tar enamel and one coat of water-resistant white wash. 
WHC-SD-WM-ER-312, Rev . 0

2.0.1.1 BY Tank Farm Waste History

B Plant metal wastes were stored in Tanks 241-BY-101 through 106 after the BX Tank Farm was full. Tanks 241-BY-107 through -110 received $B$ Plant first-cycle waste and $U$ Plant waste. Tanks 241BY-111 and -112 were used to store metal waste for a short time. Tanks 241-BY-101, -103 , and -111 received ferrocyanide wastes from the other BY tank transfers. Tank 241-BY-112 received a direct transfer from U Plant in 1957.

The sluicing operations program began in January 1954 and was completed by August 1955. The sluicing campaign had two objectives: uranium recovery and in-tank solidification preparation. Tanks within the BY Tank Farm were used as feed staging or evaporator bottoms waste receiving tanks for the in-tank solidification program. The in-tank solidification program operated as an in-tank evaporation system to concentrate the waste. Tanks 241-BY-101 through -106, -111, and -112 were involved in sluicing operations between 1954 and 1955 .

The in-tank solidification program operated in two stages. stage one involved placing an in-tank solidification unit in Tank 241-BY-102. The first in-tank solidification unit operated from March 1945 until August 1971 when it became a cooler for unit 2 . The second in-tank solidification unit was used in conjunction with Unit 1 as a cooler for Tank 241-BY-112 from August 1971 until both units were shut down in June 1974. Because of the large amount of saltcake waste created from the in-tank solidification units in the BY Tank Farm, saltwell pumping was performed on all tanks in the BY Tank Farm.

Ferrocyanide scavenging waste was sent to the BY Tank Farm. The campaign was designed to recover strontium and cesium from metal waste. Tanks 241-BY-106, -107, -108, and -110 were used as primary settling tanks for scavenged waste, and Tanks 241-BY-104 and -105 were used as settling tanks for accumulated scavenged sludge.

\subsubsection{BY Tank Farm Temperature History}

Most tanks in the BY Tank Farm have at least one thermocouple tree with the exception of Tanks 241-BY-102 and -109. Tanks 241BY-104, -105, -110, -111, and -112 have two thermocouple trees. Temperature data for Tanks 241-BY-102 and -109 are not available between 1980 and 1991. The riser configuration document indicates that thermocouple trees do not exist in these two tanks which may indicate that the thermocouple trees were removed; however, readings resume in late 1991 and 1992. Tanks 241-BY-101, -103 through -108, and -110 through -112. are former or current Ferrocyanide Watch List tanks and have a weekly temperature monitoring requirement. The other tanks have a semiannual temperature monitoring requirement. 


\subsubsection{BY Tank Farm Integrity}

The twelve 750,000-gal tanks in the BY Tank Farm are out of service. As of July 1993, seven tanks were categorized as sound (i.e., Tanks 241-BY-101, -102, -104, -110, -111, and -112), and five were categorized as assumed leakers (i.e., Tanks 241-BY-103, $-105,-106,-107,-108)$. Liquid observation wells, surface level measurements, and leak detection drywells were the methods used to monitor tank integrity. If a discrepancy is observed as a result of the surveillance .. activities; notification, reporting, documentation, and investigation of the surveillance anomaly (data outside the criteria limits, changing trend, or departure from steady state) is conducted in accordance with the appropriate procedure. Generally, the first plan of action is to determine if the discrepancy is caused by something other than a tank leak or intrusions.

With the exception of Tank 241-BY-108, the tanks in the BY Tank Farm have liquid observation wells to monitor the interstitial liquid level. The interstitial liquid levels are monitored weekly with a neutron probe and on request with a gamma probe. The maximum deviations from the established baseline are a $0.3-f t$ increase and a 0.4 -ft decrease.

Surface level monitoring with manual measurement devices in the BY Tank Farm is conducted daily or quarterly, depending on the tank. The maximum allowable decrease from the established baseline is 2.0-in. for Tanks 241-BY-102 and -109. The other tanks have no criterion for a decrease. The maximum allowable increase from the baseline ranges from 2 to 3 in., depending on the tank. The no criterion designation was established for waste tanks containing sludge with little or no solution. For these tanks, a surface level decrease is not a valid means of leak detection because the level may decrease due to sludge slumpage (especially during saltwell pumping), tape flushes, and perforation of the exposed sludge at the point of probe contact. Tanks that have a saltcake surface and that are equipped with an automatic Food Instrument Corporation gauge may have a 10-in. decrease criteria which was established to alert the operating personnel if the gauge unwinds in search of a conductive liquid surface.

The BY Tank Farm has 70 leak detection wells (i.e., drywells) that were drilled from 1949 to 1974. Five are not tank-specific and drywells 22-00-02 and 22-00-03 currently have readings greater than the $50 \mathrm{c} / \mathrm{s}$ background radiation. The drywells in the BY Tank Farm are monitored on a monthly, yearly, or on request basis with scintillation or Geiger-Mueller probes. Increased activity action criteria for monitoring conducted with scintillation and shielded scintillation probes are as follows: if the normal readings are less than $200 \mathrm{c} / \mathrm{s}$, the readings must double and exceed $200 \mathrm{c} / \mathrm{s}$; if the normal readings are greater than $200 \mathrm{c} / \mathrm{s}$, the readings must double. Increased activity action criteria for monitoring conducted with Geiger-Mueller probes are as follows: if the normal readings are between 16 and $1,000 \mathrm{c} / \mathrm{s}$, the readings must triple. 
WHC-SD-WM-ER-312， Rev. 0

If the normal readings are greater than $1,000 \mathrm{c} / \mathrm{s}$, the readings must double. Drywells associated with assumed leaking tanks are monitored for migration of contamination and the criteria limits do not apply except where new radiation peaks occur. If increasing drywell activity has not exceeded the action criteria stated above, appropriate personnel will determine if increased monitoring is required.

The BY Tank Farm layout includes the peak drywell information, tank integrity, and tank status information. The drywell information is based on summarized data prior to 1987 and from data collected from January 1990 to the present. Graphical representations of the currently active drywells mentioned above are in Appendix F. Each graph includes the peak c/s, the depth at which the peak occurred, and the date of the reading.

2.0.2 BY Tank Farm Historical Sample Analysis Data

Historically, single-shell tank waste samples have been analyzed to characterize the supernatant, sludge, and saltcake in each tank. Data for this report were compiled from samples obtained from the late 1950 s to september 1989.

\subsubsection{Current status of BY Tank Farm}

Nine tanks in the BY Tank Farm are identified on the Ferrocyanide Watch List: Tanks 241-BY-103, -104, -105, -106, -107, $-108,-110,-111$, and -112 were added to the list officially in January 1991 and have been declared unreviewed safety questions because their explosion potential exceeds previously reported safety analysis consequences. Ferrocyanide Watch List tanks are monitored for temperature on a weekly basis. Tanks 241-BY-101, 102, and -109 are low-heat load tanks ( $<40,000 \mathrm{Btu} / \mathrm{hr}$ ) and are monitored for temperature on a semiannual basis. Tank 241-BY-101 was recently removed from the Ferrocyanide watch List; therefore, the temperature is monitored weekly. The total volume of waste in the BY Tank Farm is 4,744,000 gal: 3,704,000 gal of saltcake; $102,000 \mathrm{gal}$ of unknown solids; and $938,000 \mathrm{gal}$ of sludge.

\subsubsection{BY Tank Farm In-Tank Photographs}

The BY Tank Farm is a battery of twelve 758,000-gal second generation tanks built in 1948-49. The BY Tank Farm was built to receive waste from the BX Tank Farm cascade system. As a result of experience from the first generation farms, the amount of equipment used to monitor and maintain the waste was increased in these tanks. The typical BY tank contains 11 to 13 risers through the tank dome. The photos are usually taken from one of the four main risers around the tank perimeter.

The latest in-tank photos for the BY Tank Farm date from November 1982 to September 1989. Most photos are an accurate representation of the tank contents because, except where noted, no pumping activity has occurred since the photos were taken. 
WHC-SD-WM-ER-312, Rev . 0

2.1 Tank 241-BY-101

2.1.1 241-BY-101 Tank History

2.1.1.1 Waste History Tank 241-BY-101

The waste history narrative in the following paragraph is from "A History of the 200 Area Tank Farms" (Anderson 1990). Tank 241BY-101 was filled with metal waste from the Tank 241-BX-103 cascade overflow between March 1950 and the first quarter of 1951. The tank cascaded to Tank 241-BY-102 from the third quarter of 1950 until the first quarter of 1951. The metal waste was sluiced and transferred to Tank 241-BY-103 and the tank was declared empty on May 17, 1954. The tank contained U Plant waste from the first quarter of 1955 until the fourth quarter of 1964. In the first two quarters of 1956, the tank contained scavenged waste awaiting rework. In the third quarter of 1957, scavenged feed was sent to the CR process vault. The tank contained coating waste and firstcycle waste from the fourth quarter of 1960 until the fourth quarter of 1964. The tank contained coating waste from the second quarter of 1965 until the first quarter of 1968. The tank received in-tank solidification boil-off from the second quarter of 1965 until the second quarter of 1966. In the fourth quarter of 1966, the tank received 28,000 gal of recovered butts. From the third quarter of 1967 until the fourth quarter of 1969, the tank was used for a solidification demonstration. From the second quarter of 1968 until the second quarter of 1976, the tank received evaporator bottoms waste. The tank was receiving solidification bottoms and recycle waste from the first quarter of 1972 until the second quarter of 1976. In the third quarter of 1976, the tank received concentrated evaporator feed which it held until the fourth quarter of 1977. The tank was saltwell pumped in the first and second quarters of 1977 and was declared inactive in the first quarter of 1978. From the first quarter of 1978 until the present, the tank waste has been classified as non-complexed.

The waste history narrative of the primary additions that follow in this paragraph are from the "Waste status and Transaction Record Summary" (Agnew 1994). Tank 241-BY-101 received wastewater during the fourth quarter of 1974.

Primary stabilization was completed in May 1980 and a level adjustment was made in April 1982. The tank was saltwell pumped, was declared interim stabilized, and had a level adjustment made in May 1984. Liquid observation well data have been plotted that represent interstitial levels. An engineering judgement was made prior to March 1986. After interim stabilization pumping, the interstitial liquid equalizes in the tank. In-tank photographs were taken on the following dates: several in 1976, several in 1977, March 1980, April 1981, February 1983, and September 1989. Sketch ES-TKS-E39, which shows a graphical representation of the Tank 241-BY-101 level history, and the historical waste level data used to make it are in Appendix C. The sketch shows two $\mathrm{pH}$ 
readings: 9.5 in May 1955 and $>14.0$ in June 1976. The current maximum temperature reading from July 1993 is $75^{\circ} \mathrm{F}$.

\subsubsection{Temperature History 241-BY-101}

The single thermocouple tree in Tank 241-BY-101 has 14 thermocouple probes to monitor the waste temperature. The thermocouples have specific elevation information and similar temperature readings that span from 1974 to the present. The temperatures general.Iy decrease. from the bottom to the top of the tank. There is a gap in temperature readings from February 1984 until October 1989, although a thermocouple tree was in the tank in September 1989. The Tank 241-BY-101 level history indicates that from 1974 to 1977, thermocouples 1 through 7 were in or near the solids level, thermocouples 8 through 12 were in or near the liquid level, and thermocouples 13 and 14 were in the vapor space. From 1977 to 1984, thermocouples 1 through 7 were still in or near the solids level and the rest were in the vapor space. Thereafter, thermocouples 1 through 6 were in or near the solids level and the rest were in the vapor space.

The mean temperature of the first daily recorded readings was $30^{\circ} \mathrm{F}$. A sum of the squares regression line fit for the data shows a temperature decrease with a moderate amount of variability. Thermocouples 1 through 6,12 , and 14 show pronounced temperature fluctuations of up to $70^{\circ} \mathrm{F}$ from October 1974 to July 1975 . There is an outlying data point of approximately $24^{\circ} \mathrm{F}$ in December 1981 for thermocouples 5 through 14. An annual temperature undulation is apparent after 1989. The median temperature is $71.2^{\circ} \mathrm{F}$ with a minimum of $43^{\circ} \mathrm{F}$ and a maximum of $115^{\circ} \mathrm{F}$. Tank $241-\mathrm{BY}-101$ is a former Ferrocyanide watch List tank and temperature readings are required weekly.

Plots of the thermocouple readings for Tank 241-BY-101 are in Appendix D.

\subsubsection{Integrity of Tank 241-BY-101}

Tank 241-BY-101 is categorized as sound and is interim stabilized with intrusion prevention completed. The Tank 241-BY-101 surface level is monitored quarterly with a manual tape through riser 5. Liquid waste volume is determined by a photographic evaluation and the solid waste volume is determined by a manual tape surface level gauge. The maximum allowable deviation from the 144.5-in. baseline is a 3 in. increase. There is no criterion for a decrease. The figure that graphically represents the surface level measurements from January 1991 to the present is in Appendix E. The Tank 241-BY-101 surface level monitoring is out of compliance. The last readings were taken in June 1993. The surface level plot before June 1993 indicates fluctuating readings between 144 and 141 -in.

The Tank 241-BY-101 liquid observation well is located in riser 9A. The tank is monitored weekly with a neutron probe and on 
WHC-SD-WM-ER-312, Rev. 0

request with a gamma probe to determine the interstitial liquid level. The maximum deviations from the established baseline are a 0.3 -ft increase and a 0.4-ft decrease. Sketch ES-TKS-E39 in Appendix $C$ is a graphical representation of the liquid observation well data.

Five drywells are identified for Tank 241-BY-101. Drywells 22-01-04 (active prior to 1990, current readings $>200 \mathrm{c} / \mathrm{s}$ ) and 2201-10 (active prior to 1990, current readings <200 c/s) have readings greater than the $50 \mathrm{c} / \mathrm{s}$ background radiation.

Graphical representations of the drywell data for each active drywell from January 1990 to the present are in Appendix F. Each graph includes the peak c/s (some wells have multiple peaks), the depth at which the peak occurred, and the date of the reading.

\subsubsection{Historical Sample Analysis Data for Tank 241-BY-101}

Two waste sample analyses were performed on Tank 241-BY-101. One sample was analyzed in December 1955 and the other was analyzed in August 1972. The 1972 sample was a clear, green solution containing a small amount of solids. Tables presenting the chemical and physical data obtained from these analyses are in Appendix $\mathrm{H}$.

\subsubsection{Current Status of Tank 241-BY-101}

Tank 241-BY-101 entered service in January 1950 and currently stores $387,000 \mathrm{gal}$ of waste. The waste is comprised of $350,000 \mathrm{gal}$ of saltcake and 37,000 gal of unknown waste with no supernatant or pumpable liquid remaining. The tank is identified as a low-heat load tank, is passively ventilated, and is categorized as sound with interim stabilization and intrusion prevention completed. Tank 24I-BY-101 is equipped to cascade to Tank 241-BY-102 and is first in the three-tank cascade series. The plan view and tank cross-section that depict the approximate waste level and riser configuration are in Appendix G. Tank 241-BY-101 has 18 risers and one 12-in. riser, no. 10A, is available for use.

\subsubsection{Waste Layer Volume Approximation for Tank 241-BY-101}

A comparative analysis of all layer approximations for the entire tank farm and the data used to derive the tank layer approximations are located in Appendix $\mathrm{K}$.

\subsubsection{In-Tank Photograph 241-BY-101}

The 1989 assembled photographs of the Tank 241-BY-101 interior indicates a yellow and grey saltcake surface with no visible liquid (see Appendix I). The waste measures approximately $11.5 \mathrm{ft}$ deep. The picture indicates solids clinging to the some of the equipment. The in-tank solidification prototype air heater circulator is visible in the top right of the layout. The debris shown next to the liquid observation well is equipment which has been cut off. 
WHC-SD-WM-ER-312, Rev. 0

Picture quality is good and all the equipment in the tank is easily identified and has been labelled. There have been no changes in the tank which would affect the waste since these photographs were taken; therefore, the picture should be representative of the current tank contents.

2.1.3 Synopsis Tank 241-BY-101

(To be completed) 
WHC-SD-WM-ER-312, Rev. 0

2.2 Tank 241-BY-102

2.2.1 241-BY-102 Tank History

2.2.1.1 Waste History Tank 241-BY-102

The waste history narrative in the following paragraph is from "A History of the 200 Area Tank Farms" (Anderson 1990). Tank 241BY-102 began operation in september 1950 by receiving metal wastes from the Tank 241-BY-101 cascade overflow. The cascade series was full by the first quarter of 1951. The tank continued to receive and transfer waste via the cascade lines through the first quarter of 1954. Tank 241-BY-102 sluicing began in January 1954 and was released as empty on July 13, 1954. Tributyl phosphate waste was transferred from Tank 241-BY-105 and filled Tank 241-BY-102 by the second quarter of 1955. The tank received a small infusion of coating waste during the third quarter of 1957. Various tributyl phosphate transfers into Tank 241-BY-102 occurred during the last two quarters of 1957 and all outgoing transfers went to trenches. There were no transfers between the first quarter of 1958 and the third quarter of 1964. From the first quarter of 1961 to the second quarter of 1965, the level measurement was taken on a sixmonth basis. A large amount of coating waste from Tank 241-C-102 was deposited in the fourth quarter of 1964 and the second quarter of 1965. Tank 241-BY-102 contained evaporator bottoms waste and was a feed and receiver tank for the in-tank solidification program from the fourth quarter of 1966 through the second quarter of 1976 .

Tank 241-BY-102 did not receive any primary waste additions according to the "Waste Status and Transaction Record Summary" (Agnew 1994) report.

The tank was deactivated in the third quarter of 1976 and is considered a sound tank awaiting interim stabilization. The tank was partially interim isolated in December 1982. The liquid was jet pumped on June 13, 1991. The draw down from pumping is noticeable in the liquid, solid, and liquid observation well waste levels. Solids level adjustments were made in June 1978, March 1982, March 1983, and April through August 1991. Currently, the tank contains $341,000 \mathrm{gal}$ of saltcake with no reported supernatant or sludge. The saltcake results from the use of the tank for InTank Solidification Unit Number 1 (ITS 1). In-tank photographs were taken on the following dates: October 1971, October 1973, April 1976, several in 1977, September 1979, and september 1987. Sketch ES-TKS-E40, which shows a graphical representation of the Tank 241-BY-102 level history, and the historical waste level data used to make it are in Appendix $C$. The sketch shows three $\mathrm{pH}$ readings: 9.4 in May 1955, >14.0 in september 1974, and 13.4 in November 1990. Currently temperature readings are unavailable due to the thermocouples being out of service. 
The single thermocouple tree in Tank 241-BY-102 had 14 thermocouple probes monitor the waste temperature. No thermocouple tree is currently available according to the riser configuration document. The thermocouples have similar temperature readings that span from 1974 to 1979. Unlike the rest of the probes, thermocouple 1 has one reading in November 1991.

The mean temperature of the first data recorded for thermocouples 1 through 10 was $47^{\circ} \mathrm{F}$. A sum of the squares regression line fit for the data shows a temperature increase with a large amount of variability. Throughout the time frame readings were taken, there were temperatures fluctuation of up to $60^{\circ} \mathrm{F}$. An out lying low temperature of approximately $17^{\circ} \mathrm{F}$ was common to all the thermocouples in May 1976. The median temperature is $71^{\circ} \mathrm{F}$ with a minimum of $25^{\circ} \mathrm{F}$ and a maximum of $126.5^{\circ} \mathrm{F}$. Tank 241-BY-102 has a semiannual temperature reading requirement in January and July.

Plots of the thermocouple readings for Tank 241-BY-102 are in Appendix D.

\subsubsection{Integrity of Tank 241-BY-102}

Tank 241-BY-102 is categorized as sound and is partially isolated. The Tank 241-BY-102 surface level is monitored daily with a manual tape through riser 5. Liquid waste volumes are determined by a manual tape and a photo evaluation; the solid waste volume is determined by a manual tape. The maximum allowable deviations from the 81-in. baseline value are a 2 -in. increase, and a 2-in. decrease. The figure that graphically represents the surface level measurements from January 1991 to the present is in Appendix E. The waste level dropped in the middle of 1991 which corresponds with the jet pumping effort conducted in June 1991. Since the current baseline was established on November 8, 1991, the surface level has remained steady between 81 and 81.25 in.

The Tank 241-BY-102 liquid observation well is located in riser 1. The tank is monitored weekly with a neutron probe and on request with a gamma probe to determine the interstitial liquid levels. The maximum deviations from the established baseline are a 0.3-ft increase or a 0.4-ft decrease. Sketch ES-TKS-E40 in Appendix $C$ has a graphical representation of the liquid observation well data.

Five drywells are identified for Tank 241-BY-102: drywells 2202-01 (active prior to 1990 , current readings $>200 \mathrm{c} / \mathrm{s}$ ) and $22-02-$ 09 (active prior to 1990, current readings < $200 \mathrm{c} / \mathrm{s}$ ) have readings greater than the $50 \mathrm{c} / \mathrm{s}$ background radiation.

An occurrence report was issued in August 1974 for drywell 22 02-01. Increased radiation levels were attributed to horizontal migration of preexisting contamination from Tank 241-BY-103. 
An off-normal occurrence 10-day report was written on July 3 , 1991. Drywells around Tanks 241-BY-103 and -108 showed an increase in radiation levels while waste was being transferred from Tanks 241-BY-102 and -109 to the double-contained receiver Tank 244-BX. The drywells detected waste in the transfer lines. Transfer line flushing verified that the lines were not leaking because the radiation levels decreased.

Graphical representations of the data for each active drywell from January 1990. to the present are in Appendix F. Each graph includes the peak c/s (some wells have multiple peaks), the depth at which the peak occurred, and the date of the reading.

\subsubsection{Historical Sample Analysis Data for Tank 241-BY-102}

An analysis of Tank 241-BY-102 was conducted in December 1955 for an in-farm scavenging laboratory study. A table presenting the data that resulted from the study is in Appendix $H$.

\subsubsection{Current status of Tank 241-BY-102}

Tank 241-BY-102 entered service in July 1950 and currently stores $341,000 \mathrm{gal}$ waste. The waste is comprised of $312,000 \mathrm{gal}$ of saltcake; 29,000 gal of unknown waste; and no supernatant or sludge with $22,000 \mathrm{gal}$ of pumpable interstitial liquid remaining. The tank is identified as a low-heat load tank, is passively ventilated, and is categorized as sound with partial interim isolation completed. Tank 241-BY-102 is equipped to cascade to Tank 241-BY-103 and is second in the three-tank cascade series. The plan view and tank cross-section that depict the approximate waste level and riser configuration are in Appendix G. Tank 241BY-102 has 17 risers and two 12-in. risers, no. 8 and no. $10 \mathrm{~A}$, are available for use.

\subsubsection{Waste Layer Volume Approximation for Tank 241-BY-102}

A comparative analysis of all layer approximations for the entire tank farm and the data used to derive the tank layer approximations are located in Appendix K.

\subsubsection{In-Tank Photograph 241-BY-102}

The 1987 montage of the Tank 24I-BY-102 interior indicates a yellow-grey to off-white saltcake surface surrounding a liquid pool in the center of the tank. The liquid has a dark brown/black color and yet it is translucent (see Appendix I). Approximately 341,000 gal of saltcake are in the tank which measures about 10 ft deep. In the top center of the layout, the in-tank solidification unit \#1, air heater circulator is visible. In 1991 Tank 241-BY-102 was jet pumped and level adjustment made; therefore, the picture may not represent the current tank interior or tank contents.

2.2.3 Synopsis Tank 241-BY-102

(To be completed) 
WHC-SD-WM-ER-312, Rev. 0

2.3 Tank 241-BY-103

2.3.1 241-BY-103 Tank History

2.3.1.1 Waste History Tank 241-BY-103

The waste history narrative in the following paragraph is from "A History of the 200 Area Tank Farms" (Anderson 1990). Tank 241BY-103 was filled with metal waste from Tank 241-BY-102 from October 1950 to March 1951. The tank contained metal waste until the first quarter of 1954. Sluicing was used for uranium recovery in the second quarter of 1954 and the metal waste was sent to Tanks 241-C-104 and -105 and 241-BY-109. The tank was declared empty on June 3, 1954. From the first quarter of 1955 until the second quarter of 1965, the tank contained U Plant waste. During the first two quarters of 1956, the waste was considered to be scavenged waste awaiting rework. In the third quarter of 1957, waste from scavenging was sent to the CR process vault. The tank also contained PUREX high-level waste and $U$ Plant waste from the fourth quarter of 1957 until the second quarter of 1965. The tank contained coating waste from the third quarter of 1965 until the fourth quarter of 1970. The tank was used for in-tank solidification decanting in 1965 and for in-tank solidification from 1967 until 1968. From the fourth quarter of 1968 until the fourth quarter of 1970 , the tank contained organic wash waste, evaporator bottoms waste, and coating waste. Beginning in the first quarter of 1971 and into the second quarter of 1974, the tank contained evaporator bottoms waste from the in-tank solidification unit no. 2 process. The tank was declared an assumed leaker in 1973 with a leak volume greater than 5,000 gal. Presently, the tank waste is classified as non-complexed.

The waste history narrative of the primary additions that follow in this paragraph are from the "Waste Status and Transaction Record Summary" (Agnew 1994). Tank 241-BY-103 received PUREX cladding waste from the third quarter of 1965 until the third quarter of 1966 .

A saltwell was installed in 1977. Primary stabilization was completed in september 1979 and a level adjustment was made in February 1980. A level adjustment was made in March 1982 and partial isolation was completed in December 1982. A major level adjustment was made in september 1987 because of jet pumping and another level adjustment was made in April 1990. Plotted liquid observation data represents the supernatant level. The tank was pumped in 1987 but only to a smaller supernatant level. In-tank photographs were taken on the following dates: December 1970, January 1972, September 1977, August 1979, January 1980, two in 1981, July 1984, July 1986, July 1987., December 1988, September 1989, and April 1990. Sketch ES-TKS-E4I, which shows a graphical representation of the Tank 241-BY-103 level history, and the historical waste level data used to make it are in Appendix C. The sketch shows four pH readings: 9.3 in May 1955, 9.2 in July 1957, 
WHC-SD-WM-ER-312， Rev. 0

13.3 in November 1990, and 13.5 in June 1991. The current maximum temperature reading from October 1993 is $81^{\circ} \mathrm{F}$.

\subsubsection{Temperature History 241-BY-103}

The single thermocouple tree in Tank 241-BY-103 has 14 thermocouple probes to monitor the waste temperature. Elevations are available for all the thermocouple probes. Temperature readings for thermocouples 1 through 12 are similar and span from 1974 to the present. Thermocouples 13 and 14 have little or no data before January 1989 but follow the same trends thereafter. There is a gap in temperature data from February 1984 until February 1987. Review of the Tank 241-BY-103 level history indicates that for the time period the readings were recorded, thermocouples 1 through 7 were located in or near the solids level and thermocouples 8 through 14 were in the vapor space.

The mean temperature of the first data recorded for thermocouples 1 through 12 was $39^{\circ} \mathrm{F}$. A sum of the squares regression line fit for thermocouples 1 through 12 shows a slight temperature increase with a large amount of variability. Pronounced temperature fluctuations are apparent from 1974 to 1980 . There is an outlying data point of approximately $27^{\circ} \mathrm{F}$ in December 1981. Annual temperature undulations are apparent in the data after January 1992. For this data set, the median temperature is $75^{\circ} \mathrm{F}$ with a minimum of $33.8^{\circ} \mathrm{F}$ and a maximum of $137^{\circ} \mathrm{F}$. Tank $241-\mathrm{BY}-$ 103 is on the Ferrocyanide Watch List and has a weekly temperature monitoring requirement.

Plots of the thermocouple readings for Tank $241-B Y-103$ are in Appendix D.

\subsubsection{Integrity of Tank 241-BY-103}

Tank 241-BY-103 is categorized as an assumed leaker and is partially isolated. The Tank 241-BY-103 surface level is monitored quarterly with a manual tape through riser 5. Liquid waste volume is determined by a photographic evaluation and manual tape, and solid waste volume is determined by a manual tape surface level gauge. The maximum allowable deviations from the 154-in. baseline

is a 2-in. increase. There is no criterion for a decrease. The figure that graphically represents the surface level measurements from January 1991 to the present is in Appendix E. The surface level plot indicates a decreasing trend with the surface level readings ranging between 151.75 and 150.75 in. during 1991 and decreasing to 149.5 to 147.75 in. during 1993.

An occurrence report was issued in January 1990 due to water intrusion caused by rapid snow melt runoff through the pump pits.

The Tank 241-BY-103 liquid observation well is located in riser 10A. The tank is monitored weekly with a neutron probe and on request with a gamma probe to determine the interstitial liquid levels. The maximum deviations from the established baseline are 
WHC-SD-WM-ER-312, Rev . 0

a 0.3-ft increase and a 0.4-ft decrease. Sketch ES-TKS-E4I in Appendix $C$ has a graphical representation of the liquid observation well data.

Eight drywells are identified for Tank 241-BY-103. Drywells 22-03-04 (active prior to 1990, current readings $<200 \mathrm{c} / \mathrm{s}$ ), 22-0305 (active prior to 1990, current readings > 200 c/s), 22-03-06 (active prior to 1990, current readings $>200 \mathrm{c} / \mathrm{s}$ ), and 22-03-09 (active prior to 1990, current readings > $200 \mathrm{c} / \mathrm{s}$ ) have readings greater than the $50 \mathrm{c} / \mathrm{s}$ background radiation.

Graphical representations of the data for each active drywell from January 1990 to the present are in Appendix F. Each graph includes the peak c/s (some wells have multiple peaks), the depth at which the peak occurred, and the date of the reading.

\subsubsection{Historical Sample Analysis Data for Tank 241-BY-103}

In December 1955, a sample from Tank 241-BY-103 was analyzed for an in-farm scavenging laboratory study. In 1971, a chemical analysis was performed as part of a cooling curve study of the supernatant. Tables presenting the results of these two studies are in Appendix $\mathrm{H}$.

\subsubsection{Current Status of Tank 241-BY-103}

The 1986 assembled photographs of the Tank 241-BX-103 interior is poor quality and waste surface features are unidentifiable (see Appendix I). It appears that the color may be black. Records indicate that 57 tons of Portland cement was added to this tank (Boomer 1990). Some equipment in the tank is visible and it has been identified and labelled. The tank contains approximately $66,000 \mathrm{gal}$ of waste which corresponds to approximately $1.5 \mathrm{ft}$ in depth. There have been no changes in the tank which would affect the waste since these photographs were taken; therefore, the picture should be representative of the current tank contents.

\subsubsection{Waste Layer Volume Approximation for Tank 241-BY-103}

A comparative analysis of all layer approximations for the entire tank farm and the data used to derive the tank layer approximations are located in Appendix $\mathrm{K}$.

\subsubsection{In-Tank Photograph 241-BY-103}

The 1989 photographic montage of the Tank 241-BY-103 interior indicates an off-white solid material around the outside of the tank and a gray mixture in the center (see Appendix I). All the colors appear to have been biased toward blue, so true colors are uncertain. Equipment is clearly shown and it has been identified and labelled. There is no evidence of any liquid remaining in the tank. The tank contains approximately 400,000 gallons of waste which measures about $12 \mathrm{ft}$ deep. Level adjustments were made in April of 1990 and August of 1991; therefore, the picture may not 
WHC-SD-WM-ER-312， Rev. 0

represent the current tank interior. Tank 241-BY-103 is on the Ferrocyanide Watch List.

2.3.3 Synopsis Tank 241-BY-103

(To be completed) 
WHC-SD-WM-ER-312, Rev. 0

2.4 Tank 241-BY-104

2.4.1 241-BY-104 Tank History

2.4.1.1 Waste History Tank 241-BY-104

The waste history narrative in the following paragraph is from "A History of the 200 Area Tank Farms" (Anderson 1990). Metal waste was introduced to the tank in the first quarter of 1950 through Tank 241-BX-106 cascade. The tank was filled with metal waste from March to June 1951. The tank cascade overflowed to Tank BY-105 during 1951. The tank contained metal waste until the first quarter of 1954 when it was sluiced for uranium recovery. From the second quarter of 1955 until the second quarter of 1967, the tank contained $U$ Plant waste. The tank was caverned in the fourth quarter of 1955. Periodically, accumulated sludge from the ferrocyanide scavenging process was sent to Tank 241-BY-104. From the first quarter of 1961 until the first quarter of 1968, the tank contained coating waste. Between the second quarter of 1968 and the first quarter of 1970, the tank contained ion exchange waste. From the second quarter of 1970 until the second quarter of 1976 , the tank received evaporator bottoms waste. The evaporator bottoms waste was from the in-tank solidification process. The tank contained evaporator feed bottoms waste from the third quarter of 1976 until the fourth quarter of 1977. Presently, the waste is classified as non-complexed.

Tank 241-BY-104 did not receive any primary waste additions according to the "Waste Status and Transaction Record Summary" (Agnew 1994) report.

A saltwell was installed in 1977 and the tank was declared inactive in 1978. The pump was removed in 1979. A level adjustment was made in April 1982. The tank was saltwell-pumped again in May 1983 and the tank was declared interim stabilized in January 1985. Intrusion prevention was completed in september 1990. Liquid observation well data have been plotted. Based on the saltwell pumping and interim stabilization dates, an engineering judgement was made that predicted levels before data were known. In-tank photographs were taken on the following dates: several in 1970, October 1973, two in 1976, september 1977, February 1981, and April 1983. Sketch ES-TKS-E42, which shows a graphical representation of the Tank 241-BY-104 level history, and the historical waste level data used to make it are in Appendix $C$. The sketch shows two pH readings: 9.3 in November 1955 and $>14.0$ in March 1976. The current maximum temperature readings from October 1993 are $128^{\circ} \mathrm{F}$ and $115^{\circ} \mathrm{F}$ for thermocouples in risers 1 and $10 \mathrm{~B}$ respectively.

\subsubsection{Temperature History 241-BY-104}

Tank 241-BY-104 has two thermocouple trees to monitor the waste temperature. Tree 1 has 12 thermocouple probes and tree 2 has 6 thermocouple probes. Elevations are available for all the 
thermocouples probes except 7 through 12 on tree 1 . Thermocouples 1 through 6 on tree 1 have similar data that span from 1974 to the present. Thermocouples 7 through 12 show the same trends but have little or no data after January 1983. On tree 2, all thermocouples have similar information that span from september 1992 to the present.

Review of the Tank 241-BY-104 level history indicates that tree 1 , thermocouples 1 through 5 were in or near the solids level and thermocoumples 6 through 12 were in the vapor space. For tree 2 , thermocouples 1 through 5 were in or near the solids level and thermocouple 6 was in the vapor space.

The mean temperature of the first recorded data from thermocouple tree 1 was $69^{\circ} \mathrm{F}$ and the mean temperature from thermocouple tree 2 was $101^{\circ} \mathrm{F}$. A sum of the squares regression line fit for all the data shows a slight temperature decrease with a moderate amount of variability. The temperature decreased as much as $100^{\circ} \mathrm{F}$ from the bottom to the top of the tank. For all the thermocouples on tree 1 , there is a significant increase in temperature of approximately $100^{\circ} \mathrm{F}$ from May to June 1975. Annual temperature fluctuations are apparent in tree 2. The median temperature is $122^{\circ} \mathrm{F}$ with a minimum of $51^{\circ} \mathrm{F}$ and a maximum of $237^{\circ} \mathrm{F}$. Tank 241-BY-104 is no longer connected to an operating exhauster for temperature control. Tank 241-BY-104 is on the Ferrocyanide Watch Iist and has a weekly temperature monitoring requirement.

Plots of the thermocouple readings for Tank 241-BY-104 are in Appendix D.

\subsubsection{Integrity of Tank 241-BY-104}

Tank 241-BY-104 is categorized as sound and is interim stabilized with intrusion prevention completed. The Tank 24I-BY-104 surface level is monitored quarterly with a manual tape through riser 5. Liquid waste volume is determined by a photographic evaluation and solid waste volume is determined with a manual tape surface level gauge. The maximum allowable increase from the 131-in. baseline is 3 in. There is no criterion for a decrease. The figure that graphically represents the surface level measurements from January 1991 to the present is in Appendix $E$. The surface level plot has fluctuating readings with a decreasing trend. The readings range from 131.75 to 131 in. during the first quarter of 1991 and decrease to 128.59 to 128.5 in. during the fourth quarter of 1993.

The Tank 241-BY-104 liquid observation well is located in riser 10C. The tank is monitored weekly with a neutron probe and on request with a gamma probe to determine the interstitial liquid level. The maximum deviations from the established baseline are a $0.3-\mathrm{ft}$ increase and a 0.4-ft decrease. Sketch ES-TKS-E42 in Appendix $C$ has a graphical representation of the liquid observation well data. 
Five drywells are identified for Tank 241-BY-104. Drywells 22-04-09 (active prior to 1990, current readings $<200 \mathrm{c} / \mathrm{s}$ ) has readings greater than the $50 \mathrm{c} / \mathrm{s}$ background radiation.

Graphical representations of the data for the active drywell from January 1990 to the present is in Appendix F. The graph includes the peak c/s, the depth at which the peak occurred, and the date of the reading.

\subsubsection{Historical Sample Analysis Data for Tank 241-BY-104}

No data are available or applicable. A letter published in November 1977 indicated that significant temperature gradients had been detected in the tank. According to historical records, Tank 241-BY-104 has been identified as the tank with the greatest amount of ferrocyanide wastes. There is a reference in current records to a sample analyzed in 1976 but the data are not available.

\subsubsection{Current Status of Tank 241-BY-104}

Tank 241-BY-104 entered service in February 1951 and currently stores $406,000 \mathrm{gal}$ of waste. The waste is comprised of $256,000 \mathrm{gal}$ of saltcake and 150,000 gal of sludge with no saltcake or pumpable liquid remaining. The tank is identified on the Ferrocyanide Watch List, is passively ventilated, and is categorized as sound with interim stabilization and intrusion prevention completed. Tank 241-BY-104 is equipped to cascade to Tank 241-BY-105 and is first in the three-tank cascade series. The plan view and tank crosssection that depict the approximate waste level and riser configuration are in Appendix G. Tank 241-BY-104 has 15 risers and two 4-in. risers, no. 3 and no. $10 \mathrm{~B}$ are available for use.

\subsubsection{Waste Layer Volume Approximation for Tank 241-BY-104}

A comparative analysis of all layer approximations for the entire tank farm and the data used to derive the tank layer approximations are located in Appendix $\mathrm{K}$.

\subsubsection{In-Tank Photograph 241-BY-104}

The 1983 montage of the Tank 241-BY-104 interior indicates an off-white to yellow solid surface interspersed with dark yellowbrown veins with a brown area at the base of the saltwell screen (see Appendix I). Three air lift circulators, a saltwell screen, a temperature probe and a manual tape have been identified and labelled. An old level measurement tape is lying on the waste surface in the center of the tank. The tank contains approximately 406,000 gal of waste deep which measures approximately $12 \mathrm{ft}$. Tank 241-BY-104 was jet pumped and interim stabilized in 1985 and it was saltwell pumped in 1984. The photographs were taken prior to the pumping and stabilization efforts; therefore, the picture does not represent the current tank contents. 
WHC-SD-WM-ER-312, Rev. 0

2.4.3 Synopsis Tank 241-BY-104

(To be completed) 
WHC-SD-WM-ER-312, Rev . 0

2.5 Tank 24I-BY-105

2.5.1 241-BY-105 Tank History

2.5.1.1 Waste History Tank 241-BY-105

The waste history narrative in the following paragraph is from "A History of the 200 Area Tank Farms" (Anderson 1990). Tank 241BY-105 was filled with metal waste from the cascade from June 1951 until the fourth quarter of 1951. The metal waste was sluiced for uranium recovery in the third quarter of 1954. The tank started receiving $U$ Plant waste from tanks 241-BY-107 and 241-BY-110 in the fourth quarter of 1954. The tank received $U$ Plant waste intermittently until the third quarter of 1966 . Coating waste was sent to the tank from the second quarter of 1962 until the fourth quarter of 1967. The tank was an in-tank solidification bottoms receiver during the first two quarters of 1967. The tank contained evaporator bottoms waste from the in-tank solidification program between the first quarter of 1968 to the second quarter of 1974. The tank was removed from service in the fourth quarter of 1974. The tank was saltwell pumped from 1975 to 1976. Sixty-three tons of Portland cement were added in 1977. The tank had questionable integrity and was primary stabilized in 1978. Presently, the tank waste is classified as non-complexed.

The waste history narrative of the primary additions that follow in this paragraph are from the "Waste status and Transaction Record Summary" (Agnew 1994). Tank 241-BY-105 received wastewater from the third quarter of 1957 until the fourth quarter of 1974.

A level adjustment was made in April 1982 and partial isolation was completed in December 1982. The tank was declared an assumed leaker in 1984 with a leak volume of approximately 8,000 gal. Iiquid observation well data were plotted. An engineering judgement was made prior to March 1986 based on pumping that started in 1975 and ended in 1976. In-tank photographs were taken on the following dates: several in 1970, April 1976, February 1981, November 1982, and July 1986. Sketch ES-TKS-E43 which shows a graphical representation of the Tank 241-BY-105 level history, and the historical waste level data used to make it are in Appendix $C$. The sketch shows three $\mathrm{pH}$ readings: 9.3 in July $1956,13.2$ in November 1990, and 13.3 in June 1991. The current maximum temperature readings from october 1993 are $120^{\circ} \mathrm{F}$ and $114^{\circ} \mathrm{F}$ for thermocouples in risers 1 and $10 \mathrm{C}$ respectively.

\subsubsection{Temperature History 241-BY-105}

Tank 241-BY-105 has two thermocouple trees to monitor the waste temperature: tree 1 has 16 thermocouple probes and tree 2 has 12 thermocouple probes. Elevations are available for all the thermocouple probes, except for probes 15 and 16 on tree 1 and probes 7 through 12 on tree 2 . No data are available on thermocouple 11 of tree 2 for this tank. Thermocouples 1 through 6 on tree 1 show similar readings that span from 1974 to the 
present. A gap in the recorded data occurs between 1982 and 1985 . Thermocouples 7 through 12 on tree 1 have similar temperature readings but have a gap in the data between 1982 and 1992. Data for thermocouples 13 through 16 on tree 1 are sparse. Thermocouples 13 and 14 each have 6 data points, thermocouple 15 has 4 data points, and thermocouple 16 has 3 data points. Data for tree 2 are similar for all thermocouples and are available only after January 1992 .

The Tank 241-BY-105 level. history indicates that from September 1974 to October 1982, thermocouples 1 through 7 were in or near the solids/liquid level, thermocouples 8 and 9 were in or near the solids level, and thermocouples 10 through 16 appear to have been in the vapor space. Thereafter, thermocouples 1 through 7 were still in or near the solids/liquid level, thermocouple 8 was in or near the solids level, and the rest appear to have beeen in the vapor space.

The mean temperature of the first recorded data was $47^{\circ} \mathrm{F}$ for thermocouple tree 1 and $97^{\circ} \mathrm{F}$ for thermocouple tree 2 . A sum of the squares regression line fit for thermocouple tree 1 shows a slight temperature decrease with a moderate amount of variability. Temperatures increase more than $50^{\circ} \mathrm{F}$ from the bottom to the top of the tank. For all the thermocouples on tree 1, a significant increase in temperature of approximately $75^{\circ} \mathrm{F}$ occurred from May to June 1975. Not enough data are available for an accurate regression analysis on thermocouple tree 2. Annual temperature fluctuations are apparent in tree 2. The median temperature is $103^{\circ} \mathrm{F}$ with a minimum of $44^{\circ} \mathrm{F}$ and a maximum of $179.6^{\circ} \mathrm{F}$. The tank is no longer connected to an operating exhauster for temperature control. Tank 241-BY-105 is on the Ferrocyanide Watch List and has weekly temperature monitoring requirements.

Plots of the thermocouple readings for Tank 241-BY-105 are in Appendix D.

\subsubsection{Integrity of Tank 241-BY-105}

Tank 24I-BY-105' is categorized as an assumed leaker and is partially isolated. The Tank 241-BY-105 surface level is monitored quarterly with a manual tape through riser 5. Liquid waste volume is determined by a photographic evaluation, and solid waste volume is determined by a manual tape surface level gauge and a photographic evaluation. The maximum allowable increase from the 182-in. baseline is 2 in. There is no criterion for a decrease. The figure that graphically represents the surface level measurements from January 1991 to the present is in Appendix E. The surface level graph shows fluctuating data with a decreasing trend. The readings range from 181.75 to 181 in. during the first quarter of 1991 and decrease to 173.25 to 172 in. during the fourth quarter of 1993.

The Tank 241-BY-105 liquid observation well is located in riser 10B. The tank is monitored weekly with a neutron probe and 
on request with a gamma probe to determine the interstitial Iiquid levels. The monitoring limits are a 0.3 -ft decrease and a 0.4 -ft increase. Sketch ES-TKS-E43 in Appendix C has a graphical representation of the liquid observation well data.

A discrepancy report was issued in June 1993 because the interstitial liquid level increased to the criteria limit. The tank has had a slow increasing trend since 1986, but the surface level readings show a decrease. This phenomena could be caused by solids dissolution or the formation of a depression in the solids beneath the plummet in conjunction with an intrusion.

Three drywells are identified for Tank 241-BY-105. Drywells 22-05-01 (not active prior to 1990, but with two erratic readings $>50 \mathrm{c} / \mathrm{s}$, and 22-05-09 (active prior to 1990, current readings < $200 \mathrm{c} / \mathrm{s}$ ) have or had readings greater than the $50 \mathrm{c} / \mathrm{s}$ background radiation.

Graphical representations of the drywell data for each active drywell from January 1990 to the present are in Appendix F. Each graph includes the peak c/s, the depth at which the peak occurred, and the date of the reading.

2.5.1.4 Historical Sample Analysis Data for Tank 241-BY-105

None available or applicable.

2.5.2 Current Status of Tank 241-BY-105

Tank 241-BY-105 entered service in June 1951 and currently stores $503,000 \mathrm{gal}$ of waste. The waste is comprised of $337,000 \mathrm{gal}$ of saltcake; $166,000 \mathrm{gal}$ of sludge; and no supernatant with 169,000 gal of pumpable interstitial liquid remaining. The tank is identified on the Ferrocyanide Watch List, is passively ventilated, and is categorized as an assumed leaker with partial isolation completed. Tank 241-BY-105 is equipped to cascade to Tank 241-BY106 and is second in the three-tank cascade series. The plan view and tank cross-section that depict the approximate waste level and riser configuration are in Appendix G. Tank 241-BY-105 has 19 risers and one 12-in. riser no. 7 is available for use.

\subsubsection{Waste Layer Volume Approximation for Tank 241-BY-105}

A comparative analysis of all layer approximations for the entire tank farm and the data used to derive the tank layer approximations are located in Appendix $\mathrm{K}$.

\subsubsection{In-Tank Photograph 241-BY-105}

The 1982 assembled photographs of the Tank 241-BY-105 interior indicates a mostly solid surface with colors ranging from white to tan to light brown (see Appendix I). The saltwell screen in the center of the photo extends down into a pool of reddish-brown 
liquid. In 1966, 63 tons of Portland cement were added to the tank (Welty 1988). The Iiquid observation well, temperature probe, and air lift circulator descend into depressions in the crust. The tank contains approximately $503,000 \mathrm{gal}$ of waste which measures about $15 \mathrm{ft}$ deep. A leak was reported in 1984; therefore, the picture may not represent the current tank contents.

2.5.3 Synopsis Tank 241-BY-105

(To be completed) 
WHC-SD-WM-ER-312, Rev. 0

2.6 Tank 241-BY-106

2.6.1 241-BY-106 Tank History

2.6.1.1 Waste History Tank 241-BY-106

The waste history narrative in the following paragraph is from "A History of the 200 Area Tank Farms" (Anderson 1990). Because Waste from Tank 241-BY-105 never overflowed into Tank 241-BY-106; therefore, Tank-241-BY-106 never contained metal waste from Tank 241-BY-105. Tank 241-BY-106 began to receive first-cycle waste in the second quarter of 1953. The tank held first-cycle waste until it was sluiced for uranium recovery in the fourth quarter of 1954. From the fourth quarter of 1954 until the first quarter of 1967, the tank intermittentIy received $U$ Plant waste. In the first quarter 1956, the tank was used as a settling tank for ferrocyanide scavenged waste. During 1956 and 1957, waste was sent to a crib and a ditch. Beginning in the fourth quarter of 1961, the tank received coating waste periodically until the second quarter of 1970. In the first quarter of 1968, the tank began to receive evaporator bottoms waste. The tank received in-tank solidification bottoms and recycle waste between the third quarter of 1970 and the second quarter of 1976. The tank received evaporator feed from the third quarter of 1976 until the fourth quarter of 1977. Activity in the tank was restricted in the third quarter of 1976. Presently, the tank waste is classified as non-complexed.

The waste history narrative of the primary additions that follow in this paragraph are from the "Waste status and Transaction Record Summary" (Agnew 1994). Tank 241-BY-106 received in-plant ferrocyanide waste from the first quarter of 1955 until the first quarter of 1957. From the third quarter of 1957 until the third quarter of 1974 , the tank received wastewater.

Saltwell pumping began in the first quarter of 1977 and the tank was declared inactive in 1977. Saltwell pumping was completed in 1978. A level adjustment was made in April 1992 and partial isolation was completed in December 1982. The tank was declared an assumed leaker in 1984 with a leak volume of approximately 8,000 gal. Liquid observation well data that have been plotted appear to be in error. In-tank photographs were taken on the following dates: December 1970, December 1971, two in 1972, two in 1977, August 1979, February 1981, and November 1982. Sketch ES-TKS-E44, which shows a graphical representation of the Tank 241-BY-106 level history, and the historical waste level data used to make it are in Appendix C. The sketch shows twelve $\mathrm{pH}$ readings: 9.4 in March 1955, 8.8 in June 1955, 9.8 in December 1955, 10.0 in February $1956,9.8$ in May 1956, 9.7 in July 1956, 8.8 in August 1956, 9.2 in October 1956, 9.6 in December 1956, 9.4 in February 1957, >14.0 in April 1972, and 13.5 in November 1990. The current maximum temperature reading from October 1993 is $129^{\circ} \mathrm{F}$. 
WHC-SD-WM-ER-312, Rev. 0

(active prior to 1990 , current readings $<200 \mathrm{c} / \mathrm{s}$ ) have or had readings greater than the $50 \mathrm{c} / \mathrm{s}$ background radiation.

Graphical representations of the data for each active drywell from January 1990 to the present are in Appendix F. Each graph includes the peak $\mathrm{c} / \mathrm{s}$ (some wells have multiple peaks), the depth at which the peak occurred, and the date of the reading.

2.6.1.4 Historical Sample Analysis Data for Tank 241-BY-106

Two analyses were conducted for Tank 241-BY-106: one in April 1972 and one in October 1971. A sample of the tank supernatant was analyzed in 1971 as part of an investigation to develop some cooling curves. In 1972, two samples were analyzed to determine if tank layering was occurring. The results showed no significant layering. Tables presenting the results of these analyses are in Appendix $\mathrm{H}$.

\subsubsection{Current Status of Tank 241-BY-106}

Tank 241-BY-106 entered service in 1950 and currently stores $642,000 \mathrm{gal}$ of waste. The waste is comprised of 544,000 gal of saltcake; 98,000 gal of sludge; and no supernatant with 213,000 gal of pumpable interstitial liquid remaining. The tank is identified on the Ferrocyanide Watch Iist, is passively ventilated, and is categorized as an assumed leaker with partial isolation completed. Tank 241-BY-106 is third in the three-tank cascade series. The plan view and tank cross-section that depict the approximate waste level and riser configuration are in Appendix G. Tank 241-BY-106 has 18 risers and two are available for use: one 4-in. riser (no. 4) and one 12-in. riser (no. 7).

\subsubsection{Waste Layer Volume Approximation for Tank 241-BY-106}

A comparative analysis of all layer approximations for the entire tank farm and the data used to derive the tank layer approximations are located in Appendix $\mathrm{K}$.

\subsubsection{In-Tank Photograph 241-BY-106}

The 1982 photographic montage of the contents of Tank 241-BY106 interior indicates a bright yellow to gold crusty material which appears to be sitting on top of or possibly mixed in with a white salt-like caked surface (see Appendix I). Throughout the surface are pools of reddish-brown liquid. Solids have formed around the air lift circulators, temperature probe, saltwell screen, and an old level measure tape. The tank contains approximately 642,000 gal of waste which measures about 19 ft deep. A leak was reported in 1984; therefore, the picture may not represent the current tank contents.

2.6.3 Synopsis Tank 241-BY-106

(To be completed) 
The single thermocouple tree in Tank 241-BY-106 has 12 thermocouple probes to monitor the waste temperature. Thermocouples 1 through 6 have similar temperature readings that span from 1974 to the present. Thermocouples 7 through 12 have readings similar to the first 6 thermocouples that span from 1974 to 1985. A distinct temperature decrease of approximately $40^{\circ} \mathrm{F}$ in thermocouple 6 occurred in January 1983. Review of the Tank 241BY-106 level history indicates that thermocouples 1 through 5 were in or near the solids level and the rest were in the vapor space.

The mean temperature for the first daily recorded temperature data was $60^{\circ} \mathrm{F}$. A sum of the squares regression line fit for thermocouples 1 through 6 shows a slight temperature decrease with a moderate amount of variability. Thermocouples 7 through 12 show no overall temperature change during a regression analysis; however, a moderate amount of variability exists in the data. There is a sharp temperature increase of approximately $90^{\circ} \mathrm{F}$ from June to July 1975. Two outlying points of approximately $52^{\circ} \mathrm{F}$ in October 1984 are common to all the thermocouples. The median temperature is $123.4^{\circ} \mathrm{F}$ with a minimum of $53^{\circ} \mathrm{F}$ and a maximum of 199.4 $4^{\circ} \mathrm{F}$. The tank is currently connected to an operating exhauster. Tank 241-BY-106 is on the Ferrocyanide watch List and weekly temperature readings are required.

Plots of the thermocouple readings for Tank 241-BY-106 are in Appendix D.

\subsubsection{Integrity of Tank 241-BY-106}

Tank 241-BY-106 is categorized as an assumed leaker and is partially isolated. The Tank 241-BY-106 surface level is monitored quarterly with a manual tape through riser 5. Liquid waste volume is determined by a photographic evaluation and solid waste volume is determined by a manual tape surface level gauge and a photographic evaluation. The maximum allowable increase from the 243.5-in. baseline is 2 in. There is no criterion for a decrease. The figure that graphically represents the surface level measurements from January 1991 to the present are in Appendix E. The surface level for the past 3 years has remained steady with readings ranging between 244.25 and 242.5 in.

The Tank 241-BY-106 liquid observation well is located in riser 10B. The tank is monitored weekly with a neutron probe and on request with a gamma probe to determine the interstitial liquid levels. The monitoring limits are a 0.3 -ft decrease and a 0.4 -ft increase. Sketch ES-TKS-E44 in Appendix $C$ has a graphical representation of the liquid observation well data.

Four drywells are identified for Tank 241-BY-106. Drywells 22-06-05 (active prior to 1990, current readings $>200 \mathrm{c} / \mathrm{s}$ ), 22-0607 (active prior to 1990 , current readings $<200 \mathrm{c} / \mathrm{s}$ ), and 22-06-11 
2.7.1 241-BY-107 Tank History

2.7.1.1 Waste History Tank 241-BY-107

The waste history narrative in the following paragraph is from "A History of the 200 Area Tank Farms" (Anderson 1990). Tank 241BY-107 was filled with first-cycle waste from september 1950 to March 1951. The tank.cascaded between 1951 and 1952 and from the first quarter of 1953 until the first quarter of 1954. The tank received first-cycle waste until the third and fourth quarters of 1952 when the tank was pumped to a heel. Between the first quarter of 1953 and the third quarter of 1956, the tank received firstcycle and $U$ Plant waste. The tank was pumped in the third quarter of 1954 and became a ferrocyanide scavenged waste receiver. From the third quarter of 1956 until the third quarter of 1968, the tank contained U Plant waste. From the fourth quarter of 1958 to the third quarter of 1969, the tank contained U Plant waste and coating waste. Between the fourth quarter of 1969 and the fourth quarter of 1974, the tank received evaporator bottoms waste from the intank solidification process. The tank integrity was questioned in 1974. Presently, the tank waste is classified as non-complexed.

The waste history narrative of the primary additions that follow in this paragraph are from the "Waste status and Transaction Record Summary" (Agnew 1994). Tank 241-BY-107 received first-cycle waste from the third quarter of 1950 until the third quarter of 1951. From the second quarter of 1953 until the first quarter of 1954, the tank received uranium recovery waste. The tank received in-plant ferrocyanide waste from the fourth quarter of 1954 until the second quarter of 1957. During the third quarter of 1958, the tank received wastewater.

A liquid observation well was installed in the tank in 1975. The tank was removed from service in the first quarter of 1976 and was saltwell pumped in the third quarter of 1976. The tank was primary stabilized in the first quarter of 1978. Saltwell pumping was completed in June 1979 and the tank was declared interim stabilized in July 1979. Intrusion prevention was completed in December 1982. The tank was declared an assumed leaker in 1984 with a leak volume of 15,100 gal. Liquid observation well data were plotted to represent the interstitial liquid level. Prior to 1986, the interstitial level was based on best engineering judgement. The tank was pumped in 1975 and pumping was completed in 1978. In-tank photographs were taken on the following dates: October 1969, two in 1970, June 1971, two in 1974, March 1975, May 1977, June 1979, January 1981, and October 1986. Sketch ES-TKSE45, which shows a graphical representation of the Tank 241-BY-107 level history, and the historical waste level data used to make it are in Appendix $C$. The sketch shows thirteen $\mathrm{pH}$ readings on the level history: 9.3 in November 1954, 9.4 in February 1955, 9.0 in April 1955, 9.9 in August 1955, 9.3 in October 1955, 10.0 in January 1956, 9.9 in March 1956, 9.6 in April 1956, 9.7 in June 
WHC-SD-WM-ER-312， Rev. 0

1956, 9.3 in October 1956, 9.4 in December 1956, 12.3 in April 1957, and $>14$ in July 1979. The current maximum temperature reading from October 1993 is $96^{\circ} \mathrm{F}$.

\subsubsection{Temperature History 241-BY-107}

The single thermocouple tree in Tank 241-BY-107 has 15 thermocouple probes to monitor the waste temperature. All the thermocouples have elevation data except thermocouple 15. Thermocouples 1 through 5 have similar temperature readings that span from 1974 to the present. For thermocouples 6 through 14, the available data are similar to the first 5 thermocouples; however, little data exist before 1990. There is a gap in temperature readings from April 1979 until November 1989, although a thermocouple tree was in the tank in October 1986. Thermocouple 15 has one data point. Review of the Tank 241-BY-107 level history indicates that from August 1974 to January 1978, thermocouples 1 through 6 were in or near the solids/liquid level and thermocouples 7 through 15 were in the vapor space. From January 1978 to April 1982, thermocouples 1 through 3 were in or near the solids/liquid level, thermocouples 4 through 6 were in or near the solids level, and the rest were in the vapor space. Due to a increase in the solids level between April 1982 and January 1984, thermocouples 1 through 3 were in or near the solids/Iiquid level, thermocouples 4 through 7 were in or near the liquid level, and the rest were in the vapor space. Thereafter, thermocouples 1 through 3 were in or near the solids/liquid level, thermocouples 4 through 6 were in or near the liquid level, and the rest were in the vapor space.

The mean temperature for the first daily recorded readings was $35^{\circ} \mathrm{F}$. A sum of the squares regression line fit for the first 10 thermocouples shows a slight temperature increase with a large amount of variability. Regression analysis shows that for thermocouples 11 through 14, there is no overall change in temperature and a moderate amount of variability. Not enough data points exist to do an accurate regression or variability analysis for thermocouple 15. Thermocouples 1 through 5 and 12 show a sharp temperature increase of approximately $70^{\circ} \mathrm{F}$ between June and July 1975. Variable temperatures are apparent for the data from 1974 to 1992. Annual temperature undulations are apparent after January 1992. The median temperature is $77.9^{\circ} \mathrm{F}$ with a minimum of $30.1^{\circ} \mathrm{F}$ and a maximum of $120.2^{\circ} \mathrm{F}$. Tank $241-\mathrm{BY}-107$ is a Ferrocyanide Watch List tank and weekly temperature readings are required.

Plots of the thermocouple readings for Tank 241-BY-107 are in Appendix D.

\subsubsection{Integrity of Tank 241-BY-107}

Tank 241-BY-107 is categorized as an assumed leaker and is interim stabilized with intrusion prevention completed. The Tank 241-BY-107 surface level is monitored quarterly with a manual tape through riser 4. Liquid waste volume is determined by a photographic evaluation and solid waste volume is determined by a 
manual tape surface level gauge and a photographic evaluation. The maximum allowable increase from the 103.5-in. baseline is 3 in. There is no criterion for a decrease. The figure that graphically represents the surface level measurements from January 1991 to the present is in Appendix $E$. The surface level for the past 3 years has remained steady with readings ranging between 104 and 103 in.

The Tank 241-BY-107 liquid observation well is located in riser 7. The tank is monitored weekly with a neutron probe and on request with a gamma probe to determine the interstitial liquid levels. The maximum deviations from the established baseline are a 0.3-ft increase and a 0.4-ft decrease. Sketch ES-TKS-E45 in Appendix $\mathrm{E}$ has a graphical representation of the liquid observation well data.

Six drywells are identified for Tank 241-BY-107. Drywells 2207-01 (active prior to 1990 , current readings $<200 \mathrm{c} / \mathrm{s}$ ), 22-07-02 (active prior to 1990, current readings < $200 \mathrm{c} / \mathrm{s}$ ), 22-07-05 (active prior to 1990, current readings < $200 \mathrm{c} / \mathrm{s}$ ), 22-07-07 (active prior to 1990, current readings < $200 \mathrm{c} / \mathrm{s}$ ), 22-07-09 (active prior to 1990, current readings > $200 \mathrm{c} / \mathrm{s}$ ), and 22-07-10 (active prior to 1990, current readings $>200 \mathrm{c} / \mathrm{s}$ ) have readings greater than the $50 \mathrm{c} / \mathrm{s}$ background radiation.

Occurrence reports were written in 1979 and 1980 due to increased activity in the drywells resulting from migration of contamination in the soil. The migration was attributed to snow melt, nearby water bibs, and condensate from a steam cleaner entering the soil.

Graphical representations of the drywell data for each active drywell from January 1990 to the present are in Appendix F. Each graph includes the peak c/s (some wells have multiple peaks), the depth at which the peak occurred, and the date of the reading.

\subsubsection{Historical Sample Analysis Data for Tank 241-BY-107}

No data are available or applicable. No units are shown on one table available.

\subsubsection{Current Status of Tank 241-BY-107}

Tank 241-BY-107 entered service in December 1950 and currently stores $266,000 \mathrm{gal}$ of waste. The waste is comprised of $149,000 \mathrm{gal}$ of saltcake and $117,000 \mathrm{gal}$ of sludge with no supernatant or pumpable liquid remaining. The tank is identified on the Ferrocyanide Watch List as of January 1991, is passively ventilated, and is categorized as an assumed leaker with interim stabilization and intrusion prevention completed. Tank 241-BY-107 is equipped to cascade to Tank 241-BY-108 and is first in the three-tank cascade series. The plan view and tank cross-section that depict the approximate waste level and riser configuration can be found in Appendix G. Tank 241-BY-107 has 17 risers and five are available for use (reference drawing H-2-73249 Rev. 2) : two 12-in. 
risers (no. 5 and no. 8) and three 18-in. risers (nos. 9B, 10A, and 12B).

2.7.2.1 Waste Layer Volume Approximation for Tank 241-BY-107

A comparative analysis of all layer approximations for the entire tank farm and the data used to derive the tank layer approximations are located in Appendix $\mathrm{K}$.

\subsubsection{In-Tank Photograph 241-BY-107}

The picture of the Tank 241-BY-107 interiors made up from 1986 photographs shows a rough saltcake surface, which is light yellow in color (see Appendix I). There are depressions in the surface where equipment currently extends into the waste or where equipment has been removed. This tank contains approximately $266,000 \mathrm{gal}$ of waste which measures about $7.5 \mathrm{ft}$ deep. There have been no changes in the tank that would affect the waste since these photographs were taken; therefore, the picture should be representative of the current tank contents.

2.7.3 Synopsis Tank 24I-BY-107

(To be completed) 
WHC-SD-WM-ER-312, Rev. 0

2.8 Tank 241-BY-108

2.8.1 241-BY-108 Tank History

2.8.1.1 Waste History Tank 241-BY-108

The waste history narrative in the following paragraph is from "A History of the 200 Area Tank Farms" (Anderson 1990). Tank 241BY-108 was filled with first-cycle waste from the Tank 241-BY-107 cascade from March 1951 until the second quarter of 1953. The tank received first-cycle waste until the first quarter of 1953. The tank received $U$ Plant waste from the second quarter of 1953 until the first quarter of 1959. The tank became a ferrocyanide scavenged waste receiver in 1954. Various transfers out of the tank went to a ditch or a crib. From the second quarter of 1959 until the first quarter of 1968, the tank contained U Plant waste and coating waste. Between the second quarter of 1968 and the second quarter of 1973 , the tank received evaporator bottoms waste. The evaporator bottoms waste was from the in-tank solidification process. The tank was declared an assumed leaker in 1972 with a leak volume greater than 5,000 gal. Presently, the tank waste is classified as non-complexed.

The waste history narrative of the primary additions that follow in this paragraph are from the "Waste status and Transaction Record Summary" (Agnew 1994). Tank 241-BY-108 received in-plant ferrocyanide waste from the fourth quarter of 1954 until the first quarter of 1957. From the third quarter of 1972 until the fourth quarter of 1975, the tank received wastewater.

Saltwell pumping started in the first quarter of 1977. The tank was primary stabilized and the pump was removed in 1978 . Partial isolation was completed in December 1982. A saltwell pump was installed in January 1984 and the tank was declared interim stabilized in February 1985. In-tank photographs were taken on the following dates: December 1968, October 1969, two in 1970, two in 1972, March 1974, October 1976, January 1980, April 1983, and October 1986. Sketch ES-TKS-E46, which shows a graphical representation of the Tank 241-BY-108 level history, and the historical waste level data used to make it are in Appendix $C$. The sketch shows thirteen $\mathrm{pH}$ readings: 9.0 in November $1954,9.4$ in February 1955, 9.1 in May 1955, 10.2 in October 1955, 9.7 in December 1955, 9.7 in February 1956, 9.6 in March 1956, 9.4 in May 1956, 9.7 in June 1956, 8.7 in August 1956, 9.1 in November 1956, 9.2 in January 1957, and 9.6 in April 1957. The current maximum temperature reading from October 1993 is $109^{\circ} \mathrm{F}$.

\subsubsection{Temperature History 241-BY-108}

The single thermocouple tree in Tank 241-BY-108 has 13 thermocouple probes to monitor the waste temperature. Elevations are available for the thermocouple probes except for probes 11 through 13. Thermocouples 1 through 10 have similar temperature readings that span from 1974 to the present. Limited data are 


$$
\text { WHC-SD-WM-ER-312, Rev . } 0
$$

available for thermocouples 11 through 13 . Thermocouple 11 has 9 disjointed data points, thermocouple 12 has 8 disjointed data points, and thermocouple 13 has 2 data points. There is a gap in temperature readings from October 1983 until November 1989, although a thermocouple tree was in the tank in October 1986. Review of the Tank 241-BY-108 level history, indicates that from September 1974 to October 1982, thermocouples 1 through 6 were in or near the solids level and thermocouples 7 through 13 were in the vapor space. Thereafter thermocouples 1 through 4 were in or near the solids level and the rest were in the vapor space.

The mean temperature for the first daily recorded readings was $42^{\circ} \mathrm{F}$. A sum of the squares regression line fit for the first 10 thermocouples shows no overall temperature change and a moderate amount of variability. From June to July 1975 , a $70^{\circ} \mathrm{F}$ temperature increase is common to the thermocouples. Variable temperatures are apparent from the data for september 1974 to October 1983. Sporadic data for thermocouples 11 through 13 render a regression and variability analysis invalid. The median temperature is $84^{\circ} \mathrm{F}$ with a minimum of $50^{\circ} \mathrm{F}$ and a maximum of $154^{\circ} \mathrm{F}$. Tank $241-\mathrm{BY}-108$ is on the Ferrocyanide Watch List and weekly temperature readings are required.

Plots of the thermocouple readings for Tank 241-BY-108 are in Appendix D.

\subsubsection{Integrity of Tank 241-BY-108}

Tank 24I-BY-108 is categorized as an assumed leaker and is interim stabilized with intrusion prevention completed. The Tank 241-BY-108 surface level is monitored quarterly with a manual tape through riser 4. Liquid waste volume is determined by a photographic evaluation and a manual tape surface level gauge. Solid waste volume is determined by a manual tape surface level gauge. The maximum allowable increase from the 88.25-in. baseline is 3 in. There is no criterion for a decrease. The figure that graphically represents the surface level measurements from January 1991 to the present is in Appendix E. The surface level for the past 3 years has remained steady and the readings range between 88.5 and 86 in.

An occurrence report was issued in January 1980 due to a $2,600-g a l$ liquid intrusion caused by rapid snowmelt into the tank through the pits.

Tank 241-BY-108 does not have a liquid observation well. Six drywells are identified for Tank 241-BY-108. Drywells 22-08-01 (active prior to 1990 , current readings $<200 \mathrm{c} / \mathrm{s}$ ), 22-08-02 (active prior to 1990, current readings > $200 \mathrm{c} / \mathrm{s}$ ), 22-08-05 (active prior to 1990, current readings < 200 c/s), 22-08-06 (active prior to 1990, current readings $<200 \mathrm{c} / \mathrm{s}$ ), and 22-08-12 (active prior to 1990 , current readings < $200 \mathrm{c} / \mathrm{s}$ ) have readings greater than the $50 \mathrm{c} / \mathrm{s}$ background radiation. 
Graphical representations of the drywell data for each active drywell from January 1990 to the present are in Appendix F. Each graph includes the peak c/s (some wells have multiple peaks), the depth at which the peak occurred, and the date of the reading.

\subsubsection{Historical Sample Analysis Data for Tank 241-BY-108}

No data are available or applicable.

\subsubsection{Current .Status of Tank 241-BY-108}

Tank 241-BY-108 entered service in April 1951 and currently stores $228,000 \mathrm{gal}$ of waste. The waste is comprised of 63,000 gal of saltcake and 165,000 gal of sludge with no supernatant or pumpable liquid remaining. The tank is identified on the Ferrocyanide Watch List, is passively ventilated, and is categorized as an assumed leaker with interim stabilization and intrusion prevention completed. Tank 241-BY-108 is equipped to cascade to Tank 241-BY-109 and is second in the three-tank cascade series. The plan view and tank cross-section that depict the approximate waste level and riser configuration are in Appendix G. Tank 241-BY-108 has 17 risers and five are available for use: three 4-in. risers (nos. 1, 2, and 5) and two 12-in. risers (nos. 9B and 12A).

\subsubsection{Waste Layer Volume Approximation for Tank 241-BY-108}

A comparative analysis of all layer approximations for the entire tank farm and the data used to derive the tank layer approximations are located in Appendix $\mathrm{K}$.

\subsubsection{In-Tank Photograph 241-BY-108}

The 1986 photographic montage of the Tank 241-BY-108 interior shows a white to light rose solid waste surface (see Appendix I). At the top center of the photo an air lift circulator, a temperature probe, and a manual tape are all clustered within a depression filled with liquid. The waste measures approximately $6.5 \mathrm{ft}$ deep. There have been no changes in the tank that would affect the waste since these photographs were taken; therefore, the picture should be representative of the current tank contents.

\subsubsection{Synopsis Tank 241-BY-108}

(To be completed) 
WHC-SD-WM-ER-312, Rev. 0

2.9 Tank 241-BY-109

2.9.1 241-BY-109 Tank History

2.9.1.1 Waste History Tank 241-BY-109

The waste history narrative in the following paragraph is from "A History of the 200 Area Tank Farms" (Anderson 1990). Tank 241BY-109 began operation on January 8, 1953 by receiving tributyl phosphate wastes from Tank 241-B-103. From the second quarter of 1953 through the second quarter of 1955, the tank received metal waste transfers from Tanks 241-BY-101, -108 , and -112 . Wastes were also transferred to Tank 241-C-104 at that time. A small transfer was returned from Tank 241-C-104 in the first quarter of 1955. Tributyl phosphate waste was transferred from Tank 241-BY-107 during the second quarter of 1956. In the second quarter of 1957, $677,000 \mathrm{gal}$ of waste were sent off to the BC-16 ditch. From the first quarter of 1961 to the second quarter of 1965, level measurements were taken every six months. Coating waste from Tanks 241-C-107 and -109 was transferred into Tank 241-BY-109 during the first half of 1962. More coating waste was added to the tank from a transfer between Tank 241-BY-112 and Tank 241-BY-101 during the first half of 1965. About $500,000 \mathrm{gal}$ of waste were transferred to Tank 241-BY-103 in the third quarter of 1966. From the fourth quarter of 1966 until the second quarter of 1967, Tank 241-BY-109 received more coating waste from Tank 241-C-102. Some waste was transferred to Tank 241-BY-103 and about the same volume was received from Tanks 241-BY-105 and -108. The tank was an in-tank solidification unit no.2 feed tank from the second half of 1968 through the second quarter of 1976. During the feed tank period, the tank received cladding waste, organic wash waste, and large volumes of evaporator bottoms waste. The tank then received concentrated feed and a saltwell was installed. In 1978, the waste was categorized as non-complexed.

The waste history narrative of the primary additions that follow in this paragraph are from the "Waste status and Transaction Record Summary" (Agnew 1994). Tank 241-BY-109 received wastewater from the third quarter of 1971 until the third quarter of 1975 .

The tank was deactivated on August 9, 1979 and a solids level adjustment was made. Another solids level adjustment was made in 1984 and the tank was partially interim isolated in June 1985. The liquid was jet pumped on June 16, 1991 and a solids level adjustment was made. The draw-down from pumping is noticeable in the liquids, solids, and liquid observation well waste levels. Intank photographs were taken on the following dates: October 1973, February 1977, February 1979, April 1983, and October 1986. Sketch ES-TKS-E47, which shows a graphical representation of the Tank 241-BY-109 level history, and the historical waste level data used to make it are in Appendix $\mathrm{C}$. Two $\mathrm{pH}$ readings are Iisted on the sketch: 13.2 in November 1990 and 13.4 in June 1991. Currently, temperature readings are unavailable due to out of service thermocouples. 


\subsubsection{Temperature History 241-BY-109}

The single thermocouple tree in Tank 241-BY-109 had 12 thermocouple probes to monitor the waste temperature. No thermocouple tree is currently available according to the riser configuration document. Limited data are available on all the thermocouples. All the thermocouples except 1 and 9 have similar temperature readings that span from 1976 to 1978. Thermocouple 1 has three additional data points taken in 1991 and thermocouple 9 has one data point.

The mean temperature for the first data recorded was $98^{\circ} \mathrm{F}$. A sum of the squares regression line fit for thermocouples 1 through 8 and 10 through 12 over the short time period shows a temperature decrease with a moderate amount of variability. Thermocouples 1 through 4 and 6 all have a sharp temperature decrease of approximately $65^{\circ} \mathrm{F}$ between June 1976 and March 1977. A regression analysis on thermocouple 9 would prove inconclusive due to lack of data. The median temperature is $66^{\circ} \mathrm{F}$ with a minimum of $51^{\circ} \mathrm{F}$ and a maximum of $138^{\circ} \mathrm{F}$. Tank 241-BY-109 currently has no thermocouple tree within the tank. No further temperature readings have been taken since December 1991, although temperatures should be monitored semiannually in January and July.

Plots of the thermocouple readings for Tank 241-BY-109 are in Appendix D.

\subsubsection{Integrity of Tank 241-BY-109}

Tank 241-BY-109 is partially interim isolated and is considered a sound tank awaiting interim stabilization. Tank 241BY-109 is categorized as sound and is partially isolated. The 241BY-109 Tank surface level is monitored daily with a Food Instrument Corporation gauge through riser 4. Liquid volumes are determined with a Food Instrument Corporation Gauge and the solids volume is determined by a photo evaluation and a sludge level measurement device. The Food Instrument Corporation gauge went out of service on July 17, 1993 and now weekly manual readings are required. If the Food Instrument Corporation gauge fails, manual field measurements will be conducted daily. The maximum allowable deviations from the 140.3-in. baseline are a 2-in. increase and a 2-in. decrease. The figure that graphically represents the surface level measurements from January 1991 to the present is in Appendix $\mathrm{E}$. The waste level drops in the middle of 1991 which corresponds with the saltwell pumping efforts that began in June 1991. Since the conclusion of the saltwell pumping, the surface level has remained steady with the readings ranging between 140.8 and 137.1 in. The graph showes erratic data points during the second, third, and fourth quarters of 1993. The erratic data were attributed to a mechanical malfunction in the automatic operation of the Food Instrument corporation gauge. When the unexpected readings were observed, the liquid observation well data and drywell data were checked to confirm the integrity of the tank. 
The Tank 241-BY-109 liquid observation well is located in riser 12B. The tank is monitored weekly with a neutron probe and on request with a gamma probe to determine the interstitial liquid levels. The maximum deviation from the established baseline is a 0.3 -ft increase or a 0.4-ft decrease. Sketch ES-TKS-E47 in Appendix $C$ contains a graphical representation of the liquid observation well data.

Six drywells are identified for Tank 241-BY-109. Drywells 2209-02 (active prior to 1990, current readings $<200 \mathrm{c} / \mathrm{s}$ ), 22-09-05 (active prior to 1990), 22-09-07 (active prior to 1990), 22-09-08 (active prior to 1990, current readings $>200 \mathrm{c} / \mathrm{s}$ ), and 22-09-11 (active prior to 1990, current readings < $200 \mathrm{c} / \mathrm{s}$ ) had or have readings greater than the $50 \mathrm{c} / \mathrm{s}$ background radiation.

An off-normal occurrence 10-day report was written on July 3 , 1991. Drywells around Tank 241-BY-103 and -108 showed an increase in radiation levels while waste was being transferred from Tanks 241-BY-102 and -109 to the double-contained receiver, Tank 244-BX. It was determined that the drywells were detecting the waste in the transfer lines. Flushing the transfer lines verified that the lines were not leaking because radiation levels decreased.

Graphical representations of the data for each active drywell from January 1990 to the present are in Appendix F. Each graph includes the peak c/s (some wells have multiple peaks), the depth at which the peak occurred, and the date of the reading.

\subsubsection{Historical Sample Analysis Data for Tank 241-BY-109}

A large number of samples were analyzed in 1972 and 1973 where supernatant samples were boiled and then cooled to increase solids content. All of the analyses included an estimate of cesium content and in a few cases the concentration of other radionuclides was also included in the data obtained. Tables presenting the data from these analyses are in Appendix $\mathrm{H}$.

\subsubsection{Current Status of Tank 241-BY-109}

Tank 241-BY-109 entered service in 1950 and currently stores 423,000 gal of non-complexed waste. The waste is comprised of $387,000 \mathrm{gal}$ of saltcake; $36,000 \mathrm{gal}$ of unknown waste; and no supernatant with 57,000 gal of pumpable interstitial liquid remaining. The tank is identified as a low-heat load tank, is passively ventilated, and is categorized as sound with partial isolation completed. Tank 241-BY-109 is third in the three-tank cascade series. The plan view and cross-section that depict the approximate waste level and riser configuration are in Appendix G. Tank 241-BY-109 has 19 risers and three 12-in. risers (nos. 9A, $10 B$, and $12 \mathrm{C}$ ) are available for use. 
WHC-SD-WM-ER-312, Rev. 0

2.9.2.1 Waste Layer Volume Approximation for Tank 241-BY-109

A comparative analysis of all layer approximations for the entire tank farm and the data used to derive the tank layer approximations are located in Appendix $\mathrm{K}$.

2.9.2.2 In-Tank Photograph 241-BY-109

The 1986 photographic montage of the Tank 241-BY-109 interior indicates a gold or blue liquid surface (difficult to determine source of coloration) over a submerged solids layer (see Appendix I). The liquid is clear enough to see the solids below the surface. The only equipment item in the tank is a saltwell screen. The waste measures approximately 12.5 ft deep. The tank was saltwell pumped in June 1991; therefore, the picture does not represent the current tank contents.

2.9.3 Synopsis Tank 241-BY-109

(To be completed) 
WHC-SD-WM-ER-312, Rev. 0

2.10 Tank 241-BY-110

2.10.1 241-BY-II0 Tank History

\subsubsection{Waste History Tank 241-BY-110}

The waste history narrative in the following paragraph is from "A History of the 200 Area Tank Farms" (Anderson 1990). Tank 241BY-110 began filling in the fourth quarter of 1951 with first-cycle waste. The tank contained first-cycle supernatant until the third quarter of 1954 when it was pumped to a ditch. The tank then received $U$ Plant waste until the third quarter of 1957. The tank was used as a ferrocyanide scavenged waste settling tank. Various transfers of waste were made to either a ditch or a trench. The tank received U-Plant waste and coating waste from the fourth quarter of 1957 until the third quarter of 1958. In the last quarter of 1968 and the first two quarters of 1969, the tank contained coating waste. From the second quarter of 1969 until the second quarter of 1976, the tank was used for storage of evaporator bottoms waste. The evaporator bottoms waste was from the in-tank solidification process. The tank contained concentrated evaporator feed bottoms waste from the third quarter of 1976 until the fourth quarter of 1977. Presently, the tank waste is classified as noncomplexed.

The waste history narrative of the primary additions that follow in this paragraph are from the "Waste status and Transaction Record Summary" (Agnew 1994). Tank 241-BY-110 received first-cycle waste from the fourth quarter of 1951 until the third quarter of 1952. During the third and fourth quarters of 1952, the tank received decontamination waste. From the fourth quarter of 1954 until the second quarter of 1957, the tank received in-plant ferrocyanide waste. During the fourth quarter of 1957, the tank received wastewater.

A saltwell was installed in 1977 and the tank was declared inactive in 1979. A level adjustment was made in september 1979. Saltwell pumping began in May 1983 and was completed in December 1984. Interim stabilization was completed in January 1985. Isiquid observation well data are plotted that represent the interstitial. liquid level. Prior to 1986, an engineering judgement was made based on pumping in 1983 and completion of pumping in 1984. Intank photographs were taken on the following dates: two in 1969, two in 1970, June 1971, March 1974, July 1975, April 1976, several in 1977, two in 1979, April 1983, and July 1984. Sketch ES-TKSE48, which shows a graphical representation of the Tank 241-BY-110 level history, and the historical waste level data used to make it are in Appendix $\mathrm{C}$. The sketch shows fifteen $\mathrm{pH}$ readings: 9.8 in October 1954, 9.1 in January 1955, 9.5 in April 1955, 9.2 in July $1955,9.3$ in September 1955, 9.8 in November 1955, 9.2 in January 1956, 9.6 in February 1956, 10.0 in April 1956, 9.8 in June 1956, 9.3 in August 1956, 9.4 in November 1956, 9.8 in January 1957, 11.9 in June 1957, and $>14.0$ in June 1976. The current maximum 
WHC-SD-WM-ER-312， Rev. 0

temperature readings from October 1993 are $118^{\circ} \mathrm{F}$ and $106^{\circ} \mathrm{F}$ for thermocouples in risers 1 and 10A respectively.

\subsubsection{Temperature History 241-BY-110}

Tank 241-BY-110 has 2 thermocouple trees to monitor the waste temperature: one has 14 thermocouple probes, and the other has 6 thermocouple probes. Elevations are available on both trees for thermocouples 1 through 6. Thermocouples 1 through 6 on tree 1 have similar temperature readings that span from 1974 to the present. Similar temperature readings are apparent for thermocouples 7 through 14 that span from 1974 to 1979 . Tree 2 thermocouple readings are similar to the first 6 thermocouples on tree 1, but only span from 1992 to the present.

Review of the Tank 241-BY-110 level history for thermocouple tree 1 indicates that from september 1974 to April 1985, probes 1 through 5 were located in or near the solids/liquid level and thermocouples 6 through 14 appear to have beeen in the vapor space. After April 1985, thermocouples 1 through 4 were in or near the solids/Iiquid level, thermocouple 5 was in or near the solids level, and the rest were in the vapor space. For tree 2, thermocouple probes 1 to 4 were in or near the solids/liquid level, thermocouple 5 was in or near the solids level, and thermocouple 6 appears to have been in the vapor space.

The mean temperature of the first daily recorded data was $45^{\circ} \mathrm{F}$ for tree 1 and $96^{\circ} \mathrm{F}$ for tree 2. A sum of the squares regression line fit of the thermocouples on tree 1 shows a temperature decrease with a moderate amount of variability. A sharp increase in temperature of up to $95^{\circ} \mathrm{F}$ is apparent for the tree between May and June 1975. The temperature decreases from the bottom to the top of the tank for a maximum difference in temperature between thermocouples 1 and 14 of $55^{\circ} \mathrm{F}$. The median temperature is $111.8^{\circ} \mathrm{F}$ with a minimum of $48^{\circ} \mathrm{F}$ and a maximum of $204.8^{\circ} \mathrm{F}$. Currently, the tank is connected to an operating exhauster. Tank 241-BY-110 is a Ferrocyanide Watch List tank and has a weekly tempurature moitoring requirement which resulted in more readings after October 1989.

Plots of the thermocouple readings for Tank 241-BY-110 are in Appendix D.

\subsubsection{Integrity of Tank 241-BY-110}

Tank 241-BY-110 is categorized as sound and is interim stabilized with intrusion prevention completed. The Tank 241-BY-110 surface level is monitored quarterly with a manual tape through riser 4. Liquid waste volume is determined by a manual tape surface level gauge, and solid waste volume is determined by a sludge level measurement device. The maximum allowable increase from the 151.75-in. baseline is 3 in. There is no criterion for a decrease. The figure that graphically represents the surface level measurements from January 1991 to the present is in Appendix $E$. 
The surface level plot indicates that most of the readings are between 90 to $100 \mathrm{in.}$ with four readings near the baseline value. These low readings do not exceed the decrease criteria and are attributed to the uneven surface of the saltcake.

The Tank 241-BY-110 liquid observation well is located in riser 3. The tank is monitored weekly with a neutron probe and on request basis with a gamma probe to determine the interstitial liquid levels. The maximum deviation from the established baseline value is a 0.3-ft increase or a 0.4-ft decrease. Sketch ES-TKS-E48 in Appendix $C$ has a graphical representation of the liquid observation well data.

Four drywells are identified for Tank 241-BY-110. None of these have or had readings greater than the $50 \mathrm{c} / \mathrm{s}$ background radiation.

\subsubsection{Historical Sample Analysis Data for Tank 241-BY-110 \\ No data are available or applicable.}

\subsubsection{Current status of Tank 241-BY-110}

Tank 241-BY-110 entered service in 1951 and currently stores 398,000 gal of waste. The waste is comprised of 208,000 gal of saltcake and $190,000 \mathrm{gal}$ of sludge with no supernatant or pumpable liquid remaining. The tank was identified on the Ferrocyanide Watch List in January 1991, is passively ventilated, and is categorized as sound with interim stabilization and intrusion prevention completed. Tank $241-B Y-110$ is equipped to cascade to Tank 241-BY-111 and is first in the three-tank cascade series. The plan view and cross-section that depict the approximate waste level and riser configuration are in Appendix G. Tank 241-BY-110 has 16 risers and one 18-in. riser (no. 10B) is available for use.

\subsubsection{Waste Layer Volume Approximation for Tank 241-BY-110}

A comparative analysis of all layer approximations for the entire tank farm and the data used to derive the tank layer approximations are located in Appendix $\mathrm{K}$.

\subsubsection{In-Tank Photograph 241-BY-110}

The 1984 photographic montage of the Tank 241-BY-110 interior indicates white chunks of waste distributed throughout a green matrix (see Appendix I). However, the entire tank is green; therefore, the colors are not true. There is no liquid visible anywhere on the waste surface. The saltwell screen, liquid observation well, and temperature probe appear to be totally imbedded in the salt cake. The tank contains approximately 398,000 gal of waste which measures about $12 \mathrm{ft}$ in depth. Tank 241-BY-110 was jet pumped and interim stabilized in 1985; therefore, the picture may not represent the current tank interior. 
WHC-SD-WM-ER-312, Rev . 0

2.10.3 Synopsis Tank 241-BY-110

(To be completed) 
WHC-SD-WM-ER-312, Rev . 0

2.11 Tank 241-BY-111

2.11.1 241-BY-111 Tank History

2.11.1.1 Waste History Tank 241-BY-111

The waste history narrative in the following paragraph is from "A History of the 200 Area Tank Farms" (Anderson 1990). Tank 241BY-I11 began filling with metal waste in the fourth quarter of 1951. The tank received metal waste, U plant waste, and high uranium TBP waste until the first quarter of 1955. The tank cascaded during 1952 and was sluiced in the first quarter of 1955. During the last three quarters of 1955 and the first quarter of 1957, the tank contained U Plant waste. From the second quarter of 1957 until the second quarter of 1965, the tank contained organic wash waste and coating waste. From the third quarter of 1965 until the first quarter of 1968, the tank contained coating waste. In the second quarter of 1968 until the second quarter of 1976, the tank stored evaporator bottoms waste from the in-tank solidification process. From the third quarter of 1976 until the fourth quarter of 1977, the tank contained evaporator feed. Presently, the waste is classified as non-complexed.

The waste history narrative of the primary additions that follow in this paragraph are from the "Waste status and Transaction Record Summary" (Agnew 1994). Tank 241-BY-111 received metal waste during the fourth quarter of 1951 and the first quarter of 1952 . During the fourth quarter of 1952 the tank received uranium recovery waste. During the fourth quarter of 1967 , the tank received PUREX cladding waste.

The tank was inactive in 1977 and was described as containing salt. A pump was installed in 1975 and the pumping was finished in 1978. Level adjustments were made in June 1980 and April 1982. After a saltwell pump was installed in September 1983 and the tank was pumped, Tank 241-BY-111 was declared interim stabilized in January 1985. Iiquid observation well data were plotted that represent the interstitial liquid level. Prior to 1986, an engineering judgement was made based on the saltwell pumping in 1983 and pumping was completed in 1985. In-tank photographs were taken on the following dates: December 1968, October 1969, March 1971, October 1973, November 1975, several in 1977, February 1978, February 1981, and October 1986. Sketch ES-TKS-E49, which shows a graphical representation of the Tank 241-BY-111 level history, and the historical waste level data used to make it are in Appendix $C$. The sketch shows one $\mathrm{pH}$ reading of 9.6 in June 1956. The current maximum temperature reading from October 1993 is $84^{\circ} \mathrm{F}$.

\subsubsection{Temperature History 241-BY-111}

Tank 241-BY-111 has 2 thermocouple trees to monitor the waste temperature: tree 1 has one thermocouple probe and tree 2 has six thermocouple probes. Thermocouple elevations are not available. Temperature readings for thermocouple tree 1 span from November 
WHC-SD-WM-ER-312, Rev . 0

1989 to the present. The thermocouples on tree 2 have similar readings that span from March 1993 to the present.

The first recorded temperature was $82^{\circ} \mathrm{F}$ for thermocouple tree 1 and $70^{\circ} \mathrm{F}$ for tree 2 . A sum of the squares regression line fit for all the data shows a slight temperature increase with a small amount of variability. The median temperature is $82^{\circ} \mathrm{F}$ with a minimum of $61^{\circ} \mathrm{F}$ and a maximum of $92^{\circ} \mathrm{F}$. Tank 241-BY-111 is a Ferrocyanide Watch List tank and has weekly temperature monitoring requirements.

Plots of the thermocouple readings for Tank 241-BY-111 are in Appendix D.

\subsubsection{Integrity of Tank 241-BY-111}

Tank 241-BY-111 is categorized as sound and is interim stabilized with intrusion prevention completed. The Tank 241-BY-1II surface level is monitored quarterly with a manual tape through riser 15. Liquid waste volume is determined by a photographic evaluation and solid waste volume is determined by a manual tape surface level gauge. The maximum allowable increase from the 164.25-in. baseline is 3 in. There is no criterion for a decrease. The following figure graphically represents the surface level measurements from January 1991 to the present. The surface level for the past 3 years has remained steady and the readings range from 163.75 to 162.75 in.

The Tank 241-BY-111 liquid observation well is located in riser 1. The tank is monitored weekly with a neutron probe and on request with a gamma probe to determine the interstitial liquid levels. The maximum deviation from the established baseline is a 0.3 -ft increase or a 0.4-ft decrease. Sketch ES-TKS-E49 in Appendix $C$ has a graphical representation of the liquid observation well data.

Four drywells are identified for Tank 241-BY-111. Drywells 22-11-01 (active prior to 1990, current readings $>200 \mathrm{c} / \mathrm{s}$ ) and 2211-09 (active prior to 1990, current readings < $200 \mathrm{c} / \mathrm{s}$ ) have readings greater than the $50 \mathrm{c} / \mathrm{s}$ background radiation.

Graphical representations of the drywell data for each active drywell from January 1990 to the present are in Appendix F. Each graph includes the peak c/s, the depth at which the peak occurred, and the date of the reading.

\subsubsection{Historical Sample Analysis Data for Tank 241-BY-111}

No data are available or applicable.

\subsubsection{Current Status of Tank 241-BY-111}

Tank 241-BY-111 entered service in 1951 and currently stores 459,000 gal of waste. The waste is comprised of 433,000 gal of 
saltcake and $26,000 \mathrm{gal}$ of sludge with no supernatant or pumpable liquid remaining. The tank is on the Ferrocyanide Watch List, is passively ventilated, and is categorized as sound with interim stabilization and intrusion prevention completed. Tank 241-BY-111 is equipped to cascade to Tank 241-BY-112 and is second in the three-tank cascade series. The plan view and cross-section that depict the approximate waste level and riser configuration are in Appendix G. Tank 241-BY-111 has 19 risers and four are available for use: two 4-in. risers (no. 5 and no. 10A), one 42-in. riser (no. 12), and one 12-in. riser (no. 12A).

\subsubsection{Waste Layer Volume Approximation for Tank 241-BY-111}

A comparative analysis of all layer approximations for the entire tank farm and the data used to derive the tank layer approximations are located in Appendix $\mathrm{K}$.

\subsubsection{In-Tank Photograph 241-BY-111}

The 1986 photographic montage of the Tank 241-BY-111 interior indicates a white to medium-grey uneven saltcake surface (see Appendix I). Several old discarded level measurement tapes are lying on the waste surface. The air lift circulators are encrusted with solids. The tank contains 459,000 gal of waste which measures about 13.5 ft deep. There have been no changes in the tank which would affect the waste since these photographs were taken; therefore, the picture should be representative of the current tank contents.

\subsubsection{Synopsis Tank 241-BY-111}

(To be completed) 
WHC-SD-WM-ER-312, Rev. 0

2.12 Tank 241-BY-112

2.12.1 24I-BY-112 Tank History

2.12.1.1 Waste History Tank 241-BY-112

The waste history narrative in the following paragraph is from "A History of the 200 Area Tank Farms" (Anderson 1990). Tank 241BY-112 was initially filled with metal waste in the second quarter of 1951. The tank received metal waste, high uranium waste from U Plant, and strontium semiworks until the second quarter of 1955 . The tank received cascade overflow from Tank 241-BY-111 during 1952. The tank was sluiced for uranium recovery in the second quarter of 1955. From the second quarter of 1956 until the second quarter of 1957, the tank received U Plant waste. In the second quarter of 1957, the waste was sent to a ditch. U Plant waste and coating waste were received and/or stored from the third quarter of 1957 until the second quarter of 1965. The tank began to receive coating waste from the second quarter of 1965 until the first quarter of 1968. Evaporator bottoms waste was sent to the tank from the second quarter of 1968 until the first quarter of 1976. The evaporator bottoms waste was from the in-tank solidification process. Presently the tank waste is classified as non-complexed.

The waste history narrative of the primary additions that follow in this paragraph are from the "Waste status and Transaction Record Summary" (Agnew 1994). Tank 241-BY-112 received metal waste from the second quarter of 1951 until the third quarter of 1952 . During the third and fourth quarters of 1952, the tank received decontamination waste. During the second quarter of 1957, the tank received in-plant ferrocyanide waste and uranium recovery waste. During the fourth quarter of 1967, the tank received PUREX cladding waste. The tank received wastewater during the second quarter of 1971.

The tank was removed from service and saltwell pumping was completed in 1976. Primary stabilization was completed in 1978 and the pump was removed in 1979. A level adjustment was made in April 1982. After saltwell pumping was completed, the tank was declared interim stabilized in June 1984. Liquid observation well data were plotted that represent the interstitial liquid level. Prior to 1986, an engineering judgement was made based on pumping started in September 1983 and completed in June 1984. In-tank photographs were taken on the following dates: two in 1966, one in 1968, October 1969, two in 1971, February 1975, several in 1976, January 1980, December 1982, October 1983, July 1984, and April 1988. Sketch ES-TKS-E50, which shows a graphical representation of the Tank 241-BY-112 level history, and the historical waste level data used to make it are in Appendix $C$. The sketch shows two $\mathrm{pH}$ readings: $>14.0$ in January 1972 and $>14.0$ in June 1976. The current maximum temperature reading from October 1993 is $90^{\circ} \mathrm{F}$. 
WHC-SD-WM-ER-312, Rev. 0

\subsubsection{Temperature History 241-BY-112}

Tank 241-BY-112 has two thermociouple trees to monitor the waste temperature: tree 1 has one thermocouple probe and tree 2 has six thermocouple probes. Thermocouple elevations are not available. Thermocouple tree 1 has readings that span from November 1989 to the present. Thermocouples tree 2 readings span from April 1993 to the present.

The first recorded temperature was $76.5^{\circ} \mathrm{F}$ for thermocouple tree 1 and $82^{\circ} \mathrm{F}$ for thermocouple tree 2 . A sum of the squares regression line fit for all the data shows a slight increase in temperature with a small amount of variability. The median temperature is $82^{0} \mathrm{~F}$ with a minimum of $63^{\circ} \mathrm{F}$ and a maximum of $90^{\circ} \mathrm{F}$. Tank 241-BY-112 is a Ferrocyanide watch List tank and temperature readings and are required weekly.

Plots of the thermocouple readings for Tank 241-BY-112 are in Appendix D.

\subsubsection{Integrity of Tank 241-BY-112}

Tank 241-BY-112 is categorized as sound and is interim stabilized with intrusion prevention completed. The Tank 241-BY-112 surface level is monitored quarterly with a manual tape through riser 19. Liquid waste volume is determined by a photographic evaluation, and solid waste volume is determined by a manual tape surface level gauge. The maximum allowable increase from the 113.25-in. baseline is 3 in. There is no criterion for a decrease. The figure that graphically represents the surface level measurements from January 1991 to the present is in Appendix E. The surface level for the past three years has remained steady with the readings ranging between 114.25 and 112.75 in.

The Tank 241-BY-112 liquid observation well is located in riser 15. The tank is monitored weekly with a neutron probe and on request with a gamma probe. Currently, the liquid observation well moitoring is out of compliance with documentation. The monitoring limits are a 0.3-ft decrease and a 0.4-ft increase. Sketch ES-TKS-E49 in Appendix $C$ has a graphical representation of the liquid observation well data.

Seven drywells are identified for Tank 241-BY-112. None of the drywells have or had readings greater than the $50 \mathrm{c} / \mathrm{s}$ background radiation.

\subsubsection{Historical Sample Analysis Data for Tank 241-BY-112}

The results of two analyses conducted in May 1971 and April 1972 for Tank 241-BY-112 are included in this report. The first analysis was for a series of solubility tests; however, a chemical analysis was also performed on sludge, crust, and supernatant samples. The second analysis of top solid, liquid, and bottom solid samples indicates that both the light and heavy solids were 
primarily $\mathrm{NaNO}_{3}$. Tables presenting the results of the two analyses discussed above are in Appendix $\mathrm{H}$.

\subsubsection{Current Status of Tank 241-BY-112}

Tank 241-BY-112 entered service in 1951 and currently stores 291,000 gal of waste. The waste is comprised of 274,000 gal of saltcake and $17,000 \mathrm{gal}$ of sludge with no supernatant or pumpable liquid remaining. The tank is on the Ferrocyanide Watch List as of January 1991, is passively ventilated, and is categorized as sound with interim stabilization and intrusion prevention completed. Tank 241-BY-1I2 is third in the three-tank cascade series. The plan view and cross-section that depict the approximate waste level and riser configuration are in Appendix G. Tank 241-BY-112 has 21 risers and five are available for use: three 4-in. risers (nos. 2, 20, and 5), one 12-in. riser (no. 10A), and one 6-in. riser (no. 18).

2.12.2.1 Waste Layer Volume Approximation for The Tank 241-BY-112

A comparative analysis of all layer approximations for the entire tank farm and the data used to derive the tank layer approximations are located in Appendix $\mathrm{K}$.

\subsubsection{In-Tank Photograph 241-BY-112}

The 1988 photographic montage of the Tank 241-BY-112 indicates a white to grey (with tinges of yellow) saltcake surface which appears to be clinging several feet up the sides of the tank (see Appendix I). All of the supernatant has been removed leaving 291,000 gallons of waste which measures approximately 8.5 ft deep. The temperature probe, liquid observation well, Food Instrument Corporation level probe, the center bottom saltwell screen and the In-Tank Solidification Unit 2, air heater circulator appear to be embedded in saltcake. There have been no changes in the tank which would affect the waste since these photographs were taken; therefore, the picture should be representative of the current tank contents.

2.12.3 Synopsis Tank 241-BY-112

(To be completed) 
WHC-SD-WM-ER-312, Rev. 0

\section{GLOSSARY}

0.17

0.33

1

IAYIN

IAZIN

IC

1CEB

1CS

1st Generation Tank
222-B

222-C

$222-T$

222-U

224

224-F

$231 Z$

242-A

242-B
Transaction flag key-monthly volumes derived from semiannual reports.

Transaction flag key-monthly volumes derived from quarterly reports.

Monthly report.

See Waste Abbreviations.

See Waste Abbreviations.

See Waste Abbreviations.

See Waste Abbreviations.

See Waste Abbreviations.

The original tank design encompassing

Tank Farms B, C, T, U (excluding the 200 series tanks), and $B X$. These tanks have an operating capacity of 530,000 gal, a 75-ft diameter, a 12-in. dish bottom, and a 4-ft knuckle. Also see Type II tanks.

See Facilities.

See Facilities.

See Facilities.

See Facilities.

See Waste Abbreviations.

See Waste Abbreviations.

See Waste Abbreviations.

See Facilities.

See Facilities. 
$242-5$

$242-T$

2AYIN

2AZIN

$2 \mathrm{C}$

2nd Generation Tank

2SYIN

3

3AWIN

3 rd Generation Tank

4th Generation Tank

5

$5-6 \#$

5AWIN

5th Generation Tank
See Facilities.

See Facilities.

See Waste Abbreviations.

See Waste Abbreviations.

See Waste Abbreviations.

Same as original tank design (1st generation, or type II) except the operating capacity was increased to 758,000 gal. Also see Type III tanks.

See Waste Abbreviations.

Quarterly report.

See Waste Abbreviations.

The first generation of the type IV tanks, contains the SX Tank Farm only. These tanks have a $1,000,000$ gal operating capacity, a 75-ft diameter, a 14.875-in. dish bottom, and no knuckle. Also see Type IV tanks.

The second generation of the type IV tanks, contains the A Tank Farm only. These tanks are the same as the $3 \mathrm{rd}$ generation except they have a flat bottom. Also see Type IV tanks.

See Waste Abbreviations.

Cells 5 and 6 from B-Plant.

See Waste Abbreviations.

The third generation of the type IV tanks, contains the AX Tank Farm only. These tanks are the same as the 4 th generation with the addition of grid drain slots beneath the steel liner bottom.

Semiannual report. 
GAWIN

A Plant (PUREX)

A1 SITCK

A2 SITSIRY

Active Tank

Active Drywel1

$A D D$

$A D J$

AGE, AGING

Aging Waste

Airlift Circulator

Annulus

AR Vault

$A R$
See Waste Abbreviations.

See Facilities.

See IANL Defined Waste List.

See LANL Defined Waste List.

A tank that contains more than 33,000 gal of waste and/or is still involved in waste management operations.

Drywell in which radiation readings of greater than 50 counts/second are detected. To be considered active, the readings must be consistent as to depth and radiation level for repeated readings.

Add primary waste from process.

Adjustment to waste amount-see CORR.

Waste Abbreviation.

High-level, first-cycle solvent extraction waste from the PUREX plant (NCAW) .

The airlift circulators are installed in tanks used to age wastes. The circulators promote mixing of the supernate and prevent agglomeration of radionuclides. By maintaining motion within the body of liquid, the circulators minimize superheat buildup and, consequently, minimize bumping.

A vessel space in the form of a ring; the space between concentric walls.

A structure containing tanks and chemical processing equipment that is used for waste processing or storage operations.

See IANI Defined Waste List. 
Assumed Leaker

Assumed Re-leaker

Assumed Leaking Tank

B SLTCK

B

B Plant (222-B)

B860N

Background Radiation

Baseline

BFSH

BI

BLEB

BLIX

BLIXB

BNW

BNW
A waste storage tank for which surveillance data, in the past, has indicated a loss of liquid attributed to a breach of integrity.

A designation that exists after a tank has been declared an "assumed leaker" and then the surveillance data indicate a new loss of liquid attributed to a breach of integrity.

In 1984, the criteria designations of "suspect leaker," "questionable integrity," "confirmed leaker," "declared leaker," "borderline," and "dormant" were merged into one category now reported as "assumed leaker."

See LANL Defined waste List.

See Waste Abbreviations.

See Facilities.

See Waste Abbreviations.

Radioactivity from naturally occurring sources; primarily radiation from cosmogenic and naturally occurring radionuclides.

A reference; for example, a specified liquid level or radiation level against which new information is compared.

See Waste Abbreviations.

See Waste Abbreviations.

See Waste Abbreviations.

See Waste Abbreviations.

See Waste Abbreviations.

Battelle Northwest Laboratory.

See Waste Abbreviations. 
BPDCC
BPDCS
BPDCV
BPFPS
BPLCS
BPLDC
BPIDN
Bumping, Tank Bump

BVCLN

BY SLTCK

C Plant (222-C)

C PLANT

Caisson

Calcine

CARB

CAS
See Waste Abbreviations.

See Waste Abbreviations.

See Waste Abbreviations.

See Waste Abbreviations.

See Waste Abbreviations.

See Waste Abbreviations.

See Waste Abbreviations.

A tank bump occurs when solids overheat in the lower portion of the tank. The hot solids rapidly transfer heat to the liquid, some of which quickly vaporizes. The sudden pressurization caused by vapor generation is called a "bump".

See Waste Abbreviations.

See LANL Defined Waste Iist.

See Facilities.

see SSW.

An underground structure used to store high-level waste; typical designs include corrugated metal or concrete cylinders, 55-gal drums welded end-toend, and vertical steel pipes below grade.

To heat a substance to a high temperature, but below its melting point, causing loss of volatile constituents such as moisture; refers also to the material produced by this process.

See Waste Abbreviations.

Cascade-see SET and END. This process filled three tanks with one pump using overflow siphoning. Normal use was with 
Cascade

CASS

Catch Tanks

CAW

CC

CCGI

CCGR

CCPI

CCPLX

$\mathrm{CCW}$

CDF

CE

CELL 23 a sequence of tanks such as 101, 102, 103 , or $110,111,112$.

Eleven of the single-shell tank farms (all except the AX Tank Farm) were equipped with overflow lines between tanks. The tanks were connected in series and were placed at different elevations creating a downhill gradient for liquids to flow from one tank to another.

Acronym for Computer Automated Surveillance System.

Small capacity single-shell tanks associated with diversion boxes and diverter stations. The tanks are designed to receive any transfer line cleanout, spills or leakage from the boxes, or leakage from the adjacent pipe encasement.

Current acid waste; this is PUREX acid HLW .

See Waste Abbreviations.

See Waste Abbreviations.

See Waste Abbreviations.

See Waste Abbreviations.

See Waste Abbreviations.

Concentrated customer waste.

Composition data file or transaction flag key-unit volume assumed to make stream active.

See Waste Abbreviations.

Waste from cell 23 at B-Plant. Cell 23 contained an evaporator.

See Waste Abbreviations. 
WHC-SD-WM-ER-312, Rev. 0

CF

CON

COND

Condensate

Conductivity Probe

Confirmed or

Storage Declared Leaker
See Waste Abbreviations.

See Waste Abbreviations.

Condensate-see EVAP, EB.

Iiquid formed from cooling vapors.

A device that completes an electrical circuit when contacted by a conductive material.

The designation of any underground waste tank where the data are considered sufficient to support a conclusion with 95 percent confidence that the tank has leaked.

COOI

CORR

CP

CPLX

CR Vault

Crib

Crust

CSFD
Change in waste volume due to cooling. See CORR.

Correction to waste amount-see $A D J$, IEAK, COOL.

See Waste Abbreviations.

See Waste Abbreviations.

Facility located adjacent to C Farm, used for scavenging campaign following uranium recovery. Ferrocyanide was added to tank supernatants in $C R$ Vault and the slurry was returned to C-Farm.

A linear excavation approximately $15 \mathrm{ft}$ in depth, with a perforated pipe in the bottom. The ditch is backfilled with broken rock or other loose material and then covered by soil and by a liquid impermeable membrane; the pipe is then used to distribute intermediate level liquid wastes along the crib.

A hard surface layer that has formed on top of the liquid in many waste tanks that contain concentrated solutions.

See Waste Abbreviations. 
CSR

CST

CSWLE

CSWLW

CTW

CW

CWP

CWP/ZR

CWP2

CWR

CX70

D

DBP

DC

DCS

DE

DE

Deliquescent

Desiccant

Diatomaceous Earth
See Waste Abbreviations.

See Waste Abbreviations.

See Waste Abbreviations.

See Waste Abbreviations.

Caustic waste for makeup.

See Waste Abbreviations

See Waste Abbreviations.

See Waste Abbreviations.

See LANL Defined Waste List.

See Waste Abbreviations.

See Waste Abbreviations.

Transaction Flag Key-Amount by difference.

Dibutyl phosphate.

See Waste Abbreviations.

Acroynm for dilute caustic solutions

Acronym for diatomaceous earth that was added to Tanks 241-BX-102, -SX-113, -TX-106, and -U-104 from 1970 to 1972 .

See LANL Defined Waste List.

A solid that is capable of absorbing moisture from the air and becoming a liquid.

A drying agent such as diatomaceous earth.

Diatomite, a light friable siliceous material, derived chiefly from diatom (algae) remains that is added to selected underground waste storage tanks

$$
\text { A-8 }
$$


$D B P$

DIL

DILFD

Ditch

Diversion Box

$\mathrm{DN} / \mathrm{PT}$

$\mathrm{DN} / \mathrm{PD}$

DN

Double-Shel I Tank

Double-shell slurry

Double-Shell Slurry Feed (DSSF)

Drywell to absorb and, thereby, immobilize residual liquids (i.e help to stabilize the tank).

Dibutyl phosphate.

Dilution

Dilute feed

A Iinearly oriented excavation often used for the temporary diversion or disposal of process waste streams.

A below grade concrete enclosure containing the remotely maintained jumpers and spare nozzles for the routing of waste solution to storage tank farms.

See Waste Abbreviations.

See Waste Abbreviations.

See Waste Abbreviations.

The newer underground waste storage tanks consisting of a concrete shell and two concentric carbon steel liners with an annular space between the liners.

Waste that exceeds the sodium aluminate saturation boundary in the evaporator without exceeding receiver tank composition limits. For reporting purposes, DSS is considered a solid.

Waste concentrated just before reaching the sodium aluminate saturation boundary in the evaporator without exceeding receiver tank composition limits. This form is not as concentrated as DSS.

A steel casing, generally 6 inches in diameter drilled into the ground to various depths, and used to insert monitoring instruments for measuring the presence of radioactivity or moisture 
content.

Drywell (in-tank)

DSS

DSSF

DUMM

DUMMY

DW

DWBIX

E

EB

EDTA

$E F$

EFD

END

Environs

EV

EVAP

EVAPF

Evaporator Feed
A sealed casing within a tank that is attached to a riser. It is used to insert experimental equipment, such as neutron or acoustical probes, to determine the level of drainable interstitial liquor.

See Waste Abbreviations.

See Waste Abbreviations.

See Waste Abbreviations.

See Waste Abbreviations.

See Waste Abbreviations.

See Waste Abbreviations.

Transaction flag key-waste transferred through evaporator.

See Waste Abbreviations.

Ethylenediaminetetraacetic acid.

See Waste Abbreviations.

See Waste Abbreviations.

Disconnect Cascaded Tanks, see CAS, Set.

surrounding area, vicinity.

Evaporation.

See Waste Abbreviations.

See Waste Abbreviations.

Any waste Iiquid that can be concentrated to form saltcake; e.g., low heat waste, dilute interstitial liquor, aged waste, and other radioactive waste solutions.

$$
A-10
$$


WHC-SD-WM-ER-312, Rev. 0

Evaporator-Crystallizer 242-A and 242-S waste concentration facilities that operate at a reduced pressure (vacuum) and are capable of producing a slurry containing about 30 volume percent solids at a specific gravity of greater than 1.6 .

EVS

Partial neutralization in the 242-S Evaporator.

EVT

HEDTA destruction in the 242-B or 242-T Evaporators.

FD

See Waste Abbreviations.

FECN

Ferrocyanide wastes created during a scavenging campaign in 1953-57. See SCAV, POO, TOO.

Ferrocyanide

An ion composed of iron and cyanide with the chemical formula of $\mathrm{Fe}(\mathrm{CN})^{-4}{ }_{6}$.

FIC

A Food Instrument Corporation automatic liquid level gauge based on a conductivity probe. At Hanford, they are electricaliy connected to a computer for data transmission, analysis, and reporting. Local readings may also be obtained from a dial.

First- And Second-Cycle waste contained 10 percent of the Decontamination Wastes original fission product activity and 2 percent of the products. By-product cake solution was mixed with product waste and neutralized with 50 percent caustic. The waste contained a mixture of suspended solids, hydroxides, carbonate and phosphate, scavenger metals, chromium, iron, sodium, and silicofluoride.

FLSH

See Waste Abbreviations.

FP

Fission Product Waste. Waste produced at $B$ Plant and Hot Semiworks in campaigns for $\mathrm{Cs}$ and $\mathrm{Sr}$ recovery during the 1960s. Cs was removed from PUREX supernatant and $\mathrm{Sr}$ was removed from 
FP

GA

Gamma Ray

GAS

GM Instrument

GROUP

Gunite

$\mathrm{H}_{2} \mathrm{O}$

HDRL

HEAT

HEDTA

Heel

Hexone

High-Level Waste

Historical Information
PUREX sludge; both were removed from acidic waste.

See Waste Abbreviations.

Gain to tank.

Electromagnetic radiation sometimes emitted by the nuclei of radioactive substances during decay, similar in nature to $\mathrm{X}$-rays.

Slurry growth as a result of gas generation.

Instrument for detecting low-level beta and gamma radiation using a GeigerMueller tube.

A group of tanks where ITS averaged the supernatant phases-see ITS.

A building material consisting of a mixture of cement, sand, and water that is sprayed onto a mold.

Water.

See Waste Abbreviations.

A tank correction, see CORR, COOL.

N hydroxy-ethylenediaminetriacetic acid.

The amount remaining in a vessel or container after most of the contents have been removed.

Methyl isobutyl ketone, an organic solvent used in the REDOX solvent extraction process. Also known as Isobutyl methyl ketone, Methyl isobutyl ketone, 4-Methyl 2-pentanone, MIBK.

Waste from the fuel reprocessing operations in separations plants.

All information obtained from material 
HLO

HLO

HLW

HOT-SEMI

HS

HS

HWVP

$I \& S$

Inactive Tank

INST

Interim Isolated

Interim Stabilized referenced in published documentation and unpublished sources. Unpublished sources include electronically retrieved data li.e. temperatures, liquid observation well, sampling, and surface level) and photographs.

See Waste Abbreviations.

Hanford Laboratory Operations.

See Waste Abbreviations.

See HS, SSW.

See Waste Abbreviations.

Hot Semi-Works. A pilot facility that had a variety of operations. C Plant.

Dilute, non-complexed waste from the vitrification plant.

Tank isolated and stabilized.

A tank that has been removed from liquid-processing service, has been pumped to less than 33,000 gal of waste, and is waiting to be, or is in the process of being, stabilized and interim isolated. Includes all tanks not in active or active-restricted categories. Otherwise inactive contingency spares that would be used if an active tank failed are also included.

Change in tank level due to change in instrumentation.

An administrative designation reflecting the completion of the physical effort required to minimize the addition of liquids into an inactive storage tank, process vault, sump, catch tank, or diversion box.

A tank which contains less than 50,000 gallons of drainable interstitial liquid 
Interstitial Liquor

Interstitial

Intrusion Mode

FIC Setting

Intrusion Prevention

ISO

ITS

IWW

IX

Jet Pump

Knuckle and has less than 5,000 gallons of supernatant liquid. If the tank was jet pumped to achieve interim stabilization, then the jet pump flow must also have been at or below 0.05 gallons per minute before interim stabilization is completed.

The liquid that fills the interstices or the void volume in the solid material in a waste tank.

The volume within a solid matrix made up of the crevices, cracks, and void spaces.

The FIC probe is positioned a short distance above the waste surface. If the surface level of the waste in the tank increases, thereby touching the probe tip, a positive indication is received.

This is an administrative designation reflecting the completion of the physical effort required to minimize the addition of liquid into an inactive storage tank, process vault, catch tank, sump, or diversion box.

Tank is interim-isolated.

The In-Tank Solidification Program was a program used to reduce waste volume directly by evaporating the wastes from inside certain tanks in the $B X$ and $B Y$ Farms. The process used both hot air (ITS-1) and an electric heater (ITS-2).

See Waste Abbreviations.

See Waste Abbreviations.

A modified commercially available low capacity jet pump used as a salt well pump.

Point where the side wall and the bottom 
L222S

I3A4A

$\operatorname{LaF}$

Lateral

Leak Detection Pit

ILEAK

LETF

LeveI Adjustment

Level History

Iiquid Observation Well (LOW)

Liquid Level Best Engineering Judgement Line curved surface of a tank meet.

See Waste Abbreviations.

See Waste Abbreviations.

See Waste Abbreviations.

Horizontal drywell under A Farm and certain SX Farm waste storage tanks.

Collection point for any leakage from $A X$ Farm tanks. The pits are equipped with radiation and liquid detection instruments.

Tank leak volume, see CORR.

Iiquid effluent treatment facility from $\mathrm{N}$-Reactor.

Any update in the waste inventory (or tank level) in a tank. The adjustments usually result from surveillance observations or historical investigations.

A diagram that shows the history of the waste level and waste level changes in a tank. The diagram also includes other related data.

Iiquid observation wells are used to monitor the interstitial liquid level (IIL) in single-shell waste storage tanks. Three probes are used to monitor changes in the ILL: acoustic, gamma, and neutron. Each method can indicate intrusions or leakage by increases or decreases in the III.

During the initial fill of certain single-shell tanks, only the liquid level was reported. To adjust for the big increase in level height, which occurred when solids were added to the record, a sloped line was used to reflect solids volume between the 
WHC-SD-WM-ER-312, Rev. 0

LO

LUNC

LW

Metal Waste (MW)

Molarity

Mole

MW

MWF

N

NCAW

NCPL

NCPLEX

NCPIX

NCRW

Neutralized PUREX Acid Waste initial fill and the time the solids data was recorded.

Loss from tank.

See Waste Abbreviations.

See Waste Abbreviations.

Waste from the extraction containing all the uranium, approximately 90 percent of the original fission product activity, and approximately 1 percent of the product. This waste was brought just to the neutral point with 50 percent caustic and then treated with an excess of sodium carbonate. This procedure yielded almost completely soluble waste at a minimum total volume. The exact composition of the carbonate compounds was not known but was assumed to be an uranium phosphate carbonate mixture.

Number of gram molecular weights (moles) per liter of solution.

The amount of a substance with weight equal to the molecular weight in grams.

See Waste Abbreviations.

See Waste Abbreviations.

See Waste Abbreviations.

See Waste Abbreviations.

See Waste Abbreviations.

See Waste Abbreviations.

See Waste Abbreviations.

See Waste Abbreviations.

The original plant in 1956 neutralized all the high-level waste and sent it to the 241-A Tank Farm. As fission product 
Neutron Probe

NFAW

NHAW

NIT

Noise At The Bottom of Well--Drywell Probe

Non-Complexed (NCPLX)

NPH

NRAW

NRP82

NRPO4

NRSO4

NTA

Nuclear reactor

Open Hole Salt Well recovery started, a portion of the waste was treated for strontium recovery and then neutralized. As of 1967, all of the high-level waste left PUREX as an acid solution for treatment at $B$ plant.

Probe equipped with a neutron source and detector. They are used in dry well monitoring to determine the moisture content of the soil as one means for detecting leaks in underground waste storage tanks or pipelines.

See Waste Abbreviations.

See Waste Abbreviations.

$\mathrm{HNO}_{3} / \mathrm{KMNO}_{4}$ solution added during evaporator operation.

Erroneous reading caused by probe coming contact with foreign objects at the bottom of a well.

General waste term applied to all Hanford Site liquors not identified as complexed (containing organics).

Normal paraffin hydrocarbon.

See Waste Abbreviations.

See Waste Abbreviations.

See Waste Abbreviations.

See Waste Abbreviations.

Nitrilotriacetic acid.

A device for creating a controlled nuclear chain reaction using atomic fuel, as for the production of energy.

A well in which a pump is inserted in solid waste. Frequently used to remove the liquid from tanks containing less than 2 feet of sludge. 
WHC-SD-WM-ER-312, ReV. 0

\begin{tabular}{|c|c|}
\hline Organic Wash Waste (OWW) & $\begin{array}{l}\text { The solvent used in PUREX was treated } \\
\text { before reuse by washing with potassium } \\
\text { permanganate and sodium carbonate, } \\
\text { followed by dilute nitric acid and then } \\
\text { a sodium carbonate wash. }\end{array}$ \\
\hline Out-of-Service-Tank & $\begin{array}{l}\text { A tank that does not meet the definition } \\
\text { of an in-service tank. Before september } \\
1988 \text {, these tanks were defined as } \\
\text { inactive. \{Note: all single-shell tanks } \\
\text { (SSTs) are out of service.\} }\end{array}$ \\
\hline OWW & See Waste Abbreviations. \\
\hline $\mathrm{P}$ & See Waste Abbreviations. \\
\hline P1: & See LANL Defined Waste Iist. \\
\hline P2: & See LANL Defined Waste List. \\
\hline PADFG & See Waste Abbreviations. \\
\hline PADWG & See Waste Abbreviations. \\
\hline $\begin{array}{l}\text { Partially Interim } \\
\text { Isolated }\end{array}$ & $\begin{array}{l}\text { The administrative designation } \\
\text { reflecting the completion of the } \\
\text { physical effort required for Interim } \\
\text { Isolation except for the isolation of } \\
\text { risers and piping that will be required } \\
\text { for jet pumping or for other methods of } \\
\text { stabilization. }\end{array}$ \\
\hline PAS & See Waste Abbreviations. \\
\hline PASF & See Waste Abbreviations. \\
\hline PAW & See Waste Abbreviations. \\
\hline PD & See Waste Abbreviations. \\
\hline PDBNG & See Waste Abbreviations. \\
\hline PDBSU & See Waste Abbreviations. \\
\hline PDBTG & See Waste Abbreviations. \\
\hline PDCSS & ee Waste Abbreviations. \\
\hline
\end{tabular}


PDL87

PDL89

PDNSG

PDS 87

PDS89

PDSLG

PDSUP

PFeCN

PFeCN1:

PFeCN2 :

PFMMS

PFP (z-Plant)

PFP

PFPGR

PEPNT

PFPPT

PFPSL

$\mathrm{pH}$

Pile

PL

PML89

PMS89

PN
See Waste Abbreviations.

See Waste Abbreviation.

See Waste Abbreviations.

See Waste Abbreviations.

See Waste Abbreviations.

See Waste Abbreviations.

See Waste Abbreviations.

See Waste Abbreviations.

See IANL Defined Waste Iist.

See LANL Defined Waste List.

See Waste Abbreviations.

see facilities.

Plutonium finishing plant waste (see $\mathrm{Z}$, 224, PRF).

See Waste Abbreviations.

See Waste Abbreviations.

See Waste Abbreviations.

See Waste Abbreviations.

A measure of the hydrogen ion concentration in a solution.

An early term used to describe a nuclear reactor.

See Waste Abbreviations.

See Waste Abbreviations.

See Waste Abbreviations.

See Waste Abbreviations. 
WHC-SD-WM-ER-312, Rev. 0

PNF

POO-P\#\#

Portland Cement

PRE

Primary Addition

Primary Stabilization

Probe

PSI

PSS

PSSF

Psychrometry

PT
See Waste Abbreviations.

In-Plant scavenging with FeCN-see SCAV, TOO-T\#\#.

A hydraulic cement made by finely pulverizing the clinker produced by calcining to incipient fusion a mixture of clay and limestone or similar materials.

Plutonium Reclaimation Facility-Type of waste generated in Z-Plant for

"finishing wastes". Solvent based extraction process using $\mathrm{CCl}_{4} / \mathrm{TBP}$.

An addition of waste from a specific plant or process vault. These additions come from the "waste status and Transaction Record Summary", WHC-SD-WMTI-614 \& -615 Rev. O DRAFT.

The condition of an inactive waste storage tank after all liquid above the solids, other than isolated surface pockets, has been removed. Isolated surface pockets of Iiquid are those not pumpable by conventional techniques.

A device used to get information about the environment. In this report, an instrument package designed to be inserted in drywells, risers, or ports to measure waste characteristics.

See Waste Abbreviations.

See Waste Abbreviations.

See Waste Abbreviations.

Determination of humidity or dew point from wet and dry bulb temperatures with the difference in the two used as a measure of dryness in the atmosphere.

See Waste Abbreviations. 


PUREX
PX86S
PXBAW
PXBSG
PXFTF
PXLOW
PXMET
PXMSC
PXNAW
QUESTionable Integrity

$\mathbf{R}$

R SLTCK:

RI :

R2 :

Radiation zone

Radiation

Readily available have

Risers
See Facilities.

See Waste Abbreviations.

See Waste Abbreviations.

See Waste Abbreviations.

See Waste Abbreviations.

See Waste Abbreviations.

See Waste Abbreviations.

See Waste Abbreviations.

See Waste Abbreviations.

Any tank that has a small decrease in liquid level or a radiation increase in an associated dry well, for which the data are insufficient to support a conclusion with 95 percent confidence that the tank is sound.

See Waste Abbreviations.

See LANL Defined Waste List.

See IANL Defined Waste Iist.

See IANL Defined Waste Iist.

An area containing radioactive materials in quantities significant enough to require control of personnel entry to the area.

Particles and electromagnetic energy emitted by nuclear transformations that are capable of producing ions when interacting with matter.

Risers located above grade which do not apparatus attached to or contained in them. 
WHC-SD-WM-ER-312, Rev . 0

REC

REDOX

Removed From service

is not (Tanks)

Rerun Drywell Data

RESD

RIX

$\mathrm{RMC}$

RSN

RSS

RTX

$S$

S-Plant (REDOX)

S1 SLTCK:

S2 SLTSLRY:

Salt Cake

Salt well

SCAV

Scavenged
Receive waste from another tank-see XFER.

See Facilities.

Any tank that is a confirmed leaker or intended for reuse.

Surveillance data which supersedes

"suspect data".

See Waste Abbreviations.

See Waste Abbreviations.

Remote Mechanical C-Iine -Process used in Z-Plant.

See Waste Abbreviations.

See Waste Abbreviations.

See Waste Abbreviations.

Transaction Flag Key-Partial neutralization (PNF).

See Facilities.

See IANL Defined Waste Iist.

See LANL Defined Waste List.

Crystalized nitrate and other salts deposited in waste tanks, usually after active measures are taken to remove moisture.

A hole drilled or sluiced into a salt cake and lined with a cylindrical screen to permit drainage and jet pumping of interstitial liquors.

Scavenging campaign with FECN on TBP, 1952-57. See TOO-T\#\#, POO-P\#\#.

Waste which has been treated with ferrocyanide to remove cesium from the 
WHC-SD-WM-ER-312, Rev. 0

supernatant by precipitating it into the sludge.

Scintillation Monitor

Self Concentrating

Self-Evaporation

SET

SIX

SI

SL3SY

Sludge

Slugs

Sluicing, or sluiced

Slurry

Sound Tank
A radiation detection instrument based on the principle that light pulses are produced in some materials when they are exposed to radiation.

High-level liquid radioactive waste whose constituent radionuclides contribute sufficient decay heat to cause the solution to boil and/or selfconcentrate.

A waste material in which moisture is being lost as the moisture changes to a vapor and diffuses into the atmosphere.

Connect cascaded tanks together-see CAS and END.

See Waste Abbreviations.

See Waste Abbreviations.

See Waste Abbreviations.

Primarily insoluble metal hydroxides and oxides precipitated from neutralized waste.

An early term for uranium fuel elements which had been machined or extruded into short cylinders which were then clad or encased in corrosion-resistant metals.

To wash with water. At Hanford, this has meant to dissolve or suspend in solution by action of a high pressure water stream.

Insoluble material suspended in water or aqueous solution.

The classification of a waste storage tank for which surveillance data indicates there has been no loss of liquid attributed to a breach of 
WHC-SD-WM-ER-312, Rev. 0

SPRG

SRR

SRS

SRS

SSW

STAB

Stabilization

Static Tank

Strontium Semi-Works

SU

Supernatant

(Supernatant Liquid)

Surveillance

Suspect Data integrity.

Sparge-transfer of water or volume

See Waste Abbreviations.

See Waste Abbreviations.

See Waste Abbreviations.

See Waste Abbreviations.

Tank stabilized by removal of liquid. Both floating suction and salt-well jet pumps used to remove liquid.

The removal, or immobilization, as completely as possible, of the Iiquid contained in a radioactive waste storage tank by salt well pumping, open hole salt well pumping, diatomaceous earth addition, etc.

A tank with no significant change in liquid level or involvement in transfer operations during a stated period of time.

Called C-Plant or Hot Semi-Works, was a pilot plant for both Redox and Purex, then reconfigured for strontium recovery.

See Waste Abbreviations.

In waste storage tanks, the liquid quantity defined by the difference between the measured liquid level and the measured average solids level in a tank.

Regular or systematic watch kept over someone or something.

Surveillance data which does not fall within the expected range and is superseded by "reruns." 
SV

SW RCR

SWLIQ

SWLQW

T-Plant $(222-T)$

T1 SLTCK:

T2 SLTCK:

Tank Farm

TBP

TBP

TCO

Terminal Liquior

TFECN

TH

Thermocouple Tree

Thermocouple,
Transaction Flag Key-Amount by difference in solids.

Salt-well Receiver.

See Waste Abbreviations.

See Waste Abbreviation.

See Facilities.

See LANL Defined Waste Iist.

See IANL Defined Waste List.

An area containing a number of storage tanks; i.e., a chemical tank farm for storage of chemicals used in a plant, or underground waste tank storage of radioactive waste.

Tributyl phosphate, a solvent used in the PUREX solvent extraction process.

See Waste Abbreviations.

See Waste Abbreviations.

The liquid product from the evaporationcrystallization process which, upon further concentration, forms an unacceptable solid for storage in single-shell tanks. Terminal liquor is characterized by a caustic concentration of approximately $5.5 \mathrm{M}$ (the caustic molarity will be lower if the aluminum salt saturation is reached first).

See Waste Abbreviations.

See Waste Abbreviations.

A group of thermocouples assembled in a pipe and inserted into a waste tank for measuring temperatures at regular

(normally 2 feet) vertical intervals.

A probe for measuring temperature, 
Thermocouple probe

Thermowell

THL

Thorium

TK

TL

TOO-T\#\#

TPLAL

TPLAN

TPLAS

$T R$

Trench

trFlag

Tributyl Phosphate (TBP) consisting of two dissimilar metal wires joined at one end (hot junction) with the free ends joined to a measuring instrument. Electrical potential changes due to temperature changes at the hot end are measured and calibrated to read out as temperature.

A well in a waste tank which contains thermocouples.

See Waste Abbreviations.

A chemical element which is also a fertile material. By fertile is meant that when subjected to radiation in a nuclear reactor it will be converted, in this case, to ${ }^{233}$ uranium, a potential fuel.

Tank. TK-17-2, however, was an early designation for B-Plant.

See Waste Abbreviations.

In-Tank scavenging with FECN, see SCAV, P\#\#.

See Waste Abbreviations.

See Waste Abbreviations.

See Waste Abbreviations.

Transfer from tank.

A deep furrow in the ground. At Hanford they are used for the disposal of solid waste.

Transaction Flag Keys-used by W-Trac-see $\mathrm{CDF}, \mathrm{D}, \mathrm{E}, \mathrm{S}, \mathrm{SV}, 1,3,6,0.17,0.33$.

A chemical compound, also an organic solvent used in the PUREX solvent extraction process. 
Type I Tank

Type II Tank

Type III Tank

Type IV Tank

Type V Tank

U-Plant (222-U)

U1U2

UNKN

UNKNOWN :

UR

UR :

Watch Iist Tank
These are the 200 series $B, C, T$, and $U$ Farm tanks. They have an operating capacity of $55,000 \mathrm{gal}$, a 20-ft diameter, a 6-in. dish bottom, and a 3-ft knuckle. There is no generation associated with type I tanks.

These are the original, Ist generation, tank designs, encompassing $B, C, T, U$ (excluding the 200 series tanks), and BX Tank Farms. Also see 1st Generation Tank.

These are the 2nd Generation tank designs, encompasing $B Y, S, T X$, and $T Y$ Tank Farms. Also see 2nd Generation Tank.

These include $3 \mathrm{rd}, 4$ th, and 5th generation tank designs, encompasing $\mathrm{SX}$, $A$, and AX Tank Farms respectively. Also see $3 r d$ Generation Tank, 4 th Generation Tank, and 5th Gneration Tank.

These are the first double shell tank designs, encompasing $A Y, A Z$, and SY Tank Farms.

See Facilities.

See Waste Abbreviations.

Unknown waste origin sink.

See I.ANL Defined Waste List.

Uranium recovery operation in 222-U, 1952-57. Created TBP (primary waste) and FECN (scavenging wastes). See TFeCN, PFeCN, POO, TOO, FeCN.

See IANL Defined Waste Iist.

An underground storage tank containing waste that requires special safety precautions because it may have a serious potential for release of highlevel radioactive waste because of 


$$
\text { WHC-SD-WM-ER-312， Rev. } 0
$$

$\begin{array}{ll}\text { WATER } & \text { Flush water from various sources. } \\ \text { WTR } & \text { Water. } \\ \text { WVP } & \text { Waste volume projections } \\ \text { XFER } & \text { Transfer of waste out of tank. } \\ \text { Z } & \text { See Waste Abbreviations } \\ \text { Z-PIant } & \text { See Facilities } \\ \text { Z-PLANT } & \text { PFP. Plutonium Finishing Plant. } \\ \text { ZAW } & \text { See Waste Abbreviations. } \\ \text { ZHIGH } & \text { See Waste Abbreviations. } \\ \text { ZLAB } & \text { See Waste Abbreviations. } \\ \text { ZLOW } & \text { See Waste Abbreviations. } \\ \text { ZPRFL } & \text { See Waste Abbreviations. } \\ \text { ZPRFS } & \text { See Waste Abbreviations. } \\ \text { ZRM } & \text { See Waste Abbreviations. } \\ \text { ZRMCL } & \text { See Waste Abbreviations. } \\ \text { ZRMCS } & \text { See Waste Abbreviations. }\end{array}$

uncontrolled increases in temperature or pressure. Special restrictions have been placed on these tanks by "Safety Measures for Waste Tanks at Hanford Nuclear Reservation," Section 3137 of the National Defense Authorization Act for Fiscal Year 1991, November 5, 1990, public Law 101-501 (also known as the Wyden Amendment).

Flush water from various sources.

See Waste Abbreviations

See Facilities

PFP. Plutonium Finishing Plant.

See Waste Abbreviations.

See Waste Abbreviations.

See Waste Abbreviations.

Waste Abbreviations. 


\section{WASTE ABBREVIATIONS}

1AYIN

IAZIN

1C

ICEB

ICS

224

$224-F$

2312

2AYIN

2AZIN

$2 \mathrm{C}$

2SYIN

3AWIN

$5-6$

5AWIN

6AWIN

AGE, AGING

B

B860N

BFSH

BL
Concentrated complex waste from 101 AY inventory

Pre 2-81 101AZ inventory

First cycle waste

1st cycle evaporator bottoms

1st cycle scavenging waste

224-U waste

224-U waste. IaF Pu finishing plant

Dilute, phosphate waste from $231 \mathrm{Z}$

laboratories

Pre 2-81 102AY inventory

Pre 2-81 concentrated complex waste from $102 \mathrm{Az}$ inventory

Second cycle waste

Pre 2-81 102Sy inventory

Pre 2-81 103AW inventory

B-Plant tank 5, 6 waste

Pre 2-81 105AW inventory

Concentrated phosphate waste in 106AW inventory

Aging waste

B-Plant high- level waste

Dilute, non-complexed waste from B Plant cell drainage

B-Plant flush waste water

B-Plant low-level waste 
WHC-SD-WM-ER-312, Rev. 0

BLEB

BLIX

BLIXB

BNW

BPDCC

BPDCS

BPDCV

BPFPS

BPICS

BPLDC

BPLDN

BVCLN

CARB

CC

CCGL

CCGR

CCPI

CCPIX
B-Plant low-level evaporator bottoms

B-Plant low level ion exchange?

B-Plant low level ion exchange bottoms?

Battelle Northwest Laboratory waste

Dilute, complexed waste from B Plant cesium processing

Dilute, complexed waste from B Plant strontium processing

Dilute, complexed waste from B Plant vessel clean-out

B Plant high Tru solids from retrieved PFP solids

Dilute, non-complexed waste from B Plant strontium processing

Dilute, complexed waste from B Plant cesium processing

Dilute, non-complexed waste from B Plant cesium processing

Dilute, non-complexed waste from B Plant vessel clean-out

PUREX organic wash waste

Complexant concentrate waste

B-Plant high Tru solids from retrieved

complexed concentrate

Dilute, non-complexed waste from

retrieved

complexed

concentrate

Complexant concentrate

Complexant concentrate-see CPLX 


\begin{tabular}{|c|c|}
\hline$C E$ & Evaporator concentrate \\
\hline CEM & Concrete-see CON \\
\hline $\mathrm{CF}$ & Cesium feed \\
\hline $\operatorname{CON}$ & Concrete-see CEM \\
\hline $\mathrm{CP}$ & $\begin{array}{l}\text { Concentrated phosphate waste from } \mathrm{N} \\
\text { Reactor decontamination }\end{array}$ \\
\hline CPLX & Complex waste \\
\hline CSFD & Cesium feed \\
\hline $\operatorname{CSR}$ & $\begin{array}{l}\text { Waste sent to B-Plant for cesium } \\
\text { recovery }\end{array}$ \\
\hline $\operatorname{CST}$ & Caustic solution \\
\hline CSWLE & Complexed salt well liquid east area \\
\hline CSWLW & Complexed salt well liquid west area \\
\hline $\mathrm{CW}$ & Coating waste \\
\hline CWP/ZR & $\begin{array}{l}\text { Coating waste (PUREX), Zirconium } \\
\text { cladding }\end{array}$ \\
\hline CWP & Coating waste (PUREX) \\
\hline CWR & Coating waste (REDOX) \\
\hline CX70 & $\begin{array}{l}\text { Dilute, complexed mixture hot-semiworks } \\
\text { Tru solids }\end{array}$ \\
\hline $\mathrm{DC}$ & Dilute complexed waste \\
\hline $\mathrm{DN} / \mathrm{PD}$ & DN with $P$ Tru solids \\
\hline DN & Dilute non-complexed waste \\
\hline $\mathrm{DN} / \mathrm{PT}$ & DN with PFP Tru solids \\
\hline DSS & Double-shell slurry \\
\hline DSSF & Double-shell slurry feed \\
\hline
\end{tabular}


WHC-SD-WM-ER-312, Rev. 0

\begin{tabular}{|c|c|}
\hline DUMM & Dummy waste \\
\hline DUMMY & Dummy waste \\
\hline DW & Decontamination waste \\
\hline DWBIX & $\begin{array}{l}\text { Decontamination waste and B-Plant ion } \\
\text { exchange }\end{array}$ \\
\hline EB & Evaporator bottoms \\
\hline $\mathrm{EF}$ & Evaporator feed \\
\hline EFD & Evaporator feed dilute \\
\hline EVAP & Evaporator feed (post 1976) \\
\hline EVAPF & $\begin{array}{l}\text { Dilute, non-complexed waste from } \\
\text { evaporator pad flush }\end{array}$ \\
\hline FD & Feed dilute \\
\hline FLSH & Flush water \\
\hline FP & Fission product waste \\
\hline HDRI & Hanford defense residual liquor \\
\hline HLO & Hanford laboratory operations waste \\
\hline $\mathrm{HLW}$ & $\begin{array}{l}\text { High level waste-generic for all Hanford } \\
\text { tank wastes }\end{array}$ \\
\hline HS & Hot semiworks waste \\
\hline IWW & PUREX \#1 acid concentrator waste \\
\hline IX & Ion exchange waste \\
\hline L222S & $\begin{array}{l}222 \mathrm{~s} \text { laboratory, dilute non-complexed } \\
\text { waste }\end{array}$ \\
\hline $\mathrm{I} 3 \mathrm{~A} 4 \mathrm{~A}$ & $\begin{array}{l}\text { Dilute non-complexed laboratory wastes } \\
\text { from } 300 \& 400 \text { areas }\end{array}$ \\
\hline LaF & $\begin{array}{l}\text { Lanthanum fluoride waste generated in } \\
\text { PFP }\end{array}$ \\
\hline
\end{tabular}


WHC-SD-WM-ER-312, Rev. 0

LUNC

LW

MW

MWF

$\mathrm{N}$

NCAW

NCPLEX

NCPLX

NCPI

NCRW

NFAW

NHAW

NRAW

NRP82

NRPO4

NRSO4

OWW

P

PADFG

PADWG
Dilute, non-complexed waste from UNC fuels fabrication

Laboratory waste

Metal waste

Metal waste feed

$\mathrm{N}$-Reactor waste

Neutralized current acid waste, primary HLW stream from PUREX process

Non-complexed waste-see NCPLX

Non-complexed waste-see NCPLEX

Non-Complexed waste

Neutralized cladding removal waste-same as CWP.

Aging waste from PUREX/PFM high level waste

Aging waste from PUREX/PFM processing of NPR fuel

Aging waste from PUREX/PFM residue acid waste

Dilute, non-complexed waste from FY82 100-N area waste transfer

Dilute, phosphate waste from $100 \mathrm{~N}$ area

Dilute, non-complexed waste from $100 \mathrm{~N}$ area

Organic wash waste

PUREX waste

Purex ammonia destruction waste, from fuels grade fuel.

Purex ammonia destruction waste, from 
WHC-SD-WM-ER-312, Rev. 0

weapons grade fuel

PAS

Purex acidified sludge

PASF

Purex ammonia scrubber feed

PAW

Purex acidified waste

$\mathrm{PD}$

Purex decladding waste

PDBNG

PDBSU

Decladding sludge (non-Tru) from B Plant processing

Dilute, non-complexed waste from B Plant decladding waste

PDBTG

B Plant aging waste solids from Purex decladding waste

PDCSS

Dilute non-complexed Purex decladding waste, FY1986 onIy

PDL 87

Purex decladding supernatant, 1987

PDL89

Purex decladding supernatant, non-Tru, spent metathesis removed

PDNSG

Non-Tru decladding sludge from Purex

PDS87

Purex decladding sludge

PDS89

Purex decladding sludge after FY89

PDSLG

Purex decladding sludge sol Purex

PDSUP

Dilute, non-complexed waste, Purex decladding waste

PFeCN

PFMMS

Ferrocyanide sludge produced by in-plant scavenging of waste from uranium recovery

Dilute, non-complexed waste from shear/leach processing of NPR fuel

PFPGR

Dilute, non-complexed waste from retrieved PFP solids 
WHC-SD-WM-ER-312, Rev. 0

PFPNT

PFPPT

PFPSI

PL

PML89

PMS89

PN

PNF

PSL

PSS

PSSF

PT

PX86S

PXBAW

PXBSG

PXFTF

PXIOW

PXMET

PXMSC

PXNAW
Non-tru sludge from the PFP sol $\mathrm{Z}$ Plant Dilute, non-complexed waste from the PFP (with TRUEX)

High-tru sludge from the PFP sol $z$ Plant PUREX low-Ievel waste

Purex spent metathesis liquid after FY89 Purex spent metathesis solids after FY89 Purex neutralized cladding waste

Partial neutralized waste

Purex sludge sluiced during recovery of strontium

PUREX sludge supernatant

Purex sludge supernatant feed

Tru solids from $200 \mathrm{~W}$

Dilute, non-complexed waste from Purex miscellaneous streams (NPR fuel) FY86

B Plant aging waste supernatant from retrieved aging waste

B Plant aging waste solids from retrieved aging waste

Dilute, non-complexed waste from Purex miscelianeous streams (FFTF)

Purex low level waste

Purex dilute, non-complexed decladding: spent metathesis

Dilute, non-complexed waste from Purex miscellaneous streams (NPR fuel)

Aging waste from Purex high level waste 
R

RESD

RIX

RSN

RSS

RTX

SIX

SL

SL3SY

SRR

SRS

SRS

SSW

SU

SWIIQ

SWLQW

TBP

TCO

TFeCN

$T H$

THL
REDOX waste

Residual evaporator liquor

REDOX ion exchange waste

REDOX supernatant

Redox sludge supernatant

Redox ion exchange

PUREX ion exchange waste

sludge

Double shell slurry from end of FY80, I03SY inventory

Waste sent to B-Plant for strontium recovery

Strontium sluage

Strontium recovery supernatant

Strontium semiworks waste

Supernatant

Dilute, non-complexed waste from east area single shell tanks

Dilute, non-complexed waste from west area single shell tanks

Tri-Butyl Phosphate

Dilute non-complexed waste from terminal cleanout

Ferrocyanide sludge produced by in-tank or in-farm scavenging

Thoria HLW or cladding waste

Thoria low level waste 
WHC-SD-WM-ER-312, Rev . 0

TL

TPLAL

TPLAN

TPLAS

U1U2

$\mathbf{Z}$

ZAW

ZHIGH

ZLAB

ZLOW

ZPREL

ZPRFS

ZRMCL

ZRMCS
Terminal liquor

Dilute, non-complexed waste from $T$ Plant

Dilute, non-complexed waste from $T$ Plant

Sludge from T Plant operations

Dilute, non-complexed waste from $U 1 / U 2$ groundwater pumping

Z-Plant waste

Purex waste stream from zirconium cladded fuel

Dilute, non-complexed waste from the PFP (without Truex)

Dilute, non-complexed waste from the PFP laboratories

Dilute, non-complexed waste from PreFY85 Z Plant operations

Dilute, non-complexed waste from PRF processing

PFP Tru solids from PRF processing

Dilute, non-complexed waste from PFP RMC processing

PFP Tru solids from PFP RMC processing 
WHC-SD-WM-ER-312, Rev. 0

A. IANL Defined Wastes

IANL DEFINED WASTE IIST

A1 SITCK

A2 SLTSLRY

$A R$

B SLTCK

BY SLTCK

CWP2

$\mathrm{DE}$

P1

$\mathrm{P} 2$

PFeCN1

PFeCN2

R SLTCK

RI

R2
Saltcake waste generated from the 242-A evaporator-crystallizer from 1977 until 1980 .

Salt slurry waste generated from the 242-A evaporator-crystallizer from 1981 until 1994.

"Washed" PUREX sludge fromthe AR vault.

Saltcake waste generated from the 242-B evaporator from 1951 until 1955.

Saltcake waste generated from in-tank solidification units 1 and 2 between 1965 and 1974.

Cladding waste-PUREX 2???

Diatomaceous earth.

PUREX high-level waste generated between 1955 and 1962 .

PUREX high-level waste generated between 1963 and 1967.

Ferrocyanide sludge generated from inplant scavenging of waste from uranium recovery.

Same as PFeCN1, except used $0.0025 \mathrm{M}$ ferrocyanide.

Salt cake waste from the REDOX concentrator.

REDOX waste generated between 1952 and 1957.

REDOX waste generated between 1958 and 1966 . 
S1 SITCK

S2 SLTSLRY

TI SLTCK

T2 SITCK

UNKNOWN

UR
Saltcake waste generated from the 242-S evaporator/crystallizer from 1973 until 1976.

Salt slurry waste generated from the 242-S evaporator-crystallizer from 1977 until 1980.

Saltcake waste generated from the 242-T evaporator from 1951 until 1955.

Saltcake waste generated from the 242-T evaporator from 1965 until 1955.

Unkown waste type.

Uranium recovery waste (also known as tributyl phosphate (TBP) waste). 
WHC-SD-WM-ER-312, Rev. 0

\section{FACILITIES}

A Plant (PUREX)

B Plant (222-B)

C Plant $(222-C)$

$S$ PIant (REDOX)

$T$ Plant $(222-T)$

$U$ Plant $(222-U)$

PFP ( 2 Plant)

$242-A$

$242-B$

$242-S$

$242-\mathrm{T}$
The facility at Hanford which contains the latest solvent extraction process for recovery of both plutonium and uranium.

On of the three original bismuthphosphate processing facilities. Later converted to a waste fractionation plant.

Initially a pilot plant for Redox, later a pilot plant for Purex and B-Plant waste partitioning.

The facility at Hanford which contains the original extraction process for recovery of both plutonium and uranium.

One of the three original bismuthphosphate processing facilities. Later converted to a decontamination facility.

One of the tiree original bismuthphosphate processing facilities. Later converted to a uranium recovery plant.

Plutonium Finishing Plant. The final operations for production of plutonium products are carried out in this facility.

A forced circulation vacuum evaporation system.

An evaporator that operates at atmospheric pressure. Also referred to as an open air type evaporator.

A forced circulation vacuum evaporation system.

An evaporator that operates at atmospheric pressure. Also referred to as an open air type evaporator. 
WHC-SD-WM-ER-312， Rev. 0

\section{REFERENCES}

Agnew, S.F., February 1994, Waste Status and Transaction Record Summary for the Northeast Quadrant, WHC-SD-WM-TI-615, REV. 0, Westinghouse Hanford Company, Richland, Washington.

Agnew, S.F., February 1994, Waste Status and Transaction Record Summary for the Southwest Quadrant, WHC-SD-WM-TI-614, REV. 0, Westinghouse Hanford Company, Richland, Washington.

Agnew, S.F. April 1994, Hanford Defined Wastes: Chemical and Radionuclide Compositions, LA-UR-94-2657, REV. 0, Los Alamos National Laboratory, Los Alamos, New Mexico.

Allen, G.K., March 1976, Estimated Inventory of Chemicals Added to Underground Waste Tanks, 1944 through 1975, ARH-CD-610B, Atlantic Richfield Hanford Company, Richland, Washington.

Alstad, A.T., September 19, 1991, Riser Configuration Document for Single-Shell Waste Tanks, WHC-SD-RE-TI-053, Rev. 8, Westinghouse Hanford Company, Richland, Washington.

Anderson, J.D., January, 1972- December 1972, Waste Status Summary - Chemical Processing Division, ARH-2456, Atlantic Richfield Hanford Company, Richland, Washington.

Anderson, J.D., January, 1973- December 1973, Waste Status Summary - Chemical Processing Division, ARH-2794, Atlantic Richfield Hanford Company, Richland, Washington.

Anderson, J.D., January, 1974- December 1974, Waste Status Summary - Operations Division, ARH-CD-133, Atlantic Richfield Hanford Company, Richland, Washington.

Anderson, J.D., January, 1974- December 1974, Waste Status Summary - Chemical Processing Division, ARH-CD-133, Atlantic Richfield Hanford Company, Richland, Washington.

Anderson, J.D., January, 1975- December 1975, Waste Status Summary - Production and Waste Management Division, ARH-CD-336, Atlantic Richfield Hanford Company, Richland, Washington.

Anderson, J.D., January, 1976-September 1976, Waste Status Summary - Production and Waste Management Division, ARH-CD-702, Atlantic Richfield Hänford Company, Richland, Washington.

Anderson, J.D., October, 1976- May 1977, Waste Status Summary Production and Waste Management Division, ARH-CD-822, Atlantic Richfield Hanford Company, Richland, Washington.

Anderson, J.D., June 1990, A History of the 200 Areas Tank Farms, WHC-MR-0132, Westinghouse Hanford Company, Richland, Washington. 
ARCHO, October, 1967, Monthly Report, 200 Areas Operation, ARH-60 DEL, Atlantic Richfield Hanford Company, Richland, Washington.

ARHCO, June, 1969, SX Tank Farm Air Cooling Safety Analysis, ARH-1291, Atlantic Richfield Hanford Company, Richland, Washington.

ARHCO, July, 1972 through December, Chemical Processing Division Waste status Summary, Quarterly Reports, ARH-2456 C and D, Atlantic Richfield Hanford Company, Richland, Washington.

ARHCO, January, 1973 through December 1973, Chemical Processing Division Waste Status Summary, Quarterly Reports, ARH-2694 A, B, C, and D, Atlantic Richfield Hanford Company, Richland, Washington.

ARHCO, January, 1973 through September 1975, Operations Division Waste Status Summary, Quarterly Reports, ARH-CD-133 A, B, C, and D, Atlantic Richfield Hanford Company, Richland, Washington.

ARCHO, September, 1976, Production and Waste Management Division Waste status Summary, Monthly Reports ARH-CD-702, Atlantic Richfield Hanford Company, Richland, Washington.

ARCHO, October, 1976 through June 1977, Production and Waste Management Division Waste Status Summary, Monthly Report, ARH-CD-822, Atlantic Richfield Hanford Company, Richland, Washington.

Babad, H., D. M. Camaioni, M. A. Iilga, W. D. Samuels, and D. M. Strachan, February 1993, Tank Waste Chemistry- A New Understanding of Waste Aging, WHC-SA-1694-FP, Westinghouse Hanford Company, Richland, Washington.

Boomer, R.D., June 1990, Functional Requirement Baseline for the Closure of Single-Shell Tanks, WHC-EP-0338, Westinghouse Hanford Company, Richland, Washington.

Borsheim, G.L., and B. C. Simpson, October 1991. An Assessment of the Inventories of the Ferrocyanide Watchlist Tanks, WHC-SD-WMER-133 Rev. 0, Westinghouse Hanford Company, Richland, Washington.

Borsheim, G.L., August 2, 1989, Single-Shell Tank Isolation Safety Analysis Repört, SD-WM-SAR-006-Rev 2, Westinghouse Hanford Company, Richland, Washington.

Borshiem, G. L. and N. W. Kirch, March 1991, Summary of Single-Shell Tank Waste Stability, WHC-EP-0347, Westinghouse Hanford Company, Richland, Washington. 
Boyles, V.C., June 1981, Safety Analysis Report: Stabilization of Single-Shell Waste Storage Tanks by Saltwell Jet Pumping, RHO-SD-WM-SAR-034, Rev.0, Rockwell Hanford Operations, Richland, Washington.

Brevick, C.H., I. A. Gaddis, and W. W. Pickett, June, 1994, Historical Tank Content Estimate Report for the Northeast Quadrant of the Hanford 200 East Area, WHC-SD-WM-ER-349, Rev.0, ICF Kaiser Hanford Company, Richland, Washington.

Brevick, C.H., L. A. Gaddis, and W. W. Pickett, June, 1994, Historical Tank Content Estimate Report for the Southwest Quadrant of the Hanford 200 West Area, WHC-SD-WM-ER-352, Rev.0, ICF Kaiser Hanford Company, Richland, Washington.

Brown, W.G., April 26.1993, Hazard Identification and Evaluation for Non-Stabilized Single Shell Tanks, SD-WM-SAR-022, Rev. 0, Westinghouse Hanford Company, Richland, Washington.

Bussell, J.H., February 3, 1992, Engineering Evaluation of Thermocouples in FeCN Watchlist Tanks, WHC-SD-WM-ER-134, Rev O-A, Westinghouse Hanford Company, Richland, Washington.

Cain, R.J., 1977, Isolation and stabilization of Special Tanks, (Internal Letter to J.C. Womack) Rockwell Hanford Company, Richland, Washington.

Carpenter, G.K., April, 1953 - June 1953, Waste Status Summary Separations Section, HW-28043 etc., General Electric Company, Richland, Washington.

Carpenter, G.K., September, 1953 - May 1954, Waste Status Summary - Separations Section, HW-29624 etc., General Electric Company, Richland, Washington.

Carter, George J., April, 1986- September 1986, Waste Status Summary, RHO-RE-SR-14, Rockwell Hanford Operations, Richland, Washington.

Cooley, C.R., and G.L. Richardson, July 17, 1963, Hot Semiworks Strontium-90 Recovery Program, HW-72666, General Electric Company, Richland, Washington.

ERDA, December, 1975, Waste Management Operations, ERDA-1538, Energy Research and:Development Administration, Richland, Washington.

Escobar, Glenn A., October, 1986- January 1987, Waste Status Summary, RHO-RE-SR-14, Rockwell Hanford Operations, Richland, Washington. 
WHC-SD-WM-ER-312, Rev. 0

Farley, W.G., May 20, 1992, Safety Assessment for Thermocouple Tree System Installation and Operation in Nonleaking Ferrocyanide Tanks, WHC-SD-WM-SAD-014, Rev. I, Westinghouse Hanford Company, Richland, Washington.

GE Co., July 10, 1951, REDOX Technical Manual, HW-18700-Del, General Electric Company, Richland, Washington.

Gerber, M.S., September, 1992, Legend and Legacy: Fifty Years of Defense Production at the Hanford Site, WHC-MR-0293, Rev 2, Westinghouse Hanford Company, Richland, Washington

Gerber, M.S., November, 1993, A Brief History of the Purex and $\mathrm{UO}_{3}$ Facilities, WHC-MR-0437, Westinghouse Hanford Company, Richland, Washington.

Gerber, M.S., September,1993, Multiple Missions: The 300 Area in Hanford Site History, WHC-MR-0440, Westinghouse Hanford Company, Richland, Washington.

Hanlon, B.M., November, 1989- December, 1990, Tank Farm Surveillance and Waste Status Summary Report, WHC-EP-0182, Westinghouse Hanford Company, Richland, Washington.

Hanlon, B.M., January, 1991-December, 1991, Tank Farm Surveillance and Waste Status Summary Report, WHC-EP-0182, Westinghouse Hanford Company, Richland, Washington.

Hanlon, B.M., January, 1992- December, 1992, Tank Farm Surveillance and Waste status Summary Report, WHC-EP-0182, Westinghouse Hanford Company, Richland, Washington.

Hanlon, B.M., July, 1993, Tank Farm Surveillance and Waste Status Summary Report for July 1993, WHC-EP-0182-64, Westinghouse Hanford Company, Richland, Washington.

Hanlon, B.M., January, 1993-October, 1993, Tank Farm Surveillance and Waste Status Summary Report, WHC-EP-0182, Westinghouse Hanford Company, Richland, Washington.

Hatch, P., December 18, 1969, Limitations for use of Underground Waste Tanks, ARH-951, Atlantic Richfield Hanford Company, Richland, Washington.

HEW, January 11, 1944, CVI, "Specifications for Construction of Composite Storage Tanks BIdg. No. 241," Project 9536, CVI 73550, Hanford Engineer Works, Richland, Washington.

Jenkins, C., M. Danielson, B. Rarig, R. Campbell, T. Ambalam, C. Kenoyer, and L. Hall, February, 1993, Engineering Evaluation of Alternatives Managing the Assumed Leak from SST 241-T-101, EEA93-1, Kaiser Engineers Hanford, Richland, Washington. 
Jungfleisch, F. M., March 1984, Preliminary Estimation of the Waste Inventories in Hanford Tanks through 1980, SD-WM-TI-057, Rev.0, Rockwell Hanford Operations, Richland, Washington.

KEH, February 1993, EEP Managing the Assumed Leak From SST, KEEA93-1, Kaiser Engineers Hanford, Richland, Washington.

Kirkman, M.J., G. I. Ritter, and P. W. Smith, March 6, 1969, BPlant Production Schedule and In-Tank Solidification

Alternatives, ARH-900, Atlantic Richfield Hanford Company, Richland, Washington.

Kreig, S.A., W.W. Jenkins, K.J. Leist, K. G. Squires, and J. F. Thompson, June 1990, Single-Shell Tank Waste Retrieval Study, WHC-EP-0352, UC-721, Westinghouse Hanford Company, Richland, Washington.

Lentz, J.E., April 1959- December 1959, Waste Status Summary Chemical Processing Department, HW-58579 etc., General Electric Company, Richland, Washington.

Lentz, J.E., January 1960- December 1960, Waste Status Summary Chemical Processing Department, HW-63896 etc., General Electric Company, Richland, Washington.

Iiverman, J.I., December 1975, Final Environmental Statement, Waste Management Operations Volume $I$ and 2, ERDA-1538, Energy Research and Development Administration, Richland, Washington.

Lucas, G.E., June 1989, Waste Types in Hanford Single-Shell Tanks, WHC-SD-ER-TI-00I, Rev. 0, Westinghouse Hanford Company, Richland, Washington.

McBride, J.P., July, 1953, Waste Status Summary - Separations Section, HW-29054, General Electric Company, Richland, Washington.

MCCann, D.C., July, 1981-October 1981, Waste Status Summary, RHO-CD-14, Rockwell Hanford Operations, Richland, Washington.

MCCann, D.C., October, 1981- December 1982, Waste Status Summary, RHO-RE-SR-14, Rockwell Hanford Operations, Richland, Washington.

MCCann, D.C., January, 1983- December 1983, Waste Status Summary, RHO-RE-SR-14, Rockwejll Hanford Operations, Richland, Washington.

McCann, D.C., January, 1984- February 1985, Waste Status Summary, RHO-RE-SR-14, Rockwell Hanford Operations, Richland, Washington.

Morganthaler, A.C., February, 1952 - March 1953, Waste Status Summary - Separations Section, HW-27897 etc., General Electric Company, Richland, Washington. 
Mudd, O.C., January, 1979- December 1979, Waste Status Summary, RHO-CD-14, Rockwell Hanford Operations, Richland, Washington.

Mudd, O.C., January, 1980- December 1980, Waste Status Summary, RHO-CD-14, Rockwell Hanford Operations, Richland, Washington.

Mudd, O.C., January, 1981- June 1981, Waste Status Summary, RHOCD-14, Rockwell Hanford Operations, Richland, Washington.

Mulvey, C.A., June, 1977- December 1977, Waste Status Summary, RHO-CD-14, Rockwell Hanford Operations, Richland, Washington.

Mulvey, C.A., January, 1978- December 1978, Waste Status Summary, RHO-CD-14, Rockwell Hanford Operations, Richland, Washington.

Peterson, D.E., June, 1954- May 1955, Waste Status Summary Separations Section, HW-32389 etc., General Electric Company, Richland, Washington.

Peterson, D.E., June, 1955- May 1956, Waste Status Summary Separations Section, HW-38000 etc., General Electric Company, Richland, Washington.

Peterson, D.E., September, 1956- December 1956, Waste Status Summary - Chemical Processing Department, HW-45738 etc., General Electric Company, Richland, Washington.

Peterson, D.E., June, 1956-August 1956, Waste Status Summary Separations Section, HW-43895 etc., General Electric Company, Richland, Washington.

Pines, A.G., January 4, 1991, 244-AR Vault Safety Analysis Report, SD-WM-SAR 018 Rev. 0, Westinghouse Hanford Company, Richland, Washington.

Prosk, W., and D.A. Smith, January 1986, Tank Isolation Safety Analysis Report, SD-WM-SAR-006 Rev. 1, Rockwell Hanford Operations, Richland, Washington.

RHO, June 1977 through October 1981, Waste Status Summary, Monthly Reports RHO-CD-14, Rockwell Hanford Operations, Richland, Washington.

RHO, November 1981 through June 1987, Waste status Summary, Monthly Reports RHÖRE-SR-0I4, Rockwell Hanford Operations, Richland, Washington.

Roberts, R.E., January, 1957- December 1957, Waste Status Summary - Chemical Processing Department, HW-48144 etc., General Electric Company, Richland, Washington.

Roberts, R.E., January, 1958- October 1958, Waste Status Summary - Chemical Processing Department, HW-54916 etc., General Electric Company, Richland, washington. 
WHC-SD-WM-ER-312, Rev. 0

Roberts, R.E., January, 1961- December 1961, Waste Status Summary - Chemical Processing Department, HW-71610 etc., General Electric Company, Richland, Washington.

Roberts, R.E., January, 1962- December 1962, Waste Status Summary - Chemical Processing Department, HW-74647 etc., General Electric Company, Richland, Washington.

Roberts, R.E., January, 1963- December 1963, Waste Status Summary - Chemical Processing Department, HW-78279 etc., General Electric Company, Richland, Washington.

Roberts, R.E., January, 1964-December 1964, Waste Status Summary - Chemical Processing Department, HW-83308 etc., General Electric Company, Richland, Washington.

Roberts, R.E., January, 1965- December 1965, Waste Status Summary - Chemical Processing Department, HW-83906 etc., General Electric Company, Richland, Washington.

Roberts, R.E., October, 1966- December 1966, Waste Status Summary - Chemical Processing Division, ISO-674, Isochem Inc., Richland, washington.

Roberts, R.E., January, 1966- September 1966, Waste Status Summary - Chemical Processing Department, HW-83906 etc., General Electric Company, Richland, Washington.

Rodenhizer, D.G., September 30, 1987, Hanford Waste Tank Sluicing History, SD-WM-TI-302, Westinghouse Hanford Company, Richland, washington.

Rutherford, M.J., August 31, 1948, Additional Waste Storage Facilities 200-East Area, Specification No. HW 3783, General Electric Company, Richland, Washington.

Scaief, C.C. III, October 19, 1993, TMACS I/O Termination Point Listing, WHC-8D-WM-TI-594, Rev. 0, Westinghouse Hanford Company, Richland, Washington.

Smith, D.A., August 1986, SAR, Hazard Identification and Evaluation for NonStabilized Single-Shell Tanks, SD-WM-SAR 022 Rev. 0, Rockwell Hanford Operations, Richland, Washington.

Stahl, S.M., Novembëŕ 22, 1993, Hanford Site Tank Farm Facilities Interim Safety Basis, Volume 1 and 2, WHC-SD-WM-ISB-001, Westinghouse Hanford Company, Richland, Washington.

Stong, F.S., September 29, 19860, Drywell Van In-Tank Liquid Observation Well Surveillance Data Interpretation, RHO-SD-WM-TI-237, Rockwell Hanford Operations, Richland, Washington. 
ThanMai, T. T., April 27, 1993, Thermocouple Status Single-Shell and Double-Shell Waste Tanks, WHC-SD-WM-TI-553, Rev. 0, Westinghouse Hanford Company, Richland, Washington.

Thress, M A., November 1958- February 1959, Waste Status Summary - Chemical Processing Department, HW-58579 etc., General Electric Company, Richland, Washington.

Thurman, Jack M., June, 1987 through December 1987, Tank Farm Surveillance and Waste Status Summary Report, Monthly Reports WHC-SP 0038, Westinghouse Hanford Company, Richland, Washington.

Thurman, Jack M., December, 1987 through June 1993, Tank Farm Surveillance and Waste Status Summary Report, Monthly Reports WHC-EP-0182-55, Westinghouse Hanford Company, Richland, Washington.

Thurman, Jack M., February, 1987- June 1987, Waste Status Summary, RHO-RE-SR-14, Rockwell Hanford Operations, Richland, Washington.

Thurman, Jack M., July, 1987- March, 1988, Waste Status Summary, WHC-SP-0038, Westinghouse Hanford. Company, Richland, Washington.

Thurman, Jack M., April, 1988- December, 1988, Tank Farm Surveillance and Waste Status Summary Report, WHC-EP-0182, Westinghouse Hanford Company, Richland, Washington.

Thurman, Jack M., January, 1989- October, 1989, Tank Farm Surveillance and Waste Status Summary Report, WHC-EP-0182, Westinghouse Hanford Company, Richland, Washington.

U.S.DOE, December 1987, EIS, Disposal of Hanford Defense High Level, Transuranic, and Tank Wastes, DOE/EIS-0113 Volume 1-5, Department of Energy, Richland, Washington.

Uebelacker, D.L., October, 1968- December 1968, Waste Status Summary - Chemical Processing Division, ARH-1061, Atlantic Richfield Hanford Company, Richland, Washington.

Uebelacker, D.L., January, 1969- December 1969, Waste Status Summary - Chemical Processing Division, ARH-1200, Atlantic Richfield Hanford Company, Richland, Washington.

Uebelacker, D.L., Jänuary, 1970- December 1970, Waste Status Summary - Chemical Processing Division, ARH-1666, Atlantic Richfield Hanford Company, Richland, Washington.

Vail, Terry S., January, 1985- March 1986, Waste Status Summary, RHO-RE-SR-14, Rockwell Hanford Operations, Richland, Washington.

Wagoner, J.D., March 18, 1993, Wyden Request, Document No. 9301156B R3, U.S. Department of Energy, Richland, Washington. 
Waite, J.I., April 1991, Tank Wastes Discharged Directly to the Soil at the Hanford Site, WHC-MR-227, Westinghouse Hanford Company, Richland, Washington.

Welty, R. K., September 1988, Waste Storage Tank Status and Leak Detection Criteria, WHC-SD-WM-TI-356, Westinghouse Hanford Company, Vol. 1 and 2, Richland, washington.

Welty, R.K. and Norma J. Vermeulen, September 12, 1989 with ECNs through November 08, 1993, Waste Storage Tank Status and Leak Detection Criteria, WHC-SD-WM-TI-357, Rev. IK, Westinghouse Hanford Company, Richland, Washington.

WHC, September, 1991, Failure to Continuously Man Control Room During Transfer Per Procedure, Occurrence Report RL--WHC--Tank Farm-1991-1019, Westinghouse Hanford Company, Richland, Washington.

WHC, July, 1991, High Radiation Readings in 24I-BY Drywells, Occurrence Report RL--WHC-Tank Farm-1991-1022, Westinghouse Hanford Company, Richland, Washington.

WHC, August 1993, Hanford Site Tank Farm Facilities Interim Safety Basis, WHC-SD-WM-ISB-001 Rev. 1, Volume 1 and Volume 2., Westinghouse Hanford Company,

WHC, March, 1993, Hanford Watchlist Tank Surveillance Trends, Ietter to the Honorable Ron Wyden, Westinghouse Hanford Company, Richland, Washington.

WHC, July, 1993, Unauthorized Water Usage at 241-BY Tank Farm Results in Exceeding Raw Water Operational Limit, Occurrence Report RL--WHC-Tank Farm-1993-0050, Westinghouse Hanford Company, Richland, Washington.

Winters, W.I., L. Jensen, I.M. Sasaki, R.L. Weiss, J.F. KeIler, A.J. Schmidt, and M.G. Woodruff, May 1989, Waste Characterization Plan for the Hanford Site Single-shell Tanks, WHC-EP-0210, Westinghouse Hanford Company, Richland, Washington. 


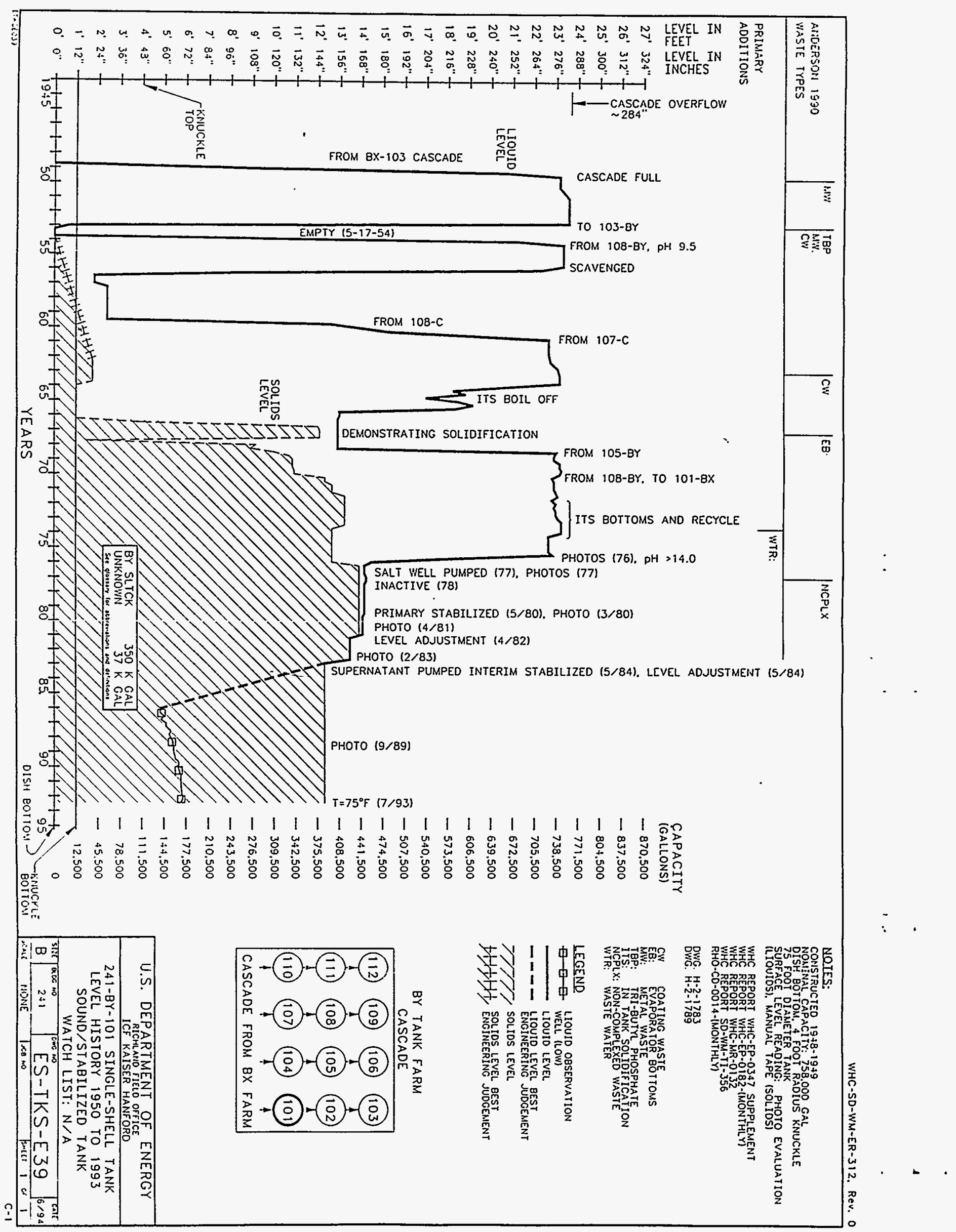




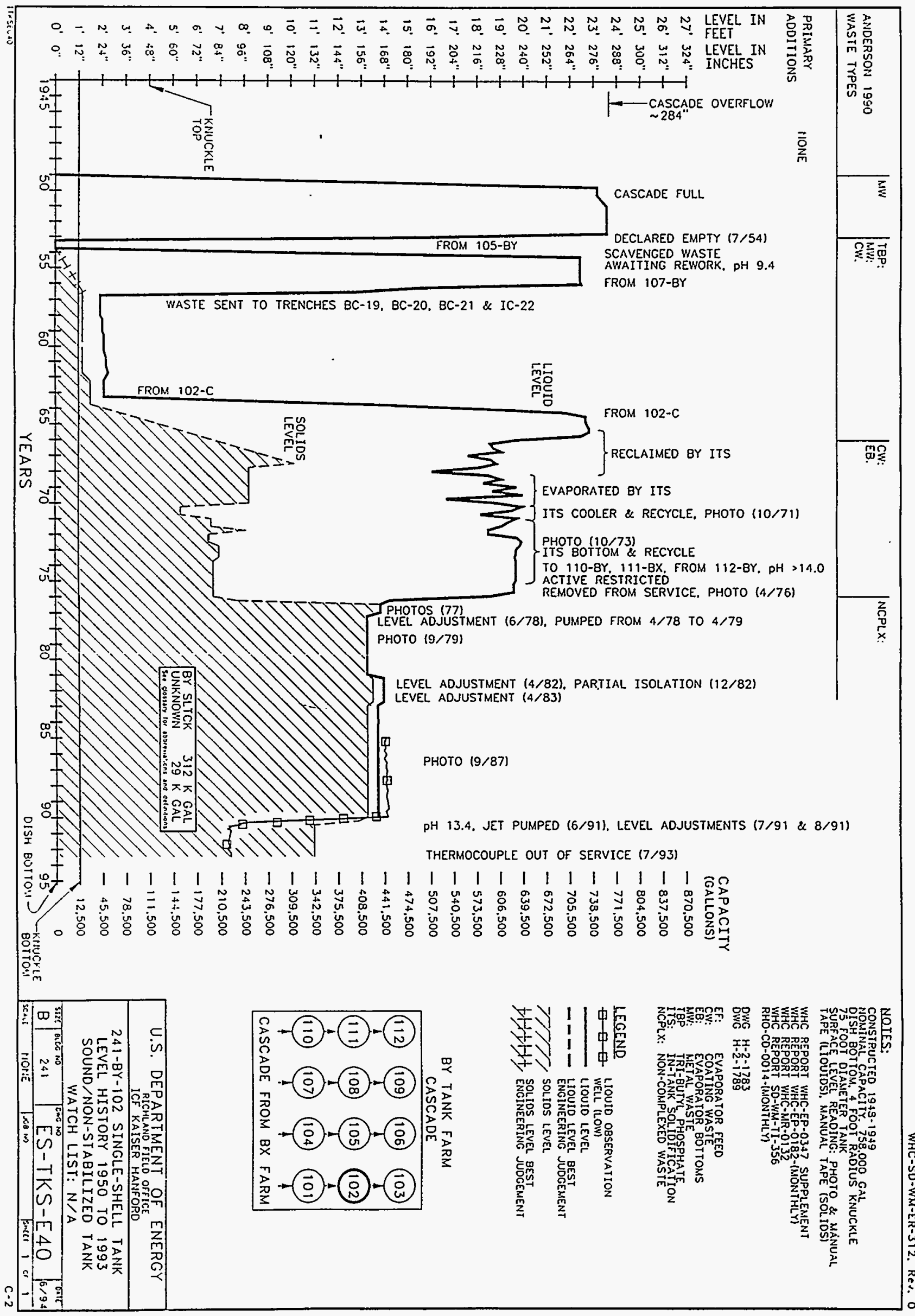




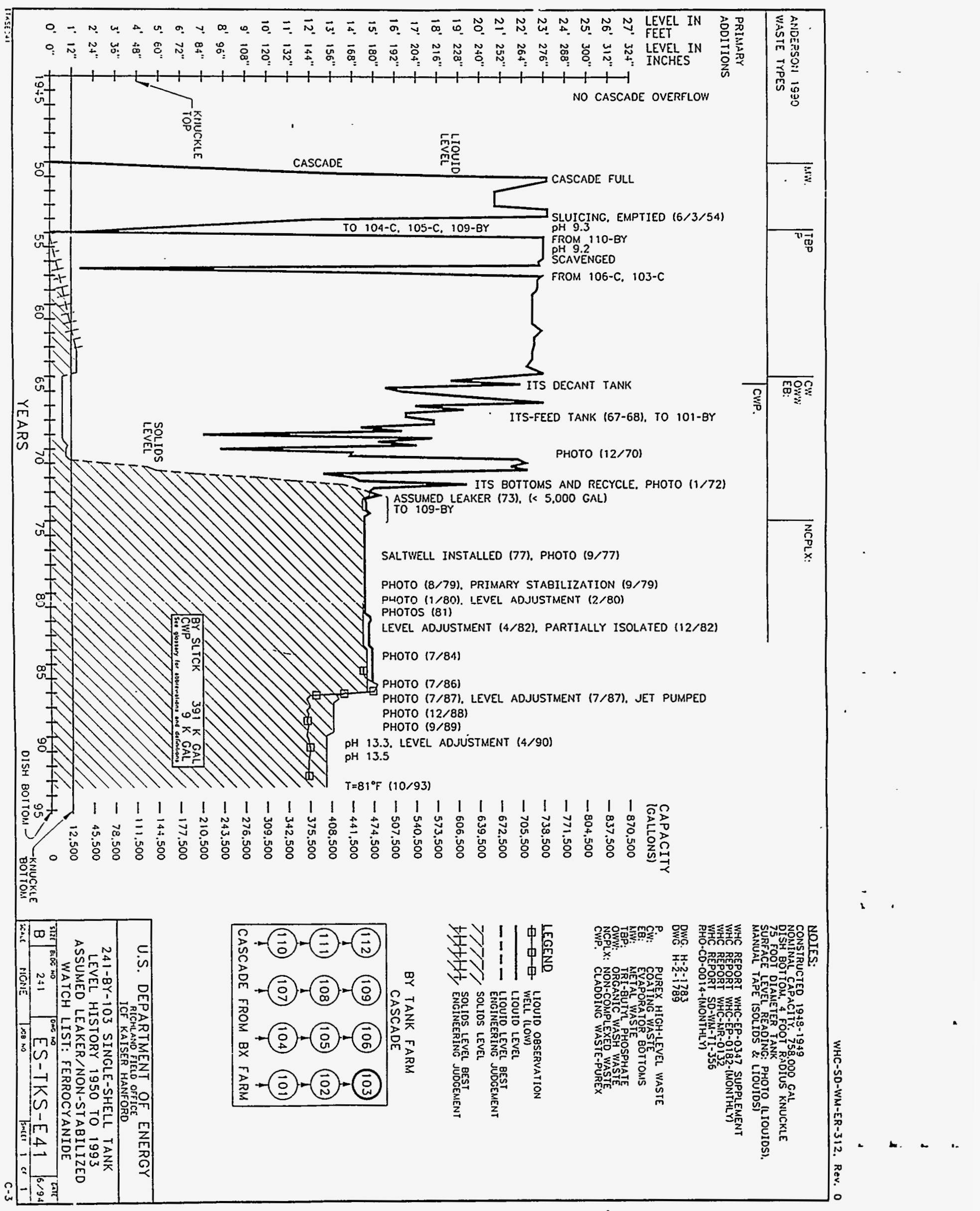




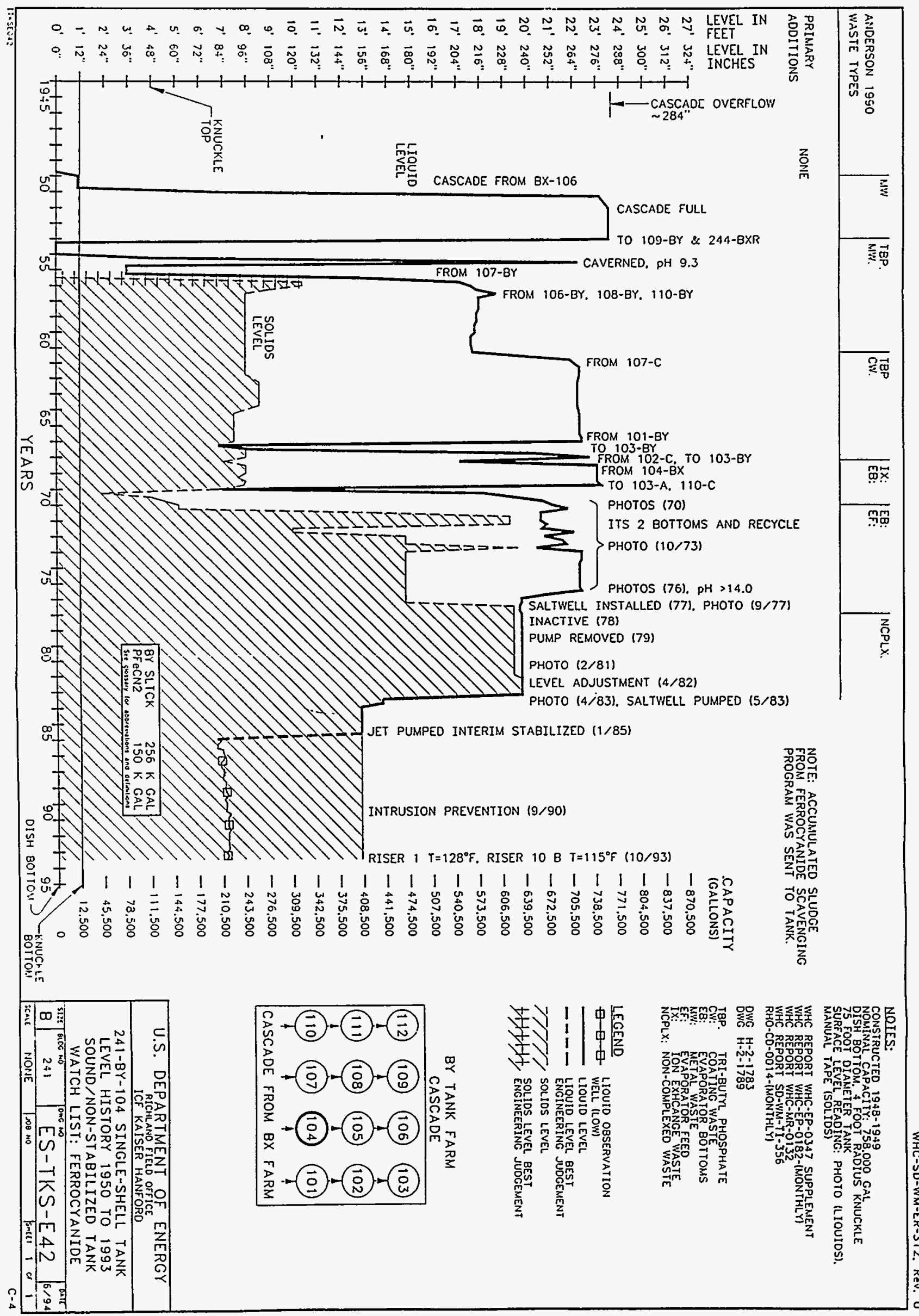




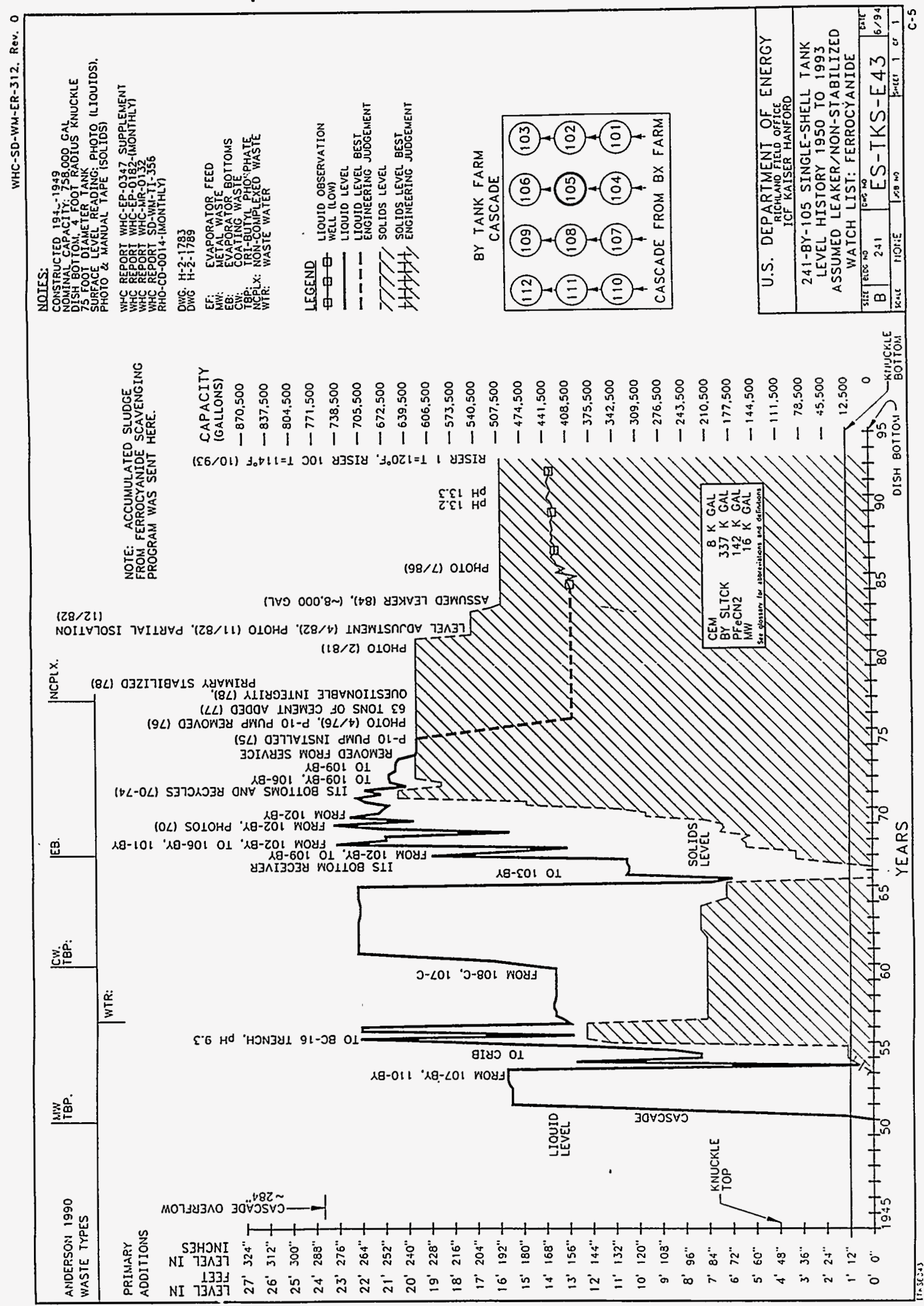




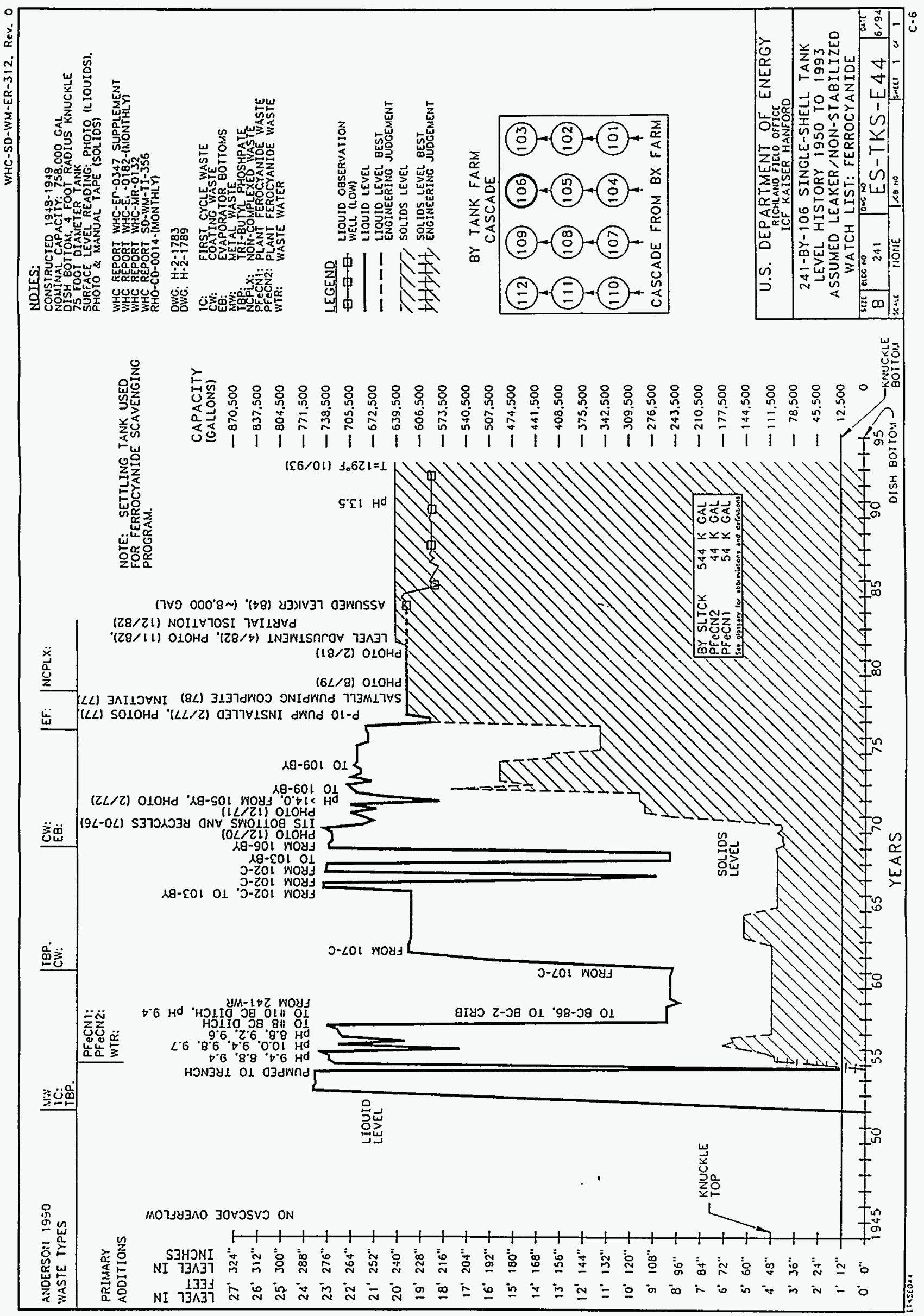


$c$

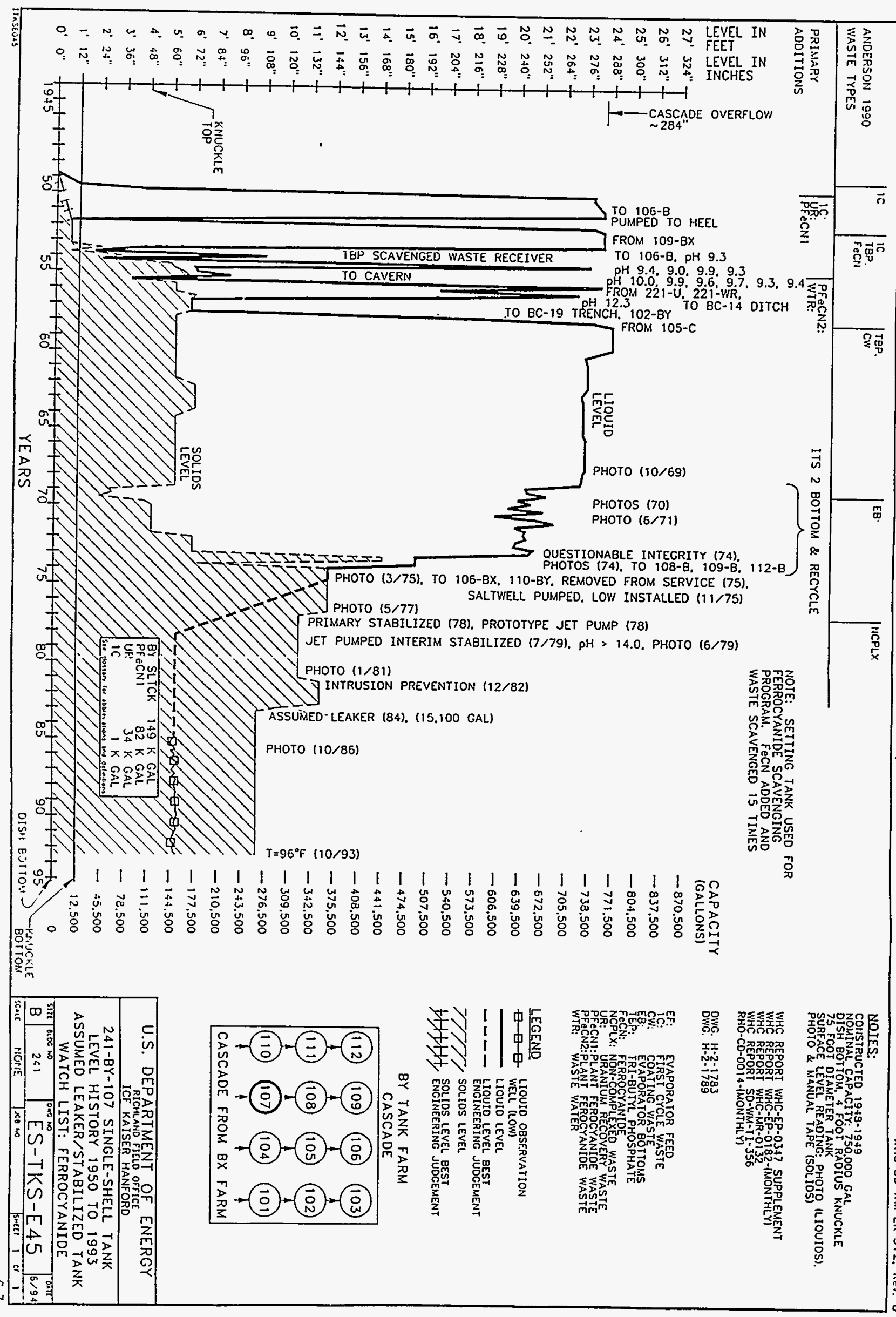




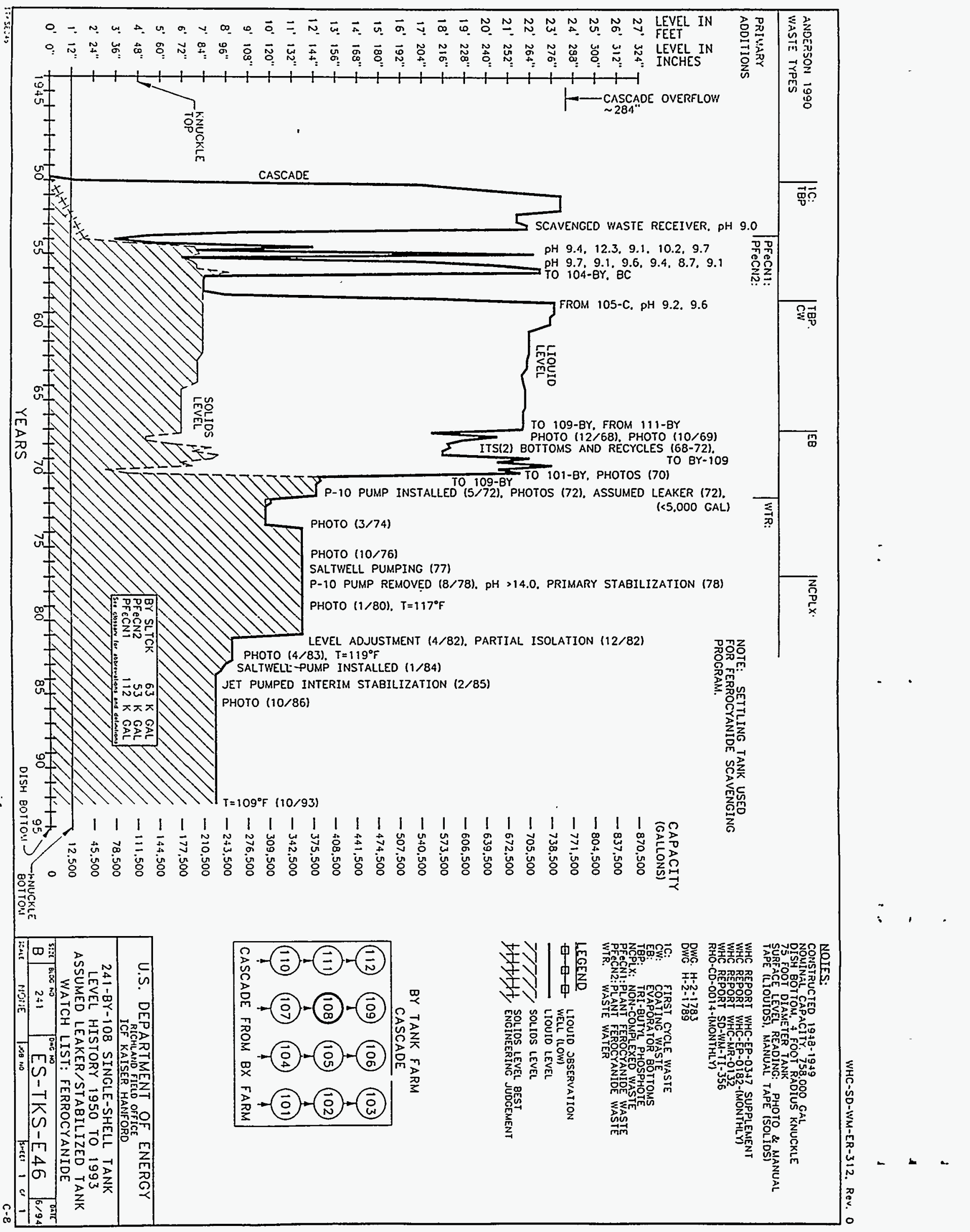




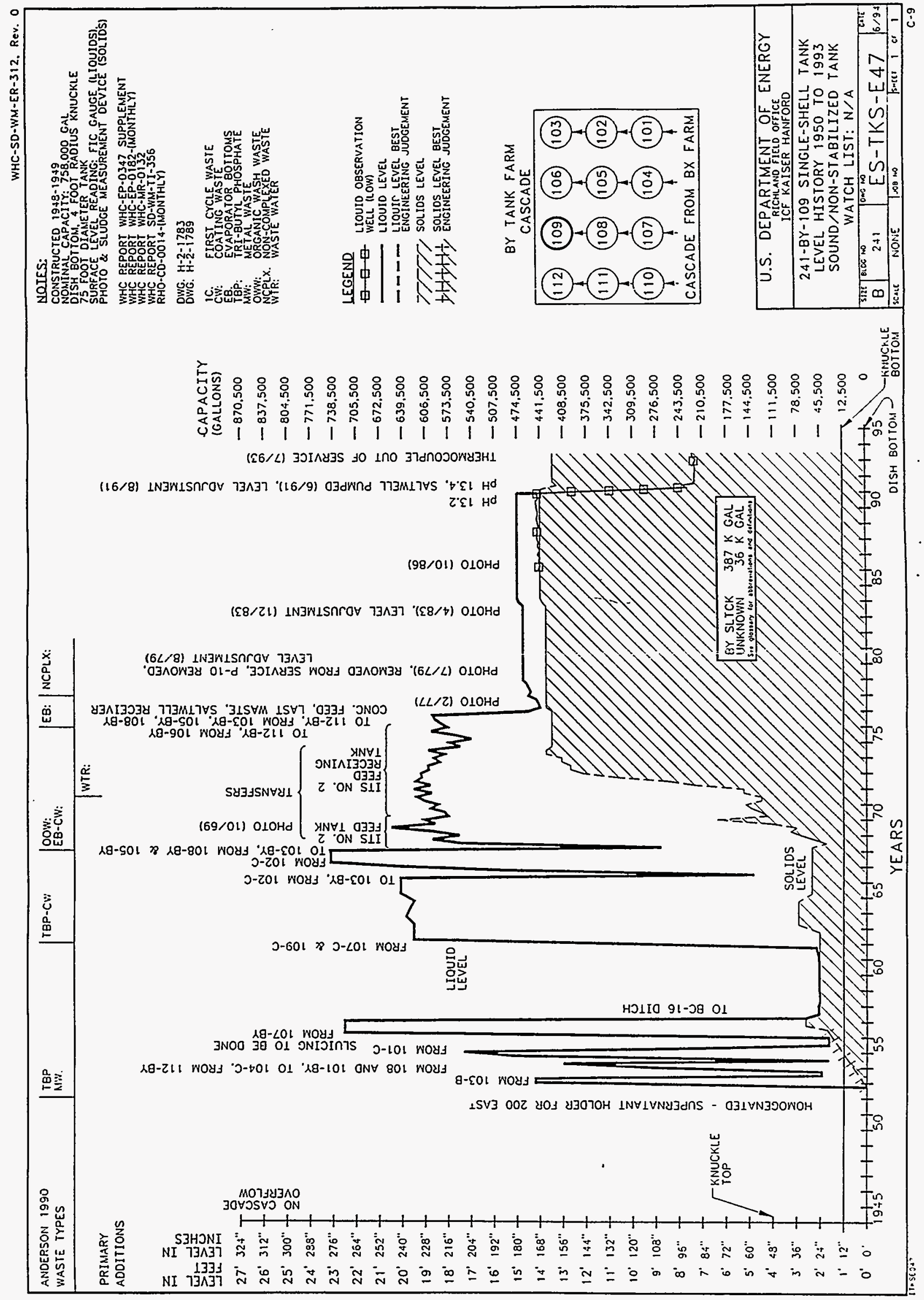




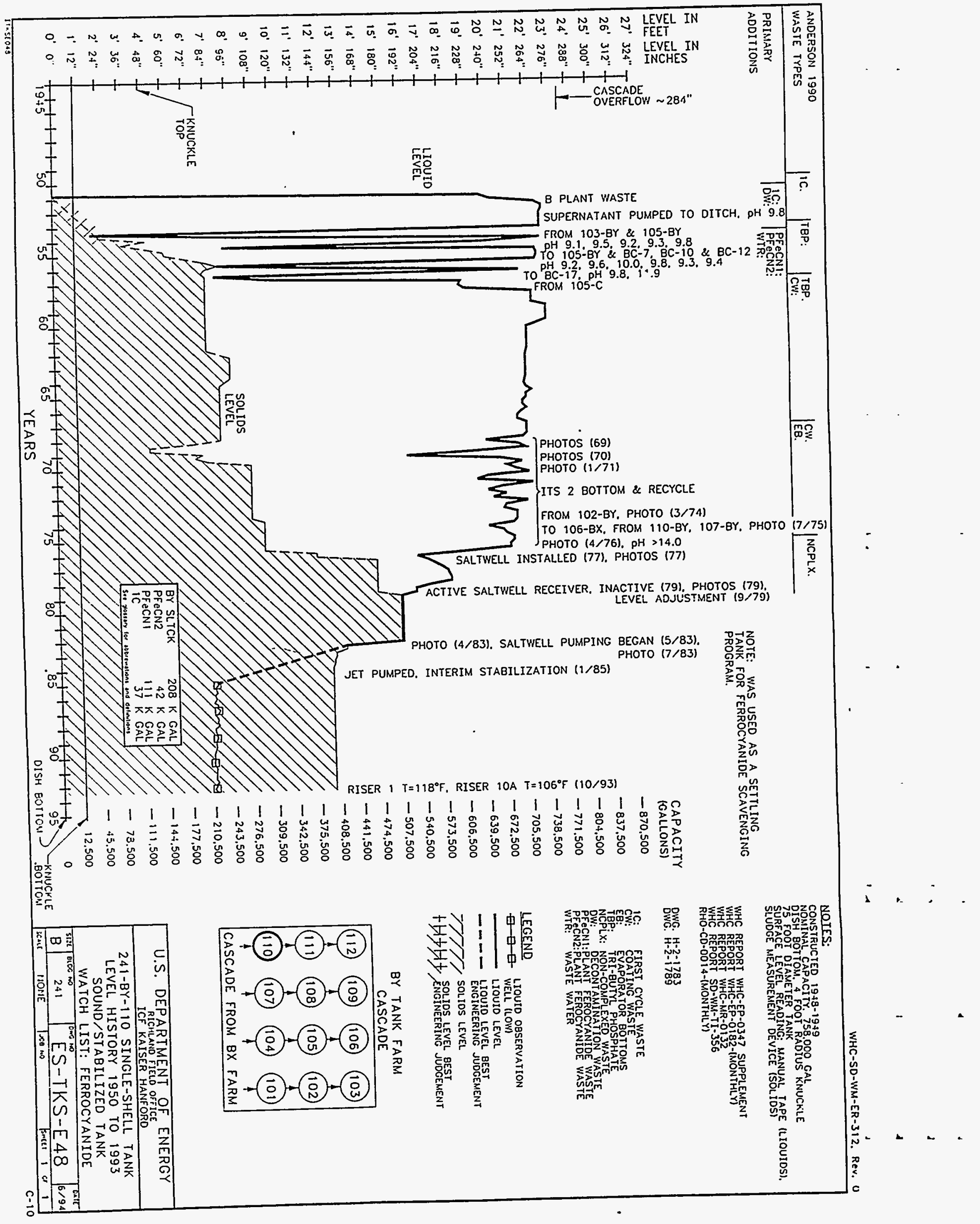




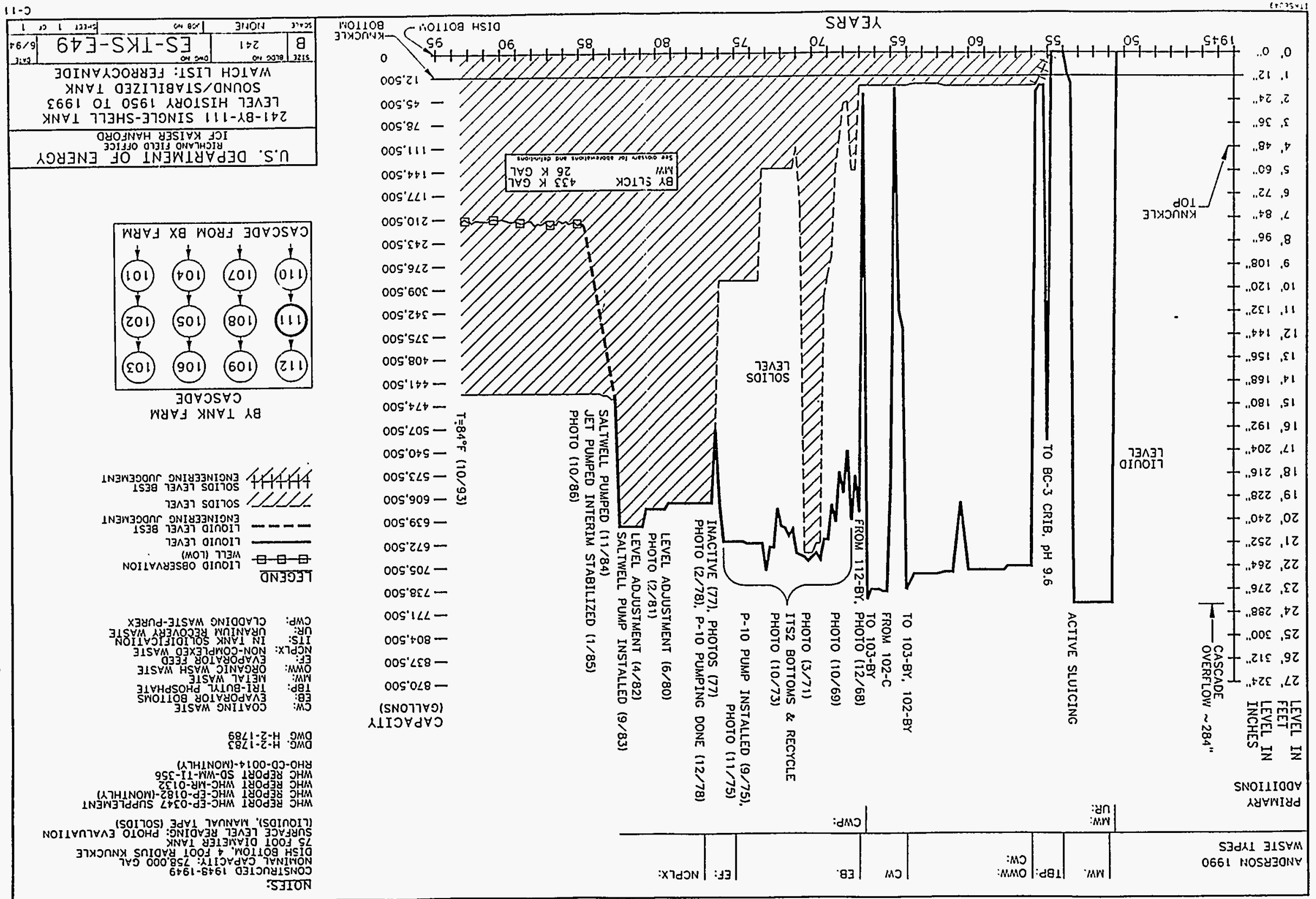




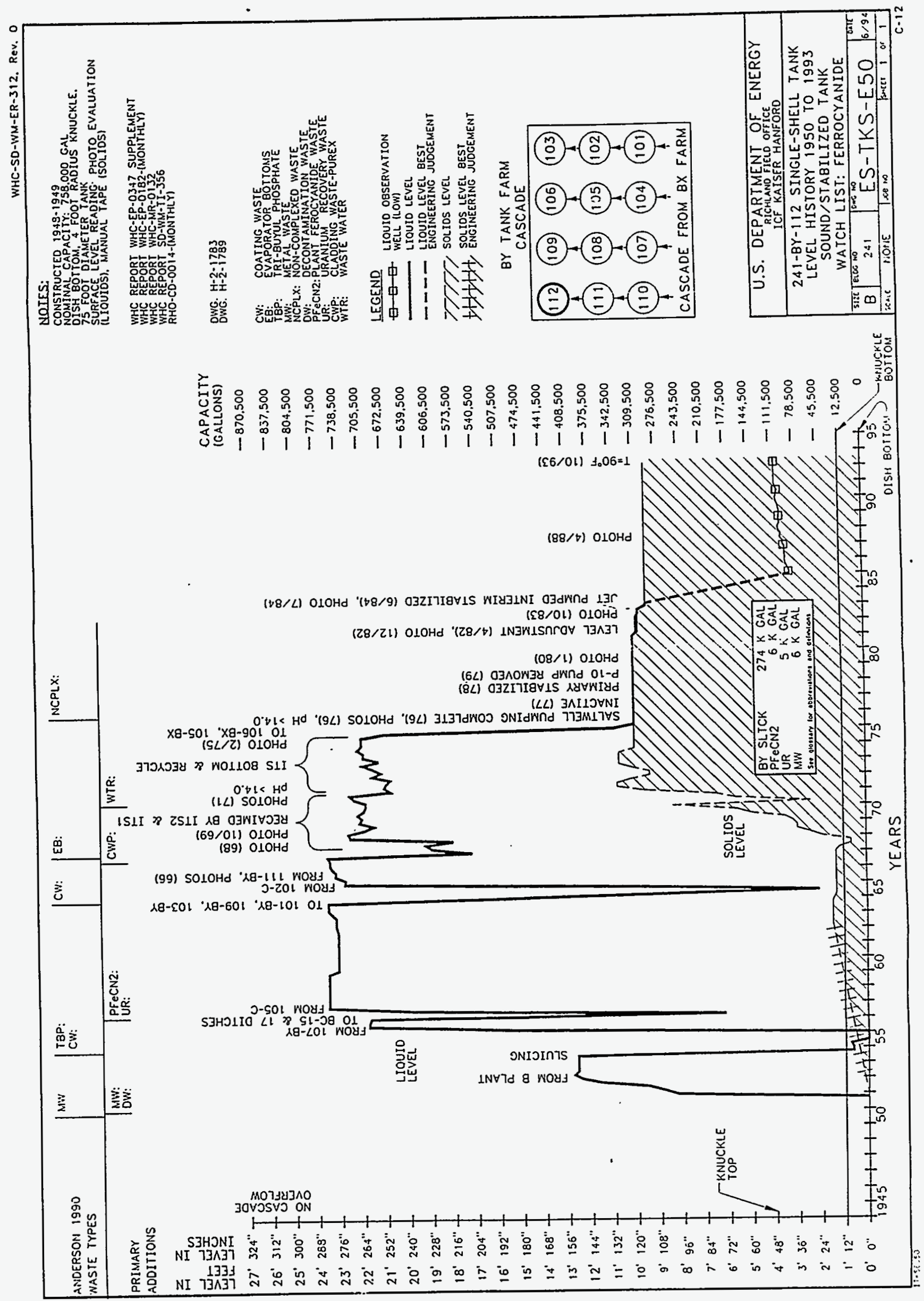


241-BY-101 SINGLE-SHELL WHC-SD-WM-ER-312, ReV.0 TANK LEVEL HISTORY

\begin{tabular}{|c|c|c|c|c|c|}
\hline Year & Total & Total & Solids & Solids & LOW \\
\hline & (K gal) & (in) & ( $\mathrm{K}$ gal) & (in) & (in) \\
\hline $1-1950$ & 290 & 112 & & & \\
\hline 2 & 661 & 248 & & & \\
\hline 3 & 744 & 278 & & & \\
\hline 4 & 744 & 278 & & & \\
\hline $1-1951$ & 744 & 278 & & & \\
\hline 2 & 744 & 278 & & & \\
\hline \multicolumn{6}{|l|}{3} \\
\hline \multicolumn{6}{|l|}{4} \\
\hline $1-1952$ & 758 & 283 & & & \\
\hline 2 & 758 & 283 & & & \\
\hline 3 & 758 & 283 & & & \\
\hline 4 & 758 & 283 & & & \\
\hline $1-1953$ & 758 & 283 & & & \\
\hline 2 & 758 & 283 & & & \\
\hline 3 & 758 & 283 & & & \\
\hline 4 & 758 & 283 & & & \\
\hline $1-1954$ & 1 & 8 & & & \\
\hline \multicolumn{6}{|l|}{2} \\
\hline \multicolumn{6}{|l|}{3} \\
\hline \multicolumn{6}{|l|}{4} \\
\hline $1-1955$ & 681 & 255 & & & \\
\hline 2 & 750 & 280 & & & \\
\hline 3 & 750 & 280 & & & \\
\hline 4 & 750 & 280 & & & \\
\hline $1-1956$ & 750 & 280 & & & \\
\hline 2 & 750 & 280 & & & \\
\hline 3 & 750 & 280 & & & \\
\hline 4 & 750 & 280 & & & \\
\hline $1-1957$ & 717 & 268 & & & \\
\hline 2 & 262 & 103 & & & \\
\hline 3 & 40 & 22 & & & \\
\hline 4 & 40 & 22 & & & \\
\hline $1-1958$ & 40 & 22 & & & \\
\hline 2 & 59 & 29 & & & \\
\hline 3 & 59 & 29 & & & \\
\hline 4 & 59 & 29 & & & \\
\hline $1-1959$ & 59 & 29 & & & \\
\hline 2 & 59 & 29 & & & \\
\hline 3 & 59 & 29 & & & 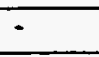 \\
\hline 4 & 60 & 29 & & & \\
\hline $1-1960$ & 60 & 29 & & & \\
\hline 2 & 60 & 29 & & & \\
\hline 3 & 60 & 29 & & & \\
\hline 4 & 394 & 151 & & & \\
\hline \multicolumn{6}{|l|}{$1-1961$} \\
\hline 2 & 483 & 183 & & & \\
\hline
\end{tabular}


241-BY-101 SINGLE-SHELL WHC-SD-WM-ER-312, ReV.0 TANK LEVEL HISTORY

\begin{tabular}{|c|c|c|c|c|c|}
\hline Year & Total & Total & Solids & Solids & LOW \\
\hline & (K gal) & (in) & ( $\mathrm{K}$ gal) & (in) & (in) \\
\hline & & & & & \\
\hline \multicolumn{6}{|l|}{3} \\
\hline 4 & 728 & 272 & & & \\
\hline \multicolumn{6}{|l|}{$1-1962$} \\
\hline 2 & 728 & 272 & & & \\
\hline \multicolumn{6}{|l|}{3} \\
\hline 4 & 728 & 272 & & & \\
\hline \multicolumn{6}{|l|}{$1-1963$} \\
\hline 2 & 730 & 273 & 37 & 21 & \\
\hline \multicolumn{6}{|l|}{3} \\
\hline 4 & 741 & 277 & 37 & 21 & \\
\hline \multicolumn{6}{|l|}{$1-1964$} \\
\hline 2 & 744 & 278 & 37 & 21 & \\
\hline \multicolumn{6}{|l|}{3} \\
\hline 4 & 744 & 278 & 37 & 21 & \\
\hline \multicolumn{6}{|l|}{$1-1965$} \\
\hline 2 & 582 & 219 & 0 & 0 & \\
\hline 3 & 601 & 226 & & & \\
\hline 4 & 541 & 204 & & & \\
\hline $1-1966$ & 590 & 222 & & & \\
\hline 2 & 612 & 230 & & & \\
\hline 3 & 585 & 220 & 0 & 0 & \\
\hline 4 & 409 & 156 & 0 & 0 & \\
\hline $1-1967$ & 409 & 156 & 0 & 0 & \\
\hline 2 & 407 & 155 & 0 & 0 & \\
\hline 3 & 407 & 155 & 109 & 47 & \\
\hline 4 & 406 & 155 & 376 & 144 & \\
\hline $1-1968$ & 406 & 155 & 378 & 145 & \\
\hline 2 & 406 & 155 & 378 & 145 & \\
\hline 3 & 407 & 155 & 378 & 145 & \\
\hline 4 & 407 & 155 & 29 & 18 & \\
\hline $1-1969$ & 407 & 155 & 281 & 110 & \\
\hline 2 & 407 & 155 & 274 & 107 & \\
\hline 3 & 739 & 276 & 310 & 120 & \\
\hline 4 & 737 & 275 & 332 & 128 & \\
\hline $1-1970$ & 737 & 275 & 337 & 130 & \\
\hline 2 & 744 & 278 & 337 & 130 & \\
\hline 3 & 743 & 278 & 337 & 130 & \\
\hline 4 & 747 & 279 & 340 & 131 & \\
\hline $1-1971$ & 745 & 278 & 340 & 131 & - \\
\hline 2 & 733 & 274 & 386 & 148 & \\
\hline 3 & 736 & 275 & 386 & 148 & \\
\hline 4 & 737 & 275 & 398 & 152 & \\
\hline \begin{tabular}{|l|}
1972 \\
\end{tabular} & 737 & 275 & 398 & 152 & \\
\hline 2 & 739 & 276 & 398 & 152 & \\
\hline 3 & 740 & 277 & 418 & 159 & \\
\hline 4 & 734 & 274 & 418 & 159 & \\
\hline
\end{tabular}


241-BY-101 SINGLE-SHELL WHC-SD-WM-ER-312, ReV.0 TANK LEVEL HISTORY

\begin{tabular}{|c|c|c|c|c|c|}
\hline Year & Total & Total & Solids & Solids & LOW \\
\hline & (K gal) & (in) & (K gal) & (in) & (in) \\
\hline 11070 & & & & & \\
\hline $1-1973$ & 738 & 276 & 418 & 159 & \\
\hline 2 & 737 & 275 & 418 & 159 & \\
\hline 3 & 738 & 276 & 418 & 159 & \\
\hline 4 & 739 & 276 & 418 & 159 & \\
\hline $1-1974$ & 740 & 277 & 418 & 159 & \\
\hline 2 & 746 & 279 & 418 & 159 & \\
\hline 3 & 748 & 279 & 418 & 159 & \\
\hline 4 & 747 & 279 & 398 & 152 & \\
\hline $1-1975$ & 747 & 279 & 398 & 152 & \\
\hline 2 & 728 & 272 & 398 & 152 & \\
\hline 3 & 728 & 272 & 398 & 152 & \\
\hline 4 & 728 & 272 & 398 & 152 & \\
\hline $1-1976$ & 728 & 272 & 398 & 152 & \\
\hline 2 & 728 & 272 & 398 & 152 & \\
\hline 3 & 733 & 274 & 398 & 152 & \\
\hline 4 & 579 & 218 & 398 & 152 & \\
\hline $1-1977$ & 458 & 174 & 398 & 152 & \\
\hline 2 & 447 & 170 & 439 & 167 & \\
\hline 3 & 447 & 170 & 439 & 167 & \\
\hline 4 & 447 & 170 & 439 & 167 & \\
\hline $1-1978$ & 450 & 171 & 439 & 167 & \\
\hline 2 & 450 & 171 & 439 & 167 & \\
\hline 3 & 447 & 170 & 439 & 167 & \\
\hline 4 & 447 & 170 & 439 & 167 & \\
\hline $1-1979$ & 447 & 170 & 439 & 167 & \\
\hline 2 & 447 & 170 & 439 & 167 & \\
\hline 3 & 447 & 170 & 439 & 167 & \\
\hline 4 & 447 & 170 & 439 & 167 & \\
\hline $1-1980$ & 447 & 170 & 439 & 167 & \\
\hline 2 & 447 & 170 & 439 & 167 & \\
\hline 3 & 447 & 170 & 439 & 167 & \\
\hline 4 & 443 & 169 & 439 & 167 & \\
\hline $1-1981$ & 443 & 169 & 439 & 167 & \\
\hline 2 & 443 & 169 & 439 & 167 & \\
\hline 3 & 443 & 169 & 439 & 167 & \\
\hline 4 & 443 & 169 & 439 & 167 & \\
\hline $1-1982$ & 443 & 169 & 439 & 167 & \\
\hline 2 & 425 & 162 & 425 & 162 & \\
\hline 3 & 425 & 162 & 425 & 162 & $\because$ \\
\hline 4 & 425 & 162 & 425 & 162 & \\
\hline $1-1983$ & 425 & 162 & 425 & 162 & \\
\hline 2 & 425 & 162 & 425 & 162 & \\
\hline 3 & 425 & 162 & 425 & 162 & \\
\hline 4 & 425 & 162 & 425 & 162 & \\
\hline $1-1984$ & 387 & 148 & 387 & 148 & \\
\hline 2 & 387 & 148 & 387 & 148 & \\
\hline
\end{tabular}


241-BY-101 SINGLE-SHELL WHC-SD-WM-ER-312, ReV. 0 TANK LEVEL HISTORY

\begin{tabular}{|c|c|c|c|c|c|}
\hline Year & Total & Total & Solids & Solids & LOW \\
\hline & (K gal) & (in) & (K gal) & (in) & (in) \\
\hline & & & & & \\
\hline 3 & 387 & 148 & 387 & 148 & \\
\hline 4 & 387 & 148 & 387 & 148 & \\
\hline $1-1985$ & 387 & 148 & 387 & 148 & \\
\hline 2 & 387 & 148 & 387 & 148 & \\
\hline 3 & 387 & 148 & 387 & 148 & \\
\hline 4 & 387 & 148 & 387 & 148 & \\
\hline $1-1986$ & 387 & 148 & 387 & 148 & \\
\hline 2 & 387 & 148 & 387 & 148 & \\
\hline 3 & 387 & 148 & 387 & 148 & \\
\hline 4 & 387 & 148 & 387 & 148 & \\
\hline $1-1987$ & 387 & 148 & 387 & 148 & \\
\hline 2 & 387 & 148 & 387 & 148 & 58 \\
\hline 3 & 387 & 148 & 387 & 148 & 58 \\
\hline 4 & 387 & 148 & 387 & 148 & 60 \\
\hline $1-1988$ & 387 & 148 & 387 & 148 & 59 \\
\hline 2 & 387 & 148 & 387 & 148 & 61 \\
\hline 3 & 387 & 148 & 387 & 148 & 63 \\
\hline 4 & 387 & 148 & 387 & 148 & 64 \\
\hline $1-1989$ & 387 & 148 & 387 & 148 & 63 \\
\hline 2 & 387 & 148 & 387 & 148 & 64 \\
\hline 3 & 387 & 148 & 387 & 148 & 65 \\
\hline 4 & 387 & 148 & 387 & 148 & 65 \\
\hline $1-1990$ & 387 & 148 & 387 & 148 & 65 \\
\hline 2 & 387 & 148 & 387 & 148 & \\
\hline 3 & 387 & 148 & 387 & 148 & 66 \\
\hline 4 & 387 & 148 & 387 & 148 & 67 \\
\hline $1-1991$ & 387 & 148 & 387 & 148 & 67 \\
\hline 2 & 387 & 148 & 387 & 148 & 68 \\
\hline 3 & 387 & 148 & 387 & 148 & 69 \\
\hline 4 & 387 & 148 & 387 & 148 & 69 \\
\hline $1-1992$ & 387 & 148 & 387 & 148 & 69 \\
\hline 2 & 387 & 148 & 387 & 148 & \\
\hline 3 & 387 & 148 & 387 & 148 & \\
\hline 4 & 387 & 148 & 387 & 148 & 70 \\
\hline $1-1993$ & 387 & 148 & 387 & 148 & 70 \\
\hline 2 & 387 & 148 & 387 & 148 & 70 \\
\hline 3 & & & & & 69 \\
\hline 4 & & & & & 70 \\
\hline
\end{tabular}


241-BY-102 SINGLE-SHELL WHC-SD-WM-ER-312, ReV.0 TANK LEVEL HISTORY

\begin{tabular}{|c|c|c|c|c|c|}
\hline Year & Total & Total & Solids & Solids & LOW \\
\hline & (K gal) & (in) & (K gal) & (in) & (in) \\
\hline & & & & & \\
\hline \multicolumn{6}{|l|}{$1-1950$} \\
\hline \multicolumn{6}{|l|}{2} \\
\hline 3 & 480 & 182 & & & \\
\hline 4 & 744 & 278 & & & \\
\hline $1-1951$ & 744 & 278 & & & \\
\hline 2 & 744 & 278 & & & \\
\hline \multicolumn{6}{|l|}{3} \\
\hline \multicolumn{6}{|l|}{4} \\
\hline $1-1952$ & 758 & 283 & & & \\
\hline 2 & 758 & 283 & & & \\
\hline 3 & 758 & 283 & & & \\
\hline 4 & 758 & 283 & & & \\
\hline $1-1953$ & 758 & 283 & & & \\
\hline 2 & 758 & 283 & & & \\
\hline 3 & 758 & 283 & & & \\
\hline 4 & 758 & 283 & & & \\
\hline $1-1954$ & 497 & 188 & & & \\
\hline 2 & 0 & 0 & & & \\
\hline \multicolumn{6}{|l|}{3} \\
\hline \multicolumn{6}{|l|}{4} \\
\hline $1-1955$ & 326 & 126 & & & \\
\hline 2 & 720 & 269 & & & \\
\hline 3 & 720 & 269 & & & \\
\hline 4 & 720 & 269 & & & \\
\hline $1-1956$ & 720 & 269 & & & \\
\hline 2 & 720 & 269 & & & \\
\hline 3 & 720 & 269 & & & \\
\hline 4 & 720 & 269 & & & \\
\hline $1-1957$ & 722 & 270 & & & \\
\hline 2 & 488 & 185 & & & \\
\hline 3 & 400 & 153 & 18 & 14 & \\
\hline 4 & 43 & 23 & 18 & 14 & \\
\hline $1-1958$ & 43 & 23 & 18 & 14 & \\
\hline 2 & 43 & 23 & 18 & 14 & \\
\hline 3 & 43 & 23 & 18 & 14 & \\
\hline 4 & 43 & 23 & 18 & 14 & \\
\hline $1-1959$ & 43 & 23 & 18 & 14 & \\
\hline 2 & 46 & 24 & 18 & 14 & \\
\hline 3 & 46 & 24 & 18 & 14 & - \\
\hline 4 & 46 & 24 & 18 & 14 & \\
\hline $1-1960$ & 46 & 24 & 18 & 14 & \\
\hline 2 & 48 & 25 & & & \\
\hline 3 & 48 & 25 & & & \\
\hline 4 & 48 & 25 & & & \\
\hline \multicolumn{6}{|l|}{$1-1961$} \\
\hline 2 & 48 & 25 & & & \\
\hline
\end{tabular}


241-BY-102 SINGLE-SHELL WHC-SD-WM-ER-312, REV.0 TANK LEVEL HISTORY

\begin{tabular}{|c|c|c|c|c|c|}
\hline Year & Total & Total & Solids & Solids & LOW \\
\hline & (K gal) & (in) & (K gal) & (in) & (in) \\
\hline \multicolumn{6}{|l|}{3} \\
\hline 4 & 51 & 26 & 18 & 14 & \\
\hline \multicolumn{6}{|l|}{$1-1962$} \\
\hline 2 & 51 & 26 & 18 & 14 & \\
\hline \multicolumn{6}{|l|}{3} \\
\hline 4 & 54 & 27 & 18 & 14 & \\
\hline \multicolumn{6}{|l|}{$1-1963$} \\
\hline 2 & 48 & 25 & 29 & 18 & \\
\hline \multicolumn{6}{|l|}{3} \\
\hline 4 & 48 & 25 & 29 & 18 & \\
\hline \multicolumn{6}{|l|}{$1-1964$} \\
\hline 2 & 48 & 25 & 29 & 18 & \\
\hline \multicolumn{6}{|l|}{3} \\
\hline 4 & 442 & 168 & 29 & 18 & \\
\hline \multicolumn{6}{|l|}{$1-1965$} \\
\hline 2 & 700 & 262 & 0 & 0 & \\
\hline 3 & 728 & 272 & 0 & 0 & \\
\hline 4 & 728 & 272 & 0 & 0 & \\
\hline $1-1966$ & 730 & 273 & 0 & 0 & \\
\hline 2 & 733 & 274 & 0 & 0 & \\
\hline 3 & 733 & 274 & 0 & 0 & \\
\hline 4 & 722 & 270 & 0 & 0 & \\
\hline $1-1967$ & 631 & 237 & 0 & 0 & \\
\hline 2 & 593 & 223 & 0 & 0 & \\
\hline 3 & 597 & 225 & 0 & 0 & \\
\hline 4 & 608 & 229 & 0 & 0 & \\
\hline $1-1968$ & 561 & 211 & 0 & 0 & \\
\hline 2 & 593 & 223 & 0 & 0 & \\
\hline 3 & 601 & 226 & 314 & 122 & \\
\hline 4 & 578 & 218 & 250 & 98 & \\
\hline $1-1969$ & 510 & 193 & 250 & 98 & \\
\hline 2 & 590 & 222 & 250 & 98 & \\
\hline 3 & 607 & 228 & 250 & 98 & \\
\hline 4 & 583 & 219 & 250 & 98 & \\
\hline $1-1970$ & 630 & 237 & 250 & 98 & \\
\hline 2 & 596 & 224 & 250 & 98 & \\
\hline 3 & 639 & 240 & 250 & 98 & \\
\hline 4 & 530 & 200 & 250 & 98 & \\
\hline $1-1971$ & 601 & 226 & 250 & 98 & - \\
\hline 2 & 637 & 239 & 155 & 64 & \\
\hline 3 & 623 & 234 & 155 & 64 & \\
\hline 4 & 579 & 218 & 155 & 64 & \\
\hline $1-1972$ & 630 & 237 & 197 & 79 & \\
\hline 2 & 618 & 232 & 197 & 79 & \\
\hline 3 & 609 & 229 & 197 & 79 & \\
\hline 4 & 590 & 222 & 246 & 97 & \\
\hline
\end{tabular}


241-BY-102 SINGLE-SHELL WHC-SD-WM-ER-312, ReV. 0 TANK LEVEL HISTORY

\begin{tabular}{|c|c|c|c|c|c|}
\hline Year & Total & Total & Solids & Solids & LOW \\
\hline & (K gal) & (in) & ( $\mathrm{K}$ gal) & (in) & (in) \\
\hline $1-1973$ & 590 & 222 & 194 & 78 & \\
\hline 2 & 631 & 237 & 194 & 78 & \\
\hline 3 & 637 & 239 & 194 & 78 & \\
\hline 4 & 635 & 238 & 208 & 83 & \\
\hline $1-1974$ & 630 & 237 & 208 & 83 & \\
\hline 2 & 629 & 236 & 208 & 83 & \\
\hline 3 & 628 & 236 & 208 & 83 & \\
\hline 4 & 629 & 236 & 200 & 80 & \\
\hline $1-1975$ & 629 & 236 & 200 & 80 & \\
\hline 2 & 629 & 236 & 200 & 80 & \\
\hline 3 & 629 & 236 & 200 & 80 & \\
\hline 4 & 629 & 236 & 200 & 80 & \\
\hline $1-1976$ & 629 & 236 & 200 & 80 & \\
\hline 2 & 626 & 235 & 200 & 80 & \\
\hline 3 & 626 & 235 & 200 & 80 & \\
\hline 4 & 626 & 235 & 200 & 80 & \\
\hline $1-1977$ & 593 & 223 & 200 & 80 & \\
\hline 2 & 450 & 171 & 233 & 92 & \\
\hline 3 & 436 & 166 & 436 & 166 & \\
\hline 4 & 436 & 166 & 436 & 166 & \\
\hline $1-1978$ & 436 & 166 & 436 & 166 & \\
\hline 2 & 417 & 159 & 417 & 159 & \\
\hline 3 & 417 & 159 & 417 & 159 & \\
\hline 4 & 417 & 159 & 417 & 159 & \\
\hline $1-1979$ & 417 & 159 & 417 & 159 & \\
\hline 2 & 417 & 159 & 417 & 159 & \\
\hline 3 & 417 & 159 & 417 & 159 & \\
\hline 4 & 417 & 159 & 417 & 159 & \\
\hline $1-1980$ & 417 & 159 & 417 & 159 & \\
\hline 2 & 417 & 159 & 417 & 159 & \\
\hline 3 & 417 & 159 & 417 & 159 & \\
\hline 4 & 417 & 159 & 417 & 159 & \\
\hline $1-1981$ & 417 & 159 & 417 & 159 & \\
\hline 2 & 417 & 159 & 417 & 159 & \\
\hline 3 & 417 & 159 & 417 & 159 & \\
\hline 4 & 417 & 159 & 417 & 159 & \\
\hline $1-1982$ & 417 & 159 & 417 & 159 & \\
\hline 2 & 440 & 167 & 425 & 162 & \\
\hline 3 & 440 & 167 & 425 & 162 & \\
\hline 4 & 440 & 167 & 425 & 162 & \\
\hline $1-1983$ & 440 & 167 & 425 & 162 & \\
\hline 2 & 440 & 167 & 425 & 162 & \\
\hline 3 & 440 & 167 & 425 & 162 & \\
\hline 4 & 440 & 167 & 425 & 162 & \\
\hline $1-1984$ & 432 & 165 & 417 & 159 & \\
\hline 2 & 432 & 165 & 417 & 159 & \\
\hline
\end{tabular}


241-BY-102 SINGLE-SHELL WHC-SD-WM-ER-312, REV.0 TANK LEVEL HISTORY

\begin{tabular}{|c|c|c|c|c|c|}
\hline Year & Total & Total & Solids & Solids & LOW \\
\hline & (K gal) & (in) & (K gal) & (in) & (in) \\
\hline & & 105 & 817 & 150 & \\
\hline 3 & 432 & 165 & 417 & 159 & \\
\hline 4 & 432 & 165 & 417 & 159 & \\
\hline $1-1985$ & 433 & 165 & 417 & 159 & \\
\hline 2 & 432 & 165 & 417 & 159 & \\
\hline 3 & 432 & 165 & 417 & 159 & \\
\hline 4 & 432 & 165 & 417 & 159 & \\
\hline $1-1986$ & 432 & 165 & 417 & 159 & 168 \\
\hline 2 & 432 & 165 & 417 & 159 & 169 \\
\hline 3 & 432 & 165 & 417 & 159 & 167 \\
\hline 4 & 432 & 165 & 417 & 159 & 168 \\
\hline $1-1987$ & 432 & 165 & 417 & 159 & 168 \\
\hline 2 & 432 & 165 & 417 & 159 & 169 \\
\hline 3 & 432 & 165 & 417 & 159 & 168 \\
\hline 4 & 432 & 165 & 417 & 159 & 169 \\
\hline $1-1988$ & 432 & 165 & 417 & 159 & 169 \\
\hline 2 & 432 & 165 & 417 & 159 & 169 \\
\hline 3 & 432 & 165 & 417 & 159 & 169 \\
\hline 4 & 432 & 165 & 417 & 159 & 169 \\
\hline $1-1989$ & 432 & 165 & 417 & 159 & 169 \\
\hline 2 & 432 & 165 & 417 & 159 & 169 \\
\hline 3 & 432 & 165 & 417 & 159 & 169 \\
\hline 4 & 432 & 165 & 417 & 159 & 169 \\
\hline $1-1990$ & 432 & 165 & 417 & 159 & 169 \\
\hline 2 & 432 & 165 & 417 & 159 & \\
\hline 3 & 432 & 165 & 417 & 159 & 170 \\
\hline 4 & 432 & 165 & 417 & 159 & 169 \\
\hline $1-1991$ & 432 & 165 & 417 & 159 & 170 \\
\hline 2 & 405 & 155 & 405 & 155 & \\
\hline 3 & 341 & 131 & 341 & 131 & 93 \\
\hline 4 & 341 & 131 & 341 & 131 & 87 \\
\hline $1-1992$ & 341 & 131 & 341 & 131 & 88 \\
\hline 2 & 341 & 131 & 341 & 131 & \\
\hline 3 & 341 & 131 & 341 & 131 & \\
\hline 4 & 341 & 131 & 341 & 131 & 87 \\
\hline $1-1993$ & 341 & 131 & 341 & 131 & 88 \\
\hline 2 & 341 & 131 & 341 & 131 & 89 \\
\hline 3 & & & & & 89 \\
\hline 4 & & & & & \\
\hline
\end{tabular}


241-BY-103 SINGLE-SHELL WHC-SD-WM-ER-312, REV.0 TANK LEVEL HISTORY

\begin{tabular}{|c|c|c|c|c|c|}
\hline Year & Total & Total & Solids & Solids & LOW \\
\hline & (K gal) & (in) & ( $\mathrm{K}$ gal) & (in) & (in) \\
\hline \multirow{2}{*}{\multicolumn{6}{|c|}{$1-1950$}} \\
\hline \multirow{2}{*}{\multicolumn{6}{|c|}{2}} \\
\hline \multirow{2}{*}{\multicolumn{6}{|c|}{3}} \\
\hline 4 & & & & & \\
\hline $1-1951$ & 425 & 163 & & & \\
\hline & 744 & 278 & & & \\
\hline 2 & 744 & 278 & & & \\
\hline \multicolumn{6}{|l|}{3} \\
\hline \multicolumn{6}{|l|}{4} \\
\hline $1-1952$ & 664 & 249 & & & \\
\hline 2 & 664 & 249 & & & \\
\hline 3 & 664 & 249 & & & \\
\hline 4 & 664 & 249 & & & \\
\hline $1-1953$ & 664 & 249 & & & \\
\hline 2 & 745 & 278 & & & \\
\hline 3 & 745 & 278 & & & \\
\hline 4 & 745 & 278 & & & \\
\hline $1-1954$ & 382 & 146 & & & \\
\hline \multicolumn{6}{|l|}{2} \\
\hline \multicolumn{6}{|l|}{3} \\
\hline \multicolumn{6}{|l|}{4} \\
\hline $1-1955$ & 128 & 54 & & & \\
\hline 2 & 739 & 276 & & & \\
\hline 3 & 739 & 276 & & & \\
\hline 4 & 739 & 276 & & & \\
\hline $1-1956$ & 739 & 276 & & & \\
\hline 2 & 739 & 276 & & & \\
\hline 3 & 739 & 276 & & & \\
\hline 4 & 739 & 276 & & & \\
\hline $1-1957$ & 732 & 274 & & & \\
\hline 2 & 733 & 274 & & & \\
\hline 3 & 26 & 17 & & & \\
\hline 4 & 447 & 170 & & & \\
\hline $1-1958$ & 736 & 275 & & & \\
\hline 2 & 730 & 273 & & & \\
\hline 3 & 730 & 273 & & & \\
\hline 4 & 730 & 273 & & & \\
\hline $1-1959$ & 728 & 272 & & & \\
\hline 2 & 722 & 270 & & & \\
\hline 3 & 722 & 270 & & & - \\
\hline 4 & 722 & 270 & & & \\
\hline $1-1960$ & 722 & 270 & & & \\
\hline 2 & 722 & 270 & & & \\
\hline 3 & 722 & 270 & & & \\
\hline 4 & 722 & 270 & & & \\
\hline \multicolumn{6}{|l|}{$1-1961$} \\
\hline 2 & 722 & 270 & & & \\
\hline
\end{tabular}


241-BY-103 SINGLE-SHELL WHC-SD-WM-ER-312, REV.0 TANK LEVEL HISTORY

\begin{tabular}{|c|c|c|c|c|c|}
\hline Year & Total & Total & Solids & Solids & LOW \\
\hline & (K gal) & (in) & (K gal) & (in) & (in) \\
\hline \multicolumn{6}{|l|}{3} \\
\hline 4 & 736 & 275 & & & \\
\hline \multicolumn{6}{|l|}{$1-1962$} \\
\hline 2 & 725 & 271 & & & \\
\hline \multicolumn{6}{|l|}{3} \\
\hline 4 & 725 & 271 & & & \\
\hline \multicolumn{6}{|l|}{$1-1963$} \\
\hline 2 & 722 & 270 & 21 & 15 & \\
\hline \multicolumn{6}{|l|}{3} \\
\hline 4 & 722 & 270 & 21 & 15 & \\
\hline \multicolumn{6}{|l|}{$1-1964$} \\
\hline 2 & 714 & 267 & 21 & 15 & \\
\hline \multicolumn{6}{|l|}{3} \\
\hline 4 & 739 & 276 & 21 & 15 & \\
\hline \multicolumn{6}{|l|}{$1-1965$} \\
\hline 2 & 596 & 224 & 0 & 0 & \\
\hline 3 & 703 & 263 & 0 & 0 & \\
\hline 4 & 494 & 187 & 0 & 0 & \\
\hline $1-1966$ & 519 & 196 & 0 & 0 & \\
\hline 2 & 590 & 222 & 0 & 0 & \\
\hline 3 & 662 & 248 & 0 & 0 & \\
\hline 4 & 739 & 276 & 0 & 0 & \\
\hline $1-1967$ & 541 & 204 & 0 & 0 & \\
\hline 2 & 615 & 231 & 0 & 0 & \\
\hline 3 & 526 & 199 & 0 & 0 & \\
\hline 4 & 526 & 199 & 0 & 0 & \\
\hline $1-1968$ & 569 & 214 & 0 & 0 & \\
\hline 2 & 569 & 214 & 0 & 0 & \\
\hline 3 & 456 & 173 & 0 & 0 & \\
\hline 4 & 519 & 196 & 0 & 0 & \\
\hline $1-1969$ & 213 & 85 & 0 & 0 & \\
\hline 2 & 566 & 213 & 0 & 0 & \\
\hline 3 & 483 & 183 & 1 & 8 & \\
\hline 4 & 542 & 205 & 8 & 10 & \\
\hline $1-1970$ & 239 & 94 & 3 & 9 & \\
\hline 2 & 442 & 168 & 4 & 9 & \\
\hline 3 & 439 & 167 & 4 & 9 & \\
\hline 4 & 700 & 262 & 9 & 11 & \\
\hline $1-1971$ & 711 & 266 & 65 & 31 & - \\
\hline 2 & 689 & 258 & 128 & 54 & \\
\hline 3 & 714 & 267 & 142 & 59 & \\
\hline 4 & 398 & 152 & 202 & 81 & \\
\hline $1-1972$ & 443 & 169 & 298 & 116 & \\
\hline 2 & 454 & 173 & 351 & 135 & \\
\hline 3 & 620 & 233 & 428 & 163 & \\
\hline 4 & 476 & 181 & 48 & 25 & \\
\hline
\end{tabular}


241-BY-103 SINGLE-SHELL WHC-SD-WM-ER-312, Rev. 0 TANK LEVEL HISTORY

\begin{tabular}{|c|c|c|c|c|c|}
\hline Year & Total & Total & Solids & Solids & LOW \\
\hline & ( $\mathrm{K}$ gal) & (in) & (K gal) & (in) & (in) \\
\hline $1-1973$ & 469 & 178 & 469 & 178 & \\
\hline 2 & 483 & 183 & 469 & 178 & \\
\hline 3 & 461 & 175 & 458 & 174 & \\
\hline 4 & 464 & 176 & 458 & 174 & \\
\hline $1-1974$ & 464 & 176 & 458 & 174 & \\
\hline 2 & 464 & 176 & 458 & 174 & \\
\hline 3 & 469 & 178 & 469 & 178 & \\
\hline 4 & 461 & 175 & 461 & 175 & \\
\hline $1-1975$ & 461 & 175 & 461 & 175 & \\
\hline 2 & 461 & 175 & 461 & 175 & \\
\hline 3 & 461 & 175 & 461 & 175 & \\
\hline 4 & 461 & 175 & 461 & 175 & \\
\hline $1-1976$ & 461 & 175 & 461 & 175 & \\
\hline 2 & 461 & 175 & 461 & 175 & \\
\hline 3 & 461 & 175 & 461 & 175 & \\
\hline 4 & 461 & 175 & 461 & 175 & \\
\hline 1.1977 & 461 & 175 & 461 & 175 & \\
\hline 2 & 461 & 175 & 461 & 175 & \\
\hline 3 & 461 & 175 & 461 & 175 & \\
\hline 4 & 461 & 175 & 461 & 175 & \\
\hline $1-1978$ & 461 & 175 & 461 & 175 & \\
\hline 2 & 461 & 175 & 461 & 175 & \\
\hline 3 & 461 & 175 & 461 & 175 & \\
\hline 4 & 461 & 175 & 461 & 175 & \\
\hline $1-1979$ & 461 & 175 & 461 & 175 & \\
\hline 2 & 461 & 175 & 461 & 175 & \\
\hline 3 & 461 & 175 & 461 & 175 & \\
\hline 4 & 461 & 175 & 461 & 175 & \\
\hline $1-1980$ & 461 & 175 & 461 & 175 & \\
\hline 2 & 461 & 175 & 461 & 175 & \\
\hline 3 & 461 & 175 & 461 & 175 & \\
\hline 4 & 461 & 175 & 458 & 174 & \\
\hline $1-1981$ & 461 & 175 & 458 & 174 & \\
\hline 2 & 461 & 175 & 458 & 174 & \\
\hline 3 & 461 & 175 & 458 & 174 & \\
\hline 4 & 470 & 178 & 458 & 174 & \\
\hline $1-1982$ & 470 & 178 & 458 & 174 & \\
\hline 2 & 467 & 177 & 459 & 174 & \\
\hline 3 & 467 & 177 & 459 & 174 & - \\
\hline 4 & 467 & 177 & 459 & 174 & \\
\hline $1-1983$ & 467 & 177 & 459 & 174 & \\
\hline 2 & 467 & 177 & 459 & 174 & \\
\hline 3 & 467 & 177 & 459 & 174 & \\
\hline 4 & 467 & 177 & 459 & 174 & \\
\hline $1-1984$ & 472 & 179 & 464 & 176 & \\
\hline 2 & 472 & 179 & 464 & 176 & \\
\hline
\end{tabular}


241-BY-103 SINGLE-SHELL WHC-SD-WM-ER-312, REV. 0 TANK LEVEL HISTORY

\begin{tabular}{|c|c|c|c|c|c|}
\hline Year & Total & Total & Solids & Solids & LOW \\
\hline & (K gal) & (in) & (K gal) & (in) & (in) \\
\hline & & & & & \\
\hline 3 & 472 & 179 & 464 & 176 & \\
\hline 4 & 472 & 179 & 464 & 176 & \\
\hline $1-1985$ & 472 & 179 & 464 & 176 & \\
\hline 2 & 472 & 179 & 464 & 176 & \\
\hline 3 & 472 & 179 & 464 & 176 & \\
\hline 4 & 472 & 179 & 464 & 176 & \\
\hline $1-1986$ & 472 & 179 & 464 & 176 & 181 \\
\hline 2 & 472 & 179 & 464 & 176 & 181 \\
\hline 3 & 472 & 179 & 464 & 176 & 181 \\
\hline 4 & 472 & 179 & 464 & 176 & 181 \\
\hline $1-1987$ & 472 & 179 & 464 & 176 & 182 \\
\hline 2 & 418 & 159 & 418 & 159 & 150 \\
\hline 3 & 419 & 160 & 419 & 160 & 144 \\
\hline 4 & 410 & 157 & 410 & 157 & 144 \\
\hline $1-1988$ & 410 & 157 & 410 & 157 & 143 \\
\hline 2 & 410 & 157 & 410 & 157 & 142 \\
\hline 3 & 410 & 157 & 410 & 157 & 141 \\
\hline 4 & 410 & 157 & 410 & 157 & 141 \\
\hline $1-1989$ & 410 & 157 & 410 & 157 & 141 \\
\hline 2 & 410 & 157 & 410 & 157 & 142 \\
\hline 3 & 410 & 157 & 410 & 157 & 141 \\
\hline 4 & 410 & 157 & 410 & 157 & 142 \\
\hline 1.1990 & 400 & 153 & 400 & 153 & 142 \\
\hline 2 & 400 & 153 & 400 & 153 & 142 \\
\hline 3 & 400 & 153 & 400 & 153 & 141 \\
\hline 4 & 400 & 153 & 400 & 153 & 142 \\
\hline $1-1991$ & 400 & 153 & 400 & 153 & 143 \\
\hline 2 & 400 & 153 & 400 & 153 & 143 \\
\hline 3 & 400 & 153 & 400 & 153 & 142 \\
\hline 4 & 400 & 153 & 400 & 153 & 142 \\
\hline 1.1992 & 400 & 153 & 400 & 153 & 142 \\
\hline 2 & 400 & 153 & 400 & 153 & \\
\hline 3 & 400 & 153 & 400 & 153 & \\
\hline 4 & 400 & 153 & 400 & 153 & 141 \\
\hline $1-1993$ & 400 & 153 & 400 & 153 & 139 \\
\hline 2 & 400 & 153 & 400 & 153 & 142 \\
\hline 3 & & & & & 142 \\
\hline 4 & & & & & \\
\hline
\end{tabular}


241-BY-104 SINGLE-SHELL WHC-SD-WM-ER-312, Rev.0 TANK LEVEL HISTORY

\begin{tabular}{|c|c|c|c|c|c|}
\hline Year & Total & Total & Solids & Solids & LOW \\
\hline & (K gal) & (in) & ( $\mathrm{K}$ gal) & (in) & (in) \\
\hline $1-1950$ & 11 & 11 & & & \\
\hline 2 & 11 & 11 & & & \\
\hline 3 & 11 & 11 & & & \\
\hline 4 & 11 & 11 & & & \\
\hline $1-1951$ & 200 & 80 & & & \\
\hline 2 & 744 & 278 & & & \\
\hline \multicolumn{6}{|l|}{3} \\
\hline \multicolumn{6}{|l|}{4} \\
\hline $1-1952$ & 758 & 283 & & & \\
\hline 2 & 758 & 283 & & & \\
\hline 3 & 758 & 283 & & & \\
\hline 4 & 758 & 283 & & & \\
\hline $1-1953$ & 758 & 283 & & & \\
\hline 2 & 758 & 283 & & & \\
\hline 3 & 758 & 283 & & & \\
\hline 4 & 758 & 283 & & & \\
\hline $1-1954$ & 758 & 283 & & & \\
\hline \multicolumn{6}{|l|}{2} \\
\hline \multicolumn{6}{|l|}{3} \\
\hline \multicolumn{6}{|l|}{4} \\
\hline \multicolumn{6}{|l|}{$1-1955$} \\
\hline 2 & 112 & 48 & & & \\
\hline 3 & 714 & 267 & & & \\
\hline 4 & 78 & 36 & & & \\
\hline $1-1956$ & 78 & 36 & & & \\
\hline 2 & 78 & 36 & & & \\
\hline 3 & 403 & 154 & 303 & 118 & \\
\hline 4 & 546 & 206 & 323 & 125 & \\
\hline $1-1957$ & 563 & 212 & 323 & 125 & \\
\hline 2 & 571 & 215 & 277 & 108 & \\
\hline 3 & 598 & 225 & 244 & 96 & \\
\hline 4 & 574 & 216 & 244 & 96 & \\
\hline $1-1958$ & 574 & 216 & 244 & 96 & \\
\hline 2 & 574 & 216 & 244 & 96 & \\
\hline 3 & 574 & 216 & 244 & 96 & \\
\hline 4 & 571 & 215 & 244 & 96 & \\
\hline $1-1959$ & 571 & 215 & 244 & 96 & \\
\hline 2 & 571 & 215 & 244 & 96 & \\
\hline 3 & 567 & 214 & 244 & 96 & $\because$ \\
\hline 4 & 567 & 214 & 244 & 96 & \\
\hline $1-1960$ & 567 & 214 & 244 & 96 & \\
\hline 2 & 563 & 212 & 244 & 96 & \\
\hline 3 & 563 & 212 & 244 & 96 & \\
\hline 4 & 563 & 212 & 244 & 96 & \\
\hline $1-1961$ & & & & & \\
\hline 2 & 565 & 213 & 244 & 96 & \\
\hline
\end{tabular}


241-BY-104 SINGLE-SHELL WHC-SD-WM-ER-312, ReV.0 TANK LEVEL HISTORY

\begin{tabular}{|c|c|c|c|c|c|}
\hline Year & Total & Total & Solids & Solids & LOW \\
\hline & (K gal) & (in) & (K gal) & (in) & (in) \\
\hline & & & & & \\
\hline \multicolumn{6}{|l|}{3} \\
\hline 4 & 703 & 263 & 244 & 96 & \\
\hline \multicolumn{6}{|l|}{$1-1962$} \\
\hline 2 & 717 & 268 & 244 & 96 & \\
\hline \multicolumn{6}{|l|}{3} \\
\hline 4 & 717 & 268 & 244 & 96 & \\
\hline \multicolumn{6}{|l|}{$1-1963$} \\
\hline 2 & 714 & 267 & 263 & 103 & \\
\hline \multicolumn{6}{|l|}{3} \\
\hline 4 & 714 & 267 & 263 & 103 & \\
\hline \multicolumn{6}{|l|}{$1-1964$} \\
\hline 2 & 714 & 267 & 263 & 103 & \\
\hline \multicolumn{6}{|l|}{3} \\
\hline 4 & 714 & 267 & 263 & 103 & \\
\hline \multicolumn{6}{|l|}{$1-1965$} \\
\hline 2 & 717 & 268 & 227 & 90 & \\
\hline 3 & 717 & 268 & 227 & 90 & \\
\hline 4 & 717 & 268 & 227 & 90 & \\
\hline $1-1966$ & 717 & 268 & 227 & 90 & \\
\hline 2 & 717 & 268 & 227 & 90 & \\
\hline 3 & 717 & 268 & 227 & 90 & \\
\hline 4 & 719 & 269 & 227 & 90 & \\
\hline 1.1967 & 719 & 269 & 227 & 90 & \\
\hline 2 & 205 & 82 & 205 & 82 & \\
\hline 3 & 249 & 98 & 244 & 96 & \\
\hline 4 & 653 & 245 & 244 & 96 & \\
\hline $1-1968$ & 730 & 273 & 244 & 96 & \\
\hline 2 & 546 & 206 & 212 & 85 & \\
\hline 3 & 741 & 277 & 244 & 96 & \\
\hline 4 & 741 & 277 & 244 & 96 & \\
\hline $1-1969$ & 741 & 277 & 244 & 96 & \\
\hline 2 & 741 & 277 & 233 & 92 & \\
\hline 3 & 740 & 277 & 244 & 96 & \\
\hline 4 & 748 & 279 & 240 & 95 & \\
\hline $1-1970$ & 211 & 84 & 206 & 82 & \\
\hline 2 & 579 & 218 & 44 & 23 & \\
\hline 3 & 623 & 234 & 112 & 48 & \\
\hline 4 & 664 & 249 & 131 & 55 & \\
\hline $1-1971$ & 677 & 254 & 150 & 62 &. \\
\hline 2 & 701 & 262 & 150 & 62 & \\
\hline 3 & 662 & 248 & 425 & 162 & \\
\hline 4 & 662 & 248 & 618 & 232 & \\
\hline $1-1972$ & 662 & 248 & 618 & 232 & \\
\hline 2 & 670 & 251 & 618 & 232 & \\
\hline 3 & 664 & 249 & 310 & 120 & \\
\hline 4 & 707 & 265 & 310 & 120 & \\
\hline
\end{tabular}


241-BY-104 SINGLE-SHELL WHC-SD-WM-ER-312, ReV. 0 TANK LEVEL HISTORY

\begin{tabular}{|c|c|c|c|c|c|}
\hline Year & Total & Total & Solids & Solids & LOW \\
\hline & ( $\mathrm{K}$ gal) & (in) & ( $\mathrm{K}$ gal) & (in) & (in) \\
\hline $1-1973$ & 668 & 250 & 469 & 178 & \\
\hline 2 & 693 & 259 & 469 & 178 & \\
\hline 3 & 697 & 261 & 469 & 178 & \\
\hline 4 & 657 & 246 & 628 & 236 & \\
\hline \begin{tabular}{|l|}
$1-1974$ \\
\end{tabular} & 718 & 269 & 469 & 178 & \\
\hline 2 & 719 & 269 & 469 & 178 & \\
\hline 3 & 718 & 269 & 469 & 178 & \\
\hline 4 & 717 & 268 & 469 & 178 & \\
\hline $1-1975$ & 717 & 268 & 469 & 178 & \\
\hline 2 & 717 & 268 & 469 & 178 & \\
\hline 3 & 717 & 268 & 469 & 178 & \\
\hline 4 & 717 & 268 & 469 & 178 & \\
\hline $1-1976$ & 717 & 268 & 469 & 178 & \\
\hline 2 & 717 & 268 & 469 & 178 & \\
\hline 3 & 719 & 269 & 469 & 178 & \\
\hline 4 & 670 & 251 & 469 & 178 & \\
\hline $1-1977$ & 634 & 238 & 469 & 178 & \\
\hline 2 & 634 & 238 & 469 & 178 & \\
\hline 3 & 631 & 237 & 623 & 234 & \\
\hline 4 & 631 & 237 & 623 & 234 & \\
\hline $1-1978$ & 634 & 238 & 623 & 234 & \\
\hline 2 & 634 & 238 & 623 & 234 & \\
\hline 3 & 634 & 238 & 623 & 234 & \\
\hline 4 & 634 & 238 & 623 & 234 & \\
\hline $1-1979$ & 634 & 238 & 623 & 234 & \\
\hline 2 & 634 & 238 & 623 & 234 & \\
\hline 3 & 634 & 238 & 623 & 234 & \\
\hline 4 & 634 & 238 & 623 & 234 & \\
\hline $1-1980$ & 634 & 238 & 623 & 234 & \\
\hline 2 & 634 & 238 & 623 & 234 & \\
\hline 3 & 634 & 238 & 623 & 234 & \\
\hline 4 & 634 & 238 & 623 & 234 & \\
\hline $1-1981$ & 634 & 238 & 623 & 234 & \\
\hline 2 & 634 & 238 & 623 & 234 & \\
\hline 3 & 634 & 238 & 623 & 234 & \\
\hline 4 & 634 & 238 & 623 & 234 & \\
\hline $1-1982$ & 634 & 238 & 623 & 234 & \\
\hline 2 & 634 & 238 & 634 & 238 & \\
\hline 3 & 634 & 238 & 634 & 238 & - \\
\hline 4 & 634 & 238 & 634 & 238 & \\
\hline $1-1983$ & 634 & 238 & 634 & 238 & \\
\hline 2 & 634 & 238 & 634 & 238 & \\
\hline 3 & 435 & 166 & 435 & 166 & \\
\hline 4 & 435 & 166 & 435 & 166 & \\
\hline $1-1984$ & 407 & 155 & 407 & 155 & \\
\hline 2 & 407 & 155 & 407 & 155 & \\
\hline
\end{tabular}


241-BY-104 SINGLE-SHELL WHC-SD-WM-ER-312, ReV. 0 TANK LEVEL HISTORY

\begin{tabular}{|c|c|c|c|c|c|}
\hline Year & Total & Total & Solids & Solids & LOW \\
\hline & (K gal) & (in) & (K gal) & (in) & (in) \\
\hline 3 & 406 & 155 & 406 & 155 & \\
\hline 4 & 406 & 155 & 406 & 155 & \\
\hline $1-1985$ & 406 & 155 & 406 & 155 & \\
\hline 2 & 406 & 155 & 406 & 155 & \\
\hline 3 & 406 & 155 & 406 & 155 & \\
\hline 4 & 406 & 155 & 406 & 155 & \\
\hline $1-1986$ & 406 & 155 & 406 & 155 & 81 \\
\hline 2 & 406 & 155 & 406 & 155 & 83 \\
\hline 3 & 406 & 155 & 406 & 155 & 81 \\
\hline 4 & 406 & 155 & 406 & 155 & 84 \\
\hline $1-1987$ & 406 & 155 & 406 & 155 & 84 \\
\hline 2 & 406 & 155 & 406 & 155 & 85 \\
\hline 3 & 406 & 155 & 406 & 155 & 83 \\
\hline 4 & 406 & 155 & 406 & 155 & 82 \\
\hline $1-1988$ & 406 & 155 & 406 & 155 & 84 \\
\hline 2 & 406 & 155 & 406 & 155 & 85 \\
\hline 3 & 406 & 155 & 406 & 155 & 84 \\
\hline 4 & 406 & 155 & 406 & 155 & 85 \\
\hline $1-1989$ & 406 & 155 & 406 & 155 & 85 \\
\hline 2 & 406 & 155 & 406 & 155 & 85 \\
\hline 3 & 406 & 155 & 406 & 155 & 87 \\
\hline 4 & 406 & 155 & 406 & 155 & 85 \\
\hline $1-1990$ & 406 & 155 & 406 & 155 & 86 \\
\hline 2 & 406 & 155 & 406 & 155 & \\
\hline 3 & 406 & 155 & 406 & 155 & 85 \\
\hline 4 & 406 & 155 & 406 & 155 & 88 \\
\hline $1-1991$ & 406 & 155 & 406 & 155 & 87 \\
\hline 2 & 406 & 155 & 406 & 155 & 86 \\
\hline 3 & 406 & 155 & 406 & 155 & 87 \\
\hline 4 & 406 & 155 & 406 & 155 & 87 \\
\hline $1-1992$ & 406 & 155 & 406 & 155 & 86 \\
\hline 2 & 406 & 155 & 406 & 155 & \\
\hline 3 & 406 & 155 & 406 & 155 & \\
\hline 4 & 406 & 155 & 406 & 155 & 87 \\
\hline $1-1993$ & 406 & 155 & 406 & 155 & 87 \\
\hline 2 & 406 & 155 & 406 & 155 & 87 \\
\hline 3 & & & & & 86 \\
\hline 4 & & & & & 87 \\
\hline
\end{tabular}


241-BY-105 SINGLE-SHELL WHC-SD-WM-ER-312, ReV. 0 TANK LEVEL HISTORY

\begin{tabular}{|c|c|c|c|c|c|}
\hline Year & Total & Total & Solids & Solids & LOW \\
\hline & ( $\mathrm{K}$ gal) & (in) & (K gal) & (in) & (in) \\
\hline \multicolumn{6}{|l|}{$1-1950$} \\
\hline \multicolumn{6}{|l|}{2} \\
\hline \multicolumn{6}{|l|}{3} \\
\hline \multicolumn{6}{|l|}{4} \\
\hline \multicolumn{6}{|l|}{$1-1951$} \\
\hline 2 & 20 & 15 & & & \\
\hline \multicolumn{6}{|l|}{3} \\
\hline \multicolumn{6}{|l|}{4} \\
\hline $1-1952$ & 491 & 186 & & & \\
\hline 2 & 491 & 186 & & & \\
\hline 3 & 491 & 186 & & & \\
\hline 4 & 491 & 186 & & & \\
\hline $1-1953$ & 491 & 186 & & & \\
\hline 2 & 495 & 187 & & & \\
\hline 3 & 497 & 188 & & & \\
\hline 4 & 497 & 188 & & & \\
\hline $1-1954$ & 497 & 188 & & & \\
\hline 2 & 497 & 188 & & & \\
\hline 3 & 1 & 8 & & & \\
\hline 4 & 399 & 153 & 14 & 13 & \\
\hline $1-1955$ & 222 & 88 & 16 & 13 & \\
\hline 2 & 222 & 88 & 16 & 13 & \\
\hline 3 & 260 & 102 & 16 & 13 & \\
\hline 4 & 371 & 142 & 16 & 13 & \\
\hline $1-1956$ & 551 & 208 & 350 & 135 & \\
\hline 2 & 708 & 265 & 384 & 147 & \\
\hline 3 & 403 & 154 & 384 & 147 & \\
\hline 4 & 706 & 264 & 384 & 147 & \\
\hline $1-1957$ & 706 & 264 & 384 & 147 & \\
\hline 2 & 409 & 156 & 384 & 147 & \\
\hline 3 & 417 & 159 & 213 & 85 & \\
\hline 4 & 428 & 163 & 213 & 85 & \\
\hline $1-1958$ & 428 & 163 & 213 & 85 & \\
\hline 2 & 431 & 164 & 213 & 85 & \\
\hline 3 & 431 & 164 & 213 & 85 & \\
\hline 4 & 429 & 163 & 213 & 85 & \\
\hline $1-1959$ & 428 & 163 & 213 & 85 & \\
\hline 2 & 428 & 163 & 213 & 85 & \\
\hline 3 & 428 & 163 & 213 & 85 & - \\
\hline 4 & 428 & 163 & 213 & 85 & \\
\hline $1-1960$ & 428 & 163 & 213 & 85 & \\
\hline 2 & 428 & 163 & 213 & 85 & \\
\hline 3 & 428 & 163 & 213 & 85 & \\
\hline 4 & 428 & 163 & 213 & 85 & \\
\hline \multicolumn{6}{|l|}{$1-1961$} \\
\hline 2 & 519 & 196 & 213 & 85 & \\
\hline
\end{tabular}


241-BY-105 SINGLE-SHELL WHC-SD-WM-ER-312, ReV.0 TANK LEVEL HISTORY

\begin{tabular}{|c|c|c|c|c|c|}
\hline Year & Total & Total & Solids & Solids & LOW \\
\hline & (K gal) & (in) & ( $\mathrm{K}$ gal) & (in) & (in) \\
\hline \multicolumn{6}{|l|}{3} \\
\hline$\frac{3}{4}$ & 711 & 266 & 213 & 85 & \\
\hline \multicolumn{6}{|l|}{$1-1962$} \\
\hline 2 & 711 & 266 & 213 & 85 & \\
\hline \multicolumn{6}{|l|}{3} \\
\hline 4 & 711 & 266 & 213 & 85 & \\
\hline \multicolumn{6}{|l|}{$1-1963$} \\
\hline 2 & 711 & 266 & 222 & 88 & \\
\hline \multicolumn{6}{|l|}{3} \\
\hline 4 & 711 & 266 & 222 & 88 & \\
\hline \multicolumn{6}{|l|}{$1-1964$} \\
\hline 2 & 708 & 265 & 222 & 88 & \\
\hline \multicolumn{6}{|l|}{3} \\
\hline 4 & 708 & 265 & 222 & 88 & \\
\hline \multicolumn{6}{|l|}{$1-1965$} \\
\hline 2 & 711 & 266 & 186 & 75 & \\
\hline 3 & 711 & 266 & 186 & 75 & \\
\hline 4 & 711 & 266 & 186 & 75 & \\
\hline $1-1966$ & 711 & 266 & 186 & 75 & \\
\hline 2 & 208 & 83 & 186 & 75 & \\
\hline 3 & 178 & 72 & 0 & 7 & \\
\hline 4 & 326 & 126 & 0 & 7 & \\
\hline $1-1967$ & 323 & 125 & 0 & 7 & \\
\hline 2 & 323 & 125 & 0 & 7 & \\
\hline 3 & 326 & 126 & 39 & 22 & \\
\hline 4 & 326 & 126 & 88 & 39 & \\
\hline $1-1968$ & 605 & 227 & 88 & 39 & \\
\hline 2 & 495 & 187 & 88 & 39 & \\
\hline 3 & 411 & 157 & 158 & 65 & \\
\hline 4 & 741 & 277 & 158 & 65 & \\
\hline $1-1969$ & 670 & 251 & 165 & 67 & \\
\hline 2 & 671 & 251 & 153 & 63 & \\
\hline 3 & 494 & 187 & 194 & 78 & \\
\hline 4 & 675 & 253 & 189 & 76 & \\
\hline $1-1970$ & 745 & 278 & 191 & 77 & \\
\hline 2 & 631 & 237 & 216 & 86 & \\
\hline 3 & 722 & 270 & 299 & 116 & \\
\hline 4 & 681 & 255 & 299 & 116 & \\
\hline $1-1971$ & 675 & 253 & 340 & 131 & $\cdot$ \\
\hline 2 & 668 & 250 & 469 & 178 & \\
\hline 3 & 700 & 262 & 469 & 178 & \\
\hline 4 & 711 & 266 & 653 & 245 & \\
\hline 1-1972 & 678 & 254 & 653 & 245 & \\
\hline 2 & 701 & 262 & 653 & 245 & \\
\hline 3 & 642 & 241 & 591 & 222 & \\
\hline 4 & 664 & 249 & 591 & 222 & \\
\hline
\end{tabular}


241-BY-105 SINGLE-SHELL WHC-SD-WM-ER-3I2, Rev. 0 TANK LEVEL HISTORY

\begin{tabular}{|c|c|c|c|c|c|}
\hline Year & Total & Total & Solids & Solids & LOW \\
\hline & ( $\mathrm{K} \mathrm{gal})$ & (in) & ( $\mathrm{K}$ gal) & (in) & (in) \\
\hline $1-1973$ & 666 & 250 & 628 & 236 & \\
\hline 2 & 666 & 250 & 628 & 236 & \\
\hline 3 & 657 & 246 & 628 & 236 & \\
\hline 4 & 657 & 246 & 628 & 236 & \\
\hline $1-1974$ & 656 & 246 & 628 & 236 & \\
\hline 2 & 652 & 245 & 628 & 236 & \\
\hline 3 & 628 & 236 & 628 & 236 & \\
\hline 4 & 626 & 235 & 626 & 235 & \\
\hline $1-1975$ & 626 & 235 & 626 & 235 & \\
\hline 2 & 626 & 235 & 626 & 235 & \\
\hline 3 & 626 & 235 & 626 & 235 & \\
\hline 4 & 626 & 235 & 626 & 235 & \\
\hline $1-1976$ & 626 & 235 & 626 & 235 & \\
\hline 2 & 626 & 235 & 626 & 235 & \\
\hline 3 & 626 & 235 & 626 & 235 & \\
\hline 4 & 626 & 235 & 626 & 235 & \\
\hline $1-1977$ & 626 & 235 & 626 & 235 & \\
\hline 2 & 626 & 235 & 626 & 235 & \\
\hline 3 & 626 & 235 & 626 & 235 & \\
\hline 4 & 626 & 235 & 626 & 235 & \\
\hline $1-1978$ & 626 & 235 & 626 & 235 & \\
\hline 2 & 626 & 235 & 626 & 235 & \\
\hline 3 & 626 & 235 & 626 & 235 & \\
\hline 4 & 626 & 235 & 626 & 235 & \\
\hline $1-1979$ & 626 & 235 & 626 & 235 & \\
\hline 2 & 626 & 235 & 626 & 235 & \\
\hline 3 & 626 & 235 & 626 & 235 & \\
\hline 4 & 626 & 235 & 626 & 235 & \\
\hline $1-1980$ & 626 & 235 & 626 & 235 & \\
\hline 2 & 626 & 235 & 626 & 235 & \\
\hline 3 & 626 & 235 & 626 & 235 & \\
\hline 4 & 626 & 235 & 626 & 235 & \\
\hline $1-1981$ & 626 & 235 & 626 & 235 & \\
\hline 2 & 626 & 235 & 626 & 235 & \\
\hline 3 & 626 & 235 & 626 & 235 & \\
\hline 4 & 626 & 235 & 626 & 235 & \\
\hline $1-1982$ & 626 & 235 & 626 & 235 & \\
\hline 2 & 546 & 206 & 546 & 206 & \\
\hline 3 & 546 & 206 & 546 & 206 & $\overline{-}$ \\
\hline 4 & 546 & 206 & 546 & 206 & \\
\hline $1-1983$ & 546 & 206 & 546 & 206 & \\
\hline 2 & 546 & 206 & 546 & 206 & \\
\hline 3 & 546 & 206 & 546 & 206 & \\
\hline 4 & 546 & 206 & 546 & 206 & \\
\hline $1-1984$ & 503 & 190 & 503 & 190 & \\
\hline 2 & 503 & 190 & 503 & 190 & \\
\hline
\end{tabular}


241-BY-105 SINGLE-SHELL WHC-SD-WM-ER-312， ReV.0 TANK LEVEL HISTORY

\begin{tabular}{|c|c|c|c|c|c|}
\hline Year & Total & Total & Solids & Solids & LOW \\
\hline & (K gal) & (in) & (K gal) & (in) & (in) \\
\hline$\overline{3}$ & 503 & 190 & 503 & 190 & \\
\hline 4 & 503 & 190 & 503 & 190 & \\
\hline $1-1985$ & 503 & 190 & 503 & 190 & \\
\hline 2 & 503 & 190 & 503 & 190 & \\
\hline 3 & 503 & 190 & 503 & 190 & \\
\hline 4 & 503 & 190 & 503 & 190 & \\
\hline $1-1986$ & 503 & 190 & 503 & 190 & 161 \\
\hline 2 & 503 & 190 & 503 & 190 & 161 \\
\hline 3 & 503 & 190 & 503 & 190 & 160 \\
\hline 4 & 503 & 190 & 503 & 190 & 161 \\
\hline $1-1987$ & 503 & 190 & 503 & 190 & 162 \\
\hline 2 & 503 & 190 & 503 & 190 & \\
\hline 3 & 503 & 190 & 503 & 190 & 161 \\
\hline 4 & 503 & 190 & 503 & 190 & 162 \\
\hline $1-1988$ & 503 & 190 & 503 & 190 & 161 \\
\hline 2 & 503 & 190 & 503 & 190 & 162 \\
\hline 3 & 503 & 190 & 503 & 190 & 162 \\
\hline 4 & 503 & 190 & 503 & 190 & 162 \\
\hline $1-1989$ & 503 & 190 & 503 & 190 & 162 \\
\hline 2 & 503 & 190 & 503 & 190 & 162 \\
\hline 3 & 503 & 190 & 503 & 190 & 162 \\
\hline 4 & 503 & 190 & 503 & 190 & 162 \\
\hline $1-1990$ & 503 & 190 & 503 & 190 & 163 \\
\hline 2 & 503 & 190 & 503 & 190 & \\
\hline 3 & 503 & 190 & 503 & 190 & 163 \\
\hline 4 & 503 & 190 & 503 & 190 & 164 \\
\hline $1-1991$ & 503 & 190 & 503 & 190 & 165 \\
\hline 2 & 503 & 190 & 503 & 190 & 165 \\
\hline 3 & 503 & 190 & 503 & 190 & 164 \\
\hline 4 & 503 & 190 & 503 & 190 & 164 \\
\hline $1-1992$ & 503 & 190 & 503 & 190 & 166 \\
\hline 2 & 503 & 190 & 503 & 190 & \\
\hline 3 & 503 & 190 & 503 & 190 & \\
\hline 4 & 503 & 190 & 503 & 190 & 164 \\
\hline $1-1993$ & 503 & 190 & 503 & 190 & 166 \\
\hline 2 & 503 & 190 & 503 & 190 & 165 \\
\hline 3 & & & & & 164 \\
\hline 4 & & & & & \\
\hline
\end{tabular}


241-BY-106 SINGLE-SHELL WHC-SD-WM-ER-312, REV. 0 TANK LEVEL HISTORY

\begin{tabular}{|c|c|c|c|c|c|}
\hline Year & Total & Total & Solids & Solids & LOW \\
\hline & ( $\mathrm{K}$ gal) & (in) & (K gal) & (in) & (in) \\
\hline \multirow{2}{*}{\multicolumn{6}{|c|}{$1-1950$}} \\
\hline & & & & & \\
\hline \multicolumn{6}{|l|}{2} \\
\hline \multicolumn{6}{|l|}{3} \\
\hline \multicolumn{6}{|l|}{4} \\
\hline \multicolumn{6}{|l|}{$1-1951$} \\
\hline \multicolumn{6}{|l|}{2} \\
\hline \multicolumn{6}{|l|}{3} \\
\hline \multicolumn{6}{|l|}{4} \\
\hline \multicolumn{6}{|l|}{$1-1952$} \\
\hline \multicolumn{6}{|l|}{2} \\
\hline \multicolumn{6}{|l|}{3} \\
\hline \multicolumn{6}{|l|}{4} \\
\hline $1-1953$ & 0 & 0 & & & \\
\hline 2 & 758 & 283 & & & \\
\hline 3 & 758 & 283 & & & \\
\hline 4 & 755 & 282 & & & \\
\hline $1-1954$ & 756 & 282 & & & \\
\hline 2 & 756 & 282 & & & \\
\hline 3 & 756 & 282 & & & \\
\hline 4 & 15 & 13 & & & \\
\hline $1-1955$ & 728 & 272 & 40 & 22 & \\
\hline 2 & 736 & 275 & 107 & 46 & \\
\hline 3 & 736 & 275 & 107 & 46 & \\
\hline 4 & 746 & 279 & 120 & 51 & \\
\hline $1-1956$ & 551 & 208 & 150 & 62 & \\
\hline 2 & 723 & 270 & 180 & 73 & \\
\hline 3 & 629 & 236 & 164 & 67 & \\
\hline 4 & 717 & 268 & 168 & 69 & \\
\hline $1-1957$ & 722 & 270 & 111 & 48 & \\
\hline 2 & 725 & 271 & 111 & 48 & \\
\hline 3 & 739 & 276 & 111 & 48 & \\
\hline 4 & 257 & 101 & 111 & 48 & \\
\hline $1-1958$ & 257 & 101 & 111 & 48 & \\
\hline 2 & 257 & 101 & 111 & 48 & \\
\hline 3 & 257 & 101 & 111 & 48 & \\
\hline 4 & 257 & 101 & 111 & 48 & \\
\hline $1-1959$ & 241 & 95 & 111 & 48 & \\
\hline 2 & 252 & 99 & 111 & 48 & \\
\hline 3 & 252 & 99 & 111 & 48 & $\cdot$ \\
\hline 4 & 252 & 99 & 111 & 48 & \\
\hline $1-1960$ & 252 & 99 & 111 & 48 & \\
\hline 2 & 252 & 99 & 111 & 48 & \\
\hline 3 & 252 & 99 & 111 & 48 & \\
\hline 4 & 252 & 99 & 111 & 48 & \\
\hline \multicolumn{6}{|l|}{$1-1961$} \\
\hline 2 & 249 & 98 & 111 & 48 & \\
\hline
\end{tabular}


241-BY-106 SINGLE-SHELL WHC-SD-WM-ER-312, REV. 0 TANK LEVEL HISTORY

\begin{tabular}{|c|c|c|c|c|c|}
\hline Year & Total & Total & Solids & Solids & LOW \\
\hline & (K gal) & (in) & (K gal) & (in) & (in) \\
\hline \multirow{2}{*}{\multicolumn{6}{|c|}{3}} \\
\hline & & & & & \\
\hline 4 & 505 & 191 & 111 & 48 & \\
\hline \multicolumn{6}{|l|}{$1-1962$} \\
\hline 2 & 623 & 234 & 111 & 48 & \\
\hline \multicolumn{6}{|l|}{3} \\
\hline 4 & 623 & 234 & 111 & 48 & \\
\hline \multicolumn{6}{|l|}{$1-1963$} \\
\hline 2 & 620 & 233 & 150 & 62 & \\
\hline \multicolumn{6}{|l|}{3} \\
\hline 4 & 620 & 233 & 150 & 62 & \\
\hline \multicolumn{6}{|l|}{ 1-1964 } \\
\hline 2 & 620 & 233 & 150 & 62 & \\
\hline \multicolumn{6}{|l|}{3} \\
\hline 4 & 620 & 233 & 150 & 62 & \\
\hline \multicolumn{6}{|l|}{$1-1965$} \\
\hline 2 & 620 & 233 & 103 & 45 & \\
\hline 3 & 620 & 233 & 103 & 45 & \\
\hline 4 & 620 & 233 & 103 & 45 & \\
\hline $1-1966$ & 620 & 233 & 103 & 45 & \\
\hline 2 & 620 & 233 & 103 & 45 & \\
\hline 3 & 744 & 278 & 103 & 45 & \\
\hline 4 & 744 & 278 & 103 & 45 & \\
\hline $1-1967$ & 395 & 151 & 103 & 45 & \\
\hline 2 & 272 & 106 & 103 & 45 & \\
\hline 3 & 741 & 277 & 103 & 45 & \\
\hline 4 & 740 & 277 & 103 & 45 & \\
\hline $1-1968$ & 739 & 276 & 103 & 45 & \\
\hline 2 & 253 & 99 & 103 & 45 & \\
\hline 3 & 253 & 99 & 103 & 45 & \\
\hline 4 & 253 & 99 & 103 & 45 & \\
\hline $1-1969$ & 736 & 275 & 105 & 46 & \\
\hline 2 & 737 & 275 & 93 & 41 & \\
\hline 3 & 732 & 274 & 96 & 42 & \\
\hline 4 & 732 & 274 & 95 & 42 & \\
\hline $1-1970$ & 733 & 274 & 103 & 45 & \\
\hline 2 & 743 & 278 & 96 & 42 & \\
\hline 3 & 689 & 258 & 98 & 43 & \\
\hline 4 & 675 & 253 & 164 & 67 & \\
\hline $1-1971$ & 689 & 258 & 249 & 98 & $\cdot$ \\
\hline 2 & 706 & 264 & 288 & 112 & \\
\hline 3 & 670 & 251 & 288 & 112 & \\
\hline 4 & 706 & 264 & 288 & 112 & \\
\hline $1-1972$ & 580 & 218 & 296 & 115 & \\
\hline 2 & 655 & 246 & 296 & 115 & \\
\hline 3 & 697 & 261 & 296 & 115 & \\
\hline 4 & 704 & 263 & 563 & 212 & \\
\hline
\end{tabular}


241-BY-106 SINGLE-SHELL WHC-SD-WM-ER-312, ReV. 0 TANK LEVEL HISTORY

\begin{tabular}{|c|c|c|c|c|c|}
\hline Year & Total & Total & Solids & Solids & LOW \\
\hline & (K gal) & (in) & ( $\mathrm{K}$ gal) & (in) & (in) \\
\hline $1-1973$ & 710 & 266 & 447 & 170 & \\
\hline 2 & 677 & 254 & 494 & 187 & \\
\hline 3 & 704 & 263 & 494 & 187 & \\
\hline 4 & 692 & 259 & 494 & 187 & \\
\hline $1-1974$ & 692 & 259 & 494 & 187 & \\
\hline 2 & 701 & 262 & 494 & 187 & \\
\hline 3 & 697 & 261 & 494 & 187 & \\
\hline 4 & 697 & 261 & 420 & 160 & \\
\hline $1-1975$ & 697 & 261 & 420 & 160 & \\
\hline 2 & 697 & 261 & 351 & 135 & \\
\hline 3 & 697 & 261 & 351 & 135 & \\
\hline 4 & 681 & 255 & 351 & 135 & \\
\hline $1-1976$ & 681 & 255 & 351 & 135 & \\
\hline 2 & 681 & 255 & 351 & 135 & \\
\hline 3 & 684 & 256 & 351 & 135 & \\
\hline 4 & 684 & 256 & 351 & 135 & \\
\hline $1-1977$ & 593 & 223 & 593 & 223 & \\
\hline 2 & 593 & 223 & 593 & 223 & \\
\hline 3 & 626 & 235 & 626 & 235 & \\
\hline 4 & 626 & 235 & 626 & 235 & \\
\hline $1-1978$ & 626 & 235 & 626 & 235 & \\
\hline 2 & 626 & 235 & 626 & 235 & \\
\hline 3 & 626 & 235 & 626 & 235 & \\
\hline 4 & 626 & 235 & 626 & 235 & \\
\hline $1-1979$ & 626 & 235 & 626 & 235 & \\
\hline 2 & 626 & 235 & 626 & 235 & \\
\hline 3 & 626 & 235 & 626 & 235 & \\
\hline 4 & 626 & 235 & 626 & 235 & \\
\hline $1-1980$ & 626 & 235 & 626 & 235 & \\
\hline 2 & 626 & 235 & 626 & 235 & \\
\hline 3 & 626 & 235 & 626 & 235 & \\
\hline 4 & 626 & 235 & 626 & 235 & \\
\hline $1-1981$ & 626 & 235 & 626 & 235 & \\
\hline 2 & 626 & 235 & 626 & 235 & \\
\hline 3 & 626 & 235 & 626 & 235 & \\
\hline 4 & 626 & 235 & 626 & 235 & \\
\hline $1-1982$ & 626 & 235 & 626 & 235 & \\
\hline 2 & 642 & 241 & 642 & 241 & \\
\hline 3 & 642 & 241 & 642 & 241 & 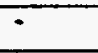 \\
\hline 4 & 642 & 241 & 642 & 241 & \\
\hline $1-1983$ & 642 & 241 & 642 & 241 & \\
\hline 2 & 642 & 241 & 642 & 241 & \\
\hline 3 & 642 & 241 & 642 & 241 & \\
\hline 4 & 642 & 241 & 642 & 241 & \\
\hline $1-1984$ & 642 & 241 & 642 & 241 & \\
\hline 2 & 642 & 241 & 642 & 241 & \\
\hline
\end{tabular}


241-BY-106 SINGLE-SHELL WHC-SD-WM-ER-312, ReV.0 TANK LEVEL HISTORY

\begin{tabular}{|l|c|c|c|c|c|}
\hline \multicolumn{1}{|c|}{ Year } & Total & Total & Solids & Solids & LOW \\
\hline & (K gal) & (in) & (K gal) & (in) & (in) \\
\hline 3 & & & & & \\
\hline 4 & 642 & 241 & 642 & 241 & \\
\hline $1-1985$ & 642 & 241 & 642 & 241 & \\
\hline 2 & 642 & 241 & 642 & 241 & \\
\hline 3 & 642 & 241 & 642 & 241 & \\
\hline 4 & 642 & 241 & 642 & 241 & \\
\hline $1-1986$ & 642 & 241 & 642 & 241 & \\
\hline 2 & 642 & 241 & 642 & 241 & \\
\hline 3 & 642 & 241 & 642 & 241 & \\
\hline 4 & 642 & 241 & 642 & 241 & 223 \\
\hline $1-1987$ & 642 & 241 & 642 & 241 & 223 \\
\hline 2 & 642 & 241 & 642 & 241 & 225 \\
\hline 3 & 642 & 241 & 642 & 241 & 224 \\
\hline 4 & 642 & 241 & 642 & 241 & 223 \\
\hline $1-1988$ & 642 & 241 & 642 & 241 & 223 \\
\hline 2 & 642 & 241 & 642 & 241 & 223 \\
\hline 3 & 642 & 241 & 642 & 241 & 222 \\
\hline 4 & 642 & 241 & 642 & 241 & 225 \\
\hline $1-1989$ & 642 & 241 & 642 & 241 & 223 \\
\hline 2 & 642 & 241 & 642 & 241 & 225 \\
\hline 3 & 642 & 241 & 642 & 241 & 225 \\
\hline 4 & 642 & 241 & 642 & 241 & 224 \\
\hline $1-1990$ & 642 & 241 & 642 & 241 & 225 \\
\hline 2 & 642 & 241 & 642 & 241 & \\
\hline 3 & 642 & 241 & 642 & 241 & 225 \\
\hline 4 & 642 & 241 & 642 & 241 & 225 \\
\hline $1-1991$ & 642 & 241 & 642 & 241 & 226 \\
\hline 2 & 642 & 241 & 642 & 241 & 226 \\
\hline 3 & 642 & 241 & 642 & 241 & 225 \\
\hline 4 & 642 & 241 & 642 & 241 & 225 \\
\hline $1-1992$ & 642 & 241 & 642 & 241 & 225 \\
\hline 2 & 642 & 241 & 642 & 241 & \\
\hline 3 & 642 & 241 & 642 & 241 & \\
\hline 4 & 642 & 241 & 642 & 241 & 225 \\
\hline $1-1993$ & 642 & 241 & 642 & 241 & 226 \\
\hline 2 & 642 & 241 & 642 & 241 & 225 \\
\hline 3 & & & & & 225 \\
\hline 4 & & & & & 225 \\
\hline
\end{tabular}


241-BY-107 SINGLE-SHELL WHC-SD-WM-ER-312, ReV.0 TANK LEVEL HISTORY

\begin{tabular}{|c|c|c|c|c|c|}
\hline Year & Total & Total & Solids & Solids & LOW \\
\hline & ( $\mathrm{K}$ gal) & (in) & (K gal) & (in) & (in) \\
\hline \multirow{2}{*}{\multicolumn{6}{|c|}{$1-1950$}} \\
\hline & & & & & \\
\hline \multicolumn{6}{|l|}{2} \\
\hline 3 & 11 & 11 & & & \\
\hline 4 & 106 & 46 & & & \\
\hline $1-1951$ & 744 & 278 & & & \\
\hline 2 & 744 & 278 & & & \\
\hline \multicolumn{6}{|l|}{3} \\
\hline \multicolumn{6}{|l|}{4} \\
\hline $1-1952$ & 758 & 283 & & & \\
\hline 2 & 758 & 283 & & & \\
\hline 3 & 334 & 129 & & & \\
\hline 4 & 1 & 8 & & & \\
\hline $1-1953$ & 743 & 278 & 1 & 8 & \\
\hline 2 & 758 & 283 & 1 & 8 & \\
\hline 3 & 758 & 283 & 1 & 8 & \\
\hline 4 & 758 & 283 & 1 & 8 & \\
\hline $1-1954$ & 758 & 283 & 1 & 8 & \\
\hline 2 & 758 & 283 & 1 & 8 & \\
\hline 3 & 101 & 44 & 44 & 23 & \\
\hline 4 & 35 & 20 & 1 & 8 & \\
\hline $1-1955$ & 276 & 108 & 72 & 34 & \\
\hline 2 & 48 & 25 & 45 & 24 & \\
\hline 3 & 739 & 276 & 142 & 59 & \\
\hline 4 & 177 & 72 & 147 & 61 & \\
\hline $1-1956$ & 180 & 73 & 160 & 66 & \\
\hline 2 & 225 & 89 & 168 & 69 & \\
\hline 3 & 87 & 39 & 87 & 39 & \\
\hline 4 & 755 & 282 & 150 & 62 & \\
\hline $1-1957$ & 524 & 198 & 150 & 62 & \\
\hline 2 & 722 & 270 & 150 & 62 & \\
\hline 3 & 486 & 184 & 178 & 72 & \\
\hline 4 & 172 & 70 & 172 & 70 & \\
\hline $1-1958$ & 172 & 70 & 172 & 70 & \\
\hline 2 & 172 & 70 & 172 & 70 & \\
\hline 3 & 172 & 70 & 172 & 70 & \\
\hline 4 & 574 & 216 & 150 & 62 & \\
\hline $1-1959$ & 744 & 278 & 150 & 62 & \\
\hline 2 & 771 & 288 & 150 & 62 & \\
\hline 3 & 771 & 288 & 150 & 62 & - \\
\hline 4 & 771 & 288 & 150 & 62 & \\
\hline $1-1960$ & 771 & 288 & 150 & 62 & \\
\hline 2 & 771 & 288 & 150 & 62 & \\
\hline 3 & 771 & 288 & 150 & 62 & \\
\hline 4 & 771 & 288 & 150 & 62 & \\
\hline \multicolumn{6}{|l|}{$1-1961$} \\
\hline 2 & 733 & 274 & 150 & 62 & \\
\hline
\end{tabular}


241-BY-107 SINGLE-SHELL WHC-SD-WM-ER-312, ReV. 0 TANK LEVEL HISTORY

\begin{tabular}{|c|c|c|c|c|c|}
\hline Year & Total & Total & Solids & Solids & LOW \\
\hline & ( $\mathrm{K}$ gal) & (in) & ( $\mathrm{K}$ gal) & (in) & (in) \\
\hline \multicolumn{6}{|l|}{3} \\
\hline 4 & 736 & 275 & 150 & 62 & \\
\hline \multicolumn{6}{|l|}{$1-1962$} \\
\hline 2 & 736 & 275 & 150 & 62 & \\
\hline \multicolumn{6}{|l|}{3} \\
\hline 4 & 736 & 275 & 150 & 62 & \\
\hline \multicolumn{6}{|l|}{$1-1963$} \\
\hline 2 & 736 & 275 & 178 & 72 & \\
\hline \multicolumn{6}{|l|}{3} \\
\hline 4 & 728 & 272 & 178 & 72 & \\
\hline \multicolumn{6}{|l|}{$1-1964$} \\
\hline 2 & 730 & 273 & 178 & 72 & \\
\hline \multicolumn{6}{|l|}{3} \\
\hline 4 & 730 & 273 & 178 & 72 & \\
\hline \multicolumn{6}{|l|}{$1-1965$} \\
\hline 2 & 730 & 273 & 150 & 62 & \\
\hline 3 & 730 & 273 & 150 & 62 & \\
\hline 4 & 730 & 273 & 150 & 62 & \\
\hline $1-1966$ & 730 & 273 & 150 & 62 & \\
\hline 2 & 730 & 273 & 150 & 62 & \\
\hline 3 & 733 & 274 & 150 & 62 & \\
\hline 4 & 733 & 274 & 150 & 62 & \\
\hline $1-1967$ & 733 & 274 & 150 & 62 & \\
\hline 2 & 733 & 274 & 150 & 62 & \\
\hline 3 & 733 & 274 & 150 & 62 & \\
\hline 4 & 733 & 274 & 150 & 62 & \\
\hline $1-1968$ & 733 & 274 & 150 & 62 & \\
\hline 2 & 733 & 274 & 150 & 62 & \\
\hline 3 & 733 & 274 & 150 & 62 & \\
\hline 4 & 730 & 273 & 150 & 62 & \\
\hline $1-1969$ & 729 & 273 & 150 & 62 & \\
\hline 2 & 728 & 272 & 150 & 62 & \\
\hline 3 & 726 & 271 & 150 & 62 & \\
\hline 4 & 649 & 243 & 150 & 62 & \\
\hline $1-1970$ & 651 & 244 & 58 & 29 & \\
\hline 2 & 678 & 254 & 61 & 30 & \\
\hline 3 & 638 & 239 & 43 & 23 & \\
\hline 4 & 656 & 246 & 106 & 46 & \\
\hline $1-1971$ & 624 & 234 & 117 & 50 & . \\
\hline 2 & 674 & 253 & 117 & 50 & \\
\hline 3 & 605 & 227 & 117 & 50 & \\
\hline 4 & 664 & 249 & 117 & 50 & \\
\hline $1-1972$ & 689 & 258 & 117 & 50 & \\
\hline 2 & 634 & 238 & 117 & 50 & \\
\hline 3 & 648 & 243 & 117 & 50 & \\
\hline 4 & 646 & 242 & 117 & 50 & \\
\hline
\end{tabular}


241-BY-107 SINGLE-SHELL WHC-SD-WM-ER-312, REV.0 TANK LEVEL HISTORY

\begin{tabular}{|c|c|c|c|c|c|}
\hline Year & Total & Total & Solids & Solids & LOW \\
\hline & (K gal) & (in) & (K gal) & (in) & (in) \\
\hline & & & & & \\
\hline $1-1973$ & 649 & 243 & 175 & 71 & \\
\hline 2 & 635 & 238 & 175 & 71 & \\
\hline 3 & 633 & 238 & 175 & 71 & \\
\hline 4 & 659 & 247 & 175 & 71 & \\
\hline $1-1974$ & 653 & 245 & 175 & 71 & \\
\hline 2 & 492 & 186 & 444 & 169 & \\
\hline 3 & 492 & 186 & 444 & 169 & \\
\hline 4 & 491 & 186 & 183 & 74 & \\
\hline $1-1975$ & 367 & 141 & 367 & 141 & \\
\hline 2 & 367 & 141 & 367 & 141 & \\
\hline 3 & 367 & 141 & 367 & 141 & \\
\hline 4 & 367 & 141 & 367 & 141 & \\
\hline $1-1976$ & 367 & 141 & 367 & 141 & \\
\hline 2 & 367 & 141 & 367 & 141 & \\
\hline 3 & 367 & 141 & 367 & 141 & \\
\hline 4 & 367 & 141 & 367 & 141 & \\
\hline $1-1977$ & 367 & 141 & 367 & 141 & \\
\hline 2 & 367 & 141 & 367 & 141 & \\
\hline 3 & 367 & 141 & 367 & 141 & \\
\hline 4 & 367 & 141 & 367 & 141 & \\
\hline $1-1978$ & 326 & 126 & 326 & 126 & \\
\hline 2 & 326 & 126 & 326 & 126 & \\
\hline 3 & 326 & 126 & 326 & 126 & \\
\hline 4 & 326 & 126 & 326 & 126 & \\
\hline $1-1979$ & 326 & 126 & 326 & 126 & \\
\hline 2 & 326 & 126 & 326 & 126 & \\
\hline 3 & 326 & 126 & 326 & 126 & \\
\hline 4 & 326 & 126 & 326 & 126 & \\
\hline $1-1980$ & 326 & 126 & 326 & 126 & \\
\hline 2 & 326 & 126 & 326 & 126 & \\
\hline 3 & 326 & 126 & 326 & 126 & \\
\hline 4 & 326 & 126 & 326 & 126 & \\
\hline $1-1981$ & 326 & 126 & 326 & 126 & \\
\hline 2 & 326 & 126 & 326 & 126 & \\
\hline 3 & 326 & 126 & 326 & 126 & \\
\hline 4 & 326 & 126 & 326 & 126 & \\
\hline $1-1982$ & 326 & 126 & 326 & 126 & \\
\hline 2 & 356 & 137 & 356 & 137 & \\
\hline 3 & 356 & 137 & 356 & 137 & \\
\hline 4 & 356 & 137 & 356 & 137 & \\
\hline $1-1983$ & 356 & 137 & 356 & 137 & \\
\hline 2 & 356 & 137 & 356 & 137 & \\
\hline 3 & 356 & 137 & 356 & 137 & \\
\hline 4 & 356 & 137 & 356 & 137 & \\
\hline $1-1984$ & 296 & 115 & 296 & 115 & \\
\hline 2 & 266 & 104 & 266 & 104 & \\
\hline
\end{tabular}


241-BY-107 SINGLE-SHELL WHC-SD-WM-ER-312, ReV. 0 TANK LEVEL HISTORY

\begin{tabular}{|c|c|c|c|c|c|}
\hline Year & Total & Total & Solids & Solids & LOW \\
\hline & (K gal) & (in) & (K gal) & (in) & (in) \\
\hline & & & 250 & 901 & \\
\hline 3 & 266 & 104 & 260 & 104 & \\
\hline 4 & 266 & 104 & 266 & 104 & \\
\hline $1-1985$ & 266 & 104 & 266 & 104 & \\
\hline 2 & 266 & 104 & 266 & 104 & \\
\hline 3 & 266 & 104 & 266 & 104 & \\
\hline 4 & 266 & 104 & 266 & 104 & \\
\hline $1-1986$ & 266 & 104 & 266 & 104 & 63 \\
\hline 2 & 266 & 104 & 266 & 104 & 61 \\
\hline 3 & 266 & 104 & 266 & 104 & 61 \\
\hline 4 & 266 & 104 & 266 & 104 & 63 \\
\hline $1-1987$ & 266 & 104 & 266 & 104 & 61 \\
\hline 2 & 266 & 104 & 266 & 104 & 63 \\
\hline 3 & 266 & 104 & 266 & 104 & 62 \\
\hline 4 & 266 & 104 & 266 & 104 & 64 \\
\hline $1-1988$ & 266 & 104 & 266 & 104 & 63 \\
\hline 2 & 266 & 104 & 266 & 104 & 61 \\
\hline 3 & 266 & 104 & 266 & 104 & 62 \\
\hline 4 & 266 & 104 & 266 & 104 & 64 \\
\hline $1-1989$ & 266 & 104 & 266 & 104 & 63 \\
\hline 2 & 266 & 104 & 266 & 104 & 63 \\
\hline 3 & 266 & 104 & 266 & 104 & 63 \\
\hline 4 & 266 & 104 & 266 & 104 & 63 \\
\hline $1-1990$ & 266 & 104 & 266 & 104 & 63 \\
\hline 2 & 266 & 104 & 266 & 104 & \\
\hline 3 & 266 & 104 & 266 & 104 & 64 \\
\hline 4 & 266 & 104 & 266 & 104 & 63 \\
\hline $1-1991$ & 266 & 104 & 266 & 104 & 64 \\
\hline 2 & 266 & 104 & 266 & 104 & 63 \\
\hline 3 & 266 & 104 & 266 & 104 & 63 \\
\hline 4 & 266 & 104 & 266 & 104 & 63 \\
\hline 1-1992 & 266 & 104 & 266 & 104 & 64 \\
\hline 2 & 266 & 104 & 266 & 104 & \\
\hline 3 & 266 & 104 & 266 & 104 & \\
\hline 4 & 266 & 104 & 266 & 104 & 61 \\
\hline $1-1993$ & 266 & 104 & 266 & 104 & 63 \\
\hline 2 & 266 & 104 & 266 & 104 & 62 \\
\hline 3 & & & & & 64 \\
\hline 4 & & & & & \\
\hline
\end{tabular}


241-BY-108 SINGLE-SHELL WHC-SD-WM-ER-312, Rev. 0 TANK LEVEL HISTORY

\begin{tabular}{|c|c|c|c|c|}
\hline Year & Total & Total & Solids & Solids \\
\hline & ( $\mathrm{K}$ gal) & (in) & (K gal) & (in) \\
\hline \multicolumn{5}{|l|}{$1-1950$} \\
\hline \multicolumn{5}{|l|}{2} \\
\hline \multicolumn{5}{|l|}{3} \\
\hline \multicolumn{5}{|l|}{4} \\
\hline $1-1951$ & 20 & 15 & & \\
\hline 2 & 538 & 203 & & \\
\hline \multicolumn{5}{|l|}{3} \\
\hline \multicolumn{5}{|l|}{4} \\
\hline $1-1952$ & 753 & 281 & & \\
\hline 2 & 753 & 281 & & \\
\hline 3 & 753 & 281 & & \\
\hline 4 & 753 & 281 & & \\
\hline $1-1953$ & 753 & 281 & & \\
\hline 2 & 687 & 257 & & \\
\hline$\overline{3}$ & 687 & 257 & & \\
\hline 4 & 687 & 257 & & \\
\hline 1.1954 & 702 & 263 & & \\
\hline 2 & 702 & 263 & & \\
\hline 3 & 266 & 104 & & \\
\hline 4 & 127 & 54 & & \\
\hline $1-1955$ & 78 & 36 & 33 & 19 \\
\hline 2 & 140 & 58 & 98 & 43 \\
\hline 3 & 376 & 144 & 197 & 79 \\
\hline 4 & 200 & 80 & 200 & 80 \\
\hline $1-1956$ & 713 & 267 & 205 & 82 \\
\hline 2 & 179 & 73 & 179 & 73 \\
\hline 3 & 523 & 198 & 194 & 78 \\
\hline 4 & 656 & 246 & 201 & 81 \\
\hline $1-1957$ & 722 & 270 & 201 & 81 \\
\hline 2 & 722 & 270 & 249 & 98 \\
\hline 3 & 213 & 85 & 213 & 85 \\
\hline 4 & 219 & 84 & 210 & 84 \\
\hline $1-1958$ & 211 & 84 & 210 & 84 \\
\hline 2 & 211 & 84 & 210 & 84 \\
\hline 3 & 211 & 84 & 210 & 84 \\
\hline 4 & 244 & 96 & 210 & 84 \\
\hline $1-1959$ & 557 & 210 & & \\
\hline 2 & 744 & 278 & & \\
\hline 3 & 744 & 278 & & \\
\hline 4 & 744 & 278 & 210 & 84 \\
\hline $1-1960$ & 744 & 278 & 210 & 84 \\
\hline 2 & 738 & 276 & 210 & 84 \\
\hline 3 & 738 & 276 & 210 & 84 \\
\hline 4 & 738 & 276 & 210 & 84 \\
\hline \multicolumn{5}{|l|}{1.1961} \\
\hline 2 & 706 & 264 & 210 & 84 \\
\hline
\end{tabular}


241-BY-108 SINGLE-SHELL WHC-SD-WM-ER-312, ReV.0 TANK LEVEL HISTORY

\begin{tabular}{|c|c|c|c|c|}
\hline Year & Total & Total & Solids & Solids \\
\hline & (K gal) & (in) & (K gal) & (in) \\
\hline \multicolumn{5}{|l|}{3} \\
\hline 4 & 706 & 264 & 210 & 84 \\
\hline \multicolumn{5}{|l|}{$1-1962$} \\
\hline 2 & 706 & 264 & 210 & 84 \\
\hline \multicolumn{5}{|l|}{3} \\
\hline 4 & 706 & 264 & 210 & 84 \\
\hline \multicolumn{5}{|l|}{$1-1963$} \\
\hline 2 & 703 & 263 & 202 & 81 \\
\hline \multicolumn{5}{|l|}{3} \\
\hline 4 & 703 & 263 & 202 & 81 \\
\hline \multicolumn{5}{|l|}{$1-1964$} \\
\hline 2 & 695 & 260 & 202 & 81 \\
\hline \multicolumn{5}{|l|}{3} \\
\hline 4 & 697 & 261 & 202 & 81 \\
\hline \multicolumn{5}{|l|}{$1-1965$} \\
\hline 2 & 700 & 262 & 178 & 72 \\
\hline 3 & 700 & 262 & 178 & 72 \\
\hline 4 & 700 & 262 & 178 & 72 \\
\hline $1-1966$ & 700 & 262 & 178 & 72 \\
\hline 2 & 700 & 262 & 178 & 72 \\
\hline 3 & 700 & 262 & 178 & 72 \\
\hline 4 & 697 & 261 & 178 & 72 \\
\hline $1-1967$ & 697 & 261 & 178 & 72 \\
\hline 2 & 697 & 261 & 178 & 72 \\
\hline 3 & 697 & 261 & 178 & 72 \\
\hline 4 & 697 & 261 & 178 & 72 \\
\hline $1-1968$ & 695 & 260 & 178 & 72 \\
\hline 2 & 557 & 210 & 178 & 72 \\
\hline 3 & 657 & 246 & 125 & 53 \\
\hline 4 & 604 & 227 & 125 & 53 \\
\hline $1-1969$ & 582 & 219 & 165 & 67 \\
\hline 2 & 585 & 220 & 222 & 88 \\
\hline 3 & 574 & 216 & 194 & 78 \\
\hline 4 & 574 & 216 & 233 & 92 \\
\hline $1-1970$ & 706 & 264 & 224 & 89 \\
\hline 2 & 657 & 246 & 176 & 71 \\
\hline 3 & 740 & 277 & 194 & 78 \\
\hline 4 & 659 & 247 & 65 & 31 \\
\hline $1-1971$ & 692 & 259 & 98 & 43 \\
\hline 2 & 385 & 147 & 385 & 147 \\
\hline 3 & 388 & 149 & 388 & 149 \\
\hline 4 & 381 & 146 & 381 & 146 \\
\hline $1-1972$ & 381 & 146 & 381 & 146 \\
\hline 2 & 381 & 146 & 381 & 146 \\
\hline 3 & 381 & 146 & 381 & 146 \\
\hline 4 & 312 & 121 & 304 & 118 \\
\hline
\end{tabular}


247-BY-108 SINGLE-SHELL WHC-SD-WM-ER-312， ReV.0 TANK LEVEL HISTORY

\begin{tabular}{|c|c|c|c|c|}
\hline Year & Total & Total & Solids & Solids \\
\hline & ( $\mathrm{K}$ gal) & (in) & (K gal) & (in) \\
\hline $1-1973$ & 312 & 121 & 304 & 118 \\
\hline 2 & 304 & 118 & 304 & 118 \\
\hline 3 & 304 & 118 & 304 & 118 \\
\hline 4 & 304 & 118 & 304 & 118 \\
\hline $1-1974$ & 304 & 118 & 304 & 118 \\
\hline 2 & 304 & 118 & 304 & 118 \\
\hline 3 & 304 & 118 & 304 & 118 \\
\hline 4 & 359 & 138 & 359 & 138 \\
\hline $1-1975$ & 359 & 138 & 359 & 138 \\
\hline 2 & 359 & 138 & 359 & 138 \\
\hline 3 & 359 & 138 & 359 & 138 \\
\hline 4 & 359 & 138 & 359 & 138 \\
\hline $1-1976$ & 359 & 138 & 359 & 138 \\
\hline 2 & 359 & 138 & 359 & 138 \\
\hline 3 & 359 & 138 & 359 & 138 \\
\hline 4 & 359 & 138 & 359 & 138 \\
\hline $1-1977$ & 359 & 138 & 359 & 138 \\
\hline 2 & 359 & 138 & 359 & 138 \\
\hline 3 & 359 & 138 & 359 & 138 \\
\hline 4 & 359 & 138 & 359 & 138 \\
\hline $1-1978$ & 359 & 138 & 359 & 138 \\
\hline 2 & 359 & 138 & 359 & 138 \\
\hline 3 & 359 & 138 & 359 & 138 \\
\hline 4 & 359 & 138 & 359 & 138 \\
\hline $1-1979$ & 359 & 138 & 359 & 138 \\
\hline 2 & 359 & 138 & 359 & 138 \\
\hline 3 & 359 & 138 & 359 & 138 \\
\hline 4 & 359 & 138 & 359 & 138 \\
\hline $1-1980$ & 359 & 138 & 359 & 138 \\
\hline 2 & 359 & 138 & 359 & 138 \\
\hline 3 & 359 & 138 & 359 & 138 \\
\hline 4 & 359 & 138 & 359 & 138 \\
\hline $1-1981$ & 359 & 138 & 359 & 138 \\
\hline 2 & 359 & 138 & 359 & 138 \\
\hline 3 & 359 & 138 & 359 & 138 \\
\hline 4 & 359 & 138 & 359 & 138 \\
\hline $1-1982$ & 359 & 138 & 359 & 138 \\
\hline 2 & 253 & 99 & 253 & 99 \\
\hline 3 & 253 & 99 & 252 & 99 \\
\hline 4 & 253 & 99 & 252 & 99 \\
\hline $1-1983$ & 253 & 99 & 252 & 99 \\
\hline 2 & 253 & 99 & 252 & 99 \\
\hline 3 & 253 & 99 & 252 & 99 \\
\hline 4 & 253 & 99 & 252 & 99 \\
\hline $1-1984$ & 239 & 94 & 239 & 94 \\
\hline 2 & 239 & 94 & 239 & 94 \\
\hline
\end{tabular}


241-BY-108 SINGLE-SHELL WHC-SD-WM-ER-312， ReV.0 TANK LEVEL HISTORY

\begin{tabular}{|c|c|c|c|c|}
\hline Year & Total & Total & Solids & Solids \\
\hline & (K gal) & (in) & (K gal) & (in) \\
\hline 3 & 228 & 90 & 228 & 90 \\
\hline 4 & 228 & 90 & 228 & 90 \\
\hline $1-1985$ & 228 & 90 & 228 & 90 \\
\hline 2 & 228 & 90 & 228 & 90 \\
\hline 3 & 228 & 90 & 228 & 90 \\
\hline 4 & 228 & 90 & 228 & 90 \\
\hline $1-1986$ & 228 & 90 & 228 & 90 \\
\hline 2 & 228 & 90 & 228 & 90 \\
\hline 3 & 228 & 90 & 228 & 90 \\
\hline 4 & 228 & 90 & 228 & 90 \\
\hline $1-1987$ & 228 & 90 & 228 & 90 \\
\hline 2 & 228 & 90 & 228 & 90 \\
\hline 3 & 228 & 90 & 228 & 90 \\
\hline 4 & 228 & 90 & 228 & 90 \\
\hline $1-1988$ & 228 & 90 & 228 & 90 \\
\hline 2 & 228 & 90 & 228 & 90 \\
\hline 3 & 228 & 90 & 228 & 90 \\
\hline 4 & 228 & 90 & 228 & 90 \\
\hline $1-1989$ & 228 & 90 & 228 & 90 \\
\hline 2 & 228 & 90 & 228 & 90 \\
\hline 3 & 228 & 90 & 228 & 90 \\
\hline 4 & 228 & 90 & 228 & 90 \\
\hline $1-1990$ & 228 & 90 & 228 & 90 \\
\hline 2 & 228 & 90 & 228 & 90 \\
\hline 3 & 228 & 90 & 228 & 90 \\
\hline 4 & 228 & 90 & 228 & 90 \\
\hline $1-1991$ & 228 & 90 & 228 & 90 \\
\hline 2 & 228 & 90 & 228 & 90 \\
\hline 3 & 228 & 90 & 228 & 90 \\
\hline 4 & 228 & 90 & 228 & 90 \\
\hline $1-1992$ & 228 & 90 & 228 & 90 \\
\hline 2 & 228 & 90 & 228 & 90 \\
\hline 3 & 228 & 90 & 228 & 90 \\
\hline 4 & 228 & 90 & 228 & 90 \\
\hline $1-1993$ & 228 & 90 & 228 & 90 \\
\hline 2 & 228 & 90 & 228 & 90 \\
\hline \multicolumn{5}{|l|}{3} \\
\hline 4 & & & & \\
\hline
\end{tabular}


241-BY-109 SINGLE-SHELL WHC-SD-WM-ER-312, ReV.0 TANK LEVEL HISTORY

\begin{tabular}{|c|c|c|c|c|c|}
\hline Year & Total & Total & Solids & Solids & LOW \\
\hline & (K gal) & (in) & ( $\mathrm{K}$ gal) & (in) & (in) \\
\hline \multirow{2}{*}{\multicolumn{6}{|c|}{$1-1950$}} \\
\hline & & & & & \\
\hline \multicolumn{6}{|c|}{2} \\
\hline \multicolumn{6}{|l|}{3} \\
\hline \multicolumn{6}{|l|}{4} \\
\hline \multicolumn{6}{|l|}{$1-1951$} \\
\hline \multicolumn{6}{|c|}{2} \\
\hline \multicolumn{6}{|l|}{3} \\
\hline \multicolumn{6}{|l|}{4} \\
\hline \multicolumn{6}{|l|}{$1-1952$} \\
\hline \multicolumn{6}{|l|}{2} \\
\hline \multicolumn{6}{|l|}{3} \\
\hline \multicolumn{6}{|l|}{4} \\
\hline $1-1953$ & 448 & 170 & & & \\
\hline 2 & 448 & 170 & & & \\
\hline 3 & 44 & 23 & & & \\
\hline 4 & 44 & 23 & & & \\
\hline $1-1954$ & 281 & 110 & & & \\
\hline 2 & 408 & 156 & & & \\
\hline 3 & 34 & 20 & & & \\
\hline 4 & 477 & 181 & & & \\
\hline $1-1955$ & 551 & 208 & & & \\
\hline 2 & 237 & 94 & & & \\
\hline 3 & 34 & 20 & & & \\
\hline 4 & 34 & 20 & & & \\
\hline $1-1956$ & 34 & 20 & & & \\
\hline 2 & 722 & 270 & & & \\
\hline 3 & 722 & 270 & 36 & 21 & \\
\hline 4 & 722 & 270 & 66 & 31 & \\
\hline $1-1957$ & 722 & 270 & 66 & 31 & \\
\hline 2 & 66 & 31 & 66 & 31 & \\
\hline 3 & 48 & 25 & 46 & 24 & \\
\hline 4 & 48 & 25 & 46 & 24 & \\
\hline $1-1958$ & 48 & 25 & 46 & 24 & \\
\hline 2 & 46 & 24 & 46 & 24 & \\
\hline 3 & 46 & 24 & 46 & 24 & \\
\hline 4 & 46 & 24 & 46 & 24 & \\
\hline $1-1959$ & 47 & 25 & 46 & 24 & \\
\hline 2 & 47 & 25 & 46 & 24 & \\
\hline 3 & 47 & 25 & 46 & 24 & - \\
\hline 4 & 47 & 25 & 46 & 24 & \\
\hline $1-1960$ & 47 & 25 & 46 & 24 & \\
\hline 2 & 47 & 25 & 46 & 24 & \\
\hline 3 & 47 & 25 & 46 & 24 & \\
\hline 4 & 47 & 25 & 46 & 24 & \\
\hline \multicolumn{6}{|l|}{$1-1961$} \\
\hline 2 & 48 & 25 & 46 & 24 & \\
\hline
\end{tabular}


241-BY-109 SINGLE-SHELL WHC-SD-WM-ER-312, REV.0 TANK LEVEL HISTORY

\begin{tabular}{|c|c|c|c|c|c|}
\hline Year & Total & Total & Solids & Solids & LOW \\
\hline & (K gal) & (in) & ( $\mathrm{K}$ gal) & (in) & (in) \\
\hline \multirow{2}{*}{\multicolumn{6}{|c|}{3}} \\
\hline & & & & & \\
\hline 4 & 51 & 26 & 46 & 24 & \\
\hline \multicolumn{6}{|l|}{$1-1962$} \\
\hline 2 & 623 & 234 & 46 & 24 & \\
\hline \multicolumn{6}{|l|}{3} \\
\hline 4 & 623 & 234 & 46 & 24 & \\
\hline \multicolumn{6}{|l|}{$1-1963$} \\
\hline 2 & 623 & 234 & 76 & 35 & \\
\hline \multicolumn{6}{|l|}{3} \\
\hline 4 & 634 & 238 & 76 & 35 & \\
\hline \multicolumn{6}{|l|}{$1-1964$} \\
\hline 2 & 629 & 236 & 76 & 35 & \\
\hline \multicolumn{6}{|l|}{3} \\
\hline 4 & 623 & 234 & 76 & 35 & \\
\hline \multicolumn{6}{|l|}{$1-1965$} \\
\hline 2 & 642 & 241 & 57 & 28 & \\
\hline 3 & 642 & 241 & 57 & 28 & \\
\hline 4 & 642 & 241 & 57 & 28 & \\
\hline $1-1966$ & 642 & 241 & 57 & 28 & \\
\hline 2 & 642 & 241 & 57 & 28 & \\
\hline 3 & 139 & 58 & 57 & 28 & \\
\hline 4 & 486 & 184 & 57 & 28 & \\
\hline $1-1967$ & 642 & 241 & 57 & 28 & \\
\hline 2 & 742 & 277 & 57 & 28 & \\
\hline 3 & 742 & 277 & 57 & 28 & \\
\hline 4 & 742 & 277 & 57 & 28 & \\
\hline $1-1968$ & 743 & 278 & 57 & 28 & \\
\hline 2 & 269 & 105 & 57 & 28 & \\
\hline 3 & 556 & 210 & 37 & 21 & \\
\hline 4 & 596 & 224 & 54 & 27 & \\
\hline $1-1969$ & 558 & 210 & 72 & 34 & \\
\hline 2 & 596 & 224 & 87 & 39 & \\
\hline 3 & 655 & 246 & 79 & 36 & \\
\hline 4 & 593 & 223 & 114 & 49 & \\
\hline $1-1970$ & 607 & 228 & 190 & 77 & \\
\hline 2 & 574 & 216 & 120 & 51 & \\
\hline 3 & 579 & 218 & 128 & 54 & \\
\hline 4 & 598 & 225 & 142 & 59 & \\
\hline $1-1971$ & 589 & 222 & 150 & 62 & - \\
\hline 2 & 608 & 229 & 128 & 54 & \\
\hline 3 & 618 & 232 & 128 & 54 & \\
\hline 4 & 598 & 225 & 153 & 63 & \\
\hline $1-1972$ & 623 & 234 & 153 & 63 & \\
\hline 2 & 600 & 226 & 222 & 88 & \\
\hline 3 & 620 & 233 & 260 & 102 & \\
\hline 4 & 612 & 230 & 334 & 129 & \\
\hline
\end{tabular}


241-BY-109 SINGLE-SHELL WHC-SD-WM-ER-312, ReV.0 TANK LEVEL HISTORY

\begin{tabular}{|c|c|c|c|c|c|}
\hline Year & Total & Total & Solids & Solids & LOW \\
\hline & (K gal) & (in) & (K gal) & (in) & (in) \\
\hline & & & & & \\
\hline $1-1973$ & 613 & 230 & 381 & 146 & \\
\hline 2 & 602 & 226 & 398 & 152 & \\
\hline 3 & 603 & 227 & 398 & 152 & \\
\hline 4 & 587 & 221 & 411 & 157 & \\
\hline $1-1974$ & 594 & 223 & 411 & 157 & \\
\hline 2 & 581 & 219 & 433 & 165 & \\
\hline 3 & 602 & 226 & 433 & 165 & \\
\hline 4 & 557 & 210 & 425 & 162 & \\
\hline $1-1975$ & 571 & 215 & 425 & 162 & \\
\hline 2 & 541 & 204 & 425 & 162 & \\
\hline 3 & 565 & 213 & 425 & 162 & \\
\hline 4 & 598 & 225 & 425 & 162 & \\
\hline $1-1976$ & 576 & 217 & 425 & 162 & \\
\hline 2 & 585 & 220 & 425 & 162 & \\
\hline 3 & 593 & 223 & 425 & 162 & \\
\hline 4 & 596 & 224 & 425 & 162 & \\
\hline $1-1977$ & 459 & 174 & 425 & 162 & \\
\hline 2 & 442 & 168 & 433 & 165 & \\
\hline 3 & 444 & 169 & 433 & 165 & \\
\hline 4 & 447 & 170 & 433 & 165 & \\
\hline $1-1978$ & 458 & 174 & 433 & 165 & \\
\hline 2 & 455 & 173 & 433 & 165 & \\
\hline 3 & 458 & 174 & 433 & 165 & \\
\hline 4 & 461 & 175 & 433 & 165 & \\
\hline $1-1979$ & 466 & 177 & 433 & 165 & \\
\hline 2 & 466 & 177 & 433 & 165 & \\
\hline 3 & 466 & 177 & 433 & 165 & \\
\hline 4 & 466 & 177 & 433 & 165 & \\
\hline $1-1980$ & 466 & 177 & 433 & 165 & \\
\hline 2 & 466 & 177 & 433 & 165 & \\
\hline 3 & 466 & 177 & 433 & 165 & \\
\hline 4 & 466 & 177 & 433 & 165 & \\
\hline $1-1981$ & 466 & 177 & 433 & 165 & \\
\hline 2 & 466 & 177 & 433 & 165 & \\
\hline 3 & 466 & 177 & 433 & 165 & \\
\hline 4 & 466 & 177 & 433 & 165 & \\
\hline $1-1982$ & 466 & 177 & 433 & 165 & \\
\hline 2 & 466 & 177 & 433 & 165 & \\
\hline 3 & 466 & 177 & 433 & 165 & $\cdot$ \\
\hline 4 & 466 & 177 & 433 & 165 & \\
\hline $1-1983$ & 466 & 177 & 433 & 165 & \\
\hline 2 & 466 & 177 & 433 & 165 & \\
\hline 3 & 466 & 177 & 433 & 165 & \\
\hline 4 & 466 & 177 & 433 & 165 & \\
\hline $1-1984$ & 474 & 180 & 441 & 168 & \\
\hline 2 & 474 & 180 & 441 & 168 & \\
\hline
\end{tabular}


241-BY-109 SINGLE-SHELL WHC-SD-WM-ER-312, ReV.0 TANK LEVEL HISTORY

\begin{tabular}{|c|c|c|c|c|c|}
\hline Year & Total & Total & Solids & Solids & LOW \\
\hline & (K gal) & (in) & ( $\mathrm{K}$ gal) & (in) & (in) \\
\hline & & & & & \\
\hline 3 & 474 & 180 & 441 & 168 & \\
\hline 4 & 474 & 180 & 441 & 168 & \\
\hline $1-1985$ & 474 & 180 & 441 & 168 & \\
\hline 2 & 474 & 180 & 441 & 168 & \\
\hline 3 & 474 & 180 & 441 & 168 & \\
\hline 4 & 474 & 180 & 441 & 168 & \\
\hline $1-1986$ & 474 & 180 & 441 & 168 & 175 \\
\hline 2 & 474 & 180 & 441 & 168 & 176 \\
\hline 3 & 474 & 180 & 441 & 168 & 176 \\
\hline 4 & 474 & 180 & 441 & 168 & 177 \\
\hline $1-1987$ & 474 & 180 & 441 & 168 & 177 \\
\hline 2 & 474 & 180 & 441 & 168 & 177 \\
\hline 3 & 474 & 180 & 441 & 168 & 176 \\
\hline 4 & 474 & 180 & 441 & 168 & 176 \\
\hline $1-1988$ & 474 & 180 & 441 & 168 & 177 \\
\hline 2 & 474 & 180 & 441 & 168 & 177 \\
\hline 3 & 474 & 180 & 441 & 168 & 177 \\
\hline 4 & 474 & 180 & 441 & 168 & 177 \\
\hline $1-1989$ & 474 & 180 & 441 & 168 & 177 \\
\hline 2 & 474 & 180 & 441 & 168 & 177 \\
\hline 3 & 474 & 180 & 441 & 168 & 177 \\
\hline 4 & 474 & 180 & 441 & 168 & 177 \\
\hline $1-1990$ & 474 & 180 & 441 & 168 & 177 \\
\hline 2 & 474 & 180 & 441 & 168 & \\
\hline 3 & 474 & 180 & 441 & 168 & 177 \\
\hline 4 & 474 & 180 & 441 & 168 & 178 \\
\hline 1-1991 & 474 & 180 & 441 & 168 & 179 \\
\hline 2 & 458 & 174 & 441 & 168 & 180 \\
\hline 3 & 423 & 161 & 423 & 161 & 153 \\
\hline 4 & 423 & 161 & 423 & 161 & 145 \\
\hline $1-1992$ & 423 & 161 & 423 & 161 & \\
\hline 2 & 423 & 161 & 423 & 161 & \\
\hline 3 & 423 & 161 & 423 & 161 & \\
\hline 4 & 423 & 161 & 423 & 161 & 136 \\
\hline $1-1993$ & 423 & 161 & 423 & 161 & 135 \\
\hline 2 & 423 & 161 & 423 & 161 & 136 \\
\hline 3 & & & & & 135 \\
\hline 4 & & & & & \\
\hline
\end{tabular}


241-BY-110 SINGLE-SHELL WHC-SD-WM-ER-312, REV. 0 TANK LEVEL HISTORY

\begin{tabular}{|c|c|c|c|c|c|}
\hline Year & Total & Total & Solids & Solids & LOW \\
\hline & ( $\mathrm{K}$ gal) & (in) & (K gal) & (in) & (in) \\
\hline & & & & & \\
\hline $1-1950$ & & & & & \\
\hline \multicolumn{6}{|l|}{2} \\
\hline \multicolumn{6}{|l|}{3} \\
\hline \multicolumn{6}{|l|}{4} \\
\hline \multicolumn{6}{|l|}{$1-1951$} \\
\hline \multicolumn{6}{|l|}{2} \\
\hline \multicolumn{6}{|l|}{3} \\
\hline \multicolumn{6}{|l|}{4} \\
\hline $1-1952$ & 638 & 239 & & & \\
\hline 2 & 653 & 245 & & & \\
\hline 3 & 722 & 270 & & & \\
\hline 4 & 732 & 274 & & & \\
\hline $1-1953$ & 732 & 274 & & & \\
\hline 2 & 731 & 273 & & & \\
\hline 3 & 731 & 273 & & & \\
\hline 4 & 731 & 273 & & & \\
\hline $1-1954$ & 731 & 273 & & & \\
\hline 2 & 731 & 273 & & & \\
\hline 3 & 37 & 21 & & & \\
\hline 4 & 261 & 102 & 51 & 26 & \\
\hline 1-1955 & 729 & 273 & 121 & 51 & \\
\hline 2 & 640 & 240 & 85 & 38 & \\
\hline 3 & 238 & 94 & & & \\
\hline 4 & 722 & 270 & 148 & 61 & \\
\hline $1-1956$ & 724 & 271 & 150 & 62 & \\
\hline 2 & 724 & 271 & & & \\
\hline 3 & 722 & 270 & 211 & 84 & \\
\hline 4 & 229 & 91 & 229 & 91 & \\
\hline $1-1957$ & 442 & 168 & 210 & 84 & \\
\hline 2 & 697 & 261 & 205 & 82 & \\
\hline 3 & 224 & 89 & 211 & 84 & \\
\hline 4 & 304 & 118 & 210 & 84 & \\
\hline $1-1958$ & 607 & 228 & 210 & 84 & \\
\hline 2 & 604 & 227 & 210 & 84 & \\
\hline 3 & 611 & 230 & 210 & 84 & \\
\hline 4 & 717 & 268 & 210 & 84 & \\
\hline $1-1959$ & 717 & 268 & 210 & 84 & \\
\hline 2 & 716 & 268 & 210 & 84 & \\
\hline 3 & 716 & 268 & 210 & 84 & $\dot{-}$ \\
\hline 4 & 739 & 276 & 210 & 84 & \\
\hline $1-1960$ & 739 & 276 & 210 & 84 & \\
\hline 2 & 739 & 276 & 210 & 84 & \\
\hline 3 & 739 & 276 & 210 & 84 & \\
\hline 4 & 739 & 276 & 210 & 84 & \\
\hline $1-1961$ & & & & & \\
\hline 2 & 708 & 265 & 210 & 84 & \\
\hline
\end{tabular}


241-BY-110 SINGLE-SHELL WHC-SD-WM-ER-312, ReV.0 TANK LEVEL HISTORY

\begin{tabular}{|c|c|c|c|c|c|}
\hline Year & Total & Total & Solids & Solids & LOW \\
\hline & (K gal) & (in) & (K gal) & (in) & (in) \\
\hline \multicolumn{6}{|l|}{3} \\
\hline 4 & 708 & 265 & 210 & 84 & \\
\hline \multicolumn{6}{|l|}{$1-1962$} \\
\hline 2 & 708 & 265 & 210 & 84 & \\
\hline \multicolumn{6}{|l|}{3} \\
\hline 4 & 708 & 265 & 210 & 84 & \\
\hline \multicolumn{6}{|l|}{$1-1963$} \\
\hline 2 & 706 & 264 & 246 & 97 & \\
\hline \multicolumn{6}{|l|}{3} \\
\hline 4 & 706 & 264 & 246 & 97 & \\
\hline \multicolumn{6}{|l|}{$1-1964$} \\
\hline 2 & 706 & 264 & 246 & 97 & \\
\hline \multicolumn{6}{|l|}{3} \\
\hline 4 & 706 & 264 & 246 & 97 & \\
\hline \multicolumn{6}{|l|}{$1-1965$} \\
\hline 2 & 703 & 263 & 230 & 91 & \\
\hline 3 & 706 & 264 & 230 & 91 & \\
\hline 4 & 713 & 267 & 230 & 91 & \\
\hline $1-1966$ & 706 & 264 & 230 & 91 & \\
\hline 2 & 706 & 264 & 230 & 91 & \\
\hline 3 & 700 & 262 & 230 & 91 & \\
\hline 4 & 700 & 262 & 230 & 91 & \\
\hline $1-1967$ & 706 & 264 & 230 & 91 & \\
\hline 2 & 697 & 261 & 230 & 91 & \\
\hline 3 & 693 & 259 & 230 & 91 & \\
\hline 4 & 693 & 259 & 230 & 91 & \\
\hline $1-1968$ & 706 & 264 & 230 & 91 & \\
\hline 2 & 706 & 264 & 230 & 91 & \\
\hline 3 & 706 & 264 & 230 & 91 & \\
\hline 4 & 706 & 264 & 230 & 91 & \\
\hline $1-1969$ & 701 & 262 & 230 & 91 & \\
\hline 2 & 642 & 241 & 189 & 76 & \\
\hline 3 & 692 & 259 & 123 & 52 & \\
\hline 4 & 703 & 263 & 123 & 52 & \\
\hline $1-1970$ & 627 & 235 & 201 & 81 & \\
\hline 2 & 519 & 196 & 190 & 77 & \\
\hline 3 & 664 & 249 & 211 & 84 & \\
\hline 4 & 697 & 261 & 277 & 108 & \\
\hline $1-1971$ & 675 & 253 & 277 & 108 & . \\
\hline 2 & 690 & 258 & 277 & 108 & \\
\hline 3 & 708 & 265 & 277 & 108 & \\
\hline 4 & 645 & 242 & 277 & 108 & \\
\hline $1-1972$ & 628 & 236 & 277 & 108 & \\
\hline 2 & 714 & 267 & 277 & 108 & \\
\hline 3 & 667 & 250 & 277 & 108 & \\
\hline 4 & 644 & 242 & 277 & 108 & \\
\hline
\end{tabular}


241-BY-110 SINGLE-SHELL WHC-SD-WM-ER-3I2, REV.0 TANK LEVEL HISTORY

\begin{tabular}{|c|c|c|c|c|c|}
\hline Year & Total & Total & Solids & Solids & LOW \\
\hline & ( $\mathrm{K}$ gall) & (in) & (K gal) & (in) & (in) \\
\hline $1-1973$ & 672 & 252 & 277 & 108 & \\
\hline 2 & 653 & 245 & 277 & 108 & \\
\hline 3 & 706 & 264 & 277 & 108 & \\
\hline 4 & 673 & 252 & 277 & 108 & \\
\hline $1-1974$ & 670 & 251 & 277 & 108 & \\
\hline 2 & 690 & 258 & 277 & 108 & \\
\hline 3 & 688 & 258 & 277 & 108 & \\
\hline 4 & 689 & 258 & 296 & 115 & \\
\hline $1-1975$ & 684 & 256 & 296 & 115 & \\
\hline 2 & 645 & 242 & 296 & 115 & \\
\hline 3 & 667 & 250 & 296 & 115 & \\
\hline 4 & 673 & 252 & 296 & 115 & \\
\hline $1-1976$ & 684 & 256 & 296 & 115 & \\
\hline 2 & 684 & 256 & 296 & 115 & \\
\hline 3 & 678 & 254 & 296 & 115 & \\
\hline 4 & 678 & 254 & 296 & 115 & \\
\hline $1-1977$ & 609 & 229 & 376 & 144 & \\
\hline 2 & 532 & 201 & 376 & 144 & \\
\hline 3 & 549 & 207 & 469 & 178 & \\
\hline 4 & 560 & 211 & 469 & 178 & \\
\hline $1-1978$ & 571 & 215 & 469 & 178 & \\
\hline 2 & 579 & 218 & 469 & 178 & \\
\hline 3 & 582 & 219 & 469 & 178 & \\
\hline 4 & 585 & 220 & 469 & 178 & \\
\hline $1-1979$ & 585 & 220 & 469 & 178 & \\
\hline 2 & 552 & 208 & 469 & 178 & \\
\hline 3 & 530 & 200 & 469 & 178 & \\
\hline 4 & 530 & 200 & 469 & 178 & \\
\hline $1-1980$ & 508 & 192 & 505 & 191 & \\
\hline 2 & 508 & 192 & 505 & 191 & \\
\hline 3 & 508 & 192 & 505 & 191 & \\
\hline 4 & 508 & 192 & 505 & 191 & \\
\hline $1-1981$ & 508 & 192 & 505 & 191 & \\
\hline 2 & 508 & 192 & 505 & 191 & \\
\hline 3 & 508 & 192 & 505 & 191 & \\
\hline 4 & 508 & 192 & 505 & 191 & \\
\hline $1-1982$ & 508 & 192 & 505 & 191 & \\
\hline 2 & 508 & 192 & 505 & 191 & \\
\hline 3 & 508 & 192 & 505 & 191 & $\cdot$ \\
\hline 4 & 508 & 192 & 505 & 191 & \\
\hline $1-1983$ & 508 & 192 & 505 & 191 & \\
\hline 2 & 507 & 192 & 505 & 191 & \\
\hline 3 & 420 & 160 & 420 & 160 & \\
\hline 4 & 420 & 160 & 420 & 160 & \\
\hline $1-1984$ & 404 & 154 & 404 & 154 & \\
\hline 2 & 404 & 154 & 404 & 154 & \\
\hline
\end{tabular}


241-BY-110 SINGLE-SHELL WHC-SD-WM-ER-312, REV. 0 TANK LEVEL HISTORY

\begin{tabular}{|l|c|c|c|c|c|}
\hline \multicolumn{1}{|c|}{ Year } & Total & Total & Solids & Solids & LOW \\
\hline & (K gal) & (in) & (K gal) & (in) & (in) \\
\hline 3 & & & & & \\
\hline 4 & 400 & 153 & 400 & 153 & \\
\hline $1-1985$ & 400 & 153 & 400 & 153 & \\
\hline 2 & 398 & 152 & 398 & 152 & \\
\hline 3 & 398 & 152 & 398 & 152 & \\
\hline 4 & 398 & 152 & 398 & 152 & \\
\hline $1-1986$ & 398 & 152 & 398 & 152 & \\
\hline 2 & 398 & 152 & 398 & 152 & 86 \\
\hline 3 & 398 & 152 & 398 & 152 & 87 \\
\hline 4 & 398 & 152 & 398 & 152 & 85 \\
\hline $1-1987$ & 398 & 152 & 398 & 152 & 87 \\
\hline 2 & 398 & 152 & 398 & 152 & 85 \\
\hline 3 & 398 & 152 & 398 & 152 & 86 \\
\hline 4 & 398 & 152 & 398 & 152 & 87 \\
\hline $1-1988$ & 398 & 152 & 398 & 152 & 86 \\
\hline 2 & 398 & 152 & 398 & 152 & 85 \\
\hline 3 & 398 & 152 & 398 & 152 & 87 \\
\hline 4 & 398 & 152 & 398 & 152 & 86 \\
\hline $1-1989$ & 398 & 152 & 398 & 152 & 87 \\
\hline 2 & 398 & 152 & 398 & 152 & 85 \\
\hline 3 & 398 & 152 & 398 & 152 & 86 \\
\hline 4 & 398 & 152 & 398 & 152 & 86 \\
\hline $1-1990$ & 398 & 152 & 398 & 152 & 85 \\
\hline 2 & 398 & 152 & 398 & 152 & \\
\hline 3 & 398 & 152 & 398 & 152 & 85 \\
\hline 4 & 398 & 152 & 398 & 152 & 85 \\
\hline $1-1991$ & 398 & 152 & 398 & 152 & 84 \\
\hline 2 & 398 & 152 & 398 & 152 & 84 \\
\hline 3 & 398 & 152 & 398 & 152 & 84 \\
\hline 4 & 398 & 152 & 398 & 152 & 84 \\
\hline $1-1992$ & 398 & 152 & 398 & 152 & 85 \\
\hline 2 & 398 & 152 & 398 & 152 & \\
\hline 3 & 398 & 152 & 398 & 152 & \\
\hline 4 & 398 & 152 & 398 & 152 & 85 \\
\hline $1-1993$ & 398 & 152 & 398 & 152 & 85 \\
\hline 2 & 398 & 152 & 398 & 152 & 84 \\
\hline 3 & & & & & 84 \\
\hline 4 & & & & & 84 \\
\hline
\end{tabular}


241-BY-111 SINGLE-SHELL WHC-SD-WM-ER-312, ReV.0 TANK LEVEL HISTORY

\begin{tabular}{|c|c|c|c|c|c|}
\hline Year & Total & Total & Solids & Solids & LOW \\
\hline & ( $\mathrm{K}$ gal) & (in) & (K gal) & (in) & (in) \\
\hline \multirow{2}{*}{\multicolumn{6}{|c|}{$1-1950$}} \\
\hline & & & & & \\
\hline \multicolumn{6}{|l|}{2} \\
\hline \multicolumn{6}{|l|}{3} \\
\hline \multicolumn{6}{|l|}{4} \\
\hline \multicolumn{6}{|l|}{$1-1951$} \\
\hline \multicolumn{6}{|l|}{2} \\
\hline \multicolumn{6}{|l|}{3} \\
\hline \multicolumn{6}{|l|}{4} \\
\hline $1-1952$ & 758 & 283 & & & \\
\hline 2 & 758 & 283 & & & \\
\hline 3 & 758 & 283 & & & \\
\hline 4 & 758 & 283 & & & \\
\hline $1-1953$ & 759 & 283 & & & \\
\hline 2 & 758 & 283 & & & \\
\hline 3 & 758 & 283 & & & \\
\hline 4 & 758 & 283 & & & \\
\hline $1-1954$ & 758 & 283 & & & \\
\hline 2 & 758 & 283 & & & \\
\hline 3 & 758 & 283 & & & \\
\hline 4 & 24 & 16 & & & \\
\hline $1-1955$ & 11 & 11 & & & \\
\hline \multicolumn{6}{|l|}{2} \\
\hline \multicolumn{6}{|l|}{3} \\
\hline \multicolumn{6}{|l|}{4} \\
\hline \multicolumn{6}{|l|}{$1-1956$} \\
\hline 2 & 526 & 199 & & & \\
\hline 3 & 26 & 17 & & & \\
\hline 4 & 26 & 17 & & & \\
\hline $1-1957$ & 35 & 20 & 26 & 17 & \\
\hline 2 & 706 & 264 & 26 & 17 & \\
\hline 3 & 706 & 264 & 26 & 17 & \\
\hline 4 & 706 & 264 & 26 & 17 & \\
\hline $1-1958$ & 706 & 264 & 26 & 17 & \\
\hline 2 & 706 & 264 & 26 & 17 & \\
\hline 3 & 706 & 264 & 26 & 17 & \\
\hline 4 & 706 & 264 & 26 & 17 & \\
\hline $1-1959$ & 711 & 266 & 26 & 17 & \\
\hline 2 & 711 & 266 & 26 & 17 & \\
\hline 3 & 711 & 266 & 26 & 17 & - \\
\hline 4 & 711 & 266 & 26 & 17 & \\
\hline $1-1960$ & 711 & 266 & 26 & 17 & \\
\hline 2 & 711 & 266 & 26 & 17 & \\
\hline 3 & 711 & 266 & 26 & 17 & \\
\hline 4 & 711 & 266 & 26 & 17 & \\
\hline \multicolumn{6}{|l|}{$1-1961$} \\
\hline 2 & 711 & 266 & 26 & 17 & \\
\hline
\end{tabular}


241-BY-111 SINGLE-SHELL WHC-SD-WM-ER-312, ReV.0 TANK LEVEL HISTORY

\begin{tabular}{|c|c|c|c|c|c|}
\hline Year & Total & Total & Solids & Solids & LOW \\
\hline & (K gal) & (in) & (K gal) & (in) & (in) \\
\hline 3 & & & & & \\
\hline 4 & 614 & 231 & 26 & 17 & \\
\hline $1-1962$ & & & & & \\
\hline 2 & 714 & 267 & 26 & 17 & \\
\hline 3 & & & & & \\
\hline 4 & 714 & 267 & 26 & 17 & \\
\hline $1-1963$ & & & & & \\
\hline 2 & 717 & 268 & 24 & 16 & \\
\hline 3 & & & & & \\
\hline 4 & 717 & 268 & 24 & 16 & \\
\hline $1-1964$ & & & & & \\
\hline 2 & 717 & 268 & 24 & 16 & \\
\hline 3 & & & & & \\
\hline 4 & 717 & 268 & 24 & 16 & \\
\hline $1-1965$ & & & & & \\
\hline 2 & 739 & 276 & 26 & 17 & \\
\hline 3 & 367 & 141 & 26 & 17 & \\
\hline 4 & 343 & 132 & 26 & 17 & \\
\hline $1-1966$ & 29 & 18 & 26 & 17 & \\
\hline 2 & 585 & 220 & 26 & 17 & \\
\hline 3 & 741 & 277 & 26 & 17 & \\
\hline 4 & 741 & 277 & 26 & 17 & \\
\hline $1-1967$ & 739 & 276 & 26 & 17 & \\
\hline 2 & 739 & 276 & 26 & 17 & \\
\hline 3 & 739 & 276 & 26 & 17 & \\
\hline 4 & 752 & 281 & 26 & 17 & \\
\hline \begin{tabular}{|l|}
$1-1968$ \\
\end{tabular} & 36 & 21 & 26 & 17 & \\
\hline 2 & 629 & 236 & 26 & 17 & \\
\hline 3 & 576 & 217 & 145 & 60 & \\
\hline 4 & 640 & 240 & 145 & 60 & \\
\hline $1-1969$ & 541 & 204 & 48 & 25 & \\
\hline 2 & 600 & 226 & 52 & 26 & \\
\hline 3 & 570 & 215 & 101 & 44 & \\
\hline 4 & 642 & 241 & 142 & 59 & \\
\hline $1-1970$ & 618 & 232 & 266 & 104 & \\
\hline 2 & 668 & 250 & 290 & 113 & \\
\hline 3 & 666 & 250 & 323 & 125 & \\
\hline 4 & 695 & 260 & 673 & 252 & \\
\hline $1-1971$ & 685 & 257 & 673 & 252 & \\
\hline 2 & 693 & 259 & 686 & 257 & \\
\hline 3 & 697 & 261 & 686 & 257 & \\
\hline 4 & 692 & 259 & 686 & 257 & \\
\hline $1-1972$ & 688 & 258 & 194 & 78 & \\
\hline 2 & 686 & 257 & 112 & 48 & \\
\hline 3 & 653 & 245 & 142 & 59 & \\
\hline 4 & 662 & 248 & 142 & 59 & \\
\hline
\end{tabular}


241-BY-111 SINGLE-SHELL WHC-SD-WM-ER-312, ReV.0 TANK LEVEL HISTORY

\begin{tabular}{|c|c|c|c|c|c|}
\hline Year & Total & Total & Solids & Solids & LOW \\
\hline & (K gal) & (in) & (K gal) & (in) & (in) \\
\hline & & & & & \\
\hline $1-1973$ & 650 & 244 & 142 & 59 & \\
\hline 2 & 649 & 243 & 142 & 59 & \\
\hline 3 & 624 & 234 & 142 & 59 & \\
\hline 4 & 677 & 254 & 142 & 59 & \\
\hline $1-1974$ & 677 & 254 & 142 & 59 & \\
\hline 2 & 711 & 266 & 142 & 59 & \\
\hline 3 & 673 & 252 & 142 & 59 & \\
\hline 4 & 673 & 252 & 299 & 116 & \\
\hline $1-1975$ & 673 & 252 & 299 & 116 & \\
\hline 2 & 673 & 252 & 299 & 116 & \\
\hline 3 & 673 & 252 & 299 & 116 & \\
\hline 4 & 670 & 251 & 299 & 116 & \\
\hline 1-1976 & 670 & 251 & 299 & 116 & \\
\hline 2 & 670 & 251 & 299 & 116 & \\
\hline 3 & 670 & 251 & 299 & 116 & \\
\hline 4 & 670 & 251 & 299 & 116 & \\
\hline $1-1977$ & 670 & 251 & 299 & 116 & \\
\hline 2 & 615 & 231 & 299 & 116 & \\
\hline 3 & 510 & 193 & 510 & 193 & \\
\hline 4 & 615 & 231 & 615 & 231 & \\
\hline $1-1978$ & 615 & 231 & 615 & 231 & \\
\hline 2 & 615 & 231 & 615 & 231 & \\
\hline 3 & 615 & 231 & 615 & 231 & \\
\hline 4 & 615 & 231 & 615 & 231 & \\
\hline $1-1979$ & 615 & 231 & 615 & 231 & \\
\hline 2 & 615 & 231 & 615 & 231 & \\
\hline 3 & 615 & 231 & 615 & 231 & \\
\hline 4 & 615 & 231 & 615 & 231 & \\
\hline $1-1980$ & 615 & 231 & 615 & 231 & \\
\hline 2 & 615 & 231 & 615 & 231 & \\
\hline 3 & 615 & 231 & 615 & 231 & \\
\hline 4 & 622 & 234 & 622 & 234 & \\
\hline $1-1981$ & 622 & 234 & 622 & 234 & \\
\hline 2 & 622 & 234 & 622 & 234 & \\
\hline 3 & 622 & 234 & 622 & 234 & \\
\hline 4 & 622 & 234 & 622 & 234 & \\
\hline $1-1982$ & 622 & 234 & 622 & 234 & \\
\hline 2 & 649 & 243 & 648 & 243 & \\
\hline 3 & 649 & 243 & 648 & 243 & $\dot{.}$ \\
\hline 4 & 649 & 243 & 648 & 243 & \\
\hline $1-1983$ & 649 & 243 & 648 & 243 & \\
\hline 2 & 649 & 243 & 648 & 243 & \\
\hline 3 & 648 & 243 & 648 & 243 & \\
\hline 4 & 648 & 243 & 648 & 243 & \\
\hline $1-1984$ & 466 & 177 & 466 & 177 & \\
\hline 2 & 466 & 177 & 466 & 177 & \\
\hline
\end{tabular}


241-BY-111 SINGLE-SHELL WHC-SD-WM-ER-312, ReV.0 TANK LEVEL HISTORY

\begin{tabular}{|l|c|c|c|c|c|}
\hline \multicolumn{1}{|c|}{ Year } & Total & Total & Solids & Solids & LOW \\
\hline & (K gal) & (in) & (K gal) & (in) & (in) \\
\hline 3 & & & & & \\
\hline 4 & 461 & 175 & 461 & 175 & \\
\hline $1-1985$ & 462 & 175 & 461 & 175 & \\
\hline 2 & 459 & 174 & 459 & 174 & \\
\hline 3 & 459 & 174 & 459 & 174 & \\
\hline 4 & 459 & 174 & 459 & 174 & \\
\hline $1-1986$ & 459 & 174 & 459 & 174 & \\
\hline 2 & 459 & 174 & 459 & 174 & 86 \\
\hline 3 & 459 & 174 & 459 & 174 & 87 \\
\hline 4 & 459 & 174 & 459 & 174 & 85 \\
\hline $1-1987$ & 459 & 174 & 459 & 174 & 87 \\
\hline 2 & 459 & 174 & 459 & 174 & 85 \\
\hline 3 & 459 & 174 & 459 & 174 & 86 \\
\hline 4 & 459 & 174 & 459 & 174 & 87 \\
\hline $1-1988$ & 459 & 174 & 459 & 174 & 88 \\
\hline 2 & 459 & 174 & 459 & 174 & 85 \\
\hline 3 & 459 & 174 & 459 & 174 & 87 \\
\hline 4 & 459 & 174 & 459 & 174 & 89 \\
\hline $1-1989$ & 459 & 174 & 459 & 174 & 87 \\
\hline 2 & 459 & 174 & 459 & 174 & 85 \\
\hline 3 & 459 & 174 & 459 & 174 & 86 \\
\hline 4 & 459 & 174 & 459 & 174 & 86 \\
\hline $1-1990$ & 459 & 174 & 459 & 174 & 85 \\
\hline 2 & 459 & 174 & 459 & 174 & \\
\hline 3 & 459 & 174 & 459 & 174 & 85 \\
\hline 4 & 459 & 174 & 459 & 174 & 85 \\
\hline $1-1991$ & 459 & 174 & 459 & 174 & 84 \\
\hline 2 & 459 & 174 & 459 & 174 & 84 \\
\hline 3 & 459 & 174 & 459 & 174 & 84 \\
\hline 4 & 459 & 174 & 459 & 174 & 84 \\
\hline $1-1992$ & 459 & 174 & 459 & 174 & 85 \\
\hline 2 & 459 & 174 & 459 & 174 & \\
\hline 3 & 459 & 174 & 459 & 174 & \\
\hline 4 & 459 & 174 & 459 & 174 & 85 \\
\hline $1-1993$ & 459 & 174 & 459 & 174 & 85 \\
\hline 2 & 459 & 174 & 459 & 174 & 84 \\
\hline 3 & & & & & 84 \\
\hline 4 & & & & & 84 \\
\hline
\end{tabular}


241-BY-112 SINGLE-SHELL WHC-SD-WM-ER-312, ReV. 0 TANK LEVEL HISTORY

\begin{tabular}{|c|c|c|c|c|c|}
\hline Year & Total & Total & Solids & Solids & LOW \\
\hline & (K gal) & (in) & (K gal) & (in) & (in) \\
\hline & & & & & \\
\hline \multicolumn{6}{|l|}{$1-1950$} \\
\hline \multicolumn{6}{|l|}{2} \\
\hline \multicolumn{6}{|l|}{3} \\
\hline \multicolumn{6}{|l|}{4} \\
\hline \multicolumn{6}{|l|}{$1-1951$} \\
\hline \multicolumn{6}{|l|}{2} \\
\hline \multicolumn{6}{|l|}{3} \\
\hline \multicolumn{6}{|l|}{4} \\
\hline $1-1952$ & 252 & 99 & & & \\
\hline 2 & 271 & 106 & & & \\
\hline 3 & 294 & 114 & & & \\
\hline 4 & 362 & 139 & & & \\
\hline $1-1953$ & 389 & 149 & & & \\
\hline 2 & 400 & 153 & & & \\
\hline 3 & 394 & 151 & & & \\
\hline 4 & 394 & 151 & & & \\
\hline $1-1954$ & 394 & 151 & & & \\
\hline 2 & 394 & 151 & & & \\
\hline 3 & 394 & 151 & & & \\
\hline 4 & 1 & 8 & & & \\
\hline $1-1955$ & 1 & 8 & & & \\
\hline 2 & 5 & 9 & & & \\
\hline \multicolumn{6}{|l|}{3} \\
\hline \multicolumn{6}{|l|}{4} \\
\hline \multicolumn{6}{|l|}{$1-1956$} \\
\hline 2 & 477 & 181 & & & \\
\hline 3 & 697 & 261 & & & \\
\hline 4 & 697 & 261 & & & \\
\hline $1-1957$ & 695 & 260 & & & \\
\hline 2 & 183 & 74 & & & \\
\hline 3 & 631 & 237 & & & \\
\hline 4 & 755 & 282 & & & \\
\hline $1-1958$ & 755 & 282 & & & \\
\hline 2 & 755 & 282 & & & \\
\hline 3 & 755 & 282 & & & \\
\hline 4 & 755 & 282 & & & \\
\hline $1-1959$ & 755 & 282 & & & \\
\hline 2 & 755 & 282 & & & \\
\hline 3 & 755 & 282 & & & 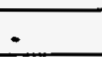 \\
\hline 4 & 755 & 282 & & & \\
\hline $1-1960$ & 755 & 282 & & & \\
\hline 2 & 741 & 277 & & & \\
\hline 3 & 741 & 277 & & & \\
\hline 4 & 741 & 277 & & & \\
\hline \multicolumn{6}{|l|}{$1-1961$} \\
\hline 2 & 741 & 277 & & & \\
\hline
\end{tabular}


241-BY-112 SINGLE-SHELL WHC-SD-WIM-ER-312, ReV.0 TANK LEVEL HISTORY

\begin{tabular}{|c|c|c|c|c|c|}
\hline Year & Total & Total & Solids & Solids & LOW \\
\hline & (K gal) & (in) & (K gal) & (in) & (in) \\
\hline 3 & & & & & \\
\hline 4 & 741 & 277 & & & \\
\hline $1-1962$ & & & & & \\
\hline 2 & 741 & 277 & & & \\
\hline 3 & & & & & \\
\hline 4 & 741 & 277 & & & \\
\hline $1-1963$ & & & & & \\
\hline 2 & 744 & 278 & 29 & 18 & \\
\hline 3 & & & & & \\
\hline 4 & 744 & 278 & 29 & 18 & \\
\hline $1-1964$ & & & & & \\
\hline 2 & 755 & 282 & 29 & 18 & \\
\hline 3 & & & & & \\
\hline 4 & 755 & 282 & 29 & 18 & \\
\hline $1-1965$ & & & & & \\
\hline 2 & 279 & 109 & 24 & 16 & \\
\hline 3 & 48 & 25 & 24 & 16 & \\
\hline 4 & 312 & 121 & 24 & 16 & \\
\hline $1-1966$ & 730 & 273 & 24 & 16 & \\
\hline 2 & 730 & 273 & 24 & 16 & \\
\hline 3 & 744 & 278 & 24 & 16 & \\
\hline 4 & 744 & 278 & 24 & 16 & \\
\hline $1-1967$ & 750 & 280 & 24 & 16 & \\
\hline 2 & 751 & 281 & 24 & 16 & \\
\hline 3 & 751 & 281 & 24 & 16 & \\
\hline 4 & 756 & 282 & 24 & 16 & \\
\hline $1-1968$ & 547 & 206 & 24 & 16 & \\
\hline 2 & 605 & 227 & 17 & 14 & \\
\hline 3 & 612 & 230 & 2 & 8 & \\
\hline 4 & 574 & 216 & 2 & 8 & \\
\hline $1-1969$ & 722 & 270 & 43 & 23 & \\
\hline 2 & 725 & 271 & 59 & 29 & \\
\hline 3 & 703 & 263 & 79 & 36 & \\
\hline 4 & 689 & 258 & 79 & 36 & \\
\hline \begin{tabular}{|l|}
$1-1970$ \\
\end{tabular} & 708 & 265 & 85 & 38 & \\
\hline 2 & 706 & 264 & 94 & 42 & \\
\hline 3 & 712 & 266 & 145 & 60 & \\
\hline 4 & 704 & 263 & 164 & 67 & \\
\hline $1-1971$ & 700 & 262 & 255 & 100 & \\
\hline 2 & 699 & 262 & 59 & 29 & \\
\hline 3 & 716 & 268 & 166 & 68 & \\
\hline 4 & 722 & 270 & 194 & 78 & \\
\hline $1-1972$ & 664 & 249 & 296 & 115 & \\
\hline 2 & 672 & 252 & 334 & 129 & \\
\hline 3 & 670 & 251 & 334 & 129 & \\
\hline 4 & 663 & 249 & 315 & 122 & \\
\hline
\end{tabular}


241-BY-112 SINGLE-SHELL WHC-SD-WM-ER-312, Rev.0 TANK LEVEL HISTORY

\begin{tabular}{|c|c|c|c|c|c|}
\hline Year & Total & Total & Solids & Solids & LOW \\
\hline & ( $\mathrm{K}$ gal) & (in) & (K gal) & (in) & (in) \\
\hline $1-1973$ & 689 & 258 & 288 & 112 & \\
\hline 2 & 679 & 254 & 288 & 112 & \\
\hline 3 & 688 & 258 & 311 & 121 & \\
\hline 4 & 703 & 263 & 331 & 128 & \\
\hline $1-1974$ & 681 & 255 & 331 & 128 & \\
\hline 2 & 702 & 263 & 331 & 128 & \\
\hline 3 & 703 & 263 & 331 & 128 & \\
\hline 4 & 711 & 266 & 310 & 120 & \\
\hline $1-1975$ & 706 & 264 & 310 & 120 & \\
\hline 2 & 706 & 264 & 310 & 120 & \\
\hline 3 & 706 & 264 & 310 & 120 & \\
\hline 4 & 673 & 252 & 310 & 120 & \\
\hline $1-1976$ & 340 & 131 & 310 & 120 & \\
\hline 2 & 310 & 120 & 310 & 120 & \\
\hline 3 & 310 & 120 & 310 & 120 & \\
\hline 4 & 310 & 120 & 310 & 120 & \\
\hline $1-1977$ & 310 & 120 & 310 & 120 & \\
\hline 2 & 310 & 120 & 310 & 120 & \\
\hline 3 & 310 & 120 & 310 & 120 & \\
\hline 4 & 310 & 120 & 310 & 120 & \\
\hline $1-1978$ & 310 & 120 & 310 & 120 & \\
\hline 2 & 310 & 120 & 310 & 120 & \\
\hline 3 & 310 & 120 & 310 & 120 & \\
\hline 4 & 310 & 120 & 310 & 120 & \\
\hline $1-1979$ & 310 & 120 & 310 & 120 & \\
\hline 2 & 310 & 120 & 310 & 120 & \\
\hline 3 & 310 & 120 & 310 & 120 & \\
\hline 4 & 310 & 120 & 310 & 120 & \\
\hline $1-1980$ & 310 & 120 & 310 & 120 & \\
\hline 2 & 310 & 120 & 310 & 120 & \\
\hline 3 & 310 & 120 & 310 & 120 & \\
\hline 4 & 310 & 120 & 310 & 120 & \\
\hline $1-1981$ & 310 & 120 & 310 & 120 & \\
\hline 2 & 310 & 120 & 310 & 120 & \\
\hline 3 & 310 & 120 & 310 & 120 & \\
\hline 4 & 310 & 120 & 310 & 120 & \\
\hline $1-1982$ & 310 & 120 & 310 & 120 & \\
\hline 2 & 304 & 118 & 301 & 117 & \\
\hline 3 & 304 & 118 & 301 & 117 & . \\
\hline 4 & 304 & 118 & 301 & 117 & \\
\hline $1-1983$ & 304 & 118 & 301 & 117 & \\
\hline 2 & 304 & 118 & 301 & 117 & \\
\hline 3 & 301 & 117 & 301 & 117 & \\
\hline 4 & 301 & 117 & 301 & 117 & \\
\hline $1-1984$ & 291 & 113 & 291 & 113 & \\
\hline 2 & 291 & 113 & 291 & 113 & \\
\hline
\end{tabular}


241-BY-112 SINGLE-SHELL WHC-SD-WM-ER-312, REV.0 TANK LEVEL HISTORY

\begin{tabular}{|c|c|c|c|c|c|}
\hline Year & Total & Total & Solids & Solids & LOW \\
\hline & (K gal) & (in) & (K gal) & (in) & (in) \\
\hline 3 & 291 & 113 & 291 & 113 & \\
\hline 4 & 291 & 113 & 291 & 113 & \\
\hline $1-1985$ & 291 & 113 & 291 & 113 & \\
\hline 2 & 291 & 113 & 291 & 113 & \\
\hline 3 & 291 & 113 & 291 & 113 & \\
\hline 4 & 291 & 113 & 291 & 113 & \\
\hline $1-1986$ & 291 & 113 & 291 & 113 & 38 \\
\hline 2 & 291 & 113 & 291 & 113 & 38 \\
\hline 3 & 291 & 113 & 291 & 113 & 38 \\
\hline 4 & 291 & 113 & 291 & 113 & 40 \\
\hline $1-1987$ & 291 & 113 & 291 & 113 & 40 \\
\hline 2 & 291 & 113 & 291 & 113 & 40 \\
\hline 3 & 291 & 113 & 291 & 113 & 40 \\
\hline 4 & 291 & 113 & 291 & 113 & 40 \\
\hline $1-1988$ & 291 & 113 & 291 & 113 & 40 \\
\hline 2 & 291 & 113 & 291 & 113 & 41 \\
\hline 3 & 291 & 113 & 291 & 113 & 42 \\
\hline 4 & 291 & 113 & 291 & 113 & 42 \\
\hline $1-1989$ & 291 & 113 & 291 & 113 & 43 \\
\hline 2 & 291 & 113 & 291 & 113 & 44 \\
\hline 3 & 291 & 113 & 291 & 113 & 43 \\
\hline 4 & 291 & 113 & 291 & 113 & 43 \\
\hline $1-1990$ & 291 & 113 & 291 & 113 & 42 \\
\hline 2 & 291 & 113 & 291 & 113 & \\
\hline 3 & 291 & 113 & 291 & 113 & 43 \\
\hline 4 & 291 & 113 & 291 & 113 & 44 \\
\hline $1-1991$ & 291 & 113 & 291 & 113 & 45 \\
\hline 2 & 291 & 113 & 291 & 113 & \\
\hline 3 & 291 & 113 & 291 & 113 & 44 \\
\hline 4 & 291 & 113 & 291 & 113 & 44 \\
\hline $1-1992$ & 291 & 113 & 291 & 113 & 45 \\
\hline 2 & 291 & 113 & 291 & 113 & \\
\hline 3 & 291 & 113 & 291 & 113 & \\
\hline 4 & 291 & 113 & 291 & 113 & 45 \\
\hline \begin{tabular}{|l|}
$1-1993$ \\
\end{tabular} & 291 & 113 & 291 & 113 & \\
\hline 2 & 291 & 113 & 291 & 113 & \\
\hline \multicolumn{6}{|l|}{3} \\
\hline 4 & & & & & \\
\hline
\end{tabular}




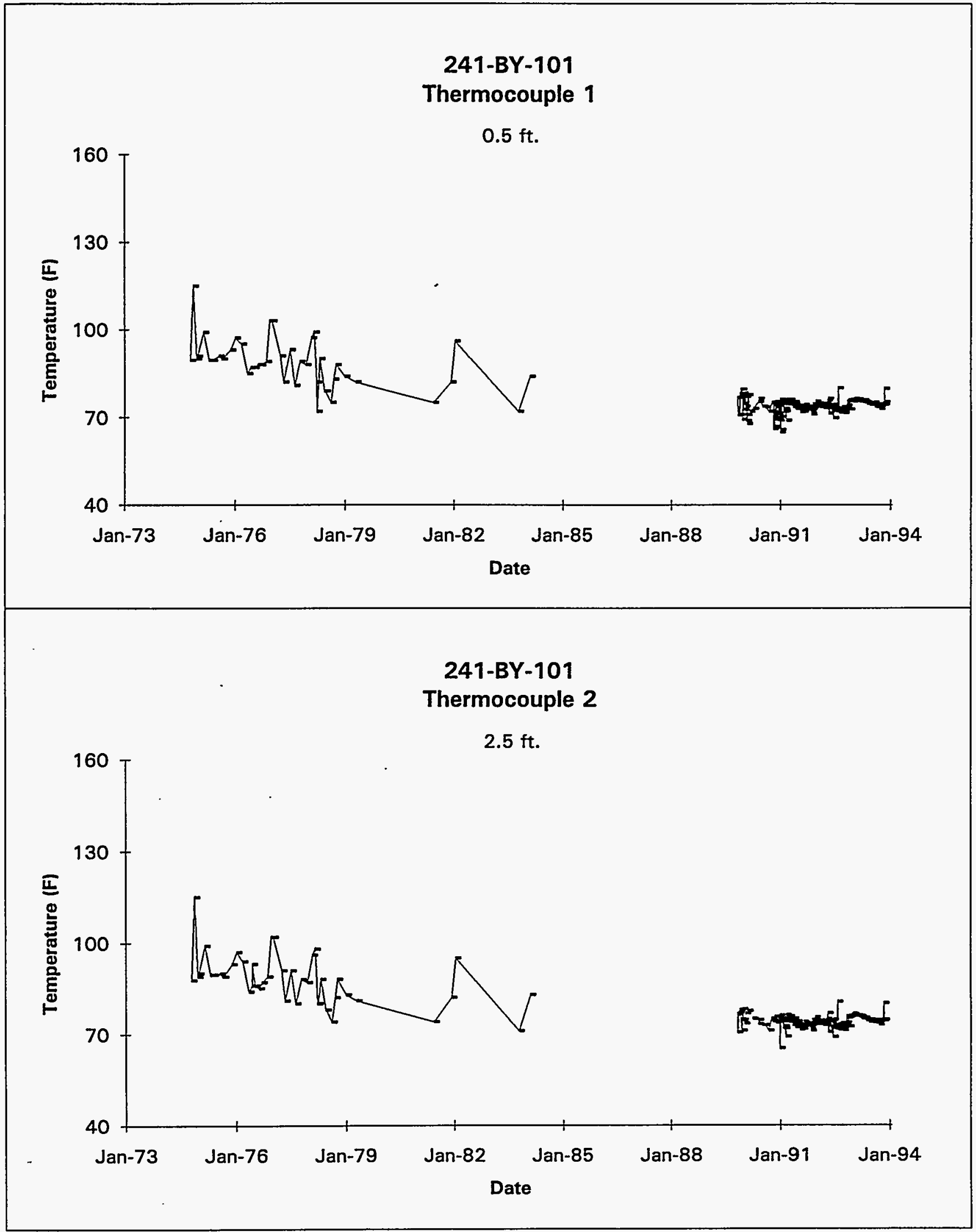

Data obtained from WHC Surveillance Analysis Computer System (SACS), November 12, 1993.

D-1 


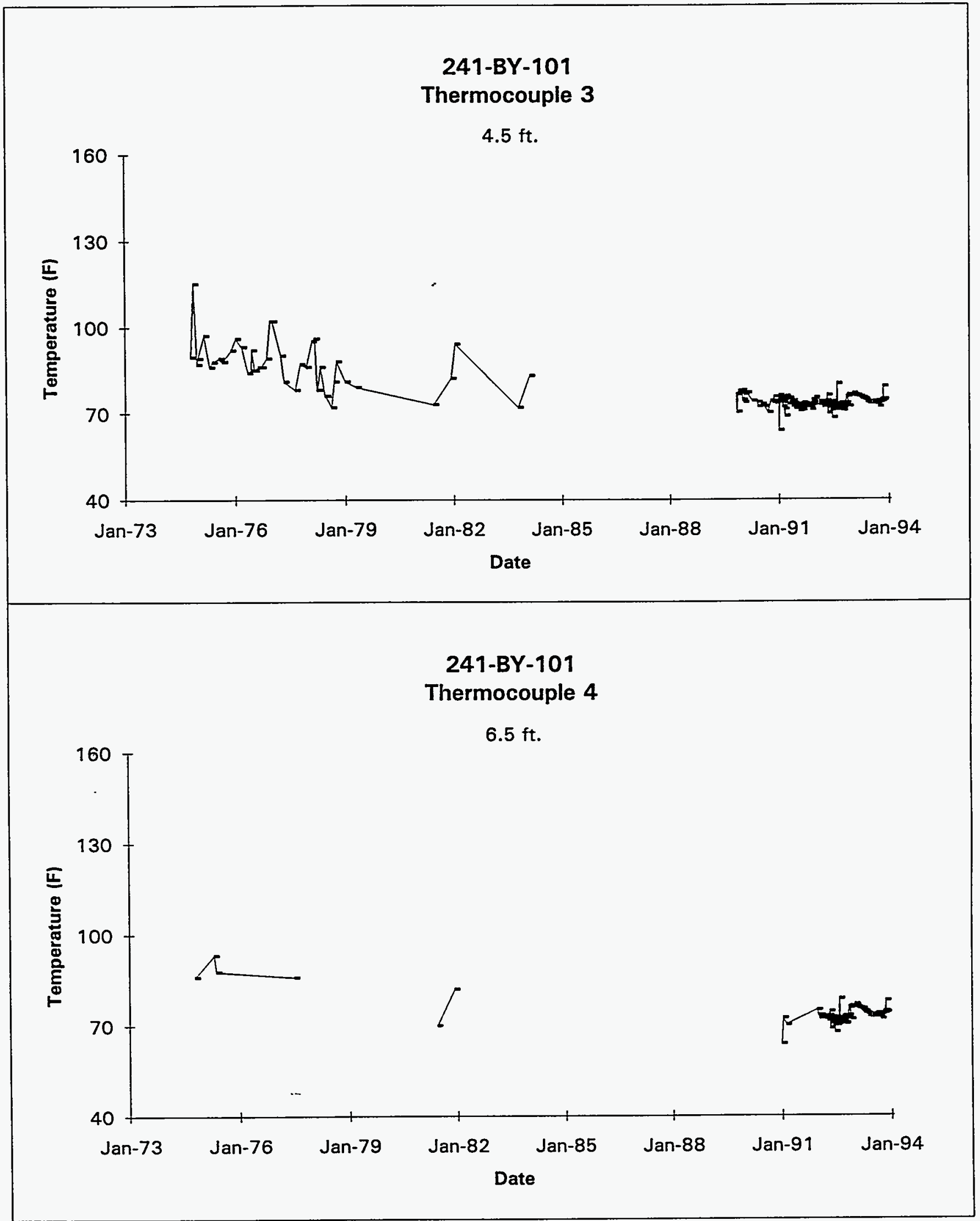

Data obtained from WHC Surveillance Analysis Computer System (SACS), November 12, 1993.

D-2 


\section{1-BY-101}

Thermocouple 5

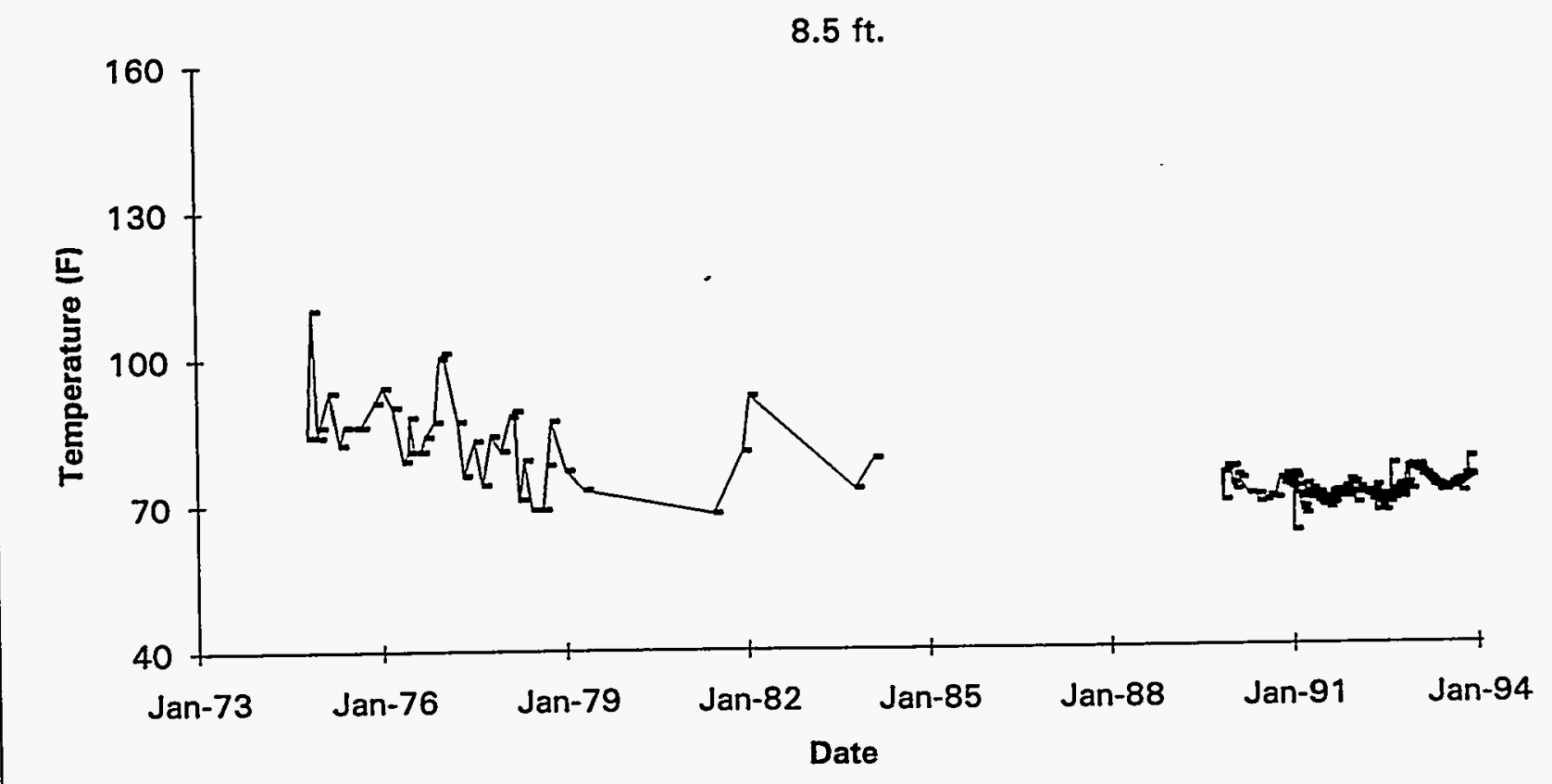

\section{1-BY-101}

Thermocouple 6

$10.5 \mathrm{ft}$.

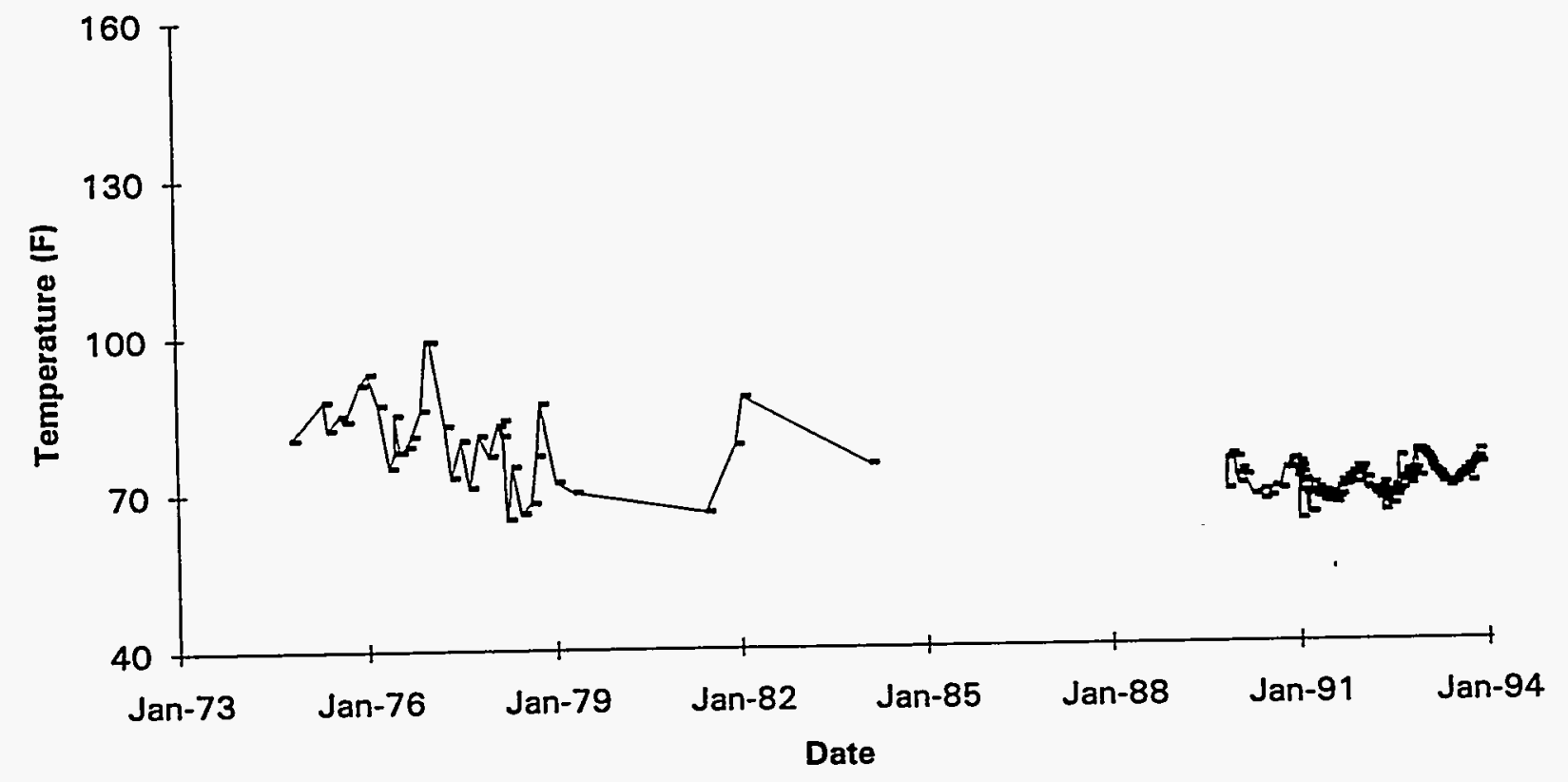

Data obtained from WHC Surveillance Analysis Computer System (SACS), November 12, 1993.

D-3 


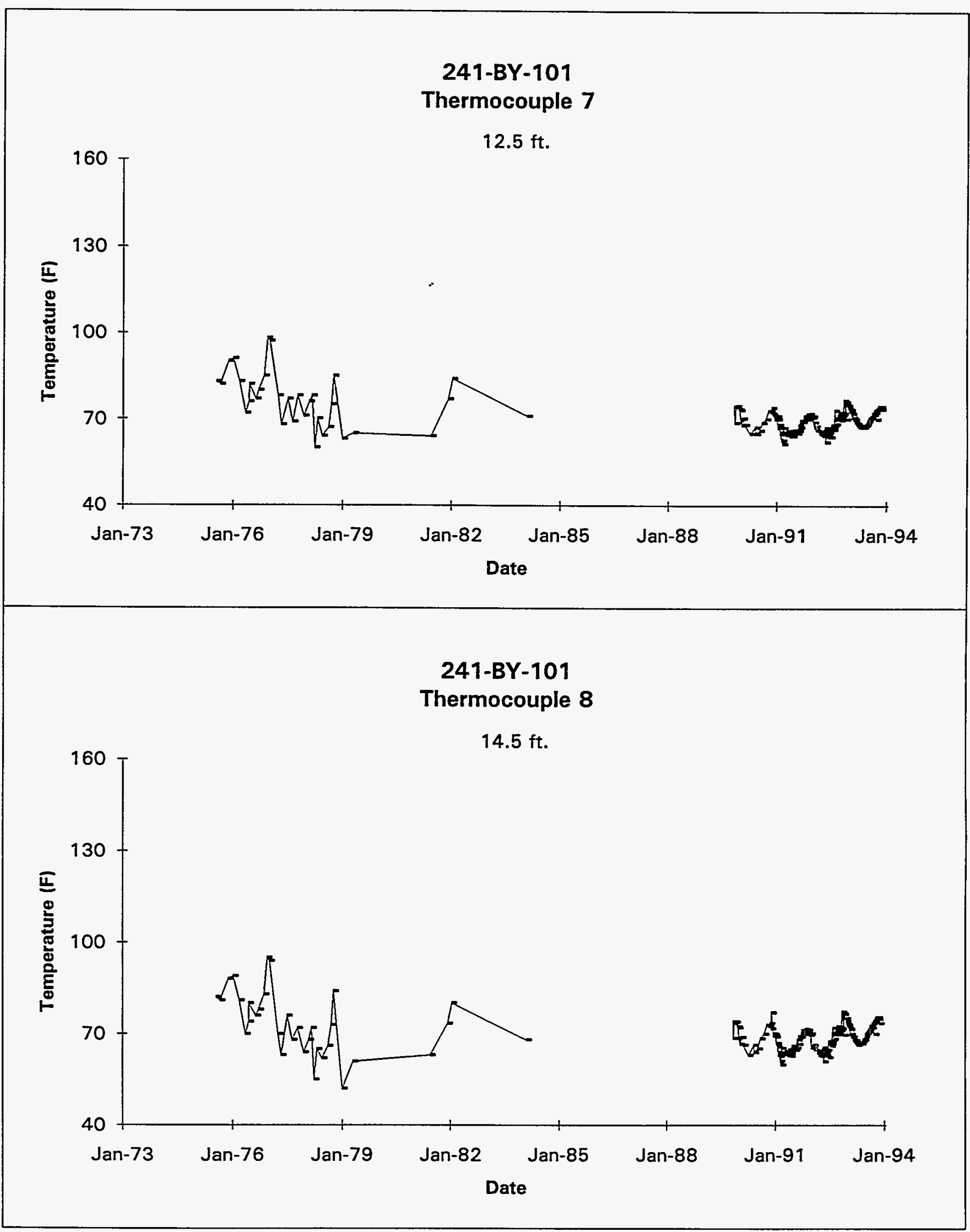

Data obtained from WHC Surveillance Analysis Computer System (SACS), November 12, 1993. 


\section{1-BY-101}

Thermocouple 9

$16.5 \mathrm{ft}$.

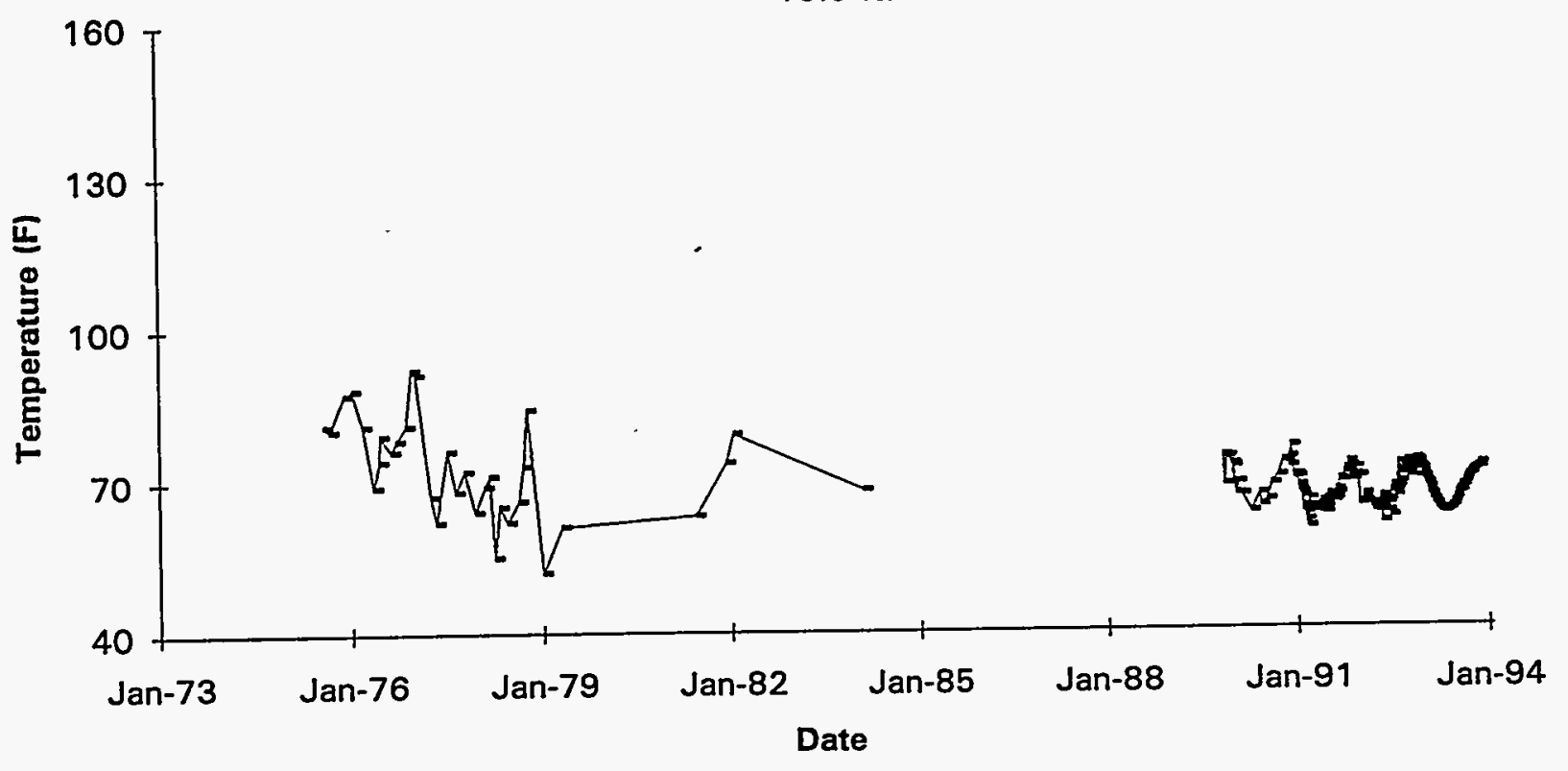

241-BY-101

Thermocouple 10

$18.5 \mathrm{ft}$.

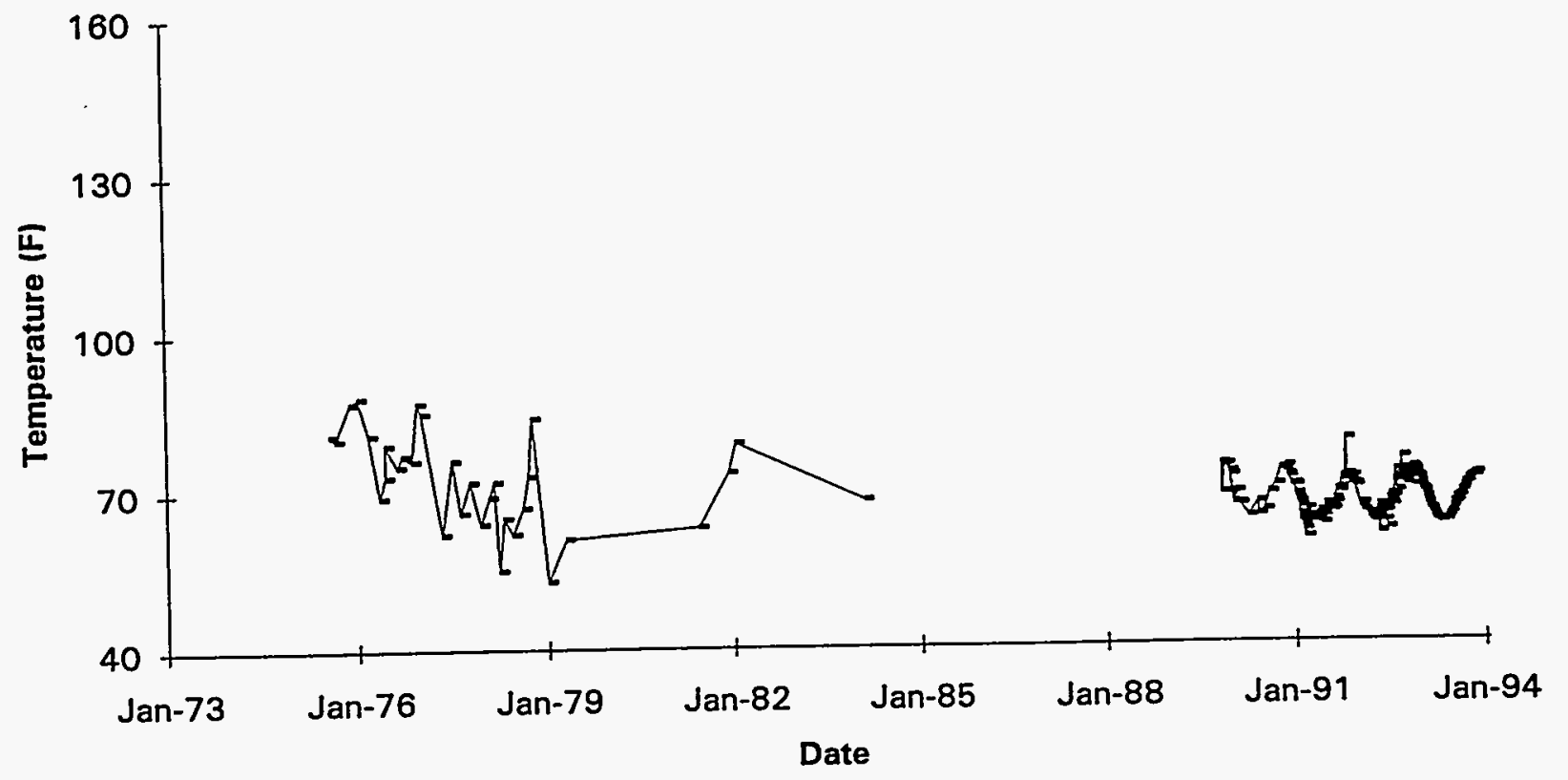

Data obtained from WHC Surveillance Analysis Computer System (SACS), November 12, 1993. 


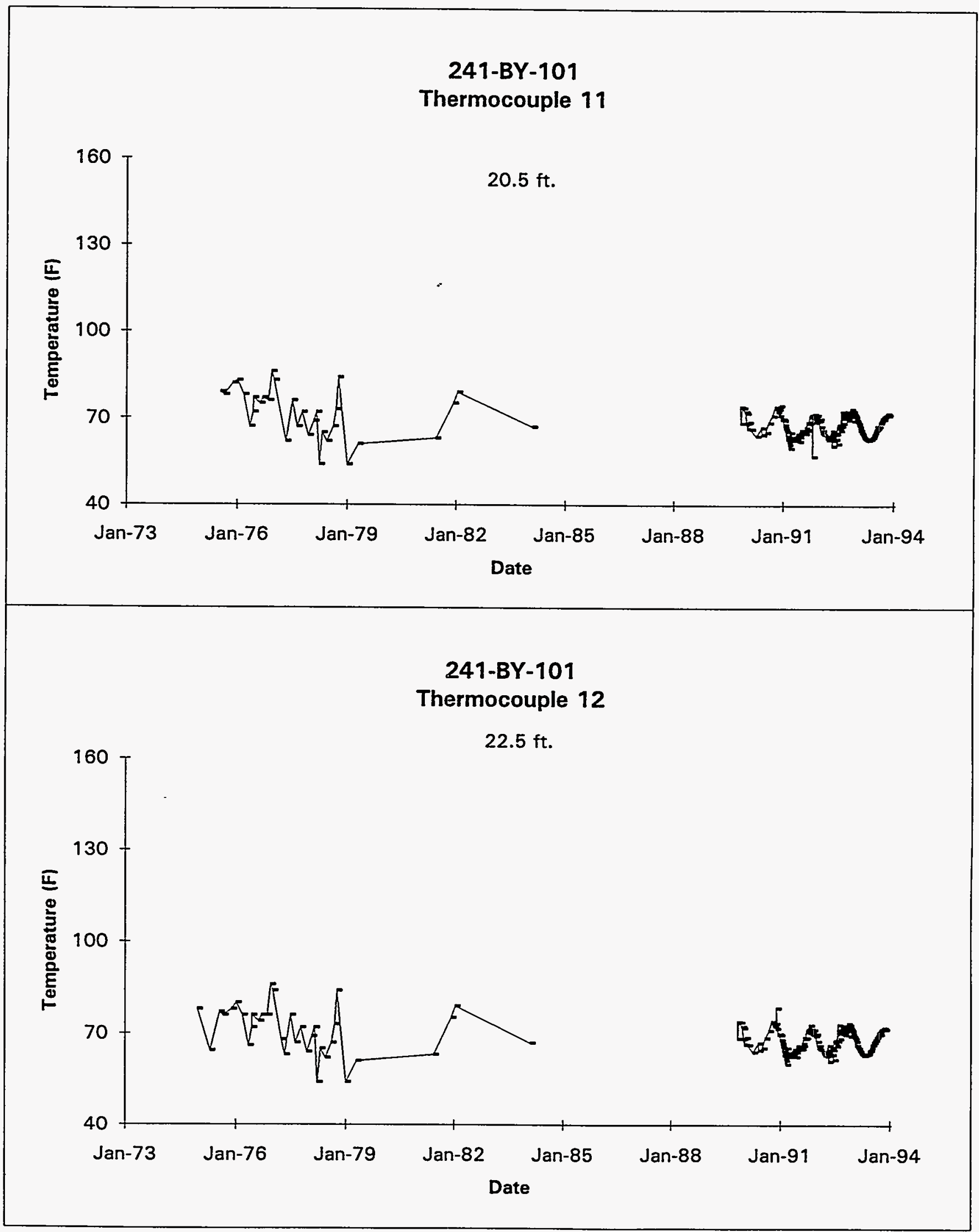

Data obtained from WHC Surveillance Analysis Computer System (SACS), November 12, 1993.

D-6 


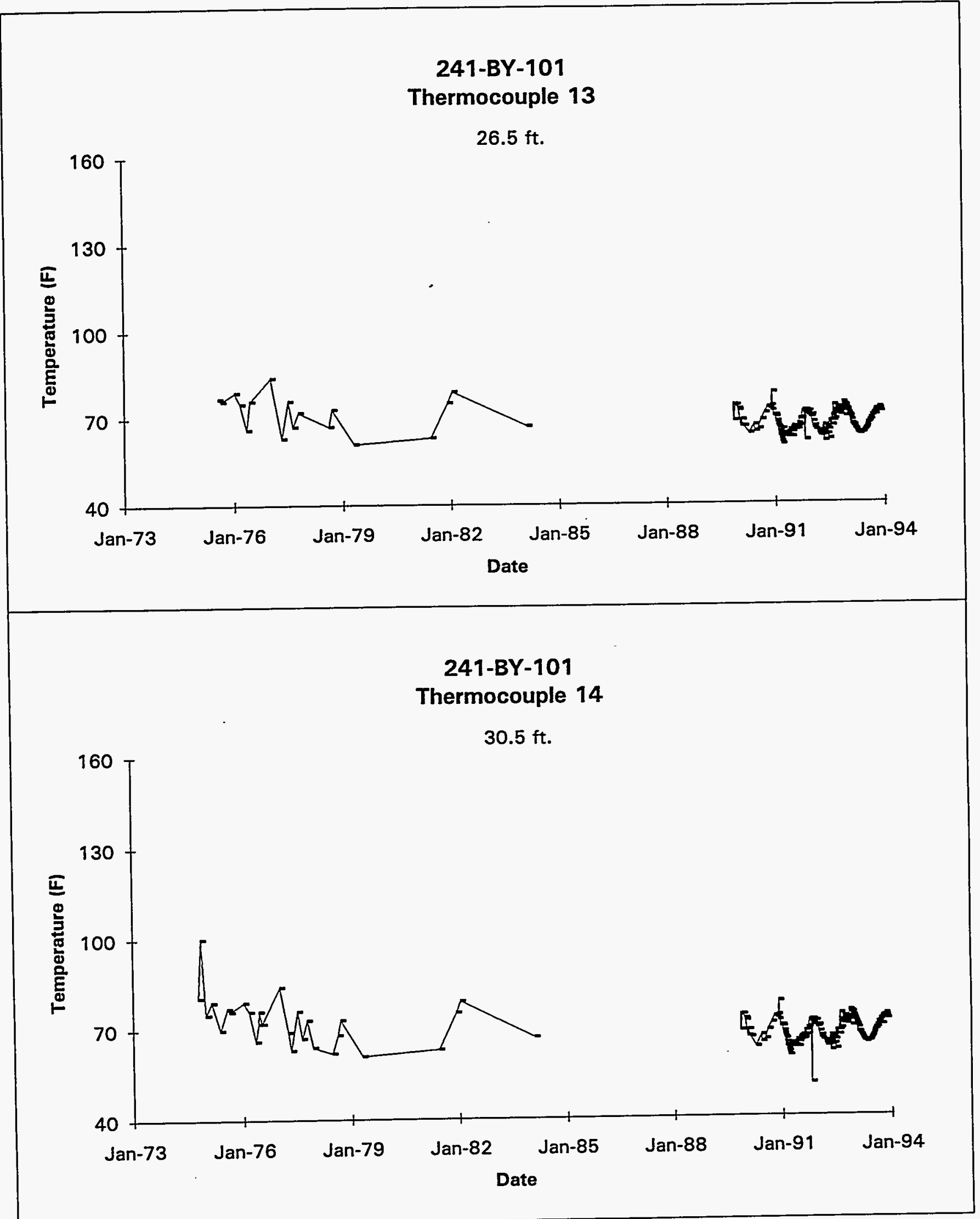

Data obtained from WHC Surveillance Analysis Computer System (SACS), November 12, 1993.

D-7 


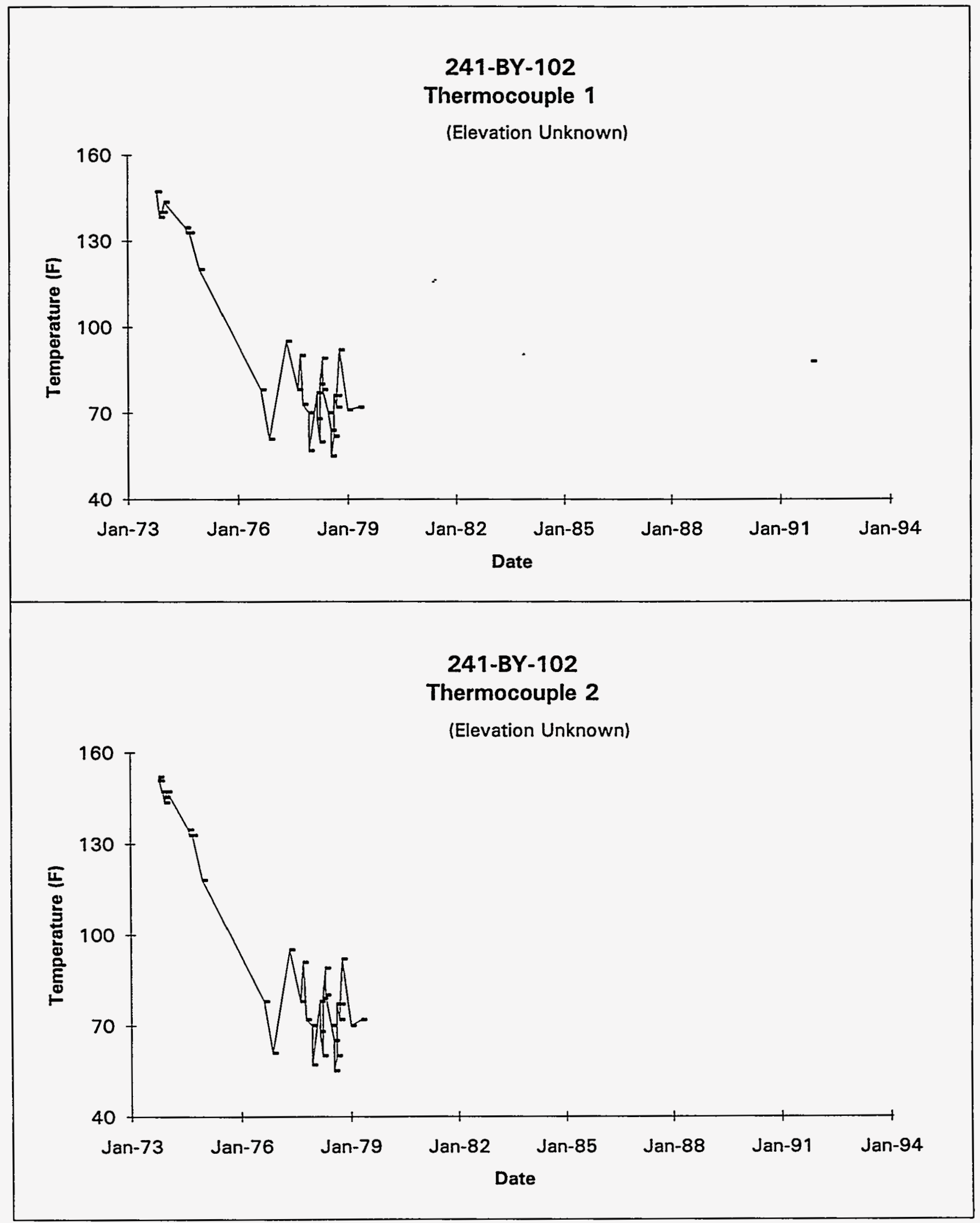

Data obtained from WHC Surveillance Analysis Computer System (SACS), November 12, 1993.

D-8 


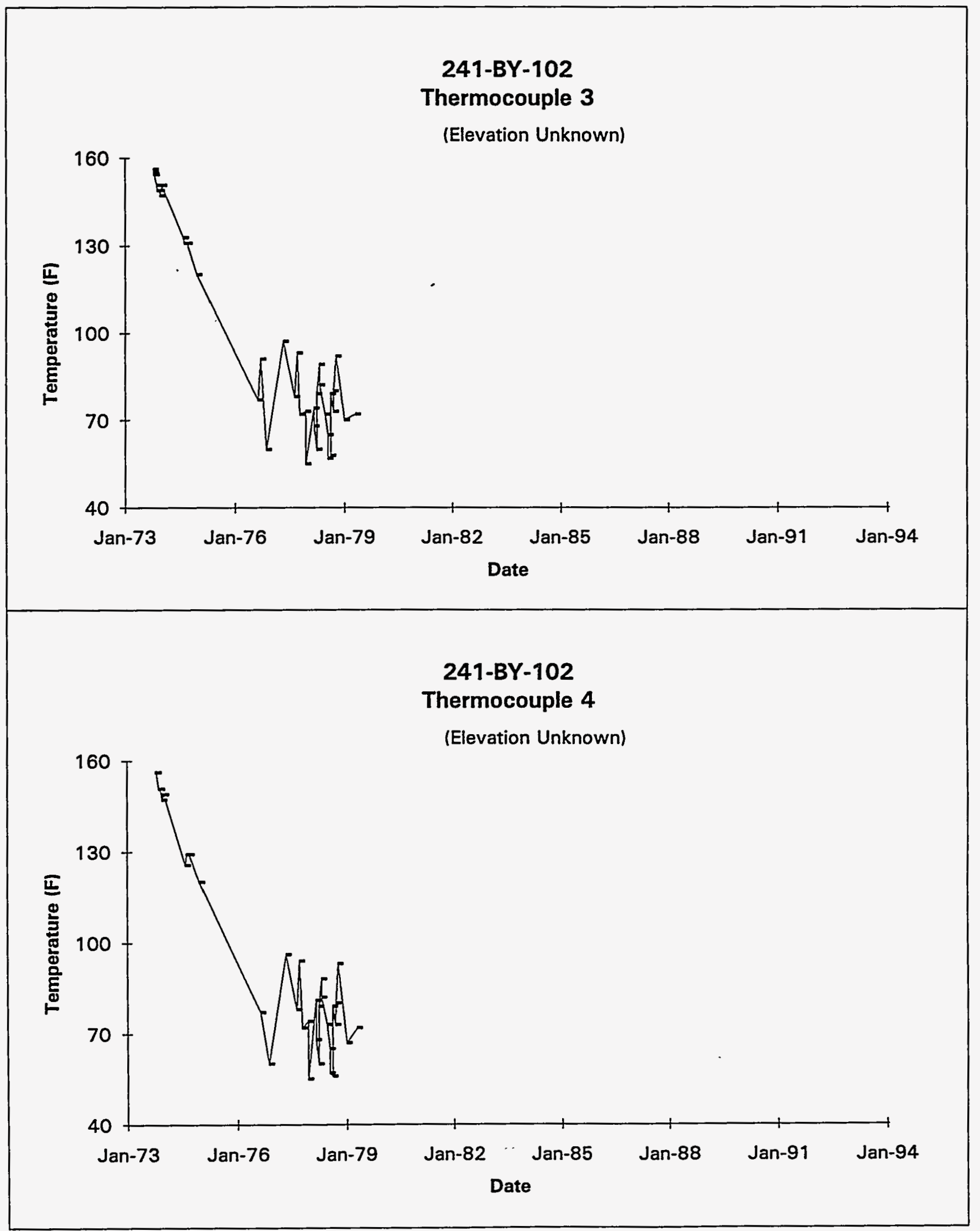

Data obtained from WHC Surveillance Analysis Computer System (SACS), November 12, 1993.

D-9 


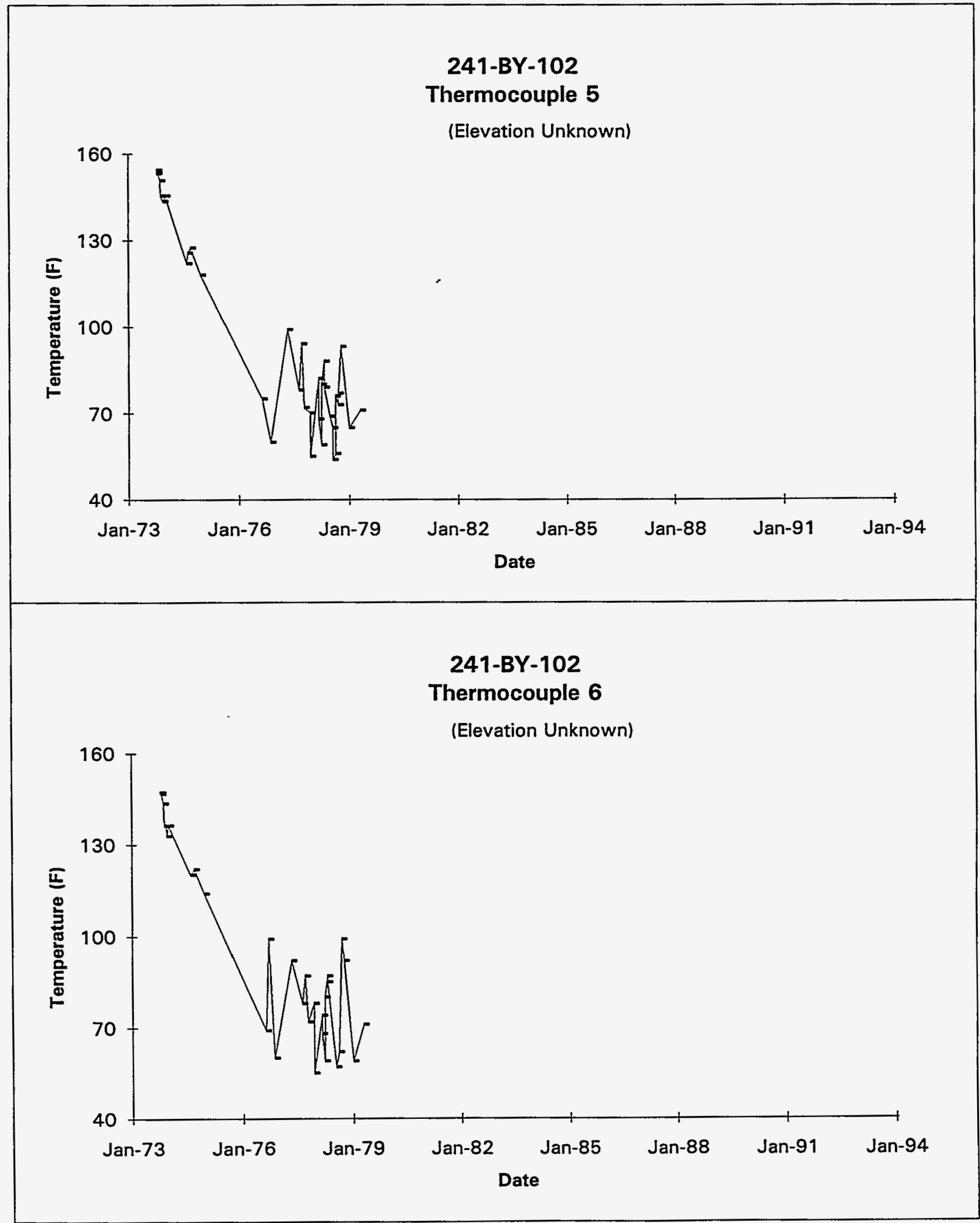

Data obtained from WHC Surveillance Analysis Computer System (SACS), November 12, 1993.

D-10 


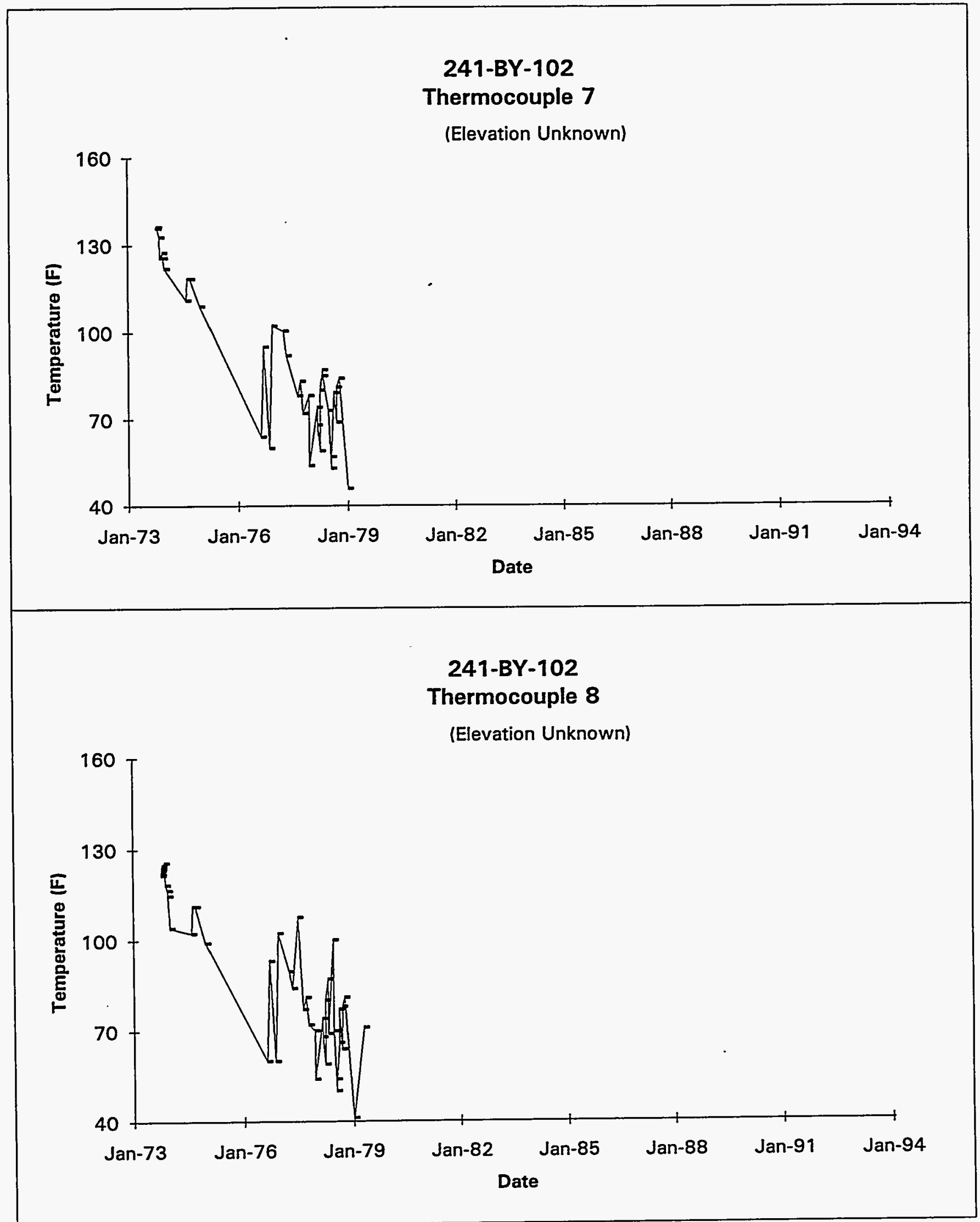

Data obtained from WHC Surveillance Analysis Computer System (SACS), November 12, 1993. 


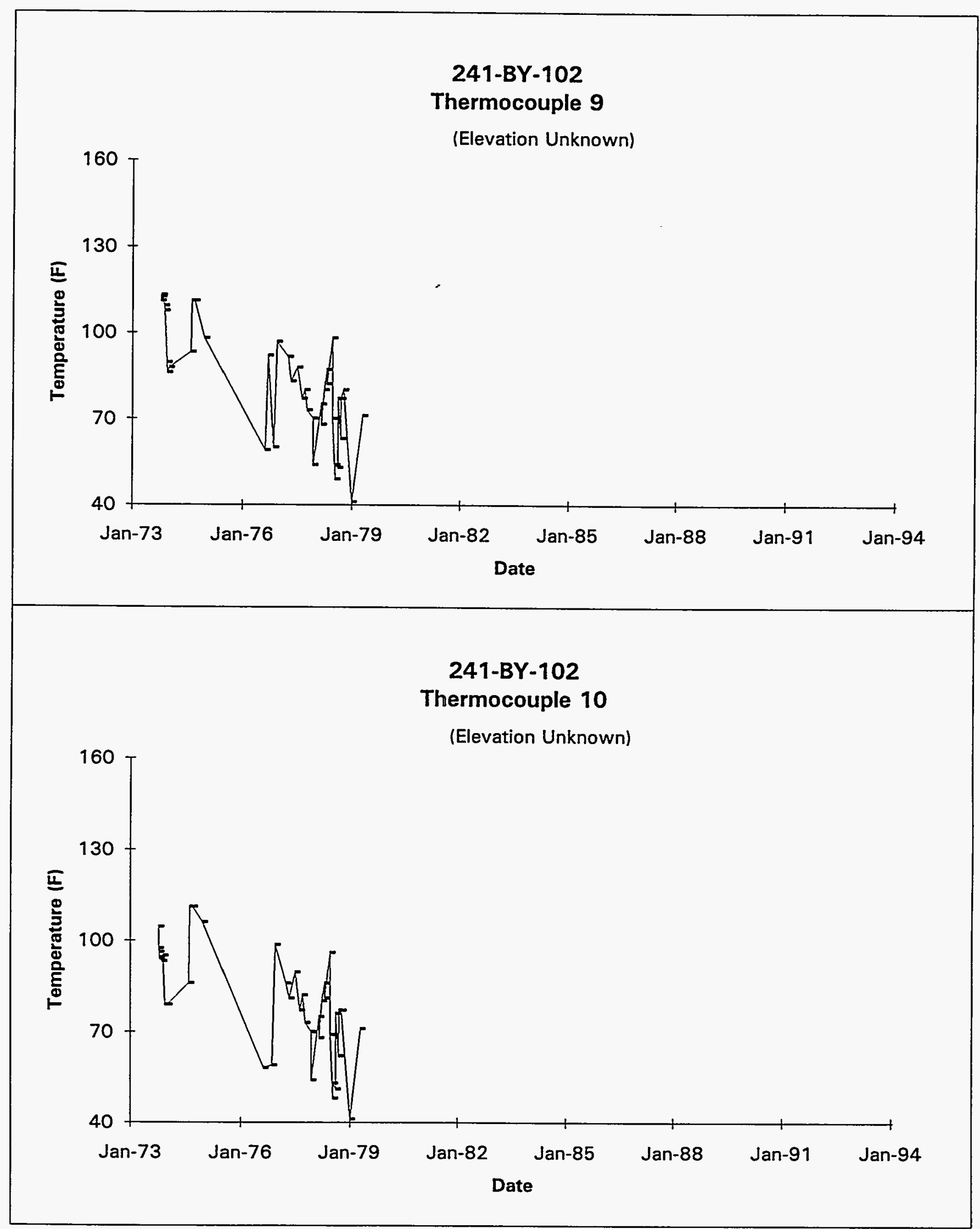

Data obtained from WHC Surveillance Analysis Computer System (SACS), November 12, 1993. 


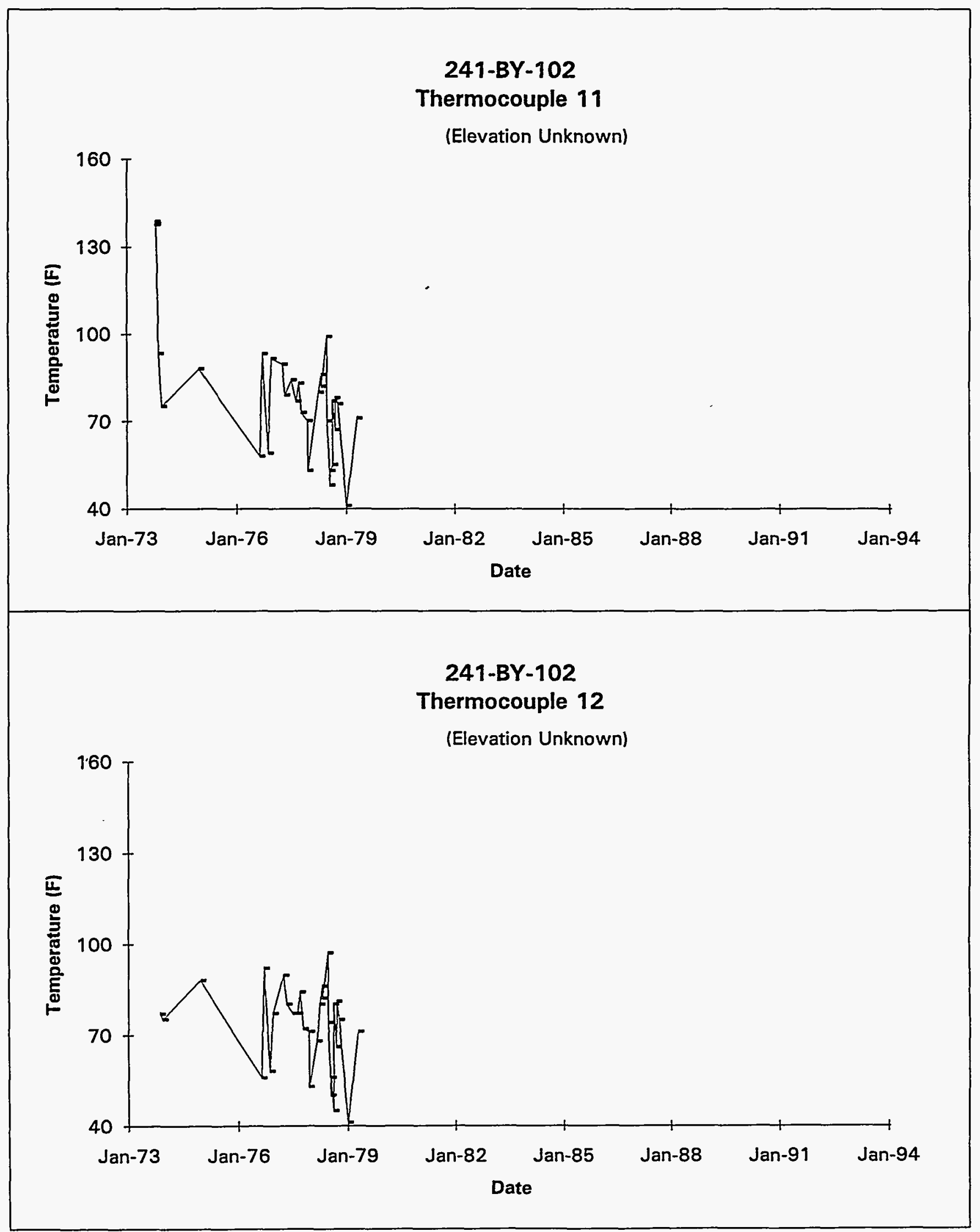

Data obtained from WHC Surveillance Analysis Computer System (SACS), November 12, 1993.

D-13 


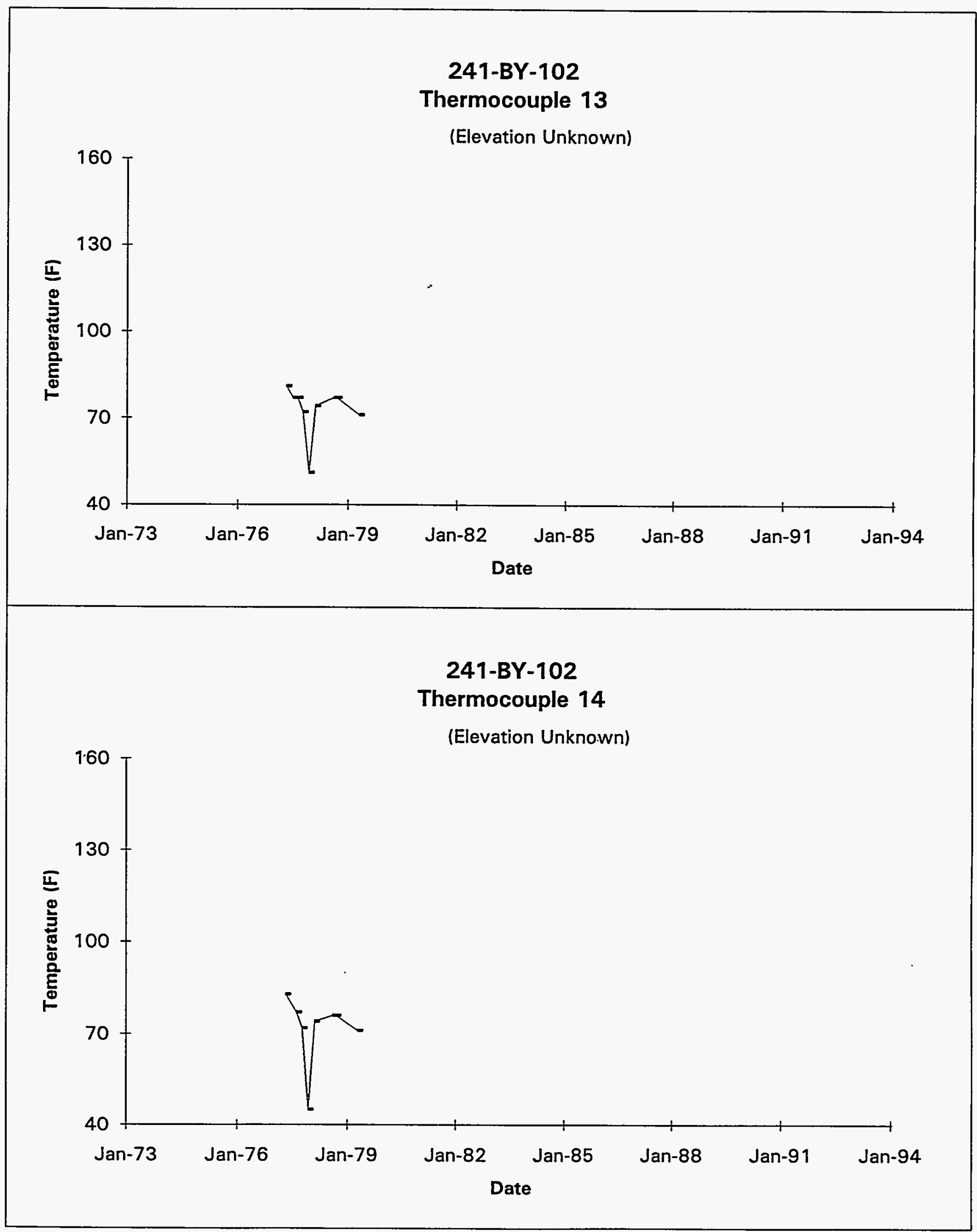

Data obtained from WHC Surveillance Analysis Computer System (SACS), November 12, 1993. 


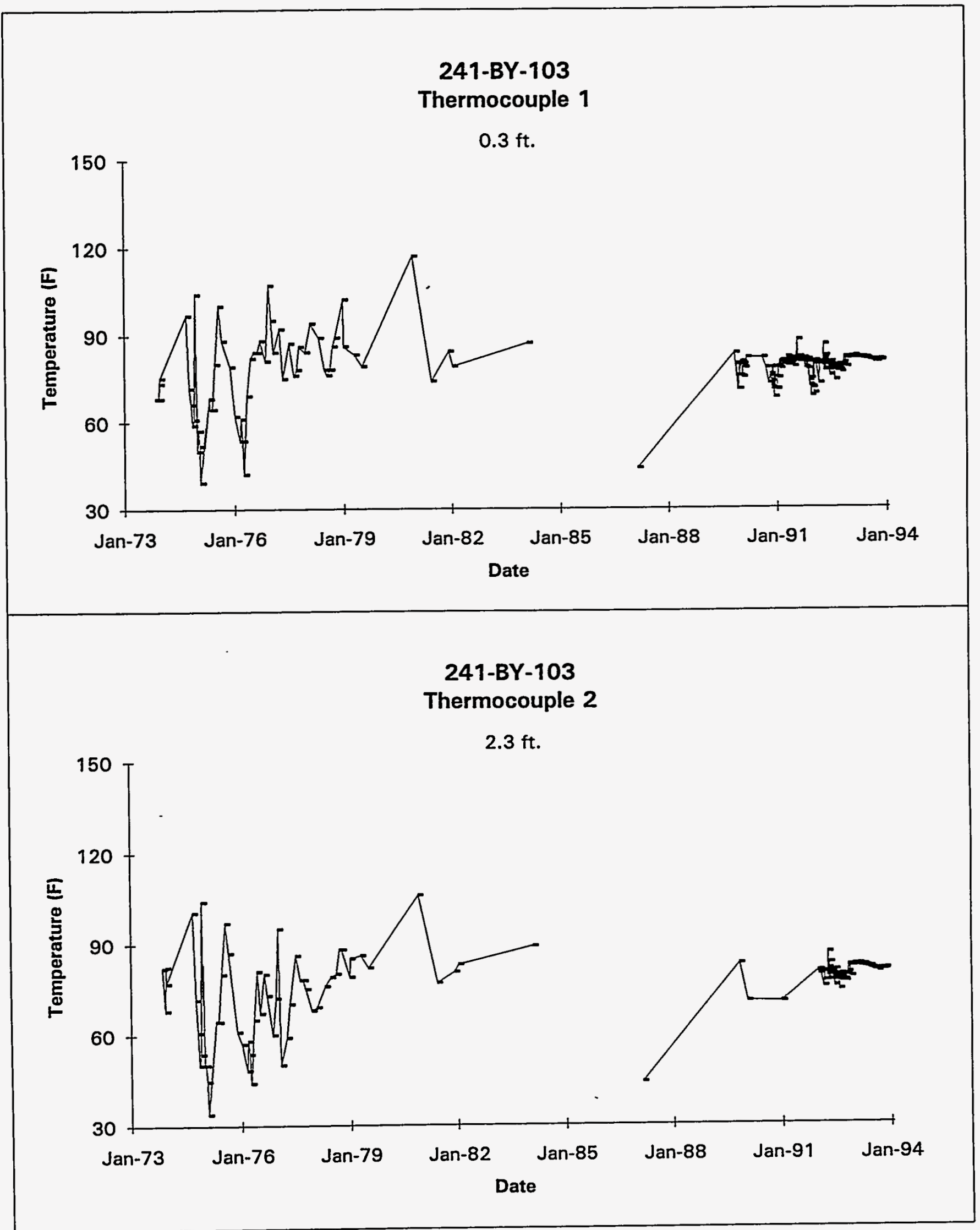

Data obtained from WHC Surveillance Analysis Computer System (SACS), November 12, 1993. 


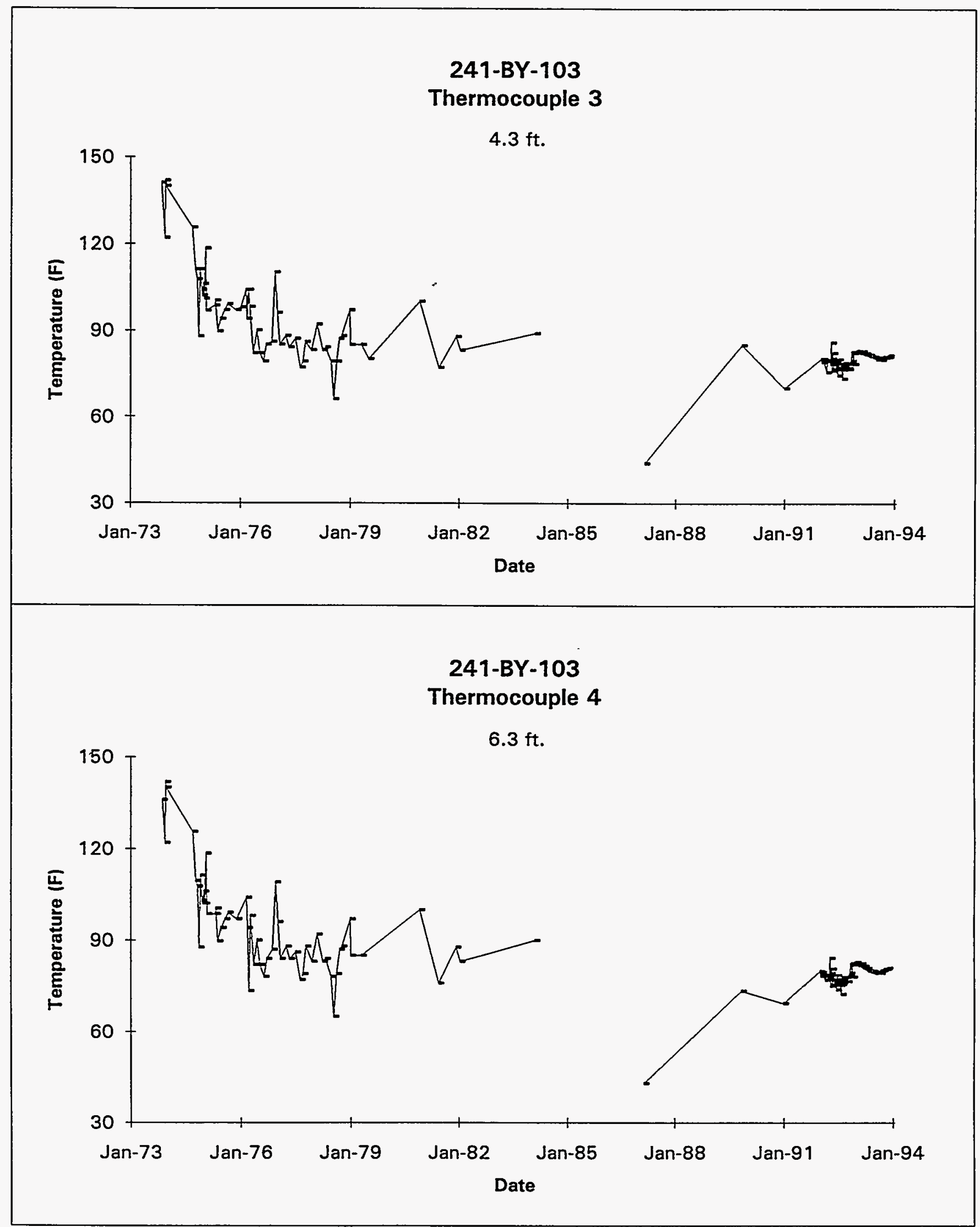

Data obtained from WHC Surveillance Analysis Computer System (SACS), November 12, 1993.

D-16 


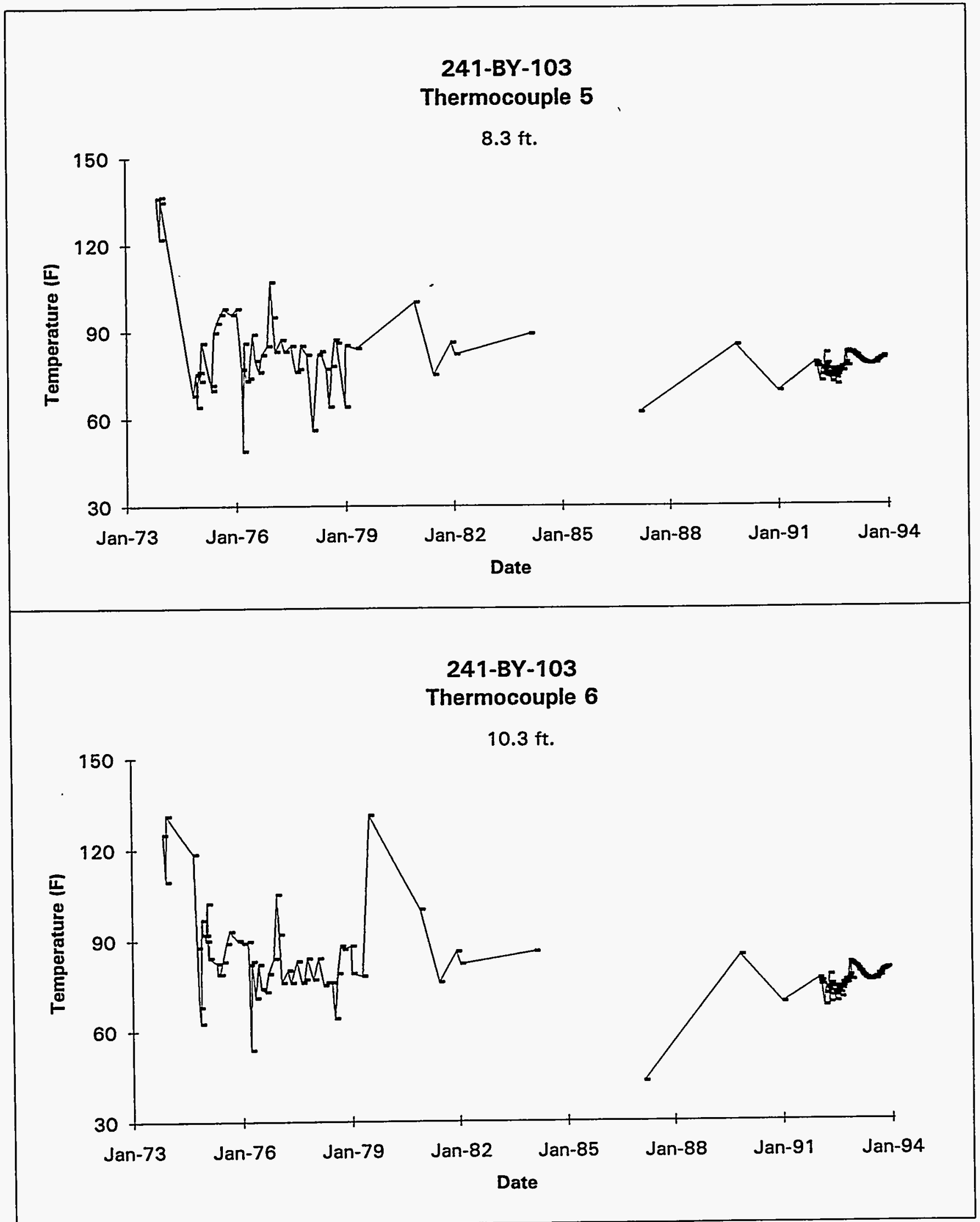

Data obtained from WHC Surveillance Analysis Computer System (SACS), November 12, 1993. 


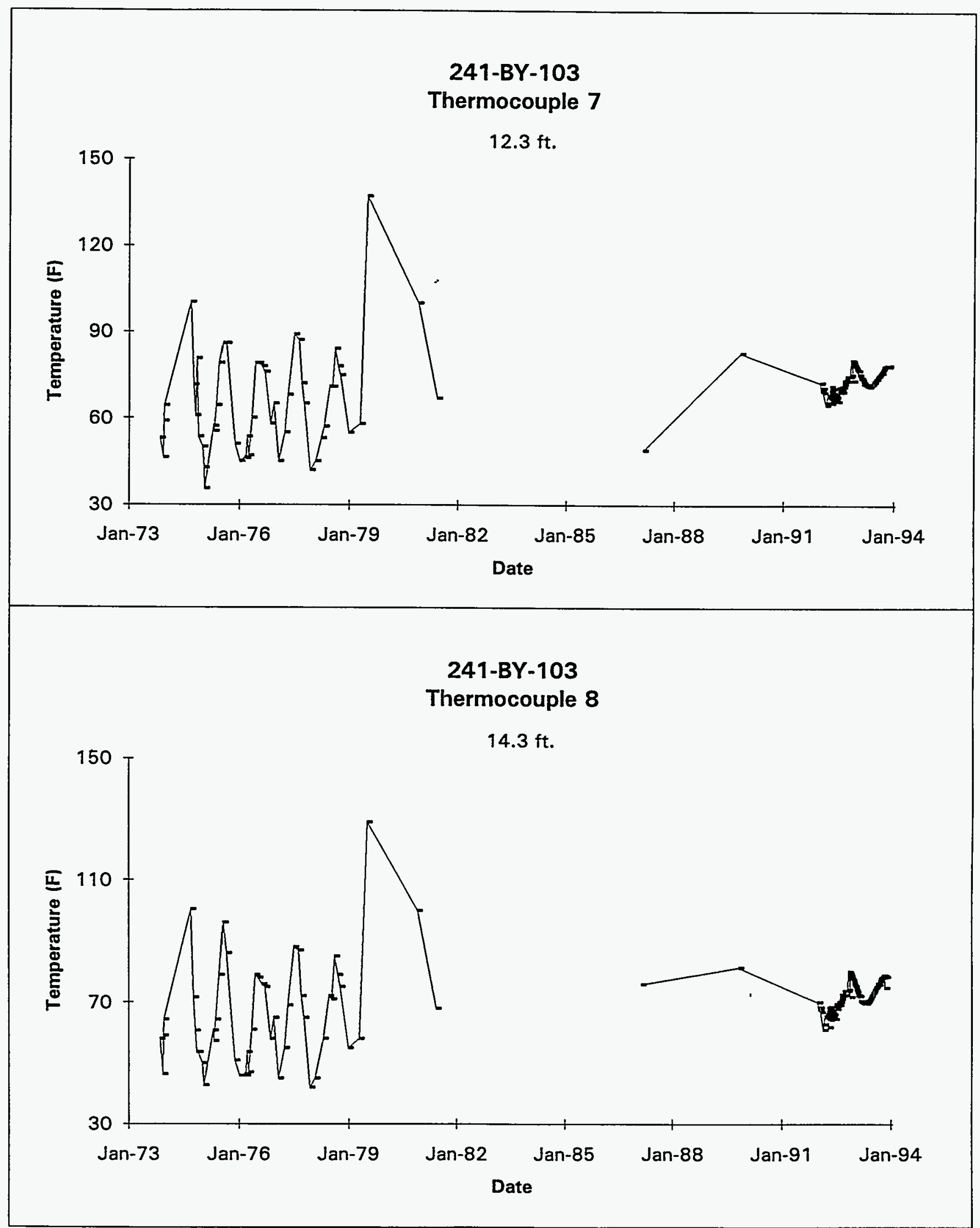

Data obtained from WHC Surveillance Analysis Computer System (SACS), November 12, 1993.

D-18 


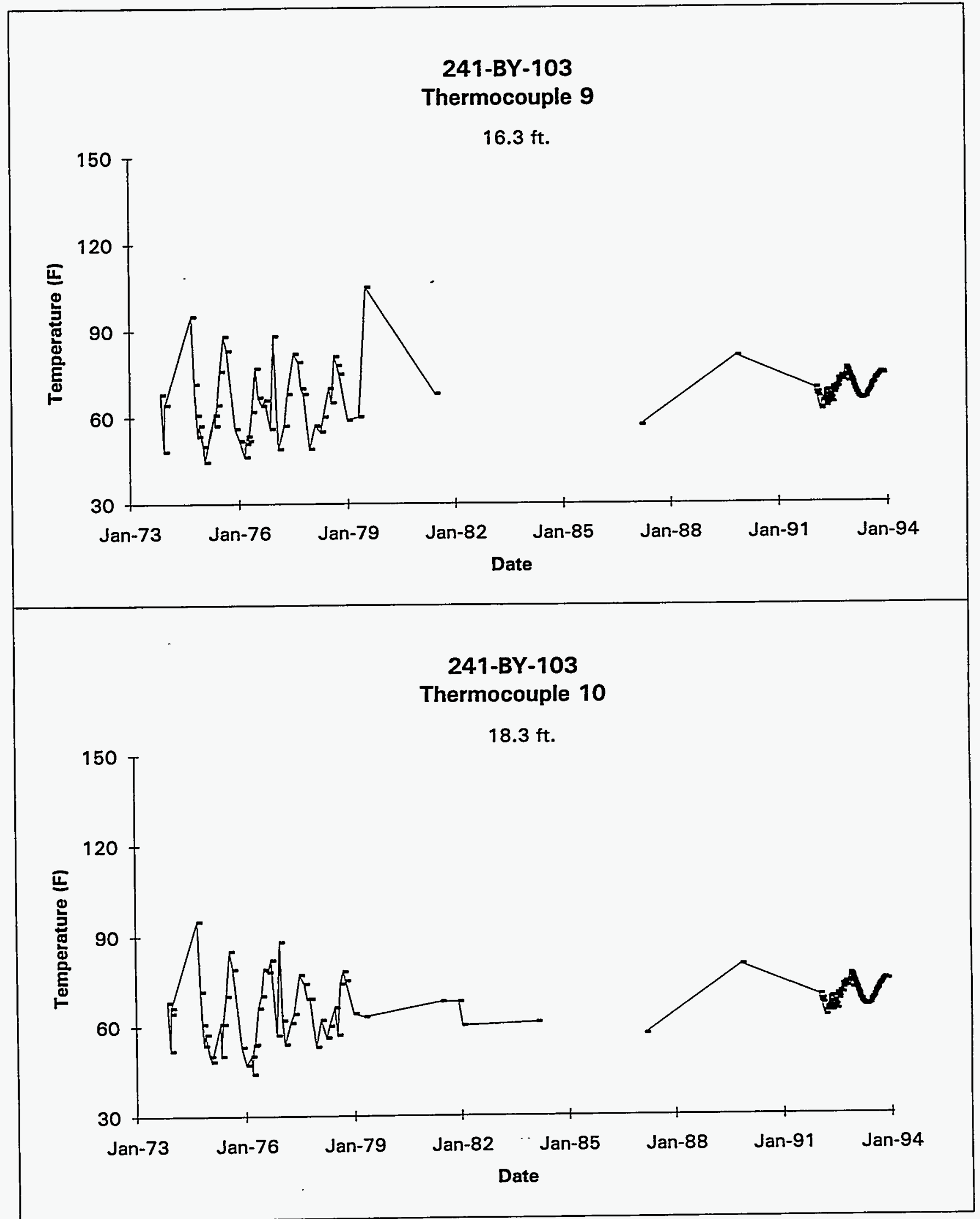

Data obtained from WHC Surveillance Analysis Computer System (SACS), November 12, 1993.

D-19 


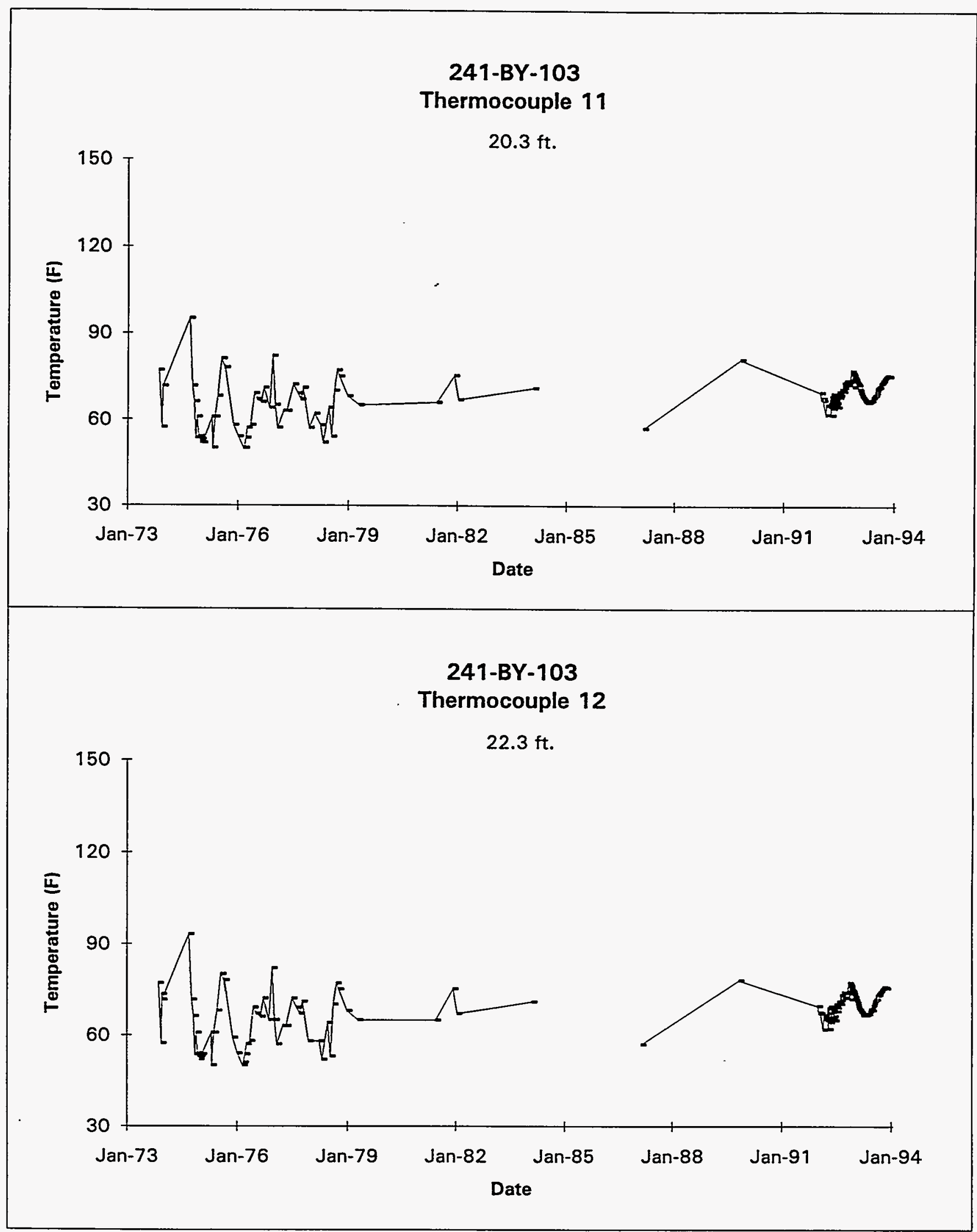

Data obtained from WHC Surveillance Analysis Computer System (SACS), November 12, 1993. 


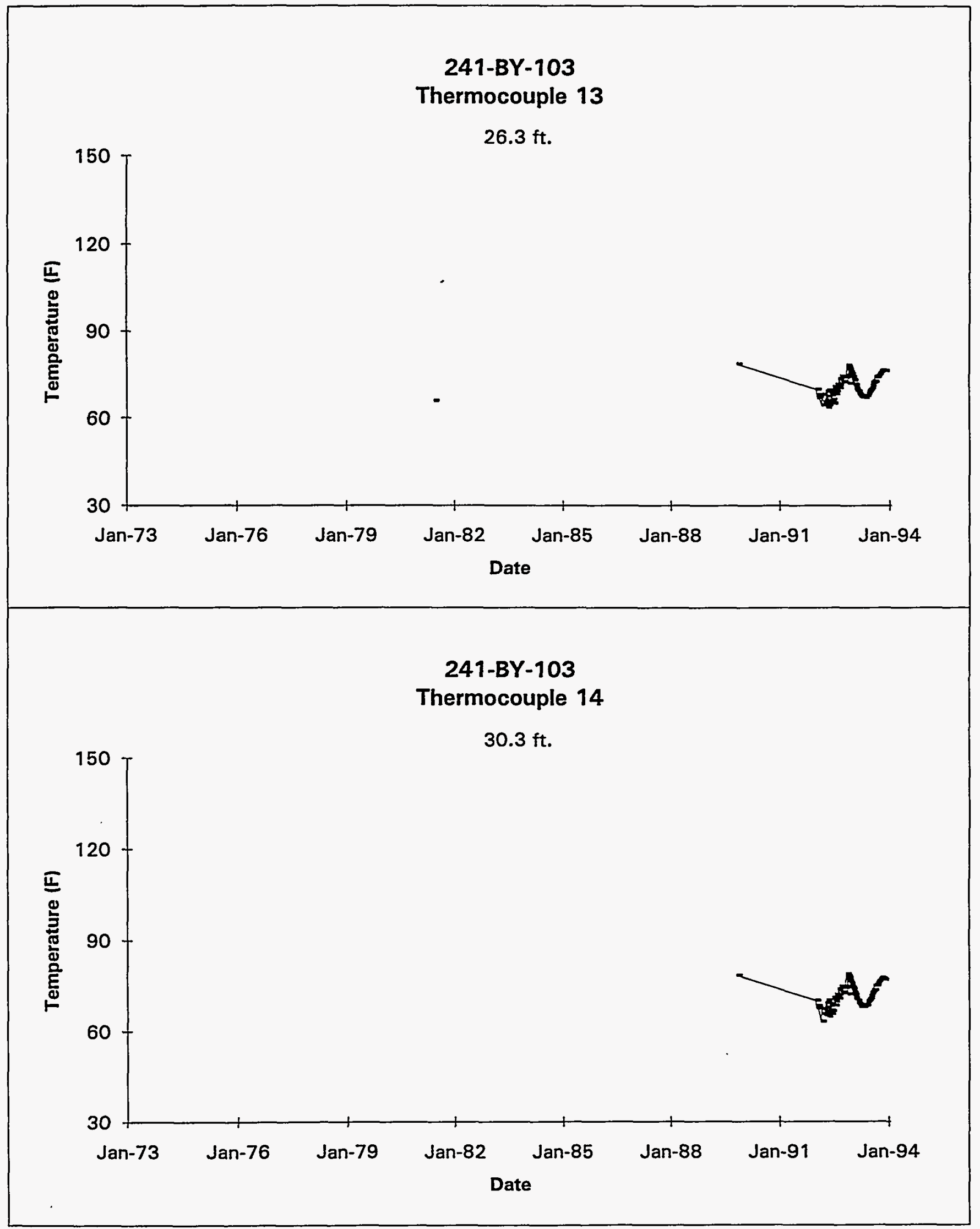

Data obtained from WHC Surveillance Analysis Computer System (SACS), November 12, 1993. 


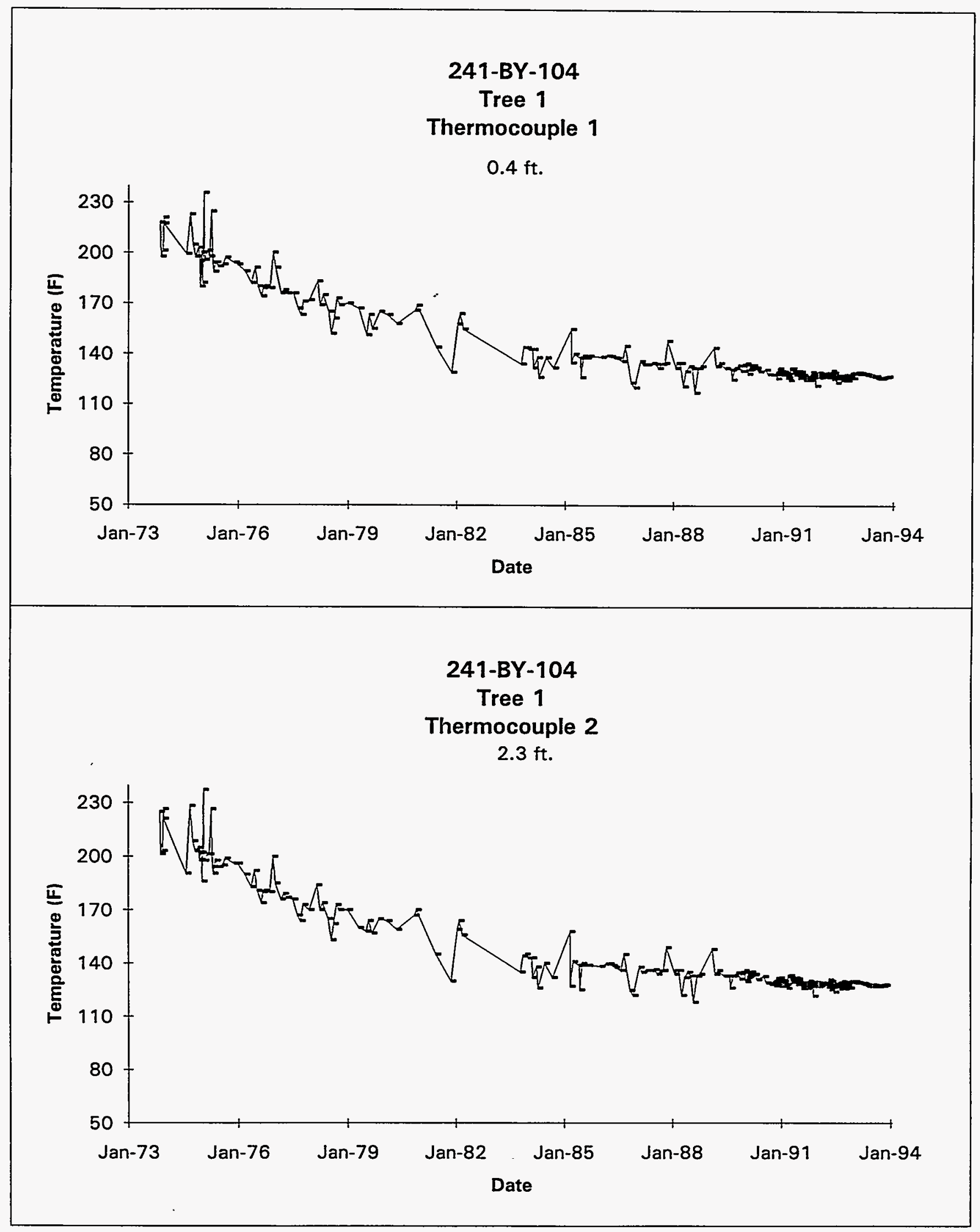

Data obtained from WHC Surveillance Analysis Computer System (SACS), November 12, 1993. 


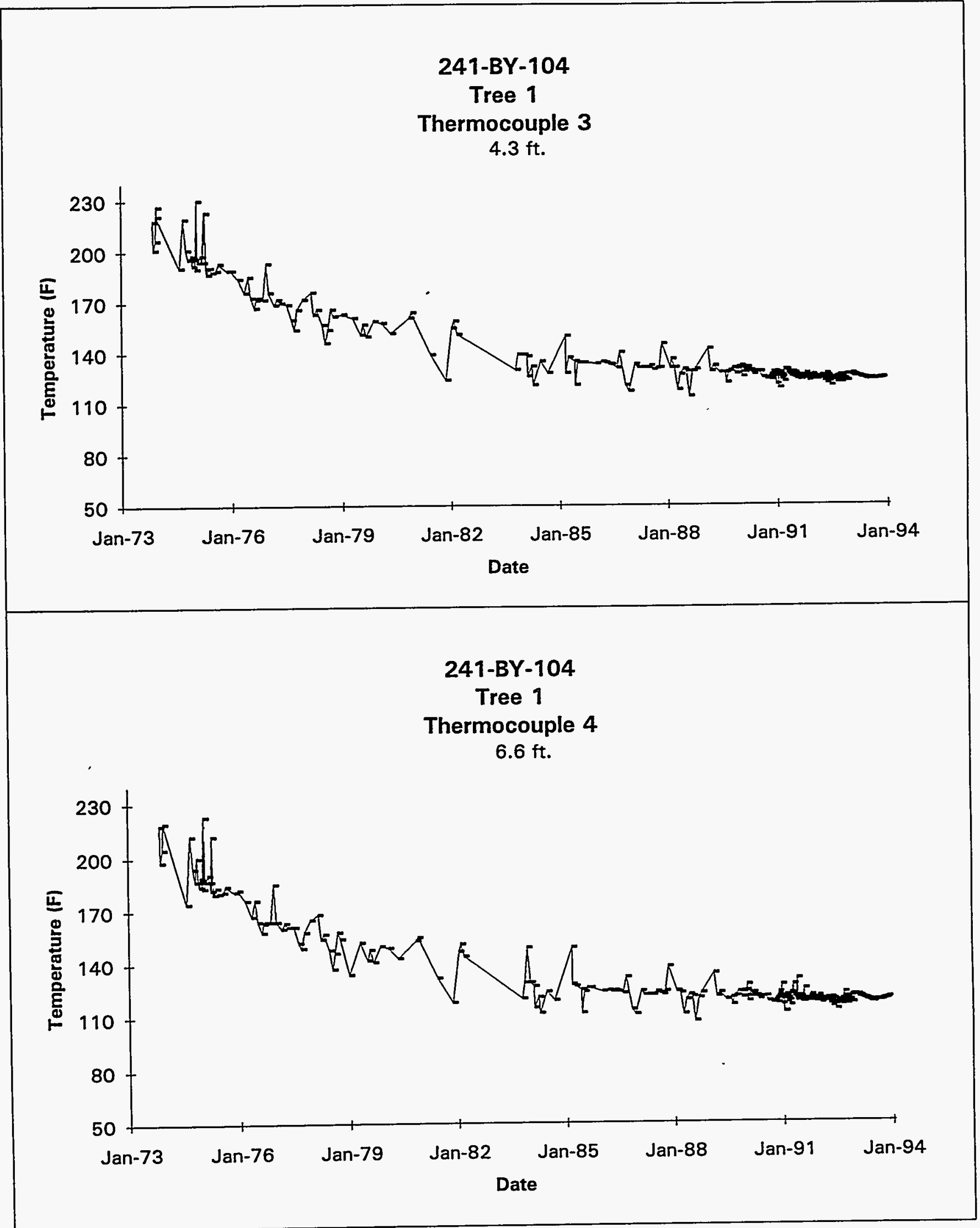

Data obtained from WHC Surveillance Analysis Computer System (SACS), November 12, 1993. 


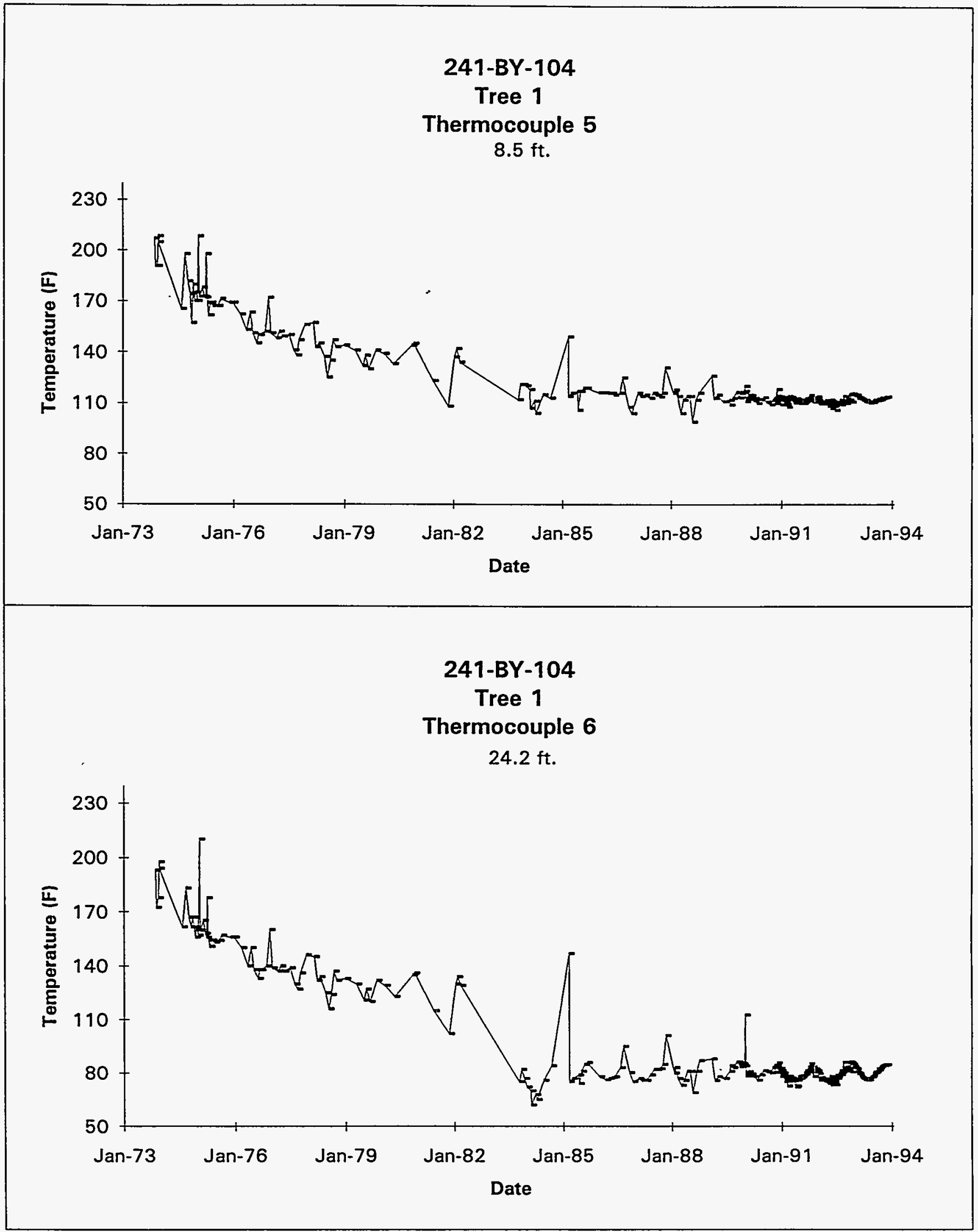

Data obtained from WHC Surveillance Analysis Computer System (SACS), November 12, 1993. 


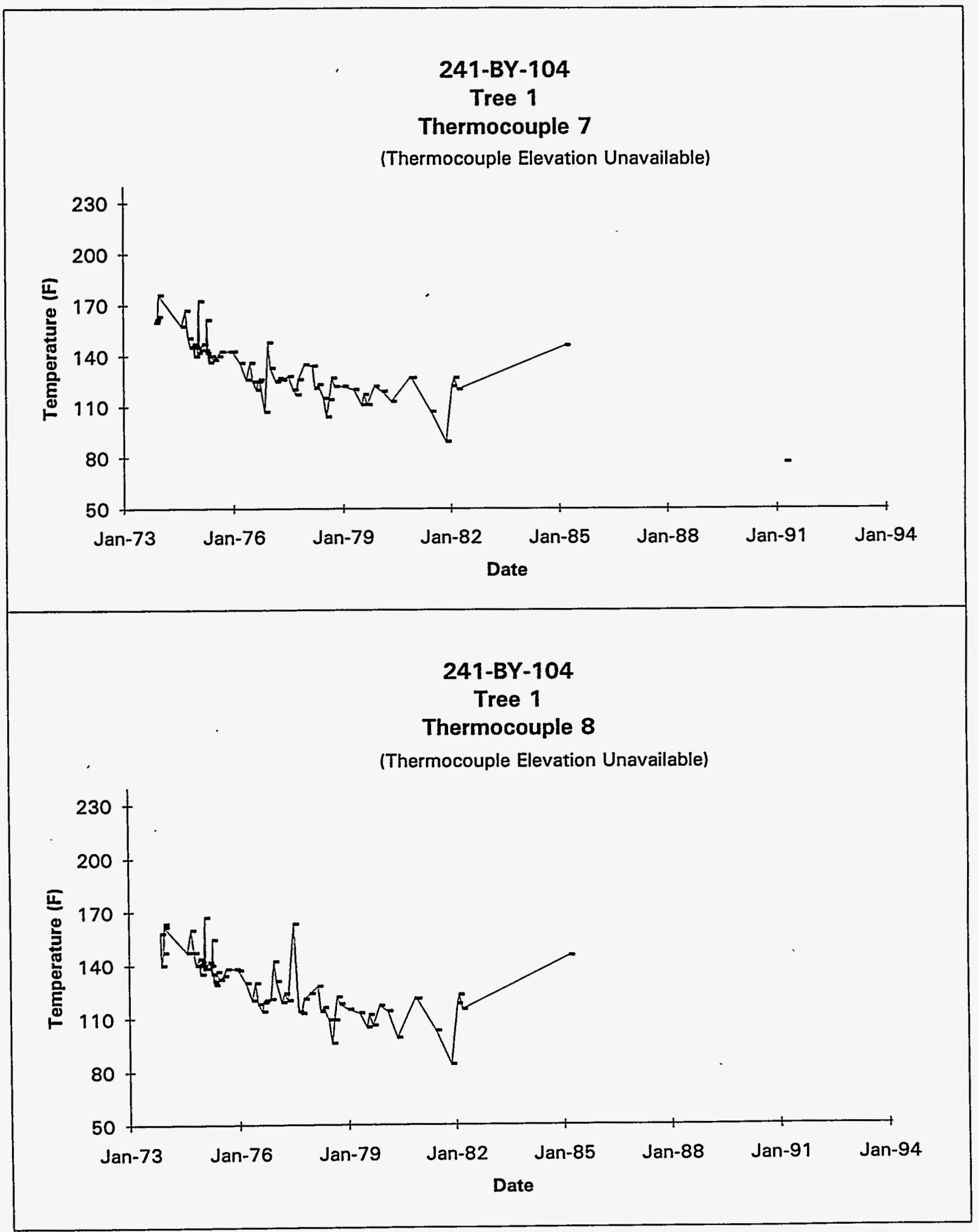

Data obtained from WHC Surveillance Analysis Computer System (SACS), November 12, 1993. 


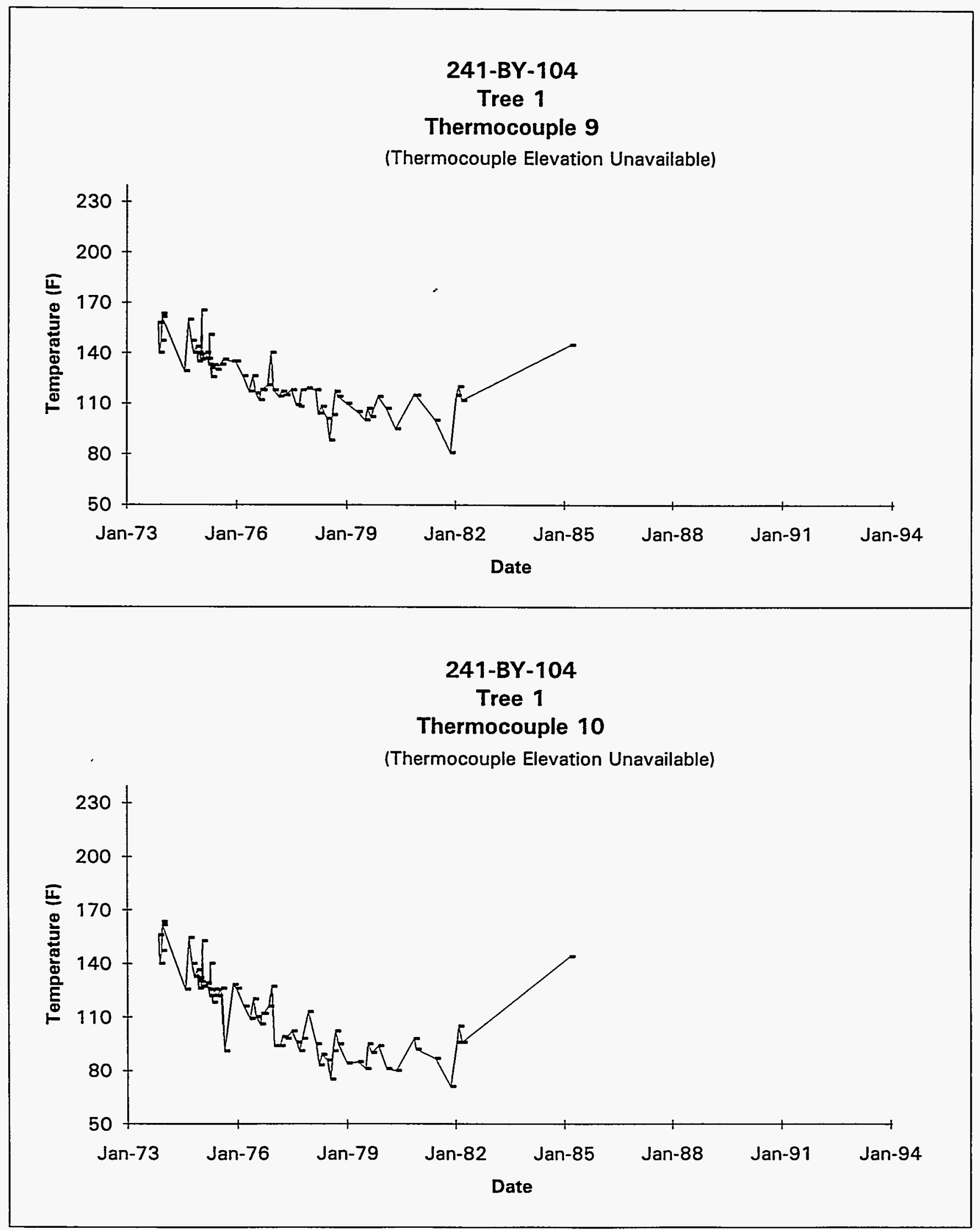

Data obtained from WHC Surveillance Analysis Computer System (SACS), November 12, 1993. 


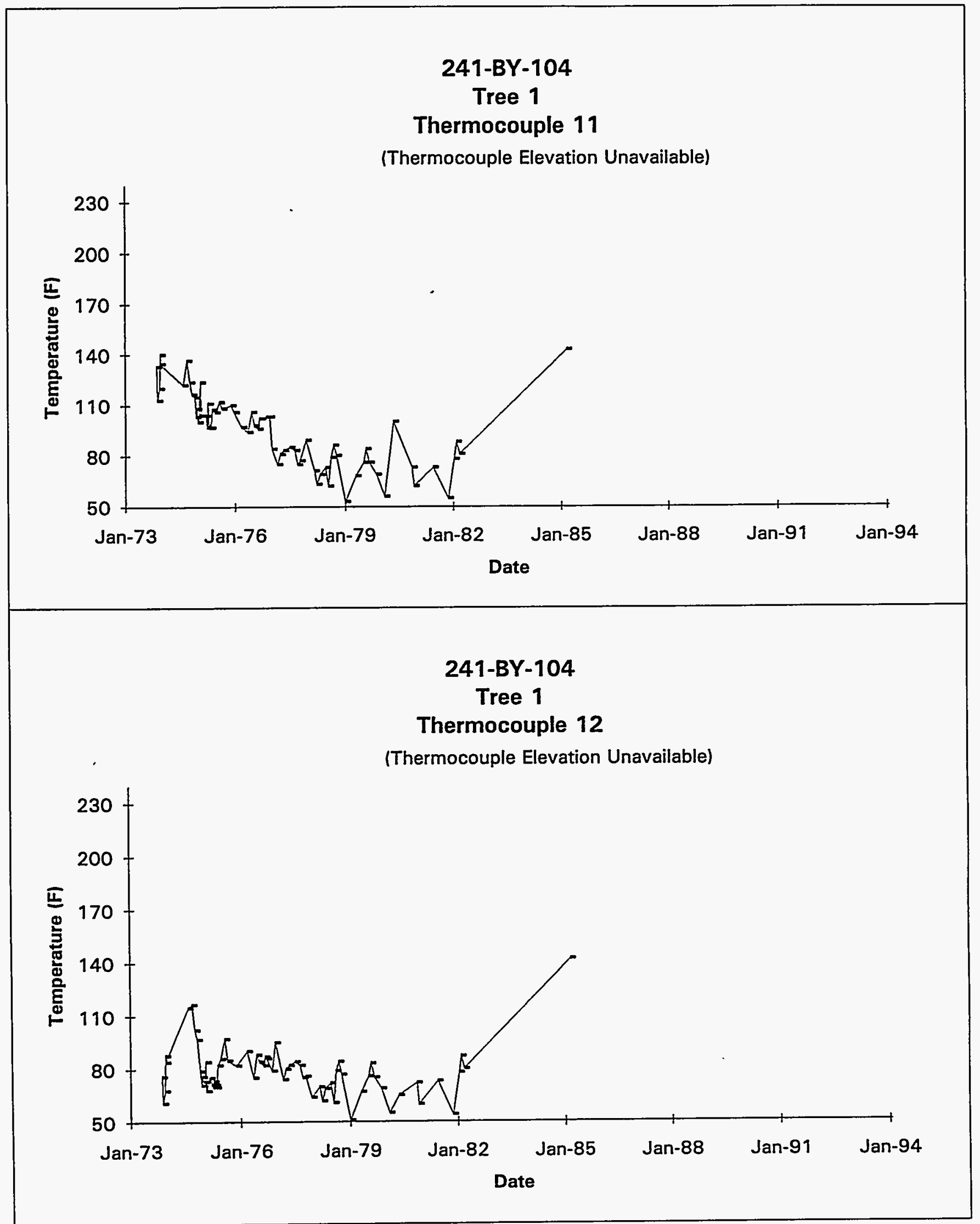

Data obtained from WHC Surveillance Analysis Computer System (SACS), November 12, 1993.

D-27 


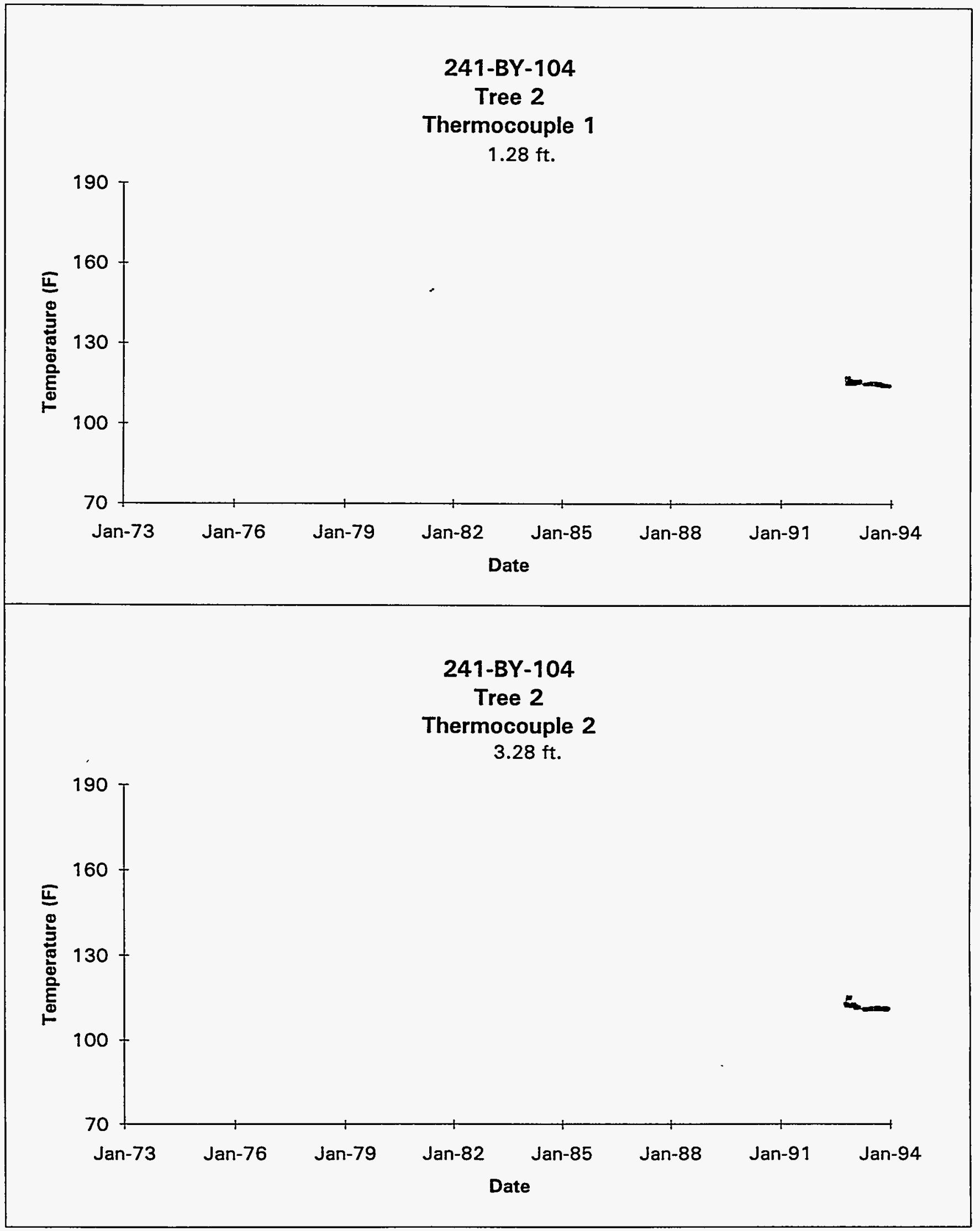

Data obtained from WHC Surveillance Analysis Computer System (SACS), November 12, 1993. 


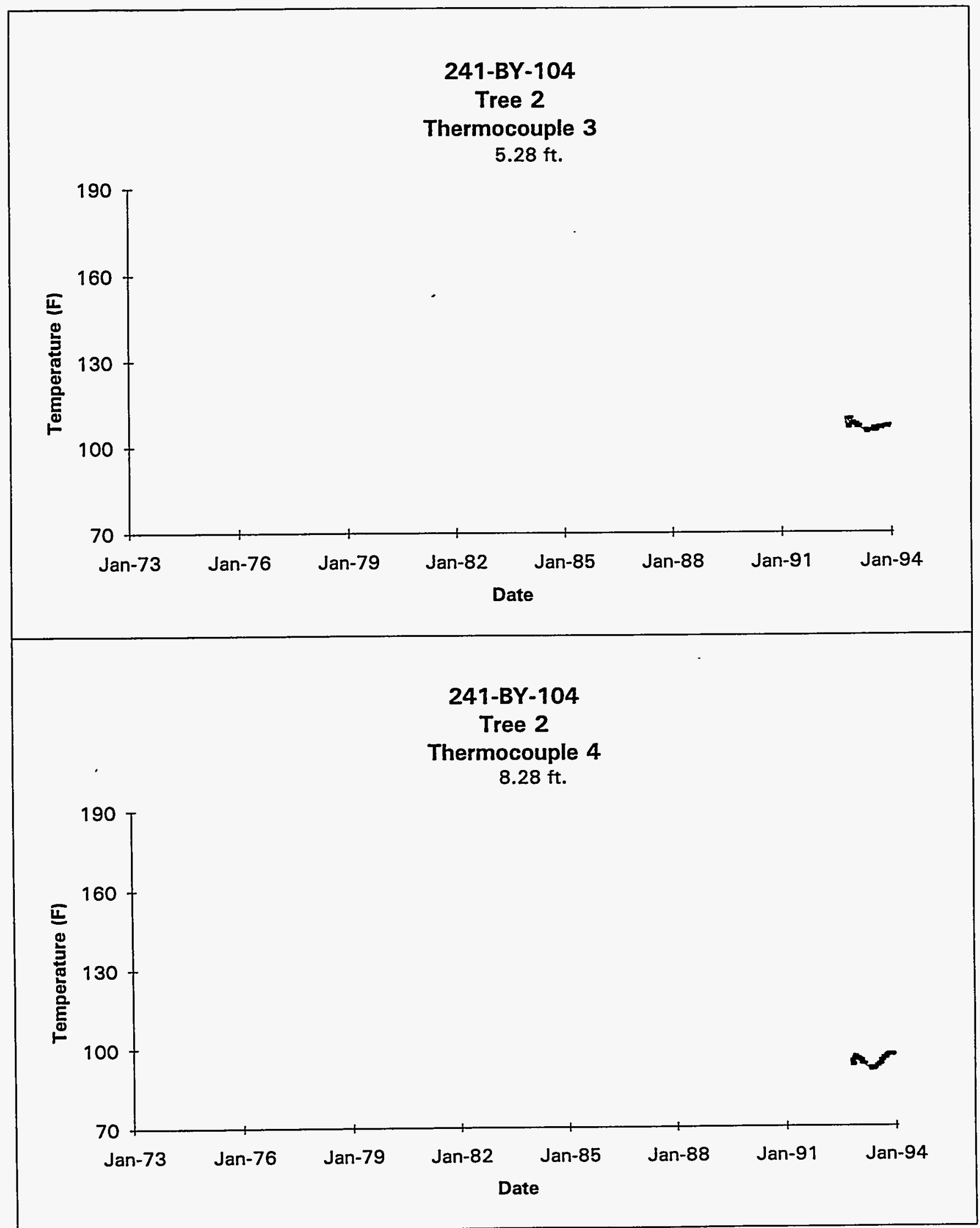

Data obtained from WHC Surveillance Analysis Computer System (SACS), November 12, 1993.

$$
\text { D-29 }
$$




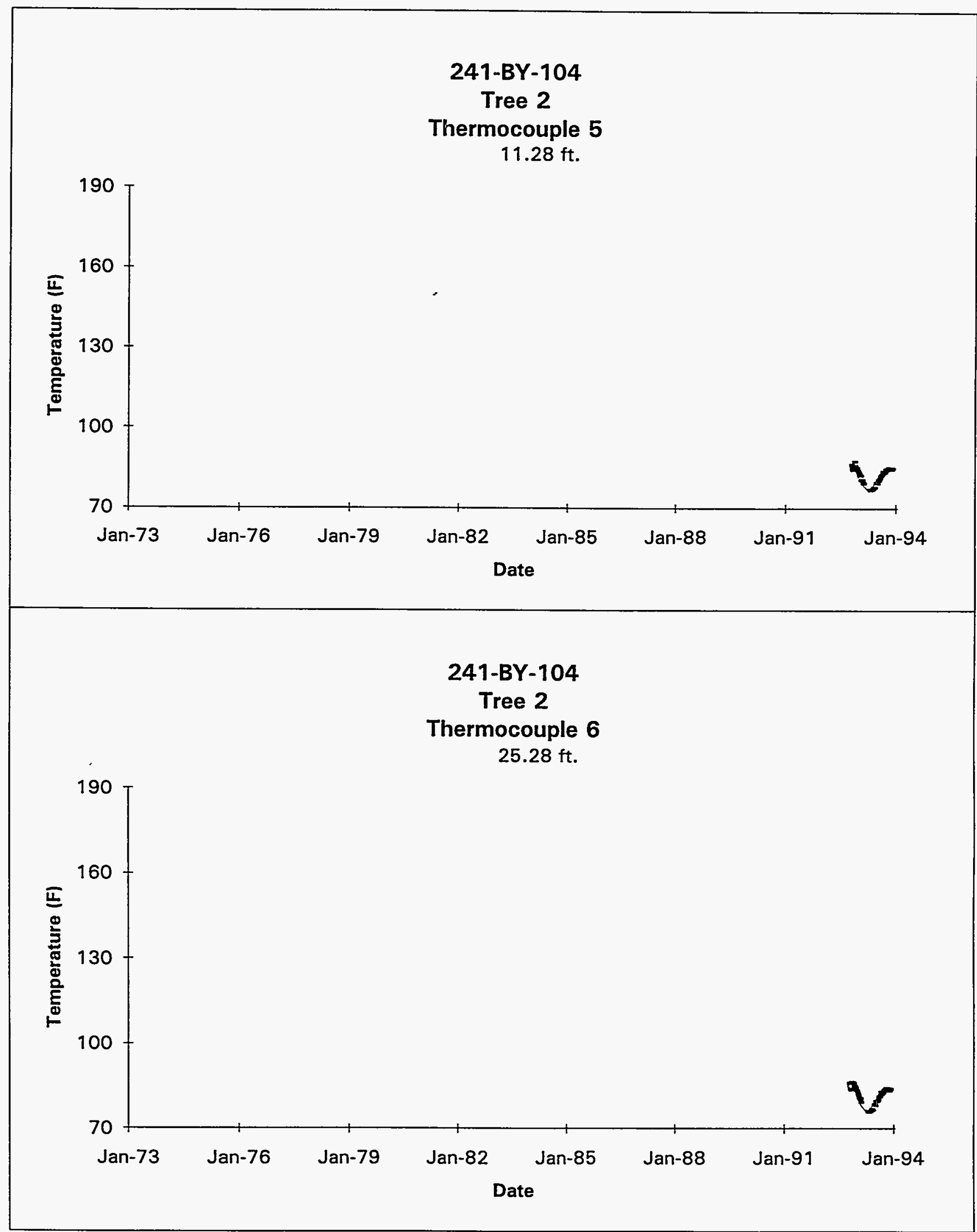

Data obtained from WHC Surveillance Analysis Computer System (SACS), November 12, 1993.

D-30 


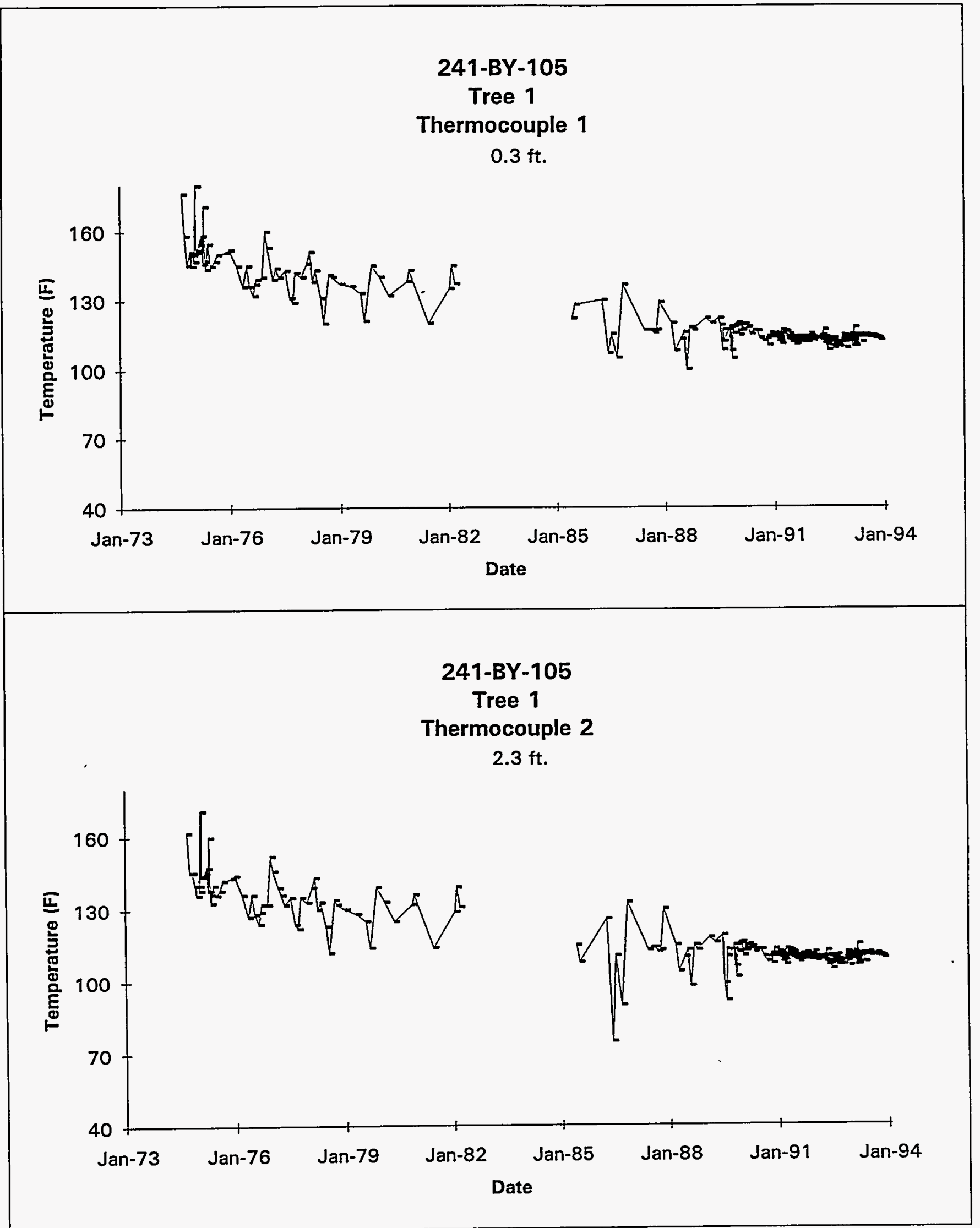

Data obtained from WHC Surveillance Analysis Computer System (SACS), November 12, 1993. 


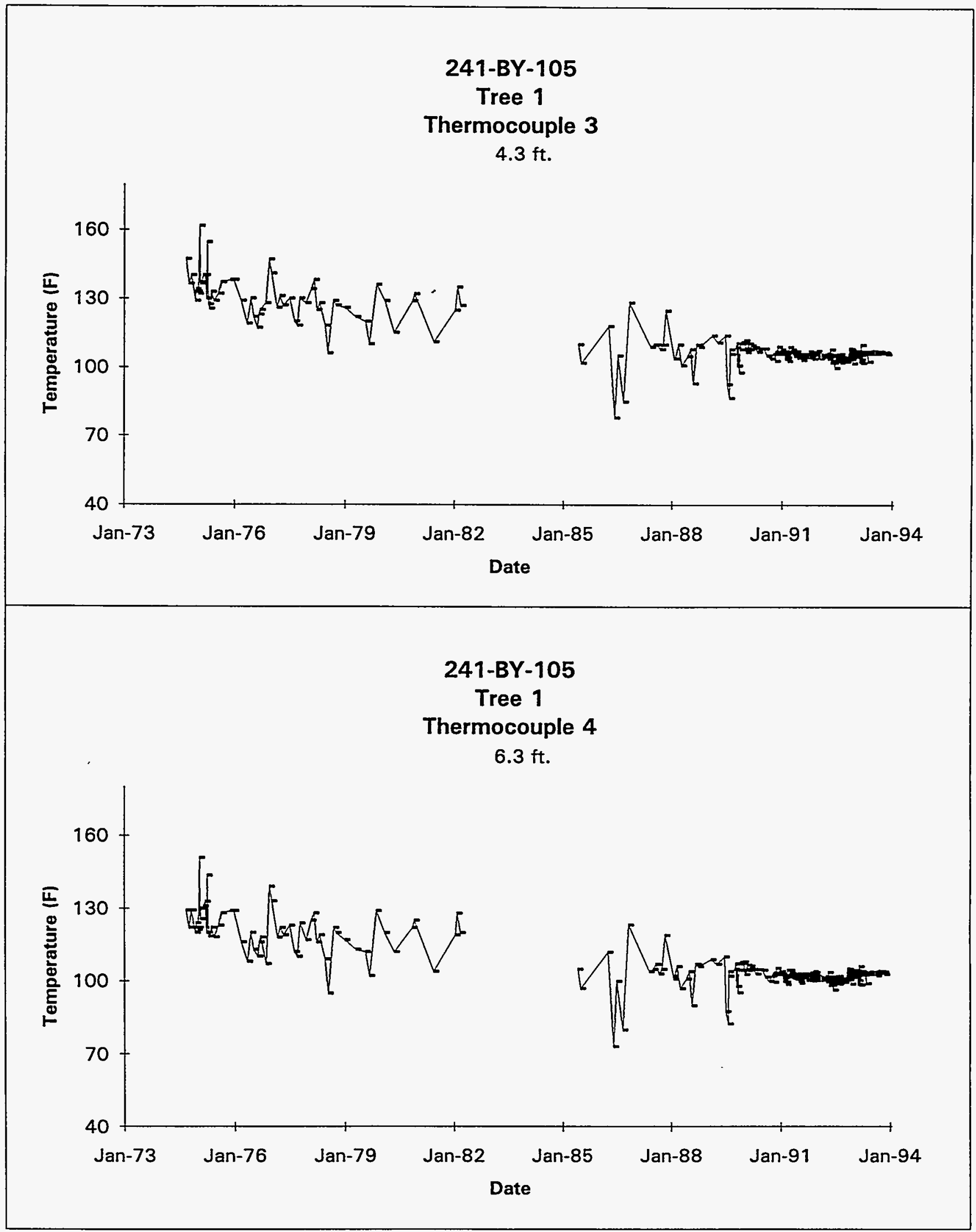

Data obtained from WHC Surveillance Analysis Computer System (SACS), November 12, 1993. 


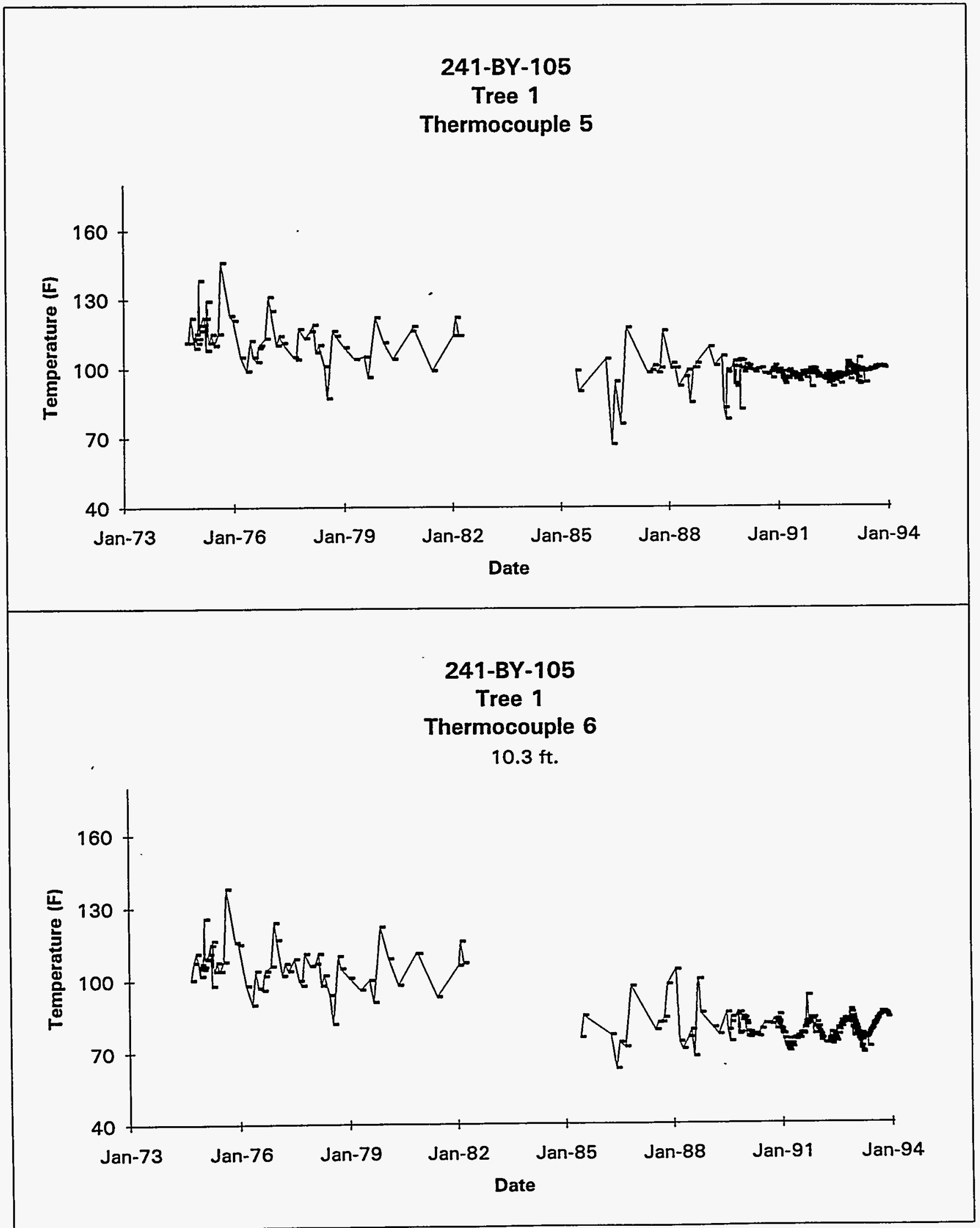

Data obtained from WHC Surveillance Analysis Computer System (SACS), November 12, 1993. 


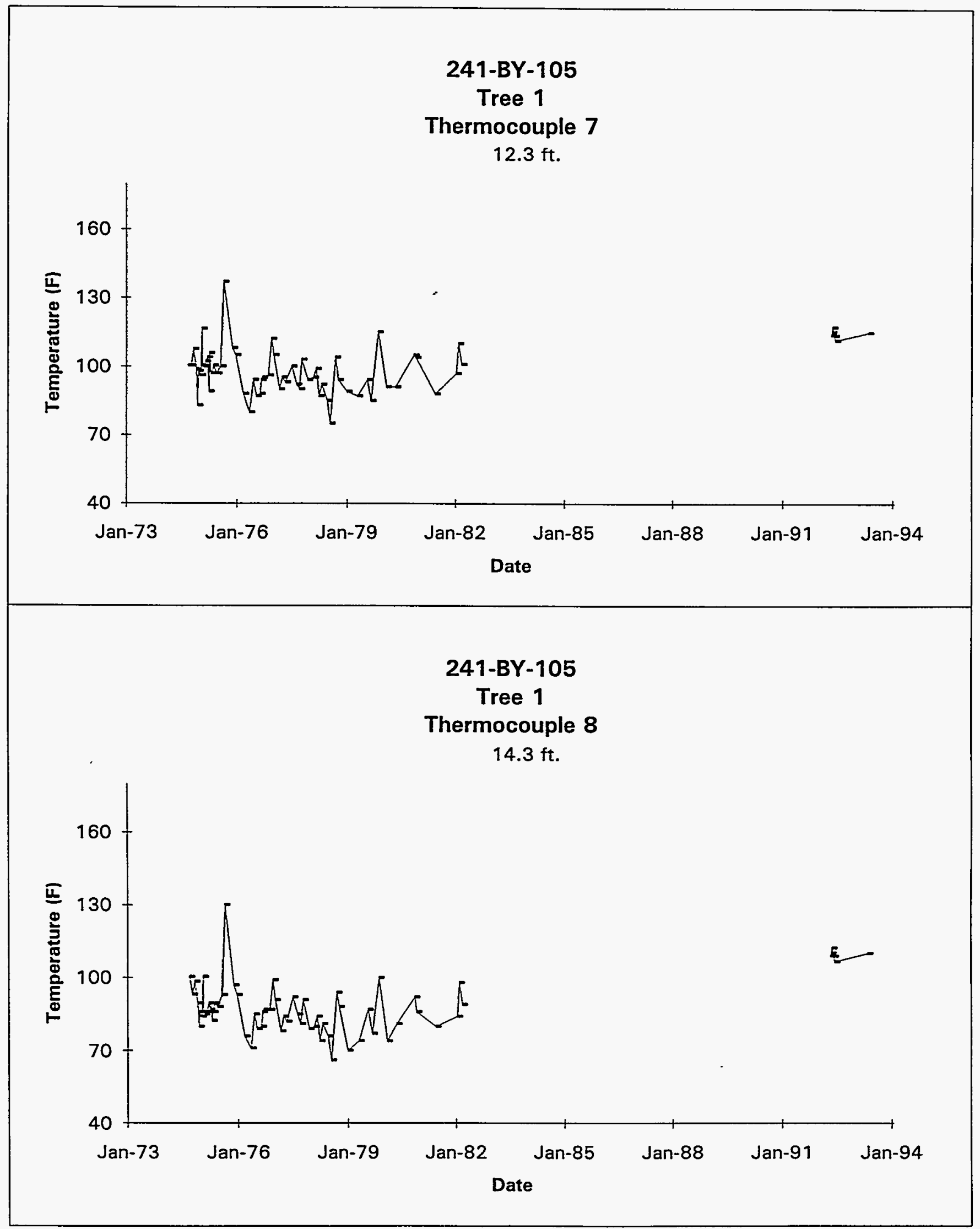

Data obtained from WHC Surveillance Analysis Computer System (SACS), November 12, 1993. 


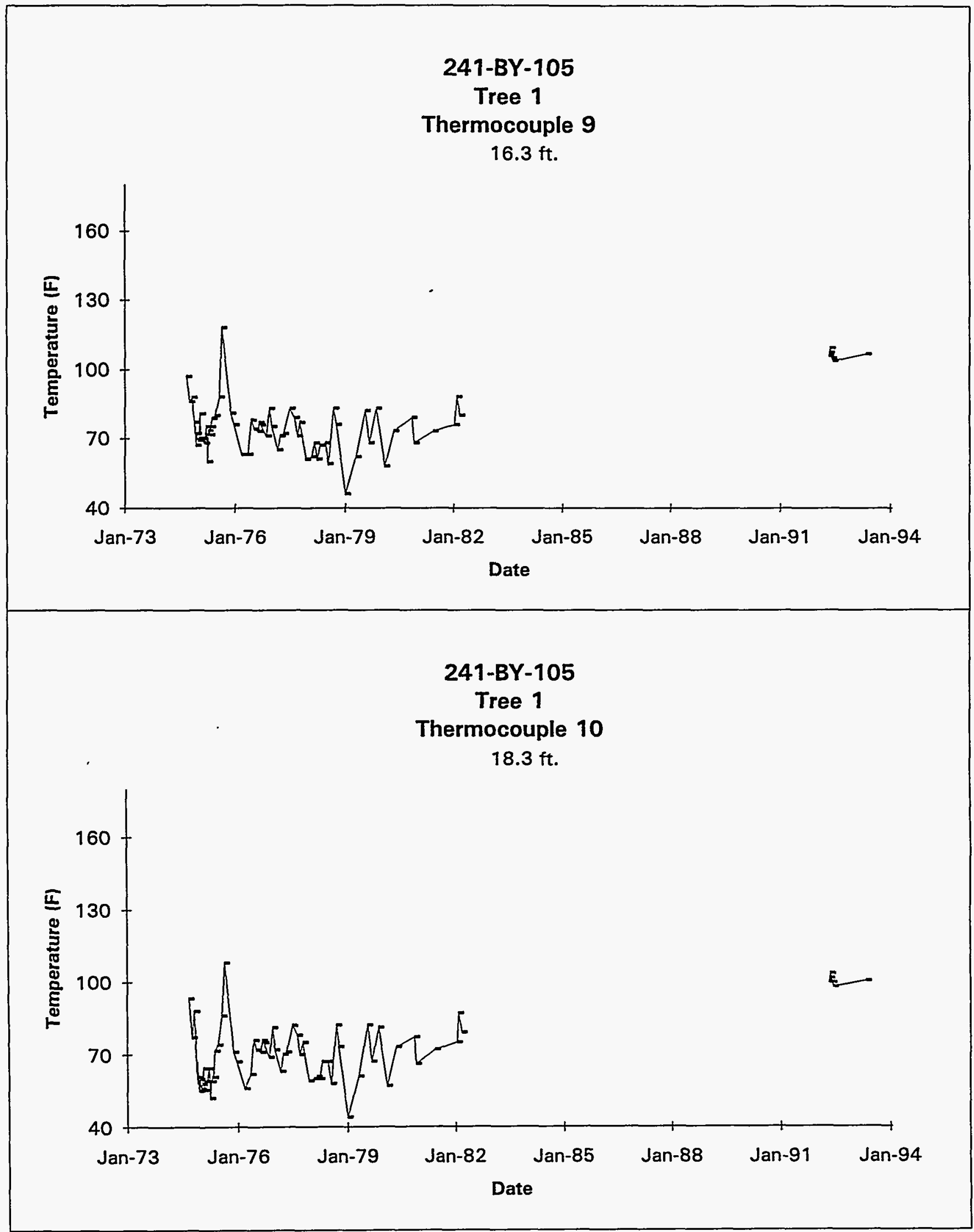

Data obtained from WHC Surveillance Analysis Computer System (SACS), November 12, 1993. 


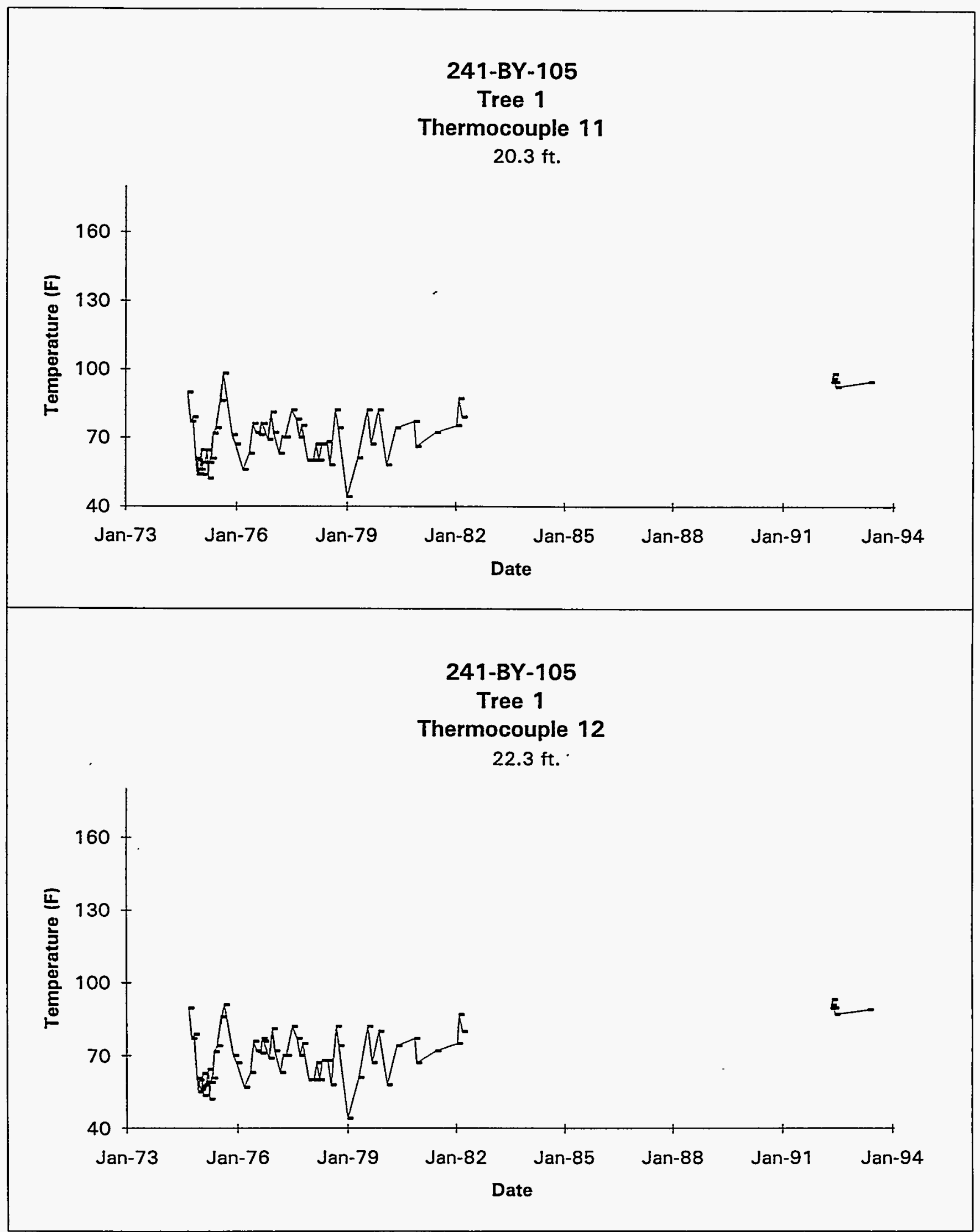

Data obtained from WHC Surveillance Analysis Computer System (SACS), November 12, 1993. 


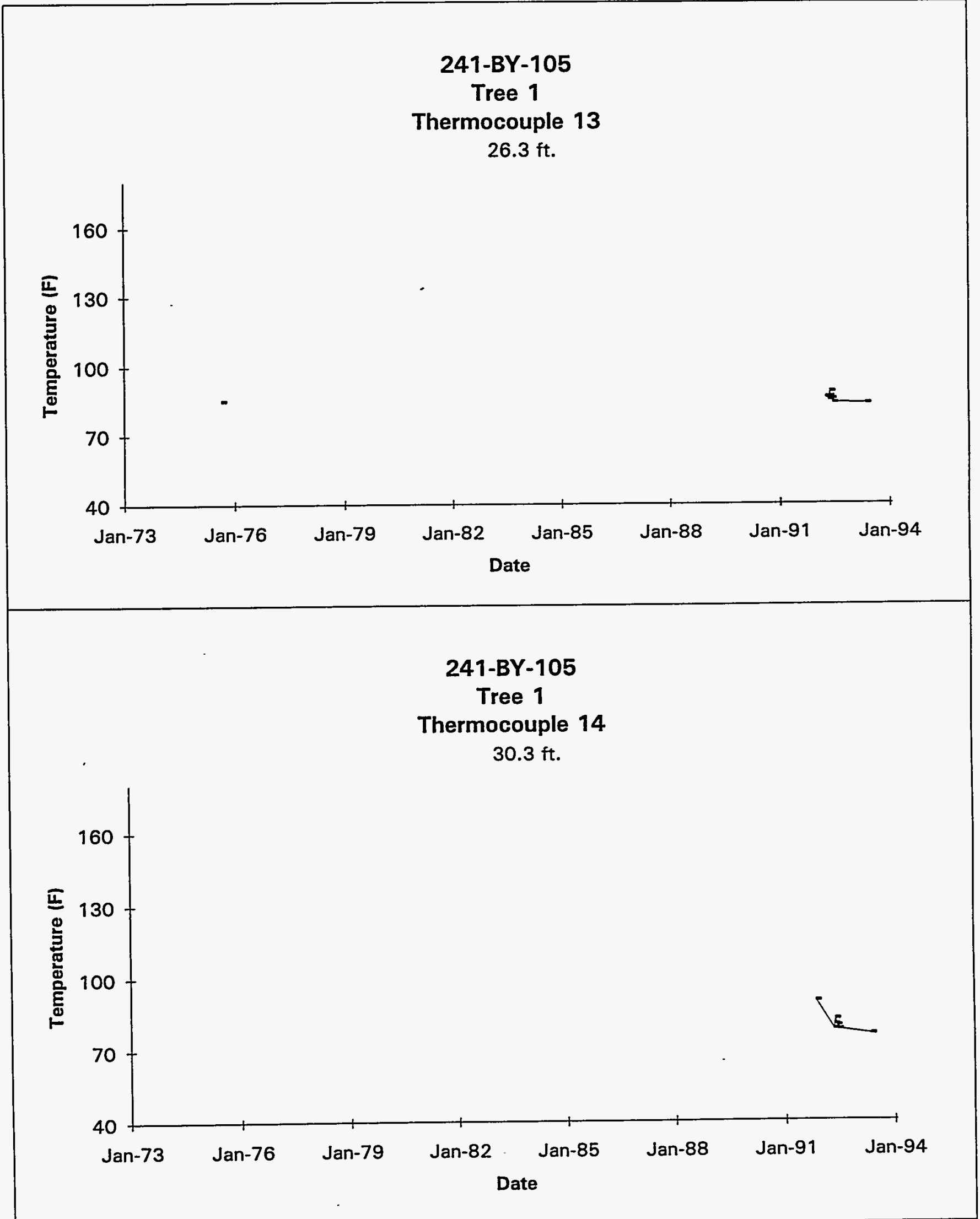

Data obtained from WHC Surveillance Analysis Computer System (SACS), November 12, 1993. 


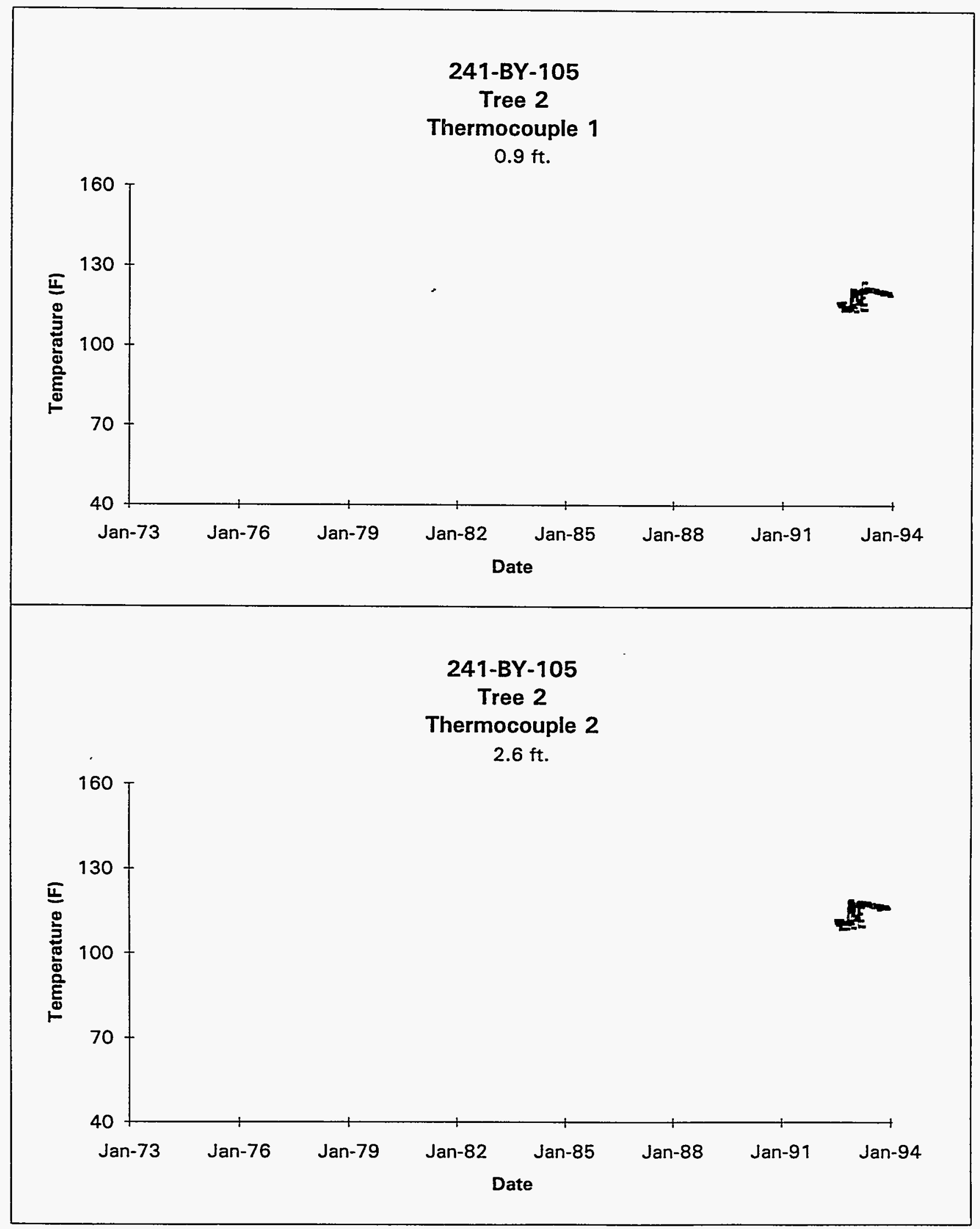

Data obtained from WHC Surveillance Analysis Computer System (SACS), November 12, 1993. 


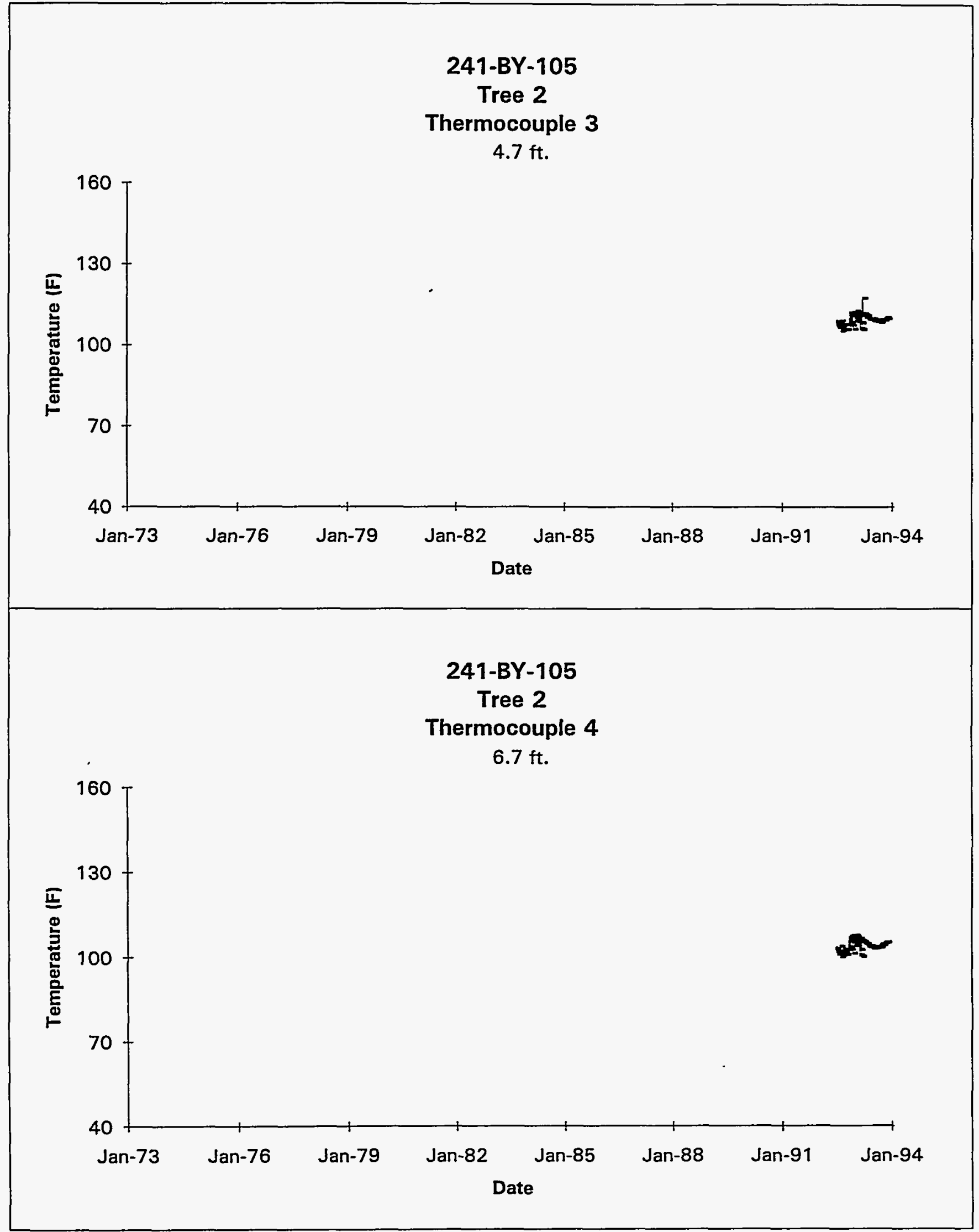

Data obtained from WHC Surveillance Analysis Computer System (SACS), November 12, 1993.

D-39 


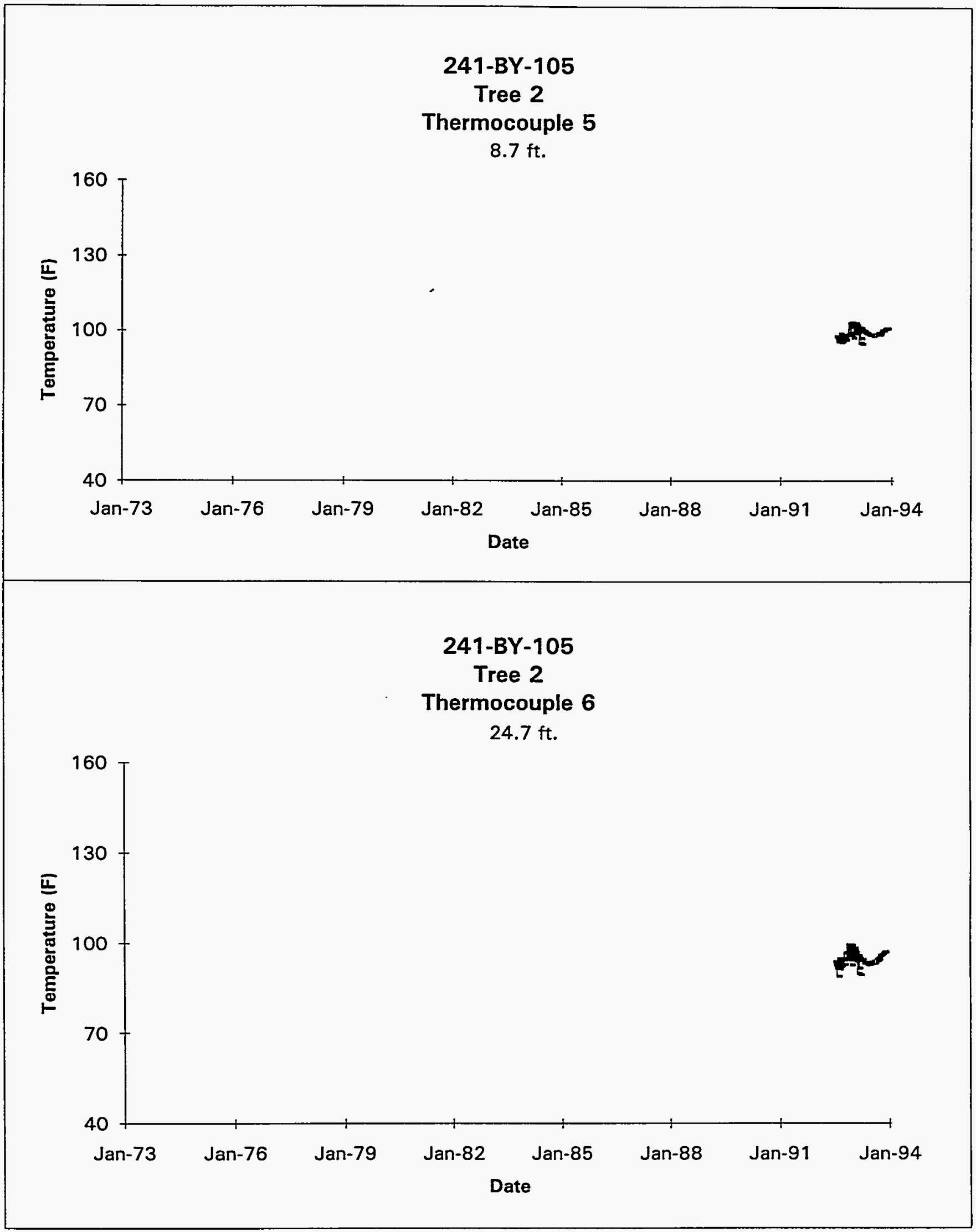

Data obtained from WHC Surveillance Analysis Computer System (SACS), November 12, 1993.

D-40 


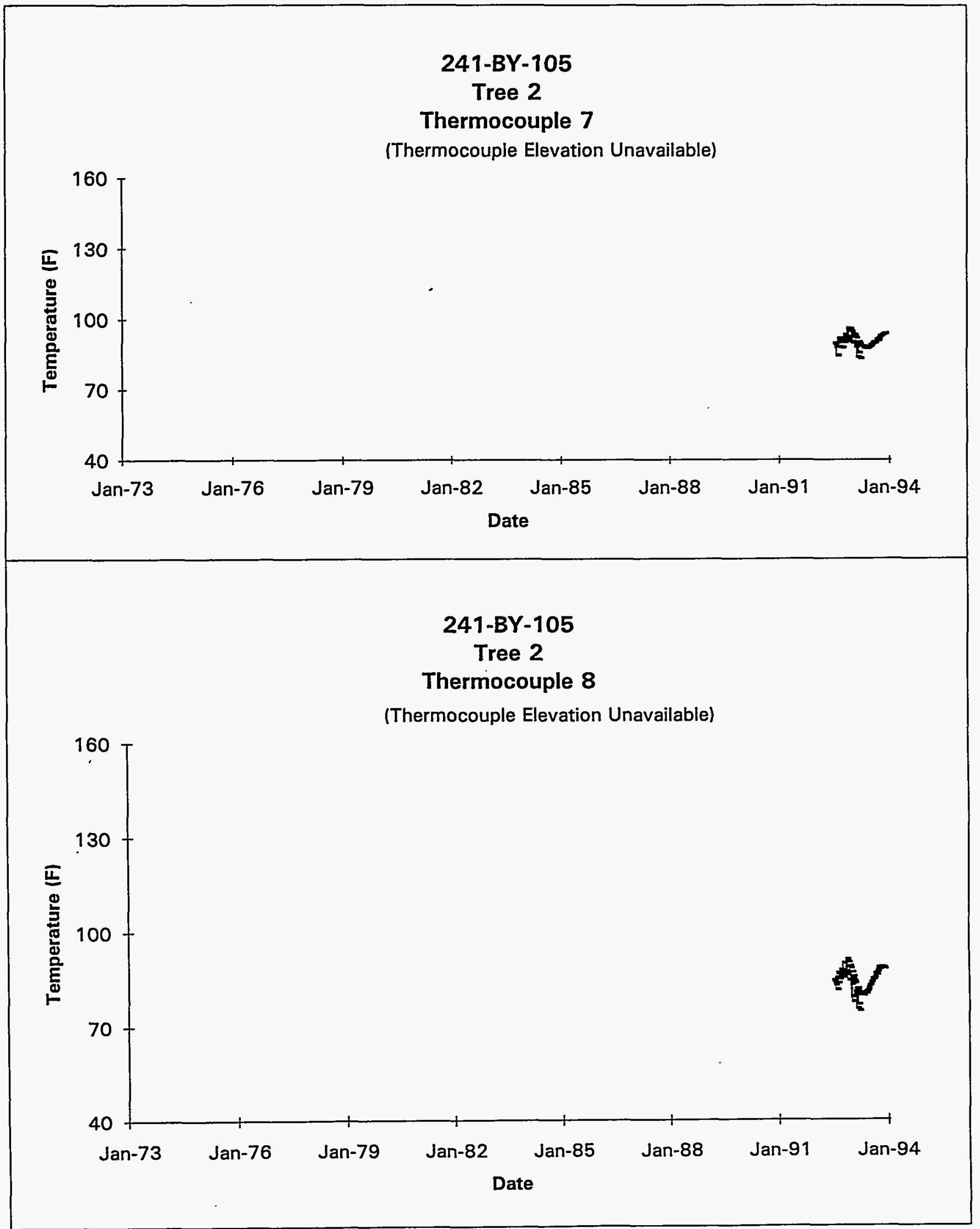

Data obtained from WHC Surveillance Analysis Computer System (SACS), November 12, 1993. 


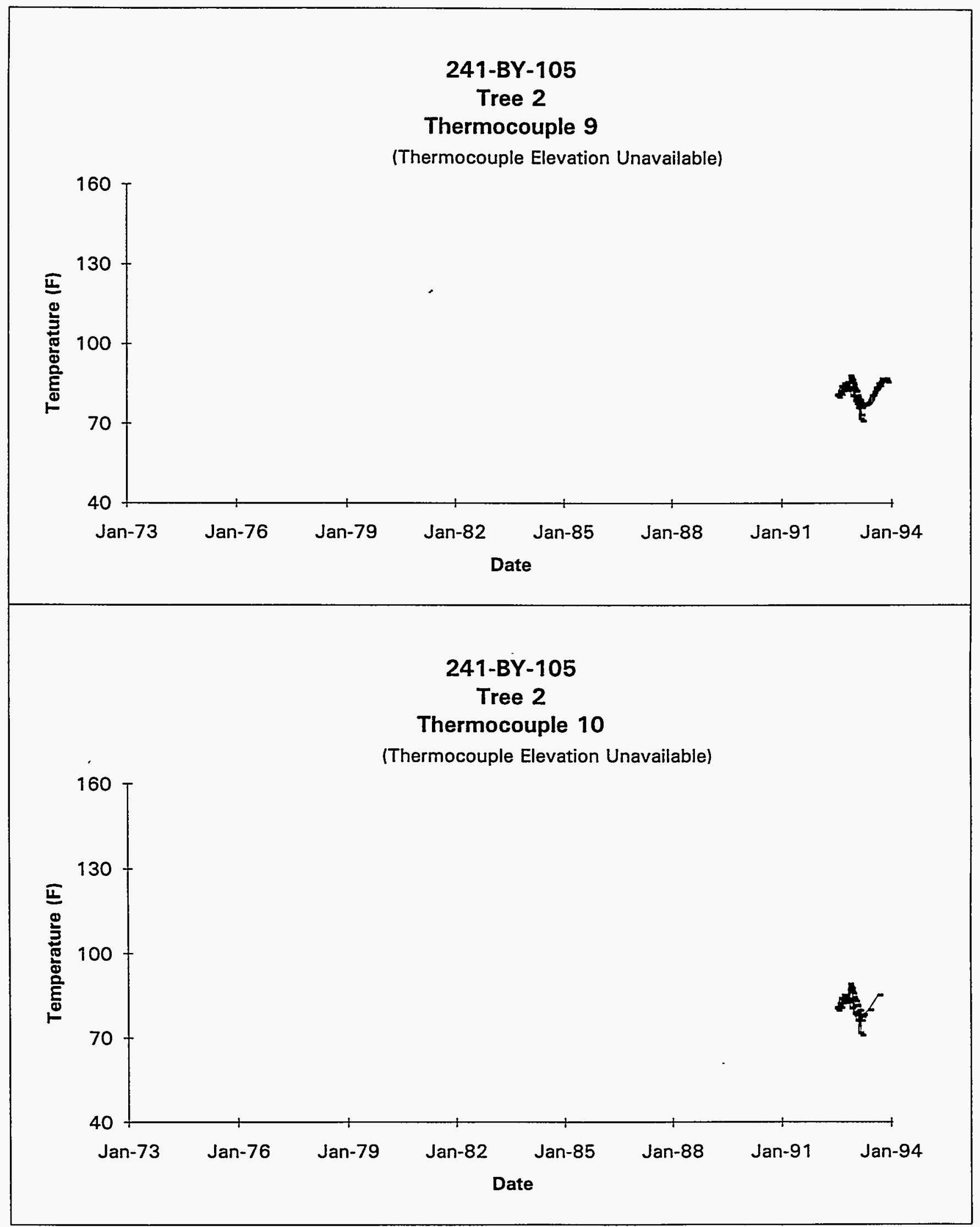

Data obtained from WHC Surveillance Analysis Computer System (SACS), November 12, 1993. 


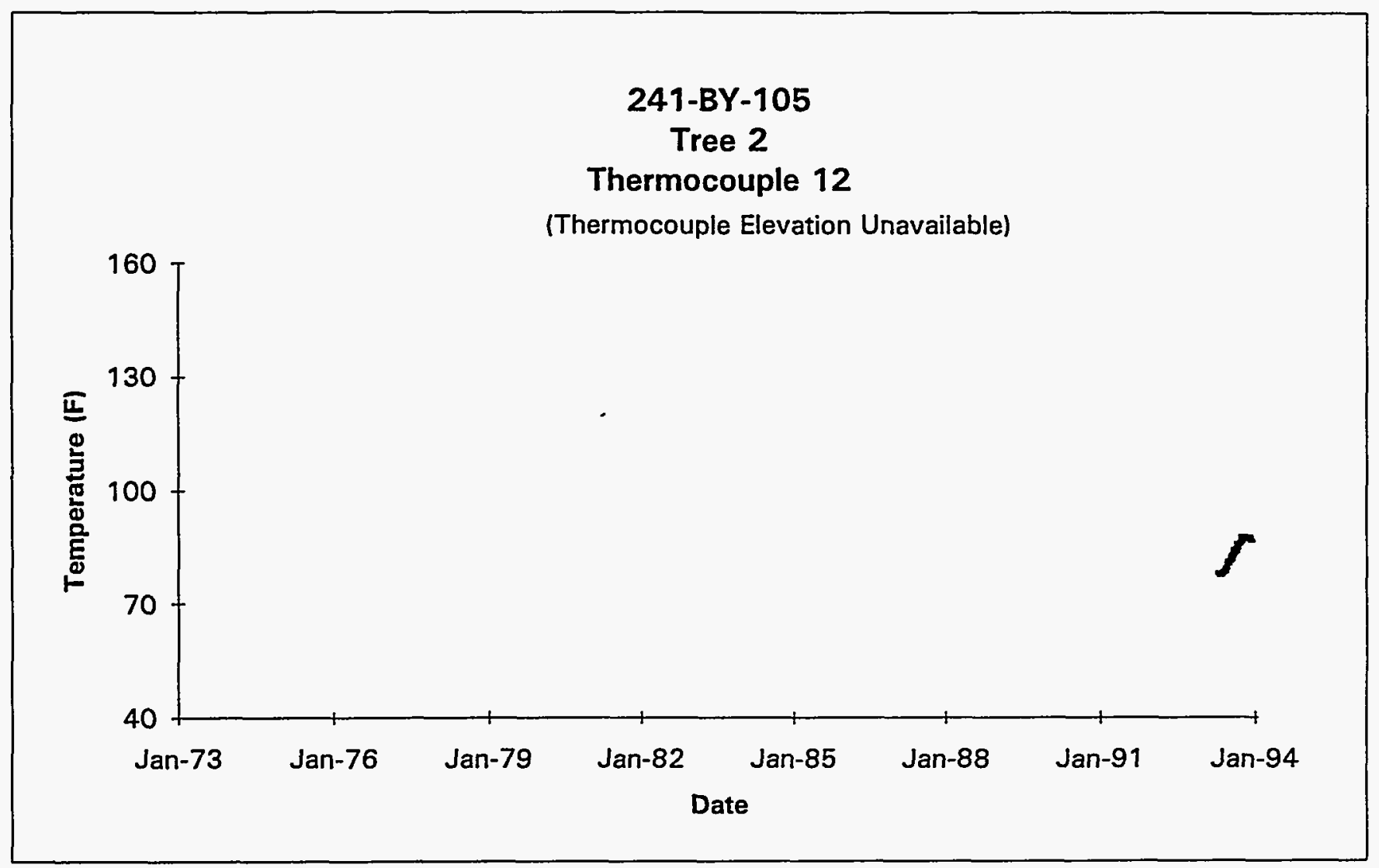

Data obtained from WHC Surveillance Analysis Computer System (SACS), November 12, 1993. 


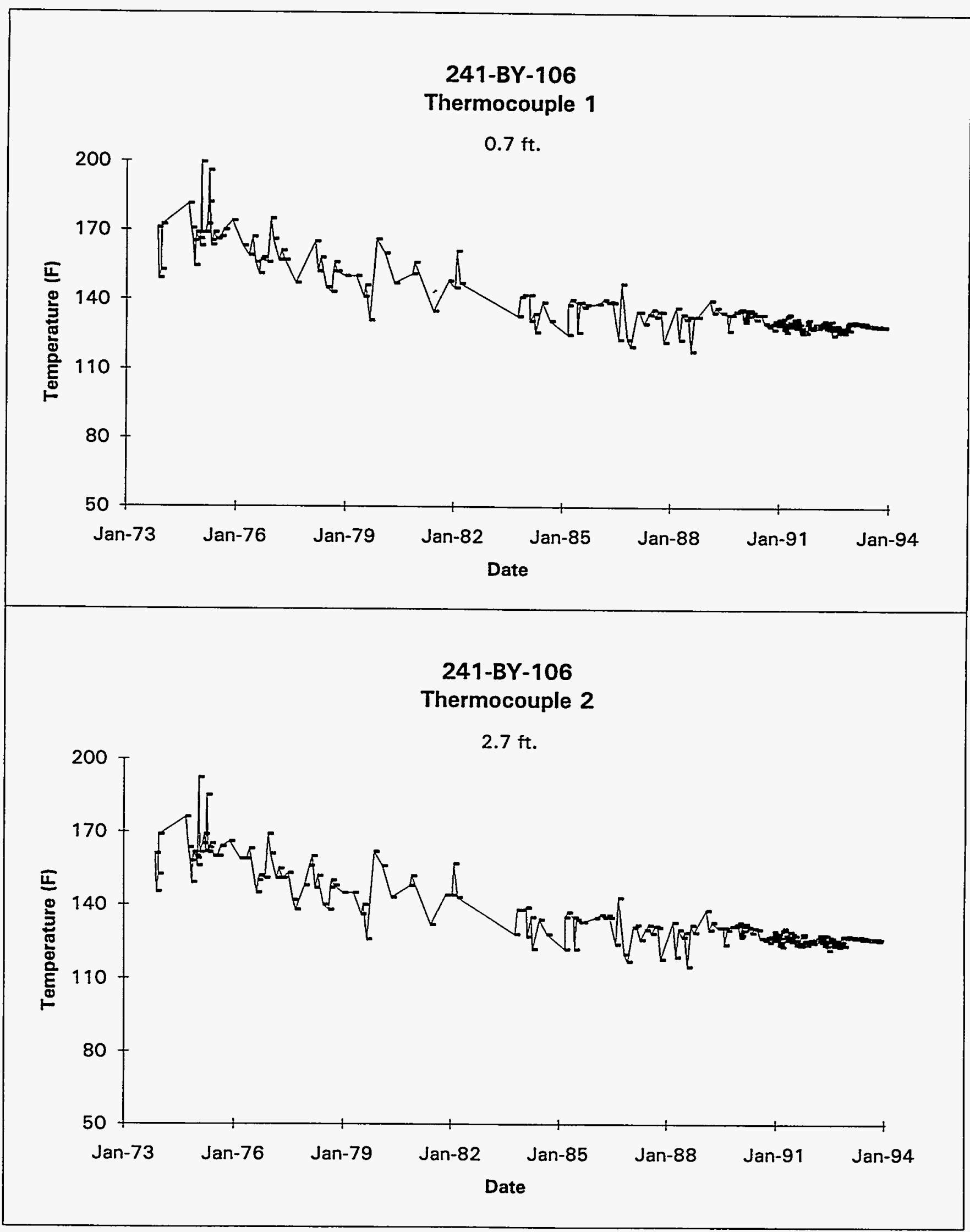

Data obtained from WHC Surveillance Analysis Computer System (SACS), November 12, 1993. 


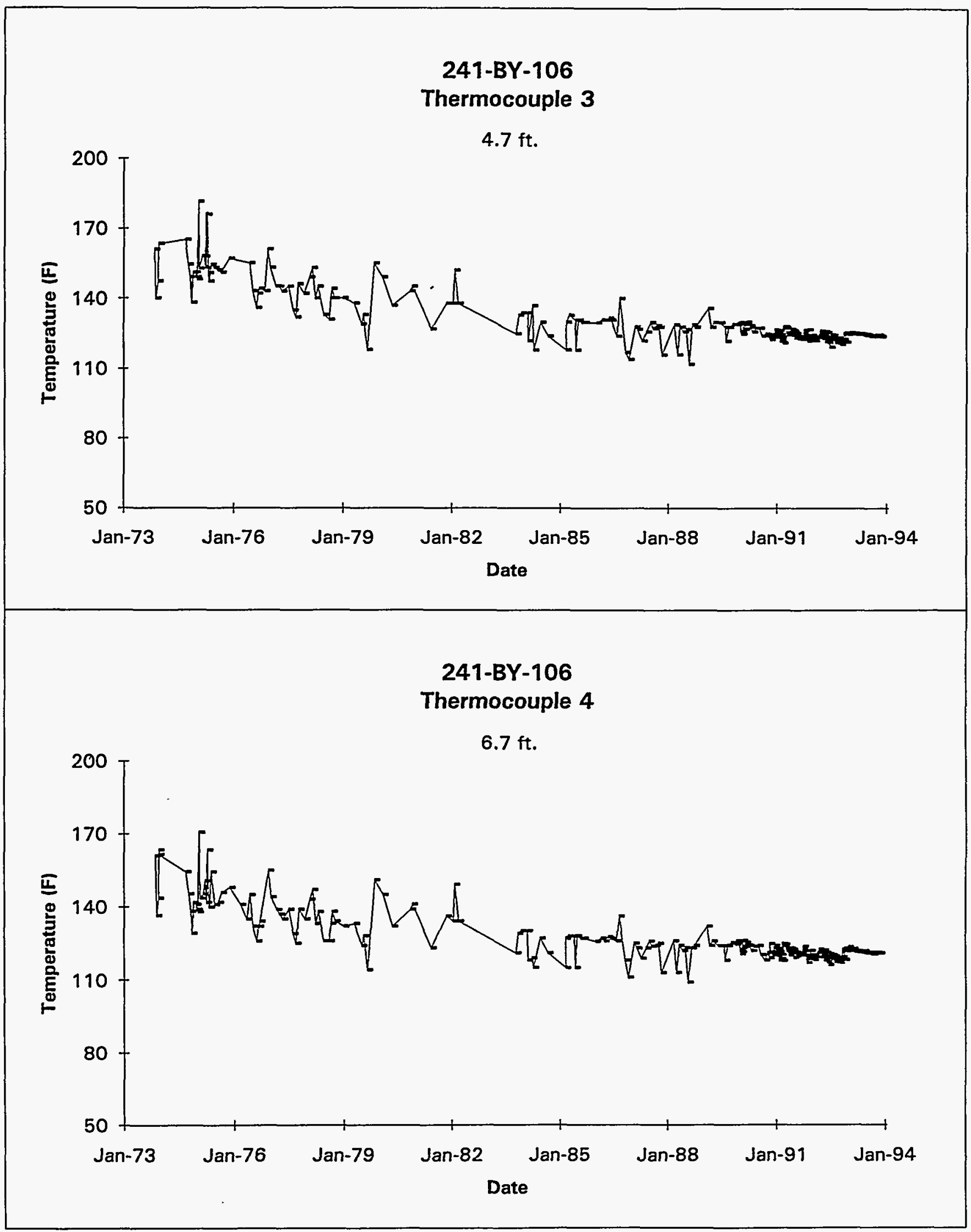

Data obtained from WHC Surveillance Analysis Computer System (SACS), November 12, 1993. 


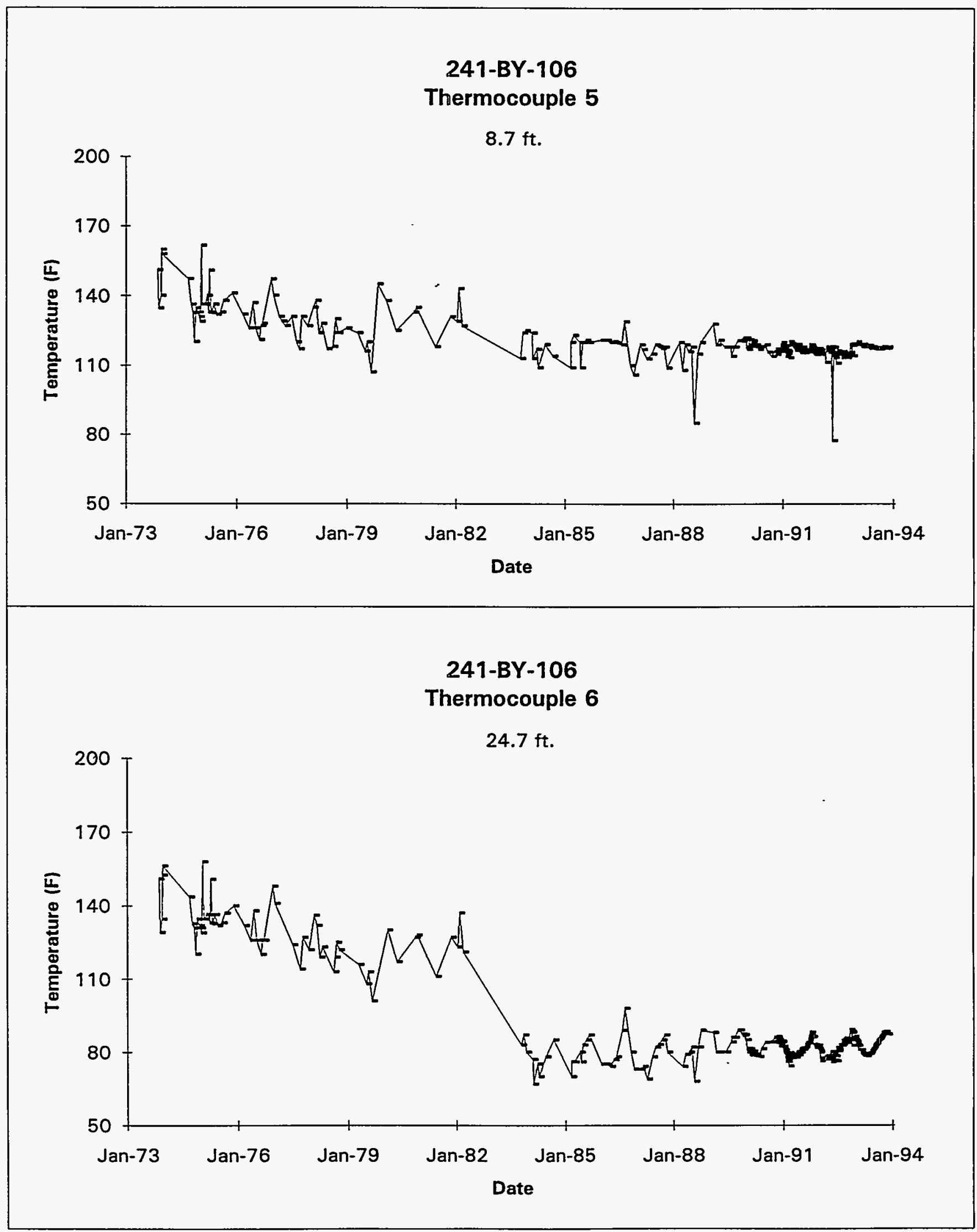

Data obtained from WHC Surveillance Analysis Computer System (SACS), November 12, 1993. 


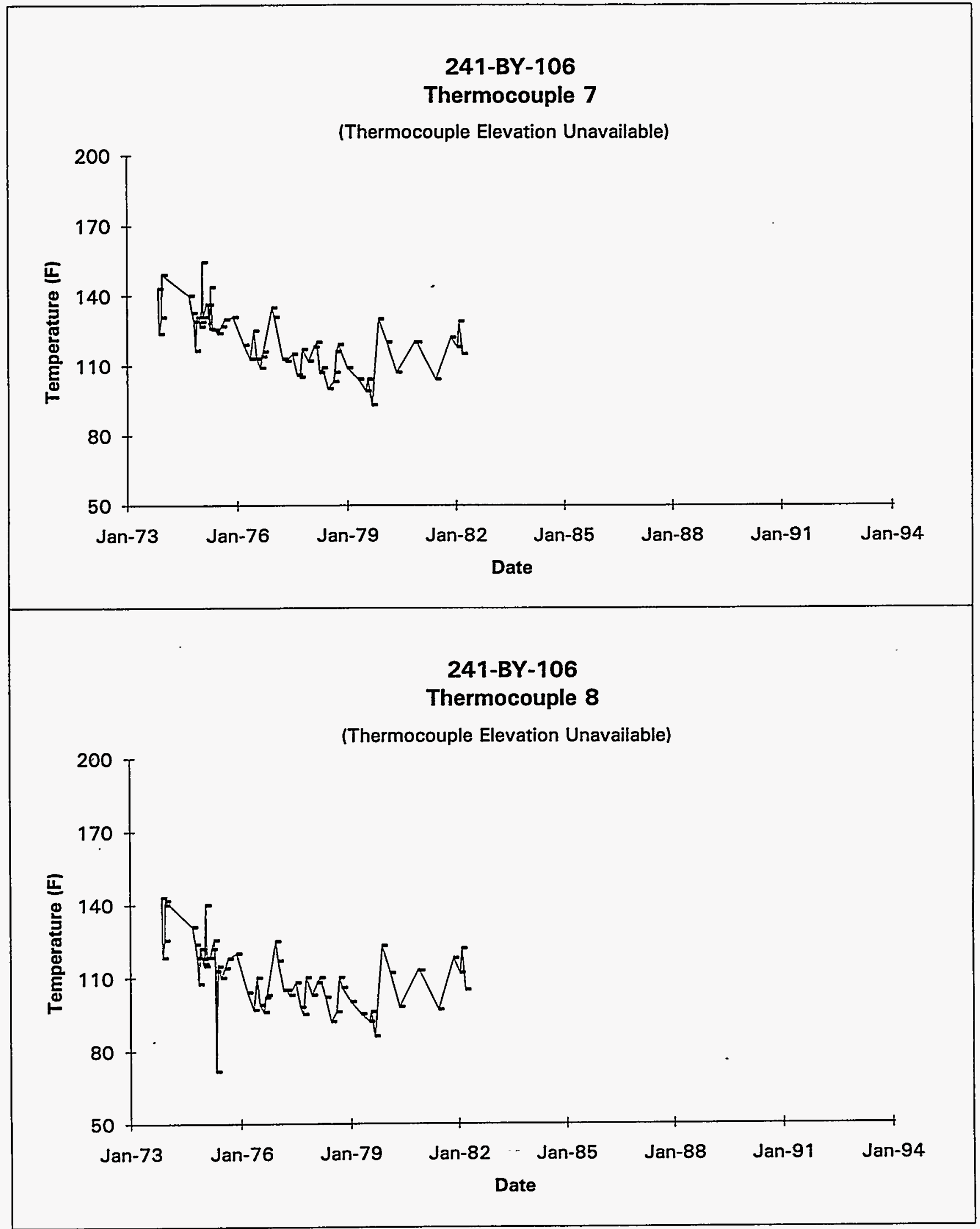

Data obtained from WHC Surveillance Analysis Computer System (SACS), November 12, 1993. 


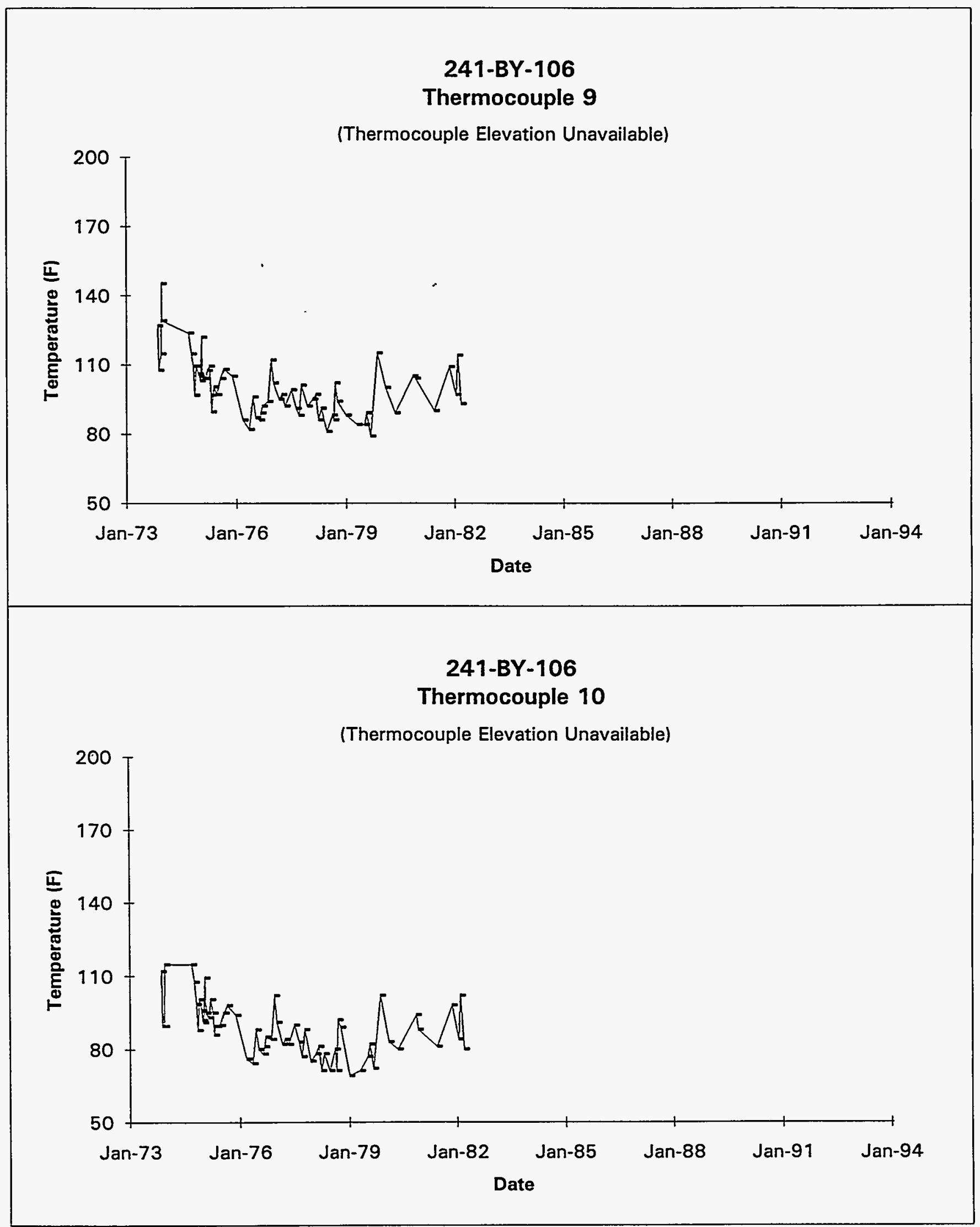

Data obtained from WHC Surveillance Analysis Computer System (SACS), November 12, 1993. 


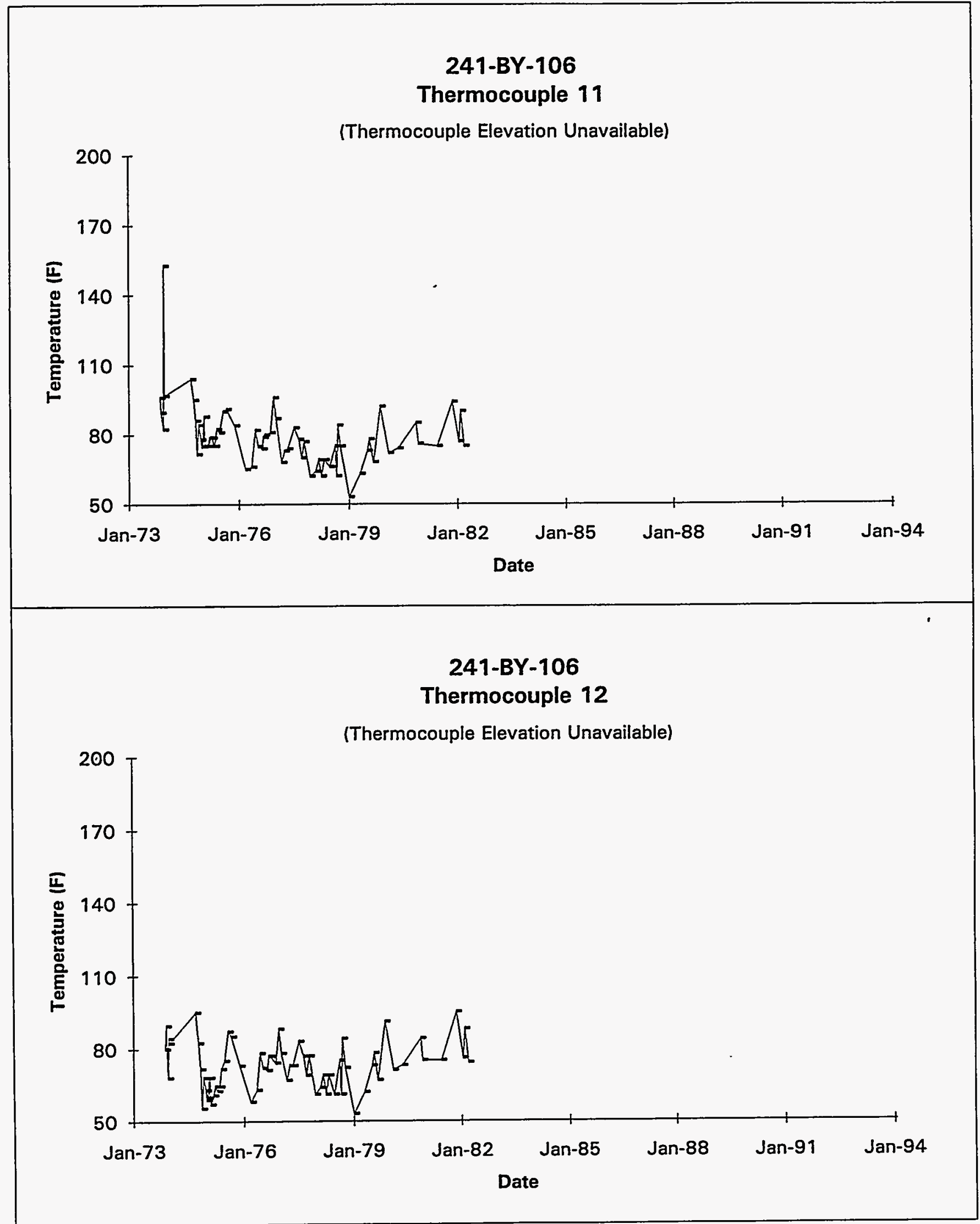

Data obtained from WHC Surveillance Analysis Computer System (SACS), November 12, 1993. 


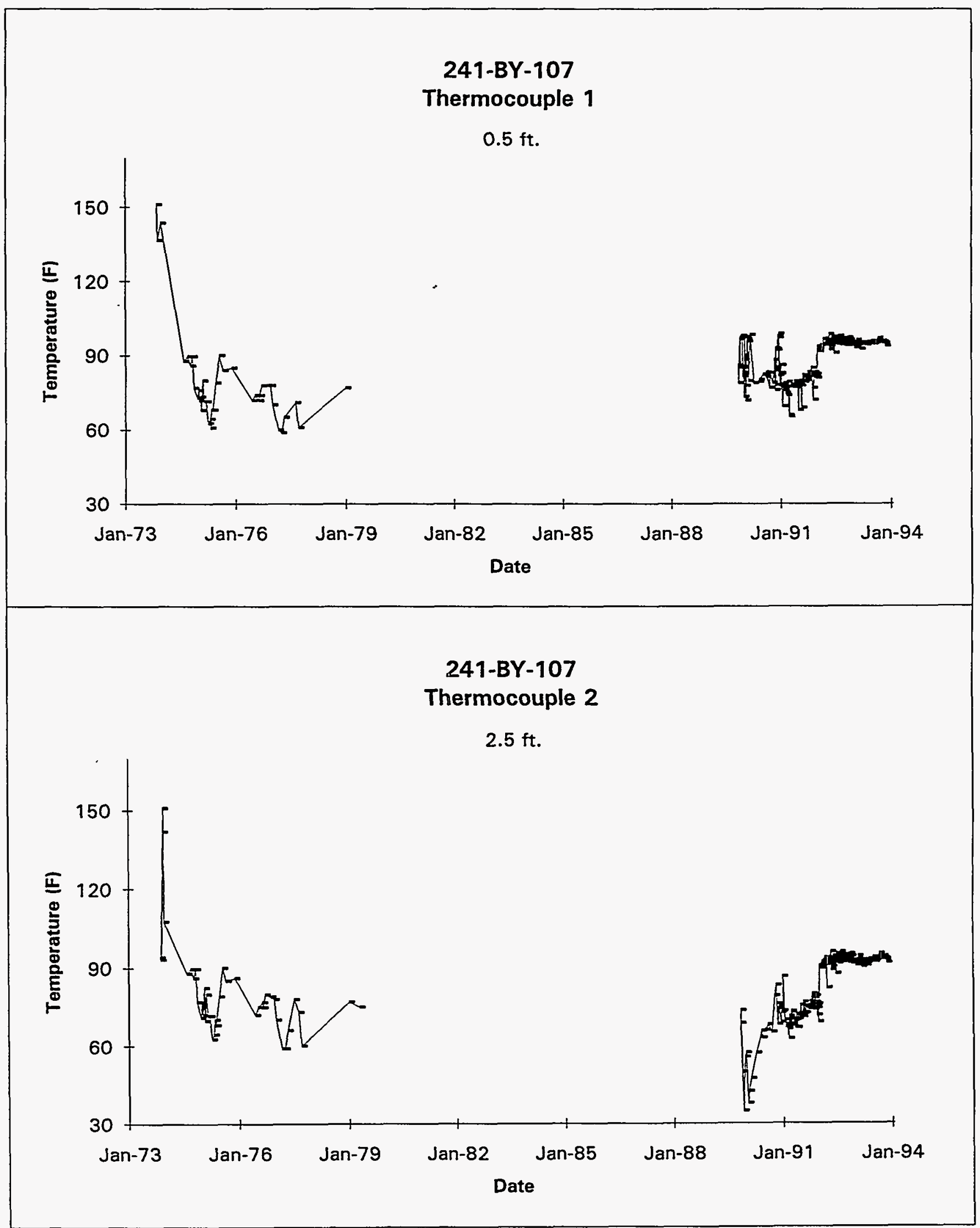

Data obtained from WHC Surveillance Analysis Computer System (SACS), November 12, 1993. 


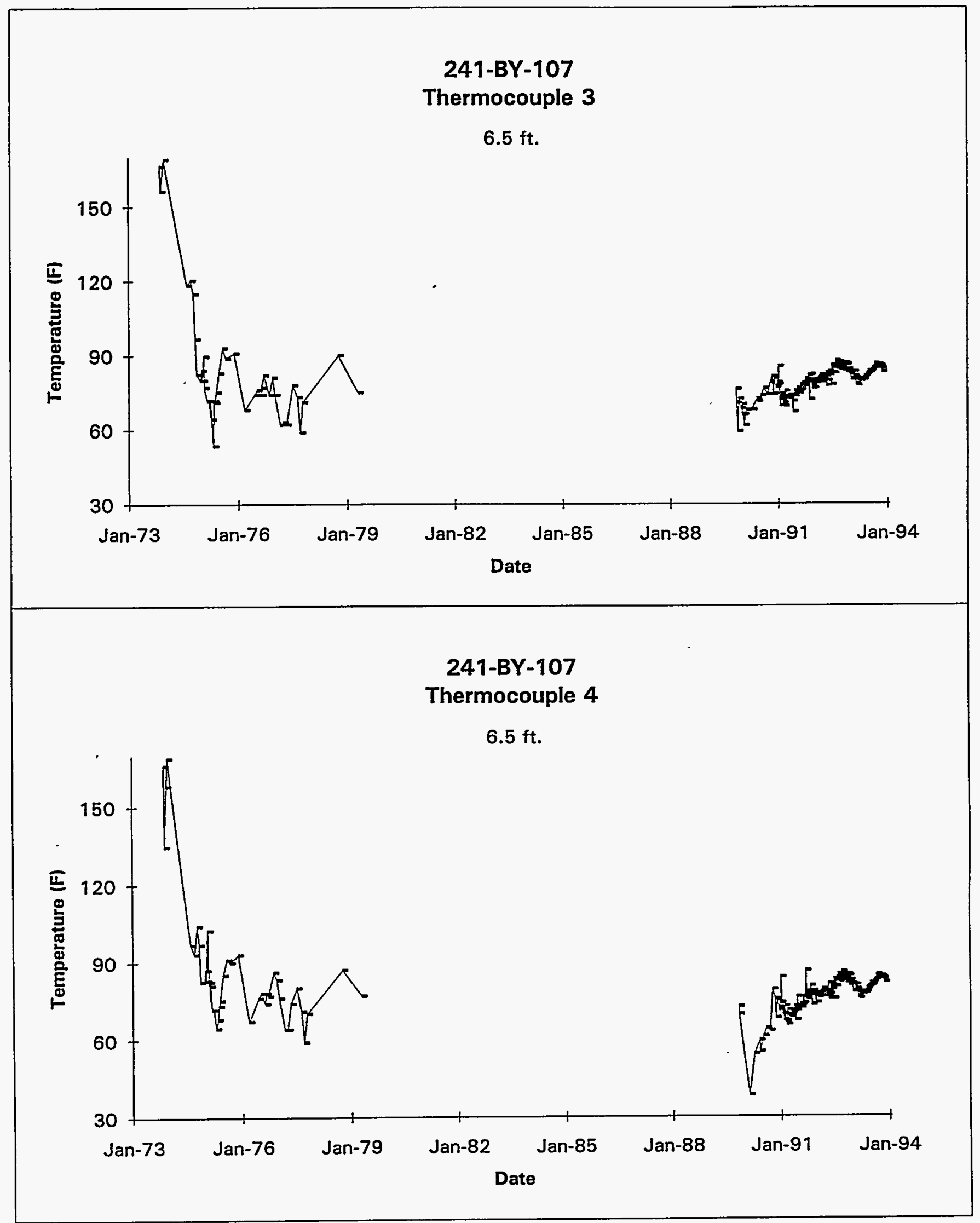

Data obtained from WHC Surveillance Analysis Computer System (SACS), November 12, 1993. 


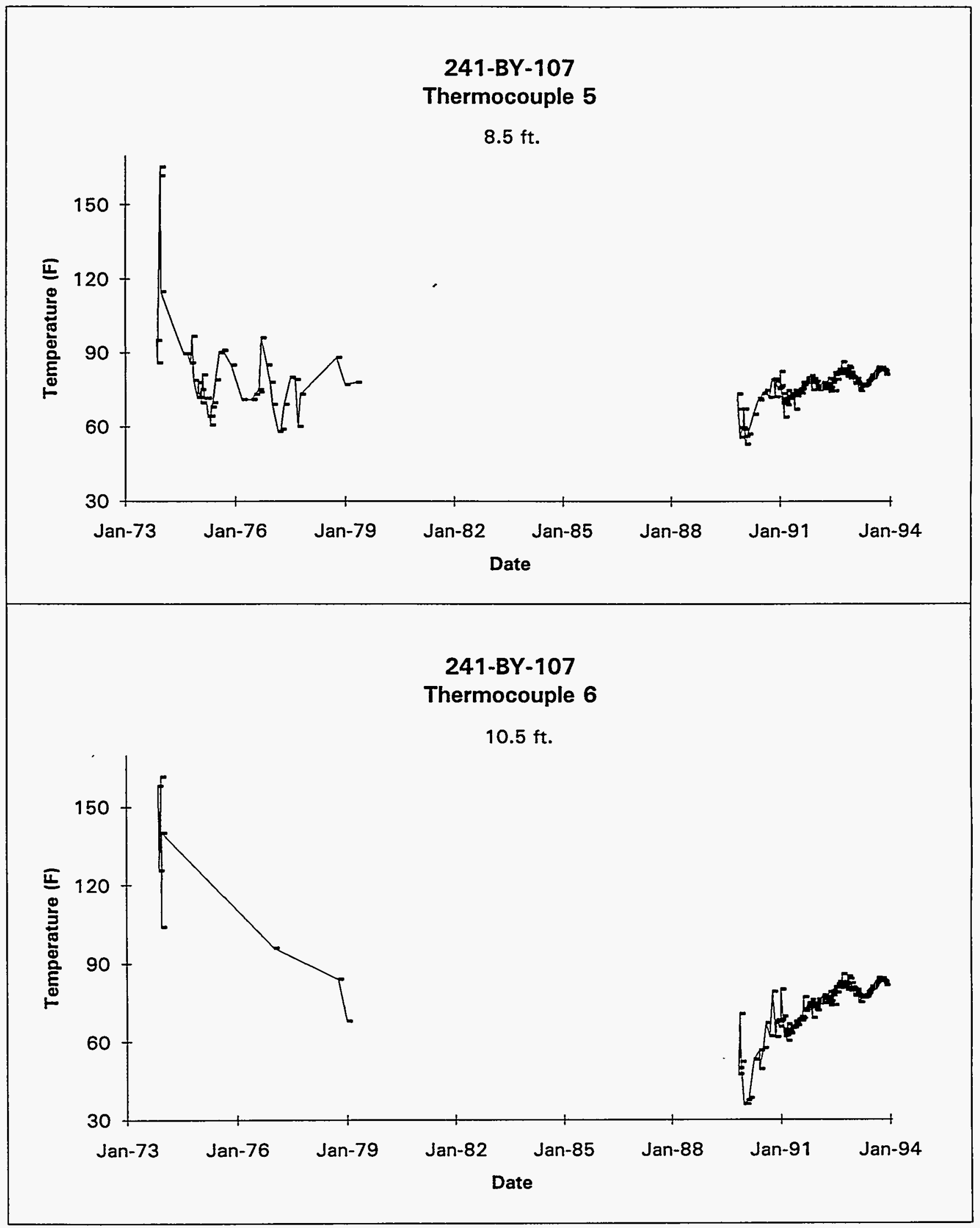

Data obtained from WHC Surveillance Analysis Computer System (SACS), November 12, 1993.

D-52 


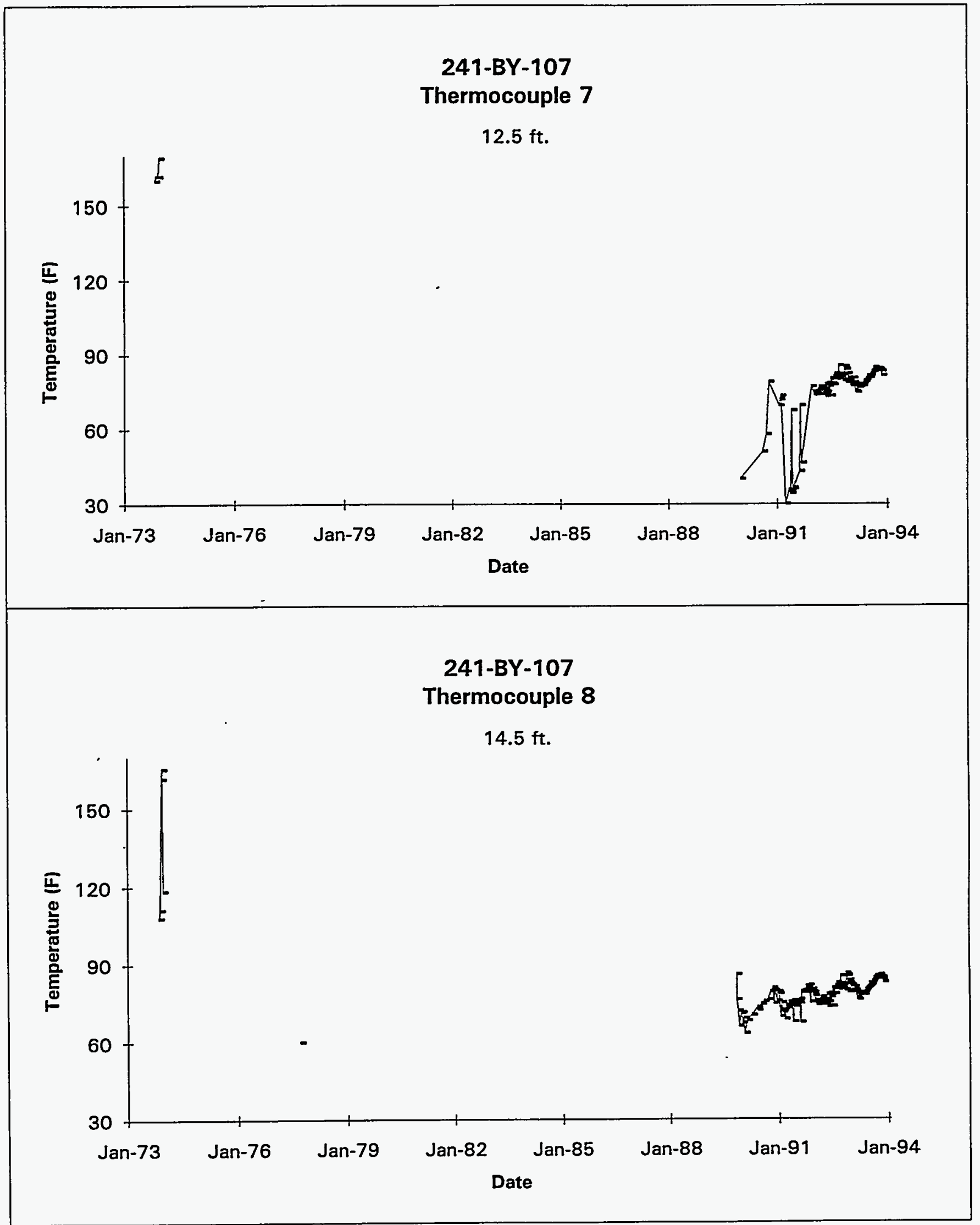

Data obtained from WHC Surveillance Analysis Computer System (SACS), November 12, 1993. 


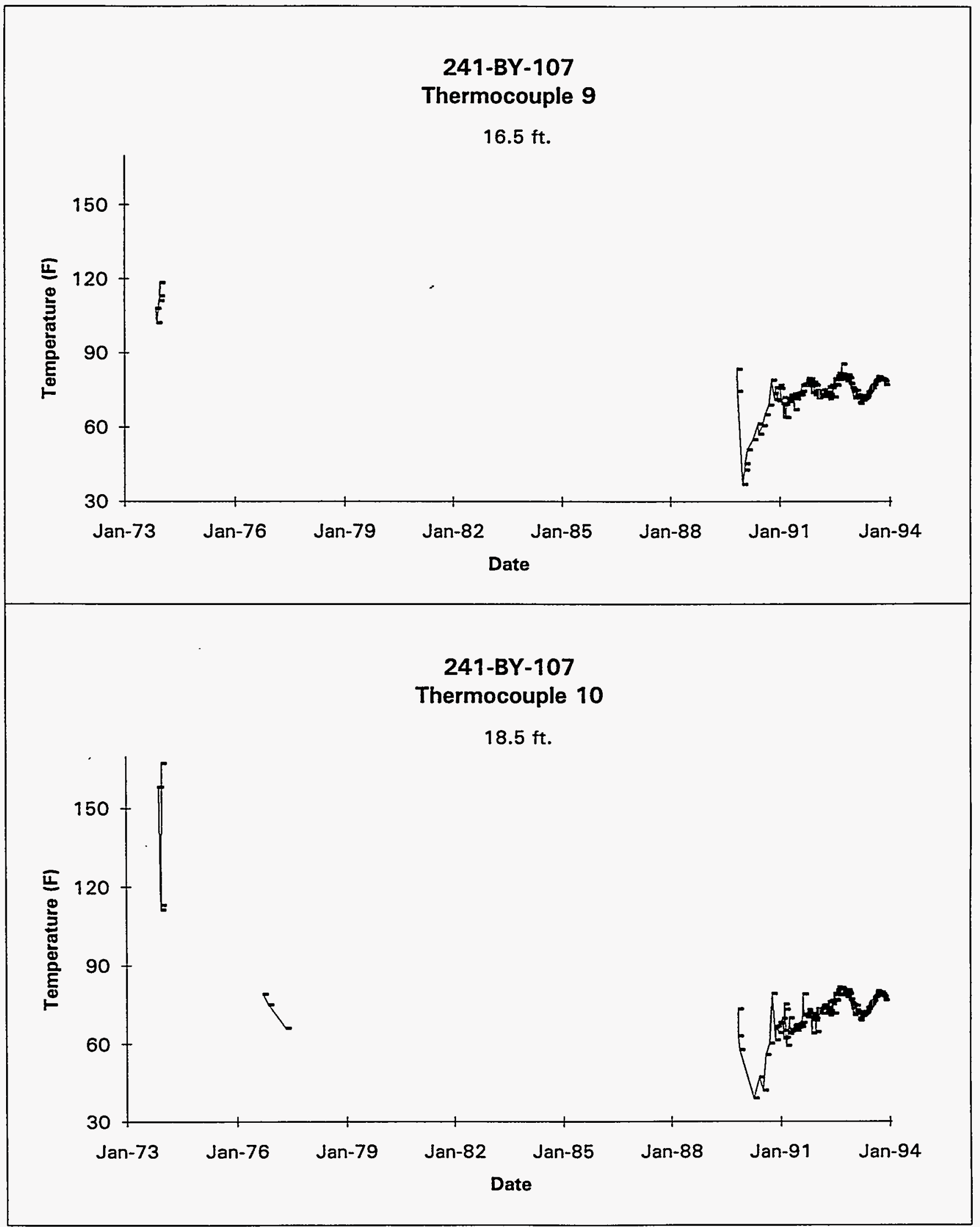

Data obtained from WHC Surveillance Analysis Computer System (SACS), November 12, 1993. 


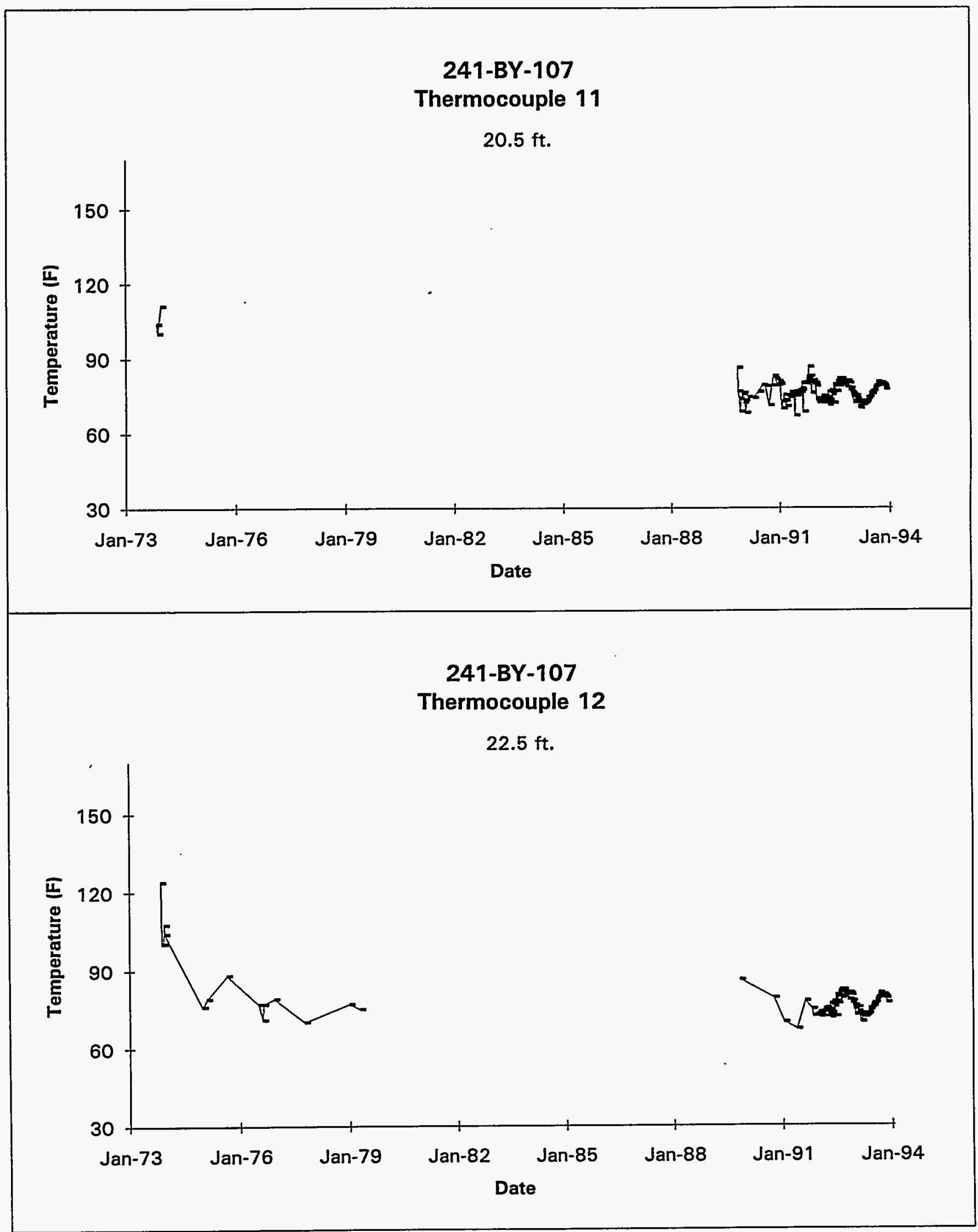

Data obtained from WHC Surveillance Analysis Computer System (SACS), November 12, 1993.

D-55 


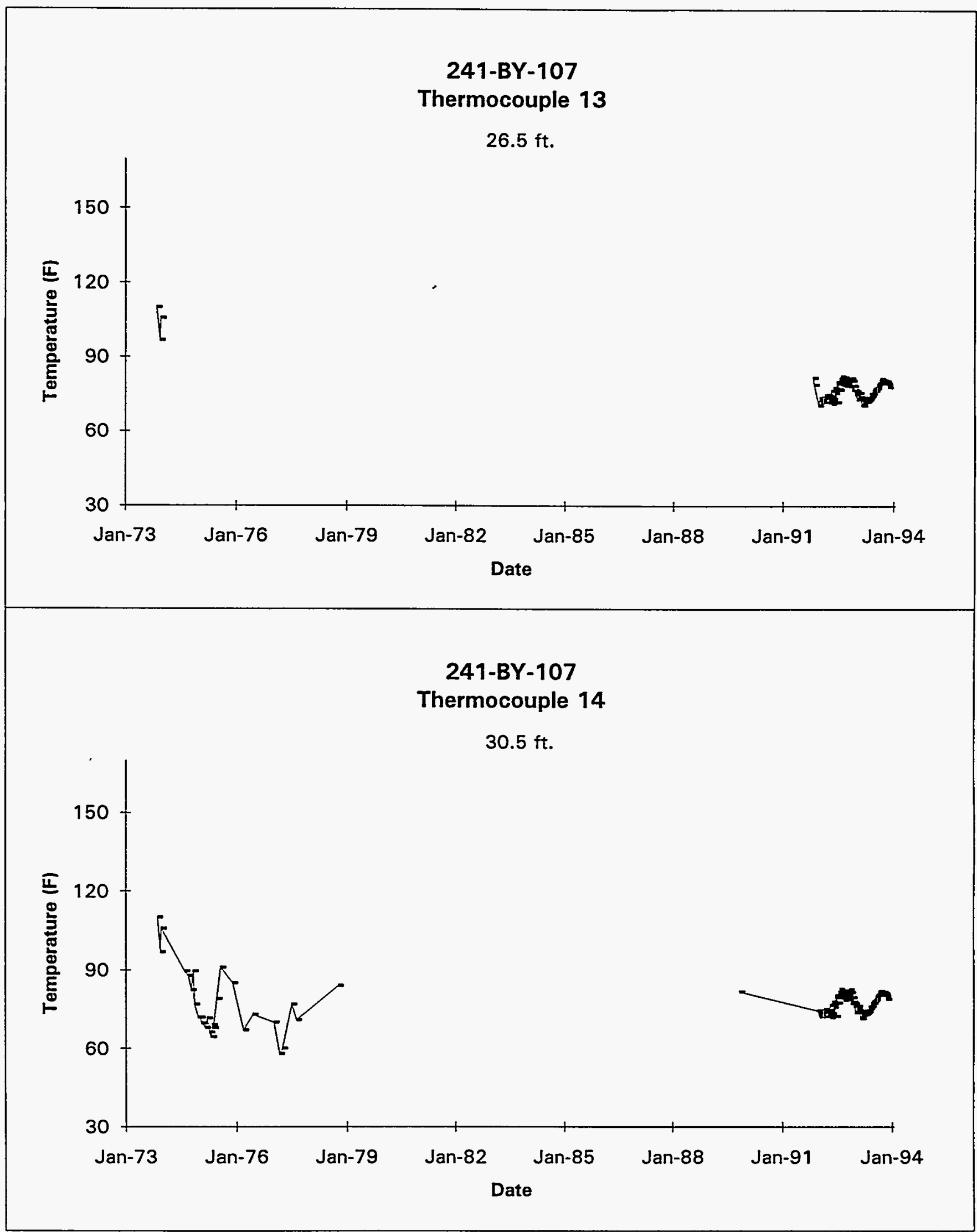

Data obtained from WHC Surveillance Analysis Computer System (SACS), November 12, 1993. 


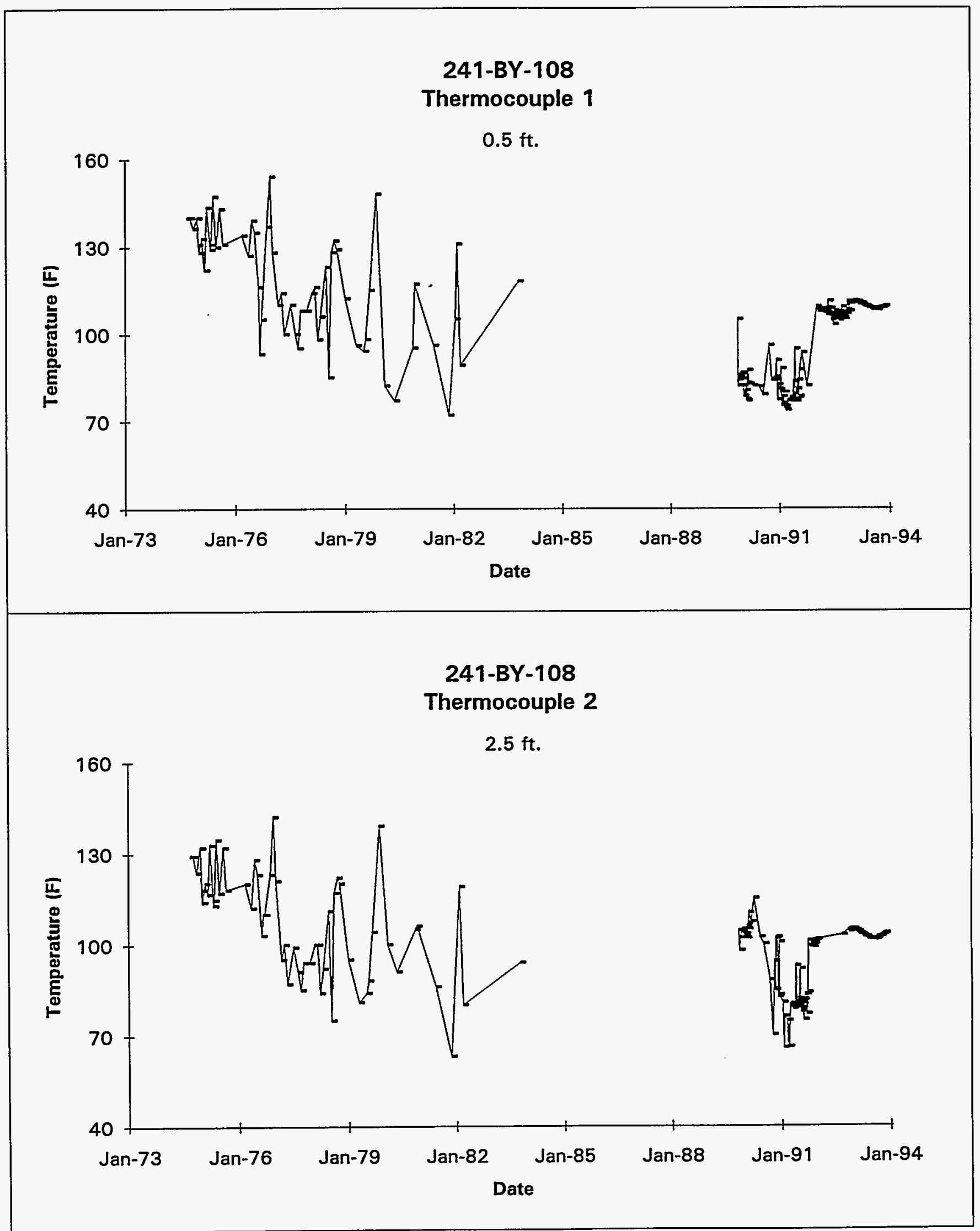

Data obtained from WHC Surveillance Analysis Computer System (SACS), November 12, 1993. 


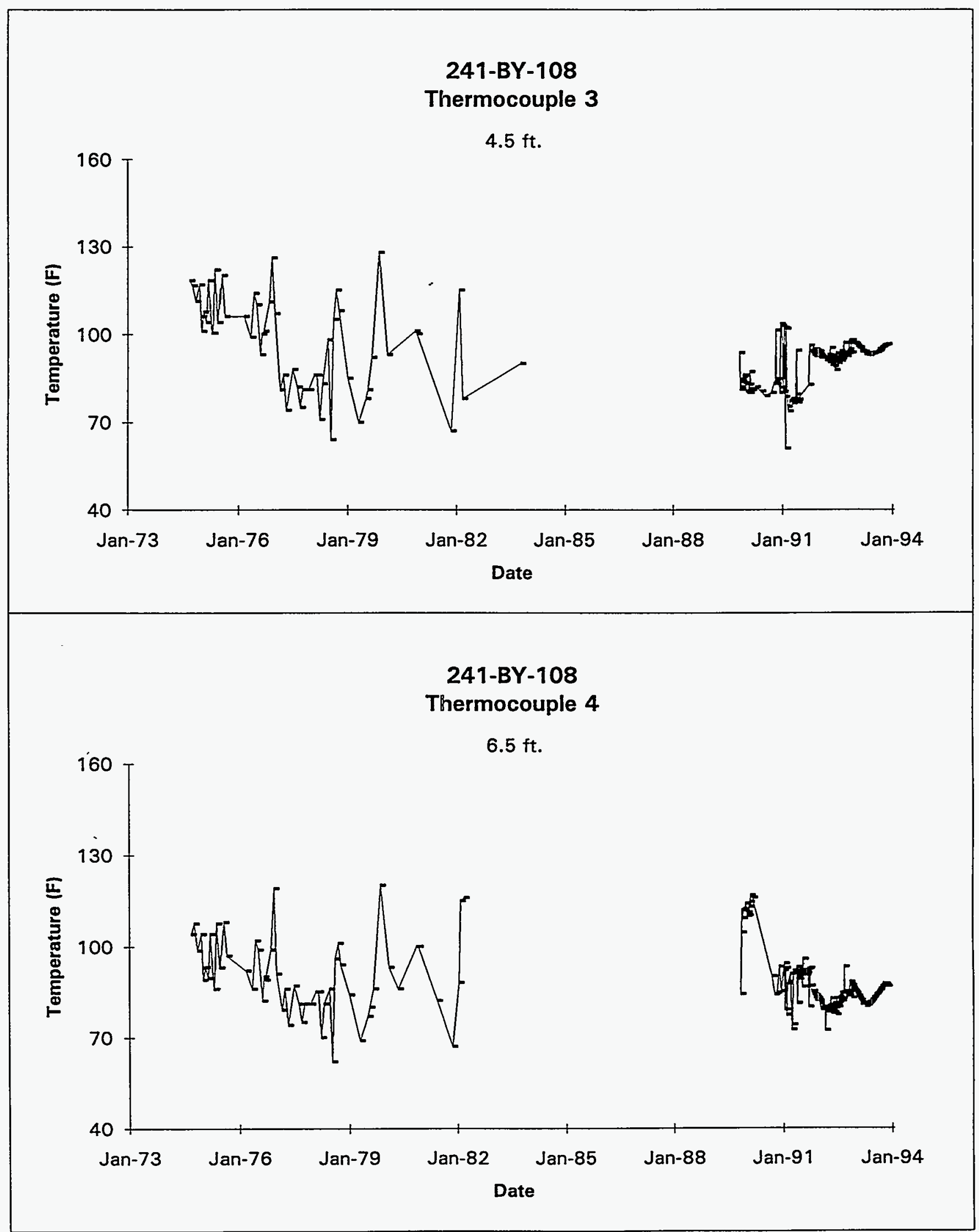

Data obtained from WHC Surveillance Analysis Computer System (SACS), November 12, 1993.

$$
\text { D-58 }
$$




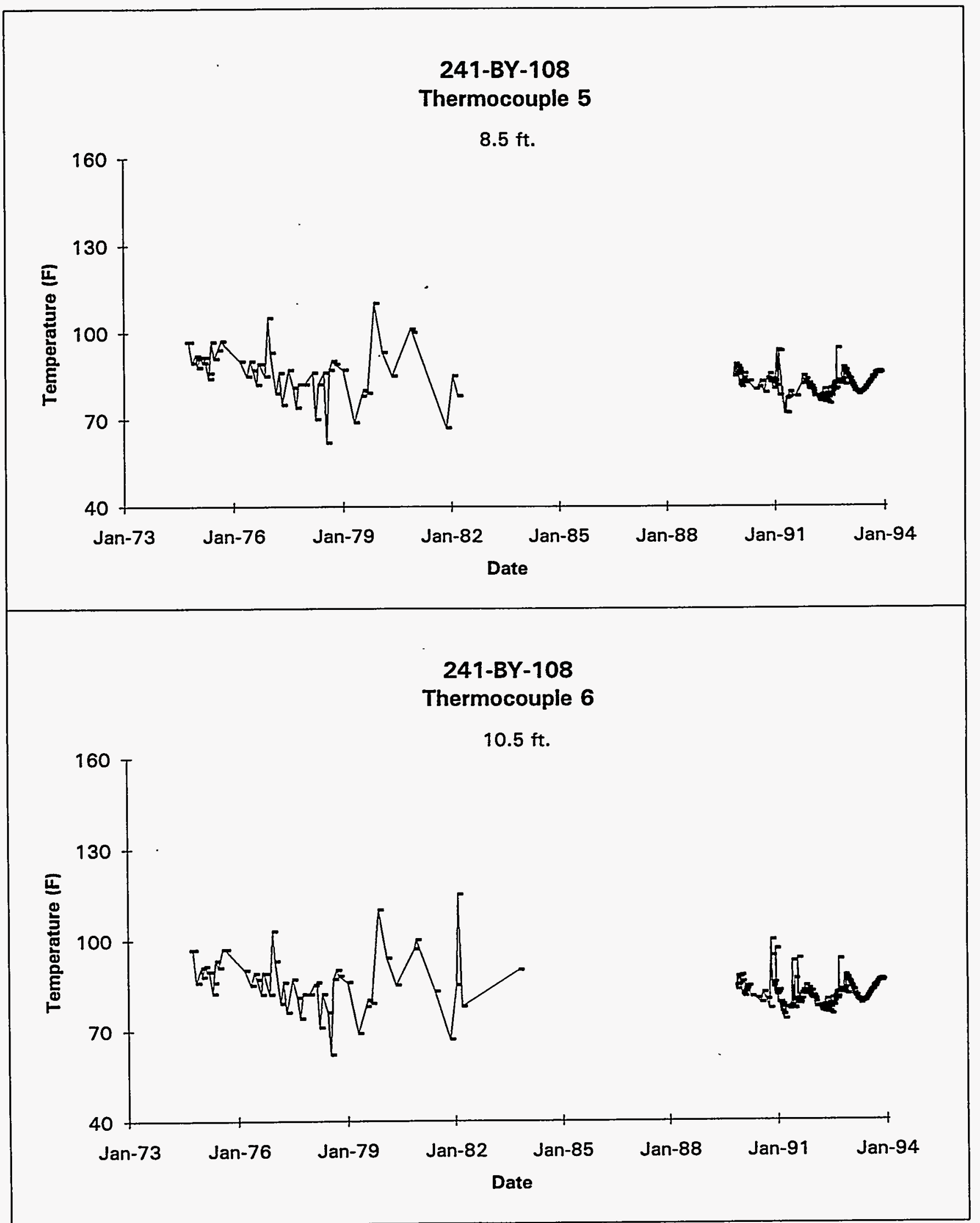

Data obtained from WHC Surveillance Analysis Computer System (SACS), November 12, 1993.

$$
\text { D-59 }
$$




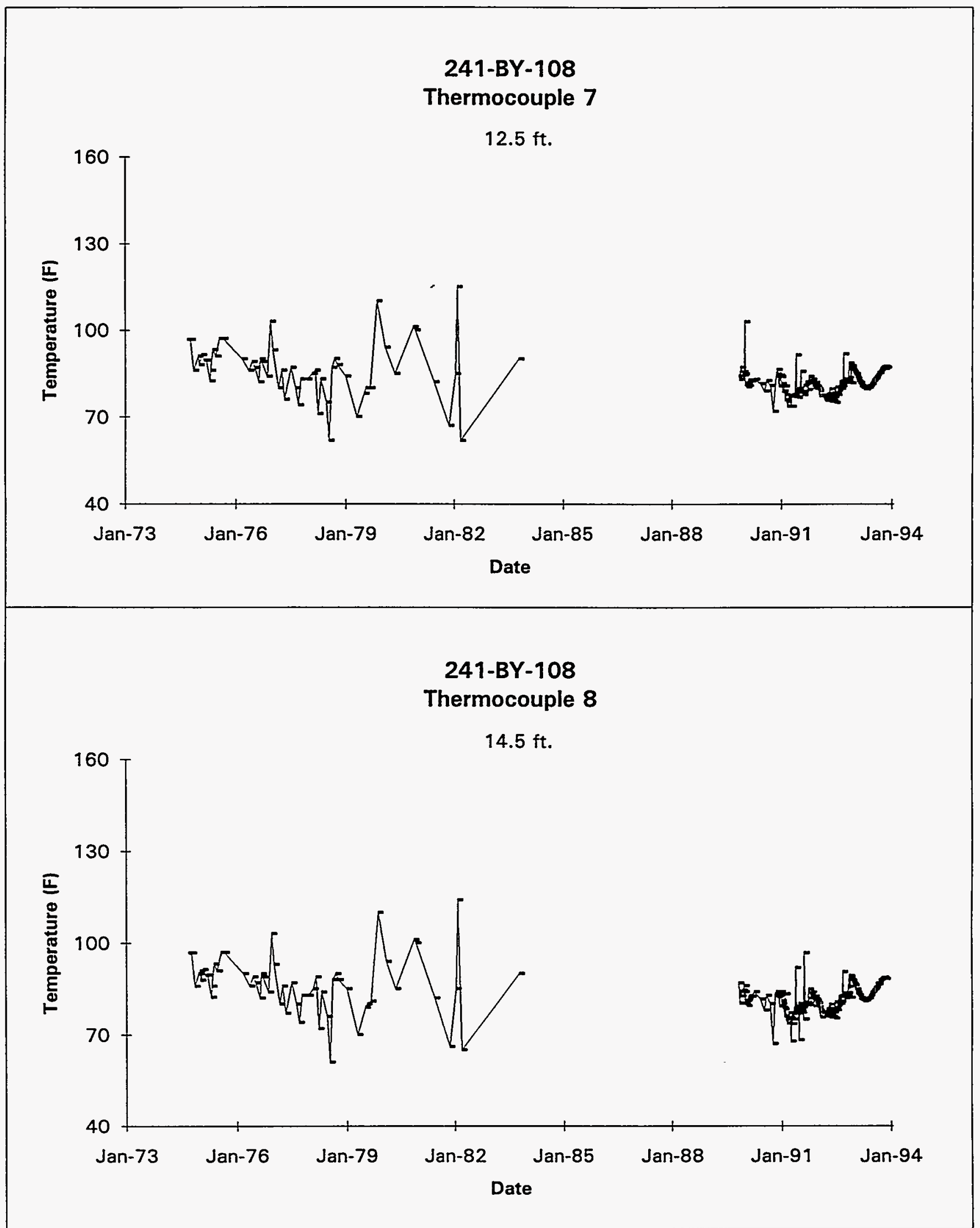

Data obtained from WHC Surveillance Analysis Computer System (SACS), November 12, 1993. 


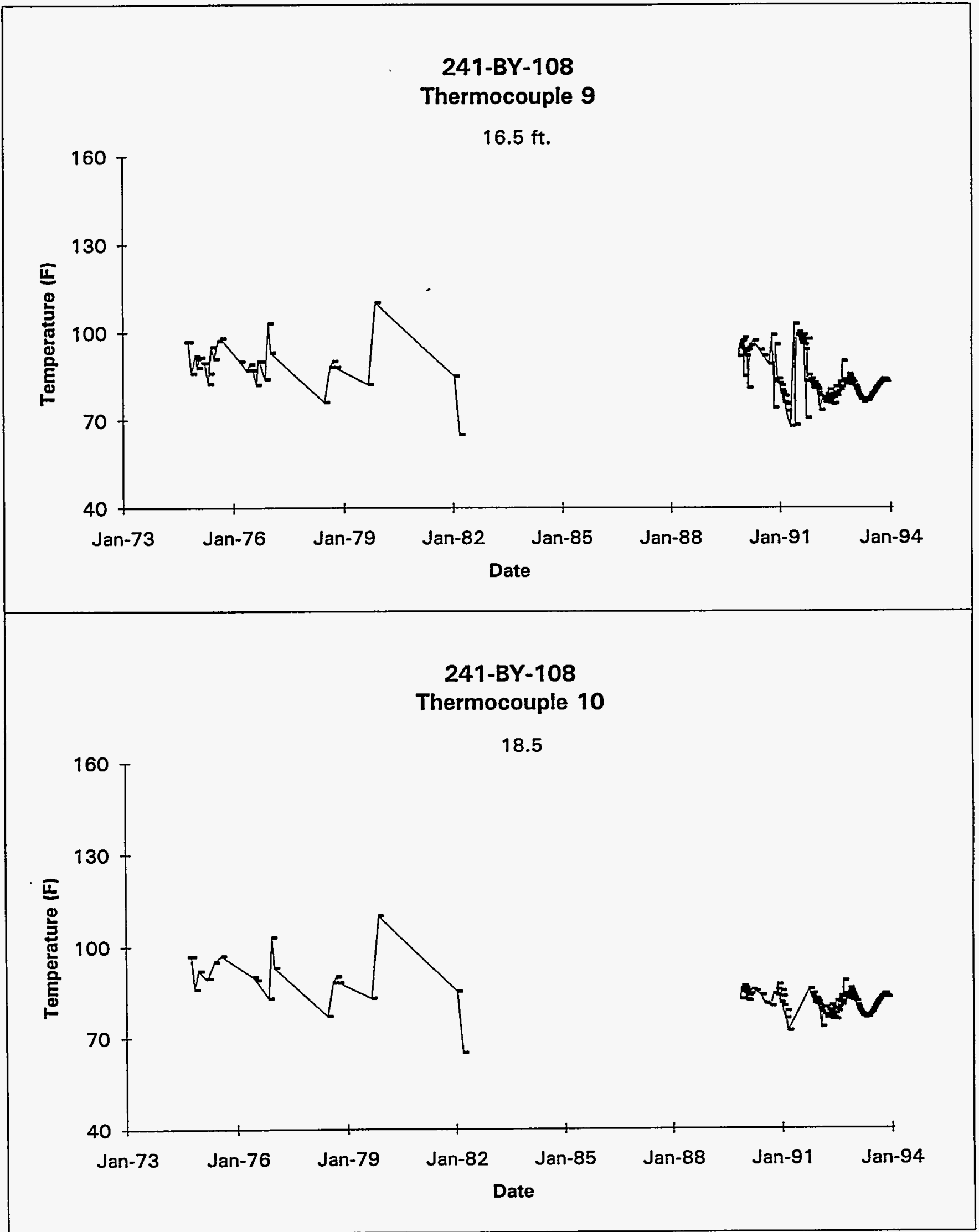

Data obtained from WHC Surveillance Analysis Computer System (SACS), November 12, 1993.

D-61 


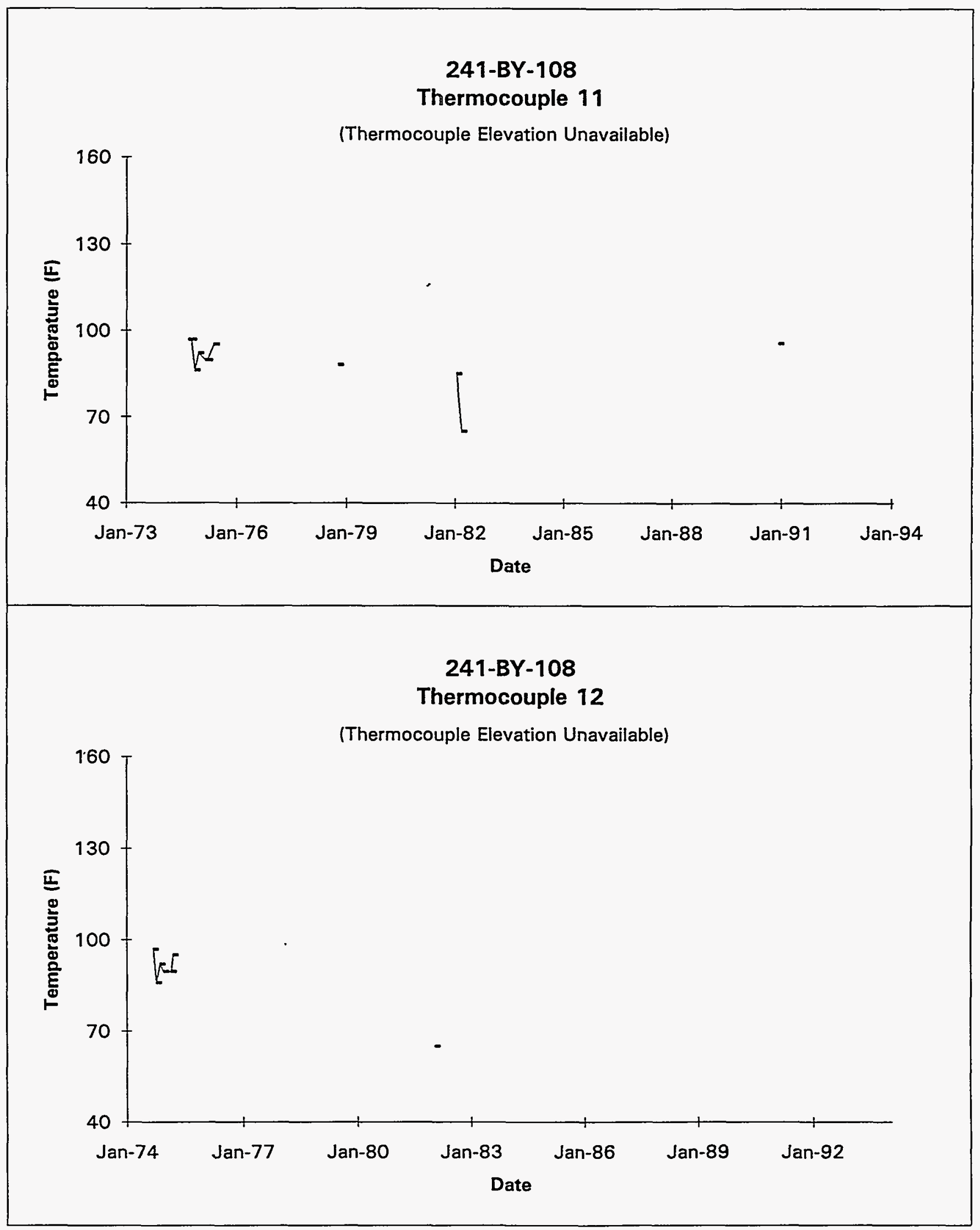

Data obtained from WHC Surveillance Analysis Computer System (SACS), November 12, 1993. 


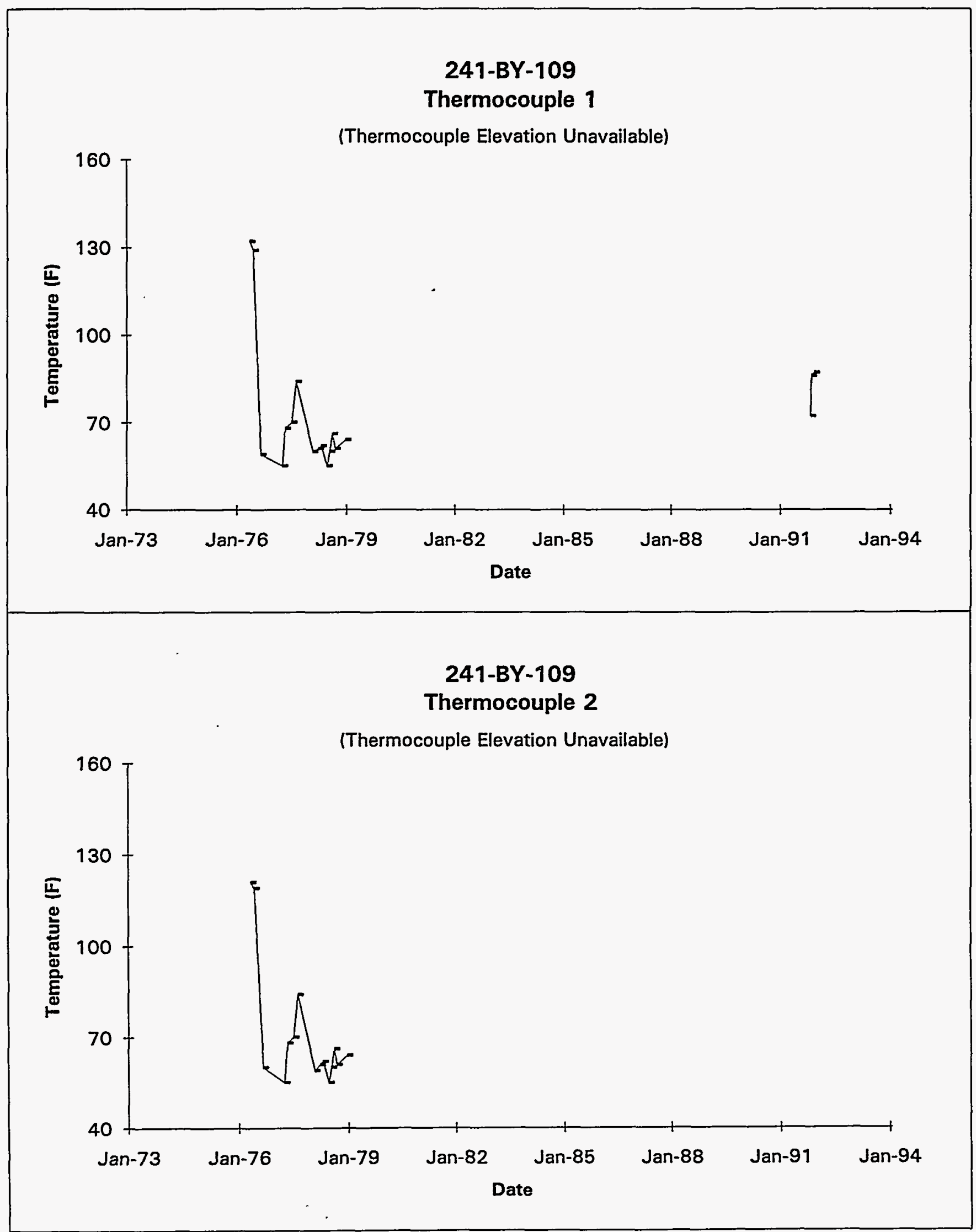

Data obtained from WHC Surveillance Analysis Computer System (SACS), November 12, 1993.

D-63 


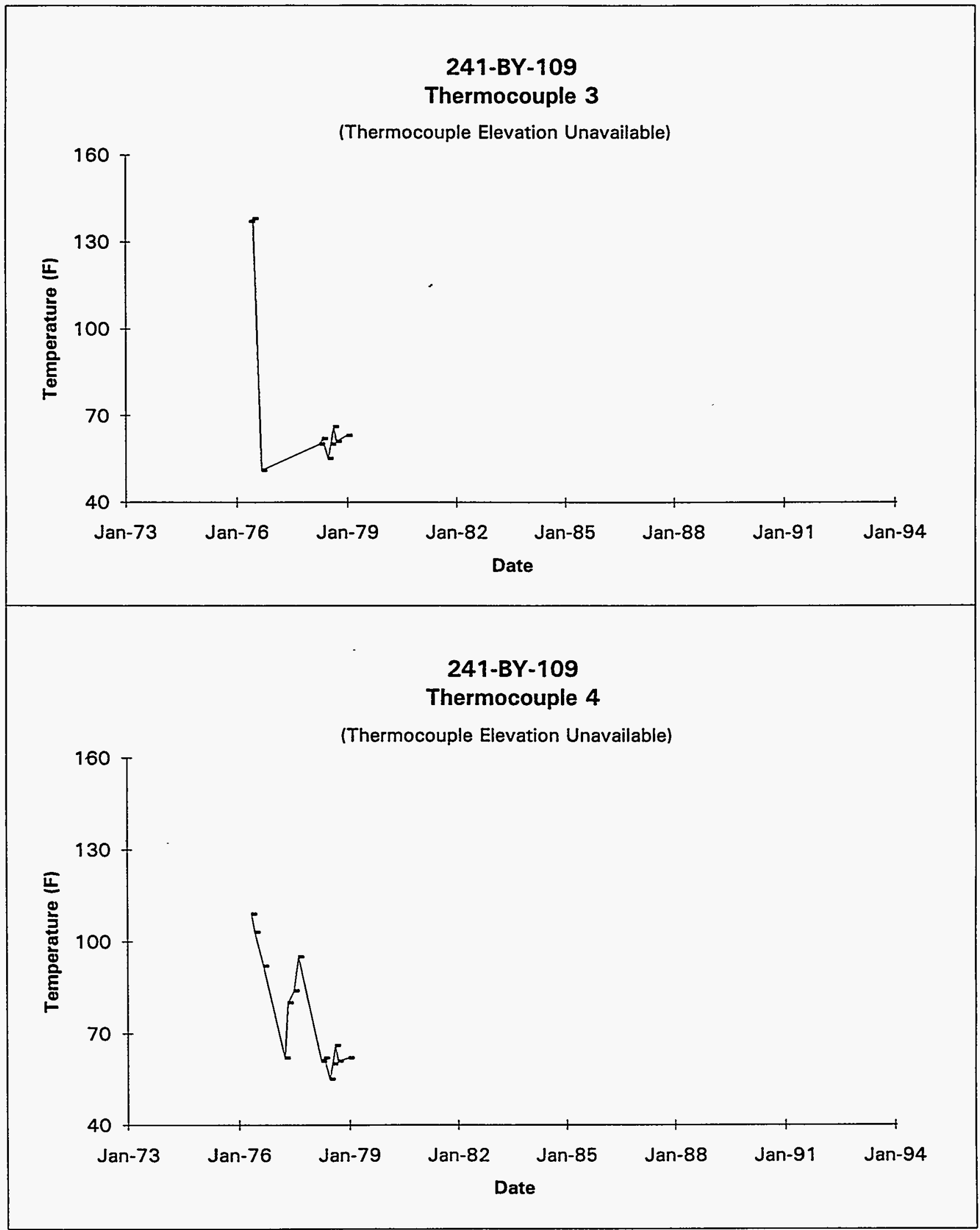

Data obtained from WHC Surveillance Analysis Computer System (SACS), November 12, 1993. 

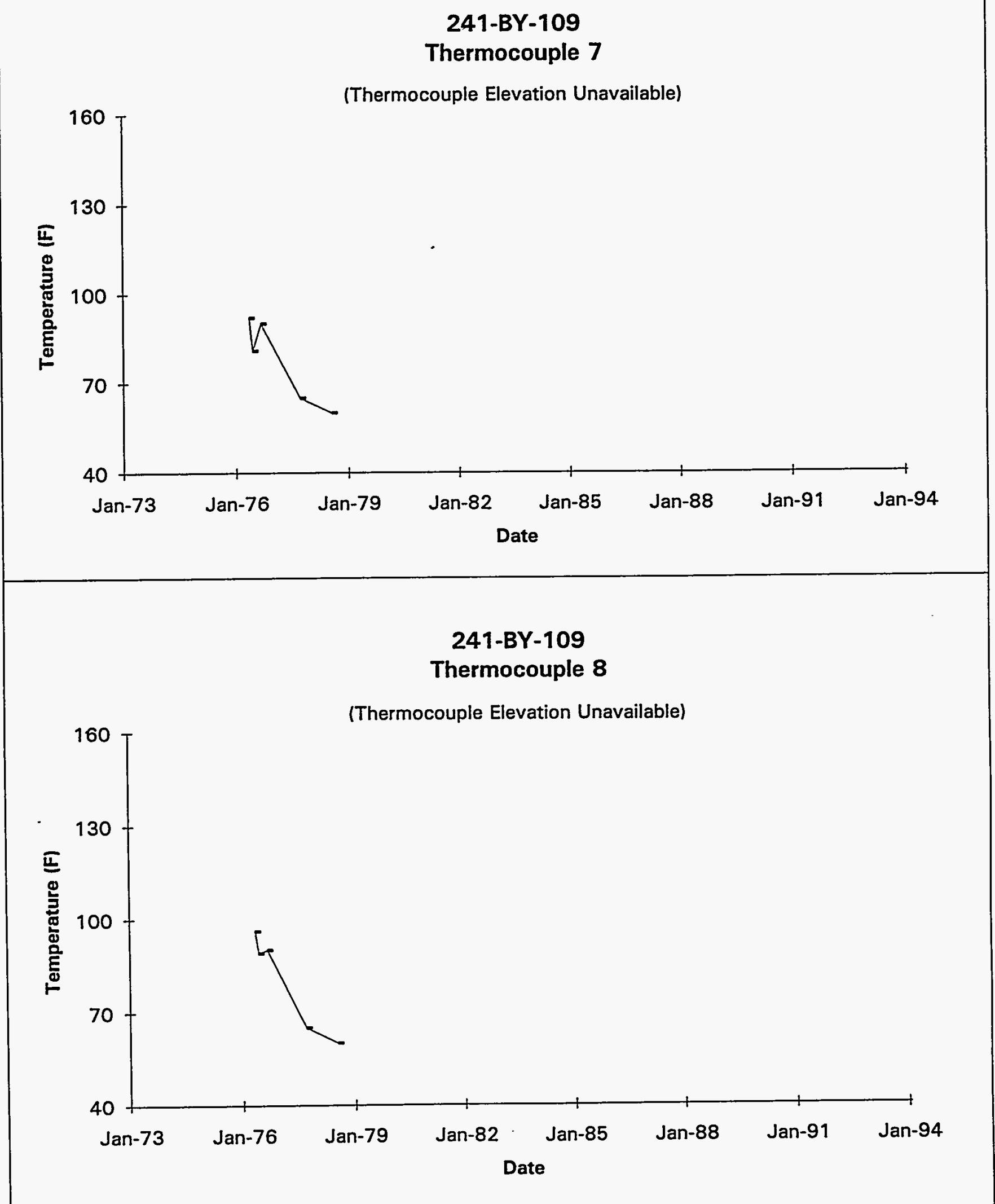

Data obtained from WHC Surveillance Analysis Computer System (SACS), November 12, 1993.

$$
\text { D-65 }
$$




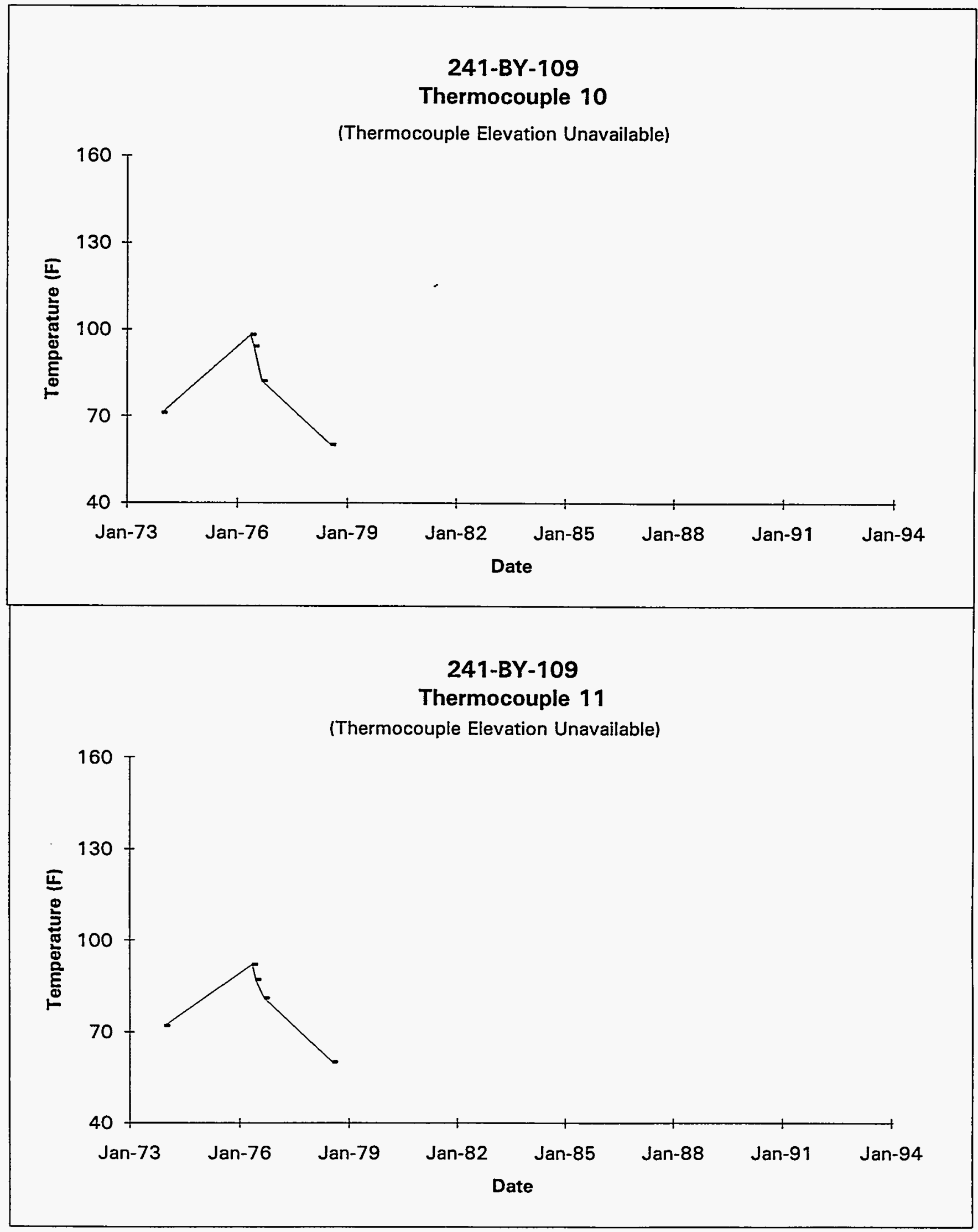

Data obtained from WHC Surveillance Analysis Computer System (SACS), November 12, 1993. 


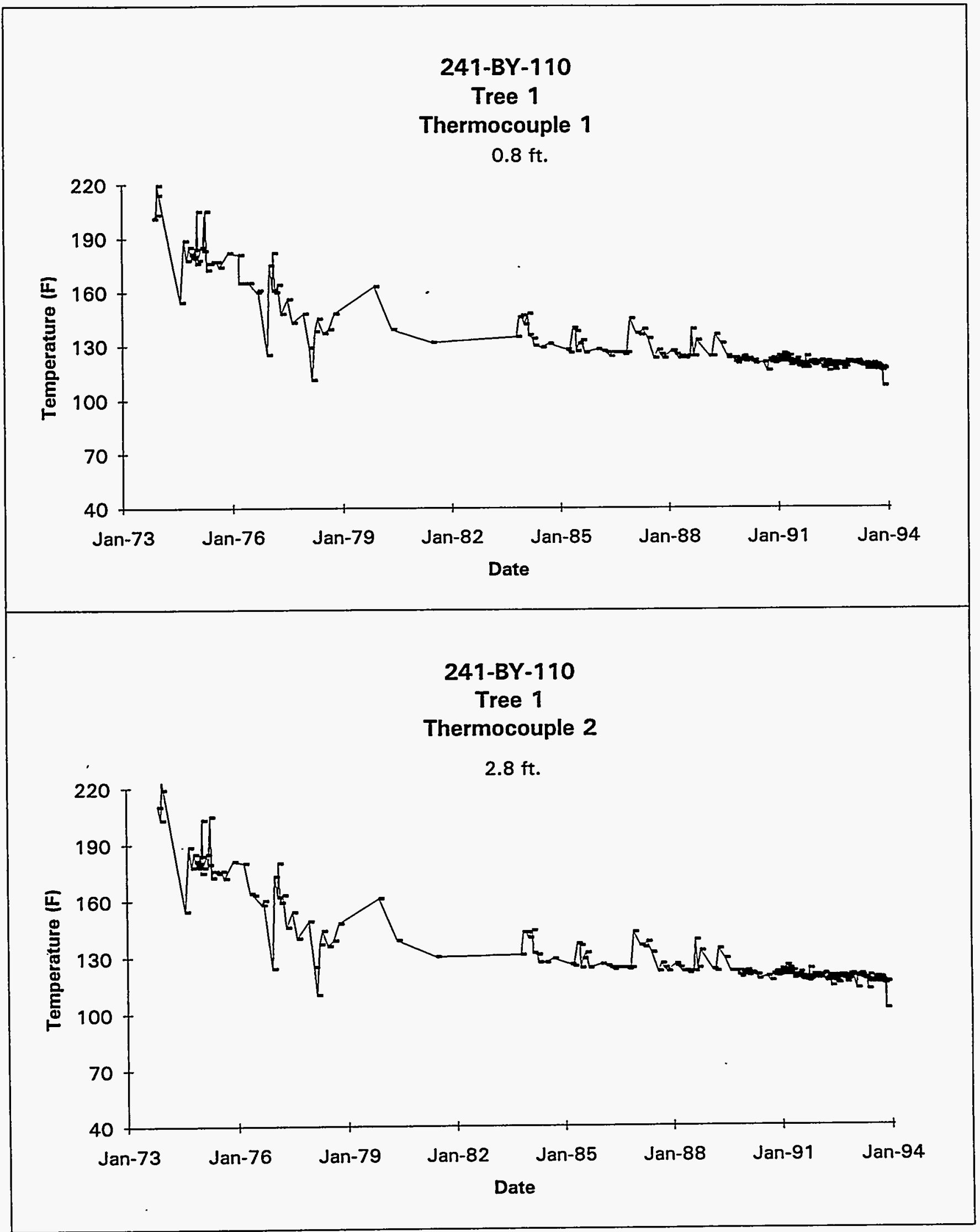

Data obtained from WHC Surveillance Analysis Computer System (SACS), November 12, 1993. 


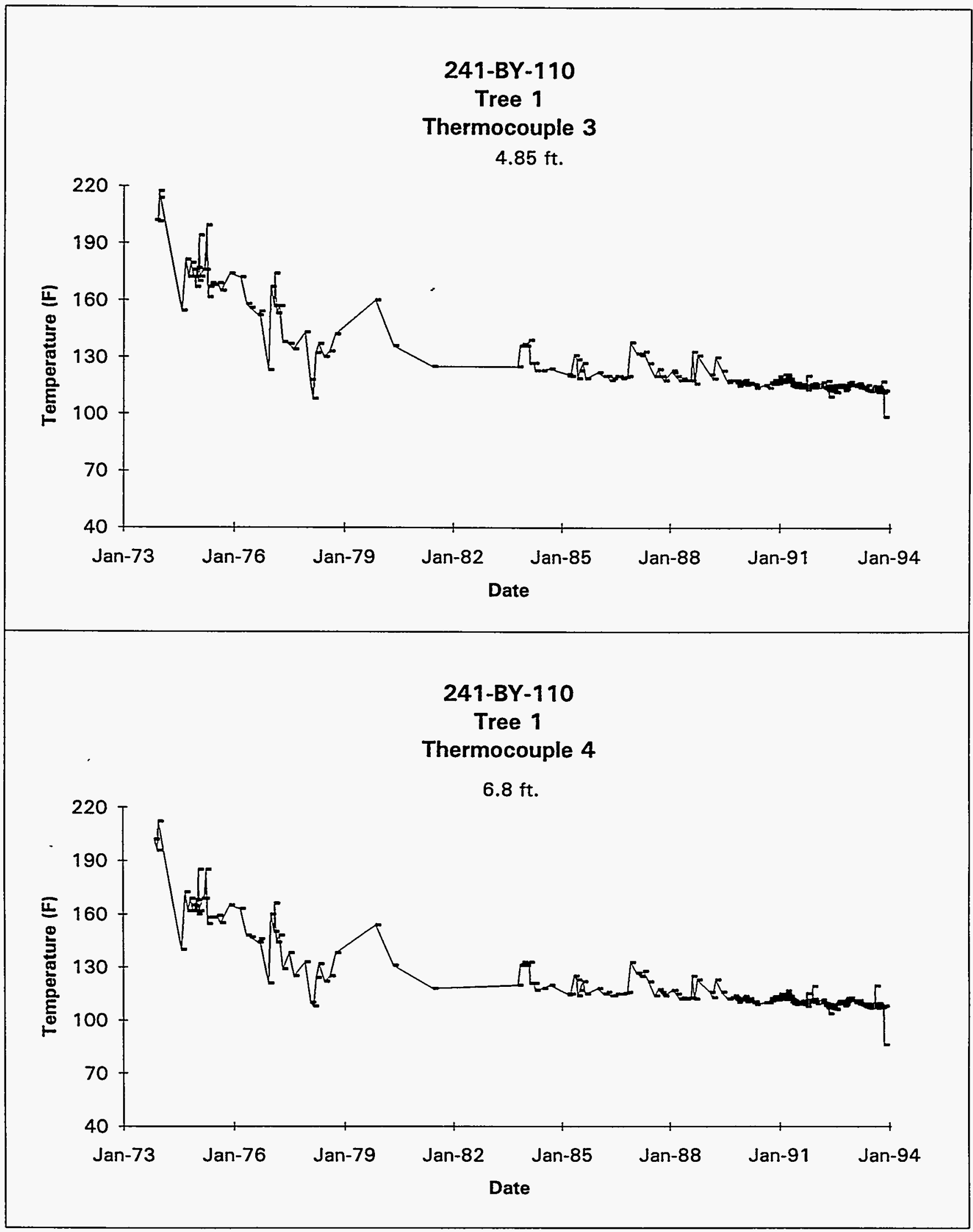

Data obtained from WHC Surveillance Analysis Computer System (SACS), November 12, 1993. 


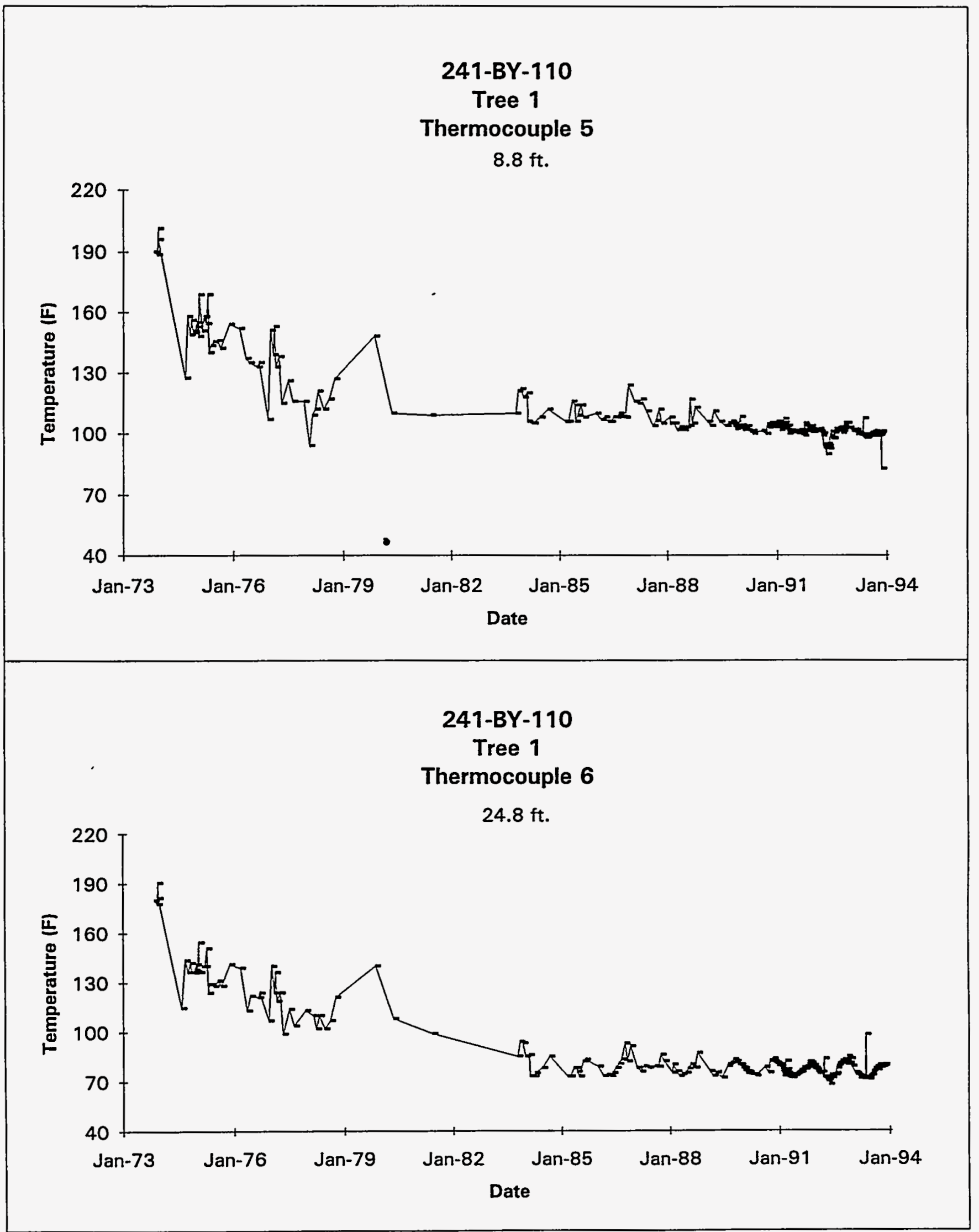

Data obtained from WHC Surveillance Analysis Computer System (SACS), November 12, 1993.

D-69 


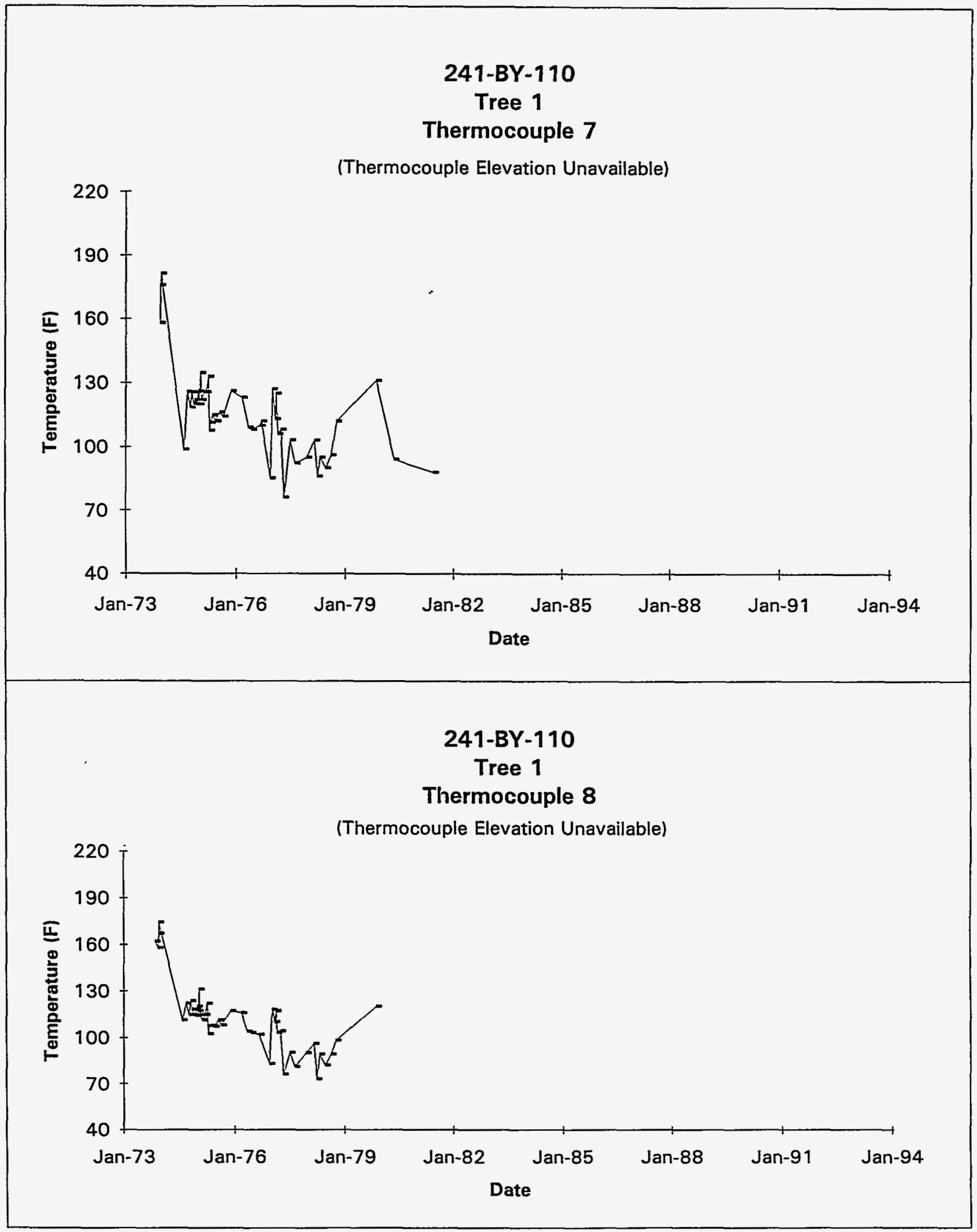

Data obtained from WHC Surveillance Analysis Computer System (SACS), November 12, 1993.

D-70 


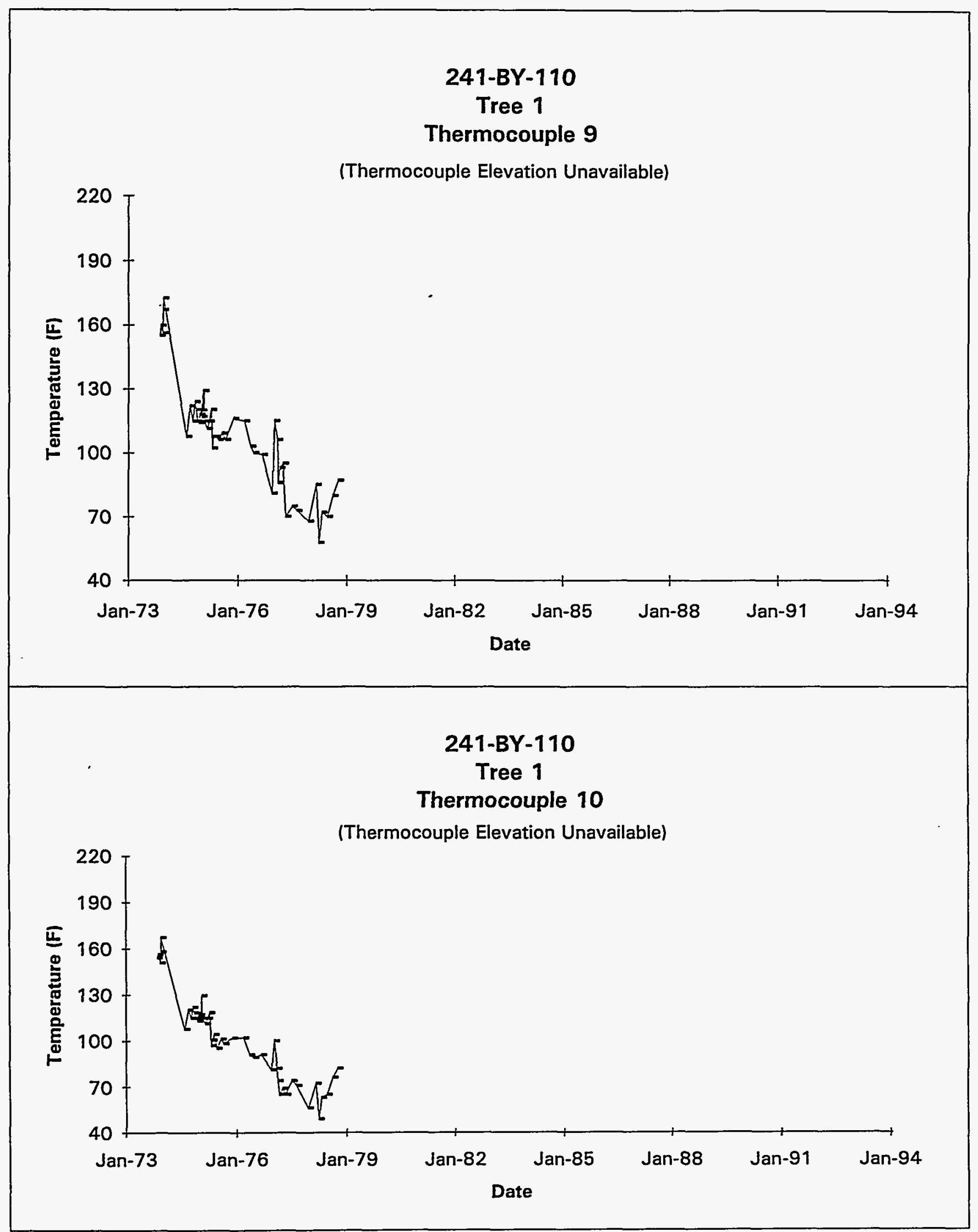

Data obtained from WHC Surveillance Analysis Computer System (SACS), November 12, 1993. 


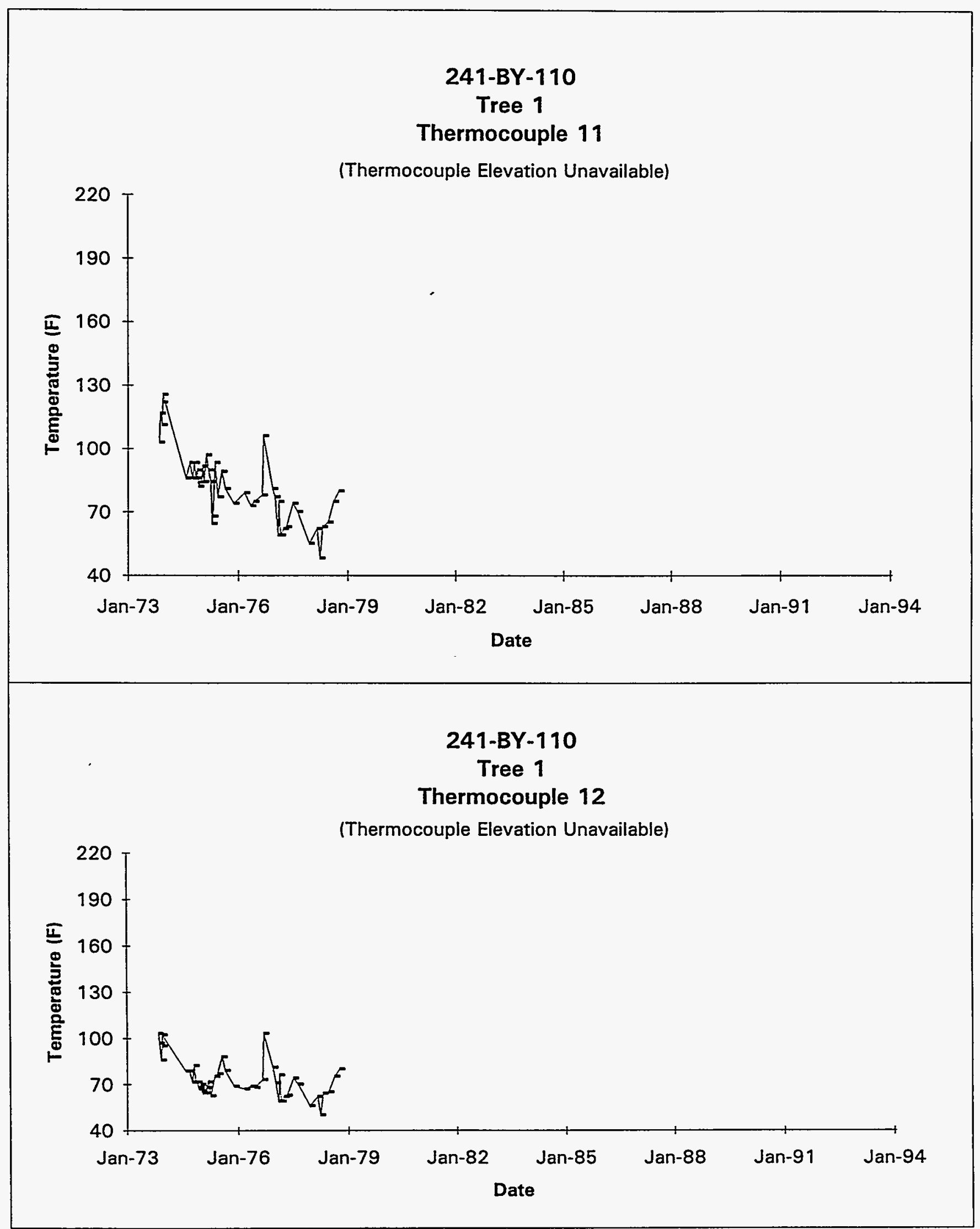

Data obtained from WHC Surveillance Analysis Computer System (SACS), November 12, 1993. 


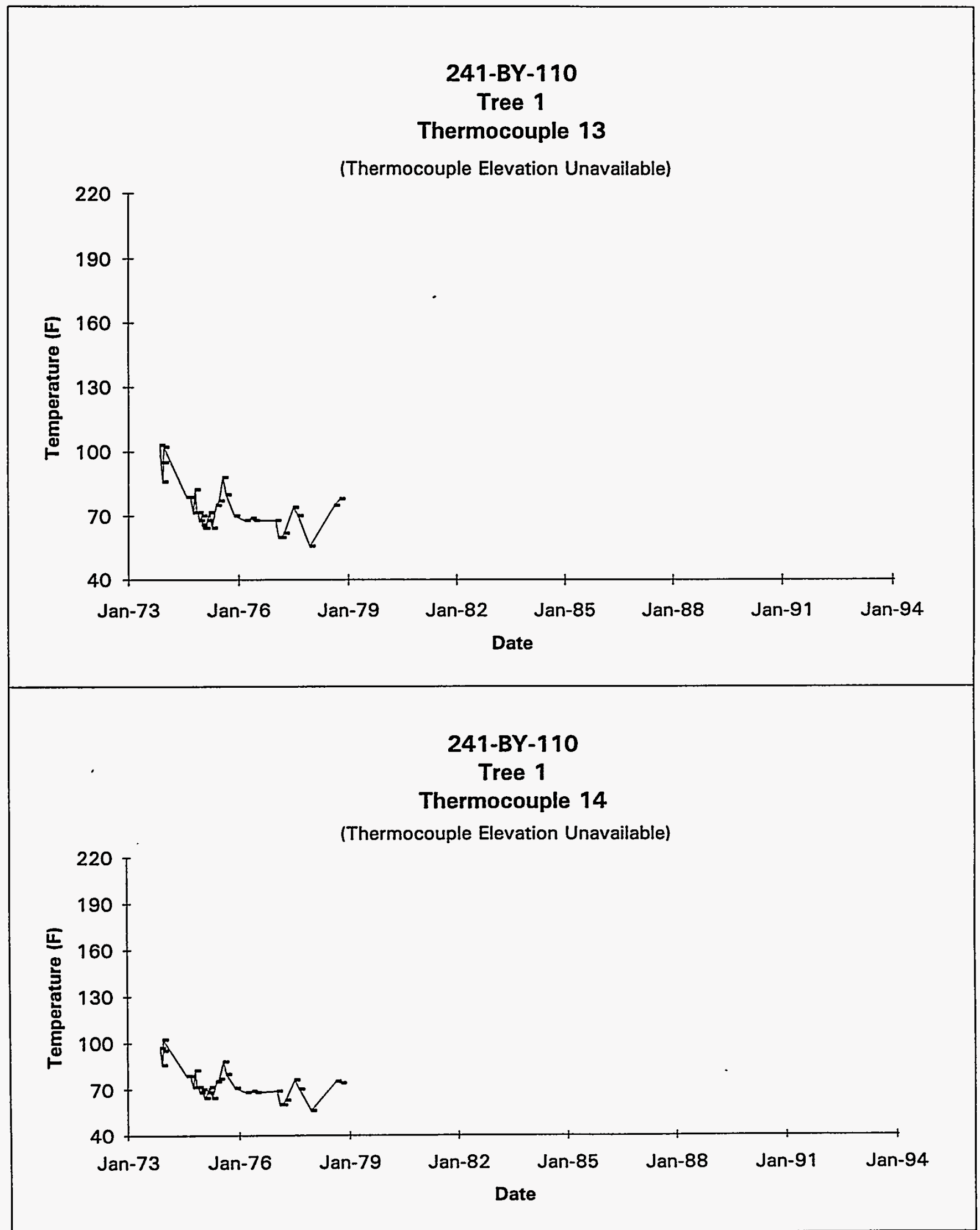

Data obtained from WHC Surveillance Analysis Computer System (SACS), November 12, 1993.

D-73 


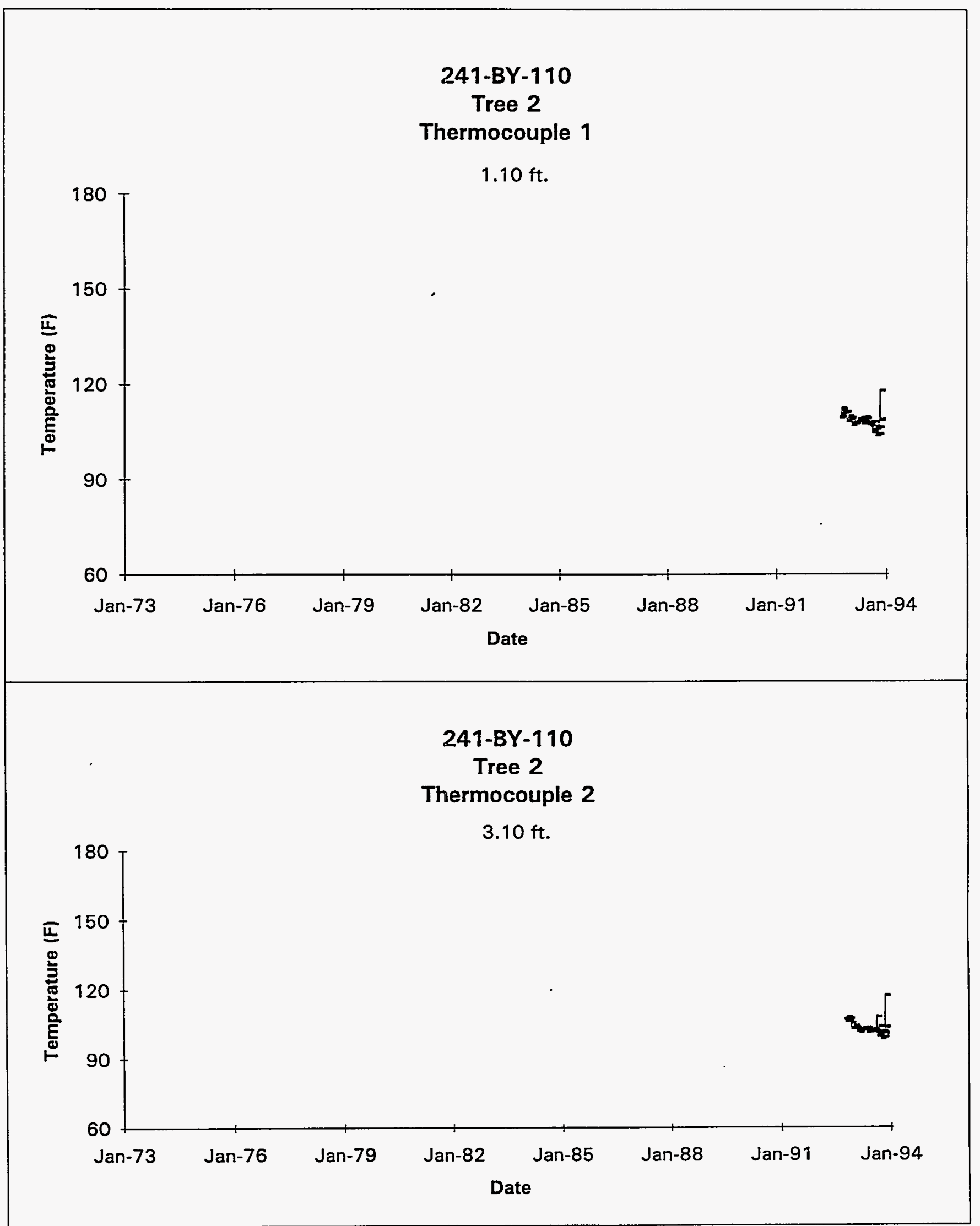

Data obtained from WHC Surveillance Analysis Computer System (SACS), November 12, 1993. 


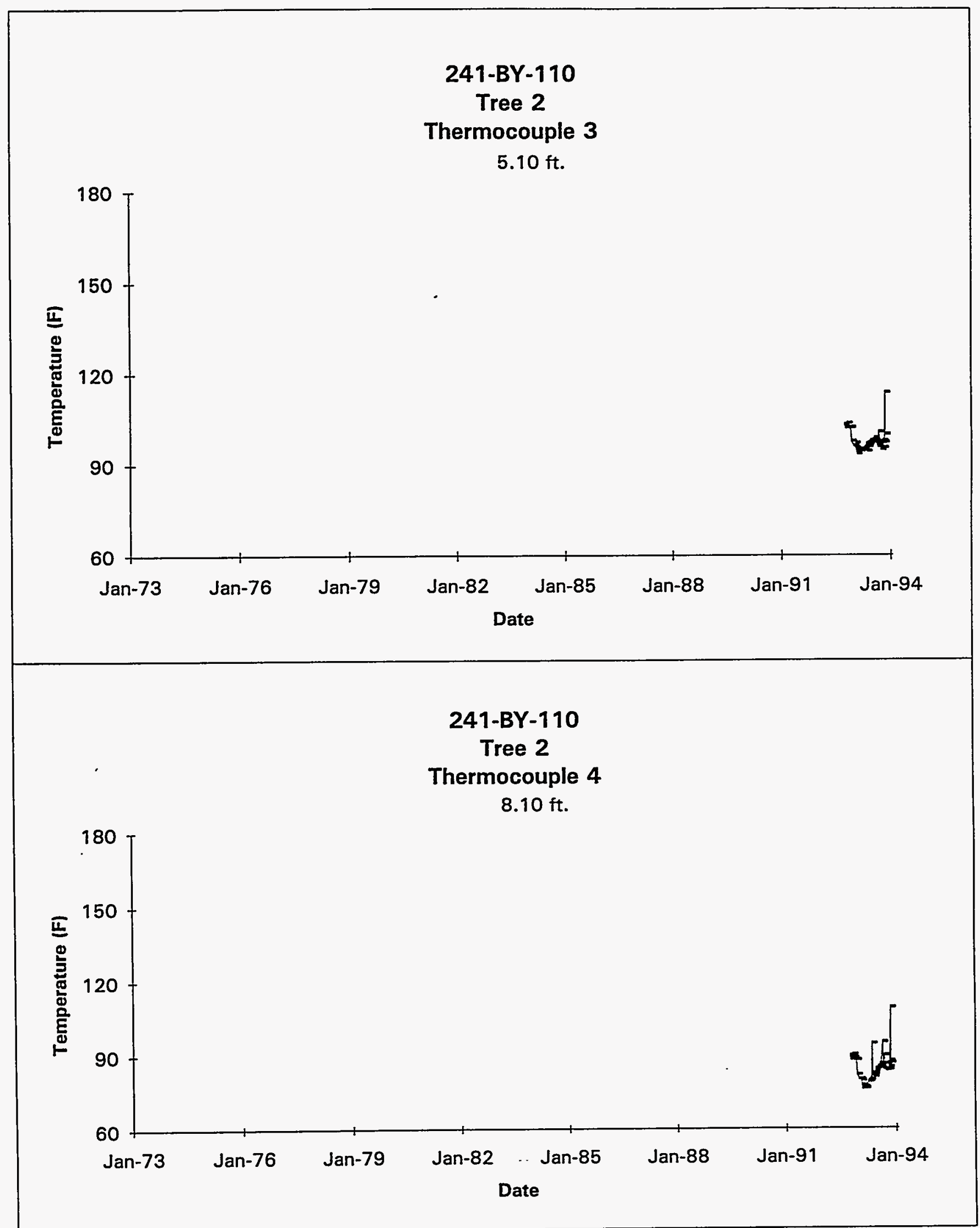

Data obtained from WHC Surveillance Analysis Computer System (SACS), November 12, 1993.

$$
\text { D-75 }
$$




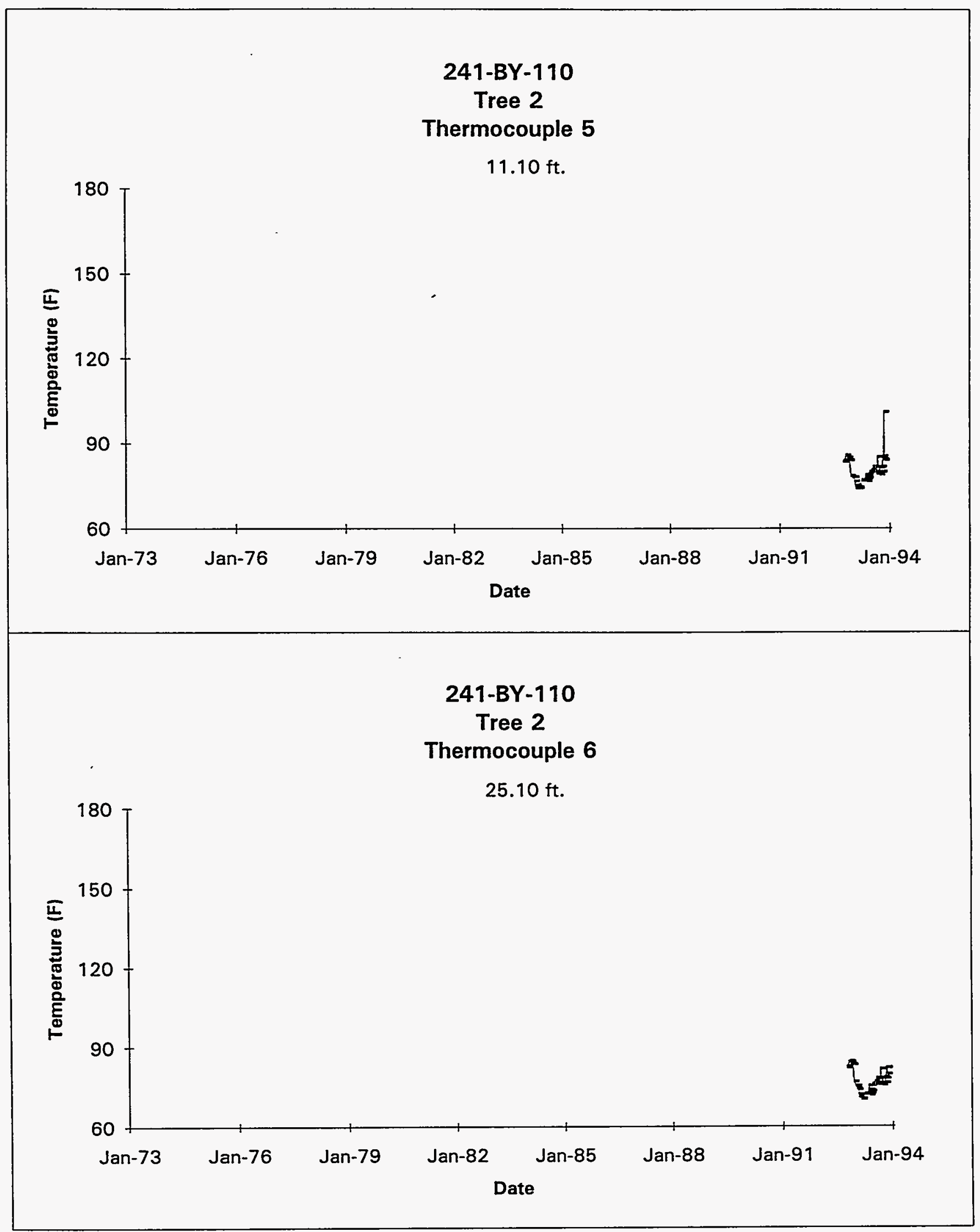

Data obtained from WHC Surveillance Analysis Computer System (SACS), November 12, 1993.

D-76 


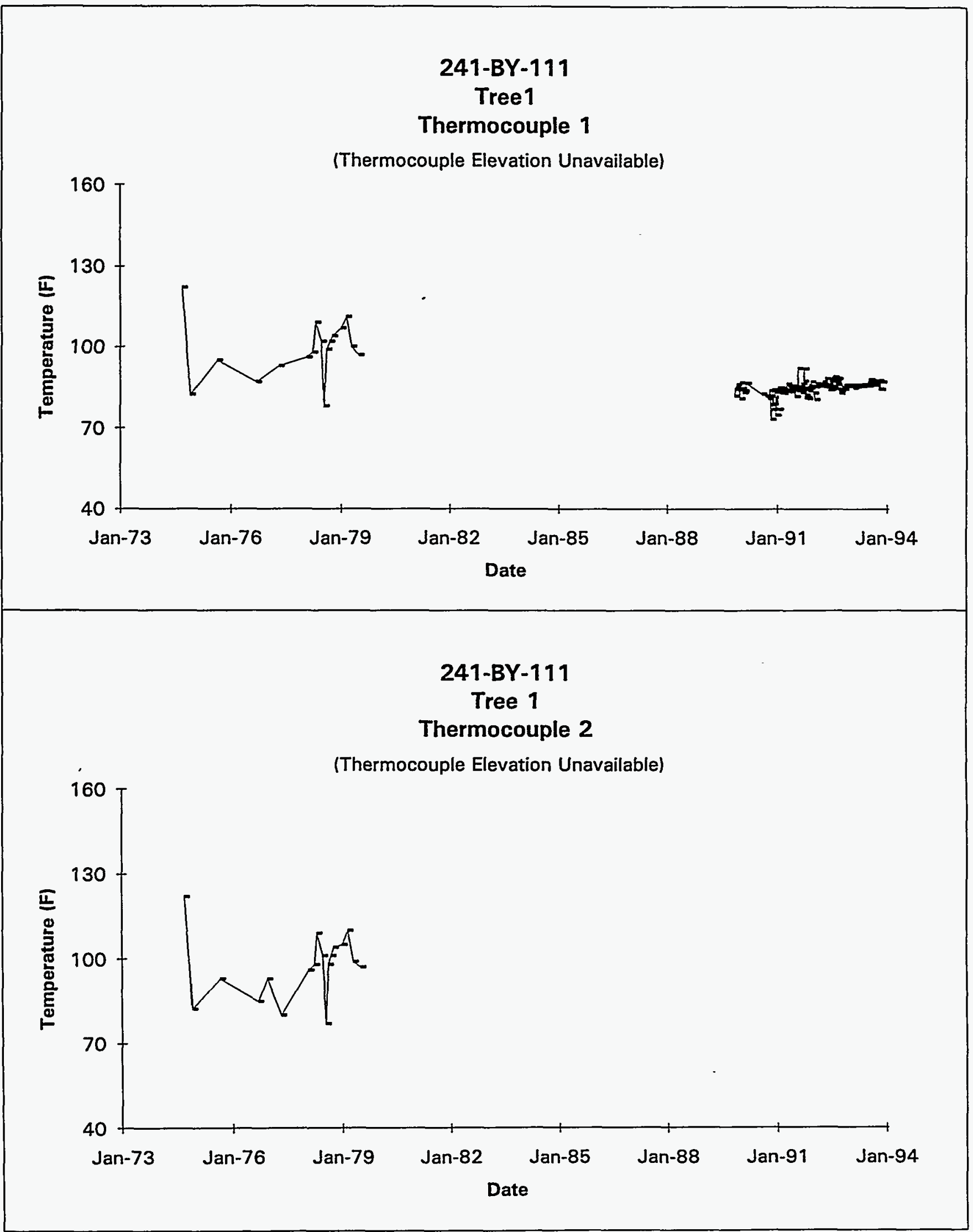

Data obtained from WHC Surveillance Analysis Computer System (SACS), November 12, 1993. 


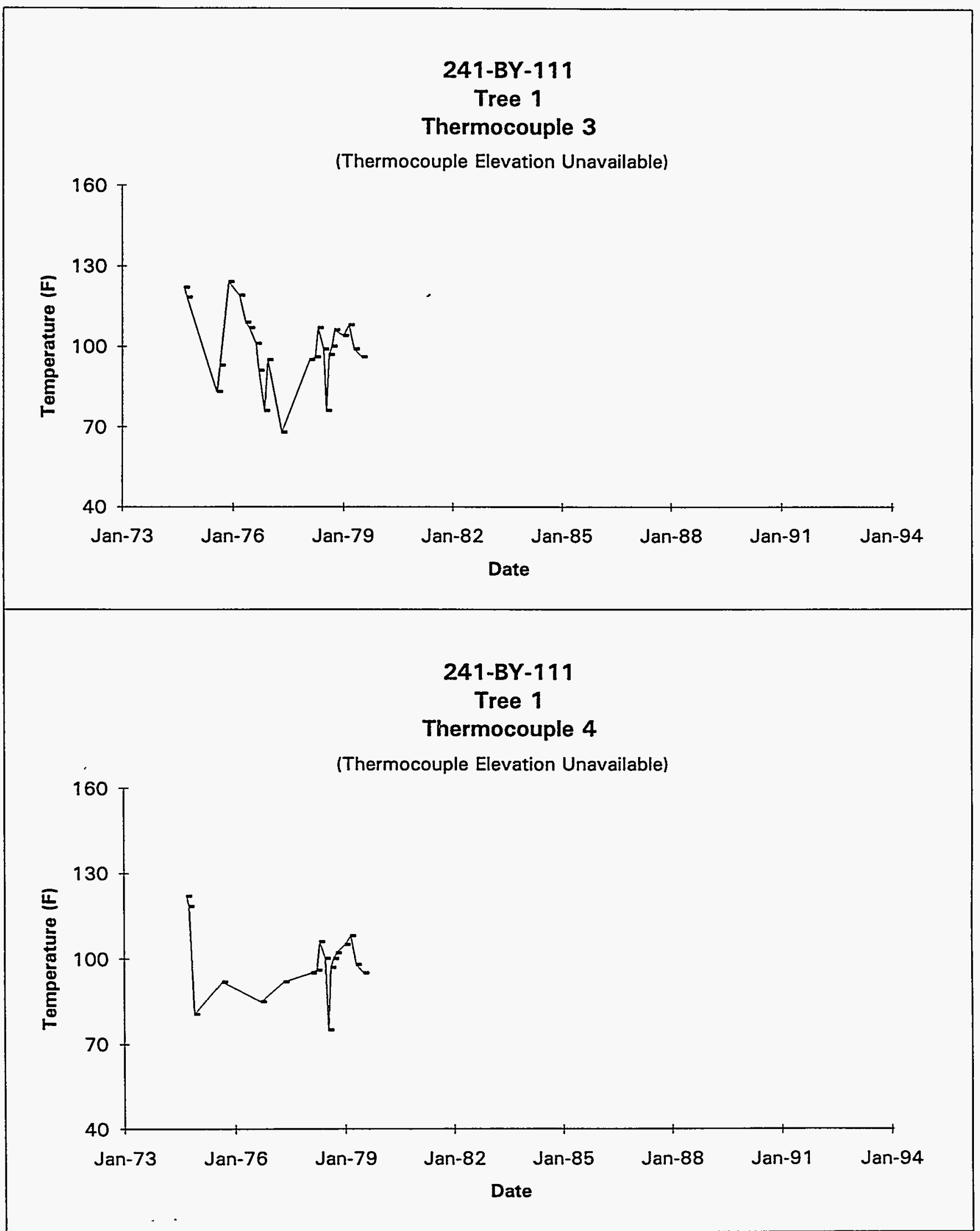

Data obtained from WHC Surveillance Analysis Computer System (SACS), November 12, 1993. 


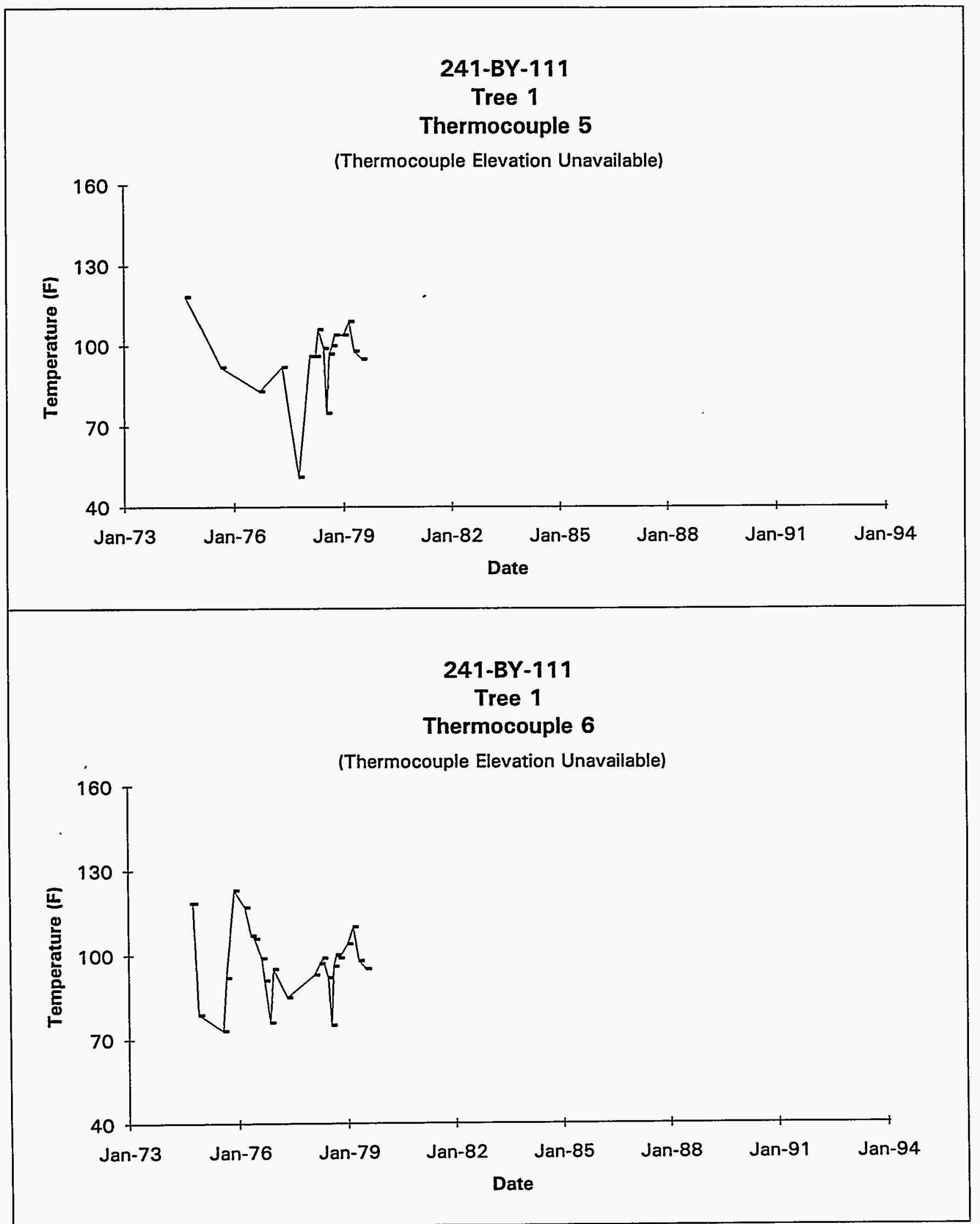

Data obtained from WHC Surveillance Analysis Computer System (SACS), November 12, 1993.

D-79 


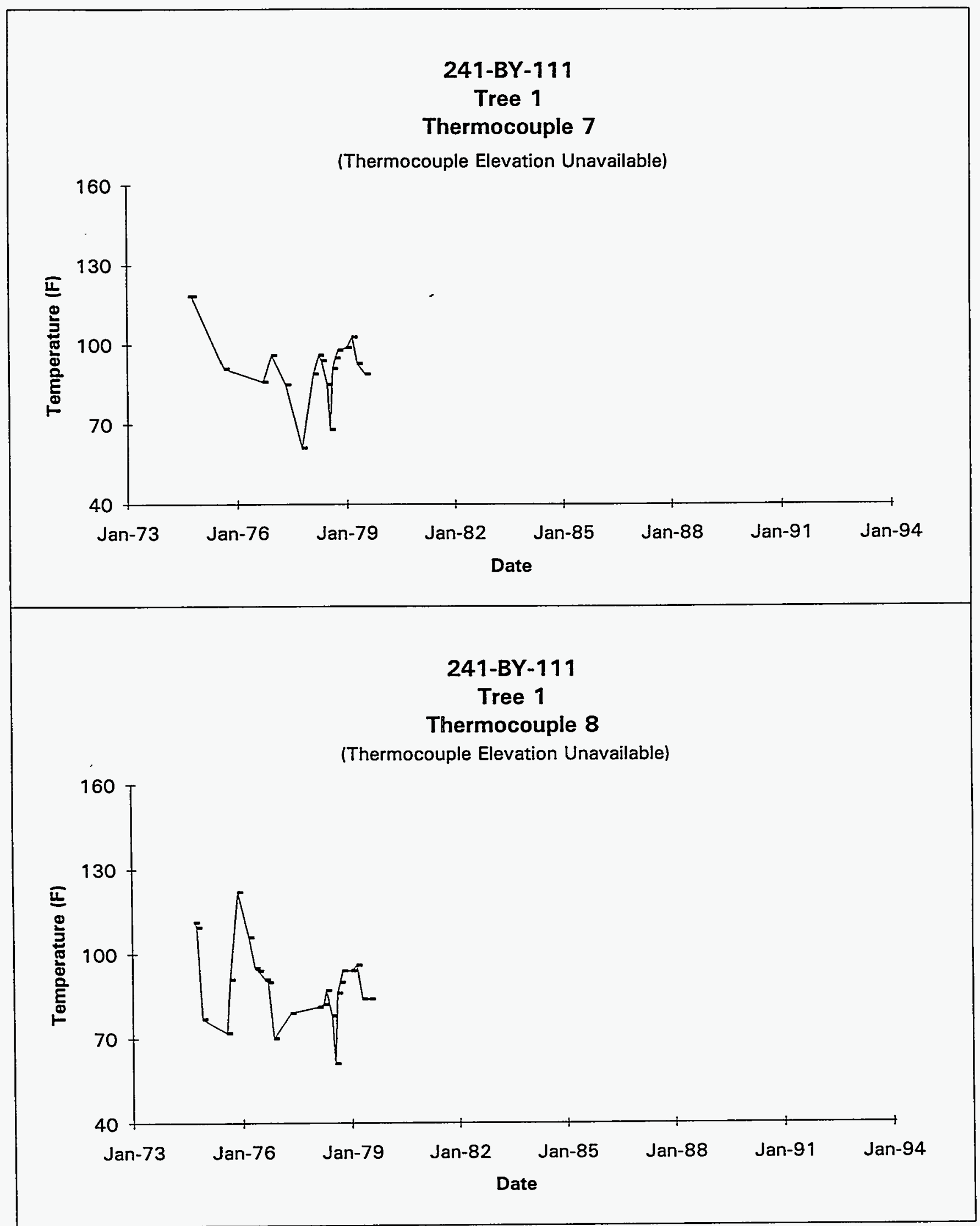

Data obtained from WHC Surveillance Analysis Computer System (SACS), November 12, 1993.

D-80 


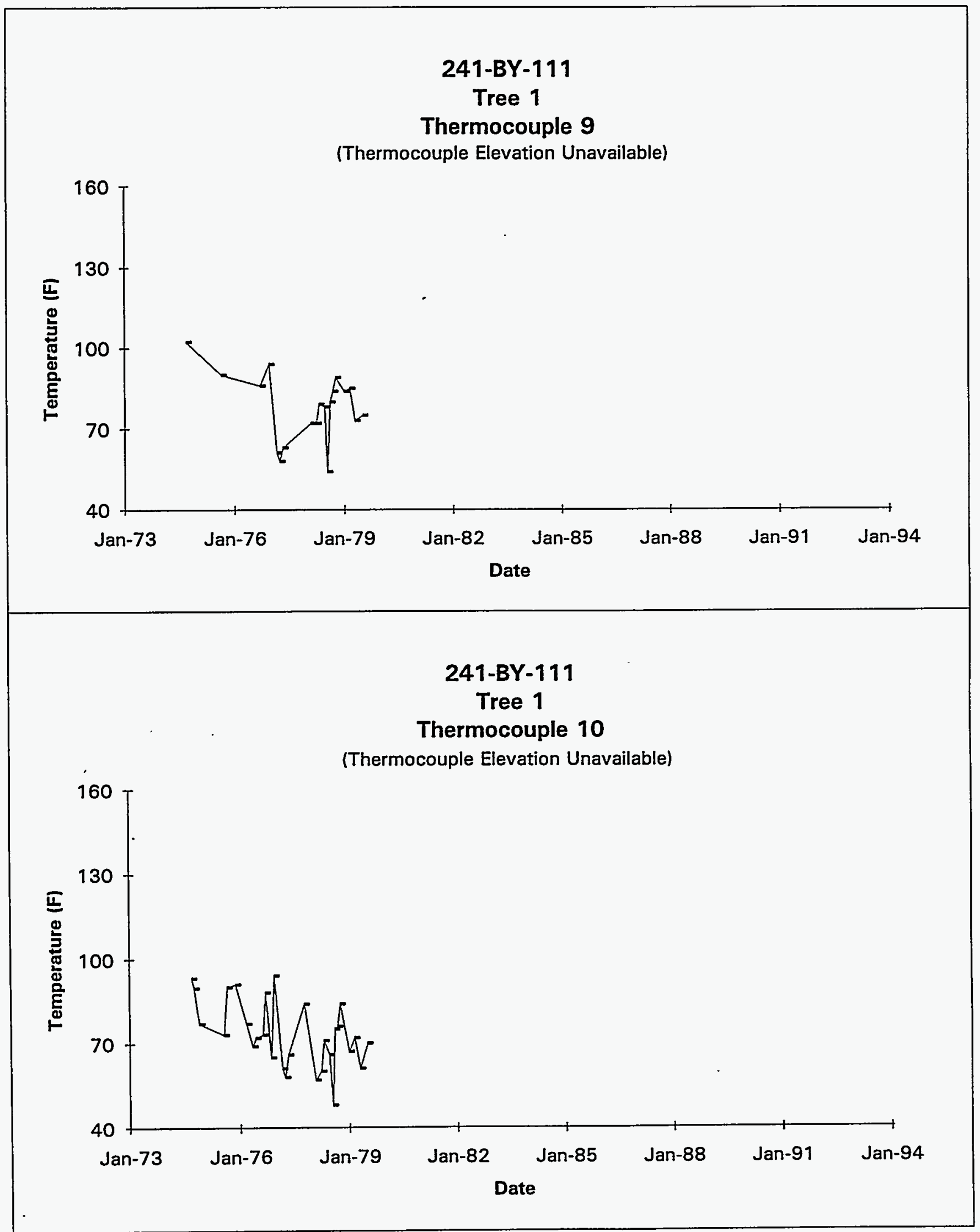

Data obtained from WHC Surveillance Analysis Computer System (SACS), November 12, 1993.

D-81 


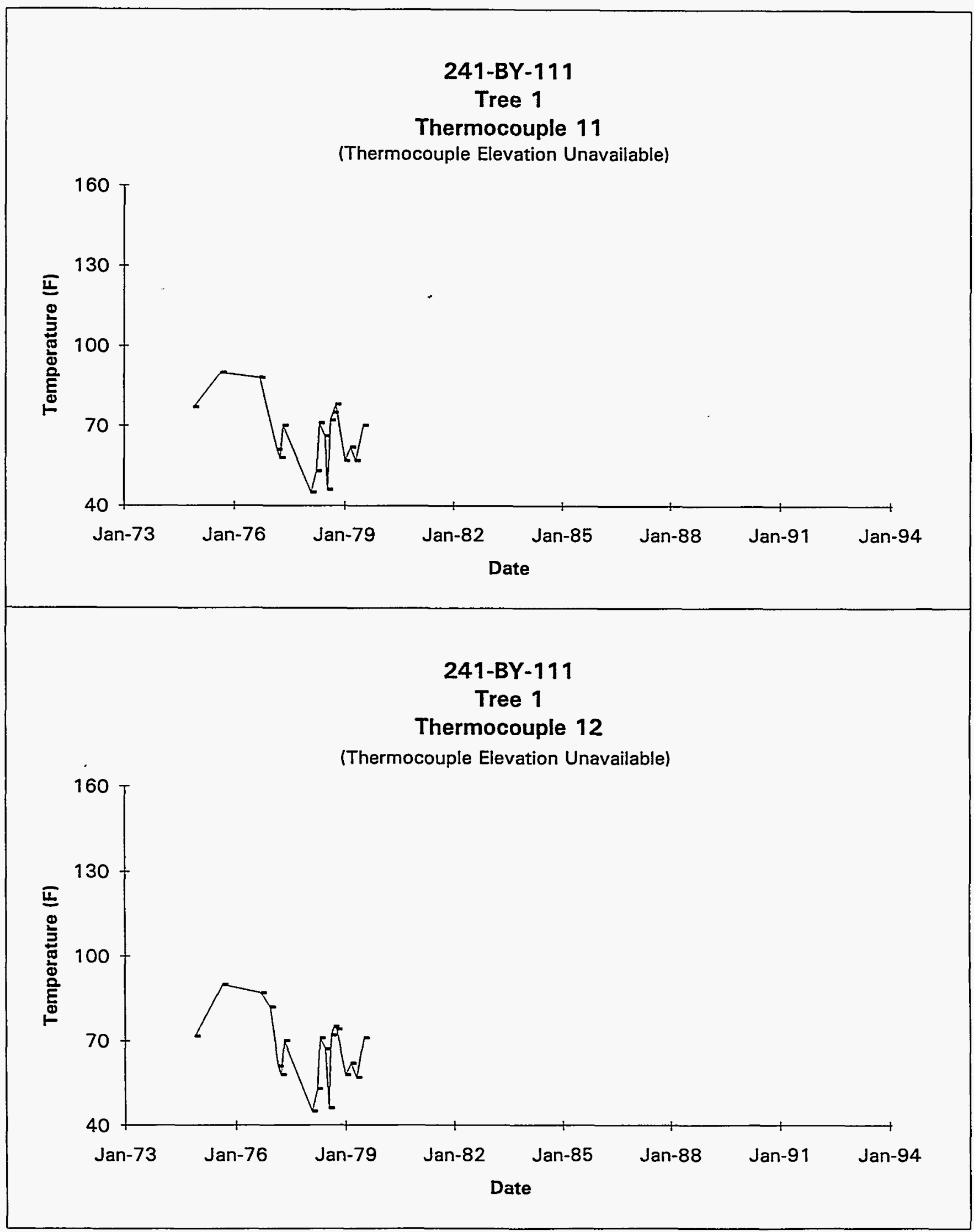

Data obtained from WHC Surveillance Analysis Computer System (SACS), November 12, 1993. 


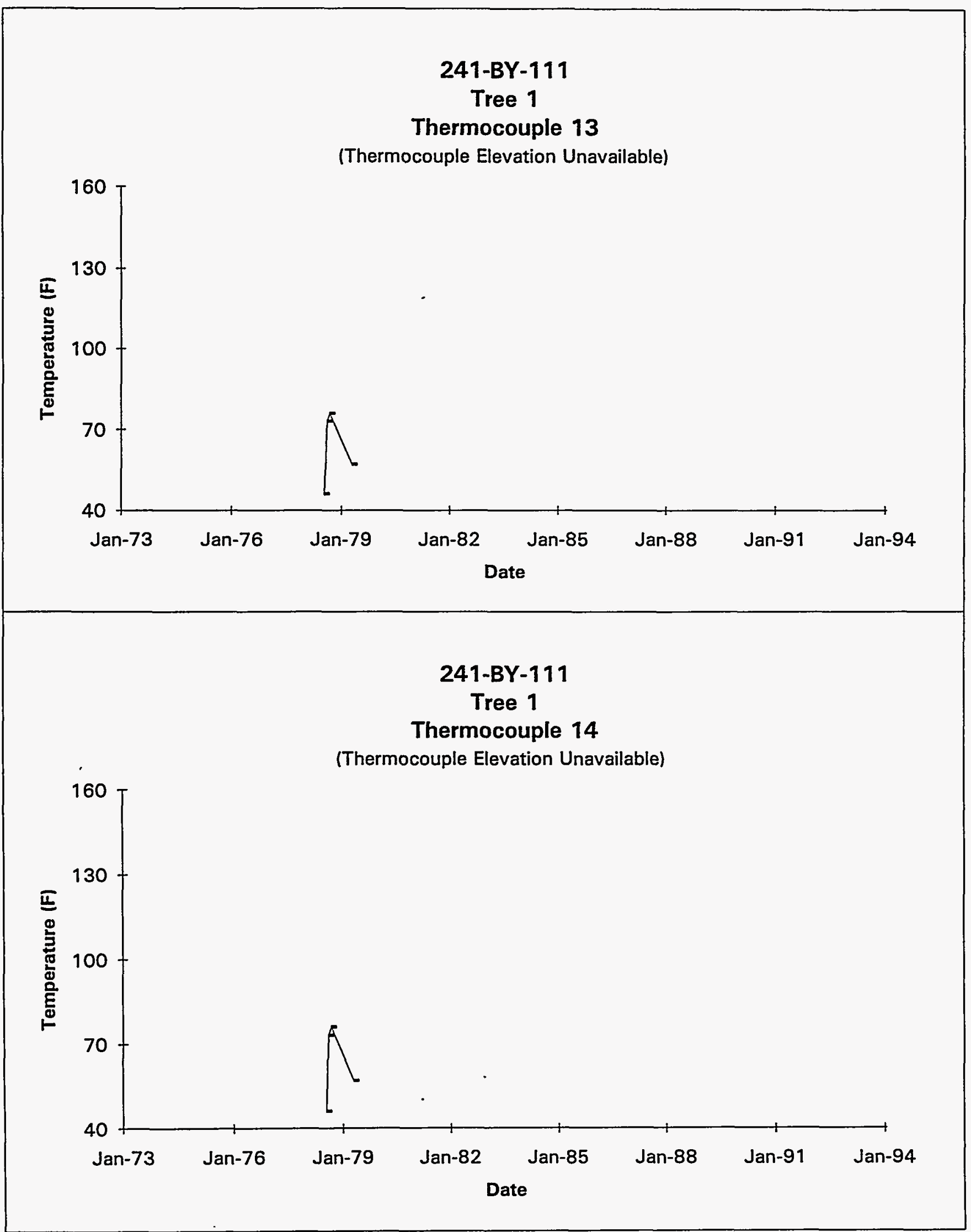

Data obtained from WHC Surveillance Analysis Computer System (SACS), November 12, 1993. 


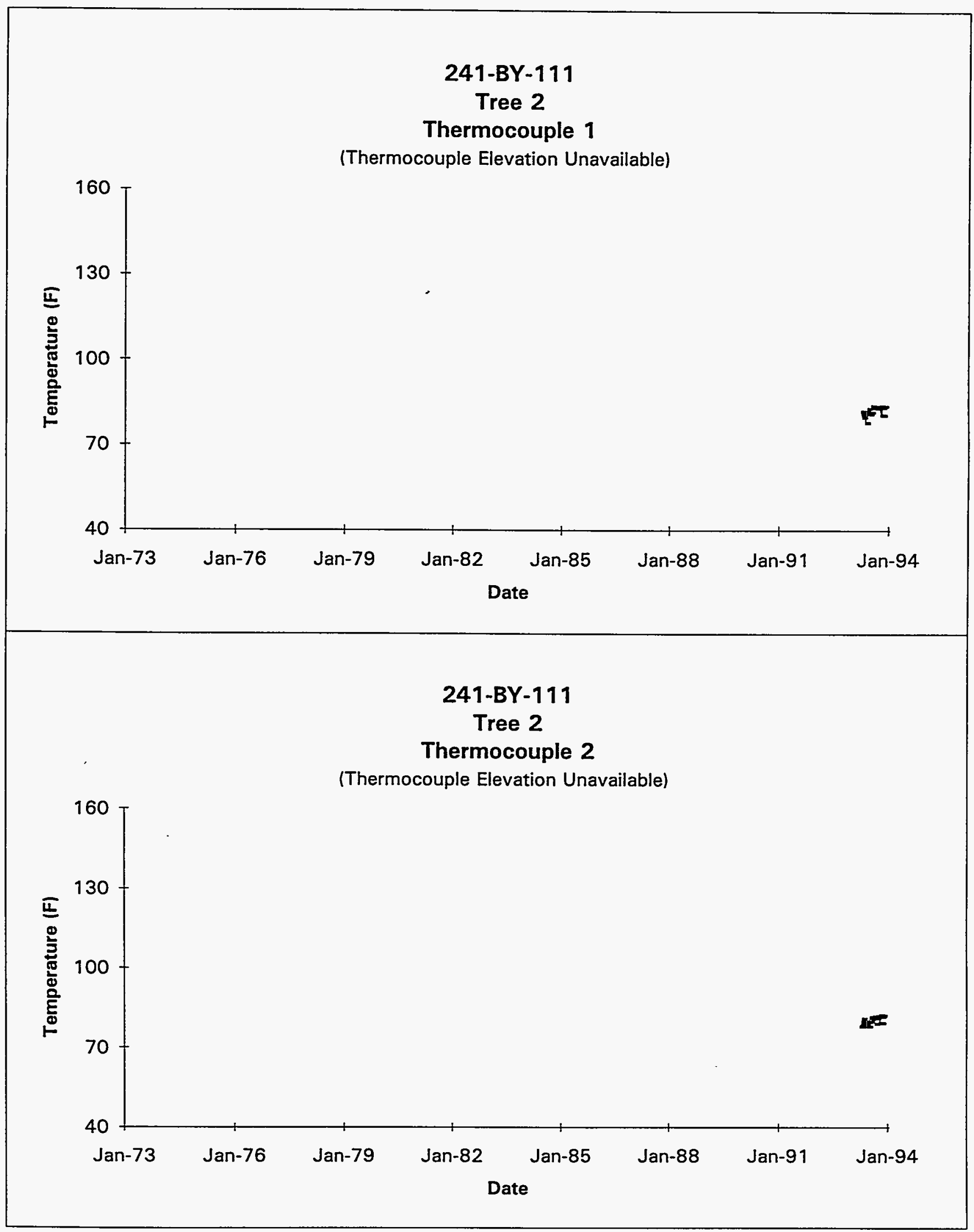

Data obtained from WHC Surveillance Analysis Computer System (SACS), November 12, 1993. 


\section{1-BY-111}

Tree 2

\section{Thermocouple 3}

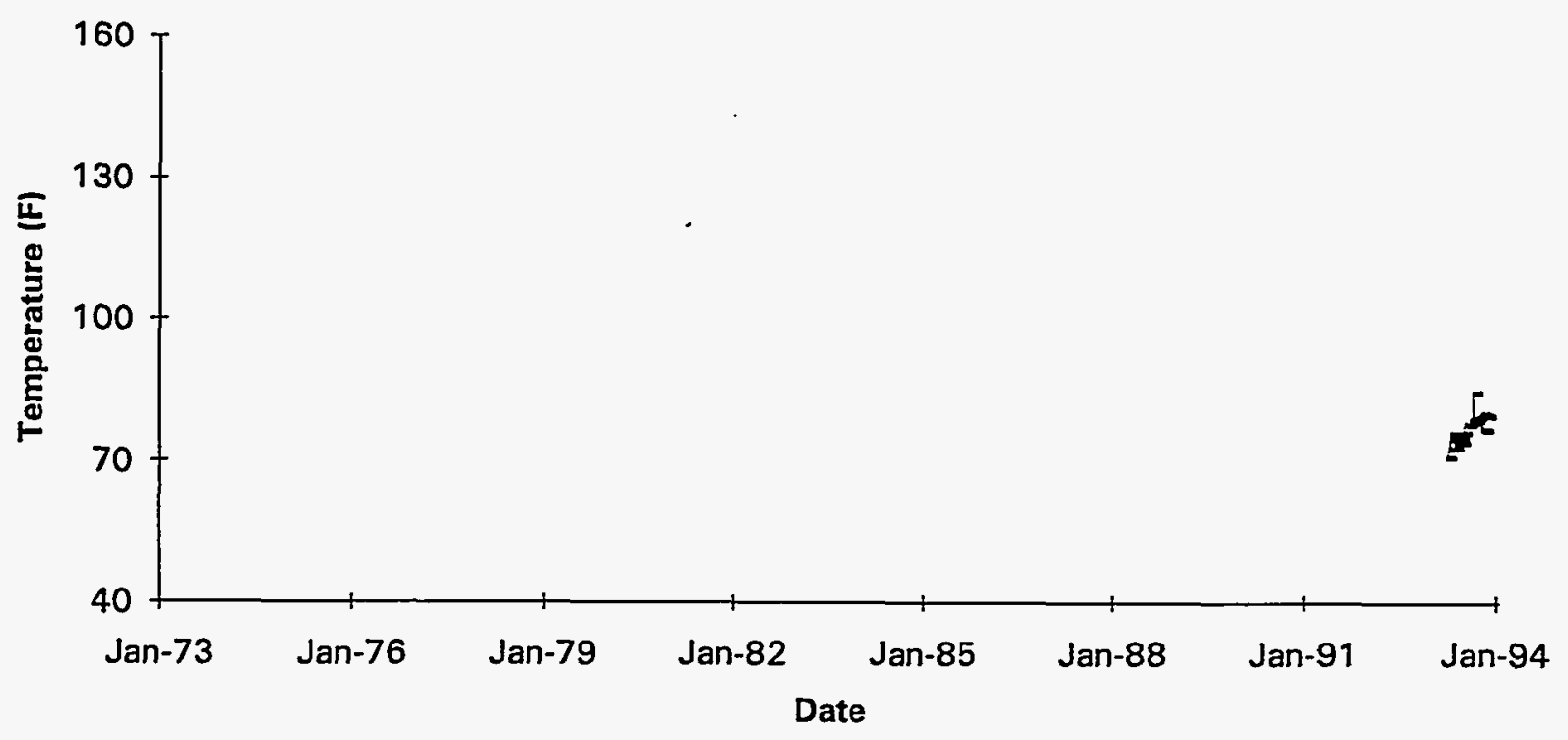

241-BY-111

Tree 2

Thermocouple 4

(Thermocouple Elevation Unavailable)

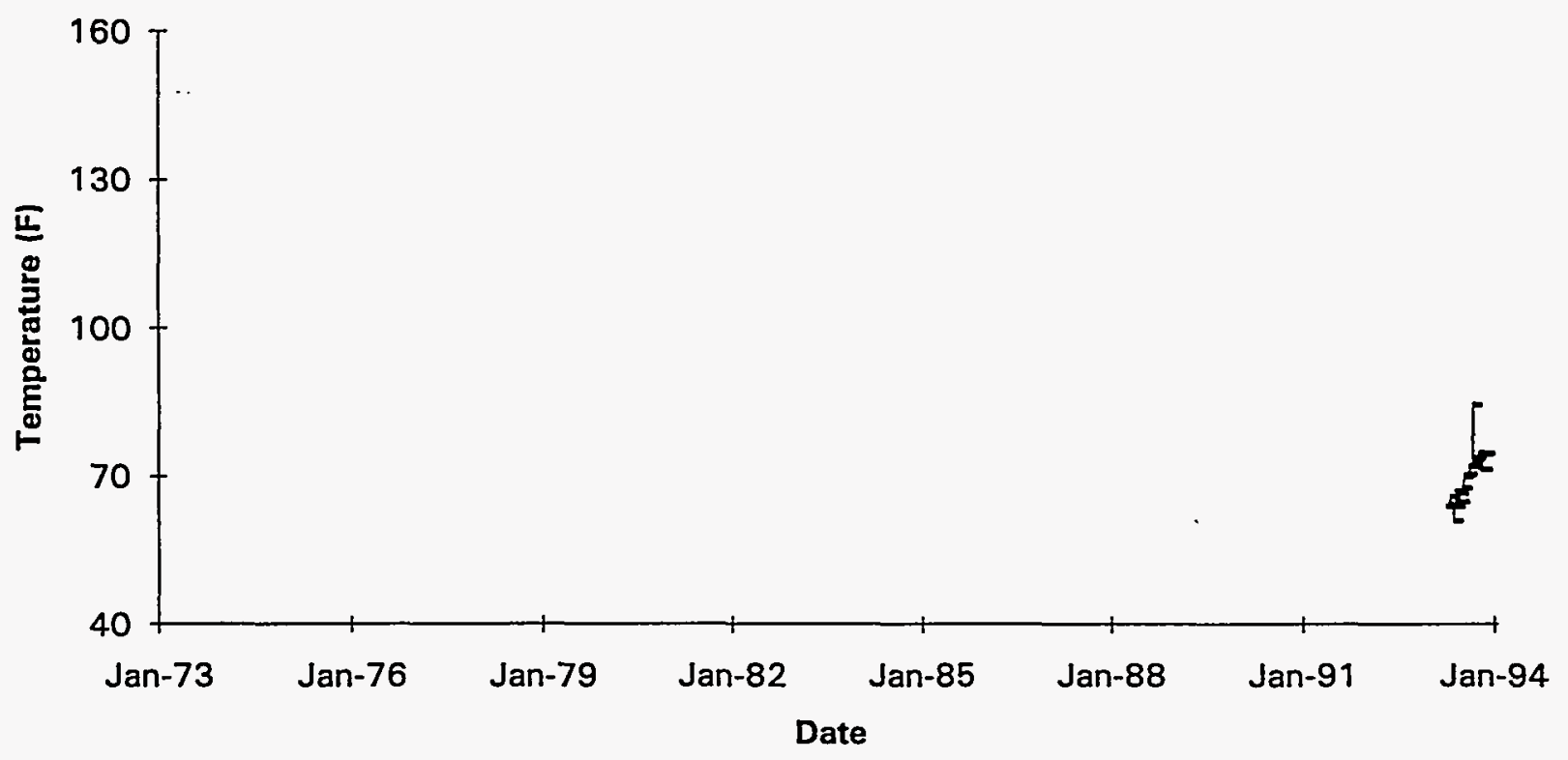

Data obtained from WHC Surveillance Analysis Computer System (SACS), November 12, 1993. 


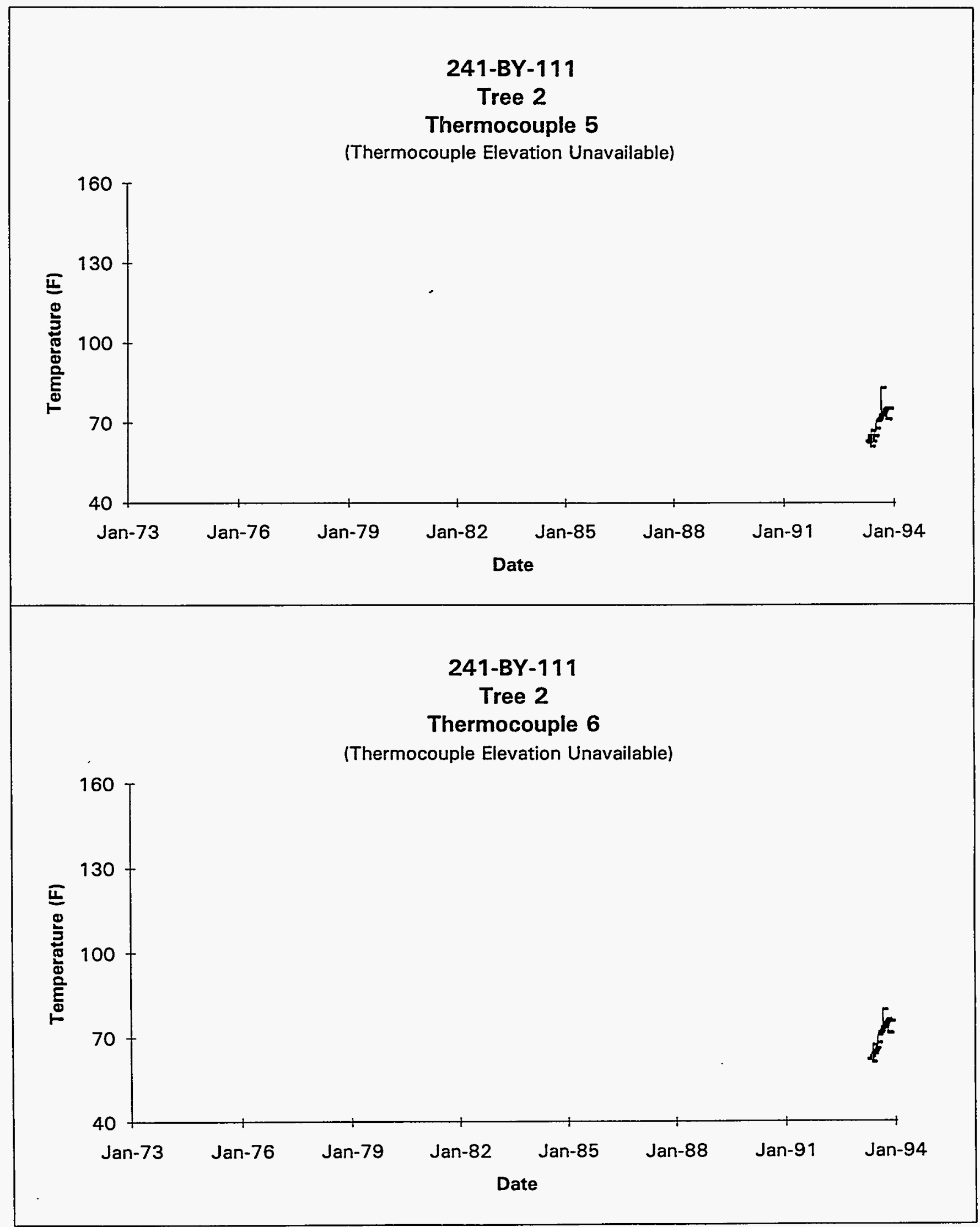

Data obtained from WHC Surveillance Analysis Computer System (SACS), November 12, 1993. 


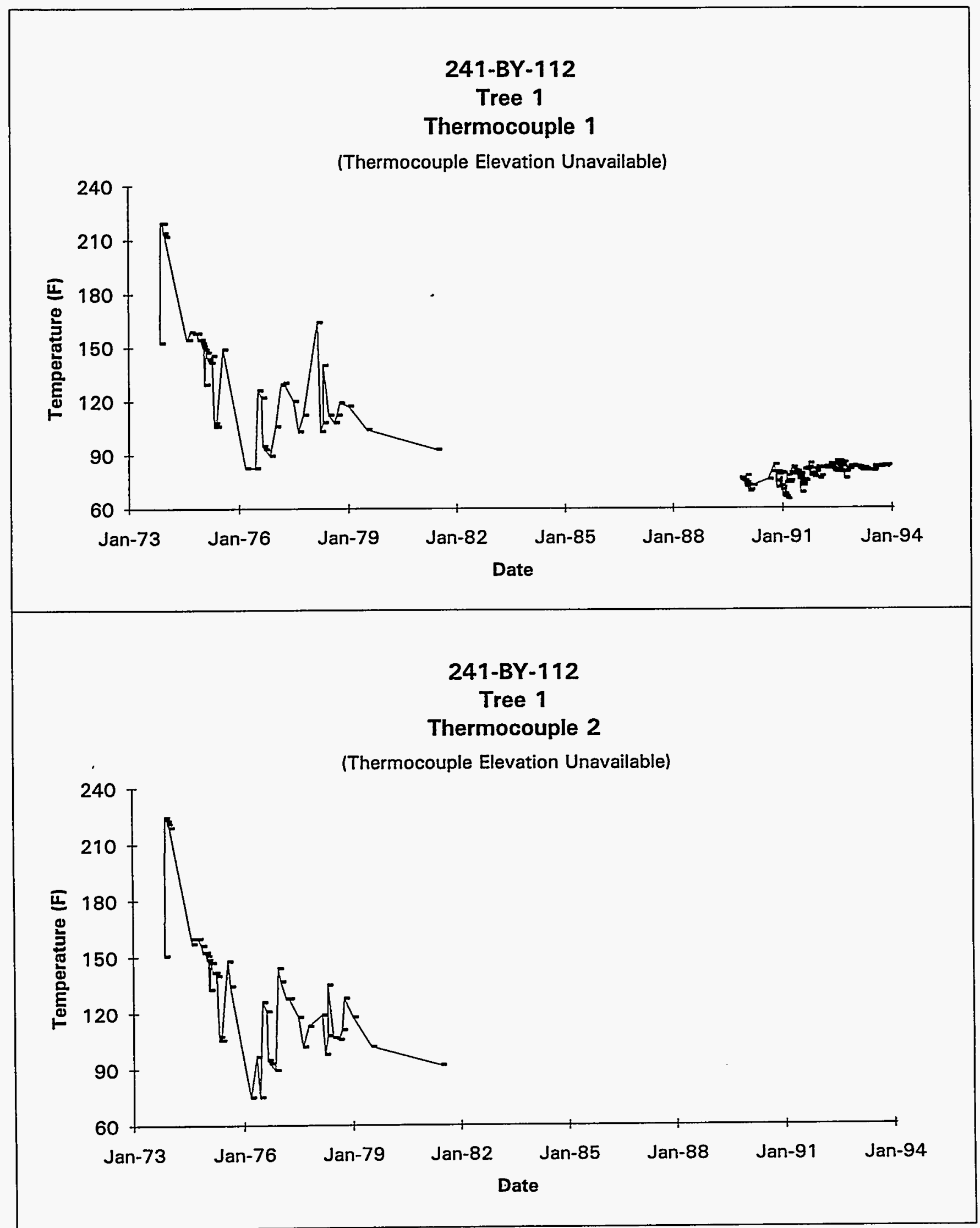

Data obtained from WHC Surveillance Analysis Computer System (SACS), November 12, 1993. 


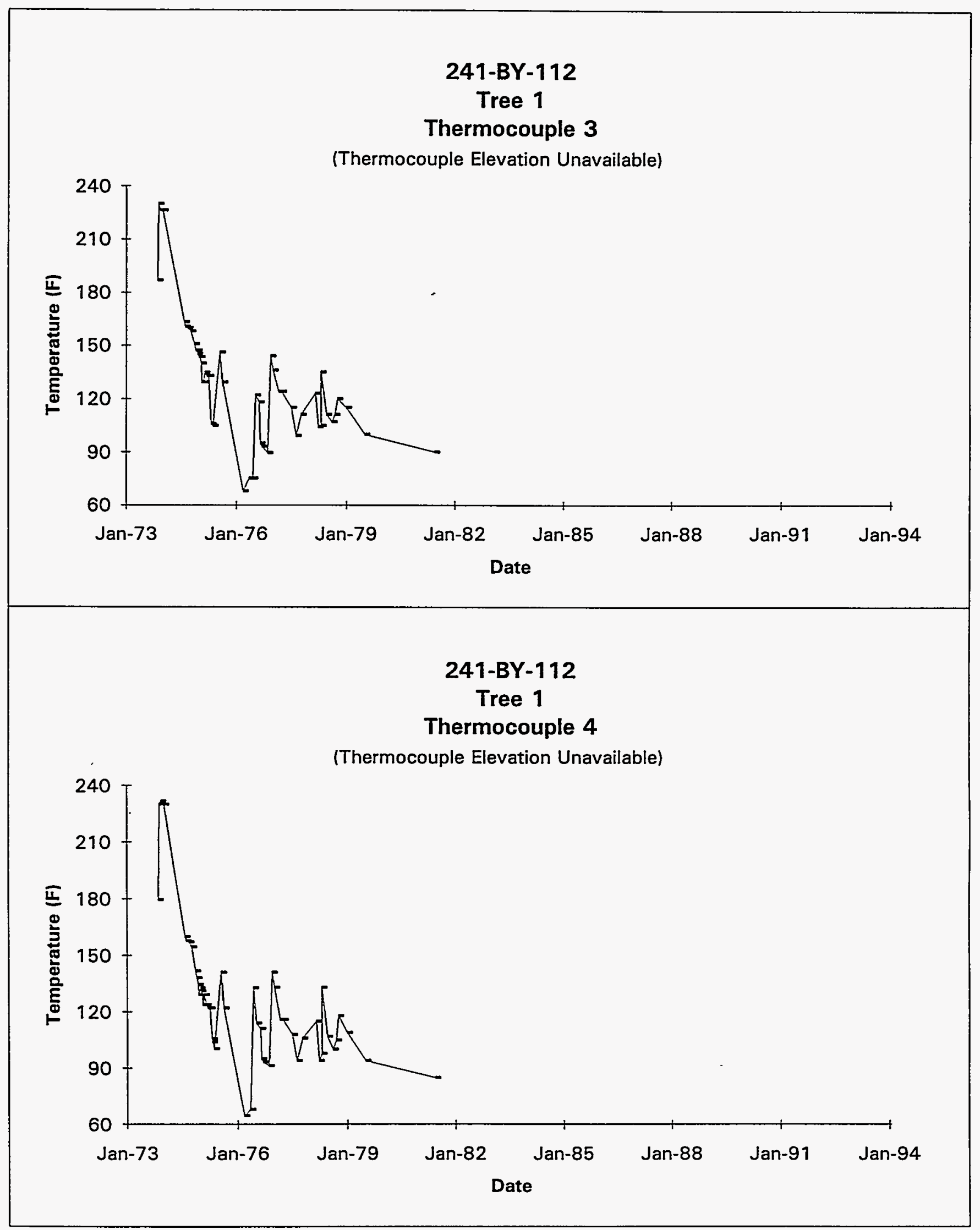

Data obtained from WHC Surveillance Analysis Computer System (SACS), November 12, 1993. 


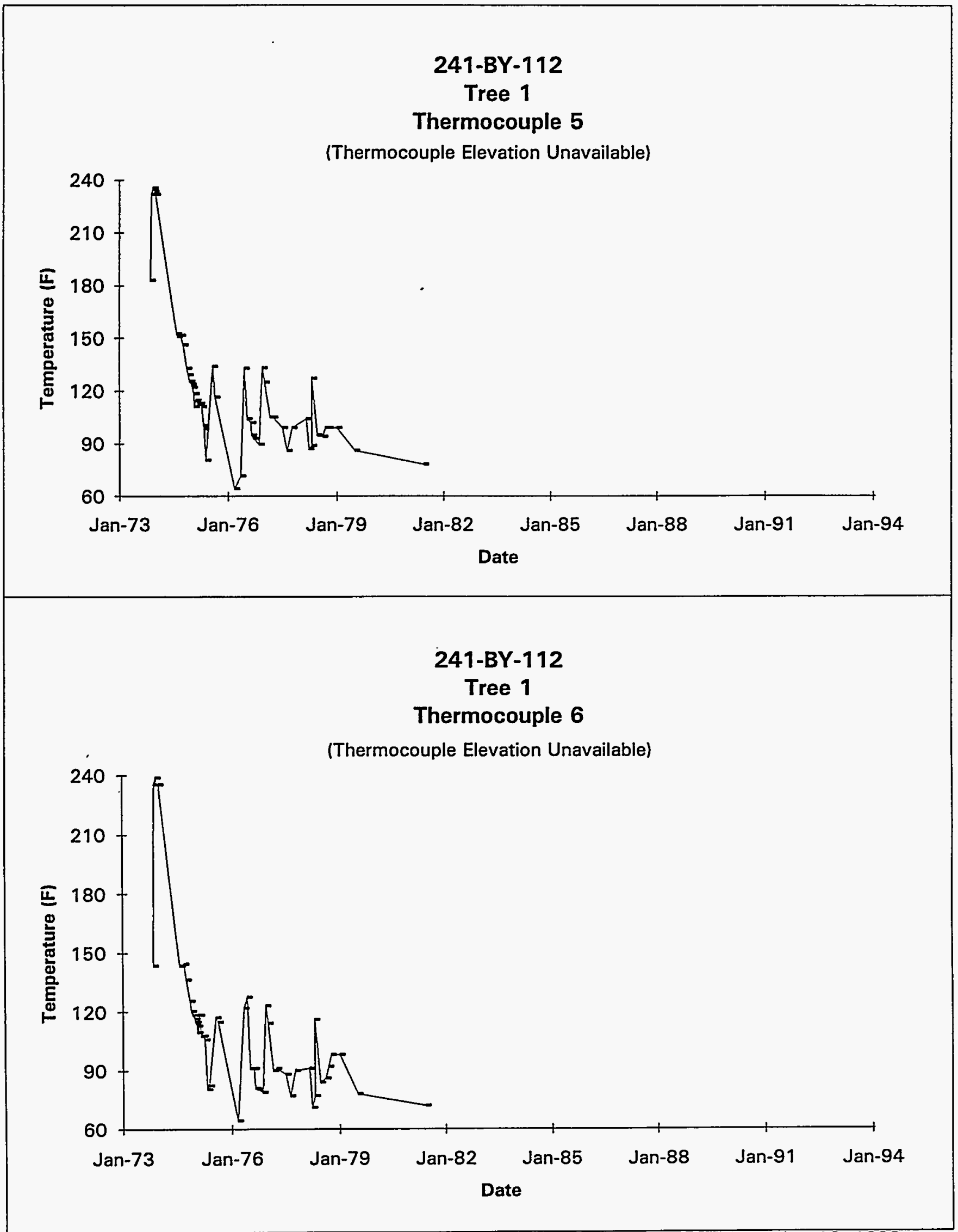

Data obtained from WHC Surveillance Analysis Computer System (SACS), November 12, 1993. 


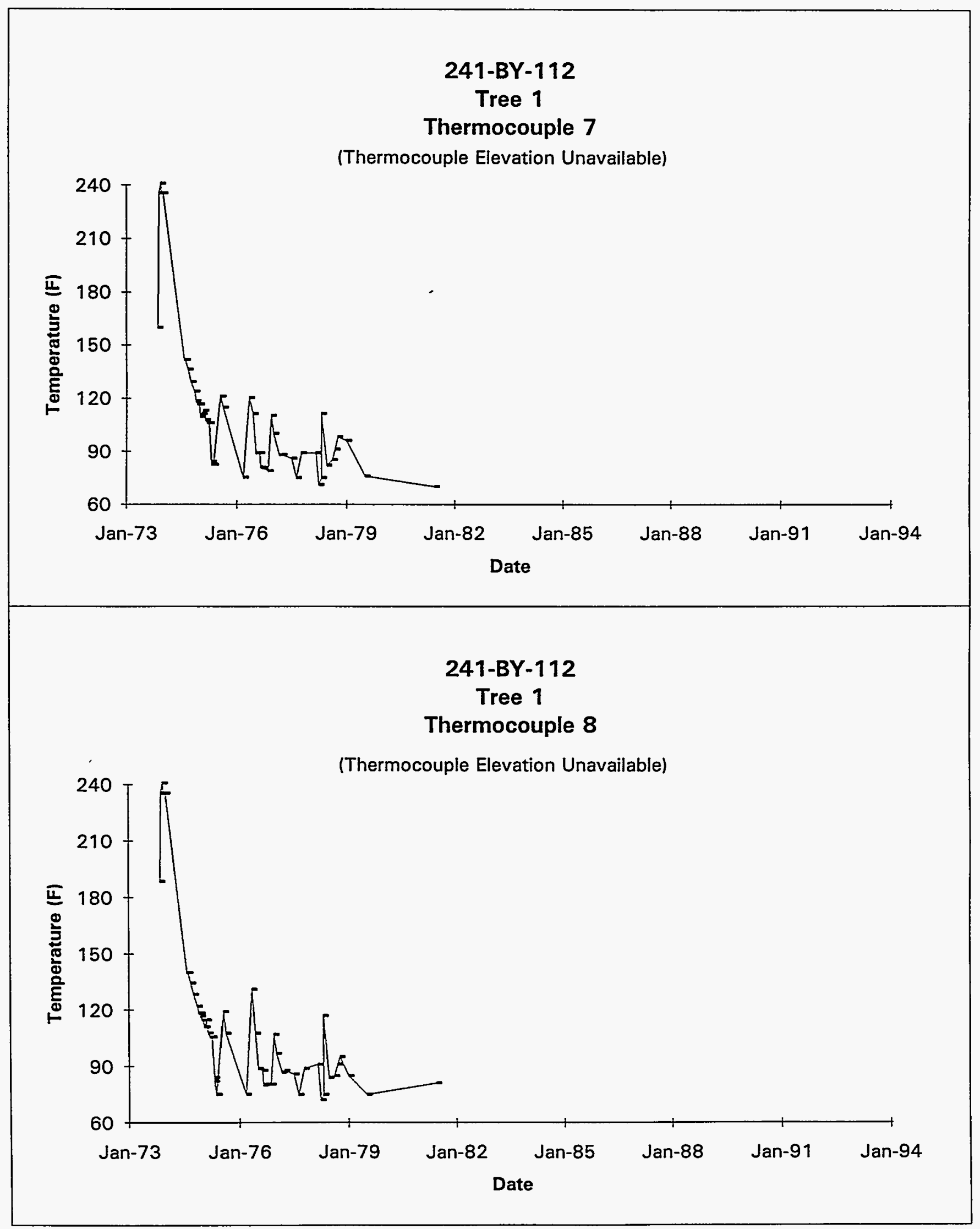

Data obtained from WHC Surveillance Analysis Computer System (SACS), November 12, 1993.

$$
\text { D-90 }
$$




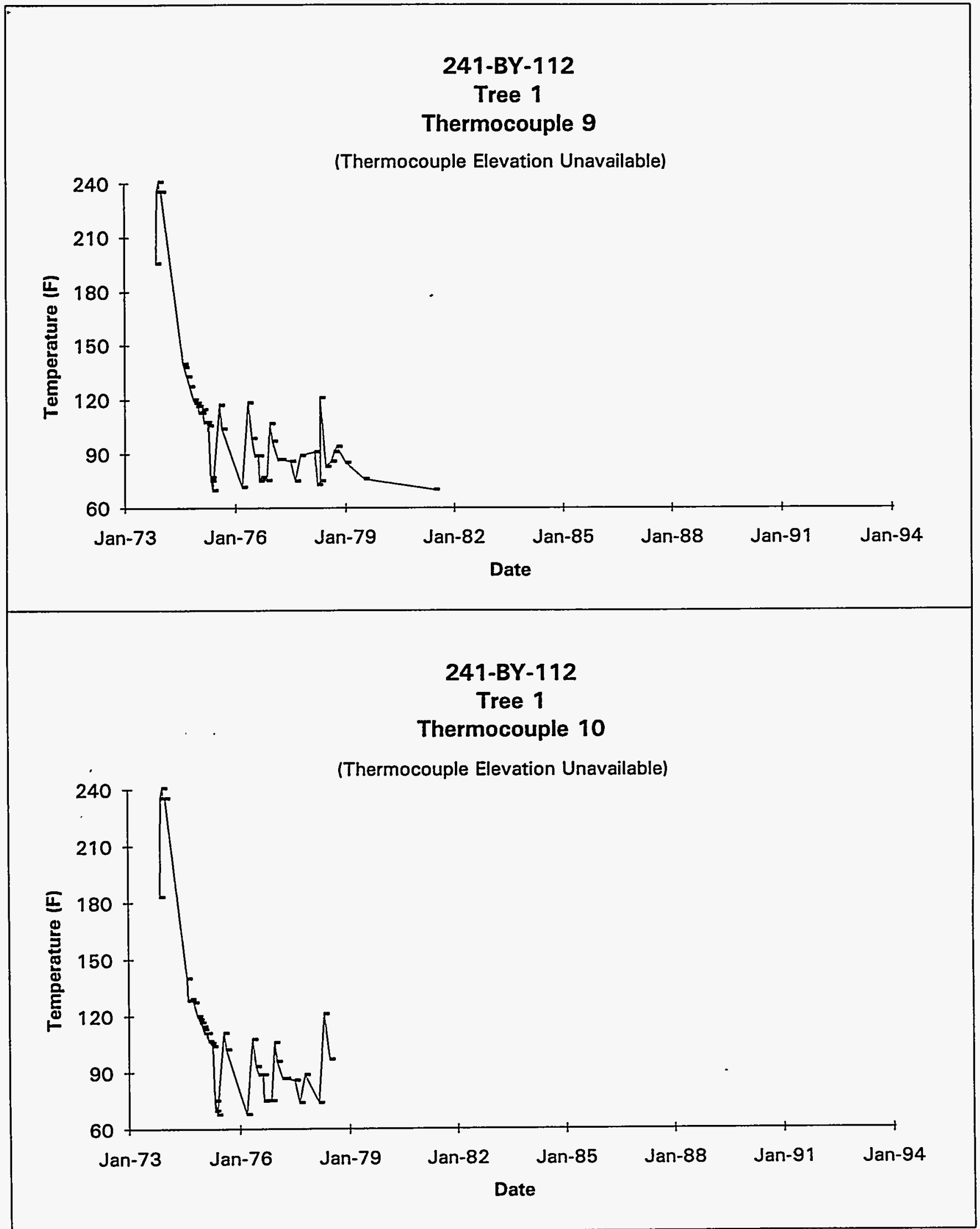

Data obtained from WHC Surveillance Analysis Computer System (SACS), November 12, 1993. 


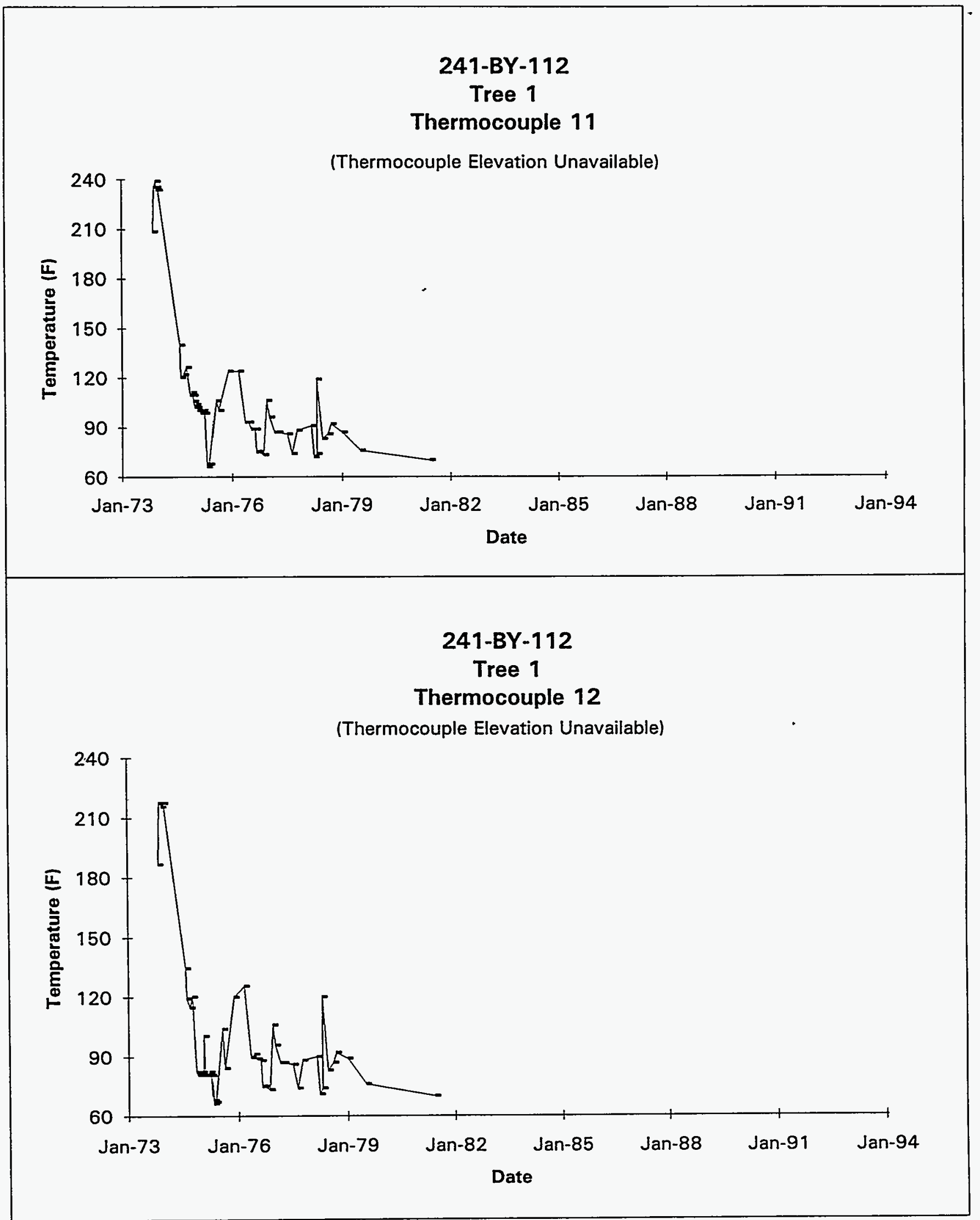

Data obtained from WHC Surveillance Analysis Computer System (SACS), November 12, 1993.

D-92 


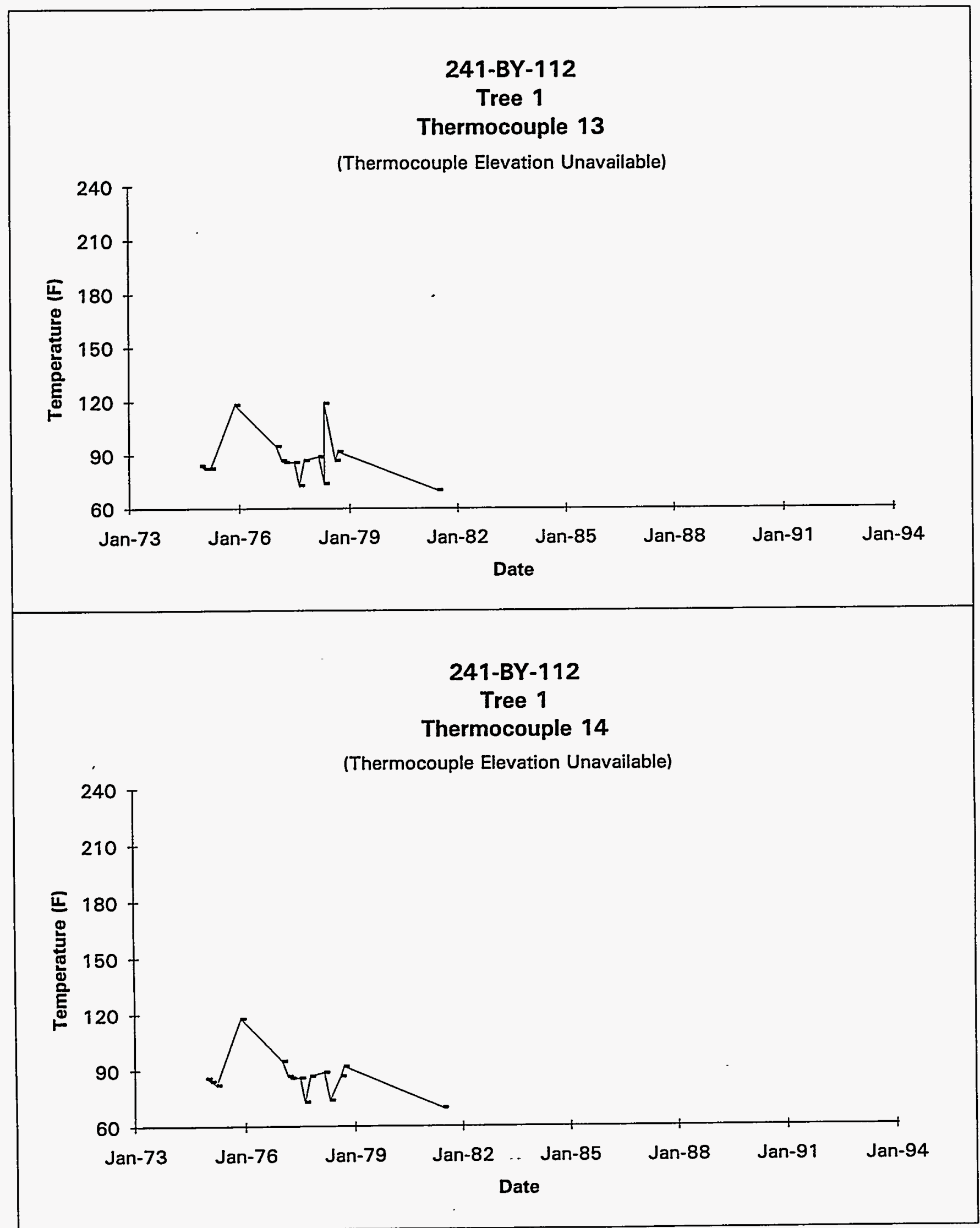

Data obtained from WHC Surveillance Analysis Computer System (SACS), November 12, 1993. 


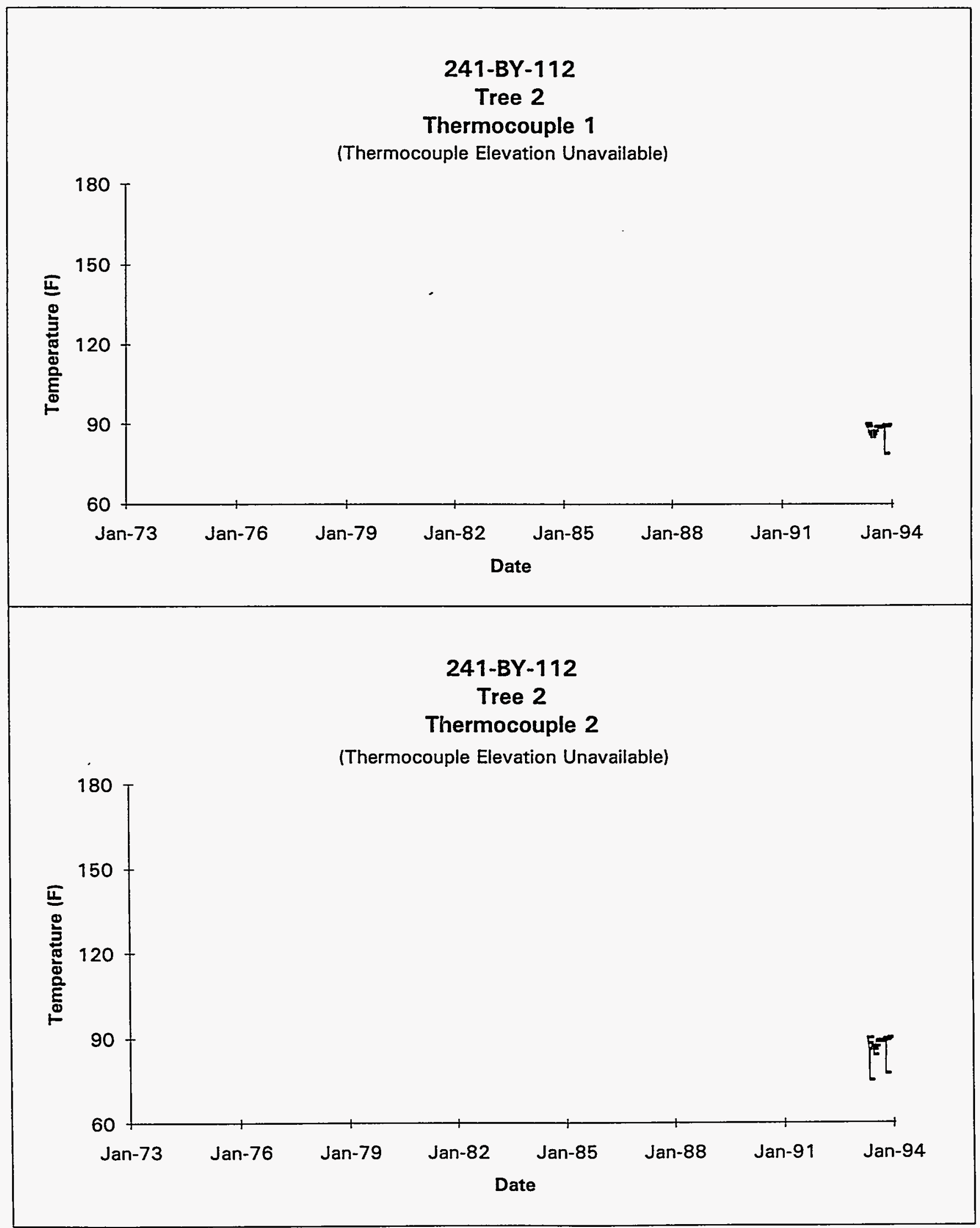

Data obtained from WHC Surveillance Analysis Computer System (SACS), November 12, 1993. D-94 


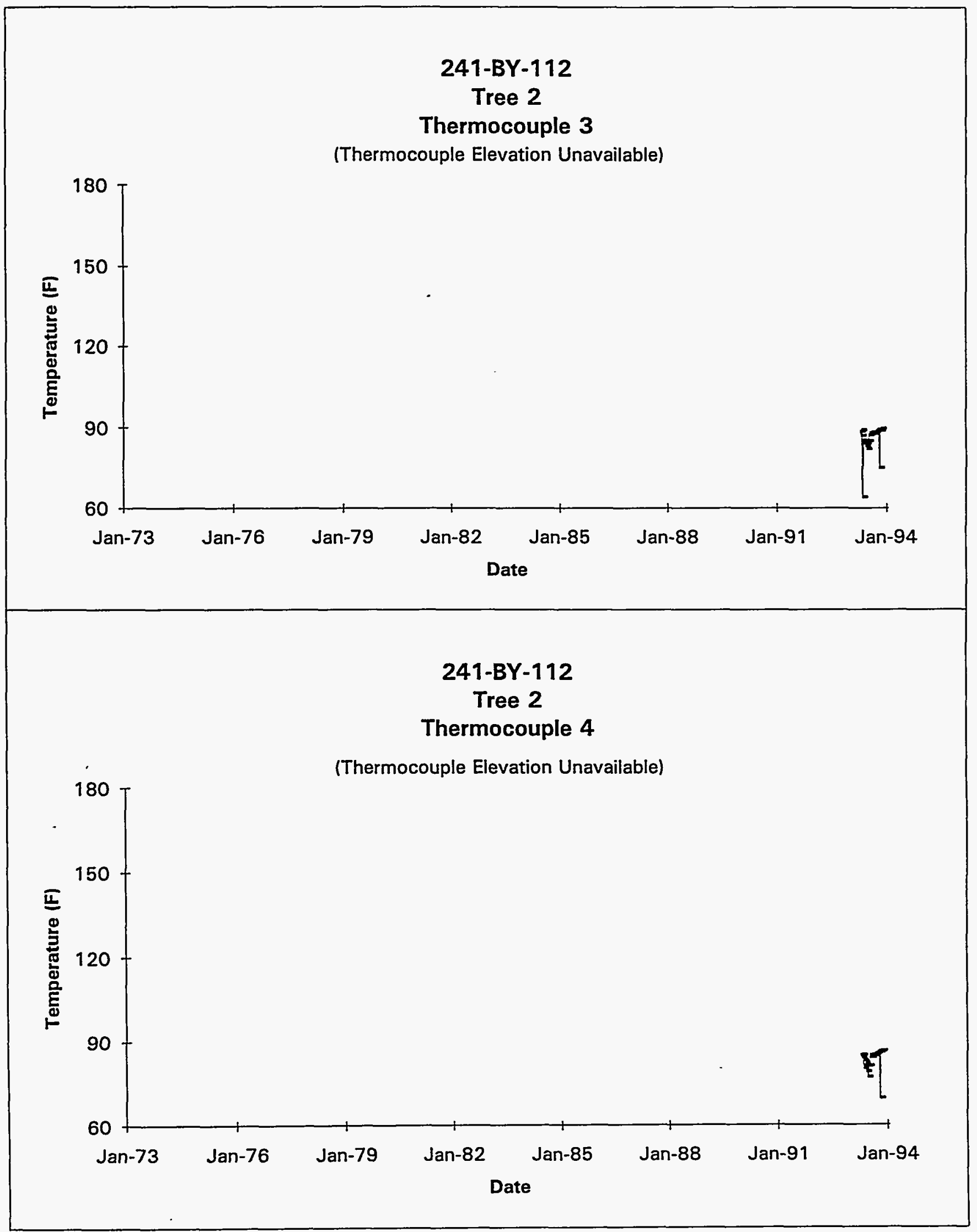

Data obtained from WHC Surveillance Analysis Computer System (SACS), November 12, 1993.

D-95 


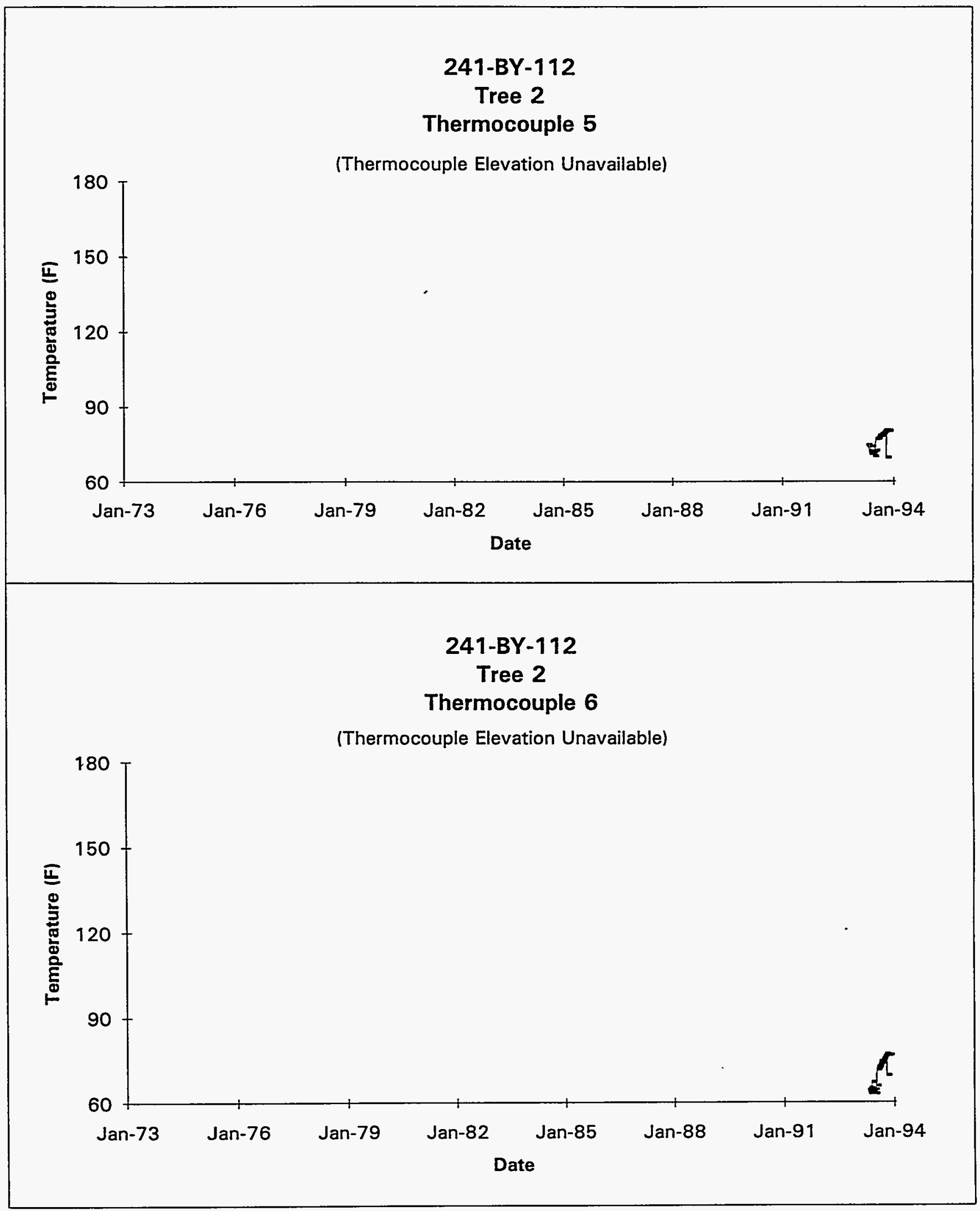

Data obtained from WHC Surveillance Analysis Computer System (SACS), November 12, 1993. 


\begin{tabular}{|c|c|c|c|c|c|c|c|}
\hline \multicolumn{8}{|c|}{ TANK 241-BY-101 } \\
\hline \multicolumn{8}{|c|}{ THERMOCOUPLE DATA } \\
\hline TREE\# & DATE & THERMO \# & TAEE SET \# & TEMP C & TEMP F & $Y=$ MAX TEMP & COMMENTS \\
\hline 1 & $10 / 18 / 74$ & 1 & 1 & 32 & 89.6 & $\mathrm{Y}$ & degres C/TWINS confirm \\
\hline 1 & $10 / 18 / 74$ & 2 & $\frac{1}{1}$ & 31 & 87.8 & $\mathbf{N}$ & degree C/TWINS confirm \\
\hline$\frac{1}{1}$ & $10 / 18 / 74$ & 3 & 1 & 32 & 89.6 & $\bar{Y}$ & degree $\mathrm{C} / \mathrm{TW}$ INS confirm \\
\hline 1 & $10 / 18 / 74$ & 4 & 1 & 30 & 86 & $\mathbf{N}$ & degree C/TWINS confirm \\
\hline 1 & $10 / 18 / 74$ & 5 & 1 & 29 & 84.2 & $\mathbf{N}$ & degree C/TWINS confirm \\
\hline 1 & $10 / 18 / 74$ & 6 & 1 & 27 & 80.6 & $\mathbf{N}$ & degree C/TWINS confirm \\
\hline 1 & $10 / 18 / 74$ & 14 & 1 & 27 & 80.6 & $\mathbf{N}$ & degree C/TWINS confirm \\
\hline 1 & $11 / 16 / 74$ & 1 & 1 & 46.11 & 114.998 & $\bar{Y}$ & degree C/TWINS confirm \\
\hline 1 & $11 / 16 / 74$ & 2 & 1 & 46.11 & 114.998 & $\bar{Y}$ & degree C/TWINS confirm \\
\hline 1 & $11 / 16 / 74$ & 3 & 1 & 46.11 & 114.998 & $\bar{Y}$ & degree C/TWINS confirm \\
\hline 1 & $11 / 16 / 74$ & 5 & 1 & 43.33 & 109.994 & $\mathbf{N}$ & degree C/TWINS confirm \\
\hline 1 & $11 / 16 / 74$ & 6 & 1 & & & $\bar{N}$ & 12.78 - suspect data (low) \\
\hline 1 & $11 / 16 / 74$ & 14 & 1 & 37.78 & 100.004 & $N$ & degree C/TWINS confirm \\
\hline 1 & $12 / 17 / 74$ & 1 & 1 & 32.22 & 89.996 & $\mathbf{Y}$ & degree C/TWINS confirm \\
\hline 1 & $12 / 17 / 74$ & 2 & $\overline{1}$ & 31.67 & 89.006 & $\bar{N}$ & degree C/TWINS confirm \\
\hline 1 & $12 / 17 / 74$ & 3 & 1 & 30.56 & 87.008 & $\mathbf{N}$ & degree C/TWINS confirm \\
\hline 1 & $12 / 17 / 74$ & 5 & 1 & 28.89 & 84.002 & $\mathbf{N}$ & degree $\mathrm{C} / \mathrm{TW}$ INS confirm \\
\hline 1 & $12 / 17 / 74$ & 6 & 1 & & & $\mathbf{N}$ & 14.44 - suspect data (low) \\
\hline$T$ & $12 / 17 / 74$ & 12 & 1 & 25.56 & 78.008 & $\mathbf{N}$ & degree $\mathrm{C} / \mathrm{TW}$ INS confirm \\
\hline 1 & $12 / 31 / 74$ & 1 & 1 & 32.78 & 97.004 & $Y$ & degree C/TWINS confirm \\
\hline 1 & $12 / 31 / 74$ & 2 & 9 & 32.22 & 89.996 & $\mathbf{N}$ & degree $\mathrm{C} / \mathrm{T}$ WINS confirm \\
\hline 9 & $12 / 31 / 74$ & 3 & 1 & 31.67 & 89.006 & $\bar{N}$ & degree C/TWINS confirm \\
\hline 9 & $12 / 31 / 74$ & 5 & 1 & 30 & 86 & $\mathbf{N}$ & degree C/TWINS confirm \\
\hline 1 & $12 / 31 / 74$ & 6 & $T$ & & & $\mathbf{N}$ & 10 - suspect data (low) \\
\hline 1 & $12 / 31 / 74$ & 14 & 1 & 23.89 & 75.002 & $N$ & degree $\mathrm{C} / \mathrm{TW}$ INS confirm \\
\hline 1 & $2 / 27 / 75$ & 1 & 1 & & 99 & $\overline{\mathbf{Y}}$ & \\
\hline 1 & $2 / 27 / 75$ & 2 & 1 & & 99 & $\bar{Y}$ & \\
\hline 9 & $2 / 27 / 75$ & 3 & 1 & & 97 & $\mathbf{N}$ & \\
\hline 1 & $2 / 27 / 75$ & 5 & 1 & & 93 & $\mathbf{N}$ & \\
\hline 7 & $2 / 27 / 75$ & 6 & 1 & & & $\mathbf{N}$ & 54 - suspect data (low) \\
\hline 1 & $2 / 27 / 75$ & 14 & 1 & & 79 & $\mathbf{N}$ & \\
\hline 9 & $4 / 22 / 75$ & 1 & 1 & 32 & 89.6 & $\mathbf{N}$ & degree $\mathrm{C} / \mathrm{TW}$ INS confirm \\
\hline 1 & $4 / 22 / 75$ & 2 & 1 & 32 & 89.6 & $\mathbf{N}$ & degree C/TWINS confirm \\
\hline 1 & $4 / 22 / 75$ & 3 & 1 & 30 & 86 & $\mathbf{N}$ & degree $\mathrm{C} / \mathrm{TW}$ INS confirm \\
\hline 1 & $4 / 22 / 75$ & 4 & 1 & 34 & 93.2 & $\bar{Y}$ & degree C/TWINS confirm \\
\hline 1 & $4 / 22 / 75$ & 5 & 1 & 28 & 82.4 & $\mathbf{N}$ & degree C/TWINS confirm \\
\hline 1 & $4 / 22 / 75$ & 6 & 1 & 31 & 87.8 & $\mathbf{N}$ & degree C/TWINS confirm \\
\hline 1 & $4 / 22 / 75$ & 12 & 1 & 18 & 64.4 & $\mathbf{N}$ & degres C/TWINS confirm \\
\hline 1 & $.5 / 20 / 75$ & 1 & 1 & 32 & 89.6 & $\mathbf{Y}$ & degree C/TWINS confirm \\
\hline 1 & $5 / 20 / 75$ & 2 & 1 & 32 & 89.6 & $\mathbf{Y}$ & degree C/TWINS confirm \\
\hline 1 & $5 / 20 / 75$ & 3 & 1 & 31 & 87.8 & $\mathbf{N}$ & degree C/TWINS confirm \\
\hline 1 & $5 / 20 / 75$ & 4 & 1 & 31 & 87.8 & $\mathbf{N}$ & degree C/TWINS confirm \\
\hline 1 & $5 / 20 / 75$ & 5 & 1 & 30 & 86 & $\mathbf{N}$ & degree C/TWINS confirm \\
\hline 1 & $5 / 20 / 75$ & 6 & 1 & 28 & 82.4 & $\mathbf{N}$ & degree C/TWINS confirm \\
\hline 1 & $5 / 20 / 75$ & 14 & 1 & 21 & 69.8 & $N$ & degrae C/TWINS confirm \\
\hline 9 & $7 / 28 / 75$ & 1 & 1 & & 91 & $\bar{Y}$ & \\
\hline 1 & $7 / 28 / 75$ & 2 & 1 & & 90 & $\mathbf{N}$ & \\
\hline 1 & $7 / 28 / 75$ & 3 & 9 & & 89 & $\mathbf{N}$ & \\
\hline 1 & $7 / 28 / 75$ & 5 & 1 & & 86 & $\mathbf{N}$ & \\
\hline 1 & $7 / 28 / 75$ & 6 & 1 & & 85 & $\mathbf{N}$ & \\
\hline 1 & $7 / 28 / 75$ & 7 & 1 & & 83 & $\mathbf{N}$ & \\
\hline$\frac{1}{1}$ & $7 / 28 / 75$ & 8 & 1 & & 82 & $\mathbf{N}$ & \\
\hline 9 & $7 / 28 / 75$ & 9 & 1 & & 81 & 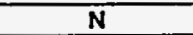 & \\
\hline$\frac{1}{1}$ & $7 / 28 / 75$ & 10 & 1 & & 81 & $\mathbf{N}$ & \\
\hline 1 & $7 / 28 / 75$ & 11 & 1 & & 79 & $\mathbf{N}$ & \\
\hline 1 & $7 / 28 / 75$ & 12 & 1 & & 77 & $\mathbf{N}$ & \\
\hline 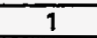 & $7 / 28 / 75$ & 13 & 1 & & 77 & $\bar{N}$ & \\
\hline$\frac{1}{1}$ & $7 / 28 / 75$ & 14 & 1 & & 77 & $N$ & \\
\hline 1 & $8 / 21 / 75$ & 1 & 1 & & 90 & $\bar{Y}$ & \\
\hline 1 & $8 / 21 / 75$ & 2 & 1 & & 89 & $\mathbf{N}$ & \\
\hline 1 & $8 / 21 / 75$ & 3 & 1 & & 88 & $\mathbf{N}$ & \\
\hline 1 & $8 / 21 / 75$ & 5 & 1 & & 86 & $\bar{N}$ & \\
\hline 1 & $8 / 21 / 75$ & 6 & 7 & & 84 & $\mathbf{N}$ & \\
\hline 9 & $8 / 21 / 75$ & 14 & 1 & & 76 & $N$ & \\
\hline 1 & $8 / 26 / 75$ & 1 & 1 & & 90 & $\bar{Y}$ & \\
\hline 1 & $8 / 26 / 75$ & 2 & 1 & & 89 & $\mathbf{N}$ & \\
\hline
\end{tabular}

Data obtained from WHC Surveillance Analysis Computer System (SACS), November 12, 1993. 
WHC-SD-WM-ER-312, Rev. 0

\begin{tabular}{|c|c|c|c|c|c|c|c|}
\hline \multicolumn{8}{|c|}{ TANK 241-BY-101 } \\
\hline \multicolumn{8}{|c|}{ THERMOCOUPLE DATA } \\
\hline & & & & & & & \\
\hline TREE\# & DATE & THERMO \# & TREE SET \# & TEMP C & TEMP F & $Y=$ MAX TEMP & COMMENTS \\
\hline 1 & $8 / 26 / 75$ & 3 & 1 & & 88 & $N$ & \\
\hline 1 & $8 / 26 / 75$ & 5 & 1 & & 86 & $\mathbf{N}$ & \\
\hline 1 & $8 / 26 / 75$ & 6 & 1 & & 84 & $\mathbf{N}$ & \\
\hline 1 & $8 / 26 / 75$ & 7 & 1 & & 82 & $\mathbf{N}$ & \\
\hline 1 & $8 / 26 / 75$ & 8 & 1 & & 81 & $\mathbf{N}$ & \\
\hline 1 & $8 / 26 / 75$ & 9 & 1 & & 80 & $\mathbf{N}$ & \\
\hline 1 & $8 / 26 / 75$ & 10 & 1 & & 80 & $\mathbf{N}$ & \\
\hline 1 & $8 / 26 / 75$ & 11 & 1 & & 78 & $\bar{N}$ & \\
\hline 1 & $8 / 26 / 75$ & 12 & 1 & & 76 & $\mathbf{N}$ & \\
\hline 1 & $8 / 26 / 75$ & 13 & 1 & & 76 & $\mathbf{N}$ & \\
\hline 1 & $8 / 26 / 75$ & 14 & 1 & & 76 & $\overline{\mathbf{N}}$ & \\
\hline 1 & $11 / 21 / 75$ & 1 & -1 & & 93 & $\bar{Y}$ & \\
\hline 1 & $19 / 29 / 75$ & 2 & 1 & & 93 & $Y$ & \\
\hline 1 & $11 / 21 / 75$ & 3 & 1 & & 92 & $N$ & \\
\hline 1 & $11 / 21 / 75$ & 5 & 1 & & 91 & $\mathbf{N}$ & \\
\hline 1 & $11 / 21 / 75$ & 6 & 1 & & 91 & $\mathbf{N}$ & \\
\hline 1 & $11 / 21 / 75$ & 7 & 1 & & 90 & $\mathrm{~N}$ & \\
\hline 1 & $11 / 21 / 75$ & 8 & 1 & & 88 & $\mathbf{N}$ & \\
\hline 1 & $11 / 21 / 75$ & 9 & 1 & & 87 & $\mathrm{~N}$ & \\
\hline 1 & $11 / 21 / 75$ & 10 & 1 & & 87 & $\mathbf{N}$ & \\
\hline 1 & $11 / 21 / 75$ & 11 & 1 & & 82 & $\bar{N}$ & \\
\hline 1 & $11 / 21 / 75$ & 12 & 1 & & 78 & $N$ & \\
\hline 1 & $1 / 7 / 76$ & 1 & 1 & & 97 & $Y$ & \\
\hline 1 & $1 / 7 / 76$ & 2 & 1 & & 97 & $\bar{Y}$ & \\
\hline 1 & $1 / 7 / 76$ & 3 & 1 & & 96 & $\mathbf{N}$ & \\
\hline 1 & $1 / 7 / 76$ & 5 & 1 & & 94 & $\mathrm{~N}$ & \\
\hline 1 & $1 / 7176$ & 6 & 1 & & 93 & $\mathrm{~N}$ & \\
\hline 1 & $9 / 7 / 76$ & 7 & 1 & & 91 & $\mathrm{~N}$ & \\
\hline 1 & $1 / 7 / 76$ & 8 & 1 & & 89 & $\mathrm{~N}$ & \\
\hline 1 & $1 / 7 / 76$ & 9 & 1 & & 88 & $\mathbf{N}$ & \\
\hline 1 & $1 / 7 / 76$ & 10 & 1 & & 88 & $N$ & \\
\hline 1 & $1 / 7 / 76$ & 11 & 1 & & 83 & $\mathbf{N}$ & \\
\hline 1 & $1 / 7 / 76$ & 12 & 1 & & 80 & $N$ & \\
\hline 1 & $1 / 7 / 76$ & 13 & 1 & & 79 & $\mathbf{N}$ & \\
\hline 1 & $1 / 7776$ & 14 & 1 & & 79 & $\mathbf{N}$ & \\
\hline 1 & $3 / 4 / 76$ & 1 & 1 & & 95 & $Y$ & \\
\hline 1 & $3 / 4 / 76$ & 2 & 1 & & 94 & $\mathrm{~N}$ & \\
\hline 1 & $3 / 4 / 76$ & 3 & 1 & & 93 & $\mathrm{~N}$ & \\
\hline 1 & $.3 / 4 / 76$ & 5 & 1 & & 90 & $N$ & \\
\hline 1 & $3 / 4 / 76$ & 6 & 1 & & 87 & $\mathbf{N}$ & \\
\hline 1 & $3 / 4 / 76$ & 7 & 1 & & 83 & $\mathbf{N}$ & \\
\hline 1 & $3 / 4 / 76$ & 8 & 1 & & 81 & $\mathbf{N}$ & \\
\hline 1 & $3 / 4 / 76$ & 9 & 1 & & 81 & $\mathbf{N}$ & \\
\hline 1 & $3 / 4 / 76$ & 10 & 1 & & 81 & $N$ & \\
\hline 1 & $3 / 4 / 76$ & 11 & 1 & & 78 & $\mathbf{N}$ & \\
\hline 1 & $3 / 4 / 76$ & 12 & 1 & & 76 & $\mathbf{N}$ & \\
\hline 1 & $3 / 4 / 76$ & 13 & 1 & & 75 & $\mathrm{~N}$ & \\
\hline 1 & $3 / 4 / 76$ & 14 & 1 & & 76 & $\mathrm{~N}$ & \\
\hline 1 & $5 / 7 / 76$ & 1 & 1 & & 85 & $\bar{Y}$ & \\
\hline 1 & $5 / 7 / 76$ & 2 & 1 & & 84 & $\mathbf{N}$ & \\
\hline 1 & $5 / 7 / 76$ & 3 & 1 & & 84 & $\mathbf{N}$ & \\
\hline 1 & $5 / 7 / 76$ & 5 & 1 & & 79 & $\mathbf{N}$ & \\
\hline 9 & $5 / 7 / 76$ & 6 & 1 & & 75 & $N$ & \\
\hline 1 & $5 / 7 / 76$ & 7 & 1 & & 72 & $\mathrm{~N}$ & \\
\hline 1 & $5 / 7 / 76$ & 8 & 1 & & 70 & $\mathrm{~N}$ & \\
\hline 9 & $5 / 7 / 76$ & 9 & 1 & & 69 & $\mathrm{~N}$ & \\
\hline 1 & $5 / 7 / 76$ & 10 & 1 & & 69 & $\bar{N}$ & \\
\hline$\frac{1}{1}$ & $5 / 7 / 76$ & 11 & 1 & & 67 & $\mathbf{N}$ & \\
\hline 1 & $5 / 7 / 76$ & 12 & 1 & & 66 & $\mathbf{N}$ & \\
\hline 1 & $5 / 7 / 76$ & 13 & 1 & & 66 & $\mathbf{N}$ & \\
\hline 1 & $5 / 7 / 76$ & 94 & 1 & & 66 & $\mathbf{N}$ & \\
\hline 1 & $6 / 7 / 76$ & 1 & 1 & & 87 & $\mathbf{Y}$ & \\
\hline 1 & $6 / 7 / 76$ & 2 & 1 & & 86 & $\mathbf{N}$ & \\
\hline 1 & $6 / 7 / 76$ & 3 & 1 & & 85 & $\mathbf{N}$ & \\
\hline 1 & $6 / 7 / 76$ & 5 & 1 & & 81 & $\mathbf{N}$ & \\
\hline 1 & $6 / 7 / 76$ & 6 & 1 & & 78 & $\mathbf{N}$ & \\
\hline
\end{tabular}

Data obtained from WHC Surveillance Analysis Computer System (SACS), November 12, 1993. 


\begin{tabular}{|c|c|c|c|c|c|c|c|}
\hline \multicolumn{8}{|c|}{ TANK 241-BY-101 } \\
\hline \multicolumn{8}{|c|}{ THERMOCOUPLE DATA } \\
\hline & & & & & & & \\
\hline TREE\# & DATE & THERMO \# & TREE SET \# & TEMP C & TEMP F & $Y=\operatorname{MAX}$ TEMP & COMMENTS \\
\hline 1 & $6 / 7 / 76$ & 7 & 1 & & 76 & $\mathbf{N}$ & \\
\hline 1 & $6 / 7 / 76$ & 8 & 1 & & 74 & $\mathbf{N}$ & \\
\hline 1 & $6 / 7 / 76$ & 9 & 1 & & 74 & $\mathbf{N}$ & \\
\hline 1 & $6 / 7 / 76$ & 10 & 1 & & 73 & $\mathbf{N}$ & \\
\hline 1 & $6 / 7 / 76$ & 11 & 1 & & 72 & $\bar{N}$ & \\
\hline 1 & $6 / 7 / 76$ & 12 & 1 & & 72 & $\mathbf{N}$ & \\
\hline 1 & $6 / 7 / 76$ & 94 & 1 & & 72 & $\mathbf{N}$ & \\
\hline 1 & $6 / 9 / 76$ & 2 & 1 & & 93 & $\bar{Y}$ & \\
\hline 9 & $6 / 9 / 76$ & 3 & 1 & & 92 & $\mathbf{N}$ & \\
\hline 1 & $6 / 9 / 76$ & 5 & 1 & & 88 & $\mathbf{N}$ & \\
\hline 1 & $6 / 9 / 76$ & 6 & 1 & & 85 & $\mathbf{N}$ & \\
\hline 1 & $6 / 9 / 76$ & 7 & 1 & & 82 & $\mathbf{N}$ & \\
\hline 1 & $6 / 9 / 76$ & 8 & 1 & & 80 & $\mathbf{N}$ & \\
\hline 1 & $6 / 9 / 76$ & 9 & 1 & & 79 & $\mathbf{N}$ & \\
\hline 1 & $6 / 9 / 76$ & 10 & 1 & & 79 & $\mathbf{N}$ & \\
\hline 1 & $6 / 9 / 76$ & 11 & 1 & & 77 & $\mathbf{N}$ & \\
\hline 1 & $6 / 9 / 76$ & 12 & 1 & & 76 & $\mathbf{N}$ & \\
\hline 1 & $6 / 9 / 76$ & 13 & 1 & & 76 & $\mathbf{N}$ & \\
\hline 1 & $6 / 9 / 76$ & 14 & 1 & & 76 & $\mathbf{N}$ & \\
\hline 1 & $7 / 7 / 76$ & 1 & 1 & & 87 & $\bar{Y}$ & \\
\hline 1 & $7 / 7 / 76$ & 2 & 1 & & 86 & $\mathrm{~N}$ & \\
\hline 1 & $7 / 7 / 76$ & 3 & 1 & & 85 & $N$ & \\
\hline 1 & 717176 & 5 & 1 & & 81 & $\mathbf{N}$ & \\
\hline 1 & $7 / 7 / 76$ & 6 & 1 & & 78 & $N$ & \\
\hline 1 & $7 / 7 / 76$ & 14 & 1 & & 72 & $\mathrm{~N}$ & \\
\hline 1 & $8 / 14 / 76$ & 1 & 1 & & 88 & $\mathbf{Y}$ & \\
\hline 1 & $8 / 14 / 76$ & 2 & 1 & & 85 & $\mathbf{N}$ & \\
\hline 1 & $8 / 14 / 76$ & 3 & 1 & & 86 & $N$ & \\
\hline 1 & $8 / 14 / 76$ & 5 & 1 & & 81 & $\mathbf{N}$ & \\
\hline 1 & $8 / 14 / 76$ & 6 & 1 & & 79 & $\mathbf{N}$ & \\
\hline 1 & $8 / 14 / 76$ & 7 & 1 & & 77 & $\mathrm{~N}$ & \\
\hline 1 & $8 / 14 / 76$ & 8 & 1 & & 76 & $\mathrm{~N}$ & \\
\hline 1 & $8 / 14 / 76$ & 9 & 1 & & 76 & $N$ & \\
\hline 1 & $8 / 14 / 76$ & 90 & 1 & & 75 & $\mathbf{N}$ & \\
\hline 1 & $8 / 14 / 76$ & 11 & 1 & & 75 & $\mathbf{N}$ & \\
\hline 1 & $8 / 14 / 76$ & 12 & 1 & & 74 & $\mathbf{N}$ & \\
\hline 1 & $9 / 13 / 76$ & 1 & 1 & & 88 & $Y$ & \\
\hline 1 & $9 / 13 / 76$ & 2 & 1 & & 87 & $N$ & \\
\hline 1 & $9 / 13 / 76$ & 3 & 1 & & 86 & $\mathbf{N}$ & \\
\hline 1 & $9 / 13 / 76$ & 5 & 1 & & 84 & $\mathbf{N}$ & \\
\hline 1 & $9 / 13 / 76$ & 6 & 1 & & 81 & $\mathbf{N}$ & \\
\hline 1 & $9 / 13 / 76$ & 7 & 1 & & 80 & $\mathbf{N}$ & \\
\hline 1 & $9 / 13 / 76$ & 8 & 9 & & 78 & $\mathbf{N}$ & \\
\hline 1 & $9 / 13 / 76$ & 9 & 1 & & 78 & $\mathrm{~N}$ & \\
\hline 1 & $9 / 13 / 76$ & 10 & 1 & & 77 & $\mathbf{N}$ & \\
\hline 1 & $9 / 13 / 76$ & 11 & 1 & & 77 & $\mathbf{N}$ & \\
\hline 1 & $9 / 13 / 76$ & 12 & 1 & & 76 & $\mathbf{N}$ & \\
\hline 1 & $11 / 7 / 76$ & 1 & 1 & & 89 & $\mathbf{Y}$ & \\
\hline 1 & $11 / 7 / 76$ & 2 & 1 & & 89 & $\mathbf{N}$ & \\
\hline 1 & $11 / 7 / 76$ & 3 & 1 & & 89 & $\mathbf{N}$ & \\
\hline 1 & $11 / 7 / 76$ & 5 & 1 & & 87 & $\mathbf{N}$ & \\
\hline 1 & $11 / 7 / 76$ & 6 & 1 & & 86 & $\mathbf{N}$ & \\
\hline 1 & $11 / 7 / 76$ & 7 & 1 & & 85 & $\mathbf{N}$ & \\
\hline 1 & $11 / 7 / 76$ & 8 & 1 & & 83 & $N$ & \\
\hline 1 & $11 / 7 / 76$ & 9 & 1 & & 81 & $N$ & \\
\hline 1 & $11 / 7 / 76$ & 70 & 1 & & 76 & $\mathbf{N}$ & \\
\hline 1 & $11 / 7 / 76$ & 11 & 1 & & 76 & $\mathrm{~N}$ & \\
\hline 1 & $11 / 7 / 76$ & 12 & 1 & & 76 & $N$ & \\
\hline 1 & $12 / 9 / 76$ & 1 & 1 & & 103 & $\bar{Y}$ & \\
\hline 1 & $12 / 9 / 76$ & 2 & 1 & & 302 & $\mathbf{N}$ & \\
\hline 1 & $12 / 9 / 76$ & 3 & 1 & & 102 & $\mathrm{~N}$ & \\
\hline 1 & $12 / 9 / 76$ & 5 & 1 & & 100 & $N$ & \\
\hline 1 & $12 / 9 / 76$ & 6 & 1 & & 99 & $\mathbf{N}$ & \\
\hline 1 & $12 / 9 / 76$ & 7 & 1 & & 98 & $\mathbf{N}$ & \\
\hline 1 & $12 / 9 / 76$ & 8 & 1 & & 95 & $\mathbf{N}$ & \\
\hline 1 & $12 / 9 / 76$ & 9 & 1 & & 92 & $\mathbf{N}$ & \\
\hline
\end{tabular}

Data obtained from WHC Surveillance Analysis Computer System (SACS), November 12, 1993. 


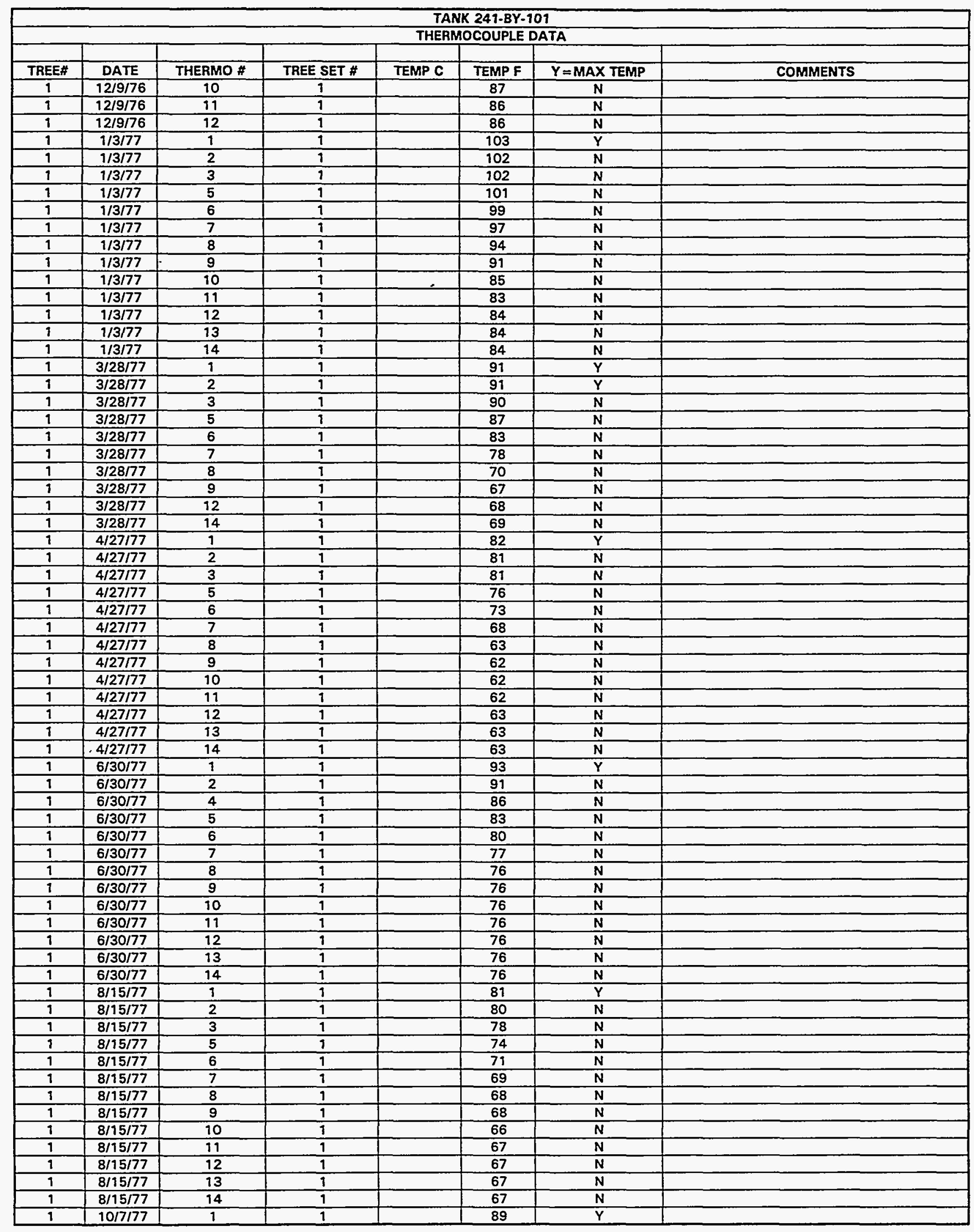

Data obtained from WHC Surveillance Analysis Computer System (SACS), November 12, 1993. 


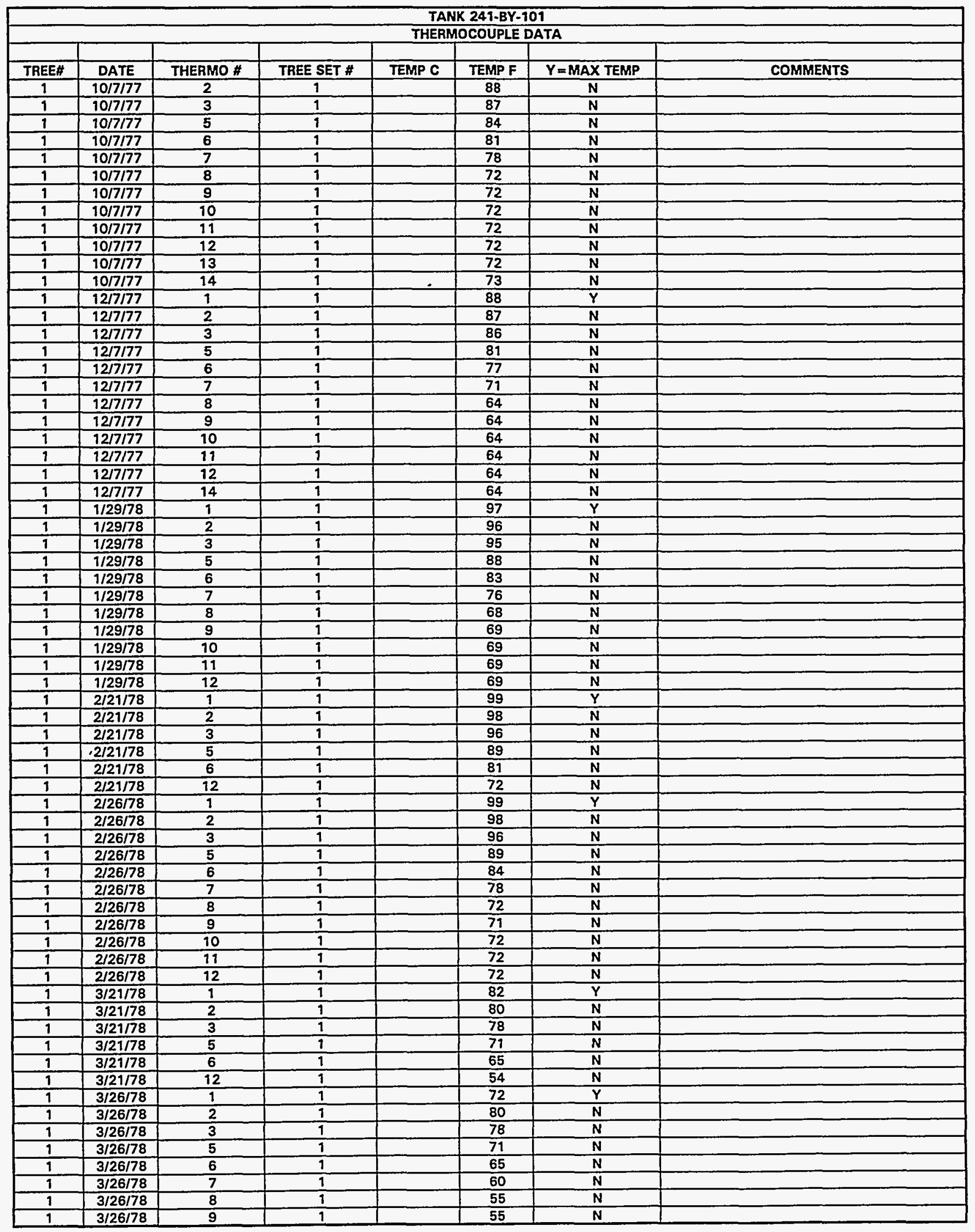

Data obtained from WHC Surveillance Analysis Computer System (SACS), November 12, 1993. 
WHC-SD-WM-ER-312, Rev. 0

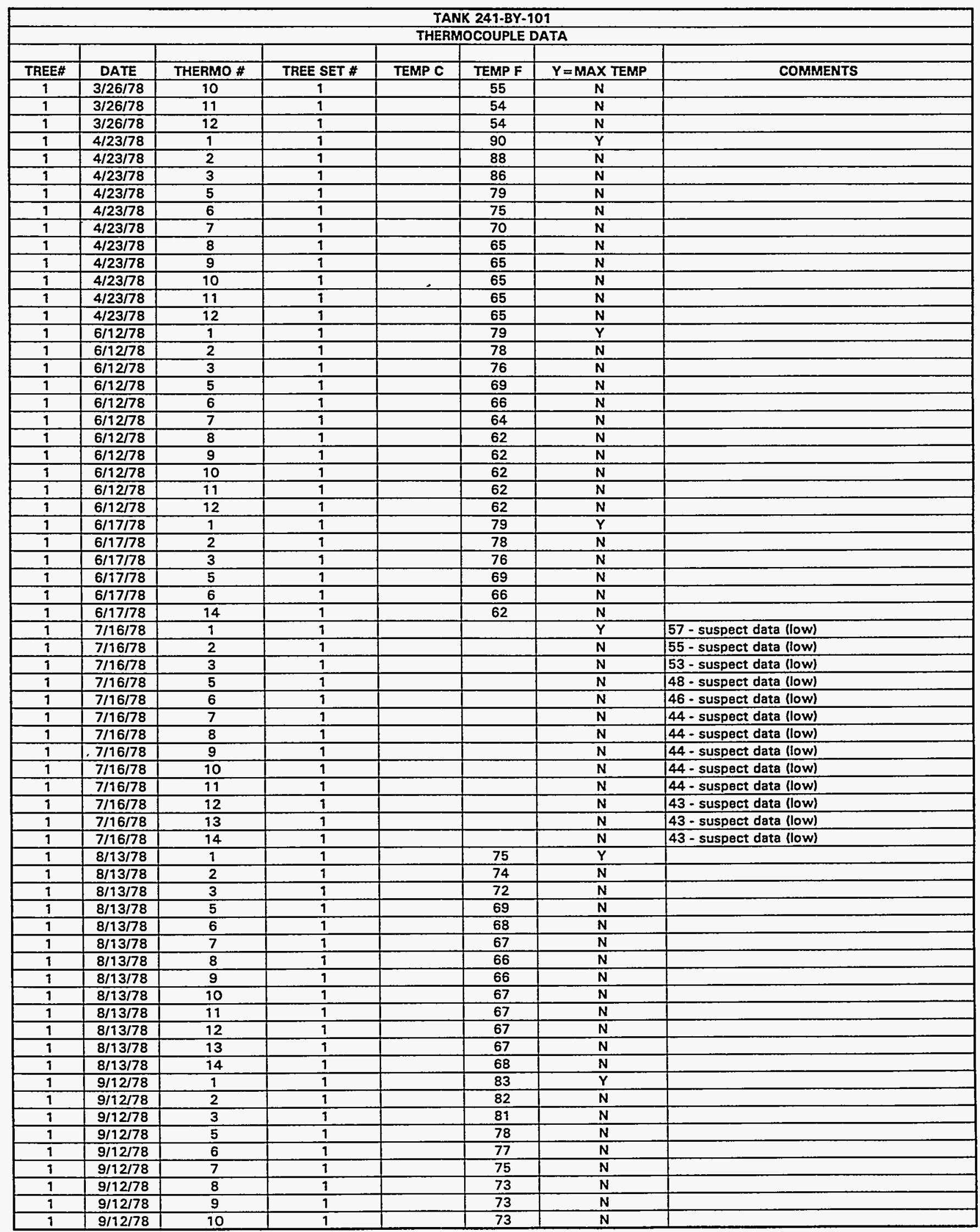

Data obtained from WHC Surveillance Analysis Computer System (SACS), November 12, 1993. 


\begin{tabular}{|c|c|c|c|c|c|c|c|}
\hline \multicolumn{8}{|c|}{ TANK 241-BY-101 } \\
\hline & & & & THERI & OCOUPLE & TAA & \\
\hline TREE\# & DATE & THERMO \# & TREE SET \# & TEMP C & TEMP F & $Y=M A X$ TEMP & COMMENTS \\
\hline 1 & $9 / 12 / 78$ & 11 & 1 & & 73 & $\mathrm{~N}$ & \\
\hline 1 & $9 / 12 / 78$ & 12 & 1 & & 73 & $\mathbf{N}$ & \\
\hline 1 & $9 / 12 / 78$ & 13 & 1 & & 73 & $\mathbf{N}$ & \\
\hline 1 & $9 / 12 / 78$ & 14 & 1 & & 73 & $\mathbf{N}$ & \\
\hline 1 & $10 / 7 / 78$ & 1 & 1 & & 88 & $\bar{Y}$ & \\
\hline 1 & $10 / 7 / 78$ & 2 & 1 & & 88 & $N$ & \\
\hline 1 & $10 / 7 / 78$ & 3 & 1 & & 88 & $N$ & \\
\hline 1 & $10 / 7 / 78$ & 5 & 1 & & 87 & $\mathbf{N}$ & \\
\hline 1 & $10 / 7 / 78$ & 6 & 1 & & 87 & $\mathrm{~N}$ & \\
\hline 1 & $10 / 7 / 78$ & 7 & 9 & & 85 & $\mathrm{~N}$ & \\
\hline 1 & $10 / 7 / 78$ & 8 & 1 & & 84 & $N$ & \\
\hline 1 & $10 / 7 / 78$ & 9 & 1 & & 84 & 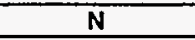 & \\
\hline 1 & $10 / 7 / 78$ & 10 & 1 & & 84 & $\mathbf{N}$ & \\
\hline 1 & $10 / 7 / 78$ & 11 & 1 & & 84 & $\mathbf{N}$ & \\
\hline 1 & $10 / 7 / 78$ & 12 & 1 & & 84 & $N$ & \\
\hline 1 & $1 / 3 / 79$ & 1 & 1 & & 84 & $\bar{Y}$ & \\
\hline 1 & $1 / 3 / 79$ & 2 & 1 & & 83 & $\mathbf{N}$ & \\
\hline 1 & $1 / 3 / 79$ & 3 & 1 & & 81 & $\mathbf{N}$ & \\
\hline 1 & $1 / 3 / 79$ & 5 & 1 & & 77 & $\mathbf{N}$ & \\
\hline 1 & $1 / 3 / 79$ & 6 & 9 & & 72 & $\mathbf{N}$ & \\
\hline 1 & $1 / 3 / 79$ & 7 & 1 & & 63 & $\mathbf{N}$ & \\
\hline 1 & $1 / 3 / 79$ & 8 & 1 & & 52 & $\mathbf{N}$ & \\
\hline 1 & $1 / 3 / 79$ & 9 & 1 & & 52 & $\bar{N}$ & \\
\hline 1 & $1 / 3 / 79$ & 10 & 1 & & 53 & $\mathbf{N}$ & \\
\hline 1 & $1 / 3 / 79$ & 11 & 1 & & 54 & $\mathbf{N}$ & \\
\hline 1 & $1 / 3 / 79$ & 12 & 1 & & 54 & $\mathbf{N}$ & \\
\hline 1 & $4 / 22 / 79$ & 1 & 1 & & 82 & $\bar{Y}$ & \\
\hline$\frac{1}{1}$ & $4 / 22 / 79$ & 2 & 1 & & 81 & $\mathbf{N}$ & \\
\hline 1 & $4 / 22 / 79$ & 3 & 1 & & 79 & $\bar{N}$ & \\
\hline 1 & $4 / 22 / 79$ & 5 & 1 & & 73 & $\mathbf{N}$ & \\
\hline 1 & $4 / 22 / 79$ & 6 & 1 & & 70 & $N$ & \\
\hline 1 & $4 / 22 / 79$ & 7 & 1 & & 65 & $\mathbf{N}$ & \\
\hline 1 & $4 / 22 / 79$ & 8 & 1 & & 61 & $\mathrm{~N}$ & \\
\hline 1 & $4 / 22 / 79$ & 9 & 1 & & 61 & $\mathrm{~N}$ & \\
\hline 1 & $4 / 22 / 79$ & 10 & 1 & & 61 & $\mathbf{N}$ & \\
\hline 1 & $4 / 22 / 79$ & 11 & 1 & & 61 & $\mathbf{N}$ & \\
\hline 1 & $4 / 22 / 79$ & 12 & 1 & & 61 & $\mathbf{N}$ & \\
\hline 1 & $4 / 22 / 79$ & 13 & 1 & & 61 & $\mathbf{N}$ & \\
\hline 1 & $.4 / 22 / 79$ & 14 & 1 & & 61 & $\mathbf{N}$ & \\
\hline 1 & $6 / 12 / 81$ & 9 & 1 & & 75 & $\mathbf{Y}$ & \\
\hline 1 & $6 / 12 / 81$ & 2 & 1 & & 74 & $\mathbf{N}$ & \\
\hline 1 & $6 / 12 / 81$ & 3 & 1 & & 73 & $\bar{N}$ & \\
\hline 1 & $6 / 12 / 81$ & 4 & 1 & & 70 & $\mathbf{N}$ & \\
\hline 1 & $6 / 12 / 81$ & 5 & 1 & & 68 & $N$ & \\
\hline 1 & $6 / 12 / 81$ & 6 & 1 & & 66 & $N$ & \\
\hline 1 & $6 / 12 / 81$ & 7 & 1 & & 64 & $\mathbf{N}$ & \\
\hline 1 & $6 / 12 / 81$ & 8 & 1 & & 63 & $\mathbf{N}$ & \\
\hline 1 & $6 / 12 / 81$ & 9 & 1 & & 63 & $N$ & \\
\hline 1 & $6 / 12 / 81$ & 10 & 1 & & 63 & $\mathbf{N}$ & \\
\hline$\frac{1}{1}$ & $6 / 12 / 81$ & 11 & 1 & & 63 & $N$ & \\
\hline 1 & $6 / 12 / 81$ & 12 & 1 & & 63 & $N$ & \\
\hline 1 & $6 / 12 / 81$ & 13 & 1 & & 63 & $\mathbf{N}$ & \\
\hline 1 & $6 / 12 / 81$ & 14 & 1 & & 63 & $\mathbf{N}$ & \\
\hline 1 & $12 / 3 / 81$ & 1 & 1 & & 82 & $\mathbf{Y}$ & \\
\hline 1 & $12 / 3 / 81$ & 2 & 1 & & 82 & $\bar{Y}$ & \\
\hline 1 & $12 / 3 / 81$ & 3 & 1 & & 82 & $\bar{Y}$ & \\
\hline 1 & $12 / 3 / 81$ & 4 & 1 & & 82 & $\mathbf{Y}$ & \\
\hline 1 & $12 / 3 / 81$ & 5 & 1 & 27 & 80.6 & $N$ & raw data - assumed degree C \\
\hline 1 & $12 / 3 / 81$ & 6 & 1 & 26 & 78.8 & $\mathbf{N}$ & raw data - assumed degree $C$ \\
\hline 1 & $12 / 3 / 81$ & 7 & 1 & 25 & 77 & N & raw data - assumed degree C \\
\hline 1 & $12 / 3 / 81$ & 8 & 1 & 23 & 73.4 & $\mathbf{N}$ & raw data - assumed degree C \\
\hline 1 & $12 / 3 / 81$ & 9 & 1 & 23 & 73.4 & $\mathbf{N}$ & raw data - essumed degree $C$ \\
\hline 1 & $12 / 3 / 81$ & 10 & 1 & 23 & 73.4 & $\mathbf{N}$ & raw data - assumed degreo C \\
\hline 1 & $12 / 3 / 81$ & 11 & 1 & 24 & 75.2 & $\mathbf{N}$ & raw data - assumed degree C \\
\hline 1 & $12 / 3 / 81$ & 12 & 1 & 24 & 75.2 & $\mathbf{N}$ & raw data - assumed degree C \\
\hline 1 & $12 / 3 / 81$ & 13 & 1 & 24 & 75.2 & $\mathbf{N}$ & raw data - assumed degree $C$ \\
\hline
\end{tabular}

Data obtained from WHC Surveillance Analysis Computer System (SACS), November 12, 1993. 
WHC-SD-WM-ER-312, Rev. 0

\begin{tabular}{|c|c|c|c|c|c|c|c|}
\hline \multicolumn{8}{|c|}{ TANK 241-BY-101 } \\
\hline \multicolumn{8}{|c|}{ THERMOCOUPLE DATA } \\
\hline & & & & & & & \\
\hline TREE\# & DATE & THERMO \# & TREE SET \# & TEMP C & TEMP F & $Y=$ MAX TEMP & COMMENTS \\
\hline 1 & $12 / 3 / 81$ & 14 & 1 & 24 & 75.2 & $\mathbf{N}$ & raw data - assumed degree C \\
\hline 1 & $1 / 14 / 82$ & 1 & 1 & & 96 & $Y$ & \\
\hline 1 & $1 / 14 / 82$ & 2 & 1 & & 95 & $\mathbf{N}$ & \\
\hline 1 & $1 / 14 / 82$ & 3 & 1 & & 94 & $\mathbf{N}$ & \\
\hline 1 & $1 / 14 / 82$ & 5 & 1 & & 92 & $\mathbf{N}$ & \\
\hline 1 & $1 / 94 / 82$ & 6 & 1 & & 88 & $\mathbf{N}$ & \\
\hline 1 & $1 / 14 / 82$ & 7 & 1 & & 84 & $\mathbf{N}$ & \\
\hline 1 & $1 / 14 / 82$ & 8 & 1 & & 80 & $\mathbf{N}$ & \\
\hline 1 & $1 / 14 / 82$ & 9 & 1 & & 79 & $\mathbf{N}$ & \\
\hline 1 & $1 / 14 / 82$ & 10 & 1 & & 79 & $\mathbf{N}$ & \\
\hline 1 & $1 / 14 / 82$ & 11 & 1 & & 79 & $\mathbf{N}$ & \\
\hline 1 & $1 / 74 / 82$ & 12 & 1 & & 79 & $\mathbf{N}$ & \\
\hline 1 & $1 / 14 / 82$ & 13 & 1 & & 79 & $\mathbf{N}$ & \\
\hline 1 & $1 / 14 / 82$ & 14 & 1 & & 79 & $\mathbf{N}$ & \\
\hline 1 & $10 / 14 / 83$ & 1 & 1 & & 72 & $\mathbf{N}$ & \\
\hline 1 & $10 / 14 / 83$ & 2 & 1 & & 71 & $\mathbf{N}$ & \\
\hline 1 & $10 / 14 / 83$ & 3 & 1 & & 72 & $\mathbf{N}$ & \\
\hline 1 & $10 / 14 / 83$ & 5 & 1 & & 73 & $Y$ & \\
\hline 1 & $2 / 7 / 84$ & 1 & 1 & & 84 & $\bar{Y}$ & \\
\hline 1 & $2 / 7 / 84$ & 2 & 1 & & 83 & $\mathbf{N}$ & \\
\hline 1 & $2 / 7 / 84$ & 3 & 1 & & 83 & $\mathbf{N}$ & \\
\hline 1 & $2 / 7 / 84$ & 5 & 1 & & 79 & $\mathbf{N}$ & \\
\hline 1 & $2 / 7 / 84$ & 6 & 1 & & 75 & $\mathrm{~N}$ & \\
\hline 1 & $2 / 7 / 84$ & 7 & 1 & & 71 & $\mathrm{~N}$ & \\
\hline 1 & $2 / 7 / 84$ & 8 & 1 & & 68 & $\mathbf{N}$ & \\
\hline 1 & $2 / 7 / 84$ & 9 & 1 & & 68 & $\mathrm{~N}$ & \\
\hline 9 & $2 / 7 / 84$ & 10 & 1 & & 68 & $N$ & \\
\hline 1 & $2 / 7 / 84$ & 11 & 1 & & 67 & $N$ & \\
\hline 1 & $2 / 7 / 84$ & 12 & 1 & & 67 & $\mathbf{N}$ & \\
\hline 1 & $2 / 7 / 84$ & 13 & 1 & & 67 & $\mathrm{~N}$ & \\
\hline 1 & $2 / 7 / 84$ & 14 & 1 & & 67 & $\mathbf{N}$ & \\
\hline 1 & $10 / 13 / 89$ & 1 & 1 & & & $\mathrm{~N}$ & $\begin{array}{c}\text { Rotory switch loose \& pushed back inside of } \\
\text { housing. }\end{array}$ \\
\hline 1 & $10 / 23 / 89$ & 1 & 1 & & & $\mathbf{N}$ & Plug missing \\
\hline 1 & $11 / 2 / 89$ & 1 & 1 & & 76.7 & $Y$ & \\
\hline 1 & $11 / 2 / 89$ & 2 & 1 & & 76.5 & $\mathrm{~N}$ & \\
\hline 1 & $11 / 2 / 89$ & 3 & 1 & & 76.3 & $\mathbf{N}$ & \\
\hline 1 & $11 / 2 / 89$ & 5 & 1 & & 75.4 & $\mathrm{~N}$ & \\
\hline 1 & $.11 / 2 / 89$ & 6 & 1 & & 75 & $\mathbf{N}$ & \\
\hline 1 & $11 / 2 / 89$ & 7 & 1 & & 74.6 & $\mathbf{N}$ & \\
\hline 1 & $11 / 2 / 89$ & 8 & 1 & & 74.2 & $\mathbf{N}$ & \\
\hline 1 & $11 / 2 / 89$ & 9 & 1 & & 74.1 & $\mathbf{N}$ & \\
\hline 1 & $11 / 2 / 89$ & 10 & 1 & & 74.1 & $\mathbf{N}$ & \\
\hline 1 & $11 / 2 / 89$ & 11 & 1 & & 74 & $\mathbf{N}$ & \\
\hline 1 & $11 / 2 / 89$ & 12 & 1 & & 73.9 & $\mathbf{N}$ & \\
\hline 1 & $11 / 2 / 89$ & 13 & 1 & & 73.9 & $\mathbf{N}$ & \\
\hline 1 & $11 / 2 / 89$ & 14 & 1 & & 73.8 & $\mathbf{N}$ & \\
\hline 1 & $11 / 4 / 89$ & 1 & 1 & & 70.7 & $Y$ & \\
\hline 1 & $11 / 4 / 89$ & 2 & 1 & & 70.4 & $N$ & \\
\hline 1 & $11 / 4 / 89$ & 3 & 1 & & 70.2 & $N$ & \\
\hline 1 & $11 / 4 / 89$ & 5 & 1 & & 69.7 & $\mathbf{N}$ & \\
\hline 1 & $11 / 4 / 89$ & 6 & 1 & & 69.3 & $N$ & \\
\hline 1 & $11 / 4 / 89$ & 7 & 1 & & 68.8 & $N$ & \\
\hline 1 & $11 / 4 / 89$ & 8 & 1 & & 68.6 & $N$ & \\
\hline 1 & $19 / 4 / 89$ & 9 & 1 & & 68.5 & $\mathbf{N}$ & \\
\hline 1 & $11 / 4 / 89$ & 10 & 1 & & 68.5 & $N$ & \\
\hline 1 & $11 / 4 / 89$ & 11 & 1 & & 68.5 & $\mathbf{N}$ & \\
\hline 1 & $11 / 4 / 89$ & 12 & 1 & & 68.5 & $\mathbf{N}$ & \\
\hline 1 & $11 / 4 / 89$ & 13 & 1 & & 68.5 & $\mathrm{~N}$ & \\
\hline 1 & $11 / 4 / 89$ & 14 & 1 & & 68.5 & $\mathbf{N}$ & \\
\hline 1 & $11 / 10 / 89$ & 1 & 1 & & & $\mathbf{N}$ & TC wires not yet in place in LOW \\
\hline 1 & $11 / 17 / 89$ & 1 & 1 & & 70.9 & $\mathbf{Y}$ & Moisture found in LOW \\
\hline 1 & $11 / 18 / 89$ & 1 & 1 & & 77.1 & $\bar{Y}$ & \\
\hline 1 & $91 / 18 / 89$ & 2 & 1 & & 76.9 & $\mathbf{N}$ & \\
\hline 1 & $11 / 18 / 89$ & 3 & 1 & & 76.7 & $\mathbf{N}$ & \\
\hline 1 & $11 / 18 / 89$ & 5 & 1 & & 76 & $\mathbf{N}$ & \\
\hline
\end{tabular}

Data obtained from WHC Surveillance Analysis Computer System (SACS), November 12, 1993. 
WHC-SD-WM-ER-312, Rev. 0

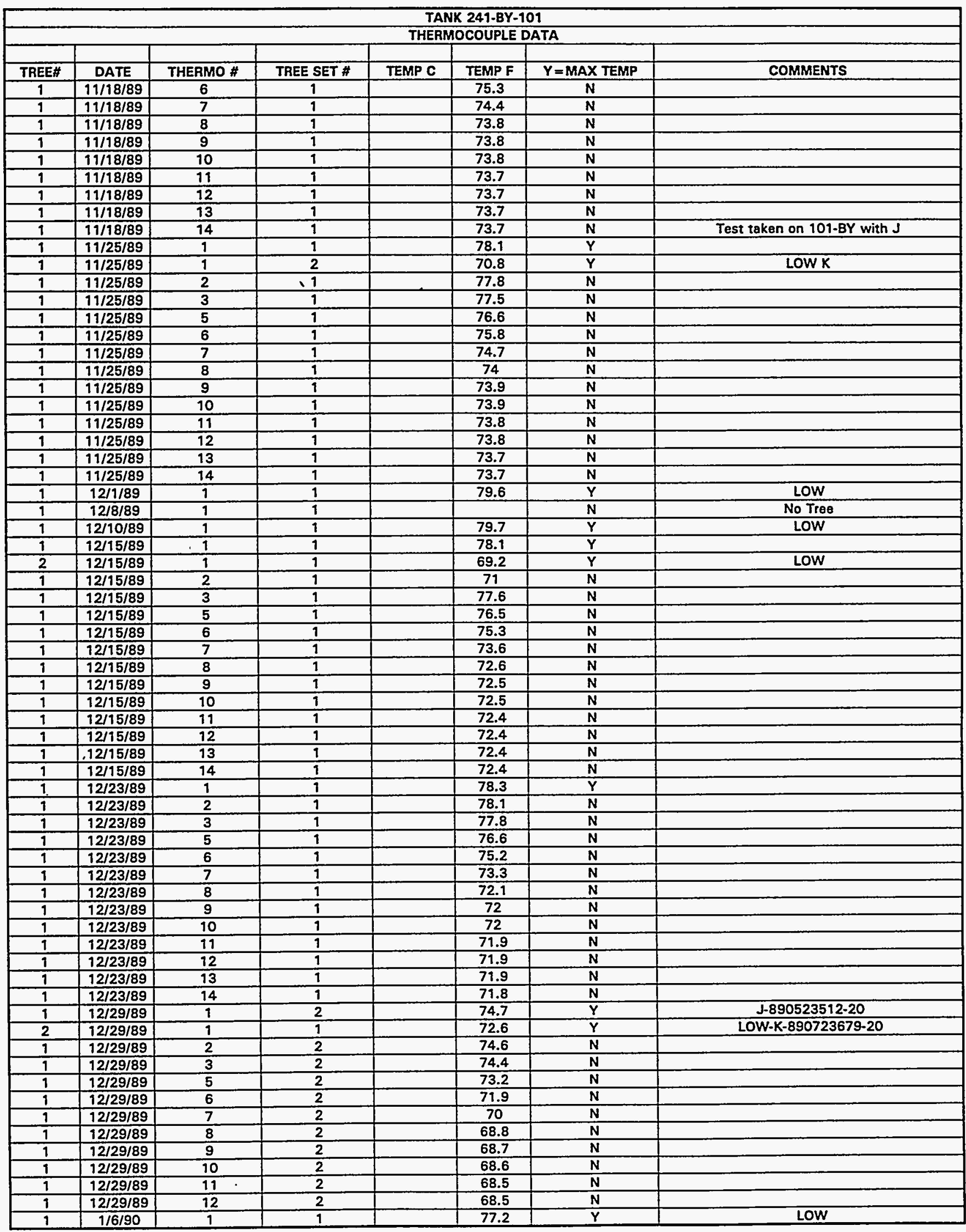

Data obtained from WHC Surveillance Analysis Computer System (SACS), November 12, 1993. 


\begin{tabular}{|c|c|c|c|c|c|c|c|}
\hline \multicolumn{8}{|c|}{ TANK 241-BY-101 } \\
\hline \multicolumn{8}{|c|}{ THERMOCOUPLE DATA } \\
\hline TREE\# & DATE & THERMO \# & TREE SET \# & TEMP C & TEMP F & $\mathrm{Y}=\mathrm{MAX}$ TEMP & COMMENTS \\
\hline 1 & $1 / 13 / 90$ & 1 & 9 & & 73.7 & $Y$ & Dimion \\
\hline 1 & $1 / 13 / 90$ & 2 & 1 & & 73.4 & $N$ & \\
\hline 1 & $1 / 13 / 90$ & 3 & 1 & & 73.5 & $N$ & \\
\hline 1 & $1 / 13 / 90$ & 5 & 1 & & 72 & $\mathbf{N}$ & \\
\hline 1 & $1 / 13 / 90$ & 6 & 1 & & 70.2 & $\bar{N}$ & \\
\hline 1 & $1 / 13 / 90$ & 7 & 1 & & 68 & $\mathbf{N}$ & \\
\hline$\frac{T}{1}$ & $1 / 13 / 90$ & 8 & $\frac{1}{1}$ & & 66.6 & $\bar{N}$ & \\
\hline 1 & $1 / 13 / 90$ & 9 & 1 & & 66.5 & $\mathbf{N}$ & \\
\hline 1 & $1 / 13 / 90$ & 10 & 1 & & 66.5 & $\mathbf{N}$ & \\
\hline$\frac{1}{1}$ & $1 / 13 / 90$ & 11 & 1 & & 66.5 & $\mathbf{N}$ & \\
\hline 1 & $1 / 13 / 90$ & 12 & 1 & & 66.5 & $N$ & \\
\hline 1 & $1 / 13 / 90$ & 13 & 1 & & 66.5 & $\mathbf{N}$ & \\
\hline 1 & $1 / 13 / 90$ & 14 & 1 & & 66.5 & $\bar{N}$ & $\mathrm{~J}$ \\
\hline 1 & $1 / 19 / 90$ & 1 & 1 & & 77.1 & $\bar{Y}$ & \\
\hline 1 & $1 / 19 / 90$ & 2 & 1 & & 76.7 & $N$ & \\
\hline 1 & $1 / 19 / 90$ & 3 & 9 & & 76.4 & $\mathbf{N}$ & \\
\hline 1 & $1 / 19 / 90$ & 5 & 1 & & 74.8 & $\mathbf{N}$ & \\
\hline 1 & $1 / 19 / 90$ & 6 & 1 & & 72.8 & $N$ & \\
\hline 1 & $1 / 19 / 90$ & 7 & 1 & & 70.3 & $N$ & \\
\hline 1 & $1 / 19 / 90$ & 8 & 1 & & 68.9 & $\bar{N}$ & \\
\hline 1 & $1 / 19 / 90$ & 9 & 1 & & 68.8 & $\mathbf{N}$ & \\
\hline 1 & $1 / 19 / 90$ & 10 & 1 & & 68.8 & $\mathrm{~N}$ & \\
\hline 1 & $1 / 79 / 90$ & 11 & 1 & & 68.7 & $\mathbf{N}$ & \\
\hline 1 & $1 / 19 / 90$ & 12 & 1 & & 68.7 & $\mathbf{N}$ & \\
\hline 1 & $1 / 19 / 90$ & 13 & 1 & & 68.6 & $\mathbf{N}$ & \\
\hline 1 & $1 / 19 / 90$ & 14 & 1 & & 68.6 & $\mathbf{N}$ & $E$ \\
\hline 1 & $1 / 27 / 90$ & 1 & 1 & & 68.7 & $\bar{Y}$ & LOW - K \\
\hline 1 & $2 / 3 / 90$ & 1 & 1 & & 70.9 & $\bar{Y}$ & $\mathrm{~K}-890723579-20$ \\
\hline 9 & $2 / 5 / 90$ & 1 & 1 & & 67.8 & $Y$ & LoW K-890723679-20 \\
\hline 1 & $2 / 11 / 90$ & 1 & 1 & & 77.7 & $Y$ & J-2E-OP-021 \\
\hline 1 & $2 / 11 / 90$ & 2 & 1 & & 77.5 & $\bar{N}$ & \\
\hline 1 & $2 / 11 / 90$ & 3 & 1 & & 76.9 & $\mathbf{N}$ & \\
\hline 1 & $2 / 11 / 90$ & 5 & 1 & & 74.2 & $\mathbf{N}$ & \\
\hline 1 & $2 / 11 / 90$ & 6 & 1 & & 71.7 & $\mathbf{N}$ & \\
\hline 1 & $2 / 11 / 90$ & 7 & 1 & & 68.2 & $\mathbf{N}$ & \\
\hline 1 & $2 / 11 / 90$ & 8 & 1 & & 66.3 & $\mathbf{N}$ & \\
\hline 1 & $2 / 11 / 90$ & 9 & 1 & & 66.3 & $\mathbf{N}$ & \\
\hline 1 & $2 / 11 / 90$ & 10 & 1 & & 66.4 & $\mathbf{N}$ & \\
\hline 1 & $2 / 11 / 90$ & 11 & 1 & & 66.3 & $\mathbf{N}$ & \\
\hline 1 & $2 / 11 / 90$ & 12 & 1 & & 66.4 & $\mathbf{N}$ & \\
\hline 1 & $2 / 11 / 90$ & 13 & 1 & & 66.3 & $\mathbf{N}$ & \\
\hline 1 & $2 / 11 / 90$ & 14 & 1 & & 66.2 & $\mathbf{N}$ & \\
\hline 1 & $3 / 2 / 90$ & 1 & 1 & & 72 & $\bar{Y}$ & K-HH-99A-K \\
\hline 1 & $3 / 2 / 90$ & 2 & 1 & & & $\mathbf{N}$ & LOW \\
\hline 1 & $4 / 8 / 90$ & 1 & 1 & & 73 & $\mathbf{N}$ & K-890723679020 \\
\hline 1 & $4 / 8 / 90$ & 2 & 1 & & 75 & $Y$ & \\
\hline 1 & $4 / 8 / 90$ & 3 & 1 & & 74 & $\mathrm{~N}$ & \\
\hline 1 & $4 / 8 / 90$ & 5 & 1 & & 71 & $\mathbf{N}$ & \\
\hline 1 & $4 / 8 / 90$ & 6 & 1 & & 68 & $\bar{N}$ & \\
\hline 1 & $4 / 8 / 90$ & 7 & 1 & & 65 & $\mathbf{N}$ & \\
\hline 1 & $4 / 8 / 90$ & 8 & 1 & & 63 & $\mathbf{N}$ & \\
\hline 1 & $4 / 8 / 90$ & 9 & 1 & & 63 & $\mathbf{N}$ & \\
\hline 1 & $4 / 8 / 90$ & 10 & 1 & & 64 & $\mathbf{N}$ & \\
\hline 1 & $4 / 8 / 90$ & 11 & 1 & & 64 & $\mathbf{N}$ & \\
\hline 1 & $4 / 8 / 90$ & 12 & 1 & & 64 & $\mathbf{N}$ & \\
\hline 1 & $4 / 8 / 90$ & 13 & 1 & & 64 & $\mathbf{N}$ & \\
\hline 1 & $4 / 8 / 90$ & 14 & 1 & & 63 & $\mathbf{N}$ & \\
\hline 1 & $5 / 5 / 90$ & 1 & 1 & & & $\mathrm{~N}$ & No Data. Discrepancy report written- \#252 \\
\hline 1 & $5 / 30 / 90$ & 1 & 1 & & 75.4 & $\mathbf{Y}$ & $\mathrm{J}-2 \mathrm{E}-\mathrm{OP}-020$ \\
\hline 1 & $5 / 30 / 90$ & 2 & 1 & & 74.5 & $\mathbf{N}$ & \\
\hline$T$ & $5 / 30 / 90$ & $\overline{3}$ & 1 & & 73.6 & $\mathbf{N}$ & \\
\hline 1 & $5 / 30 / 90$ & 5 & 1 & & 70.8 & $\mathbf{N}$ & \\
\hline 1 & $5 / 30 / 90$ & 6 & $\frac{1}{1}$ & & 68.9 & $\mathbf{N}$ & \\
\hline 1 & $5 / 30 / 90$ & 7 & 1 & & 67.1 & $N$ & \\
\hline 1 & $5 / 30 / 90$ & 8 & 9 & & 66.3 & $\mathrm{~N}$ & \\
\hline 1 & $5 / 30 / 90$ & 9 & 1 & & 66.5 & $\mathrm{~N}$ & \\
\hline
\end{tabular}

Data obtained from WHC Surveillance Analysis Computer System (SACS), November 12, 1993. 


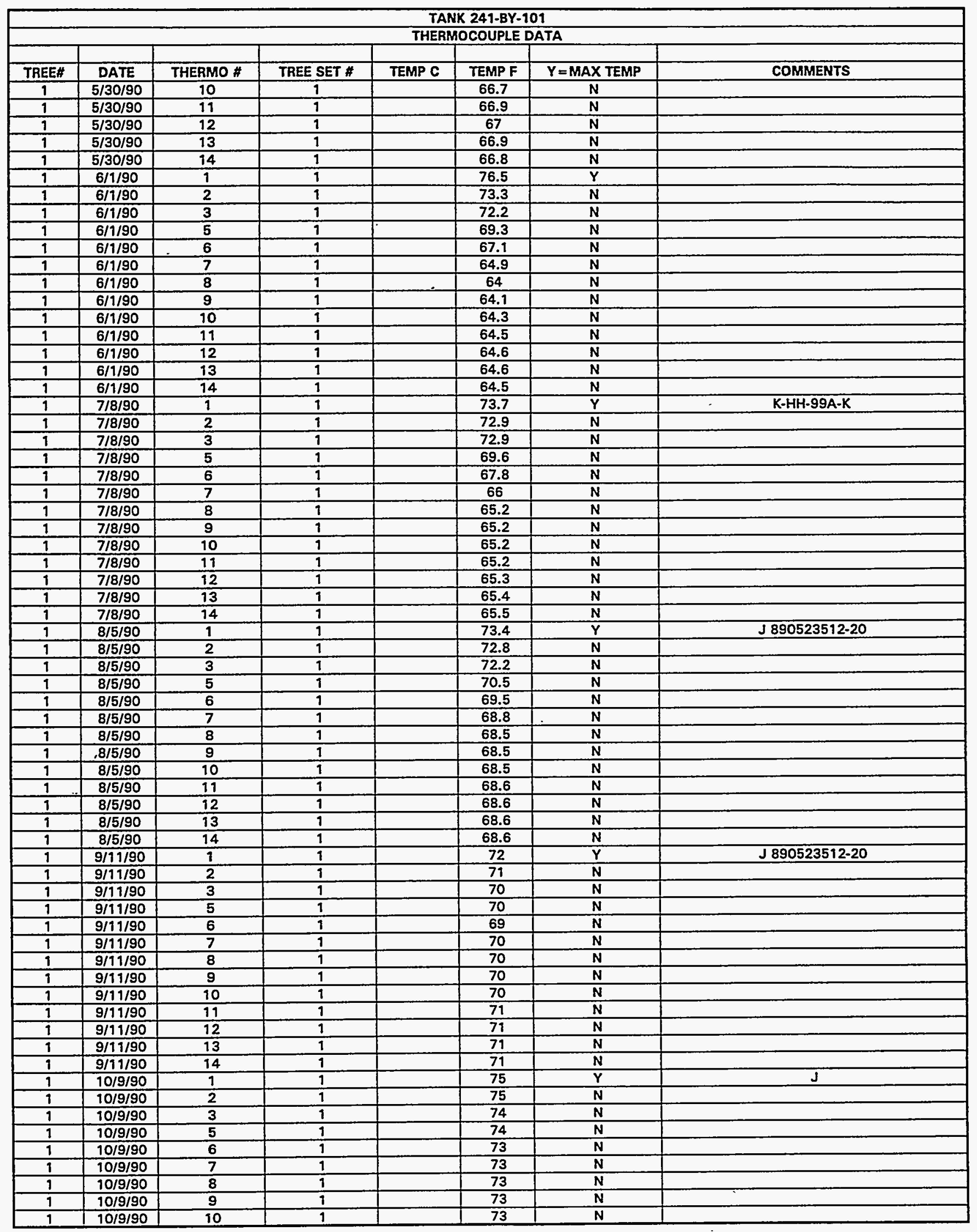

Data obtained from WHC Surveillance Analysis Computer System (SACS), November 12, 1993. 
WHC-SD-WM-ER-312, Rev. 0

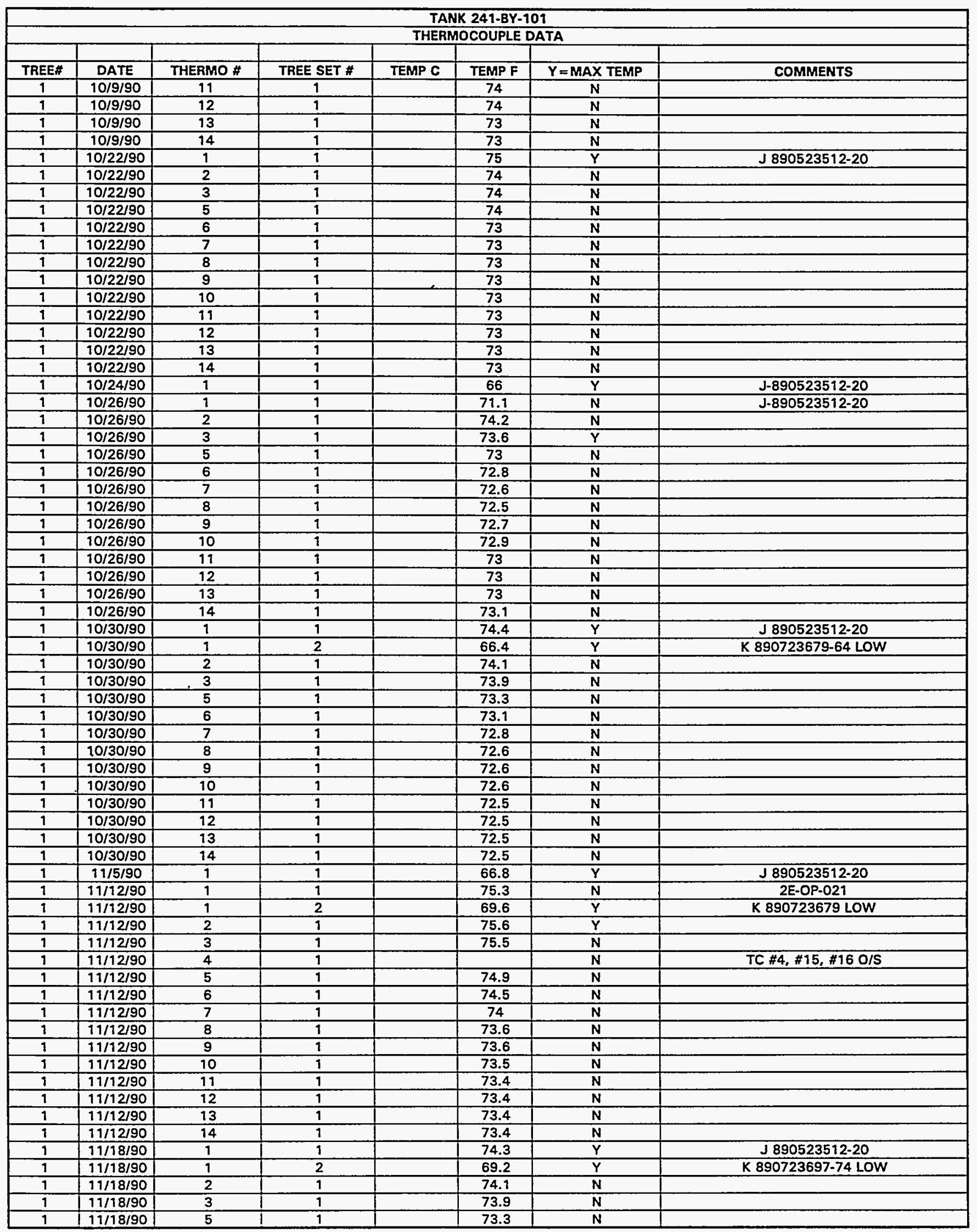

Data obtained from WHC Surveillance Analysis Computer System (SACS). November 12, 1993. 
WHC-SD-WM-ER-312, Rev. 0

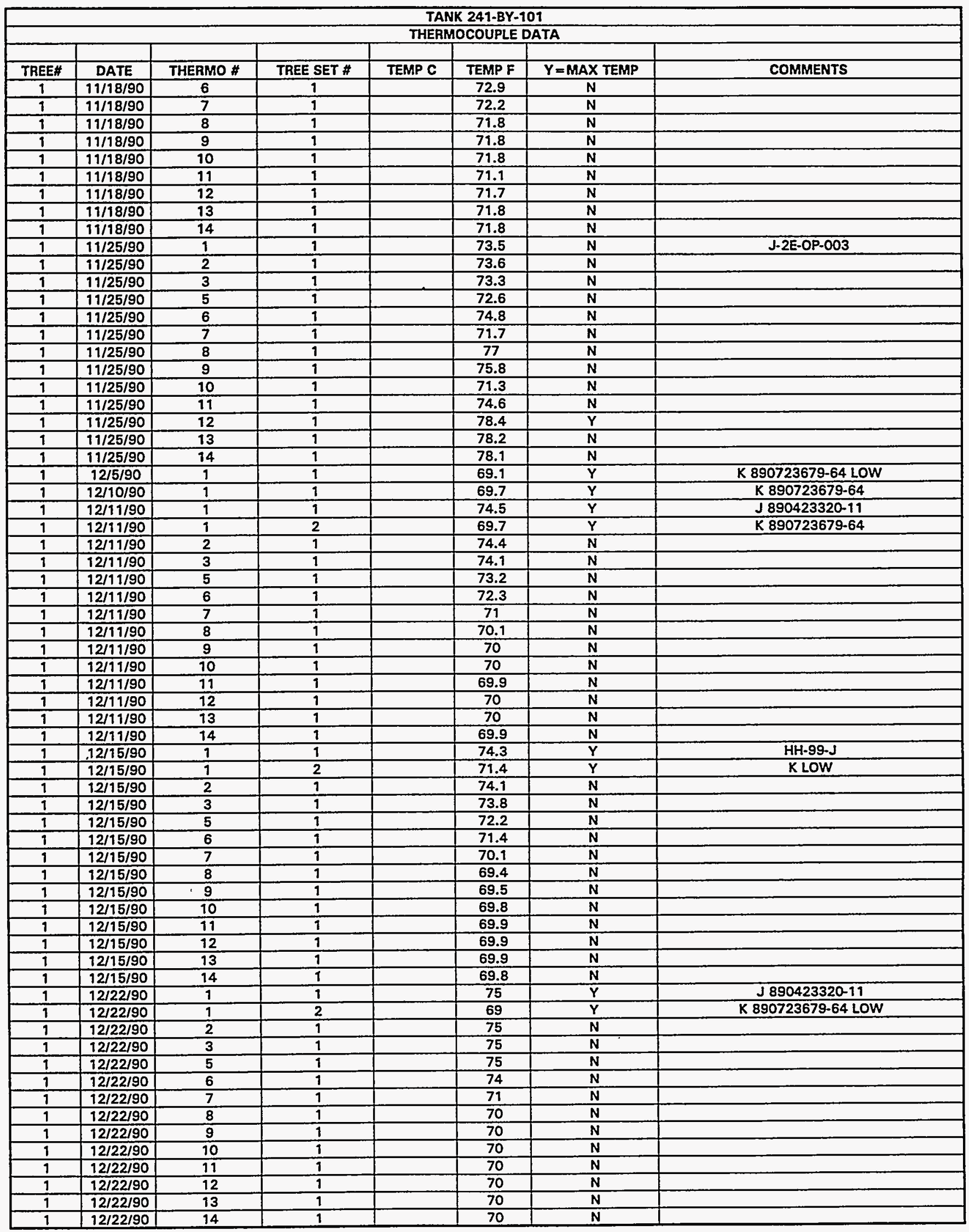

Data obtained from WHC Surveillance Analysis Computer System (SACS), November 12, 1993. 
WHC-SD-WM-ER-312, Rev. 0

\begin{tabular}{|c|c|c|c|c|c|c|c|}
\hline \multicolumn{8}{|c|}{ TANK 241-8Y-101 } \\
\hline \multicolumn{8}{|c|}{ THERMOCOUPLE DATA } \\
\hline & & & & & & & \\
\hline TREE\# & DATE & THERMO \# & TREE SET \# & TEMP C & TEMP F & $Y=$ MAX TEMP & COMMENTS \\
\hline 1 & $12 / 30 / 90$ & 1 & 1 & & 76.1 & $\mathbf{Y}$ & J 2E-OP-021 \\
\hline 1 & $12 / 30 / 90$ & 1 & 2 & & 65 & $\mathbf{N}$ & K 890723679-64 LOW(6 LOW?) \\
\hline 1 & $12 / 30 / 90$ & 2 & 1 & & 76 & $\mathbf{N}$ & \\
\hline$\frac{1}{1}$ & $12 / 30 / 90$ & 2 & 2 & & 65.3 & $\mathbf{Y}$ & \\
\hline 1 & $12 / 30 / 90$ & 3 & 1 & & 75.9 & $\mathbf{N}$ & \\
\hline 1 & $12 / 30 / 90$ & 3 & 2 & & 63.9 & $\bar{N}$ & \\
\hline 1 & $12 / 30 / 90$ & 4 & 2 & & 63.6 & $\mathbf{N}$ & \\
\hline 1 & $12 / 30 / 90$ & 5 & 1 & & 74.6 & $\mathbf{N}$ & \\
\hline 1 & $12 / 30 / 90$ & 5 & 2 & & 63.4 & $\mathbf{N}$ & \\
\hline 1 & $12 / 30 / 90$ & 6 & 1 & & 73.2 & $\mathbf{N}$ & \\
\hline 1 & $12 / 30 / 90$ & 6 & 2 & & 63.4 & $\mathbf{N}$ & \\
\hline 1 & $12 / 30 / 90$ & 7 & 1 & & 71.2 & $\mathbf{N}$ & \\
\hline 1 & $12 / 30 / 90$ & 8 & 1 & & 69.9 & $\mathbf{N}$ & \\
\hline 1 & $12 / 30 / 90$ & 9 & 1 & & 69.8 & $\mathbf{N}$ & \\
\hline 1 & $12 / 30 / 90$ & 10 & 1 & & 69.8 & $\mathbf{N}$ & \\
\hline 9 & $12 / 30 / 90$ & 11 & 1 & & 69.6 & $\mathbf{N}$ & \\
\hline 1 & $12 / 30 / 90$ & 12 & 1 & & 69.7 & $\mathbf{N}$ & \\
\hline 1 & $12 / 30 / 90$ & 13 & 1 & & 69.6 & $\mathbf{N}$ & \\
\hline 1 & $12 / 30 / 90$ & 14 & 1 & & 69.6 & $\mathbf{N}$ & \\
\hline 1 & $1 / 6 / 91$ & 1 & 1 & & 75 & $\mathbf{Y}$ & $\mathrm{J}$ \\
\hline 1 & $1 / 6 / 91$ & 1 & 2 & & 65.8 & $\mathbf{Y}$ & K LOW \\
\hline 9 & 1/6/91 & 2 & 1 & & 75 & $\mathbf{N}$ & \\
\hline 1 & $1 / 6 / 91$ & 3 & 1 & & 75 & $\mathbf{N}$ & \\
\hline 1 & $1 / 6 / 91$ & 5 & 1 & & 74 & $\mathbf{N}$ & \\
\hline 1 & $1 / 6 / 91$ & 6 & 1 & & 72 & $\mathbf{N}$ & \\
\hline 1 & $1 / 6 / 91$ & 7 & 1 & & 70 & $\mathbf{N}$ & \\
\hline 1 & $1 / 6 / 91$ & 8 & 1 & & 69 & $\bar{N}$ & \\
\hline 9 & $1 / 6 / 91$ & 9 & 9 & & 68 & $\mathbf{N}$ & \\
\hline 1 & $1 / 6 / 91$ & 10 & 1 & & 68 & $\mathbf{N}$ & \\
\hline 1 & $1 / 6 / 91$ & 11 & 1 & & 68 & $\mathbf{N}$ & \\
\hline 1 & $1 / 6 / 91$ & 12 & 1 & & 68 & $\mathbf{N}$ & \\
\hline 1 & $1 / 6 / 91$ & 13 & 1 & & 68 & $\mathbf{N}$ & \\
\hline 1 & $1 / 6 / 91$ & 94 & 1 & & 68 & $\mathbf{N}$ & \\
\hline 9 & $1 / 9 / 91$ & 1 & 1 & & 76 & $\bar{Y}$ & K 890723679-64 LOW \\
\hline 1 & $1 / 13 / 91$ & 1 & 1 & & 74.8 & $Y$ & $\mathrm{~J} 890423320-11$ \\
\hline 1 & $1 / 13 / 91$ & 1 & 2 & & 70.2 & $\bar{Y}$ & K 890723679-64 \\
\hline 1 & $1 / 13 / 91$ & 2 & 1 & & 74.5 & $\mathbf{N}$ & \\
\hline 1 & $1 / 13 / 91$ & 4 & 1 & & 72.2 & $\mathbf{N}$ & \\
\hline 1 & $.1 / 13 / 91$ & 5 & 1 & & 70.9 & $\mathbf{N}$ & \\
\hline 1 & $1 / 13 / 91$ & 6 & 1 & & 68.4 & $N$ & \\
\hline 1 & $1 / 13 / 91$ & 7 & 1 & & 67 & $\mathrm{~N}$ & \\
\hline 1 & $1 / 13 / 91$ & 8 & 1 & & 67 & $\mathbf{N}$ & \\
\hline 1 & $1 / 13 / 91$ & 9 & 1 & & 67 & $\mathbf{N}$ & \\
\hline 1 & $1 / 13 / 91$ & 10 & 1 & & 67 & $\mathbf{N}$ & \\
\hline 1 & $1 / 13 / 91$ & 11 & 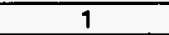 & & 67 & $\mathbf{N}$ & \\
\hline 1 & $1 / 13 / 91$ & 12 & 1 & & 67 & $\mathbf{N}$ & \\
\hline 1 & $1 / 13 / 91$ & 13 & 1 & & 66.9 & $\mathbf{N}$ & \\
\hline 1 & $1 / 13 / 91$ & 14 & $\frac{1}{1}$ & & 67 & $\mathbf{N}$ & \\
\hline 1 & $1 / 19 / 91$ & 1 & 1 & & 74.7 & $\mathbf{N}$ & J HH99A-J \\
\hline 1 & $1 / 19 / 91$ & 2 & 1 & & 74.8 & $\bar{Y}$ & \\
\hline$\frac{1}{1}$ & $1 / 19 / 91$ & 3 & 1 & & 74.6 & $\mathbf{N}$ & \\
\hline 1 & $1 / 19 / 91$ & 5 & 1 & & 72.5 & $\mathbf{N}$ & \\
\hline 1 & $1 / 19 / 91$ & 6 & 1 & & 70.7 & $\mathbf{N}$ & \\
\hline 1 & $1 / 19 / 91$ & 7 & 1 & & 68.2 & $\mathbf{N}$ & \\
\hline 1 & $1 / 19 / 91$ & 8 & 1 & & 66.7 & $\mathbf{N}$ & \\
\hline 1 & $1 / 19 / 91$ & 9 & 1 & & 66.6 & $\mathbf{N}$ & \\
\hline 1 & $1 / 19 / 99$ & 10 & 1 & & 66.5 & $\mathbf{N}$ & \\
\hline 1 & $1 / 19 / 91$ & 11 & 1 & & 66.2 & $\mathbf{N}$ & \\
\hline 1 & $1 / 19 / 91$ & 12 & 1 & & 66.1 & $\mathbf{N}$ & \\
\hline 1 & $1 / 19 / 91$ & 13 & 1 & & 66 & $\mathbf{N}$ & \\
\hline 1 & $1 / 19 / 91$ & 14 & 1 & & 65.9 & $\mathbf{N}$ & \\
\hline 1 & $1 / 29 / 91$ & 1 & 1 & & 74.7 & $\bar{Y}$ & HH-99A-J \\
\hline 1 & $1 / 29 / 91$ & 2 & 1 & & 74.5 & $\mathbf{N}$ & \\
\hline 9 & $1 / 29 / 91$ & 3 & 1 & & 74.2 & $\bar{N}$ & \\
\hline 1 & $1 / 29 / 91$ & 5 & 1 & & 72.3 & $\mathbf{N}$ & \\
\hline 1 & $1 / 29 / 91$ & 6 & 1 & & 70.2 & $\mathbf{N}$ & \\
\hline
\end{tabular}

Data obtained from WHC Surveillance Analysis Computer System (SACS), November 12, 1993. 
WHC-SD-WM-ER-312, Rev. 0

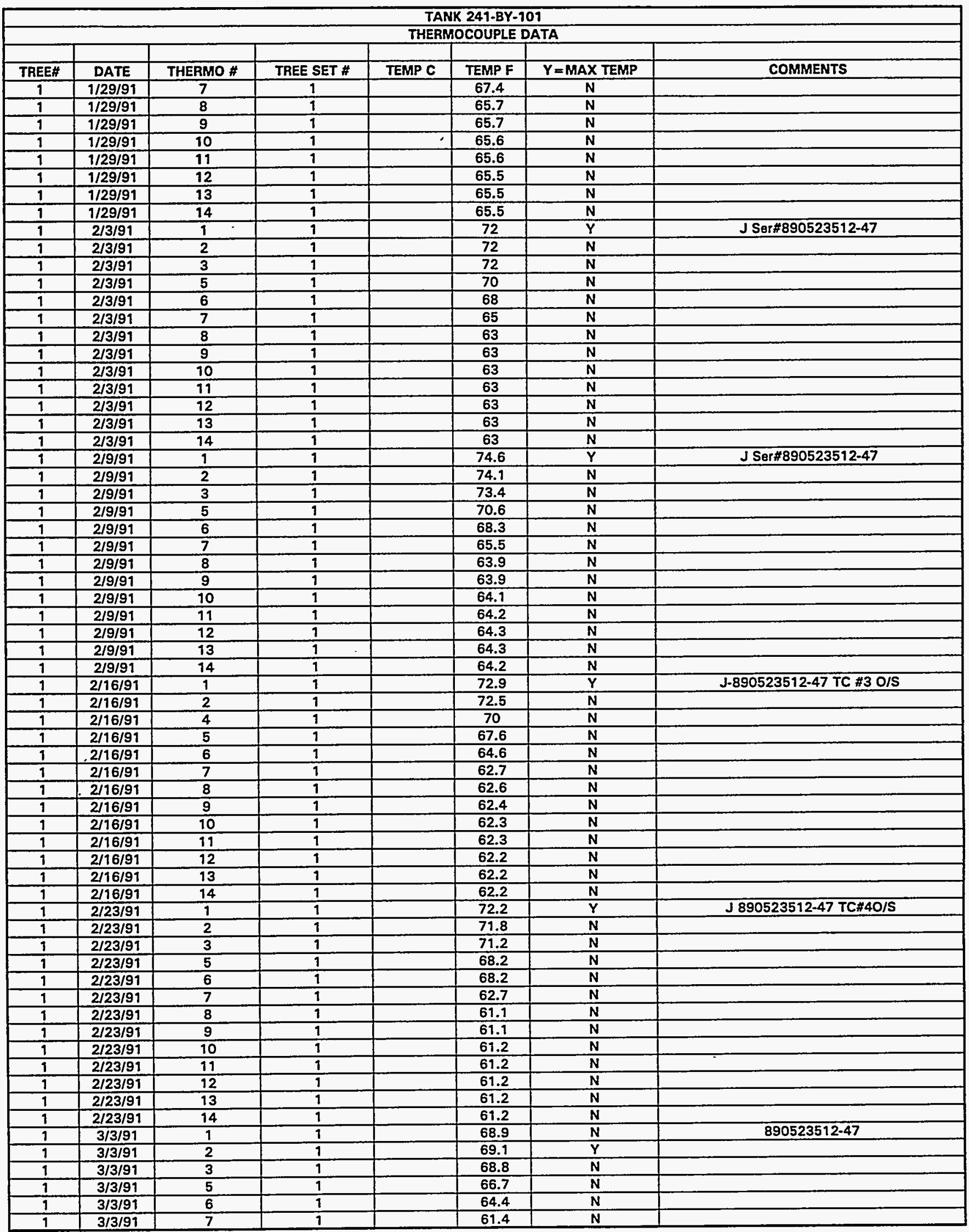

Data obtained from WHC Surveillance Analysis Computer System (SACS), November 12, 1993. 


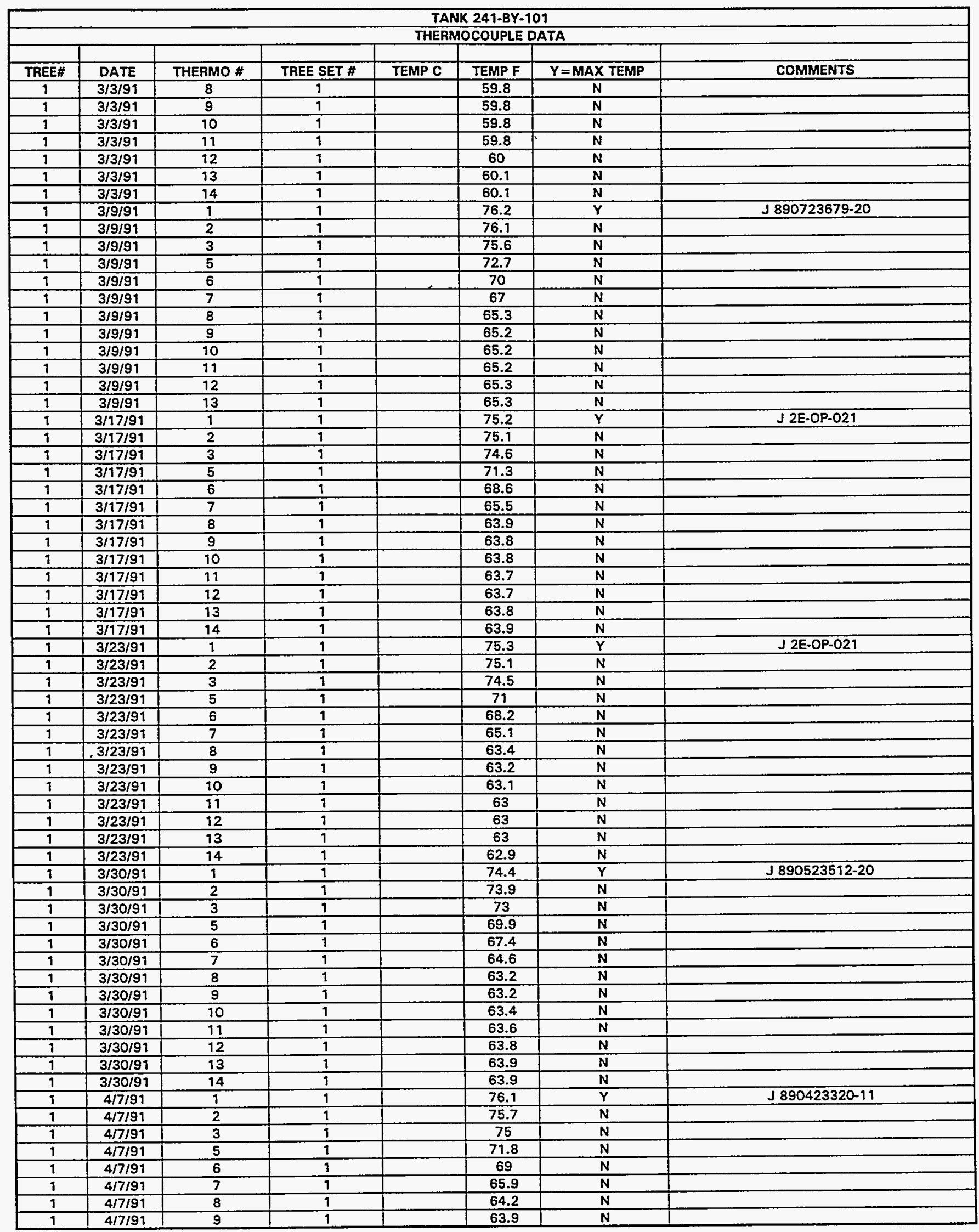

Data obtained from WHC Surveillance Analysis Computer System (SACS), November 12, 1993. 


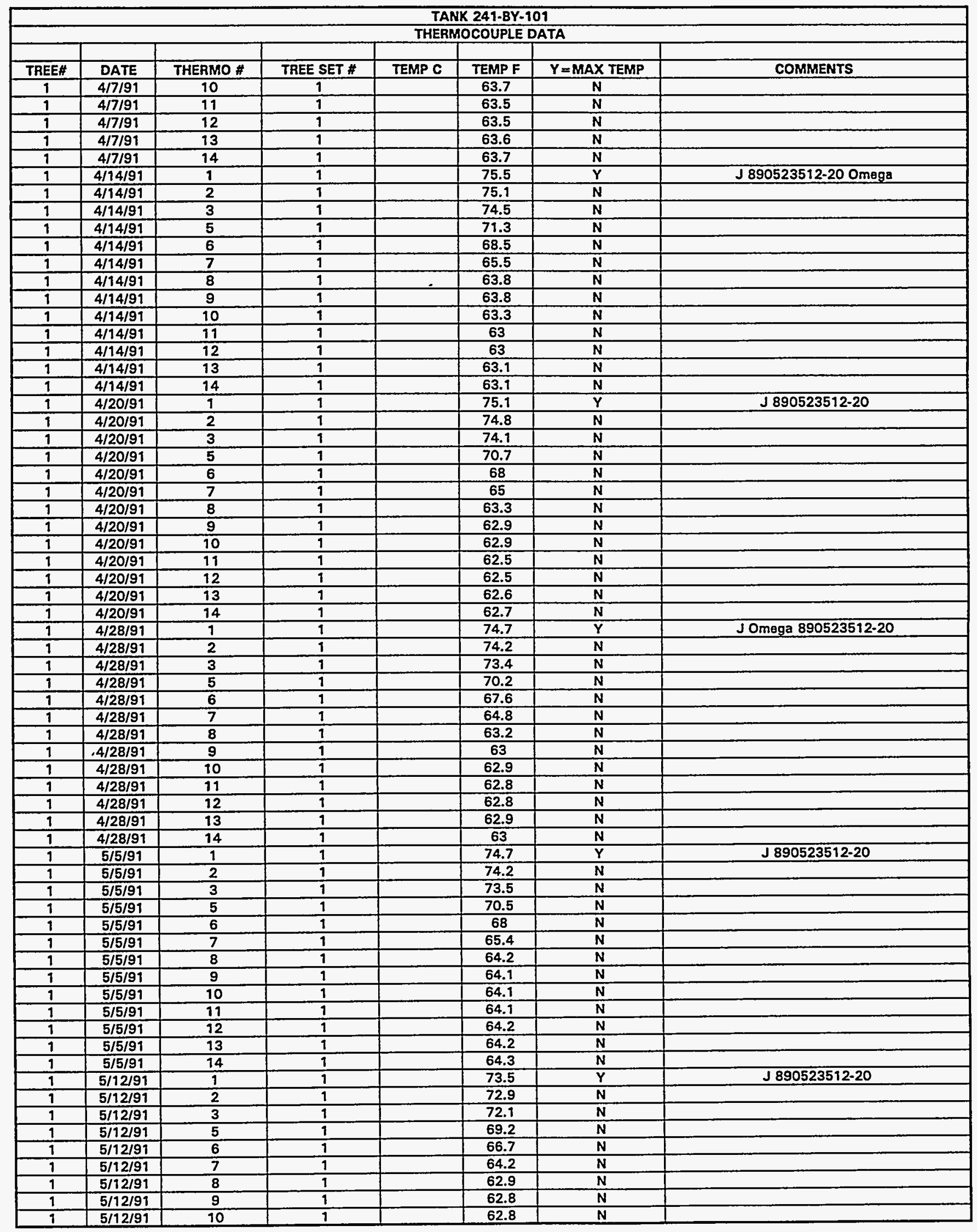

Data obtained from WHC Surveillance Analysis Computer System (SACS), November 12, 1993. 


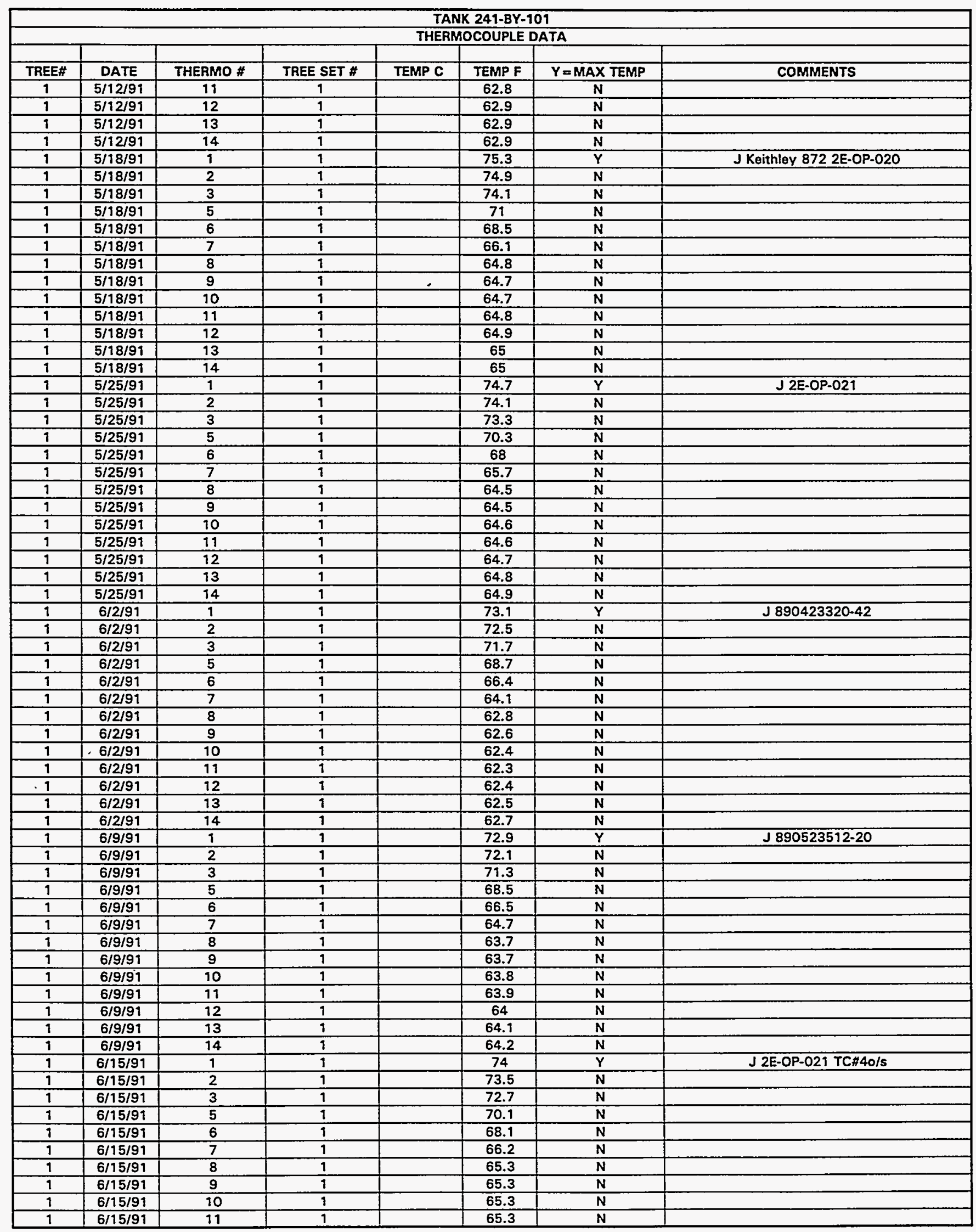

Data obtained from WHC Surveillance Analysis Computer System (SACS), November 12, 1993. 


\begin{tabular}{|c|c|c|c|c|c|c|c|}
\hline \multicolumn{8}{|c|}{ TANK 241-BY-101 } \\
\hline & & & & THER & DCOUPLE & TA & \\
\hline TREE\# & DATE & THERMO \# & TREE SET \# & TEMP C & TEMP F & $Y=$ MAX TEMP & COMMENTS \\
\hline 1 & $6 / 15 / 91$ & 12 & 1 & & 65.4 & $\mathbf{N}$ & \\
\hline 1 & $6 / 15 / 91$ & 13 & 1 & & 65.5 & $\mathbf{N}$ & \\
\hline 1 & $6 / 15 / 91$ & 14 & 1 & & 65.6 & $\mathbf{N}$ & \\
\hline 1 & $6 / 23 / 91$ & 1 & 1 & & 74 & $\bar{Y}$ & J 2E-OP-021 \\
\hline 1 & $6 / 23 / 91$ & 2 & 1 & & 74 & $\mathbf{N}$ & \\
\hline 1 & $6 / 23 / 91$ & 3 & 1 & & 73 & $\bar{N}$ & \\
\hline 1 & $6 / 23 / 91$ & 5 & 1 & & 70 & $\mathbf{N}$ & \\
\hline 1 & $6 / 23 / 91$ & 6 & 1 & & 68 & $\mathbf{N}$ & \\
\hline 1 & $6 / 23 / 91$ & 7 & 1 & & 66 & $\mathbf{N}$ & \\
\hline 1 & $6 / 23 / 91$ & 8 & 1 & & 66 & $\mathbf{N}$ & \\
\hline 1 & $6 / 23 / 91$ & 9 & 1 & & 66 & $N$ & \\
\hline 1 & $6 / 23 / 91$ & 10 & 1 & & 66 & $\bar{N}$ & \\
\hline 1 & $6 / 23 / 91$ & 11 & 1 & & 66 & $\mathbf{N}$ & \\
\hline 1 & $6 / 23 / 91$ & 12 & 1 & & 66 & $\mathbf{N}$ & \\
\hline 1 & $6 / 23 / 91$ & 13 & 1 & & 66 & $\mathbf{N}$ & \\
\hline 1 & $6 / 23 / 91$ & 14 & 1 & & 66 & $\mathbf{N}$ & \\
\hline 1 & $6 / 29 / 91$ & 1 & 1 & & 73 & $\mathbf{Y}$ & J 890423320-42 TC\#4,15,16NG \\
\hline 1 & $6 / 29 / 91$ & 2 & 9 & & 72.4 & $\mathbf{N}$ & \\
\hline 1 & $6 / 29 / 91$ & 3 & 1 & & 71.7 & $\mathbf{N}$ & \\
\hline 1 & $6 / 29 / 91$ & 5 & 1 & & 69 & $\mathbf{N}$ & \\
\hline 1 & $6 / 29 / 91$ & 6 & 1 & & 67.4 & $\mathbf{N}$ & \\
\hline 1 & $6 / 29 / 91$ & 7 & 1 & & 65.7 & $\mathbf{N}$ & \\
\hline 1 & $6 / 29 / 91$ & 8 & 1 & & 64.9 & $\mathbf{N}$ & \\
\hline 1 & $6 / 29 / 91$ & 9 & 1 & & 64.9 & $\bar{N}$ & \\
\hline 1 & $6 / 29 / 91$ & 10 & 1 & & 64.9 & $\mathbf{N}$ & \\
\hline 1 & $6 / 29 / 91$ & 11 & 1 & & 64.9 & $\bar{N}$ & \\
\hline 1 & $6 / 29 / 91$ & 12 & 1 & & 64.9 & $\mathbf{N}$ & \\
\hline 1 & $6 / 29 / 91$ & 13 & 1 & & 64.9 & $\mathrm{~N}$ & \\
\hline 1 & $6 / 29 / 91$ & 14 & 1 & & 65 & $\mathbf{N}$ & \\
\hline$\frac{1}{1}$ & $7 / 6 / 91$ & 1 & 1 & & 73.4 & $\bar{Y}$ & J $910126 K 143-42$ \\
\hline 1 & $7 / 6 / 91$ & 2 & 1 & & 72.7 & $\mathbf{N}$ & \\
\hline 1 & $7 / 6 / 91$ & 3 & 1 & & 71.9 & $\mathbf{N}$ & \\
\hline 1 & $7 / 6 / 91$ & 5 & 1 & & 69.1 & $\mathbf{N}$ & \\
\hline 1 & 7/6/91 & 6 & 1 & & 67.4 & $\mathbf{N}$ & \\
\hline 1 & $7 / 6 / 91$ & 7 & 9 & & 65.9 & $\mathbf{N}$ & \\
\hline 1 & $7 / 6 / 91$ & 8 & 1 & & 65.2 & $\mathbf{N}$ & \\
\hline 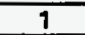 & $7 / 6 / 91$ & 9 & 1 & & 65.3 & $\mathbf{N}$ & \\
\hline 1 & $7 / 6 / 91$ & 10 & 1 & & 65.5 & $\mathbf{N}$ & \\
\hline 1 & $7 / 6 / 91$ & 11 & 1 & & 65.6 & $\bar{N}$ & \\
\hline 1 & $7 / 6 / 91$ & 12 & 1 & & 65.8 & $\mathbf{N}$ & \\
\hline 1 & $7 / 6 / 91$ & 13 & 1 & & 65.9 & $\mathbf{N}$ & \\
\hline 1 & $7 / 6 / 91$ & 14 & 9 & & 66.1 & $N$ & \\
\hline 1 & $7 / 13 / 91$ & 1 & 1 & & 73.5 & $\mathbf{Y}$ & J910126143-42 TC\$4,15,16NG \\
\hline 1 & $7 / 13 / 91$ & 2 & 1 & & 72.7 & $\mathbf{N}$ & \\
\hline 1 & $7 / 13 / 91$ & 3 & 1 & & 72 & $\mathbf{N}$ & \\
\hline 1 & $7 / 13 / 91$ & 5 & 1 & & 69.3 & $\mathbf{N}$ & \\
\hline 1 & $7 / 13 / 91$ & 6 & $\frac{1}{1}$ & & 67.4 & $\mathbf{N}$ & \\
\hline 1 & $7 / 13 / 91$ & 7 & 1 & & 65.8 & $\mathbf{N}$ & \\
\hline 1 & $7 / 13 / 91$ & 8 & 1 & & 65.2 & $\mathbf{N}$ & \\
\hline$\frac{1}{1}$ & $7 / 13 / 91$ & 9 & 1 & & 65.3 & $\mathbf{N}$ & \\
\hline 1 & $7 / 13 / 91$ & 10 & 1 & & 65.5 & $\mathbf{N}$ & \\
\hline 1 & $7 / 13 / 91$ & 11 & 1 & & 65.6 & $\mathbf{N}$ & \\
\hline 1 & $7 / 13 / 91$ & 12 & 1 & & 65.9 & $\mathbf{N}$ & \\
\hline 1 & $7 / 13 / 91$ & 13 & 1 & & 66 & $\mathbf{N}$ & \\
\hline 1 & $7 / 13 / 91$ & 14 & 1 & & 66.5 & $N$ & \\
\hline 1 & $7 / 20 / 91$ & 1 & 1 & & 71.8 & $\bar{Y}$ & J910126143-64 \\
\hline 1 & $7 / 20 / 91$ & 2 & 1 & & 71.3 & $\mathbf{N}$ & \\
\hline 1 & $7 / 20 / 91$ & 3 & 1 & & 70.5 & $N$ & \\
\hline 1 & $7 / 20 / 91$ & 5 & 1 & & 67.8 & $\mathbf{N}$ & \\
\hline 1 & $7 / 20 / 91$ & 6 & 1 & & 66.2 & $\mathbf{N}$ & \\
\hline 1 & $7 / 20 / 91$ & 7 & 1 & & 65.1 & $\bar{N}$ & \\
\hline 1 & $7 / 20 / 91$ & 8 & 1 & & 64.7 & $\mathbf{N}$ & \\
\hline 1 & $7 / 20 / 91$ & 9 & 1 & & 64.7 & $\mathbf{N}$ & \\
\hline 1 & $7 / 20 / 91$ & 10 & 1 & & 64.9 & $N$ & \\
\hline 1 & $7 / 20 / 91$ & 11 & 1 & & 65 & $\mathbf{N}$ & \\
\hline 7 & $7 / 20 / 91$ & 12 & 1 & & 65.1 & $\mathbf{N}$ & \\
\hline
\end{tabular}

Data obtained from WHC Surveillance Analysis Computer System (SACS), November 12, 1993. 


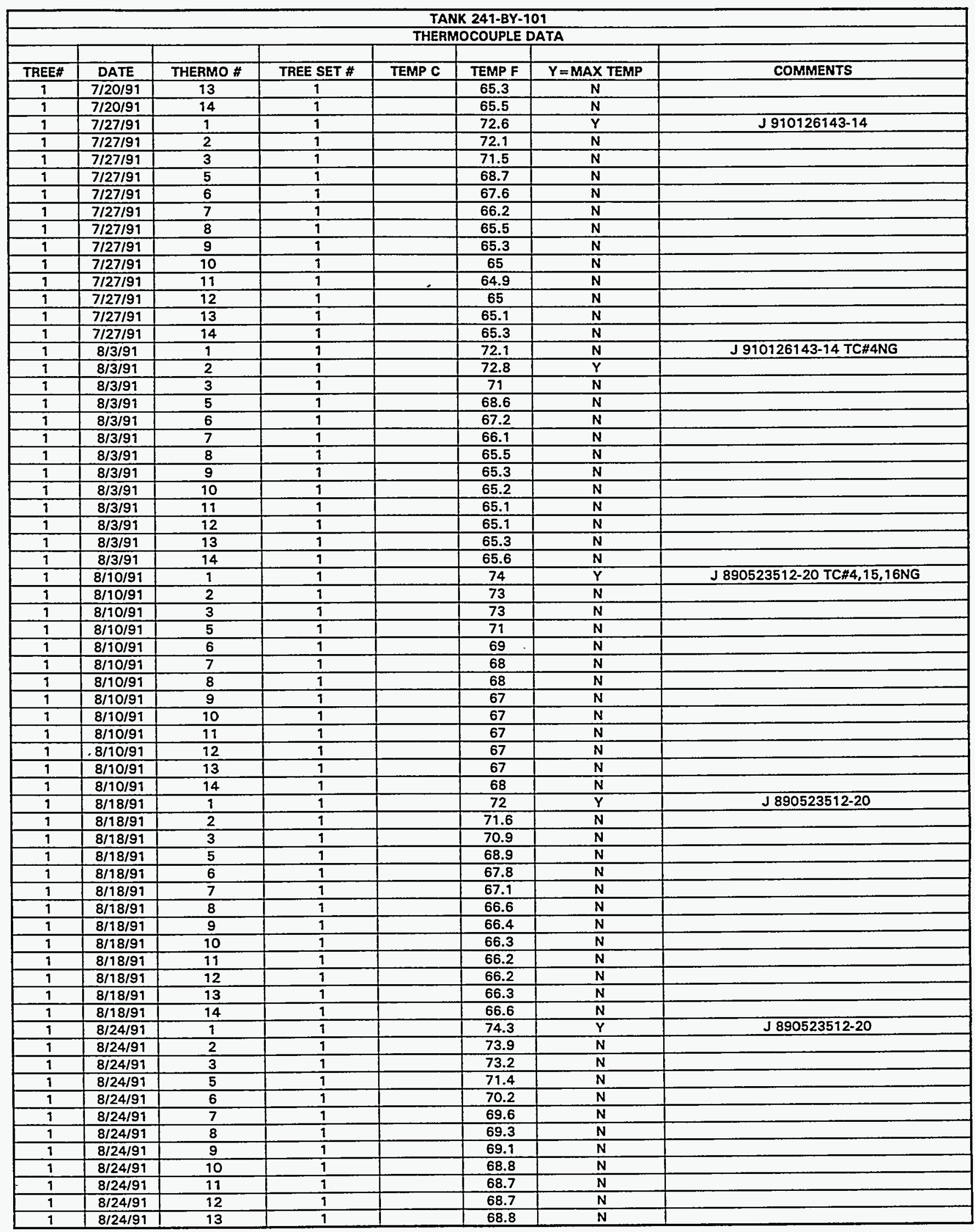

Data obtained from WHC Surveillance Analysis Computer System (SACS), November 12, 1993. 
WHC-SD-WM-ER-312, Rev. 0

\begin{tabular}{|c|c|c|c|c|c|c|c|}
\hline \multicolumn{8}{|c|}{ TANK 241-BY-101 } \\
\hline & & & & THER & COUPLE & ATA & \\
\hline TREE\# & DATE & THERMO \# & TREE SET \# & TEMP C & TEMP F & $Y=$ MAX TEMP & COMMENTS \\
\hline 1 & $8 / 31 / 91$ & 1 & 1 & & 73.1 & $\mathbf{Y}$ & J-890423320-42 \\
\hline 1 & $8 / 31 / 91$ & 2 & 9 & & 72.8 & $\mathbf{N}$ & \\
\hline 1 & $8 / 31 / 91$ & 3 & 1 & & 72.2 & $\mathbf{N}$ & \\
\hline 1 & $8 / 31 / 91$ & 5 & 1 & & 70.3 & $\mathbf{N}$ & \\
\hline$\overline{1}$ & $8 / 31 / 91$ & 6 & 1 & & 69.5 & $\bar{N}$ & \\
\hline 1 & $8 / 31 / 91$ & 7 & 1 & & 69.1 & $\mathbf{N}$ & \\
\hline 1 & $8 / 31 / 91$ & 8 & 1 & & 68.9 & $\mathbf{N}$ & \\
\hline$\frac{1}{1}$ & $8 / 31 / 91$ & 9 & $T$ & & 68.7 & $\mathbf{N}$ & \\
\hline 9 & $8 / 31 / 91$ & 10 & 1 & & 68.5 & $\mathbf{N}$ & \\
\hline 1 & $8 / 31 / 91$ & 11 & 1 & & 68.5 & $N$ & \\
\hline 1 & $8 / 31 / 91$ & 12 & 1 & & 68.5 & $\mathbf{N}$ & \\
\hline 1 & $8 / 31 / 91$ & 13 & 1 & & 68.6 & $\mathbf{N}$ & \\
\hline 1 & $8 / 31 / 91$ & 14 & 1 & & 68.9 & $\mathbf{N}$ & \\
\hline 1 & $9 / 8 / 91$ & 1 & 1 & & 73.2 & $\bar{Y}$ & J.890423320-42 \\
\hline 9 & $9 / 8 / 91$ & 2 & 1 & & 72.9 & $\mathbf{N}$ & \\
\hline 1 & $9 / 8 / 91$ & 3 & 1 & & 72.2 & $N$ & \\
\hline 1 & $9 / 8 / 91$ & 5 & 1 & & 70.3 & $\mathbf{N}$ & \\
\hline 1 & $9 / 8 / 91$ & 6 & 1 & & 69.5 & $N$ & \\
\hline 1 & $9 / 8 / 91$ & 7 & 1 & & 69.1 & $\mathrm{~N}$ & \\
\hline 1 & $9 / 8 / 91$ & 8 & 1 & & 68.9 & $\mathbf{N}$ & \\
\hline 1 & $9 / 8 / 91$ & 9 & $T$ & & 68.7 & $\mathbf{N}$ & \\
\hline 1 & $9 / 8 / 91$ & 10 & 1 & & 68.5 & $\mathbf{N}$ & \\
\hline 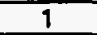 & $9 / 8 / 91$ & 11 & 1 & & 68.5 & $\mathbf{N}$ & \\
\hline$T$ & $9 / 8 / 91$ & 12 & 1 & & 68.6 & $\mathbf{N}$ & \\
\hline 1 & $9 / 8 / 91$ & 13 & 1 & & 68.6 & $\mathbf{N}$ & \\
\hline 1 & $9 / 8 / 91$ & 94 & 1 & & 68.9 & $\mathbf{N}$ & \\
\hline 1 & $9 / 15 / 91$ & 1 & 9 & & 72.6 & $\mathbf{Y}$ & J-910126143.75 \\
\hline 1 & $9 / 15 / 91$ & 2 & 1 & & 72.5 & $\mathbf{N}$ & \\
\hline$T$ & $9 / 15 / 91$ & 3 & 1 & & 72 & $\mathbf{N}$ & \\
\hline 1 & $9 / 15 / 91$ & 5 & 1 & & 70.1 & $\mathbf{N}$ & \\
\hline 1 & $9 / 15 / 91$ & 6 & 1 & & 69.5 & $\mathbf{N}$ & \\
\hline 1 & $9 / 15 / 91$ & 7 & 1 & & 69.4 & $\mathbf{N}$ & \\
\hline 1 & $9 / 15 / 91$ & 8 & 1 & & 69.3 & $\mathbf{N}$ & \\
\hline 1 & $9 / 15 / 91$ & 9 & 7 & & 69.1 & $N$ & \\
\hline 1 & $9 / 15 / 91$ & 10 & 1 & & 69.1 & $\mathbf{N}$ & \\
\hline 1 & $9 / 15 / 91$ & 11 & 1 & & 68.9 & $\mathbf{N}$ & \\
\hline 1 & $9 / 15 / 91$ & 12 & 1 & & 68.8 & $\mathbf{N}$ & \\
\hline 1 & $9 / 15 / 91$ & 13 & 1 & & 68.9 & $N$ & \\
\hline$\frac{1}{1}$ & $.9 / 15 / 91$ & 14 & $T$ & & 69.2 & $\overline{\mathbf{N}}$ & \\
\hline$\frac{1}{1}$ & $9 / 22 / 91$ & 9 & 7 & & 72.7 & $\bar{Y}$ & $J-910126143-75$ \\
\hline 1 & $9 / 22 / 91$ & 2 & 1 & & 72.5 & $\mathbf{N}$ & \\
\hline$\overline{1}$ & $9 / 22 / 91$ & 3 & 1 & & 71.9 & $\mathbf{N}$ & \\
\hline 1 & $9 / 22 / 91$ & 5 & 1 & & 70.1 & $\mathbf{N}$ & \\
\hline$\frac{1}{1}$ & $9 / 22 / 91$ & 6 & 9 & & 69.6 & $\mathbf{N}$ & \\
\hline 1 & $9 / 22 / 91$ & 7 & $\frac{1}{1}$ & & 69.9 & $\mathbf{N}$ & \\
\hline 1 & $9 / 22 / 91$ & 8 & 1 & & 70.2 & $\mathbf{N}$ & \\
\hline 1 & $9 / 22 / 91$ & 9 & 1 & & 70.4 & $\mathbf{N}$ & \\
\hline 1 & $9 / 22 / 91$ & 10 & 1 & & 70.6 & $N$ & \\
\hline 1 & $9 / 22 / 91$ & 11 & 1 & & 70.8 & $\mathbf{N}$ & \\
\hline 1 & $9 / 22 / 91$ & 12 & 1 & & 70.9 & $\mathbf{N}$ & \\
\hline 1 & $9 / 22 / 91$ & 13 & 1 & & 71.1 & $\mathbf{N}$ & \\
\hline 1 & $9 / 22 / 91$ & 14 & 1 & & 71.3 & $\mathbf{N}$ & \\
\hline 1 & $9 / 28 / 91$ & 1 & 1 & & 73.5 & $\mathbf{Y}$ & J-KEITHLY 872 \#T1-059 \\
\hline 1 & $9 / 28 / 91$ & 2 & 1 & & 73.4 & $\mathbf{N}$ & \\
\hline 1 & $9 / 28 / 91$ & 3 & 1 & & 73 & $\mathbf{N}$ & \\
\hline 1 & $9 / 28 / 91$ & 5 & 1 & & 71.5 & $\mathbf{N}$ & \\
\hline 1 & $9 / 28 / 97$ & 6 & 1 & & 71 & $N$ & \\
\hline 1 & $9 / 28 / 91$ & 7 & 1 & & 71.1 & $\mathbf{N}$ & \\
\hline 1 & $9 / 28 / 91$ & 8 & 1 & & 71.1 & $\bar{N}$ & \\
\hline 1 & $9 / 28 / 91$ & 9 & 1 & & 71.1 & $\mathrm{~N}$ & \\
\hline 1 & $9 / 28 / 91$ & 10 & 9 & & 71.1 & $\mathbf{N}$ & \\
\hline 1 & $9 / 28 / 91$ & 11 & 1 & & 71.1 & $\mathbf{N}$ & \\
\hline$\cdot 1$ & $9 / 28 / 91$ & 12 & 1 & & 71.1 & $\mathbf{N}$ & \\
\hline 1 & $9 / 28 / 91$ & 13 & 1 & & 71.2 & $\mathbf{N}$ & \\
\hline 1 & $9 / 28 / 91$ & 14 & 1 & & 71.4 & $\mathbf{N}$ & \\
\hline 1 & $10 / 5 / 91$ & 1 & 1 & & 73.7 & $\mathbf{Y}$ & J.910126143-42 \\
\hline
\end{tabular}

Data obtained from WHC Surveillance Analysis Computer System (SACS), November 12, 1993. 
WHC-SD-WM-ER-312, Rev. 0

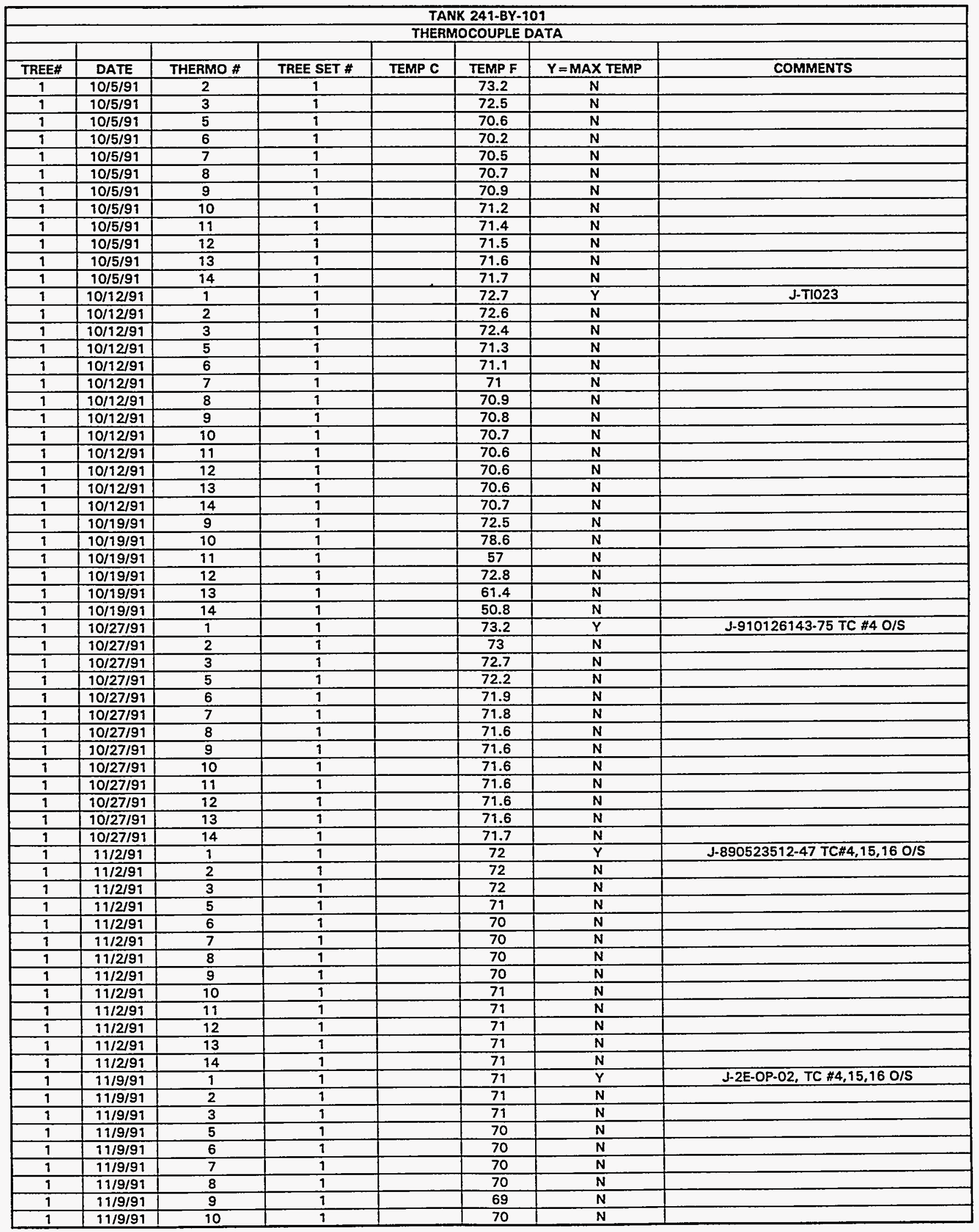

Data obtained from WHC Surveillance Analysis Computer System (SACS), November 12, 1993. 


\begin{tabular}{|c|c|c|c|c|c|c|c|}
\hline \multicolumn{8}{|c|}{ TANK 241-BY-101 } \\
\hline \multicolumn{8}{|c|}{ THERMOCOUPLE DATA } \\
\hline TREE\# & DATE & THERMO \# & TREE SET \# & TEMP C & TEMP F & $Y=$ MAX TEMP & COMMENTS \\
\hline 1 & $11 / 9 / 91$ & 11 & 1 & & 69 & $N$ & \\
\hline 1 & $11 / 9 / 91$ & 12 & 1 & & 70 & $\mathbf{N}$ & \\
\hline 1 & $11 / 9 / 91$ & 13 & 1 & & 70 & $\mathbf{N}$ & \\
\hline 1 & $11 / 9 / 91$ & 14 & 1 & & 70 & $\bar{N}$ & \\
\hline 1 & $11 / 17 / 91$ & 1 & 1 & & 73 & $\mathbf{Y}$ & J-910126143-75, TC \#4 O/S \\
\hline 1 & $11 / 17 / 91$ & 2 & 1 & & 72.8 & $\mathbf{N}$ & \\
\hline 1 & $11 / 17 / 91$ & 3 & 1 & & 72.6 & $\bar{N}$ & \\
\hline 1 & $11 / 17 / 91$ & 5 & 1 & & 71.4 & $\overline{\mathbf{N}}$ & \\
\hline 1 & $11 / 17 / 91$ & 6 & 1 & & 71.5 & $\mathbf{N}$ & \\
\hline 1 & $11 / 17 / 91$ & 7 & 1 & & 70.7 & $\mathbf{N}$ & \\
\hline 1 & $11 / 17 / 91$ & 8 & 1 & & 70.2 & $\mathbf{N}$ & \\
\hline 1 & $11 / 17 / 91$ & 9 & 1 & & 70.1 & $\mathbf{N}$ & \\
\hline 1 & $11 / 17 / 91$ & 10 & 1 & & 70.1 & $\mathbf{N}$ & \\
\hline 1 & $11 / 17 / 91$ & 11 & 1 & & 70.1 & $\mathbf{N}$ & \\
\hline 1 & $11 / 17 / 91$ & 12 & 1 & & 70.1 & $\mathbf{N}$ & \\
\hline 1 & $11 / 17 / 91$ & 13 & 1 & & 70 & $\mathbf{N}$ & \\
\hline 1 & $11 / 17 / 91$ & 94 & 9 & & 70 & $\bar{N}$ & \\
\hline 1 & $11 / 24 / 91$ & 1 & 1 & & 74.6 & $\bar{Y}$ & $\mathrm{~J}-910126143-75, \mathrm{TC} \# 415-16 \mathrm{O} / \mathrm{S}$ \\
\hline 1 & $11 / 24 / 91$ & 2 & 1 & & 74.5 & $N$ & \\
\hline 1 & $11 / 24 / 91$ & 3 & $T$ & & 74.3 & $\mathbf{N}$ & \\
\hline 1 & $11 / 24 / 91$ & 5 & 1 & & 73.6 & $\mathbf{N}$ & \\
\hline 1 & $11 / 24 / 91$ & 6 & 1 & & 73 & $\mathbf{N}$ & \\
\hline 1 & $11 / 24 / 91$ & 7 & 1 & & 72 & $\mathbf{N}$ & \\
\hline 1 & $11 / 24 / 91$ & 8 & 1 & & 71.4 & $\mathbf{N}$ & \\
\hline 1 & $11 / 24 / 91$ & 9 & 1 & & 71.4 & $\mathbf{N}$ & \\
\hline 1 & $11 / 24 / 91$ & 10 & 1 & & 71.4 & $\mathbf{N}$ & \\
\hline 1 & $11 / 24 / 91$ & 11 & 1 & & 71.3 & $\mathbf{N}$ & \\
\hline 1 & $11 / 24 / 91$ & 12 & 1 & & 71.3 & $\mathbf{N}$ & \\
\hline 1 & $11 / 24 / 91$ & 13 & 1 & & 71.3 & $\mathbf{N}$ & \\
\hline 1 & $11 / 24 / 91$ & 14 & 1 & & 71.3 & $\mathbf{N}$ & \\
\hline 1 & $12 / 1 / 91$ & 1 & 1 & & 73.7 & $\mathbf{Y}$ & J-910126143-14, TC \#4 O/S \\
\hline 1 & $12 / 1 / 91$ & 2 & 1 & & 73.6 & $\mathbf{N}$ & \\
\hline 1 & $12 / 1 / 91$ & 3 & 1 & & 73.4 & $\mathbf{N}$ & \\
\hline 1 & $12 / 1 / 91$ & 5 & 1 & & 72.6 & $\mathbf{N}$ & \\
\hline 1 & $12 / 1 / 91$ & 6 & 1 & & 71.9 & $\mathbf{N}$ & \\
\hline 1 & $12 / 1 / 91$ & 7 & 1 & & 70.7 & $\mathbf{N}$ & \\
\hline 1 & $12 / 1 / 91$ & 8. & 1 & & 69.9 & $\mathbf{N}$ & \\
\hline 1 & $12 / 1 / 91$ & 9 & 1 & & 69.8 & $\mathbf{N}$ & \\
\hline 1 & $.12 / 1 / 91$ & 10 & 1 & & 69.8 & $\mathbf{N}$ & \\
\hline 1 & $12 / 1 / 91$ & 11 & 1 & & 69.8 & $\mathbf{N}$ & \\
\hline 1 & $12 / 1 / 91$ & 12 & 1 & & 69.8 & $\mathbf{N}$ & \\
\hline 9 & $12 / 1 / 91$ & 13 & 1 & & 69.6 & $\bar{N}$ & \\
\hline 1 & $12 / 1 / 91$ & 14 & 1 & & 69.7 & $\mathbf{N}$ & \\
\hline 1 & 12/6/91 & 1 & 1 & & 74.6 & $\mathbf{Y}$ & $\begin{array}{c}\text { J-910126143-29, TC } \# 3-6 \text { O/S TC } 7-14 \text { NO } \\
\text { READINGS }\end{array}$ \\
\hline 1 & $12 / 6 / 91$ & 2 & 1 & & 74.5 & $\mathbf{N}$ & \\
\hline 1 & $12 / 8 / 91$ & 1 & 1 & & 74.1 & $\mathbf{Y}$ & J-910126143-48 TC \#4 NO READINGS \\
\hline 1 & $12 / 8 / 91$ & 2 & 1 & & 74 & $\mathbf{N}$ & \\
\hline 1 & $12 / 8 / 91$ & 3 & 1 & & 73.8 & $\mathbf{N}$ & \\
\hline 1 & $12 / 8 / 91$ & 5 & 1 & & 72.9 & $\mathbf{N}$ & \\
\hline 1 & $12 / 8 / 91$ & 6 & 1 & & 72 & $\mathbf{N}$ & \\
\hline 1 & $12 / 8 / 91$ & 7 & 1 & & 70.6 & $\mathbf{N}$ & \\
\hline 1 & $12 / 8 / 91$ & 8 & 1 & & 69.7 & $\mathbf{N}$ & \\
\hline 1 & $12 / 8 / 91$ & 9 & 7 & & 69.6 & $\bar{N}$ & \\
\hline 1 & $12 / 8 / 91$ & 10 & 1 & & 69.5 & $\mathbf{N}$ & \\
\hline 1 & $12 / 8 / 91$ & 71 & 1 & & 69.4 & $\mathbf{N}$ & \\
\hline 1 & $12 / 8 / 91$ & 12 & 1 & & 69.4 & $\mathbf{N}$ & \\
\hline 1 & $12 / 8 / 91$ & 13 & 1 & & 69.4 & $\mathbf{N}$ & \\
\hline 1 & $12 / 8 / 91$ & 14 & 1 & & 69.3 & $\mathbf{N}$ & \\
\hline 1 & $12 / 14 / 91$ & 1 & 1 & & 74.7 & $\mathbf{Y}$ & J-910126143-29 \\
\hline 1 & $12 / 14 / 91$ & 2 & 1 & & 74.6 & $\mathbf{N}$ & \\
\hline 1 & $12 / 14 / 91$ & 3 & 1 & & 74.4 & $\mathbf{N}$ & \\
\hline 1 & $12 / 14 / 91$ & 5 & 1 & & 73.1 & $\mathbf{N}$ & \\
\hline 1 & $12 / 14 / 91$ & 6 & 1 & & 72.2 & $\mathbf{N}$ & \\
\hline 1 & $12 / 14 / 91$ & 7 & 1 & & 70.8 & $\mathbf{N}$ & \\
\hline 1 & $12 / 14 / 91$ & 8 & 1 & & 69.8 & $\mathbf{N}$ & \\
\hline
\end{tabular}

Data obtained from WHC Surveillance Analysis Computer System (SACS), November 12, 1993. 


\begin{tabular}{|c|c|c|c|c|c|c|c|}
\hline \multicolumn{8}{|c|}{ TANK 241-BY-101 } \\
\hline \multicolumn{8}{|c|}{ THERMOCOUPLE DATA } \\
\hline TREE\# & DATE & THERMO \# & TREE SET \# & TEMP C & TEMP F & $Y=$ MAX TEMP & COMMENTS \\
\hline 1 & $12 / 14 / 91$ & 9 & 1 & & 69.8 & $\mathrm{~N}$ & \\
\hline 1 & $12 / 14 / 91$ & 10 & 1 & & 69.7 & $\mathbf{N}$ & \\
\hline 1 & $12 / 14 / 91$ & 11 & 1 & & 69.5 & $\mathbf{N}$ & \\
\hline 1 & $12 / 14 / 91$ & 12 & 1 & & 69.5 & $\mathbf{N}$ & \\
\hline 1 & $12 / 14 / 91$ & 13 & 1 & & 69.5 & $\mathbf{N}$ & \\
\hline 1 & $12 / 14 / 91$ & 14 & 1 & & 69.5 & $\bar{N}$ & \\
\hline 1 & $12 / 20 / 91$ & 1 & 1 & & & $\mathbf{N}$ & NO READINGS \\
\hline 1 & $12 / 22 / 91$ & 1 & 1 & & 75.5 & $\bar{Y}$ & J-900925741 OMEGA TC \#10 BAD DATA \\
\hline 1 & $12 / 22 / 91$ & 2 & 1 & & 75.4 & $\mathbf{N}$ & \\
\hline 1 & $12 / 22 / 91$ & 3 & 1 & & 75.2 & $\mathbf{N}$ & \\
\hline 1 & $12 / 22 / 91$ & 4 & 1 & & 74.9 & $\mathbf{N}$ & \\
\hline 1 & $12 / 22 / 91$ & 5 & 1 & & 71 & $\mathbf{N}$ & \\
\hline 1 & $12 / 22 / 91$ & 6 & 1 & & 73 & $\mathbf{N}$ & \\
\hline 1 & $12 / 22 / 91$ & 7 & 1 & & 71.2 & $\mathbf{N}$ & \\
\hline 1 & $12 / 22 / 91$ & 8 & 1 & & 70.1 & $\bar{N}$ & \\
\hline 1 & $12 / 22 / 91$ & 9 & 1 & & 64.1 & $\mathbf{N}$ & \\
\hline 1 & $12 / 22 / 91$ & 11 & 1 & & 70 & $\mathbf{N}$ & \\
\hline 1 & $12 / 22 / 91$ & 12 & 1 & & 70 & $\mathbf{N}$ & \\
\hline 1 & $12 / 22 / 91$ & 13 & 9 & & 70 & $\mathbf{N}$ & \\
\hline 1 & $12 / 22 / 91$ & 14 & 1 & & 69.9 & $\mathbf{N}$ & \\
\hline 1 & $12 / 29 / 91$ & 1 & 1 & & 74.6 & $\mathrm{Y}$ & J-910126143-29 TC \#10 BAD DATA \\
\hline 1 & $12 / 29 / 91$ & 2 & 9 & & 74.2 & $\mathbf{N}$ & \\
\hline 1 & $12 / 29 / 91$ & 3 & 1 & & 73.8 & $\mathbf{N}$ & \\
\hline 1 & $12 / 29 / 91$ & 4 & 1 & & 73.2 & $\mathbf{N}$ & \\
\hline 1 & $12 / 29 / 91$ & 5 & $\frac{1}{1}$ & & 68.8 & $\mathbf{N}$ & \\
\hline 1 & $12 / 29 / 91$ & 6 & 1 & & 70.9 & $\mathrm{~N}$ & \\
\hline 1 & $12 / 29 / 91$ & 7 & 1 & & 68.9 & $\mathbf{N}$ & \\
\hline 1 & $12 / 29 / 91$ & 8 & 9 & & 65.5 & $\mathbf{N}$ & \\
\hline 1 & $12 / 29 / 91$ & 9 & 1 & & 64.3 & $\mathbf{N}$ & \\
\hline 1 & $12 / 29 / 91$ & 11 & 1 & & 67.4 & $\bar{N}$ & \\
\hline 1 & $12 / 29 / 91$ & 12 & 1 & & 67.5 & $\mathrm{~N}$ & \\
\hline 1 & $12 / 29 / 91$ & 13 & 1 & & 67.7 & $\bar{N}$ & \\
\hline 1 & $12 / 29 / 91$ & 14 & 1 & & 67.8 & $\mathbf{N}$ & \\
\hline$\frac{1}{1}$ & $1 / 4 / 92$ & 1 & 1 & & & $\mathbf{N}$ & TCs not available \\
\hline 1 & $1 / 11 / 92$ & 1 & 1 & & 73.5 & $\mathbf{Y}$ & J-910126143-14 \\
\hline 1 & $1 / 19 / 92$ & 2 & 1 & & 73.1 & $\mathbf{N}$ & \\
\hline 1 & $1 / 11 / 92$ & 3 & 1 & & 72.7 & $\mathrm{~N}$ & \\
\hline 1 & $1 / 19 / 92$ & 4 & 1 & & 72.1 & $\mathbf{N}$ & \\
\hline 1 & $1 / 11 / 92$ & 5 & 1 & & 70.9 & $\mathbf{N}$ & \\
\hline$\frac{1}{1}$ & $1 / 11 / 92$ & 6 & 1 & & 69.3 & $\mathbf{N}$ & \\
\hline 1 & $1 / 11 / 92$ & 7 & 1 & & 67.1 & $\mathbf{N}$ & \\
\hline$\frac{1}{1}$ & $9 / 11 / 92$ & 8 & 1 & & 66 & $\mathbf{N}$ & \\
\hline 1 & $1 / 11 / 92$ & 9 & 1 & & 65.5 & $N$ & \\
\hline 1 & $1 / 11 / 92$ & 10 & 1 & & 65.4 & $N$ & \\
\hline 1 & $1 / 11 / 92$ & 11 & 1 & & 65.3 & $\mathbf{N}$ & \\
\hline 1 & $1 / 11 / 92$ & 12 & 1 & & 65.5 & $N$ & \\
\hline 1 & $1 / 11 / 92$ & 13 & 1 & & 65.6 & $\mathrm{~N}$ & \\
\hline 1 & $1 / 11 / 92$ & 14 & 1 & & 65.7 & $N$ & \\
\hline 1 & $1 / 16 / 92$ & 1 & 1 & & 74.8 & $Y$ & J $910126143-32$ \\
\hline 1 & $1 / 16 / 92$ & 2 & 1 & & 74.3 & $N$ & \\
\hline$\frac{1}{1}$ & $1 / 16 / 92$ & 3 & 1 & & 73.8 & $\mathbf{N}$ & \\
\hline 1 & $1 / 16 / 92$ & 4 & 9 & & 73.1 & $\mathbf{N}$ & \\
\hline 1 & $1 / 16 / 92$ & 5 & 1 & & 72 & $N$ & \\
\hline 1 & $1 / 16 / 92$ & 6 & 1 & & 70.7 & $\mathbf{N}$ & \\
\hline 1 & $1 / 16 / 92$ & 7 & 1 & & 67.7 & $\mathrm{~N}$ & \\
\hline 1 & $1 / 16 / 92$ & 8 & 1 & & 66.3 & $\mathbf{N}$ & \\
\hline 1 & $1 / 16 / 92$ & 9 & 1 & & 66 & $\mathrm{~N}$ & \\
\hline 1 & $1 / 16 / 92$ & 10 & 1 & & 66 & $\mathrm{~N}$ & \\
\hline 1 & $1 / 16 / 92$ & 11 & 1 & & 66 & $N$ & \\
\hline 1 & $1 / 16 / 92$ & 12 & 1 & & 66.2 & $N$ & \\
\hline 1 & $1 / 16 / 92$ & 13 & 1 & & 66.2 & $\mathbf{N}$ & \\
\hline 1 & $1 / 16 / 92$ & 14 & 1 & & 66.4 & $\mathbf{N}$ & \\
\hline 1 & $1 / 25 / 92$ & 1 & 1 & & 73.6 & $\mathrm{Y}$ & J-910126143-14 \\
\hline 1 & $1 / 25 / 92$ & 2 & 1 & & 73.3 & $\mathbf{N}$ & \\
\hline 1 & $1 / 25 / 92$ & 3 & 1 & & 72.9 & $\mathrm{~N}$ & \\
\hline 1 & $1 / 25 / 92$ & 4 & 9 & & 72.2 & $\mathbf{N}$ & \\
\hline
\end{tabular}

Data obtained from WHC Surveillance Analysis Computer System (SACS), November 12, 1993. 


\begin{tabular}{|c|c|c|c|c|c|c|c|}
\hline \multicolumn{8}{|c|}{ TANK 241-BY-101 } \\
\hline & & & & THER & COUPLE & & \\
\hline TREE\# & & 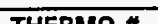 & TREF SET \# & TFMPC & & $Y=M A X$ TFMP & \\
\hline IMELH & DATE & THERMO & IREE SEI \# & TEMPC & TEMPF & $Y=$ MAX TEMP & COMMENTS \\
\hline 1 & $1 / 25 / 92$ & 5 & 1 & & 70.9 & $\mathbf{N}$ & \\
\hline 1 & $1 / 25 / 92$ & 6 & 1 & & 68.8 & $\mathrm{~N}$ & \\
\hline 1 & $1 / 25 / 92$ & 7 & 1 & & 66.2 & $N$ & \\
\hline 1 & $1 / 25 / 92$ & 8 & 1 & & 64.8 & $\mathbf{N}$ & \\
\hline 1 & $1 / 25 / 92$ & 9 & 1 & & 64.6 & $\mathbf{N}$ & \\
\hline 1 & $1 / 25 / 92$ & 10 & 1 & & 64.7 & $\mathrm{~N}$ & \\
\hline 1 & $1 / 25 / 92$ & 11 & 1 & & 64.8 & $N$ & \\
\hline 1 & $1 / 25 / 92$ & 12 & 1 & & 65 & $\mathbf{N}$ & \\
\hline 1 & $1 / 25 / 92$ & 13 & 1 & & 65 & $\mathbf{N}$ & \\
\hline 1 & $1 / 25 / 92$ & 14 & 1 & & 65.2 & $\mathbf{N}$ & \\
\hline 1 & $1 / 28 / 92^{\circ}$ & 1 & 1 & & & N & $\begin{array}{c}\text { ACCESS RESTRICTED } 1 / 28-2 / 22 \text { DUE TO } \\
\text { BATTERY FUME INCIDENT }\end{array}$ \\
\hline 1 & $2 / 24 / 92$ & 1 & 1 & & 74.7 & $\bar{Y}$ & $J-900925741-79$ \\
\hline 1 & $2 / 24 / 92$ & 2 & 1 & & 74.3 & $\mathbf{N}$ & \\
\hline 1 & $2 / 24 / 92$ & 3 & 1 & & 73.7 & $\mathbf{N}$ & \\
\hline 1 & $2 / 24 / 92$ & 4 & 1 & & 72.8 & $\mathbf{N}$ & \\
\hline 1 & $2 / 24 / 92$ & 5 & 1 & & 71.2 & $\mathbf{N}$ & \\
\hline 1 & $2 / 24 / 92$ & 6 & 1 & & 68.8 & $\mathbf{N}$ & \\
\hline 1 & $2 / 24 / 92$ & 7 & 1 & & 65.9 & $\mathbf{N}$ & \\
\hline 1 & $2 / 24 / 92$ & 8 & 1 & & 64.4 & $\mathbf{N}$ & \\
\hline 1 & $2 / 24 / 92$ & 9 & 1 & & 64.1 & $\mathbf{N}$ & \\
\hline 1 & $2 / 24 / 92$ & 10 & 1 & & 64.1 & $\mathbf{N}$ & \\
\hline 1 & $2 / 24 / 92$ & 11 & 1 & & 64.2 & $\mathbf{N}$ & \\
\hline 1 & $2 / 24 / 92$ & 12 & 1 & & 64.3 & $\mathbf{N}$ & \\
\hline 1 & $2 / 24 / 92$ & 13 & 1 & & 64.4 & $\mathbf{N}$ & \\
\hline 1 & $2 / 24 / 92$ & 14 & 1 & & 64.5 & $\mathbf{N}$ & \\
\hline 1 & $2 / 29 / 92$ & 1 & 9 & & 74.5 & $\bar{Y}$ & J-91012614332 \\
\hline 1 & $2 / 29 / 92$ & 2 & 1 & & 74 & $\mathbf{N}$ & \\
\hline 9 & $2 / 29 / 92$ & 3 & 1 & & 73.3 & $\mathbf{N}$ & \\
\hline 1 & $2 / 29 / 92$ & 4 & 1 & & 72.4 & $\mathbf{N}$ & \\
\hline 1 & $2 / 29 / 92$ & 5 & 1 & & 70.6 & $N$ & \\
\hline 9 & $2 / 29 / 92$ & 6 & 1 & & 68.2 & $\mathbf{N}$ & \\
\hline 1 & $2 / 29 / 92$ & 7 & 1 & & 65.3 & $\mathbf{N}$ & \\
\hline 1 & $2 / 29 / 92$ & 8 & 1 & & 63.8 & $\mathbf{N}$ & \\
\hline 1 & $2 / 29 / 92$ & 9 & 1 & & 63.5 & $\mathbf{N}$ & \\
\hline 1 & $2 / 29 / 92$ & 10 & 1 & & 63.5 & $\mathbf{N}$ & \\
\hline 1 & $2 / 29 / 92$ & 11 & 1 & & 63.5 & $\mathbf{N}$ & \\
\hline 1 & $2 / 29 / 92$ & 12 & 1 & & 63.6 & $\mathbf{N}$ & \\
\hline 1 & $2 / 29 / 92$ & 13 & 1 & & 63.8 & $\mathbf{N}$ & \\
\hline 1 & $2 / 29 / 92$ & 14 & 1 & & 64 & $\mathbf{N}$ & \\
\hline 1 & $3 / 8 / 92$ & 1 & 1 & & 73.2 & $\mathbf{Y}$ & J910126143-48 \\
\hline 1 & $3 / 8 / 92$ & 2 & 1 & & 72.8 & $\mathbf{N}$ & \\
\hline 1 & $3 / 8 / 92$ & 3 & 1 & & 72 & $\mathbf{N}$ & \\
\hline 1 & $3 / 8 / 92$ & 4 & 1 & & 72 & $\mathbf{N}$ & \\
\hline 1 & $3 / 8 / 92$ & 5 & 1 & & 70.4 & $\mathbf{N}$ & \\
\hline 1 & $3 / 8 / 92$ & 6 & 1 & & 67.9 & $\mathbf{N}$ & \\
\hline 1 & $3 / 8 / 92$ & 7 & 1 & & 65 & $\mathbf{N}$ & \\
\hline 1 & $3 / 8 / 92$ & 8 & 1 & & 63.5 & $\mathbf{N}$ & \\
\hline 1 & $3 / 8 / 92$ & 9 & 1 & & 63.4 & $\mathbf{N}$ & \\
\hline 1 & $3 / 8 / 92$ & 10 & 1 & & 63.4 & $\mathbf{N}$ & \\
\hline 9 & $3 / 8 / 92$ & 91 & 1 & & 63.3 & $\mathbf{N}$ & \\
\hline 1 & $3 / 8 / 92$ & 12 & 1 & & 63.4 & $\mathbf{N}$ & \\
\hline 1 & $3 / 8 / 92$ & 13 & 1 & & 63.4 & $N$ & \\
\hline 1 & $3 / 8 / 92$ & 14 & 1 & & 63.4 & $\mathrm{~N}$ & \\
\hline 1 & $3 / 15 / 92$ & 1 & 1 & & 73.9 & $\bar{Y}$ & J-910126143-32 \\
\hline 1 & $3 / 15 / 92$ & 2 & 1 & & 73.5 & $N$ & \\
\hline 1 & $3 / 15 / 92$ & 3 & 1 & & 72.9 & $\mathbf{N}$ & \\
\hline 1 & $3 / 15 / 92$ & 4 & 1 & & 72.1 & $\mathbf{N}$ & \\
\hline 1 & $3 / 15 / 92$ & 5 & 1 & & 70.3 & $\mathbf{N}$ & \\
\hline 1 & $3 / 15 / 92$ & 6 & 1 & & 67.8 & $\mathbf{N}$ & \\
\hline 1 & $3 / 15 / 92$ & 7 & 1 & & 64.8 & $N$ & \\
\hline 1 & $3 / 15 / 92$ & 8 & 1 & & 63.1 & $\mathbf{N}$ & \\
\hline 1 & $3 / 15 / 92$ & 9 & 1 & & 62.9 & $\mathbf{N}$ & \\
\hline 9 & $3 / 15 / 92$ & 10 & 1 & & 62.9 & $\mathrm{~N}$ & \\
\hline 1 & $3 / 15 / 92$ & 11 & 1 & & 62.9 & $\mathbf{N}$ & \\
\hline 1 & $3 / 15 / 92$ & 12 & 1 & & 63 & $\mathbf{N}$ & \\
\hline
\end{tabular}

Data obtained from WHC Surveillance Analysis Computer System (SACS), November 12, 1993. 
WHC-SD-WM-ER-312, Rev. 0

\begin{tabular}{|c|c|c|c|c|c|c|c|}
\hline \multicolumn{8}{|c|}{ TANK 241-BY-101 } \\
\hline \multicolumn{8}{|c|}{ THERMOCOUPLE DATA } \\
\hline TREE\# & DATE & THERMO \# & TREE SET \# & TEMP C & TEMP F & $Y=$ MAX TEMP & COMMENTS \\
\hline 9 & $3 / 15 / 92$ & 13 & 1 & & 63 & $\mathrm{~N}$ & \\
\hline 1 & $3 / 15 / 92$ & 14 & 1 & & 63.1 & $\ddot{N}$ & \\
\hline 1 & $3 / 20 / 92$ & 1 & 1 & & 74 & $Y$ & $\mathrm{~J} 91012614332$ \\
\hline 1 & $3 / 20 / 92$ & $\overline{2}$ & 1 & & 73.6 & $\mathbf{N}$ & \\
\hline 1 & $3 / 20 / 92$ & 3 & 1 & & 73 & $\mathbf{N}$ & \\
\hline 1 & $3 / 20 / 92$ & 4 & $\overline{1}$ & & 72.1 & $N$ & \\
\hline 1 & $3 / 20 / 92$ & 5 & 1 & & 70.3 & $\mathbf{N}$ & \\
\hline 9 & $3 / 20 / 92$ & 6 & 1 & & 67.9 & $\mathbf{N}$ & \\
\hline 1 & $3 / 20 / 92$ & 7 & 1 & & 64.9 & $N$ & \\
\hline 1 & $3 / 20 / 92$ & 8 & 1 & & 63.4 & $\bar{N}$ & \\
\hline 1 & $3 / 20 / 92$ & 9 & 1 & & 63.2 & $\bar{N}$ & \\
\hline 1 & $3 / 20 / 92$ & 10 & 1 & & 63.2 & $\mathbf{N}$ & \\
\hline 1 & $3 / 20 / 92$ & 11 & 1 & & 63.1 & $\mathbf{N}$ & \\
\hline 1 & $3 / 20 / 92$ & 12 & 1 & & 63.2 & $\mathbf{N}$ & \\
\hline 1 & $3 / 20 / 92$ & 14 & $\frac{1}{1}$ & & 63.2 & $\bar{N}$ & \\
\hline 1 & $3 / 29 / 92$ & 1 & 1 & & 74.5 & $\bar{Y}$ & $J 910126143-66$ \\
\hline 1 & $3 / 29 / 92$ & 2 & 1 & & 73.9 & $\mathbf{N}$ & \\
\hline 1 & $3 / 29 / 92$ & 3 & 1 & & 73.2 & $\bar{N}$ & \\
\hline 1 & $3 / 29 / 92$ & 4 & 1 & & 72.3 & $\mathbf{N}$ & \\
\hline 1 & $3 / 29 / 92$ & 5 & $\overline{1}$ & & 70.5 & $N$ & \\
\hline 1 & $3 / 29 / 92$ & 6 & 1 & & 68 & $\mathbf{N}$ & \\
\hline$\frac{1}{1}$ & $3 / 29 / 92$ & 7 & 1 & & 65.1 & $\mathrm{~N}$ & \\
\hline 1 & $3 / 29 / 92$ & 8 & 1 & & 63.6 & $N$ & \\
\hline 1 & $3 / 29 / 92$ & 9 & 1 & & 63.3 & $\mathbf{N}$ & \\
\hline 1 & $3 / 29 / 92$ & 10 & 1 & & 63.3 & $\mathbf{N}$ & \\
\hline 1 & $3 / 29 / 92$ & 11 & 1 & & 63.3 & $\mathbf{N}$ & \\
\hline 1 & $3 / 29 / 92$ & 12 & 9 & & 63.4 & $\mathbf{N}$ & \\
\hline 1 & $3 / 29 / 92$ & 13 & $\overline{1}$ & & 63.4 & $\mathbf{N}$ & \\
\hline 1 & $3 / 29 / 92$ & 14 & 1 & & 63.6 & $\mathbf{N}$ & \\
\hline 1 & $4 / 3 / 92$ & 1 & 1 & & 73.1 & $\bar{Y}$ & J 910126143-14 \\
\hline 1 & $4 / 3 / 92$ & 2 & 1 & & 72.8 & $N$ & \\
\hline 1 & $4 / 3 / 92$ & 3 & $\overline{1}$ & & 72.1 & $\mathrm{~N}$ & \\
\hline 1 & $4 / 3 / 92$ & 4 & 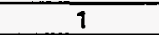 & & 71.3 & $\mathbf{N}$ & \\
\hline 1 & $4 / 3 / 92$ & 5 & 1 & & 69.4 & $\mathbf{N}$ & \\
\hline 1 & $4 / 3 / 92$ & 6 & 1 & & 67 & $\mathbf{N}$ & \\
\hline 9 & $4 / 3 / 92$ & 7 & 1 & & 64.3 & $\mathrm{~N}$ & \\
\hline 1 & $4 / 3 / 92$ & 8 & 1 & & 62.8 & $\mathbf{N}$ & \\
\hline 1 & $4 / 3 / 92$ & 9 & 7 & & 62.8 & $\mathbf{N}$ & \\
\hline 1 & $4 / 3 / 92$ & 10 & $\frac{1}{1}$ & & 62.8 & $\bar{N}$ & \\
\hline 1 & $4 / 3 / 92$ & 11 & 1 & & 62.9 & $\mathbf{N}$ & \\
\hline 1 & $4 / 3 / 92$ & 12 & 1 & & 62.8 & $\mathbf{N}$ & \\
\hline 1 & $4 / 3 / 92$ & 13 & $T$ & & 62.9 & $N$ & \\
\hline 1 & $4 / 3 / 92$ & 94 & 1 & & 63 & $\mathbf{N}$ & \\
\hline 1 & $4 / 11 / 92$ & 1 & 1 & & 74.4 & $Y$ & J $910126143-64$ \\
\hline 1 & $4 / 11 / 92$ & 2 & 9 & & 73.9 & $\mathbf{N}$ & \\
\hline 1 & $4 / 11 / 92$ & 3 & 1 & & 73.2 & $N$ & \\
\hline 1 & $4 / 11 / 92$ & 4 & 1 & & 72.1 & $\bar{N}$ & \\
\hline 1 & $4 / 11 / 92$ & 5 & 1 & & 70.2 & $\mathbf{N}$ & \\
\hline 1 & $4 / 11 / 92$ & 6 & 1 & & 67.7 & $N$ & \\
\hline 1 & $4 / 11 / 92$ & 7 & 1 & & 65 & $N$ & \\
\hline 1 & $4 / 11 / 92$ & 8 & 1 & & 63.6 & $\mathbf{N}$ & \\
\hline 1 & $4 / 11 / 92$ & 9 & 9 & & 63.4 & $\mathbf{N}$ & \\
\hline 9 & $4 / 11 / 92$ & 10 & 1 & & 63.5 & $\bar{N}$ & \\
\hline 1 & $4 / 11 / 92$ & 11 & 1 & & 63.6 & $\mathbf{N}$ & \\
\hline 1 & $4 / 11 / 92$ & 12 & $\frac{1}{1}$ & & 63.8 & $\mathbf{N}$ & \\
\hline$\frac{1}{1}$ & $4 / 11 / 92$ & 13 & 1 & & 64 & $\mathbf{N}$ & \\
\hline 1 & $4 / 11 / 92$ & 14 & $\frac{1}{1}$ & & 64.3 & $\mathbf{N}$ & \\
\hline 1 & $4 / 17 / 92$ & 1 & 1 & & 75.6 & $\bar{Y}$ & J 89052351220 \\
\hline 1 & $4 / 17 / 92$ & 2 & 1 & & 74.9 & $\bar{N}$ & \\
\hline 1 & $4 / 17 / 92$ & 3 & 1 & & 74.1 & $\mathrm{~N}$ & \\
\hline 1 & $4 / 17 / 92$ & 4 & 1 & & 73 & $\mathbf{N}$ & \\
\hline 9 & $4 / 17 / 92$ & 5 & 1 & & 71.2 & $\mathbf{N}$ & \\
\hline 1 & $4 / 17 / 92$ & 6 & 1 & & 68.7 & $\mathbf{N}$ & \\
\hline 1 & $4 / 17 / 92$ & 7 & 1 & & 66 & $N$ & \\
\hline 1 & $4 / 17 / 92$ & 8 & $\frac{1}{1}$ & & 64.8 & $\mathbf{N}$ & \\
\hline$\overline{1}$ & $4 / 17 / 92$ & 9 & 1 & & 64.5 & $\bar{N}$ & \\
\hline
\end{tabular}

Data obtained from WHC Surveillance Analysis Computer System (SACS), November 12, 1993. 
WHC-SD-WM-ER-312, Rev. 0

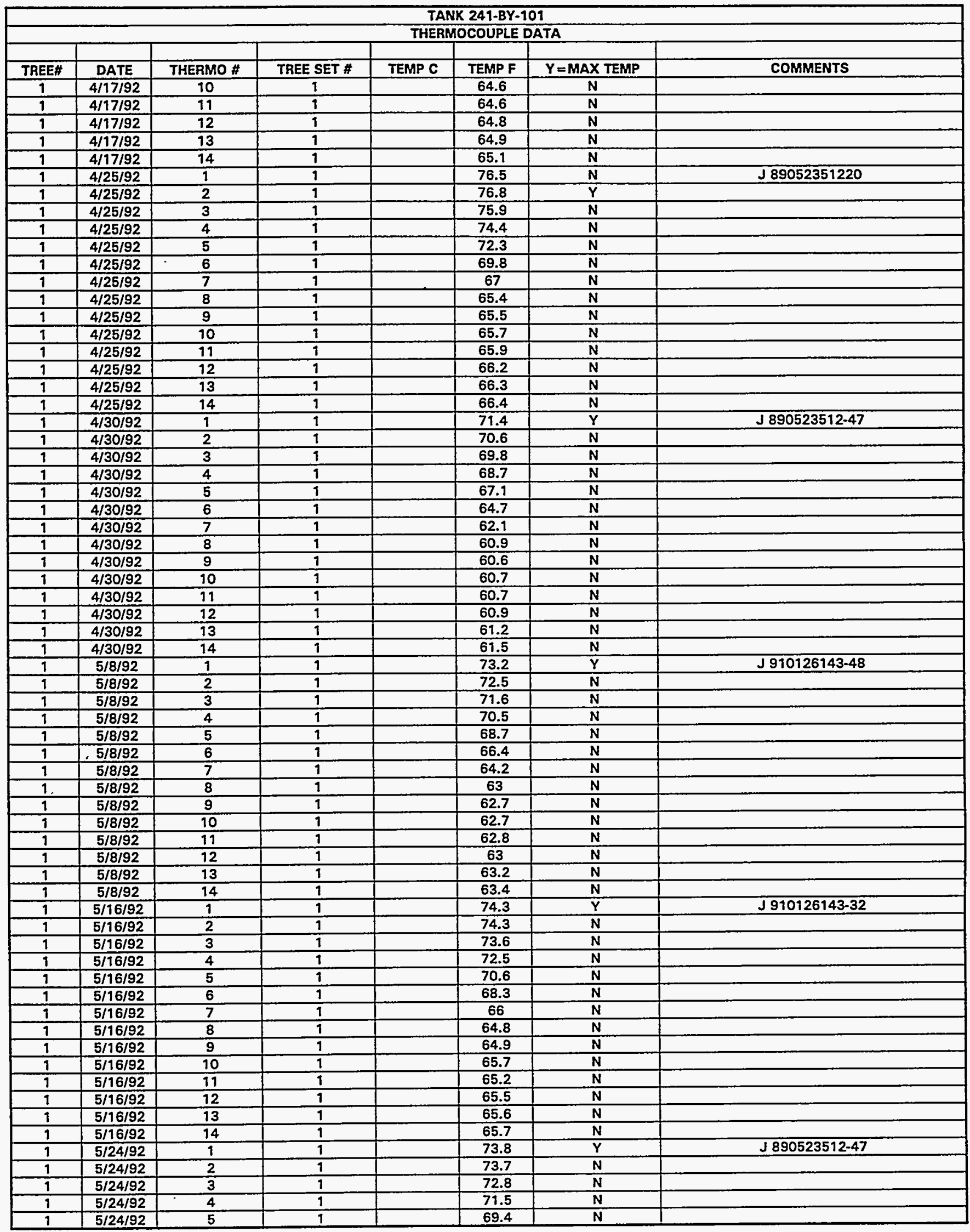

Data obtained from WHC Surveillance Analysis Computer System (SACS), November 12, 1993. 


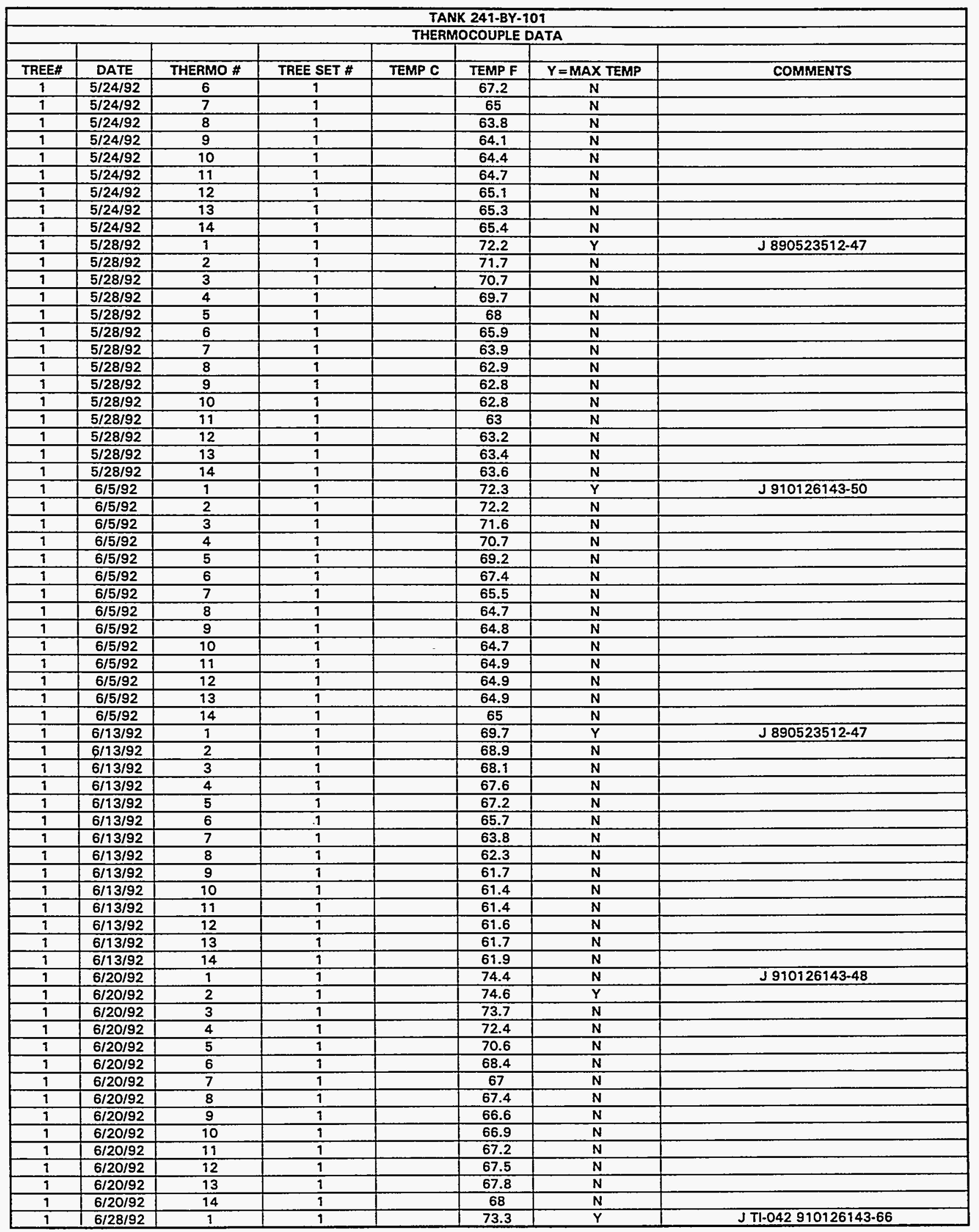

Data obtained from WHC Surveillance Analysis Computer System (SACS), November 12, 1993. 


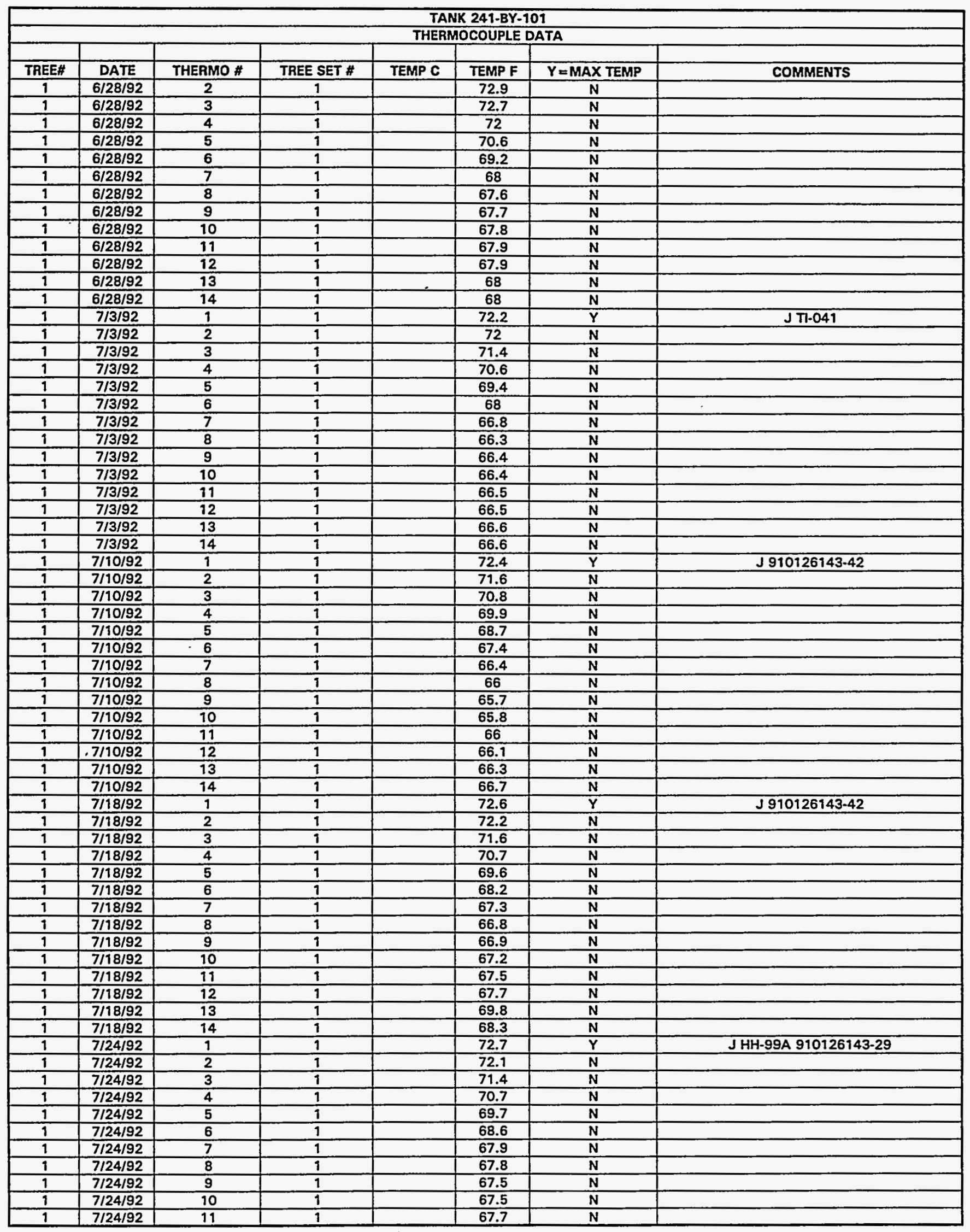

Data obtained from WHC Surveillance Analysis Computer System (SACS), November 12, 1993. 


\begin{tabular}{|c|c|c|c|c|c|c|c|}
\hline \multicolumn{8}{|c|}{ TANK 241-BY-101 } \\
\hline & & & & THERI & DCOUPLE & & \\
\hline TREE\# & DATE & THERMO $\#$ & TREE SET \# & TEMP C & TEMP F & $Y=M A X$ TEMP & COMMENTS \\
\hline 1 & $7 / 24 / 92$ & 12 & 1 & & 67.8 & $\mathrm{~N}$ & \\
\hline 1 & $7 / 24 / 92$ & 13 & 1 & & 67.9 & $\bar{N}$ & \\
\hline 1 & $7 / 24 / 92$ & 14 & 1 & & 68.1 & $\mathrm{~N}$ & \\
\hline 1 & $8 / 1 / 92$ & 1 & 1 & & 80.1 & $\mathbf{N}$ & $\mathrm{J} T 1-041$ \\
\hline 1 & $8 / 1 / 92$ & 2 & 1 & & 80.5 & $Y$ & \\
\hline 1 & $8 / 9 / 92$ & 3 & 1 & & 79.9 & $\mathbf{N}$ & \\
\hline 1 & $8 / 1 / 92$ & 4 & 1 & & 78.5 & $\mathbf{N}$ & \\
\hline 1 & $8 / 1 / 92$ & 5 & 1 & & 76.8 & $\mathbf{N}$ & \\
\hline 1 & $8 / 1 / 92$ & 6 & 1 & & 75 & $\mathbf{N}$ & \\
\hline 1 & $8 / 1 / 92$ & 7 & 1 & & 73.1 & $\mathbf{N}$ & \\
\hline 1 & $8 / 1 / 92$ & 8 & 1 & & 72 & $\mathbf{N}$ & \\
\hline 1 & $8 / 1 / 92$ & 9 & 1 & & 72.1 & $\bar{N}$ & \\
\hline 1 & $8 / 1 / 92$ & 10 & 1 & & 72.4 & $\mathbf{N}$ & \\
\hline 1 & $8 / 1 / 92$ & 11 & 1 & & 72.5 & $N$ & \\
\hline 1 & $8 / 1 / 92$ & 12 & 1 & & 73 & $\mathbf{N}$ & \\
\hline 1 & $8 / 1 / 92$ & 13 & 1 & & 73.4 & $N$ & \\
\hline 1 & $8 / 1 / 92$ & 14 & 1 & & 73.8 & $\mathbf{N}$ & \\
\hline 1 & $8 / 7 / 92$ & 1 & 1 & & 71.6 & $Y$ & $\mathrm{~J} 890423320-42$ \\
\hline 1 & $8 / 7 / 92$ & 2 & 1 & & 71.4 & $N$ & \\
\hline 1 & $8 / 7 / 92$ & 3 & 1 & & 70.9 & $\mathrm{~N}$ & \\
\hline 1 & $8 / 7 / 92$ & 4 & 1 & & 70.3 & $\mathbf{N}$ & \\
\hline 1 & $8 / 7 / 92$ & 5 & 1 & & 69.6 & $\mathbf{N}$ & \\
\hline 1 & $8 / 7 / 92$ & 6 & 1 & & 68.8 & $\mathrm{~N}$ & \\
\hline 1 & $8 / 7 / 92$ & 7 & 1 & & 68.4 & $\mathrm{~N}$ & \\
\hline 1 & $8 / 7 / 92$ & 8 & 1 & & 68.3 & $\mathbf{N}$ & \\
\hline 1 & $8 / 7 / 92$ & 9 & 1 & & 68.4 & $N$ & \\
\hline 1 & $8 / 7 / 92$ & 10 & 1 & & 68.4 & $\bar{N}$ & \\
\hline 1 & $8 / 7 / 92$ & 11 & 1 & & 68.6 & $\mathrm{~N}$ & \\
\hline 1 & $8 / 7 / 92$ & 12 & 1 & & 68.5 & $\mathbf{N}$ & \\
\hline 1 & $8 / 7 / 92$ & 13 & 1 & & 68.6 & $\mathrm{~N}$ & \\
\hline 1 & $8 / 7 / 92$ & 14 & 1 & & 68.7 & $N$ & \\
\hline 1 & $8 / 15 / 92$ & 1 & 1 & & 72.9 & $\mathrm{~N}$ & $\mathrm{~J} 890423320$ \\
\hline 1 & $8 / 15 / 92$ & 2 & 1 & & 73.1 & $\bar{Y}$ & \\
\hline 1 & $8 / 15 / 92$ & 3 & 1 & & 72.9 & $\mathbf{N}$ & \\
\hline 1 & $8 / 15 / 92$ & 4 & 1 & & 72.3 & $\mathrm{~N}$ & \\
\hline 1 & $8 / 15 / 92$ & 5 & 1 & & 71.5 & $\mathbf{N}$ & \\
\hline 1 & $8 / 15 / 92$ & 6 & 1 & & 70.9 & $\mathbf{N}$ & \\
\hline 1 & $8 / 15 / 92$ & 7 & 1 & & 70.6 & $\mathbf{N}$ & \\
\hline 1 & $8 / 15 / 92$ & 8 & 9 & & 70.5 & $\mathbf{N}$ & \\
\hline 1 & $8 / 15 / 92$ & 9 & 1 & & 70.9 & $\mathbf{N}$ & \\
\hline 1 & $8 / 15 / 92$ & 10 & 1 & & 71.1 & $\mathbf{N}$ & \\
\hline 1 & $8 / 15 / 92$ & 11 & 9 & & 71.3 & $\mathbf{N}$ & \\
\hline 1 & $8 / 15 / 92$ & 12 & 1 & & 71.3 & $\mathbf{N}$ & \\
\hline 1 & $8 / 15 / 92$ & 13 & 1 & & 71.5 & $\mathbf{N}$ & \\
\hline 1 & $8 / 15 / 92$ & 14 & 1 & & 71.5 & $\mathbf{N}$ & \\
\hline 1 & $8 / 21 / 92$ & 1 & 1 & & 72.2 & $Y$ & J $890423320-42$ \\
\hline 9 & $8 / 21 / 92$ & 2 & 1 & & 72.1 & $\mathbf{N}$ & \\
\hline 1 & $8 / 21 / 92$ & 3 & 1 & & 71.8 & $\mathrm{~N}$ & \\
\hline 1 & $8 / 21 / 92$ & 4 & 1 & & 71.4 & $\mathbf{N}$ & \\
\hline 1 & $8 / 21 / 92$ & 5 & 1 & & 70.7 & $\bar{N}$ & \\
\hline 1 & $8 / 21 / 92$ & 6 & 1 & & 70.1 & $N$ & \\
\hline 1 & $8 / 21 / 92$ & 7 & 1 & & 69.9 & $\mathrm{~N}$ & \\
\hline 1 & $8 / 21 / 92$ & 8 & 1 & & 69.9 & $\mathrm{~N}$ & \\
\hline 1 & $8 / 21 / 92$ & 9 & 1 & & 70 & $\mathbf{N}$ & \\
\hline 1 & $8 / 21 / 92$ & 10 & 1 & & 70.2 & $\mathrm{~N}$ & \\
\hline 1 & $8 / 21 / 92$ & 11 & 1 & & 70.3 & $\mathbf{N}$ & \\
\hline 1 & $8 / 21 / 92$ & 12 & 1 & & 70.3 & $\mathbf{N}$ & \\
\hline 1 & $8 / 21 / 92$ & 13 & 1 & & 70.4 & $\mathbf{N}$ & \\
\hline 1 & $8 / 21 / 92$ & 14 & 1 & & 70.5 & $N$ & \\
\hline 9 & $8 / 30 / 92$ & 1 & 1 & & 73.2 & $\mathbf{N}$ & J $890423320-42$ \\
\hline 9 & $8 / 30 / 92$ & 2 & 1 & & 73.4 & $\bar{Y}$ & \\
\hline 1 & $8 / 30 / 92$ & 3 & 1 & & 73.2 & $\mathbf{N}$ & \\
\hline 1 & $8 / 30 / 92$ & 4 & 1 & & 72.7 & $\mathbf{N}$ & \\
\hline 1 & $8 / 30 / 92$ & 5 & 1 & & 72 & $N$ & \\
\hline 1 & $8 / 30 / 92$ & 6 & 1 & & 71.6 & $\mathbf{N}$ & \\
\hline 1 & $8 / 30 / 92$ & 7 & 1 & & 71.5 & $\mathrm{~N}$ & \\
\hline
\end{tabular}

Data obtained from WHC Surveillance Analysis Computer System (SACS), November 12, 1993. 


\begin{tabular}{|c|c|c|c|c|c|c|c|}
\hline \multicolumn{8}{|c|}{ TANK 241-BY-101 } \\
\hline \multicolumn{8}{|c|}{ THERMOCOUPLE DATA } \\
\hline TAEEH & DATE & THERMO \# & TREE SET \# & TEMP C & TEMP F & $Y=M A X$ TEMP & COMMENTS \\
\hline 1 & $8 / 30 / 92$ & 8 & 1 & & 71.5 & $\mathbf{N}$ & \\
\hline$T$ & $8 / 30 / 92$ & 9 & 1 & & 72 & $\mathbf{N}$ & \\
\hline 1 & $8 / 30 / 92$ & 10 & 1 & & 72.3 & $\mathbf{N}$ & \\
\hline$\frac{1}{1}$ & $8 / 30 / 92$ & 11 & 1 & & 72.5 & $\mathbf{N}$ & \\
\hline 1 & $8 / 30 / 92$ & 12 & 1 & & 72.7 & $\mathbf{N}$ & \\
\hline 9 & $8 / 30 / 92$ & 13 & 1 & & 72.8 & $\mathbf{N}$ & \\
\hline 1 & $8 / 30 / 92$ & 14 & 1 & & 72.9 & $\mathbf{N}$ & \\
\hline 1 & $9 / 6 / 92$ & 1 & 1 & & 72.1 & $\bar{Y}$ & J 890423320-42 \\
\hline 1 & $9 / 6 / 92$ & 2 & 1 & & 72 & $\bar{N}$ & \\
\hline 1 & $9 / 6 / 92$ & 3 & 1 & & 71.7 & $\mathbf{N}$ & \\
\hline 1 & $9 / 6 / 92$ & 4 & 1 & & 71.4 & $\mathrm{~N}$ & \\
\hline$T$ & $9 / 6 / 92$ & 5 & $T$ & & 70.5 & $\mathbf{N}$ & \\
\hline 1 & $9 / 6 / 92$ & 6 & 1 & & 70.5 & $\mathbf{N}$ & \\
\hline 1 & $9 / 6 / 92$ & 7 & $\overline{1}$ & & 70.8 & $\mathbf{N}$ & \\
\hline 1 & $9 / 6 / 92$ & 8 & 1 & & 70.8 & $\mathbf{N}$ & \\
\hline 1 & $9 / 6 / 92$ & 9 & 1 & & 70.5 & $\mathbf{N}$ & \\
\hline$\frac{1}{1}$ & $9 / 6 / 92$ & 10 & 1 & & 75 & $\mathbf{N}$ & \\
\hline 1 & $9 / 6 / 92$ & 11 & 7 & & 71.1 & $\mathbf{N}$ & \\
\hline 1 & $9 / 6 / 92$ & 12 & 1 & & 71 & $N$ & \\
\hline 1 & $9 / 6 / 92$ & 13 & 1 & & 71.1 & $\mathbf{N}$ & \\
\hline$T$ & $9 / 6 / 92$ & 14 & 1 & & 71.2 & $\mathbf{N}$ & \\
\hline 1 & $9 / 12 / 92$ & 1 & 1 & & 73.5 & $\bar{Y}$ & J $890423320-42$ \\
\hline 1 & $9 / 12 / 92$ & 2 & 7 & & 73.3 & $\mathbf{N}$ & \\
\hline 1 & $9 / 12 / 92$ & 3 & 9 & & 73.1 & $\mathbf{N}$ & \\
\hline$\frac{1}{1}$ & $9 / 12 / 92$ & 4 & 1 & & 72.8 & $\mathbf{N}$ & \\
\hline$T$ & $9 / 12 / 92$ & 5 & 1 & & 72.5 & $\overline{\mathbf{N}}$ & \\
\hline 1 & $9 / 12 / 92$ & 6 & 1 & & 72.3 & $\mathbf{N}$ & \\
\hline 1 & $9 / 12 / 92$ & 7 & 1 & & 72.4 & $\mathbf{N}$ & \\
\hline 1 & $9 / 12 / 92$ & 8 & 1 & & 72.5 & $\mathbf{N}$ & \\
\hline 1 & $9 / 12 / 92$ & 9 & 1 & & 72.6 & $\mathbf{N}$ & \\
\hline 1 & $9 / 12 / 92$ & 10 & 1 & & 72.6 & $\mathbf{N}$ & \\
\hline 1 & $9 / 12 / 92$ & 11 & 1 & & 72.6 & $\bar{N}$ & \\
\hline 1 & $9 / 12 / 92$ & 12 & $\overline{1}$ & & 72.6 & $N$ & \\
\hline 1 & $9 / 12 / 92$ & 13 & 1 & & 72.6 & $\mathbf{N}$ & \\
\hline 1 & $9 / 12 / 92$ & 14 & 1 & & 72.7 & $\mathbf{N}$ & \\
\hline 1 & $9 / 20 / 92$ & 1 & 1 & & 73.3 & $\bar{Y}$ & J910126143-64 \\
\hline 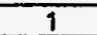 & $9 / 20 / 92$ & 2 & 1 & & 73.1 & $\overline{\mathbf{N}}$ & \\
\hline 1 & $9 / 20 / 92$ & 3 & 1 & & 72.9 & $\bar{N}$ & \\
\hline 1 & $.9 / 20 / 92$ & 4 & 1 & & 72.6 & $\mathbf{N}$ & \\
\hline 1 & $9 / 20 / 92$ & 5 & 1 & & 72.3 & $\mathbf{N}$ & \\
\hline 1 & $9 / 20 / 92$ & 6 & $T$ & & 72.2 & $\mathbf{N}$ & \\
\hline 1 & $9 / 20 / 92$ & 7 & 1 & & 72.3 & $\mathbf{N}$ & \\
\hline 9 & $9 / 20 / 92$ & 8 & 1 & & 72.4 & $\bar{N}$ & \\
\hline 1 & $9 / 20 / 92$ & 9 & 7 & & 72.4 & $\mathrm{~N}$ & \\
\hline 1 & $9 / 20 / 92$ & 10 & 1 & & 72.5 & $\mathbf{N}$ & \\
\hline 1 & $9 / 20 / 92$ & 91 & 1 & & 72.5 & $\bar{N}$ & \\
\hline 1 & $9 / 20 / 92$ & 12 & 1 & & 72.5 & $\mathbf{Y}$ & \\
\hline$\frac{1}{1}$ & $9 / 20 / 92$ & 13 & 1 & & 72.5 & $\bar{N}$ & \\
\hline 1 & $9 / 20 / 92$ & 14 & $\frac{1}{1}$ & & 72.5 & $\mathbf{N}$ & \\
\hline 1 & $9 / 26 / 92$ & 1 & 1 & & 71.5 & $\bar{Y}$ & J 910126143-64 \\
\hline 1 & $9 / 26 / 92$ & 2 & 1 & & 71.1 & $N$ & \\
\hline 1 & $9 / 26 / 92$ & 3 & 1 & & 70.7 & $\mathbf{N}$ & \\
\hline 1 & $9 / 26 / 92$ & 4 & 1 & & 70.2 & $\mathbf{N}$ & \\
\hline$T$ & $9 / 26 / 92$ & 5 & 1 & & 69.9 & $\mathbf{N}$ & \\
\hline 1 & $9 / 26 / 92$ & 6 & 1 & & 69.7 & $\mathbf{N}$ & \\
\hline 1 & $9 / 26 / 92$ & 7 & 9 & & 69.7 & $\mathbf{N}$ & \\
\hline$\frac{1}{1}$ & $9 / 26 / 92$ & 8 & 1 & & 69.8 & $\bar{N}$ & \\
\hline 1 & $9 / 26 / 92$ & 9 & $T$ & & 69.6 & $\mathbf{N}$ & \\
\hline 1 & $9 / 26 / 92$ & 10 & 1 & & 69.7 & $\mathbf{N}$ & \\
\hline 1 & $9 / 26 / 92$ & 11 & 1 & & 69.8 & $\mathbf{N}$ & \\
\hline 1 & $9 / 26 / 92$ & 12 & $T$ & & 69.9 & $\mathbf{N}$ & \\
\hline 1 & $9 / 26 / 92$ & 13 & 1 & & 70 & $\mathbf{N}$ & \\
\hline 1 & $9 / 26 / 92$ & 14 & 1 & & 70.3 & $\mathbf{N}$ & \\
\hline$\frac{1}{1}$ & $10 / 1 / 92$ & 1 & 9 & & 72 & $\bar{Y}$ & J $890423320-42$ \\
\hline 9 & $10 / 1 / 92$ & 2 & 1 & & 72 & $\mathbf{N}$ & \\
\hline 1 & $10 / 1 / 92$ & 3 & 1 & & 71.6 & $\mathbf{N}$ & \\
\hline
\end{tabular}

Data obtained from WHC Surveillance Analysis Computer System (SACS), November 12, 1993. 


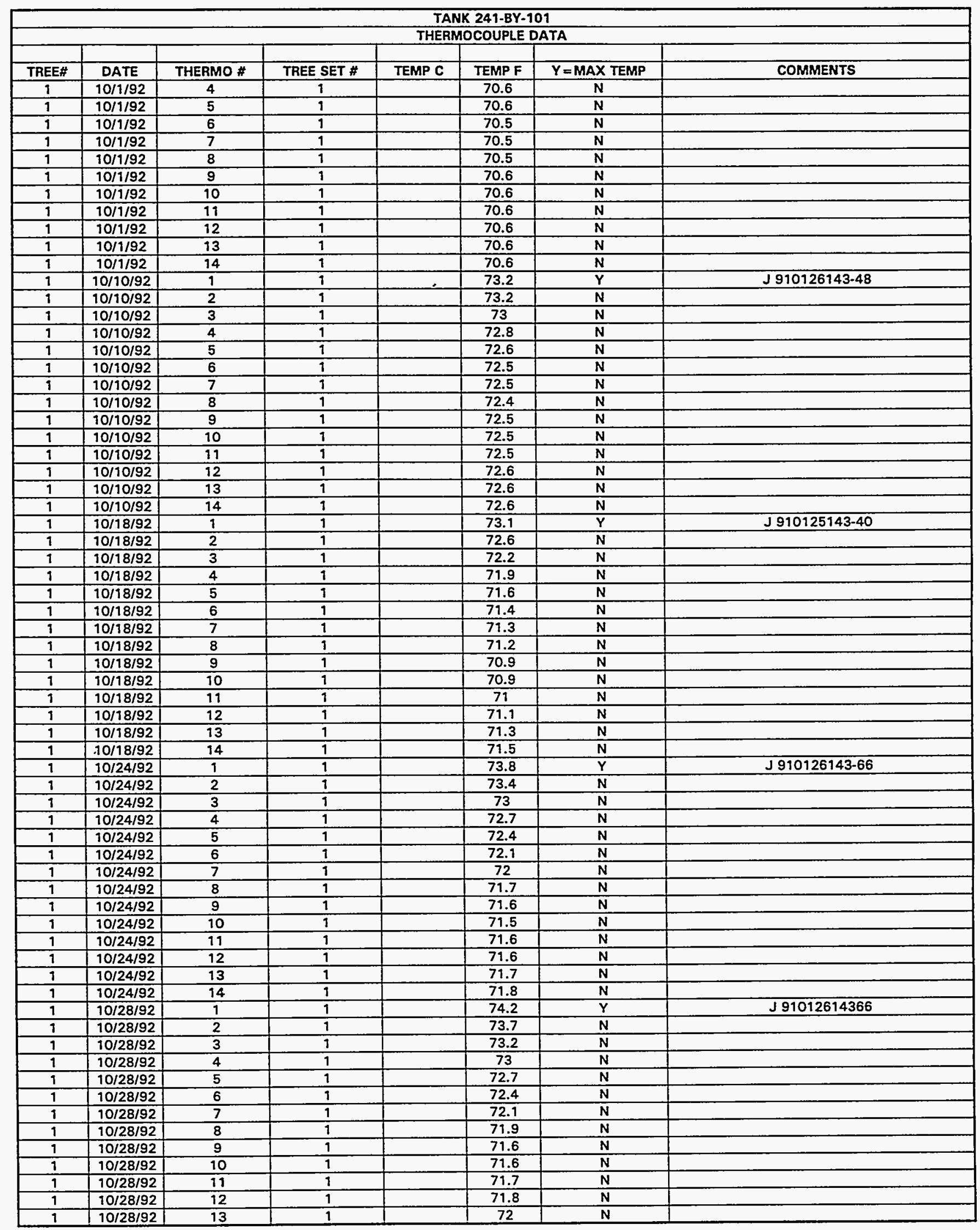

Data obtained from WHC Surveillance Analysis Computer System (SACS), November 12, 1993. 


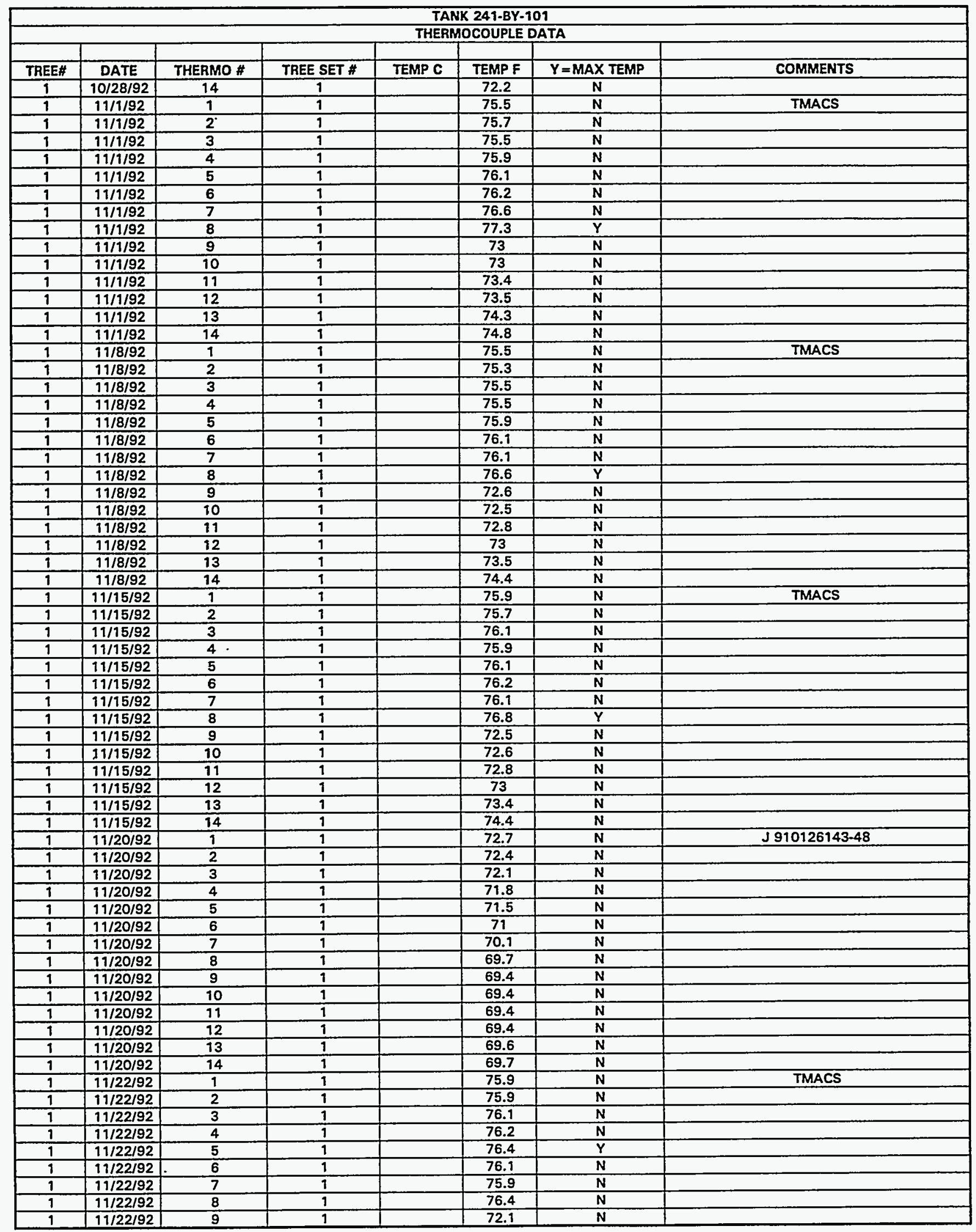

Data obtained from WHC Surveillance Analysis Computer System (SACS), November 12, 1993. 
WHC-SD-WM-ER-312, Rev. 0

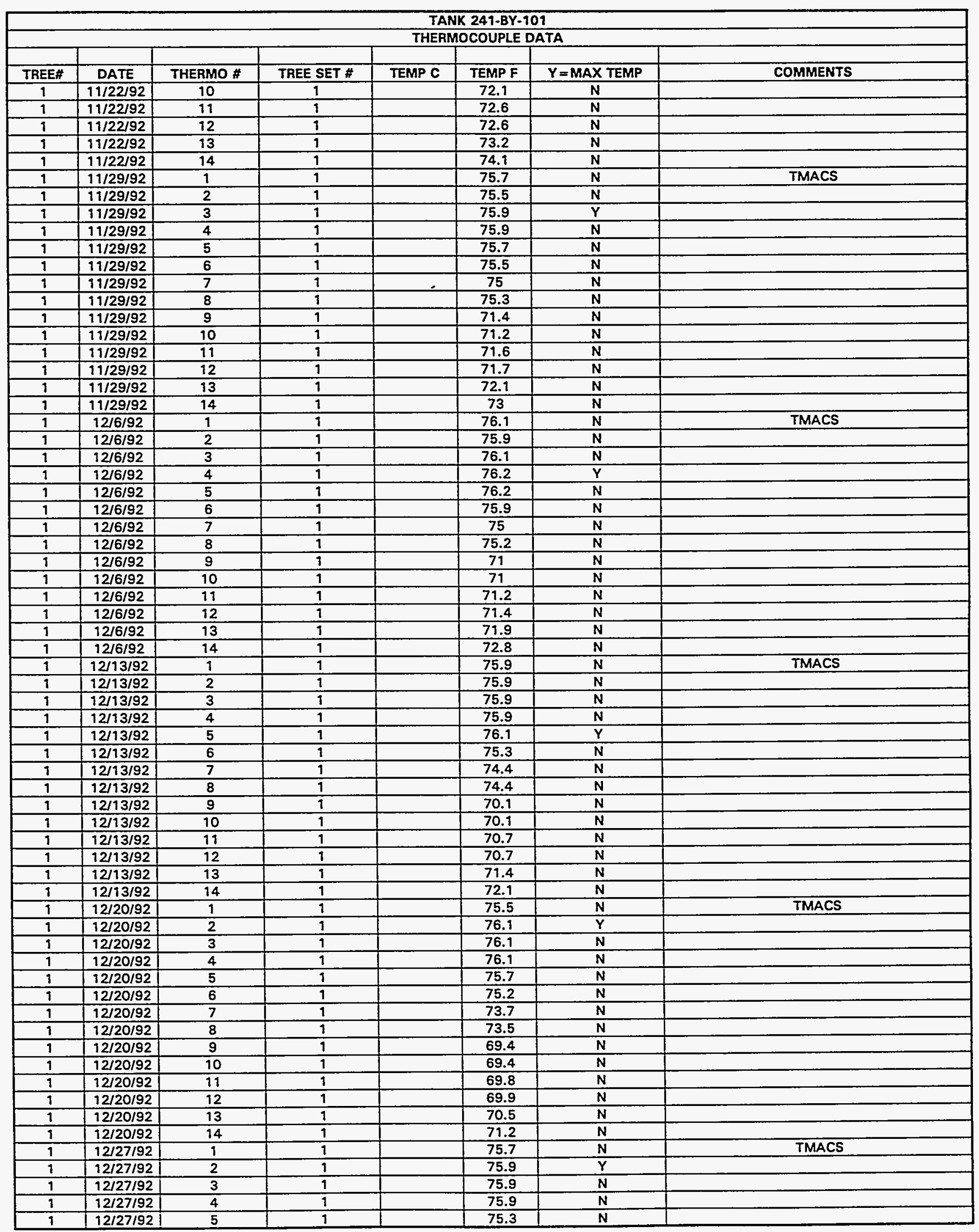

Data obtained from WHC Surveillance Analysis Computer System (SACS), November 12, 1993. 


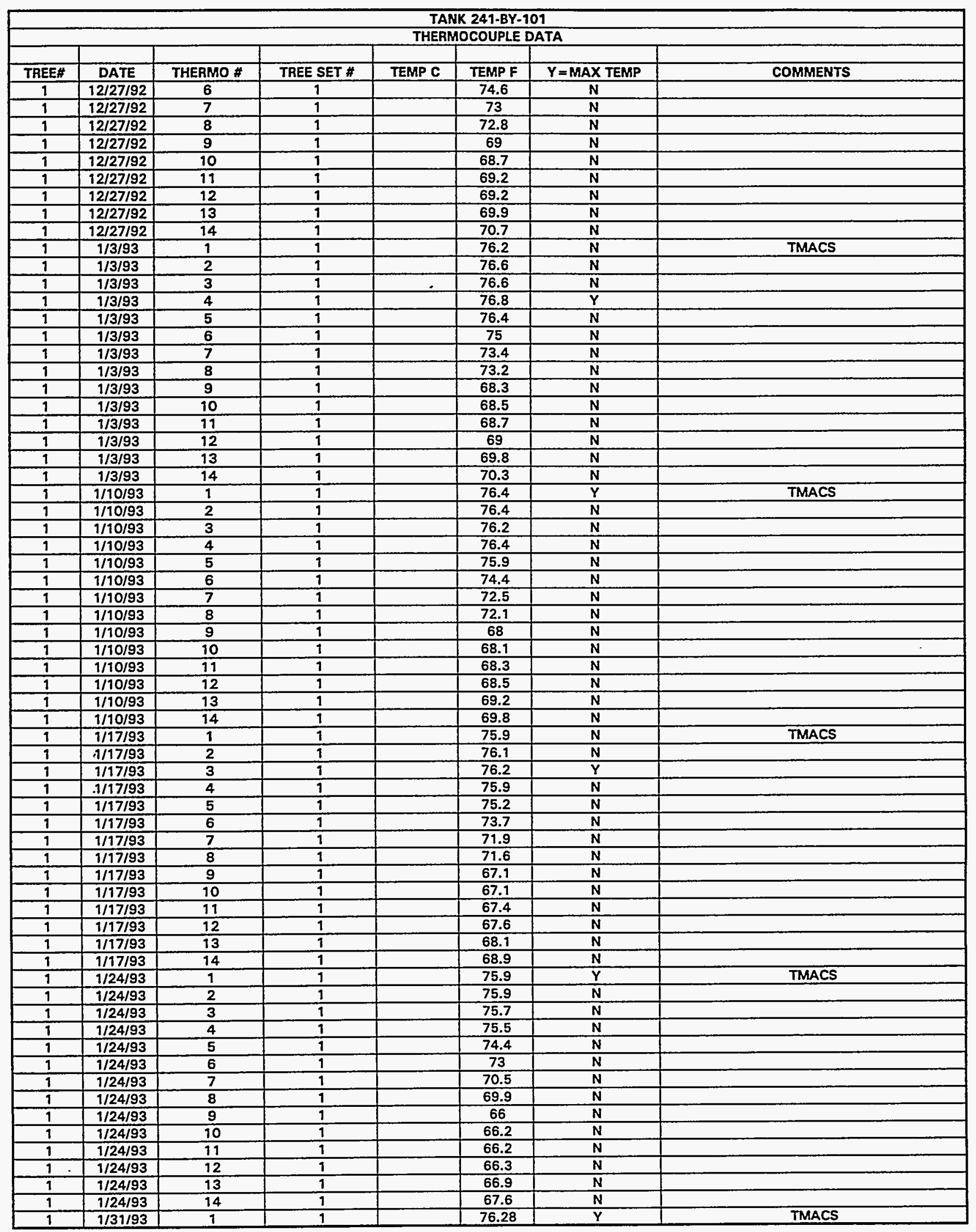

Data obtained from WHC Surveillance Analysis Computer System (SACS), November 12, 1993. 


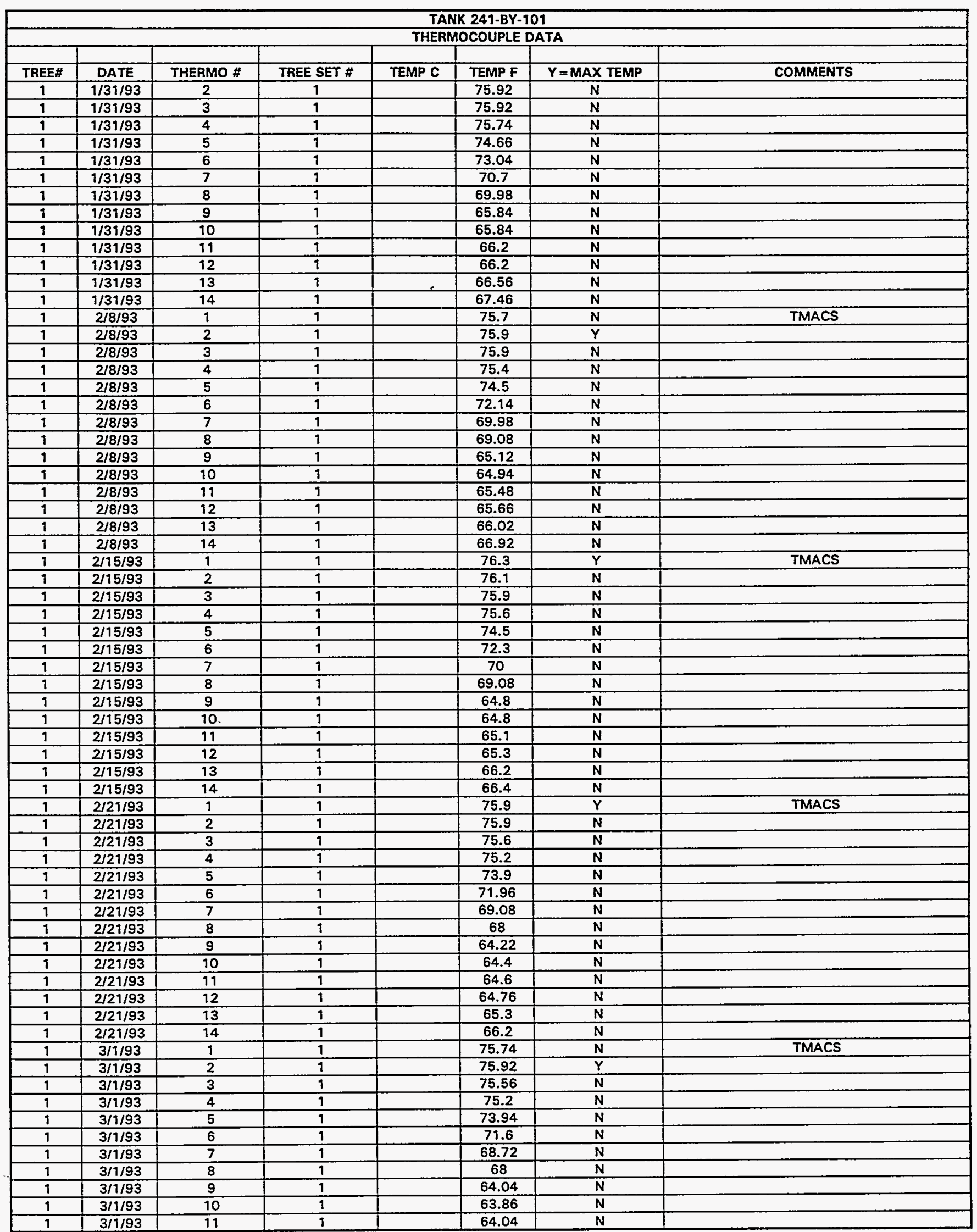

Data obtained from WHC Surveillance Analysis Computer System (SACS), November 12, 1993. 


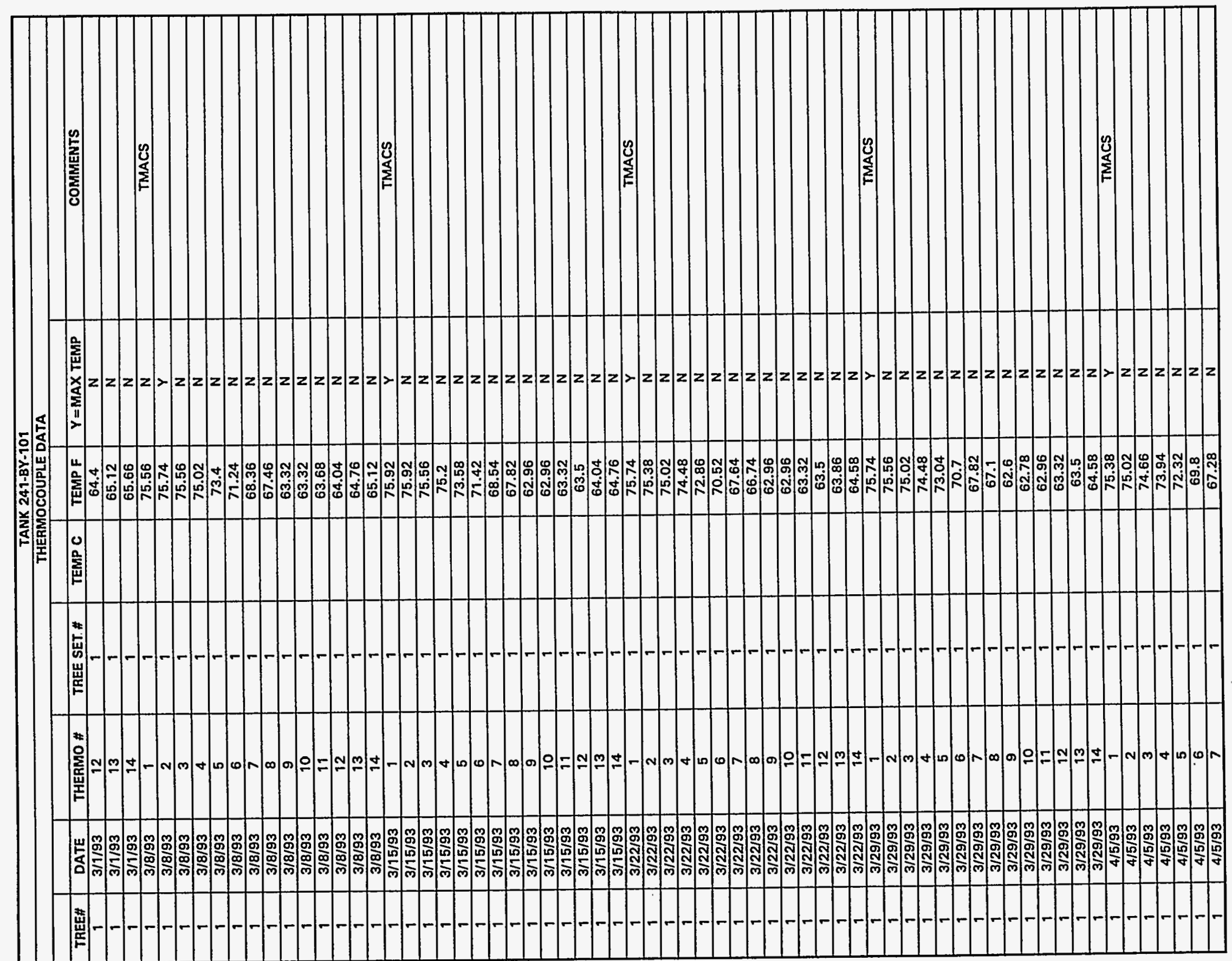

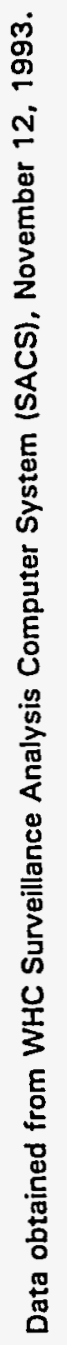

용 案 
WHC-SD-WM-ER-312, Rev. 0

\begin{tabular}{|c|c|c|c|c|c|c|c|}
\hline \multicolumn{8}{|c|}{ TANK 241-BY-101 } \\
\hline \multicolumn{8}{|c|}{ THERMOCOUPLE DATA } \\
\hline & & & & & & & \\
\hline TREE\# & DATE & THERMO \# & TREE SET \# & TEMP C & TEMP F & $Y=M A X$ TEMP & COMMENTS \\
\hline 1 & $4 / 5 / 93$ & 8 & 1 & & 66.38 & $\mathbf{N}$ & \\
\hline 1 & $4 / 5 / 93$ & 9 & 1 & & 62.6 & $\mathrm{~N}$ & \\
\hline 9 & $4 / 5 / 93$ & 10 & 9 & & 62.6 & $\mathrm{~N}$ & \\
\hline 1 & $4 / 5 / 93$ & 11 & 1 & & 62.96 & $\mathbf{N}$ & \\
\hline 1 & $4 / 5 / 93$ & 12 & 1 & & 62.96 & $\mathbf{N}$ & \\
\hline 9 & $4 / 5 / 93$ & 13 & 1 & & 63.68 & $\mathbf{N}$ & \\
\hline 1 & $4 / 5 / 93$ & 14 & 1 & & 64.4 & $\mathrm{~N}$ & \\
\hline 1 & $4 / 12 / 93$ & 1 & 1 & & 75.92 & $\ddot{Y}$ & TMAC \\
\hline 9 & $4 / 12 / 93$ & 2 & 1 & & 75.2 & $\mathrm{~N}$ & \\
\hline 1 & $4 / 12 / 93$ & 3 & 1 & & 75.02 & $\bar{N}$ & \\
\hline 1 & $4 / 12 / 93$ & 4 & 1 & & 74.3 & $\mathrm{~N}$ & \\
\hline 1 & $4 / 12 / 93$ & 5 & 1 & & 72.68 & $\mathbf{N}$ & \\
\hline 1 & $4 / 12 / 93$ & 6 & 1 & & 70.16 & $\bar{N}$ & \\
\hline 1 & $4 / 12 / 93$ & 7 & 1 & & 67.1 & $\bar{N}$ & \\
\hline 1 & $4 / 12 / 93$ & 8 & 1 & & 66.56 & $\mathbf{N}$ & \\
\hline 9 & $4 / 12 / 93$ & 9 & 1 & & 62.6 & $\mathbf{N}$ & \\
\hline 1 & $4 / 12 / 93$ & 10 & 1 & & 62.42 & $\mathbf{N}$ & \\
\hline 9 & $4 / 12 / 93$ & 11 & 1 & & 62.78 & $\mathbf{N}$ & \\
\hline 1 & $4 / 12 / 93$ & 12 & 1 & & 62.96 & $\mathbf{N}$ & \\
\hline 1 & $4 / 12 / 93$ & 13 & 1 & & 63.5 & $\mathbf{N}$ & \\
\hline 1 & $4 / 12 / 93$ & 14 & 1 & & 64.04 & $\mathbf{N}$ & \\
\hline 9 & $4 / 19 / 93$ & 1 & 9 & & 75.56 & $\mathrm{Y}$ & TMACS \\
\hline 1 & $4 / 19 / 93$ & 2 & 1 & & 75.2 & $\bar{N}$ & \\
\hline 1 & $4 / 19 / 93$ & 3 & 1 & & 74.66 & N & \\
\hline 1 & $4 / 19 / 93$ & 4 & 1 & & 73.94 & $\bar{N}$ & \\
\hline 1 & $4 / 19 / 93$ & 5 & 1 & & 72.32 & $N$ & \\
\hline 1 & $4 / 19 / 93$ & 6 & 1 & & 69.98 & $\mathbf{N}$ & \\
\hline 1 & $4 / 19 / 93$ & 7 & 1 & & 67.1 & $\mathbf{N}$ & \\
\hline 1 & $4 / 19 / 93$ & 8 & 9 & & 66.74 & $\bar{N}$ & \\
\hline 1 & $4 / 19 / 93$ & 9 & 1 & & 62.42 & $\mathrm{~N}$ & \\
\hline 1 & $4 / 19 / 93$ & 10 & 1 & & 62.6 & $N$ & \\
\hline 1 & $4 / 19 / 93$ & 11 & 1 & & 62.78 & $\mathbf{N}$ & \\
\hline 1 & $4 / 19 / 93$ & 12 & 1 & & 62.96 & $\bar{N}$ & \\
\hline 1 & $4 / 19 / 93$ & 13 & 9 & & 63.32 & $N$ & \\
\hline 1 & $4 / 19 / 93$ & 14 & 9 & & 64.04 & $\bar{N}$ & \\
\hline 1 & $4 / 25 / 93$ & 1 & 1 & & 75.2 & $Y$ & TMACS \\
\hline 1 & $4 / 25 / 93$ & 2 & 1 & & 75.02 & $\mathrm{~N}$ & \\
\hline 1 & $4 / 25 / 93$ & 3 & 1 & & 74.3 & $\mathrm{~N}$ & \\
\hline 1 & $4 / 25 / 93$ & 4 & 1 & & 73.76 & $\mathrm{~N}$ & \\
\hline 1 & $4 / 25 / 93$ & 5 & 1 & & 71.96 & $\mathbf{N}$ & \\
\hline 1 & $4 / 25 / 93$ & 6 & 1 & & 69.8 & $\mathrm{~N}$ & \\
\hline 1 & $4 / 25 / 93$ & 7 & 1 & & 67.1 & $\mathrm{~N}$ & \\
\hline 9 & $4 / 25 / 93$ & 8 & 1 & & 66.74 & $\mathrm{~N}$ & \\
\hline 1 & $4 / 25 / 93$ & 9 & 1 & & 62.96 & $\mathrm{~N}$ & \\
\hline 1 & $4 / 25 / 93$ & 10 & 1 & & 62.78 & $\mathbf{N}$ & \\
\hline 1 & $4 / 25 / 93$ & 11 & 1 & & 62.96 & $N$ & \\
\hline 9 & $4 / 25 / 93$ & 12 & 1 & & 63.32 & $\mathrm{~N}$ & \\
\hline 9 & $4 / 25 / 93$ & 13 & 1 & & 63.86 & $\mathrm{~N}$ & \\
\hline 1 & $4 / 25 / 93$ & 14 & 1 & & 64.58 & $\mathbf{N}$ & \\
\hline 9 & $5 / 3 / 93$ & 1 & 1 & & 75.56 & $Y$ & TMACS \\
\hline 9 & $5 / 3 / 93$ & 2 & 1 & & 75.2 & $\mathbf{N}$ & \\
\hline 1 & $5 / 3 / 93$ & 3 & 1 & & 74.66 & $\mathbf{N}$ & \\
\hline 1 & $5 / 3 / 93$ & 4 & 1 & & 73.58 & $\mathbf{N}$ & \\
\hline 9 & $5 / 3 / 93$ & 5 & 1 & & 72.5 & $\mathrm{~N}$ & \\
\hline 1 & $5 / 3 / 93$ & 6 & 9 & & 69.98 & $\mathbf{N}$ & \\
\hline 1 & $5 / 3 / 93$ & 7 & 1 & & 67.64 & $\mathbf{N}$ & \\
\hline 1 & $5 / 3 / 93$ & 8 & 1 & & 67.1 & $\mathbf{N}$ & \\
\hline 1 & $5 / 3 / 93$ & 9 & 9 & & 62.78 & $\mathbf{N}$ & \\
\hline 1 & $5 / 3 / 93$ & 10 & 1 & & 62.78 & $\mathbf{N}$ & \\
\hline 1 & $5 / 3 / 93$ & 11 & 1 & & 63.32 & $\mathrm{~N}$ & \\
\hline 1 & $5 / 3 / 93$ & 12 & 1 & & 63.32 & $N$ & \\
\hline 1 & $5 / 3 / 93$ & 13 & 9 & & 64.04 & 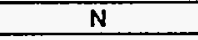 & \\
\hline 1 & $5 / 3 / 93$ & 14 & 1 & & 64.76 & $N$ & \\
\hline 1 & $5 / 10 / 93$ & 1 & 1 & & 75.2 & $\mathrm{~N}$ & TMACS \\
\hline 1 & $5 / 10 / 93$ & 2 & 1 & & 74.66 & $\bar{Y}$ & \\
\hline 1 & $5 / 10 / 93$ & 3 & 1 & & 74.3 & $\mathbf{N}$ & \\
\hline
\end{tabular}

Data obtained from WHC Surveillance Analysis Computer System (SACS), November 12, 1993. 


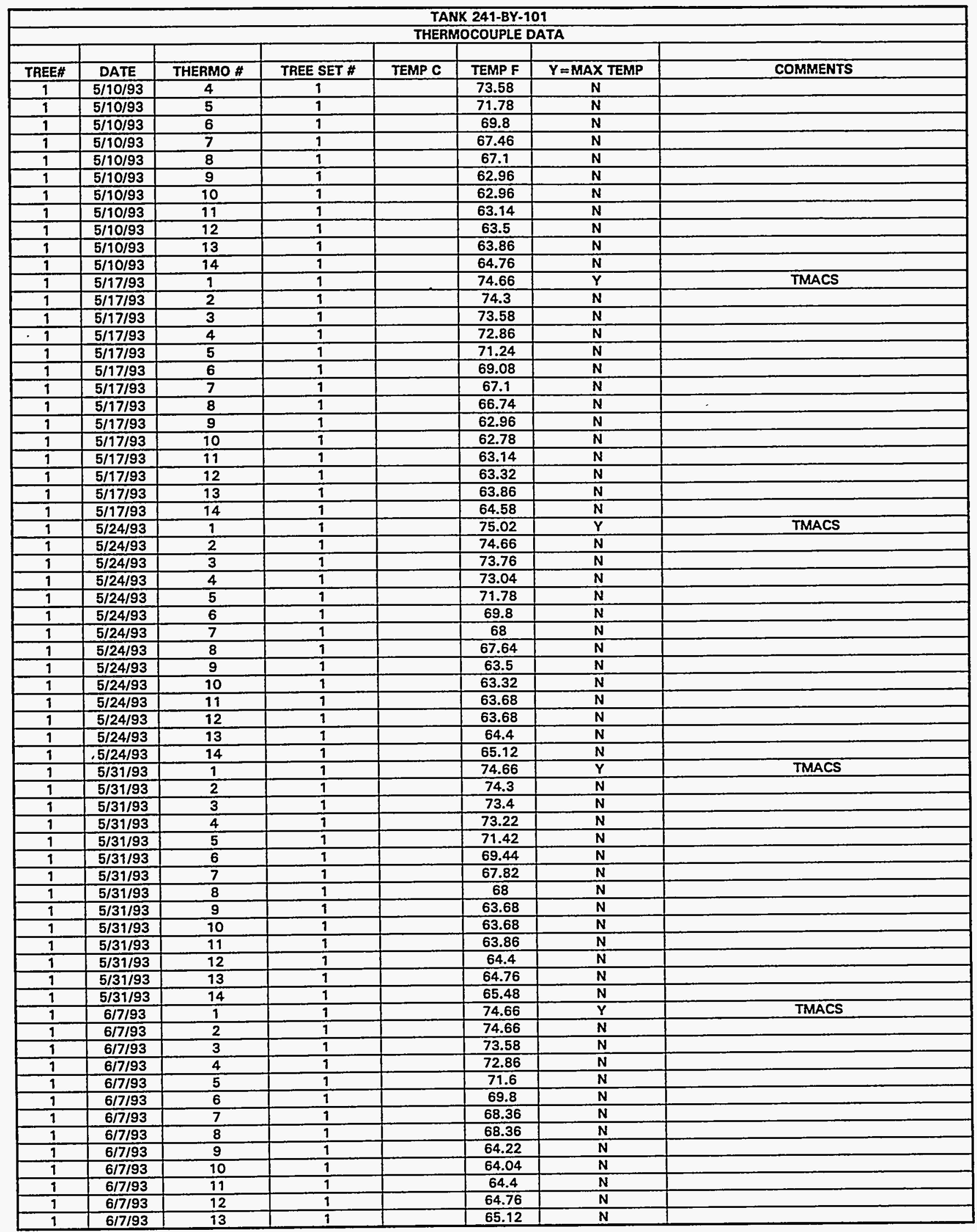

Data obtained from WHC Surveillance Analysis Computer System (SACS), November 12, 1993. 


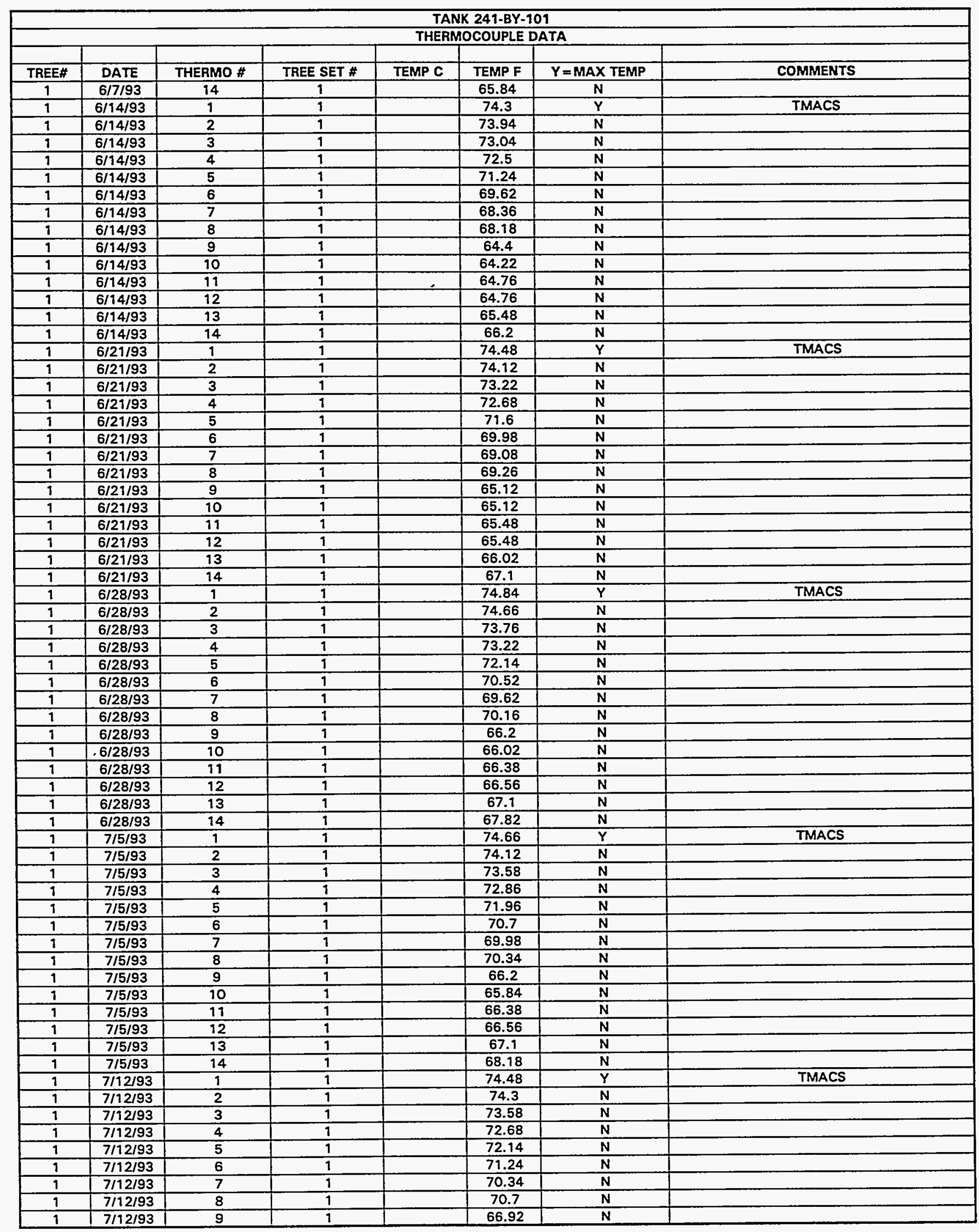

Data obtained from WHC Surveillance Analysis Computer System (SACS), November 12, 1993. 


\begin{tabular}{|c|c|c|c|c|c|c|c|}
\hline \multicolumn{8}{|c|}{ TANK 241-BY-101 } \\
\hline & & & & THER & COUPLE & ITA & \\
\hline TREEH & DATE & THERMO \# & TREE SET \# & TEMP C & TEMP F & $Y=M A X$ TEMP & COMMENTS \\
\hline 1 & $7 / 12 / 93$ & 10 & 1 & & 66.92 & $\mathrm{~N}$ & \\
\hline$\frac{1}{1}$ & $7 / 12 / 93$ & 11 & 1 & & 67.46 & $N$ & \\
\hline 1 & $7 / 12 / 93$ & 12 & 1 & & 67.46 & $\mathbf{N}$ & \\
\hline 1 & $7 / 12 / 93$ & 13 & 1 & & 68 & $\bar{N}$ & \\
\hline 1 & $7 / 12 / 93$ & 14 & 1 & & 68.72 & $\bar{N}$ & \\
\hline 1 & $7 / 18 / 93$ & 1 & 1 & & 74.3 & $\mathbf{Y}$ & TMACS \\
\hline 1 & $7 / 18 / 93$ & 2 & 1 & & 73.76 & $\mathbf{N}$ & \\
\hline 1 & $7 / 18 / 93$ & 3 & 1 & & 73.4 & $\bar{N}$ & \\
\hline$\frac{1}{1}$ & $7 / 18 / 93$ & 4 & $\frac{1}{1}$ & & 72.5 & $\bar{N}$ & \\
\hline 1 & $7 / 18 / 93$ & 5 & 1 & & 71.78 & $\bar{N}$ & \\
\hline 1 & $7 / 18 / 93$ & 6 & 1 & & 70.88 & $N$ & \\
\hline 1 & $7 / 18 / 93$ & 7 & 1 & . & 70.52 & $\mathbf{N}$ & \\
\hline$\frac{T}{1}$ & $7 / 18 / 93$ & 8 & 9 & & 70.88 & $\bar{N}$ & \\
\hline 1 & $7 / 18 / 93$ & 9 & 1 & & 66.56 & $\mathbf{N}$ & \\
\hline$\frac{1}{1}$ & $7 / 18 / 93$ & 10 & $\frac{1}{1}$ & & 66.38 & $\mathbf{N}$ & \\
\hline 1 & $7 / 18 / 93$ & 11 & $\frac{1}{1}$ & & 66.92 & $\mathbf{N}$ & \\
\hline 1 & $7 / 18 / 93$ & 12 & 1 & & 67.28 & $\mathbf{N}$ & \\
\hline 1 & $7 / 18 / 93$ & 13 & 1 & & 67.64 & $\mathbf{N}$ & \\
\hline 1 & $7 / 18 / 93$ & 14 & $\frac{1}{1}$ & & 68.54 & $\bar{N}$ & \\
\hline 1 & $7 / 26 / 93$ & 1 & 1 & & 75.2 & $\bar{Y}$ & TMACS \\
\hline 1 & $7 / 26 / 93$ & 2 & 1 & & 74.48 & $\mathbf{N}$ & \\
\hline 1 & $7 / 26 / 93$ & 3 & 1 & & 73.76 & $\bar{N}$ & \\
\hline$\frac{1}{1}$ & $7 / 26 / 93$ & 4 & 1 & & 73.4 & $\mathbf{N}$ & \\
\hline 1 & $7 / 26 / 93$ & 5 & 1 & & 72.68 & $\mathbf{N}$ & \\
\hline 1 & $7 / 26 / 93$ & 6 & 1 & & 71.42 & $\mathbf{N}$ & \\
\hline 1 & $7 / 26 / 93$ & 7 & 9 & & 70.88 & $\mathbf{N}$ & \\
\hline 1 & $7 / 26 / 93$ & 8 & 1 & & 71.42 & $\mathrm{~N}$ & \\
\hline$\frac{1}{1}$ & $7 / 26 / 93$ & 9 & 1 & & 67.64 & $\mathbf{N}$ & \\
\hline 1 & $7 / 26 / 93$ & 10 & 1 & & 67.46 & $\mathbf{N}$ & \\
\hline 1 & $7 / 26 / 93$ & 11 & 1 & & 67.46 & $\mathbf{N}$ & \\
\hline 1 & $7 / 26 / 93$ & 12 & 1 & & 68.18 & $\bar{N}$ & \\
\hline$\frac{T}{1}$ & $7 / 26 / 93$ & 13 & 1 & & 68.72 & $\bar{N}$ & \\
\hline 1 & $7 / 26 / 93$ & 14 & 1 & & 69.44 & $\mathbf{N}$ & \\
\hline$i$ & $8 / 1 / 93$ & 1 & 1 & & 73.76 & $\bar{Y}$ & TMACS \\
\hline 1 & $8 / 1 / 93$ & 2 & 1 & & 73.76 & $N$ & \\
\hline 1 & $8 / 1 / 93$ & 3 & 1 & & 73.04 & $\mathbf{N}$ & \\
\hline 9 & $8 / 1 / 93$ & 4. & 1 & & 72.86 & $\mathbf{N}$ & \\
\hline 1 & $8 / 1 / 93$ & 5 & 1 & & 72.14 & $\mathbf{N}$ & \\
\hline 1 & $.8 / 1 / 93$ & 6 & 1 & & 71.24 & $N$ & \\
\hline 1 & $8 / 1 / 93$ & 7 & 1 & & 70.88 & $N$ & \\
\hline$\frac{T}{1}$ & $8 / 1 / 93$ & 8 & 1 & & 71.42 & $\mathbf{N}$ & \\
\hline 1 & $8 / 1 / 93$ & 9 & 1 & & 67.46 & $\mathbf{N}$ & \\
\hline 1 & $8 / 1 / 93$ & 10 & 1 & & 67.28 & $\mathbf{N}$ & \\
\hline 9 & $8 / 1 / 93$ & 19 & 1 & & 67.46 & $\mathbf{N}$ & \\
\hline 1 & $8 / 1 / 93$ & 12 & 1 & & 68 & $\overline{\mathbf{N}}$ & \\
\hline 1 & $8 / 1 / 93$ & 13 & 1 & & 68.36 & $\mathbf{N}$ & \\
\hline 1 & $8 / 1 / 93$ & 14 & 1 & & 69.08 & $\mathbf{N}$ & \\
\hline 1 & $8 / 9 / 93$ & 1 & 1 & & 74.3 & $\bar{Y}$ & TTMACS \\
\hline 1 & $8 / 9 / 93$ & 2 & 1 & & 74.3 & $\mathbf{N}$ & \\
\hline 9 & $8 / 9 / 93$ & 3 & 1 & & 73.76 & $\mathrm{~N}$ & \\
\hline$\frac{1}{1}$ & $8 / 9 / 93$ & 4 & 1 & & 73.76 & $\mathbf{N}$ & \\
\hline 1 & $8 / 9 / 93$ & 5 & 1 & & 73.04 & $\mathbf{N}$ & \\
\hline 1 & $8 / 9 / 93$ & 6 & 1 & & 72.14 & $\mathbf{N}$ & \\
\hline 1 & $8 / 9 / 93$ & 7 & 1 & & 72.14 & $\mathbf{N}$ & \\
\hline 1 & $8 / 9 / 93$ & 8 & 1 & & 72.68 & $\mathbf{N}$ & \\
\hline 1 & $8 / 9 / 93$ & 9 & 1 & & 68.36 & $\mathbf{N}$ & \\
\hline 1 & $8 / 9 / 93$ & 10 & 1 & & 68.36 & $\mathbf{N}$ & \\
\hline 1 & $8 / 9 / 93$ & 11 & 1 & & 68.72 & $\mathbf{N}$ & \\
\hline 1 & $8 / 9 / 93$ & 12 & 1 & & 68.9 & $\mathbf{N}$ & \\
\hline 9 & $8 / 9 / 93$ & 13 & 1 & & 69.8 & $\mathbf{N}$ & \\
\hline 1 & $8 / 9 / 93$ & 14 & 1 & & 70.7 & $\mathbf{N}$ & \\
\hline 1 & $8 / 15 / 93$ & 1 & 1 & & 74.3 & $\bar{Y}$ & tmacs \\
\hline 1 & $8 / 15 / 93$ & 2 & 1 & & 73.94 & $\mathbf{N}$ & \\
\hline 1 & $8 / 15 / 93$ & 3 & 1 & & 73.58 & $\mathbf{N}$ & \\
\hline 1 & $8 / 15 / 93$ & 4 & 7 & & 73.22 & $\mathrm{~N}$ & \\
\hline 1 & $8 / 15 / 93$ & 5 & 1 & & 72.68 & $\mathbf{N}$ & \\
\hline
\end{tabular}

Data obtained from WHC Surveillance Analysis Computer System (SACS), November 12, 1993. 


\begin{tabular}{|c|c|c|c|c|c|c|c|}
\hline \multicolumn{8}{|c|}{ TANK 241-BY-101 } \\
\hline & & & & THER & COUPLE & ATA & \\
\hline TREE\# & DATE & THERMO \# & TREE SET \# & TEMP C & TEMP F & $Y=M A X$ TEMP & COMMENTS \\
\hline 1 & $8 / 15 / 93$ & 6 & 1 & & 72.14 & $\mathbf{N}$ & \\
\hline 1 & $8 / 15 / 93$ & 7 & 1 & & 71.78 & $\mathbf{N}$ & \\
\hline 1 & $8 / 15 / 93$ & 8 & 1 & & 72.5 & $\mathbf{N}$ & \\
\hline 1 & $8 / 15 / 93$ & 9 & 1 & & 68.54 & $\mathbf{N}$ & \\
\hline 1 & $8 / 15 / 93$ & 10 & 1 & & 68.36 & $\mathbf{N}$ & \\
\hline 1 & $8 / 15 / 93$ & 11 & 1 & & 68.72 & $\mathrm{~N}$ & \\
\hline 1 & $8 / 15 / 93$ & 12 & 1 & & 69.08 & $N$ & \\
\hline 1 & $8 / 15 / 93$ & 13 & 1 & & 69.62 & $\mathbf{N}$ & \\
\hline 1 & $8 / 15 / 93$ & 14 & 1 & & 70.16 & $\mathbf{N}$ & \\
\hline 1 & $8 / 23 / 93$ & 1 & 1 & & 73.58 & $Y$ & TMACS \\
\hline 1 & $8 / 23 / 93$ & 2 & 1 & & 73.58 & $\mathbf{N}$ & \\
\hline 1 & $8 / 23 / 93$ & 3 & 1 & - & 73.04 & $\mathbf{N}$ & \\
\hline 1 & $8 / 23 / 93$ & 4 & 1 & & 72.86 & $\mathbf{N}$ & \\
\hline 1 & $8 / 23 / 93$ & 5 & 1 & & 72.5 & $\bar{N}$ & \\
\hline 1 & $8 / 23 / 93$ & 6 & 1 & & 71.6 & $\mathbf{N}$ & \\
\hline 1 & $8 / 23 / 93$ & 7 & 1 & & 71.6 & $\mathbf{N}$ & \\
\hline 1 & $8 / 23 / 93$ & 8 & 1 & & 72.32 & $\mathbf{N}$ & \\
\hline 1 & $8 / 23 / 93$ & 9 & 1 & & 69.08 & $N$ & \\
\hline 1 & $8 / 23 / 93$ & 10 & 1 & & 69.08 & $\overline{\mathbf{N}}$ & $=$ \\
\hline 1 & $8 / 23 / 93$ & 11 & 1 & & 69.26 & $\mathbf{N}$ & \\
\hline 1 & $8 / 23 / 93$ & 12 & 1 & & 69.44 & $\mathbf{N}$ & \\
\hline 1 & $8 / 23 / 93$ & 13 & 1 & & 70.16 & $\mathrm{~N}$ & \\
\hline 1 & $8 / 23 / 93$ & 14 & 1 & & 70.7 & $\mathrm{~N}$ & \\
\hline 1 & $8 / 26 / 93$ & 1 & 1 & & 73.6 & $\bar{Y}$ & J TYPE \\
\hline 1 & $8 / 26 / 93$ & 2 & 1 & & 73.6 & $\mathbf{N}$ & \\
\hline 1 & $8 / 26 / 93$ & 3 & 1 & & 73 & $\mathbf{N}$ & \\
\hline 1 & $8 / 26 / 93$ & 4 & 1 & & 72.9 & $\mathbf{N}$ & \\
\hline 1 & $8 / 26 / 93$ & 5 & 1 & & 72.3 & $\mathbf{N}$ & \\
\hline 1 & $8 / 26 / 93$ & 6 & 1 & & 71.4 & $\mathbf{N}$ & \\
\hline 1 & $8 / 26 / 93$ & 7 & 1 & & 79.6 & $\mathbf{N}$ & \\
\hline 1 & $8 / 26 / 93$ & 8 & $T$ & & 72.1 & $N$ & \\
\hline 1 & $8 / 26 / 93$ & 9 & 1 & & 69.4 & $N$ & \\
\hline 1 & $8 / 26 / 93$ & 10 & 1 & & 69.7 & $\mathbf{N}$ & \\
\hline 1 & $8 / 26 / 93$ & 11 & 1 & & 69.6 & $\mathbf{N}$ & \\
\hline 1 & $8 / 26 / 93$ & 12 & 1 & & 69.6 & $\mathbf{N}$ & \\
\hline 1 & $8 / 26 / 93$ & 13 & 1 & & 70.2 & $\overline{\mathbf{N}}$ & \\
\hline 1 & $8 / 26 / 93$ & 14 & 1 & & 71.1 & $\mathbf{N}$ & \\
\hline 1 & $8 / 30 / 93$ & 1 & 1 & & 74.48 & $Y$ & TMACS \\
\hline 1 & $.8 / 30 / 93$ & 2 & 1 & & 74.48 & $\mathbf{N}$ & \\
\hline 1 & $8 / 30 / 93$ & 3 & 1 & & 73.94 & $\mathbf{N}$ & \\
\hline 1 & $8 / 30 / 93$ & 4 & 1 & & 73.76 & $\mathbf{N}$ & \\
\hline 1 & $8 / 30 / 93$ & 5 & 1 & & 73.22 & $\mathbf{N}$ & \\
\hline 1 & $8 / 30 / 93$ & 6 & 1 & & 72.86 & $\mathbf{N}$ & \\
\hline 1 & $8 / 30 / 93$ & 7 & 1 & & 72.86 & $\mathbf{N}$ & \\
\hline 1 & $8 / 30 / 93$ & 8 & 1 & & 73.58 & $\mathbf{N}$ & \\
\hline 1 & $8 / 30 / 93$ & 9 & 1 & & 69.8 & $\mathbf{N}$ & \\
\hline 1 & $8 / 30 / 93$ & 10 & 1 & & 69.8 & $\mathbf{N}$ & \\
\hline 9 & $8 / 30 / 93$ & 11 & 1 & & 70.16 & $\mathbf{N}$ & \\
\hline 1 & $8 / 30 / 93$ & 12 & 1 & & 70.34 & $\mathbf{N}$ & \\
\hline 1 & $8 / 30 / 93$ & 13 & 1 & & 70.52 & $\mathbf{N}$ & \\
\hline 1 & $8 / 30 / 93$ & 14 & 1 & & 71.78 & $\mathbf{N}$ & \\
\hline 1 & $9 / 6 / 93$ & 1 & 1 & & 73.76 & $Y$ & TMACS \\
\hline 1 & $9 / 6 / 93$ & 2 & 1 & & 73.58 & $\mathbf{N}$ & \\
\hline 1 & $9 / 6 / 93$ & 3 & 1 & & 73.22 & $\mathrm{~N}$ & \\
\hline 1 & $9 / 6 / 93$ & 4 & 1 & & 72.86 & $\mathbf{N}$ & \\
\hline 1 & $9 / 6 / 93$ & 5 & 1 & & 72.86 & $\mathbf{N}$ & \\
\hline 1 & $9 / 6 / 93$ & 6 & 1 & & 72.32 & $N$ & \\
\hline 1 & $9 / 6 / 93$ & 7 & 1 & & 72.5 & $N$ & \\
\hline 1 & 9/6/93 & 8 & 1 & & 73.4 & $\mathrm{~N}$ & \\
\hline 1 & $9 / 6 / 93$ & 9 & 1 & & 69.69 & $\mathbf{N}$ & \\
\hline 1 & $9 / 6 / 93$ & 10 & 1 & & 69.62 & $\mathbf{N}$ & \\
\hline 1 & $9 / 6 / 93$ & 11 & 1 & & 70.16 & $N$ & \\
\hline 1 & $9 / 6 / 93$ & 12 & 1 & & 70.52 & $\mathbf{N}$ & \\
\hline 1 & $9 / 6 / 93$ & 13 & 1 & & 70.88 & $\mathbf{N}$ & \\
\hline 1 & $9 / 6 / 93$ & 14 & 1 & & 71.6 & $\mathrm{~N}$ & \\
\hline 1 & $9 / 12 / 93$ & 1 & 1 & & 74.3 & $\bar{Y}$ & TMACS \\
\hline
\end{tabular}

Data obtained from WHC Surveillance Analysis Computer System (SACS), November 12, 1993. 
WHC-SD-WM-ER-312, Rev. 0

\begin{tabular}{|c|c|c|c|c|c|c|c|}
\hline \multicolumn{8}{|c|}{ TANK 241-BY-101 } \\
\hline \multicolumn{8}{|c|}{ THERMOCOUPLE DATA } \\
\hline & & & & & & & \\
\hline TREE\# & DATE & THERMO \# & TREE SET \# & TEMP C & TEMP F & $Y=$ MAX TEMP & COMMENTS \\
\hline 1 & $9 / 12 / 93$ & 2 & 1 & & 73.94 & $N$ & \\
\hline 1 & $9 / 12 / 93$ & 3 & 1 & & 73.76 & $\mathbf{N}$ & \\
\hline$\frac{1}{1}$ & $9 / 12 / 93$ & 4 & 1 & & 73.58 & $\mathbf{N}$ & \\
\hline 1 & $9 / 12 / 93$ & 5 & 1 & & 73.4 & $N$ & \\
\hline 1 & $9 / 12 / 93$ & 6 & 1 & & 73.04 & $N$ & \\
\hline 1 & $9 / 12 / 93$ & 7 & 1 & & 73.4 & $\mathbf{N}$ & \\
\hline 1 & $9 / 12 / 93$ & 8 & 1 & & 74.12 & $\mathbf{N}$ & \\
\hline 1 & $9 / 12 / 93$ & 9 & 1 & & 70.16 & $\mathbf{N}$ & \\
\hline 1 & $9 / 12 / 93$ & 10 & 1 & & 70.16 & $\mathbf{N}$ & \\
\hline 1 & $9 / 12 / 93$ & 11 & 1 & & 70.52 & $\mathbf{N}$ & \\
\hline 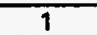 & $9 / 12 / 93$ & 12 & 1 & & 70.7 & $\mathbf{N}$ & \\
\hline 1 & $9 / 12 / 93$ & 13 & 1 & & 71.24 & $\mathbf{N}$ & \\
\hline 1 & $9 / 12 / 93$ & 14 & 1 & & 72.14 & $\mathbf{N}$ & \\
\hline 1 & $9 / 17 / 93$ & 1 & 1 & & 73 & $\mathrm{Y}$ & J 777-13-20-010 \\
\hline 1 & $9 / 17 / 93$ & 2 & 1 & & 73 & $\mathbf{N}$ & \\
\hline 1 & $9 / 17 / 93$ & 3 & 1 & & 72 & $\mathrm{~N}$ & \\
\hline 1 & $9 / 17 / 93$ & 4 & 1 & & 72 & $\mathbf{N}$ & \\
\hline 1 & $9 / 17 / 93$ & 5 & 1 & & 71 & $\mathbf{N}$ & \\
\hline 1 & $9 / 17 / 93$ & 6 & 1 & & 70 & $\mathbf{N}$ & \\
\hline$T$ & $9 / 17 / 93$ & 7 & 1 & & 70 & $\mathbf{N}$ & \\
\hline 1 & $9 / 17 / 93$ & 8 & 1 & & 70 & $\mathbf{N}$ & \\
\hline 1 & $9 / 17 / 93$ & 9 & 1 & & 70 & $\mathbf{N}$ & \\
\hline 1 & $9 / 17 / 93$ & 10 & 1 & & 70 & $\mathbf{N}$ & \\
\hline 1 & $9 / 17 / 93$ & 11 & 1 & & 70 & $\bar{N}$ & \\
\hline 1 & $9 / 17 / 93$ & 12 & 1 & & 70 & $\mathbf{N}$ & \\
\hline 1 & $9 / 17 / 93$ & 13 & 9 & & 70 & $\mathbf{N}$ & \\
\hline 1 & $9 / 17 / 93$ & 14 & 1 & & 70 & $\mathbf{N}$ & \\
\hline 1 & $9 / 19 / 93$ & 1 & 1 & & 74.48 & $Y$ & TMACS \\
\hline 1 & $9 / 19 / 93$ & 2 & 1 & & 74.3 & $\bar{N}$ & \\
\hline 1 & $9 / 19 / 93$ & 3 & 1 & & 73.58 & $\mathbf{N}$ & \\
\hline 1 & $9 / 19 / 93$ & 4 & 1 & & 73.4 & $\mathbf{N}$ & \\
\hline 1 & $9 / 19 / 93$ & 5 & 1 & & 73.22 & $\mathbf{N}$ & \\
\hline 1 & $9 / 19 / 93$ & 6 & 1 & & 73.04 & $\mathbf{N}$ & \\
\hline 1 & $9 / 19 / 93$ & 7 & 1 & & 73.4 & $\mathbf{N}$ & \\
\hline 1 & $9 / 19 / 93$ & 8 & 1 & & 74.3 & $\mathbf{N}$ & \\
\hline 1 & $9 / 19 / 93$ & 9 & 1 & & 70.52 & $\mathbf{N}$ & \\
\hline 1 & $9 / 19 / 93$ & 10 & 1 & & 70.52 & $\bar{N}$ & \\
\hline 1 & $9 / 19 / 93$ & 11 & 1 & & 70.88 & $\mathbf{N}$ & \\
\hline 1 & $.9 / 19 / 93$ & 12 & 1 & & 71.24 & $N$ & \\
\hline 1 & $9 / 19 / 93$ & 13 & 1 & & 71.78 & $\mathrm{~N}$ & \\
\hline 1 & $9 / 19 / 93$ & 14 & 1 & & 72.5 & $\mathrm{~N}$ & \\
\hline 1 & $9 / 26 / 93$ & 1 & 1 & & 73.94 & $\bar{N}$ & TMACS \\
\hline 9 & $9 / 26 / 93$ & 2 & 1 & & 74.12 & $\mathbf{N}$ & \\
\hline$T$ & $9 / 26 / 93$ & 3 & 1 & & 73.58 & $\mathbf{N}$ & \\
\hline 1 & $9 / 26 / 93$ & 4 & 1 & & 73.76 & $\mathbf{N}$ & \\
\hline 1 & $9 / 26 / 93$ & 5 & 1 & & 73.4 & $N$ & \\
\hline 1 & $9 / 26 / 93$ & 6 & 1 & & 73.22 & $N$ & \\
\hline 1 & $9 / 26 / 93$ & 7 & 1 & & 73.58 & $N$ & \\
\hline 1 & $9 / 26 / 93$ & 8 & 1 & & 74.66 & $Y$ & \\
\hline 1 & $9 / 26 / 93$ & 9 & 1 & & 70.7 & $N$ & \\
\hline 1 & $9 / 26 / 93$ & 10 & 1 & & 70.88 & $N$ & \\
\hline 1 & $9 / 26 / 93$ & 71 & 1 & & 70.88 & $N$ & \\
\hline 1 & $9 / 26 / 93$ & 12 & 1 & & 71.42 & $\mathbf{N}$ & \\
\hline 1 & $9 / 26 / 93$ & 13 & 1 & & 71.78 & $\mathbf{N}$ & \\
\hline 1 & $9 / 26 / 93$ & 14 & 1 & & 72.86 & $\mathbf{N}$ & \\
\hline 1 & $10 / 3 / 93$ & 1 & 1 & & 74.3 & $\mathbf{N}$ & TMACS \\
\hline 1 & $10 / 3 / 93$ & 2 & 1 & & 74.3 & $\mathbf{N}$ & \\
\hline 1 & $10 / 3 / 93$ & 3 & 1 & & 73.76 & $\mathbf{N}$ & \\
\hline 1 & $10 / 3 / 93$ & 4 & 1 & & 73.76 & $\mathbf{N}$ & \\
\hline 1 & $10 / 3 / 93$ & 5 & 1 & & 73.58 & $\mathbf{N}$ & \\
\hline 1 & $10 / 3 / 93$ & 6 & 1 & & 73.76 & $\mathbf{N}$ & \\
\hline 1 & $10 / 3 / 93$ & 7 & 1 & & 73.94 & $\mathbf{N}$ & \\
\hline 1 & $10 / 3 / 93$ & 8 & 1 & & 75.2 & $\bar{Y}$ & \\
\hline 1 & $10 / 3 / 93$ & 9 & 1 & & 70.88 & $\mathrm{~N}$ & \\
\hline 1 & $10 / 3 / 93$ & 10 & 1 & & 70.88 & $\mathrm{~N}$ & \\
\hline 1 & $10 / 3 / 93$ & 11 & 1 & & 71.24 & $\mathbf{N}$ & \\
\hline
\end{tabular}

Data obtained from WHC Surveillance Analysis Computer System (SACS), November 12, 1993. 


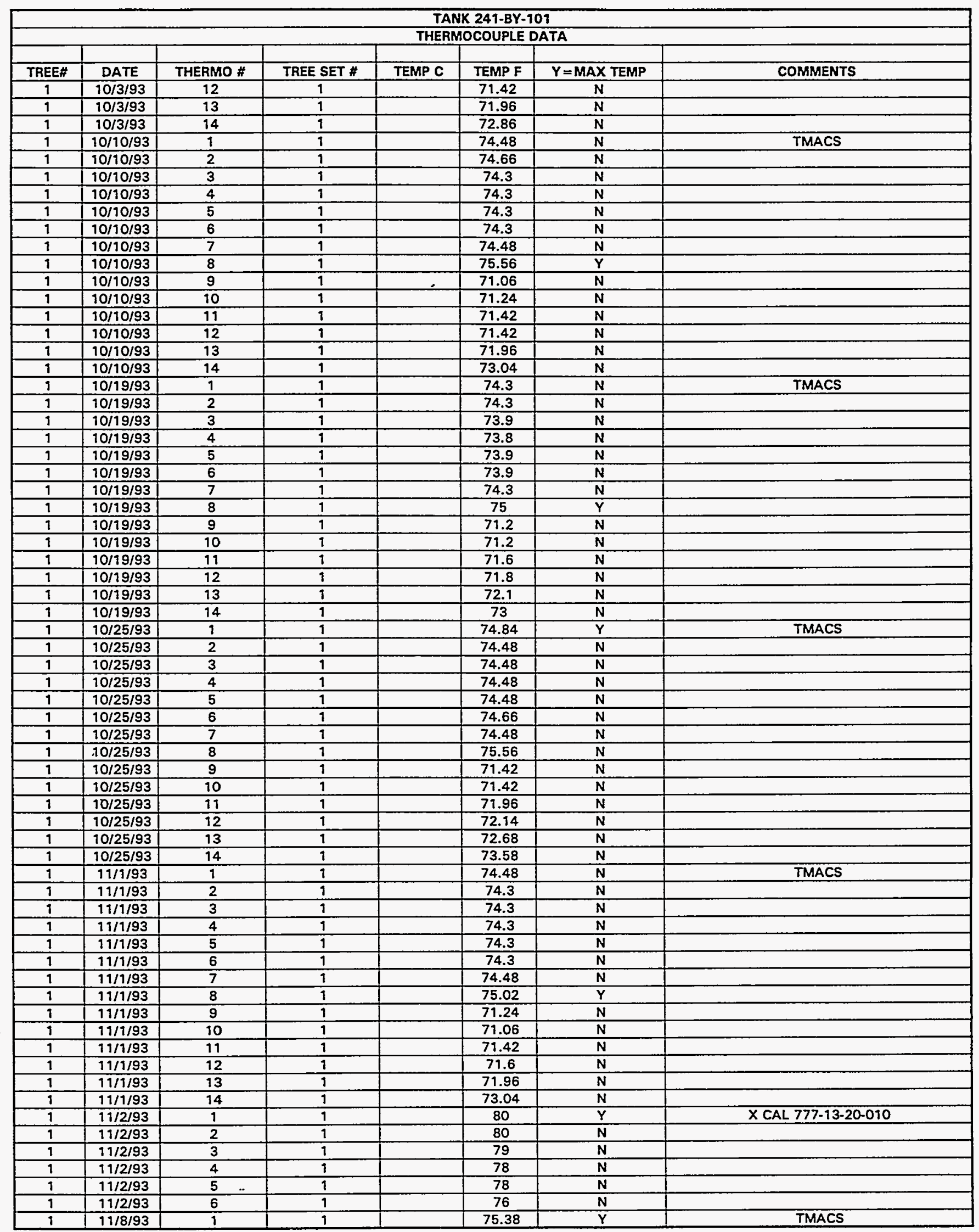

Data obtained from WHC Surveiliance Analysis Computer System (SACS), November 12, 1993. 
WHC-SD-WM-ER-312, Rev. 0

\begin{tabular}{|c|c|c|c|c|c|c|c|}
\hline \multicolumn{8}{|c|}{ TANK 241-BY-101 } \\
\hline \multicolumn{8}{|c|}{ THERMOCOUPLE DATA } \\
\hline & & & & & & & \\
\hline TREEH & DATE & THERMO \# & TREE SET \# & TEMP C & TEMP F & $Y=M A X$ TEMP & COMMENTS \\
\hline 1 & $11 / 8 / 93$ & 2 & 1 & & 74.66 & $\mathbf{N}$ & \\
\hline 1 & $11 / 8 / 93$ & 3 & 1 & & 74.48 & $\mathbf{N}$ & \\
\hline 1 & $11 / 8 / 93$ & 4 & 1 & & 74.12 & $\mathbf{N}$ & \\
\hline 1 & $11 / 8 / 93$ & 5 & 1 & & 74.12 & $\mathbf{N}$ & \\
\hline 1 & $11 / 8 / 93$ & 6 & 1 & & 73.58 & $\mathbf{N}$ & \\
\hline 1 & $11 / 8 / 93$ & 7 & 1 & & 73.58 & $\mathbf{N}$ & \\
\hline 1 & $11 / 8 / 93$ & 8 & 1 & & 73.58 & $\mathbf{N}$ & \\
\hline 1 & $11 / 8 / 93$ & 9 & 1 & & 71.96 & $\mathbf{N}$ & \\
\hline 1 & $11 / 8 / 93$ & 10 & 1 & & 71.6 & $\mathbf{N}$ & \\
\hline 1 & $11 / 8 / 93$ & 11 & 1 & & 71.6 & $\mathbf{N}$ & \\
\hline$T$ & $11 / 8 / 93$ & 12 & 1 & & 71.6 & $\mathbf{N}$ & \\
\hline 1 & $11 / 8 / 93$ & 13 & 1 & & 71.24 & $N$ & \\
\hline 1 & $11 / 8 / 93$ & 14 & 1 & & 71.96 & $N$ & \\
\hline
\end{tabular}

Data obtained from WHC Surveillance Analysis Computer System (SACS), November 12, 1993.

D-141 
WHC-SD-WM-ER-312, Rev. 0

\begin{tabular}{|c|c|c|c|c|c|c|c|}
\hline \multicolumn{8}{|c|}{ TANK 241-BY-102 } \\
\hline \multicolumn{8}{|c|}{ THERMOCOUPLE DATA } \\
\hline TREE \# & DATE & THERMO \# & TREE SET \# & TEMP (C) & TEMP (F) & $Y=$ MAX TEMP & COMMENTS \\
\hline & $10 / 13 / 73$ & 1 & & 64 & 147.2 & & raw data - assumed degreee C \\
\hline & $10 / 13 / 73$ & 2 & & 66 & 150.8 & & raw data - assumed degrees C \\
\hline & $10 / 13 / 73$ & 3 & & 69 & 156.2 & & raw data - assumed degreee C \\
\hline & $10 / 13 / 73$ & 4 & & 69 & 156.2 & & raw data - assumed degreee C \\
\hline & $10 / 13 / 73$ & 5 & & 68 & 154.4 & & raw data - assumed degreee C \\
\hline & $10 / 13 / 73$ & 6 & & 64 & 147.2 & & raw data - assumed degreee C \\
\hline & $10 / 13 / 73$ & 7 & & 57.7 & 135.86 & & raw data - assumed degreoe C \\
\hline & $10 / 13 / 73$ & 8 & & 49.7 & 121.46 & & raw data - assumed degreee $C$ \\
\hline & $10 / 13 / 73$ & 9 & & 44 & 111.2 & & raw data - assumed degreee $C$ \\
\hline & $10 / 13 / 73$ & 10 & & 40.3 & 104.54 & & raw data - assumed degreee C \\
\hline & $10 / 13 / 73$ & 11 & & 59.2 & 138.56 & & raw data - assumed degreee C \\
\hline & $10 / 14 / 73$ & 1 & & 64. & 147.2 & & raw data - assumed degreee C \\
\hline & $10 / 14 / 73$ & 2 & & 66 & 150.8 & & raw data - assumed degrees C \\
\hline & $10 / 14 / 73$ & 3 & & 68.7 & 155.66 & & raw data - assumed degreee $C$ \\
\hline & $10 / 14 / 73$ & 4 & & 69 & 156.2 & & raw data - assumed degreee C \\
\hline & $10 / 14 / 73$ & 5 & & 68 & 154.4 & & raw data - assumed degreee C \\
\hline & $10 / 14 / 73$ & 6 & & 64 & 147.2 & & raw data - assumed degreae $C$ \\
\hline & $10 / 14 / 73$ & 7 & & 57.7 & 135.86 & & raw data - assumed degreee C \\
\hline & $10 / 14 / 73$ & 8 & & 50 & 122 & & raw data - assumed degreee $C$ \\
\hline & $10 / 14 / 73$ & 9 & & 44 & 111.2 & & raw data - assumed degreee $C$ \\
\hline & $10 / 14 / 73$ & 10 & & 36.3 & 97.34 & & raw data - assumed degreee C \\
\hline & $10 / 14 / 73$ & 11 & & 59.1 & 138.38 & & raw data - assumed degreee C \\
\hline & $10 / 15 / 73$ & 1 & & 64 & 147.2 & & raw data - assumed degreee $C$ \\
\hline & $10 / 15 / 73$ & 2 & & 66 & 150.8 & & raw data - assumed degreee $C$ \\
\hline & $10 / 15 / 73$ & 3 & & 68 & 154.4 & & raw data - assumed degreee $C$ \\
\hline & $10 / 15 / 73$ & 4 & & 69 & 156.2 & & raw data - assumed degreee C \\
\hline & $10 / 15 / 73$ & 5 & & 67.3 & 153.14 & & raw data - assumed degreee C \\
\hline & $10 / 15 / 73$ & 6 & & 64 & 147.2 & & raw data - assumed degreee C \\
\hline & $10 / 15 / 73$ & 7 & & 57.7 & 135.86 & & raw data - assumed degreee C \\
\hline & $10 / 15 / 73$ & 8 & & 50 & 122 & & raw data - assumed degreee C \\
\hline & $10 / 15 / 73$ & 9 & & 44.7 & 192.46 & & raw data - assumed degreee C \\
\hline & $10 / 15 / 73$ & 10 & & 35.7 & 96.26 & & raw data - assumed degreee C \\
\hline & $10 / 75 / 73$ & 11 & & 58.6 & 137.48 & & raw data - assumed degreee C \\
\hline & $10 / 16 / 73$ & 1 & & 64 & 147.2 & & raw data - assumed degreee C \\
\hline & $10 / 16 / 73$ & 2 & & 66 & 150.8 & & raw data - assumed degreee C \\
\hline & $10 / 16 / 73$ & 3 & & 68 & 154.4 & & raw data - assumed degreee C \\
\hline & $10 / 16 / 73$ & 4 & & 69 & 156.2 & & raw data - assumed degrees C \\
\hline & $10 / 16 / 73$ & 5 & & 68 & 154.4 & & raw data - assumed degreee C \\
\hline & $10 / 16 / 73$ & 6 & & 64 & 147.2 & & raw data - assumed degreee C \\
\hline & $10 / 16 / 73$ & 7 & & 57.7 & 135.86 & & raw data - assumed degreee C \\
\hline & $10 / 16 / 73$ & 8 & & 50.7 & 123.26 & & raw data - assumed degreee C \\
\hline & $10 / 16 / 73$ & 9 & & 44.7 & 112.46 & & raw data - assumed degreee C \\
\hline & $10 / 16 / 73$ & 10 & & 35.7 & 96.26 & & raw data - assumed degreee C \\
\hline & $10 / 16 / 73$ & 11 & & 58.6 & 137.48 & & raw data - assumed degreeo C \\
\hline & $10 / 17 / 73$ & 1 & & 64 & 147.2 & & raw data - assumed degreee C \\
\hline & $10 / 17 / 73$ & 2 & & 66.7 & 152.06 & & raw data - assumed degreee $C$ \\
\hline & $10 / 17 / 73$ & 3 & & 68.3 & 154.94 & & raw data - assumed degreee C \\
\hline & $10 / 17 / 73$ & 4 & & 69 & 156.2 & & raw data - assumed degreos C \\
\hline & $10 / 17 / 73$ & 5 & & 68 & 154.4 & & raw data - assumed degreee $C$ \\
\hline & $10 / 17 / 73$ & 6 & & 64 & 147.2 & & raw data - assumed degreee C \\
\hline & $10 / 17 / 73$ & 7 & & 58 & 136.4 & & raw data - assumed degreee C \\
\hline & $10 / 17 / 73$ & 8 & & 51 & 123.8 & & raw data - assumed degreee C \\
\hline & $10 / 17 / 73$ & 9 & & 44.7 & 112.46 & & raw data - assumed degreee C \\
\hline & $10 / 17 / 73$ & 10 & & 35.7 & 96.26 & & raw data - assumed degrees C \\
\hline & $10 / 17 / 73$ & 11 & & 58.9 & 138.02 & & raw data - assumed degreee C \\
\hline & $10 / 18 / 73$ & 1 & & 64 & 147.2 & & raw data - assumed degreee C \\
\hline & $10 / 18 / 73$ & 2 & & 66 & 150.8 & & raw data - assumed degreeo $\mathrm{C}$ \\
\hline & $10 / 18 / 73$ & 3 & & 68 & 154.4 & & raw data - assumed degreee C \\
\hline & $10 / 18 / 73$ & $\overline{4}$ & & 69 & 156.2 & & raw data - assumed degreee C \\
\hline & $10 / 18 / 73$ & 5 & & 68 & 154.4 & & raw data - assumed degreee C \\
\hline & $10 / 18 / 73$ & 6 & & 64 & 147.2 & & fraw data - assumed degreee $\mathrm{C}$ \\
\hline & $10 / 18 / 73$ & 7 & & 58 & 136.4 & & raw data - assumed degreee C \\
\hline & $10 / 18 / 73$ & 8 & & 51 & 123.8 & & raw data - assumed degreee C \\
\hline & $10 / 18 / 73$ & 9 & & 44.7 & 112.46 & & raw data - assumed degreee C \\
\hline & $10 / 18 / 73$ & 10 & & 34.7 & 94.46 & & raw data - assumed degreee C \\
\hline & $10 / 18 / 73$ & 11 & & 58.7 & 137.66 & & raw data - assumed degraes C \\
\hline
\end{tabular}

Data obtained from WHC Surveillance Analysis Computer System (SACS), June 28, 1993. 


\begin{tabular}{|c|c|c|c|c|c|c|c|}
\hline \multicolumn{8}{|c|}{ TANK 241-BY-102 } \\
\hline \multicolumn{8}{|c|}{ THERMOCOUPLE DATA } \\
\hline & & THERMO \# & TREFET \# & TEMP (C) & TEMP (F) & $Y=$ MAX TEMP & \\
\hline TREE \# & $\frac{\text { DATE }}{10 / 19 / 73}$ & $\frac{1}{1}$ & IHEE SEI H & 64 & 147.2 & & \begin{tabular}{|c} 
ColhMENTS \\
raw data - assumed degreee C
\end{tabular} \\
\hline & $10 / 19 / 73$ & 2 & & 66 & 150.8 & & raw data - assumed degrees C \\
\hline & $10 / 19 / 73$ & 3 & & 68 & 154.4 & & raw data - assumed degrees C \\
\hline & $10 / 19 / 73$ & 4 & & 69 & 156.2 & & raw data - assumed degreee C \\
\hline & $10 / 19 / 73$ & 5 & & 67.7 & 153.86 & & raw data - assumed degreee C \\
\hline & $10 / 19 / 73$ & 6 & & 63.7 & 146.66 & & raw data - assumed degree日 C \\
\hline & $10 / 19 / 73$ & 7 & & 58 & 136.4 & & raw data - assumed degreee C \\
\hline & $10 / 19 / 73$ & 8 & & 51 & 123.8 & & raw data - assumed degrees C \\
\hline & $10 / 19 / 73$ & 9 & & 45 & 113 & & raw data - assumed degreee C \\
\hline & $10 / 19 / 73$ & 10 & & 34.7 & 94.46 & & raw data - assumed degraee C \\
\hline & $10 / 19 / 73$ & 11 & & 58.7 & 137.66 & & raw data - assumed degreee C \\
\hline & $10 / 20 / 73$ & 1 & & 64. & 147.2 & & raw data - assumed degreee $\mathrm{C}$ \\
\hline & $10 / 20 / 73$ & 2 & & 66 & 150.8 & & raw data - assumed degreee C \\
\hline & $10 / 20 / 73$ & 3 & & 68 & 154.4 & & raw data - assumed degreee C \\
\hline & $10 / 20 / 73$ & 4 & & 69 & 156.2 & & raw data - assumed degreee C \\
\hline & $10 / 20 / 73$ & 5 & & 67.5 & 153.5 & & raw data - assumed degrees C \\
\hline & $10 / 20 / 73$ & 6 & & 64 & 147.2 & & raw data - assumed degreee C \\
\hline & $10 / 20 / 73$ & 7 & & 58 & 136.4 & & raw data - assumed degreee C \\
\hline & $10 / 20 / 73$ & 8 & & 51.5 & 124.7 & & raw data - assumed degreee $C$ \\
\hline & $10 / 20 / 73$ & 9 & & 45 & 113 & & raw data - assumed degreee C \\
\hline & $10 / 20 / 73$ & 10 & & 34.5 & 94.1 & & raw data - assumed degreee C \\
\hline & $10 / 20 / 73$ & 11 & & 58.8 & 137.84 & & raw data - assumed degreee $\mathrm{C}$ \\
\hline & $11 / 12 / 73$ & 1 & & 60 & 140 & & raw data - assumed degreee C \\
\hline & $11 / 12 / 73$ & 2 & & 64 & 147.2 & & raw data - assumed degreee C \\
\hline & $11 / 12 / 73$ & 3 & & 66 & 150.8 & & raw data - assumed degrese C \\
\hline & $11 / 12 / 73$ & 4 & & 66 & 150.8 & & raw data - assumed degreee C \\
\hline & $11 / 12 / 73$ & 5 & & 66 & 150.8 & & raw data - assumed degreee C \\
\hline & $11 / 12 / 73$ & 6 & & 62 & 143.6 & & raw data - assumed degreee C \\
\hline & $11 / 12 / 73$ & 7 & & 56 & 132.8 & & raw data - assumed degreee C \\
\hline & $11 / 12 / 73$ & 8 & & 52 & 125.6 & & raw data - assumed degreee $C$ \\
\hline & $11 / 12 / 73$ & 9 & & 43 & 109.4 & & raw data - assumed degreee C \\
\hline & $11 / 12 / 73$ & 10 & & 34 & 93.2 & & raw data - assumed degreee C \\
\hline & $11 / 12 / 73$ & 11 & & 34 & 93.2 & & raw data - assumed degrees C \\
\hline & $11 / 12 / 73$ & 12 & & 25 & 77 & & raw data - assumed degreee $C$ \\
\hline & $11 / 21 / 73$ & 1 & & 59 & 138.2 & & raw data - assumed degreee C \\
\hline & $11 / 21 / 73$ & 2 & & 64 & 147.2 & & raw data - assumed degreee $C$ \\
\hline & $11 / 21 / 73$ & 3 & & 65 & 149 & & raw data - assumed degreee C \\
\hline & $11 / 21 / 73$ & 4 & & 66 & 150.8 & & raw data - assumed degreee C \\
\hline & $11 / 21 / 73$ & 5 & & 63 & 145.4 & & raw data - assumed degreee C \\
\hline & $11 / 21 / 73$ & 6 & & 58 & 136.4 & & raw data - assumed degrees C \\
\hline & $11 / 21 / 73$ & 7 & & 52 & 125.6 & & raw data - assumed degrees C \\
\hline & $11 / 21 / 73$ & 8 & & 48 & 118.4 & & raw data - assumed degreee C \\
\hline & $11 / 21 / 73$ & 9 & & 42 & 107.6 & & raw data - assumed degreee C \\
\hline & $11 / 21 / 73$ & 10 & & 35 & 95 & & raw data - assumed degreee C \\
\hline & $12 / 10 / 73$ & 1 & & 60 & 140 & & raw data - assumed degrees C \\
\hline & $12 / 10 / 73$ & 2 & & 62 & 143.6 & & raw data - assumed degrees C \\
\hline & $12 / 10 / 73$ & 3 & & 65 & 149 & & raw data - assumed degrees C \\
\hline & $12 / 10 / 73$ & 4 & & 65 & 149 & & raw data - assumed degreee C \\
\hline & $12 / 10 / 73$ & 5 & & 62 & 143.6 & & raw data - assumed degreee C \\
\hline & $12 / 10 / 73$ & 6 & & 58 & 136.4 & & raw data - assumed degreee C \\
\hline & $12 / 10 / 73$ & 7 & & 53 & 127.4 & & raw data - assumed degreee C \\
\hline & $12 / 10 / 73$ & 8 & & 47 & 116.6 & & raw data - assumed degreee C \\
\hline & $12 / 10 / 73$ & 9 & & 32 & 89.6 & & raw data - assumed degreee C \\
\hline & $12 / 10 / 73$ & 10 & & 26 & 78.8 & & raw data - assumed degrees C \\
\hline & $12 / 10 / 73$ & 11 & & 24 & 75.2 & & raw data - assumed degreee C \\
\hline & $12 / 10 / 73$ & 12 & & 24 & 75.2 & & raw data - assumed degreee C \\
\hline & $12 / 17 / 73$ & 1 & & 60 & 140 & & raw data - assumed degreee $C$ \\
\hline & $12 / 17 / 73$ & 2 & & 63 & 145.4 & & raw data - assumed degreee C \\
\hline & $12 / 17 / 73$ & 3 & & 64 & 147.2 & & raw data - assumed degreee C \\
\hline & $12 / 17 / 73$ & 4 & & 64 & 147.2 & & raw data - assumed degreee C \\
\hline & $12 / 17 / 73$ & 5 & & 62 & 143.6 & & raw data - assumed degreee C \\
\hline & $12 / 17 / 73$ & 6 & & 56 & 132.8 & & raw data - assumed degreee C \\
\hline & $12 / 17 / 73$ & 7 & & 52 & 125.6 & & raw data - assumed degreee C \\
\hline & $12 / 17 / 73$ & 8 & & 46 & 114.8 & & raw data - assumed degreae C \\
\hline & $12 / 17 / 73$ & 9 & & 30 & 86 & & raw data - assumed degreee C \\
\hline & $12 / 17 / 73$ & 10 & & 26 & 78.8 & & raw data - assumed degreee C \\
\hline
\end{tabular}

Data obtained from WHC Surveillance Analysis Computer System (SACS), June 28, 1993. 
WHC-SD-WM-ER-312, Rev. 0

\begin{tabular}{|c|c|c|c|c|c|c|c|}
\hline \multicolumn{8}{|c|}{ TANK 241-BY-102 } \\
\hline \multicolumn{8}{|c|}{ THERMOCOUPLE DATA } \\
\hline TREE \# & DATE & THERMO \# & TREE SET \# & TEMP (C) & TEMP (F) & $Y=$ MAX TEMP & COMMENTS \\
\hline & $1 / 4 / 74$ & 1 & & 62 & 143.6 & & raw data - assumed degreee C \\
\hline & $1 / 4 / 74$ & 2 & & 64 & 147.2 & & raw data - assumed degreee C \\
\hline & $1 / 4 / 74$ & 3 & & 66 & 150.8 & & raw data - assumed degreea C \\
\hline & $1 / 4 / 74$ & 4 & & 65 & 149 & & raw data - assumed degreee C \\
\hline & $1 / 4 / 74$ & 5 & & 63 & 145.4 & & raw data - assumed degrees C \\
\hline & $1 / 4 / 74$ & 6 & & 58 & 136.4 & & raw date - assumed degreee C \\
\hline & $1 / 4 / 74$ & 7 & & 50 & 122 & & raw data - assumed degrees $\mathrm{C}$ \\
\hline & $1 / 4 / 74$ & 8 & & 40 & 104 & & raw data - assumed degreee C \\
\hline & $1 / 4 / 74$ & 9 & & 31 & 87.8 & & raw data - assumed degreee C \\
\hline & $1 / 4 / 74$ & 10 & & 26 & 78.8 & & raw data - assumed degreee C \\
\hline 1 & $8 / 5 / 74$ & 1 & 1 & 57 & 134.6 & $\overline{\mathrm{Y}}$ & raw data - assumed degreee C \\
\hline 1 & $8 / 5 / 74$ & 2 & 1 & 57 & 134.6 & $\overline{\mathbf{N}}$ & raw data - assumed degreee C \\
\hline 1 & $8 / 5 / 74$ & 3 & 1 & 56 & 132.8 & $\mathbf{N}$ & raw data - assumed degreoe C \\
\hline 1 & $8 / 5 / 74$ & 4 & 1 & 52 & 125.6 & $\mathbf{N}$ & raw data - assumed degreee C \\
\hline 1 & $8 / 5 / 74$ & 5 & 1 & 50 & 122 & $\mathbf{N}$ & raw data - assumed degreee C \\
\hline 1 & $8 / 5 / 74$ & 6 & 1 & 49 & 120.2 & $\mathbf{N}$ & raw data - assumed degreee C \\
\hline 1 & $8 / 5 / 74$ & 7 & 1 & 44 & 111.2 & $\mathbf{N}$ & raw data - assumed degrees C \\
\hline 1 & $8 / 5 / 74$ & 8 & 1 & 39 & 102.2 & $\mathbf{N}$ & raw data - assumed degreee C \\
\hline 1 & $8 / 5 / 74$ & 9 & 1 & 34 & 93.2 & $\mathbf{N}$ & raw data - assumed degrees C \\
\hline 1 & $8 / 5 / 74$ & 10 & 1 & 30 & 86 & $\mathbf{N}$ & raw data - assumed degreee C \\
\hline 1 & $8 / 15 / 74$ & 1 & 1 & 56 & 132.8 & $\bar{Y}$ & raw data - assumed degreee C \\
\hline 1 & $8 / 15 / 74$ & 2 & 1 & 56 & 132.8 & $\mathbf{N}$ & raw data - assumed degrees C \\
\hline 1 & $8 / 15 / 74$ & 3 & 1 & 55 & 131 & $\mathbf{N}$ & raw data - assumed degreee C \\
\hline 1 & $8 / 15 / 74$ & 4 & 1 & 54 & 129.2 & $\mathbf{N}$ & raw data - assumed degreee C \\
\hline 1 & $8 / 15 / 74$ & 5 & 1 & 52 & 125.6 & $\mathbf{N}$ & raw data - assumed degreee C \\
\hline 1 & $8 / 15 / 74$ & 6 & 1 & 49 & 120.2 & $\mathbf{N}$ & raw data - assumed degree $\mathrm{C}$ \\
\hline 1 & $8 / 15 / 74$ & 7 & 1 & 48 & 918.4 & $\overline{\mathbf{N}}$ & raw data - assumed degreee C \\
\hline 1 & $8 / 15 / 74$ & 8 & 1 & 44 & 111.2 & $\mathbf{N}$ & raw data - assumed degreee C \\
\hline 1 & $8 / 15 / 74$ & 9 & 1 & 44 & 111.2 & $\mathbf{N}$ & raw data - assumed degreee C \\
\hline 1 & $8 / 15 / 74$ & 10 & 1 & 44 & 111.2 & $N$ & raw data - assumed degreee $\mathrm{C}$ \\
\hline 1 & $9 / 12 / 74$ & 1 & 1 & 56 & 132.8 & $\mathbf{Y}$ & raw data - assumed degrees C \\
\hline 1 & $9 / 12 / 74$ & 2 & 1 & 56 & 132.8 & $\mathbf{N}$ & raw data - assumed degreee C \\
\hline 1 & $9 / 12 / 74$ & 3 & 1 & 55 & 131 & $\mathbf{N}$ & raw data - assumed degreee C \\
\hline 1 & $9 / 12 / 74$ & 4 & 1 & 54 & 129.2 & $\mathrm{~N}$ & raw data - assumed degrees C \\
\hline 1 & $9 / 12 / 74$ & 5 & 9 & 53 & 127.4 & $N$ & raw data - assumed degreee $C$ \\
\hline 1 & $9 / 12 / 74$ & 6 & 1 & 50 & 122 & $\mathbf{N}$ & raw data - assumed degreee $C$ \\
\hline 1 & $9 / 12 / 74$ & 7 & 1 & 48 & 118.4 & $\mathbf{N}$ & raw data - assumed degreee $C$ \\
\hline 1 & $9 / 12 / 74$ & 8 & 1 & 44 & 111.2 & $\mathrm{~N}$ & raw data - assumed degreee C \\
\hline 1 & $.9 / 12 / 74$ & 9 & 1 & 44 & 111.2 & $\mathbf{N}$ & raw data - assumed degreee $C$ \\
\hline 1 & $9 / 12 / 74$ & 10 & 1 & 44 & 111.2 & $\mathbf{N}$ & raw data - assumed degreee C \\
\hline 9 & $11 / 16 / 74$ & 9 & 1 & & & $\bar{Y}$ & 67.78 - suspect data (low) \\
\hline 1 & $11 / 16 / 74$ & 2 & 1 & & & $\mathbf{N}$ & 67.78 - suspect data (low) \\
\hline 1 & $11 / 16 / 74$ & 3 & 1 & & & $\mathbf{N}$ & 68.33 - suspect data (low) \\
\hline 1 & $11 / 16 / 74$ & 4 & 1 & & & $\mathbf{N}$ & 67.78 - suspect data (low) \\
\hline 1 & $11 / 16 / 74$ & 5 & 1 & & & $\bar{N}$ & 67.78 - suspect data (low) \\
\hline 1 & $11 / 16 / 74$ & 6 & 1 & & & $\mathbf{N}$ & 65.56 - suspect data (low) \\
\hline 1 & $11 / 16 / 74$ & 7 & 1 & & & $\mathbf{N}$ & 58.89 - suspect data (low) \\
\hline 1 & $11 / 16 / 74$ & 8 & 1 & & & $\mathbf{N}$ & 54.44 - suspect data (low) \\
\hline 9 & $11 / 16 / 74$ & 9 & 1 & & & $\mathbf{N}$ & 51.67 - suspect data (low) \\
\hline 1 & $11 / 16 / 74$ & 10 & 1 & & & $\mathbf{N}$ & 51.67 - suspect date (low) \\
\hline 1 & $11 / 16 / 74$ & 11 & 1 & & & $\mathbf{N}$ & 46.11 - suspect data (low) \\
\hline 1 & $11 / 16 / 74$ & 12 & 1 & & & $\mathbf{N}$ & 46.11 - suspect data (low) \\
\hline 1 & $12 / 17 / 74$ & 1 & 1 & 48.89 & 120.002 & $\bar{Y}$ & raw data - assumed degreee C \\
\hline 1 & $12 / 17 / 74$ & 2 & 1 & 47.78 & 118.004 & $\mathbf{N}$ & raw data - assumed degreee C \\
\hline 1 & $12 / 17 / 74$ & 3 & 1 & 48.89 & 120.002 & $\mathbf{N}$ & raw data - assumed degrees C \\
\hline 1 & $12 / 17 / 74$ & 4 & 1 & 48.89 & 120.002 & $\mathbf{N}$ & raw data - assumed degreae C \\
\hline 1 & $12 / 17 / 74$ & 5 & 1 & 47.78 & 118.004 & $\mathbf{N}$ & raw data - assumed degreee C \\
\hline 1 & $12 / 17 / 74$ & 6 & 1 & 45.56 & 114.008 & $\mathbf{N}$ & raw data - assumed degreee C \\
\hline 1 & $12 / 17 / 74$ & 7 & 1 & 42.78 & 109.004 & $\mathbf{N}$ & raw data - assumed degrees C \\
\hline 1 & $12 / 17 / 74$ & 8 & 1 & 37.22 & 98.996 & $\mathbf{N}$ & raw data - assumed degreae C \\
\hline 1 & $12 / 17 / 74$ & 9 & 1 & 36.67 & 98.006 & $\mathbf{N}$ & raw data - assumed degreee C \\
\hline 1 & $12 / 17 / 74$ & 10 & 1 & 41.11 & 105.998 & $\mathbf{N}$ & raw data - assumed degreee C \\
\hline 1 & $12 / 17 / 74$ & 11 & 1 & 31.11 & 87.998 & $\mathbf{N}$ & raw data - assumed degreee C \\
\hline 1 & $12 / 17 / 74$ & 12 & 1 & 31.11 & 87.998 & $\mathbf{N}$ & raw data - assumed degreee C \\
\hline 1 & $12 / 31 / 74$ & 1 & 1 & & & $\mathrm{~N}$ & $0 / 5$ \\
\hline 9 & 1/8/75 & 1 & 1 & & & $\mathrm{~N}$ & $0 / 5$ \\
\hline
\end{tabular}

Data obtained from WHC Surveillance Analysis Computer System (SACS), June 28, 1993. 


\begin{tabular}{|c|c|c|c|c|c|c|c|}
\hline \multicolumn{8}{|c|}{$\begin{array}{l}\text { TANK 241-BY-102 } \\
\text { THERMOCOUPLE DATA }\end{array}$} \\
\hline & & & & & & & \\
\hline TREE \# & DATE & THERMO \# & TREE SET \# & TEMP (C) & TEMP (F) & $Y=$ MAX TEMP & COMMENTS \\
\hline 1 & $5 / 20 / 76$ & 1 & 1 & & & $\mathrm{~N}$ & 5 - suspect data (low) \\
\hline 1 & $5 / 20 / 76$ & 2 & 1 & & & $\mathbf{N}$ & 7 - suspact data (low) \\
\hline 1 & $5 / 20 / 76$ & 3 & 1 & & & $\mathbf{N}$ & 6 - suspect data (low) \\
\hline$T$ & $5 / 20 / 76$ & 4 & 1 & & & $\bar{N}$ & 6 - suspect data (low) \\
\hline 1 & $5 / 20 / 76$ & 5 & 1 & & & $\mathbf{N}$ & 6- suspect data (low) \\
\hline 1 & $5 / 20 / 76$ & 6 & 1 & & & $\mathbf{N}$ & 10 - suspect data (low) \\
\hline 9 & $5 / 20 / 76$ & 7 & 1 & & & $\bar{N}$ & 13 - suspect data (low) \\
\hline 1 & $5 / 20 / 76$ & 8 & 1 & & & $\mathbf{N}$ & 18 - suspect data (low) \\
\hline 9 & $5 / 20 / 76$ & 9 & 1 & & & $\mathbf{N}$ & 20 - suspect data (low) \\
\hline 1 & $5 / 20 / 76$ & 10 & 1 & & & $\mathbf{N}$ & 20 - suspect data (low) \\
\hline 1 & $5 / 20 / 76$ & 11 & 1 & & & $\mathbf{N}$ & 20 - suspect data (low) \\
\hline$\overline{1}$ & $5 / 20 / 76$ & 12 & 1 & & & $\mathbf{N}$ & 20 - suspect data (low) \\
\hline 1 & $5 / 20 / 76$ & 13 & 1 & & & $\mathbf{N}$ & 20 - suspect data (low) \\
\hline 1 & $5 / 20 / 76$ & 14 & 1 & & & $\mathbf{N}$ & 20 - suspect data (low) \\
\hline 1 & $8 / 14 / 76$ & 1 & 1 & & 78 & $\mathbf{N}$ & raw data - assume reverse TC order \\
\hline 1 & $8 / 14 / 76$ & 2 & 1 & & 78 & $\mathbf{Y}$ & raw data - assume reverse TC order \\
\hline 1 & $8 / 14 / 76$ & 3 & 1 & & 77 & $\mathbf{N}$ & raw data - assume reverse TC order \\
\hline 1 & $8 / 14 / 76$ & 4 & 1 & & 77 & $\mathbf{N}$ & raw data - assume reverse TC order \\
\hline 1 & $8 / 14 / 76$ & 5 & 1 & & 75 & $\mathbf{N}$ & raw data - assume reverse TC order \\
\hline 1 & $8 / 14 / 76$ & 6 & 1 & & 69 & $\mathbf{N}$ & raw data - assume reverse TC order \\
\hline 1 & $8 / 14 / 76$ & 7 & 1 & & 64 & $\mathbf{N}$ & raw data - assume reverse TC order \\
\hline 1 & $8 / 14 / 76$ & 8 & 1 & & 60 & $\mathbf{N}$ & raw data - assume reverse TC order \\
\hline 1 & $8 / 14 / 76$ & 9 & 1 & & 59 & $\mathbf{N}$ & Iraw data - assume reverse TC order \\
\hline 1 & $8 / 14 / 76$ & 10 & 1 & & 58 & $\mathbf{N}$ & raw data - assume reverse TC order \\
\hline 1 & $8 / 14 / 76$ & 11 & 1 & & 58 & $\mathbf{N}$ & raw data - assume reverse TC order \\
\hline 1 & $8 / 14 / 76$ & 12 & 1 & & 56 & $N$ & raw data - assume reverse TC order \\
\hline 9 & $9 / 13 / 76$ & 1 & 1 & & & $\mathbf{N}$ & 105 - suspect data (high), reverse TC order \\
\hline 1 & $9 / 13 / 76$ & 2 & 1 & & & $\mathbf{N}$ & 105 - suspect data (high), reverse TC order \\
\hline 1 & $9 / 13 / 76$ & 3 & 1 & & & $\mathbf{N}$ & 104 - suspect data (high), reverse TC order \\
\hline 1 & $9 / 13 / 76$ & 4 & 1 & & & $\mathbf{Y}$ & 105 - suspect data (high), reverse TC order \\
\hline 1 & $9 / 13 / 76$ & 5 & 1 & & & $\mathbf{N}$ & 103 - suspect data (high), revarse TC order \\
\hline 1 & $9 / 13 / 76$ & 6 & 1 & & 99 & $\bar{N}$ & raw data - assume reverse TC order \\
\hline 1 & $9 / 13 / 76$ & 7 & 1 & & 95 & $\mathbf{N}$ & raw data - assume reverse TC order \\
\hline 1 & $9 / 13 / 76$ & 8 & 1 & & 93 & $\mathbf{N}$ & raw data - assume reverse TC order \\
\hline 1 & $9 / 13 / 76$ & 9 & 1 & & 92 & $\mathbf{N}$ & raw data - assume reverse TC order \\
\hline 1 & $9 / 13 / 76$ & 10 & 1 & & $\overline{91}$ & $\mathbf{N}$ & raw data - assume reverse TC order \\
\hline 1 & $9 / 13 / 76$ & 11 & 1 & & 93 & $\mathrm{~N}$ & raw date - assume reverse TC order \\
\hline 1 & $9 / 13 / 76$ & 12 & 1 & & 92 & $\mathbf{N}$ & raw deta - assume revarse TC order \\
\hline 1 & $.11 / 7 / 76$ & 1 & 1 & & 61 & $\mathbf{N}$ & raw data - assume reverse TC order \\
\hline 1 & $11 / 7 / 76$ & 2 & 1 & & 61 & $\bar{Y}$ & raw data - assume reverse TC order \\
\hline$\frac{1}{1}$ & $11 / 7 / 76$ & 3 & 1 & & 60 & $\mathbf{N}$ & raw data - assume reverse TC order \\
\hline 1 & $19 / 7 / 76$ & 4 & 1 & & 60 & $\mathbf{N}$ & raw data - assume reverse TC order \\
\hline 1 & $11 / 7 / 76$ & 5 & 1 & & 60 & $\mathbf{N}$ & raw data - assume reverse TC order \\
\hline 1 & $11 / 7 / 76$ & 6 & 1 & & 60 & $\bar{N}$ & raw data - assume reverse TC order \\
\hline 1 & $11 / 7 / 76$ & 7 & 1 & & 60 & $\mathbf{N}$ & raw data - assume reverse TC order \\
\hline 1 & $11 / 7 / 76$ & 8 & $\frac{1}{1}$ & & 60 & $N$ & raw data - assume reverse TC order \\
\hline 1 & $11 / 7 / 76$ & 9 & 1 & & 60 & $\mathbf{N}$ & raw data - assume reverse TC order \\
\hline 1 & $11 / 7 / 76$ & 10 & 1 & & 59 & $\mathbf{N}$ & raw data - assume reverse TC order \\
\hline 1 & $11 / 7 / 76$ & 11 & 1 & & 59 & $\mathbf{N}$ & raw data - assume reverse TC order \\
\hline 1 & $11 / 7 / 76$ & 12 & 1 & & 58 & $\mathbf{N}$ & raw data - assume reverse TC order \\
\hline 1 & $12 / 9 / 76$ & 7 & 1 & 39 & 102.2 & $\mathbf{N}$ & raw data - assume degree $C \&$ reverse TC order \\
\hline 1 & $12 / 9 / 76$ & 8 & 1 & 39 & 102.2 & Y & raw data - assume degree $C \&$ reverse TC order \\
\hline 1 & $12 / 9 / 76$ & 9 & 1 & 36 & 96.8 & $\mathrm{~N}$ & raw data - assume degree $C \&$ reverse TC order \\
\hline 1 & $12 / 9 / 76$ & 10 & 1 & 37 & 98.6 & $N$ & raw data - assume degree $C \&$ reverse TC order \\
\hline 1 & $12 / 9 / 76$ & 11 & 1 & 33 & 91.4 & $\mathbf{N}$ & raw data - assume degree $C \&$ reverse TC order \\
\hline 1 & $12 / 9 / 76$ & 12 & 1 & 25 & 77 & $\mathbf{N}$ & raw data - assume degree $C \&$ reverse TC order \\
\hline 1 & $9 / 3 / 77$ & 1 & $\frac{1}{1}$ & & & $\mathbf{N}$ & $0 / 5$ \\
\hline 1 & $3 / 28 / 77$ & 1 & $i$ & & & $\mathbf{N}$ & 54 - suspect data (low), reverse TC order \\
\hline 1 & $3 / 28 / 77$ & 2 & 1 & & & $\bar{Y}$ & 54 - suspect data (low), reverse TC order \\
\hline 1 & $3 / 28 / 77$ & 3 & $\frac{1}{1}$ & & & $\mathbf{N}$ & 49 - suspect data (low), reverse TC order \\
\hline
\end{tabular}

Data obtained from WHC Surveillance Analysis Computer System (SACS), June 28, 1993. 
WHC-SD-WM-ER-312, Rev. 0

\begin{tabular}{|c|c|c|c|c|c|c|c|}
\hline \multicolumn{8}{|c|}{ TANK 241-BY-102 } \\
\hline \multicolumn{8}{|c|}{ THERMOCOUPLE DATA } \\
\hline TREE \# & DATE & THERMO \# & TREE SET \# & TEMP (C) & TEMP (F) & $Y=\operatorname{MAX}$ TEMP & COMMENTS \\
\hline 1 & $3 / 28 / 77$ & 4 & 1 & & & $\mathbf{N}$ & 51 - suspect data (low), reverse TC order \\
\hline 1 & $3 / 28 / 77$ & 5 & 1 & & & $\mathbf{N}$ & 50 - suspect data (low), reverse TC order \\
\hline 1 & $3 / 28 / 77$ & 6 & 1 & & & $\mathbf{N}$ & 42 - suspect data (low), reverse TC order \\
\hline 1 & $3 / 28 / 77$ & 7 & 1 & 38 & 100.4 & $\mathbf{N}$ & raw data - assume degree C \& reverse TC order \\
\hline 1 & $3 / 28 / 77$ & 8 & 1 & 32 & 89.6 & $\mathbf{N}$ & raw data - assume degree C \& reverse TC order \\
\hline 1 & $3 / 28 / 77$ & 9 & 1. & 33 & 91.4 & $\mathbf{N}$ & raw data - assume degree $C \&$ reverse TC order \\
\hline 1 & $3 / 28 / 77$ & 10 & 1 & 30 & 86 & $\mathrm{~N}$ & raw data - assume degree C \& reverse TC order \\
\hline 1 & $3 / 28 / 77$ & 11 & 1 & $32^{\circ}$ & 89.6 & $\mathbf{N}$ & raw data - assume degree $C \&$ reverse TC order \\
\hline 1 & $3 / 28 / 77$ & 12 & 1 & 32 & 89.6 & $\mathrm{~N}$ & raw data - assume degree $C \&$ reverse TC order \\
\hline 1 & $4 / 27 / 77$ & 1 & 1 & & 95 & $\mathbf{N}$ & raw data - assume reverse TC order \\
\hline 1 & $4 / 27 / 77$ & 2 & 1 & & 95 & $\mathrm{~N}$ & raw data - assume reverse TC order \\
\hline 1 & $4 / 27 / 77$ & 3 & 1 & & 97 & $\mathbf{N}$ & raw data - assume reverse TC order \\
\hline 1 & $4 / 27 / 77$ & 4 & 1 & & 96 & $N$ & raw data - assume reverse TC order \\
\hline 1 & $4 / 27 / 77$ & 5 & 1 & & 99 & $\bar{Y}$ & raw data - assume reverse TC order \\
\hline 1 & $4 / 27 / 77$ & 6 & 1 & & 92 & $N$ & raw data - assume reverse TC order \\
\hline 1 & $4 / 27 / 77$ & 7 & 1 & & 92 & $\mathrm{~N}$ & raw data - assume reverse TC order \\
\hline 1 & $4 / 27 / 77$ & 8 & 1 & & 84 & $\mathbf{N}$ & raw data - assume reverse TC order \\
\hline 1 & $4 / 27 / 77$ & 9 & 1 & & 83 & $\mathrm{~N}$ & raw data - assume reverse TC order \\
\hline 1 & $4 / 27 / 77$ & 10 & 1 & & 81 & $\mathbf{N}$ & raw data - assume reverse TC order \\
\hline 1 & $4 / 27 / 77$ & 11 & 1 & & 79 & $N$ & raw data - assume reverse TC order \\
\hline 1 & $4 / 27 / 77$ & 12 & 1 & & 80 & $\mathbf{N}$ & raw data - assume reverse TC order \\
\hline 1 & $4 / 27 / 77$ & 13 & 1 & & 81 & $\mathbf{N}$ & raw data - assume reverse TC order \\
\hline 1 & $4 / 27 / 77$ & 14 & 1 & & 83 & $\mathbf{N}$ & raw data - assume reverse TC order \\
\hline 1 & $6 / 30 / 77$ & 1 & 1 & & & $\mathbf{N}$ & 52 - suspect data (low), reverse TC order \\
\hline 1 & $6 / 30 / 77$ & 2 & 1 & & & $\mathbf{N}$ & 52 - suspect data (low), reverse TC order \\
\hline 9 & $6 / 30 / 77$ & 3 & 1 & & & $\mathbf{N}$ & 52 - suspect data (low), reverse TC order \\
\hline 1 & $6 / 30 / 7.7$ & 4 & 1 & & & $\mathbf{N}$ & 52 - suspect data (low), reverse TC order \\
\hline 1 & $6 / 30 / 77$ & 5 & 1 & & & $\mathbf{N}$ & 52 - suspect data (low), reverse TC order \\
\hline 1 & $6 / 30 / 77$ & 6 & 1 & & & $\mathbf{N}$ & 52 - suspect data (low), reverse TC order \\
\hline 1 & $6 / 30 / 77$ & 7 & 1 & & & $\mathbf{N}$ & 51 - suspect data (low), reverse TC order \\
\hline 1 & $6 / 30 / 77$ & 8 & 1 & 42 & 107.6 & $\mathbf{N}$ & raw data - assume reverse TC order \\
\hline 1 & $6 / 30 / 77$ & 9 & 1 & 31 & 87.8 & $\mathbf{N}$ & raw data - assume reverse TC order \\
\hline 1 & $.6 / 30 / 77$ & 10 & 1 & 32 & 89.6 & $\mathbf{N}$ & raw data - assume reverse TC order \\
\hline 1 & $6 / 30 / 77$ & 11 & 1 & 29 & 84.2 & $\mathbf{N}$ & raw data - assume reverse TC order \\
\hline 1 & $6 / 30 / 77$ & 12 & 1 & 25 & 77 & $\mathbf{N}$ & raw data - assume reverse TC order \\
\hline 1 & $6 / 30 / 77$ & 13 & 1 & 25 & 77 & $\mathbf{N}$ & raw data - assume reverse TC order \\
\hline 1 & $8 / 15 / 77$ & 1 & 1 & & 78 & $\mathbf{N}$ & raw data - assume reverse TC order \\
\hline 1 & $8 / 15 / 77$ & 2 & 1 & & 78 & $\mathbf{N}$ & raw data - assume reverse TC order \\
\hline 1 & $8 / 15 / 77$ & 3 & 1 & & 78 & $N$ & raw data - assume reverse TC order \\
\hline 1 & $8 / 15 / 77$ & 4 & 1 & & 78 & $\mathbf{N}$ & raw data - assume reverse TC order \\
\hline 1 & $8 / 15 / 77$ & 5 & 1 & & 78 & $\mathbf{N}$ & raw data - assume reverse TC order \\
\hline 1 & $8 / 15 / 77$ & 6 & 1 & & 78 & $\mathbf{N}$ & raw data - assume reverse TC order \\
\hline 1 & $8 / 15 / 77$ & 7 & 1 & & 78 & $\bar{Y}$ & raw data - assume reverse TC order \\
\hline 1 & $8 / 15 / 77$ & 8 & 1 & & 77 & $\mathbf{N}$ & raw data - assume reverse TC order \\
\hline 1 & $8 / 15 / 77$ & 9 & 1 & & 77 & $\mathbf{N}$ & raw data - assume reverse TC order \\
\hline 1 & $8 / 15 / 77$ & 10 & 1 & & 77 & $\mathbf{N}$ & raw data - assume reverse TC order \\
\hline 9 & $8 / 15 / 77$ & 11 & 1 & & 77 & $\mathbf{N}$ & raw data - assume reverse TC order \\
\hline 1 & $8 / 15 / 77$ & 12 & 1 & & 77 & $\mathbf{N}$ & raw data - assume reverse TC order \\
\hline 1 & $8 / 15 / 77$ & 13 & 1 & & 77 & $\mathbf{N}$ & raw data - assume reverse TC order \\
\hline 1 & $8 / 15 / 77$ & 14 & 1 & & 77 & $\mathbf{N}$ & raw data - assume reverse TC order \\
\hline 1 & $9 / 12 / 77$ & 1 & 1 & & 90 & $\mathbf{N}$ & raw data - assume reverse TC order \\
\hline 1 & $9 / 12 / 77$ & 2 & 1 & & 91 & $\mathbf{N}$ & raw data - assume reverse TC order \\
\hline 1 & $9 / 12 / 77$ & 3 & 1 & & 93 & $\mathbf{N}$ & raw data - assume reverse TC order \\
\hline 1 & $9 / 12 / 77$ & 4 & 1 & & 94 & $\mathbf{N}$ & raw data - assume reverse TC order \\
\hline 1 & $9 / 12 / 77$ & 5 & 1 & & 94 & $\bar{Y}$ & raw data - assume reverse TC order \\
\hline 1 & $9 / 12 / 77$ & 6 & 1 & & 87 & $\mathrm{~N}$ & raw data - assume reverse TC order \\
\hline 1 & $9 / 12 / 77$ & 7 & 1 & & 83 & $\mathbf{N}$ & raw data - assume reverse TC order \\
\hline 1 & $9 / 12 / 77$ & 8 & 1 & & 81 & $\mathbf{N}$ & raw data - assume reverse TC order \\
\hline 1 & $9 / 12 / 77$ & 9 & 1 & & 80 & $\mathrm{~N}$ & raw data - assume reverse TC order \\
\hline 1 & $9 / 12 / 77$ & 10 & 1 & & 82 & $\mathbf{N}$ & raw data - assume reverse TC order \\
\hline
\end{tabular}

Data obtained from WHC Surveillance Analysis Computer System (SACS), June 28, 1993. 


\begin{tabular}{|c|c|c|c|c|c|c|c|}
\hline \multicolumn{8}{|c|}{ TANK 241-BY-102 } \\
\hline \multicolumn{8}{|c|}{ THERMOCOUPLE DATA } \\
\hline TREE \# & DATE & THERMO \# & TREE SET \# & TEMP (C) & TEMP (F) & $Y=$ MAX TEMP & COMMENTS \\
\hline 1 & $9 / 12 / 77$ & 11 & 1 & & 83 & $\mathbf{N}$ & raw data - assume reverse TC order \\
\hline 1 & $9 / 12 / 77$ & 12 & 1 & & 84 & $\mathbf{N}$ & raw data - assume reverse TC order \\
\hline 1 & $10 / 7 / 77$ & 1 & 1 & & 73 & $\mathbf{N}$ & raw data - assume reverse TC order \\
\hline 1 & $10 / 7 / 77$ & 2 & 1 & & 72 & $\mathbf{N}$ & raw data - assume reverse TC order \\
\hline 1 & $10 / 7 / 77$ & 3 & 1 & & 72 & $\mathbf{N}$ & raw data - assume reverse TC order \\
\hline 1 & $10 / 7 / 77$ & 4 & 9 & & $\overline{72}$ & $\mathbf{N}$ & raw data - assume reverse TC order \\
\hline 1 & $10 / 7 / 77$ & 5 & 1 & & 72 & $\mathbf{N}$ & raw data - assume reverse TC order \\
\hline 1 & $10 / 7 / 77$ & 6 & 1 & & 72 & $\mathbf{N}$ & raw data - assume reverse TC order \\
\hline 1 & $10 / 7 / 77$ & 7 & 1 & & 72 & $\bar{N}$ & raw data - assume reverse TC order \\
\hline 1 & $10 / 7 / 77$ & 8 & 1 & & 72 & $\mathbf{N}$ & raw data - assume reverse TC order \\
\hline 1 & $10 / 7 / 77$ & 9 & 1 & & 73 & $\mathbf{N}$ & raw data - assume reverse TC order \\
\hline 1 & $10 / 7 / 77$ & 10 & 1 & 0 & 73 & $\mathbf{N}$ & raw data - assume reverse TC order \\
\hline 1 & $10 / 7 / 77$ & 19 & 1 & & 73 & $\mathbf{Y}$ & raw data - assume reverse TC order \\
\hline 1 & $10 / 7 / 77$ & 12 & 1 & & 72 & $\mathbf{N}$ & raw data - assume reverse TC order \\
\hline 1 & $10 / 7 / 77$ & 13 & 1 & & 72 & $\mathbf{N}$ & raw data - assume reverse TC order \\
\hline 1 & $10 / 7 / 77$ & 14 & 1 & & 72 & $\bar{N}$ & raw data - assume reverse TC order \\
\hline 1 & $12 / 7 / 77$ & 1 & 1 & & 70 & $\mathbf{N}$ & \\
\hline 1 & $12 / 7 / 77$ & 1 & 2 & & 57 & $\mathbf{N}$ & raw data - assume reverse TC order \\
\hline 1 & $12 / 7 / 77$ & 2 & 1 & & 70 & $\mathbf{N}$ & \\
\hline$T$ & $12 / 7 / 77$ & 2 & 2 & & 57 & $\bar{Y}$ & raw data - assume reverse TC order \\
\hline 1 & $12 / 7 / 77$ & 3 & 1 & & 73 & $\mathbf{N}$ & \\
\hline 1 & $12 / 7 / 77$ & 3 & 2 & & 55 & $\mathbf{N}$ & raw data - assume reverse TC order \\
\hline 1 & $12 / 7 / 77$ & 4 & 1 & & 74 & $N$ & \\
\hline 1 & $12 / 7 / 77$ & 4 & 2 & & 55 & $\mathbf{N}$ & raw data - assume reverse TC order \\
\hline 1 & $12 / 7 / 77$ & 5 & 1 & & 70 & $\mathbf{N}$ & \\
\hline 1 & $12 / 7 / 77$ & 5 & 2 & & 55 & $\mathbf{N}$ & raw data - assume reverse TC order \\
\hline 1 & $12 / 7 / 77$ & 6 & 1 & & 78 & $\mathbf{Y}$ & \\
\hline 1 & $12 / 7 / 77$ & 6 & 2 & & 55 & $\mathbf{N}$ & raw data - assume reverse TC order \\
\hline 1 & $12 / 7 / 77$ & 7 & 1 & & 78 & $\mathbf{N}$ & \\
\hline 1 & $12 / 7 / 77$ & 7 & 2 & & 54 & $\mathbf{N}$ & raw data - assume reverse TC order \\
\hline 1 & $12 / 7 / 77$ & 8 & 1 & & 70 & $\mathbf{N}$ & \\
\hline 1 & $12 / 7 / 77$ & 8 & 2 & & 54 & $\mathbf{N}$ & raw data - assume reverse TC order \\
\hline 1 & $12 / 7 / 77$ & 9 & 1 & & 70 & $\mathbf{N}$ & \\
\hline 1 & $12 / 7 / 77$ & 9 & 2 & & 54 & $\mathbf{N}$ & raw data - assume reverse TC order \\
\hline 1 & $12 / 7 / 77$ & 10 & 1 & & 70 & $\mathbf{N}$ & \\
\hline 1 & $12 / 7 / 77$ & 10 & 2 & & 54 & $\mathbf{N}$ & raw data - assume reverse TC order \\
\hline 1 & $12 / 7 / 77$ & 11. & 1 & & 70 & $\mathbf{N}$ & \\
\hline 1 & $12 / 7 / 77$ & 11 & 2 & & 53 & $\mathbf{N}$ & raw data - assume reverse TC order \\
\hline 1 & $.12 / 7 / 77$ & 12 & 1 & & 71 & $\mathbf{N}$ & \\
\hline 1 & $12 / 7 / 77$ & 12 & 2 & & 53 & $\mathbf{N}$ & raw data - assume reverse TC order \\
\hline 1 & $12 / 7 / 77$ & 13 & 2 & & 51 & $\mathbf{N}$ & raw data - assume reverse TC order \\
\hline 1 & $12 / 7 / 77$ & 14 & 2 & & 45 & $\mathbf{N}$ & raw data - assume reverse TC ordar \\
\hline 1 & $2 / 12 / 78$ & 13 & 1 & & 74 & $\mathbf{N}$ & raw data - assume reverse TC order \\
\hline 1 & $2 / 12 / 78$ & 14 & 1 & & 74 & $\mathbf{N}$ & raw data - assume reverse TC order \\
\hline 1 & $2 / 26 / 78$ & 1 & 1 & & 77 & $\mathbf{N}$ & \\
\hline 1 & $2 / 26 / 78$ & 1 & 2 & & 68 & $\bar{Y}$ & \\
\hline 1 & $2 / 26 / 78$ & 2 & 1 & & 78 & $\mathbf{N}$ & \\
\hline 1 & $2 / 26 / 78$ & 2 & 2 & & 68 & $\mathbf{N}$ & \\
\hline 1 & $2 / 26 / 78$ & 3 & 1 & & 74 & $\mathbf{N}$ & \\
\hline 1 & $2 / 26 / 78$ & 3 & 2 & & 68 & $\mathbf{N}$ & \\
\hline 1 & $2 / 26 / 78$ & 4 & 1 & & 81 & $\mathbf{N}$ & \\
\hline 1 & $2 / 26 / 78$ & 4 & 2 & & 68 & $\mathbf{N}$ & \\
\hline 1 & $2 / 26 / 78$ & 5 & 1 & & 82 & $Y$ & \\
\hline 1 & $2 / 26 / 78$ & 5 & 2 & & 68 & $N$ & \\
\hline 1 & $2 / 26 / 78$ & 6 & 1 & & 74 & $\mathbf{N}$ & \\
\hline 1 & $2 / 26 / 78$ & 6 & 2 & & 68 & $\mathbf{N}$ & \\
\hline 1 & $2 / 26 / 78$ & 7 & 1 & & 74 & $\mathbf{N}$ & \\
\hline 1 & $2 / 26 / 78$ & 7 & 2 & & 68 & $N$ & \\
\hline 1 & $2 / 26 / 78$ & 8 & 1 & & 74 & $\mathbf{N}$ & \\
\hline 1 & $2 / 26 / 78$ & 8 & 2 & & 68 & $N$ & \\
\hline 1 & $2 / 26 / 78$ & 9 & 1 & & 75 & $\mathbf{N}$ & \\
\hline 1 & $2 / 26 / 78$ & 9 & 2 & & 68 & $\mathbf{N}$ & \\
\hline 1 & $2 / 26 / 78$ & 10 & 1 & & 75 & $\mathbf{N}$ & \\
\hline 1 & $2 / 26 / 78$ & 10 & 2 & & 68 & $\mathrm{~N}$ & \\
\hline 1 & $2 / 26 / 78$ & 12 & 2 & & 68 & $N$ & \\
\hline 1 & $3 / 26 / 78$ & 1 & 1 & & 60 & $Y$ & \\
\hline
\end{tabular}

Data obtained from WHC Surveillance Analysis Computer System (SACS), June 28, 1993. 


\begin{tabular}{|c|c|c|c|c|c|c|c|}
\hline \multicolumn{8}{|c|}{ TANK 241-BY-102 } \\
\hline \multicolumn{8}{|c|}{ THERMOCOUPLE DATA } \\
\hline TREE \# & DATE & THERMO \# & TREE SET \# & TEMP (C) & TEMP (F) & $Y=M A X$ TEMP & COMMENTS \\
\hline 1 & $3 / 26 / 78$ & 1 & 2 & & 80 & $Y$ & \\
\hline 1 & $3 / 26 / 78$ & 2 & 1 & & 60 & $\mathbf{N}$ & \\
\hline 1 & $3 / 26 / 78$ & 2 & 2 & & 79 & $\mathbf{N}$ & \\
\hline 1 & $3 / 26 / 78$ & 3 & 1 & & 60 & $\mathbf{N}$ & \\
\hline 1 & $3 / 26 / 78$ & 3 & 2 & & 79 & $\mathbf{N}$ & \\
\hline 9 & $3 / 26 / 78$ & 4 & 1 & & 60 & $\mathbf{N}$ & \\
\hline 1 & $3 / 26 / 78$ & 4 & 2 & & 79 & $\mathbf{N}$ & \\
\hline 1 & $3 / 26 / 78$ & 5 & 1 & & 59 & $\mathbf{N}$ & \\
\hline 1 & $3 / 26 / 78$ & 5 & 2 & & 80 & $\mathbf{N}$ & \\
\hline 1 & $3 / 26 / 78$ & 6 & 1 & & 59 & $\mathbf{N}$ & \\
\hline 1 & $3 / 26 / 78$ & 6 & 2 & & 80 & $\bar{N}$ & \\
\hline 1 & $3 / 26 / 78$ & 7 & 1 & $\therefore$ & 59 & $\mathbf{N}$ & \\
\hline 1 & $3 / 26 / 78$ & 7 & 2 & & 80 & $\mathbf{N}$ & \\
\hline 1 & $3 / 26 / 78$ & 8 & 1 & & 59 & $\mathbf{N}$ & \\
\hline 1 & $3 / 26 / 78$ & 8 & 2 & & 80 & $\mathrm{~N}$ & \\
\hline 1 & $3 / 26 / 78$ & 9 & 2 & & 80 & $\mathbf{N}$ & \\
\hline 1 & $3 / 26 / 78$ & 10 & 2 & & 80 & $\mathbf{N}$ & \\
\hline 9 & $3 / 26 / 78$ & 19 & 2 & & 80 & $\mathbf{N}$ & \\
\hline 1 & $3 / 26 / 78$ & 12 & 2 & & 80 & $\mathbf{N}$ & \\
\hline$\overline{1}$ & $4 / 23 / 78$ & 1 & 1 & & 89 & $Y$ & \\
\hline 1 & $4 / 23 / 78$ & 1 & 2 & & 78 & $\mathbf{N}$ & \\
\hline 1 & $4 / 23 / 78$ & 2 & 1 & & 89 & $\mathrm{~N}$ & \\
\hline 1 & $4 / 23 / 78$ & 2 & 2 & & 80 & $N$ & \\
\hline 1 & $4 / 23 / 78$ & 3 & 1 & & 89 & $\mathrm{~N}$ & \\
\hline 1 & $4 / 23 / 78$ & 3 & 2 & & 82 & $\mathrm{~N}$ & \\
\hline 1 & $4 / 23 / 78$ & 4 & 1 & & 88 & $\mathrm{~N}$ & \\
\hline 1 & $4 / 23 / 78$ & 4 & 2 & & 82 & $\mathbf{N}$ & \\
\hline 1 & $4 / 23 / 78$ & 5 & 1 & & 88 & $N$ & \\
\hline 1 & $4 / 23 / 78$ & 5 & 2 & & 79 & $\mathbf{N}$ & \\
\hline 1 & $4 / 23 / 78$ & 6 & 1 & & 87 & $\mathbf{N}$ & \\
\hline 1 & $4 / 23 / 78$ & 6 & 2 & & 85 & $Y$ & \\
\hline 1 & $4 / 23 / 78$ & 7 & 1 & & 87 & $\bar{N}$ & \\
\hline 1 & $4 / 23 / 78$ & 7 & 2 & & 85 & $\mathbf{N}$ & \\
\hline 1 & $4 / 23 / 78$ & 8 & 1 & & 87 & $\mathrm{~N}$ & \\
\hline 1 & $4 / 23 / 78$ & 8 & 2 & & 69 & $\bar{N}$ & \\
\hline 1 & $4 / 23 / 78$ & 9 & 1 & & 87 & $\mathbf{N}$ & \\
\hline 1 & $4 / 23 / 78$ & 9 & 2 & & 82 & $\mathbf{N}$ & \\
\hline$\frac{1}{1}$ & $4 / 23 / 78$ & 10 & 1 & & 86 & $\bar{N}$ & \\
\hline 1 & $.4 / 23 / 78$ & 10 & 2 & & 81 & $\mathrm{~N}$ & \\
\hline 1 & $4 / 23 / 78$ & 11 & 1 & & 86 & $\mathbf{N}$ & \\
\hline 1 & $4 / 23 / 78$ & 11 & 2 & & 82 & $\mathbf{N}$ & \\
\hline 1 & $4 / 23 / 78$ & 12 & 1 & & 86 & $\mathbf{N}$ & \\
\hline 1 & $4 / 23 / 78$ & 12 & 2 & & 82 & $\mathbf{N}$ & \\
\hline 1 & $6 / 17 / 78$ & 1 & 1 & & & $\mathbf{N}$ & 115 - suspect date (high), reverse TC order \\
\hline 9 & $6 / 17 / 78$ & 1 & 2 & & 70 & $\mathbf{N}$ & \\
\hline 1 & $6 / 17 / 78$ & 2 & 1 & & & $\mathbf{N}$ & 114 - suspect data (high), reverse TC order \\
\hline 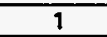 & $6 / 17 / 78$ & 2 & 2 & & 70 & $\mathbf{N}$ & \\
\hline 9 & $6 / 17 / 78$ & 3 & 1 & & & $\mathbf{N}$ & 117 - suspect data (high), reverse TC order \\
\hline 1 & $6 / 17 / 78$ & 3 & 2 & & 72 & $\mathbf{N}$ & \\
\hline 1 & $6 / 17 / 78$ & 4 & 1 & & & $\mathbf{N}$ & 117 - suspect data (high), reverse TC order \\
\hline 1 & $6 / 17 / 78$ & 4 & 2 & & 73 & $\mathbf{N}$ & \\
\hline 1 & $6 / 17 / 78$ & 5 & $\frac{1}{1}$ & & & $\bar{Y}$ & 117 - suspect data (high), reverse TC order \\
\hline 1 & $6 / 17 / 78$ & 5 & 2 & & 69 & $\mathbf{N}$ & \\
\hline 1 & $6 / 17 / 78$ & 6 & 1 & & & $\mathbf{N}$ & 111 - suspect data (high), reverse TC order \\
\hline 1 & $6 / 17 / 78$ & 7 & 1 & & & $\mathbf{N}$ & 104 - suspect data (high), reverse TC order \\
\hline 1 & $6 / 17 / 78$ & 7 & 2 & & 73 & $\mathbf{N}$ & \\
\hline 1 & $6 / 17 / 78$ & 8 & 1 & & 100 & $\mathbf{N}$ & raw data - assume reverse TC order \\
\hline 1 & $6 / 17 / 78$ & 8 & 2 & & 70 & $\mathbf{N}$ & \\
\hline 1 & $6 / 17 / 78$ & 9 & 1 & & 98 & $\mathbf{N}$ & raw data - assume reverse $T C$ order \\
\hline 1 & $6 / 17 / 78$ & 9 & 2 & & 70 & $\mathbf{N}$ & \\
\hline 9 & $6 / 17 / 78$ & 10 & 1 & & 96 & $\mathbf{N}$ & raw data - assume reverse TC order \\
\hline 1 & $6 / 17 / 78$ & 10 & 2 & & 69 & $\mathbf{N}$ & \\
\hline 1 & $6 / 17 / 78$ & 11 & 1 & & 99 & $\mathbf{N}$ & raw data - assume reverse TC order \\
\hline 1 & $6 / 17 / 78$ & 11 & 2 & & 70 & $\mathbf{N}$ & \\
\hline 1 & $6 / 17 / 78$ & 12 & 1 & & 97 & $\mathrm{~N}$ & raw data - assume reverse TC order \\
\hline 1 & $6 / 17 / 78$ & 12 & 2 & & 74 & $\mathbf{N}$ & \\
\hline
\end{tabular}

Data obtained from WHC Surveillance Analysis Computer System (SACS), June 28, 1993. 


\begin{tabular}{|c|c|c|c|c|c|c|c|}
\hline \multicolumn{8}{|c|}{ TANK 241-BY-102 } \\
\hline \multicolumn{8}{|c|}{ THERMOCOUPLE DATA } \\
\hline TREE \# & DATE & THERMO \# & TREE SET \# & TEMP (C) & TEMP (F) & $Y=$ MAX TEMP & COMMENTS \\
\hline 1 & $7 / 16 / 78$ & 1 & 1 & & 64 & $\mathbf{N}$ & raw data - assume reverse TC order \\
\hline 1 & $7 / 16 / 78$ & 1 & 2 & & 55 & $\mathbf{N}$ & \\
\hline 1 & $7 / 16 / 78$ & 2 & 1 & & 65 & $\mathbf{N}$ & raw data - assume reverse TC order \\
\hline 1 & $7 / 16 / 78$ & 2 & 2 & & 55 & $\mathbf{N}$ & \\
\hline 1 & $7 / 16 / 78$ & 3 & 1 & & 65 & $\mathbf{N}$ & raw data - assume reverse TC order \\
\hline 1 & $7 / 16 / 78$ & 3 & 2 & & 57 & $N$ & \\
\hline 1 & $7 / 16 / 78$ & 4 & 1 & & 65 & $\mathbf{N}$ & raw data - assume reverse TC order \\
\hline 1 & $7 / 16 / 78$ & 4 & 2 & & 57 & $N$ & \\
\hline 1 & $7 / 16 / 78$ & 5 & 1 & & 65 & $Y$ & raw data - assume reverse TC order \\
\hline 1 & $7 / 16 / 78$ & 5 & 2 & & 54 & N & \\
\hline 1 & $7 / 16 / 78$ & 6 & 1 & & 57 & $\mathbf{N}$ & raw data - assume reverse TC order \\
\hline 1 & $7 / 16 / 78$ & 7 & 1 & $\div$ & 53 & $\mathbf{N}$ & raw data - assume reverse TC order \\
\hline 1 & $7 / 16 / 78$ & 7 & 2 & & 57 & $\mathbf{N}$ & \\
\hline 1 & $7 / 16 / 78$ & 8 & 1 & & 50 & $\mathbf{N}$ & raw data - assume reverse TC order \\
\hline 1 & $7 / 16 / 78$ & 8 & 2 & & 54 & $\mathbf{N}$ & \\
\hline 1 & $7 / 16 / 78$ & 9 & 1 & & 49 & $\mathbf{N}$ & raw data - assume reverse TC order \\
\hline 1 & $7 / 16 / 78$ & 9 & 2 & & 54 & $\mathbf{N}$ & \\
\hline 1 & $7 / 16 / 78$ & 10 & 1 & & 48 & $\mathbf{N}$ & raw data - assume reverse TC order \\
\hline 1 & $7 / 16 / 78$ & 10 & 2 & & 53 & $\mathbf{N}$ & \\
\hline 1 & $7 / 16 / 78$ & 11 & 1 & & 48 & $\mathbf{N}$ & raw data - assume reverse TC order \\
\hline 1 & $7 / 16 / 78$ & 11 & 2 & & 53 & $\mathbf{N}$ & \\
\hline 1 & $7 / 16 / 78$ & 12 & 1 & & 50 & $\mathbf{N}$ & raw data - assume reverse TC order \\
\hline 9 & $7 / 16 / 78$ & 12 & 2 & & 56 & $\mathbf{N}$ & \\
\hline 1 & $8 / 13 / 78$ & 1 & 1 & & 62 & $\mathrm{~N}$ & raw data - assume reverse TC order \\
\hline 1 & $8 / 13 / 78$ & 1 & 2 & & 76 & $\mathbf{N}$ & \\
\hline 1 & $8 / 13 / 78$ & 2 & 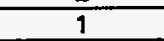 & & 60 & $\mathbf{N}$ & raw data - assume raverse TC order \\
\hline 1 & $8 / 13 / 78$ & 2 & 2 & & 77 & $\mathbf{N}$ & \\
\hline 1 & $8 / 13 / 78$ & 3 & 1 & & 58 & $\mathbf{N}$ & raw data - assume reverse TC order \\
\hline 1 & $8 / 13 / 78$ & 3 & 2 & & 79 & $\mathbf{N}$ & \\
\hline 1 & $8 / 13 / 78$ & 4 & 1 & & 56 & $\mathbf{N}$ & raw data - assume reverse TC order \\
\hline 1 & $8 / 13 / 78$ & 4 & 2 & & 79 & $\mathbf{N}$ & \\
\hline 1 & $8 / 13 / 78$ & 5 & 1 & & 56 & $\mathbf{N}$ & raw data - assume reverse TC order \\
\hline 1 & $8 / 13 / 78$ & 5 & 2 & & 76 & $\mathbf{N}$ & \\
\hline 1 & $8 / 13 / 78$ & 6 & 1 & & 62 & $\mathbf{N}$ & raw data - assume reverse TC order \\
\hline 1 & $8 / 13 / 78$ & 7 & 2 & & 79 & $N$ & \\
\hline 1 & $8 / 13 / 78$ & 8 & 1 & & 66 & $N$ & raw data - assume reverse TC order \\
\hline 1 & $8 / 13 / 78$ & 8 & 2 & & 77 & $\mathbf{N}$ & \\
\hline 1 & $8 / 13 / 78$ & 9 & 1 & & 53 & $\mathbf{N}$ & raw data - assume reverse TC order \\
\hline 1 & $.8 / 13 / 78$ & 9 & 2 & & 77 & $\mathbf{N}$ & \\
\hline 1 & $8 / 13 / 78$ & 10 & 9 & & 51 & $\mathbf{N}$ & raw data - assume reverse TC order \\
\hline 1 & $8 / 13 / 78$ & 10 & 2 & & 76 & $\mathbf{N}$ & \\
\hline 1 & $8 / 13 / 78$ & 19 & 1 & & 55 & $\mathbf{N}$ & raw data - assume reverse TC order \\
\hline 1 & $8 / 13 / 78$ & 11 & 2 & & 77 & $\mathbf{N}$ & \\
\hline 1 & $8 / 13 / 78$ & 12 & 1 & & 45 & $\mathbf{N}$ & raw data - assume reverse TC order \\
\hline 1 & $8 / 13 / 78$ & 12 & 2 & & 80 & $\mathbf{N}$ & \\
\hline 1 & $8 / 13 / 78$ & 13 & 2 & & 77 & $\mathbf{N}$ & \\
\hline 1 & $8 / 13 / 78$ & 14 & 2 & & 76 & $N$ & \\
\hline 1 & $9 / 10 / 78$ & 1 & 1 & & 72 & $\mathbf{N}$ & raw data - assume reverse TC order \\
\hline 1 & $9 / 10 / 78$ & 2 & 1 & & 72 & $\mathbf{N}$ & raw data - assume reverse TC order \\
\hline 1 & $9 / 10 / 78$ & 3 & 1 & & 73 & $\mathbf{N}$ & raw data - assume reverse TC order \\
\hline 1 & $9 / 10 / 78$ & 4 & 1 & & 73 & $\mathbf{N}$ & raw data - assume reverse TC order \\
\hline 1 & $9 / 10 / 78$ & 5 & 1 & & 73 & $\bar{Y}$ & raw data - assume reverse TC order \\
\hline 1 & $9 / 10 / 78$ & 7 & 1 & & 69 & $\mathbf{N}$ & raw data - assume reverse TC order \\
\hline 9 & $9 / 10 / 78$ & 8 & 1 & & 64 & $\mathbf{N}$ & raw data - assume reverse TC order \\
\hline$\frac{1}{1}$ & $9 / 10 / 78$ & 9 & 1 & & 63 & $\mathbf{N}$ & raw data - assume reverse TC order \\
\hline 1 & $9 / 10 / 78$ & 10 & 1 & & 62 & $\mathbf{N}$ & raw data - assume reverse TC order \\
\hline 1 & $9 / 10 / 78$ & 11 & 1 & & 67 & $\mathbf{N}$ & raw data - assume reverse TC order \\
\hline 1 & $9 / 10 / 78$ & 12 & 1 & & 66 & $\mathbf{N}$ & raw data - assume reverse TC order \\
\hline 1 & $9 / 12 / 78$ & 1 & 1 & & 76 & $\mathbf{N}$ & \\
\hline 1 & $9 / 12 / 78$ & 2 & 1 & & 77 & $\mathbf{N}$ & \\
\hline 1 & $9 / 12 / 78$ & 3 & 9 & & 80 & $\mathbf{N}$ & \\
\hline 1 & $9 / 12 / 78$ & 4 & 1 & & 80 & $\mathbf{N}$ & \\
\hline 1 & $9 / 12 / 78$ & 5 & 1 & & 77 & $\mathrm{~N}$ & \\
\hline 1 & $9 / 12 / 78$ & 6 & 1 & & 99 & $Y$ & \\
\hline 1 & $9 / 12 / 78$ & 7 & 1 & & 81 & $\mathbf{N}$ & \\
\hline 1 & $9 / 12 / 78$ & 8 & 1 & & 78 & $\mathbf{N}$ & \\
\hline
\end{tabular}

Data obtained from WHC Surveillance Analysis Computer System (SACS), June 28, 1993. 
WHC-SD-WM-ER-312, Rev. 0

\begin{tabular}{|c|c|c|c|c|c|c|c|}
\hline \multicolumn{8}{|c|}{ TANK 241-BY-102 } \\
\hline \multicolumn{8}{|c|}{ THEAMOCOUPLE DATA } \\
\hline TREE \# & DATE & THERMO \# & TREE SET \# & TEMP (C) & TEMP (F) & $Y=M A X$ TEMP & COMMENTS \\
\hline 1 & $9 / 12 / 78$ & 9 & 1 & & 77 & $\mathbf{N}$ & \\
\hline 1 & $9 / 12 / 78$ & 10 & 1 & & 77 & $\mathbf{N}$ & \\
\hline 1 & $9 / 12 / 78$ & 11 & 1 & & 78 & $\mathbf{N}$ & \\
\hline 1 & $9 / 12 / 78$ & 12 & 1 & & 81 & $\mathbf{N}$ & \\
\hline 1 & $9 / 12 / 78$ & 13 & 1 & & 77 & $\mathbf{N}$ & \\
\hline 1 & $9 / 12 / 78$ & 14 & 1 & & 76 & $\mathbf{N}$ & \\
\hline 1 & $10 / 7 / 78$ & 1 & 1 & & 92 & $\mathbf{N}$ & raw data - assume reverse TC order \\
\hline 1 & $10 / 7 / 78$ & 2 & 1 & & 92 & $\mathbf{N}$ & raw data - assume reverse TC order \\
\hline 1 & $10 / 7 / 78$ & 3 & 1 & & 92 & $\mathbf{N}$ & raw data - assume reverse TC order \\
\hline 1 & $10 / 7 / 78$ & 4 & 1 & & 93 & $\mathbf{N}$ & raw data - assume reverse TC order \\
\hline 1 & $10 / 7 / 78$ & 5 & 1 & & 93 & $\mathbf{Y}$ & raw data - assume reverse TC order \\
\hline 1 & $10 / 7 / 78$ & 6 & 1 & 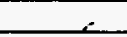 & 92 & $\mathbf{N}$ & raw data - assume reverse TC order \\
\hline 1 & $10 / 7 / 78$ & 7 & 1 & & 84 & $\mathbf{N}$ & raw data - assume reverse TC order \\
\hline 1 & $10 / 7 / 78$ & 8 & 1 & & 81 & $\mathbf{N}$ & raw data - assume reverse TC order \\
\hline 1 & $10 / 7 / 78$ & 9 & 1 & & 80 & $\mathbf{N}$ & raw data - assume reverse TC order \\
\hline 1 & $10 / 7 / 78$ & 10 & 1 & & 77 & $\mathbf{N}$ & raw data - assume reverse TC order \\
\hline 1 & $10 / 7 / 78$ & 11 & 1 & & 76 & $\mathbf{N}$ & raw data - assume reverse TC order \\
\hline 1 & $10 / 7 / 78$ & 12 & 1 & & 75 & $\mathbf{N}$ & raw data - assume reverse TC order \\
\hline 1 & $1 / 3 / 79$ & 1 & 1 & & 71 & $\mathbf{Y}$ & \\
\hline 1 & $1 / 3 / 79$ & 2 & 1 & & 70 & $\mathbf{N}$ & \\
\hline 1 & $1 / 3 / 79$ & 3 & 1 & & 70 & $\mathbf{N}$ & \\
\hline 1 & $1 / 3 / 79$ & 4 & 1 & & 67 & $\mathbf{N}$ & \\
\hline 1 & $1 / 3 / 79$ & 5 & 1 & & 65 & $\mathbf{N}$ & \\
\hline 1 & $1 / 3 / 79$ & 6 & 1 & & 59 & $\mathbf{N}$ & \\
\hline 1 & $1 / 3 / 79$ & 7 & 1 & & 46 & $\mathbf{N}$ & \\
\hline 1 & $1 / 3 / 79$ & 8 & 1 & & 41 & $\mathbf{N}$ & \\
\hline 1 & $1 / 3 / 79$ & 9 & 1 & & 41 & $\mathbf{N}$ & \\
\hline 1 & $1 / 3 / 79$ & 10 & 1 & & 41 & $\mathbf{N}$ & \\
\hline 1 & $1 / 3 / 79$ & 11 & 1 & & 41 & $\mathbf{N}$ & \\
\hline 1 & $1 / 3 / 79$ & 12 & 1 & & 41 & $\mathbf{N}$ & \\
\hline 1 & $4 / 22 / 79$ & 1 & 1 & & 72 & $Y$ & \\
\hline 1 & $4 / 22 / 79$ & 2 & 1 & & 72 & $\mathbf{N}$ & \\
\hline 1 & $4 / 22 / 79$ & 3 & 1 & & 72 & $\mathbf{N}$ & \\
\hline 1 & $4 / 22 / 79$ & 4 & 1 & & 72 & $\mathbf{N}$ & \\
\hline 1 & $4 / 22 / 79$ & 5 & 1 & & 71 & $\mathbf{N}$ & \\
\hline 1 & $4 / 22 / 79$ & 6 & 1 & & 71 & $\mathbf{N}$ & \\
\hline 1 & $4 / 22 / 79$ & 8 & 1 & & 71 & $\mathbf{N}$ & \\
\hline 1 & $4 / 22 / 79$ & 9 & 1 & & 71 & $\mathbf{N}$ & \\
\hline 1 & $4 / 22 / 79$ & 10 & 1 & & 71 & $\mathbf{N}$ & \\
\hline 1 & $4 / 22 / 79$ & 11 & 1 & & 71 & $\mathbf{N}$ & \\
\hline 1 & $4 / 22 / 79$ & 12 & 1 & & 71 & $\mathbf{N}$ & \\
\hline 1 & $4 / 22 / 79$ & 13 & 1 & & 71 & $\mathbf{N}$ & \\
\hline 1 & $4 / 22 / 79$ & 14 & 1 & & 71 & $\mathbf{N}$ & \\
\hline 1 & $11 / 2 / 91$ & 1 & 1 & & 88 & $\mathbf{Y}$ & $K-890523512-47$ \\
\hline 1 & $11 / 19 / 91$ & 1 & 1 & & & $\mathbf{N}$ & O/S NO TC TREE \\
\hline 1 & $7 / 2 / 92$ & 1 & 1 & & & $\mathbf{N}$ & O/S NO TC TREE \\
\hline 1 & $1 / 1 / 93$ & 1 & 1 & & & $\mathbf{N}$ & OIS NO TC TREE \\
\hline
\end{tabular}

Data obtained from WHC Surveillance Anaiysis Computer System (SACS), June 28, 1993. 
WHC-SD-WM-ER-312, Rev. 0

\begin{tabular}{|c|c|c|c|c|c|c|c|}
\hline \multicolumn{8}{|c|}{ TANK 241-BY-103 } \\
\hline & & & & THER & COUPLE & ATA & \\
\hline TREE \# & DATE & THERMO \# & TREE SET \# & TEMP C & TEMPF & $Y=$ MAX TEMP & COMMENTS \\
\hline 1 & $11 / 12 / 73$ & 1 & & & 68 & & \\
\hline 1 & $111 / 12 / 73$ & 2 & & & 82 & & \\
\hline 1 & $11 / 12 / 73$ & 3 & & & 141 & & \\
\hline 1 & $11 / 12 / 73$ & 4 & & & 136 & & \\
\hline 1 & $11 / 12 / 73$ & 5 & & & 136 & & \\
\hline 1 & $11 / 12 / 73$ & 6 & & & 125 & & \\
\hline 1 & $11 / 12 / 73$ & 7 & & & 53 & & \\
\hline 1 & $11 / 12 / 73$ & 8 & & & 58 & & \\
\hline 1 & $11 / 12 / 73$ & 9 & & & 68 & & \\
\hline 1 & $11 / 12 / 73$ & 10 & & & 68 & & \\
\hline 1 & $11 / 12 / 73$ & 11 & & & 77 & & \\
\hline 1 & $11 / 12 / 73$ & 12 & & $\therefore$ & 77 & & \\
\hline 1 & $11 / 21 / 73$ & 1 & & & & & 10 - suspect data (low) \\
\hline 1 & $11 / 21 / 73$ & 2 & & & & & 16 - suspect data (low) \\
\hline 1 & $11 / 21 / 73$ & 3 & & & & & 48 - suspect data (low) \\
\hline 1 & $11 / 21 / 73$ & 4 & & & & & 48 - suspect data (low) \\
\hline 1 & $11 / 21 / 73$ & 5 & & & & & 45 - suspect data (low) \\
\hline 1 & $11 / 21 / 73$ & 6 & & & & & 40 - suspect data (low) \\
\hline 1 & $11 / 21 / 73$ & 7 & & & & & 0 - suspect data (low) \\
\hline 1 & $11 / 21 / 73$ & 8 & & & & & 0-suspect data (low) \\
\hline 1 & $11 / 21 / 73$ & 9 & & & & & 4-suspect data (low) \\
\hline 1 & $11 / 21 / 73$ & 10 & & & & & 8 - suspect data (low) \\
\hline 1 & $11 / 21 / 73$ & 11 & & & & & 11 - suspect data (low) \\
\hline 1 & $11 / 21 / 73$ & 12 & & & & & 11 - suspect data (low) \\
\hline 1 & $12 / 10 / 73$ & 1 & & 20 & 68 & & raw data - assumed degree C \\
\hline 1 & $12 / 10 / 73$ & 2 & & 20 & 68 & & raw data - assumed degreo C \\
\hline 1 & $12 / 10 / 73$ & 3 & & 50 & 122 & & raw data - assumed degreo C \\
\hline 1 & $12 / 10 / 73$ & 4 & & 50 & 122 & & raw data - assumed degree C \\
\hline 1 & $12 / 10 / 73$ & 5 & & 50 & 122 & & raw data - assumed degree C \\
\hline 1 & $12 / 10 / 73$ & 6 & & 43 & 109.4 & & raw data - assumed dagree C \\
\hline 1 & $12 / 10 / 73$ & 7 & & 8 & 46.4 & & raw data - assumed degree C \\
\hline 1 & $12 / 10 / 73$ & 8 & & 8 & 46.4 & & raw data - assumed degree C \\
\hline 1 & $12 / 10 / 73$ & 9 & & 9 & 48.2 & & raw data - assumed degree C \\
\hline 1 & $12 / 10 / 73$ & 10 & & 11 & 51.8 & & raw data - assumed degres C \\
\hline 1 & $12 / 10 / 73$ & 11 & & 14 & 57.2 & & raw data - assumed degree C \\
\hline 1 & $12 / 10 / 73$ & 12 & & 14 & 57.2 & & raw data - assumed degree C \\
\hline 1 & $12 / 17 / 73$ & 1 & & 23 & 73.4 & & raw data - assumed degree C \\
\hline 1 & $12 / 17 / 73$ & 2 & & 28 & 82.4 & & raw data - assumed degree C \\
\hline 1 & $12 / 17 / 73$ & 3 & & 61 & 141.8 & & raw data - assumed degree C \\
\hline 1 & $12 / 17 / 73$ & 4 & & 61 & 141.8 & & raw data - assumed degree C \\
\hline 1 & $12 / 17 / 73$ & 5 & & 58 & 136.4 & & raw data - assumed degree C \\
\hline 1 & $12 / 17 / 73$ & 6 & & 55 & 131 & & raw data - assumed degree C \\
\hline 1 & $12 / 17 / 73$ & 7 & & 15 & 59 & & raw data - assumed degree C \\
\hline 1 & $12 / 17 / 73$ & 8 & & 15 & 59 & & raw data - assumed degree C \\
\hline 1 & $12 / 17 / 73$ & 9 & & 18 & 64.4 & & raw data - assumed degree C \\
\hline 9 & $12 / 17 / 73$ & 10 & & 18 & 64.4 & & raw data - assumed degree $C$ \\
\hline 1 & $12 / 17 / 73$ & 11 & & 22 & 71.6 & & raw data - assumed degree C \\
\hline 1 & $12 / 17 / 73$ & 12 & & 23 & 73.4 & & raw data - assumed degree C \\
\hline 1 & $12 / 20 / 73$ & 1 & & 24 & 75.2 & & raw data - assumed degree C \\
\hline$\frac{1}{1}$ & $12 / 20 / 73$ & 2 & & 25 & 77 & & raw data - assumed degree C \\
\hline 1 & $12 / 20 / 73$ & 3 & & 60 & 140 & & raw data - assumed degree C \\
\hline 1 & $12 / 20 / 73$ & 4 & & 60 & 140 & & raw data - assumed degree C \\
\hline 1 & $12 / 20 / 73$ & 5 & & 57 & 134.6 & & raw data - assumed degree C \\
\hline 1 & $12 / 20 / 73$ & 6 & & 55 & 131 & & raw data - assumed degree C \\
\hline 1 & $12 / 20 / 73$ & 7 & & 18 & 64.4 & & raw data - assumed degres C \\
\hline 1 & $12 / 20 / 73$ & 8 & & 18 & 64.4 & & raw data - assumed degree C \\
\hline 1 & $12 / 20 / 73$ & 9 & & 18 & 64.4 & & raw data - assumed degree C \\
\hline 1 & $12 / 20 / 73$ & 10 & & 19 & 66.2 & & raw data - assumed degree C \\
\hline 1 & $12 / 20 / 73$ & 71 & & 22 & 71.6 & & raw data - assumed degree C \\
\hline 1 & $12 / 20 / 73$ & 12 & & 22 & 71.6 & & raw data - assumed degree C \\
\hline 1 & $12 / 20 / 73$ & 75 & & 38 & 100.4 & & raw data - assumed degree C \\
\hline 1 & $9 / 12 / 74$ & 1 & 1 & 36 & 96.8 & $\mathbf{N}$ & degree C/TWINS confirm \\
\hline 1 & $9 / 12 / 74$ & 2 & 1 & 38 & 100.4 & $\mathbf{N}$ & degree C/TWINS confirm \\
\hline 1 & $9 / 12 / 74$ & 3 & 1 & 52 & 925.6 & $\bar{Y}$ & degree C/TWINS confirm \\
\hline 1 & $9 / 12 / 74$ & $\overline{4}$ & 1 & 52 & 125.6 & $\mathbf{N}$ & degree C/TWINS confirm \\
\hline 1 & $9 / 12 / 74$ & 6 & 1 & 48 & 118.4 & $\mathbf{N}$ & degree C/TWINS confirm \\
\hline
\end{tabular}

Data obtained from WHC Surveillance Analysis Computer System (SACS), June 2, 1993. 


\begin{tabular}{|c|c|c|c|c|c|c|c|}
\hline \multicolumn{8}{|c|}{ TANK 241-BY-103 } \\
\hline \multicolumn{8}{|c|}{ THERMOCOUPLE DATA } \\
\hline TREE \# & DATE & THERMO \# & TREE SET \# & TEMP C & TEMP F & $Y=$ MAX TEMP & COMMENTS \\
\hline 1 & $9 / 12 / 74$ & 7 & 1 & 38 & 100.4 & $\mathrm{~N}$ & degree C/TWINS confirm \\
\hline 1 & $9 / 12 / 74$ & 8 & 1 & 38 & 100.4 & $\mathrm{~N}$ & degree C/TWINS confirm \\
\hline 1 & $9 / 12 / 74$ & 9 & 1 & 35 & 95 & $\mathbf{N}$ & degree C/TWINS confirm \\
\hline 1 & $9 / 12 / 74$ & 10 & 1 & 35 & 95 & $\mathbf{N}$ & degree $\mathrm{C} / \mathrm{TW}$ INS confirm \\
\hline 1 & $9 / 12 / 74$ & 11 & 1 & 35 & 95 & $\mathbf{N}$ & degree $\mathrm{C} / \mathrm{TW}$ INS confirm \\
\hline 1 & $9 / 12 / 74$ & 12 & 9 & 34 & 93.2 & $\mathbf{N}$ & degree $C / T$ WINS confirm \\
\hline 1 & $10 / 8 / 74$ & 1 & 1 & 22 & 71.6 & $N$ & degree $\mathrm{C} / \mathrm{TWINS}$ confirm \\
\hline 1 & $10 / 8 / 74$ & 2 & 1 & 22 & 71.6 & $\mathbf{N}$ & degree $\mathrm{C} / \mathrm{TW}$ WNS confirm \\
\hline$T$ & $10 / 8 / 74$ & 3 & 9 & 44 & 911.2 & $\bar{Y}$ & degree C/TWINS confirm \\
\hline 1 & $10 / 8 / 74$ & 4 & 1 & 43 & 109.4 & $\mathbf{N}$ & degree C/TWINS confirm \\
\hline 1 & $10 / 8 / 74$ & 6 & 1 & 31 & 87.8 & $\bar{N}$ & degree C/TWINS confirm \\
\hline 1 & $10 / 8 / 74$ & 7 & 9 & 22 & 71.6 & $\mathbf{N}$ & degree C/TWINS confirm \\
\hline 1 & $10 / 8 / 74$ & 8 & 1 & 22 & 71.6 & $\mathbf{N}$ & degree C/TWINS confirm \\
\hline 9 & $10 / 8 / 74$ & 9 & 1 & 22 & 71.6 & $N$ & degree $\mathrm{C} / \mathrm{T}$ WINS confirm \\
\hline 1 & $10 / 8 / 74$ & 10 & 1 & 22 & 71.6 & $\bar{N}$ & degree C/TWINS confirm \\
\hline 1 & $10 / 8 / 74$ & 11 & 1 & 22 & 71.6 & $\mathbf{N}$ & degree C/TWINS confirm \\
\hline 1 & $10 / 8 / 74$ & 12 & 1 & 22 & 79.6 & $\mathbf{N}$ & degree C/TWINS confirm \\
\hline 1 & $10 / 29 / 74$ & 1 & 1 & 19 & 66.2 & $\mathbf{N}$ & degree C/TWINS confirm \\
\hline 1 & $10 / 29 / 74$ & 2 & 1 & 16 & 60.8 & $N$ & degree C/TWINS confirm \\
\hline 1 & $90 / 29 / 74$ & 3 & 1 & 42 & 107.6 & $\bar{Y}$ & degree C/TWINS confirm \\
\hline 1 & $10 / 29 / 74$ & 4 & 1 & 42 & 107.6 & $\bar{N}$ & degree C/TWINS confirm \\
\hline 1 & $10 / 29 / 74$ & 5 & 1 & 20 & 68 & $\mathbf{N}$ & degree C/TWINS confirm \\
\hline 1 & $10 / 29 / 74$ & 6 & 1 & 20 & 68 & $\bar{N}$ & degree C/TWINS confirm \\
\hline 1 & $10 / 29 / 74$ & 7 & 1 & 16 & 60.8 & $\mathbf{N}$ & degree C/TWINS confirm \\
\hline 1 & $10 / 29 / 74$ & 8 & 1 & 16 & 60.8 & $N$ & degree C/TWINS confirm \\
\hline 1 & $10 / 29 / 74$ & 9 & 1 & 16 & 60.8 & $\mathbf{N}$ & degree $\mathrm{C} / \mathrm{TW}$ WNS confirm \\
\hline 1 & $10 / 29 / 74$ & 10 & 1 & 16 & 60.8 & $\mathbf{N}$ & degree C/TWINS confirm \\
\hline 1 & $10 / 29 / 74$ & 11 & 1 & 19 & 66.2 & $\mathbf{N}$ & degree C/TWINS confirm \\
\hline 1 & $10 / 29 / 74$ & 12 & 1 & 19 & 66.2 & $\mathbf{N}$ & degree C/TWINS confirm \\
\hline 1 & $11 / 12 / 74$ & 1 & 1 & 15 & 59 & $\mathbf{N}$ & degree C/TWINS confirm \\
\hline 1 & $11 / 12 / 74$ & 2 & 1 & 10 & 50 & $\mathbf{N}$ & degree C/TWINS confirm \\
\hline 1 & $11 / 12 / 74$ & 3 & 1 & 31 & 87.8 & $\bar{Y}$ & degree C/TWINS confirm \\
\hline$\frac{1}{1}$ & $11 / 12 / 74$ & 4 & $\frac{1}{1}$ & 31 & 87.8 & $\mathbf{N}$ & degree C/TWINS confirm \\
\hline 1 & $11 / 12 / 74$ & 6 & 1 & 17 & 62.6 & $\mathbf{N}$ & degree C/TWINS confirm \\
\hline 1 & $11 / 12 / 74$ & 7 & 1 & 27 & 80.6 & $\overline{\mathbf{N}}$ & degree $\mathrm{C} / \mathrm{TW}$ INS confirm \\
\hline 1 & $11 / 12 / 74$ & 8 & 1 & 12 & 53.6 & $\mathbf{N}$ & degree C/TWINS confirm \\
\hline 1 & $11 / 12 / 74$ & 9 & 1 & 12 & 53.6 & $\bar{N}$ & degree C/TWINS confirm \\
\hline 9 & $11 / 12 / 74$ & 10 & 1 & 12 & 53.6 & $\mathbf{N}$ & degree C/TWINS confirm \\
\hline 1 & $.11 / 12 / 74$ & 11 & 1 & 12 & 53.6 & $\mathbf{N}$ & degree C/TWINS confirm \\
\hline 1 & $11 / 12 / 74$ & 12 & 1 & 12 & 53.6 & $\mathbf{N}$ & degree $\mathrm{C} / \mathrm{TW}$ INS confirm \\
\hline 1 & $11 / 26 / 74$ & 9 & 1 & 16 & 60.8 & $\mathbf{N}$ & degree $\mathrm{C} / \mathrm{T}$ WINS confirm \\
\hline 1 & $11 / 26 / 74$ & 2 & 1 & 12 & 53.6 & $\mathbf{N}$ & degree $\mathrm{C} / \mathrm{T}$ WiNS confirm \\
\hline 1 & $11 / 26 / 74$ & 3 & 1 & 44 & 111.2 & $\mathrm{Y}$ & degree $\mathrm{C} / \mathrm{TW}$ INS confirm \\
\hline 9 & $11 / 26 / 74$ & 4 & 1 & 44 & 111.2 & $\mathbf{N}$ & degree C/TWINS confirm \\
\hline 1 & $11 / 26 / 74$ & 5 & 1 & 24 & 75.2 & $\mathbf{N}$ & degree C/TWINS confirm \\
\hline 1 & $11 / 26 / 74$ & 6 & 1 & 36 & 96.8 & $\mathbf{N}$ & degree C/TWINS confirm \\
\hline 1 & $11 / 26 / 74$ & 7 & 9 & 12 & 53.6 & $\mathbf{N}$ & degree C/TWINS confirm \\
\hline 1 & $11 / 26 / 74$ & 8 & 1 & 12 & 53.6 & $\mathbf{N}$ & degree C/TWINS confirm \\
\hline$i$ & $11 / 26 / 74$ & 9 & 1 & 14 & 57.2 & $\mathbf{N}$ & degree C/TWINS confirm \\
\hline 1 & $11 / 26 / 74$ & 10 & 1 & 14 & 57.2 & $\mathbf{N}$ & degrea C/TWINS confirm \\
\hline 1 & $11 / 26 / 74$ & 11 & 1 & 16 & 60.8 & $\mathbf{N}$ & degree C/TWINS confirm \\
\hline 1 & $11 / 26 / 74$ & 12 & 1 & 16 & 60.8 & $\mathbf{N}$ & degree C/TWINS confirm \\
\hline 1 & $12 / 11 / 74$ & 1 & 9 & 40 & 104 & $\bar{Y}$ & degree C/TWINS confirm \\
\hline 1 & $12 / 11 / 74$ & 2 & 1 & 40 & 104 & $\mathbf{N}$ & degree C/TWINS confirm \\
\hline 9 & $12 / 11 / 74$ & 3 & 1 & 40 & 104 & $\mathbf{N}$ & degree C/TWINS confirm \\
\hline 9 & $12 / 11 / 74$ & 4 & 9 & 39.44 & 102.992 & $N$ & degree $\mathrm{C} / \mathrm{TW}$ INS confirm \\
\hline 1 & $12 / 11 / 74$ & 5 & 1 & 17.78 & 64.004 & $N$ & degree C/TWINS confirm \\
\hline 1 & $12 / 11 / 74$ & 6 & 1 & 33.33 & 91.994 & $N$ & degree C/TWINS confirm \\
\hline 1 & $12 / 11 / 74$ & 11 & 1 & 12.22 & 53.996 & $\mathbf{N}$ & degree C/TWINS confirm \\
\hline 1 & $12 / 11 / 74$ & 12 & 9 & 91.67 & 53.006 & $\mathbf{N}$ & degree $\mathrm{C} / \mathrm{TW}$ WINS confirm \\
\hline 1 & $12 / 17 / 74$ & 3 & 1 & 38.89 & 102.002 & $\bar{Y}$ & degree $\mathrm{C} / \mathrm{TW}$ INS confirm \\
\hline 1 & $12 / 17 / 74$ & 4 & 1 & 38.89 & 102.002 & $\mathrm{~N}$ & degree C/TWINS confirm \\
\hline 1 & $12 / 17 / 74$ & 5 & 1 & 24.44 & 75.992 & $\mathbf{N}$ & degree C/TWINS confirm \\
\hline 1 & $12 / 17 / 74$ & 6 & 1 & 33.33 & 91.994 & $\mathbf{N}$ & degree C/TWINS confirm \\
\hline 1 & $12 / 17 / 74$ & 11 & 1 & 12.22 & 53.996 & $\mathrm{~N}$ & degree $C / T$ WINS confirm \\
\hline 1 & $12 / 17 / 74$ & 12 & 1 & 12.22 & 53.996 & $\mathbf{N}$ & degroe C/TWINS confirm \\
\hline
\end{tabular}

Data obtained from WHC Surveillance Analysis Computer System (SACS), June 2, 1993. 


\begin{tabular}{|c|c|c|c|c|c|c|c|}
\hline \multicolumn{8}{|c|}{ TANK 249-BY-103 } \\
\hline \multicolumn{8}{|c|}{ THERMOCOUPLE DATA } \\
\hline TREE \# & DATE & THERMO \# & TREE SET \# & TEMP C & TEMP F & $Y=$ MAX TEMP & COMMENTS \\
\hline 1 & $12 / 31 / 74$ & 1 & 1 & 10 & 50 & $\mathbf{N}$ & degree $\mathrm{C} / \mathrm{TW}$ WNS confirm \\
\hline 1 & $12 / 31 / 74$ & 2 & 1 & 10 & 50 & $\bar{N}$ & degree $\mathrm{C} / \mathrm{TW}$ iNS confirm \\
\hline 1 & $12 / 31 / 74$ & 3 & $T$ & 41.11 & 105.998 & $\bar{Y}$ & degres C/TWINS confirm \\
\hline 1 & $12 / 31 / 74$ & 4 & 1 & 41.11 & 105.998 & $\mathbf{N}$ & degree C/TWINS confirm \\
\hline 1 & $12 / 31 / 74$ & 5 & 1 & 24.44 & 75.992 & $\mathbf{N}$ & degree CITWINS confirm \\
\hline 1 & $12 / 31 / 74$ & 6 & 1 & 33.33 & 91.994 & $\mathbf{N}$ & degree $\mathrm{C} / \mathrm{TW}$ INS confirm \\
\hline 1 & $12 / 31 / 74$ & 11 & 1 & 11.11 & 51.998 & $\bar{N}$ & degree $\mathrm{C} / \mathrm{TW}$ INS confirm \\
\hline 1 & $12 / 31 / 74$ & 12 & 1 & 11.11 & 51.998 & $\mathbf{N}$ & degree C/TWINS confirm \\
\hline 1 & $1 / 8 / 75$ & 1 & 1 & 13.89 & 57.002 & $\bar{N}$ & degree $\mathrm{C} / \mathrm{TW}$ INS confirm \\
\hline 1 & $1 / 8 / 75$ & 2 & 1 & 10 & 50 & $\mathbf{N}$ & degree C/TWINS confirm \\
\hline 1 & $1 / 8 / 75$ & 3 & 1 & 38.33 & 100.994 & $\mathbf{N}$ & degree C/TWINS confirm \\
\hline 1 & $1 / 8 / 75$ & 4 & 1 & 38.89 & 102.002 & $\mathbf{Y}$ & degree C/TWINS confirm \\
\hline 1 & $1 / 8 / 75$ & 5 & 1 & 22.78 & 73.004 & $\mathbf{N}$ & degree CrTWiNS confirm \\
\hline 1 & $1 / 8 / 75$ & 6 & 1 & 32.22 & 89.996 & $\mathbf{N}$ & degree $\mathrm{C} / \mathrm{TW}$ INS confirm \\
\hline 1 & $1 / 8 / 75$ & 7 & 1 & 10 & 50 & $\mathbf{N}$ & degree C/TWINS confirm \\
\hline 1 & $1 / 8 / 75$ & 8 & 1 & 10 & 50 & $\mathbf{N}$ & degree C/TWINS confirm \\
\hline 9 & $1 / 8 / 75$ & 9 & 1 & 10 & 50 & $\mathbf{N}$ & degree C/TWINS confirm \\
\hline 1 & $1 / 8 / 75$ & 10 & 1 & 10 & 50 & $\mathbf{N}$ & degree C/TWINS confirm \\
\hline 1 & $1 / 8 / 75$ & 11 & 1 & 11.67 & 53.006 & $\bar{N}$ & degree C/TWINS confirm \\
\hline 1 & $1 / 8 / 75$ & 12 & 9 & 11.67 & 53.006 & $\mathbf{N}$ & degree CrTWINS confirm \\
\hline 1 & $1 / 21 / 75$ & 1 & 1 & 11 & 51.8 & $\mathbf{N}$ & degree C/TWINS confirm \\
\hline 1 & 1/21/75 & 2 & 1 & 7 & 44.6 & $\mathbf{N}$ & degree C/TWINS confirm \\
\hline 1 & $1 / 21 / 75$ & 3 & 1 & 48 & 118.4 & $\bar{Y}$ & degree C/TWINS confirm \\
\hline 1 & $1 / 21 / 75$ & 4 & 1 & 48 & 118.4 & $\mathbf{N}$ & degree $\mathrm{C} / \mathrm{TW}$ INS confirm \\
\hline 1 & $1 / 21 / 75$ & 5 & 1 & 30 & 86 & $\mathbf{N}$ & degree $\mathrm{C} / \mathrm{TW}$ INS confirm \\
\hline 1 & $1 / 21 / 75$ & 6 & 1 & 39 & 102.2 & $\mathbf{N}$ & degree C/TWINS confirm \\
\hline 1 & $1 / 21 / 75$ & 7 & 1 & 6 & 42.8 & $\mathbf{N}$ & degree C/TWINS confirm \\
\hline 1 & $1 / 21 / 75$ & 8 & 1 & 6 & 42.8 & $\mathbf{N}$ & degree C/TWINS confirm \\
\hline 1 & $1 / 21 / 75$ & 9 & 1 & 7 & 44.6 & $N$ & degree $\mathrm{C} / \mathrm{TW}$ INS confirm \\
\hline 1 & $1 / 21 / 75$ & 10 & 1 & 9 & 48.2 & $\mathbf{N}$ & degree $\mathrm{C} T$ TWINS confirm \\
\hline 1 & $1 / 21 / 75$ & 11 & 1 & 11 & 51.8 & $\mathbf{N}$ & degree C/TWINS confirm \\
\hline 1 & $1 / 21 / 75$ & 12 & 1 & 12 & 53.6 & $\mathbf{N}$ & degree C/TWINS confirm \\
\hline 1 & $1 / 28 / 75$ & 1 & 1 & 4 & 39.2 & $\mathbf{N}$ & degree C/TWINS confirm \\
\hline 1 & $1 / 28 / 75$ & 2 & 1 & 1 & 33.8 & $\mathbf{N}$ & degree C/TWINS confirm \\
\hline 1 & $1 / 28 / 75$ & 3 & 1 & 36 & 96.8 & $N$ & degree CTWINS confirm \\
\hline 1 & $1 / 28 / 75$ & 4 & 1 & 37 & 98.6 & $\mathbf{Y}$ & degree CTWINS confirm \\
\hline 1 & $1 / 28 / 75$ & 5 & 1 & 30 & 86 & $\mathbf{N}$ & degree C/TWINS confirm \\
\hline 1 & $1 / 28 / 75$ & 6 & 1 & 29 & 84.2 & $\mathbf{N}$ & degree C/TWINS confirm \\
\hline 1 & $.1 / 28 / 75$ & 7 & 1 & 2 & 35.6 & $\mathbf{N}$ & degree $\mathrm{C} / \mathrm{TW}$ inS confirm \\
\hline 1 & $4 / 22 / 75$ & 1 & 1 & 20 & 68 & $\mathbf{N}$ & degree C/TWINS confirm \\
\hline 1 & $4 / 22 / 75$ & 2 & 1 & 18 & 64.4 & $\mathbf{N}$ & degree C/TWinS confirm \\
\hline 9 & $4 / 22 / 75$ & 3 & 1 & 37 & 98.6 & $\mathbf{Y}$ & degree C/TWINS confirm \\
\hline 1 & $4 / 22 / 75$ & 4 & 1 & 37 & 98.6 & $\mathbf{N}$ & degree C/TWINS confirm \\
\hline$T$ & $4 / 22 / 75$ & 5 & 1 & 22 & 71.6 & $\mathbf{N}$ & degree $\mathrm{C}$ TWWINS confirm \\
\hline 1 & $4 / 22 / 75$ & 6 & 1 & 28 & 82.4 & $\mathbf{N}$ & degree C/TWINS confirm \\
\hline 1 & $4 / 22 / 75$ & 7 & 1 & 14 & 57.2 & $\mathbf{N}$ & degree C/TWINS confirm \\
\hline 1 & $4 / 22 / 75$ & 8 & 1 & 16 & 60.8 & $\mathbf{N}$ & degree $\mathrm{C} / \mathrm{TW}$ WNS confirm \\
\hline 1 & $4 / 22 / 75$ & 9 & 1 & 16 & 60.8 & $N$ & degree C/TWINS confirm \\
\hline 1 & $4 / 22 / 75$ & 10 & 1 & 16 & 60.8 & $\mathbf{N}$ & degree $\mathrm{C} / \mathrm{TW}$ INS confirm \\
\hline 1 & $4 / 22 / 75$ & 11 & 1 & 16 & 60.8 & $\mathrm{~N}$ & degree $\mathrm{C} / \mathrm{TW}$ INS confirm \\
\hline 1 & $4 / 22 / 75$ & 12 & 1 & 16 & 60.8 & $\mathbf{N}$ & degree $\mathrm{C} T$ TWINS confirm \\
\hline 1 & $4 / 29 / 75$ & 1 & 9 & 18 & 64.4 & $\mathbf{N}$ & degree C/TWINS confirm \\
\hline 1 & $4 / 29 / 75$ & 2 & 1 & 18 & 64.4 & $N$ & degree CTWWINS confirm \\
\hline 1 & $4 / 29 / 75$ & 3 & 1 & 38 & 100.4 & $\bar{Y}$ & degree C/TWINS confirm \\
\hline 1 & $4 / 29 / 75$ & 4 & 1 & 38 & 100.4 & $\mathrm{~N}$ & degree C/TWINS confirm \\
\hline 1 & $4 / 29 / 75$ & 5 & 1 & 29 & 69.8 & $\mathbf{N}$ & degree $\mathrm{C} / \mathrm{TW}$ INS confirm \\
\hline 1 & $4 / 29 / 75$ & 6 & 1 & 26 & 78.8 & $\mathbf{N}$ & degree C/TWINS confirm \\
\hline 1 & $4 / 29 / 75$ & 7 & 1 & 13 & 55.4 & $\mathbf{N}$ & degree C/TWINS confirm \\
\hline 1 & $4 / 29 / 75$ & 8 & 1 & 14 & 57.2 & $\mathbf{N}$ & degree C/TWINS confirm \\
\hline 1 & $4 / 29 / 75$ & 9 & 1 & 14 & 57.2 & $\mathbf{N}$ & degree C/TWINS confirm \\
\hline 1 & $4 / 29 / 75$ & 10 & 1 & 10 & 50 & $\mathbf{N}$ & degree C/TWINS confirm \\
\hline 1 & $4 / 29 / 75$ & 19 & 1 & 10 & 50 & $\mathrm{~N}$ & degree C/TWINS confirm \\
\hline 1 & $4 / 29 / 75$ & 12 & 1 & 10 & 50 & $N$ & degree C/TWINS confirm \\
\hline 1 & $5 / 20 / 75$ & 1 & 1 & 18 & 64.4 & $N$ & degree C/TWINS confirm \\
\hline 1 & $5 / 20 / 75$ & 2 & 1 & 18 & 64.4 & $\mathrm{~N}$ & degree C/TWINS confirm \\
\hline 1 & $5 / 20 / 75$ & 3 & 1 & 32 & 89.6 & $\bar{Y}$ & degree C/TWINS confirm \\
\hline
\end{tabular}

Data obtained from WHC Surveillance Analysis Computer System (SACS), June 2, 1993. 


\begin{tabular}{|c|c|c|c|c|c|c|c|}
\hline \multicolumn{8}{|c|}{$\begin{array}{l}\text { TANK 241-BY-103 } \\
\text { THERMOCOUPLE DATA }\end{array}$} \\
\hline & & & & THERI & COUPLE D & & \\
\hline TREE \# & DATE & THERMO \# & TREE SET \# & TEMP C & TEMP F & $Y=$ MAX TEMP & COMMENTS \\
\hline 1 & $5 / 20 / 75$ & 4 & 1 & 32 & 89.6 & $\mathrm{~N}$ & degree C/TWINS confirm \\
\hline 1 & $5 / 20 / 75$ & 5 & 1 & 32 & 89.6 & $\mathbf{N}$ & degree C/TWINS confirm \\
\hline 1 & $5 / 20 / 75$ & 6 & 1 & 26 & 78.8 & $\mathbf{N}$ & degree C/TWINS confirm \\
\hline 1 & $5 / 20 / 75$ & 7 & 1 & 18 & 64.4 & $\mathbf{N}$ & degree C/TWINS confirm \\
\hline 1 & $5 / 20 / 75$ & 8 & 1 & 18 & 64.4 & $\mathbf{N}$ & degree C/TWINS confirm \\
\hline 1 & $5 / 20 / 75$ & 9 & 1 & 18 & 64.4 & $\mathbf{N}$ & degree C/TWINS confirm \\
\hline 1 & $5 / 20 / 75$ & 10 & 1 & 16 & 60.8 & $\mathbf{N}$ & degree C/TWINS confirm \\
\hline 1 & $5 / 20 / 75$ & 11 & 1 & 16 & 60.8 & $\mathbf{N}$ & degree C/TWINS confirm \\
\hline 1 & $5 / 20 / 75$ & 12 & 1 & 16 & 60.8 & $\mathbf{N}$ & degree C/TWINS confitm \\
\hline 1 & $6 / 17 / 75$ & 1 & 1 & & 80 & $\mathbf{N}$ & \\
\hline 1 & $6 / 17 / 75$ & 2 & 1 & & 80 & $\mathbf{N}$ & \\
\hline 1 & $6 / 17 / 75$ & 3 & 1 & $\therefore$ & 94 & $\bar{Y}$ & \\
\hline 1 & $6 / 17 / 75$ & 4 & 1 & & 94 & $\mathbf{N}$ & \\
\hline 1 & $6 / 17 / 75$ & 5 & 1 & & 93 & $\mathbf{N}$ & \\
\hline 1 & $6 / 17 / 75$ & 6 & 1 & & 83 & $\mathbf{N}$ & \\
\hline 1 & $6 / 17 / 75$ & 7 & 1 & & 79 & $\mathbf{N}$ & \\
\hline 1 & $6 / 17 / 75$ & 8 & 1 & & 79 & $\bar{N}$ & \\
\hline 1 & $6 / 17 / 75$ & 9 & 1 & & 76 & $\mathbf{N}$ & \\
\hline 1 & $6 / 17 / 75$ & 10 & 1 & & 70 & $\mathbf{N}$ & \\
\hline 1 & $6 / 17 / 75$ & 11 & 1 & & 68 & $\mathbf{N}$ & \\
\hline 1 & $6 / 17 / 75$ & 12 & 1 & & 68 & $\mathbf{N}$ & \\
\hline 1 & $7 / 28 / 75$ & 1 & 1 & & 100 & $\bar{Y}$ & \\
\hline 1 & $7 / 28 / 75$ & 2 & 1 & & 97 & $\mathrm{~N}$ & \\
\hline 1 & $7 / 28 / 75$ & 3 & 1 & & 97 & $\mathbf{N}$ & \\
\hline 1 & $7 / 28 / 75$ & 4 & 1 & & 97 & $N$ & \\
\hline 1 & $7 / 28 / 75$ & 5 & 1 & & 96 & $\mathrm{~N}$ & \\
\hline 1 & $7 / 28 / 75$ & 6 & 1 & & 89 & $N$ & \\
\hline 1 & $7 / 28 / 75$ & 7 & 1 & & 86 & $N$ & \\
\hline 1 & $7 / 28 / 75$ & 8 & 1 & & 96 & $\mathrm{~N}$ & \\
\hline 1 & $7 / 28 / 75$ & 9 & 1 & & 88 & $N$ & \\
\hline 1 & $7 / 28 / 75$ & 10 & 1 & & 85 & $\mathbf{N}$ & \\
\hline 1 & $7 / 28 / 75$ & 11 & 1 & & 81 & $\mathbf{N}$ & \\
\hline 1 & $7 / 28 / 75$ & 12 & 1 & & 80 & $\mathrm{~N}$ & \\
\hline 1 & $8 / 26 / 75$ & 1 & 1 & & 88 & $\mathrm{~N}$ & \\
\hline 1 & $8 / 26 / 75$ & 2 & 1 & & 87 & $\bar{N}$ & \\
\hline 1 & $8 / 26 / 75$ & 3 & 1 & & 99 & $\bar{Y}$ & \\
\hline 1 & $8 / 26 / 75$ & 4 & 1 & & 99 & $\mathbf{N}$ & \\
\hline 1 & $8 / 26 / 75$ & 5 & 1 & & 98 & $\mathbf{N}$ & \\
\hline 1 & $.8 / 26 / 75$ & 6 & 1 & & 93 & $\mathbf{N}$ & \\
\hline 1 & $8 / 26 / 75$ & 7 & 1 & & 86 & $\mathbf{N}$ & \\
\hline 1 & $8 / 26 / 75$ & 8 & 1 & & 86 & $\mathbf{N}$ & \\
\hline 1 & $8 / 26 / 75$ & 9 & 1 & & 83 & $\bar{N}$ & \\
\hline 1 & $8 / 26 / 75$ & 10 & 1 & & 79 & $\mathbf{N}$ & \\
\hline 1 & $8 / 26 / 75$ & 11 & 1 & & 78 & $\bar{N}$ & \\
\hline 1 & $8 / 26 / 75$ & 12 & 1 & & 78 & $\mathrm{~N}$ & \\
\hline 1 & $11 / 21 / 75$ & 1 & 1 & & 79 & $\mathbf{N}$ & \\
\hline 1 & $11 / 21 / 75$ & 2 & 1 & & 61 & $\mathbf{N}$ & \\
\hline 1 & $11 / 21 / 75$ & 3 & 1 & & 97 & $\mathbf{Y}$ & \\
\hline 1 & $11 / 21 / 75$ & 4 & 1 & & 97 & $\mathbf{N}$ & \\
\hline 1 & $11 / 21 / 75$ & 5 & 1 & & 96 & $\mathbf{N}$ & \\
\hline 1 & $11 / 21 / 75$ & 6 & 1 & & 90 & $\mathbf{N}$ & \\
\hline 1 & $11 / 21 / 75$ & 7 & 1 & & 51 & $\mathbf{N}$ & \\
\hline 1 & $11 / 21 / 75$ & 8 & 1 & & 51 & $\mathbf{N}$ & \\
\hline 1 & $11 / 21 / 75$ & 9 & 1 & & 56 & $\mathbf{N}$ & \\
\hline 1 & $11 / 21 / 75$ & 10 & 1 & & 53 & $\mathbf{N}$ & \\
\hline 1 & $11 / 21 / 75$ & 11 & 1 & & 58 & $\mathbf{N}$ & \\
\hline 1 & $11 / 21 / 75$ & 12 & 1 & & 59 & $\mathbf{N}$ & \\
\hline 1 & $1 / 7 / 76$ & 1 & 1 & & 62 & $\mathbf{N}$ & \\
\hline 1 & $1 / 7 / 76$ & 2 & 1 & & 57 & $\mathbf{N}$ & \\
\hline 1 & $1 / 7 / 76$ & 3 & 1 & & 98 & $Y$ & \\
\hline 1 & $1 / 7 / 76$ & 5 & 1 & & 98 & $\mathbf{N}$ & \\
\hline 1 & $1 / 7 / 76$ & 6 & 1 & & 89 & $N$ & \\
\hline 1 & $1 / 7 / 76$ & 7 & 1 & & 45 & $\bar{N}$ & \\
\hline 1 & $1 / 7 / 76$ & 8 & 1 & & 46 & $\bar{N}$ & \\
\hline 1 & $1 / 7 / 76$ & 9 & 1 & & 52 & $N$ & \\
\hline 1 & $1 / 7 / 76$ & 10 & 1 & & 47 & $N$ & \\
\hline
\end{tabular}

Data obtained from WHC Surveillance Analysis Computer System (SACS), June 2, 1993. 


\begin{tabular}{|c|c|c|c|c|c|c|c|}
\hline \multicolumn{8}{|c|}{ TANK 241-BY-103 } \\
\hline \multicolumn{8}{|c|}{ THERMOCOUPLE DATA } \\
\hline TAEE \# & DATE & THERMO \# & TREE SET \# & TEMP C & TEMP F & $Y=M A X$ TEMP & COMMENTS \\
\hline 1 & $1 / 7 / 76$ & 11 & 1 & & 54 & $\mathrm{~N}$ & - \\
\hline 1 & $1 / 7 / 76$ & 12 & 1 & & 54 & $\mathbf{N}$ & \\
\hline 1 & $2 / 27 / 76$ & 1 & 1 & 12 & 53.6 & $\mathbf{N}$ & \\
\hline 1 & $2 / 27 / 76$ & 2 & 1 & 9 & 48.2 & $\mathbf{N}$ & \\
\hline 1 & $2 / 27 / 76$ & 3 & 1 & 40 & 104 & $Y$ & \\
\hline 1 & $2 / 27 / 76$ & 4 & 1 & 40 & 104 & $\mathbf{N}$ & \\
\hline 1 & $2 / 27 / 76$ & 5 & 1 & 25 & 77 & $\mathbf{N}$ & \\
\hline 1 & $2 / 27 / 76$ & 6 & $T$ & 32 & 89.6 & $\bar{N}$ & \\
\hline 1 & $2 / 27 / 76$ & 7 & 9 & 8 & 46.4 & $\overline{\mathbf{N}}$ & \\
\hline 1 & $2 / 27 / 76$ & 8 & $\frac{1}{1}$ & 8 & 46.4 & $N$ & \\
\hline 1 & $2 / 27 / 76$ & 9 & 1 & 8 & 46.4 & $\mathbf{N}$ & \\
\hline 1 & $2 / 27 / 76$ & 10 & 9 & 10. & 50 & $N$ & \\
\hline$T$ & $2 / 27 / 76$ & 11 & 9 & 10 & 50 & $\mathbf{N}$ & \\
\hline 1 & $2 / 27 / 76$ & 12 & 1 & 10 & 50 & $\mathbf{N}$ & \\
\hline 9 & $3 / 4 / 76$ & 1 & $\frac{9}{1}$ & & 61 & $N$ & \\
\hline 1 & $3 / 4 / 76$ & 2 & 1 & & 58 & $\mathbf{N}$ & \\
\hline 1 & $3 / 4 / 76$ & 3 & 1 & & 94 & $\bar{Y}$ & \\
\hline 1 & $3 / 4 / 76$ & 4 & 1 & & 94 & $\mathbf{N}$ & \\
\hline 1 & $3 / 4 / 76$ & 5 & 1 & & 49 & $\mathbf{N}$ & \\
\hline 9 & $3 / 4 / 76$ & 6 & $\frac{1}{1}$ & & 82 & $\mathrm{~N}$ & \\
\hline 1 & $3 / 4 / 76$ & 7 & 1 & & 46 & $\mathbf{N}$ & \\
\hline 1 & $3 / 4 / 76$ & 8 & 1 & & 46 & $\mathbf{N}$ & \\
\hline$\frac{1}{1}$ & $3 / 4 / 76$ & 9 & 9 & & 51 & $\mathbf{N}$ & \\
\hline 1 & $3 / 4 / 76$ & 10 & 1 & & 44 & N & \\
\hline 1 & $3 / 4 / 76$ & 11 & 1 & & 50 & $N$ & \\
\hline 1 & $3 / 4 / 76$ & 12 & 1 & & 51 & $\mathbf{N}$ & \\
\hline 1 & $3 / 18 / 76$ & 1 & 1 & 12 & 53.6 & $\mathbf{N}$ & raw data - assumed degree C \\
\hline 1 & $3 / 18 / 76$ & 2 & 1 & 12 & 53.6 & $N$ & raw data - assumed degree C \\
\hline 1 & $3 / 18 / 76$ & 3 & 1 & 40 & 104 & $\bar{Y}$ & raw data - assumed degree C \\
\hline 1 & $3 / 18 / 76$ & 4 & 1 & 23 & 73.4 & $\mathbf{N}$ & raw data - assumed degree C \\
\hline 1 & $3 / 18 / 76$ & 5 & 1 & 30 & 86 & $\mathrm{~N}$ & raw data - assumed degree C \\
\hline 9 & $3 / 78 / 76$ & 6 & $T$ & 12 & 53.6 & $\overline{\mathbf{N}}$ & raw data - assumed degree C \\
\hline 1 & $3 / 18 / 76$ & 7 & 1 & 12 & 53.6 & $\mathbf{N}$ & raw data - assumed degree C \\
\hline 9 & $3 / 18 / 76$ & 8 & 1 & 12 & 53.6 & $\mathbf{N}$ & raw data - assumed degree C \\
\hline 9 & $3 / 18 / 76$ & 9 & 1 & 12 & 53.6 & $\mathbf{N}$ & raw data - assumed degree C \\
\hline 1 & $3 / 18 / 76$ & 10 & 1 & 12 & 53.6 & $N$ & raw data - assumed degree C \\
\hline 1 & $3 / 18 / 76$ & 11 & 1 & 12 & 53.6 & $\mathrm{~N}$ & raw data - assumed degreo C \\
\hline 9 & $3 / 18 / 76$ & 12 & $\overline{1}$ & $\overline{12}$ & 53.6 & $\mathbf{N}$ & raw data - assumed degree C \\
\hline 9 & $.4 / 1 / 76$ & 1 & 1 & 5.56 & 42.008 & $\mathrm{~N}$ & raw data - assumed degree C \\
\hline 1 & $4 / 1 / 76$ & 2 & 1 & 6.67 & 44.006 & $\mathbf{N}$ & raw data - assumed degree C \\
\hline 1 & $4 / 1 / 76$ & 3 & 1 & 36.67 & 98.006 & $\mathbf{Y}$ & raw data - assumed degree C \\
\hline 1 & $4 / 1 / 76$ & 4 & 1 & 36.67 & 98.006 & $\mathbf{N}$ & raw data - assumed degree C \\
\hline 1 & $4 / 1 / 76$ & 5 & 1 & 22.78 & 73.004 & $\mathbf{N}$ & raw data - assumed degreo C \\
\hline$\frac{1}{9}$ & $4 / 1 / 76$ & 6 & $\frac{1}{1}$ & 28.33 & 82.994 & $\mathbf{N}$ & raw data - assumed degree C \\
\hline 1 & $4 / 1 / 76$ & 7 & 1 & 8.33 & 46.994 & $\bar{N}$ & raw data - assumed degree $C$ \\
\hline 1 & $4 / 1 / 76$ & 8 & 1 & 8.33 & 46.994 & $\mathbf{N}$ & raw data - assumed degree C \\
\hline 1 & $4 / 1 / 76$ & 9 & 1 & 11.11 & 51.998 & $\mathbf{N}$ & raw data - assumed degreo C \\
\hline$T$ & $4 / 1 / 76$ & 10 & 1 & 12.22 & 53.996 & $\mathbf{N}$ & raw data - assumed degree C \\
\hline 9 & $4 / 1176$ & 11 & 1 & 13.89 & 57.002 & $\mathbf{N}$ & raw data - assumed degree C \\
\hline 1 & $4 / 1 / 76$ & 12 & 1 & 13.89 & 57.002 & $\mathbf{N}$ & raw data - assumed degree $C$ \\
\hline$\frac{1}{1}$ & $5 / 7 / 76$ & 1 & 1 & & 69 & $\mathbf{N}$ & \\
\hline 1 & $5 / 7 / 76$ & 2 & 1 & & 65 & $\mathbf{N}$ & \\
\hline$\frac{\pi}{1}$ & $5 / 7 / 76$ & 3 & 1 & & 82 & $\bar{Y}$ & \\
\hline 1 & $5 / 7 / 76$ & 4 & 9 & & 82 & $\mathbf{N}$ & \\
\hline 1 & $5 / 7 / 76$ & 5 & 1 & & 74 & $\mathbf{N}$ & \\
\hline 1 & $5 / 7 / 76$ & 6 & 1 & & 71 & $\mathbf{N}$ & \\
\hline$\frac{1}{1}$ & $5 / 7 / 76$ & 7 & 1 & & 60 & $\mathbf{N}$ & \\
\hline 1 & $5 / 7 / 76$ & 8 & 1 & & 61 & $\mathbf{N}$ & \\
\hline 1 & $5 / 7 / 76$ & 9 & 9 & & 62 & $\mathbf{N}$ & \\
\hline 1 & $5 / 7 / 76$ & 10 & 1 & & 66 & $N$ & \\
\hline 9 & $5 / 7 / 76$ & 11 & 1 & & 58 & $N$ & \\
\hline 1 & $5 / 7 / 76$ & 12 & 9 & & 58 & $\mathbf{N}$ & \\
\hline 1 & $6 / 9 / 76$ & 1 & 9 & & 82 & $\mathrm{~N}$ & \\
\hline 1 & $6 / 9 / 76$ & 2 & 1 & & 81 & $N$ & \\
\hline 1 & $6 / 9 / 76$ & 3 & 7 & & 90 & $\bar{Y}$ & \\
\hline 1 & $6 / 9 / 76$ & 4 & 1 & & 90 & $N$ & \\
\hline
\end{tabular}

Data obtained from WHC Surveillance Analysis Computer System (SACS), June 2, 1993. 


\begin{tabular}{|c|c|c|c|c|c|c|c|}
\hline \multicolumn{8}{|c|}{ TANK 241-BY-103 } \\
\hline \multicolumn{8}{|c|}{ THERMOCOUPLE DATA } \\
\hline TREE \# & DATE & THERMO \# & TREE SET \# & TEMP C & TEMP F & $Y=$ MAX TEMP & COMMENTS \\
\hline 1 & $6 / 9 / 76$ & 5 & 1 & & 89 & $\mathbf{N}$ & \\
\hline 1 & $6 / 9 / 76$ & 6 & 1 & & 82 & $\mathbf{N}$ & \\
\hline 1 & $6 / 9 / 76$ & 7 & 1 & & 79 & $N$ & \\
\hline 1 & $6 / 9 / 76$ & 8 & $T$ & & 79 & $\mathbf{N}$ & \\
\hline 1 & $6 / 9 / 76$ & 9 & 1 & & 77 & $\bar{N}$ & \\
\hline 1 & $6 / 9 / 76$ & 10 & 1 & & 70 & $\mathbf{N}$ & \\
\hline 1 & $6 / 9 / 76$ & 11 & 1 & & 69 & $\mathbf{N}$ & \\
\hline 1 & $6 / 9 / 76$ & 12 & 1 & & 69 & $\mathbf{N}$ & \\
\hline 1 & $7 / 7 / 76$ & 1 & 1 & & 84 & $\bar{Y}$ & \\
\hline 1 & $7 / 7 / 76$ & 2 & 1 & & 67 & $\mathrm{~N}$ & \\
\hline 1 & $7 / 7 / 76$ & 3 & 1 & & 82 & $\bar{N}$ & \\
\hline 1 & $7 / 7 / 76$ & 4 & 1 & $\therefore$ & 82 & $N$ & \\
\hline 1 & $7 / 7 / 76$ & 5 & 1 & & 80 & $N$ & \\
\hline 1 & $7 / 7 / 76$ & 6 & 1 & & 74 & $N$ & \\
\hline 1 & $7 / 7 / 76$ & 7 & 1 & & 79 & $\bar{N}$ & \\
\hline 1 & $7 / 7 / 76$ & 8 & 1 & & 78 & $\mathbf{N}$ & \\
\hline 1 & $7 / 7 / 76$ & 9 & 1 & & 67 & $\mathbf{N}$ & \\
\hline 1 & $7 / 7 / 76$ & 10 & 1 & & 79 & $N$ & \\
\hline 1 & $7 / 7 / 76$ & 11 & 1 & & 67 & $\mathbf{N}$ & \\
\hline 1 & $7 / 7 / 76$ & 12 & 1 & & 67 & $\mathbf{N}$ & \\
\hline 1 & $8 / 14 / 76$ & 1 & 1 & & 84 & $\bar{Y}$ & \\
\hline 1 & $8 / 14 / 76$ & 2 & 1 & & 80 & $N$ & \\
\hline 1 & $8 / 14 / 76$ & 3 & 1 & & 79 & $\mathrm{~N}$ & \\
\hline 1 & $8 / 14 / 76$ & 4 & 1 & & 78 & $\mathbf{N}$ & \\
\hline 1 & $8 / 14 / 76$ & 5 & 1 & & 76 & $N$ & \\
\hline 1 & $8 / 14 / 76$ & 6 & 1 & & 73 & $\mathbf{N}$ & \\
\hline 1 & $8 / 14 / 76$ & 7 & 1 & & 78 & $N$ & \\
\hline 1 & $8 / 14 / 76$ & 8 & 1 & & 76 & $\bar{N}$ & \\
\hline 1 & $8 / 14 / 76$ & 9 & 1 & & 64 & $\mathbf{N}$ & \\
\hline 1 & $8 / 14 / 76$ & 10 & 1 & & 78 & $\mathbf{N}$ & \\
\hline 1 & $8 / 14 / 76$ & 11 & 1 & & 66 & $\mathbf{N}$ & \\
\hline 1 & $8 / 14 / 76$ & 12 & 1 & & 66 & $\mathrm{~N}$ & \\
\hline 1 & $9 / 13 / 76$ & 1 & 1 & & 88 & $\bar{Y}$ & \\
\hline 1 & $9 / 13 / 76$ & 2 & 1 & & 73 & $\mathbf{N}$ & \\
\hline 1 & $9 / 13 / 76$ & 3 & 1 & & 85 & $\bar{N}$ & \\
\hline 1 & $9 / 13 / 76$ & 4 & 1 & & 84 & $\mathbf{N}$ & \\
\hline 1 & $9 / 13 / 76$ & 5 & 1 & & 82 & $\mathbf{N}$ & \\
\hline 1 & $9 / 13 / 76$ & 6 & 1 & & 79 & $\mathbf{N}$ & \\
\hline 1 & $.9 / 13 / 76$ & 7 & 1 & & 76 & $\overline{\mathbf{N}}$ & \\
\hline 1 & $9 / 13 / 76$ & 8 & 1 & & 75 & $\mathrm{~N}$ & \\
\hline 1 & $9 / 13 / 76$ & 9 & 1 & & 66 & $\mathbf{N}$ & \\
\hline 1 & $9 / 13 / 76$ & 10 & 1 & & 82 & $\mathbf{N}$ & \\
\hline 1 & $9 / 13 / 76$ & 11 & 1 & & 71 & $\mathbf{N}$ & \\
\hline 1 & $9 / 13 / 76$ & 12 & 1 & & 72 & $\mathbf{N}$ & \\
\hline 1 & $11 / 7 / 76$ & 1 & 1 & & 81 & $\mathrm{~N}$ & \\
\hline 1 & $11 / 7 / 76$ & 2 & 1 & & 60 & $\mathbf{N}$ & \\
\hline$\frac{1}{1}$ & $11 / 7 / 76$ & 3 & 1 & & 86 & $\mathrm{~N}$ & \\
\hline 1 & $11 / 7 / 76$ & 4 & 1 & & 87 & $\mathbf{N}$ & \\
\hline 1 & $11 / 7 / 76$ & 5 & 1 & & 85 & $\mathrm{~N}$ & \\
\hline 1 & $11 / 7 / 76$ & 6 & 1 & & 84 & $\mathrm{~N}$ & \\
\hline 1 & $11 / 7 / 76$ & 7 & 1 & & 58 & $\mathbf{N}$ & \\
\hline 1 & $11 / 7 / 76$ & 8 & 1 & & 58 & $\mathbf{N}$ & \\
\hline 1 & $11 / 7 / 76$ & 9 & $\frac{1}{1}$ & & 56 & $\mathrm{~N}$ & \\
\hline 1 & $11 / 7 / 76$ & 10 & 1 & & 57 & $\mathrm{~N}$ & \\
\hline 1 & $11 / 7 / 76$ & 11 & 1 & & 64 & $\mathbf{N}$ & \\
\hline 9 & $19 / 7 / 76$ & 12 & 9 & & 65 & $\mathbf{N}$ & \\
\hline 1 & $12 / 9 / 76$ & 1 & 1 & & 107 & $N$ & \\
\hline 1 & $12 / 9 / 76$ & 2 & 1 & & 72 & $N$ & \\
\hline 1 & $12 / 9 / 76$ & 3 & 1 & & 110 & $\bar{Y}$ & \\
\hline 1 & $12 / 9 / 76$ & 4 & 1 & & 109 & $\mathrm{~N}$ & \\
\hline 1 & $12 / 9 / 76$ & 5 & $\frac{1}{1}$ & & 107 & $\mathrm{~N}$ & \\
\hline 1 & $12 / 9 / 76$ & 6 & 1 & & 105 & $\mathrm{~N}$ & \\
\hline 1 & $12 / 9 / 76$ & 7 & 7 & & 65 & $\mathbf{N}$ & \\
\hline 1 & $12 / 9 / 76$ & 8 & 1 & & 65 & $\mathbf{N}$ & \\
\hline 1 & $12 / 9 / 76$ & 9 & 1 & & 88 & $N$ & \\
\hline 1 & $12 / 9 / 76$ & 10 & 1 & & 88 & $\mathbf{N}$ & \\
\hline
\end{tabular}

Data obtained from WHC Surveillance Analysis Computer System (SACS), June 2, 1993. 


\begin{tabular}{|c|c|c|c|c|c|c|c|}
\hline \multicolumn{8}{|c|}{ TANK 241-BY-103 } \\
\hline & & & & THERI & COUPLE & ITA & \\
\hline TREE \# & DATE & THERMO \# & TREE SET \# & TEMP C & TEMP F & $Y=M A X$ TEMP & COMMENTS \\
\hline 1 & $12 / 9 / 76$ & 11 & 1 & & 82 & $N$ & \\
\hline 1 & $12 / 9 / 76$ & 12 & 9 & & 82 & $\bar{N}$ & \\
\hline 1 & $1 / 3 / 77$ & $\frac{1}{1}$ & 9 & & 95 & $\bar{N}$ & \\
\hline 1 & $1 / 3 / 77$ & 2 & 1 & & 95 & $\mathbf{N}$ & \\
\hline$\frac{1}{1}$ & $1 / 3 / 77$ & 3 & 1 & & 96 & $\bar{Y}$ & \\
\hline 1 & $1 / 3 / 77$ & 4 & 1 & & 96 & $\mathbf{N}$ & \\
\hline 1 & $1 / 3 / 77$ & 5 & 9 & & 95 & $\mathbf{N}$ & \\
\hline 1 & $1 / 3 / 77$ & 6 & 1 & & 92 & $\mathbf{N}$ & \\
\hline 1 & $1 / 3 / 77$ & 10 & 1 & & 62 & $\mathbf{N}$ & \\
\hline 1 & $1 / 3 / 77$ & 11 & 1 & & 65 & $\mathbf{N}$ & \\
\hline$\frac{1}{1}$ & $1 / 3 / 77$ & 12 & 1 & & 65 & $\bar{N}$ & \\
\hline 1 & $1 / 26 / 77$ & 1 & 9 & $\vec{c}$ & 84 & $\bar{N}$ & \\
\hline 1 & $1 / 26 / 77$ & 2 & 1 & & 50 & $\mathbf{N}$ & \\
\hline 1 & $1 / 26 / 77$ & 3 & 1 & & 85 & $\bar{Y}$ & \\
\hline 1 & $1 / 26 / 77$ & 4 & 9 & & 84 & $\bar{N}$ & \\
\hline 1 & $1 / 26 / 77$ & 5 & 1 & & 83 & $\mathbf{N}$ & \\
\hline 1 & $1 / 26 / 77$ & 6 & 1 & & 76 & $\mathbf{N}$ & \\
\hline 1 & $1 / 26 / 77$ & 7 & 1 & & 45 & $N$ & \\
\hline 1 & $1 / 26 / 77$ & 8 & 1 & & 45 & $\bar{N}$ & \\
\hline 1 & $1 / 26 / 77$ & 9 & 1 & & 49 & $\mathbf{N}$ & \\
\hline 1 & $1 / 26 / 77$ & 10 & 9 & & 54 & $\overline{\mathbf{N}}$ & \\
\hline 1 & $1 / 26 / 77$ & 11 & 1 & & 57 & $\mathbf{N}$ & \\
\hline 1 & $1 / 26 / 77$ & 12 & 1 & & 57 & $\bar{N}$ & \\
\hline 1 & $3 / 28 / 77$ & 1 & $\frac{1}{1}$ & & 92 & $\overline{\mathbf{Y}}$ & \\
\hline 1 & $3 / 28 / 77$ & 2 & 1 & & 59 & $\mathbf{N}$ & \\
\hline 1 & $3 / 28 / 77$ & 3 & 1 & & 88 & $\bar{N}$ & \\
\hline 1 & $3 / 28 / 77$ & 4 & 9 & & 88 & $\mathbf{N}$ & \\
\hline 1 & $3 / 28 / 77$ & 5 & 1 & & 87 & $\mathrm{~N}$ & \\
\hline 1 & $3 / 28 / 77$ & 6 & 1 & & 80 & $\mathbf{N}$ & \\
\hline 1 & $3 / 28 / 77$ & 7 & 1 & & 55 & $\mathbf{N}$ & \\
\hline 1 & $3 / 28 / 77$ & 8 & 1 & & 55 & $\mathrm{~N}$ & \\
\hline 1 & $3 / 28 / 77$ & 9 & 1 & & 57 & $\mathbf{N}$ & \\
\hline 1 & $3 / 28 / 77$ & 10 & 1 & & 61 & $\mathbf{N}$ & \\
\hline 1 & $3 / 28 / 77$ & 17 & 1 & & 63 & $\overline{\mathbf{N}}$ & \\
\hline 1 & $3 / 28 / 77$ & 12 & 1 & & 63 & $\mathrm{~N}$ & \\
\hline 1 & $4 / 27 / 77$ & 1 & 1 & & 75 & $\mathbf{N}$ & \\
\hline 1 & $4 / 27 / 77$ & 2 & 1 & & 70 & $\mathbf{N}$ & \\
\hline 1 & $4 / 27 / 77$ & 3 & 9 & & 84 & $\bar{Y}$ & \\
\hline 1 & $.4 / 27 / 77$ & 4 & 1 & & 84 & $\mathbf{N}$ & \\
\hline 1 & $4 / 27 / 77$ & 5 & 1 & & 83 & $\bar{N}$ & \\
\hline T & $4 / 27 / 77$ & 6 & 1 & & 76 & $\mathbf{N}$ & \\
\hline 1 & $4 / 27 / 77$ & 7 & 1 & & 68 & $\mathrm{~N}$ & \\
\hline 1 & $4 / 27 / 77$ & 8 & 1 & & 69 & $\mathbf{N}$ & \\
\hline 1 & $4 / 27 / 77$ & 9 & 1 & & 68 & $\mathbf{N}$ & \\
\hline 1 & $4 / 27 / 77$ & 10 & 1 & & 64 & $\mathrm{~N}$ & \\
\hline 1 & $4 / 27 / 77$ & 11 & 1 & & 63 & $\mathbf{N}$ & \\
\hline 1 & $4 / 27 / 77$ & 12 & 1 & & 63 & $\mathbf{N}$ & \\
\hline$\frac{1}{1}$ & $4 / 27 / 77$ & 13 & 1 & & & $\mathbf{N}$ & o/s \\
\hline 1 & $4 / 27 / 77$ & 14 & 1 & & & $\mathbf{N}$ & $0 / 5$ \\
\hline 9 & $6 / 30 / 77$ & 1 & 1 & & 87 & $\mathbf{N}$ & \\
\hline 1 & $6 / 30 / 77$ & 2 & 1 & & 86 & $\mathbf{N}$ & \\
\hline 1 & $6 / 30 / 77$ & 3 & 1 & & 87 & $\overline{\mathbf{N}}$ & \\
\hline 1 & $6 / 30 / 77$ & 4 & 1 & & 86 & $\mathbf{N}$ & \\
\hline 9 & $6 / 30 / 77$ & 5 & 1 & & 85 & $\mathbf{N}$ & \\
\hline 1 & $6 / 30 / 77$ & 6 & 9 & & 83 & $\mathbf{N}$ & \\
\hline 1 & $6 / 30 / 77$ & 7 & 1 & & 89 & $\bar{Y}$ & \\
\hline 9 & $6 / 30 / 77$ & 8 & 1 & & 88 & $\mathbf{N}$ & \\
\hline 1 & $6 / 30 / 77$ & 9 & 1 & & 82 & $\mathbf{N}$ & \\
\hline 1 & $6 / 30 / 77$ & 10 & 1 & & 77 & $N$ & \\
\hline 1 & $6 / 30 / 77$ & 11 & 1 & & 72 & $\mathbf{N}$ & \\
\hline 9 & $6 / 30 / 77$ & 12 & 1 & & 72 & $\mathbf{N}$ & \\
\hline 1 & $8 / 15 / 77$ & 1 & 9 & & 76 & $\mathbf{N}$ & \\
\hline 1 & $8 / 15 / 77$ & 2 & 1 & & 78 & $\bar{N}$ & \\
\hline 1 & $8 / 15 / 77$ & 3 & 1 & & 77 & $N$ & \\
\hline 9 & $8 / 15 / 77$ & 4 & 9 & & 77 & $\mathbf{N}$ & \\
\hline 1 & $8 / 15 / 77$ & 5 & 1 & & 76 & $\mathbf{N}$ & \\
\hline
\end{tabular}

Data obtained from WHC Surveillance Analysis Computer System (SACS), June 2, 1993. 


\begin{tabular}{|c|c|c|c|c|c|c|c|}
\hline \multicolumn{8}{|c|}{ TANK 241-BY-103 } \\
\hline & & & & THER & COUPLE D & TTA & \\
\hline TREE \# & DATE & THERMO \# & TREE SET \# & TEMP C & TEMP F & $Y=$ MAX TEMP & COMMENTS \\
\hline 1 & $8 / 15 / 77$ & 6 & 1 & & 76 & $\mathrm{~N}$ & \\
\hline 1 & $8 / 15 / 77$ & 7 & 1 & & 87 & $Y$ & \\
\hline 1 & $8 / 15 / 77$ & 8 & 1 & & 87 & $\mathbf{N}$ & \\
\hline 1 & $8 / 15 / 77$ & 9 & 1 & & 79 & $\mathbf{N}$ & \\
\hline 1 & $8 / 15 / 77$ & 10 & 1 & & 74 & $\mathbf{N}$ & \\
\hline 1 & $8 / 15 / 77$ & 11 & 1 & & 69 & $N$ & \\
\hline 1 & $8 / 15 / 77$ & 12 & 1 & & 69 & $N$ & \\
\hline 1 & $9 / 12 / 77$ & 1 & 1 & & 78 & $\mathrm{~N}$ & \\
\hline 1 & $9 / 12 / 77$ & 2 & 1 & & 78 & $N$ & \\
\hline 1 & $9 / 12 / 77$ & 3 & 1 & & 79 & $\bar{Y}$ & \\
\hline$i$ & $9 / 12 / 77$ & 4 & 1 & & 79 & $\mathbf{N}$ & \\
\hline 1 & $9 / 12 / 77$ & 5 & 1 & & 77 & $N$ & \\
\hline 1 & $9 / 12 / 77$ & 6 & 1 & & 77 & $N$ & \\
\hline 1 & $9 / 12 / 77$ & 7 & 1 & & 72 & $N$ & \\
\hline 1 & $9 / 12 / 77$ & 8 & 1 & & 72 & $\mathbf{N}$ & \\
\hline 1 & $9 / 12 / 77$ & 9 & 1 & & 70 & $\mathbf{N}$ & \\
\hline 1 & $9 / 12 / 77$ & 10 & 1 & & 69 & $\mathrm{~N}$ & \\
\hline 1 & $9 / 12 / 77$ & 11 & 1 & & 67 & $\mathbf{N}$ & \\
\hline 1 & $9 / 12 / 77$ & 12 & 1 & & 67 & $\mathbf{N}$ & \\
\hline 1 & $10 / 7 / 77$ & 1 & 1 & & 86 & $\mathbf{N}$ & \\
\hline 1 & $10 / 7 / 77$ & 2 & 1 & & 75 & $\mathbf{N}$ & \\
\hline 1 & $10 / 7 / 77$ & 3 & 1 & & 86 & $\mathbf{N}$ & \\
\hline 1 & $10 / 7 / 77$ & 4 & 1 & & 88 & $Y$ & \\
\hline 1 & $10 / 7 / 77$ & 5 & 1 & & 85 & $\mathrm{~N}$ & \\
\hline 1 & $10 / 7 / 77$ & 6 & 1 & & 84 & $N$ & \\
\hline 1 & $10 / 7 / 77$ & 7 & 1 & & 65 & $\mathrm{~N}$ & \\
\hline 1 & $10 / 7 / 77$ & 8 & 1 & & 65 & $\bar{N}$ & \\
\hline 1 & $10 / 7 / 77$ & 9 & 1 & & 68 & $\mathbf{N}$ & \\
\hline 1 & $10 / 7 / 77$ & 10 & 1 & & 69 & $\mathbf{N}$ & \\
\hline 1 & $10 / 7 / 77$ & 11 & 1 & & 71 & $\mathbf{N}$ & \\
\hline 1 & $10 / 7 / 77$ & 12 & 1 & & 71 & $\mathbf{N}$ & \\
\hline 1 & $12 / 7 / 77$ & 1 & 1 & & 84 & $Y$ & \\
\hline 1 & $12 / 7 / 77$ & 2 & 1 & & 68 & $\mathbf{N}$ & \\
\hline 1 & $12 / 7 / 77$ & 3 & 1 & & 83 & $\bar{N}$ & \\
\hline 1 & $12 / 7 / 77$ & 4 & 1 & & 83 & $\mathbf{N}$ & \\
\hline 1 & $12 / 7 / 77$ & 5 & 1 & & 82 & $\mathbf{N}$ & \\
\hline 1 & $12 / 7 / 77$ & 6 & 1 & & 77 & $\mathbf{N}$ & \\
\hline 1 & $12 / 7 / 77$ & 7 & 1 & & 42 & $\mathbf{N}$ & \\
\hline 1 & $.12 / 7 / 77$ & 8 & $\frac{1}{1}$ & & 42 & $\mathbf{N}$ & \\
\hline 1 & $12 / 7 / 77$ & 9 & 1 & & 49 & $\mathbf{N}$ & \\
\hline 1 & $12 / 7 / 77$ & 10 & 1 & & 53 & $\mathbf{N}$ & \\
\hline 1 & $12 / 7 / 77$ & 11 & 1 & & 57 & $\mathbf{N}$ & \\
\hline 1 & $12 / 7 / 77$ & 12 & 1 & & 58 & $\mathbf{N}$ & \\
\hline 1 & $1 / 29 / 78$ & 1 & 1 & & 94 & $Y$ & \\
\hline 1 & $1 / 29 / 78$ & 2 & 1 & & 69 & $\mathbf{N}$ & \\
\hline 1 & $1 / 29 / 78$ & 3 & 1 & & 92 & $\mathbf{N}$ & \\
\hline 1 & $1 / 29 / 78$ & 4 & 1 & & 92 & $N$ & \\
\hline 1 & $1 / 29 / 78$ & 5 & 1 & & 56 & $\bar{N}$ & \\
\hline 1 & $1 / 29 / 78$ & 6 & 1 & & 84 & $\mathbf{N}$ & \\
\hline 1 & $1 / 29 / 78$ & 7 & 1 & & 45 & $\mathrm{~N}$ & \\
\hline 1 & $9 / 29 / 78$ & 8 & 1 & & 45 & $\bar{N}$ & \\
\hline 1 & $1 / 29 / 78$ & 9 & 1 & & 57 & $\mathbf{N}$ & \\
\hline 1 & $9 / 29 / 78$ & 10 & 9 & & 62 & $\mathbf{N}$ & \\
\hline 1 & $1 / 29 / 78$ & 11 & 1 & & 62 & $\mathrm{~N}$ & \\
\hline 1 & $3 / 26 / 78$ & 3 & 1 & & 83 & $\mathbf{N}$ & \\
\hline 1 & $3 / 26 / 78$ & 4 & 1 & & 83 & $\overline{\mathbf{N}}$ & \\
\hline 1 & $3 / 26 / 78$ & 5 & 1 & & 82 & $\mathbf{N}$ & \\
\hline 1 & $3 / 26 / 78$ & 6 & 1 & & 75 & $\mathbf{N}$ & \\
\hline 1 & $3 / 26 / 78$ & 7 & 1 & & 53 & $\mathbf{N}$ & \\
\hline 1 & $3 / 26 / 78$ & 9 & 1 & & 55 & $\mathbf{N}$ & \\
\hline 1 & $3 / 26 / 78$ & 10 & 1 & & 56 & $\mathbf{N}$ & \\
\hline 1 & $3 / 26 / 78$ & 11 & 9 & & 58 & $\mathbf{N}$ & \\
\hline 1 & $3 / 26 / 78$ & 12 & 1 & & 58 & $\mathbf{N}$ & \\
\hline 9 & $4 / 23 / 78$ & 1 & 1 & & 89 & $Y$ & \\
\hline 1 & $4 / 23 / 78$ & 2 & 1 & & 76 & $\mathrm{~N}$ & \\
\hline 1 & $4 / 23 / 78$ & 3 & 9 & & 84 & $\mathbf{N}$ & \\
\hline
\end{tabular}

Data obtained from WHC Surveillance Analysis Computer System (SACS), June 2, 1993. 


\begin{tabular}{|c|c|c|c|c|c|c|c|}
\hline \multicolumn{8}{|c|}{ TANK 241-BY-103 } \\
\hline & & & & THERI & COUPLE D & TTA & \\
\hline TREE \# & DATE & THERMO \# & TREE SET \# & TEMP C & TEMP F & $\mathrm{Y}=$ MAX TEMP & COMMENTS \\
\hline 1 & $4 / 23 / 78$ & 4 & 1 & & 84 & $\mathbf{N}$ & \\
\hline 1 & $4 / 23 / 78$ & 5 & 1 & & 83 & $\mathbf{N}$ & \\
\hline 1 & $4 / 23 / 78$ & 6 & 1 & & 76 & $N$ & \\
\hline 1 & $4 / 23 / 78$ & 7 & 1 & & 57 & $N$ & \\
\hline 1 & $4 / 23 / 78$ & 8 & 1 & & 58 & $\mathbf{N}$ & \\
\hline 1 & $4 / 23 / 78$ & 9 & 1 & & 60 & $\mathbf{N}$ & \\
\hline 1 & $4 / 23 / 78$ & 10 & 1 & & 60 & $N$ & \\
\hline 1 & $4 / 23 / 78$ & 11 & 1 & & 52 & $\mathbf{N}$ & \\
\hline 1 & $4 / 23 / 78$ & 12 & 1 & & 52 & $\mathbf{N}$ & \\
\hline 1 & $6 / 17 / 78$ & 1 & 1 & & 78 & $\mathbf{N}$ & \\
\hline 1 & $6 / 17 / 78$ & 2 & 9 & & 79 & $\bar{Y}$ & \\
\hline 1 & $6 / 17 / 78$ & 3 & 1 & $=$ & 79 & $\mathbf{N}$ & \\
\hline 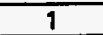 & $6 / 17 / 78$ & 4 & $\frac{1}{1}$ & & 78 & $\mathbf{N}$ & \\
\hline 1 & $6 / 17 / 78$ & 5 & $T$ & & 77 & $\mathbf{N}$ & \\
\hline$T$ & $6 / 17 / 78$ & 6 & 1 & & 76 & $\mathbf{N}$ & \\
\hline 1 & $6 / 17 / 78$ & 7 & 1 & & 71 & $\mathbf{N}$ & \\
\hline 1 & $6 / 17 / 78$ & 8 & 1 & & 72 & $\mathbf{N}$ & \\
\hline 1 & $6 / 17 / 78$ & 9 & 1 & & 70 & $\mathbf{N}$ & \\
\hline 1 & $6 / 17 / 78$ & 10 & 1 & & 66 & $\bar{N}$ & \\
\hline 1 & $6 / 17 / 78$ & 11 & 1 & & 64 & $\mathbf{N}$ & \\
\hline 1 & $6 / 17 / 78$ & 12 & 9 & & 64 & $\mathbf{N}$ & \\
\hline 1 & $7 / 16 / 78$ & 1 & 1 & & 76 & $\bar{Y}$ & \\
\hline 1 & $7 / 16 / 78$ & 3 & 1 & & 66 & $\bar{N}$ & \\
\hline 1 & $7 / 16 / 78$ & 4 & 1 & & 65 & $\mathbf{N}$ & \\
\hline 1 & $7 / 16 / 78$ & 5 & 1 & & 64 & $\mathbf{N}$ & \\
\hline 1 & $7 / 16 / 78$ & 6 & 9 & & 64 & $\mathrm{~N}$ & \\
\hline 1 & $7 / 16 / 78$ & 7 & 1 & & 71 & $\mathbf{N}$ & \\
\hline 1 & $7 / 16 / 78$ & 8 & 1 & & 71 & $\mathbf{N}$ & \\
\hline 1 & $7 / 16 / 78$ & 9 & 1 & & 65 & $\mathbf{N}$ & \\
\hline 1 & $7 / 16 / 78$ & 10 & 1 & & 57 & $\mathbf{N}$ & \\
\hline 1 & $7 / 16 / 78$ & 11 & 1 & & 54 & $\mathbf{N}$ & \\
\hline 1 & $7 / 16 / 78$ & 12 & 1 & & 53 & $\mathbf{N}$ & \\
\hline 1 & $8 / 13 / 78$ & 1 & 1 & & 78 & $\mathbf{N}$ & \\
\hline 1 & $8 / 13 / 78$ & 2 & 1 & & 80 & $\mathrm{~N}$ & \\
\hline 1 & $8 / 13 / 78$ & 3 & 1 & & 79 & $\mathbf{N}$ & \\
\hline 1 & $8 / 13 / 78$ & 4 & 1 & & 79 & $\mathbf{N}$ & \\
\hline 1 & $8 / 13 / 78$ & 5 & 1 & & 78 & $\mathbf{N}$ & \\
\hline 1 & $8 / 13 / 78$ & 6 & 1 & & 79 & $N$ & \\
\hline 1 & $8 / 13 / 78$ & 7 & 1 & & 84 & $\mathbf{N}$ & \\
\hline 1 & $8 / 13 / 78$ & 8 & 1 & & 85 & $Y$ & \\
\hline$\frac{1}{1}$ & $8 / 13 / 78$ & 9 & 1 & & 81 & $\mathbf{N}$ & \\
\hline 1 & $8 / 13 / 78$ & 10 & 1 & & 74 & $\mathbf{N}$ & \\
\hline 1 & $8 / 13 / 78$ & 11 & 1 & & 70 & $\mathbf{N}$ & \\
\hline 1 & $8 / 13 / 78$ & 12 & 7 & & 70 & $\mathbf{N}$ & \\
\hline 1 & $9 / 12 / 78$ & 1 & 1 & & 86 & $\mathbf{N}$ & \\
\hline 1 & $9 / 12 / 78$ & 2 & 1 & & 88 & $\bar{Y}$ & \\
\hline 1 & $9 / 12 / 78$ & 3 & 1 & & 87 & $\mathbf{N}$ & \\
\hline 1 & $9 / 12 / 78$ & 4 & 1 & & 87 & $\mathbf{N}$ & \\
\hline 1 & $9 / 12 / 78$ & 5 & 1 & & 87 & $\mathbf{N}$ & \\
\hline 1 & $9 / 12 / 78$ & 6 & 1 & & 88 & $\mathbf{N}$ & \\
\hline 1 & $9 / 12 / 78$ & 7 & 1 & & 78 & $N$ & \\
\hline 1 & $9 / 12 / 78$ & 8 & 1 & & 79 & $\mathbf{N}$ & \\
\hline 1 & $9 / 12 / 78$ & 9 & 1 & & 78 & $\mathbf{N}$ & \\
\hline 1 & $9 / 12 / 78$ & 10 & 1 & & 78 & $N$ & \\
\hline 1 & $9 / 12 / 78$ & 11 & 1 & & 77 & $N$ & \\
\hline 1 & $9 / 12 / 78$ & 12 & 1 & & 77 & $\mathbf{N}$ & \\
\hline 1 & $10 / 7 / 78$ & 1 & 1 & & 89 & $\bar{Y}$ & \\
\hline 1 & $10 / 7 / 78$ & 2 & 1 & & 88 & $\mathbf{N}$ & \\
\hline 1 & $10 / 7 / 78$ & 3 & 1 & & 88 & $\mathbf{N}$ & \\
\hline 1 & $10 / 7 / 78$ & 4 & 1 & & 88 & $\mathbf{N}$ & \\
\hline 1 & $10 / 7 / 78$ & 5 & 1 & & 86 & $\mathbf{N}$ & \\
\hline 1 & $10 / 7 / 78$ & 6 & 1 & & 87 & $\mathbf{N}$ & \\
\hline 1 & $10 / 7 / 78$ & 7 & 1 & & 75 & $N$ & \\
\hline 1 & $10 / 7 / 78$ & 8 & 1 & & 75 & $\mathbf{N}$ & \\
\hline 1 & $10 / 7 / 78$ & 9 & 1 & & 75 & $\mathbf{N}$ & \\
\hline 1 & $10 / 7 / 78$ & 10 & 1 & & 75 & $N$ & \\
\hline
\end{tabular}

Data obtained from WHC Surveillance Analysis Computer System (SACS), June 2, 1993. 


\begin{tabular}{|c|c|c|c|c|c|c|c|}
\hline \multicolumn{8}{|c|}{ TANK 247-BY-103 } \\
\hline & & & & THERI & COUPLE & TA & \\
\hline & & & & & & & \\
\hline TAEE \# & DATE & THERMO \# & TREE SET \# & TEMP C & TEMP F & $Y=M A X$ TEMP & COMMENTS \\
\hline 1 & $10 / 7 / 78$ & 11 & 1 & & 75 & $\mathbf{N}$ & \\
\hline 1 & $10 / 7 / 78$ & 12 & 1 & & 75 & $\mathbf{N}$ & \\
\hline 1 & $12 / 28 / 78$ & 1 & 1 & & 102 &. $\mathrm{Y}$ &. \\
\hline 1 & $12 / 28 / 78$ & 2 & 1 & & 79 & $\mathrm{~N}$ & \\
\hline 1 & $12 / 28 / 78$ & 3 & 1 & & 97 & $\mathrm{~N}$ & \\
\hline 1 & $12 / 28 / 78$ & 4 & 1 & & 97 & $\mathbf{N}$ & \\
\hline 1 & $12 / 28 / 78$ & 5 & 1 & & 64 & $\mathbf{N}$ & \\
\hline 1 & $12 / 28 / 78$ & 6 & 1 & & 88 & $\mathrm{~N}$ & \\
\hline 1 & $12 / 28 / 78$ & 7 & 1 & & 55 & $\mathbf{N}$ & \\
\hline 1 & $12 / 28 / 78$ & 8 & 1 & & 55 & $\bar{N}$ & \\
\hline 1 & $12 / 28 / 78$ & 9 & 1 & & 59 & $N$ & \\
\hline 1 & $12 / 28 / 78$ & 10 & 1 & $\because$ & 64 & $N$ & \\
\hline 9 & $12 / 28 / 78$ & 11 & 1 & & 68 & $N$ & \\
\hline 9 & $12 / 28 / 78$ & 12 & 1 & & 68 & $N$ & \\
\hline 1 & $1 / 3 / 79$ & 1 & 1 & & 86 & $\bar{Y}$ & \\
\hline 1 & $1 / 3 / 79$ & 2 & 1 & & 85 & $\mathbf{N}$ & \\
\hline 9 & $1 / 3 / 79$ & 3 & 1 & & 85 & $\mathbf{N}$ & \\
\hline 9 & $1 / 3 / 79$ & 4 & 1 & & 85 & $\bar{N}$ & \\
\hline 1 & $1 / 3 / 79$ & 5 & 1 & & 85 & $\mathrm{~N}$ & \\
\hline 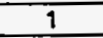 & $1 / 3 / 79$ & 6 & 1 & & 79 & $\mathbf{N}$ & \\
\hline 1 & $4 / 22 / 79$ & 1 & 1 & & 83 & $\mathbf{N}$ & \\
\hline 1 & $4 / 22 / 79$ & 2 & 1 & & 86 & $\bar{Y}$ & \\
\hline 1 & $4 / 22 / 79$ & 3 & 1 & & 85 & $\mathrm{~N}$ & \\
\hline 9 & $4 / 22 / 79$ & 4 & 1 & & 85 & $\bar{N}$ & \\
\hline 7 & $4 / 22 / 79$ & 5 & T & & 84 & $\mathbf{N}$ & \\
\hline 1 & $4 / 22 / 79$ & 6 & 1 & & 78 & $\mathrm{~N}$ & \\
\hline 9 & $4 / 22 / 79$ & 7 & 1 & & 58 & $N$ & \\
\hline 1 & $4 / 22 / 79$ & 8 & 1 & & 58 & $\mathrm{~N}$ & \\
\hline 9 & $4 / 22 / 79$ & 9 & 1 & & 60 & $\mathrm{~N}$ & \\
\hline 1 & $4 / 22 / 79$ & 10 & 1 & & 63 & $\mathbf{N}$ & \\
\hline 9 & $4 / 22 / 79$ & 11 & 1 & & 65 & $\mathbf{N}$ & \\
\hline 9 & $4 / 22 / 79$ & 12 & 1 & & 65 & $\bar{N}$ & \\
\hline 1 & $7 / 7 / 79$ & 1 & 1 & & 79 & $\mathrm{~N}$ & \\
\hline 1 & $7 / 7 / 79$ & 2 & 1 & & 82 & $\mathbf{N}$ & \\
\hline 1 & $7 / 7 / 79$ & 3 & 1 & & 80 & $\mathbf{N}$ & \\
\hline 9 & $7 / 7179$ & 6 & 1 & & 131 & $\mathbf{N}$ & \\
\hline 9 & $7 / 7 / 79$ & 7 & 1 & & 137 & $\bar{Y}$ & \\
\hline 7 & $7 / 7 / 79$ & 8 & 1 & & 129 & $\mathbf{N}$ & \\
\hline 1 & .717179 & 9 & 1 & & 105 & $\mathbf{N}$ & \\
\hline 9 & $12 / 1 / 80$ & 1 & 1 & & 117 & $\mathbf{Y}$ & \\
\hline 9 & $12 / 1 / 80$ & 2 & 1 & & 106 & N & \\
\hline 9 & $12 / 1 / 80$ & 3 & 1 & & 100 & $\mathbf{N}$ & \\
\hline 9 & $12 / 1 / 80$ & 4 & 1 & & 100 & $\mathbf{N}$ & \\
\hline 1 & $12 / 1 / 80$ & 5 & 1 & & 100 & $\mathbf{N}$ & \\
\hline 9 & $12 / 1 / 80$ & 6 & 1 & & 100 & $\mathbf{N}$ & \\
\hline 1 & $12 / 1 / 80$ & 7 & 1 & & 100 & $\mathbf{N}$ & \\
\hline 1 & $12 / 1 / 80$ & 8 & 9 & & 100 & $\mathbf{N}$ & \\
\hline 1 & $6 / 12 / 81$ & 9 & 9 & & 74 & $\mathrm{~N}$ & \\
\hline 1 & $6 / 12 / 81$ & 2 & 1 & & 77 & $Y$ & \\
\hline 1 & $6 / 12 / 81$ & 3 & 1 & & 77 & $N$ & \\
\hline 1 & $6 / 12 / 81$ & 4 & 1 & & 76 & $\mathbf{N}$ & \\
\hline 1 & $6 / 12 / 81$ & 5 & 9 & & 75 & $\mathbf{N}$ & \\
\hline 1 & $6 / 12 / 81$ & 6 & 9 & & 76 & $\mathrm{~N}$ & \\
\hline 1 & $6 / 12 / 81$ & 7 & 1 & & 67 & $\mathrm{~N}$ & \\
\hline 1 & $6 / 12 / 81$ & 8 & 9 & & 68 & N & \\
\hline 1 & $6 / 12 / 81$ & 9 & 9 & & 68 & $\mathbf{N}$ & \\
\hline 1 & $6 / 12 / 81$ & 90 & 9 & & 68 & $\mathrm{~N}$ & \\
\hline 1 & $6 / 12 / 81$ & 11 & 1 & & 66 & $\mathrm{~N}$ & \\
\hline 1 & $6 / 12 / 81$ & 12 & 1 & & 65 & $\mathrm{~N}$ & \\
\hline 1 & $6 / 12 / 81$ & 13 & 1 & & 66 & $\mathbf{N}$ & \\
\hline 1 & $12 / 3 / 81$ & 1 & 1 & 29 & 84.2 & $N$ & raw data - assumed degree C \\
\hline 1 & $12 / 3 / 81$ & 2 & 1 & 27 & 80.6 & $N$ & raw data - assumed degree C \\
\hline 1 & $12 / 3 / 81$ & 3 & 1 & 31 & 87.8 & $\bar{Y}$ & raw data - assumed degree C \\
\hline 1 & $12 / 3 / 81$ & 4 & 1 & 31 & 87.8 & $N$ & raw data - assumed degree C \\
\hline 1 & $12 / 3 / 81$ & 5 & & 30 & 86 & $\mathbf{N}$ & raw data - assumed degree $\mathrm{C}$ \\
\hline
\end{tabular}

Data obtained from WHC Surveillance Analysis Computer System (SACS), June 2, 1993. 


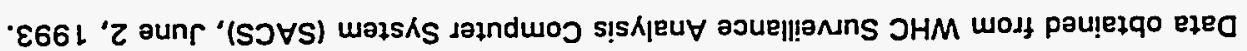

\begin{tabular}{|c|c|c|c|c|c|c|c|}
\hline & $\lambda$ & $I \cdot G L$ & & 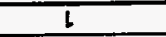 & $T$ & $06 / 6 ! / L$ & $I$ \\
\hline Mo7X & $\lambda$ & $\varepsilon .08$ & & $\downarrow$ & 1 & $06 / \varepsilon \downarrow / l$ & $l$ \\
\hline MOT & $\lambda$ & 2.08 & & $t$ & 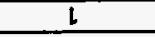 & $06 / 9 / 6$ & 1 \\
\hline \multirow{2}{*}{$0 z-6 \angle 9 \varepsilon z \angle 068-x-M 07$} & $\lambda$ & 2.08 & & 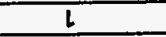 & $l$ & $68 / 62 / Z 1$ & $t$ \\
\hline & $\lambda$ & $9^{\circ} \subseteq L$ & & 1 & $I$ & $68 / \varepsilon Z / 21$ & $t$ \\
\hline X MOT & $\lambda$ & $9.6 L$ & & 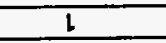 & 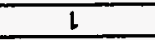 & $68 / 9 L / 2 L$ & 1 \\
\hline MO7 & $\lambda$ & $L L$ & & $\downarrow$ & I & $68 / 0 \mathrm{~L} / \mathrm{LL}$ & 1 \\
\hline MO7 & $\lambda$ & $k$ & & 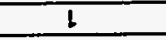 & $t$ & $68 / L / Z !$ & I \\
\hline MO7 & 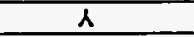 & $\angle \cdot 6 L$ & & $I$ & $L$ & 68/SZ/LL & 1 \\
\hline 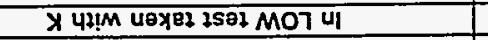 & $\lambda$ & $\varepsilon^{*} 6 L$ & & 1 & I & $68 / 8 L / L L$ & 1 \\
\hline MOT u! punof ainisiow & $\lambda$ & $9 . G L$ & & $l$ & $l$ & $68 / \angle L / L L$ & $t$ \\
\hline \multirow{47}{*}{ 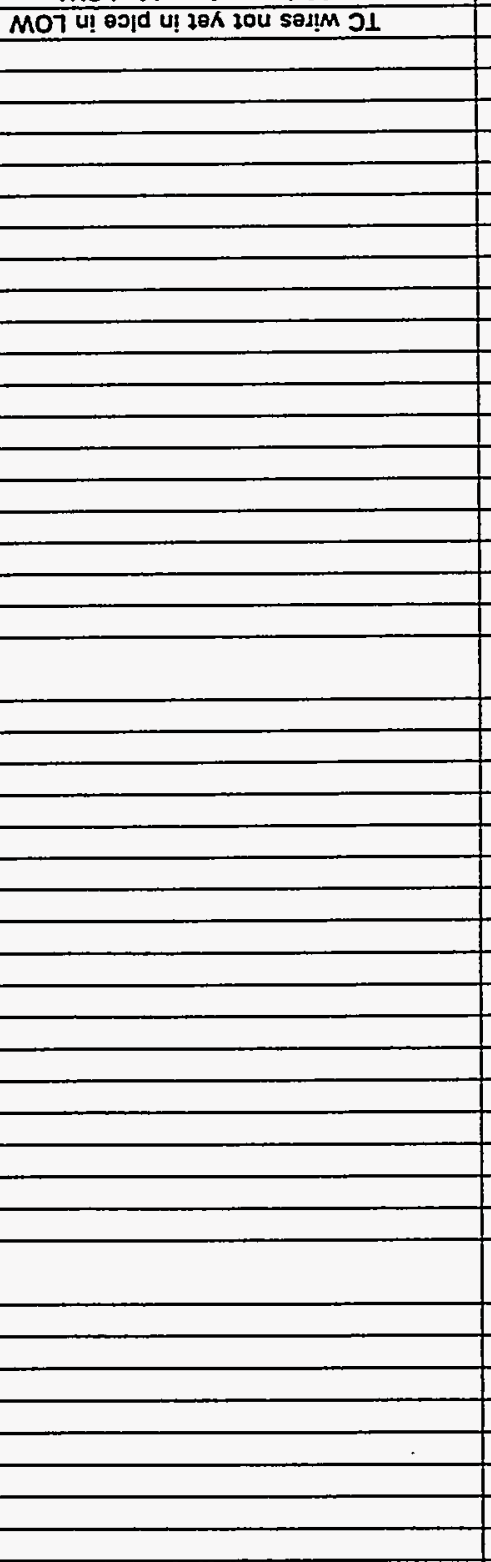 } & $N$ & & & 1 & $T$ & $68 / 0 \mathrm{l} / \mathrm{LL}$ & 1 \\
\hline & $\mathbf{N}$ & $2.6 L$ & & $t$ & 91 & $68 / 2 / L L$ & 1 \\
\hline & $N$ & $1.6 L$ & & $\mathbf{L}$ & $\underline{\text { sl }}$ & $68 / Z / L L$ & $I$ \\
\hline & $\mathbf{N}$ & 7.82 & & 1 & $t i$ & 68/Z/LL & $I$ \\
\hline & $\mathbf{N}$ & 9.86 & & $t$ & $\varepsilon l$ & 6B/Z/LL & $I$ \\
\hline & $N$ & $8 L$ & & 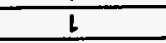 & $\overline{Z l}$ & $68 / 2 / L L$ & 1 \\
\hline & $\mathbf{N}$ & 6.08 & & 1 & It & $68 / Z / L L$ & $L$ \\
\hline & $\mathbf{N}$ & $8.6 L$ & & $t$ & $\mathrm{OL}$ & $68 / 2 / L 1$ & 1 \\
\hline & $\mathbf{N}$ & 18 & & $l$ & 6 & $68 / 2 / L L$ & $l$ \\
\hline & $\mathbf{N}$ & $\varepsilon \cdot 18$ & & 1 & 8 & $68 / 2 / 11$ & $t$ \\
\hline & $N$ & 8.28 & & L & $L$ & $68 / 2 / L 1$ & 1 \\
\hline & $\mathbf{N}$ & $9^{\circ} \bullet 8$ & & 1 & 9 & $68 / 2 / L L$ & $t$ \\
\hline & $\lambda$ & 58 & & $t$ & $\underline{s}$ & $68 / 2 / L !$ & $I$ \\
\hline & $\mathbf{N}$ & $t^{\circ} \varepsilon L$ & & 1. & $t$ & $68 / 2 / 6 !$ & 1 \\
\hline & $\mathbf{N}$ & $6^{\circ}+8$ & & 1 & $\varepsilon$ & $68 / 2 / L \downarrow$ & 1 \\
\hline & $\mathbf{N}$ & $\varepsilon 8$ & & 1 & 2 & $68 / Z / L i$ & 1 \\
\hline & $\mathbf{N}$ & $7^{\circ} \varepsilon 8$ & & $b$ & $l$ & $68 / 2 / L / 6$ & 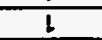 \\
\hline & $\mathbf{N}$ & $\angle S$ & & $I$ & $\overline{Z L}$ & $\angle 8 / 92 / 2$ & 1 \\
\hline & $\mathbf{N}$ & $\angle S$ & & $t$ & LL & $\angle 8 / 9 Z / 2$ & 1 \\
\hline & $\mathrm{N}$ & $\angle G$ & & 1 & OL & $\angle 8 / 92 / 2$ & $t$ \\
\hline & $\mathbf{N}$ & $\angle G$ & & 1 & 6 & $\angle 8 / 9 Z / Z$ & 1 \\
\hline & $\lambda$ & $9 L$ & & $I$ & 8 & $\angle 8 / 9 Z / Z$ & $t$ \\
\hline & $\mathbf{N}$ & $6 t$ & & $I$ & $L$ & $\angle 8 / 9 Z / Z$ & $l$ \\
\hline & $\mathbf{N}$ & $\varepsilon t$ & & $l$ & 9 & $\angle 8 / 9 Z / 2$ & 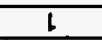 \\
\hline & $\mathbf{N}$ & 29 & & 1 & S & $\angle 8 / 9 Z / Z$ & 1 \\
\hline & $N$ & $\varepsilon t$ & & I & $t$ & $\angle 8 / 92 / 2$ & $\frac{T}{1}$ \\
\hline & $\mathbf{N}$ & $\phi$ & & $l$ & $\varepsilon$ & $\angle 8 / 92 / 2$ & $t$ \\
\hline & $N$ & + & & $l$ & $z$ & $\angle 8 / 92 / 2$ & $I$ \\
\hline & $\mathbf{N}$ & 60 & & 1 & 1 & $\angle 8 / 9 Z / Z$ & $l$ \\
\hline & $\mathbf{N}$ & $I L$ & & 1 & $2 L$ & $+8 / L / 2$ & 1 \\
\hline & $\mathbf{N}$ & $L L$ & & 1 & 11 & $+8 / L \sqrt{2}$ & 1 \\
\hline & $\mathbf{N}$ & 19 & & 1 & $O L$ & $+8 / L / 2$ & 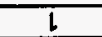 \\
\hline & $\mathbf{N}$ & 98 & & 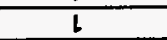 & 9 & $\$ 8 / L / 2$ & $L$ \\
\hline & $\mathbf{N}$ & 68 & & $i$ & G & $\$ 8 / L / 2$ & l \\
\hline & $\lambda$ & 06 & & 1 & $t$ & $\$ 8 / L / 2$ & $l$ \\
\hline & $\mathbf{N}$ & 68 & & 1 & $\varepsilon$ & $78 / L / Z$ & $t$ \\
\hline & $\bar{N}$ & 68 & & 1 & 2 & $+8 / L / 2$ & 1 \\
\hline & $N$ & $\angle 8$ & & $b$ & $l$ & $68 / L \sqrt{Z}$ & 1 \\
\hline & $N$ & $\angle 9$ & & $b$ & ZI & $28 / t L / L$ & $\mathfrak{l}$ \\
\hline & $\mathbf{N}$ & $\angle 9$ & & $\downarrow$ & IL & $28 / 6 / / 6$ & L \\
\hline & $\bar{N}$ & 09 & 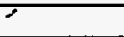 & l & OL & $28 / 61 / 1$ & I \\
\hline & $\mathbf{N}$ & 28 & & $\mathfrak{l}$ & 9 & $28 / 6 ! / 1$ & $t$ \\
\hline & $\mathbf{N}$ & 28 & & 1 & 9 & $28 / 61 / 6$ & $I$ \\
\hline & $\mathbf{N}$ & $\varepsilon 8$ & & $l$ & 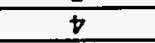 & $28 / 61 / 6$ & $b$ \\
\hline & $\mathbf{N}$ & $\varepsilon 8$ & & 1 & $\varepsilon$ & $28 / 6 ! / 1$ & b \\
\hline & $\lambda$ & $\varepsilon 8$ & & l & 2 & $28 / 51 / 6$ & 1 \\
\hline & $\mathbf{N}$ & $6 L$ & & 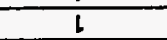 & 1 & $28 / t h / 1$ & $t$ \\
\hline J өө16ep pownsse - elep MEJ & $\mathbf{N}$ & $\tau \cdot g L$ & $\nabla 2$ & $l$ & $2 l$ & $18 / \varepsilon / Z !$ & 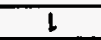 \\
\hline כ өөנ6өp pounsse - eiвp MEJ & $\mathbf{N}$ & $2 \cdot 9 L$ & $t 2$ & 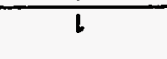 & HI & $18 / \varepsilon / Z \downarrow$ & 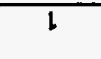 \\
\hline כ 8016әp pounsse - 81Rp MEd & $\mathbf{N}$ & 89 & OZ & $I$ & $O L$ & $18 / \varepsilon / 2 !$ & $L$ \\
\hline כ өөנ6өp pounSSB - вłEp MEd & $\mathbf{N}$ & 98 & $0 \varepsilon$ & 1 & 9 & $18 / \varepsilon / Z \downarrow$ & 1 \\
\hline 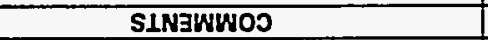 & $d W \exists \perp X V W=\lambda$ & $\pm d W I \perp$ & O dWII & \# $1 \exists S \exists \exists 41$ & \# OWY & ב1Va & $\# \exists \exists \forall 1$ \\
\hline & $\forall 18$ & ヨาdกัว & Iप्षझHI & & & & \\
\hline & & $L-19-t+2$ & & & & & \\
\hline
\end{tabular}




\begin{tabular}{|c|c|c|c|c|c|c|c|}
\hline \multicolumn{8}{|c|}{ TANK 241-BY-103 } \\
\hline \multicolumn{8}{|c|}{ THERMOCOUPLE DATA } \\
\hline TREE \# & DATE & THERMO \# & TREE SET \# & TEMP C & TEMP F & $Y=$ MAX TEMP & COMMENTS \\
\hline 1 & $1 / 19 / 90$ & 2 & 1 & & 70.6 & $\mathbf{N}$ & $\begin{array}{c}\text { (Taken twice on same day using } \mathrm{J} \% \mathrm{E} \\
\text { instruments.) }\end{array}$ \\
\hline 1 & $1 / 27 / 90$ & 1 & 1 & & 79.5 & $\bar{Y}$ & LOW $-K$ \\
\hline 1 & $2 / 5 / 90$ & 1 & 1 & & 78.3 & $\bar{Y}$ & LOW K-890723679-20 \\
\hline 1 & $2 / 11 / 90$ & 1 & 1 & & & $\mathbf{N}$ & $\begin{array}{l}\text { K-890723679-20 Unable to locate } \\
\text { thermocouple. }\end{array}$ \\
\hline 1 & $3 / 2 / 90$ & 1 & 1 & & 81.8 & $\bar{Y}$ & $K-H H-99 A-K$ \\
\hline 9 & $3 / 2 / 90$ & 2 & 1 & & & $\mathbf{N}$ & LOW \\
\hline 1 & $4 / 8 / 90$ & 1 & 1 & & & $\mathbf{N}$ & No Data - SSTPE notified \\
\hline 1 & $5 / 5 / 90$ & 1 & 1 & & & $\mathbf{N}$ & Not taken per PM 90-049 \\
\hline 1 & $6 / 1 / 90$ & 1 & 1 & & & $N$ & O/S Not taken per PM 90-051 \\
\hline 1 & $8 / 5 / 90$ & 1 & 1 & $\therefore$ & & N & J1 written to ro-install LOW \\
\hline 1 & $8 / 7 / 90$ & 1 & 1 & & 81.8 & $\bar{Y}$ & LOW \\
\hline 1 & $9 / 11 / 90$ & 1 & 1 & & 78.3 & $\bar{Y}$ & LOW \\
\hline 1 & $10 / 9 / 90$ & 9 & 1 & & 73 & $\bar{Y}$ & LOW \\
\hline 1 & $10 / 24 / 90$ & 1 & 1 & & & $\mathbf{Y}$ & $\begin{array}{c}\text { No Data - Requested shift office obtain } \\
\text { readings } 10 / 26\end{array}$ \\
\hline 1 & $10 / 26 / 90$ & 1 & 1 & & 75.9 & $\bar{Y}$ & J-890523512-20 \\
\hline 1 & $10 / 30 / 90$ & 1 & 2 & & 75.4 & $\bar{Y}$ & K 890723679-64 LOW \\
\hline 7 & $11 / 5 / 90$ & 1 & 1 & & 73.6 & $\bar{Y}$ & $J 890523512-20$ \\
\hline 1 & $11 / 14 / 90$ & 1 & 1 & & 71 & $\bar{Y}$ & K 890723679-64 LOW \\
\hline 1 & $11 / 18 / 90$ & 1 & 1 & & 78.2 & $\bar{Y}$ & K 890723697.74 LOW \\
\hline$T$ & $11 / 25 / 90$ & 1 & 1 & & 78.4 & $\bar{Y}$ & $\mathrm{~K}-890723679-64$ \\
\hline 1 & $12 / 5 / 90$ & 1 & 1 & & 68 & $\dot{Y}$ & K 890723679-64 LOW \\
\hline 1 & $12 / 10 / 90$ & 1 & 1 & & 75 & $Y$ & K 890723679-64 \\
\hline 1 & $12 / 11 / 90$ & 1 & 2 & & 75 & $\bar{Y}$ & K 890723679-64 \\
\hline 1 & $12 / 15 / 90$ & $\frac{1}{1}$ & 2 & & 74.9 & $\mathbf{Y}$ & K LOW \\
\hline 1 & $12 / 22 / 90$ & 9 & 1 & & 71 & $\bar{Y}$ & K 890723679.64 LOW \\
\hline 1 & $12 / 30 / 90$ & 1 & 1 & & 70.8 & $\bar{Y}$ & K LOW $890723679-646$ readings on LOW \\
\hline 1 & $12 / 30 / 90$ & 2 & 1 & & 70.4 & $\mathbf{N}$ & \\
\hline 1 & $12 / 30 / 90$ & 3 & 1 & & 69.9 & $\mathbf{N}$ & \\
\hline 1 & $12 / 30 / 90$ & 4 & 1 & & 69.3 & $\mathbf{N}$ & \\
\hline 1 & $12 / 30 / 90$ & 5 & 1 & & 69.2 & $\mathbf{N}$ & \\
\hline 1 & $12 / 30 / 90$ & 6 & 1 & & 68.9 & $\mathbf{N}$ & \\
\hline 1 & $1 / 6 / 91$ & 1 & 1 & & & $\mathbf{N}$ & K LOW Probe not in well - called shift office. \\
\hline 1 & $1 / 13 / 91$ & 9 & 1 & & 74.7 & $\bar{Y}$ & K 890723679-64 \\
\hline 1 & $1 / 19 / 91$ & 1 & 1 & & 78.8 & $\bar{Y}$ & K HH99A-K \\
\hline 1 & $.1 / 29 / 91$ & 1 & 1 & & 80 & $\bar{Y}$ & K HH-99A LOW \\
\hline 1 & $2 / 3 / 91$ & 1 & 1 & & 78 & $\bar{Y}$ & K Ser\#896723679-64 \\
\hline 1 & $2 / 9 / 91$ & 1 & 1 & & 80.5 & $\bar{Y}$ & K890723679-64 \\
\hline 1 & $2 / 16 / 91$ & 1 & 1 & & 80.1 & $\bar{Y}$ & $K-890723679-64$ \\
\hline 1 & $2 / 23 / 91$ & 1 & 1 & & 80.6 & $\bar{Y}$ & K 890723679-64 LOW \\
\hline 1 & $.3 / 3 / 91$ & 1 & 1 & & 79.4 & $\mathrm{Y}$ & LOW $890923679-64$ \\
\hline 1 & $3 / 9 / 91$ & 1 & 1 & & 79.8 & $\bar{Y}$ & K 890723679-20 \\
\hline 1 & $3 / 17 / 91$ & 1 & 1 & & 79.3 & $\bar{Y}$ & LOW 890923679-64 \\
\hline 1 & $3 / 23 / 91$ & 1 & 1 & & 80.2 & $\bar{Y}$ & LOW 890923679-64 \\
\hline 1 & $3 / 30 / 91$ & 1 & 1 & & 79.1 & $\bar{Y}$ & LOW 890923679-64 \\
\hline 1 & $4 / 7 / 91$ & 1 & 1 & & 81.9 & $\bar{Y}$ & LOW 890623567-12 \\
\hline 1 & $4 / 14 / 91$ & 1 & 1 & & 81.3 & $\bar{Y}$ & K 890623567-12 LOW \\
\hline 1 & $4 / 20 / 91$ & 1 & 1 & & 81.6 & $\bar{Y}$ & K 890723633-64 LOW \\
\hline 1 & $4 / 28 / 91$ & 1 & 1 & & 81.3 & $\bar{Y}$ & LOW $890623567-12$ \\
\hline 1 & $5 / 5 / 91$ & 1 & 1 & & 80 & $\bar{Y}$ & K 690723633.64 LOW \\
\hline 1 & $5 / 12 / 91$ & 1 & 1 & & 79.1 & $\mathbf{N}$ & K 890723679.20 Low \\
\hline 1 & $5 / 18 / 91$ & 1 & 1 & & 80.4 & $Y$ & K 890723633-64 LOW \\
\hline 1 & $5 / 25 / 91$ & 1 & 1 & & 79.8 & $\bar{Y}$ & K 890723633-64 LOW \\
\hline 1 & $6 / 2 / 91$ & 1 & 9 & & 80.3 & $\bar{Y}$ & K 890623567-12 LOW \\
\hline 1 & 6/9/91 & 1 & 1 & & 81.5 & $\bar{Y}$ & K 890623567-12 LOW \\
\hline 1 & $6 / 15 / 91$ & 1 & 1 & & 78.6 & $\bar{Y}$ & K 890723633-64 LOW \\
\hline 1 & $6 / 23 / 91$ & 1 & 9 & & 80.9 & $\bar{Y}$ & LOW 890623567-12 \\
\hline 1 & $6 / 29 / 91$ & 1 & 1 & & 81.2 & $\bar{Y}$ & K 890623567-12 LOW \\
\hline 1 & $7 / 6 / 91$ & 7 & 1 & & 81.7 & $\bar{Y}$ & K 890623567.12 LOW \\
\hline 1 & $7 / 13 / 91$ & 7 & 1 & & 82 & $\bar{Y}$ & K 890623567.12 LOW \\
\hline 1 & $7 / 20 / 91$ & 1 & 1 & & 87.8 & $\bar{Y}$ & K 890623567-12 LOW \\
\hline 1 & $7 / 27 / 91$ & $T$ & 1 & & 80.7 & $\bar{Y}$ & K 890725813 LOW \\
\hline 1 & $8 / 3 / 91$ & 1 & 1 & & 80.1 & $\bar{Y}$ & K 890723679-20 LOW \\
\hline
\end{tabular}

Data obtained from WHC Surveillance Analysis Computer System (SACS), June 2, 1993. 


\begin{tabular}{|c|c|c|c|c|c|c|c|}
\hline \multicolumn{8}{|c|}{ TANK 241-BY-103 } \\
\hline \multicolumn{8}{|c|}{ THERMOCOUPLE DATA } \\
\hline TPEF \# & 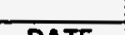 & THFo" 4 & TREF & TFMP C & TTMO E & & \\
\hline 1 & $8 / 10 / 91$ & 1 & 1 & & 82 & $Y$ & K 890723699-20 LOW \\
\hline 1 & $8 / 18 / 91$ & 1 & 1 & & 81.1 & $\bar{Y}$ & K 890623567-12 LOW \\
\hline 9 & $8 / 24 / 91$ & 1 & 1 & & 80.5 & $\bar{Y}$ & K 910226246-56 LOW \\
\hline 9 & $8 / 31 / 91$ & 1 & 1 & & 80.2 & $\bar{Y}$ & K-910226246-45 LOW \\
\hline 1 & $9 / 8 / 91$ & 1 & 1 & & 80.2 & $\bar{Y}$ & K-910226246-45 LOW \\
\hline 1 & $9 / 15 / 91$ & 1 & 1 & & 81.2 & $\bar{N}$ & $\mathrm{~K}-890623567-12$ \\
\hline 1 & $9 / 22 / 91$ & 1 & 1 & & 81.4 & $\bar{Y}$ & K-890723679-64 LOW \\
\hline 1 & $9 / 28 / 91$ & 1 & 1 & & 81.3 & $\bar{Y}$ & K-890723679-64 Omega LOW \\
\hline 9 & $10 / 5 / 91$ & 1 & 9 & & 78.2 & $\overline{\mathbf{Y}}$ & K-910226246-45 LOW \\
\hline 1 & $10 / 12 / 91$ & 1 & 1 & & 81.2 & $\bar{Y}$ & $K-8962356712$ \\
\hline 1 & $10 / 19 / 91$ & 1 & 1 & & 80.8 & $\bar{Y}$ & K-890723633-71 LOW \\
\hline 1 & $10 / 27 / 91$ & 9 & 1 & & 77.8 & $\bar{Y}$ & $k-910226246-45$ \\
\hline 1 & $11 / 2 / 91$ & 1 & 1 & & 81 & $\bar{Y}$ & K-890523512-47 \\
\hline 1 & $11 / 9 / 91$ & 9 & 1 & & 80 & $\bar{Y}$ & K-890723633-71 LOW \\
\hline 1 & $11 / 17 / 91$ & 9 & 1 & & 73.8 & $\bar{Y}$ & K-890723679-64 LOW \\
\hline 1 & $11 / 24 / 91$ & 1 & 1 & & 72 & $\bar{Y}$ & K-890723679-64 \\
\hline 1 & $12 / 1 / 91$ & 9 & 1 & & 74.4 & $\bar{Y}$ & K-890723679-64 LOW \\
\hline 1 & $12 / 6 / 91$ & 1 & $\frac{1}{1}$ & & 68.6 & $\bar{Y}$ & K-91022624634 LOW \\
\hline 1 & $12 / 14 / 91$ & 1 & 1 & & 71.5 & $\bar{Y}$ & K-910226246-68 LOW \\
\hline 1 & $12 / 20 / 91$ & 1 & 1 & & 71.3 & $\bar{Y}$ & $k-910426539-39$ \\
\hline 1 & $12 / 29 / 91$ & 1 & 1 & & 80.4 & $\bar{Y}$ & J.910126143-29 \\
\hline 9 & $12 / 29 / 91$ & 2 & 1 & & 80.4 & $\mathbf{N}$ & \\
\hline 1 & $12 / 29 / 91$ & 3 & 1 & & 80.2 & $\mathbf{N}$ & \\
\hline 9 & $12 / 29 / 91$ & 4 & 1 & & 79.8 & $\mathbf{N}$ & \\
\hline 1 & $12 / 29 / 91$ & 5 & 1 & & 78.7 & $\mathbf{N}$ & \\
\hline 9 & $12 / 29 / 91$ & 6 & 1 & & 76.5 & $\mathbf{N}$ & \\
\hline 1 & $12 / 29 / 91$ & 7 & 1 & & 72.5 & $\mathbf{N}$ & \\
\hline 1 & $12 / 29 / 91$ & 8 & 1 & & 70.1 & $\mathbf{N}$ & \\
\hline 1 & $12 / 29 / 91$ & 9 & 1 & & 69.6 & $\mathbf{N}$ & \\
\hline 1 & $12 / 29 / 91$ & 10 & 1 & & 69.6 & N & \\
\hline$T$ & $12 / 29 / 91$ & 11 & 1 & & 69.7 & $\mathbf{N}$ & \\
\hline 9 & $12 / 29 / 91$ & 12 & 9 & & 69.7 & $\mathbf{N}$ & \\
\hline 1 & $12 / 29 / 91$ & 13 & 9 & & 69.9 & $\mathbf{N}$ & \\
\hline 1 & $12 / 29 / 91$ & 14 & 1 & & 70 & $\mathbf{N}$ & \\
\hline 1 & $7 / 4 / 92$ & 1 & 1 & & 69.3 & $\bar{Y}$ & 890623567.12 HH.99A-K LOW \\
\hline 1 & $1 / 9 / 92$ & 1 & 1 & & 79.5 & $\bar{Y}$ & $0126143-14$ \\
\hline 1 & $1 / 9 / 92$ & 2 & 1 & & 79.2 & $\mathbf{N}$ & \\
\hline 1 & $1 / 9 / 92$ & 3 & 1 & & 79 & $\mathbf{N}$ & \\
\hline 1 & $1 / 9 / 92$ & 4 & 1 & & 78.4 & $\mathbf{N}$ & \\
\hline 9 & $1 / 9 / 92$ & 5 & 1 & & 77.2 & $\mathbf{N}$ & \\
\hline 1 & $1 / 9 / 92$ & 6 & 1 & & 74.6 & $\mathbf{N}$ & \\
\hline 1 & $1 / 9 / 92$ & 7 & 1 & & 69.9 & $\mathbf{N}$ & \\
\hline 9 & $1 / 9 / 92$ & 8 & 9 & & 67.8 & $\bar{N}$ & \\
\hline 9 & $1 / 9 / 92$ & 9 & 1 & & 67.4 & $\mathbf{N}$ & \\
\hline 9 & 1/9/92 & 10 & 1 & & 67.4 & $\mathbf{N}$ & \\
\hline 1 & $1 / 9 / 92$ & 11 & 1 & & 67.5 & $\mathbf{N}$ & \\
\hline 1 & $1 / 9 / 92$ & 12 & 1 & & 67.5 & $\mathbf{N}$ & \\
\hline 1 & $1 / 9 / 92$ & 13 & 1 & & 67.7 & $N$ & \\
\hline 1 & $1 / 9 / 92$ & 14 & 1 & & 67.9 & $\mathbf{N}$ & \\
\hline 1 & $1 / 11 / 92$ & 1 & 1 & & 79.3 & $\bar{Y}$ & $J-910126143-14$ \\
\hline 1 & $1 / 19 / 92$ & 2 & 1 & & 79.2 & $\mathbf{N}$ & \\
\hline 1 & $1 / 11 / 92$ & 3 & 1 & & 79 & $\mathbf{N}$ & \\
\hline$\frac{1}{1}$ & $1 / 11 / 92$ & 4 & 1 & & 78.3 & $\mathbf{N}$ & \\
\hline 1 & $1 / 11 / 92$ & 5 & 1 & & 77.1 & $\mathbf{N}$ & \\
\hline$\frac{1}{1}$ & $1 / 11 / 92$ & 6 & 1 & & 74.4 & $\mathbf{N}$ & \\
\hline 1 & $1 / 11 / 92$ & 7 & 1 & & 69.6 & $\mathbf{N}$ & \\
\hline 1 & $1 / 11 / 92$ & 8 & 9 & & 67.5 & $\mathbf{N}$ & \\
\hline 1 & $1 / 11 / 92$ & 9 & 1 & & 67.2 & $\bar{N}$ & \\
\hline 1 & $1 / 11 / 92$ & 10 & 9 & & 67.2 & $\bar{N}$ & \\
\hline 1 & $1 / 11 / 92$ & 11 & 7 & & 67.2 & $\mathrm{~N}$ & \\
\hline 1 & $1 / 11 / 92$ & 12 & 1 & & 67.2 & $\mathbf{N}$ & \\
\hline 1 & $1 / 19 / 92$ & 13 & 1 & & 67.1 & $N$ & \\
\hline$T$ & $1 / 11 / 92$ & 14 & 1 & & 67.6 & $\mathbf{N}$ & \\
\hline 1 & $1 / 16 / 92$ & 1 & 1 & & 80.5 & $Y$ & J910126143-32 \\
\hline 1 & $1 / 16 / 92$ & 2 & 1 & & 80.5 & $N$ & \\
\hline
\end{tabular}

Data obtained from WHC Surveillance Analysis Computer System (SACS), June 2, 1993. 
WHC-SD-WM-ER-312, Rev. 0

\begin{tabular}{|c|c|c|c|c|c|c|c|}
\hline \multicolumn{8}{|c|}{ TANK 241-BY-103 } \\
\hline \multicolumn{8}{|c|}{ THERMOCOUPLE DATA } \\
\hline TREE \# & DATE & THERMO \# & TREE SET \# & TEMP C & TEMP F & $\mathrm{Y}=$ MAX TEMP & COMMENTS \\
\hline 1 & $1 / 16 / 92$ & 3 & 1 & & 80.2 & $N$ & \\
\hline 1 & $1 / 16 / 92$ & 4 & 1 & & 79.6 & $\mathrm{~N}$ & \\
\hline 1 & $1 / 16 / 92$ & 5 & 1 & & 78.3 & $\mathbf{N}$ & \\
\hline 1 & $1 / 16 / 92$ & 6 & 1 & & 75.6 & $\mathbf{N}$ & \\
\hline 1 & $1 / 16 / 92$ & 7 & 1 & & 70.7 & $\mathbf{N}$ & \\
\hline 1 & $1 / 16 / 92$ & 8 & 1 & & 68.4 & $\mathrm{~N}$ & \\
\hline 1 & $1 / 16 / 92$ & 9 & 1 & & 68.1 & $\mathrm{~N}$ & \\
\hline 1 & $1 / 16 / 92$ & 10 & 1 & & 68.1 & $\mathbf{N}$ & \\
\hline 1 & $1 / 16 / 92$ & 11 & 1 & & 67.8 & $\mathbf{N}$ & \\
\hline 1 & $1 / 16 / 92$ & 12 & 1 & & 67.6 & $\bar{N}$ & \\
\hline 1 & $1 / 16 / 92$ & 13 & 1 & & 68.1 & $\mathbf{N}$ & \\
\hline 1 & $1 / 16 / 92$ & 14 & 9 & 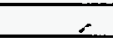 & 68.2 & $\mathbf{N}$ & \\
\hline 1 & $1 / 25 / 92$ & 1 & 1 & & 79.7 & $\mathbf{N}$ & $\mathrm{J}-910126143-14$ \\
\hline 1 & $1 / 25 / 92$ & 2 & 1 & & 79.8 & $\mathbf{Y}$ & \\
\hline 1 & $1 / 25 / 92$ & 3 & 1 & & 79.5 & $\mathbf{N}$ & \\
\hline 1 & $1 / 25 / 92$ & 4 & 1 & & 78.7 & $\bar{N}$ & \\
\hline 1 & $1 / 25 / 92$ & 5 & 1 & & 77.4 & $\bar{N}$ & \\
\hline 1 & $1 / 25 / 92$ & 6 & 1 & & 74.5 & N & \\
\hline 1 & $1 / 25 / 92$ & 7 & 1 & & 69.2 & $\mathbf{N}$ & \\
\hline 1 & $1 / 25 / 92$ & 8 & 1 & & 66.9 & $\mathbf{N}$ & \\
\hline 1 & $1 / 25 / 92$ & 9 & 1 & & 66.6 & $\mathbf{N}$ & \\
\hline 1 & $1 / 25 / 92$ & 10 & 1 & & 66.8 & $\mathbf{N}$ & \\
\hline 1 & $1 / 25 / 92$ & 11 & 1 & & 67 & $\mathbf{N}$ & \\
\hline 1 & $1 / 25 / 92$ & 12 & 1 & & 67.2 & $\mathbf{N}$ & \\
\hline 1 & $1 / 25 / 92$ & 13 & 1 & & 67.4 & $\mathbf{N}$ & \\
\hline 1 & $1 / 25 / 92$ & 14 & 1 & & 67.5 & $\mathbf{N}$ & \\
\hline 1 & $1 / 28 / 92$ & 1 & 1 & & & $\mathbf{N}$ & $\begin{array}{c}\text { ACCESS RESTRICTED } 1 / 28-2 / 22 \text { DUE TO } \\
\text { BATTERY FUME INCIDENT }\end{array}$ \\
\hline 1 & $2 / 24 / 92$ & 1 & 1 & & 72.8 & $\mathrm{~N}$ & J-900925741.79 \\
\hline 1 & $2 / 24 / 92$ & 2 & 1 & & 75.2 & $\mathbf{N}$ & \\
\hline 1 & $2 / 24 / 92$ & 3 & 1 & & 75.6 & $\bar{N}$ & \\
\hline 1 & $2 / 24 / 92$ & 4 & 1 & & 77 & $\bar{Y}$ & \\
\hline 1 & $2 / 24 / 92$ & 5 & 1 & & 72.4 & N & \\
\hline 1 & $2 / 24 / 92$ & 6 & 1 & & 67.7 & $\bar{N}$ & \\
\hline 1 & $2 / 24 / 92$ & 7 & 1 & & 64.9 & $\mathbf{N}$ & \\
\hline 1 & $2 / 24 / 92$ & 8 & 1 & & 61 & $\mathbf{N}$ & \\
\hline 1 & $2 / 24 / 92$ & 9 & 1 & & 62.2 & $\mathbf{N}$ & \\
\hline 1 & $2 / 24 / 92$ & 10 & 1 & & 62.7 & $\mathbf{N}$ & \\
\hline 1 & $.2 / 24 / 92$ & 11 & 1 & & 61.9 & $\mathbf{N}$ & \\
\hline 1 & $2 / 24 / 92$ & 12 & 1 & & 61.9 & $\mathbf{N}$ & \\
\hline 1 & $2 / 24 / 92$ & 13 & 1 & & 64.6 & $\mathbf{N}$ & \\
\hline 1 & $2 / 24 / 92$ & 14 & 1 & & 63.1 & $\mathbf{N}$ & \\
\hline 1 & $2 / 29 / 92$ & 1 & 1 & & 79.4 & $\overline{\mathbf{N}}$ & $K+1-030 / 9104265397$ \\
\hline 1 & $2 / 29 / 92$ & 2 & 1 & & 77 & $\mathbf{N}$ & \\
\hline 1 & $2 / 29 / 92$ & 3 & 1 & & 79.9 & $\bar{Y}$ & \\
\hline 1 & $2 / 29 / 92$ & 4 & 1 & & 78.3 & $\bar{N}$ & \\
\hline 1 & $2 / 29 / 92$ & 5 & 1 & & 74.5 & $\mathbf{N}$ & \\
\hline 1 & 2/29/92 & 6 & 1 & & 71.4 & $\mathbf{N}$ & \\
\hline 1 & $2 / 29 / 92$ & 7 & 1 & & 65.6 & $\mathbf{N}$ & \\
\hline 1 & $2 / 29 / 92$ & 8 & 1 & & 62.8 & $\mathbf{N}$ & \\
\hline 1 & $2 / 29 / 92$ & 9 & 1 & & 62.6 & $\overline{\mathbf{N}}$ & \\
\hline 1 & $2 / 29 / 92$ & 10 & 1 & & 64 & $\mathbf{N}$ & \\
\hline 1 & $2 / 29 / 92$ & 11 & 1 & & 65.3 & $\mathbf{N}$ & \\
\hline 1 & $2 / 29 / 92$ & 12 & 1 & & 66.6 & $\mathbf{N}$ & \\
\hline 1 & $2 / 29 / 92$ & 13 & 1 & & 68.2 & $\mathbf{N}$ & \\
\hline 1 & $2 / 29 / 92$ & 14 & 1 & & 67.2 & $\mathbf{N}$ & \\
\hline 1 & $2 / 29 / 92$ & 15 & 1 & & 62.2 & $\overline{\mathbf{N}}$ & \\
\hline 1 & $3 / 15 / 92$ & 1 & 1 & & 80 & $\mathbf{Y}$ & $J-910126143-32$ \\
\hline 1 & $3 / 15 / 92$ & 2 & 9 & & 80 & $\mathbf{N}$ & \\
\hline 1 & $3 / 15 / 92$ & 3 & 1 & & 79.5 & $\mathbf{N}$ & \\
\hline 1 & $3 / 15 / 92$ & 4 & 1 & & 78.6 & $\mathbf{N}$ & \\
\hline 1 & $3 / 15 / 92$ & 5 & 1 & & 76.8 & $\mathrm{~N}$ & \\
\hline 1 & $3 / 15 / 92$ & 6 & 1 & & 73.5 & $\mathbf{N}$ & \\
\hline 1 & $3 / 15 / 92$ & 7 & 1 & & 68 & $\mathbf{N}$ & \\
\hline 1 & $3 / 15 / 92$ & 8 & 1 & & 65.5 & $\mathbf{N}$ & \\
\hline
\end{tabular}

Data obtained from WHC Surveillance Analysis Computer System (SACS), June 2, 1993. 


\begin{tabular}{|c|c|c|c|c|c|c|c|}
\hline \multicolumn{8}{|c|}{ TANK 241-BY-103 } \\
\hline \multicolumn{8}{|c|}{ THERMOCOUPLE DATA } \\
\hline TREE \# & DATE & THEAMO \# & TAEE SET \# & TEMP C & TEMP F & $Y=$ MAX TEMP & COMMENTS \\
\hline 1 & $3 / 15 / 92$ & 9 & 1 & & 65.2 & $\mathbf{N}$ & \\
\hline 1 & $3 / 15 / 92$ & 10 & 1 & & 65.2 & $\mathbf{N}$ & \\
\hline 1 & $3 / 15 / 92$ & 12 & 1 & & 65.4 & $\mathbf{N}$ & \\
\hline 1 & $3 / 15 / 92$ & 13 & 1 & & 65.5 & $\mathbf{N}$ & \\
\hline 1 & $3 / 15 / 92$ & 14 & 1 & & 65.5 & $\mathbf{N}$ & \\
\hline 1 & $3 / 20 / 92$ & 1 & 1 & & 79.8 & $\bar{Y}$ & J 91012614332 \\
\hline 1 & $3 / 20 / 92$ & 2 & 1 & & 79.7 & $\mathbf{N}$ & \\
\hline 1 & $3 / 20 / 92$ & 3 & 1 & & 79.3 & $N$ & \\
\hline 1 & $3 / 20 / 92$ & 4 & 1 & & 78.1 & $\mathbf{N}$ & \\
\hline 1 & $3 / 20 / 92$ & 5 & 1 & & 76.4 & $\mathbf{N}$ & \\
\hline 1 & $3 / 20 / 92$ & 6 & 1 & & 73.1 & $N$ & \\
\hline 1 & $3 / 20 / 92$ & 7 & 1 & $\therefore$ & 67.7 & $\mathbf{N}$ & \\
\hline 1 & $3 / 20 / 92$ & 8 & 1 & & 65.3 & $\mathbf{N}$ & \\
\hline 1 & $3 / 20 / 92$ & 9 & 1 & & 65 & $\mathbf{N}$ & \\
\hline 1 & $3 / 20 / 92$ & 10 & 1 & & 65.1 & $\mathbf{N}$ & \\
\hline 1 & $3 / 20 / 92$ & 11 & 1 & & 65.1 & $\mathbf{N}$ & \\
\hline 1 & $3 / 20 / 92$ & 12 & 1 & & 65.2 & $\mathbf{N}$ & \\
\hline 1 & $3 / 20 / 92$ & 13 & 1 & & 65.3 & $\mathbf{N}$ & \\
\hline 1 & $3 / 20 / 92$ & 14 & 1 & & 65.5 & $\mathbf{N}$ & \\
\hline 1 & $3 / 29 / 92$ & 1 & 1 & & 80.2 & $Y$ & $\mathrm{~J} 910126143-66$ \\
\hline 1 & $3 / 29 / 92$ & 2 & 1 & & 80 & $\mathbf{N}$ & \\
\hline 1 & $3 / 29 / 92$ & 3 & 1 & & 79.5 & $\mathbf{N}$ & \\
\hline 1 & $3 / 29 / 92$ & 4 & 1 & & 78.5 & $\mathbf{N}$ & \\
\hline 1 & $3 / 29 / 92$ & 5 & 1 & & 76.7 & $N$ & \\
\hline 1 & $3 / 29 / 92$ & 6 & 1 & & 73.5 & $\mathbf{N}$ & \\
\hline 1 & $3 / 29 / 92$ & 7 & 1 & & 68.3 & $\mathbf{N}$ & \\
\hline 1 & $3 / 29 / 92$ & 8 & 1 & & 66.1 & $N$ & \\
\hline 1 & $3 / 29 / 92$ & 9 & 1 & & 65.8 & $\mathbf{N}$ & \\
\hline 1 & $3 / 29 / 92$ & 10 & 1 & & 65.8 & $\mathbf{N}$ & \\
\hline 1 & $3 / 29 / 92$ & 11 & 1 & & 65.9 & $N$ & \\
\hline 1 & $3 / 29 / 92$ & 12 & 1 & & 65.9 & $\mathbf{N}$ & \\
\hline 1 & $3 / 29 / 92$ & 13 & 1 & & 66.1 & $\mathbf{N}$ & \\
\hline 1 & $3 / 29 / 92$ & 14 & 1 & & 66.2 & $\mathbf{N}$ & \\
\hline 1 & $4 / 3 / 92$ & 1 & 1 & & 79.1 & $Y$ & J 910126143-14 \\
\hline 1 & $4 / 3 / 92$ & 2 & 1 & & 78.9 & $\mathbf{N}$ & \\
\hline 1 & $4 / 3 / 92$ & 3 & 1 & & 78.4 & $\mathbf{N}$ & \\
\hline 1 & $4 / 3 / 92$ & 4. & 1 & & 77.3 & $\mathbf{N}$ & \\
\hline 1 & $4 / 3 / 92$ & 5 & 1 & & 75.4 & $\mathbf{N}$ & \\
\hline 1 & $4 / 3 / 92$ & 6 & 1 & & 72.2 & $\mathbf{N}$ & \\
\hline 1 & $4 / 3 / 92$ & 7 & 1 & & 67.2 & $\mathbf{N}$ & \\
\hline 1 & $4 / 3 / 92$ & 8 & 1 & & 65 & $\mathbf{N}$ & \\
\hline 1 & $4 / 3 / 92$ & 9 & 1 & & 64.8 & $\mathbf{N}$ & \\
\hline 1 & $4 / 3 / 92$ & 10 & 1 & & 64.8 & $\mathbf{N}$ & \\
\hline 1 & $4 / 3 / 92$ & 11 & 1 & & 64.9 & $N$ & \\
\hline 1 & $4 / 3 / 92$ & 12 & 1 & & 65 & $N$ & \\
\hline 1 & $4 / 3 / 92$ & 73 & 1 & & 65.1 & $\mathbf{N}$ & \\
\hline 1 & $4 / 3 / 92$ & 14 & 1 & & 65.3 & $\mathbf{N}$ & \\
\hline 1 & 4/11/92 & 1 & 1 & & 86.3 & $\mathbf{N}$ & $\begin{array}{c}\text { K 890723679-80 Will retake temperatures and } \\
\text { double check e }\end{array}$ \\
\hline 1 & $4 / 11 / 92$ & 2 & 1 & & 86.5 & $\mathbf{Y}$ & \\
\hline 1 & $4 / 11 / 92$ & 3 & 1 & & 86 & $\mathbf{N}$ & \\
\hline 1 & $4 / 11 / 92$ & 4 & 1 & & 84.3 & $\mathbf{N}$ & \\
\hline 1 & $4 / 11 / 92$ & 5 & 1 & & 81.9 & $\mathbf{N}$ & \\
\hline 1 & $4 / 11 / 92$ & 6 & 1 & & 77.8 & $\mathbf{N}$ & \\
\hline 1 & $4 / 11 / 92$ & 7 & 1 & & 71.4 & $\mathbf{N}$ & \\
\hline 1 & $4 / 11 / 92$ & 8 & 1 & & 68.3 & $\mathbf{N}$ & \\
\hline 1 & $4 / 11 / 92$ & 9 & 1 & & 68.3 & $\mathbf{N}$ & \\
\hline 1 & $4 / 11 / 92$ & 10 & 1 & & 68.4 & $\mathbf{N}$ & \\
\hline 1 & $4 / 11 / 92$ & 11 & 1 & & 68.2 & $\mathbf{N}$ & \\
\hline 1 & $4 / 11 / 92$ & 12 & 1 & & 68.9 & $\mathbf{N}$ & \\
\hline 1 & $4 / 11 / 92$ & 13 & 1 & & 69.1 & $\mathbf{N}$ & \\
\hline 1 & $4 / 11 / 92$ & 14 & 1 & & 69.2 & $\mathbf{N}$ & \\
\hline 1 & $4 / 13 / 92$ & 1 & 1 & & 77.4 & $\mathbf{Y}$ & Rechecked \\
\hline 1 & $4 / 13 / 92$ & 2 & 1 & & 77.1 & $\mathbf{N}$ & \\
\hline 1 & $4 / 13 / 92$ & 3 & 1 & & 76 & $\mathbf{N}$ & \\
\hline 1 & $4 / 13 / 92$ & 4 & 1 & & 75 & $\mathbf{N}$ & \\
\hline
\end{tabular}

Data obtained from WHC Surveillance Analysis Computer System (SACS), June 2, 1993. 
WHC-SD-WM-ER-312, Rev. 0

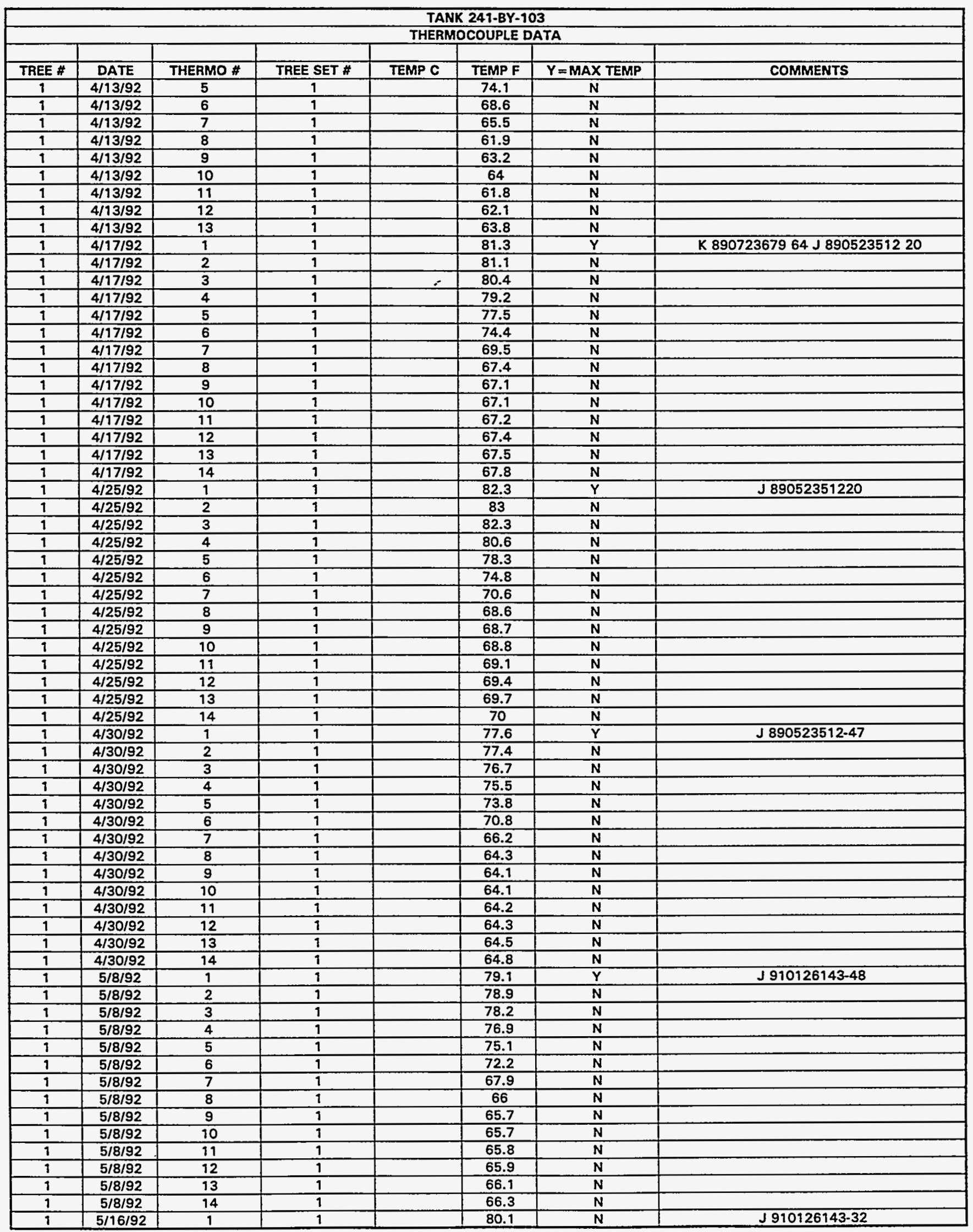

Data obtained from WHC Surveillance Analysis Computer System (SACS), June 2, 1993. 


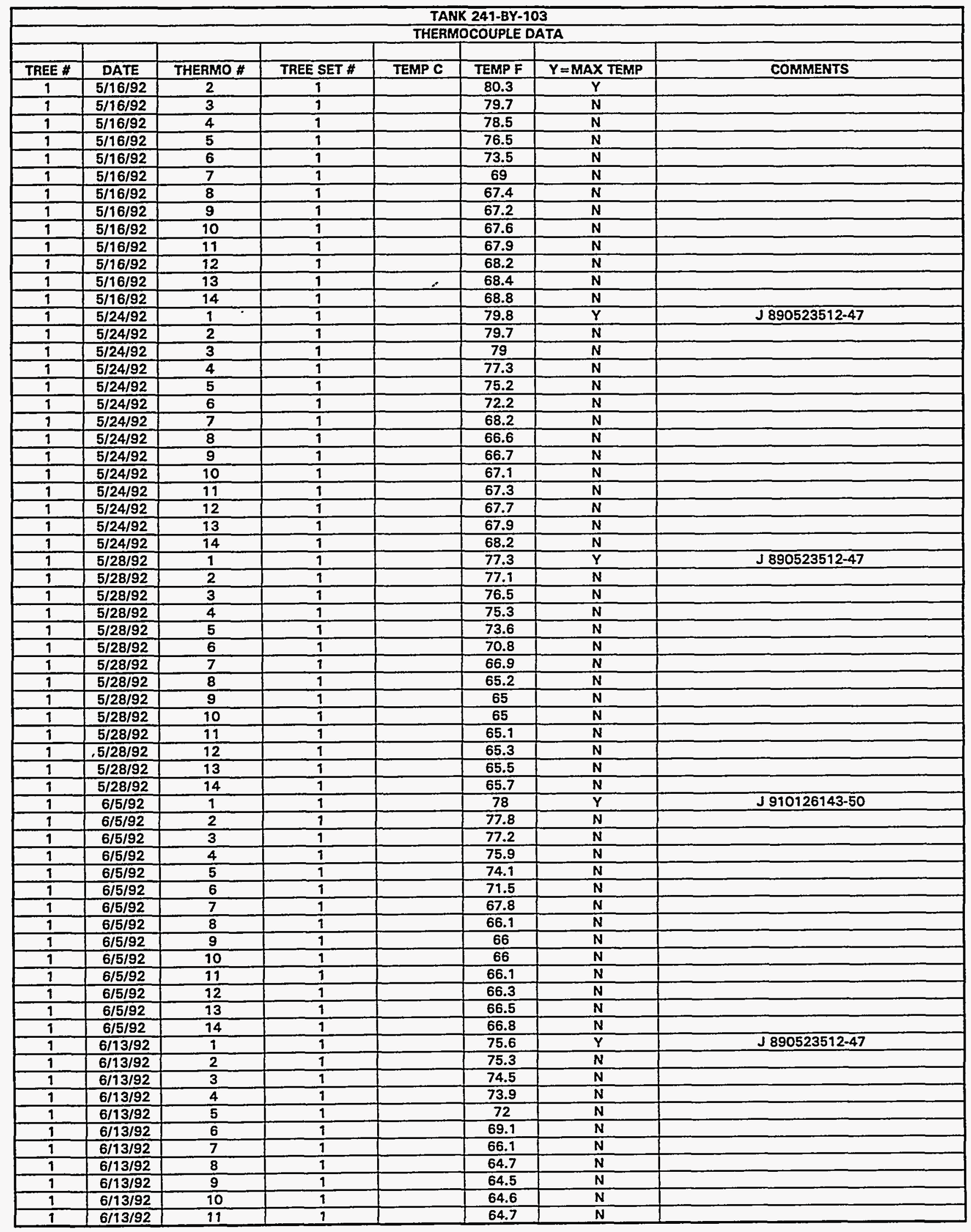

Data obtained from WHC Surveillance Analysis Computer System (SACS), June 2, 1993. 
WHC-SD-WM-ER-312, Rev. 0

\begin{tabular}{|c|c|c|c|c|c|c|c|}
\hline \multicolumn{8}{|c|}{ TANK 241-BY-103 } \\
\hline & & & & THER & COUPLE I & TTA & \\
\hline TREE \# & DATE & THERMO \# & TREE SET \# & TEMP C & TEMP F & $Y=$ MAX TEMP & COMMENTS \\
\hline 1 & $6 / 13 / 92$ & 12 & 1 & & 65 & $\mathbf{N}$ & \\
\hline 1 & $6 / 13 / 92$ & 13 & 1 & & 65.2 & $\mathbf{N}$ & \\
\hline 1 & $6 / 20 / 92$ & 1 & 1 & & 80.2 & $\mathbf{N}$ & J 910126143.48 \\
\hline 1 & $6 / 20 / 92$ & 2 & 1 & & 80.6 & $\bar{Y}$ & \\
\hline 1 & $6 / 20 / 92$ & 3 & 1 & & 80 & $\mathbf{N}$ & \\
\hline 1 & $6 / 20 / 92$ & 4 & 1 & & 78.5 & $\bar{N}$ & \\
\hline 1 & $6 / 20 / 92$ & 5 & 1 & & 76.4 & $\mathbf{N}$ & \\
\hline 1 & $6 / 20 / 92$ & 6 & 1 & & 73.9 & $\mathbf{N}$ & \\
\hline 1 & $6 / 20 / 92$ & 7 & 1 & & 70.9 & $\mathbf{N}$ & \\
\hline 1 & $6 / 20 / 92$ & 8 & 1 & & 69.5 & $\mathbf{N}$ & \\
\hline 1 & $6 / 20 / 92$ & 9 & 1 & & 69.6 & $\mathbf{N}$ & \\
\hline 1 & $6 / 20 / 92$ & 10 & 1 & & 69.8 & $\mathbf{N}$ & \\
\hline 1 & $6 / 20 / 92$ & 11 & 1 & & 70 & $\mathbf{N}$ & \\
\hline 1 & $6 / 20 / 92$ & 12 & 1 & & 70.3 & $\mathbf{N}$ & \\
\hline 1 & $6 / 20 / 92$ & 13 & 1 & & 70.7 & $\mathbf{N}$ & \\
\hline 1 & $6 / 20 / 92$ & 14 & 1 & & 71 & $\mathbf{N}$ & \\
\hline 1 & $6 / 28 / 92$ & 1 & 1 & & 79.1 & $\mathbf{Y}$ & $\mathrm{J} \mathrm{T} 1-042910126143-66$ \\
\hline 1 & $6 / 28 / 92$ & 2 & 1 & & 79.1 & $\mathbf{N}$ & \\
\hline 1 & $6 / 28 / 92$ & 3 & 1 & & 78.5 & $\mathbf{N}$ & \\
\hline 1 & $6 / 28 / 92$ & 4 & 1 & & 77.4 & $\mathbf{N}$ & \\
\hline 1 & $6 / 28 / 92$ & 5 & 1 & & 75.7 & $\bar{N}$ & \\
\hline 1 & $6 / 28 / 92$ & 6 & 1 & & 73.4 & $\mathbf{N}$ & \\
\hline 1 & $6 / 28 / 92$ & 7 & 1 & & 70.5 & $\mathbf{N}$ & \\
\hline 1 & $6 / 28 / 92$ & 8 & 1 & & 69 & $\mathbf{N}$ & \\
\hline 1 & $6 / 28 / 92$ & 9 & 1 & & 68.9 & $\mathbf{N}$ & \\
\hline 1 & $6 / 28 / 92$ & 10 & 1 & & 69.2 & $\mathbf{N}$ & \\
\hline 1 & $6 / 28 / 92$ & 11 & 1 & & 69.4 & $\mathbf{N}$ & \\
\hline 1 & $6 / 28 / 92$ & 12 & 1 & & 69.7 & $\mathbf{N}$ & \\
\hline 1 & $6 / 28 / 92$ & 13 & 1 & & 70.1 & $\mathbf{N}$ & \\
\hline 1 & $6 / 28 / 92$ & 14 & 1 & & 70.4 & $\mathbf{N}$ & \\
\hline 1 & $7 / 3 / 92$ & 1 & 1 & & 77.8 & $\bar{Y}$ & $\mathrm{~J} \mathrm{Tl}-041$ \\
\hline 1 & $7 / 3 / 92$ & 2 & 1 & & 77.5 & $\mathbf{N}$ & \\
\hline 1 & $7 / 3 / 92$ & 3 & 1 & & 76.9 & $\mathbf{N}$ & \\
\hline 1 & $7 / 3 / 92$ & 4 & 1 & & 75.8 & $\mathbf{N}$ & \\
\hline 1 & $7 / 3 / 92$ & 5 & 1 & & 74.3 & $\mathbf{N}$ & \\
\hline 1 & $7 / 3 / 92$ & 6 & 1 & & 72.2 & $\mathbf{N}$ & \\
\hline 1 & $7 / 3 / 92$ & 7 & 1 & & 69.4 & $\mathbf{N}$ & \\
\hline 1 & $7 / 3 / 92$ & 8 & 1 & & 68 & $\mathbf{N}$ & \\
\hline 1 & $7 / 3 / 92$ & 9 & 1 & & 67.8 & $\mathbf{N}$ & \\
\hline 1 & $7 / 3 / 92$ & 10 & 1 & & 67.9 & $\mathbf{N}$ & \\
\hline 1 & $7 / 3 / 92$ & 91 & 1 & & 68 & $\mathbf{N}$ & \\
\hline 1 & $7 / 3 / 92$ & 12 & 1 & & 68 & $\mathbf{N}$ & \\
\hline 1 & $7 / 3 / 92$ & 13 & 1 & & 68.3 & $\mathbf{N}$ & \\
\hline 1 & $7 / 3 / 92$ & 14 & 1 & & 68.6 & $\mathbf{N}$ & \\
\hline 1 & $7 / 10 / 92$ & 1 & 1 & & 78.4 & $\mathrm{Y}$ & J910126143-42 \\
\hline 1 & $7 / 10 / 92$ & 2 & 1 & & 78.1 & $\mathbf{N}$ & \\
\hline 1 & $7 / 10 / 92$ & 3 & 1 & & 77.5 & $\mathbf{N}$ & \\
\hline 1 & $7 / 10 / 92$ & 4 & 1 & & 76.3 & $\mathbf{N}$ & \\
\hline 1 & $7 / 10 / 92$ & 5 & 1 & & 74.9 & $\mathbf{N}$ & \\
\hline 1 & $7 / 10 / 92$ & 6 & 1 & & 72.9 & $\mathbf{N}$ & \\
\hline 1 & $7 / 10 / 92$ & 7 & 1 & & 70.3 & $\mathbf{N}$ & \\
\hline 1 & $7 / 10 / 92$ & 8 & 1 & & 69 & $\mathbf{N}$ & \\
\hline 1 & $7 / 10 / 92$ & 9 & 1 & & 68.7 & $\mathbf{N}$ & \\
\hline 1 & $7 / 10 / 92$ & 10 & 1 & & 68.8 & $\mathbf{N}$ & \\
\hline 1 & $7 / 10 / 92$ & 11 & 1 & & 68.9 & $\mathbf{N}$ & \\
\hline 1 & $7 / 10 / 92$ & 12 & 1 & & 69.2 & $\mathbf{N}$ & \\
\hline 1 & $7 / 10 / 92$ & 13 & 1 & & 69.5 & $\mathbf{N}$ & \\
\hline 1 & $7 / 10 / 92$ & 14 & 1 & & 69.8 & $\mathbf{N}$ & \\
\hline 1 & $7 / 18 / 92$ & 1 & 1 & & 78.8 & $\mathbf{N}$ & J $910126143-42$ \\
\hline 1 & $7 / 18 / 92$ & 2 & 1 & & 79 & $Y$ & \\
\hline 1 & $7 / 18 / 92$ & 3 & 1 & & 78.5 & $\mathbf{N}$ & \\
\hline 1 & $7 / 18 / 92$ & 4 & 1 & & 77.4 & $\mathbf{N}$ & \\
\hline 1 & $7 / 18 / 92$ & 5 & 1 & & 75.8 & $\mathbf{N}$ & \\
\hline 1 & $7 / 18 / 92$ & 6 & 1 & & 73.6 & $\mathbf{N}$ & \\
\hline 1 & $7 / 18 / 92$ & 7 & 1 & & 71.3 & $N$ & \\
\hline 1 & $7 / 18 / 92$ & 8 & 1 & & 70 & $\mathrm{~N}$ & \\
\hline
\end{tabular}

Data obtained from WHC Surveillance Analysis Computer System (SACS), June 2, 1993. 


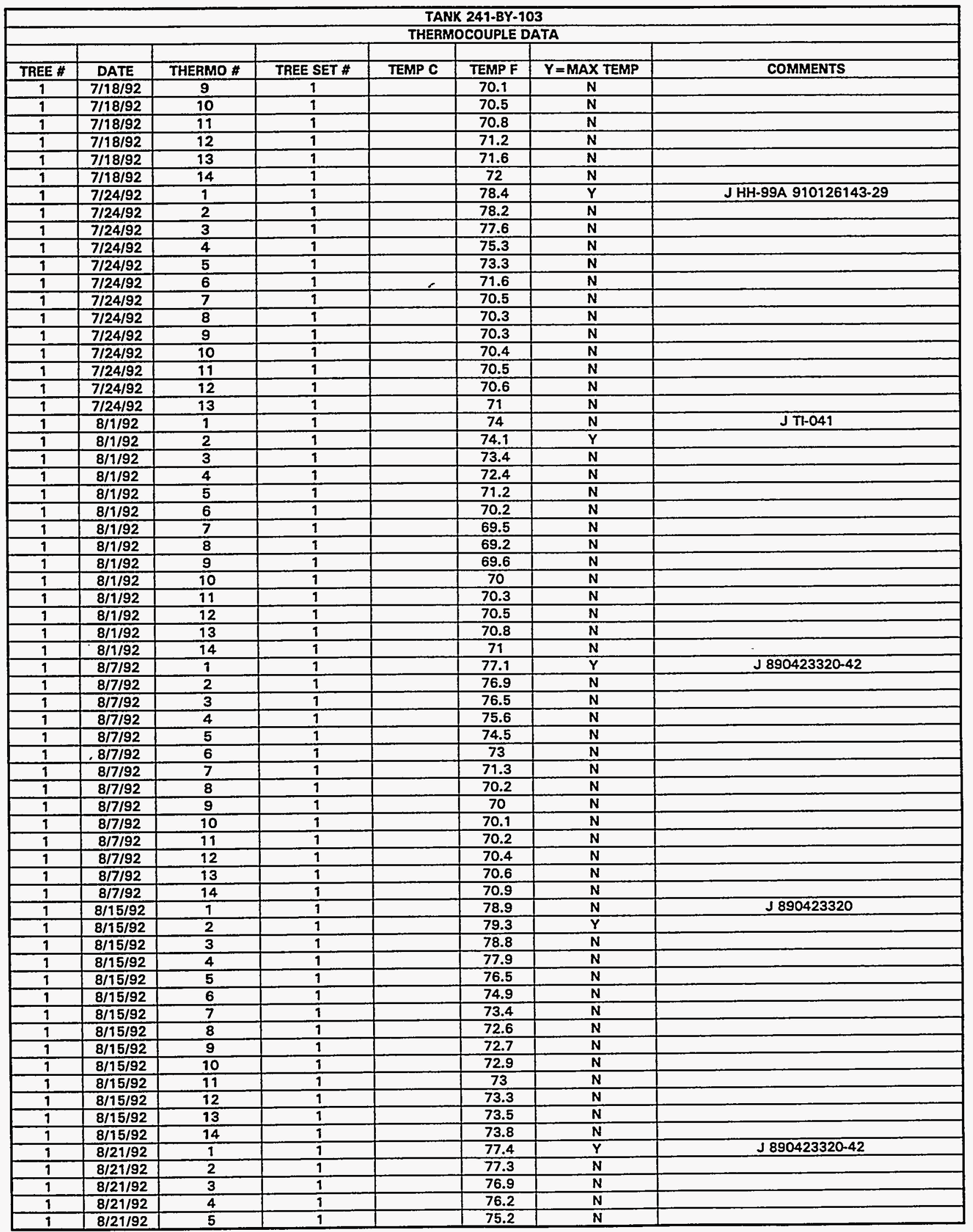

Data obtained from WHC Surveillance Analysis Computer System (SACS), June 2, 1993. 


\begin{tabular}{|c|c|c|c|c|c|c|c|}
\hline \multicolumn{8}{|c|}{ TANK 241-BY-103 } \\
\hline \multicolumn{8}{|c|}{ THERMOCOUPLE DATA } \\
\hline TREE \# & DATE & THERMO \# & TREE SET \# & TEMP C & TEMP F & $Y=$ MAX TEMP & COMMENTS \\
\hline 1 & $8 / 21 / 92$ & 6 & 1 & & 73.8 & $\mathrm{~N}$ & \\
\hline 1 & $8 / 21 / 92$ & 7 & 1 & & 72.2 & $\mathbf{N}$ & \\
\hline 1 & $8 / 21 / 92$ & 8 & 1 & & 71.3 & $\mathbf{N}$ & \\
\hline 1 & $8 / 21 / 92$ & 9 & 1 & & 71.2 & $\mathbf{N}$ & \\
\hline 1 & $8 / 21 / 92$ & 10 & 1 & & 71.5 & $\mathbf{N}$ & \\
\hline 1 & $8 / 21 / 92$ & 11 & 1 & & 71.4 & $\bar{N}$ & \\
\hline 1 & $8 / 21 / 92$ & 12 & 1 & & 71.5 & $\bar{N}$ & \\
\hline 1 & $8 / 21 / 92$ & 13 & 1 & & 71.8 & $\bar{N}$ & \\
\hline 1 & $8 / 21 / 92$ & 14 & 1 & & 72.1 & $\bar{N}$ & \\
\hline$\frac{1}{1}$ & $8 / 30 / 92$ & 1 & 1 & & 79.2 & $Y$ & J $890423320-42$ \\
\hline 1 & $8 / 30 / 92$ & 2 & 1 & & 79.1 & $\dot{N}$ & \\
\hline 1 & $8 / 30 / 92$ & 3 & 1 & & 78.7 & $\mathbf{N}$ & \\
\hline 1 & $8 / 30 / 92$ & 4 & $\frac{1}{1}$ & & 77.8 & $\mathbf{N}$ & \\
\hline 1 & $8 / 30 / 92$ & 5 & 1 & & 76.6 & $\mathbf{N}$ & \\
\hline 1 & $8 / 30 / 92$ & 6 & 1 & & 75.2 & $\mathbf{N}$ & \\
\hline 1 & $8 / 30 / 92$ & 7 & 1 & & 73.6 & $\mathrm{~N}$ & \\
\hline 1 & $8 / 30 / 92$ & 8 & 1 & & 72.6 & $\mathbf{N}$ & \\
\hline 1 & $8 / 30 / 92$ & 9 & 1 & & 72.9 & $\overline{\mathbf{N}}$ & \\
\hline$\frac{1}{1}$ & $8 / 30 / 92$ & 10 & 9 & & 73.3 & $\mathbf{N}$ & \\
\hline 1 & $8 / 30 / 92$ & 91 & 1 & & 73.6 & $\bar{N}$ & \\
\hline 1 & $8 / 30 / 92$ & 12 & 1 & & 74.1 & $\mathbf{N}$ & \\
\hline 1 & $8 / 30 / 92$ & 13 & 1 & & 74.4 & $\mathbf{N}$ & \\
\hline 1 & $8 / 30 / 92$ & 14 & 1 & & 74.8 & $\mathbf{N}$ & \\
\hline 1 & $9 / 6 / 92$ & 1 & 1 & & 77.5 & $\bar{Y}$ & J 890423320.42 \\
\hline 1 & $9 / 6 / 92$ & 2 & 1 & & 77.5 & $\mathbf{N}$ & \\
\hline 1 & $9 / 6 / 92$ & 3 & 1 & & 77.2 & $\mathbf{N}$ & \\
\hline 1 & $9 / 6 / 92$ & 4 & 1 & & 76.5 & $\mathbf{N}$ & \\
\hline 1 & $9 / 6 / 92$ & 5 & 1 & & 75.7 & $\mathbf{N}$ & \\
\hline 1 & $9 / 6 / 92$ & 6 & 1 & & 74.6 & $\mathbf{N}$ & \\
\hline 1 & $9 / 6 / 92$ & 7 & 1 & & 73.2 & $\mathbf{N}$ & \\
\hline 1 & $9 / 6 / 92$ & 8 & 1 & & 72.3 & $\mathbf{N}$ & \\
\hline 1 & $9 / 6 / 92$ & 9 & 1 & & 72.2 & $\mathbf{N}$ & \\
\hline 1 & $9 / 6 / 92$ & 10 & 1 & & 72.2 & $\mathbf{N}$ & \\
\hline 1 & $9 / 6 / 92$ & 19 & 1 & & 72.3 & $\mathbf{N}$ & \\
\hline 1 & $9 / 6 / 92$ & 12 & $\frac{1}{1}$ & & 72.5 & $\mathbf{N}$ & \\
\hline 1 & $9 / 6 / 92$ & 13 & 1 & & 72.7 & $\mathbf{N}$ & \\
\hline 1 & $9 / 6 / 92$ & 14 & 1 & & 72.9 & $\mathbf{N}$ & \\
\hline 1 & $9 / 12 / 92$ & $\frac{1}{1}$ & 1 & & 78.6 & $\overline{\mathbf{N}}$ & J $890423320-42$ \\
\hline 1 & $.9 / 12 / 92$ & 2 & 1 & & 78.7 & $\bar{Y}$ & \\
\hline 1 & $9 / 12 / 92$ & 3 & 1 & & 78.4 & $\mathbf{N}$ & \\
\hline 1 & $9 / 12 / 92$ & 4 & 1 & & 77.9 & $\mathbf{N}$ & \\
\hline 1 & $9 / 12 / 92$ & 5 & 1 & & 77.1 & $\mathbf{N}$ & \\
\hline 1 & $9 / 12 / 92$ & 6 & 1 & & 76 & $\mathbf{N}$ & \\
\hline 1 & $9 / 12 / 92$ & 7 & 1 & & 74.7 & $\mathbf{N}$ & \\
\hline 1 & $9 / 12 / 92$ & 8 & 1 & & 73.8 & $\mathbf{N}$ & \\
\hline 1 & $9 / 12 / 92$ & 9 & 1 & & 73.6 & $\mathbf{N}$ & \\
\hline 1 & $9 / 12 / 92$ & 10 & 1 & & 73.7 & $\mathbf{N}$ & \\
\hline 1 & $9 / 12 / 92$ & 11 & 1 & & 73.8 & $N$ & \\
\hline 1 & $9 / 12 / 92$ & 12 & 1 & & 73.9 & $N$ & \\
\hline 1 & $9 / 12 / 92$ & 13 & 1 & & 74.1 & $\mathbf{N}$ & \\
\hline 1 & $9 / 12 / 92$ & 14 & 1 & & 74.5 & $\mathbf{N}$ & \\
\hline 1 & $9 / 20 / 92$ & 1 & 1 & & 78.9 & $Y$ & J910126143-64 \\
\hline 1 & $9 / 20 / 92$ & 2 & $\overline{1}$ & & 78.7 & $\mathrm{~N}$ & \\
\hline 1 & $9 / 20 / 92$ & 3 & 1 & & 78.4 & $\mathrm{~N}$ & \\
\hline 9 & $9 / 20 / 92$ & 4 & $\frac{1}{1}$ & & 77.9 & $\mathbf{N}$ & \\
\hline 1 & $9 / 20 / 92$ & 5 & 1 & & 77.1 & $\mathrm{~N}$ & \\
\hline 1 & $9 / 20 / 92$ & 6 & 1 & & 76 & $\mathbf{N}$ & \\
\hline 1 & $9 / 20 / 92$ & 7 & 1 & & 74.7 & $N$ & \\
\hline 9 & $9 / 20 / 92$ & 8 & 1 & & 73.8 & $\mathrm{~N}$ & \\
\hline 1 & $9 / 20 / 92$ & 9 & 1 & & 73.7 & $\mathbf{N}$ & \\
\hline 9 & $9 / 20 / 92$ & 10 & 1 & & 73.7 & $\mathbf{N}$ & \\
\hline 1 & $9 / 20 / 92$ & 11 & 1 & & 73.8 & $N$ & \\
\hline 1 & $9 / 20 / 92$ & 12 & 1 & & 74 & $\mathbf{N}$ & \\
\hline 1 & $9 / 20 / 92$ & 13 & 1 & & 74.3 & $\overline{\mathrm{N}}$ & \\
\hline 9 & $9 / 20 / 92$ & 14 & 1 & & 74.5 & $\mathbf{N}$ & \\
\hline$\frac{1}{1}$ & $9 / 26 / 92$ & 1 & 1 & & 77.4 & $Y$ & J $910126143-64$ \\
\hline
\end{tabular}

Data obtained from WHC Surveillance Analysis Computer System (SACS), June 2, 1993. 


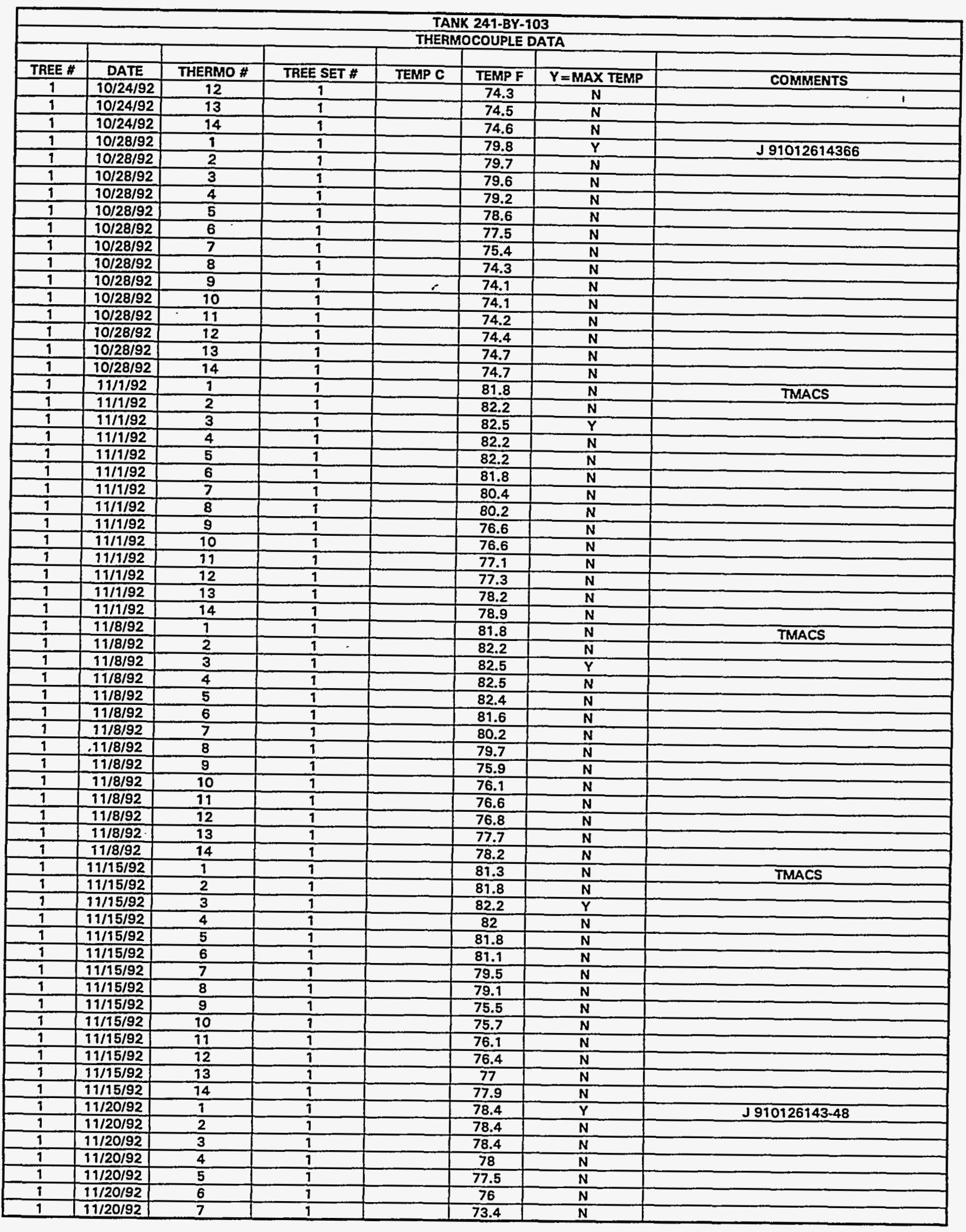

Data obtained from WHC Surveillance Analysis Computer System (SACS), June 2, 1993. 
WHC-SD-WM-ER-312, Rev. 0

\begin{tabular}{|c|c|c|c|c|c|c|c|}
\hline \multicolumn{8}{|c|}{ TANK 241-BY-103 } \\
\hline \multicolumn{8}{|c|}{ THERMOCOUPLE DATA } \\
\hline TREE \# & DATE & THERMO \# & TREE SET \# & TEMP C & TEMPF & $Y=$ MAX TEMP & COMMENTS \\
\hline 1 & $9 / 26 / 92$ & 2 & 1 & & 77.4 & $N$ & \\
\hline 1 & $9 / 26 / 92$ & 3 & 9 & & 77.1 & $\mathbf{N}$ & \\
\hline 1 & $9 / 26 / 92$ & 4 & 9 & & 76.5 & $\ddot{N}$ & \\
\hline 1 & $9 / 26 / 92$ & 5 & 1 & & 75.8 & $\ddot{N}$ & \\
\hline 1 & $9 / 26 / 92$ & 6 & 1 & & 74.9 & N & \\
\hline 1 & $9 / 26 / 92$ & 7 & 1 & & 73.4 & $\ddot{N}$ & \\
\hline 1 & $9 / 26 / 92$ & 8 & 1 & & 72.6 & $N$ & \\
\hline 1 & $9 / 26 / 92$ & 9 & 1 & & 72.3 & $N$ & \\
\hline 1 & $9 / 26 / 92$ & 10 & 1 & & 72.4 & $\mathbf{N}$ & \\
\hline 1 & $9 / 26 / 92$ & 11 & 1 & & 72.5 & $\ddot{N}$ & \\
\hline 1 & $9 / 26 / 92$ & 12 & 1 & & 72.6 & $\mathbf{N}$ & \\
\hline 1 & $9 / 26 / 92$ & 13 & 1 & & 72.8 & $N$ & \\
\hline 1 & $9 / 26 / 92$ & 14 & 1 & & 73 & $\mathbf{N}$ & \\
\hline 1 & $10 / 1 / 92$ & 1 & 1 & & 76.7 & $\mathbf{N}$ & $\mathrm{J} 890423320-42$ \\
\hline 1 & $10 / 1 / 92$ & 2 & 1 & & 76.8 & $Y$ & \\
\hline 1 & $10 / 1 / 92$ & 3 & 1 & & 76.7 & $\mathbf{N}$ & \\
\hline 1 & $10 / 1 / 92$ & 4 & 1 & & 76.5 & $\bar{N}$ & \\
\hline 1 & $10 / 1 / 92$ & 5 & 1 & & 75.9 & $N$ & \\
\hline 1 & $10 / 1 / 92$ & 6 & $\frac{1}{1}$ & & 74.9 & $\mathbf{N}$ & \\
\hline 1 & $10 / 1 / 92$ & 7 & 1 & & 73.4 & $\mathbf{N}$ & \\
\hline 1 & $10 / 1 / 92$ & 8 & 1 & & 72.5 & $\mathbf{N}$ & \\
\hline 1 & $10 / 1 / 92$ & 9 & 1 & & 72.2 & $\ddot{N}$ & \\
\hline 1 & $10 / 1 / 92$ & 10 & 1 & & 72.3 & $\ddot{N}$ & \\
\hline 7 & $10 / 1 / 92$ & 11 & 1 & & 72.3 & $N$ & \\
\hline 1 & $10 / 1 / 92$ & 12 & 1 & & 72.4 & $\mathbf{N}$ & \\
\hline 1 & $10 / 1 / 92$ & 13 & 1 & & 72.6 & $\mathbf{N}$ & \\
\hline 1 & $10 / 1 / 92$ & 14 & 1 & & 72.7 & $N$ & \\
\hline 1 & $10 / 10 / 92$ & 1 & 1 & & 78.6 & $\bar{Y}$ & J $910126143-48$ \\
\hline 1 & $10 / 10 / 92$ & 2 & 1 & & 78.6 & $N$ & \\
\hline 1 & $10 / 10 / 92$ & 3 & 1 & & 78.4 & $N$ & \\
\hline 1 & $10 / 10 / 92$ & 4 & 1 & & 78 & $N$ & \\
\hline 9 & $10 / 10 / 92$ & 5 & 1 & & 77.4 & $\mathbf{N}$ & \\
\hline 1 & $10 / 10 / 92$ & 6 & 1 & & 76.3 & $\mathbf{N}$ & \\
\hline 1 & $10 / 10 / 92$ & 7 & 1 & & 74.7 & $N$ & \\
\hline 1 & $10 / 10 / 92$ & 8 & 1 & & 73.8 & $N$ & \\
\hline 1 & $10 / 10 / 92$ & 9 & 1 & & 73.5 & $\mathbf{N}$ & \\
\hline 1 & $10 / 10 / 92$ & 10 & 1 & & 73.6 & $\bar{N}$ & \\
\hline$i$ & $10 / 10 / 92$ & 11 & 1 & & 73.7 & $\mathbf{N}$ & \\
\hline 1 & $.10 / 10 / 92$ & 12 & 1 & & 73.8 & $N$ & \\
\hline 1 & $10 / 10 / 92$ & 13 & 1 & & 74.1 & $\mathbf{N}$ & \\
\hline 1 & $10 / 10 / 92$ & 14 & 1 & & 74.3 & $N$ & \\
\hline$\frac{1}{1}$ & $10 / 18 / 92$ & 1 & 1 & & 79.1 & $\bar{Y}$ & J 910125143-40 \\
\hline 1 & $10 / 18 / 92$ & 2 & 1 & & 78.9 & $\mathbf{N}$ & \\
\hline 1 & $10 / 18 / 92$ & 3 & 1 & & 78.7 & $\mathbf{N}$ & \\
\hline 1 & $10 / 18 / 92$ & 4 & 1 & & 78.3 & $N$ & \\
\hline 1 & $10 / 18 / 92$ & 5 & 1 & & 77.7 & $\mathbf{N}$ & \\
\hline 1 & $10 / 18 / 92$ & 6 & $\frac{1}{1}$ & & 76.6 & $\mathbf{N}$ & \\
\hline 1 & $10 / 18 / 92$ & 7 & 1 & & 74.8 & $\bar{N}$ & \\
\hline 1 & $10 / 18 / 92$ & 8 & 1 & & 73.8 & $\mathrm{~N}$ & \\
\hline 1 & $10 / 18 / 92$ & 9 & 1 & & 73.6 & $\bar{N}$ & \\
\hline$\frac{1}{1}$ & $10 / 18 / 92$ & 10 & 1 & & 73.6 & $\mathbf{N}$ & \\
\hline 1 & $10 / 18 / 92$ & 11 & 1 & & 73.7 & $N$ & \\
\hline 1 & $10 / 18 / 92$ & 12 & 1 & & 73.9 & $N$ & \\
\hline 1 & $10 / 18 / 92$ & 13 & 1 & & 74.1 & $\mathbf{N}$ & \\
\hline 1 & $10 / 18 / 92$ & 14 & 1 & & 74.3 & $\mathbf{N}$ & \\
\hline 1 & $10 / 24 / 92$ & 1 & 1 & & 79.6 & $Y$ & \\
\hline$\frac{\pi}{1}$ & $10 / 24 / 92$ & 2 & 1 & & 79.5 & $\mathbf{N}$ & \\
\hline 1 & $10 / 24 / 92$ & 3 & 1 & & 79.2 & $\mathbf{N}$ & \\
\hline 1 & $10 / 24 / 92$ & 4 & 1 & & 78.8 & $N$ & \\
\hline 1 & $10 / 24 / 92$ & 5 & 1 & & 78.1 & N & \\
\hline 1 & $10 / 24 / 92$ & 6 & 1 & & 77.1 & $\mathbf{N}$ & \\
\hline 1 & $10 / 24 / 92$ & 7 & 1 & & 75.3 & $\mathbf{N}$ & \\
\hline 1 & $10 / 24 / 92$ & 8 & 1 & & 74.2 & $\mathrm{~N}$ & \\
\hline 1 & $10 / 24 / 92$ & 9 & 1 & & 74.1 & $\mathbf{N}$ & \\
\hline 9 & $10 / 24 / 92$ & 10 & 1 & & 74.1 & $\mathbf{N}$ & \\
\hline 1 & $10 / 24 / 92$ & 11 & 1 & & 74.2 & $\mathbf{N}$ & \\
\hline
\end{tabular}

Data obtained from WHC Surveillance Analysis Computer System (SACS), June 2, 1993. 


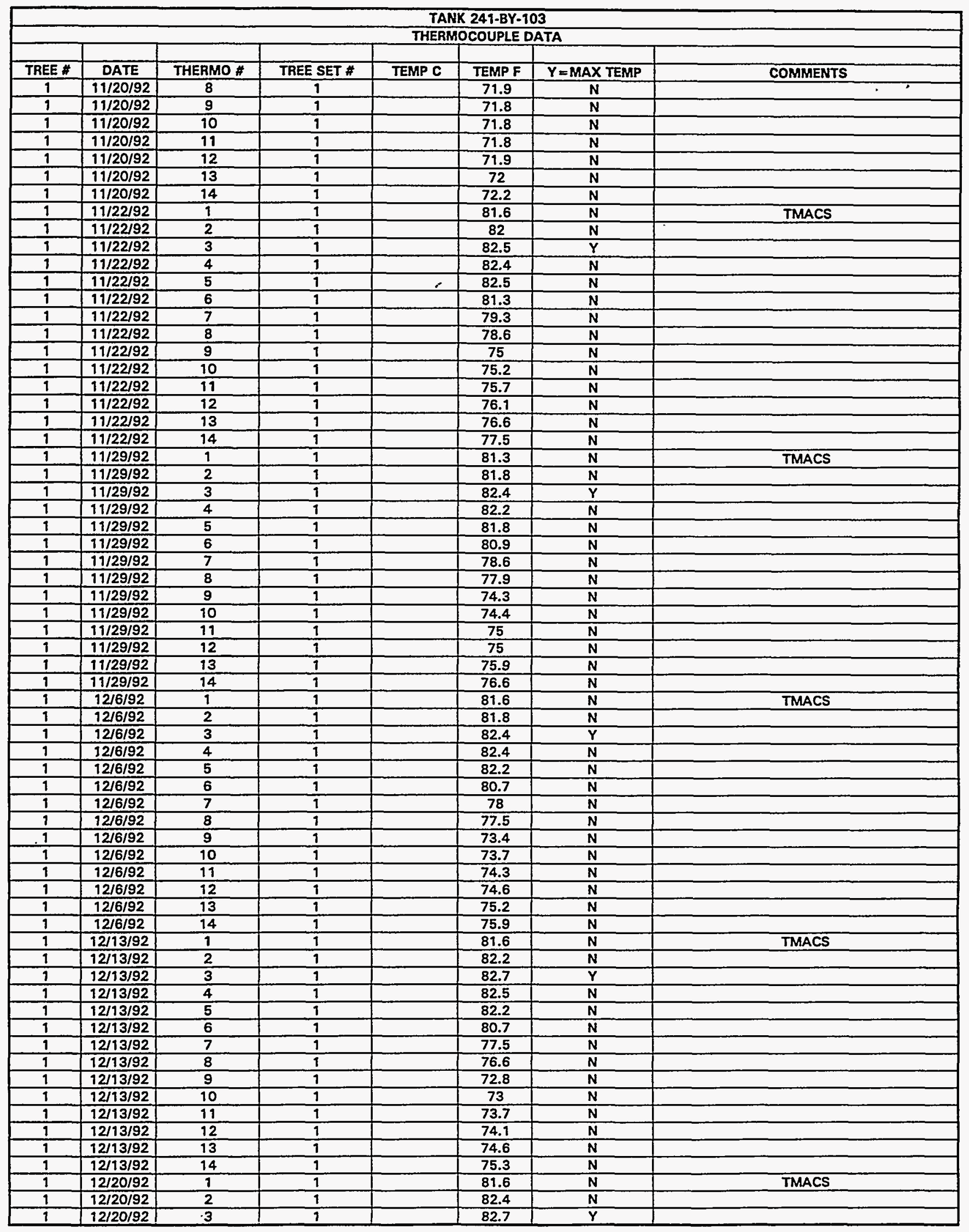

Data obtained from WHC Surveillance Analysis Computer System (SACS), June 2, 1993. 


\begin{tabular}{|c|c|c|c|c|c|c|c|}
\hline \multicolumn{8}{|c|}{ TANK 241-BY-103 } \\
\hline \multicolumn{8}{|c|}{ THERMOCOUPLE DATA } \\
\hline TREE \# & DATE & THERMO \# & TREE SET \# & TEMP C & TEMP F & $Y=M A X$ TEMP & COMMENTS \\
\hline 1 & $12 / 20 / 92$ & 4 & 1 & & 82.7 & $N$ & \\
\hline 1 & $12 / 20 / 92$ & 5 & 1 & & 82.2 & $\bar{N}$ & \\
\hline 1 & $12 / 20 / 92$ & 6 & 1 & & 80.6 & $\mathbf{N}$ & \\
\hline 1 & $12 / 20 / 92$ & 7 & 1 & & 77.1 & $\mathbf{N}$ & \\
\hline 1 & $12 / 20 / 92$ & 8 & 1 & & 76.1 & $\mathbf{N}$ & \\
\hline 1 & $12 / 20 / 92$ & 9 & 1 & & 72.5 & $\bar{N}$ & \\
\hline 1 & $12 / 20 / 92$ & 10 & 1 & & 72.6 & $\mathbf{N}$ & \\
\hline 1 & $12 / 20 / 92$ & 11 & 1 & & 73.2 & $\mathbf{N}$ & \\
\hline 1 & $12 / 20 / 92$ & 12 & 1 & & 73.5 & $\mathbf{N}$ & \\
\hline 1 & $12 / 20 / 92$ & 13 & 1 & & 74.3 & $\mathbf{N}$ & \\
\hline 1 & $12 / 20 / 92$ & 14 & 1 & & 74.6 & $\mathbf{N}$ & \\
\hline 1 & $12 / 27 / 92$ & 1 & 1 & $\bar{r}$ & 82 & $\mathbf{N}$ & TMACS \\
\hline 1 & $12 / 27 / 92$ & 2 & 1 & & 82.5 & $\mathbf{N}$ & \\
\hline 1 & $12 / 27 / 92$ & 3 & 9 & & 83.1 & $\bar{Y}$ & \\
\hline 1 & $12 / 27 / 92$ & 4 & 1 & & 82.7 & $\mathbf{N}$ & \\
\hline 1 & $12 / 27 / 92$ & 5 & 1 & & 82.2 & $\mathbf{N}$ & \\
\hline 1 & $12 / 27 / 92$ & 6 & 1 & & 80.4 & $\mathbf{N}$ & \\
\hline 1 & $12 / 27 / 92$ & 7 & 1 & & 76.8 & $\mathbf{N}$ & \\
\hline 1 & $12 / 27 / 92$ & 8 & 1 & & 75.7 & $\mathbf{N}$ & \\
\hline 1 & $12 / 27 / 92$ & 9 & 1 & & 72.1 & $\mathbf{N}$ & \\
\hline 1 & $12 / 27 / 92$ & 10 & 1 & & 72.1 & $\mathbf{N}$ & \\
\hline 1 & $12 / 27 / 92$ & 11 & 1 & & 72.8 & $\mathbf{N}$ & \\
\hline 1 & $12 / 27 / 92$ & 12 & 1 & & 72.8 & $\mathbf{N}$ & \\
\hline 1 & $12 / 27 / 92$ & 13 & 1 & & 73.5 & $\mathbf{N}$ & \\
\hline 1 & $12 / 27 / 92$ & 14 & 1 & & 74.3 & $\mathbf{N}$ & \\
\hline 1 & $1 / 3 / 93$ & 1 & 1 & & 81.8 & $\mathbf{N}$ & TMACS \\
\hline 1 & $1 / 3 / 93$ & 2 & 1 & & 82.5 & $\mathbf{N}$ & \\
\hline 1 & $1 / 3 / 93$ & 3 & 1 & & 82.9 & $Y$ & \\
\hline 9 & $1 / 3 / 93$ & 4 & 1 & & 82.9 & $\mathbf{N}$ & \\
\hline 1 & $1 / 3 / 93$ & 5 & 1 & & 82 & $\mathbf{N}$ & \\
\hline 1 & $1 / 3 / 93$ & 6 & 1 & & 80.4 & $\mathbf{N}$ & \\
\hline 9 & $1 / 3 / 93$ & 7 & 1 & & 76.8 & $\mathbf{N}$ & \\
\hline 1 & $1 / 3 / 93$ & 8 & 1 & & 75.3 & $\mathbf{N}$ & \\
\hline 1 & $1 / 3 / 93$ & 9 & 1 & & 71.4 & $\mathbf{N}$ & \\
\hline 9 & $1 / 3 / 93$ & 10 & 1 & & 71.4 & $\mathbf{N}$ & \\
\hline 1 & $1 / 3 / 93$ & 11 & 1 & & 72.3 & $\mathbf{N}$ & \\
\hline 1 & $1 / 3 / 93$ & 12 & 1 & & 72.5 & $\mathbf{N}$ & \\
\hline 9 & $1 / 3 / 93$ & 13 & 1 & & 73.2 & $\mathbf{N}$ & \\
\hline 1 & $.1 / 3 / 93$ & 14 & 1 & & 73.9 & $\mathbf{N}$ & \\
\hline 1 & $1 / 10 / 93$ & 1 & 1 & & 81.8 & $\mathbf{N}$ & TMACS \\
\hline$\frac{1}{1}$ & $1 / 10 / 93$ & 2 & 1 & & 82.4 & $\mathbf{N}$ & \\
\hline 1 & $1 / 10 / 93$ & 3 & 1 & & 82.5 & $\bar{Y}$ & \\
\hline 1 & $1 / 10 / 93$ & 4 & 1 & & 82.2 & $\mathbf{N}$ & \\
\hline$\frac{1}{1}$ & $1 / 10 / 93$ & 5 & 1 & & 81.6 & $\mathbf{N}$ & \\
\hline 9 & $1 / 10 / 93$ & 6 & 1 & & 79.7 & $\mathbf{N}$ & \\
\hline 1 & $1 / 10 / 93$ & 7 & 1 & & 75.5 & $\mathbf{N}$ & \\
\hline 1 & $1 / 10 / 93$ & 8 & 1 & & 74.3 & $\mathbf{N}$ & \\
\hline 1 & $1 / 10 / 93$ & 9 & 1 & & 70.3 & $\mathbf{N}$ & \\
\hline 1 & $1 / 10 / 93$ & 10 & 1 & & 70.5 & $\mathbf{N}$ & \\
\hline 7 & $9 / 10 / 93$ & 11 & 1 & & 79 & $\bar{N}$ & \\
\hline 1 & $1 / 10 / 93$ & 12 & 1 & & 71.6 & $\mathbf{N}$ & \\
\hline$\frac{1}{1}$ & $1 / 10 / 93$ & 13 & 1 & & 71.9 & $\mathbf{N}$ & \\
\hline 1 & $1 / 10 / 93$ & 14 & 1 & & 72.8 & $\mathbf{N}$ & \\
\hline 1 & $1 / 17 / 93$ & 1 & 1 & & 82 & $\mathbf{N}$ & TMACS \\
\hline$\frac{1}{1}$ & $1 / 17 / 93$ & 2 & 1 & & 82.4 & $\bar{N}$ & \\
\hline 1 & $1 / 17 / 93$ & 3 & 1 & & 82.5 & $\bar{Y}$ & \\
\hline 1 & $1 / 17 / 93$ & 4 & 1 & & 82.4 & $\mathbf{N}$ & \\
\hline 1 & $1 / 17 / 93$ & 5 & 9 & & 81.5 & $\bar{N}$ & \\
\hline 1 & $1 / 17 / 93$ & 6 & 1 & & 79.5 & $N$ & \\
\hline 1 & $1 / 17 / 93$ & 7 & 1 & & 75.2 & $\mathrm{~N}$ & \\
\hline 1 & $1 / 17 / 93$ & 8 & 1 & & 73.5 & $\mathbf{N}$ & \\
\hline 1 & $1 / 17 / 93$ & 9 & 1 & & 69.8 & $\mathbf{N}$ & \\
\hline 1 & $1 / 17 / 93$ & 10 & 1 & & 69.9 & $\mathbf{N}$ & \\
\hline 1 & $1 / 17 / 93$ & 11 & 1 & & 70.5 & $\mathbf{N}$ & \\
\hline 1 & $1 / 17 / 93$ & 12 & 1 & & 70.8 & $\bar{N}$ & \\
\hline 1 & $1 / 17 / 93$ & 13 & 1 & & 71.6 & $\mathbf{N}$ & \\
\hline
\end{tabular}

Data obtained from WHC Surveiliance Analysis Computer System (SACS), June 2, 1993. 


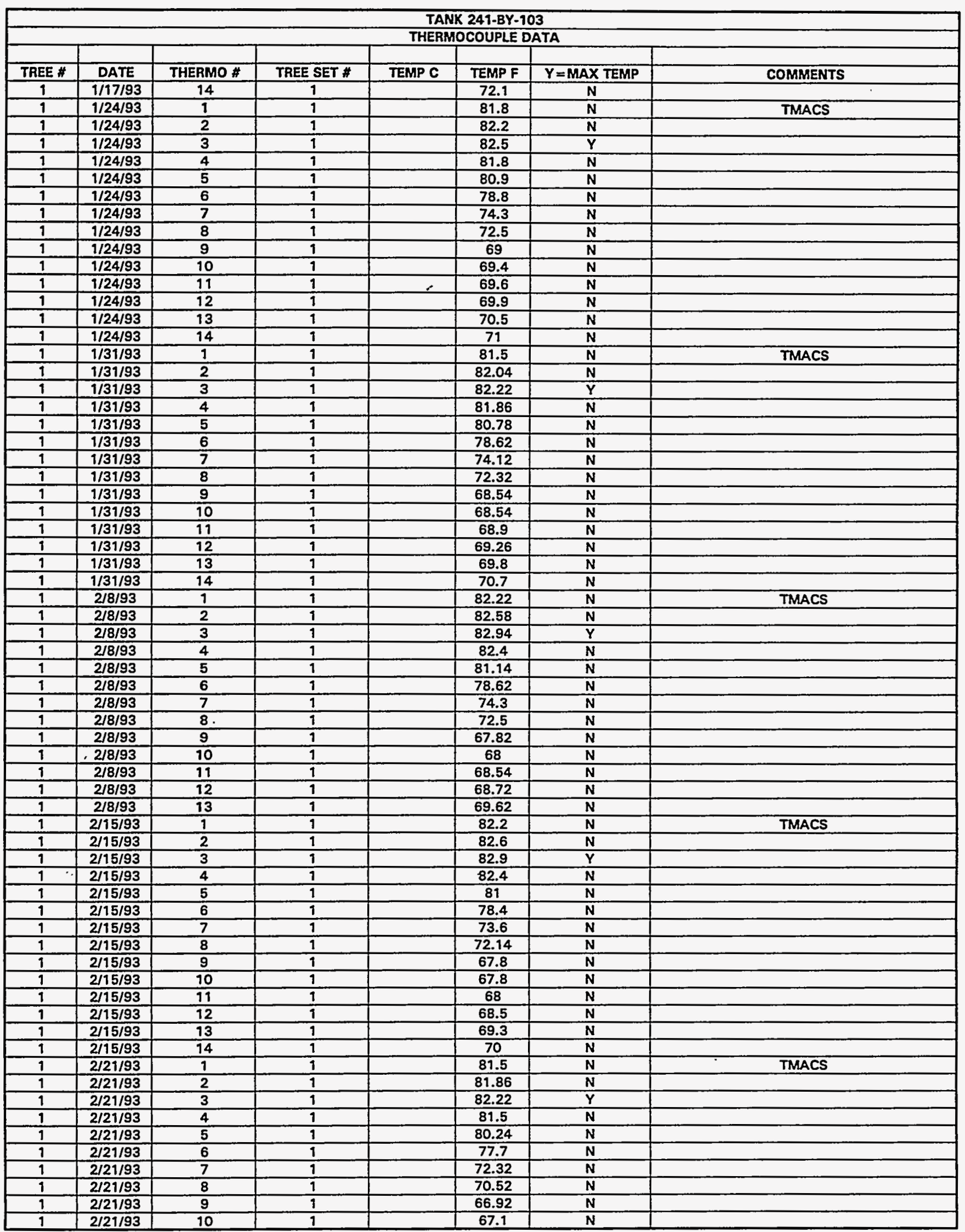

Data obtained from WHC Surveillance Analysis Computer System (SACS), June 2, 1993. 
WHC-SD-WM-ER-312, Rev. 0

\begin{tabular}{|c|c|c|c|c|c|c|c|}
\hline \multicolumn{8}{|c|}{ TANK 241-BY-103 } \\
\hline \multicolumn{8}{|c|}{ THERMOCOUPLE DATA } \\
\hline TREE \# & DATE & THERMO \# & TREE SET \# & TEMP C & TEMP F & $Y=$ MAX TEMP & COMMENTS \\
\hline 1 & $2 / 21 / 93$ & 11 & 1 & & 67.82 & $N$ & \\
\hline 1 & $2 / 21 / 93$ & 12 & 1 & & 68 & $\mathbf{N}$ & \\
\hline 1 & $2 / 21 / 93$ & 13 & 1 & & 68.72 & $\mathbf{N}$ & \\
\hline 1 & $2 / 21 / 93$ & 14 & 1 & & 69.26 & $\mathbf{N}$ & \\
\hline 1 & $3 / 1 / 93$ & 1 & 1 & & 81.86 & $N$ & TMACS \\
\hline 1 & $3 / 1 / 93$ & 2 & 1 & & 82.22 & $\bar{Y}$ & \\
\hline 1 & $3 / 1 / 93$ & 3 & 1 & & 82.22 & $\mathbf{N}$ & \\
\hline$\frac{1}{1}$ & $3 / 1 / 93$ & 4 & 1 & & 81.68 & $\mathbf{N}$ & \\
\hline 1 & $3 / 1 / 93$ & 5 & 1 & & 80.24 & $\mathbf{N}$ & \\
\hline 1 & $3 / 1 / 93$ & 6 & 1 & & 77.54 & $\mathrm{~N}$ & \\
\hline 9 & $3 / 9 / 93$ & 7 & 1 & & 72.86 & $N$ & \\
\hline 1 & $3 / 1 / 93$ & 8 & 1 &. & 70.52 & $\mathbf{N}$ & \\
\hline 1 & $3 / 1 / 93$ & 9 & 1 & & 66.74 & $\mathbf{N}$ & \\
\hline 1 & $3 / 1 / 93$ & 10 & 1 & & 66.74 & $\mathbf{N}$ & \\
\hline 1 & $3 / 1 / 93$ & 11 & 1 & & 67.46 & $\bar{N}$ & \\
\hline 1 & $3 / 1 / 93$ & 12 & 1 & & 67.82 & $\mathbf{N}$ & \\
\hline 1 & $3 / 1 / 93$ & 13 & 1 & & 68.36 & $\mathbf{N}$ & \\
\hline 1 & $3 / 9 / 93$ & 14 & 1 & & 69.08 & $\mathbf{N}$ & \\
\hline 1 & $3 / 8 / 93$ & 1 & 1 & & 81.86 & $\mathbf{N}$ & TMACS \\
\hline 1 & $3 / 8 / 93$ & 2 & 1 & & 82.22 & $\bar{Y}$ & \\
\hline 1 & $3 / 8 / 93$ & 3 & 1 & & 82.04 & $\mathrm{~N}$ & \\
\hline 1 & $3 / 8 / 93$ & 4 & 1 & & 81.14 & $\mathbf{N}$ & \\
\hline 1 & $3 / 8 / 93$ & 5 & 1 & & 80.06 & $\mathbf{N}$ & \\
\hline 1 & $3 / 8 / 93$ & 6 & 1 & & 77.18 & $\mathbf{N}$ & \\
\hline 1 & $3 / 8 / 93$ & 7 & $\frac{1}{1}$ & & 72.14 & $N$ & \\
\hline 1 & $3 / 8 / 93$ & 8 & 1 & & 70.16 & $\mathbf{N}$ & \\
\hline 1 & $3 / 8 / 93$ & 9 & 1 & & 66.38 & $\mathbf{N}$ & \\
\hline 1 & $3 / 8 / 93$ & 10 & 1 & & 66.56 & $\bar{N}$ & \\
\hline 1 & $3 / 8 / 93$ & 11 & 1 & & 67.1 & $\mathbf{N}$ & \\
\hline 1 & $3 / 8 / 93$ & 12 & 1 & & 67.46 & $\mathbf{N}$ & \\
\hline 1 & $3 / 8 / 93$ & 13 & 1 & & 68 & $\bar{N}$ & \\
\hline 9 & $3 / 8 / 93$ & 14 & 1 & & 68.72 & $\mathbf{N}$ & \\
\hline 1 & $3 / 15 / 93$ & 1 & 1 & & 81.68 & $\mathbf{N}$ & TMACS \\
\hline 1 & $3 / 15 / 93$ & 2 & 1 & & 81.86 & $Y$ & \\
\hline 1 & $3 / 15 / 93$ & 3 & 1 & & 81.86 & $\mathbf{N}$ & \\
\hline 1 & $3 / 15 / 93$ & 4 & 1 & & 81.14 & $\mathbf{N}$ & \\
\hline 1 & $3 / 15 / 93$ & 5 & 1 & & 79.7 & $\mathrm{~N}$ & \\
\hline 1 & $3 / 15 / 93$ & 6 & 1 & & 77 & $\mathbf{N}$ & \\
\hline 1 & $.3 / 15 / 93$ & 7 & 1 & & 72.14 & $\mathbf{N}$ & \\
\hline 1 & $3 / 15 / 93$ & 8 & 1 & & 69.98 & $\mathbf{N}$ & \\
\hline 1 & $3 / 15 / 93$ & 9 & 1 & & 66.2 & $\mathbf{N}$ & \\
\hline 1 & $3 / 15 / 93$ & 10 & 1 & & 66.2 & $\mathbf{N}$ & \\
\hline 1 & $3 / 15 / 93$ & 11 & 1 & & 66.56 & $\mathbf{N}$ & \\
\hline 1 & $3 / 15 / 93$ & 32 & 9 & & 66.56 & $\mathbf{N}$ & \\
\hline 1 & $3 / 15 / 93$ & 13 & 1 & & 67.64 & $\mathbf{N}$ & \\
\hline 1 & $3 / 15 / 93$ & 14 & 1 & & 68 & $\mathbf{N}$ & \\
\hline 1 & $3 / 22 / 93$ & 1 & 1 & & 81.86 & $\mathbf{N}$ & TMACS \\
\hline$\frac{1}{1}$ & $3 / 22 / 93$ & 2 & 1 & & 82.04 & $\mathbf{N}$ & \\
\hline 1 & $3 / 22 / 93$ & 3 & $\frac{1}{1}$ & & 82.22 & $Y$ & \\
\hline 1 & $3 / 22 / 93$ & 4 & 1 & & 81.5 & $\mathbf{N}$ & \\
\hline 1 & $3 / 22 / 93$ & 5 & 1 & & 79.7 & $\bar{N}$ & \\
\hline 1 & $3 / 22 / 93$ & 6 & 1 & & 77.18 & $\mathbf{N}$ & \\
\hline 9 & $3 / 22 / 93$ & 7 & 1 & & 71.96 & $\mathbf{N}$ & \\
\hline 1 & $3 / 22 / 93$ & 8 & $\frac{1}{1}$ & & 70.52 & $\mathbf{N}$ & \\
\hline 1 & $3 / 22 / 93$ & 9 & 1 & & 66.2 & $N$ & \\
\hline 9 & $3 / 22 / 93$ & 10 & 1 & & 66.38 & $\mathbf{N}$ & \\
\hline 1 & $3 / 22 / 93$ & 11 & 1 & & 66.92 & $\mathbf{N}$ & \\
\hline 1 & $3 / 22 / 93$ & 12 & 1 & & 67.28 & 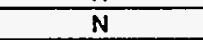 & \\
\hline 1 & $3 / 22 / 93$ & 13 & 1 & & 67.82 & $\mathrm{~N}$ & \\
\hline 1 & $3 / 22 / 93$ & 14 & 1 & & 68.72 & $\bar{N}$ & \\
\hline 1 & $3 / 29 / 93$ & 1 & 1 & & 81.68 & $N$ & TMACS \\
\hline 1 & $3 / 29 / 93$ & 2 & 1 & & 81.68 & $\mathbf{N}$ & \\
\hline 1 & $3 / 29 / 93$ & 3 & 1 & & 81.86 & $Y$ & \\
\hline 9 & $3 / 29 / 93$ & 4 & 1 & & 80.78 & $\mathrm{~N}$ & \\
\hline 1 & $3 / 29 / 93$ & 5 & 1 & & 79.34 & $\mathbf{N}$ & \\
\hline 1 & $3 / 29 / 93$ & 6 & 1 & & 76.46 & $\mathrm{~N}$ & \\
\hline
\end{tabular}

Data obtained from WHC Surveillance Analysis Computer System (SACS), June 2, 1993. 
WHC-SD-WM-ER-312, Rev. 0

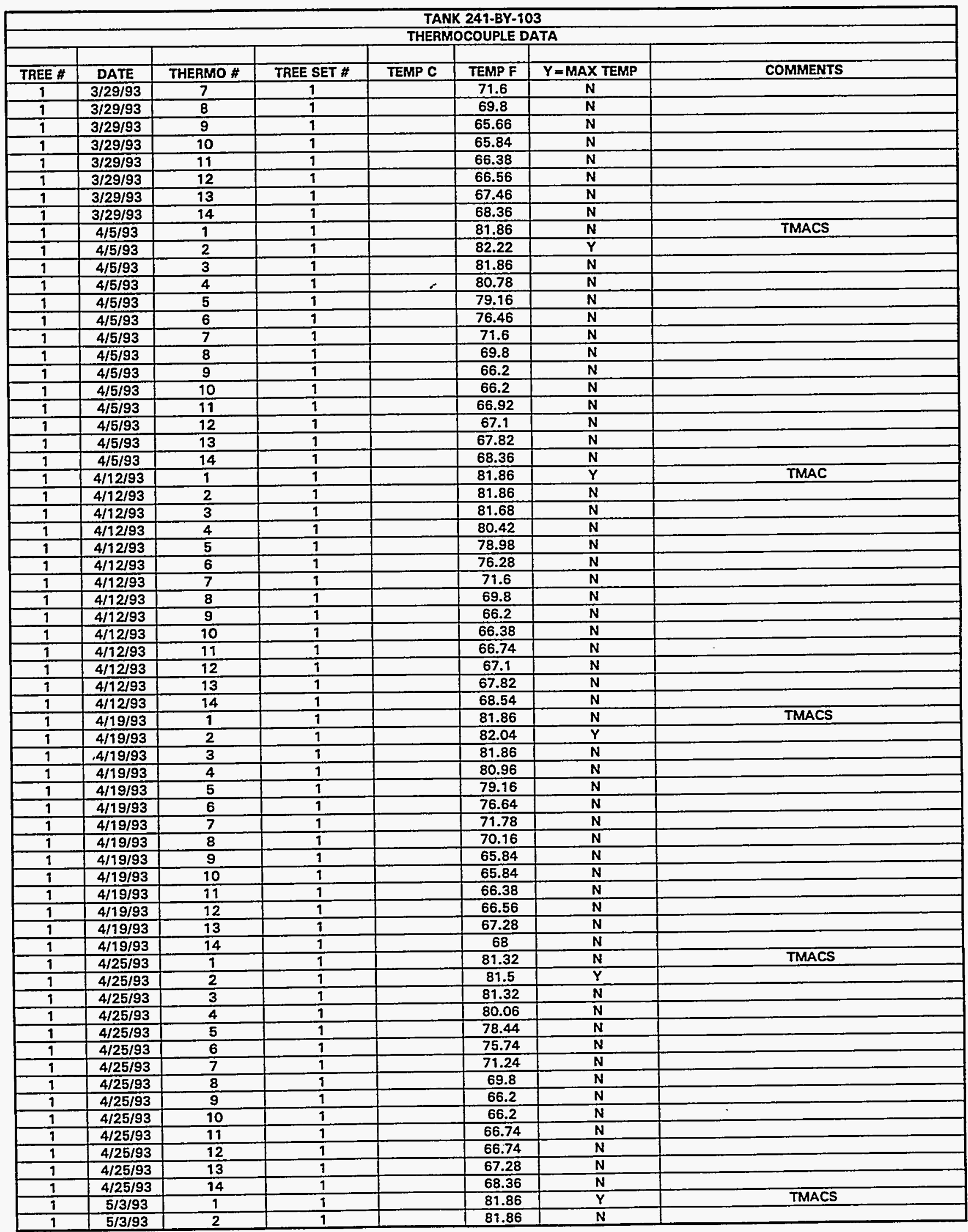

Data abtained from WHC Surveillance Analysis Computer System (SACS), June 2, 1993. 
WHC-SD-WM-ER-312, Rev. 0

\begin{tabular}{|c|c|c|c|c|c|c|c|}
\hline \multicolumn{8}{|c|}{ TANK 241-BY-103 } \\
\hline \multicolumn{8}{|c|}{ THERMOCOUPLE DATA } \\
\hline TREE \# & DATE & THERMO \# & TREE SET $\#$ & TEMP C & TEMP F & $Y=$ MAX TEMP & COMMENTS \\
\hline 1 & $5 / 3 / 93$ & 3 & 1 & & 81.68 & $N$ & \\
\hline 1 & $5 / 3 / 93$ & 4 & 1 & & 80.24 & $\mathbf{N}$ & \\
\hline 1 & $5 / 3 / 93$ & 5 & 1 & & 78.8 & $\mathrm{~N}$ & \\
\hline 1 & $5 / 3 / 93$ & 6 & 1 & & 76.1 & $\mathbf{N}$ & \\
\hline 9 & $5 / 3 / 93$ & 7 & 1 & & 71.96 & $\mathbf{N}$ & \\
\hline 1 & $5 / 3 / 93$ & 8 & 1 & & 70.7 & $\mathbf{N}$ & \\
\hline 1 & $5 / 3 / 93$ & 9 & 1 & & 66.2 & $\mathbf{N}$ & \\
\hline 1 & $5 / 3 / 93$ & 10 & 1 & & 66.2 & $\bar{N}$ & \\
\hline 1 & $5 / 3 / 93$ & 11 & 1 & & 66.56 & $\mathbf{N}$ & \\
\hline 1 & $5 / 3 / 93$ & 12 & 9 & & 66.92 & $\mathrm{~N}$ & \\
\hline 1 & $5 / 3 / 93$ & 13 & 1 & & 67.82 & $\mathbf{N}$ & \\
\hline 1 & $5 / 3 / 93$ & 14 & 1 & $r$ & 68.36 & $\mathbf{N}$ & \\
\hline 1 & $5 / 10 / 93$ & 1 & 1 & & 81.5 & $\bar{Y}$ & TMACS \\
\hline 1 & $5 / 10 / 93$ & 2 & 1 & & 81.5 & $\mathbf{N}$ & \\
\hline 1 & $5 / 10 / 93$ & 3 & 1 & & 81.14 & $\bar{N}$ & \\
\hline 1 & $5 / 10 / 93$ & 4 & 1 & & 80.06 & $\mathbf{N}$ & \\
\hline 1 & $5 / 10 / 93$ & 5 & 1 & & 78.62 & $\mathbf{N}$ & \\
\hline 1 & $5 / 10 / 93$ & 6 & 1 & & 75.92 & $\bar{N}$ & \\
\hline 1 & $5 / 10 / 93$ & 7 & 1 & & 71.96 & $\mathbf{N}$ & \\
\hline 1 & $5 / 10 / 93$ & 8 & 1 & & 70.34 & $\mathbf{N}$ & \\
\hline 1 & $5 / 10 / 93$ & 9 & 1 & & 66.38 & $\mathbf{N}$ & \\
\hline 1 & $5 / 10 / 93$ & 10 & 1 & & 66.38 & $N$ & \\
\hline 1 & $5 / 10 / 93$ & 11 & 1 & & 66.92 & $\mathrm{~N}$ & \\
\hline 1 & $5 / 10 / 93$ & 12 & 1 & & 67.1 & $\mathbf{N}$ & \\
\hline 1 & $5 / 10 / 93$ & 13 & 1 & & 67.82 & $\bar{N}$ & \\
\hline 1 & $5 / 10 / 93$ & 14 & 1 & & 68.36 & $\mathbf{N}$ & \\
\hline 1 & $5 / 17 / 93$ & 1 & 1 & & 81.5 & $\mathbf{Y}$ & TMACS \\
\hline 1 & $5 / 17 / 93$ & 2 & 1 & & 81.32 & $\mathbf{N}$ & \\
\hline 1 & $5 / 17 / 93$ & 3 & 1 & & 81.14 & $\mathbf{N}$ & \\
\hline 1 & $5 / 17 / 93$ & 4 & 1 & & 80.06 & $\mathbf{N}$ & \\
\hline 1 & $5 / 17 / 93$ & 5 & 1 & & 78.26 & $\bar{N}$ & \\
\hline$\therefore 1$ & $5 / 17 / 93$ & 6 & 1 & & 75.92 & $\mathbf{N}$ & \\
\hline 1 & $5 / 17 / 93$ & 7 & 1 & & 72.14 & $N$ & \\
\hline 1 & $5 / 17 / 93$ & 8 & 1 & & 70.88 & $\bar{N}$ & \\
\hline 1 & $5 / 17 / 93$ & 9 & 1 & & 66.38 & $\mathbf{N}$ & \\
\hline 1 & $5 / 17 / 93$ & 10 & 1 & & 66.38 & $\mathbf{N}$ & \\
\hline 1 & $5 / 17 / 93$ & 11 & 1 & & 66.92 & $\mathbf{N}$ & \\
\hline 1 & $5 / 17 / 93$ & 12 & 1 & & 67.1 & $\mathbf{N}$ & \\
\hline 9 & $.5 / 17 / 93$ & 13 & 1 & & 67.82 & $\mathbf{N}$ & \\
\hline 1 & $5 / 17 / 93$ & 14 & 1 & & 68.72 & $\mathbf{N}$ & \\
\hline 1 & $5 / 24 / 93$ & 1 & 1 & & 81.14 & $\mathbf{N}$ & TMACS \\
\hline 1 & $5 / 24 / 93$ & 2 & 1 & & 81.32 & $\bar{Y}$ & \\
\hline 1 & $5 / 24 / 93$ & 3 & 1 & & 81.14 & $\mathbf{N}$ & \\
\hline 1 & $5 / 24 / 93$ & 4 & 1 & & 79.88 & $\mathbf{N}$ & \\
\hline 9 & $5 / 24 / 93$ & 5 & 9 & & 78.08 & $\mathbf{N}$ & \\
\hline 1 & $5 / 24 / 93$ & 6 & 1 & & 75.74 & $\mathrm{~N}$ & \\
\hline 1 & $5 / 24 / 93$ & 7 & 1 & & 72.14 & $\mathbf{N}$ & \\
\hline 1 & $5 / 24 / 93$ & 8 & 1 & & 71.24 & $\mathrm{~N}$ & \\
\hline 1 & $5 / 24 / 93$ & 9 & 1 & & 67.46 & $N$ & \\
\hline 1 & $5 / 24 / 93$ & 10 & 1 & & 67.28 & $\mathbf{N}$ & \\
\hline 1 & $5 / 24 / 93$ & 11 & 1 & & 67.82 & $\mathbf{N}$ & \\
\hline 1 & $5 / 24 / 93$ & 12 & 1 & & 68 & $\mathbf{N}$ & \\
\hline 1 & $5 / 24 / 93$ & 13 & 1 & & 68.72 & $\mathrm{~N}$ & \\
\hline 1 & $5 / 24 / 93$ & 94 & 1 & & 69.62 & $\mathrm{~N}$ & \\
\hline 1 & $5 / 31 / 93$ & 1 & 1 & & 81.14 & $Y$ & TMACS \\
\hline 1 & $5 / 31 / 93$ & 2 & 1 & & 81.14 & $\mathrm{~N}$ & \\
\hline 1 & $5 / 31 / 93$ & 3 & 1 & & 80.96 & $\mathrm{~N}$ & \\
\hline 1 & $5 / 31 / 93$ & 4 & 1 & & 79.7 & $N$ & \\
\hline 1 & $5 / 31 / 93$ & 5 & 1 & & 78.08 & $\mathbf{N}$ & \\
\hline 1 & $5 / 31 / 93$ & 6 & 1 & & 75.74 & $\mathrm{~N}$ & \\
\hline 1 & $5 / 31 / 93$ & 7 & 1 & & 72.68 & $\mathbf{N}$ & \\
\hline 1 & $5 / 31 / 93$ & 8 & 1 & & 71.6 & $\mathbf{N}$ & \\
\hline 1 & $5 / 31 / 93$ & 9 & 1 & & 67.82 & $N$ & \\
\hline 1 & $5 / 31 / 93$ & 90 & 9 & & 67.82 & $\mathbf{N}$ & \\
\hline 1 & $5 / 31 / 93$ & 91 & 1 & & 68 & $\mathrm{~N}$ & \\
\hline 1 & $5 / 31 / 93$ & 12 & 1 & & 68.54 & $\mathbf{N}$ & \\
\hline
\end{tabular}

Data obtained from WHC Surveillance Analysis Computer System (SACS), June 2, 1993. 


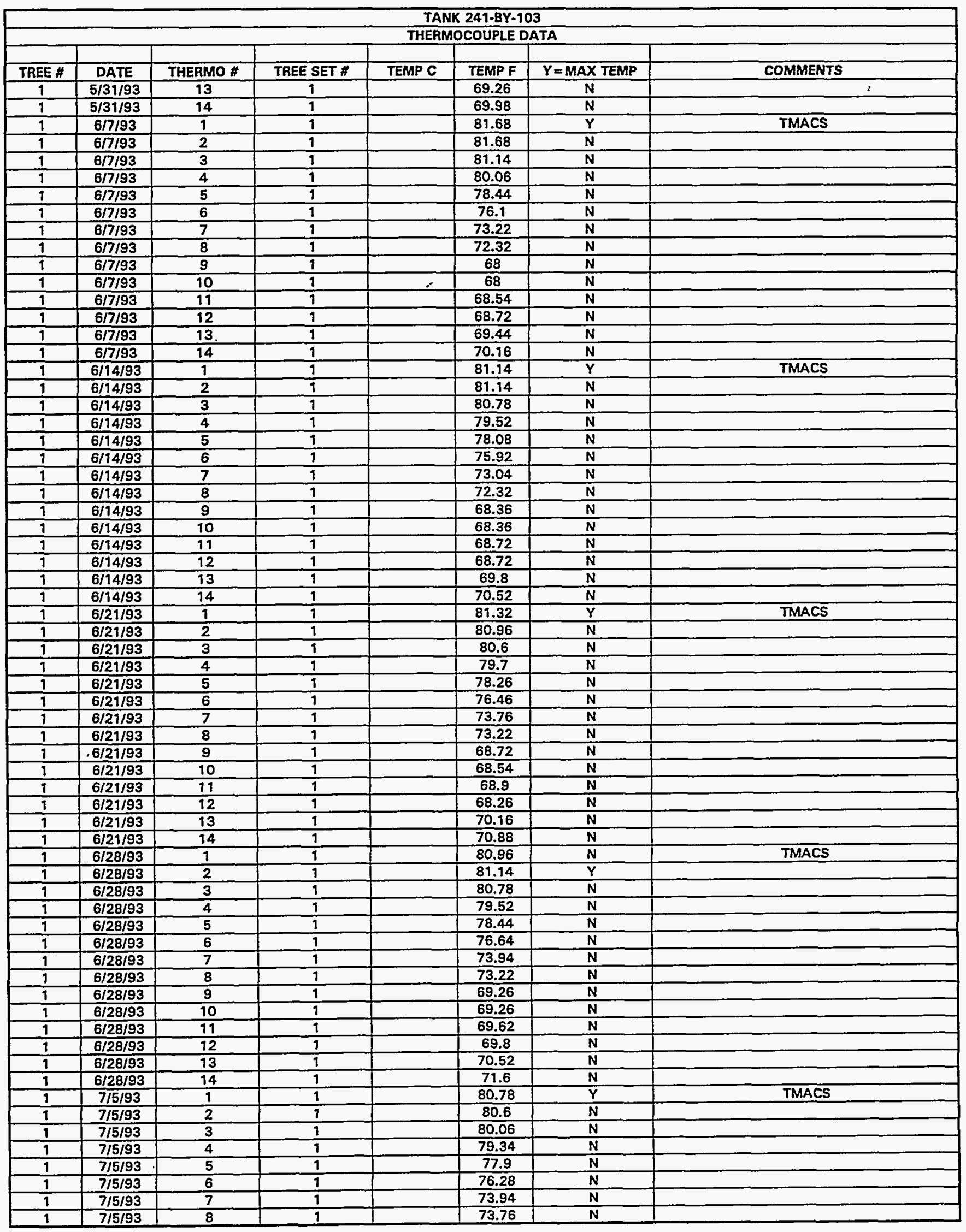

Data obtained from WHC Surveillance Analysis Computer System (SACS), June 2, 1993. 
WHC-SD-WM-ER-312, Rev. 0

\begin{tabular}{|c|c|c|c|c|c|c|c|}
\hline \multicolumn{8}{|c|}{ TANK 241-BY-103 } \\
\hline \multicolumn{8}{|c|}{ THERMOCOUPLE DATA } \\
\hline TREE \# & DATE & THERMO \# & TREE SET \# & TEMP C & TEMP F & $Y=M A X$ TEMP & COMMENTS \\
\hline 1 & $7 / 5 / 93$ & 9 & 1 & & 69.98 & $\bar{N}$ & \\
\hline 1 & $7 / 5 / 93$ & 10 & 1 & & 69.98 & $\mathbf{N}$ & \\
\hline 1 & $7 / 5 / 93$ & 11 & 1 & & 70.52 & $\mathrm{~N}$ & \\
\hline$i$ & $7 / 5 / 93$ & 12 & 1 & & 70.52 & $\bar{N}$ & \\
\hline 1 & $7 / 5 / 93$ & 13 & 1 & & 71.42 & $\mathrm{~N}$ & \\
\hline 1 & $7 / 5 / 93$ & 14 & 1 & & 72.32 & $\mathbf{N}$ & \\
\hline 1 & $7 / 12 / 93$ & 1 & 1 & & 80.78 & $\mathbf{Y}$ & TMACS \\
\hline 1 & $7 / 12 / 93$ & 2 & 1 & & 80.6 & $N$ & \\
\hline 1 & $7 / 12 / 93$ & 3 & 1 & & 80.6 & $\mathbf{N}$ & \\
\hline 1 & $7 / 12 / 93$ & 4 & 1 & & 79.52 & $\ddot{N}$ & \\
\hline 1 & $7 / 12 / 93$ & 5 & 9 & & 78.26 & $\mathbf{N}$ & \\
\hline 1 & $7 / 12 / 93$ & 6 & 1 & $\therefore$ & 76.82 & $\mathrm{~N}$ & \\
\hline 1 & $7 / 12 / 93$ & 7 & 9 & & 74.66 & $\bar{N}$ & \\
\hline 1 & $7 / 12 / 93$ & 8 & 1 & & 74.3 & $\mathbf{N}$ & \\
\hline 1 & $7 / 12 / 93$ & 9 & 1 & & 70.7 & $\mathrm{~N}$ & \\
\hline 1 & $7 / 12 / 93$ & 10 & 1 & & 70.52 & $\ddot{N}$ & \\
\hline 1 & $7 / 12 / 93$ & 91 & 9 & & 71.24 & $\bar{N}$ & \\
\hline 1 & $7 / 12 / 93$ & 12 & 1 & & 71.24 & $\mathbf{N}$ & \\
\hline 1 & $7 / 12 / 93$ & 13 & 1 & & 72.32 & $\bar{N}$ & \\
\hline 1 & $7 / 12 / 93$ & 14 & 1 & & 72.86 & $\ddot{N}$ & \\
\hline 1 & $7 / 18 / 93$ & 1 & 1 & & 80.96 & $\mathrm{Y}$ & TMACS \\
\hline 1 & $7 / 18 / 93$ & 2 & 1 & & 80.96 & $\dot{N}$ & \\
\hline 1 & $7 / 18 / 93$ & 3 & 1 & & 80.24 & $\bar{N}$ & \\
\hline 1 & $7 / 18 / 93$ & 4 & 1 & & 79.34 & $\mathrm{~N}$ & \\
\hline 1 & $7 / 18 / 93$ & 5 & 1 & & 78.44 & $\mathbf{N}$ & \\
\hline 1 & $7 / 18 / 93$ & 6 & 1 & & 76.1 & $\mathrm{~N}$ & \\
\hline 1 & $7 / 18 / 93$ & 7 & 1 & & 75.02 & $\mathrm{~N}$ & \\
\hline 1 & $7 / 18 / 93$ & 8 & 1 & & 75.02 & $\overline{\mathbf{N}}$ & \\
\hline 1 & $7 / 18 / 93$ & 9 & 1 & & 70.88 & $\mathrm{~N}$ & \\
\hline$\frac{1}{1}$ & $7 / 18 / 93$ & 10 & 1 & & 70.7 & $\mathbf{N}$ & \\
\hline 1 & $7 / 18 / 93$ & 11 & 1 & & 71.24 & $\bar{N}$ & \\
\hline 1 & $7 / 18 / 93$ & 12 & 1 & & 71.6 & $\mathrm{~N}$ & \\
\hline 1 & $7 / 18 / 93$ & 13 & 1 & & 72.32 & $\mathrm{~N}$ & \\
\hline 1 & $7 / 18 / 93$ & 14 & 1 & & 73.04 & $\mathrm{~N}$ & \\
\hline 1 & $7 / 26 / 93$ & 1 & 1 & & 80.96 & $\bar{Y}$ & TMACS \\
\hline 1 & $7 / 26 / 93$ & 2 & 1 & & 80.6 & $\mathrm{~N}$ & \\
\hline 1 & $7 / 26 / 93$ & 3 & 1 & & 80.24 & $\mathrm{~N}$ & \\
\hline 9 & $7 / 26 / 93$ & 4 & 1 & & 79.34 & $\mathbf{N}$ & \\
\hline 1 & $7 / 26 / 93$ & 5 & 1 & & 78.26 & $\mathbf{N}$ & \\
\hline 1 & $7 / 26 / 93$ & 6 & 1 & & 76.82 & $\mathbf{N}$ & \\
\hline 1 & $7 / 26 / 93$ & 7 & 1 & & 75.02 & $\mathbf{N}$ & \\
\hline 1 & $7 / 26 / 93$ & 8 & 1 & & 74.84 & $\mathbf{N}$ & \\
\hline 1 & $7 / 26 / 93$ & 9 & 1 & & 71.42 & $\mathbf{N}$ & \\
\hline 9 & $7 / 26 / 93$ & 10 & 1 & & 71.24 & $\mathbf{N}$ & \\
\hline 1 & $7 / 26 / 93$ & 11 & 1 & & 71.78 & $\mathbf{N}$ & \\
\hline 1 & $7 / 26 / 93$ & 12 & 1 & & 71.96 & $\bar{N}$ & \\
\hline 1 & $7 / 26 / 93$ & 13 & 1 & & 72.86 & $\mathbf{N}$ & \\
\hline 1 & $7 / 26 / 93$ & 14 & 1 & & 73.58 & $\mathbf{N}$ & \\
\hline 1 & $8 / 1 / 93$ & 9 & 1 & & 80.6 & $\bar{Y}$ & TAMCS \\
\hline 1 & $8 / 1 / 93$ & 2 & 1 & & 80.6 & $N$ & \\
\hline 1 & $8 / 1 / 93$ & 3 & 1 & & 80.24 & $\mathbf{N}$ & \\
\hline 1 & $8 / 7 / 93$ & 4 & 9 & & 79.34 & $\mathbf{N}$ & \\
\hline 1 & $8 / 1 / 93$ & 5 & 1 & & 78.26 & $\mathbf{N}$ & \\
\hline 1 & $8 / 1 / 93$ & 6 & 1 & & 76.82 & $\mathbf{N}$ & \\
\hline 1 & $8 / 1 / 93$ & 7 & 1 & & 75.56 & $\bar{N}$ & \\
\hline 9 & $8 / 1 / 93$ & 8 & 1 & & 75.38 & $\mathbf{N}$ & \\
\hline 1 & $8 / 1 / 93$ & 9 & 1 & & 71.24 & $\mathbf{N}$ & \\
\hline 1 & $8 / 1 / 93$ & 10 & 1 & & 71.24 & $\mathbf{N}$ & \\
\hline 1 & $8 / 1 / 93$ & 11 & 1 & & 71.6 & $\mathbf{N}$ & \\
\hline 9 & $8 / 1 / 93$ & 12 & 9 & & 71.96 & $\bar{N}$ & \\
\hline 1 & $8 / 1 / 93$ & 13 & 9 & & 72.68 & $\mathbf{N}$ & \\
\hline 1 & $8 / 1 / 93$ & 14 & 1 & & 73.58 & $\mathbf{N}$ & \\
\hline 1 & $8 / 9 / 93$ & 1 & 9 & & 80.6 & $Y$ & TMACS \\
\hline 9 & $8 / 9 / 93$ & 2 & 1 & & 80.42 & $\bar{N}$ & \\
\hline 1 & $8 / 9 / 93$ & 3 & 1 & & 80.24 & $\mathbf{N}$ & \\
\hline 1 & $8 / 9 / 93$ & 4 & 9 & & 79.52 & $\mathbf{N}$ & \\
\hline
\end{tabular}

Data obtained from WHC Surveillance Analysis Computer System (SACS), June 2, 1993. 


\begin{tabular}{|c|c|c|c|c|c|c|c|}
\hline \multicolumn{8}{|c|}{ TANK 241-BY-103 } \\
\hline \multicolumn{8}{|c|}{ THERMOCOUPLE DATA } \\
\hline TREE \# & DATE & THEAMO \# & TREE SET \# & TEMP C & TEMP F & $Y=M A X$ TEMP & COMMENTS \\
\hline 1 & $8 / 9 / 93$ & 5 & 1 & & 78.8 & $N$ & \\
\hline 1 & $8 / 9 / 93$ & 6 & 1 & & 77.9 & $\mathbf{N}$ & \\
\hline 1 & $8 / 9 / 93$ & 7 & 1 & & 76.1 & $\mathbf{N}$ & \\
\hline 1 & $8 / 9 / 93$ & 8 & 1 & & 76.28 & $\mathbf{N}$ & \\
\hline 1 & $8 / 9 / 93$ & 9 & 1 & & 72.5 & $\mathbf{N}$ & \\
\hline 1 & $8 / 9 / 93$ & 10 & 1 & & 72.14 & $\mathbf{N}$ & \\
\hline 1 & $8 / 9 / 93$ & 11 & 1 & & 72.86 & $\mathbf{N}$ & \\
\hline 1 & $8 / 9 / 93$ & 12 & 1 & & 73.04 & $\mathbf{N}$ & \\
\hline 1 & $8 / 9 / 93$ & 13 & 1 & & 74.3 & $\mathbf{N}$ & \\
\hline 1 & $8 / 9 / 93$ & 14 & 1 & & 74.84 & $\mathbf{N}$ & \\
\hline 1 & $8 / 15 / 93$ & 1 & 1 & & 80.6 & $Y$ & tmacs \\
\hline 1 & $8 / 15 / 93$ & 2 & 1 & $\leftarrow$ & 80.42 & $\mathbf{N}$ & \\
\hline 1 & $8 / 15 / 93$ & 3 & 1 & & 80.06 & $\mathbf{N}$ & \\
\hline 1 & $8 / 15 / 93$ & 4 & 1 & & 79.34 & $\mathbf{N}$ & \\
\hline 1 & $8 / 15 / 93$ & 5 & 1 & & 78.62 & $\mathbf{N}$ & \\
\hline 1 & $8 / 15 / 93$ & 6 & 1 & & 77.54 & $\mathbf{N}$ & \\
\hline 1 & $8 / 15 / 93$ & 7 & 1 & & 76.1 & $\mathbf{N}$ & \\
\hline 1 & $8 / 15 / 93$ & 8 & 1 & & 76.28 & $\mathbf{N}$ & \\
\hline 1 & $8 / 15 / 93$ & 9 & 1 & & 72.5 & $\mathbf{N}$ & 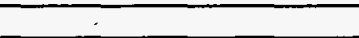 \\
\hline 1 & $8 / 15 / 93$ & 10 & 1 & & 72.86 & $\mathbf{N}$ & \\
\hline 1 & $8 / 15 / 93$ & 11 & 1 & & 72.86 & $\mathbf{N}$ & \\
\hline 1 & $8 / 15 / 93$ & 12 & 1 & & 73.22 & $\mathbf{N}$ & \\
\hline 1 & $8 / 15 / 93$ & 13 & 1 & & 74.12 & $\mathbf{N}$ & \\
\hline 1 & $8 / 15 / 93$ & 14 & 1 & & 74.84 & $\mathbf{N}$ & \\
\hline 1 & $8 / 23 / 93$ & 1 & 1 & & 80.6 & $\mathbf{Y}$ & TMACS \\
\hline 1 & $8 / 23 / 93$ & 2 & 1 & & 80.6 & $\mathbf{N}$ & \\
\hline 1 & $8 / 23 / 93$ & 3 & 1 & & 80.06 & $\mathbf{N}$ & \\
\hline 1 & $8 / 23 / 93$ & 4 & 1 & & 79.52 & $\mathbf{N}$ & \\
\hline 1 & $8 / 23 / 93$ & 5 & 1 & & 78.8 & $\mathbf{N}$ & \\
\hline 1 & $8 / 23 / 93$ & 6 & 1 & & 77.54 & $\mathbf{N}$ & \\
\hline 1 & $8 / 23 / 93$ & 7 & 1 & & 76.46 & $\mathbf{N}$ & \\
\hline 1 & $8 / 23 / 93$ & 8 & 1 & & 76.46 & $\mathbf{N}$ & \\
\hline 1 & $8 / 23 / 93$ & 9 & 1 & & 73.22 & $\mathbf{N}$ & \\
\hline 1 & $8 / 23 / 93$ & 10 & 1 & & 72.68 & $\mathbf{N}$ & \\
\hline 1 & $8 / 23 / 93$ & 11 & 1 & & 73.22 & $\mathbf{N}$ & \\
\hline 1 & $8 / 23 / 93$ & 12 & 1 & & 73.76 & $\mathbf{N}$ & \\
\hline 1 & $8 / 23 / 93$ & 13 & 1 & & 74.48 & $\mathbf{N}$ & \\
\hline 1 & $8 / 23 / 93$ & 14 & 1 & & 75.2 & $\mathbf{N}$ & \\
\hline 1 & $.8 / 26 / 93$ & 1 & 1 & & 80.2 & $\mathbf{Y}$ & J TYPE \\
\hline 1 & $8 / 26 / 93$ & 2 & 1 & & 80.1 & $\mathbf{N}$ & \\
\hline 1 & $8 / 26 / 93$ & 3 & $t$ & & 79.9 & $\mathbf{N}$ & \\
\hline 1 & $8 / 26 / 93$ & 4 & 1 & & 79.2 & $\mathbf{N}$ & \\
\hline 1 & $8 / 26 / 93$ & 5 & 1 & & 78.3 & $\mathbf{N}$ & \\
\hline 1 & $8 / 26 / 93$ & 6 & 1 & & 77.4 & $\mathbf{N}$ & \\
\hline 1 & $8 / 26 / 93$ & 7 & 1 & & 76.1 & $\mathbf{N}$ & \\
\hline 1 & $8 / 26 / 93$ & 8 & 1 & & 75.9 & $\mathbf{N}$ & \\
\hline 1 & $8 / 26 / 93$ & 9 & 1 & & 72.9 & $\mathbf{N}$ & \\
\hline 1 & $8 / 26 / 93$ & 10 & 1 & & 72.9 & $\mathbf{N}$ & \\
\hline 1 & $8 / 26 / 93$ & 11 & 1 & & 73.2 & $\mathbf{N}$ & \\
\hline 1 & $8 / 26 / 93$ & 12 & 1 & & 73.4 & $\mathbf{N}$ & \\
\hline 1 & $8 / 26 / 93$ & 13 & 1 & & 74.3 & $\overline{\mathbf{N}}$ & \\
\hline 1 & $8 / 26 / 93$ & 14 & 1 & & 75 & $\mathbf{N}$ & \\
\hline 1 & $8 / 30 / 93$ & 1 & 1 & & 80.96 & $Y$ & TMACS \\
\hline 1 & $8 / 30 / 93$ & 2 & 1 & & 80.78 & $\mathbf{N}$ & \\
\hline 1 & $8 / 30 / 93$ & 3 & 1 & & 80.6 & $\mathbf{N}$ & \\
\hline 1 & $8 / 30 / 93$ & 4 & 1 & & 80.06 & $\mathbf{N}$ & \\
\hline 1 & $8 / 30 / 93$ & 5 & 1 & & 79.34 & $\mathbf{N}$ & \\
\hline 1 & $8 / 30 / 93$ & 6 & 1 & & 78.26 & $\mathbf{N}$ & \\
\hline 1 & $8 / 30 / 93$ & 7 & 1 & & 77.18 & $\mathbf{N}$ & \\
\hline 1 & $8 / 30 / 93$ & 8 & 1 & & 77.18 & $\mathbf{N}$ & \\
\hline 1 & $8 / 30 / 93$ & 9 & 1 & & 73.22 & $\mathbf{N}$ & \\
\hline 1 & $8 / 30 / 93$ & 10 & 1 & & 73.22 & $N$ & \\
\hline 1 & $8 / 30 / 93$ & 11 & 1 & & 73.76 & $\mathbf{N}$ & \\
\hline 1 & $8 / 30 / 93$ & 12 & 1 & & 73.94 & $N$ & \\
\hline 1 & $8 / 30 / 93$ & 13 & 1 & & 74.66 & $\mathbf{N}$ & \\
\hline 1 & $8 / 30 / 93$ & 14 & 1 & & 75.56 & $N$ & \\
\hline
\end{tabular}

Data obtained from WHC Surveillance Analysis Computer System (SACS), June 2, 1993. 


\begin{tabular}{|c|c|c|c|c|c|c|c|}
\hline \multicolumn{8}{|c|}{ TANK 241-BY-103 } \\
\hline \multicolumn{8}{|c|}{ THERMOCOUPLE DATA } \\
\hline TREE \# & DATE & THERMO \# & TREE SET \# & TEMP C & TEMP F & $Y=$ MAX TEMP & COMMENTS \\
\hline 1 & $9 / 6 / 93$ & 1 & $\frac{1}{1}$ & & 80.78 & $\mathbf{N}$ & TMACS \\
\hline 1 & $9 / 6 / 93$ & 2 & 1 & & 80.96 & $\bar{Y}$ & \\
\hline 1 & $9 / 6 / 93$ & 3 & 1 & & 80.6 & $N$ & \\
\hline 1 & $9 / 6 / 93$ & 4 & 1 & & 79.88 & $\mathrm{~N}$ & \\
\hline 1 & $9 / 6 / 93$ & 5 & 1 & & 79.16 & $\mathbf{N}$ & \\
\hline 1 & $9 / 6 / 93$ & 6 & 1 & & 78.44 & $\mathbf{N}$ & \\
\hline 1 & $9 / 6 / 93$ & 7 & 1 & & 77.54 & $\mathbf{N}$ & \\
\hline 1 & $9 / 6 / 93$ & 8 & 1 & & 77.72 & $\mathbf{N}$ & \\
\hline 1 & $9 / 6 / 93$ & 9 & 1 & & 73.58 & $\mathbf{N}$ & \\
\hline 1 & $9 / 6 / 93$ & 10 & 1 & & 73.4 & $\mathbf{N}$ & \\
\hline 1 & $9 / 6 / 93$ & 11 & 1 & & 73.76 & $N$ & \\
\hline 1 & $9 / 6 / 93$ & 12 & 1 & & 74.3 & $N$ & \\
\hline 1 & $9 / 6 / 93$ & 13 & 1 & & 75.02 & $\mathbf{N}$ & \\
\hline 1 & $9 / 6 / 93$ & 14 & 1 & & 75.92 & $\mathbf{N}$ & \\
\hline 1 & $9 / 12 / 93$ & 1 & 1 & & 80.96 & $\mathbf{Y}$ & TMACS \\
\hline 1 & $9 / 12 / 93$ & 2 & 1 & & 80.96 & $\mathbf{N}$ & \\
\hline 1 & $9 / 12 / 93$ & 3 & 1 & & 80.96 & $\mathbf{N}$ & \\
\hline 1 & $9 / 12 / 93$ & 4 & 1 & & 80.24 & $\mathbf{N}$ & \\
\hline 1 & $9 / 12 / 93$ & 5 & 1 & & 79.52 & $\mathbf{N}$ & \\
\hline 1 & $9 / 12 / 93$ & 6 & 1 & & 79.16 & $\mathrm{~N}$ & \\
\hline 1 & $9 / 12 / 93$ & 7 & 1 & & 78.08 & $\mathbf{N}$ & \\
\hline 1 & $9 / 12 / 93$ & 8 & 1 & & 78.26 & $\mathrm{~N}$ & \\
\hline 1 & $9 / 12 / 93$ & 9 & 1 & & 73.76 & $N$ & \\
\hline 1 & $9 / 12 / 93$ & 10 & 1 & & 73.76 & $\mathbf{N}$ & \\
\hline 1 & $9 / 12 / 93$ & 11 & 1 & & 74.48 & $\bar{N}$ & \\
\hline 1 & $9 / 12 / 93$ & 12 & 1 & & 74.66 & $\bar{N}$ & \\
\hline 1 & $9 / 12 / 93$ & 13 & 1 & & 75.38 & $\mathrm{~N}$ & \\
\hline 1 & $9 / 12 / 93$ & 14 & 1 & & 76.46 & $\mathrm{~N}$ & \\
\hline 1 & $9 / 19 / 93$ & 1 & 1 & & 80.78 & $\mathrm{~N}$ & TMACS \\
\hline 1 & 9/19/93 & 2 & 1 & & 80.78 & $\mathrm{~N}$ & \\
\hline 1 & $9 / 19 / 93$ & 3 & 1 & & 80.96 & $\bar{Y}$ & \\
\hline 1 & $9 / 19 / 93$ & 4 & 1 & & 80.24 & $\bar{N}$ & \\
\hline 1 & 9/19/93 & 5 & 1 & & 79.52 & $N$ & \\
\hline$\frac{1}{1}$ & $9 / 19 / 93$ & 6 & 1 & & 78.98 & $\mathrm{~N}$ & \\
\hline 9 & $9 / 19 / 93$ & 7 & 1 & & 78.26 & $\mathrm{~N}$ & \\
\hline 1 & 9/19/93 & 8 & 1 & & 78.26 & $\mathrm{~N}$ & \\
\hline 9 & $9 / 19 / 93$ & 9 & 1 & & 73.94 & $\mathrm{~N}$ & \\
\hline 1 & $9 / 19 / 93$ & 10 & 1 & & 74.12 & $\mathrm{~N}$ & \\
\hline 1 & 9/19/93 & 11 & 1 & & 74.66 & $\mathbf{N}$ & \\
\hline 1 & $9 / 19 / 93$ & 12 & 9 & & 74.84 & $\mathbf{N}$ & \\
\hline 1 & $9 / 19 / 93$ & 13 & 1 & & 75.56 & $\mathrm{~N}$ & \\
\hline 1 & 9/19/93 & 14 & 1 & & 76.64 & $\mathbf{N}$ & \\
\hline 1 & $9 / 26 / 93$ & 1 & 1 & & 80.24 & $\mathbf{N}$ & TMACS \\
\hline 1 & $9 / 26 / 93$ & 2 & 1 & & 80.6 & $\bar{Y}$ & \\
\hline 1 & $9 / 26 / 93$ & 3 & 1 & & 80.6 & $\bar{N}$ & \\
\hline 9 & $9 / 26 / 93$ & 4 & 1 & & 80.24 & $\mathbf{N}$ & \\
\hline 1 & $9 / 26 / 93$ & 5 & 1 & & 79.7 & $\mathbf{N}$ & \\
\hline 1 & $9 / 26 / 93$ & 6 & 1 & & 79.16 & $\mathbf{N}$ & \\
\hline 1 & $9 / 26 / 93$ & 7 & 1 & & 78.26 & $\mathbf{N}$ & \\
\hline 1 & $9 / 26 / 93$ & 8 & 1 & & 78.26 & $\mathbf{N}$ & \\
\hline 1 & $9 / 26 / 93$ & 9 & 1 & & 74.48 & $\mathrm{~N}$ & \\
\hline 1 & $9 / 26 / 93$ & 10 & 1 & & 74.66 & $\overline{\mathbf{N}}$ & \\
\hline 1 & $9 / 26 / 93$ & 11 & 1 & & 75.02 & $\mathbf{N}$ & \\
\hline 1 & $9 / 26 / 93$ & 12 & $\frac{1}{1}$ & & 75.38 & $\mathbf{N}$ & \\
\hline 1 & $9 / 26 / 93$ & 13 & 1 & & 76.1 & $\mathbf{N}$ & \\
\hline 1 & $9 / 26 / 93$ & 14 & 1 & & 77 & $N$ & \\
\hline 1 & $10 / 3 / 93$ & 1 & 1 & & 80.6 & $\mathrm{~N}$ & TMACS \\
\hline 1 & $10 / 3 / 93$ & 2 & 1 & & 80.78 & $\mathrm{~N}$ & \\
\hline 1 & $10 / 3 / 93$ & 3 & 1 & & 80.96 & $\mathbf{Y}$ & \\
\hline 1 & $10 / 3 / 93$ & 4 & $\frac{1}{1}$ & & 80.6 & $\mathbf{N}$ & \\
\hline 1 & $10 / 3 / 93$ & 5 & 1 & & 80.06 & $\mathbf{N}$ & \\
\hline 1 & $10 / 3 / 93$ & 6 & 1 & & 79.52 & $\mathbf{N}$ & \\
\hline 1 & $10 / 3 / 93$ & 7 & 1 & & 78.44 & $\mathbf{N}$ & \\
\hline 1 & $10 / 3 / 93$ & 8 & 1 & & 78.8 & $\mathbf{N}$ & \\
\hline 1 & $10 / 3 / 93$ & 9 & 1 & & 74.66 & $\mathbf{N}$ & \\
\hline 1 & $10 / 3 / 93$ & 10 & 1 & & 74.66 & $\mathbf{N}$ & \\
\hline
\end{tabular}

Data obtained from WHC Surveillance Analysis Computer System (SACS), June 2, 1993. 


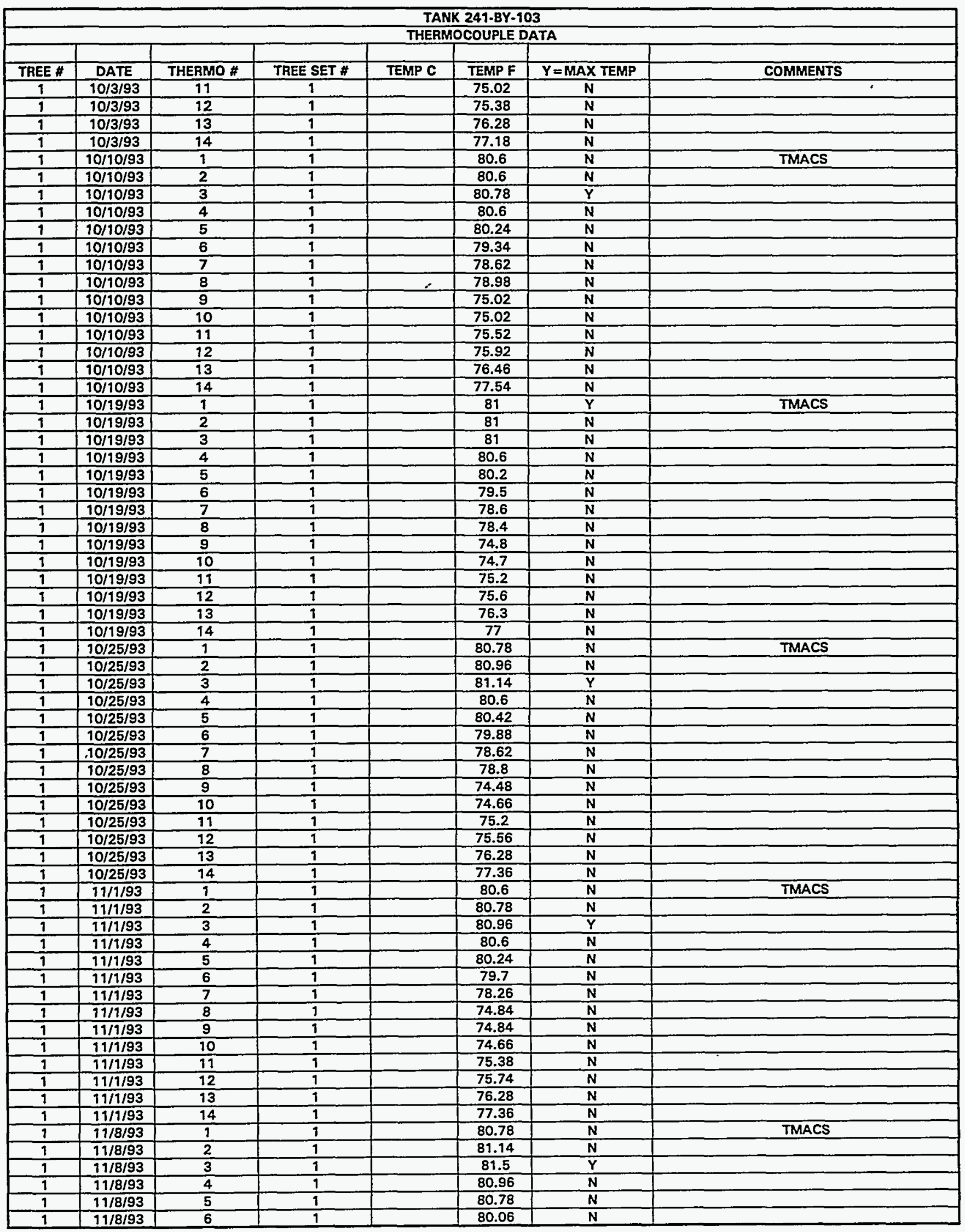

Data obtained from WHC Surveillance Analysis Computer System (SACS), June 2, 1993. 
WHC-SD-WM-ER-312, Rev. 0

\begin{tabular}{|c|c|c|c|c|c|c|c|}
\hline \multicolumn{9}{|c|}{ TANK 241-BY-103 } \\
\hline & & & & & & \\
\hline TREE \# & DATE & THERMO \# & TREE SET \# & TEMP C & TEMP F & Y=MAX TEMP & COMMENTS \\
\hline 1 & $11 / 8 / 93$ & 7 & 1 & & 78.62 & $\mathrm{~N}$ & \\
\hline 1 & $11 / 8 / 93$ & 8 & 1 & & 78.44 & $\mathrm{~N}$ & \\
\hline 1 & $11 / 8 / 93$ & 9 & 1 & & 74.48 & $\mathrm{~N}$ & \\
\hline 1 & $11 / 8 / 93$ & 10 & 1 & & 74.66 & $\mathrm{~N}$ & \\
\hline 1 & $11 / 8 / 93$ & 11 & 1 & & 75.2 & $\mathrm{~N}$ & \\
\hline 1 & $11 / 8 / 93$ & 12 & 1 & & 75.56 & $\mathrm{~N}$ & \\
\hline 1 & $11 / 8 / 93$ & 13 & 1 & & 76.28 & $\mathrm{~N}$ & \\
\hline 1 & $11 / 8 / 93$ & 14 & 1 & & 77 & $\mathrm{~N}$ & \\
\hline
\end{tabular}

Data obtained from WHC Surveillance Analysis Computer System (SACS), June 2, 1993.

D-184 


\begin{tabular}{|c|c|c|c|c|c|c|c|}
\hline \multicolumn{8}{|c|}{ TANK 241-BY-104 } \\
\hline \multicolumn{8}{|c|}{ THERMOCOUPLE DATA } \\
\hline TREE: & DATE & THERMO\# & TREE SET\# & TEMP C & TEMP F & $Y=$ MAXTEMP & COMMENTS \\
\hline 1 & $11 / 12 / 73$ & 1 & & & 218 & & \\
\hline 1 & $11 / 12 / 73$ & 2 & & & 225 & & \\
\hline 1 & $11 / 12 / 73$ & 3 & & & 218 & & \\
\hline 1 & $11 / 12 / 73$ & 4 & & & 218 & & \\
\hline 1 & $11 / 12 / 73$ & 5 & & & 207 & & \\
\hline 1 & $11 / 12 / 73$ & 6 & & & 193 & & \\
\hline 1 & $11 / 12 / 73$ & 7 & & & 160 & & \\
\hline 1 & $11 / 12 / 73$ & 8 & & & 158 & & \\
\hline 1 & $11 / 12 / 73$ & 9 & & & 158 & & \\
\hline 1 & $11 / 12 / 73$ & 10 & & & 156 & & \\
\hline 1 & $11 / 12 / 73$ & 11 & & & 133 & & \\
\hline 1 & $11 / 12 / 73$ & 12 & & $r$ & 76 & & \\
\hline 1 & $11 / 21 / 73$ & 1 & & 92 & 197.6 & & raw data - assumed degree C \\
\hline 1 & $11 / 21 / 73$ & 2 & & 94 & 201.2 & & raw data - assumed degree $C$ \\
\hline 1 & $11 / 21 / 73$ & 3 & & 94 & 201.2 & & raw data - assumed degree C \\
\hline 1 & $11 / 21 / 73$ & 4 & & 92 & 197.6 & & raw data - assumed degree C \\
\hline 1 & $11 / 21 / 73$ & 5 & & 88 & 190.4 & & raw data - assumed degree C \\
\hline 1 & $11 / 21 / 73$ & 6 & & 78 & 172.4 & & raw data - assumed degree C \\
\hline 1 & $11 / 21 / 73$ & 7 & & 72 & 161.6 & & raw data - assumed degree $\mathrm{C}$ \\
\hline 1 & $11 / 21 / 73$ & 8 & & 60 & 140 & & raw data - assumed degree $C$ \\
\hline 1 & $11 / 21 / 73$ & 9 & & 60 & 140 & & raw data - assumed degree $C$ \\
\hline 1 & $11 / 21 / 73$ & 10 & & 60 & 140 & & raw data - assumed degree C \\
\hline 1 & $11 / 21 / 73$ & 91 & & 45 & 113 & & raw data - assumed degree C \\
\hline 1 & $11 / 21 / 73$ & 12 & & 16 & 60.8 & & raw data - assumed degree C \\
\hline 1 & $12 / 10 / 73$ & 1 & & 94 & 201.2 & & raw data - assumed degree $C$ \\
\hline 1 & $12 / 10 / 73$ & 2 & & 95 & 203 & & raw data - assumed degree C \\
\hline 1 & $12 / 10 / 73$ & 3 & & 97 & 206.6 & & raw data - assumed degree C \\
\hline 1 & $12 / 10 / 73$ & 4 & & 96 & 204.8 & & raw data - assumed degree $C$ \\
\hline 1 & $12 / 10 / 73$ & 5 & & 88 & 190.4 & & raw data - assumed degree C \\
\hline 1 & $12 / 10 / 73$ & 6 & & 81 & 177.8 & & raw data - assumed degree $C$ \\
\hline 1 & $12 / 10 / 73$ & 7 & & 73 & 163.4 & & raw data - assumed degree $C$ \\
\hline 1 & $12 / 10 / 73$ & 8 & & 64 & 147.2 & & raw data - assumed degree $C$ \\
\hline 1 & $12 / 10 / 73$ & 9 & & 64 & 147.2 & & raw data - assumed degree $C$ \\
\hline 1 & $12 / 10 / 73$ & 10 & & 64 & 147.2 & & raw data - assumed degree C \\
\hline 1 & $12 / 10 / 73$ & 11 & & 49. & 120.2 & & raw data - assumed degree C \\
\hline 1 & $12 / 10 / 73$ & 12 & & 20 & 68 & & raw data - assumed degree C \\
\hline 1 & $12 / 17 / 73$ & 1 & & 105 & 221 & & raw data - assumed degree $C$ \\
\hline 1 & $.12 / 17 / 73$ & 2 & & 108 & 226.4 & & raw data - assumed degree $C$ \\
\hline 1 & $12 / 17 / 73$ & 3 & & 108 & 226.4 & & raw data - assumed degree C \\
\hline 1 & $12 / 17 / 73$ & 4 & & 104 & 219.2 & & raw data - assumed degree C \\
\hline 1 & $12 / 17 / 73$ & 5 & & 98 & 208.4 & & raw data - assumed degree C \\
\hline 1 & $12 / 17 / 73$ & 6 & & 92 & 197.6 & & raw data - assumed degree C \\
\hline 9 & $12 / 17 / 73$ & 7 & & 80 & 176 & & raw data - assumed degree C \\
\hline 1 & $12 / 17 / 73$ & 8 & & 73 & 163.4 & & raw data - assumed degree $C$ \\
\hline 1 & $12 / 17 / 73$ & 9 & & 73 & 163.4 & & raw data - assumed degree $C$ \\
\hline 1 & $12 / 17 / 73$ & 10 & & 73 & 163.4 & & raw data - assumed degree C \\
\hline 1 & $12 / 17 / 73$ & 11 & & 60 & 140 & & raw data - assumed degree C \\
\hline 1 & $12 / 17 / 73$ & 12 & & 31 & 87.8 & & raw data - assumed degree C \\
\hline 1 & $12 / 20 / 73$ & 1 & & 103 & 217.4 & & raw data - assumed degree $C$ \\
\hline 1 & $12 / 20 / 73$ & 2 & & 105 & 221 & & raw data - assumed degree C \\
\hline 1 & $12 / 20 / 73$ & 3 & & 105 & 221 & & raw data - assumed degree C \\
\hline 1 & $12 / 20 / 73$ & 4 & & 104 & 219.2 & & raw data - assumed degree C \\
\hline 1 & $12 / 20 / 73$ & 5 & & 96 & 204.8 & & raw data - assumed degree C \\
\hline 1 & $12 / 20 / 73$ & 6 & & 90 & 194 & & raw data - assumed degree C \\
\hline 1 & $12 / 20 / 73$ & 7 & & 80 & 176 & & raw data - assumed degree $C$ \\
\hline 1 & $12 / 20 / 73$ & 8 & & 72 & 161.6 & & raw data - assumed degree $C$ \\
\hline 1 & $12 / 20 / 73$ & 9 & & 72 & 161.6 & & raw data - assumed degree C \\
\hline 1 & $12 / 20 / 73$ & 10 & & 72 & 161.6 & & raw data - assumed degree C \\
\hline 1 & $12 / 20 / 73$ & 11 & & 57 & 134.6 & & raw data - assumed degree C \\
\hline 1 & $12 / 20 / 73$ & 12 & & 29 & 84.2 & & raw data - assumed degree $C$ \\
\hline 1 & $12 / 20 / 73$ & 15 & & 90 & 194 & & raw data - assumed degree C \\
\hline 1 & $8 / 5 / 74$ & 1 & 1 & 93 & 199.4 & $\bar{Y}$ & degree C/TWINS confirm \\
\hline 1 & $8 / 5 / 74$ & 2 & 1 & 88 & 190.4 & $N$ & degree C/TWINS confirm \\
\hline 1 & $8 / 5 / 74$ & 3 & 1 & BB & 190.4 & $\mathbf{N}$ & degree $\mathrm{C} / \mathrm{TW}$ INS confirm \\
\hline 1 & $8 / 5 / 74$ & 4 & 1 & 79 & 174.2 & $\mathbf{N}$ & degree C/TWINS confirm \\
\hline
\end{tabular}

Data obtained from WHC Surveillance Analysis Computer System (SACS), November 12, 1993. 
WHC-SD-WM-ER-312, Rev. 0

\begin{tabular}{|c|c|c|c|c|c|c|c|}
\hline \multicolumn{8}{|c|}{ TANK 241-BY-104 } \\
\hline \multicolumn{8}{|c|}{ THERMOCOUPLE DATA } \\
\hline TREE\# & DATE & THERMO\# & TREE SET\# & TEMP C & TEMPF & $Y=M A X T E M P$ & COMMENTS \\
\hline 9 & $8 / 5 / 74$ & 5 & 1 & 74 & 165.2 & $N$ & degree C/TWINS confirm \\
\hline 9 & $8 / 5 / 74$ & 6 & 1 & 72 & 161.6 & $N$ & degree C/TWINS confirm \\
\hline 1 & $8 / 5 / 74$ & 7 & 9 & 70 & 158 & $N$ & degree C/TWINS confirm \\
\hline$\frac{1}{1}$ & $8 / 5 / 74$ & 8 & $\overline{1}$ & 64 & 147.2 & $\bar{N}$ & degree C/TWINS confirm \\
\hline 1 & $8 / 5 / 74$ & 9 & 1 & 54 & 129.2 & $N$ & degree C/TWINS confirm \\
\hline 1 & $8 / 5 / 74$ & 10 & 1 & 52 & 125.6 & $N$ & degree C/TWINS confirm \\
\hline 1 & $8 / 5 / 74$ & 11 & 1 & 50 & 122 & $N$ & degree C/TWINS confirm \\
\hline 1 & $8 / 5 / 74$ & 12 & 9 & 46 & 114.8 & $\mathbf{N}$ & degree C/TWINS confirm \\
\hline 1 & $9 / 12 / 74$ & 1 & 9 & 106 & 222.8 & $\bar{N}$ & degree C/TWINS confirm \\
\hline 1 & $9 / 12 / 74$ & 2 & 9 & 109 & 228.2 & $\mathbf{Y}$ & degree C/TWINS confirm \\
\hline 1 & $9 / 12 / 74$ & 3 & 1 & 104 & 219.2 & $\bar{N}$ & degree C/TWINS confirm \\
\hline 1 & $9 / 12 / 74$ & 4 & 1 & $100 r$ & 212 & $\mathbf{N}$ & degree C/TWINS confirm \\
\hline 1 & $9 / 12 / 74$ & 5 & 9 & 92 & 197.6 & $\mathbf{N}$ & degree C/TWINS confirm \\
\hline 9 & $9 / 12 / 74$ & 6 & 1 & 84 & 183.2 & $\mathbf{N}$ & degree C/TWINS confirm \\
\hline 1 & $9 / 12 / 74$ & 7 & 1 & 75 & 167 & $\mathbf{N}$ & degree C/TWINS confirm \\
\hline 1 & $9 / 12 / 74$ & 8 & 1 & 71 & 159.8 & $\mathbf{N}$ & degree $\mathrm{C} / \mathrm{TW}$ INS confirm \\
\hline 1 & $9 / 12 / 74$ & 9 & 1 & 71 & 159.8 & $\mathbf{N}$ & degree $\mathrm{C} / \mathrm{TW}$ INS confirm \\
\hline 1 & $9 / 12 / 74$ & 10 & 1 & 68 & 154.4 & $\mathbf{N}$ & degree $\mathrm{C} / \mathrm{TWINS}$ confirm \\
\hline 1 & $9 / 12 / 74$ & 11 & 1 & 58 & 136.4 & $\mathrm{~N}$ & degree C/TWINS confirm \\
\hline 1 & $9 / 12 / 74$ & 12 & 1 & 47 & 116.6 & $\mathbf{N}$ & degree C/TWINS confirm \\
\hline 1 & $10 / 8 / 74$ & 1 & $T$ & 96 & 204.8 & $N$ & degree C/TWINS confirm \\
\hline 1 & $10 / 8 / 74$ & 2 & 1 & 98 & 208.4 & $\overline{\mathbf{Y}}$ & degree $\mathrm{C} / \mathrm{TW} I N S$ confirm \\
\hline 1 & $10 / 8 / 74$ & 3 & 1 & 94 & 201.2 & $\mathbf{N}$ & degree $\mathrm{C} / \mathrm{TWINS}$ confirm \\
\hline 1 & $10 / 8 / 74$ & 4 & 1 & 90 & 194 & $\bar{N}$ & degree C/TWINS confirm \\
\hline 1 & $10 / 8 / 74$ & 5 & 1 & 83 & 181.4 & $N$ & degree $\mathrm{C} / \mathrm{TW}$ INS confirm \\
\hline 9 & $10 / 8 / 74$ & 6 & 1 & 75 & 167 & $\bar{N}$ & degree $\mathrm{C} / \mathrm{TWINS}$ confirm \\
\hline 1 & $10 / 8 / 74$ & 7 & 1 & 66 & 150.8 & $\mathbf{N}$ & degree $\mathrm{C} / \mathrm{TW}$ INS confirm \\
\hline 1 & $10 / 8 / 74$ & 8 & 1 & 64 & 147.2 & $\bar{N}$ & degree C/TWINS confirm \\
\hline 1 & $10 / 8 / 74$ & 9 & 1 & 64 & 147.2 & $\mathbf{N}$ & degree C $/$ TWINS confirm \\
\hline 1 & $10 / 8 / 74$ & 10 & 1 & 60 & 140 & $\mathbf{N}$ & degree C/TWINS confirm \\
\hline 1 & $10 / 8 / 74$ & 11 & 1 & 51 & 123.8 & $\mathbf{N}$ & degree $\mathrm{C} / \mathrm{TW}$ INS confirm \\
\hline 1 & $10 / 8 / 74$ & 12 & 1 & 39 & 102.2 & $\mathbf{N}$ & degree $\mathrm{C} / \mathrm{TWINS}$ confirm \\
\hline 1 & $10 / 29 / 74$ & 1 & 1 & 92 & 197.6 & $\mathbf{N}$ & degree $C / T W I N S$ confirm \\
\hline 1 & $10 / 29 / 74$ & 2 & 1 & 95 & 203 & $\bar{Y}$ & degree $\mathrm{C} / \mathrm{TW}$ INS confirm \\
\hline 1 & $10 / 29 / 74$ & 3 & 1 & 91 & 195.8 & $\mathbf{N}$ & degree $\mathrm{C} / \mathrm{TWINS}$ confirm \\
\hline 1 & $10 / 29 / 74$ & 4 & 1 & 86 & 186.8 & $\mathbf{N}$ & degree $\mathrm{C} / \mathrm{TW}$ INS confirm \\
\hline 1 & $10 / 29 / 74$ & 5 & 1 & 79 & 174.2 & $\bar{N}$ & degree C/TWINS confirm \\
\hline 1 & $.10 / 29 / 74$ & 6 & 1 & 72 & 161.6 & $N$ & degree $\mathrm{C} / \mathrm{TW}$ INS confirm \\
\hline 1 & $10 / 29 / 74$ & 7 & 1 & 63 & 145.4 & $N$ & degree $\mathrm{C} / \mathrm{TW}$ INS confirm \\
\hline 1 & $10 / 29 / 74$ & 8 & 1 & 60 & 140 & $\mathbf{N}$ & degree $\mathrm{C} / \mathrm{TW}$ INS confirm \\
\hline 1 & $10 / 29 / 74$ & 9 & 1 & 60 & 140 & $\mathbf{N}$ & degree $\mathrm{C} / \mathrm{TW}$ INS confirm \\
\hline 1 & $10 / 29 / 74$ & 10 & 1 & 56 & 132.8 & $\mathbf{N}$ & degree $\mathrm{C} / \mathrm{TWINS}$ confirm \\
\hline 1 & $.10 / 29 / 74$ & 17 & 9 & 47 & 116.6 & $\mathbf{N}$ & degree $\mathrm{C} / \mathrm{TW}$ INS confirm \\
\hline 1 & $10 / 29 / 74$ & 12 & 1 & 36 & 96.8 & $\mathrm{~N}$ & degree C/TWINS confirm \\
\hline 1 & $11 / 12 / 74$ & 4 & 1 & 93.33 & 199.994 & $\bar{Y}$ & degree C/TWINS confirm \\
\hline 9 & $11 / 12 / 74$ & 5 & 1 & 69.44 & 156.992 & $\mathbf{N}$ & degree $\mathrm{C} / \mathrm{TW}$ INS confirm \\
\hline$\frac{9}{1}$ & $11 / 12 / 74$ & 6 & 1 & 75 & 167 & $\mathbf{N}$ & degree $\mathrm{C} / \mathrm{TWINS}$ confirm \\
\hline 1 & $11 / 26 / 74$ & 1 & 1 & 95 & 203 & $N$ & degree C/TWINS confirm \\
\hline 1 & $11 / 26 / 74$ & 2 & 1 & 96 & 204.8 & $\bar{Y}$ & degree C/TWINS confirm \\
\hline 1 & $11 / 26 / 74$ & 3 & 1 & 92 & 197.6 & $\mathbf{N}$ & degree $\mathrm{C} / \mathrm{TW}$ INS confirm \\
\hline 1 & $11 / 26 / 74$ & 4 & 1 & 86 & 186.8 & $\mathbf{N}$ & degree $\mathrm{C} / \mathrm{TW}$ INS confirm \\
\hline 1 & $11 / 26 / 74$ & 5 & 1 & 82 & 179.6 & $\mathbf{N}$ & degree $\mathrm{C} / \mathrm{TW} I N S$ confirm \\
\hline 1 & $11 / 26 / 74$ & 6 & 1 & 72 & 161.6 & $\mathbf{N}$ & degree $\mathrm{C} /$ TWINS confirm \\
\hline 1 & $11 / 26 / 74$ & 7 & 1 & 64 & 147.2 & $\mathbf{N}$ & degree C/TWINS confirm \\
\hline 1 & $11 / 26 / 74$ & 8 & 1 & 62 & 143.6 & $\mathbf{N}$ & degree C/TWINS confirm \\
\hline 1 & $11 / 26 / 74$ & 9 & 9 & 62 & 943.6 & $\mathbf{N}$ & degree C/TWINS confirm \\
\hline 1 & $11 / 26 / 74$ & 10 & $\frac{1}{1}$ & 58 & 136.4 & $\mathbf{N}$ & degree $\mathrm{C} / \mathrm{TW}$ INS confirm \\
\hline 1 & $11 / 26 / 74$ & 11 & 1 & 46 & 114.8 & $\mathbf{N}$ & degree C/TWINS confirm \\
\hline 1 & $11 / 26 / 74$ & 12 & 1 & 26 & 78.8 & $\mathbf{N}$ & degree $\mathrm{C} / \mathrm{TW}$ INS confirm \\
\hline 1 & $12 / 11 / 74$ & 1 & 1 & 90.56 & 195.008 & $\mathbf{N}$ & degree C/TWINS confirm \\
\hline 1 & $12 / 11 / 74$ & 2 & 1 & 92.22 & 197.996 & $\overline{\mathrm{Y}}$ & degree C/TWINS confirm \\
\hline 1 & $12 / 11 / 74$ & 3 & 1 & 88.89 & 192.002 & $\bar{N}$ & degree C/TWINS confirm \\
\hline 1 & $12 / 11 / 74$ & 4 & 1 & 84.44 & 183.992 & $\mathbf{N}$ & degree C/TWINS confirm \\
\hline 1 & $12 / 11 / 74$ & 5 & 1 & 76.67 & 170.006 & $\bar{N}$ & degree C/TWINS confirm \\
\hline 9 & $12 / 11 / 74$ & 6 & 1 & 68.89 & 156.002 & $\mathbf{N}$ & degree C/TWINS confirm \\
\hline
\end{tabular}

Data obtained from WHC Surveillance Analysis Computer System (SACS), November 12, 1993. 


\begin{tabular}{|c|c|c|c|c|c|c|c|}
\hline \multicolumn{8}{|c|}{$\begin{array}{l}\text { TANK 241-BY-104 } \\
\text { THERMOCOUPLE DATA }\end{array}$} \\
\hline & & & & THER & MOCOUPLE & DATA & \\
\hline TREE\# & DATE & THERMO\# & TREE SET\# & TEMP C & TEMP F & $Y=$ MAXTEMP & COMMENTS \\
\hline 1 & $12 / 11 / 74$ & 7 & 1 & 60 & 140 & $\mathbf{N}$ & degree C/TWINS confirm \\
\hline 1 & $12 / 11 / 74$ & 8 & 1 & 57.22 & 134.996 & $\mathbf{N}$ & degree C/TWINS confirm \\
\hline 1 & $12 / 11 / 74$ & 9 & 1 & 57.22 & 134.996 & $\mathbf{N}$ & degree $\mathrm{C} / \mathrm{TW}$ WNS confirm \\
\hline 1 & $12 / 11 / 74$ & 10 & 1 & 52.22 & 125.996 & $\mathbf{N}$ & degree C/TWINS confirm \\
\hline 1 & $12 / 11 / 74$ & 11 & 1 & 39.44 & 102.992 & $\mathbf{N}$ & degree C/TWINS confirm \\
\hline 1 & $12 / 11 / 74$ & 12 & 1 & 21.67 & 71.006 & $\mathbf{N}$ & degree C/TWINS confirm \\
\hline 1 & $12 / 17 / 74$ & 1 & 1 & 82.22 & 179.996 & $\mathbf{N}$ & degree C/TWINS confirm \\
\hline 1 & $12 / 17 / 74$ & 2 & 1 & 94.44 & 201.992 & $\mathbf{Y}$ & degree C/TWINS confirm \\
\hline 1 & $12 / 17 / 74$ & 3 & 1 & 91.56 & 196.808 & $\mathbf{N}$ & degree C/TWINS confirm \\
\hline 1 & $12 / 17 / 74$ & 4 & 1 & 87.22 & 188.996 & $\mathbf{N}$ & degree C/TWINS confirm \\
\hline 1 & $12 / 17 / 74$ & 5 & 1 & 79.44 & 174.992 & $\mathbf{N}$ & degree C/TWINS confirm \\
\hline 1 & $12 / 17 / 74$ & 6 & 1 & 71.11 & 159.998 & $\mathbf{N}$ & degree $\mathrm{C} / \mathrm{TW}$ INS confirm \\
\hline 1 & $12 / 17 / 74$ & 7 & 1 & 63.33 & 145.994 & $N$ & degree C/TWINS confirm \\
\hline 1 & $12 / 17 / 74$ & 8 & 1 & 61.19 & 141.998 & $N$ & degree C/TWINS confirm \\
\hline 1 & $12 / 17 / 74$ & 9 & 1 & 60 & 140 & $\mathbf{N}$ & degree C/TWINS confirm \\
\hline 1 & $12 / 17 / 74$ & 10 & 1 & 55.56 & 132.008 & $\mathbf{N}$ & degree C/TWINS confirm \\
\hline 1 & $12 / 17 / 74$ & 11 & 1 & 42.22 & 107.996 & $\mathbf{N}$ & degree C/TWINS confirm \\
\hline 1 & $12 / 17 / 74$ & 12 & 1 & 24.44 & 75.992 & $N$ & degree C/TWINS confirm \\
\hline 1 & $12 / 31 / 74$ & 1 & 1 & 93.33 & 199.994 & $\mathbf{N}$ & degree C/TWINS confirm \\
\hline 1 & $12 / 31 / 74$ & 2 & 1 & 94.56 & 202.208 & $Y$ & degree C/TWINS confirm \\
\hline 1 & $12 / 31 / 74$ & 3 & 1 & 91.11 & 195.998 & $N$ & degree C/TWINS confirm \\
\hline 1 & $12 / 31 / 74$ & 4 & 1 & 86.67 & 188.006 & $\mathrm{~N}$ & degree C/TWINS confirm \\
\hline 1 & $12 / 31 / 74$ & 5 & 1 & 79.44 & 174.992 & $\mathbf{N}$ & degree C/TWINS confirm \\
\hline 1 & $12 / 31 / 74$ & 6 & 1 & 71.11 & 159.998 & $\mathbf{N}$ & degree C/TWINS confirm \\
\hline 1 & $12 / 31 / 74$ & 7 & 1 & 62.78 & 145.004 & $\mathrm{~N}$ & degree $\mathrm{C} / \mathrm{TW}$ INS confirm \\
\hline 1 & $12 / 31 / 74$ & 8 & 1 & 60 & 140 & $\mathbf{N}$ & degree C/TWINS confirm \\
\hline 1 & $12 / 31 / 74$ & 9 & 1 & 59.44 & 138.992 & $\mathbf{N}$ & degree C/TWINS confirm \\
\hline 1 & $12 / 31 / 74$ & 10 & 1 & 54.44 & 129.992 & $\mathbf{N}$ & degree C/TWINS confirm \\
\hline 1 & $12 / 31 / 74$ & 11 & 1 & 37.78 & 100.004 & $\mathbf{N}$ & degree C/TWINS confirm \\
\hline 1 & $12 / 31 / 74$ & 12 & 1 & 22.78 & 73.004 & $\mathbf{N}$ & degree C/TWINS confirm \\
\hline 1 & $1 / 8 / 75$ & 1 & 1 & 83.33 & 181.994 & $\mathbf{N}$ & degree C/TWINS confirm \\
\hline 1 & $1 / 8 / 75$ & 2 & 1 & 85.56 & 186.008 & $\mathbf{N}$ & degree C/TWINS confirm \\
\hline 1 & $1 / 8 / 75$ & 3 & 1 & 87.78 & 190.004 & $\mathbf{Y}$ & degree C/TWINS confirm \\
\hline 1 & $1 / 8 / 75$ & 4 & 1 & 83.89 & 183.002 & $\mathbf{N}$ & degree C/TWINS confirm \\
\hline 1 & $1 / 8 / 75$ & 5 & 1 & 76.67 & 170.006 & $\mathbf{N}$ & degree C/TWINS confirm \\
\hline 1 & $1 / 8 / 75$ & 6 & 1 & 69.44 & 156.992 & $\mathbf{N}$ & degree C/TWINS confirm \\
\hline 1 & $1 / 8 / 75$ & 7 & 1 & 61.11 & 141.998 & $\mathbf{N}$ & degree C/TWINS confirm \\
\hline 1 & $.1 / 8 / 75$ & 8 & 1 & 58.89 & 138.002 & $\mathbf{N}$ & degree C/TWINS confirm \\
\hline 1 & $1 / 8 / 75$ & 9 & 1 & 57.78 & 136.004 & $\mathbf{N}$ & degree C/TWINS confirm \\
\hline 1 & $9 / 8 / 75$ & 10 & 1 & 52.78 & 127.004 & $\mathbf{N}$ & degree $\mathrm{C} / \mathrm{TW}$ WNS confirm \\
\hline 1 & $1 / 8 / 75$ & 11 & 1 & 40 & 104 & $\mathbf{N}$ & degree $\mathrm{C} / \mathrm{TW}$ INS confirm \\
\hline 1 & $1 / 8 / 75$ & 12 & 1 & 22.78 & 73.004 & $N$ & degree C/TWINS confirm \\
\hline 1 & $1 / 21 / 75$ & 1 & 1 & 113 & 235.4 & $\mathbf{N}$ & degree C/TWINS confirm \\
\hline 1 & $1 / 21 / 75$ & 2 & 1 & 114 & 237.2 & $\mathbf{Y}$ & degree C/TWINS confirm \\
\hline 1 & $1 / 21 / 75$ & 3 & 1 & 110 & 230 & $\mathbf{N}$ & degree C/TWINS confirm \\
\hline 1 & $1 / 21 / 75$ & 4 & 1 & 106 & 222.8 & $N$ & degree $\mathrm{C} / \mathrm{TW}$ INS confirm \\
\hline 1 & $1 / 21 / 75$ & 5 & 1 & 98 & 208.4 & $\mathbf{N}$ & degree C/TWINS confirm \\
\hline 1 & $1 / 21 / 75$ & 6 & 1 & 99 & 210.2 & $\mathbf{N}$ & degree C/TWINS confirm \\
\hline 1 & $1 / 21 / 75$ & 7 & 1 & 78 & 172.4 & $\mathbf{N}$ & degree $\mathrm{C} / \mathrm{TW}$ INS confirm \\
\hline 1 & $1 / 21 / 75$ & 8 & 1 & 75 & 167 & $\mathbf{N}$ & degree $\mathrm{C} / \mathrm{TW}$ WNS confirm \\
\hline 1 & $1 / 21 / 75$ & 9 & 1 & 74 & 165.2 & $N$ & degree C/TWINS confirm \\
\hline 9 & $9 / 29 / 75$ & 10 & 1 & 67 & 152.6 & $\mathbf{N}$ & degree C/TWINS confirm \\
\hline 1 & $1 / 21 / 75$ & 11 & 1 & 51 & 123.8 & $\mathbf{N}$ & degree C/TWINS confirm \\
\hline 1 & $1 / 21 / 75$ & 12 & 1 & 29 & 84.2 & $\mathbf{N}$ & degree C/TWINS confirm \\
\hline 1 & $1 / 28 / 75$ & 1 & 1 & 91 & 195.8 & $\mathbf{N}$ & degree C/TWINS confirm \\
\hline 1 & $1 / 28 / 75$ & 2 & 1 & 92 & 197.6 & $\mathbf{Y}$ & degree C/TWINS confirm \\
\hline 1 & $1 / 28 / 75$ & 3 & 1 & 90 & 194 & $\mathbf{N}$ & degree C/TWINS confirm \\
\hline 1 & $1 / 28 / 75$ & 4 & 1 & 86 & 186.8 & $\mathrm{~N}$ & degree C/TWINS confirm \\
\hline 1 & $1 / 28 / 75$ & 5 & 1 & 78 & 172.4 & $\mathbf{N}$ & degree $\mathrm{C} / \mathrm{TW}$ INS confirm \\
\hline 1 & $1 / 28 / 75$ & 6 & 1 & 71 & 159.8 & $\mathbf{N}$ & degree $\mathrm{C} / \mathrm{TW}$ INS confirm \\
\hline 1 & $1 / 28 / 75$ & 7 & 1 & 62 & 143.6 & $\mathbf{N}$ & degree $\mathrm{C} / \mathrm{TW}$ INS confirm \\
\hline 1 & $1 / 28 / 75$ & 8 & 1 & 59 & 138.2 & $\mathbf{N}$ & degree $\mathrm{C} / \mathrm{TW}$ INS confirm \\
\hline 1 & $1 / 28 / 75$ & 9 & 1 & 58 & 136.4 & $\mathbf{N}$ & degree C/TWINS confirm \\
\hline 1 & $1 / 28 / 75$ & 10 & 1 & 53 & 127.4 & $\mathbf{N}$ & degree C/TWINS confirm \\
\hline 1 & $1 / 28 / 75$ & 11 & 1 & 40 & 104 & $\mathbf{N}$ & degree C/TWINS confirm \\
\hline
\end{tabular}

Data obtained from WHC Surveillance Analysis Computer System (SACS), November 12, 1993. 


\begin{tabular}{|c|c|c|c|c|c|c|c|}
\hline \multicolumn{8}{|c|}{ TANK 241-BY-104 } \\
\hline \multicolumn{8}{|c|}{ THERMOCOUPLE DATA } \\
\hline TREE\# & DATE & THERMO\# & TREE SET \# & TEMP C & TEMP F & $\mathbf{Y}=$ MAXTEMP & COMMENTS \\
\hline 1 & $1 / 28 / 75$ & 12 & 1 & 20 & 68 & $N$ & degree CRWINS confirm \\
\hline 9 & $2 / 27 / 75$ & 1 & 1 & 94 & 201.2 & $\ddot{N}$ & degree C/TWINS confirm \\
\hline 1 & $2 / 27 / 75$ & 2 & 1 & 94 & 201.2 & $\bar{Y}$ & degree $\mathrm{C} / \mathrm{TW}$ INS confirm \\
\hline 1 & $2 / 27 / 75$ & 3 & 1 & 92 & 197.6 & $\mathbf{N}$ & degree C/TWINS confirm \\
\hline 1 & $2 / 27 / 75$ & 4 & 1 & 88 & 190.4 & $\mathbf{N}$ & degree $\mathrm{C} / \mathrm{TW}$ INS confirm \\
\hline 1 & $2 / 27 / 75$ & 5 & 1 & 81 & 177.8 & $\mathbf{N}$ & degree C/TWINS confirm \\
\hline 1 & $2 / 27 / 75$ & 6 & 1 & 74 & 165.2 & $\mathbf{N}$ & degree C/TWINS confirm \\
\hline 1 & $2 / 27 / 75$ & 7 & 1 & 64 & 147.2 & $\mathbf{N}$ & degree C/TWINS confirm \\
\hline 1 & $2 / 27 / 75$ & 8 & 1 & 61 & 141.8 & $N$ & degree C/TWINS confirm \\
\hline 1 & $2 / 27 / 75$ & 9 & 1 & 60 & 140 & $\mathbf{N}$ & degree C/TWINS confirm \\
\hline 1 & $2 / 27 / 75$ & 10 & 1 & 54 & 129.2 & $\mathbf{N}$ & degree C/TWINS confirm \\
\hline 1 & $2 / 27 / 75$ & 11 & 1 & 40 & 104 & $\mathbf{N}$ & degree C/TWINS confirm \\
\hline 1 & $2 / 27 / 75$ & 12 & 9 & 24 & 75.2 & $\mathbf{N}$ & degree $\mathrm{C}$ TWWINS confirm \\
\hline 1 & $3 / 18 / 75$ & 1 & 1 & 92 & 197.6 & $N$ & degree C/TWINS confirm \\
\hline 1 & $3 / 18 / 75$ & 2 & 1 & 94 & 201.2 & $\mathrm{Y}$ & degree $\mathrm{C} / \mathrm{TWINS}$ confirm \\
\hline 1 & $3 / 18 / 75$ & 3 & 1 & 90 & 194 & $\mathbf{N}$ & degree C/TWINS confirm \\
\hline 1 & $3 / 18 / 75$ & 4 & 1 & 86 & 186.8 & $\mathbf{N}$ & degree $\mathrm{C} / \mathrm{TWINS}$ confirm \\
\hline 1 & $3 / 18 / 75$ & 5 & 1 & 78 & 172.4 & $\mathbf{N}$ & degree $\mathrm{C} / \mathrm{TWINS}$ confirm \\
\hline 1 & $3 / 18 / 75$ & 6 & 1 & 70 & 158 & $N$ & degree $\mathrm{C} / \mathrm{TW}$ INS confirm \\
\hline 1 & $3 / 18 / 75$ & 7 & 1 & 62 & 143.6 & $\mathbf{N}$ & degree $\mathrm{C} / \mathrm{TW}$ WINS confirm \\
\hline 1 & $3 / 18 / 75$ & 8 & 1 & 60 & 140 & $N$ & degree $\mathrm{C} / \mathrm{TWINS}$ confirm \\
\hline 1 & $3 / 18 / 75$ & 9 & 1 & 58 & 136.4 & $\mathbf{N}$ & degree $\mathrm{C} / \mathrm{TW}$ INS confirm \\
\hline 1 & $3 / 18 / 75$ & 10 & 1 & 52 & 125.6 & $\mathbf{N}$ & degree $\mathrm{C} / \mathrm{TW}$ INS confirm \\
\hline 1 & $3 / 18 / 75$ & 11 & 1 & 40 & 104 & $\mathbf{N}$ & degree C/TWINS confirm \\
\hline 1 & $3 / 18 / 75$ & 12 & 1 & 22 & 71.6 & $\mathbf{N}$ & degree C/TWINS confirm \\
\hline 1 & $4 / 1 / 75$ & 4 & 1 & 83.33 & 181.994 & $\mathbf{N}$ & degree C/TWINS confirm \\
\hline 1 & $4 / 1 / 75$ & 5 & 1 & 77.78 & 172.004 & $\mathbf{N}$ & degree $\mathrm{C} / \mathrm{TW}$ INS confirm \\
\hline 1 & $4 / 1 / 75$ & 6 & 1 & 68.89 & 156.002 & $N$ & degree C/TWINS confirm \\
\hline 1 & $4 / 1 / 75$ & 7 & 1 & 61.11 & 141.998 & $N$ & degree C/TWINS confirm \\
\hline 1 & $4 / 1 / 75$ & 8 & 1 & 57.22 & 134.996 & $\mathbf{N}$ & degree C/TWINS confirm \\
\hline 1 & $4 / 1 / 75$ & 9 & 1 & 56.11 & 132.998 & $N$ & degree C/TWINS confirm \\
\hline 1 & $4 / 1 / 75$ & 10 & 1 & 50 & 122 & $\mathbf{N}$ & degree C/TWINS confirm \\
\hline 1 & $4 / 1 / 75$ & 11 & 1 & 36.11 & 96.998 & $\mathrm{~N}$ & degree C/TWINS confirm \\
\hline 1 & $4 / 1 / 75$ & 12 & 1 & 21.11 & 69.998 & $\mathbf{N}$ & degree $\mathrm{C} / \mathrm{TW}$ WINS confirm \\
\hline 1 & $4 / 8 / 75$ & 1 & 1 & 107 & 224.6 & $\bar{N}$ & degree C/TWINS confirm \\
\hline 9 & $4 / 8 / 75$ & 2 & 1 & 108 & 226.4 & $\mathbf{Y}$ & degree C/TWINS confirm \\
\hline 1 & $4 / 8 / 75$ & 3 & 1 & 106 & 222.8 & $\mathbf{N}$ & degree C/TWINS confirm \\
\hline 1 & $4 / 8 / 75$ & 4 & 1 & 100 & 212 & $\mathbf{N}$ & degree $\mathrm{C} / \mathrm{TW}$ WNS confirm \\
\hline 1 & $4 / 8 / 75$ & 5 & 1 & 92 & 197.6 & $\mathbf{N}$ & degree $\mathrm{C}$ TWWINS confirm \\
\hline 1 & $4 / 8 / 75$ & 6 & 1 & 81 & 177.8 & $\mathbf{N}$ & degree C/TWINS confirm \\
\hline 1 & $4 / 8 / 75$ & 7 & 1 & 72 & 161.6 & $\mathbf{N}$ & degree $\mathrm{C} / \mathrm{TW}$ INS confirm \\
\hline 1 & $4 / 8 / 75$ & 8 & 1 & 68 & 154.4 & $\bar{N}$ & degree $\mathrm{C} / \mathrm{TW}$ WNS confirm \\
\hline 1 & $4 / 8 / 75$ & 9 & 1 & 66 & 150.8 & $\bar{N}$ & degree C/TWINS confirm \\
\hline 9 & $4 / 8 / 75$ & 10 & 1 & 60 & 140 & $N$ & degree $\mathrm{C} / \mathrm{TW}$ WNS confirm \\
\hline 1 & $4 / 8 / 75$ & 11 & 1 & 44 & 111.2 & $\bar{N}$ & degree C/TWINS confirm \\
\hline 1 & $4 / 8 / 75$ & 12 & 1 & 23 & 73.4 & $\bar{N}$ & degree C/TWINS confirm \\
\hline 1 & $4 / 22 / 75$ & 1 & 1 & 90 & 194 & $\mathbf{N}$ & degree $\mathrm{C} / \mathrm{TWINS}$ confirm \\
\hline 1 & $4 / 22 / 75$ & 2 & 1 & 90 & 194 & $\bar{Y}$ & degree C/TWINS confirm \\
\hline 9 & $4 / 22 / 75$ & 3 & 1 & 88 & 190.4 & $\mathbf{N}$ & degree $\mathrm{C} / \mathrm{TW}$ INS confirm \\
\hline 1 & $4 / 22 / 75$ & 4 & 1 & 82 & 179.6 & $\mathbf{N}$ & degree $\mathrm{C} / \mathrm{TW}$ INS confirm \\
\hline 1 & $4 / 22 / 75$ & 5 & $\frac{1}{1}$ & 76 & 168.8 & $\mathbf{N}$ & degree $\mathrm{C} / \mathrm{TW}$ WNS confirm \\
\hline$\frac{1}{1}$ & $4 / 22 / 75$ & 6 & 1 & 68 & 154.4 & $\mathbf{N}$ & degree C/TWINS confirm \\
\hline 1 & $4 / 22 / 75$ & 7 & 1 & 60 & 140 & $\bar{N}$ & degree C/TWINS confirm \\
\hline$\frac{1}{1}$ & $4 / 22 / 75$ & 8 & 1 & 55 & 131 & $\mathbf{N}$ & degree $\mathrm{C} / \mathrm{TW}$ WINS confirm \\
\hline 1 & $4 / 22 / 75$ & 9 & 1 & 55 & 131 & $\mathbf{N}$ & degree $\mathrm{C} / \mathrm{TW}$ WNS confirm \\
\hline 1 & $4 / 22 / 75$ & 10 & 9 & 50 & 122 & $N$ & degree $\mathrm{C} / \mathrm{TW}$ INS confirm \\
\hline 1 & $4 / 22 / 75$ & 11 & 1 & 36 & 96.8 & $\mathbf{N}$ & degree $\mathrm{C} / \mathrm{TW}$ WINS confirm \\
\hline 1 & $4 / 22 / 75$ & 12 & 1 & 22 & 71.6 & $\mathbf{N}$ & degree $\mathrm{C} / \mathrm{TW}$ INS confirm \\
\hline 1 & $4 / 29 / 75$ & 1 & 1 & 87 & 188.6 & $\mathbf{N}$ & degree $\mathrm{C} / \mathrm{TW}$ WNS confirm \\
\hline 1 & $4 / 29 / 75$ & 2 & 9 & 88 & 190.4 & $\bar{Y}$ & degree $\mathrm{C} /$ TWINS confirm \\
\hline 1 & $4 / 29 / 75$ & 3 & $\frac{1}{1}$ & 86 & 186.8 & $\mathbf{N}$ & degree $\mathrm{C} / \mathrm{TW}$ INS confirm \\
\hline 9 & $4 / 29 / 75$ & 4 & 1 & 82 & 179.6 & $\mathbf{N}$ & degree C/TWINS confirm \\
\hline 1 & $4 / 29 / 75$ & 5 & 1 & 72 & 161.6 & $\mathbf{N}$ & degree $\mathrm{C} / \mathrm{TW}$ WNS confirm \\
\hline 1 & $4 / 29 / 75$ & 6 & 1 & 66 & 150.8 & $\bar{N}$ & degree C/TWINS confirm \\
\hline 1 & $4 / 29 / 75$ & 7 & 1 & 58 & 136.4 & $\mathrm{~N}$ & degree C/TWINS confirm \\
\hline
\end{tabular}

Data obtained from WHC Surveillance Analysis Computer System (SACS), November 12, 1993. 


\begin{tabular}{|c|c|c|c|c|c|c|c|}
\hline \multicolumn{8}{|c|}{ TANK 241-8Y-104 } \\
\hline \multicolumn{8}{|c|}{ THERMOCOUPLE DATA } \\
\hline TREE\# & DATE & THERMO\# & TREE SET\# & TEMP C & TEMPF & $Y=$ MAXTEMP & COMMENTS \\
\hline$T$ & $4 / 29 / 75$ & 8 & 1 & 54 & 129.2 & $\mathbf{N}$ & degree CTWWINS confirm \\
\hline 1 & $4 / 29 / 75$ & 9 & 9 & 52 & 125.6 & $\mathbf{N}$ & degree C/TWINS confirm \\
\hline$\frac{1}{1}$ & $4 / 29 / 75$ & 10 & 1 & 48 & 118.4 & $\mathbf{N}$ & degree C/TWINS confirm \\
\hline 1 & $4 / 29 / 75$ & 11 & 1 & 36 & 96.8 & $\bar{N}$ & degree C/TWINS confirm \\
\hline 1 & $4 / 29 / 75$ & 12 & 1 & 21 & 69.8 & $\mathrm{~N}$ & degree $\mathrm{C} / \mathrm{TW}$ INS confirm \\
\hline 1 & $5 / 20 / 75$ & 1 & 1 & 90 & 194 & $N$ & degree $\mathrm{C} / \mathrm{TW}$ INS confirm \\
\hline 1 & $5 / 20 / 75$ & 2 & 1 & 92 & 197.6 & $\mathbf{Y}$ & degree $\mathrm{C} / \mathrm{T}$ WINS confirm \\
\hline 1 & $5 / 20 / 75$ & 3 & 1 & 88 & 190.4 & $\mathbf{N}$ & degree C/TWINS confirm \\
\hline 1 & $5 / 20 / 75$ & 4 & 1 & 84 & 183.2 & $N$ & degree C/TWINS confirm \\
\hline$\frac{1}{1}$ & $5 / 20 / 75$ & 5 & 1 & 76 & 168.8 & $\mathbf{N}$ & degree C/TWINS confirm \\
\hline 1 & $5 / 20 / 75$ & 6 & 1 & 68 & 154.4 & $\mathbf{N}$ & degree C/TWINS confirm \\
\hline 1 & $5 / 20 / 75$ & 7 & 1 & $60 \div$ & 140 & $\mathbf{N}$ & degree C/TWINS confirm \\
\hline 1 & $5 / 20 / 75$ & 8 & 1 & 58 & 136.4 & $\bar{N}$ & degree $\mathrm{C}$ TWWINS confirm \\
\hline 1 & $5 / 20 / 75$ & 9 & 1 & 56 & 132.8 & $\mathbf{N}$ & degree C/TWINS confirm \\
\hline 1 & $5 / 20 / 75$ & 10 & 1 & 52 & 125.6 & $\mathbf{N}$ & degree $\mathrm{C} T$ WWINS confirm \\
\hline 1 & $5 / 20 / 75$ & 11 & 1 & 42 & 107.6 & $\mathbf{N}$ & degree C/TWINS confirm \\
\hline 1 & $5 / 20 / 75$ & 12 & 1 & 28 & 82.4 & $\mathbf{N}$ & degree C/TWINS confirm \\
\hline 1 & $6 / 17 / 75$ & 1 & 1 & & 192 & $\bar{N}$ & \\
\hline 1 & $6 / 17 / 75$ & 2 & 1 & & 194 & $\bar{Y}$ & \\
\hline 1 & $6 / 17 / 75$ & 3 & 1 & & 188 & $\mathbf{N}$ & \\
\hline 1 & $6 / 17 / 75$ & 4 & 1 & & 180 & $\mathbf{N}$ & \\
\hline 1 & $6 / 17775$ & 5 & 1 & & 167 & $\bar{N}$ & \\
\hline 9 & $6 / 17 / 75$ & 6 & 1 & & 153 & $\mathbf{N}$ & \\
\hline 1 & $6 / 17 / 75$ & 7 & 1 & & 138 & $\mathbf{N}$ & \\
\hline 1 & $6 / 17 / 75$ & 8 & 1 & & 132 & $\mathbf{N}$ & \\
\hline 1 & $6 / 17 / 75$ & 9 & 1 & & 130 & $\mathbf{N}$ & \\
\hline 1 & $6 / 17 / 75$ & 10 & 1 & & 122 & $\mathbf{N}$ & \\
\hline$\frac{1}{1}$ & $6 / 17 / 75$ & 11 & 1 & & 106 & $\mathbf{N}$ & \\
\hline 1 & $6 / 17 / 75$ & 12 & 1 & & 86 & $\mathbf{N}$ & \\
\hline 1 & $7 / 28 / 75$ & 9 & 1 & & 193 & $\mathbf{N}$ & \\
\hline 1 & $7 / 28 / 75$ & 2 & 1 & & 195 & $Y$ & \\
\hline 1 & $7 / 28 / 75$ & 3 & 1 & & 189 & $\mathbf{N}$ & \\
\hline 1 & $7 / 28 / 75$ & 4 & 1 & & 181 & $\mathbf{N}$ & \\
\hline$\frac{1}{1}$ & $7 / 28 / 75$ & 5 & 1 & & 167 & $\mathbf{N}$ & \\
\hline 1 & $7 / 28 / 75$ & 6 & 1 & & 154 & $\mathbf{N}$ & \\
\hline 1 & $7 / 28 / 75$ & 7 & 1 & & 140 & $\mathbf{N}$ & \\
\hline 1 & $7 / 28 / 75$ & 8 & 1 & & 134 & $\mathbf{N}$ & \\
\hline 1 & $7 / 28 / 75$ & 9 & 1 & & 133 & $\mathbf{N}$ & \\
\hline 1 & $7 / 28 / 75$ & 10 & 1 & & 126 & $\mathbf{N}$ & \\
\hline 9 & $7 / 28 / 75$ & 11 & 1 & & 112 & $\mathrm{~N}$ & \\
\hline 1 & $7 / 28 / 75$ & 12 & 1 & & 97 & $\mathbf{N}$ & \\
\hline 1 & $8 / 26 / 75$ & 1 & 1 & & 197 & $\mathbf{N}$ & \\
\hline 9 & $8 / 26 / 75$ & 2 & 1 & & 199 & $Y$ & \\
\hline 1 & $8 / 26 / 75$ & 3 & 1 & & 193 & $\mathbf{N}$ & \\
\hline 1 & $8 / 26 / 75$ & 4 & 1 & & 184 & $\mathrm{~N}$ & \\
\hline 9 & $8 / 26 / 75$ & 5 & 1 & & 171 & $\mathrm{~N}$ & \\
\hline 1 & $8 / 26 / 75$ & 6 & 1 & & 157 & $\mathbf{N}$ & \\
\hline 1 & $8 / 26 / 75$ & 7 & 1 & & 143 & $\mathbf{N}$ & \\
\hline 1 & $8 / 26 / 75$ & 8 & 1 & & 138 & $\mathbf{N}$ & \\
\hline 1 & $8 / 26 / 75$ & 9 & 1 & & 136 & $\mathbf{N}$ & \\
\hline 1 & $8 / 26 / 75$ & 10 & 1 & & 91 & $\mathbf{N}$ & \\
\hline 1 & $8 / 26 / 75$ & 11 & 1 & & 108 & $\mathrm{~N}$ & \\
\hline 1 & $8 / 26 / 75$ & 12 & 1 & & 85 & $\mathbf{N}$ & \\
\hline 1 & $11 / 21 / 75$ & 1 & 1 & & 194 & $\mathbf{N}$ & \\
\hline 1 & $11 / 21 / 75$ & 2 & 1 & & 196 & $Y$ & . \\
\hline 1 & $11 / 21 / 75$ & 3 & 1 & & 189 & $N$ & \\
\hline 1 & $11 / 21 / 75$ & 4 & 1 & & 181 & $\mathbf{N}$ & \\
\hline 1 & $11 / 21 / 75$ & 5 & 1 & & 169 & $\mathbf{N}$ & \\
\hline 1 & $11 / 21 / 75$ & 6 & 1 & & 156 & $\mathbf{N}$ & \\
\hline 1 & $11 / 21 / 75$ & 7 & 1 & & 143 & $\mathbf{N}$ & \\
\hline 1 & $11 / 21 / 75$ & 8 & 1 & & 138 & $\mathrm{~N}$ & \\
\hline 1 & $11 / 21 / 75$ & 9 & 1 & & 135 & $\mathbf{N}$ & \\
\hline 1 & $11 / 21 / 75$ & 10 & 1 & & 128 & $\mathbf{N}$ & \\
\hline 9 & $11 / 21 / 75$ & 11 & 1 & & 110 & $\mathbf{N}$ & \\
\hline 1 & $11 / 21 / 75$ & 12 & 1 & & 82 & $\mathbf{N}$ & \\
\hline
\end{tabular}

Data obtained from WHC Surveillance Analysis Computer System (SACSI, November 12, 1993. 


\begin{tabular}{|c|c|c|c|c|c|c|c|}
\hline \multicolumn{8}{|c|}{ TANK 241-BY-104 } \\
\hline & & & & THEF & AOCOUPL & DATA & \\
\hline TREE\# & DATE & THERMO\# & TREE SET\# & TEMP C & TEMP F & $Y=$ MAXTEMP & COMMENTS \\
\hline 1 & $12 / 22 / 75$ & 1 & 1 & & 193 & $\mathbf{N}$ & \\
\hline 1 & $12 / 22 / 75$ & 2 & 1 & & 196 & $Y$ & \\
\hline 1 & $12 / 22 / 75$ & 3 & 1 & & 189 & $\mathrm{~N}$ & \\
\hline 1 & $12 / 22 / 75$ & 4 & 1 & & 182 & $\mathbf{N}$ & \\
\hline 1 & $12 / 22 / 75$ & 5 & 1 & & 169 & $\mathbf{N}$ & \\
\hline 1 & $12 / 22 / 75$ & 6 & 1 & & 156 & $\mathbf{N}$ & \\
\hline 1 & $12 / 22 / 75$ & 7 & 1 & & 143 & $\mathbf{N}$ & \\
\hline 1 & $12 / 22 / 75$ & 8 & 1 & & 137 & $\mathbf{N}$ & \\
\hline 1 & $12 / 22 / 75$ & 9 & 1 & & 135 & $\mathbf{N}$ & \\
\hline 1 & $12 / 22 / 75$ & 10 & 1 & & 126 & $\bar{N}$ & \\
\hline 1 & $12 / 22 / 75$ & 11 & 1 & & 106 & $N$ & \\
\hline 1 & $3 / 4 / 76$ & 1 & 1 & - & 189 & $\mathbf{N}$ & \\
\hline 1 & $3 / 4 / 76$ & 2 & 1 & & 190 & $\mathbf{Y}$ & \\
\hline 1 & $3 / 4 / 76$ & 3 & 1 & & 184 & $\mathbf{N}$ & \\
\hline 1 & $3 / 4 / 76$ & 4 & 1 & & 176 & $\mathbf{N}$ & \\
\hline 1 & $3 / 4 / 76$ & 5 & 1 & & 162 & $\bar{N}$ & \\
\hline 1 & $3 / 4 / 76$ & 6 & 1 & & 150 & $N$ & \\
\hline 1 & $3 / 4 / 76$ & 7 & 1 & & 136 & $\mathrm{~N}$ & \\
\hline 1 & $3 / 4 / 76$ & 8 & 1 & & 130 & $\mathbf{N}$ & \\
\hline 1 & $3 / 4 / 76$ & 9 & 1 & & 126 & $\mathbf{N}$ & \\
\hline 1 & $3 / 4 / 76$ & 10 & 1 & & 116 & $N$ & \\
\hline 1 & $3 / 4 / 76$ & 11 & 1 & & 97 & $\mathbf{N}$ & \\
\hline 1 & $3 / 4 / 76$ & 12 & 1 & & 90 & $\mathbf{N}$ & \\
\hline 1 & $5 \Pi \pi \Pi 6$ & 1 & 1 & & 182 & $\bar{N}$ & \\
\hline 1 & $5 / 776$ & 2 & 1 & & 183 & $\bar{Y}$ & \\
\hline 1 & $5 / 7 / 76$ & 3 & 1 & & 176 & $\mathbf{N}$ & \\
\hline 1 & $5 \Pi 76$ & 4 & 1 & & 167 & $\mathbf{N}$ & \\
\hline 1 & $5 \Pi 7 \Pi 6$ & 5 & 1 & & 153 & $\mathrm{~N}$ & \\
\hline 1 & $5 / 7 / 76$ & 6 & 1 & & 140 & $\mathbf{N}$ & \\
\hline 1 & $5 \Pi 7 \Pi 6$ & 7 & 1 & & 126 & $N$ & \\
\hline$\frac{1}{1}$ & $5 \Pi 7 \Pi 76$ & 8 & 1 & & 120 & $\mathbf{N}$ & \\
\hline 1 & $5 / 7 / 76$ & 9 & 1 & & 117 & $\bar{N}$ & \\
\hline 1 & $5 \Pi 7 \Pi 6$ & 10 & 1 & & 109 & $\mathrm{~N}$ & \\
\hline 1 & $5 / 7 / 76$ & 11 & 1 & & 94 & $\mathbf{N}$ & \\
\hline 1 & $5 / 7 \pi 76$ & 12 & 1 & & 75 & $\mathbf{N}$ & \\
\hline 1 & $6 / 9 / 76$ & 1 & 1 & & 191 & $\overline{\mathbf{N}}$ & \\
\hline 9 & $6 / 9776$ & 2 & 9 & & 192 & $\bar{Y}$ & \\
\hline 1 & $.6 / 9 / 76$ & 3 & 1 & & 185 & $\mathrm{~N}$ & \\
\hline 1 & $6 / 9 / 76$ & 4 & 1 & & 176 & $\mathbf{N}$ & \\
\hline 1 & $6 / 9 / 76$ & 5 & 1 & & 163 & $\mathbf{N}$ & \\
\hline$\frac{1}{1}$ & $6 / 9 / 76$ & 6 & 9 & & 150 & $\mathbf{N}$ & \\
\hline 1 & $6 / 9 / 76$ & 7 & 1 & & 136 & $N$ & \\
\hline 1 & $6 / 9 / 76$ & 8 & 1 & & 130 & $\mathbf{N}$ & \\
\hline 1 & $6 / 9 / 76$ & 9 & 1 & & 126 & $\mathbf{N}$ & \\
\hline 1 & $6 / 9 / 76$ & 10 & 1 & & 120 & $\mathbf{N}$ & \\
\hline 1 & $6 / 9 / 76$ & 11 & 1 & & 106 & $\mathrm{~N}$ & \\
\hline 9 & $6 / 9 / 76$ & 12 & 1 & & 88 & $\mathbf{N}$ & \\
\hline$i$ & $7 \pi \Pi 76$ & 1 & 1 & & 180 & $\mathbf{N}$ & \\
\hline 1 & $7 \pi 776$ & 2 & 1 & & 181 & $Y$ & \\
\hline 9 & $7 \Pi 7 \pi 6$ & 3 & 1 & & 173 & $\mathbf{N}$ & \\
\hline 1 & $7 \Pi \Pi 76$ & 4 & 1 & & 164 & $\mathbf{N}$ & \\
\hline 1 & $7 / 7776$ & 5 & 1 & & 151 & $\mathbf{N}$ & \\
\hline 1 & $7 / 7776$ & 6 & 1 & & 138 & $\mathbf{N}$ & \\
\hline 1 & $7 \pi 776$ & 7 & 1 & & 125 & $N$ & \\
\hline 1 & $7 \pi \Pi 6$ & 8 & 1 & & 118 & $\mathbf{N}$ & \\
\hline 1 & $7 \pi \Pi 66$ & 9 & 1 & & 116 & $\mathrm{~N}$ & \\
\hline 1 & $7 / 7 \Pi 6$ & 10 & 1 & & 110 & $\mathbf{N}$ & \\
\hline 1 & 717176 & 11 & 1 & & 98 & $N$ & \\
\hline 1 & $7 / 7 / 76$ & 12 & 1 & & 84 & $N$ & \\
\hline 1 & $8 / 14 / 76$ & 1 & 1 & & 174 & $\mathrm{~N}$ & \\
\hline 1 & $8 / 14 / 76$ & 2 & 1 & & 174 & $Y$ & \\
\hline 1 & $8 / 14 / 76$ & 3 & 1 & & 167 & $N$ & \\
\hline 1 & $8 / 14 / 76$ & 4 & 1 & & 158 & $\mathrm{~N}$ & \\
\hline 1 & $8 / 14 / 76$ & 5 & 1 & & 145 & $\mathbf{N}$ & \\
\hline 1 & $8 / 14 / 76$ & 6 & 1 & & 133 & $\mathrm{~N}$ & \\
\hline
\end{tabular}

Data obtained from WHC Surveillance Analysis Computer System (SACS), November 12, 1993. 


\begin{tabular}{|c|c|c|c|c|c|c|c|}
\hline \multicolumn{8}{|c|}{ TANK 241-BY-104 } \\
\hline & & & & THER & 1OCOUPL & $\overline{A T A}$ & \\
\hline TREE\# & DATE & THERMO\# & TREE SET\# & TEMP C & TEMP F & $Y=M A X T E M P$ & COMMENTS \\
\hline 1 & $8 / 14 / 76$ & 7 & 1 & & 120 & $\mathrm{~N}$ & \\
\hline 1 & $8 / 14 / 76$ & 8 & 1 & & 114 & $\mathrm{~N}$ & \\
\hline 1 & $8 / 14 / 76$ & 9 & 1 & & 112 & $\mathbf{N}$ & \\
\hline$\frac{1}{1}$ & $8 / 14 / 76$ & 10 & 1 & & 106 & $\mathbf{N}$ & \\
\hline 1 & $8 / 14 / 76$ & 11 & 1 & & 96 & $\mathbf{N}$ & \\
\hline 1 & $8 / 74 / 76$ & 12 & 1 & & 82 & $\bar{N}$ & \\
\hline 1 & $8 / 28 \pi 76$ & 1 & 1 & & 179 & $\mathbf{N}$ & \\
\hline 1 & $8 / 28 / 76$ & 2 & 1 & & 180 & $\mathbf{Y}$ & \\
\hline 1 & $8 / 28 / 76$ & 3 & 1 & & 172 & $N$ & \\
\hline 9 & $8 / 28 / 76$ & 4 & 1 & & 163 & $\bar{N}$ & \\
\hline 9 & $8 / 28 / 76$ & 5 & 1 & & 150 & $\mathbf{N}$ & \\
\hline 1 & $8 / 28 / 76$ & 6 & 1 & & 138 & $\mathbf{N}$ & \\
\hline 1 & $8 / 28 / 76$ & 7 & 1 & & 125 & $\mathbf{N}$ & \\
\hline$\frac{1}{1}$ & $8 / 28 / 76$ & 8 & 1 & & 119 & $\mathbf{N}$ & \\
\hline 9 & $8 / 28 / 76$ & 9 & 1 & & 118 & $\bar{N}$ & \\
\hline 1 & $8 / 28 / 76$ & 10 & 1 & & 912 & $\mathbf{N}$ & \\
\hline 1 & $8 / 28 / 76$ & 11 & 1 & & 102 & $\mathbf{N}$ & \\
\hline 1 & $8 / 28 / 76$ & 92 & 1 & & 87 & $\mathbf{N}$ & \\
\hline$\frac{1}{1}$ & $9 / 13 / 76$ & 9 & $T$ & & 180 & $\bar{N}$ & \\
\hline 1 & $9 / 13 / 76$ & 2 & 1 & & 181 & $\bar{Y}$ & \\
\hline 1 & $9 / 13 / 76$ & 3 & 1 & & 173 & $\mathbf{N}$ & \\
\hline 1 & $9 / 13 / 76$ & 4 & 1 & & 164 & $\mathbf{N}$ & \\
\hline$\frac{1}{1}$ & $9 / 13 / 76$ & 5 & 1 & & 150 & $\mathbf{N}$ & \\
\hline 1 & $9 / 13 / 76$ & 6 & 1 & & 138 & $\bar{N}$ & \\
\hline 1 & $9 / 13 / 76$ & 7 & 1 & & 126 & $\mathbf{N}$ & \\
\hline 1 & $9 / 13 / 76$ & 8 & 1 & & 120 & $\mathbf{N}$ & \\
\hline$\frac{1}{1}$ & $9 / 13 / 76$ & 9 & 1 & & 118 & $\mathbf{N}$ & \\
\hline 1 & $9 / 13 / 76$ & 10 & 1 & & 112 & $\mathbf{N}$ & it \\
\hline 1 & $9 / 13 / 76$ & 11 & 1 & & 102 & $\mathbf{N}$ & $\therefore$ \\
\hline 1 & $9 / 13 / 76$ & 12 & 1 & & 86 & $\mathbf{N}$ & \\
\hline 1 & $11 / 7 / 76$ & 1 & 1 & & 179 & $\mathbf{N}$ & \\
\hline$\frac{1}{1}$ & $11 / 7 / 76$ & 2 & $\frac{1}{1}$ & & 180 & $\bar{Y}$ & \\
\hline 1 & $11 / 7 / 76$ & 3 & 1 & & 172 & $\mathbf{N}$ & \\
\hline 1 & $11 / 7 / 76$ & 4 & 1 & & 164 & $\mathbf{N}$ & \\
\hline 1 & $11 / 7 / 76$ & 5 & 1 & & 152 & $\mathbf{N}$ & \\
\hline 1 & $11 / 7 / 76$ & 6 & 1 & & 140 & $\mathbf{N}$ & \\
\hline 1 & $11 / 7 / 76$ & 7 & 1 & & 107 & $\mathbf{N}$ & \\
\hline 1 & $.11 / 7 / 76$ & 8 & 1 & & 121 & $\mathbf{N}$ & \\
\hline$\frac{1}{1}$ & $11 / 7 / 76$ & 9 & 9 & & 121 & $\mathrm{~N}$ & \\
\hline 1 & $11 / 7 \pi 6$ & 10 & 1 & & 116 & $\mathbf{N}$ & \\
\hline 1 & $11 / 7 / 76$ & 11 & 1 & & 103 & $N$ & \\
\hline 1 & $11 / 7 / 76$ & 12 & 1 & & 79 & $\mathrm{~N}$ & \\
\hline 1 & $12 / 9 / 76$ & 1 & 1 & & 200 & $\mathbf{N}$ & \\
\hline 1 & $12 / 9 / 76$ & 2 & 1 & & 200 & $Y$ & \\
\hline 1 & $12 / 9 / 76$ & 3 & 1 & & 193 & $\mathbf{N}$ & \\
\hline 1 & $12 / 9 / 76$ & 4 & 1 & & 185 & $\mathbf{N}$ & \\
\hline 1 & $12 / 9 / 76$ & 5 & 1 & & 172 & $N$ & \\
\hline 1 & $12 / 9 / 76$ & 6 & 1 & & 160 & $\mathbf{N}$ & \\
\hline 1 & $12 / 9 / 76$ & 7 & 1 & & 148 & $\mathbf{N}$ & \\
\hline 1 & $12 / 9 / 76$ & 8 & 9 & & 142 & $\mathbf{N}$ & \\
\hline 1 & $12 / 9 / 76$ & 9 & 1 & & 140 & $\mathbf{N}$ & \\
\hline 1 & $12 / 9 / 76$ & 10 & 1 & & 127 & $\bar{N}$ & \\
\hline 1 & $12 / 9 / 76$ & 11 & 1 & & 103 & $\mathbf{N}$ & \\
\hline 1 & $12 / 9 / 76$ & 12 & 1 & & 95 & $\mathbf{N}$ & \\
\hline 1 & $1 / 3 / 77$ & 1 & 1 & & 991 & $\bar{Y}$ & \\
\hline 1 & $1 / 3 / 77$ & 2 & 1 & & 185 & $\mathbf{N}$ & \\
\hline 1 & $1 / 3 / 77$ & 3 & 1 & & 176 & $\mathbf{N}$ & \\
\hline 1 & $1 / 3 / 77$ & 4 & 1 & & 164 & $\mathbf{N}$ & \\
\hline$T$ & $1 / 3 / 77$ & 5 & 1 & & 151 & $N$ & \\
\hline 1 & $1 / 3 / 77$ & 6 & 1 & & 139 & $\mathbf{N}$ & \\
\hline 9 & $1 / 3 / 77$ & 7 & 1 & & 133 & $\mathrm{~N}$ & \\
\hline 1 & $1 / 3 / 77$ & 8 & 1 & & 131 & $\mathbf{N}$ & \\
\hline 1 & $1 / 3 / 77$ & 9 & 1 & & 118 & $\mathbf{N}$ & \\
\hline 1 & $1 / 3 / 77$ & 10 & 1 & & 94 & $\mathrm{~N}$ & \\
\hline 1 & $1 / 3 / 77$ & 11 & 1 & & 84 & $N$ & \\
\hline
\end{tabular}

Data obtained from WHC Surveillance Analysis Computer System (SACS), November 12, 1993. 


\begin{tabular}{|c|c|c|c|c|c|c|c|}
\hline \multicolumn{8}{|c|}{ TANK 241-BY-104 } \\
\hline \multicolumn{8}{|c|}{ THERMOCOUPLE DATA } \\
\hline TREE\# & DATE & THERMO\# & TREE SET\# & TEMP C & TEMP F & $Y=$ MAXTEMP & COMMENTS \\
\hline 1 & $1 / 3 / 77$ & 12 & 1 & & & $\mathrm{~N}$ & \\
\hline 1 & $2 / 26 / 77$ & 1 & 1 & & 176 & $Y$ & \\
\hline 1 & $2 / 26 / 77$ & 2 & 1 & & 176 & $\mathbf{N}$ & \\
\hline 1 & $2 / 26 / 77$ & 3 & 1 & & 169 & $\mathbf{N}$ & \\
\hline 1 & $2 / 26 / 77$ & 4 & 1 & & 160 & $\mathbf{N}$ & \\
\hline 9 & $2 / 26 / 77$ & 5 & 1 & & 148 & $\mathrm{~N}$ & \\
\hline 9 & $2 / 26 / 77$ & 6 & 1 & & 137 & $\bar{N}$ & \\
\hline 1 & $2 / 26 / 77$ & 7 & 1 & & 125 & $\mathbf{N}$ & \\
\hline 1 & $2 / 26 / 77$ & 8 & 1 & & 119 & $\mathbf{N}$ & \\
\hline 1 & $2 / 26 / 77$ & 9 & 1 & & 114 & $\mathbf{N}$ & \\
\hline 1 & $2 / 26 / 77$ & 10 & 1 & & 94 & $\mathbf{N}$ & \\
\hline 1 & $2 / 26 / 77$ & 11 & 1 & & 75 & $\bar{N}$ & \\
\hline 1 & $2 / 26 / 77$ & 12 & 1 & & 74 & $\mathrm{~N}$ & \\
\hline 1 & $3 / 28 / 77$ & 1 & 1 & & 178 & $\mathrm{~N}$ & \\
\hline 1 & $3 / 28 / 77$ & 2 & 1 & & 179 & $\bar{Y}$ & \\
\hline 1 & $3 / 28 / 77$ & 3 & 1 & & 172 & $\mathbf{N}$ & \\
\hline 1 & $3 / 28 / 77$ & 4 & 1 & & 163 & $\mathrm{~N}$ & \\
\hline 9 & $3 / 28 / 77$ & 5 & 1 & & 152 & $\mathbf{N}$ & \\
\hline 1 & $3 / 28 / 77$ & 6 & 1 & & 140 & $\bar{N}$ & \\
\hline 1 & $3 / 28 / 77$ & 7 & 1 & & 127 & $\mathbf{N}$ & \\
\hline 1 & $3 / 28 / 77$ & 8 & 1 & & 124 & $\mathbf{N}$ & \\
\hline 1 & $3 / 28 / 77$ & 9 & 1 & & 117 & $\mathrm{~N}$ & \\
\hline 1 & $3 / 28 / 77$ & 10 & 1 & & 99 & $\mathbf{N}$ & \\
\hline 1 & $3 / 28 / 77$ & 11 & 1 & & 81 & $N$ & \\
\hline 1 & $3 / 28 / 77$ & 12 & 1 & & 80 & $\mathbf{N}$ & \\
\hline 1 & $4 / 27 / 77$ & 1 & 1 & & 176 & $\mathbf{N}$ & \\
\hline 1 & $4 / 27 / 77$ & 2 & 1 & & 177 & $Y$ & \\
\hline 1 & $4 / 27 / 77$ & 3 & 1 & & 170 & $\dot{N}$ & \\
\hline 1 & $4 / 27 / 77$ & 4 & 1 & & 161 & $\mathbf{N}$ & \\
\hline 1 & $4 / 27 / 77$ & 5 & 1 & & 149 & $\mathrm{~N}$ & \\
\hline 1 & $4 / 27 / 77$ & 6 & 1 & & 137 & $\mathrm{~N}$ & \\
\hline 1 & $4 / 27 / 77$ & 7 & 1 & & 126 & $\mathrm{~N}$ & \\
\hline 1 & $4 / 27 / 77$ & 8 & 1 & & 120 & $\mathrm{~N}$ & \\
\hline 1 & $4 / 27 / 77$ & 9 & 1 & & 115 & $\mathrm{~N}$ & \\
\hline 1 & $4 / 27 / 77$ & 10 & 1 & & 98 & $N$ & \\
\hline 1 & $4 / 27 / 77$ & 1.1 & 1 & & 83 & $N$ & \\
\hline 1 & $4 / 27 / 77$ & 12 & 1 & & 82 & $\mathbf{N}$ & \\
\hline 1 & $6 / 30 / 77$ & 1 & 1 & & 976 & $\mathrm{~N}$ & \\
\hline$\frac{1}{1}$ & $6 / 30 / 77$ & 2 & 1 & & 176 & $Y$ & \\
\hline 1 & $6 / 30 / 77$ & 3 & 1 & & 169 & $\bar{N}$ & \\
\hline 1 & $6 / 30 / 77$ & 4 & 1 & & 161 & $\mathbf{N}$ & \\
\hline 1 & $6 / 30 / 77$ & 5 & 1 & & 150 & $\bar{N}$ & \\
\hline 1 & $6 / 30 / 77$ & 6 & 1 & & 139 & $\mathbf{N}$ & \\
\hline 1 & $6 / 30 / 77$ & 7 & 1 & & 128 & $\bar{N}$ & \\
\hline 1 & $6 / 30 / 77$ & 8 & 1 & & 163 & $\mathbf{N}$ & \\
\hline 1 & $6 / 30 / 77$ & 9 & 1 & & 118 & $\mathbf{N}$ & \\
\hline 1 & $6 / 30 / 77$ & 10 & 1 & & 102 & $\mathbf{N}$ & \\
\hline 1 & $6 / 30 / 77$ & 11 & 1 & & 85 & $\mathbf{N}$ & \\
\hline 1 & $6 / 30 / 77$ & 12 & 1 & & 84 & $\mathbf{N}$ & \\
\hline 1 & $8 / 15 / 77$ & 1 & 1 & & 167 & $Y$ & \\
\hline$\frac{1}{1}$ & $8 / 15 / 77$ & 2 & $\frac{1}{1}$ & & 167 & $\mathbf{N}$ & \\
\hline 1 & $8 / 15 / 77$ & 3 & 1 & & 160 & $\mathbf{N}$ & \\
\hline 1 & $8 / 15 / 77$ & 4 & 1 & & 152 & $N$ & \\
\hline 1 & $8 / 15 / 77$ & 5 & 1 & & 141 & $\mathrm{~N}$ & \\
\hline$\frac{1}{1}$ & $8 / 15 / 77$ & 6 & $\frac{1}{1}$ & & 130 & $\mathbf{N}$ & \\
\hline 1 & $8 / 15 / 77$ & 7 & $\frac{1}{1}$ & & 120 & $\overline{\mathbf{N}}$ & \\
\hline 1 & $8 / 15 / 77$ & 8 & 1 & & 114 & $\mathbf{N}$ & \\
\hline 1 & $8 / 15 / 77$ & 9 & 1 & & 109 & $\mathbf{N}$ & \\
\hline 1 & $8 / 15 / 77$ & 10 & 1 & & 96 & $N$ & \\
\hline$\frac{1}{1}$ & $8 / 15 / 77$ & 11 & 1 & & 83 & $\mathbf{N}$ & \\
\hline 1 & $8 / 15 \pi 7$ & 12 & 1 & & 82 & $\mathbf{N}$ & \\
\hline 1 & $9 / 12 \pi 7$ & 1 & 1 & & 163 & $\mathbf{N}$ & \\
\hline 1 & $9 / 12 / 77$ & 2 & 1 & & 164 & $\bar{Y}$ & \\
\hline 1 & $9 / 12 / 77$ & 3 & 1 & & 154 & $N$ & \\
\hline 1 & $9 / 12 / 77$ & 4 & 1 & & 149 & $N$ & \\
\hline
\end{tabular}

Data obtained from WHC Surveillance Analysis Computer System (SACS), November 12, 1993. 


\begin{tabular}{|c|c|c|c|c|c|c|c|}
\hline \multicolumn{8}{|c|}{ TANK 241-BY-104 } \\
\hline & & & & InE & OOCOUPL & & \\
\hline TREE\# & DATE & THERMO\# & TREE SET \# & TEMP C & TEMP F & $Y=$ MAXTEMP & COMMENTS \\
\hline 1 & $9 / 12 / 77$ & 5 & 1 & & 138 & $\mathbf{N}$ & \\
\hline 1 & $9 / 12 / 77$ & 6 & 1 & & 127 & $\mathbf{N}$ & \\
\hline 1 & $9 / 12 / 77$ & 7 & 1 & & 117 & $\mathbf{N}$ & \\
\hline 1 & $9 / 12 / 77$ & 8 & 1 & & 113 & $\bar{N}$ & \\
\hline 1 & $9 / 12 / 77$ & 9 & 1 & & 108 & $\mathbf{N}$ & \\
\hline 1 & $9 / 12 / 77$ & 10 & 1 & & 91 & $N$ & \\
\hline 1 & $9 / 12 / 77$ & 11 & 1 & & 75 & $\mathrm{~N}$ & \\
\hline 1 & $9 / 12 / 77$ & 12 & 1 & & 75 & $\mathbf{N}$ & \\
\hline 1 & $10 / 7 / 77$ & 1 & 1 & & 171 & $\mathbf{N}$ & \\
\hline 1 & $10 / 7 / 77$ & 2 & 9 & & 173 & $\bar{Y}$ & \\
\hline 1 & $10 / 7 / 77$ & 3 & 1 & & 166 & $\mathbf{N}$ & \\
\hline 1 & $10 / 7 / 77$ & 4 & 1 & 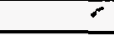 & 158 & $\mathbf{N}$ & \\
\hline 1 & $10 / 7 / 77$ & 5 & 1 & & 147 & $\mathbf{N}$ & \\
\hline 1 & $10 / 7 / 77$ & 6 & 1 & & 136 & $\bar{N}$ & \\
\hline 1 & $10 / 7177$ & 7 & 1 & & 126 & $\mathbf{N}$ & \\
\hline 1 & $10 / 7 / 77$ & 8 & 1 & & 121 & $\mathbf{N}$ & \\
\hline 1 & $10 / 7 / 77$ & 9 & 1 & & 118 & $N$ & \\
\hline 1 & $10 / 7 / 77$ & 10 & 1 & & 98 & $\mathbf{N}$ & \\
\hline 1 & $10 / 7 / 77$ & 11 & 9 & & 77 & $N$ & \\
\hline 1 & $10 / 7 / 77$ & 12 & 1 & & 76 & $\mathbf{N}$ & \\
\hline 1 & $12 / 7 / 77$ & 1 & 1 & & 172 & $\bar{Y}$ & \\
\hline 1 & $12 / 7 / 77$ & 2 & 1 & & 170 & $\mathbf{N}$ & \\
\hline 1 & $12 / 7 / 77$ & 3 & 1 & & 172 & $\mathbf{N}$ & \\
\hline 1 & $12 \sqrt{777}$ & 4 & 1 & & 165 & $\bar{N}$ & \\
\hline 1 & $12 / 7 / 77$ & 5 & 1 & & 156 & $\mathbf{N}$ & \\
\hline 1 & $12 / 7 / 77$ & 6 & 1 & & 146 & $N$ & \\
\hline 1 & $12 / 7 / 77$ & 7 & 1 & & 135 & $N$ & \\
\hline 1 & $12 / 7 / 77$ & 8 & 1 & & 124 & $\mathbf{N}$ & \\
\hline 1 & $12 / 7 / 77$ & 9 & 1 & & 119 & $\overline{\mathbf{N}}$ & \\
\hline 1 & $12 / 7 / 77$ & 10 & 1 & & 113 & $\mathbf{N}$ & \\
\hline 1 & $12 / 7 / 77$ & 11 & 1 & & 89 & $N$ & \\
\hline 1 & $12 / 7 / 77$ & 12 & 1 & & 64 & $\mathbf{N}$ & \\
\hline 1 & $2 / 26 / 78$ & 1 & 1 & & 183 & $N$ & \\
\hline 1 & $2 / 26 / 78$ & 2 & 1 & & 184 & $\mathbf{Y}$ & \\
\hline 9 & $2 / 26 / 78$ & 3 & 1 & & 176 & $\mathbf{N}$ & \\
\hline 1 & $2 / 26 / 78$ & 4. & 1 & & 168 & $\mathbf{N}$ & \\
\hline 1 & $2 / 26 / 78$ & 5 & 1 & & 157 & $\mathbf{N}$ & \\
\hline 1 & $.2 / 26 / 78$ & 6 & 1 & & 145 & $\mathbf{N}$ & \\
\hline$\frac{1}{1}$ & $2 / 26 / 78$ & 7 & 1 & & 134 & $\mathbf{N}$ & \\
\hline 9 & $2 / 26 / 78$ & 8 & 1 & & 128 & $\overline{\mathbf{N}}$ & \\
\hline 1 & $2 / 26 / 78$ & 9 & 1 & & 118 & $\mathbf{N}$ & \\
\hline 1 & $2 / 26 / 78$ & 10 & 1 & & 95 & $\mathbf{N}$ & \\
\hline 1 & $2 / 26 / 78$ & 11 & 1 & & 71 & $N$ & \\
\hline 1 & $2 / 26 / 78$ & 12 & 1 & & 70 & $\mathbf{N}$ & \\
\hline 1 & $3 / 24 / 78$ & 1 & 1 & & 169 & $N$ & \\
\hline 1 & $3 / 24 / 78$ & 2 & 1 & & 170 & $\bar{Y}$ & \\
\hline 1 & $3 / 24 / 78$ & 3 & 1 & & 163 & $\bar{N}$ & \\
\hline 1 & $3 / 24 / 78$ & 4 & 1 & & 154 & $\mathbf{N}$ & \\
\hline 1 & $3 / 24 / 78$ & 5 & 1 & & 143 & $\mathbf{N}$ & \\
\hline$\frac{1}{1}$ & $3 / 24 / 78$ & 6 & 1 & & 132 & $\mathbf{N}$ & \\
\hline 1 & $3 / 24 / 78$ & 7 & 1 & & 121 & $\mathbf{N}$ & \\
\hline 1 & $3 / 24 / 78$ & 8 & 1 & & 114 & $\mathbf{N}$ & \\
\hline 1 & $3 / 24 / 78$ & 9 & 1 & & 104 & $\mathbf{N}$ & \\
\hline 1 & $3 / 24 / 78$ & 10 & 1 & & 83 & $\mathbf{N}$ & \\
\hline 1 & $3 / 24 / 78$ & 11 & 1 & & 63 & $\mathbf{N}$ & \\
\hline 1 & $3 / 24 / 78$ & 12 & 1 & & 62 & $\mathbf{N}$ & \\
\hline 9 & $4 / 23 / 78$ & 1 & 1 & & 175 & $\bar{Y}$ & \\
\hline 1 & $4 / 23 / 78$ & 2 & 1 & & 174 & $\mathbf{N}$ & \\
\hline 1 & $4 / 23 / 78$ & 3 & 1 & & 166 & $\mathbf{N}$ & \\
\hline 1 & $4 / 23 / 78$ & 4 & 9 & & 157 & $\mathrm{~N}$ & \\
\hline 1 & $4 / 23 / 78$ & 5 & 1 & & 145 & $\mathbf{N}$ & \\
\hline 1 & $4 / 23 / 78$ & 6 & 1 & & 134 & $\mathbf{N}$ & \\
\hline 1 & $4 / 23 / 78$ & 7 & 1 & & 123 & $\bar{N}$ & \\
\hline 1 & $4 / 23 / 78$ & 8 & 1 & & 116 & $\mathrm{~N}$ & \\
\hline 1 & $4 / 23 / 78$ & 9 & 9 & & 108 & $N$ & \\
\hline
\end{tabular}

Data obtained from WHC Surveillance Analysis Computer System (SACS), November 12, 1993. 


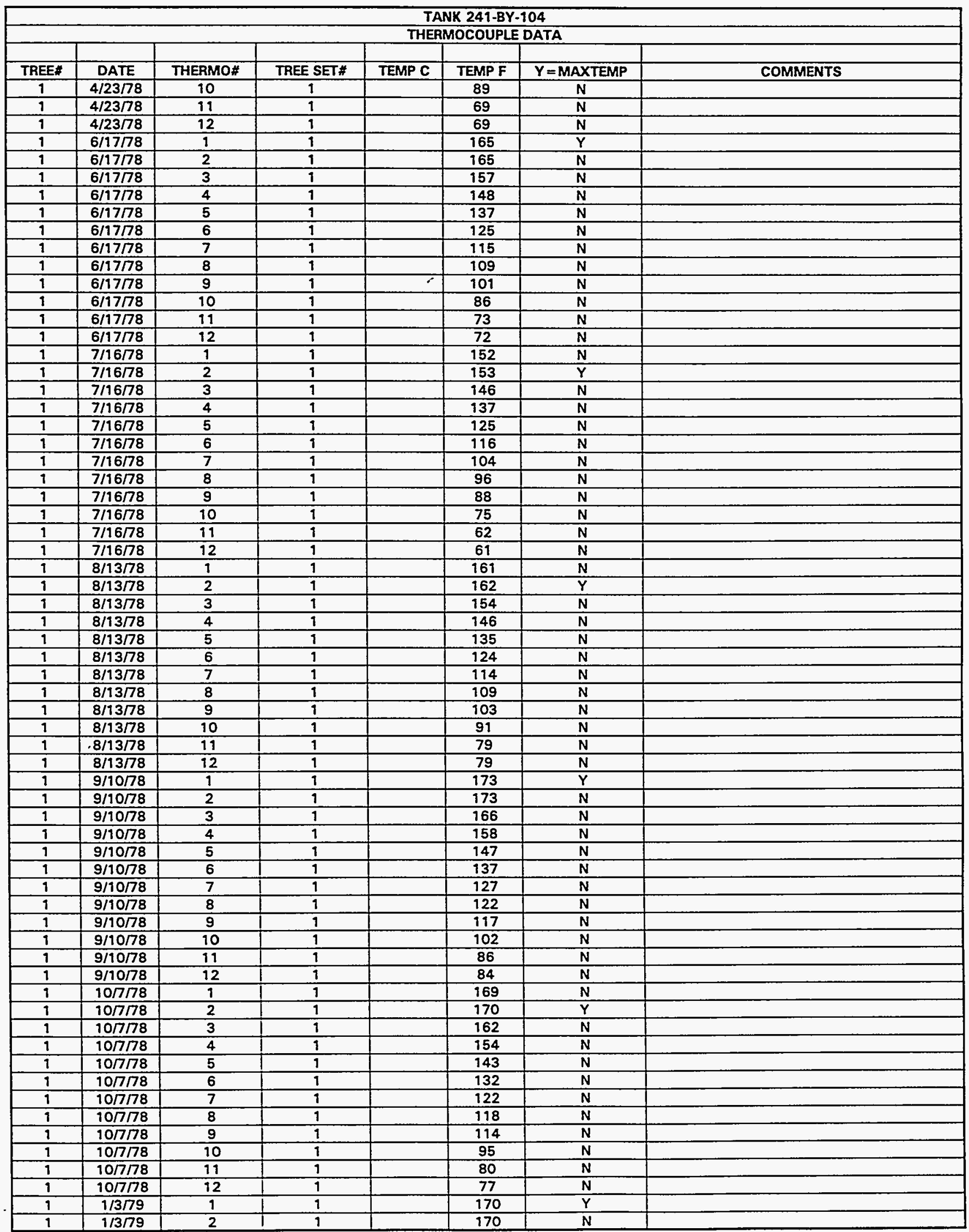

Data obtained from WHC Surveillance Analysis Computer System (SACS), November 12, 1993. 


\begin{tabular}{|c|c|c|c|c|c|c|c|}
\hline \multicolumn{8}{|c|}{ TANK 241-BY-104 } \\
\hline & & & & THER & AOCOUPL & DATA & \\
\hline TREE\# & DATE & THERMO\# & TREE SET\# & TEMP C & TEMP F & $Y=$ MAXTEMP & COMMENTS \\
\hline 1 & $1 / 3 / 79$ & 3 & 1 & & 163 & $N$ & \\
\hline 1 & $1 / 3 / 79$ & 4 & 9 & & 134 & $\mathrm{~N}$ & \\
\hline 1 & $1 / 3 / 79$ & 5 & 1 & & 144 & $\mathbf{N}$ & \\
\hline 1 & $1 / 3 / 79$ & 6 & 1 & & 133 & $\overline{\mathbf{N}}$ & \\
\hline 1 & $1 / 3 / 79$ & 7 & 1 & & 122 & $\mathbf{N}$ & \\
\hline 1 & $1 / 3 / 79$ & 8 & 9 & & 115 & $N$ & \\
\hline 1 & $1 / 3 / 79$ & 9 & 1 & & 110 & $N$ & \\
\hline 1 & $1 / 3 / 79$ & 10 & 1 & & 84 & $\mathbf{N}$ & \\
\hline 1 & $1 / 3 / 79$ & 11 & 9 & & 53 & $\bar{N}$ & \\
\hline 1 & $1 / 3 / 79$ & 12 & 1 & & 51 & $\mathbf{N}$ & \\
\hline 1 & $4 / 22 / 79$ & 1 & 1 & & 167 & $\bar{Y}$ & \\
\hline 1 & $4 / 22 / 79$ & 2 & 1 & $\therefore$ & 160 & $\mathbf{N}$ & \\
\hline 1 & $4 / 22 / 79$ & 3 & 1 & & 161 & $\mathbf{N}$ & \\
\hline 1 & $4 / 22 / 79$ & 4 & 1 & & 152 & $\mathbf{N}$ & \\
\hline 1 & $4 / 22 / 79$ & 5 & 1 & & 141 & $\bar{N}$ & \\
\hline 1 & $4 / 22 / 79$ & 6 & 1 & & 130 & $\mathbf{N}$ & \\
\hline 1 & $4 / 22 / 79$ & 7 & 1 & & 120 & $\mathrm{~N}$ & \\
\hline 1 & $4 / 22 / 79$ & 8 & 1 & & 113 & $\mathrm{~N}$ & \\
\hline 1 & $4 / 22 / 79$ & 9 & 1 & & 105 & $\mathbf{N}$ & \\
\hline 1 & $4 / 22 / 79$ & 10 & 1 & & 85 & $N$ & \\
\hline 1 & $4 / 22 / 79$ & 11 & 1 & & 68 & $N$ & \\
\hline 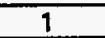 & $4 / 22 / 79$ & 12 & 1 & & 67 & $\mathrm{~N}$ & \\
\hline 1 & $7 \pi / 79$ & 1 & 1 & & 151 & $\mathbf{N}$ & \\
\hline 1 & $7 / 7 / 79$ & 2 & 1 & & 158 & $\bar{Y}$ & \\
\hline 1 & $7 \pi \Pi 79$ & 3 & 1 & & 151 & $N$ & \\
\hline 1 & $7 \Pi 779$ & 4 & 1 & & 142 & $\mathrm{~N}$ & \\
\hline 1 & $7 / 7 / 79$ & 5 & 1 & & 132 & $\mathbf{N}$ & \\
\hline 1 & 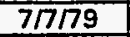 & 6 & 1 & & 121 & $\mathbf{N}$ & \\
\hline 1 & $7 / 7 / 79$ & 7 & 1 & & 111 & $N$ & \\
\hline 1 & $7 / 7 / 79$ & 8 & 1 & & 105 & $\mathrm{~N}$ & \\
\hline 1 & $7 / 7 / 79$ & 9 & 1 & & 100 & $\mathrm{~N}$ & \\
\hline 1 & $7 / 7 / 79$ & 10 & 1 & & 81 & $\mathbf{N}$ & \\
\hline 1 & $7 / 7 / 79$ & 11 & 1 & & 76 & $N$ &. \\
\hline 1 & $7 / 779$ & 12 & 1 & & 76 & $\mathbf{N}$ & \\
\hline 9 & $8 / 1 / 79$ & 1 & 1 & & 163 & $\mathbf{N}$ & \\
\hline 1 & $8 / 1 / 79$ & 2 & 1 & & 164 & $Y$ & \\
\hline 1 & $8 / 1 / 79$ & 3 & $\overline{1}$ & & 157 & $\mathbf{N}$ & \\
\hline 1 & $8 / 1 / 79$ & 4 & 1 & & 148 & $\mathbf{N}$ & \\
\hline 1 & $8 / 1 / 79$ & 5 & 1 & & 138 & $\mathbf{N}$ & \\
\hline 1 & $8 / 1 / 79$ & 6 & 1 & & 127 & $\mathbf{N}$ & \\
\hline 1 & $8 / 1 / 79$ & 7 & 1 & & 117 & $\mathbf{N}$ & \\
\hline 1 & $8 / 1 / 79$ & 8 & 1 & & 112 & $\mathbf{N}$ & \\
\hline 1 & $8 / 1 / 79$ & 9 & 1 & & 107 & $\mathbf{N}$ & \\
\hline 1 & $8 / 1 / 79$ & 10 & 1 & & 95 & $\mathbf{N}$ & \\
\hline 1 & $8 / 1 / 79$ & 11 & 1 & & 84 & $\mathbf{N}$ & \\
\hline 1 & $8 / 1 / 79$ & 12 & 1 & & 83 & $\bar{N}$ & \\
\hline 1 & $9 / 4 / 79$ & 1 & 1 & & 155 & $N$ & \\
\hline 1 & $9 / 4 / 79$ & 2 & 1 & & 157 & $\bar{Y}$ & \\
\hline 1 & $9 / 4 / 79$ & 3 & 1 & & 150 & $\mathbf{N}$ & \\
\hline 1 & $9 / 4 / 79$ & 4 & 1 & & 141 & $\mathbf{N}$ & \\
\hline 1 & $9 / 4 / 79$ & 5 & 1 & & 130 & $\mathbf{N}$ & \\
\hline 1 & $9 / 4 / 79$ & 6 & 1 & & 120 & $N$ & \\
\hline 1 & $9 / 4 / 79$ & 7 & 1 & & 111 & $N$ & \\
\hline 1 & $9 / 4 / 79$ & 8 & 1 & & 106 & $\mathrm{~N}$ & \\
\hline 1 & $9 / 4 / 79$ & 9 & 1 & & 102 & $\mathbf{N}$ & \\
\hline 1 & $9 / 4 / 79$ & 10 & 1 & & 90 & $\mathbf{N}$ & \\
\hline 1 & $9 / 4 / 79$ & 11 & 1 & & 76 & $\mathbf{N}$ & \\
\hline 1 & $9 / 4 / 79$ & 12 & 1 & & 75 & $\mathbf{N}$ & \\
\hline 1 & $11 / 15 / 79$ & 1 & 1 & & 165 & $\mathbf{Y}$ & \\
\hline 1 & $11 / 15 / 79$ & 2 & 1 & & 165 & $\mathbf{N}$ & \\
\hline 1 & $11 / 15 / 79$ & 3 & 1 & & 159 & $\mathbf{N}$ & \\
\hline 1 & $11 / 15 / 79$ & 4 & 1 & & 150 & $\mathrm{~N}$ & \\
\hline 1 & $11 / 15 / 79$ & 5 & 1 & & 141 & $\mathbf{N}$ & \\
\hline 1 & $11 / 15 / 79$ & 6 & 1 & & 132 & $N$ & \\
\hline 1 & $11 / 15 / 79$ & 7 & 1 & & 122 & $\mathbf{N}$ & \\
\hline
\end{tabular}

Data obtained from WHC Surveillance Analysis Computer System (SACS), November 12, 1993. 


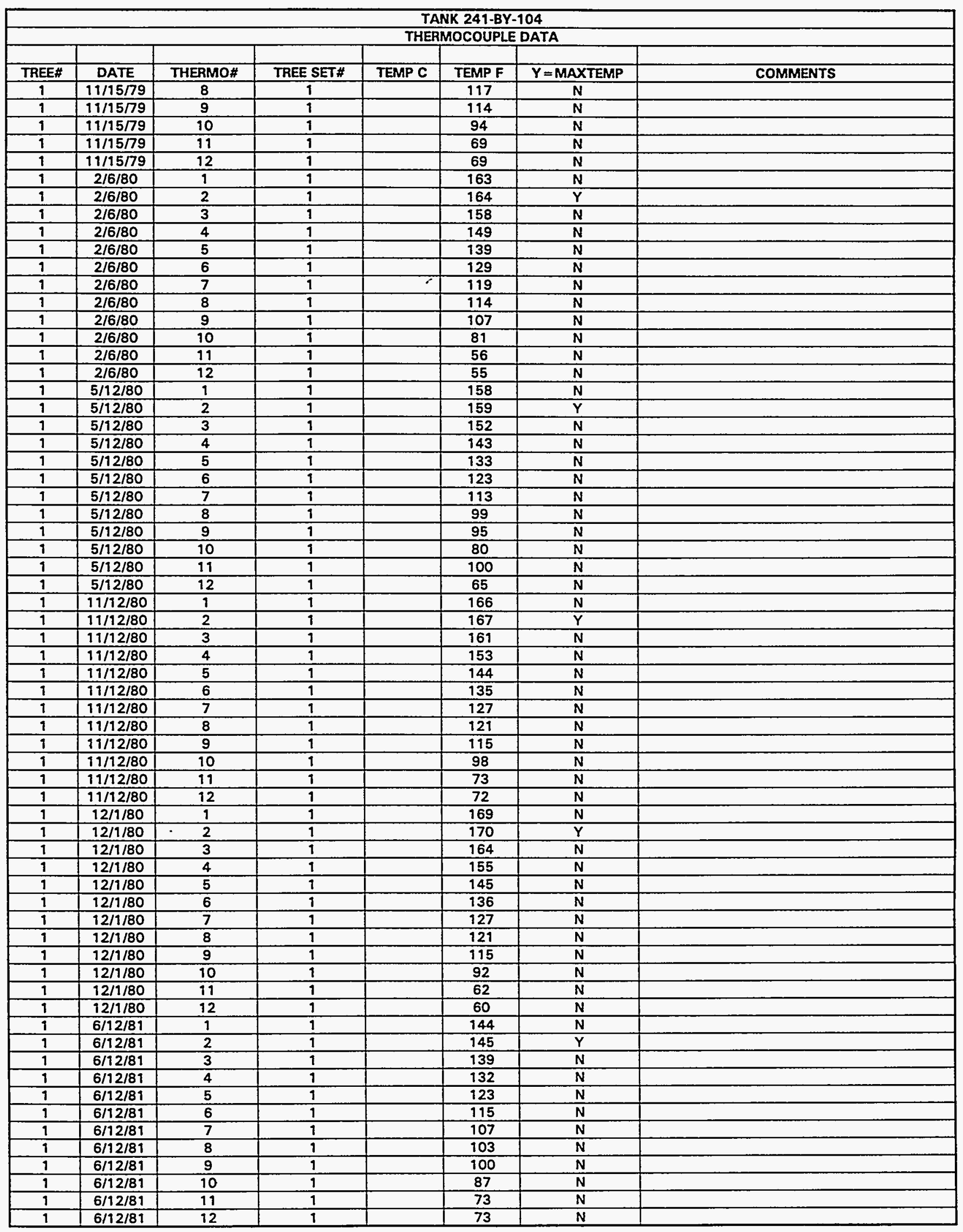

Data obtained from WHC Surveillance Analysis Computer System (SACS), November 12, 1993. 
WHC-SD-WM-ER-312, Rev. 0

\begin{tabular}{|c|c|c|c|c|c|c|c|}
\hline \multirow{2}{*}{\multicolumn{8}{|c|}{ TANK 241-BY-104 }} \\
\hline & & & & & & & \\
\hline & & & & & & & \\
\hline TREE\# & DATE & THERMO\# & TREE SET\# & TEMPC & TEMPF & $\mathrm{Y}=\mathrm{MAXTEMP}$ & COMMENTS \\
\hline 1 & $11 / 11 / 81$ & 1 & 1 & & 129 & $N$ & \\
\hline 1 & $11 / 11 / 81$ & 2 & 1 & & 130 & $\bar{Y}$ & \\
\hline 1 & $11 / 111 / 81$ & 3 & 1 & & 124 & $\mathrm{~N}$ & \\
\hline 1 & $11 / 11 / 81$ & 4 & 1 & & 118 & $\mathbf{N}$ & \\
\hline 1 & $11 / 11 / 81$ & 5 & 1 & & 108 & $\mathbf{N}$ & 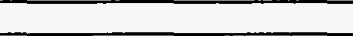 \\
\hline 1 & $11 / 11 / 81$ & 6 & 1 & & 102 & $\mathbf{N}$ & \\
\hline 1 & $11 / 11 / 81$ & 7 & 1 & & 89 & $\mathrm{~N}$ & \\
\hline 1 & $11 / 11 / 81$ & 8 & 1 & & 84 & $\mathbf{N}$ & \\
\hline 1 & $11 / 11 / 81$ & 9 & 1 & & 81 & $\bar{N}$ & \\
\hline 1 & $11 / 11 / 81$ & 10 & 1 & & 79 & $\mathrm{~N}$ & \\
\hline$\frac{1}{1}$ & $11 / 11 / 81$ & 19 & $\frac{1}{1}$ & & 55 & $\mathbf{N}$ & \\
\hline 1 & $11 / 11 / 81$ & 12 & 1 & $\div$ & 54 & $N$ & \\
\hline 1 & $1 / 14 / 82$ & 1 & 1 & & 158 & $\mathrm{~N}$ & \\
\hline 1 & $1 / 14 / 82$ & 2 & 1 & & 159 & $\bar{Y}$ & \\
\hline 1 & $1 / 14 / 82$ & 3 & 1 & & 155 & $\mathbf{N}$ & \\
\hline 1 & $1 / 14 / 82$ & 4 & 1 & & 147 & $\mathbf{N}$ & \\
\hline 1 & $1 / 14 / 82$ & 5 & 1 & & 137 & $N$ & \\
\hline 1 & $1 / 14 / 82$ & 6 & 1 & & 130 & $\mathbf{N}$ & \\
\hline 1 & $1 / 14 / 82$ & 7 & 1 & & 122 & $N$ & \\
\hline$\frac{1}{1}$ & $1 / 14 / 82$ & 8 & 9 & & 118 & $N$ & \\
\hline 1 & $1 / 14 / 82$ & 9 & 1 & & 115 & $\mathbf{N}$ & \\
\hline 1 & $1 / 14 / 82$ & 10 & 1 & & 96 & $\mathbf{N}$ & \\
\hline 1 & 1/14/82 & 11 & 1 & & 78 & $\mathbf{N}$ & \\
\hline 1 & $1 / 14 / 82$ & 12 & 1 & & 78 & $\mathbf{N}$ & \\
\hline 1 & $2 / 4 / 82$ & 9 & 1 & & 164 & $\bar{Y}$ & \\
\hline 1 & $2 / 4 / 82$ & 2 & 1 & & 164 & $\mathrm{~N}$ & \\
\hline 1 & $2 / 4 / 82$ & 3 & 1 & & 159 & $N$ & \\
\hline 1 & $2 / 4 / 82$ & 4 & 1 & & 151 & $\mathrm{~N}$ & \\
\hline 1 & $2 / 4 / 82$ & 5 & 1 & & 142 & $\mathrm{~N}$ & \\
\hline 1 & $2 / 4 / 82$ & 6 & 1 & & 134 & $\mathbf{N}$ & \\
\hline 1 & $2 / 4 / 82$ & 7 & 9 & & 127 & $\mathrm{~N}$ & \\
\hline 1 & $2 / 4 / 82$ & 8 & 1 & & 123 & $\mathrm{~N}$ & \\
\hline 1 & $2 / 4 / 82$ & 9 & 1 & & 120 & $\mathbf{N}$ & \\
\hline 1 & $2 / 4 / 82$ & 10 & 1 & & 105 & $\mathrm{~N}$ & \\
\hline 1 & $2 / 4 / 82$ & 11 & 1 & & 88 & $\mathbf{N}$ & \\
\hline 1 & $2 / 4 / 82$ & 12 & 1 & & 87 & $\mathbf{N}$ & \\
\hline 9 & $3 / 10 / 82$ & 1 & 9 & & 155 & $\mathbf{N}$ & \\
\hline 1 & $3 / 10 / 82$ & 2 & 1 & & 156 & $\bar{Y}$ & \\
\hline 1 & $3 / 10 / 82$ & 3 & 1 & & 151 & $\mathbf{N}$ & \\
\hline 1 & $3 / 10 / 82$ & 4 & 1 & & 144 & $\mathbf{N}$ & \\
\hline 1 & $3 / 10 / 82$ & 5 & 1 & & 134 & $\mathbf{N}$ & \\
\hline 1 & $3 / 10 / 82$ & 6 & 1 & & 129 & $\mathbf{N}$ & \\
\hline 1 & $3 / 10 / 82$ & 7 & 1 & & 120 & $N$ & \\
\hline 1 & $3 / 10 / 82$ & 8 & 1 & & 115 & $N$ & \\
\hline 1 & $3 / 10 / 82$ & 9 & 1 & & 112 & $\mathbf{N}$ & \\
\hline 1 & $3 / 10 / 82$ & 10 & 1 & & 96 & $\mathbf{N}$ & \\
\hline 1 & $3 / 10 / 82$ & 19 & 1 & & 81 & $\mathbf{N}$ & \\
\hline 1 & $3 / 10 / 82$ & 12 & 1 & & 80 & $\mathbf{N}$ & \\
\hline 1 & $10 / 14 / 83$ & 1 & 1 & & 134 & $\mathbf{N}$ & \\
\hline 1 & \begin{tabular}{|l}
$10 / 14 / 83$ \\
\end{tabular} & 2 & 1 & & 135 & $\bar{Y}$ & \\
\hline 1 & $10 / 14 / 83$ & 3 & 1 & & 130 & $N$ & \\
\hline 1 & \begin{tabular}{|c|}
$10 / 14 / 83$ \\
\end{tabular} & 4 & 1 & & 120 & $\mathbf{N}$ & \\
\hline 1 & $10 / 14 / 83$ & 5 & 1 & & 112 & $N$ & \\
\hline 1 & $10 / 14 / 83$ & 6 & 1 & & 75 & $\mathbf{N}$ & \\
\hline 1 & $19 / 8 / 83$ & 1 & 1 & & 144 & $N$ & \\
\hline 1 & $111 / 8 / 83$ & 2 & 1 & & 144 & $\bar{Y}$ & \\
\hline$\frac{1}{1}$ & $11 / 8 / 83$ & 3 & 1 & & 139 & $N$ & \\
\hline 1 & $11 / 8 / 83$ & 4 & 1 & & 129 & $\mathbf{N}$ & \\
\hline 1 & $11 / 8 / 83$ & 5 & 1 & & 121 & $\mathbf{N}$ & \\
\hline 1 & $11 / 8 / 83$ & 6 & 1 & & 82 & $\mathrm{~N}$ & \\
\hline 1 & $12 / 6 / 83$ & 1 & 1 & & 144 & $\mathbf{N}$ & \\
\hline 1 & $12 / 6 / 83$ & 2 & 1 & & 145 & $\mathrm{~N}$ & \\
\hline 1 & $12 / 6 / 83$ & 3 & 1 & & 139 & $\mathbf{N}$ & \\
\hline 7 & $12 / 6 / 83$ & 4 & 1 & & 149 & $Y$ & \\
\hline 1 & $12 / 6 / 83$ & 5 & 1 & & 121 & $\mathbf{N}$ & \\
\hline
\end{tabular}

Data obtained from WHC Surveillance Analysis Computer System (SACS), November 12, 1993. 
WHC-SD-WM-ER-312, Rev. 0

\begin{tabular}{|c|c|c|c|c|c|c|c|}
\hline \multirow{2}{*}{\multicolumn{8}{|c|}{$\begin{array}{c}\text { TANK 241-BY-104 } \\
\text { THERMOCOUPLEDATA }\end{array}$}} \\
\hline & & & & & & DATA & \\
\hline TREE\# & DATE & THERMO\# & TREE SET\# & TEMP C & TEMP F & $Y=$ MAXTEMP & COMMENTS \\
\hline 1 & $12 / 6 / 83$ & 6 & 1 & & 77 & $N$ & \\
\hline 1 & $1 / 3 / 84$ & 1 & 1 & & 143 & $\mathbf{N}$ & \\
\hline 1 & $1 / 3 / 84$ & 2 & 1 & & 143 & $\bar{Y}$ & \\
\hline 1 & $1 / 3 / 84$ & 3 & 1 & & 139 & $\mathrm{~N}$ & \\
\hline 1 & $1 / 3 / 84$ & 4 & 9 & & 129 & $\mathrm{~N}$ & \\
\hline 1 & $1 / 3 / 84$ & 5 & 9 & & 120 & $\bar{N}$ & \\
\hline 1 & $1 / 3 / 84$ & 6 & 1 & & 72 & $\mathrm{~N}$ & \\
\hline 9 & $2 / 7 / 84$ & 1 & 1 & & 143 & $\mathbf{N}$ & \\
\hline 1 & $2 \pi / 84$ & 2 & 1 & & 143 & $\bar{Y}$ & \\
\hline 1 & $2 / 7 / 84$ & 3 & 1 & & 138 & $\mathbf{N}$ & \\
\hline 1 & $2 / 7 / 84$ & 4 & 1 & & 127 & $\mathbf{N}$ & \\
\hline 1 & $2 \pi / 84$ & 5 & 9 & 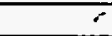 & 118 & $\mathbf{N}$ & \\
\hline 1 & $2 \pi / 84$ & 6 & 1 & & 70 & $N$ & \\
\hline 1 & $2 / 14 / 84$ & 1 & 1 & & 132 & $\mathrm{~N}$ & \\
\hline 1 & $2 / 14 / 84$ & 2 & $\frac{1}{1}$ & & 132 & $\bar{Y}$ & \\
\hline 1 & $2 / 14 / 84$ & 3 & 9 & & 126 & $\mathbf{N}$ & \\
\hline 1 & $2 / 14 / 84$ & 4 & 1 & & 115 & $\mathbf{N}$ & \\
\hline 1 & $2 / 14 / 84$ & 5 & 7 & & 107 & $\mathbf{N}$ & \\
\hline 1 & $2 / 14 / 84$ & 6 & 1 & & 62 & $\mathrm{~N}$ & \\
\hline 1 & $3 / 26 / 84$ & 9 & 9 & & 138 & $\mathbf{N}$ & \\
\hline 1 & $3 / 26 / 84$ & 2 & 1 & & 138 & $\bar{Y}$ & \\
\hline 1 & $3 / 26 / 84$ & 3 & 9 & & 132 & $\mathrm{~N}$ & \\
\hline 1 & $3 / 26 / 84$ & 4 & 1 & & 121 & $\mathrm{~N}$ & \\
\hline 1 & $3 / 26 / 84$ & 5 & 7 & & 911 & $\bar{N}$ & \\
\hline 1 & $3 / 26 / 84$ & 6 & 1 & & 68 & $\mathbf{N}$ & \\
\hline 1 & $4 / 7 / 84$ & 1 & 1 & & 126 & $\mathrm{~N}$ & \\
\hline 1 & $4 / 7 / 84$ & 2 & 1 & & 126 & $\mathrm{Y}$ & \\
\hline 9 & $4 / 7 / 84$ & 3 & 9 & & 121 & $\mathbf{N}$ & \\
\hline 1 & $4 / 7 / 84$ & 4 & 1 & & 112 & $\mathbf{N}$ & \\
\hline 1 & $4 / 7 / 84$ & 5 & 1 & & 104 & $\mathrm{~N}$ & \\
\hline 1 & $4 / 7 / 84$ & 6 & 1 & & 65 & $\mathbf{N}$ & \\
\hline 1 & $6 / 19 / 84$ & 9 & $\frac{1}{1}$ & & 138 & $\bar{N}$ & \\
\hline$\frac{1}{1}$ & $6 / 19 / 84$ & $\frac{1}{2}$ & $\frac{1}{1}$ & & 140 & $\frac{Y}{y}$ & \\
\hline 1 & $6 / 19 / 84$ & 3 & 1 & & 135 & $\mathbf{N}$ & \\
\hline 1 & $6 / 19 / 84$ & 4 & 1 & & 124 & $\mathbf{N}$ & \\
\hline 1 & $6 / 19 / 84$ & 5 & 1 & & 115 & $\mathbf{N}$ & \\
\hline 1 & $6 / 19 / 84$ & 6 & 1 & & 76 & $\mathrm{~N}$ & \\
\hline 1 & $9 / 3 / 84$ & 1 & 1 & & 132 & $\bar{N}$ & \\
\hline 1 & $9 / 3 / 84$ & 2 & 1 & & 132 & $Y$ & \\
\hline 1 & $9 / 3 / 84$ & 3 & 1 & & 128 & $\mathbf{N}$ & \\
\hline 1 & $9 / 3 / 84$ & 4 & 1 & & 119 & $\mathbf{N}$ & \\
\hline 1 & $9 / 3 / 84$ & 5 & 1 & & 113 & $\mathbf{N}$ & \\
\hline 1 & $9 / 3 / 84$ & 6 & 1 & & 84 & $\mathrm{~N}$ & \\
\hline 9 & $3 / 3 / 85$ & 9 & 1 & & 155 & $\mathrm{~N}$ & \\
\hline 1 & $3 / 3 / 85$ & 2 & 1 & & 158 & $\bar{Y}$ & \\
\hline 7 & $3 / 3 / 85$ & 3 & 1 & & 150 & $\bar{N}$ & \\
\hline 1 & $3 / 3 / 85$ & 4 & 1 & & 149 & $\mathrm{~N}$ & \\
\hline 1 & $3 / 3 / 85$ & 5 & 1 & & 149 & $\mathrm{~N}$ & \\
\hline 1 & $3 / 3 / 85$ & 6 & 1 & & 147 & $\bar{N}$ & \\
\hline 1 & $3 / 3 / 85$ & 7 & 1 & & 146 & $N$ & \\
\hline 1 & $3 / 3 / 85$ & 8 & 1 & & 945 & $\mathrm{~N}$ & \\
\hline 1 & $3 / 3 / 85$ & 9 & 1 & & 145 & $\mathrm{~N}$ & \\
\hline 1 & $3 / 3 / 85$ & 10 & 1 & & 144 & $\mathbf{N}$ & \\
\hline 1 & $3 / 3 / 85$ & 11 & 1 & & 143 & $N$ & \\
\hline 1 & $3 / 3 / 85$ & 12 & 9 & & 142 & $\mathrm{~N}$ & \\
\hline 1 & $3 / 5 / 85$ & 1 & 1 & & 135 & $\bar{Y}$ & \\
\hline 1 & $3 / 5 / 85$ & 2 & 1 & & 127 & $\mathrm{~N}$ & \\
\hline 1 & $3 / 5 / 85$ & 3 & 1 & & 128 & $N$ & \\
\hline 1 & $3 / 5 / 85$ & 4 & 1 & & 128 & $\mathbf{N}$ & \\
\hline 1 & $3 / 5 / 85$ & 5 & 1 & & 114 & $\mathbf{N}$ & \\
\hline 1 & $3 / 5 / 85$ & 6 & 1 & & 75 & $\mathbf{N}$ & \\
\hline 1 & $4 / 1 / 85$ & 1 & 1 & & 140 & $\mathbf{N}$ & \\
\hline 1 & $4 / 1 / 85$ & 2 & 1 & & 141 & $\bar{Y}$ & \\
\hline 1 & $4 / 1 / 85$ & 3 & 1 & & 137 & $\mathbf{N}$ & \\
\hline 1 & $4 / 1 / 85$ & 4 & 1 & & 127 & $N$ & \\
\hline
\end{tabular}

Data obtained from WHC Surveiliance Analysis Computer System (SACS), November 12, 1993. 


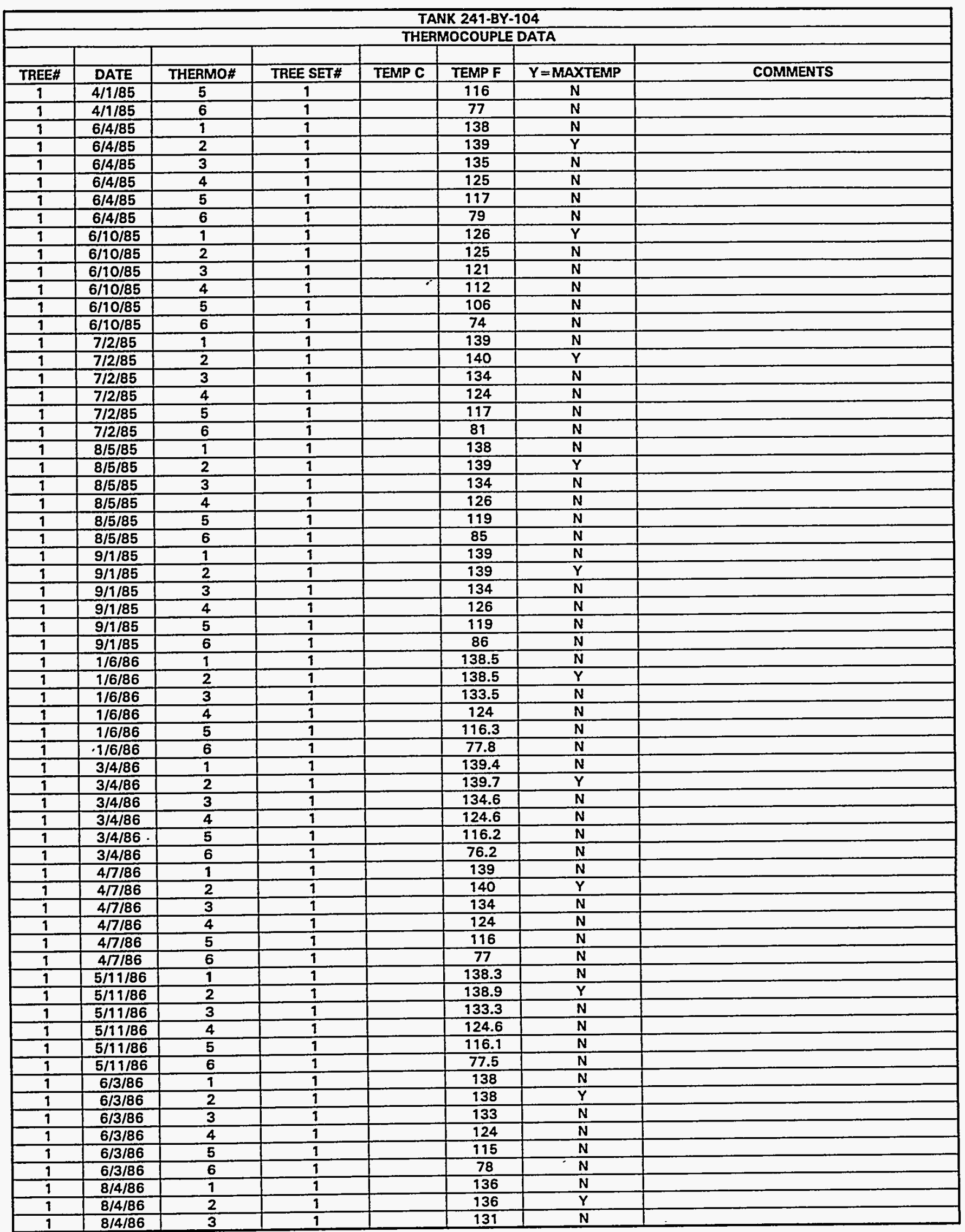

Data obtained from WHC Surveillance Analysis Computer System (SACS), November 12, 1993. 


\begin{tabular}{|c|c|c|c|c|c|c|c|}
\hline \multicolumn{8}{|c|}{ TANK 241-BY-104 } \\
\hline & & & & THEF & 1OCOUPL & DATA & \\
\hline TREE\# & DATE & THERMO\# & TREE SET\# & TEMP C & TEMP F & $Y=$ MAXTEMP & COMMENTS \\
\hline 1 & $8 / 4 / 86$ & 4 & 1 & & 123 & $\mathbf{N}$ & \\
\hline 1 & $8 / 4 / 86$ & 5 & 1 & & 116 & $\overline{\mathbf{N}}$ & \\
\hline 1 & $8 / 4 / 86$ & 6 & 1 & & 83 & $\mathbf{N}$ & \\
\hline 1 & $9 / 1 / 86$ & 1 & 1 & & 145 & $\mathbf{N}$ & \\
\hline 1 & $9 / 1 / 86$ & 2 & 1 & & 145 & $\mathbf{Y}$ & \\
\hline 1 & $9 / 1 / 86$ & 3 & 1 & & 140 & $\mathbf{N}$ & \\
\hline 1 & $9 / 1 / 86$ & 4 & 1 & & 132 & $\bar{N}$ & \\
\hline 1 & $9 / 1 / 86$ & 5 & 1 & & 125 & $\bar{N}$ & \\
\hline 1 & $9 / 1 / 86$ & 6 & 1 & & 95 & $\bar{N}$ & \\
\hline 1 & $11 / 2 / 86$ & 1 & 1 & & 123.1 & $\mathbf{N}$ & \\
\hline 1 & $11 / 2 / 86$ & 2 & 1 & & 124.7 & $\bar{Y}$ & \\
\hline 1 & $11 / 2 / 86$ & 3 & 1 & $\%$ & 120.7 & $\mathrm{~N}$ & \\
\hline 1 & $11 / 2 / 86$ & 4 & $\frac{1}{1}$ & & 113.8 & $\mathbf{N}$ & \\
\hline 9 & $11 / 2 / 86$ & 5 & 1 & & 107.7 & $\mathbf{N}$ & \\
\hline 1 & $11 / 2 / 86$ & 6 & 1 & & 80.27 & $\mathbf{N}$ & \\
\hline 1 & $12 / 3 / 86$ & 1 & 1 & & 120 & $\mathbf{N}$ & \\
\hline 1 & $12 / 3 / 86$ & 2 & 1 & & 122 & $\mathbf{Y}$ & \\
\hline 1 & $12 / 3 / 86$ & 3 & 1 & & 117 & $\mathbf{N}$ & \\
\hline 1 & $12 / 3 / 86$ & 4 & 1 & & 111 & $N$ & \\
\hline 9 & $12 / 3 / 86$ & 5 & 1 & & 104 & $N$ & \\
\hline 1 & $12 / 3 / 86$ & 6 & 1 & & 75 & $\mathbf{N}$ & \\
\hline 1 & $2 / 3 / 87$ & 1 & 1 & & 136 & $\mathbf{N}$ & \\
\hline 1 & $2 / 3 / 87$ & 2 & 1 & & 138 & $\bar{Y}$ & \\
\hline 9 & $2 / 3 / 87$ & 3 & 1 & & 133 & $\bar{N}$ & \\
\hline 1 & $2 / 3 / 87$ & 4 & 1 & & 124 & $\bar{N}$ & \\
\hline 1 & $2 / 3 / 87$ & 5 & 1 & & 116 & $\mathbf{N}$ & \\
\hline 1 & $2 / 3 / 87$ & 6 & 1 & & 77 & $\mathbf{N}$ & \\
\hline 1 & $3 / 2 / 87$ & 1 & 1 & & 134 & $\mathbf{N}$ & \\
\hline 1 & $3 / 2 / 87$ & 2 & 1 & & 135 & $\bar{Y}$ & \\
\hline 9 & $3 / 2 / 87$ & 3 & 1 & & 131 & $\mathbf{N}$ & \\
\hline 9 & $3 / 2 / 87$ & 4 & 1 & & 122 & $\mathbf{N}$ & \\
\hline 1 & $3 / 2 / 87$ & 5 & 1 & & 114 & $\mathbf{N}$ & \\
\hline 1 & $3 / 2 / 87$ & 6 & 1 & & 76 & $\mathbf{N}$ & \\
\hline 1 & $4 / 13 / 87$ & 1 & 1 & & 134 & $N$ & \\
\hline 1 & $4 / 13 / 87$ & 2 & 1 & & 136 & $\bar{Y}$ & \\
\hline 1 & $4 / 13 / 87$ & 3 & 1 & & 131 & $\mathbf{N}$ & \\
\hline 1 & $4 / 13 / 87$ & 4 & 1 & & 122 & $\mathrm{~N}$ & \\
\hline 1 & $.4 / 13 / 87$ & 5 & 1 & & 115 & $\mathbf{N}$ & \\
\hline 1 & $4 / 13 / 87$ & 6 & 1 & & 76 & $\mathbf{N}$ & \\
\hline 1 & $6 / 1 / 87$ & 1 & 1 & & 135 & $\mathbf{N}$ & \\
\hline 1 & $6 / 1 / 87$ & 2 & 1 & & 136 & $\bar{Y}$ & \\
\hline 1 & $6 / 1 / 87$ & 3 & 1 & & 131 & $\mathbf{N}$ & \\
\hline 1 & $6 / 1 / 87$ & 4 & 1 & & 122 & $N$ & \\
\hline 1 & $6 / 1 / 87$ & 5 & 1 & & 113 & $\mathrm{~N}$ & \\
\hline 1 & $6 / 9 / 87$ & 6 & 1 & & 79 & $\mathbf{N}$ & \\
\hline 1 & $7 / 6 / 87$ & 1 & 1 & & 134.3 & $\mathrm{~N}$ & \\
\hline 1 & $7 / 6 / 87$ & 2 & 1 & & 136.7 & $\bar{Y}$ & \\
\hline 1 & $7 / 6 / 87$ & 3 & 1 & & 132.2 & $\mathbf{N}$ & \\
\hline 1 & $7 / 6 / 87$ & 4 & 1 & & 123.8 & $\mathrm{~N}$ & \\
\hline 1 & $7 / 6 / 87$ & 5 & 1 & & 116 & $N$ & \\
\hline 1 & $7 / 6 / 87$ & 6 & 1 & & 82 & $\bar{N}$ & \\
\hline 1 & $8 / 4 / 87$ & 1 & 1 & & 132 & $\mathbf{N}$ & \\
\hline 1 & $8 / 4 / 87$ & 2 & 1 & & 134 & $Y$ & \\
\hline 1 & $8 / 4 / 87$ & 3 & 1 & & 130 & $\mathbf{N}$ & \\
\hline 1 & $8 / 4 / 87$ & 4 & 1 & & 123 & $\mathbf{N}$ & \\
\hline 1 & $8 / 4 / 87$ & 5 & 1 & & 115 & $\mathbf{N}$ & \\
\hline 1 & $8 / 4 / 87$ & 6 & 1 & & 82 & $\mathbf{N}$ & \\
\hline 1 & $9 / 7 / 87$ & 1 & 1 & & 134.4 & $\mathbf{N}$ & \\
\hline 1 & $9 / 7 / 87$ & 2 & 1 & & 135.9 & $\bar{Y}$ & \\
\hline 1 & $9 / 7 / 87$ & 3 & 1 & & 130.7 & $\mathbf{N}$ & \\
\hline 1 & $9 / 7 / 87$ & 4 & 1 & & 122.2 & $N$ & \\
\hline 1 & $9 / 7 / 87$ & 5 & 1 & & 113.8 & $\mathrm{~N}$ & \\
\hline 1 & $9 / 7 / 87$ & 6 & 1 & & 82.5 & $\bar{N}$ & \\
\hline 1 & $10 / 5 / 87$ & 1 & 1 & & 135 & $N$ & \\
\hline 1 & $10 / 5 / 87$ & 2 & 1 & & 136 & $\bar{Y}$ & \\
\hline
\end{tabular}

Data obtained from WHC Surveillance Analysis Computer System (SACS), November 12, 1993. 


\begin{tabular}{|c|c|c|c|c|c|c|c|}
\hline \multicolumn{8}{|c|}{ TANK 241-BY-104 } \\
\hline & & & & THEF & IOCOUPLE & DATA & \\
\hline TREE\# & DATE & THERMO\# & TREE SET\# & TEMP C & TEMP F & $Y=$ MAXTEMP & COMMENTS \\
\hline 1 & $10 / 5 / 87$ & 3 & 1 & & 131 & $\mathbf{N}$ & \\
\hline 1 & $10 / 5 / 87$ & 4 & 1 & & 124 & $\mathbf{N}$ & \\
\hline 1 & $10 / 5 / 87$ & 5 & 1 & & 116 & $\mathbf{N}$ & \\
\hline 1 & $10 / 5 / 87$ & 6 & 1 & & 85 & $\mathbf{N}$ & \\
\hline 1 & $11 / 3 / 87$ & 1 & 1 & & 148 & $\mathbf{N}$ & \\
\hline 1 & $11 / 3 / 87$ & 2 & 1 & & 149 & $\mathbf{Y}$ & \\
\hline 1 & $11 / 3 / 87$ & 3 & 1 & & 145 & $\mathbf{N}$ & \\
\hline 1 & $11 / 3 / 87$ & 4 & 1 & & 138 & $\mathbf{N}$ & \\
\hline 1 & $11 / 3 / 87$ & 5 & 1 & & 131 & $\mathbf{N}$ & \\
\hline 1 & $11 / 3 / 87$ & 6 & 1 & & 101 & $\overline{\mathbf{N}}$ & \\
\hline 1 & $1 / 25 / 88$ & 1 & 1 & & 132 & $\mathbf{N}$ & \\
\hline$\frac{1}{1}$ & $1 / 25 / 88$ & 2 & 1 & $\circ$ & 134 & $\bar{Y}$ & \\
\hline 1 & $1 / 25 / 88$ & 3 & 1 & & 131 & $\overline{\mathbf{N}}$ & \\
\hline 1 & $9 / 25 / 88$ & 4 & 1 & & 124 & $\mathbf{N}$ & \\
\hline 1 & $1 / 25 / 88$ & 5 & 1 & & 116 & $N$ & \\
\hline 1 & $1 / 25 / 88$ & 6 & 1 & & 80 & $\mathbf{N}$ & \\
\hline 1 & $2 / 1 / 88$ & 1 & 1 & & 135 & $\bar{N}$ & \\
\hline 1 & $2 / 1 / 88$ & 2 & 1 & & 136 & $\bar{Y}$ & \\
\hline 1 & $2 / 1 / 88$ & 3 & 1 & & 136 & $\bar{N}$ & \\
\hline 1 & $2 / 1 / 8 B$ & 4 & 1 & & 124 & $N$ & \\
\hline 1 & $2 / 1 / 88$ & 5 & 1 & & 118 & $\mathbf{N}$ & \\
\hline 1 & $2 / 1 / 88$ & 6 & 1 & & 83 & $N$ & \\
\hline 1 & $3 / 7 / 88$ & 1 & 1 & & 135 & $N$ & \\
\hline 1 & $3 / 7 / 88$ & 2 & 1 & & 136 & $\bar{Y}$ & \\
\hline 1 & $3 / 7 / 88$ & 3 & 1 & & 131 & $\mathrm{~N}$ & \\
\hline 1 & $3 / 7 / 88$ & 4 & 1 & & 123 & $\mathrm{~N}$ & \\
\hline 1 & $3 / 7 / 88$ & 5 & 1 & & 114 & $\mathbf{N}$ & \\
\hline 1 & $3 / 7 / 88$ & 6 & 1 & & 77 & $\bar{N}$ & \\
\hline 1 & $4 / 6 / 88$ & 1 & 1 & & 121 & $\mathbf{N}$ & \\
\hline 1 & $4 / 6 / 88$ & 2 & 1 & & 122 & $\bar{Y}$ & \\
\hline 1 & $4 / 6 / 88$ & 3 & 1 & & 118 & $\mathbf{N}$ & \\
\hline$\frac{1}{1}$ & $4 / 6 / 88$ & 4 & 1 & & 191 & $\mathbf{N}$ & \\
\hline 1 & $4 / 6 / 88$ & 5 & 1 & & 104 & $\mathbf{N}$ & \\
\hline 1 & $4 / 6 / 88$ & 6 & $\frac{1}{1}$ & & 73 & $\mathbf{N}$ & \\
\hline 1 & $5 / 2 / 88$ & 1 & 1 & & 130 & $\mathrm{~N}$ & \\
\hline 1 & $5 / 2 / 88$ & 2 & 1 & & 132 & $\bar{Y}$ & \\
\hline$\frac{1}{1}$ & $5 / 2 / 88$ & 3 & 1 & & 127 & $\mathbf{N}$ & \\
\hline 1 & $.5 / 2 / 88$ & 4 & 1 & & 119 & $N$ & \\
\hline 1 & $5 / 2 / 88$ & 5 & 1 & & 112 & $N$ & \\
\hline 1 & $5 / 2 / 88$ & 6 & 1 & & 76 & $\mathbf{N}$ & \\
\hline 1 & $5 / 3 / 88$ & 1 & 1 & & 130 & $\mathbf{N}$ & \\
\hline 1 & $5 / 3 / 88$ & 2 & 1 & & 132 & $\bar{Y}$ & \\
\hline 1 & $5 / 3 / 88$ & 3 & 1 & & 127 & $\mathbf{N}$ & \\
\hline 9 & $5 / 3 / 88$ & 4 & 9 & & 119 & $N$ & \\
\hline 1 & $5 / 3 / 88$ & 5 & 1 & & 112 & $\mathbf{N}$ & \\
\hline 1 & $5 / 3 / 88$ & 6 & 9 & & 76 & $\mathbf{N}$ & \\
\hline 1 & $6 / 7 / 88$ & 1 & 1 & & 133 & $\bar{N}$ & \\
\hline 1 & $6 / 7 / 88$ & 2 & 1 & & 135 & $\bar{Y}$ & \\
\hline 1 & $6 / 7 / 88$ & 3 & 1 & & 130 & $\mathbf{N}$ & \\
\hline 1 & $6 / 7 / 88$ & 4 & 1 & & 122 & $\mathbf{N}$ & \\
\hline 1 & $6 / 7 / 88$ & 5 & 1 & & 114 & $\mathbf{N}$ & \\
\hline 1 & $6 / 7 / 88$ & 6 & 1 & & 81 & $\mathbf{N}$ & \\
\hline 1 & $7 / 4 / 88$ & 1 & 1 & & 132 & $\mathbf{N}$ & \\
\hline 1 & $7 / 4 / 88$ & 2 & 1 & & 133 & $Y$ & \\
\hline 1 & $7 / 4 / 88$ & 3 & 1 & & 129 & $\mathbf{N}$ & \\
\hline 1 & $7 / 4 / 8 B$ & 4 & 1 & & 121 & $\mathrm{~N}$ & \\
\hline$T$ & $7 / 4 / 88$ & 5 & 1 & & 114 & $N$ & \\
\hline 1 & $7 / 4 / 88$ & 6 & 1 & & 81 & $N$ & \\
\hline 1 & $7 / 7 / 88$ & 1 & 1 & & & $\mathbf{N}$ & 81 - suspect data (low) \\
\hline 1 & $8 / 1 / 88$ & 1 & 1 & & 117 & $N$ & \\
\hline 1 & $8 / 1 / 88$ & 2 & 1 & & 118 & $Y$ & \\
\hline 1 & $8 / 1 / 88$ & 3 & 9 & & 114 & $\mathbf{N}$ & \\
\hline 1 & $8 / 1 / 88$ & 4 & 1 & & 107 & $N$ & \\
\hline 1 & $8 / 1 / 88$ & 5 & 1 & & 99 & $\mathbf{N}$ & \\
\hline 7 & $8 / 1 / 88$ & 6 & 1 & & 69 & $\mathrm{~N}$ & \\
\hline
\end{tabular}

Data obtained from WHC Surveillance Analysis Computer System (SACS), November 12, 1993. 


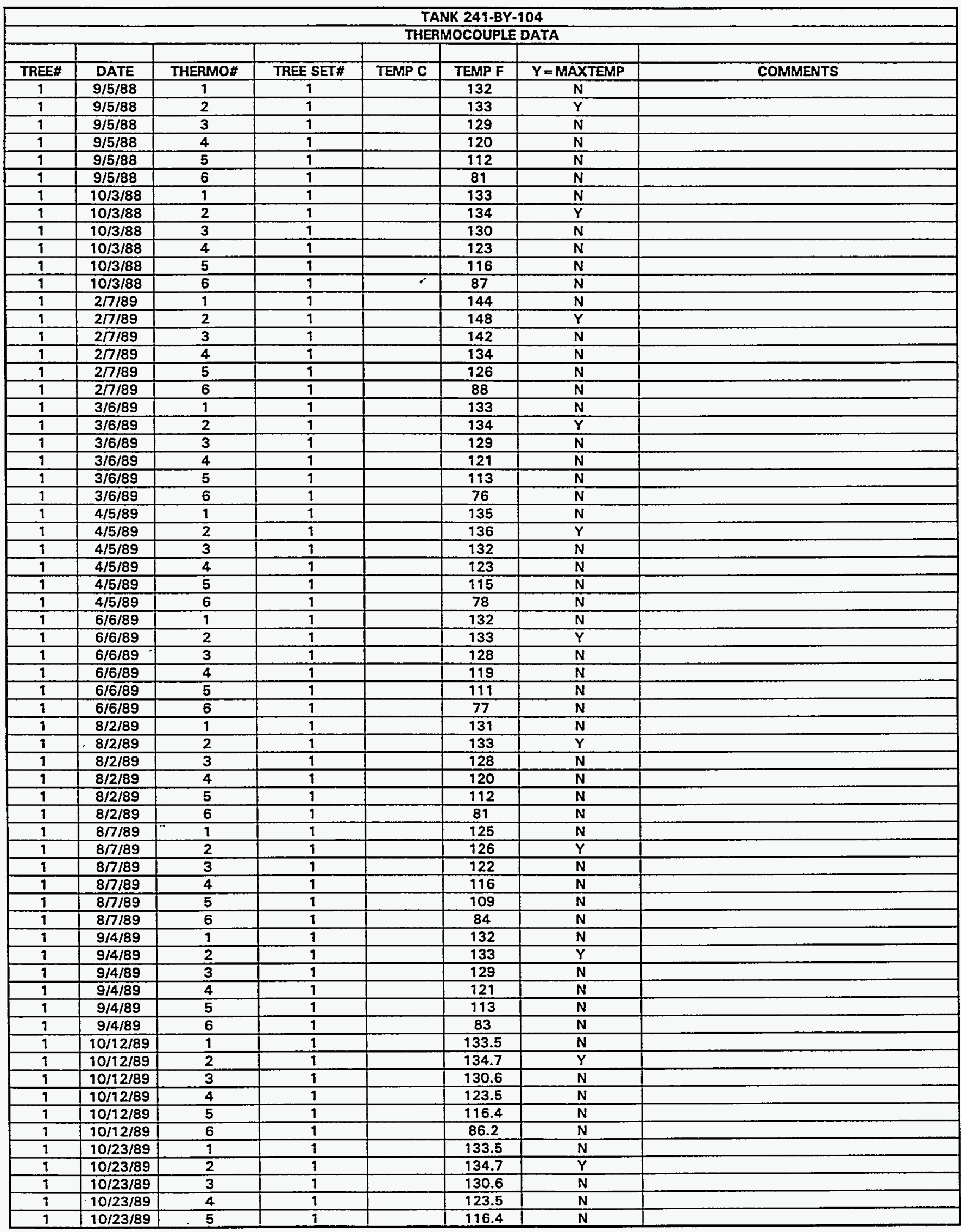

Data obtained from WHC Surveillance Analysis Computer System (SACS), November 12, 1993. 


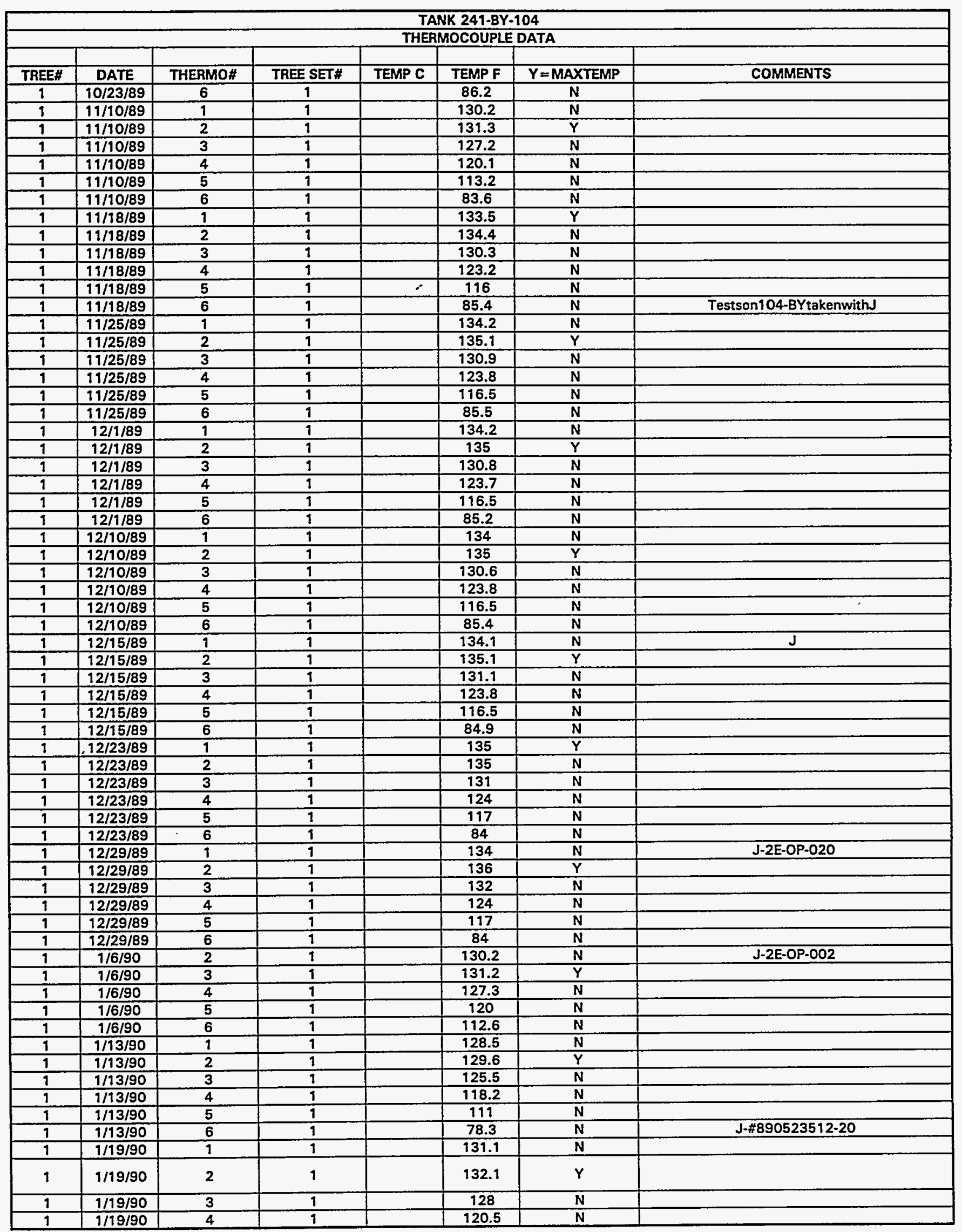

Data obtained from WHC Surveillance Analysis Computer System (SACS), November 12, 1993. 
WHC-SD-WM-ER-312, Rev. 0

\begin{tabular}{|c|c|c|c|c|c|c|c|}
\hline \multicolumn{8}{|c|}{ TANK 241-BY-104 } \\
\hline \multicolumn{8}{|c|}{ THERMOCOUPLE DATA } \\
\hline TREE\# & DATE & THERMO\# & TREE SET\# & TEMP C & TEMPF & $\mathrm{Y}=$ MAXTEMP & COMMENTS \\
\hline 1 & $1 / 19 / 90$ & 5 & 1 & & 113 & $N$ & \\
\hline 1 & $1 / 19 / 90$ & 6 & 1 & & 80.1 & $\mathrm{~N}$ & \\
\hline 1 & $1 / 27 / 90$ & 1 & 1 & & 131.2 & $\mathrm{~N}$ & J-2E-OP-020 \\
\hline 1 & $1 / 27 / 90$ & 2 & 1 & & 132.3 & $Y$ & \\
\hline 1 & $1 / 27 / 90$ & 3 & 1 & & 128 & $\mathbf{N}$ & \\
\hline 1 & $1 / 27 / 90$ & 4 & 1 & & 120.4 & $N$ & \\
\hline 1 & $1 / 27 / 90$ & 5 & 1 & & 112.6 & $\mathbf{N}$ & \\
\hline 1 & $1 / 27 / 90$ & 6 & 1 & & 78.9 & $\mathbf{N}$ & \\
\hline 1 & $2 / 3 / 90$ & 1 & 1 & & 133.8 & $\mathbf{N}$ & \\
\hline 1 & $2 / 3 / 90$ & 2 & 1 & & 134.8 & $\bar{Y}$ & \\
\hline 1 & $2 / 3 / 90$ & 3 & 1 & & 130.5 & $\bar{N}$ & \\
\hline 1 & $2 / 3 / 90$ & 4 & 1 & 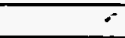 & 122.8 & $\mathbf{N}$ & \\
\hline 1 & $2 / 3 / 90$ & 5 & 1 & & 115 & $\mathbf{N}$ & \\
\hline 1 & $2 / 3 / 90$ & 6 & 1 & & 80.7 & $\mathbf{N}$ & J-2E-OP-02O \\
\hline 1 & $2 / 5 / 90$ & 1 & 1 & & 131.2 & $\mathbf{N}$ & J-2E-OP-021 \\
\hline 1 & $2 / 5 / 90$ & 2 & 1 & & 132.4 & $Y$ & \\
\hline 1 & $2 / 5 / 90$ & 3 & 1 & & 128.1 & $\mathbf{N}$ & \\
\hline 1 & $2 / 5 / 90$ & 4 & 1 & & 120.4 & $\mathbf{N}$ & \\
\hline 1 & $2 / 5 / 90$ & 5 & 1 & & 112.5 & $N$ & \\
\hline 1 & $2 / 5 / 90$ & 6 & 1 & & 78.4 & $\mathbf{N}$ & \\
\hline 1 & $2 / 11 / 90$ & 1 & 1 & & 131.8 & $N$ & J-2E-OP-021 \\
\hline 1 & $2 / 11 / 90$ & 2 & 1 & & 133 & $\bar{Y}$ & \\
\hline 1 & $2 / 11 / 90$ & 3 & 1 & & 128.6 & $\mathbf{N}$ & \\
\hline 1 & $2 / 11 / 90$ & 4 & 1 & & 120.7 & $\mathbf{N}$ & \\
\hline 1 & $2 / 11 / 90$ & 5 & 1 & & 112.4 & $\mathbf{N}$ & \\
\hline 1 & $2 / 11 / 90$ & 6 & 1 & & 78.1 & $N$ & \\
\hline 1 & $3 / 2 / 90$ & 1 & 1 & & 133.7 & $\mathbf{N}$ & \\
\hline 1 & $3 / 2 / 90$ & 2 & 1 & & 135.3 & $Y$ & \\
\hline 1 & $3 / 2 / 90$ & 3 & 1 & & 131 & $N$ & \\
\hline 1 & $3 / 2 / 90$ & 4 & 1 & & 122.8 & $N$ & \\
\hline 1 & $3 / 2 / 90$ & 5 & 1 & & 114.7 & $N$ & \\
\hline 1 & $3 / 2 / 90$ & 6 & 1 & & 79.1 & $\mathbf{N}$ & \\
\hline 1 & $3 / 5 / 90$ & 1 & 1 & & 133.5 & $\mathbf{N}$ & TypeJ-2E-OP-020 \\
\hline 1 & $3 / 5 / 90$ & 2 & 1 & & 134.6 & $\mathrm{Y}$ & \\
\hline 1 & $3 / 5 / 90$ & 3 & 1 & & 130.3 & $\mathbf{N}$ & \\
\hline 1 & $3 / 5 / 90$ & 4 & 1 & & 122.3 & $\mathbf{N}$ & \\
\hline 1 & $3 / 5 / 90$ & 5 & 1 & & 114.3 & $\mathbf{N}$ & \\
\hline 1 & $.3 / 5 / 90$ & 6 & 1 & & 79.5 & $\mathrm{~N}$ & \\
\hline 1 & $4 / 3 / 90$ & 1 & 1 & & 132.5 & $\mathbf{N}$ & Typel2E-OP-020 \\
\hline 1 & $4 / 3 / 90$ & 2 & $T$ & & 133.7 & $\bar{Y}$ & \\
\hline 1 & $4 / 3 / 90$ & 3 & 1 & & 129.2 & $\mathbf{N}$ & \\
\hline 1 & $4 / 3 / 90$ & 4 & 1 & & 120.5 & $\mathbf{N}$ & \\
\hline 1 & $4 / 3 / 90$ & 5 & 1 & & 111.7 & $\mathbf{N}$ & \\
\hline 1 & $4 / 8 / 90$ & 1 & 1 & & 132 & $\mathbf{N}$ & \\
\hline 1 & $4 / 8 / 90$ & 2 & 1 & & 134 & $Y$ & \\
\hline 1 & $4 / 8 / 90$ & 3 & 1 & & 129 & $\mathbf{N}$ & \\
\hline 1 & $4 / 8 / 90$ & 4 & 1 & & 121 & $\mathbf{N}$ & \\
\hline 1 & $4 / 8 / 90$ & 5 & 1 & & 113 & $\mathrm{~N}$ & \\
\hline 1 & $4 / 8 / 90$ & 6 & 1 & & 78 & $\mathrm{~N}$ & \\
\hline 1 & $5 / 5 / 90$ & 1 & 1 & & 130 & $N$ & \\
\hline 1 & $5 / 5 / 90$ & 2 & 1 & & 131 & $\bar{Y}$ & \\
\hline 1 & $5 / 5 / 90$ & 3 & 1 & & 127 & $N$ & \\
\hline 1 & $5 / 5 / 90$ & 4 & 1 & & 119 & $\bar{N}$ & \\
\hline 1 & $5 / 5 / 90$ & 5 & 1 & & 110 & $\mathbf{N}$ & \\
\hline 1 & $5 / 5 / 90$ & 6 & 1 & & 76 & $\mathbf{N}$ & \\
\hline 1 & $6 / 1 / 90$ & 3 & 1 & & 128.7 & $Y$ & \\
\hline 1 & $6 / 1 / 90$ & 4 & 1 & & 120.4 & $N$ & \\
\hline 1 & $6 / 1 / 90$ & 5 & 1 & & 112.1 & $\mathbf{N}$ & \\
\hline 1 & $6 / 1 / 90$ & 6 & 1 & & 79 & $N$ & $\begin{array}{c}\text { TC\#1 2inconsistentwithnorm.Discrep.rpt \#263iss } \\
\text { ued }\end{array}$ \\
\hline 1 & $7 / 8 / 90$ & 1 & 1 & & 131 & $\mathbf{N}$ & J-2E-OP-020 \\
\hline 1 & $7 / 8 / 90$ & 2 & 9 & & 132.7 & $\bar{Y}$ & \\
\hline 1 & $7 / 8 / 90$ & 3 & 1 & & 128.4 & $\mathrm{~N}$ & \\
\hline 1 & $7 / 8 / 90$ & 4 & 1 & & 120.7 & $N$ & \\
\hline 1 & $7 / 8 / 90$ & 5 & 1 & & 113.3 & $N$ & \\
\hline
\end{tabular}

Data obtained from WHC Surveillance Analysis Computer System (SACS), November 12, 1993. 
WHC-SD-WM-ER-312, Rev. 0

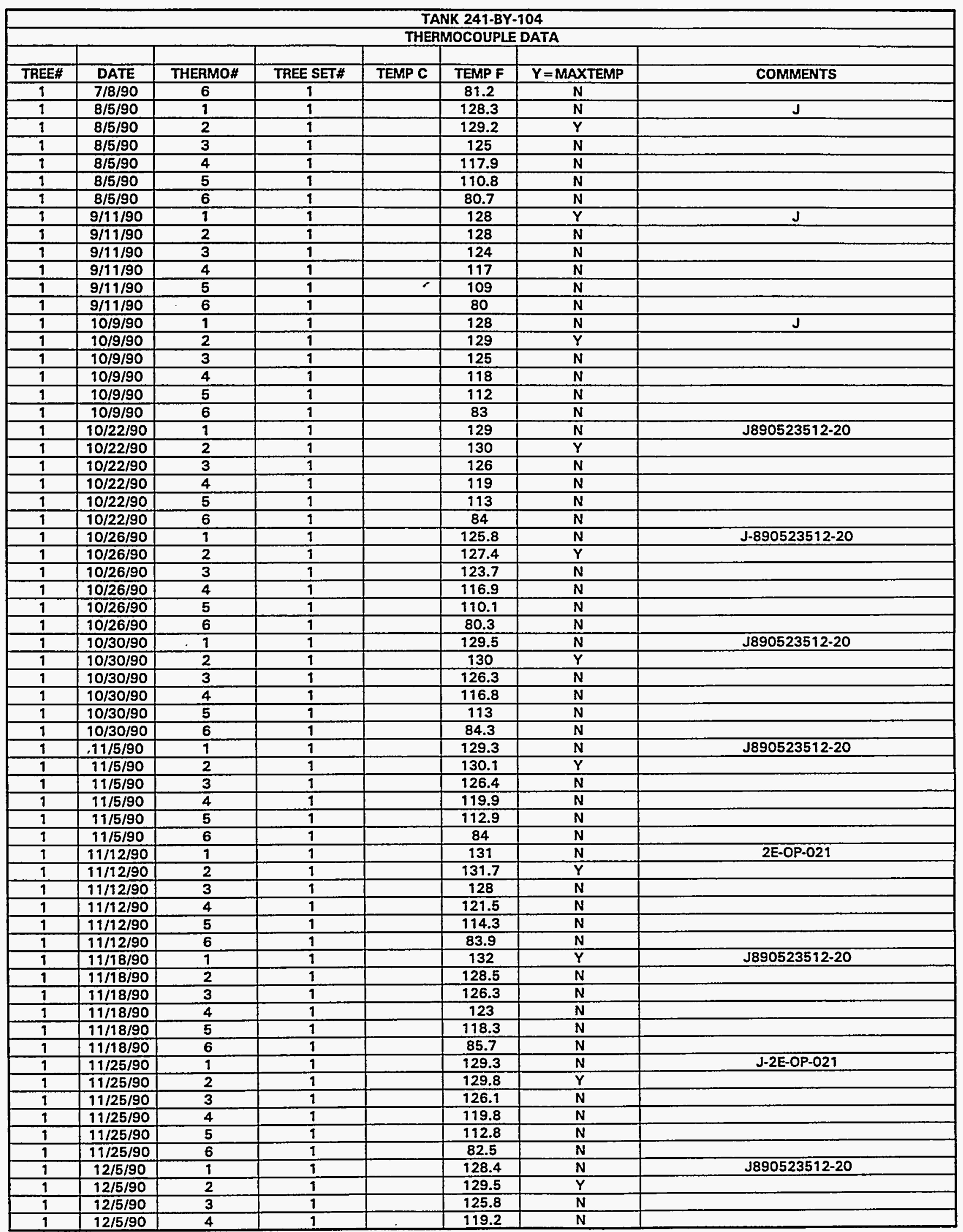

Data obtained from WHC Surveillance Analysis Computer System (SACS), November 12, 1993. 


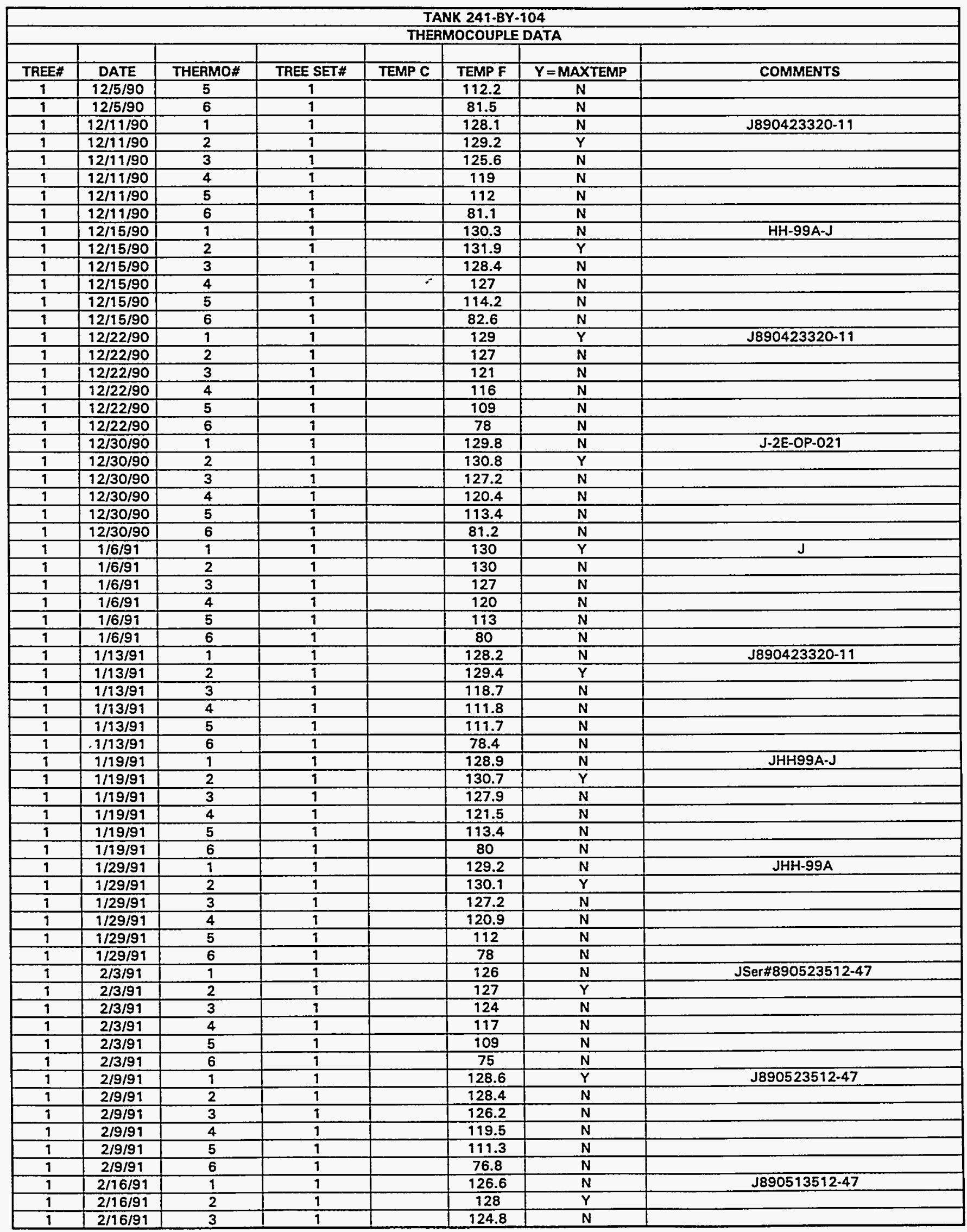

Data obtained from WHC Surveillance Analysis Computer System (SACS), November 12, 1993. 


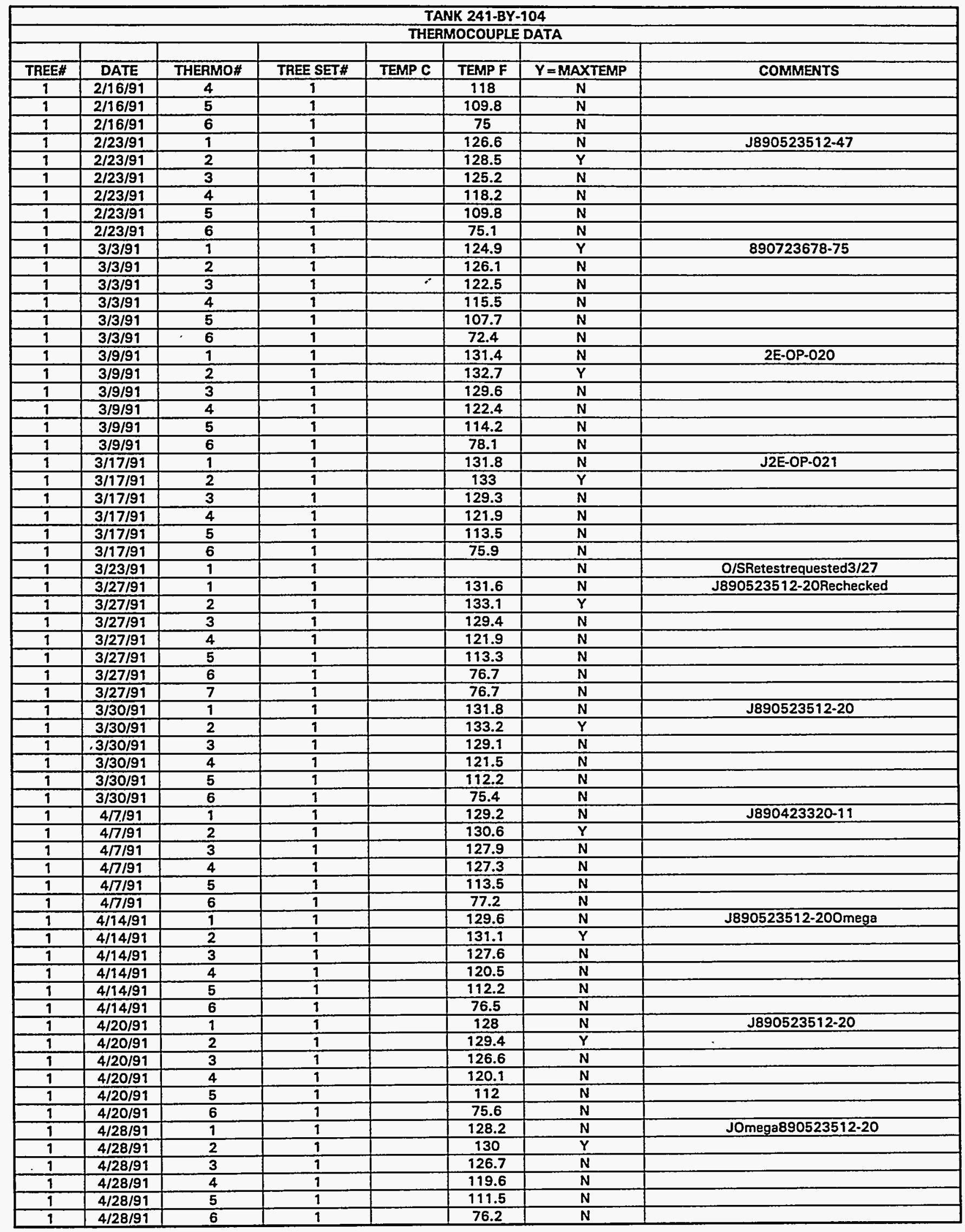

Data obtained from WHC Surveillance Analysis Computer System (SACS), November 12, 1993. 


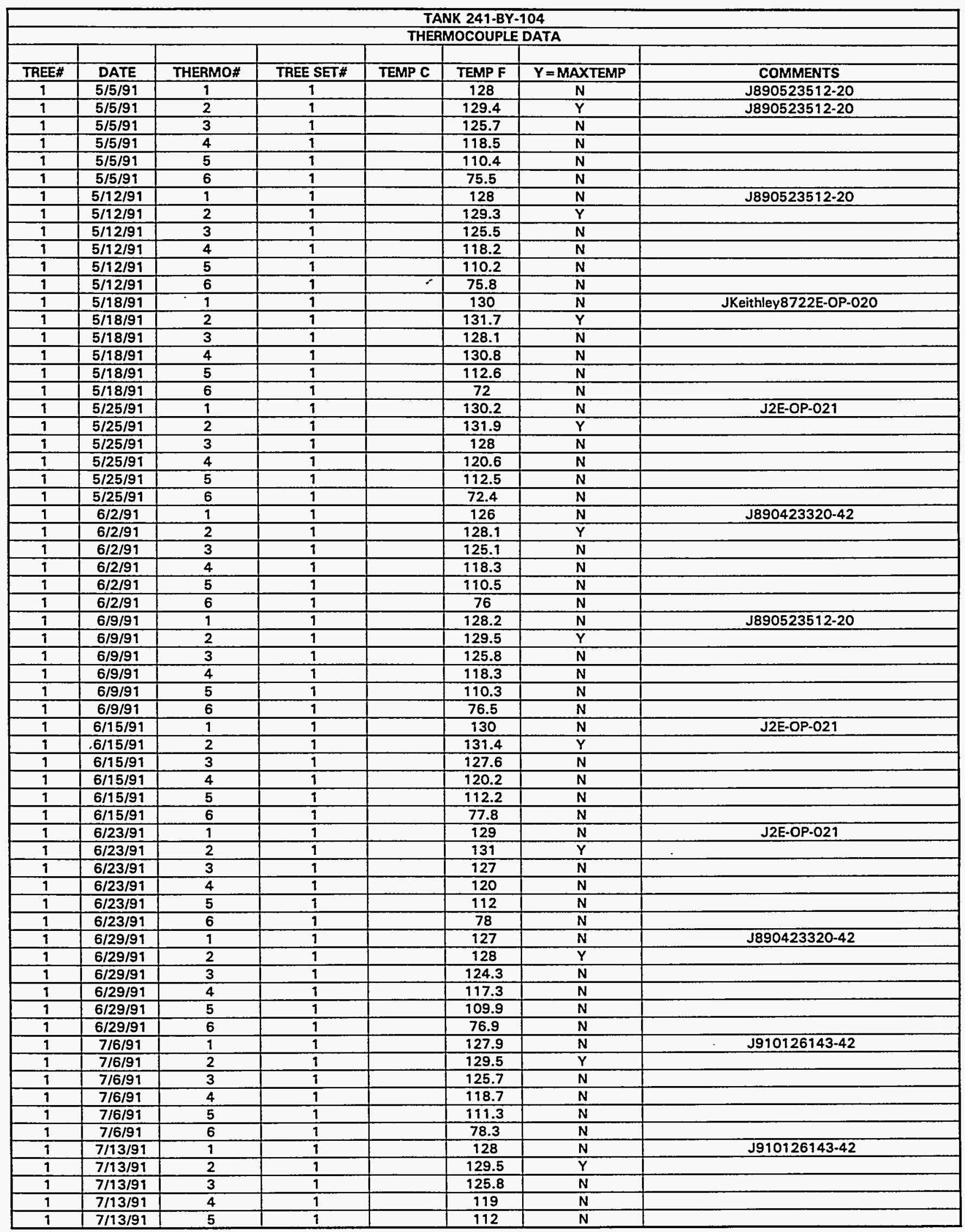

Data obtained from WHC Surveillance Analysis Computer System (SACS), November 12, 1993. 
WHC-SD-WM-ER-312, Rev. 0

\begin{tabular}{|c|c|c|c|c|c|c|c|}
\hline \multicolumn{8}{|c|}{ TANK 241-BY-104 } \\
\hline \multicolumn{8}{|c|}{ THERMOCOUPLE DATA } \\
\hline TREE\# & DATE & THERMO\# & TREE SET\# & TEMP C & TEMP F & $Y=$ MAXTEMP & COMMENTS \\
\hline 1 & $7 / 13 / 91$ & 6 & 1 & & 78.4 & $N$ & \\
\hline 1 & $7 / 20 / 91$ & 1 & 1 & & 126.4 & $N$ & J910126143.64 \\
\hline 1 & $7 / 20 / 91$ & 2 & 1 & & 128.1 & $\bar{Y}$ & \\
\hline$T$ & $7 / 20 / 91$ & 3 & 1 & & 124.2 & $\mathbf{N}$ & \\
\hline 1 & $7 / 20 / 91$ & 4 & 1 & & 117.4 & $\mathbf{N}$ & \\
\hline 1 & $7 / 20 / 91$ & 5 & 1 & & 110 & $\mathbf{N}$ & \\
\hline 1 & $7 / 20 / 91$ & 6 & 1 & & 77.7 & $\mathbf{N}$ & \\
\hline 1 & $7 / 27 / 91$ & 1 & 1 & & 125.1 & $\mathbf{N}$ & J910126143-14 \\
\hline 1 & $7 / 27 / 91$ & 2 & 1 & & 125.8 & $\bar{Y}$ & \\
\hline 1 & $7 / 27 / 91$ & 3 & $T$ & & 123.9 & $\mathbf{N}$ & \\
\hline 1 & $7 / 27 / 91$ & 4 & 1 & & 125.1 & $\mathbf{N}$ & \\
\hline 1 & $7 / 27 / 91$ & 5 & 1 & 5 & 110.9 & $\mathbf{N}$ & \\
\hline 1 & $8 / 3 / 91$ & 1 & 1 & & 124.9 & $\mathbf{N}$ & $\mathrm{J} 910126143-14$ \\
\hline 1 & $8 / 3 / 91$ & 2 & 1 & & 126.3 & $\bar{Y}$ & \\
\hline 1 & $8 / 3 / 91$ & 3 & 1 & & 123.6 & $\mathbf{N}$ & \\
\hline 1 & $8 / 3 / 91$ & 4 & 1 & & 117.9 & $\mathbf{N}$ & \\
\hline 1 & $8 / 3 / 91$ & 5 & 1 & & 110.1 & $N$ & \\
\hline 1 & $8 / 3 / 91$ & 6 & 1 & & 78.4 & $\mathbf{N}$ & \\
\hline 1 & $8 / 10 / 91$ & 1 & 1 & & 127 & $\mathbf{N}$ & $J 890523512-20$ \\
\hline 1 & $8 / 10 / 91$ & 2 & 1 & & 129 & $\bar{Y}$ & 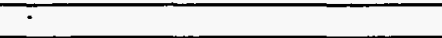 \\
\hline 1 & $8 / 10 / 91$ & 3 & 1 & & 126 & $\mathbf{N}$ & \\
\hline 1 & $8 / 10 / 91$ & 4 & 1 & & 119 & $N$ & \\
\hline 1 & $8 / 10 / 91$ & 5 & 1 & & 112 & $N$ & \\
\hline 1 & $8 / 10 / 91$ & 6 & 1 & & 80 & $\mathbf{N}$ & \\
\hline 1 & $8 / 18 / 91$ & 1 & 1 & & 124.8 & $\mathbf{N}$ & $J 890523512-20$ \\
\hline 1 & $8 / 18 / 91$ & 2 & 1 & & 126.5 & $\bar{Y}$ & \\
\hline 1 & $8 / 18 / 91$ & 3 & 1 & & 123.7 & $\mathbf{N}$ & \\
\hline 1 & $8 / 18 / 91$ & 4 & 1 & & 117.8 & $\mathbf{N}$ & \\
\hline 1 & $8 / 18 / 91$ & 5 & 1 & & 110.8 & $\mathbf{N}$ & \\
\hline 1 & $8 / 18 / 91$ & 6 & 1 & & 79.2 & $\mathbf{N}$ & \\
\hline 1 & $8 / 24 / 91$ & 1 & 1 & & 125.8 & $\mathbf{N}$ & $5890523512-20$ \\
\hline 1 & $8 / 24 / 91$ & 2 & 9 & & 127.4 & $\bar{Y}$ & \\
\hline 1 & $8 / 24 / 91$ & 3 & 1 & & 124.8 & $\bar{N}$ & \\
\hline 1 & $8 / 24 / 91$ & 4 & 1 & & 119.2 & $\mathbf{N}$ & \\
\hline 1 & $8 / 24 / 91$ & 5 & 1 & & 112.7 & $\mathbf{N}$ & \\
\hline 1 & $8 / 24 / 91$ & 6 & 1 & & 81.2 & $\mathbf{N}$ & \\
\hline 1 & $8 / 31 / 91$ & 1 & 1 & & 124.8 & $\mathbf{N}$ & $\mathrm{J}-890423320-42$ \\
\hline 1 & $.8 / 31 / 91$ & 2 & $T$ & & 126.3 & $\bar{Y}$ & \\
\hline 1 & $8 / 31 / 91$ & 3 & 1 & & 124.1 & $\mathbf{N}$ & \\
\hline 1 & $8 / 31 / 91$ & 4 & 1 & & 118.4 & $\mathbf{N}$ & \\
\hline$\overline{1}$ & $8 / 31 / 91$ & 5 & 1 & & 111.7 & $\mathbf{N}$ & \\
\hline 1 & $8 / 31 / 91$ & 6 & 9 & & 80.4 & $\mathbf{N}$ & \\
\hline 1 & $9 / 8 / 91$ & 1 & 1 & & 124.8 & $\mathbf{N}$ & $J-890423320-42$ \\
\hline 1 & $9 / 8 / 91$ & 2 & 1 & & 126.2 & $\mathbf{Y}$ & \\
\hline 1 & $9 / 8 / 91$ & 3 & 1 & & 124.1 & $\mathbf{N}$ & \\
\hline 1 & $9 / 8 / 91$ & 4 & 1 & & 118.2 & $\bar{N}$ & \\
\hline 1 & $9 / 8 / 91$ & 5 & 1 & & 111.7 & $\mathbf{N}$ & \\
\hline 1 & $9 / 8 / 91$ & 6 & 1 & & 80.4 & $\mathbf{N}$ & \\
\hline 1 & $9 / 15 / 91$ & 9 & 1 & & 125.3 & $\mathbf{N}$ & $\mathrm{J}-910126143-75$ \\
\hline$\frac{1}{1}$ & $9 / 15 / 91$ & 2 & 1 & & 127.1 & $Y$ & \\
\hline 1 & $9 / 15 / 91$ & 3 & 1 & & 124.3 & $\mathbf{N}$ & \\
\hline 1 & $9 / 15 / 91$ & 4 & 1 & & 118.9 & $\mathbf{N}$ & \\
\hline$T$ & $9 / 15 / 91$ & 5 & 1 & & 112.4 & $N$ & \\
\hline 1 & $9 / 15 / 91$ & 6 & 1 & & 82 & $N$ & \\
\hline 1 & $9 / 22 / 91$ & 2 & 1 & & 129.5 & $\bar{Y}$ & $\mathrm{~J}-910126143-75 \mathrm{TC} \# 10 / \mathrm{S}$ \\
\hline 1 & $9 / 22 / 91$ & 3 & 1 & & 126 & $\bar{N}$ & \\
\hline 1 & $9 / 22 / 91$ & 4 & 1 & & 120 & $\mathbf{N}$ & \\
\hline 1 & $9 / 22 / 91$ & 5 & 1 & & 113.1 & $\bar{N}$ & \\
\hline 1 & $9 / 22 / 91$ & 6 & 1 & & 83.4 & $\mathbf{N}$ & \\
\hline 1 & $9 / 28 / 91$ & 1 & 1 & & & $\mathbf{N}$ & SuppliedAirAccessOnly \\
\hline 1 & $10 / 1 / 91$ & 9 & 1 & & 127.9 & $\mathbf{N}$ & $J-910126143.75$ \\
\hline 1 & $10 / 1 / 91$ & 2 & 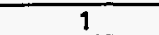 & & 128.6 & $\bar{Y}$ & \\
\hline 1 & $10 / 1 / 91$ & 3 & 1 & & 124.4 & $\mathbf{N}$ & \\
\hline 1 & $10 / 1 / 91$ & 4 & 1 & & 117.9 & $N$ & \\
\hline 1 & $10 / 1 / 91$ & 5 & 1 & & 111.6 & $\mathbf{N}$ & \\
\hline
\end{tabular}

Data obtained from WHC Surveillance Analysis Computer System (SACS), November 12, 1993. 


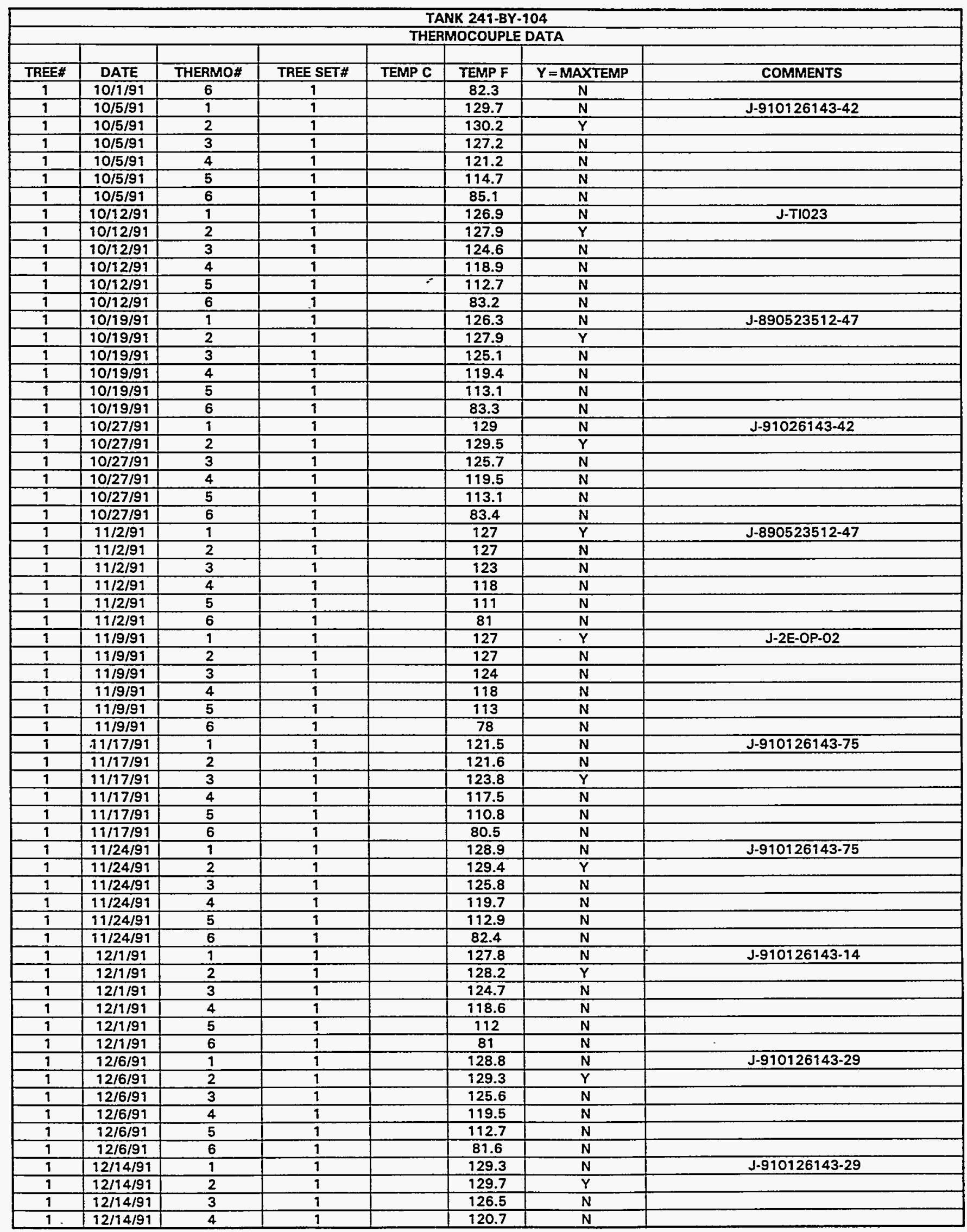

Data obtained from WHC Surveillance Analysis Computer System (SACS), November 12, 1993. 
WHC-SD-WM-ER-312, Rev. 0

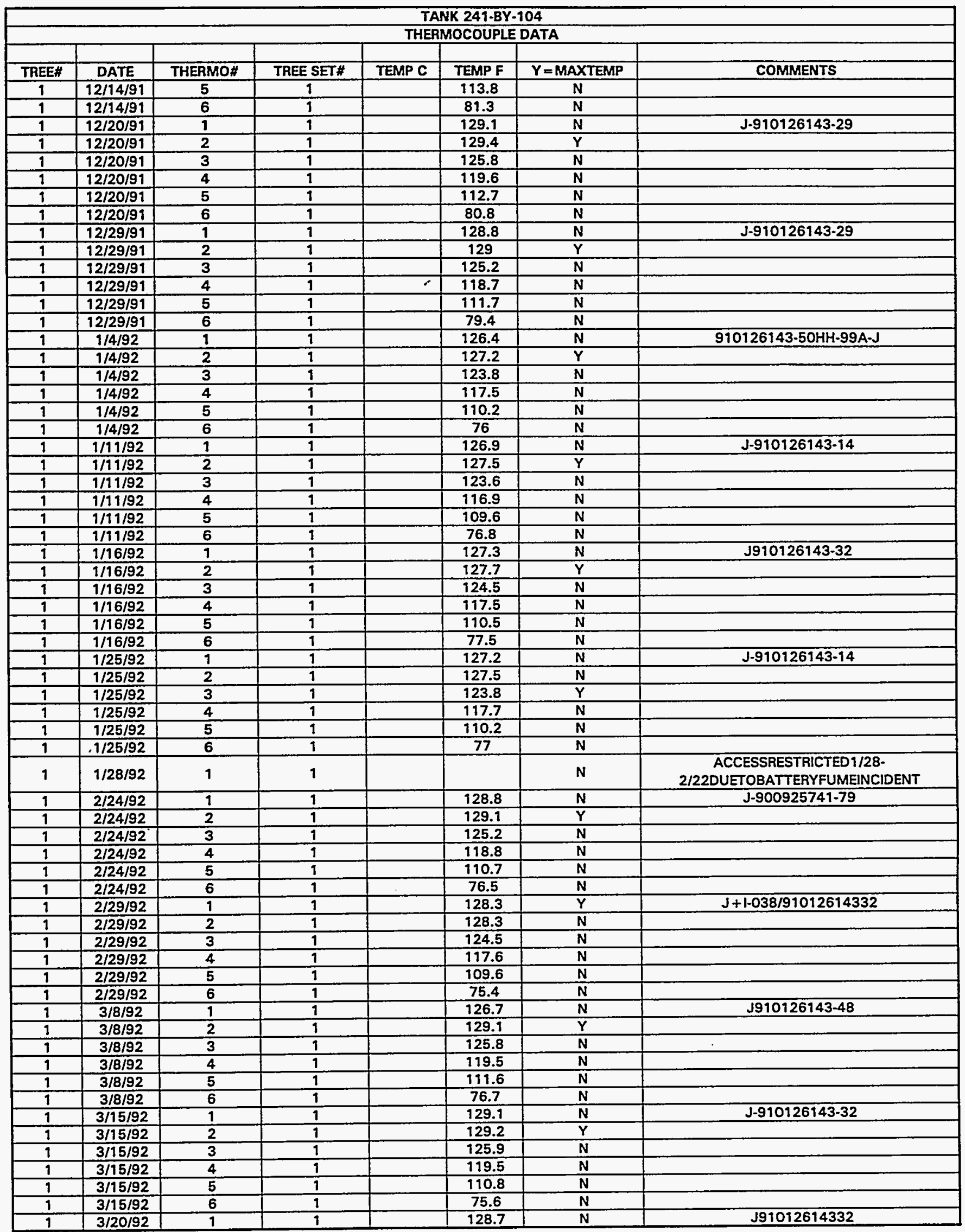

Data obtained from WHC Surveillance Analysis Computer System (SACS), November 12, 1993. 


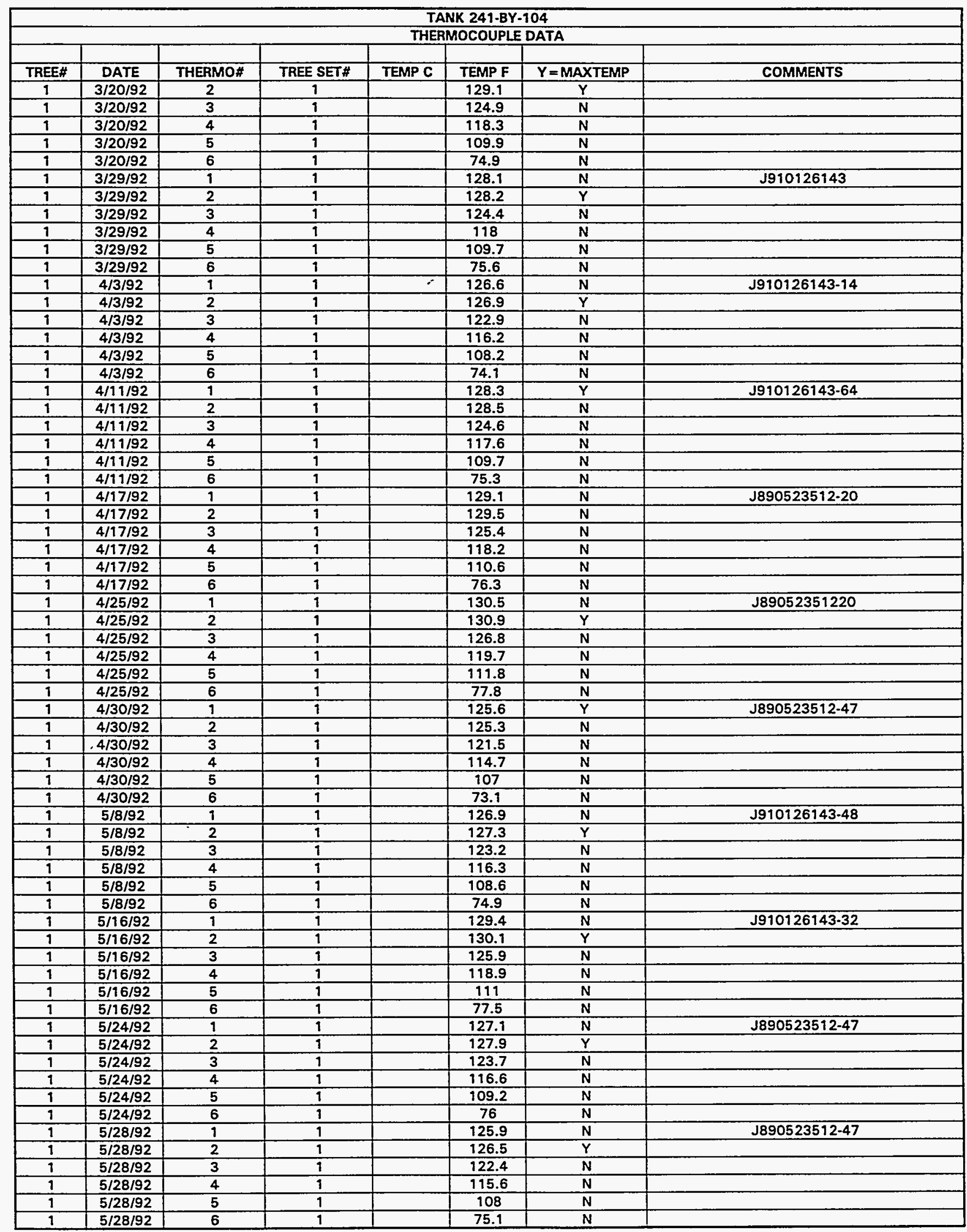

Data obtained from WHC Surveillance Analysis Computer System (SACS), November 12, 1993. 


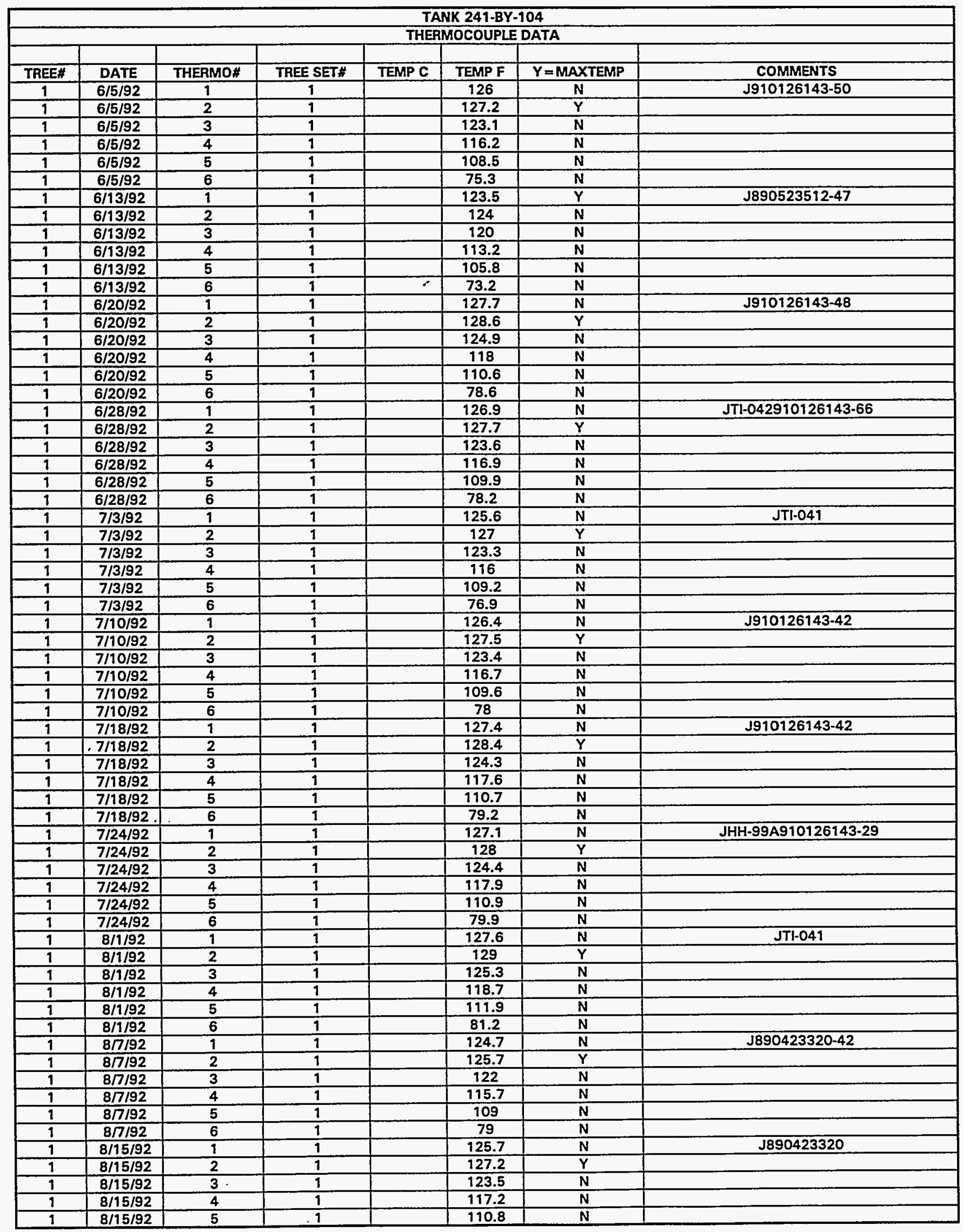

Data obtained from WHC Surveillance Analysis Computer System (SACS), November 12, 1993. 
WHC-SD-WM-ER-312, Rev. 0

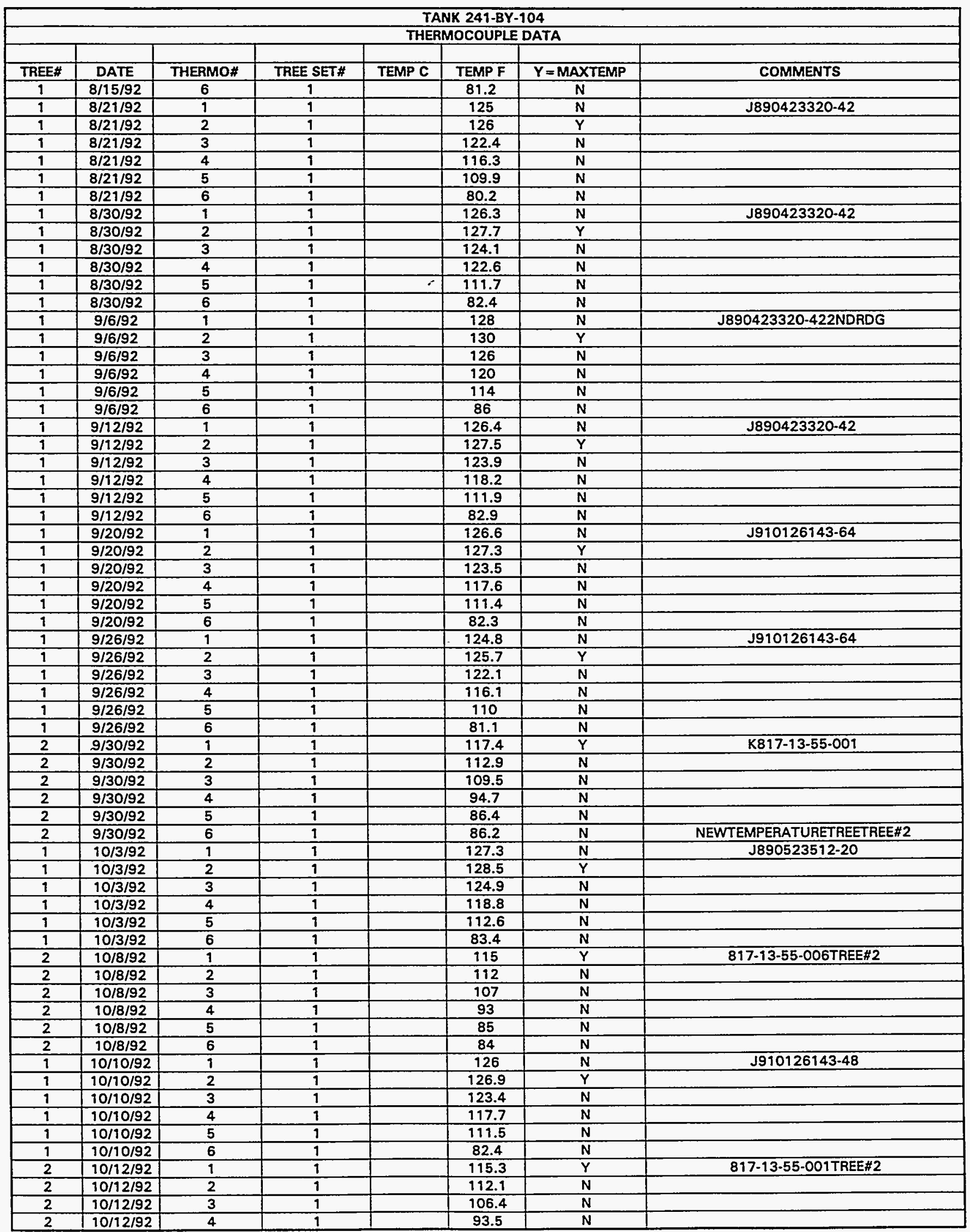

Data obtained from WHC Surveillance Analysis Computer System (SACS), November 12, 1993. 
WHC-SD-WM-ER-312, Rev. 0

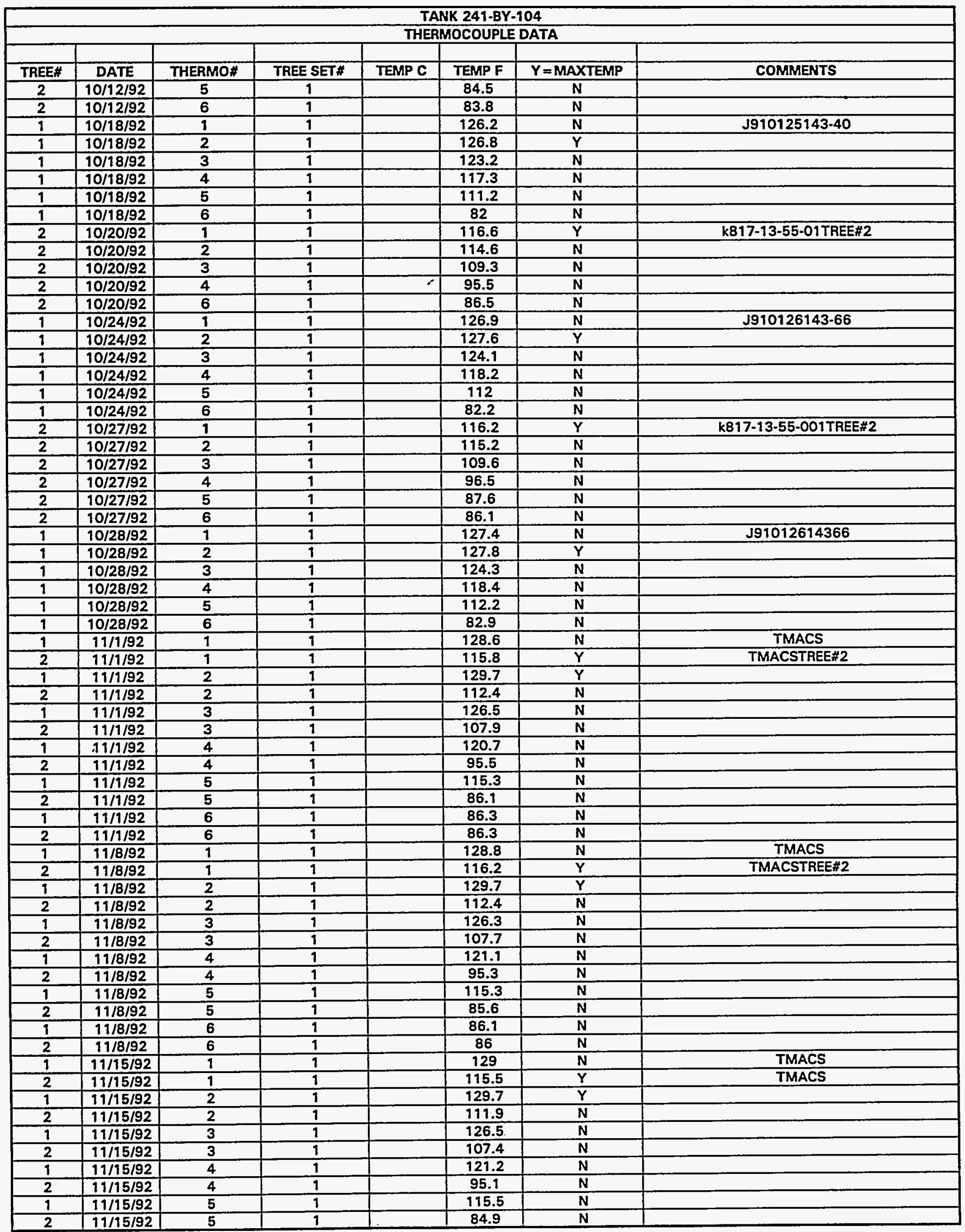

Data obtained from WHC Surveillance Analysis Computer System (SACS), November 12, 1993. 
WHC-SD-WM-ER-312, Rev. 0

\begin{tabular}{|c|c|c|c|c|c|c|c|}
\hline \multicolumn{8}{|c|}{ TANK 241-BY-104 } \\
\hline \multicolumn{8}{|c|}{ THERMOCOUPLE DATA } \\
\hline TREE\# & DATE & THERMO\# & TREE SET\# & TEMP C & TEMP F & $Y=$ MAXTEMP & COMMENTS \\
\hline 1 & $11 / 15 / 92$ & 6 & 1 & & 86 & $\mathbf{N}$ & \\
\hline 2 & $11 / 15 / 92$ & 6 & 1 & & 85.2 & $\mathbf{N}$ & \\
\hline 1 & $11 / 20 / 92$ & 1 & 1 & & 126.1 & $\mathbf{N}$ & $J 910126143-48$ \\
\hline 1 & $11 / 20 / 92$ & 2 & 1 & & 126.4 & $\bar{Y}$ & \\
\hline 1 & $11 / 20 / 92$ & 3 & 1 & & 122.8 & $\mathbf{N}$ & \\
\hline 1 & $11 / 20 / 92$ & 4 & 1 & & 116.9 & $\mathrm{~N}$ & \\
\hline 1 & $11 / 20 / 92$ & 5 & 1 & & 110.7 & $\mathbf{N}$ & \\
\hline 1 & $11 / 20 / 92$ & 6 & 1 & & 80.4 & $\mathbf{N}$ & \\
\hline 1 & $11 / 22 / 92$ & 1 & 1 & & 129.2 & $\mathbf{N}$ & TMACS \\
\hline 2 & $11 / 22 / 92$ & 1 & 1 & & 116.2 & $\bar{Y}$ & TMACS \\
\hline 1 & $11 / 22 / 92$ & 2 & 1 & & 129.9 & $\bar{Y}$ & \\
\hline 2 & $11 / 22 / 92$ & 2 & 1 & 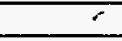 & 112.4 & $\mathbf{N}$ & \\
\hline 1 & $11 / 22 / 92$ & 3 & 1 & & 126.6 & $\mathbf{N}$ & \\
\hline 2 & $11 / 22 / 92$ & 3 & 1 & & 108.1 & $\mathbf{N}$ & \\
\hline 1 & $11 / 22 / 92$ & 4 & 9 & & 121.2 & $\mathbf{N}$ & \\
\hline 2 & $11 / 22 / 92$ & 4 & 1 & & 95.7 & $\mathbf{N}$ & \\
\hline 1 & $11 / 22 / 92$ & 5 & 1 & & 115.5 & $\mathbf{N}$ & \\
\hline 2 & $11 / 22 / 92$ & 5 & 1 & & 85.2 & $\mathbf{N}$ & \\
\hline 1 & $11 / 22 / 92$ & 6 & 1 & & 85.6 & $N$ & \\
\hline 2 & $11 / 22 / 92$ & 6 & 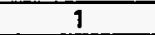 & & 85.1 & $\mathbf{N}$ & \\
\hline 1 & $11 / 29 / 92$ & 1 & 1 & & 129.2 & $\mathbf{N}$ & TMACS \\
\hline 2 & $11 / 29 / 92$ & 1 & 1 & & 114.9 & $\mathbf{N}$ & TMACS \\
\hline 1 & $11 / 29 / 92$ & 2 & 1 & & 129.9 & $Y$ & \\
\hline 2 & $11 / 29 / 92$ & 2 & 1 & & 112.4 & $\mathbf{N}$ & \\
\hline 1 & $11 / 29 / 92$ & 3 & 1 & & 126.6 & $\mathbf{N}$ & \\
\hline 2 & $11 / 29 / 92$ & 3 & 1 & & 107.6 & $\mathbf{N}$ & \\
\hline 1 & $11 / 29 / 92$ & 4 & 1 & & 121.4 & $\mathbf{N}$ & \\
\hline 2 & $11 / 29 / 92$ & 4 & 1 & & 95.5 & $\mathbf{N}$ & \\
\hline 1 & $11 / 29 / 92$ & 5 & 1 & & 115.7 & $N$ & \\
\hline 2 & $11 / 29 / 92$ & 5 & 1 & & 84.3 & $N$ & \\
\hline 1 & $11 / 29 / 92$ & 6 & 1 & & 85.2 & $\mathbf{N}$ & \\
\hline 2 & $11 / 29 / 92$ & 6 & 1 & & 84.5 & $\mathbf{N}$ & \\
\hline 1 & $12 / 6 / 92$ & 1 & 1 & & 129.2 & $\mathbf{N}$ & TMACSRISER1 \\
\hline 2 & $12 / 6 / 92$ & 1 & 1 & & 115.8 & $Y$ & TMACSRISER10B \\
\hline 1 & $12 / 6 / 92$ & 2 & 1 & & 129.9 & $\bar{Y}$ & \\
\hline 2 & $12 / 6 / 92$ & 2 & 1 & & 112.6 & $\mathbf{N}$ & \\
\hline 1 & $12 / 6 / 92$ & 3 & 1 & & 126.6 & $\mathbf{N}$ & \\
\hline 2 & $.12 / 6 / 92$ & 3 & 1 & & 107.7 & $\mathbf{N}$ & \\
\hline 1 & $12 / 6 / 92$ & 4 & 1 & & 121.4 & $\mathrm{~N}$ & \\
\hline 2 & $12 / 6 / 92$ & 4 & 1 & & 95.1 & $N$ & \\
\hline 1 & $12 / 6 / 92$ & 5 & 1 & & 115.5 & $\mathrm{~N}$ & \\
\hline 2 & $12 / 6 / 92$ & 5 & 1 & & 83.8 & $\mathbf{N}$ & \\
\hline 1 & $12 / 6 / 92$ & 6 & 1 & & 84.9 & $\mathbf{N}$ & \\
\hline 2 & $12 / 6 / 92$ & 6 & 1 & & 83.8 & $\mathbf{N}$ & \\
\hline 1 & $12 / 13 / 92$ & 1 & $\frac{1}{1}$ & & 129.2 & $\mathbf{N}$ & TMACS \\
\hline 2 & $12 / 13 / 92$ & 1 & 1 & & 115.5 & $\bar{Y}$ & TMACS \\
\hline 1 & $12 / 13 / 92$ & 2 & 1 & & 129.7 & $\bar{Y}$ & \\
\hline 2 & $12 / 13 / 92$ & 2 & 1 & & 112.1 & $\mathbf{N}$ & \\
\hline 1 & $12 / 13 / 92$ & 3 & 1 & & 126.6 & $\mathbf{N}$ & \\
\hline 2 & $12 / 13 / 92$ & 3 & 1 & & 107.4 & $\mathbf{N}$ & \\
\hline 1 & $12 / 13 / 92$ & 4 & 1 & & 121.2 & $N$ & \\
\hline 2 & $12 / 13 / 92$ & 4 & 1 & & 94.8 & $\mathbf{N}$ & \\
\hline 1 & $12 / 13 / 92$ & 5 & 1 & & 115.3 & $\mathbf{N}$ & \\
\hline 2 & $12 / 13 / 92$ & 5 & $i$ & & 83.3 & $\mathrm{~N}$ & \\
\hline 1 & $12 / 13 / 92$ & 6 & 1 & & 84.2 & $N$ & \\
\hline 2 & $12 / 13 / 92$ & 6 & 1 & & 83.4 & $\mathbf{N}$ & \\
\hline 1 & $12 / 20 / 92$ & 1 & 1 & & 129.2 & $\mathbf{N}$ & TMACS \\
\hline 2 & $12 / 20 / 92$ & 1 & 1 & & 115.7 & $\bar{Y}$ & TMACS \\
\hline 1 & $12 / 20 / 92$ & 2 & 1 & & 129.7 & $Y$ & \\
\hline 2 & $12 / 20 / 92$ & 2 & 1 & & 112.1 & $N$ & \\
\hline 1 & $12 / 20 / 92$ & 3 & 1 & & 126.6 & $\mathrm{~N}$ & \\
\hline 2 & $12 / 20 / 92$ & 3 & 1 & & 107.7 & $\mathbf{N}$ & \\
\hline 1 & $12 / 20 / 92$ & 4 & 1 & & 121.1 & $\mathbf{N}$ & \\
\hline 2 & $12 / 20 / 92$ & 4 & 1 & & 95 & $\mathbf{N}$ & \\
\hline 1 & $12 / 20 / 92$ & 5 & 1 & & 114.9 & $\mathbf{N}$ & \\
\hline
\end{tabular}

Data obtained from WHC Surveillance Analysis Computer System (SACS), November 12, 1993. 


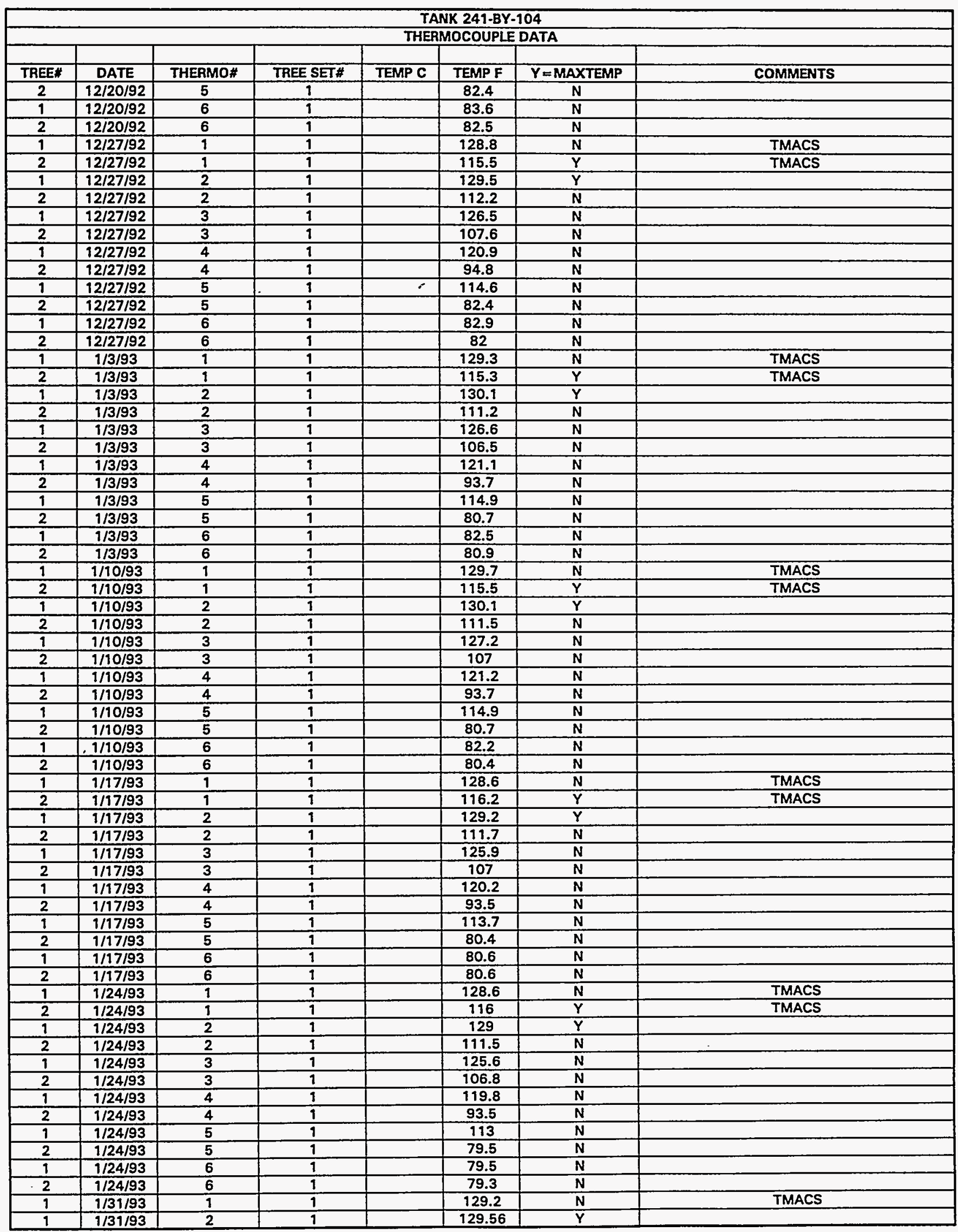

Data obtained from WHC Surveillance Analysis Computer System (SACS), November 12, 1993. 


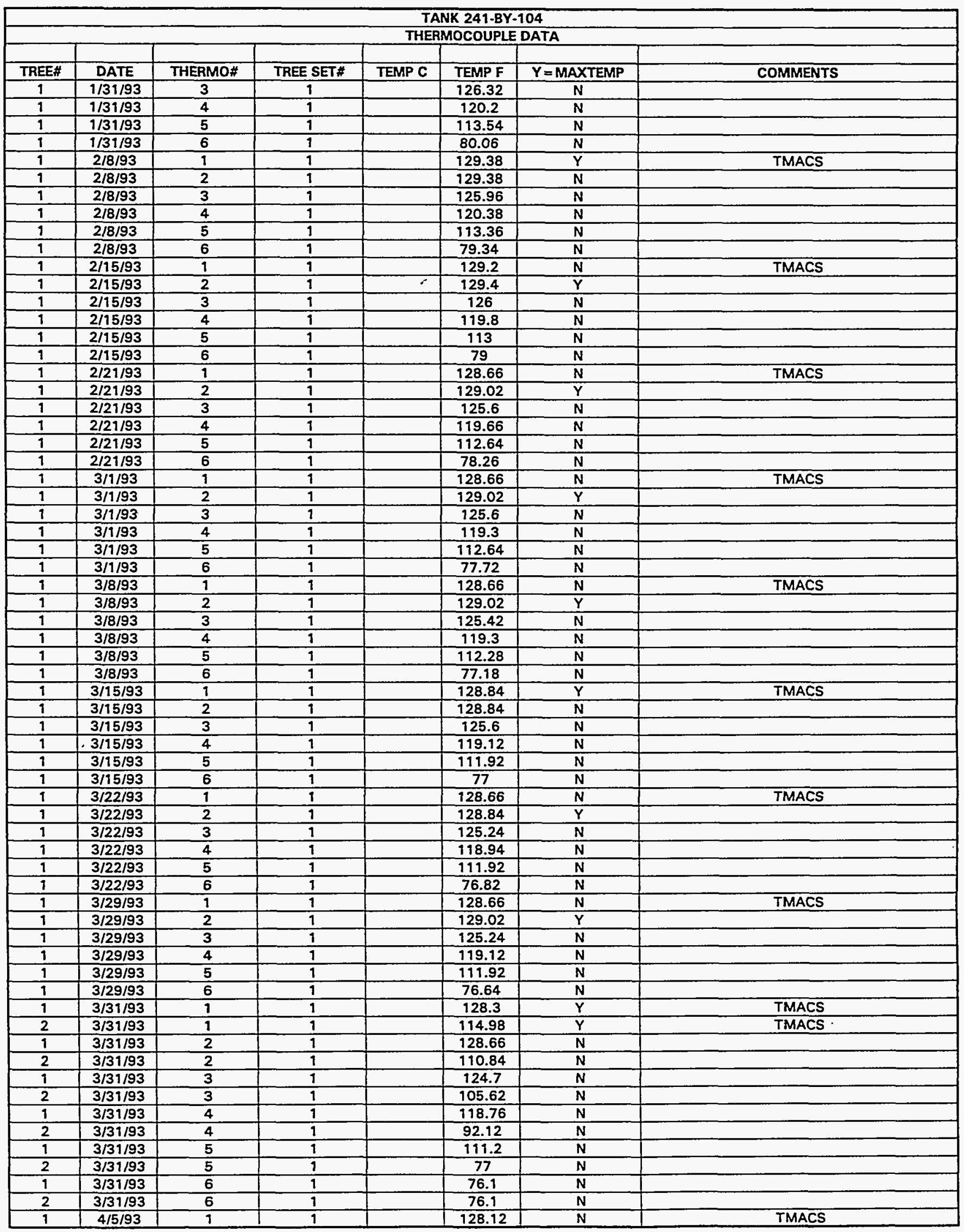

Data obtained from WHC Surveillance Analysis Computer System (SACS), November 12, 1993. 


\begin{tabular}{|c|c|c|c|c|c|c|c|}
\hline \multicolumn{8}{|c|}{ TANK 241-BY-104 } \\
\hline \multicolumn{8}{|c|}{ THERMOCOUPLE DATA } \\
\hline TREE\# & DATE & THERMO\# & TREE SET\# & TEMP C & TEMP F & $Y=$ MAXTEMP & COMMENTS \\
\hline 2 & $4 / 5 / 93$ & 1 & 1 & & 114.98 & $Y$ & TMACS \\
\hline 1 & $4 / 5 / 93$ & 2 & 1 & & 128.48 & $\bar{Y}$ & \\
\hline 2 & $4 / 5 / 93$ & 2 & $\frac{1}{1}$ & & 111.02 & $\mathbf{N}$ & \\
\hline 1 & $4 / 5 / 93$ & 3 & $\frac{1}{1}$ & & 124.88 & $\mathrm{~N}$ & \\
\hline 2 & $4 / 5 / 93$ & 3 & 1 & & 105.44 & $\mathbf{N}$ & \\
\hline$T$ & $4 / 5 / 93$ & 4 & 1 & & 118.4 & $\mathbf{N}$ & \\
\hline 2 & $4 / 5 / 93$ & 4 & 1 & & 91.76 & $\mathbf{N}$ & \\
\hline 1 & $4 / 5 / 93$ & 5 & 1 & & 111.56 & $\mathbf{N}$ & \\
\hline 2 & $4 / 5 / 93$ & 5 & 1 & & 76.82 & $\mathbf{N}$ & \\
\hline 1 & $4 / 5 / 93$ & 6 & 1 & & 76.1 & $\mathbf{N}$ & \\
\hline 2 & $4 / 5 / 93$ & 6 & 1 & & 76.1 & N & \\
\hline 1 & $4 / 12 / 93$ & 1 & 1 & 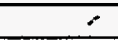 & 128.66 & $\mathbf{N}$ & TMAC \\
\hline 2 & $4 / 12 / 93$ & 1 & 9 & & 114.8 & $\bar{Y}$ & TMAC \\
\hline 1 & $4 / 12 / 93$ & 2 & 1 & & 128.84 & $\bar{Y}$ & \\
\hline 2 & $4 / 12 / 93$ & 2 & $\frac{1}{1}$ & & 110.48 & $\mathbf{N}$ & \\
\hline 1 & $4 / 12 / 93$ & 3 & 1 & & 125.06 & $\mathbf{N}$ & \\
\hline 2 & $4 / 12 / 93$ & 3 & 1 & & 104.72 & $\mathbf{N}$ & \\
\hline 1 & $4 / 12 / 93$ & 4 & 1 & & 118.94 & $\mathbf{N}$ & \\
\hline 2 & $4 / 12 / 93$ & 4 & 1 & & 91.22 & $\mathbf{N}$ & \\
\hline 1 & $4 / 12 / 93$ & 5 & 1 & & 111.74 & $\mathbf{N}$ & \\
\hline 2 & $4 / 12 / 93$ & 5 & 1 & & 77 & $\mathbf{N}$ & \\
\hline 9 & $4 / 12 / 93$ & 6 & 1 & & 76.46 & $\bar{N}$ & \\
\hline 2 & $4 / 12 / 93$ & 6 & 1 & & 75.74 & $\mathbf{N}$ & \\
\hline T & $4 / 19 / 93$ & 1 & 1 & & 128.12 & $\mathbf{N}$ & TMACS \\
\hline 2 & $4 / 19 / 93$ & 1 & 1 & & 114.98 & $\bar{Y}$ & TMACS \\
\hline 1 & $4 / 19 / 93$ & 2 & 1 & & 128.48 & $\bar{Y}$ & \\
\hline 2 & $4 / 19 / 93$ & 2 & 1 & & 110.66 & $\mathbf{N}$ & \\
\hline$T$ & $4 / 19 / 93$ & $\overline{3}$ & 1 & & 124.7 & $\mathbf{N}$ & \\
\hline 2 & $4 / 19 / 93$ & 3 & 1 & & 105.26 & $\mathbf{N}$ & \\
\hline 1 & $4 / 19 / 93$ & 4 & 1 & & 118.4 & $\mathbf{N}$ & \\
\hline 2 & $4 / 19 / 93$ & 4 & 1 & & 91.94 & $\mathbf{N}$ & \\
\hline 9 & $4 / 19 / 93$ & 5 & 1 & & 111.2 & $\overline{\mathbf{N}}$ & \\
\hline 2 & $4 / 19 / 93$ & 5 & 1 & & 77 & $\mathrm{~N}$ & \\
\hline 1 & $4 / 19 / 93$ & 6 & 1 & & 76.1 & $\mathbf{N}$ & \\
\hline 2 & $4 / 19 / 93$ & 6 & 1 & & 76.1 & $\mathbf{N}$ & \\
\hline 1 & $4 / 25 / 93$ & 1 & 1 & & 128.12 & $\mathbf{N}$ & TMACS \\
\hline 2 & $4 / 25 / 93$ & 1 & 1 & & 114.98 & $\bar{Y}$ & TMACS \\
\hline 1 & $4 / 25 / 93$ & 2 & 1 & & 128.48 & $\bar{Y}$ & \\
\hline 2 & $4 / 25 / 93$ & 2 & 1 & & 110.66 & $\mathbf{N}$ & \\
\hline 1 & $4 / 25 / 93$ & 3 & 1 & & 124.7 & $\mathbf{N}$ & \\
\hline 2 & $4 / 25 / 93$ & 3 & 1 & & 105.26 & $\bar{N}$ & \\
\hline 1 & $4 / 25 / 93$ & 4 & 1 & & 118.22 & $\mathrm{~N}$ & \\
\hline 2 & $4 / 25 / 93$ & 4 & 1 & & 91.77 & $\mathbf{N}$ & \\
\hline 1 & $4 / 25 / 93$ & 5 & 1 & & 110.84 & $\mathbf{N}$ & \\
\hline 2 & $4 / 25 / 93$ & 5 & 1 & & 77.18 & $\mathbf{N}$ & \\
\hline 1 & $4 / 25 / 93$ & 6 & 1 & & 75.92 & $\mathbf{N}$ & \\
\hline 2 & $4 / 25 / 93$ & 6 & 1 & & 75.92 & $\mathbf{N}$ & \\
\hline 1 & $5 / 3 / 93$ & 1 & 1 & & 127.76 & $\mathbf{N}$ & TMACS \\
\hline 2 & $5 / 3 / 93$ & 1 & 1 & & 114.98 & $Y$ & TMACS \\
\hline 1 & $5 / 3 / 93$ & 2 & 1 & & 127.94 & $Y$ & \\
\hline 2 & $5 / 3 / 93$ & 2 & 1 & & 110.66 & $\mathbf{N}$ & \\
\hline 1 & $5 / 3 / 93$ & 3 & 1 & & 124.34 & $\mathbf{N}$ & \\
\hline 2 & $5 / 3 / 93$ & 3 & 1 & & 105.44 & $N$ & \\
\hline 1 & $5 / 3 / 93$ & 4 & 1 & & 117.86 & $\mathbf{N}$ & \\
\hline 2 & $5 / 3 / 93$ & 4 & 1 & & 91.4 & $N$ & \\
\hline 1 & $5 / 3 / 93$ & 5 & 1 & & 111.2 & $\mathrm{~N}$ & \\
\hline 2 & $5 / 3 / 93$ & 5 & 1 & & 77.54 & $\mathbf{N}$ & \\
\hline$\frac{1}{1}$ & $5 / 3 / 93$ & 6 & $\frac{1}{1}$ & & 75.92 & $\mathbf{N}$ & \\
\hline 2 & $5 / 3 / 93$ & 6 & 1 & & 76.46 & $\mathbf{N}$ & \\
\hline 1 & $5 / 10 / 93$ & 1 & 1 & & 128.12 & $\mathbf{N}$ & TMACS \\
\hline 2 & $5 / 10 / 93$ & 1 & 1 & & 115.16 & $Y$ & TMACS \\
\hline$\frac{4}{1}$ & $5 / 10 / 93$ & 2 & 1 & & 128.48 & $\dot{Y}$ & \\
\hline 2 & $5 / 10 / 93$ & 2 & 1 & & 111.02 & $\mathbf{N}$ & \\
\hline 1 & $5 / 10 / 93$ & 3 & 1 & & 124.7 & $\mathbf{N}$ & \\
\hline 2 & $5 / 10 / 93$ & 3 & 1 & & 105.44 & $N$ & \\
\hline
\end{tabular}

Data obtained from WHC Surveillance Analysis Computer System (SACS), November 12, 1993. 


\begin{tabular}{|c|c|c|c|c|c|c|c|}
\hline \multicolumn{8}{|c|}{ TANK 241-BY-104 } \\
\hline & & & & THEI & 10 COUPL & DATA & \\
\hline TREE\# & DATE & THERMO\# & TREE SET\# & TEMP C & TEMPF & $Y=M A X T E M P$ & COMMENTS \\
\hline 1 & $5 / 10 / 93$ & 4 & 9 & & 118.22 & $N$ & \\
\hline 2 & $5 / 10 / 93$ & 4 & 1 & & 91.94 & $\bar{N}$ & \\
\hline 1 & $5 / 10 / 93$ & 5 & 1 & & 111.2 & $\mathbf{N}$ & \\
\hline 2 & $5 / 10 / 93$ & 5 & 1 & & 77.9 & $\mathbf{N}$ & \\
\hline 1 & $5 / 10 / 93$ & 6 & 1 & & 76.82 & $N$ & \\
\hline 2 & $5 / 10 / 93$ & 6 & 1 & & 76.82 & $\bar{N}$ & \\
\hline 1 & $5 / 17 / 93$ & 1 & 1 & & 127.4 & $\bar{Y}$ & TMACS \\
\hline 2 & $5 / 17 / 93$ & 1 & 1 & & 114.98 & $\bar{Y}$ & TMACS \\
\hline 1 & $5 / 17 / 93$ & 2 & 1 & & 127.4 & $\mathbf{N}$ & \\
\hline 2 & $5 / 17 / 93$ & 2 & 1 & & 110.84 & $N$ & \\
\hline 1 & $5 / 17 / 93$ & 3 & 1 & & 123.98 & $\mathbf{N}$ & \\
\hline 2 & $5 / 17 / 93$ & 3 & 1 & $\because$ & 105.26 & $\mathbf{N}$ & \\
\hline 1 & $5 / 17 / 93$ & 4 & 1 & & 117.32 & $N$ & \\
\hline 2 & $5 / 17 / 93$ & 4 & 1 & & 91.94 & $\mathbf{N}$ & \\
\hline 1 & $5 / 17 / 93$ & 5 & 1 & & 110.3 & $\mathbf{N}$ & \\
\hline 2 & $5 / 17 / 93$ & 5 & 1 & & 77.9 & $N$ & \\
\hline 1 & $5 / 17 / 93$ & 6 & 1 & & 76.1 & $\mathrm{~N}$ & \\
\hline 2 & $5 / 17 / 93$ & 6 & 1 & & 76.64 & $\mathbf{N}$ & \\
\hline 1 & $6 / 7 / 93$ & 1 & 1 & & 127.4 & $\mathbf{N}$ & TMACS \\
\hline 2 & $6 / 7 / 93$ & 1 & 1 & & 115.52 & $\bar{Y}$ & TMACS \\
\hline 1 & $6 / 7 / 93$ & 2 & 1 & & 127.76 & $Y$ & \\
\hline 2 & $6 / 7 / 93$ & 2 & 1 & & 111.2 & $\mathbf{N}$ & \\
\hline 1 & $6 / 7 / 93$ & 3 & 1 & & 124.34 & $N$ & \\
\hline 2 & $6 / 7 / 93$ & 3 & 1 & & 105.62 & $\mathbf{N}$ & \\
\hline 1 & $6 / 7 / 93$ & 4 & 1 & & 118.04 & $N$ & \\
\hline 2 & $6 / 7 / 93$ & 4 & 1 & & 92.84 & $N$ & \\
\hline 1 & $6 / 7 / 93$ & 5 & 1 & & 111.02 & $\mathbf{N}$ & \\
\hline 2 & $6 / 7 / 93$ & 5 & 1 & & 79.7 & $\mathrm{~N}$ & \\
\hline 1 & $6 / 7 / 93$ & 6 & 1 & & 77.72 & $N$ & \\
\hline 2 & $6 / 7 / 93$ & 6 & 1 & & 78.44 & $\mathbf{N}$ & \\
\hline 1 & $6 / 14 / 93$ & 1 & 1 & & 126.86 & $\bar{N}$ & TMACS \\
\hline 2 & $6 / 14 / 93$ & 1 & 1 & & 114.8 & $\bar{Y}$ & TMACS \\
\hline 1 & $6 / 14 / 93$ & 2 & 1 & & 127.22 & $\bar{Y}$ & \\
\hline 2 & $6 / 14 / 93$ & 2 & 1 & & 110.66 & $\mathbf{N}$ & \\
\hline 1 & $6 / 14 / 93$ & 3 & 1 & & 123.62 & $N$ & \\
\hline 2 & $6 / 14 / 93$ & 3 & $\frac{1}{1}$ & & 105.26 & $\mathbf{N}$ & \\
\hline 1 & $6 / 14 / 93$ & 4 & 1 & & 117.14 & $\mathbf{N}$ & \\
\hline 2 & $.6 / 14 / 93$ & 4 & 1 & & 92.66 & $\mathrm{~N}$ & \\
\hline 1 & $6 / 14 / 93$ & 5 & 1 & & 110.48 & $\mathbf{N}$ & \\
\hline 2 & $6 / 14 / 93$ & 5 & 1 & & 79.52 & $\mathrm{~N}$ & \\
\hline$\frac{1}{1}$ & $6 / 14 / 93$ & 6 & 1 & & 77.54 & $\mathbf{N}$ & \\
\hline 2 & $6 / 14 / 93$ & 6 & 1 & & 78.26 & $\mathbf{N}$ & \\
\hline 1 & $6 / 21 / 93$ & 1 & 1 & & 127.04 & $\mathbf{N}$ & TMACS \\
\hline 2 & $6 / 21 / 93$ & 1 & 1 & & 115.52 & $\bar{Y}$ & TMACS \\
\hline 1 & $6 / 21 / 93$ & 2 & 9 & & 127.4 & $\bar{Y}$ & \\
\hline 2 & $6 / 21 / 93$ & 2 & 1 & & 111.38 & $\mathbf{N}$ & \\
\hline 1 & $6 / 21 / 93$ & 3 & 1 & & 123.62 & $\mathbf{N}$ & \\
\hline 2 & $6 / 21 / 93$ & 3 & 1 & & 106.34 & $\mathbf{N}$ & \\
\hline 1 & $6 / 21 / 93$ & 4 & 1 & & 117.32 & $\mathbf{N}$ & \\
\hline 2 & $6 / 21 / 93$ & 4 & 9 & & 93.56 & $\mathbf{N}$ & \\
\hline 1 & $6 / 21 / 93$ & 5 & 1 & & 110.84 & $\mathbf{N}$ & \\
\hline 2 & $6 / 21 / 93$ & 5 & $\frac{1}{1}$ & & 80.78 & $\bar{N}$ & \\
\hline 1 & $6 / 21 / 93$ & 6 & $\frac{1}{1}$ & & 78.08 & $\mathrm{~N}$ & \\
\hline 2 & $6 / 21 / 93$ & 6 & 1 & & 79.7 & $\mathbf{N}$ & \\
\hline$\frac{1}{1}$ & $6 / 28 / 93$ & 1 & 1 & & 127.04 & $\mathrm{~N}$ & TMACS \\
\hline 2 & $6 / 28 / 93$ & 1 & 9 & & 114.98 & $\bar{Y}$ & TMACS \\
\hline 1 & $6 / 28 / 93$ & 2 & 1 & & 127.76 & $\bar{Y}$ & \\
\hline 2 & $6 / 28 / 93$ & 2 & 9 & & 110.84 & $\mathrm{~N}$ & \\
\hline 1 & $6 / 28 / 93$ & 3 & 1 & & 124.16 & $N$ & \\
\hline 2 & $6 / 28 / 93$ & 3 & 1 & & 105.8 & $\mathrm{~N}$ & \\
\hline 1 & $6 / 28 / 93$ & 4 & 1 & & 117.86 & $N$ & \\
\hline 2 & $6 / 28 / 93$ & 4 & 1 & & 93.56 & $\mathbf{N}$ & \\
\hline 1 & $6 / 28 / 93$ & 5 & 1 & & 111.38 & $N$ & \\
\hline 2 & $6 / 28 / 93$ & 5 & 1 & & 80.6 & $\mathbf{N}$ & \\
\hline 1 & $6 / 28 / 93$ & 6 & 1 & & 78.98 & $N$ & \\
\hline
\end{tabular}

Data obtained from WHC Surveillance Analysis Computer System (SACS), November 12, 1993. 


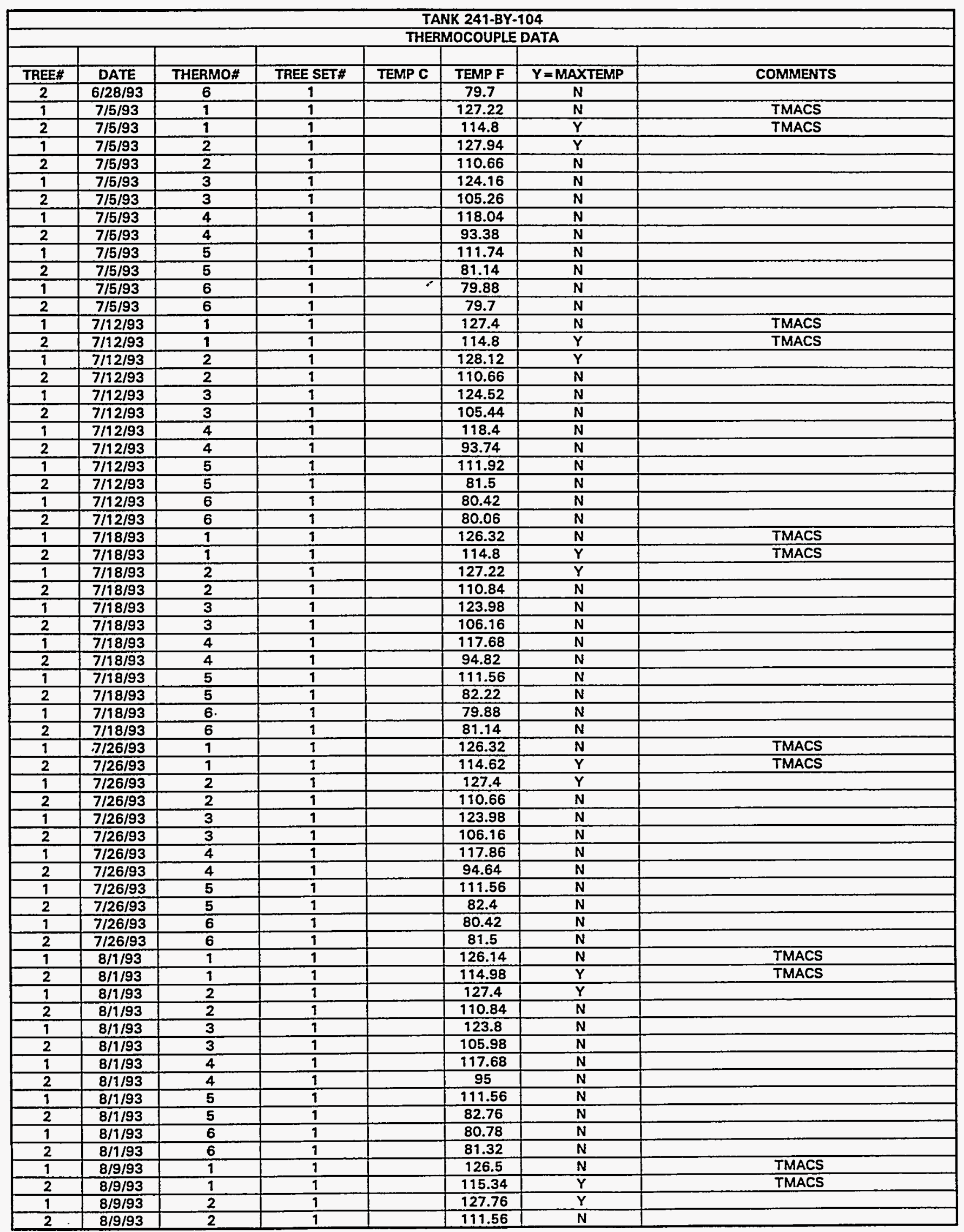

Data obtained from WHC Surveillance Analysis Computer System (SACS), November 12, 1993. 


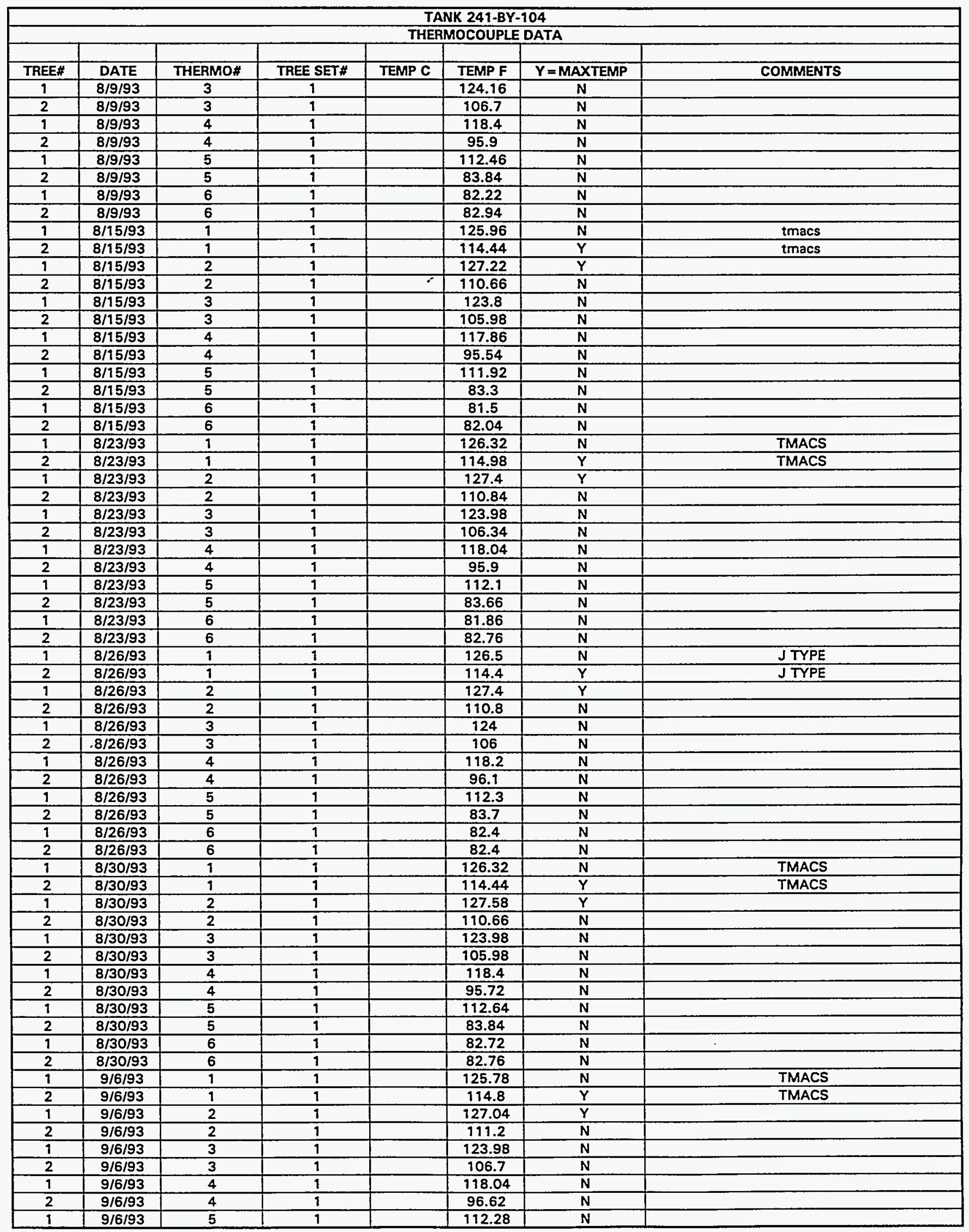

Data obtained from WHC Surveillance Analysis Computer System (SACS), November 12, 1993. 


\begin{tabular}{|c|c|c|c|c|c|c|c|}
\hline \multirow{2}{*}{\multicolumn{8}{|c|}{$\begin{array}{l}\text { TANK 241-BY-104 } \\
\text { THEPM }\end{array}$}} \\
\hline & & & & & & DATA & \\
\hline TREE\#\# & DATE & THERMO\# & TREE SET\# & TEMP C & TEMP F & $Y=$ MAXTEMP & COMMENTS \\
\hline 2 & $9 / 6 / 93$ & 5 & 1 & & 84.74 & $N$ & \\
\hline 1 & $9 / 6 / 93$ & 6 & 1 & & 82.98 & $\mathrm{~N}$ & \\
\hline 2 & $9 / 6 / 93$ & 6 & 1 & & 83.48 & $\mathbf{N}$ & \\
\hline 1 & $9 / 12 / 93$ & 1 & 1 & & 126.32 & $\mathrm{~N}$ & TMACS \\
\hline 2 & $9 / 12 / 93$ & 1 & 1 & & 194.44 & $\bar{Y}$ & TMACS \\
\hline 1 & $9 / 12 / 93$ & 2 & 1 & & 127.76 & $\bar{Y}$ & \\
\hline 2 & $9 / 12 / 93$ & 2 & 1 & & 111.02 & $\mathbf{N}$ & \\
\hline 1 & $9 / 12 / 93$ & 3 & 1 & & 124.16 & $\bar{N}$ & \\
\hline 2 & $9 / 12 / 93$ & 3 & 1 & & 106.52 & $N$ & \\
\hline 1 & $9 / 12 / 93$ & 4 & 1 & & 118.76 & $\mathrm{~N}$ & \\
\hline 2 & $9 / 12 / 93$ & 4 & 1 & & 96.44 & $\mathbf{N}$ & \\
\hline 1 & $9 / 12 / 93$ & 5 & $\frac{\pi}{1}$ & & 113.18 & $\mathrm{~N}$ & \\
\hline 2 & $9 / 12 / 93$ & 5 & 1 & & 84.74 & $\bar{N}$ & \\
\hline 9 & $9 / 12 / 93$ & 6 & 1 & & 83.66 & $\mathrm{~N}$ & \\
\hline 2 & $9 / 12 / 93$ & $\frac{5}{6}$ & 1 & & 83.66 & $\mathbf{N}$ & \\
\hline 1 & $9 / 19 / 93$ & 1 & 1 & & 126.5 & $\mathbf{N}$ & TMACS \\
\hline 2 & $9 / 19 / 93$ & 1 & 1 & & 114.08 & $Y$ & TMACS \\
\hline 1 & $9 / 19 / 93$ & 2 & 1 & & 127.58 & $\mathbf{N}$ & \\
\hline 2 & $9 / 19 / 93$ & $\frac{2}{2}$ & $i$ & & 110.84 & $\mathrm{~N}$ & \\
\hline 1 & 9/19/93 & 3 & 1 & & 123.98 & $\mathbf{N}$ & \\
\hline 2 & $9 / 19 / 93$ & 3 & 1 & & 106.52 & $\mathrm{~N}$ & \\
\hline 1 & 9/19/93 & 4 & 1 & & 118.58 & $\mathbf{N}$ & \\
\hline 2 & $9 / 19 / 93$ & 4 & 9 & & 96.98 & $\mathrm{~N}$ & \\
\hline 1 & $9 / 19 / 93$ & 5 & 1 & & 113 & $\mathrm{~N}$ & \\
\hline 2 & $9 / 19 / 93$ & 5 & 1 & & 84.56 & $\mathbf{N}$ & \\
\hline 1 & $9 / 19 / 93$ & 6 & 1 & & 83.84 & $N$ & \\
\hline 2 & 9/19/93 & 6 & 9 & & 83.66 & $\mathbf{N}$ & \\
\hline 1 & $9 / 26 / 93$ & 1 & 1 & & 126.5 & $\mathrm{~N}$ & TMACS \\
\hline 2 & $9 / 26 / 93$ & 1 & 9 & & 114.62 & $\bar{Y}$ & TMACS \\
\hline 1 & $9 / 26 / 93$ & 2 & 1 & & 127.58 & $\bar{Y}$ & \\
\hline 2 & $9 / 26 / 93$ & 2 & 1 & & 111.2 & $\mathbf{N}$ & \\
\hline 1 & $9 / 26 / 93$ & 3 & 1 & & 124.16 & $\mathrm{~N}$ & \\
\hline 2 & $9 / 26 / 93$ & 3 & 9 & & 106.7 & $\mathbf{N}$ & \\
\hline 1 & $9 / 26 / 93$ & 4 & 9 & & 118.76 & $\mathbf{N}$ & \\
\hline$\frac{1}{2}$ & $9 / 26 / 93$ & 4 & 9 & & 97.16 & $\mathrm{~N}$ & \\
\hline 1 & $9 / 26 / 93$ & 5 & 1 & & 113 & $\mathbf{N}$ & \\
\hline 2 & $9 / 26 / 93$ & 5 & 1 & & 84.92 & $\mathbf{N}$ & \\
\hline 1 & $9 / 26 / 93$ & 6 & 9 & & 84.02 & $\mathrm{~N}$ & \\
\hline 2 & $9 / 26 / 93$ & 6 & 9 & & 84.2 & $\mathrm{~N}$ & \\
\hline 1 & $10 / 3 / 93$ & 1 & 1 & & 126.68 & $\mathrm{~N}$ & TMACS \\
\hline$\frac{1}{2}$ & $10 / 3 / 93$ & $\frac{1}{1}$ & 1 & & 114.62 & $\frac{Y}{Y}$ & TMACS \\
\hline 1 & $10 / 3 / 93$ & 2 & 1 & & 127.76 & $\bar{Y}$ & \\
\hline 2 & $10 / 3 / 93$ & 2 & 1 & & 111.02 & $\mathbf{N}$ & \\
\hline 1 & $10 / 3 / 93$ & 3 & 1 & & 124.34 & $\mathrm{~N}$ & \\
\hline 2 & $10 / 3 / 93$ & 3 & 1 & & 106.88 & $\mathbf{N}$ & \\
\hline 1 & $10 / 3 / 93$ & 4 & 1 & & 119.12 & $N$ & \\
\hline 2 & $10 / 3 / 93$ & 4 & 1 & & 97.34 & $\mathbf{N}$ & \\
\hline 1 & $10 / 3 / 93$ & 5 & 1 & & 113.36 & $\mathbf{N}$ & \\
\hline$\frac{1}{2}$ & $10 / 3 / 93$ & $\frac{5}{5}$ & $\frac{1}{1}$ & & 85.28 & $\mathrm{~N}$ & \\
\hline 1 & $10 / 3 / 93$ & 6 & 1 & & 84.2 & $\mathbf{N}$ & \\
\hline 2 & $10 / 3 / 93$ & 6 & 1 & & 84.38 & $\mathbf{N}$ & \\
\hline$\frac{2}{9}$ & $10 / 10 / 93$ & $\frac{\pi}{1}$ & 1 & & 126.68 & $\mathrm{~N}$ & TMACS \\
\hline 2 & $10 / 10 / 93$ & 1 & 1 & & 114.26 & $\bar{Y}$ & TMACS \\
\hline 9 & $10 / 10 / 93$ & 2 & 1 & & 127.76 & $Y$ & \\
\hline 2 & $10 / 10 / 93$ & 2 & $\frac{1}{1}$ & & 110.84 & N & \\
\hline 9 & $10 / 10 / 93$ & 3 & 1 & & 124.16 & $\mathbf{N}$ & \\
\hline 2 & $10 / 10 / 93$ & 3 & 1 & & 106.7 & $\mathbf{N}$ & \\
\hline 1 & $10 / 10 / 93$ & 4 & 1 & & 118.94 & $\mathbf{N}$ & \\
\hline 2 & $10 / 10 / 93$ & 4 & 9 & & 97.16 & $N$ & \\
\hline 9 & $10 / 10 / 93$ & 5 & 1 & & 113.36 & $\mathrm{~N}$ & \\
\hline$\frac{1}{2}$ & $10 / 10 / 93$ & $\frac{\pi}{5}$ & $\frac{1}{1}$ & & 85.1 & $\mathrm{~N}$ & \\
\hline 1 & $10 / 10 / 93$ & 6 & 1 & & 84.38 & $\mathbf{N}$ & \\
\hline 2 & $10 / 10 / 93$ & 6 & 1 & & 84.02 & $N$ & \\
\hline$\frac{2}{1}$ & $10 / 19 / 93$ & $\frac{0}{1}$ & 1 & & 126.7 & $N$ & TMACS \\
\hline 2 & $10 / 19 / 93$ & 1 & 1 & & 114.1 & $Y$ & TMACS \\
\hline
\end{tabular}

Data obtained from WHC Surveillance Analysis Computer System (SACS), November 12, 1993. 


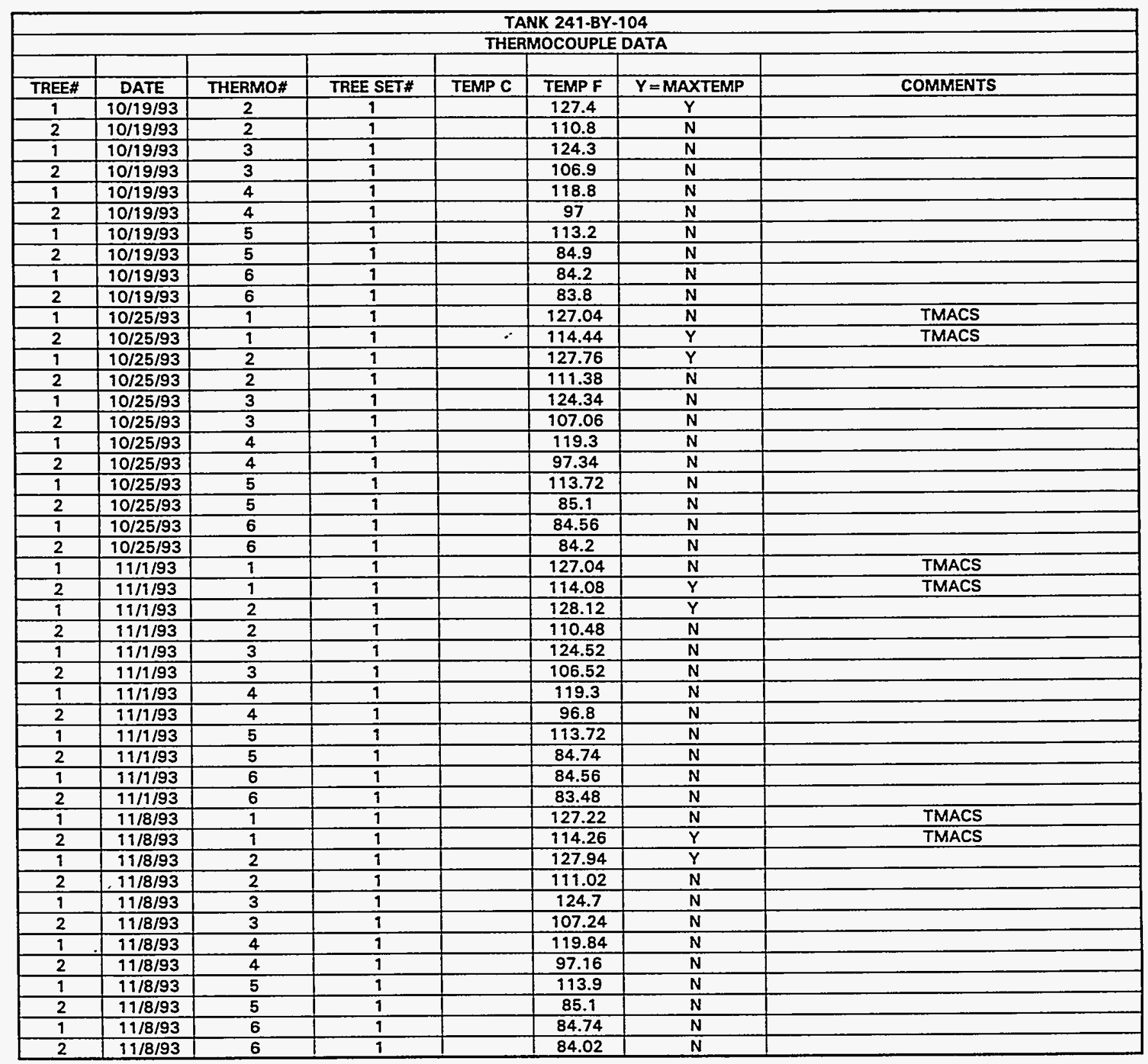

Data obtained from WHC Surveillance Analysis Computer System (SACS), November 12, 1993. 


\begin{tabular}{|c|c|c|c|c|c|c|c|}
\hline \multicolumn{8}{|c|}{ TANK 251-BY-105 } \\
\hline \multicolumn{8}{|c|}{ THERMOCOUPLE DATA } \\
\hline TREE \# & DATE & THERMO \# & TREE SET \# & TEMP C & TEMP F & $Y=M A X$ TEMP & COMMENTS \\
\hline 1 & $9 / 12 / 74$ & 1 & 1 & 80 & 176 & $Y$ & degree C/TWINS confirm \\
\hline 1 & $9 / 12 / 74$ & 2 & 9 & 72 & 161.6 & $\mathbf{N}$ & degree C/TWINS confirm \\
\hline 1 & $9 / 12 / 74$ & 3 & 1 & 64 & 147.2 & $\mathbf{N}$ & degree CrTWINS confirm \\
\hline 1 & $9 / 12 / 74$ & 4 & 1 & 54 & 129.2 & $N$ & degree $\mathrm{C} / T$ WINS confirm \\
\hline 1 & $9 / 12 / 74$ & 5 & 1 & 44 & 111.2 & $\mathbf{N}$ & degree C/TWINS confirm \\
\hline 1 & $9 / 12 / 74$ & 6 & 1 & 38 & 100.4 & $\mathbf{N}$ & degree C/TWINS confirm \\
\hline 1 & $9 / 12 / 74$ & 7 & 1 & 38 & 100.4 & $\mathbf{N}$ & degree C/TWINS confirm \\
\hline 1 & $9 / 12 / 74$ & 8 & 1 & 38 & 100.4 & $\mathbf{N}$ & degree C/TWINS confirm \\
\hline 1 & $9 / 12 / 74$ & 9 & 1 & 36 & 96.8 & $\mathbf{N}$ & degrae C/TWINS confirm \\
\hline 1 & $9 / 12 / 74$ & 10 & 1 & 34 & 93.2 & $\mathbf{N}$ & degree C/TWINS confirm \\
\hline 1 & $9 / 12 / 74$ & 11 & 1 & 32 & 89.6 & $\bar{N}$ & degree C/TWINS confirm \\
\hline 1 & $9 / 12 / 74$ & 12 & 1 & 32 & 89.6 & $\mathbf{N}$ & degree $\mathrm{C} / \mathrm{TW}$ INS confirm \\
\hline 1 & $10 / 8 / 74$ & 1 & 1 & 70 & 158 & $\bar{Y}$ & degree C/TWINS confirm \\
\hline 9 & $10 / 8 / 74$ & 2 & 1 & 63 & 145.4 & $\mathbf{N}$ & degree C/TWINS confirm \\
\hline 9 & $10 / 8 / 74$ & 3 & 1 & 58 & 136.4 & $\mathbf{N}$ & degree C/TWINS confirm \\
\hline$\frac{1}{1}$ & $10 / 8 / 74$ & 4 & 1 & 50 & 122 & $\mathbf{N}$ & degree $\mathrm{C} / \mathrm{TW}$ INS confirm \\
\hline 1 & $10 / 8 / 74$ & 5 & 1 & 44 & 111.2 & $\mathbf{N}$ & degree C/TWINS confirm \\
\hline 1 & $10 / 8 / 74$ & 6 & 1 & 42 & 107.6 & $\mathbf{N}$ & degree $\mathrm{C} / \mathrm{TW}$ INS confirm \\
\hline 1 & $10 / 8 / 74$ & 7 & 1 & 38 & 100.4 & $\mathbf{N}$ & degree C/TWINS confirm \\
\hline 1 & $10 / 8 / 74$ & 8 & 1 & 34 & 93.2 & $\bar{N}$ & degree C/TWINS confirm \\
\hline 1 & $10 / 8 / 74$ & 9 & 1 & 30 & 86 & $\mathbf{N}$ & degree $\mathrm{C} / \mathrm{TW}$ INS confirm \\
\hline 1 & $10 / 8 / 74$ & 10 & 1 & 25 & 77 & $\mathbf{N}$ & degree $\mathrm{C} / \mathrm{TW}$ INS confirm \\
\hline 1 & $10 / 8 / 74$ & 11 & 1 & 25 & 77 & $\mathbf{N}$ & degree $\mathrm{C} / \mathrm{T}$ WINS confirm \\
\hline$\frac{1}{1}$ & $10 / 8 / 74$ & 12 & 1 & 25 & 77 & $N$ & degree C/TWINS confirm \\
\hline 1 & $10 / 29 / 74$ & 1 & 1 & 63 & 145.4 & $\bar{Y}$ & degree $\mathrm{C} / \mathrm{TW}$ INS confirm \\
\hline 1 & $10 / 29 / 74$ & 2 & 1 & 63 & 145.4 & $\mathbf{N}$ & degres C/TWINS confirm \\
\hline 1 & $10 / 29 / 74$ & 3 & 1 & 60 & 140 & $\bar{N}$ & degree C/TWINS confirm \\
\hline 1 & $10 / 29 / 74$ & 4 & 1 & 54 & 129.2 & $N$ & degree C/TWINS confirm \\
\hline 1 & $10 / 29 / 74$ & 5 & 1 & 50 & 122 & $N$ & degree C/TWINS confirm \\
\hline 1 & $10 / 29 / 74$ & 6 & 1 & 44 & 111.2 & $\mathbf{N}$ & degree C/TWINS confirm \\
\hline 1 & $10 / 29 / 74$ & 7 & 7 & 42 & 107.6 & $\mathbf{N}$ & degree $\mathrm{C} / \mathrm{TW}$ INS confirm \\
\hline 1 & $10 / 29 / 74$ & 8 & 1 & 37 & 98.6 & $\mathbf{N}$ & degree C/TWINS confirm \\
\hline 1 & $10 / 29 / 74$ & 9 & 1 & 31 & 87.8 & $\mathrm{~N}$ & degree C/TWINS confirm \\
\hline 1 & $10 / 29 / 74$ & 10 & 1 & 31 & 87.8 & $N$ & degree C/TWINS confirm \\
\hline 1 & $10 / 29 / 74$ & 11 & 1 & 26 & 78.8 & $\mathbf{N}$ & degree C/TWINS confirm \\
\hline 1 & $10 / 29 / 74$ & 12 & 1 & 26 & 78.8 & $\mathbf{N}$ & degree C/TWINS confirm \\
\hline 1 & $11 / 26 / 74$ & 1 & 1 & 66 & 150.8 & $\bar{Y}$ & degree C/TWINS confirm \\
\hline 1 & $11 / 26 / 74$ & 2 & 1 & 60 & 140 & $\mathbf{N}$ & degree $\mathrm{C} / \mathrm{TW}$ INS confirm \\
\hline 1 & $11 / 26 / 74$ & 3 & 1 & 56 & 132.8 & $\mathbf{N}$ & degree C/TWINS confirm \\
\hline 1 & $11 / 26 / 74$ & 4 & 1 & 50 & 122 & $\mathrm{~N}$ & degree $\mathrm{C} / \mathrm{TW}$ INS confirm \\
\hline 1 & $11 / 26 / 74$ & 5 & 1 & 45 & 113 & $\mathrm{~N}$ & degree C/TWINS confirm \\
\hline 1 & $11 / 26 / 74$ & 6 & 1 & 41 & 105.8 & $\mathbf{N}$ & degree C/TWINS confirm \\
\hline 1 & $11 / 26 / 74$ & 7 & 1 & 37 & 98.6 & $\mathbf{N}$ & degree C/TWINS confirm \\
\hline 1 & $11 / 26 / 74$ & 8 & 1 & 32 & 89.6 & $\mathbf{N}$ & degree C/TWINS confirm \\
\hline 1 & $11 / 26 / 74$ & 9 & 1 & 25 & 77 & $\mathbf{N}$ & degree C/TWINS confirm \\
\hline 1 & $11 / 26 / 74$ & 10 & 1 & 16 & 60.8 & $\mathbf{N}$ & degree C/TWINS confirm \\
\hline 1 & $11 / 26 / 74$ & 11 & 1 & 16 & 60.8 & $\mathbf{N}$ & degree C/TWINS confirm \\
\hline 1 & $11 / 26 / 74$ & 12 & 1 & 16 & 60.8 & $\mathbf{N}$ & degree C/TWINS confirm \\
\hline 1 & $12 / 11 / 74$ & 1 & 1 & 62.78 & 145.004 & $\bar{Y}$ & degree C/TWINS confirm \\
\hline 1 & $12 / 11 / 74$ & 2 & 1 & 57.78 & 136.004 & $\mathbf{N}$ & degree C/TWINS confirm \\
\hline 1 & $12 / 11 / 74$ & 3 & 1 & 53.89 & 129.002 & $\mathbf{N}$ & degree C/TWINS confirm \\
\hline 1 & $12 / 11 / 74$ & 4 & 1 & 48.89 & 120.002 & $\mathbf{N}$ & degree C/TWINS confirm \\
\hline 1 & $12 / 11 / 74$ & 5 & 1 & 42.78 & 109.004 & $\mathbf{N}$ & degree C/TWINS confirm \\
\hline 1 & $12 / 11 / 74$ & 6 & 1 & 38.89 & 102.002 & $\mathbf{N}$ & degree CTTWINS confirm \\
\hline 1 & $12 / 11 / 74$ & 7 & 1 & 28.33 & 82.994 & $\mathbf{N}$ & degree CTTWINS confirm \\
\hline 1 & $12 / 11 / 74$ & 8 & 1 & 26.67 & 80.006 & $\mathbf{N}$ & degree C/TWINS confirm \\
\hline 1 & $12 / 11 / 74$ & 9 & 1 & 19.44 & 66.992 & $\mathbf{N}$ & degree C/TWINS confirm \\
\hline 1 & $12 / 11 / 74$ & 10 & 1 & 12.78 & 55.004 & $\mathbf{N}$ & degree C/TWINS confirm \\
\hline 1 & $12 / 111 / 74$ & 11 & 1 & 12.22 & 53.996 & $\mathbf{N}$ & degree C/TWINS confirm \\
\hline 1 & $12 / 11 / 74$ & 12 & 1 & 12.78 & 55.004 & $\mathbf{N}$ & degree C/TWINS confirm \\
\hline 1 & $12 / 17 / 74$ & 1 & 1 & 65.56 & 150.008 & $\bar{Y}$ & degree C/TWINS confirm \\
\hline 1 & $12 / 17 / 74$ & 2 & 1 & 60 & 140 & $\mathbf{N}$ & degree C/TWINS confirm \\
\hline 9 & $12 / 17 / 74$ & 3 & 1 & 56.67 & 134.006 & $\mathbf{N}$ & degree C/TWINS confirm \\
\hline$\frac{1}{1}$ & $12 / 17 / 74$ & 4 & 1 & 51.11 & 123.998 & $\mathbf{N}$ & degree C/TWINS confirm \\
\hline 1 & $12 / 17 / 74$ & 5 & 1 & 46.11 & 114.998 & $\mathrm{~N}$ & degree C/TWINS confirm \\
\hline 1 & $12 / 17 / 74$ & 6 & 1 & 41.67 & 107.006 & $\mathbf{N}$ & degree C/TWINS confirm \\
\hline 1 & $12 / 17 / 74$ & 7 & 1 & 36.67 & 98.006 & $\mathbf{N}$ & degree C/TWINS confirm \\
\hline 1 & $12 / 17 / 74$ & 8 & 1 & 30 & 86 & $\mathrm{~N}$ & degree C/TWINS confirm \\
\hline
\end{tabular}

Data obtained from WHC Surveillance Analysis Computer System (SACS), June 2, 1993. 
WHC-SD-WM-ER-312, Rev. 0

\begin{tabular}{|c|c|c|c|c|c|c|c|}
\hline \multicolumn{8}{|c|}{ TANK 251-BY-105 } \\
\hline & & & & THEF & COUPLE D & & \\
\hline TREE \# & DATE & THERMO \# & TREE SET \# & TEMP C & TEMP F & $Y=\operatorname{MAX}$ TEMP & COMMENTS \\
\hline 1 & $12 / 17 / 74$ & 9 & 1 & 22.22 & 71.996 & $N$ & degree $\mathrm{C} / \mathrm{TW}$ INS confirm \\
\hline 1 & $12 / 17 / 74$ & 10 & 1 & 15.56 & 60.008 & $\mathbf{N}$ & degree C/TWINS confirm \\
\hline 1 & $12 / 17 / 74$ & 11 & 1 & 15.56 & 60.008 & $N$ & degree C/TWINS confirm \\
\hline 1 & $12 / 17 / 74$ & 12 & 1 & 15.56 & 60.008 & $\mathrm{~N}$ & degree $\mathrm{C} / \mathrm{TW}$ WNS confirm \\
\hline 9 & $12 / 31 / 74$ & 1 & 1 & 65.56 & 150.008 & $\bar{Y}$ & degree C/TWINS confirm \\
\hline 1 & $12 / 31 / 74$ & 2 & 1 & 60 & 140 & $\mathbf{N}$ & degree C/TWINS confirm \\
\hline$\overline{1}$ & $12 / 31 / 74$ & 3 & 1 & 56.11 & 132.998 & $\mathbf{N}$ & degree C/TWINS confirm \\
\hline 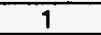 & $12 / 31 / 74$ & 4 & 1 & 49.44 & 120.992 & $\mathbf{N}$ & degree $\mathrm{C} / \mathrm{TW}$ INS confirm \\
\hline 1 & $12 / 31 / 74$ & 5 & 1 & 43.89 & 111.002 & $\mathbf{N}$ & degree C/TWINS confirm \\
\hline 1 & $12 / 31 / 74$ & 6 & 1 & 40.56 & 105.008 & $N$ & degree C/TWINS confirm \\
\hline 1 & $12 / 31 / 74$ & 7 & 1 & 35.56 & 96.008 & $\mathbf{N}$ & degree C/TWINS confirm \\
\hline 1 & $12 / 31 / 74$ & 8 & 1 & 28.89 & 84.002 & $\mathbf{N}$ & degree C/TWINS confirm \\
\hline 7 & $12 / 31 / 74$ & 9 & 1 & 20.56 & 69.008 & $\mathbf{N}$ & degree C/TWINS confirm \\
\hline 1 & $12 / 31 / 74$ & 10 & 1 & 13.33 & 55.994 & $\mathbf{N}$ & degree C/TWINS confirm \\
\hline 1 & $12 / 31 / 74$ & 11 & 1 & 12.22 & 53.996 & $\mathbf{N}$ & degree $\mathrm{C} / \mathrm{TW}$ INS confirm \\
\hline 1 & $12 / 31 / 74$ & 12 & 1 & 13.33 & 55.994 & $\mathbf{N}$ & degree C/TWINS confirm \\
\hline 1 & $1 / 8 / 75$ & 1 & 1 & 63.89 & 147.002 & $\mathbf{Y}$ & degree C/TWINS confirm \\
\hline 1 & $1 / 8 / 75$ & 2 & 1 & 58.89 & 138.002 & $\mathbf{N}$ & degree C/TWINS confirm \\
\hline 1 & $1 / 8 / 75$ & 3 & 1 & 55.56 & 132.008 & $\mathbf{N}$ & degree $\mathrm{C} / \mathrm{TWW}$ INS confirm \\
\hline 1 & $1 / 8 / 75$ & 4 & 1 & 50 & 122 & $\mathbf{N}$ & degree C/TWINS confirm \\
\hline 1 & $1 / 8 / 75$ & 5 & 1 & 45 & 113 & $\mathbf{N}$ & degree C/TWINS confirm \\
\hline 1 & $1 / 8 / 75$ & 6 & 1 & 41.11 & 105.998 & $\mathbf{N}$ & degree C/TWINS confirm \\
\hline 1 & $1 / 8 / 75$ & 7 & 1 & 35.56 & 96.008 & $\mathbf{N}$ & degree C/TWINS confirm \\
\hline 1 & $1 / 8 / 75$ & 8 & 1 & 28.89 & 84.002 & $\mathbf{N}$ & degree C/TWINS confirm \\
\hline 1 & $1 / 8 / 75$ & 9 & 1 & 21.11 & 69.998 & $\mathbf{N}$ & degree $\mathrm{C} / \mathrm{TW}$ INS confirm \\
\hline 1 & $1 / 8 / 75$ & 10 & 1 & 14.44 & 57.992 & $\mathbf{N}$ & degree C/TWINS confirm \\
\hline 1 & $1 / 8 / 75$ & 11 & 1 & 13.33 & 55.994 & $\mathbf{N}$ & degree C/TWINS confirm \\
\hline 1 & $1 / 8 / 75$ & 12 & 1 & 13.89 & 57.002 & $\overline{\mathbf{N}}$ & degree C/TWINS confirm \\
\hline 1 & $1 / 21 / 75$ & 9 & 1 & 82 & 179.6 & $\mathbf{Y}$ & degree C/TWINS confirm \\
\hline 1 & $1 / 21 / 75$ & 2 & 9 & 77 & 170.6 & $\mathbf{N}$ & degree $\mathrm{C} / \mathrm{T}$ WINS confirm \\
\hline 1 & $1 / 21 / 75$ & 3 & 1 & 72 & 161.6 & $\mathbf{N}$ & degree $\mathrm{C} / \mathrm{TW}$ iNS confirm \\
\hline 1 & $1 / 21 / 75$ & 4 & 1 & 66 & 150.8 & $\mathbf{N}$ & degree C/TWINS confirm \\
\hline 1 & $1 / 21 / 75$ & 5 & 1 & 59 & 138.2 & $\mathbf{N}$ & degree C/TWINS confirm \\
\hline 1 & $1 / 21 / 75$ & 6 & 1 & 52 & 125.6 & $\mathbf{N}$ & degree C/TWINS confirm \\
\hline 1 & $1 / 21 / 75$ & 7 & 1 & 47 & 116.6 & $\mathrm{~N}$ & degree C/TWINS confirm \\
\hline$\frac{1}{1}$ & $1 / 21 / 75$ & 8 & 7 & 38 & 100.4 & $\bar{N}$ & degree C/TWINS confirm \\
\hline 1 & $1 / 21 / 75$ & 9 & 1 & 27 & 80.6 & $\mathbf{N}$ & degree C/TWINS confirm \\
\hline 1 & $1 / 21 / 75$ & 10 & 1 & 18 & 64.4 & $\mathbf{N}$ & degree C/TWINS confirm \\
\hline 1 & $1 / 21 / 75$ & 11 & 1 & 18 & 64.4 & $\mathbf{N}$ & degree C/TWINS confirm \\
\hline 1 & $1 / 21 / 75$ & 12 & 1 & 17 & 62.6 & $\mathbf{N}$ & degree CrTWINS confirm \\
\hline 1 & $1 / 28 / 75$ & 1 & 1 & 66 & 150.8 & $\mathbf{Y}$ & degree C/TWINS confirm \\
\hline 9 & $1 / 28 / 75$ & 2 & 1 & 62 & 143.6 & $\mathbf{N}$ & degree C/TWINS confirm \\
\hline 1 & $1 / 28 / 75$ & 3 & 1 & 58 & 136.4 & $\mathbf{N}$ & degree C/TWINS confirm \\
\hline 1 & $1 / 28 / 75$ & 4 & 1 & 52 & 125.6 & $\mathbf{N}$ & degree C/TWINS confirm \\
\hline$\frac{1}{1}$ & $1 / 28 / 75$ & 5 & 1 & 47 & 116.6 & $\mathbf{N}$ & degree C/TWINS confirm \\
\hline 1 & $1 / 28 / 75$ & 6 & 1 & 43 & 109.4 & $\mathbf{N}$ & degree $\mathrm{C} / \mathrm{TW}$ INS confirm \\
\hline 1 & $1 / 28 / 75$ & 7 & 1 & 38 & 100.4 & $\mathbf{N}$ & degree C/TWINS confirm \\
\hline 1 & $1 / 28 / 75$ & 8 & 1 & 30 & 86 & $\mathbf{N}$ & degree C/TWINS confirm \\
\hline 1 & $1 / 28 / 75$ & 9 & 1 & 21 & 69.8 & $\mathbf{N}$ & degree C/TWINS confirm \\
\hline 1 & $1 / 28 / 75$ & 10 & 1 & 13 & 55.4 & $\mathrm{~N}$ & degree C/TWINS confirm \\
\hline 1 & $1 / 28 / 75$ & 11 & 1 & 12 & 53.6 & $\bar{N}$ & degree C/TWINS confirm \\
\hline 1 & $1 / 28 / 75$ & 12 & 1 & 12 & 53.6 & $\mathrm{~N}$ & degree C/TWINS confirm \\
\hline 1 & $2 / 5 / 75$ & 1 & 1 & 66.67 & 152.006 & $Y$ & degree C/TWINS confirm \\
\hline 1 & $2 / 5 / 75$ & 2 & 1 & 62.22 & 143.996 & $\mathbf{N}$ & degree $\mathrm{C} / \mathrm{TW}$ INS confirm \\
\hline 1 & $2 / 5 / 75$ & 3 & 1 & 58.33 & 136.994 & $\mathbf{N}$ & degree C/TWINS confirm \\
\hline 1. & $2 / 5 / 75$ & 4 & 1 & 54.44 & 129.992 & $\mathbf{N}$ & degree $\mathrm{C} / \mathrm{TW}$ INS confirm \\
\hline 1 & $2 / 5 / 75$ & 5 & 1 & 48.33 & 118.994 & $\mathbf{N}$ & degree C/TWINS confirm \\
\hline 1 & $2 / 5 / 75$ & 6 & 1 & 42.78 & 109.004 & $\bar{N}$ & degree C/TWINS confirm \\
\hline 1 & $2 / 5 / 75$ & 7 & 1 & 37.78 & 100.004 & $\mathbf{N}$ & degree C/TWINS confirm \\
\hline 1 & $2 / 5 / 75$ & 8 & 1 & 29.44 & 84.992 & $\mathrm{~N}$ & degree C/TWiNS confirm \\
\hline 1 & $2 / 5 / 75$ & 9 & 1 & 21.11 & 69.998 & $\mathbf{N}$ & degree C/TWINS confirm \\
\hline 1 & $2 / 5 / 75$ & 10 & 1 & 15 & 59 & $\mathbf{N}$ & degree C/TWINS confirm \\
\hline 1 & $2 / 5 / 75$ & 11 & 1 & 15 & 59 & $\mathbf{N}$ & degree C/TWINS confirm \\
\hline 1 & $2 / 5 / 75$ & 12 & 1 & 14.44 & 57.992 & $\mathbf{N}$ & degree C/TWINS confirm \\
\hline 1 & $2 / 27 / 75$ & 1 & 1 & 68 & 154.4 & $\bar{Y}$ & degree $\mathrm{C} / \mathrm{TW}$ INS confitm \\
\hline 1 & $2 / 27 / 75$ & 2 & 1 & 63 & 145.4 & $\mathbf{N}$ & degree C/TWINS confirm \\
\hline 1 & $2 / 27 / 75$ & 3 & 1 & 60 & 140 & $\mathbf{N}$ & degree C/TWINS confirm \\
\hline 1 & $2 / 27 / 75$ & 4 & 1 & 55 & 131 & $\mathrm{~N}$ & Idegree C/TWINS confirm \\
\hline
\end{tabular}

Data obtained from WHC Surveillance Analysis Computer System (SACS), June 2, 1993. 


\begin{tabular}{|c|c|c|c|c|c|c|c|}
\hline \multicolumn{8}{|c|}{ TANK 251-BY-105 } \\
\hline \multicolumn{8}{|c|}{ THERMOCOUPLE DATA } \\
\hline TREE \# & DATE & THERMO \# & TREE SET \# & TEMP C & TEMP F & $Y=$ MAX TEMP & COMMENTS \\
\hline 1 & $2 / 27 / 75$ & 5 & 1 & 50 & 122 & $\bar{N}$ & degree C/TWINS confirm \\
\hline 1 & $2 / 27 / 75$ & 6 & 1 & 44 & 111.2 & $\mathbf{N}$ & degree C/TWINS confirm \\
\hline 1 & $2 / 27 / 75$ & 7 & 1 & 39 & 102.2 & $\mathbf{N}$ & degrae C/TWiNS confirm \\
\hline 1 & $2 / 27 / 75$ & 8 & 1 & 30 & 86 & $\mathbf{N}$ & degree $\mathrm{C} / \mathrm{TW}$ INS confirm \\
\hline 1 & $2 / 27 / 75$ & 9 & 1 & 20 & 68 & $\bar{N}$ & degree $\mathrm{C} / \mathrm{TW}$ INS confirm \\
\hline 9 & $2 / 27 / 75$ & 10 & 1 & 15 & 59 & $\mathbf{N}$ & degree C/TWINS confirm \\
\hline 1 & $2 / 27 / 75$ & 11 & 1 & 15 & 59 & $\mathbf{N}$ & degree C/TWINS confirm \\
\hline 1 & $2 / 27 / 75$ & 12 & 1 & 15 & 59 & $\mathbf{N}$ & degree C/TWiNS confirm \\
\hline 9 & $3 / 18 / 75$ & 1 & 9 & 70 & 158 & $\bar{Y}$ & degree C/TWINS confirm \\
\hline 1 & $3 / 18 / 75$ & 2 & 1 & 64 & 147.2 & $\mathbf{N}$ & degree C/TWINS confirm \\
\hline 1 & $3 / 18 / 75$ & 3 & 1 & 60 & 140 & $\mathbf{N}$ & degree C/TWINS confirm \\
\hline 1 & $3 / 18 / 75$ & 4 & 1 & 56 & 132.8 & $\mathbf{N}$ & degree $\mathrm{C} / \mathrm{TW}$ iNS confirm \\
\hline 1 & $3 / 18 / 75$ & 5 & 1 & 50 & 122 & $\mathbf{N}$ & degree C/TWINS confirm \\
\hline$\frac{1}{1}$ & $3 / 18 / 75$ & 6 & 1 & 46 & 114.8 & $\mathbf{N}$ & degree C/TWINS confirm \\
\hline 1 & $3 / 18 / 75$ & 7 & 1 & 40 & 104 & $\mathbf{N}$ & degree C/TWINS confirm \\
\hline 1 & $3 / 18 / 75$ & 8 & 1 & 32 & 89.6 & $\bar{N}$ & degree C/TWINS confirm \\
\hline 9 & $3 / 18 / 75$ & 9 & 1 & 24 & 75.2 & $\mathbf{N}$ & degree $\mathrm{C} / \mathrm{TW}$ INS confirm \\
\hline 1 & $3 / 18 / 75$ & 10 & 1 & 18 & 64.4 & $N$ & degree C/TWINS confirm \\
\hline 9 & $3 / 18 / 75$ & 19 & 1 & 18 & 64.4 & $\mathbf{N}$ & degree C/TWINS confirm \\
\hline 1 & $3 / 18 / 75$ & 12 & 1 & 18 & 64.4 & $\mathbf{N}$ & degree C/TWINS confirm \\
\hline 1 & $4 / 1 / 75$ & 1 & 1 & 63.33 & 145.994 & $\mathbf{Y}$ & degree C/TWINS confirm \\
\hline 1 & $4 / 1 / 75$ & 2 & 1 & 58.89 & 138.002 & $\mathbf{N}$ & degree C/TWINS confirm \\
\hline 1 & $4 / 1 / 75$ & 3 & 1 & 54.44 & 129.992 & $\mathrm{~N}$ & degree $\mathrm{C} / \mathrm{T}$ WINS confirm \\
\hline 1 & $4 / 1 / 75$ & 4 & 9 & 48.89 & 120.002 & $\mathbf{N}$ & degree C/TWINS confirm \\
\hline 1 & $4 / 1 / 75$ & 5 & 1 & 42.22 & 107.996 & $\mathbf{N}$ & degree C/TWINS confirm \\
\hline 1 & $4 / 1 / 75$ & 6 & 1 & 36.67 & 98.006 & $\mathbf{N}$ & degree $\mathrm{C} / \mathrm{TW}$ INS confirm \\
\hline 1 & $4 / 4 / 75$ & 7 & 1 & 31.67 & 89.006 & $\mathbf{N}$ & degree $\mathrm{C} / \mathrm{T}$ WINS confirm \\
\hline 1 & $4 / 1 / 75$ & 8 & 9 & 30.56 & 87.008 & $\mathbf{N}$ & degree C/TWINS confirm \\
\hline$\overline{1}$ & $4 / 1 / 75$ & 9 & $\frac{1}{1}$ & 15.56 & 60.008 & $\mathbf{N}$ & degree C/TWINS confirm \\
\hline 1 & $4 / 1 / 75$ & 10 & 1 & 11.11 & 51.998 & $\mathbf{N}$ & degree C/TWINS confirm \\
\hline 1 & $4 / 1 / 75$ & 11 & 1 & 11.11 & 51.998 & $\mathbf{N}$ & degree C/TWINS confirm \\
\hline 1 & $4 / 1 / 75$ & 12 & 1 & 11.11 & 51.998 & $\mathrm{~N}$ & degree C/TWINS confirm \\
\hline 1 & $4 / 8 / 75$ & 1 & 1 & 77 & 170.6 & $\bar{Y}$ & degree C/TWINS confirm \\
\hline 1 & $4 / 8 / 75$ & 2 & 1 & 71 & 159.8 & $\mathbf{N}$ & degree C/TWINS confirm \\
\hline$\frac{1}{1}$ & $4 / 8 / 75$ & 3 & 1 & 68 & 154.4 & $\mathbf{N}$ & degree C/TWINS confirm \\
\hline 1 & $4 / 8 / 75$ & 4 & 1 & 62 & 143.6 & $N$ & degree C/TWINS confirm \\
\hline 1 & $4 / 8 / 75$ & 5 & 1 & 54 & 129.2 & $\mathbf{N}$ & degree C/TWINS confirm \\
\hline 1 & $4 / 8 / 75$ & 6 & 1 & 47 & 116.6 & $\mathbf{N}$ & degree $\mathrm{C} / \mathrm{TW}$ INS confirm \\
\hline 1 & $4 / 8 / 75$ & 7 & 1 & 41 & 105.8 & $\mathrm{~N}$ & degree C/TWINS confirm \\
\hline 1 & $.4 / 8 / 75$ & 8 & 1 & 32 & 89.6 & $\mathbf{N}$ & degree C/TWINS confirm \\
\hline 9 & $4 / 8 / 75$ & 9 & 1 & 23 & 73.4 & $\mathbf{N}$ & degree C/TWINS confirm \\
\hline 1 & $4 / 8 / 75$ & 10 & 1 & 15 & 59 & $\mathbf{N}$ & degree CTWWINS confirm \\
\hline$\frac{1}{1}$ & $4 / 8 / 75$ & 11 & 1 & 15 & 59 & $\mathbf{N}$ & degree $\mathrm{C} / \mathrm{TW}$ INS confirm \\
\hline 1 & $4 / 8 / 75$ & 12 & 1 & 15 & 59 & $\mathbf{N}$ & degrea CTTWINS confirm \\
\hline 1 & $4 / 22 / 75$ & 1 & 1 & 64 & 147.2 & $\mathbf{Y}$ & degree C/TWINS confirm \\
\hline 9 & $4 / 22 / 75$ & 2 & 1 & 58 & 136.4 & $\mathbf{N}$ & degree C/TWINS confirm \\
\hline 1 & $4 / 22 / 75$ & 3 & 1 & 53 & 127.4 & $\mathbf{N}$ & degree C/TWINS confirm \\
\hline 1 & $4 / 22 / 75$ & 4 & 1 & 48 & 118.4 & $\mathbf{N}$ & degree C/TWINS confirm \\
\hline 1 & $4 / 22 / 75$ & 5 & 1 & 44 & 111.2 & $N$ & degree C/TWINS confirm \\
\hline 1 & $4 / 22 / 75$ & 6 & 1 & 40 & 104 & $\mathbf{N}$ & degree C/TWINS confirm \\
\hline 1 & $4 / 22 / 75$ & 7 & 1 & 36 & 96.8 & $\mathbf{N}$ & degree CITWINS confirm \\
\hline 1 & $4 / 22 / 75$ & 8 & 1 & 28 & 82.4 & $\bar{N}$ & degree C/TWINS confirm \\
\hline 1 & $4 / 22 / 75$ & 9 & 9 & 22 & 71.6 & $\mathbf{N}$ & degree C/TWINS confism \\
\hline 9 & $4 / 22 / 75$ & 10 & 1 & 16 & 60.8 & $\mathbf{N}$ & degree C/TWINS confirm \\
\hline 1 & $4 / 22 / 75$ & 11 & 1 & 16 & 60.8 & $N$ & degree CTTWINS confirm \\
\hline 1 & $4 / 22 / 75$ & 12 & 1 & 16 & 60.8 & $\mathbf{N}$ & degree C/TWINS confirm \\
\hline 1 & $4 / 29 / 75$ & 1 & 1 & 62 & 143.6 & $\mathbf{Y}$ & degree C/TWiNS confirm \\
\hline 1 & $4 / 29 / 75$ & 2 & 1 & 56 & 132.8 & $\mathbf{N}$ & degree C/TWINS confirm \\
\hline 1 & $4 / 29 / 75$ & 3 & 1 & 52 & 125.6 & $\mathbf{N}$ & degree CTTWINS confirm \\
\hline 1 & $4 / 29 / 75$ & 4 & 1 & 48 & 118.4 & $\mathbf{N}$ & degree C/TWINS confirm \\
\hline 1 & $4 / 29 / 75$ & 5 & 1 & 44 & 111.2 & $\bar{N}$ & degree C/TWINS confirm \\
\hline 1 & $4 / 29 / 75$ & 6 & 1 & 40 & 104 & $\mathbf{N}$ & degree C/TWINS confirm \\
\hline 1 & $4 / 29 / 75$ & 7 & 1 & 36 & 96.8 & $\mathrm{~N}$ & degree CITWINS confirm \\
\hline 1 & $4 / 29 / 75$ & 8 & 1 & 30 & 86 & $\mathbf{N}$ & degree $\mathrm{C} / \mathrm{TWW}$ INS confirm \\
\hline 1 & $4 / 29 / 75$ & 9 & 1 & 24 & 75.2 & $\mathbf{N}$ & degree C/TWINS confirm \\
\hline 9 & $4 / 29 / 75$ & 10 & 1 & 16 & 60.8 & $\mathbf{N}$ & degree C/TWINS confirm \\
\hline 1 & $4 / 29 / 75$ & 11 & 9 & 16 & 60.8 & $\mathbf{N}$ & degree C/TWINS confirm \\
\hline 1 & $4 / 29 / 75$ & 12 & 1 & 16 & 60.8 & $N$ & degree C/TWINS confirm \\
\hline
\end{tabular}

Data obtained from WHC Surveillance Analysis Computer System (SACS), June 2, 1993. 


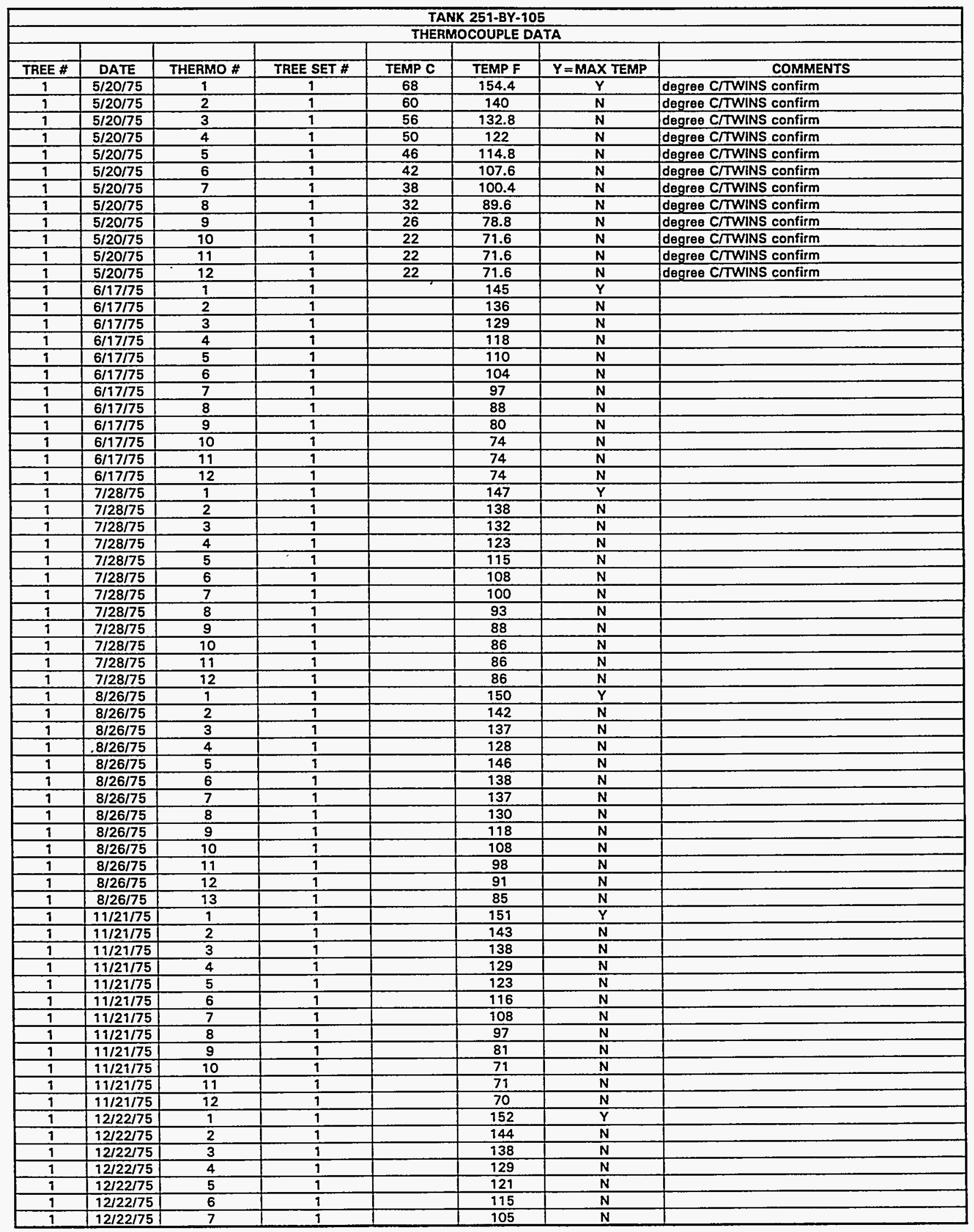

Data obtained from WHC Surveillance Analysis Computer System (SACS), June 2, 1993. 


\begin{tabular}{|c|c|c|c|c|c|c|c|}
\hline \multicolumn{8}{|c|}{ TANK 251-BY-105 } \\
\hline \multicolumn{8}{|c|}{ THERMOCOUPLE DATA } \\
\hline TREE \# & DATE & THERMO \# & TREE SET \# & TEMP C & TEMP F & $Y=M A X$ TEMP & COMMENTS \\
\hline 1 & $12 / 22 / 75$ & 8 & 1 & & 93 & $\mathrm{~N}$ & \\
\hline 1 & $12 / 22 / 75$ & 9 & 1 & & 76 & $\mathbf{N}$ & \\
\hline 9 & $12 / 22 / 75$ & 10 & 1 & & 67 & $\mathbf{N}$ & \\
\hline 1 & $12 / 22 / 75$ & 11 & 1 & & 67 & $\mathbf{N}$ & \\
\hline 9 & $12 / 22 / 75$ & 12 & 1 & & 67 & $\mathbf{N}$ & \\
\hline 1 & $3 / 4 / 76$ & 1 & 1 & & 145 & $\mathbf{Y}$ & \\
\hline 1 & $3 / 4 / 76$ & 2 & 1 & & 136 & $\mathbf{N}$ & \\
\hline 1 & $3 / 4 / 76$ & 3 & 1 & & 129 & $N$ & \\
\hline 1 & $3 / 4 / 76$ & 4 & 9 & & 116 & $N$ & \\
\hline$\frac{1}{1}$ & $3 / 4 / 76$ & 5 & 1 & & 105 & $\mathbf{N}$ & \\
\hline 1 & $3 / 4 / 76$ & 6 & 1 & & 98 & $\mathbf{N}$ & \\
\hline 9 & $3 / 4 / 76$ & 7 & 1 & & 88 & $\mathbf{N}$ & \\
\hline 1 & $3 / 4 / 76$ & 8 & 1 & & 76 & $\mathrm{~N}$ & \\
\hline 1 & $3 / 4 / 76$ & 9 & 1 & & 63 & $N$ & \\
\hline 1 & $3 / 4 / 76$ & 10 & 1 & & 56 & $\mathbf{N}$ & \\
\hline 1 & $3 / 4 / 76$ & 11 & 1 & & 56 & $N$ & \\
\hline$\frac{1}{1}$ & $3 / 4 / 76$ & 12 & 1 & & 57 & $\mathbf{N}$ & \\
\hline 1 & $5 / 7 / 76$ & 1 & 9 & & 136 & $\bar{Y}$ & \\
\hline 1 & $5 / 7 / 76$ & 2 & 1 & & 127 & $\mathbf{N}$ & \\
\hline 1 & $5 / 7 / 76$ & 3 & 1 & & 119 & $\mathbf{N}$ & \\
\hline$\frac{1}{1}$ & $5 / 7 / 76$ & 4 & 1 & & 108 & $\mathbf{N}$ & \\
\hline 1 & $5 / 7 / 76$ & 5 & 1 & & 99 & $\mathbf{N}$ & \\
\hline 1 & $5 / 7 / 76$ & 6 & 9 & & 90 & $\mathbf{N}$ & \\
\hline 1 & $5 / 7 / 76$ & 7 & 1 & & 80 & $\mathbf{N}$ & \\
\hline 1 & $5 / 7 / 76$ & 8 & 1 & & 71 & $N$ & \\
\hline$\frac{1}{1}$ & $5 / 7 / 76$ & 9 & 1 & & 63 & $N$ & \\
\hline 1 & $5 / 7 / 76$ & 10 & 1 & & 62 & $\mathbf{N}$ & \\
\hline 1 & $5 / 7 / 76$ & 11 & 1 & & 63 & $\mathbf{N}$ & \\
\hline 1 & $5 / 7 / 76$ & 12 & 1 & & 63 & $N$ & \\
\hline 1 & $6 / 9 / 76$ & 1 & 1 & & 145 & $Y$ & \\
\hline 1 & $6 / 9 / 76$ & 2 & 1 & & 136 & N & \\
\hline 1 & $6 / 9 / 76$ & 3 & $\frac{1}{1}$ & & 130 & $\mathbf{N}$ & \\
\hline 1 & $6 / 9 / 76$ & 4 & 1 & & 120 & $\mathbf{N}$ & \\
\hline 1 & $6 / 9 / 76$ & 5 & 1 & & 112 & $\mathbf{N}$ & \\
\hline 1 & $6 / 9 / 76$ & 6 & 1 & & 104 & $\mathbf{N}$ & \\
\hline 1 & $6 / 9 / 76$ & 7 & 1 & & 94 & $\bar{N}$ & \\
\hline 1 & $6 / 9 / 76$ & 8 & 1 & & 85 & $\mathbf{N}$ & \\
\hline 1 & $6 / 9 / 76$ & 9 & 1 & & 78 & $\mathbf{N}$ & \\
\hline 1 & $6 / 9 / 76$ & 10 & 1 & & 76 & $N$ & \\
\hline 1 & $.6 / 9 / 76$ & 11 & 1 & & 76 & $\mathbf{N}$ & \\
\hline 1 & $6 / 9 / 76$ & 12 & 1 & & 76 & $N$ & \\
\hline 1 & $7 / 7176$ & 1 & 1 & & 136 & $\mathrm{Y}$ & \\
\hline 1 & $.7 / 7 / 76$ & 2 & 1 & & 128 & $\mathbf{N}$ & \\
\hline 1 & $7 / 7 / 76$ & 3 & 1 & & 122 & $\mathbf{N}$ & \\
\hline 1 & $7 / 7 / 76$ & 4 & 1 & & 113 & $\mathbf{N}$ & \\
\hline 1 & $7 / 7 / 76$ & 5 & 1 & & 105 & $\mathbf{N}$ & \\
\hline 1 & $7 / 7 / 76$ & 6 & 1 & & 97 & $\mathrm{~N}$ & \\
\hline 1 & $7 / 7 / 76$ & 7 & 9 & & 87 & $\mathbf{N}$ & \\
\hline 1 & $7 / 7 / 76$ & 8 & 1 & & 79 & $\mathbf{N}$ & \\
\hline 1 & $7 / 7 / 76$ & 9 & 1 & & 74 & $\mathbf{N}$ & \\
\hline 1 & $7 / 7 / 76$ & 10 & 1 & & 72 & $\mathbf{N}$ & \\
\hline 1 & $7 / 7 / 76$ & 11 & 1 & & 72 & $\mathbf{N}$ & \\
\hline 1 & $7 / 7 / 76$ & 12 & 1 & & 72 & $N$ & \\
\hline 1 & $8 / 14 / 76$ & 9 & 1 & & 132 & $\bar{Y}$ & \\
\hline 1 & $8 / 14 / 76$ & 2 & 1 & & 124 & $\mathbf{N}$ & \\
\hline 1 & $8 / 14 / 76$ & 3 & 1 & & 117 & $N$ & \\
\hline 1 & $8 / 14 / 76$ & 4 & 1 & & 110 & $N$ & \\
\hline 1 & $8 / 14 / 76$ & 5 & 1 & & 103 & $\mathbf{N}$ & \\
\hline 1 & $8 / 14 / 76$ & 6 & 1 & & 96 & $\mathbf{N}$ & \\
\hline 7 & $8 / 14 / 76$ & 7 & 1 & & 88 & $\mathbf{N}$ & \\
\hline 1 & $8 / 14 / 76$ & 8 & 1 & & 80 & $N$ & \\
\hline 1 & $8 / 14 / 76$ & 9 & 1 & & 73 & $\mathbf{N}$ & \\
\hline 1 & $8 / 14 / 76$ & 10 & 1 & & 71 & $\mathbf{N}$ & \\
\hline 1 & $8 / 14 / 76$ & 19 & 1 & & 71 & $\mathbf{N}$ & \\
\hline 1 & $8 / 14 / 76$ & 12 & $\frac{1}{1}$ & & 71 & $N$ & \\
\hline 1 & $8 / 28 / 76$ & 1 & 1 & & 137 & $\bar{Y}$ & \\
\hline 1 & $8 / 28 / 76$ & 2 & 1 & & 129 & $\mathbf{N}$ & \\
\hline 1 & $8 / 28 / 76$ & 3 & 1 & & 123 & $\mathbf{N}$ & \\
\hline
\end{tabular}

Data obtained from WHC Surveillance Analysis Computer System (SACS), June 2, 1993. 


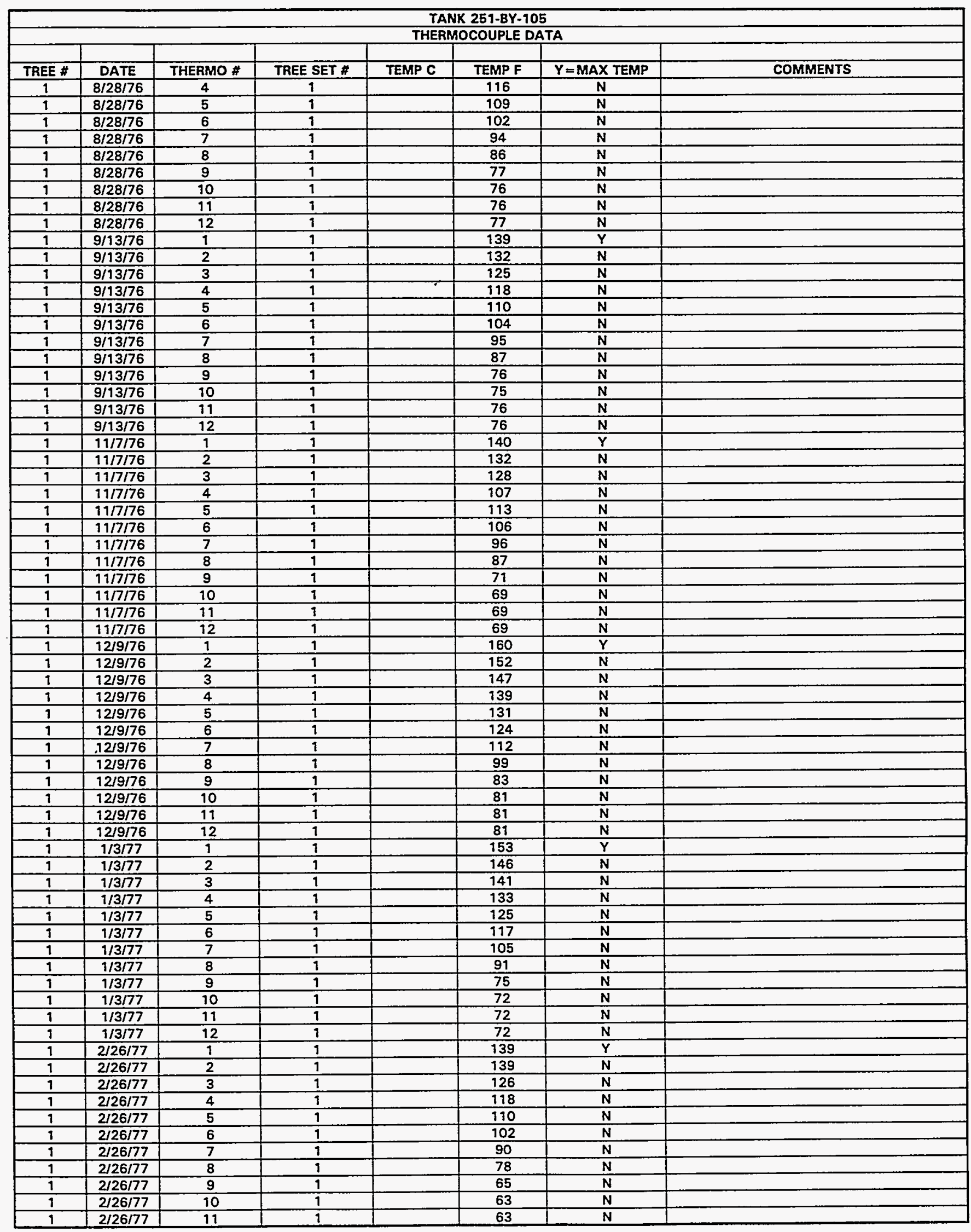

Data obtained from WHC Surveillance Analysis Computer System (SACS), June 2, 1993. 


\begin{tabular}{|c|c|c|c|c|c|c|c|}
\hline \multicolumn{8}{|c|}{ TANK 251-BY-105 } \\
\hline & & & & THER & COUPLE & & \\
\hline TREE \# & DATE & THERMO \# & TREE SET \# & TEMP C & TEMP F & $Y=$ MAX TEMP & COMMENTS \\
\hline 1 & $2 / 26 / 77$ & 12 & 1 & & 63 & $\mathbf{N}$ & \\
\hline$\frac{1}{1}$ & $3 / 28 / 77$ & 1 & 1 & & 144 & $\mathbf{Y}$ & \\
\hline 1 & $3 / 28 / 77$ & 2 & $\frac{1}{1}$ & & 136 & $\mathbf{N}$ & \\
\hline 1 & $3 / 28 / 77$ & 3 & 1 & & 131 & $\mathbf{N}$ & \\
\hline$T$ & $3 / 28 / 77$ & 4 & 1 & & 122 & $\mathbf{N}$ & \\
\hline$\frac{1}{1}$ & $3 / 28 / 77$ & 5 & 1 & & 114 & $\bar{N}$ & \\
\hline 1 & $3 / 28 / 77$ & 6 & 1 & & 107 & $\mathbf{N}$ & \\
\hline 9 & $3 / 28 / 77$ & 7 & 9 & & 95 & $\mathbf{N}$ & \\
\hline 1 & $3 / 28 / 77$ & 8 & 1 & & 84 & $\mathbf{N}$ & \\
\hline 1 & $3 / 28 / 77$ & 9 & 1 & & 71 & $\mathbf{N}$ & \\
\hline 1 & $3 / 28 / 77$ & 10 & 1 & & 70 & $\mathbf{N}$ & \\
\hline 1 & $3 / 28 / 77$ & 11 & 1 & & 70 & $\mathbf{N}$ & \\
\hline 1 & $3 / 28 / 77$ & 12 & 1 & & 70 & $\mathbf{N}$ & \\
\hline 1 & $4 / 27 / 77$ & 1 & 1 & & 140 & $Y$ & \\
\hline 1 & $4 / 27 / 77$ & 2 & 1 & & 132 & $\mathbf{N}$ & \\
\hline 1 & $4 / 27 / 77$ & 3 & 1 & & 127 & $N$ & \\
\hline 1 & $4 / 27 / 77$ & 4 & 1 & & 119 & $\mathbf{N}$ & \\
\hline 9 & $4 / 27 / 77$ & 5 & 1 & & 111 & $\mathbf{N}$ & \\
\hline 1 & $4 / 27 / 77$ & 6 & 1 & & 104 & $\mathbf{N}$ & \\
\hline$\frac{1}{1}$ & $4 / 27 / 77$ & 7 & 9 & & 93 & $\mathbf{N}$ & \\
\hline 1 & $4 / 27 / 77$ & 8 & 1 & & 82 & $\mathbf{N}$ & \\
\hline 1 & $4 / 27 / 77$ & 9 & 9 & & 72 & $\bar{N}$ & \\
\hline 1 & $4 / 27 / 77$ & 10 & 1 & & 71 & $\mathbf{N}$ & \\
\hline 1 & $4 / 27 / 77$ & 11 & 1 & & 70 & $\mathbf{N}$ & \\
\hline 9 & $4 / 27 / 77$ & 12 & 1 & & 70 & $\mathbf{N}$ & \\
\hline 1 & $6 / 30 / 77$ & 1 & 1 & & 143 & $\mathbf{Y}$ & \\
\hline 1 & $6 / 30 / 77$ & 2 & 9 & & 135 & $\mathbf{N}$ & \\
\hline 1 & $6 / 30 / 77$ & 3 & 1 & & 130 & $\mathbf{N}$ & \\
\hline 1 & $6 / 30 / 77$ & 4 & 1 & & 123 & $\mathbf{N}$ & \\
\hline 1 & $6 / 30 / 77$ & 5 & 1 & & & $\mathbf{N}$ & 175 - suspect data (high) \\
\hline 1 & $6 / 30 / 77$ & 6 & 1 & & 109 & $\mathbf{N}$ & \\
\hline 1 & $6 / 30 / 77$ & 7 & 1 & & 100 & $\mathbf{N}$ & \\
\hline$\frac{1}{1}$ & $6 / 30 / 77$ & 8 & 7 & & 92 & $\mathbf{N}$ & \\
\hline 1 & $6 / 30 / 77$ & 9 & 1 & & 83 & $N$ & \\
\hline 1 & $6 / 30 / 77$ & 10 & 1 & & 82 & $\mathbf{N}$ & \\
\hline 1 & $6 / 30 / 77$ & 11 & 1 & & 82 & $\mathbf{N}$ & \\
\hline$\frac{1}{1}$ & $6 / 30 / 77$ & 12 & $T$ & & 82 & $\mathbf{N}$ & \\
\hline 1 & $8 / 15 / 77$ & 1 & 1 & & 131 & $\mathbf{Y}$ & \\
\hline$\frac{1}{1}$ & $8 / 15 / 77$ & 2 & 1 & & 124 & $\mathbf{N}$ & \\
\hline 1 & $.8 / 15 / 77$ & 3 & 1 & & 120 & $\mathbf{N}$ & \\
\hline 1 & $8 / 15 / 77$ & 4 & 9 & & 112 & $\bar{N}$ & \\
\hline 1 & $8 / 15 / 77$ & 5 & 1 & & 105 & $\mathbf{N}$ & \\
\hline 1 & $8 / 15 / 77$ & 6 & 1 & & 100 & $\mathbf{N}$ & \\
\hline 1 & $8 / 15 / 77$ & 7 & 1 & & 92 & $\mathbf{N}$ & \\
\hline 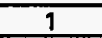 & $8 / 15 / 77$ & 8 & 1 & & 85 & $\mathbf{N}$ & \\
\hline 1 & $8 / 15 / 77$ & 9 & 1 & & 79 & $\mathbf{N}$ & \\
\hline$\frac{1}{1}$ & $8 / 15 / 77$ & 10 & $T$ & & 78 & $\mathbf{N}$ & \\
\hline 1 & $8 / 15 / 77$ & 11 & 1 & & 78 & $\mathbf{N}$ & \\
\hline 1 & $8 / 15 / 77$ & 12 & 1 & & 77 & $\mathbf{N}$ & \\
\hline 1 & $9 / 12 / 77$ & 1 & 1 & & 129 & $\mathbf{Y}$ & \\
\hline 1 & $9 / 12 / 77$ & 2 & 1 & & 122 & $\mathbf{N}$ & \\
\hline 1 & $9 / 12 / 77$ & 3 & 1 & & 118 & $\mathbf{N}$ & \\
\hline 1 & $9 / 12 / 77$ & 4 & 1 & & 110 & N & \\
\hline 1 & $9 / 12 / 77$ & 5 & 1 & & 104 & $\mathrm{~N}$ & \\
\hline 1 & $9 / 12 / 77$ & 6 & 1 & & 98 & $\mathbf{N}$ & \\
\hline 1 & $9 / 12 / 77$ & 7 & 1 & & 90 & $\mathbf{N}$ & \\
\hline 1 & $9 / 12 / 77$ & 8 & 1 & & 81 & $\mathbf{N}$ & \\
\hline 1 & $9 / 12 / 77$ & 9 & 1 & & 71 & $N$ & \\
\hline 1 & $9 / 12 / 77$ & 10 & 1 & & 70 & $\mathbf{N}$ & \\
\hline 1 & $9 / 12 / 77$ & 11 & 1 & & 70 & $\mathbf{N}$ & \\
\hline$\frac{1}{1}$ & $9 / 12 / 77$ & 12 & 1 & & 70 & $\mathbf{N}$ & \\
\hline 1 & $10 / 7 / 77$ & 1 & 1 & & 142 & $Y$ & \\
\hline 1 & $10 / 7 / 77$ & 2 & 1 & & 135 & $N$ & \\
\hline 1 & $10 / 7 / 77$ & 3 & 1 & & 130 & $N$ & \\
\hline 1 & $10 / 7 / 77$ & 4 & 1 & & 124 & $\mathbf{N}$ & \\
\hline 1 & $10 / 7 / 77$ & 5 & 1 & & 117 & $N$ & \\
\hline 1 & $10 / 7 / 77$ & 6 & 1 & & 111 & $N$ & \\
\hline 1 & $10 / 7 / 77$ & 7 & 1 & & 103 & $\mathrm{~N}$ & \\
\hline
\end{tabular}

Data obtained from WHC Surveillance Analysis Computer System (SACS), June 2, 1993. 


\begin{tabular}{|c|c|c|c|c|c|c|c|}
\hline \multicolumn{8}{|c|}{ TANK 251-BY-105 } \\
\hline \multicolumn{8}{|c|}{ THERMOCOUPLE DATA } \\
\hline TREE \# & DATE & THERMO \# & TREE SET \# & TEMP C & TEMP F & $Y=$ MAX TEMP & COMMENTS \\
\hline 1 & $10 / 7 / 77$ & 8 & 1 & & 91 & $\mathbf{N}$ & \\
\hline 1 & $10 / 7 / 77$ & 9 & 1 & & 77 & $\mathbf{N}$ & \\
\hline 1 & $10 / 7 / 77$ & 10 & 1 & & 75 & $\mathbf{N}$ & \\
\hline 1 & $10 / 7 / 77$ & 11 & 7 & & 75 & $\mathbf{N}$ & \\
\hline 1 & $10 / 7 / 77$ & 12 & 1 & & 75 & $\mathbf{N}$ & \\
\hline 1 & $12 / 7 / 77$ & 1 & 1 & & 140 & $\bar{Y}$ & \\
\hline$T$ & $12 / 7 / 77$ & 2 & 1 & & 133 & $\mathbf{N}$ & \\
\hline 1 & $12 / 7 / 77$ & 3 & 1 & & 128 & $\mathbf{N}$ & \\
\hline 1 & $12 / 7 / 77$ & 4 & 1 & & 917 & $\mathbf{N}$ & \\
\hline 1 & $12 / 7 / 77$ & 5 & 1 & & 113 & $\mathbf{N}$ & \\
\hline 1 & $12 / 7 / 77$ & 6 & 1 & & 106 & $\mathbf{N}$ & \\
\hline 1 & $12 / 7 / 77$ & 7 & 1 & & 94 & $\mathbf{N}$ & \\
\hline 1 & $12 / 7 / 77$ & 8 & 1 & & 79 & $\mathbf{N}$ & \\
\hline 1 & $12 / 7 / 77$ & 9 & 1 & & 61 & $\mathbf{N}$ & \\
\hline 1 & $12 / 7 / 77$ & 10 & 1 & & 59 & $\mathbf{N}$ & \\
\hline 1 & $12 / 7 / 77$ & 11 & 1 & & 60 & $N$ & \\
\hline 1 & $12 / 7 / 77$ & 12 & 1 & & 60 & $\mathbf{N}$ & \\
\hline 1 & $1 / 29 / 78$ & 1 & 1 & & 146 & $Y$ & \\
\hline 1 & $1 / 29 / 78$ & 2 & 1 & & 139 & $N$ & \\
\hline 1 & $1 / 29 / 78$ & 3 & 1 & & 134 & $N$ & \\
\hline 1 & $1 / 29 / 78$ & 4 & 1 & & 125 & $\mathbf{N}$ & \\
\hline 1 & $1 / 29 / 78$ & 5 & 1 & & 116 & $\mathbf{N}$ & \\
\hline 9 & $1 / 29 / 78$ & 6 & 9 & & 107 & $\mathbf{N}$ & \\
\hline 1 & $1 / 29 / 78$ & 7 & 1 & & 95 & $\mathbf{N}$ & \\
\hline 1 & $1 / 29 / 78$ & 8 & 1 & & 80 & $N$ & \\
\hline 1 & $1 / 29 / 78$ & 9 & 1 & & 62 & $\mathbf{N}$ & \\
\hline 1 & $1 / 29 / 78$ & 10 & 1 & & 60 & $\mathbf{N}$ & \\
\hline 1 & $1 / 29 / 78$ & 11 & 9 & & 60 & $\mathbf{N}$ & \\
\hline 1 & $1 / 29 / 78$ & 12 & 9 & & 60 & $\mathrm{~N}$ & \\
\hline 1 & $2 / 26 / 78$ & 1 & 1 & & 151 & $\bar{Y}$ & \\
\hline 1 & $2 / 26 / 78$ & 2 & 1 & & 143 & $\mathbf{N}$ & \\
\hline 1 & $2 / 26 / 78$ & 3 & 1 & & 138 & $\mathbf{N}$ & \\
\hline 1 & $2 / 26 / 78$ & 4 & 7 & & 128 & $\bar{N}$ & \\
\hline 1 & $2 / 26 / 78$ & 5 & 1 & & 119 & $\mathbf{N}$ & \\
\hline 1 & $2 / 26 / 78$ & 6 & 1 & & 111 & $\mathbf{N}$ & \\
\hline 1 & $2 / 26 / 78$ & 7 & 1 & & 99 & $\mathbf{N}$ & \\
\hline 1 & $2 / 26 / 78$ & 8 & 1 & & 84 & $\bar{N}$ & \\
\hline 1 & $2 / 26 / 78$ & 9 & 1 & & 68 & $\mathbf{N}$ & \\
\hline 1 & $2 / 26 / 78$ & 10 & 1 & & 61 & $N$ & \\
\hline 1 & $.2 / 26 / 78$ & 11 & 1 & & 67 & $\bar{N}$ & \\
\hline 1 & $2 / 26 / 78$ & 12 & 1 & & 67 & $\mathbf{N}$ & \\
\hline 1 & $3 / 26 / 78$ & 1 & 1 & & 138 & $\bar{Y}$ & \\
\hline 1 & $3 / 26 / 78$ & 2 & 1 & & 130 & $\mathbf{N}$ & \\
\hline 1 & $3 / 26 / 78$ & 3 & 1 & & 125 & $\mathbf{N}$ & \\
\hline 1 & $3 / 26 / 78$ & 4 & 1 & & 116 & $\mathbf{N}$ & \\
\hline 9 & $3 / 26 / 78$ & 5 & 1 & & 107 & $\mathrm{~N}$ & \\
\hline 1 & $3 / 26 / 78$ & 6 & 1 & & 98 & $\mathrm{~N}$ & \\
\hline 1 & $3 / 26 / 78$ & 7 & 1 & & 87 & $\mathrm{~N}$ & \\
\hline 1 & $3 / 26 / 78$ & 8 & 1 & & 74 & $\mathbf{N}$ & \\
\hline 9 & $3 / 26 / 78$ & 9 & 1 & & 61 & $N$ & \\
\hline 9 & $3 / 26 / 78$ & 10 & 1 & & 60 & $\mathbf{N}$ & \\
\hline 1 & $3 / 26 / 78$ & 19 & 1 & & 60 & $\mathbf{N}$ & \\
\hline 1 & $3 / 26 / 78$ & 12 & 7 & & 60 & $\mathbf{N}$ & \\
\hline 1 & $4 / 23 / 78$ & 1 & 1 & & 143 & $Y$ & \\
\hline 1 & $4 / 23 / 78$ & 2 & 1 & & 133 & $N$ & \\
\hline 1 & $4 / 23 / 78$ & 3 & 1 & & 128 & $\mathbf{N}$ & \\
\hline 1 & $4 / 23 / 78$ & 4 & 1 & & 119 & $N$ & \\
\hline 9 & $4 / 23 / 78$ & 5 & 1 & & 110 & $\mathrm{~N}$ & \\
\hline 1 & $4 / 23 / 78$ & 6 & 1 & & 102 & $\mathrm{~N}$ & \\
\hline 1 & $4 / 23 / 78$ & 7 & 1 & & 92 & $\mathrm{~N}$ & \\
\hline 1 & $4 / 23 / 78$ & 8 & 1 & & 81 & $\mathbf{N}$ & \\
\hline 1 & $4 / 23 / 78$ & 9 & 1 & & 67 & $\mathbf{N}$ & \\
\hline 1 & $4 / 23 / 78$ & 10 & 1 & & 67 & $\mathbf{N}$ & \\
\hline 1 & $4 / 23 / 78$ & 11 & 1 & & 67 & $\mathbf{N}$ & \\
\hline 1 & $4 / 23 / 78$ & 12 & 1 & & 68 & $\mathbf{N}$ & \\
\hline 1 & $6 / 17 / 78$ & 1 & 1 & & 131 & $Y$ & \\
\hline 1 & $6 / 17 / 78$ & 2 & 1 & & 123 & $\mathrm{~N}$ & \\
\hline 1 & $6 / 17 / 78$ & 3 & 1 & & 118 & $N$ & \\
\hline
\end{tabular}

Data obtained from WHC Surveillance Analysis Computer System (SACS), June 2, 1993. 


\begin{tabular}{|c|c|c|c|c|c|c|c|}
\hline \multicolumn{8}{|c|}{ TANK 251-BY-105 } \\
\hline & & & & THER & COUPLE & & \\
\hline TREE \# & DATE & THERMO \# & TREE SET \# & TEMP C & TEMP F & $Y=$ MAX TEMP & COMMENTS \\
\hline 1 & $6 / 17 / 78$ & 4 & 1 & & 109 & $\mathbf{N}$ & \\
\hline 1 & $6 / 17 / 78$ & 5 & 1 & & 101 & $\mathbf{N}$ & \\
\hline 1 & $6 / 17 / 78$ & 6 & 1 & & 94 & $\mathbf{N}$ & \\
\hline 1 & $6 / 17 / 78$ & 7 & 1 & & 85 & $\mathbf{N}$ & \\
\hline 1 & $6 / 17 / 78$ & 8 & 1 & & 76 & $\mathbf{N}$ & \\
\hline$\frac{T}{1}$ & $6 / 17 / 78$ & 9 & 1 & & 68 & $\mathbf{N}$ & \\
\hline$\frac{1}{1}$ & $6 / 17 / 78$ & 10 & 1 & & 67 & $\mathbf{N}$ & \\
\hline 1 & $6 / 17 / 78$ & 11 & 1 & & 68 & $N$ & \\
\hline 1 & $6 / 17 / 78$ & 12 & 1 & & 68 & $\mathbf{N}$ & \\
\hline 1 & $7 / 16 / 78$ & 1 & 1 & & 120 & $\bar{Y}$ & \\
\hline 1 & $7 / 16 / 78$ & 2 & 1 & & 112 & $\mathbf{N}$ & \\
\hline 1 & $7 / 16 / 78$ & 3 & 1 & & 106 & $N$ & \\
\hline 1 & $7 / 16 / 78$ & 4 & 1 & & 95 & $\mathbf{N}$ & \\
\hline 1 & $7 / 16 / 78$ & 5 & 1 & & 87 & $\mathbf{N}$ & \\
\hline 1 & $7 / 16 / 78$ & 6 & 1 & & 82 & $\bar{N}$ & \\
\hline 1 & $7 / 16 / 78$ & 7 & 1 & & 75 & $N$ & \\
\hline 1 & $7 / 16 / 78$ & 8 & 1 & & 66 & $\mathbf{N}$ & \\
\hline 1 & $7 / 16 / 78$ & 9 & 1 & & 59 & $N$ & \\
\hline 1 & $7 / 16 / 78$ & 10 & 1 & & 58 & $\mathrm{~N}$ & \\
\hline 1 & $7 / 16 / 78$ & 11 & 1 & & 58 & $\mathbf{N}$ & \\
\hline 1 & $7 / 16 / 78$ & 12 & 1 & & 58 & N & \\
\hline 1 & $9 / 12 / 78$ & 1 & 1 & & 941 & $\bar{Y}$ & \\
\hline 1 & $9 / 12 / 78$ & 2 & 1 & & 134 & $\mathbf{N}$ & \\
\hline 1 & $9 / 12 / 78$ & 3 & 1 & & 129 & $\mathbf{N}$ & \\
\hline 1 & $9 / 12 / 78$ & 4 & 1 & & 122 & $\mathbf{N}$ & \\
\hline 1 & $9 / 12 / 78$ & 5 & 1 & & 116 & $\bar{N}$ & \\
\hline 1 & $9 / 12 / 78$ & 6 & 1 & & 110 & $\mathbf{N}$ & \\
\hline 1 & $9 / 12 / 78$ & 7 & 1 & & 104 & $\mathbf{N}$ & \\
\hline 1 & $9 / 12 / 78$ & 8 & 1 & & 94 & $\mathbf{N}$ & \\
\hline 1 & $9 / 12 / 78$ & 9 & 1 & & 83 & $\mathbf{N}$ & \\
\hline$\frac{1}{1}$ & $9 / 12 / 78$ & 10 & 1 & & 82 & $\mathbf{N}$ & \\
\hline 1 & $9 / 12 / 78$ & 11 & 1 & & 82 & $\mathbf{N}$ & \\
\hline 1 & $9 / 12 / 78$ & 12 & 1 & & 82 & $\mathbf{N}$ & \\
\hline 1 & $10 / 7 / 78$ & 1 & 1 & & 140 & $Y$ & \\
\hline 1 & $10 / 7 / 78$ & 2 & 1 & & 132 & $\mathbf{N}$ & \\
\hline 1 & $10 / 7 / 78$ & 3 & 1 & & 127 & $\mathbf{N}$ & \\
\hline 1 & $10 / 7 / 78$ & 4 & 1 & & 120 & $\bar{N}$ & \\
\hline 1 & $10 / 7 / 78$ & 5 & 1 & & 114 & $\mathbf{N}$ & \\
\hline 1 & $10 / 7 / 78$ & 6 & 1 & & 105 & $\mathbf{N}$ & \\
\hline 1 & $10 / 7 / 78$ & 7 & 1 & & 94 & $\mathbf{N}$ & \\
\hline$\frac{1}{1}$ & $10 / 7 / 78$ & 8 & 1 & & 88 & $\mathbf{N}$ & \\
\hline 1 & $10 / 7 / 78$ & 9 & 1 & & 76 & $N$ & \\
\hline 1 & $10 / 7 / 78$ & 10 & 1 & & 73 & $N$ & \\
\hline 9 & $10 / 7 / 78$ & 11 & 1 & & 74 & $\mathbf{N}$ & \\
\hline 1 & $10 / 7 / 78$ & 12 & 9 & & 74 & $\mathbf{N}$ & \\
\hline 9 & $1 / 3 / 79$ & 1 & 1 & & 137 & $\mathrm{Y}$ & \\
\hline 1 & $1 / 3 / 79$ & 2 & 1 & & 130 & $\mathbf{N}$ & \\
\hline 1 & $1 / 3 / 79$ & 3 & 1 & & 126 & $\mathbf{N}$ & \\
\hline 1 & $1 / 3 / 79$ & 4 & 1 & & 117 & $\mathbf{N}$ & \\
\hline 1 & $1 / 3 / 79$ & 5 & 1 & & 109 & $\mathbf{N}$ & \\
\hline 1 & $1 / 3 / 79$ & 6 & 1 & & 101 & $\mathbf{N}$ & \\
\hline 1 & $1 / 3 / 79$ & 7 & 1 & & 89 & $\mathbf{N}$ & \\
\hline$\frac{1}{1}$ & $1 / 3 / 79$ & 8 & 1 & & 70 & $\mathbf{N}$ & \\
\hline 1 & $1 / 3 / 79$ & 9 & 1 & & 46 & $\mathbf{N}$ & \\
\hline 1 & $1 / 3 / 79$ & 10 & 1 & & 44 & $\mathbf{N}$ & \\
\hline 1 & $1 / 3 / 79$ & 11 & 1 & & 44 & $\mathbf{N}$ & \\
\hline 1 & $1 / 3 / 79$ & 12 & 1 & & 44 & $N$ & \\
\hline 1 & $4 / 22 / 79$ & 1 & 1 & & 136 & $\mathbf{Y}$ & - \\
\hline 1 & $4 / 22 / 79$ & 2 & 9 & & 128 & $\mathbf{N}$ & \\
\hline 1 & $4 / 22 / 79$ & 3 & 1 & & 122 & $\mathrm{~N}$ & \\
\hline 1 & $4 / 22 / 79$ & 4 & 1 & & 113 & $\mathrm{~N}$ & \\
\hline$\frac{T}{1}$ & $4 / 22 / 79$ & 5 & 1 & & 104 & $\mathbf{N}$ & \\
\hline 1 & $4 / 22 / 79$ & 6 & 1 & & 96 & $\bar{N}$ & \\
\hline 1 & $4 / 22 / 79$ & 7 & 1 & & 87 & $\mathbf{N}$ & \\
\hline 1 & $4 / 22 / 79$ & 8 & 1 & & 74 & $\mathbf{N}$ & \\
\hline 1 & $4 / 22 / 79$ & 9 & 1 & & 62 & $N$ & \\
\hline 1 & $4 / 22 / 79$ & 10 & 1 & & 61 & $N$ & \\
\hline 1 & $4 / 22 / 79$ & 11 & 1 & & 61 & $N$ & \\
\hline
\end{tabular}

Data obtained from WHC Surveillance Analysis Computer System (SACS), June 2, 1993. 


\begin{tabular}{|c|c|c|c|c|c|c|c|}
\hline \multicolumn{8}{|c|}{ TANK 251-BY-105 } \\
\hline & & & & THEF & COUPLE L & & \\
\hline TREE \# & DATE & THERMO \# & TREE SET \# & TEMP C & TEMP F & $Y=M A X$ TEMP & COMMENTS \\
\hline 1 & $4 / 22 / 79$ & 12 & 1 & & 61 & $\mathbf{N}$ & \\
\hline 1 & $7 / 25 / 79$ & 9 & 9 & & 133 & $\mathrm{Y}$ & \\
\hline 9 & $7 / 25 / 79$ & 2 & 1 & & 125 & $\mathbf{N}$ & \\
\hline 1 & $7 / 25 / 79$ & 3 & 1 & & 120 & $\mathbf{N}$ & \\
\hline 1 & $7 / 25 / 79$ & 4 & 1 & & 112 & $\mathbf{N}$ & \\
\hline 1 & $7 / 25 / 79$ & 5 & 1 & & 105 & $\mathbf{N}$ & \\
\hline 1 & $7 / 25 / 79$ & 6 & 1 & & 100 & $\mathbf{N}$ & \\
\hline 1 & $7 / 25 / 79$ & 7 & 1 & & 94 & $\mathbf{N}$ & \\
\hline 1 & $7 / 25 / 79$ & 8 & 9 & & 87 & $\mathrm{~N}$ & \\
\hline 1 & $7 / 25 / 79$ & 9 & 1 & & 82 & $\mathbf{N}$ & \\
\hline 1 & $7 / 25 / 79$ & 10 & 1 & & 82 & $\mathbf{N}$ & \\
\hline 1 & $7 / 25 / 79$ & 11 & 1 & & 82 & $\mathbf{N}$ & \\
\hline 1 & $7 / 25 / 79$ & 12 & 1 & & 82 & $\mathbf{N}$ & \\
\hline 1 & $9 / 4 / 79$ & 1 & 1 & & 121 & $\mathbf{Y}$ & \\
\hline 1 & $9 / 4 / 79$ & 2 & 1 & & 114 & $\mathbf{N}$ & \\
\hline 1 & $9 / 4 / 79$ & 3 & 1 & & 110 & $\mathbf{N}$ & \\
\hline 1 & $9 / 4 / 79$ & 4 & 1 & & 102 & $\mathbf{N}$ & \\
\hline 1 & $9 / 4 / 79$ & 5 & 1 & & 96 & $\mathbf{N}$ & \\
\hline 1 & $9 / 4 / 79$ & 6 & 1 & & 91 & $\mathbf{N}$ & \\
\hline 1 & $9 / 4 / 79$ & 7 & 1 & & 85 & $\mathbf{N}$ & \\
\hline 9 & $9 / 4 / 79$ & 8 & 1 & & 77 & $\mathbf{N}$ & \\
\hline 1 & $9 / 4 / 79$ & 9 & 1 & & 68 & $\mathbf{N}$ & \\
\hline 1 & $9 / 4 / 79$ & 10 & 1 & & 67 & $\mathbf{N}$ & \\
\hline 1 & $9 / 4 / 79$ & 11 & 1 & & 67 & $\mathbf{N}$ & \\
\hline 1 & $9 / 4 / 79$ & 12 & 1 & & 67 & $\mathbf{N}$ & \\
\hline 1 & $11 / 15 / 79$ & 1 & 1 & & 145 & $\bar{Y}$ & \\
\hline$\frac{1}{1}$ & $11 / 15 / 79$ & 2 & 1 & & 139 & $\mathrm{~N}$ & \\
\hline 1 & $11 / 15 / 79$ & 3 & 1 & & 136 & $\mathbf{N}$ & \\
\hline 1 & $11 / 15 / 79$ & 4 & 1 & & 129 & $\mathrm{~N}$ & \\
\hline 1 & $11 / 15 / 79$ & 5 & 1 & & 122 & $\mathbf{N}$ & \\
\hline 1 & $11 / 15 / 79$ & 6 & 1 & & 122 & $\mathbf{N}$ & \\
\hline 1 & $11 / 15 / 79$ & 7 & 1 & & 115 & $\mathbf{N}$ & \\
\hline 1 & $11 / 15 / 79$ & 8 & 1 & & 100 & $\mathbf{N}$ & \\
\hline 1 & $11 / 15 / 79$ & 9 & 1 & & 83 & $\mathbf{N}$ & \\
\hline 1 & $11 / 15 / 79$ & 10 & 1 & & 81 & $\mathrm{~N}$ & \\
\hline 1 & $11 / 15 / 79$ & 11 & 1 & & 82 & $\bar{N}$ & \\
\hline 1 & $11 / 15 / 79$ & 12 & 1 & & 80 & $\mathrm{~N}$ & \\
\hline 1 & $2 / 6 / 80$ & 1 & 1 & & 140 & $\bar{Y}$ & \\
\hline 9 & $2 / 6 / 80$ & 2 & 1 & & 133 & $\bar{N}$ & \\
\hline 1 & $2 / 6 / 80$ & 3 & 1 & & 129 & $\bar{N}$ & \\
\hline 1 & $2 / 6 / 80$ & 4 & 1 & & 120 & $\mathrm{~N}$ & \\
\hline 1 & $2 / 6 / 80$ & 5 & 1 & & 111 & $\mathbf{N}$ & \\
\hline 1 & $2 / 6 / 80$ & 6 & 1 & & 109 & $\mathrm{~N}$ & \\
\hline 1 & $2 / 6 / 80$ & 7 & 1 & & 91 & $\mathbf{N}$ & \\
\hline 1 & $2 / 6 / 80$ & 8 & 1 & & 74 & $\mathbf{N}$ & \\
\hline 1 & $2 / 6 / 80$ & 9 & 1 & & 58 & $\mathbf{N}$ & \\
\hline 1 & $2 / 6 / 80$ & 10 & 1 & & 57 & $\mathbf{N}$ & \\
\hline 1 & $2 / 6 / 80$ & 11 & 1 & & 58 & $\mathbf{N}$ & \\
\hline 1 & $2 / 6 / 80$ & 12 & 1 & & 58 & $\mathrm{~N}$ & \\
\hline 1 & $5 / 12 / 80$ & 1 & 1 & & 132 & $\bar{Y}$ & \\
\hline 1 & $5 / 12 / 80$ & 2 & 9 & & 125 & $\mathbf{N}$ & \\
\hline 1 & $5 / 12 / 80$ & 3 & 1 & & 115 & $\mathbf{N}$ & \\
\hline 1 & $5 / 12 / 80$ & 4 & 1 & & 112 & $\mathbf{N}$ & \\
\hline 1 & $5 / 12 / 80$ & 5 & 1 & & 104 & $\mathbf{N}$ & \\
\hline 1 & $5 / 12 / 80$ & 6 & 1 & & 98 & $\mathbf{N}$ & \\
\hline 1 & $5 / 12 / 80$ & 7 & 1 & & 91 & $\mathrm{~N}$ & \\
\hline 1 & $5 / 12 / 80$ & 8 & 1 & & 81 & $\mathbf{N}$ & \\
\hline 1 & $5 / 12 / 80$ & 9 & 1 & & 73 & $\mathrm{~N}$ & \\
\hline 1 & $5 / 12 / 80$ & 10 & 1 & & 73 & $\mathbf{N}$ & \\
\hline 1 & $5 / 12 / 80$ & 11 & 1 & & 74 & $\mathrm{~N}$ & \\
\hline 1 & $5 / 12 / 80$ & 12 & 1 & & 74 & $\mathbf{N}$ & \\
\hline 1 & $11 / 12 / 80$ & 1 & 1 & & 138 & $Y$ & \\
\hline 1 & $111 / 12 / 80$ & 2 & 1 & & 132 & $\mathbf{N}$ & \\
\hline 1 & $11 / 12 / 80$ & 3 & 1 & & 129 & $\mathbf{N}$ & \\
\hline 1 & $11 / 12 / 80$ & 4 & 1 & & 122 & $\mathbf{N}$ & \\
\hline 1 & $11 / 12 / 80$ & 5 & 1 & & 116 & $N$ & \\
\hline 1 & $11 / 12 / 80$ & 6 & 1 & & 111 & $\mathbf{N}$ & \\
\hline 1 & $11 / 12 / 80$ & 7 & 1 & & 105 & $N$ & \\
\hline
\end{tabular}

Data obtained from WHC Surveillance Analysis Computer System (SACS), June 2, 1993. 


\begin{tabular}{|c|c|c|c|c|c|c|c|}
\hline \multirow{2}{*}{\multicolumn{8}{|c|}{$\begin{array}{l}\text { TANK 251-BY-105 } \\
\text { THERMOCOUPLE DATA }\end{array}$}} \\
\hline & & & & & & & \\
\hline TREE \# & DATE & THERMO \# & TREE SET \# & TEMP C & TEMP F & $Y=M A X$ TEMP & COMMENTS \\
\hline 1 & $11 / 12 / 80$ & 8 & 1 & & 92 & $\mathbf{N}$ & \\
\hline 9 & $11 / 12 / 80$ & 9 & 1 & & 79 & $\mathbf{N}$ & \\
\hline 1 & $11 / 12 / 80$ & 10 & 1 & & 77 & $\mathbf{N}$ & \\
\hline 1 & $11 / 12 / 80$ & 11 & 1 & & 77 & $\mathbf{N}$ & \\
\hline 1 & $11 / 12 / 80$ & 12 & 1 & & 77 & $\mathbf{N}$ & \\
\hline 1 & $12 / 1 / 80$ & 1 & 1 & & 143 & $Y$ & \\
\hline 1 & $12 / 1 / 80$ & 2 & 1 & & 136 & $\mathrm{~N}$ & \\
\hline 1 & $12 / 1 / 80$ & 3 & 1 & & 132 & $\mathbf{N}$ & \\
\hline 1 & $12 / 1 / 80$ & 4 & 1. & & 125 & $\mathbf{N}$ & \\
\hline 1 & $12 / 1 / 80$ & 5 & 1 & & 918 & $\mathbf{N}$ & \\
\hline 1 & $12 / 1 / 80$ & 6 & 1 & & 111 & $N$ & \\
\hline 1 & $12 / 1 / 80$ & 7 & 1 & & 104 & $\mathbf{N}$ & \\
\hline 1 & $12 / 1 / 80$ & 8 & 1 & & 86 & $\mathbf{N}$ & \\
\hline 1 & $12 / 1 / 80$ & 9 & 1 & & 68 & $\mathbf{N}$ & \\
\hline 1 & $12 / 1 / 80$ & 10 & 1 & & 66 & $\mathbf{N}$ & \\
\hline 1 & $12 / 1 / 80$ & 11 & 1 & & 66 & $N$ & \\
\hline 1 & $12 / 1 / 80$ & 12 & 1 & & 67 & $\mathbf{N}$ & \\
\hline 1 & $6 / 12 / 81$ & 1 & 9 & & 120 & $\bar{Y}$ & \\
\hline 1 & $6 / 12 / 81$ & 2 & 1 & & 114 & $\mathbf{N}$ & \\
\hline 1 & $6 / 12 / 81$ & 3 & 1 & & 111 & $\mathrm{~N}$ & \\
\hline 1 & $6 / 12 / 81$ & 4 & 1 & & 104 & $N$ & \\
\hline 1 & $6 / 12 / 81$ & 5 & 1 & & 99 & $\mathbf{N}$ & \\
\hline 1 & $6 / 12 / 81$ & 6 & 1 & & 93 & $\mathbf{N}$ & \\
\hline 1 & $6 / 12 / 81$ & 7 & 1 & & 88 & $\mathbf{N}$ & \\
\hline 1 & $6 / 12 / 81$ & 8 & 1 & & 80 & $\mathbf{N}$ & \\
\hline 1 & $6 / 12 / 81$ & 9 & 1 & & 73 & $\mathrm{~N}$ & \\
\hline 1 & $6 / 12 / 81$ & 10 & 1 & & 72 & $N$ & \\
\hline 1 & $6 / 12 / 81$ & 11 & 1 & & 72 & $\mathbf{N}$ & \\
\hline 1 & $6 / 12 / 81$ & 12 & 1 & & 72 & $\mathbf{N}$ & \\
\hline 1 & $1 / 14 / 82$ & 1 & 1 & & 135 & $Y$ & \\
\hline 1 & $1 / 14 / 82$ & 2 & 1 & & 129 & $\mathbf{N}$ & \\
\hline 9 & $1 / 14 / 82$ & 3 & 1 & & 125 & $\mathbf{N}$ & \\
\hline 1 & $1 / 14 / 82$ & 4 & 1 & & 119 & $\mathbf{N}$ & \\
\hline 1 & $1 / 14 / 82$ & 5 & 1 & & 114 & $N$ & \\
\hline 1 & $1 / 14 / 82$ & 6 & 1 & & 106 & $\mathrm{~N}$ & \\
\hline 1 & $1 / 14 / 82$ & 7 & 1 & & 97 & $N$ & \\
\hline 1 & $1 / 14 / 82$ & 8 & 1 & & 84 & $N$ & \\
\hline 1 & $1 / 14 / 82$ & 9 & 1 & & 76 & $\mathbf{N}$ & \\
\hline 1 & $1 / 14 / 82$ & 10 & 1 & & 75 & $\mathbf{N}$ & \\
\hline 1 & $.9 / 14 / 82$ & 11 & 1 & & 75 & $\mathbf{N}$ & \\
\hline 1 & $1 / 14 / 82$ & 12 & 1 & & 75 & $\mathbf{N}$ & \\
\hline 1 & $2 / 4 / 82$ & 1 & 1 & & 145 & $\mathbf{Y}$ & \\
\hline 1 & $2 / 4 / 82$ & 2 & 1 & & 139 & $\mathbf{N}$ & \\
\hline 1 & $2 / 4 / 82$ & 3 & 1 & & 135 & $\mathbf{N}$ & \\
\hline 1 & $2 / 4 / 82$ & 4 & 1 & & 128 & $N$ & \\
\hline 1 & $2 / 4 / 82$ & 5 & 1 & & 122 & $\mathbf{N}$ & \\
\hline 1 & $2 / 4 / 82$ & 6 & 1 & & 116 & $\mathrm{~N}$ & \\
\hline 1 & $2 / 4 / 82$ & 7 & 1 & & 110 & $N$ & \\
\hline 1 & $2 / 4 / 82$ & 8 & 1 & & 98 & $N$ & \\
\hline 1 & $2 / 4 / 82$ & 9 & 1 & & 88 & $\mathbf{N}$ & \\
\hline 1 & $2 / 4 / 82$ & 10 & 1 & & 87 & $\mathbf{N}$ & \\
\hline 1 & $2 / 4 / 82$ & 11 & 1 & & 87 & $\mathbf{N}$ & \\
\hline 1 & $2 / 4 / 82$ & 12 & 1 & & 87 & $\mathbf{N}$ & \\
\hline 1 & $3 / 10 / 82$ & 1 & 1 & & 137 & $\bar{Y}$ & \\
\hline 1 & $3 / 10 / 82$ & 2 & 1 & & 131 & $N$ & \\
\hline 1 & $3 / 10 / 82$ & 3 & 1 & & 127 & $\mathbf{N}$ & \\
\hline 1 & $3 / 10 / 82$ & 4 & 1 & & 120 & $N$ & \\
\hline 1 & $3 / 10 / 82$ & 5 & 1 & & 114 & $\mathbf{N}$ & \\
\hline 1 & $3 / 10 / 82$ & 6 & 1 & & 107 & $\mathbf{N}$ & \\
\hline 1 & $3 / 70 / 82$ & 7 & 1 & & 101 & $\mathbf{N}$ & \\
\hline 1 & $3 / 10 / 82$ & 8 & 1 & & 89 & $\mathbf{N}$ & \\
\hline 1 & $3 / 10 / 82$ & 9 & 1 & & 80 & $\mathbf{N}$ & \\
\hline 1 & $3 / 10 / 82$ & 10 & 1 & & 79 & $\bar{N}$ & \\
\hline 1 & $3 / 10 / 82$ & 11 & 1 & & 79 & $\bar{N}$ & \\
\hline 1 & $3 / 10 / 82$ & 12 & 1 & & 80 & $\mathbf{N}$ & \\
\hline 1 & $6 / 4 / 85$ & 1 & 1 & & 122 & $\bar{Y}$ & \\
\hline 1 & $6 / 4 / 85$ & 2 & 9 & & 115 & $N$ & \\
\hline 1 & $6 / 4 / 85$ & 3 & 1 & & 110 & $N$ & \\
\hline
\end{tabular}

Data obtained from WHC Surveillance Analysis Computer System (SACS), June 2, 1993. 


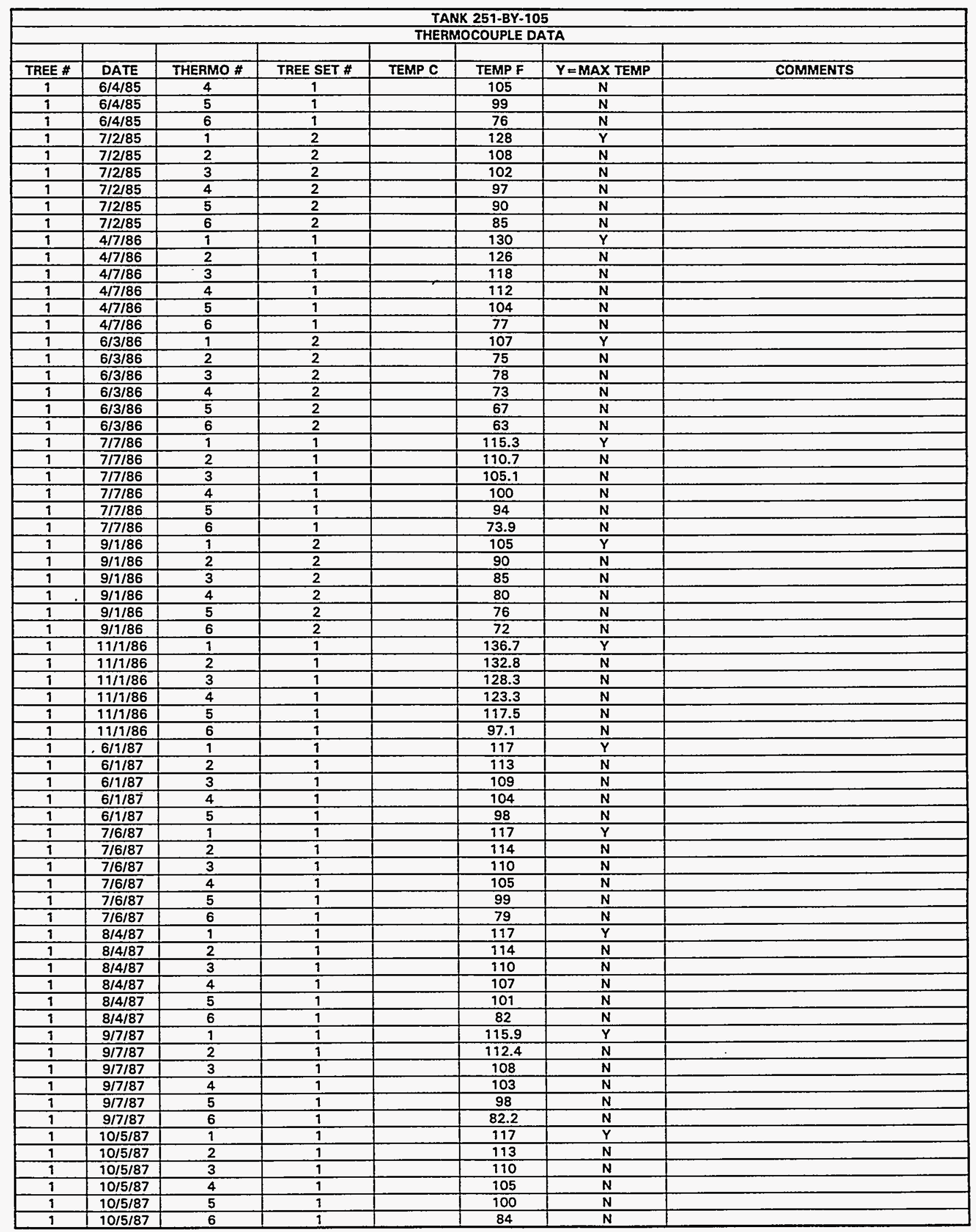

Data obtained from WHC Surveillance Analysis Computer System (SACS). June 2, 1993. 


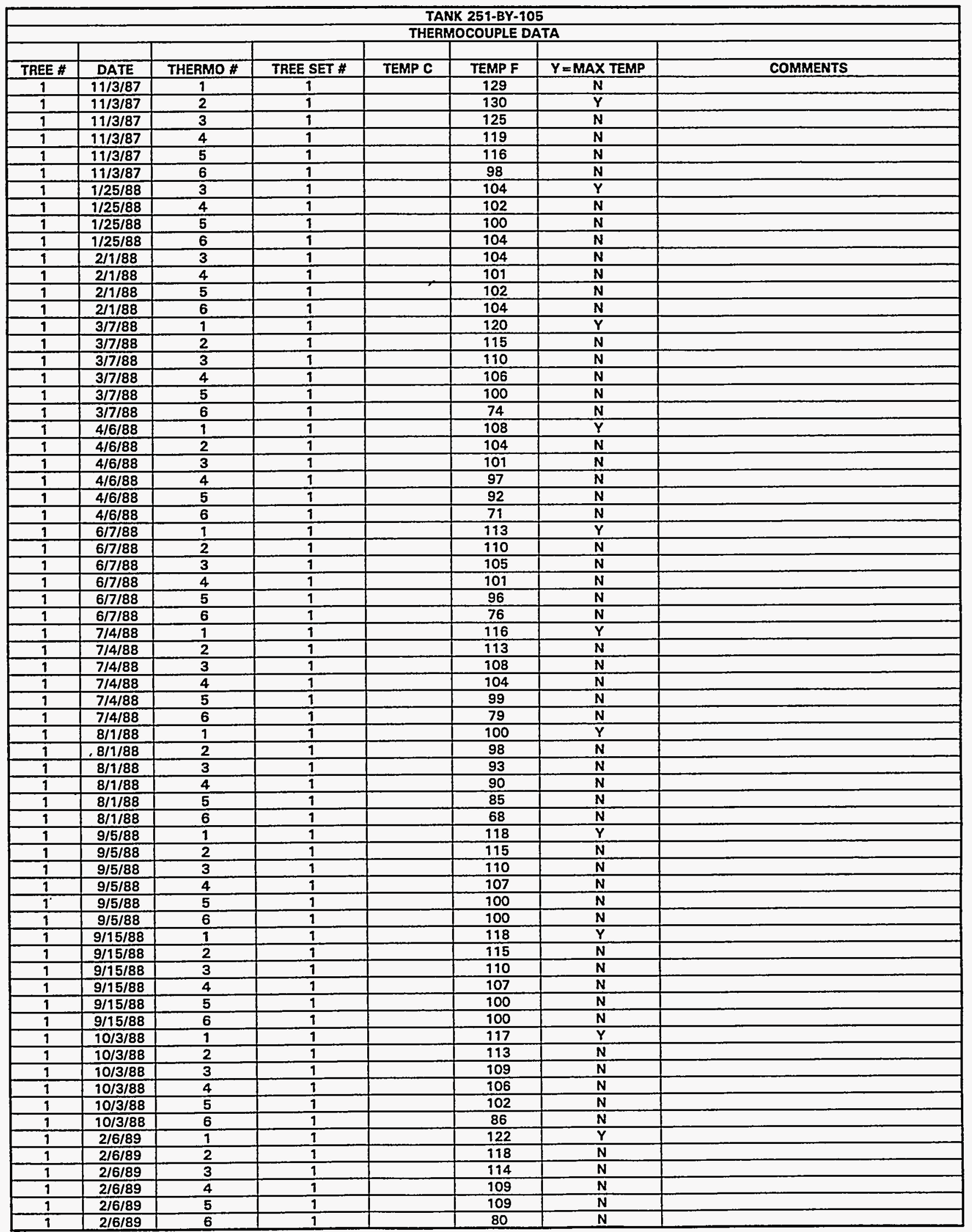

Data obtained from WHC Surveillance Analysis Computer System (SACS), June 2, 1993. 
WHC-SD-WM-ER-312, Rev. 0

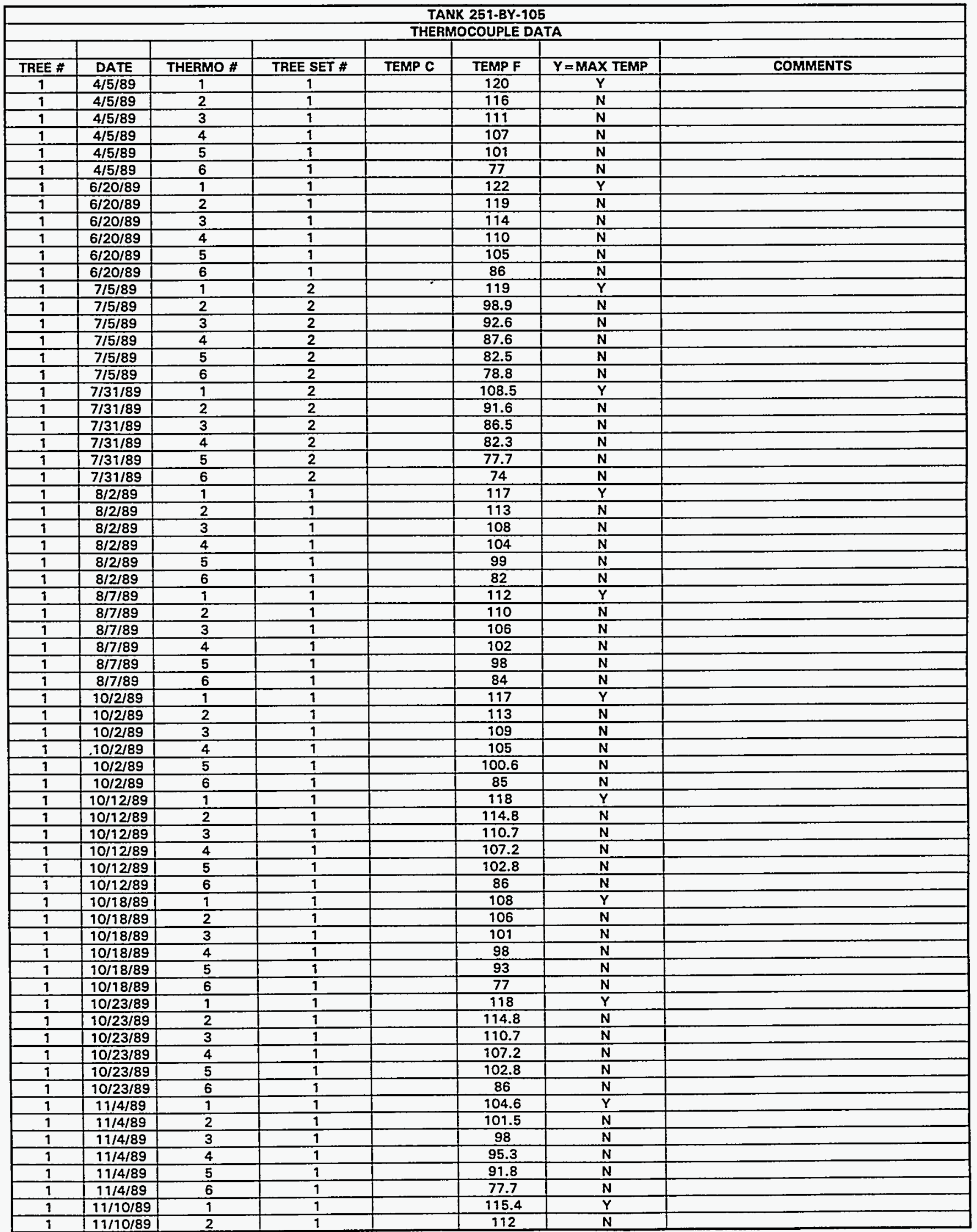

Data obtained from WHC Surveillance Analysis Computer System (SACS), June 2, 1993. 


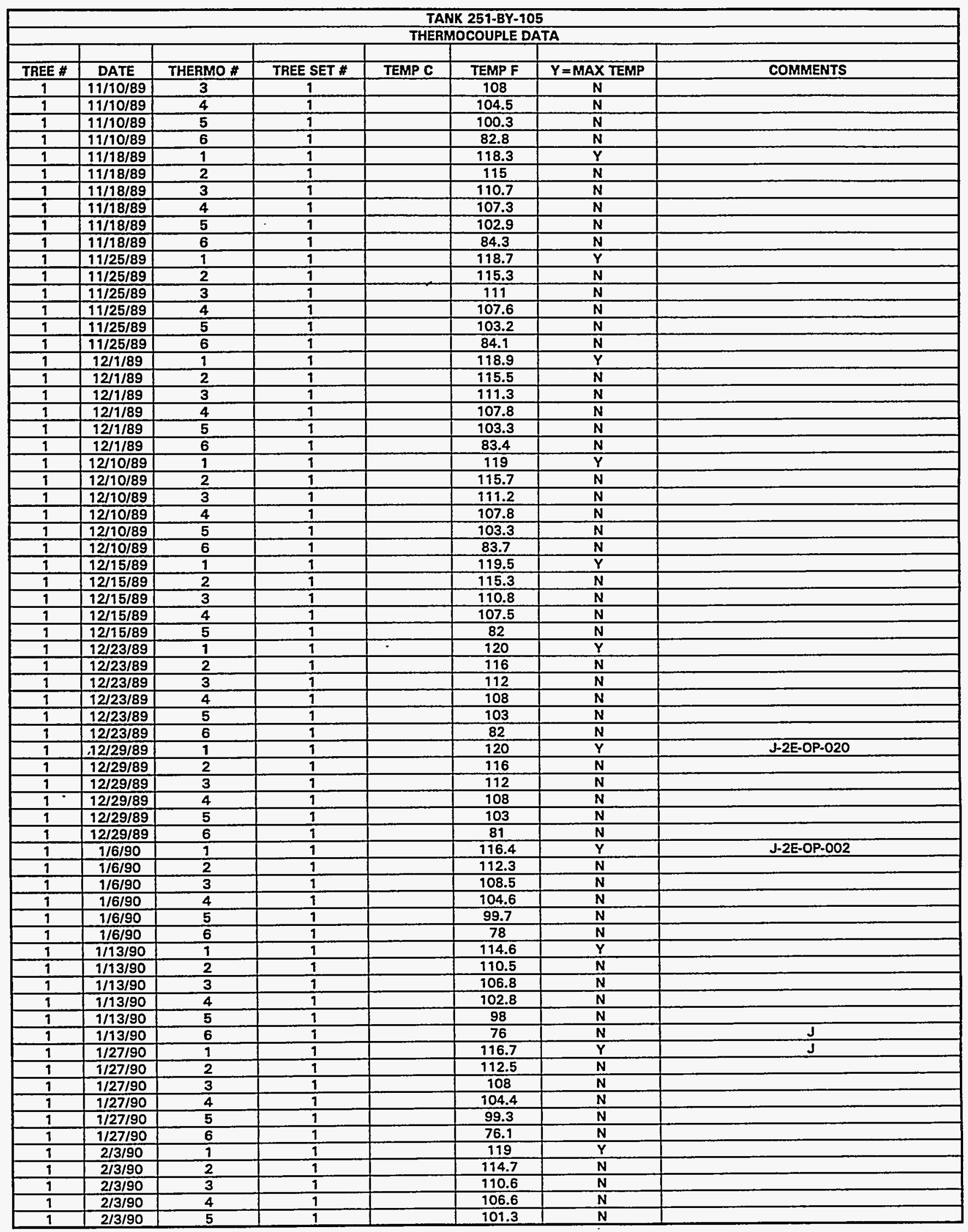

Data obtained from WHC Surveillance Analysis Computer System (SACS), June 2, 1993. 


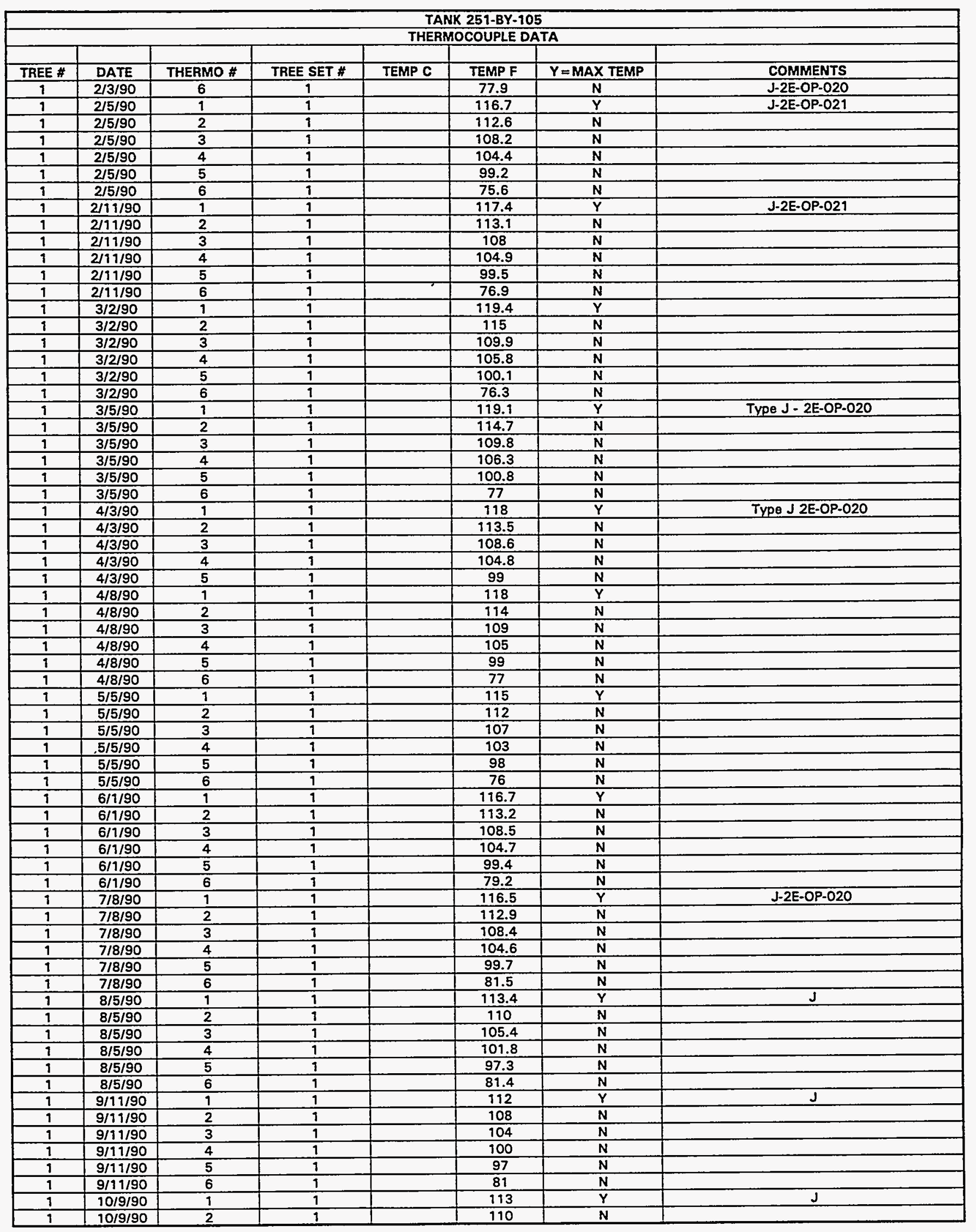

Data obtained from WHC Surveillance Analysis Computer System (SACS), June 2, 1993. 
WHC-SD-WM-ER-312, Rev. 0

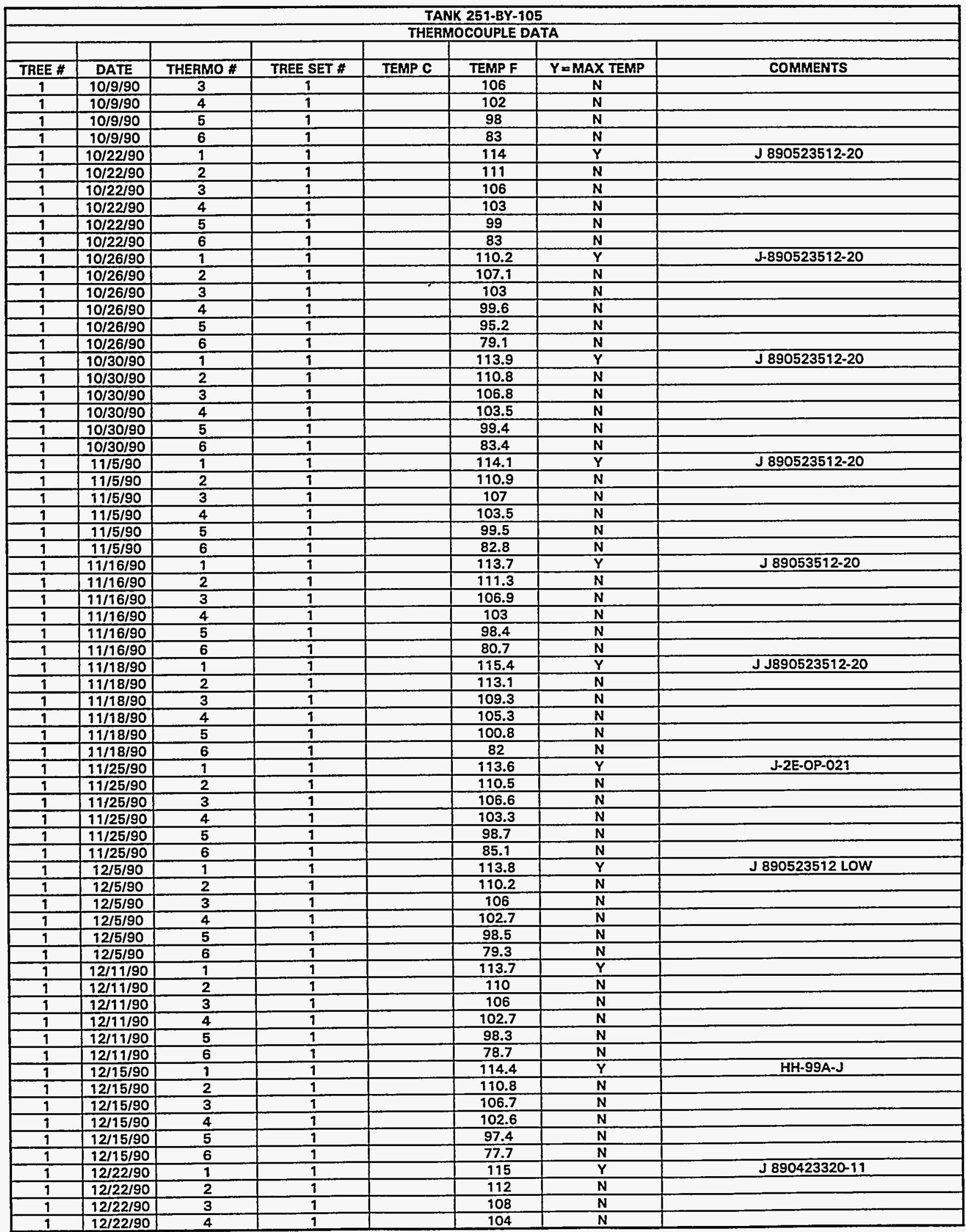

Data obtained from WHC Surveillance Analysis Computer System (SACS), June 2, 1993. 


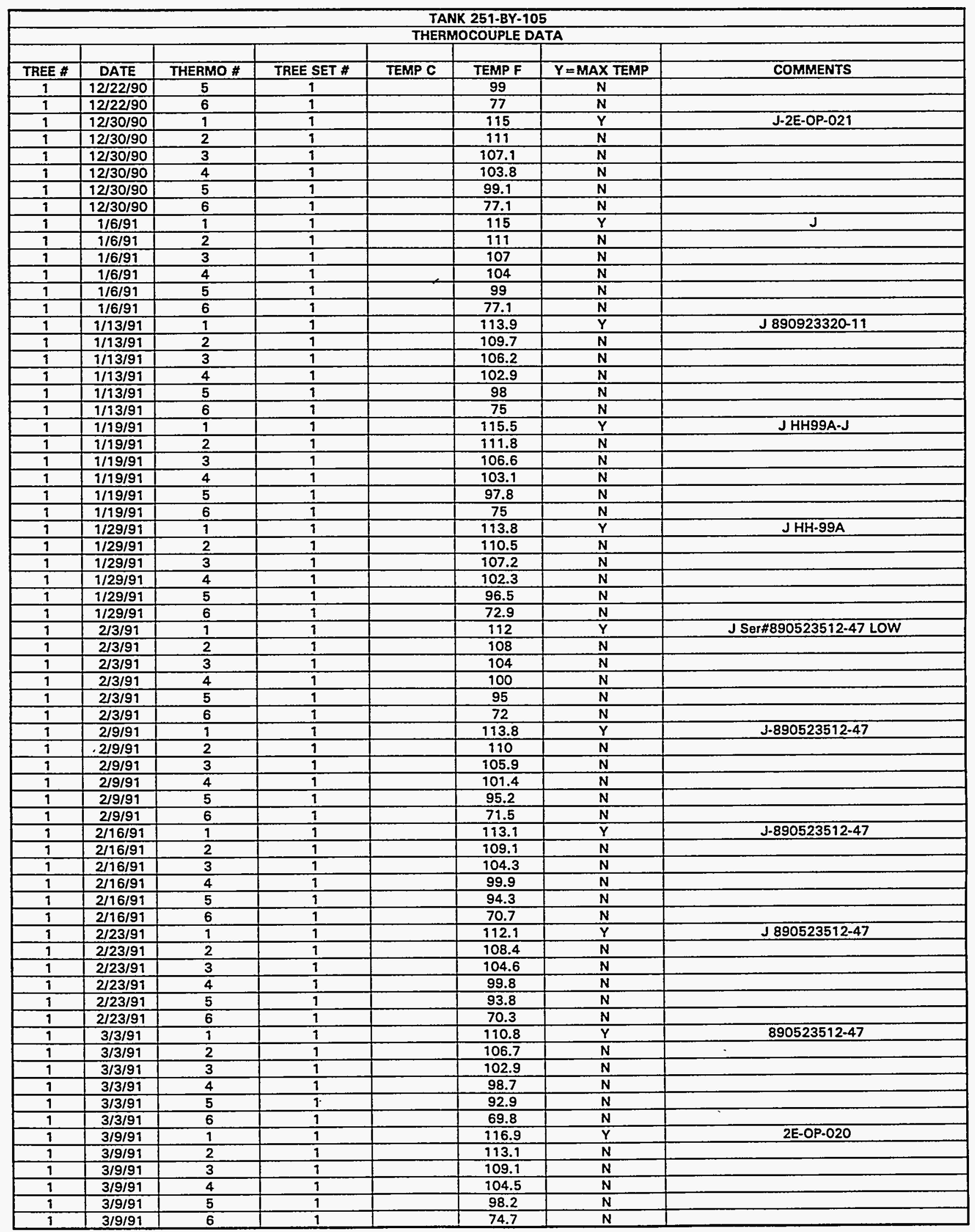

Data obtained from WHC Surveillance Analysis Computer System (SACS), June 2, 1993. 
WHC-SD-WM-ER-312, Rev. 0

\begin{tabular}{|c|c|c|c|c|c|c|c|}
\hline \multicolumn{8}{|c|}{ TANK 251-BY-105 } \\
\hline \multicolumn{8}{|c|}{ THERMOCOUPLE DATA } \\
\hline TAEE \# & $\overline{\text { DATE }}$ & THERMO \# & TREE SET \# & TEMP C & TEMP F & $Y=M A X$ TEMP & COMMENTS \\
\hline 1 & $3 / 17 / 91$ & 1 & 1 & & 115.2 & $\mathrm{Y}$ & $J 2 E-0 P-021$ \\
\hline 1 & $3 / 17 / 91$ & 2 & 1 & & 110 & $\mathbf{N}$ & \\
\hline 1 & $3 / 17 / 91$ & 3 & 1 & & 106.8 & $\mathbf{N}$ & \\
\hline 1 & $3 / 17 / 91$ & 4 & 1 & & 101.7 & $\mathbf{N}$ & \\
\hline 1 & $3 / 17 / 91$ & 5 & 1 & & 94.8 & $\mathbf{N}$ & \\
\hline$\frac{1}{1}$ & $3 / 17 / 91$ & 6 & 1 & & 72.1 & $\mathrm{~N}$ & \\
\hline 1 & $3 / 27 / 91$ & 1 & 1 & & 116 & $\mathbf{Y}$ & J 890523512-20 Rechocked \\
\hline 1 & $3 / 27 / 91$ & 2 & 1 & & 111.8 & $\mathbf{N}$ & \\
\hline$\frac{1}{1}$ & $3 / 27 / 91$ & 3 & 9 & & 107.4 & $\mathbf{N}$ & \\
\hline 1 & $3 / 27 / 91$ & 4 & 1 & & 102.5 & $\overline{\mathbf{N}}$ & \\
\hline 1 & $3 / 27 / 91$ & 5 & 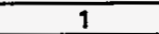 & & 95.7 & $\mathbf{N}$ & \\
\hline 1 & $3 / 27 / 91$ & 6 & 1 & & 72.8 & $\mathbf{N}$ & \\
\hline$T$ & $3 / 30 / 91$ & 1 & 1 & & 116 & $\bar{Y}$ & J 890523512-20 \\
\hline 1 & $3 / 30 / 91$ & 2 & 1 & & 111.1 & $\mathbf{N}$ & \\
\hline$T$ & $3 / 30 / 91$ & 3 & 1 & & 106.9 & $\mathbf{N}$ & \\
\hline 1 & $3 / 30 / 91$ & 4 & 1 & & 101.7 & $\mathbf{N}$ & \\
\hline 1 & $3 / 30 / 91$ & 5 & 1 & & 94.8 & $\mathbf{N}$ & \\
\hline 1 & $3 / 30 / 91$ & 6 & 1 & & 71.6 & $\mathbf{N}$ & \\
\hline 1 & $4 / 7 / 91$ & 1 & 1 & & 116.5 & $\mathbf{Y}$ & J $890423320-11$ \\
\hline 1 & $4 / 7 / 91$ & 2 & 1 & & 112.7 & $\mathbf{N}$ & \\
\hline$\frac{1}{1}$ & $4 / 7 / 91$ & 3 & 1 & & 108 & $\bar{N}$ & \\
\hline 1 & $4 / 7 / 91$ & 4 & 1 & & 104.6 & $N$ & \\
\hline 1 & $4 / 7 / 91$ & 5 & 1 & & 98.7 & $\mathbf{N}$ & \\
\hline$T$ & $4 / 7 / 91$ & 6 & 1 & & 75 & $\mathbf{N}$ & \\
\hline 1 & $4 / 14 / 91$ & 1 & 1 & & 116.2 & $\bar{Y}$ & J 890523512-20 Omega \\
\hline 9 & $4 / 14 / 91$ & 2 & 1 & & 112.6 & $\mathbf{N}$ & \\
\hline 1 & $4 / 14 / 91$ & 3 & 1 & & 107.6 & $\bar{N}$ & \\
\hline 9 & $4 / 14 / 91$ & 4 & 1 & & 103.7 & $\bar{N}$ & \\
\hline$T$ & $4 / 14 / 91$ & 5 & 1 & & 97.8 & $\mathbf{N}$ & \\
\hline$\frac{1}{1}$ & $4 / 14 / 91$ & 6 & 1 & & 74.7 & $\mathbf{N}$ & \\
\hline$\frac{1}{1}$ & $4 / 20 / 91$ & 9 & $T$ & & 116 & $\bar{Y}$ & $J 890523512-20$ \\
\hline 1 & $4 / 20 / 91$ & 2 & $\frac{1}{1}$ & & 112.4 & $\mathbf{N}$ & \\
\hline 1 & $4 / 20 / 91$ & 3 & 1 & & 106.6 & $\mathbf{N}$ & \\
\hline 1 & $4 / 20 / 91$ & 4 & 1 & & 103.2 & $\mathbf{N}$ & \\
\hline 1 & $4 / 20 / 91$ & 5 & 1 & & 97.6 & $N$ & \\
\hline 1 & $4 / 20 / 91$ & 6 & 1 & & 75.3 & $\mathbf{N}$ & \\
\hline 1 & $4 / 28 / 91$ & 1 & 1 & & 114 & $\mathbf{Y}$ & J Omaga $890523512-20$ \\
\hline 1 & $4 / 28 / 91$ & 2 & 1 & & 110.7 & $\mathbf{N}$ & \\
\hline 1 & $4 / 28 / 91$ & 3 & 9 & & 106.1 & $\mathbf{N}$ & \\
\hline 1 & $.4 / 28 / 91$ & 4 & 1 & & 102.1 & $\mathbf{N}$ & \\
\hline 1 & $4 / 28 / 91$ & 5 & 1 & & 96.4 & $\mathbf{N}$ & \\
\hline 1 & $4 / 28 / 91$ & 6 & 1 & & 74.8 & $\mathbf{N}$ & \\
\hline 1 & $5 / 5 / 91$ & 1 & $\frac{1}{1}$ & & 112.9 & $\bar{Y}$ & J $890523512-20$ \\
\hline 1 & $5 / 5 / 91$ & 2 & 9 & & 109.7 & $\mathbf{N}$ & \\
\hline 1 & $5 / 5 / 91$ & 3 & 1 & & 105.2 & $\mathbf{N}$ & \\
\hline 1 & $5 / 5 / 91$ & 4 & 1 & & 101.5 & $\mathbf{N}$ & \\
\hline 1 & $5 / 5 / 91$ & 5 & 1 & & 95.8 & $\mathbf{N}$ & \\
\hline 1 & $5 / 5 / 91$ & 6 & 1 & & 74.6 & $\mathbf{N}$ & \\
\hline 1 & $5 / 12 / 91$ & 1 & 1 & & 113.3 & $\bar{Y}$ & J 890523512-20 \\
\hline 1 & $5 / 12 / 91$ & 2 & 1 & & 109.8 & $\mathbf{N}$ & \\
\hline 1 & $5 / 12 / 91$ & 3 & 1 & & 105.2 & $\mathbf{N}$ & \\
\hline 1 & $5 / 12 / 91$ & 4 & 1 & & 101.3 & $\mathbf{N}$ & \\
\hline 1 & $5 / 12 / 91$ & 5 & 1 & & 95.8 & $\mathbf{N}$ & \\
\hline 1 & $5 / 12 / 91$ & 6 & 1 & & 74.9 & $\mathbf{N}$ & \\
\hline 1 & $5 / 18 / 91$ & 1 & 1 & & 115 & $\mathbf{Y}$ & J Kiethly 872 2E-OP-020 \\
\hline$\frac{1}{1}$ & $5 / 18 / 91$ & 2 & 1 & & 111.8 & $\mathbf{N}$ & \\
\hline 9 & $5 / 18 / 91$ & 3 & 1 & & 107.3 & $\mathbf{N}$ & \\
\hline 9 & $5 / 18 / 91$ & 4 & $\frac{1}{1}$ & & 103.1 & $\mathbf{N}$ & \\
\hline $\bar{T}$ & $5 / 18 / 91$ & 5 & 1 & & 97.5 & $\mathbf{N}$ & \\
\hline 1 & $5 / 18 / 91$ & 6 & 1 & & 76 & $\mathbf{N}$ & \\
\hline 1 & $5 / 25 / 91$ & 1 & 1 & & 914.4 & $\bar{Y}$ & J 2E-OP-021 \\
\hline 1 & $5 / 25 / 91$ & 2 & 1 & & 111,3 & $\mathbf{N}$ & \\
\hline 1 & $5 / 25 / 91$ & 3 & 1 & & 106.9 & $\bar{N}$ & \\
\hline$\frac{9}{1}$ & $5 / 25 / 91$ & 4 & 1 & & 102.7 & $\mathbf{N}$ & \\
\hline 1 & $5 / 25 / 91$ & 5 & 1 & & 97.1 & $N$ & \\
\hline 1 & $5 / 25 / 91$ & 6 & 1 & & 76.2 & $\mathbf{N}$ & \\
\hline 1 & $6 / 2 / 91$ & 1 & 1 & & 112.4 & $Y$ & $\mathrm{~J} 890423320-42$ \\
\hline 1 & $6 / 2 / 91$ & 2 & 1 & & 109.1 & $\mathbf{N}$ & \\
\hline
\end{tabular}

Data obtained from WHC Surveillance Analysis Computer System (SACS), June 2, 1993. 


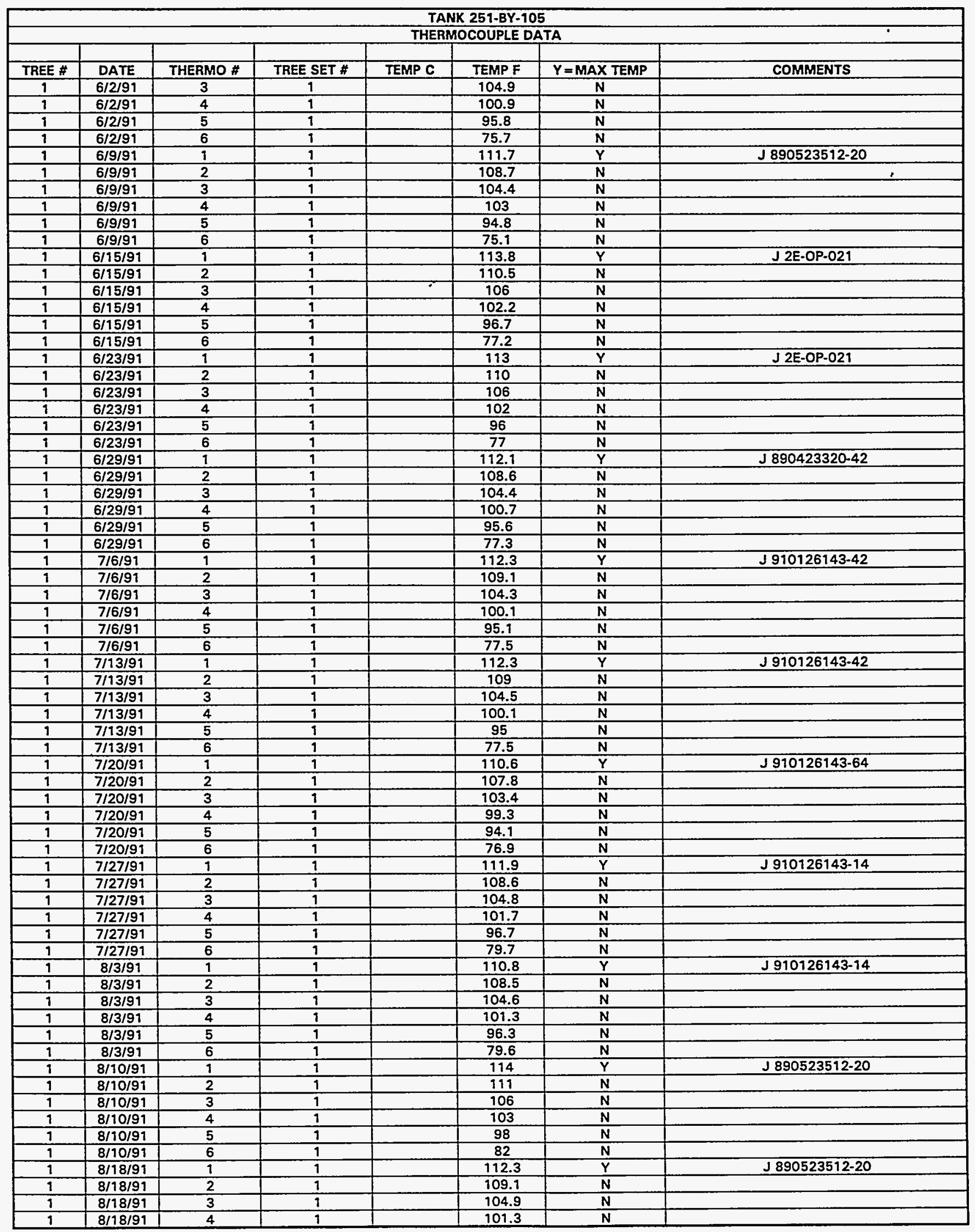

Data obtained from WHC Surveillance Analysis Computer System (SACS), June 2, 1993. 
WHC-SD-WM-ER-312, Rev. 0

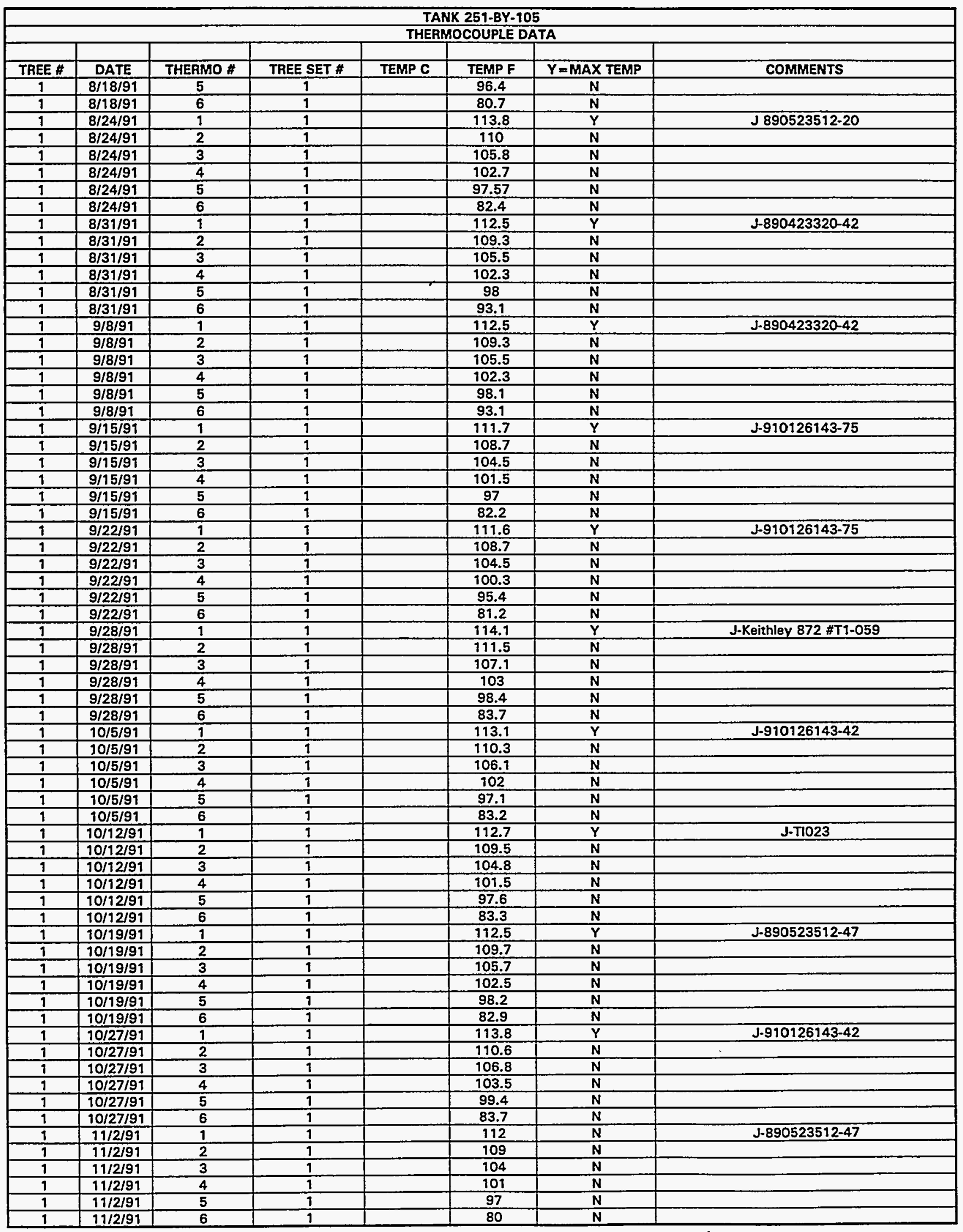

Data obtained from WHC Surveillance Analysis Computer System (SACS), June 2, 1993. 
WHC-SD-WM-ER-312, Rev. 0

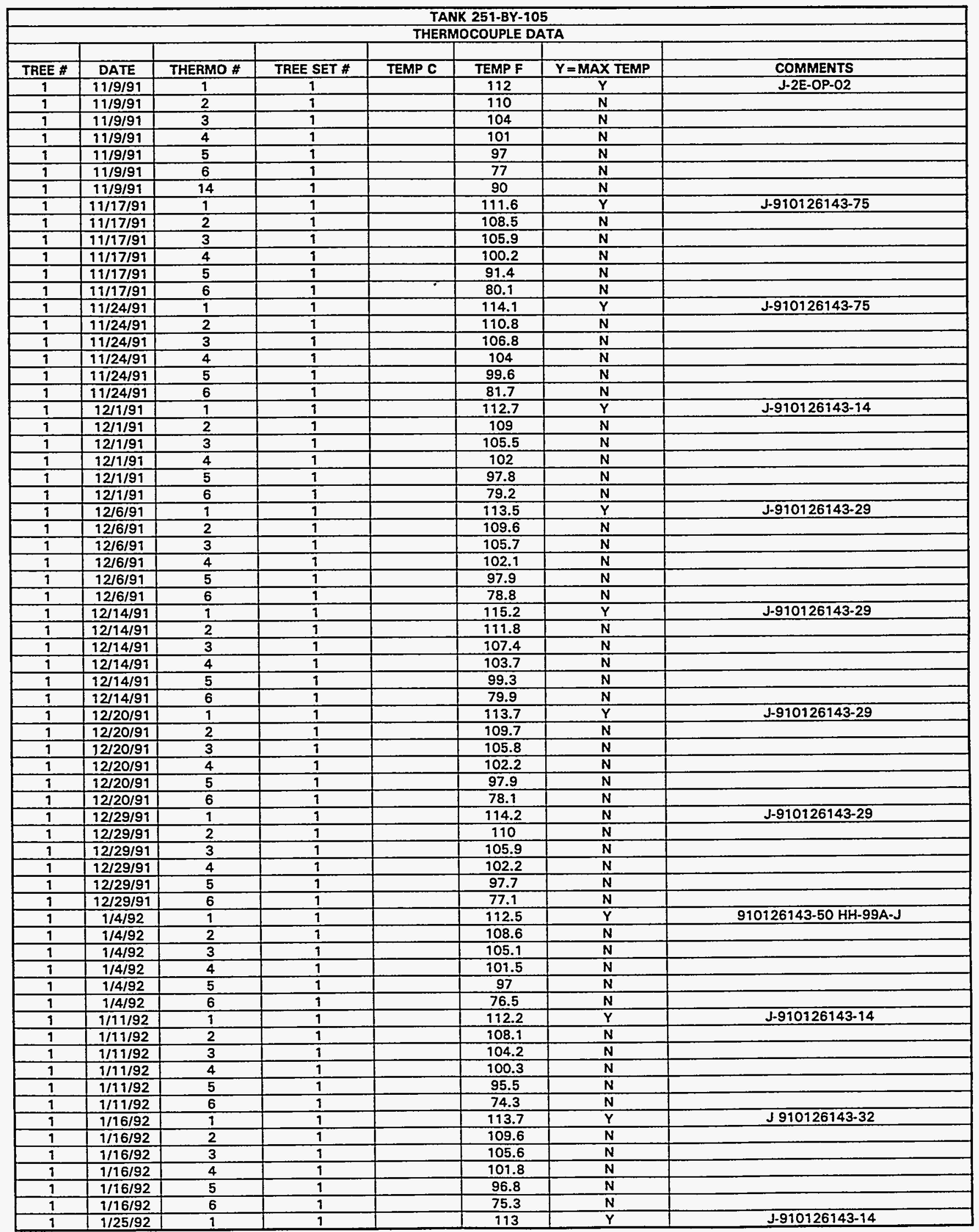

Data obtained from WHC Surveillance Analysis Computer System (SACS), June 2, 1993. 
WHC-SD-WM-ER-312, Rev. 0

\begin{tabular}{|c|c|c|c|c|c|c|c|}
\hline \multicolumn{8}{|c|}{ TANK 251-BY-105 } \\
\hline \multicolumn{8}{|c|}{ THERMOCOUPLE DATA } \\
\hline TREE \# & DATE & THERMO \# & TREE SET \# & TEMP C & TEMP F & $Y=$ MAX TEMP & COMMENTS \\
\hline 1 & $1 / 25 / 92$ & 2 & 1 & & 109.1 & $N$ & \\
\hline 1 & $1 / 25 / 92$ & 3 & 1 & & 105.3 & $\mathbf{N}$ & \\
\hline 1 & $1 / 25 / 92$ & 4 & 1 & & 101.5 & $\mathbf{N}$ & \\
\hline$\frac{1}{1}$ & $1 / 25 / 92$ & 5 & 1 & & 96.5 & $\mathbf{N}$ & \\
\hline 1 & $1 / 25 / 92$ & 6 & 1 & & 74.9 & $\mathbf{N}$ & \\
\hline 1 & $1 / 28 / 92$ & 1 & 1 & & & N & $\begin{array}{l}\text { ACCESS RESTRICTED } 1 / 28-2 / 22 \text { DUE TO } \\
\text { BATTERY FUME INCIDENT }\end{array}$ \\
\hline 1 & $2 / 24 / 92$ & 1 & 1 & & 113.2 & $\mathbf{N}$ & J-900925741-79 \\
\hline 1 & $2 / 24 / 92$ & 2 & 1 & & 108.7 & $\mathbf{N}$ & \\
\hline 1 & $2 / 24 / 92$ & 3 & 1 & & 104.8 & $\mathbf{N}$ & \\
\hline$\frac{1}{1}$ & $2 / 24 / 92$ & 4 & 1 & & 100.6 & $\mathbf{N}$ & \\
\hline 1 & $2 / 24 / 92$ & 5 & 1 & & 95.5 & $\mathbf{N}$ & \\
\hline 1 & $2 / 24 / 92$ & $\overline{6}$ & 1 & & 73.3 & $\mathbf{N}$ & \\
\hline 1 & $2 / 29 / 92$ & 1 & 1 & & 113.5 & $\mathbf{N}$ & $J+1.038 / 912012614332$ \\
\hline 1 & $2 / 29 / 92$ & 2 & 1 & & 109.1 & $\mathbf{N}$ & \\
\hline 1 & $2 / 29 / 92$ & 3 & 1 & & 105 & $\bar{N}$ & \\
\hline 1 & $2 / 29 / 92$ & 4 & 1 & & 100.8 & $\mathbf{N}$ & \\
\hline 1 & $2 / 29 / 92$ & 5 & 1 & & 95.6 & $\mathbf{N}$ & \\
\hline 1 & $2 / 29 / 92$ & 6 & 1 & & 73.3 & $\mathbf{N}$ & \\
\hline 1 & $3 / 8 / 92$ & 1 & 1 & & 113.5 & $\bar{Y}$ & J $910126143-48$ \\
\hline 1 & $3 / 8 / 92$ & 2 & 1 & & 109.9 & $\mathbf{N}$ & \\
\hline 1 & $3 / 8 / 92$ & 3 & 1 & & 105.8 & $\mathbf{N}$ & \\
\hline 1 & $3 / 8 / 92$ & 4 & 1 & & 101.4 & $\mathbf{N}$ & \\
\hline 1 & $3 / 8 / 92$ & 5 & 1 & & 95.5 & $\mathbf{N}$ & \\
\hline 1 & $3 / 8 / 92$ & 6 & 1 & & 73 & $\mathbf{N}$ & \\
\hline 1 & $3 / 15 / 92$ & 1 & 1 & & 113.3 & $\bar{Y}$ & J-910126143-32 \\
\hline$\frac{1}{1}$ & $3 / 15 / 92$ & 2 & $\frac{1}{1}$ & & 109.5 & $\bar{N}$ & \\
\hline 1 & $3 / 15 / 92$ & 3 & 1 & & 105.5 & $\mathbf{N}$ & \\
\hline$\frac{1}{1}$ & $3 / 15 / 92$ & 4 & 1 & & 100.8 & $\mathbf{N}$ & \\
\hline 1 & $3 / 15 / 92$ & 5 & 1 & & 94.9 & $\mathbf{N}$ & \\
\hline 1 & $3 / 15 / 92$ & 6 & 1 & & 73.2 & $\mathbf{N}$ & \\
\hline 1 & $3 / 20 / 92$ & 1 & 1 & & 173.6 & $\mathbf{N}$ & $\mathrm{J} 91012614332$ \\
\hline 1 & $3 / 20 / 92$ & 2 & 1 & & 109.1 & $\mathbf{N}$ & \\
\hline 1 & $3 / 20 / 92$ & 3 & 1 & & 105 & N & \\
\hline 1 & $3 / 20 / 92$ & 4 & 1 & & 100.8 & $\mathbf{N}$ & \\
\hline 1 & $3 / 20 / 92$ & 5 & 1 & & 95.3 & $\mathbf{N}$ & \\
\hline 1 & $3 / 20 / 92$ & 6 & 1 & & 73.2 & $\mathbf{N}$ & \\
\hline 1 & $3 / 29 / 92$ & 1 & 1 & & 114.2 & $\mathbf{N}$ & $\mathrm{J} 910126143-66$ \\
\hline 9 & $3 / 29 / 92$ & 2 & $\frac{1}{1}$ & & 110.1 & $\mathbf{N}$ & \\
\hline 1 & $.3 / 29 / 92$ & 3 & 1 & & 106.1 & $\mathbf{N}$ & \\
\hline 1 & $3 / 29 / 92$ & 4 & 1 & & 101.9 & $\mathbf{N}$ & \\
\hline 1 & $3 / 29 / 92$ & 5 & 1 & & 96.6 & $\mathbf{N}$ & \\
\hline 1 & $3 / 29 / 92$ & 6 & 1 & & 75 & $N$ & \\
\hline 1 & $4 / 3 / 92$ & 1 & 1 & & 112.6 & $\mathbf{N}$ & J910126143-14 \\
\hline$\frac{T}{1}$ & $4 / 3 / 92$ & 2 & 1 & & 108 & $\bar{N}$ & \\
\hline 1 & $4 / 3 / 92$ & 3 & 1 & & 104 & $\bar{N}$ & \\
\hline$\frac{1}{1}$ & $4 / 3 / 92$ & 4 & 9 & & 99.8 & $\mathbf{N}$ & \\
\hline$\frac{1}{1}$ & $4 / 3 / 92$ & 5 & $\frac{1}{1}$ & & 94.5 & $\mathbf{N}$ & \\
\hline$\frac{1}{1}$ & $4 / 3 / 92$ & 6 & 1 & & 73.3 & $\mathbf{N}$ & \\
\hline 1 & $4 / 3 / 92$ & 13 & 1 & & 86 & $\mathbf{N}$ & \\
\hline 1 & $4 / 11 / 92$ & 1 & 1 & & 113.7 & $\bar{Y}$ & $J 910126143064$ \\
\hline$\frac{1}{1}$ & $4 / 11 / 92$ & 2 & 1 & & 107.7 & $\mathbf{N}$ & \\
\hline$\frac{1}{1}$ & $4 / 11 / 92$ & 3 & 1 & & 105 & $N$ & \\
\hline 1 & $4 / 11 / 92$ & 4 & 1 & & 100.9 & $N$ & \\
\hline 1 & $4 / 11 / 92$ & 5 & 1 & & 95.5 & $N$ & \\
\hline 1 & $4 / 11 / 92$ & 6 & 1 & & 74.1 & $\mathbf{N}$ & \\
\hline 1 & $4 / 17 / 92$ & 1 & 1 & & 114.7 & $\mathbf{Y}$ & J890523512 20 \\
\hline 1 & $4 / 17 / 92$ & 2 & $\overline{1}$ & & 110 & $\mathbf{N}$ & $\cdot$ \\
\hline$\frac{T}{1}$ & $4 / 17 / 92$ & 3 & 1 & & 105.8 & $N$ & \\
\hline$\frac{1}{1}$ & $4 / 17 / 92$ & 4 & 1 & & 101.7 & $\mathbf{N}$ & \\
\hline$\frac{1}{1}$ & $4 / 17 / 92$ & 5 & 1 & & 96.3 & $\mathbf{N}$ & \\
\hline 1 & $4 / 17 / 92$ & 6 & 1 & & 75.4 & $N$ & \\
\hline$\frac{1}{1}$ & $4 / 25 / 92$ & 1 & 1 & & 116.8 & $\bar{Y}$ & $J 89052351220$ \\
\hline 1 & $4 / 25 / 92$ & 2 & 1 & & 112.4 & $\mathbf{N}$ & \\
\hline 1 & $4 / 25 / 92$ & 3 & 1 & & 108.1 & $\mathbf{N}$ & \\
\hline$\frac{1}{1}$ & $4 / 25 / 92$ & 4 & 1 & & 103.6 & $\mathbf{N}$ & \\
\hline$\frac{1}{1}$ & $4 / 25 / 92$ & 5 & $\overline{1}$ & & 98 & $N$ & \\
\hline 1 & $4 / 25 / 92$ & 6 & 1 & & 77.4 & $\mathbf{N}$ & \\
\hline
\end{tabular}

Data obtained from WHC Surveillance Analysis Computer System (SACS), June 2, 1993. 


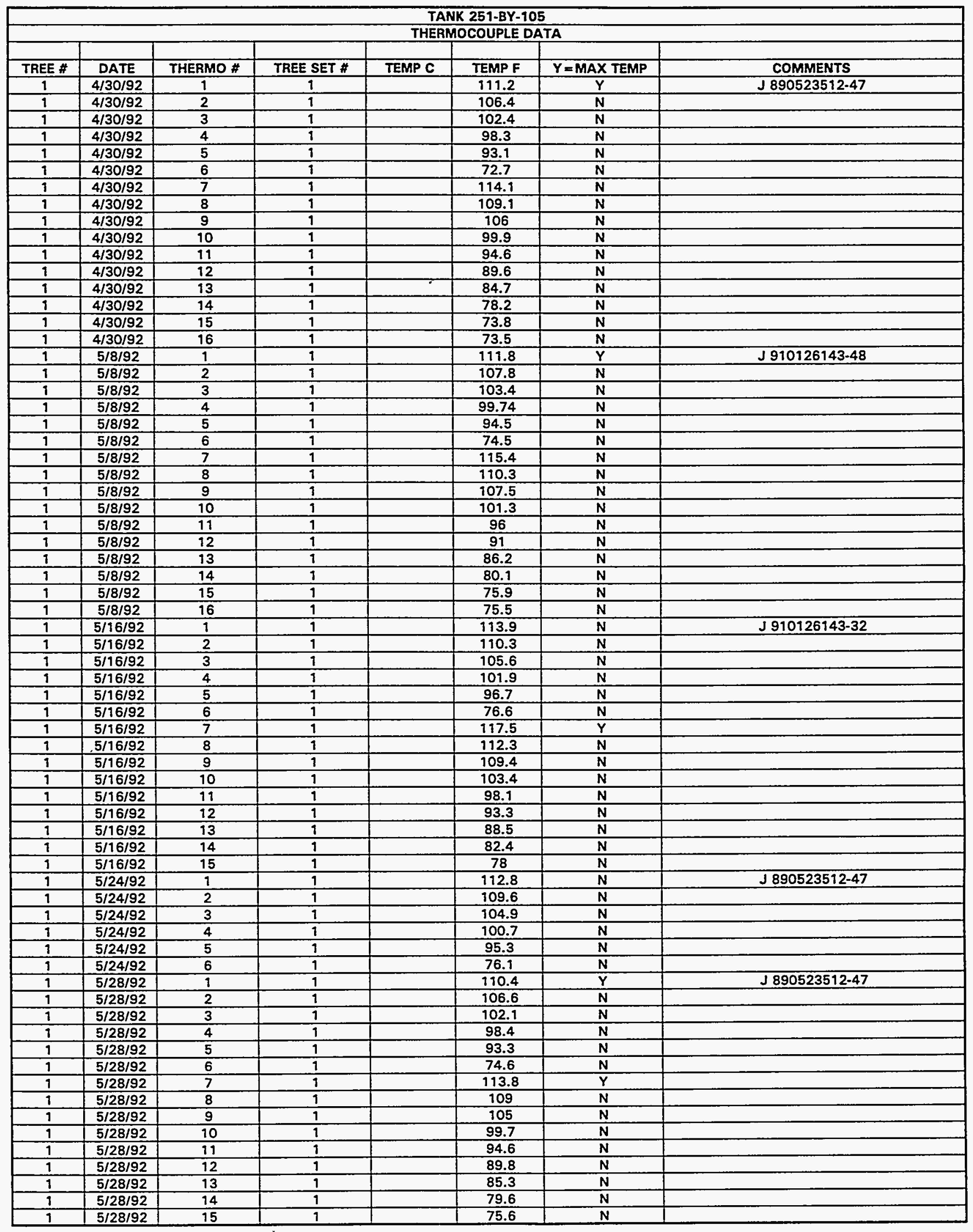

Data obtained from WHC Surveillance Analysis Computer System (SACS), June 2, 1993. 


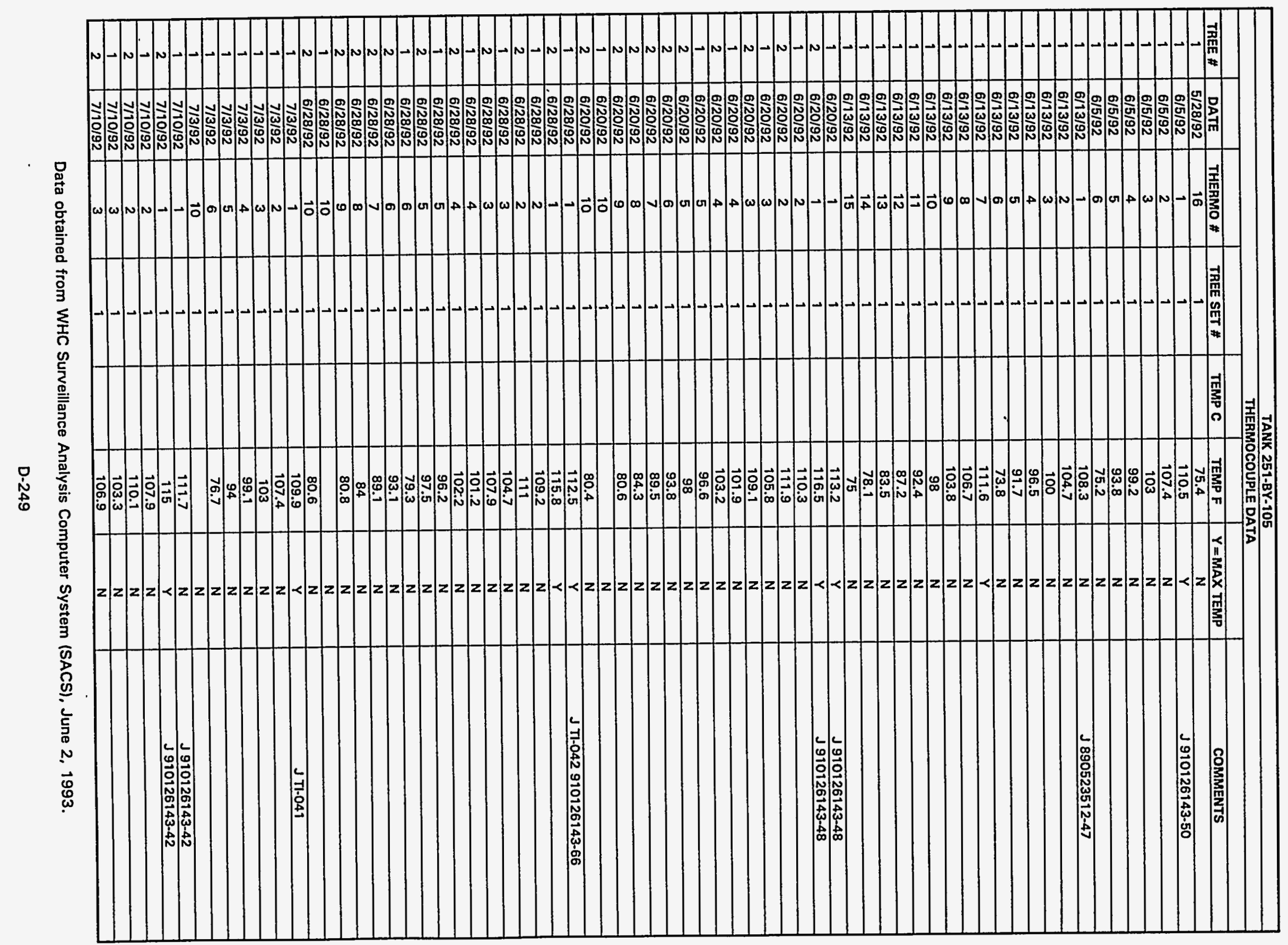


WHC-SD-WM-ER-312, Rev. 0

\begin{tabular}{|c|c|c|c|c|c|c|c|}
\hline \multicolumn{8}{|c|}{ TANK 251-BY-105 } \\
\hline & & & & THER! & COUPLE D & & \\
\hline TREE \# & DATE & THERMO \# & TREE SET \# & TEMP C & TEMP F & $Y=M A X$ TEMP & COMMENTS \\
\hline 1 & $7 / 10 / 92$ & 4 & 1 & & 99.7 & $\mathbf{N}$ & \\
\hline 2 & $7 / 10 / 92$ & 4 & 1 & & 101.1 & $\mathbf{N}$ & \\
\hline$\frac{1}{1}$ & $7 / 10 / 92$ & 5 & 1 & & 94.9 & $\mathbf{N}$ & \\
\hline 2 & $7 / 10 / 92$ & 5 & 1 & & 96 & $\mathbf{N}$ & \\
\hline 1 & $7 / 10 / 92$ & 6 & 1 & & 78.5 & $\mathbf{N}$ & \\
\hline 2 & $7 / 10 / 92$ & 6 & 1 & & 91.5 & $N$ & \\
\hline 2 & $7 / 10 / 92$ & 7 & 1 & & 87.8 & $\mathbf{N}$ & \\
\hline 2 & $7 / 10 / 92$ & 8 & 1 & & 82.9 & $\mathbf{N}$ & \\
\hline 2 & $7 / 10 / 92$ & 9 & 1 & & 79.9 & $\mathbf{N}$ & \\
\hline 9 & $7 / 10 / 92$ & 10 & 1 & & & $\mathbf{N}$ & \\
\hline 2 & $7 / 10 / 92$ & 10 & 1 & & 79.6 & $\mathbf{N}$ & \\
\hline 1 & $7 / 18 / 92$ & 1 & 1 & & 112.6 & $\mathbf{Y}$ & J $910126143-42$ \\
\hline 2 & $7 / 18 / 92$ & 1 & 1 & & 115.7 & $\bar{Y}$ & J $910126143-42$ \\
\hline 1 & $7 / 18 / 92$ & 2 & 1 & & 109.3 & $\mathbf{N}$ & \\
\hline 2 & $7 / 18 / 92$ & 2 & 1 & & 110.9 & $\mathbf{N}$ & \\
\hline 1 & $7 / 18 / 92$ & 3 & 1 & & 105 & $\mathbf{N}$ & \\
\hline 2 & $7 / 18 / 92$ & 3 & 1 & & 107.9 & $\mathbf{N}$ & \\
\hline 1 & $7 / 18 / 92$ & 4 & 1 & & 101.5 & $\mathbf{N}$ & \\
\hline 2 & $7 / 18 / 92$ & 4 & 1 & & 102.3 & $\mathrm{~N}$ & \\
\hline 1 & $7 / 18 / 92$ & 5 & 1 & & 96.8 & $N$ & \\
\hline 2 & $7 / 18 / 92$ & 5 & 1 & & 97.6 & $\bar{N}$ & \\
\hline 1 & $7 / 18 / 92$ & 6 & 1 & & 80.5 & $\mathbf{N}$ & \\
\hline 2 & $7 / 18 / 92$ & 6 & 1 & & 93.4 & $\mathbf{N}$ & \\
\hline 2 & $7 / 18 / 92$ & 7 & 1 & & 89.6 & $\mathbf{N}$ & \\
\hline 2 & $7 / 18 / 92$ & 8 & 1 & & 85 & $\mathbf{N}$ & \\
\hline 2 & $7 / 18 / 92$ & 9 & 1 & & 82 & $\mathrm{~N}$ & \\
\hline 1 & $7 / 18 / 92$ & 10 & 1 & & & $\mathrm{~N}$ & \\
\hline 2 & $7 / 18 / 92$ & 10 & 1 & & 81.9 & $\mathrm{~N}$ & \\
\hline 1 & $7 / 24 / 92$ & 1 & 1 & & 112.3 & $\bar{Y}$ & J HH-99A 910126143-29 \\
\hline 2 & $7 / 24 / 92$ & 1 & 1 & & 115.6 & $\bar{Y}$ & J HH-99A 910126143-29 \\
\hline 1 & $7 / 24 / 92$ & 2 & 1 & & 108.6 & $\mathbf{N}$ & \\
\hline$\overline{2}$ & $7 / 24 / 92$ & 2 & 9 & & 110.8 & $\mathbf{N}$ & \\
\hline 1 & $7 / 24 / 92$ & 3 & 1 & & 104.1 & $\mathbf{N}$ & \\
\hline 2 & $7 / 24 / 92$ & 3 & 1 & & 107.7 & $\mathbf{N}$ & \\
\hline 1 & $7 / 24 / 92$ & 4 & 1 & & 100.6 & $\mathbf{N}$ & \\
\hline 2 & $7 / 24 / 92$ & 4 & 1 & & 101.8 & $\mathbf{N}$ & \\
\hline 1 & $7 / 24 / 92$ & 5 & 1 & & 96.1 & $\mathbf{N}$ & \\
\hline 2 & $7 / 24 / 92$ & 5 & 1 & & 96.8 & $\mathbf{N}$ & \\
\hline 1 & $7 / 24 / 92$ & 6 & 1 & & 80.5 & $\mathbf{N}$ & \\
\hline 2 & $7 / 24 / 92$ & 6 & 1 & & 88.9 & $N$ & \\
\hline 2 & $7 / 24 / 92$ & 7 & 1 & & 84.3 & $\mathbf{N}$ & \\
\hline 2 & $7 / 24 / 92$ & 8 & 1 & & 81.4 & $\mathbf{N}$ & \\
\hline 2 & $7 / 24 / 92$ & 9 & 1 & & 81.1 & $\mathbf{N}$ & \\
\hline 1 & $7 / 24 / 92$ & 10 & 1 & & & $\mathbf{N}$ & \\
\hline 2 & $7 / 24 / 92$ & 10 & 1 & & 81 & $\mathbf{N}$ & \\
\hline 1 & $8 / 1 / 92$ & 1 & 1 & & 113.3 & $\mathbf{Y}$ & J T1-041 \\
\hline 2 & $8 / 1 / 92$ & 1 & 1 & & 116.6 & $\bar{Y}$ & $\mathrm{JTT}-041$ \\
\hline 1 & $8 / 1 / 92$ & 2 & 1 & & 110.4 & $\mathbf{N}$ & \\
\hline 2 & $8 / 1 / 92$ & 2 & 1 & & 111.9 & $\mathbf{N}$ & \\
\hline 1 & $8 / 1 / 92$ & 3 & 1 & & 106 & $\mathbf{N}$ & \\
\hline 2 & $8 / 1 / 92$ & 3 & 1 & & 109.2 & $\mathbf{N}$ & \\
\hline 1 & $8 / 1 / 92$ & 4 & 1 & & 102.1 & $\mathbf{N}$ & \\
\hline 2 & $8 / 1 / 92$ & 4 & 9 & & 103.8 & $\mathbf{N}$ & \\
\hline 1 & $8 / 1 / 92$ & 5 & 1 & & 97.4 & $\mathbf{N}$ & \\
\hline 2 & $8 / 1 / 92$ & 5 & 1 & & 99.1 & $\mathbf{N}$ & \\
\hline 1 & $8 / 1 / 92$ & 6 & 1 & & 82.1 & $\mathbf{N}$ & \\
\hline 2 & $8 / 1 / 92$ & 6 & 1 & & 94.8 & $\mathrm{~N}$ & \\
\hline 2 & $8 / 1 / 92$ & 7 & 1 & & 91.5 & $\mathbf{N}$ & \\
\hline 2 & $8 / 1 / 92$ & 8 & 1 & & 86.7 & $\mathbf{N}$ & \\
\hline 2 & $8 / 1 / 92$ & 9 & 1 & & 83.9 & $\mathbf{N}$ & \\
\hline 1 & $8 / 1 / 92$ & 10 & 1 & & & $\mathbf{N}$ & \\
\hline 2 & $8 / 1 / 92$ & 10 & 1 & & 83.8 & $\mathbf{N}$ & \\
\hline 1 & $8 / 7 / 92$ & 1 & 1 & & 109 & $\bar{Y}$ & $J 890423320-42$ \\
\hline 2 & $8 / 7 / 92$ & 1 & 1 & & 113.5 & $Y$ & $J 890423320-42$ \\
\hline 9 & $8 / 7 / 92$ & 2 & 1 & & 106.5 & $\mathbf{N}$ & \\
\hline 2 & $8 / 7 / 92$ & 2 & 1 & & 108.7 & $\mathbf{N}$ & \\
\hline 1 & $8 / 7 / 92$ & 3 & 1 & & 102.3 & $\mathbf{N}$ & \\
\hline 2 & $8 / 7 / 92$ & 3 & 1 & & 105.6 & $N$ & \\
\hline
\end{tabular}

Data obtained from WHC Surveillance Analysis Computer System (SACS), June 2, 1993. 


\begin{tabular}{|c|c|c|c|c|c|c|c|}
\hline & & & & $T A$ & 251-BY-1 & & \\
\hline & & & & THER & COUPLE I & & \\
\hline TREE \# & DATE & THERMO \# & TREE SET \# & TEMP C & TEMP F & $Y=$ MAX TEMP & COMMENTS \\
\hline 1 & $8 / 7 / 92$ & 4 & 1 & & 98.9 & $\mathbf{N}$ & \\
\hline 2 & $8 / 7 / 92$ & 4 & 1 & & 100.1 & $\mathbf{N}$ & \\
\hline 1 & $8 / 7 / 92$ & 5 & 1 & & 94.6 & $\mathbf{N}$ & \\
\hline 2 & $8 / 7 / 92$ & 5 & 1 & & 95.5 & $\mathbf{N}$ & \\
\hline 9 & $8 / 7 / 92$ & 6 & 1 & & 80 & $\mathbf{N}$ & \\
\hline 2 & $8 / 7 / 92$ & 6 & 1 & & 91.4 & $\mathbf{N}$ & \\
\hline 2 & $8 / 7 / 92$ & 7 & 1 & & 88.1 & $\mathbf{N}$ & \\
\hline 2 & $8 / 7 / 92$ & 8 & 1 & & 83.6 & $\mathrm{~N}$ & \\
\hline 2 & $8 / 7 / 92$ & 9 & 1 & & 81 & $\mathbf{N}$ & \\
\hline 9 & $8 / 7 / 92$ & 10 & 1 & & & $\mathbf{N}$ & \\
\hline 2 & $8 / 7 / 92$ & 10 & 1 & & 80.6 & $\mathbf{N}$ & \\
\hline 9 & $8 / 15 / 92$ & 1 & $\overline{1}$ & & 111.8 & $\mathrm{Y}$ & J 890423320 \\
\hline 2 & $8 / 15 / 92$ & 1 & 1 & & 115 & $\bar{Y}$ & \\
\hline 1 & $8 / 15 / 92$ & 2 & 1 & & 108.8 & $\mathbf{N}$ & \\
\hline 2 & $8 / 15 / 92$ & 2 & 1 & & 110.4 & $\mathbf{N}$ & \\
\hline 1 & $8 / 15 / 92$ & 3 & 1 & & 104.7 & $\mathbf{N}$ & \\
\hline$\overline{2}$ & $8 / 15 / 92$ & 3 & 1 & & 107.8 & $\mathbf{N}$ & \\
\hline 1 & $8 / 15 / 92$ & 4 & 1 & & 101.1 & $N$ & \\
\hline 2 & $8 / 15 / 92$ & 4 & 1 & & 102.4 & $\mathbf{N}$ & \\
\hline 1 & $8 / 15 / 92$ & 5 & 1 & & 96.5 & $\mathbf{N}$ & \\
\hline 2 & $8 / 15 / 92$ & 5 & 1 & & 98 & $\mathbf{N}$ & \\
\hline 1 & $8 / 15 / 92$ & 6 & 1 & & 82.1 & $\bar{N}$ & \\
\hline 2 & $8 / 15 / 92$ & 6 & 1 & & 93.9 & $\mathbf{N}$ & \\
\hline 2 & $8 / 15 / 92$ & 7 & 1 & & 90.6 & $\mathbf{N}$ & \\
\hline 2 & $8 / 15 / 92$ & 8 & 1 & & 86.1 & $\mathbf{N}$ & \\
\hline 2 & $8 / 15 / 92$ & 9 & 1 & & 83.4 & $\mathbf{N}$ & \\
\hline 1 & $8 / 15 / 92$ & 10 & 1 & & & $\mathbf{N}$ & \\
\hline 2 & $8 / 15 / 92$ & 10 & 1 & & 83.3 & $\mathbf{N}$ & \\
\hline 1 & $8 / 21 / 92$ & 1 & 1 & & 110.4 & $\mathbf{Y}$ & J $890423320-42$ \\
\hline 2 & $8 / 21 / 92$ & 1 & $T$ & & 113.7 & $\mathbf{Y}$ & J $890423320-42$ \\
\hline 1 & $8 / 21 / 92$ & 2 & 1 & & 106.9 & $\mathbf{N}$ & \\
\hline 2 & $8 / 21 / 92$ & 2 & 1 & & 108.8 & $\mathbf{N}$ & \\
\hline 1 & $8 / 21 / 92$ & 3 & 1 & & 102.8 & $\mathbf{N}$ & \\
\hline 2 & $8 / 21 / 92$ & 3 & 1 & & 105.9 & $N$ & \\
\hline 1 & $8 / 21 / 92$ & 4 & 1 & & 99.4 & $\mathbf{N}$ & \\
\hline 2 & $8 / 21 / 92$ & 4 & 9 & & 100.5 & $\mathbf{N}$ & \\
\hline 1 & $8 / 21 / 92$ & 5 & 1 & & 95.1 & $\mathbf{N}$ & \\
\hline 2 & $8 / 21 / 92$ & 5 & 1 & & 96 & $\bar{N}$ & \\
\hline 1 & $8 / 21 / 92$ & 6 & 1 & & 81.2 & $\mathbf{N}$ & \\
\hline 2 & $8 / 21 / 92$ & 6 & 1 & & 92.1 & $\mathbf{N}$ & \\
\hline 2 & $8 / 21 / 92$ & 7 & 1 & & 89.9 & $\mathbf{N}$ & \\
\hline 2 & $8 / 21 / 92$ & 8 & 1 & & 84.8 & $\bar{N}$ & \\
\hline 2 & $8 / 21 / 92$ & 9 & 1 & & 82.2 & $\mathbf{N}$ & \\
\hline$\frac{1}{1}$ & $8 / 21 / 92$ & 10 & 1 & & & $\mathbf{N}$ & \\
\hline 2 & $8 / 21 / 92$ & 10 & 1 & & 82 & $\mathbf{N}$ & \\
\hline 1 & $8 / 30 / 92$ & 1 & 1 & & 110.8 & $\mathbf{N}$ & J 890423320-42 \\
\hline 2 & $8 / 30 / 92$ & 1 & 1 & & 114.9 & $\mathbf{Y}$ & $\mathrm{J} 890423320-42$ \\
\hline 1 & $8 / 30 / 92$ & 2 & 1 & & 108.3 & $\mathbf{N}$ & \\
\hline 2 & $8 / 30 / 92$ & 2 & 1 & & 111.2 & $\mathbf{N}$ & \\
\hline 1 & $8 / 30 / 92$ & 3 & 1 & & 104.5 & $\mathbf{N}$ & \\
\hline 2 & $8 / 30 / 92$ & 3 & 1 & & 107.9 & $\mathbf{N}$ & \\
\hline 1 & $8 / 30 / 92$ & 4 & 1 & & 101.2 & $\mathbf{N}$ & \\
\hline 2 & $8 / 30 / 92$ & 4 & 1 & & 102.8 & $\mathbf{N}$ & \\
\hline 1 & $8 / 30 / 92$ & 5 & 1 & & 92.8 & $\mathbf{N}$ & \\
\hline 2 & $8 / 30 / 92$ & 5 & 1 & & 98.5 & $\mathbf{N}$ & \\
\hline 1 & $8 / 30 / 92$ & 6 & 1 & & 83.2 & $\mathbf{N}$ & \\
\hline 2 & $8 / 30 / 92$ & 6 & 1 & & 94.9 & $\mathbf{N}$ & \\
\hline 2 & $8 / 30 / 92$ & 7 & 1 & & 91.7 & $\mathbf{N}$ & \\
\hline 2 & $8 / 30 / 92$ & 8 & 1 & & 87.6 & $\mathbf{N}$ & \\
\hline 2 & $8 / 30 / 92$ & 9 & 1 & & 84.9 & $N$ & \\
\hline 1 & $8 / 30 / 92$ & 10 & 1 & & & $\mathbf{N}$ & \\
\hline 2 & $8 / 30 / 92$ & 10 & 1 & & 85 & $N$ & \\
\hline 1 & 9/6/92 & 1 & 1 & & 110.5 & Y & $\begin{array}{l}\mathrm{J} 890423320-42 \text { LOW RDGS, RECHECK } \\
\text { SHOWS IT IS CORRECT }\end{array}$ \\
\hline 2 & $9 / 6 / 92$ & 1 & 1 & & 113.8 & $\mathbf{Y}$ & $\begin{array}{l}\text { J 890423320-42 LOW RDGS, RECHECK } \\
\text { SHOWS IT IS CORRECT }\end{array}$ \\
\hline 1 & $9 / 6 / 92$ & 2 & 1 & & 106.5 & $\mathbf{N}$ & \\
\hline 2 & $9 / 6 / 92$ & 2 & 1 & & 108.7 & $N$ & \\
\hline
\end{tabular}

Data obtained from WHC Surveillance Analysis Computer System (SACS), June 2, 1993. 


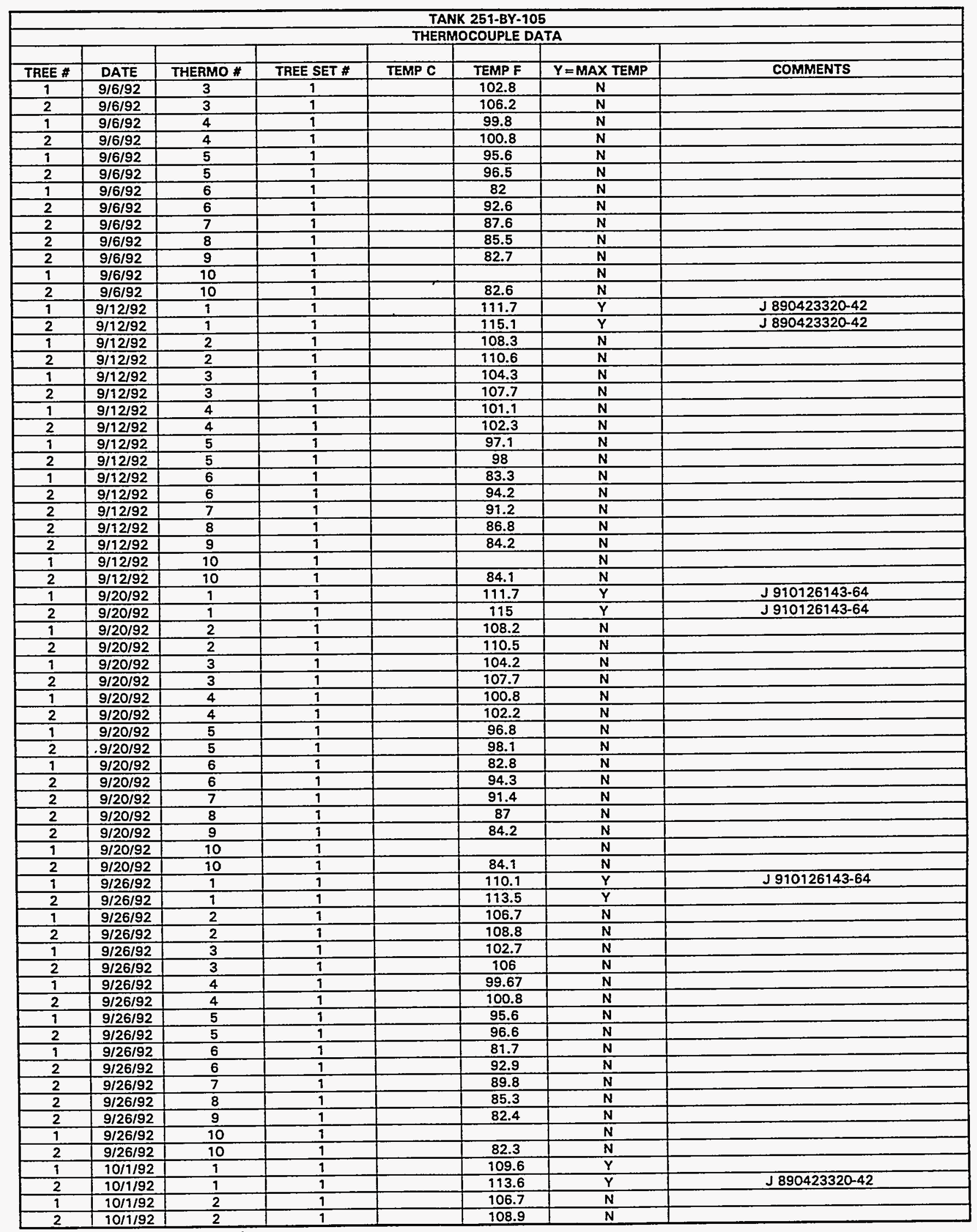

Data obtained from WHC Surveillance Analysis Computer System (SACS), June 2, 1993. 


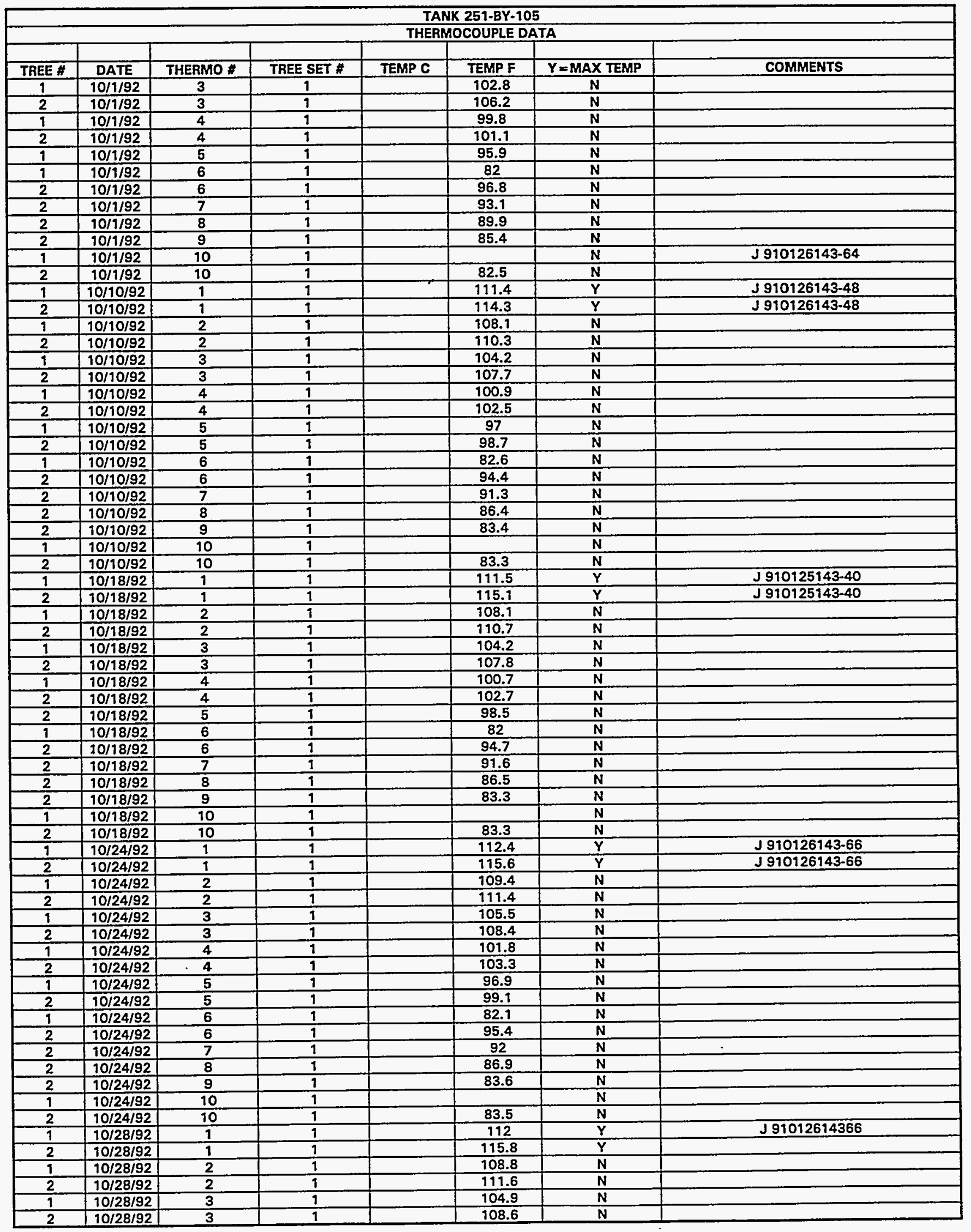

Data obtained from WHC Surveillance Analysis Computer System (SACS), June 2, 1993. 
WHC-SD-WM-ER-312, Rev. 0

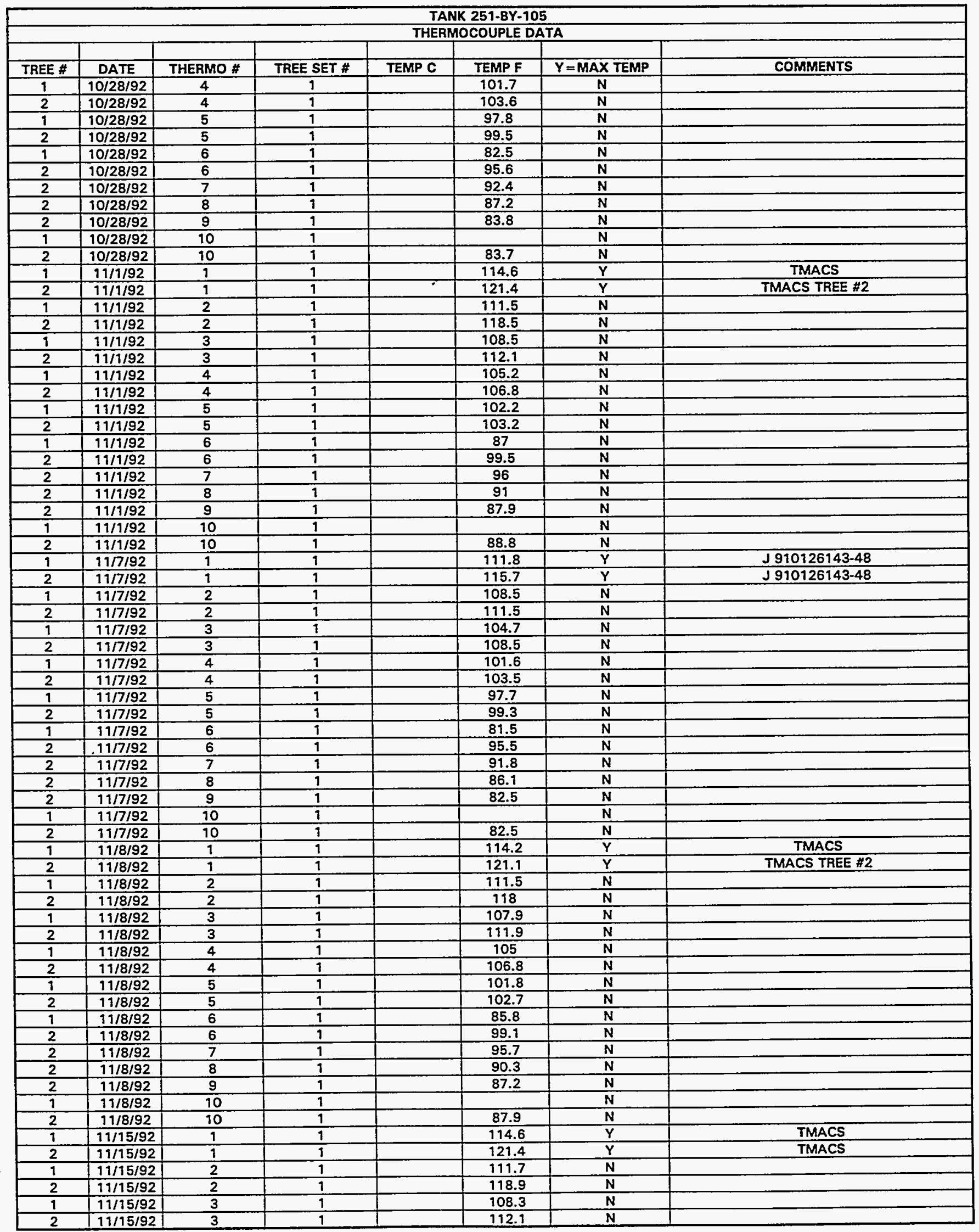

Data obtained from WHC Surveillance Analysis Computer System (SACS), June 2, 1993. 
WHC-SD-WM-ER-312, Rev. 0

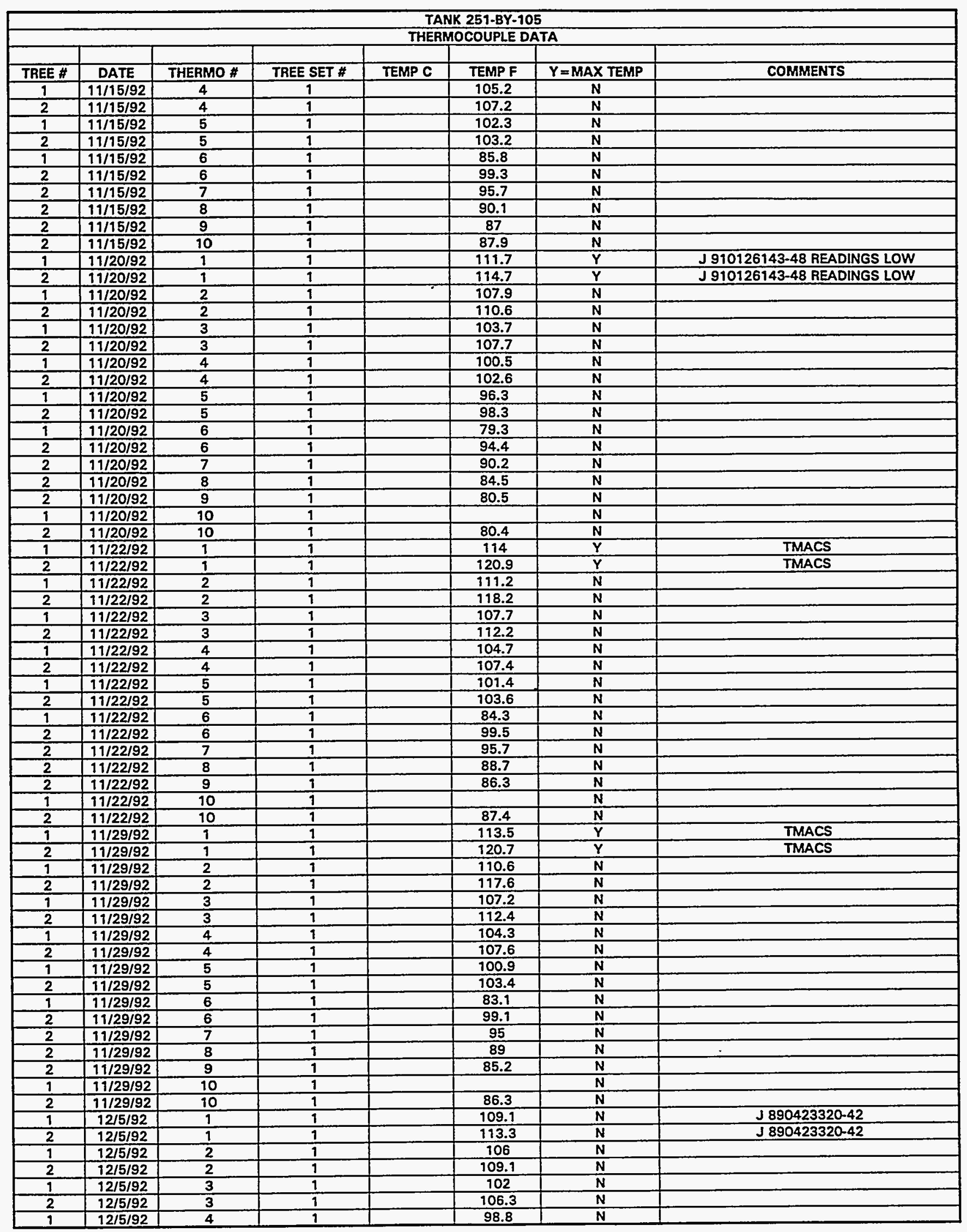

Data obtained from WHC Surveillance Analysis Computer System (SACS), June 2, 1993. 
WHC-SD-WM-ER-312, Rev. 0

\begin{tabular}{|c|c|c|c|c|c|c|c|}
\hline \multicolumn{8}{|c|}{ TANK 251-BY-105 } \\
\hline & & & & THEF & COUPLE & & \\
\hline TREE \# & DATE & THERMO \# & TREE SET \# & TEMP C & TEMP F & $Y=\operatorname{MAX} T E M P$ & COMMENTS \\
\hline 2 & $12 / 5 / 92$ & 4 & 1 & & 101.3 & $\mathbf{N}$ & \\
\hline 1 & $12 / 5 / 92$ & 5 & 1 & & 94.7 & $\mathbf{N}$ & \\
\hline 2 & $12 / 5 / 92$ & 5 & 1 & & 97.33 & $\mathbf{N}$ & \\
\hline 9 & $12 / 5 / 92$ & 6 & 9 & & 75.9 & $\mathrm{~N}$ & \\
\hline 2 & $12 / 5 / 92$ & 6 & 1 & & 92.7 & $\mathbf{N}$ & \\
\hline 1 & $12 / 5 / 92$ & 10 & 1 & & & $\mathbf{N}$ & \\
\hline 2 & $12 / 5 / 92$ & 10 & 1 & & & $\mathrm{~N}$ & \\
\hline 1 & $12 / 6 / 92$ & $\frac{1}{1}$ & 1 & & 113.1 & $\mathrm{Y}$ & TMACS RISER $10 \mathrm{C}$ \\
\hline 2 & $12 / 6 / 92$ & 1 & 1 & & 120.3 & $\mathbf{Y}$ & TMACS RISER 1 \\
\hline 1 & $12 / 6 / 92$ & 2 & 1 & & 110.3 & $\mathbf{N}$ & \\
\hline 2 & $12 / 6 / 92$ & 2 & 1 & & 117.3 & $\mathbf{N}$ & \\
\hline 1 & $12 / 6 / 92$ & 3 & 1 & & 106.7 & $\mathbf{N}$ & \\
\hline 2 & $12 / 6 / 92$ & 3 & 1 & & 112.2 & $\mathbf{N}$ & \\
\hline 1 & $12 / 6 / 92$ & 4 & 9 & & 104.7 & $\mathbf{N}$ & \\
\hline 2 & $12 / 6 / 92$ & 4 & 1 & & 107.6 & $\mathbf{N}$ & \\
\hline 1 & $12 / 6 / 92$ & 5 & 1 & & 100.5 & $\mathbf{N}$ & \\
\hline 2 & $12 / 6 / 92$ & 5 & 1 & & 103.2 & $\mathbf{N}$ & \\
\hline 1 & $12 / 6 / 92$ & 6 & 1 & & 81.8 & $\mathbf{N}$ & \\
\hline 2 & $12 / 6 / 92$ & 6 & 1 & & 99.3 & $\mathbf{N}$ & \\
\hline 2 & $12 / 6 / 92$ & 7 & 1 & & 94.6 & $\mathbf{N}$ & \\
\hline 2 & $12 / 6 / 92$ & 8 & 1 & & 88.3 & $\mathbf{N}$ & \\
\hline 2 & $12 / 6 / 92$ & 9 & 1 & & 84.5 & $\mathbf{N}$ & \\
\hline 9 & $12 / 6 / 92$ & 10 & 1 & & & $\mathbf{N}$ & \\
\hline 2 & $12 / 6 / 92$ & 10 & 1 & & 85.6 & $\bar{N}$ & \\
\hline 1 & $12 / 13 / 92$ & 1 & 1 & & 113.3 & $\bar{Y}$ & TMACS \\
\hline 2 & $12 / 13 / 92$ & 1 & 1 & & 120.7 & $\bar{Y}$ & TMACS \\
\hline 1 & $12 / 13 / 92$ & 2 & 1 & & 110.4 & $\mathbf{N}$ & \\
\hline 2 & $12 / 13 / 92$ & 2 & 1 & & 117.6 & $\mathbf{N}$ & \\
\hline 1 & $12 / 13 / 92$ & 3 & 1 & & 106.8 & $\mathbf{N}$ & \\
\hline 2 & $12 / 13 / 92$ & 3 & 1 & & 111.9 & $\mathbf{N}$ & \\
\hline 1 & $12 / 13 / 92$ & 4 & 1 & & 104.3 & $\mathbf{N}$ & \\
\hline 2 & $12 / 13 / 92$ & 4 & 1 & & 107 & $\mathbf{N}$ & \\
\hline$\frac{1}{1}$ & $12 / 13 / 92$ & 5 & 9 & & 100.5 & $\mathbf{N}$ & \\
\hline 2 & $12 / 13 / 92$ & 5 & 1 & & 102.9 & $\mathbf{N}$ & \\
\hline$\frac{1}{1}$ & $12 / 13 / 92$ & 6 & $\overline{1}$ & & 81.1 & $\mathbf{N}$ & \\
\hline 2 & $12 / 13 / 92$ & 5 & 1 & & 98.2 & $\mathbf{N}$ & \\
\hline 2 & $12 / 13 / 92$ & 7 & 9 & & 93.7 & $N$ & \\
\hline 2 & $12 / 13 / 92$ & 8 & 1 & & 87 & $\mathbf{N}$ & \\
\hline 2 & $12 / 13 / 92$ & 9 & 9 & & 83.3 & $\mathbf{N}$ & \\
\hline 1 & $.12 / 13 / 92$ & 10 & 1 & & & $\mathbf{N}$ & \\
\hline 2 & $12 / 13 / 92$ & 10 & 1 & & 84.2 & $\mathbf{N}$ & \\
\hline 1 & $12 / 19 / 92$ & 1 & 1 & & 112 & $\bar{Y}$ & $J 910126143-14$ \\
\hline 2 & $12 / 19 / 92$ & 1 & 1 & & 116.1 & $\mathbf{Y}$ & $J 910126143-14$ \\
\hline 1 & $12 / 19 / 92$ & 2 & 1 & & 108.7 & $\mathbf{N}$ & \\
\hline 2 & $12 / 19 / 92$ & 2 & $\frac{1}{1}$ & & 112 & $\mathbf{N}$ & \\
\hline$\frac{1}{1}$ & $12 / 19 / 92$ & 3 & 1 & & 104.8 & $\mathrm{~N}$ & \\
\hline 2 & $12 / 19 / 92$ & 3 & 1 & & 109.1 & $\mathbf{N}$ & \\
\hline$\frac{1}{1}$ & $12 / 19 / 92$ & 4 & 1 & & 101.6 & $\mathbf{N}$ & \\
\hline 2 & $12 / 19 / 92$ & 4 & 1 & & 103.9 & $\mathbf{N}$ & \\
\hline$\frac{1}{1}$ & $12 / 19 / 92$ & 5 & 1 & & 97.2 & $N$ & \\
\hline 2 & $12 / 19 / 92$ & 5 & 1 & & 99.4 & $\mathrm{~N}$ & \\
\hline 9 & $12 / 19 / 92$ & 6 & 1 & & 77.1 & $\mathbf{N}$ & \\
\hline 2 & $12 / 19 / 92$ & 6 & 1 & & 94.8 & $\bar{N}$ & \\
\hline 2 & $12 / 19 / 92$ & 7 & 1 & & 89.7 & $\mathbf{N}$ & \\
\hline 2 & $12 / 19 / 92$ & 8 & 1 & & 83 & $\bar{N}$ & \\
\hline 2 & $12 / 19 / 92$ & 9 & 1 & & 78.6 & $\mathbf{N}$ & \\
\hline 1 & $12 / 19 / 92$ & 10 & 1 & & & $\mathbf{N}$ & \\
\hline 2 & $12 / 19 / 92$ & 10 & 1 & & 78.5 & $\mathbf{N}$ & \\
\hline 1 & $12 / 20 / 92$ & 1 & 1 & & 113.1 & $\mathrm{Y}$ & TMACS \\
\hline 2 & $12 / 20 / 92$ & 1 & 1 & & 120.3 & $Y$ & TMACS \\
\hline 9 & $12 / 20 / 92$ & 2 & 1 & & 110.1 & $\mathrm{~N}$ & \\
\hline 2 & $12 / 20 / 92$ & 2 & 1 & & 117.3 & $\mathbf{N}$ & \\
\hline 1 & $12 / 20 / 92$ & 3 & 1 & & 106.7 & 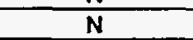 & \\
\hline 2 & $12 / 20 / 92$ & 3 & 1 & & 112.2 & $\mathrm{~N}$ & \\
\hline 1 & $12 / 20 / 92$ & 4 & 1 & & 103.6 & $\mathrm{~N}$ & \\
\hline 2 & \begin{tabular}{|l|}
$12 / 20 / 92$ \\
\end{tabular} & 4 & 1 & & 107.2 & $\mathbf{N}$ & \\
\hline 1 & $12 / 20 / 92$ & 5 & 1 & & 100.2 & $\mathbf{N}$ & \\
\hline 2 & $12 / 20 / 92$ & 5 & 1 & & 102.9 & $\bar{N}$ & \\
\hline
\end{tabular}

Data obtained from WHC Surveillance Analysis Computer System (SACS), June 2, 1993. 
WHC-SD-WM-ER-312, Rev. 0

\begin{tabular}{|c|c|c|c|c|c|c|c|}
\hline \multicolumn{8}{|c|}{ TANK 251-BY-105 } \\
\hline \multicolumn{8}{|c|}{ THERMOCOUPLE DATA } \\
\hline TREE \# & DATE & THERMO \# & TREE SET \# & TEMP C & TEMP F & $Y=M A X$ TEMP & COMMENTS \\
\hline 1 & $12 / 20 / 92$ & 6 & 1 & & 80.2 & $\mathbf{N}$ & \\
\hline 2 & $12 / 20 / 92$ & 6 & 1 & & 98.2 & $\mathbf{N}$ & \\
\hline 2 & $12 / 20 / 92$ & 7 & 9 & & 93.3 & $\mathbf{N}$ & \\
\hline 2 & $12 / 20 / 92$ & 8 & 1 & & 86.9 & $\mathbf{N}$ & \\
\hline 2 & $12 / 20 / 92$ & 9 & 1 & & 82.7 & $\mathbf{N}$ & \\
\hline 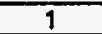 & $12 / 20 / 92$ & 10 & 1 & & & $\mathbf{N}$ & \\
\hline 2 & $12 / 20 / 92$ & 10 & 1 & & 83.4 & $\mathbf{N}$ & \\
\hline 1 & $12 / 25 / 92$ & 1 & 1 & & 114.2 & $\mathbf{Y}$ & J $890523512-20$ \\
\hline 2 & $12 / 25 / 92$ & 1 & 1 & & 117.7 & $\bar{Y}$ & J 890523512-20 \\
\hline 1 & $12 / 25 / 92$ & 2 & 1 & & 110.5 & $\mathbf{N}$ & \\
\hline 2 & $12 / 25 / 92$ & 2 & 9 & & 113.4 & $\mathbf{N}$ & \\
\hline 1 & $12 / 25 / 92$ & 3 & 1 & & 106.2 & $\overline{\mathbf{N}}$ & \\
\hline 2 & $12 / 25 / 92$ & 3 & 1 & & 110.6 & $\mathbf{N}$ & \\
\hline 1 & $12 / 25 / 92$ & 4 & 1 & & 103 & $\bar{N}$ & \\
\hline 2 & $12 / 25 / 92$ & 4 & 9 & & 105.3 & $\mathbf{N}$ & \\
\hline$\frac{1}{1}$ & $12 / 25 / 92$ & 5 & $\frac{1}{1}$ & & 98.3 & $\mathrm{~N}$ & \\
\hline 2 & $12 / 25 / 92$ & 5 & 1 & & 100.4 & $\mathbf{N}$ & \\
\hline 1 & $12 / 25 / 92$ & 6 & 1 & & 77.7 & $\mathbf{N}$ & \\
\hline 2 & $12 / 25 / 92$ & 6 & 1 & & 95.7 & $\mathbf{N}$ & \\
\hline 2 & $12 / 25 / 92$ & 7 & 9 & & 90.2 & $\mathbf{N}$ & \\
\hline 2 & $12 / 25 / 92$ & 8 & 1 & & 79.1 & $\mathbf{N}$ & \\
\hline 2 & $12 / 25 / 92$ & 9 & 1 & & 79.1 & $\mathbf{N}$ & \\
\hline 1 & $12 / 25 / 92$ & 10 & 9 & & & $\mathbf{N}$ & \\
\hline 2 & $12 / 25 / 92$ & 10 & 1 & & 78.8 & $\mathbf{N}$ & \\
\hline 9 & $12 / 27 / 92$ & 9 & $\frac{1}{1}$ & & 113.7 & $\mathrm{Y}$ & TMACS \\
\hline 2 & $12 / 27 / 92$ & 1 & 1 & & 120.9 & $\bar{Y}$ & TMACS \\
\hline$\frac{1}{1}$ & $12 / 27 / 92$ & 2 & 1 & & 110.6 & $\mathbf{N}$ & \\
\hline 2 & $12 / 27 / 92$ & 2 & 1 & & 117.8 & $\mathbf{N}$ & \\
\hline 1 & $12 / 27 / 92$ & 3 & 9 & & 107.2 & $\mathbf{N}$ & \\
\hline 2 & $12 / 27 / 92$ & 3 & 9 & & 111.9 & $\mathbf{N}$ & \\
\hline 1 & $12 / 27 / 92$ & 4 & 1 & & 104.3 & $\mathbf{N}$ & \\
\hline 2 & $12 / 27 / 92$ & $\overline{4}$ & 1 & & 106.8 & $\mathbf{N}$ & \\
\hline 1 & $12 / 27 / 92$ & 5 & 1 & & 100.4 & $\bar{N}$ & \\
\hline 2 & $12 / 27 / 92$ & 5 & 9 & & 102.5 & $\mathrm{~N}$ & \\
\hline 1 & $12 / 27 / 92$ & 6 & 1 & & 79.8 & $\mathbf{N}$ & \\
\hline 2 & $12 / 27 / 92$ & 6 & 1 & & 97.8 & $\mathrm{~N}$ & \\
\hline 2 & $12 / 27 / 92$ & 7 & 1 & & 93 & $\mathrm{~N}$ & \\
\hline 2 & $12 / 27 / 92$ & 8 & 1 & & 85.1 & $\mathbf{N}$ & \\
\hline 2 & $12 / 27 / 92$ & 9 & 1 & & 82 & $\mathbf{N}$ & \\
\hline 9 & $12 / 27 / 92$ & 10 & 1 & & & $N$ & \\
\hline 2 & $12 / 27 / 92$ & 10 & 1 & & 82.9 & $N$ & \\
\hline 1 & $1 / 1 / 93$ & 1 & 1 & & 112 & $\mathbf{Y}$ & $\mathrm{J} 890523512-47$ \\
\hline 2 & $1 / 1 / 93$ & 1 & 1 & & 116.3 & $\bar{Y}$ & J $890523512-47$ \\
\hline 1 & $1 / 1 / 93$ & 2 & 1 & & 109.8 & $\mathbf{N}$ & \\
\hline 2 & 1/1/93 & 2 & 1 & & 113.5 & $\mathbf{N}$ & \\
\hline$\frac{1}{1}$ & $1 / 1 / 93$ & 3 & $T$ & & 105.8 & $\bar{N}$ & \\
\hline 2 & $1 / 1 / 93$ & 3 & 9 & & 109.9 & $\mathbf{N}$ & \\
\hline 1 & $1 / 1 / 93$ & 4 & 1 & & 102.7 & $\mathbf{N}$ & \\
\hline 2 & $1 / 1 / 93$ & 4 & 1 & & 105 & $\mathbf{N}$ & \\
\hline 1 & $1 / 1 / 93$ & 5 & 1 & & 98.2 & $N$ & \\
\hline 2 & $1 / 1 / 93$ & 5 & 1 & & 99.9 & $\mathbf{N}$ & \\
\hline 1 & $1 / 1 / 93$ & 6 & 1 & & 76.3 & $\mathbf{N}$ & \\
\hline 2 & $1 / 1 / 93$ & 6 & 1 & & 94.7 & $\mathbf{N}$ & \\
\hline 2 & 1/1/93 & 7 & 1 & & 89.8 & $\mathbf{N}$ & \\
\hline 2 & $1 / 1 / 93$ & 8 & 1 & & 77.6 & $\mathbf{N}$ & \\
\hline 2 & $1 / 1 / 93$ & 9 & 1 & & 77.6 & $\mathbf{N}$ & \\
\hline 1 & $1 / 1 / 93$ & 10 & 1 & & & $\mathbf{N}$ & \\
\hline 2 & $1 / 1 / 93$ & 10 & 1 & & 78 & $\mathbf{N}$ & \\
\hline 1 & $1 / 3 / 93$ & 1 & 1 & & 113 & $Y$ & TMACS \\
\hline 2 & $1 / 3 / 93$ & 1 & 1 & & 120.7 & $\mathbf{Y}$ & TMACS \\
\hline 7 & $1 / 3 / 93$ & 2 & 1 & & 110.1 & $\mathbf{N}$ & \\
\hline 2 & $1 / 3 / 93$ & 2 & 1 & & 117.6 & $\mathbf{N}$ & \\
\hline 1 & $1 / 3 / 93$ & 3 & 1 & & 106.5 & $\mathbf{N}$ & \\
\hline 2 & $1 / 3 / 93$ & 3 & 1 & & 112.8 & $\bar{N}$ & \\
\hline 1 & $1 / 3 / 93$ & 4 & 1 & & 103.6 & $\mathbf{N}$ & \\
\hline 2 & $1 / 3 / 93$ & 4 & 1 & & 107.7 & $\mathbf{N}$ & \\
\hline 1 & $1 / 3 / 93$ & 5 & 1 & & 99.8 & $\mathrm{~N}$ & \\
\hline 2 & $1 / 3 / 93$ & 5 & 1 & & 103.2 & $\mathbf{N}$ & \\
\hline
\end{tabular}

Data obtained from WHC Surveillance Analysis Computer System (SACS), June 2, 1993. 
WHC-SD-WM-ER-312, Rev. 0

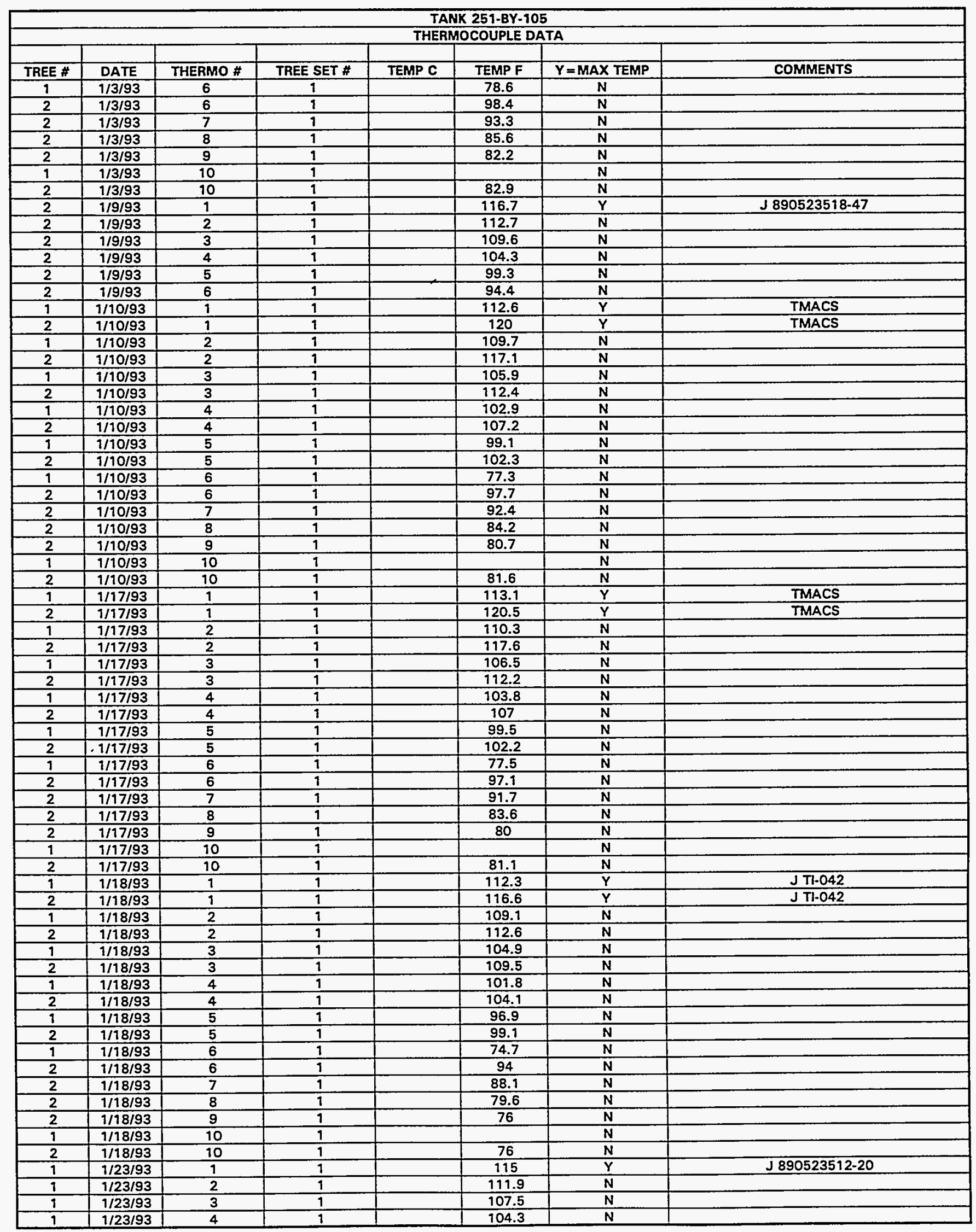

Data obtained from WHC Surveillance Analysis Computer System (SACS), June 2, 1993. 
WHC-SD-WM-ER-312, Rev. 0

\begin{tabular}{|c|c|c|c|c|c|c|c|}
\hline \multicolumn{8}{|c|}{ TANK 251-BY-105 } \\
\hline & & & & THER & COUPLE D & & \\
\hline TREE \# & DATE & THERMO \# & TREE SET \# & TEMP C & TEMP F & $Y=$ MAX TEMP & COMMENTS \\
\hline 1 & $1 / 23 / 93$ & 5 & 1 & & 99.4 & $\mathbf{N}$ & \\
\hline 1 & $1 / 23 / 93$ & 6 & $\frac{1}{1}$ & & 76.6 & $\mathbf{N}$ & \\
\hline 1 & $1 / 24 / 93$ & 1 & 1 & & 114 & $\mathbf{Y}$ & TMACS \\
\hline 2 & $1 / 24 / 93$ & 1 & 1 & & 121.2 & $\bar{Y}$ & TMACS \\
\hline 1 & $1 / 24 / 93$ & 2 & 1 & & 111.2 & $\mathbf{N}$ & \\
\hline 2 & $1 / 24 / 93$ & 2 & 1 & & 117.8 & $\mathbf{N}$ & \\
\hline 1 & $1 / 24 / 93$ & 3 & 9 & & 107.2 & $\mathbf{N}$ & \\
\hline 2 & $1 / 24 / 93$ & 3 & 1 & & 111.5 & $\mathbf{N}$ & \\
\hline$\frac{1}{1}$ & $1 / 24 / 93$ & 4 & 1 & & 104.3 & $\mathbf{N}$ & \\
\hline 2 & $1 / 24 / 93$ & 4 & 1 & & 106.1 & $\mathbf{N}$ & \\
\hline 1 & $1 / 24 / 93$ & 5 & 1 & & 99.8 & $\mathbf{N}$ & \\
\hline 2 & $1 / 24 / 93$ & 5 & 1 & & 101.3 & $\mathbf{N}$ & \\
\hline 1 & $1 / 24 / 93$ & 6 & 1 & & 77.1 & $\mathbf{N}$ & \\
\hline 2 & $1 / 24 / 93$ & 6 & 1 & & 95.9 & $\mathbf{N}$ & \\
\hline 2 & $1 / 24 / 93$ & 7 & 1 & & 89.6 & $\mathbf{N}$ & \\
\hline 2 & $1 / 24 / 93$ & 8 & 1 & & 80.7 & $\mathbf{N}$ & \\
\hline 2 & $1 / 24 / 93$ & 9 & 1 & & 78.2 & $\mathbf{N}$ & \\
\hline 2 & $1 / 24 / 93$ & 10 & 1 & & 78.8 & $\mathbf{N}$ & \\
\hline 1 & $1 / 31 / 93$ & 1 & 1 & & 111.9 & $\mathbf{Y}$ & J $910126143-40$ \\
\hline 1 & $1 / 31 / 93$ & 1 & 2 & & 114.08 & $\mathbf{Y}$ & TMACS \\
\hline 2 & $1 / 31 / 93$ & 1 & 1 & & 121.28 & $\mathbf{Y}$ & TMACS \\
\hline 1 & $1 / 31 / 93$ & 2 & 1 & & 108.8 & $\mathbf{N}$ & \\
\hline$\frac{1}{1}$ & $1 / 31 / 93$ & 2 & 2 & & 110.84 & $\mathbf{N}$ & \\
\hline 2 & $1 / 31 / 93$ & 2 & 1 & & 118.22 & $\mathbf{N}$ & \\
\hline 1 & $1 / 31 / 93$ & 3 & 1 & & 104.8 & $\mathbf{N}$ & \\
\hline 1 & $1 / 31 / 93$ & 3 & 2 & & 107.24 & $N$ & \\
\hline 2 & $1 / 31 / 93$ & 3 & 1 & & 112.1 & $N$ & \\
\hline 1 & $1 / 31 / 93$ & 4 & 1 & & 101.6 & $\mathbf{N}$ & \\
\hline 1 & $1 / 31 / 93$ & 4 & 2 & & 104.54 & $\mathbf{N}$ & \\
\hline 2 & $1 / 31 / 93$ & 4 & 1 & & 106.52 & $\mathbf{N}$ & \\
\hline 1 & $1 / 31 / 93$ & 5 & 1 & & 96.5 & $\mathbf{N}$ & \\
\hline 1 & $1 / 31 / 93$ & 5 & 2 & & 99.86 & $\mathbf{N}$ & \\
\hline 2 & $1 / 31 / 93$ & 5 & 1 & & 101.48 & $\mathbf{N}$ & \\
\hline 1 & $1 / 31 / 93$ & 6 & 1 & & 73.8 & $\mathbf{N}$ & \\
\hline 1 & $1 / 31 / 93$ & 6 & 2 & & 77.18 & $\mathbf{N}$ & \\
\hline 2 & $1 / 31 / 93$ & 6 & 1 & & 95.9 & $\mathbf{N}$ & \\
\hline 2 & $1 / 31 / 93$ & 7 & 1 & & 89.96 & $\mathbf{N}$ & \\
\hline 2 & $1 / 31 / 93$ & 8. & 1 & & 81.68 & $\mathbf{N}$ & \\
\hline 2 & $1 / 31 / 93$ & 9 & 1 & & 78.98 & $\mathbf{N}$ & \\
\hline 1 & $.1 / 31 / 93$ & 10 & 1 & & & $\mathbf{N}$ & \\
\hline 1 & $1 / 31 / 93$ & 10 & 2 & & & $\mathbf{N}$ & \\
\hline 2 & $1 / 31 / 93$ & 10 & 1 & & 79.7 & $\mathbf{N}$ & \\
\hline 1 & $2 / 5 / 93$ & 1 & 1 & & 114.2 & $\mathbf{Y}$ & J $910126143-50$ \\
\hline 2 & $2 / 5 / 93$ & 1 & 1 & & 118.5 & $\mathbf{Y}$ & J $910126143-50$ \\
\hline 1 & $2 / 5 / 93$ & 2 & 1 & & 111.1 & $\mathbf{N}$ & \\
\hline 2 & $2 / 5 / 93$ & 2 & 1 & & 114.2 & $\mathbf{N}$ & \\
\hline 1 & $2 / 5 / 93$ & 3 & 1 & & 106.6 & $\mathbf{N}$ & \\
\hline 2 & $2 / 5 / 93$ & 3 & 1 & & 111.1 & $\mathbf{N}$ & \\
\hline 1 & $2 / 5 / 93$ & 4 & 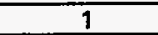 & & 103.3 & $\mathbf{N}$ & \\
\hline 2 & $2 / 5 / 93$ & 4 & 1 & & 105.4 & $\mathbf{N}$ & \\
\hline 1 & $2 / 5 / 93$ & 5 & 1 & & 98.1 & $\mathbf{N}$ & \\
\hline 2 & $2 / 5 / 93$ & 5 & 1 & & 100.2 & $\mathbf{N}$ & \\
\hline 1 & $2 / 5 / 93$ & 6 & 1 & & 74.8 & $\mathrm{~N}$ & \\
\hline 2 & $2 / 5 / 93$ & 6 & 1 & & 94.9 & $\mathbf{N}$ & \\
\hline 2 & $2 / 5 / 93$ & 7 & 1 & & 88.3 & $\mathbf{N}$ & \\
\hline 2 & $2 / 5 / 93$ & 8 & 1 & & 79.8 & $\mathbf{N}$ & \\
\hline 2 & $2 / 5 / 93$ & 9 & 1 & & 76.4 & $\mathbf{N}$ & \\
\hline 1 & $2 / 5 / 93$ & 10 & 1 & & & $\mathbf{N}$ & \\
\hline 2 & $2 / 5 / 93$ & 10 & 1 & & 76.3 & $\mathbf{N}$ & \\
\hline 1 & $2 / 8 / 93$ & 1 & 1 & & 114.26 & $\mathbf{Y}$ & TMACS \\
\hline 2 & $2 / 8 / 93$ & 1 & 1 & & 121.46 & $\mathbf{Y}$ & TMACS \\
\hline 1 & 2/8/93 & 2 & 1 & & 111.02 & $\mathbf{N}$ & \\
\hline 2 & $2 / 8 / 93$ & 2 & 1 & & 118.4 & $\mathbf{N}$ & \\
\hline 1 & $2 / 8 / 93$ & 3 & 1 & & 106.88 & $\mathbf{N}$ & \\
\hline 2 & $2 / 8 / 93$ & 3 & 1 & & 111.92 & $\mathbf{N}$ & \\
\hline 1 & $2 / 8 / 93$ & 4 & 1 & & 104.36 & $\mathbf{N}$ & \\
\hline 2 & $2 / 8 / 93$ & 4 & 1 & & 106.52 & $\mathbf{N}$ & \\
\hline 1 & $2 / 8 / 93$ & 5 & 1 & & 99.68 & $\mathbf{N}$ & \\
\hline
\end{tabular}

Data obtained from WHC Surveillance Analysis Computer System (SACS), June 2, 1993. 


\begin{tabular}{|c|c|c|c|c|c|c|c|}
\hline \multicolumn{8}{|c|}{ TANK 251-BY-105 } \\
\hline & & & & THEF & COUPLE & & \\
\hline TREE \# & DATE & THERMO \# & TREE SET \# & TEMP C & TEMP F & $Y=$ MAX TEMP & COMMENTS \\
\hline 2 & $2 / 8 / 93$ & 5 & 1 & & 101.48 & $\mathbf{N}$ & \\
\hline 1 & $2 / 8 / 93$ & 6 & 1 & & 76.82 & $\mathbf{N}$ & \\
\hline 2 & $2 / 8 / 93$ & 6 & 1 & & 95.9 & $\bar{N}$ & \\
\hline 2 & $2 / 8 / 93$ & 7 & 1 & & 89.96 & $\mathbf{N}$ & \\
\hline 2 & $2 / 8 / 93$ & 8 & 1 & & 81.68 & $\mathbf{N}$ & \\
\hline 2 & $2 / 8 / 93$ & 9 & 1 & & 78.8 & $\mathbf{N}$ & \\
\hline 1 & $2 / 8 / 93$ & 10 & 9 & & & $\overline{\mathbf{N}}$ & \\
\hline 2 & $2 / 8 / 93$ & 10 & 1 & & 79.34 & $\bar{N}$ & \\
\hline 1 & $2 / 12 / 93$ & 1 & 1 & & 110 & $\bar{Y}$ & J $890423320-42$ \\
\hline 2 & $2 / 12 / 93$ & 1 & 1 & & 113.9 & $\bar{Y}$ & J $890423320-42$ \\
\hline 1 & $2 / 12 / 93$ & 2 & 1 & & 106.5 & $\mathbf{N}$ & \\
\hline 2 & $2 / 12 / 93$ & 2 & 1 & & 109.6 & $\mathbf{N}$ & \\
\hline 1 & $2 / 12 / 93$ & 3 & 1 & & 102.7 & $\mathrm{~N}$ & \\
\hline 2 & $2 / 12 / 93$ & 3 & 9 & & 106.5 & $\mathrm{~N}$ & \\
\hline 1 & $2 / 12 / 93$ & 4 & 1 & & 98.8 & $N$ & \\
\hline 2 & $2 / 12 / 93$ & 4 & 9 & & 100.7 & $\mathbf{N}$ & \\
\hline 1 & $2 / 12 / 93$ & 5 & 1 & & 93.7 & $\bar{N}$ & \\
\hline 2 & $2 / 12 / 93$ & 5 & 1 & & 95.3 & $\overline{\mathbf{N}}$ & \\
\hline$\frac{1}{1}$ & $2 / 12 / 93$ & 6 & 1 & & 70.8 & $\mathrm{~N}$ & \\
\hline 2 & $2 / 12 / 93$ & 6 & 1 & & 89.9 & $N$ & \\
\hline 2 & $2 / 12 / 93$ & 7 & 1 & & 83.7 & $\mathrm{~N}$ & \\
\hline 2 & $2 / 12 / 93$ & 8 & 1 & & 75.4 & $\bar{N}$ & \\
\hline 2 & $2 / 12 / 93$ & 9 & 1 & & 71.9 & $\mathrm{~N}$ & \\
\hline 1 & $2 / 12 / 93$ & 10 & 1 & & & $\mathrm{~N}$ & \\
\hline 2 & $2 / 12 / 93$ & 10 & 1 & & 71.7 & $\mathbf{N}$ & \\
\hline 1 & $2 / 15 / 93$ & 1 & 1 & & 113.7 & $\bar{Y}$ & TMACS \\
\hline 2 & $2 / 15 / 93$ & 1 & 1 & & 120.7 & $\bar{Y}$ & TMACS \\
\hline 1 & $2 / 15 / 93$ & 2 & $T$ & & 110.7 & $N$ & \\
\hline 2 & $2 / 15 / 93$ & 2 & 9 & & 197.7 & $\mathbf{N}$ & \\
\hline 1 & $2 / 15 / 93$ & 3 & 1 & & 106.7 & $\bar{N}$ & \\
\hline 2 & $2 / 15 / 93$ & 3 & 1 & & 111.9 & $\mathbf{N}$ & \\
\hline 1 & $2 / 15 / 93$ & 4 & 1 & & 103.3 & $\bar{N}$ & \\
\hline 2 & $2 / 15 / 93$ & 4 & 1 & & 106.5 & 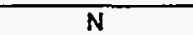 & \\
\hline 1 & $2 / 15 / 93$ & 5 & 1 & & 99.14 & $N$ & \\
\hline 2 & $2 / 15 / 93$ & 5 & 9 & & 101.3 & $\mathbf{N}$ & \\
\hline 1 & $2 / 15 / 93$ & 6 & $\frac{1}{1}$ & & 76.14 & $N$ & \\
\hline 2 & $2 / 15 / 93$ & 6 & 1 & & 95.7 & $\bar{N}$ & \\
\hline 2 & $2 / 15 / 93$ & 7 & 1 & & 90 & $\mathbf{N}$ & \\
\hline 2 & $2 / 15 / 93$ & 8 & 1 & & 81.68 & $\bar{N}$ & \\
\hline 2 & $.2 / 15 / 93$ & 9 & 1 & & 78.62 & $N$ & \\
\hline 9 & $2 / 15 / 93$ & 10 & 1 & & & $N$ & \\
\hline 2 & $2 / 15 / 93$ & 10 & 1 & & 79.34 & $N$ & \\
\hline 1 & $2 / 21 / 93$ & 1 & 1 & & 111.9 & $\bar{Y}$ & J $910126143-48$ \\
\hline 1 & $2 / 21 / 93$ & $\frac{1}{1}$ & 2 & & 113 & $\bar{Y}$ & TMACS \\
\hline 2 & $2 / 21 / 93$ & 1 & 1 & & 915.9 & $\bar{Y}$ & \\
\hline 2 & $2 / 21 / 93$ & 1 & 2 & & 120.38 & $\mathbf{Y}$ & TMACS \\
\hline 9 & $2 / 21 / 93$ & 2 & 1 & & 108.2 & $\mathbf{N}$ & \\
\hline 1 & $2 / 21 / 93$ & 2 & 2 & & 109.76 & $\mathrm{~N}$ & \\
\hline 2 & $2 / 21 / 93$ & 2 & 1 & & 111.8 & $\mathrm{~N}$ & \\
\hline 2 & $2 / 21 / 93$ & 2 & 2 & & 116.78 & $\bar{N}$ & \\
\hline 1 & $2 / 21 / 93$ & 3 & 1 & & 103.7 & $\mathbf{N}$ & \\
\hline 9 & $2 / 21 / 93$ & 3 & 2 & & 105.98 & $\mathbf{N}$ & \\
\hline 2 & $2 / 21 / 93$ & 3 & 1 & & 108.5 & $\mathbf{N}$ & \\
\hline 2 & $2 / 21 / 93$ & 3 & 2 & & 111.74 & $\bar{N}$ & \\
\hline$\frac{1}{1}$ & $2 / 21 / 93$ & 4 & 1 & & 100.3 & $\mathrm{~N}$ & \\
\hline 1 & $2 / 21 / 93$ & 4 & 2 & & 102.74 & $\mathrm{~N}$ & \\
\hline 2 & $2 / 21 / 93$ & 4 & 1 & & 102.6 & $\mathrm{~N}$ & \\
\hline 2 & $2 / 21 / 93$ & 4 & 2 & & 105.98 & $\mathbf{N}$ & \\
\hline 1 & $2 / 21 / 93$ & 5 & 1 & & 95.2 & $N$ & \\
\hline 1 & $2 / 21 / 93$ & 5 & 2 & & 98.42 & $\mathrm{~N}$ & \\
\hline 2 & $2 / 21 / 93$ & 5 & 9 & & 97.2 & $\mathbf{N}$ & \\
\hline 2 & $2 / 21 / 93$ & 5 & 2 & & 100.76 & $\mathbf{N}$ & \\
\hline 1 & $2 / 21 / 93$ & 6 & 1 & & 71.8 & $\mathbf{N}$ & \\
\hline 1 & $2 / 21 / 93$ & 6 & 2 & & 74.84 & $\mathbf{N}$ & \\
\hline 2 & $2 / 21 / 93$ & 6 & 1 & & 91.7 & $N$ & \\
\hline 2 & $2 / 21 / 93$ & 6 & 2 & & 95.18 & $\mathbf{N}$ & \\
\hline 2 & $2 / 21 / 93$ & 7 & 9 & & 85.4 & $\mathbf{N}$ & \\
\hline 2 & $2 / 21 / 93$ & 7 & 2 & & 89.6 & $\mathbf{N}$ & \\
\hline
\end{tabular}

Data obtained from WHC Surveillance Analysis Computer System (SACS), June 2, 1993. 


\begin{tabular}{|c|c|c|c|c|c|c|c|}
\hline \multicolumn{8}{|c|}{ TANK 251-BY-105 } \\
\hline & & & & THEF & COUPLE D & & \\
\hline TREE \# & DATE & THERMO \# & TREE SET \# & TEMP C & TEMP F & $Y=$ MAX TEMP & COMMENTS \\
\hline 2 & $2 / 21 / 93$ & 8 & 1 & & 76.7 & $N$ & \\
\hline$\frac{2}{2}$ & $2 / 21 / 93$ & 8 & 2 & & 80.6 & $\mathbf{N}$ & \\
\hline 2 & $2 / 21 / 93$ & 9 & 1 & & 73.2 & $\mathbf{N}$ & \\
\hline 2 & $2 / 21 / 93$ & 9 & 2 & & 77.54 & $\mathbf{N}$ & \\
\hline 1 & $2 / 21 / 93$ & 10 & 1 & & & $\mathbf{N}$ & \\
\hline 1 & $2 / 21 / 93$ & 10 & 2 & & & $\mathbf{N}$ & \\
\hline 2 & $2 / 21 / 93$ & 10 & $\frac{1}{1}$ & & 78 & $\mathbf{N}$ & \\
\hline 2 & $2 / 21 / 93$ & 10 & 2 & & 78.08 & $\mathbf{N}$ & \\
\hline 1 & $2 / 27 / 93$ & 1 & 1 & & 118 & $\bar{Y}$ & J $900925741-79$ \\
\hline 2 & $2 / 27 / 93$ & 1 & 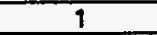 & & 124 & $\bar{Y}$ & \\
\hline 1 & $2 / 27 / 93$ & 2 & 1 & & 115 & $\mathbf{N}$ & \\
\hline 2 & $2 / 27 / 93$ & 2 & 9 & & 118 & $\mathbf{N}$ & \\
\hline 1 & $2 / 27 / 93$ & 3 & 1 & & 110 & $\mathbf{N}$ & \\
\hline 2 & $2 / 27 / 93$ & 3 & 9 & & 112 & $\mathbf{N}$ & \\
\hline 1 & $2 / 27 / 93$ & 4 & 9 & & 106 & $\mathbf{N}$ & \\
\hline 2 & $2 / 27 / 93$ & 4 & 1 & & 106 & $\mathbf{N}$ & \\
\hline 1 & $2 / 27 / 93$ & 5 & 1 & & 104 & $N$ & \\
\hline 2 & $2 / 27 / 93$ & 5 & 1 & & 101 & $\mathbf{N}$ & \\
\hline 9 & $2 / 27 / 93$ & 6 & 1 & & 77 & $\bar{N}$ & \\
\hline 2 & $2 / 27 / 93$ & 6 & 1 & & 95 & $N$ & \\
\hline 2 & $2 / 27 / 93$ & 7 & 9 & & 89 & $N$ & \\
\hline 2 & $2 / 27 / 93$ & 8 & 1 & & 80 & $N$ & \\
\hline 2 & $2 / 27 / 93$ & 9 & 1 & & 76 & $\mathbf{N}$ & \\
\hline$\frac{1}{1}$ & $2 / 27 / 93$ & 10 & 1 & & & $\mathbf{N}$ & \\
\hline 2 & $2 / 27 / 93$ & 10 & 1 & & 76 & $\mathbf{N}$ & \\
\hline 1 & $3 / 1 / 93$ & 1 & 1 & & 112.82 & $\bar{Y}$ & TMACS \\
\hline 2 & $3 / 1 / 93$ & 1 & 1 & & 120.2 & $\bar{Y}$ & TMACS \\
\hline$\frac{1}{1}$ & $3 / 1 / 93$ & 2 & 1 & & 109.58 & $\mathbf{N}$ & \\
\hline 2 & $3 / 1 / 93$ & 2 & 1 & & 116.42 & $\mathbf{N}$ & \\
\hline 9 & $3 / 1 / 93$ & 3 & 1 & & 105.8 & $\mathbf{N}$ & \\
\hline 2 & $3 / 1 / 93$ & 3 & 1 & & 111.56 & $\mathbf{N}$ & \\
\hline$\frac{1}{1}$ & $3 / 1 / 93$ & 4 & 1 & & 102.56 & $\mathbf{N}$ & \\
\hline 2 & $3 / 1 / 93$ & 4 & 1 & & 105.8 & $\mathbf{N}$ & \\
\hline 1 & $3 / 1 / 93$ & 5 & 1 & & 97.88 & $\mathbf{N}$ & \\
\hline 2 & $3 / 1 / 93$ & 5 & 1 & & 100.58 & $\mathbf{N}$ & \\
\hline 1 & $3 / 1 / 93$ & 6 & 9 & & 74.3 & $\mathrm{~N}$ & \\
\hline 2 & $3 / 1 / 93$ & 6 & 1 & & 94.82 & $\mathbf{N}$ & \\
\hline 2 & $3 / 1 / 93$ & 7 & 1 & & 88.88 & $\bar{N}$ & \\
\hline 2 & $3 / 1 / 93$ & 8 & 1 & & 80.24 & $\mathbf{N}$ & \\
\hline 2 & $3 / 1 / 93$ & 9 & 1 & & 76.82 & $\bar{N}$ & \\
\hline 1 & $3 / 1 / 93$ & 10 & 1 & & & $\mathbf{N}$ & \\
\hline 2 & $3 / 1 / 93$ & 10 & $\frac{1}{1}$ & & 77.54 & $\mathrm{~N}$ & \\
\hline 1 & $3 / 5 / 93$ & 1 & 1 & & 110.4 & $\bar{Y}$ & J 900925741-79 \\
\hline 2 & $3 / 5 / 93$ & 1 & 1 & & 113.8 & $\bar{Y}$ & J 900925741-79 \\
\hline 1 & $3 / 5 / 93$ & 2 & 1 & & 106.6 & $\mathbf{N}$ & \\
\hline 2 & $3 / 5 / 93$ & 2 & 1 & & 109.6 & $\mathbf{N}$ & \\
\hline 1 & $3 / 5 / 93$ & 3 & 1 & & 102.2 & $\mathbf{N}$ & \\
\hline 2 & $3 / 5 / 93$ & 3 & 1 & & 106.1 & $\mathrm{~N}$ & \\
\hline 1 & $3 / 5 / 93$ & 4 & $T$ & & 98.6 & $\mathbf{N}$ & \\
\hline 2 & $3 / 5 / 93$ & 4 & 1 & & 100.3 & $\mathbf{N}$ & \\
\hline 1 & $3 / 5 / 93$ & 5 & 1 & & 93.2 & $\mathbf{N}$ & \\
\hline 2 & $3 / 5 / 93$ & 5 & 1 & & 94.9 & $\mathbf{N}$ & \\
\hline$\frac{1}{1}$ & $3 / 5 / 93$ & 6 & 1 & & 69.6 & $\mathbf{N}$ & \\
\hline 2 & $3 / 5 / 93$ & 6 & 1 & & 89.4 & $\mathbf{N}$ & \\
\hline 2 & $3 / 5 / 93$ & 7 & 1 & & 83.1 & $\mathbf{N}$ & \\
\hline 2 & $3 / 5 / 93$ & 8 & 1 & & 74.7 & $\mathrm{~N}$ & \\
\hline 2 & $3 / 5 / 93$ & 9 & 1 & & 70.9 & $\mathbf{N}$ & \\
\hline 1 & $3 / 5 / 93$ & 10 & 1 & & & $\mathbf{N}$ & \\
\hline 2 & $3 / 5 / 93$ & 10 & 1 & & 70.8 & $\mathbf{N}$ & \\
\hline 1 & $3 / 8 / 93$ & 1 & 1 & & 114.26 & $\bar{Y}$ & TMACS \\
\hline 2 & $3 / 8 / 93$ & 1 & 1 & & 121.46 & $\bar{Y}$ & TMACS \\
\hline 1 & $3 / 8 / 93$ & 2 & 1 & & 110.84 & $\mathbf{N}$ & \\
\hline 2 & $3 / 8 / 93$ & 2 & 1 & & 118.22 & $\mathrm{~N}$ & \\
\hline$\frac{1}{1}$ & $3 / 8 / 93$ & 3 & 1 & & 106.88 & $\bar{N}$ & \\
\hline 2 & $3 / 8 / 93$ & 3 & 1 & & 111.2 & $\mathbf{N}$ & \\
\hline 1 & $3 / 8 / 93$ & 4 & 1 & & 103.82 & $N$ & \\
\hline 2 & $3 / 8 / 93$ & 4 & 1 & & 105.26 & $\mathrm{~N}$ & \\
\hline 1 & $3 / 8 / 93$ & 5 & 1 & & 98.96 & 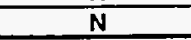 & \\
\hline
\end{tabular}

Data obtained from WHC Surveillance Analysis Computer System (SACS), June 2, 1993. 


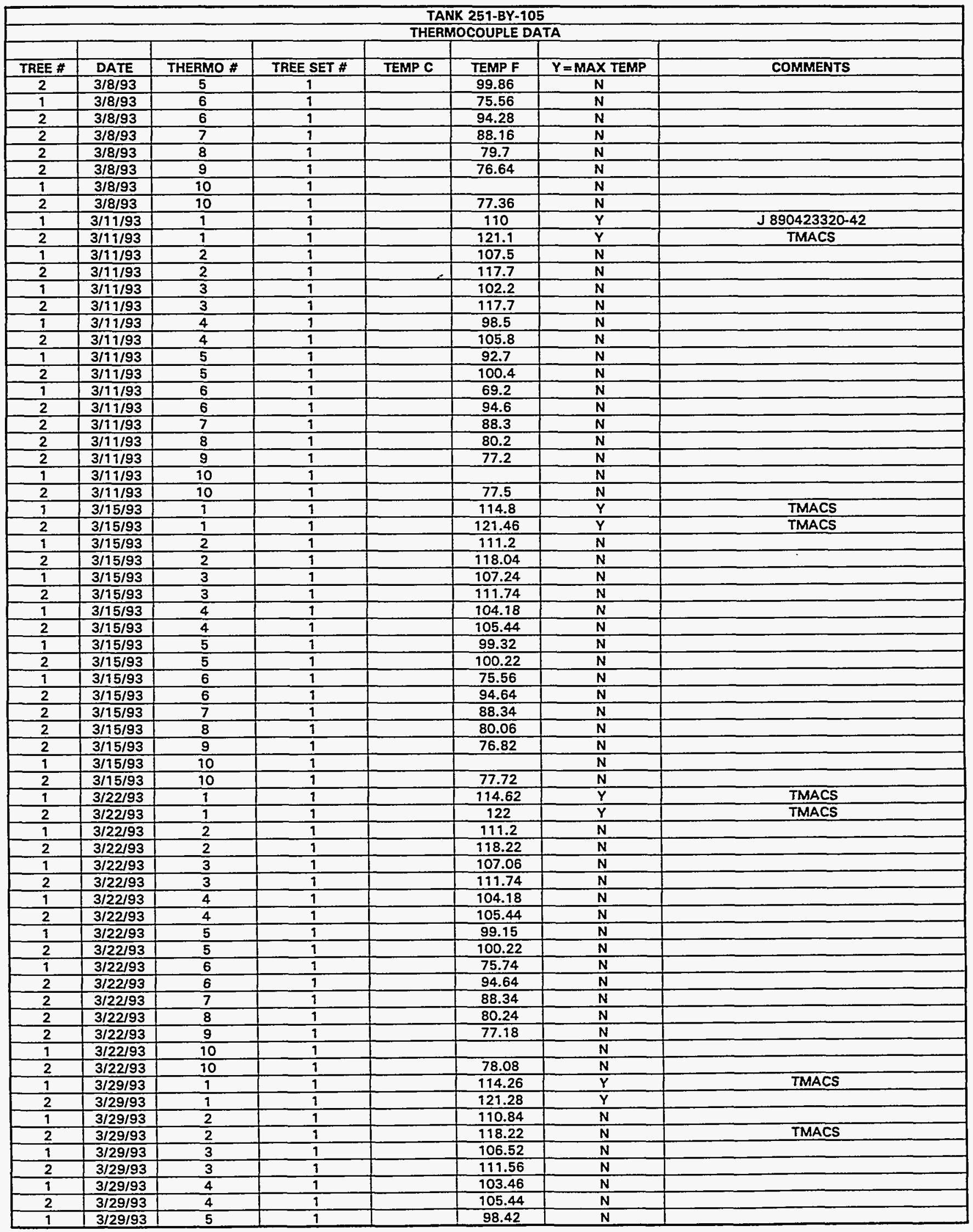

Data obtained from WHC Surveillance Analysis Computer System (SACS), June 2, 1993. 


\begin{tabular}{|c|c|c|c|c|c|c|c|}
\hline \multicolumn{8}{|c|}{ TANK 251-8Y.105 } \\
\hline & & & & & & & \\
\hline TREE \# & DATE & THERMO \# & TREE SET \# & TEMP C & TEMP F & $Y=$ MAX TEMP & COMMENTS \\
\hline 2 & $3 / 29 / 93$ & 5 & 1 & & 100.04 & $N$ & \\
\hline 1 & $3 / 29 / 93$ & 6 & 1 & & 75.38 & $\overline{\mathbf{N}}$ & \\
\hline 2 & $3 / 29 / 93$ & 6 & 1 & & 94.46 & $\mathbf{N}$ & \\
\hline 1 & $3 / 29 / 93$ & 7 & 1 & & & $\mathbf{N}$ & \\
\hline 2 & $3 / 29 / 93$ & 7 & 1 & & 88.34 & $\mathbf{N}$ & \\
\hline$\frac{1}{1}$ & $3 / 29 / 93$ & 8 & $\frac{1}{1}$ & & & $\mathbf{N}$ & \\
\hline 2 & $3 / 29 / 93$ & 8 & 1 & & 80.42 & $\mathbf{N}$ & \\
\hline 1 & $3 / 29 / 93$ & 9 & 1 & & & $\mathbf{N}$ & \\
\hline 2 & $3 / 29 / 93$ & 9 & 1 & & 77.54 & $N$ & \\
\hline 1 & $3 / 29 / 93$ & 10 & 1 & & & $N$ & \\
\hline$\frac{1}{1}$ & $3 / 29 / 93$ & 11 & 1 & & & $\mathbf{N}$ & \\
\hline 1 & $3 / 29 / 93$ & 12 & 9 & $\circ$ & & $\mathbf{N}$ & \\
\hline 2 & $3 / 29 / 93$ & 12 & 1 & & 78.08 & $\mathbf{N}$ & \\
\hline 1 & $3 / 29 / 93$ & 13 & $\frac{1}{1}$ & & & $\mathbf{N}$ & \\
\hline 1 & $3 / 29 / 93$ & 14 & 1 & & & $\mathbf{N}$ & \\
\hline 1 & $3 / 29 / 93$ & 15 & 1 & & & $\mathbf{N}$ & \\
\hline 1 & $3 / 29 / 93$ & 16 & 1 & & & $\overline{\mathbf{N}}$ & \\
\hline 1 & $4 / 5 / 93$ & 1 & 1 & & 113.9 & $Y$ & TMACS \\
\hline 2 & $4 / 5 / 93$ & 1 & 9 & & 120.74 & $\bar{Y}$ & TMACS \\
\hline 1 & $4 / 5 / 93$ & 2 & $\frac{1}{1}$ & & 110.3 & $\mathbf{N}$ & \\
\hline 2 & $4 / 5 / 93$ & 2 & 1 & & 117.14 & $\bar{N}$ & \\
\hline 1 & $4 / 5 / 93$ & 3 & 1 & & 106.16 & $\mathbf{N}$ & \\
\hline 2 & $4 / 5 / 93$ & 3 & 1 & & 110.66 & $\mathbf{N}$ & \\
\hline 1 & $4 / 5 / 93$ & 4 & 1 & & 103.28 & $\mathbf{N}$ & \\
\hline 2 & $4 / 5 / 93$ & 4 & 1 & & 104.9 & $\mathbf{N}$ & \\
\hline 1 & $4 / 5 / 93$ & 5 & 1 & & 98.06 & $\bar{N}$ & \\
\hline 2 & $4 / 5 / 93$ & 5 & 1 & & 99.5 & $\mathbf{N}$ & \\
\hline$\frac{1}{1}$ & $4 / 5 / 93$ & 6 & 1 & & 75.2 & $\mathbf{N}$ & \\
\hline 2 & $4 / 5 / 93$ & 6 & 1 & & 93.38 & $\mathrm{~N}$ & \\
\hline 1 & $4 / 5 / 93$ & 7 & 1 & & & $N$ & \\
\hline 2 & $4 / 5 / 93$ & 7 & 1 & & 87.44 & $N$ & \\
\hline 1 & $4 / 5 / 93$ & 8 & 1 & & & $\mathbf{N}$ & \\
\hline 2 & $4 / 5 / 93$ & 8 & 1 & & 80.06 & $\mathbf{N}$ & \\
\hline 1 & $4 / 5 / 93$ & 9 & 1 & & & $N$ & \\
\hline 2 & $4 / 5 / 93$ & 9 & 1 & & 77 & $N$ & \\
\hline 1 & $4 / 5 / 93$ & 10 & 1 & & & 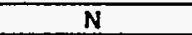 & \\
\hline 1 & $4 / 5 / 93$ & 11 & 1 & & & $N$ & \\
\hline 1 & $4 / 5 / 93$ & 12 & 1 & & & $N$ & \\
\hline 2 & $4 / 5 / 93$ & 12 & 1 & & 77.72 & $\bar{N}$ & \\
\hline 1 & $.4 / 5 / 93$ & 13 & 1 & & & $N$ & \\
\hline 1 & $4 / 5 / 93$ & 14 & 1 & & & $N$ & \\
\hline 1 & $4 / 5 / 93$ & 15 & 1 & & & $\mathbf{N}$ & \\
\hline 1 & $4 / 5 / 93$ & 16 & 1 & & & $\mathbf{N}$ & \\
\hline 1 & $4 / 12 / 93$ & 1 & 1 & & 114.26 & $\mathbf{Y}$ & TMAC \\
\hline 2 & $4 / 12 / 93$ & 1 & 1 & & 121.46 & $\mathbf{Y}$ & TMAC \\
\hline 1 & $4 / 72 / 93$ & 2 & 1 & & 111.02 & $N$ & \\
\hline 2 & $4 / 12 / 93$ & 2 & 1 & & 117.86 & $\mathbf{N}$ & \\
\hline 1 & $4 / 12 / 93$ & 3 & 1 & & 106.7 & $\mathbf{N}$ & \\
\hline 2 & $4 / 12 / 93$ & 3 & 1 & & 111.2 & $\mathbf{N}$ & \\
\hline 1 & $4 / 12 / 93$ & $\sqrt{4}$ & 1 & & 103.46 & $\bar{N}$ & \\
\hline 2 & $4 / 12 / 93$ & 4 & 1 & & 104.72 & $\mathbf{N}$ & \\
\hline 1 & $4 / 12 / 93$ & 5 & 1 & & 98.42 & $\mathbf{N}$ & \\
\hline 2 & $4 / 12 / 93$ & 5 & 1 & & 99.32 & $\mathbf{N}$ & \\
\hline 9 & $4 / 12 / 93$ & 6 & 1 & & 75.92 & $\mathbf{N}$ & \\
\hline 2 & $4 / 12 / 93$ & 6 & 1 & & 93.74 & $\mathbf{N}$ & \\
\hline 1 & $4 / 12 / 93$ & 7 & 1 & & & $\mathrm{~N}$ & \\
\hline 2 & $4 / 12 / 93$ & 7 & 1 & & 87.62 & $N$ & \\
\hline 1 & $4 / 12 / 93$ & 8 & 1 & & & $\mathrm{~N}$ & \\
\hline 2 & $4 / 72 / 93$ & 8 & 1 & & 79.7 & $\mathbf{N}$ & \\
\hline 1 & $4 / 12 / 93$ & 9 & 1 & & & $\mathbf{N}$ & \\
\hline 2 & $4 / 12 / 93$ & 9 & 1 & & 77 & $\mathbf{N}$ & \\
\hline 1 & $4 / 12 / 93$ & 10 & 1 & & & $\mathbf{N}$ & \\
\hline 1 & $4 / 12 / 93$ & 11 & 1 & & & $\mathbf{N}$ & \\
\hline 1 & $4 / 12 / 93$ & 12 & 1 & & & $N$ & \\
\hline 2 & $4 / 12 / 93$ & 12 & 1 & & 77.54 & $\mathbf{N}$ & \\
\hline 1 & $4 / 12 / 93$ & 13 & 1 & & & $\mathbf{N}$ & \\
\hline 9 & $4 / 12 / 93$ & 14 & 1 & & & $\mathbf{N}$ & \\
\hline 9 & $4 / 12 / 93$ & 15 & 1 & & & $\mathbf{N}$ & \\
\hline
\end{tabular}

Data obtained from WHC Surveillance Analysis Computer System (SACS), June 2, 1993. 
WHC-SD-WM-ER-312, Rev. 0

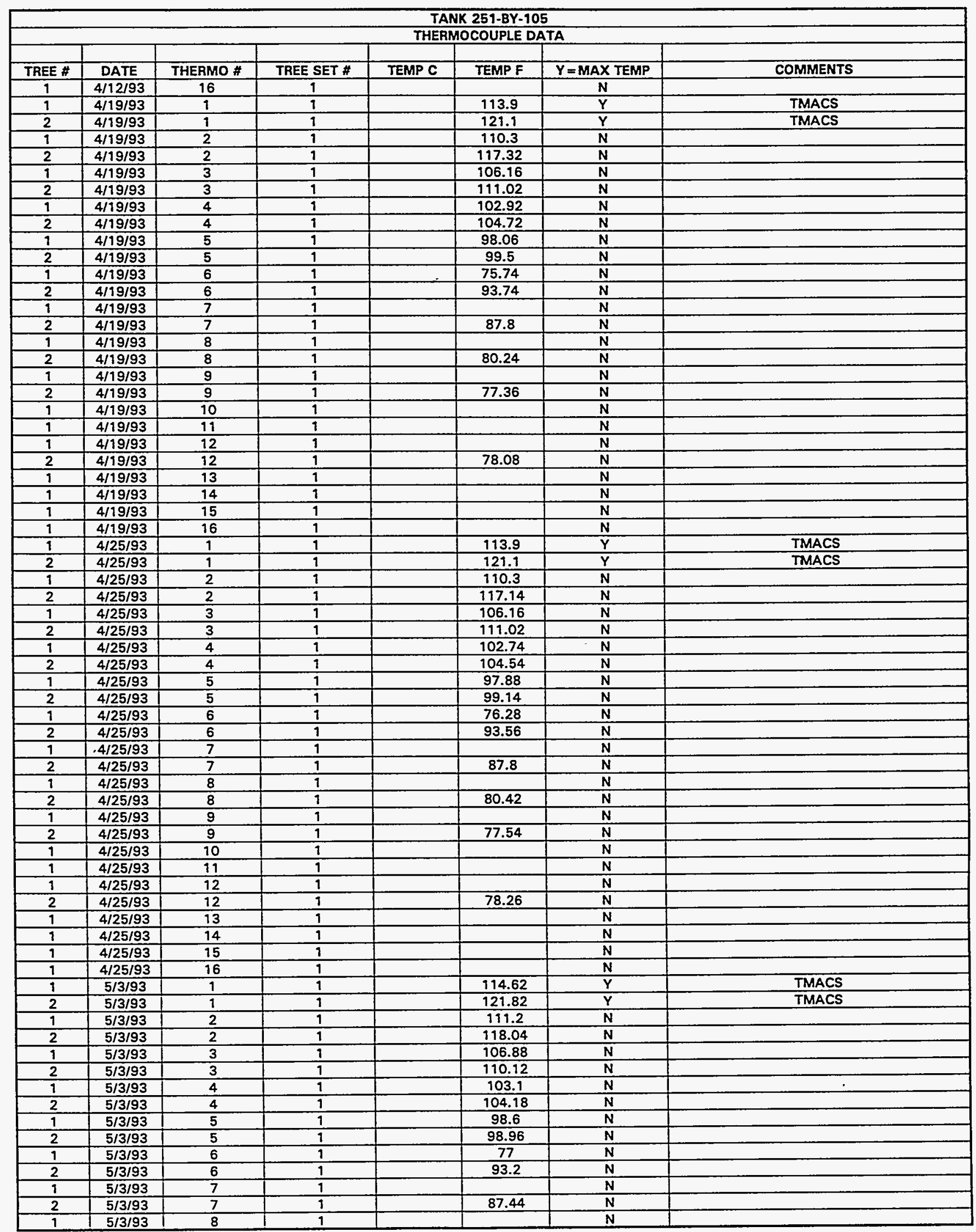

Data obtained from WHC Surveillance Analysis Computer System (SACS), June 2, 1993. 
WHC-SD-WM-ER-312, Rev. 0

\begin{tabular}{|c|c|c|c|c|c|c|c|}
\hline \multicolumn{8}{|c|}{ TANK 251-BY-105 } \\
\hline & & & & THEF & COUPLE & & \\
\hline TAEE \# & DATE & THERMO \# & TREE SET \# & TEMP C & TEMP F & $Y=$ MAX TEMP & COMMENTS \\
\hline 2 & $5 / 3 / 93$ & 8 & 1 & & 80.6 & $\mathrm{~N}$ & \\
\hline 1 & $5 / 3 / 93$ & 9 & 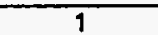 & & & $\mathbf{N}$ & \\
\hline 2 & $5 / 3 / 93$ & 9 & 1 & & 77.9 & $\mathbf{N}$ & \\
\hline 1 & $5 / 3 / 93$ & 10 & 1 & & & $\mathbf{N}$ & \\
\hline$\frac{1}{1}$ & $5 / 3 / 93$ & 11 & 1 & & & $\mathbf{N}$ & \\
\hline 1 & $5 / 3 / 93$ & 12 & 1 & & & $\mathbf{N}$ & \\
\hline 2 & $5 / 3 / 93$ & 12 & 1 & & 78.62 & $\mathbf{N}$ & \\
\hline 1 & $5 / 3 / 93$ & 13 & 1 & & & $\mathbf{N}$ & \\
\hline 1 & $5 / 3 / 93$ & 14 & 1 & & & $\mathbf{N}$ & \\
\hline 1 & $5 / 3 / 93$ & 15 & 9 & & & $\mathbf{N}$ & \\
\hline 1 & $5 / 3 / 93$ & 16 & 1 & & & $\mathbf{N}$ & \\
\hline 1 & $5 / 8 / 93$ & 1 & 1 & & 111.6 & $\bar{Y}$ & $\mathrm{~J} 910126143-50$ \\
\hline$\frac{1}{1}$ & $5 / 8 / 93$ & 2 & 1 & & 107.6 & $\mathbf{N}$ & \\
\hline 1 & $5 / 8 / 93$ & 3 & 1 & & 102.7 & $\mathbf{N}$ & \\
\hline 1 & $5 / 8 / 93$ & 4 & 1 & & 99 & $\mathbf{N}$ & \\
\hline 1 & $5 / 8 / 93$ & 5 & 1 & & 93.3 & $\mathbf{N}$ & \\
\hline$T$ & $5 / 8 / 93$ & 6 & 1 & & 71.6 & $\mathbf{N}$ & \\
\hline 9 & $5 / 8 / 93$ & 7 & 1 & & 115 & $\mathbf{N}$ & \\
\hline 1 & $5 / 8 / 93$ & 8 & 1 & & 110 & $\mathbf{N}$ & \\
\hline 1 & $5 / 8 / 93$ & 9 & 1 & & 106.8 & $\mathbf{N}$ & \\
\hline 1 & $5 / 8 / 93$ & 10 & 1 & & 100.4 & $\mathbf{N}$ & \\
\hline 1 & $5 / 8 / 93$ & 11 & 1 & & 94.7 & $\mathbf{N}$ & \\
\hline 1 & $5 / 8 / 93$ & 12 & 1 & & 89.1 & $\mathbf{N}$ & \\
\hline 1 & $5 / 8 / 93$ & 13 & 1 & & 83.3 & $\mathbf{N}$ & \\
\hline 1 & $5 / 8 / 93$ & 14 & 1 & & 76.2 & $\mathbf{N}$ & \\
\hline$\frac{T}{1}$ & $5 / 8 / 93$ & 15 & 1 & & 72.9 & $\mathbf{N}$ & \\
\hline 1 & $5 / 8 / 93$ & 16 & 1 & & 72.6 & $\mathbf{N}$ & \\
\hline 1 & $5 / 10 / 93$ & 1 & 1 & & 114.62 & $\bar{Y}$ & TMACS \\
\hline 2 & $5 / 10 / 93$ & 1 & 1 & & 121.46 & $\bar{Y}$ & TMACS \\
\hline 1 & $5 / 10 / 93$ & 2 & 1 & & 111.02 & $\mathbf{N}$ & \\
\hline 2 & $5 / 10 / 93$ & 2 & 1 & & 117.68 & $\mathbf{N}$ & \\
\hline$\frac{1}{1}$ & $5 / 10 / 93$ & 3 & 1 & & 106.88 & $\mathbf{N}$ & \\
\hline 2 & $5 / 10 / 93$ & 3 & 1 & & 109.94 & $\mathbf{N}$ & \\
\hline 1 & $5 / 10 / 93$ & 4 & 1 & & 103.46 & $\mathbf{N}$ & \\
\hline 2 & $5 / 10 / 93$ & 4 & 1 & & 103.82 & $\mathbf{N}$ & \\
\hline 1 & $5 / 70 / 93$ & 5 & 1 & & 98.42 & $\mathbf{N}$ & \\
\hline 2 & $5 / 10 / 93$ & 5 & 1 & & 98.6 & $\mathbf{N}$ & \\
\hline 1 & $5 / 10 / 93$ & 6 & 1 & & 77.36 & $\mathbf{N}$ & \\
\hline 2 & $5 / 10 / 93$ & 6 & 1 & & 92.84 & $\mathbf{N}$ & \\
\hline 1 & $.5 / 10 / 93$ & 7 & 1 & & & $\mathbf{N}$ & \\
\hline 2 & $5 / 10 / 93$ & 7 & 9 & & 87.26 & $\mathbf{N}$ & \\
\hline 1 & $5 / 10 / 93$ & 8 & 1 & & & $\mathbf{N}$ & \\
\hline 2 . & $5 / 10 / 93$ & 8 & 1 & & 80.24 & $\mathbf{N}$ & \\
\hline 1 & $5 / 10 / 93$ & 9 & 1 & & & $\mathbf{N}$ & \\
\hline 2 & $5 / 10 / 93$ & 9 & 1 & & 77.9 & $\mathbf{N}$ & \\
\hline 1 & $5 / 10 / 93$ & 10 & 1 & & & $\mathbf{N}$ & \\
\hline$T$ & $5 / 10 / 93$ & 11 & 1 & & & $\mathrm{~N}$ & \\
\hline 1 & $5 / 10 / 93$ & 12 & 1 & & & $N$ & \\
\hline 2 & $5 / 10 / 93$ & 12 & 1 & & 78.44 & $\mathbf{N}$ & \\
\hline 1 & $5 / 10 / 93$ & 13 & 1 & & & $\mathbf{N}$ & \\
\hline 1 & $5 / 10 / 93$ & 14 & 1 & & & $\mathbf{N}$ & \\
\hline 1 & $5 / 10 / 93$ & 15 & 1 & & & $N$ & \\
\hline 1 & $5 / 10 / 93$ & 16 & 1 & & & $\mathbf{N}$ & \\
\hline 1 & $5 / 17 / 93$ & 1 & 1 & & 114.8 & $\bar{Y}$ & TMACS \\
\hline 2 & $5 / 17 / 93$ & 1 & 1 & & 121.64 & $\bar{Y}$ & TMACS \\
\hline 1 & $5 / 17 / 93$ & 2 & 1 & & 111.2 & $\mathbf{N}$ & \\
\hline 2 & $5 / 17 / 93$ & 2 & 1 & & 117.68 & $\mathbf{N}$ & \\
\hline 1 & $5 / 17 / 93$ & 3 & 1 & & 107.24 & $\mathbf{N}$ & \\
\hline 2 & $5 / 17 / 93$ & 3 & 1 & & 110.12 & $\mathbf{N}$ & \\
\hline 1 & $5 / 17 / 93$ & $\frac{\pi}{4}$ & 1 & & 103.64 & $N$ & \\
\hline 2 & $5 / 17 / 93$ & 4 & 1 & & 103.82 & $\mathbf{N}$ & \\
\hline 1 & $5 / 17 / 93$ & 5 & 1 & & 98.78 & $\mathbf{N}$ & \\
\hline 2 & $5 / 17 / 93$ & 5 & 1 & & 98.6 & $\mathrm{~N}$ & \\
\hline 1 & $5 / 17 / 93$ & 6 & 1 & & 78.26 & $\mathbf{N}$ & \\
\hline 2 & $5 / 17 / 93$ & 6 & 1 & & 92.84 & $\mathbf{N}$ & \\
\hline 1 & $5 / 17 / 93$ & 7 & 1 & & & $\mathbf{N}$ & \\
\hline 2 & $5 / 17 / 93$ & 7 & 1 & & 87.62 & $\mathbf{N}$ & \\
\hline 9 & $5 / 17 / 93$ & 8 & 1 & & & $\mathbf{N}$ & \\
\hline
\end{tabular}

Data obtained from WHC Surveillance Analysis Computer System (SACS), June 2, 1993. 


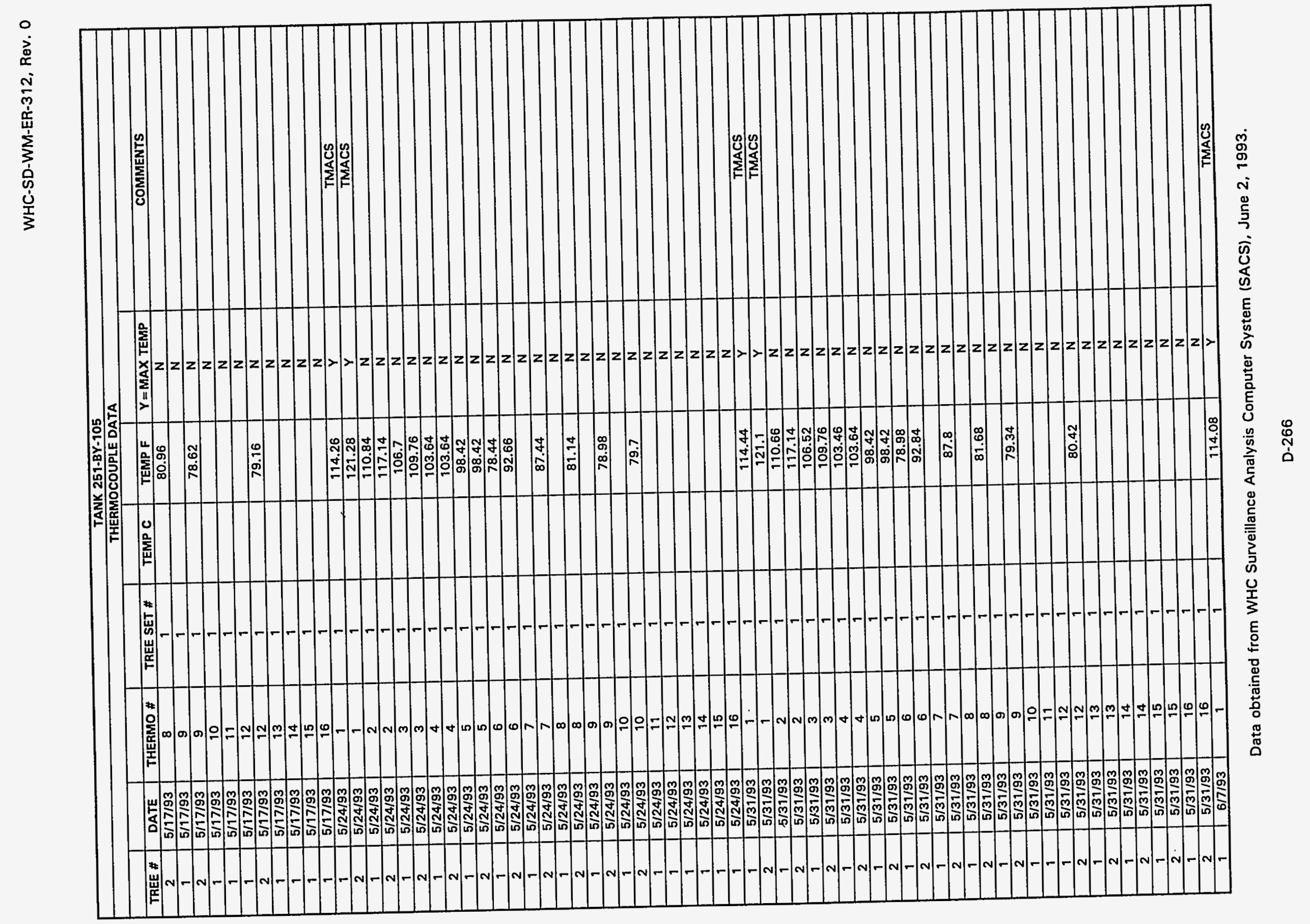


WHC-SD-WM-ER-312, Rev. 0

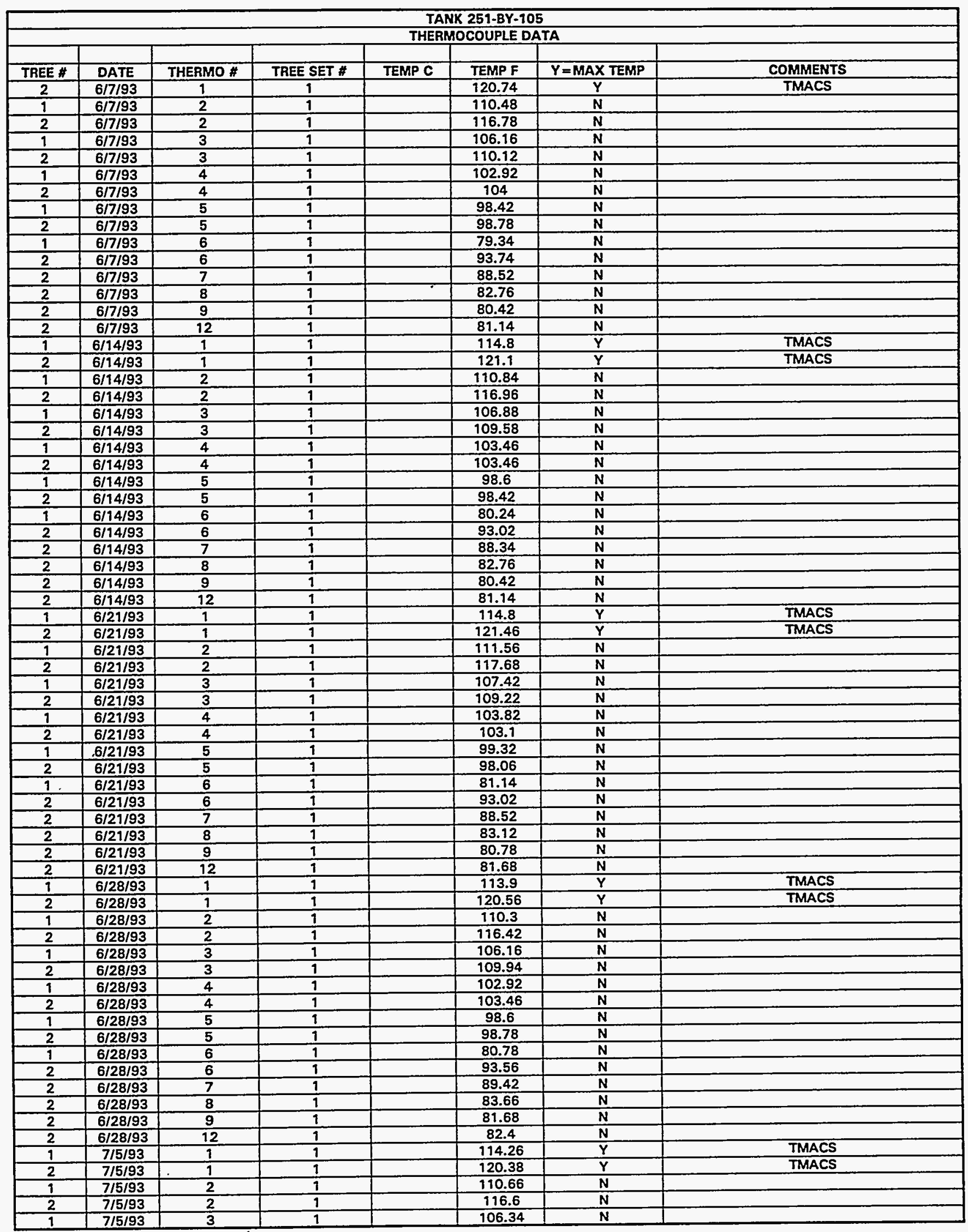

Data obtained from WHC Surveillance Analysis Computer System (SACS), June 2, 1993. 


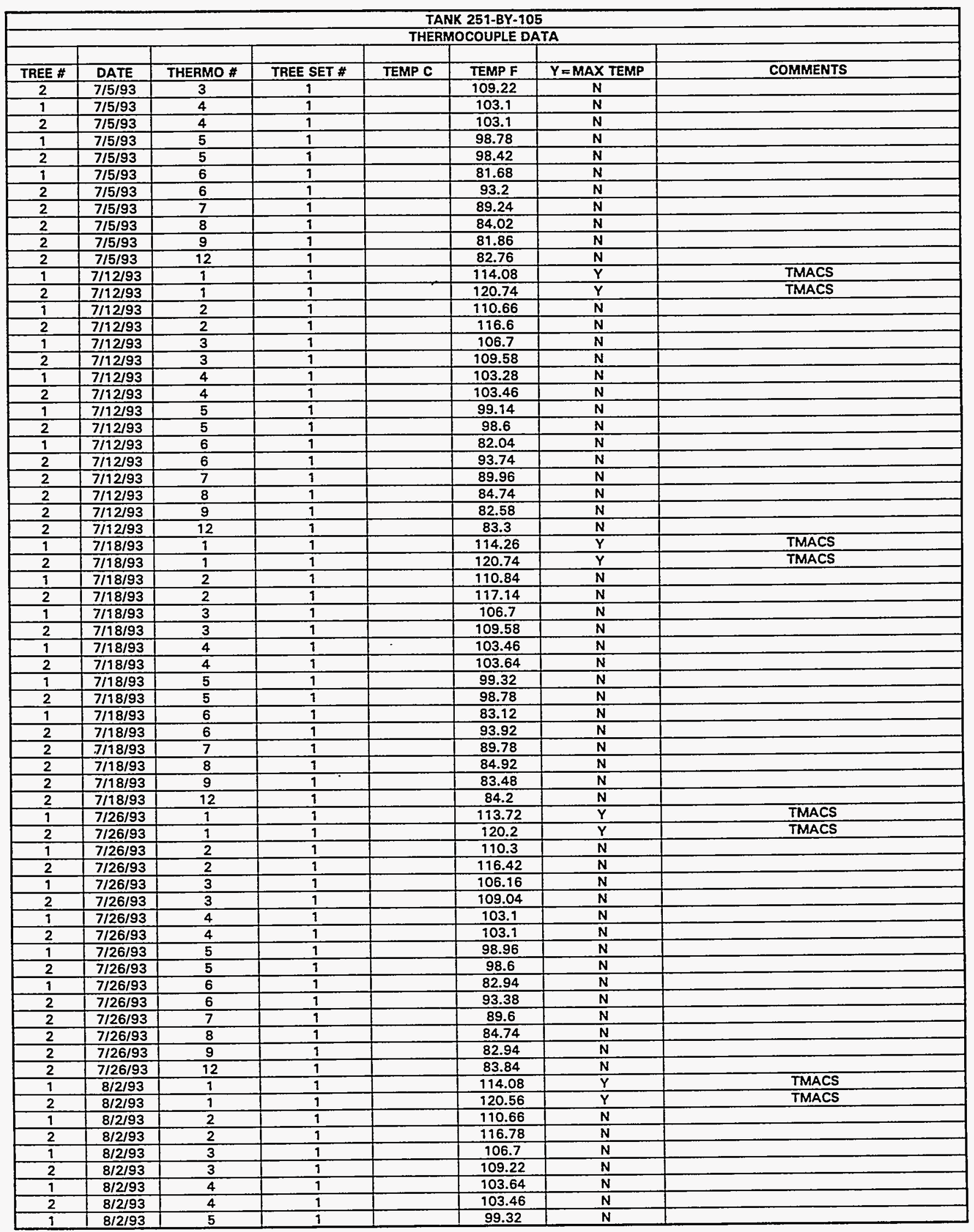

Data obtained from WHC Surveillance Analysis Computer System (SACS), June 2, 1993. 
WHC-SD-WM-ER-312, Rev. 0

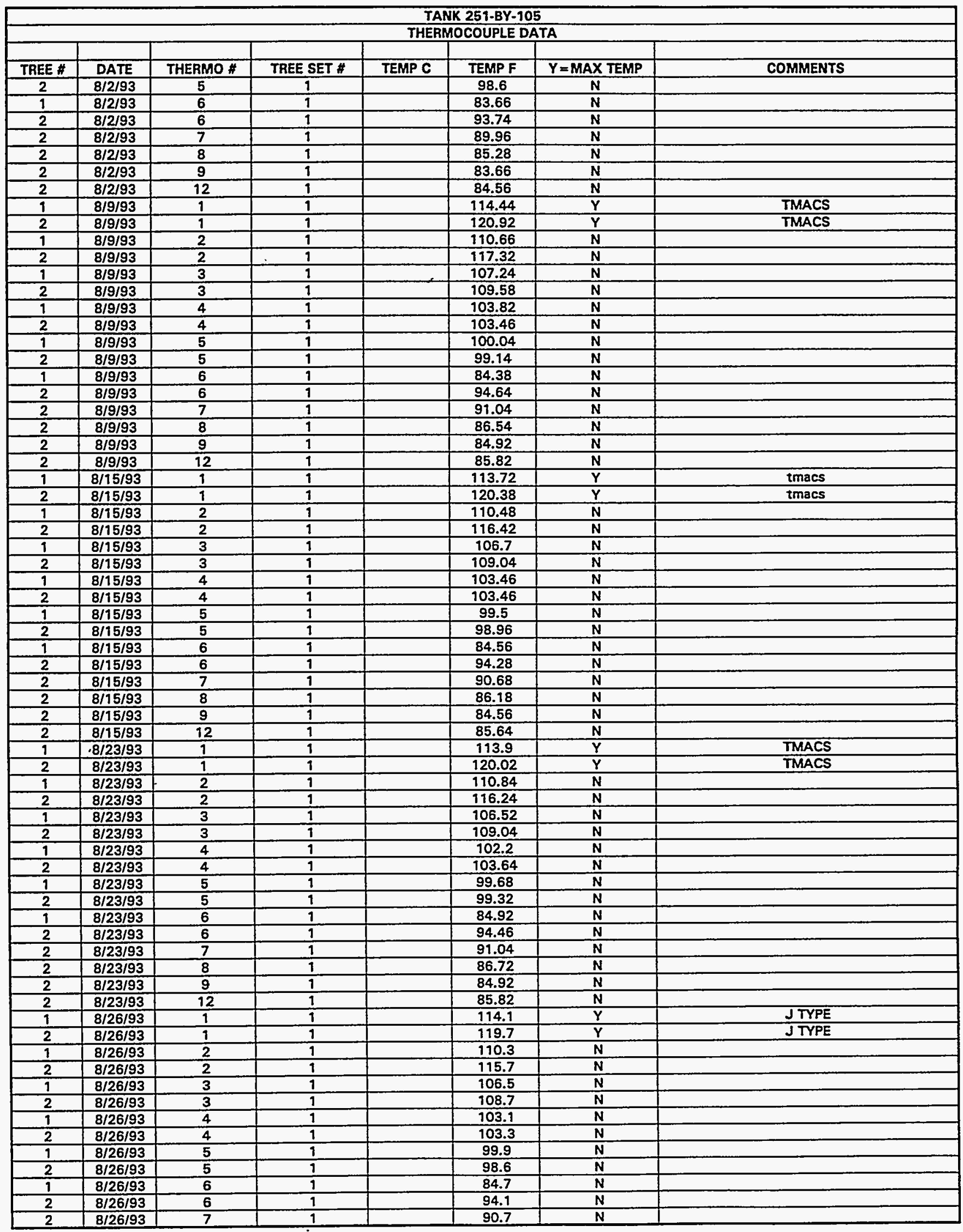

Data obtained from WHC Surveillance Analysis Computer System (SACS), June 2, 1993. 


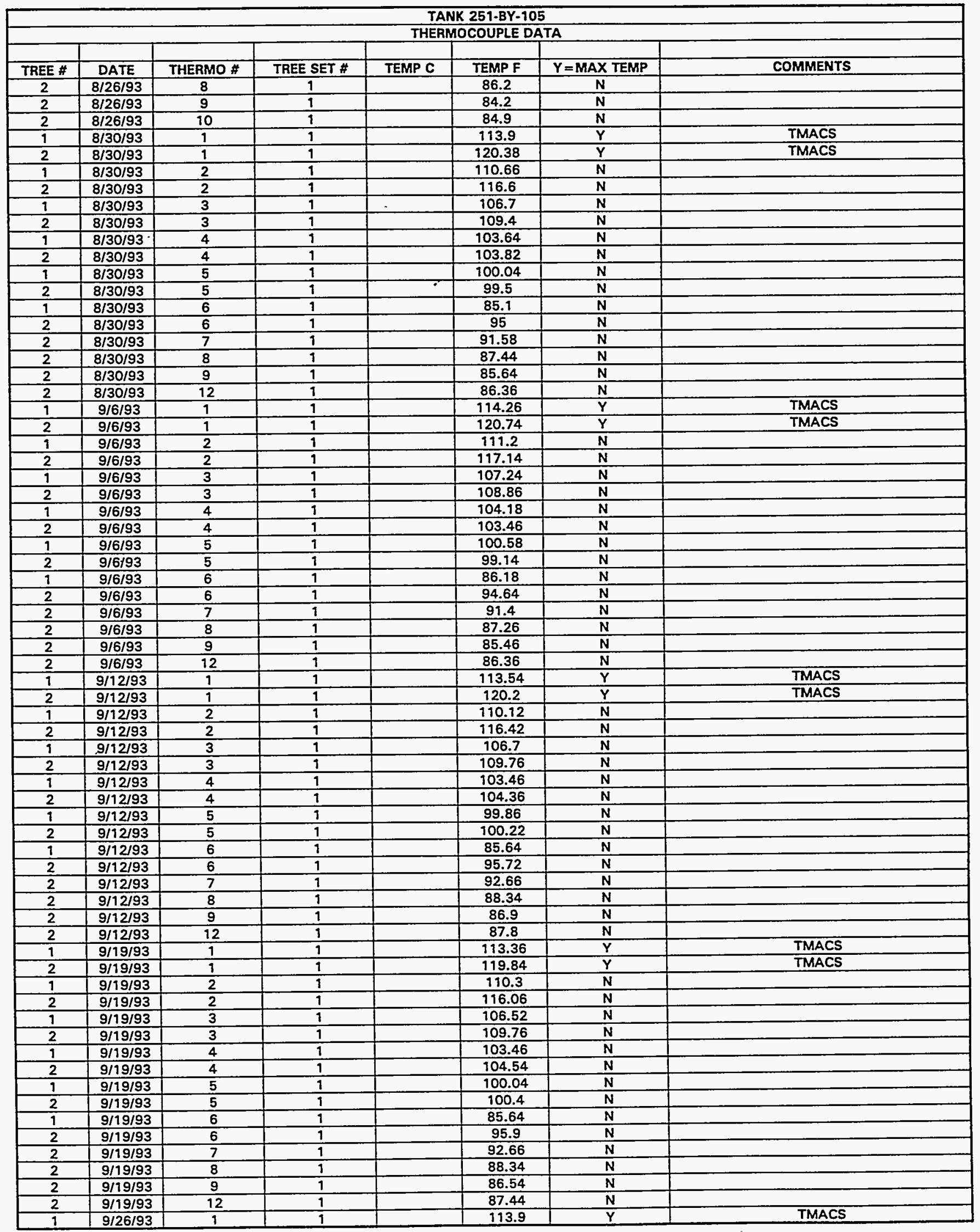

Data obtained from WHC Surveillance Analysis Computer System (SACS), June 2, 1993. 


\begin{tabular}{|c|c|c|c|c|c|c|c|}
\hline \multicolumn{8}{|c|}{ TANK 251-BY-105 } \\
\hline & & & & THER & COUPLE & & \\
\hline TREE \# & DATE & THERMO \# & TREE SET \# & TEMP C & TEMP F & $Y=M A X$ TEMP & COMMENTS \\
\hline 2 & $9 / 26 / 93$ & 1 & 1 & & 120.56 & $\mathrm{Y}$ & TMACS \\
\hline 1 & $9 / 26 / 93$ & 2 & 9 & & 110.66 & $\bar{N}$ & \\
\hline 2 & $9 / 26 / 93$ & 2 & 1 & & 116.78 & $\mathbf{N}$ & \\
\hline 1 & $9 / 26 / 93$ & 3 & 1 & & 106.88 & $\mathbf{N}$ & \\
\hline 2 & $9 / 26 / 93$ & 3 & 1 & & 109.76 & $\mathbf{N}$ & \\
\hline 1 & $9 / 26 / 93$ & 4 & 1 & & 104.18 & $\mathbf{N}$ & \\
\hline 2 & $9 / 26 / 93$ & 4 & 1 & & 104.18 & $\mathbf{N}$ & \\
\hline 1 & $9 / 26 / 93$ & 5 & 1 & & 100.58 & $\mathbf{N}$ & \\
\hline 2 & $9 / 26 / 93$ & 5 & 1 & & 100.22 & $\mathbf{N}$ & \\
\hline 1 & $9 / 26 / 93$ & 6 & 1 & & 86.18 & $\bar{N}$ & \\
\hline 2 & $9 / 26 / 93$ & 6 & 1 & & 95.9 & $\mathbf{N}$ & \\
\hline 2 & $9 / 26 / 93$ & 7 & 1 & & 92.66 & $\mathbf{N}$ & \\
\hline 2 & $9 / 26 / 93$ & 8 & 1 & & 87.98 & $\mathbf{N}$ & \\
\hline 2 & $9 / 26 / 93$ & 9 & 1 & & 86.54 & $\mathbf{N}$ & \\
\hline 2 & $9 / 26 / 93$ & 12 & 1 & & 87.62 & $\mathbf{N}$ & \\
\hline 1 & $10 / 3 / 93$ & 1 & 1 & & 113.72 & $\mathbf{Y}$ & TMACS \\
\hline 2 & $10 / 3 / 93$ & $T$ & 1 & & 120.2 & $\bar{Y}$ & TMACS \\
\hline 1 & $10 / 3 / 93$ & 2 & 1 & & 110.48 & $\mathbf{N}$ & \\
\hline 2 & $10 / 3 / 93$ & 2 & 1 & & 116.42 & $\mathbf{N}$ & \\
\hline 1 & $10 / 3 / 93$ & 3 & 1 & & 106.88 & $\mathbf{N}$ & \\
\hline 2 & $10 / 3 / 93$ & 3 & 1 & & 109.76 & $\mathbf{N}$ & \\
\hline 1 & $10 / 3 / 93$ & 4 & 1 & & 103.82 & $\mathbf{N}$ & \\
\hline 2 & $10 / 3 / 93$ & 4 & 1 & & 104.36 & $\mathbf{N}$ & \\
\hline$T$ & $10 / 3 / 93$ & 5 & $\frac{1}{1}$ & & 100.22 & $\mathbf{N}$ & \\
\hline 2 & $10 / 3 / 93$ & 5 & 1 & & 100.22 & $\mathbf{N}$ & \\
\hline$\frac{1}{1}$ & $10 / 3 / 93$ & 6 & 1 & & 86 & $N$ & \\
\hline 2 & $10 / 3 / 93$ & 6 & 9 & & 96.26 & $N$ & \\
\hline 2 & $10 / 3 / 93$ & 7 & 1 & & 93.2 & $N$ & \\
\hline 2 & $10 / 3 / 93$ & 8 & 1 & & 88.7 & $\mathbf{N}$ & \\
\hline 2 & $10 / 3 / 93$ & 9 & 1 & & 86.9 & $\bar{N}$ & \\
\hline 2 & $10 / 3 / 93$ & 12 & 1 & & 87.8 & $N$ & \\
\hline 1 & $10 / 10 / 93$ & 1 & 1 & & 113.36 & $\bar{Y}$ & TMACS \\
\hline 2 & $10 / 10 / 93$ & 1 & 9 & & 119.84 & $\bar{Y}$ & TMACS \\
\hline 1 & $10 / 10 / 93$ & 2 & 1 & & 110.12 & $\bar{N}$ & \\
\hline 2 & $10 / 10 / 93$ & 2 & 1 & & 116.42 & $\mathbf{N}$ & \\
\hline$\frac{1}{1}$ & $10 / 10 / 93$ & 3 & 1 & & 106.52 & $N$ & \\
\hline 2 & $10 / 10 / 93$ & 3 & 1 & & 109.58 & $\mathbf{N}$ & \\
\hline 1 & $10 / 10 / 93$ & 4 & 1 & & 103.64 & $\mathbf{N}$ & \\
\hline 2 & $10 / 10 / 93$ & 4 & 1 & & 104.58 & $\mathbf{N}$ & \\
\hline 1 & $10 / 10 / 93$ & 5 & 1 & & 100.22 & $N$ & \\
\hline 2 & $10 / 10 / 93$ & 5 & 1 & & 100.76 & $\mathbf{N}$ & \\
\hline 1 & $10 / 10 / 93$ & 6 & 1 & & 85.64 & $\mathbf{N}$ & \\
\hline 2 & $10 / 10 / 93$ & 6 & 1 & & 96.26 & $\mathbf{N}$ & \\
\hline 2 & $10 / 10 / 93$ & 7 & 1 & & 93.2 & $N$ & \\
\hline 2 & $10 / 10 / 93$ & 8 & 1 & & 88.34 & $\mathrm{~N}$ & \\
\hline 2 & $10 / 10 / 93$ & 9 & 1 & & 86.72 & $N$ & \\
\hline 2 & $10 / 10 / 93$ & 12 & 1 & & 87.62 & $\mathbf{N}$ & \\
\hline 1 & $10 / 19 / 93$ & 1 & 1 & & 113.4 & $\bar{Y}$ & TMACS \\
\hline 2 & $10 / 19 / 93$ & 1 & 1 & & 119.8 & $\bar{Y}$ & TMACS \\
\hline 1 & $10 / 19 / 93$ & 2 & 1 & & 110.3 & $\mathbf{N}$ & \\
\hline 2 & $10 / 19 / 93$ & 2 & 1 & & 116.2 & $\mathbf{N}$ & \\
\hline 1 & $10 / 19 / 93$ & 3 & 1 & & 106.7 & $N$ & \\
\hline 2 & $10 / 19 / 93$ & 3 & 1 & & 110.1 & $\mathbf{N}$ & \\
\hline 1 & $10 / 19 / 93$ & 4 & 1 & & 104 & $\mathbf{N}$ & \\
\hline 2 & $10 / 19 / 93$ & 4 & 1 & & 104.9 & $\mathbf{N}$ & \\
\hline 1 & $10 / 19 / 93$ & 5 & 1 & & 100.2 & $\mathbf{N}$ & \\
\hline 2 & $10 / 19 / 93$ & 5 & 1 & & 100.9 & $\mathbf{N}$ & \\
\hline 1 & $10 / 19 / 93$ & 6 & 1 & & 85.6 & $\mathbf{N}$ & \\
\hline 2 & $10 / 19 / 93$ & 6 & 1 & & 96.8 & $\mathbf{N}$ & \\
\hline 2 & $10 / 19 / 93$ & 7 & 1 & & 93.4 & $\mathbf{N}$ & \\
\hline 2 & $10 / 19 / 93$ & 8 & 1 & & 88.3 & $\mathbf{N}$ & \\
\hline 2 & $10 / 19 / 93$ & 9 & 1 & & 86.5 & $\mathbf{N}$ & \\
\hline 2 & $10 / 19 / 93$ & 12 & 1 & & 87.4 & $\mathbf{N}$ & \\
\hline 9 & $10 / 25 / 93$ & 1 & 1 & & 113.36 & $\bar{Y}$ & TMACS \\
\hline 2 & $10 / 25 / 93$ & 1 & 1 & & 120.2 & $Y$ & TMACS \\
\hline 1 & $10 / 25 / 93$ & 2 & 1 & & 110.12 & $\mathbf{N}$ & \\
\hline 2 & $10 / 25 / 93$ & 2 & 1 & & 116.78 & $\mathbf{N}$ & \\
\hline 1 & $10 / 25 / 93$ & 3 & 1 & & 106.7 & $\mathbf{N}$ & \\
\hline
\end{tabular}

Data obtained from WHC Surveillance Analysis Computer System (SACS), June 2, 1993. 


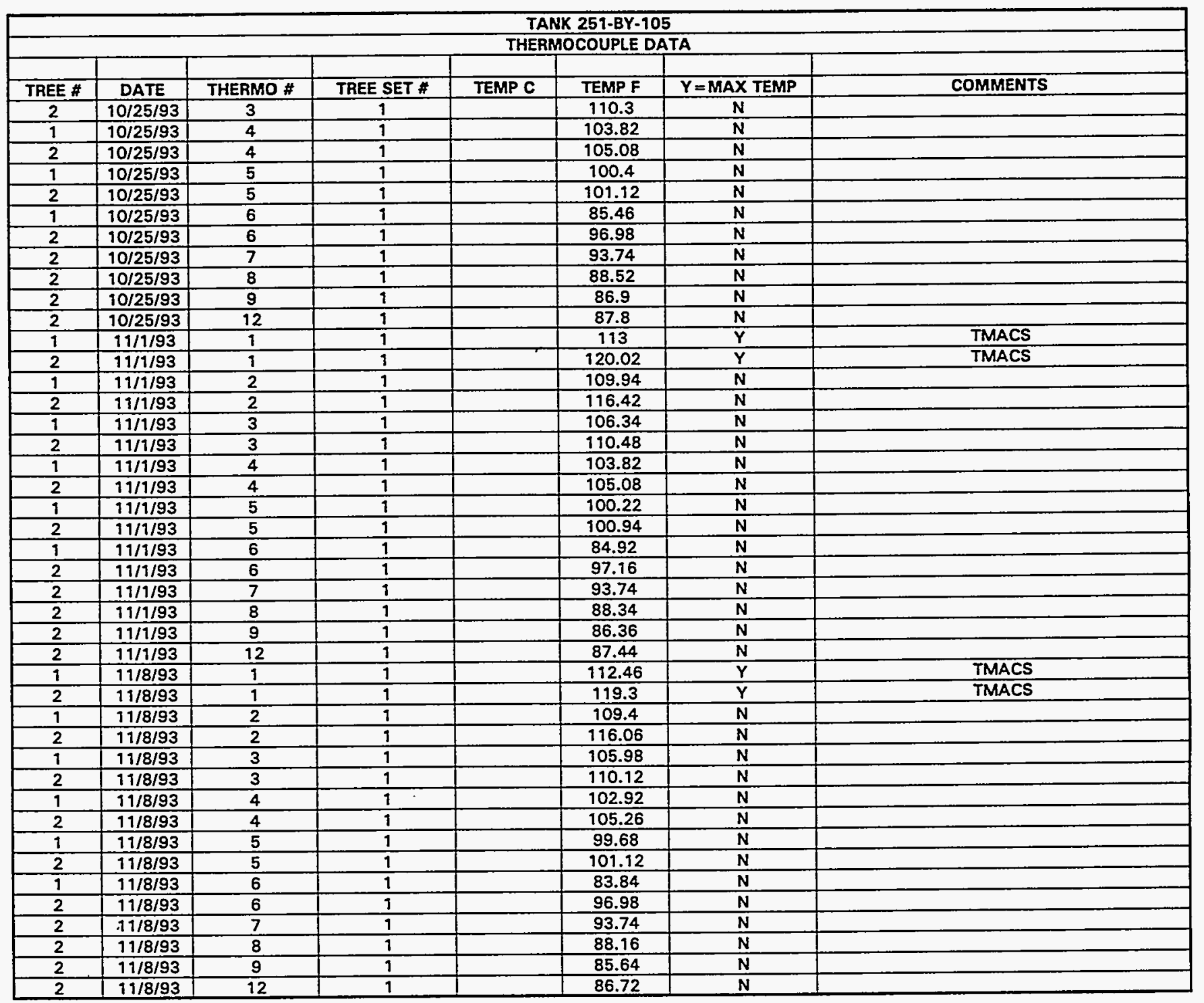

Data obtained from WHC Surveillance Analysis Computer System (SACS), June 2, 1993. 
WHC-SD-WM-ER-312, Rev. 0

\begin{tabular}{|c|c|c|c|c|c|c|c|}
\hline \multicolumn{8}{|c|}{ TANK 241-8Y-106 } \\
\hline \multicolumn{8}{|c|}{ THERMOCOUPLE DATA } \\
\hline TREE \# & DATE & THERMO \# & TREE SET \# & TEMP C & TEMP F & $Y=$ MAX TEMP & COMMENTS \\
\hline 1 & $11 / 12 / 73$ & 1 & & & 171 & & \\
\hline 1 & $11 / 12 / 73$ & 2 & & & 161 & & \\
\hline 1 & $11 / 12 / 73$ & 3 & & & 161 & & \\
\hline 1 & $11 / 12 / 73$ & 4 & & & 161 & & \\
\hline 1 & $11 / 12 / 73$ & 5 & & & 151 & & \\
\hline 1 & $11 / 12 / 73$ & 6 & & & 151 & & \\
\hline 1 & $11 / 12 / 73$ & 7 & & & 143 & & \\
\hline 1 & $11 / 12 / 73$ & 8 & & & 143 & & \\
\hline 1 & $11 / 12 / 73$ & 9 & & & 127 & & \\
\hline 1 & $11 / 12 / 73$ & 10 & & & 112 & & \\
\hline 1 & $11 / 12 / 73$ & 11 & & & 96 & & \\
\hline 1 & $11 / 12 / 73$ & 12 & & $\therefore$ & 80 & & \\
\hline 1 & $11 / 21 / 73$ & 1 & & 65 & 149 & & raw data - assumed degree C \\
\hline 1 & $11 / 21 / 73$ & 2 & & 63 & 145.4 & & raw data - assumed degree C \\
\hline 1 & $11 / 21 / 73$ & 3 & & 60 & 140 & & raw data - assumed degreo C \\
\hline 1 & $11 / 21 / 73$ & 4 & & 58 & 136.4 & & raw data - assumed degree C \\
\hline 1 & $11 / 21 / 73$ & 5 & & 57 & 134.6 & & raw data - assumed degree C \\
\hline 9 & $11 / 21 / 73$ & 6 & & 54 & 129.2 & & raw data - assumed degree C \\
\hline 1 & $11 / 21 / 73$ & 7 & & 51 & 123.8 & & raw data - assumed degree C \\
\hline 1 & $11 / 21 / 73$ & 8 & & 48 & 118.4 & & raw data - assumed degree C \\
\hline 1 & $11 / 21 / 73$ & 9 & & 42 & 107.6 & & raw data - assumed degree C \\
\hline 1 & $11 / 21 / 73$ & 10 & & 32 & 89.6 & & raw data - assumed degree C \\
\hline 1 & $11 / 21 / 73$ & 11 & & 32 & 89.6 & & raw data - assumed degree C \\
\hline 1 & $11 / 21 / 73$ & 12 & & 32 & 89.6 & & raw data - assumed degree C \\
\hline 1 & $12 / 10 / 73$ & 1 & & 67 & 152.6 & & raw data - assumed degreo C \\
\hline 1 & $12 / 10 / 73$ & 2 & & 67 & 152.6 & & raw data - assumed degree C \\
\hline 1 & $12 / 10 / 73$ & 3 & & 64 & 147.2 & & raw data - assumed degree C \\
\hline 1 & $12 / 10 / 73$ & 4 & & 62 & 143.6 & & raw data - assumed degree C \\
\hline 1 & $12 / 10 / 73$ & 5 & & 60 & 140 & & raw data - assumed degree C \\
\hline 1 & $12 / 10 / 73$ & 6 & & 57 & 134.6 & & raw data - assumed degree C \\
\hline 1 & $12 / 10 / 73$ & 7 & & 55 & 131 & & raw data - assumed degree C \\
\hline 1 & $12 / 10 / 73$ & 8 & & 52 & 125.6 & & raw data - assumed degree C \\
\hline 1 & $12 / 10 / 73$ & 9 & & 46 & 114.8 & & raw data - assumed degree C \\
\hline 1 & $12 / 10 / 73$ & 10 & & 32 & 89.6 & & raw data - assumed degree C \\
\hline 1 & $12 / 10 / 73$ & 11 & & 28 & 82.4 & & raw data - assumed degree C \\
\hline 1 & $12 / 10 / 73$ & 12 & & 20 & 68 & & raw data - assumed degree C \\
\hline 1 & $12 / 17 / 73$ & 1 & & 78 & 172.4 & & raw data - assumed degree C \\
\hline 1 & $12 / 17 / 73$ & 2 & & 76 & 168.8 & & raw data - assumed degree C \\
\hline 1 & $.12 / 17 / 73$ & 3 & & 73 & 163.4 & & raw data - assumed degree C \\
\hline 1 & $12 / 17 / 73$ & 4 & & 73 & 163.4 & & raw data - assumed degree C \\
\hline 1 & $12 / 17 / 73$ & 5 & & 71 & 159.8 & & raw data - assumed degree C \\
\hline 1 & $12 / 17 / 73$ & 6 & & 67 & 152.6 & & raw data - assumed degree C \\
\hline 1 & $12 / 17 / 73$ & 7 & & 65 & 149 & & raw data - assumed degree C \\
\hline 1 & $12 / 17 / 73$ & 8 & & 60 & 140 & & raw data - assumed degreo C \\
\hline 1 & $12 / 17 / 73$ & 9 & & 63 & 145.4 & & raw data - assumed degree C \\
\hline 1 & $12 / 17 / 73$ & 10 & & 46 & 114.8 & & raw data - assumed degree C \\
\hline 1 & $12 / 17 / 73$ & 11 & & 67 & 152.6 & & raw data - assumed degree C \\
\hline 1 & $12 / 17 / 73$ & 12 & & 29 & 84.2 & & raw data - assumed degres C \\
\hline 1 & $12 / 20 / 73$ & 1 & & 78 & 172.4 & & raw data - assumed degree C \\
\hline 1 & $12 / 20 / 73$ & 2 & & 76 & 168.8 & & raw data - assumed degree C \\
\hline 1 & $12 / 20 / 73$ & 3 & & 73 & 163.4 & & raw data - assumed degree C \\
\hline 1 & $12 / 20 / 73$ & 4 & & 72 & 161.6 & & raw data - assumed degreo C \\
\hline 1 & $12 / 20 / 73$ & 5 & & 70 & 158 & & raw data - assumed degreo C \\
\hline 1 & $12 / 20 / 73$ & 6 & & 69 & 156.2 & & raw data - assumed degree C \\
\hline 1 & $12 / 20 / 73$ & 7 & & 65 & 149 & & raw data - assumed degree C \\
\hline 1 & $12 / 20 / 73$ & 8 & & 61 & 141.8 & & raw data - assumed degree C \\
\hline 1 & $12 / 20 / 73$ & 9 & & 54 & 129.2 & & raw data - assumed degree C \\
\hline 1 & $12 / 20 / 73$ & 10 & & 46 & 194.8 & & raw data - assumed degree C \\
\hline 1 & $12 / 20 / 73$ & 11 & & 36 & 96.8 & & raw data - assumed degree C \\
\hline 1 & $12 / 20 / 73$ & 72 & & 28 & 82.4 & & raw data - assumed degree C \\
\hline 1 & $12 / 20 / 73$ & 15 & & 70 & 158 & & raw data - assumed degree C \\
\hline 1 & $8 / 5 / 74$ & 1 & & & & $\bar{Y}$ & 113 - suspect data (low) \\
\hline 1 & $8 / 5 / 74$ & 2 & & & & $\mathbf{N}$ & 114 - suspect data (low) \\
\hline 1 & $8 / 5 / 74$ & 3 & & & & $\bar{N}$ & 112 - suspect data (low) \\
\hline 1 & $8 / 5 / 74$ & 4 & & & & $\mathbf{N}$ & 108 - suspect data (low) \\
\hline 9 & $8 / 5 / 74$ & 5 & & & & $\mathrm{~N}$ & 100 - suspect data (low) \\
\hline
\end{tabular}

Data obtained from WHC Surveillance Analysis Computer System (SACS), June 2, 1993. 
WHC-SD-WM-ER-312, Rev. 0

\begin{tabular}{|c|c|c|c|c|c|c|c|}
\hline \multicolumn{8}{|c|}{ TANK 241-BY-106 } \\
\hline \multicolumn{8}{|c|}{ THEAMOCOUPLE DATA } \\
\hline & & & & TFMP C & TEMP F & $\bar{Y}=$ MAX TEMP & COMMENTS \\
\hline 1 & $8 / 5 / 74$ & 6 & & & & $\begin{array}{l}\mathbf{N} \\
N\end{array}$ & $\mid \frac{92 \text { - suspect data (low) }}{76 \text { - suspect data (low) }}$ \\
\hline 1 & $8 / 5 / 74$ & 7 & & & & $\mathrm{~N}$ & \\
\hline 1 & $8 / 5 / 74$ & 8 & & & & $\mathbf{N}$ & 78 - suspect data (low) \\
\hline 1 & $8 / 5 / 74$ & 9 & & & & $\mathbf{N}$ & 78 - suspect data (low) \\
\hline 1 & $8 / 5 / 74$ & 10 & & & & $\mathbf{N}$ & 75 - suspect data (low) \\
\hline 1 & $8 / 5 / 74$ & 11 & & & & $N$ & 66 - suspoct data (low) \\
\hline 1 & $8 / 5 / 74$ & 12 & & & & $\mathbf{N}$ & 54 - suspect data (low) \\
\hline 1 & $9 / 12 / 74$ & 1 & 1 & 83 & 181.4 & $\bar{Y}$ & degree C/TWINS confirm \\
\hline 1 & $9 / 12 / 74$ & 2 & 1 & 80 & 176 & $N$ & degree C/TWINS confirm \\
\hline 1 & $9 / 12 / 74$ & 3 & 1 & 74 & 165.2 & $\mathbf{N}$ & degree C/TWINS confirm \\
\hline 1 & $9 / 12 / 74$ & 4 & 1 & 68 & 154.4 & $\mathbf{N}$ & degree C/TWINS confirm \\
\hline 1 & $9 / 12 / 74$ & 5 & 1 & 64 & 147.2 & $\mathbf{N}$ & degree C/TWINS confirm \\
\hline 1 & $9 / 12 / 74$ & 6 & 1 & 62 & 143.6 & $\mathbf{N}$ & degree C/TWINS confirm \\
\hline 1 & $9 / 12 / 74$ & 7 & 1 & 60 & 140 & $\mathbf{N}$ & degree C/TWiNS confirm \\
\hline 1 & $9 / 12 / 74$ & 8 & 1 & 55 & 131 & $\mathbf{N}$ & degree C/TWINS confirm \\
\hline 1 & $9 / 12 / 74$ & 9 & 1 & 51 & 123.8 & $\mathbf{N}$ & degree C/TWINS confirm \\
\hline 1 & $9 / 12 / 74$ & 10 & 1 & 46 & 114.8 & $\mathbf{N}$ & degree C/TWINS confirm \\
\hline 1 & $9 / 12 / 74$ & 11 & 1 & 40 & 104 & $\mathbf{N}$ & degree C/TWINS confirm \\
\hline 1 & $9 / 12 / 74$ & 12 & 1 & 35 & 95 & $\mathbf{N}$ & degree C/TWINS confirm \\
\hline 1 & $10 / 8 / 74$ & 1 & 1 & 77 & 170.6 & $\mathbf{Y}$ & degree C/TWINS confirm \\
\hline 1 & $10 / 8 / 74$ & 2 & 1 & 73 & 163.4 & $\mathbf{N}$ & degree C/TWINS confirm \\
\hline 1 & $10 / 8 / 74$ & 3 & 1 & 68 & 154.4 & $\mathbf{N}$ & degree C/TWINS confirm \\
\hline 1 & $10 / 8 / 74$ & 4 & 1 & 63 & 145.4 & $\mathbf{N}$ & degree C/TWINS confirm \\
\hline 1 & $10 / 8 / 74$ & 5 & 1 & 58 & 136.4 & $\mathrm{~N}$ & degree C/TWINS confirm \\
\hline 1 & $10 / 8 / 74$ & 6 & 1 & 56 & 132.8 & $\mathbf{N}$ & degree C/TWINS confirm \\
\hline 1 & $10 / 8 / 74$ & 7 & 1 & 56 & 132.8 & $\mathbf{N}$ & degree $\mathrm{C} / \mathrm{TW}$ INS confirm \\
\hline 1 & $10 / 8 / 74$ & 8 & 1 & 51 & 123.8 & $\mathrm{~N}$ & degree C/TWINS confirm \\
\hline 1 & $10 / 8 / 74$ & 9 & 1 & 46 & 114.8 & $N$ & degree C/TWINS confirm \\
\hline 1 & $10 / 8 / 74$ & 10 & 1 & 42 & 107.6 & $\mathrm{~N}$ & degree C/TWINS confirm \\
\hline 1 & $10 / 8 / 74$ & 11 & 1 & 35 & 95 & $N$ & degree C/TWINS confirm \\
\hline 1 & $10 / 8 / 74$ & 12 & 1 & 28 & 82.4 & $\mathbf{N}$ & degree C/TWINS confirm \\
\hline 1 & $10 / 29 / 74$ & 1 & 1 & 74 & 165.2 & $\mathbf{Y}$ & degree C/TWINS confirm \\
\hline 1 & $10 / 29 / 74$ & 2 & 1 & 70 & 158 & $\mathrm{~N}$ & degree C/TWINS confirm \\
\hline 1 & $10 / 29 / 74$ & 3 & 1 & 65 & 149 & $\mathbf{N}$ & degree C/TWINS confirm \\
\hline 1 & $10 / 29 / 74$ & 4 & 1 & 59 & 138.2 & $\overline{\mathbf{N}}$ & degree C/TWINS confirm \\
\hline 9 & $10 / 29 / 74$ & 5 & 1 & 56 & 132.8 & $\mathbf{N}$ & degree C/TWINS confirm \\
\hline 1 & $10 / 29 / 74$ & 6 & 1 & 55 & 131 & $\mathbf{N}$ & degree C/TWINS confirm \\
\hline 1 & $10 / 29 / 74$ & 7 & 1 & 54 & 129.2 & $\mathbf{N}$ & degree C/TWINS confirm \\
\hline 1 & $10 / 29 / 74$ & 8 & 1 & 48 & 118.4 & $\mathbf{N}$ & degree C/TWINS confirm \\
\hline 1 & \begin{tabular}{|l|}
$10 / 29 / 74$ \\
\end{tabular} & 9 & 1 & 43 & 109.4 & $\mathbf{N}$ & degree C/TWINS confirm \\
\hline 1 & $10 / 29 / 74$ & 10 & 1 & 37 & 98.6 & $\mathbf{N}$ & degree $\mathrm{C} / \mathrm{TW}$ INS confirm \\
\hline 1 & $10 / 29 / 74$ & 19 & 1 & 30 & 86 & $\mathbf{N}$ & degree C/TWINS confirm \\
\hline 1 & $10 / 29 / 74$ & 12 & 1 & 22 & 71.6 & $\mathrm{~N}$ & degree C/TWINS confirm \\
\hline 1 & $19 / 12 / 74$ & 1 & 1 & 68 & 154.4 & $\mathbf{Y}$ & degree C/TWINS confirm \\
\hline 1 & $11 / 12 / 74$ & 2 & 1 & 65 & 149 & $\mathrm{~N}$ & degree C/TWINS confirm \\
\hline 1 & $11 / 12 / 74$ & 3 & 1 & 59 & 138.2 & $\mathbf{N}$ & degree $\mathrm{C} / \mathrm{TW}$ INS confirm \\
\hline 1 & $11 / 12 / 74$ & 4 & 1 & 54 & 129.2 & $\mathbf{N}$ & degree C/TWINS confirm \\
\hline 1 & $11 / 12 / 74$ & 5 & 1 & 49 & 120.2 & $\mathrm{~N}$ & degree $\mathrm{C} T$ TWINS confirm \\
\hline 1 & $11 / 12 / 74$ & 6 & 1 & 49 & 120.2 & $N$ & degree C/TWINS confirm \\
\hline 1 & $11 / 12 / 74$ & 7 & 1 & 47 & 116.6 & $\mathbf{N}$ & degree C/TWINS confirm \\
\hline 1 & $11 / 12 / 74$ & 8 & 1 & 42 & 107.6 & $\mathbf{N}$ & degree C/TWINS confirm \\
\hline 1 & $11 / 12 / 74$ & 9 & 1 & 36 & 96.8 & $\mathbf{N}$ & degree C/TWINS confirm \\
\hline 1 & $11 / 12 / 74$ & 10 & 1 & 39 & 87.8 & $\mathbf{N}$ & degree $\mathrm{C} / \mathrm{TWINS}$ confirm \\
\hline 1 & $11 / 12 / 74$ & 19 & 1 & 22 & 71.6 & $\mathrm{~N}$ & degree CrTWINS confirm \\
\hline 1 & $11 / 12 / 74$ & 12 & 1 & 13 & 55.4 & $\mathbf{N}$ & degree C/TWINS confirm \\
\hline 1 & $11 / 26 / 74$ & 1 & 1 & 76 & 168.8 & $\mathbf{Y}$ & degree C/TWiNS confirm \\
\hline 1 & $19 / 26 / 74$ & 2 & 1 & 72 & 161.6 & $\mathbf{N}$ & degree C/TWINS confirm \\
\hline 1 & $11 / 26 / 74$ & 3 & 1 & 66 & 150.8 & $\mathrm{~N}$ & degree C/TWINS confirm \\
\hline 1 & $11 / 26 / 74$ & 4 & 1 & 61 & 141.8 & $\mathbf{N}$ & degree CITWINS confirm \\
\hline 1 & $11 / 26 / 74$ & 5 & 1 & 57 & 134.6 & $N$ & degree C/TWINS confirm \\
\hline 1 & $11 / 26 / 74$ & 6 & 1 & 57 & 134.6 & $\mathrm{~N}$ & degree $\mathrm{C} / \mathrm{TWINS}$ confirm \\
\hline 1 & $11 / 26 / 74$ & 7 & 1 & 55 & 131 & $\mathrm{~N}$ & degree C/TWINS confirm \\
\hline 1 & $11 / 26 / 74$ & 8 & 1 & 50 & 122 & $\mathbf{N}$ & degree C/TWINS confirm \\
\hline 1 & $11 / 26 / 74$ & 9 & 1 & 43 & 109.4 & $\mathbf{N}$ & degree C/TWINS confirm \\
\hline 1 & $11 / 26 / 74$ & 10 & 1 & 38 & 100.4 & $\mathbf{N}$ & degree C/TWINS confirm \\
\hline 9 & $11 / 26 / 74$ & 11 & 1 & 29 & 84.2 & $\mathbf{N}$ & degree C/TWINS confirm \\
\hline
\end{tabular}

Data obtained from WHC Surveillance Analysis Computer System (SACS), June 2, 1993. 


\begin{tabular}{|c|c|c|c|c|c|c|c|}
\hline \multicolumn{8}{|c|}{$\begin{array}{l}\text { TANK 241-BY-106 } \\
\text { THERMOCOUPLE DATA }\end{array}$} \\
\hline & & & & THERA & COUPLE D & & \\
\hline TREE \# & DATE & THERMO \# & TREE SET \# & TEMP C & TEMP F & $Y=$ MAX TEMP & COMMENTS \\
\hline 1 & $11 / 26 / 74$ & 12 & 1 & 20 & 68 & $\mathbf{N}$ & degree C/TWINS confirm \\
\hline 1 & $12 / 17 / 74$ & 1 & 1 & 74.44 & 165.992 & $\mathrm{Y}$ & degree C/TWINS confirm \\
\hline 1 & $12 / 17 / 74$ & 2 & 1 & 71.11 & 159.998 & $N$ & degres C/TWINS confirm \\
\hline 1 & $12 / 17 / 74$ & 3 & 1 & 66.11 & 150.998 & $\mathbf{N}$ & degree C/TWINS confirm \\
\hline 1 & $12 / 17 / 74$ & 4 & 1 & 60.56 & 141.008 & $\mathbf{N}$ & degree C/TWINS confitm \\
\hline 1 & $12 / 17 / 74$ & 5 & 1 & 56.11 & 132.998 & $\bar{N}$ & degree C/TWINS confirm \\
\hline 1 & $12 / 17 / 74$ & 6 & 1 & 55.56 & 132.008 & $\mathbf{N}$ & degree C/TWINS confirm \\
\hline 1 & $12 / 17 / 74$ & 7 & 1 & 55 & 131 & $\mathbf{N}$ & degree C/TWINS confirm \\
\hline 1 & $12 / 17 / 74$ & 8 & 1 & 47.78 & 118.004 & $\mathbf{N}$ & degree $\mathrm{C} / \mathrm{TW}$ INS confirm \\
\hline 1 & $12 / 17 / 74$ & 9 & 1 & 41.11 & 105.998 & $\mathbf{N}$ & degree C/TWINS confirm \\
\hline 1 & $12 / 17 / 74$ & 10 & 1 & 35.56 & 96.008 & $\mathbf{N}$ & degree C/TWINS confirm \\
\hline 1 & $12 / 17 / 74$ & 11 & 1 & 25.56 . & 78.008 & $\mathbf{N}$ & degree C/TWINS confirm \\
\hline 1 & $12 / 17 / 74$ & 12 & 1 & 17.22 & 62.996 & $N$ & degree C/TWINS confirm \\
\hline 1 & $12 / 31 / 74$ & 1 & 1 & 74.44 & 165.992 & $\bar{Y}$ & degree C/TWINS confirm \\
\hline 1 & $12 / 31 / 74$ & 2 & 1 & 70.56 & 159.008 & $\mathbf{N}$ & degree C/TWINS confirm \\
\hline 1 & $12 / 31 / 74$ & 3 & 9 & 65 & 149 & $\bar{N}$ & degree C/TWINS confirm \\
\hline 1 & $12 / 31 / 74$ & 4 & 1 & 59.44 & 138.992 & $\mathbf{N}$ & degree $\mathrm{C} / \mathrm{TW}$ INS confirm \\
\hline 1 & $12 / 31 / 74$ & 5 & 1 & 55 & 131 & $\mathbf{N}$ & degree C/TWINS confirm \\
\hline 1 & $12 / 31 / 74$ & 6 & 1 & 55 & 131 & $\mathbf{N}$ & degrea $\mathrm{C} / \mathrm{TW}$ INS confirm \\
\hline 1 & $12 / 31 / 74$ & 7 & 1 & 52.78 & 127.004 & $\mathbf{N}$ & degree C/TWINS confirm \\
\hline 1 & $12 / 31 / 74$ & 8 & 1 & 46.67 & 116.006 & $\mathbf{N}$ & degree C/TWiNs confirm \\
\hline 1 & $12 / 31 / 74$ & 9 & 1 & 40.56 & 105.008 & $N$ & degree C/TWINS confirm \\
\hline 1 & $12 / 31 / 74$ & 10 & 1 & 33.33 & 91.994 & $\mathbf{N}$ & degree C/TWINS confirm \\
\hline 1 & $12 / 31 / 74$ & 11 & 1 & 23.89 & 75.002 & $\mathbf{N}$ & degree C/TWINS confirm \\
\hline 1 & $12 / 31 / 74$ & 12 & 1 & 15 & 59 & $\mathbf{N}$ & degree $\mathrm{C} / \mathrm{TW}$ INS confirm \\
\hline 1 & $1 / 8 / 75$ & 1 & 1 & 72.78 & 163.004 & $\bar{Y}$ & degree C/TWINS confirm \\
\hline 1 & $1 / 8 / 75$ & 2 & 1 & 68.89 & 156.002 & $\mathbf{N}$ & degree C/TWINS confirm \\
\hline 1 & $1 / 8 / 75$ & 3 & 1 & 64.44 & 147.992 & $\mathbf{N}$ & degree C/TWINS confirm \\
\hline 1 & $1 / 8 / 75$ & 4 & 1 & 58.89 & 138.002 & $\mathbf{N}$ & degree C/TWINS confirm \\
\hline 1 & $1 / 8 / 75$ & 5 & 1 & 53.89 & 129.002 & $\mathrm{~N}$ & degree $\mathrm{C} / \mathrm{TW}$ INS confirm \\
\hline 1 & $1 / 8 / 75$ & 6 & 1 & 53.89 & 129.002 & $\mathbf{N}$ & degree C/TWINS confirm \\
\hline 1 & $1 / 8 / 75$ & 7 & 1 & 53.89 & 129.002 & $N$ & degree C/TWINS confirm \\
\hline 1 & $1 / 8 / 75$ & 8 & 1 & 46.11 & 114.998 & $\mathbf{N}$ & degree C/TWINS confirm \\
\hline 1 & $1 / 8 / 75$ & 9 & 1 & 39.44 & 102.992 & $\mathbf{N}$ & degree C/TWINS confirm \\
\hline 1 & $1 / 8 / 75$ & 10 & 1 & 32.78 & 91.004 & $\mathbf{N}$ & degree C/TWINS confirm \\
\hline 1 & $1 / 8 / 75$ & 11 & 1 & 23.89 & 75.002 & $\mathbf{N}$ & degree C/TWINS confirm \\
\hline 1 & $1 / 8 / 75$ & 12 & 1 & 15.56 & 60.008 & $\mathbf{N}$ & degree C/TWINS confirm \\
\hline 1 & $1 / 21 / 75$ & 1 & 1 & 93 & 199.4 & $\bar{Y}$ & degree C/TWINS confirm \\
\hline 1 & $.1 / 21 / 75$ & 2 & 1 & 89 & 192.2 & $N$ & degree C/TWINS confirm \\
\hline 1 & $1 / 21 / 75$ & 3 & 1 & 83 & 181.4 & $\mathbf{N}$ & degree C/TWINS confirm \\
\hline 1 & $1 / 21 / 75$ & 4 & 1 & 77 & 170.6 & $\mathbf{N}$ & degree C/TWINS confirm \\
\hline 1 & $1 / 21 / 75$ & 5 & 1 & 72 & 161.6 & $\mathbf{N}$ & degree C/TWINS confirm \\
\hline 1 & $1 / 21 / 75$ & 6 & 1 & 70 & 158 & $\mathbf{N}$ & degree C/TWINS confirm \\
\hline 1 & $1 / 21 / 75$ & 7 & 1 & 68 & 154.4 & $\mathbf{N}$ & degree C/TWINS confirm \\
\hline 1 & $1 / 21 / 75$ & 8 & 1 & 60 & 140 & $\mathbf{N}$ & degree C/TWINS confirm \\
\hline 1 & $1 / 21 / 75$ & 9 & 1 & 50 & 122 & $\mathbf{N}$ & degree C/TWINS confirm \\
\hline 1 & $1 / 21 / 75$ & 10 & 1 & 43 & 109.4 & $\mathbf{N}$ & degree C/TWINS confirm \\
\hline 1 & $1 / 21 / 75$ & 11 & 1 & 31 & 87.8 & $\mathbf{N}$ & degree C/TWINS confirm \\
\hline 1 & $1 / 21 / 75$ & 12 & 1 & 20 & 68 & $\mathbf{N}$ & degree C/TWINS confirm \\
\hline 1 & $1 / 28 / 75$ & 1 & 1 & 76 & 168.8 & $\mathbf{Y}$ & degree C/TWINS confirm \\
\hline 1 & $1 / 28 / 75$ & 2 & 1 & 72 & 161.6 & $\mathbf{N}$ & degree CrTWINS confirm \\
\hline 1 & $1 / 28 / 75$ & 3 & 1 & 67 & 152.6 & $\mathbf{N}$ & degrae C/TWINS confirm \\
\hline 1 & $1 / 28 / 75$ & 4 & 1 & 62 & 143.6 & $\mathbf{N}$ & degree C/TWINS confirm \\
\hline 1 & $1 / 28 / 75$ & 5 & 1 & 58 & 136.4 & $\mathbf{N}$ & degree C/TWINS confirm \\
\hline 1 & $1 / 28 / 75$ & 6 & 1 & 57 & 134.6 & $\mathbf{N}$ & degree C/TWINS confirm \\
\hline 1 & $1 / 28 / 75$ & 7 & 1 & 55 & 131 & $N$ & degree C/TWINS confirm \\
\hline 1 & $1 / 28 / 75$ & 8 & 1 & 48 & 118.4 & $\mathbf{N}$ & degree C/TWINS confirm \\
\hline 1 & $1 / 28 / 75$ & 9 & 1 & 40 & 104 & $\mathrm{~N}$ & degree C/TWINS confirm \\
\hline 1 & $1 / 28 / 75$ & 10 & 1 & 35 & 95 & $\mathbf{N}$ & degree C/TWINS confirm \\
\hline 1 & $1 / 28 / 75$ & 11 & 1 & 24 & 75.2 & $N$ & degree $\mathrm{C} / \mathrm{TW}$ INS confirm \\
\hline 1 & $1 / 28 / 75$ & 12 & 1 & 14 & 57.2 & $\mathbf{N}$ & degree $\mathrm{C} / \mathrm{TWW}$ INS confirm \\
\hline 1 & $2 / 27 / 75$ & 1 & 1 & 76 & 168.8 & $\bar{Y}$ & degree $\mathrm{C} / \mathrm{TW}$ INS confirm \\
\hline 1 & $2 / 27 / 75$ & 2 & 1 & 74 & 165.2 & $\mathbf{N}$ & degree C/TWINS confirm \\
\hline 1 & $2 / 27 / 75$ & 3 & 1 & 70 & 158 & $\mathrm{~N}$ & degree $\mathrm{C} / \mathrm{TW}$ WNS confirm \\
\hline 1 & $2 / 27 / 75$ & 4 & 1 & 63 & 145.4 & $\mathbf{N}$ & degree C/TWINS confirm \\
\hline 1 & $2 / 27 / 75$ & 5 & 1 & 58 & 136.4 & $\mathbf{N}$ & degree C/TWINS confirm \\
\hline
\end{tabular}

Data obtained from WHC Surveillance Analysis Computer System (SACS), June 2, 1993. 


\begin{tabular}{|c|c|c|c|c|c|c|c|}
\hline \multicolumn{8}{|c|}{ TANK 241-BY-106 } \\
\hline \multicolumn{8}{|c|}{ THERMOCOUPLE DATA } \\
\hline & & & & & & & \\
\hline TREE \# & DATE & THERMO \# & TREE SET \# & TEMP C & TEMP F & $Y=M A X$ TEMP & COMMENTS \\
\hline 1 & $2 / 27 / 75$ & 6 & 1 & 58 & 136.4 & $\mathbf{N}$ & degree C/TWINS confirm \\
\hline 1 & $2 / 27 / 75$ & 7 & 1 & 58 & 136.4 & $\mathbf{N}$ & degree C/TWINS confirm \\
\hline 1 & $2 / 27 / 75$ & 8 & 1 & 48 & 118.4 & $\mathbf{N}$ & degree C/TWINS confirm \\
\hline 1 & $2 / 27 / 75$ & 9 & 1 & 40 & 104 & $\mathbf{N}$ & degree C/TWINS confirm \\
\hline 1 & $2 / 27 / 75$ & 10 & 1 & 34 & 93.2 & $\mathbf{N}$ & degree C/TWINS confirm \\
\hline 1 & $2 / 27 / 75$ & 11 & 1 & 24 & 75.2 & $\mathbf{N}$ & degree C/TWINS confirm \\
\hline 1 & $2 / 27 / 75$ & 12 & 1 & 16 & 60.8 & $\mathbf{N}$ & degree C/TWINS confirm \\
\hline 9 & $3 / 18 / 75$ & 1 & 1 & 78 & 172.4 & $Y$ & degree C/TWINS confirm \\
\hline 1 & $3 / 18 / 75$ & 2 & 1 & 76 & 168.8 & $\mathbf{N}$ & degree C/TWINS confirm \\
\hline 1 & $3 / 18 / 75$ & 3 & 1 & 70 & 158 & $\mathbf{N}$ & degree C/TWINS confirm \\
\hline 1 & $3 / 18 / 75$ & 4 & 1 & 66 & 150.8 & $\mathbf{N}$ & degree C/TWINS confirm \\
\hline 1 & $3 / 18 / 75$ & 5 & 1 & 60 & 140 & $\mathbf{N}$ & degree C/TWINS confirm \\
\hline 1 & $3 / 18 / 75$ & 6 & 1 & 58 & 136.4 & $\mathbf{N}$ & degree C/TWINS confirm \\
\hline 1 & $3 / 18 / 75$ & 7 & 1 & 58 & 136.4 & $\mathbf{N}$ & degree C/TWINS confirm \\
\hline 1 & $3 / 18 / 75$ & 8 & 1 & 50 & 122 & $\mathbf{N}$ & degree CTTWINS confirm \\
\hline 1 & $3 / 18 / 75$ & 9 & 1 & 42 & 107.6 & $\mathrm{~N}$ & degree C/TWINS confirm \\
\hline 1 & $3 / 18 / 75$ & 10 & 1 & 38 & 100.4 & $\bar{N}$ & degree C/TWINS confirm \\
\hline 1 & $3 / 18 / 75$ & 11 & 1 & 26 & 78.8 & $N$ & degree C/TWiNS confirm \\
\hline 1 & $3 / 18 / 75$ & 12 & 1 & 18 & 64.4 & $\mathbf{N}$ & degree C/TWINS confirm \\
\hline 1 & $4 / 1 / 75$ & 1 & 1 & 83.33 & 181.994 & $Y$ & degree C/TWINS confirm \\
\hline 1 & $4 / 1 / 75$ & 2 & 1 & 72.22 & 161.996 & $\mathbf{N}$ & degree C/TWINS confirm \\
\hline 1 & $4 / 1 / 75$ & 3 & 1 & 67.22 & 152.996 & $N$ & degree C/TWINS confirm \\
\hline 1 & $4 / 1 / 75$ & 4 & 1 & 61.11 & 141.998 & $\mathbf{N}$ & degree C/TWINS confirm \\
\hline 1 & $4 / 1 / 75$ & 5 & 1 & 56.11 & 132.998 & $N$ & degree C/TWINS confirm \\
\hline 1 & $4 / 1 / 75$ & 6 & 1 & 56.11 & 132.998 & $\mathbf{N}$ & degree $\mathrm{C} / \mathrm{TW}$ INS confirm \\
\hline 1 & $4 / 1 / 75$ & 7 & 1 & 52.22 & 125.996 & $\mathbf{N}$ & degres C/TWINS confirm \\
\hline 1 & $4 / 8 / 75$ & 1 & 1 & 91 & 195.8 & $\bar{Y}$ & degree C/TWINS confirm \\
\hline 1 & $4 / 8 / 75$ & 2 & 1 & 85 & 185 & $\mathbf{N}$ & degree C/TWINS confirm \\
\hline 1 & $4 / 8 / 75$ & 3 & 1 & 80 & 176 & $\mathbf{N}$ & degree C/TWINS confirm \\
\hline 1 & $4 / 8 / 75$ & 4 & 1 & 73 & 163.4 & $\mathbf{N}$ & degree C/TWINS confirm \\
\hline 1 & $4 / 8 / 75$ & 5 & 1 & 66 & 150.8 & $\mathbf{N}$ & degree C/TWINS confirm \\
\hline 1 & $4 / 8 / 75$ & 6 & 1 & 66 & 150.8 & $\bar{N}$ & degree C/TWINS confirm \\
\hline 1 & $4 / 8 / 75$ & 7 & 1 & 62 & 143.6 & $\mathbf{N}$ & degree C/TWINS confirm \\
\hline 1 & $4 / 8 / 75$ & 8 & 1 & 52 & 125.6 & $\mathbf{N}$ & degree C/TWINS confirm \\
\hline 1 & $4 / 8 / 75$ & 9 & 1 & 43 & 109.4 & $\mathbf{N}$ & degree C/TWINS confirm \\
\hline 1 & $4 / 8 / 75$ & 10 & 1 & 35 & 95 & $\bar{N}$ & degree C/TWINS confirm \\
\hline 1 & $4 / 8 / 75$ & 11. & 1 & 26 & 78.8 & $\mathbf{N}$ & degree $\mathrm{C} / \mathrm{TW}$ INS confirm \\
\hline 1 & $4 / 8 / 75$ & 12 & 1 & 17 & 62.6 & $\mathrm{~N}$ & degree C/TWINS confirm \\
\hline 1 & $.4 / 22 / 75$ & 1 & 1 & 74 & 165.2 & $\mathrm{Y}$ & degree C/TWINS confirm \\
\hline 1 & $4 / 22 / 75$ & 2 & 1 & 72 & 161.6 & $\mathbf{N}$ & degree CrTWINS confirm \\
\hline 1 & $4 / 22 / 75$ & 3 & 1 & 66 & 150.8 & $\mathrm{~N}$ & degres C/TWINS confirm \\
\hline 1 & $4 / 22 / 75$ & 4 & 1 & 60 & 140 & $\mathbf{N}$ & degree C/TWINS confirm \\
\hline 1 & $4 / 22 / 75$ & 5 & 1 & 56 & 132.8 & $\mathbf{N}$ & degree $\mathrm{C} / \mathrm{T}$ WINS confirm \\
\hline 1 & $4 / 22 / 75$ & 6 & 1 & 56 & 132.8 & $N$ & degree C/TWINS confirm \\
\hline 1 & $4 / 22 / 75$ & 7 & 9 & 52 & 125.6 & $\mathbf{N}$ & degree C/TWINS confirm \\
\hline 1 & $4 / 22 / 75$ & 8 & 1 & 45 & 113 & $\mathbf{N}$ & degree C/TWINS confirm \\
\hline 1 & $4 / 22 / 75$ & 9 & 1 & 36 & 96.8 & $\mathrm{~N}$ & degree C/TWiNS confirm \\
\hline 1 & $4 / 22 / 75$ & 10 & 1 & 30 & 86 & $\mathrm{~N}$ & degree CrTWINS confirm \\
\hline 9 & $4 / 22 / 75$ & 11 & 1 & 24 & 75.2 & $\mathbf{N}$ & degree C/TWINS confirm \\
\hline 1 & $4 / 22 / 75$ & 12 & 1 & 18 & 64.4 & $\mathbf{N}$ & degrae C/TWINS confirm \\
\hline 1 & $4 / 29 / 75$ & 1 & 1 & 73 & 163.4 & $Y$ & degree C/TWINS confirm \\
\hline 1 & $4 / 29 / 75$ & 2 & 1 & 73 & 163.4 & $\mathbf{N}$ & degree $\mathrm{C} / \mathrm{TW}$ INS confirm \\
\hline 1 & $4 / 29 / 75$ & 3 & 1 & 64 & 147.2 & $\mathbf{N}$ & degree $\mathrm{C} / \mathrm{TWW}$ INS confirm \\
\hline 1 & $4 / 29 / 75$ & 4 & 1 & 60 & 140 & $\mathbf{N}$ & degres C/TWINS confirm \\
\hline 1 & $4 / 29 / 75$ & 5 & 1 & 56 & 132.8 & $\mathbf{N}$ & degree C/TWINS confirm \\
\hline 1 & $4 / 29 / 75$ & 6 & 1 & 56 & 132.8 & $\mathbf{N}$ & degree C/TWINS confirm \\
\hline 1 & $4 / 29 / 75$ & 7 & 1 & 52 & 125.6 & $\bar{N}$ & degree C/TWINS confirm \\
\hline 1 & $4 / 29 / 75$ & 8 & 1 & 22 & 71.6 & $N$ & degree C/TWINS confirm \\
\hline 1 & $4 / 29 / 75$ & 9 & 1 & 32 & 89.6 & $\mathbf{N}$ & degree C/TWINS confirm \\
\hline 1 & $4 / 29 / 75$ & 10 & 1 & 32 & 89.6 & $\mathbf{N}$ & degree C/TWINS confirm \\
\hline 1 & $4 / 29 / 75$ & 91 & 1 & 24 & 75.2 & $\mathbf{N}$ & degree C/TWINS confirm \\
\hline 1 & $4 / 29 / 75$ & 12 & 1 & 18 & 64.4 & $N$ & degree C/TWINS confirm \\
\hline 1 & $5 / 20 / 75$ & 1 & 1 & 76 & 168.8 & $\mathrm{Y}$ & degree C/TWINS confirm \\
\hline 1 & $5 / 20 / 75$ & 2 & 1 & 74 & 165.2 & $\mathbf{N}$ & degree C/TWINS confirm \\
\hline 1 & $5 / 20 / 75$ & 3 & 1 & 68 & 154.4 & $\mathbf{N}$ & degree C/TWINS confirm \\
\hline 1 & $5 / 20 / 75$ & 4 & 1 & 68 & 154.4 & $\mathbf{N}$ & degree $\mathrm{C} / \mathrm{TWW}$ INS confirm \\
\hline
\end{tabular}

Data obtained from WHC Surveillance Analysis Computer System (SACS), June 2, 1993. 


\begin{tabular}{|c|c|c|c|c|c|c|c|}
\hline \multicolumn{8}{|c|}{ TANK 241-BY-106 } \\
\hline \multicolumn{8}{|c|}{ THERMOCOUPLE DATA } \\
\hline TREE \# & DATE & THERMO \# & TREE SET \# & TEMP C & TEMP F & $Y=$ MAX TEMP & COMMENTS \\
\hline 1 & $5 / 20 / 75$ & 5 & 1 & 58 & 136.4 & $\mathbf{N}$ & degree C/TWINS confirm \\
\hline 1 & $5 / 20 / 75$ & 6 & 1 & 58 & 136.4 & $\mathbf{N}$ & degree C/TWINS confirm \\
\hline 1 & $5 / 20 / 75$ & 7 & 1 & 52 & 125.6 & $\mathbf{N}$ & degree CITWINS confirm \\
\hline 1 & $5 / 20 / 75$ & 8 & 1 & 46 & 114.8 & $\mathbf{N}$ & degree C/TWINS confirm \\
\hline 1 & $5 / 20 / 75$ & 9 & 1 & 38 & 100.4 & $N$ & degree C/TWINS confirm \\
\hline$\frac{1}{1}$ & $5 / 20 / 75$ & 10 & 9 & 32 & 89.6 & $\mathrm{~N}$ & degree C/TWINS confirm \\
\hline 1 & $5 / 20 / 75$ & 11 & 1 & 28 & 82.4 & $\mathbf{N}$ & degree $\mathrm{C} / \mathrm{TW}$ WNS confirm \\
\hline 1 & $5 / 20 / 75$ & 12 & 1 & 22 & 71.6 & $\mathbf{N}$ & degree C $/$ TWINS confirm \\
\hline 1 & $6 / 17 / 75$ & 1 & 1 & & 166 & $\bar{Y}$ & \\
\hline 1 & $6 / 17 / 75$ & 2 & 1 & & 160 & $\mathbf{N}$ & \\
\hline 1 & $6 / 17 / 75$ & 3 & 1 & & 153 & $\mathbf{N}$ & \\
\hline 1 & $6 / 17 / 75$ & 4 & 1 & & 141 & N & \\
\hline 1 & $6 / 17 / 75$ & 5 & 1 & & 132 & $N$ & \\
\hline 1 & $6 / 17 / 75$ & 6 & 1 & & 132 & $\mathbf{N}$ & \\
\hline 1 & $6 / 17 / 75$ & 7 & 1 & & 124 & $\mathbf{N}$ & \\
\hline 1 & $6 / 17 / 75$ & 8 & 1 & & 110 & $N$ & \\
\hline 1 & $6 / 17 / 75$ & 9 & 1 & & 97 & $\mathbf{N}$ & \\
\hline 1 & $6 / 17 / 75$ & 10 & 1 & & 90 & $N$ & \\
\hline 1 & $6 / 17 / 75$ & 11 & 1 & & 81 & $\mathbf{N}$ & \\
\hline 1 & $6 / 17 / 75$ & 12 & 1 & & 75 & $N$ & \\
\hline 1 & $7 / 28 / 75$ & 1 & 1 & & 167 & $\bar{Y}$ & \\
\hline 1 & $7 / 28 / 75$ & 2 & 1 & & 160 & $N$ & \\
\hline 1 & $7 / 28 / 75$ & 3 & 1 & & 152 & N & \\
\hline 1 & $7 / 28 / 75$ & 4 & 1 & & 142 & $\mathbf{N}$ & \\
\hline 1 & $7 / 28 / 75$ & 5 & 1 & & 133 & $\mathrm{~N}$ & \\
\hline$T$ & $7 / 28 / 75$ & 6 & 1 & & 133 & $\bar{N}$ & \\
\hline 1 & $7 / 28 / 75$ & 7 & 1 & & 127 & $\bar{N}$ & \\
\hline 1 & $7 / 28 / 75$ & 8 & 1 & & 114 & $\mathrm{~N}$ & \\
\hline$T$ & $7 / 28 / 75$ & 9 & 1 & & 104 & N & \\
\hline 1 & $7 / 28 / 75$ & 10 & 1 & & 95 & $N$ & \\
\hline 1 & $7 / 28 / 75$ & 11 & 1 & & 90 & $\mathbf{N}$ & \\
\hline 1 & $7 / 28 / 75$ & 12 & 1 & & 87 & $\mathbf{N}$ & \\
\hline 1 & $8 / 26 / 75$ & 1 & 1 & & 170 & $Y$ & \\
\hline 1 & $8 / 26 / 75$ & 2 & 1 & & 164 & $\mathbf{N}$ & \\
\hline 1 & $8 / 26 / 75$ & 3 & 1 & & 151 & $\mathrm{~N}$ & \\
\hline 1 & $8 / 26 / 75$ & 4 & 1 & & 146 & $\mathbf{N}$ & \\
\hline 1 & $8 / 26 / 75$ & 5. & 1 & & 138 & $\mathbf{N}$ & \\
\hline 1 & $8 / 26 / 75$ & 6 & 1 & & 137 & $\mathbf{N}$ & \\
\hline 9 & $.8 / 26 / 75$ & 7 & 1 & & 130 & $\mathbf{N}$ & \\
\hline 1 & $8 / 26 / 75$ & 8 & 9 & & 118 & $\mathbf{N}$ & \\
\hline 1 & $8 / 26 / 75$ & 9 & 1 & & 108 & $\bar{N}$ & \\
\hline 1 & $8 / 26 / 75$ & 10 & 1 & & 98 & $\mathbf{N}$ & \\
\hline 1 & $8 / 26 / 75$ & 11 & 1 & & 91 & $\mathbf{N}$ & \\
\hline 1 & $8 / 26 / 75$ & 12 & 1 & & 85 & $\mathbf{N}$ & \\
\hline 1 & $11 / 21 / 75$ & 1 & 1 & & 174 & $\bar{Y}$ & \\
\hline 1 & $11 / 21 / 75$ & 2 & 1 & & 166 & $\mathbf{N}$ & \\
\hline 1 & $11 / 21 / 75$ & 3 & 1 & & 157 & $\mathbf{N}$ & \\
\hline 1 & $11 / 21 / 75$ & 4 & 1 & & 148 & $\mathbf{N}$ & \\
\hline 1 & $11 / 21 / 75$ & 5 & 1 & & 141 & $\mathbf{N}$ & \\
\hline 1 & $11 / 21 / 75$ & 6 & 1 & & 140 & $\mathbf{N}$ & \\
\hline 1 & $11 / 21 / 75$ & 7 & 1 & & 131 & $\mathbf{N}$ & \\
\hline 1 & $11 / 21 / 75$ & 8 & 1 & & 120 & $\mathbf{N}$ & \\
\hline 1 & $11 / 21 / 75$ & 9 & 1 & & 105 & $\mathbf{N}$ & \\
\hline 1 & $11 / 21 / 75$ & 10 & 1 & & 94 & $\mathbf{N}$ & \\
\hline 1 & $11 / 21 / 75$ & 11 & 1 & & 84 & $\mathbf{N}$ & \\
\hline 1 & $11 / 21 / 75$ & 12 & 1 & & 73 & $\mathbf{N}$ & \\
\hline 1 & $3 / 4 / 76$ & 1 & 1 & & 163 & $Y$ & \\
\hline 1 & $3 / 4 / 76$ & 2 & 1 & & 159 & $\bar{N}$ & \\
\hline 1 & $3 / 4 / 76$ & 4 & 1 & & 141 & $N$ & \\
\hline 1 & $3 / 4 / 76$ & 5 & 1 & & 132 & $N$ & \\
\hline 1 & $3 / 4 / 76$ & 6 & 1 & & 132 & $\mathbf{N}$ & \\
\hline 1 & $3 / 4 / 76$ & 7 & 1 & & 119 & $\mathbf{N}$ & \\
\hline 1 & $3 / 4 / 76$ & 8 & 1 & & 104 & $\mathbf{N}$ & \\
\hline 1 & $3 / 4 / 76$ & 9 & 1 & & 86 & $\mathrm{~N}$ & \\
\hline 1 & $3 / 4 / 76$ & 10 & 7 & & 76 & $\mathbf{N}$ & \\
\hline 1 & $3 / 4 / 76$ & 11 & 1 & & 65 & $\mathbf{N}$ & \\
\hline
\end{tabular}

Data obtained from WHC Surveillance Analysis Computer System (SACS), June 2, 1993. 


\begin{tabular}{|c|c|c|c|c|c|c|c|}
\hline \multicolumn{8}{|c|}{ TANK 241-BY-106 } \\
\hline \multicolumn{8}{|c|}{ THERMOCOUPLE DATA } \\
\hline TREE \# & DATE & THERMO \# & TREE SET \# & TEMP C & TEMP F & $\bar{Y}=$ MAX TEMP & COMMENTS \\
\hline 1 & $3 / 4 / 76$ & 12 & 1 & & 58 & $N$ & \\
\hline 1 & $5 / 7 / 76$ & 1 & 1 & & 159 & $\bar{Y}$ & \\
\hline 9 & $5 / 7 / 76$ & 2 & 1 & & 159 & $\mathbf{N}$ & \\
\hline 1 & $5 / 7 / 76$ & 4 & 1 & & 135 & $N$ & \\
\hline 1 & $5 / 7 / 76$ & 5 & 1 & & 126 & $\mathbf{N}$ & \\
\hline 1 & $5 / 7 / 76$ & 6 & 1 & & 126 & $\mathbf{N}$ & \\
\hline 1 & $5 / 7 / 76$ & 7 & 1 & & 113 & $\mathbf{N}$ & \\
\hline 1 & $5 / 7 / 76$ & 8 & 1 & & 97 & $\overline{\mathbf{N}}$ & \\
\hline 9 & $5 / 7 / 76$ & 9 & 1 & & 82 & $\mathbf{N}$ & \\
\hline 1 & $5 / 7 / 76$ & 10 & 1 & & 74 & $\mathbf{N}$ & \\
\hline 1 & $5 / 7 / 76$ & 11 & 1 & & 66 & $\overline{\mathbf{N}}$ & \\
\hline 1 & $5 / 7 / 76$ & 12 & 1 & $\therefore$ & 63 & $\mathbf{N}$ & \\
\hline 9 & $6 / 9 / 76$ & 1 & 9 & & 167 & $\bar{Y}$ & \\
\hline 1 & $6 / 9 / 76$ & 2 & 1 & & 163 & $\bar{N}$ & \\
\hline 1 & $6 / 9 / 76$ & 3 & 1 & & 155 & $\mathbf{N}$ & \\
\hline 1 & $6 / 9 / 76$ & 4 & 1 & & 145 & $\overline{\mathbf{N}}$ & \\
\hline 1 & $6 / 9 / 76$ & 5 & 1 & & 137 & $\bar{N}$ & \\
\hline 1 & $6 / 9 / 76$ & 6 & 1 & & 138 & $\mathbf{N}$ & \\
\hline 9 & $6 / 9 / 76$ & 7 & 1 & & 125 & $\bar{N}$ & \\
\hline 1 & $6 / 9 / 76$ & 8 & 1 & & 110 & $\overline{\mathrm{N}}$ & \\
\hline 1 & $6 / 9 / 76$ & 9 & 1 & & 96 & $\mathbf{N}$ & \\
\hline 1 & $6 / 9 / 76$ & 10 & 9 & & 88 & $\mathbf{N}$ & \\
\hline 1 & $6 / 9 / 76$ & 11 & 1 & & 82 & $\mathbf{N}$ & \\
\hline 1 & $6 / 9 / 76$ & 12 & 1 & & 78 & $\mathbf{N}$ & \\
\hline 9 & $7 / 7 / 76$ & 1 & 1 & & 156 & $\bar{Y}$ & \\
\hline 1 & $7 / 7 / 76$ & 3 & 1 & & 143 & $\mathbf{N}$ & \\
\hline 1 & $7 / 7 / 76$ & 4 & 1 & & 132 & $\mathbf{N}$ & \\
\hline 9 & $7 / 7 / 76$ & 5 & 1 & & 126 & $\bar{N}$ & \\
\hline 1 & $7 / 7 / 76$ & 6 & 1 & & 126 & $\mathbf{N}$ & \\
\hline 1 & $7 / 7 / 76$ & 7 & 1 & & 113 & $\mathbf{N}$ & \\
\hline 9 & $7 / 7 / 76$ & 8 & 1 & & 99 & $\mathbf{N}$ & \\
\hline 1 & $7 / 7 / 76$ & 9 & 1 & & 87 & $\mathbf{N}$ & \\
\hline 1 & $7 / 7 / 76$ & 10 & 1 & & 80 & $\mathbf{N}$ & \\
\hline 1 & 717176 & 19 & 1 & & 75 & $\mathbf{N}$ & \\
\hline 1 & $7 / 7 / 76$ & 12 & 1 & & 72 & $\mathrm{~N}$ & \\
\hline 1 & $8 / 14 / 76$ & 1 & 1 & & 151 & $\mathrm{Y}$ & \\
\hline 1 & $8 / 14 / 76$ & 2 & 1 & & 945 & $\bar{N}$ & \\
\hline 1 & $8 / 14 / 76$ & 3 & 1 & & 136 & $\mathbf{N}$ & \\
\hline 1 & $8 / 14 / 76$ & 4 & 1 & & 126 & $\mathbf{N}$ & \\
\hline 1 & $8 / 14 / 76$ & 5 & 1 & & 121 & $\mathbf{N}$ & \\
\hline 1 & $8 / 14 / 76$ & 6 & 1 & & 120 & $\mathbf{N}$ & \\
\hline 1 & $8 / 14 / 76$ & 7 & 1 & & 109 & $\mathbf{N}$ & \\
\hline 1 & $8 / 14 / 76$ & 8 & 1 & & 96 & $\mathrm{~N}$ & \\
\hline 1 & $8 / 74 / 76$ & 9 & 1 & & 86 & $\mathbf{N}$ & \\
\hline 1 & $8 / 14 / 76$ & 10 & 1 & & 78 & $\mathbf{N}$ & \\
\hline 1 & $8 / 14 / 76$ & 11 & 1 & & 74 & $\mathbf{N}$ & \\
\hline 1 & $8 / 74 / 76$ & 12 & 1 & & 71 & $\mathbf{N}$ & \\
\hline 1 & $8 / 28 / 76$ & 1 & 1 & & 157 & $\bar{Y}$ & \\
\hline 1 & $8 / 28 / 76$ & 2 & 1 & & 150 & $\mathbf{N}$ & \\
\hline 1 & $8 / 28 / 76$ & 3 & 1 & & 142 & $\mathbf{N}$ & \\
\hline 1 & $8 / 28 / 76$ & 4 & 1 & & 132 & $\mathbf{N}$ & \\
\hline 1 & $8 / 28 / 76$ & 5 & 1 & & 127 & $\mathbf{N}$ & \\
\hline 1 & $8 / 28 / 76$ & 6 & 1 & & 126 & $\mathbf{N}$ & \\
\hline 1 & $8 / 28 / 76$ & 7 & $\frac{1}{1}$ & & 114 & $\mathbf{N}$ & \\
\hline 1 & $8 / 28 / 76$ & 8 & 1 & & 102 & $\mathbf{N}$ & \\
\hline 1 & $8 / 28 / 76$ & 9 & 1 & & 89 & $\mathbf{N}$ & \\
\hline 1 & $8 / 28 / 76$ & 10 & 1 & & 81 & $\mathbf{N}$ & \\
\hline 1 & $8 / 28 / 76$ & 11 & 1 & & 79 & $\mathbf{N}$ & \\
\hline 1 & $8 / 28 / 76$ & 12 & 1 & & 77 & $\mathbf{N}$ & \\
\hline 1 & $9 / 13 / 76$ & 1 & 1 & & 158 & $Y$ & \\
\hline 1 & $9 / 13 / 76$ & 2 & 1 & & 152 & $N$ & \\
\hline 9 & $9 / 13 / 76$ & 3 & 1 & & 144 & $\mathrm{~N}$ & \\
\hline 1 & $9 / 13 / 76$ & 4 & 1 & & 134 & $N$ & \\
\hline 1 & $9 / 13 / 76$ & 5 & 1 & & 128 & $\mathbf{N}$ & \\
\hline 1 & $9 / 13 / 76$ & 6 & 1 & & 126 & $N$ & \\
\hline 1 & $9 / 13 / 76$ & 7 & 1 & & 116 & $\mathrm{~N}$ & \\
\hline
\end{tabular}

Data obtained from WHC Surveillance Analysis Computer System (SACS), June 2, 1993. 
WHC-SD-WM-ER-312, Rev. 0

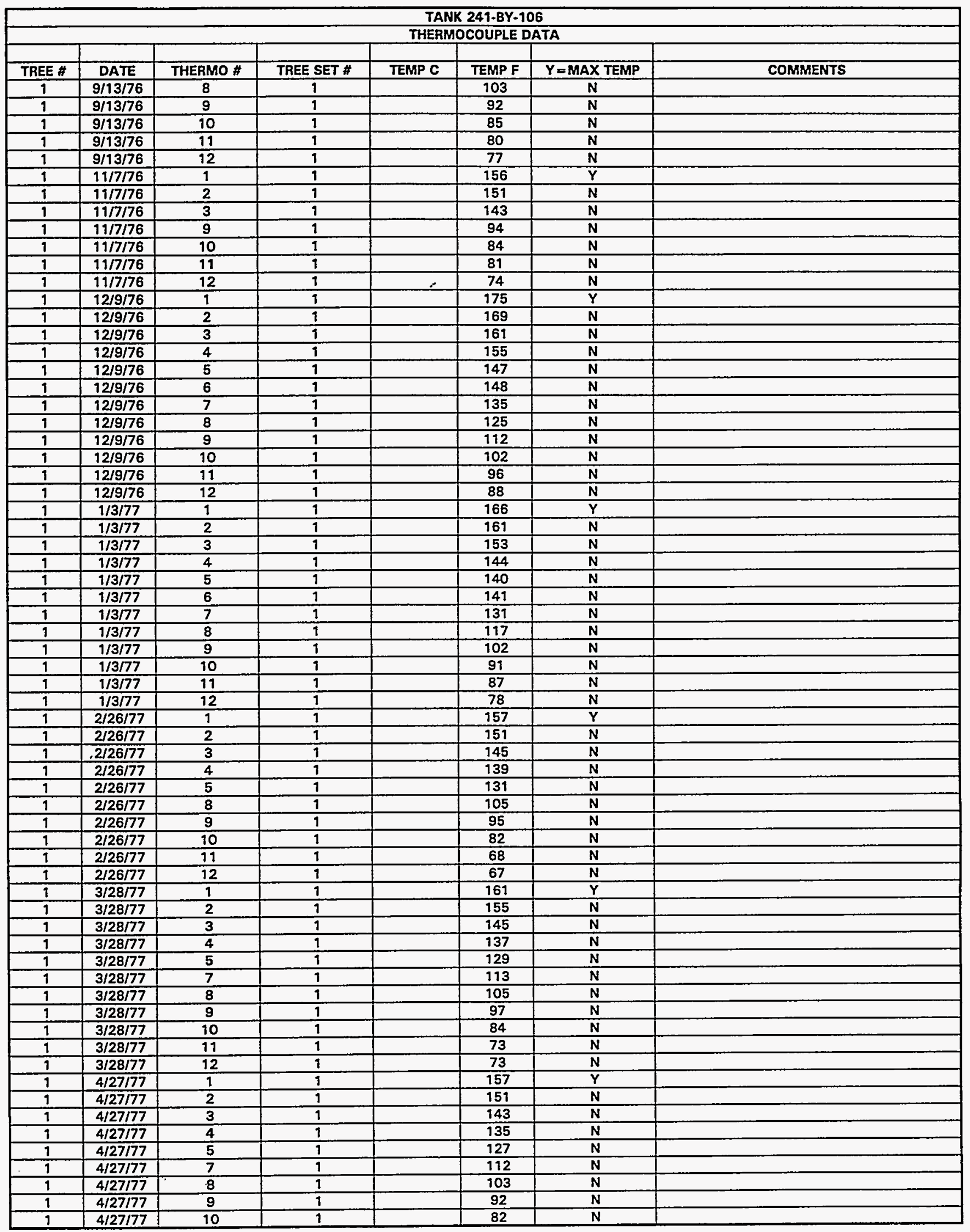

Data obtained from WHC Surveillance Analysis Computer System (SACS), June 2, 1993. 
WHC-SD-WM-ER-312, Rev. 0

\begin{tabular}{|c|c|c|c|c|c|c|c|}
\hline \multicolumn{8}{|c|}{ TANK 241-BY-106 } \\
\hline & & & & THERh & COUPLE D & ITA & \\
\hline TREE \# & DATE & THERMO \# & TREE SET \# & TEMP C & TEMP F & $Y=$ MAX TEMP & COMMENTS \\
\hline 1 & $4 / 27 / 77$ & 11 & 1 & & 74 & $\mathrm{~N}$ & \\
\hline 1 & $4 / 27 / 77$ & 12 & 1 & & 73 & $\mathrm{~N}$ & \\
\hline 1 & $6 / 30 / 77$ & 2 & 1 & & 153 & $\bar{Y}$ & \\
\hline 1 & $6 / 30 / 77$ & 3 & $\frac{1}{1}$ & & 145 & $\mathbf{N}$ & \\
\hline 1 & $6 / 30 / 77$ & 4 & 1 & & 139 & $\mathrm{~N}$ & \\
\hline 1 & $6 / 30 / 77$ & 5 & 1 & & 131 & $\mathbf{N}$ & \\
\hline 1 & $6 / 30 / 77$ & 6 & 7 & & 124 & $N$ & \\
\hline 1 & $6 / 30 / 77$ & 7 & 1 & & 115 & $N$ & \\
\hline 1 & $6 / 30 / 77$ & 8 & 9 & & 108 & $\mathbf{N}$ & \\
\hline 1 & $6 / 30 / 77$ & 9 & 1 & & 99 & $\mathbf{N}$ & \\
\hline 1 & $6 / 30 / 77$ & 10 & 1 & & 90 & $\mathbf{N}$ & \\
\hline 1 & $6 / 30 / 77$ & 11 & 1 & 5 & 83 & $N$ & \\
\hline 1 & $6 / 30 / 77$ & 12 & 9 & & 83 & $\mathrm{~N}$ & \\
\hline 1 & $8 / 15 / 77$ & 1 & 1 & & 147 & $\bar{Y}$ & \\
\hline 1 & $8 / 15 / 77$ & 2 & 1 & & 142 & $\overline{\mathbf{N}}$ & \\
\hline 1 & $8 / 15 / 77$ & 3 & 9 & & 135 & $\bar{N}$ & \\
\hline 1 & $8 / 15 / 77$ & 4 & 1 & & 129 & $\mathbf{N}$ & \\
\hline 1 & $8 / 15 / 77$ & 5 & 1 & & 120 & $\mathbf{N}$ & \\
\hline 1 & $8 / 15 / 77$ & 7 & 1 & & 106 & $\mathbf{N}$ & \\
\hline 1 & $8 / 15 / 77$ & 8 & 1 & & 98 & $\mathbf{N}$ & \\
\hline 1 & $8 / 15 / 77$ & 9 & 1 & & 91 & $\mathbf{N}$ & \\
\hline 1 & $8 / 15 / 77$ & 10 & 9 & & 83 & $\mathbf{N}$ & \\
\hline 1 & $8 / 15 / 77$ & 11 & 9 & & 78 & $\mathbf{N}$ & \\
\hline 1 & $8 / 15 / 77$ & 12 & 1 & & 77 & $\mathrm{~N}$ & \\
\hline 1 & $9 / 12 / 77$ & 2 & 1 & & 138 & $\bar{Y}$ & \\
\hline 1 & $9 / 12 / 77$ & 3 & 1 & & 132 & $\mathbf{N}$ & \\
\hline 1 & $9 / 12 / 77$ & 4 & 1 & & 125 & $\mathbf{N}$ & \\
\hline 1 & $9 / 12 / 77$ & 5 & 1 & & 117 & $\mathbf{N}$ & \\
\hline 1 & $9 / 12 / 77$ & 6 & 9 & & 114 & $\mathrm{~N}$ & \\
\hline 9 & $9 / 12 / 77$ & 7 & 1 & & 105 & $\mathrm{~N}$ & \\
\hline 1 & $9 / 12 / 77$ & 8 & 1 & & 95 & $N$ & \\
\hline 1 & $9 / 12 / 77$ & 9 & 1 & & 88 & $\mathrm{~N}$ & \\
\hline 1 & $9 / 12 / 77$ & 10 & 1 & & 77 & $\bar{N}$ & \\
\hline 1 & $9 / 12 / 77$ & 11 & 1 & & 70 & $\mathbf{N}$ & \\
\hline 1 & $9 / 12 / 77$ & 12 & 1 & & 69 & $\bar{N}$ & \\
\hline 1 & $10 / 7 / 77$ & 3 & 1 & & 146 & $\bar{Y}$ & \\
\hline 1 & $10 / 7 / 77$ & 4 & 1 & & 139 & $\mathbf{N}$ & \\
\hline 1 & $10 / 7 / 77$ & 5 & 1 & & 131 & $\mathbf{N}$ & \\
\hline$\frac{1}{1}$ & $10 / 7 / 77$ & 6 & 9 & & 127 & $N$ & \\
\hline 1 & $10 / 7 / 77$ & 7 & 1 & & 117 & $\mathbf{N}$ & \\
\hline 1 & $10 / 7 / 77$ & 8 & 1 & & 110 & $\bar{N}$ & \\
\hline 1 & $90 / 7 / 77$ & 9 & 1 & & 101 & $N$ & \\
\hline 1 & $10 / 7 / 77$ & 10 & 1 & & 88 & $\bar{N}$ & \\
\hline 1 & $10 / 7 / 77$ & 11 & 1 & & 77 & $\mathrm{~N}$ & \\
\hline 9 & $10 / 7 / 77$ & 12 & $\frac{1}{1}$ & & 77 & $\mathbf{N}$ & \\
\hline 1 & $12 / 7 / 77$ & 2 & 1 & & 148 & $Y$ & \\
\hline 1 & $12 / 7 / 77$ & 3 & 1 & & 142 & $\mathbf{N}$ & \\
\hline 9 & $12 / 7 / 77$ & 4 & 9 & & 135 & $\mathbf{N}$ & \\
\hline 1 & $12 / 7 / 77$ & 5 & 1 & & 127 & $\mathrm{~N}$ & \\
\hline 1 & $12 / 7 / 77$ & 6 & 1 & & 122 & $\mathbf{N}$ & \\
\hline 1 & $12 / 7 / 77$ & 7 & 9 & & 112 & $N$ & \\
\hline 1 & $12 / 7 / 77$ & 8 & 1 & & 103 & $N$ & \\
\hline 1 & $12 / 7 / 77$ & 9 & 1 & & 92 & $\mathrm{~N}$ & \\
\hline$\frac{1}{1}$ & $12 / 7 / 77$ & 10 & 1 & & 75 & $\mathbf{N}$ & \\
\hline 1 & $12 / 7 / 77$ & 11 & 9 & & 62 & $\mathbf{N}$ & \\
\hline 1 & $12 / 7 / 77$ & 12 & 1 & & 61 & $\mathrm{~N}$ & \\
\hline 1 & $1 / 29 / 78$ & 2 & 1 & & 156 & $Y$ & \\
\hline 1 & $1 / 29 / 78$ & 3 & 9 & & 149 & $\mathbf{N}$ & \\
\hline 9 & $1 / 29 / 78$ & 4 & 1 & & 143 & $\bar{N}$ & \\
\hline 1 & $1 / 29 / 78$ & 5 & 1 & & 135 & $\mathbf{N}$ & \\
\hline 9 & $1 / 29 / 78$ & 6 & 1 & & 136 & $\mathbf{N}$ & \\
\hline 9 & $1 / 29 / 78$ & 7 & 1 & & 118 & $N$ & \\
\hline 1 & $1 / 29 / 78$ & 8 & 1 & & 108 & $\mathbf{N}$ & \\
\hline 9 & $7 / 29 / 78$ & 9 & $\frac{1}{1}$ & & 95 & $\mathbf{N}$ & \\
\hline$\frac{1}{1}$ & $1 / 29 / 78$ & 10 & 1 & & 78 & $N$ & \\
\hline$\frac{1}{1}$ & $1 / 29 / 78$ & 11 & 1 & & 64 & $\mathbf{N}$ & \\
\hline
\end{tabular}

Data obtained from WHC Surveillance Analysis Computer System (SACS), June 2, 1993. 


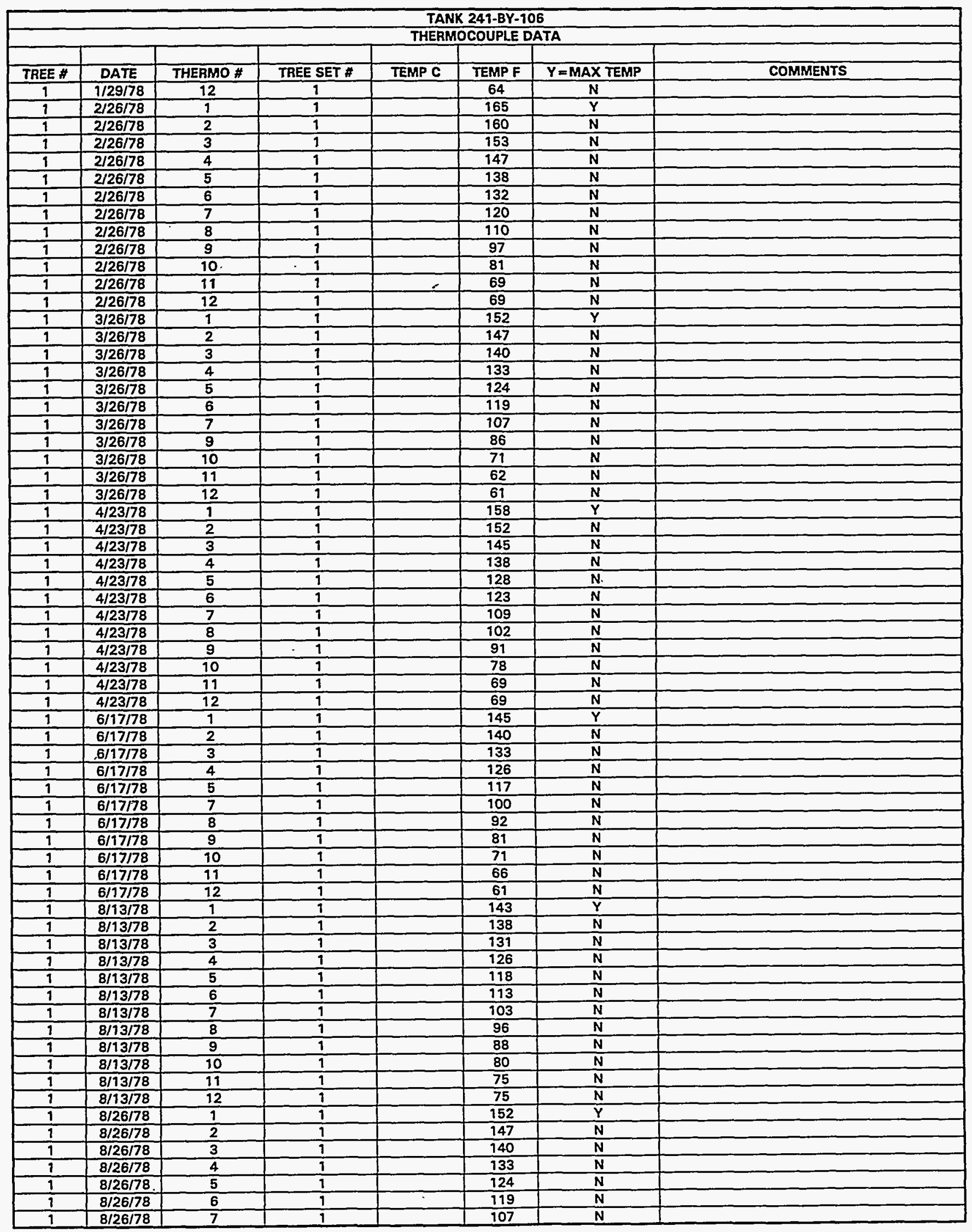

Data obtained from WHC Surveillance Analysis Computer System (SACS), June 2, 1993. 
WHC-SD-WM-ER-312, Rev. 0

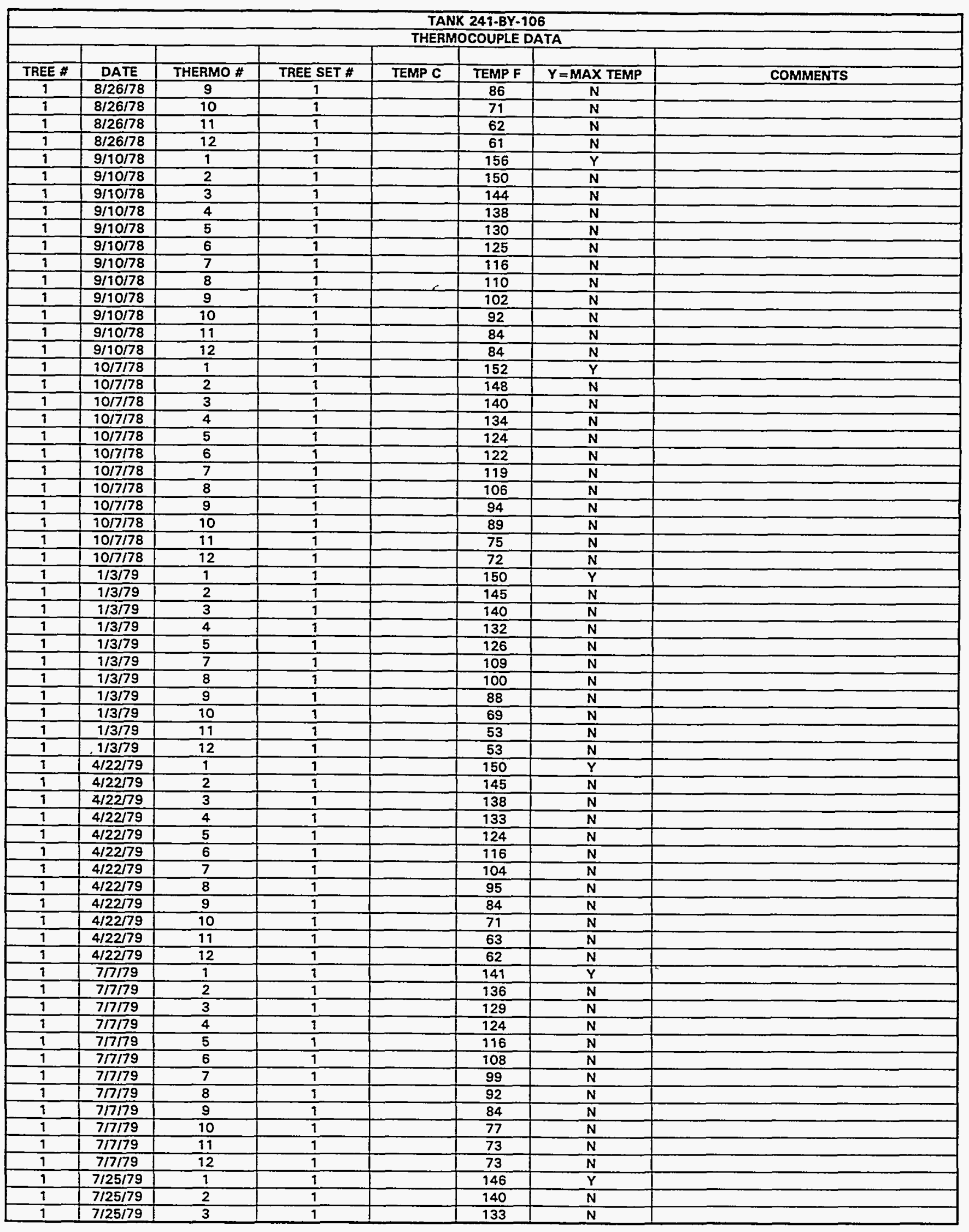

Data obtained from WHC Surveillance Analysis Computer System (SACS), June 2, 1993. 


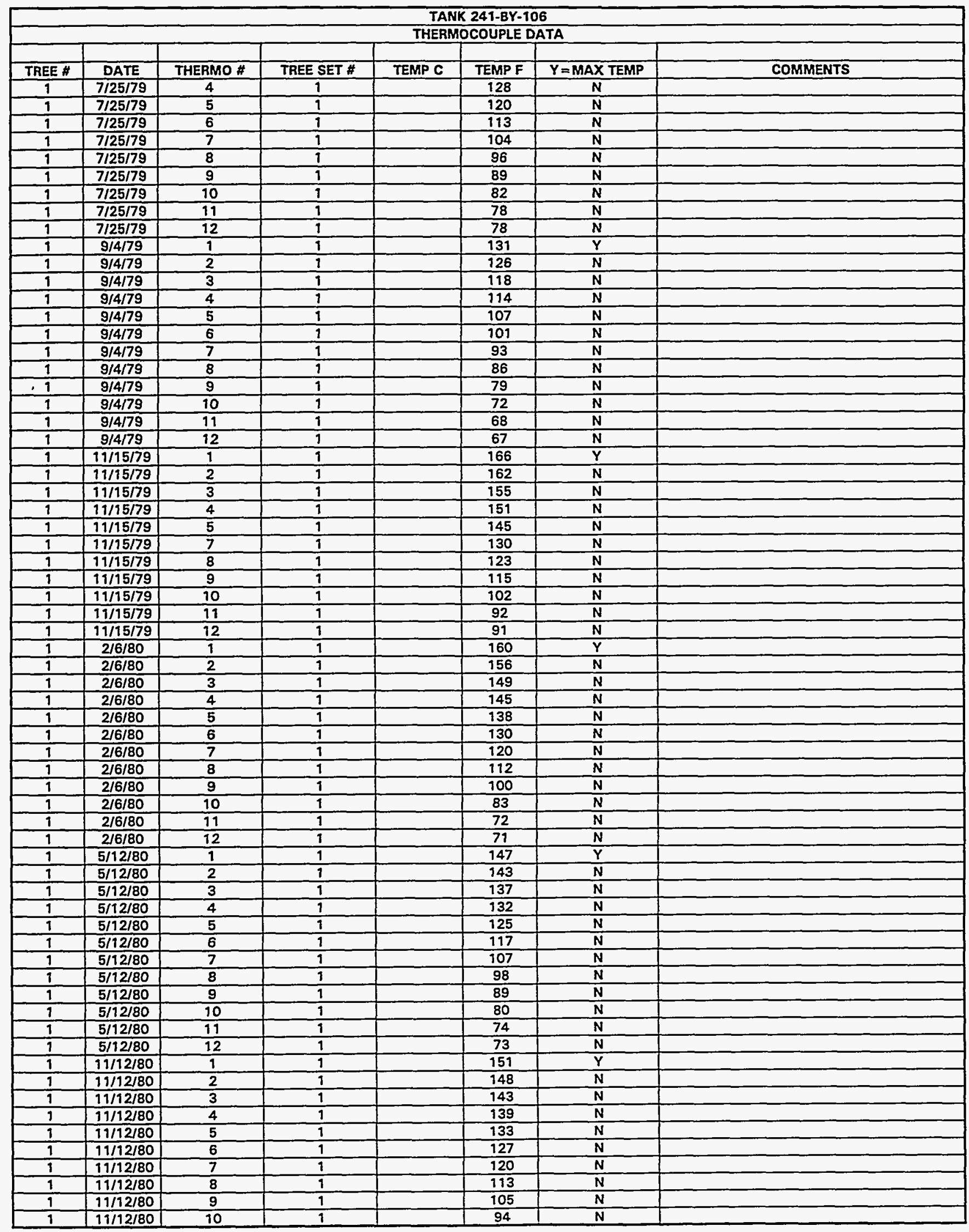

Data obtained from WHC Surveillance Analysis Computer System (SACS), June 2, 1993. 


\begin{tabular}{|c|c|c|c|c|c|c|c|}
\hline \multicolumn{8}{|c|}{ TANK 241-BY-106 } \\
\hline & & & & THERN & COUPLE D & TA & \\
\hline TREE \# & DATE & THERMO \# & TREE SET \# & TEMP C & TEMP F & $Y=M A X$ TEMP & COMMENTS \\
\hline 1 & $11 / 12 / 80$ & 11 & 1 & & 85 & $\mathbf{N}$ & \\
\hline 1 & $11 / 12 / 80$ & 12 & 1 & & 84 & $\mathbf{N}$ & \\
\hline 1 & $12 / 1 / 80$ & 1 & 9 & & 156 & $\bar{Y}$ & \\
\hline 1 & $12 / 1 / 80$ & 2 & 1 & & 152 & $\bar{N}$ & \\
\hline 1 & $12 / 1 / 80$ & 3 & 1 & & 145 & $\mathbf{N}$ & \\
\hline 1 & $12 / 1 / 80$ & 4 & 1 & & 141 & $\bar{N}$ & \\
\hline 1 & $12 / 1 / 80$ & 5 & 1 & & 135 & $\mathbf{N}$ & \\
\hline 1 & $12 / 1 / 80$ & 6 & 1 & & 128 & $\mathrm{~N}$ & \\
\hline 1 & $12 / 1 / 80$ & 7 & 1 & & 120 & $\mathbf{N}$ & \\
\hline 1 & $12 / 1 / 80$ & 8 & 1 & & 113 & $\mathrm{~N}$ & \\
\hline 9 & $12 / 1 / 80$ & 9 & 1 & & 104 & $\mathrm{~N}$ & \\
\hline 1 & $12 / 1 / 80$ & 10 & 1 & & 88 & $\mathbf{N}$ & \\
\hline 1 & $12 / 1 / 80$ & 11 & 1 & & 76 & $\mathbf{N}$ & \\
\hline 1 & $12 / 1 / 80$ & 12 & 1 & & 75 & $\mathbf{N}$ & \\
\hline 1 & $6 / 12 / 81$ & 1 & 1 & & 135 & $\bar{Y}$ & \\
\hline 1 & $6 / 12 / 81$ & 2 & 1 & & 132 & N & \\
\hline 1 & $6 / 12 / 81$ & 3 & 1 & & 127 & $\mathbf{N}$ & \\
\hline 1 & $6 / 12 / 81$ & 4 & 1 & & 123 & $\mathrm{~N}$ & \\
\hline 1 & $6 / 12 / 81$ & 5 & 1 & & 118 & $\mathrm{~N}$ & \\
\hline 1 & $6 / 12 / 81$ & 6 & 1 & & 111 & $\mathbf{N}$ & \\
\hline 1 & $6 / 12 / 81$ & 7 & 1 & & 104 & $\mathrm{~N}$ & \\
\hline 1 & $6 / 12 / 81$ & 8 & 1 & & 97 & $N$ & \\
\hline 1 & $6 / 12 / 81$ & 9 & 1 & & 90 & $\mathrm{~N}$ & \\
\hline 1 & $6 / 12 / 81$ & 10 & 1 & & 81 & $N$ & \\
\hline 1 & $6 / 12 / 81$ & 11 & 1 & & 75 & $N$ & \\
\hline 1 & $6 / 12 / 81$ & 12 & 1 & & 75 & $\mathrm{~N}$ & \\
\hline 1 & $11 / 11 / 81$ & 1 & 1 & & 148 & $\bar{Y}$ & \\
\hline 1 & $91 / 11 / 81$ & 2 & 1 & & 144 & $\mathrm{~N}$ & \\
\hline 1 & $11 / 11 / 81$ & 3 & 1 & & 138 & $N$ & \\
\hline 1 & $11 / 11 / 81$ & 4 & 9 & & 136 & $N$ & \\
\hline 1 & $11 / 11 / 81$ & 5 & 1 & & 131 & $\mathbf{N}$ & \\
\hline 1 & $11 / 11 / 81$ & 6 & 1 & & 127 & $\mathbf{N}$ & \\
\hline 1 & $11 / 11 / 81$ & 7 & 1 & & 122 & $N$ & \\
\hline 1 & $11 / 11 / 81$ & 8 & 1 & & 118 & $\mathbf{N}$ & \\
\hline 1 & $11 / 11 / 81$ & 9 & 1 & & 109 & $\mathrm{~N}$ & \\
\hline 1 & $11 / 11 / 81$ & 10 & 1 & & 98 & $\mathbf{N}$ & \\
\hline 1 & $11 / 11 / 81$ & 11 & 1 & & 94 & $\mathbf{N}$ & \\
\hline 1 & $11 / 11 / 81$ & 12 & 1 & & 95 & $\mathbf{N}$ & \\
\hline 1 & $1 / 14 / 82$ & 1 & 1 & & 145 & $\bar{Y}$ & \\
\hline 9 & $1 / 14 / 82$ & 2 & 1 & & 144 & $\mathbf{N}$ & \\
\hline 1 & $1 / 14 / 82$ & 3 & 9 & & 138 & $\mathbf{N}$ & \\
\hline 1 & $1 / 14 / 82$ & 4 & 1 & & 134 & $N$ & \\
\hline 1 & $1 / 14 / 82$ & 5 & 1 & & 129 & $\mathbf{N}$ & \\
\hline 1 & $1 / 14 / 82$ & 6 & 1 & & 123 & $\mathbf{N}$ & \\
\hline 1 & $1 / 14 / 82$ & 7 & 1 & & 118 & $\mathbf{N}$ & \\
\hline 1 & $1 / 14 / 82$ & 8 & 9 & & 112 & $N$ & \\
\hline 1 & $1 / 14 / 82$ & 9 & 1 & & 97 & $\mathbf{N}$ & \\
\hline 1 & $1 / 14 / 82$ & 10 & 9 & & 84 & $\bar{N}$ & \\
\hline 1 & $9 / 14 / 82$ & 11 & 1 & & 77 & $\bar{N}$ & \\
\hline 1 & $1 / 14 / 82$ & 12 & 1 & & 76 & $\mathrm{~N}$ & \\
\hline 1 & $2 / 4 / 82$ & 9 & 9 & & 161 & $\bar{Y}$ & \\
\hline 1 & $2 / 4 / 82$ & 2 & 1 & & 157 & $N$ & \\
\hline 1 & $2 / 4 / 82$ & 3 & 1 & & 152 & $\mathrm{~N}$ & \\
\hline 1 & $2 / 4 / 82$ & 4 & 9 & & 149 & $N$ & \\
\hline 1 & $2 / 4 / 82$ & 5 & 1 & & 143 & $\mathbf{N}$ & \\
\hline 1 & $2 / 4 / 82$ & 6 & 1 & & 137 & $N$ & \\
\hline 1 & $2 / 4 / 82$ & 7 & 1 & & 129 & $\mathbf{N}$ & \\
\hline 1 & $2 / 4 / 82$ & 8 & 1 & & 122 & $\mathrm{~N}$ & \\
\hline 1 & $2 / 4 / 82$ & 9 & 1 & & 114 & $\mathrm{~N}$ & \\
\hline 1 & $2 / 4 / 82$ & 10 & 9 & & 102 & $\bar{N}$ & \\
\hline 1 & $2 / 4 / 82$ & 11 & 1 & & 90 & $\mathrm{~N}$ & \\
\hline 1 & $2 / 4 / 82$ & 12 & 9 & & 88 & $\bar{N}$ & \\
\hline 1 & $3 / 10 / 82$ & 1 & 1 & & 147 & $\bar{Y}$ & \\
\hline 1 & $3 / 10 / 82$ & 2 & 1 & & 143 & $\mathrm{~N}$ & \\
\hline 1 & $3 / 10 / 82$ & 3 & 9 & & 138 & $\bar{N}$ & \\
\hline 1 & $3 / 10 / 82$ & 4 & 1 & & 134 & $\mathrm{~N}$ & \\
\hline
\end{tabular}

Data obtained from WHC Surveillance Analysis Computer System (SACS), June 2, 1993. 
WHC-SD-WM-ER-312, Rev. 0

\begin{tabular}{|c|c|c|c|c|c|c|c|}
\hline \multicolumn{8}{|c|}{ TANK 241-BY-106 } \\
\hline \multicolumn{8}{|c|}{ THERMOCOUPLE DATA } \\
\hline TREE \# & DATE & THERMO \# & TREE SET \# & TEMP C & TEMP F & $Y=$ MAX TEMP & COMMENTS \\
\hline 1 & $3 / 10 / 82$ & 5 & 1 & & 127 & $\mathrm{~N}$ & \\
\hline 9 & $3 / 10 / 82$ & 6 & 9 & & 121 & $\mathrm{~N}$ & \\
\hline 9 & $3 / 10 / 82$ & 7 & 1 & & 115 & $\mathbf{N}$ & \\
\hline 1 & $3 / 10 / 82$ & 8 & 1 & & 105 & N & \\
\hline 1 & $3 / 10 / 82$ & 9 & 1 & & 93 & N & \\
\hline 1 & $3 / 10 / 82$ & 10 & 1 & & 80 & $\mathbf{N}$ & \\
\hline 1 & $3 / 10 / 82$ & 11 & 1 & & 75 & $\mathbf{N}$ & \\
\hline 1 & $3 / 10 / 82$ & 12 & 1 & & 74 & $\mathbf{N}$ & \\
\hline 1 & $10 / 14 / 83$ & 1 & 1 & & 133 & $\mathbf{Y}$ & \\
\hline 1 & $10 / 14 / 83$ & 2 & 1 & & 128 & $\mathbf{N}$ & \\
\hline 1 & $10 / 14 / 83$ & 3 & 1 & & 125 & $\mathbf{N}$ & \\
\hline 1 & $10 / 14 / 83$ & 4 & 1 & & 121 & $\mathbf{N}$ & \\
\hline 1 & $10 / 14 / 83$ & 5 & 1 & & 113 & $\bar{N}$ & \\
\hline 1 & $10 / 14 / 83$ & 6 & 9 & & 83 & $\bar{N}$ & \\
\hline 1 & $11 / 8 / 83$ & 1 & 1 & & 141 & $Y$ & \\
\hline 1 & $11 / 8 / 83$ & 2 & 1 & & 138 & N & \\
\hline 1 & $11 / 8 / 83$ & 3 & 1 & & 133 & $\mathbf{N}$ & \\
\hline 1 & $11 / 8 / 83$ & 4 & 1 & & 129 & $N$ & \\
\hline 9 & $11 / 8 / 83$ & 5 & 1 & & 124 & $N$ & \\
\hline 1 & $11 / 8 / 83$ & 6 & 1 & & 87 & $\mathrm{~N}$ & \\
\hline 1 & $12 / 6 / 83$ & 1 & 1 & & 142 & $\bar{Y}$ & \\
\hline 1 & $12 / 6 / 83$ & 2 & $\frac{1}{1}$ & & 138 & $\bar{N}$ & \\
\hline 1 & $12 / 6 / 83$ & 3 & 9 & & 134 & $\mathbf{N}$ & \\
\hline 1 & $12 / 6 / 83$ & 4 & 1 & & 130 & $N$ & \\
\hline 1 & $12 / 6 / 83$ & 5 & 1 & & 125 & $N$ & \\
\hline 1 & $12 / 6 / 83$ & 6 & 1 & & 80 & $\bar{N}$ & \\
\hline 1 & $2 / 7 / 84$ & 1 & 1 & & 142 & $\bar{Y}$ & \\
\hline 1 & $2 / 7 / 84$ & 2 & 1 & & 139 & $\mathbf{N}$ & \\
\hline 1 & $2 / 7 / 84$ & 3 & 1 & & 134 & $\mathbf{N}$ & \\
\hline 1 & $2 / 7 / 84$ & 4 & 1 & & 130 & $\mathbf{N}$ & \\
\hline 9 & $2 / 7 / 84$ & 5 & 1 & & 124 & $N$ & \\
\hline 1 & $2 / 7 / 84$ & 6 & 1 & & 77 & $\mathrm{~N}$ & \\
\hline 1 & $2 / 14 / 84$ & 1 & $\frac{1}{1}$ & & 131 & $\bar{Y}$ & \\
\hline 1 & $2 / 14 / 84$ & 2 & 1 & & 127 & $\mathbf{N}$ & \\
\hline 1 & $2 / 14 / 84$ & 3 & 1 & & 122 & $N$ & \\
\hline 1 & $2 / 14 / 84$ & 4 & 1 & & 118 & $\bar{N}$ & \\
\hline 1 & $2 / 14 / 84$ & 5 & 1 & & 113 & $\mathbf{N}$ & \\
\hline 1 & $2 / 14 / 84$ & 6 & 1 & & 67 & $N$ & \\
\hline 1 & $3 / 26 / 84$ & 1 & 7 & & 134 & $\bar{Y}$ & \\
\hline 1 & $3 / 26 / 84$ & 2 & 9 & & 135 & $\mathrm{~N}$ & \\
\hline 9 & $3 / 26 / 84$ & 3 & 1 & & 137 & $N$ & \\
\hline 1 & $3 / 26 / 84$ & 4 & 1 & & 119 & $\mathrm{~N}$ & \\
\hline 1 & $3 / 26 / 84$ & 5 & 1 & & 117 & $\mathbf{N}$ & \\
\hline 1 & $3 / 26 / 84$ & 6 & 1 & & 75 & $N$ & \\
\hline 1 & $4 / 7 / 84$ & 1 & 1 & & 126 & $Y$ & \\
\hline 1 & $4 / 7 / 84$ & 2 & 1 & & 122 & $\mathbf{N}$ & \\
\hline 1 & $4 / 7 / 84$ & 3 & 1 & & 118 & $\mathbf{N}$ & \\
\hline 1 & $4 / 7 / 84$ & 4 & 1 & & 915 & $\mathbf{N}$ & \\
\hline 1 & $4 / 7 / 84$ & 5 & 1 & & 109 & $N$ & \\
\hline 9 & $4 / 7 / 84$ & 6 & 1 & & 70 & $\mathrm{~N}$ & \\
\hline 9 & $6 / 19 / 84$ & 1 & 1 & & 139 & $Y$ & \\
\hline 1 & $6 / 19 / 84$ & 2 & 1 & & 134 & $\mathbf{N}$ & \\
\hline 1 & $6 / 19 / 84$ & 3 & 1 & & 130 & $N$ & \\
\hline 1 & $6 / 19 / 84$ & 4 & 1 & & 127 & $\mathbf{N}$ & \\
\hline 1 & $6 / 19 / 84$ & 5 & 1 & & 119 & $\mathbf{N}$ & \\
\hline 1 & $6 / 19 / 84$ & 6 & 1 & & 78 & $\mathbf{N}$ & \\
\hline 9 & $9 / 3 / 84$ & 1 & 1 & & 131 & $Y$ & \\
\hline 1 & $9 / 3 / 84$ & 2 & 1 & & 128 & $N$ & \\
\hline$\frac{1}{1}$ & $9 / 3 / 84$ & 3 & 1 & & 124 & $\mathbf{N}$ & \\
\hline 1 & $9 / 3 / 84$ & 4 & 1 & & 121 & $N$ & \\
\hline 1 & $9 / 3 / 84$ & 5 & 1 & & 114 & $\mathbf{N}$ & \\
\hline 1 & $9 / 3 / 84$ & 6 & 1 & & 85 & $\mathbf{N}$ & \\
\hline 1 & $10 / 8 / 84$ & 1 & 1 & & & $\bar{Y}$ & 77 - suspect data (low) \\
\hline 1 & $10 / 8 / 84$ & 2 & 1 & & & $\mathbf{N}$ & 73 - suspect data (low) \\
\hline 1 & $10 / 8 / 84$ & 3 & 1 & & & $N$ & 68 - suspect data (low) \\
\hline 1 & $10 / 8 / 84$ & 4 & 1 & & & $\bar{N}$ & 68 - suspect data (10w) \\
\hline
\end{tabular}

Data obtained from WHC Surveillance Analysis Computer System (SACS), June 2, 1993. 


\begin{tabular}{|c|c|c|c|c|c|c|c|}
\hline \multirow{2}{*}{\multicolumn{8}{|c|}{$\begin{array}{l}\text { TANK 247-BY-106 } \\
\text { THERMOCOUPLE DATA }\end{array}$}} \\
\hline & & & & & & & \\
\hline TREE \# & DATE & THERMO \# & TREE SET \# & TEMP C & TEMP F & $Y=$ MAX TEMP & COMMENTS \\
\hline 1 & $10 / 8 / 84$ & 5 & 1 & & & $\mathbf{N}$ & 58 - suspect data (low) \\
\hline 1 & $10 / 8 / 84$ & 6 & 1 & & & $\mathrm{~N}$ & 56 - suspect data (low) \\
\hline 1 & $10 / 8 / 84$ & 7 & 1 & & & $\mathrm{~N}$ & 56 - suspect data (low) \\
\hline 1 & $10 / 8 / 84$ & 8 & 1 & & & $\mathbf{N}$ & 51 - suspect data (low) \\
\hline 1 & $10 / 8 / 84$ & 9 & 1 & & & $\mathbf{N}$ & 46 - suspect data (low) \\
\hline 1 & $10 / 8 / 84$ & 10 & 1 & & & $\mathbf{N}$ & 35 - suspect data (low) \\
\hline 1 & $10 / 8 / 84$ & 11 & 1 & & & $\mathbf{N}$ & 35 - suspect data (low) \\
\hline 1 & $10 / 29 / 84$ & 1 & 1 & & & $\mathbf{Y}$ & 74 - suspect data (low) \\
\hline 1 & $10 / 29 / 84$ & 2 & 1 & & & $\mathbf{N}$ & 70 - suspect data (low) \\
\hline 1 & $10 / 29 / 84$ & 3 & 1 & & & $\bar{N}$ & 65 - suspect data (low) \\
\hline 1 & $10 / 29 / 84$ & 4 & 1 & & & $\mathbf{N}$ & 59 - suspect data (low) \\
\hline 1 & $10 / 29 / 84$ & 5 & 1 & & & $\mathbf{N}$ & 56 - suspect data (low) \\
\hline 1 & $10 / 29 / 84$ & 6 & 1 & & & $\mathbf{N}$ & 55 - suspect data (low) \\
\hline 1 & $10 / 29 / 84$ & 7 & 1 & & & $\mathbf{N}$ & 54 - suspect data (low) \\
\hline 1 & $10 / 29 / 84$ & 8 & 1 & & & N & 48 - suspect data (low) \\
\hline 1 & $10 / 29 / 84$ & 9 & 1 & & & $\mathbf{N}$ & 43 - suspect data (low) \\
\hline 1 & $10 / 29 / 84$ & 10 & 1 & & & $\mathbf{N}$ & 37 - suspect data (low) \\
\hline 1 & $10 / 29 / 84$ & 11 & 1 & & & $\mathbf{N}$ & 30 - suspect data (low) \\
\hline 1 & $10 / 29 / 84$ & 12 & 1 & & & $\mathbf{N}$ & 22 - suspect data (low) \\
\hline 1 & $3 / 3 / 85$ & 1 & 1 & & 125 & $\mathrm{Y}$ & \\
\hline 1 & $3 / 3 / 85$ & 2 & 1 & & 122 & $\mathbf{N}$ & \\
\hline 1 & $3 / 3 / 85$ & 3 & 1 & & 118 & $\mathrm{~N}$ & \\
\hline 1 & $3 / 3 / 85$ & 4 & 1 & & 115 & $\mathbf{N}$ & \\
\hline 1 & $3 / 3 / 85$ & 5 & 1 & & 109 & $\mathrm{~N}$ & \\
\hline 1 & $3 / 3 / 85$ & 6 & 1 & & 70 & $\mathrm{~N}$ & \\
\hline 1 & $3 / 5 / 85$ & 1 & 1 & & 138 & $\bar{Y}$ & \\
\hline 1 & $3 / 5 / 85$ & 2 & 1 & & 135 & $\mathrm{~N}$ & \\
\hline 1 & $3 / 5 / 85$ & 3 & 9 & & 130 & $\mathrm{~N}$ & \\
\hline 1 & $3 / 5 / 85$ & 4 & 1 & & 127 & $\mathrm{~N}$ & \\
\hline 1 & $3 / 5 / 85$ & 5 & 1 & & 120 & $\ddot{N}$ & \\
\hline 1 & $3 / 5 / 85$ & 6 & 1 & & 76 & $\mathrm{~N}$ & \\
\hline 1 & $4 / 1 / 85$ & 1 & 1 & & 140 & $\bar{Y}$ & \\
\hline 1 & $4 / 1 / 85$ & 2 & 1 & & 137 & $\mathrm{~N}$ & \\
\hline 1 & $4 / 1 / 85$ & 3 & 1 & & 133 & $N$ & \\
\hline 1 & $4 / 1 / 85$ & 4 & 1 & & 128 & $\mathrm{~N}$ & \\
\hline 9 & $4 / 1 / 85$ & 5 & 1 & & 123 & $\mathbf{N}$ & \\
\hline 1 & $4 / 1 / 85$ & 6 & 1 & & 76 & $\mathrm{~N}$ & \\
\hline 1 & $6 / 4 / 85$ & 1 & 1 & & 139 & $\ddot{Y}$ & \\
\hline 1 & $6 / 4 / 85$ & 2 & 1 & & 135 & $\bar{N}$ & \\
\hline 1 & $6 / 4 / 85$ & 3 & 1 & & 131 & $\mathrm{~N}$ & \\
\hline 1 & $6 / 4 / 85$ & 4 & 1 & & 128 & $\mathrm{~N}$ & \\
\hline 1 & $6 / 4 / 85$ & 5 & 1 & & 120 & $\mathbf{N}$ & \\
\hline 1 & $6 / 4 / 85$ & 6 & 1 & & 80 & $N$ & \\
\hline 1 & $6 / 10 / 85$ & 1 & 1 & & 126 & $\bar{Y}$ & \\
\hline 1 & $6 / 10 / 85$ & 2 & 1 & & 122 & $\mathrm{~N}$ & \\
\hline 1 & $6 / 10 / 85$ & 3 & 1 & & 118 & $\mathbf{N}$ & \\
\hline 1 & $6 / 10 / 85$ & 4 & 1 & & 115 & $\mathrm{~N}$ & \\
\hline 9 & $6 / 10 / 85$ & 5 & 1 & & 109 & $N$ & \\
\hline 1 & $6 / 10 / 85$ & 6 & 1 & & 76 & $N$ & \\
\hline 1 & $7 / 2 / 85$ & 1 & 1 & & 139 & $Y$ & \\
\hline 1 & $7 / 2 / 85$ & 2 & 1 & & 134 & $\mathbf{N}$ & \\
\hline 1 & $7 / 2 / 85$ & 3 & 1 & & 131 & $N$ & \\
\hline 1 & $7 / 2 / 85$ & 4 & 1 & & 128 & $\mathrm{~N}$ & \\
\hline 1 & $7 / 2 / 85$ & 5 & 1 & & 120 & $\mathbf{N}$ & \\
\hline 1 & $7 / 2 / 85$ & 6 & 1 & & 83 & $\mathrm{~N}$ & \\
\hline 1 & $8 / 5 / 85$ & 1 & 1 & & 137 & $\bar{Y}$ & \\
\hline 1 & $8 / 5 / 85$ & 2 & 1 & & 133 & $\mathbf{N}$ & \\
\hline 1 & $8 / 5 / 85$ & 3 & 1 & & 130 & $\mathbf{N}$ & \\
\hline 1 & $8 / 5 / 85$ & 4 & 1 & & 127 & $\mathbf{N}$ & \\
\hline 1 & $8 / 5 / 85$ & 5 & 1 & & 121 & $\mathbf{N}$ & \\
\hline 1 & $8 / 5 / 85$ & 6 & 1 & & 85 & $\mathrm{~N}$ & \\
\hline 1 & $9 / 1 / 85$ & 1 & 1 & & 138 & $\bar{Y}$ & \\
\hline 1 & $9 / 1 / 85$ & 2 & 1 & & 133 & $\mathbf{N}$ & \\
\hline 1 & $9 / 1 / 85$ & 3 & 1 & & 130 & $\mathbf{N}$ & \\
\hline 1 & $9 / 1 / 85$ & 4 & 1 & & 127 & $\mathbf{N}$ & \\
\hline 1 & $9 / 1 / 85$ & 5 & 1 & & 120 & $\mathbf{N}$ & \\
\hline
\end{tabular}

Data obtained from WHC Surveillance Analysis Computer System (SACS), June 2, 1993. 


\begin{tabular}{|c|c|c|c|c|c|c|c|}
\hline \multicolumn{8}{|c|}{ TANK 241-BY-106 } \\
\hline & & & & THER & COUPLE & ITA & \\
\hline TAEE \# & DATE & THERMO & TREE SET \# & TEMP C & TEMP F & $Y=M A X$ TEMP & COMMENTS \\
\hline 1 & $9 / 1 / 85$ & 6 & 1 & & 87 & $N$ & \\
\hline 1 & $1 / 6 / 86$ & $\frac{1}{1}$ & 1 & & 138.3 & $\bar{Y}$ & \\
\hline 1 & $1 / 6 / 86$ & 2 & 1 & & 134.8 & $N$ & \\
\hline 9 & $1 / 6 / 86$ & 3 & 1 & & 129.8 & $\bar{N}$ & \\
\hline 1 & $1 / 6 / 86$ & 4 & 1 & & 125.7 & $N$ & \\
\hline 1 & $1 / 6 / 86$ & 5 & 1 & & 121 & $\bar{N}$ & \\
\hline 1 & $1 / 6 / 86$ & 6 & 1 & & 75 & $\mathbf{N}$ & \\
\hline 1 & $3 / 4 / 86$ & 1 & 1 & & 140 & $\bar{Y}$ & \\
\hline 7 & $3 / 4 / 86$ & 2 & 1 & & 136 & $\mathbf{N}$ & \\
\hline 1 & $3 / 4 / 86$ & 3 & 9 & & 131 & $\bar{N}$ & \\
\hline 1 & $3 / 4 / 86$ & 4 & 1 & & 127 & $\bar{N}$ & \\
\hline 1 & $3 / 4 / 86$ & 5 & 1 & & 121 & $N$ & \\
\hline 1 & $3 / 4 / 86$ & 6 & 1 & & 75 & $\bar{N}$ & \\
\hline 1 & $4 / 7 / 86$ & 1 & 1 & & 139 & $\bar{Y}$ & \\
\hline 1 & $4 / 7 / 86$ & 2 & 1 & & 135 & $\mathbf{N}$ & \\
\hline 1 & $4 / 7 / 86$ & 3 & 1 & & 139 & $\bar{N}$ & \\
\hline$\frac{1}{1}$ & $4 / 7 / 86$ & 4 & 1 & & 126 & $\mathbf{N}$ & \\
\hline 1 & $4 / 7 / 86$ & 5 & 1 & & 120 & $\bar{N}$ & \\
\hline 7 & $4 / 7 / 86$ & 6 & 1 & & 74 & $\bar{N}$ & \\
\hline 1 & $5 / 11 / 86$ & 9 & 1 & & 139.3 & $\bar{Y}$ & \\
\hline 1 & $5 / 11 / 86$ & 2 & 1 & & 135.9 & $\overline{\mathbf{N}}$ & \\
\hline 1 & $5 / 11 / 86$ & 3 & 1 & & 131.8 & $\mathbf{N}$ & \\
\hline 1 & $5 / 11 / 86$ & 4 & 1 & & 127.6 & $N$ & \\
\hline 1 & $5 / 11 / 86$ & 5 & 1 & & 120.3 & $\mathbf{N}$ & \\
\hline 1 & $5 / 11 / 86$ & 6 & 1 & & 77 & $\bar{N}$ & \\
\hline 1 & $6 / 3 / 86$ & 1 & 1 & & 139 & $\bar{Y}$ & \\
\hline 7 & $6 / 3 / 86$ & 2 & 1 & & 135 & $\mathrm{~N}$ & \\
\hline$\overline{1}$ & $6 / 3 / 86$ & 3 & 1 & & 131 & $\mathbf{N}$ & \\
\hline 7 & $6 / 3 / 86$ & 4 & 1 & & 127 & $\mathrm{~N}$ & \\
\hline 1 & $6 / 3 / 86$ & 5 & 1 & & 120 & $\mathbf{N}$ & \\
\hline 1 & $6 / 3 / 86$ & 6 & 1 & & 78 & $\mathbf{N}$ & \\
\hline 1 & $8 / 4 / 86$ & 1 & 1 & & 123 & $\bar{Y}$ & \\
\hline 1 & $8 / 4 / 86$ & 2 & 1 & & 124 & $N$ & \\
\hline 1 & $8 / 4 / 86$ & 3 & 1 & & 124 & $\mathbf{N}$ & \\
\hline 1 & $8 / 4 / 86$ & 4 & 9 & & 126 & $\mathbf{N}$ & \\
\hline 1 & $8 / 4 / 86$ & 5 & 1 & & 119 & $\mathbf{N}$ & \\
\hline 1 & $8 / 4 / 86$ & 6. & 1 & & 89 & $\bar{N}$ & \\
\hline 1 & $9 / 1 / 86$ & 1 & 1 & & 147 & $\bar{Y}$ & \\
\hline 1 & $9 / 1 / 86$ & 2 & 1 & & 143 & $\mathbf{N}$ & \\
\hline 1 & $9 / 1 / 86$ & 3 & $\frac{T}{1}$ & & 140 & $N$ & \\
\hline 1 & $9 / 1 / 86$ & 4 & 1 & & 136 & $\mathbf{N}$ & \\
\hline 1 & $9 / 1 / 86$ & 5 & 1 & & 129 & $\mathbf{N}$ & \\
\hline 1 & $9 / 1 / 86$ & 6 & 1 & & 98 & $\mathbf{N}$ & \\
\hline 1 & $11 / 1 / 86$ & 1 & 1 & & 123 & $\bar{Y}$ & \\
\hline 1 & $11 / 1 / 86$ & 2 & 7 & & 120 & $N$ & \\
\hline 1 & $11 / 1 / 86$ & 3 & 1 & & 117 & $\mathbf{N}$ & \\
\hline 9 & $11 / 1 / 86$ & 4 & 1 & & 118 & $N$ & \\
\hline 1 & $11 / 1 / 86$ & 5 & 1 & & 110 & $\mathrm{~N}$ & \\
\hline 1 & $11 / 1 / 86$ & 6 & 1 & & 80 & $\mathbf{N}$ & \\
\hline 1 & $12 / 3 / 86$ & 1 & 1 & & 120 & $\bar{Y}$ & \\
\hline 1 & $12 / 3 / 86$ & 2 & 1 & & 117 & $\mathbf{N}$ & \\
\hline 1 & $12 / 3 / 86$ & 3 & 1 & & 114 & $N$ & \\
\hline 1 & $12 / 3 / 86$ & 4 & 1 & & 111 & $N$ & \\
\hline 1 & $12 / 3 / 86$ & 5 & 1 & & 106 & $N$ & \\
\hline 1 & $12 / 3 / 86$ & 6 & 1 & & 73 & $N$ & \\
\hline 1 & $2 / 3 / 87$ & 7 & 1 & & 135 & $\bar{Y}$ & \\
\hline 1 & $2 / 3 / 87$ & 2 & 1 & & 131 & $\mathbf{N}$ & \\
\hline 1 & $2 / 3 / 87$ & 3 & 1 & & 128 & $\mathbf{N}$ & \\
\hline 1 & $2 / 3 / 87$ & 4 & 1 & & 125 & $N$ & \\
\hline 1 & $2 / 3 / 87$ & 5 & 1 & & 119 & $N$ & \\
\hline 1 & $2 / 3 / 87$ & 6 & 1 & & 73 & $\mathbf{N}$ & \\
\hline 1 & $3 / 2 / 87$ & 1 & 1 & & 135 & $\bar{Y}$ & \\
\hline 1 & $3 / 2 / 87$ & 2 & 1 & & 132 & $\mathbf{N}$ & \\
\hline 1 & $3 / 2 / 87$ & 3 & $i$ & & 127 & $N$ & \\
\hline 1 & $3 / 2 / 87$ & 4 & 9 & & 123 & $\bar{N}$ & \\
\hline 9 & $3 / 2 / 87$ & 5 & 1 & & 117 & $N$ & \\
\hline
\end{tabular}

Data obtained from WHC Surveillance Analysis Computer System (SACS), June 2, 1993. 
WHC-SD-WM-ER-312, Rev. 0

\begin{tabular}{|c|c|c|c|c|c|c|c|}
\hline \multicolumn{8}{|c|}{ TANK 241-BY-106 } \\
\hline \multicolumn{8}{|c|}{ THERMOCOUPLE DATA } \\
\hline TREE \# & DATE & THERMO \# & TREE SET \# & TEMP C & TEMP F & $Y=$ MAX TEMP & COMMENTS \\
\hline 1 & $3 / 2 / 87$ & 6 & 1 & & 74 & $\mathrm{~N}$ & \\
\hline 1 & $4 / 13 / 87$ & 1 & 1 & & 130 & $\mathrm{Y}$ & \\
\hline 1 & $4 / 13 / 87$ & 2 & 1 & & 126 & $\mathbf{N}$ & \\
\hline 1 & $4 / 13 / 87$ & 3 & 1 & & 122 & $\mathbf{N}$ & \\
\hline 1 & $4 / 13 / 87$ & 4 & 1 & & 119 & $\mathbf{N}$ & \\
\hline 1 & $4 / 13 / 87$ & 5 & 1 & & 113 & $\mathbf{N}$ & \\
\hline 1 & $4 / 13 / 87$ & 6 & 1 & & 69 & $\mathbf{N}$ & \\
\hline 1 & $6 / 1 / 87$ & 1 & 1 & & 134 & $Y$ & \\
\hline 1 & $6 / 9 / 87$ & 2 & 1 & & 130 & $\bar{N}$ & \\
\hline 1 & $6 / 1 / 87$ & 3 & 1 & & 126 & $\mathbf{N}$ & \\
\hline 1 & $6 / 1 / 87$ & 4 & 1 & & 123 & $\mathbf{N}$ & \\
\hline 1 & $6 / 1 / 87$ & 5 & 1 & & 115 & $\mathbf{N}$ & \\
\hline 1 & $6 / 9 / 87$ & 6 & 1 & & 78 & $\mathbf{N}$ & \\
\hline 1 & $7 / 6 / 87$ & 1 & 1 & & 136 & $Y$ & \\
\hline 1 & $7 / 6 / 87$ & 2 & 1 & & 132 & $\mathbf{N}$ & \\
\hline 1 & $7 / 6 / 87$ & 3 & 1 & & 130 & $\mathbf{N}$ & \\
\hline 1 & $7 / 6 / 87$ & 4 & 1 & & 126 & $\mathbf{N}$ & \\
\hline 1 & $7 / 6 / 87$ & 5 & 1 & & 119 & $\mathbf{N}$ & \\
\hline 1 & $7 / 6 / 87$ & 6 & 1 & & 82 & $\mathbf{N}$ & \\
\hline 1 & $8 / 4 / 87$ & $\frac{1}{1}$ & $\frac{1}{1}$ & & 132.9 & $Y$ & \\
\hline 1 & $8 / 4 / 87$ & 2 & 1 & & 128.6 & $\mathrm{~N}$ & \\
\hline 1 & $8 / 4 / 87$ & 3 & 1 & & 127.3 & $\mathbf{N}$ & \\
\hline 1 & $8 / 4 / 87$ & 4 & 1 & & 123.8 & $\mathbf{N}$ & \\
\hline 1 & $8 / 4 / 87$ & 5 & 1 & & 118.3 & $\mathbf{N}$ & \\
\hline 1 & $8 / 4 / 87$ & 6 & 1 & & 83.1 & $\mathbf{N}$ & \\
\hline 1 & $9 / 7 / 87$ & 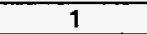 & 1 & & 135.1 & $Y$ & \\
\hline 1 & $9 / 7 / 87$ & 2 & 1 & & 131.6 & $\mathbf{N}$ & \\
\hline 1 & $9 / 7 / 87$ & 3 & 1 & & 128.7 & $\mathbf{N}$ & \\
\hline 1 & $9 / 7 / 87$ & 4 & 1 & & 124.2 & $\mathbf{N}$ & \\
\hline 1 & $9 / 7 / 87$ & 5 & 1 & & 117.3 & $\mathbf{N}$ & \\
\hline 1 & $9 / 7 / 87$ & 6 & 1 & & 85.1 & $\mathbf{N}$ & \\
\hline 1 & $10 / 5 / 87$ & 1 & 1 & & 135 & $\bar{Y}$ & \\
\hline 1 & $10 / 5 / 87$ & 2 & 1 & & 131 & $\mathbf{N}$ & \\
\hline 1 & $10 / 5 / 87$ & 3 & 1 & & 128 & $\mathbf{N}$ & \\
\hline 1 & $10 / 5 / 87$ & 4 & 1 & & 125 & $\mathbf{N}$ & \\
\hline 9 & $10 / 5 / 87$ & 5 & 1 & & 118 & $\mathrm{~N}$ & \\
\hline 1 & $10 / 5 / 87$ & 6 & 1 & & 87 & $\mathbf{N}$ & \\
\hline 1 & $11 / 3 / 87$ & 1 & 1 & & 122 & $\bar{Y}$ & \\
\hline 1 & $11 / 3 / 87$ & 2 & 1 & & 118 & $\mathbf{N}$ & \\
\hline 1 & $11 / 3 / 87$ & 3 & 1 & & 116 & $\mathbf{N}$ & \\
\hline 1 & $11 / 3 / 87$ & 4 & 9 & & 113 & $\mathbf{N}$ & \\
\hline 1 & $11 / 3 / 87$ & 5 & 1 & & 109 & $\mathbf{N}$ & \\
\hline 1 & $11 / 3 / 87$ & 6 & 1 & & 80 & $\mathbf{N}$ & \\
\hline 1 & $3 / 7 / 88$ & 1 & $\frac{1}{1}$ & & 137 & $\bar{Y}$ & \\
\hline 1 & $3 / 7 / 88$ & 2 & 1 & & 133 & $\mathrm{~N}$ & \\
\hline 1 & $3 / 7 / 88$ & 3 & 1 & & 129 & $\mathbf{N}$ & \\
\hline 1 & $3 / 7 / 88$ & 4 & 1 & & 126 & $\mathbf{N}$ & \\
\hline 1 & $3 / 7 / 88$ & 5 & 1 & & 120 & $\mathbf{N}$ & \\
\hline 1 & $4 / 6 / 88$ & 1 & 1 & & 123 & $\bar{Y}$ & \\
\hline 1 & $4 / 6 / 88$ & 2 & 1 & & 119 & $\mathbf{N}$ & \\
\hline 1 & $4 / 6 / 88$ & 3 & 1 & & 116 & $\mathrm{~N}$ & \\
\hline 1 & $4 / 6 / 88$ & 4 & 1 & & 113 & $\mathbf{N}$ & \\
\hline 1 & $4 / 6 / 88$ & 5 & 9 & & 108 & $\mathrm{~N}$ & \\
\hline 1 & $4 / 6 / 88$ & 6 & 1 & & 74 & $\mathrm{~N}$ & \\
\hline 1 & $5 / 2 / 88$ & 1 & 1 & & 134 & $Y$ & \\
\hline 1 & $5 / 2 / 88$ & 2 & 1 & & 130 & $N$ & \\
\hline 1 & $5 / 2 / 88$ & 3 & 1 & & 128 & $\bar{N}$ & \\
\hline 1 & $5 / 2 / 88$ & 4 & 1 & & 124 & $\mathrm{~N}$ & \\
\hline 1 & $5 / 2 / 88$ & 5 & 1 & & 119 & $\mathrm{~N}$ & \\
\hline 1 & $5 / 2 / 88$ & 6 & 1 & & 79 & $\bar{N}$ & \\
\hline 1 & $5 / 3 / 88$ & 1 & 1 & & 134 & $Y$ & \\
\hline 1 & $5 / 3 / 88$ & 2 & 1 & & 130 & $N$ & \\
\hline 1 & $5 / 3 / 88$ & 3 & $\frac{1}{1}$ & & 928 & $\mathrm{~N}$ & \\
\hline 1 & $5 / 3 / 88$ & 4 & 1 & & 124 & $\mathrm{~N}$ & \\
\hline 1 & $5 / 3 / 88$ & 5 & 1 & & 119 & $\mathbf{N}$ & \\
\hline 1 & $5 / 3 / 88$ & 6 & 1 & & 79 & $\mathrm{~N}$ & \\
\hline
\end{tabular}

Data obtained from WHC Surveillance Analysis Computer System (SACS), June 2, 1993. 


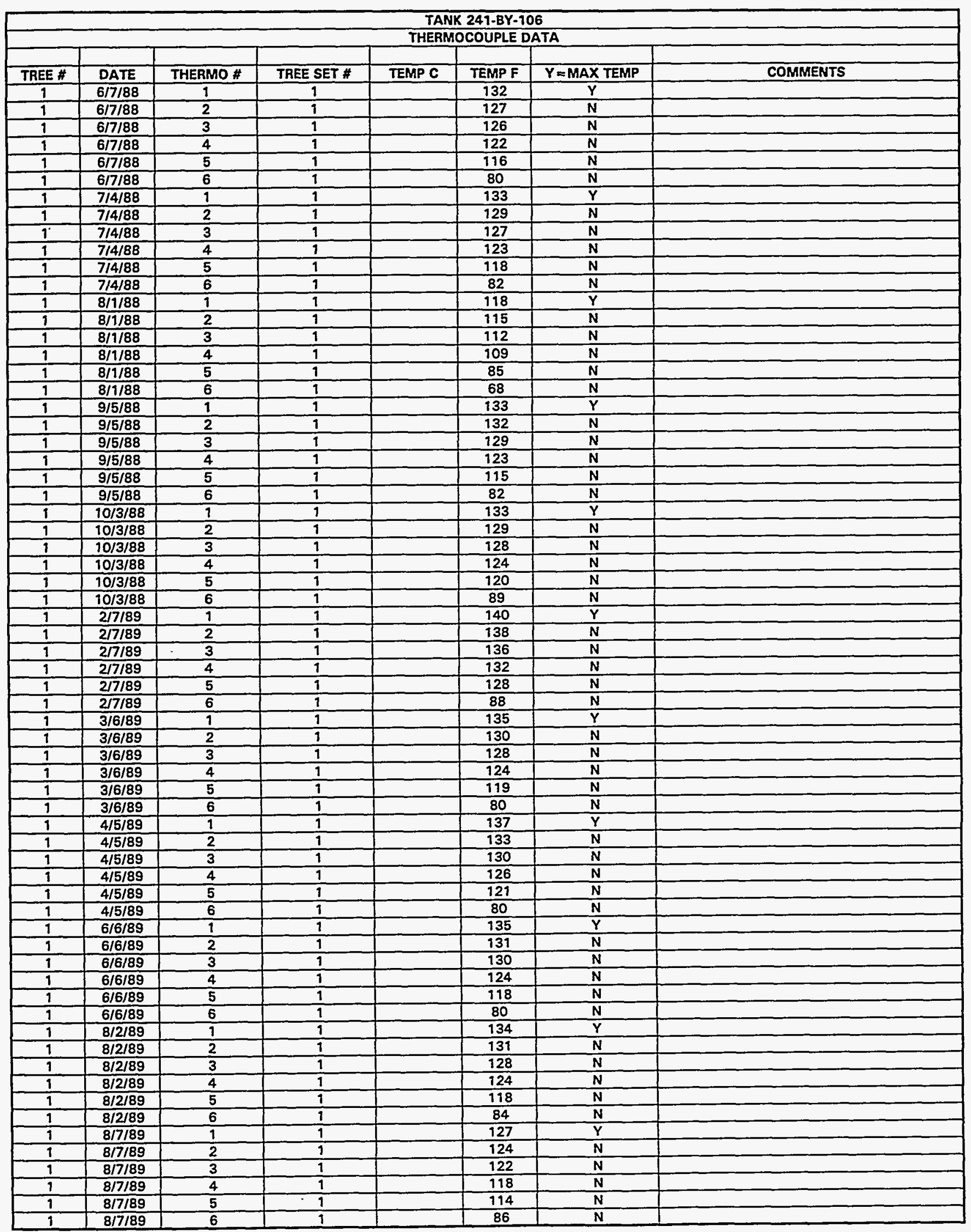

Data obtained from WHC Surveillance Analysis Computer System (SACS), June 2, 1993. 
WHC-SD-WM-ER-312, Rev. 0

\begin{tabular}{|c|c|c|c|c|c|c|c|}
\hline \multicolumn{8}{|c|}{ TANK 241-BY-106 } \\
\hline & & & & THERI & COUPLE I & TA & \\
\hline TREE \# & DATE & THERMO \# & TREE SET \# & TEMP C & TEMPF & & \\
\hline 1 & $9 / 4 / 89$ & 1 & 1 & Denir 6 & $\frac{1 \text { LMPF }}{134}$ & 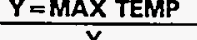 & COMMENTS \\
\hline 1 & $9 / 4 / 89$ & 2 & 1 & & & $Y$ & \\
\hline 1 & $9 / 4 / 89$ & 3 & 1 & & 130 & $\mathbf{N}$ & \\
\hline 1 & & 4 & $\frac{1}{1}$ & & 128 & $\mathbf{N}$ & \\
\hline 1 & $\begin{array}{l}9 / 4 / 89 \\
9 / 4 / 89\end{array}$ & 5 & $\frac{1}{1}$ & & 124 & $\mathbf{N}$ & \\
\hline 1 & $\begin{array}{l}9 / 4 / 89 \\
9 / 4 / 89\end{array}$ & 6 & $\frac{1}{1}$ & & 118 & $\mathbf{N}$ & \\
\hline 1 & $10 / 12 / 89$ & 1 & $\frac{1}{1}$ & & 86 & $\mathrm{~N}$ & \\
\hline 1 & $10 / 12 / 89$ & 2 & 1 & & $\frac{135.4}{131.5}$ & $Y$ & \\
\hline 1 & $10 / 12 / 89$ & 3 & 1 & & $\begin{array}{l}131.5 \\
129.1\end{array}$ & $\frac{N}{N}$ & \\
\hline 1 & $10 / 12 / 89$ & 4 & 1 & & 125.1 & $\frac{N}{N}$ & \\
\hline 1 & $10 / 12 / 89$ & 5 & 1 & & 120.7 & $\mathbf{N}$ & \\
\hline 1 & $10 / 12 / 89$ & 6 & 1 & & 88.9 & $\mathbf{N}$ & \\
\hline 1 & $10 / 23 / 89$ & 9 & 1 & & 135.4 & $\mathrm{Y}$ & \\
\hline 1 & $10 / 23 / 89$ & 2 & 1 & & 131.5 & $\mathbf{N}$ & \\
\hline 1 & $10 / 23 / 89$ & 3 & 1 & & 129.1 & $\mathbf{N}$ & \\
\hline 1 & $10 / 23 / 89$ & 4 & 1 & & 125.1 & $\mathbf{N}$ & \\
\hline 1 & $10 / 23 / 89$ & 5 & 1 & & 120.7 & $\mathbf{N}$ & \\
\hline 1 & $10 / 23 / 89$ & 6 & 1 & & 88.9 & $\mathbf{N}$ & \\
\hline 1 & $11 / 18 / 89$ & 1 & 9 & & 135.8 & $\mathrm{Y}$ & \\
\hline 9 & $11 / 18 / 89$ & 2 & 1 & & 131.6 & $\mathbf{N}$ & \\
\hline 1 & $11 / 18 / 89$ & 3 & 1 & & 129.2 & $\mathbf{N}$ & \\
\hline 1 & $11 / 18 / 89$ & 4 & 1 & & 124.8 & $\bar{N}$ & \\
\hline 1 & $11 / 18 / 89$ & 5 & 1 & & 121 & $\mathrm{~N}$ & \\
\hline 1 & $11 / 18 / 89$ & 6 & 1 & & 87.5 & $N$ & Tests on 106-BY taken with J \\
\hline 1 & $11 / 25 / 89$ & 1 & 1 & & 135.6 & $\bar{Y}$ & \\
\hline 1 & $11 / 25 / 89$ & 2 & 1 & & 131.9 & $\bar{N}$ & \\
\hline 9 & $11 / 25 / 89$ & 3 & 1 & & 129.2 & $\mathbf{N}$ & \\
\hline 1 & $11 / 25 / 89$ & 4 & 1 & & 124.5 & $\mathrm{~N}$ & \\
\hline 1 & $11 / 25 / 89$ & 5 & 1 & & 120.5 & $N$ & \\
\hline 1 & $11 / 25 / 89$ & 6 & 1 & & 87 & $\mathrm{~N}$ & \\
\hline 1 & $12 / 1 / 89$ & 1 & 1 & & 136.3 & $\bar{Y}$ & \\
\hline 1 & $12 / 1 / 89$ & 2 & 1 & & 132.4 & $\mathbf{N}$ & \\
\hline 1 & $12 / 1 / 89$ & 3 & 1 & & 129.5 & $N$ & \\
\hline 1 & $12 / 1 / 89$ & 4 & 1 & & 126 & $\mathrm{~N}$ & \\
\hline 1 & $12 / 1 / 89$ & 5 & 1 & & 121.6 & $\bar{N}$ & \\
\hline 1 & $12 / 1 / 89$ & 6 & 1 & & 86.7 & $\mathrm{~N}$ & \\
\hline 1 & $12 / 10 / 89$ & 1 & 1 & & 136.3 & $\bar{Y}$ & \\
\hline 1 & $12 / 10 / 89$ & 2 & 1 & & 132.4 & $\mathbf{N}$ & \\
\hline 1 & $12 / 10 / 89$ & 3 & 1 & & 129.8 & $\mathbf{N}$ & \\
\hline 1 & $12 / 10 / 89$ & 4 & 9 & & 125.9 & $\mathrm{~N}$ & \\
\hline 1 & $12 / 10 / 89$ & 5 & 1 & & 122 & $N$ & \\
\hline 1 & $12 / 10 / 89$ & 6 & 1 & & 87 & $N$ & \\
\hline 1 & $12 / 15 / 89$ & 1 & 1 & & 136 & $Y$ & \\
\hline 1 & $12 / 15 / 89$ & 2 & 1 & & 132.3 & $\mathbf{N}$ & \\
\hline 1 & $12 / 15 / 89$ & 3 & 1 & & 129.6 & $\mathbf{N}$ & \\
\hline 1 & $12 / 15 / 89$ & 4 & 1 & & 125.6 & $\mathbf{N}$ & \\
\hline 1 & $12 / 15 / 89$ & 5 & 1 & & 121.3 & $N$ & \\
\hline 1 & $12 / 23 / 89$ & 1 & 1 & & 136 & $Y$ & \\
\hline 1 & $12 / 23 / 89$ & 2 & 1 & & 132 & $N$ & \\
\hline 1 & $12 / 23 / 89$ & 3 & 1 & & 130 & $\mathrm{~N}$ & \\
\hline 1 & $12 / 23 / 89$ & 4 & 1 & & 126 & $N$ & \\
\hline 1 & $12 / 23 / 89$ & 5 & 1 & & 122 & $\mathbf{N}$ & \\
\hline 1 & $12 / 23 / 89$ & 6 & 1 & & 85 & $N$ & \\
\hline 1 & $12 / 29 / 89$ & 1 & 1 & & 136 & $\bar{Y}$ & J-2E-OP-2O \\
\hline 1 & $12 / 29 / 89$ & 2 & 1 & & 133 & $\mathbf{N}$ & \\
\hline 1 & $12 / 29 / 89$ & 3 & 1 & & 130 & $\mathrm{~N}$ & \\
\hline 1 & $12 / 29 / 89$ & 4 & 1 & & 126 & $\mathbf{N}$ & \\
\hline 1 & $12 / 29 / 89$ & 5 & 1 & & 122 & $\mathrm{~N}$ & \\
\hline 1 & $12 / 29 / 89$ & 6 & 1 & & 85 & $\mathbf{N}$ & \\
\hline 1 & $1 / 6 / 90$ & 1 & 1 & & 132.6 & $\bar{Y}$ & \\
\hline 1 & $1 / 6 / 90$ & 2 & 1 & & 128.7 & $N$ & \\
\hline 1 & $1 / 6 / 90$ & 3 & 1 & & 126.2 & $\mathbf{N}$ & \\
\hline 1 & $1 / 6 / 90$ & 4 & 1 & & 122.4 & $\mathbf{N}$ & \\
\hline 1 & $1 / 6 / 90$ & 5 & 1 & & 118 & $\mathbf{N}$ & \\
\hline 1 & $1 / 6 / 90$ & 6 & 1 & & 81.5 & $\mathrm{~N}$ & \\
\hline 1 & $1 / 13 / 90$ & 1 & 1 & & 131 & $\bar{Y}$ & \\
\hline
\end{tabular}

Data obtained from WHC Surveillance Analysis Computer System (SACS), June 2, 1993. 


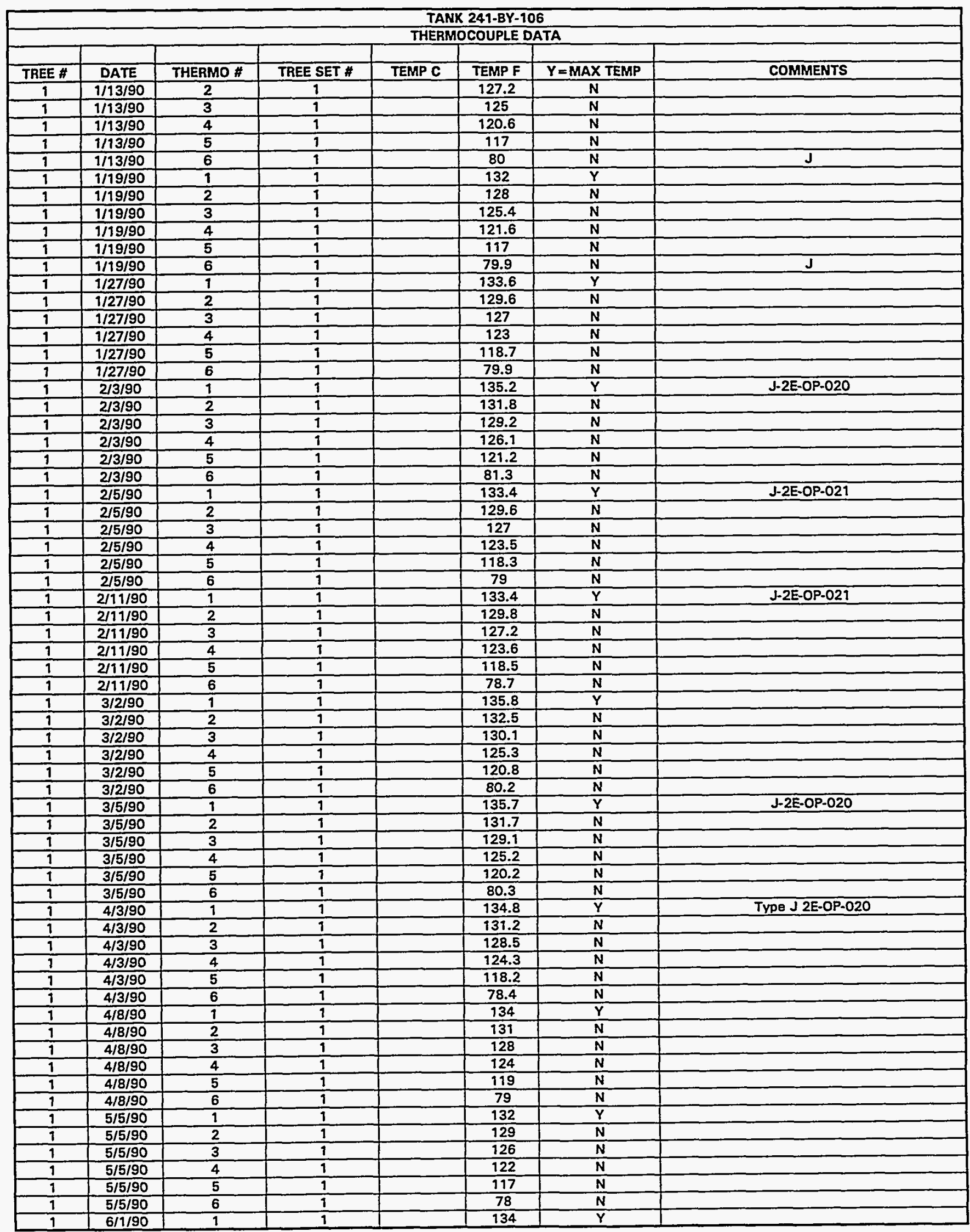

Data obtained from WHC Surveillance Analysis Computer System (SACS), June 2, 1993. 
WHC-SD-WM-ER-312, Rev. 0

\begin{tabular}{|c|c|c|c|c|c|c|c|}
\hline \multicolumn{8}{|c|}{ TANK 241-BY-106 } \\
\hline \multicolumn{8}{|c|}{ THERMOCOUPLE DATA } \\
\hline TREE \# & DATE & THERMO \# & TREE SET \# & TEMP C & TEMP F & $\mathrm{Y}=$ MAX TEMP & COMMENTS \\
\hline 1 & $6 / 1 / 90$ & 2 & 1 & & 130.6 & 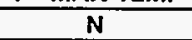 & \\
\hline 1 & $6 / 1 / 90$ & 3 & 1 & & 127.6 & $\mathbf{N}$ & \\
\hline 1 & $6 / 1 / 90$ & 4 & 1 & & 123.7 & $\mathbf{N}$ & \\
\hline 1 & $6 / 1 / 90$ & 5 & 1 & & 118.2 & $\bar{N}$ & \\
\hline 1 & $6 / 1 / 90$ & 6 & 1 & & 81.2 & $\mathbf{N}$ & \\
\hline 1 & $7 / 8 / 90$ & 1 & 1 & & 133.9 & $\mathrm{Y}$ & J-2E-OP-02O \\
\hline 1 & $7 / 8 / 90$ & 2 & 1 & & 130.2 & $\mathbf{N}$ & \\
\hline 1 & $7 / 8 / 90$ & 3 & 1 & & 127.6 & $N$ & \\
\hline 1 & $7 / 8 / 90$ & 4 & 1 & & 124 & 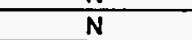 & \\
\hline 1 & $7 / 8 / 90$ & 5 & 1 & & 119 & $N$ & \\
\hline 1 & $7 / 8 / 90$ & 6 & 1 & & 83.9 & $\mathrm{~N}$ & \\
\hline 1 & $8 / 5 / 90$ & 1 & 1 & & 130.3 & $Y$ & $J$ \\
\hline 1 & $8 / 5 / 90$ & 2 & 1 & & 126.6 & $\bar{N}$ & \\
\hline 1 & $8 / 5 / 90$ & 3 & 1 & & 124.1 & $\mathrm{~N}$ & \\
\hline 1 & $8 / 5 / 90$ & 4 & 1 & & 120.2 & $\frac{N}{N}$ & \\
\hline 1 & $8 / 5 / 90$ & 5 & 1 & & 115.8 & $N$ & \\
\hline 1 & $8 / 5 / 90$ & 6 & 1 & & 83.9 & $\bar{N}$ & \\
\hline 1 & $9 / 11 / 90$ & 1 & 1 & & 129 & $Y$ & $J$ \\
\hline 1 & $9 / 11 / 90$ & 2 & 1 & & 126 & $\mathbf{N}$ & \\
\hline 1 & $9 / 11 / 90$ & 3 & 1 & & 125 & $\mathrm{~N}$ & \\
\hline 1 & $9 / 11 / 90$ & 4 & 1 & & 118 & $N$ & \\
\hline 1 & $9 / 11 / 90$ & 5 & 1 & & 919 & $\ddot{N}$ & \\
\hline 1 & $9 / 11 / 90$ & 6 & 1 & & 84 & $\mathrm{~N}$ & \\
\hline 1 & $10 / 9 / 90$ & 1 & 1 & & 130 & $Y$ & $J$ \\
\hline 1 & $10 / 9 / 90$ & 2 & 1 & & 127 & $\bar{N}$ & \\
\hline 1 & $10 / 9 / 90$ & 3 & 9 & & 124 & $N$ & \\
\hline 1 & $10 / 9 / 90$ & 4 & 1 & & 121 & $\bar{N}$ & \\
\hline 1 & $10 / 9 / 90$ & 5 & 1 & & 116 & $\mathrm{~N}$ & \\
\hline 1 & $10 / 9 / 90$ & 6 & 1 & & 86 & $\ddot{N}$ & \\
\hline 1 & $10 / 26 / 90$ & 1 & $\frac{1}{1}$ & & 127.6 & $Y$ & J-890523512-20 \\
\hline 1 & $10 / 26 / 90$ & 2 & 1 & & 124.9 & $\mathbf{N}$ & \\
\hline 1 & $10 / 26 / 90$ & 3 & 9 & & 122.6 & $N$ & \\
\hline 1 & $10 / 26 / 90$ & 4 & 1 & & 119 & $\bar{N}$ & \\
\hline 1 & $10 / 26 / 90$ & 5 & 1 & & 114.8 & $N$ & \\
\hline 1 & $10 / 26 / 90$ & 6 & 1 & & 83.8 & $N$ & \\
\hline 1 & $10 / 30 / 90$ & 1 & 1 & & 130.1 & $Y$ & J 89052312-20 \\
\hline 1 & $10 / 30 / 90$ & 2 & $T$ & & 126.9 & $\bar{N}$ & \\
\hline 1 & $10 / 30 / 90$ & 3 & 1 & & 124.7 & $\mathrm{~N}$ & \\
\hline 1 & $10 / 30 / 90$ & 4 & 1 & & 124.7 & $\bar{N}$ & \\
\hline 1 & $10 / 30 / 90$ & 5 & 1 & & 117.2 & $\bar{N}$ & \\
\hline 1 & $10 / 30 / 90$ & 6 & 1 & & 86.3 & $\mathrm{~N}$ & \\
\hline 1 & $11 / 5 / 90$ & 1 & $\frac{1}{1}$ & & 131.1 & $\bar{Y}$ & J 890523512-20 \\
\hline 1 & $11 / 5 / 90$ & 2 & 1 & & 127.4 & $\bar{N}$ & \\
\hline 1 & $11 / 5 / 90$ & 3 & 1 & & 124.7 & $N$ & \\
\hline 1 & $11 / 5 / 90$ & 4 & 1 & & 121.2 & $\mathrm{~N}$ & \\
\hline 1 & $11 / 5 / 90$ & 5 & 1 & & 116.8 & $\mathbf{N}$ & \\
\hline 1 & $11 / 5 / 90$ & 6 & 1 & & 86 & $\mathrm{~N}$ & \\
\hline 1 & $19 / 12 / 90$ & 1 & 1 & & 129.9 & $\bar{Y}$ & $2 \mathrm{E}-\mathrm{OP}-021$ \\
\hline 1 & $11 / 12 / 90$ & 2 & 1 & & 126.5 & $\mathbf{N}$ & \\
\hline 1 & $19 / 12 / 90$ & 3 & 9 & & 124.5 & $N$ & \\
\hline 1 & $11 / 12 / 90$ & 4 & 1 & & 121.2 & $\mathrm{~N}$ & \\
\hline 1 & $11 / 12 / 90$ & 5 & 1 & & 117.1 & $\mathrm{~N}$ & \\
\hline 1 & $11 / 12 / 90$ & 6 & $\frac{1}{1}$ & & 85.2 & $\mathbf{N}$ & \\
\hline$\overline{1}$ & $11 / 18 / 90$ & 1 & $\frac{T}{1}$ & & 130.5 & $\bar{Y}$ & $J 890523512-20$ \\
\hline 1 & $11 / 18 / 90$ & 2 & 1 & & 126.8 & $\mathbf{N}$ & \\
\hline 1 & $11 / 18 / 90$ & 3 & 1 & & 124.7 & $\mathbf{N}$ & \\
\hline 1 & $11 / 18 / 90$ & 4 & 1 & & 121.4 & $\mathbf{N}$ & \\
\hline 1 & $11 / 18 / 90$ & 5 & 1 & & 116.5 & $\mathbf{N}$ & \\
\hline 1 & $11 / 18 / 90$ & 6 & 1 & & 85 & $\mathbf{N}$ & \\
\hline 1 & $11 / 25 / 90$ & 1 & 1 & & 130.3 & $\bar{Y}$ & J 2E-OP-029 \\
\hline 9 & $11 / 25 / 90$ & 2 & 1 & & 126.9 & $\bar{N}$ & \\
\hline 1 & $11 / 25 / 90$ & 3 & 1 & & 124.4 & $N$ & \\
\hline 1 & $11 / 25 / 90$ & 4 & 1 & & 120.3 & $\mathbf{N}$ & \\
\hline 1 & $11 / 25 / 90$ & 5 & 1 & & 116.5 & $\mathbf{N}$ & \\
\hline 1 & $11 / 25 / 90$ & 6 & 1 & & 82.4 & $\bar{N}$ & \\
\hline 1 & $12 / 5 / 90$ & 1 & 1 & & 130.2 & $Y$ & J 890523512-20 \\
\hline
\end{tabular}

Data obtained from WHC Surveillance Analysis Computer System (SACS), June 2, 1993. 
WHC-SD-WM-ER-312, Rev. 0

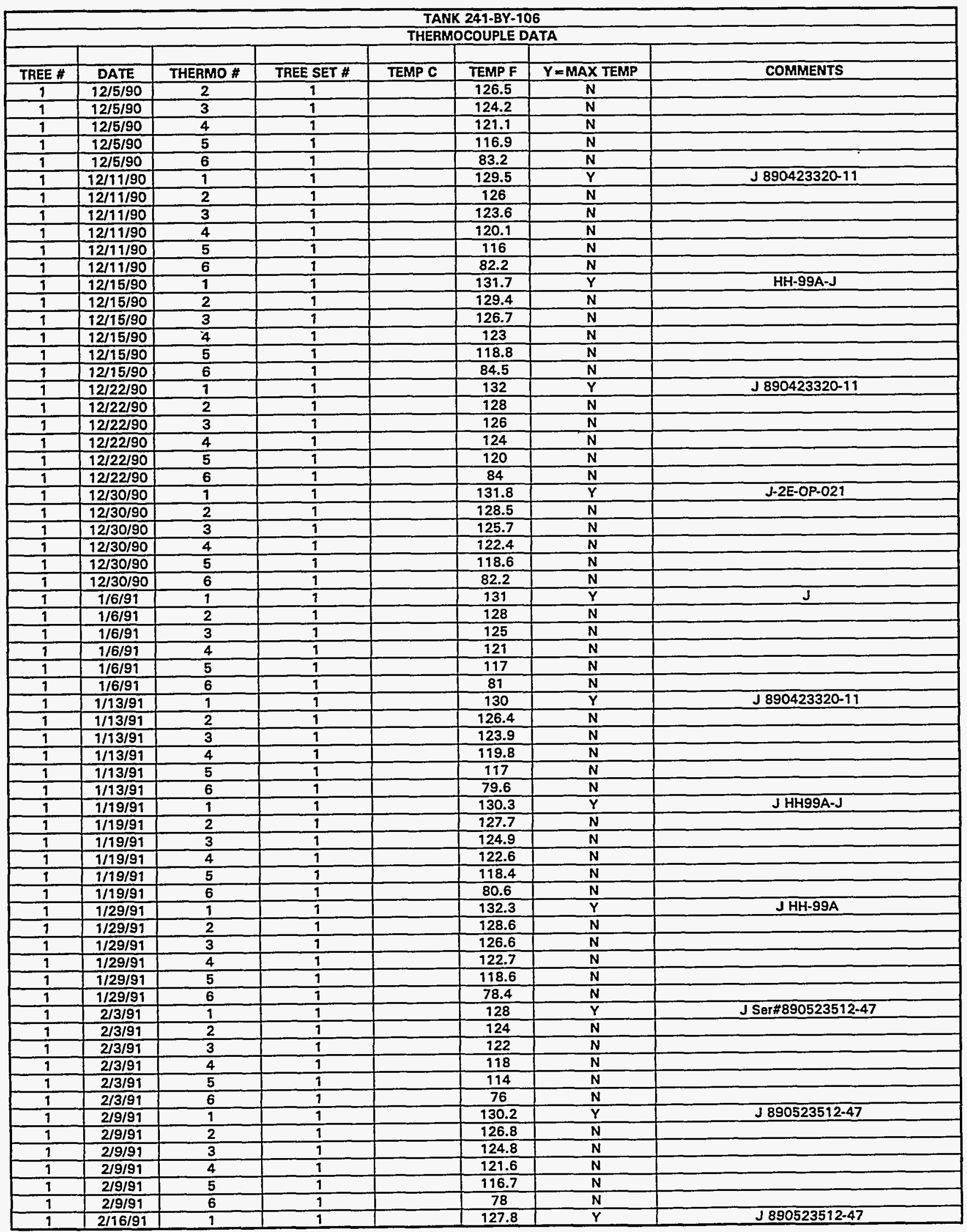

Data obtained from WHC Surveillance Analysis Computer System (SACS), June 2, 1993. 
WHC-SD-WM-ER-312, ReV. 0

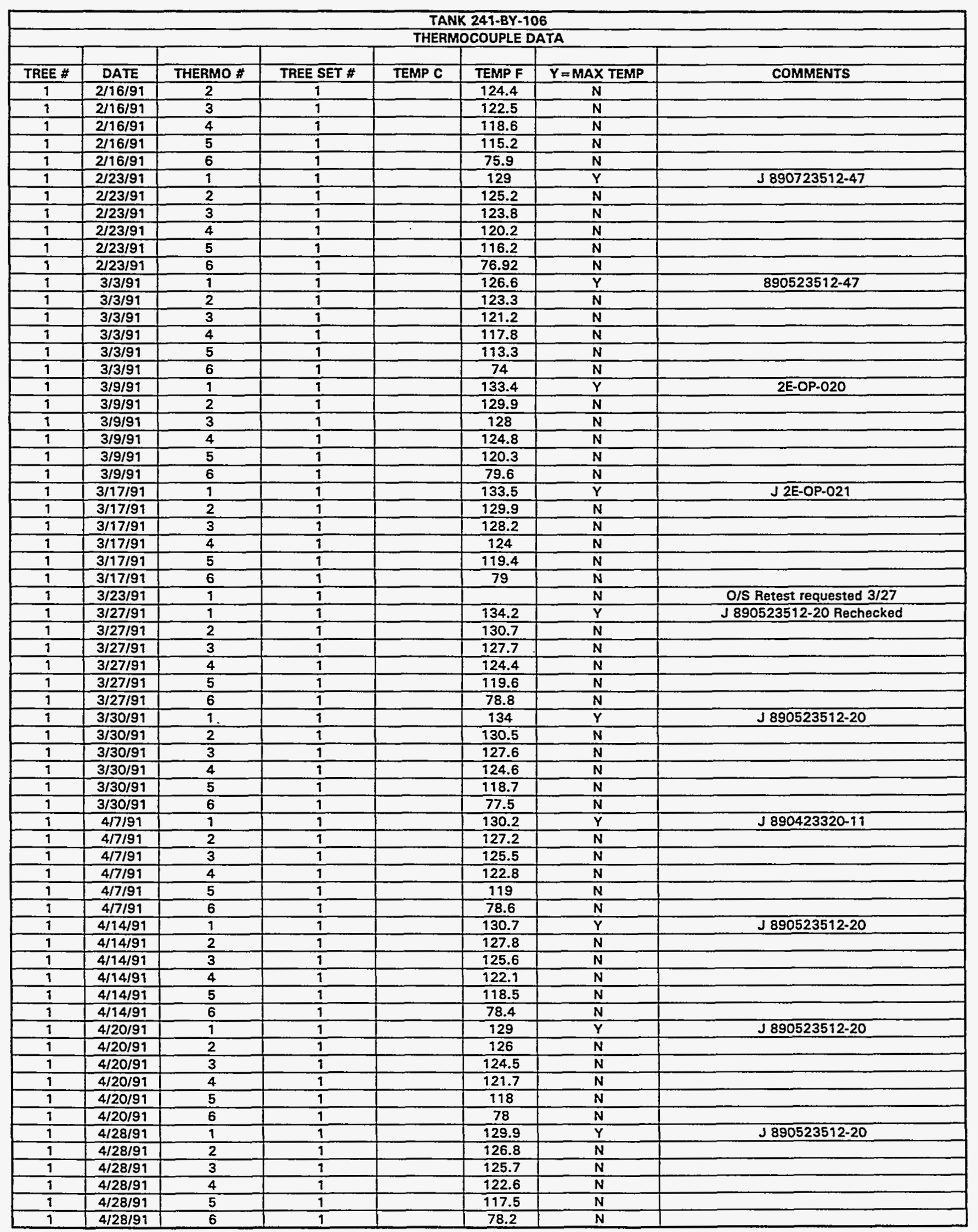

Data obtained from WHC Surveillance Analysis Computer System (SACS), June 2, 1993. 
WHC-SD-WM-ER-312, Rev. 0

\begin{tabular}{|c|c|c|c|c|c|c|c|}
\hline \multicolumn{8}{|c|}{ TANK 241-BY-106 } \\
\hline \multicolumn{8}{|c|}{ THERMOCOUPLE DATA } \\
\hline TREE \# & DATE & THERMO \# & TREE SET \# & TEMP C & TEMP F & $Y=M A X T E M P$ & COMMENTS \\
\hline 1 & $5 / 5 / 91$ & 1 & 1 & & 130 & $Y$ & J $890523512-20$ \\
\hline 1 & $5 / 5 / 91$ & 2 & 1 & & 126.7 & $N$ & \\
\hline 1 & $5 / 5 / 91$ & 3 & $\overline{1}$ & & 124.8 & $\bar{N}$ & \\
\hline 1 & $5 / 5 / 91$ & 4 & 1 & & 120.8 & $\bar{N}$ & \\
\hline 1 & $5 / 5 / 91$ & 5 & 1 & & 116.5 & $\mathbf{N}$ & \\
\hline 1 & $5 / 5 / 91$ & 6 & 1 & & 77.7 & $N$ & \\
\hline 1 & $5 / 12 / 91$ & 1 & 1 & & 130.3 & $\bar{Y}$ & J 890523512 \\
\hline 7 & $5 / 12 / 91$ & 2 & 1 & & 126.7 & $\mathbf{N}$ & \\
\hline 1 & $5 / 12 / 91$ & 3 & 1 & & 124.7 & $\mathrm{~N}$ & \\
\hline 1 & $5 / 12 / 91$ & 4 & 1 & & 120.3 & $\mathbf{N}$ & \\
\hline 1 & $5 / 12 / 91$ & 5 & 1 & & 116.5 & $\mathbf{N}$ & \\
\hline 1 & $5 / 12 / 91$ & 6 & 1 & & 78.2 & $\mathbf{N}$ & \\
\hline 1 & $5 / 18 / 91$ & 1 & 1 & & 132 & $\bar{Y}$ & J Keithley 872 2E-OP-020 \\
\hline 1 & $5 / 18 / 91$ & 2 & 1 & & 129.1 & $\mathrm{~N}$ & \\
\hline 1 & $5 / 18 / 91$ & 3 & 1 & & 127.1 & $\mathbf{N}$ & \\
\hline 1 & $5 / 18 / 91$ & 4 & 1 & & 123.1 & $\mathbf{N}$ & \\
\hline 1 & $5 / 18 / 91$ & 5 & 1 & & 119.1 & $\mathbf{N}$ & \\
\hline 1 & $5 / 18 / 91$ & 6 & 1 & & 79.6 & $\mathbf{N}$ & \\
\hline 1 & $5 / 25 / 91$ & 1 & 1 & & 132.2 & $\bar{Y}$ & J 2E-OP-021 \\
\hline 1 & $5 / 25 / 91$ & 2 & 1 & & 129.3 & $N$ & \\
\hline 1 & $5 / 25 / 91$ & 3 & 1 & & 127 & $\bar{N}$ & \\
\hline 1 & $5 / 25 / 91$ & 4 & 1 & & 123 & $N$ & \\
\hline 1 & $5 / 25 / 91$ & 5 & 1 & & 118.9 & $\mathbf{N}$ & \\
\hline 1 & $5 / 25 / 91$ & 6 & 1 & & 79.9 & $\bar{N}$ & \\
\hline 1 & $6 / 2 / 91$ & $\frac{1}{1}$ & 1 & & 128.3 & $Y$ & J 890423320-42 \\
\hline 1 & $6 / 2 / 91$ & 2 & $\frac{1}{1}$ & & 125.4 & $\mathbf{N}$ & \\
\hline 1 & $6 / 2 / 91$ & 3 & 1 & & 124.5 & $N$ & \\
\hline 1 & $6 / 2 / 91$ & 4 & 1 & & 121.5 & $\mathrm{~N}$ & \\
\hline 1 & $6 / 2 / 91$ & 5 & 1 & & 116.6 & $\mathbf{N}$ & \\
\hline$T$ & $6 / 2 / 91$ & 6 & 1 & & 78.6 & $N$ & \\
\hline 1 & $6 / 9 / 91$ & 1 & 1 & & 129.6 & $\bar{Y}$ & J $890523512-20$ \\
\hline 1 & $6 / 9 / 91$ & 2 & 1 & & 127 & $\mathbf{N}$ & \\
\hline 1 & $6 / 9 / 91$ & 3 & 1 & & 124.9 & $\mathbf{N}$ & \\
\hline 7 & $6 / 9 / 91$ & 4 & 1 & & 121 & $\mathrm{~N}$ & \\
\hline 1 & $6 / 9 / 91$ & 5 & 1 & & 116.4 & $\bar{N}$ & \\
\hline 1 & $6 / 9 / 91$ & 6 & 1 & & 79.4 & $N$ & \\
\hline 1 & $6 / 15 / 91$ & 1 & 1 & & 132.1 & $\bar{Y}$ & J 2E-OP-021 \\
\hline$\overline{9}$ & $6 / 15 / 91$ & 2 & 1 & & 128.6 & $\mathbf{N}$ & \\
\hline 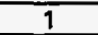 & $6 / 15 / 91$ & 3 & 1 & & 126.6 & $\bar{N}$ & \\
\hline$\frac{1}{9}$ & $6 / 15 / 91$ & 4 & 1 & & 122.6 & $\mathrm{~N}$ & \\
\hline 1 & $6 / 15 / 91$ & 5 & 1 & & 117.9 & $\bar{N}$ & \\
\hline 1 & $6 / 15 / 91$ & 6 & 1 & & 80.5 & N & \\
\hline 1 & $6 / 23 / 91$ & 1 & 1 & & 131 & $\bar{Y}$ & J 2E-OP-021 \\
\hline 1 & $6 / 23 / 91$ & 2 & 1 & & 128 & $\mathbf{N}$ & \\
\hline 9 & $6 / 23 / 91$ & 3 & 1 & & 126 & $\bar{N}$ & \\
\hline 1 & $6 / 23 / 91$ & 4 & 1 & & 122 & $\mathbf{N}$ & \\
\hline$\frac{9}{1}$ & $6 / 23 / 91$ & 5 & 1 & & 118 & $\mathbf{N}$ & \\
\hline 1 & $6 / 23 / 91$ & 6 & 9 & & 81 & $\mathbf{N}$ & \\
\hline$\overline{1}$ & $6 / 29 / 91$ & 1 & 1 & & 129 & $Y$ & J 890423220-42 \\
\hline$\frac{1}{1}$ & $6 / 29 / 91$ & 2 & 1 & & 125.7 & $\mathbf{N}$ & \\
\hline 1 & $6 / 29 / 91$ & 3 & 1 & & 123.4 & $\mathbf{N}$ & \\
\hline 1 & $6 / 29 / 91$ & 4 & 1 & & 119.2 & $\mathbf{N}$ & \\
\hline 1 & $6 / 29 / 91$ & 5 & 1 & & 115.6 & $\mathbf{N}$ & \\
\hline 1 & $6 / 29 / 91$ & 6 & 1 & & 79.8 & $\mathbf{N}$ & \\
\hline 1 & $7 / 6 / 91$ & 1 & 1 & & 130 & $\bar{Y}$ & J $910126143-42$ \\
\hline 1 & $7 / 6 / 91$ & 2 & 1 & & 127.6 & $N$ & \\
\hline 1 & $7 / 6 / 91$ & 3 & 1 & & 125.4 & $\overline{\mathbf{N}}$ & \\
\hline 1 & $7 / 6 / 91$ & 4 & 1 & & 121.4 & $\mathbf{N}$ & \\
\hline 1 & $7 / 6 / 91$ & 5 & 1 & & 117.3 & $\mathbf{N}$ & \\
\hline 1 & $7 / 6 / 91$ & 6 & 1 & & 81.4 & $N$ & \\
\hline$\frac{1}{1}$ & $7 / 13 / 91$ & 1 & 1 & & 130 & $\bar{Y}$ & J $910126143-42$ \\
\hline 1 & $7 / 13 / 91$ & 2 & 1 & & 127.6 & $\mathbf{N}$ & \\
\hline 9 & $7 / 13 / 91$ & 3 & 1 & & 125.4 & $\mathbf{N}$ & \\
\hline 1 & $7 / 13 / 91$ & 4 & 1 & & 121.4 & $\mathbf{N}$ & \\
\hline 1 & $7 / 13 / 91$ & 5 & 1 & & 118 & $\bar{N}$ & \\
\hline 1 & $7 / 13 / 91$ & 6 & 1 & & 81.4 & $\mathbf{N}$ & \\
\hline
\end{tabular}

Data obtained from WHC Surveillance Analysis Computer System (SACS), June 2, 1993. 


\begin{tabular}{|c|c|c|c|c|c|c|c|}
\hline \multicolumn{8}{|c|}{ TANK 241-BY-106 } \\
\hline \multicolumn{8}{|c|}{ THERMOCOUPLE DATA } \\
\hline TREE \# & DATE & THERMO \# & TREE SET \# & TEMP C & TEMP F & $Y=$ MAX TEMP & COMMENTS \\
\hline 1 & $7 / 20 / 91$ & 1 & 1 & & 128.3 & $Y$ & J910126143-64 \\
\hline 1 & $7 / 20 / 91$ & 2 & 1 & & 125.9 & $\mathbf{N}$ & \\
\hline 1 & $7 / 20 / 91$ & 3 & 1 & & 124.1 & $\mathbf{N}$ & \\
\hline 1 & $7 / 20 / 91$ & 4 & 1 & & 119.7 & $\mathbf{N}$ & \\
\hline 1 & $7 / 20 / 91$ & 5 & 1 & & 115.7 & $\mathrm{~N}$ & \\
\hline 1 & $7 / 20 / 91$ & 6 & 1 & & 80.8 & $\mathbf{N}$ & \\
\hline 1 & $7 / 27 / 91$ & 1 & 1 & & 126.7 & $\mathbf{Y}$ & $J 910126143-14$ \\
\hline 1 & $7 / 27 / 91$ & 2 & 1 & & 124.3 & $\mathbf{N}$ & \\
\hline 1 & $7 / 27 / 91$ & 3 & $\overline{1}$ & & 122.9 & $\mathbf{N}$ & \\
\hline 1 & $7 / 27 / 91$ & 4 & 1 & & 120.3 & $\bar{N}$ & \\
\hline$\frac{1}{1}$ & $7 / 27 / 91$ & 5 & 1 & & 115.3 & $\mathbf{N}$ & \\
\hline 1 & $7 / 27 / 91$ & 6 & 1 & & 81.1 & $\mathbf{N}$ & \\
\hline 1 & $8 / 3 / 91$ & 1 & 1 & & 126.3 & $\bar{Y}$ & J $910126143-14$ \\
\hline$\frac{1}{1}$ & $8 / 3 / 91$ & 2 & 1 & & 124.2 & $\bar{N}$ & \\
\hline 1 & $8 / 3 / 91$ & 3 & 1 & & 123.4 & $\mathbf{N}$ & \\
\hline 1 & $8 / 3 / 91$ & 4 & 1 & & 120.4 & $\mathrm{~N}$ & \\
\hline 1 & $8 / 3 / 91$ & 5 & 1 & & 116.2 & $\mathbf{N}$ & \\
\hline 1 & $8 / 3 / 91$ & 6 & 1 & & 81.6 & $\overline{\mathbf{N}}$ & \\
\hline 1 & $8 / 10 / 91$ & 1 & 1 & & 126 & $Y$ & $J 890523512-20$ \\
\hline$\frac{1}{1}$ & $8 / 10 / 91$ & 2 & 1 & & 124 & $\mathbf{N}$ & \\
\hline 1 & $8 / 10 / 91$ & 3 & 1 & & 124 & $\mathbf{N}$ & \\
\hline 1 & $8 / 10 / 91$ & 4 & $T$ & & 121 & $\mathbf{N}$ & \\
\hline$\frac{1}{1}$ & $8 / 10 / 91$ & 5 & 1 & & 117 & $\mathbf{N}$ & \\
\hline 1 & $8 / 10 / 91$ & 6 & 1 & & 82 & $\mathbf{N}$ & \\
\hline 1 & $8 / 18 / 91$ & 1 & 1 & & 126.5 & $Y$ & J $890523512-20$ \\
\hline$\frac{1}{1}$ & $8 / 18 / 91$ & 2 & 1 & & 124.3 & $\mathbf{N}$ & \\
\hline 1 & $8 / 18 / 91$ & 3 & 1 & & 123.5 & $\mathbf{N}$ & \\
\hline 1 & $8 / 18 / 91$ & 4 & 1 & & 120.7 & $\mathbf{N}$ & \\
\hline 1 & $8 / 18 / 91$ & 5 & 1 & & 116.5 & $\mathrm{~N}$ & \\
\hline 1 & $8 / 18 / 91$ & 6 & 1 & & 83 & $\bar{N}$ & \\
\hline 1 & $8 / 24 / 91$ & 1 & 1 & & 127.2 & $Y$ & $J 890523512-20$ \\
\hline 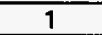 & $8 / 24 / 91$ & 2 & $\frac{1}{1}$ & & 124.8 & $\mathbf{N}$ & \\
\hline 1 & $8 / 24 / 91$ & 3 & 1 & & 923.7 & $\overline{\mathbf{N}}$ & \\
\hline 1 & $8 / 24 / 91$ & 4 & $T$ & & 120.9 & $\mathbf{N}$ & \\
\hline 1 & $8 / 24 / 91$ & 5 & 1 & & 116.9 & $\mathbf{N}$ & \\
\hline 1 & $8 / 24 / 91$ & 6 & 1 & & 83.8 & $\bar{N}$ & \\
\hline 1 & $8 / 31 / 91$ & 1 & 1 & & 126.2 & $\mathrm{Y}$ & $J .890423320-42$ \\
\hline$\frac{1}{1}$ & $8 / 31 / 91$ & 2 & 1 & & 123.6 & $\mathbf{N}$ & \\
\hline 1 & $8 / 31 / 91$ & 3 & 1 & & 122.7 & $\mathbf{N}$ & \\
\hline 9 & $8 / 31 / 91$ & 4 & 1 & & 120 & $\mathbf{N}$ & \\
\hline 1 & $8 / 31 / 91$ & 5 & 1 & & 115.7 & $\mathbf{N}$ & \\
\hline 1 & $8 / 31 / 91$ & 6 & 1 & & 83.5 & $\mathbf{N}$ & \\
\hline 1 & $9 / 8 / 91$ & 1 & 9 & & 126.2 & $\bar{Y}$ & J-890423320-42 \\
\hline 1 & $9 / 8 / 91$ & 2 & 1 & & 123.6 & $\mathbf{N}$ & \\
\hline 1 & $9 / 8 / 91$ & 3 & 1 & & 122.7 & $\mathbf{N}$ & \\
\hline 1 & $9 / 8 / 91$ & 4 & 1 & & 120.1 & $\mathrm{~N}$ & \\
\hline 1 & $9 / 8 / 91$ & 5 & 1 & & 115.7 & $\mathbf{N}$ & \\
\hline 1 & 9/8/91 & 6 & 1 & & 83.5 & $\mathbf{N}$ & \\
\hline 1 & $9 / 15 / 91$ & 1 & 1 & & 126.4 & $\bar{Y}$ & J-910126143-75 \\
\hline 1 & $9 / 15 / 91$ & 2 & 1 & & 123.9 & $\mathbf{N}$ & \\
\hline 1 & $9 / 15 / 91$ & 3 & 1 & & 123.2 & $\mathbf{N}$ & \\
\hline 1 & $9 / 15 / 91$ & 4 & 1 & & 120.9 & $\mathbf{N}$ & \\
\hline 1 & $9 / 15 / 91$ & 5 & 1 & & 116.2 & $\mathrm{~N}$ & \\
\hline$\frac{1}{1}$ & $9 / 15 / 91$ & 6 & 1 & & 85 & $\mathbf{N}$ & \\
\hline 1 & $9 / 22 / 91$ & 1 & 1 & & 131.4 & $\bar{Y}$ & J-910126143-75 \\
\hline 9 & $9 / 22 / 91$ & 2 & 1 & & 128.2 & $N$ & \\
\hline 1 & $9 / 22 / 91$ & 3 & 1 & & 125.2 & $\mathbf{N}$ & \\
\hline 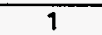 & $9 / 22 / 91$ & 4 & 1 & & 121.8 & $\mathbf{N}$ & \\
\hline 1 & $9 / 22 / 91$ & 5 & 1 & & 117.7 & $\mathbf{N}$ & \\
\hline 1 & $9 / 22 / 91$ & 6 & 1 & & 86.4 & $N$ & \\
\hline 1 & $9 / 28 / 91$ & 1 & 1 & & 130.2 & $\bar{Y}$ & J-Keithley $872 \# T 1.059$ \\
\hline 1 & $9 / 28 / 91$ & 2 & 1 & & 127.4 & $\mathbf{N}$ & \\
\hline 1 & $9 / 28 / 91$ & 3 & 1 & & 126.3 & $\bar{N}$ & \\
\hline 1 & $9 / 28 / 91$ & 4 & 1 & & 123.2 & $\mathbf{N}$ & \\
\hline 1 & $9 / 28 / 91$ & 5 & 1 & & 119 & $\overline{\mathbf{N}}$ & \\
\hline 1 & $9 / 28 / 91$ & 6 & 1 & & 86.7 & $\mathbf{N}$ & \\
\hline
\end{tabular}

Data obtained from WHC Surveillance Analysis Computer System (SACS), June 2, 1993. 
WHC-SD-WM-ER-312, Rev. 0

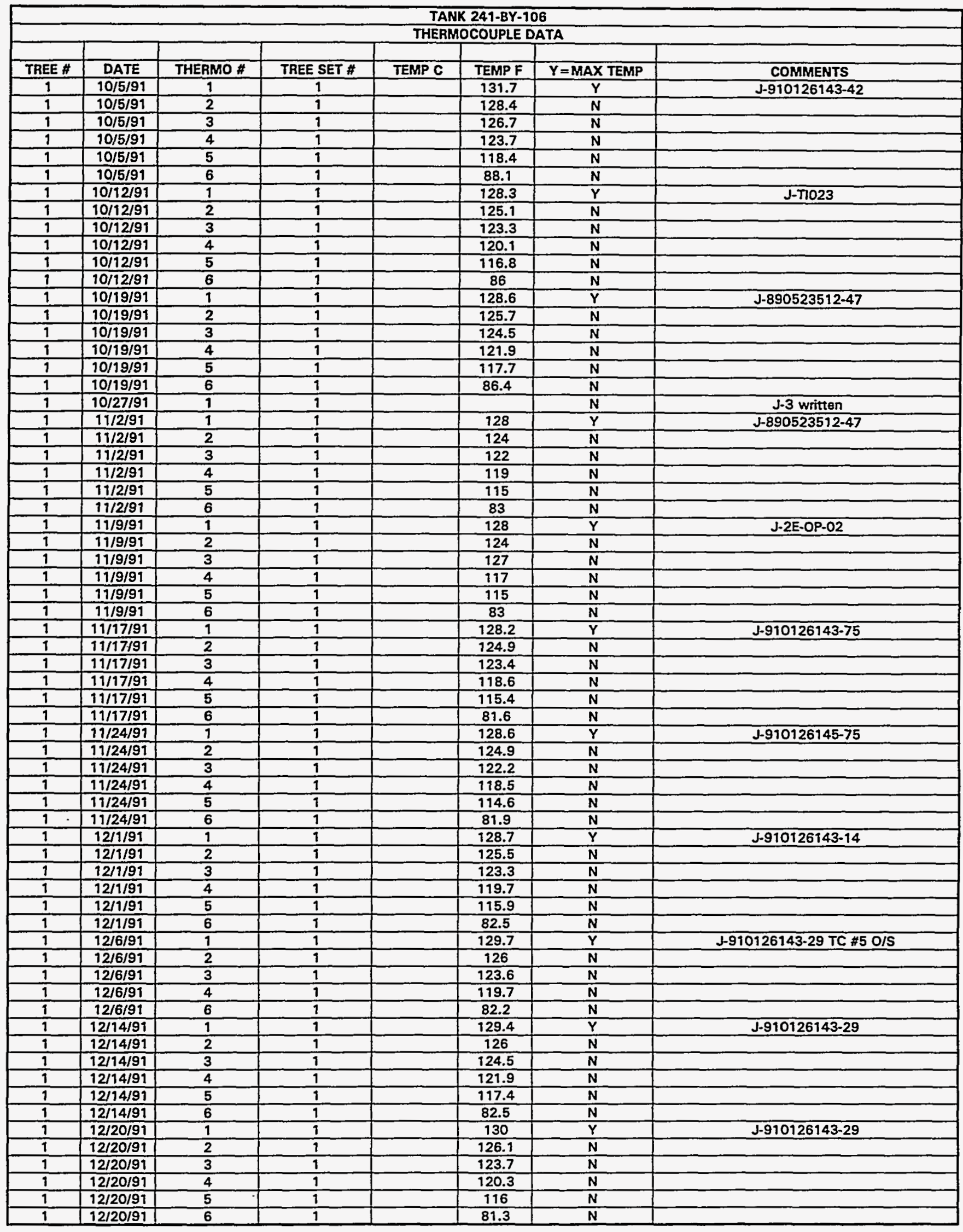

Data obtained from WHC Surveillance Analysis Computer System (SACS), June 2, 1993. 


\begin{tabular}{|c|c|c|c|c|c|c|c|}
\hline \multicolumn{8}{|c|}{ TANK 241-BY-106 } \\
\hline \multicolumn{8}{|c|}{ THERMOCOUPLE DATA } \\
\hline TREE \# & DATE & THERMO \# & TREE SET \# & TEMP C & TEMPF & $Y=$ MAX TEMP & COMMENTS \\
\hline 1 & $12 / 29 / 91$ & 1 & 1 & & 129.8 & $\mathrm{Y}$ & $J-910126143-29$ \\
\hline 1 & $12 / 29 / 91$ & 2 & 1 & & 126.2 & $\mathbf{N}$ & \\
\hline 1 & $12 / 29 / 91$ & 3 & 1 & & 123.7 & $N$ & \\
\hline 1 & $12 / 29 / 91$ & 4 & 1 & & 119.6 & $\mathbf{N}$ & \\
\hline 1 & $12 / 29 / 91$ & 5 & 1 & & 116.2 & $\mathbf{N}$ & \\
\hline 1 & $12 / 29 / 91$ & 6 & 1 & & 80.4 & $\mathbf{N}$ & \\
\hline 1 & $1 / 4 / 92$ & 1 & 1 & & 129 & $\bar{Y}$ & $910126143-50 \mathrm{HH}-99 \mathrm{~A}-\mathrm{J}$ \\
\hline 1 & $1 / 4 / 92$ & 2 & 1 & & 125.7 & $\mathbf{N}$ & \\
\hline 1 & $1 / 4 / 92$ & 3 & 1 & & 123.4 & $\mathbf{N}$ & \\
\hline 1 & $1 / 4 / 92$ & 4 & 1 & & 119.5 & $\bar{N}$ & \\
\hline 1 & $1 / 4 / 92$ & 5 & 1 & & 116.8 & $\mathbf{N}$ & \\
\hline 1 & $1 / 4 / 92$ & 6 & 1 & & 79.8 & $\mathrm{~N}$ & \\
\hline 1 & $1 / 11 / 92$ & 1 & 1 & & 128.3 & $\bar{Y}$ & $910126143-14$ \\
\hline 1 & $1 / 11 / 92$ & 2 & 1 & & 124.8 & $\bar{N}$ & \\
\hline 1 & $1 / 11 / 92$ & 3 & 1 & & 122.2 & $\mathbf{N}$ & \\
\hline 1 & $1 / 11 / 92$ & 4 & 1 & & 118.2 & $\bar{N}$ & \\
\hline 1 & $1 / 11 / 92$ & 5 & 1 & & 114.7 & $N$ & \\
\hline 1 & $1 / 11 / 92$ & 6 & 1 & & 76.1 & $\mathbf{N}$ & \\
\hline 1 & $1 / 16 / 92$ & 1 & 1 & & 130 & $\bar{Y}$ & J $910126143-32$ \\
\hline 1 & $1 / 16 / 92$ & 2 & 1 & & 126.2 & $\mathbf{N}$ & \\
\hline 1 & $1 / 16 / 92$ & 3 & 1 & & 123.5 & $\mathbf{N}$ & \\
\hline 1 & $1 / 16 / 92$ & 4 & 1 & & 120 & $N$ & \\
\hline 1 & $1 / 16 / 92$ & 5 & 1 & & 116.2 & $\mathbf{N}$ & \\
\hline 1 & $1 / 16 / 92$ & 6 & 1 & & 77.3 & $\mathbf{N}$ & \\
\hline 1 & $1 / 28 / 92$ & 1 & 1 & & & $\mathbf{N}$ & $\begin{array}{l}\text { ACCESS RESTRICTED } 1 / 28-2 / 22 \text { DUE TO } \\
\text { BATTERY FUME INCIDENT }\end{array}$ \\
\hline 1 & $2 / 24 / 92$ & 1 & 1 & & 130.5 & $\bar{Y}$ & J-900925741.79 \\
\hline 1 & $2 / 24 / 92$ & 2 & 1 & & 126.6 & $\mathbf{N}$ & \\
\hline 1 & $2 / 24 / 92$ & 3 & 1 & & 124.4 & $\mathbf{N}$ & \\
\hline 1 & $2 / 24 / 92$ & 4 & 1 & & 121.3 & $\mathbf{N}$ & \\
\hline 1 & $2 / 24 / 92$ & 5 & 1 & & 116.3 & $\bar{N}$ & \\
\hline 1 & $2 / 24 / 92$ & 6 & 1 & & 77.9 & $\mathbf{N}$ & \\
\hline 1 & $2 / 29 / 92$ & 1 & 1 & & 129 & $\bar{Y}$ & $J+1-038 / 91012614332$ \\
\hline 1 & $2 / 29 / 92$ & 2 & 1 & & 125.8 & $\mathbf{N}$ & \\
\hline 1 & $2 / 29 / 92$ & 3 & 1 & & 123.6 & $\mathbf{N}$ & \\
\hline 1 & $2 / 29 / 92$ & 4 & 1 & & 119.3 & $\mathbf{N}$ & \\
\hline 1 & $2 / 29 / 92$ & 5 & 1 & & 111.3 & $\mathbf{N}$ & \\
\hline 1 & $2 / 29 / 92$ & 6 & 1 & & 77 & $\mathbf{N}$ & \\
\hline 1 & $3 / 8 / 92$ & 1 & 1 & & 129.5 & $\bar{Y}$ & $J 910126143-48$ \\
\hline 1 & $3 / 8 / 92$ & 2 & 1 & & 126.7 & $\mathbf{N}$ & \\
\hline 1 & $3 / 8 / 92$ & 3 & 1 & & 125.7 & $\mathrm{~N}$ & \\
\hline 1 & $3 / 8 / 92$ & 4 & 1 & & 122.5 & $\mathbf{N}$ & \\
\hline 1 & $3 / 8 / 92$ & 5 & 1 & & 117.8 & $\mathbf{N}$ & \\
\hline 1 & $3 / 8 / 92$ & 6 & 1 & & 78.3 & $\mathbf{N}$ & \\
\hline 7 & $3 / 15 / 92$ & 1 & 1 & & 131.3 & $\bar{Y}$ & J-910126143-32 \\
\hline 1 & $3 / 15 / 92$ & 2 & 1 & & 128.1 & $\mathbf{N}$ & \\
\hline 1 & $3 / 15 / 92$ & 3 & 1 & & 126.3 & $\mathbf{N}$ & \\
\hline 9 & $3 / 15 / 92$ & 4 & 1 & & 122.2 & $\mathbf{N}$ & \\
\hline 1 & $3 / 15 / 92$ & 5 & 1 & & 117.9 & $\mathbf{N}$ & \\
\hline 1 & $3 / 15 / 92$ & 6 & 1 & & 78 & $\mathbf{N}$ & \\
\hline 1 & $3 / 20 / 92$ & 1 & 1 & & 130.5 & $\mathbf{N}$ & \\
\hline 1 & $3 / 20 / 92$ & 2 & 1 & & 126.6 & $\mathbf{N}$ & \\
\hline 1 & $3 / 20 / 92$ & 3 & 1 & & 124.6 & $\mathbf{N}$ & \\
\hline 1 & $3 / 20 / 92$ & 4 & 1 & & 121 & $\mathbf{N}$ & \\
\hline 1 & $3 / 20 / 92$ & 5 & 1 & & 116.4 & $\mathbf{N}$ & \\
\hline 1 & $3 / 20 / 92$ & 6 & 1 & & 76.9 & $\mathbf{N}$ & \\
\hline 1 & $3 / 29 / 92$ & 1 & 1 & & 130.5 & $\bar{Y}$ & J910126143-66 \\
\hline 1 & $3 / 29 / 92$ & 2 & 1 & & 127.3 & $\mathbf{N}$ & \\
\hline 1 & $3 / 29 / 92$ & 3 & 1 & & 124.9 & $\mathbf{N}$ & \\
\hline 1 & $3 / 29 / 92$ & 4 & 1 & & 121.4 & $\mathbf{N}$ & \\
\hline 1 & $3 / 29 / 92$ & 5 & 1 & & 117.2 & $\bar{N}$ & \\
\hline 1 & $3 / 29 / 92$ & 6 & 1 & & 78.2 & $N$ & \\
\hline 1 & $4 / 3 / 92$ & 1 & 1 & & 128.2 & $Y$ & J910126143-14 \\
\hline 1 & $4 / 3 / 92$ & 2 & 1 & & 125.2 & $\mathbf{N}$ & \\
\hline 1 & $4 / 3 / 92$ & 3 & 1 & & 123.2 & $\mathbf{N}$ & \\
\hline 1 & $4 / 3 / 92$ & 4 & 1 & & 119.7 & $\mathbf{N}$ & \\
\hline
\end{tabular}

Data obtained from WHC Surveillance Analysis Computer System (SACS), June 2, 1993. 


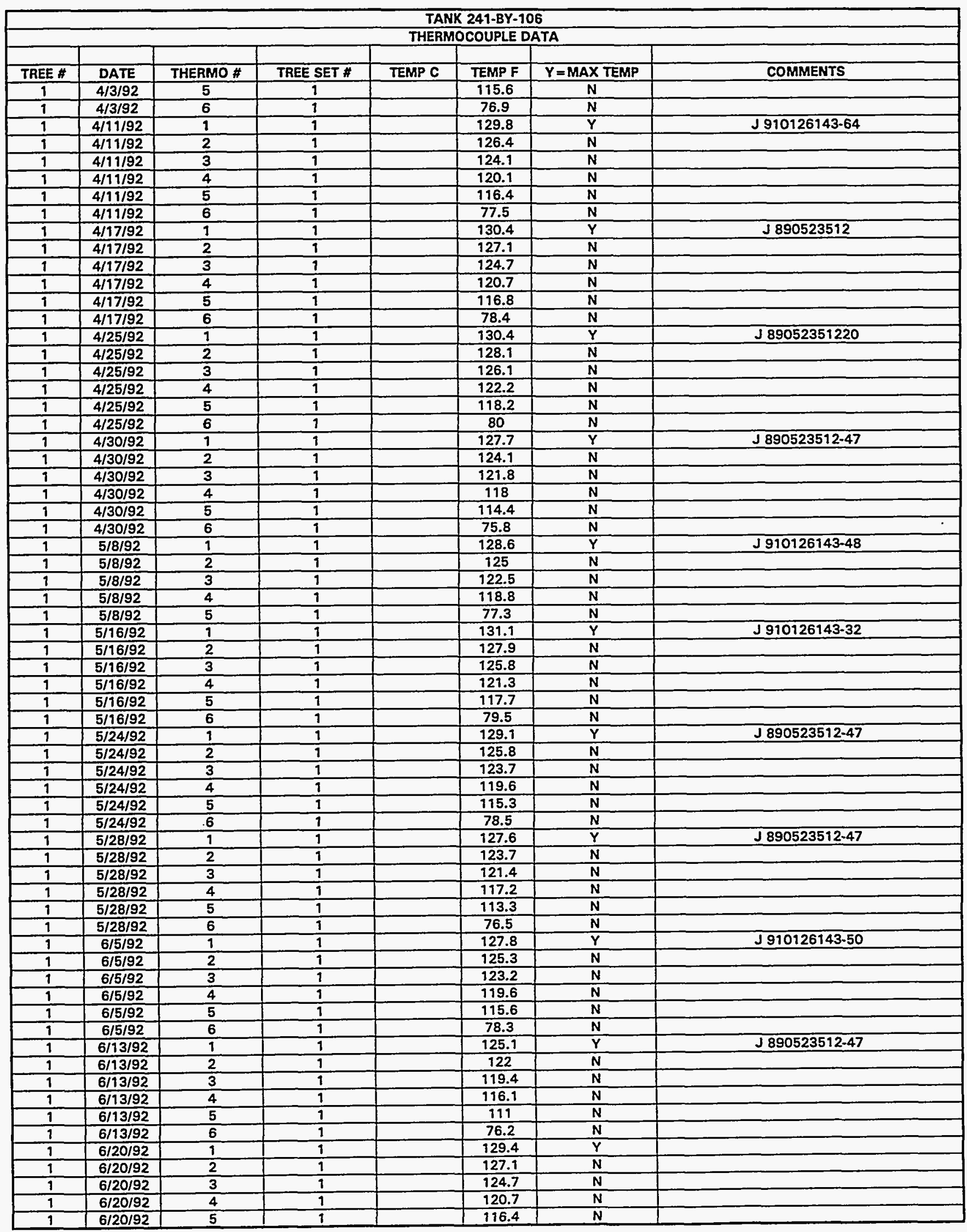

Data obtained from WHC Surveillance Analysis Computer System (SACS), June 2, 1993. 
WHC-SD-WM-ER-312, Rev. 0

\begin{tabular}{|c|c|c|c|c|c|c|c|}
\hline \multicolumn{8}{|c|}{ TANK 241-BY-106 } \\
\hline \multicolumn{8}{|c|}{ THERMOCOUPLE DATA } \\
\hline TREE \# & DATE & THERMO \# & TREE SET \# & TEMP C & TEMP F & $Y=M A X$ TEMP & COMMENTS \\
\hline 1 & $6 / 20 / 92$ & 6 & 1 & & 81.6 & $N$ & \\
\hline 1 & $6 / 28 / 92$ & 1 & 1 & & 128.7 & $\bar{Y}$ & J T1-042 910126143-66 \\
\hline 1 & $6 / 28 / 92$ & 2 & 1 & & 125.9 & $\mathbf{N}$ & \\
\hline 1 & $6 / 28 / 92$ & 3 & 1 & & 123.5 & $\mathbf{N}$ & \\
\hline 1 & $6 / 28 / 92$ & 4 & 1 & & 119.8 & $\mathrm{~N}$ & \\
\hline 1 & $6 / 28 / 92$ & 5 & 1 & & 114.9 & $\mathbf{N}$ & \\
\hline 1 & $6 / 28 / 92$ & 6 & 1 & & 81.59 & $\mathbf{N}$ & \\
\hline 1 & $7 / 3 / 92$ & 1 & 1 & & 127.7 & $\mathbf{Y}$ & $J T I-041$ \\
\hline 1 & $7 / 3 / 92$ & 2 & 1 & & 125.5 & $\mathbf{N}$ & \\
\hline 1 & $7 / 3 / 92$ & 3 & 1 & & 123.2 & $\mathbf{N}$ & \\
\hline 1 & $7 / 3 / 92$ & 4 & 1 & & 119.7 & $\mathbf{N}$ & \\
\hline 1 & $7 / 3 / 92$ & 5 & 1 & & 114.8 & $\mathbf{N}$ & \\
\hline 1 & $7 / 3 / 92$ & 6 & 1 & & 80.3 & $\bar{N}$ & \\
\hline 1 & $7 / 10 / 92$ & 1 & 1 & & 128.5 & $\mathbf{Y}$ & J910126143-42 \\
\hline 1 & $7 / 10 / 92$ & 2 & 1 & & 125.4 & $\mathbf{N}$ & \\
\hline 1 & $7 / 10 / 92$ & 3 & 1 & & 122.7 & $\mathbf{N}$ & \\
\hline 1 & $7 / 10 / 92$ & 4 & 1 & & 118.6 & $\mathbf{N}$ & \\
\hline 1 & $7 / 10 / 92$ & 5 & 1 & & 114.7 & $\mathbf{N}$ & \\
\hline 1 & $7 / 10 / 92$ & 6 & 1 & & 81 & $\mathbf{N}$ & \\
\hline 1 & $7 / 18 / 92$ & 1 & 1 & & 129.4 & $\bar{Y}$ & J 910126143.42 \\
\hline 1 & $7 / 18 / 92$ & 2 & 1 & & 126.7 & $\mathbf{N}$ & \\
\hline 1 & $7 / 18 / 92$ & 3 & 1 & & 124.5 & $\mathbf{N}$ & \\
\hline 1 & $7 / 18 / 92$ & 4 & 1 & & 120.2 & $\mathbf{N}$ & \\
\hline 1 & $7 / 18 / 92$ & 5 & 1 & & 115.9 & $\mathbf{N}$ & \\
\hline 1 & $7 / 18 / 92$ & 6 & 1 & & 82.8 & $\mathbf{N}$ & \\
\hline 1 & $7 / 24 / 92$ & 1 & 1 & & 128.8 & $\mathbf{Y}$ & J HH-99A 910126143-29 \\
\hline 1 & $7 / 24 / 92$ & 2 & 1 & & 125.9 & $\mathbf{N}$ & \\
\hline 1 & $7 / 24 / 92$ & 3 & 1 & & 123.3 & $\mathbf{N}$ & \\
\hline 1 & $7 / 24 / 92$ & 4 & 1 & & 119.8 & $\mathbf{N}$ & \\
\hline 1 & $7 / 24 / 92$ & 5 & 1 & & 115.1 & $\mathbf{N}$ & \\
\hline 1 & $7 / 24 / 92$ & 6 & 1 & & 82.6 & $\mathbf{N}$ & \\
\hline 1 & $8 / 1 / 92$ & 1 & 1 & & 126.1 & $\bar{Y}$ & $\mathrm{JTI}-041$ \\
\hline 1 & $8 / 1 / 92$ & 2 & 1 & & 123.6 & $\mathbf{N}$ & \\
\hline 1 & $8 / 1 / 92$ & 3 & 1 & & 122.6 & $\mathbf{N}$ & \\
\hline 1 & $8 / 1 / 92$ & 4 & 1 & & 119.8 & $\bar{N}$ & \\
\hline 1 & $8 / 1 / 92$ & 5 & 1 & & 116 & $\mathbf{N}$ & \\
\hline 1 & $8 / 1 / 92$ & 6 & 1 & & 82.8 & $\mathbf{N}$ & \\
\hline 1 & $8 / 7 / 92$ & 9 & 1 & & 126.4 & $\bar{Y}$ & $J 890423320-42$ \\
\hline 1 & $8 / 7 / 92$ & 2 & 1 & & 123.6 & $\mathbf{N}$ & \\
\hline 1 & $8 / 7 / 92$ & 3 & 1 & & 121.4 & $\mathbf{N}$ & \\
\hline 1 & $8 / 7 / 92$ & 4 & 1 & & 117.5 & $\mathbf{N}$ & \\
\hline 1 & $8 / 7 / 92$ & 5 & 1 & & 113.4 & $\mathbf{N}$ & \\
\hline 1 & $8 / 7 / 92$ & 6 & 1 & & 82.5 & $\mathbf{N}$ & \\
\hline 1 & $8 / 15 / 92$ & 9 & 1 & & 128 & $\bar{Y}$ & J 890423320 \\
\hline 1 & $8 / 15 / 92$ & 2 & 1 & & 125.8 & $\mathbf{N}$ & \\
\hline 1 & $8 / 15 / 92$ & 3 & 1 & & 123.4 & $\mathrm{~N}$ & \\
\hline 9 & $8 / 15 / 92$ & 4 & 1 & & 919.7 & $\mathbf{N}$ & \\
\hline 1 & $8 / 15 / 92$ & 5 & 1 & & 115.9 & $\mathbf{N}$ & \\
\hline 1 & $8 / 15 / 92$ & 6 & 1 & & 84.7 & $\mathbf{N}$ & \\
\hline 1 & $8 / 21 / 92$ & 1 & 9 & & 126.5 & $\bar{Y}$ & J $890423320-42$ \\
\hline 1 & $8 / 21 / 92$ & 2 & 1 & & 123.6 & $\mathrm{~N}$ & \\
\hline 1 & $8 / 21 / 92$ & 3 & 1 & & 121.3 & $\mathbf{N}$ & \\
\hline 9 & $8 / 21 / 92$ & 4 & 1 & & 117.2 & $\mathrm{~N}$ & \\
\hline 1 & $8 / 21 / 92$ & 5 & 1 & & 113.6 & $\mathbf{N}$ & \\
\hline 1 & $8 / 21 / 92$ & 6 & 1 & & 83.1 & $\mathbf{N}$ & \\
\hline 1 & $8 / 30 / 92$ & 9 & 9 & & 128.1 & $\bar{Y}$ & $\mathrm{~J} 890423320-42$ \\
\hline 1 & $8 / 30 / 92$ & 2 & 1 & & 125.7 & $\mathbf{N}$ & \\
\hline 1 & $8 / 30 / 92$ & 3 & 1 & & 123.3 & $\mathbf{N}$ & \\
\hline 1 & $8 / 30 / 92$ & 4 & 1 & & 119.2 & $\mathbf{N}$ & \\
\hline 1 & $8 / 30 / 92$ & 5 & 1 & & 115.2 & $\mathbf{N}$ & \\
\hline 1 & $8 / 30 / 92$ & 6 & 9 & & 85.2 & $\mathbf{N}$ & \\
\hline 1 & $9 / 6 / 92$ & 1 & 1 & & 127 & $\mathbf{Y}$ & $\mathrm{J} 890423320-42$ \\
\hline 1 & $9 / 6 / 92$ & 2 & 1 & & 123.7 & $\mathbf{N}$ & \\
\hline 1 & $9 / 6 / 92$ & 3 & 1 & & 121.2 & $\mathrm{~N}$ & \\
\hline 1 & $9 / 6 / 92$ & 4 & 1 & & 118.1 & $\mathbf{N}$ & \\
\hline 1 & $9 / 6 / 92$ & 5 & 1 & & 113.5 & $\mathbf{N}$ & \\
\hline
\end{tabular}

Data obtained from WHC Surveiliance Analysis Computer System (SACS), June 2, 1993. 


\begin{tabular}{|c|c|c|c|c|c|c|c|}
\hline \multicolumn{8}{|c|}{ TANK 241-BY-106 } \\
\hline \multicolumn{8}{|c|}{ THERMOCOUPLE DATA } \\
\hline TREE \# & DATE & THERMO \# & TREE SET \# & TEMP C & TEMP F & $Y=M A X$ TEMP & COMMENTS \\
\hline 1 & $9 / 6 / 92$ & 6 & 1 & & 84.2 & $\mathbf{N}$ & \\
\hline 1 & $9 / 12 / 92$ & 1 & 1 & & 127.9 & $\bar{Y}$ & J $890423320-42$ \\
\hline 1 & $9 / 12 / 92$ & 2 & 1 & & 124.9 & $N$ & \\
\hline 1 & $9 / 12 / 92$ & 3 & 1 & & 122.5 & $\mathbf{N}$ & \\
\hline$\frac{1}{1}$ & $9 / 12 / 92$ & 4 & 1 & & 118.8 & $\mathbf{N}$ & \\
\hline 1 & $9 / 12 / 92$ & 5 & 1 & & 115.4 & $\mathbf{N}$ & \\
\hline 1 & $9 / 12 / 92$ & 6 & 1 & & 85.6 & $\mathbf{N}$ & \\
\hline 1 & $9 / 20 / 92$ & 1 & 1 & & 127.9 & $\mathbf{Y}$ & $\mathrm{J} 910126143-64$ \\
\hline 1 & $9 / 20 / 92$ & 2 & 1 & & 125 & $\mathbf{N}$ & \\
\hline 1 & $9 / 20 / 92$ & 3 & 1 & & 122.4 & $\mathbf{N}$ & \\
\hline 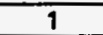 & $9 / 20 / 92$ & 4 & 1 & & 118.4 & $\mathbf{N}$ & \\
\hline 1 & $9 / 20 / 92$ & 5 & 1 & & 114.8 & $\overline{\mathbf{N}}$ & \\
\hline 1 & $9 / 20 / 92$ & 6 & 1 & & 85.3 & $\mathbf{N}$ & \\
\hline 1 & $9 / 26 / 92$ & 1 & 1 & & 126.4 & $\bar{Y}$ & J910126143-64 \\
\hline 1 & $9 / 26 / 92$ & 2 & 1 & & 123.2 & $\bar{N}$ & \\
\hline 1 & $9 / 26 / 92$ & 3 & 1 & & 120.7 & $\mathbf{N}$ & \\
\hline 1 & $9 / 26 / 92$ & 4 & 1 & & 117.7 & $\mathbf{N}$ & \\
\hline 1 & $9 / 26 / 92$ & 5 & 1 & & 113.3 & $\mathbf{N}$ & \\
\hline 1 & $9 / 26 / 92$ & 6 & 1 & & 83.8 & $\mathbf{N}$ & \\
\hline 1 & $10 / 1 / 92$ & 1 & 1 & & 126.1 & $\bar{Y}$ & J $890423320-42$ \\
\hline 1 & $10 / 1 / 92$ & 2 & 1 & & 123.4 & $\mathbf{N}$ & \\
\hline 1 & $10 / 1 / 92$ & 3 & 1 & & 120.6 & $\mathbf{N}$ & \\
\hline 1 & $10 / 1 / 92$ & 4 & 1 & & 117.1 & $\mathbf{N}$ & \\
\hline 1 & $10 / 1 / 92$ & 5 & 1 & & 113.9 & $\mathbf{N}$ & \\
\hline 1 & $10 / 1 / 92$ & 6 & 1 & & 84.1 & $\mathbf{N}$ & \\
\hline 1 & $10 / 10 / 92$ & 1 & 1 & & 127.9 & $\bar{Y}$ & J $910126143-48$ \\
\hline 1 & $10 / 10 / 92$ & 2 & 1 & & 124.5 & $\mathbf{N}$ & \\
\hline 1 & $10 / 10 / 92$ & 3 & 1 & & 121.9 & $\mathbf{N}$ & \\
\hline 1 & $10 / 10 / 92$ & 4 & 1 & & 118.7 & $\overline{\mathbf{N}}$ & \\
\hline 1 & $10 / 10 / 92$ & 5 & 1 & & 114.8 & $\mathbf{N}$ & \\
\hline$\frac{1}{1}$ & $10 / 10 / 92$ & 6 & 7 & & 85 & $\overline{\mathbf{N}}$ & \\
\hline 1 & $10 / 18 / 92$ & 1 & 1 & & 128.2 & $\bar{Y}$ & $\mathrm{~J} 910125143-40$ \\
\hline 1 & $10 / 18 / 92$ & 2 & 1 & & 124.5 & $\mathbf{N}$ & \\
\hline 1 & $10 / 18 / 92$ & 3 & 1 & & 121.9 & $\mathbf{N}$ & \\
\hline 1 & $10 / 18 / 92$ & 4 & 1 & & 118.5 & $\mathbf{N}$ & \\
\hline 1 & $10 / 18 / 92$ & 5 & 1 & & 114.8 & $\mathbf{N}$ & \\
\hline 1 & $10 / 18 / 92$ & 6. & 1 & & 84.9 & $\mathbf{N}$ & \\
\hline 1 & $10 / 24 / 92$ & 1 & 1 & & 128.4 & $Y$ & $J 910126143-66$ \\
\hline 1 & $10 / 24 / 92$ & 2 & 1 & & 124.9 & $\mathbf{N}$ & \\
\hline 1 & $10 / 24 / 92$ & 3 & 1 & & 122.5 & $\mathbf{N}$ & \\
\hline 1 & $10 / 24 / 92$ & 4 & 1 & & 119.4 & $N$ & \\
\hline 1 & $10 / 24 / 92$ & 5 & 1 & & 115.3 & $\mathbf{N}$ & \\
\hline 1 & $10 / 24 / 92$ & 6 & 1 & & 85.3 & $\mathbf{N}$ & \\
\hline 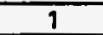 & $10 / 28 / 92$ & 1 & 1 & & 128.6 & $\mathbf{Y}$ & J91012614366 \\
\hline 9 & $10 / 28 / 92$ & 2 & 1 & & 125.1 & $\mathbf{N}$ & \\
\hline$\frac{T}{1}$ & $10 / 28 / 92$ & 3 & $\frac{1}{1}$ & & 122.8 & $\mathbf{N}$ & \\
\hline 1 & $10 / 28 / 92$ & 4 & 1 & & 119.4 & $\bar{N}$ & \\
\hline 1 & $10 / 28 / 92$ & 5 & 1 & & 115.5 & $\mathbf{N}$ & \\
\hline 1 & $10 / 28 / 92$ & 6 & 1 & & 85.3 & $\mathbf{N}$ & \\
\hline 1 & $11 / 1 / 92$ & 1 & 1 & & 129.9 & $\bar{Y}$ & TMACS \\
\hline 1 & $11 / 1 / 92$ & 2 & 1 & & 127.2 & $\mathbf{N}$ & \\
\hline$T$ & $11 / 1 / 92$ & 3 & 1 & & 125.2 & $\mathrm{~N}$ & \\
\hline 1 & $11 / 1 / 92$ & 4 & 1 & & 122.3 & $\mathbf{N}$ & \\
\hline 1 & $11 / 1 / 92$ & 5 & 1 & & 118.9 & $\mathbf{N}$ & \\
\hline 1 & $11 / 1 / 92$ & 6 & 1 & & 89.2 & $\mathbf{N}$ & \\
\hline 1 & $19 / 8 / 92$ & 1 & 1 & & 130.1 & $Y$ & TMACS \\
\hline 1 & $11 / 8 / 92$ & 2 & 1 & & 127.4 & $\mathbf{N}$ & \\
\hline 1 & $11 / 8 / 92$ & $\overline{3}$ & 1 & & 125 & $N$ & \\
\hline 1 & $11 / 8 / 92$ & 4 & 1 & & 121.8 & $\mathrm{~N}$ & \\
\hline 1 & $11 / 8 / 92$ & 5 & 1 & & 118.5 & $N$ & \\
\hline 1 & $11 / 8 / 92$ & 6 & 1 & & 88.3 & $N$ & \\
\hline 1 & $11 / 15 / 92$ & 1 & 1 & & 130.8 & $\bar{Y}$ & TMACS \\
\hline 7 & $19 / 15 / 92$ & 2 & 1 & & 127.2 & $\mathbf{N}$ & \\
\hline 1 & $11 / 15 / 92$ & 3 & 1 & & 125.6 & $\mathrm{~N}$ & \\
\hline 1 & $11 / 15 / 92$ & 4 & 1 & & 122.7 & $\mathbf{N}$ & \\
\hline 1 & $11 / 15 / 92$ & 5 & 1 & & 118.5 & $\mathbf{N}$ & \\
\hline
\end{tabular}

Data obtained from WHC Surveillance Analysis Computer System (SACS), June 2, 1993. 
WHC-SD-WM-ER-312, Rev. 0

\begin{tabular}{|c|c|c|c|c|c|c|c|}
\hline \multicolumn{8}{|c|}{ TANK 241-BY-106 } \\
\hline \multicolumn{8}{|c|}{ THERMOCOUPLE DATA } \\
\hline TREE \# & DATE & THERMO \# & TREE SET \# & TEMP C & TEMP F & $Y=$ MAX TEMP & COMMENTS \\
\hline$\frac{1}{1}$ & $11 / 15 / 92$ & 6 & $\frac{9}{1}$ & & 88.5 & $\mathbf{N}$ & \\
\hline 1 & $11 / 20 / 92$ & 1 & 1 & & 127.3 & $Y$ & J $910126143-48$ \\
\hline 1 & $11 / 20 / 92$ & 2 & 1 & & 123.6 & $\mathbf{N}$ & \\
\hline$\frac{1}{1}$ & $11 / 20 / 92$ & 3 & $\frac{1}{1}$ & & 121.5 & $\bar{N}$ & \\
\hline 1 & $11 / 20 / 92$ & 4 & 1 & & 118.2 & $\mathbf{N}$ & \\
\hline$\frac{1}{1}$ & $11 / 20 / 92$ & 5 & 1 & & 114.2 & $\mathbf{N}$ & \\
\hline 1 & $11 / 20 / 92$ & 6 & 1 & & 82.5 & $\overline{\mathbf{N}}$ & \\
\hline 1 & $11 / 22 / 92$ & 1 & 1 & & 130.2 & $\mathbf{Y}$ & TMACS \\
\hline 1 & $11 / 22 / 92$ & 2 & 1 & & 127.2 & $\mathbf{N}$ & \\
\hline 1 & $11 / 22 / 92$ & 3 & 1 & & 125.2 & $\mathbf{N}$ & \\
\hline 1 & $11 / 22 / 92$ & 4 & 1 & & 121.8 & $\mathbf{N}$ & \\
\hline 1 & $11 / 22 / 92$ & 5 & 1 & & 119.3 & $\mathbf{N}$ & \\
\hline 1 & $11 / 22 / 92$ & 6 & 1 & & 87.9 & $\mathbf{N}$ & \\
\hline 1 & $11 / 29 / 92$ & 1 & 1 & & 130.6 & $\bar{Y}$ & TMACS \\
\hline 1 & $11 / 29 / 92$ & 2 & 1 & & 127.2 & $\mathbf{N}$ & \\
\hline 1 & $11 / 29 / 92$ & 3 & 9 & & 125.6 & $\overline{\mathbf{N}}$ & \\
\hline 1 & $11 / 29 / 92$ & 4 & 1 & & 122.9 & $\mathbf{N}$ & \\
\hline 1 & $11 / 29 / 92$ & 5 & 1 & & 119.4 & $\mathbf{N}$ & \\
\hline 1 & $11 / 29 / 92$ & 6 & 1 & & 85.8 & $\mathbf{N}$ & \\
\hline 1 & $12 / 6 / 92$ & 1 & 1 & & 130.1 & $\bar{Y}$ & TMACS \\
\hline 1 & $12 / 6 / 92$ & 2 & 1 & & 127 & $\mathbf{N}$ & \\
\hline 1 & $12 / 6 / 92$ & 3 & 9 & & 925.2 & $\mathbf{N}$ & \\
\hline 1 & $12 / 6 / 92$ & 4 & 1 & & 121.8 & $\mathbf{N}$ & \\
\hline 1 & $12 / 6 / 92$ & 5 & 1 & & 118.9 & $\mathbf{N}$ & \\
\hline 1 & $12 / 6 / 92$ & 6 & 1 & & 86.3 & $\mathbf{N}$ & \\
\hline 1 & $12 / 13 / 92$ & 1 & 1 & & 129.9 & $\bar{Y}$ & TMACS \\
\hline 1 & $12 / 13 / 92$ & 2 & 1 & & 126.8 & $\mathbf{N}$ & \\
\hline 1 & $12 / 13 / 92$ & 3 & 1 & & 125 & $\mathbf{N}$ & \\
\hline 1 & $12 / 13 / 92$ & 4 & 1 & & 122.5 & $\mathbf{N}$ & \\
\hline 1 & $12 / 13 / 92$ & 5 & 1 & & 119.1 & $\mathbf{N}$ & \\
\hline 1 & $12 / 13 / 92$ & 6 & 1 & & 85.2 & $\mathbf{N}$ & \\
\hline 1 & $12 / 20 / 92$ & 1 & 1 & & 130.6 & $\bar{Y}$ & TMACS \\
\hline 1 & $12 / 20 / 92$ & 2 & 1 & & 127 & $\mathbf{N}$ & \\
\hline 1 & $12 / 20 / 92$ & 3 & 1 & & 125.6 & $\bar{N}$ & \\
\hline 1 & $12 / 20 / 92$ & 4 & 1 & & 122.5 & $\mathbf{N}$ & \\
\hline 1 & $12 / 20 / 92$ & 5 & 1 & & 118.9 & $\mathbf{N}$ & \\
\hline 1 & $12 / 20 / 92$ & 6 & 1 & & 84.9 & $\mathbf{N}$ & \\
\hline 1 & $12 / 27 / 92$ & 1 & 1 & & 130.4 & $\bar{Y}$ & TMACS \\
\hline 1 & $12 / 27 / 92$ & 2 & 1 & & 127.2 & $\mathbf{N}$ & \\
\hline 1 & $12 / 27 / 92$ & 3 & 1 & & 125.4 & $\bar{N}$ & \\
\hline 1 & $12 / 27 / 92$ & 4 & 1 & & 122.1 & $\bar{N}$ & \\
\hline 1 & $12 / 27 / 92$ & 5 & 1 & & 119.4 & $\mathrm{~N}$ & \\
\hline 1 & $12 / 27 / 92$ & 6 & 1 & & 84.5 & $\bar{N}$ & \\
\hline 1 & $1 / 3 / 93$ & 9 & 1 & & 131 & $\mathbf{Y}$ & TMACS \\
\hline 1 & $1 / 3 / 93$ & 2 & 1 & & 127.7 & $N$ & \\
\hline 1 & $1 / 3 / 93$ & 3 & 1 & & 125.7 & $\bar{N}$ & \\
\hline 1 & $1 / 3 / 93$ & 4 & 1 & & 123.4 & $N$ & \\
\hline 1 & $1 / 3 / 93$ & 5 & 9 & & 120.2 & $\mathbf{N}$ & \\
\hline 1 & $1 / 3 / 93$ & 6 & 1 & & 84 & $\mathrm{~N}$ & \\
\hline 1 & $1 / 10 / 93$ & 1 & 1 & & 130.4 & $\bar{Y}$ & TMACS \\
\hline 1 & $1 / 10 / 93$ & 2 & 1 & & 127.2 & $\mathbf{N}$ & \\
\hline 1 & $1 / 10 / 93$ & 3 & 1 & & 125.2 & $\mathrm{~N}$ & \\
\hline 1 & $1 / 10 / 93$ & 4 & 1 & & 122.1 & $\bar{N}$ & \\
\hline 1 & $1 / 10 / 93$ & 5 & 1 & & 119.1 & $\mathbf{N}$ & \\
\hline 1 & $1 / 10 / 93$ & 6 & 1 & & 82.9 & $\mathbf{N}$ & \\
\hline 1 & $1 / 17 / 93$ & 1 & 1 & & 130.2 & $\bar{Y}$ & TMACS \\
\hline 1 & $1 / 17 / 93$ & 2 & 1 & & 127 & $\mathbf{N}$ & \\
\hline 1 & $1 / 17 / 93$ & 3 & 1 & & 125.2 & $\mathbf{N}$ & \\
\hline 1 & $1 / 17 / 93$ & 4 & 1 & & 122.3 & $\mathbf{N}$ & \\
\hline 1 & $1 / 17 / 93$ & 5 & 1 & & 118.9 & $\mathbf{N}$ & \\
\hline 1 & $1 / 17 / 93$ & 6 & 1 & & 82.4 & $\mathbf{N}$ & \\
\hline 1 & $1 / 24 / 93$ & 1 & 1 & & 130.6 & $\bar{Y}$ & TMACS \\
\hline 1 & $1 / 24 / 93$ & 2 & 1 & & 127 & $N$ & \\
\hline 1 & $1 / 24 / 93$ & 3 & 1 & & 125.2 & $\bar{N}$ & \\
\hline 1 & $1 / 24 / 93$ & 4 & 1 & & 122.5 & $\bar{N}$ & \\
\hline 1 & $1 / 24 / 93$ & 5 & 1 & & 119.1 & $N$ & \\
\hline
\end{tabular}

Data obtained from WHC Surveillance Analysis Computer System (SACS), June 2, 1993. 
WHC-SD-WM-ER-312, Rev. 0

\begin{tabular}{|c|c|c|c|c|c|c|c|}
\hline \multicolumn{8}{|c|}{ TANK 241-8Y-106 } \\
\hline \multicolumn{8}{|c|}{ THERMOCOUPLE DATA } \\
\hline TREE \# & DATE & THERMO \# & TREE SET \# & TEMP C & TEMP F & $\bar{Y}=$ MAX TEMP & COMMENTS \\
\hline 1 & $1 / 24 / 93$ & 6 & 1 & & 80.9 & $\mathbf{N}$ & \\
\hline 1 & $1 / 31 / 93$ & 1 & 1 & & 130.46 & $\bar{Y}$ & TMACS \\
\hline 1 & $1 / 31 / 93$ & 2 & 1 & & 127.04 & $\mathbf{N}$ & \\
\hline 1 & $1 / 31 / 93$ & 3 & 1 & & 125.24 & $\overline{\mathbf{N}}$ & \\
\hline 9 & $1 / 31 / 93$ & 4 & 1 & & 122.72 & $N$ & \\
\hline 1 & $1 / 31 / 93$ & 5 & 1 & & 118.94 & $N$ & \\
\hline 1 & $1 / 31 / 93$ & 6 & 1 & & 80.96 & $\mathrm{~N}$ & \\
\hline$\frac{1}{1}$ & $2 / 8 / 93$ & 1 & 1 & & 130.46 & $\mathbf{Y}$ & TMACS \\
\hline 1 & $2 / 8 / 93$ & 2 & 1 & & 127.04 & $\mathbf{N}$ & \\
\hline 1 & $2 / 8 / 93$ & 3 & 1 & & 125.24 & $\bar{N}$ & \\
\hline 9 & $2 / 8 / 93$ & 4 & 1 & & 922 & $\bar{N}$ & \\
\hline 1 & $2 / 8 / 93$ & 5 & 1 & & 118.94 & $\bar{N}$ & \\
\hline 1 & $2 / 8 / 93$ & 6 & 1 & & 80.96 & $\mathbf{N}$ & \\
\hline 9 & $2 / 15 / 93$ & 1 & 1 & & 130.3 & $Y$ & TMACS \\
\hline$\frac{T}{1}$ & $2 / 15 / 93$ & 2 & 1 & & 127.2 & N & \\
\hline 1 & $2 / 15 / 93$ & 3 & 9 & & 125.6 & $\mathbf{N}$ & \\
\hline 9 & $2 / 15 / 93$ & 4 & 1 & & 122.7 & $\mathbf{N}$ & \\
\hline 1 & $2 / 15 / 93$ & 5 & 1 & & 118.9 & $\bar{N}$ & \\
\hline 1 & $2 / 15 / 93$ & 6 & 1 & & 80.6 & $N$ & \\
\hline 1 & $2 / 21 / 93$ & 1 & 1 & & 130.1 & $\mathbf{Y}$ & TMACS \\
\hline 1 & $2 / 21 / 93$ & 2 & 1 & & 126.68 & $\mathbf{N}$ & \\
\hline 1 & $2 / 21 / 93$ & 3 & 1 & & 125.24 & $\mathbf{N}$ & \\
\hline$\overline{1}$ & $2 / 21 / 93$ & 4 & 1 & & 122.04 & $\mathbf{N}$ & \\
\hline 1 & $2 / 21 / 93$ & 5 & 1 & & 118.22 & $\bar{N}$ & \\
\hline 1 & $2 / 21 / 93$ & 6 & 1 & & 79.52 & $\mathbf{N}$ & \\
\hline 1 & $3 / 1 / 93$ & $T$ & 1 & & 130.28 & $\bar{Y}$ & TMACS \\
\hline$i$ & $3 / 1 / 93$ & 2 & 1 & & 126.68 & $\dot{N}$ & \\
\hline 1 & $3 / 1 / 93$ & 3 & 1 & & 125.06 & $\mathbf{N}$ & \\
\hline 1 & $3 / 1 / 93$ & 4 & 1 & & 121.64 & $\bar{N}$ & \\
\hline 1 & $3 / 1 / 93$ & 5 & 1 & & 118.76 & $\mathbf{N}$ & \\
\hline 1 & $3 / 1 / 93$ & 6 & 1 & & 78.98 & $\mathbf{N}$ & \\
\hline 9 & $3 / 8 / 93$ & 1 & 1 & & 130.28 & $\bar{Y}$ & TMACS \\
\hline 1 & $3 / 8 / 93$ & 2 & 1 & & 127.04 & $N$ & \\
\hline 1 & $3 / 8 / 93$ & 3 & 1 & & 124.88 & $\mathbf{N}$ & \\
\hline 9 & $3 / 8 / 93$ & 4 & 1 & & 121.64 & $\bar{N}$ & \\
\hline 9 & $3 / 8 / 93$ & 5 & 1 & & 119.12 & $\overline{\mathbf{N}}$ & \\
\hline 9 & $3 / 8 / 93$ & 6 & 9 & & 78.98 & $\mathbf{N}$ & \\
\hline 9 & $3 / 15 / 93$ & 7 & 1 & & 129.74 & $\mathbf{Y}$ & TMACS \\
\hline 9 & $3 / 15 / 93$ & 2 & 1 & & 126.68 & $\mathbf{N}$ & \\
\hline 1 & $3 / 15 / 93$ & 3 & 1 & & 125.24 & $\mathbf{N}$ & \\
\hline 1 & $3 / 15 / 93$ & 4 & 1 & & 121.64 & $\mathbf{N}$ & \\
\hline 1 & $3 / 15 / 93$ & 5 & 9 & & 117.86 & $\mathbf{N}$ & \\
\hline 1 & $3 / 15 / 93$ & 6 & 1 & & 78.63 & $\mathbf{N}$ & \\
\hline$\frac{1}{1}$ & $3 / 22 / 93$ & 1 & 1 & & 130.46 & $\bar{Y}$ & TMACS \\
\hline 9 & $3 / 22 / 93$ & 2 & 1 & & 127.04 & $\mathbf{N}$ & \\
\hline 1 & $3 / 22 / 93$ & 3 & 9 & & 125.24 & $\mathbf{N}$ & \\
\hline 1 & $3 / 22 / 93$ & 4 & 9 & & 122 & $\mathbf{N}$ & \\
\hline 1 & $3 / 22 / 93$ & 5 & 1 & & 118.4 & $\mathbf{N}$ & \\
\hline 1 & $3 / 22 / 93$ & 6 & 9 & & 78.44 & $\mathbf{N}$ & \\
\hline 9 & $3 / 29 / 93$ & 1 & 1 & & 130.1 & $Y$ & TMACS \\
\hline 9 & $3 / 29 / 93$ & 2 & 1 & & 126.68 & $\mathrm{~N}$ & \\
\hline 9 & $3 / 29 / 93$ & 3 & 1 & & 125.24 & $\mathbf{N}$ & \\
\hline 9 & $3 / 29 / 93$ & 4 & 1 & & 121.46 & $\mathbf{N}$ & \\
\hline 1 & $3 / 29 / 93$ & 5 & 1 & & 118.4 & $\mathbf{N}$ & \\
\hline$\frac{1}{1}$ & $3 / 29 / 93$ & 6 & 1 & & 78.62 & $\mathbf{N}$ & \\
\hline 1 & $4 / 5 / 93$ & 1 & 9 & & 129.56 & $\mathbf{Y}$ & TMACS \\
\hline 1 & $4 / 5 / 93$ & 2 & 1 & & 126.86 & $\bar{N}$ & \\
\hline 1 & $4 / 5 / 93$ & 3 & 1 & & 125.06 & $N$ & \\
\hline 1 & $4 / 5 / 93$ & 4 & 1 & & 121.64 & $\mathrm{~N}$ & \\
\hline 1 & $4 / 5 / 93$ & 5 & 1 & & 118.4 & $\mathbf{N}$ & \\
\hline 1 & $4 / 5 / 93$ & 6 & 1 & & 78.62 & $\bar{N}$ & \\
\hline 1 & $4 / 12 / 93$ & 1 & 1 & & 130.1 & $\mathrm{~N}$ & TMACS \\
\hline 1 & $4 / 12 / 93$ & 2 & 1 & & 126.86 & $N$ & \\
\hline 1 & $4 / 12 / 93$ & 3 & 1 & & 125.06 & $\mathbf{N}$ & \\
\hline 1 & $4 / 12 / 93$ & 4 & 1 & & 121.82 & $\mathbf{N}$ & \\
\hline 1 & $4 / 12 / 93$ & 5 & 9 & & 118.04 & $\bar{N}$ & \\
\hline
\end{tabular}

Data obtained from WHC Surveillance Analysis Computer System (SACS), June 2, 1993. 
WHC-SD-WM-ER-312, Rev. 0

\begin{tabular}{|c|c|c|c|c|c|c|c|}
\hline \multicolumn{8}{|c|}{ TANK 241-BY-106 } \\
\hline \multicolumn{8}{|c|}{ THERMOCOUPLE DATA } \\
\hline TREE \# & DATE & THERMO \# & TREE SET \# & TEMP C & TEMP F & $Y=$ MAX TEMP & COMMENTS \\
\hline 1 & $4 / 12 / 93$ & 6 & 1 & & 78.26 & $\mathbf{N}$ & \\
\hline 1 & $4 / 19 / 93$ & 9 & 1 & & 130.1 & $\mathrm{Y}$ & TMACS \\
\hline 1 & $4 / 19 / 93$ & 2 & 1 & & 126.86 & $\mathbf{N}$ & \\
\hline 1 & $4 / 19 / 93$ & 3 & 1 & & 125.42 & $\mathbf{N}$ & \\
\hline 1 & $4 / 19 / 93$ & 4 & 1 & & 122 & $\mathbf{N}$ & \\
\hline 1 & $4 / 19 / 93$ & 5 & 1 & & 118.4 & $\bar{N}$ & \\
\hline 1 & $4 / 19 / 93$ & 6 & 1 & & 78.62 & $\mathrm{~N}$ & \\
\hline 1 & $4 / 25 / 93$ & 1 & 1 & & 130.28 & $\mathbf{Y}$ & TMACS \\
\hline 1 & $4 / 25 / 93$ & 2 & 1 & & 127.22 & $\mathbf{N}$ & \\
\hline 1 & $4 / 25 / 93$ & 3 & 1 & & 125.06 & $\mathbf{N}$ & \\
\hline 1 & $4 / 25 / 93$ & 4 & 1 & & 121.82 & $\mathbf{N}$ & \\
\hline 1 & $4 / 25 / 93$ & 5 & 1 & & 118.58 & $\mathbf{N}$ & \\
\hline 1 & $4 / 25 / 93$ & 6 & 1 & & 79.34 & $\mathbf{N}$ & \\
\hline 1 & $5 / 3 / 93$ & 1 & 1 & & 129.92 & $\mathbf{Y}$ & TMACS \\
\hline 1 & $5 / 3 / 93$ & 2 & 1 & & 127.04 & $\mathbf{N}$ & \\
\hline 1 & $5 / 3 / 93$ & 3 & 1 & & 125.24 & $\mathbf{N}$ & \\
\hline 1 & $5 / 3 / 93$ & 4 & 9 & & 121.82 & $\mathbf{N}$ & \\
\hline$\frac{1}{1}$ & $5 / 3 / 93$ & 5 & 1 & & 118.76 & $\mathbf{N}$ & \\
\hline 1 & $5 / 3 / 93$ & 6 & 1 & & 79.34 & $\mathbf{N}$ & \\
\hline 1 & $5 / 10 / 93$ & 1 & 1 & & 129.38 & $\bar{Y}$ & TMACS \\
\hline 1 & $5 / 10 / 93$ & 2 & 1 & & 126.68 & $\mathbf{N}$ & \\
\hline 1 & $5 / 10 / 93$ & 3 & 1 & & 124.7 & $\mathbf{N}$ & \\
\hline 1 & $5 / 10 / 93$ & 4 & 1 & & 122 & $\mathbf{N}$ & \\
\hline 1 & $5 / 10 / 93$ & 5 & 1 & & 117.5 & $\mathbf{N}$ & \\
\hline 1 & $5 / 10 / 93$ & 6 & 1 & & 79.16 & $\mathbf{N}$ & \\
\hline 1 & $5 / 17 / 93$ & 1 & 1 & & 129.2 & $Y$ & TMACS \\
\hline 1 & $5 / 17 / 93$ & 2 & 9 & & 126.14 & $\mathbf{N}$ & \\
\hline 1 & $5 / 17 / 93$ & 3 & 1 & & 124.7 & $\mathbf{N}$ & \\
\hline 1 & $5 / 17 / 93$ & 4 & 1 & & 121.28 & $\mathbf{N}$ & \\
\hline 1 & $5 / 17 / 93$ & 5 & 1 & & 117.32 & $\mathrm{~N}$ & \\
\hline 1 & $5 / 17 / 93$ & 6 & 1 & & 79.34 & $\mathrm{~N}$ & \\
\hline 1 & $5 / 24 / 93$ & 1 & 1 & & 129.2 & $\bar{Y}$ & TMACS \\
\hline 1 & $5 / 24 / 93$ & 2 & 1 & & 126.32 & $N$ & \\
\hline 1 & $5 / 24 / 93$ & 3 & 1 & & 124.52 & $\mathbf{N}$ & \\
\hline 1 & $5 / 24 / 93$ & 4 & 1 & & 121.46 & $\mathbf{N}$ & \\
\hline 1 & $5 / 24 / 93$ & 5 & 1 & & 117.68 & $\mathrm{~N}$ & \\
\hline 1 & $5 / 24 / 93$ & 6 & 1 & & 79.88 & $\mathbf{N}$ & \\
\hline 1 & $5 / 31 / 93$ & 1 & 1 & & 129.2 & $Y$ & TMACS \\
\hline 1 & $5 / 31 / 93$ & 2 & 1 & & 126.32 & $\mathbf{N}$ & \\
\hline 1 & $5 / 31 / 93$ & 3 & 1 & & 124.88 & $N$ & \\
\hline 1 & $5 / 31 / 93$ & 4 & 1 & & 121.82 & $\mathrm{~N}$ & \\
\hline 1 & $5 / 31 / 93$ & 5 & 1 & & 117.68 & $N$ & \\
\hline 1 & $5 / 31 / 93$ & 6 & 1 & & 80.42 & $\mathrm{~N}$ & \\
\hline 1 & $6 / 7 / 93$ & 1 & 1 & & 129.56 & $Y$ & TMACS \\
\hline 1 & $6 / 7 / 93$ & 2 & 1 & & 126.86 & $\mathbf{N}$ & \\
\hline 1 & $6 / 7 / 93$ & 3 & 1 & & 125.06 & $\mathbf{N}$ & \\
\hline 1 & $6 / 7 / 93$ & 4 & 1 & & 122 & $N$ & \\
\hline 1 & $6 / 7 / 93$ & 5 & 1 & & 118.22 & $\mathbf{N}$ & \\
\hline 1 & $6 / 7 / 93$ & 6 & 1 & & 81.14 & $\mathbf{N}$ & \\
\hline 1 & $6 / 14 / 93$ & 1 & 1 & & 129.2 & $Y$ & TMACS \\
\hline 1 & $6 / 14 / 93$ & 2 & 1 & & 126.32 & $\mathbf{N}$ & \\
\hline 1 & $6 / 14 / 93$ & 3 & 1 & & 124.52 & $\mathrm{~N}$ & \\
\hline 1 & $6 / 14 / 93$ & 4 & 1 & & 121.46 & $\mathbf{N}$ & \\
\hline 1 & $6 / 14 / 93$ & 5 & 1 & & 117.32 & $\mathbf{N}$ & \\
\hline 1 & $6 / 14 / 93$ & 6 & 1 & & 81.32 & $\mathbf{N}$ & \\
\hline 1 & $6 / 21 / 93$ & 1 & 1 & & 129.2 & 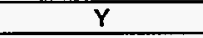 & TMACS \\
\hline 1 & $6 / 21 / 93$ & 2 & 1 & & 126.32 & $\mathrm{~N}$ & \\
\hline 1 & $6 / 21 / 93$ & 3 & 1 & & 124.88 & $\mathbf{N}$ & \\
\hline 1 & $6 / 21 / 93$ & 4 & 1 & & 121.1 & $\mathbf{N}$ & \\
\hline 1 & $6 / 21 / 93$ & 5 & 1 & & 117.5 & $\mathbf{N}$ & \\
\hline 1 & $6 / 21 / 93$ & 6 & 1 & & 82.04 & $\mathrm{~N}$ & \\
\hline 1 & $6 / 28 / 93$ & 9 & 1 & & 129.38 & $Y$ & TMACS \\
\hline 1 & $6 / 28 / 93$ & 2 & 1 & & 126.68 & $N$ & \\
\hline 1 & $6 / 28 / 93$ & 3 & 1 & & 124.7 & $\mathbf{N}$ & \\
\hline 1 & $6 / 28 / 93$ & 4 & 1 & & 121.28 & $\mathrm{~N}$ & \\
\hline 1 & $6 / 28 / 93$ & 5 & 1 & & 117.68 & $\mathrm{~N}$ & \\
\hline
\end{tabular}

Data obtained from WHC Surveillance Analysis Computer System (SACS), June 2, 1993. 


\begin{tabular}{|c|c|c|c|c|c|c|c|}
\hline \multicolumn{8}{|c|}{ TANK 241-BY-106 } \\
\hline & & & & THERM & COUPLE & TA & \\
\hline TREE \# & DATE & THERMO \# & TREE SET \# & TEMP C & TEMP F & $Y=M A X$ TEMP & COMMENTS \\
\hline 1 & $6 / 28 / 93$ & 6 & 1 & & 82.76 & $\mathbf{N}$ & \\
\hline 1 & $7 / 5 / 93$ & 1 & 1 & & 128.84 & $\mathbf{Y}$ & TMACS \\
\hline 1 & $7 / 5 / 93$ & 2 & 1 & & 126.5 & $\bar{N}$ & \\
\hline 1 & $7 / 5 / 93$ & 3 & 1 & & 124.52 & $\mathbf{N}$ & \\
\hline 1 & $7 / 5 / 93$ & 4 & 1 & & 120.74 & $\bar{N}$ & \\
\hline 1 & $7 / 5 / 93$ & 5 & 1 & & 117.14 & $\mathbf{N}$ & \\
\hline 1 & $7 / 5 / 93$ & 6 & 1 & & 82.94 & $\mathbf{N}$ & \\
\hline 1 & $7 / 12 / 93$ & 1 & 1 & & 129.38 & $\bar{Y}$ & TMACS \\
\hline 1 & $7 / 12 / 93$ & 2 & 1 & & 126.5 & $\mathbf{N}$ & \\
\hline 1 & $7 / 12 / 93$ & 3 & 1 & & 124.34 & $\mathrm{~N}$ & \\
\hline 1 & $7 / 12 / 93$ & 4 & 1 & & 121.46 & $\mathbf{N}$ & \\
\hline 1 & $7 / 12 / 93$ & 5 & 1 & & 117.5 & $\mathbf{N}$ & \\
\hline 1 & $7 / 12 / 93$ & 6 & 1 & & 83.48 & $\mathbf{N}$ & \\
\hline 1 & $7 / 19 / 93$ & 1 & 1 & & 128.66 & $\mathbf{Y}$ & TMACS \\
\hline 1 & $7 / 19 / 93$ & 2 & 1 & & 126.14 & $\mathbf{N}$ & \\
\hline 1 & $7 / 19 / 93$ & 3 & 1 & & 124.16 & N & \\
\hline 1 & $7 / 19 / 93$ & 4 & 1 & & 120.74 & $N$ & \\
\hline 1 & $7 / 19 / 93$ & 5 & 1 & & 117.14 & $\mathbf{N}$ & \\
\hline 1 & $7 / 19 / 93$ & 6 & 1 & & 83.66 & $N$ & \\
\hline 1 & $7 / 26 / 93$ & 1 & 1 & & 129.02 & $\bar{Y}$ & TMACS \\
\hline 1 & $7 / 26 / 93$ & 2 & 1 & & 126.32 & $\mathbf{N}$ & \\
\hline 1 & $7 / 26 / 93$ & 3 & 1 & & 124.52 & $\mathbf{N}$ & \\
\hline 1 & $7 / 26 / 93$ & 4 & 1 & & 121.46 & $\mathbf{N}$ & \\
\hline 1 & $7 / 26 / 93$ & 5 & 1 & & 117.5 & $\mathbf{N}$ & \\
\hline 1 & $7 / 26 / 93$ & 6 & 1 & & 84.38 & $\mathbf{N}$ & \\
\hline 1 & $8 / 2 / 93$ & 1 & 9 & & 128.84 & $\bar{Y}$ & TMACS \\
\hline 1 & $8 / 2 / 93$ & 2 & 1 & & 126.32 & $\mathbf{N}$ & \\
\hline 1 & $8 / 2 / 93$ & 3 & 1 & & 124.52 & $\mathbf{N}$ & \\
\hline 1 & $8 / 2 / 93$ & 4 & 1 & & 121.1 & $\mathbf{N}$ & \\
\hline 1 & $8 / 2 / 93$ & 5 & 1 & & 116.96 & $\mathbf{N}$ & \\
\hline 1 & $8 / 2 / 93$ & 6 & 1 & & 84.92 & $\mathbf{N}$ & \\
\hline 1 & $8 / 9 / 93$ & 1 & 1 & & 128.84 & $\bar{Y}$ & TMACS \\
\hline 1 & $8 / 9 / 93$ & 2 & 1 & & 126.32 & $\mathbf{N}$ & \\
\hline 1 & $8 / 9 / 93$ & 3 & 1 & & 124.16 & $\mathrm{~N}$ & \\
\hline 1 & $8 / 9 / 93$ & 4 & 1 & & 120.74 & $N$ & \\
\hline 1 & $8 / 9 / 93$ & 5 & 1 & & 116.96 & $\mathbf{N}$ & \\
\hline 1 & $8 / 9 / 93$ & 6 & 1 & & 85.28 & $\mathbf{N}$ & \\
\hline 1 & $8 / 15 / 93$ & 1 & 1 & & 128.84 & $Y$ & tmacs \\
\hline 1 & $8 / 15 / 93$ & 2 & 1 & & 125.96 & $\mathbf{N}$ & \\
\hline 1 & $8 / 15 / 93$ & 3 & 1 & & 123.98 & N & \\
\hline 1 & $8 / 15 / 93$ & 4 & 1 & & 121.1 & $\mathbf{N}$ & \\
\hline 1 & $8 / 15 / 93$ & 5 & 1 & & 116.96 & $\mathbf{N}$ & \\
\hline 1 & $8 / 15 / 93$ & 6 & 1 & & 85.46 & $\overline{\mathbf{N}}$ & \\
\hline 1. & $8 / 23 / 93$ & 1 & 1 & & 129.02 & $\bar{Y}$ & TMACS \\
\hline 1 & $8 / 23 / 93$ & 2 & 1 & & 126.14 & $\mathbf{N}$ & \\
\hline 1 & $8 / 23 / 93$ & 3 & 1 & & 124.16 & $\mathbf{N}$ & \\
\hline 1 & $8 / 23 / 93$ & 4 & 1 & & 120.56 & $\mathbf{N}$ & \\
\hline 1 & $8 / 23 / 93$ & 5 & 1 & & 117.14 & $N$ & \\
\hline 1 & $8 / 23 / 93$ & 6 & 1 & & 86.18 & $N$ & \\
\hline 1 & $8 / 26 / 93$ & 1 & 1 & & 128.7 & $\bar{Y}$ & J TYPE \\
\hline 1 & $8 / 26 / 93$ & 2 & 1 & & 126 & $\mathbf{N}$ & \\
\hline 1 & $8 / 26 / 93$ & 3 & 1 & & 124.2 & $\mathbf{N}$ & \\
\hline 1 & $8 / 26 / 93$ & 4 & 1 & & 121.1 & $\mathbf{N}$ & \\
\hline 1 & $8 / 26 / 93$ & 5 & 9 & & 117.3 & $\mathrm{~N}$ & \\
\hline 1 & $8 / 26 / 93$ & 6 & 1 & & 86 & $\mathbf{N}$ & \\
\hline 1 & $8 / 30 / 93$ & 1 & 1 & & 128.84 & $\bar{Y}$ & TMACS \\
\hline 1 & $8 / 30 / 93$ & 2 & 1 & & 125.96 & $\mathbf{N}$ & \\
\hline 9 & $8 / 30 / 93$ & 3 & 1 & & 124.34 & $N$ & \\
\hline 1 & $8 / 30 / 93$ & 4 & 1 & & 120.74 & $\mathbf{N}$ & \\
\hline 1 & $8 / 30 / 93$ & 5 & 1 & & 117.32 & $\mathbf{N}$ & \\
\hline 1 & $8 / 30 / 93$ & 6 & 1 & & 86.54 & $N$ & \\
\hline 1 & $9 / 6 / 93$ & 1 & 1 & & 128.48 & $\bar{Y}$ & TMACS \\
\hline 1 & $9 / 6 / 93$ & 2 & 1 & & 125.96 & $\bar{N}$ & \\
\hline 1 & $9 / 6 / 93$ & 3 & 1 & & 124.16 & $\mathbf{N}$ & \\
\hline 1 & $9 / 6 / 93$ & 4 & 1 & & 120.38 & $\mathrm{~N}$ & \\
\hline 1 & $9 / 6 / 93$ & 5 & 1 & & 117.32 & $\mathbf{N}$ & \\
\hline
\end{tabular}

Data obtained from WHC Surveillance Analysis Computer System (SACS), June 2, 1993. 
WHC-SD-WM-ER-312, Rev. 0

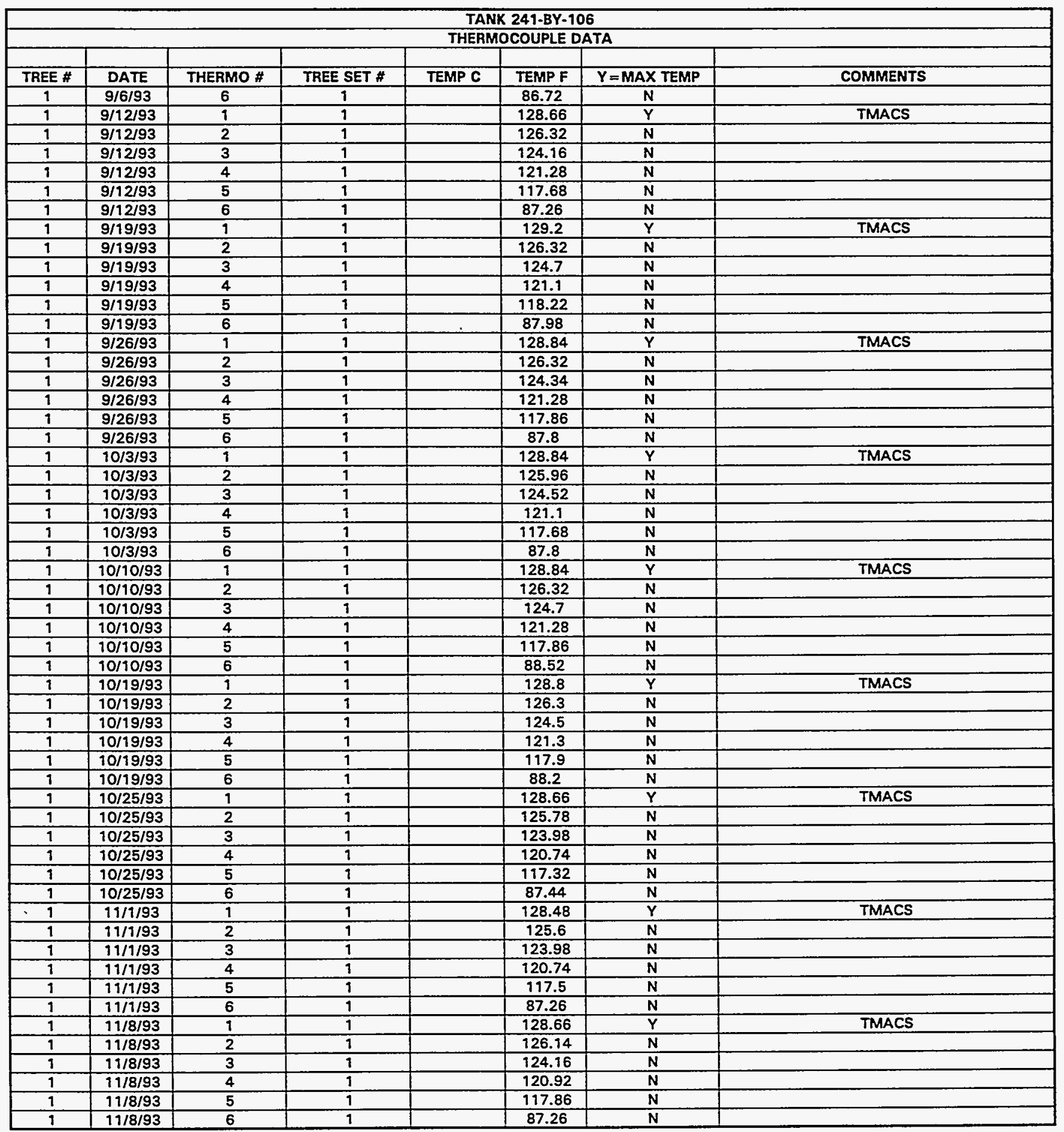

Data obtained from WHC Surveillance Analysis Computer System (SACS), June 2, 1993. 


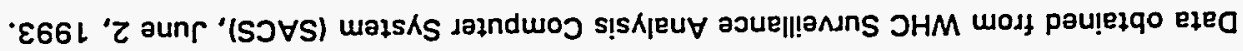

\begin{tabular}{|c|c|c|c|c|c|c|c|}
\hline J eөl6op peunsse - Elep MeJ & & 8.901 & $\frac{15}{b}$ & & $\overline{D L}$ & $\varepsilon L / O Z / Z !$ & 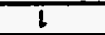 \\
\hline J өөנ6өp pounnsse - вlep $M \theta 1$ & & 8.901 & 10 & & $\varepsilon L$ & $\varepsilon L / 0 Z / Z L$ & $L$ \\
\hline כ aoj6op pounnsse - elep MEJ & & 701 & Ot & & $2 L$ & $\varepsilon \angle / O Z / Z L$ & I \\
\hline 2 adibap pounsse - elep Med & & $Z \cdot L L L$ & $t$ & & LL & $\varepsilon \angle / O Z / Z L$ & 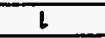 \\
\hline כ өөנ6өp pounsse - 81ep мes & & $\angle 91$ & GL & & 01 & $\varepsilon \angle / O Z / Z L$ & 1 \\
\hline כ 2әлбар paunsse - elep Med & & 7.811 & $8 t$ & & 6 & $\varepsilon \angle / O Z / Z L$ & $t$ \\
\hline J aol6ep pounsse - elep Mef & & $6.8 L L$ & 86 & & 8 & $\varepsilon L / O Z / Z \downarrow$ & $I$ \\
\hline 2 อod6ap pounsSe - elep ME1 & & 8.896 & $9 L$ & & $L$ & $\varepsilon \angle / O Z / Z L$ & 1 \\
\hline כ вә16өp pounsSe - elep MEI & & ODL & 09 & & 9 & $\varepsilon L / O Z / Z L$ & $!$ \\
\hline J өอג6ap pounsss - вlep Mes & & $8+11$ & 96 & & $\mathbf{G}$ & $\varepsilon \angle / O Z / Z L$ & $\bar{L}$ \\
\hline כ จә16аp pownsse - elep Med & & 8.896 & $9 L$ & & $t$ & $\varepsilon \angle 102 / 2 L$ & $t$ \\
\hline J eә16日p paunSSB - elep MES & & 8.891 & $\overline{9 L}$ & & $\bar{\varepsilon}$ & EL/OZIRL & $I$ \\
\hline 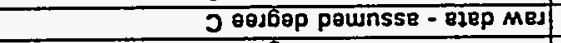 & & $9 \cdot \mathrm{LOl}$ & $2 \emptyset$ & & $\bar{z}$ & $\varepsilon \angle / O Z / Z L$ & I \\
\hline כ 0อдbep pawnsse - elep MEI & & $9 \cdot \varepsilon+1$ & 29 & & 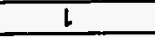 & $\varepsilon L / O Z / Z L$ & 1 \\
\hline J өอ160p peunsse - в3ер MEA & & $8^{\circ} \mathrm{GOL}$ & 16 & & $\nabla L$ & $\varepsilon L I L L / Z L$ & $\mathfrak{l}$ \\
\hline כ өөנ6өp peunsse - вдер Mes & & $8^{\circ} \mathrm{GOL}$ & Lt & & $\varepsilon \mathrm{E}$ & $\varepsilon L / L L / Z L$ & $\mathbf{l}$ \\
\hline כ өөנbop pounSSE - elep MRI & & $9^{\circ} \angle O L$ & $2 t$ & & $\overline{\mathbf{Z l}}$ & $\varepsilon L I L L / Z L$ & 1 \\
\hline (46!U) ezep 100dsns - $t<$ & & & & & LL & $\varepsilon L I L I / Z !$ & $\mathfrak{L}$ \\
\hline J oes6ep pounsse - вlep Mes & & $\varepsilon \mathfrak{E L}$ & St & & OL & $\varepsilon \angle L L L / Z L$ & $\mathrm{l}$ \\
\hline J өө16өp pounsse - вlep MEA & & $\varepsilon \downarrow$ & St & & 6 & $\varepsilon L I L L / Z L$ & $I$ \\
\hline J өөנ6өp pawnSse - elep MeI & & 2991 & $\forall L$ & & 8 & $\varepsilon \angle / \angle L / Z l$ & $t$ \\
\hline (46! & & & & & $L$ & $\varepsilon \angle / L L / Z L$ & $\bar{L}$ \\
\hline J eөs6ep paunsse - 8qep Mes & & tOL & ot & & 9 & $\varepsilon \angle I \angle L / Z L$ & $I$ \\
\hline 2 өอ16өp pounsse - в1еp Mej & & $Z \cdot 991$ & $\not L$ & & $\mathbf{g}$ & $\varepsilon \angle I L L / Z L$ & 1 \\
\hline (MOl) 81Ep 200dsns - $\varepsilon \varepsilon$ & & & & & $t$ & $\varepsilon L / L L / Z L$ & 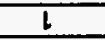 \\
\hline (MO|) Elep 200dSns - $9 \varepsilon$ & & & & & $\bar{\varepsilon}$ & $\varepsilon \angle I \angle L / Z L$ & $\mathrm{l}$ \\
\hline J eəs6ap pounssa - elep med & & $8^{\circ} 091$ & 99 & & $z$ & $\varepsilon L / \angle L / Z L$ & L \\
\hline ว өod6əp pounsse - elep мed & & 8.96 & $9 \varepsilon$ & & thl & $\varepsilon L / O L / Z L$ & 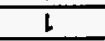 \\
\hline כ вә16ар pounsse - elep MeI & & $8 \cdot 96$ & $9 \varepsilon$ & & $\overline{\varepsilon l}$ & $E L / O L / Z L$ & I \\
\hline כ өal6ap paunsSe - ełep MeJ & & 7001 & $8 \varepsilon$ & & $Z l$ & $E L / O L / Z L$ & $I$ \\
\hline (46!4) elep loadsns - 04 & & & & & IL & $\varepsilon L / O L / Z L$ & 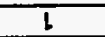 \\
\hline 5 god6əp paunsse - elep Med & & $2 \cdot 111$ & to & & $\mathbf{O L}$ & $\varepsilon \angle / O L / Z L$ & I \\
\hline כ oәd6ар paunsse - elep Mes & & $\overline{Z L L L}$ & $t \phi$ & & 6 & $\varepsilon L / O L / Z L$ & 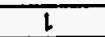 \\
\hline 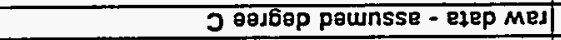 & & $9 \cdot 191$ & $\overline{Z L}$ & & 8 & $\varepsilon L / O L / Z L$ & 1 \\
\hline J өอ168p pounsse - elep MeJ & & $9 \cdot 191$ & $Z L$ & & $L$ & ELIOL/ZL & $l$ \\
\hline ว ваנ6вp pounsse - ezвp Mes & & 9.191 & $2 L$ & & 9 & $\varepsilon \angle L O L / L L$ & $\downarrow$ \\
\hline 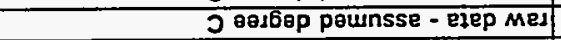 & & 9.191 & $Z L$ & & $\underline{s}$ & $\varepsilon L I O L / Z L$ & $\downarrow$ \\
\hline ว өәر6өр pөшnsse - едер MEs & & $89 L$ & $O L$ & & $t$ & $\varepsilon L / O L / Z L$ & 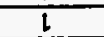 \\
\hline (mol) elep Loadsns - 8Z & & & & & $\bar{\varepsilon}$ & $\varepsilon \angle / O L / Z L$ & $I$ \\
\hline J өel6ep pawnsse - ejep Mes & & $8 \cdot 1+1$ & 19 & & 2 & $\varepsilon L / O L / Z L$ & $\mathrm{l}$ \\
\hline J өә1Бар pownsse - exep Med & & 7.001 & $8 \varepsilon$ & & $2 !$ & $\varepsilon L / L Z / L$ & l \\
\hline J өә16өp pounsse - 818p Med & & $\$ 001$ & $8 \varepsilon$ & & IL & $\varepsilon L / L Z / L L$ & 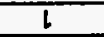 \\
\hline J өอsбеp pounsse - elep Mes & & 896 & $O L$ & & OL & $\varepsilon L / L Z / L L$ & 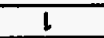 \\
\hline כ ваנбөр pownsse - еqер Med & & $2 \cdot 201$ & $6 \varepsilon$ & & 6 & $\varepsilon L / L Z / L L$ & $i$ \\
\hline כ әөJ6əp pQUnSSE - Elep MEJ & & Z゙LL & 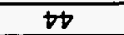 & & 8 & $\varepsilon L / L Z / L !$ & $\mathrm{L}$ \\
\hline J aes6ap paunsSE - elep MEJ & & 9.196 & $Z L$ & & $L$ & $\varepsilon \angle / L Z / L /$ & $b$ \\
\hline ว өอ16ер pounsse - elep Med & & $9^{\circ} \mathrm{GZL}$ & 29 & & 9 & $\varepsilon L / L Z / L L$ & $\bar{I}$ \\
\hline כ әөנ6әр pounsse - elep MEJ & & 98 & $0 \varepsilon$ & & $\mathbf{s}$ & $\varepsilon L / L Z / L L$ & $l$ \\
\hline J eөsбөp pownsse - elep MES & & $9^{\circ}+\varepsilon L$ & $\angle S$ & & $t$ & $\varepsilon L / L Z / L L$ & $b$ \\
\hline ว өөנ6өp pəunsse - elep Mes & & $2 \cdot 991$ & 69 & & $\varepsilon$ & $\varepsilon \angle / L Z / L L$ & $t$ \\
\hline כ ooj6op paunsse - elep Med & & $\tau \cdot \varepsilon 6$ & $\nabla \varepsilon$ & & $z$ & $\varepsilon L / L Z / L L$ & $T$ \\
\hline 2 8016өp pounsse - elep Mej & & $t^{\circ} 9 \varepsilon l$ & 89 & & $\bar{t}$ & $\varepsilon L / L Z / L L$ & 1 \\
\hline כ 8016op paWnSSE - elep MES & & OLL & & & $\rightarrow l$ & $\varepsilon L / Z L / L L$ & $T$ \\
\hline J әө16өp pawnsse - eqер MEs & & OLl & & & $\varepsilon l$ & $\varepsilon L / Z L / L L$ & $t$ \\
\hline ว өอנ6өp pownSS8 - ejep ME1 & & $\Rightarrow Z L$ & & & $\mathbf{Z L}$ & $\varepsilon L / Z L / L L$ & $I$ \\
\hline 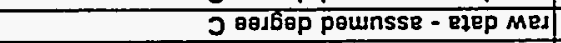 & & tOL & & & LL & $E L / Z L / L L$ & $l$ \\
\hline J өetBep pounsse - elep Med & & 891 & & & OL & $\varepsilon \angle / Z L / L L$ & l \\
\hline 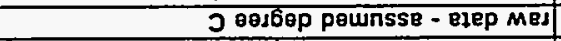 & & 801 & & & 6 & $\varepsilon L / Z L / L !$ & 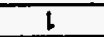 \\
\hline 5 өeibep pownsse - elep MEJ & & 801 & & & 8 & $\varepsilon L / Z L / L L$ & I \\
\hline כ өөt6op paunsse - elep MEd & & 091 & & & $L$ & $\varepsilon L / Z L / L L$ & l \\
\hline ว өө16өр pqunsse - elep MEd & & $89 !$ & & & 9 & $\varepsilon L / Z L / L$ & $l$ \\
\hline ว өөנ6ар pөunsse - 81ер м81 & & 96 & & & $\mathbf{S}$ & $\varepsilon L / Z \backslash / L !$ & $I$ \\
\hline 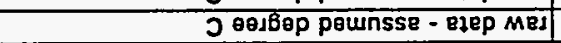 & & 991 & & & $t$ & $\varepsilon L / Z\rfloor / L \downarrow$ & $l$ \\
\hline כ вел6вр pounsse - elep MEd & & 991 & & & $\varepsilon$ & $\varepsilon L / Z\lfloor/ L L$ & 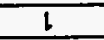 \\
\hline J 8016өp peunsse - elep Med & & 76 & & & $\bar{z}$ & $\varepsilon L / Z \downarrow / L L$ & 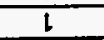 \\
\hline כ 8өנ6өp pownSSE - 83Bp MEJ & & IS! & & & $t$ & $\varepsilon L / Z L / L L$ & 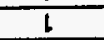 \\
\hline SLN3WWOO & $d W \exists \perp X \forall W=\lambda$ & $\exists d W \exists \perp$ & O dWZI & \# 1ヨS 3381 & \# OWYG & $\exists \perp \forall a$ & 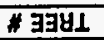 \\
\hline \multicolumn{8}{|c|}{$\forall \perp \forall 0$ 3าdก०วОWy } \\
\hline & & $l-18-l+2$ & $\Delta \forall 1$ & & & & \\
\hline
\end{tabular}




\begin{tabular}{|c|c|c|c|c|c|c|c|}
\hline \multicolumn{8}{|c|}{ TANK 241-BY-107 } \\
\hline \multicolumn{8}{|c|}{ THERMOCOUPLE DATA } \\
\hline & & & & & & & \\
\hline TREE \# & DATE & THERMO \# & TREE SET \# & TEMP C & TEMP F & $Y=M A X$ TEMP & $\begin{array}{l}\text { COMMENTS } \\
\end{array}$ \\
\hline 1 & $12 / 20 / 73$ & 15 & & 48 & 118.4 & & raw data - assumed degree $C$ \\
\hline 1 & $8 / 5 / 74$ & 1 & 1 & 31 & 87.8 & $\mathbf{N}$ & degree C/TWINS confirm \\
\hline 1 & $8 / 5 / 74$ & 2 & 1 & 31 & 87.8 & $\mathbf{N}$ & degree C/TWINS confirm \\
\hline 1 & $8 / 5 / 74$ & 3 & 1 & 48 & 118.4 & $Y$ & degree C/TWINS confirm \\
\hline 1 & $8 / 5 / 74$ & 4 & 1 & 36 & 96.8 & $N$ & degree C/TWINS confirm \\
\hline 1 & $8 / 5 / 74$ & 5 & 1 & 32 & 89.6 & $\mathbf{N}$ & degree C/TWINS confirm \\
\hline 1 & $8 / 5 / 74$ & 14 & 1 & 32 & 89.6 & $\mathbf{N}$ & degree C/TWINS confirm \\
\hline 1 & $9 / 12 / 74$ & 1 & 1 & 32 & 89.6 & $\mathrm{~N}$ & degree C/TWINS confirm \\
\hline 1 & $9 / 12 / 74$ & 2 & 1 & 32 & 89.6 & $\mathbf{N}$ & degree C/TWINS confirm \\
\hline 1 & $9 / 12 / 74$ & 3 & 1 & 49 & 120.2 & $\bar{Y}$ & degree C/TWINS confirm \\
\hline 1 & $9 / 12 / 74$ & 4 & 1 & 34 & 93.2 & $\mathbf{N}$ & degree C/TWINS confirm \\
\hline 1 & $9 / 12 / 74$ & 5 & 1 & 32 & 89.6 & $\mathbf{N}$ & degres C/TWINS confirm \\
\hline 1 & $9 / 12 / 74$ & 14 & 1 & 31 & 87.8 & $\mathbf{N}$ & degree C/TWINS confirm \\
\hline 1 & $10 / 8 / 74$ & 1 & 1 & 30 & 86 & $\mathbf{N}$ & degree C/TWINS confirm \\
\hline 1 & $10 / 8 / 74$ & 2 & 1 & 30 & 86 & $\mathbf{N}$ & degree C/TWINS confirm \\
\hline 1 & $10 / 8 / 74$ & 3 & 1 & 46 & 114.8 & $Y$ & degree C/TWINS confirm \\
\hline 1 & $10 / 8 / 74$ & 4 & 1 & 40 & 104 & $\mathbf{N}$ & degree C/TWINS confirm \\
\hline 1 & $10 / 8 / 74$ & 5 & 1 & 30 & 86 & $\mathbf{N}$ & degree C/TWINS confirm \\
\hline 1 & $10 / 8 / 74$ & 14 & 1 & 28 & 82.4 & $\mathbf{N}$ & degree C/TWINS confirm \\
\hline 1 & $10 / 29 / 74$ & 1 & 1 & 32 & 89.6 & $\mathbf{N}$ & degree C/TWINS confirm \\
\hline 1 & $10 / 29 / 74$ & 2 & 1 & 32 & 89.6 & $\mathbf{N}$ & degree C/TWINS confirm \\
\hline 1 & $10 / 29 / 74$ & 3 & 1 & 36 & 96.8 & $\bar{Y}$ & degree C/TWINS confirm \\
\hline 1 & $10 / 29 / 74$ & 4 & 1 & 36 & 96.8 & $Y$ & degree C/TWINS confirm \\
\hline 1 & $10 / 29 / 74$ & 5 & 1 & 36 & 96.8 & $Y$ & degree C/TWINS confirm \\
\hline 1 & $10 / 29 / 74$ & 14 & 1 & 32 & 89.6 & $\mathbf{N}$ & degrae C/TWINS confirm \\
\hline 1 & $19 / 12 / 74$ & 1 & 1 & 25 & 77 & $\mathbf{N}$ & degree C/TWINS confirm \\
\hline 1 & $11 / 12 / 74$ & 2 & 1 & 25 & 77 & $\mathbf{N}$ & degree C/TWINS confirm \\
\hline 1 & $11 / 12 / 74$ & 3 & 1 & 28 & 82.4 & $\bar{Y}$ & degree C/TWINS confirm \\
\hline 1 & $11 / 12 / 74$ & 4 & 1 & 28 & 82.4 & $Y$ & degree C/TWINS confirm \\
\hline 1 & $11 / 12 / 74$ & 5 & 1 & 26 & 78.8 & $\mathbf{N}$ & degree C/TWINS confirm \\
\hline 1 & $11 / 12 / 74$ & 14 & 1 & 25 & 77 & $\mathbf{N}$ & degree C/TWINS confirm \\
\hline 1 & $12 / 17 / 74$ & 1 & 1 & 22.78 & 73.004 & $\mathbf{N}$ & degree C/TWINS confirm \\
\hline 1 & $12 / 17 / 74$ & 2 & 1 & 21.67 & 71.006 & $\mathbf{N}$ & degree $\mathrm{C} / \mathrm{TW}$ INS confirm \\
\hline 1 & $12 / 17 / 74$ & 3 & 1 & 26.67 & 80.006 & $\mathrm{~N}$ & degree $\mathrm{C} / \mathrm{TW}$ INS confirm \\
\hline 1 & $12 / 17 / 74$ & 4 & 1 & 28.33 & 82.994 & $Y$ & degree C/TWINS confirm \\
\hline 1 & $12 / 17 / 74$ & 5 & 1 & 22.22 & 71.996 & $N$ & degree C/TWINS confirm \\
\hline 1 & $12 / 17 / 74$ & 14 & 1 & 22.22 & 71.996 & $\bar{N}$ & degree C/TWINS confirm \\
\hline 1 & $12 / 31 / 74$ & 1 & 1 & 24.44 & 75.992 & $\mathbf{N}$ & degree C/TWINS confirm \\
\hline 1 & $12 / 31 / 74$ & 2 & 1 & 24.44 & 75.992 & $\mathbf{N}$ & degree CITWINS confirm \\
\hline 1 & $12 / 31 / 74$ & 3 & 1 & 28.89 & 84.002 & $\mathbf{N}$ & degree C/TWINS confirm \\
\hline 1 & $12 / 31 / 74$ & 4 & 1 & 30.56 & 87.008 & $Y$ & degree C/TWINS confirm \\
\hline 1 & $12 / 31 / 74$ & 5 & 1 & 25.56 & 78.008 & $\mathbf{N}$ & degree C/TWINS confirm \\
\hline 1 & $12 / 31 / 74$ & 12 & 1 & 24.44 & 75.992 & $\mathbf{N}$ & degree C/TWINS confirm \\
\hline 1 & $-1 / 8 / 75$ & 1 & 1 & 22.22 & 71.996 & $\mathbf{N}$ & degree C/TWINS confirm \\
\hline 1 & $1 / 8 / 75$ & 2 & 1 & 22.22 & 71.996 & $\mathbf{N}$ & degree C/TWINS confirm \\
\hline 1 & $1 / 8 / 75$ & 3 & 1 & 26.67 & 80.006 & $\mathbf{N}$ & degree C/TWINS confirm \\
\hline 1 & $1 / 8 / 75$ & 4 & 1 & 28.33 & 82.994 & $Y$ & degree C/TWINS confirm \\
\hline 1 & $1 / 8 / 75$ & 5 & 1 & 22.22 & 71.996 & $\mathbf{N}$ & degree C/TWINS confirm \\
\hline 1 & $1 / 8 / 75$ & 14 & 1 & 22.22 & 71.996 & $\mathbf{N}$ & degree C/TWINS confirm \\
\hline 1 & $1 / 21 / 75$ & 1 & 1 & 23 & 73.4 & $\mathbf{N}$ & degree C/TWINS confirm \\
\hline 1 & $1 / 21 / 75$ & 2 & 1 & 28 & 82.4 & $\mathbf{N}$ & degree $\mathrm{C} / \mathrm{TW}$ INS confirm \\
\hline 1 & $1 / 21 / 75$ & 3 & 1 & 32 & 89.6 & $\mathbf{N}$ & degree C/TWINS confirm \\
\hline 1 & $1 / 21 / 75$ & 4 & 1 & 39 & 102.2 & $\bar{Y}$ & degree $\mathrm{C} / \mathrm{TW}$ INS confirm \\
\hline 1 & $1 / 21 / 75$ & 5 & 1 & 24 & 75.2 & $\mathbf{N}$ & degree C/TWINS confirm \\
\hline 1 & $1 / 21 / 75$ & 14 & 1 & 21 & 69.8 & $\mathbf{N}$ & degree $\mathrm{C} / \mathrm{TW}$ INS confirm \\
\hline 1 & $1 / 28 / 75$ & 1 & 1 & 20 & 68 & $\mathbf{N}$ & degree C/TWINS confirm \\
\hline 1 & $1 / 28 / 75$ & 2 & 1 & 21 & 69.8 & $\mathbf{N}$ & degree C/TWINS confirm \\
\hline 1 & $1 / 28 / 75$ & 3 & 1 & 25 & 77 & $\mathbf{N}$ & degree C/TWINS confirm \\
\hline 1 & $1 / 28 / 75$ & 4 & 1 & 28 & 82.4 & $\bar{Y}$ & degree C/TWINS confirm \\
\hline 1 & $1 / 28 / 75$ & 5 & 1 & 21 & 69.8 & $\mathbf{N}$ & degree CrTWINS contirm \\
\hline 1 & $1 / 28 / 75$ & 14 & 1 & 21 & 69.8 & $\mathbf{N}$ & degree C/TWINS confirm \\
\hline 1 & $2 / 7 / 75$ & 1 & 1 & 26.67 & 80.006 & $\mathbf{N}$ & degree C/TWINS confirm \\
\hline 1 & $2 / 7 / 75$ & 2 & 1 & 26.67 & 80.006 & $\mathbf{N}$ & degree C/TWINS confirm \\
\hline 1 & $2 / 7 / 75$ & 4 & 1 & 27.22 & 80.996 & $\mathbf{Y}$ & degree C/TWINS confirm \\
\hline 1 & $2 / 7 / 75$ & 5 & 1 & 27.22 & 80.996 & $Y$ & degree $\mathrm{C} / \mathrm{TW}$ WINS confirm \\
\hline 1 & $2 / 7 / 75$ & 12 & 1 & 26.11 & 78.998 & $\mathbf{N}$ & degree C/TWINS confirm \\
\hline
\end{tabular}

Data obtained from WHC Surveillance Analysis Computer System (SACS), June 2, 1993. 


\begin{tabular}{|c|c|c|c|c|c|c|c|}
\hline \multicolumn{8}{|c|}{ TANK 241-BY-107 } \\
\hline \multicolumn{8}{|c|}{ THERMOCOUPLE DATA } \\
\hline TREE \# & DATE & THERMO \# & TREE SET \# & TEMP C & TEMP F & $Y=$ MAX TEMP & COMMENTS \\
\hline 1 & $2 / 27 / 75$ & 1 & 1 & 22 & 71.6 & $\mathbf{Y}$ & degree C/TWINS confirm \\
\hline 1 & $2 / 27 / 75$ & 2 & 1 & 22 & 71.6 & $\mathbf{Y}$ & degree C/TWINS confirm \\
\hline 1 & $2 / 27 / 75$ & 3 & 1 & 22 & 71.6 & $\mathbf{Y}$ & degree C/TWINS confirm \\
\hline 1 & $2 / 27 / 75$ & 4 & 1 & 22 & 71.6 & $\bar{Y}$ & degree C/TWINS confirm \\
\hline 1 & $2 / 27 / 75$ & 5 & 1 & 22 & 71.6 & $\mathbf{Y}$ & degree C/TWINS confirm \\
\hline 1 & $2 / 27 / 75$ & 14 & 1 & 20 & 68 & $N$ & degree C/TWINS confirm \\
\hline 1 & $3 / 18 / 75$ & 1 & 1 & 22 & 71.6 & $\mathbf{Y}$ & degree C/TWINS confirm \\
\hline 1 & $3 / 18 / 75$ & 2 & 1 & 22 & 71.6 & $\mathbf{Y}$ & degree C/TWINS confirm \\
\hline 1 & $3 / 18 / 75$ & 3 & 1 & 22 & 71.6 & $\mathbf{Y}$ & degree C/TWINS confirm \\
\hline 1 & $3 / 18 / 75$ & 4 & 1 & 22 & 71.6 & $Y$ & degree C/TWINS confirm \\
\hline 1 & $3 / 18 / 75$ & 5 & 1 & 22 & 71.6 & $\mathrm{Y}$ & degreo C/TWINS confirm \\
\hline 1 & $3 / 18 / 75$ & 14 & 1 & 22 & 71.6 & $\mathbf{Y}$ & degree C/TWINS confirm \\
\hline 1 & $4 / 8 / 75$ & 1 & 1 & 17 & 62.6 & $\mathbf{N}$ & degree C/TWINS confirm \\
\hline 1 & $4 / 8 / 75$ & 2 & 1 & 17 & 62.6 & $\mathbf{N}$ & degree C/TWINS confirm \\
\hline 1 & $4 / 8 / 75$ & 3 & 1 & 18 & 64.4 & $N$ & degree C/TWINS confirm \\
\hline 1 & $4 / 8 / 75$ & 4 & 1 & 18 & 64.4 & $\mathbf{N}$ & degree $\mathrm{C} / \mathrm{TW}$ WINS confirm \\
\hline 1 & $4 / 8 / 75$ & 5 & 1 & 18 & 64.4 & $\mathbf{N}$ & degree C/TWINS confirm \\
\hline 1 & $4 / 8 / 75$ & 14 & 1 & 19 & 66.2 & $\bar{Y}$ & degree C/TWINS confirm \\
\hline 1 & $4 / 22 / 75$ & 1 & 1 & 18 & 64.4 & $\mathbf{N}$ & degree C/TWINS confirm \\
\hline 1 & $4 / 22 / 75$ & 3 & 1 & 12 & 53.6 & $\mathbf{N}$ & degree C/TWINS confirm \\
\hline 1 & $4 / 22 / 75$ & 5 & 1 & 18 & 64.4 & $\mathbf{N}$ & degree C/TWINS confirm \\
\hline 1 & $4 / 22 / 75$ & 14 & 1 & 18 & 64.4 & $\mathbf{N}$ & degree C/TWINS confirm \\
\hline 1 & $4 / 29 / 75$ & 1 & 1 & 16 & 60.8 & $\mathbf{N}$ & degree C/TWINS confirm \\
\hline 1 & $4 / 29 / 75$ & 2 & 1 & 18 & 64.4 & $\mathbf{N}$ & degree C/TWINS confirm \\
\hline 1 & $4 / 29 / 75$ & 3 & 1 & 22 & 71.6 & $\mathbf{N}$ & degree C/TWINS confirm \\
\hline 1 & $4 / 29 / 75$ & 4 & 1 & 20 & 68 & $\mathbf{N}$ & degree C/TWINS confirm \\
\hline 1 & $4 / 29 / 75$ & 5 & 1 & 16 & 60.8 & $\mathbf{N}$ & degree C/TWINS confirm \\
\hline 1 & $4 / 29 / 75$ & 14 & 1 & 18 & 64.4 & $\mathbf{N}$ & degree C/TWINS confirm \\
\hline 1 & $5 / 7 / 75$ & 1 & 1 & & 68 & $N$ & \\
\hline 1 & $5 / 7 / 75$ & 2 & 1 & & 70 & $\mathbf{N}$ & \\
\hline 1 & $5 / 7 / 75$ & 3 & 1 & & 71 & $\mathbf{N}$ & \\
\hline 1 & $5 / 7 / 75$ & 4 & 1 & & 73 & $Y$ & \\
\hline 1 & $5 / 7 / 75$ & 5 & 1 & & 68 & $\mathbf{N}$ & \\
\hline 1 & $5 / 7 / 75$ & 14 & 1 & & 69 & $\mathbf{N}$ & \\
\hline 1 & $5 / 20 / 75$ & 1 & 1 & 20 & 68 & $\mathbf{N}$ & degree C/TWINS confirm \\
\hline 1 & $5 / 20 / 75$ & 2 & 1 & 20 & 68 & $\mathbf{N}$ & degree C/TWINS confirm \\
\hline 1 & $5 / 20 / 75$ & 3 & 1 & 24 & 75.2 & $\mathbf{Y}$ & degree C/TWINS confirm \\
\hline 1 & $5 / 20 / 75$ & 4 & 1 & 24 & 75.2 & $\mathbf{Y}$ & degree C/TWINS confirm \\
\hline 1 & $5 / 20 / 75$ & 5 & 1 & 21 & 69.8 & $\mathbf{N}$ & degree C/TWINS confirm \\
\hline 1 & $5 / 20 / 75$ & 14 & 1 & 20 & 68 & $\mathbf{N}$ & degree C/TWINS confirm \\
\hline 1 & $6 / 17 / 75$ & 1 & 1 & & 79 & $\mathbf{N}$ & \\
\hline 1 & $6 / 17 / 75$ & 2 & 1 & & 79 & $\mathbf{N}$ & \\
\hline 1 & $6 / 17 / 75$ & 3 & 1 & & 83 & $\mathbf{N}$ & \\
\hline 1 & $6 / 17 / 75$ & 4 & 1 & & 85 & $Y$ & \\
\hline 1 & $6 / 17 / 75$ & 5 & 1 & & 79 & $\mathbf{N}$ & \\
\hline 1 & $6 / 17 / 75$ & 14 & 1 & & 79 & $\mathbf{N}$ & \\
\hline 1 & $7 / 28 / 75$ & 1 & 1 & & 90 & $\mathbf{N}$ & \\
\hline 1 & $7 / 28 / 75$ & 2 & 1 & & 90 & $\mathbf{N}$ & \\
\hline 1 & $7 / 28 / 75$ & 3 & 1 & & 93 & $Y$ & \\
\hline 1 & $7 / 28 / 75$ & 4 & 1 & & 91 & $\mathbf{N}$ & \\
\hline 1 & $7 / 28 / 75$ & 5 & 1 & & 90 & $\mathbf{N}$ & \\
\hline 1 & $7 / 28 / 75$ & 14 & 1 & & 91 & $\mathbf{N}$ & \\
\hline 1 & $8 / 26 / 75$ & 1 & 1 & & 84 & $N$ & \\
\hline 1 & $8 / 26 / 75$ & 2 & 1 & & 85 & $\mathbf{N}$ & \\
\hline 1 & $8 / 26 / 75$ & 3 & 1 & & 89 & $\mathbf{N}$ & \\
\hline 1 & $8 / 26 / 75$ & 4 & 1 & & 90 & $\mathbf{N}$ & \\
\hline 1 & $8 / 26 / 75$ & 5 & 1 & & 91 & $N$ & \\
\hline 1 & $8 / 26 / 75$ & 12 & 1 & & 88 & $\mathbf{N}$ & \\
\hline 1 & $11 / 21 / 75$ & 1 & 1 & & 85 & $N$ & \\
\hline 1 & $11 / 21 / 75$ & 2 & 1 & & 86 & $\mathbf{N}$ & \\
\hline 1 & $11 / 21 / 75$ & 3 & 1 & & 91 & $\mathbf{N}$ & \\
\hline 1 & $11 / 21 / 75$ & 4 & 1 & & 93 & $\mathbf{N}$ & \\
\hline 1 & $11 / 21 / 75$ & 5 & 1 & & 85 & $\mathbf{N}$ & \\
\hline 1 & $11 / 21 / 75$ & 14 & 1 & & 85 & $\mathbf{N}$ & \\
\hline 1 & $3 / 4 / 76$ & 3 & 1 & & 68 & $N$ & \\
\hline 1 & $3 / 4 / 76$ & 4 & 1 & & 67 & $\mathbf{N}$ & \\
\hline
\end{tabular}

Data obtained from WHC Surveillance Analysis Computer System (SACS), June 2, 1993. 


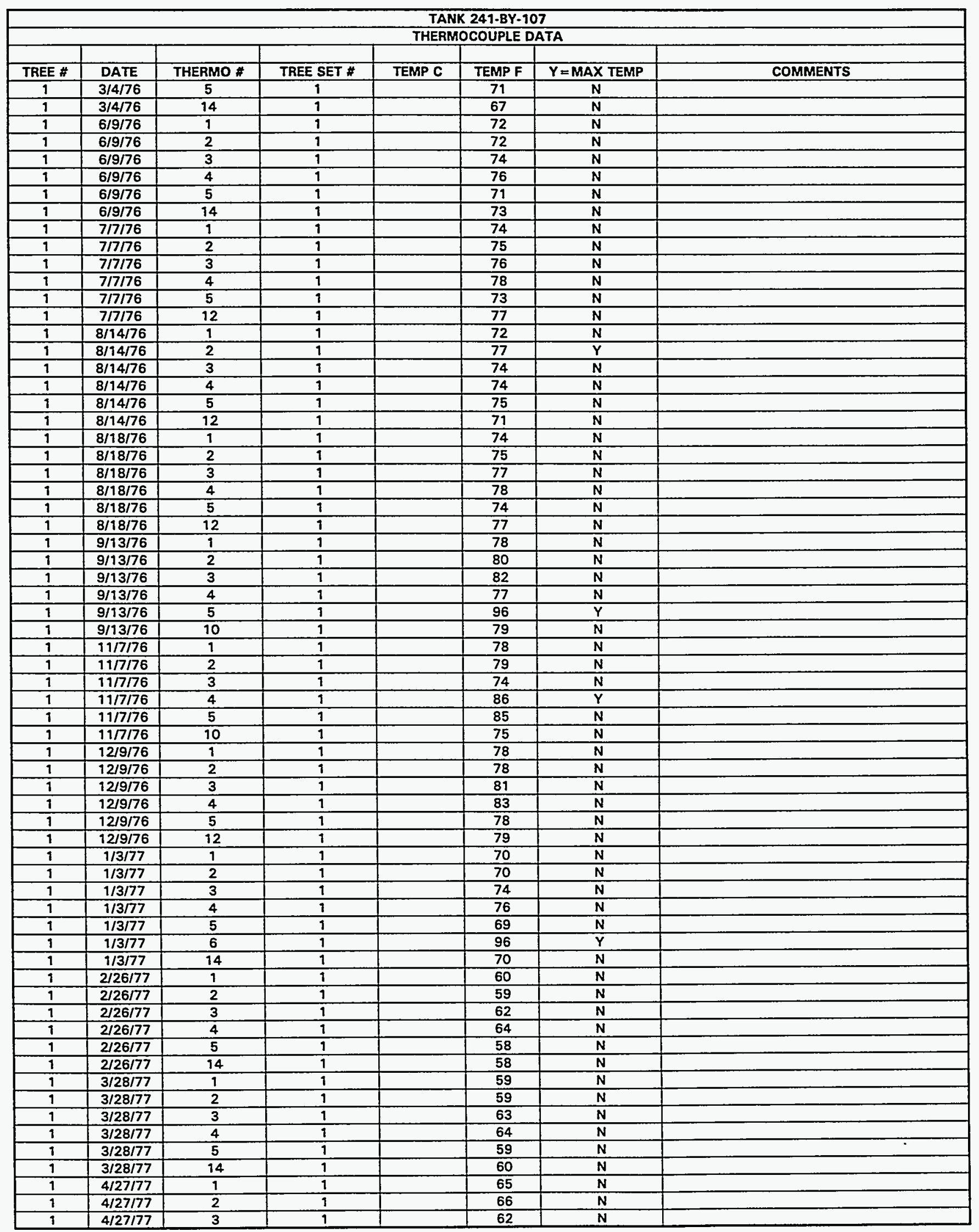

Data obtained from WHC Surveillance Analysis Computer System (SACS), June 2, 1993. 


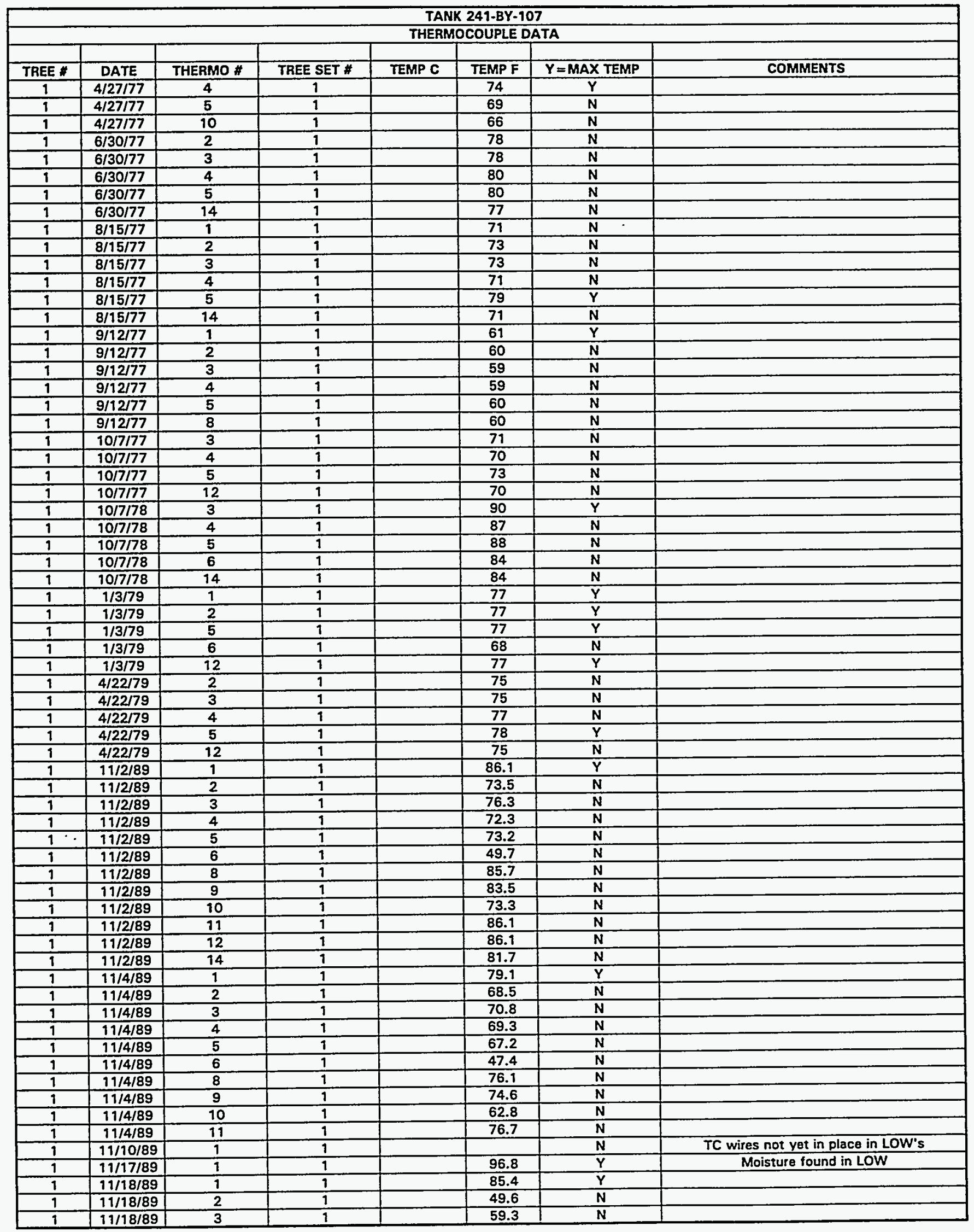

Data obtained from WHC Surveillance Analysis Computer System (SACS), June 2, 1993. 
WHC-SD-WM-ER-312, Rev. 0

\begin{tabular}{|c|c|c|c|c|c|c|c|}
\hline \multicolumn{8}{|c|}{ TANK 241-BY-107 } \\
\hline \multicolumn{8}{|c|}{ THERMOCOUPLE DATA } \\
\hline TREE \# & DATE & THERMO \# & TREE SET \# & TEMP C & TEMP F & $Y=$ MAX TEMP & COMMENTS \\
\hline 1 & $11 / 18 / 89$ & 5 & 1 & & 59.7 & $N$ & \\
\hline 1 & $11 / 18 / 89$ & 6 & 1 & & 70.5 & $\mathbf{N}$ & \\
\hline 1 & $11 / 18 / 89$ & 8 & $\frac{1}{1}$ & & 71.6 & $\mathbf{N}$ & \\
\hline 1 & $11 / 18 / 89$ & 10 & 1 & & 57.5 & $\mathbf{N}$ & \\
\hline 1 & $11 / 18 / 89$ & 11 & 1 & & 73.7 & $\mathbf{N}$ & Tests taken on 107-BY with J \\
\hline 1 & $11 / 25 / 89$ & 1 & 1 & & 85.2 & $\bar{Y}$ & suspect $6 / 28 / 94$ ej \\
\hline 2 & $11 / 25 / 89$ & 1 & 1 & & 97.4 & $\mathbf{N}$ & LOW K \\
\hline 1 & $11 / 25 / 89$ & 2 & 1 & & 34.8 & $\mathbf{N}$ & \\
\hline 9 & $11 / 25 / 89$ & 3 & 1 & & 72.4 & $\mathbf{N}$ & \\
\hline 1 & $11 / 25 / 89$ & 5 & 1 & & 55.8 & $\bar{N}$ & \\
\hline 1 & $11 / 25 / 89$ & 6 & 1 & & 52.1 & $\mathbf{N}$ & \\
\hline 1 & $11 / 25 / 89$ & 8 & 1 & & 65.8 & $\overline{\mathbf{N}}$ & \\
\hline 1 & $11 / 25 / 89$ & 9 & 1 & & & $\mathbf{N}$ & 22.1 - suspect dats (low) \\
\hline 1 & $11 / 25 / 89$ & 10 & 1 & & & $\mathbf{N}$ & 19.3 - suspect data (low) \\
\hline 1 & $11 / 25 / 89$ & 11 & $\frac{1}{1}$ & & 68.6 & $\mathbf{N}$ & \\
\hline 2 & $12 / 1 / 89$ & 1 & 1 & & 97.8 & $\mathbf{Y}$ & LOW \\
\hline 2 & $12 / 10 / 89$ & 1 & 1 & & 98 & $\bar{Y}$ & Low \\
\hline 1 & $12 / 15 / 89$ & 1 & $\frac{1}{1}$ & & 81.8 & $\bar{Y}$ & $\bar{K}$ \\
\hline 1 & $12 / 15 / 89$ & 1 & 2 & & 85.4 & $Y$ & LOW \\
\hline 1 & $12 / 15 / 89$ & 2 & 1 & & 55.4 & $\mathbf{N}$ & \\
\hline 1 & $12 / 15 / 89$ & 3 & 1 & & 68.6 & $\mathbf{N}$ & \\
\hline 1 & $12 / 15 / 89$ & 5 & 1 & & 59.7 & $\mathbf{N}$ & \\
\hline 1 & $12 / 15 / 89$ & 6 & 1 & & & $\mathbf{N}$ & 31.9 - suspect data (low) \\
\hline 1 & $12 / 15 / 89$ & 8 & 1 & & 70.8 & $\bar{N}$ & \\
\hline 1 & $12 / 15 / 89$ & 11 & 1 & & 75.4 & $\mathbf{N}$ & \\
\hline 1 & $12 / 23 / 89$ & 1 & 1 & & 83 & $\mathbf{Y}$ & \\
\hline 1 & $12 / 23 / 89$ & 2 & $\frac{1}{1}$ & & 57 & $\mathbf{N}$ & \\
\hline 1 & $12 / 23 / 89$ & 3 & 9 & & 70 & $\mathbf{N}$ & \\
\hline 1 & $12 / 23 / 89$ & 5 & 1 & & 59 & $\mathbf{N}$ & \\
\hline 1 & $12 / 23 / 89$ & 6 & $T$ & & 36 & $\mathbf{N}$ & \\
\hline 1 & $12 / 23 / 89$ & 7 & 1 & & 40 & $\mathbf{N}$ & \\
\hline 1 & $12 / 23 / 89$ & 8 & 1 & & 71 & $\mathrm{~N}$ & \\
\hline 1 & $12 / 23 / 89$ & 9 & 1 & & 37 & $N$ & \\
\hline 1 & $12 / 23 / 89$ & 11 & 1 & & 76 & $\mathrm{~N}$ & \\
\hline 1 & $12 / 29 / 89$ & 1 & 2 & & 78 & $\bar{Y}$ & $J-890523512-209-20$ \\
\hline 2 & $12 / 29 / 89$ & 1 & 1 & & 73.5 & $Y$ & LOW-K-890723679-20 \\
\hline 1 & $12 / 29 / 89$ & 3 & 2 & & 66 & $\mathbf{N}$ & \\
\hline 1 & $12 / 29 / 89$ & 5 & 2 & & 67.1 & $\mathbf{N}$ & \\
\hline 1 & $12 / 29 / 89$ & 8 & 2 & & 67.1 & $\mathbf{N}$ & \\
\hline$\frac{1}{1}$ & $12 / 29 / 89$ & 11 & 2 & & 72.1 & $\mathbf{N}$ & \\
\hline 1 & $1 / 6 / 90$ & 1 & 1 & & 97.5 & $\bar{Y}$ & LOW \\
\hline 1 & $1 / 13 / 90$ & 1 & 1 & & 77.9 & $\bar{Y}$ & \\
\hline 1 & $1 / 13 / 90$ & 2 & 1 & & 37.7 & $\mathbf{N}$ & \\
\hline 1 & $1 / 13 / 90$ & 3 & 1 & & 66.2 & $\mathbf{N}$ & \\
\hline 1 & $1 / 13 / 90$ & 4 & 1 & & & $\mathbf{N}$ & 26.8 - suspect data (low) \\
\hline 1 & $1 / 13 / 90$ & 5 & 1 & & 56.2 & $\mathbf{N}$ & \\
\hline 1 & $1 / 13 / 90$ & 6 & 1 & & 35.9 & $\mathbf{N}$ & \\
\hline 1 & $1 / 13 / 90$ & 8 & 1 & & 68.8 & $N$ & \\
\hline$\frac{1}{1}$ & $1 / 13 / 90$ & 9 & $\frac{1}{1}$ & & 42.7 & $\bar{N}$ & \\
\hline 1 & $1 / 13 / 90$ & 10 & 1 & & & $\bar{N}$ & 15.4 - suspect data (low) \\
\hline 1 & $1 / 13 / 90$ & 11 & 1 & & 73.1 & $\mathbf{N}$ & J \\
\hline 9 & $1 / 19 / 90$ & 1 & $\frac{1}{1}$ & & 71.9 & $\bar{Y}$ & \\
\hline 1 & $1 / 19 / 90$ & 2 & 1 & & 42.3 & $\mathbf{N}$ & \\
\hline 1 & $1 / 19 / 90$ & 3 & $\overline{1}$ & & 61.8 & $\mathbf{N}$ & \\
\hline 9 & $1 / 19 / 90$ & 4 & 1 & & & $\mathbf{N}$ & 31.3 - suspect data (low) \\
\hline 1 & $1 / 19 / 90$ & 5 & 1 & & 53 & $\mathbf{N}$ & \\
\hline 1 & $1 / 19 / 90$ & 6 & 1 & & 37.3 & $\mathbf{N}$ & \\
\hline 9 & $1 / 19 / 90$ & 8 & 9 & & 63 & $\mathbf{N}$ & \\
\hline 1 & $1 / 19 / 90$ & 9 & 1 & & 45.4 & $\mathbf{N}$ & \\
\hline 1 & $1 / 19 / 90$ & 10 & 1 & & & $\mathbf{N}$ & 18.2 - suspect data (low) \\
\hline 1 & $1 / 19 / 90$ & 11 & 1 & & 68 & $\mathbf{N}$ & $E$ \\
\hline 2 & $1 / 27 / 90$ & 1 & 1 & & 96.9 & $Y$ & LOW-K \\
\hline 1 & $2 / 3 / 90$ & 1 & 1 & & 96.5 & $\bar{Y}$ & LOW K-890723579-20 \\
\hline 2 & $2 / 3 / 90$ & 1 & 1 & & 96.5 & $\mathbf{Y}$ & LOW K-890723579-20 \\
\hline 2 & $2 / 5 / 90$ & 1 & 1 & & 95.9 & $\bar{Y}$ & LOW J-2E-OP-021 \\
\hline 1 & $2 / 11 / 90$ & $T$ & 1 & & 79.9 & $\bar{Y}$ & J-2E-OP-021 \\
\hline
\end{tabular}

Data obtained from WHC Surveillance Analysis Computer System (SACS), June 2, 1993. 
WHC-SD-WM-ER-312, Rev. 0

\begin{tabular}{|c|c|c|c|c|c|c|c|}
\hline \multicolumn{8}{|c|}{ TANK 241-BY-107 } \\
\hline \multicolumn{8}{|c|}{ THERMOCOUPLE DATA } \\
\hline TREE \# & DATE & THERMO \# & TREE SET \# & TEMP C & TEMP F & $Y=M A X$ TEMP & COMMENTS \\
\hline 1 & $2 / 11 / 90$ & 2 & 1 & & 47.2 & $N$ & \\
\hline 1 & $2 / 11 / 90$ & 3 & 1 & & 67.8 & 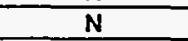 & \\
\hline 1 & $2 / 11 / 90$ & 4 & 1 & & 38 & $\ddot{N}$ & \\
\hline 1 & $2 / 11 / 90$ & 5 & 1 & & 57 & $\ddot{N}$ & \\
\hline 1 & $2 / 11 / 90$ & 6 & 1 & & 38.2 & $\mathbf{N}$ & \\
\hline 1 & $2 / 11 / 90$ & 8 & 7 & & 68 & $\bar{N}$ & \\
\hline 1 & $2 / 11 / 90$ & 9 & 1 & & 51 & $\mathbf{N}$ & \\
\hline 1 & $2 / 11 / 90$ & 10 & 1 & & & $N$ & 23.4 - suspect data (low) \\
\hline 1 & $2 / 11 / 90$ & 11 & $T$ & & 74.4 & $\mathbf{N}$ & \\
\hline 2 & $3 / 2 / 90$ & 1 & 1 & & 98.5 & $\mathbf{Y}$ & K-HH-99A-K \\
\hline 1 & $4 / 8 / 90$ & 1 & 1 & & 79 & $\bar{Y}$ & K-890723679-20 \\
\hline 1 & $4 / 8 / 90$ & 2 & 1 & & 57 & $\mathbf{N}$ & \\
\hline 1 & $4 / 8 / 90$ & 3 & 1 & & 68 & $\mathbf{N}$ & \\
\hline 1 & $4 / 8 / 90$ & 4 & 1 & & 54 & $\mathbf{N}$ & \\
\hline 1 & $4 / 8 / 90$ & 5 & 1 & & 65 & $N$ & \\
\hline 1 & $4 / 8 / 90$ & 6 & 1 & & 53 & $\overline{\mathbf{N}}$ & \\
\hline 1 & $4 / 8 / 90$ & 8 & 1 & & 70 & $\mathbf{N}$ & \\
\hline 1 & $4 / 8 / 90$ & 9 & $T$ & & 55 & $\mathbf{N}$ & \\
\hline 1 & $4 / 8 / 90$ & 10 & 1 & & 39 & $\mathbf{N}$ & \\
\hline 1 & $4 / 8 / 90$ & 11 & 1 & & 74 & $\mathbf{N}$ & \\
\hline 1 & $5 / 5 / 90$ & 1 & 1 & & & $\mathbf{N}$ & No Data. Discrepancy Report written- $\# 252$ \\
\hline 1 & $5 / 30 / 90$ & 1 & 1 & & 79.6 & $\bar{Y}$ & J-2E-OP-020 \\
\hline 1 & $5 / 30 / 90$ & 2 & 1 & & 65.5 & $\mathbf{N}$ & \\
\hline$\frac{1}{1}$ & $5 / 30 / 90$ & 3 & 1 & & 72.3 & $\mathbf{N}$ & \\
\hline 1 & $5 / 30 / 90$ & 4 & 7 & & 59.4 & $\mathbf{N}$ & \\
\hline 1 & $5 / 30 / 90$ & 5 & 1 & & 71.5 & $\mathbf{N}$ & \\
\hline 1 & $5 / 30 / 90$ & 6 & 1 & & 56.5 & $\mathbf{N}$ & \\
\hline 1 & $5 / 30 / 90$ & 8 & 1 & & 73 & $\mathbf{N}$ & \\
\hline 1 & $5 / 30 / 90$ & 9 & 1 & & 61.3 & $\mathrm{~N}$ & \\
\hline 1 & $5 / 30 / 90$ & 10 & 1 & & 47 & $N$ & \\
\hline 1 & $5 / 30 / 90$ & 11 & 1 & & 76.5 & $N$ & \\
\hline 1 & $6 / 1 / 90$ & 1 & 1 & & 80.4 & $\bar{Y}$ & \\
\hline 1 & $6 / 1 / 90$ & 2 & 1 & & 62.9 & $\mathbf{N}$ & \\
\hline 1 & $6 / 1 / 90$ & 3 & 1 & & 71.4 & $\bar{N}$ & \\
\hline 1 & $6 / 1 / 90$ & 4 & 1 & & 55 & $\mathbf{N}$ & \\
\hline 1 & $6 / 1 / 90$ & 5 & 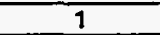 & & 70.8 & $N$ & \\
\hline 1 & $6 / 1 / 90$ & 6 & 1 & & 49.3 & $N$ & \\
\hline 1 & $6 / 1 / 90$ & 8 & 1 & & 72.1 & $\mathbf{N}$ & \\
\hline 1 & $6 / 7 / 90$ & 9 & 1 & & 57.2 & $\mathbf{N}$ & \\
\hline 1 & $6 / 1 / 90$ & 11 & 1 & & 76.7 & $\mathbf{N}$ & \\
\hline 1 & $7 / 8 / 90$ & 1 & 1 & & 82.5 & $\bar{Y}$ & HH-99A-K \\
\hline 1 & $7 / 8 / 90$ & 2 & 1 & & 65.9 & $\mathbf{N}$ & \\
\hline 1 & $7 / 8 / 90$ & 3 & 1 & & 73.7 & $N$ & \\
\hline 1 & $7 / 8 / 90$ & 4 & 1 & & 60.9 & $\mathbf{N}$ & \\
\hline 1 & $7 / 8 / 90$ & 5 & 1 & & 73.5 & $\mathbf{N}$ & \\
\hline 1 & $7 / 8 / 90$ & 6 & 1 & & 57.4 & $\bar{N}$ & \\
\hline 1 & $7 / 8 / 90$ & 8 & 1 & & 74.3 & $\mathbf{N}$ & \\
\hline 1 & $7 / 8 / 90$ & 9 & 1 & & 60.6 & $\mathbf{N}$ & \\
\hline 1 & $7 / 8 / 90$ & 10 & 1 & & 41.9 & $N$ & \\
\hline 1 & $7 / 8 / 90$ & 11 & 1 & & 79.2 & $\mathbf{N}$ & \\
\hline 1 & $8 / 5 / 90$ & 1 & 1 & & 81.4 & $Y$ & $\mathrm{~J}$ \\
\hline 2 & $8 / 5 / 90$ & 1 & 1 & & 83.1 & $\bar{Y}$ & LOW \\
\hline 1 & $8 / 5 / 90$ & 2 & 1 & & 68 & $\mathbf{N}$ & \\
\hline 1 & $8 / 5 / 90$ & 3 & 1 & & 76.7 & $\bar{N}$ & \\
\hline 1 & $8 / 5 / 90$ & 4 & 1 & & 64 & $N$ & \\
\hline 1 & $8 / 5 / 90$ & 5 & 1 & & 74.8 & $\mathbf{N}$ & \\
\hline 1 & $8 / 5 / 90$ & 6 & 1 & & 67 & $\mathbf{N}$ & \\
\hline 1 & $8 / 5 / 90$ & 7 & 1 & & 51 & $\mathbf{N}$ & \\
\hline 1 & $8 / 5 / 90$ & 8 & 1 & & 75.3 & $\mathbf{N}$ & \\
\hline 1 & $8 / 5 / 90$ & 9 & 1 & & 65 & $N$ & \\
\hline$\frac{1}{1}$ & $8 / 5 / 90$ & 10 & 1 & & 55.6 & $\mathbf{N}$ & \\
\hline 1 & $8 / 5 / 90$ & 11 & 1 & & 78.9 & $\mathbf{N}$ & \\
\hline 1 & $9 / 11 / 90$ & 1 & 1 & & 77 & $\bar{Y}$ & $\mathrm{~J}$ \\
\hline 2 & $9 / 11 / 90$ & 9 & 1 & & 83 & $Y$ & Low \\
\hline 1 & $9 / 111 / 90$ & 2 & 1 & & 65 & $\mathbf{N}$ & \\
\hline 1 & $9 / 11 / 90$ & 3 & 1 & & 74 & $\mathbf{N}$ & \\
\hline
\end{tabular}

Data obtained from WHC Surveillance Analysis Computer System (SACS), June 2, 1993. 


\begin{tabular}{|c|c|c|c|c|c|c|c|}
\hline \multicolumn{8}{|c|}{ TANK 241-BY-107 } \\
\hline \multicolumn{8}{|c|}{ THERMOCOUPLE DATA } \\
\hline TREE \# & DATE & THERMO \# & TREE SET \# & TEMP C & TEMP F & $\mathrm{Y}=\mathrm{MAX}$ TEMP & COMMFNTS \\
\hline 1 & $9 / 19 / 90$ & 4 & 1 & & 63 & $\mathbf{N}$ & \\
\hline 1 & $9 / 11 / 90$ & 5 & 1 & & 72 & $\mathbf{N}$ & \\
\hline 1 & $9 / 11 / 90$ & 6 & 1 & & 62 & $\mathbf{N}$ & \\
\hline 1 & $9 / 11 / 90$ & 7 & 1 & & 58 & $\mathbf{N}$ & \\
\hline 9 & $9 / 11 / 90$ & 8 & 1 & & 76 & $\mathbf{N}$ & \\
\hline 1 & $9 / 11 / 90$ & 9 & 1 & & 69 & $\mathbf{N}$ & \\
\hline 9 & $9 / 11 / 90$ & 10 & 1 & & 60 & $\overline{\mathbf{N}}$ & \\
\hline 1 & $9 / 11 / 90$ & 11 & 1 & & 71 & $\mathbf{N}$ & \\
\hline 1 & $10 / 9 / 90$ & 1 & 1 & & 79 & $\bar{Y}$ & $\mathrm{~J}$ \\
\hline 9 & $10 / 9 / 90$ & $\overline{2}$ & 9 & & 79 & $\mathbf{N}$ & \\
\hline 1 & $10 / 9 / 90$ & 3 & 1 & & 79 & $\mathbf{N}$ & \\
\hline 1 & $10 / 9 / 90$ & 4 & 1 & & 79 & $\bar{N}$ & \\
\hline 1 & $10 / 9 / 90$ & 5 & 1 & & 79 & $\mathbf{N}$ & \\
\hline 1 & $10 / 9 / 90$ & 6 & 1 & & 79 & $N$ & \\
\hline 1 & $10 / 9 / 90$ & 7 & 1 & & 79 & $\mathbf{N}$ & \\
\hline 9 & $10 / 9 / 90$ & 8 & 9 & & 79 & $\overline{\mathbf{N}}$ & \\
\hline 1 & $10 / 9 / 90$ & 9 & 1 & & 79 & $\mathbf{N}$ & \\
\hline$T$ & $10 / 9 / 90$ & 10 & 1 & & 79 & $\mathbf{N}$ & \\
\hline 1 & $10 / 9 / 90$ & 11 & 9 & & 79 & $\mathbf{N}$ & \\
\hline 1 & $10 / 9 / 90$ & 12 & 1 & & 79 & $\bar{N}$ & \\
\hline 1 & $10 / 22 / 90$ & 2 & 1 & & 83 & $\mathbf{Y}$ & $\begin{array}{l}\mathrm{J} 890723678-75 \text { Reading on TC } \# 2 \text { only. J-1 } \\
\text { written }\end{array}$ \\
\hline 1 & $10 / 26 / 90$ & 1 & 1 & & 83.9 & $\bar{Y}$ & $\mathrm{~J}-890523512-20 \mathrm{~J}-1$ written \\
\hline 1 & $10 / 26 / 90$ & 2 & 1 & & 75.9 & $\mathbf{N}$ & \\
\hline 1 & $10 / 30 / 90$ & 1 & 9 & & 85.1 & $\bar{Y}$ & $J 890523512-20$ \\
\hline 1 & $10 / 30 / 90$ & 1 & 2 & & 88.1 & $\mathbf{Y}$ & K 890723679-64 LOW \\
\hline 1 & $10 / 30 / 90$ & 2 & 1 & & 73.9 & $\mathbf{N}$ & \\
\hline 1 & $10 / 30 / 90$ & 3 & 1 & & 81.5 & $\bar{N}$ & \\
\hline 1 & $10 / 30 / 90$ & 4 & 1 & & 75.3 & $\mathrm{~N}$ & \\
\hline 1 & $10 / 30 / 90$ & 5 & 1 & & 79.4 & $\mathbf{N}$ & \\
\hline 1 & $10 / 30 / 90$ & 6 & 1 & & 67.4 & $\mathbf{N}$ & \\
\hline 1 & $10 / 30 / 90$ & 8 & 1 & & 80.4 & $\mathbf{N}$ & \\
\hline 1 & $10 / 30 / 90$ & 9 & 1 & & 73.7 & $\mathbf{N}$ & \\
\hline 1 & $10 / 30 / 90$ & 10 & 1 & & 66.2 & $\mathbf{N}$ & \\
\hline 1 & $10 / 30 / 90$ & 11 & 1 & & 82.7 & $\mathbf{N}$ & \\
\hline 1 & $11 / 5 / 90$ & 1 & 1 & & 76 & $\bar{Y}$ & $J 890523512-20$ \\
\hline 1 & $11 / 12 / 90$ & 1 & 1 & & 78.1 & $\bar{Y}$ & $2 E-O P-021$ \\
\hline 1 & $11 / 12 / 90$ & 1 & 2 & & 92.8 & $\mathbf{Y}$ & K 890723679 LOW \\
\hline$\frac{1}{1}$ & $11 / 12 / 90$ & 2 & 1 & & 67.9 & $\mathbf{N}$ & \\
\hline 1 & $11 / 12 / 90$ & 3 & 1 & & 74.3 & $\mathbf{N}$ & \\
\hline 1 & $11 / 12 / 90$ & 4 & 1 & & 67.9 & $\mathbf{N}$ & \\
\hline 1 & $11 / 12 / 90$ & 5 & 1 & & 72.2 & $\mathbf{N}$ & \\
\hline 1 & $11 / 12 / 90$ & 6 & 1 & & 61.7 & $\mathbf{N}$ & \\
\hline 1 & $11 / 12 / 90$ & 8 & 1 & & 74.7 & $\mathbf{N}$ & TC \#7,\#11,\#120/S \\
\hline 1 & $11 / 12 / 90$ & 9 & 1 & & 71.2 & $\mathbf{N}$ & \\
\hline 1 & $11 / 12 / 90$ & 10 & 1 & & 61.3 & $\mathbf{N}$ & \\
\hline 1 & $11 / 18 / 90$ & 1 & 1 & & 85.3 & $\bar{Y}$ & $J 890523512-20$ \\
\hline 1 & $11 / 18 / 90$ & 1 & 2 & & 92.6 & $Y$ & K 890723697-74 LOW \\
\hline 1 & $11 / 18 / 90$ & 2 & 1 & & 74.7 & $\mathbf{N}$ & \\
\hline 1 & $11 / 18 / 90$ & 3 & 1 & & 80.7 & $\mathbf{N}$ & \\
\hline 1 & $11 / 18 / 90$ & 4 & 1 & & 74.8 & $\mathbf{N}$ & \\
\hline 1 & $11 / 18 / 90$ & 5 & 1 & & 78.4 & $\mathbf{N}$ & \\
\hline 1 & $11 / 18 / 90$ & 6 & 9 & & 68.2 & $\mathbf{N}$ & \\
\hline 1 & $11 / 18 / 90$ & 8 & 1 & & 79.5 & $\mathbf{N}$ & \\
\hline 1 & $19 / 18 / 90$ & 9 & 1 & & 76 & $\mathbf{N}$ & \\
\hline 1 & $11 / 18 / 90$ & 10 & 1 & & 66.5 & $\mathbf{N}$ & \\
\hline 1 & $11 / 18 / 90$ & 11 & 1 & & 81.6 & $\mathbf{N}$ & \\
\hline 1 & $11 / 25 / 90$ & 1 & 1 & & 92.2 & $\bar{Y}$ & K-890723679-64 LOW \\
\hline 1 & $12 / 5 / 90$ & 1 & 1 & & 97.8 & $\bar{Y}$ & K 890723679-64 LOW \\
\hline 1 & $12 / 10 / 90$ & 1 & 1 & & 97.4 & $Y$ & K 890723679-64 \\
\hline 1 & $12 / 11 / 90$ & 1 & 1 & & 82 & $\bar{Y}$ & $J 890423320-11$ \\
\hline 1 & $12 / 11 / 90$ & 1 & 2 & & 97.4 & $\bar{Y}$ & K 890723679-64 \\
\hline 1 & $12 / 11 / 90$ & 2 & 1 & & 68.9 & $\mathbf{N}$ & \\
\hline 1 & $12 / 11 / 90$ & 3 & 1 & & 77 & $\mathbf{N}$ & \\
\hline 1 & $12 / 11 / 90$ & 4 & 1 & & 71 & $\mathbf{N}$ & \\
\hline 1 & $12 / 19 / 90$ & 5 & 1 & & 75.5 & $\mathbf{N}$ & \\
\hline
\end{tabular}

Data obtained from WHC Surveillance Analysis Computer System (SACS), June 2, 1993. 
WHC-SD-WM-ER-312, Rev. 0

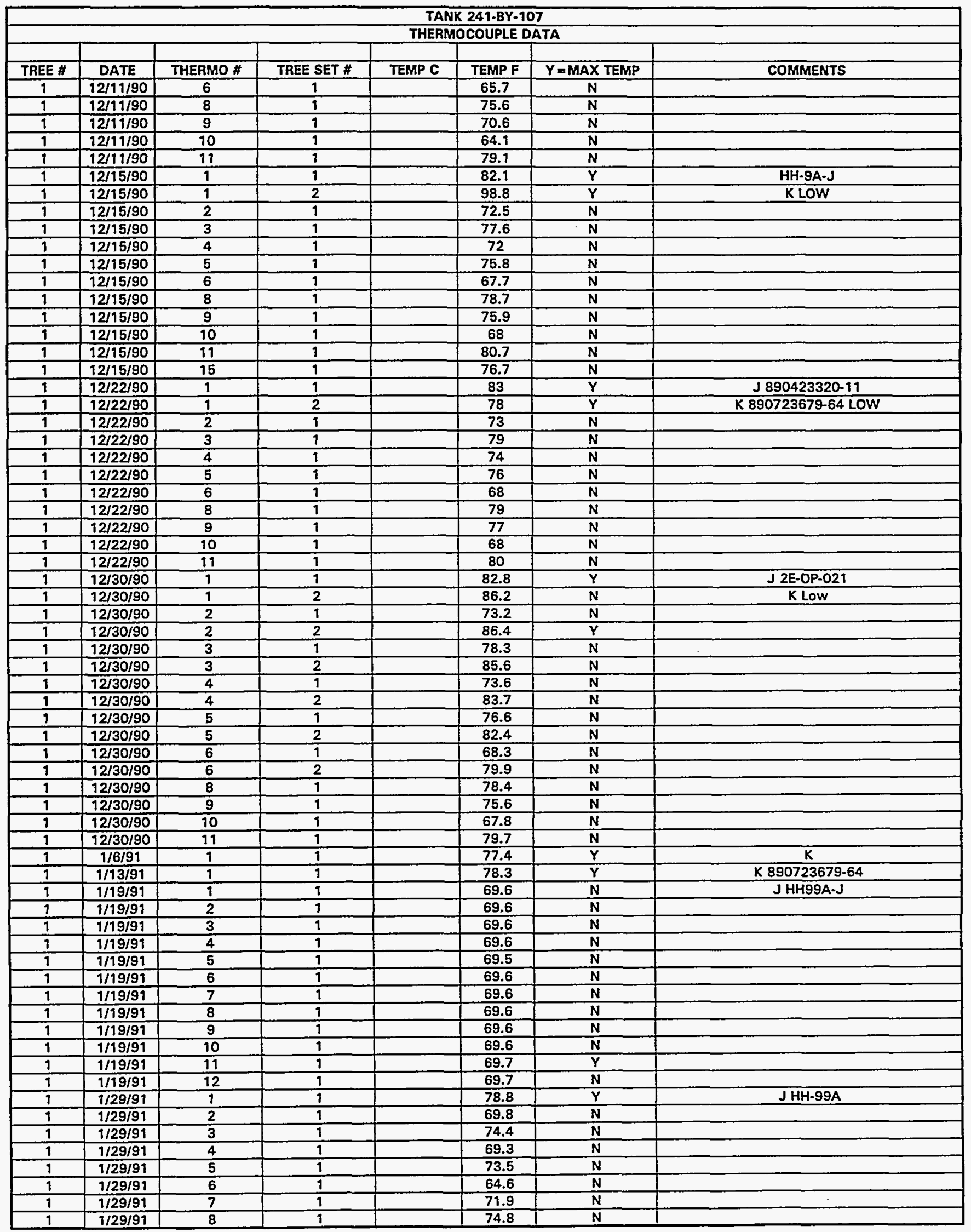

Data obtained from WHC Surveillance Analysis Computer System (SACS), June 2, 1993. 
WHC-SD-WM-ER-312, Rev. 0

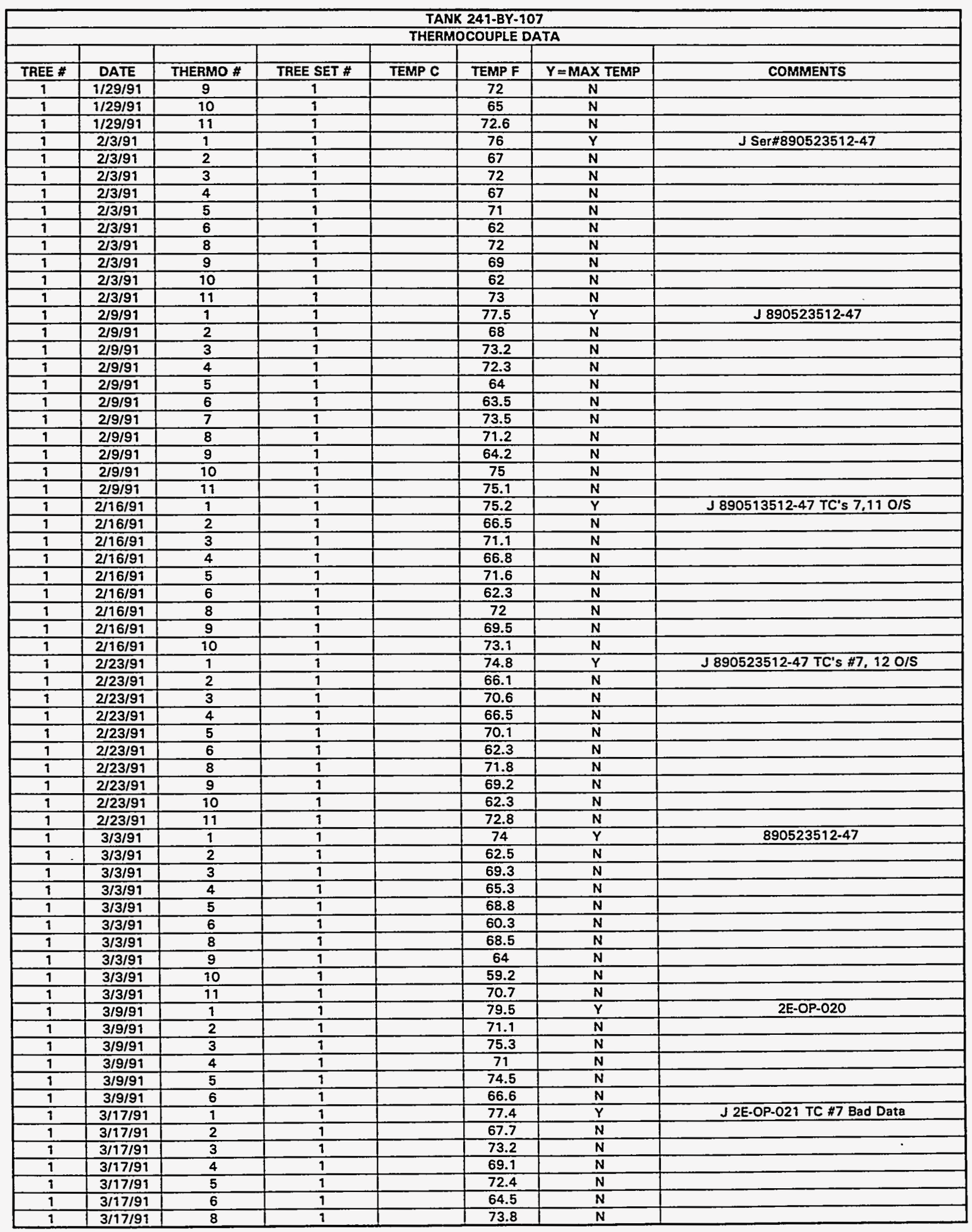

Data obtained from WHC Surveillance Analysis Computer System (SACS), June 2, 1993. 
WHC-SD-WM-ER-312, Rev. 0

\begin{tabular}{|c|c|c|c|c|c|c|c|}
\hline \multicolumn{8}{|c|}{ TANK 241-BY-107 } \\
\hline \multicolumn{8}{|c|}{ THERMOCOUPLE DATA } \\
\hline TREE \# & DATE & THERMO \# & TREE SET \# & TEMP C & TEMPF & $Y=$ MAX TEMP & COMMENTS \\
\hline 1 & $3 / 17 / 91$ & 9 & 1 & & 71.6 & $\mathbf{N}$ & \\
\hline 1 & $3 / 17 / 91$ & 10 & 1 & & 64.3 & $\mathbf{N}$ & \\
\hline 9 & $3 / 17 / 91$ & 11 & 1 & & 74.7 & $N$ & \\
\hline 1 & $3 / 27 / 91$ & 1 & 1 & & 66 & $\mathbf{N}$ & J 890523512-20 Rechecked \\
\hline 1 & $3 / 27 / 91$ & 1 & 2 & & 77.3 & $\bar{Y}$ & J 890523512-20 Set $\# 2$ \\
\hline 1 & $3 / 27 / 91$ & 2 & 1 & & 73.1 & $\bar{Y}$ & \\
\hline 1 & $3 / 27 / 91$ & 2 & 2 & & 68.1 & $\mathbf{N}$ & \\
\hline 1 & $3 / 27 / 91$ & 3 & 2 & & 73.1 & $\mathbf{N}$ & \\
\hline 1 & $3 / 27 / 91$ & 4 & 2 & & 68.6 & $\mathbf{N}$ & \\
\hline 1 & $3 / 27 / 91$ & 5 & $\overline{2}$ & & 72 & $\mathbf{N}$ & \\
\hline 1 & $3 / 27 / 91$ & 6 & 2 & & 63.7 & $\mathbf{N}$ & \\
\hline 1 & $3 / 27 / 91$ & 7 & 2 & & 30.1 & $\mathbf{N}$ & \\
\hline 1 & $3 / 27 / 91$ & 8 & 2 & & 73.4 & $\mathbf{N}$ & \\
\hline 1 & $3 / 27 / 91$ & 9 & 2 & & 70.8 & $\mathbf{N}$ & \\
\hline 1 & $3 / 27 / 97$ & 10 & 2 & & 63.8 & $\mathbf{N}$ & \\
\hline 1 & $3 / 27 / 91$ & 19 & 2 & & 74.9 & $\mathbf{N}$ & \\
\hline 1 & $3 / 30 / 91$ & 1 & 7 & & 65.5 & $\mathbf{N}$ & J 890523512-20 Lower \\
\hline 1 & $3 / 30 / 91$ & 1 & 2 & & 77.3 & $\bar{Y}$ & J 890523512-20 Set \#2 Upper \\
\hline 1 & $3 / 30 / 91$ & 2 & 1 & & 72.7 & $\mathbf{Y}$ & \\
\hline 1 & $3 / 30 / 91$ & 2 & 2 & & 68 & $\mathbf{N}$ & \\
\hline 1 & $3 / 30 / 91$ & 3 & 2 & & 72.6 & $\mathbf{N}$ & \\
\hline 1 & $3 / 30 / 91$ & 4 & 2 & & 68.3 & $\mathbf{N}$ & \\
\hline 1 & $3 / 30 / 91$ & 5 & 2 & & 71.5 & $\mathbf{N}$ & \\
\hline 1 & $3 / 30 / 91$ & 6 & 2 & & 63.1 & $\mathbf{N}$ & \\
\hline 1 & $3 / 30 / 91$ & 8 & 2 & & 72.8 & $\mathbf{N}$ & \\
\hline 1 & $3 / 30 / 91$ & 9 & 2 & & 70.3 & $\mathbf{N}$ & \\
\hline 1 & $3 / 30 / 91$ & 10 & 2 & & 69.7 & $\mathbf{N}$ & \\
\hline 1 & $3 / 30 / 91$ & 11 & 2 & & 74.2 & $\mathbf{N}$ & \\
\hline 1 & $4 / 7 / 91$ & 1 & 1 & & 78.1 & $\bar{Y}$ & J 890423320-11 \\
\hline 1 & $4 / 7 / 91$ & 2 & 1 & & 70 & $\mathbf{N}$ & \\
\hline 1 & $4 / 7 / 91$ & 3 & 1 & & 73.9 & $\mathbf{N}$ & \\
\hline 9 & $4 / 7 / 91$ & 4 & 1 & & 70.3 & $\mathbf{N}$ & \\
\hline 1 & $4 / 7 / 91$ & 5 & 1 & & 73.4 & $\mathbf{N}$ & \\
\hline 1 & $4 / 7 / 91$ & 6 & 1 & & 65.6 & $\mathbf{N}$ & \\
\hline 9 & 4/7/91 & 8 & 1 & & 75 & $\mathbf{N}$ & \\
\hline 1 & $4 / 7 / 91$ & 9 & 1 & & 72.8 & $\mathbf{N}$ & \\
\hline 1 & $4 / 7 / 91$ & 10 & 1 & & 65.4 & $\mathbf{N}$ & \\
\hline 1 & $4 / 7 / 91$ & 11 & 1 & & 76.2 & $\mathbf{N}$ & \\
\hline 1 & $4 / 14 / 91$ & 1 & 1 & & 78.3 & $Y$ & J 890523512-20 Omega \\
\hline 1 & $4 / 14 / 91$ & 2 & 1 & & 70 & $\mathbf{N}$ & \\
\hline 1 & $4 / 14 / 91$ & 3 & 1 & & 73.8 & $\mathbf{N}$ & \\
\hline 1 & $4 / 14 / 99$ & 4 & 1 & & 70.4 & $\mathbf{N}$ & \\
\hline 1 & $4 / 14 / 91$ & 5 & 1 & & 73.2 & $\mathbf{N}$ & \\
\hline 1 & 4/14/91 & 6 & 1 & & 65.5 & $\mathbf{N}$ & \\
\hline 9 & $4 / 14 / 91$ & 8 & 1 & & 74.2 & $\mathbf{N}$ & \\
\hline 1 & $4 / 14 / 91$ & 9 & 1 & & 71.8 & $\mathbf{N}$ & \\
\hline 1 & $4 / 14 / 91$ & 10 & $\frac{1}{1}$ & & 64.6 & $\bar{N}$ & \\
\hline 1 & $4 / 14 / 91$ & 11 & 1 & & 75.4 & $\mathbf{N}$ & \\
\hline 1 & $4 / 20 / 91$ & 1 & 1 & & 77.5 & $\mathbf{Y}$ & $J 890523512-20$ \\
\hline 1 & $4 / 20 / 91$ & 2 & 1 & & 69.4 & $\bar{N}$ & \\
\hline 1 & $4 / 20 / 91$ & 3 & 1 & & 73.1 & $\mathbf{N}$ & \\
\hline 1 & $4 / 20 / 91$ & 4 & 1 & & 69.5 & $\mathbf{N}$ & \\
\hline 1 & $4 / 20 / 91$ & 5 & 1 & & 72.6 & $\mathbf{N}$ & \\
\hline 1 & $4 / 20 / 91$ & 6 & 1 & & 65.2 & $\mathbf{N}$ & \\
\hline 1 & $4 / 20 / 91$ & 8 & 1 & & 74.1 & $\mathbf{N}$ & \\
\hline 1 & $4 / 20 / 91$ & 9 & 1 & & 71.9 & $\mathbf{N}$ & \\
\hline 1 & $4 / 20 / 91$ & 10 & $i$ & & 64.9 & $\mathbf{N}$ & \\
\hline 1 & $4 / 20 / 91$ & 11 & 1 & & 75.4 & $\mathbf{N}$ & \\
\hline 1 & $4 / 28 / 91$ & 1 & 1 & & 77.8 & $Y$ & J Omega 890523512.20 \\
\hline 1 & $4 / 28 / 91$ & 2 & 1 & & 69.6 & $\mathbf{N}$ & \\
\hline 1 & $4 / 28 / 91$ & 3 & 1 & & 73.5 & $\mathbf{N}$ & \\
\hline 1 & $4 / 28 / 91$ & 4 & 1 & & 70.2 & $\mathbf{N}$ & \\
\hline 1 & $4 / 28 / 91$ & 5 & 1 & & 72.9 & $\mathbf{N}$ & \\
\hline 1 & $4 / 28 / 91$ & 6 & 1 & & 65.6 & $\mathbf{N}$ & \\
\hline 1 & $4 / 28 / 91$ & 7 & 1 & & 35.6 & $\mathbf{N}$ & \\
\hline 1 & $4 / 28 / 91$ & 8 & 1 & & 74 & $\mathbf{N}$ & \\
\hline
\end{tabular}

Data obtained from WHC Surveillance Analysis Computer System (SACS), June 2, 1993. 


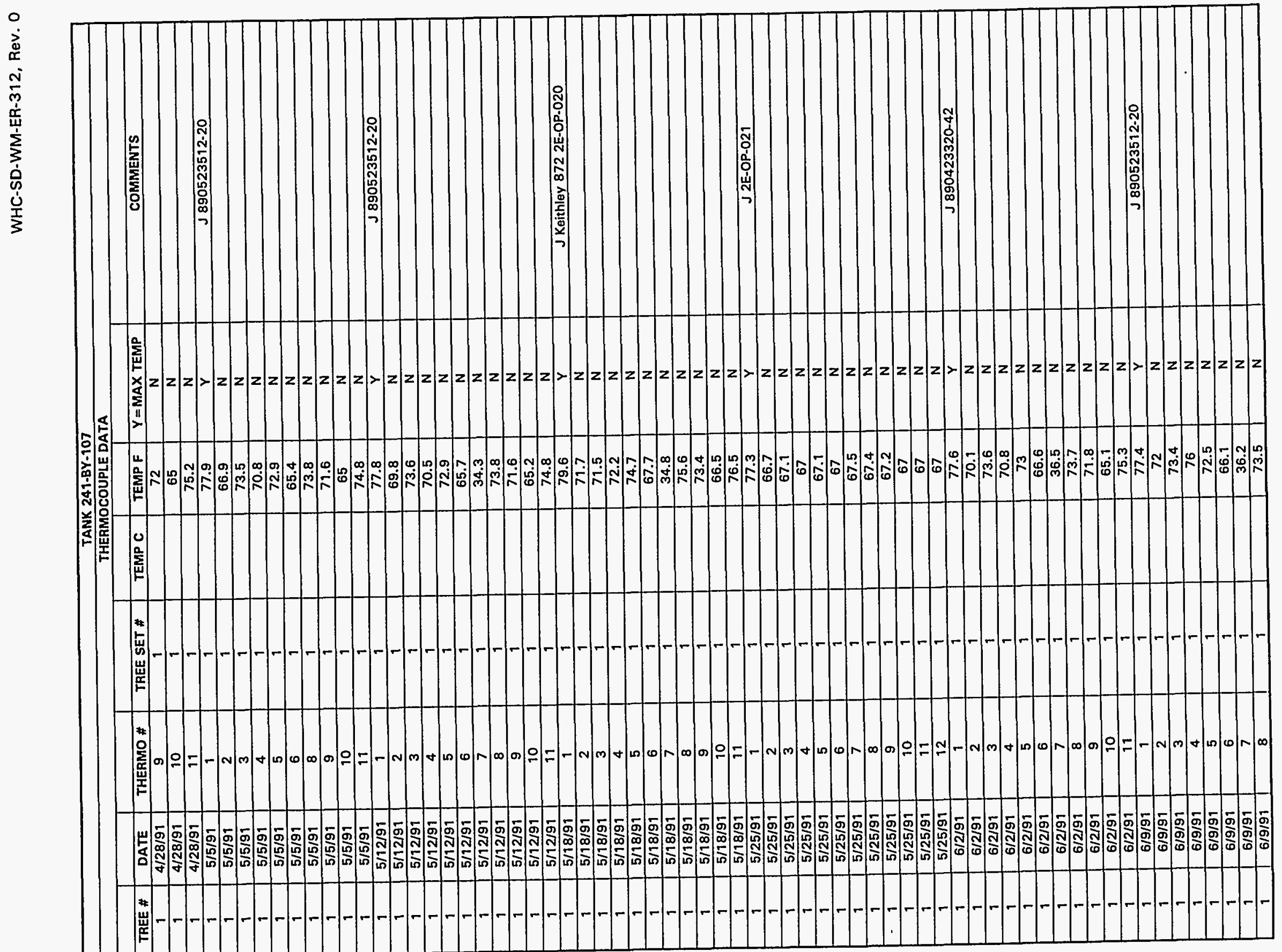




\begin{tabular}{|c|c|c|c|c|c|c|c|}
\hline \multicolumn{8}{|c|}{ TANK 247-BY-107 } \\
\hline \multicolumn{8}{|c|}{ THERMOCOUPLE DATA } \\
\hline TREE \# & DATE & THERMO \# & TREE SET \# & TEMP C & TEMP F & $Y=$ MAX TEMP & COMMENTS \\
\hline 1 & $6 / 9 / 91$ & 9 & 1 & & 71.5 & $\mathbf{N}$ & \\
\hline 1 & 6/9/91 & 10 & 1 & & 64.9 & $\mathbf{N}$ & \\
\hline 1 & 6/9/91 & 11 & 1 & & 74.9 & $\mathbf{N}$ & \\
\hline 1 & $6 / 15 / 91$ & 1 & 9 & & 79.4 & $\overline{\mathrm{Y}}$ & J 2E-OP-021 TCH7 Bad data \\
\hline 1 & $6 / 15 / 91$ & 2 & 1 & & 72.1 & $\mathbf{N}$ & \\
\hline 1 & $6 / 15 / 91$ & 3 & 1 & & 75.5 & $\mathbf{N}$ & \\
\hline 9 & $6 / 15 / 91$ & 4 & 1 & & 72.5 & $\mathbf{N}$ & \\
\hline 1 & $6 / 15 / 91$ & 5 & 1 & & 74.8 & $\mathbf{N}$ & \\
\hline 1 & $6 / 15 / 91$ & 6 & 1 & & 68.3 & $\mathbf{N}$ & \\
\hline 1 & $6 / 15 / 91$ & 8 & 9 & & 75.6 & $\mathbf{N}$ & \\
\hline 1 & $6 / 15 / 91$ & 9 & 1 & & 73.6 & $\mathbf{N}$ & \\
\hline 1 & $6 / 15 / 91$ & 10 & 1 & & 66.8 & $\mathbf{N}$ & \\
\hline 1 & $6 / 15 / 91$ & 11 & 1 & & 76.6 & $\mathbf{N}$ & \\
\hline 1 & $6 / 23 / 91$ & 1 & 1 & & 79 & $\bar{Y}$ & J 2E-OP-021 \\
\hline 1 & $6 / 23 / 91$ & 2 & 1 & & 71 & $\mathbf{N}$ & \\
\hline 1 & $6 / 23 / 91$ & 3 & 1 & & 75 & $\mathbf{N}$ & \\
\hline 9 & $6 / 23 / 91$ & 4 & 1 & & 72 & $\mathbf{N}$ & \\
\hline 1 & $6 / 23 / 91$ & 5 & 1 & & 74 & $\mathbf{N}$ & \\
\hline 1 & $6 / 23 / 91$ & 6 & 1 & & 68 & $\mathbf{N}$ & \\
\hline 1 & $6 / 23 / 91$ & 8 & 9 & & 75 & $\mathbf{N}$ & \\
\hline 1 & $6 / 23 / 91$ & 9 & 1 & & 73 & $\mathbf{N}$ & \\
\hline 1 & $6 / 23 / 91$ & 10 & 1 & & 66 & $\mathbf{N}$ & \\
\hline 1 & $6 / 23 / 91$ & 19 & 9 & & 76 & $\mathbf{N}$ & \\
\hline 1 & $6 / 29 / 91$ & 1 & 1 & & 68.1 & $\mathbf{N}$ & J $890423320-42$ TC\#3,7,12NG \\
\hline 1 & $6 / 29 / 91$ & 2 & 1 & & 75.3 & $\bar{Y}$ & \\
\hline 1 & $6 / 29 / 91$ & 4 & 9 & & 72.4 & $\mathbf{N}$ & \\
\hline 1 & $6 / 29 / 91$ & 5 & 1 & & 74.5 & $\mathbf{N}$ & \\
\hline 1 & $6 / 29 / 91$ & 6 & 1 & & 68.4 & $\mathbf{N}$ & \\
\hline 1 & $6 / 29 / 91$ & 8 & 1 & & 75.2 & $\mathbf{N}$ & \\
\hline 1 & $6 / 29 / 91$ & 9 & 1 & & 72.9 & $\mathbf{N}$ & \\
\hline 1 & $6 / 29 / 91$ & 10 & 1 & & 66.2 & $\mathbf{N}$ & \\
\hline 1 & $6 / 29 / 91$ & 11 & 1 & & 76.2 & $\mathbf{N}$ & \\
\hline 1 & $7 / 6 / 91$ & 1 & 1 & & 79.1 & $\bar{Y}$ & J $910126143 \mathrm{TC} \# 7,12 \mathrm{NG}$ \\
\hline 1 & $7 / 6 / 91$ & 2 & 1 & & 71.9 & $\mathbf{N}$ & \\
\hline 1 & 7/6/91 & 3 & 1 & & 75.5 & $\mathbf{N}$ & \\
\hline 1 & $7 / 6 / 91$ & 4 & 1 & & 72.6 & $\mathbf{N}$ & \\
\hline 1 & $7 / 6 / 91$ & 5 & 1 & & 74.6 & $\mathbf{N}$ & \\
\hline 1 & $7 / 6 / 91$ & 6 & 1 & & 68.6 & $\mathbf{N}$ & \\
\hline 1 & $7 / 6 / 91$ & 8 & 1 & & 75.6 & $\mathbf{N}$ & \\
\hline 1 & 7/6/91 & 9 & 1 & & 73.7 & $\bar{N}$ & \\
\hline 1 & $7 / 6 / 91$ & 10 & 1 & & 67.4 & $\mathbf{N}$ & \\
\hline 1 & $7 / 6 / 91$ & 11 & 1 & & 77.1 & $\bar{N}$ & \\
\hline 1 & $7 / 13 / 91$ & 1 & 1 & & 79.9 & $\bar{Y}$ & J 910126143-42(upper)TC\#7Bad data \\
\hline 1 & $7 / 13 / 91$ & 2 & 1 & & 72.5 & $\mathbf{N}$ & \\
\hline 1 & $7 / 13 / 91$ & 3 & 1 & & 76.7 & $\mathbf{N}$ & \\
\hline 1 & $7 / 13 / 91$ & 4 & 1 & & 73.3 & $\mathbf{N}$ & \\
\hline 1 & $7 / 13 / 91$ & 5 & 1 & & 75.3 & $\mathbf{N}$ & \\
\hline 1 & $7 / 13 / 91$ & 6 & 1 & & 69.4 & $\mathbf{N}$ & \\
\hline 1 & $7 / 13 / 91$ & 8 & 1 & & 75.7 & $\mathbf{N}$ & \\
\hline$\frac{7}{1}$ & $7 / 13 / 91$ & 9 & 1 & & 74 & $\mathbf{N}$ & \\
\hline 1 & $7 / 13 / 91$ & 10 & 1 & & 67.5 & $\mathbf{N}$ & \\
\hline 1 & $7 / 13 / 91$ & 19 & 1 & & 77.2 & $\bar{N}$ & \\
\hline 1 & $7 / 20 / 91$ & 1 & 1 & & 78 & $\bar{Y}$ & J 910126143-64 TCH7o/s \\
\hline 9 & $7 / 20 / 91$ & 2 & 1 & & 70.9 & $\mathbf{N}$ & \\
\hline 1 & $7 / 20 / 91$ & 3 & 1 & & 74.6 & $\mathbf{N}$ & \\
\hline 1 & $7 / 20 / 91$ & 4 & 1 & & 71.8 & $\mathbf{N}$ & \\
\hline 1 & $7 / 20 / 91$ & 5 & 1 & & 73.7 & $\mathbf{N}$ & \\
\hline 1 & $7 / 20 / 91$ & 6 & 1 & & 68 & $\mathbf{N}$ & \\
\hline 1 & $7 / 20 / 91$ & 8 & 1 & & 74.7 & $\mathbf{N}$ & \\
\hline 1 & $7 / 20 / 91$ & 9 & 1 & & 73 & $\bar{N}$ & \\
\hline 1 & $7 / 20 / 91$ & 10 & 1 & & 66.4 & $\bar{N}$ & \\
\hline 1 & $7 / 20 / 91$ & 11 & 1 & & 76.2 & $N$ & \\
\hline 1 & $7 / 27 / 91$ & 1 & 1 & & 68.9 & $\mathbf{N}$ & $\mathrm{J} 910126143-14 \mathrm{TC} \# 3,7,12 \mathrm{NG}$ \\
\hline 1 & $7 / 27 / 91$ & 2 & 1 & & 76.6 & $\mathbf{N}$ & \\
\hline 1 & $7 / 27 / 91$ & 4 & 1 & & 73.2 & $\mathbf{N}$ & \\
\hline 1 & $7 / 27 / 91$ & 5 & 1 & & 75.2 & $\mathbf{N}$ & \\
\hline
\end{tabular}

Data obtained from WHC Surveillance Analysis Computer System (SACS), June 2, 1993. 


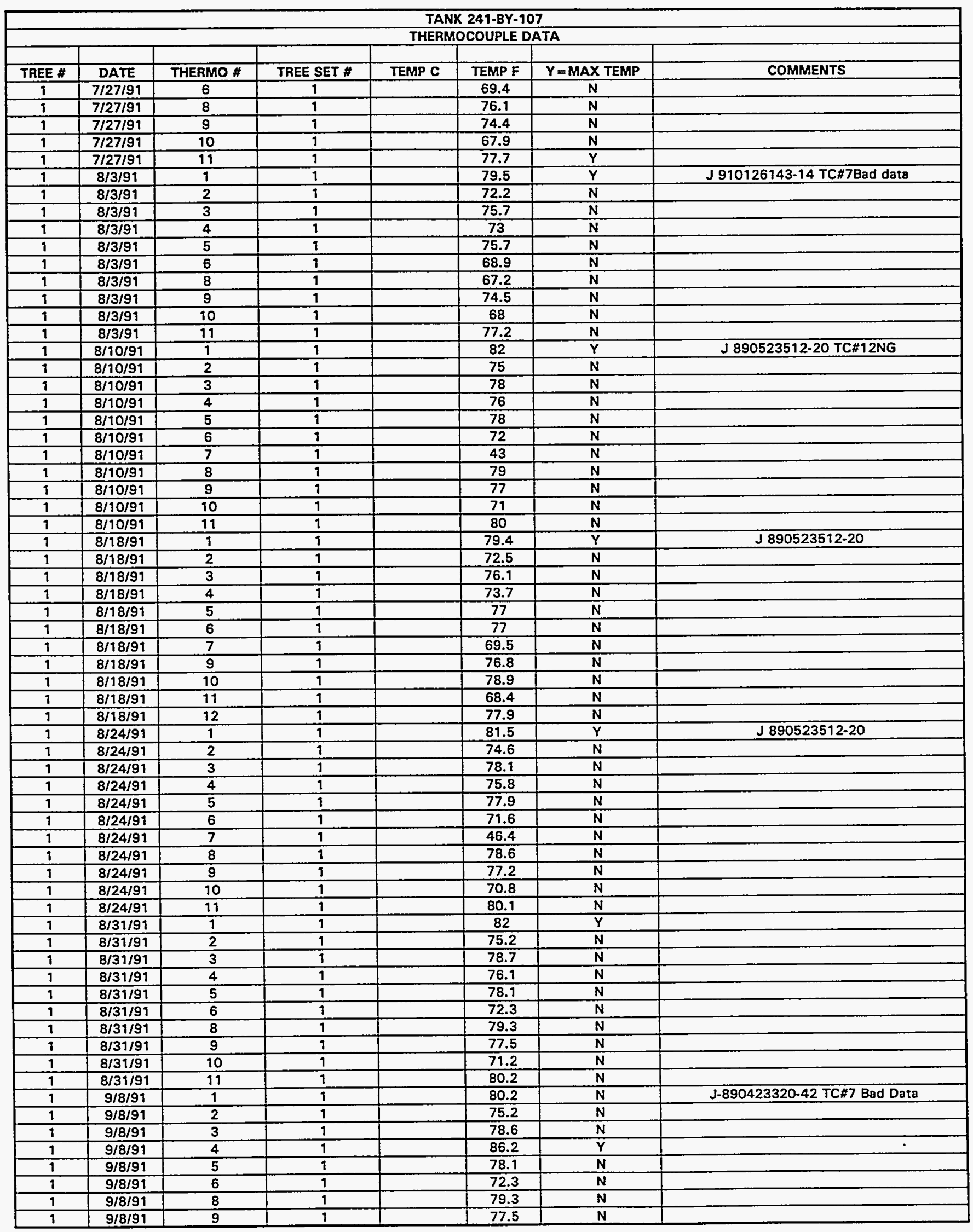

Data obtained from WHC Surveillance Analysis Computer System (SACS), June 2, 1993. 


\begin{tabular}{|c|c|c|c|c|c|c|c|}
\hline \multicolumn{8}{|c|}{ TANK 241-BY-107 } \\
\hline \multicolumn{8}{|c|}{ THERMOCOUPLE DATA } \\
\hline & & & & & & & $\cdot$ \\
\hline TREE : & DATE & THERMO * & TREE SET & TEMP C & TEMP F & $Y=M A X$ TEMP & COMMENTS \\
\hline 1 & $9 / 8 / 91$ & 10 & 1 & & 71.2 & $\mathbf{N}$ & \\
\hline$\frac{1}{1}$ & $8 / 8 / 97$ & 11 & 1 & & 80.2 & $\mathbf{N}$ & \\
\hline 1 & $9 / 15 / 91$ & 1 & 1 & & 81 & $\bar{Y}$ & $\mathrm{~J}-910126143-75$ \\
\hline$\frac{1}{1}$ & $9 / 15 / 91$ & 2 & 1 & & 74 & $\mathbf{N}$ & \\
\hline$\frac{7}{1}$ & $9 / 16 / 91$ & $\overline{3}$ & 1 & & 77.3 & $\mathbf{N}$ & \\
\hline 1 & $9 / 15 / 91$ & 4 & 1 & & 75.1 & $\bar{N}$ & . \\
\hline$\frac{1}{1}$ & $9 / 16 / 91$ & 5 & 1 & & 77.9 & $\mathbf{N}$ & \\
\hline 1 & $9 / 15 / 91$ & 6 & 1 & & 72.7 & $\ddot{N}$ & \\
\hline 1 & $9 / 15 / 91$ & 8 & 1 & & 78.7 & $\mathbf{N}$ & \\
\hline 1 & $9 / 15 / 91$ & 9 & 1 & & 76.8 & $\mathbf{N}$ & \\
\hline 1 & $9 / 15 / 91$ & 10 & 1 & & 70.1 & $\mathbf{N}$ & \\
\hline 1 & $9 / 16 / 91$ & 11 & 1 & & 79.8 & $\mathbf{N}$ & \\
\hline 1 & $9 / 22 / 91$ & 1 & 1 & & 81.9 & $\bar{Y}$ & $\mathrm{~J}-910126143-75$ \\
\hline 1 & $9 / 22 / 91$ & 2 & 1 & & 75.2 & $\mathbf{N}$ & \\
\hline 1 & $9 / 22 / 91$ & 3 & 1 & & 79.2 & $\mathbf{N}$ & \\
\hline 1 & $9 / 22 / 91$ & 4 & 1 & & 77 & $\mathbf{N}$ & \\
\hline 1 & $9 / 22 / 91$ & 5 & 1 & & 78.8 & $\mathbf{N}$ & \\
\hline$\frac{1}{1}$ & $9 / 22 / 91$ & $\frac{5}{6}$ & 1 & & 73.6 & $N$ & \\
\hline 9 & $9 / 22 / 91$ & 8 & 1 & & 79.8 & $\mathbf{N}$ & \\
\hline 1 & $9 / 22 / 91$ & 9 & 1 & & 78.8 & $\mathbf{N}$ & \\
\hline 1 & $9 / 22 / 91$ & 10 & $\frac{1}{1}$ & & 71.5 & $\mathbf{N}$ & \\
\hline 1 & $9 / 22 / 91$ & 11 & 1 & & 81.2 & $\ddot{N}$ & \\
\hline 1 & $9 / 28 / 91$ & 1 & 1 & & 83.3 & $\mathbf{Y}$ & J-Keithley 872 T1-059 TC 7 Bad Data \\
\hline 1 & $9 / 28 / 91$ & 2 & 1 & & 76.7 & $\mathbf{N}$ & \\
\hline 1 & $9 / 28 / 91$ & 3 & 1 & & 80.4 & $\bar{N}$ & \\
\hline 1 & $9 / 28 / 91$ & 4 & 1 & & 78.2 & $\mathbf{N}$ & \\
\hline 1 & $9 / 28 / 91$ & 5 & 1 & & 79.9 & $\mathbf{N}$ & \\
\hline 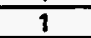 & $9 / 28 / 91$ & 6 & 1 & & 74.7 & $\mathbf{N}$ & \\
\hline 1 & $9 / 28 / 91$ & 8 & 1 & & 81.2 & $\mathbf{N}$ & \\
\hline 1 & $9 / 28 / 91$ & 9 & 1 & & 79.8 & $\mathbf{N}$ & \\
\hline 1 & $9 / 28 / 91$ & 10 & 1 & & 72.9 & $\mathbf{N}$ & \\
\hline$\frac{1}{1}$ & $9 / 28 / 91$ & 11 & 1 & & 82.4 & $\mathbf{N}$ & \\
\hline 1 & $10 / 5 / 91$ & 9 & 1 & & 82.5 & $\bar{Y}$ & J.910126143-42 TC \&7 Bad Data \\
\hline$\frac{1}{1}$ & $10 / 5 / 91$ & 2 & 1 & & 75.9 & $\mathbf{N}$ & \\
\hline 1 & $10 / 5 / 91$ & 3 & 1 & & 79.7 & $N$ & \\
\hline 1 & $10 / 5 / 91$ & 4 & 1 & & 77.8 & $\mathbf{N}$ & \\
\hline 1 & $10 / 5 / 91$ & 5 & 9 & & 79.2 & $\mathbf{N}$ & \\
\hline 1 & $10 / 6 / 91$ & $\frac{6}{6}$ & 1 & & 73.9 & $\mathbf{N}$ & \\
\hline 1 & $10 / 5 / 91$ & 8 & 1 & & 81.2 & $\mathbf{N}$ & \\
\hline$i$ & $10 / 5 / 91$ & 9 & 1 & & 79.7 & $\mathbf{N}$ & \\
\hline 1 & $10 / 5 / 91$ & 10 & 1 & & 72.5 & $\mathbf{N}$ & \\
\hline 1 & $10 / 5 / 91$ & 11 & 1 & & 82.5 & $\mathbf{N}$ & \\
\hline 1 & $10 / 12 / 91$ & 1 & 1 & & 82.7 & $\mathbf{N}$ & J-T1023 TC $\$ 7$ Bad Data \\
\hline 1 & $10 / 12 / 91$ & 2 & 1 & & 76.3 & $\mathbf{N}$ & \\
\hline 1 & $10 / 12 / 91$ & 3 & 1 & & 79.8 & $\mathbf{N}$ & \\
\hline 1 & $10 / 12 / 91$ & 4 & 1 & & 78 & $\bar{N}$ & \\
\hline 1 & $10 / 12 / 91$ & 5 & 1 & & 79.6 & $\mathbf{N}$ & \\
\hline 1 & $10 / 12 / 91$ & 6 & 1 & & 74.5 & $\mathbf{N}$ & \\
\hline 1 & $10 / 12 / 91$ & 8 & 1 & & 80.6 & $\mathbf{N}$ & \\
\hline 9 & $10 / 12 / 91$ & 9 & 1 & & 78.6 & $N$ & \\
\hline 1 & $10 / 12 / 91$ & 10 & $\frac{1}{1}$ & & 70.1 & $\mathbf{N}$ & \\
\hline 1 & $10 / 12 / 91$ & 11 & 1 & & 86.5 & $\mathbf{Y}$ & \\
\hline 1 & $10 / 19 / 91$ & 1 & 1 & & 82.8 & $\bar{Y}$ & J-890523512-47 TC:7 Bad Data \\
\hline 1 & $10 / 19 / 91$ & 2 & 1 & & 79.8 & $\dot{N}$ & \\
\hline 9 & $10 / 19 / 91$ & 3 & 1 & & 79.3 & $\mathbf{N}$ & \\
\hline 1 & $10 / 19 / 91$ & 4 & 1 & & 77.6 & $\bar{N}$ & \\
\hline 1 & $10 / 19 / 91$ & $\frac{T}{5}$ & 1 & & 78.8 & $\mathbf{N}$ & $\dot{-}$ \\
\hline 1 & $10 / 19 / 91$ & 8 & 1 & & 73.4 & $\mathbf{N}$ & \\
\hline$\frac{1}{1}$ & $10 / 19 / 91$ & 8 & 1 & & 79.3 & $\bar{N}$ & \\
\hline 1 & $10 / 19 / 91$ & 9 & 1 & & 77.6 & $\mathbf{N}$ & \\
\hline 1 & $10 / 19 / 91$ & 10 & 1 & & 70 & $\mathbf{N}$ & \\
\hline 1 & $10 / 19 / 91$ & 11 & 1 & & 80.5 & $N$ & \\
\hline$\frac{\dot{1}}{1}$ & $10 / 27 / 91$ & 1 & 1 & & 85.1 & $\bar{Y}$ & J-910126143-42 TC \#7 Bad Data \\
\hline 1 & $10 / 27 / 91$ & 2 & 1 & & 78.3 & $\mathbf{N}$ & \\
\hline 1 & $10 / 27 / 91$ & 3 & 1 & & 81.8 & $N$ & \\
\hline 1 & $10 / 27 / 91$ & 4 & 1 & & 80.3 & $\mathbf{N}$ & \\
\hline
\end{tabular}

Data obtained from WHC Surveillance Analysis Computer System (SACS), June 2, 1993. 


\begin{tabular}{|c|c|c|c|c|c|c|c|}
\hline \multicolumn{8}{|c|}{ TANK 241-BY-107 } \\
\hline \multicolumn{8}{|c|}{ THERMOCOUPLE DATA } \\
\hline & & & & & & & \\
\hline TREE \# & DATE & THERMO \# & TREE SET \# & TEMP C & TEMP F & $Y=$ MAX TEMP & COMMENTS \\
\hline 1 & $10 / 27 / 91$ & 5 & 1 & & 80.8 & $\mathbf{N}$ & \\
\hline 1 & $10 / 27 / 91$ & 6 & 1 & & 75.8 & $\mathbf{N}$ & \\
\hline 1 & $10 / 27 / 91$ & 8 & 1 & & 81.6 & $\mathbf{N}$ & \\
\hline 1 & $10 / 27 / 91$ & 9 & 9 & & 79.7 & $N$ & \\
\hline 1 & $10 / 27 / 91$ & 10 & 1 & & 71.5 & $N$ & \\
\hline 1 & $10 / 27 / 91$ & 19 & 1 & & 82.8 & $\mathbf{N}$ & \\
\hline 1 & $10 / 27 / 91$ & 12 & 1 & & 74.6 & $\mathbf{N}$ & \\
\hline 1 & $10 / 27 / 91$ & 13 & 1 & & 81.8 & $\mathbf{N}$ & \\
\hline 1 & $11 / 2 / 91$ & 1 & 1 & & 82 & $\bar{Y}$ & J-890523512.47, TC \#7 BAD DATA \\
\hline 1 & $11 / 2 / 91$ & 2 & 1 & & 75 & $\mathbf{N}$ & \\
\hline 1 & $11 / 2 / 91$ & 3 & 1 & & 79 & $\mathbf{N}$ & \\
\hline 1 & $11 / 2 / 91$ & 4 & 1 & & 77 & $\mathbf{N}$ & \\
\hline 1 & $11 / 2 / 91$ & 5 & 1 & & 78 & $\mathbf{N}$ & \\
\hline 1 & $11 / 2 / 91$ & 6 & 1 & & 73 & $\overline{\mathbf{N}}$ & \\
\hline 1 & $11 / 2 / 91$ & 8 & 1 & & 79 & $\mathbf{N}$ & \\
\hline 1 & $11 / 2 / 91$ & 9 & 1 & & 78 & $\mathbf{N}$ & \\
\hline 1 & $11 / 2 / 91$ & 10 & 1 & & 69 & $\mathbf{N}$ & \\
\hline 1 & $11 / 2 / 91$ & 11 & 1 & & 81 & $\mathbf{N}$ & \\
\hline 1 & $11 / 2 / 91$ & 12 & 1 & & 72 & $\mathbf{N}$ & \\
\hline 1 & $11 / 2 / 91$ & 13 & 1 & & 79 & $\mathbf{N}$ & \\
\hline 1 & $11 / 9 / 91$ & 1 & 1 & & 77 & $\bar{Y}$ & J-2E-OP-02, TC $\# 7,12,13$ O/S \\
\hline 1 & $11 / 9 / 91$ & 2 & 1 & & 74 & $\mathrm{~N}$ & \\
\hline 1 & $11 / 9 / 91$ & 3 & 1 & & 72 & $N$ & \\
\hline 1 & $11 / 9 / 91$ & 4 & 1 & & 73 & $\mathbf{N}$ & \\
\hline 1 & $11 / 9 / 91$ & 5 & 1 & & 75 & $\mathbf{N}$ & \\
\hline 1 & $11 / 9 / 91$ & 6 & 1 & & 69 & $\mathbf{N}$ & \\
\hline 1 & $11 / 9 / 91$ & 8 & 1 & & 75 & $\mathrm{~N}$ & \\
\hline 1 & $11 / 9 / 91$ & 9 & 1 & & 74 & $\mathbf{N}$ & \\
\hline 1 & $11 / 9 / 91$ & 10 & 9 & & 64 & $\mathrm{~N}$ & \\
\hline 1 & $11 / 9 / 91$ & 11 & 1 & & 76 & $\mathbf{N}$ & \\
\hline 9 & $11 / 17 / 91$ & 9 & 1 & & 72.2 & $N$ & J-91012643-75 TC \#7 BAD DATA \\
\hline 1 & $11 / 17 / 91$ & 2 & 1 & & 79.1 & $\mathbf{N}$ & \\
\hline 1 & $11 / 17 / 91$ & 3 & 1 & & 82.2 & $\bar{Y}$ & \\
\hline 1 & $11 / 17 / 91$ & 4 & 1 & & 77.4 & $\mathbf{N}$ & \\
\hline 1 & $11 / 17 / 91$ & 5 & 1 & & 78.4 & $\mathbf{N}$ & \\
\hline 1 & $11 / 17 / 91$ & 6 & 1 & & 73.5 & $\mathbf{N}$ & \\
\hline 1 & $11 / 17 / 91$ & 8. & 1 & & 78.9 & $\mathbf{N}$ & \\
\hline 1 & $11 / 17 / 91$ & 9 & 1 & & 76.9 & $\mathbf{N}$ & \\
\hline 1 & $11 / 17 / 91$ & 10 & 1 & & 69.7 & $\mathbf{N}$ & \\
\hline 1 & $11 / 17 / 91$ & 11 & 1 & & 79.8 & $\mathbf{N}$ & \\
\hline 9 & $11 / 24 / 91$ & 1 & 1 & & 83.4 & $\bar{Y}$ & J-910126143-75, TC \#7 BAD DATA \\
\hline 1 & $11 / 24 / 91$ & 2 & 1 & & 76.5 & $\mathbf{N}$ & \\
\hline 1 & $11 / 24 / 91$ & 3 & 1 & & 80 & $\mathbf{N}$ & \\
\hline .1 & $11 / 24 / 91$ & 4 & 1 & & 78.4 & $\mathbf{N}$ & \\
\hline 1 & $11 / 24 / 91$ & 5 & 1 & & 79.7 & $\bar{N}$ & \\
\hline 1 & $11 / 24 / 91$ & 6 & 1 & & 74.7 & $\mathrm{~N}$ & \\
\hline 1 & $11 / 24 / 91$ & 8 & 1 & & 80 & $\mathbf{N}$ & \\
\hline 1 & $11 / 24 / 91$ & 9 & 1 & & 78.3 & $\mathbf{N}$ & \\
\hline 1 & $11 / 24 / 99$ & 10 & 1 & & 71.4 & $\mathbf{N}$ & \\
\hline 1 & $11 / 24 / 91$ & 11 & 9 & & 89.1 & $\bar{N}$ & \\
\hline$i$ & $12 / 1 / 91$ & 1 & 1 & & 82.7 & $\bar{Y}$ & J-910126143-14 TC \#7 BAD DATA \\
\hline 1 & $12 / 1 / 91$ & 2 & 9 & & 75.5 & $\mathbf{N}$ & \\
\hline 1 & $12 / 1 / 91$ & 3 & 1 & & 78.8 & $\mathbf{N}$ & \\
\hline 1 & $12 / 1 / 91$ & 4 & 1 & & 77.4 & $\mathrm{~N}$ & \\
\hline 1 & $12 / 1 / 91$ & 5 & 1 & & 78.4 & $\mathbf{N}$ & \\
\hline 1 & $12 / 1 / 91$ & 6 & 1 & & 73.5 & $\mathbf{N}$ & \\
\hline 1 & $12 / 1 / 91$ & 8 & 1 & & 78.8 & $\mathbf{N}$ & \\
\hline 1 & $12 / 1 / 91$ & 9 & 1 & & 77 & $\mathbf{N}$ & \\
\hline 1 & $12 / 1 / 91$ & 10 & 1 & & 70.3 & $\mathbf{N}$ & \\
\hline 1 & $12 / 1 / 91$ & 11 & 1 & & 79.8 & $\mathbf{N}$ & \\
\hline 1 & $12 / 6 / 91$ & 1 & 1 & & 81.5 & $\bar{Y}$ & \\
\hline 1 & $12 / 6 / 91$ & 2 & 1 & & 71.6 & $\mathbf{N}$ & \\
\hline 1 & $12 / 6 / 91$ & 3 & 1 & & 76.6 & $\mathbf{N}$ & \\
\hline 1 & $12 / 6 / 91$ & 4 & 1 & & 76.9 & $\mathbf{N}$ & \\
\hline 1 & $12 / 6 / 91$ & 5 & 1 & & 78.1 & $\mathrm{~N}$ & \\
\hline 1 & $12 / 6 / 91$ & 6 & 1 & & 72.2 & $\mathrm{~N}$ & \\
\hline
\end{tabular}

Data obtained from WHC Surveillance Analysis Computer System (SACS), June 2, 1993. 


\begin{tabular}{|c|c|c|c|c|c|c|c|}
\hline \multicolumn{8}{|c|}{ TANK 247-BY-107 } \\
\hline \multicolumn{8}{|c|}{ THERMOCOUPLE DATA } \\
\hline TREE \# & DATE & THERMO \# & TREE SET \# & TEMP C & TEMPF & $Y=M A X$ TEMP & COMMENTS \\
\hline 1 & $12 / 6 / 91$ & 7 & .1 & & 77.2 & $\mathbf{N}$ & \\
\hline 1 & $12 / 6 / 91$ & 8 & 1 & & 77.6 & $\mathbf{N}$ & \\
\hline 1 & $12 / 6 / 91$ & 9 & 1 & & 73 & $\mathbf{N}$ & \\
\hline 1 & $12 / 6 / 91$ & 10 & 1 & & 68.9 & $\mathbf{N}$ & \\
\hline 1 & $12 / 6 / 91$ & 11 & 1 & & 79.7 & $\mathbf{N}$ & \\
\hline 1 & $12 / 8 / 91$ & 1 & 1 & & 81.2 & $\bar{Y}$ & J-910126143-48 TC \#7 BAD DATA \\
\hline 1 & $12 / 8 / 91$ & 2 & 1 & & 74.5 & $\mathbf{N}$ & \\
\hline 1 & $12 / 8 / 91$ & 3 & 1 & & 77.9 & $\mathbf{N}$ & \\
\hline 1 & $12 / 8 / 91$ & 4 & 1 & & 76.4 & $\bar{N}$ & \\
\hline 1 & $12 / 8 / 91$ & 5 & 1 & & 77.8 & $N$ & \\
\hline 9 & $12 / 8 / 91$ & 6 & 1 & & 72.5 & $\mathbf{N}$ & \\
\hline 1 & $12 / 8 / 91$ & 8 & 1 & & 78.8 & $\mathbf{N}$ & \\
\hline 1 & $12 / 8 / 91$ & 9 & 1 & & 77.3 & $\bar{N}$ & \\
\hline 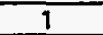 & $12 / 8 / 91$ & 10 & $\frac{1}{1}$ & & 70.5 & $\mathbf{N}$ & \\
\hline 1 & $12 / 8 / 91$ & 11 & 1 & & 79.9 & $\mathbf{N}$ & \\
\hline 1 & $12 / 14 / 91$ & 1 & 1 & & 82.6 & $\mathbf{Y}$ & $\begin{array}{c}\mathrm{J}-910126143-29, \text { TC \#7 BAD DATA TC } \# 12 \\
\text { O/S }\end{array}$ \\
\hline 1 & $12 / 14 / 91$ & 2 & 1 & & 75.9 & $N$ & \\
\hline 1 & $12 / 14 / 91$ & 3 & 1 & & 79.9 & $\mathbf{N}$ & \\
\hline 1 & $12 / 14 / 91$ & 4 & 1 & & 77.1 & $\bar{N}$ & \\
\hline 1 & $12 / 14 / 91$ & 5 & 1 & & 78.5 & $\mathbf{N}$ & \\
\hline 1 & $12 / 14 / 91$ & 6 & 1 & & 73.3 & $N$ & \\
\hline 1 & $12 / 14 / 91$ & 8 & 1 & & 78.7 & $N$ & \\
\hline 1 & $12 / 14 / 91$ & 9 & 9 & & 77.1 & $\bar{N}$ & \\
\hline 1 & $12 / 14 / 91$ & 10 & 1 & & 69.8 & $\mathbf{N}$ & \\
\hline 1 & $12 / 14 / 91$ & 11 & 1 & & 80 & $\mathbf{N}$ & \\
\hline 1 & $12 / 20 / 91$ & 1 & 9 & & & $\mathbf{N}$ & NO READINGS \\
\hline 1 & $12 / 22 / 91$ & 1 & 1 & & 81.1 & $\mathbf{Y}$ & $\begin{array}{l}\text { J-900925741 OMEGA TC } \# 7,12 \\
\text { BAD DATA }\end{array}$ \\
\hline 1 & $12 / 22 / 91$ & 2 & 1 & & 69.1 & $\bar{N}$ & \\
\hline 1 & $12 / 22 / 91$ & 3 & 1 & & 76.8 & $\mathbf{N}$ & \\
\hline 9 & $12 / 22 / 91$ & 4 & 1 & & 73.7 & $\mathbf{N}$ & \\
\hline 1 & $12 / 22 / 91$ & 5 & 1 & & 76.5 & $\mathbf{N}$ & \\
\hline 1 & $12 / 22 / 91$ & 6 & 1 & & 71.8 & $\mathbf{N}$ & \\
\hline 9 & $12 / 22 / 91$ & 8 & 1 & & 77.5 & $\mathbf{N}$ & \\
\hline 1 & $12 / 22 / 91$ & 9 & 1 & & 74.9 & $\mathbf{N}$ & \\
\hline 1 & $12 / 22 / 91$ & 10 & 1 & & 64.4 & $N$ & \\
\hline$\frac{T}{1}$ & $12 / 22 / 91$ & 11 & 1 & & 78.9 & $\mathbf{N}$ & \\
\hline 1 & $12 / 22 / 91$ & 13 & 1 & & 70.7 & $\mathbf{N}$ & \\
\hline 1 & $12 / 22 / 91$ & 14 & 1 & & 74.4 & $\bar{N}$ & \\
\hline 1 & $12 / 29 / 91$ & 1 & 1 & & 93.4 & $\mathbf{Y}$ & $\begin{array}{l}\text { OVER SURVEILLANCE LIMIT RETEST REO } \\
1 / 2 / 30 / 91\end{array}$ \\
\hline 1 & $12 / 29 / 91$ & 2 & 1 & & 90.4 & $\mathbf{N}$ & \\
\hline 1 & $12 / 29 / 91$ & 3 & 1 & & 80.2 & $\bar{N}$ & \\
\hline 1 & $12 / 29 / 91$ & 4 & 1 & & 77.5 & $\bar{N}$ & \\
\hline 1 & $12 / 29 / 91$ & 5 & 1 & & 76.5 & $\mathrm{~N}$ & \\
\hline 9 & $12 / 29 / 91$ & 6 & 1 & & 76.1 & $\bar{N}$ & \\
\hline 1 & $12 / 29 / 91$ & 7 & 1 & & 75 & $\mathbf{N}$ & \\
\hline 1 & $12 / 29 / 91$ & 8 & 1 & & 75.7 & $\mathbf{N}$ & \\
\hline$\frac{9}{1}$ & $12 / 29 / 91$ & 9 & 1 & & 73.7 & $N$ & \\
\hline 1 & $12 / 29 / 91$ & 10 & 1 & & 73.2 & $\bar{N}$ & \\
\hline 1 & $12 / 29 / 91$ & 11 & 1 & & 73.8 & $\mathbf{N}$ & \\
\hline$\frac{1}{1}$ & $12 / 29 / 91$ & 12 & 1 & & 73.4 & $\mathbf{N}$ & \\
\hline 9 & $12 / 29 / 91$ & 13 & 1 & & 73.7 & $\mathbf{N}$ & \\
\hline 1 & $12 / 29 / 91$ & 14 & $\frac{1}{1}$ & & 73.9 & $\mathbf{N}$ & \\
\hline 1 & $12 / 30 / 91$ & 1 & 1 & & 93.5 & $\bar{Y}$ & $\mathrm{~J}-910126143-50$ \\
\hline$\frac{7}{1}$ & $12 / 30 / 91$ & 2 & 1 & & 90.3 & $\bar{N}$ & \\
\hline 1 & $12 / 30 / 91$ & 3 & 9 & & 80 & $\mathbf{N}$ & \\
\hline 1 & $12 / 30 / 91$ & 4 & 9 & & 77.4 & $\mathbf{N}$ & \\
\hline 9 & $12 / 30 / 91$ & 5 & 1 & & 76.3 & $\mathbf{N}$ & \\
\hline 1 & $12 / 30 / 91$ & 6 & 1 & & 76 & $\mathbf{N}$ & \\
\hline 1 & $12 / 30 / 91$ & 7 & 9 & & 75 & $\mathbf{N}$ & \\
\hline 1 & $12 / 30 / 91$ & 8 & 1 & & 75.6 & $\mathbf{N}$ & \\
\hline$\overline{1}$ & $12 / 30 / 91$ & 9 & 1 & & 73.6 & $\mathbf{N}$ & \\
\hline 1 & $12 / 30 / 91$ & 10 & 1 & & 73 & $N$ & \\
\hline 1 & $12 / 30 / 91$ & 11 & 1 & & 73.6 & $\overline{\mathbf{N}}$ & \\
\hline
\end{tabular}

Data obtained from WHC Surveillance Analysis Computer System (SACS), June 2, 1993. 


\begin{tabular}{|c|c|c|c|c|c|c|c|}
\hline \multicolumn{8}{|c|}{ TANK 241-BY-107 } \\
\hline \multicolumn{8}{|c|}{ THERMOCOUPLE DATA } \\
\hline TREE \# & DATE & THERMO \# & TREE SET \# & TEMP C & TEMP F & $\mathrm{Y}=$ MAX TEMP & COMMENTS \\
\hline 1 & $12 / 30 / 91$ & 12 & 1 & & 73.2 & $N$ & \\
\hline 1 & $12 / 30 / 91$ & 13 & 1 & & 73.6 & $\mathrm{~N}$ & \\
\hline 1 & $12 / 30 / 91$ & 14 & 1 & & 73.8 & $\mathbf{N}$ & \\
\hline 1 & $1 / 3 / 92$ & 1 & 1 & & 93 & $\bar{Y}$ & J-910126143-50 \\
\hline 1 & $1 / 3 / 92$ & 2 & 1 & & 90 & $\mathbf{N}$ & \\
\hline 1 & $1 / 3 / 92$ & 3 & 1 & & 80 & $\bar{N}$ & \\
\hline 1 & $1 / 3 / 92$ & 4 & 1 & & 77 & $\mathbf{N}$ & \\
\hline 1 & $1 / 3 / 92$ & 5 & 1 & & 76 & $\mathbf{N}$ & \\
\hline 1 & $1 / 3 / 92$ & 6 & 1 & & 75 & $\bar{N}$ & \\
\hline 1 & $1 / 3 / 92$ & 7 & 1 & & 74 & $\mathbf{N}$ & \\
\hline$\frac{1}{1}$ & $9 / 3 / 92$ & 8 & 1 & & 75 & $\mathbf{N}$ & \\
\hline 1 & $1 / 3 / 92$ & 9 & 1 & & 72 & $\mathbf{N}$ & \\
\hline 1 & $1 / 3 / 92$ & 10 & 1 & & 72 & $\mathbf{N}$ & \\
\hline 1 & $1 / 3 / 92$ & 11 & 1 & & 73 & $\bar{N}$ & \\
\hline 1 & $1 / 3 / 92$ & 12 & 1 & & 72 & $\mathbf{N}$ & \\
\hline 1 & $1 / 3 / 92$ & 13 & 1 & & 73 & $\mathbf{N}$ & \\
\hline 1 & $1 / 3 / 92$ & 14 & 1 & & 73 & $\mathbf{N}$ & \\
\hline 1 & $1 / 7 / 92$ & 1 & 1 & & & N & $\begin{array}{l}\text { TEMP INCREASE DUE TO THERMOCOUPLE } \\
\text { REPAIR;DISCREP\#TFSA-92-431 }\end{array}$ \\
\hline 1 & $1 / 11 / 92$ & 1 & 1 & & 91.8 & $Y$ & $910126143-14$ \\
\hline 1 & $1 / 11 / 92$ & 2 & 1 & & 89.6 & $N$ & \\
\hline 1 & $1 / 11 / 92$ & 3 & 1 & & 78.7 & $\mathbf{N}$ & \\
\hline 1 & $1 / 11 / 92$ & 4 & 1 & & 75.9 & $N$ & \\
\hline 1 & $1 / 11 / 92$ & 5 & 1 & & 75 & $\bar{N}$ & \\
\hline 1 & $1 / 11 / 92$ & 6 & 1 & & 74.4 & $\mathbf{N}$ & \\
\hline 1 & $1 / 11 / 92$ & 7 & 1 & & 73.8 & $\mathrm{~N}$ & \\
\hline 1 & $1 / 11 / 92$ & 8 & 1 & & 74 & $\mathbf{N}$ & \\
\hline 1 & $1 / 11 / 92$ & 9 & 1 & & 79.8 & $\mathrm{~N}$ & \\
\hline 1 & $1 / 11 / 92$ & 10 & 1 & & 71.4 & $N$ & \\
\hline 1 & $1 / 11 / 92$ & 11 & 9 & & 72.1 & $\mathrm{~N}$ & \\
\hline 1 & $1 / 11 / 92$ & 12 & 1 & & 71.7 & $\mathbf{N}$ & \\
\hline 1 & $1 / 11 / 92$ & 13 & 1 & & 72 & $\mathrm{~N}$ & \\
\hline 1 & $1 / 11 / 92$ & 14 & 1 & & 72.2 & $\mathbf{N}$ & \\
\hline 1 & $1 / 16 / 92$ & 1 & 1 & & 93 & $\mathbf{Y}$ & J 910126143-32 \\
\hline 1 & $1 / 16 / 92$ & 2 & 1 & & 91.1 & $\mathrm{~N}$ & \\
\hline 1 & $1 / 16 / 92$ & 3 & 1 & & 79.5 & $\mathbf{N}$ & \\
\hline 1 & $1 / 16 / 92$ & 4 & 1 & & 77.2 & $\mathbf{N}$ & \\
\hline 1 & $1 / 16 / 92$ & 5 & 1 & & 76.2 & $\mathrm{~N}$ & \\
\hline 1 & $1 / 16 / 92$ & 6 & 1 & & 75.1 & $\bar{N}$ & \\
\hline 1 & $1 / 16 / 92$ & 7 & 1 & & 75.1 & $\mathbf{N}$ & \\
\hline 1 & $1 / 16 / 92$ & 8 & 1 & & 75.3 & $\mathbf{N}$ & \\
\hline 1 & $1 / 16 / 92$ & 9 & 1 & & 73 & $\mathbf{N}$ & \\
\hline 1 & $1 / 16 / 92$ & 10 & 1 & & 72.6 & $\mathbf{N}$ & \\
\hline 1 & $1 / 16 / 92$ & 11 & 1 & & 73.2 & $\mathbf{N}$ & \\
\hline 1 & $1 / 16 / 92$ & 12 & 1 & & 72.8 & $\mathbf{N}$ & \\
\hline 1 & $1 / 16 / 92$ & 13 & 1 & & 73.1 & $\mathrm{~N}$ & \\
\hline 1 & $1 / 16 / 92$ & 14 & 1 & & 73.2 & $\mathbf{N}$ & \\
\hline 1 & $1 / 25 / 92$ & 1 & 1 & & 94.1 & $\bar{Y}$ & J-910126143-14 \\
\hline 1 & $1 / 25 / 92$ & 2 & 1 & & 92 & $\bar{N}$ & \\
\hline 1 & $1 / 25 / 92$ & 3 & 1 & & 80.4 & $\mathbf{N}$ & \\
\hline 1 & $1 / 25 / 92$ & 4 & 1 & & 77.7 & $\mathrm{~N}$ & \\
\hline 1 & $1 / 25 / 92$ & 5 & 1 & & 76.6 & $\mathbf{N}$ & \\
\hline 1 & $1 / 25 / 92$ & 6 & 1 & & 76 & $N$ & \\
\hline 1 & $1 / 25 / 92$ & 7 & 1 & & 75.3 & $\mathbf{N}$ & \\
\hline 1 & $1 / 25 / 92$ & 8 & 1 & & 75.5 & $\mathbf{N}$ & \\
\hline 1 & $1 / 25 / 92$ & 9 & 1 & & 73.2 & $\mathbf{N}$ & \\
\hline 1 & $1 / 25 / 92$ & 10 & 1 & & 73.1 & $N$ & \\
\hline 1 & $1 / 25 / 92$ & 11 & 1 & & 73.7 & $\mathbf{N}$ & \\
\hline 1 & $1 / 25 / 92$ & 12 & 1 & & 73.6 & $\mathbf{N}$ & \\
\hline 1 & $1 / 25 / 92$ & 13 & 1 & & 73.8 & $\mathbf{N}$ & \\
\hline 1 & $1 / 25 / 92$ & 14 & 1 & & 74.2 & $\mathbf{N}$ & \\
\hline 1 & $1 / 28 / 92$ & 1 & 1 & & & N & $\begin{array}{l}\text { ACCESS RESTRICTED } 1 / 28-2 / 22 \text { DUE TO } \\
\text { BATTERY FUME INCIDENT }\end{array}$ \\
\hline 1 & $2 / 24 / 92$ & 1 & 1 & & 96.6 & $\bar{Y}$ & J-900925741-79 \\
\hline 1 & $2 / 24 / 92$ & 2 & 1 & & 93.7 & $N$ & \\
\hline
\end{tabular}

Data obtained from WHC Surveillance Analysis Computer System (SACS), June 2, 1993. 


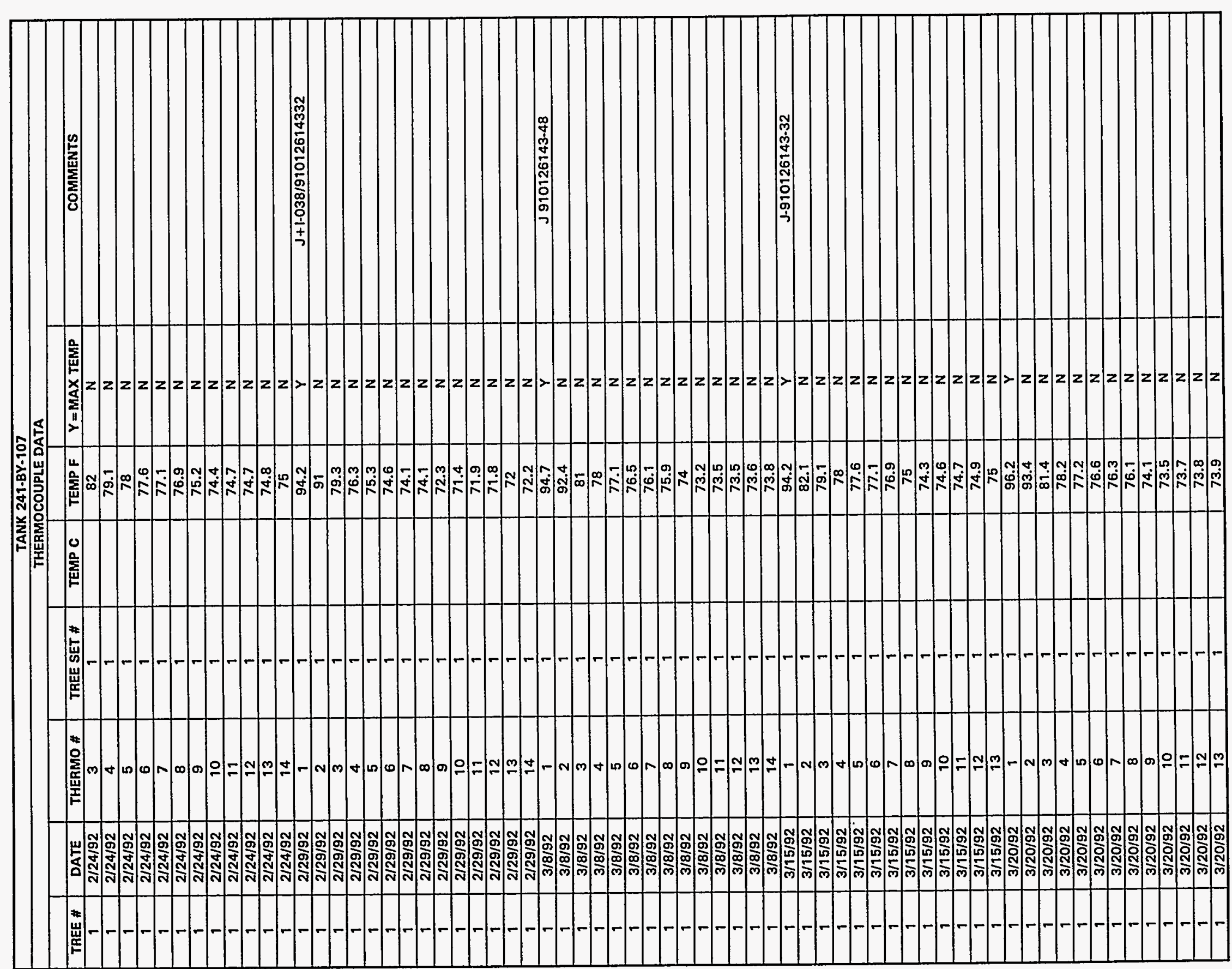




\begin{tabular}{|c|c|c|c|c|c|c|c|}
\hline \multicolumn{8}{|c|}{ TANK 241-BY-107 } \\
\hline \multicolumn{8}{|c|}{ THERMOCOUPLE DATA } \\
\hline TREE \# & DATE & THERMO \# & TREE SET \# & TEMP C & TEMP F & $Y=$ MAX TEMP & COMMENTS \\
\hline 1 & $3 / 20 / 92$ & 14 & 1 & & 74 & $N$ & \\
\hline 1 & $3 / 29 / 92$ & 1 & 1 & & 94.9 & $\bar{Y}$ & J 910126143.66 \\
\hline 1 & $3 / 29 / 92$ & 2 & 1 & & 92.4 & $\mathbf{N}$ & \\
\hline 1 & $3 / 29 / 92$ & 3 & $\frac{1}{1}$ & & 80.8 & $\mathbf{N}$ & \\
\hline 1 & $3 / 29 / 92$ & 4 & 1 & & 77.9 & $\mathbf{N}$ & \\
\hline 1 & $3 / 29 / 92$ & 5 & 1 & & 76.8 & $\mathbf{N}$ & \\
\hline 1 & $3 / 29 / 92$ & 6 & 1 & & 76.1 & $\mathbf{N}$ & \\
\hline 1 & $3 / 29 / 92$ & 7 & 1 & & 75.6 & $\mathbf{N}$ & \\
\hline 1 & $3 / 29 / 92$ & 8 & 1 & & 75.6 & $\mathbf{N}$ & \\
\hline 1 & $3 / 29 / 92$ & 9 & 1 & & 73.8 & $\overline{\mathrm{N}}$ & \\
\hline 1 & $3 / 29 / 92$ & 10 & 1 & & 73.4 & $\mathbf{N}$ & \\
\hline 1 & $3 / 29 / 92$ & 11 & 9 & & 74 & $\mathbf{N}$ & \\
\hline 1 & $3 / 29 / 92$ & 12 & 1 & & 74 & $\mathbf{N}$ & \\
\hline 1 & $3 / 29 / 92$ & 13 & 1 & & 74.3 & $\mathbf{N}$ & \\
\hline 1 & $3 / 29 / 92$ & 14 & 1 & & 74.5 & $\mathbf{N}$ & \\
\hline 1 & $4 / 3 / 92$ & 1 & 1 & & 94.9 & $Y$ & $J 910126143.14$ \\
\hline 1 & $4 / 3 / 92$ & 2 & 1 & & 91.6 & $\mathbf{N}$ & \\
\hline 1 & $4 / 3 / 92$ & 3 & 1 & & 80.2 & $\mathbf{N}$ & \\
\hline 1 & $4 / 3 / 92$ & 4 & 1 & & 77.4 & $\mathbf{N}$ & \\
\hline 1 & $4 / 3 / 92$ & 5 & 1 & & 76.4 & $\mathbf{N}$ & \\
\hline 1 & $4 / 3 / 92$ & 6 & 1 & & 75.8 & $\mathbf{N}$ & \\
\hline 1 & $4 / 3 / 92$ & 7 & 1 & & 75.5 & $\mathbf{N}$ & \\
\hline 1 & $4 / 3 / 92$ & 8 & 1 & & 75.3 & $\bar{N}$ & \\
\hline 1 & $4 / 3 / 92$ & 9 & 1 & & 73.2 & $\mathbf{N}$ & \\
\hline 1 & $4 / 3 / 92$ & 10 & $\frac{1}{1}$ & & 72.8 & $\mathbf{N}$ & \\
\hline$\frac{T}{1}$ & $4 / 3 / 92$ & 11 & 1 & & 73.1 & $\mathbf{N}$ & \\
\hline 1 & $4 / 3 / 92$ & 12 & 1 & & 73.1 & $\mathbf{N}$ & \\
\hline$\frac{1}{1}$ & $4 / 3 / 92$ & 13 & 1 & & 73.2 & $\mathbf{N}$ & \\
\hline 1 & $4 / 3 / 92$ & 14 & 1 & & 73.5 & $\mathbf{N}$ & \\
\hline 1 & $4 / 11 / 92$ & 1 & 1 & & 94.2 & $\bar{Y}$ & J $910125143-64$ \\
\hline 1 & $4 / 11 / 92$ & 2 & 1 & & 91.1 & $\mathbf{N}$ & \\
\hline 1 & $4 / 11 / 92$ & 3 & 1 & & 79.8 & $\mathbf{N}$ & \\
\hline 1 & $4 / 11 / 92$ & 4 & 1 & & 76.6 & $\mathbf{N}$ & \\
\hline 1 & $4 / 11 / 92$ & 5 & 1 & & 75.7 & $\mathbf{N}$ & \\
\hline 1 & $4 / 11 / 92$ & 6 & 1 & & 75 & $\mathbf{N}$ & \\
\hline 1 & $4 / 11 / 92$ & 7 & 1 & & 74.5 & $N$ & \\
\hline 1 & $4 / 11 / 92$ & 8 & 1 & & 74.5 & $\mathbf{N}$ & \\
\hline 1 & $4 / 11 / 92$ & 9 & 1 & & 72.4 & $\mathbf{N}$ & \\
\hline 1 & $4 / 11 / 92$ & 10 & 1 & & 72 & $\mathbf{N}$ & \\
\hline 1 & $4 / 11 / 92$ & 11 & 1 & & 72.5 & $\mathbf{N}$ & \\
\hline 1 & $4 / 11 / 92$ & 12 & 1 & & 72.5 & $\mathbf{N}$ & \\
\hline 1 & $4 / 11 / 92$ & 13 & 1 & & 72.7 & $\mathrm{~N}$ & \\
\hline 1 & $4 / 11 / 92$ & 14 & 1 & & 73 & $\mathbf{N}$ & \\
\hline 1 & $4 / 17 / 92$ & 1 & 1 & & 95.9 & $\bar{Y}$ & J 8905235220 \\
\hline 1 & $4 / 17 / 92$ & 2 & 1 & & 92.3 & $\mathbf{N}$ & \\
\hline 1 & $4 / 17 / 92$ & 3 & 1 & & 81.4 & $\mathbf{N}$ & \\
\hline 1 & $4 / 17 / 92$ & 4 & 1 & & 78.3 & $\mathbf{N}$ & \\
\hline 1 & $4 / 17 / 92$ & 5 & 1 & & 77.3 & $\mathbf{N}$ & \\
\hline 1 & $4 / 17 / 92$ & 6 & $\frac{1}{1}$ & & 76.6 & $N$ & \\
\hline 1 & $4 / 17 / 92$ & 7 & 1 & & 76.1 & $\bar{N}$ & \\
\hline 1 & $4 / 17 / 92$ & 8 & 1 & & 76.1 & $\mathbf{N}$ & \\
\hline 1 & $4 / 17 / 92$ & 9 & 1 & & 74.2 & $\mathbf{N}$ & \\
\hline 1 & $4 / 17 / 92$ & 10 & 1 & & 73.7 & $\mathbf{N}$ & \\
\hline 1 & $4 / 17 / 92$ & 11 & 1 & & 74.2 & $\mathbf{N}$ & \\
\hline$\frac{1}{1}$ & $4 / 17 / 92$ & 12 & 7 & & 74.2 & $\mathbf{N}$ & \\
\hline 1 & $4 / 17 / 92$ & 13 & 1 & & 74.4 & $\mathbf{N}$ & \\
\hline 1 & $4 / 17 / 92$ & 14 & 1 & & 74.7 & $\mathbf{N}$ & \\
\hline 1 & $4 / 25 / 92$ & 1 & 1 & & 98.5 & $Y$ & $\mathrm{~J} 89052351220$ \\
\hline 1 & $4 / 25 / 92$ & 2 & 1 & & 95.7 & $\hat{N}$ & \\
\hline 1 & $4 / 25 / 92$ & 3 & 1 & & 83 & $\mathrm{~N}$ & \\
\hline 7 & $4 / 25 / 92$ & 4 & 1 & & 81.2 & $\mathbf{N}$ & \\
\hline 1 & $4 / 25 / 92$ & 5 & 1 & & 79.7 & $\mathbf{N}$ & \\
\hline 1 & $4 / 25 / 92$ & 6 & 1 & & 78.8 & $\mathbf{N}$ & \\
\hline 1 & $4 / 25 / 92$ & 7 & 1 & & 78.1 & $\mathbf{N}$ & \\
\hline 1 & $4 / 25 / 92$ & 8 & 1 & & 78 & $\mathbf{N}$ & \\
\hline 1 & $4 / 25 / 92$ & 9 & $\frac{1}{1}$ & & 76.3 & $N$ & \\
\hline
\end{tabular}

Data obtained from WHC Surveillance Analysis Computer System (SACS), June 2, 1993. 
WHC-SD-WM-ER-312, Rev. 0

\begin{tabular}{|c|c|c|c|c|c|c|c|}
\hline \multicolumn{8}{|c|}{ TANK 241-BY.107 } \\
\hline \multicolumn{8}{|c|}{ THERMOCOUPLE DATA } \\
\hline TREE \# & DATE & THERMO \# & TREE SET \# & TEMP C & TEMP F & $Y=$ MAX TEMP & COMMENTS \\
\hline 1 & $4 / 25 / 92$ & 10 & 1 & & 75.8 & $\mathbf{N}$ & \\
\hline 1 & $4 / 25 / 92$ & 11 & 1 & & 76.5 & $\mathbf{N}$ & \\
\hline 9 & $4 / 25 / 92$ & 12 & 1 & & 76.6 & $\mathbf{N}$ & \\
\hline 1 & $4 / 25 / 92$ & 13 & 1 & & 76.7 & $\mathbf{N}$ & \\
\hline 1 & $4 / 25 / 92$ & 14 & 1 & & 76.6 & $\mathbf{N}$ & \\
\hline 1 & $4 / 30 / 92$ & 1 & 1 & & 92.2 & $\mathbf{Y}$ & $J 890523512-47$ \\
\hline 1 & $4 / 30 / 92$ & 2 & 1 & & 88.9 & $\mathbf{N}$ & \\
\hline 1 & $4 / 30 / 92$ & 3 & 1 & & 77.8 & $\mathbf{N}$ & \\
\hline 1 & $4 / 30 / 92$ & 4 & 1 & & 75.4 & $\mathbf{N}$ & \\
\hline 9 & $4 / 30 / 92$ & 5 & 1 & & 74.4 & $\mathbf{N}$ & \\
\hline 1 & $4 / 30 / 92$ & 6 & 1 & & 73.8 & $\bar{N}$ & \\
\hline 1 & $4 / 30 / 92$ & 7 & 1 & & 73.3 & $\mathbf{N}$ & \\
\hline 9 & $4 / 30 / 92$ & 8 & 1 & & 73.2 & $\mathbf{N}$ & \\
\hline 1 & $4 / 30 / 92$ & 9 & 1 & & 71.5 & $\mathbf{N}$ & \\
\hline 1 & $4 / 30 / 92$ & 10 & 1 & & 70.8 & $\mathbf{N}$ & \\
\hline 1 & $4 / 30 / 92$ & 11 & 1 & & 71.3 & $\mathbf{N}$ & \\
\hline 1 & $4 / 30 / 92$ & 12 & 1 & & 71.3 & $\bar{N}$ & \\
\hline 1 & $4 / 30 / 92$ & 13 & 1 & & 71.5 & $\mathbf{N}$ & \\
\hline 1 & $4 / 30 / 92$ & 14 & 1 & & 71.8 & $\mathbf{N}$ & \\
\hline 1 & $5 / 8 / 92$ & 1 & 1 & & 93.8 & $\bar{Y}$ & J910126143-48 \\
\hline 1 & $5 / 8 / 92$ & 2 & 1 & & 90.3 & $\mathbf{N}$ & \\
\hline 1 & $5 / 8 / 92$ & 3 & 1 & & 79.6 & $\mathbf{N}$ & \\
\hline 1 & $5 / 8 / 92$ & 4 & 1 & & 77.1 & $\mathbf{N}$ & \\
\hline 1 & $5 / 8 / 92$ & 5 & 1 & & 76 & $\mathbf{N}$ & \\
\hline 1 & $5 / 8 / 92$ & 6 & 1 & & 75.4 & $\bar{N}$ & \\
\hline 1 & $5 / 8 / 92$ & 7 & 1 & & 75 & $\mathbf{N}$ & . \\
\hline 1 & $5 / 8 / 92$ & 8 & 1 & & 74.9 & $\mathbf{N}$ & \\
\hline 1 & $5 / 8 / 92$ & 9 & 1 & & 73.3 & $\mathbf{N}$ & \\
\hline 1 & $5 / 8 / 92$ & 10 & 1 & & 72.6 & $\mathbf{N}$ & \\
\hline 1 & $5 / 8 / 92$ & 11 & 1 & & 73 & $\mathbf{N}$ & \\
\hline 1 & $5 / 8 / 92$ & 12 & 1 & & 73 & $\mathbf{N}$ & \\
\hline 1 & $5 / 8 / 92$ & 13 & 1 & & 73.2 & $\mathbf{N}$ & \\
\hline 1 & $5 / 8 / 92$ & 14 & 1 & & 73.5 & $\mathbf{N}$ & \\
\hline 1 & $5 / 16 / 92$ & 1 & 1 & & 97.2 & $\bar{Y}$ & J910126143-32 \\
\hline 1 & $5 / 16 / 92$ & 2 & 1 & & 94.1 & $\mathbf{N}$ & \\
\hline 1 & $5 / 16 / 92$ & 3 & 1 & & 83.3 & $\mathbf{N}$ & \\
\hline 1 & $5 / 16 / 92$ & 4 & 1 & & 80.7 & $\mathbf{N}$ & \\
\hline$\frac{1}{1}$ & $5 / 16 / 92$ & 5 & 1 & & 79.6 & $\mathbf{N}$ & \\
\hline 1 & $5 / 16 / 92$ & 6 & 1 & & 79 & $\mathbf{N}$ & \\
\hline 1 & $5 / 16 / 92$ & 7 & 1 & & 78.5 & $\mathbf{N}$ & \\
\hline 1 & $5 / 16 / 92$ & 8 & 1 & & 78.3 & $\mathbf{N}$ & \\
\hline 1 & $5 / 16 / 92$ & 9 & 1 & & 77.1 & $\mathbf{N}$ & \\
\hline 1 & $5 / 16 / 92$ & 10 & 1 & & 76.5 & $\mathbf{N}$ & \\
\hline 1 & $5 / 16 / 92$ & 11 & 1 & & 77.1 & $\mathbf{N}$ & \\
\hline 1 & $5 / 16 / 92$ & 12 & 1 & & 77.4 & $\mathbf{N}$ & \\
\hline 1 & $5 / 16 / 92$ & 13 & 1 & & 77.8 & $\mathbf{N}$ & \\
\hline 1 & $5 / 16 / 92$ & 14 & 1 & & 77.8 & $\mathbf{N}$ & \\
\hline 1 & $5 / 24 / 92$ & 1 & 1 & & 96.1 & $\mathbf{Y}$ & J 890523512-47 \\
\hline 1 & $5 / 24 / 92$ & 2 & 1 & & 93.4 & $\mathbf{N}$ & \\
\hline 1 & $5 / 24 / 92$ & 3 & 1 & & 83.4 & $\mathbf{N}$ & \\
\hline 1 & $5 / 24 / 92$ & 4 & 1 & & 80 & $\mathbf{N}$ & \\
\hline 1 & $5 / 24 / 92$ & .5 & 1 & & 78.6 & $\mathbf{N}$ & \\
\hline 1 & $5 / 24 / 92$ & 6 & 1 & & 77.9 & $\mathbf{N}$ & \\
\hline 1 & $5 / 24 / 92$ & 7 & 1 & & 77.5 & $\mathbf{N}$ & \\
\hline 1 & $5 / 24 / 92$ & 8 & 1 & & 77.5 & $\mathbf{N}$ & \\
\hline 1 & $5 / 24 / 92$ & 9 & 1 & & 76.4 & $\mathbf{N}$ & \\
\hline 1 & $5 / 24 / 92$ & 10 & 1 & & 76 & $N$ & \\
\hline 1 & $5 / 24 / 92$ & 19 & 1 & & 76.5 & $\mathbf{N}$ & \\
\hline 1 & $5 / 24 / 92$ & 12 & 1 & & 76.8 & $\mathbf{N}$ & \\
\hline$T$ & $5 / 24 / 92$ & 13 & 1 & & 77.2 & $\mathbf{N}$ & \\
\hline 1 & $5 / 24 / 92$ & 14 & 1 & & 77.5 & $\mathbf{N}$ & \\
\hline 1 & $5 / 28 / 92$ & 1 & 9 & & 94.9 & $\bar{Y}$ & J 890523512.47 \\
\hline 1 & $5 / 28 / 92$ & 2 & 1 & & 99.7 & $\mathbf{N}$ & \\
\hline 1 & $5 / 28 / 92$ & 3 & 1 & & 82 & $\mathbf{N}$ & \\
\hline 1 & $5 / 28 / 92$ & 4 & 1 & & 79.1 & $\mathbf{N}$ & \\
\hline 1 & $5 / 28 / 92$ & 5 & 1 & & 78 & $\mathbf{N}$ & \\
\hline
\end{tabular}

Data obtained from WHC Surveillance Analysis Computer System (SACS), June 2, 1993. 
WHC-SD-WM-ER-312, Rev. 0

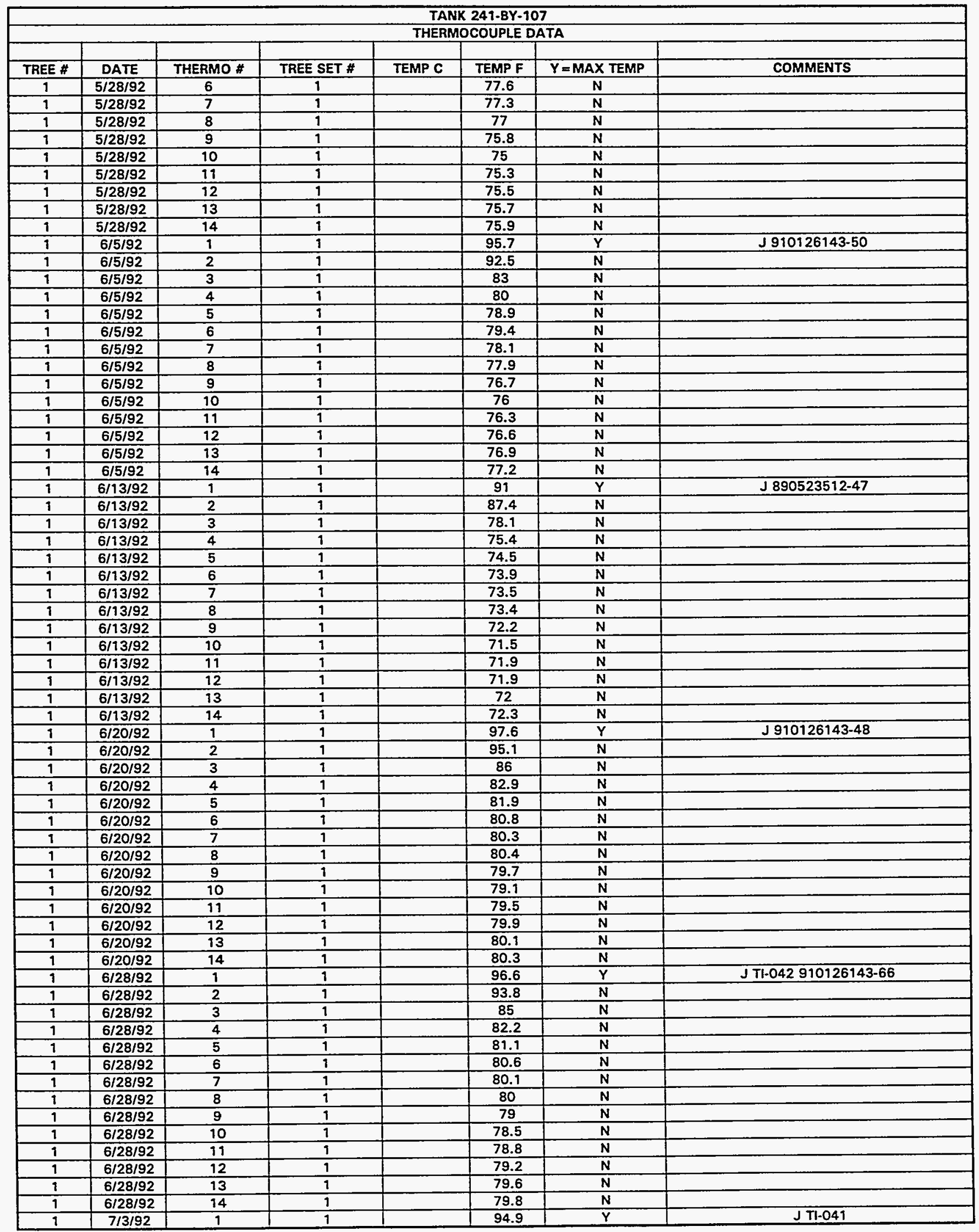

Data obtained from WHC Surveillance Analysis Computer System (SACS), June 2, 1993. 


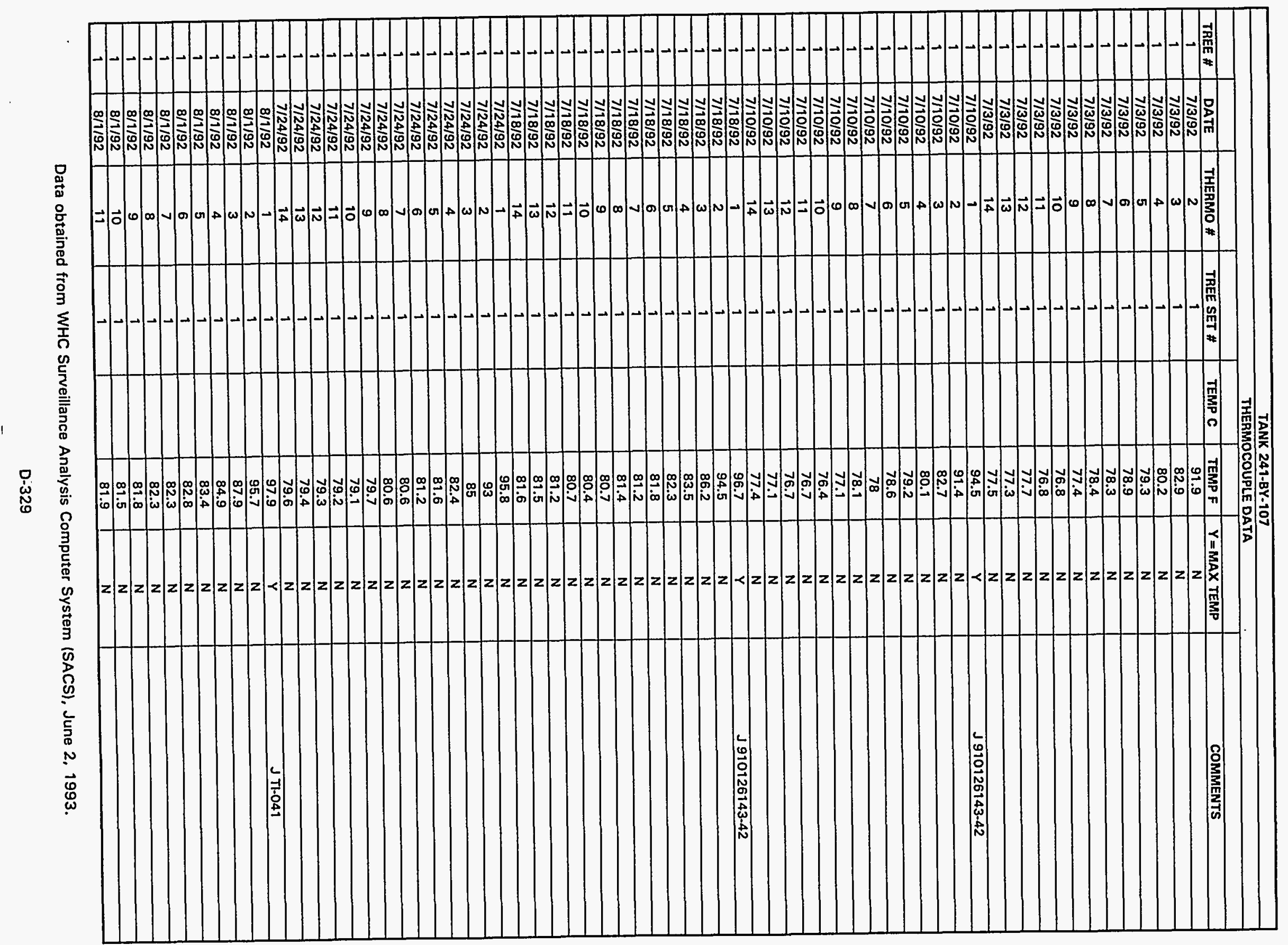

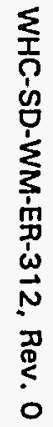




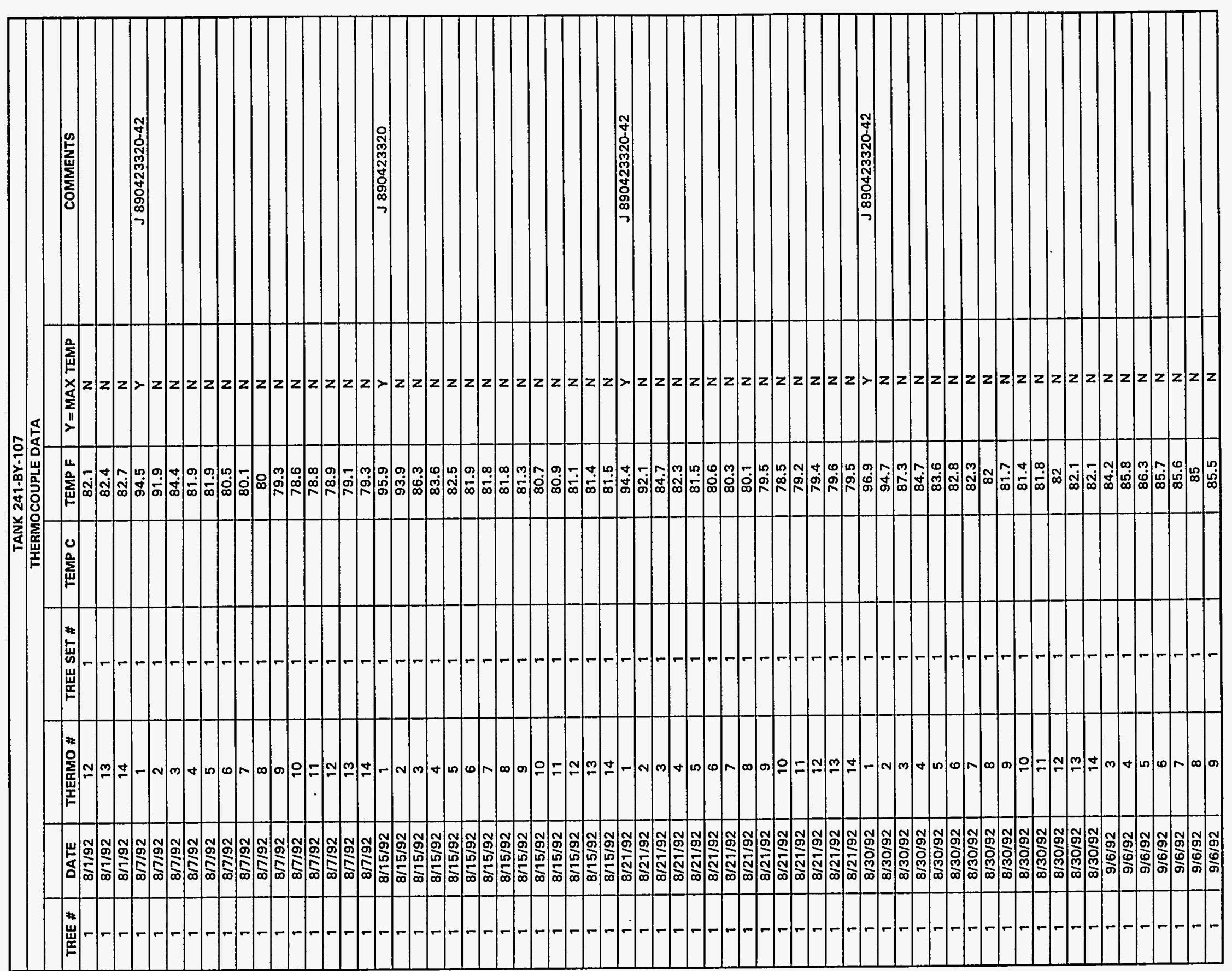

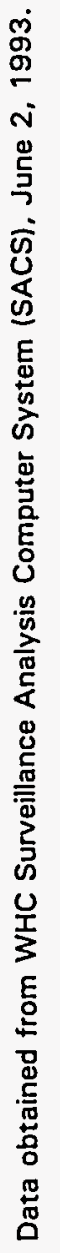




\begin{tabular}{|c|c|c|c|c|c|c|c|}
\hline \multicolumn{8}{|c|}{ TANK 241-BY-107 } \\
\hline \multicolumn{8}{|c|}{ THERMOCOUPLE DATA } \\
\hline TREE \# & DATE & THERMO \# & TAEE SET \# & TEMP C & TEMP F & $Y=M A X$ TEMP & COMMENTS \\
\hline 1 & $9 / 6 / 92$ & 10 & 1 & & 81 & $N$ & \\
\hline 1 & $9 / 6 / 92$ & 11 & 1 & & 79.8 & $N$ & \\
\hline 1 & $9 / 6 / 92$ & 12 & 1 & & 79.5 & $\mathbf{N}$ & \\
\hline 1 & $9 / 6 / 92$ & 13 & 1 & & 78.7 & $\mathbf{N}$ & \\
\hline 1 & $9 / 6 / 92$ & 14 & 1 & & 78.5 & $\overline{\mathbf{N}}$ & \\
\hline 1 & $9 / 12 / 92$ & 1 & 1 & & 95.8 & $\mathbf{Y}$ & J 890423320-42 \\
\hline 1 & $9 / 12 / 92$ & 2 & 1 & & 93.4 & $\mathbf{N}$ & \\
\hline 1 & $9 / 12 / 92$ & 3 & 1 & & 86.2 & $\mathbf{N}$ & \\
\hline 1 & $9 / 12 / 92$ & 4 & 1 & & 84.1 & $\mathbf{N}$ & \\
\hline 1 & $9 / 12 / 92$ & 5 & 1 & & 83.2 & $\bar{N}$ & \\
\hline 1 & $9 / 12 / 92$ & 6 & 1 & & 82.7 & $\mathbf{N}$ & \\
\hline 1 & $9 / 12 / 92$ & 7 & 1 & & 82.3 & $N$ & \\
\hline 1 & $9 / 12 / 92$ & 8 & 1 & & 82.1 & $\mathbf{N}$ & \\
\hline 1 & $9 / 12 / 92$ & 9 & 1 & & 81.5 & $\mathbf{N}$ & \\
\hline 1 & $9 / 12 / 92$ & 10 & 1 & & 80.8 & $\mathbf{N}$ & \\
\hline 1 & $9 / 12 / 92$ & 11 & 1 & & 81.1 & $\mathbf{N}$ & \\
\hline 1 & $9 / 12 / 92$ & 12 & 1 & & 81.1 & $\mathbf{N}$ & \\
\hline 1 & $9 / 12 / 92$ & 13 & 1 & & 81.3 & $\mathbf{N}$ & \\
\hline 1 & $9 / 12 / 92$ & 14 & 1 & & 81.5 & $\mathbf{N}$ & \\
\hline 1 & $9 / 20 / 92$ & 1 & 1 & & 95.1 & $\mathbf{Y}$ & J $910126143-64$ \\
\hline 1 & $9 / 20 / 92$ & 2 & 1 & & 92.8 & $\mathbf{N}$ & \\
\hline 1 & $9 / 20 / 92$ & 3 & 1 & & 85.3 & $\mathbf{N}$ & \\
\hline 1 & $9 / 20 / 92$ & 4 & 1 & & 82.9 & $\mathbf{N}$ & \\
\hline 1 & $9 / 20 / 92$ & 5 & 1 & & 82 & $\mathbf{N}$ & \\
\hline 1 & $9 / 20 / 92$ & 6 & 1 & & 81.6 & $\mathbf{N}$ & \\
\hline 1 & $9 / 20 / 92$ & 7 & 1 & & 81.2 & $\mathbf{N}$ & \\
\hline 1 & $9 / 20 / 92$ & 8 & 1 & & 81.1 & $\mathbf{N}$ & \\
\hline 1 & $9 / 20 / 92$ & 9 & 1 & & 80.3 & $\mathbf{N}$ & \\
\hline 1 & $9 / 20 / 92$ & 10 & 1 & & 79.7 & $\mathbf{N}$ & \\
\hline 1 & $9 / 20 / 92$ & 11 & 1 & & 80 & $\mathbf{N}$ & \\
\hline 1 & $9 / 20 / 92$ & 12 & 1 & & 80 & $\mathbf{N}$ & \\
\hline 1 & $9 / 20 / 92$ & 13 & 1 & & 80.2 & $\mathbf{N}$ & \\
\hline 1 & $9 / 20 / 92$ & 14 & 1 & & 80.5 & $\mathbf{N}$ & \\
\hline 1 & $9 / 26 / 92$ & 1 & 1 & & 94.8 & $\mathbf{Y}$ & $\mathrm{J} 910126143-64$ \\
\hline 1 & $9 / 26 / 92$ & 2 & 1 & & 92.5 & $\mathbf{N}$ & \\
\hline 1 & $9 / 26 / 92$ & 3 & 1 & & 85.2 & $\mathbf{N}$ & \\
\hline 1 & $9 / 26 / 92$ & 4 & 1 & & 82.9 & $\mathbf{N}$ & \\
\hline 1 & $9 / 26 / 92$ & 5 & 1 & & 82.1 & $\mathbf{N}$ & \\
\hline 1 & $9 / 26 / 92$ & 6 & 1 & & 81.7 & $\mathbf{N}$ & \\
\hline 1 & $9 / 26 / 92$ & $\overline{7}$ & 1 & & 81.4 & $\mathbf{N}$ & \\
\hline 1 & $9 / 26 / 92$ & 8 & 1 & & 81.1 & $\mathbf{N}$ & \\
\hline 1 & $9 / 26 / 92$ & 9 & 1 & & 80.3 & $\mathbf{N}$ & \\
\hline 1 & $9 / 26 / 92$ & 10 & 1 & & 79.8 & $\mathbf{N}$ & \\
\hline 1 & $9 / 26 / 92$ & 11 & 1 & & 80 & $\mathbf{N}$ & \\
\hline 1 & $9 / 26 / 92$ & 12 & 1 & & 80.8 & $\mathbf{N}$ & \\
\hline 1 & $9 / 26 / 92$ & 13 & 1 & & 80.1 & $\mathbf{N}$ & \\
\hline 1 & $9 / 26 / 92$ & 14 & 1 & & 80.3 & $\mathbf{N}$ & \\
\hline 1 & $10 / 1 / 92$ & 1 & 1 & & 94.2 & $\mathbf{Y}$ & $\mathrm{J} 890423320-42$ \\
\hline 1 & $10 / 1 / 92$ & 2 & 1 & & 92.4 & $\mathbf{N}$ & \\
\hline 1 & $10 / 1 / 92$ & 3 & 1 & & 84.9 & $\mathbf{N}$ & \\
\hline 1 & $10 / 1 / 92$ & 4 & 1 & & 82.7 & $\mathbf{N}$ & \\
\hline 1 & $10 / 1 / 92$ & 5 & 1 & & 81.9 & $\mathbf{N}$ & \\
\hline 1 & $10 / 1 / 92$ & 6 & 1 & & 81.6 & $\mathbf{N}$ & \\
\hline 1 & $10 / 1 / 92$ & 7 & 1 & & 81.3 & $\mathbf{N}$ & \\
\hline 1 & $10 / 1 / 92$ & 8 & 1 & & 80.9 & $\mathbf{N}$ & \\
\hline 1 & $10 / 1 / 92$ & 9 & 1 & & 80.2 & $\mathbf{N}$ & \\
\hline 1 & $10 / 1 / 92$ & 10 & 1 & & 79.7 & $\mathbf{N}$ & \\
\hline 1 & $10 / 1 / 92$ & 11 & 1 & & 79.8 & $\mathbf{N}$ & \\
\hline 1 & $10 / 1 / 92$ & 12 & 1 & & 79.9 & $\mathbf{N}$ & \\
\hline 1 & $10 / 1 / 92$ & 13 & 1 & & 80 & $\mathbf{N}$ & \\
\hline 1 & $10 / 1 / 92$ & 14 & 1 & & 80.1 & $N$ & \\
\hline 1 & $10 / 10 / 92$ & 1 & 1 & & 95.8 & $\mathbf{Y}$ & $\mathrm{J} 910126143.48$ \\
\hline 1 & $10 / 10 / 92$ & 2 & 1 & & 94 & $\mathbf{N}$ & \\
\hline 1 & $10 / 10 / 92$ & 3 & 1 & & 86.1 & $N$ & \\
\hline 1 & $10 / 10 / 92$ & 4 & 1 & & 83.9 & $\mathbf{N}$ & 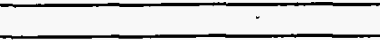 \\
\hline 1 & $10 / 10 / 92$ & 5 & 1 & & 83 & $\mathbf{N}$ & \\
\hline
\end{tabular}

Data obtained from WHC Surveillance Analysis Computer System (SACS), June 2, 1993. 


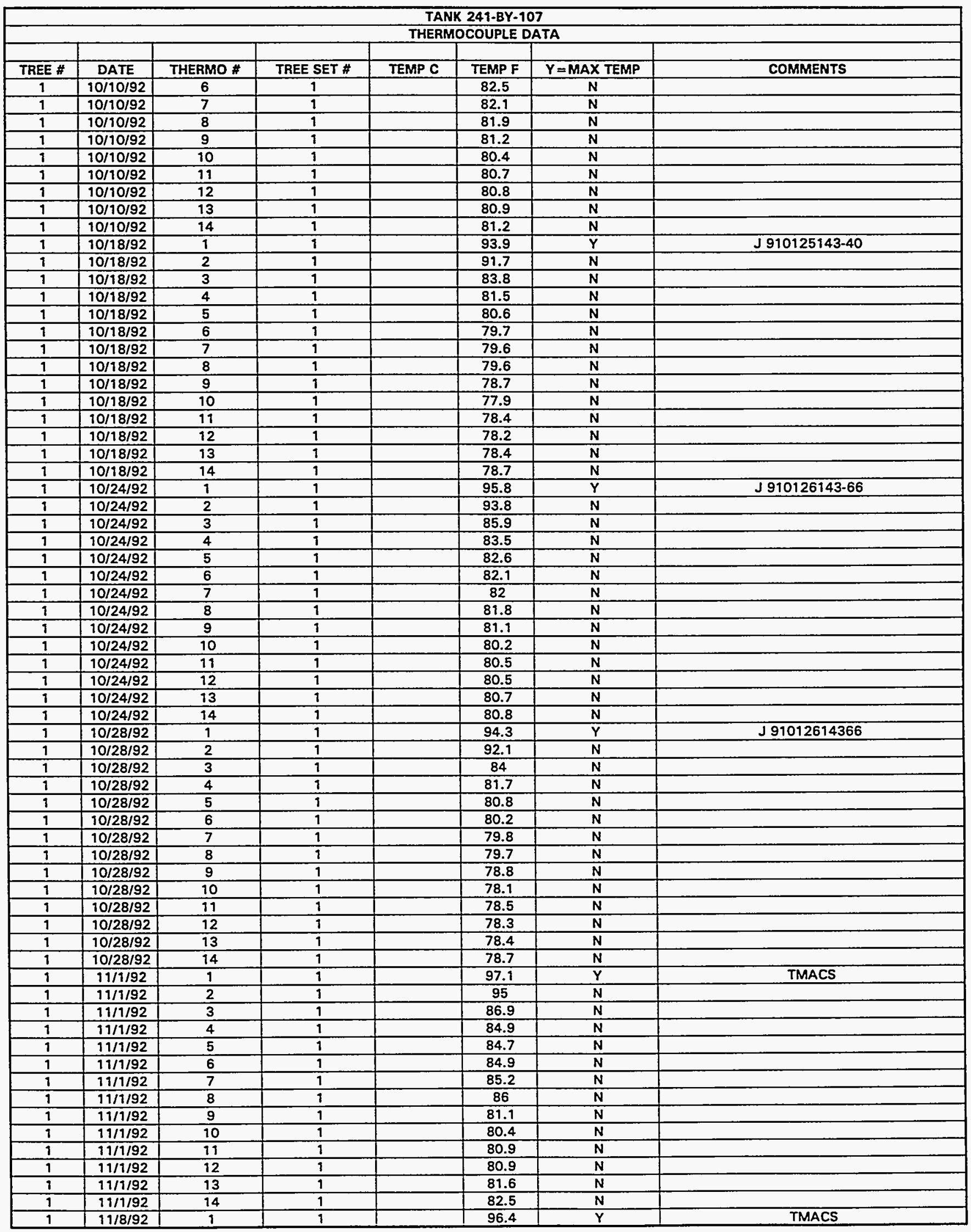

Data obtained from WHC Surveillance Analysis Computer System (SACS), June 2, 1993. 
WHC-SD-WM-ER-312, Rev. 0

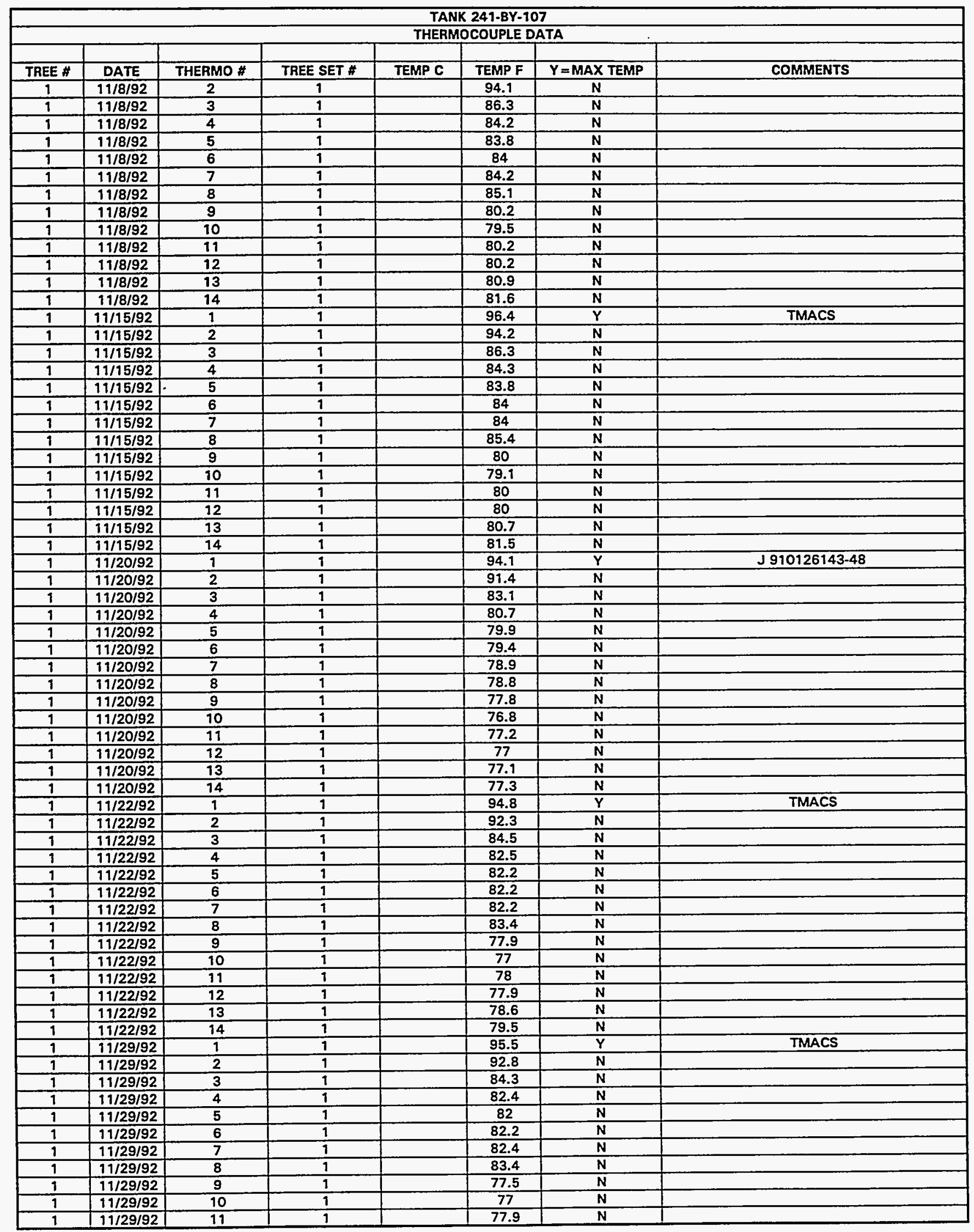

Data obtained from WHC Surveillance Analysis Computer System (SACS), June 2, 1993. 


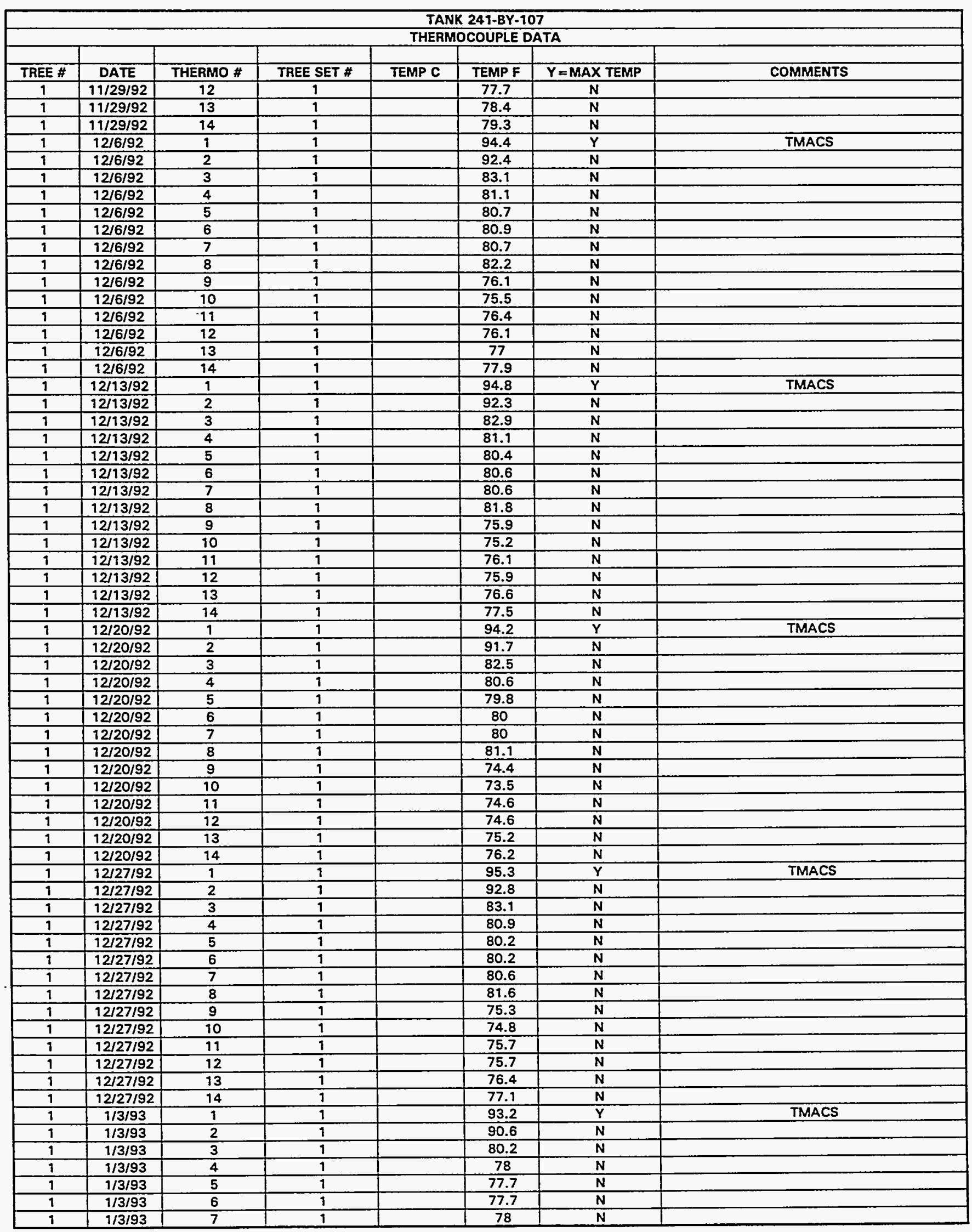

Data obtained from WHC Surveillance Analysis Computer System (SACS), June 2, 1993. 


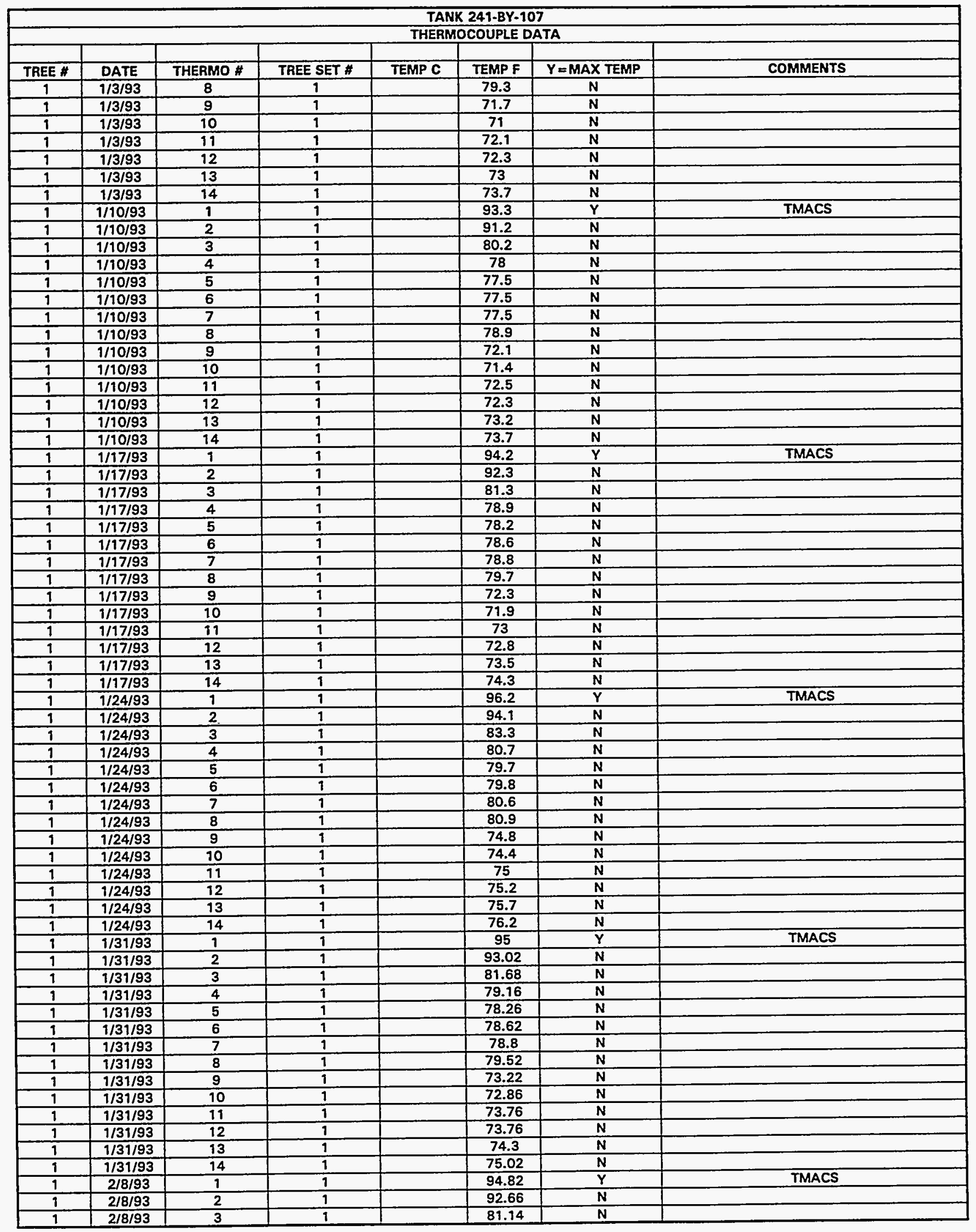

Data obtained from WHC Surveillance Analysis Computer System (SACS), June 2, 1993. 


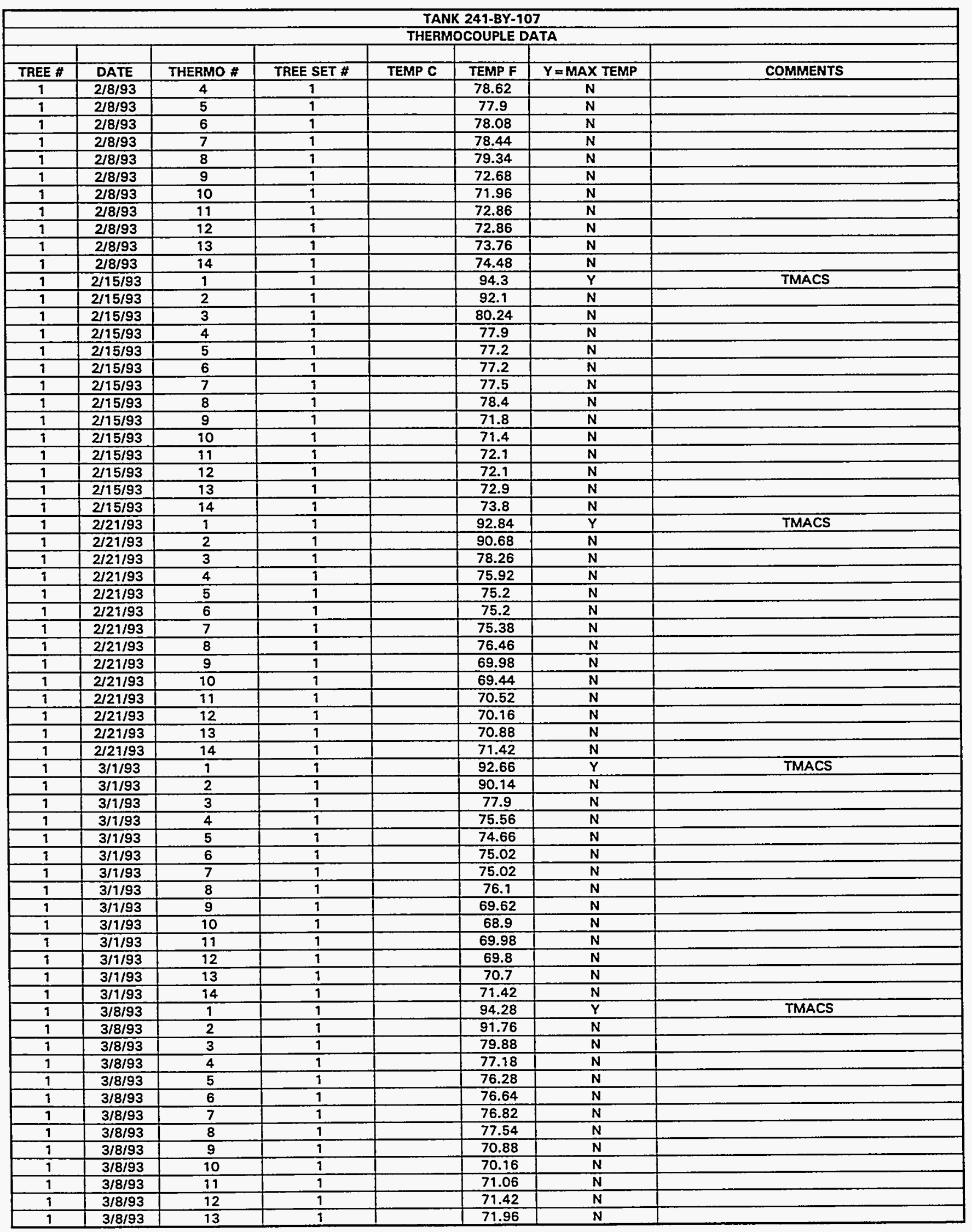

Data obtained from WHC Surveillance Analysis Computer System (SACS), June 2, 1993. 


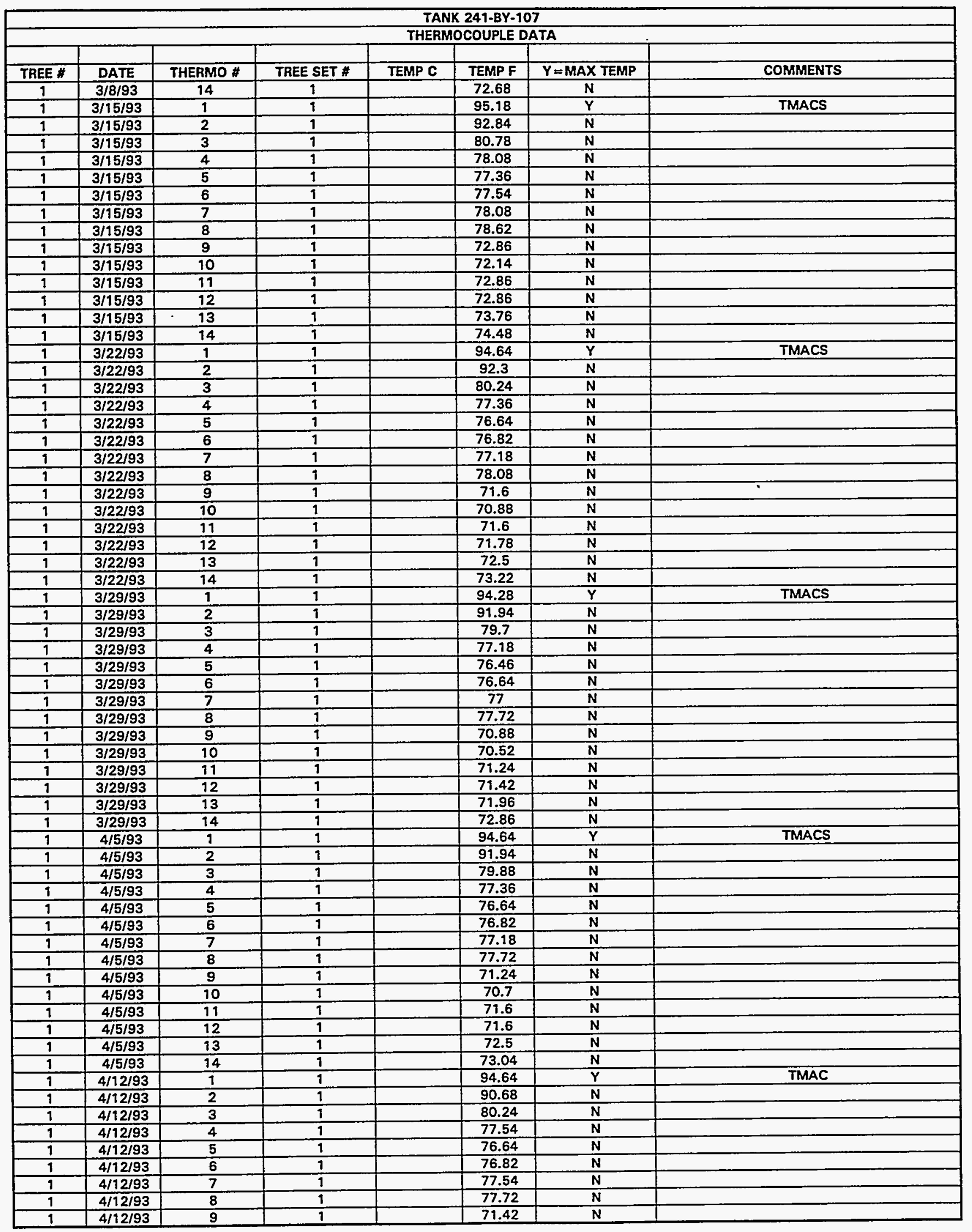

Data obtained from WHC Surveillance Analysis Computer System (SACS), June 2, 1993. 


\begin{tabular}{|c|c|c|c|c|c|c|c|}
\hline \multicolumn{8}{|c|}{ TANK 241-BY-107 } \\
\hline \multicolumn{8}{|c|}{ THERMOCOUPLE DATA } \\
\hline TREE \# & DATE & THERMO \# & TREE SET \# & TEMP C & TEMP F & $Y=M A X$ TEMP & COMMENTS \\
\hline 1 & $4 / 12 / 93$ & 10 & 1 & & 71.06 & $N$ & \\
\hline 1 & $4 / 12 / 93$ & 11 & $\frac{1}{1}$ & & 71.6 & $\bar{N}$ & \\
\hline 1 & $4 / 12 / 93$ & 12 & 1 & & 71.96 & $\mathbf{N}$ & \\
\hline 1 & $4 / 12 / 93$ & 13 & 1 & & 72.32 & $\mathbf{N}$ & \\
\hline 1 & $4 / 12 / 93$ & 14 & 1 & & 73.22 & $\mathbf{N}$ & \\
\hline 1 & $4 / 19 / 93$ & 1 & 1 & & 94.64 & $\mathbf{Y}$ & TMACS \\
\hline 1 & $4 / 19 / 93$ & 2 & 1 & & 91.04 & $\mathbf{N}$ & \\
\hline 1 & $4 / 19 / 93$ & 3 & 1 & & 80.24 & $\mathbf{N}$ & \\
\hline 1 & $4 / 19 / 93$ & 4 & 1 & & 77.9 & $\mathbf{N}$ & \\
\hline 1 & $4 / 19 / 93$ & 5 & 1 & & 76.82 & $\mathbf{N}$ & \\
\hline 9 & $4 / 19 / 93$ & 6 & 9 & & 77 & $\mathbf{N}$ & \\
\hline 1 & $4 / 19 / 93$ & 7 & 1 & & 77.54 & $\mathbf{N}$ & \\
\hline 1 & $4 / 19 / 93$ & 8 & 1 & & 77.9 & $\mathbf{N}$ & \\
\hline 1 & $4 / 19 / 93$ & 9 & 1 & & 71.6 & $\mathbf{N}$ & \\
\hline 1 & $4 / 19 / 93$ & 10 & 1 & & 71.24 & $\mathbf{N}$ & \\
\hline 1 & $4 / 19 / 93$ & 11 & 1 & & 71.96 & $\mathbf{N}$ & \\
\hline 1 & $4 / 19 / 93$ & 12 & $T$ & & 71.96 & $\bar{N}$ & \\
\hline 1 & $4 / 19 / 93$ & 13 & 1 & & 72.68 & $\mathbf{N}$ & \\
\hline 1 & $4 / 19 / 93$ & 14 & 1 & & 73.4 & $\mathbf{N}$ & \\
\hline 1 & $4 / 25 / 93$ & 1 & 1 & & 94.82 & $\mathbf{Y}$ & TMACS \\
\hline 1 & $4 / 25 / 93$ & 2 & 1 & & 91.94 & $\mathbf{N}$ & \\
\hline 1 & $4 / 25 / 93$ & 3 & 1 & & 80.6 & $\mathbf{N}$ & \\
\hline 1 & $4 / 25 / 93$ & 4 & 1 & & 78.08 & $\mathbf{N}$ & \\
\hline 1 & $4 / 25 / 93$ & 5 & 1 & & 77.18 & $\mathbf{N}$ & \\
\hline 1 & $4 / 25 / 93$ & 6 & 1 & & 77.36 & $\mathbf{N}$ & \\
\hline 1 & $4 / 25 / 93$ & 7 & 1 & & 77.9 & $\mathbf{N}$ & \\
\hline 1 & $4 / 25 / 93$ & 8 & 1 & & 78.62 & $\mathbf{N}$ & \\
\hline 1 & $4 / 25 / 93$ & 9 & 1 & & 72.14 & $\mathbf{N}$ & \\
\hline 1 & $4 / 25 / 93$ & 10 & 1 & & 71.6 & $\mathbf{N}$ & \\
\hline 1 & $4 / 25 / 93$ & 11 & 1 & & 72.14 & $\mathbf{N}$ & \\
\hline 1 & $4 / 25 / 93$ & 12 & 1 & & 72.5 & $\mathbf{N}$ & \\
\hline 1 & $4 / 25 / 93$ & 13 & 1 & & 73.22 & $\mathbf{N}$ & \\
\hline 1 & $4 / 25 / 93$ & 14 & 1 & & 73.76 & $\mathbf{N}$ & \\
\hline 1 & $5 / 3 / 93$ & 1 & 1 & & 94.28 & $\bar{Y}$ & TMACS \\
\hline 1 & $5 / 3 / 93$ & 2 & 1 & & 91.76 & $\mathbf{N}$ & \\
\hline 1 & $5 / 3 / 93$ & 3 & 1 & & 80.06 & $\mathbf{N}$ & \\
\hline 1 & $5 / 3 / 93$ & 4 & 1 & & 77.54 & $\mathbf{N}$ & \\
\hline 1 & $5 / 3 / 93$ & 5 & 1 & & 77 & $\mathbf{N}$ & \\
\hline 1 & $5 / 3 / 93$ & 6 & 1 & & 77.18 & $\mathbf{N}$ & \\
\hline 1 & $5 / 3 / 93$ & 7 & 1 & & 77.54 & $\mathbf{N}$ & \\
\hline 1 & $5 / 3 / 93$ & 8 & 1 & & 78.08 & $\mathbf{N}$ & \\
\hline 1 & $5 / 3 / 93$ & 9 & 1 & & 71.96 & $N$ & \\
\hline 1 & $5 / 3 / 93$ & 10 & 1 & & 71.6 & $\mathbf{N}$ & \\
\hline 1 & $5 / 3 / 93$ & 11 & 1 & & 72.32 & $\mathbf{N}$ & \\
\hline 1 & $5 / 3 / 93$ & 12 & 1 & & 72.5 & $\mathbf{N}$ & \\
\hline 1 & $5 / 3 / 93$ & 13 & 1 & & 73.04 & $\mathbf{N}$ & \\
\hline$\frac{1}{1}$ & $5 / 3 / 93$ & 94 & 1 & & 73.76 & $\mathrm{~N}$ & \\
\hline 1 & $5 / 10 / 93$ & 1 & 1 & & 94.82 & $Y$ & TMACS \\
\hline 1 & $5 / 10 / 93$ & 2 & 1 & & 92.3 & $N$ & \\
\hline 7 & $5 / 10 / 93$ & 3 & 9 & & 81.14 & $N$ & \\
\hline 1 & $5 / 10 / 93$ & 4 & 1 & & 78.26 & $\mathbf{N}$ & \\
\hline 1 & $5 / 10 / 93$ & 5 & 1 & & 77.54 & $N$ & \\
\hline 9 & $5 / 10 / 93$ & 6 & 1 & & 77.9 & $\mathbf{N}$ & \\
\hline 1 & $5 / 10 / 93$ & 7 & 1 & & 78.44 & $\mathbf{N}$ & \\
\hline 1 & $5 / 10 / 93$ & 8 & 1 & & 78.98 & $\mathbf{N}$ & \\
\hline 9 & $5 / 10 / 93$ & 9 & $\frac{1}{1}$ & & 72.68 & $N$ & \\
\hline 1 & $5 / 10 / 93$ & 10 & 1 & & 72.14 & $\mathbf{N}$ & \\
\hline 1 & $5 / 10 / 93$ & 11 & 1 & & 72.86 & $\mathbf{N}$ & \\
\hline 1 & $5 / 10 / 93$ & 12 & 1 & & 73.04 & $N$ & \\
\hline 1 & $5 / 70 / 93$ & 13 & 1 & & 73.76 & $\mathrm{~N}$ & \\
\hline 1 & $5 / 10 / 93$ & 14 & 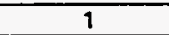 & & 74.3 & $\mathbf{N}$ & \\
\hline 1 & $5 / 17 / 93$ & 1 & 1 & & 95.54 & $\mathbf{Y}$ & TMACS \\
\hline 1 & $5 / 17 / 93$ & 2 & 1 & & 93.2 & $\mathbf{N}$ & \\
\hline 1 & $5 / 17 / 93$ & 3 & 1 & & 81.68 & $\bar{N}$ & \\
\hline 1 & $5 / 17 / 93$ & 4 & 1 & & 79.34 & $\mathbf{N}$ & \\
\hline 1 & $5 / 17 / 93$ & 5 & 1 & & 78.62 & $\mathbf{N}$ & \\
\hline
\end{tabular}

Data obtained from WHC Surveillance Analysis Computer System (SACS), June 2, 1993. 
WHC-SD-WM-ER-312, Rev. 0

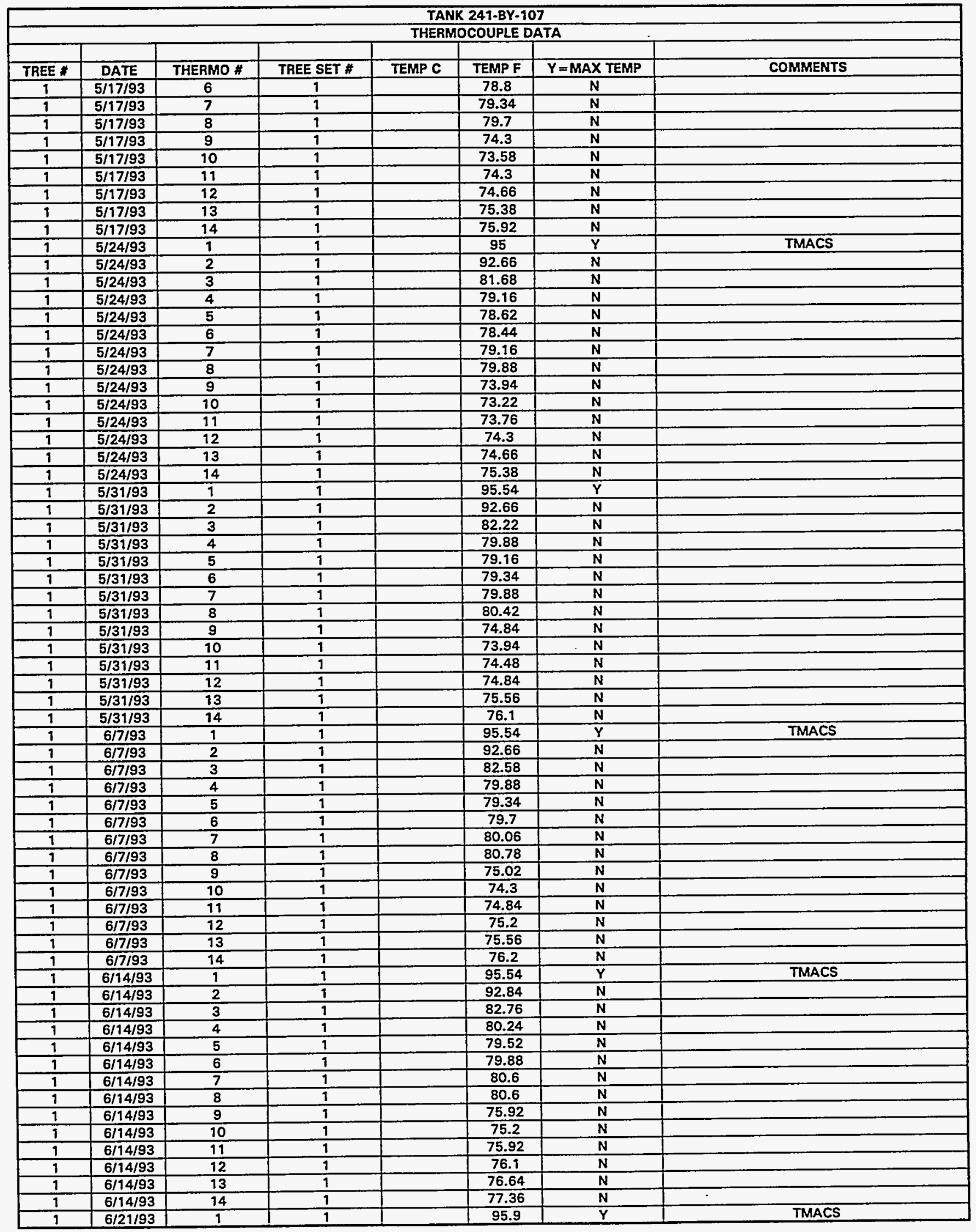

Data obtained from WHC Surveillance Analysis Computer System (SACS), June 2, 1993. 
WHC-SD-WM-ER-312, Rev. 0

\begin{tabular}{|c|c|c|c|c|c|c|c|}
\hline \multicolumn{8}{|c|}{ TANK 241-BY-107 } \\
\hline \multicolumn{8}{|c|}{ THERMOCOUPLE DATA } \\
\hline TREE \# & DATE & THERMO \# & TREE SET \# & TEMP C & TEMP F & $Y=$ MAX TEMP & COMMENTS \\
\hline 1 & $6 / 21 / 93$ & 2 & 1 & & 93.74 & $N$ & \\
\hline 1 & $6 / 21 / 93$ & 3 & 1 & & 83.66 & $\mathbf{N}$ & \\
\hline 1 & $6 / 21 / 93$ & 4 & 1 & & 81.32 & $\mathrm{~N}$ & \\
\hline 1 & $6 / 21 / 93$ & 5 & 1 & & 80.96 & $\mathbf{N}$ & \\
\hline 1 & $6 / 21 / 93$ & 6 & 1 & & 80.96 & $\mathbf{N}$ & \\
\hline 1 & $6 / 21 / 93$ & 7 & 1 & & 81.5 & $\mathbf{N}$ & \\
\hline 1 & $6 / 21 / 93$ & 8 & 1 & & 82.22 & $\mathbf{N}$ & \\
\hline 1 & $6 / 21 / 93$ & 9 & 1 & & 76.64 & $\mathbf{N}$ & \\
\hline 1 & $6 / 21 / 93$ & 10 & 1 & & 75.92 & $N$ & \\
\hline 1 & $6 / 21 / 93$ & 11 & 1 & & 76.46 & $\mathbf{N}$ & \\
\hline 9 & $6 / 21 / 93$ & 12 & 1 & & 76.82 & $\mathbf{N}$ & \\
\hline 1 & $6 / 21 / 93$ & 13 & 1 & & 77.36 & $\mathbf{N}$ & \\
\hline 1 & $6 / 21 / 93$ & 14 & 1 & & 78.08 & $\bar{N}$ & \\
\hline 1 & $6 / 28 / 93$ & 1 & 1 & & 94.82 & $Y$ & TMACS \\
\hline 1 & $6 / 28 / 93$ & 2 & 1 & & 92.3 & $\mathrm{~N}$ & \\
\hline 1 & $6 / 28 / 93$ & 3 & 1 & & 83.12 & $\bar{N}$ & \\
\hline 1 & $6 / 28 / 93$ & 4 & 1 & & 80.42 & $\mathbf{N}$ & \\
\hline 1 & $6 / 28 / 93$ & 5 & 1 & & 79.88 & $\mathbf{N}$ & \\
\hline 1 & $6 / 28 / 93$ & 6 & 1 & & 80.06 & $N$ & \\
\hline 1 & $6 / 28 / 93$ & 7 & 1 & & 80.78 & $\mathrm{~N}$ & \\
\hline 1 & $6 / 28 / 93$ & 8 & 1 & & 81.14 & $\mathbf{N}$ & \\
\hline 1 & $6 / 28 / 93$ & 9 & 1 & & 75.92 & $\mathbf{N}$ & \\
\hline 1 & $6 / 28 / 93$ & 10 & 1 & & 75.02 & $\mathbf{N}$ & \\
\hline 1 & $6 / 28 / 93$ & 11 & 1 & & 75.74 & $\mathbf{N}$ & \\
\hline 1 & $6 / 28 / 93$ & 12 & 1 & & 75.74 & $\mathbf{N}$ & \\
\hline 1 & $6 / 28 / 93$ & 13 & 1 & & 76.64 & $\mathrm{~N}$ & \\
\hline 1 & $6 / 28 / 93$ & 14 & 1 & & 77.36 & $N$ & \\
\hline 1 & $7 / 5 / 93$ & 1 & 1 & & 95 & $\bar{Y}$ & TMACS \\
\hline 1 & $7 / 5 / 93$ & 2 & 1 & & 92.48 & $\mathrm{~N}$ & \\
\hline 1 & $7 / 5 / 93$ & 3 & 1 & & 83.12 & $\mathbf{N}$ & \\
\hline 1 & $7 / 5 / 93$ & 4 & 1 & & 80.78 & $\bar{N}$ & \\
\hline 1 & $7 / 5 / 93$ & 5 & 1 & & 80.24 & $\mathrm{~N}$ & \\
\hline 1 & $7 / 5 / 93$ & 6 & 1 & & 80.42 & $\mathbf{N}$ & \\
\hline 1 & $7 / 5 / 93$ & 7 & 1 & & 80.96 & $\mathrm{~N}$ & \\
\hline 1 & $7 / 5 / 93$ & 8 & 1 & & 81.86 & $N$ & \\
\hline 1 & $7 / 5 / 93$ & 9 & 1 & & 77 & $\mathbf{N}$ & \\
\hline 1 & $7 / 5 / 93$ & 10 & 1 & & 75.92 & $\mathrm{~N}$ & \\
\hline 1 & $7 / 5 / 93$ & 11 & 1 & & 76.64 & $\mathrm{~N}$ & \\
\hline 1 & $7 / 5 / 93$ & 12 & 1 & & 76.82 & $\mathbf{N}$ & \\
\hline 1 & $7 / 5 / 93$ & 13 & 1 & & 77.54 & $\bar{N}$ & \\
\hline 1 & $7 / 5 / 93$ & 14 & 1 & & 78.26 & $\mathbf{N}$ & \\
\hline 1 & $7 / 12 / 93$ & 1 & 1 & & 94.64 & $Y$ & TMACS \\
\hline 1 & $7 / 12 / 93$ & 2 & 1 & & 92.12 & $\mathbf{N}$ & \\
\hline 1 & $7 / 12 / 93$ & 3 & 1 & & 83.12 & $\mathbf{N}$ & \\
\hline 1 & $7 / 12 / 93$ & 4 & 1 & & 80.78 & $\mathbf{N}$ & \\
\hline 1 & $7 / 12 / 93$ & 5 & 1 & & 80.42 & $\mathbf{N}$ & \\
\hline 1 & $7 / 12 / 93$ & 6 & 1 & & 80.78 & $\mathbf{N}$ & \\
\hline 1 & $7 / 12 / 93$ & 7 & 1 & & 80.96 & $\mathbf{N}$ & \\
\hline 1 & $7 / 12 / 93$ & 8 & 1 & & 81.68 & $\mathbf{N}$ & \\
\hline 1 & $7 / 12 / 93$ & 9 & 1 & & 77.18 & $\mathbf{N}$ & \\
\hline 1 & $7 / 12 / 93$ & 10 & 1 & & 76.28 & $\mathbf{N}$ & \\
\hline 1 & $7 / 12 / 93$ & 11 & 1 & & 76.82 & $\mathbf{N}$ & \\
\hline 1 & $7 / 12 / 93$ & 12 & 1 & & 76.82 & $\mathbf{N}$ & \\
\hline 1 & $7 / 12 / 93$ & 13 & 1 & & 77.72 & $\mathbf{N}$ & \\
\hline 1 & $7 / 12 / 93$ & 14 & 1 & & 78.44 & $\mathbf{N}$ & \\
\hline 1 & $7 / 19 / 93$ & 1 & 1 & & 95 & $Y$ & TMACS \\
\hline 1 & $7 / 19 / 93$ & 2 & 1 & & 92.66 & $\mathbf{N}$ & \\
\hline 1 & $7 / 19 / 93$ & 3 & 1 & & 83.84 & $\mathbf{N}$ & \\
\hline 1 & $7 / 19 / 93$ & 4 & 1 & & 81.5 & $\mathbf{N}$ & \\
\hline 1 & $7 / 19 / 93$ & 5 & 1 & & 81.14 & $\mathbf{N}$ & \\
\hline 1 & $7 / 19 / 93$ & 6 & 1 & & 81.14 & $\mathrm{~N}$ & \\
\hline 1 & $7 / 19 / 93$ & 7 & 1 & & 81.68 & $\mathrm{~N}$ & \\
\hline 1 & $7 / 19 / 93$ & 8 & 1 & & 82.58 & $\mathbf{N}$ & \\
\hline 1 & $7 / 19 / 93$ & 9 & 1 & & 77.36 & $\mathbf{N}$ & \\
\hline 1 & $7 / 19 / 93$ & 10 & 1 & & 76.46 & $\mathrm{~N}$ & \\
\hline 1 & $7 / 19 / 93$ & 11 & 1 & & 77 & $\mathrm{~N}$ & \\
\hline
\end{tabular}

Data obtained from WHC Surveillance Analysis Computer System (SACS), June 2, 1993. 


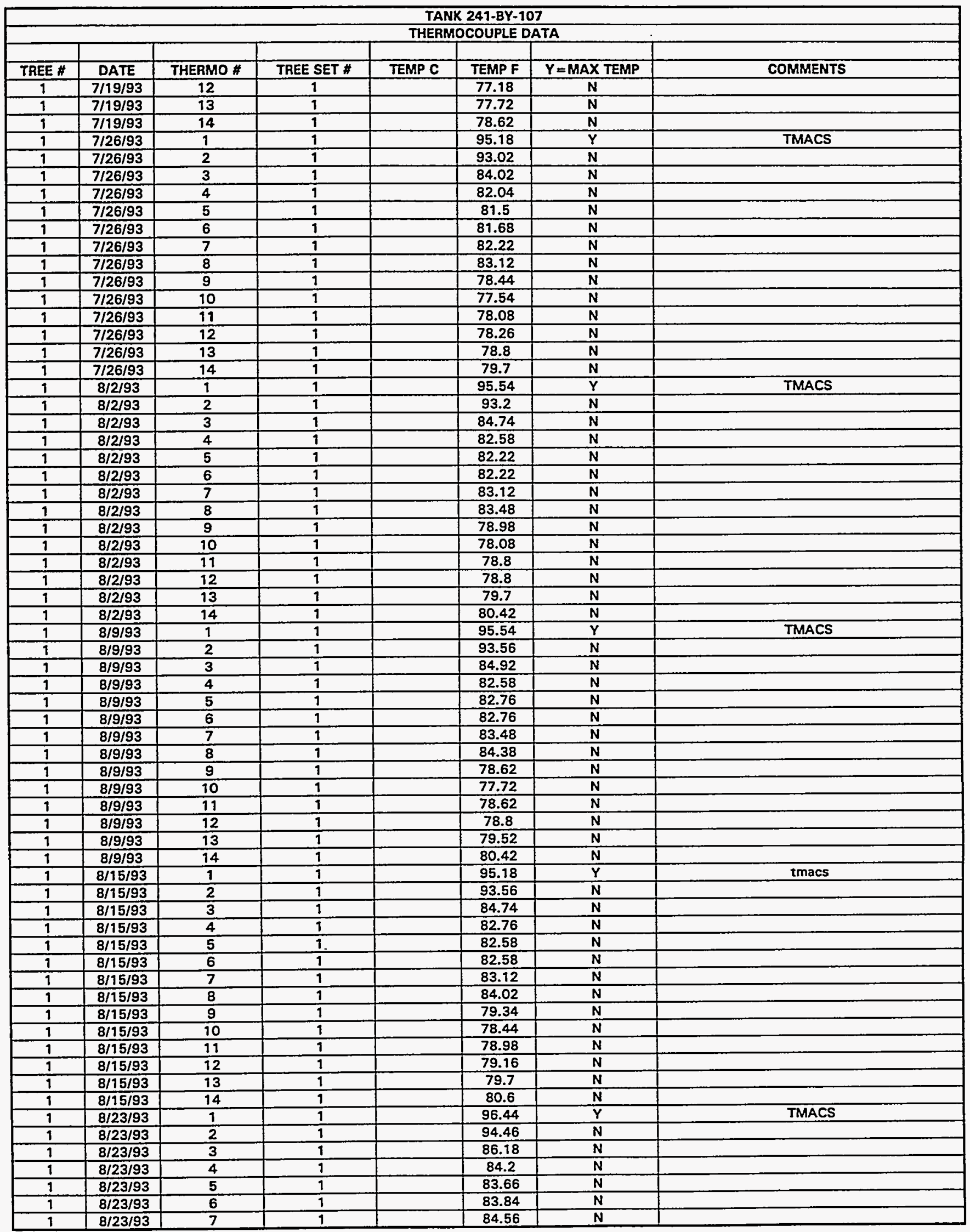

Data obtained from WHC Surveillance Analysis Computer System (SACS), June 2, 1993. 


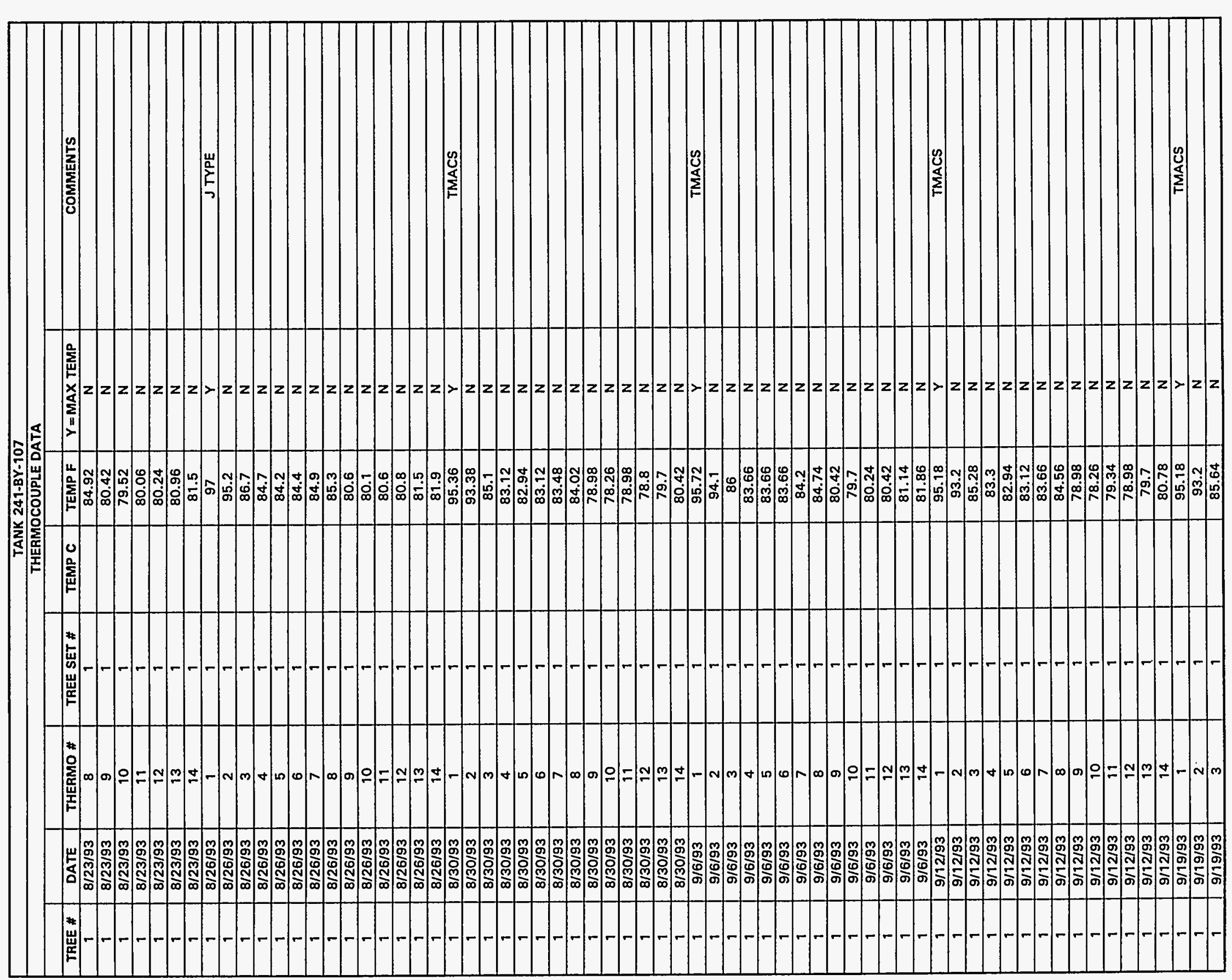

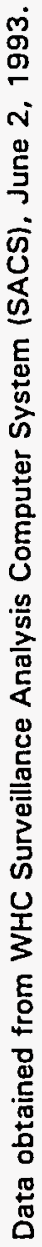


WHC-SD-WM-ER-312, Rev. 0

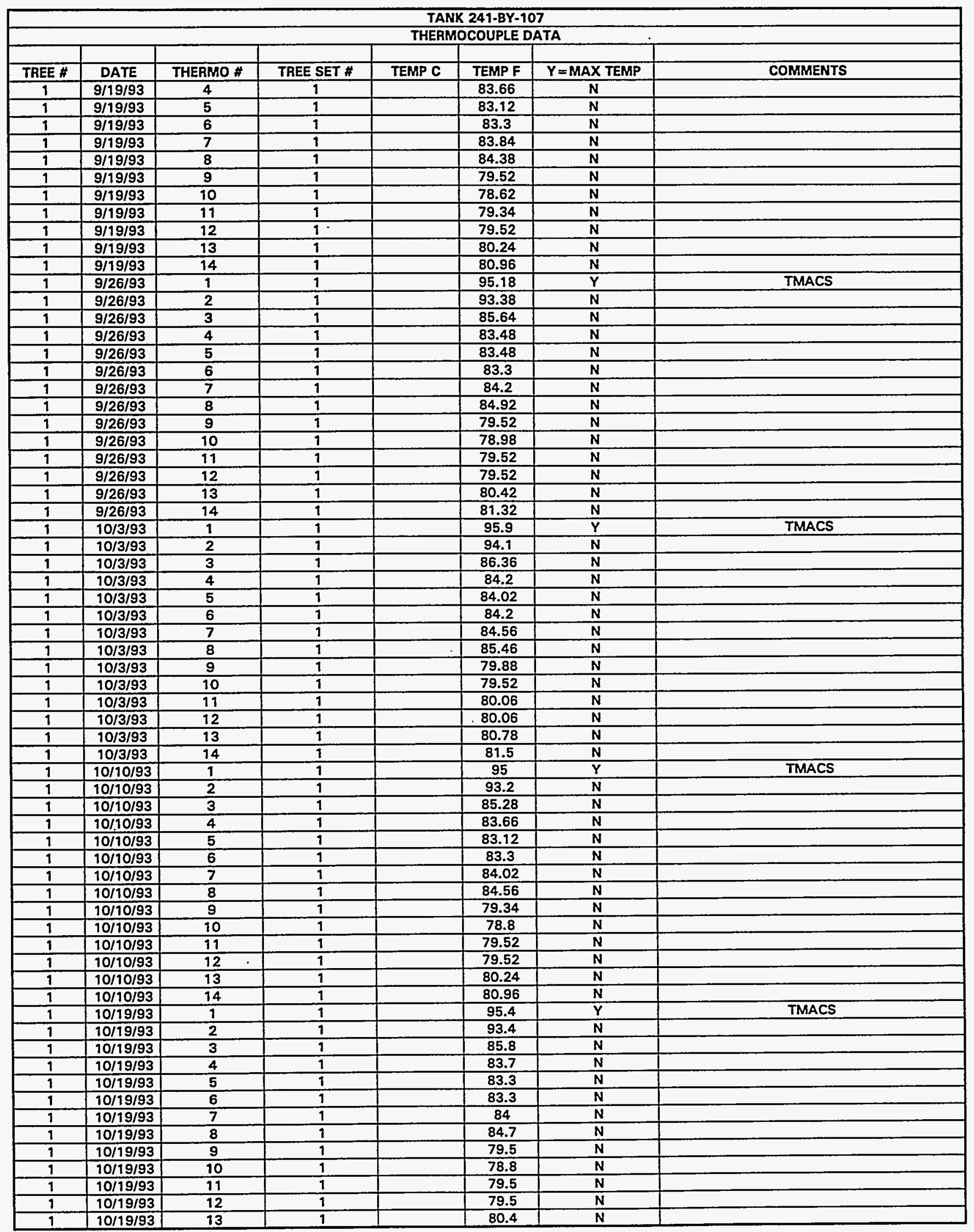

Data obtained from WHC Surveillance Analysis Computer System (SACS), June 2, 1993. 


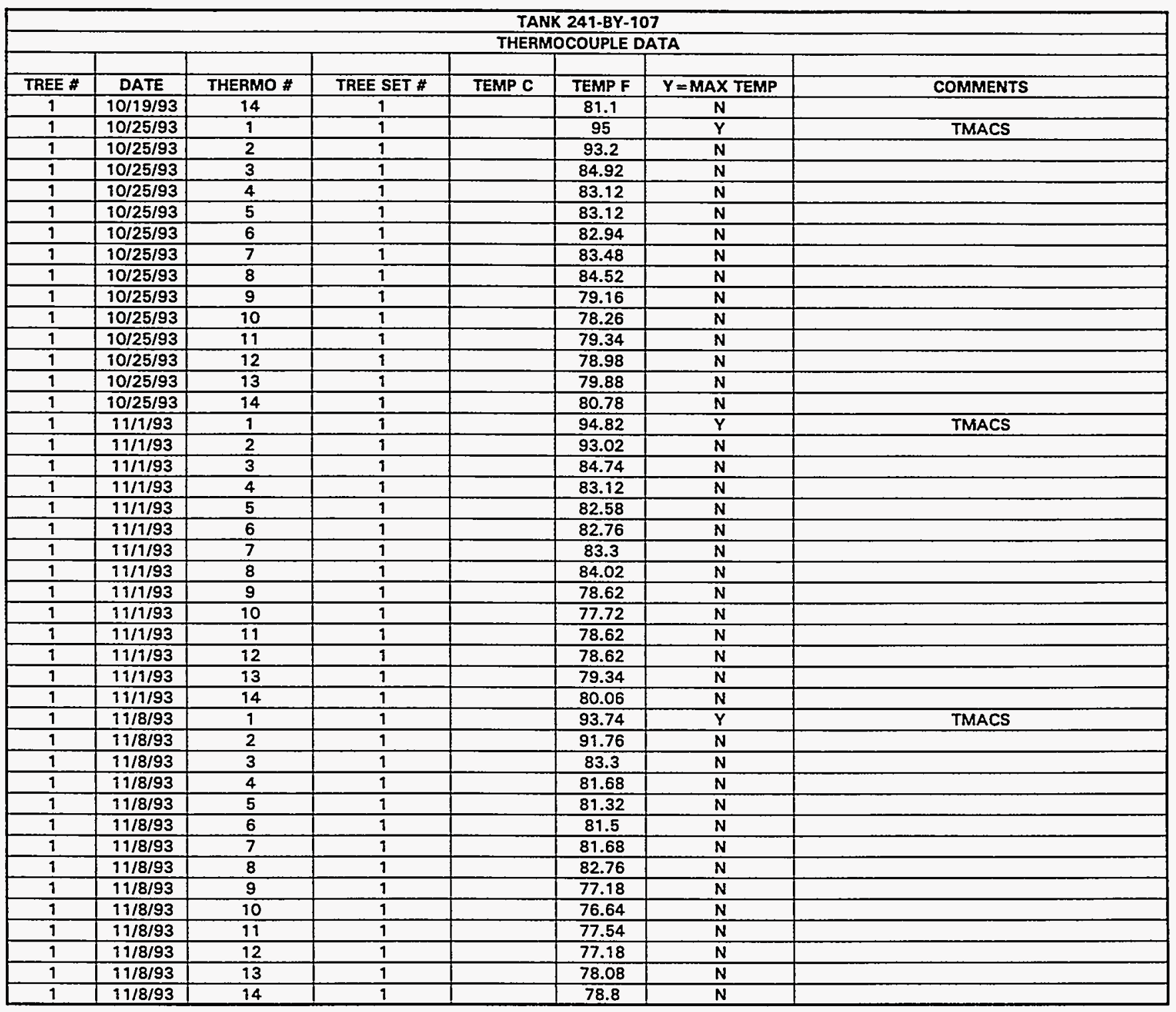

Data obtained from WHC Surveillance Analysis Computer System (SACS), June 2, 1993. 
WHC-SD-WM-ER-312, Rev. 0

\begin{tabular}{|c|c|c|c|c|c|c|c|}
\hline \multicolumn{8}{|c|}{ TANK 241-BY-108 } \\
\hline \multicolumn{8}{|c|}{ THERMOCOUPLE DATA } \\
\hline TREE \# & DATE & THERMO \# & TREE SET \# & TEMP C & TEMP F & $Y=$ MAX TEMP & COMMENTS \\
\hline 1 & $9 / 12 / 74$ & 1 & 1 & 60 & 140 & $Y$ & degree C/TWINS confirm \\
\hline 1 & $9 / 12 / 74$ & 2 & 1 & 54 & 129.2 & $\mathbf{N}$ & degree C/TWINS confirm \\
\hline 1 & $9 / 12 / 74$ & 3 & $\overline{1}$ & 48 & 118.4 & $\mathbf{N}$ & degree C/TWINS confirm \\
\hline 1 & $9 / 12 / 74$ & 4 & 1 & 40 & 104 & $\bar{N}$ & degree C/TWINS confirm \\
\hline$T$ & $9 / 12 / 74$ & 5 & 1 & 36 & 96.8 & $\mathbf{N}$ & degree C/TWINS confirm \\
\hline 1 & $9 / 12 / 74$ & 6 & 1 & 36 & 96.8 & $\mathbf{N}$ & degree C/TWINS confirm \\
\hline 1 & $9 / 12 / 74$ & 7 & 1 & 36 & 96.8 & $\mathbf{N}$ & degree C/TWINS confirm \\
\hline 1 & $9 / 12 / 74$ & 8 & $\overline{1}$ & 36 & 96.8 & $\mathbf{N}$ & degree C/TWINS confirm \\
\hline$\overline{1}$ & $9 / 12 / 74$ & 9 & $T$ & 36 & 96.8 & $\overline{\mathbf{N}}$ & degree C/TWINS confirm \\
\hline 1 & $9 / 12 / 74$ & 10 & 1 & 36 & 96.8 & $N$ & degree C/TWINS confirm \\
\hline 1 & $9 / 12 / 74$ & 11 & 1 & 36 & 96.8 & $\mathbf{N}$ & degree C/TWINS confirm \\
\hline 1 & $9 / 12 / 74$ & 12 & 1 & 36 & 96.8 & $\mathbf{N}$ & degree C/TWINS confirm \\
\hline 1 & $10 / 8 / 74$ & 1 & 1 & 60 & 140 & $\mathbf{Y}$ & degree $\mathrm{C} / \mathrm{TW}$ INS confirm \\
\hline 1 & $10 / 8 / 74$ & 2 & 1 & 54 & 129.2 & $\mathbf{N}$ & degree C/TWINS confirm \\
\hline 1 & $10 / 8 / 74$ & 3 & 1 & 47 & 116.6 & $\mathbf{N}$ & degree C/TWINS confirm \\
\hline 1 & $10 / 8 / 74$ & 4 & 1 & 42 & 107.6 & $\mathbf{N}$ & degree C/TWINS confirm \\
\hline$\frac{1}{1}$ & $10 / 8 / 74$ & 5 & 1 & 36 & 96.8 & $\mathbf{N}$ & degree C/TWINS confirm \\
\hline 1 & $10 / 8 / 74$ & 6 & 1 & 36 & 96.8 & $\bar{N}$ & degree C/TWINS confirm \\
\hline 1 & $10 / 8 / 74$ & 7 & 1 & 36 & 96.8 & $\mathbf{N}$ & degrae C/TWINS confirm \\
\hline 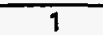 & $10 / 8 / 74$ & 8 & 1 & 36 & 96.8 & $\mathbf{N}$ & degree C/TWINS confirm \\
\hline$T$ & $10 / 8 / 74$ & 9 & 1 & 36 & 96.8 & $\mathbf{N}$ & degree C/TWiNS confirm \\
\hline 1 & $10 / 8 / 74$ & 10 & 1 & 36 & 96.8 & $\mathbf{N}$ & degree C/TWINS confirm \\
\hline 1 & $10 / 8 / 74$ & 11 & 1 & 36 & 96.8 & $\mathbf{N}$ & degree C/TWINS confirm \\
\hline$\frac{1}{1}$ & $10 / 8 / 74$ & 12 & $T$ & 36 & 96.8 & $\mathbf{N}$ & degree C/TWINS confirm \\
\hline 1 & $11 / 12 / 74$ & 1 & 1 & 58 & 136.4 & $\mathbf{Y}$ & degree C/TWINS confirm \\
\hline 1 & $11 / 12 / 74$ & 2 & 1 & 51 & 123.8 & $\mathbf{N}$ & degree C/TWINS confirm \\
\hline$\overline{1}$ & $11 / 12 / 74$ & 3 & $\frac{1}{1}$ & 44 & 111.2 & $\mathbf{N}$ & degree C/TWINS confirm \\
\hline 1 & $11 / 12 / 74$ & 4 & 1 & 37 & 98.6 & $\mathbf{N}$ & degree C/TWINS confirm \\
\hline 1 & $11 / 12 / 74$ & 5 & 1 & 32 & 89.6 & $\mathrm{~N}$ & degree C/TWINS confitm \\
\hline 1 & $11 / 12 / 74$ & 6 & 1 & 30 & 86 & $\mathbf{N}$ & degree C/TWINS confirm \\
\hline 1 & $11 / 12 / 74$ & 7 & 1 & 30 & 86 & $\mathbf{N}$ & degree C/TWINS confirm \\
\hline 1 & $11 / 12 / 74$ & 8 & 1 & 30 & 86 & $\mathbf{N}$ & degree CITWINS confirm \\
\hline 1 & $11 / 12 / 74$ & 9 & 1 & 30 & 86 & $\mathbf{N}$ & degreo C/TWiNS confirm \\
\hline 1 & $11 / 12 / 74$ & 10 & 1 & 30 & 86 & $\mathbf{N}$ & degree C/TWINS confirm \\
\hline 1 & $11 / 12 / 74$ & 11 & 1 & 30 & 86 & $\mathbf{N}$ & degres C/TWiNS confirm \\
\hline 7 & $11 / 12 / 74$ & 12 & 1 & 30 & 86 & $\mathbf{N}$ & degree CTTWINS confirm \\
\hline 1 & $12 / 17 / 74$ & 1 & 1 & 60 & 140 & $\bar{Y}$ & degree C/TWINS confirm \\
\hline 1 & $12 / 17 / 74$ & 2 & 1 & 55.56 & 132.008 & $\mathbf{N}$ & degree C/TWINS confirm \\
\hline 1 & $12 / 17 / 74$ & 3 & 1 & 47.22 & 116.996 & $\mathbf{N}$ & degree C/TWINS confirm \\
\hline 9 & $12 / 17 / 74$ & 4 & 1 & 40 & 104 & $\mathbf{N}$ & degree C/TWINS confirm \\
\hline 1 & $12 / 17 / 74$ & 5 & 1 & 33.33 & 91.994 & $\mathbf{N}$ & degree C/TWINS confirm \\
\hline 1 & $12 / 17 / 74$ & $\overline{8}$ & 1 & 32.22 & 89.996 & $\mathbf{N}$ & degree C/TWINS confirm \\
\hline 1 & $12 / 17 / 74$ & 9 & 1 & 33.33 & 91.994 & $\mathbf{N}$ & degree CTWINS confirm \\
\hline 1 & $12 / 17 / 74$ & 10 & 1 & 33.33 & 91.994 & $\mathbf{N}$ & degree CITWINS confirm \\
\hline 1 & $12 / 17 / 74$ & 11 & 1 & 33.33 & 91.994 & $\ddot{N}$ & degrae CrTWINS confirm \\
\hline 1 & $12 / 17 / 74$ & 12 & 1 & 33.33 & 91.994 & $\mathbf{N}$ & degree C/TWINS confirm \\
\hline 1 & $12 / 31 / 74$ & 1 & 1 & 55 & 131 & $\mathbf{Y}$ & degree C/TWINS confirm \\
\hline 1 & $12 / 31 / 74$ & 2 & $\frac{1}{1}$ & 47.78 & 118.004 & $\mathbf{N}$ & degree C/TWINS confirm \\
\hline 9 & $12 / 31 / 74$ & $\overline{3}$ & 1 & 41.11 & 105.998 & $\mathbf{N}$ & degree CTTWiNS confirm \\
\hline 1 & $12 / 31 / 74$ & 4 & 1 & 33.89 & 93.002 & $\mathbf{N}$ & degree C/TWINS confirm \\
\hline$\frac{1}{1}$ & $12 / 31 / 74$ & 5 & 1 & 32.78 & 91.004 & $\bar{N}$ & degree C/TWINS confirm \\
\hline 1 & $12 / 31 / 74$ & 6 & 1 & 32.78 & 91.004 & $\mathbf{N}$ & degree CTTWINS confirm \\
\hline 1 & $12 / 31 / 74$ & 7 & 1 & 32.78 & 91.004 & $\mathbf{N}$ & degree C/TWINS confirm \\
\hline 1 & $12 / 31 / 74$ & 8 & 1 & 32.78 & 91.004 & $\mathbf{N}$ & degree C/TWINS confirm \\
\hline$\frac{1}{1}$ & $12 / 31 / 74$ & 9 & 1 & 32.78 & 91.004 & $\mathbf{N}$ & degree C/TWINS confirm \\
\hline 1 & $1 / 8 / 75$ & 1 & 1 & 53.33 & 127.994 & $\bar{Y}$ & degree C/TWINS confirm \\
\hline 1 & $1 / 8 / 75$ & 2 & 9 & 45.56 & 114.008 & $\bar{N}$ & degree C/TWINS confirm \\
\hline 1 & $1 / 8 / 75$ & 3 & 1 & 38.33 & 100.994 & $\mathbf{N}$ & degree C/TWINS confirm \\
\hline$T$ & $9 / 8 / 75$ & 4 & 1 & 31.67 & 89.006 & $N$ & degree C/TWINS confirm \\
\hline 1 & $1 / 8 / 75$ & 5 & 1 & 31.11 & 87.998 & $\mathbf{N}$ & degree C/TWINS confirm \\
\hline 1 & $1 / 8 / 75$ & 6 & 1 & 31.11 & 87.998 & $\mathbf{N}$ & degree C/TWINS confirm \\
\hline 1 & $1 / 8 / 75$ & 7 & $\overline{1}$ & 31.11 & 87.998 & $\mathbf{N}$ & degree C/TWINS confirm \\
\hline 1 & $1 / 8 / 75$ & 8 & 1 & 31.11 & 87.998 & $\mathbf{N}$ & degree C/TWINS confirm \\
\hline 1 & $1 / 8 / 75$ & 9 & 1 & 37.11 & 87.998 & $N$ & degree C/TWINS confirm \\
\hline 1 & $1 / 28 / 75$ & 1 & 1 & 56 & 132.8 & $\bar{Y}$ & degree $\mathrm{C} / \mathrm{TW}$ INS confirm \\
\hline 9 & $1 / 28 / 75$ & 2 & 1 & 49 & 120.2 & $\mathbf{N}$ & Idegree C/TWINS confirm \\
\hline
\end{tabular}

Data obtained from WHC Surveillance Analysis Computer System (SACS), June 2, 1993. 


\begin{tabular}{|c|c|c|c|c|c|c|c|}
\hline \multicolumn{8}{|c|}{ TANK 241-BY-108 } \\
\hline \multicolumn{8}{|c|}{ THERMOCOUPLE DATA } \\
\hline TREE & DATE & THERMO \# & TREE SET \# & TEMP C & TEMP F & $Y=$ MAX TEMP & COMMENTS \\
\hline 1 & $1 / 28 / 75$ & 3 & 1 & 42 & 107.6 & $N$ & degree C/TWINS confirm \\
\hline 1 & $1 / 28 / 75$ & 4 & 1 & 34 & 93.2 & $\mathrm{~N}$ & degree C/TWINS confirm \\
\hline 1 & $1 / 28 / 75$ & 5 & 1 & 33 & 91.4 & $\mathbf{N}$ & degree C/TWINS confirm \\
\hline 1 & $1 / 28 / 75$ & 6 & 1 & 33 & 91.4 & $\mathbf{N}$ & degree C/TWINS confirm \\
\hline 1 & $1 / 28 / 75$ & 7 & 1 & 33 & 91.4 & $\mathbf{N}$ & degree C/TWINS confirm \\
\hline 1 & $1 / 28 / 75$ & 8 & 1 & 33 & 91.4 & $\mathbf{N}$ & degree C/TWINS confirm \\
\hline 1 & $1 / 28 / 75$ & 9 & 1 & 33 & 91.4 & $\bar{N}$ & degree $\mathrm{C} / \mathrm{TW}$ WNS confirm \\
\hline 1 & $2 / 27 / 75$ & 1 & 1 & 50 & 122 & $\bar{Y}$ & degree C/TWiNS confirm \\
\hline 1 & $2 / 27 / 75$ & 2 & 1 & 47 & 116.6 & $\mathbf{N}$ & degree C/TWiNS confirm \\
\hline 1 & $2 / 27 / 75$ & 3 & 1 & 40 & 104 & $\mathbf{N}$ & degree $\mathrm{C} / \mathrm{T}$ WINS confirm \\
\hline 1 & $2 / 27 / 75$ & 4 & 1 & 32 & 89.6 & $\mathbf{N}$ & degree C/TWINS confirm \\
\hline 1 & $2 / 27 / 75$ & 5 & 1 & 32 & 89.6 & $\mathbf{N}$ & degree C/TWINS confirm \\
\hline 1 & $2 / 27 / 75$ & 6 & 1 & 32 & 89.6 & $\mathbf{N}$ & degree C/TWiNS confirm \\
\hline 1 & $2 / 27 / 75$ & 7 & 1 & 32 & 89.6 & $\mathbf{N}$ & degree C/TWiNS confirm \\
\hline 1 & $2 / 27 / 75$ & 8 & 1 & 32 & 89.6 & $\overline{\mathbf{N}}$ & degree C/TWINS confirm \\
\hline 1 & $2 / 27 / 75$ & 9 & 1 & 32 & 89.6 & $\mathbf{N}$ & degree C/TWINS confirm \\
\hline 1 & $2 / 27 / 75$ & 10 & 1 & 32 & 89.6 & $\mathbf{N}$ & degree C/TWINS confirm \\
\hline 1 & $2 / 27 / 75$ & 11 & 1 & 32 & 89.6 & $\mathbf{N}$ & degree C/TWINS confirm \\
\hline 1 & $2 / 27 / 75$ & 12 & 1 & 32 & 89.6 & $\mathbf{N}$ & degree C/TWINS confirm \\
\hline 1 & $3 / 18 / 75$ & 1 & 1 & 62 & 143.6 & $\bar{Y}$ & degree C/TWINS confirm \\
\hline 1 & $3 / 18 / 75$ & 2 & 1 & 56 & 132.8 & $\mathbf{N}$ & degree $\mathrm{C} / \mathrm{TW}$ INS confirm \\
\hline 1 & $3 / 18 / 75$ & 3 & 1 & 48 & 118.4 & $\mathbf{N}$ & degree C/TWINS confirm \\
\hline 1 & $3 / 18 / 75$ & 4 & 1 & 40 & 104 & $\mathbf{N}$ & degree C/TWINS confirm \\
\hline 1 & $3 / 18 / 75$ & 5 & 1 & 33 & 91.4 & $\mathbf{N}$ & degree C/TWINS confirm \\
\hline 1 & $3 / 18 / 75$ & 6 & $\frac{1}{1}$ & 32 & 89.6 & $\mathbf{N}$ & degree C/TWINS confirm \\
\hline 9 & $3 / 18 / 75$ & 7 & 1 & 32 & 89.6 & $\mathbf{N}$ & degree C/TWINS confirm \\
\hline 1 & $3 / 18 / 75$ & 8 & 1 & 32 & 89.6 & $\mathbf{N}$ & degree C/TWINS confirm \\
\hline 1 & $3 / 18 / 75$ & 9 & 1 & 32 & 89.6 & $\mathbf{N}$ & degree C/TWINS confirm \\
\hline 1 & $3 / 18 / 75$ & 10 & 1 & 32 & 89.6 & $\mathbf{N}$ & degree $\mathrm{C} / \mathrm{TW}$ WNS confirm \\
\hline 1 & $3 / 18 / 75$ & 11 & 1 & 32 & 89.6 & $\mathbf{N}$ & degree C/TWiNS confirm \\
\hline 1 & $3 / 18 / 75$ & 12 & 1 & 32 & 89.6 & $\mathbf{N}$ & degree C/TWINS confirm \\
\hline 1 & $4 / 22 / 75$ & 1 & 1 & 54 & 129.2 & $\bar{Y}$ & degree C/TWINS confirm \\
\hline 1 & $4 / 22 / 75$ & 2 & 1 & 45 & 113 & $\mathbf{N}$ & degree C/TWINS confirm \\
\hline 1 & $4 / 22 / 75$ & 3 & 1 & 38 & 100.4 & $\mathbf{N}$ & degree $\mathrm{C} / \mathrm{TW}$ iNS confirm \\
\hline 1 & $4 / 22 / 75$ & 4 & 1 & 30 & 86 & $\mathbf{N}$ & degree C/TWINS confirm \\
\hline 1 & $4 / 22 / 75$ & 5 & 1 & 29 & 84.2 & $\mathbf{N}$ & degree $\mathrm{C} / \mathrm{T}$ WINS confirm \\
\hline 1 & $4 / 22 / 75$ & 6 & 1 & 28 & 82.4 & $\mathbf{N}$ & degree $\mathrm{C} / \mathrm{TWW}$ iNS confirm \\
\hline 1 & $4 / 22 / 75$ & 7 & 1 & 28 & 82.4 & $\mathbf{N}$ & degree C/TWINS confirm \\
\hline 1 & $4 / 22 / 75$ & 8 & 1 & 28 & 82.4 & $\mathbf{N}$ & degree C/TWINS confirm \\
\hline 1 & $4 / 22 / 75$ & 9 & 1 & 28 & 82.4 & $\mathbf{N}$ & degree C/TWINS confirm \\
\hline 1 & $4 / 29 / 75$ & 1 & 1 & 55 & 131 & $\bar{Y}$ & degree C/TWINS confirm \\
\hline 1 & $4 / 29 / 75$ & 2 & 1 & 46 & 114.8 & $\mathbf{N}$ & degree $\mathrm{C} / \mathrm{TW}$ INS confirm \\
\hline 1 & $4 / 29 / 75$ & 3 & 1 & 38 & 100.4 & $\mathbf{N}$ & degree C/TWINS confirm \\
\hline 1 & $4 / 29 / 75$ & 4 & 1 & 30 & 86 & $\mathbf{N}$ & degree $\mathrm{C} / \mathrm{TW}$ WiNS confirm \\
\hline 1 & $4 / 29 / 75$ & 5 & 1 & 30 & 86 & $\mathbf{N}$ & degree C/TWINS confirm \\
\hline 1 & $4 / 29 / 75$ & 6 & 1 & 30 & 86 & $\mathbf{N}$ & degree C/TWINS confirm \\
\hline 1 & $4 / 29 / 75$ & 7 & 1 & 30 & 86 & $\mathbf{N}$ & degrae C/TWINS confirm \\
\hline 1 & $4 / 29 / 75$ & 8 & 1 & 30 & 86 & $\mathbf{N}$ & degree C/TWINS confirm \\
\hline 1 & $4 / 29 / 75$ & 9 & 1 & 30 & 86 & $\mathrm{~N}$ & degree C/TWINS confirm \\
\hline 1 & $5 / 20 / 75$ & 1 & 1 & 64 & 147.2 & $\mathbf{Y}$ & degree C/TWINS confirm \\
\hline 9 & $5 / 20 / 75$ & 2 & 1 & 57 & 134.6 & $\mathbf{N}$ & degree C/TWINS confirm \\
\hline 1 & $5 / 20 / 75$ & 3 & 1 & 50 & 122 & $\mathbf{N}$ & degree $\mathrm{C} / \mathrm{TW}$ INS contirm \\
\hline 1 & $5 / 20 / 75$ & 4 & 1 & 42 & 107.6 & $\mathbf{N}$ & degree C/TWINS confirm \\
\hline 9 & $5 / 20 / 75$ & 5 & 1 & 36 & 96.8 & $\mathbf{N}$ & degree C/TWINS confirm \\
\hline 1 & $5 / 20 / 75$ & 6 & 1 & 34 & 93.2 & $\mathbf{N}$ & degree C/TWINS confirm \\
\hline 1 & $5 / 20 / 75$ & 7 & 1 & 34 & 93.2 & $\mathbf{N}$ & degree C/TWINS confirm \\
\hline 1 & $5 / 20 / 75$ & 8 & 1 & 34 & 93.2 & $\mathbf{N}$ & degree C/TWINS confirm \\
\hline 1 & $5 / 20 / 75$ & 9 & 1 & 35 & 95 & $\mathbf{N}$ & degree $\mathrm{C} / \mathrm{TW}$ INS confirm \\
\hline 1 & $5 / 20 / 75$ & 10 & 1 & 35 & 95 & $\mathbf{N}$ & degree $\mathrm{C} / \mathrm{TW}$ INS confirm \\
\hline 1 & $5 / 20 / 75$ & 19 & 1 & 35 & 95 & $\mathbf{N}$ & degree C/TWINS confirm \\
\hline 9 & $5 / 20 / 75$ & 12 & 1 & 35 & 95 & $\mathbf{N}$ & degree C/TWINS confirm \\
\hline 1 & $6 / 17 / 75$ & 1 & 1 & & 130 & $Y$ & \\
\hline 1 & $6 / 17 / 75$ & 2 & 1 & & 117 & $\mathbf{N}$ & \\
\hline 1 & $6 / 17 / 75$ & 3 & 1 & & 104 & $\mathrm{~N}$ & \\
\hline 1 & $6 / 97 / 75$ & 4 & 1 & & 93 & $N$ & \\
\hline 1 & $6 / 17 / 75$ & 5 & 1 & & 99 & $\mathbf{N}$ & \\
\hline
\end{tabular}

Data obtained from WHC Surveillance Analysis Computer System (SACS), June 2, 1993. 


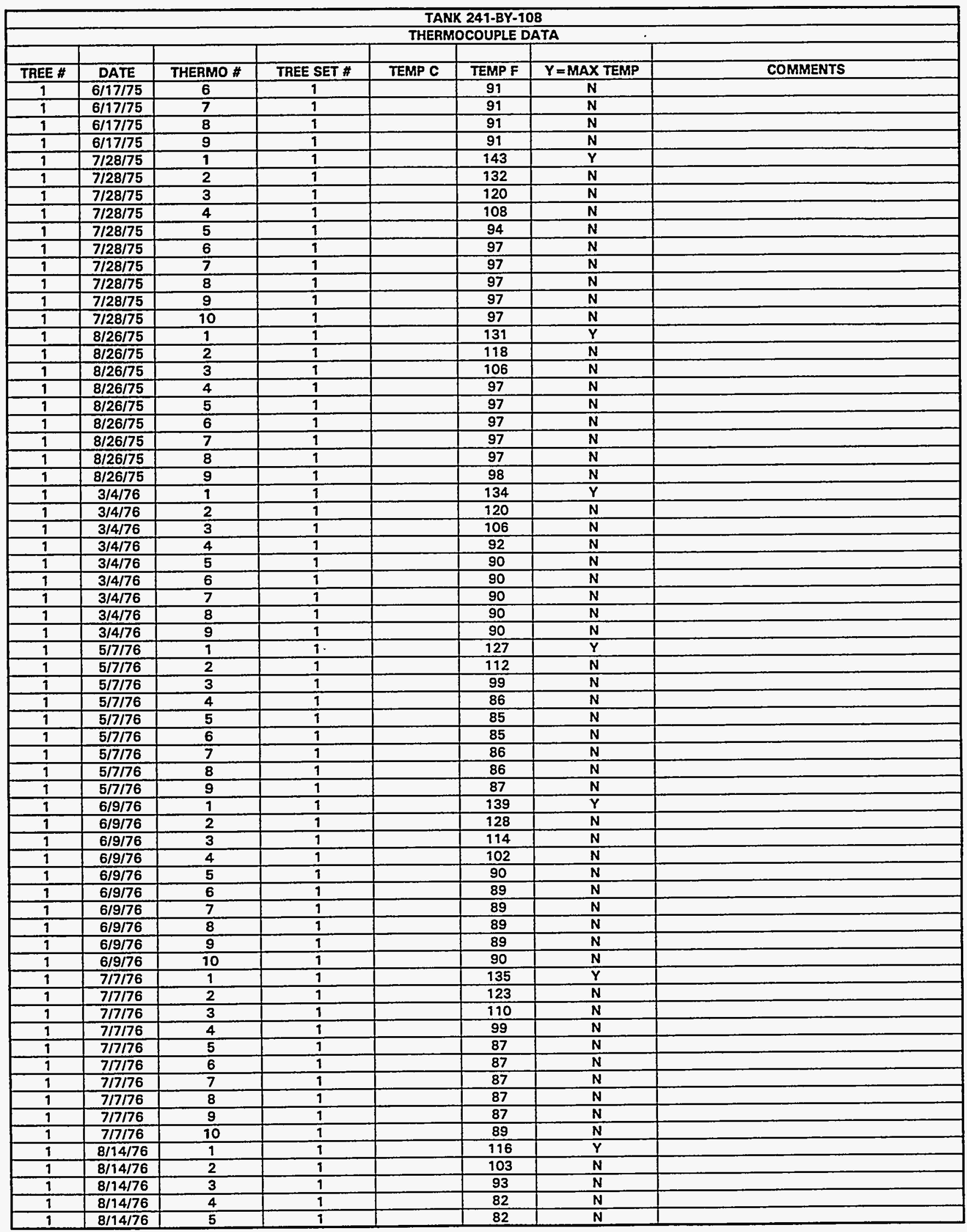

Data obtained from WHC Surveillance Analysis Computer System (SACS), June 2, 1993. 
WHC-SD-WM-ER-312, Rev. 0

\begin{tabular}{|c|c|c|c|c|c|c|c|}
\hline \multicolumn{8}{|c|}{ TANK 241-BY-108 } \\
\hline & & & & THERM & COUPLE D & ATA & \\
\hline TREE \# & DATE & THERMO \# & TREE SET \# & TEMP C & TEMP F & $\bar{Y}=$ MAX TEMP & COMMENTS \\
\hline 1 & $8 / 14 / 76$ & 6 & 1 & & 82 & $\frac{N}{N}$ & \\
\hline 1 & $8 / 14 / 76$ & 7 & 1 & & 82 & $\mathrm{~N}$ & \\
\hline 9 & $8 / 14 / 76$ & 8 & 1 & & 82 & $N$ & \\
\hline 1 & $8 / 14 / 76$ & 9 & 1 & & 82 & $\bar{N}$ & \\
\hline 9 & $8 / 28 / 76$ & 1 & 1 & & 93 & $\mathbf{N}$ & \\
\hline 1 & $8 / 28 / 76$ & 2 & 1 & & 110 & $\mathrm{Y}$ & \\
\hline 1 & $8 / 28 / 76$ & 3 & 1 & & 100 & $\mathrm{~N}$ & \\
\hline 1 & $8 / 28 / 76$ & 4 & 1 & & 90 & $\mathrm{~N}$ & \\
\hline 1 & $8 / 28 / 76$ & 5 & 1 & & 89 & $\mathbf{N}$ & \\
\hline 1 & $8 / 28 / 76$ & 6 & 1 & & 89 & $\bar{N}$ & \\
\hline 1 & $8 / 28 / 76$ & 7 & 1 & & 90 & $\mathrm{~N}$ & \\
\hline 1 & $8 / 28 / 76$ & 8 & 9 & & 90 & $\mathbf{N}$ & \\
\hline 1 & $8 / 28 / 76$ & 9 & 1 & & 90 & $\mathbf{N}$ & \\
\hline 1 & $9 / 13 / 76$ & 1 & 1 & & 105 & $\mathbf{N}$ & \\
\hline 1 & $9 / 13 / 76$ & 2 & 1 & & 110 & $\bar{Y}$ & \\
\hline 1 & $9 / 13 / 76$ & 3 & 1 & & 109 & $\mathbf{N}$ & \\
\hline 1 & $9 / 13 / 76$ & 4 & 1 & & 89 & $N$ & \\
\hline 1 & $9 / 13 / 76$ & 5 & 1 & & 89 & $\mathrm{~N}$ & \\
\hline 1 & $9 / 13 / 76$ & 6 & 1 & & 89 & $\mathbf{N}$ & \\
\hline 1 & $9 / 13 / 76$ & 7 & 1 & & 89 & $\mathbf{N}$ & \\
\hline 1 & $9 / 13 / 76$ & 8 & 1 & & 89 & $\mathbf{N}$ & \\
\hline 1 & $9 / 13 / 76$ & 9 & 1 & & 90 & $\mathbf{N}$ & \\
\hline 1 & $11 / 7 / 76$ & 1 & 1 & & 137 & $Y$ & \\
\hline 1 & $11 / 7 / 76$ & 2 & 1 & & 123 & $\mathbf{N}$ & \\
\hline 1 & $11 / 7 / 76$ & 3 & 1 & & 111 & $\mathbf{N}$ & \\
\hline 1 & $11 / 7 / 76$ & 4 & 1 & & 99 & $\mathbf{N}$ & \\
\hline 1 & $11 / 7 / 76$ & 5 & 1 & & 85 & $N$ & \\
\hline 1 & $11 / 7 / 76$ & 6 & 1 & & 82 & $\mathrm{~N}$ & \\
\hline 1 & $11 / 7 / 76$ & 7 & 1 & & 84 & $N$ & \\
\hline 1 & $11 / 7 / 76$ & 8 & 1 & & 84 & $\mathbf{N}$ & \\
\hline 1 & $11 / 7 / 76$ & 9 & 1 & & 84 & $\mathrm{~N}$ & \\
\hline 1 & $11 / 7 / 76$ & 10 & 1 & & 83 & $N$ & \\
\hline 1 & $12 / 9 / 76$ & 1 & 1 & & 154 & $Y$ & \\
\hline 1 & $12 / 9 / 76$ & 2 & 1 & & 142 & $\mathrm{~N}$ & \\
\hline 1 & $12 / 9 / 76$ & 3 & 1 & & 126 & N & \\
\hline 1 & $12 / 9 / 76$ & 4 & 1 & & 119 & $N$ & \\
\hline 1 & $12 / 9 / 76$ & 5 & 1 & & 105 & $N$ & \\
\hline 1 & $12 / 9 / 76$ & 6 & 1 & & 103 & $\mathbf{N}$ & \\
\hline 1 & $12 / 9 / 76$ & 7 & 1 & & 103 & $\mathbf{N}$ & \\
\hline 1 & $12 / 9 / 76$ & 8 & 1 & & 103 & $\mathbf{N}$ & \\
\hline 1 & $12 / 9 / 76$ & 9 & 1 & & 103 & $\mathbf{N}$ & \\
\hline 1 & $12 / 9 / 76$ & 10 & 1 & & 103 & $\mathbf{N}$ & \\
\hline 9 & $1 / 3 / 77$ & 1 & 1 & & 128 & $Y$ & \\
\hline 1 & $1 / 3 / 77$ & 2 & 1 & & 121 & $\mathbf{N}$ & \\
\hline 1 & $1 / 3 / 77$ & 3 & 1 & & 107 & $\mathbf{N}$ & \\
\hline 1 & $1 / 3 / 77$ & 4 & 1 & & 91 & $\mathbf{N}$ & \\
\hline 1 & $1 / 3 / 77$ & 5 & 1 & & 93 & $\mathrm{~N}$ & \\
\hline 1 & $1 / 3 / 77$ & 6 & 1 & & 93 & $\mathbf{N}$ & \\
\hline 1 & $1 / 3 / 77$ & 7 & 1 & & 93 & $\mathbf{N}$ & \\
\hline 1 & $1 / 3 / 77$ & 8 & 1 & & 93 & $\mathbf{N}$ & \\
\hline 1 & $1 / 3 / 77$ & 9 & 1 & & 93 & $\mathbf{N}$ & \\
\hline 1 & $1 / 3 / 77$ & 10 & 1 & & 93 & $\mathbf{N}$ & \\
\hline 1 & $2 / 26 / 77$ & 1 & 1 & & 110 & $\bar{Y}$ & \\
\hline 1 & $2 / 26 / 77$ & 2 & 1 & & 95 & $\mathbf{N}$ & \\
\hline 1 & $2 / 26 / 77$ & 3 & 1 & & 81 & $\mathbf{N}$ & \\
\hline 1 & $2 / 26 / 77$ & 4 & 1 & & 79 & $N$ & \\
\hline 1 & $2 / 26 / 77$ & 5 & 1 & & 79 & $\mathbf{N}$ & \\
\hline 1 & $2 / 26 / 77$ & 6 & 1 & & 79 & $N$ & \\
\hline 1 & $2 / 26 / 77$ & 7 & 1 & & 80 & $\mathbf{N}$ & \\
\hline 1 & $2 / 26 / 77$ & 8 & 1 & & 80 & $\mathbf{N}$ & \\
\hline 1 & $3 / 28 / 77$ & 1 & 1 & & 194 & $Y$ & \\
\hline 1 & $3 / 28 / 77$ & 2 & 1. & & 100 & $\mathbf{N}$ & \\
\hline 1 & $3 / 28 / 77$ & 3 & 1 & & 86 & $N$ & \\
\hline 1 & $3 / 28 / 77$ & 4 & 1 & & 86 & $\mathbf{N}$ & \\
\hline 1 & $3 / 28 / 77$ & 5 & 1 & & 86 & $\mathbf{N}$ & \\
\hline 1 & $3 / 28 / 77$ & 6 & 1 & & 86 & $N$ & \\
\hline
\end{tabular}

Data obtained from WHC Surveillance Analysis Computer System (SACS), June 2, 1993. 


\begin{tabular}{|c|c|c|c|c|c|c|c|}
\hline \multicolumn{8}{|c|}{ TANK 241-BY-108 } \\
\hline & & & & THERM & COUPLE D & ITA & \\
\hline TREE \# & DATE & THERMO \# & TREE SET \# & TEMP C & TEMP F & $Y=$ MAX TEMP & COMMENTS \\
\hline 1 & $3 / 28 / 77$ & 7 & 1 & Limin & 86 & $\mathrm{~N}$ & \\
\hline 1 & $3 / 28 / 77$ & 8 & 1 & & 86 & $\mathbf{N}$ & \\
\hline 1 & $4 / 27 / 77$ & 1 & 1 & & 100 & $\mathbf{Y}$ & \\
\hline 1 & $4 / 27 / 77$ & 2 & 1 & & 87 & $\mathbf{N}$ & \\
\hline 1 & $4 / 27 / 77$ & 3 & 1 & & 74 & $\mathbf{N}$ & \\
\hline 1 & $4 / 27 / 77$ & 4 & 1 & & 74 & $\mathbf{N}$ & \\
\hline 1 & $4 / 27 / 77$ & 5 & 1 & & 75 & $\mathbf{N}$ & \\
\hline 1 & $4 / 27 / 77$ & 6 & 1 & & 76 & $\mathbf{N}$ & \\
\hline 1 & $4 / 27 / 77$ & 7 & 1 & & 76 & $\mathbf{N}$ & \\
\hline 1 & $4 / 27 / 77$ & 8 & 1 & & 77 & $\mathrm{~N}$ & \\
\hline 1 & $6 / 30 / 77$ & 1 & 1 & & 110 & $\bar{Y}$ & \\
\hline 1 & $6 / 30 / 77$ & 2 & 1 & & 99 & $\bar{N}$ & \\
\hline 1 & $6 / 30 / 77$ & 3 & 1 & & 88 & $\mathbf{N}$ & \\
\hline 1 & $6 / 30 / 77$ & 4 & 1 & & 87 & $\mathbf{N}$ & \\
\hline 1 & $6 / 30 / 77$ & 5 & 1 & & 87 & $\mathbf{N}$ & \\
\hline 1 & $6 / 30 / 77$ & 6 & 1 & & 87 & $\mathrm{~N}$ & \\
\hline 1 & $6 / 30 / 77$ & 7 & 1 & & 87 & $\mathbf{N}$ & \\
\hline 1 & $6 / 30 / 77$ & 8 & 1 & & 87 & $\mathbf{N}$ & \\
\hline 9 & $8 / 15 / 77$ & 1 & 1 & & 100 & $\bar{Y}$ & \\
\hline 1 & $8 / 15 / 77$ & 2 & 1 & & 91 & $N$ & $\therefore$ \\
\hline 1 & $8 / 15 / 77$ & 3 & 1 & & 82 & $\mathbf{N}$ & \\
\hline 1 & $8 / 15 / 77$ & 4 & 1 & & 81 & $\mathbf{N}$ & \\
\hline 1 & $8 / 15 / 77$ & 5 & 1 & & 81 & $\mathrm{~N}$ & \\
\hline 1 & $8 / 15 / 77$ & 6 & 1 & & 81 & $\mathbf{N}$ & \\
\hline 1 & $8 / 15 / 77$ & 7 & 1 & & 80 & $\mathbf{N}$ & \\
\hline 1 & $8 / 15 / 77$ & 8 & 1 & & 80 & $\mathbf{N}$ & \\
\hline 1 & $9 / 12 / 77$ & 1 & 1 & & 95 & $\bar{Y}$ & \\
\hline 1 & $9 / 12 / 77$ & 2 & 1 & & 85 & $\mathbf{N}$ & \\
\hline 1 & $9 / 12 / 77$ & 3 & 1 & & 75 & $\mathbf{N}$ & \\
\hline 1 & $9 / 12 / 77$ & 4 & 1 & & 75 & $\mathrm{~N}$ & \\
\hline 1 & $9 / 12 / 77$ & 5 & 1 & & 74 & $\mathbf{N}$ & \\
\hline 1 & $9 / 12 / 77$ & 6 & 1 & & 74 & $\mathbf{N}$ & \\
\hline 1 & $9 / 12 / 77$ & 7 & 1 & & 74 & $N$ & \\
\hline 1 & $9 / 12 / 77$ & 8 & 1 & & 74 & $\mathbf{N}$ & \\
\hline 1 & $10 / 7 / 77$ & 1 & 1 & & 108 & $\mathbf{Y}$ & \\
\hline 1 & $10 / 7 / 77$ & 2 & 1 & & 94 & $N$ & \\
\hline 1 & $10 / 7 / 77$ & 3 & 1 & & 81 & $\mathbf{N}$ & \\
\hline 1 & $10 / 7 / 77$ & 4 & 1 & & 81 & $N$ & \\
\hline 1 & $10 / 7 / 77$ & 5 & 1 & & 82 & $\mathbf{N}$ & \\
\hline 1 & $10 / 7 / 77$ & 6 & 1 & & 82 & $\mathbf{N}$ & \\
\hline 1 & $10 / 7 / 77$ & 7 & 1 & & 83 & $N$ & \\
\hline 1 & $10 / 7 / 77$ & 8 & 1 & & 83 & $\mathbf{N}$ & \\
\hline 1 & $12 / 7 / 77$ & 1 & 1 & & 108 & $\bar{Y}$ & \\
\hline 1 & $12 / 7 / 77$ & 2 & 1 & & 94 & $\mathbf{N}$ & \\
\hline 1 & $12 / 7 / 77$ & 3 & 1 & & 81 & $\mathbf{N}$ & \\
\hline 1 & $12 / 7 / 77$ & 4 & 1 & & 81 & $\mathbf{N}$ & \\
\hline 1 & $12 / 7 / 77$ & 5 & 1 & & 82 & $\mathbf{N}$ & \\
\hline 1 & $12 / 7 / 77$ & 6 & 1 & & 82 & $\mathbf{N}$ & \\
\hline 1 & $12 / 7 / 77$ & 7 & 1 & & 83 & $\mathbf{N}$ & \\
\hline 1 & $12 / 7 / 77$ & 8 & 1 & & 83 & $\mathbf{N}$ & \\
\hline 1 & $1 / 29 / 78$ & 1 & 1 & & 114 & $\begin{array}{r}\mathbf{Y} \\
\end{array}$ & \\
\hline 1 & $1 / 29 / 78$ & 2 & 1 & & 100 & $\mathbf{N}$ & \\
\hline 1 & $1 / 29 / 78$ & 3 & 1 & & 86 & $\mathbf{N}$ & \\
\hline 1 & $1 / 29 / 78$ & 4 & 1 & & 85 & $N$ & \\
\hline 1 & $1 / 29 / 78$ & 6 & 1 & & 85 & $\mathbf{N}$ & \\
\hline 1 & $1 / 29 / 78$ & 7 & 1 & & 85 & $\mathbf{N}$ & \\
\hline 1 & $1 / 29 / 78$ & 8 & 1 & & 85 & $N$ & \\
\hline 1 & $2 / 26 / 78$ & 1 & 1 & & 116 & $Y$ & \\
\hline 1 & $2 / 26 / 78$ & 2 & 1 & & 100 & $\mathbf{N}$ & \\
\hline 1 & $2 / 26 / 78$ & 3 & 1 & & 86 & $\mathbf{N}$ & \\
\hline 1 & $2 / 26 / 78$ & 4 & 1 & & 85 & $\mathbf{N}$ & \\
\hline 1 & $2 / 26 / 78$ & 5 & 1 & & 86 & $N$ & \\
\hline 1 & $2 / 26 / 78$ & 6 & 1 & & 86 & $\mathbf{N}$ & \\
\hline 1 & $2 / 26 / 78$ & 7 & 1 & & 86 & $\mathbf{N}$ & \\
\hline 1 & $2 / 26 / 78$ & 8 & 1 & & 89 & $\mathbf{N}$ & \\
\hline 1 & $3 / 26 / 78$ & 1 & 1 & & 98 & $\bar{Y}$ & \\
\hline
\end{tabular}

Data obtained from WHC Surveillance Analysis Computer System (SACS), June 2, 1993. 
WHC-SD-WM-ER-312, Rev. 0

\begin{tabular}{|c|c|c|c|c|c|c|c|}
\hline \multicolumn{8}{|c|}{ TANK 241-BY-108 } \\
\hline & & & & & & & \\
\hline TREE \# & $\overline{D A T E}$ & THERMO \# & TREE SET \# & TEMP C & TEMP F & $Y=$ MAX TEMP & COMMENTS \\
\hline$T$ & $3 / 26 / 78$ & 2 & 1 & & 84 & $\mathbf{N}$ & \\
\hline 1 & $3 / 26 / 78$ & 3 & 1 & & 71 & $\bar{N}$ & \\
\hline$\overline{1}$ & $3 / 26 / 78$ & 4 & 1 & & 70 & $\mathbf{N}$ & \\
\hline 1 & $3 / 26 / 78$ & 5 & 1 & & 70 & $\mathbf{N}$ & \\
\hline 1 & $3 / 26 / 78$ & 6 & 1 & & 71 & $\bar{N}$ & \\
\hline 1 & $3 / 26 / 78$ & 7 & $\frac{1}{1}$ & & 71 & $N$ & \\
\hline 9 & $3 / 26 / 78$ & 8 & 1 & & 72 & $\mathrm{~N}$ & \\
\hline 1 & $4 / 23 / 78$ & 1 & 1 & & 106 & $\bar{Y}$ & \\
\hline 1 & $4 / 23 / 78$ & 2 & 1 & & 92 & $\mathbf{N}$ & \\
\hline 1 & $4 / 23 / 78$ & 3 & 1 & & 83 & $\mathbf{N}$ & \\
\hline 1 & $4 / 23 / 78$ & 4 & 1 & & 81 & $\mathbf{N}$ & \\
\hline 1 & $4 / 23 / 78$ & 5 & 1 & & 82 & $\mathbf{N}$ & \\
\hline 1 & $4 / 23 / 78$ & 6 & 1 & & 82 & $\mathrm{~N}$ & \\
\hline 1 & $4 / 23 / 78$ & 7 & 1 & & 83 & $\mathbf{N}$ & \\
\hline 1 & $4 / 23 / 78$ & 8 & 1 & & 84 & $\mathbf{N}$ & \\
\hline 1 & $6 / 17 / 78$ & 1 & 1 & & 123 & $\bar{Y}$ & \\
\hline 1 & $6 / 17 / 78$ & 2 & 1 & & 111 & $\mathbf{N}$ & \\
\hline 1 & $6 / 17 / 78$ & 3 & 1 & & 98 & $\mathbf{N}$ & \\
\hline 1 & $6 / 17 / 78$ & 4 & 1 & & 86 & $\mathbf{N}$ & \\
\hline 1 & $6 / 17 / 78$ & 5 & 1 & & 86 & $\mathbf{N}$ & \\
\hline 1 & $6 / 17 / 78$ & 6 & 1 & & 76 & $\mathbf{N}$ & \\
\hline 1 & $6 / 17 / 78$ & 7 & 1 & & 75 & $\mathrm{~N}$ & \\
\hline 1 & $6 / 17 / 78$ & 8 & 1 & & 76 & $\mathbf{N}$ & \\
\hline 1 & $6 / 17 / 78$ & 9 & 1 & & 76 & $\bar{N}$ & \\
\hline 9 & $6 / 17 / 78$ & 10 & 1 & & 77 & $\mathbf{N}$ & \\
\hline 1 & $7 / 16 / 78$ & 1 & 1 & & 85 & $\bar{Y}$ & \\
\hline 1 & $7 / 16 / 78$ & 2 & 1 & & 75 & $\mathbf{N}$ & \\
\hline 1 & $7 / 16 / 78$ & 3 & 1 & & 64 & $\mathbf{N}$ & \\
\hline 1 & $7 / 16 / 78$ & 4 & 1 & & 62 & $\mathbf{N}$ & \\
\hline 1 & $7 / 16 / 78$ & 5 & 1 & & 62 & $\mathbf{N}$ & \\
\hline 1 & $7 / 16 / 78$ & 6 & 1 & & 62 & $\mathbf{N}$ & \\
\hline 1 & $7 / 16 / 78$ & 7 & 1 & & 62 & $\mathbf{N}$ & \\
\hline 1 & $7 / 16 / 78$ & 8 & 1 & & 61 & $\mathbf{N}$ & \\
\hline$\frac{1}{1}$ & $8 / 13 / 78$ & 1 & 1 & & 128 & $Y$ & \\
\hline 1 & $8 / 13 / 78$ & 2 & 1 & & 117 & $\bar{N}$ & \\
\hline 1 & $8 / 13 / 78$ & 3 & 1 & & 105 & $\mathbf{N}$ & \\
\hline$\frac{1}{1}$ & $8 / 13 / 78$ & 4. & 1 & & 96 & $\mathbf{N}$ & \\
\hline 1 & $8 / 13 / 78$ & 5 & 1 & & 87 & $\mathrm{~N}$ & \\
\hline 1 & $8 / 13 / 78$ & 6 & 1 & & 87 & $\mathbf{N}$ & \\
\hline 1 & $8 / 13 / 78$ & 7 & 1 & & 87 & $\mathrm{~N}$ & \\
\hline 1 & $8 / 13 / 78$ & 8 & 1 & & 88 & $\mathbf{N}$ & \\
\hline 1 & $8 / 13 / 78$ & 9 & 1 & & 88 & $\mathbf{N}$ & \\
\hline 1 & $8 / 13 / 78$ & 10 & 1 & & 88 & $N$ & \\
\hline 1 & $9 / 12 / 78$ & 1 & 1 & & 132 & $\bar{Y}$ & \\
\hline 1 & $9 / 12 / 78$ & 2 & 9 & & 122 & $\mathbf{N}$ & \\
\hline 1 & $9 / 12 / 78$ & 3 & 1 & & 115 & $\mathbf{N}$ & \\
\hline 1 & $9 / 12 / 78$ & 4 & 1 & & 101 & $\mathbf{N}$ & \\
\hline 1 & $9 / 12 / 78$ & 5 & 1 & & 90 & $\mathbf{N}$ & \\
\hline 1 & $9 / 12 / 78$ & 6 & 1 & & 90 & $\bar{N}$ & \\
\hline 1 & $9 / 12 / 78$ & 7 & 1 & & 90 & $\mathbf{N}$ & \\
\hline 1 & $9 / 12 / 78$ & 8 & 1 & & 90 & $\mathbf{N}$ & \\
\hline 1 & $9 / 12 / 78$ & 9 & 1 & & 90 & $\mathbf{N}$ & \\
\hline 1 & $9 / 12 / 78$ & 10 & 1 & & 90 & $N$ & \\
\hline 1 & $10 / 7 / 78$ & 1 & 1 & & 129 & $\bar{Y}$ & \\
\hline 1 & $10 / 7 / 78$ & 2 & 1 & & 120 & $\bar{N}$ & \\
\hline 1 & $10 / 7 / 78$ & 3 & 1 & & 108 & $\mathbf{N}$ & \\
\hline 1 & $10 / 7 / 78$ & 4 & 1 & & 94 & $\mathbf{N}$ & \\
\hline 1 & $10 / 7 / 78$ & 5 & 1 & & 89 & $\mathbf{N}$ & \\
\hline 1 & $10 / 7 / 78$ & 6 & 1 & & 88 & $\mathbf{N}$ & \\
\hline 1 & $10 / 7 / 78$ & 7 & 1 & & 88 & $\bar{N}$ & \\
\hline 1 & $10 / 7 / 78$ & 8 & 1 & & 88 & $\mathbf{N}$ & \\
\hline 1 & $10 / 7 / 78$ & 9 & 1 & & 88 & $\mathbf{N}$ & \\
\hline 1 & $10 / 7 / 78$ & 10 & 1 & & 88 & $\mathbf{N}$ & \\
\hline 1 & $10 / 7 / 78$ & 11 & 1 & & 88 & $\mathbf{N}$ & \\
\hline 1 & $10 / 7 / 78$ & 12 & 9 & & 88 & $\bar{N}$ & \\
\hline 1 & $1 / 3 / 79$ & 1 & 1 & & 112 & $\bar{Y}$ & \\
\hline
\end{tabular}

Data obtained from WHC Surveillance Analysis Computer System (SACS), June 2, 1993. 
WHC-SD-WM-ER-312, Rev. 0

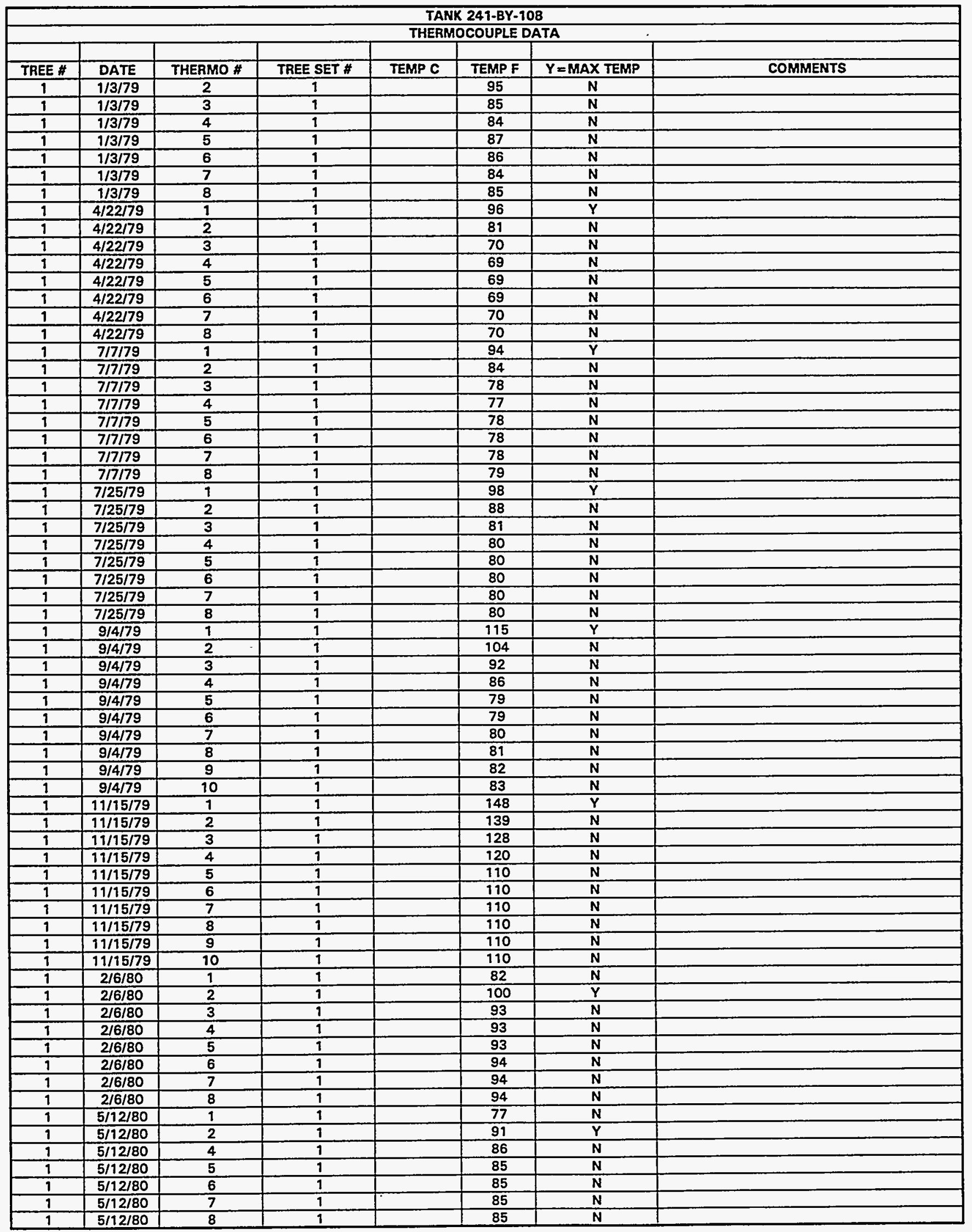

Data obtained from WHC Surveillance Analysis Computer System (SACS), June 2, 1993. 
WHC-SD-WM-ER-312, Rev. 0

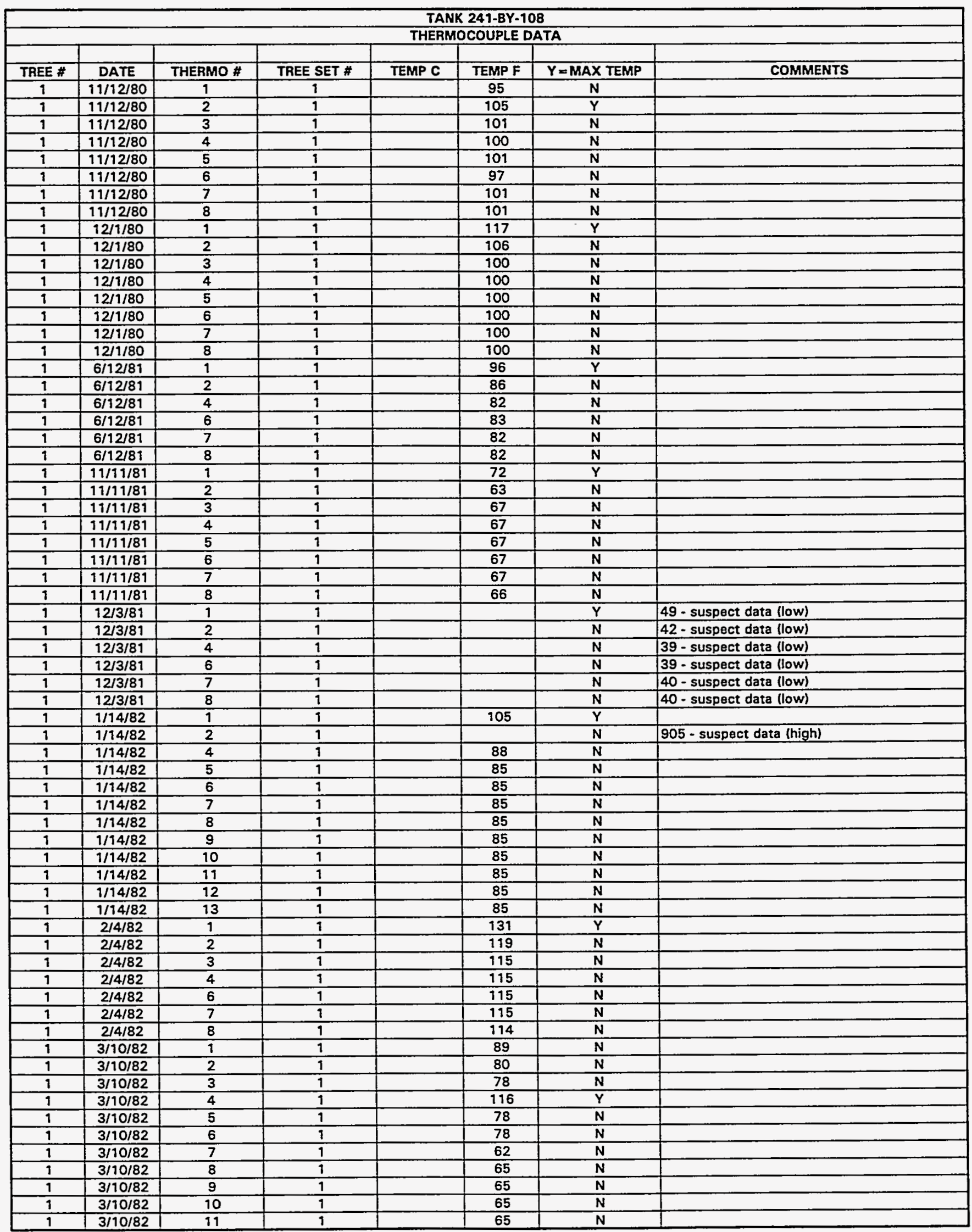

Data obtained from WHC Surveiliance Analysis Computer System (SACS), June 2, 1993. 


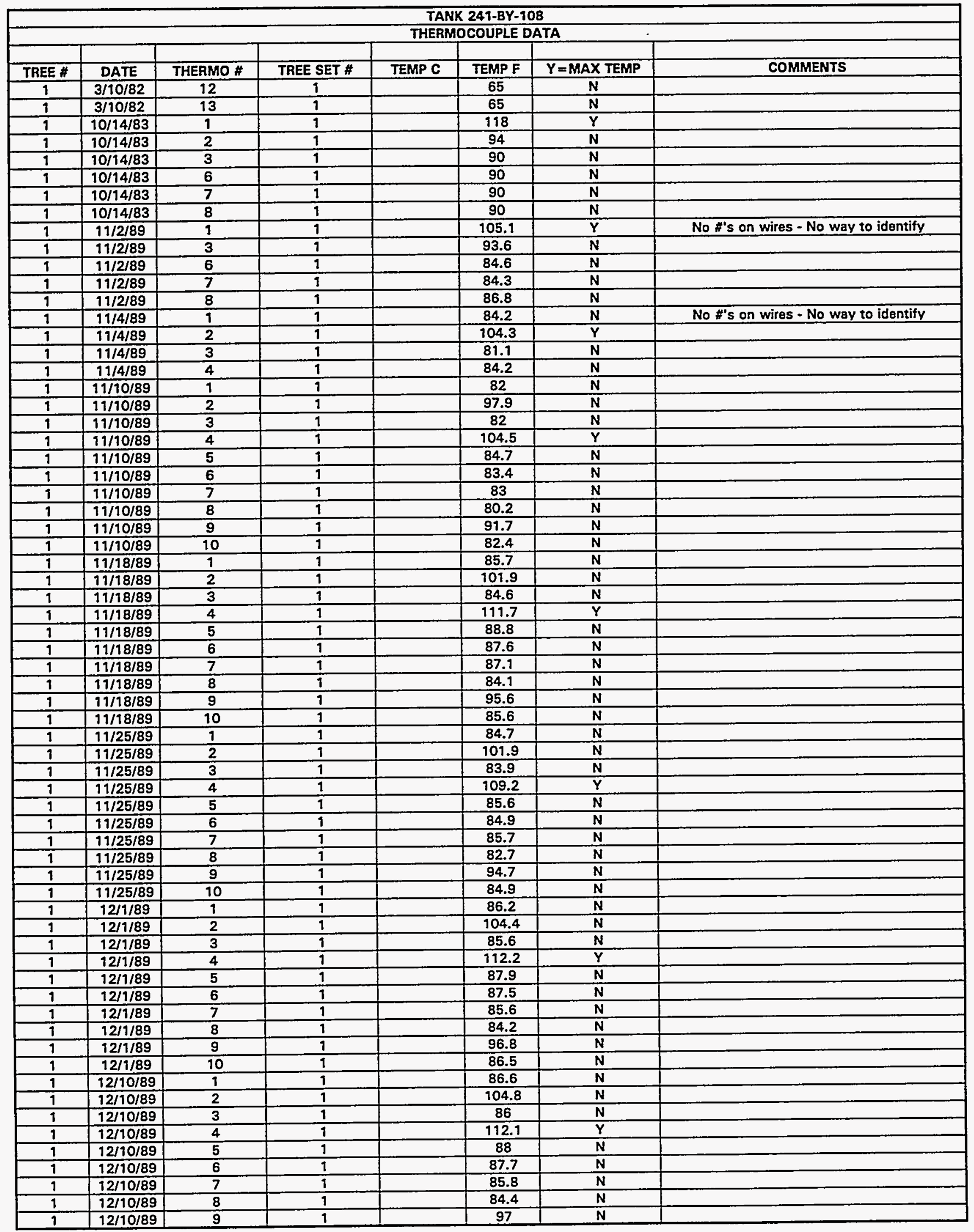

Data obtained from WHC Surveillance Analysis Computer System (SACS), June 2, 1993. 
WHC-SD-WM-ER-312, Rev. 0

\begin{tabular}{|c|c|c|c|c|c|c|c|}
\hline \multicolumn{8}{|c|}{ TANK 241-BY-108 } \\
\hline \multicolumn{8}{|c|}{ THERMOCOUPLE DATA } \\
\hline TREE \# & DATE & THERMO \# & TREE SET \# & TEMP C & TEMP F & $Y=$ MAX TEMP & COMMENTS \\
\hline 1 & $12 / 10 / 89$ & 10 & 1 & & 86.6 & $N$ & \\
\hline 1 & $12 / 15 / 89$ & 1 & 1 & & 84.4 & $N$ & \\
\hline 1 & $12 / 15 / 89$ & 2 & 1 & & 103.8 & $\mathbf{N}$ & \\
\hline 1 & $12 / 15 / 89$ & 3 & 1 & & 83.4 & $N$ & \\
\hline 1 & $12 / 15 / 89$ & $\overline{4}$ & 1 & & 111.8 & $\bar{Y}$ & \\
\hline 1 & $12 / 15 / 89$ & 5 & 1 & & 85.3 & $\mathbf{N}$ & \\
\hline 1 & $12 / 15 / 89$ & 6 & 1 & & 85.5 & $\overline{\mathbf{N}}$ & \\
\hline 1 & $12 / 15 / 89$ & 7 & 1 & & 84.4 & $\mathbf{N}$ & \\
\hline 1 & $12 / 15 / 89$ & 8 & 1 & & 83.9 & $N$ & \\
\hline 1 & $12 / 15 / 89$ & 9 & 1 & & 94 & $\mathbf{N}$ & \\
\hline 1 & $12 / 15 / 89$ & 10 & 1 & & 84.4 & $\overline{\mathbf{N}}$ & \\
\hline 1 & $12 / 23 / 89$ & 9 & 1 & & 86 & $N$ & \\
\hline 1 & $12 / 23 / 89$ & 2 & 1 & & 105 & $\mathbf{N}$ & \\
\hline 1 & $12 / 23 / 89$ & 3 & 9 & & 86 & $\mathbf{N}$ & \\
\hline 1 & $12 / 23 / 89$ & 4 & 1 & & 114 & $Y$ & \\
\hline 1 & $12 / 23 / 89$ & 5 & 1 & & 87 & $\mathbf{N}$ & \\
\hline 1 & $12 / 23 / 89$ & 6 & 1 & & 88 & $\mathbf{N}$ & \\
\hline$T$ & $12 / 23 / 89$ & 7 & 1 & & 85 & $\bar{N}$ & \\
\hline 1 & $12 / 23 / 89$ & 8 & 1 & & 86 & $\mathbf{N}$ & \\
\hline$T$ & \begin{tabular}{|l|}
$12 / 23 / 89$ \\
\end{tabular} & 9 & 1 & & 98 & $\bar{N}$ & \\
\hline 1 & $12 / 23 / 89$ & 10 & 1 & & 86 & $\mathrm{~N}$ & \\
\hline 1 & \begin{tabular}{|l|}
$12 / 29 / 89$ \\
\end{tabular} & 1 & 1 & & 82 & $\mathbf{N}$ & J-2E-OP-020 \\
\hline 1 & \begin{tabular}{|l|}
$12 / 29 / 89$ \\
\end{tabular} & 2 & 9 & & 102 & $\mathbf{N}$ & \\
\hline 1 & $12 / 29 / 89$ & 3 & 1 & & 83 & $\mathrm{~N}$ & \\
\hline 1 & $12 / 29 / 89$ & 4 & 1 & & 114 & $\bar{Y}$ & \\
\hline 1 & $12 / 29 / 89$ & 5 & 9 & & 86 & $\mathbf{N}$ & \\
\hline 1 & $12 / 29 / 89$ & 6 & 1 & & 86 & $\bar{N}$ & \\
\hline 1 & \begin{tabular}{|l|}
$12 / 29 / 89$ \\
\end{tabular} & 7 & 1 & & 103 & $\mathbf{N}$ & \\
\hline 1 & $12 / 29 / 89$ & 8 & 1 & & 84 & $\mathbf{N}$ & \\
\hline 1 & $12 / 29 / 89$ & 9 & 1 & & 85 & $N$ & \\
\hline 1 & $12 / 29 / 89$ & 10 & 1 & & 85 & $\mathbf{N}$ & \\
\hline 1 & $1 / 6 / 90$ & 9 & 1 & & 78.5 & $N$ & J-2E-OP-O02 \\
\hline$\frac{1}{1}$ & $1 / 6 / 90$ & 2 & 1 & & 102.6 & $N$ & \\
\hline 1 & $1 / 6 / 90$ & 3 & 1 & & 80.5 & $\mathbf{N}$ & \\
\hline 1 & $1 / 6 / 90$ & 4 & 1 & & 109.9 & $\bar{Y}$ & \\
\hline 1 & $1 / 6 / 90$ & 5 & 9 & & 81.8 & $\mathbf{N}$ & \\
\hline 1 & $1 / 6 / 90$ & 6 & 1 & & 81.8 & $\mathbf{N}$ & \\
\hline 1 & $1 / 6 / 90$ & 7 & 1 & & 81.1 & $\mathbf{N}$ & \\
\hline 1 & $1 / 6 / 90$ & 8 & 9 & & 80 & $\bar{N}$ & \\
\hline 1 & $1 / 6 / 90$ & 9 & 9 & & 93.9 & $N$ & \\
\hline 1 & $1 / 6 / 90$ & 10 & 1 & & 82 & $\mathbf{N}$ & \\
\hline 1 & $9 / 13 / 90$ & 2 & 9 & & 103.3 & $\mathrm{~N}$ & \\
\hline 1 & $1 / 13 / 90$ & 3 & 1 & & 80.4 & $\mathrm{~N}$ & \\
\hline 1 & $1 / 13 / 90$ & 4 & $\overline{1}$ & & 111.2 & $\mathbf{N}$ & \\
\hline 1 & $9 / 13 / 90$ & 5 & 1 & & 82.8 & $N$ & \\
\hline 1 & $1 / 13 / 90$ & 6 & 1 & & 82.2 & $\mathbf{N}$ & \\
\hline 1 & $1 / 13 / 90$ & 7 & 1 & & 81.7 & $\mathbf{N}$ & \\
\hline 1 & $1 / 13 / 90$ & 8 & 1 & & 81.2 & $\bar{N}$ & \\
\hline 1 & $1 / 13 / 90$ & 9 & 1 & & 93.6 & $\mathbf{N}$ & \\
\hline 1 & $1 / 13 / 90$ & 10 & 1 & & 83.8 & $\mathbf{N}$ & \\
\hline 1 & $1 / 19 / 90$ & 1 & 1 & & 80.4 & $N$ & \\
\hline 1 & $7 / 19 / 90$ & 2 & 1 & & 102 & $\mathbf{N}$ & \\
\hline 1 & $1 / 19 / 90$ & 3 & 1 & & 80 & $\bar{N}$ & \\
\hline 1 & $1 / 19 / 90$ & 4 & 1 & & 110.3 & $\bar{Y}$ & \\
\hline 1 & $1 / 79 / 90$ & 5 & 1 & & 81.1 & $\overline{\mathbf{N}}$ & \\
\hline 1 & $1 / 19 / 90$ & 6 & 1 & & 81.1 & $\mathbf{N}$ & \\
\hline 1 & $1 / 19 / 90$ & 7 & 1 & & 80.5 & $\mathbf{N}$ & \\
\hline 1 & $1 / 19 / 90$ & 8 & 1 & & 79.5 & $\mathbf{N}$ & \\
\hline 7 & $1 / 19 / 90$ & 9 & 1 & & 92 & $\mathbf{N}$ & \\
\hline 1 & $1 / 19 / 90$ & 10 & 1 & & 81.9 & $\mathbf{N}$ & $\mathrm{JJ}-1$ \& J-4 requested \\
\hline$\frac{1}{1}$ & $1 / 27 / 90$ & 1 & 1 & & 77.4 & $\mathbf{N}$ & \\
\hline 1 & $1 / 27 / 90$ & 2 & $\frac{1}{1}$ & & 105.8 & $\mathbf{N}$ & \\
\hline 1 & $1 / 27 / 90$ & 3 & 1 & & 81.4 & $\mathbf{N}$ & \\
\hline 1 & $1 / 27 / 90$ & 4 & 1 & & 113.1 & $\bar{Y}$ & \\
\hline 1 & $1 / 27 / 90$ & 5 & $\frac{1}{1}$ & & 82.7 & $\bar{N}$ & \\
\hline 9 & $1 / 27 / 90$ & 6 & 1 & & 82.6 & $\mathbf{N}$ & \\
\hline
\end{tabular}

Data obtained from WHC Surveillance Analysis Computer System (SACS), June 2, 1993. 
WHC-SD-WM-ER-312, Rev. O

\begin{tabular}{|c|c|c|c|c|c|c|c|}
\hline \multicolumn{8}{|c|}{ TANK 241-BY-108 } \\
\hline \multicolumn{8}{|c|}{ THERMOCOUPLE DATA } \\
\hline TREE \# & DATE & THERMO \# & TREE SET \# & TEMP C & TEMP F & $Y=M A X$ TEMP & COMMENTS \\
\hline 1 & $1 / 27 / 90$ & 7 & 1 & & 82.1 & $\mathbf{N}$ & \\
\hline $\bar{T}$ & $1 / 27 / 90$ & 8 & 1 & & 81.2 & $\mathbf{N}$ & \\
\hline 1 & $1 / 27 / 90$ & 9 & 1 & & 93.8 & $\mathbf{N}$ & \\
\hline 1 & $1 / 27 / 90$ & 10 & 1 & & 83.6 & $\mathbf{N}$ & \\
\hline 1 & $2 / 3 / 90$ & $T$ & 1 & & 83 & $\bar{N}$ & J-2E-OP-020 \\
\hline 1 & $2 / 3 / 90$ & 2 & 1 & & 106.7 & $\mathbf{N}$ & \\
\hline$\overline{1}$ & $2 / 3 / 90$ & 3 & 1 & & 82.9 & $\mathbf{N}$ & \\
\hline 1 & $2 / 3 / 90$ & $\overline{4}$ & 1 & & 114.7 & $\bar{Y}$ & \\
\hline 1 & $2 / 3 / 90$ & 5 & 1 & & 84.1 & $\mathbf{N}$ & \\
\hline 1 & $2 / 3 / 90$ & 6 & 1 & & 84.4 & $\mathbf{N}$ & \\
\hline 1 & $2 / 3 / 90$ & 7 & 1 & & 89 & $\mathbf{N}$ & \\
\hline 1 & $2 / 3 / 90$ & 8 & 1 & & 81.8 & $\mathbf{N}$ & \\
\hline 1 & $2 / 3 / 90$ & 9 & 1 & & 94.6 & $\bar{N}$ & \\
\hline 1 & $2 / 3 / 90$ & 10 & 1 & & 84.2 & $\mathbf{N}$ & \\
\hline 1 & $2 / 5 / 90$ & $\frac{1}{1}$ & 1 & & 76.9 & $\mathbf{N}$ & J-2E-OP-021 \\
\hline$\frac{T}{1}$ & $2 / 5 / 90$ & 2 & 1 & & 104.9 & $\bar{N}$ & \\
\hline$T$ & $2 / 5 / 90$ & 3 & 1 & & 80.1 & $\mathbf{N}$ & \\
\hline 1 & $2 / 5 / 90$ & 4 & 1 & & 112.9 & $\mathbf{Y}$ & \\
\hline 1 & $2 / 5 / 90$ & 5 & 1 & & 83 & $N$ & \\
\hline 9 & $2 / 5 / 90$ & $\overline{6}$ & 1 & & 82.9 & $\bar{N}$ & \\
\hline 1 & $2 / 5 / 90$ & 7 & 1 & & 82.7 & $\mathbf{N}$ & \\
\hline$\frac{T}{1}$ & $2 / 5 / 90$ & 8 & 1 & & 82 & 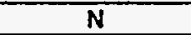 & \\
\hline 1 & $2 / 5 / 90$ & 9 & 1 & & 94 & $\mathbf{N}$ & \\
\hline 9 & $2 / 5 / 90$ & 10 & 1 & & 84.1 & $\mathbf{N}$ & \\
\hline 1 & $2 / 11 / 90$ & 1 & 1 & & 87.3 & $\mathbf{N}$ & J-2E-OP-021 \\
\hline 1 & $2 / 11 / 90$ & 2 & 1 & & 110.4 & $\mathbf{N}$ & \\
\hline 1 & $2 / 11 / 90$ & 3 & 1 & & 87.2 & $\mathbf{N}$ & \\
\hline 1 & $2 / 11 / 90$ & 4 & 1 & & 116.7 & $\bar{Y}$ & \\
\hline 1 & $2 / 11 / 90$ & 5 & 9 & & 85.5 & $\bar{N}$ & \\
\hline 1 & $2 / 11 / 90$ & 6 & 1 & & 83.6 & $\bar{N}$ & \\
\hline 1 & $2 / 11 / 90$ & 7 & 1 & & 82.8 & $\mathbf{N}$ & \\
\hline 1 & $2 / 11 / 90$ & 8 & $\frac{T}{1}$ & & 82.7 & $\mathbf{N}$ & \\
\hline 1 & $2 / 11 / 90$ & 9 & 1 & & 81 & $\mathbf{N}$ & \\
\hline 1 & $2 / 11 / 90$ & 10 & 1 & & 84 & $\mathbf{N}$ & \\
\hline 9 & $3 / 2 / 90$ & 1 & $T$ & & 82.7 & $\overline{\mathbf{N}}$ & J-2E-OP-020 \\
\hline 1 & $3 / 2 / 90$ & 2 & 1 & & 107.3 & $\mathbf{N}$ & \\
\hline 1 & $3 / 2 / 90$ & 3 & 1 & & 81 & $\mathbf{N}$ & \\
\hline 1 & $3 / 2 / 90$ & 4 & 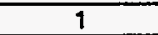 & & 116 & $\bar{Y}$ & \\
\hline 1 & $3 / 2 / 90$ & 5 & 1 & & 82.3 & $\mathbf{N}$ & \\
\hline$T$ & $3 / 2 / 90$ & 6 & 1 & & 84.5 & $\mathbf{N}$ & \\
\hline 9 & $3 / 2 / 90$ & 7 & 1 & & 82.4 & $\overline{\mathbf{N}}$ & \\
\hline 1 & $3 / 2 / 90$ & 8 & 1 & & 82.5 & $N$ & \\
\hline 1 & $3 / 2 / 90$ & 9 & 1 & & 95.5 & $N$ & \\
\hline 1 & $3 / 2 / 90$ & 10 & 1 & & 85.5 & $\mathbf{N}$ & \\
\hline 1 & $4 / 8 / 90$ & 1 & 1 & & 82 & $\mathbf{N}$ & J-2E-OP-020 \\
\hline 1 & $4 / 8 / 90$ & 2 & 1 & & 115 & $\bar{Y}$ & \\
\hline$\frac{1}{1}$ & $4 / 8 / 90$ & 3 & 1 & & 82 & $\mathbf{N}$ & \\
\hline 1 & $4 / 8 / 90$ & 5 & 1 & & 83 & $\mathbf{N}$ & \\
\hline 1 & $4 / 8 / 90$ & 6 & 1 & & 81 & $\mathbf{N}$ & \\
\hline 1 & $4 / 8 / 90$ & 7 & 1 & & 83 & $\mathbf{N}$ & \\
\hline 1 & $4 / 8 / 90$ & 8 & 1 & & 84 & $\mathbf{N}$ & \\
\hline 1 & $4 / 8 / 90$ & 9 & 1 & & 97 & $\bar{N}$ & \\
\hline 1 & $4 / 8 / 90$ & 10 & 1 & & 85 & $\mathbf{N}$ & \\
\hline 1 & $6 / 1 / 90$ & 1 & 1 & & & $\mathbf{N}$ & $\begin{array}{l}\text { No data. Reqd Shift Office, Ahrens, to obtain } \\
\text { reading } 6 / 4 / 90\end{array}$ \\
\hline 1 & $6 / 4 / 90$ & 1 & 1 & & 81.7 & $\mathbf{N}$ & \\
\hline$\frac{1}{1}$ & $6 / 4 / 90$ & 2 & 1 & & 102.3 & $\mathbf{Y}$ & \\
\hline 1 & $6 / 4 / 90$ & 3 & 1 & & 80.5 & $\mathbf{N}$ & \\
\hline$\frac{1}{1}$ & $6 / 4 / 90$ & 5 & 1 & & 80.2 & $\mathbf{N}$ & \\
\hline 1 & $6 / 4 / 90$ & 6 & 1 & & 80.4 & $\mathbf{N}$ & \\
\hline 1 & $6 / 4 / 90$ & 7 & 1 & & 81.6 & $\mathrm{~N}$ & \\
\hline 1 & $6 / 4 / 90$ & 8 & 9 & & 81.6 & $\mathbf{N}$ & \\
\hline 1 & $6 / 4 / 90$ & 9 & 1 & & 93.9 & $N$ & \\
\hline 1 & $6 / 4 / 90$ & 10 & 1 & & 83.7 & $\mathbf{N}$ & \\
\hline 1 & $7 / 13 / 90$ & 1 & 1 & & 79 & $\mathbf{N}$ & J-2E-OP-02O \\
\hline$\frac{T}{1}$ & $7 / 13 / 90$ & 2 & 1 & & 100 & $\bar{Y}$ & \\
\hline
\end{tabular}

Data obtained from WHC Surveillance Analysis Computer System (SACS), June 2, 1993. 


\begin{tabular}{|c|c|c|c|c|c|c|c|}
\hline \multicolumn{8}{|c|}{ TANK 241-BY-10B } \\
\hline & & & & THERN & COUPLE D & TA & \\
\hline & & & & & & & \\
\hline$\frac{\text { TREE \# }}{1}$ & DATE & THERMO \# & TREE SET \# & TEMP C & TEMP F & $Y=M A X$ TEMP & COMMENTS \\
\hline$\frac{1}{1}$ & $7 / 13 / 90$ & 3 & 1 & & 79 & $\mathbf{N}$ & \\
\hline 1 & $7 / 13 / 90$ & 5 & 1 & & 81 & $\mathrm{~N}$ & \\
\hline 1 & $7 / 13 / 90$ & 6 & 1 & & 79 & $\mathbf{N}$ & \\
\hline 1 & $7 / 13 / 90$ & 7 & 1 & & 79 & $\bar{N}$ & \\
\hline 1 & $7 / 13 / 90$ & 8 & 1 & & 78 & $\mathbf{N}$ & \\
\hline 1 & $7 / 13 / 90$ & 9 & 1 & & 92 & $\mathbf{N}$ & \\
\hline 1 & $7 / 13 / 90$ & 10 & 1 & & 81 & $\mathbf{N}$ & \\
\hline 1 & $8 / 5 / 90$ & 5 & 1 & & 82.9 & $\bar{Y}$ & $J-T C 1,2,3,4$ wires not marked \\
\hline 1 & $8 / 5 / 90$ & 6 & 1 & & 82.3 & $\mathbf{N}$ & \\
\hline 1 & $8 / 5 / 90$ & 7 & 1 & & 82.5 & $\mathbf{N}$ & \\
\hline 1 & $8 / 5 / 90$ & 8 & 1 & & 82.8 & $\mathbf{N}$ & \\
\hline 1 & $9 / 11 / 90$ & 9 & 1 & & 96 & $\bar{Y}$ & $\mathrm{~J}$ \\
\hline 1 & $9 / 11 / 90$ & 2 & 1 & & 88 & $\mathbf{N}$ & \\
\hline 1 & $9 / 11 / 90$ & 3 & 1 & & 80 & $\overline{\mathbf{N}}$ & \\
\hline 1 & $9 / 11 / 90$ & 4 & 1 & & 90 & $\mathbf{N}$ & \\
\hline 1 & $9 / 11 / 90$ & 5 & 1 & & 79 & $\mathbf{N}$ & \\
\hline 1 & $9 / 11 / 90$ & 6 & 1 & & 80 & $\mathrm{~N}$ & \\
\hline 1 & $9 / 11 / 90$ & 7 & 1 & & 81 & $N$ & \\
\hline 1 & $9 / 11 / 90$ & 8 & 1 & & 80 & $\mathbf{N}$ & \\
\hline 1 & $9 / 11 / 90$ & 9 & 9 & & 89 & $N$ & \\
\hline 1 & $9 / 11 / 90$ & 10 & 1 & & 80 & $\bar{N}$ & \\
\hline 1 & $10 / 9 / 90$ & 9 & 1 & & 84 & $\bar{N}$ & $\mathrm{~J}$ \\
\hline 1 & $10 / 9 / 90$ & 2 & 1 & & 70 & $\mathrm{~N}$ & \\
\hline 1 & $10 / 9 / 90$ & 3 & 1 & & 83 & $\mathbf{N}$ & \\
\hline 1 & $10 / 9 / 90$ & 4 & 1 & & 84 & $\overline{\mathbf{N}}$ & \\
\hline 1 & $10 / 9 / 90$ & 5 & 1 & & 84 & $\mathbf{N}$ & \\
\hline 1 & $10 / 9 / 90$ & 6 & 1 & & 77 & $\mathbf{N}$ & \\
\hline 1 & $10 / 9 / 90$ & 7 & 1 & & 72 & $\mathbf{N}$ & \\
\hline 1 & $10 / 9 / 90$ & 8 & 1 & & 67 & $\mathbf{N}$ & \\
\hline 1 & $10 / 9 / 90$ & 9 & 1 & & 99 & $Y$ & \\
\hline 9 & $10 / 9 / 90$ & 10 & 1 & & 84 & $\mathbf{N}$ & \\
\hline 1 & $10 / 22 / 90$ & 1 & 1 & & & $\mathbf{N}$ & J $890523512-20$ No readings. J-1 written \\
\hline 1 & $10 / 26 / 90$ & 1 & 1 & & 84.6 & $\bar{N}$ & J-890523512-20 \\
\hline 1 & $10 / 26 / 90$ & 2 & 9 & & 94.2 & $\mathbf{N}$ & \\
\hline 1 & $10 / 26 / 90$ & 3 & 1 & & 84.3 & $\mathbf{N}$ & \\
\hline 1 & $10 / 26 / 90$ & 4 & 1 & & 84.4 & $\mathbf{N}$ & \\
\hline 1 & $10 / 26 / 90$ & 5 & 1 & & 82.9 & $\mathrm{~N}$ & \\
\hline 1 & $10 / 26 / 90$ & 6 & 1 & & 99.9 & $\bar{Y}$ & \\
\hline 1 & $10 / 26 / 90$ & 8 & 1 & & 82.6 & $\mathbf{N}$ & \\
\hline 1 & $10 / 26 / 90$ & 9 & 1 & & 83 & $\mathbf{N}$ & \\
\hline 1 & $10 / 30 / 90$ & 1 & 1 & & 84.2 & $\mathbf{N}$ & $J 890523512-20$ \\
\hline 1 & $10 / 30 / 90$ & 2 & 1 & & 85.1 & $\overline{\mathbf{N}}$ & \\
\hline 1 & $10 / 30 / 90$ & 3 & 1 & & 101.3 & $\bar{Y}$ & \\
\hline 1 & $10 / 30 / 90$ & 4 & 1 & & 84.7 & $\mathbf{N}$ & \\
\hline 1 & $10 / 30 / 90$ & 5 & 1 & & 85.4 & $\mathbf{N}$ & \\
\hline 1 & $10 / 30 / 90$ & 6 & 1 & & 94.5 & $\mathbf{N}$ & \\
\hline 1 & $10 / 30 / 90$ & 8 & 1 & & 83.2 & $\mathbf{N}$ & \\
\hline 1 & $10 / 30 / 90$ & 9 & 1 & & 74.1 & $\mathbf{N}$ & \\
\hline 1 & $11 / 5 / 90$ & 2 & 1 & & 84.7 & $\mathbf{N}$ & \\
\hline 1 & $11 / 5 / 90$ & 3 & 1 & & 83 & $\mathbf{N}$ & \\
\hline 1 & $11 / 5 / 90$ & 5 & 1 & & 82.7 & $\mathbf{N}$ & \\
\hline 1 & $11 / 5 / 90$ & 6 & 1 & & 84.2 & $\mathbf{N}$ & \\
\hline 1 & $11 / 5 / 90$ & 7 & 1 & & 84.1 & $\mathbf{N}$ & \\
\hline 1 & $11 / 5 / 90$ & 9 & 1 & & 83.6 & $N$ & \\
\hline 1 & $11 / 5 / 90$ & 10 & 1 & & 83.7 & $\mathbf{N}$ & \\
\hline 1 & $11 / 12 / 90$ & 1 & 1 & & 84 & $\mathbf{N}$ & 2E-OP-021 \\
\hline 1 & $11 / 12 / 90$ & 2 & 1 & & 101.8 & $\mathbf{Y}$ & \\
\hline 1 & $11 / 12 / 90$ & 3 & 1 & & 84.8 & $\mathbf{N}$ & \\
\hline 1 & $11 / 12 / 90$ & 5 & 1 & & 83.6 & $\mathbf{N}$ & TC\#4 O/S \\
\hline 1 & $11 / 12 / 90$ & 6 & 1 & & 84.6 & $\mathbf{N}$ & \\
\hline 1 & $11 / 12 / 90$ & 7 & 1 & & 85 & $N$ & \\
\hline 1 & $11 / 12 / 90$ & 8 & 1 & & 84 & $\mathrm{~N}$ & \\
\hline 1 & $11 / 12 / 90$ & 9 & 1 & & 95.8 & $\mathrm{~N}$ & \\
\hline 1 & $11 / 12 / 90$ & 10 & 1 & & 85.5 & $\mathbf{N}$ & \\
\hline 1 & $11 / 18 / 90$ & 1 & 1 & & 85.1 & $N$ & J890523512-20 \\
\hline 1 & $11 / 18 / 90$ & 2 & 1 & & 102.1 & $\bar{Y}$ & \\
\hline
\end{tabular}

Data obtained from WHC Surveillance Analysis Computer System (SACS), June 2, 1993. 
WHC-SD-WM-ER-312, Rev. 0

\begin{tabular}{|c|c|c|c|c|c|c|c|}
\hline \multicolumn{8}{|c|}{ TANK 241-BY-108 } \\
\hline \multicolumn{8}{|c|}{ THERMOCOUPLE DATA } \\
\hline & & & & & & & \\
\hline TREE \# & DATE & THERMO \# & TREE SET \# & TEMP C & TEMPF & $Y=$ MAX TEMP & COMMENTS \\
\hline 1 & $11 / 18 / 90$ & 5 & 1 & & 83.2 & $N$ & \\
\hline$T$ & $11 / 18 / 90$ & 6 & 1 & & 85.6 & $\mathbf{N}$ & \\
\hline 1 & $11 / 18 / 90$ & 7 & 1 & & 86.4 & $\mathbf{N}$ & \\
\hline 9 & $11 / 18 / 90$ & 8 & 1 & & 82.9 & $\mathbf{N}$ & \\
\hline 1 & $11 / 18 / 90$ & 9 & 9 & & & $\mathbf{N}$ & 57.8 - suspect data (low) \\
\hline 1 & $11 / 18 / 90$ & 10 & 1 & & 87.2 & $\mathbf{N}$ & \\
\hline 1 & $11 / 25 / 90$ & 9 & 1 & & 90.6 & $\mathbf{N}$ & J-2E-OP-003 \\
\hline 1 & $11 / 25 / 90$ & 2 & 1 & & 102.3 & $\mathbf{Y}$ & \\
\hline 1 & $11 / 25 / 90$ & 4 & 1 & & 93.2 & $\mathbf{N}$ & \\
\hline 1 & $11 / 25 / 90$ & 5 & 1 & & 83.6 & $\mathbf{N}$ & \\
\hline 9 & $11 / 25 / 90$ & 7 & 1 & & 84.4 & $\mathbf{N}$ & \\
\hline 1 & $11 / 25 / 90$ & 8 & 1 & & 82.3 & $N$ & \\
\hline 1 & $12 / 5 / 90$ & 1 & 1 & & 82.4 & $N$ & J 890523512-20 TC \#4 Bad Data \\
\hline 1 & $12 / 5 / 90$ & 2 & 1 & & 82.5 & $\mathbf{N}$ & \\
\hline 1 & $12 / 5 / 90$ & 3 & 1 & & 81.8 & $\mathbf{N}$ & \\
\hline 1 & $12 / 5 / 90$ & 5 & 1 & & 82.4 & $\mathbf{N}$ & \\
\hline 1 & $12 / 5 / 90$ & 6 & 1 & & 82 & $\mathbf{N}$ & \\
\hline 1 & $12 / 5 / 90$ & 8 & 1 & & $82 . \overline{6}$ & $\bar{N}$ & \\
\hline 1 & $12 / 5 / 90$ & 9 & 1 & & 84 & $\mathbf{N}$ & \\
\hline 1 & $12 / 5 / 90$ & 11 & 9 & & 95.5 & $\mathbf{N}$ & \\
\hline 1 & $12 / 11 / 90$ & 1 & 1 & & 77.2 & $\mathbf{N}$ & $J 890423320-11$ \\
\hline 9 & $12 / 11 / 90$ & 2 & 1 & & 100.6 & $\bar{Y}$ & \\
\hline 1 & $12 / 11 / 90$ & 3 & 1 & & 80.1 & $\mathbf{N}$ & \\
\hline 1 & $12 / 11 / 90$ & 5 & 1 & & 80.5 & $\mathbf{N}$ & \\
\hline 1 & $12 / 11 / 90$ & 6 & 1 & & 81.5 & $\mathbf{N}$ & \\
\hline 1 & $12 / 11 / 90$ & 7 & 1 & & 80.8 & $\mathbf{N}$ & \\
\hline 1 & $12 / 11 / 90$ & 8 & 9 & & 79.3 & $\mathrm{~N}$ & \\
\hline 1 & $12 / 11 / 90$ & 9 & 9 & & & $\mathbf{N}$ & 45.7 - suspect data (low) \\
\hline 1 & $12 / 11 / 90$ & 10 & 1 & & 81.2 & $\bar{N}$ & \\
\hline 1 & $12 / 15 / 90$ & 1 & 9 & & 82.4 & $\mathbf{N}$ & HH-99A-J \\
\hline 1 & $12 / 15 / 90$ & 2 & 1 & & 83.1 & $\mathbf{N}$ & \\
\hline 1 & $12 / 15 / 90$ & 3 & 9 & & 103.6 & $\bar{Y}$ & \\
\hline 1 & $12 / 15 / 90$ & 4 & 1 & & 85 & $\bar{N}$ & \\
\hline 1 & $12 / 15 / 90$ & 5 & 1 & & 83.2 & $\mathbf{N}$ & \\
\hline 1 & $12 / 15 / 90$ & 6 & 1 & & 96.8 & $\mathbf{N}$ & \\
\hline 1 & $12 / 15 / 90$ & 7 & 1 & & 84.5 & $\mathbf{N}$ & \\
\hline 1 & $12 / 15 / 90$ & 8 & 1 & & 82.9 & $\mathbf{N}$ & \\
\hline 1 & $12 / 15 / 90$ & 9 & 1 & & 82.5 & $\bar{N}$ & \\
\hline 1 & $12 / 22 / 90$ & $T$ & 9 & & 89 & $\overline{\mathbf{N}}$ & J $890423320-11$ \\
\hline 1 & $12 / 22 / 90$ & 5 & 1 & & 83 & $\mathbf{N}$ & \\
\hline 1 & $12 / 22 / 90$ & 6 & 1 & & 82 & $\mathbf{N}$ & \\
\hline$\frac{1}{1}$ & $12 / 22 / 90$ & 7 & 9 & & 84 & $\mathbf{N}$ & \\
\hline 1 & $12 / 22 / 90$ & 8 & 1 & & 84 & $\mathbf{N}$ & \\
\hline 1 & $12 / 22 / 90$ & 9 & 9 & & & $\mathbf{N}$ & 32 - suspect data (low) \\
\hline 1 & $12 / 22 / 90$ & 10 & 9 & & 85 & $\mathbf{N}$ & \\
\hline 1 & $12 / 30 / 90$ & 1 & 1 & & 80.6 & $\bar{N}$ & J-2E-OP-021 \\
\hline 1 & $12 / 30 / 90$ & 3 & 1 & & 103 & $\bar{Y}$ & \\
\hline 1 & $12 / 30 / 90$ & 5 & 1 & & 81.4 & $\mathbf{N}$ & \\
\hline 1 & $12 / 30 / 90$ & 6 & 1 & & 82.9 & $\mathbf{N}$ & \\
\hline$T$ & $12 / 30 / 90$ & 7 & 1 & & 81.6 & $\bar{N}$ & \\
\hline 1 & $12 / 30 / 90$ & 8 & 9 & & 81 & $\mathbf{N}$ & \\
\hline 1 & $12 / 30 / 90$ & 9 & 1 & & 81.7 & $\mathbf{N}$ & \\
\hline 1 & $12 / 30 / 90$ & 10 & 1 & & 83.3 & $\mathbf{N}$ & \\
\hline 1 & $1 / 6 / 91$ & 7 & 1 & & 80 & $\overline{\mathbf{N}}$ & $J$ \\
\hline 9 & $1 / 6 / 91$ & 4 & 1 & & 92 & $\mathbf{Y}$ & \\
\hline 9 & $1 / 6 / 91$ & 6 & 1 & & 79 & $\mathbf{N}$ & \\
\hline 1 & $1 / 6 / 91$ & 7 & 1 & & 81 & $\mathbf{N}$ & \\
\hline 1 & $1 / 6 / 91$ & B & 9 & & 80 & $\mathbf{N}$ & \\
\hline$\frac{T}{1}$ & $1 / 6 / 91$ & 9 & 1 & & 80 & $\mathbf{N}$ & \\
\hline 1 & 1/6/91 & 10 & 1 & & 80 & $\mathbf{N}$ & \\
\hline 1 & $1 / 13 / 91$ & 1 & 1 & & 88 & $\bar{N}$ & $J 890423320-11$ \\
\hline 1 & $1 / 13 / 91$ & 3 & 1 & & 80.3 & $\mathbf{N}$ & \\
\hline 9 & $1 / 13 / 91$ & 4 & 1 & & 94.2 & $\bar{Y}$ & \\
\hline$\frac{1}{1}$ & $1 / 13 / 91$ & 5 & 1 & & & $\bar{N}$ & 50 - suspect data (low) \\
\hline 1 & $1 / 13 / 91$ & 6 & 1 & & 78.9 & $\mathbf{N}$ & \\
\hline 9 & $1 / 13 / 91$ & 7 & 1 & & 78.9 & $\mathbf{N}$ & \\
\hline
\end{tabular}

Data obtained from WHC Surveillance Analysis Computer System (SACS), June 2, 1993. 


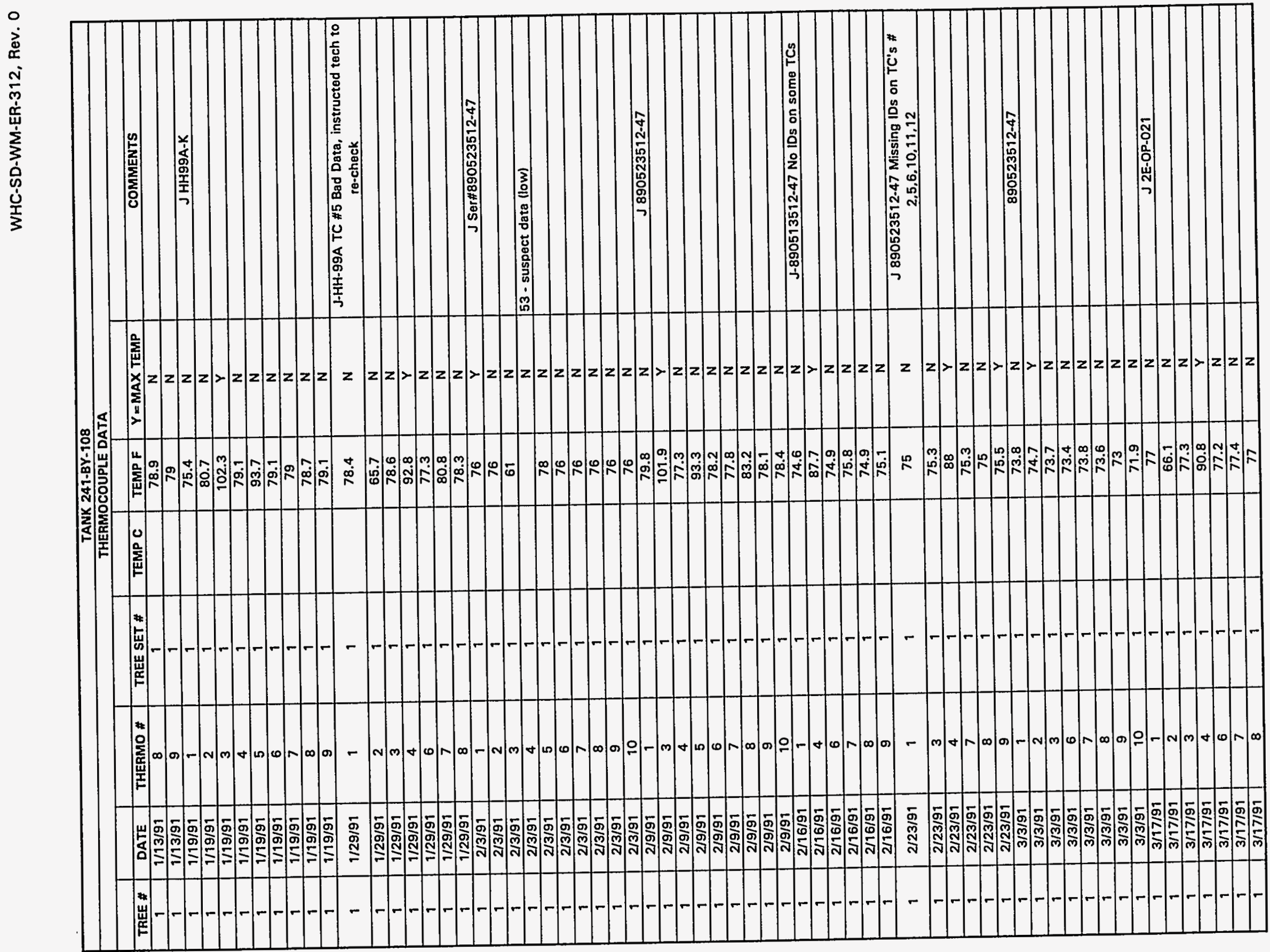




\begin{tabular}{|c|c|c|c|c|c|c|c|}
\hline \multicolumn{8}{|c|}{ TANK 241-BY-108 } \\
\hline & & & & THER & COUPLE & TA & \\
\hline TREE \# & DATE & THERMO \# & TREE SET \# & TEMP C & TEMPF & $Y=$ MAX TEMP & COMMENTS \\
\hline 1 & $3 / 30 / 91$ & 1 & 1 & & 77.2 & $\mathbf{N}$ & J $890523512-20$ \\
\hline 1 & $3 / 30 / 91$ & 2 & 9 & & 79.8 & $\overline{\mathbf{Y}}$ & \\
\hline 1 & $3 / 30 / 91$ & 3 & 1 & & 77.5 & $\mathbf{N}$ & \\
\hline 1 & $3 / 30 / 91$ & 4 & 1 & & 72.4 & $\mathbf{N}$ & \\
\hline 9 & $3 / 30 / 91$ & 5 & 1 & & 72.1 & $\overline{\mathbf{N}}$ & \\
\hline 1 & $3 / 30 / 91$ & 6 & 1 & & 77.2 & $\mathbf{N}$ & \\
\hline 1 & $3 / 30 / 91$ & 7 & 1 & & 77.3 & $\mathbf{N}$ & \\
\hline$\frac{1}{1}$ & $3 / 30 / 91$ & 8 & 1 & & 75.5 & $\mathbf{N}$ & \\
\hline 1 & $4 / 7 / 91$ & 1 & 1 & & 78 & $\bar{N}$ & J 890423320-11 \\
\hline 1 & $4 / 7 / 91$ & 2 & 1 & & 80.2 & $\bar{Y}$ & \\
\hline$\frac{1}{1}$ & $4 / 7 / 91$ & 3 & 1 & & 78 & $\mathbf{N}$ & \\
\hline 1 & $4 / 7 / 91$ & 4 & 1 & & 74 & $\mathbf{N}$ & \\
\hline 1 & $4 / 7 / 91$ & 6 & 1 & & 77.4 & $\mathbf{N}$ & \\
\hline 1 & $4 / 7 / 91$ & 7 & 1 & & 73.7 & $\overline{\mathbf{N}}$ & \\
\hline 1 & $4 / 7 / 91$ & 8 & 1 & & 67.8 & $\mathbf{N}$ & \\
\hline 1 & 4/7/91 & 9 & 1 & & 67.8 & $\mathbf{N}$ & \\
\hline 1 & $4 / 14 / 91$ & 1 & 1 & & 76.7 & $\mathbf{N}$ & J 890523512-20 \\
\hline 1 & $4 / 14 / 91$ & 2 & 1 & & 79.2 & $\overline{\mathbf{Y}}$ & \\
\hline 1 & $4 / 14 / 91$ & 3 & 1 & & 76.4 & $\mathbf{N}$ & \\
\hline 1 & $4 / 14 / 91$ & 4 & 1 & & 91.8 & $\mathbf{N}$ & \\
\hline 1 & $4 / 14 / 91$ & 5 & 1 & & 77 & $\mathbf{N}$ & \\
\hline 1 & $4 / 14 / 91$ & 6 & 1 & & 76.8 & $\mathbf{N}$ & \\
\hline 9 & $4 / 14 / 91$ & 7 & 9 & & 77.2 & $\mathbf{N}$ & \\
\hline 1 & $4 / 14 / 91$ & 8 & 1 & & 76.7 & $\overline{\mathbf{N}}$ & \\
\hline 1 & $4 / 20 / 91$ & 1 & 1 & & 77.1 & $\mathbf{N}$ & $\mathrm{J} 890523512-20$ \\
\hline 1 & $4 / 20 / 91$ & 2 & 1 & & 79.8 & $\mathbf{N}$ & \\
\hline 1 & $4 / 20 / 91$ & 3 & 1 & & 77.5 & $\mathbf{N}$ & \\
\hline 1 & $4 / 20 / 91$ & 4 & 9 & & 91.9 & $\overline{\mathbf{Y}}$ & \\
\hline 1 & $4 / 20 / 91$ & 5 & 1 & & 72 & $\overline{\mathbf{N}}$ & \\
\hline 1 & $4 / 20 / 91$ & 6 & 1 & & 77.5 & $\mathbf{N}$ & \\
\hline 1 & $4 / 20 / 91$ & 7 & 1 & & 77.7 & $\mathbf{N}$ & \\
\hline 1 & $4 / 20 / 91$ & 8 & 1 & & 73.4 & $\mathbf{N}$ & \\
\hline 1 & $4 / 28 / 91$ & 1 & 1 & & 77.1 & $\mathbf{N}$ & J 890523512-20 TC\#9 Bad data \\
\hline 1 & $4 / 28 / 91$ & 2 & 1 & & 78.6 & $\mathbf{N}$ & \\
\hline 1 & $4 / 28 / 91$ & 3 & 1 & & 77.1 & $\mathbf{N}$ & \\
\hline 1 & $4 / 28 / 91$ & 4 & 1 & & 90.9 & $\overline{\mathbf{Y}}$ & \\
\hline 1 & $4 / 28 / 91$ & 5 & 1 & & 77.3 & $\mathbf{N}$ & \\
\hline 1 & $4 / 28 / 91$ & 6 & 1 & & 77.4 & $\mathbf{N}$ & \\
\hline 1 & $4 / 28 / 91$ & 7 & 1 & & 77.1 & $\mathbf{N}$ & \\
\hline 9 & $4 / 28 / 91$ & 8 & 1 & & 75 & $\mathbf{N}$ & \\
\hline 1 & $5 / 5 / 91$ & 1 & 1 & & 77.4 & $\mathbf{N}$ & J 890523512-20 TC\#9 Bad data \\
\hline 1 & $5 / 5 / 91$ & 2 & 1 & & 80.1 & $\mathbf{N}$ & \\
\hline 1 & $5 / 5 / 91$ & 3 & 1 & & 77.6 & $\mathbf{N}$ & \\
\hline 1 & $5 / 5 / 91$ & 4 & 1 & & 91.5 & $\bar{Y}$ & \\
\hline 1 & $5 / 5 / 91$ & 6 & 1 & & 78 & $\mathbf{N}$ & \\
\hline 1 & $5 / 5 / 91$ & 7 & 1 & & 77.8 & $\mathbf{N}$ & \\
\hline 1 & $5 / 5 / 91$ & 8 & 1 & & 77.9 & $\mathbf{N}$ & \\
\hline 1 & $5 / 12 / 91$ & 1 & 1 & & 83.5 & $\mathbf{N}$ & J 890523512 \\
\hline 9 & $5 / 12 / 91$ & 2 & 1 & & 79.5 & $\mathbf{N}$ & \\
\hline 1 & $5 / 12 / 91$ & 3 & 1 & & 78 & $\mathbf{N}$ & \\
\hline 1 & $5 / 12 / 91$ & 4 & 1 & & 91.7 & $\overline{\mathbf{Y}}$ & \\
\hline 1 & $5 / 12 / 91$ & 5 & 1 & & 77.5 & $\mathbf{N}$ & \\
\hline 1 & $5 / 12 / 91$ & 6 & 1 & & 77.5 & $\mathbf{N}$ & \\
\hline 1 & $5 / 12 / 91$ & 7 & 1 & & 78.1 & $\bar{N}$ & \\
\hline 1 & $5 / 12 / 91$ & 8 & 1 & & 78.6 & $\mathbf{N}$ & \\
\hline 1 & $5 / 18 / 91$ & 1 & 1 & & 79.2 & $\mathbf{N}$ & K Keithley 872 2E-OP-020 \\
\hline 1 & $5 / 18 / 91$ & 2 & 1 & & 79 & $\mathbf{N}$ & \\
\hline 1 & $5 / 18 / 91$ & 3 & 1 & & 79.4 & $\mathbf{N}$ & \\
\hline 1 & $5 / 18 / 91$ & 4 & 1 & & 93.1 & $\bar{Y}$ & \\
\hline 1 & $5 / 18 / 91$ & 5 & 1 & & 79.2 & $\mathbf{N}$ & \\
\hline 1 & $5 / 18 / 91$ & 6 & 1 & & 79.5 & $\mathbf{N}$ & \\
\hline 1 & $5 / 18 / 91$ & 7 & 1 & & 79.4 & $\mathbf{N}$ & \\
\hline 1 & $5 / 18 / 91$ & 8 & 1 & & 79.2 & $\mathbf{N}$ & \\
\hline 1 & $5 / 18 / 91$ & 9 & 1 & & 102.6 & $\mathbf{N}$ & TC\# 9 SUSPECT \\
\hline 1 & $5 / 25 / 91$ & 1 & 1 & & 94.7 & $\bar{Y}$ & J 2E-OP-021 \\
\hline 1 & $5 / 25 / 91$ & 2 & 1 & & 92.8 & $N$ & \\
\hline
\end{tabular}

Data obtained from WHC Surveillance Analysis Computer System (SACS), June 2, 1993. 


\begin{tabular}{|c|c|c|c|c|c|c|c|}
\hline \multicolumn{8}{|c|}{ TANK 241-BY-108 } \\
\hline & & & & THERA & COUPLE D & & \\
\hline & & & & & & & \\
\hline TREE \# & DATE & THERMO \# & TREE SET \# & TEMP C & TEMP F & $Y=M A X$ TEMP & COMMENTS \\
\hline 1 & $5 / 25 / 91$ & 3 & 1 & & 94.4 & $\mathbf{N}$ & \\
\hline 1 & $5 / 25 / 91$ & 4 & 1 & & 81.1 & $\mathbf{N}$ & \\
\hline 1 & $5 / 25 / 91$ & 6 & 1 & & 92.8 & $\mathbf{N}$ & \\
\hline 1 & $5 / 25 / 91$ & 7 & 1 & & 91.5 & $\mathbf{N}$ & \\
\hline 1 & $5 / 25 / 91$ & 8 & 1 & & 91.9 & $N$ & \\
\hline 1 & $5 / 25 / 91$ & 9 & 1 & & 68.1 & $\mathrm{~N}$ & \\
\hline 1 & $6 / 2 / 91$ & 1 & 1 & & 76.8 & $\mathbf{N}$ & $\mathrm{J} 890423320-42$ \\
\hline 1 & $6 / 2 / 91$ & 2 & 1 & & 80.1 & $\mathbf{N}$ & \\
\hline 1 & $6 / 2 / 91$ & 3 & 1 & & 76.8 & $\mathrm{~N}$ & \\
\hline 1 & $6 / 2 / 91$ & 4 & 1 & & 90.1 & $\mathbf{N}$ & \\
\hline 1 & $6 / 2 / 91$ & 6 & 1 & & 77.2 & $\mathbf{N}$ & \\
\hline 1 & $6 / 2 / 91$ & 7 & 1 & & 77 & $\mathrm{~N}$ & \\
\hline 1 & $6 / 2 / 91$ & 8 & 1 & & 76.9 & $\mathbf{N}$ & \\
\hline 1 & $6 / 2 / 91$ & 9 & 1 & & 99.1 & $Y$ & \\
\hline 1 & $6 / 9 / 91$ & 1 & 1 & & 77.4 & $\mathrm{~N}$ & $J 890523512-20$ \\
\hline 1 & $6 / 9 / 91$ & 2 & 1 & & 79.1 & $N$ & \\
\hline 1 & $6 / 9 / 91$ & 3 & 1 & & 77.7 & $\mathbf{N}$ & \\
\hline 1 & $6 / 9 / 91$ & 4 & 1 & & 89.2 & $\bar{Y}$ & \\
\hline 1 & $6 / 9 / 91$ & 6 & 1 & & 76.9 & $\mathbf{N}$ & \\
\hline 1 & $6 / 9 / 91$ & 7 & 1 & & 76.8 & $\mathbf{N}$ & \\
\hline 1 & $6 / 9 / 91$ & 8 & 1 & & 77.1 & $\mathrm{~N}$ & \\
\hline 1 & $6 / 9 / 91$ & 9 & 1 & & 99 & $\mathbf{N}$ & \\
\hline 1 & $6 / 15 / 91$ & 1 & 1 & & 81 & $\mathbf{N}$ & $\begin{array}{l}\text { J 2E-OP-021 TC\#3,10 Bad data; \#9 Bad data in } \\
\text { vapor space }\end{array}$ \\
\hline 1 & $6 / 15 / 91$ & 2 & 1 & & 81.9 & $\mathbf{N}$ & \\
\hline 1 & $6 / 15 / 91$ & 4 & 1 & & 91.4 & $\bar{Y}$ & \\
\hline 1 & $6 / 15 / 91$ & 6 & 1 & & 86.9 & $\mathrm{~N}$ & \\
\hline 1 & $6 / 15 / 91$ & 7 & 1 & & 79.6 & $\mathrm{~N}$ & \\
\hline 1 & $6 / 15 / 91$ & 8 & 1 & & 78.8 & $\mathrm{~N}$ & \\
\hline 1 & $6 / 23 / 91$ & 1 & 1 & & 84 & $\mathrm{~N}$ & J 2E-OP-021 \\
\hline 1 & $6 / 23 / 91$ & 2 & 1 & & 81 & $N$ & \\
\hline 1 & $6 / 23 / 91$ & 4 & 1 & & 92 & $\mathrm{~N}$ & \\
\hline 1 & $6 / 23 / 91$ & 6 & 1 & & 80 & $\mathbf{N}$ & \\
\hline 1 & $6 / 23 / 91$ & 7 & 1 & & 80 & $N$ & \\
\hline 1 & $6 / 23 / 91$ & 8 & 1 & & 80 & $\mathbf{N}$ & \\
\hline 1 & $6 / 23 / 91$ & 9 & 1 & & 100 & $Y$ & \\
\hline 1 & $6 / 29 / 91$ & 1 & 1 & & 78.1 & $\mathrm{~N}$ & J 890423320-42 TC\#3,5,10 Broken wire \\
\hline 1 & $6 / 29 / 91$ & 2 & 1 & & 80.6 & $\mathbf{N}$ & \\
\hline 1 & $6 / 29 / 91$ & 4 & 1 & & 90.8 & $\bar{Y}$ & \\
\hline 1 & $6 / 29 / 91$ & 6 & 1 & & 78.5 & $\mathbf{N}$ & \\
\hline 1 & $6 / 29 / 91$ & 7 & 1 & & 78.6 & $\mathbf{N}$ & \\
\hline 1 & $6 / 29 / 91$ & 8 & 1 & & 68.1 & $\mathbf{N}$ & \\
\hline 1 & $6 / 29 / 91$ & 9 & 1 & & 98.5 & $\mathbf{N}$ & \\
\hline 1 & $7 / 6 / 91$ & 1 & 1 & & 78.4 & $\mathbf{N}$ & J910126143-42 TC\#3,5,10NG \\
\hline 1 & $7 / 6 / 91$ & 2 & 1 & & 91.7 & $\mathbf{N}$ & \\
\hline 1 & $7 / 6 / 91$ & 4 & 1 & & 90.8 & $\mathbf{N}$ & \\
\hline 1 & $7 / 6 / 91$ & 6 & 1 & & 78.9 & $\mathbf{N}$ & \\
\hline 1 & $7 / 6 / 91$ & 7 & 1 & & 78.9 & $\mathbf{N}$ & \\
\hline 1 & $7 / 6 / 91$ & 8 & 1 & & 78.7 & $\mathbf{N}$ & \\
\hline 1 & $7 / 6 / 91$ & 9 & 1 & & 97.3 & $Y$ & \\
\hline 1 & $7 / 13 / 91$ & 1 & 1 & & 87.5 & $\mathbf{N}$ & J910126143-42 TC\#3,5,10NG \\
\hline 1 & $7 / 13 / 91$ & 2 & 1 & & 77.7 & $\mathbf{N}$ & \\
\hline 1 & $7 / 13 / 91$ & 4 & 1 & & 86.5 & $\mathbf{N}$ & \\
\hline 1 & $7 / 13 / 91$ & 6 & 1 & & 79.1 & $\mathbf{N}$ & \\
\hline 1 & $7 / 13 / 91$ & 7 & 1 & & 85.7 & $\mathbf{N}$ & \\
\hline 1 & $7 / 13 / 91$ & 8 & 1 & & 77.9 & $\mathrm{~N}$ & \\
\hline 1 & $7 / 13 / 91$ & 9 & 1 & & 97.5 & $Y$ & \\
\hline 1 & $7 / 20 / 91$ & 2 & 1 & & 78.9 & $N$ & J $910126143-64$ TC\#1,3,10o/s \\
\hline 1 & $7 / 20 / 91$ & 4 & 1 & & 95.7 & $\mathbf{N}$ & \\
\hline 1 & $7 / 20 / 91$ & 5 & 1 & & 77.7 & $\mathbf{N}$ & \\
\hline 1 & $7 / 20 / 91$ & 6 & 1 & & 93.6 & $\mathrm{~N}$ & \\
\hline 1 & $7 / 20 / 91$ & 7 & 1 & & 78.6 & $\mathbf{N}$ & \\
\hline 1 & $7 / 20 / 91$ & 8 & 1 & & 77.2 & $\mathbf{N}$ & \\
\hline 1 & $7 / 20 / 91$ & 9 & 1 & & 96.1 & $Y$ & \\
\hline 1 & $7 / 27 / 91$ & 2 & 1 & & 80.8 & $\mathbf{N}$ & J $910126143-14$ TC\#1,3,5,10 \\
\hline 1 & $7 / 27 / 91$ & 4 & 1 & & 90.8 & $\mathrm{~N}$ & \\
\hline
\end{tabular}

Data obtained from WHC Surveillance Analysis Computer System (SACS), June 2, 1993. 
WHC-SD-WM-ER-312, Rev. 0

\begin{tabular}{|c|c|c|c|c|c|c|c|}
\hline \multicolumn{8}{|c|}{ TANK 241-BY-108 } \\
\hline & & & & THERI & COUPLE I & TA & \\
\hline TREE \# & DATE & THERMO \# & TAEE SET \# & TEMP C & TEMPF & $Y=$ MAX TEMP & COMMENTS \\
\hline 1 & $7 / 27 / 91$ & 6 & 1 & & 78.6 & $\mathbf{N}$ & \\
\hline 1 & $7 / 27 / 91$ & 7 & 1 & & 77.7 & $\mathbf{N}$ & \\
\hline 1 & $7 / 27 / 91$ & 8 & 1 & & 78.9 & $\mathbf{N}$ & \\
\hline 1 & $7 / 27 / 91$ & 9 & 1 & & 97.8 & $\bar{Y}$ & \\
\hline 1 & $8 / 3 / 91$ & 1 & 1 & & 93.4 & $\bar{N}$ & J 910126143-14 TC\#3,5,10N/G \\
\hline 1 & $8 / 3 / 91$ & 2 & 1 & & 80.3 & $\mathbf{N}$ & \\
\hline 1 & $8 / 3 / 91$ & 4 & 1 & & 90.5 & $N$ & \\
\hline 1 & $8 / 3 / 91$ & 6 & 1 & & 79.2 & $N$ & \\
\hline 1 & $8 / 3 / 91$ & 7 & 1 & & 78.8 & $\mathbf{N}$ & \\
\hline 1 & $8 / 3 / 91$ & 8 & 1 & & 79.9 & $\mathbf{N}$ & \\
\hline 1 & $8 / 3 / 91$ & $\overline{9}$ & 1 & & 96.6 & $\bar{Y}$ & \\
\hline 1 & $8 / 10 / 91$ & 2 & 1 & & 75 & $\mathbf{N}$ & J 890523512-20 TC\#1,3,5,11NG \\
\hline 1 & $8 / 10 / 91$ & 4 & 1 & & 92 & $\mathbf{N}$ & \\
\hline 1 & $8 / 10 / 91$ & 6 & 1 & & 82 & $\mathbf{N}$ & \\
\hline 1 & $8 / 10 / 91$ & 7 & 1 & & 81 & $\mathbf{N}$ & \\
\hline 1 & $8 / 10 / 91$ & 8 & 1 & & 75 & $N$ & \\
\hline 1 & $8 / 10 / 91$ & 9 & 1 & & 99 & $Y$ & \\
\hline 1 & $8 / 18 / 91$ & 2 & 1 & & 81.5 & $N$ & J 890523512-20 \\
\hline 1 & $8 / 18 / 91$ & 4 & 1 & & 90.5 & $\mathbf{N}$ & \\
\hline 1 & $8 / 18 / 91$ & 6 & 1 & & 80 & $\bar{N}$ & \\
\hline 1 & $8 / 18 / 91$ & 8 & 1 & & 96.7 & $Y$ & \\
\hline 1 & $8 / 18 / 91$ & 9 & 1 & & 95.7 & $\mathbf{N}$ & \\
\hline 9 & $8 / 24 / 91$ & 2 & 1 & & 83.3 & $\mathbf{N}$ & J 890523512-20 \\
\hline 1 & $8 / 24 / 91$ & 4 & 1 & & 91.3 & $\mathbf{N}$ & \\
\hline 1 & $8 / 24 / 91$ & 6 & 1 & & 81.8 & $\mathbf{N}$ & \\
\hline 1 & $8 / 24 / 91$ & 7 & 1 & & 81.6 & $\mathbf{N}$ & \\
\hline 1 & $8 / 24 / 91$ & 8 & 1 & & 80 & $\mathbf{N}$ & \\
\hline 1 & $8 / 24 / 91$ & 9 & 1 & & 94 & $\bar{Y}$ & \\
\hline 1 & $8 / 31 / 91$ & 2 & 1 & & 83.3 & $\mathbf{N}$ & $J-890423320-42$ \\
\hline 1 & $8 / 31 / 91$ & 4 & 1 & & 92.5 & $Y$ & \\
\hline 1 & $8 / 31 / 91$ & 6 & 1 & & 82.8 & $\mathbf{N}$ & \\
\hline 1 & $8 / 31 / 91$ & 7 & 1 & & 82.1 & $\mathbf{N}$ & \\
\hline 1 & $8 / 31 / 91$ & 8 & 1 & & 80.5 & $\mathbf{N}$ & \\
\hline 1 & $8 / 31 / 91$ & 9 & 1 & & 83.2 & $\mathbf{N}$ & \\
\hline 1 & $9 / 8 / 91$ & 2 & 1 & & 83.3 & $\mathbf{N}$ & J-890423320-42 \\
\hline 1 & $9 / 8 / 91$ & 4 & 1 & & 92.5 & $\bar{Y}$ & \\
\hline 1 & $9 / 8 / 91$ & 6 & 1 & & 82.6 & $\mathbf{N}$ & \\
\hline 1 & 9/8/91 & 7 & 1 & & 82 & $\mathbf{N}$ & \\
\hline 1 & $9 / 8 / 91$ & 8 & 1 & & 80.5 & $\mathbf{N}$ & \\
\hline 1 & $9 / 8 / 91$ & 9 & 1 & & 83.2 & $\mathbf{N}$ & \\
\hline 1 & $9 / 15 / 91$ & 2 & 1 & & 76.9 & $\mathbf{N}$ & J-910126143-75 NO MAX FLAGGED \\
\hline 1 & $9 / 15 / 91$ & 4 & 1 & & 80 & $\mathbf{N}$ & \\
\hline 1 & $9 / 15 / 91$ & 6 & 1 & & 81.6 & $\mathbf{N}$ & \\
\hline 1 & $9 / 15 / 91$ & 7 & 1 & & 79.5 & $N$ & \\
\hline 1 & $9 / 15 / 91$ & 8 & 1 & & 79.6 & $\mathbf{N}$ & \\
\hline 1 & $9 / 15 / 91$ & 9 & 1 & & 70.5 & $\mathbf{N}$ & \\
\hline 1 & $9 / 22 / 91$ & 1 & 1 & & 82.1 & $\mathbf{N}$ & $1-910126143-75$ \\
\hline 1 & $9 / 22 / 91$ & 2 & 1 & & 84 & $\mathbf{N}$ & \\
\hline 1 & $9 / 22 / 91$ & 3 & 1 & & 82.8 & $\mathbf{N}$ & \\
\hline 1 & $9 / 22 / 91$ & 4 & 1 & & 92.7 & $\mathbf{N}$ & \\
\hline 1 & $9 / 22 / 91$ & 5 & 1 & & 82 & $\mathrm{~N}$ & \\
\hline 1 & $9 / 22 / 91$ & 6 & 1 & & 82.5 & $N$ & \\
\hline 1 & $9 / 22 / 91$ & 7 & 1 & & 82.2 & $\mathbf{N}$ & \\
\hline 1 & $9 / 22 / 91$ & 8 & 1 & & 81.8 & $\mathbf{N}$ & \\
\hline 1 & $9 / 22 / 91$ & 9 & 1 & & 97.5 & $\mathbf{Y}$ & \\
\hline 1 & $9 / 28 / 91$ & 2 & 1 & & 101 & $\bar{Y}$ & \\
\hline 1 & $9 / 28 / 91$ & 3 & 1 & & 96.2 & $\mathbf{N}$ & \\
\hline 1 & $9 / 28 / 91$ & 4 & 1 & & 86.9 & $\mathbf{N}$ & \\
\hline 1 & $9 / 28 / 91$ & 5 & 1 & & 84.8 & $\mathbf{N}$ & \\
\hline 1 & $9 / 28 / 91$ & 6 & 1 & & 84.5 & $\mathbf{N}$ & \\
\hline 1 & $9 / 28 / 91$ & 7 & 1 & & 84 & $\mathbf{N}$ & \\
\hline 1 & $9 / 28 / 91$ & 8 & 1 & & 84.8 & $\mathbf{N}$ & \\
\hline 1 & $9 / 28 / 91$ & 9 & 1 & & 85.2 & $\mathbf{N}$ & \\
\hline 1 & $9 / 28 / 91$ & 10 & 1 & & 85.6 & $N$ & \\
\hline 1 & $10 / 5 / 91$ & 1 & 1 & & & $\mathbf{N}$ & O/S No Readings \\
\hline 1 & $10 / 12 / 91$ & 2 & 1 & & 99.3 & $Y$ & \\
\hline
\end{tabular}

Data obtained from WHC Surveillance Analysis Computer System (SACS), June 2, 1993. 


\begin{tabular}{|c|c|c|c|c|c|c|c|}
\hline \multicolumn{8}{|c|}{ TANK 241-BY-108 } \\
\hline & & & & & & & \\
\hline TREE \# & DATE & THERMO \# & TREE SET \# & TEMP C & TEMP F & $Y=$ MAX TEMP & COMMENTS \\
\hline 1 & $10 / 12 / 91$ & 3 & 1 & & 94.3 & $\mathbf{N}$ & \\
\hline 1 & $10 / 12 / 91$ & 4 & 1 & & 85 & $\mathbf{N}$ & \\
\hline 1 & $10 / 12 / 91$ & 5 & 1 & & 83.1 & $\mathbf{N}$ & \\
\hline 1 & $10 / 12 / 91$ & 6 & 1 & & 82.9 & $\mathbf{N}$ & \\
\hline 1 & $10 / 12 / 91$ & 7 & 1 & & 82.5 & $\mathbf{N}$ & \\
\hline 1 & $10 / 12 / 91$ & 8 & 1 & & 83.4 & $\mathrm{~N}$ & \\
\hline 1 & $10 / 12 / 91$ & 9 & 1 & & 83.6 & $\mathbf{N}$ & \\
\hline 1 & $10 / 12 / 91$ & 10 & 1 & & 83.8 & $\mathbf{N}$ & \\
\hline 1 & $10 / 20 / 91$ & 2 & 1 & & 99.1 & $\mathbf{Y}$ & \\
\hline 1 & $10 / 20 / 91$ & 3 & 1 & & 93.8 & $N$ & \\
\hline 1 & $10 / 20 / 91$ & 4 & 1 & & 84.2 & $\bar{N}$ & \\
\hline 1 & $10 / 20 / 91$ & 5 & 1 & & 82.4 & $\mathbf{N}$ & \\
\hline 1 & $10 / 20 / 91$ & 6 & 1 & & 82.3 & $\mathbf{N}$ & \\
\hline 1 & $10 / 20 / 91$ & 7 & 1 & & 81.9 & $N$ & \\
\hline 1 & $10 / 20 / 91$ & 8 & 1 & & 82.7 & $\mathbf{N}$ & \\
\hline 1 & $10 / 20 / 91$ & 9 & 1 & & 82.8 & $\mathrm{~N}$ & \\
\hline 1 & $10 / 20 / 91$ & 10 & 1 & & 83 & $\mathbf{N}$ & \\
\hline 1 & $10 / 27 / 91$ & 2 & 1 & & 100.3 & $Y$ & \\
\hline 1 & $10 / 27 / 91$ & 3 & 1 & & 94.9 & $\mathbf{N}$ & \\
\hline 1 & $10 / 27 / 91$ & 4 & 1 & & 85.1 & $\mathbf{N}$ & \\
\hline 1 & $10 / 27 / 91$ & 5 & 1 & & 83.6 & $\mathbf{N}$ & \\
\hline 1 & $10 / 27 / 91$ & 6 & 1 & & 83.5 & $\mathbf{N}$ & \\
\hline 1 & $10 / 27 / 91$ & 7 & 1 & & 83 & $\mathbf{N}$ & \\
\hline 1 & $10 / 27 / 91$ & 8 & 1 & & 83.8 & $\mathbf{N}$ & \\
\hline 1 & $10 / 27 / 91$ & 9 & 1 & & 84 & $N$ & \\
\hline 1 & $10 / 27 / 99$ & 10 & 1 & & 84.1 & $\mathbf{N}$ & \\
\hline 1 & $111 / 2 / 91$ & 2 & 1 & & 99 & $\bar{Y}$ & \\
\hline 1 & $11 / 2 / 91$ & 3 & 1 & & 93 & $\mathbf{N}$ & \\
\hline 9 & $11 / 2 / 91$ & 4 & 9 & & 83 & $\mathrm{~N}$ & \\
\hline 1 & $11 / 2 / 91$ & 5 & 1 & & 82 & $\mathrm{~N}$ & \\
\hline 9 & $11 / 2 / 91$ & 6 & 1 & & 82 & $\mathbf{N}$ & \\
\hline 1 & $11 / 2 / 91$ & 7 & 1 & & 81 & $\mathrm{~N}$ & \\
\hline 1 & $11 / 2 / 91$ & 8 & 1 & & 82 & $N$ & \\
\hline 9 & $11 / 2 / 91$ & 9 & 9 & & 82 & $\mathbf{N}$ & \\
\hline 1 & $11 / 2 / 91$ & 10 & 1 & & 82 & $\mathrm{~N}$ & \\
\hline 1 & $11 / 9 / 91$ & 1 & 1 & & & $\mathbf{N}$ & $\begin{array}{c}51 \text { - suspect data (low) J-2E-OP-02, TC \#1 } \\
\text { BAD DATA }\end{array}$ \\
\hline 1 & $11 / 9 / 91$ & 2 & 1 & & 99 & $Y$ & \\
\hline 1 & $11 / 9 / 91$ & 3 & 1 & & 93 & $N$ & \\
\hline 1 & $11 / 9 / 91$ & 4 & 1 & & 84 & $\mathbf{N}$ & \\
\hline 1 & $11 / 9 / 91$ & 5 & 1 & & 82 & $N$ & \\
\hline 1 & $11 / 9 / 91$ & 6 & 1 & & 81 & $\mathbf{N}$ & \\
\hline 1 & $11 / 9 / 91$ & 7 & 1 & & 81 & $\mathbf{N}$ & \\
\hline 1 & $11 / 9 / 91$ & 8 & 1 & & 82 & $\mathbf{N}$ & \\
\hline 1 & $11 / 9 / 91$ & 9 & 1 & & 81 & $\mathrm{~N}$ & \\
\hline 1 & $11 / 9 / 91$ & 10 & 1 & & 81 & $\mathbf{N}$ & \\
\hline 9 & $11 / 17 / 91$ & 2 & 1 & & 98.9 & $Y$ & \\
\hline 1 & $11 / 17 / 91$ & 3 & 1 & & 92.6 & $\mathbf{N}$ & \\
\hline 1 & $11 / 17 / 91$ & 4 & 1 & & 82.2 & $\mathbf{N}$ & \\
\hline 1 & $11 / 17 / 91$ & 5 & 1 & & 80.4 & $\mathbf{N}$ & \\
\hline 1 & $11 / 17 / 91$ & 6 & 1 & & 80.5 & $\mathbf{N}$ & \\
\hline 9 & $11 / 17 / 91$ & 7 & 1 & & 80.1 & $\mathbf{N}$ & \\
\hline 1 & $11 / 17 / 91$ & 8 & 1 & & 79.3 & N & \\
\hline 1 & $11 / 17 / 91$ & 9 & 1 & & 81.1 & $\mathbf{N}$ & \\
\hline 1 & $11 / 17 / 91$ & 10 & 1 & & 81.1 & $\mathbf{N}$ & \\
\hline 1 & $11 / 24 / 91$ & 2 & 1 & & 101.1 & $Y$ & \\
\hline 1 & $11 / 24 / 91$ & 3 & 1 & & 94.8 & $\mathbf{N}$ & \\
\hline 1 & $11 / 24 / 91$ & 4 & 1 & & 83.7 & $N$ & \\
\hline 1 & $11 / 24 / 91$ & 5 & 1 & & 82.2 & $\mathbf{N}$ & \\
\hline 1 & $11 / 24 / 91$ & 6 & 1 & & 82.6 & $\mathbf{N}$ & \\
\hline 1 & $11 / 24 / 91$ & 7 & 1 & & 81.9 & $\mathbf{N}$ & \\
\hline 1 & $11 / 24 / 91$ & 8 & 1 & & 82.5 & $\mathbf{N}$ & \\
\hline 1 & $11 / 24 / 91$ & 9 & 1 & & 82.6 & $\mathbf{N}$ & \\
\hline 1 & $111 / 24 / 91$ & 10 & 1 & & 82.5 & $\mathrm{~N}$ & \\
\hline 1 & $12 / 1 / 99$ & 2 & 1 & & 100 & $Y$ & \\
\hline 1 & $12 / 1 / 91$ & 3 & 1 & & 93.3 & $N$ & \\
\hline
\end{tabular}

Data obtained from WHC Surveillance Analysis Computer System (SACS), June 2, 1993. 


\begin{tabular}{|c|c|c|c|c|c|c|c|}
\hline \multicolumn{8}{|c|}{ TANK 241-BY-108 } \\
\hline & & & & THER & COUPLE & & \\
\hline TREE \# & DATE & THERMO \# & TREE SET \# & TEMP C & TEMPF & $\bar{Y}=$ MAX TEMP & COMMENTS \\
\hline 1 & $12 / 1 / 91$ & 4 & $\frac{1}{1}$ & & 82 & $\mathbf{N}$ & \\
\hline 9 & $12 / 1 / 91$ & 5 & 1 & & 80.2 & $\ddot{N}$ & \\
\hline 1 & $12 / 1 / 91$ & 6 & 1 & & 80.2 & $\ddot{N}$ & \\
\hline 1 & $12 / 1 / 91$ & 7 & $\frac{1}{1}$ & & 79.8 & N & \\
\hline 1 & $12 / 1 / 91$ & 8 & 1 & & 80.6 & $\ddot{N}$ & \\
\hline 1 & $12 / 1 / 91$ & 9 & 1 & & 80.8 & $\mathbf{N}$ & \\
\hline 1 & $12 / 1 / 91$ & 10 & 1 & & 80.7 & $N$ & \\
\hline 1 & $12 / 6 / 91$ & 2 & 1 & & 100.9 & $\bar{Y}$ & \\
\hline 1 & $12 / 6 / 91$ & 3 & $\frac{T}{1}$ & & 94.5 & $\mathbf{N}$ & \\
\hline 9 & $12 / 6 / 91$ & 4 & 1 & & 83.1 & $\mathbf{N}$ & \\
\hline 1 & $12 / 6 / 91$ & 5 & 1 & & 81.2 & $\mathrm{~N}$ & \\
\hline 1 & $12 / 6 / 91$ & 6 & 1 & & 81.2 & $N$ & \\
\hline 1 & $12 / 6 / 91$ & 7 & 1 & & 80.7 & $N$ & \\
\hline 1 & $12 / 6 / 91$ & 8 & 1 & & 81.5 & $\ddot{N}$ & \\
\hline 1 & $12 / 6 / 91$ & 9 & 1 & & 81.8 & $\mathbf{N}$ & \\
\hline 1 & $12 / 6 / 91$ & 10 & 1 & & 81.9 & $\bar{N}$ & \\
\hline 1 & $12 / 14 / 91$ & 2 & 1 & & 101.5 & $\ddot{Y}$ & \\
\hline 1 & $12 / 14 / 91$ & 3 & 1 & & 94.3 & $N$ & \\
\hline$\frac{1}{1}$ & $12 / 14 / 91$ & $\overline{4}$ & $T$ & & 82.7 & $\mathbf{N}$ & \\
\hline 1 & $12 / 14 / 91$ & 5 & 1 & & 80.9 & $\mathbf{N}$ & \\
\hline 1 & $12 / 14 / 91$ & 6 & 1 & & 80.8 & $N$ & \\
\hline$\frac{1}{1}$ & $12 / 14 / 91$ & 7 & 1 & & 80.4 & $\mathbf{N}$ & \\
\hline 1 & $12 / 14 / 91$ & 8 & 1 & & 81.1 & $\mathbf{N}$ & \\
\hline 1 & $12 / 14 / 91$ & 9 & 1 & & 81.3 & $N$ & \\
\hline 1 & $12 / 14 / 91$ & 10 & 1 & & 81.3 & $\mathbf{N}$ & \\
\hline 1 & $12 / 20 / 91$ & 2 & 1 & & 101.3 & $Y$ & \\
\hline 1 & $12 / 20 / 91$ & 3 & 1 & & 93.9 & $\mathbf{N}$ & \\
\hline$\frac{1}{1}$ & $12 / 20 / 91$ & 4 & 1 & & 82 & $\bar{N}$ & \\
\hline 1 & $12 / 20 / 91$ & 5 & 1 & & 80.2 & $\ddot{N}$ & \\
\hline 1 & $12 / 20 / 91$ & 6 & 1 & & 80.3 & $\mathbf{N}$ & \\
\hline 1 & $12 / 20 / 91$ & 7 & 1 & & 80 & $\mathbf{N}$ & \\
\hline 1 & $12 / 20 / 91$ & 8 & 1 & & 80.5 & $\mathbf{N}$ & \\
\hline 1 & $12 / 20 / 91$ & 9 & 1 & & 80.7 & $\mathbf{N}$ & \\
\hline 1 & $12 / 20 / 91$ & 10 & 1 & & 80.6 & $\mathbf{N}$ & \\
\hline 1 & $12 / 29 / 91$ & 1 & 1 & & 109.2 & $\bar{Y}$ & \\
\hline 1 & $12 / 29 / 91$ & 2 & 1 & & 101.5 & $N$ & \\
\hline 7 & $12 / 29 / 91$ & 3 & 1 & & 94.2 & $N$ & \\
\hline 1 & $12 / 29 / 91$ & 4 & 1 & & 81.9 & $\mathbf{N}$ & \\
\hline 1 & $12 / 29 / 91$ & $\overline{5}$ & 1 & & 80 & $\mathbf{N}$ & \\
\hline 1 & $12 / 29 / 91$ & 6 & 1 & & 80 & $\mathbf{N}$ & \\
\hline 1 & $12 / 29 / 91$ & 7 & 1 & & 79.6 & $\mathbf{N}$ & \\
\hline 1 & $12 / 29 / 91$ & 8 & 1 & & 80.2 & $\mathbf{N}$ & \\
\hline 1 & $12 / 29 / 91$ & 9 & 1 & & 80.3 & $\bar{N}$ & \\
\hline 1 & $12 / 29 / 91$ & 10 & 1 & & 80.2 & N & \\
\hline 1 & $1 / 3 / 92$ & 1 & 1 & & 109 & $\bar{Y}$ & $\mathrm{~J}-910126143-50 \mathrm{TC} \# 2,11-14 \mathrm{O} / \mathrm{S}$ \\
\hline 1 & $1 / 3 / 92$ & 3 & 1 & & 94 & $\mathbf{N}$ & \\
\hline 1 & $1 / 3 / 92$ & 4 & 1 & & 81 & $\mathbf{N}$ & \\
\hline 1 & $1 / 3 / 92$ & 5 & 1 & & 79 & $\mathbf{N}$ & \\
\hline 1 & $1 / 3 / 92$ & 6 & 1 & & 79 & $\mathbf{N}$ & \\
\hline 1 & $1 / 3 / 92$ & 7 & 1 & & 79 & $\mathbf{N}$ & \\
\hline 1 & $1 / 3 / 92$ & 8 & 1 & & 79 & $\mathbf{N}$ & \\
\hline 1 & $1 / 3 / 92$ & 9 & 1 & & 79 & $\mathbf{N}$ & \\
\hline 1 & $1 / 3 / 92$ & 10 & $\frac{1}{1}$ & & 79 & $\mathbf{N}$ & \\
\hline$T$ & $1 / 11 / 92$ & 1 & 1 & & 107.6 & $\bar{Y}$ & $910126143-14$ \\
\hline 1 & $1 / 11 / 92$ & 3 & 1 & & 92.1 & $\dot{N}$ & \\
\hline 1 & $1 / 11 / 92$ & 4 & 1 & & 79.5 & $\mathbf{N}$ & \\
\hline 1 & $1 / 11 / 92$ & 5 & 1 & & 77.7 & $\mathbf{N}$ & \\
\hline 1 & $1 / 11 / 92$ & $\frac{6}{6}$ & $\overline{1}$ & & 77.6 & $\mathrm{~N}$ & \\
\hline 1 & $1 / 11 / 92$ & 7 & 1 & & 77.3 & $\mathbf{N}$ & \\
\hline 1 & $1 / 11 / 92$ & 8 & 1 & & 77.9 & $\mathbf{N}$ & \\
\hline 1 & $1 / 11 / 92$ & 9 & 1 & & 78 & $\mathbf{N}$ & \\
\hline 1 & $1 / 11 / 92$ & 10 & 1 & & 78 & $\mathbf{N}$ & \\
\hline 1 & $1 / 16 / 92$ & 1 & 1 & & 108.2 & $\bar{Y}$ & J910126143-32 \\
\hline 1 & $1 / 16 / 92$ & 3 & 1 & & 93 & $\mathbf{N}$ & \\
\hline 1 & $1 / 16 / 92$ & 4 & $\overline{1}$ & & 79 & $\mathbf{N}$ & \\
\hline 1 & $1 / 16 / 92$ & 5 & $T$ & & 77.7 & $\mathbf{N}$ & \\
\hline
\end{tabular}

Data obtained from WHC Surveillance Analysis Computer System (SACS), June 2, 1993. 
WHC-SD-WM-ER-312, Rev. 0

\begin{tabular}{|c|c|c|c|c|c|c|c|}
\hline \multicolumn{8}{|c|}{ TANK 241-BY-108 } \\
\hline \multicolumn{8}{|c|}{ THERMOCOUPLE DATA } \\
\hline TREE \# & DATE & THERMO \# & TREE SET \# & TEMP C & TEMP F & $\mathbf{Y}=\mathbf{M A X}$ TEMP & COMMENTS \\
\hline 1 & $1 / 16 / 92$ & 6 & 1 & & 77.6 & $\mathbf{N}$ & \\
\hline 1 & $1 / 16 / 92$ & 7 & 1 & & 77.4 & $\mathbf{N}$ & \\
\hline$\frac{1}{1}$ & $1 / 16 / 92$ & 8 & 1 & & 77.4 & $\mathbf{N}$ & \\
\hline 1 & $1 / 16 / 92$ & 9 & 1 & & 78 & $\bar{N}$ & \\
\hline$\frac{1}{1}$ & $1 / 16 / 92$ & 10 & $\frac{1}{1}$ & & 77.9 & $\mathbf{N}$ & \\
\hline 1 & $1 / 25 / 92$ & 1 & 1 & & 107.9 & $\bar{Y}$ & J-910126143-14 \\
\hline 1 & $1 / 25 / 92$ & 2 & 1 & & & $\mathbf{N}$ & \\
\hline 9 & $1 / 25 / 92$ & 3 & 9 & & 92.6 & $\mathbf{N}$ & \\
\hline 1 & $1 / 25 / 92$ & 4 & 1 & & 80 & $\mathrm{~N}$ & \\
\hline 1 & $1 / 25 / 92$ & 5 & 1 & & 77.9 & $\mathbf{N}$ & \\
\hline 1 & $1 / 25 / 92$ & 6 & 1 & & 77.8 & $\mathbf{N}$ & \\
\hline 1 & $1 / 25 / 92$ & 7 & 1 & & 77.5 & $\mathbf{N}$ & \\
\hline 9 & $1 / 25 / 92$ & 8 & 9 & & 75.5 & $\bar{N}$ & \\
\hline 1 & $1 / 25 / 92$ & 9 & 1 & & 73.2 & $\mathbf{N}$ & \\
\hline 1 & $1 / 25 / 92$ & 10 & 1 & & 73.1 & $\mathbf{N}$ & \\
\hline 1 & $1 / 25 / 92$ & 15 & 1 & & & $\overline{\mathbf{N}}$ & \\
\hline 1 & $1 / 28 / 92$ & 1 & 1 & & & $\mathbf{N}$ & $\begin{array}{c}\text { ACCESS RESTRICTED } 1 / 28-2 / 22 \text { DUE TO } \\
\text { BATTERY FUME INCIDENT }\end{array}$ \\
\hline 1 & $2 / 24 / 92$ & 1 & 1 & & 108.4 & $\bar{Y}$ & J-900925741-79 \\
\hline 1 & $2 / 24 / 92$ & 2 & 1 & & & $\mathbf{N}$ & \\
\hline 1 & $2 / 24 / 92$ & 3 & 1 & & 92.1 & $\mathbf{N}$ & \\
\hline 1 & $2 / 24 / 92$ & 4 & 1 & & 79.4 & $\mathbf{N}$ & \\
\hline 1 & $2 / 24 / 92$ & 5 & $\frac{1}{1}$ & & 77.2 & $\mathbf{N}$ & \\
\hline 1 & $2 / 24 / 92$ & 6 & 1 & & 77.2 & $\mathbf{N}$ & \\
\hline 1 & $2 / 24 / 92$ & 7 & 1 & & 76.8 & $\mathbf{N}$ & \\
\hline 9 & $2 / 24 / 92$ & 8 & 1 & & 77.3 & $\mathbf{N}$ & \\
\hline 1 & $2 / 24 / 92$ & 9 & 1 & & 77.5 & $\mathbf{N}$ & \\
\hline 1 & $2 / 24 / 92$ & 10 & 1 & & 79.5 & $\mathbf{N}$ & \\
\hline 1 & $2 / 29 / 92$ & 1 & 1 & & 108.4 & $\mathbf{Y}$ & $J+1.038 / 91012614332$ \\
\hline 1 & $2 / 29 / 92$ & 3 & 1 & & 92 & $\mathbf{N}$ & \\
\hline 1 & $2 / 29 / 92$ & 4 & 1 & & 72.2 & $\mathbf{N}$ & \\
\hline 1 & $2 / 29 / 92$ & 5 & $T$ & & 76.9 & $\mathbf{N}$ & \\
\hline 1 & $2 / 29 / 92$ & 6 & 1 & & 76.8 & $\mathbf{N}$ & \\
\hline 1 & $2 / 29 / 92$ & 7 & 1 & & 76.5 & $\mathbf{N}$ & \\
\hline 1 & $2 / 29 / 92$ & 8 & 1 & & 77 & $\mathbf{N}$ & \\
\hline 1 & $2 / 29 / 92$ & 9 & 1 & & 77.2 & $\mathbf{N}$ & \\
\hline 1 & $2 / 29 / 92$ & 10 & $T$ & & 77.1 & $\mathbf{N}$ & \\
\hline 1 & $3 / 8 / 92$ & 1 & 1 & & 107.3 & $\mathbf{Y}$ & $J 910126143.48$ \\
\hline 1 & $3 / 8 / 92$ & 3 & 1 & & 91.1 & $\mathbf{N}$ & \\
\hline 1 & $3 / 8 / 92$ & 4 & 1 & & 78.3 & $\mathbf{N}$ & \\
\hline 1 & $3 / 8 / 92$ & 5 & 1 & & 76.1 & $\mathbf{N}$ & \\
\hline 1 & $3 / 8 / 92$ & 6 & 1 & & 76.1 & $\mathbf{N}$ & \\
\hline 1 & $3 / 8 / 92$ & 7 & 1 & & 75.8 & $\mathbf{N}$ & \\
\hline 1 & $3 / 8 / 92$ & 8 & 1 & & 76.1 & $\mathbf{N}$ & \\
\hline 1 & $3 / 8 / 92$ & 9 & 1 & & 76.2 & $\mathbf{N}$ & \\
\hline 1 & $3 / 8 / 92$ & 10 & 1 & & 76.2 & $\mathbf{N}$ & \\
\hline 1 & $3 / 15 / 92$ & 1 & 1 & & 108.5 & $Y$ & J-910126143-32 \\
\hline 1 & $3 / 15 / 92$ & 2 & 1 & & & $\mathbf{N}$ & $T C \# 2,100 / \mathrm{S}$ \\
\hline 1 & $3 / 15 / 92$ & 3 & 1 & & 92 & $\mathbf{N}$ & \\
\hline 9 & $3 / 15 / 92$ & 4 & $\frac{1}{1}$ & & 79.1 & $\mathbf{N}$ & \\
\hline 1 & $3 / 15 / 92$ & 5 & 1 & & 76.7 & $\mathbf{N}$ & \\
\hline 1 & $3 / 15 / 92$ & 6 & 1 & & 76.6 & $\mathbf{N}$ & \\
\hline 1 & $3 / 15 / 92$ & 7 & 1 & & 76.3 & $\mathbf{N}$ & \\
\hline 1 & $3 / 15 / 92$ & 8 & 1 & & 76.6 & $\mathbf{N}$ & \\
\hline 1 & $3 / 15 / 92$ & 9 & 1 & & 76.7 & $\mathbf{N}$ & \\
\hline 1 & $3 / 15 / 92$ & 10 & 1 & & 76.7 & $\mathbf{N}$ & \\
\hline 1 & $3 / 20 / 92$ & 1 & 1 & & 108.1 & $\bar{Y}$ & J 91012614332 TC $\# 20 / 5$ \\
\hline 1 & $3 / 20 / 92$ & 3 & 1 & & 91.7 & $\mathrm{~N}$ & \\
\hline 1 & $3 / 20 / 92$ & 4 & 1 & & 78.6 & $\mathbf{N}$ & \\
\hline 9 & $3 / 20 / 92$ & 5 & 1 & & 76.3 & $\mathbf{N}$ & \\
\hline 1 & $3 / 20 / 92$ & 6 & 1 & & 76.3 & $\mathbf{N}$ & \\
\hline 1 & $3 / 20 / 92$ & 7 & 1 & & 76 & $\mathbf{N}$ & \\
\hline 1 & $3 / 20 / 92$ & 8 & 1 & & 76.3 & $\mathbf{N}$ & \\
\hline 1 & $3 / 20 / 92$ & 9 & 1 & & 76.5 & $\bar{N}$ & \\
\hline 1 & $3 / 20 / 92$ & 10 & 1 & & 76.4 & $\mathbf{N}$ & \\
\hline 1 & $3 / 29 / 92$ & 9 & 1 & & 108.6 & $\bar{Y}$ & J910126143.66 TC $\# 20 / \mathrm{S}$ \\
\hline
\end{tabular}

Data obtained from WHC Surveillance Analysis Computer System (SACS), June 2, 1993. 
WHC-SD-WM-ER-312, ReV. 0

\begin{tabular}{|c|c|c|c|c|c|c|c|}
\hline \multicolumn{8}{|c|}{ TANK 241-BY-108 } \\
\hline \multicolumn{8}{|c|}{ THERMOCOUPLE DATA } \\
\hline TREF \# & DATF & THERMO \# & TREE SET \# & TFMP C & TFMP $E$ & $Y=M A X$ TFMD & COMMENTS \\
\hline 1 & $3 / 29 / 92$ & 3 & $\frac{1}{1}$ & & 92.9 & $N$ & CUMMIETS \\
\hline$\frac{1}{1}$ & $3 / 29 / 92$ & 4 & 1 & & 80.3 & $\ddot{N}$ & \\
\hline 1 & $3 / 29 / 92$ & 5 & 1 & & 78.2 & $\mathbf{N}$ & \\
\hline 1 & $3 / 29 / 92$ & 6 & 1 & & 78.1 & $\mathbf{N}$ & \\
\hline 1 & $3 / 29 / 92$ & 7 & 1 & & 77.9 & $\mathbf{N}$ & \\
\hline 1 & $3 / 29 / 92$ & 8 & 1 & & 78.2 & $\mathbf{N}$ & \\
\hline 1 & $3 / 29 / 92$ & 9 & 1 & & 78.5 & $\mathbf{N}$ & \\
\hline 1 & $3 / 29 / 92$ & 10 & 1 & & 78.6 & $\mathbf{N}$ & \\
\hline$T$ & $4 / 3 / 92$ & 1 & 1 & & 106.5 & $\mathbf{Y}$ & J 910126143-14 \\
\hline$T$ & $4 / 3 / 92$ & 3 & 1 & & 90.5 & $\mathbf{N}$ & \\
\hline 1 & $4 / 3 / 92$ & 4 & 1 & & 78.2 & $\mathbf{N}$ & \\
\hline 1 & $4 / 3 / 92$ & 5 & 1 & & 75.9 & $\mathbf{N}$ & \\
\hline 1 & $4 / 3 / 92$ & 6 & 1 & & 75.9 & $\mathbf{N}$ & \\
\hline 1 & $4 / 3 / 92$ & 7 & 1 & & 75.6 & $\mathbf{N}$ & \\
\hline 1 & $4 / 3 / 92$ & 8 & 1 & & 75.8 & $\mathbf{N}$ & \\
\hline 1 & $4 / 3 / 92$ & 9 & 1 & & 76 & $\mathbf{N}$ & \\
\hline 1 & $4 / 3 / 92$ & 10 & 1 & & 76.1 & $\overline{\mathbf{N}}$ & \\
\hline 1 & $4 / 11 / 92$ & 1 & 1 & & 107.6 & $Y$ & J 910126143-64 \\
\hline 1 & $4 / 11 / 92$ & 3 & 1 & & 91.7 & $\mathbf{N}$ & \\
\hline 1 & $4 / 11 / 92$ & 4 & 1 & & 79.2 & $\mathrm{~N}$ & \\
\hline 9 & $4 / 11 / 92$ & 5 & 1 & & 77.1 & $N$ & \\
\hline 1 & $4 / 11 / 92$ & 6 & 1 & & 77 & $\mathbf{N}$ & \\
\hline 1 & $4 / 11 / 92$ & 7 & 1 & & 76.7 & $\mathbf{N}$ & \\
\hline 9 & $4 / 11 / 92$ & 8 & 1 & & 77.2 & $\mathbf{N}$ & \\
\hline 1 & $4 / 11 / 92$ & 9 & 1 & & 77.4 & $\mathbf{N}$ & \\
\hline 1 & $4 / 11 / 92$ & 10 & 1 & & 77.4 & $\mathbf{N}$ & \\
\hline 1 & $4 / 17 / 92$ & 1 & $\frac{1}{1}$ & & 108.9 & $\bar{Y}$ & \\
\hline 1 & $4 / 17 / 92$ & 3 & 1 & & 92.8 & $\mathbf{N}$ & \\
\hline 1 & $4 / 17 / 92$ & 4 & 1 & & 80.5 & $\mathbf{N}$ & \\
\hline 1 & $4 / 17 / 92$ & 5 & 1 & & 78.3 & $\mathbf{N}$ & \\
\hline$\frac{1}{1}$ & $4 / 17 / 92$ & 6 & $T$ & & 78.2 & $\mathbf{N}$ & \\
\hline 1 & $4 / 17 / 92$ & 7 & 1 & & 78.1 & $\mathbf{N}$ & \\
\hline 1 & $4 / 17 / 92$ & 8 & 1 & & 78.5 & $\mathbf{N}$ & \\
\hline 1 & $4 / 17 / 92$ & 9 & 1 & & 78.6 & $\mathbf{N}$ & \\
\hline 1 & $4 / 17 / 92$ & 10 & 1 & & 78.6 & $\mathbf{N}$ & \\
\hline 1 & $4 / 25 / 92$ & 1 & 1 & & 111.1 & $\mathbf{Y}$ & $\mathrm{J} 89052351220$ \\
\hline 1 & $4 / 25 / 92$ & 3 & 1 & & 95.31 & $\mathbf{N}$ & \\
\hline 1 & $4 / 25 / 92$ & 4 & 1 & & 82.6 & $\mathbf{N}$ & \\
\hline 9 & $4 / 25 / 92$ & 5 & 1 & & 80.1 & $\mathbf{N}$ & \\
\hline 1 & $4 / 25 / 92$ & 6 & 1 & & 79.9 & $\mathbf{N}$ & \\
\hline 1 & $4 / 25 / 92$ & 7 & 1 & & 79.6 & $\mathbf{N}$ & \\
\hline 1 & $4 / 25 / 92$ & 8 & 1 & & 79.8 & $\mathrm{~N}$ & \\
\hline 1 & $4 / 25 / 92$ & 9 & 1 & & 80.1 & $\mathbf{N}$ & \\
\hline 1 & $4 / 25 / 92$ & 10 & 7 & & 80.2 & $\mathbf{N}$ & \\
\hline 1 & $4 / 30 / 92$ & 3 & 1 & & 89.6 & $\mathbf{N}$ & J 890523512-47 NO MAX FLAGGED \\
\hline 1 & $4 / 30 / 92$ & 4 & 1 & & 77.9 & $\mathrm{~N}$ & \\
\hline 1 & $4 / 30 / 92$ & 5 & 9 & & 75.6 & $N$ & \\
\hline 1 & $4 / 30 / 92$ & 6 & 1 & & 75.5 & $\mathbf{N}$ & \\
\hline 1 & $4 / 30 / 92$ & 7 & 1 & & 75.3 & $\mathbf{N}$ & \\
\hline 1 & $4 / 30 / 92$ & 8 & 1 & & 75.5 & $\mathbf{N}$ & \\
\hline 1 & $4 / 30 / 92$ & 9 & 1 & & 75.7 & $\bar{N}$ & \\
\hline 1 & $4 / 30 / 92$ & 10 & 1 & & 75.7 & $\mathrm{~N}$ & \\
\hline 1 & $5 / 8 / 92$ & 1 & 1 & & 106.4 & $Y$ & J 910126143-48 TC \#2 O/S \\
\hline 1 & $5 / 8 / 92$ & 3 & $\frac{1}{1}$ & & 90.6 & $\mathbf{N}$ & \\
\hline 1 & $5 / 8 / 92$ & 4 & 1 & & 79 & $\mathbf{N}$ & \\
\hline 1 & $5 / 8 / 92$ & 5 & 1 & & 76.7 & $\mathbf{N}$ & \\
\hline 1 & $5 / 8 / 92$ & 6 & 1 & & 76.6 & $\mathbf{N}$ & \\
\hline 1 & $5 / 8 / 92$ & 7 & 1 & & 76.4 & $N$ & \\
\hline 1 & $5 / 8 / 92$ & 8 & 1 & & 76.7 & $\mathbf{N}$ & \\
\hline 1 & $5 / 8 / 92$ & 9 & 1 & & 76.9 & $\mathbf{N}$ & \\
\hline 1 & $5 / 8 / 92$ & 10 & 1 & & 76.9 & $\mathrm{~N}$ & \\
\hline 1 & $5 / 16 / 92$ & 1 & 1 & & 108.5 & $\bar{Y}$ & $J 910126143.32$ \\
\hline 1 & $5 / 16 / 92$ & 3 & 1 & & 92.7 & $\mathrm{~N}$ & \\
\hline 1 & $5 / 16 / 92$ & 4 & 1 & & 81.2 & $\mathbf{N}$ & \\
\hline 1 & $5 / 16 / 92$ & 5 & 9 & $\bar{r}$ & 78.7 & $\mathbf{N}$ & \\
\hline 9 & $5 / 16 / 92$ & 6 & 1 & & 78.7 & N & \\
\hline
\end{tabular}

Data obtained from "WHC Surveillance Analysis Computer System (SACS), June 2, 1993. 
WHC-SD-WM-ER-312, Rev. 0

\begin{tabular}{|c|c|c|c|c|c|c|c|}
\hline \multicolumn{8}{|c|}{ TANK 241-BY-108 } \\
\hline \multicolumn{8}{|c|}{ THERMOCOUPLE DATA } \\
\hline TREE \# & DATE & THERMO \# & TREE SET \# & TEMP C & TEMP F & $Y=$ MAX TEMP & COMMENTS \\
\hline 1 & $5 / 16 / 92$ & 7 & 1 & & 78.4 & $\mathbf{N}$ & \\
\hline 1 & $5 / 16 / 92$ & 8 & 1 & & 78.6 & $\mathbf{N}$ & \\
\hline 1 & $5 / 16 / 92$ & 9 & 1 & & 78.8 & $\mathbf{N}$ & \\
\hline 1 & $5 / 16 / 92$ & 10 & 1 & & 79.2 & $\overline{\mathbf{N}}$ & \\
\hline 1 & $5 / 24 / 92$ & 1 & 1 & & 106.9 & $\bar{Y}$ & J 890523512-47 \\
\hline 1 & $5 / 24 / 92$ & 3 & 1 & & 91.9 & $\mathbf{N}$ & \\
\hline 1 & $5 / 24 / 92$ & 4 & 1 & & 80.2 & $\mathbf{N}$ & \\
\hline 1 & $5 / 24 / 92$ & 5 & 1 & & 77.9 & $\mathbf{N}$ & \\
\hline 1 & $5 / 24 / 92$ & 6 & 1 & & 77.9 & $N$ & \\
\hline 1 & $5 / 24 / 92$ & 7 & 1 & & 77.6 & $\mathrm{~N}$ & \\
\hline 1 & $5 / 24 / 92$ & 8 & 1 & & 77.9 & $\mathbf{N}$ & \\
\hline 1 & $5 / 24 / 92$ & 9 & 1 & & 78.4 & $\mathbf{N}$ & \\
\hline 1 & $5 / 28 / 92$ & 1 & 1 & & 104.7 & $\bar{Y}$ & J $890523512-47$ \\
\hline 9 & $5 / 28 / 92$ & 3 & 1 & & 89.1 & $\mathbf{N}$ & \\
\hline 1 & $5 / 28 / 92$ & 4 & 1 & & 78.1 & $\mathbf{N}$ & \\
\hline 1 & $5 / 28 / 92$ & 5 & 1 & & 75.9 & $\mathbf{N}$ & \\
\hline 1 & $5 / 28 / 92$ & 6 & 1 & & 75.8 & $\mathbf{N}$ & \\
\hline 1 & $5 / 28 / 92$ & 7 & 1 & & 75.7 & $\mathbf{N}$ & \\
\hline 1 & $5 / 28 / 92$ & 8 & 1 & & 75.9 & $\mathbf{N}$ & \\
\hline 1 & $5 / 28 / 92$ & 9 & 1 & & 75.1 & $\mathbf{N}$ & \\
\hline 1 & $5 / 28 / 92$ & 10 & 1 & & 76.2 & $\mathbf{N}$ & \\
\hline 1 & $6 / 5 / 92$ & 1 & 1 & & 106.1 & $\bar{Y}$ & $J 910126143.50$ \\
\hline 1 & $6 / 5 / 92$ & 3 & 1 & & 90.6 & $\mathbf{N}$ & \\
\hline 1 & $6 / 5 / 92$ & 4 & 1 & & 79.6 & $\mathbf{N}$ & \\
\hline 1 & $6 / 5 / 92$ & 5 & 1 & & 77.3 & $\mathbf{N}$ & \\
\hline 1 & $6 / 5 / 92$ & 6 & 1 & & 77.2 & $\mathbf{N}$ & $\div$ \\
\hline 1 & $6 / 5 / 92$ & 7 & 1 & & 76.9 & $\mathbf{N}$ & \\
\hline 1 & $6 / 5 / 92$ & 8 & 1 & & 77.2 & $\mathbf{N}$ & \\
\hline 1 & $6 / 5 / 92$ & 9 & 1 & & 77.5 & $\mathbf{N}$ & \\
\hline 1 & $6 / 5 / 92$ & 10 & 1 & & 77.5 & $\mathrm{~N}$ & \\
\hline 1 & $6 / 13 / 92$ & 1 & 1 & & 103.1 & $\bar{Y}$ & J 890523512-47 \\
\hline 1 & $6 / 13 / 92$ & 3 & 1 & & 87.8 & $N$ & \\
\hline 1 & $6 / 13 / 92$ & 4 & 1 & & 77.4 & $N$ & \\
\hline 1 & $6 / 13 / 92$ & 5 & 1 & & 75.2 & $N$ & \\
\hline 1 & $6 / 13 / 92$ & 6 & 1 & & 75.1 & $\mathbf{N}$ & \\
\hline 1 & $6 / 13 / 92$ & 7 & 1 & & 75 & $\mathbf{N}$ & \\
\hline 1 & $6 / 13 / 92$ & 8 & 1 & & 75.3 & $\mathbf{N}$ & \\
\hline 1 & $6 / 13 / 92$ & 9 & 1 & & 75.5 & $\mathbf{N}$ & \\
\hline 9 & $6 / 13 / 92$ & 10 & 1 & & 75.5 & $\mathbf{N}$ & \\
\hline 1 & $6 / 20 / 92$ & 1 & 1 & & 107.7 & $\bar{Y}$ & $J 910126143-48$ \\
\hline 1 & $6 / 20 / 92$ & 3 & 1 & & 93.4 & $\mathbf{N}$ & \\
\hline 1 & $6 / 20 / 92$ & 4 & 1 & & 82.8 & $\mathbf{N}$ & \\
\hline 1 & $6 / 20 / 92$ & 5 & 1 & & 80.5 & $\mathbf{N}$ & \\
\hline 1 & $6 / 20 / 92$ & 6 & 1 & & 80.4 & $\mathbf{N}$ & \\
\hline 1 & $6 / 20 / 92$ & 7 & 1 & & 80.3 & $\mathrm{~N}$ & \\
\hline 1 & $6 / 20 / 92$ & 8 & 1 & & 80.5 & $\mathbf{N}$ & \\
\hline 1 & $6 / 20 / 92$ & 9 & 1 & & 81.2 & $\mathbf{N}$ & \\
\hline 9 & $6 / 20 / 92$ & 10 & 1 & & 81.5 & $\mathbf{N}$ & \\
\hline 1 & $6 / 28 / 92$ & 9 & 1 & & 105.5 & $\bar{Y}$ & $\mathrm{~J} \mathrm{T1}-042910126143-66$ \\
\hline 1 & $6 / 28 / 92$ & 3 & 9 & & 90.6 & $\mathbf{N}$ & \\
\hline 1 & $6 / 28 / 92$ & 4 & 1 & & 80.5 & $\mathbf{N}$ & \\
\hline 1 & $6 / 28 / 92$ & 5 & 1 & & 78.5 & $\bar{N}$ & \\
\hline 1 & $6 / 28 / 92$ & 6 & 1 & & 78.5 & $\mathbf{N}$ & \\
\hline 1 & $6 / 28 / 92$ & 7 & 1 & & 78.4 & $\mathbf{N}$ & \\
\hline 9 & $6 / 28 / 92$ & 8 & 1 & & 78.7 & $\mathbf{N}$ & \\
\hline 1 & $6 / 28 / 92$ & 9 & 1 & & 79 & $\mathrm{~N}$ & \\
\hline 1 & $6 / 28 / 92$ & 10 & 1 & & 79.1 & $\mathbf{N}$ & \\
\hline 1 & $7 / 3 / 92$ & 1 & 1 & & 105.2 & $\bar{Y}$ & $\mathrm{JTH}-042$ \\
\hline 1 & $7 / 3 / 92$ & 3 & 1 & & 90.12 & $\mathbf{N}$ & \\
\hline 1 & $7 / 3 / 92$ & 4 & 1 & & 80 & $N$ & \\
\hline 1 & $7 / 3 / 92$ & 5 & 1 & & 77.9 & $\mathbf{N}$ & \\
\hline 1 & $7 / 3 / 92$ & 6 & 1 & & 77.9 & $\mathbf{N}$ & \\
\hline 1 & $7 / 3 / 92$ & 7 & 1 & & 77.8 & $\bar{N}$ & \\
\hline 1 & $7 / 3 / 92$ & 8 & 1 & & 78 & $\mathbf{N}$ & \\
\hline 1 & $7 / 3 / 92$ & 9 & 1 & & 78.2 & $N$ & \\
\hline 1 & $7 / 3 / 92$ & 10 & 1 & & 78.3 & $\mathbf{N}$ & \\
\hline
\end{tabular}

Data obtained from WHC Surveillance Analysis Computer System (SACS), June 2, 1993. 
WHC-SD-WM-ER-312, Rev. 0

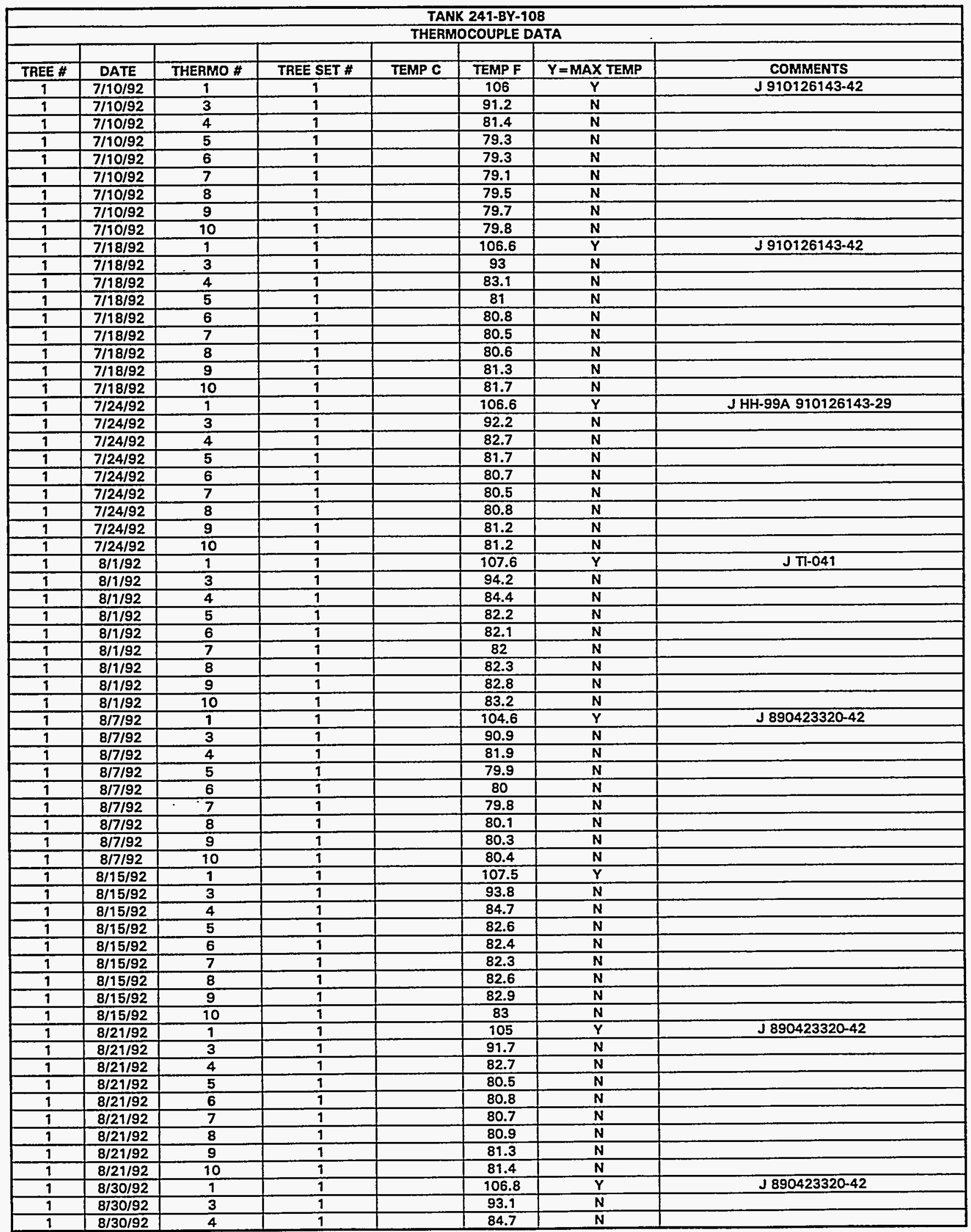

Data obtained from WHC Surveillance Analysis Computer System (SACS), June 2, 1993. 


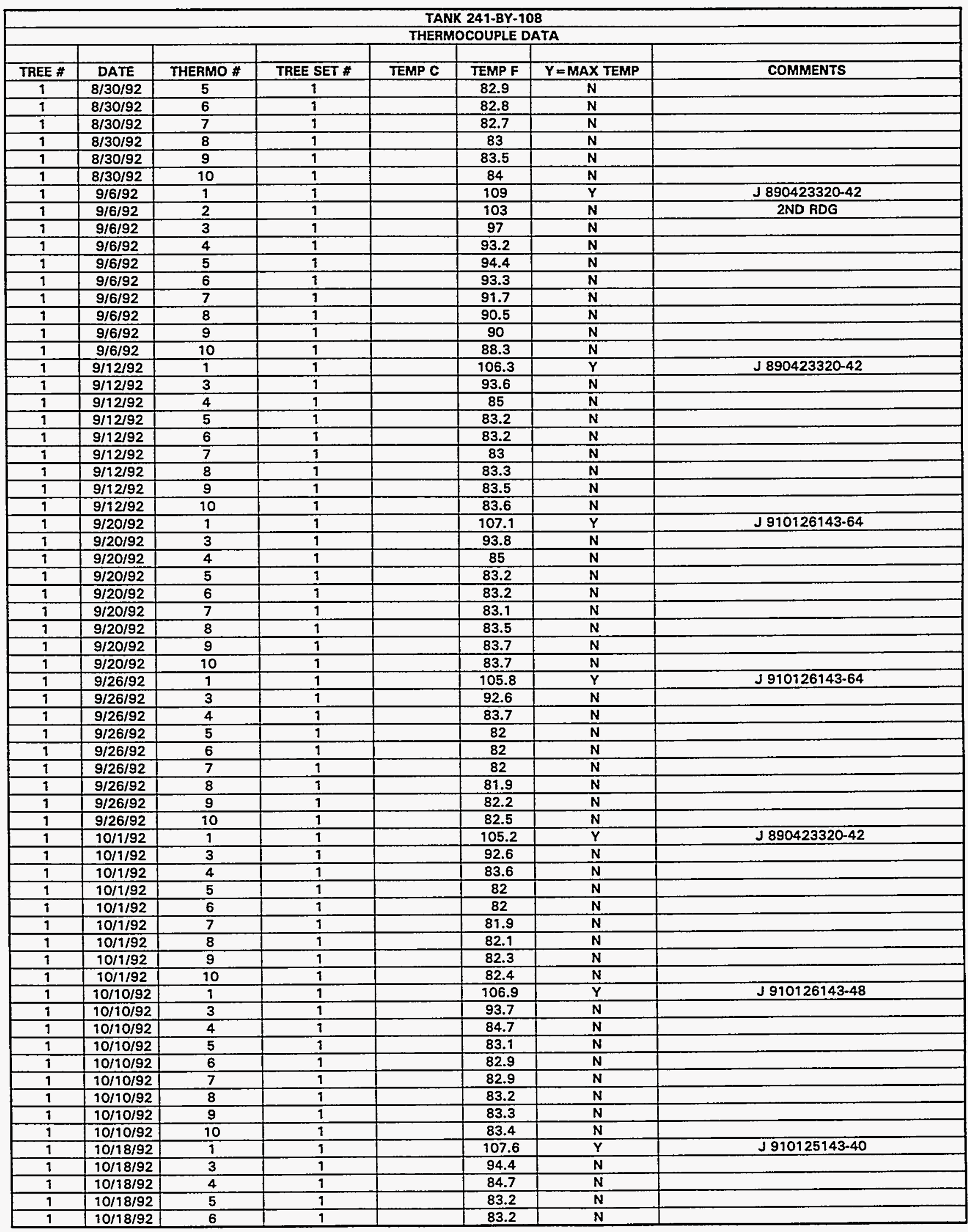

Data obtained from WHC Surveillance Analysis Computer System (SACS), June 2, 1993. 
WHC-SD-WM-ER-312, Rev. 0

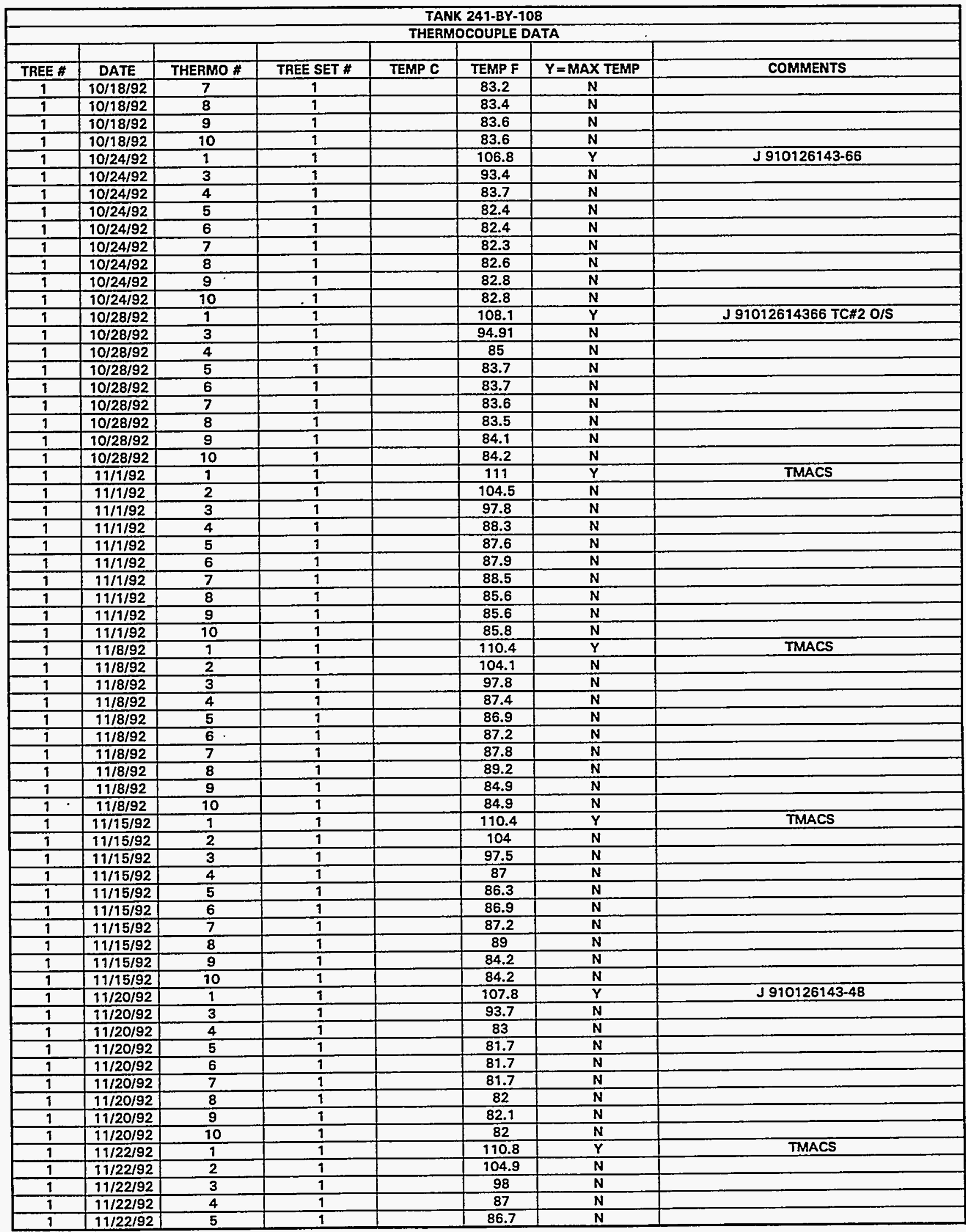

Data obtained from WHC Surveillance Analysis Computer System (SACS), June 2, 1993. 
WHC-SD-WM-ER-312, Rev. 0

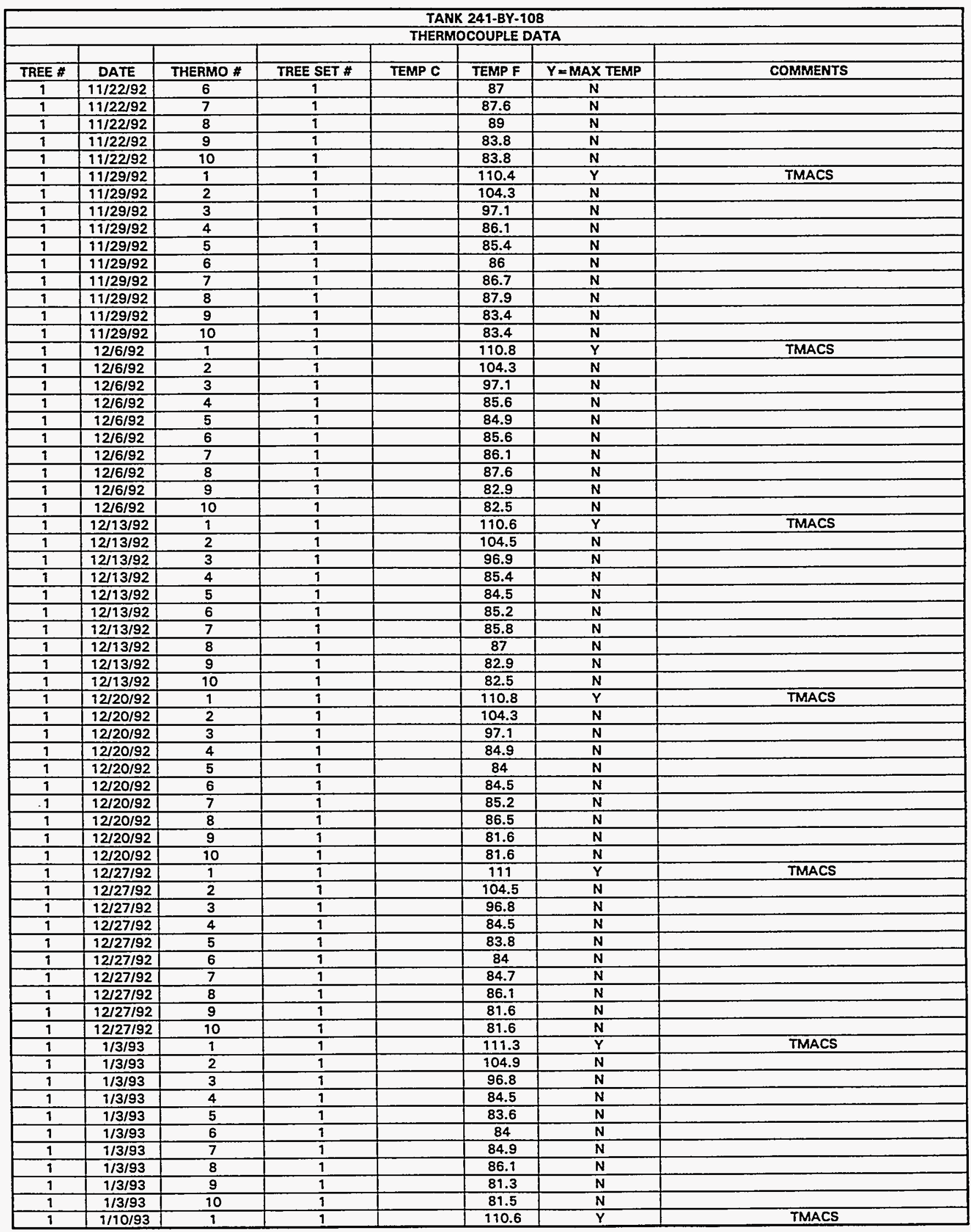

Data obtained from WHC Surveillance Analysis Computer System (SACS), June 2, 1993. 
WHC-SD-WM-ER-312, Rev. 0

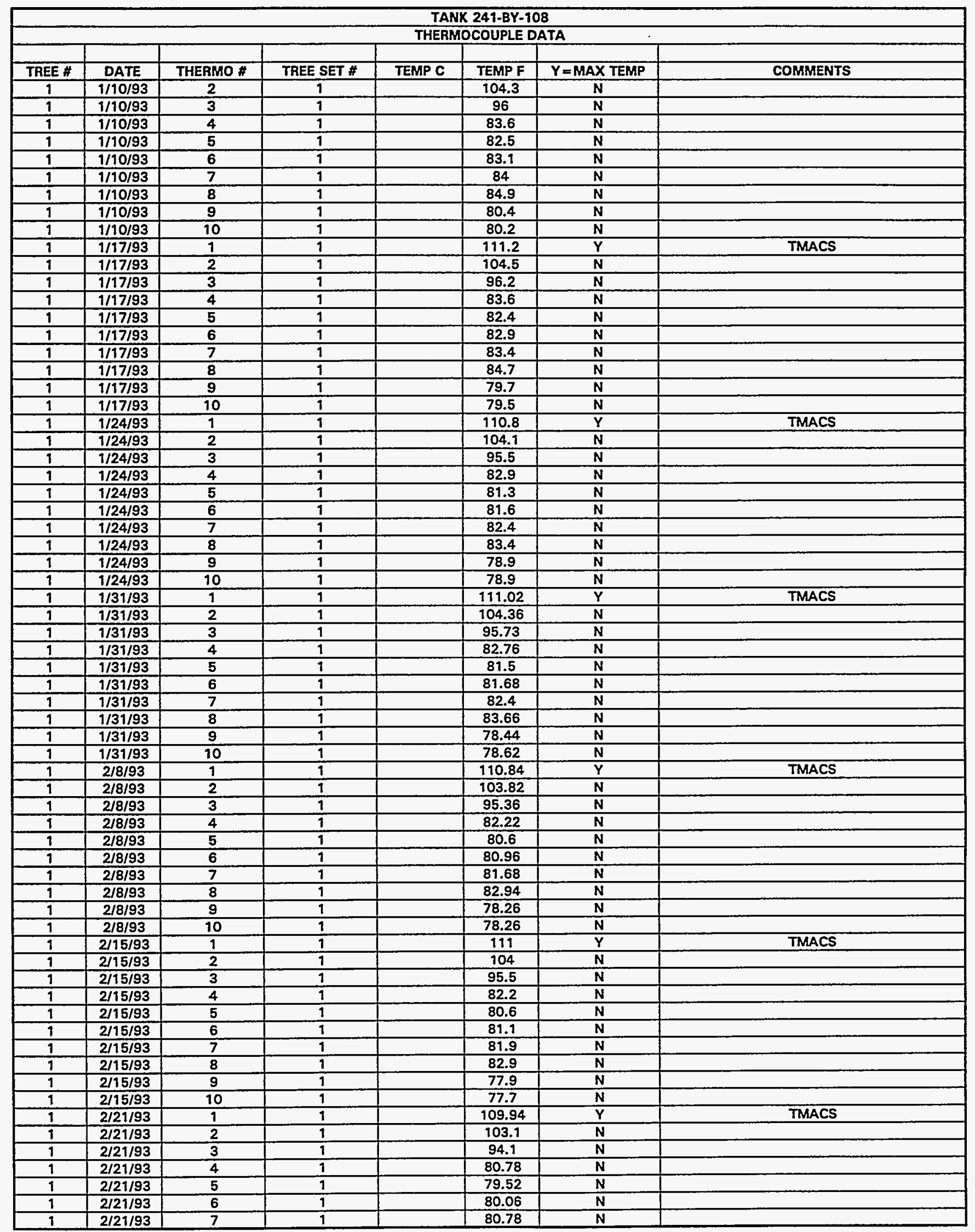

Data obtained from WHC Surveillance Analysis Computer System (SACS), June 2, 1993. 
WHC-SD-WM-ER-312, Rev. 0

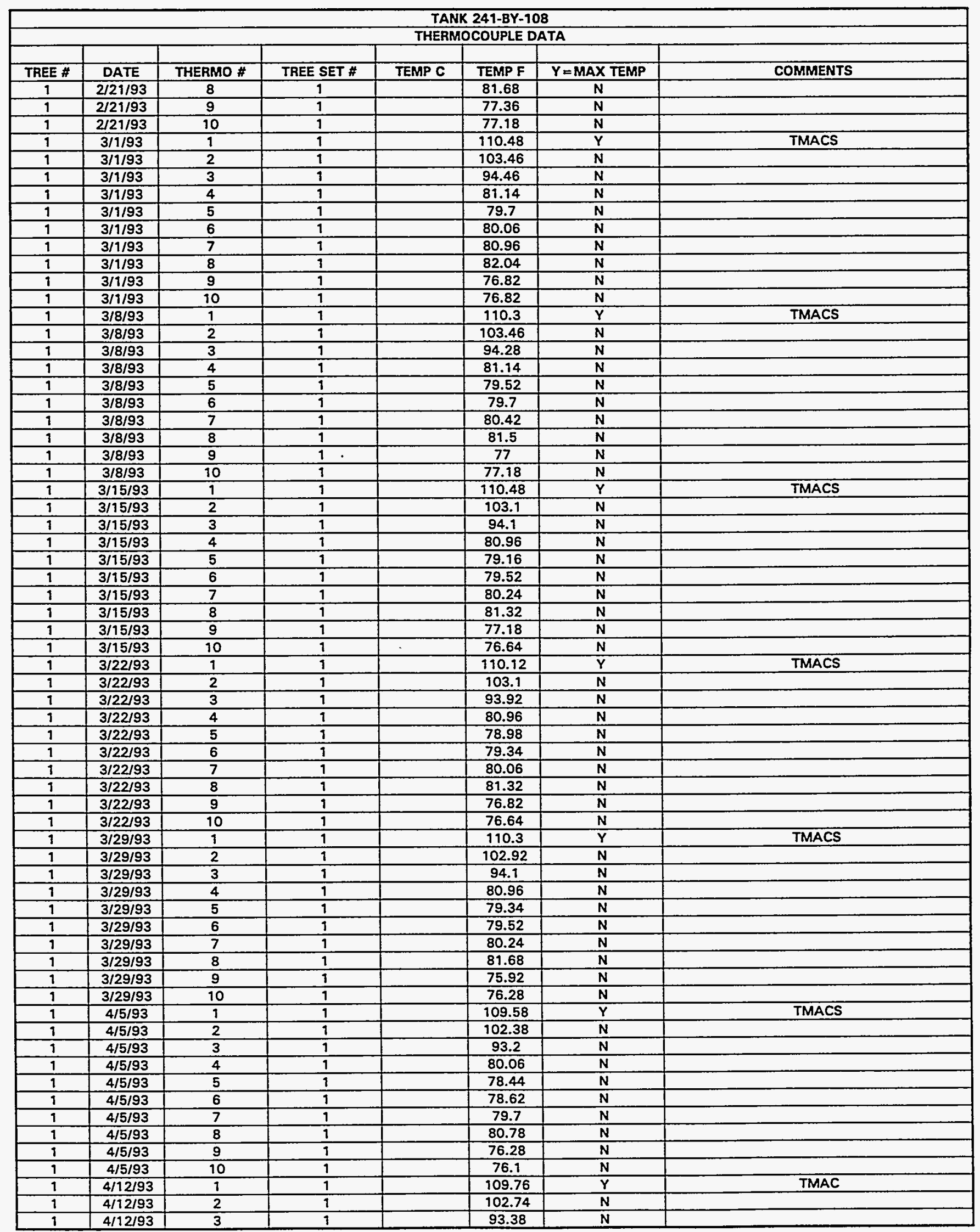

Data obtained from WHC Surveillance Analysis Computer System (SACS), June 2, 1993. 


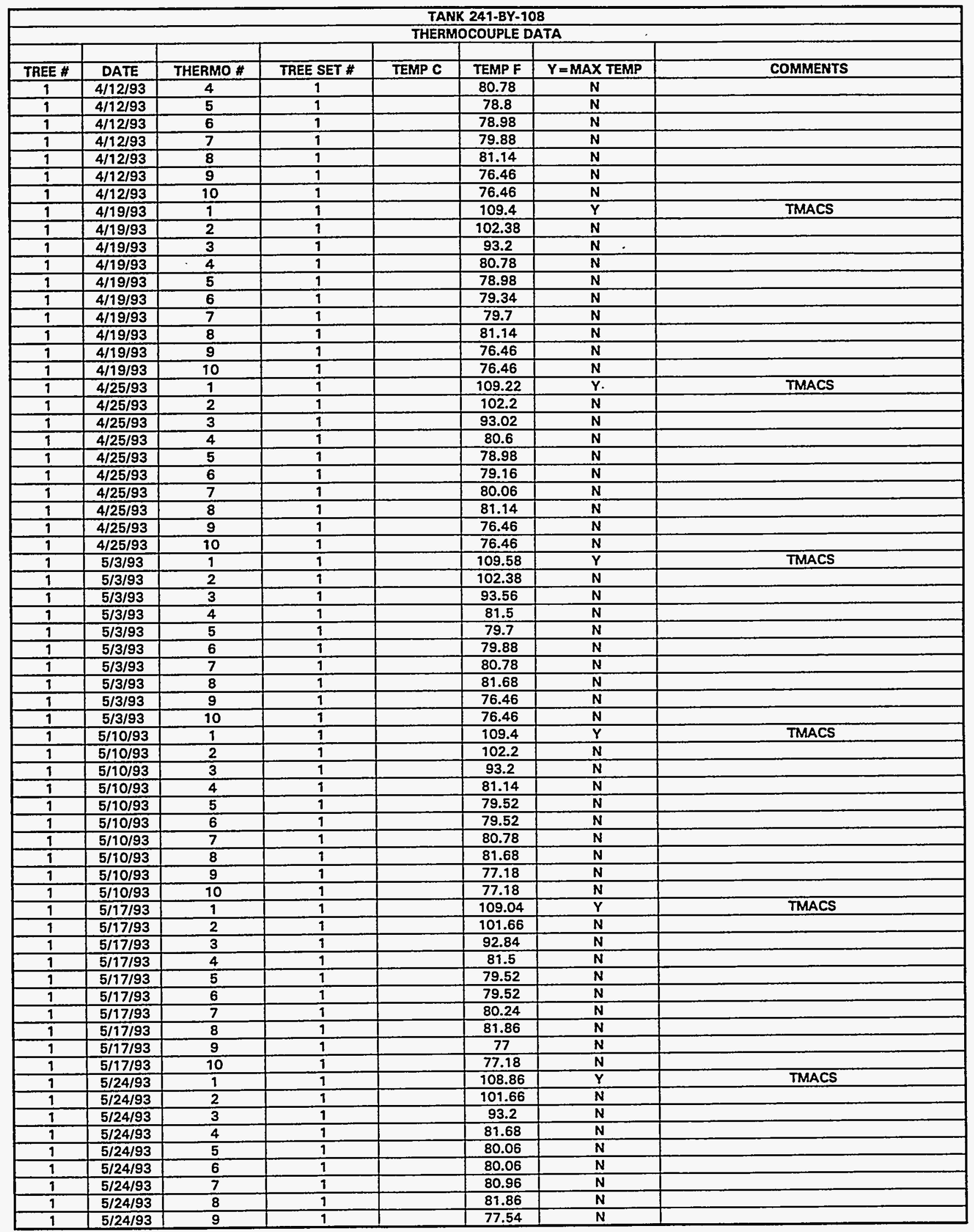

Data obtained from WHC Surveillance Analysis Computer System (SACS), June 2, 1993. 


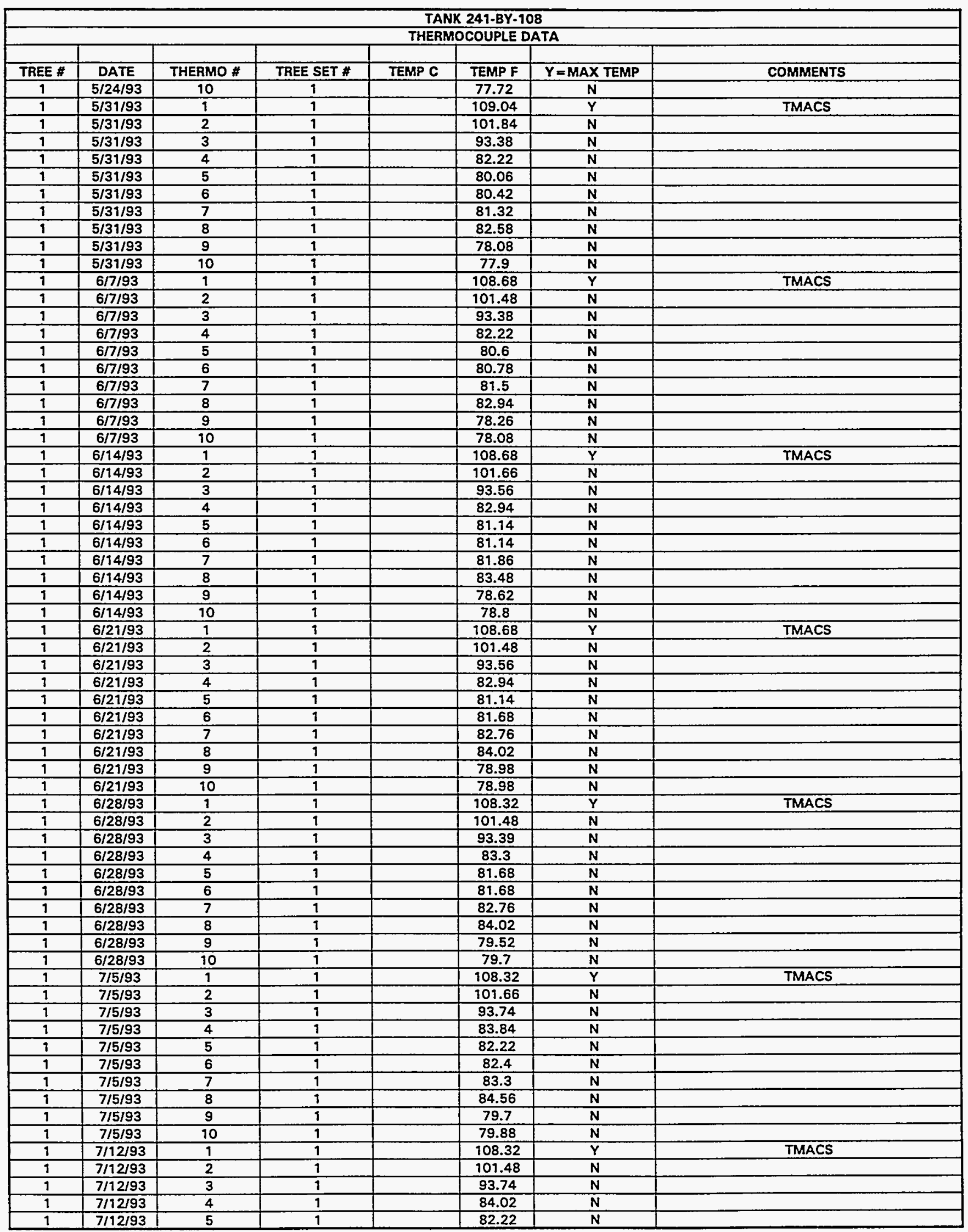

Data obtained from WHC Surveillance Analysis Computer System (SACS), June 2, 1993. 


\begin{tabular}{|c|c|c|c|c|c|c|c|}
\hline \multicolumn{8}{|c|}{ TANK 241-BY-108 } \\
\hline & & & & THER & COUPLE I & TA & \\
\hline TREE \# & DATE & THERMO \# & TREE SET \# & TEMP C & TEMPF & $Y=$ MAX TEMP & COMMENTS \\
\hline 1 & $7 / 12 / 93$ & 6 & 1 & & 82.58 & $\mathbf{N}$ & \\
\hline 1 & $7 / 12 / 93$ & 7 & 1 & & 83.3 & $\ddot{N}$ & \\
\hline 1 & $7 / 12 / 93$ & 8 & 1 & & 84.56 & $\mathbf{N}$ & \\
\hline 1 & $7 / 12 / 93$ & 9 & 1 & & 80.42 & $\mathbf{N}$ & \\
\hline 1 & $7 / 12 / 93$ & 10 & 1 & & 80.6 & $\mathbf{N}$ & \\
\hline 9 & $7 / 19 / 93$ & 1 & 1 & & 108.32 & $\bar{Y}$ & TMACS \\
\hline 1 & $7 / 19 / 93$ & 2 & $\frac{1}{1}$ & & 101.66 & $\dot{N}$ & \\
\hline 9 & $7 / 19 / 93$ & 3 & 1 & & 93.74 & $\mathbf{N}$ & \\
\hline$\frac{1}{1}$ & $7 / 19 / 93$ & 4 & $\overline{1}$ & & 84.38 & $\mathbf{N}$ & \\
\hline$\frac{1}{1}$ & $7 / 19 / 93$ & 5 & $\frac{\pi}{1}$ & & 82.76 & $\mathbf{N}$ & \\
\hline$\frac{1}{1}$ & $7 / 19 / 93$ & 6 & 9 & & 83.12 & $\mathbf{N}$ & \\
\hline$T$ & $7 / 19 / 93$ & 7 & 9 & & 84.02 & $\mathbf{N}$ & \\
\hline 1 & $7 / 19 / 93$ & 8 & 1 & & 85.28 & N & \\
\hline 1 & $7 / 19 / 93$ & 9 & 9 & & 80.42 & $\mathbf{N}$ & \\
\hline 1 & $7 / 19 / 93$ & 10 & 9 & & 80.6 & $\mathbf{N}$ & \\
\hline 1 & $7 / 26 / 93$ & 1 & 1 & & 108.32 & $\bar{Y}$ & TMACS \\
\hline 1 & $7 / 26 / 93$ & 2 & 1 & & 101.3 & $\bar{N}$ & \\
\hline$\frac{1}{1}$ & $7 / 26 / 93$ & 3 & 1 & & 93.74 & $\bar{N}$ & \\
\hline 1 & $7 / 26 / 93$ & 4 & 9 & & 84.38 & $\mathbf{N}$ & \\
\hline 9 & $7 / 26 / 93$ & 5 & 1 & & 82.94 & $\mathbf{N}$ & \\
\hline$\overline{1}$ & $7 / 26 / 93$ & 6 & 1 & & 82.94 & $\mathbf{N}$ & \\
\hline 1 & $7 / 26 / 93$ & 7 & 1 & & 83.84 & $\ddot{N}$ & \\
\hline 1 & $7 / 26 / 93$ & 8 & 1 & & 84.92 & $\mathrm{~N}$ & \\
\hline 1 & $7 / 26 / 93$ & 9 & 1 & & 81.14 & $\mathrm{~N}$ & \\
\hline 1 & $7 / 26 / 93$ & 10 & 1 & & 81.14 & $\mathbf{N}$ & \\
\hline$\frac{1}{1}$ & $8 / 2 / 93$ & 1 & 1 & & 108.32 & $\bar{Y}$ & TMACS \\
\hline$\frac{1}{1}$ & $8 / 2 / 93$ & 2 & 9 & & 101.84 & $\mathbf{N}$ & \\
\hline 1 & $8 / 2 / 93$ & 3 & 1 & & 94.1 & $\mathbf{N}$ & \\
\hline$\frac{1}{1}$ & $8 / 2 / 93$ & 4 & 1 & & 84.74 & $\mathbf{N}$ & \\
\hline$\frac{T}{1}$ & $8 / 2 / 93$ & 5 & 1 & & 83.12 & $\mathbf{N}$ & \\
\hline 1 & $8 / 2 / 93$ & 6 & 1 & & 83.3 & $\mathrm{~N}$ & \\
\hline 1 & $8 / 2 / 93$ & 7 & 1 & & 84.56 & $\mathrm{~N}$ & \\
\hline 1 & $8 / 2 / 93$ & 8 & 1 & & 85.46 & $\mathbf{N}$ & \\
\hline$\frac{1}{1}$ & $8 / 2 / 93$ & 9 & 1 & & 81.14 & $\bar{N}$ & \\
\hline 1 & $8 / 2 / 93$ & 10 & 1 & & 81.68 & $\mathbf{N}$ & \\
\hline 1 & $8 / 9 / 93$ & 1 & 1 & & 108.5 & $\bar{Y}$ & TMACS \\
\hline 1 & $8 / 9 / 93$ & 2 & 1 & & 101.84 & $\mathrm{~N}$ & \\
\hline 1 & $8 / 9 / 93$ & 3 & 1 & & 94.28 & $\mathbf{N}$ & \\
\hline 9 & $8 / 9 / 93$ & 4 & 9 & & 85.1 & $\mathrm{~N}$ & \\
\hline 1 & $8 / 9 / 93$ & 5 & 1 & & 83.84 & $\mathbf{N}$ & \\
\hline 1 & $8 / 9 / 93$ & 6 & 1 & & 84.2 & $\mathbf{N}$ & \\
\hline 9 & $8 / 9 / 93$ & 7 & 9 & & 85.28 & $\mathrm{~N}$ & \\
\hline 1 & $8 / 9 / 93$ & 8 & 1 & & 86.72 & $\ddot{N}$ & \\
\hline 1 & $8 / 9 / 93$ & 9 & 1 & & 81.68 & $\mathrm{~N}$ & \\
\hline 1 & $8 / 9 / 93$ & 10 & 1 & & 81.68 & $\mathbf{N}$ & \\
\hline 9 & $8 / 15 / 93$ & 1 & 1 & & 108.68 & $\bar{Y}$ & tmacs \\
\hline 1 & $8 / 15 / 93$ & 2 & 1 & & 102.2 & $N$ & \\
\hline 1 & $8 / 15 / 93$ & 3 & 1 & & 94.82 & $\mathbf{N}$ & \\
\hline$\frac{1}{1}$ & $8 / 75 / 93$ & 4 & $\frac{1}{1}$ & & 85.82 & $\mathbf{N}$ & \\
\hline 1 & $8 / 15 / 93$ & 5 & 9 & & 84.38 & $\bar{N}$ & \\
\hline 1 & $8 / 15 / 93$ & 6 & 1 & & 84.56 & $\mathbf{N}$ & \\
\hline 9 & $8 / 15 / 93$ & 7 & 1 & & 85.46 & $\mathbf{N}$ & \\
\hline 1 & $8 / 15 / 93$ & 8 & 1 & & 86.72 & $\overline{\mathbf{N}}$ & \\
\hline$\frac{1}{1}$ & $8 / 15 / 93$ & 9 & 1 & & 82.04 & $\mathrm{~N}$ & \\
\hline 9 & $8 / 15 / 93$ & 10 & 1 & & 82.04 & $\mathbf{N}$ & \\
\hline 1 & $8 / 23 / 93$ & 1 & 1 & & 108.14 & $Y$ & TMACS \\
\hline 1 & $8 / 23 / 93$ & 2 & 1 & & 101.66 & $\mathbf{N}$ & \\
\hline 1 & $8 / 23 / 93$ & 3 & 1 & & 94.46 & $\mathbf{N}$ & \\
\hline 9 & $8 / 23 / 93$ & 4 & 1 & & 85.46 & $\mathbf{N}$ & \\
\hline 9 & $8 / 23 / 93$ & 5 & 1 & & 83.84 & $\mathbf{N}$ & \\
\hline 1 & $8 / 23 / 93$ & 6 & 1 & & 84.02 & $\mathbf{N}$ & \\
\hline 1 & $8 / 23 / 93$ & 7 & 1 & & 85.28 & $\mathbf{N}$ & \\
\hline 1 & $8 / 23 / 93$ & 8 & $\frac{9}{1}$ & & 86.36 & $\mathrm{~N}$ & \\
\hline 1 & $8 / 23 / 93$ & 9 & 1 & & 82.22 & $\mathbf{N}$ & \\
\hline 1 & $8 / 23 / 93$ & 10 & 1 & & 82.22 & $\mathbf{N}$ & \\
\hline 1 & $8 / 26 / 93$ & 1 & 1 & & 108.1 & $Y$ & JTYPE \\
\hline
\end{tabular}

Data obtained from WHC Surveillance Analysis Computer System (SACS), June 2, 1993. 


\begin{tabular}{|c|c|c|c|c|c|c|c|}
\hline \multicolumn{8}{|c|}{ TANK 241-BY-108 } \\
\hline & & & & THERI & COUPLE D & & \\
\hline TREE \# & DATE & THERMO \# & TREE SET \# & TEMP C & TEMP F & $Y=M A X$ TEMP & COMMENTS \\
\hline 1 & $8 / 26 / 93$ & 2 & 1 & & 101.8 & $\mathbf{N}$ & \\
\hline 1 & $8 / 26 / 93$ & 3 & 1 & & 94.5 & $\mathbf{N}$ & \\
\hline 1 & $8 / 26 / 93$ & 4 & 1 & & 85.6 & $\mathbf{N}$ & \\
\hline 1 & $8 / 26 / 93$ & 5 & 1 & & 84.2 & $\bar{N}$ & \\
\hline 1 & $8 / 26 / 93$ & 6 & 1 & & 84.4 & $\mathbf{N}$ & \\
\hline 1 & $8 / 26 / 93$ & 7 & 1 & & 85.3 & $\mathbf{N}$ & \\
\hline 1 & $8 / 26 / 93$ & 8 & 1 & & 86.4 & $\mathbf{N}$ & \\
\hline 1 & $8 / 26 / 93$ & 9 & 1 & & 82.2 & $\mathbf{N}$ & \\
\hline 1 & $8 / 26 / 93$ & 10 & 1 & & 82.4 & $\mathrm{~N}$ & \\
\hline 9 & $8 / 30 / 93$ & 1 & 1 & & 108.32 & $\bar{Y}$ & TMACS \\
\hline 1 & $8 / 30 / 93$ & 2 & 1 & & 102.02 & $\mathbf{N}$ & \\
\hline 1 & $8 / 30 / 93$ & 3 & 1 & & 94.82 & $\mathbf{N}$ & \\
\hline 1 & $8 / 30 / 93$ & 4 & 1 & & 86 & $\mathbf{N}$ & \\
\hline 1 & $8 / 30 / 93$ & 5 & 1 & & 84.56 & $\mathbf{N}$ & \\
\hline 9 & $8 / 30 / 93$ & 6 & 1 & & 84.74 & $\mathbf{N}$ & \\
\hline 1 & $8 / 30 / 93$ & 7 & 1 & & 85.82 & $\mathbf{N}$ & \\
\hline 1 & $8 / 30 / 93$ & 8 & 1 & & 86.72 & $\mathbf{N}$ & \\
\hline 1 & $8 / 30 / 93$ & 9 & 1 & & 82.76 & $N$ & \\
\hline 1 & $8 / 30 / 93$ & 10 & 1 & & 82.94 & $N$ & \\
\hline 1 & $9 / 6 / 93$ & 1 & 1 & & 108.86 & $Y$ & TMACS \\
\hline 1 & $9 / 6 / 93$ & 2 & 1 & & 102.38 & $\mathbf{N}$ & \\
\hline 1 & $9 / 6 / 93$ & 3 & 1 & & 95.36 & $\mathbf{N}$ & \\
\hline 1 & $9 / 6 / 93$ & 4 & $\frac{1}{1}$ & & 86.54 & $\mathbf{N}$ & \\
\hline 1 & $9 / 6 / 93$ & 5 & 1 & & 84.74 & $\mathbf{N}$ & \\
\hline 1 & $9 / 6 / 93$ & 6 & 1 & & 85.1 & $\bar{N}$ & \\
\hline 1 & $9 / 6 / 93$ & 7 & 1 & & 86.36 & $\mathbf{N}$ & \\
\hline 1 & $9 / 6 / 93$ & 8 & 1 & & 87.62 & $\mathbf{N}$ & \\
\hline 1 & $9 / 6 / 93$ & 9 & 9 & & 82.76 & $\bar{N}$ & \\
\hline 1 & $9 / 6 / 93$ & 10 & 1 & & 82.94 & $\mathbf{N}$ & \\
\hline 1 & $9 / 12 / 93$ & 1 & 1 & & 108.68 & $\bar{Y}$ & TMACS \\
\hline 1 & $9 / 12 / 93$ & 2 & 9 & & 102.38 & $\bar{N}$ & \\
\hline 1 & $9 / 12 / 93$ & 3 & 1 & & 95.54 & $N$ & \\
\hline 1 & $9 / 12 / 93$ & 4 & 9 & & 86.9 & $\mathbf{N}$ & \\
\hline 1 & $9 / 12 / 93$ & 5 & 1 & & 85.64 & $N$ & \\
\hline 1 & $9 / 12 / 93$ & 6 & 1 & & 85.64 & $N$ & \\
\hline 1 & $9 / 12 / 93$ & 7 & 9 & & 86.9 & $\mathbf{N}$ & \\
\hline 1 & $9 / 12 / 93$ & 8 & 1 & & 87.98 & $N$ & \\
\hline 1 & $9 / 12 / 93$ & 9 & 1 & & 83.12 & $\mathbf{N}$ & \\
\hline 1 & $9 / 12 / 93$ & 10 & 1 & & 83.3 & $\mathrm{~N}$ & \\
\hline 1 & $9 / 19 / 93$ & 1 & 1 & & 108.68 & $\bar{Y}$ & TMACS \\
\hline 1 & $9 / 19 / 93$ & 2 & 1 & & 102.56 & $\bar{N}$ & \\
\hline 9 & $9 / 79 / 93$ & 3 & 9 & & 95.72 & $\mathbf{N}$ & \\
\hline 1 & $9 / 19 / 93$ & 4 & 1 & & 86.9 & $\mathbf{N}$ & \\
\hline 9 & $9 / 19 / 93$ & 5 & 1 & & 85.64 & $\mathbf{N}$ & \\
\hline 1 & $9 / 19 / 93$ & 6 & 1 & & 85.64 & $\bar{N}$ & \\
\hline 1 & $9 / 19 / 93$ & 7 & 1 & & 86.72 & $\mathrm{~N}$ & \\
\hline 9 & $9 / 79 / 93$ & 8 & 1 & & 87.98 & $\mathbf{N}$ & \\
\hline 1 & $9 / 19 / 93$ & 9 & 1 & & 83.3 & $\bar{N}$ & \\
\hline 1 & $9 / 19 / 93$ & 10 & 1 & & 83.3 & $\mathbf{N}$ & \\
\hline 9 & $9 / 26 / 93$ & 1 & 1 & & 109.04 & $\bar{Y}$ & TMACS \\
\hline 1 & $9 / 26 / 93$ & 2 & 1 & & 102.74 & $\mathbf{N}$ & \\
\hline 1 & $9 / 26 / 93$ & 3 & 1 & & 96.08 & $\mathbf{N}$ & \\
\hline 1 & $9 / 26 / 93$ & 4 & 1 & & 87.62 & $\mathbf{N}$ & \\
\hline 1 & $9 / 26 / 93$ & 5 & 1 & & 86 & $\mathbf{N}$ & \\
\hline 1 & $9 / 26 / 93$ & 6 & 1 & & 86.18 & $\mathbf{N}$ & \\
\hline 1 & $9 / 26 / 93$ & 7 & 1 & & 87.26 & $\mathbf{N}$ & \\
\hline 1 & $9 / 26 / 93$ & 8 & 1 & & 88.34 & $\mathbf{N}$ & \\
\hline 1 & $9 / 26 / 93$ & 9 & 1 & & 83.48 & $N$ & \\
\hline 1 & $9 / 26 / 93$ & 10 & 1 & & 83.48 & $\bar{N}$ & \\
\hline 1 & $10 / 3 / 93$ & 1 & 1 & & 108.86 & $Y$ & TMACS \\
\hline 1 & $10 / 3 / 93$ & 2 & 9 & & 102.56 & $N$ & \\
\hline 1 & $10 / 3 / 93$ & 3 & 1 & & 95.9 & $N$ & \\
\hline 1 & $10 / 3 / 93$ & 4 & 1 & & 87.26 & $N$ & \\
\hline 9 & $10 / 3 / 93$ & 5 & 1 & & 85.64 & $\mathbf{N}$ & \\
\hline 1 & $10 / 3 / 93$ & 6 & 1 & & 86 & $\mathbf{N}$ & \\
\hline 1 & $10 / 3 / 93$ & 7 & 1 & & 87.08 & $N$ & \\
\hline
\end{tabular}

Data obtained from WHC Surveillance Analysis Computer System (SACS), June 2, 1993. 


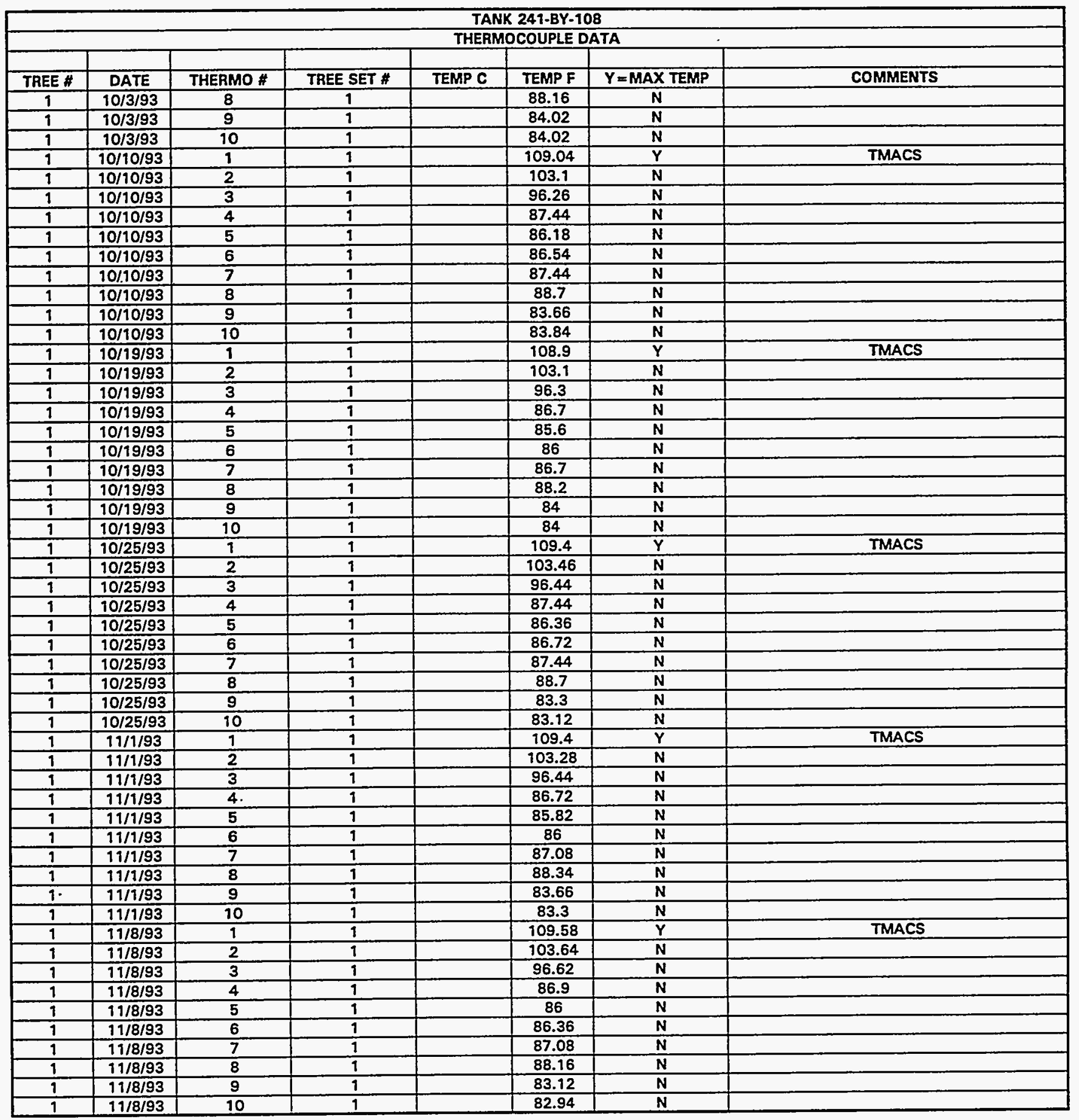

Data obtained from WHC Surveillance Analysis Computer System (SACS), June 2, 1993. 


\begin{tabular}{|c|c|c|c|c|c|c|c|}
\hline \multicolumn{8}{|c|}{ TANK 241-BY-109 } \\
\hline \multicolumn{8}{|c|}{ THERMOCOUPLE DATA } \\
\hline & & & & & & & \\
\hline TREE \# & DATE & THERMO \# & TREE SET \# & TEMP C & TEMP F & $Y=\operatorname{MAX} T E M P$ & COMMENTS \\
\hline 1 & $11 / 12 / 73$ & 17 & & & 46 & & \\
\hline 1 & $11 / 12 / 73$ & 18 & & & 59 & & \\
\hline 1 & $11 / 12 / 73$ & 19 & & & 79 & & \\
\hline 9 & $11 / 21 / 73$ & 17 & & & 190 & & \\
\hline 1 & $11 / 21 / 73$ & 18 & & & 80 & & \\
\hline 1 & $12 / 10 / 73$ & 17 & & & 179 & & \\
\hline 1 & $12 / 10 / 73$ & 98 & & & 75 & & \\
\hline 1 & $12 / 10 / 73$ & 19 & & & 73 & & \\
\hline 1 & $12 / 17 / 73$ & 8 & & & & & 186 - suspect data (high) \\
\hline 1 & $12 / 17 / 73$ & 9 & & & 78 & & \\
\hline 1 & $12 / 17 / 73$ & 10 & & & 71 & & \\
\hline 1 & $12 / 20 / 73$ & 8 & & & & & 178 - suspect data (high) \\
\hline 1 & $12 / 20 / 73$ & 9 & & & 70 & & \\
\hline 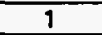 & $12 / 20 / 73$ & 11 & & & 72 & & \\
\hline 1 & $12 / 20 / 73$ & 15 & & & 75 & & \\
\hline 1 & $1 / 4 / 74$ & 17 & & & 167 & & \\
\hline 1 & $1 / 4 / 74$ & 18 & & & 64 & & \\
\hline 1 & $1 / 4 / 74$ & 19 & & & 65 & & \\
\hline 1 & $5 / 7 / 76$ & 1 & 9 & & 132 & $\mathbf{N}$ & \\
\hline$\overline{1}$ & $5 / 7 / 76$ & 2 & 9 & & 121 & $\mathbf{N}$ & \\
\hline 1 & $5 / 7 / 76$ & 3 & $\overline{1}$ & & 137 & $\bar{Y}$ & \\
\hline 1 & $5 / 7 / 76$ & 4 & $\overline{1}$ & & 909 & $\mathbf{N}$ & \\
\hline 1 & $5 / 7 / 76$ & 5 & 9 & & 91 & $\mathbf{N}$ & \\
\hline 1 & $5 / 7 / 76$ & 6 & 1 & & 126 & $\bar{N}$ & \\
\hline 1 & $5 \longdiv { 7 / 7 6 }$ & 7 & 9 & & 92 & $\bar{N}$ & \\
\hline 1 & $5 / 7 / 76$ & 8 & 1 & & 96 & $\mathbf{N}$ & \\
\hline 1 & $5 / 7 / 76$ & 10 & 1 & & 98 & $N$ & \\
\hline 1 & $5 / 7 / 76$ & 11 & 1 & & 92 & $\mathbf{N}$ & \\
\hline 1 & $5 / 7 / 76$ & 12 & 1 & & 84 & $N$ & \\
\hline 1 & $6 / 9 / 76$ & 1 & 1 & & 129 & $\mathbf{N}$ & \\
\hline 9 & $6 / 9 / 76$ & 2 & 9 & & 119 & $\mathrm{~N}$ & \\
\hline 1 & $6 / 9 / 76$ & 3 & 1 & & 138 & $\bar{Y}$ & \\
\hline 9 & $6 / 9 / 76$ & 4 & 9 & & 103 & $\mathbf{N}$ & \\
\hline 1 & $6 / 9 / 76$ & 5 & 1 & & 78 & $\mathbf{N}$ & \\
\hline 1 & $6 / 9 / 76$ & 6 & 9 & & 111 & $\bar{N}$ & \\
\hline 1 & $6 / 9 / 76$ & 7 & 1 & & 81 & $\bar{N}$ & \\
\hline 1 & $6 / 9 / 76$ & .8 & 1 & & 89 & $\mathbf{N}$ & \\
\hline$\frac{1}{1}$ & $6 / 9 / 76$ & 10 & $\frac{1}{1}$ & & 94 & $\bar{N}$ & \\
\hline 1 & $6 / 9 / 76$ & 11 & 1 & & 87 & $\bar{N}$ & \\
\hline 9 & $6 / 9 / 76$ & 12 & 1 & & 74 & $\mathbf{N}$ & \\
\hline 1 & $8 / 28 / 76$ & 1 & 1 & & 59 & $\mathbf{N}$ & \\
\hline 1 & $8 / 28 / 76$ & 2 & 1 & & 60 & $\mathbf{N}$ & \\
\hline 1 & $8 / 28 / 76$ & 3 & 1 & & 51 & $\mathbf{N}$ & \\
\hline 1 & $8 / 28 / 76$ & 4 & 1 & & 92 & $\bar{Y}$ & \\
\hline 1 & $8 / 28 / 76$ & 5 & 1 & & 86 & $\mathbf{N}$ & \\
\hline 1 & $8 / 28 / 76$ & 6 & 1 & & 79 & $\mathbf{N}$ & \\
\hline 1 & $8 / 28 / 76$ & 7 & 1 & & 90 & $\bar{N}$ & \\
\hline 1 & $8 / 28 / 76$ & 8 & 1 & & 90 & $\overline{\mathbf{N}}$ & \\
\hline 1 & $8 / 28 / 76$ & 10 & 1 & & 82 & $\mathbf{N}$ & \\
\hline 1 & $8 / 28 / 76$ & 11 & 1 & & 81 & $N$ & \\
\hline 1 & $8 / 28 / 76$ & 12 & 1 & & 93 & $\mathbf{N}$ & \\
\hline 1 & $9 / 13 / 76$ & 1 & 1 & & & $\bar{N}$ & $0 / 5$ \\
\hline 1 & $11 / 7 / 76$ & 9 & 1 & & & $\mathbf{N}$ & $0 / 5$ \\
\hline 1 & $12 / 9 / 76$ & 1 & 1 & & & $\mathbf{N}$ & $\mathrm{O} / \mathrm{S}$ \\
\hline 1 & $1 / 3 / 77$ & 1 & 1 & & & $\bar{N}$ & $0 / 5$ \\
\hline 1 & $3 / 28 / 77$ & 1 & 1 & & 55 & $N$ & \\
\hline 1 & $3 / 28 / 77$ & 2 & 9 & & 55 & $\mathbf{N}$ & \\
\hline 1 & $3 / 28 / 77$ & 4 & 1 & & 62 & $\mathbf{N}$ & \\
\hline 9 & $4 / 27 / 77$ & 1 & 1 & & 68 & $\mathbf{N}$ & \\
\hline 1 & $4 / 27 / 77$ & 2 & 9 & & 68 & $\mathbf{N}$ & \\
\hline 1 & $4 / 27 / 77$ & 4 & 1 & & 80 & $\mathbf{N}$ & \\
\hline 1 & $6 / 30 / 77$ & $\frac{1}{1}$ & 1 & & 70 & $\mathbf{N}$ & \\
\hline 1 & $6 / 30 / 77$ & 2 & 1 & & 70 & $\bar{N}$ & \\
\hline 1 & $6 / 30 / 77$ & 4 & 1 & & 84 & $N$ & \\
\hline 1 & $8 / 15 / 77$ & 1 & 9 & & 84 & $\mathbf{N}$ & \\
\hline 1 & $8 / 15 / 77$ & 2 & 9 & & 84 & $N$ & \\
\hline
\end{tabular}

Data obtained from WHC Surveillance Analysis Computer System (SACS), June 28, 1993. 


\begin{tabular}{|c|c|c|c|c|c|c|c|}
\hline \multicolumn{8}{|c|}{ TANK 241-BY-109 } \\
\hline & & & & THEF & DCOUPLE & & \\
\hline TREE \# & DATE & THERMO \# & TREE SET \# & TEMP C & TEMP F & $Y=$ MAX TEMP & COMMENTS \\
\hline 1 & $8 / 15 / 77$ & 3 & 1 & & & $\bar{Y}$ & 110 - suspect date (high) \\
\hline 1 & $8 / 15 / 77$ & 4 & 1 & & 95 & $Y$ & \\
\hline 9 & $9 / 12 / 77$ & 7 & 1 & & 65 & $\mathbf{N}$ & \\
\hline$T$ & $9 / 12 / 77$ & 8 & 1 & & 65 & $\mathbf{N}$ & \\
\hline 1 & $9 / 12 / 77$ & 10 & 1 & & & $\bar{N}$ & \\
\hline 1 & $10 / 7 / 77$ & 1 & 1 & & & $\mathbf{N}$ & $0 / 5$ \\
\hline 1 & $12 / 7 / 77$ & 1 & 1 & & & $\mathbf{N}$ & $0 / 5$ \\
\hline 1 & $1 / 29 / 78$ & 1 & 1 & & 60 & $\bar{Y}$ & \\
\hline 9 & $1 / 29 / 78$ & 2 & 1 & & 59 & $\mathbf{N}$ & \\
\hline 1 & $3 / 26 / 78$ & 1 & 1 & & 61 & $\bar{Y}$ & \\
\hline 1 & $3 / 26 / 78$ & 2 & 1 & & 61 & $\mathbf{N}$ & \\
\hline 1 & $3 / 26 / 78$ & 3 & 1 & & 60 & $\mathbf{N}$ & \\
\hline 1 & $3 / 26 / 78$ & 4 & 1 & & 61 & $\mathbf{N}$ & \\
\hline$T$ & $4 / 23 / 78$ & 1 & 1 & & 62 & $\bar{Y}$ & \\
\hline 1 & $4 / 23 / 78$ & 2 & 1 & & 62 & $\mathbf{N}$ & \\
\hline 1 & $4 / 23 / 78$ & 3 & 1 & & 62 & $\bar{N}$ & \\
\hline 1 & $4 / 23 / 78$ & 4 & 1 & & 62 & $\mathbf{N}$ & \\
\hline$T$ & $6 / 17 / 78$ & 1 & 1 & & 55 & $\bar{Y}$ & \\
\hline 1 & $6 / 17 / 78$ & 2 & 1 & & 55 & $\bar{N}$ & \\
\hline 1 & $6 / 17 / 78$ & 3 & 1 & & 55 & $\overline{\mathbf{N}}$ & \\
\hline 9 & $6 / 17 / 78$ & 4 & 9 & & 55 & $\bar{N}$ & \\
\hline$T$ & $7 / 16 / 78$ & 7 & 1 & & 60 & $\bar{Y}$ & \\
\hline 1 & $7 / 16 / 78$ & 2 & $T$ & & 60 & $N$ & \\
\hline 1 & $7 / 16 / 78$ & 3 & 1 & & 60 & $\mathbf{N}$ & \\
\hline 1 & $7 / 16 / 78$ & 4 & 1 & & 60 & $\bar{N}$ & \\
\hline 1 & $7 / 16 / 78$ & 5 & 1 & & 60 & $\mathbf{N}$ & \\
\hline 1 & $7 / 16 / 78$ & 6 & 1 & & 60 & $\mathbf{N}$ & \\
\hline 1 & $7 / 16 / 78$ & 7 & 1 & & 60 & $\mathbf{N}$ & \\
\hline 1 & $7 / 16 / 78$ & 8 & 1 & & 60 & $\mathbf{N}$ & \\
\hline 1 & $7 / 16 / 78$ & 9 & 1 & & 60 & $\mathrm{~N}$ & \\
\hline 1 & $7 / 16 / 78$ & 10 & 1 & & 60 & $N$ & \\
\hline 1 & $7 / 16 / 78$ & 11 & 1 & & 60 & $\mathbf{N}$ & \\
\hline$T$ & $7 / 16 / 78$ & 12 & 9 & & 60 & $\mathbf{N}$ & \\
\hline 1 & $8 / 13 / 78$ & 1 & 1 & & 66 & $Y$ & \\
\hline 1 & $8 / 13 / 78$ & $\overline{2}$ & 1 & & 66 & $\mathbf{N}$ & \\
\hline 1 & $8 / 13 / 78$ & 3 & 1 & & 66 & $N$ & \\
\hline 9 & $8 / 13 / 78$ & 4 & 1 & & 66 & $\bar{N}$ & \\
\hline 1 & $9 / 10 / 78$ & 1 & 1 & & 61 & $\bar{Y}$ & \\
\hline 1 & $9 / 10 / 78$ & 2 & 1 & & 61 & $\mathbf{N}$ & \\
\hline 1 & $9 / 10 / 78$ & 3 & 1 & & 61 & $\mathbf{N}$ & \\
\hline 1 & $9 / 10 / 78$ & 4 & 1 & & 61 & $N$ & \\
\hline 9 & $12 / 3 / 78$ & 9 & 1 & & & $N$ & $0 / 5$ \\
\hline 1 & $12 / 26 / 78$ & 7 & 1 & & 64 & $\bar{Y}$ & \\
\hline 1 & $12 / 26 / 78$ & 2 & 1 & & 64 & $\mathbf{N}$ & \\
\hline 1 & $12 / 26 / 78$ & 3 & 1 & & 63 & $\mathbf{N}$ & \\
\hline 1 & $12 / 26 / 78$ & 4 & 1 & & 62 & $\mathbf{N}$ & \\
\hline 1 & $10 / 27 / 91$ & 1 & 1 & & 72.3 & $\overline{\mathbf{Y}}$ & J-910126143-42 LOW \\
\hline 1 & $11 / 2 / 91$ & $\frac{1}{1}$ & 1 & & 86 & $\bar{Y}$ & \\
\hline 1 & $11 / 19 / 91$ & 1 & 1 & & & $\mathbf{N}$ & O/S NO TC TREEE \\
\hline 1 & $12 / 1 / 91$ & 1 & 1 & & 87.2 & $\bar{Y}$ & LOW K-890723679-64 \\
\hline 9 & $7 / 2 / 92$ & $\frac{T}{1}$ & 1 & & & $\mathbf{N}$ & O/S NO TC TREE \\
\hline 1 & $1 / 1 / 93$ & 1 & 1 & & & $\mathbf{N}$ & OIS NO TC TREE \\
\hline
\end{tabular}

Data obtained from WHC Surveillance Analysis Computer System (SACS), June 28, 1993. 
WHC-SD-WM-ER-312, Rev. 0

\begin{tabular}{|c|c|c|c|c|c|c|c|}
\hline \multicolumn{8}{|c|}{ TANK 241-BY-110 } \\
\hline \multicolumn{8}{|c|}{ THERMOCOUPLE DATA } \\
\hline TREE\# & DATE & THERMO \# & TREE SET \# & TEMP C & TEMP F & $Y=$ MAX TEMP & COMMENTS \\
\hline 1 & $11 / 12 / 73$ & 1 & & & 201 & & \\
\hline 1 & $11 / 12 / 73$ & 2 & & & 210 & & \\
\hline 1 & $11 / 12 / 73$ & 3 & & & 202 & & \\
\hline 1 & $11 / 12 / 73$ & 4 & & & 202 & & \\
\hline$T$ & $11 / 12 / 73$ & 5 & & & 190 & & \\
\hline 1 & $11 / 12 / 73$ & 6 & & & 180 & & \\
\hline 1 & $11 / 12 / 73$ & 7 & & & & & 88 - suspect data (low) \\
\hline 1 & $11 / 12 / 73$ & 8 & & & 162 & & \\
\hline 1 & $11 / 12 / 73$ & 9 & & & 155 & & \\
\hline 9 & $11 / 12 / 73$ & 10 & & & 154 & & \\
\hline 1 & $11 / 12 / 73$ & 11 & & & 103 & & \\
\hline 1 & $11 / 12 / 73$ & 12 & & & 103 & & \\
\hline$\frac{1}{1}$ & $11 / 12 / 73$ & 13 & & & 103 & & \\
\hline 1 & $11 / 21 / 73$ & 9 & & 71 & 159.8 & & raw data - assumed degree C \\
\hline 1 & $11 / 21 / 73$ & 10 & & 69 & 156.2 & & raw data - assumed degree $C$ \\
\hline 1 & $11 / 21 / 73$ & 11 & & 47 & 116.6 & & raw date - assumed degree C \\
\hline 1 & $11 / 21 / 73$ & 12 & & 36 & 96.8 & & raw data - assumed degree C \\
\hline 9 & $11 / 21 / 73$ & 13 & & 35 & 95 & & raw data - assumed degree C \\
\hline 1 & $11 / 21 / 73$ & 14 & & 36 & 96.8 & & raw data - assumed degree C \\
\hline 1 & $12 / 10 / 73$ & 1 & & 95 & 203 & & raw data - assumed degree C \\
\hline 1 & $12 / 10 / 73$ & 2 & & 95 & 203 & & raw data - assumed degree C \\
\hline 1 & $12 / 10 / 73$ & 3 & & 94 & 201.2 & & raw data - assumed degree C \\
\hline 1 & $12 / 10 / 73$ & 4 & & 91 & 195.8 & & raw data - assumed degree C \\
\hline 1 & $12 / 10 / 73$ & 5 & & 87 & 188.6 & & raw data - assumed degree C \\
\hline 1 & $12 / 10 / 73$ & 6 & & 81 & 177.8 & & raw data - assumed degree C \\
\hline 1 & $12 / 10 / 73$ & 7 & & 70 & 158 & & raw data - assumed degree $C$ \\
\hline T & $12 / 10 / 73$ & 8 & & 70 & 158 & & raw data - assumed degree C \\
\hline$\frac{1}{1}$ & $12 / 10 / 73$ & 9 & & 69 & 156.2 & & raw data - assumed degree C \\
\hline 1 & $12 / 10 / 73$ & 10 & & 66 & 150.8 & & raw data - assumed degree $C$ \\
\hline 1 & $12 / 10 / 73$ & 11 & & 44 & 111.2 & & raw data - assumed degree C \\
\hline 1 & $12 / 10 / 73$ & 12 & & 30 & 86 & & raw data - assumed degree C \\
\hline 1 & $12 / 10 / 73$ & 13 & & 30 & 86 & & raw data - assumed degree $C$ \\
\hline 1 & $12 / 10 / 73$ & 14 & & 30 & 86 & & raw data - essumed degree C \\
\hline 1 & $12 / 17 / 73$ & 1 & & 101 & 213.8 & & raw data - assumed degree $C$ \\
\hline 1 & $12 / 17 / 73$ & 2 & & 104 & 219.2 & & raw data - assumed degree $C$ \\
\hline 1 & $12 / 17 / 73$ & 3 & & 101 & 213.8 & & raw data - assumed degree C \\
\hline 1 & $12 / 17 / 73$ & 4 & & 100 & 212 & & raw data - assumed degree C \\
\hline 1 & $12 / 17 / 73$ & 5 & & 94 & 201.2 & & raw data - assumed degrea C \\
\hline 1 & $12 / 17 / 73$ & 6 & & 88 & 190.4 & & raw data - assumed degree C \\
\hline 1 & $12 / 17 / 73$ & 7 & & 80 & 176 & & raw data - assumed degree C \\
\hline 1 & $12 / 17 / 73$ & 8 & & 75 & 167 & & raw data - assumed degree C \\
\hline 1 & $12 / 17 / 73$ & 9 & & 75 & 167 & & raw data - assumed degree C \\
\hline 1 & $12 / 17 / 73$ & 10 & & 70 & 158 & & raw data - assumed degree C \\
\hline 1 & $12 / 17 / 73$ & 11 & & 50 & 122 & & raw data - assumed degree C \\
\hline 1 & $12 / 17 / 73$ & 12 & & 35 & 95 & & raw data - assumed degree C \\
\hline 1 & $12 / 17 / 73$ & 13 & & 35 & 95 & & raw data - assumed degree C \\
\hline 1 & $12 / 17 / 73$ & 14 & & 35 & 95 & & raw data - assumed degree C \\
\hline 1 & $12 / 20 / 73$ & 1 & & 104 & 219.2 & & raw data - assumed degree $C$ \\
\hline 1 & $12 / 20 / 73$ & 2 & & 107 & 224.6 & & raw data - assumed degree C \\
\hline 1 & $12 / 20 / 73$ & 3 & & 103 & 217.4 & & raw data - assumed degree C \\
\hline 1 & $12 / 20 / 73$ & 4 & & 100 & 212 & & raw data - assumed degree $C$ \\
\hline 1 & $12 / 20 / 73$ & 5 & & 91 & 195.8 & & raw data - assumed degree C \\
\hline 1 & $12 / 20 / 73$ & 6 & & 83 & 181.4 & & raw data - assumed degree C \\
\hline 1 & $12 / 20 / 73$ & 7 & & 83 & 181.4 & & raw data - assumed degree $C$ \\
\hline 1 & $12 / 20 / 73$ & 8 & & 79 & 174.2 & & raw data - assumed degree C \\
\hline 1 & $12 / 20 / 73$ & 9 & & 78 & 172.4 & & raw data - assumed degrea C \\
\hline 9 & $12 / 20 / 73$ & 10 & & 75 & 167 & & raw data - assumed degree C \\
\hline 1 & $12 / 20 / 73$ & 11 & & 52 & 725.6 & & raw data - assumed degres C \\
\hline 1 & $12 / 20 / 73$ & 12 & & 39 & 102.2 & & raw data - assumed degree C \\
\hline 1 & $12 / 20 / 73$ & 13 & & 39 & 102.2 & & raw data - assumed degree C \\
\hline 9 & $12 / 20 / 73$ & 14 & & 39 & 102.2 & & raw date - assumed degreo C \\
\hline 1 & $12 / 20 / 73$ & 15 & & 83 & 181.4 & & raw data - assumed degree $C$ \\
\hline 1 & $8 / 5 / 74$ & 1 & 1 & 68 & 154.4 & $Y$ & degree C/TWINS confirm \\
\hline 1 & $8 / 5 / 74$ & 2 & 1 & 68 & 154.4 & $\mathbf{N}$ & degree C/TWINS confirm \\
\hline 1 & $8 / 5 / 74$ & 3 & 1 & 68 & 154.4 & $\mathbf{N}$ & degree C/TWINS confirm \\
\hline 1 & $8 / 5 / 74$ & 4 & 1 & 60 & 140 & $\mathbf{N}$ & degree C/TWINS confirm \\
\hline
\end{tabular}

Data obtained from WHC Surveillance Analysis Computer System (SACS), November 12, 1993. 
WHC-SD-WM-ER-312, Rev. 0

\begin{tabular}{|c|c|c|c|c|c|c|c|}
\hline \multicolumn{8}{|c|}{ TANK 241-BY-110 } \\
\hline & & & & THER & COUPLE & & \\
\hline TREE\# & DATE & THERMO \# & TREE SET \# & TEMP C & TEMPF & $Y=M A X$ TEMP & COMMENTS \\
\hline 1 & $8 / 5 / 74$ & 5 & 1 & 53 & 127.4 & $\mathbf{N}$ & degree $\mathrm{C} / \mathrm{TW}$ INS confirm \\
\hline 1 & $8 / 5 / 74$ & 6 & 1 & 46 & 114.8 & $\mathbf{N}$ & degree C/TWINS confirm \\
\hline 1 & $8 / 5 / 74$ & 7 & 1 & & & N & 37 - suspect data (low) \\
\hline 1 & $8 / 5 / 74$ & 8 & 1 & 44 & 111.2 & $\mathbf{N}$ & degree $\mathrm{C} / \mathrm{T}$ WINS confirm \\
\hline 1 & $8 / 5 / 74$ & 9 & 1 & 42 & 107.6 & $N$ & degree C/TWINS confirm \\
\hline 1 & $8 / 5 / 74$ & 10 & 1 & 42 & 107.6 & $\mathbf{N}$ & degree CTTWINS confirm \\
\hline 1 & $8 / 5 / 74$ & 11 & 1 & 30 & 86 & $\mathrm{~N}$ & degree C/TWINS confirm \\
\hline 1 & $8 / 5 / 74$ & 12 & 1 & 26 & 78.8 & $\mathbf{N}$ & degree C/TWINS confirm \\
\hline 9 & $8 / 5 / 74$ & 13 & 1 & 26 & 78.8 & $\mathbf{N}$ & degree C/TWINS confirm \\
\hline 1 & $8 / 5 / 74$ & 14 & 1 & 26 & 78.8 & $\mathbf{N}$ & degreo C/TWINS confirm \\
\hline 9 & $9 / 12 / 74$ & 1 & 1 & 87 & 188.6 & $\mathbf{Y}$ & degree C/TWINS confirm \\
\hline 1 & $9 / 12 / 74$ & 2 & 1 & 87 & 188.6 & $\mathbf{N}$ & degree C/TWINS confirm \\
\hline 1 & $9 / 12 / 74$ & 3 & 1 & 83 & 181.4 & $\mathbf{N}$ & degree $\mathrm{C} / \mathrm{T}$ WiNS confirm \\
\hline 1 & $9 / 12 / 74$ & 4 & 1 & 78 & 172.4 & $\mathbf{N}$ & degres C/TWINS confirm \\
\hline 1 & $9 / 12 / 74$ & 5 & 1 & 70 & 158 & $\mathbf{N}$ & degree C/TWINS confirm \\
\hline 1 & $9 / 12 / 74$ & 6 & 1 & 62 & 143.6 & $\mathbf{N}$ & degree C/TWINS confirm \\
\hline 1 & $9 / 12 / 74$ & 7 & 1 & 52 & 125.6 & $N$ & degree C/TWINS confirm \\
\hline 1 & $9 / 12 / 74$ & 8 & 1 & 50 & 122 & $\bar{N}$ & degree CITWINS confirm \\
\hline 1 & $9 / 12 / 74$ & 9 & 1 & 50 & 122 & $\mathbf{N}$ & degree C/TWINS confirm \\
\hline 1 & $9 / 12 / 74$ & 10 & 1 & 49 & 120.2 & $\mathbf{N}$ & degree C/TWINS confirm \\
\hline 1 & $9 / 12 / 74$ & 11 & 1 & 34 & 93.2 & $N$ & degree C/TWINS confirm \\
\hline 1 & $9 / 12 / 74$ & 12 & 1 & 26 & 78.8 & $\mathbf{N}$ & degree C/TWINS confirm \\
\hline 1 & $9 / 12 / 74$ & 13 & 1 & 26 & 78.8 & $\mathbf{N}$ & degree $\mathrm{C} / \mathrm{TW}$ WNS confirm \\
\hline 1 & $9 / 12 / 74$ & 14 & 1 & 26 & 78.8 & $\mathbf{N}$ & degree C/TWINS confirm \\
\hline 1 & $10 / 8 / 74$ & 1 & 1 & 81 & 177.8 & $Y$ & degree C/TWINS confirm \\
\hline 1 & $10 / 8 / 74$ & 2 & 1 & 81 & 177.8 & $\mathbf{N}$ & degree C/TWINS confirm \\
\hline 1 & $10 / 8 / 74$ & 3 & 1 & 78 & 172.4 & $N$ & degree C/TWINS confirm \\
\hline 1 & $10 / 8 / 74$ & 4 & 1 & 72 & 161.6 & $\mathbf{N}$ & degres C/TWINS confirm \\
\hline 1 & $10 / 8 / 74$ & 5 & 1 & 65 & 149 & $\mathbf{N}$ & degree C/TWINS confirm \\
\hline 1 & $10 / 8 / 74$ & 6 & 1 & 58 & 136.4 & $\mathbf{N}$ & degreo C/TWINS confirm \\
\hline 1 & $10 / 8 / 74$ & 7 & 1 & 48 & 118.4 & $\mathbf{N}$ & degree CrTWINS confirm \\
\hline 1 & $10 / 8 / 74$ & 8 & 1 & 46 & 114.8 & $N$ & degree C/TWINS confirm \\
\hline 1 & $10 / 8 / 74$ & 9 & 1 & 46 & 114.8 & $\mathbf{N}$ & degree C/TWINS confirm \\
\hline 1 & $10 / 8 / 74$ & 10 & 1 & 46 & 114.8 & $\mathbf{N}$ & degree C/TWINS confirm \\
\hline 1 & $10 / 8 / 74$ & 11 & 1 & 30 & 86 & $\mathbf{N}$ & degree C/TWINS confirm \\
\hline 1 & $10 / 8 / 74$ & 12 & 1 & 22 & 71.6 & $\mathbf{N}$ & degree C/TWINS confirm \\
\hline 1 & $10 / 8 / 74$ & 13 & 1 & 22 & 71.6 & $\mathbf{N}$ & degree C/TWINS confirm \\
\hline 1 & $10 / 8 / 74$ & 14 & 1 & 22 & 71.6 & $\mathbf{N}$ & degree C/TWINS confirm \\
\hline 1 & $10 / 29 / 74$ & 1 & 1 & 85 & 185 & $Y$ & degree C/TWINS confirm \\
\hline 1 & $10 / 29 / 74$ & 2 & 9 & 85 & 185 & $\mathbf{N}$ & degres CITWINS confirm \\
\hline 1 & $10 / 29 / 74$ & 3 & 1 & 82 & 179.6 & $\mathbf{N}$ & degree C/TWINS confirm \\
\hline 1 & $10 / 29 / 74$ & 4 & 1 & 76 & 168.8 & $\mathbf{N}$ & degree CTWWNS confirm \\
\hline 1 & $10 / 29 / 74$ & 5 & 1 & 69 & 156.2 & $N$ & degree CrTWINS confirm \\
\hline 1 & $10 / 29 / 74$ & 6 & 1 & 61 & 141.8 & $\mathbf{N}$ & degree C/TWINS confirm \\
\hline 1 & $10 / 29 / 74$ & 7 & 1 & 52 & 125.6 & $\mathbf{N}$ & degree $\mathrm{C} / \mathrm{TW}$ INS confirm \\
\hline 1 & $10 / 29 / 74$ & 8 & 1 & 51 & 123.8 & $\mathbf{N}$ & degree C/TWINS confirm \\
\hline 1 & $10 / 29 / 74$ & 9 & 1 & 51 & 123.8 & $\mathbf{N}$ & degree CTWWINS canfirm \\
\hline 1 & $10 / 29 / 74$ & 10 & 1 & 50 & 122 & $\mathbf{N}$ & degree $\mathrm{C} / \mathrm{TW}$ INS confirm \\
\hline 1 & $10 / 29 / 74$ & 11 & 1 & 34 & 93.2 & N & degree C/TWINS canfirm \\
\hline 1 & $10 / 29 / 74$ & 12 & 1 & 28 & 82.4 & $\mathbf{N}$ & degree C/TWiNS confirm \\
\hline 1 & $10 / 29 / 74$ & 13 & 9 & 28 & 82.4 & $\mathbf{N}$ & degree C/TWINS confirm \\
\hline 9 & $10 / 29 / 74$ & 14 & 9 & 28 & 82.4 & $\mathbf{N}$ & degree C/TWINS confirm \\
\hline 1 & $11 / 12 / 74$ & 1 & 1 & 83 & 181.4 & $\bar{Y}$ & degree C/TWINS confirm \\
\hline 1 & $11 / 12 / 74$ & 2 & 1 & 83 & 181.4 & $\mathbf{N}$ & degree $\mathrm{C} / \mathrm{TW}$ INS confirm \\
\hline 1 & $11 / 12 / 74$ & 3 & 1 & 80 & 176 & $\mathbf{N}$ & degree $\mathrm{C} / \mathrm{TW}$ WNS confirm \\
\hline 1 & $11 / 12 / 74$ & 4 & 1 & 74 & 165.2 & $\mathbf{N}$ & degree C/TWiNS confirm \\
\hline 1 & $11 / 12 / 74$ & 5 & 1 & 66 & 150.8 & $\mathbf{N}$ & degree C/TWINS confirm \\
\hline 1 & $11 / 12 / 74$ & 6 & 1 & 58 & 136.4 & $\bar{N}$ & degree C/TWINS confirm \\
\hline 1 & $11 / 12 / 74$ & 7 & 1 & 49 & 120.2 & $\bar{N}$ & degree C $T$ TWINS confirm \\
\hline 9 & $11 / 12 / 74$ & 8 & 1 & 48 & 118.4 & $\mathbf{N}$ & degree C/TWINS confirm \\
\hline 1 & $11 / 12 / 74$ & 9 & 1 & 49 & 120.2 & $\mathbf{N}$ & degree C/TWINS confirm \\
\hline 1 & $11 / 12 / 74$ & 10 & 1 & 48 & 118.4 & $\mathbf{N}$ & degrae $\mathrm{C} / \mathrm{TW}$ INS confirm \\
\hline 1 & $11 / 12 / 74$ & 11 & 1 & 30 & 86 & $\mathbf{N}$ & degree C/TWINS confirm \\
\hline 1 & $11 / 12 / 74$ & 12 & 1 & 22 & 71.6 & $\mathbf{N}$ & degree C/TWINS confirm \\
\hline 1 & $11 / 12 / 74$ & 13 & 1 & 22 & 71.6 & $\mathbf{N}$ & degree C/TWINS confirm \\
\hline 1 & $11 / 12 / 74$ & 14 & 1 & 22 & 71.6 & $\mathbf{N}$ & degree C/TWINS confirm \\
\hline
\end{tabular}

Data obtained from WHC Surveillance Analysis Computer System (SACS), November 12, 1993. 


\begin{tabular}{|c|c|c|c|c|c|c|c|}
\hline \multicolumn{8}{|c|}{ TANK 241-BY-110 } \\
\hline \multicolumn{8}{|c|}{ THERMOCOUPLE DATA } \\
\hline TREE\# & DATE & THERMO \# & TREE SET : & TEMP C & TEMP F & $Y=$ MAX TEMP & COMMENTS \\
\hline 1 & $11 / 26 / 74$ & 1 & 1 & 82 & 179.6 & $\mathbf{Y}$ & degrea CrTwiNs confirm \\
\hline 1 & $11 / 26 / 74$ & 2 & 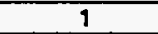 & 82 & 179.6 & $\mathbf{N}$ & degree C/TWins confirm \\
\hline 1 & $11 / 26 / 74$ & 3 & 1 & 78 & 172.4 & $N$ & degree C/TWNNS confirm \\
\hline 1 & $11 / 26 / 74$ & 4 & 1 & 72 & 161.6 & $N$ & degree C/TWINS confirm \\
\hline 1 & $11 / 26 / 74$ & 5 & 1 & 66 & 150.8 & $\mathbf{N}$ & degree C/TWINS confirm \\
\hline 1 & $11 / 26 / 74$ & 6 & 1 & 58 & 136.4 & $\mathbf{N}$ & degree C/TWINS confirm \\
\hline 1 & $11 / 26 / 74$ & 7 & 1 & 50 & 122 & $\mathbf{N}$ & degree C/TWINS confirm \\
\hline 1 & $11 / 26 / 74$ & 8 & 1 & 46 & 114.8 & $\mathbf{N}$ & degree C/TWINS confirm \\
\hline 1 & $11 / 26 / 74$ & 9 & 1 & 46 & 194.8 & $N$ & degree C/TWMNS confirm \\
\hline 1 & $11 / 26 / 74$ & 10 & 1 & 46 & 194.8 & $\mathbf{N}$ & degree C/TWINS confirm \\
\hline 1 & $11 / 26 / 74$ & 11 & 1 & 32 & 89.6 & $\mathbf{N}$ & degree $\mathrm{C} / \mathrm{TW}$ INS confirm \\
\hline 1 & $11 / 26 / 74$ & 12 & 1 & 22 & 71.6 & $\mathbf{N}$ & degree $\mathrm{C} / \mathrm{TW}$ INS confirm \\
\hline 1 & $11 / 26 / 74$ & 13 & 1 & 22 & 71.6 & $\mathbf{N}$ & degree C/TWINS confirm \\
\hline 1 & $11 / 26 / 74$ & 14 & 1 & 22 & 71.6 & $\mathbf{N}$ & degree C/TWiNS confirm \\
\hline 1 & $12 / 11 / 74$ & 1 & 1 & 81.67 & 179.006 & $\mathbf{Y}$ & degree C/TWINS confirm \\
\hline 1 & $12 / 11 / 74$ & 2 & 1 & 81.11 & 177.998 & $\mathbf{N}$ & degree CTTWINS confirm \\
\hline 1 & $12 / 11 / 74$ & 3 & 1 & 77.78 & 172.004 & $\mathbf{N}$ & degree $\mathrm{C} / \mathrm{TW} / \mathrm{NS}$ confirm \\
\hline 1 & $12 / 19 / 74$ & 4 & 1 & 72.22 & 161.996 & $\mathbf{N}$ & degree C/TWINS confirm \\
\hline 1 & $12 / 11 / 74$ & 5 & 1 & 65.56 & 150.008 & $\mathbf{N}$ & degree C/TWNSS confirm \\
\hline 1 & $12 / 11 / 74$ & 6 & 1 & 57.78 & 136.004 & $\mathbf{N}$ & degree C/TWINS confirm \\
\hline 1 & $12 / 11 / 74$ & 7 & 1 & 48.89 & 120.002 & $\mathbf{N}$ & degree C/TWINS confirm \\
\hline 1 & $12 / 11 / 74$ & 8 & 1 & 45.56 & 114.008 & $\mathbf{N}$ & degreo CITWINS confirm \\
\hline 1 & $12 / 11 / 74$ & 9 & 1 & 45.56 & 114.008 & $N$ & degree CTTWINS confirm \\
\hline 9 & $12 / 11 / 74$ & 10 & 9 & 45 & 113 & $\bar{N}$ & degree C/TWINS confirm \\
\hline 9 & $12 / 11 / 74$ & 11 & 9 & 27.78 & 82.004 & $\mathbf{N}$ & degree $\mathrm{C} / \mathrm{T}$ WiNS confirm \\
\hline 1 & $12 / 11 / 74$ & 12 & 1 & 19.44 & 66.992 & $\mathbf{N}$ & degree CTWWNS confirm \\
\hline$\frac{T}{1}$ & $12 / 11 / 74$ & 13 & 1 & 20 & 68 & $\mathbf{N}$ & degree C/TWINS confirm \\
\hline 1 & $12 / 11 / 74$ & 14 & 1 & 20 & 68 & $\mathbf{N}$ & degree $\mathrm{C} / \mathrm{TW}$ NS confirm \\
\hline 1 & $12 / 17 / 74$ & 1 & 1 & 82.22 & 179.996 & $\bar{Y}$ & degree C/TWINS confirm \\
\hline 1 & $12 / 17 / 74$ & 2 & 1 & 82.22 & 179.996 & $\mathbf{N}$ & degree C/TWINS confirm \\
\hline 1 & $12 / 17 / 74$ & 3 & 1 & 75 & 167 & $\mathbf{N}$ & degree C/TWINS confirm \\
\hline 1 & $12 / 17 / 74$ & 4 & 1 & 73.33 & 163.994 & $\mathbf{N}$ & degree C/TWINS confirm \\
\hline 1 & $12 / 17 / 74$ & 5 & 1 & 67.22 & 152.996 & $\bar{N}$ & degree C/TWWNS confirm \\
\hline 1 & $12 / 17 / 74$ & 6 & 1 & 58.89 & 138.002 & $\mathbf{N}$ & degree C/TWINS confirm \\
\hline 1 & $12 / 17 / 74$ & 7 & 1 & 50 & 122 & $\mathbf{N}$ & degree C/TWINS confirm \\
\hline 1 & $12 / 17 / 74$ & 8 & 1 & 47.78 & 118.004 & $\mathbf{N}$ & degree C/TWINS confirm \\
\hline 1 & $12 / 17 / 74$ & 9 & 1 & 47.78 & 118.004 & $\mathbf{N}$ & degree CrTWNS confirm \\
\hline 1 & $12 / 17 / 74$ & 10 & 1 & 46.67 & 116.006 & $\mathbf{N}$ & degree C/TWiNS confirm \\
\hline 1 & $12 / 17 / 74$ & 19 & 1 & 28.89 & 84.002 & $\mathbf{N}$ & degree C/TWNNS confirm \\
\hline 1 & $12 / 17 / 74$ & 12 & 1 & 20 & 68 & $\mathbf{N}$ & degree C/TWINS confirm \\
\hline 1 & $12 / 31 / 74$ & 1 & 1 & 84.44 & 183.992 & $\mathbf{Y}$ & degree C/TWINS confirm \\
\hline 1 & $12 / 31 / 74$ & 2 & 9 & 84.44 & 183.992 & $\mathbf{N}$ & degree C/TWINS confirm \\
\hline 1 & $12 / 31 / 74$ & 3 & 1 & 80.56 & 177.008 & $\mathbf{N}$ & degree C/TWINS confirm \\
\hline 9 & $12 / 31 / 74$ & 4 & 1 & 75.56 & 168.008 & $\mathbf{N}$ & degreo C/TWINS confirm \\
\hline 1 & $12 / 31 / 74$ & 5 & 1 & 68.33 & 154.994 & $\mathbf{N}$ & degree C/TWINS confirm \\
\hline 1 & $12 / 31 / 74$ & 6 & 1 & 60.56 & 141.008 & $\mathbf{N}$ & degree CrTwiNS confirm \\
\hline 1 & $12 / 31 / 74$ & 7 & 1 & 52.22 & 125.996 & $\mathbf{N}$ & degree C/TWiNS confirm \\
\hline 1 & $12 / 31 / 74$ & 8 & 1 & 48.89 & 120.002 & $\mathbf{N}$ & degree C/TWINS confirm \\
\hline 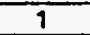 & $12 / 31 / 74$ & 9 & 9 & 48.89 & 120.002 & $\mathbf{N}$ & degree C/TWINS confirm \\
\hline 1 & $12 / 31 / 74$ & 10 & 1 & 47.22 & 116.996 & $\mathbf{N}$ & degree C/TWINS confirm \\
\hline 1 & $12 / 31 / 74$ & 11 & 1 & 28.89 & 84.002 & $\mathbf{N}$ & degree C/TWINS confirm \\
\hline 1 & $12 / 31 / 74$ & 12 & 1 & 21.11 & 69.998 & $\mathbf{N}$ & degree C/TWINS confirm \\
\hline 1 & $12 / 31 / 74$ & 13 & 1 & 21.11 & 69.998 & $\mathbf{N}$ & degree C/TWINS confirm \\
\hline 1 & $12 / 31 / 74$ & 14 & 1 & 21.11 & 69.998 & $\mathbf{N}$ & degree $\mathrm{C} / \mathrm{TW}$ INS confirm \\
\hline 1 & $1 / 8 / 75$ & 1 & 1 & 80 & 176 & $Y$ & degree C/TWINS confirm \\
\hline 1 & $1 / 8 / 75$ & 2 & 1 & 79.44 & 174.992 & $\mathbf{N}$ & degree C/TWINS confirm \\
\hline 1 & $1 / 8 / 75$ & 3 & $\frac{1}{1}$ & 76.67 & 170.006 & $\bar{N}$ & degree C/TWINS confirm \\
\hline 1 & $1 / 8 / 75$ & 4 & 1 & 79.11 & 159.998 & $\mathbf{N}$ & degree C/TWINS confirm \\
\hline 1 & $1 / 8 / 75$ & 5 & 1 & 64.44 & 147.992 & $\mathbf{N}$ & degree C/TWINS confirm \\
\hline 1 & $9 / 8 / 75$ & 6 & 1 & 58.33 & 136.994 & $\mathbf{N}$ & degree C/TWINS confirm \\
\hline 1 & $1 / 8 / 75$ & 7 & 1 & 48.89 & 120.002 & $\mathbf{N}$ & degree C/TWINS confirm \\
\hline 1 & $1 / 8 / 75$ & 8 & 1 & 47.22 & 116.996 & $\bar{N}$ & degree C/TWINS confirm \\
\hline 1 & $1 / 8 / 75$ & 9 & 1 & 47.22 & 116.996 & $\mathbf{N}$ & degree C/TWINS confirm \\
\hline 1 & $1 / 8 / 75$ & 10 & 1 & 46.11 & 114.998 & $\mathbf{N}$ & degree C/TWINS confirm \\
\hline 1 & $1 / 8 / 75$ & 11 & 1 & 28.89 & 84.002 & $\mathbf{N}$ & degree C/TWINS confirm \\
\hline 1 & $1 / 8 / 75$ & 12 & 1 & 18.89 & 66.002 & $\mathbf{N}$ & degree C/TWINS confirm \\
\hline
\end{tabular}

Data obtained from WHC Surveillance Analysis Computer System (SACS), November 12, 1993. 


\begin{tabular}{|c|c|c|c|c|c|c|c|}
\hline \multicolumn{8}{|c|}{ TANK 241-BY-110 } \\
\hline \multicolumn{8}{|c|}{ THERMOCOUPLE DATA } \\
\hline TREE\# & DATE & THERMO \# & TREE SET \# & TEMP C & TEMP F & $Y=$ MAX TEMP & COMMENTS \\
\hline 1 & $1 / 8 / 75$ & 13 & 1 & 18.89 & 66.002 & $\mathbf{N}$ & degree CrTWINS confirm \\
\hline 1 & $1 / 21 / 75$ & 1 & 1 & 96 & 204.8 & $\mathbf{Y}$ & degree C/TWINS confirm \\
\hline 1 & $1 / 21 / 75$ & 2 & 1 & 95 & 203 & $\mathbf{N}$ & degree C/TWINS confirm \\
\hline 1 & $1 / 21 / 75$ & 3 & 1 & 90 & 194 & $\mathbf{N}$ & degree $\mathrm{C} / \mathrm{TW}$ WNS confirm \\
\hline 1 & $1 / 21 / 75$ & 4 & 1 & 85. & 185 & $\mathbf{N}$ & degree C/TWINS confirm \\
\hline 1 & $1 / 21 / 75$ & 5 & 1 & 76 & 168.8 & $\mathbf{N}$ & degree C/TWINS confirm \\
\hline 1 & $1 / 21 / 75$ & 6 & 1 & 68 & 154.4 & $\mathbf{N}$ & degree $\mathrm{C} / \mathrm{TW}$ INS confirm \\
\hline 1 & $1 / 21 / 75$ & 7 & 1 & 57 & 134.6 & $\mathbf{N}$ & degree $\mathrm{C} / \mathrm{TW}$ INS confirm \\
\hline 1 & $1 / 21 / 75$ & 8 & 1 & 55 & 131 & $\mathbf{N}$ & degree $\mathrm{C} / \mathrm{TW}$ inS confirm \\
\hline 1 & $1 / 21 / 75$ & 9 & 1 & 54 & 129.2 & $\mathbf{N}$ & degree C/TWINS confirm \\
\hline 1 & $1 / 21 / 75$ & 10 & 1 & 54 & 129.2 & $\mathbf{N}$ & degree C/TWINS confirm \\
\hline 1 & $1 / 21 / 75$ & 11 & 1 & 33 & 91.4 & $N$ & degree C/TWINS confirm \\
\hline 1 & $1 / 21 / 75$ & 12 & 1 & 18 & 64.4 & $\mathbf{N}$ & degree C/TWINS confirm \\
\hline 1 & $1 / 21 / 75$ & 13 & $T$ & 18 & 64.4 & $\mathbf{N}$ & degree C/TWINS confirm \\
\hline 1 & $1 / 28 / 75$ & 1 & 1 & 81 & 177.8 & $Y$ & degreo C/TWINS confirm \\
\hline 1 & $1 / 28 / 75$ & 2 & 1 & 81 & 177.8 & $N$ & degree C/TWINS confitm \\
\hline 1 & $1 / 28 / 75$ & 3 & 1 & 78 & 172.4 & $\mathbf{N}$ & degree C/TWINS confirm \\
\hline 1 & $1 / 28 / 75$ & 4 & 1 & 72 & 161.6 & $\mathbf{N}$ & degree C/TWINS confirm \\
\hline 1 & $1 / 28 / 75$ & 5 & 1 & 66 & 150.8 & $\mathbf{N}$ & degree C/TWINS confirm \\
\hline 1 & $1 / 28 / 75$ & 6 & 1 & 58 & 136.4 & $\mathbf{N}$ & degree C/TWINS confirm \\
\hline 1 & $1 / 28 / 75$ & 7 & 1 & 50 & 122 & $\mathbf{N}$ & degree $\mathrm{C}$ TWWINS confirm \\
\hline 1 & $1 / 28 / 75$ & 8 & 1 & 46 & 114.8 & $\mathbf{N}$ & degree C/TWINS confirm \\
\hline 1 & $1 / 28 / 75$ & 9 & 1 & 46 & 114.8 & $\mathbf{N}$ & degree C/TWINS confirm \\
\hline 1 & $1 / 28 / 75$ & 10 & 1 & 46 & 114.8 & $\mathbf{N}$ & degree C/TWINS confirm \\
\hline 1 & $1 / 28 / 75$ & 11 & 1 & 29 & 84.2 & $\mathbf{N}$ & degree C/TWINS confitm \\
\hline 1 & $1 / 28 / 75$ & 12 & 1 & 18 & 64.4 & $\mathbf{N}$ & degree $\mathrm{C} / \mathrm{TW} W \mathrm{NS}$ confirm \\
\hline 1 & $1 / 28 / 75$ & 13 & 1 & 18 & 64.4 & $\mathbf{N}$ & degree C/TWINS confirm \\
\hline 1 & $1 / 28 / 75$ & 14 & 1 & 18 & 64.4 & $\mathbf{N}$ & degree C/TWINS confirm \\
\hline 1 & $2 / 27 / 75$ & 1 & 1 & 85 & 185 & $\bar{Y}$ & degree C/TWINS confirm \\
\hline 1 & $2 / 27 / 75$ & 2 & 1 & 85 & 185 & $\mathbf{N}$ & degree C/TWINS confirm \\
\hline 1 & $2 / 27 / 75$ & 3 & 1 & 80 & 176 & $\mathbf{N}$ & degree C/TWINS confirm \\
\hline 1 & $2 / 27 / 75$ & 4 & 1 & 76 & 168.8 & $\mathbf{N}$ & degree C/TWINS confirm \\
\hline 1 & $2 / 27 / 75$ & 5 & 1 & 70 & 158 & $\mathbf{N}$ & degree C/TWINS confirm \\
\hline 1 & $2 / 27 / 75$ & 6 & 1 & 60 & 140 & $\mathbf{N}$ & degrea C/TWINS confirm \\
\hline 1 & $2 / 27 / 75$ & 7 & 1 & 52 & 125.6 & $\mathbf{N}$ & degree C/TWINS confirm \\
\hline 1 & $2 / 27 / 75$ & 8 & 1 & 44 & 111.2 & $\mathbf{N}$ & degree C/TWINS confirm \\
\hline 1 & $2 / 27 / 75$ & 9 & 1 & 44 & 111.2 & $\mathbf{N}$ & degree C/TWINS confirm \\
\hline 1 & $2 / 27 / 75$ & 10 & 1 & 44 & 111.2 & $N$ & degree C/TWINS confirm \\
\hline 1 & $2 / 27 / 75$ & 11 & 1 & 36 & 96.8 & $\mathbf{N}$ & degree C/TWINS confirm \\
\hline 1 & $2 / 27 / 75$ & 12 & 1 & 20 & 68 & $\mathbf{N}$ & degree C/TWiNS confirm \\
\hline 1 & $2 / 27 / 75$ & 13 & 1 & 20 & 68 & $\mathbf{N}$ & degree C/TWINS confirm \\
\hline 1 & $2 / 27 / 75$ & 14 & 1 & 20 & 68 & $\mathbf{N}$ & degree C/TWINS confirm \\
\hline 9 & $3 / 18 / 75$ & 1 & 1 & 84 & 183.2 & $\bar{Y}$ & degree C/TWINS confirm \\
\hline 1 & $3 / 18 / 75$ & 2 & 1 & 82 & 179.6 & $\mathbf{N}$ & degree C/TWINS confirm \\
\hline 1 & $3 / 18 / 75$ & 3 & 1 & 80 & 176 & $\mathbf{N}$ & degree C/TWINS confirm \\
\hline 1 & $3 / 18 / 75$ & 4 & 1 & 76 & 168.8 & $\mathbf{N}$ & degree C/TWINS confirm \\
\hline 1 & $3 / 18 / 75$ & 5 & 1 & 68 & 154.4 & $\mathbf{N}$ & degree C/TWINS confirm \\
\hline 1 & $3 / 18 / 75$ & 6 & 1 & 60 & 140 & $\mathbf{N}$ & degree $\mathrm{C} / \mathrm{TW}$ INS confirm \\
\hline 1 & $3 / 18 / 75$ & 7 & 1 & 52 & 125.6 & $\mathbf{N}$ & degree C/TWINS confirm \\
\hline 1 & $3 / 18 / 75$ & 8 & 1 & 46 & 114.8 & $\mathbf{N}$ & degree C/TWINS confirm \\
\hline 1 & $3 / 18 / 75$ & 9 & 1 & 46 & 114.8 & $\mathbf{N}$ & degrae C/TWINS confirm \\
\hline 1 & $3 / 78 / 75$ & 10 & 1 & 46 & 114.8 & $\mathbf{N}$ & degree C/TWINS confirm \\
\hline 1 & $3 / 18 / 75$ & 11 & 1 & 32 & 89.6 & $\mathbf{N}$ & degree C/TWINS confirm \\
\hline 1 & $3 / 18 / 75$ & 12 & 1 & 22 & 71.6 & $\mathbf{N}$ & degree C/TWINS confirm \\
\hline 1 & $3 / 18 / 75$ & 13 & 1 & 22 & 71.6 & $\mathbf{N}$ & degree CrTWINS confirm \\
\hline 1 & $3 / 18 / 75$ & 14 & 1 & 22 & 71.6 & $\mathbf{N}$ & degree C/TWINS confirm \\
\hline 1 & $4 / 8 / 75$ & 1 & 1 & 96 & 204.8 & $\bar{Y}$ & degree $\mathrm{C} / \mathrm{TW}$ INS confirm \\
\hline 1 & $4 / 8 / 75$ & 2 & 1 & 96 & 204.8 & $\mathbf{N}$ & degree C/TWINS confirm \\
\hline 9 & $4 / 8 / 75$ & 3 & 1 & 93 & 199.4 & $\mathbf{N}$ & degree C/TWINS confirm \\
\hline 1 & $4 / 8 / 75$ & 4 & 1 & 85 & 185 & $\mathbf{N}$ & degree C/TWINS confirm \\
\hline 1 & $4 / 8 / 75$ & 5 & 1 & 76 & 168.8 & $\mathbf{N}$ & degree C/TWINS confirm \\
\hline 1 & $4 / 8 / 75$ & 6 & 1 & 66 & 150.8 & $\mathbf{N}$ & degree C/TWINS confirm \\
\hline 1 & $4 / 8 / 75$ & 7 & 1 & 56 & 132.8 & $\mathbf{N}$ & degree C/TWINS confirm \\
\hline 1 & $4 / 8 / 75$ & 8 & 9 & 50 & 122 & $\mathbf{N}$ & degree C/TWins confirm \\
\hline 1 & $4 / 8 / 75$ & 9 & 1 & 49 & 120.2 & $\mathbf{N}$ & degree C/TWINS confirm \\
\hline 1 & $4 / 8 / 75$ & 10 & 1 & 48 & 118.4 & $\mathbf{N}$ & degree C/TWINS confirm \\
\hline
\end{tabular}

Data obtained from WHC Surveillance Analysis Computer System (SACS), November 12, 1993. 


\begin{tabular}{|c|c|c|c|c|c|c|c|}
\hline \multicolumn{8}{|c|}{ TANK 241-BY-110 } \\
\hline \multicolumn{8}{|c|}{ THERMOCOUPLE DATA } \\
\hline TREE\# & DATE & THERMO \# & TREE SET \# & TEMP C & TEMP F & $Y=$ MAX TEMP & COMMENTS \\
\hline 1 & $4 / 8 / 75$ & 11 & 1 & 29 & 84.2 & $\mathbf{N}$ & degree C/TWINS confirm \\
\hline 1 & $4 / 8 / 75$ & 12 & 1 & 17 & 62.6 & $\mathbf{N}$ & degree $\mathrm{C} / \mathrm{TW}$ INS confirm \\
\hline 1 & $4 / 8 / 75$ & 13 & 1 & 18 & 64.4 & $\mathbf{N}$ & degree $\mathrm{C} / \mathrm{TW}$ INS confirm \\
\hline 1 & $4 / 8 / 75$ & 14 & 1 & 18 & 64.4 & $\mathbf{N}$ & degree $\mathrm{C} / \mathrm{TW}$ INS confirm \\
\hline 1 & $4 / 22 / 75$ & 1 & 1 & 78 & 172.4 & $Y$ & degree C/TWINS confirm \\
\hline 1 & $4 / 22 / 75$ & 2 & 1 & 78 & 172.4 & $\mathbf{N}$ & degree C/TWINS confirm \\
\hline 1 & $4 / 22 / 75$ & 3 & 1 & 72 & 161.6 & $\mathbf{N}$ & degree C/TWINS confirm \\
\hline 1 & $4 / 22 / 75$ & 4 & 1 & 68 & 154.4 & $\overline{\mathbf{N}}$ & degree C/TWINS confirm \\
\hline 1 & $4 / 22 / 75$ & 5 & 1 & 60 & 140 & $N$ & degree C/TWINS confirm \\
\hline 1 & $4 / 22 / 75$ & 6 & 1 & 51 & 123.8 & $\mathrm{~N}$ & degree C/TWINS confirm \\
\hline 1 & $4 / 22 / 75$ & 7 & 1 & 42 & 107.6 & $\bar{N}$ & degree C/TWINS confirm \\
\hline 1 & $4 / 22 / 75$ & 8 & 1 & 39 & 102.2 & $\mathbf{N}$ & degree C/TWINS contirm \\
\hline 1 & $4 / 22 / 75$ & 9 & 1 & 39 & 102.2 & $N$ & degree C/TWINS confism \\
\hline 1 & $4 / 22 / 75$ & 10 & 1 & 36 & 96.8 & $\mathbf{N}$ & degrae C/TWINS confirm \\
\hline 1 & $4 / 22 / 75$ & 11 & 1 & 18 & 64.4 & $\mathbf{N}$ & degree C/TWINS confirm \\
\hline 1 & $4 / 29 / 75$ & 1 & 1 & 80 & 176 & $\bar{Y}$ & degree $\mathrm{C} / \mathrm{TW}$ iNS confirm \\
\hline 1 & $4 / 29 / 75$ & 2 & 1 & 80 & 176 & $N$ & degree C/TWINS confirm \\
\hline 1 & $4 / 29 / 75$ & 3 & 1 & 75 & 167 & $\mathbf{N}$ & degree C/TWINS confirm \\
\hline 1 & $4 / 29 / 75$ & 4 & 1 & 70 & 158 & $\mathbf{N}$ & degree C/TWINS confirm \\
\hline 9 & $4 / 29 / 75$ & 5 & 1 & 62 & 143.6 & $\mathbf{N}$ & degree C/TWINS confirm \\
\hline 1 & $4 / 29 / 75$ & 6 & 1 & 54 & 129.2 & $\mathbf{N}$ & degree C/TWINS confirm \\
\hline 1 & $4 / 29 / 75$ & 7 & 1 & 44 & 111.2 & $N$ & degree C/TWINS confirm \\
\hline 1 & $4 / 29 / 75$ & 8 & 1 & 42 & 107.6 & $\mathbf{N}$ & degree C/TWINS confirm \\
\hline 1 & $4 / 29 / 75$ & 9 & 1 & 42 & 107.6 & $\mathbf{N}$ & degree C/TWINS confirm \\
\hline 1 & $4 / 29 / 75$ & 10 & 1 & 38 & 100.4 & $\ddot{N}$ & degree C/TWINS confirm \\
\hline 1 & $4 / 29 / 75$ & 11 & 1 & 20 & 68 & $\mathbf{N}$ & degree C/TWINS confirm \\
\hline 1 & $5 / 20 / 75$ & 1 & 1 & 80 & 176 & $\bar{Y}$ & degree $\mathrm{C} / \mathrm{TW}$ INS confirm \\
\hline 1 & $5 / 20 / 75$ & 2 & 1 & 80 & 176 & $\mathbf{N}$ & degree $\mathrm{C} / \mathrm{TW}$ INS confirm \\
\hline 1 & $5 / 20 / 75$ & 3 & 1 & 76 & 168.8 & $\mathbf{N}$ & degree $\mathrm{C} / \mathrm{TW}$ INS confitm \\
\hline 1 & $5 / 20 / 75$ & 4 & 1 & 70 & 158 & $\ddot{N}$ & degree $\mathrm{C} / \mathrm{TW}$ INS confirm \\
\hline 1 & $5 / 20 / 75$ & 5 & 1 & 63 & 145.4 & $\mathbf{N}$ & degree C/TWINS confirm \\
\hline 1 & $5 / 20 / 75$ & 6 & 1 & 54 & 129.2 & N & degree C/TWINS confirm \\
\hline 1 & $5 / 20 / 75$ & 7 & 1 & 46 & 114.8 & $\mathbf{N}$ & degree $\mathrm{C} / \mathrm{TW}$ INS confirm \\
\hline 1 & $5 / 20 / 75$ & 8 & 1 & 42 & 107.6 & $N$ & degree $\mathrm{C} / \mathrm{TW}$ WINS confirm \\
\hline 1 & $5 / 20 / 75$ & 9 & 1 & 42 & 107.6 & $\mathrm{~N}$ & degree C/TWINS confirm \\
\hline 1 & $5 / 20 / 75$ & 10 & 1 & 40 & 104 & $\mathrm{~N}$ & degree $\mathrm{C} /$ TWINS confirm \\
\hline 1 & $5 / 20 / 75$ & 11 & 1 & 34 & 93.2 & $\mathbf{N}$ & degree C/TWINS confirm \\
\hline 1 & $5 / 20 / 75$ & 12 & 1 & 24 & 75.2 & $N$ & degree C/TWINS confirm \\
\hline 1 & $5 / 20 / 75$ & 13 & 1 & 24 & 75.2 & $\mathbf{N}$ & degree C/TWINS confirm \\
\hline 1 & $5 / 20 / 75$ & 14 & 1 & 24 & 75.2 & $\mathbf{N}$ & degree C/TWINS confirm \\
\hline 1 & $6 / 17 / 75$ & 1 & 1 & & 177 & $\bar{Y}$ & \\
\hline 1 & $6 / 17 / 75$ & 2 & 9 & & 175 & $\mathbf{N}$ & \\
\hline 1 & $6 / 17 / 75$ & 3 & 1 & & 168 & $\mathbf{N}$ & \\
\hline 1 & $6 / 17 / 75$ & 4 & 1 & & 158 & $\mathrm{~N}$ & \\
\hline 1 & $6 / 17 / 75$ & 5 & 1 & & 145 & $\mathbf{N}$ & \\
\hline 1 & $6 / 17 / 75$ & 6 & 1 & & 128 & $\mathbf{N}$ & \\
\hline 1 & $6 / 17 / 75$ & 7 & 1 & & 112 & $\mathbf{N}$ & \\
\hline 1 & $6 / 17 / 75$ & 8 & 1 & & 107 & $\mathbf{N}$ & \\
\hline 1 & $6 / 17 / 75$ & 9 & 1 & & 106 & $\mathbf{N}$ & \\
\hline 1 & $6 / 17 / 75$ & 10 & 1 & & 95 & $N$ & \\
\hline 1 & $6 / 17 / 75$ & 11 & 1 & & 77 & $\mathbf{N}$ & \\
\hline 1 & $6 / 17 / 75$ & 12 & 1 & & 77 & $N$ & \\
\hline 1 & $6 / 17 / 75$ & 13 & 1 & & 77 & $\mathbf{N}$ & \\
\hline 1 & $6 / 17 / 75$ & 14 & 1 & & 77 & $N$ & \\
\hline 1 & $7 / 28 / 75$ & 1 & 1 & & 177 & $Y$ & \\
\hline 1 & $7 / 28 / 75$ & 2 & 1 & & 176 & $\mathbf{N}$ & \\
\hline 1 & $7 / 28 / 75$ & 3 & 1 & & 169 & $\mathbf{N}$ & \\
\hline 1 & $7 / 28 / 75$ & 4 & 1 & & 159 & $N$ & \\
\hline 1 & $7 / 28 / 75$ & 5 & 1 & & 146 & $N$ & \\
\hline 1 & $7 / 28 / 75$ & 6 & 1 & & 131 & $N$ & \\
\hline 1 & $7 / 28 / 75$ & 7 & 1 & & 116 & $\mathbf{N}$ & \\
\hline 1 & $7 / 28 / 75$ & 8 & 1 & & 111 & $\mathbf{N}$ & \\
\hline 1 & $7 / 28 / 75$ & 9 & 1 & & 109 & $\mathbf{N}$ & \\
\hline 1 & $7 / 28 / 75$ & 10 & 1 & & 101 & $\mathbf{N}$ & \\
\hline 1 & $7 / 28 / 75$ & 11 & 1 & & 89 & $\mathbf{N}$ & \\
\hline 1 & $7 / 28 / 75$ & 12 & 1 & & 88 & $\mathbf{N}$ & \\
\hline
\end{tabular}

Data obtained from WHC Surveillance Analysis Computer System (SACS), November 12, 1993. 


\begin{tabular}{|c|c|c|c|c|c|c|c|}
\hline \multicolumn{8}{|c|}{$\begin{array}{l}\text { TANK 241-BY-110 } \\
\text { THERMOCOUPIE DATA }\end{array}$} \\
\hline & & & & THER & COUPLE & & \\
\hline TREE\# & DATE & THERMO \# & TREE SET \# & TEMP C & TEMP F & $Y=$ MAX TEMP & COMMENTS \\
\hline 1 & $7 / 28 / 75$ & 13 & 1 & & 88 & $\mathbf{N}$ & \\
\hline 1 & $7 / 28 / 75$ & 14 & 1 & & 88 & $\overline{\mathbf{N}}$ & \\
\hline 1 & $8 / 26 / 75$ & 1 & 1 & & 174 & $\overline{\mathbf{Y}}$ & \\
\hline 1 & $8 / 26 / 75$ & 2 & 1 & & 172 & $\mathbf{N}$ & \\
\hline 1 & $8 / 26 / 75$ & 3 & 1 & & 165 & $\mathbf{N}$ & \\
\hline 1 & $8 / 26 / 75$ & 4 & 1 & & 155 & $\mathbf{N}$ & \\
\hline 1 & $8 / 26 / 75$ & 5 & 1 & & 142 & $\mathbf{N}$ & \\
\hline 1 & $8 / 26 / 75$ & 6 & 1 & & 128 & $\mathbf{N}$ & \\
\hline 1 & $8 / 26 / 75$ & 7 & 1 & & 114 & $\mathbf{N}$ & \\
\hline 1 & $8 / 26 / 75$ & 8 & 9 & & 108 & $N$ & \\
\hline 1 & $8 / 26 / 75$ & 9 & 1 & & 106 & $\bar{N}$ & \\
\hline 1 & $8 / 26 / 75$ & 10 & 1 & & 98 & $\mathbf{N}$ & \\
\hline 1 & $8 / 26 / 75$ & 11 & 9 & & 81 & $\overline{\mathbf{N}}$ & \\
\hline 1 & $8 / 26 / 75$ & 12 & 1 & & 79 & $\bar{N}$ & \\
\hline 1 & $8 / 26 / 75$ & 13 & 1 & & 80 & $\mathbf{N}$ & \\
\hline 1 & $8 / 26 / 75$ & 14 & 1 & & 80 & $\mathbf{N}$ & \\
\hline 1 & $11 / 21 / 75$ & 1 & 1 & & 182 & $\bar{Y}$ & \\
\hline 1 & $11 / 21 / 75$ & 2 & 1 & & 181 & $\mathbf{N}$ & \\
\hline 9 & $19 / 21 / 75$ & 3 & 1 & & 174 & $\mathbf{N}$ & \\
\hline 1 & $11 / 21 / 75$ & 4 & 1 & & 165 & $\mathbf{N}$ & \\
\hline 1 & $11 / 21 / 75$ & 5 & 1 & & 154 & $\mathbf{N}$ & \\
\hline 1 & $11 / 21 / 75$ & 6 & 1 & & 141 & $\bar{N}$ & \\
\hline 1 & $11 / 21 / 75$ & 7 & 1 & & 126 & $\mathbf{N}$ & \\
\hline 1 & $11 / 21 / 75$ & 8 & 1 & & 117 & $\mathbf{N}$ & \\
\hline 1 & $11 / 21 / 75$ & 9 & 9 & & 116 & $\mathbf{N}$ & \\
\hline 1 & $11 / 21 / 75$ & 10 & 1 & & 102 & $\mathbf{N}$ & \\
\hline 1 & $11 / 21 / 75$ & 17 & 1 & & 74 & $\mathbf{N}$ & \\
\hline 1 & $11 / 21 / 75$ & 12 & 1 & & 69 & $\mathrm{~N}$ & \\
\hline 1 & $11 / 21 / 75$ & 13 & 1 & & 70 & $\mathrm{~N}$ & \\
\hline 1 & $11 / 21 / 75$ & 14 & 1 & & 71 & $\mathbf{N}$ & \\
\hline 1 & $3 / 4 / 76$ & 1 & 1 & & 181 & $\bar{Y}$ & \\
\hline 1 & $3 / 4 / 76$ & 2 & 1 & & 180 & $N$ & \\
\hline 1 & $3 / 4 / 76$ & 3 & 1 & & 172 & $\mathbf{N}$ & \\
\hline 1 & $3 / 4 / 76$ & 4 & 1 & & 163 & $\mathbf{N}$ & \\
\hline 1 & $3 / 4 / 76$ & 5 & 1 & & 152 & $\mathbf{N}$ & \\
\hline 1 & $3 / 4 / 76$ & 6 & 1 & & 139 & $N$ & \\
\hline 1 & $3 / 4 / 76$ & 7 & 9 & & 123 & $\bar{N}$ & \\
\hline 1 & $3 / 4 / 76$ & 8 & 1 & & 116 & $\mathbf{N}$ & \\
\hline 1 & $3 / 4 / 76$ & 9 & 1 & & 115 & $\mathbf{N}$ & \\
\hline 1 & $3 / 4 / 76$ & 10 & 1 & & 102 & $N$ & \\
\hline 1 & $3 / 4 / 76$ & 11 & 1 & & 79 & $\mathbf{N}$ & \\
\hline 1 & $3 / 4 / 76$ & 12 & 1 & & 67 & $\mathbf{N}$ & \\
\hline 9 & $3 / 4 / 76$ & 13 & 1 & & 68 & $\mathbf{N}$ & \\
\hline 1 & $3 / 4 / 76$ & 14 & 1 & & 68 & $\mathbf{N}$ & \\
\hline 1 & $3 / 7 / 76$ & 1 & 1 & & 165 & $\bar{Y}$ & \\
\hline 1 & $5 / 7 / 76$ & 1 & 1 & & 165 & $\mathbf{Y}$ & \\
\hline 1 & $5 / 7 / 76$ & 2 & 1 & & 164 & $\mathbf{N}$ & \\
\hline 1 & $5 / 7 / 76$ & 3 & 1 & & 158 & $N$ & \\
\hline 9 & $5 / 7 / 76$ & 4 & 1 & & 148 & $\mathbf{N}$ & \\
\hline 1 & $5 / 7 / 76$ & 5 & 9 & & 137 & $\mathbf{N}$ & \\
\hline 1 & $5 / 7 / 76$ & 6 & 9 & & 113 & $\mathrm{~N}$ & \\
\hline 9 & $5 / 7 / 76$ & 7 & 1 & & 109 & $\mathbf{N}$ & \\
\hline 1 & $5 / 7 / 76$ & 8 & 1 & & 104 & $\mathbf{N}$ & \\
\hline$T$ & $5 / 7 / 76$ & 9 & 1 & & 103 & $\mathbf{N}$ & \\
\hline 9 & $5 / 7 / 76$ & 10 & 1 & & 91 & $\mathrm{~N}$ & \\
\hline 1 & $5 / 7 / 76$ & 11 & 1 & & 73 & $\mathbf{N}$ & \\
\hline 1 & $5 / 7 / 76$ & 12 & 1 & & 69 & $\mathbf{N}$ & \\
\hline 1 & $5 / 7 / 76$ & 13 & 1 & & 69 & $\mathrm{~N}$ & \\
\hline$T$ & $5 / 7 / 76$ & 14 & 1 & & 69 & $\mathbf{N}$ & \\
\hline 1 & $6 / 9 / 76$ & 1 & 9 & & 165 & $\bar{Y}$ & \\
\hline 1 & $6 / 9 / 76$ & 2 & 1 & & 163 & $\mathbf{N}$ & \\
\hline 1 & $6 / 9 / 76$ & 3 & 1 & & 156 & $\mathbf{N}$ & \\
\hline 1 & $6 / 9 / 76$ & 4 & 1 & & 147 & $\mathbf{N}$ & \\
\hline 1 & $6 / 9 / 76$ & 5 & 1 & & 135 & $\mathbf{N}$ & \\
\hline 1 & $6 / 9 / 76$ & 6 & 1 & & 122 & $\mathbf{N}$ & \\
\hline 1 & $6 / 9 / 76$ & 7 & 1 & & 108 & $\mathbf{N}$ & \\
\hline
\end{tabular}

Data obtained from WHC Surveillance Analysis Computer System (SACS), November 12, 1993. 


\begin{tabular}{|c|c|c|c|c|c|c|c|}
\hline \multicolumn{8}{|c|}{ TANK 241-BY-110 } \\
\hline \multicolumn{8}{|c|}{ THERMOCOUPLE DATA } \\
\hline & & & & & & & \\
\hline TREE\# & DATE & THERMO \# & TREE SET \# & TEMP C & TEMP F & $Y=$ MAX TEMP & COMMENTS \\
\hline 1 & $6 / 9 / 76$ & 8 & 1 & & 103 & $\mathbf{N}$ & \\
\hline 1 & $6 / 9 / 76$ & 9 & 1 & & 100 & $N$ & \\
\hline 1 & $6 / 9 / 76$ & 10 & 1 & & 89 & $N$ & \\
\hline 1 & $6 / 9 / 76$ & 11 & 1 & & 75 & $\mathbf{N}$ & \\
\hline 1 & $6 / 9 / 76$ & 12 & 1 & & 68 & $\mathbf{N}$ & \\
\hline 1 & $6 / 9 / 76$ & 13 & 1 & & 68 & $N$ & \\
\hline 1 & $6 / 9 / 76$ & 14 & 1 & & 68 & $\mathbf{N}$ & \\
\hline 1 & $8 / 28 / 76$ & 1 & 1 & & 160 & $Y$ & \\
\hline 1 & $8 / 28 / 76$ & 2 & 1 & & 158 & $\mathrm{~N}$ & \\
\hline 1 & $8 / 28 / 76$ & 3 & 1 & & 152 & $\mathbf{N}$ & \\
\hline 1 & $8 / 28 / 76$ & 4 & 1 & & 144 & $\mathbf{N}$ & \\
\hline 1 & $8 / 28 / 76$ & 5 & 1 & & 133 & $\mathbf{N}$ & \\
\hline 1 & $8 / 28 / 76$ & 6 & 1 & & 121 & $\mathbf{N}$ & \\
\hline 1 & $8 / 28 / 76$ & 7 & 1 & & 110 & $N$ & \\
\hline 1 & $8 / 28 / 76$ & 8 & 1 & & 102 & $\mathbf{N}$ & \\
\hline 1 & $8 / 28 / 76$ & 9 & 8 & & 99 & $\mathbf{N}$ & \\
\hline 1 & $8 / 28 / 76$ & 10 & 1 & & 99 & $\mathrm{~N}$ & \\
\hline 1 & $8 / 28 / 76$ & 11 & 1 & & 78 & $\mathbf{N}$ & \\
\hline 1 & $8 / 28 / 76$ & 12 & 1 & & 73 & $\mathbf{N}$ & \\
\hline 1 & $9 / 13 / 76$ & 1 & 1 & & 161 & $\bar{Y}$ & \\
\hline 1 & $9 / 13 / 76$ & 2 & 1 & & 160 & $\mathbf{N}$ & \\
\hline 1 & $9 / 13 / 76$ & 3 & 1 & & 154 & $\mathbf{N}$ & \\
\hline 1 & $9 / 13 / 76$ & 4 & 1 & & 146 & $\mathbf{N}$ & \\
\hline 1 & $9 / 13 / 76$ & 5 & 1 & & 135 & $\mathbf{N}$ & \\
\hline 1 & $9 / 13 / 76$ & 6 & 1 & & 124 & $\mathbf{N}$ & \\
\hline 1 & $9 / 13 / 76$ & 7 & 1 & & 112 & $\mathbf{N}$ & \\
\hline 1 & $9 / 13 / 76$ & 81 & 1 & & 106 & $\mathbf{N}$ & \\
\hline 1 & $9 / 13 / 76$ & 12 & 1 & & 103 & $\mathbf{N}$ & \\
\hline 1 & $12 / 9 / 76$ & 1 & 1 & & 125 & $Y$ & \\
\hline 1 & $12 / 9 / 76$ & 2 & 1 & & 124 & $\bar{N}$ & \\
\hline 1 & $12 / 9 / 76$ & 3 & 1 & & 123 & $\mathrm{~N}$ & \\
\hline 1 & $12 / 9 / 76$ & 4 & 1 & & 121 & $\mathbf{N}$ & \\
\hline 1 & $12 / 9 / 76$ & 5 & 1 & & 107 & $\mathbf{N}$ & \\
\hline 1 & $12 / 9 / 76$ & 6 & 1 & & 107 & $N$ & \\
\hline 1 & $12 / 9 / 76$ & 7 & 1 & & 85 & $N$ & \\
\hline 1 & $12 / 9 / 76$ & 8 & 1 & & 83 & $N$ & \\
\hline 1 & $12 / 9 / 76$ & 9 & 1 & & 81 & $\mathrm{~N}$ & \\
\hline 1 & $12 / 9 / 76$ & 10 & 1 & & 81 & $\mathrm{~N}$ & \\
\hline 1 & $12 / 9 / 76$ & 11 & 1 & & 81 & $N$ & \\
\hline 1 & $12 / 9 / 76$ & 12 & 1 & & 89 & $N$ & \\
\hline 1 & $1 / 3 / 77$ & 1 & 1 & & 175 & $\bar{Y}$ & \\
\hline 1 & $1 / 3 / 77$ & 2 & 1 & & 173 & $\mathbf{N}$ & \\
\hline 1 & $1 / 3 / 77$ & 3 & 9 & & 167 & $N$ & \\
\hline 1 & $1 / 3 / 77$ & 4 & 1 & & 160 & $\mathbf{N}$ & \\
\hline 1 & $1 / 3 / 77$ & 5 & 1 & & 151 & $\mathbf{N}$ & \\
\hline 1 & $1 / 3 / 77$ & 6 & 1 & & 140 & $\mathbf{N}$ & \\
\hline 1 & $1 / 3 / 77$ & 7 & 1 & & 127 & $\mathbf{N}$ & \\
\hline 1 & $1 / 3 / 77$ & 8 & 1 & & 118 & $\mathbf{N}$ & \\
\hline 1 & $1 / 3 / 77$ & 9 & 1 & & 115 & $\mathbf{N}$ & \\
\hline 1 & $1 / 3 / 77$ & 10 & 1 & & 100 & $\mathbf{N}$ & \\
\hline 1 & $9 / 3 / 77$ & 11 & 1 & & 77 & $\mathbf{N}$ & \\
\hline 1 & $1 / 3 / 77$ & 12 & 1 & & 71 & $\mathbf{N}$ & \\
\hline 1 & $1 / 3 / 77$ & 13 & 1 & & 68 & $\mathbf{N}$ & \\
\hline 1 & $1 / 3 / 77$ & 14 & 1 & & 69 & $N$ & \\
\hline 1 & $2 / 2 / 77$ & 1 & 1 & & 161 & $\mathrm{~N}$ & \\
\hline 1 & $2 / 2 / 77$ & 2 & 1 & & 162 & $\bar{Y}$ & \\
\hline 1 & $2 / 2 / 77$ & 3 & 1 & & 157 & $\mathbf{N}$ & \\
\hline 1 & $2 / 2 / 77$ & 4 & 1 & & 150 & $\mathbf{N}$ & \\
\hline 1 & $2 / 2 / 77$ & 5 & 1 & & 139 & $\mathbf{N}$ & \\
\hline 1 & $2 / 2 / 77$ & 6 & 1 & & 124 & $N$ & \\
\hline 9 & $2 / 2 / 77$ & 7 & 1 & & 113 & $\mathbf{N}$ & \\
\hline 1 & $2 / 2 / 77$ & 8 & 1 & & 110 & $\mathbf{N}$ & \\
\hline 1 & $2 / 2 / 77$ & 9 & 1 & & 106 & $N$ & \\
\hline 1 & $2 / 2 / 77$ & 10 & 1 & & 82 & $\mathbf{N}$ & \\
\hline 1 & $2 / 2 / 77$ & 11 & 1 & & 59 & $\mathbf{N}$ & \\
\hline 1 & $2 / 2 / 77$ & 12 & 1 & & 59 & $N$ & \\
\hline
\end{tabular}

Data obtained from WHC Surveillance Analysis Computer System (SACS), November 12, 1993. 


\begin{tabular}{|c|c|c|c|c|c|c|c|}
\hline \multicolumn{8}{|c|}{ TANK 241-BY-110 } \\
\hline & & & & THEF & COUPLE & $\overline{\text { TA }}$ & \\
\hline TREE\# & DATE & THERMO \# & TREE SET \# & TEMP C & TEMP F & $Y=$ MAX TEMP & COMMENTS \\
\hline 1 & $2 / 2 / 77$ & 13 & 1 & & 60 & $\mathbf{N}$ & \\
\hline 1 & $2 / 2 / 77$ & 14 & 1 & & 60 & $\mathbf{N}$ & \\
\hline$T$ & $2 / 9 / 77$ & $T$ & $\frac{T}{1}$ & & 182 & $\bar{Y}$ & \\
\hline 1 & $2 / 9 / 77$ & 2 & 1 & & 180 & $N$ & \\
\hline 1 & $2 / 9 / 77$ & 3 & 1 & & 174 & $N$ & \\
\hline 1 & $2 / 9 / 77$ & $\overline{4}$ & 1 & & 166 & $\mathbf{N}$ & \\
\hline 1 & $2 / 9 / 77$ & 5 & 9 & & 153 & $\mathbf{N}$ & \\
\hline 1 & $2 / 9 / 77$ & 6 & 1 & & 136 & $\mathbf{N}$ & \\
\hline 1 & $2 / 9 / 77$ & 7 & 1 & & 125 & $\mathbf{N}$ & \\
\hline 1 & $2 / 9 / 77$ & 8 & 1 & & 117 & $\mathbf{N}$ & \\
\hline 1 & $2 / 9 / 77$ & 9 & 1 & & 86 & $\mathbf{N}$ & \\
\hline 1 & $2 / 9 / 77$ & 10 & 1 & & 74 & $\mathbf{N}$ & \\
\hline 1 & $2 / 9 / 77$ & 11 & 1 & & 75 & $\mathbf{N}$ & \\
\hline 1 & $2 / 9 / 77$ & 12 & 1 & & 76 & $N$ & \\
\hline 1 & $2 / 26 / 77$ & 1 & 1 & & 160 & $\mathrm{Y}$ & \\
\hline 1 & $2 / 26 / 77$ & 2 & 1 & & 159 & $\mathrm{~N}$ & \\
\hline 1 & $2 / 26 / 77$ & 3 & $T$ & & 153 & $\mathbf{N}$ & \\
\hline 1 & $2 / 26 / 77$ & 4 & 1 & & 144 & $\mathbf{N}$ & \\
\hline 1 & $2 / 26 / 77$ & 5 & 1 & & 133 & $\mathbf{N}$ & \\
\hline 1 & $2 / 26 / 77$ & 6 & 1 & & 119 & $\mathbf{N}$ & \\
\hline 1 & $2 / 26 / 77$ & 7 & 1 & & 106 & $\mathbf{N}$ & \\
\hline 1 & $2 / 26 / 77$ & 8 & 1 & & 103 & $\mathbf{N}$ & \\
\hline 1 & $2 / 26 / 77$ & 9 & 1 & & 93 & $\mathrm{~N}$ & \\
\hline 1 & $2 / 26 / 77$ & 10 & 1 & & 65 & $\bar{N}$ & \\
\hline 1 & $2 / 26 / 77$ & 11 & 1 & & 59 & $\mathbf{N}$ & \\
\hline 1 & $2 / 26 / 77$ & 12 & 1 & & 59 & $\mathrm{~N}$ & \\
\hline 1 & $2 / 26 / 77$ & 73 & 1 & & 60 & $\mathbf{N}$ & \\
\hline 1 & $2 / 26 / 77$ & 14 & 1 & & 60 & $\mathbf{N}$ & \\
\hline 1 & $3 / 28 / 77$ & 1 & 1 & & 164 & $\bar{Y}$ & \\
\hline 1 & $3 / 28 / 77$ & 2 & 1 & & 163 & $\mathbf{N}$ & \\
\hline 1 & $3 / 28 / 77$ & 3 & 1 & & 157 & $\mathbf{N}$ & \\
\hline 1 & $3 / 28 / 77$ & 4 & 1 & & $14 \overline{8}$ & $\mathbf{N}$ & \\
\hline 1 & $3 / 28 / 77$ & 5 & 1 & & 138 & $\bar{N}$ & \\
\hline 1 & $3 / 28 / 77$ & 6 & 1 & & 124 & $\bar{N}$ & \\
\hline 1 & $3 / 28 / 77$ & 7 & 1 & & 108 & $\mathbf{N}$ & \\
\hline 1 & $3 / 28 / 77$ & 8 & 1 & & 104 & $\mathbf{N}$ & \\
\hline 1 & $3 / 28 / 77$ & 9 & 1 & & 95 & $N$ & \\
\hline 9 & $3 / 28 / 77$ & 10 & 1 & & 69 & $N$ & \\
\hline 1 & $3 / 28 / 77$ & 11 & 9 & & 62 & $\mathbf{N}$ & \\
\hline 1 & $3 / 28 / 77$ & 12 & 1 & & 62 & $\mathbf{N}$ & \\
\hline 1 & $3 / 28 / 77$ & 13 & 1 & & 62 & $N$ & \\
\hline 1 & $3 / 28 / 77$ & 14 & 1 & & 63 & $\mathbf{N}$ & \\
\hline 1 & $4 / 27 / 77$ & $\frac{1}{1}$ & 1 & & 148 & $\bar{Y}$ & \\
\hline 1 & $4 / 27 / 77$ & 2 & 1 & & 146 & $\mathbf{N}$ & \\
\hline 1 & $4 / 27 / 77$ & 3 & 1 & & 138 & $N$ & \\
\hline 9 & $4 / 27 / 77$ & 4 & $T$ & & 129 & $\mathbf{N}$ & \\
\hline 1 & $4 / 27 / 77$ & 5 & 1 & & 115 & $\bar{N}$ & \\
\hline 1 & $4 / 27 / 77$ & 6 & 1 & & 99 & $\mathbf{N}$ & \\
\hline 7 & $4 / 27 / 77$ & 7 & 9 & & 76 & $\mathbf{N}$ & \\
\hline 1 & $4 / 27 / 77$ & 8 & 1 & & 76 & $N$ & \\
\hline 1 & $4 / 27 / 77$ & 9 & 1 & & 70 & $\mathbf{N}$ & \\
\hline 1 & $4 / 27 / 77$ & 10 & 1 & & 65 & $\mathbf{N}$ & \\
\hline 1 & $4 / 27 / 77$ & 11 & 9 & & 63 & $\mathbf{N}$ & \\
\hline 1 & $4 / 27 / 77$ & 12 & 1 & & 63 & $\mathbf{N}$ & \\
\hline 1 & $6 / 30 / 77$ & 1 & 1 & & 156 & $Y$ & \\
\hline 1 & $6 / 30 / 77$ & 2 & 1 & & 154 & $\mathbf{N}$ & \\
\hline 1 & $6 / 30 / 77$ & 3 & 1 & & 137 & $\mathbf{N}$ & \\
\hline 1 & $6 / 30 / 77$ & 4 & 1 & & 138 & $N$ & \\
\hline 1 & $6 / 30 / 77$ & 5 & 1 & & 126 & $N$ & \\
\hline 1 & $6 / 30 / 77$ & 6 & 1 & & 114 & $\mathbf{N}$ & \\
\hline 1 & $6 / 30 / 77$ & 7 & 1 & & 103 & $N$ & \\
\hline 1 & $6 / 30 / 77$ & 8 & 1 & & 90 & $N$ & \\
\hline 1 & $6 / 30 / 77$ & 9 & 1 & & 75 & $\mathbf{N}$ & \\
\hline 1 & $6 / 30 / 77$ & 10 & 1 & & 74 & $\bar{N}$ & \\
\hline 1 & $6 / 30 / 77$ & 11 & 1 & & 74 & $\mathbf{N}$ & \\
\hline 1 & $6 / 30 / 77$ & 12 & 1 & & 74 & $\mathbf{N}$ & \\
\hline
\end{tabular}

Data obtained from WHC Surveillance Analysis Computer System (SACS), November 12, 1993. 
WHC-SD-WM-ER-312, Rev. 0

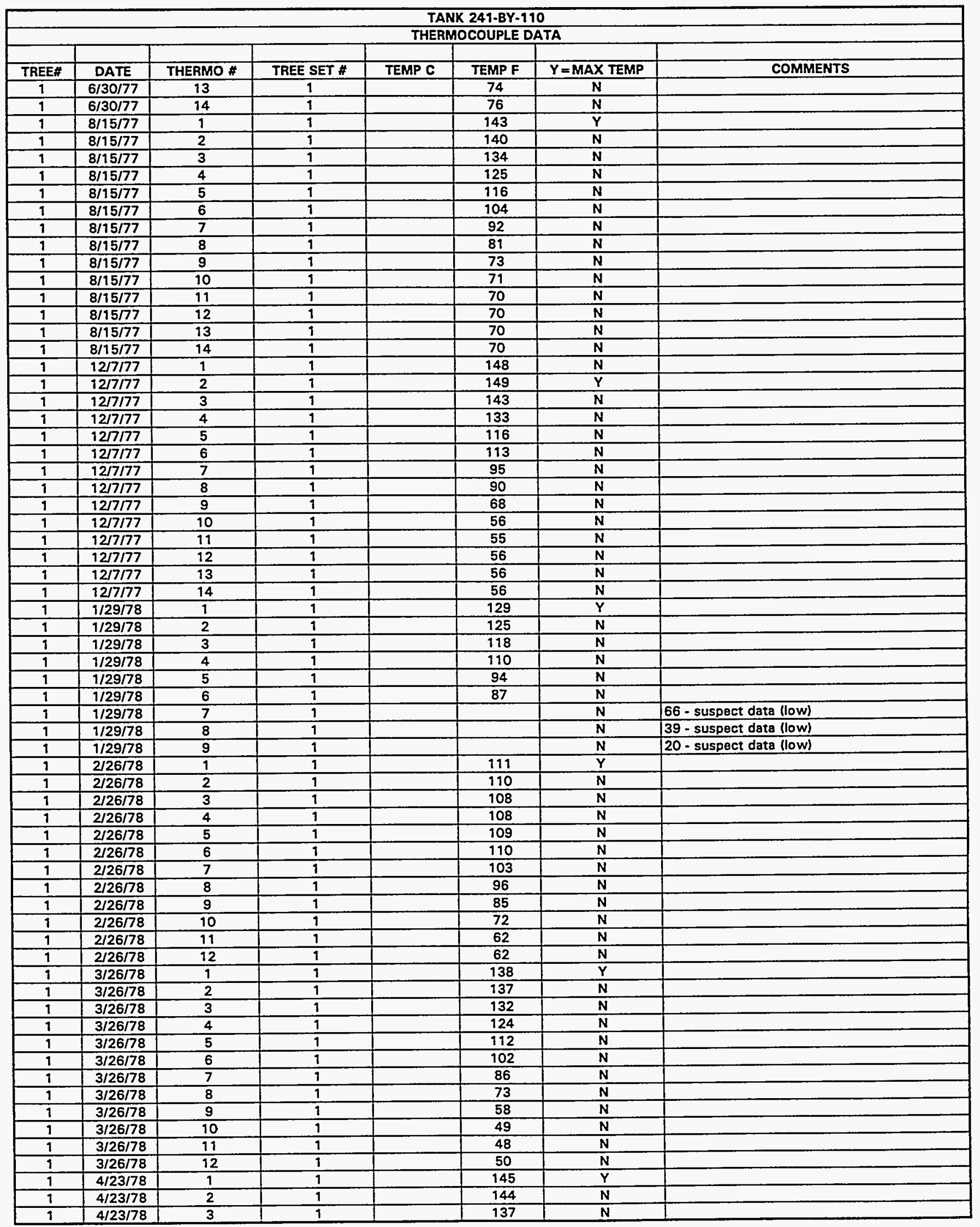

Data obtained from WHC Surveillance Analysis Computer System (SACS), November 12, 1993. 


\begin{tabular}{|c|c|c|c|c|c|c|c|}
\hline \multicolumn{8}{|c|}{ TANK 241-BY-110 } \\
\hline & & & & THEF & COUPLE & & \\
\hline TREE\# & DATE & THERMO \# & TREE SET \# & TEMP C & TEMP F & $Y=$ MAX TEMP & COMMENTS \\
\hline 1 & $4 / 23 / 78$ & 4 & 1 & & 132 & $\mathbf{N}$ & \\
\hline$T$ & $4 / 23 / 78$ & 5 & 1 & & 121 & $\bar{N}$ & \\
\hline 9 & $4 / 23 / 78$ & 6 & $T$ & & 110 & $\mathbf{N}$ & \\
\hline 1 & $4 / 23 / 78$ & 7 & 1 & & 95 & $\mathbf{N}$ & \\
\hline 1 & $4 / 23 / 78$ & 8 & 1 & & 89 & $N$ & \\
\hline 9 & $4 / 23 / 78$ & 9 & 1 & & 72 & $\mathbf{N}$ & \\
\hline 1 & $4 / 23 / 78$ & 10 & 1 & & 63 & $\mathbf{N}$ & \\
\hline 1 & $4 / 23 / 78$ & 11 & 1 & & 63 & $\bar{N}$ & \\
\hline$\frac{T}{1}$ & $4 / 23 / 78$ & 12 & 1 & & 64 & $\mathbf{N}$ & \\
\hline 1 & $6 / 17 / 78$ & $T$ & 1 & & 137 & $\bar{Y}$ & \\
\hline 1 & $6 / 17 / 78$ & 2 & 1 & & 136 & $\mathbf{N}$ & \\
\hline 1 & $6 / 17 / 78$ & 3 & 1 & & 130 & $\mathbf{N}$ & \\
\hline 1 & $6 / 17 / 78$ & 4 & 1 & & 122 & $\mathbf{N}$ & \\
\hline 9 & $6 / 17 / 78$ & 5 & 1 & & 912 & $\bar{N}$ & \\
\hline 1 & $6 / 17 / 78$ & 6 & 1 & & 102 & $\mathbf{N}$ & \\
\hline$T$ & $6 / 17 / 78$ & 7 & 1 & & 90 & $\mathbf{N}$ & \\
\hline 1 & $6 / 17 / 78$ & 8 & 1 & & 82 & $\mathbf{N}$ & \\
\hline 1 & $6 / 17 / 78$ & 9 & 1 & & 70 & $\mathbf{N}$ & \\
\hline 1 & $6 / 17 / 78$ & 10 & 1 & & 65 & $\mathbf{N}$ & \\
\hline$T$ & $6 / 17 / 78$ & 11 & 1 & & 65 & $\bar{N}$ & \\
\hline 1 & $6 / 17 / 78$ & 12 & $\overline{1}$ & & 65 & $\mathbf{N}$ & \\
\hline 1 & $8 / 13 / 78$ & 1 & 1 & & 139 & $Y$ & \\
\hline 1 & $8 / 13 / 78$ & 2 & 1 & & 139 & $\mathbf{N}$ & \\
\hline 1 & $8 / 13 / 78$ & 3 & 1 & & 133 & $N$ & \\
\hline 1 & $8 / 13 / 78$ & 4 & 1 & & 125 & $\mathbf{N}$ & \\
\hline 1 & $8 / 13 / 78$ & 5 & 1 & & 117 & $\mathrm{~N}$ & \\
\hline 1 & $8 / 13 / 78$ & 6 & 7 & & 107 & $\mathbf{N}$ & \\
\hline 9 & $8 / 13 / 78$ & 7 & 1 & & 96 & $\mathbf{N}$ & \\
\hline$\frac{1}{1}$ & $8 / 13 / 78$ & 8 & 1 & & 89 & $N$ & \\
\hline 9 & $8 / 13 / 78$ & 9 & 1 & & 80 & $\mathrm{~N}$ & \\
\hline 1 & $8 / 13 / 78$ & 10 & 1 & & 76 & $\mathbf{N}$ & \\
\hline$T$ & $8 / 13 / 78$ & 11 & 1 & & 75 & $N$ & \\
\hline$T$ & $8 / 13 / 78$ & 12 & 1 & & 75 & $N$ & \\
\hline 1 & $8 / 13 / 78$ & 13 & 1 & & 75 & $\mathbf{N}$ & \\
\hline 1 & $8 / 13 / 78$ & 14 & 9 & & 75 & $\mathbf{N}$ & \\
\hline$T$ & $10 / 7 / 78$ & 1 & 1 & & 148 & $\bar{Y}$ & \\
\hline 9 & $10 / 7 / 78$ & 2 & $\frac{T}{1}$ & & 148 & $\bar{N}$ & \\
\hline 1 & $10 / 7 / 78$ & 3 & 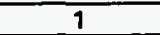 & & 142 & $\mathbf{N}$ & \\
\hline 1 & $10 / 7 / 78$ & 4 & 1 & & 138 & $\mathbf{N}$ & \\
\hline 9 & $10 / 7 / 78$ & 5 & 9 & & 127 & $N$ & \\
\hline 1 & $.10 / 7 / 78$ & 6 & 1 & & 121 & $\mathbf{N}$ & \\
\hline 9 & $10 / 7 / 78$ & 7 & 1 & & 112 & $\mathbf{N}$ & \\
\hline 9 & $10 / 7 / 78$ & 8 & 9 & & 98 & $\mathbf{N}$ & \\
\hline 1 & $10 / 7 / 78$ & 9 & 1 & & 87 & $\mathbf{N}$ & \\
\hline 9 & $10 / 7 / 78$ & 10 & $T$ & & 82 & $\mathbf{N}$ & \\
\hline 1 & $10 / 7 / 78$ & 11 & 1 & & 80 & $N$ & \\
\hline 1 & $10 / 7 / 78$ & 12 & 1 & & 80 & $\mathbf{N}$ & \\
\hline 1 & $10 / 7 / 78$ & 13 & 1 & & 78 & $N$ & \\
\hline 1 & $10 / 7 / 78$ & 14 & 1 & & 74 & $\mathbf{N}$ & \\
\hline 1 & $11 / 15 / 79$ & 1 & 9 & & 163 & $\bar{Y}$ & \\
\hline 1 & $11 / 15 / 79$ & 2 & 1 & & 161 & $\mathbf{N}$ & \\
\hline 1 & $11 / 15 / 79$ & 3 & 1 & & 160 & $\mathbf{N}$ & \\
\hline 1 & $11 / 15 / 79$ & 4 & 1 & & 154 & $N$ & \\
\hline 1 & $11 / 15 / 79$ & 5 & 1 & & 148 & $\bar{N}$ & \\
\hline$\frac{1}{1}$ & $11 / 15 / 79$ & 6 & 1 & & 140 & $\mathbf{N}$ & \\
\hline 1 & $71 / 15 / 79$ & 7 & 1 & & 131 & $\mathbf{N}$ & \\
\hline 1 & $11 / 15 / 79$ & 8 & 1 & & 120 & $\mathrm{~N}$ & \\
\hline 1 & $5 / 12 / 80$ & 1 & 1 & & 139 & $\bar{Y}$ & \\
\hline 1 & $5 / 12 / 80$ & 2 & 1 & & 139 & $\mathbf{N}$ & \\
\hline 9 & $5 / 12 / 80$ & 3 & 1 & & 136 & $\mathbf{N}$ & \\
\hline 1 & $5 / 12 / 80$ & 4 & 1 & & 131 & $\mathbf{N}$ & \\
\hline 1 & $5 / 12 / 80$ & 5 & 1 & & 110 & $\mathbf{N}$ & \\
\hline 1 & $5 / 12 / 80$ & 6 & 1 & & 108 & $\mathbf{N}$ & \\
\hline 1 & $5 / 12 / 80$ & 7 & 9 & & 94 & $\mathbf{N}$ & \\
\hline 1 & $6 / 12 / 81$ & 1 & 1 & & 132 & $\bar{Y}$ & \\
\hline 1 & $6 / 12 / 81$ & 2 & 1 & & 130 & $\mathbf{N}$ & \\
\hline
\end{tabular}

Data obtained from WHC Surveillance Analysis Computer System (SACS), November 12, 1993. 
WHC-SD-WM-ER-312, Rev. 0

\begin{tabular}{|c|c|c|c|c|c|c|c|}
\hline \multicolumn{8}{|c|}{ TANK 241-BY-110 } \\
\hline & & & & THER & COUPLE & & \\
\hline & $\pi T$ & & & & $T$ THO 5 & & \\
\hline$\frac{\text { TREE\# }}{1}$ & DATE & THERMO \# & TREE SEI $\pi$ & TEMP C & TEMP F & $Y=$ MAX TEMP & COMMENTS \\
\hline 1 & $6 / 12 / 81$ & 3 & 1 & & 125 & $\mathbf{N}$ & \\
\hline 1 & $6 / 12 / 81$ & 4 & 1 & & 118 & $\mathbf{N}$ & \\
\hline 9 & $6 / 12 / 81$ & 5 & 1 & & 109 & $\mathbf{N}$ & \\
\hline 1 & $6 / 12 / 81$ & 6 & 1 & & 99 & $\mathbf{N}$ & \\
\hline 1 & $6 / 12 / 81$ & 7 & 1 & & 88 & $\bar{N}$ & \\
\hline 1 & $10 / 14 / 83$ & 1 & 1 & & 135 & $\mathbf{Y}$ & \\
\hline$T$ & $10 / 14 / 83$ & 2 & 1 & & 931 & $\mathbf{N}$ & \\
\hline 1 & $10 / 14 / 83$ & 3 & 1 & & 125 & $\mathrm{~N}$ & \\
\hline 1 & $10 / 14 / 83$ & 4 & 1 & & 120 & $\mathbf{N}$ & \\
\hline 1 & $10 / 14 / 83$ & 5 & 1 & & 110 & $N$ & \\
\hline 1 & $10 / 14 / 83$ & 6 & 1 & & 85 & $N$ & \\
\hline 1 & $11 / 8 / 83$ & 1 & 1 & & 146 & $\bar{Y}$ & \\
\hline 1 & $11 / 8 / 83$ & 2 & 1 & & 143 & $\mathbf{N}$ & \\
\hline 1 & $11 / 8 / 83$ & 3 & 1 & & 136 & $\mathbf{N}$ & \\
\hline 1 & $11 / 8 / 83$ & 4 & 1 & & 131 & $\bar{N}$ & \\
\hline 1 & $11 / 8 / 83$ & 5 & 1 & & 121 & $\mathbf{N}$ & \\
\hline 1 & $11 / 8 / 83$ & 6 & 1 & & 94 & $\bar{N}$ & \\
\hline 1 & $12 / 6 / 83$ & 1 & 1 & & 147 & $\bar{Y}$ & \\
\hline 1 & $12 / 6 / 83$ & 2 & 1 & & 143 & $\mathrm{~N}$ & \\
\hline 1 & $12 / 6 / 83$ & 3 & 1 & & 137 & $\mathrm{~N}$ & \\
\hline 1 & $12 / 6 / 83$ & 4 & 1 & & 133 & $\bar{N}$ & \\
\hline 1 & $12 / 6 / 83$ & 5 & 1 & & 122 & $\mathbf{N}$ & \\
\hline 1 & $12 / 6 / 83$ & 6 & 1 & & 93 & $\mathbf{N}$ & \\
\hline 1 & $1 / 3 / 84$ & 1 & 1 & & 142 & $\bar{Y}$ & \\
\hline 1 & $1 / 3 / 84$ & 2 & 1 & & 140 & $\mathbf{N}$ & \\
\hline 1 & $1 / 3 / 84$ & 3 & 1 & & 136 & $\mathbf{N}$ & \\
\hline 1 & $1 / 3 / 84$ & 4 & $\frac{1}{1}$ & & 131 & $\mathbf{N}$ & \\
\hline 1 & $1 / 3 / 84$ & 5 & 1 & & 118 & $N$ & \\
\hline 1 & $1 / 3 / 84$ & 6 & 1 & & 85 & $\bar{N}$ & \\
\hline 1 & $2 / 7 / 84$ & 1 & 9 & & 148 & $\bar{Y}$ & \\
\hline 1 & $2 / 7 / 84$ & 2 & 1 & & 144 & $\bar{N}$ & \\
\hline 1 & $2 / 7 / 84$ & 3 & 1 & & 139 & $N$ & \\
\hline 1 & $2 / 7 / 84$ & 4 & 1 & & 133 & $\bar{N}$ & \\
\hline 1 & $2 / 7 / 84$ & 5 & 1 & & 120 & $\mathrm{~N}$ & \\
\hline 1 & $2 / 7 / 84$ & 6 & 1 & & 86 & $\mathbf{N}$ & \\
\hline 1 & $2 / 14 / 84$ & 1 & 1 & & 136 & $\bar{Y}$ & \\
\hline 1 & $2 / 14 / 84$ & 2 & 1 & & 132 & $\mathbf{N}$ & \\
\hline 1 & $2 / 14 / 84$ & 3 & 1 & & 127 & $\mathbf{N}$ & \\
\hline 1 & $2 / 14 / 84$ & 4 & 9 & & 121 & $\bar{N}$ & \\
\hline 1 & $2 / 14 / 84$ & 5 & 1 & & 106 & $\mathrm{~N}$ & \\
\hline 1 & $2 / 14 / 84$ & 6 & 1 & & 73 & $\mathbf{N}$ & \\
\hline 1 & $3 / 26 / 84$ & 1 & 9 & & 134 & $\bar{Y}$ & \\
\hline 1 & $3 / 26 / 84$ & 2 & 1 & & 131 & $\mathbf{N}$ & \\
\hline 1 & $3 / 26 / 84$ & 3 & 1 & & 127 & $\mathbf{N}$ & \\
\hline 1 & $3 / 26 / 84$ & 4 & 1 & & 121 & $\mathbf{N}$ & \\
\hline 1 & $3 / 26 / 84$ & 5 & 1 & & 105 & $\mathbf{N}$ & \\
\hline 1 & $3 / 26 / 84$ & 6 & 1 & & 73 & $\mathbf{N}$ & \\
\hline 1 & $4 / 7 / 84$ & 1 & 1 & & 130 & $\mathbf{Y}$ & \\
\hline 1 & $4 / 7 / 84$ & 2 & $\frac{1}{1}$ & & 127 & $\bar{N}$ & \\
\hline 1 & $4 / 7 / 84$ & 3 & 1 & & 123 & $\mathbf{N}$ & \\
\hline 1 & $4 / 7 / 84$ & 4 & 1 & & 117 & $N$ & \\
\hline 1 & $4 / 7 / 84$ & 5 & 9 & & 105 & $\mathbf{N}$ & \\
\hline 1 & $4 / 7 / 84$ & 6 & 1 & & 75 & $\mathbf{N}$ & \\
\hline 1 & $6 / 19 / 84$ & 1 & 1 & & 129 & $Y$ & \\
\hline 1 & $6 / 19 / 84$ & 2 & 1 & & 927 & $\mathbf{N}$ & \\
\hline 1 & $6 / 19 / 84$ & 3 & 1 & & 123 & $\mathbf{N}$ & \\
\hline 1 & $6 / 19 / 84$ & 4 & 1 & & 118 & $\mathbf{N}$ & \\
\hline 1 & $6 / 19 / 84$ & 5 & 1 & & 108 & $\bar{N}$ & \\
\hline 1 & $6 / 19 / 84$ & 6 & 1 & & 78 & $\mathbf{N}$ & \\
\hline 1 & $9 / 3 / 84$ & 1 & 1 & & 131 & $Y$ & \\
\hline 1 & $9 / 3 / 84$ & 2 & 1 & & 129 & $\mathrm{~N}$ & \\
\hline 1 & $9 / 3 / 84$ & 3 & 1 & & 124 & $\mathbf{N}$ & \\
\hline 1 & $9 / 3 / 84$ & 4 & 1 & & 120 & $\mathrm{~N}$ & \\
\hline 1 & $9 / 3 / 84$ & 5 & 1 & & 112 & $\mathrm{~N}$ & \\
\hline 1 & $9 / 3 / 84$ & 6 & 1 & & 85 & $\mathbf{N}$ & \\
\hline 1 & $3 / 3 / 85$ & 1 & 1 & & 128 & $Y$ & \\
\hline
\end{tabular}

Data obtained from WHC Surveillance Analysis Computer System (SACS), November 12, 1993. 


\begin{tabular}{|c|c|c|c|c|c|c|c|}
\hline \multicolumn{8}{|c|}{ TANK 241-BY-110 } \\
\hline & & & & THEF & COUPLE & & \\
\hline TREE\# & DATE & THERMO \# & TREE SET \# & TEMP C & TEMP F & $Y=M A X$ TEMP & COMMENTS \\
\hline 1 & $3 / 3 / 85$ & 2 & 1 & & 126 & $\mathbf{N}$ & \\
\hline 1 & $3 / 3 / 85$ & 3 & 1 & & 121 & $\mathbf{N}$ & \\
\hline 1 & $3 / 3 / 85$ & 4 & 1 & & 115 & $\mathbf{N}$ & \\
\hline 1 & $3 / 3 / 85$ & 5 & 1 & & 106 & $\mathrm{~N}$ & \\
\hline 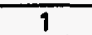 & $3 / 3 / 85$ & 6 & 1 & & 73 & $\mathbf{N}$ & \\
\hline 1 & $3 / 5 / 85$ & 1 & 9 & & 127.6 & $\mathbf{Y}$ & \\
\hline 1 & $3 / 5 / 85$ & 2 & 9 & & 125.7 & $\mathbf{N}$ & \\
\hline 9 & $3 / 5 / 85$ & 3 & 1 & & 120.5 & $\mathbf{N}$ & \\
\hline 1 & $3 / 5 / 85$ & 4 & 1 & & 114.5 & $\mathbf{N}$ & \\
\hline 1 & $3 / 5 / 85$ & 5 & 1 & & 105.8 & $\mathbf{N}$ & \\
\hline 7 & $3 / 5 / 85$ & 6 & 1 & & 73 & $\mathbf{N}$ & \\
\hline 1 & $4 / 1 / 85$ & 1 & 1 & & 126 & $\bar{Y}$ & \\
\hline$T$ & $4 / 1 / 85$ & 2 & $\frac{1}{1}$ & & 125 & $\mathbf{N}$ & \\
\hline 1 & $4 / 1 / 85$ & 3 & 1 & & 120 & $\mathbf{N}$ & \\
\hline 1 & $4 / 1 / 85$ & 4 & 1 & & 115 & $\mathbf{N}$ & \\
\hline 1 & $4 / 1 / 85$ & 5 & 1 & & 106 & $N$ & \\
\hline 1 & $4 / 1 / 85$ & 6 & 1 & & 73 & $\mathbf{N}$ & \\
\hline 1 & $5 / 7 / 85$ & 1 & 1 & & 140 & $\bar{Y}$ & \\
\hline 1 & $5 / 7 / 85$ & 2 & 1 & & 137 & $\mathbf{N}$ & \\
\hline$\frac{1}{1}$ & $5 / 7 / 85$ & 3 & 7 & & 131 & $\mathbf{N}$ & \\
\hline$\frac{1}{1}$ & $5 / 7 / 85$ & 4 & 9 & & 125 & $\mathbf{N}$ & \\
\hline 1 & $5 / 7 / 85$ & 5 & 1 & & 116 & $\mathbf{N}$ & \\
\hline$\frac{1}{1}$ & $5 / 7 / 85$ & 6 & 1 & & 78 & $N$ & \\
\hline 1 & $6 / 4 / 85$ & 1 & 1 & & 138 & $\bar{Y}$ & \\
\hline 1 & $6 / 4 / 85$ & 2 & 1 & & 136 & $\mathbf{N}$ & \\
\hline 1 & $6 / 4 / 85$ & 3 & 7 & & 129 & $\mathbf{N}$ & \\
\hline 1 & $6 / 4 / 85$ & 4 & 1 & & 123 & $\mathbf{N}$ & \\
\hline 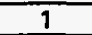 & $6 / 4 / 85$ & 5 & 1 & & 114 & $\mathbf{N}$ & \\
\hline$\frac{T}{1}$ & $6 / 4 / 85$ & 6 & 7 & & 78 & $\mathbf{N}$ & \\
\hline 1 & $6 / 10 / 85$ & 1 & 1 & & 127 & $\bar{Y}$ & \\
\hline 1 & $6 / 10 / 85$ & 2 & 1 & & 124 & $\mathbf{N}$ & \\
\hline 1 & $6 / 10 / 85$ & 3 & 9 & & 119 & $\bar{N}$ & \\
\hline 1 & $6 / 10 / 85$ & 4 & 1 & & 114 & $\mathbf{N}$ & \\
\hline 1 & $6 / 10 / 85$ & 5 & 1 & & 106 & $\mathbf{N}$ & \\
\hline 9 & $6 / 70 / 85$ & 6 & 1 & & 76 & $\mathbf{N}$ & \\
\hline$\frac{1}{1}$ & $7 / 2 / 85$ & 1 & 1 & & 131 & $\bar{Y}$ & \\
\hline 1 & $7 / 2 / 85$ & 2 & 9 & & 129 & $\mathbf{N}$ & \\
\hline 1 & $7 / 2 / 85$ & 3 & 1 & & 123 & $\mathbf{N}$ & \\
\hline 1 & $7 / 2 / 85$ & 4 & 1 & & 117 & $\bar{N}$ & \\
\hline 1 & $7 / 2 / 85$ & 5 & 1 & & 109 & $\mathbf{N}$ & \\
\hline 1 & $7 / 2 / 85$ & 6 & 1 & & 73 & $\mathbf{N}$ & \\
\hline 1 & $8 / 5 / 85$ & 1 & 1 & & 133 & $\bar{Y}$ & \\
\hline 9 & $8 / 5 / 85$ & 2 & 1 & & 132 & $\mathbf{N}$ & \\
\hline 1 & $8 / 5 / 85$ & 3 & 9 & & 127 & $\mathbf{N}$ & \\
\hline 1 & $8 / 5 / 85$ & 4 & 1 & & 122 & N & \\
\hline 1 & $8 / 5 / 85$ & 5 & 1 & & 114 & $\bar{N}$ & \\
\hline 1 & $8 / 5 / 85$ & 6 & 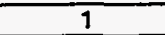 & & 82 & $\mathbf{N}$ & \\
\hline 1 & $9 / 1 / 85$ & 1 & 1 & & 126 & $\mathbf{Y}$ & \\
\hline 1 & $9 / 1 / 85$ & 2 & 1 & & 124 & $\mathbf{N}$ & \\
\hline 1 & $9 / 1 / 85$ & 3 & 1 & & 119 & $\mathrm{~N}$ & \\
\hline 1 & $9 / 1 / 85$ & 4 & 1 & & 115 & $\bar{N}$ & \\
\hline 1 & $9 / 1 / 85$ & 5 & 1 & & 108 & $N$ & \\
\hline 1 & $9 / 1 / 85$ & 6 & 1 & & 83 & $\bar{N}$ & \\
\hline 1 & $1 / 6 / 86$ & 1 & 1 & & 128 & $\bar{Y}$ & \\
\hline 1 & $1 / 6 / 86$ & 2 & 1 & & 126 & $\mathbf{N}$ & \\
\hline 1 & $1 / 6 / 86$ & 3 & 1 & & 122 & $\mathbf{N}$ & \\
\hline 1 & $1 / 6 / 86$ & 4 & 1 & & 118 & $N$ & \\
\hline 1 & $1 / 6 / 86$ & 5 & 1 & & 110 & $\bar{N}$ & \\
\hline 1 & $1 / 6 / 86$ & 6 & 1 & & 79 & $\mathbf{N}$ & \\
\hline 1 & $3 / 4 / 86$ & 9 & 1 & & 127 & $\bar{Y}$ & \\
\hline 1 & $3 / 4 / 86$ & 2 & 1 & & 125 & $\bar{N}$ & \\
\hline 1 & $3 / 4 / 86$ & 3 & 1 & & 120 & $\mathbf{N}$ & \\
\hline 1 & $3 / 4 / 86$ & 4 & 1 & & 115 & $\mathbf{N}$ & \\
\hline 1 & $3 / 4 / 86$ & 5 & 1 & & 107 & $N$ & \\
\hline$\frac{1}{1}$ & $3 / 4 / 86$ & 6 & 1 & & 73 & $\bar{N}$ & \\
\hline 1 & $4 / 7 / 86$ & 1 & 1 & & 126 & $Y$ & \\
\hline
\end{tabular}

Data obtained from WHC Surveillance Analysis Computer System (SACS), November 12, 1993. 


\begin{tabular}{|c|c|c|c|c|c|c|c|}
\hline \multicolumn{8}{|c|}{ TANK 241-BY-110 } \\
\hline \multicolumn{8}{|c|}{ THERMOCOUPLE DATA } \\
\hline & & & & & & & \\
\hline TREE\# & DATE & THERMO \# & TREE SET \# & TEMP C & TEMP F & $Y=$ MAX TEMP & COMMENTS \\
\hline 1 & $4 / 7 / 86$ & 2 & 1 & & 124 & $\mathbf{N}$ & \\
\hline 1 & $4 / 7 / 86$ & 3 & 1 & & 120 & $\mathbf{N}$ & \\
\hline 1 & $4 / 7 / 86$ & 4 & 1 & & 116 & $\mathbf{N}$ & \\
\hline 1 & $4 / 7 / 86$ & 5 & 1 & & 108 & $\mathbf{N}$ & \\
\hline 1 & $4 / 7 / 86$ & 6 & 1 & & 74 & $\mathbf{N}$ & \\
\hline 1 & $5 / 11 / 86$ & 9 & 1 & & 124 & $Y$ & \\
\hline 1 & $5 / 11 / 86$ & 2 & 1 & & 123 & $\mathbf{N}$ & \\
\hline 1 & $5 / 11 / 86$ & 3 & 1 & & 118 & $\mathbf{N}$ & \\
\hline 1 & $5 / 11 / 86$ & 4 & 1 & & 114 & $N$ & \\
\hline 1 & $5 / 11 / 86$ & 5 & 1 & & 106 & $\mathrm{~N}$ & \\
\hline 1 & $5 / 11 / 86$ & 6 & 1 & & 73 & $\mathbf{N}$ & - \\
\hline 1 & $6 / 3 / 86$ & 1 & 1 & & 126 & $Y$ & \\
\hline 1 & $6 / 3 / 86$ & 2 & 1 & & 124 & $\mathbf{N}$ & \\
\hline 1 & $6 / 3 / 86$ & 3 & 1 & & 119 & $\mathbf{N}$ & \\
\hline 1 & $6 / 3 / 86$ & 4 & 1 & & 114 & $N$ & \\
\hline 1 & $6 / 3 / 86$ & 5 & 1 & & 106 & $\mathbf{N}$ & \\
\hline 1 & $6 / 3 / 86$ & 6 & 1 & & 75 & $N$ & \\
\hline 1 & $7 / 7 / 86$ & 1 & 1 & & 126 & $Y$ & \\
\hline 1 & $7 / 7 / 86$ & 2 & 1 & & 124 & $\mathbf{N}$ & \\
\hline 1 & $7 / 7 / 86$ & 3 & 1 & & 120 & $\mathbf{N}$ & \\
\hline 1 & $7 / 7 / 86$ & 4 & 1 & & 115 & $\mathbf{N}$ & \\
\hline 1 & $7 / 7 / 86$ & 5 & 1 & & 108 & $\mathbf{N}$ & \\
\hline 1 & $7 / 7 / 86$ & 6 & 1 & & 78 & $\mathbf{N}$ & \\
\hline 1 & $8 / 4 / 86$ & 1 & 1 & & 126 & $\bar{Y}$ & \\
\hline 1 & $8 / 4 / 86$ & 2 & 1 & & 124 & $\mathbf{N}$ & \\
\hline 1 & $8 / 4 / 86$ & 3 & 1 & & 120 & $\mathbf{N}$ & \\
\hline 1 & $8 / 4 / 86$ & 4 & 1 & & 115 & $\mathbf{N}$ & \\
\hline 1 & $8 / 4 / 86$ & 5 & 1 & & 108 & $\mathbf{N}$ & \\
\hline 1 & $8 / 4 / 86$ & 6 & 1 & & 80.7 & $\mathrm{~N}$ & \\
\hline 1 & $9 / 2 / 86$ & 1 & 1 & & 126 & $Y$ & \\
\hline 1 & $9 / 2 / 86$ & 2 & 1 & & 124 & $N$ & \\
\hline 1 & $9 / 2 / 86$ & 3 & 1 & & 119 & $\mathbf{N}$ & \\
\hline 1 & $9 / 2 / 86$ & 4 & 1 & & 115 & $\mathbf{N}$ & \\
\hline 1 & $9 / 2 / 86$ & 5 & 1 & & 110 & $\mathrm{~N}$ & \\
\hline 1 & $9 / 2 / 86$ & 6 & 1 & & 83 & $N$ & \\
\hline 1 & $10 / 6 / 86$ & 1 & 1 & & 125.2 & $\bar{Y}$ & \\
\hline 1 & $10 / 6 / 86$ & 2 & 1 & & 123.3 & $\mathbf{N}$ & \\
\hline 1 & $10 / 6 / 86$ & 3 & 1 & & 119.6 & $N$ & \\
\hline 1 & $10 / 6 / 86$ & 4 & 1 & & 115.4 & $\mathbf{N}$ & \\
\hline 1 & $10 / 6 / 86$ & 5 & 1 & & 108.2 & $\mathrm{~N}$ & \\
\hline 9 & $10 / 6 / 86$ & 6 & 1 & & 92.8 & $\mathbf{N}$ & \\
\hline 1 & $11 / 1 / 86$ & 1 & 1 & & 126 & $\bar{Y}$ & \\
\hline 1 & $11 / 1 / 86$ & 2 & 1 & & 124 & $\mathbf{N}$ & \\
\hline 1 & $11 / 1 / 86$ & 3 & 1 & & 120 & $\mathbf{N}$ & \\
\hline 1 & $11 / 1 / 86$ & 4 & 1 & & 116 & $\mathbf{N}$ & \\
\hline 1 & $11 / 1 / 86$ & 5 & 1 & & 108 & $\mathbf{N}$ & \\
\hline 1 & $11 / 1 / 86$ & 6 & 1 & & 82 & $\mathbf{N}$ & \\
\hline 1 & $12 / 2 / 86$ & 1 & 9 & & 145 & $\mathbf{Y}$ & \\
\hline 1 & $12 / 2 / 86$ & 2 & 1 & & 143 & $\mathbf{N}$ & \\
\hline 1 & $12 / 2 / 86$ & 3 & 1 & & 138 & $\mathbf{N}$ & \\
\hline 1 & $12 / 2 / 86$ & 4 & 1 & & 133 & $\mathbf{N}$ & \\
\hline 1 & $12 / 2 / 86$ & 5 & 1 & & 124 & $\mathbf{N}$ & \\
\hline 1 & $12 / 2 / 86$ & 6 & 1 & & 91 & $\mathbf{N}$ & \\
\hline 1 & $2 / 3 / 87$ & 1 & 1 & & 137 & $Y$ & \\
\hline 1 & $2 / 3 / 87$ & 2 & 1 & & 136 & $\mathrm{~N}$ & \\
\hline 1 & $2 / 3 / 87$ & 3 & 1 & & 132 & $\mathbf{N}$ & \\
\hline 1 & $2 / 3 / 87$ & 4 & $\begin{array}{r}1 \\
\end{array}$ & & 127 & $\mathbf{N}$ & \\
\hline 1 & $2 / 3 / 87$ & 5 & 1 & & 116 & $\mathbf{N}$ & \\
\hline 1 & $2 / 3 / 87$ & 6 & 1 & & 78 & $\mathrm{~N}$ & \\
\hline 1 & $3 / 12 / 87$ & 9 & 1 & & 136 & $Y$ & \\
\hline 1 & $3 / 12 / 87$ & 2 & 1 & & 135 & $N$ & \\
\hline 9 & $3 / 12 / 87$ & 3 & 1 & & 131 & $\mathbf{N}$ & \\
\hline 1 & $3 / 12 / 87$ & 4 & 1 & & 125 & $\mathbf{N}$ & \\
\hline 1 & $3 / 12 / 87$ & 5 & 1 & & 115 & $\mathbf{N}$ & \\
\hline 1 & $3 / 12 / 87$ & 6 & 1 & & 76 & $\mathbf{N}$ & \\
\hline 1 & $4 / 13 / 87$ & 1 & 1 & & 139 & $Y$ & \\
\hline
\end{tabular}

Data obtained from WHC Surveillance Analysis Computer System (SACS), November 12, 1993. 


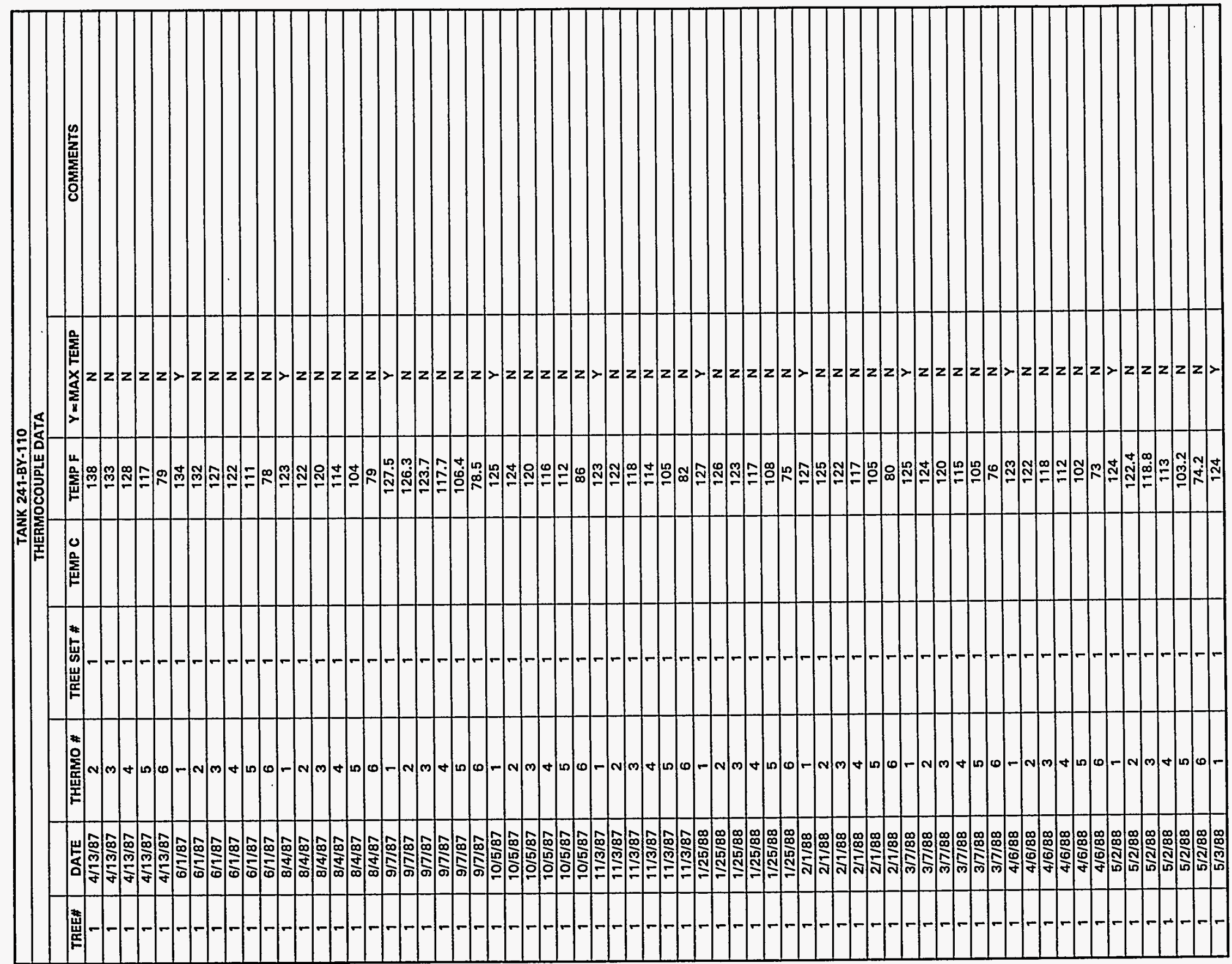

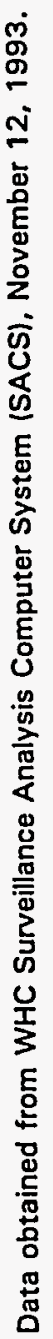

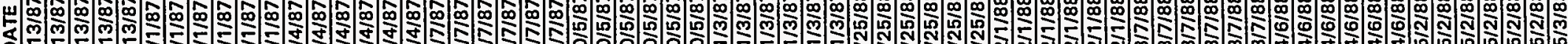

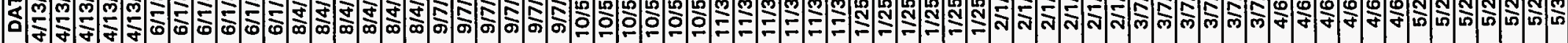




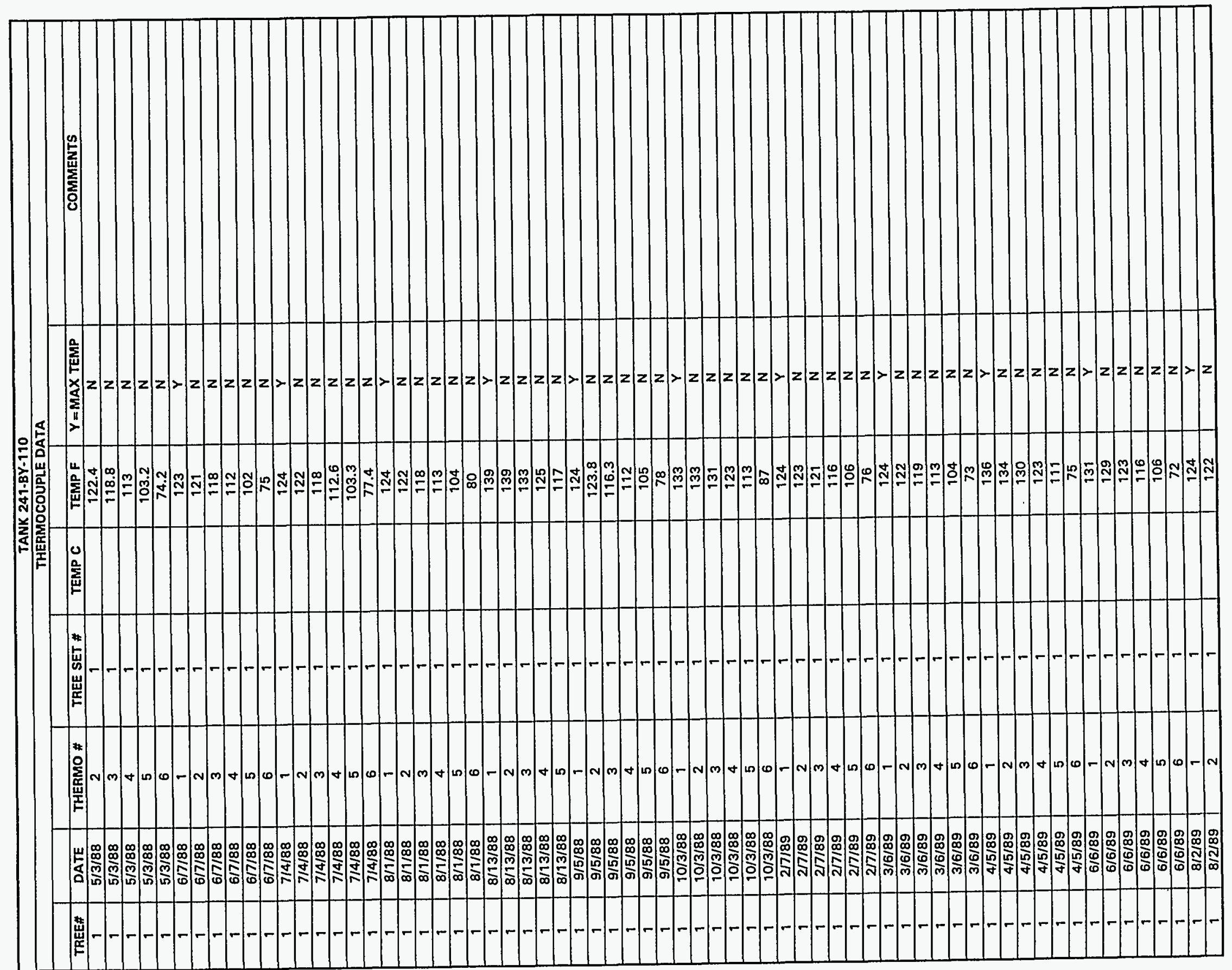




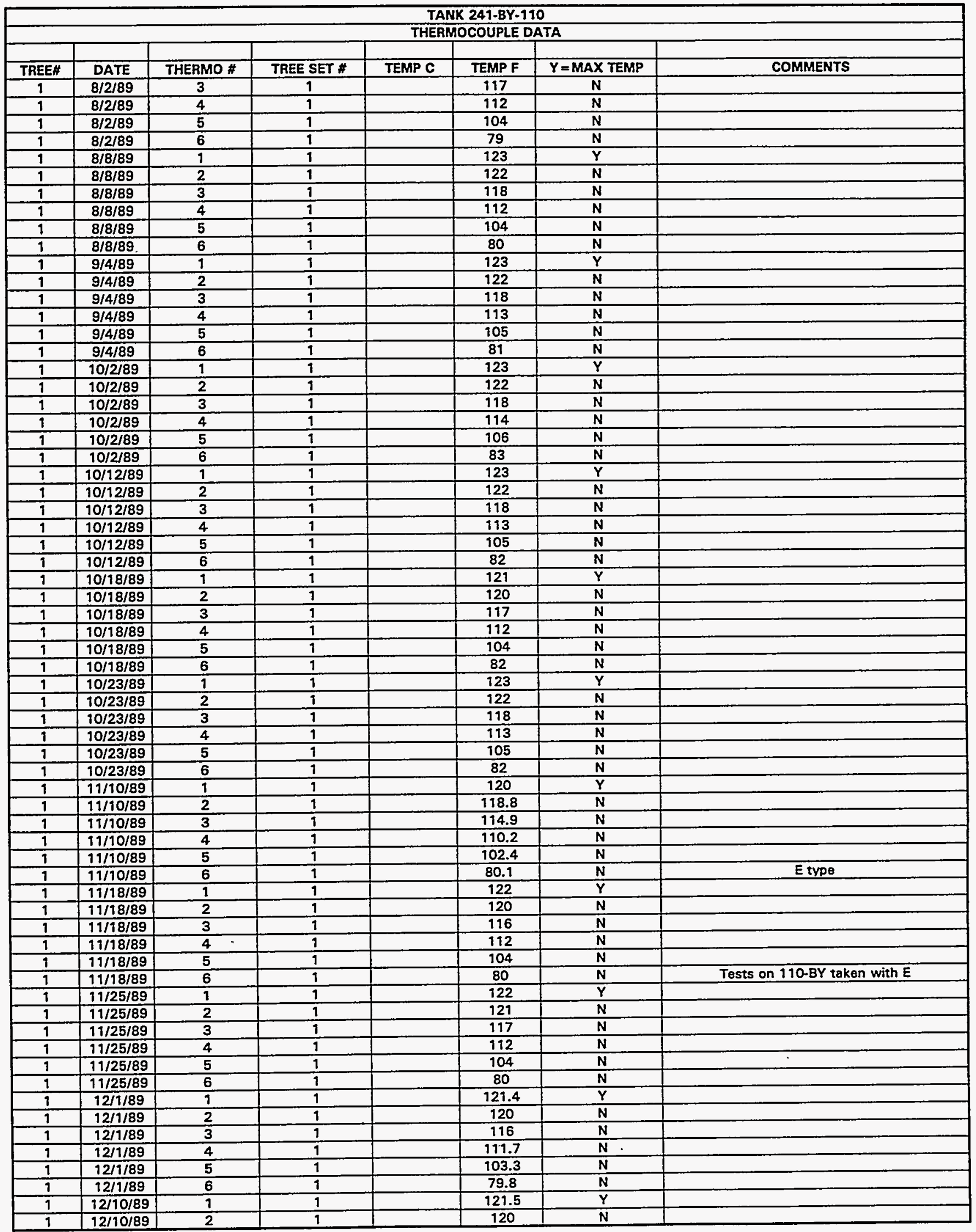

Data obtained from WHC Surveillance Analysis Computer System (SACS), November 12, 1993. 


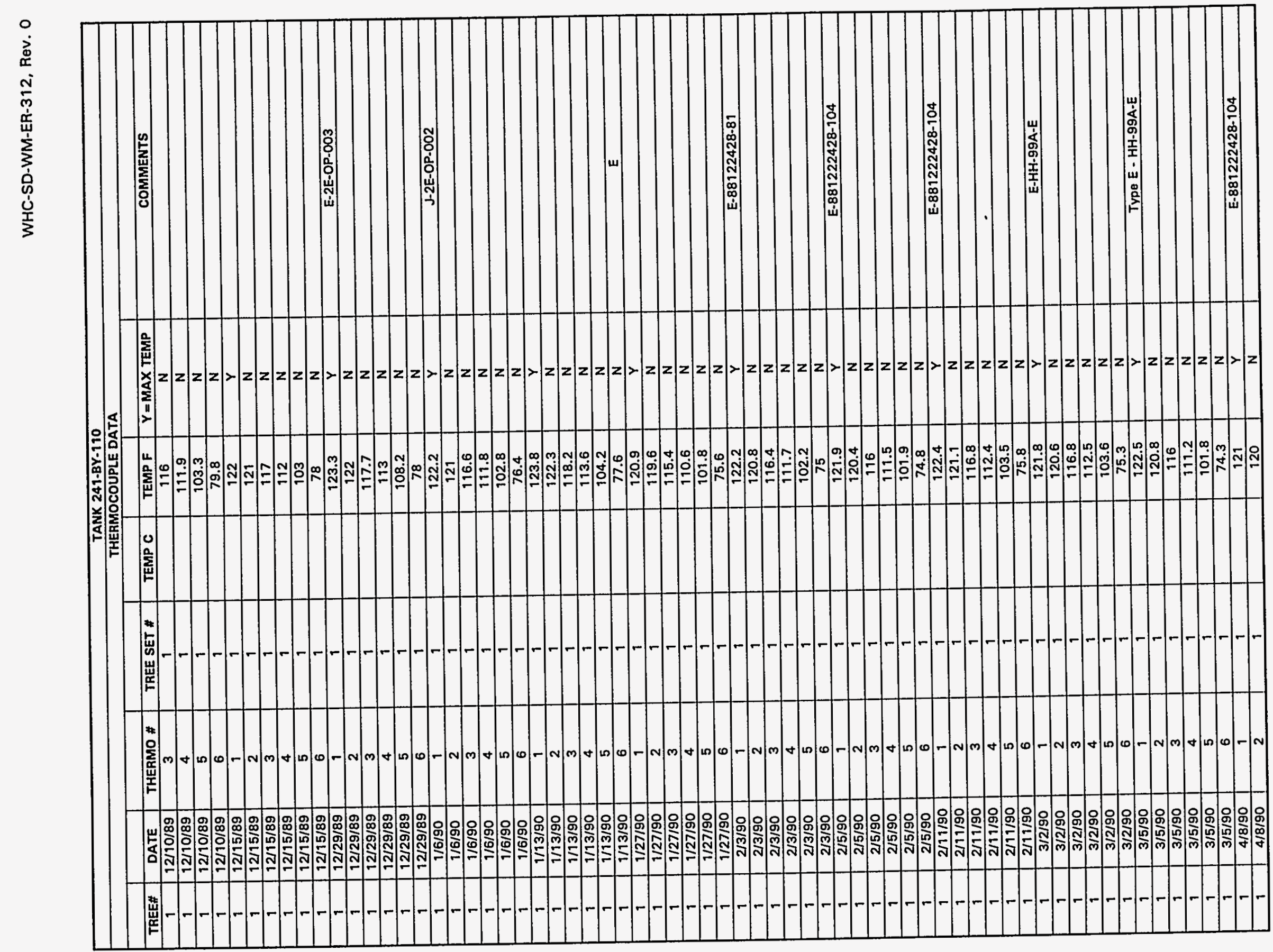




\begin{tabular}{|c|c|c|c|c|c|c|c|}
\hline \multicolumn{8}{|c|}{ TANK 247-BY-110 } \\
\hline & & & & THER & COUPLE & & \\
\hline TREE\# & DATE & THERMO \# & TREE SET \# & TEMP C & TEMP F & $Y=$ MAX TEMP & COMMENTS \\
\hline 1 & $4 / 8 / 90$ & 3 & 1 & & 116 & $N$ & \\
\hline 1 & $4 / 8 / 90$ & 4 & 1 & & 110 & $N$ & \\
\hline 1 & $4 / 8 / 90$ & 5 & 1 & & 101 & $\mathbf{N}$ & \\
\hline 1 & $4 / 8 / 90$ & 6 & 1 & & 74 & $\mathbf{N}$ & \\
\hline 1 & $4 / 26 / 90$ & 1 & 1 & & 121.4 & $\bar{Y}$ & HH-99A-E, SER \# 881222428-83 \\
\hline$T$ & $4 / 26 / 90$ & 2 & 1 & & 119.8 & $\mathbf{N}$ & \\
\hline 1 & $4 / 26 / 90$ & 3 & 1 & & 115.8 & $\mathbf{N}$ & \\
\hline 1 & $4 / 26 / 90$ & 4 & 1 & & 110.6 & $\mathbf{N}$ & \\
\hline 1 & $4 / 26 / 90$ & 5 & 1 & & 101.4 & $\mathbf{N}$ & \\
\hline 9 & $4 / 26 / 90$ & 6 & 1 & & 74 & $\mathbf{N}$ & \\
\hline 1 & $5 / 5 / 90$ & 1 & 1 & & 120 & $\bar{Y}$ & \\
\hline 1 & $5 / 5 / 90$ & 2 & 1 & & 118 & $\mathbf{N}$ & \\
\hline 9 & $5 / 5 / 90$ & 3 & 1 & & 114 & $N$ & \\
\hline 1 & $5 / 5 / 90$ & 4 & 1 & & 109 & $\mathbf{N}$ & \\
\hline 1 & $5 / 5 / 90$ & 5 & 1 & & 100 & $\mathbf{N}$ & \\
\hline 1 & $5 / 5 / 90$ & 6 & 1 & & 73 & $\mathbf{N}$ & \\
\hline 9 & $8 / 5 / 90$ & 1 & 1 & & 120.5 & $\bar{Y}$ & $E$ \\
\hline$T$ & $8 / 5 / 90$ & 2 & 1 & & 119.1 & $\mathbf{N}$ & \\
\hline 1 & $8 / 5 / 90$ & 3 & 1 & & 115.6 & $\mathbf{N}$ & \\
\hline 1 & $8 / 5 / 90$ & 4 & 1 & & 110.1 & $\mathbf{N}$ & \\
\hline 9 & $8 / 5 / 90$ & 5 & 1 & & 901.4 & $\mathbf{N}$ & \\
\hline 1 & $8 / 5 / 90$ & 6 & 1 & & 78.3 & $N$ & \\
\hline 1 & $9 / 11 / 90$ & 1 & 1 & & 116 & $\mathbf{N}$ & $E$ \\
\hline$T$ & $9 / 11 / 90$ & 2 & 1 & & 917 & $\bar{Y}$ & \\
\hline 1 & $9 / 11 / 90$ & 3 & 1 & & 114 & $\mathbf{N}$ & \\
\hline 1 & $9 / 19 / 90$ & 4 & 1 & & 110 & $\mathbf{N}$ & \\
\hline 1 & $9 / 11 / 90$ & 5 & 1 & & 100 & $\mathbf{N}$ & \\
\hline 1 & $9 / 11 / 90$ & 6 & 1 & & 75 & $\mathbf{N}$ & \\
\hline 1 & $9 / 28 / 90$ & 1 & 1 & & 121.9 & $\bar{Y}$ & $E$ \\
\hline 1 & $9 / 28 / 90$ & 2 & 1 & & 320.4 & $\mathbf{N}$ & \\
\hline 1 & $9 / 28 / 90$ & 3 & 1 & & 117 & $\mathbf{N}$ & \\
\hline 1 & $9 / 28 / 90$ & 4 & 1 & & 112.5 & N & \\
\hline 1 & $9 / 28 / 90$ & 5 & 1 & & 104.6 & $\mathrm{~N}$ & \\
\hline 1 & $9 / 28 / 90$ & 6 & 1 & & 82.2 & $\mathbf{N}$ & \\
\hline 1 & $10 / 9 / 90$ & 1 & 1 & & 121 & $\bar{Y}$ & $E$ \\
\hline 1 & $10 / 9 / 90$ & 2 & 1 & & 120 & $\mathbf{N}$ & \\
\hline 1 & $10 / 9 / 90$ & 3 & 1 & & 116 & $\mathbf{N}$ & \\
\hline 1 & $10 / 9 / 90$ & 4 & 1 & & 111 & $\mathbf{N}$ & \\
\hline 1 & $10 / 9 / 90$ & 5 & 1 & & 103 & $\mathbf{N}$ & \\
\hline 1 & $10 / 9 / 90$ & 6 & 1 & & 82 & $\mathrm{~N}$ & \\
\hline 1 & $10 / 19 / 90$ & 1 & 1 & & 120.5 & $\bar{Y}$ & $E$ \\
\hline 1 & $10 / 19 / 90$ & 2 & 9 & & 119.6 & $\mathbf{N}$ & \\
\hline 1 & $10 / 19 / 90$ & 3 & 1 & & 116.2 & $\mathbf{N}$ & \\
\hline 1 & $10 / 19 / 90$ & 4 & 1 & & 111.5 & $\mathbf{N}$ & \\
\hline 1 & $10 / 19 / 90$ & 5 & 1 & & 103.5 & $\mathbf{N}$ & \\
\hline 1 & $10 / 19 / 90$ & 6 & 1 & & 82.2 & $\mathbf{N}$ & \\
\hline 1 & $10 / 22 / 90$ & 1 & 1 & & 122 & $\bar{Y}$ & E 890723678-75 \\
\hline 1 & $10 / 22 / 90$ & 2 & 1 & & 121 & $\mathbf{N}$ & \\
\hline 1 & $10 / 22 / 90$ & 3 & 1 & & 117 & $\mathbf{N}$ & \\
\hline 1 & $10 / 22 / 90$ & 4 & 1 & & 112 & $\mathbf{N}$ & \\
\hline 1 & $10 / 22 / 90$ & 5 & 1 & & 105 & $\mathbf{N}$ & \\
\hline 1 & $10 / 22 / 90$ & 6 & 1 & & 83 & $\mathbf{N}$ & \\
\hline 1 & $10 / 26 / 90$ & 1 & 1 & & 121.4 & $\mathbf{Y}$ & $E-890723678-75$ \\
\hline 1 & $10 / 26 / 90$ & 2 & 1 & & 120.5 & $\mathbf{N}$ & \\
\hline 1 & $10 / 26 / 90$ & 3 & 1 & & 117.2 & $\mathbf{N}$ & \\
\hline 1 & $10 / 26 / 90$ & 4 & 1 & & 112.4 & $\mathbf{N}$ & \\
\hline 1 & $10 / 26 / 90$ & 5 & 1 & & 104.3 & $\mathbf{N}$ & \\
\hline 1 & $10 / 26 / 90$ & 6 & 1 & & 82.2 & $\mathbf{N}$ & \\
\hline 1 & $10 / 30 / 90$ & 9 & 1 & & 122.7 & $Y$ & $3890523512-20$ \\
\hline 1 & $10 / 30 / 90$ & 2 & 1 & & 121.6 & $\mathbf{N}$ & \\
\hline 1 & $10 / 30 / 90$ & 3 & 1 & & 118.2 & $\mathbf{N}$ & \\
\hline 7 & $10 / 30 / 90$ & 4 & 1 & & 113.5 & $\mathbf{N}$ & \\
\hline 1 & $10 / 30 / 90$ & 5 & 1 & & 105.4 & $\mathbf{N}$ & \\
\hline 1 & $10 / 30 / 90$ & 6 & 1 & & 83.5 & $\mathbf{N}$ & \\
\hline 1 & $11 / 2 / 90$ & 1 & 1 & & 121.3 & $\bar{Y}$ & E 2E-OP-003 \\
\hline 9 & $11 / 2 / 90$ & 2 & 1 & & 120.1 & $\mathrm{~N}$ & \\
\hline
\end{tabular}

Data obtained from WHC Surveillance Analysis Computer System (SACS). November 12, 1993. 
WHC-SD-WM-ER-312, Rev. 0

\begin{tabular}{|c|c|c|c|c|c|c|c|}
\hline \multicolumn{8}{|c|}{ TANK 241-BY-110 } \\
\hline \multicolumn{8}{|c|}{ THERMOCOUPLE DATA } \\
\hline & & & & & & & \\
\hline TREE\# & DATE & THERMO \# & TREE SET \# & TEMP C & TEMP F & $Y=$ MAX TEMP & COMMENTS \\
\hline 1 & $11 / 2 / 90$ & 3 & 1 & & 116.6 & $\mathbf{N}$ & \\
\hline 1 & $11 / 2 / 90$ & 4 & 1 & & 111.8 & $\mathbf{N}$ & \\
\hline 1 & $11 / 2 / 90$ & 5 & 1 & & 103.8 & $\mathbf{N}$ & \\
\hline 1 & $11 / 2 / 90$ & 6 & 1 & & 81.5 & $\mathbf{N}$ & \\
\hline 1 & $11 / 5 / 90$ & 1 & 1 & & 120.6 & $\mathbf{Y}$ & E 2E-OP-003 \\
\hline 1 & $11 / 5 / 90$ & 2 & 1 & & 120 & $\mathbf{N}$ & \\
\hline 1 & $11 / 5 / 90$ & 3 & 1 & & 116 & $\mathbf{N}$ & \\
\hline 1 & $11 / 5 / 90$ & 4 & 1 & & 111.5 & $\mathbf{N}$ & \\
\hline 1 & $11 / 5 / 90$ & 5 & 1 & & 103.7 & $\mathbf{N}$ & \\
\hline 1 & $11 / 5 / 90$ & 6 & 1 & & 81.3 & $\mathrm{~N}$ & \\
\hline 1 & $11 / 13 / 90$ & 1 & 1 & & 121.6 & $\bar{Y}$ & $E$ \\
\hline 1 & $11 / 13 / 90$ & 2 & 1 & & 120.5 & $\mathbf{N}$ & \\
\hline 1 & $11 / 13 / 90$ & 3 & 1 & & 117.3 & $\mathbf{N}$ & \\
\hline 1 & $11 / 13 / 90$ & 4 & 1 & & 112.6 & $\mathbf{N}$ & \\
\hline 1 & $11 / 13 / 90$ & 5 & 1 & & 104.7 & $\mathbf{N}$ & \\
\hline 1 & $11 / 13 / 90$ & 6 & 1 & & 81.8 & $\mathbf{N}$ & \\
\hline 1 & $11 / 18 / 90$ & 1 & 1 & & 119.8 & $Y$ & 2E-OP-003 \\
\hline 1 & $11 / 18 / 90$ & 2 & 1 & & 119.4 & $\mathrm{~N}$ & \\
\hline 1 & $11 / 18 / 90$ & 3 & 1 & & 118.1 & $\mathbf{N}$ & \\
\hline 1 & $11 / 18 / 90$ & 4 & 1 & & 113.7 & $\mathrm{~N}$ & \\
\hline 1 & $11 / 18 / 90$ & 5 & 1 & & 104.8 & $\bar{N}$ & \\
\hline 1 & $11 / 18 / 90$ & 6 & 1 & & 80.9 & $N$ & \\
\hline 1 & $11 / 25 / 90$ & 1 & 1 & & 121.4 & $\bar{Y}$ & E-20-003 \\
\hline 1 & $11 / 25 / 90$ & 2 & 1 & & 120.4 & $\mathbf{N}$ & \\
\hline 1 & $11 / 25 / 90$ & 3 & 1 & & 117.5 & $\bar{N}$ & \\
\hline 1 & $11 / 25 / 90$ & 4 & 1 & & 112.9 & $\mathbf{N}$ & \\
\hline 1 & $11 / 25 / 90$ & 5 & 1 & & 104.8 & $\mathbf{N}$ & \\
\hline 1 & $11 / 25 / 90$ & 6 & 1 & & 80.7 & $\mathbf{N}$ & \\
\hline 1 & $12 / 5 / 90$ & 1 & 1 & & 122.1 & $Y$ & E 890236678-75 \\
\hline 1 & $12 / 5 / 90$ & 2 & 1 & & 121.2 & $\mathbf{N}$ & \\
\hline 1 & $12 / 5 / 90$ & 3 & 1 & & 117.9 & $\mathbf{N}$ & \\
\hline 1 & $12 / 5 / 90$ & 4 & 1 & & 113.1 & $\mathbf{N}$ & \\
\hline 1 & $12 / 5 / 90$ & 5 & 1 & & 105 & $\mathbf{N}$ & \\
\hline 1 & $12 / 5 / 90$ & 6 & 1 & & 80.8 & $\mathbf{N}$ & \\
\hline 1 & $12 / 11 / 90$ & 1 & 1 & & 120.7 & $Y$ & E 890723678-75 \\
\hline 1 & $12 / 11 / 90$ & 2 & 1 & & 119.9 & $\mathbf{N}$ & \\
\hline 1 & $12 / 11 / 90$ & 3 & 1 & & 916.3 & $\mathrm{~N}$ & \\
\hline 1 & $12 / 11 / 90$ & 4 & 1 & & 111.7 & $N$ & \\
\hline 1 & $12 / 11 / 90$ & 5 & 1 & & 103.3 & $\mathrm{~N}$ & \\
\hline 1 & $12 / 11 / 90$ & 6 & 1 & & 79.1 & $\mathbf{N}$ & \\
\hline 1 & $12 / 15 / 90$ & 1 & 1 & & 121.6 & $\bar{Y}$ & HH-99A-E \\
\hline 1 & $12 / 15 / 90$ & 2 & 1 & & 120.6 & $\mathrm{~N}$ & \\
\hline 1 & $12 / 15 / 90$ & 3 & 1 & & 118.2 & $\mathbf{N}$ & \\
\hline 1 & $12 / 15 / 90$ & 4 & 1 & & 112.9 & $\mathbf{N}$ & \\
\hline 1 & $12 / 15 / 90$ & 5 & 1 & & 104.3 & $N$ & \\
\hline 1 & $12 / 15 / 90$ & 6 & 1 & & 79.1 & $\mathbf{N}$ & \\
\hline 1 & $12 / 22 / 90$ & 1 & 2 & & 124 & $Y$ & $\begin{array}{c}\text { Recheck of temperatures taken on } 12 / 22 / 90 \\
\text { requested }\end{array}$ \\
\hline 1 & $12 / 22 / 90$ & 2 & 2 & & 123 & $N$ & \\
\hline 1 & $12 / 22 / 90$ & 3 & 2 & & 120 & $\mathrm{~N}$ & \\
\hline 1 & $12 / 30 / 90$ & 1 & 1 & & 124 & $\bar{Y}$ & $E 703147$ \\
\hline 1 & $12 / 30 / 90$ & 2 & 1 & & 123 & $\mathbf{N}$ & \\
\hline 1 & $12 / 30 / 90$ & 3 & 1 & & 119 & $\mathbf{N}$ & \\
\hline 1 & $12 / 30 / 90$ & 4 & 1 & & 115 & $N$ & \\
\hline 1 & $12 / 30 / 90$ & 5 & 1 & & 106 & $N$ & \\
\hline 1 & $12 / 30 / 90$ & 6 & 1 & & 80 & $\mathbf{N}$ & \\
\hline 1 & $1 / 6 / 91$ & 1 & 1 & & 123 & $Y$ & \\
\hline 1 & $1 / 6 / 91$ & 2 & 1 & & 122 & $\mathbf{N}$ & \\
\hline 1 & $1 / 6 / 91$ & 3 & 1 & & 118 & $\mathrm{~N}$ & \\
\hline 1 & $1 / 6 / 91$ & 4 & 1 & & 114 & $N$ & \\
\hline 1 & $1 / 6 / 91$ & 5 & 1 & & 105 & $N$ & \\
\hline 1 & $1 / 6 / 91$ & 6 & 1 & & 79 & $\mathbf{N}$ & \\
\hline 1 & $1 / 13 / 91$ & 1 & 1 & & 122 & $Y$ & E 743010 \\
\hline 1 & $1 / 13 / 91$ & 2 & 1 & & 121 & $\mathbf{N}$ & \\
\hline 1 & $1 / 13 / 91$ & 3 & 1 & & 118 & $\overline{\mathbf{N}}$ & \\
\hline 1 & $1 / 13 / 91$ & 4 & 1 & & 115 & $\mathbf{N}$ & \\
\hline
\end{tabular}

Data obtained from WHC Surveillance Analysis Computer System (SACS), November 12, 1993. 
WHC-SD-WM-ER-312, Rev. 0

\begin{tabular}{|c|c|c|c|c|c|c|c|}
\hline \multicolumn{8}{|c|}{ TANK 241-BY-110 } \\
\hline & & & & THEI & COUPLE & & \\
\hline TREE\#\# & DATE & THERMO \# & TREE SET \# & TEMP C & TEMP F & $Y=$ MAX TEMP & COMMENTS \\
\hline 1 & $1 / 13 / 91$ & 5 & 1 & & 106 & $\bar{N}$ & \\
\hline 1 & $1 / 13 / 91$ & 6 & 1 & & 78 & $\bar{N}$ & \\
\hline 1 & $1 / 19 / 91$ & 1 & 1 & & 122.6 & $Y$ & E Plug Broken \\
\hline 1 & $1 / 19 / 91$ & 2 & 1 & & 122 & $\dot{N}$ & \\
\hline 1 & $1 / 19 / 91$ & 3 & 1 & & 118.6 & $\mathbf{N}$ & \\
\hline 1 & $1 / 19 / 91$ & 4 & 1 & & 114.5 & $\mathbf{N}$ & \\
\hline 1 & $1 / 19 / 91$ & 5 & 1 & & 105 & $\mathbf{N}$ & \\
\hline 1 & $1 / 19 / 91$ & 6 & 1 & & 76.1 & $\mathbf{N}$ & \\
\hline$\frac{1}{1}$ & $1 / 29 / 91$ & 1 & 1 & & 122 & $\bar{Y}$ & E Omega 450 AET \\
\hline 1 & $1 / 29 / 91$ & 2 & 1 & & 121 & $\bar{N}$ & \\
\hline 1 & $1 / 29 / 91$ & 3 & 1 & & 118 & $\mathbf{N}$ & \\
\hline 1 & $1 / 29 / 91$ & 4 & 1 & & 114 & $\mathrm{~N}$ & \\
\hline 1 & $1 / 29 / 91$ & 5 & 1 & & 105 & $N$ & \\
\hline 1 & $1 / 29 / 91$ & 6 & 1 & & 76 & $\bar{N}$ & \\
\hline 1 & $2 / 3 / 91$ & 1 & 1 & & 121 & $Y$ & E Ser:703147 \\
\hline 9 & $2 / 3 / 91$ & 2 & 1 & & 120 & $\mathbf{N}$ & \\
\hline 1 & $2 / 3 / 91$ & 3 & 1 & & 117 & $\mathbf{N}$ & \\
\hline 1 & $2 / 3 / 91$ & 4 & 1 & & 112 & $N$ & \\
\hline 9 & $2 / 3 / 91$ & 5 & 1 & & 102 & $\mathbf{N}$ & \\
\hline 1 & $2 / 3 / 91$ & 6 & 1 & & 73 & $\bar{N}$ & \\
\hline 1 & $2 / 9 / 91$ & 9 & 1 & & 125.5 & $Y$ & E890723678-75 \\
\hline 1 & $2 / 9 / 91$ & 2 & 1 & & 124.9 & $\mathbf{N}$ & \\
\hline 1 & $2 / 9 / 91$ & 3 & 1 & & 121 & $N$ & \\
\hline$\frac{1}{1}$ & $2 / 9 / 91$ & 4 & 1 & & 114.3 & $\mathbf{N}$ & \\
\hline 1 & $2 / 9 / 91$ & 5 & 1 & & 104.3 & $\mathbf{N}$ & \\
\hline$\frac{9}{1}$ & $2 / 9 / 91$ & 6 & $\frac{\pi}{1}$ & & 74.6 & $\mathbf{N}$ & \\
\hline 9 & $2 / 16 / 91$ & 1 & 1 & & 122 & $\ddot{\gamma}$ & $E-703147$ \\
\hline 1 & $2 / 16 / 91$ & 2 & 1 & & 120.6 & $\dot{N}$ & \\
\hline$T$ & $2 / 16 / 91$ & 3 & 1 & & 117.2 & $\mathbf{N}$ & \\
\hline 1 & $2 / 16 / 91$ & 4 & $T$ & & 111.9 & $\mathbf{N}$ & \\
\hline 1 & $2 / 16 / 91$ & 5 & 1 & & 102.5 & $N$ & \\
\hline 1 & $2 / 16 / 91$ & 6 & 1 & & 73.6 & $\mathbf{N}$ & \\
\hline 1 & $2 / 23 / 91$ & 1 & 1 & & 122.8 & $\bar{Y}$ & E 703147 \\
\hline$\frac{T}{1}$ & $2 / 23 / 91$ & 2 & 1 & & 121.8 & $N$ & \\
\hline 1 & $2 / 23 / 91$ & 3 & 1 & & 118.8 & $N$ & \\
\hline 1 & $2 / 23 / 91$ & $\overline{4}$ & $\frac{1}{1}$ & & 113.6 & $\mathbf{N}$ & \\
\hline 1 & $2 / 23 / 91$ & 5 & 1 & & 103.8 & $N$ & \\
\hline$\frac{1}{1}$ & $2 / 23 / 91$ & 6 & 1 & & 74 & $\mathrm{~N}$ & \\
\hline$\frac{1}{1}$ & $3 / 3 / 91$ & 1 & 9 & & 124.7 & $Y$ & $890723678-75$ \\
\hline 1 & $3 / 3 / 91$ & 2 & 1 & & 123.4 & $\mathbf{N}$ & \\
\hline 9 & $3 / 3 / 91$ & 3 & 1 & & 120 & $\mathbf{N}$ & \\
\hline 1 & $3 / 3 / 91$ & 4 & 1 & & 114.6 & $\mathbf{N}$ & \\
\hline 1 & $3 / 3 / 91$ & 5 & 1 & & 104.7 & $\mathbf{N}$ & \\
\hline 1 & $3 / 3 / 91$ & 6 & 1 & & 81.9 & $\mathbf{N}$ & \\
\hline 1 & $3 / 9 / 91$ & 1 & 9 & & 124.2 & $\bar{Y}$ & $2 E-O P \cdot 020$ \\
\hline 1 & $3 / 9 / 91$ & 2 & 1 & & 123.3 & $\mathbf{N}$ & \\
\hline 1 & $3 / 9 / 91$ & 3 & 1 & & 121.2 & $\mathbf{N}$ & \\
\hline 1 & $3 / 9 / 91$ & 4 & 1 & & 116.8 & $\mathbf{N}$ & \\
\hline 1 & $3 / 9 / 91$ & 5 & 1 & & 107.4 & $N$ & \\
\hline 1 & $3 / 9 / 91$ & 6 & 1 & & 77 & $\bar{N}$ & \\
\hline 1 & $3 / 17 / 91$ & $\frac{1}{1}$ & 1 & & 922.8 & $\bar{Y}$ & E 890723678-75 \\
\hline 1 & $3 / 17 / 91$ & 2 & 1 & & 122.3 & $\mathbf{N}$ & \\
\hline 1 & $3 / 17 / 91$ & 3 & 1 & & 120.1 & $\mathbf{N}$ & \\
\hline 1 & $3 / 17 / 91$ & 4 & 1 & & 115.1 & $N$ & \\
\hline 1 & $3 / 17 / 91$ & 5 & 1 & & 105.5 & $\mathbf{N}$ & \\
\hline 1 & $3 / 17 / 91$ & 6 & 1 & & 75 & $\mathbf{N}$ & \\
\hline$T$ & $3 / 23 / 91$ & 1 & 1 & & 121.3 & $\bar{Y}$ & $E 703147$ \\
\hline 1 & $3 / 23 / 91$ & 2 & 1 & & 120.4 & $\mathbf{N}$ & \\
\hline 1 & $3 / 23 / 91$ & 3 & 1 & & 117.4 & $N$ & \\
\hline 1 & $3 / 23 / 91$ & 4 & 1 & & 112 & $\mathbf{N}$ & \\
\hline 7 & $3 / 23 / 91$ & 5 & 1 & & 102.6 & $\mathbf{N}$ & \\
\hline 1 & $3 / 23 / 91$ & 6 & 1 & & 72.3 & $N$ & \\
\hline 1 & $3 / 30 / 91$ & 1 & 1 & & 121 & $\bar{Y}$ & J890523512-20 \\
\hline$\frac{1}{1}$ & $3 / 30 / 91$ & 2 & 1 & & 120 & $\mathbf{N}$ & \\
\hline 1 & $3 / 30 / 91$ & 3 & $\frac{1}{1}$ & & 118 & $\mathbf{N}$ & \\
\hline 1 & $3 / 30 / 91$ & 4 & 1 & & 112 & $\mathbf{N}$ & \\
\hline
\end{tabular}

Data obtained from WHC Surveillance Analysis Computer System (SACS), November 12, 1993. 
WHC-SD-WM-ER-312, Rev. 0

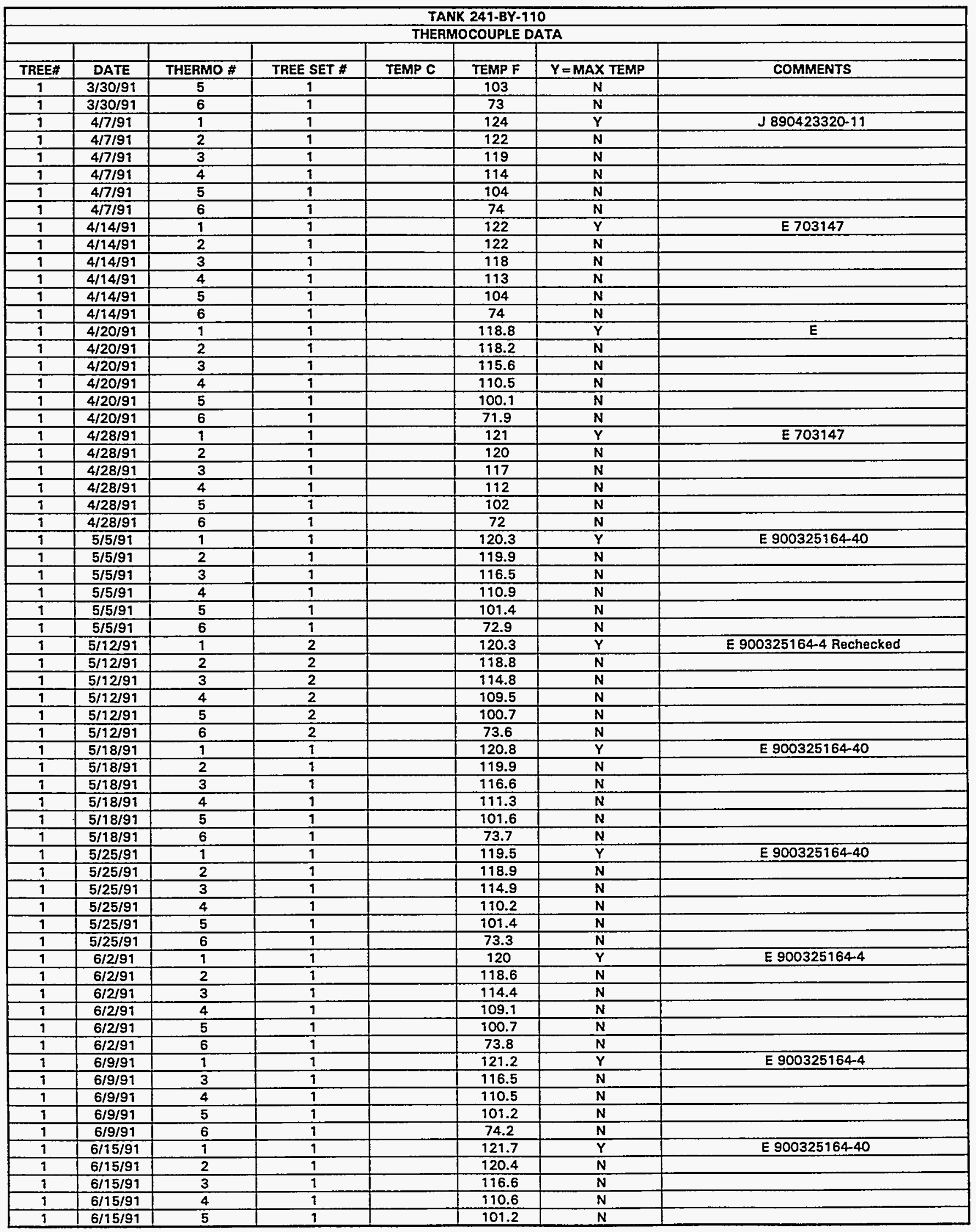

Data obtained from WHC Surveillance Analysis Computer System (SACS), November 12, 1993. 
WHC-SD-WM-ER-312, Rev. 0

\begin{tabular}{|c|c|c|c|c|c|c|c|}
\hline \multicolumn{8}{|c|}{ TANK 241-BY-110 } \\
\hline & & & & THEF & COUPLE & TA & \\
\hline TREE\# & DATE & THERMO \# & TREE SET \# & TEMP C & TEMP F & $Y=$ MAX TEMP & COMMENTS \\
\hline 1 & $6 / 15 / 91$ & 6 & 1 & & 74.8 & $\mathrm{~N}$ & \\
\hline 1 & $6 / 23 / 91$ & 1 & 1 & & 122.6 & $\bar{Y}$ & J 2E-OP-021 \\
\hline 1 & $6 / 23 / 91$ & 2 & 1 & & 121.3 & $\mathbf{N}$ & \\
\hline 1 & 6/23/91 & 3 & 1 & & 116.8 & $\mathbf{N}$ & \\
\hline 1 & $6 / 23 / 91$ & 4 & 1 & & 110 & $\overline{\mathbf{N}}$ & \\
\hline 1 & $6 / 23 / 91$ & 5 & 1 & & 100.9 & $\mathbf{N}$ & \\
\hline 1 & $6 / 23 / 91$ & 6 & 1 & & 74.6 & $\mathbf{N}$ & \\
\hline 1 & $6 / 29 / 91$ & 1 & 1 & & 120.5 & $\bar{Y}$ & E 900325164-4 \\
\hline 1 & $6 / 29 / 91$ & 2 & 1 & & 119.2 & $\mathbf{N}$ & \\
\hline 1 & $6 / 29 / 91$ & 3 & 1 & & 115.1 & $\mathbf{N}$ & \\
\hline 1 & $6 / 29 / 91$ & 4 & 1 & & 109.6 & $\mathbf{N}$ & \\
\hline 1 & $6 / 29 / 91$ & 5 & 1 & & 101.1 & $\mathbf{N}$ & \\
\hline 1 & $6 / 29 / 91$ & 6 & 1 & & 75.3 & $N$ & \\
\hline 1 & $7 / 6 / 91$ & 1 & 1 & & 118.3 & $\mathbf{Y}$ & E $900325164-40$ \\
\hline 1 & $7 / 6 / 91$ & 2 & 1 & & 117.9 & $\mathbf{N}$ & \\
\hline 1 & $7 / 6 / 91$ & 3 & 1 & & 114.8 & $\mathbf{N}$ & \\
\hline 1 & $7 / 6 / 91$ & 4 & 1 & & 109.7 & $\mathbf{N}$ & \\
\hline 1 & $7 / 6 / 91$ & 5 & 1 & & 100.9 & $\mathbf{N}$ & \\
\hline 1 & $7 / 6 / 91$ & 6 & 1 & & 75.2 & $\mathbf{N}$ & \\
\hline 1 & $7 / 13 / 91$ & 1 & 1 & & 120.4 & $\mathbf{Y}$ & E 900325164-40 \\
\hline 1 & $7 / 13 / 91$ & 2 & 1 & & 119.2 & $\overline{\mathbf{N}}$ & \\
\hline 1 & $7 / 13 / 91$ & 3 & 1 & & 115.4 & $\mathbf{N}$ & \\
\hline 1 & $7 / 13 / 91$ & 4 & 1 & & 110.2 & $\mathbf{N}$ & \\
\hline 1 & $7 / 13 / 91$ & 5 & 1 & & 101.5 & $\mathbf{N}$ & \\
\hline 1 & $7 / 13 / 91$ & 6 & 1 & & 76.4 & $\mathbf{N}$ & \\
\hline 1 & $7 / 20 / 91$ & 1 & 1 & & 118 & $\mathbf{Y}$ & $E 900325164-40$ \\
\hline 1 & $7 / 20 / 91$ & 2 & 1 & & 117.4 & $\mathbf{N}$ & \\
\hline 1 & $7 / 20 / 91$ & 3 & 1 & & 114 & $\mathbf{N}$ & \\
\hline 1 & $7 / 20 / 91$ & 4 & 1 & & 109.2 & $\mathbf{N}$ & \\
\hline 1 & $7 / 20 / 91$ & 5 & 1 & & 100.3 & $\bar{N}$ & \\
\hline 1 & $7 / 20 / 91$ & 6 & 1 & & 75.3 & $\mathbf{N}$ & \\
\hline 1 & $7 / 27 / 91$ & 1 & 1 & & 119.1 & $\mathbf{Y}$ & $E$ \\
\hline 1 & $7 / 27 / 91$ & 2 & 1 & & 118 & $\mathbf{N}$ & \\
\hline 1 & $7 / 27 / 91$ & 3 & 1 & & 114.7 & $\mathbf{N}$ & \\
\hline 1 & $7 / 27 / 91$ & 4 & 1 & & 109.5 & $\mathbf{N}$ & \\
\hline 1 & $7 / 27 / 91$ & 5 & 1 & & 100.3 & $\mathbf{N}$ & \\
\hline 1 & $7 / 27 / 91$ & 6 & 1 & & 75.7 & $\mathbf{N}$ & \\
\hline 1 & $8 / 3 / 91$ & 1 & 1 & & 118.6 & $\mathbf{Y}$ & E $900325164-40$ \\
\hline 1 & $8 / 3 / 91$ & 2 & 1 & & 118 & $\mathbf{N}$ & \\
\hline 1 & $8 / 3 / 91$. & 3 & 1 & & 114.5 & $\mathbf{N}$ & \\
\hline 1 & $8 / 3 / 91$ & 4 & 1 & & 109.4 & $\mathbf{N}$ & \\
\hline 1 & $8 / 3 / 91$ & 5 & 1 & & 100.5 & $\mathbf{N}$ & \\
\hline 1 & $8 / 3 / 91$ & 6 & 1 & & 76.8 & $\mathbf{N}$ & \\
\hline 1 & $8 / 10 / 91$ & 1 & 1 & & 120 & $\mathbf{Y}$ & $E$ \\
\hline 1 & $8 / 10 / 91$ & 2 & 1 & & 119 & $\mathbf{N}$ & \\
\hline 1 & $8 / 10 / 91$ & 3 & 1 & & 116 & $\mathbf{N}$ & \\
\hline 1 & $8 / 10 / 91$ & 4 & 1 & & 111 & $\mathbf{N}$ & \\
\hline 1 & $8 / 10 / 91$ & 5 & 1 & & 102 & $\mathbf{N}$ & \\
\hline 1 & $8 / 10 / 91$ & 6 & 1 & & 78 & $\mathbf{N}$ & \\
\hline 1 & $8 / 18 / 91$ & 1 & 1 & & 117.4 & $\mathbf{Y}$ & E 900325164-40 \\
\hline 1 & $8 / 18 / 91$ & 2 & 1 & & 117.2 & $N$ & \\
\hline 1 & $8 / 18 / 91$ & 3 & 1 & & 114.2 & $N$ & \\
\hline 1 & $8 / 18 / 91$ & 4 & 1 & & 109.4 & $\mathbf{N}$ & \\
\hline 1 & $8 / 18 / 91$ & 5 & 1 & & 100.2 & $\mathbf{N}$ & \\
\hline 1 & $8 / 18 / 91$ & 6 & 1 & & 77.1 & $\mathbf{N}$ & \\
\hline 1 & $8 / 24 / 91$ & 1 & 1 & & 118.3 & $\mathbf{Y}$ & E $900325164-4$ \\
\hline 1 & $8 / 24 / 91$ & 2 & 1 & & 117.7 & $\mathbf{N}$ & \\
\hline 1 & $8 / 24 / 91$ & 3 & 1 & & 114.5 & $\mathbf{N}$ & \\
\hline 1 & $8 / 24 / 91$ & 4 & 1 & & 109.6 & $N$ & \\
\hline 1 & $8 / 24 / 91$ & 5 & 1 & & 100.8 & $\mathbf{N}$ & \\
\hline 1 & $8 / 24 / 91$ & 6 & 1 & & 77.7 & $\mathbf{N}$ & \\
\hline 1 & $8 / 31 / 91$ & 1 & 1 & & 117.7 & $\mathbf{Y}$ & $E-900325164-4$ \\
\hline 1. & $8 / 31 / 91$ & 2 & 1 & & 117.3 & $\mathbf{N}$ & \\
\hline 1 & $8 / 31 / 91$ & 3 & 1 & & 114.2 & $\mathbf{N}$ & \\
\hline 1 & $8 / 31 / 91$ & 4 & 1 & & 109.1 & $\mathbf{N}$ & \\
\hline 1 & $8 / 31 / 91$ & 5 & 1 & & 99.8 & $N$ & \\
\hline
\end{tabular}

Data obtained from WHC Surveillance Analysis Computer System (SACS), November 12, 1993.

D-401 
WHC-SD-WM-ER-312, Rev. 0

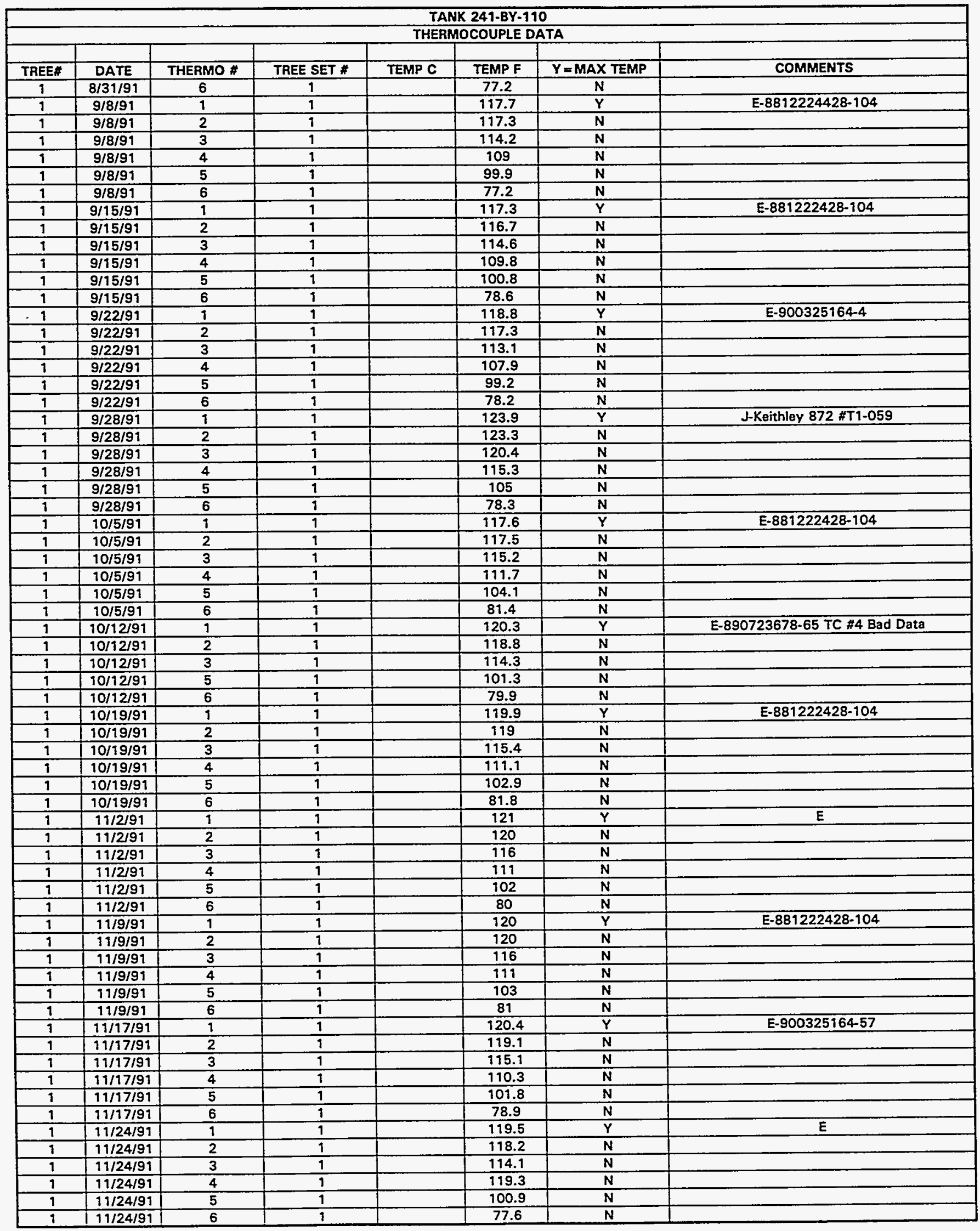

Data obtained from WHC Surveillance Analysis Computer System (SACS), November 12, 1993. 
WHC-SD-WM-ER-312, Rev. 0

\begin{tabular}{|c|c|c|c|c|c|c|c|}
\hline \multicolumn{8}{|c|}{ TANK 249-BY-110 } \\
\hline & & & & THER & COUPLE & & \\
\hline TREE\# & DATE & THERMO \# & TREE SET \# & TEMP C & TEMP F & $Y=M A X$ TEMP & COMMENTS \\
\hline 1 & $12 / 1 / 91$ & 1 & 1 & & 121.1 & $Y$ & $E-703147$ \\
\hline 1 & $12 / 1 / 91$ & 2 & 1 & & 120.2 & $\bar{N}$ & \\
\hline 1 & $12 / 1 / 91$ & 3 & 1 & & 116.5 & $\bar{N}$ & \\
\hline 1 & $12 / 1 / 91$ & 4 & 1 & & 112 & $\mathbf{N}$ & \\
\hline 9 & $12 / 1 / 91$ & 5 & 1 & & 103.7 & $\mathbf{N}$ & \\
\hline$T$ & $12 / 1 / 91$ & 6 & 1 & & 79.6 & $N$ & \\
\hline 1 & $12 / 8 / 91$ & 1 & 1 & & 120 & $Y$ & $E-900325164-89$ \\
\hline 1 & $12 / 8 / 91$ & 2 & 1 & & 199.2 & $\mathbf{N}$ & \\
\hline 9 & $12 / 8 / 91$ & 3 & 1 & & 115.2 & $\mathbf{N}$ & \\
\hline 1 & $12 / 8 / 91$ & 4 & 1 & & 110.3 & $\mathbf{N}$ & \\
\hline 9 & $12 / 8 / 91$ & 5 & 1 & & 101.8 & $\mathbf{N}$ & \\
\hline 1 & $12 / 8 / 91$ & 6 & 1 & & 77.5 & $\bar{N}$ & \\
\hline$T$ & $12 / 14 / 91$ & 1 & 1 & & 118.8 & $\bar{Y}$ & $E-900325164-89$ \\
\hline 1 & $12 / 14 / 91$ & 2 & 1 & & 118.1 & $\mathbf{N}$ & \\
\hline 1 & $12 / 14 / 91$ & 3 & 1 & & 114.8 & $\mathbf{N}$ & \\
\hline 1 & $12 / 14 / 91$ & 4 & 1 & & 110.9 & $N$ & \\
\hline 1 & $12 / 14 / 91$ & 5 & 1 & & 102.4 & $\mathbf{N}$ & \\
\hline$\frac{1}{1}$ & $12 / 14 / 91$ & 6 & 1 & & 77.2 & $\mathbf{N}$ & \\
\hline 1 & $12 / 20 / 91$ & 1 & 1 & & 119.8 & $Y$ & $E-900325164-70$ \\
\hline 1 & $12 / 20 / 91$ & 2 & 1 & & 118.9 & $\bar{N}$ & \\
\hline 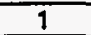 & $12 / 20 / 91$ & 3 & 1 & & 115.2 & $\mathbf{N}$ & \\
\hline 1 & $12 / 20 / 91$ & 4 & 1 & & 110.6 & $\mathbf{N}$ & \\
\hline 1 & $12 / 20 / 91$ & 5 & 1 & & 102.2 & $\mathbf{N}$ & \\
\hline 9 & $12 / 20 / 91$ & 6 & 1 & & 77.8 & $\mathbf{N}$ & \\
\hline 1 & $12 / 29 / 91$ & 1 & 1 & & 119.5 & $Y$ & $\mathrm{~J}-910126143-29$ \\
\hline 1 & $12 / 29 / 91$ & 2 & 1 & & 118.3 & $\overline{\mathbf{N}}$ & \\
\hline 1 & $12 / 29 / 91$ & 3 & 1 & & 114.3 & $\mathbf{N}$ & \\
\hline 1 & $12 / 29 / 91$ & 4 & 1 & & 109.6 & $\mathbf{N}$ & \\
\hline 1 & $12 / 29 / 91$ & 5 & $\frac{1}{1}$ & & 101.1 & $\mathbf{N}$ & \\
\hline 1 & $12 / 29 / 91$ & 6 & 1 & & 75.9 & $\mathbf{N}$ & \\
\hline 1 & $1 / 4 / 92$ & 1 & 1 & & 119.7 & $\bar{Y}$ & $900325164-70$ \\
\hline 1 & $1 / 4 / 92$ & 2 & 1 & & 118.2 & $\mathbf{N}$ & \\
\hline 9 & $1 / 4 / 92$ & 3 & 1 & & 114.6 & $\mathbf{N}$ & \\
\hline 9 & $1 / 4 / 92$ & 4 & 9 & & 110.5 & $\mathbf{N}$ & \\
\hline 1 & $1 / 4 / 92$ & 5 & 1 & & 101.6 & $\mathbf{N}$ & \\
\hline 9 & $1 / 4 / 92$ & 6 & $\frac{1}{1}$ & & 76.6 & $\mathbf{N}$ & \\
\hline 1 & $1 / 11 / 92$ & 1 & 1 & & 120.1 & $\mathbf{Y}$ & $900325164-89$ \\
\hline 1 & $1 / 11 / 92$ & 2 & $\frac{1}{1}$ & & 179.1 & $\mathbf{N}$ & \\
\hline 1 & $1 / 11 / 92$ & 3 & 1 & & 115 & $\bar{N}$ & \\
\hline 1 & $1 / 11 / 92$ & 4 & 1 & & 110.1 & $\mathbf{N}$ & \\
\hline 1 & $1 / 11 / 92$ & 5 & 1 & & 101.3 & $\mathbf{N}$ & \\
\hline 1 & $1 / 11 / 92$ & 6 & 1 & & 74.9 & $\mathbf{N}$ & \\
\hline 1 & $1 / 16 / 92$ & 1 & 1 & & 120.8 & $\bar{Y}$ & J $910126143-32$ \\
\hline 1 & $1 / 16 / 92$ & 2 & 1 & & 119.7 & $\mathbf{N}$ & \\
\hline 1 & $1 / 16 / 92$ & 3 & 1 & & 115.3 & $\mathbf{N}$ & \\
\hline 1 & $1 / 16 / 92$ & 4 & 1 & & 110.7 & $\mathbf{N}$ & \\
\hline 1 & $1 / 16 / 92$ & 5 & 1 & & 101.8 & $\mathbf{N}$ & \\
\hline 1 & $1 / 16 / 92$ & 6 & $T$ & & 75.3 & $\mathbf{N}$ & \\
\hline 1 & $1 / 25 / 92$ & 1 & 1 & & 120.6 & $\bar{Y}$ & $E-900325164-89$ \\
\hline 1 & $1 / 25 / 92$ & 2 & 1 & & 119.9 & $\mathbf{N}$ & \\
\hline 1 & $1 / 25 / 92$ & 3 & 1 & & 115.7 & $\mathbf{N}$ & \\
\hline 1 & $9 / 25 / 92$ & 4 & 1 & & 110.6 & $\mathbf{N}$ & \\
\hline 9 & $1 / 25 / 92$ & 5 & 1 & & 101.7 & $\mathbf{N}$ & \\
\hline 1 & $1 / 25 / 92$ & 6 & 1 & & 74.5 & $\mathbf{N}$ & \\
\hline 1 & $1 / 28 / 92$ & 1 & 1 & & & $\mathbf{N}$ & $\begin{array}{l}\text { ACCESS RESTRICTED } 1 / 28-2 / 22 \text { DUE TO } \\
\text { BATTERY FUME INCIDENT }\end{array}$ \\
\hline 1 & $2 / 24 / 92$ & 1 & 1 & & 121.5 & $Y$ & $E-881222428-104$ \\
\hline 1 & $2 / 24 / 92$ & 2 & 1 & & 120.7 & $\bar{N}$ & \\
\hline 1 & $2 / 24 / 92$ & 3 & 1 & & 117 & $\mathbf{N}$ & \\
\hline 1 & $2 / 24 / 92$ & 4 & 1 & & 111.7 & $\mathbf{N}$ & \\
\hline 1 & $2 / 24 / 92$ & 5 & 1 & & 102.4 & $\mathbf{N}$ & \\
\hline 1 & $2 / 24 / 92$ & 6 & 1 & & 75.4 & $\bar{N}$ & \\
\hline 1 & $3 / 8 / 92$ & 1 & 1 & & 118 & $\bar{Y}$ & E 900325164-47 \\
\hline 1 & $3 / 8 / 92$ & 2 & 1 & & 117.4 & $\mathbf{N}$ & \\
\hline 1 & $3 / 8 / 92$ & 3 & 1 & & 994.6 & $N$ & \\
\hline 1 & $3 / 8 / 92$ & 4 & 1 & & 110.1 & $\mathbf{N}$ & \\
\hline
\end{tabular}

Data obtained from WHC Surveillance Analysis Computer System (SACS), November 12, 1993. 


\begin{tabular}{|c|c|c|c|c|c|c|c|}
\hline \multicolumn{8}{|c|}{ TANK 241-BY-110 } \\
\hline \multicolumn{8}{|c|}{ THERMOCOUPLE DATA } \\
\hline TREE\# & $\overline{\text { DATE }}$ & THERMO \# & TREE SET \# & TEMP C & TEMP F & $Y=M A X$ TEMP & COMMENTS \\
\hline 1 & $3 / 8 / 92$ & 5 & 1 & & 101.2 & $\mathrm{~N}$ & \\
\hline 1 & $3 / 8 / 92$ & 6 & 1 & & 72.5 & $\mathrm{~N}$ & \\
\hline 1 & $3 / 15 / 92$ & 1 & 1 & & 117.3 & $\mathrm{Y}$ & E 900325164-47 \\
\hline 1 & $3 / 15 / 92$ & 2 & 1 & & 116.6 & $\mathbf{N}$ & \\
\hline 1 & $3 / 15 / 92$ & 3 & 1 & & 114.2 & $\mathbf{N}$ & \\
\hline 1 & $3 / 15 / 92$ & 4 & $\frac{1}{1}$ & & 109.7 & $\mathbf{N}$ & \\
\hline 1 & $3 / 15 / 92$ & 5 & 1 & & 100.3 & $N$ & \\
\hline 1 & $3 / 15 / 92$ & 6 & 1 & & 83.4 & $\mathbf{N}$ & \\
\hline 1 & $3 / 20 / 92$ & 1 & 1 & & 119.2 & $\bar{Y}$ & E 90032516447 \\
\hline 1 & $3 / 20 / 92$ & 2 & 1 & & 118 & $\mathbf{N}$ & \\
\hline 1 & $3 / 20 / 92$ & 3 & 1 & & 114.1 & $N$ & \\
\hline 9 & $3 / 20 / 92$ & 4 & 1 & & 108.4 & $\mathbf{N}$ & \\
\hline 1 & $3 / 20 / 92$ & 5 & 9 & & 99 & $\bar{N}$ & \\
\hline 1 & $3 / 20 / 92$ & 6 & 1 & & 71.9 & $\mathbf{N}$ & \\
\hline 1 & $3 / 29 / 92$ & 1 & 1 & & 120.1 & $\bar{Y}$ & E $900325164-18$ \\
\hline 1 & $3 / 29 / 92$ & 2 & 1 & & 119.4 & $\mathbf{N}$ & \\
\hline 1 & $3 / 29 / 92$ & 3 & 1 & & 115.2 & $\mathbf{N}$ & \\
\hline 1 & $3 / 29 / 92$ & 4 & 1 & & 109.7 & $\mathrm{~N}$ & \\
\hline 1 & $3 / 29 / 92$ & 5 & 1 & & 100.2 & $\mathbf{N}$ & \\
\hline 1 & $3 / 29 / 92$ & 6 & 1 & & 72.5 & $\mathbf{N}$ & \\
\hline 1 & $4 / 3 / 92$ & 1 & 1 & & 117.9 & $\bar{Y}$ & $J 910126143-14$ \\
\hline 1 & $4 / 3 / 92$ & 2 & 1 & & 117.2 & $N$ & \\
\hline 1 & $4 / 3 / 92$ & 3 & 1 & & 113.6 & $\mathrm{~N}$ & \\
\hline 1 & $4 / 3 / 92$ & 4 & 1 & & 108.8 & N & \\
\hline 1 & $4 / 3 / 92$ & 5 & 1 & & 94.8 & $\mathrm{~N}$ & \\
\hline 1 & $4 / 3 / 92$ & 6 & 1 & & 71 & $\mathrm{~N}$ & \\
\hline 1 & $4 / 11 / 92$ & 1 & 9 & & 118.2 & $\bar{Y}$ & E 900325164-18 \\
\hline 1 & $4 / 19 / 92$ & 2 & 9 & & 117.2 & $\bar{N}$ & \\
\hline 1 & $4 / 11 / 92$ & 3 & 1 & & 117.8 & $\overline{\mathbf{N}}$ & \\
\hline 1 & $4 / 11 / 92$ & 4 & 1 & & 107.3 & $\bar{N}$ & \\
\hline 1 & $4 / 11 / 92$ & 5 & 1 & & 93.2 & $\mathbf{N}$ & \\
\hline 1 & $4 / 11 / 92$ & 6 & 1 & & 70.1 & $\bar{N}$ & \\
\hline 1 & $4 / 17 / 92$ & 1 & 1 & & 119.5 & $\bar{Y}$ & E 90032516418 \\
\hline 1 & $4 / 17 / 92$ & 2 & 1 & & 117.2 & $\mathbf{N}$ & \\
\hline 1 & $4 / 17 / 92$ & 3 & 1 & & 112.7 & $\mathbf{N}$ & \\
\hline 1 & $4 / 17 / 92$ & 4 & 1 & & 107.1 & $\mathrm{~N}$ & \\
\hline 1 & $4 / 17 / 92$ & 5 & 1 & & 93.1 & $\mathrm{~N}$ & \\
\hline 1 & $4 / 17 / 92$ & 6 & 1 & & 70.1 & $\mathbf{N}$ & \\
\hline 1 & $4 / 25 / 92$ & 1 & 1 & & 121.3 & $\bar{Y}$ & E 9003251641B \\
\hline 1 & $4 / 25 / 92$ & 2 & 1 & & 119.7 & $\mathbf{N}$ & \\
\hline 1 & $4 / 25 / 92$ & 3 & 1 & & 115.2 & $\mathbf{N}$ & \\
\hline 1 & $4 / 25 / 92$ & 4 & 1 & & 109.2 & $\mathrm{~N}$ & \\
\hline 1 & $4 / 25 / 92$ & 5 & 1 & & 94.7 & $\bar{N}$ & \\
\hline 1 & $4 / 25 / 92$ & 6 & 1 & & 71.5 & $\mathbf{N}$ & \\
\hline 1 & $4 / 30 / 92$ & 1 & 1 & & 118.3 & $\bar{Y}$ & $E 300325164-18$ \\
\hline 1 & $4 / 30 / 92$ & 2 & 1 & & 117 & $\bar{N}$ & \\
\hline 1 & $4 / 30 / 92$ & 3 & 1 & & 112.6 & $\bar{N}$ & \\
\hline 1 & $4 / 30 / 92$ & 4 & 1 & & 107.1 & $\mathbf{N}$ & \\
\hline 1 & $4 / 30 / 92$ & 5 & 1 & & 93 & $\mathbf{N}$ & \\
\hline 1 & $4 / 30 / 92$ & 6 & 1 & & 70.8 & $\mathbf{N}$ & \\
\hline 1 & $5 / 8 / 92$ & 1 & 1 & & 115.7 & $Y$ & E 900325164-47 \\
\hline 1 & $5 / 8 / 92$ & 2 & 1 & & 114 & $\mathbf{N}$ & \\
\hline 1 & $5 / 8 / 92$ & 3 & 1 & & 109.5 & $\mathbf{N}$ & \\
\hline 1 & $5 / 8 / 92$ & 4 & 1 & & 103.9 & $\mathbf{N}$ & \\
\hline 1 & $5 / 8 / 92$ & 5 & 1 & & 90 & $\mathbf{N}$ & \\
\hline 1 & $5 / 8 / 92$ & 6 & 1 & & 68 & $\mathbf{N}$ & \\
\hline 1 & $5 / 16 / 92$ & 1 & 1 & & 119.8 & $\bar{Y}$ & E 900325164-85 \\
\hline 1 & $5 / 16 / 92$ & 2 & 1 & & 918.8 & $\mathbf{N}$ & \\
\hline 1 & $5 / 16 / 92$ & 3 & 1 & & 114.6 & $\mathbf{N}$ & \\
\hline 1 & $5 / 16 / 92$ & 4 & 1 & & 109.1 & $\mathbf{N}$ & \\
\hline 1 & $5 / 16 / 92$ & 5 & 9 & & 95 & $N$ & \\
\hline 1 & $5 / 16 / 92$ & 6 & 1 & & 73.6 & $\mathrm{~N}$ & \\
\hline 9 & $5 / 24 / 92$ & 1 & 1 & & 118.9 & $\bar{Y}$ & $E 90032516418$ \\
\hline 1 & $5 / 24 / 92$ & 2 & 1 & & 118.4 & $\mathrm{~N}$ & \\
\hline 1 & $5 / 24 / 92$ & 3 & 9 & & 114.2 & $\mathbf{N}$ & \\
\hline 7 & $5 / 24 / 92$ & 4 & 1 & & 108.3 & $\mathbf{N}$ & \\
\hline
\end{tabular}

Data obtained from WHC Surveillance Analysis Computer System (SACS), November 12, 1993. 


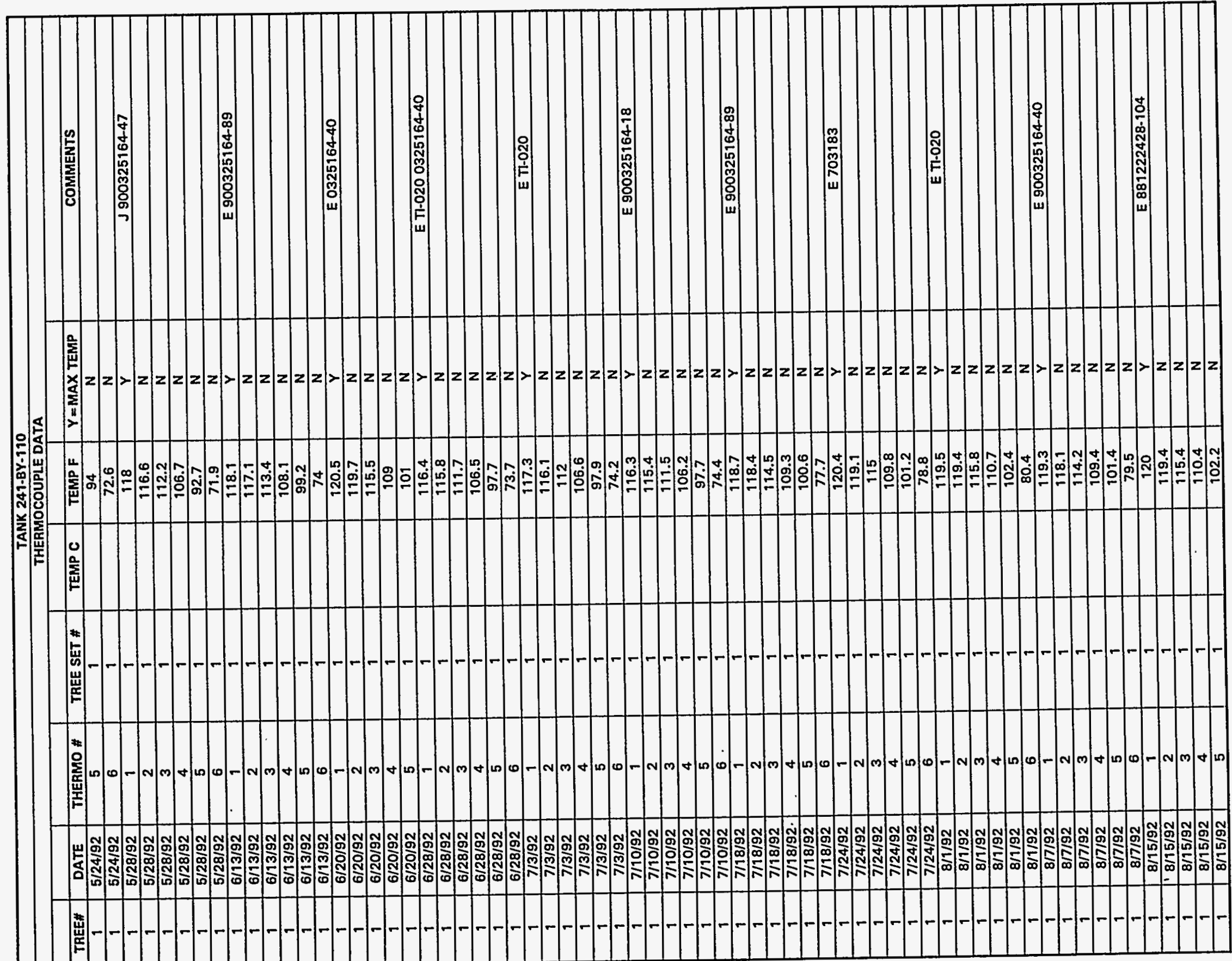

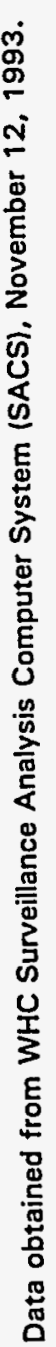




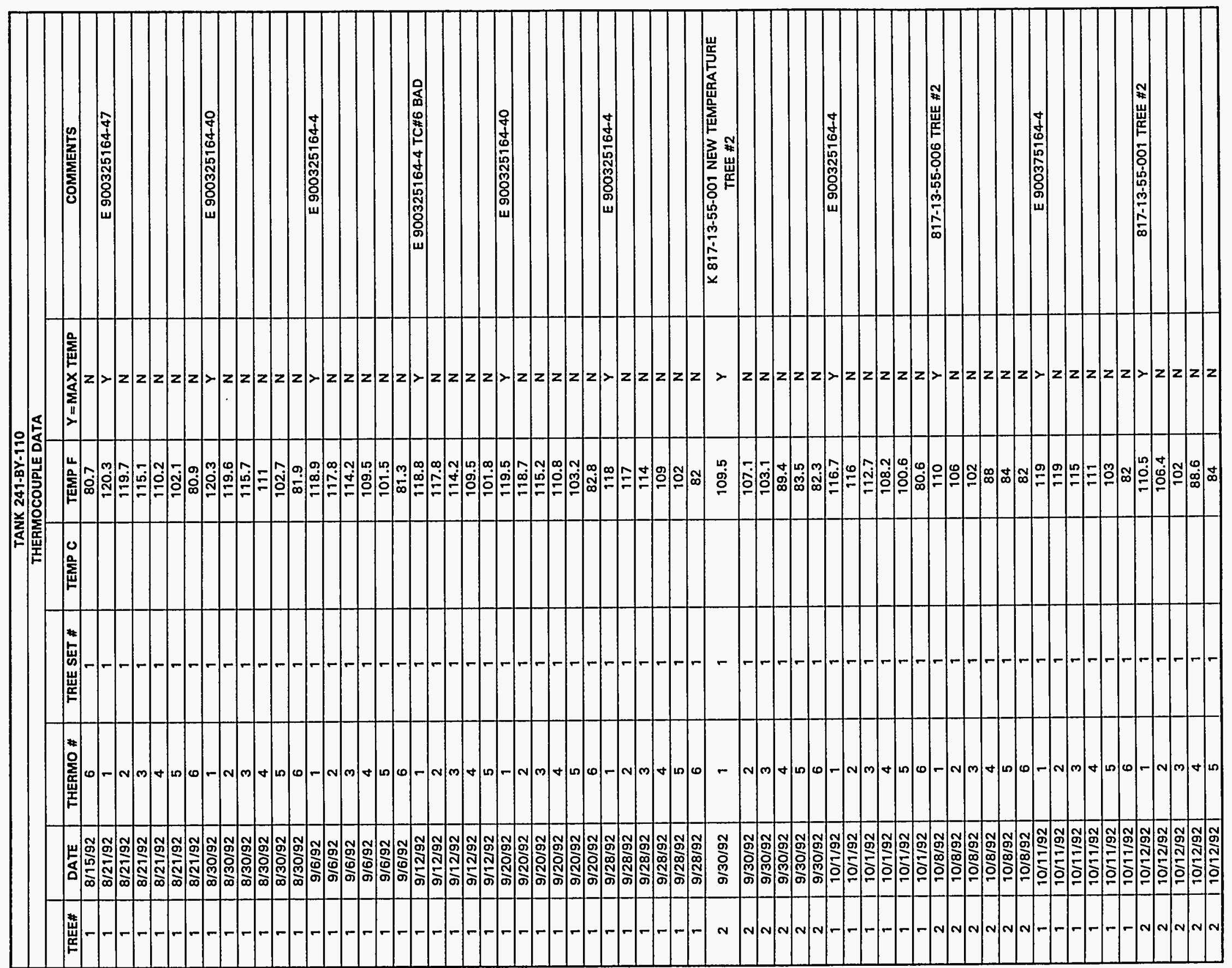

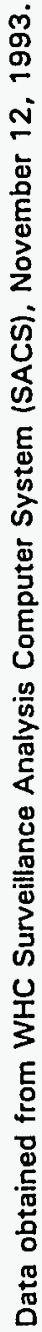

 


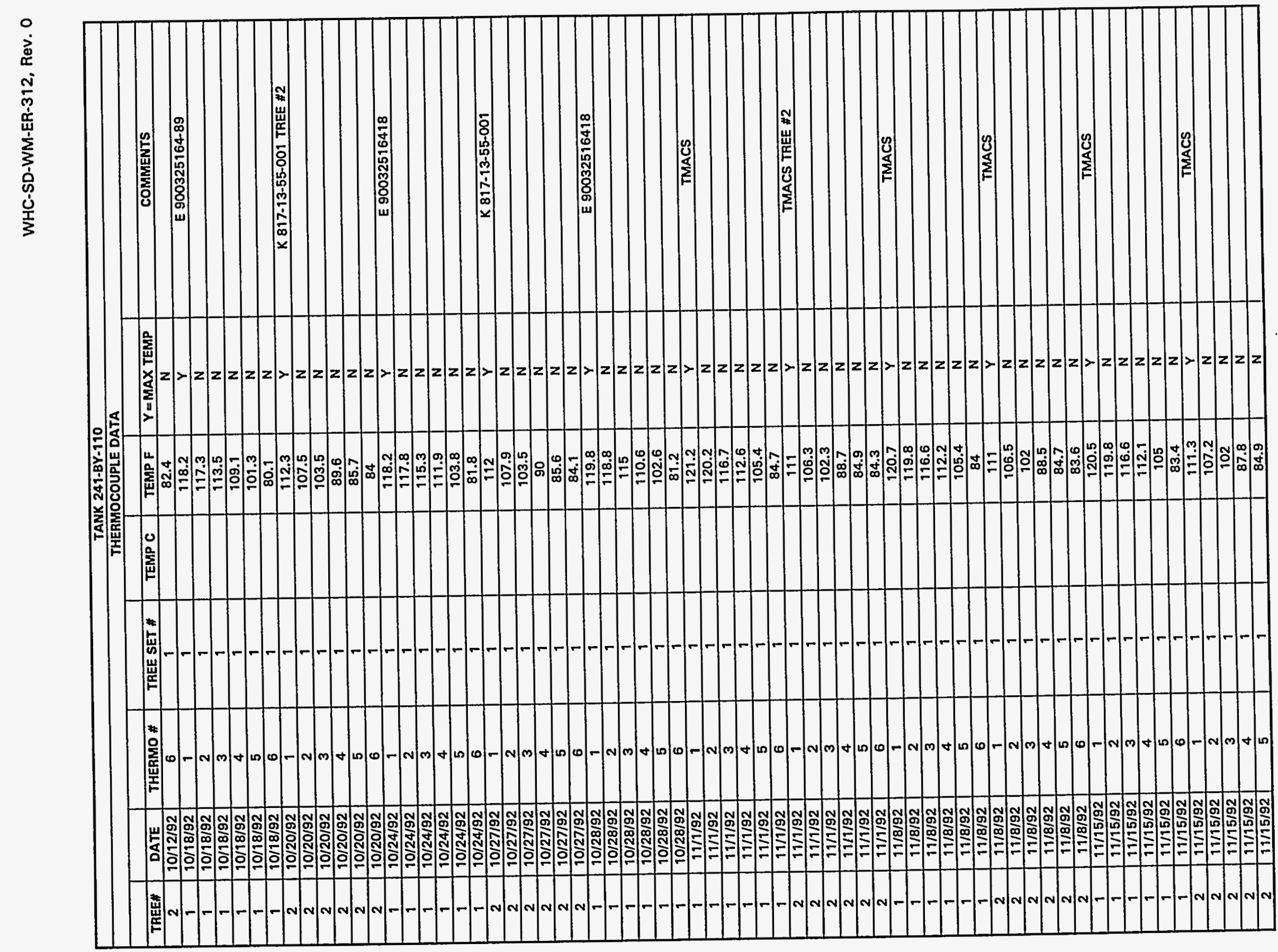

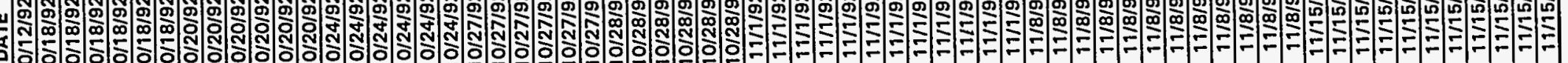
: 
WHC-SD-WM-ER-312, Rev. 0

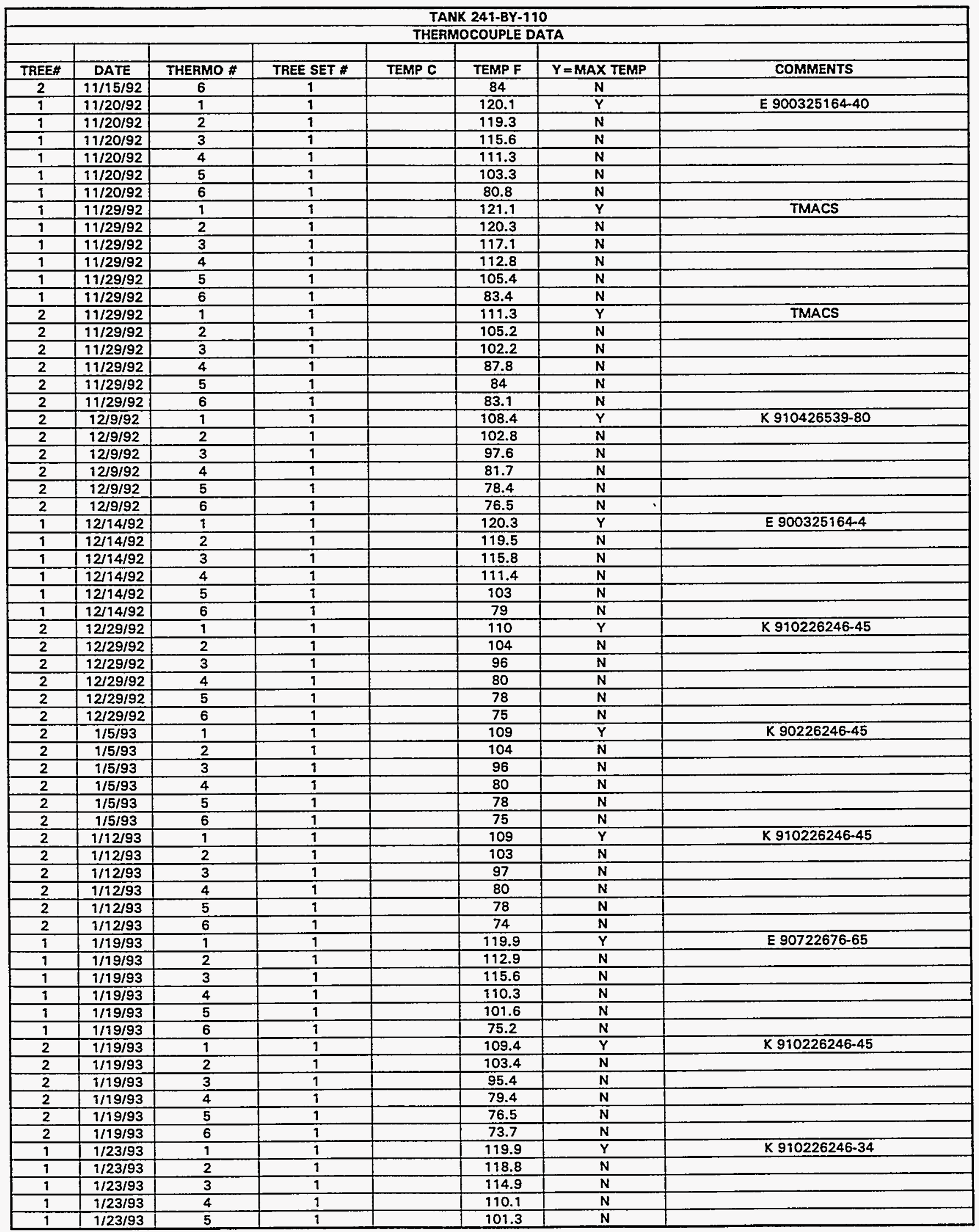

Data obtained from WHC Surveillance Analysis Computer System (SACS), November 12, 1993. 
WHC-SD-WM-ER-312, Rev. 0

\begin{tabular}{|c|c|c|c|c|c|c|c|}
\hline \multirow{2}{*}{\multicolumn{8}{|c|}{$\begin{array}{l}\text { TANK 241-BY-110 } \\
\text { THERMOCOUPLE DATA }\end{array}$}} \\
\hline & & & & & & & \\
\hline TREE\# & DATE & THERMO \# & TREE SET \# & TEMP C & TEMP F & $Y=M A X$ TEMP & COMMENTS \\
\hline 1 & $1 / 23 / 93$ & 6 & 1 & & 74.4 & $\mathbf{N}$ & \\
\hline 1 & $1 / 31 / 93$ & 1 & 1 & & 720.5 & $\bar{Y}$ & E $88222428-104$ \\
\hline 1 & $1 / 31 / 93$ & 2 & 1 & & 119.3 & $\mathbf{N}$ & \\
\hline 1 & $1 / 31 / 93$ & 3 & 1 & & 115.1 & $\mathbf{N}$ & \\
\hline$T$ & $1 / 31 / 93$ & 4 & 1 & & 110.1 & $\mathbf{N}$ & \\
\hline 1 & $1 / 31 / 93$ & 5 & 1 & & 101.2 & $N$ & \\
\hline 1 & $1 / 31 / 93$ & 6 & 1 & & 73.9 & $\mathrm{~N}$ & \\
\hline 2 & $1 / 31 / 93$ & 1 & 1 & & 107 & $Y$ & K8907236373-64 \\
\hline 2 & $1 / 31 / 93$ & 2 & 1 & & 101.6 & $N$ & \\
\hline 2 & $1 / 31 / 93$ & 3 & 1 & & 93.5 & $\bar{N}$ & \\
\hline 2 & $1 / 31 / 93$ & 4 & 7 & & 76.2 & $\mathrm{~N}$ & \\
\hline 2 & $1 / 31 / 93$ & 5 & 1 & & 74.1 & $\mathbf{N}$ & \\
\hline 2 & $1 / 31 / 93$ & 6 & 1 & & 70.8 & $N$ & \\
\hline 1 & $2 / 5 / 93$ & 1 & 1 & & 120.9 & $Y$ & E 881222428-104 \\
\hline$\frac{1}{1}$ & $2 / 5 / 93$ & 2 & $T$ & & 119.9 & $\mathbf{N}$ & \\
\hline 1 & $2 / 5 / 93$ & 3 & 1 & & 116.1 & $\mathbf{N}$ & \\
\hline 1 & $2 / 5 / 93$ & 4 & 1 & & 111.4 & $\mathbf{N}$ & \\
\hline 1 & $2 / 5 / 93$ & 5 & 1 & & 102.1 & $\mathbf{N}$ & \\
\hline 1 & $2 / 5 / 93$ & 6 & 1 & & 74.6 & $\mathbf{N}$ & \\
\hline 2 & $2 / 5 / 93$ & 1 & 9 & & 107.8 & $Y$ & K 890723679-64 \\
\hline 2 & $2 / 5 / 93$ & 2 & 1 & & 102.7 & $\mathbf{N}$ & \\
\hline 2 & $2 / 5 / 93$ & 3 & 1 & & 94.4 & $\mathbf{N}$ & \\
\hline 2 & $2 / 5 / 93$ & 4 & $\frac{1}{1}$ & & 77.5 & $\mathbf{N}$ & \\
\hline 2 & $2 / 5 / 93$ & 5 & 1 & & 75.2 & $\mathrm{~N}$ & \\
\hline 2 & $2 / 5 / 93$ & 6 & 1 & & 71.8 & $N$ & \\
\hline 2 & $2 / 21 / 93$ & 1 & $\frac{1}{1}$ & & 107.7 & $\bar{Y}$ & $K 890723679-84$ \\
\hline 2 & $2 / 21 / 93$ & 2 & 1 & & 101.2 & $\mathbf{N}$ & \\
\hline 2 & $2 / 21 / 93$ & 3 & 9 & & 95 & $N$ & \\
\hline 2 & $2 / 21 / 93$ & 4 & 1 & & 76.9 & $N$ & \\
\hline 2 & $2 / 21 / 93$ & 5 & 1 & & 74 & $N$ & \\
\hline 2 & $2 / 21 / 93$ & 6 & 1 & & 70.2 & $\mathbf{N}$ & \\
\hline 1 & $2 / 22193$ & 1 & 1 & & 121.1 & $Y$ & $E 90032516455$ \\
\hline 1 & $2 / 22 / 93$ & 2 & 1 & & 120.3 & $N$ & \\
\hline 1 & $2 / 22 / 93$ & 3 & 1 & & 116.5 & $\mathbf{N}$ & \\
\hline 1 & $2 / 22 / 93$ & 4 & 1 & & 911.3 & $\mathbf{N}$ & \\
\hline 1 & $2 / 22 / 93$ & 5 & 1 & & 101.9 & $\mathbf{N}$ & \\
\hline 1 & $2 / 22 / 93$ & 6 & 1 & & 73.3 & $\bar{N}$ & \\
\hline 1 & $2 / 27 / 93$ & 1 & 1 & & 119.4 & $\bar{Y}$ & E $90325164-47$ \\
\hline 1 & $2 / 27 / 93$ & 2 & 1 & & 118.5 & $\mathbf{N}$ & \\
\hline 1 & $2 / 27 / 93$ & 3 & 1 & & 114.3 & $\mathbf{N}$ & \\
\hline 1 & $2 / 27 / 93$ & 4 & 1 & & 109.2 & $\mathbf{N}$ & \\
\hline 1 & $2 / 27 / 93$ & 5 & 1 & & 99.9 & $N$ & \\
\hline 9 & $2 / 27 / 93$ & 6 & 1 & & 71.8 & $\mathbf{N}$ & \\
\hline 2 & $2 / 27 / 93$ & 1 & 1 & & 108 & $Y$ & K910426539-80 \\
\hline 2 & $2 / 27 / 93$ & 2 & $T$ & & 102 & $N$ & \\
\hline 2 & $2 / 27 / 93$ & 3 & 1 & & 94.6 & $\mathbf{N}$ & \\
\hline 2 & $2 / 27 / 93$ & 4 & 1 & & 76.4 & $\mathbf{N}$ & \\
\hline 2 & $2 / 27 / 93$ & 5 & 1 & & 74.6 & $\mathbf{N}$ & \\
\hline 2 & $2 / 27 / 93$ & 6 & 1 & & 70 & $N$ & \\
\hline 1 & $3 / 5 / 93$ & $\frac{1}{1}$ & 1 & & 120.5 & $\bar{Y}$ & E 90325164-47 \\
\hline$T$ & $3 / 5 / 93$ & $\overline{2}$ & 1 & & 119.5 & $\mathbf{N}$ & \\
\hline 1 & $3 / 5 / 93$ & 3 & 1 & & 115.5 & $\mathbf{N}$ & \\
\hline 1 & $3 / 5 / 93$ & 4 & 1 & & 110.1 & $\mathbf{N}$ & \\
\hline 1 & $3 / 5 / 93$ & 5 & 1 & & 100.4 & $\mathbf{N}$ & \\
\hline 1 & $3 / 5 / 93$ & 6 & 1 & & 71.9 & $\mathbf{N}$ & \\
\hline 2 & $3 / 5 / 93$ & 1 & 1 & & 107.7 & $\bar{Y}$ & K910426539.80 \\
\hline 2 & $3 / 5 / 93$ & 2 & 1 & & 102.2 & $\mathbf{N}$ & \\
\hline 2 & $3 / 5 / 93$ & 3 & 1 & & 94.3 & $N$ & \\
\hline 2 & $3 / 5 / 93$ & 4 & 1 & & 76.6 & $\mathbf{N}$ & \\
\hline 2 & $3 / 5 / 93$ & 5 & 1 & & 74.2 & $N$ & \\
\hline 2 & $3 / 5 / 93$ & 6 & 1 & & 70.1 & $\bar{N}$ & \\
\hline 1 & $3 / 8 / 93$ & 1 & 1 & & 119.66 & $Y$ & TMACS \\
\hline 1 & $3 / 8 / 93$ & 2 & 7 & & 118.4 & $\bar{N}$ & \\
\hline 1 & $3 / 8 / 93$ & 3 & 1 & & 114.62 & $\mathbf{N}$ & \\
\hline 1 & $3 / 8 / 93$ & 4 & 1 & & 109.4 & $\mathbf{N}$ & \\
\hline 1 & $3 / 8 / 93$ & 5 & 9 & & 100.76 & $\mathbf{N}$ & \\
\hline
\end{tabular}

Data obtained from WHC Surveillance Analysis Computer System (SACS), November 12, 1993. 


\begin{tabular}{|c|c|c|c|c|c|c|c|}
\hline \multicolumn{8}{|c|}{ TANK 241-BY-110 } \\
\hline \multicolumn{8}{|c|}{ THERMOCOUPLE DATA } \\
\hline TREE\# & DATE & THERMO \# & TREE SET \# & TEMP C & TEMPF & $Y=$ MAX TEMP & COMMENTS \\
\hline 1 & $3 / 8 / 93$ & 6 & 1 & & 72.87 & $\mathbf{N}$ & \\
\hline$\frac{1}{1}$ & $3 / 15 / 93$ & 9 & 9 & & 119.3 & $\bar{Y}$ & TMACS \\
\hline 1 & $3 / 15 / 93$ & 2 & 1 & & 118.4 & $\mathbf{N}$ & \\
\hline 1 & $3 / 15 / 93$ & 3 & 1 & & 114.62 & $\mathbf{N}$ & \\
\hline$\frac{1}{1}$ & $3 / 15 / 93$ & 4 & 9 & & 109.4 & $\mathbf{N}$ & \\
\hline 1 & $3 / 15 / 93$ & 5 & 1 & & 100.58 & $\mathbf{N}$ & \\
\hline 1 & $3 / 22 / 93$ & 1 & 1 & & 919.48 & $\mathbf{Y}$ & TMACS \\
\hline 1 & $3 / 22 / 93$ & 2 & 1 & & 118.4 & $\mathbf{N}$ & \\
\hline 1 & $3 / 22 / 93$ & 3 & 1 & & 114.62 & $\mathbf{N}$ & \\
\hline 1 & $3 / 22 / 93$ & 4 & 1 & & 109.4 & $\mathbf{N}$ & \\
\hline 1 & $3 / 22 / 93$ & 5 & 1 & & 100.58 & $\mathbf{N}$ & \\
\hline 1 & $3 / 22 / 93$ & 6 & 9 & & 72.5 & $\mathbf{N}$ & \\
\hline 1 & $3 / 29 / 93$ & 7 & 1 & & 118.94 & $\overline{\mathrm{Y}}$ & TMACS \\
\hline 1 & $3 / 29 / 93$ & 2 & 1 & & 117.86 & $\mathbf{N}$ & \\
\hline 1 & $3 / 29 / 93$ & 3 & 1 & & 114.26 & $\mathbf{N}$ & \\
\hline 1 & $3 / 29 / 93$ & 4 & 1 & & 108.86 & $\mathrm{~N}$ & \\
\hline 1 & $3 / 29 / 93$ & 5 & 1 & & 100.22 & $\mathbf{N}$ & \\
\hline 9 & $3 / 29 / 93$ & 6 & 9 & & 71.78 & $\mathbf{N}$ & \\
\hline 1 & $3 / 31 / 93$ & 1 & 9 & & 118.94 & $\bar{Y}$ & TMACS \\
\hline 1 & $3 / 31 / 93$ & 2 & 1 & & 118.04 & $N$ & \\
\hline 1 & $3 / 31 / 93$ & 3 & 1 & & 114.08 & $\mathbf{N}$ & \\
\hline 1 & $3 / 31 / 93$ & 4 & 1 & & 108.68 & $N$ & \\
\hline 1 & $3 / 31 / 93$ & 5 & 9 & & 99.68 & $\bar{N}$ & \\
\hline 1 & $3 / 31 / 93$ & 6 & 1 & & 71.42 & $\mathbf{N}$ & \\
\hline 2 & $3 / 31 / 93$ & 1 & 9 & & 109 & $\bar{Y}$ & \\
\hline 2 & $3 / 31 / 93$ & 2 & 1 & & 103 & $\mathbf{N}$ & \\
\hline 2 & $3 / 31 / 93$ & 3 & 1 & & 95 & $\mathbf{N}$ & \\
\hline 2 & $3 / 31 / 93$ & 4 & 7 & & 79 & $\bar{N}$ & \\
\hline 2 & $3 / 31 / 93$ & 5 & 1 & & 77 & $N$ & \\
\hline 2 & $3 / 31 / 93$ & 6 & 1 & & 72 & $N$ & \\
\hline 9 & $4 / 5 / 93$ & 1 & 1 & & 118.58 & $\bar{Y}$ & TMACS \\
\hline 1 & $4 / 5 / 93$ & 2 & 1 & & 117.68 & N & \\
\hline 9 & $4 / 5 / 93$ & 3 & 1 & & 113.9 & $\mathbf{N}$ & \\
\hline 9 & $4 / 5 / 93$ & 4 & 9 & & 108.68 & $\bar{N}$ & \\
\hline 1 & $4 / 5 / 93$ & 5 & 1 & & 99.68 & $\mathbf{N}$ & \\
\hline 1 & $4 / 5 / 93$ & 6 & 1 & & 71.6 & $\mathbf{N}$ & \\
\hline 2 & $4 / 5 / 93$ & 1 & 1 & & 108.86 & $\bar{Y}$ & TMACS \\
\hline 2 & $4 / 5 / 93$ & 2 & 1 & & 103.1 & $\mathbf{N}$ & \\
\hline 2 & $4 / 5 / 93$ & 3 & 1 & & 95.18 & $N$ & \\
\hline 2 & $4 / 5 / 93$ & 4 & 9 & & 79.34 & $\bar{N}$ & \\
\hline 2 & $4 / 5 / 93$ & 5 & 1 & & 77 & $\bar{N}$ & \\
\hline 2 & $4 / 5 / 93$ & 6 & 1 & & 72.14 & $N$ & \\
\hline 9 & $4 / 12 / 93$ & 1 & 1 & & 119.3 & $\bar{Y}$ & TMAC \\
\hline 1 & $4 / 12 / 93$ & 2 & 1 & & 118.04 & $\mathbf{N}$ & \\
\hline 1 & $4 / 12 / 93$ & 3 & 1 & & 114.08 & $N$ & \\
\hline 1 & $4 / 12 / 93$ & 4 & 1 & & 108.86 & $N$ & \\
\hline 1 & $4 / 12 / 93$ & 5 & 1 & & 99.86 & $\mathbf{N}$ & \\
\hline 1 & $4 / 12 / 93$ & 6 & 1 & & 71.6 & $N$ & \\
\hline 2 & $4 / 12 / 93$ & 1 & 1 & & 108.86 & $\bar{Y}$ & TMAC \\
\hline 2 & $4 / 12 / 93$ & 2 & 1 & & 102.92 & $\mathbf{N}$ & \\
\hline 2 & $4 / 12 / 93$ & 3 & 1 & & 95.18 & $\mathbf{N}$ & \\
\hline 2 & $4 / 12 / 93$ & 4 & 1 & & 79.34 & $N$ & \\
\hline 2 & $4 / 12 / 93$ & 5 & 1 & & 76.82 & $\mathbf{N}$ & \\
\hline 2 & $4 / 12 / 93$ & 6 & 1 & & 72.14 & $\mathrm{~N}$ & \\
\hline 1 & $4 / 19 / 93$ & 1 & 1 & & 118.76 & $\bar{Y}$ & TMACS \\
\hline 1 & $4 / 19 / 93$ & 2 & 1 & & 117.68 & $\bar{N}$ & \\
\hline 1 & $4 / 19 / 93$ & 3 & 1 & & 113.72 & $\mathbf{N}$ & \\
\hline 1 & $4 / 19 / 93$ & 4 & 1 & & 108.32 & $\mathbf{N}$ & \\
\hline 1 & $4 / 19 / 93$ & 5 & 1 & & 99.68 & $\bar{N}$ & \\
\hline 1 & $4 / 19 / 93$ & 6 & 1 & & 71.6 & $\bar{N}$ & \\
\hline 2 & $4 / 19 / 93$ & 9 & 1 & & 108.5 & $\bar{Y}$ & TMACS \\
\hline 2 & $4 / 19 / 93$ & 2 & 1 & & 102.38 & $\overline{\mathbf{N}}$ & \\
\hline 2 & $4 / 19 / 93$ & 3 & 1 & & 95.54 & $\mathbf{N}$ & \\
\hline 2 & $4 / 19 / 93$ & 4 & 1 & & 79.7 & $\mathbf{N}$ & \\
\hline 2 & $4 / 19 / 93$ & 5 & 1 & & 77 & $\mathbf{N}$ & \\
\hline 2 & $4 / 19 / 93$ & 6 & 1 & & 71.96 & $\mathbf{N}$ & \\
\hline
\end{tabular}

Data obtained from WHC Surveillance Analysis Computer System (SACS), November 12, 1993. 


\begin{tabular}{|c|c|c|c|c|c|c|c|}
\hline \multicolumn{8}{|c|}{ TANK 241-BY-110 } \\
\hline & & & & THEF & COUPLE & & \\
\hline TREE\# & DATE & THERMO \# & TREE SET \# & TEMP C & TEMP F & $Y=M A X$ TEMP & COMMENTS \\
\hline$T$ & $4 / 25 / 93$ & 1 & 1 & & 118.76 & $\mathbf{Y}$ & TMACS \\
\hline 1 & $4 / 25 / 93$ & 2 & 1 & & 117.5 & $N$ & \\
\hline 1 & $4 / 25 / 93$ & 3 & 1 & & 113.72 & $\mathbf{N}$ & \\
\hline 1 & $4 / 25 / 93$ & 4 & 1 & & 108.32 & $\mathrm{~N}$ & \\
\hline 1 & $4 / 25 / 93$ & 5 & 1 & & 99.32 & $N$ & \\
\hline 1 & $4 / 25 / 93$ & 6 & 1 & & 71.78 & $\mathbf{N}$ & \\
\hline 2 & $4 / 25 / 93$ & 1 & 1 & & 108.32 & $\bar{Y}$ & TMACS \\
\hline 2 & $4 / 25 / 93$ & 2 & 1 & & 102.2 & $\mathbf{N}$ & \\
\hline 2 & $4 / 25 / 93$ & 3 & 1 & & 95.54 & $N$ & \\
\hline 2 & $4 / 25 / 93$ & 4 & 1 & & 80.06 & $\mathrm{~N}$ & \\
\hline 2 & $4 / 25 / 93$ & 5 & 1 & & 77 & $\bar{N}$ & \\
\hline 2 & $4 / 25 / 93$ & 6 & $T$ & & 71.96 & $\mathbf{N}$ & \\
\hline 1 & $5 / 3 / 93$ & 1 & 1 & & 118.22 & $\mathbf{Y}$ & TMACS \\
\hline 1 & $5 / 3 / 93$ & 2 & 1 & & 117.14 & $\mathrm{~N}$ & \\
\hline 1 & $5 / 3 / 93$ & 3 & 1 & & 113 & $\mathbf{N}$ & \\
\hline 1 & $5 / 3 / 93$ & 4 & 1 & & 107.6 & $\mathbf{N}$ & \\
\hline$\overline{1}$ & $5 / 3 / 93$ & 5 & 1 & & 99.14 & $\mathbf{N}$ & \\
\hline$\frac{1}{1}$ & $5 / 3 / 93$ & 6 & 7 & & 71.6 & $\mathbf{N}$ & \\
\hline 2 & $5 / 3 / 93$ & 1 & 1 & & 109.22 & $\bar{Y}$ & TMACS \\
\hline 2 & $5 / 3 / 93$ & 2 & 1 & & 102.92 & $\mathbf{N}$ & \\
\hline 2 & $5 / 3 / 93$ & 3 & 1 & & 96.62 & $\mathbf{N}$ & \\
\hline 2 & $5 / 3 / 93$ & 4 & 1 & & 81.5 & $\mathrm{~N}$ & \\
\hline$\frac{2}{2}$ & $5 / 3 / 93$ & 5 & $\frac{1}{1}$ & & 77.9 & $\mathbf{N}$ & \\
\hline 2 & $5 / 3 / 93$ & 6 & 1 & & 73.22 & $\mathbf{N}$ & \\
\hline 1 & $5 / 8 / 93$ & 1 & 7 & & 118.6 & $Y$ & E 90032516485 \\
\hline 1 & $5 / 8 / 93$ & 2 & 1 & & 112.3 & $\mathbf{N}$ & \\
\hline 1 & $5 / 8 / 93$ & 3 & 1 & & 113.1 & $\mathbf{N}$ & \\
\hline 1 & $5 / 8 / 93$ & 4 & 1 & & 107.6 & $\mathbf{N}$ & \\
\hline 1 & $5 / 8 / 93$ & 5 & 1 & & 107.6 & $\mathbf{N}$ & \\
\hline 1 & $5 / 8 / 93$ & 6 & 1 & & 98 & $\bar{N}$ & \\
\hline 2 & $5 / 8 / 93$ & 9 & 1 & & 107.6 & $\bar{Y}$ & K 910426539-7 \\
\hline 2 & $5 / 8 / 93$ & 2 & 1 & & 101.2 & $\mathbf{N}$ & \\
\hline 2 & $5 / 8 / 93$ & 3 & 1 & & 94.4 & $\mathbf{N}$ & \\
\hline 2 & $5 / 8 / 93$ & 4 & 1 & & 94.4 & $\mathbf{N}$ & \\
\hline 2 & $5 / 8 / 93$ & 5 & 1 & & 78.8 & $\mathbf{N}$ & \\
\hline 2 & $5 / 8 / 93$ & 6 & 1 & & 75.1 & $\mathbf{N}$ & \\
\hline 1 & $5 / 10 / 93$ & 1 & $T$ & & 118.22 & $\bar{Y}$ & TMACS \\
\hline 1 & $5 / 10 / 93$ & 2 & 1 & & 117.14 & $\mathbf{N}$ & \\
\hline 1 & $5 / 10 / 93$ & 3 & 1 & & 113 & $\mathbf{N}$ & \\
\hline 1 & $5 / 10 / 93$ & 4 & 1 & & 107.6 & $\mathbf{N}$ & \\
\hline 9 & $5 / 10 / 93$ & 5 & 1 & & 98.78 & $\mathbf{N}$ & \\
\hline 1 & $5 / 10 / 93$ & 6 & 1 & & 71.6 & $\mathbf{N}$ & \\
\hline 2 & $5 / 10 / 93$ & $\overline{1}$ & 1 & & 109.4 & $\bar{Y}$ & TMACS \\
\hline 2 & $5 / 10 / 93$ & 2 & 1 & & 103.28 & $\bar{N}$ & \\
\hline 2 & $5 / 10 / 93$ & 3 & 1 & & 96.62 & $\mathbf{N}$ & \\
\hline 2 & $5 / 10 / 93$ & 4 & 1 & & 81.68 & $\mathbf{N}$ & \\
\hline 2 & $5 / 10 / 93$ & 5 & 9 & & 78.08 & $\mathbf{N}$ & \\
\hline 2 & $5 / 10 / 93$ & 6 & 1 & & 73.22 & $\mathbf{N}$ & \\
\hline 1 & $5 / 17 / 93$ & 1 & $T$ & & 119.9 & $\bar{Y}$ & $E 900325164-85$ \\
\hline 1 & $5 / 17 / 93$ & 2 & 1 & & 179.6 & $\mathbf{N}$ & \\
\hline 9 & $5 / 17 / 93$ & 3 & 1 & & 115.4 & $\mathbf{N}$ & \\
\hline 1 & $5 / 17 / 93$ & 4 & 1 & & 109.6 & $N$ & \\
\hline 1 & $5 / 17 / 93$ & 5 & 1 & & 99.9 & $\mathbf{N}$ & \\
\hline 7 & $5 / 17 / 93$ & 6 & 7 & & 72.2 & $\mathbf{N}$ & \\
\hline 2 & $5 / 17 / 93$ & 1 & 1 & & 108 & $\bar{Y}$ & k910426539-7 \\
\hline 2 & $5 / 17 / 93$ & 2 & 1 & & 102.5 & $\mathbf{N}$ & \\
\hline 2 & $5 / 17 / 93$ & 3 & 7 & & 97 & $\mathbf{N}$ & \\
\hline 2 & $5 / 17 / 93$ & 4 & 1 & & 82.4 & $\mathbf{N}$ & \\
\hline 2 & $5 / 17 / 93$ & 5 & 1 & & 78.2 & $\mathbf{N}$ & \\
\hline 2 & $5 / 17 / 93$ & 6 & 1 & & 73.7 & $\mathbf{N}$ & \\
\hline 9 & $5 / 23 / 93$ & 1 & 1 & & 116.9 & $\bar{Y}$ & E 900325164-40 \\
\hline 1 & $5 / 23 / 93$ & 2 & 1 & & 116 & $\mathbf{N}$ & \\
\hline 1 & $5 / 23 / 93$ & 3 & 1 & & 112.8 & $\mathbf{N}$ & \\
\hline 1 & $5 / 23 / 93$ & 4 & 1 & & 107.5 & $\mathbf{N}$ & \\
\hline 1 & $5 / 23 / 93$ & 5 & 1 & & 97.9 & $\bar{N}$ & \\
\hline 1 & $5 / 23 / 93$ & 6 & 1 & & 71.4 & $\mathbf{N}$ & \\
\hline
\end{tabular}

Data obtained from WHC Surveillance Analysis Computer System (SACS), November 12, 1993. 


\begin{tabular}{|c|c|c|c|c|c|c|c|}
\hline \multicolumn{8}{|c|}{ TANK 241-BY-110 } \\
\hline & & & & THER & COUPLE D & & \\
\hline TREE\# & $\overline{\text { DATE }}$ & THERMO \# & TREE SET \# & TEMP C & TEMP F & $\bar{Y}=$ MAX TEMP & COMMENTS \\
\hline 2 & $5 / 23 / 93$ & 1 & 1 & & 107.8 & $Y$ & K890723633-71 \\
\hline 2 & $5 / 23 / 93$ & 2 & 1 & & 101.4 & $\mathrm{~N}$ & \\
\hline 2 & $5 / 23 / 93$ & 3 & 1 & & 95.8 & $\mathbf{N}$ & \\
\hline 2 & $5 / 23 / 93$ & 4 & 1 & & 80.9 & $\mathrm{~N}$ & \\
\hline 2 & $5 / 23 / 93$ & 5 & 1 & & 76.6 & $\mathbf{N}$ & \\
\hline$\overline{2}$ & $5 / 23 / 93$ & 6 & 1 & & 71.6 & $N$ & \\
\hline 1 & $5 / 30 / 93$ & 1 & 1 & & 118 & $Y$ & E $900325164-85$ \\
\hline 1 & $5 / 30 / 93$ & 2 & 1 & & 116.8 & $\bar{N}$ & \\
\hline 1 & $5 / 30 / 93$ & 3 & 1 & & 112.6 & $\mathrm{~N}$ & \\
\hline 1 & $5 / 30 / 93$ & 4 & 1 & & 107.2 & $\mathbf{N}$ & \\
\hline 1 & $5 / 30 / 93$ & 5 & 1 & & 97.9 & $\mathbf{N}$ & \\
\hline 1 & $5 / 30 / 93$ & 6 & 1 & & 71.3 & $\mathbf{N}$ & \\
\hline 2 & $5 / 30 / 93$ & 1 & 1 & & 107.9 & $\mathrm{Y}$ & K 890723679.64 \\
\hline 2 & $5 / 30 / 93$ & 2 & 1 & & 101.6 & $\mathbf{N}$ & \\
\hline 2 & $5 / 30 / 93$ & 3 & 1 & & 96.4 & $\mathbf{N}$ & \\
\hline 2 & $5 / 30 / 93$ & 4 & 1 & & 81.8 & $\mathbf{N}$ & \\
\hline 2 & $5 / 30 / 93$ & 5 & 1 & & 77.6 & $\mathbf{N}$ & \\
\hline 2 & $5 / 30 / 93$ & 6 & 9 & & 72.5 & $\mathbf{N}$ & \\
\hline 1 & $6 / 4 / 93$ & 1 & 1 & & 118 & $Y$ & E $900325164-85$ \\
\hline 1 & $6 / 4 / 93$ & 2 & 1 & & 117.2 & $\mathbf{N}$ & \\
\hline 1 & $6 / 4 / 93$ & 3 & 1 & & 113 & $\mathbf{N}$ & \\
\hline 1 & $6 / 4 / 93$ & 4 & 1 & & 107.6 & $\mathbf{N}$ & \\
\hline 1 & $6 / 4 / 93$ & 5 & 1 & & 98 & $N$ & \\
\hline 1 & $6 / 4 / 93$ & 6 & 1 & & 71.4 & $\bar{N}$ & \\
\hline 2 & $6 / 4 / 93$ & 1 & 1 & & 108.1 & $Y$ & K890723679-64 \\
\hline 2 & $6 / 4 / 93$ & 2 & 1 & & 101.5 & $\mathrm{~N}$ & \\
\hline 2 & $6 / 4 / 93$ & 3 & 1 & & 96.6 & $\bar{N}$ & \\
\hline 2 & $6 / 4 / 93$ & 4 & 1 & & 82.5 & $\mathbf{N}$ & \\
\hline 2 & $6 / 4 / 93$ & 5 & 1 & & 78 & $\bar{N}$ & \\
\hline 2 & $6 / 4 / 93$ & 6 & 1 & & 73.1 & $\mathbf{N}$ & \\
\hline 1 & $6 / 7 / 93$ & 1 & 1 & & 117.86 & $\bar{Y}$ & TMACS \\
\hline 9 & $6 / 7 / 93$ & 2 & 1 & & 116.78 & $\mathrm{~N}$ & \\
\hline 1 & $6 / 7 / 93$ & 3 & 1 & & 112.46 & $\mathrm{~N}$ & \\
\hline 1 & $6 / 7 / 93$ & 4 & 1 & & 107.24 & $\mathbf{N}$ & \\
\hline 1 & $6 / 7 / 93$ & 5 & 1 & & 98.78 & $\mathrm{~N}$ & \\
\hline 1 & $6 / 7 / 93$ & 6 & 1 & & 73.04 & $\mathbf{N}$ & \\
\hline 2 & $6 / 7 / 93$ & 1 & 1 & & 109.04 & $Y$ & TMACS \\
\hline 2 & $6 / 7 / 93$ & 2 & 1 & & 102.02 & $\mathbf{N}$ & \\
\hline 2 & $6 / 7 / 93$ & 3 & 1 & & 97.34 & $\mathbf{N}$ & \\
\hline 2 & $6 / 7 / 93$ & 4 & 1 & & 83.3 & $\mathbf{N}$ & \\
\hline 2 & $6 / 7 / 93$ & 5 & 1 & & 78.98 & $\mathbf{N}$ & \\
\hline 2 & $6 / 7 / 93$ & 6 & 1 & & 75.02 & $\mathrm{~N}$ & \\
\hline 1 & $6 / 14 / 93$ & 1 & 1 & & 117.5 & $\bar{Y}$ & TMACS \\
\hline 1 & $6 / 14 / 93$ & 2 & 1 & & 116.24 & $\mathbf{N}$ & \\
\hline 1 & $6 / 14 / 93$ & 3 & 1 & & 112.28 & $\mathbf{N}$ & \\
\hline 1 & $6 / 14 / 93$ & 4 & 1 & & 107.06 & $\mathbf{N}$ & \\
\hline 1 & $6 / 14 / 93$ & 5 & 1 & & 98.42 & $\bar{N}$ & \\
\hline 1 & $6 / 14 / 93$ & 6 & 9 & & 73.22 & $N$ & \\
\hline 2 & $6 / 14 / 93$ & 1 & 9 & & 109.58 & $\bar{Y}$ & TMACS \\
\hline 2 & $6 / 14 / 93$ & 2 & 1 & & 102.2 & $\bar{N}$ & \\
\hline 2 & $6 / 14 / 93$ & 3 & 1 & & 97.7 & $\mathbf{N}$ & \\
\hline 2 & $6 / 14 / 93$ & 4 & 1 & & 84.02 & $N$ & \\
\hline 2 & $6 / 14 / 93$ & 5 & 1 & & 79.7 & $\mathbf{N}$ & \\
\hline 2 & $6 / 14 / 93$ & 6 & 1 & & 75.74 & $\bar{N}$ & \\
\hline 1 & $6 / 21 / 93$ & 1 & 1 & & 117.5 & $\bar{Y}$ & TMACS \\
\hline 1 & $6 / 21 / 93$ & 2 & 1 & & 116.42 & $\bar{N}$ & \\
\hline 1 & $6 / 21 / 93$ & 3 & 1 & & 112.46 & $\mathbf{N}$ & \\
\hline 1 & $6 / 21 / 93$ & 4 & 1 & & 107.24 & $\mathbf{N}$ & \\
\hline 1 & $6 / 21 / 93$ & 5 & 1 & & 98.78 & $\mathbf{N}$ & \\
\hline 1 & $6 / 21 / 93$ & 6 & 1 & & 73.94 & $\mathbf{N}$ & \\
\hline 2 & $6 / 21 / 93$ & 1 & 1 & & 109.04 & $\mathbf{N}$ & \\
\hline 2 & $6 / 21 / 93$ & 2 & 1 & & 102.38 & $N$ & \\
\hline 2 & $6 / 21 / 93$ & 3 & 1 & & 97.7 & $N$ & \\
\hline 2 & $6 / 21 / 93$ & 4 & 1 & & 84.38 & $\mathrm{~N}$ & \\
\hline 2 & $6 / 21 / 93$ & 5 & 1 & & 79.88 & $\bar{N}$ & \\
\hline 2 & $6 / 21 / 93$ & 6 & 1 & & 75.74 & $\mathrm{~N}$ & \\
\hline
\end{tabular}

Data obtained from WHC Surveillance Analysis Computer System (SACS), November 12, 1993. 


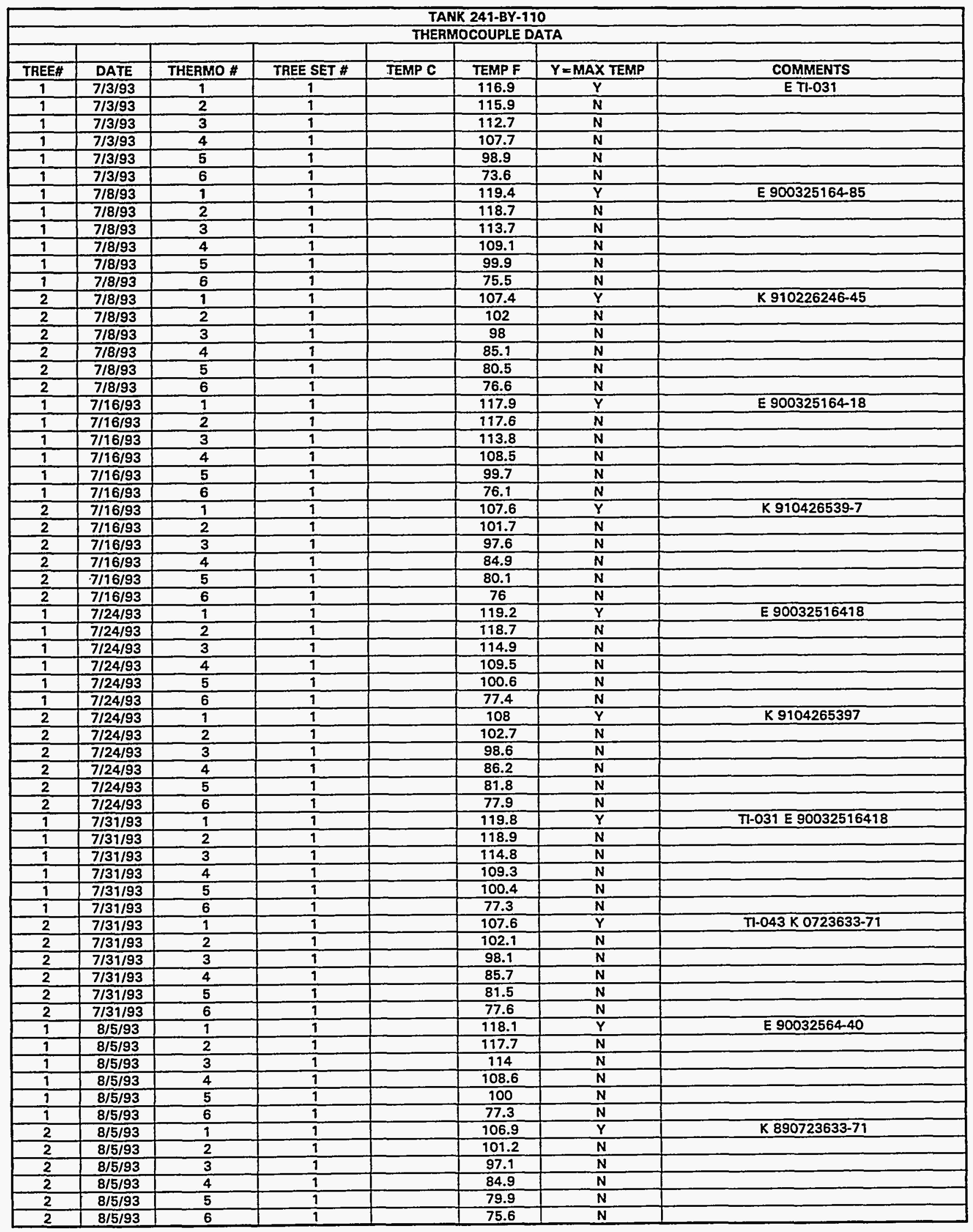

Data obtained from WHC Surveillance Analysis Computer System (SACS), November 12, 1993. 


\begin{tabular}{|c|c|c|c|c|c|c|c|}
\hline \multicolumn{8}{|c|}{ TANK 241-BY-110 } \\
\hline \multicolumn{8}{|c|}{ THEAMOCOUPLE DATA } \\
\hline TREE\# & DATE & THERMO \# & TREE SET \# & TEMP C & TEMP F & $Y=$ MAX TEMP & COMMENTS \\
\hline 1 & $8 / 13 / 93$ & 1 & 1 & & 119 & $\mathbf{N}$ & $E 900325164-40$ \\
\hline 1 & $8 / 13 / 93$ & 2 & 1 & & 118 & $\mathrm{~N}$ & \\
\hline 1 & $8 / 13 / 93$ & 3 & 1 & & 114.9 & $\bar{N}$ & \\
\hline 1 & $8 / 13 / 93$ & 4 & 1 & & 119.7 & $Y$ & \\
\hline 1 & $8 / 13 / 93$ & 5 & 1 & & 101.1 & $\mathbf{N}$ & \\
\hline 1 & $8 / 13 / 93$ & 6 & 1 & & 78.6 & $\mathbf{N}$ & \\
\hline 2 & $8 / 13 / 93$ & 1 & 1 & & 108.1 & $Y$ & K9104265-39-7 \\
\hline 2 & $8 / 13 / 93$ & 2 & 1 & & 108 & $\mathbf{N}$ & \\
\hline 2 & $8 / 13 / 93$ & 3 & 1 & & & $\mathbf{N}$ & 107.9 - suspect data (high) \\
\hline 2 & $8 / 13 / 93$ & 4 & 1 & & & $\mathbf{N}$ & 107.8 - suspect data (high) \\
\hline 2 & $8 / 13 / 93$ & 5 & 1 & & & $N$ & 107.6 - suspect data (high) \\
\hline 2 & $8 / 13 / 93$ & 6 & 1 & & & $\mathbf{N}$ & 107.6 - suspect data (high) \\
\hline 1 & $8 / 20 / 93$ & 1 & 1 & & 117.2 & $\mathbf{Y}$ & E $900325164-4 \mathrm{TC} 7 \mathrm{O} / \mathrm{S}$ \\
\hline 1 & $8 / 20 / 93$ & 2 & 1 & & 116.2 & $\mathbf{N}$ & \\
\hline 1 & $8 / 20 / 93$ & 3 & 1 & & 112.5 & $\mathbf{N}$ & \\
\hline 1 & $8 / 20 / 93$ & 4 & 1 & & 107.6 & $\mathbf{N}$ & \\
\hline 1 & $8 / 20 / 93$ & 5 & 1 & & 99.5 & $\bar{N}$ & \\
\hline 1 & $8 / 20 / 93$ & 6 & 1 & & 77.2 & $\mathbf{N}$ & \\
\hline 2 & $8 / 20 / 93$ & 1 & 1 & & 104.7 & $\bar{Y}$ & K 890723633-71 \\
\hline 2 & $8 / 20 / 93$ & 2 & 1 & & 100 & $\mathbf{N}$ & \\
\hline 2 & $8 / 20 / 93$ & 3 & 1 & & 96.3 & $\mathbf{N}$ & \\
\hline 2 & $8 / 20 / 93$ & 4 & 1 & & 95 & $\mathbf{N}$ & \\
\hline 2 & $8 / 20 / 93$ & 5 & 1 & & 80 & $\mathbf{N}$ & \\
\hline 2 & $8 / 20 / 93$ & 6 & 1 & & 76.3 & $N$ & \\
\hline 1 & $8 / 26 / 93$ & 1 & 1 & & 116.5 & $\bar{Y}$ & E 900325164-40 \\
\hline 1 & $8 / 26 / 93$ & 2 & 1 & & 116.3 & $\mathbf{N}$ & \\
\hline 1 & $8 / 26 / 93$ & 3 & 1 & & 111.9 & $\mathrm{~N}$ & \\
\hline 1 & $8 / 26 / 93$ & 4 & 1 & & 107.8 & $\mathrm{~N}$ & \\
\hline 1 & $8 / 26 / 93$ & 5 & 1 & & 99.3 & $\mathbf{N}$ & \\
\hline 1 & $8 / 26 / 93$ & 6 & 1 & & 76.6 & $\mathbf{N}$ & \\
\hline 1 & $8 / 28 / 93$ & 1 & 1 & & 117.1 & $Y$ & E 900325164-18 \\
\hline 1 & $8 / 28 / 93$ & 2 & 1 & & 115.7 & $\mathbf{N}$ & \\
\hline 1 & $8 / 28 / 93$ & 3 & 1 & & 111.8 & $\bar{N}$ & \\
\hline 1 & $8 / 28 / 93$ & 4 & 1 & & 107 & $\mathrm{~N}$ & \\
\hline 1 & $8 / 28 / 93$ & 5 & 1 & & 98.9 & $\mathrm{~N}$ & \\
\hline 1 & $8 / 28 / 93$ & 6 & 1 & & 77.2 & $\mathbf{N}$ & \\
\hline 1 & $8 / 28 / 93$ & 1 & 2 & & 117.5 & $\bar{Y}$ & E 900325164-18 \\
\hline 1 & $8 / 28 / 93$ & 2 & 2 & & 116.2 & $\mathbf{N}$ & \\
\hline 1 & $8 / 28 / 93$ & 3 & 2 & & 112.3 & $\mathbf{N}$ & \\
\hline 1 & $8 / 28 / 93$ & 4 & 2 & & 107.5 & $\mathbf{N}$ & \\
\hline 1 & $8 / 28 / 93$ & 5 & 2 & & 99.7 & $\mathbf{N}$ & \\
\hline 1 & $8 / 28 / 93$ & 6 & 2 & & 77.7 & $\mathbf{N}$ & \\
\hline 2 & $8 / 28 / 93$ & 1 & 1 & & 105.4 & $Y$ & K $890723633-71$ \\
\hline 2 & $8 / 28 / 93$ & 2 & 1 & & 99.8 & $\mathbf{N}$ & \\
\hline 2 & $8 / 28 / 93$ & 3 & 1 & & 95.7 & $\mathbf{N}$ & \\
\hline 2 & $8 / 28 / 93$ & 4 & 1 & & 84.2 & $\mathbf{N}$ & \\
\hline 2 & $8 / 28 / 93$ & 5 & 1 & & 79.3 & $\mathbf{N}$ & \\
\hline 2 & $8 / 28 / 93$ & 6 & 1 & & 75.6 & $\mathbf{N}$ & \\
\hline 1 & $9 / 3 / 93$ & 1 & 1 & & 119.1 & $\bar{Y}$ & E $900325164-18$ \\
\hline 1 & $9 / 3 / 93$ & 2 & 1 & & 118.7 & $\mathbf{N}$ & \\
\hline 1 & $9 / 3 / 93$ & 3 & 1 & & 114.9 & $\mathbf{N}$ & \\
\hline 1 & 9/3/93 & 4 & 1 & & 109.8 & $\mathbf{N}$ & \\
\hline 1 & $9 / 3 / 93$ & 5 & 1 & & 101.4 & $\mathbf{N}$ & \\
\hline 1 & $9 / 3 / 93$ & 6 & 1 & & 79.8 & $\mathbf{N}$ & \\
\hline 2 & $9 / 3 / 93$ & 1 & 1 & & 108.1 & $Y$ & K910426539-7 \\
\hline 2 & $9 / 3 / 93$ & 2 & 1 & & 103.8 & $N$ & \\
\hline 2 & $9 / 3 / 93$ & 3 & 1 & & 100.6 & $\mathbf{N}$ & \\
\hline 2 & $9 / 3 / 93$ & 4 & 1 & & 89.6 & $\mathrm{~N}$ & \\
\hline 2 & $9 / 3 / 93$ & 5 & 1 & & 85.1 & $N$ & \\
\hline 2 & $9 / 3 / 93$ & 6 & 1 & & 81.5 & $\mathbf{N}$ & \\
\hline 1 & $9 / 9 / 93$ & 1 & 1 & & 118.7 & $Y$ & $E 881222428-104$ \\
\hline 1 & $9 / 9 / 93$ & 2 & 1 & & 118.1 & $\mathbf{N}$ & \\
\hline 1 & $9 / 9 / 93$ & 3 & 1 & & 114.5 & $\mathbf{N}$ & \\
\hline 9 & 9/9/93 & 4 & 1 & & 109.4 & $\mathbf{N}$ & \\
\hline 1 & 9/9/93 & 5 & 1 & & 109.1 & $\mathbf{N}$ & \\
\hline 1 & $9 / 9 / 93$ & 6 & 1 & & 79.6 & $\mathbf{N}$ & \\
\hline
\end{tabular}

Data obtained from WHC Surveillance Analysis Computer System (SACS), November 12, 1993. 


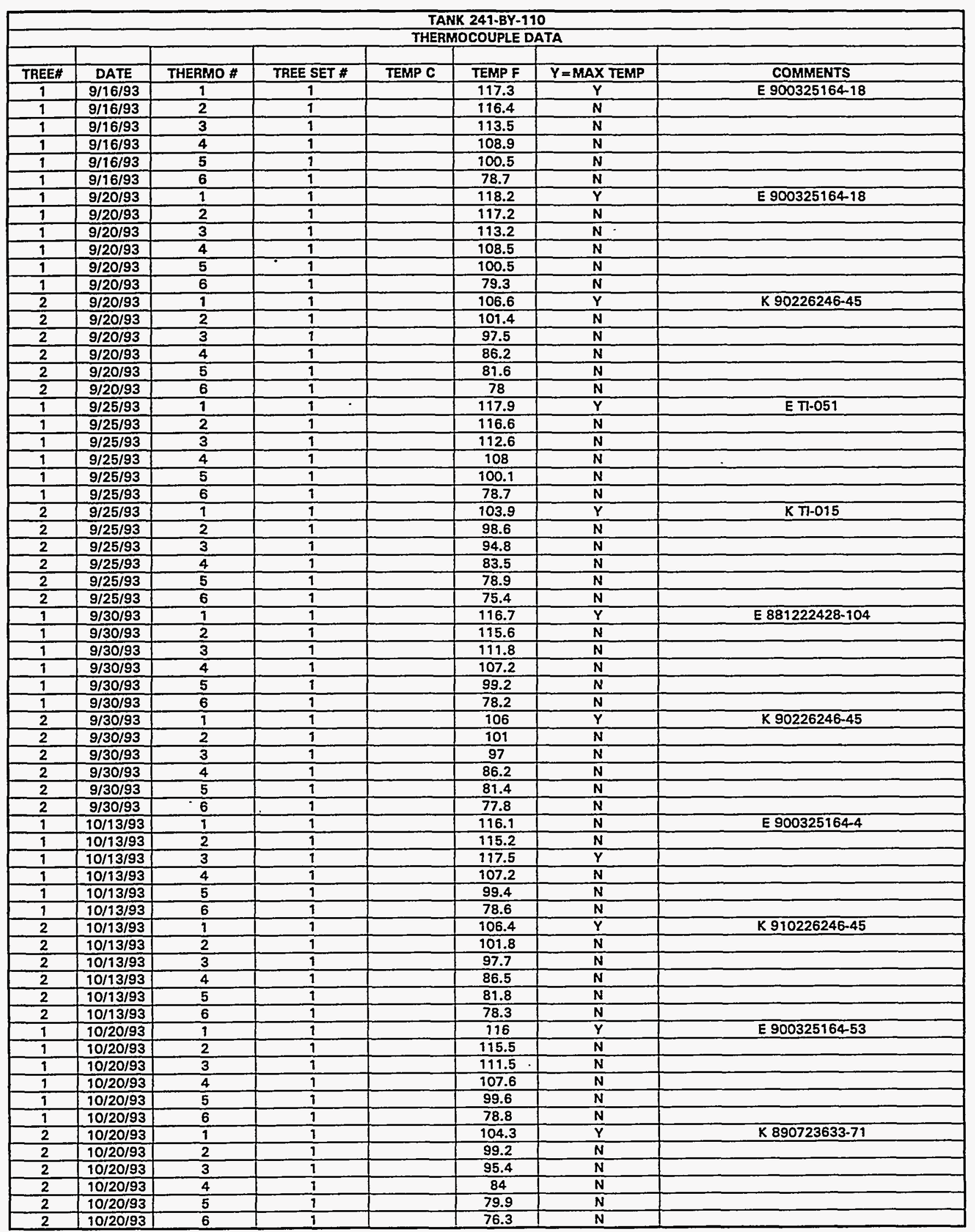

Data obtained from WHC Surveillance Analysis Computer System (SACS), November 12, 1993. 
WHC-SD-WM-ER-312, Rev. 0

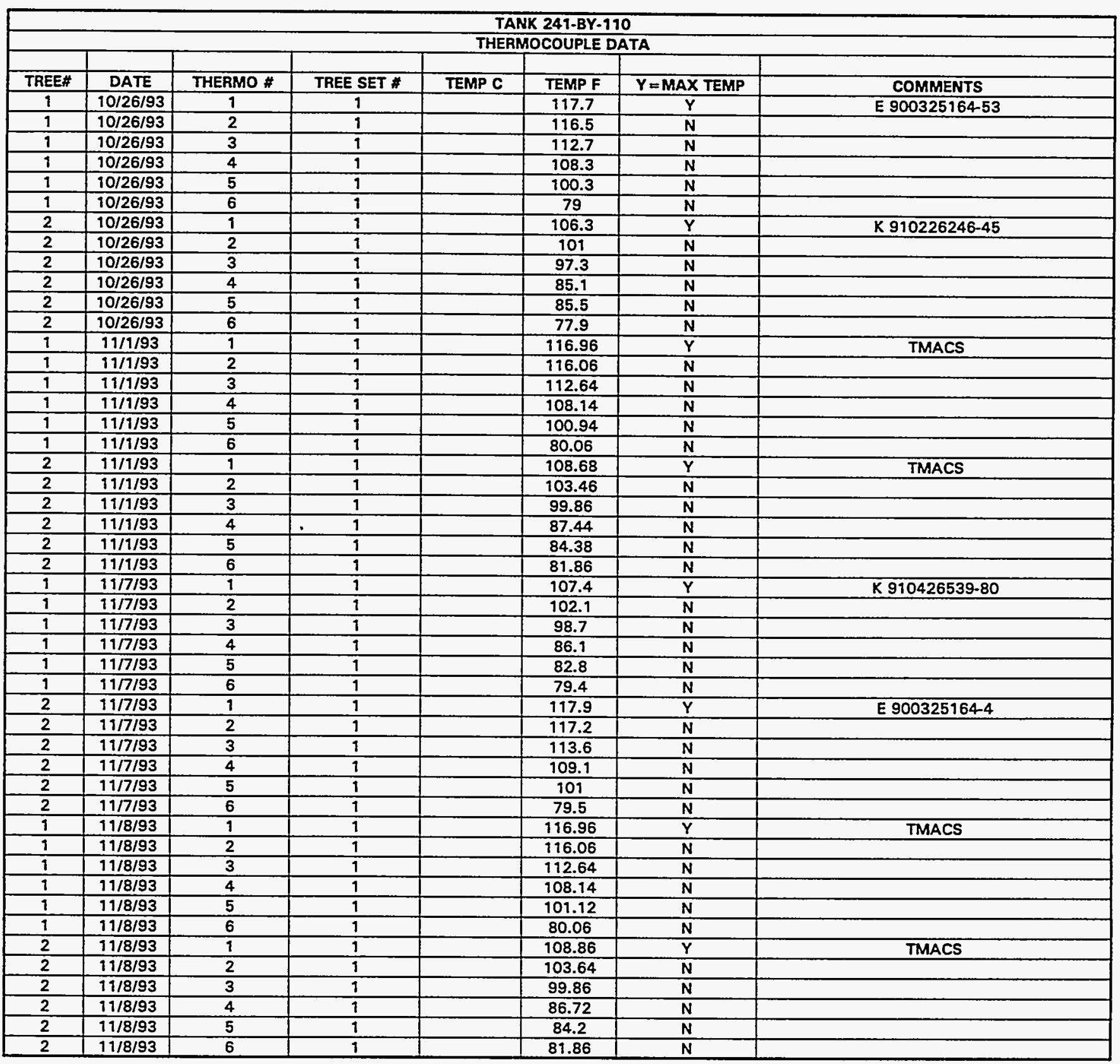

Data obtained from WHC Surveillance Analysis Computer System (SACS), November 12, 1993. 


\begin{tabular}{|c|c|c|c|c|c|c|c|}
\hline \multicolumn{8}{|c|}{ TANK 241-BY-119 } \\
\hline & & & & & & & \\
\hline TREE\# & DATE & THERMO \# & TREE SET \# & TEMPC & TEMPF & $Y=\operatorname{MAX}$ TEMP & COMMENTS \\
\hline 1 & $9 / 12 / 74$ & 9 & & 50 & 122 & & raw data - assumed degree $C$ \\
\hline 1 & $9 / 12 / 74$ & 2 & & 50 & 922 & & raw data - essumed degree $\mathrm{C}$ \\
\hline 1 & $9 / 12 / 74$ & 3 & & 50 & 122 & & raw data - assumed degree $\mathrm{C}$ \\
\hline 1 & $9 / 12 / 74$ & $\overline{4}$ & & 50 & 122 & & raw data - assumed degree $C$ \\
\hline 1 & $9 / 12 / 74$ & 5 & & 48 & 118.4 & & raw data - assumed degree $\mathrm{C}$ \\
\hline$i$ & $9 / 12 / 74$ & 6 & & 48 & 118.4 & & raw data - assumed degree $\mathrm{C}$ \\
\hline 9 & $9 / 12 / 74$ & 7 & & 48 & 118.4 & & raw data - assumed degree $C$ \\
\hline 1 & $9 / 12 / 74$ & 8 & & 44 & 111.2 & & raw data - assumed degree $\mathrm{C}$ \\
\hline 1 & $9 / 12 / 74$ & 9 & & 39 & 102.2 & & raw data - assumed degree $\mathrm{C}$ \\
\hline 1 & $9 / 12 / 74$ & 10 & & 34 & 93.2 & & raw data - assumed degree $\mathrm{C}$ \\
\hline 1 & $10 / 8 / 74$ & 3 & & 48 & 118.4 & & raw data - assumed degree $C$ \\
\hline 1 & $10 / 8 / 74$ & 4 & & 48 & 118.4 & & raw data - assumed degree C \\
\hline 1 & $10 / 8 / 74$ & 6 & & 48 & 118.4 & & raw data - assumed degree $C$ \\
\hline 1 & $10 / 8 / 74$ & $\frac{5}{7}$ & & 48 & 118.4 & & raw data - assumed degree $\mathrm{C}$ \\
\hline 1 & $10 / 8 / 74$ & 8 & & 43 & 109.4 & & raw data - assumed degree $\mathrm{C}$ \\
\hline$\frac{1}{1}$ & $10 / 8 / 74$ & 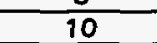 & & 32 & 89.6 & & raw data - assumed degree $C$ \\
\hline 1 & $11 / 26 / 74$ & 1 & & 28 & 82.4 & & raw data - assumed degree $\mathrm{C}$ \\
\hline 1 & $11 / 26 / 74$ & 2 & & 28 & 82.4 & & raw data - assumed degree $C$ \\
\hline$\frac{i}{1}$ & $11 / 26 / 74$ & $\frac{2}{4}$ & & 27 & 80.6 & & raw data - assumed degree $C$ \\
\hline 1 & $11 / 26 / 74$ & 6 & & 26 & 78.8 & & raw data - assumed degree $C$ \\
\hline 1 & $11 / 26 / 74$ & 8 & & 25 & 77 & & raw data - assumed degree $C$ \\
\hline 1 & $11 / 26 / 74$ & 10 & & 25 & 77 & & raw data - assumed degree $C$ \\
\hline 1 & $11 / 26 / 74$ & 11 & & 25 & 77 & & raw data - assumed degree C \\
\hline$\frac{1}{1}$ & $11 / 26 / 74$ & 12 & & $\frac{25}{22}$ & 71.6 & & raw data - assumed degree $C$ \\
\hline 1 & $12 / 17 / 74$ & 1 & & & & & 13.33 - suspect data (low) \\
\hline 1 & $12 / 17 / 74$ & 2 & & & & & 14.44 - suspect data (low) \\
\hline$\frac{1}{1}$ & $12 / 17 / 74$ & $\frac{1}{3}$ & & & & & 14.44 - suspect data (low) \\
\hline 1 & $12 / 17 / 74$ & 4 & & & & & 13.33 - suspect data (low) \\
\hline 1 & $12 / 17774$ & 5 & & & & & 13.89 - suspect data (low) \\
\hline$\frac{1}{1}$ & $12 / 17 / 74$ & 6 & & & & & 14.44 - suspect data (low) \\
\hline 1 & $12 / 17 / 74$ & 7 & & & & & 13.89 - suspect data (low) \\
\hline$\frac{1}{1}$ & $12 / 17 / 74$ & 8 & & & & & 14.44 - suspect data (low) \\
\hline 1 & $12 / 17 / 74$ & 9 & & & & & 13.89 - suspect data (low) \\
\hline 1 & $12 / 17 / 74$ & 10 & & & & & 13.33 - suspect data (low) \\
\hline$\frac{1}{1}$ & $12 / 17 / 74$ & 11 & & & & & 13.33 - suspect data (low) \\
\hline 1 & $12 / 17 / 74$ & 12 & & & & & 13.33 - suspect data (low) \\
\hline$\frac{1}{1}$ & $\frac{12 / 10 / 75}{6 / 10 / 7}$ & $\frac{12}{1}$ & & & & & 58 - suspect data (low) \\
\hline$\frac{1}{1}$ & $6 / 10 / 75$ & 2 & & & & & 58 - suspect data (low) \\
\hline 1 & $6 / 10 / 75$ & 3 & & & & & 58 - suspect data (low) \\
\hline 1 & $6 / 10 / 75$ & $\overline{4}$ & & & & & 58 - suspect data (low) \\
\hline 1 & $6 / 10 / 75$ & 5 & & & & & 58 - suspect data (low) \\
\hline$\frac{1}{1}$ & $6 / 10 / 75$ & $\frac{5}{6}$ & & & & & 58 - suspect data (low) \\
\hline 1 & $6 / 10 / 75$ & 7 & & & & & 58 - suspect data (low) \\
\hline 1 & $6 / 10 / 75$ & 8 & & & & & 58 - suspect data (low) \\
\hline 1 & $6 / 10 / 75$ & 9 & & & & & 58 - suspect data (low) \\
\hline$i$ & $6 / 10 / 75$ & 10 & & & & & 58 - suspect data (low) \\
\hline 1 & $6 / 10 / 75$ & 11 & & & & & 60- suspect data (low) \\
\hline$\frac{1}{1}$ & $6 / 10 / 75$ & 12 & & & & & 60- suspect data (low) \\
\hline$\frac{1}{1}$ & $6 / 10 / 75$ & $\frac{12}{13}$ & & & & & 60 - suspect data (low) \\
\hline 1 & $6 / 10 / 75$ & 14 & & & & & 60- suspect data (low) \\
\hline 1 & $7 / 28 / 75$ & 3 & & & 83 & & \\
\hline 1 & $7 / 28 / 75$ & 6 & & & 73 & & \\
\hline 9 & $7 / 28 / 75$ & $\frac{5}{8}$ & & & 72 & & \\
\hline$\frac{1}{1}$ & $7 / 28 / 75$ & $\frac{50}{10}$ & & & 73 & & \\
\hline$\frac{1}{1}$ & $8 / 26 / 75$ & 1 & & & 95 & & \\
\hline$\frac{1}{1}$ & $\frac{0.120170}{8 / 26 / 75}$ & $\frac{1}{2}$ & & & $\frac{93}{3}$ & & \\
\hline 1 & $8 / 26 / 75$ & 3 & & & 93 & & \\
\hline$\frac{1}{1}$ & 80 & $\frac{3}{4}$ & & & $\frac{95}{35}$ & & \\
\hline 1 & $8 / 26 / 75$ & 5 & & & 92 & & \\
\hline 1 & $8 / 26 / 75$ & 6 & & & 92 & & \\
\hline$\frac{1}{9}$ & $8 / 26 / 75$ & $\frac{5}{7}$ & & & 91 & & \\
\hline 1 & $8 / 26 / 75$ & 8 & & & 91 & & \\
\hline 1 & $8 / 26 / 75$ & 9 & & & 90 & & \\
\hline 9 & $8 / 26 / 75$ & 10 & & & 90 & & \\
\hline 1 & $8 / 26 / 75$ & 11 & & & 90 & & \\
\hline
\end{tabular}

Data obtained from WHC Surveillance Analysis Computer System (SACS), November 12, 1993. 
WHC-SD-WM-ER-312, Rev. 0

\begin{tabular}{|c|c|c|c|c|c|c|c|}
\hline \multicolumn{8}{|c|}{ TANK 241-BY-111 } \\
\hline & & & & THER & OCOUPLE & ATA & \\
\hline TREE\# & DATE & THERMO \# & TREE SET \# & TEMP C & TEMP F & $Y=M A X$ TEMP & COMMENTS \\
\hline 1 & $8 / 26 / 75$ & 12 & & & 90 & & \\
\hline 1 & $11 / 21 / 75$ & 3 & & & 124 & & \\
\hline$i$ & $11 / 21 / 75$ & 6 & & & 123 & & \\
\hline 1 & $11 / 21 / 75$ & 8 & & & 122 & & \\
\hline 1 & $11 / 21 / 75$ & 10 & & & 91 & & \\
\hline 1 & $3 / 4 / 76$ & 3 & & & 119 & & \\
\hline 1 & $3 / 4 / 76$ & 6 & & & 117 & & \\
\hline 1 & $3 / 4 / 76$ & 8 & & & 106 & & \\
\hline 1 & $3 / 4 / 76$ & 10 & & & 77 & & \\
\hline 1 & $5 / 7 / 76$ & 3 & & & 109 & & \\
\hline 1 & $5 \longdiv { 7 \Pi 6 }$ & 6 & & & 107 & & \\
\hline 1 & $5 / 7 / 76$ & 8 & & & 95 & & \\
\hline 1 & $5 / 7 / 76$ & 10 & & & 69 & & \\
\hline 1 & $6 / 9 / 76$ & 3 & & & 107 & & \\
\hline 1 & $6 / 9 / 76$ & 6 & & & 106 & & \\
\hline 1 & $6 / 9 / 76$ & 8 & & & 94 & & \\
\hline 1 & $6 / 9 / 76$ & 10 & & & 72 & & \\
\hline 1 & $8 / 14 / 76$ & 3 & & & 101 & & \\
\hline 1 & $8 / 14 / 76$ & 6 & & & 99 & & \\
\hline 1 & $8 / 14 / 76$ & 8 & & & 91 & & \\
\hline 1 & $8 / 14 / 76$ & 10 & & & 73 & & \\
\hline 1 & $8 / 14 / 76$ & 3 & & & 101 & & \\
\hline 1 & $8 / 14 / 76$ & 6 & & & 99 & & \\
\hline 1 & $8 / 14 / 76$ & 8 & & & 91 & & \\
\hline 1 & $8 / 14 / 76$ & 10 & & & 73 & & \\
\hline 1 & $9 / 13 / 76$ & 1 & & & 87 & & \\
\hline 1 & $9 / 13 / 76$ & 2 & & & 85 & & \\
\hline 1 & $9 / 13 / 76$ & 3 & & & 91 & & \\
\hline 1 & $9 / 13 / 76$ & 4 & & & 85 & & \\
\hline 1 & $9 / 13 / 76$ & 5 & & & 83 & & \\
\hline 1 & $9 / 13 / 76$ & 6 & & & 91 & & \\
\hline 1 & $9 / 13 / 76$ & 7 & & & 86 & & \\
\hline 1 & $9 / 13 / 76$ & 8 & & & 90 & & \\
\hline 1 & $9 / 13 / 76$ & 9 & & & 86 & & \\
\hline 1 & $9 / 13 / 76$ & 10 & & & 88 & & \\
\hline 1 & $9 / 13 / 76$ & 11 & & & 88 & & \\
\hline 1 & $9 / 13 / 76$ & 12 & & & 87 & & \\
\hline 1 & $11 / 7 / 76$ & 3 & & & 76 & & \\
\hline 1 & $11 / 7 / 76$ & 6 & & & 76 & & \\
\hline 1 & $11 / 7 / 76$ & 8 & & & 70 & & \\
\hline 1 & $11 / 7 / 76$ & 10 & & & 65 & & \\
\hline 9 & $12 / 9 / 76$ & 2 & & & 93 & & \\
\hline 1 & $12 / 9 / 76$ & 3 & & & 95 & & \\
\hline 1 & $12 / 9 / 76$ & 6 & & & 95 & & \\
\hline 1 & $12 / 9 / 76$ & 7 & & & 96 & & \\
\hline 1 & $12 / 9 / 76$ & 9 & & & 94 & & \\
\hline 1 & $12 / 9 / 76$ & 10 & & & 94 & & \\
\hline 1 & $12 / 9 / 76$ & 12 & & & 82 & & \\
\hline 1 & $1 / 3 / 77$ & 1 & & & & & 36 - suspect data (low) \\
\hline 1 & $1 / 3 / 77$ & 2 & & & & & 35 - suspect data (low) \\
\hline 1 & $1 / 3 / 77$ & 3 & & & & & 38 - suspect data (low) \\
\hline 1 & $1 / 3 / 77$ & 4 & & & & & 35 - suspect data (low) \\
\hline 9 & $1 / 3 / 77$ & 5 & & & & & 35 - suspect data (low) \\
\hline 1 & $1 / 3 / 77$ & 6 & & & & & 37 - suspect data (low) \\
\hline 1 & $1 / 3 / 77$ & 7 & & & & & 35 - suspect data (low) \\
\hline 1 & $1 / 3 / 77$ & 8 & & & & & 38 - suspect data (low) \\
\hline 7 & $1 / 3 / 77$ & 9 & & & & & 35 - suspect data (low) \\
\hline 1 & $1 / 3 / 77$ & 10 & & & & & 36 - suspect data (low) \\
\hline 1 & $1 / 3 / 77$ & 11 & & & & & 35 - suspect data (low) \\
\hline 1 & $1 / 3 / 77$ & 12 & & & & & 36 - suspect data (low) \\
\hline 1 & $2 / 26 / 77$ & 1 & & & & & 60 - suspect data (low) \\
\hline 1 & $2 / 26 / 77$ & 2 & & & & & 61 - suspect data (low) \\
\hline 1 & $2 / 26 / 77$ & 3 & & & & & 61 - suspect data (low) \\
\hline 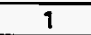 & $2 / 26 / 77$ & 4 & & & & & 61 - suspect data (low) \\
\hline 1 & $2 / 26 / 77$ & 5 & & & & & 61 - suspect data (low) \\
\hline
\end{tabular}

Data obtained from WHC Surveillance Analysis Computer System (SACS), November 12, 1993. 


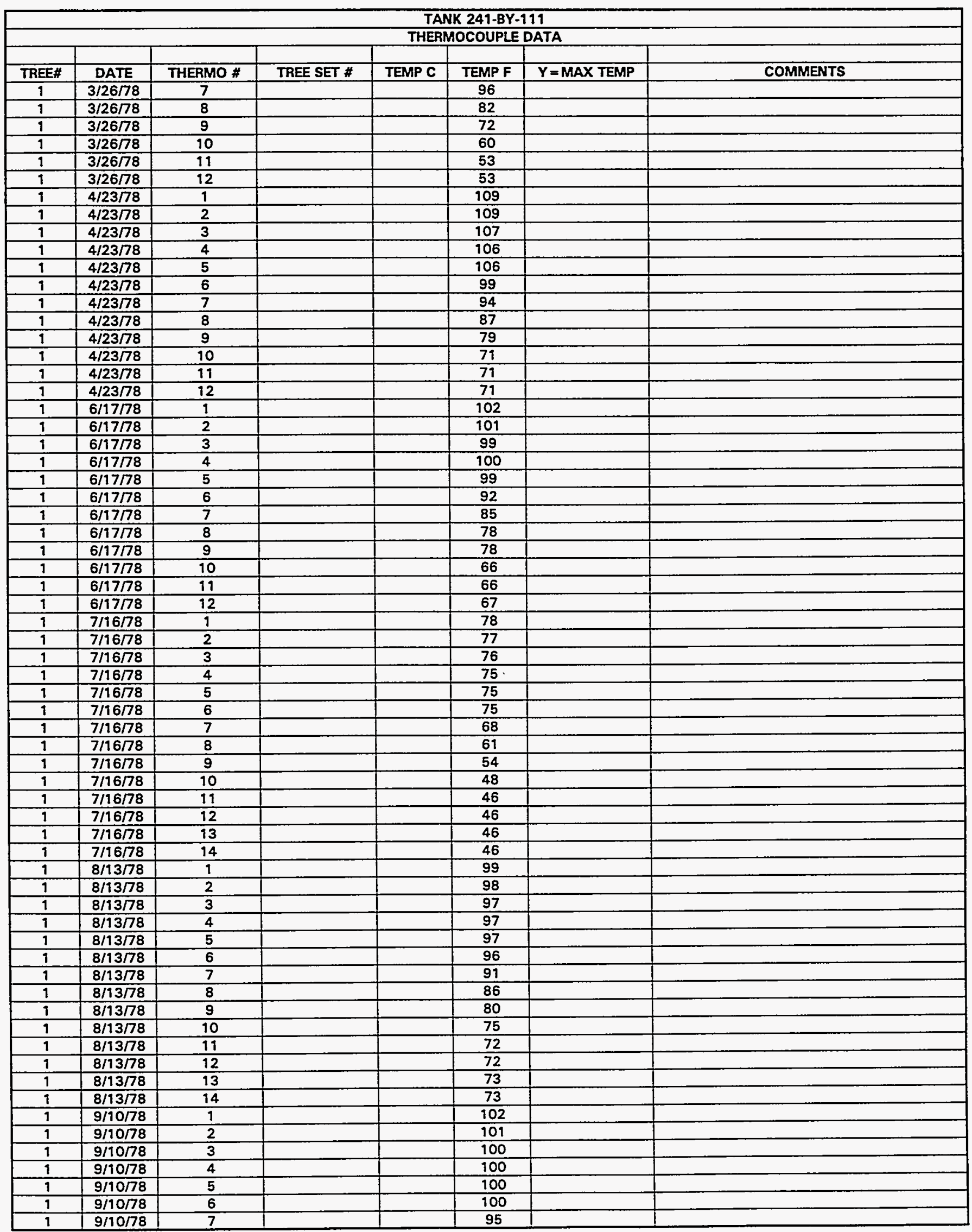

Data obtained from WHC Surveillance Analysis Computer System (SACS), November 12, 1993. 
WHC-SD-WM-ER-312, Rev. 0

\begin{tabular}{|c|c|c|c|c|c|c|c|}
\hline \multicolumn{8}{|c|}{ TANK 241-BY-111 } \\
\hline \multicolumn{8}{|c|}{ THERMOCOUPLE DATA } \\
\hline TREE\# & DATE & THERMO \# & TREE SET \# & TEMP C & TEMPF & $Y=$ MAX TEMP & COMMENTS \\
\hline 1 & $9 / 10 / 78$ & 8 & & & 90 & & \\
\hline 1 & $9 / 10 / 78$ & 9 & & & 84 & & \\
\hline$T$ & $9 / 10 / 78$ & 10 & & & 76 & & \\
\hline 1 & $9 / 10 / 78$ & 11 & & & 75 & & \\
\hline 1 & $9 / 10 / 78$ & 12 & & & 75 & & \\
\hline 1 & $9 / 10 / 78$ & 13 & & & 76 & & \\
\hline 1 & $9 / 10 / 78$ & 14 & & & 76 & & \\
\hline 1 & $10 / 7 / 78$ & 1 & & & 104 & & \\
\hline$T$ & $10 / 7 / 78$ & 2 & & & 104 & & \\
\hline 1 & $10 / 7 / 78$ & 3 & & & 106 & & \\
\hline 1 & $10 / 7 / 78$ & 4 & & & 102 & & \\
\hline$T$ & $10 / 7 / 78$ & 5 & & & 104 & & \\
\hline 1 & $10 / 7 / 78$ & 6 & & & 99 & & \\
\hline 1 & $10 / 7 / 78$ & 7 & & & 98 & & \\
\hline 1 & $10 / 7 / 78$ & 8 & & & 94 & & \\
\hline 1 & $10 / 7 / 78$ & 9 & & & 89 & & \\
\hline 1 & $10 / 7 / 78$ & 10 & & & 84 & & \\
\hline 1 & $10 / 7 / 78$ & 11 & & & 78 & & \\
\hline 1 & $10 / 778$ & 12 & & & 74 & & \\
\hline$\overline{1}$ & $1 / 3 / 79$ & 1 & & & 107 & & \\
\hline 1 & $1 / 3 / 79$ & 2 & & & 105 & & \\
\hline 9 & $1 / 3 / 79$ & 3 & & & 104 & & \\
\hline 1 & $1 / 3 / 79$ & 4 & & & 105 & & \\
\hline 1 & $1 / 3 / 79$ & 5 & & & 104 & & \\
\hline 1 & $1 / 3 / 79$ & 6 & & & 104 & & \\
\hline 1 & $1 / 3 / 79$ & 7 & & & 99 & & \\
\hline 1 & $1 / 3 / 79$ & 8 & & & 94 & & \\
\hline 1 & $1 / 3 / 79$ & 9 & & & 84 & & \\
\hline 1 & $1 / 3 / 79$ & 10 & & & 67 & & \\
\hline 1 & $1 / 3 / 79$ & 11 & & & 57 & & \\
\hline 1 & $1 / 3 / 79$ & 12 & & & 58 & & \\
\hline 1 & $4 / 22 / 79$ & 1 & & & 100 & & \\
\hline 1 & $4 / 22 / 79$ & 2 & & & 99 & & \\
\hline 1 & $4 / 22 / 79$ & 3 & & & 99 & & \\
\hline 1 & $4 / 22 / 79$ & 4 & & & 98 & & \\
\hline 9 & $4 / 22 / 79$ & 5 & & & 98 & & \\
\hline 1 & $4 / 22 / 79$ & 6 & & & 98 & & \\
\hline 1 & $4 / 22 / 79$ & 7 & & & 93 & & \\
\hline 1 & $4 / 22 / 79$ & 8 & & & 84 & & \\
\hline 1 & $4 / 22 / 79$ & 9 & & & 73 & & \\
\hline$\frac{1}{1}$ & $4 / 22 / 7 \theta$ & 10 & & & 61 & & \\
\hline 1 & $4 / 22 / 79$ & 11 & & & 57 & & \\
\hline 1 & $4 / 22 / 79$ & 12 & & & 57 & & \\
\hline 1 & $4 / 22 / 79$ & 13 & & & 57 & & \\
\hline 1 & $4 / 22 / 79$ & 14 & & & 57 & & \\
\hline 1 & $7 / 7 \pi 9$ & 1 & & & 97 & & \\
\hline 9 & $7 / 7 / 79$ & 2 & & & 97 & & \\
\hline 1 & $7 \pi / 79$ & 3 & & & 96 & & \\
\hline 1 & $7 \Pi 779$ & 4 & & & 95 & & \\
\hline 1 & $7 \pi / 79$ & 5 & & & 95 & & \\
\hline 1 & $7 \Pi 7 / 79$ & 6 & & & 95 & & \\
\hline 1 & 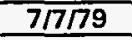 & 7 & & & 89 & & \\
\hline 1 & $7 \pi / 79$ & 8 & & & 84 & & \\
\hline 1 & $7 / 7 / 79$ & 9 & & & 75 & & \\
\hline 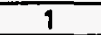 & 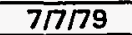 & 10 & & & 70 & & \\
\hline 1 & $7 \Pi 7 / 79$ & 71 & & & 70 & & \\
\hline 1 & $7 / 7 / 79$ & 12 & & & 71 & & \\
\hline 1 & $10 / 13 / 89$ & 1 & 1 & & & $\mathbf{N}$ & $\begin{array}{c}\text { Could not find a manual reading station of } \\
\text { housing. }\end{array}$ \\
\hline 1 & $11 / 3 / 89$ & 1 & 1 & & 82 & $Y$ & $R-1,1^{\circ}$ off bottom of LOW \\
\hline 1 & $11 / 10 / 89$ & 1 & 2 & & 84.5 & $\bar{Y}$ & $\ln$ LOW \\
\hline 1 & $11 / 11 / 89$ & 1 & 1 & & 84.5 & $\bar{Y}$ & K type of LOW on 11-11-89 84.5 \\
\hline 1 & $11 / 18 / 89$ & 1 & 1 & & 85 & $\bar{Y}$ & In LOW Test taken with $K$ \\
\hline$T$ & $11 / 25 / 89$ & 1 & 1 & & 86.1 & $\bar{Y}$ & LOW \\
\hline 1 & $12 / 1 / 89$ & 9 & 1 & & 84.6 & $Y$ & LOW \\
\hline
\end{tabular}

Data obtained from WHC Surveillance Analysis Computer System (SACS), November 12, 1993. 


\begin{tabular}{|c|c|c|c|c|c|c|c|}
\hline \multicolumn{8}{|c|}{ TANK 241-BY-111 } \\
\hline \multicolumn{8}{|c|}{ THERMOCOUPLE DATA } \\
\hline TREE\# & DATE & THERMO \# & TREE SET \# & TEMP C & TEMP F & $Y=$ MAX TEMP & COMMENTS \\
\hline 1 & $12 / 10 / 89$ & 1 & 1 & & 84.6 & $\mathrm{Y}$ & LOW \\
\hline 1 & $12 / 15 / 89$ & 1 & 1 & & 84.2 & $Y$ & LOW \\
\hline 1 & $12 / 23 / 89$ & 1 & 1 & & 81 & $Y$ & \\
\hline$\frac{1}{1}$ & $12 / 29 / 89$ & 1 & 1 & & 86.9 & $\bar{Y}$ & LOW-K-890723679-20 \\
\hline 1 & $1 / 6 / 90$ & 1 & 1 & & 84.4 & $\bar{Y}$ & LOW \\
\hline 1 & $1 / 13 / 90$ & 1 & 1 & & 84.8 & $\bar{Y}$ & K LOW \\
\hline 1 & $1 / 27 / 90$ & 1 & 1 & & 83.2 & $\bar{Y}$ & LOW - K \\
\hline 1 & $2 / 3 / 90$ & 1 & 1 & & 83.7 & $\bar{Y}$ & LOW K-890723579-20 \\
\hline 1 & $2 / 5 / 90$ & 1 & 1 & & 84 & $\bar{Y}$ & LOW K-890723679-20 \\
\hline 1 & $2 / 11 / 90$ & 1 & 1 & & & $N$ & Unable to locate thermocouple. \\
\hline 1 & $3 / 2 / 90$ & 1 & 1 & & 86.7 & $\bar{Y}$ & K-HH-99A-K LOW \\
\hline 1 & $4 / 8 / 90$ & 1 & 1 & & & $\mathbf{N}$ & No data - SSTPE notified \\
\hline 1 & $5 / 5 / 90$ & $\frac{1}{1}$ & 1 & & & $\mathrm{~N}$ & Not taken per PM 90-049 \\
\hline 1 & $6 / 1 / 90$ & 1 & 1 & & & $\mathbf{N}$ & Not taken per PM 90-051 \\
\hline 1 & $8 / 5 / 90$ & 1 & 1 & & 82.7 & $Y$ & LOW \\
\hline 1 & $8 \Pi / 90$ & 1 & 1 & & & $\mathbf{N}$ & LOW to be re-instated after cleanout \\
\hline 1 & $9 / 11 / 90$ & 1 & 1 & & 82 & $\bar{Y}$ & LOW \\
\hline 1 & $10 / 9 / 90$ & 1 & 1 & & 81 & $\bar{Y}$ & LOW \\
\hline$i$ & $10 / 24 / 90$ & 1 & 1 & & 84 & $Y$ & K-HH-99A-K \\
\hline 1 & $10 / 26 / 90$ & 9 & 1 & & 82 & $\bar{Y}$ & $\mathrm{~J}-890523512-20$ \\
\hline 1 & $10 / 30 / 90$ & 1 & 2 & & 78.8 & $\mathrm{Y}$ & K 890723679-64 LOW \\
\hline 1 & $11 / 5 / 90$ & 1 & 1 & & 73.4 & $\bar{Y}$ & $\mathrm{~J} 890523512-20$ \\
\hline 1 & $11 / 14 / 90$ & 1 & 1 & & 77 & $\bar{Y}$ & K 890723679-64 LOW \\
\hline 1 & $11 / 18 / 90$ & 1 & 1 & & 84.3 & $\bar{Y}$ & K 890723697-74 LOW \\
\hline 1 & $11 / 25 / 90$ & 1 & 1 & & 79.1 & $\bar{Y}$ & K-890723679-64 \\
\hline 1 & $12 / 10 / 90$ & 1 & 1 & & 83.4 & $\bar{Y}$ & K 890723679-64 \\
\hline 1 & $12 / 11 / 90$ & 1 & 2 & & 83.4 & $\bar{Y}$ & K 890723679-64 \\
\hline 1 & $12 / 15 / 90$ & 1 & 2 & & 83.5 & $\bar{Y}$ & K LOW \\
\hline 1 & $12 / 22 / 90$ & 1 & 2 & & 75 & $\bar{Y}$ & K 890723679-64 LOW \\
\hline 1 & $12 / 30 / 90$ & 1 & 1 & & 83.8 & $\bar{Y}$ & K 890723679-64 LOW 6 readings on LOW? \\
\hline 1 & $1 / 6 / 91$ & 1 & 1 & & & $\mathbf{N}$ & K LOW Probe not in well - called shift office. \\
\hline 1 & $1 / 9 / 91$ & 1 & 1 & & 77.1 & $Y$ & K 890723679-64 LOW \\
\hline 1 & $1 / 13 / 91$ & 1 & 1 & & 84.9 & $Y$ & K 890723679-64 \\
\hline 1 & $1 / 19 / 91$ & 1 & 1 & & 83.4 & $\bar{Y}$ & K HH99A (Low) \\
\hline 1 & $1 / 29 / 91$ & 1 & 1 & & 84.2 & $\mathbf{Y}$ & K HH-99A LOW \\
\hline 1 & $2 / 3 / 91$ & 1 & 1 & & 83 & $\bar{Y}$ & K Ser \#896723679-64 LOW \\
\hline 1 & $2 / 9 / 91$ & 1 & 1 & & 84.4 & $\bar{Y}$ & K 890723679-64 LOW \\
\hline 1 & $2 / 16 / 91$ & 1 & 1 & & 85 & $\bar{Y}$ & K-890723679-64 LOW \\
\hline 1 & $2 / 23 / 91$ & 1 & 1 & & 84.8 & $Y$ & K Ser\#896723679-64 LOW \\
\hline 1 & $3 / 3 / 91$ & 1 & 1 & & 83 & $Y$ & LOW 890923679.64 \\
\hline 1 & $3 / 9 / 91$ & 1 & 1 & & 84.4 & $Y$ & K 890723679-20 \\
\hline 1 & $3 / 17 / 91$ & 1 & 1 & & 84.4 & $Y$ & LOW 890923679.64 \\
\hline 1 & $3 / 23 / 91$ & 1 & 1 & & 84.1 & $Y$ & LOW 890923679.64 \\
\hline 1 & $3 / 30 / 91$ & 1 & 1 & & 83.7 & $\bar{Y}$ & LOW 890923679-64 \\
\hline 1 & $4 / 7 / 91$ & 1 & 1 & & 86.5 & $\mathbf{Y}$ & LOW $890623567-12$ \\
\hline 1 & $4 / 14 / 91$ & 1 & 1 & & & $N$ & K LOW No readings obtained-LOW full of water \\
\hline 1 & $4 / 20 / 91$ & 1 & 1 & & 85.8 & $Y$ & K 890723633-64 LOW \\
\hline 1 & $4 / 28 / 91$ & 9 & 1 & & 85.6 & $\bar{Y}$ & LOW $890623567-12$ \\
\hline 1 & $5 / 5 / 91$ & 1 & 1 & & 84.4 & $\bar{Y}$ & K 690723633-64 LOW \\
\hline 1 & $5 / 12 / 91$ & 1 & 1 & & 83.5 & $\bar{Y}$ & K 890723679-20 LOW \\
\hline 1 & $5 / 18 / 91$ & 1 & 1 & & 84.8 & $\bar{Y}$ & K 890723633-64 LOW \\
\hline 1 & $5 / 25 / 91$ & 1 & 1 & & 85.7 & $\bar{Y}$ & K 890723633-64 LOW \\
\hline 1 & $6 / 2 / 91$ & 1 & 1 & & 85.7 & $\bar{Y}$ & K 890623567-12 LOW \\
\hline 1 & $6 / 9 / 91$ & 1 & 1 & & 85.8 & $Y$ & K 890623567-12 LOW \\
\hline 1 & $6 / 15 / 91$ & 1 & 1 & & 84.4 & $\bar{Y}$ & K 890723633.64 LOW \\
\hline 1 & $6 / 23 / 91$ & 1 & 1 & & & $\mathbf{N}$ & No reading. Work Order written. \\
\hline 1 & $6 / 29 / 91$ & 1 & 1 & & 81.8 & $Y$ & K 890623567-12LOW \\
\hline 1 & $7 / 6 / 91$ & 1 & 1 & & 84.5 & $\bar{Y}$ & K 890623567-12 LOW \\
\hline 1 & $7 / 13 / 91$ & 1 & 1 & & 84.2 & $\mathbf{Y}$ & K 890623567-12 LOW \\
\hline 1 & $7 / 20 / 91$ & 1 & 1 & & 83.9 & 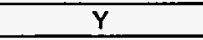 & K 890623567.12 LOW \\
\hline 1 & $7 / 27 / 91$ & 1 & 1 & & 92.2 & $Y$ & K 890725813 \\
\hline 1 & $8 / 3 / 91$ & 1 & 1 & & 85.4 & $\mathbf{Y}$ & K 890723679.20 LOW \\
\hline 1 & $8 / 10 / 91$ & 1 & 1 & & 85 & $Y$ & K 890723699.20 LOW \\
\hline 1 & $8 / 18 / 91$ & 1 & 1 & & 85.5 & $Y$ & K 890623567-12 LOW \\
\hline 1 & $8 / 24 / 91$ & 1 & 1 & & 83.3 & $Y$ & K 910226246-56 LOW \\
\hline
\end{tabular}

Data obtained from WHC Surveillance Analysis Computer System (SACS), November 12, 1993. 
WHC-SD-WM-ER-312, Rev. 0

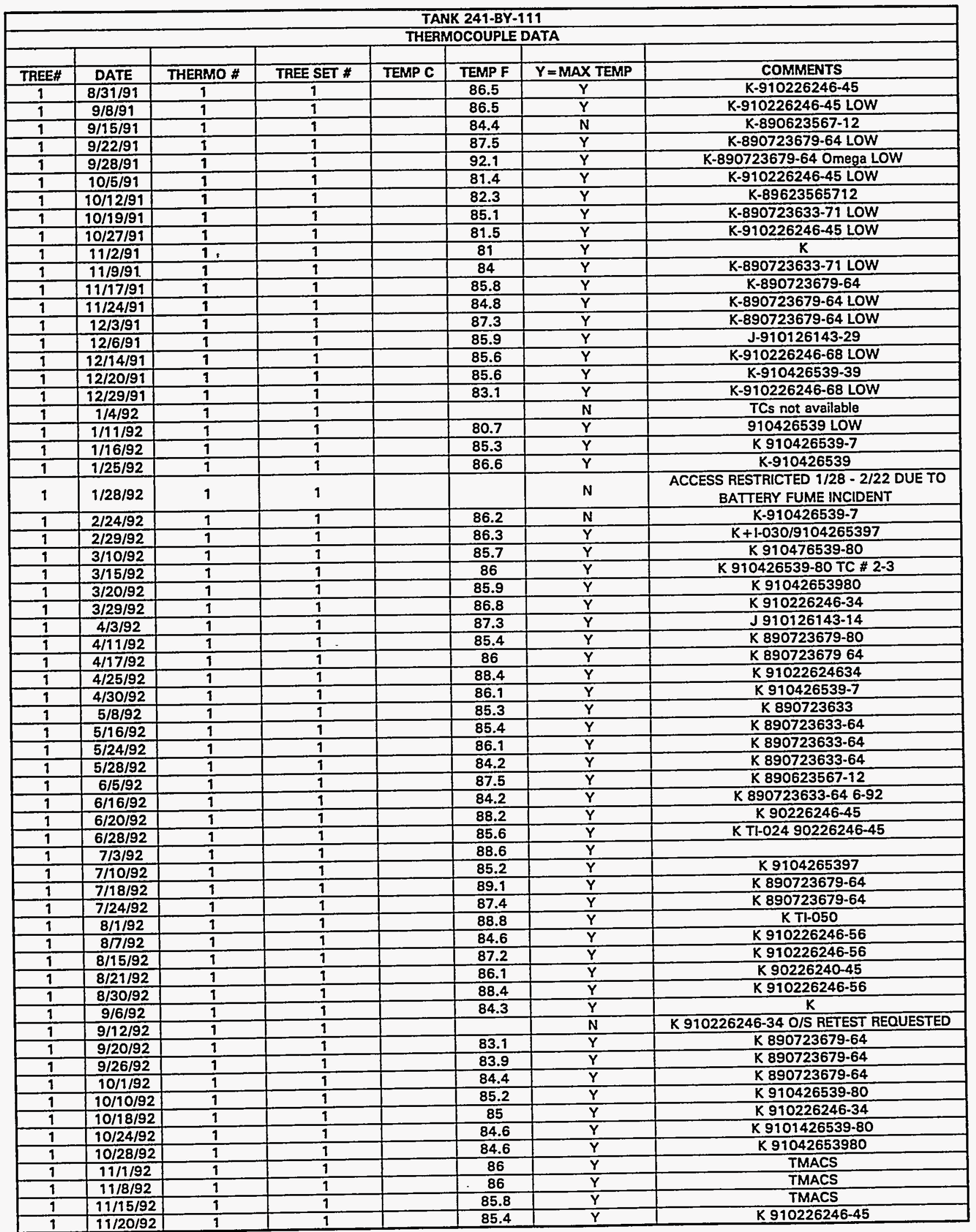

Data obtained from WHC Surveillance Analysis Computer System (SACS), November 12, 1993. 


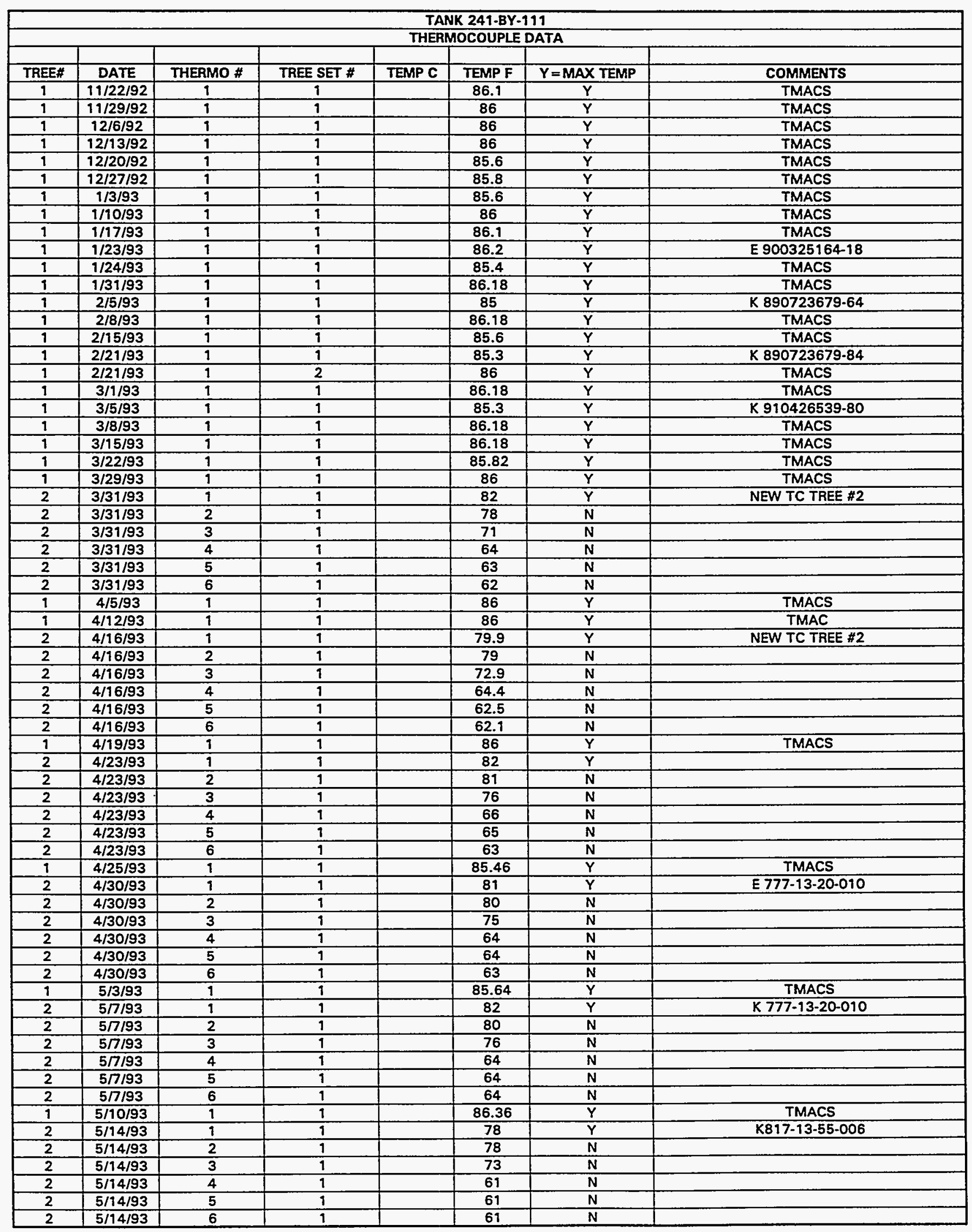

Data obtained from WHC Surveillance Analysis Computer System (SACS), November 12, 1993. 
WHC-SD-WM-ER-312, Rev. 0

\begin{tabular}{|c|c|c|c|c|c|c|c|}
\hline & & & & & 241-BY & & \\
\hline & & & & THER & OCOUPLI & ATA & \\
\hline TREE\# & DATE & THERMO \# & TREE SET \# & TEMP C & TEMP F & $Y=M A X X$ TEMP & COMMENTS \\
\hline 1 & $5 / 17 / 93$ & 1 & 1 & & 85.64 & $Y$ & TMACS \\
\hline 2 & $5 / 21 / 93$ & 1 & 1 & & 81 & $\bar{Y}$ & $K$ \\
\hline 2 & $5 / 21 / 93$ & 2 & 9 & & 79 & $\mathbf{N}$ & \\
\hline 2 & $5 / 21 / 93$ & 3 & 1 & & 75 & $\mathbf{N}$ & \\
\hline 2 & $5 / 21 / 93$ & 4 & 1 & & 67 & $\mathbf{N}$ & \\
\hline 2 & $5 / 21 / 93$ & 5 & 1 & & 67 & $\mathbf{N}$ & \\
\hline 2 & $5 / 21 / 93$ & 6 & 1 & & 67 & $\mathbf{N}$ & \\
\hline 1 & $5 / 24 / 93$ & 1 & 1 & & 86.36 & $\mathbf{Y}$ & TMACS \\
\hline 2 & $5 / 27 / 93$ & $T$ & 1 & & 81 & $\overline{\mathbf{Y}}$ & K 817-13.55.006 \\
\hline 2 & $5 / 27 / 93$ & 2 & 1 & & 79 & $\mathbf{N}$ & \\
\hline 2 & $5 / 27 / 93$ & 3 & 1 & & 74 & $\bar{N}$ & \\
\hline 2 & $5 / 27 / 93$ & 4 & 1 & & 64 & $\mathbf{N}$ & \\
\hline 2 & $5 / 27 / 93$ & 5 & 1 & & 63 & $\mathbf{N}$ & \\
\hline 2 & $5 / 27 / 93$ & 6 & 1 & & 64 & $\mathbf{N}$ & \\
\hline 7 & $5 / 31 / 93$ & 9 & 9 & & 86 & $\bar{Y}$ & TMACS \\
\hline 2 & $6 / 4 / 93$ & 1 & 1 & & 83 & $\overline{\mathbf{Y}}$ & K817.13.55.006 \\
\hline 2 & $6 / 4 / 93$ & 2 & 1 & & 80 & $\mathbf{N}$ & \\
\hline 2 & $6 / 4 / 93$ & 3 & 1 & & 76 & $\mathbf{N}$ & \\
\hline 2 & $6 / 4 / 93$ & 4 & 1 & & 65 & $\mathbf{N}$ & \\
\hline 2 & $6 / 4 / 93$ & 5 & 1 & & 65 & $\bar{N}$ & \\
\hline 2 & $6 / 4 / 93$ & 6 & 1 & & 65 & $\mathbf{N}$ & \\
\hline 1 & $6 / 7 / 93$ & 1 & 1 & & 85.64 & $\bar{Y}$ & TMACS \\
\hline 1 & $6 / 14 / 93$ & 1 & 1 & & 85.64 & $\bar{Y}$ & \\
\hline 2 & $6 / 14 / 93$ & 1 & 1 & & 81.4 & $\bar{Y}$ & K 817-13-55-001 \\
\hline 2 & $6 / 14 / 93$ & 2 & 1 & & 79.6 & $\bar{N}$ & \\
\hline 2 & $6 / 14 / 93$ & 3 & 1 & & 75.1 & $\mathbf{N}$ & \\
\hline 2 & $6 / 14 / 93$ & 4 & 1 & & 66.6 & $\bar{N}$ & \\
\hline 2 & $6 / 14 / 93$ & 5 & 1 & & 64.9 & $\mathbf{N}$ & \\
\hline 2 & $6 / 14 / 93$ & 6 & 1 & & 65.6 & $\mathbf{N}$ & \\
\hline 2 & $6 / 18 / 93$ & 1 & 2 & & 81 & $\bar{Y}$ & $K 777-13-20-10$ \\
\hline 2 & $6 / 18 / 93$ & 2 & 2 & & 78 & $N$ & \\
\hline 2 & $6 / 18 / 93$ & 3 & 2 & & 74 & $\mathbf{N}$ & \\
\hline 2 & $6 / 18 / 93$ & 4 & 2 & & 65 & $\mathbf{N}$ & \\
\hline 2 & $6 / 18 / 93$ & 5 & 2 & & 65 & $\mathbf{N}$ & \\
\hline 2 & $6 / 18 / 93$ & 6 & 2 & & 66 & $\mathbf{N}$ & \\
\hline 1 & $6 / 21 / 93$ & 1 & 9 & & 85.64 & $\bar{Y}$ & TMACS \\
\hline 1 & $6 / 27 / 93$ & 1 & 1 & & 87.08 & $\bar{Y}$ & TMACS. REQ. MAN. RDGS. \\
\hline 9 & $7 / 3 / 93$ & 7 & 1 & & 85.9 & $\bar{Y}$ & KTI-024 \\
\hline 2 & $7 / 3 / 93$ & 1 & 9 & & 81.7 & $\bar{Y}$ & K T1.024 \\
\hline 2 & $7 / 3 / 93$ & 2 & 1 & & 79.9 & $\mathbf{N}$ & \\
\hline 2 & $7 / 3 / 93$ & 3 & 1 & & 76.2 & $\mathbf{N}$ & \\
\hline 2 & $7 / 3 / 93$ & 4 & 9 & & 67.7 & $\mathbf{N}$ & \\
\hline 2 & $7 / 3 / 93$ & 5 & 1 & & 67.8 & $\mathbf{N}$ & \\
\hline 2 & $7 / 3 / 93$ & 6 & 1 & & 67.9 & $\mathrm{~N}$ & \\
\hline 1 & $7 / 5 / 93$ & 1 & 1 & & 88.16 & $\bar{Y}$ & TMACS \\
\hline 1 & $7 / 12 / 93$ & 1 & 1 & & 87.44 & $\bar{Y}$ & TMACS \\
\hline 2 & $7 / 12 / 93$ & 1 & 1 & & 84.02 & $\bar{Y}$ & TMACS \\
\hline 2 & $7 / 12 / 93$ & 2 & 1 & & 81.5 & $N$ & \\
\hline 2 & $7 / 12 / 93$ & 3 & 9 & & 78.08 & $\mathbf{N}$ & \\
\hline 2 & $7 / 12 / 93$ & 4 & 1 & & 69.98 & $\mathbf{N}$ & \\
\hline 2 & $7 / 12 / 93$ & 5 & 1 & & 70.52 & $N$ & \\
\hline 2 & $7 / 12 / 93$ & 6 & 1 & & 70.7 & $\mathbf{N}$ & \\
\hline 9 & $7 / 19 / 93$ & 1 & 1 & & 88.16 & $\bar{Y}$ & TMACS \\
\hline 2 & $7 / 19 / 93$ & 1 & 1 & & 83.66 & $\mathbf{Y}$ & TMACS \\
\hline 2 & $7 / 19 / 93$ & 2 & 1 & & 81.32 & $\mathrm{~N}$ & \\
\hline 2 & $7 / 19 / 93$ & 3 & 1 & & 77.9 & $\mathbf{N}$ & \\
\hline 2 & $7 / 19 / 93$ & 4 & 1 & & 70.34 & $\mathbf{N}$ & \\
\hline 2 & $7 / 19 / 93$ & 5 & 1 & & 70.88 & $\mathbf{N}$ & \\
\hline 2 & $7 / 19 / 93$ & 6 & 1 & & 71.42 & $\mathbf{N}$ & \\
\hline 1 & $7 / 26 / 93$ & 9 & 1 & & 87.44 & $\bar{Y}$ & TMACS \\
\hline 2 & $7 / 26 / 93$ & 1 & 1 & & 83.12 & $\bar{Y}$ & TMACS \\
\hline 2 & $7 / 26 / 93$ & 2 & 1 & & 80.96 & $N$ & \\
\hline 2 & $7 / 26 / 93$ & 3 & 1 & & 77.72 & $\mathrm{~N}$ & \\
\hline 2 & $7 / 26 / 93$ & 4 & 1 & & 70.34 & $\mathbf{N}$ & \\
\hline 2 & $7 / 26 / 93$ & 5 & 1 & & 71.06 & $\mathbf{N}$ & \\
\hline
\end{tabular}

Data obtained from WHC Surveillance Analysis Computer System (SACS), November 12, 1993. 


\begin{tabular}{|c|c|c|c|c|c|c|c|}
\hline \multicolumn{8}{|c|}{ TANK 241-BY-111 } \\
\hline \multicolumn{8}{|c|}{ THERMOCOUPLE DATA } \\
\hline TREE\# & DATE & THERMO \# & TREE SET \# & TEMP C & TEMP F & $Y=M A X$ TEMP & COMMENTS \\
\hline 2 & $7 / 26 / 93$ & 6 & 1 & & 71.42 & $N$ & \\
\hline 1 & $8 / 2 / 93$ & 1 & 1 & & 87.62 & $\bar{Y}$ & TMACS \\
\hline 2 & $8 / 2 / 93$ & 1 & 1 & & 83.48 & $\bar{Y}$ & TMACS \\
\hline 2 & $8 / 2 / 93$ & 2 & 1 & & 81.14 & 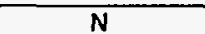 & \\
\hline 2 & $8 / 2 / 93$ & 3 & 1 & & 78.08 & $\mathbf{N}$ & \\
\hline 2 & $8 / 2 / 93$ & 4 & 1 & & 70.7 & $\mathbf{N}$ & \\
\hline 2 & $8 / 2 / 93$ & 5 & 1 & & 71.6 & $\mathbf{N}$ & \\
\hline 2 & $8 / 2 / 93$ & 6 & 1 & & 72.14 & $\mathbf{N}$ & \\
\hline$\frac{1}{1}$ & $8 / 9 / 93$ & 1 & 1 & & 87.8 & $\bar{Y}$ & TMACS \\
\hline 2 & $8 / 9 / 93$ & 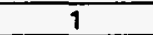 & 9 & & 83.48 & $\bar{Y}$ & TMACS \\
\hline 2 & $8 / 9 / 93$ & 2 & 1 & & 81.68 & $\bar{N}$ & \\
\hline 2 & $8 / 9 / 93$ & 3 & 1 & & 78.8 & $\mathbf{N}$ & \\
\hline 2 & $8 / 9 / 93$ & 4 & 9 & & 71.96 & $N$ & \\
\hline 2 & $8 / 9 / 93$ & 5 & 1 & & 72.5 & $\mathrm{~N}$ & \\
\hline 2 & $8 / 9 / 93$ & 6 & 1 & & 73.04 & $\mathbf{N}$ & \\
\hline 1 & $8 / 15 / 93$ & 1 & 1 & & 87.44 & $\mathrm{Y}$ & tmacs \\
\hline 2 & $8 / 15 / 93$ & 1 & 1 & & 83.84 & $Y$ & tmacs \\
\hline 2 & $8 / 15 / 93$ & 2 & 1 & & 81.86 & $\mathbf{N}$ & \\
\hline 2 & $8 / 15 / 93$ & 3 & 9 & & 78.98 & $\mathbf{N}$ & \\
\hline 2 & $8 / 15 / 93$ & 4 & 1 & & 72.32 & $\bar{N}$ & \\
\hline 2 & $8 / 15 / 93$ & 5 & $\frac{1}{1}$ & & 72.86 & $\mathbf{N}$ & \\
\hline 2 & $8 / 15 / 93$ & 6 & 1 & & 73.22 & $N$ & \\
\hline 1 & $8 / 23 / 93$ & 1 & 1 & & 87.44 & $\bar{Y}$ & TMACS \\
\hline 2 & $8 / 23 / 93$ & 1 & 1 & & 83.12 & $\bar{Y}$ & TMACS \\
\hline 2 & $8 / 23 / 93$ & 2 & 1 & & 81.5 & $N$ & \\
\hline 2 & $8 / 23 / 93$ & 3 & 1 & & 78.26 & N & \\
\hline 2 & $8 / 23 / 93$ & 4 & 1 & & 71.96 & $\mathbf{N}$ & \\
\hline 2 & $8 / 23 / 93$ & 5 & 1 & & 72.68 & $\bar{N}$ & \\
\hline 2 & $8 / 23 / 93$ & 6 & 1 & & 73.22 & $\mathbf{N}$ & \\
\hline 1 & $8 / 26 / 93$ & 1 & 1 & & 86.01 & $\mathrm{Y}$ & K TYPE \\
\hline 2 & $8 / 26 / 93$ & 1 & 1 & & 83.3 & $\bar{Y}$ & K TYPE \\
\hline 2 & $8 / 26 / 93$ & 2 & 1 & & 81.1 & $\mathbf{N}$ & \\
\hline 2 & $8 / 26 / 93$ & 3 & 9 & & 78.6 & $\bar{N}$ & \\
\hline 2 & $8 / 26 / 93$ & 4 & 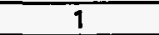 & & 72.1 & $\mathrm{~N}$ & \\
\hline 2 & $8 / 26 / 93$ & 5 & 1 & & 72.7 & $\bar{N}$ & \\
\hline 2 & $8 / 26 / 93$ & 6 & 1 & & 73.2 & $N$ & \\
\hline 2 & $8 / 28 / 93$ & 1 & 1 & & 83.8 & $\mathbf{N}$ & K89072363371 \\
\hline 2 & $8 / 28 / 93$ & 2 & $\frac{1}{1}$ & & 79.1 & $\mathrm{~N}$ & \\
\hline 2 & $8 / 28 / 93$ & 3 & 1 & & 84.7 & $\bar{Y}$ & \\
\hline 2 & $8 / 28 / 93$ & 4 & 1 & & 84.6 & $\mathbf{N}$ & \\
\hline 2 & $8 / 28 / 93$ & 5 & 7 & & 83 & $N$ & \\
\hline 2 & $8 / 28 / 93$ & 6 & 1 & & 79.5 & $\mathbf{N}$ & \\
\hline 1 & $8 / 30 / 93$ & 1 & 1 & & 87.62 & $\bar{Y}$ & TMACS \\
\hline 2 & $8 / 30 / 93$ & 1 & 1 & & 83.84 & $Y$ & TMACS \\
\hline 2 & $8 / 30 / 93$ & 2 & 9 & & 81.68 & $\mathbf{N}$ & \\
\hline 2 & $8 / 30 / 93$ & 3 & 1 & & 79.16 & $\mathbf{N}$ & \\
\hline 2 & $8 / 30 / 93$ & 4 & 1 & & 73.04 & $N$ & \\
\hline 2 & $8 / 30 / 93$ & 5 & 1 & & 73.58 & $\mathbf{N}$ & \\
\hline 2 & $8 / 30 / 93$ & 6 & 1 & & 73.94 & $\mathrm{~N}$ & \\
\hline 1 & $9 / 6 / 93$ & 1 & 1 & & 87.26 & $\bar{Y}$ & TMACS \\
\hline 2 & $9 / 6 / 93$ & 1 & 1 & & 83.48 & $Y$ & TMACS \\
\hline 2 & $9 / 6 / 93$ & 2 & 1 & & 81.14 & $\mathbf{N}$ & \\
\hline 2 & $9 / 6 / 93$ & 3 & 1 & & 78.8 & $\mathbf{N}$ & \\
\hline 2 & $9 / 6 / 93$ & 4 & 1 & & 73.04 & $N$ & \\
\hline 2 & $9 / 6 / 93$ & 5 & 1 & & 73.4 & $\mathbf{N}$ & \\
\hline 2 & $9 / 6 / 93$ & 6 & 1 & & 74.3 & $\mathbf{N}$ & \\
\hline 1 & $9 / 72 / 93$ & 1 & 1 & & 87.26 & $\bar{Y}$ & TMACS \\
\hline 2 & $9 / 12 / 93$ & 1 & 1 & & 83.84 & $Y$ & TMACS \\
\hline 2 & $9 / 12 / 93$ & 2 & 1 & & 82.04 & $\bar{N}$ & \\
\hline 2 & $9 / 12 / 93$ & 3 & 1 & & 79.34 & $\mathrm{~N}$ & \\
\hline 2 & $9 / 12 / 93$ & 4 & 1 & & 73.94 & $N$ & \\
\hline 2 & $9 / 12 / 93$ & 5 & 1 & & 74.66 & $\mathrm{~N}$ & \\
\hline 2 & $9 / 12 / 93$ & 6 & 1 & & 74.84 & $\mathbf{N}$ & \\
\hline$\frac{1}{1}$ & $9 / 19 / 93$ & 9 & 1 & & 87.62 & $\bar{Y}$ & TMACS \\
\hline 2 & $9 / 19 / 93$ & 1 & 1 & & 83.48 & $Y$ & TMACS \\
\hline
\end{tabular}

Data obtained from WHC Surveillance Analysis Computer System (SACS), November 12, 1993. 


\begin{tabular}{|c|c|c|c|c|c|c|c|}
\hline \multicolumn{8}{|c|}{ TANK 241-BY-111 } \\
\hline \multicolumn{8}{|c|}{ THERMOCOUPLE DATA } \\
\hline TREE\# & DATE & THERMO \# & TREE SET \# & TEMP C & TFMP F & $Y=$ MAX TEMP & \\
\hline 2 & $9 / 19 / 93$ & 2 & 1 & & 81.68 & $N$ & 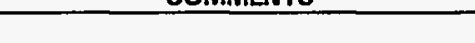 \\
\hline 2 & $9 / 19 / 93$ & 3 & 9 & & 79.34 & $\mathbf{N}$ & \\
\hline 2 & $9 / 19 / 93$ & 4 & 1 & & 73.76 & $\mathbf{N}$ & \\
\hline 2 & $9 / 19 / 93$ & 5 & 1 & & 74.48 & $\mathbf{N}$ & \\
\hline 2 & $9 / 19 / 93$ & 6 & 1 & & 74.66 & $\mathbf{N}$ & \\
\hline 1 & $9 / 26 / 93$ & 1 & 1 & & 87.26 & $\mathbf{Y}$ & TMACS \\
\hline 2 & $9 / 26 / 93$ & 1 & 1 & & 83.84 & $\bar{Y}$ & TMACS \\
\hline 2 & $9 / 26 / 93$ & 2 & 1 & & 81.68 & $\mathbf{N}$ & \\
\hline 2 & $9 / 26 / 93$ & 3 & 1 & & 79.52 & $\mathbf{N}$ & \\
\hline 2 & $9 / 26 / 93$ & 4 & 1 & & 74.12 & $N$ & \\
\hline 2 & $9 / 26 / 93$ & 5 & 1 & & 74.84 & $\mathbf{N}$ & \\
\hline 2 & $9 / 26 / 93$ & 6 & 1 & & 75.38 & $N$ & \\
\hline 1 & $10 / 3 / 93$ & 1 & 1 & & 87.62 & $\bar{Y}$ & TMACS \\
\hline 2 & $10 / 3 / 93$ & 1 & 1 & & 83.84 & $\bar{Y}$ & TMACS \\
\hline 2 & $10 / 3 / 93$ & 2 & 1 & & 82.04 & $\mathbf{N}$ & \\
\hline 2 & $10 / 3 / 93$ & 3 & 1 & & 79.7 & $\mathbf{N}$ & \\
\hline 2 & $10 / 3 / 93$ & 4 & 1 & & 74.66 & $\mathrm{~N}$ & \\
\hline 2 & $10 / 3 / 93$ & 5 & 1 & & 75.2 & $\mathbf{N}$ & \\
\hline 2 & $10 / 3 / 93$ & 6 & 1 & & 75.74 & $N$ & \\
\hline 1 & $10 / 10 / 93$ & 1 & 1 & & 87.8 & $\bar{Y}$ & TMACS \\
\hline 2 & $10 / 10 / 93$ & 1 & 1 & & 84.02 & $\bar{Y}$ & TMACS \\
\hline 2 & $10 / 10 / 93$ & 2 & 1 & & 82.4 & $\mathbf{N}$ & \\
\hline 2 & $10 / 10 / 93$ & 3 & 1 & & 80.24 & $\mathrm{~N}$ & \\
\hline 2 & $10 / 10 / 93$ & 4 & 1 & & 74.84 & $\mathrm{~N}$ & \\
\hline 2 & $10 / 10 / 93$ & 5 & 1 & & 75.38 & $\mathbf{N}$ & \\
\hline 2 & $10 / 10 / 93$ & 6 & 1 & & 76.1 & $\mathbf{N}$ & \\
\hline 1 & $10 / 13 / 93$ & 1 & 1 & & 84.7 & $\bar{Y}$ & K 910226246-45 \\
\hline 2 & $10 / 13 / 93$ & 1 & 1 & & 80.8 & $\bar{Y}$ & $K 910226246-45$ \\
\hline 2 & $10 / 13 / 93$ & 2 & 1 & & 79.5 & $N$ & \\
\hline 2 & $10 / 13 / 93$ & 3 & 1 & & 76.8 & $N$ & \\
\hline 2 & $10 / 13 / 93$ & 4 & 1 & & 71.5 & $\bar{N}$ & \\
\hline 2 & $10 / 13 / 93$ & 5 & 1 & & 71.4 & $\mathrm{~N}$ & \\
\hline 2 & $10 / 13 / 93$ & 6 & 1 & & 71.5 & $\mathrm{~N}$ & \\
\hline 1 & $10 / 19 / 93$ & 1 & 1 & & 87.4 & $\bar{Y}$ & TMACS \\
\hline 2 & $10 / 19 / 93$ & 1 & 1 & & 83.7 & $\bar{Y}$ & TMACS \\
\hline 2 & $10 / 19 / 93$ & 2 & 1 & & 81.7 & $\mathbf{N}$ & \\
\hline 2 & $10 / 19 / 93$ & 3 & 1 & & 79.9 & $N$ & \\
\hline 2 & $10 / 19 / 93$ & 4 & 1 & & 74.7 & $\bar{N}$ & \\
\hline 2 & $10 / 19 / 93$ & 5 & 1 & & 75.2 & $\mathrm{~N}$ & \\
\hline 2 & $10 / 19 / 93$ & 6 & 1 & & 75.7 & $N$ & \\
\hline 2 & $10 / 25 / 93$ & 1 & 9 & & 83.3 & $\bar{Y}$ & TMACS \\
\hline 2 & $10 / 25 / 93$ & 2 & 1 & & 81.68 & $\mathbf{N}$ & \\
\hline 2 & $10 / 25 / 93$ & 3 & 1 & & 79.7 & $N$ & \\
\hline 2 & $10 / 25 / 93$ & 4 & 1 & & 74.48 & $\mathbf{N}$ & \\
\hline 2 & $10 / 25 / 93$ & 5 & 1 & & 75.2 & $N$ & \\
\hline 2 & $10 / 25 / 93$ & 6 & 1 & & 75.74 & $\mathbf{N}$ & \\
\hline 1 & $10 / 26 / 93$ & 1 & 1 & & 84.5 & $Y$ & K 910226246-45 RETAKE FOR $10 / 24$ \\
\hline 2 & $10 / 26 / 93$ & 1 & 1 & & 80.6 & $\bar{Y}$ & $K 910226246-45$ \\
\hline 2 & $10 / 26 / 93$ & 2 & 1 & & 79.2 & $\mathbf{N}$ & \\
\hline 2 & $10 / 26 / 93$ & 3 & 1 & & 76.7 & $\mathbf{N}$ & \\
\hline 2 & $10 / 26 / 93$ & 4 & 1 & & 71.4 & $\mathbf{N}$ & \\
\hline 2 & $10 / 26 / 93$ & 5 & 1 & & 71.2 & $\mathbf{N}$ & \\
\hline 2 & $10 / 26 / 93$ & 6 & 1 & & 71.3 & $\mathrm{~N}$ & \\
\hline 1 & $11 / 1 / 93$ & 1 & 1 & & 87.26 & $y$ & TMACS \\
\hline 2 & $11 / 1 / 93$ & 1 & 1 & & 83.84 & $\bar{Y}$ & TMACS \\
\hline 2 & $11 / 1 / 93$ & 2 & 1 & & 81.86 & $\mathbf{N}$ & \\
\hline 2 & $11 / 1 / 93$ & 3 & 1 & & 79.88 & $N$ & \\
\hline 2 & $11 / 1 / 93$ & 4 & 1 & & 74.66 & $N$ & \\
\hline 2 & $11 / 1 / 93$ & 5 & 1 & & 75.38 & $N$ & \\
\hline 2 & $11 / 1 / 93$ & 6 & 1 & & 75.74 & $\mathbf{N}$ & \\
\hline 1 & $11 / 8 / 93$ & 1 & 1 & & 87.26 & $Y$ & TMACS \\
\hline 2 & $11 / 8 / 93$ & 1 & 1 & & 83.84 & $Y$ & TMACS \\
\hline 2 & $11 / 8 / 93$ & 2 & 1 & & 82.22 & $\bar{N}$ & \\
\hline 2 & $11 / 8 / 93$ & 3 & 1 & & 79.7 & $\mathbf{N}$ & \\
\hline 2 & $11 / 8 / 93$ & 4 & 1 & & 74.66 & $N$ & \\
\hline
\end{tabular}

Data obtained from WHC Surveillance Analysis Computer System (SACS), November 12, 1993. 
WHC-SD-WM-ER-312, Rev. 0

TANK 241-BY-111

\begin{tabular}{|c|c|c|c|c|c|c|c|}
\hline \multicolumn{9}{|c|}{ TANK 241-BY-111 } \\
\hline & & & & & \\
\hline TREE\# & DATE & THERMO \# & TREE SET \# & TEMP C & TEMP F & Y=MAX TEMP & COMMENTS \\
\hline 2 & $11 / 8 / 93$ & 5 & 1 & & 75.2 & $\mathrm{~N}$ & \\
\hline 2 & $11 / 8 / 93$ & 6 & 1 & & 75.56 & $\mathrm{~N}$ & \\
\hline
\end{tabular}

Data obtained from WHC Surveillance Analysis Computer System (SACS), November 12, 1993. 


\begin{tabular}{|c|c|c|c|c|c|c|c|}
\hline \multicolumn{8}{|c|}{ TANK 241-BY-112 } \\
\hline \multicolumn{8}{|c|}{ THERMOCOUPLE DATA } \\
\hline TREE\# & DATE & THERMO \# & TREE SET \# & TEMP C & TEMP F & $Y=M A X$ TEMP & COMMENTS \\
\hline 1 & $11 / 12 / 73$ & 1 & & 67 & 152.6 & & raw data - assumed degree C \\
\hline 1 & $11 / 12 / 73$ & 2 & & 66 & 150.8 & & raw data - assumed degree C \\
\hline 1 & $11 / 12 / 73$ & 3 & & 86 & 186.8 & & raw data - assumed degree C \\
\hline 1 & $11 / 12 / 73$ & 4 & & 82 & 179.6 & & raw data - assumed degree C \\
\hline 1 & $11 / 12 / 73$ & 5 & & 84 & 183.2 & & raw data - assumed degree C \\
\hline 1 & $11 / 12 / 73$ & 6 & & 62 & 143.6 & & raw data - assumed degree C \\
\hline 1 & $11 / 12 / 73$ & 7 & & 71 & 159.8 & & raw data - assumed degree C \\
\hline 1 & $11 / 12 / 73$ & 8 & & 87 & 188.6 & & raw data - assumed degree C \\
\hline 1 & $11 / 12 / 73$ & 9 & & 91 & 195.8 & & raw data - assumed degree C \\
\hline 1 & $11 / 12 / 73$ & 10 & & 84 & 183.2 & & raw data - assumed degree C \\
\hline 1 & $11 / 12 / 73$ & 19 & & 98 & 208.4 & & raw dats - assumed degree C \\
\hline 1 & $11 / 12 / 73$ & 12 & & 86 & 186.8 & & raw data - assumed degree C \\
\hline 1 & $11 / 21 / 73$ & 1 & & 104 & 219.2 & & raw data - assumed degree C \\
\hline 1 & $11 / 21 / 73$ & 2 & & 107 & 224.6 & & raw data - assumed degree C \\
\hline 1 & $11 / 21 / 73$ & 3 & & 110 & 230 & & raw data - assumed degree C \\
\hline 1 & $11 / 21 / 73$ & 4 & & 110 & 230 & & raw data - assumed degree $C$ \\
\hline 9 & $11 / 21 / 73$ & 5 & & 111 & 231.8 & & raw data - assumed degree C \\
\hline 1 & $11 / 21 / 73$ & 6 & & 113 & 235.4 & & raw data - assumed degree C \\
\hline 1 & $11 / 21 / 73$ & 7 & & 113 & 235.4 & & raw data - assumed degree $C$ \\
\hline$\frac{1}{1}$ & $11 / 21 / 73$ & 8 & & 113 & 235.4 & & raw data - assumed degree $C$ \\
\hline 1 & $11 / 21 / 73$ & 9 & & 113 & 235.4 & & raw data - assumed degree $\mathrm{C}$ \\
\hline 1 & $11 / 21 / 73$ & 10 & & 113 & 235.4 & & raw data - assumed degree C \\
\hline 1 & $11 / 21 / 73$ & 11 & & 113 & 235.4 & & raw data - assumed degree C \\
\hline 1 & $11 / 21 / 73$ & 12 & & 103 & 217.4 & & raw data - assumed degree C \\
\hline 1 & $12 / 10 / 73$ & 1 & & 104 & 219.2 & & raw data - assumed degree C \\
\hline 1 & $12 / 10 / 73$ & 2 & & 106 & 222.8 & & raw data - assumed degree C \\
\hline 1 & $12 / 10 / 73$ & 3 & & 108 & 226.4 & & raw data - assumed degree $C$ \\
\hline 1 & $12 / 10 / 73$ & 4 & & 111 & 231.8 & & raw data - assumed degree $C$ \\
\hline 1 & $12 / 10 / 73$ & 5 & & 113 & 235.4 & & raw data - assumed degree $C$ \\
\hline 1 & $12 / 10 / 73$ & 6 & & 115 & 239 & & raw data - assumed degree C \\
\hline 1 & $12 / 10 / 73$ & 7 & & 116 & 240.8 & & raw data - assumed degree C \\
\hline 1 & $12 / 10 / 73$ & 8 & & 116 & 240.8 & & raw data - assumed degree $C$ \\
\hline 1 & $12 / 10 / 73$ & 9 & & 116 & 240.8 & & raw data - assumed degree $C$ \\
\hline 1 & $12 / 10 / 73$ & 10 & & 116 & 240.8 & & raw data - assumed degree C \\
\hline 1 & $12 / 10 / 73$ & 11 & & 115 & 239 & & raw data - assumed degree $\mathrm{C}$ \\
\hline 1 & $12 / 10 / 73$ & 12 & & 103 & 217.4 & & raw data - assumed degree $\mathrm{C}$ \\
\hline 1 & $12 / 17 / 73$ & 1 & & 101 & 213.8 & & raw data - assumed degree C \\
\hline 1 & $12 / 17 / 73$ & 2 & & 105 & 221 & & raw data - assumed degree $C$ \\
\hline 1 & $12 / 17 / 73$ & 3 & & 108 & 226.4 & & raw data - assumed degree $C$ \\
\hline 1 & $12 / 17 / 73$ & 4 & & 110 & 230 & & raw data - assumed degree $C$ \\
\hline 1 & $12 / 17 / 73$ & 5 & & 112 & 233.6 & & raw data - assumed degree $C$ \\
\hline 1 & $12 / 17 / 73$ & 6 & & 113 & 235.4 & & raw data - assumed degree $C$ \\
\hline 1 & $12 / 17 / 73$ & 7 & & 113 & 235.4 & & raw data - assumed degree $\mathrm{C}$ \\
\hline 1 & $12 / 17 / 73$ & 8 & & 113 & 235.4 & & raw data - assumed degree $\mathrm{C}$ \\
\hline 1 & $12 / 17 / 73$ & 9 & & 113 & 235.4 & & raw data - assumed degree $C$ \\
\hline 1 & $12 / 17 / 73$ & 10 & & 113 & 235.4 & & raw data - assumed degree C \\
\hline 1 & $12 / 17 / 73$ & 11 & & 112 & 233.6 & & raw data - assumed degree C \\
\hline 1 & $12 / 17 / 73$ & 12 & & 102 & 215.6 & & raw data - assumed degree C \\
\hline 1 & $12 / 20 / 73$ & 1 & & 101 & 213.8 & & raw data - assumed degree $C$ \\
\hline 1 & $12 / 20 / 73$ & 2 & & 105 & 221 & & raw data - assumed degree C \\
\hline 1 & $12 / 20 / 73$ & 3 & & 108 & 226.4 & & raw data - assumed degree C \\
\hline 1 & $12 / 20 / 73$ & 4 & & 110 & 230 & & raw data - assumed degree C \\
\hline 1 & $12 / 20 / 73$ & 5 & & 111 & 231.8 & & raw data - assumed degree C \\
\hline 1 & $12 / 20 / 73$ & 6 & & 113 & 235.4 & & raw data - assumed degree $C$ \\
\hline$\frac{1}{1}$ & $12 / 20 / 73$ & 7 & & 113 & 235.4 & & raw data - assumed degree $C$ \\
\hline 1 & $12 / 20 / 73$ & 8 & & 113 & 235.4 & & raw data - assumed degree $C$ \\
\hline 1 & $12 / 20 / 73$ & 9 & & 113 & 235.4 & & raw data - assumed degree $C$ \\
\hline 1 & $12 / 20 / 73$ & 10 & & 113 & 235.4 & & raw data - assumed degree C \\
\hline 1 & $12 / 20 / 73$ & 11 & & 113 & 235.4 & & raw data - assumed degree C \\
\hline 1 & $12 / 20 / 73$ & $\overline{12}$ & & 102 & 215.6 & & raw data - assumed degree C \\
\hline 1 & $12 / 20 / 73$ & 15 & & 110 & 230 & & raw data - assumed degree C \\
\hline 1 & $1 / 4 / 74$ & 1 & & 100 & 212 & & raw data - assumed degree $C$ \\
\hline 1 & $9 / 4 / 74$ & 2 & & 104 & 219.2 & & raw data - assumed degree C \\
\hline 1 & $1 / 4 / 74$ & 3 & & 108 & 226.4 & & raw data - assumed degree C \\
\hline 1 & $1 / 4 / 74$ & 4 & & 110 & 230 & & raw data - assumed degree C \\
\hline
\end{tabular}

Data obtained from WHC Surveiliance Analysis Computer System (SACS), November 12, 1993. 


\begin{tabular}{|c|c|c|c|c|c|c|c|}
\hline \multicolumn{8}{|c|}{ TANK 241-BY-112 } \\
\hline \multicolumn{8}{|c|}{ THERMOCOUPLE DATA } \\
\hline TREE\# & DATE & THERMO \# & TREE SET \# & TEMP C & TEMP F & $Y=$ MAX TEMP & COMMENTS \\
\hline 1 & $1 / 4 / 74$ & 5 & MEL SEI & 111 & 231.8 & & raw data-assumed degree $C$ \\
\hline 1 & $1 / 4 / 74$ & 6 & & 113 & 235.4 & & raw data - assumed degree C \\
\hline 1 & $1 / 4 / 74$ & 7 & & 113 & 235.4 & & raw data - assumed degree C \\
\hline 1 & $1 / 4 / 74$ & 8 & & 113 & 235.4 & & raw data - assumed degree $C$ \\
\hline 1 & $1 / 4 / 74$ & 9 & & 113 & 235.4 & & raw data - assumed degree C \\
\hline 1 & $1 / 4 / 74$ & 10 & & 113 & 235.4 & & raw data - assumed degree C \\
\hline 1 & $1 / 4 \Pi 74$ & 11 & & 112 & 233.6 & & raw data - assumed degree C \\
\hline 1 & $1 / 4 / 74$ & 12 & & 103 & 217.4 & & raw data - assumed degree C \\
\hline 1 & $8 / 5 / 74$ & 1 & & 68 & 154.40 & & raw data - assumed degree C \\
\hline 1 & $8 / 5 / 74$ & 2 & & 71 & 159.80 & & raw data - assumed degree $C$ \\
\hline 1 & $8 / 5 / 74$ & 3 & & 73 & 163.40 & & raw data - assumed degree C \\
\hline 1 & $8 / 5 / 74$ & 4 & & 71 & 159.80 & & raw data - assumed degree C \\
\hline 1 & $8 / 5 / 74$ & 5 & & 67 & 152.60 & & raw data - assumed degree C \\
\hline 1 & $8 / 5 / 74$ & 6 & & 62 & 143.60 & & raw data - assumed degree C \\
\hline 1 & $8 / 5 / 74$ & 7 & & 61 & 141.80 & & raw data - assumed degree C \\
\hline 1 & $8 / 5 / 74$ & 8 & & 60 & 140.00 & & raw data - assumed degree C \\
\hline 1 & $8 / 5 / 74$ & 9 & & 60 & 140.00 & & raw data - assumed degree C \\
\hline 1 & $8 / 5 / 74$ & 10 & & 60 & 140.00 & & raw data - assumed degree $C$ \\
\hline 1 & $8 / 5 / 74$ & 11 & & 60 & 140.00 & & raw data - assumed degree C \\
\hline 1 & $8 / 5 / 74$ & 12 & & 57 & 134.60 & & raw data - assumed degree C \\
\hline 1 & $8 / 15 / 74$ & 1 & & 68 & 154.40 & & raw data - assumed degree C \\
\hline 1 & $8 / 15 / 74$ & 2 & & 69.5 & 157.10 & & raw data - assumed degree C \\
\hline 1 & $8 / 15 / 74$ & 3 & & 71.5 & 160.70 & & raw data - assumed degree $C$ \\
\hline 1 & $8 / 15 / 74$ & 4 & & 70 & 158.00 & & raw data - assumed degree C \\
\hline 1 & $8 / 15 / 74$ & 5 & & 66 & 150.80 & & raw data - assumed degree C \\
\hline 1 & $8 / 75 / 74$ & 6 & & 62 & 143.60 & & raw data - assumed degree C \\
\hline 1 & $8 / 15 / 74$ & 7 & & 61 & 141.80 & & raw data - assumed degree $C$ \\
\hline 1 & $8 / 15 / 74$ & 8 & & 60 & 140.00 & & raw data - assumed degree $C$ \\
\hline 1 & $8 / 15 / 74$ & 9 & & 59 & 138.20 & & raw data - assumed degree $C$ \\
\hline 1 & $8 / 15 / 74$ & 10 & & 53.5 & 128.30 & & raw data - assumed degree $\mathrm{C}$ \\
\hline 1 & $8 / 15 / 74$ & 11 & & 49 & 120.20 & & raw data - assumed degree C \\
\hline 1 & $8 / 15 / 74$ & 12 & & 48.5 & 119.30 & & raw data - assumed degree C \\
\hline 9 & $9 / 12 / 74$ & 1 & & 70.5 & 158.90 & & raw data - assumed degree C \\
\hline 1 & $9 / 12 / 74$ & 2 & & 71 & 159.80 & & raw data - assumed degree C \\
\hline 1 & $9 / 12 / 74$ & 3 & & 71 & 159.80 & & raw data - assumed degree $C$ \\
\hline 1 & $9 / 12 / 74$ & 4 & & 69.5 & 157.10 & & raw data - assumed degree C \\
\hline$\frac{1}{1}$ & $9 / 12 / 74$ & 5 & & 66.5 & 151.70 & & raw data - assumed degree C \\
\hline 1 & $9 / 12 / 74$ & 6 & & 62.5 & 144.50 & & raw data - assumed degree $\mathrm{C}$ \\
\hline 1 & $9 / 12 / 74$ & 7 & & 58 & 136.40 & & raw data - assumed degree C \\
\hline 1 & $9 / 92 / 74$ & 8 & & 57 & 134.60 & & raw data - assumed degree C \\
\hline 1 & $9 / 12 / 74$ & 9 & & 56 & 132.80 & & raw data - assumed degree C \\
\hline 1 & $9 / 12 / 74$ & 10 & & 54 & 129.20 & & raw data - assumed degree $C$ \\
\hline 1 & $9 / 12 / 74$ & 11 & & 50 & 122.00 & & raw data - assumed degree C \\
\hline 1 & $9 / 12 / 74$ & 12 & & 46 & 114.80 & & raw data - assumed degree C \\
\hline 1 & $10 / 8 / 74$ & 1 & & 70 & 158.00 & & raw data - assumed degree C \\
\hline 1 & $10 / 8 / 74$ & 2 & & 71 & 159.80 & & raw data - assumed degree $C$ \\
\hline 1 & $10 / 8 / 74$ & 3 & & 70 & 158.00 & & raw data - assumed degree $C$ \\
\hline 1 & $10 / 8 / 74$ & 4 & & 68 & 154.40 & & raw data - assumed degree $C$ \\
\hline 1 & $10 / 8 / 74$ & 5 & & 63.5 & 146.30 & & raw data - assumed degree C \\
\hline 1 & $10 / 8 / 74$ & 6 & & 58 & 136.40 & & Iraw data - assumed degree C \\
\hline 1 & $10 / 8 / 74$ & 7 & & 54 & 129.20 & & raw data - assumed degree $C$ \\
\hline 1 & $10 / 8 / 74$ & 8 & & 53.5 & 128.30 & & raw data - assumed degree $\mathrm{C}$ \\
\hline 1 & $10 / 8 / 74$ & 9 & & 53 & 127.40 & & raw data - assumed degree $C$ \\
\hline 1 & $10 / 8 / 74$ & 10 & & 53 & 127.40 & & raw data - assumed degree C \\
\hline 1 & $10 / 8 / 74$ & 11 & & 52.5 & 126.50 & & raw data - assumed degree $C$ \\
\hline 1 & $10 / 8 / 74$ & 12 & & 49 & 120.20 & & raw data - assumed degree $\mathrm{C}$ \\
\hline 1 & $11 / 12 / 74$ & 1 & & 70 & 158.00 & & raw data - assumed degree C \\
\hline 1 & $11 / 12 / 74$ & 2 & & 69 & 156.20 & & raw data - assumed degree $C$ \\
\hline 1 & $11 / 12 / 74$ & 3 & & 66 & 150.80 & & raw data - assumed degree C \\
\hline 1 & $11 / 12 / 74$ & 4 & & 61 & 141.80 & & raw data - assumed degree $C$ \\
\hline 1 & $11 / 12 / 74$ & 5 & & 56 & 132.80 & & raw data - assumed degree $C$ \\
\hline 1 & $11 / 12 / 74$ & 6 & & 52 & 125.60 & & raw data - assumed degree $C$ \\
\hline 1 & $19 / 92 / 74$ & 7 & & 51 & 123.80 & & raw data - assumed degree C \\
\hline 1 & $11 / 12 / 74$ & 8 & & 50 & 122.00 & & raw data - assumed degree C \\
\hline 1 & $11 / 12 / 74$ & 9 & & 49 & 120.20 & & Iraw data - assumed degree $C$ \\
\hline
\end{tabular}

Data obtained from WHC Surveillance Analysis Computer System (SACS), November 12, 1993. 
WHC-SD-WM-ER-312, Rev. 0

\begin{tabular}{|c|c|c|c|c|c|c|c|}
\hline \multicolumn{8}{|c|}{ TANK 241-BY-112 } \\
\hline & & & & THERI & OCOUPLE & ATA & \\
\hline TREE\# & DATE & THERMO \# & TREE SET \# & TEMP C & TEMP F & $Y=$ MAX TEMP & COMMENTS \\
\hline 1 & $11 / 12 / 74$ & 10 & & 49 & 120.20 & & raw data - assumed degree C \\
\hline 1 & $11 / 12 / 74$ & 11 & & 43 & 109.40 & & raw data - assumed degree C \\
\hline 1 & $11 / 12 / 74$ & 12 & & 28 & 82.40 & & raw data - assumed degree C \\
\hline 9 & $11 / 26 / 74$ & 1 & & 68 & 154.40 & & raw data - assumed degree $C$ \\
\hline 1 & $11 / 26 / 74$ & 2 & & 67 & 152.60 & & raw data - assumed degree C \\
\hline 1 & $11 / 26 / 74$ & 3 & & 64 & 147.20 & & raw data - assumed degree $C$ \\
\hline 1 & $11 / 26 / 74$ & 4 & & 59 & 138.20 & & raw data - assumed degree C \\
\hline 1 & $11 / 26 / 74$ & 5 & & 54 & 129.20 & & raw data - assumed degree $C$ \\
\hline 1 & $11 / 26 / 74$ & 6 & & 49 & 120.20 & & raw data - assumed degree C \\
\hline 1 & $11 / 26 / 74$ & 7 & & 48 & 118.40 & & raw data - assumed degree C \\
\hline 1 & $11 / 26 / 74$ & 8 & & 48 & 118.40 & & raw data - assumed degree C \\
\hline 1 & $11 / 26 / 74$ & 9 & & 48 & 118.40 & & raw data - assumed degree C \\
\hline 1 & $11 / 26 / 74$ & 10 & & 48 & 118.40 & & raw data - assumed degree C \\
\hline 1 & $11 / 26 / 74$ & 11 & & 44 & 111.20 & & raw data - assumed degree C \\
\hline 9 & $11 / 26 / 74$ & 12 & & 27 & 80.60 & & raw data - assumed degree $C$ \\
\hline 1 & $12 / 11 / 74$ & 1 & & 68 & 154.40 & & raw data - assumed degree C \\
\hline 1 & $12 / 11 / 74$ & 2 & & 67 & 152.60 & & raw data - assumed degree $C$ \\
\hline 1 & $12 / 11 / 74$ & 3 & & 64 & 147.20 & & raw data - assumed degree C \\
\hline 1 & $12 / 11 / 74$ & 4 & & 57 & 134.60 & & raw data - assumed degree C \\
\hline 1 & $12 / 11 / 74$ & 5 & & 52 & 125.60 & & raw data - assumed degree $C$ \\
\hline 1 & $12 / 11 / 74$ & 6 & & 48 & 118.40 & & raw data - assumed degree C \\
\hline$\frac{1}{1}$ & $12 / 11 / 74$ & 7 & & 47 & 116.60 & & raw data - assumed degree C \\
\hline 1 & $12 / 11 / 74$ & 8 & & 48 & 118.40 & & raw data - assumed degree C \\
\hline 1 & $12 / 91 / 74$ & 9 & & 48 & 118.40 & & raw data - assumed degree $\mathrm{C}$ \\
\hline 1 & $12 / 11 / 74$ & 10 & & 47 & 116.60 & & raw data - assumed degree C \\
\hline 1 & $12 / 11 / 74$ & 11 & & 43 & 109.40 & & raw data - assumed degree C \\
\hline 1 & $12 / 11 / 74$ & 12 & & 28 & 82.40 & & raw data - assumed degree $\mathrm{C}$ \\
\hline 1 & $12 / 11 / 74$ & 13 & & 29 & 84.20 & & raw data - assumed degree C \\
\hline 1 & $12 / 11 / 74$ & 14 & & 30 & 86.00 & & raw data - assumed degree C \\
\hline 1 & $12 / 11 / 74$ & 15 & & 28 & 82.40 & & raw data - assumed degree C \\
\hline 1 & $12 / 11 / 74$ & 16 & & 26 & 78.80 & & raw data - assumed degree C \\
\hline 1 & $12 / 17 / 74$ & 1 & & 68 & 154.40 & & raw data - assumed degree C \\
\hline 1 & $12 / 17 / 74$ & 2 & & 67 & 152.60 & & raw data - assumed degree $\mathrm{C}$ \\
\hline 1 & $12 / 17 / 74$ & 3 & & 63 & 145.40 & & raw data - assumed degree $\mathrm{C}$ \\
\hline 1 & $12 / 17 / 74$ & 4 & & 54 & 129.20 & & raw data - assumed degree C \\
\hline 1 & $12 / 17 / 74$ & 5 & & 52 & 125.60 & & raw data - assumed degree $\mathrm{C}$ \\
\hline 1 & $12 / 17 / 74$ & 6 & & 48 & 118.40 & & raw data - assumed degree C \\
\hline 1 & $12 / 17 / 74$ & 7 & & 47 & 116.60 & & raw data - assumed degree C \\
\hline 1 & $12 / 17 / 74$ & 8 & & 47 & 116.60 & & raw data - assumed degree C \\
\hline 1 & $12 / 17 / 74$ & 9 & & 47 & 116.60 & & raw data - assumed degree C \\
\hline 1 & $12 / 17 / 74$ & 10 & & 47 & 116.60 & & raw data - assumed degree $\mathrm{C}$ \\
\hline 1 & $12 / 17 / 74$ & 11 & & 41 & 105.80 & & raw data - assumed degree $C$ \\
\hline 1 & $12 / 17 / 74$ & 12 & & 28 & 82.40 & & raw data - assumed degree C \\
\hline 1 & $12 / 31 / 74$ & 1 & & 67 & 152.60 & & raw data - assumed degree C \\
\hline 1 & $12 / 31 / 74$ & 2 & & 66 & 150.80 & & raw data - assumed degree $C$ \\
\hline 9 & $12 / 31 / 74$ & 3 & & 62 & 143.60 & & raw data - assumed degree C \\
\hline 1 & $12 / 31 / 74$ & 4 & & 56 & 132.80 & & raw data - assumed degree C \\
\hline 1 & $12 / 31 / 74$ & 5 & & 51 & 123.80 & & raw data - assumed degree C \\
\hline 1 & $12 / 31 / 74$ & 6 & & 47 & 116.60 & & raw data - assumed degree C \\
\hline 1 & $12 / 31 / 74$ & 7 & & 47 & 116.60 & & raw data - assumed degree C \\
\hline 1 & $12 / 31 / 74$ & 8 & & 46 & 114.80 & & raw data - assumed degree C \\
\hline 1 & $12 / 31 / 74$ & 9 & & 47 & 116.60 & & raw data - assumed degree C \\
\hline 1 & $12 / 31 / 74$ & 10 & & 46 & 114.80 & & raw data - assumed degree C \\
\hline 1 & $12 / 31 / 74$ & 11 & & 39 & 102.20 & & raw deta - assumed degree C \\
\hline 1 & $12 / 31 / 74$ & 12 & & 28 & 82.40 & & raw data - assumed degree C \\
\hline 1 & $1 / 8 / 75$ & 1 & & 66 & 150.80 & & raw data - assumed degree C \\
\hline 1 & $1 / 8 / 75$ & 2 & & 65 & 149.00 & & raw data - assumed degree C \\
\hline 1 & $1 / 8 / 75$ & 3 & & 62 & 143.60 & & raw data - assumed degree C \\
\hline 9 & $1 / 8 / 75$ & 4 & & 55 & 131.00 & & raw data - assumed degree C \\
\hline 1 & $1 / 8 / 75$ & 5 & & 50 & 122.00 & & raw data - assumed degree C \\
\hline 1 & $1 / 8 / 75$ & 6 & & 46 & 114.80 & & raw data - assumed degree C \\
\hline 1 & $1 / 8 / 75$ & 7 & & 43 & 109.40 & & raw data - assumed degree C \\
\hline 1 & $1 / 8 / 75$ & 8 & & 46 & 114.80 & & raw data - assumed degree $C$ \\
\hline 1 & $1 / 8 / 75$ & 9 & & 45 & 113.00 & & raw data - assumed degree C \\
\hline 1 & $1 / 8 / 75$ & 10 & & 45 & 113.00 & & raw data - assumed degree C \\
\hline
\end{tabular}

Data obtained from WHC Surveillance Analysis Computer System (SACS), November 12, 1993. 


\begin{tabular}{|c|c|c|c|c|c|c|c|}
\hline \multicolumn{8}{|c|}{ TANK 241-BY-112 } \\
\hline \multicolumn{8}{|c|}{ THERMOCOUPLE DATA } \\
\hline TREE\# & DATE & THERMO \# & TREE SET \# & TEMP C & TEMPF & $Y=$ MAX TEMP & COMMENTS \\
\hline 1 & $1 / 8 / 75$ & 11 & & 40 & 104.00 & & raw data - assumed degree $C$ \\
\hline 1 & $1 / 8 / 75$ & 12 & & 28 & 82.40 & & raw data - assumed degree C \\
\hline 1 & $1 / 21 / 75$ & 1 & & 65 & 149.00 & & raw data - assumed degree C \\
\hline 1 & $1 / 21 / 75$ & 2 & & 64 & 147.20 & & raw data - assumed degree C \\
\hline 1 & $1 / 21 / 75$ & 3 & & 60 & 140.00 & & raw data - assumed degree $\mathrm{C}$ \\
\hline 1 & $1 / 21 / 75$ & 4 & & 54 & 129.20 & & raw data - assumed degree C \\
\hline 1 & $1 / 21 / 75$ & 5 & & 48 & 118.40 & & raw data - assumed degree $C$ \\
\hline 1 & $1 / 21 / 75$ & 6 & & 45 & 113.00 & & raw data - assumed degree C \\
\hline 1 & $1 / 21 / 75$ & 7 & & 44 & 111.20 & & raw data - assumed degree C \\
\hline 1 & $1 / 21 / 75$ & 8 & & 44 & 111.20 & & raw data - assumed degree $C$ \\
\hline 1 & $1 / 21 / 75$ & 9 & & 45 & 113.00 & & raw data - assumed degree C \\
\hline 1 & $1 / 21 / 75$ & 10 & & 44 & 111.20 & & raw data - assumed degree C \\
\hline 1 & $1 / 21 / 75$ & 11 & & 39 & 102.20 & & raw data - assumed degree $C$ \\
\hline 1 & $1 / 21 / 75$ & 12 & & 27 & 80.60 & & raw data - assumed degree C \\
\hline 1 & $1 / 21 / 75$ & 13 & & 28 & 82.40 & & raw data - assumed degree C \\
\hline 1 & $1 / 21 / 75$ & 14 & & 29 & 84.20 & & raw data - assumed degree C \\
\hline 1 & $1 / 28 / 75$ & 1 & & 54 & 129.20 & & raw data - assumed degree C \\
\hline 1 & $1 / 28 / 75$ & 2 & & 56 & 132.80 & & raw data - assumed degree C \\
\hline 1 & $1 / 28 / 75$ & 3 & & 54 & 129.20 & & raw data - assumed degree C \\
\hline 1 & $1 / 28 / 75$ & 4 & & 51 & 123.80 & & raw data - assumed degree C \\
\hline 1 & $1 / 28 / 75$ & 5 & & 44 & 111.20 & & raw data - assumed degree $C$ \\
\hline 1 & $1 / 28 / 75$ & 6 & & 43 & 109.40 & & raw data - assumed degree C \\
\hline 1 & $1 / 28 / 75$ & 7 & & 44 & 111.20 & & raw data - assumed degree C \\
\hline 1 & $1 / 28 / 75$ & 8 & & 44 & 111.20 & & raw data - assumed degree C \\
\hline 1 & $1 / 28 / 75$ & 9 & & 45 & 113.00 & & raw data - assumed degree C \\
\hline 1 & $1 / 28 / 75$ & 10 & & 44 & 111.20 & & raw data - assumed degree $C$ \\
\hline 1 & $1 / 28 / 75$ & 11 & & 38 & 100.40 & & raw data - assumed degree C \\
\hline 1 & $1 / 28 / 75$ & 12 & & 38 & 100.40 & & raw data - assumed degree C \\
\hline 1 & $2 / 12 / 75$ & 1 & & 64 & 147.20 & & raw data - assumed degree C \\
\hline 1 & $2 / 12 / 75$ & 2 & & 64 & 147.20 & & raw data - assumed degree C \\
\hline 1 & $2 / 12 / 75$ & 3 & & 54 & 129.20 & & raw data - assumed degree C \\
\hline 1 & $2 / 12 / 75$ & 4 & & 54 & 129.20 & & raw data - assumed degree C \\
\hline 1 & $2 / 12 / 75$ & 5 & & 46 & 114.80 & & raw data - assumed degree C \\
\hline 1 & $2 / 12 / 75$ & 6 & & 48 & 118.40 & & raw data - assumed degree C \\
\hline 1 & $2 / 12 / 75$ & 7 & & 45 & 113.00 & & raw data - assumed degree $\mathrm{C}$ \\
\hline 1 & $2 / 12 / 75$ & 8 & & 46 & 114.80 & & raw data - assumed degree $C$ \\
\hline 1 & $2 / 12 / 75$ & 9 & & 46 & 114.80 & & raw data - assumed degree C \\
\hline 1 & $2 / 12 / 75$ & 10 & & 44 & 111.20 & & raw data - assumed degree C \\
\hline 1 & $2 / 12 / 75$ & 11 & & 38 & 100.40 & & raw data - assumed degree $C$ \\
\hline 1 & $2 / 12 / 75$ & 12 & & 27 & 80.60 & & raw data - assumed degree C \\
\hline 1 & $2 / 27 / 75$ & 1 & & 62 & 143.60 & & raw data - assumed degree C \\
\hline 1 & $2 / 27 / 75$ & 2 & & 61 & 141.80 & & raw data - assumed degree C \\
\hline 1 & $2 / 27 / 75$ & 3 & & 57 & 134.60 & & raw data - assumed degree C \\
\hline 1 & $2 / 27 / 75$ & 4 & & 51 & 123.80 & & raw data - assumed degree C \\
\hline 1 & $2 / 27 / 75$ & 5 & & 45 & 113.00 & & raw data - assumed degree C \\
\hline 1 & $2 / 27 / 75$ & 6 & & 42 & 107.60 & & raw data - assumed degree C \\
\hline 1 & $2 / 27 / 75$ & 7 & & 42 & 107.60 & & raw data - assumed degree C \\
\hline 1 & $2 / 27 / 75$ & 8 & & 42 & 107.60 & & raw data - assumed degree C \\
\hline 1 & $2 / 27 / 75$ & 9 & & 42 & 107.60 & & raw data - assumed degree C \\
\hline 1 & $2 / 27 / 75$ & 10 & & 41.5 & 106.70 & & raw data - assumed degree C \\
\hline 1 & $2 / 27 / 75$ & 11 & & 37 & 98.60 & & raw data - assumed degree C \\
\hline 1 & $2 / 27 / 75$ & 12 & & 27 & 80.60 & & raw data - assumed degree $C$ \\
\hline 1 & $3 / 18 / 75$ & 1 & & 61 & 141.80 & & raw data - assumed degree C \\
\hline 1 & $3 / 18 / 75$ & 2 & & 61 & 141.80 & & raw data - assumed degree $C$ \\
\hline 1 & $3 / 18 / 75$ & 3 & & 56 & 132.80 & & raw data - assumed degree C \\
\hline 1 & $3 / 18 / 75$ & 4 & & 50 & 122.00 & & raw data - assumed degree $C$ \\
\hline 1 & $3 / 18 / 75$ & 5 & & 45 & 113.00 & & raw data - essumed degree $C$ \\
\hline 1 & $3 / 18 / 75$ & 6 & & 42 & 107.60 & & raw data - assumed degree C \\
\hline 1 & $3 / 18 / 75$ & 7 & & 41 & 105.80 & & raw data - assumed degree $C$ \\
\hline 1 & $3 / 18 / 75$ & 8 & & 41 & 105.80 & & raw data - assumed degree $\mathrm{C}$ \\
\hline 1 & $3 / 18 / 75$ & 9 & & 42 & 107.60 & & raw data - assumed degree $C$ \\
\hline 1 & $3 / 18 / 75$ & 10 & & 41 & 105.80 & & raw data - assumed degree $C$ \\
\hline 1 & $3 / 18 / 75$ & 91 & & 38 & 100.40 & & raw data - assumed degree $C$ \\
\hline 1 & $3 / 18 / 75$ & 12 & & 28 & 82.40 & & raw data - assumed degree C \\
\hline 1 & $3 / 18 / 75$ & 13 & & 28 & 82.40 & & raw data - assumed degree $C$ \\
\hline
\end{tabular}

Data obtained from WHC Surveillance Analysis Computer System (SACS), November 12, 1993. 


\begin{tabular}{|c|c|c|c|c|c|c|c|}
\hline \multicolumn{8}{|c|}{ TANK 241-BY-112 } \\
\hline & & & & & & & \\
\hline TREE\# & DATE & THERMO \# & TREE SET \# & TEMP C & TEMP F & $Y=$ MAX TEMP & COMMENTS \\
\hline 1 & $3 / 18 / 75$ & 14 & & 28 & 82.40 & & raw data - assumed degree C \\
\hline 1 & $4 / 8 / 75$ & 1 & & 63 & 145.40 & & raw data - assumed degree C \\
\hline 1 & $4 / 8 / 75$ & 2 & & 60 & 140.00 & & raw data - assumed degree C \\
\hline 1 & $4 / 8 / 75$ & 3 & & 56 & 132.80 & & raw data - assumed degree C \\
\hline 1 & $4 / 8 / 75$ & 4 & & 50 & 122.00 & & raw data - assumed degree C \\
\hline 1 & $4 / 8 / 75$ & 5 & & 44 & 111.20 & & raw data - assumed degree $C$ \\
\hline 1 & $4 / 8 / 75$ & 6 & & 41 & 105.80 & & raw data - assumed degree C \\
\hline 1 & $4 / 8 / 75$ & 7 & & 41 & 105.80 & & raw data - assumed degree $C$ \\
\hline 1 & $4 / 8 / 75$ & 8 & & 41 & 105.80 & & raw data - assumed degree $C$ \\
\hline 1 & $4 / 8 / 75$ & 9 & & 41 & 105.80 & & raw data - assumed degree C \\
\hline 1 & $4 / 8 / 75$ & 10 & & 40 & 104.00 & & raw data - sssumed degree $\mathrm{C}$ \\
\hline 1 & $4 / 8 / 75$ & 11 & & 37 & 98.60 & & raw data - sssumed degree $\mathrm{C}$ \\
\hline 1 & $4 / 8 / 75$ & 12 & & 27 & 80.60 & & raw data - assumed degree $C$ \\
\hline 1 & $4 / 29 / 75$ & 1 & & 41 & 105.80 & & raw data - assumed degree C \\
\hline 1 & $4 / 29 / 75$ & 2 & & 41 & 105.80 & & raw data - assumed degree C \\
\hline 1 & $4 / 29 / 75$ & 3 & & 41 & 105.80 & & raw data - assumed degree C \\
\hline 1 & $4 / 29 / 75$ & 4 & & 40 & 104.00 & & raw data - assumed degree C \\
\hline 1 & $4 / 29 / 75$ & 5 & & 37 & 98.60 & & raw data - assumed degree $\mathrm{C}$ \\
\hline 1 & $4 / 29 / 75$ & 6 & & 27 & 80.60 & & raw data - assumed degree C \\
\hline 1 & $4 / 29 / 75$ & 7 & & 28 & 82.40 & & raw data - assumed degree C \\
\hline 1 & $4 / 29 / 75$ & 8 & & 29 & 84.20 & & raw data - assumed degree C \\
\hline 1 & $4 / 29 / 75$ & 9 & & 24 & 75.20 & & raw data - assumed degree C \\
\hline 1 & $4 / 29 / 75$ & 10 & & 21 & 69.80 & & raw data - assumed degree C \\
\hline 1 & $4 / 29 / 75$ & 11 & & 19 & 66.20 & & raw data - assumed degree C \\
\hline 1 & $4 / 29 / 75$ & 12 & & 19 & 66.20 & & raw data - assumed degree C \\
\hline 1 & $5 / 1 / 75$ & 1 & & 42 & 107.60 & & raw data - assumed degree $C$ \\
\hline 1 & $5 / 1 / 75$ & 2 & & 42 & 107.60 & & raw data - assumed degree C \\
\hline 1 & $5 / 1 / 75$ & 3 & & 41 & 105.80 & & raw data - assumed degree C \\
\hline 1 & $5 / 1 / 75$ & 4 & & 41 & 105.80 & & raw data - assumed degree C \\
\hline 1 & $5 / 1 / 75$ & 5 & & 38 & 100.40 & & raw data - assumed degree C \\
\hline 1 & $5 / 1 / 75$ & 6 & & 28 & 82.40 & & raw data - assumed degree C \\
\hline 1 & $5 / 1 / 75$ & 7 & & 29 & 84.20 & & raw data - assumed degree C \\
\hline 1 & $5 / 1 / 75$ & 8 & & 28 & 82.40 & & raw data - assumed degree $C$ \\
\hline 1 & $5 / 1 / 75$ & 9 & & 25 & 77.00 & & raw data - assumed degree $C$ \\
\hline 1 & $5 / 1 / 75$ & 10 & & 24 & 75.20 & & raw data - assumed degree $C$ \\
\hline 1 & $5 / 1 / 75$ & 11. & & 20 & 68.00 & & raw data - assumed degree $C$ \\
\hline 1 & $5 / 1 / 75$ & 12 & & 20 & 68.00 & & raw data - assumed degree C \\
\hline 1 & $5 / 20 / 75$ & 1 & & 41 & 105.80 & & raw data - assumed degree $C$ \\
\hline 1 & $5 / 20 / 75$ & 2 & & 41 & 105.80 & & raw data - assumed degree $C$ \\
\hline 1 & $5 / 20 / 75$ & 3 & & 40.5 & 104.90 & & raw data - assumed degree C \\
\hline 1 & $5 / 20 / 75$ & 4 & & 38 & 100.40 & & raw data - assumed degree C \\
\hline 1 & $5 / 20 / 75$ & 5 & & 27 & 80.60 & & raw data - assumed degree C \\
\hline 1 & $5 / 20 / 75$ & 6 & & 28 & 82.40 & & raw data - assumed degree C \\
\hline 1 & $5 / 20 / 75$ & 7 & & 28 & 82.40 & & raw data - assumed degree C \\
\hline 1 & $5 / 20 / 75$ & 8 & & 24 & 75.20 & & raw data - assumed degree $C$ \\
\hline 1 & $5 / 20 / 75$ & 9 & & 21 & 69.80 & & raw data - assumed degree C \\
\hline 1 & $5 / 20 / 75$ & 10 & & 20 & 68.00 & & raw data - assumed degree C \\
\hline 1 & $5 / 20 / 75$ & 11 & & 20 & 68.00 & & raw data - assumed degree C \\
\hline 1 & $5 / 20 / 75$ & 12 & & 19.5 & 67.10 & & raw data - assumed degree C \\
\hline 1 & $7 / 28 / 75$ & 1 & & & 149 & & \\
\hline 1 & $7 / 28 / 75$ & 2 & & & 148 & & \\
\hline 1 & $7 / 28 / 75$ & 3 & & & 146 & & \\
\hline 1 & $7 / 28 / 75$ & 4 & & & 141 & & \\
\hline 1 & $7 / 28 / 75$ & 5 & & & 134 & & \\
\hline 1 & $7 / 28 / 75$ & 6 & & & 117 & & \\
\hline 1 & $7 / 28 / 75$ & 7 & & & 121 & & \\
\hline 1 & $7 / 28 / 75$ & 8 & & & 119 & & \\
\hline 9 & $7 / 28 / 75$ & 9 & & & 117 & & \\
\hline 1 & $7 / 28 / 75$ & 10 & & & 111 & & \\
\hline 1 & $7 / 28 / 75$ & 11 & & & 106 & & \\
\hline 1 & $7 / 28 / 75$ & 12 & & & 104 & & \\
\hline 1 & $8 / 20 / 75$ & 1 & & & & & 20 - suspect data (low) \\
\hline 1 & $8 / 20 / 75$ & 2 & & 57 & 134.60 & & raw data - assumed degree C \\
\hline 1 & $8 / 20 / 75$ & 3 & & 54 & 129.20 & & raw data - assumed degree $C$ \\
\hline 1 & $8 / 20 / 75$ & 4 & & 50 & 122.00 & & raw data - assumed degree $C$ \\
\hline
\end{tabular}

Data obtained from WHC Surveillance Analysis Computer System (SACS), November 12, 1993. 


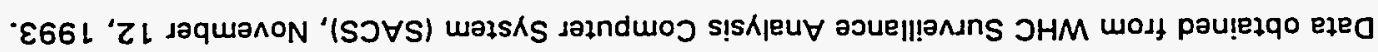

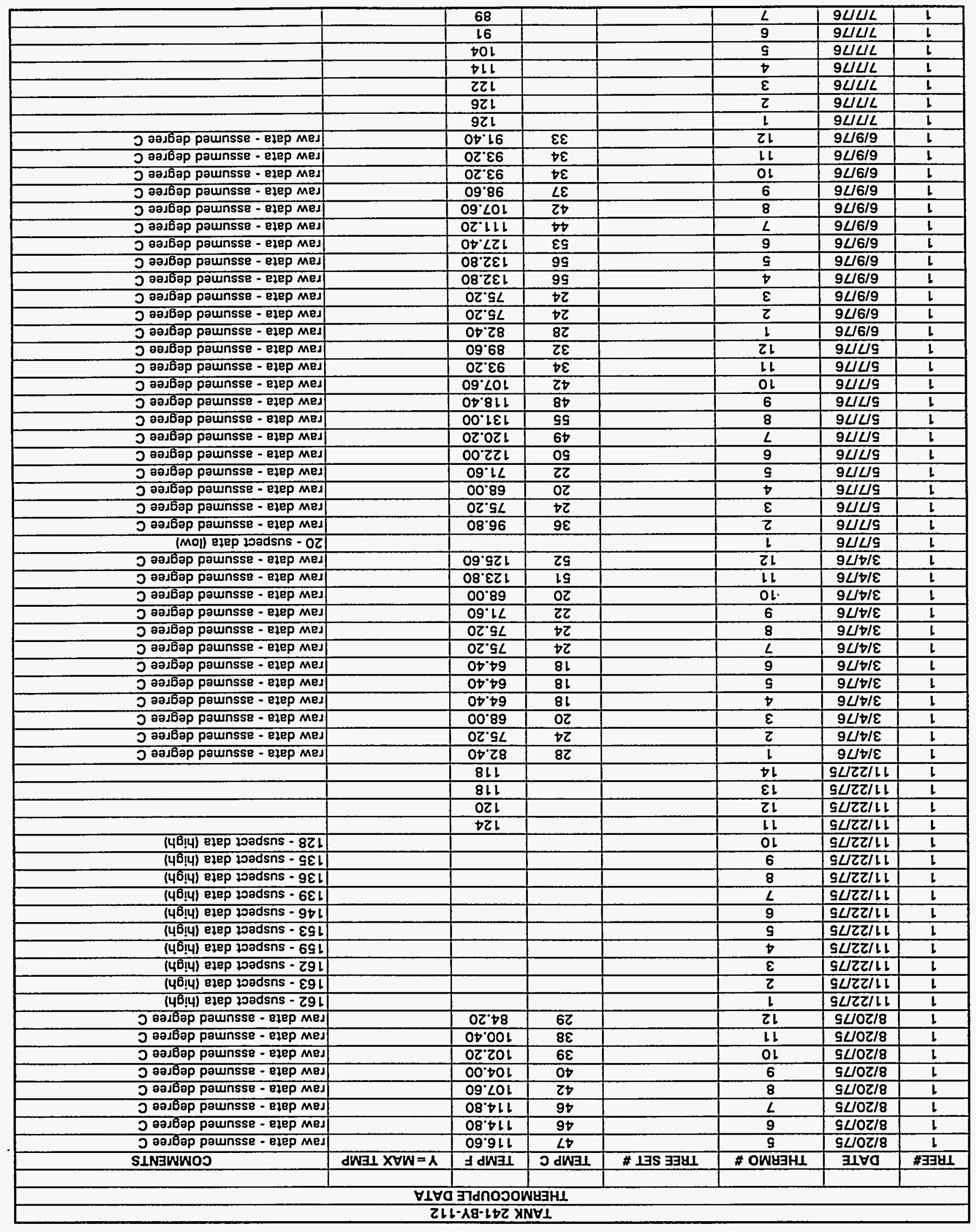




\begin{tabular}{|c|c|c|c|c|c|c|c|}
\hline \multicolumn{8}{|c|}{ TANK 241-BY-112 } \\
\hline \multicolumn{8}{|c|}{ THERMOCOUPLE DATA } \\
\hline TREE\# & DATE & THERMO \# & TREE SET \# & TEMP C & TEMP F & $Y=M A X$ TEMP & COMMENTS \\
\hline$\frac{1}{1}$ & $7 / 7 / 76$ & 8 & & & 89 & & \\
\hline 1 & $7 / 7 / 76$ & 9 & & & 89 & & \\
\hline 1 & $7 / 7 / 76$ & 10 & & & 89 & & \\
\hline 1 & $7 / 7 / 76$ & 11 & & & 89 & & \\
\hline 1 & $7 / 7 / 76$ & 12 & & & 89 & & \\
\hline 1 & $8 / 14 / 76$ & 9 & & & 122 & & \\
\hline$\overline{1}$ & $8 / 14 / 76$ & 2 & & & 121 & & \\
\hline 1 & $8 / 14 / 76$ & 3 & & & 118 & & \\
\hline 1 & $8 / 14 / 76$ & 4 & & & 111 & & \\
\hline 1 & $8 / 14 / 76$ & 5 & & & 102 & & \\
\hline 1 & $8 / 14 / 76$ & 6 & & & 91 & & \\
\hline 1 & $8 / 14 \sqrt[76]{ }$ & 7 & & & 89 & & \\
\hline 1 & $8 / 14 / 76$ & 8 & & & 88 & & \\
\hline 1 & $8 / 14 / 76$ & 9 & & & 89 & & \\
\hline 1 & $8 / 14 / 76$ & 10 & & & 89 & & \\
\hline$\frac{1}{1}$ & $8 / 14 / 76$ & 11 & & & 89 & & \\
\hline 1 & $8 / 14 / 76$ & 12 & & & 88 & & \\
\hline 1 & $8 / 26 / 76$ & 1 & & & 95 & & \\
\hline 1 & $8 / 26 / 76$ & 2 & & & 95 & & \\
\hline 7 & $8 / 26 / 76$ & 3 & & & 95 & & \\
\hline 1 & $8 / 26 / 76$ & 4 & & & 95 & & \\
\hline 1 & $8 / 26 / 76$ & 5 & & & 95 & & \\
\hline 1 & $8 / 26 / 76$ & 6 & & & 81 & & \\
\hline 9 & $8 / 26 / 76$ & 7 & & & 81 & & \\
\hline 1 & $8 / 26 / 76$ & 8 & & & 80 & & \\
\hline 1 & $8 / 26 / 76$ & 9 & & & 75 & & \\
\hline 1 & $8 / 26 / 76$ & 10 & & & 75 & & \\
\hline 1 & $8 / 26 / 76$ & 11 & & & 75 & & \\
\hline 9 & $8 / 26 / 76$ & 12 & & & 75 & & \\
\hline 1 & $9 / 13 / 76$ & 1 & & 34 & 93.20 & & |raw data - assumed degree $C$ \\
\hline 1 & $9 / 13 / 76$ & 2 & & 34 & 93.20 & & raw data - assumed degree $C$ \\
\hline 1 & $9 / 13 / 76$ & 3 & & 34 & 93.20 & & raw data - assumed degree $C$ \\
\hline 1 & $9 / 13 / 76$ & 4 & & 34 & 93.20 & & raw data - assumed degree C \\
\hline 1 & $9 / 13 / 76$ & 5 & & 34 & 93.20 & & raw data - assumed degree C \\
\hline 1 & $9 / 13 / 76$ & 6 & & 27 & 80.60 & & raw data - assumed degree $C$ \\
\hline 1 & $9 / 13 / 76$ & 7 & & 27 & 80.60 & & raw data - assumed degree C \\
\hline 1 & $9 / 13 / 76$ & 8 & & 27 & 80.60 & & raw data - assumed degree $C$ \\
\hline 1 & $9 / 13 / 76$ & 9 & & 25 & 77.00 & & raw data - assumed degree C \\
\hline 1 & $9 / 13 / 76$ & 10 & & 24 & 75.20 & & raw data - assumed degree C \\
\hline 1 & $9 / 13 / 76$ & 19 & & 24 & 75.20 & & raw data - assumed degree C \\
\hline 1 & $9 / 13 / 76$ & 12 & & 24 & 75.20 & & raw data - assumed degree C \\
\hline 1 & $11 / 7 / 76$ & 1 & & 32 & 89.60 & & raw data - assumed degree C \\
\hline 1 & $11 / 7 / 76$ & 2 & & 32 & 89.60 & & raw data - assumed degree C \\
\hline 1 & $11 / 7 / 76$ & 3 & & 32 & 89.60 & & raw data - assumed degree C \\
\hline 1 & $11 / 7 / 76$ & 4 & & 33 & 91.40 & & raw data - assumed degree $C$ \\
\hline 1 & $11 / 7 / 76$ & 5 & & 32 & 89.60 & & raw data - assumed degree C \\
\hline 1 & $11 / 7 / 76$ & 6 & & 26 & 78.80 & & raw data - assumed degree $C$ \\
\hline 1 & $11 / 7 / 76$ & 7 & & 26 & 78.80 & & raw data - assumed degree C \\
\hline 1 & $11 / 7 / 76$ & 8 & & 27 & 80.60 & & raw data - assumed degree C \\
\hline 1 & $11 / 7 / 76$ & 9 & & 24 & 75.20 & & raw data - assumed degree C \\
\hline 1 & $11 / 7 / 76$ & 10 & & 24 & 75.20 & & raw data - assumed degree C \\
\hline 1 & $11 / 7 / 76$ & 11 & & 23 & 73.40 & & raw data - assumed degree C \\
\hline 1 & $11 / 7 / 76$ & 12 & & 23 & 73.40 & & raw data - assumed degree C \\
\hline 1 & $12 / 9 / 76$ & 2 & & & 144 & & \\
\hline 1 & $12 / 9 / 76$ & 3 & & & 144 & & \\
\hline 1 & $12 / 9 / 76$ & 4 & & & 141 & & \\
\hline 9 & $12 / 9 / 76$ & 5 & & & 133 & & \\
\hline 1 & $12 / 9 / 76$ & 6 & & & 123 & & \\
\hline 1 & $12 / 9 / 76$ & 7 & & & 110 & & \\
\hline 1 & $12 / 9 / 76$ & 8 & & & 107 & & \\
\hline 1 & $12 / 9 / 76$ & 9 & & & 107 & & \\
\hline 1 & $12 / 9 / 76$ & 10 & & & 106 & & \\
\hline 1 & $12 / 9 / 76$ & 11 & & & 106 & & \\
\hline 1 & $12 / 9 / 76$ & 12 & & & 106 & & \\
\hline 1 & $1 / 3 / 77$ & 1 & & & 106 & & \\
\hline
\end{tabular}

Data obtained from WHC Surveillance Analysis Computer System (SACS), November 12, 1993. 


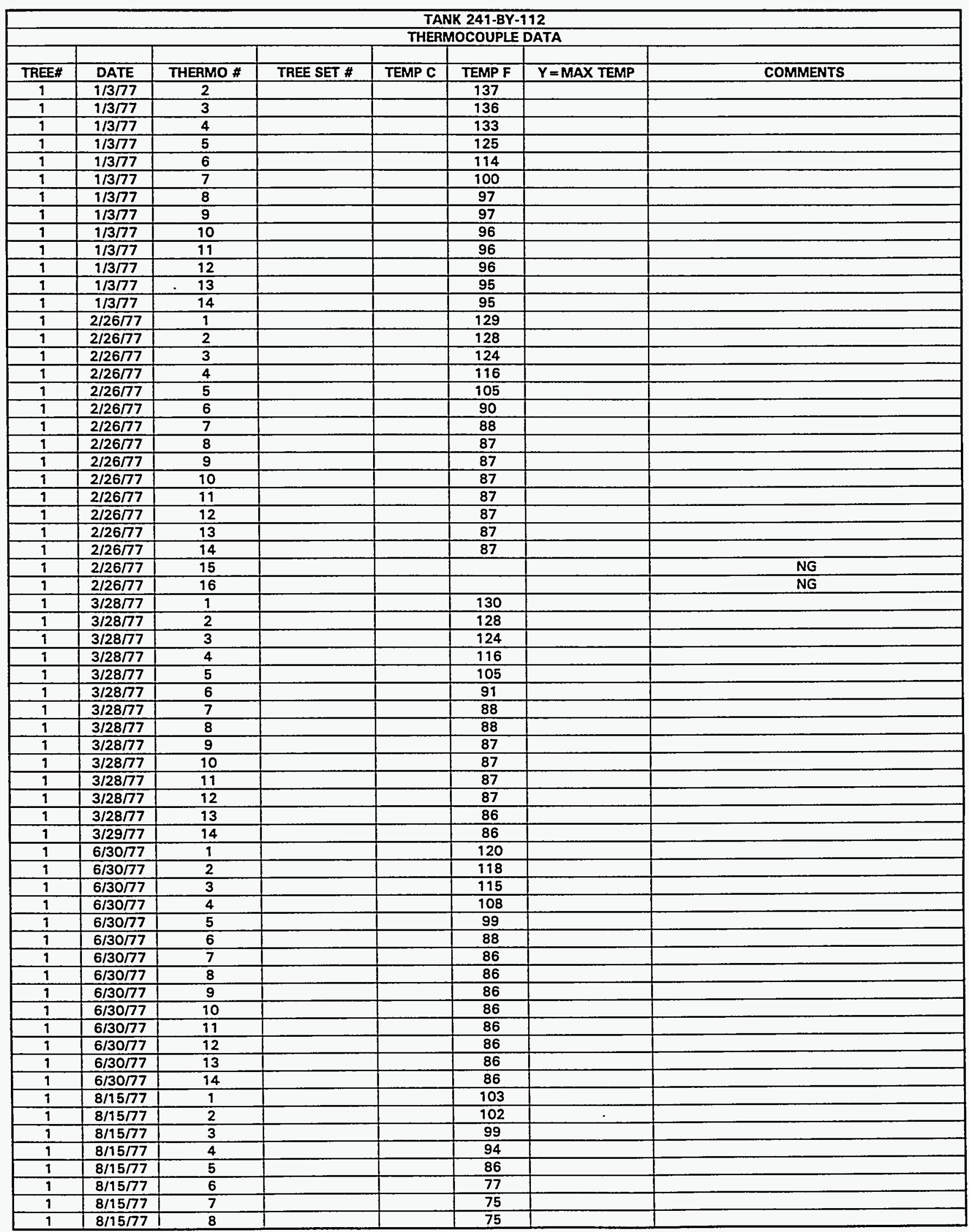

Data obtained from WHC Surveillance Analysis Computer System (SACS), November 12, 1993. 


\begin{tabular}{|c|c|c|c|c|c|c|c|}
\hline \multicolumn{8}{|c|}{ TANK 241-BY-112 } \\
\hline \multicolumn{8}{|c|}{ THERMOCOUPLE DATA } \\
\hline TREE\# & DATE & THERMO \# & TREE SET \# & TEMP C & TEMP F & $Y=M A X$ TEMP & COMMENTS \\
\hline 1 & $8 / 15 / 77$ & 9 & & & 75 & & \\
\hline 1 & $8 / 15 / 77$ & 10 & & & 74 & & \\
\hline 1 & $8 / 1 5 \longdiv { 7 7 }$ & 11 & & & 74 & & \\
\hline 1 & $8 / 15 / 77$ & 12 & & & 74 & & \\
\hline 1 & $8 / 15 / 77$ & 13 & & & 73 & & \\
\hline 1 & $8 / 15 / 77$ & 14 & & & 73 & & \\
\hline 1 & $10 / 7 / 77$ & 1 & & & 112 & & \\
\hline 1 & $10 / 7 / 77$ & 2 & & & 113 & & \\
\hline 1 & $10 / 7 / 77$ & 3 & & & 111 & & \\
\hline 1 & $10 / 7 / 77$ & 4 & & & 106 & & \\
\hline 1 & $10 / 7 / 77$ & 5 & & & 99 & & \\
\hline 1 & $10 / 7 / 77$ & 6 & & & 90 & & \\
\hline 1 & $10 / 7 / 77$ & 7 & $\cdot$ & & 89 & & \\
\hline 1 & $10 / 7 / 77$ & 8 & & & 89 & & \\
\hline 9 & $10 / 7 / 77$ & 9 & & & 89 & & \\
\hline 1 & 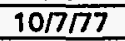 & 10 & & & 89 & & \\
\hline 1 & $10 / 7 / 77$ & 11 & & & 88 & & \\
\hline 1 & $10 / 7 / 77$ & 12 & & & 88 & & \\
\hline 1 & $10 / 7 \sqrt[77]{ }$ & 13 & & & 87 & & \\
\hline 1 & $10 / 7 \pi 7$ & 14 & & & 87 & & \\
\hline 1 & $12 / 7 / 77$ & 1 & & & & & 70 - suspect data (low) \\
\hline 1 & $1 2 \longdiv { 7 7 7 }$ & 2 & & & & & 69 - suspect data (low) \\
\hline 1 & $12 / 7 / 77$ & 3 & & & & & NG \\
\hline 1 & $12 \pi / 77$ & 4 & & & & & NG \\
\hline 9 & $12 / 7 / 77$ & 5 & & & & & NG \\
\hline 1 & $12 / 7 / 77$ & 6 & & & & & NG \\
\hline 1 & $12 / 7 / 77$ & 7 & & & & & NG \\
\hline 1 & $12 / 7 / 77$ & 8 & & & & & NG \\
\hline 1 & $12 / 7 / 77$ & 9 & & & & & NG \\
\hline 1 & $12 \sqrt[7]{777}$ & 10 & & & & & NG \\
\hline 1 & $12 / 7 / 77$ & 11 & & & & & NG \\
\hline 1 & $12 / 7 / 77$ & 12 & & & & & NG \\
\hline 1 & $12 / 7 / 77$ & 13 & & & & & NG \\
\hline 1 & $12 / 7 / 77$ & 14 & & & & & NG \\
\hline 1 & $2 / 26 / 78$ & 1 & & & 164 & & \\
\hline 1 & $2 / 26 / 78$ & 2 & & & 119 & & \\
\hline 1 & $2 / 26 / 78$ & 3 & & & 123 & & \\
\hline 1 & $2 / 26 / 78$ & 4 & & & 115 & & \\
\hline 1 & $2 / 26 / 78$ & 5 & & & 104 & & \\
\hline 1 & $2 / 26 / 78$ & 6 & & & 91 & & \\
\hline 1 & $2 / 26 / 78$ & 7 & & & 89 & & \\
\hline 1 & $2 / 26 / 78$ & 8 & & & 91 & & \\
\hline$\frac{1}{1}$ & $2 / 26 / 78$ & 9 & & & 91 & & \\
\hline 1 & $2 / 26 / 78$ & 10 & & & 74 & & \\
\hline 1 & $2 / 26 / 78$ & 11 & & & 91 & & \\
\hline 1 & $2 / 26 / 78$ & 12 & & & 90 & & \\
\hline$\frac{1}{1}$ & $2 / 26 / 78$ & 13 & & & 89 & & \\
\hline 1 & $2 / 26 / 78$ & 14 & & & 89 & & \\
\hline 1 & $3 / 26 / 78$ & 1 & & & 103 & & \\
\hline 9 & $3 / 26 / 78$ & 2 & & & 98 & & \\
\hline 1 & $3 / 26 / 78$ & 3 & & & 104 & & \\
\hline 1 & $3 / 26 / 78$ & 4 & & & 94 & & \\
\hline 1 & $3 / 26 / 78$ & 5 & & & 87 & & \\
\hline$\frac{1}{1}$ & $3 / 26 / 78$ & 6 & & & 71 & & \\
\hline 1 & $3 / 26 / 78$ & 7 & & & 71 & & \\
\hline 1 & $3 / 26 / 78$ & 8 & & & 72 & & \\
\hline 1 & $3 / 26 / 78$ & 9 & & & 73 & & \\
\hline 1 & $3 / 26 / 78$ & 10 & & & & & 53 - suspect data (low) \\
\hline 1 & $3 / 26 / 78$ & 11 & & & 72 & & \\
\hline 1 & $3 / 26 / 78$ & 12 & & & 71 & & \\
\hline 1 & $4 / 22 / 78$ & 1 & & & 108 & & \\
\hline 1 & $4 / 22 / 78$ & 2 & & & 108 & & \\
\hline 1 & $4 / 22 / 78$ & 3 & & & 105 & & \\
\hline 1 & $4 / 22 / 78$ & 4 & & & 98 & & \\
\hline 1 & $4 / 22 / 78$ & 5 & & & 89 & & \\
\hline
\end{tabular}

Data obtained from WHC Surveillance Analysis Computer System (SACS), November 12, 1993. 


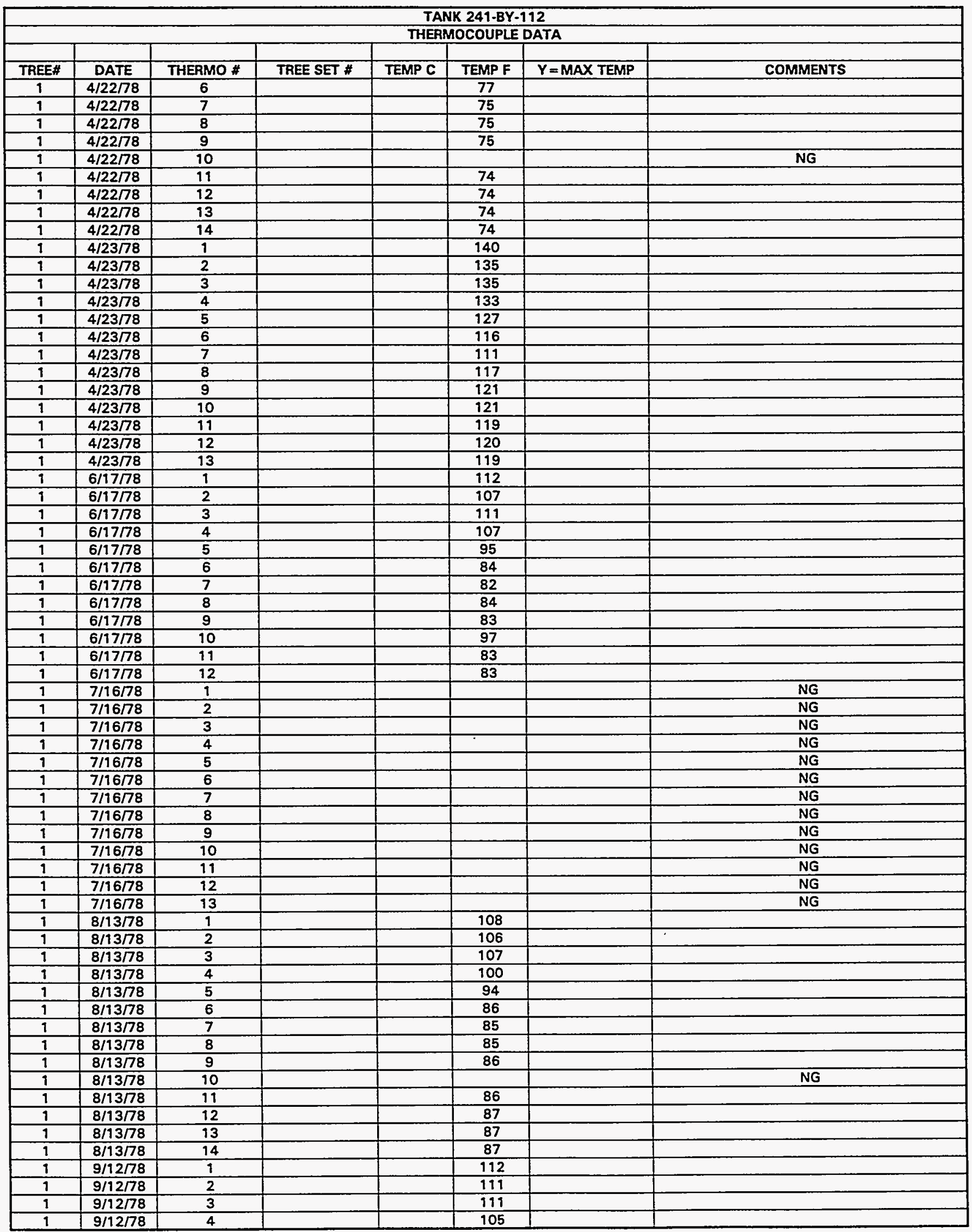

Data obtained from WHC Surveillance Analysis Computer System (SACS), November 12, 1993. 


\begin{tabular}{|c|c|c|c|c|c|c|c|}
\hline \multicolumn{8}{|c|}{ TANK 241-BY-112 } \\
\hline \multicolumn{8}{|c|}{ THERMOCOUPLE DATA } \\
\hline TAEE\# & DATE & THERMO \# & TREE SET $\#$ & TEMP C & TEMPF & $Y=M A X$ TEMP & COMMENTS \\
\hline 1 & $9 / 12 / 78$ & 5 & & & 99 & & \\
\hline 1 & $9 / 12 / 78$ & 6 & & & 92 & & \\
\hline 1 & $9 / 12 / 78$ & 7 & & & 99 & & \\
\hline 1 & $9 / 12 / 78$ & 8 & & & 91 & & \\
\hline 1 & $9 / 12 / 78$ & 9 & & & 91 & & \\
\hline 1 & $9 / 12 / 78$ & 10 & & & & & NG \\
\hline 1 & $9 / 12 / 78$ & 11 & & & 92 & & \\
\hline 1 & $9 / 12 / 78$ & 12 & & & 92 & & \\
\hline 1 & $9 / 12 / 78$ & 13 & & & 92 & & \\
\hline 1 & $9 / 12 / 78$ & 14 & & & 92 & & \\
\hline 1 & $1 0 \longdiv { 7 } 7 8$ & 1 & & & 119 & & \\
\hline 1 & $10 / 7 / 78$ & 2 & & & 128 & & \\
\hline 1 & $10 / 7 / 78$ & 3 & & & 120 & & \\
\hline 1 & $10 / 7 / 78$ & 4 & & & 118 & & \\
\hline 1 & $10 / 7 / 78$ & 5 & & & 99 & & \\
\hline 1 & $10 / 7 / 78$ & 6 & & & 98 & & \\
\hline 1 & $10 / 7 / 78$ & 7 & & & 98 & & \\
\hline 1 & $10 / 7 / 78$ & 8 & & & 95 & & \\
\hline 1 & $10 / 7 / 78$ & 9 & & & 94 & & \\
\hline 1 & $1 / 3 / 79$ & 1 & & & 117 & & \\
\hline 1 & $1 / 3 / 79$ & 2 & & & 118 & & \\
\hline 1 & $1 / 3 / 79$ & 3 & & & 115 & & \\
\hline 1 & $1 / 3 / 79$ & 4 & & & 109 & & \\
\hline 1 & $1 / 3 / 79$ & 5 & & & 99 & & \\
\hline 1 & $1 / 3 / 79$ & 6 & & & 98 & & \\
\hline 1 & $1 / 3 / 79$ & 7 & & & 96 & & \\
\hline 1 & $1 / 3 / 79$ & 8 & & & 85 & & \\
\hline 1 & $1 / 3 / 79$ & 9 & & & 85 & & \\
\hline 1 & $1 / 3 / 79$ & 10 & & & & & NG \\
\hline 1 & $1 / 3 / 79$ & 11 & & & 87 & & \\
\hline 1 & $1 / 3 / 79$ & 12 & & & 89 & & \\
\hline 1 & $7 / 7 / 79$ & 1 & & & 104 & & \\
\hline 1 & $7 / 7 / 79$ & 2 & & & 102 & & \\
\hline 1 & $7 / 7 / 79$ & 3 & & & 100 & & \\
\hline 1 & $7 / 7 / 79$ & 4 & & & 94 & & \\
\hline 1 & $7 / 7779$ & 5 & & & 86 & & \\
\hline 1 & $7 / 7 / 79$ & 6 & & & 78 & & \\
\hline 9 & $7 / 7 / 79$ & 7 & & & 76 & & \\
\hline 1 & $7 / 7 / 79$ & 8 & & & 75 & & \\
\hline 1 & $7 / 7 / 79$ & 9 & & & 76 & & \\
\hline 9 & $7 \Pi 7 \Pi 9$ & 10 & & & & & NG \\
\hline 1 & 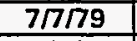 & 11 & & & 76 & & \\
\hline 1 & $7 / 7 / 79$ & 12 & & & 76 & & \\
\hline 1 & $6 / 12 / 81$ & 1 & & & 93 & & \\
\hline 1 & $6 / 12 / 81$ & 2 & & & 92 & & \\
\hline 9 & $6 / 12 / 81$ & 3 & & & 90 & & \\
\hline 1 & $6 / 12 / 81$ & 4 & & & 85 & & \\
\hline 9 & $6 / 12 / 81$ & 5 & & & 78 & & \\
\hline 1 & $6 / 12 / 81$ & 6 & & & 72 & & \\
\hline 1 & $6 / 12 / 81$ & 7 & & & 70 & & \\
\hline 1 & $6 / 12 / 81$ & 8 & & & 81 & & \\
\hline 1 & $6 / 12 / 81$ & 9 & & & 70 & & \\
\hline 1 & $6 / 12 / 81$ & 11 & & & 70 & & \\
\hline 1 & $6 / 12 / 81$ & 12 & & & 70 & & \\
\hline 1 & $6 / 12 / 81$ & 13 & & & 70 & & \\
\hline 1 & $6 / 12 / 81$ & 14 & & & 70 & & \\
\hline 1 & $10 / 13 / 89$ & 1 & 1 & & & $\mathbf{N}$ & $\begin{array}{c}\text { Could not find a manual reading station of } \\
\text { housing. }\end{array}$ \\
\hline 1 & $11 / 3 / 89$ & 1 & 1 & & 76.5 & $\mathbf{Y}$ & \\
\hline 1 & $11 / 11 / 89$ & 1 & 1 & & 75.7 & $\bar{Y}$ & K type of LOW on $11-11-8975.7$ \\
\hline 1 & $11 / 18 / 89$ & 1 & 1 & & 76.2 & $\mathbf{Y}$ & In LOW Test taken with $K$ \\
\hline 1 & $11 / 25 / 89$ & 1 & 1 & & 75 & $\bar{Y}$ & LOW \\
\hline 1 & $12 / 1 / 89$ & 1 & 1 & & 74.9 & $\mathbf{Y}$ & LOW \\
\hline 1 & $12 / 10 / 89$ & 1 & 1 & & 75 & $Y$ & LOW \\
\hline 1 & $12 / 15 / 89$ & 1 & 1 & & 73.5 & $\bar{Y}$ & LOW \\
\hline
\end{tabular}

Data obtained from WHC Surveillance Analysis Computer System (SACS), November 12, 1993. 


\begin{tabular}{|c|c|c|c|c|c|c|c|}
\hline \multicolumn{8}{|c|}{ TANK 241-BY-112 } \\
\hline & & & & THER & OCOUPLE & ATA & \\
\hline TREE\# & DATE & THERMO \# & TREE SET \# & TEMP C & TEMP F & $Y=M A X$ TEMP & COMMENTS \\
\hline 1 & $12 / 23 / 89$ & 1 & 1 & & 72 & $\mathrm{Y}$ & \\
\hline 1 & $12 / 29 / 89$ & 1 & 1 & & 74.1 & $Y$ & LOW-K-890723679-20 \\
\hline 1 & $12 / 29 / 89$ & 1 & 2 & & 78 & $\bar{Y}$ & $J-890523512-20$ \\
\hline 1 & $1 / 6 / 90$ & 1 & 1 & & 72.4 & $\bar{Y}$ & LOW \\
\hline 1 & $1 / 13 / 90$ & 1 & 1 & & 72.8 & $\mathbf{Y}$ & K LOW \\
\hline 1 & $1 / 27 / 90$ & 1 & 1 & & 69.2 & $\mathbf{Y}$ & LOW - K \\
\hline$\overline{1}$ & $2 / 3 / 90$ & 1 & 1 & & 70.1 & $\bar{Y}$ & LOW K-890723579-20 \\
\hline 1 & $2 / 5 / 90$ & 1 & 1 & & 70.1 & $\mathrm{Y}$ & LOW K-890723679-20 \\
\hline 1 & $2 / 11 / 90$ & 1 & 1 & & & $\mathbf{N}$ & Unable to locate thermocouple. \\
\hline 1 & $3 / 2 / 90$ & 9 & 1 & & 72.3 & $\mathbf{Y}$ & K-HH-99A-K \\
\hline 1 & $5 / 5 / 90$ & 1 & 1 & & & $\mathbf{N}$ & Not taken per PM 90-049 \\
\hline 1 & $6 / 1 / 90$ & 1 & 1 & & & $\mathrm{~N}$ & Not taken per PM 90-051 \\
\hline 1 & $8 / 5 / 90$ & 1 & $\mathbf{i}$ & & & $N$ & J1 written to re-instate LOW \\
\hline 1 & $8 / 7 / 90$ & 1 & 1 & & 75.9 & $Y$ & K LOW \\
\hline 1 & $9 / 11 / 90$ & 1 & 1 & & 80 & $Y$ & LOW K-890723679-64 \\
\hline 1 & $10 / 9 / 90$ & 1 & 1 & & 84 & $\bar{Y}$ & LOW \\
\hline 1 & $10 / 24 / 90$ & 1 & 1 & & 80 & $\bar{Y}$ & K-HH-99A-K \\
\hline 1 & $10 / 26 / 90$ & 1 & 1 & & 79.3 & $\mathrm{Y}$ & $\mathrm{J}-890523512-20$ \\
\hline 1 & $10 / 30 / 90$ & 1 & 2 & & 74.8 & $\bar{Y}$ & K 890723679-64 LOW \\
\hline 1 & $11 / 5 / 90$ & 1 & 1 & & 71.4 & $\bar{Y}$ & $\mathrm{~J} 890523512-20$ \\
\hline 1 & $11 / 12 / 90$ & 1 & 2 & & 80 & $\bar{Y}$ & K 890723679 LOW \\
\hline 1 & $11 / 18 / 90$ & 1 & 1 & & 79.6 & $\bar{Y}$ & K 890723697.74 LOW \\
\hline 1 & $11 / 25 / 90$ & 1 & 1 & & 78.7 & $\bar{Y}$ & K-890723679-64 \\
\hline 1 & $12 / 5 / 90$ & 1 & 1 & & 72.1 & $Y$ & K 890723679-64 LOW \\
\hline 1 & $12 / 10 / 90$ & 1 & 1 & & 72.1 & $\bar{Y}$ & K 890723679-64 \\
\hline 1 & $12 / 11 / 90$ & 1 & 2 & & 72.1 & $\bar{Y}$ & K 890723679-64 \\
\hline 1 & $12 / 15 / 90$ & 1 & 1 & & 79.4 & $Y$ & K HH-99-A-K \\
\hline 1 & $12 / 22 / 90$ & 1 & 2 & & 70 & $Y$ & K 890723679-64 LOW \\
\hline 1 & $12 / 30 / 90$ & 1 & 1 & & 79.1 & $\bar{Y}$ & K $890723679-64$ LOW-6 readings LOW? \\
\hline 1 & $1 / 6 / 91$ & 1 & 1 & & 66.8 & $\bar{Y}$ & $K$ \\
\hline 1 & $1 / 13 / 91$ & 1 & 1 & & 67.8 & $\bar{Y}$ & K 890723679-64 \\
\hline 1 & $1 / 19 / 91$ & 1 & 1 & & 65.8 & $Y$ & K HH99A LOW \\
\hline 1 & $1 / 29 / 91$ & 1 & 1 & & 66.1 & $\bar{Y}$ & K HH-99A LOW \\
\hline 1 & $2 / 3 / 91$ & 1 & 1 & & 74 & $\bar{Y}$ & K 896723679-64 LOW \\
\hline 1 & $2 / 9 / 91$ & 1 & 1 & & 64.9 & $\bar{Y}$ & K 890723679-64 LOW \\
\hline 1 & $2 / 16 / 91$ & 1 & 1 & & 77.8 & $\bar{Y}$ & K-890723679-64 LOW \\
\hline 1 & $2 / 23 / 91$ & 1 & 1 & & 74.7 & $\bar{Y}$ & K 896723679-64 LOW \\
\hline 1 & $3 / 3 / 91$ & 1 & 1 & & 73.9 & $\bar{Y}$ & $890923679-64$ LOW \\
\hline 1 & $3 / 9 / 91$ & 1 & 1 & & 75.1 & $\bar{Y}$ & K 890723679-20 \\
\hline 1 & $3 / 17 / 91$ & 1 & 1 & & 79.4 & $Y$ & $890923679-64$ LOW \\
\hline 1 & $3 / 23 / 91$ & 1 & 1 & & 78.7 & $\bar{Y}$ & $890923679-64$ LOW \\
\hline 1 & $3 / 30 / 91$ & 1 & 1 & & 79.2 & $\bar{Y}$ & LOW $890923679-64$ \\
\hline 1 & $4 / 7 / 91$ & 1 & 1 & & 82.4 & $\bar{Y}$ & LOW $890623567-12$ \\
\hline 1 & $4 / 14 / 91$ & 1 & 1 & & & $\mathbf{N}$ & K LOW-NR obtained \\
\hline 1 & $4 / 20 / 91$ & 1 & 1 & & 80.8 & $\bar{Y}$ & K 890723633-64 LOW \\
\hline 1 & $4 / 28 / 91$ & 1 & 1 & & 80.4 & $\mathbf{Y}$ & $890623567-12$ LOW \\
\hline 1 & $5 / 5 / 91$ & 1 & 1 & & 79.5 & $Y$ & K 690723633-64 LOW \\
\hline 1 & $5 / 12 / 91$ & 1 & 1 & & 78.7 & $Y$ & K 890723679-20 LOW \\
\hline 1 & $5 / 18 / 91$ & 1 & 1 & & 80.3 & $\mathbf{Y}$ & K 890723633-64 LOW \\
\hline 1 & $5 / 25 / 91$ & 1 & 1 & & 79.5 & $Y$ & K 890723633-64 LOW \\
\hline 1 & $6 / 2 / 91$ & 1 & 1 & & 76.1 & $\bar{Y}$ & K 890623567-12 LOW \\
\hline 1 & $6 / 9 / 91$ & 1 & 1 & & 77.5 & $\bar{Y}$ & K 890623567-12 LOW \\
\hline 1 & $6 / 15 / 91$ & 1 & 1 & & 77.3 & $Y$ & K 890723633.64 LOW \\
\hline 1 & $6 / 23 / 91$ & 1 & 1 & & 79 & $\mathbf{Y}$ & LOW $890623567-12$ \\
\hline 1 & $6 / 29 / 91$ & 9 & 1 & & 68.6 & $\bar{Y}$ & K 890623567-12LOW \\
\hline 1 & $7 / 6 / 91$ & 1 & 1 & & 74.4 & $Y$ & K 890623567.12 LOW \\
\hline 1 & $7 / 13 / 91$ & 1 & 1 & & 72.3 & $Y$ & K 890623567-12 LOW \\
\hline 1 & $7 / 20 / 91$ & 1 & 1 & & 73.5 & $\bar{Y}$ & K 890623567-12 LOW \\
\hline 1 & $7 / 27 / 91$ & 1 & 1 & & 81.3 & $Y$ & K 890725813 LOW \\
\hline 1 & $8 / 3 / 91$ & 1 & 1 & & 75.5 & $\bar{Y}$ & K 890723679-20 LOW \\
\hline 1 & $8 / 10 / 91$ & 1 & 1 & & 75 & $\bar{Y}$ & K 890723699-20 LOW \\
\hline 1 & $8 / 18 / 91$ & $i$ & 1 & & 81.8 & $\bar{Y}$ & K 890623567-12 LOW \\
\hline 1 & $8 / 24 / 91$ & 1 & 1 & & 81 & $Y$ & K 910226246-56 LOW \\
\hline 9 & $8 / 31 / 91$ & 1 & 1 & & 82 & 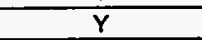 & K-910226246-45 \\
\hline
\end{tabular}

Data obtained from WHC Surveillance Analysis Computer System (SACS), November 12, 1993. 
WHC-SD-WM-ER-312, Rev. 0

\begin{tabular}{|c|c|c|c|c|c|c|c|}
\hline \multicolumn{8}{|c|}{ TANK 241-BY-112 } \\
\hline \multicolumn{8}{|c|}{ THERMOCOUPLE DATA } \\
\hline & & & & & & & \\
\hline TREE\# & DATE & THERMO \# & TREE SET \# & TEMP C & TEMP F & $Y=\operatorname{MAX}$ TEMP & $\begin{array}{c}\text { COMMENTS } \\
\text { K-910226246-45 }\end{array}$ \\
\hline 1 & 9/8/91 & 1 & 1 & & 82 & $Y$ & K-910226246-45 LOW \\
\hline 1 & $9 / 15 / 91$ & 1 & 1 & & 82.6 & $\mathbf{N}$ & $\mathrm{K}-890623567-12$ \\
\hline 1 & $9 / 22 / 91$ & 1 & 1 & & 84.6 & $\bar{Y}$ & K-890723679.64 LOW \\
\hline 1 & $9 / 28 / 91$ & 1 & 1 & & & $\mathbf{N}$ & $\mathrm{TC} \# 1 \mathrm{O} / \mathrm{S}$ \\
\hline 1 & $10 / 1 / 91$ & 1 & 1 & & 77.8 & $\bar{Y}$ & K-910226246-45 LOW \\
\hline 1 & $10 / 5 / 91$ & 1 & 1 & & 77.9 & $\bar{Y}$ & K-910226246-45 LOW \\
\hline 1 & $10 / 92 / 91$ & 1 & 1 & & 81.4 & $\bar{Y}$ & $K-89623565712$ \\
\hline 1 & $10 / 19 / 91$ & 1 & 1 & & 78.6 & $\bar{Y}$ & K-890723633-71 LOW \\
\hline 1 & $10 / 27 / 91$ & 1 & 1 & & 79.5 & $\bar{Y}$ & K-910226246.45 LOW \\
\hline 1 & $11 / 2 / 91$ & 1 & 1 & & 77 & $Y$ & $\mathrm{~K}$ \\
\hline$\frac{T}{1}$ & $11 / 9 / 91$ & 1 & 1 & & 78 & $Y$ & K-890723633-71 LOW \\
\hline 1 & $11 / 17 / 91$ & 1 & 1 & & & $\mathbf{N}$ & NO READINGS J-3 \\
\hline 1 & $11 / 24 / 91$ & 1 & 1 & & 79.8 & $\mathbf{Y}$ & K-890723679-64 LOW \\
\hline 1 & $12 / 1 / 91$ & 1 & 1 & & 80.3 & $\mathbf{Y}$ & K-890723679-64 LOW \\
\hline 1 & $12 / 8 / 91$ & 1 & 1 & & 82.4 & $\bar{Y}$ & K-89062357-12 LOW \\
\hline 9 & $12 / 14 / 91$ & 1 & 1 & & 82.1 & $\mathbf{N}$ & K-910226246-68 LOW \\
\hline 1 & $12 / 20 / 91$ & 1 & 1 & & 82.4 & $\bar{Y}$ & K-910426539-39 \\
\hline 1 & $12 / 29 / 91$ & 1 & 1 & & 76.3 & $\bar{Y}$ & K-910226246-68 LOW \\
\hline 1 & $1 / 4 / 92$ & 1 & 1 & & & $\mathrm{~N}$ & TCs not available \\
\hline 1 & $1 / 11 / 92$ & 1 & 1 & & 77.5 & $Y$ & 910426539 LOW \\
\hline 1 & $1 / 16 / 92$ & 1 & 1 & & 82 & $Y$ & K 910426539-7 \\
\hline 1 & $1 / 28 / 92$ & 1 & 1 & & & $N$ & $\begin{array}{l}\text { ACCESS RESTRICTED } 1 / 28-2 / 22 \text { DUE TO } \\
\text { BATTERY FUME INCIDENT }\end{array}$ \\
\hline 1 & $2 / 24 / 92$ & 1 & 1 & & 82.4 & $N$ & \\
\hline 1 & $2 / 29 / 92$ & 1 & 1 & & 82.8 & $\bar{Y}$ & $K+1-030 / 9104265397$ \\
\hline 1 & $3 / 10 / 92$ & 1 & 1 & & 81.7 & $Y$ & K910476539-80 \\
\hline 1 & $3 / 15 / 92$ & 1 & 1 & & 82 & $\bar{Y}$ & K 910426539-80 \\
\hline 1 & $3 / 20 / 92$ & 1 & 1 & & 81.9 & $\bar{Y}$ & K 91042653980 \\
\hline 1 & $3 / 29 / 92$ & 1 & 1 & & 82.8 & $\bar{Y}$ & K 910226246-34 \\
\hline 1 & $4 / 3 / 92$ & 1 & 1 & & 83 & $Y$ & J 910126143-14 \\
\hline 1 & $4 / 11 / 92$ & 1 & 1 & & 84.3 & $Y$ & K 890723679-80 \\
\hline 1 & $4 / 17 / 92$ & 1 & 1 & & 81.7 & $\bar{Y}$ & K 89072367964 \\
\hline 1 & $4 / 25 / 92$ & 1 & 1 & & 84.1 & $\bar{Y}$ & K91022624634 \\
\hline 1 & $4 / 30 / 92$ & 1 & 1 & & 81.9 & $\bar{Y}$ & K 910426539-7 \\
\hline 1 & $5 / 16 / 92$ & 1 & 1 & & 81.4 & $\bar{Y}$ & K890723633-64 \\
\hline 1 & $5 / 24 / 92$ & 1 & 1 & & 80.4 & $Y$ & K 890723633-64 \\
\hline 1 & $5 / 28 / 92$ & 1 & 1 & & 80.3 & $\dot{Y}$ & K 890723633-64 \\
\hline 1 & $6 / 5 / 92$ & 1 & 1 & & 83.8 & $\bar{Y}$ & K 890623567-12 \\
\hline 1 & $6 / 13 / 92$ & 1 & 1 & & 86 & $Y$ & K 910426539-7 \\
\hline 1 & $6 / 16 / 92$ & 1 & 1 & & 80 & $Y$ & K 890723633.64 6-92 \\
\hline 1 & $6 / 20 / 92$ & 1 & 1 & & 84.8 & $\bar{Y}$ & K 90226246-45 \\
\hline 1 & $6 / 28 / 92$ & $\frac{1}{1}$ & 1 & & 85.1 & $\bar{Y}$ & KTI-02490226246.45 \\
\hline 1 & $7 / 3 / 92$ & 1 & 1 & & 82.6 & $\bar{Y}$ & \\
\hline 1 & $7 / 10 / 92$ & 1 & 1 & & 81.4 & $\mathbf{Y}$ & K9104265397 \\
\hline 1 & $7 / 18 / 92$ & 1 & 1 & & 85.9 & $\bar{Y}$ & K 890723679-64 \\
\hline 1 & $7 / 24 / 92$ & 1 & 1 & & 83.5 & $\dot{Y}$ & K 890723679-64 \\
\hline 1 & $8 / 1 / 92$ & 1 & 1 & & 85.5 & $\bar{Y}$ & K TI-050 \\
\hline 1 & $8 / 7 / 92$ & 1 & 1 & & 79.6 & $Y$ & K 910226246-56 \\
\hline 1 & $8 / 15 / 92$ & 1 & 1 & & 84.4 & $\bar{Y}$ & K 910226246-56 \\
\hline 1 & $8 / 21 / 92$ & 1 & 1 & & 82.7 & $\bar{Y}$ & K90226240-45 \\
\hline 1 & $8 / 30 / 92$ & 1 & 1 & & 85.2 & $Y$ & K 910226246.56 \\
\hline 1 & $9 / 6 / 92$ & 1 & 1 & & 80.5 & $\bar{Y}$ & $\mathbf{K}$ \\
\hline 1 & $9 / 12 / 92$ & 1 & 1 & & 76.3 & $\bar{Y}$ & K 910226246-34 \\
\hline 1 & $9 / 20 / 92$ & 1 & 1 & & 79.8 & $Y$ & K 890723679-64 \\
\hline 1 & $9 / 26 / 92$ & 9 & 1 & & 80.6 & $Y$ & K $890723679-64$ \\
\hline 1 & $10 / 1 / 92$ & 1 & 1 & & 81.2 & $\mathbf{Y}$ & K 890723679-64 \\
\hline 1 & $10 / 18 / 92$ & 1 & 1 & & 81.9 & $\bar{Y}$ & K 910226246-34 \\
\hline 1 & $10 / 24 / 92$ & 1 & 1 & & 83 & $\mathrm{Y}$ & K 9101426539.80 \\
\hline 1 & $10 / 28 / 92$ & 1 & 1 & & 81.5 & $\mathrm{Y}$ & K91042653980 \\
\hline 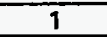 & $11 / 1 / 92$ & 1 & 1 & & 83.1 & $Y$ & TMACS \\
\hline 1 & $11 / 8 / 92$ & 1 & 1 & & 82.9 & $\bar{Y}$ & TMACS \\
\hline 9 & $11 / 15 / 92$ & 1 & 1 & & 82.7 & $Y$ & TMACS \\
\hline 1 & $11 / 20 / 92$ & 1 & 1 & & 82.7 & $\bar{Y}$ & K 910226246-45 \\
\hline 1 & $11 / 22 / 92$ & 1 & 1 & & 83.1 & $\mathrm{Y}$ & TMACS \\
\hline 1 & $11 / 29 / 92$ & 1 & 1 & & 83.1 & $Y$ & TMACS \\
\hline
\end{tabular}

Data obtained from WHC Surveillance Analysis Computer System (SACS), November 12, 1993. 
WHC-SD-WM-ER-312, Rev. 0

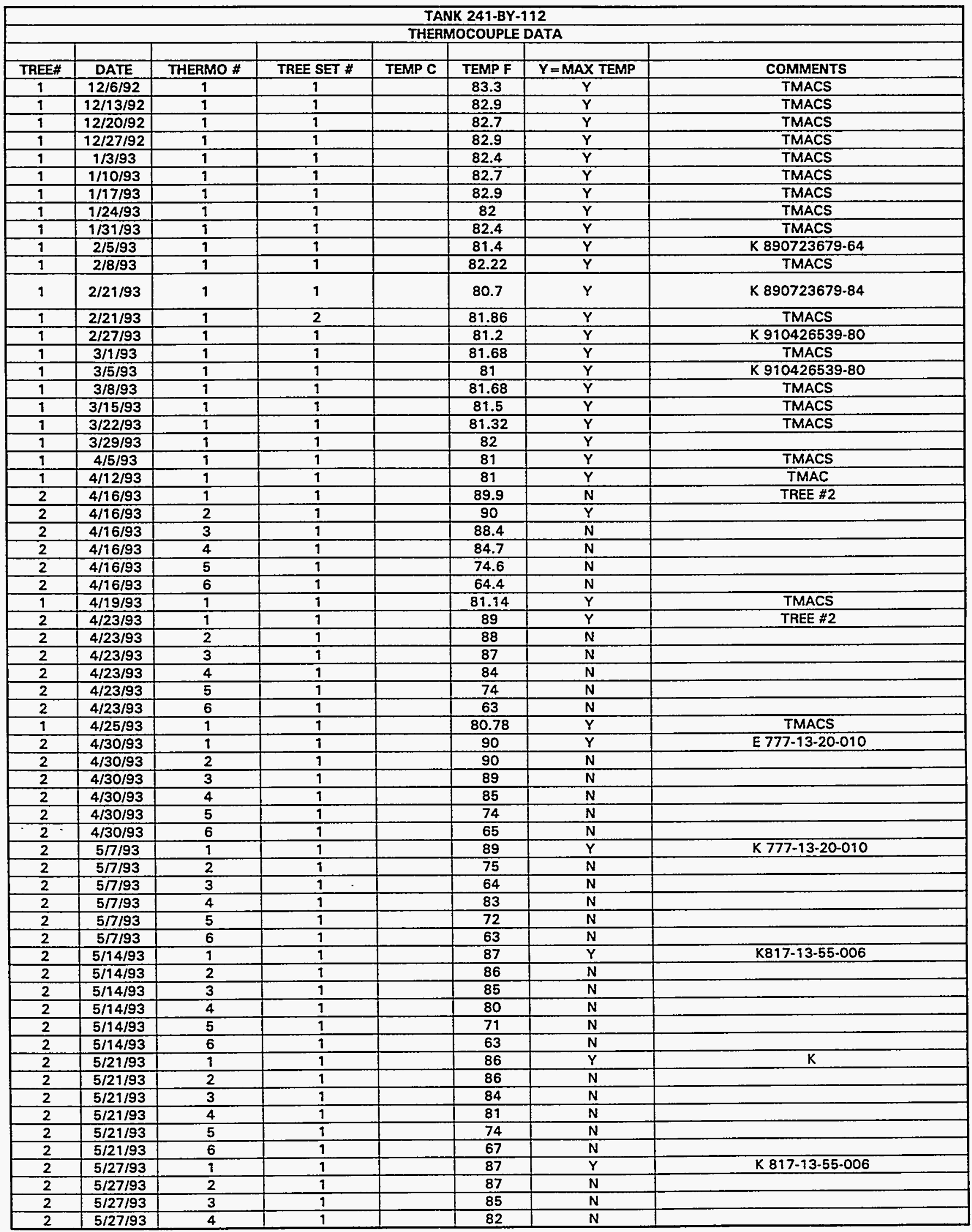

Data obtained from WHC Surveillance Analysis Computer System (SACS), November 12, 1993. 


\begin{tabular}{|c|c|c|c|c|c|c|c|}
\hline \multicolumn{8}{|c|}{ TANK 241-BY-112 } \\
\hline \multicolumn{8}{|c|}{ THERMOCOUPLE DATA } \\
\hline & & & & & & & \\
\hline TREE\# & DATE & THERMO \# & TREE SET \# & TEMP C & TEMP F & $Y=M A X$ TEMP & COMMENTS \\
\hline 2 & $5 / 27 / 93$ & 5 & 1 & & 71 & $N$ & \\
\hline 2 & $5 / 27 / 93$ & 6 & 1 & & 64 & $\mathbf{N}$ & \\
\hline$\overline{2}$ & $6 / 4 / 93$ & 1 & 1 & & 85 & $\bar{Y}$ & $K 817-13-55-006$ \\
\hline 2 & $6 / 4 / 93$ & 2 & 1 & & 87 & $\mathbf{N}$ & \\
\hline 2 & $6 / 4 / 93$ & 3 & 1 & & 83 & $N$ & \\
\hline 2 & $6 / 4 / 93$ & 4 & 1 & & 79 & $\mathrm{~N}$ & \\
\hline 2 & $6 / 4 / 93$ & 5 & 1 & & 71 & $\mathbf{N}$ & \\
\hline 2 & $6 / 4 / 93$ & 6 & 1 & & 63 & $\mathbf{N}$ & \\
\hline 2 & $6 / 14 / 93$ & 1 & 1 & & 87.1 & $\bar{Y}$ & $K 817-13-55-001$ \\
\hline 2 & $6 / 14 / 93$ & 2 & 1 & & 86 & $\mathbf{N}$ & \\
\hline 2 & $6 / 14 / 93$ & 3 & 1 & & 84 & $\mathbf{N}$ & \\
\hline 2 & $6 / 14 / 93$ & 4 & 1 & & 80.9 & $\mathbf{N}$ & \\
\hline 2 & $6 / 14 / 93$ & 5 & 1 & & 71.7 & $\mathbf{N}$ & \\
\hline 2 & $6 / 14 / 93$ & 6 & 1 & & 64.5 & $\mathrm{~N}$ & \\
\hline 2 & $6 / 18 / 93$ & 1 & 1 & & 86 & $\mathbf{Y}$ & $k 777-13-20-010$ \\
\hline 2 & $6 / 18 / 93$ & 2 & 1 & & 84 & $\mathrm{~N}$ & \\
\hline 2 & $6 / 18 / 93$ & 3 & 1 & & 82 & $\mathbf{N}$ & \\
\hline 2 & $6 / 18 / 93$ & 4 & 1 & & 77 & $\mathbf{N}$ & \\
\hline 2 & $6 / 18 / 93$ & 5 & 1 & & 70 & $\mathbf{N}$ & \\
\hline 2 & $6 / 18 / 93$ & 6 & 1 & & 63 & $\mathbf{N}$ & \\
\hline 1 & $6 / 19 / 93$ & 1 & 1 & & 80.5 & $Y$ & $\begin{array}{l}\text { LOWERED TEMP PROBE TO BOTTOM OF } \\
\text { LOW. K } 910426539-80\end{array}$ \\
\hline 1 & $6 / 19 / 93$ & 3 & 1 & & & $\mathbf{N}$ & \\
\hline 1 & $6 / 19 / 93$ & 4 & 1 & & & $N$ & \\
\hline 1 & $6 / 19 / 93$ & 5 & 1 & & & $N$ & \\
\hline 1 & $6 / 19 / 93$ & 6 & 1 & & & $\mathbf{N}$ & \\
\hline 1 & $6 / 21 / 93$ & 1 & 1 & & 80.78 & $\mathbf{Y}$ & TMACS \\
\hline 1 & $6 / 27 / 93$ & 1 & 1 & & 82.4 & $\mathbf{Y}$ & TMACS. down req. man. rdgs. \\
\hline 1 & $7 / 3 / 93$ & 1 & 9 & & 81.3 & $\dot{Y}$ & man. rdg. \\
\hline 2 & $7 / 3 / 93$ & 1 & 1 & & 87.3 & $\bar{Y}$ & KT1-024 \\
\hline 2 & $7 / 3 / 93$ & 2 & 9 & & 87 & $\mathbf{N}$ & \\
\hline 2 & $7 / 3 / 93$ & 3 & 1 & & 84.9 & $\mathbf{N}$ & \\
\hline 2 & $7 / 3 / 93$ & 4 & 1 & & 81 & $N$ & \\
\hline 2 & $7 / 3 / 93$ & 5 & 1 & & 72.3 & $N$ & \\
\hline 2 & $7 / 3 / 93$ & 6 & 1 & & 65.7 & $N$ & \\
\hline 1 & $7 / 5 / 93$ & 1 & 1 & & 83.12 & $\bar{Y}$ & TMACS \\
\hline 1 & $7 / 12 / 93$ & 1 & 1 & & 82.4 & $Y$ & TMACS \\
\hline 2 & $7 / 12 / 93$ & 1 & 1 & & 88.88 & $\bar{Y}$ & TMACS \\
\hline 2 & $7 / 12 / 93$ & 2 & 1 & & 88.7 & $\mathbf{N}$ & \\
\hline 2 & $7 / 12 / 93$ & 3 & 1 & & 87.08 & $\mathbf{N}$ & \\
\hline 2 & $7 / 12 / 93$ & 4 & 1 & & 84.02 & $\mathbf{N}$ & \\
\hline 2 & $7 / 12 / 93$ & 5 & 1 & & 76.82 & $\mathbf{N}$ & \\
\hline 2 & $7 / 12 / 93$ & 6 & 1 & & 71.42 & $\mathbf{N}$ & \\
\hline 1 & $7 / 19 / 93$ & 1 & 1 & & 82.76 & $\mathbf{Y}$ & TMACS \\
\hline 2 & $7 / 19 / 93$ & 1 & 1 & & 88.88 & $\mathbf{N}$ & TMACS \\
\hline 2 & $7 / 19 / 93$ & 2 & 1 & & 89.06 & $\bar{Y}$ & \\
\hline 2 & $7 / 19 / 93$ & 3 & 9 & & 87.8 & $\mathbf{N}$ & \\
\hline 2 & $7 / 19 / 93$ & 4 & 1 & & 84.74 & $N$ & \\
\hline 2 & $7 / 19 / 93$ & 5 & 1 & & 77.36 & $\mathrm{~N}$ & \\
\hline 2 & $7 / 19 / 93$ & 6 & 1 & & 72.5 & $\mathbf{N}$ & \\
\hline 1 & $7 / 26 / 93$ & 1 & 1 & & 82.58 & $\bar{Y}$ & TMACS \\
\hline 2 & $7 / 26 / 93$ & 1 & 1 & & 88.7 & $Y$ & TMACS \\
\hline 2 & $7 / 26 / 93$ & 2 & 1 & & 88.7 & $\mathbf{N}$ & \\
\hline 2 & $7 / 26 / 93$ & 3 & 1 & & 87.44 & $\mathbf{N}$ & \\
\hline 2 & $7 / 26 / 93$ & 4 & 1 & & 84.02 & $\mathrm{~N}$ & \\
\hline 2 & $7 / 26 / 93$ & 5 & 1 & & 77.18 & $\mathbf{N}$ & \\
\hline 2 & $7 / 26 / 93$ & 6 & 1 & & 72.32 & $\mathbf{N}$ & \\
\hline 1 & $8 / 2 / 93$ & $\frac{1}{1}$ & 1 & & 83.12 & $\bar{Y}$ & TMACS \\
\hline 2 & $8 / 2 / 93$ & 1 & 1 & & 89.06 & $Y$ & TMACS \\
\hline 2 & $8 / 2 / 93$ & 2 & 1 & & 89.06 & $\mathrm{~N}$ & \\
\hline 2 & $8 / 2 / 93$ & 3 & 1 & & 87.62 & $\mathbf{N}$ & \\
\hline 2 & $8 / 2 / 93$ & 4 & 1 & & 84.74 & $\mathbf{N}$ & \\
\hline 2 & $8 / 2 / 93$ & 5 & 1 & & 78.08 & $\mathbf{N}$ & \\
\hline 2 & $8 / 2 / 93$ & 6 & 1 & & 72.86 & $N$ & \\
\hline$\frac{T}{1}$ & $8 / 9 / 93$ & 1 & 1 & & 83.12 & $\bar{Y}$ & TMACS \\
\hline
\end{tabular}

Data obtained from WHC Surveillance Analysis Computer System (SACS), November 12, 1993. 


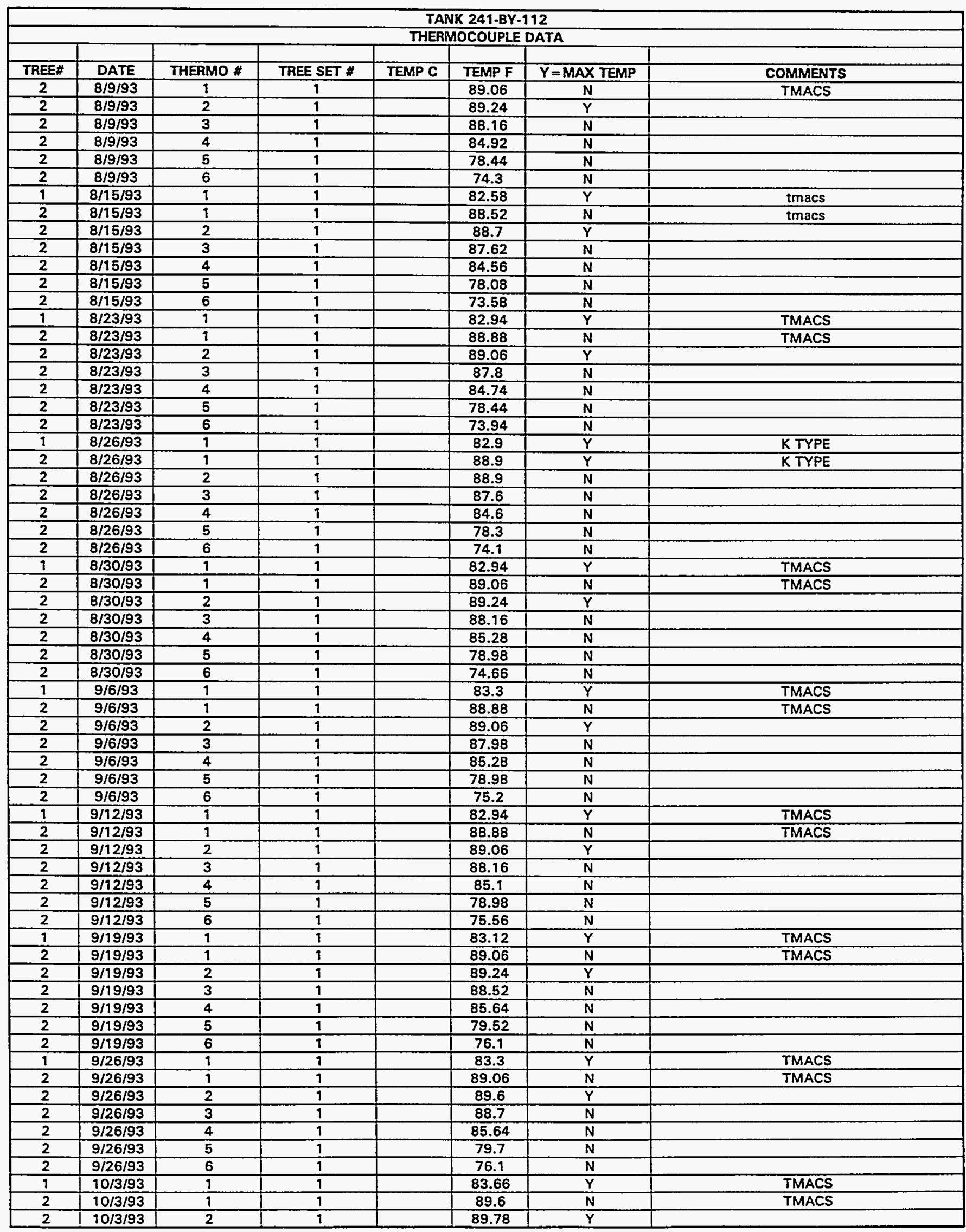

Data obtained from WHC Surveillance Analysis Computer System (SACS), November 12, 1993. 


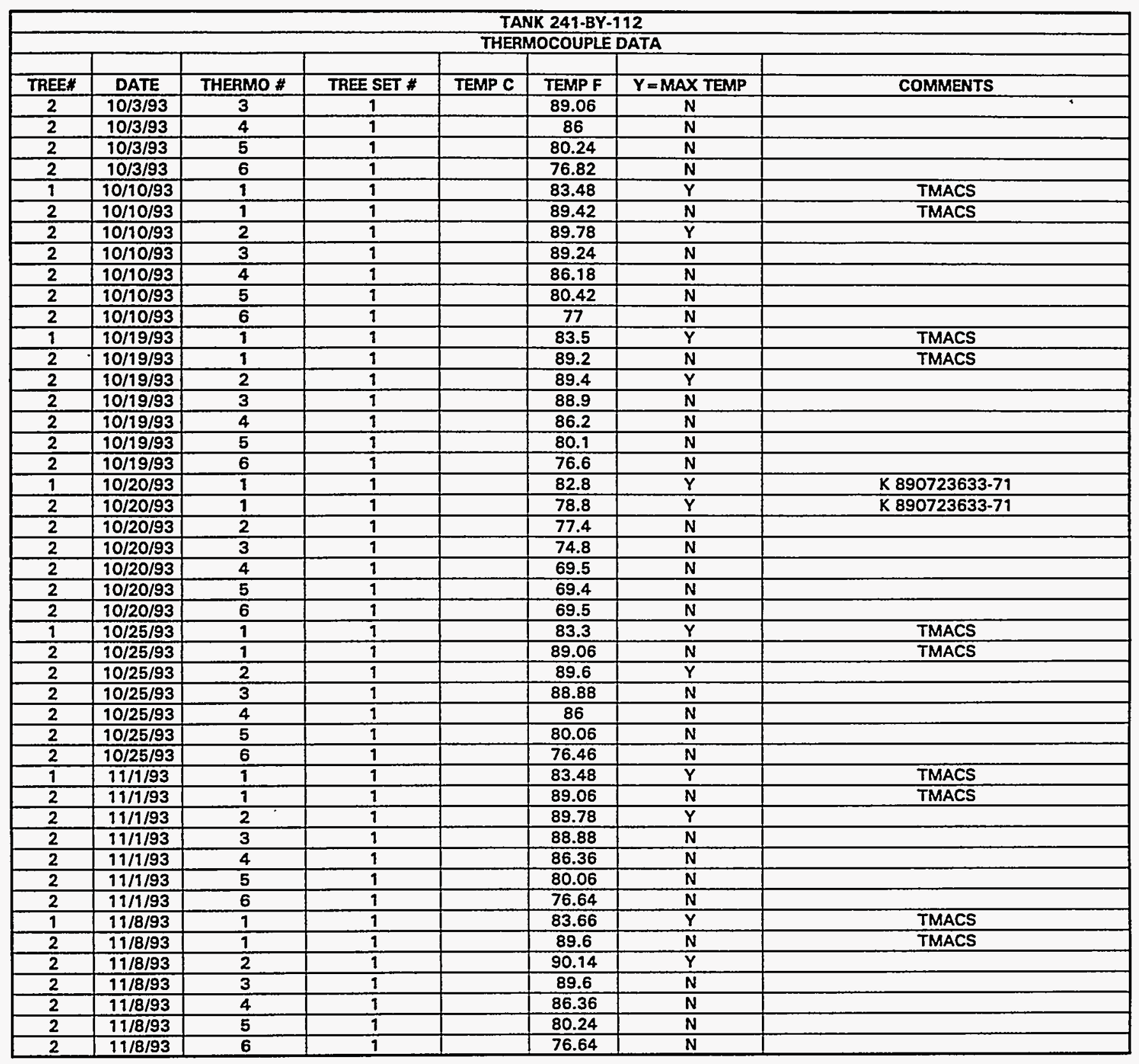

Data obtained from WHC Surveillance Analysis Computer System (SACS), November 12, 1993. 
WHC-SD-WM-ER-312, Rev. 0

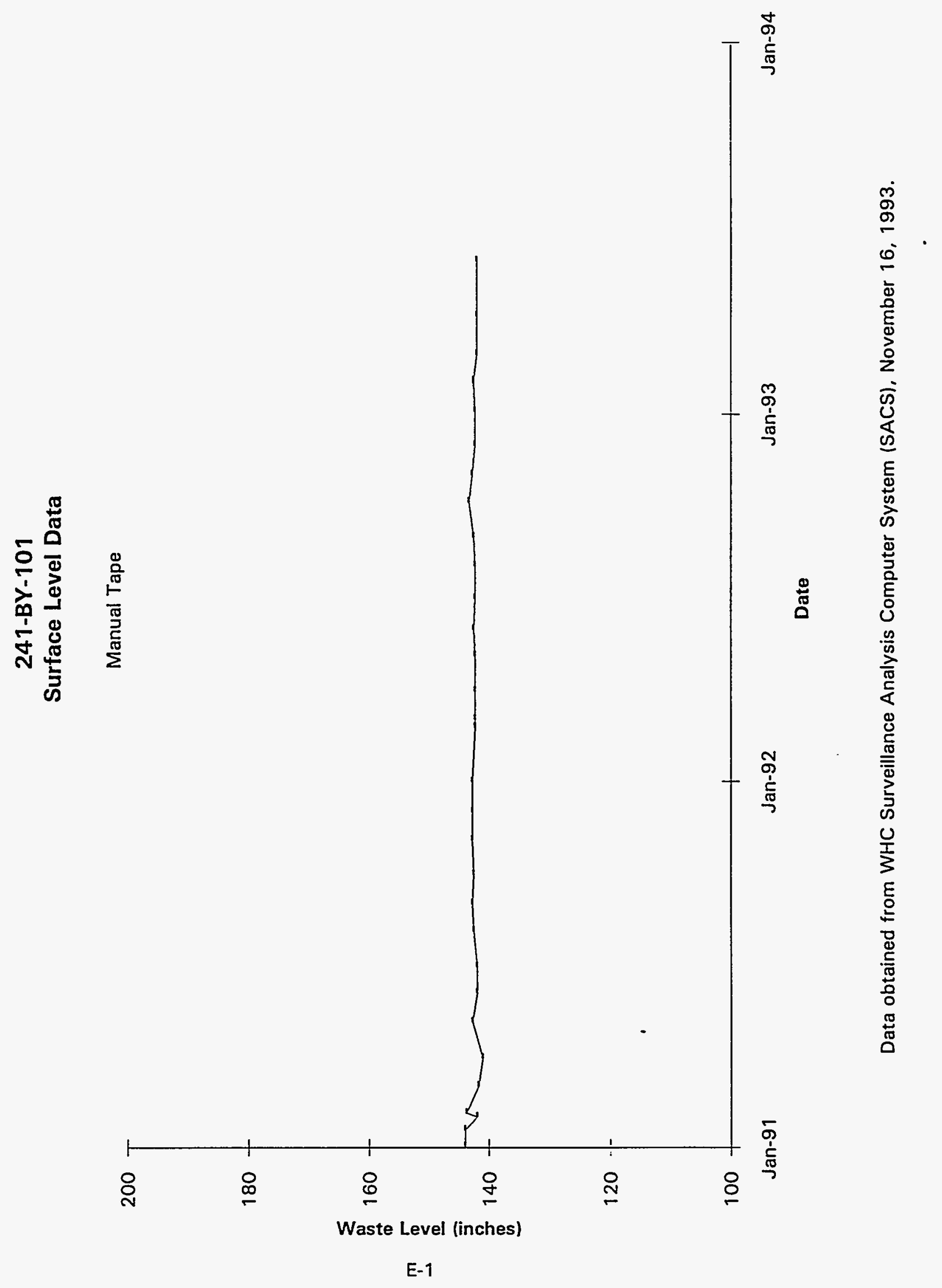




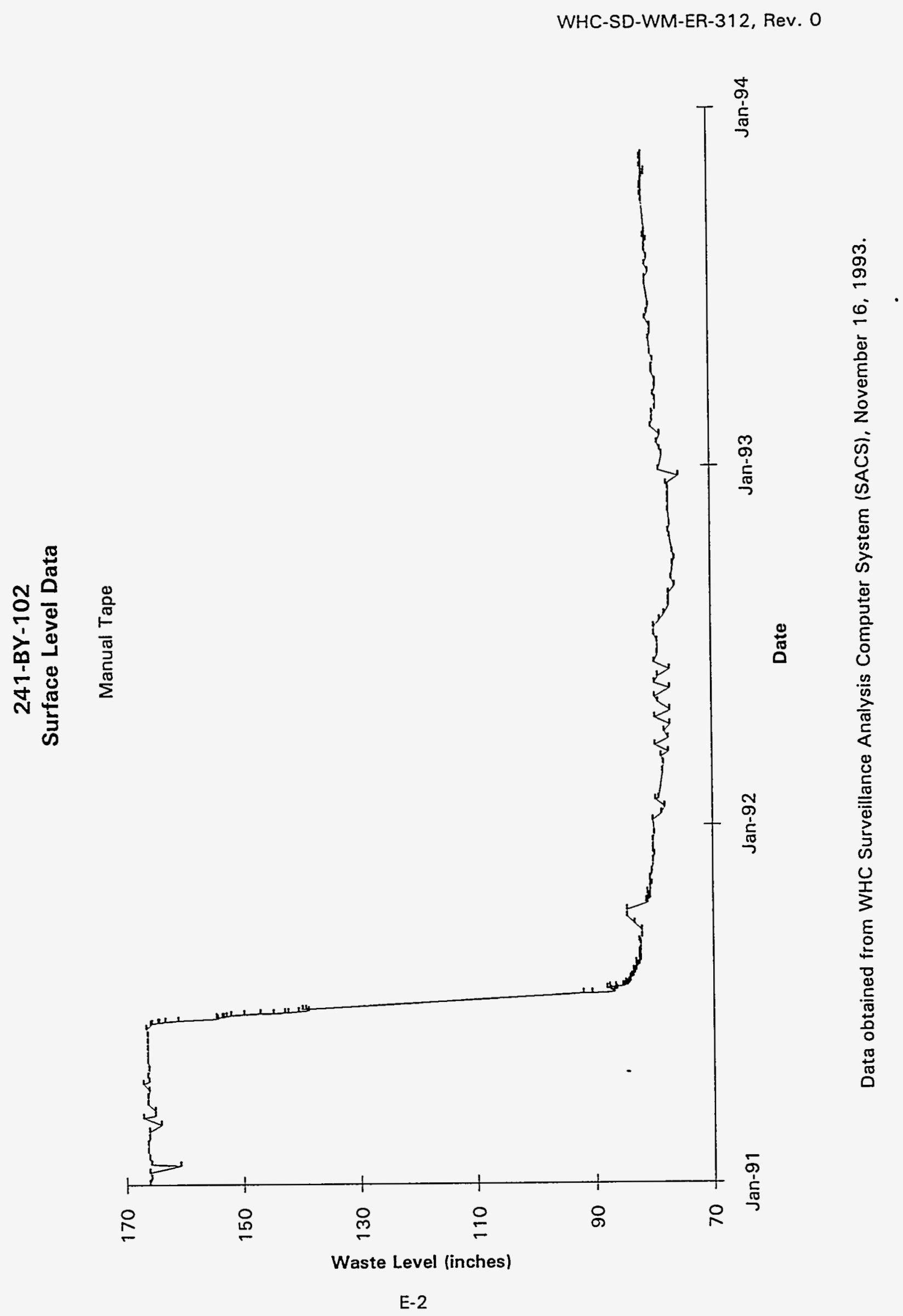



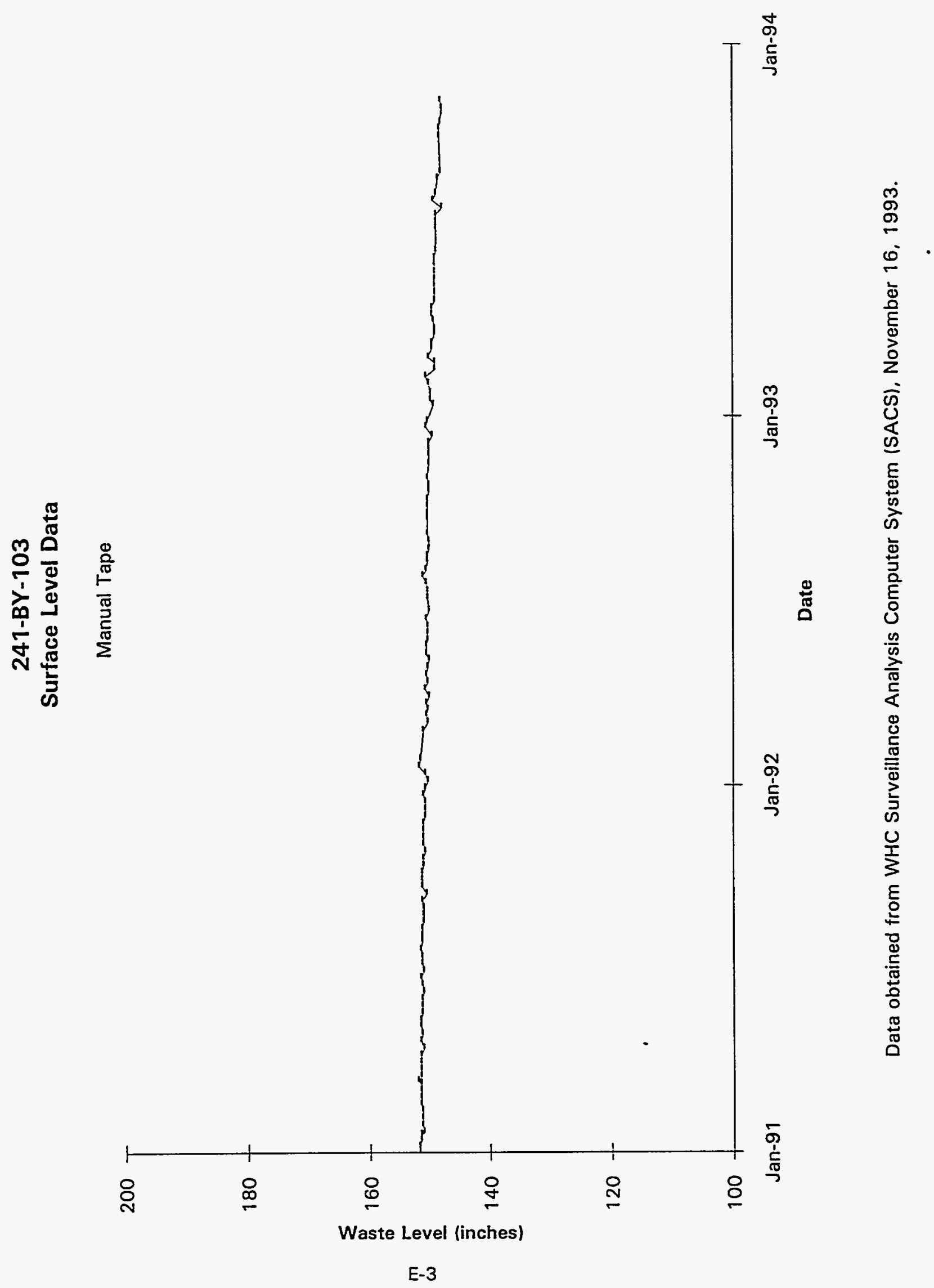
WHC-SD-WM-ER-312, Rev. 0

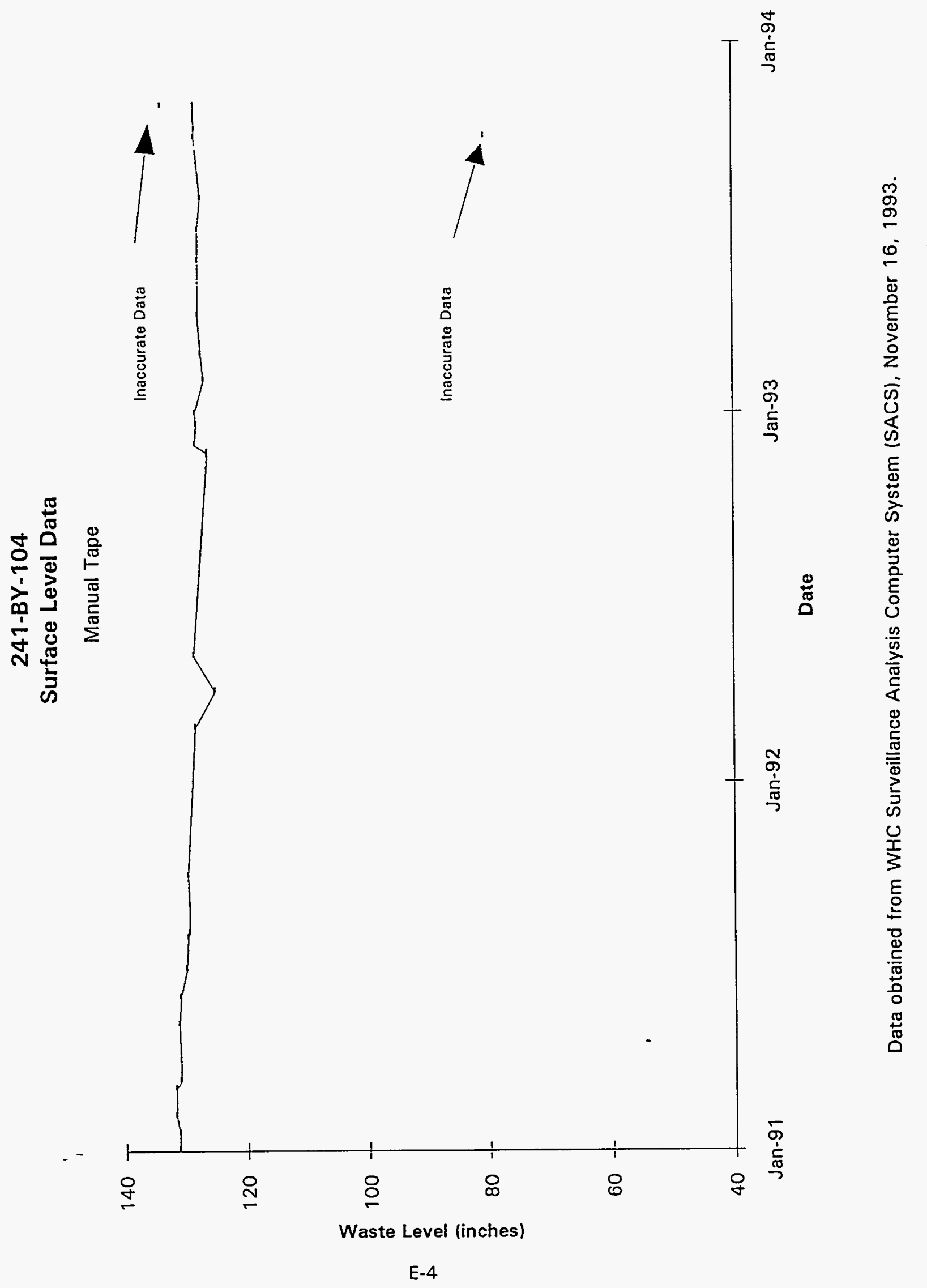


WHC-SD-WM-ER-312, Rev. 0

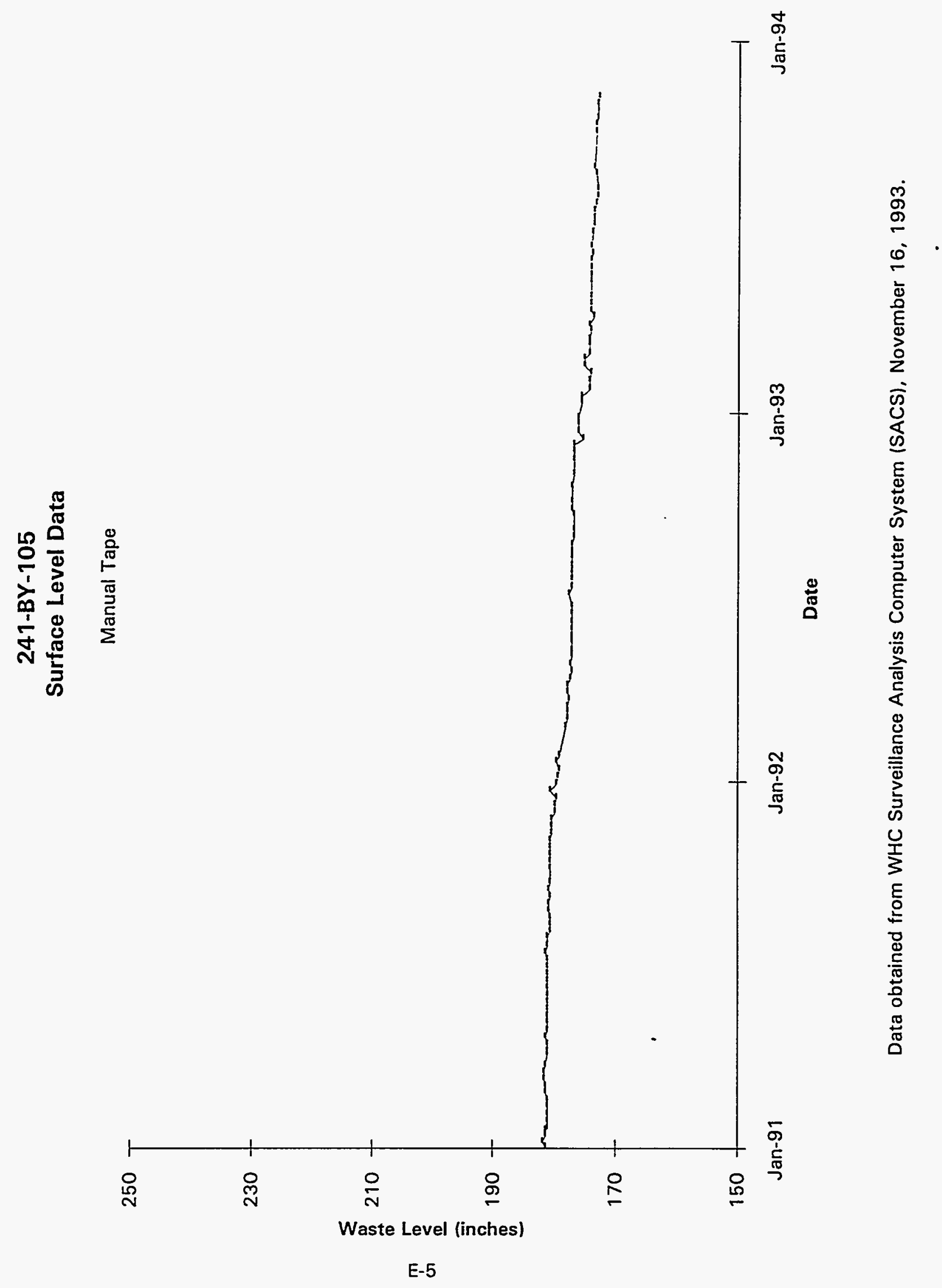


WHC-SD-WM-ER-312, Rev. O
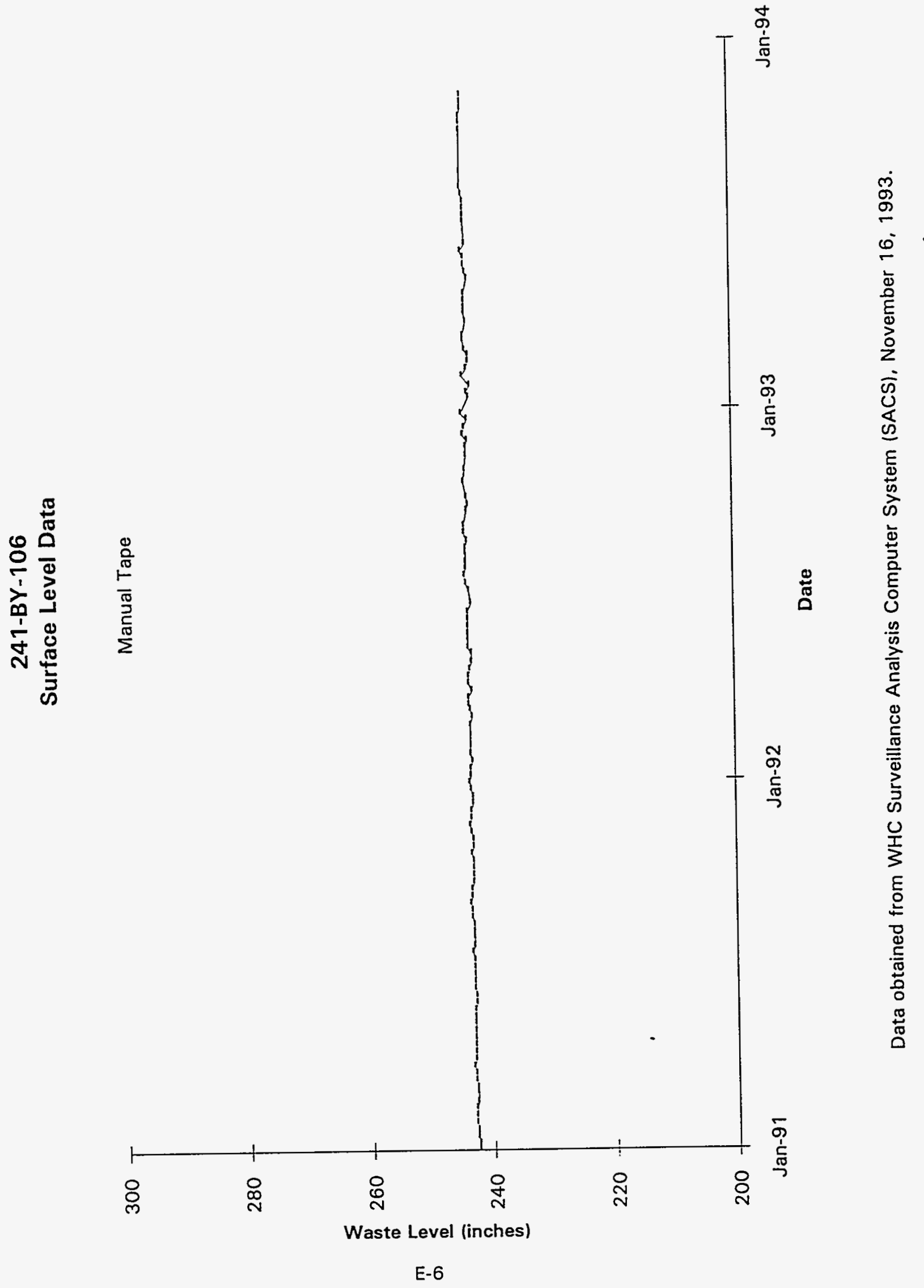
WHC-SD-WM-ER-312, Rev. 0

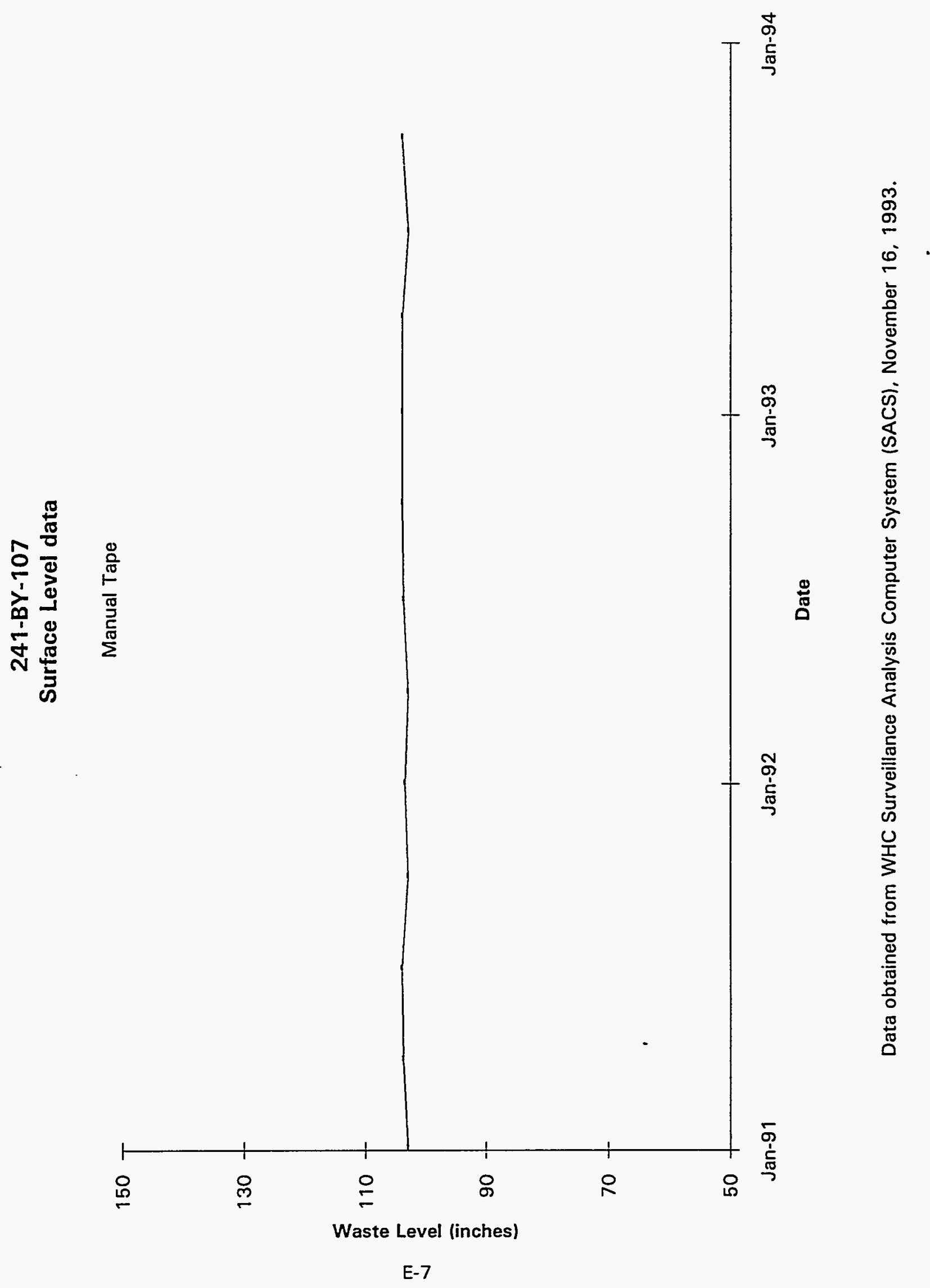


241-BY-108

\section{Surface Level Data}

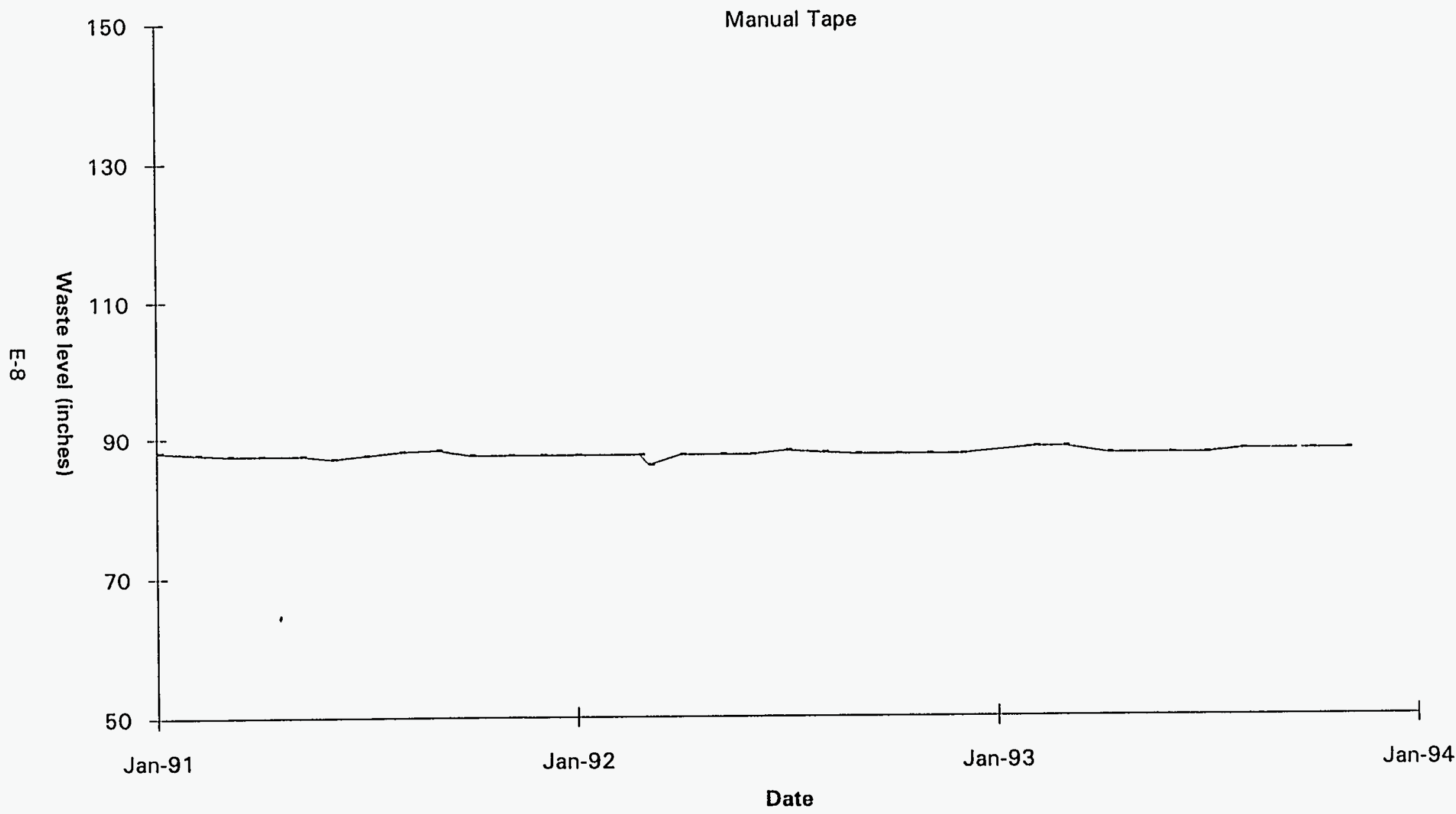

Data obtained from WHC Surveillance Analysis Computer System (SACS), November 16, 1993. 
WHC-SD-WM-ER-312, Rev. 0

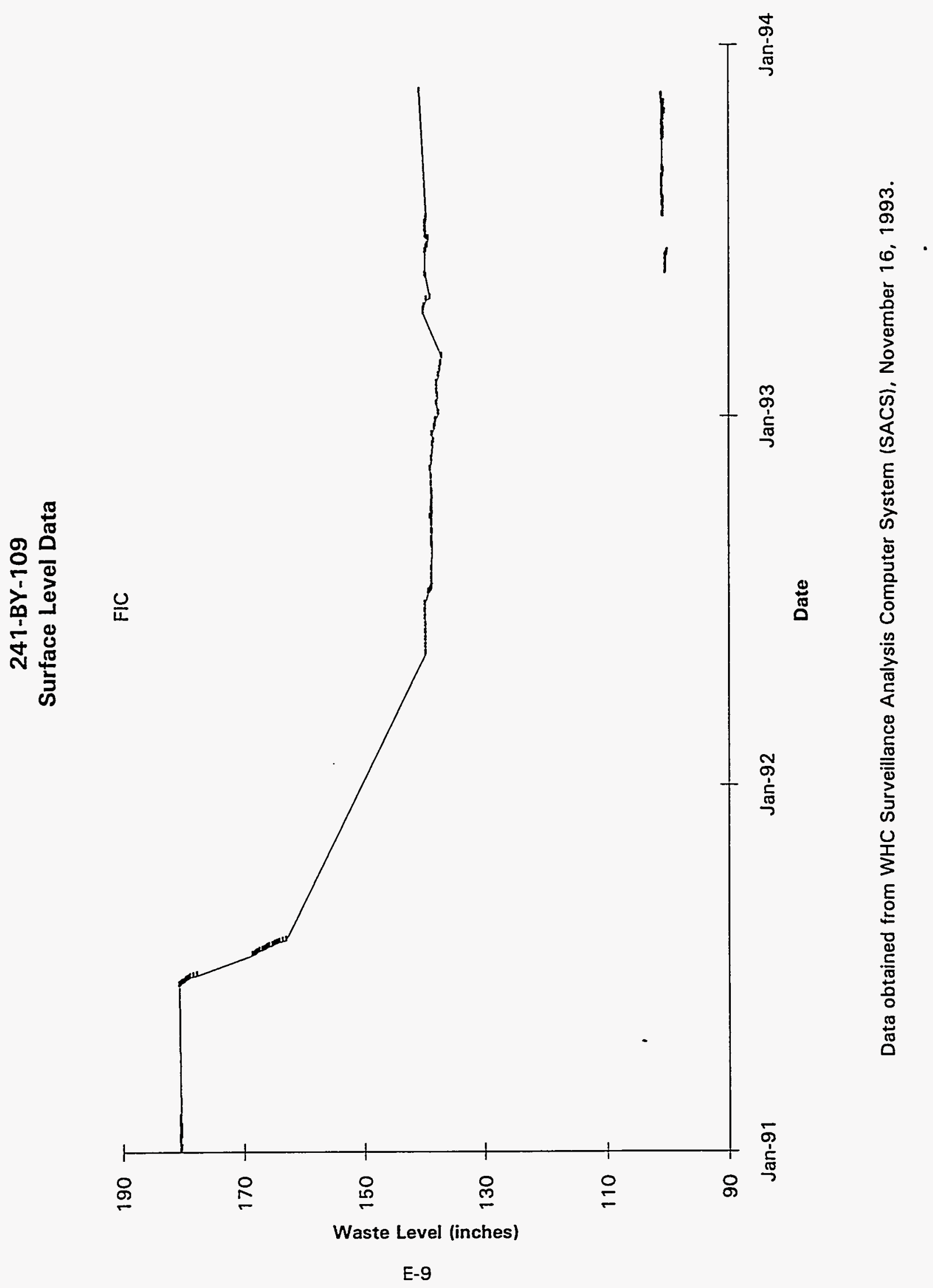


WHC-SD-WM-ER-312, Rev. O

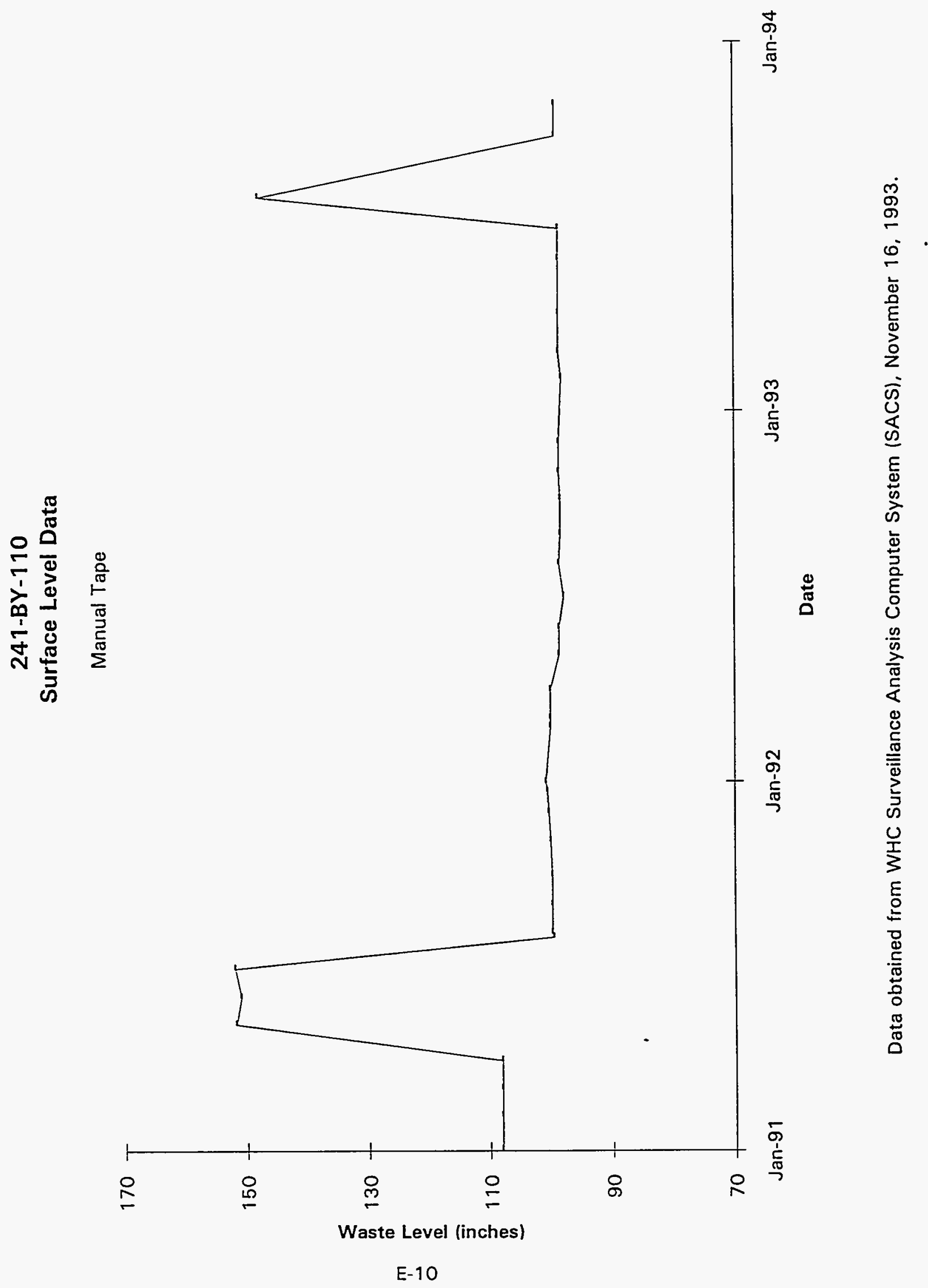


WHC-SD-WM-ER-312, Rev. 0

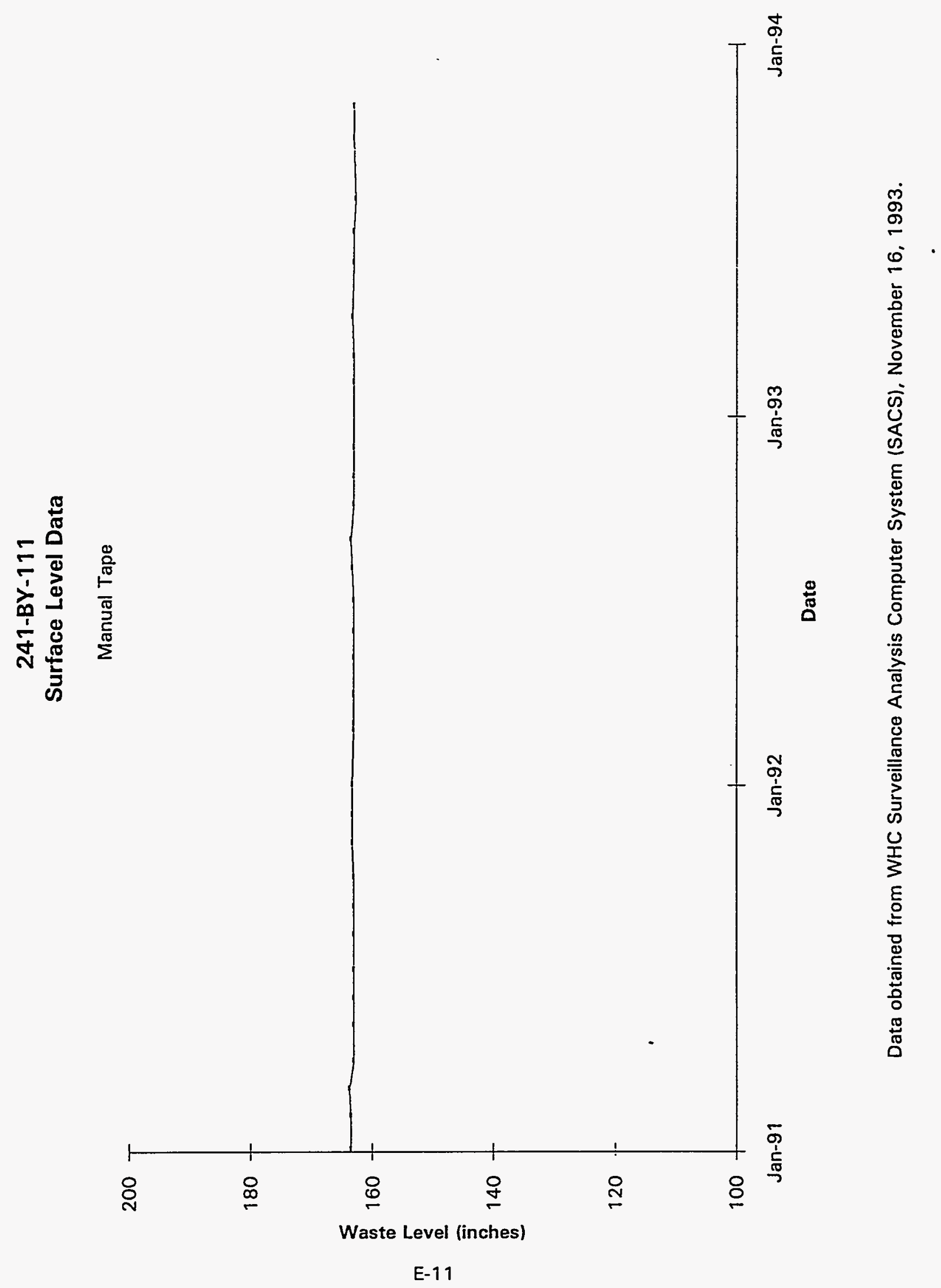


WHC-SD-WM-ER-312, Rev. 0

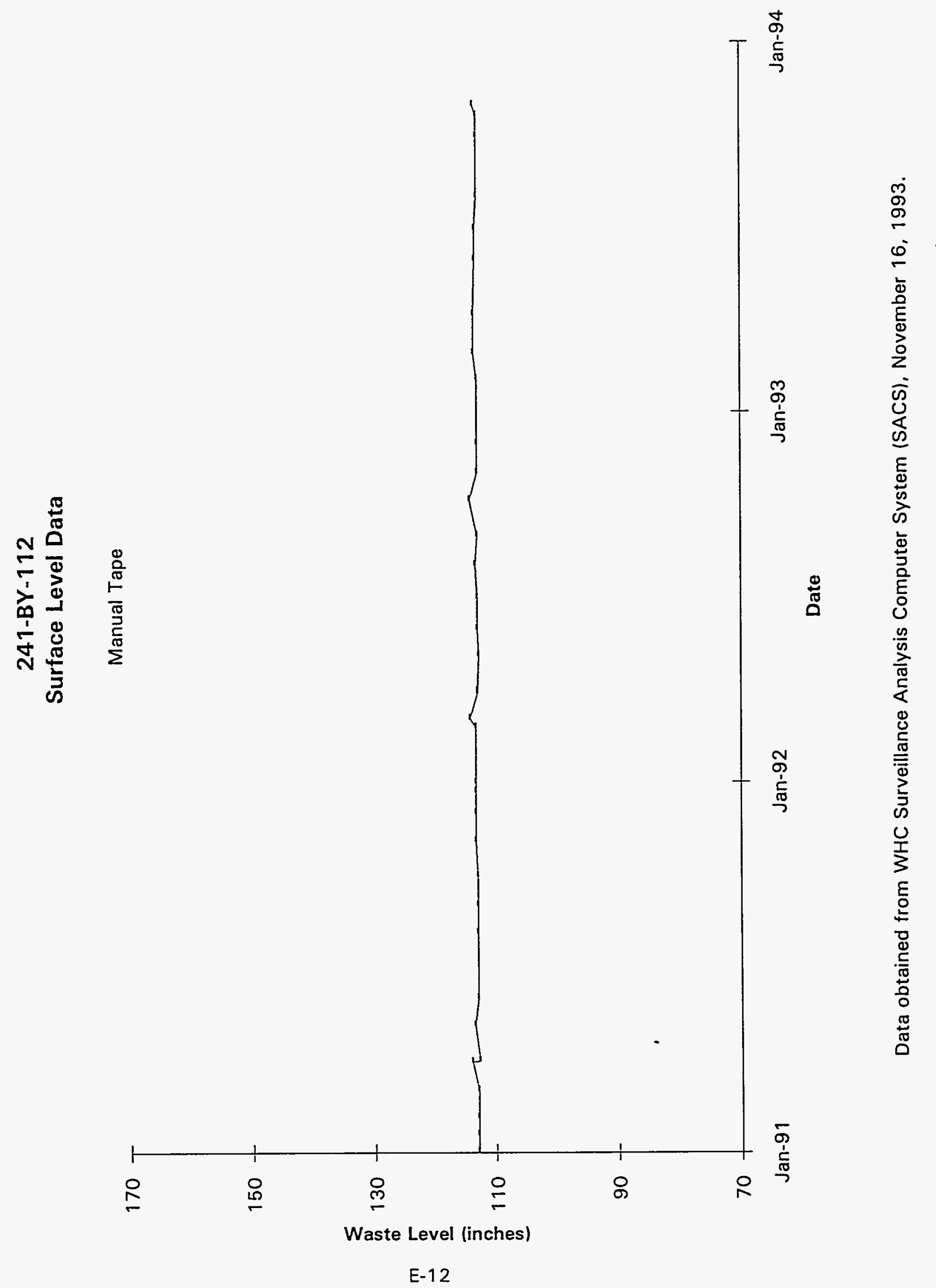




\begin{tabular}{|c|c|c|c|c|c|}
\hline \multicolumn{6}{|c|}{ Surface Level Data } \\
\hline \multicolumn{6}{|c|}{ Manual Tape } \\
\hline Year & Month & Day & Hour & Minutes & Surface Level \\
\hline 91 & 1 & 2 & 15 & 25 & 144 \\
\hline 91 & 1 & 19 & 15 & 25 & 144 \\
\hline 91 & 2 & 1 & 10 & 0 & 142 \\
\hline 91 & 2 & 5 & 8 & 20 & 143.75 \\
\hline 91 & 3 & 4 & 14 & 56 & 141.75 \\
\hline 91 & 4 & 1 & 15 & 24 & 141 \\
\hline 91 & 5 & 7 & 13 & 24 & 142.75 \\
\hline 91 & 6 & 5 & 9 & 55 & 142 \\
\hline 91 & 7 & 1 & 9 & 55 & 142 \\
\hline 91 & 8 & 6 & 20 & 17 & 142.5 \\
\hline 91 & 9 & 2 & 10 & 0 & 142.75 \\
\hline 91 & 10 & 1 & 10 & 0 & 142.5 \\
\hline 91 & 11 & 4 & 10 & 0 & 142.75 \\
\hline 91 & 12 & 2 & 10 & 0 & 142.75 \\
\hline 92 & 1 & 1 & 10 & 0 & 142.75 \\
\hline 92 & 2 & 25 & 15 & 15 & 142.25 \\
\hline 92 & 3 & 4 & 8 & 37 & 142.25 \\
\hline 92 & 4 & 1 & 14 & 57 & 142.25 \\
\hline 92 & 5 & 6 & 7 & 52 & 142.25 \\
\hline 92 & 6 & 7 & 15 & 28 & 142.5 \\
\hline 92 & 7 & 2 & 15 & 33 & 142.25 \\
\hline 92 & 8 & 3 & 17 & 27 & 142.25 \\
\hline 92 & 9 & 1 & 15 & 25 & 142.5 \\
\hline 92 & 10 & 6 & 6 & 53 & 143.25 \\
\hline 92 & 11 & 2 & 15 & 20 & 142.75 \\
\hline 92 & 12 & 1 & 14 & 43 & 142.25 \\
\hline 92 & 12 & 1 & 18 & 2 & 142.25 \\
\hline 93 & 1 & 4 & 10 & 0 & 142.25 \\
\hline 93 & 2 & 1 & 10 & 0 & 142.5 \\
\hline 93 & 2 & 3 & 2 & 28 & 142.5 \\
\hline 93 & 3 & 1 & 10 & 0 & 142 \\
\hline 93 & 3 & 1 & 17 & 22 & 142 \\
\hline 93 & 4 & 8 & 0 & 44 & 142 \\
\hline 93 & 6 & 1 & 14 & 48 & 142 \\
\hline & & & & & \\
\hline & & & & & \\
\hline & & & & & \\
\hline & & & & & \\
\hline & & & & & \\
\hline & & & & & \\
\hline & & & & & \\
\hline & & & & & \\
\hline & & & & & \\
\hline & & & & & \\
\hline & & & & & \\
\hline
\end{tabular}

Data obtained from WHC Surveillance Analysis Computer System (SACS), November 16, 1993. E-13 


\begin{tabular}{|c|c|c|c|c|c|}
\hline \multicolumn{6}{|c|}{ Surface Level Data } \\
\hline \multicolumn{6}{|c|}{ Manual Tape } \\
\hline Year & Month & Day & Hour & Minutes & Surface Level \\
\hline 91 & 1 & 2 & 8 & 11 & 166 \\
\hline 91 & 1 & 7 & 14 & 33 & 165.75 \\
\hline 91 & 1 & 14 & 15 & 11 & 166 \\
\hline 91 & 1 & 21 & 1 & 31 & 160.75 \\
\hline 91 & 1 & 22 & 7 & 59 & 165.75 \\
\hline 91 & 1 & 28 & 15 & 8 & 166 \\
\hline 91 & 2 & 4 & 15 & 28 & 166.25 \\
\hline 91 & 2 & 12 & 16 & 39 & 166.25 \\
\hline 91 & 2 & 18 & 18 & 25 & 166 \\
\hline 91 & 2 & 25 & 15 & 12 & 166 \\
\hline 91 & 3 & 4 & 14 & 56 & 164 \\
\hline 91 & 3 & 11 & 16 & 42 & 167 \\
\hline 91 & 3 & 13 & 15 & 27 & 165 \\
\hline 91 & 3 & 18 & 17 & 25 & 165 \\
\hline 91 & 3 & 25 & 15 & 1 & 166.25 \\
\hline 91 & 4 & 1 & 15 & 24 & 166.25 \\
\hline 91 & 4 & 8 & 17 & 53 & 166 \\
\hline 91 & 4 & 15 & 15 & 23 & 167 \\
\hline 91 & 4 & 17 & 7 & 35 & 166 \\
\hline 91 & 4 & 22 & 15 & 3 & 166.25 \\
\hline 91 & 4 & 29 & 17 & 17 & 166 \\
\hline 91 & 5 & 7 & 13 & 24 & 166.25 \\
\hline 91 & 5 & 13 & 14 & 54 & 166.25 \\
\hline 91 & 5 & 20 & 14 & 57 & 166.25 \\
\hline 91 & 5 & 27 & 14 & 41 & 166.25 \\
\hline 91 & 6 & 3 & 14 & 56 & 166.25 \\
\hline 91 & 6 & 10 & 14 & 55 & 166.5 \\
\hline 91 & 6 & 14 & 15 & 34 & 165.75 \\
\hline 91 & 6 & 15 & 15 & 12 & 165.5 \\
\hline 91 & 6 & 16 & 6 & 37 & 164.5 \\
\hline 91 & 6 & 16 & 15 & 26 & 164.25 \\
\hline 91 & 6 & 17 & 4 & 10 & 163.25 \\
\hline 91 & 6 & 18 & 2 & 12 & 161 \\
\hline 91 & 6 & 20 & 16 & 30 & 154.25 \\
\hline 91 & 6 & 21 & 6 & 8 & 154.5 \\
\hline 91 & 6 & 21 & 16 & 47 & 153.5 \\
\hline 91 & 6 & 21 & 22 & 33 & 153.25 \\
\hline 91 & 6 & 22 & 15 & 12 & 152.75 \\
\hline 91 & 6 & 22 & 16 & 25 & 152.75 \\
\hline 91 & 6 & 23 & 17 & 18 & 152 \\
\hline 91 & 6 & 24 & 18 & 8 & 149.75 \\
\hline 91 & 6 & 25 & 15 & 17 & 147 \\
\hline 91 & 6 & 25 & 23 & 8 & 144.75 \\
\hline 91 & 6 & 26 & 7 & 20 & 142.75 \\
\hline 91 & 6 & 26 & 18 & 6 & 142.25 \\
\hline 91 & 6 & 27 & 17 & 29 & 140.5 \\
\hline
\end{tabular}

Data obtained from WHC Surveillance Analysis Computer System (SACS), November 16, 1993. 


\begin{tabular}{|c|c|c|c|c|c|}
\hline Year & Month & Day & Hour & Minutes & Surface Level \\
\hline 91 & 6 & 28 & 19 & 17 & 138.75 \\
\hline 91 & 6 & 29 & 1 & 23 & 139.25 \\
\hline 91 & 6 & 29 & 9 & 55 & 139.75 \\
\hline 91 & 7 & 14 & 8 & 47 & 92 \\
\hline 91 & 7 & 14 & 23 & 18 & 90.5 \\
\hline 91 & 7 & 15 & 14 & 23 & 86.75 \\
\hline 91 & 7 & 15 & 14 & 39 & 86.75 \\
\hline 91 & 7 & 16 & 13 & 34 & 86.75 \\
\hline 91 & 7 & 17 & 2 & 22 & 87.25 \\
\hline 91 & 7 & 18 & 15 & 11 & 86.5 \\
\hline 91 & 7 & 18 & 17 & 8 & 86.25 \\
\hline 91 & 7 & 19 & 23 & 22 & 88 \\
\hline 91 & 7 & 20 & 15 & 6 & 87.5 \\
\hline 91 & 7 & 20 & 20 & 59 & 86.5 \\
\hline 91 & 7 & 21 & 15 & 0 & 85.25 \\
\hline 91 & 7 & 21 & 18 & 55 & 85 \\
\hline 91 & 7 & 22 & 7 & 18 & 84.75 \\
\hline 91 & 7 & 22 & 21 & 21 & 84.5 \\
\hline 91 & 7 & 23 & 7 & 25 & 84.5 \\
\hline 91 & 7 & 23 & 15 & 49 & 84.5 \\
\hline 91 & 7 & 24 & 7 & 24 & 84.75 \\
\hline 91 & 7 & 24 & 15 & 3 & 84.25 \\
\hline 91 & 7 & 24 & 20 & 12 & 84.75 \\
\hline 91 & 7 & 25 & 8 & 37 & 84.25 \\
\hline 91 & 7 & 26 & 7 & 20 & 84 \\
\hline 91 & 7 & 26 & 14 & 38 & 84 \\
\hline 91 & 7 & 27 & 7 & 29 & 84 \\
\hline 91 & 7 & 27 & 14 & 55 & 84 \\
\hline 91 & 7 & 27 & 17 & 22 & 84 \\
\hline 91 & 7 & 28 & 7 & 17 & 84 \\
\hline 91 & 7 & 28 & 14 & 29 & 84 \\
\hline 91 & 7 & 29 & 2 & 38 & 84 \\
\hline 91 & 7 & 29 & 21 & 3 & 84 \\
\hline 91 & 7 & 30 & 23 & 1 & 83.75 \\
\hline 91 & 7 & 31 & 0 & 29 & 83.75 \\
\hline 91 & 7 & 31 & 15 & 19 & 83.5 \\
\hline 91 & 7 & 31 & 22 & 52 & 83.75 \\
\hline 91 & 8 & 2 & 6 & 36 & 83.5 \\
\hline 91 & 8 & 2 & 15 & 20 & 83.5 \\
\hline 91 & 8 & 3 & 6 & 51 & 83.25 \\
\hline 91 & 8 & 3 & 15 & 25 & 83.5 \\
\hline 91 & 8 & 3 & 23 & 18 & 83.5 \\
\hline 91 & 8 & 4 & 7 & 4 & 83.25 \\
\hline 91 & 8 & 4 & 22 & 39 & 83.5 \\
\hline 91 & 8 & 5 & 15 & 6 & 83.25 \\
\hline 91 & 8 & 5 & 18 & 12 & 83.25 \\
\hline 91 & 8 & 6 & 14 & 50 & 83 \\
\hline 91 & 8 & 6 & 23 & 4 & 83.5 \\
\hline
\end{tabular}

Data obtained from WHC Surveillance Analysis Computer System (SACS), November 16, 1993. 


\begin{tabular}{|c|c|c|c|c|c|}
\hline Year & Month & Day & Hour & Minutes & Surface Level \\
\hline 91 & 8 & 7 & 17 & 24 & 83 \\
\hline 91 & 8 & 8 & 17 & 30 & 83 \\
\hline 91 & 8 & 9 & 7 & 26 & 83 \\
\hline 91 & 8 & 9 & 17 & 20 & 83 \\
\hline 91 & 8 & 10 & 1 & 10 & 83 \\
\hline 91 & 8 & 10 & 10 & 17 & 82.75 \\
\hline 91 & 8 & 10 & 23 & 3 & 83 \\
\hline 91 & 8 & 11 & 1 & 30 & 83 \\
\hline 91 & 8 & 11 & 15 & 19 & 82.75 \\
\hline 91 & 8 & 12 & 7 & 8 & 82.5 \\
\hline 91 & 8 & 12 & 13 & 51 & 83 \\
\hline 91 & 8 & 13 & 5 & 18 & 83 \\
\hline 91 & 8 & 13 & 11 & 34 & 83 \\
\hline 91 & 8 & 14 & 2 & 25 & 82.5 \\
\hline 91 & 8 & 14 & 15 & 17 & 83 \\
\hline 91 & 8 & 14 & 18 & 45 & 83 \\
\hline 91 & 8 & 14 & 23 & 1 & 82.5 \\
\hline 91 & 8 & 15 & 1 & 51 & 82.5 \\
\hline 91 & 8 & 15 & 14 & 5 & 82.5 \\
\hline 91 & 8 & 15 & 16 & 59 & 82.5 \\
\hline 91 & 8 & 16 & 5 & 21 & 82.5 \\
\hline 91 & 8 & 16 & 14 & 30 & 82.5 \\
\hline 91 & 8 & 16 & 23 & 3 & 82.25 \\
\hline 91 & 8 & 17 & 7 & 9 & 82.5 \\
\hline 91 & 8 & 17 & 14 & 3 & 82.5 \\
\hline 91 & 8 & 17 & 17 & 13 & 82.25 \\
\hline 91 & 8 & 18 & 1 & 21 & 82.25 \\
\hline 91 & 8 & 18 & 14 & 49 & 82.25 \\
\hline 91 & 8 & 18 & 18 & 10 & 82.25 \\
\hline 91 & 8 & 19 & 7 & 14 & 82.5 \\
\hline 91 & 8 & 19 & 14 & 22 & 82.25 \\
\hline 91 & 8 & 19 & 17 & 15 & 82.25 \\
\hline 91 & 8 & 20 & 22 & 38 & 82.25 \\
\hline 91 & 8 & 21 & 9 & 53 & 82.25 \\
\hline 91 & 8 & 21 & 15 & 7 & 82.25 \\
\hline 91 & 8 & 21 & 16 & 44 & 82.25 \\
\hline 91 & 8 & 22 & 7 & 34 & 82.25 \\
\hline 91 & 8 & 22 & 10 & 53 & 82.25 \\
\hline 91 & 8 & 22 & 17 & 15 & 82.25 \\
\hline 91 & 8 & 23 & 7 & 24 & 82.25 \\
\hline 91 & 8 & 23 & 14 & 43 & 82.25 \\
\hline 91 & 8 & 24 & 12 & 24 & 82.5 \\
\hline 91 & 8 & 25 & 8 & 20 & 82.25 \\
\hline 91 & 8 & 25 & 13 & 22 & 82.25 \\
\hline 91 & 8 & 25 & 23 & 16 & 82.25 \\
\hline 91 & 8 & 26 & 1 & 14 & 82.25 \\
\hline 91 & 9 & 2 & 14 & 57 & 82.25 \\
\hline 91 & 9 & 5 & 14 & 57 & 82.5 \\
\hline
\end{tabular}

Data obtained from WHC Surveillance Analysis Computer System (SACS), November 16, 1993. 


\begin{tabular}{|c|c|c|c|c|c|}
\hline Year & Month & Day & Hour & Minutes & Surface Level \\
\hline 91 & 9 & 9 & 14 & 57 & 82 \\
\hline 91 & 9 & 16 & 14 & 57 & 82 \\
\hline 91 & 9 & 23 & 14 & 57 & 83.25 \\
\hline 91 & 9 & 30 & 14 & 57 & 84.5 \\
\hline 91 & 10 & 7 & 7 & 20 & 84.5 \\
\hline 91 & 10 & 14 & 7 & 20 & 81 \\
\hline 91 & 10 & 15 & 7 & 20 & 81.25 \\
\hline 91 & 10 & 16 & 7 & 20 & 81.25 \\
\hline 91 & 10 & 17 & 7 & 22 & 81 \\
\hline 91 & 10 & 18 & 7 & 22 & 80.75 \\
\hline 91 & 10 & 19 & 1 & 8 & 80.5 \\
\hline 91 & 10 & 20 & 1 & 8 & 80.5 \\
\hline 91 & 10 & 21 & 1 & 8 & 80.5 \\
\hline 91 & 10 & 22 & 1 & 8 & 80.5 \\
\hline 91 & 10 & 23 & 1 & 8 & 81 \\
\hline 91 & 10 & 24 & 1 & 8 & 80.5 \\
\hline 91 & 10 & 25 & 1 & 8 & 81 \\
\hline 91 & 10 & 26 & 1 & 8 & 80.5 \\
\hline 91 & 10 & 27 & 1 & 8 & 80.5 \\
\hline 91 & 10 & 28 & 1 & 8 & 80.5 \\
\hline 91 & 10 & 29 & 1 & 34 & 80.5 \\
\hline 91 & 10 & 30 & 7 & 25 & 80.5 \\
\hline 91 & 11 & 1 & 7 & 26 & 80.5 \\
\hline 91 & 11 & 2 & 7 & 13 & 80.25 \\
\hline 91 & 11 & 3 & 6 & 56 & 80.5 \\
\hline 91 & 11 & 4 & 3 & 17 & 80.5 \\
\hline 91 & 11 & 5 & 2 & 37 & 80.5 \\
\hline 91 & 11 & 6 & 5 & 12 & 80.5 \\
\hline 91 & 11 & 7 & 1 & 10 & 80.5 \\
\hline 91 & 11 & 8 & 1 & 48 & 80.5 \\
\hline 91 & 11 & 13 & 6 & 43 & 80.25 \\
\hline 91 & 11 & 14 & 1 & 15 & 80.25 \\
\hline 91 & 11 & 15 & 6 & 13 & 80.25 \\
\hline 91 & 11 & 25 & 6 & 53 & 80 \\
\hline 91 & 11 & 26 & 6 & 28 & 80 \\
\hline 91 & 12 & 2 & 6 & 43 & 79.75 \\
\hline 91 & 12 & 5 & 6 & 43 & 80 \\
\hline 91 & 12 & 9 & 6 & 43 & 80 \\
\hline 91 & 12 & 16 & 6 & 43 & 80 \\
\hline 91 & 12 & 23 & 6 & 43 & 79.75 \\
\hline 92 & 1 & 6 & 15 & 15 & 80 \\
\hline 92 & 1 & 13 & 15 & 15 & 78.5 \\
\hline 92 & 1 & 20 & 15 & 15 & 78 \\
\hline 92 & 1 & 27 & 15 & 15 & 79.5 \\
\hline 92 & 1 & 28 & 15 & 15 & 79 \\
\hline 92 & 2 & 25 & 15 & 15 & 78.25 \\
\hline 92 & 3 & 4 & 8 & 37 & 78.12 \\
\hline 92 & 3 & 11 & 8 & 23 & 78.5 \\
\hline
\end{tabular}

Data obtained from WHC Surveillance Analysis Computer System (SACS), November 16, 1993. 


\begin{tabular}{|c|c|c|c|c|c|}
\hline Year & Month & Day & Hour & Minutes & Surface Level \\
\hline 92 & 3 & 16 & 16 & 33 & 77.25 \\
\hline 92 & 3 & 23 & 15 & 29 & 79.5 \\
\hline 92 & 3 & 30 & 15 & 33 & 77.25 \\
\hline 92 & 4 & 6 & 17 & 57 & 78 \\
\hline 92 & 4 & 13 & 15 & 39 & 77 \\
\hline 92 & 4 & 20 & 16 & 26 & 79.5 \\
\hline 92 & 4 & 27 & 22 & 5 & 77 \\
\hline 92 & 4 & 28 & 6 & 8 & 77 \\
\hline 92 & 5 & 6 & 7 & 52 & 79 \\
\hline 92 & 5 & 11 & 15 & 27 & 79.5 \\
\hline 92 & 5 & 20 & 8 & 15 & 77 \\
\hline 92 & 5 & 25 & 15 & 27 & 79.5 \\
\hline 92 & 6 & 1 & 15 & 28 & 79 \\
\hline 92 & 6 & 8 & 15 & 25 & 77 \\
\hline 92 & 6 & 15 & 15 & 13 & 79.5 \\
\hline 92 & 6 & 24 & 8 & 32 & 79 \\
\hline 92 & 7 & 6 & 16 & 47 & 79 \\
\hline 92 & 7 & 13 & 15 & 21 & 79.5 \\
\hline 92 & 7 & 21 & 15 & 19 & 79.5 \\
\hline 92 & 7 & 28 & 14 & 31 & 78.5 \\
\hline 92 & 8 & 3 & 17 & 27 & 77.75 \\
\hline 92 & 8 & 12 & 8 & 33 & 77 \\
\hline 92 & 8 & 17 & 15 & 30 & 77 \\
\hline 92 & 8 & 24 & 15 & 2 & 77 \\
\hline 92 & 9 & 1 & 15 & 25 & 76 \\
\hline 92 & 9 & 8 & 23 & 5 & 76.5 \\
\hline 92 & 9 & 23 & 14 & 20 & 76.25 \\
\hline 92 & 9 & 28 & 14 & 43 & 76 \\
\hline 92 & 10 & 5 & 14 & 20 & 76.25 \\
\hline 92 & 10 & 21 & 7 & 51 & 76.75 \\
\hline 92 & 10 & 26 & 19 & 18 & 77 \\
\hline 92 & 11 & 2 & 15 & 20 & 76.75 \\
\hline 92 & 11 & 16 & 19 & 44 & 77 \\
\hline 92 & 11 & 25 & 7 & 57 & 77 \\
\hline 92 & 12 & 1 & 14 & 43 & 77 \\
\hline 92 & 12 & 9 & 15 & 46 & 77 \\
\hline 92 & 12 & 14 & 14 & 4 & 77.25 \\
\hline 92 & 12 & 22 & 20 & 48 & 75.25 \\
\hline 92 & 12 & 28 & 22 & 13 & 78.5 \\
\hline 93 & 1 & 13 & 15 & 2 & 78 \\
\hline 93 & 1 & 18 & 20 & 43 & 78.25 \\
\hline 93 & 1 & 25 & 22 & 57 & 78.75 \\
\hline 93 & 2 & 2 & 15 & 58 & 78.25 \\
\hline 93 & 2 & 10 & 9 & 55 & 79.75 \\
\hline 93 & 2 & 17 & 15 & 17 & 79.5 \\
\hline 93 & 2 & 24 & 14 & 19 & 79.5 \\
\hline 93 & 3 & 1 & 17 & 22 & 79 \\
\hline 93 & 3 & 10 & 8 & 36 & 79 \\
\hline
\end{tabular}

Data obtained from WHC Surveillance Analysis Computer System (SACS), November 16, 1993. 


\begin{tabular}{|c|c|c|c|c|c|}
\hline Year & Month & Day & Hour & Minutes & Surface Level \\
\hline 93 & 3 & 15 & 17 & 24 & 79.25 \\
\hline 93 & 3 & 24 & 9 & 49 & 79 \\
\hline 93 & 3 & 29 & 14 & 18 & 79 \\
\hline 93 & 4 & 7 & 8 & 4 & 79.5 \\
\hline 93 & 4 & 12 & 15 & 15 & 79.5 \\
\hline 93 & 4 & 19 & 13 & 43 & 79.25 \\
\hline 93 & 4 & 26 & 19 & 2 & 79.75 \\
\hline 93 & 5 & 11 & 15 & 14 & 80 \\
\hline 93 & 5 & 17 & 14 & 58 & 79.75 \\
\hline 93 & 5 & 24 & 14 & 14 & 79.75 \\
\hline 93 & 6 & 1 & 14 & 48 & 80.5 \\
\hline 93 & 6 & 7 & 17 & 0 & 80.25 \\
\hline 93 & 6 & 14 & 14 & 51 & 80 \\
\hline 93 & 7 & 6 & 11 & 2 & 80.5 \\
\hline 93 & 7 & 12 & 22 & 7 & 80.5 \\
\hline 93 & 7 & 19 & 14 & 40 & 80 \\
\hline 93 & 7 & 26 & 15 & 1 & 80.5 \\
\hline 93 & 8 & 2 & 14 & 39 & 80.25 \\
\hline 93 & 8 & 9 & 14 & 56 & 80.5 \\
\hline 93 & 8 & 11 & 13 & 44 & 80.5 \\
\hline 93 & 8 & 12 & 13 & 32 & 80.5 \\
\hline 93 & 8 & 13 & 14 & 6 & 80.5 \\
\hline 93 & 8 & 14 & 15 & 18 & 80.5 \\
\hline 93 & 8 & 15 & 17 & 4 & 80.5 \\
\hline 93 & 8 & 17 & 13 & 51 & 80.5 \\
\hline 93 & 8 & 18 & 14 & 47 & 80.5 \\
\hline 93 & 8 & 19 & 14 & 20 & 80.5 \\
\hline 93 & 8 & 20 & 11 & 18 & 80.25 \\
\hline 93 & 8 & 21 & 13 & 37 & 80.5 \\
\hline 93 & 8 & 22 & 14 & 21 & 80.5 \\
\hline 93 & 8 & 23 & 12 & 28 & 80.75 \\
\hline 93 & 8 & 24 & 15 & 50 & 80.75 \\
\hline 93 & 8 & 25 & 17 & 5 & 80.5 \\
\hline 93 & 8 & 26 & 15 & 50 & 80.5 \\
\hline 93 & 8 & 27 & 18 & 40 & 80.5 \\
\hline 93 & 8 & 28 & 15 & 27 & 80.5 \\
\hline 93 & 9 & 29 & 16 & 38 & 81 \\
\hline 93 & 9 & 30 & 21 & 35 & 81 \\
\hline 93 & 10 & 1 & 20 & 50 & 81 \\
\hline 93 & 10 & 1 & 20 & 56 & 81 \\
\hline 93 & 10 & 2 & 22 & 58 & 81 \\
\hline 93 & 10 & 4 & 23 & 24 & 81 \\
\hline 93 & 10 & 5 & 13 & 46 & 81.25 \\
\hline 93 & 10 & 6 & 19 & 5 & 81 \\
\hline 93 & 10 & 8 & 20 & 30 & 81 \\
\hline 93 & 10 & 9 & 21 & 15 & 81 \\
\hline 93 & 10 & 10 & 22 & 18 & 81 \\
\hline 93 & 10 & 12 & 0 & 19 & 81.25 \\
\hline
\end{tabular}

Data obtained from WHC Surveillance Analysis Computer System (SACS), November 16, 1993. 


\begin{tabular}{|c|c|c|c|c|c|}
\hline Year & Month & Day & Hour & Minutes & Surface Level \\
\hline 93 & 10 & 13 & 21 & 54 & 81 \\
\hline 93 & 10 & 14 & 17 & 39 & 81.25 \\
\hline 93 & 10 & 15 & 20 & 3 & 81.25 \\
\hline 93 & 10 & 16 & 20 & 14 & 81 \\
\hline 93 & 10 & 17 & 20 & 55 & 81 \\
\hline 93 & 10 & 18 & 21 & 13 & 81 \\
\hline 93 & 10 & 19 & 21 & 19 & 81 \\
\hline 93 & 10 & 20 & 21 & 44 & 81 \\
\hline 93 & 10 & 21 & 21 & 52 & 81 \\
\hline 93 & 10 & 22 & 20 & 36 & 81 \\
\hline 93 & 10 & 23 & 20 & 54 & 81 \\
\hline 93 & 10 & 26 & 21 & 32 & 80.5 \\
\hline 93 & 10 & 27 & 21 & 20 & 81 \\
\hline 93 & 10 & 28 & 15 & 22 & 81 \\
\hline 93 & 10 & 30 & 14 & 23 & 80.5 \\
\hline 93 & 11 & 1 & 15 & 44 & 81 \\
\hline 93 & 11 & 3 & 16 & 58 & 81.25 \\
\hline 93 & 11 & 4 & 17 & 35 & 81 \\
\hline 93 & 11 & 5 & 14 & 31 & 81 \\
\hline 93 & 11 & 6 & 14 & 15 & 81 \\
\hline 93 & 11 & 7 & 13 & 57 & 81 \\
\hline 93 & 11 & 8 & 14 & 38 & 81 \\
\hline 93 & 11 & 9 & 14 & 51 & 81 \\
\hline 93 & 11 & 10 & 14 & 33 & 81 \\
\hline 93 & 11 & 11 & 15 & 39 & 81.25 \\
\hline 93 & 11 & 13 & 15 & 2 & 81.25 \\
\hline 93 & 11 & 14 & 14 & 32 & 81 \\
\hline 93 & 11 & 15 & 15 & 4 & 81 \\
\hline & & & & & \\
\hline & & & & & \\
\hline & & & & & \\
\hline & & & & & \\
\hline & & & & & \\
\hline & & & & & \\
\hline & & & & & \\
\hline & & & & & \\
\hline & & & & & \\
\hline & & & & & \\
\hline & & & & & \\
\hline & & & & & \\
\hline & & & & & \\
\hline & & & & & \\
\hline & & & & & \\
\hline & & & & & \\
\hline & & & & & \\
\hline & & & & & \\
\hline & & & & & \\
\hline
\end{tabular}

Data obtained from WHC Surveillance Analysis Computer System (SACS), November 16, 1993. 


\begin{tabular}{|c|c|c|c|c|c|}
\hline \multicolumn{6}{|c|}{ Surface Level Data } \\
\hline \multicolumn{6}{|c|}{ Manual Tape } \\
\hline Year & Month & Day & Hour & Minutes & Surface Level \\
\hline 91 & 1 & 2 & 8 & 11 & 151.75 \\
\hline 91 & 1 & 7 & 14 & 33 & 151.75 \\
\hline 91 & 1 & 14 & 15 & 11 & 151.5 \\
\hline 91 & 1 & 19 & 8 & 11 & 151.5 \\
\hline 91 & 1 & 21 & 1 & 31 & 151 \\
\hline 91 & 1 & 28 & 15 & 8 & 151.25 \\
\hline 91 & 2 & 4 & 15 & 28 & 151.25 \\
\hline 91 & 2 & 12 & 16 & 39 & 151.25 \\
\hline 91 & 2 & 18 & 18 & 25 & 151.5 \\
\hline 91 & 2 & 25 & 15 & 12 & 151.5 \\
\hline 91 & 3 & 4 & 14 & 56 & 151.5 \\
\hline 91 & 3 & 11 & 16 & 42 & 151.5 \\
\hline 91 & 3 & 13 & 15 & 27 & 152 \\
\hline 91 & 3 & 18 & 17 & 25 & 151.5 \\
\hline 91 & 3 & 25 & 15 & 1 & 151.5 \\
\hline 91 & 4 & 1 & 15 & 24 & 151.5 \\
\hline 91 & 4 & 8 & 17 & 53 & 151.5 \\
\hline 91 & 4 & 15 & 15 & 23 & 151 \\
\hline 91 & 4 & 22 & 15 & 3 & 151.5 \\
\hline 91 & 4 & 29 & 17 & 17 & 151.25 \\
\hline 91 & 5 & 7 & 13 & 24 & 151.5 \\
\hline 91 & 5 & 13 & 14 & 54 & 151.5 \\
\hline 91 & 5 & 20 & 14 & 57 & 151.25 \\
\hline 91 & 5 & 27 & 14 & 41 & 151.25 \\
\hline 91 & 6 & 3 & 14 & 56 & 151.25 \\
\hline 91 & 6 & 10 & 14 & 55 & 151 \\
\hline 91 & 6 & 17 & 15 & 8 & 151.25 \\
\hline 91 & 6 & 24 & 18 & 8 & 151.5 \\
\hline 91 & 7 & 1 & 14 & 23 & 151 \\
\hline 91 & 7 & 8 & 14 & 23 & 151.25 \\
\hline 91 & 7 & 15 & 14 & 23 & 151.25 \\
\hline 91 & 7 & 22 & 21 & 50 & 151.5 \\
\hline 91 & 7 & 31 & 8 & 15 & 151.25 \\
\hline 91 & 8 & 5 & 18 & 12 & 151.25 \\
\hline 91 & 8 & 12 & 15 & 17 & 151.25 \\
\hline 91 & 8 & 19 & 14 & 58 & 151 \\
\hline 91 & 8 & 26 & 14 & 58 & 151 \\
\hline 91 & 9 & 2 & 14 & 58 & 151 \\
\hline 91 & 9 & 9 & 14 & 58 & 151.25 \\
\hline 91 & 9 & 16 & 14 & 58 & 150.5 \\
\hline 91 & 9 & 23 & 14 & 57 & 151.25 \\
\hline 91 & 9 & 30 & 14 & 58 & 151.25 \\
\hline 91 & 10 & 7 & 14 & 57 & 151.25 \\
\hline 91 & 10 & 14 & 14 & 57 & 151 \\
\hline 91 & 10 & 21 & 14 & 57 & 151 \\
\hline 91 & 10 & 28 & 14 & 57 & 150.75 \\
\hline
\end{tabular}

Data obtained from WHC Surveillance Analysis Computer System (SACS), November 16, 1993. 


\begin{tabular}{|c|c|c|c|c|c|}
\hline Year & Month & Day & Hour & Minutes & Surface Level \\
\hline 91 & 11 & 4 & 14 & 57 & 151 \\
\hline 91 & 11 & 11 & 14 & 57 & 151 \\
\hline 91 & 11 & 18 & 14 & 57 & 151 \\
\hline 91 & 11 & 25 & 14 & 57 & 151 \\
\hline 91 & 12 & 2 & 14 & 57 & 150.75 \\
\hline 91 & 12 & 9 & 14 & 57 & 150.75 \\
\hline 91 & 12 & 16 & 14 & 57 & 150.75 \\
\hline 91 & 12 & 23 & 14 & 57 & 151 \\
\hline 91 & 12 & 30 & 14 & 57 & 150.75 \\
\hline 92 & 1 & 6 & 14 & 57 & 150.25 \\
\hline 92 & 1 & 13 & 14 & 57 & 150.75 \\
\hline 92 & 1 & 20 & 14 & 57 & 151.75 \\
\hline 92 & 1 & 27 & 14 & 57 & 151.5 \\
\hline 92 & 2 & 25 & 15 & 15 & 151 \\
\hline 92 & 3 & 4 & 8 & 37 & 150.25 \\
\hline 92 & 3 & 11 & 8 & 23 & 150.5 \\
\hline 92 & 3 & 17 & 15 & 18 & 150.25 \\
\hline 92 & 3 & 23 & 15 & 29 & 150.5 \\
\hline 92 & 3 & 30 & 15 & 33 & 150 \\
\hline 92 & 4 & 6 & 17 & 57 & 150.75 \\
\hline 92 & 4 & 13 & 15 & 39 & 150.25 \\
\hline 92 & 4 & 20 & 16 & 26 & 150.5 \\
\hline 92 & 4 & 28 & 6 & 8 & 150.25 \\
\hline 92 & 5 & 6 & 7 & 52 & 150 \\
\hline 92 & 5 & 11 & 15 & 27 & 150.5 \\
\hline 92 & 5 & 20 & 8 & 15 & 150.5 \\
\hline 92 & 5 & 25 & 15 & 27 & 150.25 \\
\hline 92 & 6 & 1 & 15 & 28 & 150.25 \\
\hline 92 & 6 & 8 & 15 & 25 & 150.25 \\
\hline 92 & 6 & 15 & 15 & 13 & 150.5 \\
\hline 92 & 6 & 24 & 8 & 32 & 150 \\
\hline 92 & 7 & 6 & 16 & 47 & 150.25 \\
\hline 92 & 7 & 13 & 15 & 21 & 150.25 \\
\hline 92 & 7 & 21 & 15 & 19 & 150.5 \\
\hline 92 & 7 & 28 & 14 & 31 & 151 \\
\hline 92 & 8 & 3 & 17 & 27 & 150.5 \\
\hline 92 & 8 & 12 & 8 & 33 & 150.25 \\
\hline 92 & 8 & 17 & 15 & 30 & 150.25 \\
\hline 92 & 8 & 24 & 15 & 2 & 150 \\
\hline 92 & 9 & 1 & 15 & 25 & 150 \\
\hline 92 & 9 & 8 & 23 & 5 & 150.25 \\
\hline 92 & 9 & 23 & 14 & 20 & 150.25 \\
\hline 92 & 9 & 28 & 14 & 43 & 150.25 \\
\hline 92 & 10 & 6 & 6 & 53 & 150.25 \\
\hline 92 & 10 & 12 & 6 & 53 & 150.25 \\
\hline 92 & 10 & 21 & 7 & 51 & 150 \\
\hline 92 & 10 & 26 & 15 & 40 & 150 \\
\hline 92 & 11 & 2 & 15 & 20 & 150.25 \\
\hline
\end{tabular}

Data obtained from WHC Surveillance Analysis Computer System (SACS), November 16, 1993. 


\begin{tabular}{|c|c|c|c|c|c|}
\hline Year & Month & Day & Hour & Minutes & Surface Level \\
\hline 92 & 11 & 16 & 19 & 44 & 150 \\
\hline 92 & 11 & 25 & 7 & 57 & 150 \\
\hline 92 & 12 & 1 & 14 & 43 & 150 \\
\hline 92 & 12 & 7 & 17 & 40 & 150 \\
\hline 92 & 12 & 14 & 14 & 4 & 149.5 \\
\hline 92 & 12 & 22 & 20 & 48 & 150.5 \\
\hline 92 & 12 & 28 & 22 & 13 & 150.25 \\
\hline 93 & 1 & 13 & 15 & 2 & 149.25 \\
\hline 93 & 1 & 18 & 20 & 43 & 149.75 \\
\hline 93 & 1 & 25 & 22 & 57 & 149.75 \\
\hline 93 & 2 & 3 & 2 & 28 & 150 \\
\hline 93 & 2 & 10 & 9 & 55 & 150.5 \\
\hline 93 & 2 & 17 & 15 & 17 & 149 \\
\hline 93 & 2 & 24 & 14 & 19 & 149 \\
\hline 93 & 3 & 1 & 17 & 22 & 150 \\
\hline 93 & 3 & 10 & 8 & 36 & 149.5 \\
\hline 93 & 3 & 15 & 17 & 24 & 149.5 \\
\hline 93 & 3 & 24 & 9 & 49 & 149 \\
\hline 93 & 3 & 29 & 14 & 18 & 149 \\
\hline 93 & $\overline{4}$ & 7 & 8 & 4 & 149.25 \\
\hline 93 & 4 & 12 & 15 & 15 & 149.5 \\
\hline 93 & 4 & 19 & 13 & 43 & 149.5 \\
\hline 93 & 4 & 26 & 19 & 2 & 149 \\
\hline 93 & 5 & 11 & 15 & 14 & 149 \\
\hline 93 & 5 & 17 & 14 & 58 & 149 \\
\hline 93 & 5 & 24 & 14 & 14 & 149 \\
\hline 93 & 6 & 1 & 14 & 48 & 149 \\
\hline 93 & 6 & 7 & 17 & 0 & 149 \\
\hline 93 & 6 & 14 & 14 & 51 & 148.75 \\
\hline 93 & 7 & 6 & 11 & 2 & 148.75 \\
\hline 93 & 7 & 12 & 22 & 7 & 148.75 \\
\hline 93 & 7 & 19 & 14 & 40 & 148.75 \\
\hline 93 & 7 & 26 & 15 & 1 & 147.75 \\
\hline 93 & 8 & 2 & 14 & 39 & 149.25 \\
\hline 93 & 8 & 9 & 14 & 56 & 148.75 \\
\hline 93 & 8 & 24 & 15 & 50 & 148.5 \\
\hline 93 & 8 & 30 & 17 & 1 & 148 \\
\hline 93 & 10 & 4 & 23 & 24 & 148.25 \\
\hline 93 & 10 & 12 & 19 & 6 & 148.25 \\
\hline 93 & 10 & 18 & 21 & 13 & 148 \\
\hline 93 & 11 & 1 & 15 & 44 & 147.75 \\
\hline 93 & 11 & 8 & 14 & 38 & 148 \\
\hline & & & & & \\
\hline & & & & & \\
\hline & & & & & \\
\hline & & & & & \\
\hline & & & & & \\
\hline
\end{tabular}

Data obtained from WHC Surveillance Analysis Computer System (SACS), November 16, 1993. 


\begin{tabular}{|c|c|c|c|c|c|}
\hline \multicolumn{6}{|c|}{ Surface Level Data } \\
\hline \multicolumn{6}{|c|}{ Manual Tape } \\
\hline Year & Month & Day & Hour & Minutes & Surface Level \\
\hline 91 & 1 & 2 & 15 & 25 & 131.25 \\
\hline 91 & 1 & 19 & 15 & 25 & 131.25 \\
\hline 91 & 2 & 5 & 8 & 20 & 131.75 \\
\hline 91 & 3 & 4 & 14 & 56 & 131.75 \\
\hline 91 & 3 & 12 & 10 & 0 & 131 \\
\hline 91 & 4 & 1 & 15 & 24 & 131 \\
\hline 91 & 5 & 7 & 13 & 24 & 131.25 \\
\hline 91 & 6 & 3 & 14 & 56 & 131 \\
\hline 91 & 7 & 1 & 10 & 0 & 130 \\
\hline 91 & 8 & 1 & 10 & 0 & 129.75 \\
\hline 91 & 8 & 5 & 18 & 12 & 129.5 \\
\hline 91 & 9 & 2 & 15 & 25 & 129.5 \\
\hline 91 & 10 & 1 & 10 & 0 & 129.75 \\
\hline 92 & 2 & 25 & 15 & 15 & 128.5 \\
\hline 92 & 4 & 1 & 14 & 57 & 125.25 \\
\hline 92 & 5 & 6 & 7 & 52 & 128.75 \\
\hline 92 & 11 & 20 & 7 & 57 & 126.5 \\
\hline 92 & 11 & 23 & 7 & 57 & 126.5 \\
\hline 92 & 12 & 1 & 14 & 43 & 128.5 \\
\hline 92 & 12 & 21 & 14 & 43 & 128.25 \\
\hline 93 & 1 & 1 & 2 & 28 & 128.5 \\
\hline 93 & 2 & 3 & 2 & 28 & 127 \\
\hline 93 & 3 & 1 & 17 & 22 & 127.5 \\
\hline 93 & 4 & 8 & 0 & 44 & 128 \\
\hline 93 & 6 & 1 & 14 & 48 & 128 \\
\hline 93 & 7 & 1 & 14 & 49 & 128 \\
\hline 93 & 8 & 2 & 14 & 39 & 127.5 \\
\hline 93 & 10 & 1 & 20 & 56 & $* 80.5$ \\
\hline 93 & 10 & 1 & 21 & 30 & 128.5 \\
\hline 93 & 11 & 1 & 15 & 44 & $* 134$ \\
\hline 93 & 11 & 1 & 18 & 23 & 128.59 \\
\hline & & & & & \\
\hline & & & & & \\
\hline & & & & & \\
\hline & & & & & \\
\hline & & & & & \\
\hline & & & & & \\
\hline & & & & & $\cdot$ \\
\hline & & & & & \\
\hline & & & & & \\
\hline & & & & & \\
\hline & & & & & \\
\hline & & & & & \\
\hline & & & & & \\
\hline & & & & & \\
\hline
\end{tabular}

Data obtained from WHC Surveillance Analysis Computer System (SACS), November 16, 1993. 


\begin{tabular}{|c|c|c|c|c|c|}
\hline \multicolumn{6}{|c|}{ Surface Level Data } \\
\hline \multicolumn{6}{|c|}{ Manual Tape } \\
\hline Year & Month & Day & Hour & Minutes & Surface Level \\
\hline 91 & 1 & 2 & 8 & 11 & 181.25 \\
\hline 91 & 1 & 7 & 14 & 33 & 181.75 \\
\hline 91 & 1 & 14 & 15 & 11 & 181.25 \\
\hline 91 & 1 & 19 & 8 & 11 & 181.25 \\
\hline 91 & 1 & 21 & 1 & 31 & 181 \\
\hline 91 & 1 & 28 & 15 & 8 & 181 \\
\hline 91 & 2 & 4 & 15 & 28 & 181 \\
\hline 91 & 2 & 12 & 16 & 39 & 181 \\
\hline 91 & 2 & 18 & 18 & 25 & 181 \\
\hline 91 & 2 & 26 & 7 & 11 & 181.25 \\
\hline 91 & 3 & 4 & 14 & 56 & 181.25 \\
\hline 91 & 3 & 11 & 16 & 42 & 181.5 \\
\hline 91 & 3 & 18 & 17 & 25 & 181.5 \\
\hline 91 & 3 & 25 & 15 & 1 & 181.25 \\
\hline 91 & 4 & 5 & 8 & 33 & 181 \\
\hline 91 & 4 & 8 & 17 & 53 & 181 \\
\hline 91 & 4 & 15 & 15 & 23 & 181 \\
\hline 91 & 4 & 22 & 15 & 3 & 181.25 \\
\hline 91 & 4 & 29 & 17 & 17 & 181 \\
\hline 91 & 5 & 7 & 13 & 24 & 181 \\
\hline 91 & 5 & 13 & 14 & 54 & 181 \\
\hline 91 & 5 & 20 & 21 & 51 & 181 \\
\hline 91 & 5 & 27 & 14 & 41 & 181 \\
\hline 91 & 6 & 3 & 14 & 56 & 181 \\
\hline 91 & 6 & 10 & 14 & 55 & 181 \\
\hline 91 & 6 & 17 & 15 & 8 & 181 \\
\hline 91 & 6 & 24 & 18 & 8 & 181 \\
\hline 91 & 7 & 1 & 21 & 50 & 181 \\
\hline 91 & 7 & 8 & 21 & 50 & 181 \\
\hline 91 & 7 & 15 & 14 & 23 & 181.25 \\
\hline 91 & 7 & 22 & 21 & 50 & 181 \\
\hline 91 & 7 & 31 & 0 & 29 & 181 \\
\hline 91 & 8 & 5 & 18 & 12 & 180.5 \\
\hline 91 & 8 & 12 & 15 & 17 & 180.5 \\
\hline 91 & 8 & 19 & 14 & 58 & 180.5 \\
\hline 91 & 8 & 26 & 14 & 58 & 180.75 \\
\hline 91 & 9 & 2 & 14 & 57 & 180.75 \\
\hline 91 & 9 & 9 & 14 & 57 & 180.5 \\
\hline 91 & 9 & 16 & 14 & 57 & 180.75 \\
\hline 91 & 9 & 23 & 14 & 57 & 180.5 \\
\hline 91 & 10 & 7 & 14 & 57 & 180.5 \\
\hline 91 & 10 & 14 & 14 & 57 & 180.5 \\
\hline 91 & 10 & 21 & 14 & 57 & 180.5 \\
\hline 91 & 10 & 28 & 14 & 57 & 180.5 \\
\hline 91 & 11 & 4 & 14 & 57 & 180.5 \\
\hline 91 & 11 & 11 & 14 & 57 & 180.25 \\
\hline
\end{tabular}

Data obtained from WHC Surveillance Analysis Computer System (SACS), November 16, 1993. 


\begin{tabular}{|c|c|c|c|c|c|}
\hline Year & Month & Day & Hour & Minutes & Surface Level \\
\hline 91 & 11 & 18 & 14 & 57 & 180.25 \\
\hline 91 & 11 & 25 & 14 & 57 & 180.25 \\
\hline 91 & 12 & 2 & 14 & 57 & 179.75 \\
\hline 91 & 12 & 9 & 14 & 57 & 179.75 \\
\hline 91 & 12 & 16 & 14 & 57 & 179.5 \\
\hline 91 & 12 & 23 & 14 & 57 & 180.5 \\
\hline 91 & 12 & 30 & 14 & 57 & 179.5 \\
\hline 92 & 1 & 6 & 14 & 57 & 179.25 \\
\hline 92 & 1 & 13 & 14 & 57 & 179 \\
\hline 92 & 1 & 21 & 14 & 57 & 179.5 \\
\hline 92 & 1 & 27 & 14 & 57 & 179 \\
\hline 92 & 2 & 25 & 15 & 15 & 178 \\
\hline 92 & 3 & 4 & 8 & 37 & 177.75 \\
\hline 92 & 3 & 11 & 8 & 23 & 177.75 \\
\hline 92 & 3 & 16 & 16 & 33 & 177.75 \\
\hline 92 & 3 & 23 & 15 & 29 & 177.5 \\
\hline 92 & 3 & 30 & 15 & 33 & 177.75 \\
\hline 92 & 4 & 6 & 17 & 57 & 177.75 \\
\hline 92 & 4 & 13 & 15 & 39 & 177.25 \\
\hline 92 & 4 & 20 & 16 & 26 & 177 \\
\hline 92 & 4 & 27 & 22 & 5 & 177.25 \\
\hline 92 & 5 & 6 & 7 & 52 & 177 \\
\hline 92 & 5 & 11 & 15 & 27 & 177 \\
\hline 92 & 5 & 20 & 8 & 15 & 177 \\
\hline 92 & 5 & 25 & 15 & 27 & 177 \\
\hline 92 & 6 & 1 & 15 & 28 & 177 \\
\hline 92 & 6 & 8 & 15 & 25 & 177 \\
\hline 92 & 6 & 17 & 8 & 1 & 177 \\
\hline 92 & 6 & 24 & 8 & 32 & 177 \\
\hline 92 & 7 & 6 & 16 & 47 & 177.5 \\
\hline 92 & 7 & 13 & 15 & 21 & 177 \\
\hline 92 & 7 & 21 & 15 & 19 & 177 \\
\hline 92 & 7 & 28 & 14 & 31 & 177 \\
\hline 92 & 8 & 3 & 17 & 27 & 177 \\
\hline 92 & 8 & 12 & 8 & 33 & 177 \\
\hline 92 & 8 & 17 & 15 & 30 & 177 \\
\hline 92 & 8 & 24 & 15 & 2 & 177 \\
\hline 92 & 9 & 1 & 15 & 25 & 176.75 \\
\hline 92 & 9 & 8 & 23 & 5 & 176.75 \\
\hline 92 & 9 & 23 & 14 & 20 & 176.75 \\
\hline 92 & 9 & 28 & 14 & 43 & 177 \\
\hline 92 & 10 & 6 & 6 & 53 & 177 \\
\hline 92 & 10 & 21 & 7 & 51 & 177 \\
\hline 92 & 11 & 2 & 15 & 20 & 176.75 \\
\hline 92 & 11 & 16 & 19 & 44 & 176.75 \\
\hline 92 & 11 & 25 & 7 & 57 & 176.75 \\
\hline 92 & 12 & 1 & 14 & 43 & 176.75 \\
\hline 92 & 12 & 7 & 17 & 40 & 175.25 \\
\hline
\end{tabular}

Data obtained from WHC Surveillance Analysis Computer System (SACS), November 16, 1993. 


\begin{tabular}{|c|c|c|c|c|c|}
\hline Year & Month & Day & Hour & Minutes & Surface Level \\
\hline 92 & 12 & 14 & 14 & 4 & 176 \\
\hline 92 & 12 & 22 & 20 & 48 & 176 \\
\hline 92 & 12 & 28 & 22 & 13 & 176 \\
\hline 93 & 1 & 12 & 10 & 37 & 175.5 \\
\hline 93 & 1 & 14 & 0 & 37 & 175.5 \\
\hline 93 & 1 & 18 & 20 & 43 & 175.5 \\
\hline 93 & 1 & 25 & 22 & 57 & 174.25 \\
\hline 93 & 2 & 2 & 15 & 58 & 174.25 \\
\hline 93 & 2 & 10 & 9 & 55 & 174 \\
\hline 93 & 2 & 17 & 15 & 17 & 175 \\
\hline 93 & 2 & 24 & 14 & 19 & 175 \\
\hline 93 & 3 & 1 & 17 & 22 & 174.25 \\
\hline 93 & 3 & 10 & 8 & 36 & 174.25 \\
\hline 93 & 3 & 15 & 17 & 24 & 174.25 \\
\hline 93 & 3 & 24 & 9 & 49 & 174 \\
\hline 93 & 3 & 29 & 14 & 18 & 174.25 \\
\hline 93 & 4 & 7 & 8 & 4 & 173.5 \\
\hline 93 & 4 & 12 & 15 & 15 & 174 \\
\hline 93 & 4 & 19 & 13 & 43 & 174 \\
\hline 93 & 4 & 26 & 19 & 2 & 174 \\
\hline 93 & 5 & 11 & 15 & 14 & 174 \\
\hline 93 & 5 & 17 & 14 & 58 & 174 \\
\hline 93 & 5 & 24 & 14 & 14 & 174 \\
\hline 93 & 6 & 1 & 14 & 48 & 174 \\
\hline 93 & 6 & 7 & 17 & 0 & 173.75 \\
\hline 93 & 6 & 14 & 14 & 51 & 174 \\
\hline 93 & 6 & 28 & 14 & 33 & 173.75 \\
\hline 93 & 7 & 6 & 11 & 2 & 173.5 \\
\hline 93 & 7 & 12 & 15 & 14 & 173.5 \\
\hline 93 & 7 & 19 & 14 & 40 & 173.5 \\
\hline 93 & 7 & 26 & 15 & 1 & 173.25 \\
\hline 93 & 8 & 2 & 14 & 39 & 173 \\
\hline 93 & 8 & 9 & 14 & 56 & 173 \\
\hline 93 & 8 & 24 & 15 & 50 & 173.25 \\
\hline 93 & 8 & 30 & 17 & 1 & 173.5 \\
\hline 93 & 10 & 4 & 23 & 24 & 173.25 \\
\hline 93 & 10 & 12 & 19 & 6 & 173.25 \\
\hline 93 & 10 & 18 & 21 & 13 & 173 \\
\hline 93 & 11 & 1 & 15 & 44 & 173 \\
\hline \multirow[t]{2}{*}{93} & 11 & 8 & 14 & 38 & 172.75 \\
\hline & & & & & - \\
\hline & & & & & \\
\hline & & & & & \\
\hline & & & & & \\
\hline & & & & & \\
\hline & & & & & \\
\hline & & & & & \\
\hline & & & & & \\
\hline
\end{tabular}

Data obtained from WHC Surveillance Analysis Computer System (SACS), November 16, 1993. 


\begin{tabular}{|c|c|c|c|c|c|}
\hline \multicolumn{6}{|c|}{ Surface Level Data } \\
\hline \multicolumn{6}{|c|}{ Manual Tape } \\
\hline Year & Month & Day & Hour & Minutes & Surface Level \\
\hline 91 & 1 & 2 & 8 & 11 & 242.5 \\
\hline 91 & 1 & 7 & 14 & 33 & 242.5 \\
\hline 91 & 1 & 14 & 15 & 11 & 242.75 \\
\hline 91 & 1 & 19 & 8 & 11 & 242.75 \\
\hline 91 & 1 & 21 & 1 & 31 & 242.75 \\
\hline 91 & 1 & 28 & 15 & 8 & 243 \\
\hline 91 & 1 & 28 & 15 & 12 & 243 \\
\hline 91 & 2 & 4 & 15 & 28 & 243 \\
\hline 91 & 2 & 12 & 16 & 39 & 243 \\
\hline 91 & 2 & 18 & 18 & 25 & 242.75 \\
\hline 91 & 2 & 27 & 7 & 33 & 242.75 \\
\hline 91 & 3 & 4 & 14 & 56 & 242.75 \\
\hline 91 & 3 & 11 & 16 & 42 & 243 \\
\hline 91 & 3 & 18 & 17 & 25 & 243 \\
\hline 91 & 3 & 25 & 15 & 1 & 243.25 \\
\hline 91 & 4 & 1 & 15 & 24 & 243 \\
\hline 91 & 4 & 8 & 17 & 53 & 243 \\
\hline 91 & 4 & 15 & 15 & 23 & 243 \\
\hline 91 & 4 & 22 & 15 & 3 & 243 \\
\hline 91 & 4 & 29 & 17 & 17 & 243 \\
\hline 91 & 5 & 7 & 13 & 24 & 243 \\
\hline 91 & 5 & 13 & 14 & 54 & 243 \\
\hline 91 & 5 & 20 & 14 & 57 & 243 \\
\hline 91 & 5 & 27 & 14 & 41 & 242.75 \\
\hline 91 & 6 & 3 & 14 & 56 & 242.75 \\
\hline 91 & 6 & 10 & 14 & 55 & 243 \\
\hline 91 & 6 & 17 & 15 & 8 & 243 \\
\hline 91 & 6 & 24 & 18 & 8 & 243 \\
\hline 91 & 7 & 1 & 21 & 50 & 243 \\
\hline 91 & 7 & 8 & 21 & 50 & 243 \\
\hline 91 & 7 & 16 & 14 & 59 & 243.25 \\
\hline 91 & 7 & 22 & 21 & 50 & 243 \\
\hline 91 & 7 & 31 & 8 & 15 & 243 \\
\hline 91 & 8 & 5 & 18 & 12 & 243 \\
\hline 91 & 8 & 12 & 15 & 17 & 243 \\
\hline 91 & 8 & 19 & 14 & 58 & 243.25 \\
\hline 91 & 8 & 26 & 14 & 58 & 243.25 \\
\hline 91 & 9 & 2 & 14 & 58 & 243.5 \\
\hline 91 & 9 & 9 & 14 & 58 & 243.25 \\
\hline 91 & 9 & 16 & 14 & 58 & 243.25 \\
\hline 91 & 9 & 23 & 14 & 57 & 243 \\
\hline 91 & 9 & 30 & 14 & 58 & 243 \\
\hline 91 & 10 & 7 & 14 & 58 & 243 \\
\hline 91 & 10 & 14 & 14 & 58 & 243 \\
\hline 91 & 10 & 21 & 14 & 58 & 243.25 \\
\hline 91 & 10 & 28 & 14 & 58 & 243 \\
\hline
\end{tabular}

Data obtained from WHC Surveillance Analysis Computer System (SACS), November 16, 1993. 


\begin{tabular}{|c|c|c|c|c|c|}
\hline Year & Month & Day & Hour & Minutes & Surface Level \\
\hline 91 & 11 & 4 & 14 & 58 & 243 \\
\hline 91 & 11 & 11 & 14 & 58 & 243.25 \\
\hline 91 & 11 & 18 & 14 & 58 & 243.5 \\
\hline 91 & 11 & 25 & 14 & 58 & 243.25 \\
\hline 91 & 12 & 2 & 14 & 58 & 243.25 \\
\hline 91 & 12 & 10 & 14 & 58 & 243 \\
\hline 97 & 12 & 16 & 14 & 58 & 243 \\
\hline 91 & 12 & 23 & 14 & 58 & 243.25 \\
\hline 91 & 12 & 30 & 14 & 58 & 243.5 \\
\hline 92 & 1 & 6 & 14 & 58 & 243.25 \\
\hline 92 & 1 & 13 & 14 & 58 & 243.25 \\
\hline 92 & 1 & 20 & 14 & 58 & 243 \\
\hline 92 & 1 & 27 & 14 & 58 & 243.25 \\
\hline 92 & 2 & 25 & 15 & 15 & 243.25 \\
\hline 92 & 3 & 4 & 8 & 37 & 243 \\
\hline 92 & 3 & 11 & 8 & 23 & 243.25 \\
\hline 92 & 3 & 16 & 16 & 33 & 243.5 \\
\hline 92 & 3 & 23 & 15 & 29 & 243.5 \\
\hline 92 & 3 & 30 & 15 & 33 & 243 \\
\hline 92 & 4 & 6 & 17 & 57 & 243.5 \\
\hline 92 & 4 & 13 & 15 & 39 & 243.5 \\
\hline 92 & 4 & 20 & 16 & 26 & 243.25 \\
\hline 92 & 4 & 27 & 22 & 5 & 243 \\
\hline 92 & 5 & 6 & 7 & 52 & 243 \\
\hline 92 & 5 & 11 & 15 & 27 & 243.5 \\
\hline 92 & 5 & 20 & 8 & 15 & 243.5 \\
\hline 92 & 5 & 25 & 15 & 27 & 243.5 \\
\hline 92 & 6 & 1 & 15 & 28 & 243.5 \\
\hline 92 & 6 & 8 & 15 & 25 & 243.5 \\
\hline 92 & 6 & 15 & 15 & 13 & 243.5 \\
\hline 92 & 6 & 24 & 8 & 32 & 243 \\
\hline 92 & 7 & 6 & 16 & 47 & 243.25 \\
\hline 92 & 7 & 13 & 15 & 21 & 243.75 \\
\hline 92 & 7 & 21 & 15 & 19 & 244 \\
\hline 92 & 7 & 28 & 14 & 31 & 243.75 \\
\hline 92 & 8 & 3 & 17 & 27 & 243.75 \\
\hline 92 & 8 & 12 & 8 & 33 & 243.75 \\
\hline 92 & 8 & 17 & 15 & 30 & 243.75 \\
\hline 92 & 8 & 24 & 15 & 2 & 243.5 \\
\hline 92 & 9 & 1 & 15 & 25 & 244 \\
\hline 92 & 9 & 8 & 23 & 5 & 244 \\
\hline 92 & 9 & 23 & 14 & 20 & 243.5 \\
\hline 92 & 9 & 28 & 14 & 43 & 243.25 \\
\hline 92 & 9 & 28 & 16 & 38 & 243.25 \\
\hline 92 & 10 & 6 & 6 & 53 & 243.5 \\
\hline 92 & 10 & 21 & 7 & 51 & 244 \\
\hline 92 & 11 & 2 & 15 & 20 & 243.75 \\
\hline 92 & 11 & 16 & 19 & 44 & 243.5 \\
\hline
\end{tabular}

Data obtained from WHC Surveillance Analysis Computer System (SACS), November 16, 1993. 


\begin{tabular}{|c|c|c|c|c|c|}
\hline Year & Month & Day & Hour & Minutes & Surface Level \\
\hline 92 & 11 & 25 & 7 & 57 & 243.5 \\
\hline 92 & 12 & 1 & 14 & 43 & 243.25 \\
\hline 92 & 12 & 7 & 17 & 40 & 244 \\
\hline 92 & 12 & 14 & 14 & 4 & 243.75 \\
\hline 92 & 12 & 22 & 20 & 48 & 243.25 \\
\hline 92 & 12 & 28 & 22 & 13 & 244.25 \\
\hline 93 & 1 & 13 & 15 & 2 & 243 \\
\hline 93 & 1 & 18 & 20 & 43 & 243.25 \\
\hline 93 & 1 & 25 & 22 & 57 & 242.75 \\
\hline 93 & 2 & 3 & 2 & 28 & 244 \\
\hline 93 & 2 & 10 & 9 & 55 & 243.25 \\
\hline 93 & 2 & 17 & 15 & 17 & 243 \\
\hline 93 & 2 & 24 & 14 & 19 & 243 \\
\hline 93 & 3 & 1 & 17 & 22 & 243.5 \\
\hline 93 & 3 & 10 & 8 & 36 & 243.75 \\
\hline 93 & 3 & 15 & 17 & 24 & 243.75 \\
\hline 93 & 3 & 29 & 14 & 18 & 243.25 \\
\hline 93 & 4 & 7 & 8 & 4 & 243.5 \\
\hline 93 & 4 & 12 & 15 & 15 & 243.5 \\
\hline 93 & 4 & 19 & 13 & 43 & 243.5 \\
\hline 93 & 4 & 26 & 19 & 2 & 243.5 \\
\hline 93 & 5 & 11 & 15 & 14 & 243 \\
\hline 93 & 5 & 17 & 14 & 58 & 243.25 \\
\hline 93 & 5 & 24 & 14 & 14 & 243.5 \\
\hline 93 & 6 & 1 & 14 & 48 & 243.5 \\
\hline 93 & 6 & 7 & 17 & 0 & 244 \\
\hline 93 & 6 & 14 & 14 & 51 & 243.25 \\
\hline 93 & 7 & 6 & 11 & 2 & 243.5 \\
\hline 93 & 7 & 12 & 15 & 14 & 243.5 \\
\hline 93 & 7 & 19 & 14 & 40 & 243.5 \\
\hline 93 & 7 & 26 & 15 & 1 & 243.5 \\
\hline 93 & 8 & 2 & 14 & 39 & 243.75 \\
\hline 93 & 8 & 9 & 14 & 56 & 244 \\
\hline 93 & 8 & 24 & 15 & 50 & 244 \\
\hline 93 & 10 & 4 & 23 & 24 & 244 \\
\hline 93 & 10 & 12 & 19 & 6 & 244 \\
\hline 93 & 10 & 18 & 21 & 13 & 244 \\
\hline 93 & 10 & 26 & 21 & 32 & 243.75 \\
\hline 93 & 11 & 1 & 15 & 44 & 243.75 \\
\hline 93 & 11 & 8 & 14 & 38 & 243.75 \\
\hline & & & & & \\
\hline & & & & & \\
\hline & & & & & \\
\hline & & & & & \\
\hline & & & & & \\
\hline & & & & & \\
\hline & & & & & \\
\hline
\end{tabular}

Data obtained from WHC Surveillance Analysis Computer System (SACS), November 16, 1993. 


\begin{tabular}{|c|c|c|c|c|c|}
\hline \multicolumn{6}{|c|}{ Surface Level Data } \\
\hline \multicolumn{6}{|c|}{ Manual Tape } \\
\hline Year & Month & Day & Hour & Minutes & Surface Level \\
\hline 91 & 1 & 2 & 15 & 25 & 103 \\
\hline 91 & 4 & 1 & 15 & 24 & 103.75 \\
\hline 91 & 7 & 1 & 15 & 24 & 104 \\
\hline 91 & 10 & 1 & 15 & 24 & 103 \\
\hline 92 & 1 & 1 & 14 & 57 & 103.5 \\
\hline 92 & 4 & 1 & 14 & 57 & 103 \\
\hline 92 & 7 & 2 & 15 & 33 & 103.75 \\
\hline 92 & 10 & 6 & 6 & 53 & 104 \\
\hline 93 & 1 & 4 & 10 & 0 & 104 \\
\hline 93 & 4 & 8 & 0 & 44 & 104 \\
\hline 93 & 7 & 1 & 14 & 49 & 103 \\
\hline 93 & 10 & 1 & 20 & 56 & 104 \\
\hline & & & & & \\
\hline & & & & & \\
\hline & & & & & \\
\hline & & & & & \\
\hline & & & & & \\
\hline & & & & & \\
\hline & & & & & \\
\hline & & & & & \\
\hline & & & & & \\
\hline & & & & & \\
\hline & & & & & \\
\hline & & & & & \\
\hline & & & & & \\
\hline & & & & & \\
\hline & & & & & \\
\hline & & & & & \\
\hline & & & & & \\
\hline & & & & & \\
\hline & & & & & \\
\hline & & & & & \\
\hline & & & & & \\
\hline & & & & & \\
\hline & & & & & \\
\hline & & & & & \\
\hline & & & & & \\
\hline & & & & & \\
\hline & & & & & \\
\hline & & & & & \\
\hline & & & & & \\
\hline & & & & & \\
\hline & & & & & \\
\hline & & & & & \\
\hline & & & & & \\
\hline
\end{tabular}

Data obtained from WHC Surveillance Analysis Computer System (SACS), November 16, 1993. E-31 


\begin{tabular}{|c|c|c|c|c|c|}
\hline \multicolumn{6}{|c|}{ Surface Level Data } \\
\hline \multicolumn{6}{|c|}{ Manual Tape } \\
\hline Year & Month & Day & Hour & Minutes & Surface Level \\
\hline 91 & 1 & 2 & 15 & 25 & 88 \\
\hline 91 & 2 & 5 & 8 & 20 & 87.75 \\
\hline 91 & 3 & 5 & 4 & 23 & 87.5 \\
\hline 91 & 4 & 1 & 15 & 24 & 87.5 \\
\hline 91 & 5 & 7 & 13 & 24 & 87.5 \\
\hline 91 & 6 & 3 & 14 & 56 & 87 \\
\hline 91 & 7 & 1 & 14 & 56 & 87.5 \\
\hline 91 & 8 & 1 & 14 & 51 & 88 \\
\hline 91 & 9 & 2 & 14 & 51 & 88.25 \\
\hline 91 & 10 & 1 & 14 & 51 & 87.5 \\
\hline 91 & 11 & 4 & 14 & 51 & 87.5 \\
\hline 91 & 12 & 2 & 14 & 51 & 87.5 \\
\hline 92 & 1 & 1 & 15 & 15 & 87.5 \\
\hline 92 & 2 & 25 & 15 & 15 & 87.5 \\
\hline 92 & 3 & 4 & 8 & 37 & 86 \\
\hline 92 & 4 & 1 & 14 & 57 & 87.5 \\
\hline 92 & 5 & 6 & 7 & 52 & 87.5 \\
\hline 92 & 6 & 1 & 15 & 28 & 87.5 \\
\hline 92 & 7 & 2 & 15 & 33 & 88 \\
\hline 92 & 8 & 3 & 17 & 27 & 87.75 \\
\hline 92 & 9 & 1 & 15 & 25 & 87.5 \\
\hline 92 & 10 & 6 & 6 & 53 & 87.5 \\
\hline 92 & 11 & 2 & 15 & 20 & 87.5 \\
\hline 92 & 12 & 1 & 14 & 43 & 87.5 \\
\hline 93 & 2 & 3 & 2 & 28 & 88.5 \\
\hline 93 & 3 & 1 & 10 & 0 & 88.5 \\
\hline 93 & 3 & 1 & 17 & 22 & 88.5 \\
\hline 93 & 4 & 8 & 0 & 44 & 87.5 \\
\hline 93 & 6 & 1 & 14 & 48 & 87.5 \\
\hline 93 & 7 & 1 & 14 & 49 & 87.5 \\
\hline 93 & 8 & 2 & 14 & 39 & 88 \\
\hline 93 & 10 & 1 & 20 & 56 & 88 \\
\hline 93 & 11 & 1 & 15 & 44 & 88 \\
\hline & & & & & \\
\hline & & & & & \\
\hline & & & & & \\
\hline & & & & & \\
\hline & & & & & \\
\hline & & & & & \\
\hline & & & & & \\
\hline & & & & & \\
\hline & & & & & \\
\hline & & & & & \\
\hline & & & & & \\
\hline & & & & & \\
\hline
\end{tabular}

Data obtained from WHC Surveillance Analysis Computer System (SACS), November 16, 1993. 


\begin{tabular}{|c|c|c|c|c|c|}
\hline \multicolumn{6}{|c|}{ Surface Level Data } \\
\hline \multicolumn{6}{|c|}{ FIC } \\
\hline Year & Month & Day & Hour & Minutes & Surface Level \\
\hline 91 & 1 & 1 & 7 & 3 & 180.5 \\
\hline 91 & 1 & 3 & 7 & 3 & 180.4 \\
\hline 91 & 1 & 5 & 7 & 2 & 180.4 \\
\hline 91 & 1 & 7 & 7 & 2 & 180.5 \\
\hline 91 & 1 & 8 & 7 & 3 & 180.5 \\
\hline 91 & 1 & 9 & 7 & 2 & 180.5 \\
\hline 91 & 1 & 10 & 7 & 2 & 180.5 \\
\hline 91 & 1 & 11 & 7 & 3 & 180.5 \\
\hline 91 & 1 & 12 & 6 & 2 & 180.6 \\
\hline 91 & 1 & 13 & 7 & 3 & 180.6 \\
\hline 91 & 1 & 14 & 7 & 4 & 180.5 \\
\hline 91 & 1 & 15 & 7 & 3 & 180.6 \\
\hline 91 & 1 & 16 & 7 & 4 & 180.4 \\
\hline 91 & 1 & 17 & 7 & 4 & 180.4 \\
\hline 91 & 1 & 18 & 7 & 4 & 180.5 \\
\hline 91 & 1 & 22 & 7 & 4 & 180.4 \\
\hline 91 & 1 & 23 & 7 & 4 & 180.5 \\
\hline 91 & 1 & 24 & 7 & 4 & 180.5 \\
\hline 91 & 1 & 25 & 7 & 3 & 180.4 \\
\hline 91 & 1 & 26 & 7 & 3 & 180.4 \\
\hline 91 & 1 & 27 & 7 & 3 & 180.5 \\
\hline 91 & 1 & 28 & 7 & 10 & 180.6 \\
\hline 91 & 1 & 29 & 7 & 2 & 180.5 \\
\hline 91 & 1 & 30 & 7 & 10 & 180.5 \\
\hline 91 & 1 & 31 & 7 & 2 & 180.5 \\
\hline 91 & 2 & 1 & 7 & 2 & 180.6 \\
\hline 91 & 2 & 2 & 7 & 4 & 180.6 \\
\hline 91 & 2 & 3 & 7 & 3 & 180.5 \\
\hline 91 & 2 & 4 & 7 & 3 & 180.5 \\
\hline 91 & 6 & 16 & 7 & 5 & 180.8 \\
\hline 91 & 6 & 17 & 7 & 5 & 180.5 \\
\hline 91 & 6 & 18 & 7 & 5 & 180.4 \\
\hline 91 & 6 & 19 & 7 & 5 & 180.1 \\
\hline 91 & 6 & 20 & 7 & 5 & 179.8 \\
\hline 91 & 6 & 21 & 7 & 6 & 179.7 \\
\hline 91 & 6 & 22 & 7 & 5 & 179.4 \\
\hline 91 & 6 & 23 & 7 & 5 & 179.1 \\
\hline 91 & 6 & 24 & 7 & 5 & 179 \\
\hline 91 & 6 & 25 & 7 & 6 & 178.4 \\
\hline 91 & 6 & 26 & 7 & 3 & 177.8 \\
\hline 91 & 7 & 16 & 7 & 6 & 168.6 \\
\hline 91 & 7 & 17 & 7 & 5 & 168.2 \\
\hline 91 & 7 & 18 & 7 & 5 & 168 \\
\hline 91 & 7 & 19 & 7 & 5 & 167.8 \\
\hline 91 & 7 & 20 & 7 & 5 & 167.4 \\
\hline 91 & 7 & 21 & 7 & 5 & 167 \\
\hline
\end{tabular}

Data obtained from WHC Surveillance Analysis Computer System (SACS), November 16, 1993. 


\begin{tabular}{|c|c|c|c|c|c|}
\hline Year & Month & Day & Hour & Minutes & Surface Level \\
\hline 91 & 7 & 22 & 7 & 5 & 166.6 \\
\hline 91 & 7 & 23 & 7 & 5 & 166.3 \\
\hline 91 & 7 & 24 & 6 & 5 & 166.1 \\
\hline 91 & 7 & 25 & 7 & 1 & 165.8 \\
\hline 91 & 7 & 26 & 7 & 2 & 165.3 \\
\hline 91 & 7 & 27 & 7 & 2 & 164.9 \\
\hline 91 & 7 & 28 & 7 & 2 & 164.5 \\
\hline 91 & 7 & 29 & 7 & 2 & 164.1 \\
\hline 91 & 7 & 30 & 7 & 2 & 163.6 \\
\hline 91 & 7 & 31 & 7 & 2 & 163 \\
\hline 92 & 5 & 11 & 10 & 3 & 139.8 \\
\hline 92 & 5 & 18 & 10 & 3 & 139.8 \\
\hline 92 & 5 & 25 & 10 & 3 & 139.8 \\
\hline 92 & 6 & 1 & 10 & 3 & 139.8 \\
\hline 92 & 6 & 8 & 10 & 3 & 139.8 \\
\hline 92 & 6 & 15 & 10 & 3 & 139.9 \\
\hline 92 & 6 & 22 & 10 & 3 & 139.9 \\
\hline 92 & 6 & 29 & 10 & 3 & 139.9 \\
\hline 92 & 7 & 11 & 7 & 6 & 139.3 \\
\hline 92 & 7 & 12 & 7 & 6 & 139.1 \\
\hline 92 & 7 & 13 & 7 & 6 & 139 \\
\hline 92 & 7 & 14 & 7 & 6 & 138.9 \\
\hline 92 & 7 & 15 & 7 & 6 & 138.9 \\
\hline 92 & 7 & 16 & 7 & 6 & 138.8 \\
\hline 92 & 7 & 17 & 7 & 6 & 138.8 \\
\hline 92 & 7 & 18 & 7 & 6 & 138.8 \\
\hline 92 & 7 & 19 & 7 & 6 & 138.8 \\
\hline 92 & 7 & 20 & 6 & 6 & 138.8 \\
\hline 92 & 7 & 21 & 10 & 3 & 138.8 \\
\hline 92 & 7 & 25 & 4 & 6 & 138.8 \\
\hline 92 & 7 & 27 & 10 & 3 & 138.8 \\
\hline 92 & 8 & 3 & 10 & 3 & 138.8 \\
\hline 92 & 8 & 26 & 7 & 3 & 138.7 \\
\hline 92 & 8 & 27 & 7 & 4 & 138.7 \\
\hline 92 & 8 & 28 & 7 & 2 & 138.8 \\
\hline 92 & 8 & 29 & 7 & 2 & 138.8 \\
\hline 92 & 8 & 30 & 7 & 2 & 138.8 \\
\hline 92 & 8 & 31 & 7 & 2 & 138.8 \\
\hline 92 & 9 & 1 & 7 & 2 & 138.8 \\
\hline 92 & 9 & 2 & 7 & 2 & 138.8 \\
\hline 92 & 9 & 9 & 6 & 3 & 138.7 \\
\hline 92 & 9 & 10 & 7 & 3 & 138.8 \\
\hline 92 & 9 & 17 & 7 & 6 & 138.8 \\
\hline 92 & 9 & 18 & 7 & 6 & 138.8 \\
\hline 92 & 9 & 21 & 7 & 6 & 138.8 \\
\hline 92 & 9 & 22 & 7 & 3 & 138.8 \\
\hline 92 & 9 & 23 & 7 & 3 & 138.9 \\
\hline 92 & 9 & 24 & 7 & 3 & 139 \\
\hline
\end{tabular}

Data obtained from WHC Surveillance Analysis Computer System (SACS), November 16, 1993. 


\begin{tabular}{|c|c|c|c|c|c|}
\hline Year & Month & Day & Hour & Minutes & Surface Level \\
\hline 92 & 9 & 25 & 7 & 3 & 138.8 \\
\hline 92 & 9 & 26 & 7 & 2 & 138.7 \\
\hline 92 & 9 & 27 & 7 & 2 & 138.7 \\
\hline 92 & 9 & 28 & 7 & 2 & 138.7 \\
\hline 92 & 9 & 29 & 7 & 3 & 138.7 \\
\hline 92 & 9 & 30 & 7 & 2 & 138.8 \\
\hline 92 & 10 & 1 & 7 & 3 & 138.9 \\
\hline 92 & 10 & 2 & 7 & 2 & 138.8 \\
\hline 92 & 10 & 3 & 7 & 3 & 138.8 \\
\hline 92 & 10 & 4 & 7 & 3 & 138.7 \\
\hline 92 & 10 & 5 & 7 & 3 & 138.7 \\
\hline 92 & 10 & 6 & 7 & 3 & 138.7 \\
\hline 92 & 10 & 8 & 7 & 2 & 138.8 \\
\hline 92 & 10 & 9 & 7 & 3 & 138.7 \\
\hline 92 & 10 & 10 & 7 & 3 & 138.7 \\
\hline 92 & 10 & 11 & 7 & 3 & 138.7 \\
\hline 92 & 10 & 14 & 7 & 3 & 138.7 \\
\hline 92 & 10 & 15 & 7 & 4 & 138.7 \\
\hline 92 & 10 & 16 & 7 & 3 & 138.7 \\
\hline 92 & 10 & 17 & 7 & 3 & 138.7 \\
\hline 92 & 10 & 18 & 7 & 3 & 138.8 \\
\hline 92 & 10 & 19 & 7 & 3 & 138.8 \\
\hline 92 & 10 & 26 & 7 & 4 & 138.9 \\
\hline 92 & 11 & 2 & 7 & 3 & 138.9 \\
\hline 92 & 11 & 10 & 7 & 3 & 139 \\
\hline 92 & 11 & 20 & 7 & 3 & 138.8 \\
\hline 92 & 12 & 4 & 7 & 3 & 138.6 \\
\hline 92 & 12 & 7 & 7 & 3 & 138.5 \\
\hline 92 & 12 & 14 & 7 & 3 & 138.7 \\
\hline 92 & 12 & 21 & 7 & 4 & 138.3 \\
\hline 92 & 12 & 23 & 7 & 4 & 138.2 \\
\hline 92 & 12 & 28 & 7 & 3 & 138.1 \\
\hline 93 & 1 & 4 & 7 & 6 & 137.6 \\
\hline 93 & 1 & 13 & 10 & 6 & 137.9 \\
\hline 93 & 1 & 21 & 10 & 2 & 137.8 \\
\hline 93 & 1 & 25 & 10 & 3 & 137.9 \\
\hline 93 & 2 & 1 & 10 & 4 & 137.9 \\
\hline 93 & 2 & 2 & 10 & 4 & 137.9 \\
\hline 93 & 2 & 10 & 7 & 4 & 137.6 \\
\hline 93 & 2 & 16 & 10 & 2 & 137.4 \\
\hline 93 & 2 & 22 & 10 & 4 & 137.2 \\
\hline 93 & 3 & 1 & 10 & 3 & 137.1 \\
\hline 93 & 4 & 13 & 14 & 6 & 140.15 \\
\hline 93 & 4 & 13 & 15 & 4 & 140.15 \\
\hline 93 & 4 & 14 & 15 & 4 & 140.15 \\
\hline 93 & 4 & 15 & 15 & 4 & 140.15 \\
\hline 93 & 4 & 16 & 15 & 4 & 140.1 \\
\hline 93 & 4 & 19 & 15 & 4 & 140 \\
\hline
\end{tabular}

Data obtained from WHC Surveillance Analysis Computer System (SACS), November 16, 1993. 


\begin{tabular}{|c|c|c|c|c|c|}
\hline Year & Month & Day & Hour & Minutes & Surface Level \\
\hline 93 & 4 & 26 & 15 & 4 & 139.6 \\
\hline 93 & 4 & 28 & 15 & 4 & 139 \\
\hline 93 & 5 & 19 & 7 & 3 & 139.7 \\
\hline 93 & 5 & 20 & 7 & 3 & 139.9 \\
\hline 93 & 5 & 22 & 7 & 3 & $* 100.4$ \\
\hline 93 & 5 & 23 & 7 & 4 & $* 100.4$ \\
\hline 93 & 5 & 24 & 7 & 3 & $* 100.3$ \\
\hline 93 & 5 & 25 & 7 & 3 & $* 100.4$ \\
\hline 93 & 5 & 26 & 7 & 3 & $* 100.4$ \\
\hline 93 & 5 & 27 & 7 & 3 & $* 100.4$ \\
\hline 93 & 5 & 28 & 7 & 3 & $* 100.4$ \\
\hline 93 & 5 & 29 & 7 & 5 & $* 100.2$ \\
\hline 93 & 5 & 30 & 7 & 5 & $* 100.2$ \\
\hline 93 & 5 & 31 & 7 & 6 & $* 100.3$ \\
\hline 93 & 6 & 1 & 7 & 6 & $* 100.3$ \\
\hline 93 & 6 & 2 & 7 & 6 & $* 100.2$ \\
\hline 93 & 6 & 3 & 7 & 6 & $* 100.2$ \\
\hline 93 & 6 & 4 & 7 & 6 & $* 100.3$ \\
\hline 93 & 6 & 5 & 7 & 6 & $* 100.4$ \\
\hline 93 & 6 & 6 & 7 & 5 & $* 100.4$ \\
\hline 93 & 6 & 7 & 7 & 5 & ${ }^{*} 100.3$ \\
\hline 93 & 6 & 8 & 7 & 5 & $* 100.2$ \\
\hline 93 & 6 & 9 & 7 & 5 & $* 100.1$ \\
\hline 93 & 6 & 10 & 7 & 4 & $* 100.1$ \\
\hline 93 & 6 & 11 & 7 & 5 & $* 100$ \\
\hline 93 & 6 & 12 & 7 & 5 & 139.9 \\
\hline 93 & 6 & 13 & 7 & 4 & 139.7 \\
\hline 93 & 6 & 14 & 7 & 5 & 139.7 \\
\hline 93 & 6 & 15 & 7 & 5 & 139.7 \\
\hline 93 & 6 & 16 & 7 & 5 & 139.7 \\
\hline 93 & 6 & 17 & 7 & 5 & 139.5 \\
\hline 93 & 6 & 18 & 7 & 6 & 139.5 \\
\hline 93 & 6 & 25 & 7 & 3 & 139.3 \\
\hline 93 & 6 & 26 & 7 & 3 & 139.6 \\
\hline 93 & 6 & 27 & 7 & 4 & 139.8 \\
\hline 93 & 6 & 28 & 7 & 3 & 139.8 \\
\hline 93 & 6 & 29 & 7 & 4 & 139.8 \\
\hline 93 & 6 & 30 & 7 & 3 & 139.7 \\
\hline 93 & 7 & 1 & 7 & 6 & 139.7 \\
\hline 93 & 7 & 2 & 7 & 6 & 139.8 \\
\hline 93 & 7 & 3 & 7 & 3 & 139.9 \\
\hline 93 & 7 & 4 & 7 & 4 & 139.9 \\
\hline 93 & 7 & 5 & 7 & 3 & 139.9 \\
\hline 93 & 7 & 6 & 7 & 3 & 139.8 \\
\hline 93 & 7 & 7 & 7 & 4 & 139.8 \\
\hline 93 & 7 & 8 & 7 & 4 & 139.8 \\
\hline 93 & 7 & 9 & 7 & 5 & 139.9 \\
\hline 93 & 7 & 10 & 7 & 3 & 139.9 \\
\hline
\end{tabular}

Data obtained from WHC Surveillance Analysis Computer System (SACS), November 16, 1993. 


\begin{tabular}{|c|c|c|c|c|c|}
\hline Year & Month & Day & Hour & Minutes & Surface Level \\
\hline 93 & 7 & 11 & 7 & 3 & 139.8 \\
\hline 93 & 7 & 12 & 7 & 2 & 139.7 \\
\hline 93 & 7 & 13 & 7 & 3 & 139.7 \\
\hline 93 & 7 & 14 & 7 & 6 & 139.7 \\
\hline 93 & 7 & 15 & 7 & 6 & 139.6 \\
\hline 93 & 7 & 16 & 7 & 6 & 139.6 \\
\hline 93 & 7 & 17 & 7 & 7 & $* 100.7$ \\
\hline 93 & 7 & 18 & 7 & 6 & $* 100.8$ \\
\hline 93 & 7 & 19 & 7 & 3 & $* 100.7$ \\
\hline 93 & 7 & 20 & 7 & 3 & $* 100.7$ \\
\hline 93 & 7 & 21 & 7 & 3 & $* 100.7$ \\
\hline 93 & 7 & 22 & 7 & 3 & ${ }^{*} 100.7$ \\
\hline 93 & 7 & 23 & 7 & 3 & $* 100.7$ \\
\hline 93 & 7 & 24 & 7 & 3 & $* 100.7$ \\
\hline 93 & 7 & 26 & 7 & 3 & $* 100.7$ \\
\hline 93 & 7 & 27 & 7 & 3 & $* 100.7$ \\
\hline 93 & 7 & 28 & 7 & 4 & $* 100.8$ \\
\hline 93 & 7 & 29 & 7 & 3 & $* 100.8$ \\
\hline 93 & 7 & 30 & 7 & 3 & $* 100.7$ \\
\hline 93 & 7 & 31 & 7 & 3 & $* 100.6$ \\
\hline 93 & 8 & 1 & 7 & 3 & $* 100.6$ \\
\hline 93 & 8 & 2 & 7 & 6 & $* 100.7$ \\
\hline 93 & 8 & 3 & 7 & 3 & $* 100.7$ \\
\hline 93 & 8 & 4 & 7 & 3 & $* 100.8$ \\
\hline 93 & 8 & 5 & 7 & 4 & ${ }^{*} 100.8$ \\
\hline 93 & 8 & 7 & 7 & 4 & $* 100.9$ \\
\hline 93 & 8 & 8 & 6 & 4 & $* 100.9$ \\
\hline 93 & 8 & 9 & 7 & 4 & $* 100.8$ \\
\hline 93 & 8 & 10 & 7 & 4 & $* 100.8$ \\
\hline 93 & 8 & 11 & 7 & 4 & $* 100.8$ \\
\hline 93 & 8 & 12 & 7 & 4 & $* 100.8$ \\
\hline 93 & 8 & 13 & 7 & 4 & $* 100.8$ \\
\hline 93 & 8 & 14 & 7 & 3 & $* 100.8$ \\
\hline 93 & 8 & 15 & 7 & 4 & $* 100.9$ \\
\hline 93 & 8 & 16 & 7 & 4 & $* 100.9$ \\
\hline 93 & 8 & 17 & 7 & 4 & $* 100.9$ \\
\hline 93 & 8 & 18 & 7 & 3 & * 100.8 \\
\hline 93 & 8 & 19 & 7 & 4 & $* 100.9$ \\
\hline 93 & 8 & 20 & 7 & 4 & $* 100.9$ \\
\hline 93 & 8 & 21 & 7 & 4 & $* 100.9$ \\
\hline 93 & 8 & 22 & 7 & 4 & $* 100.8$ \\
\hline 93 & 8 & 23 & 6 & 3 & $* 100.9$ \\
\hline 93 & 8 & 24 & 7 & 4 & $* 100.8$ \\
\hline 93 & 8 & 25 & 7 & 4 & $* 100.7$ \\
\hline 93 & 8 & 26 & 7 & 4 & ${ }^{*} 100.7$ \\
\hline 93 & 8 & 27 & 7 & 5 & $* 100.7$ \\
\hline 93 & 8 & 28 & 7 & 4 & $* 100.7$ \\
\hline 93 & 8 & 29 & 7 & 3 & $* 100.7$ \\
\hline
\end{tabular}

Data obtained from WHC Surveillance Analysis Computer System (SACS), November 16, 1993. 


\begin{tabular}{|c|c|c|c|c|c|}
\hline Year & Month & Day & Hour & Minutes & Surface Level \\
\hline 93 & 8 & 30 & 7 & 4 & *100.6 \\
\hline 93 & 8 & 31 & 7 & 4 & $* 100.8$ \\
\hline 93 & 9 & 1 & 7 & 4 & $* 100.8$ \\
\hline 93 & 9 & 30 & 7 & 3 & $* 100.8$ \\
\hline 93 & 10 & 1 & 7 & 6 & * 100.7 \\
\hline 93 & 10 & 2 & 7 & 5 & $* 100.6$ \\
\hline 93 & 10 & 3 & 7 & 6 & $* 100.6$ \\
\hline 93 & 10 & 4 & 7 & 6 & $* 100.8$ \\
\hline 93 & 10 & 5 & 7 & 6 & $* 100.9$ \\
\hline 93 & 10 & 6 & 7 & 6 & $* 100.9$ \\
\hline 93 & 10 & 7 & 7 & 5 & $* 100.7$ \\
\hline 93 & 10 & 8 & 7 & 4 & $* 100.6$ \\
\hline 93 & 10 & 9 & 7 & 3 & $* 100.6$ \\
\hline 93 & 10 & 10 & 7 & 10 & $* 100.7$ \\
\hline 93 & 10 & 11 & 7 & 3 & $* 100.8$ \\
\hline 93 & 10 & 12 & 7 & 4 & $* 100.7$ \\
\hline 93 & 10 & 13 & 7 & 3 & $* 100.7$ \\
\hline 93 & 10 & 14 & 7 & 4 & $* 100.8$ \\
\hline 93 & 10 & 15 & 7 & 4 & $* 100.9$ \\
\hline 93 & 10 & 16 & 7 & 4 & $* 100.8$ \\
\hline 93 & 10 & 17 & 7 & 3 & $* 100.8$ \\
\hline 93 & 10 & 18 & 7 & 4 & $* 100.8$ \\
\hline 93 & 10 & 19 & 7 & 4 & $* 100.7$ \\
\hline 93 & 10 & 20 & 7 & 3 & $* 100.6$ \\
\hline 93 & 10 & 21 & 7 & 4 & $* 100.7$ \\
\hline 93 & 10 & 22 & 7 & 4 & $* 100.7$ \\
\hline 93 & 10 & 23 & 7 & 4 & $* 100.8$ \\
\hline 93 & 10 & 24 & 7 & 4 & $* 100.7$ \\
\hline 93 & 10 & 26 & 7 & 10 & $* 100.4$ \\
\hline 93 & 10 & 27 & 7 & 4 & $\$ 100.4$ \\
\hline 93 & 10 & 28 & 7 & 4 & $* 100.8$ \\
\hline 93 & 10 & 29 & 7 & 10 & $* 100.6$ \\
\hline 93 & 10 & 30 & 7 & 4 & $* 100.6$ \\
\hline 93 & 10 & 31 & 7 & 6 & $* 100.8$ \\
\hline 93 & 11 & 1 & 7 & 6 & $* 100.6$ \\
\hline 93 & 11 & 3 & 7 & 6 & ${ }^{*} 100.8$ \\
\hline 93 & 11 & 5 & 7 & 6 & $* 100.5$ \\
\hline 93 & 11 & 6 & 7 & 6 & $* 100.9$ \\
\hline 93 & 11 & 7 & 7 & 6 & $* 100.9$ \\
\hline 93 & 11 & 8 & 7 & 6 & ${ }^{*} 100.9$ \\
\hline 93 & 11 & 9 & 7 & 3 & $* 100.9$ \\
\hline 93 & 11 & 10 & 7 & 4 & $* 100.9$ \\
\hline 93 & 11 & 11 & 7 & 4 & $* 101$ \\
\hline 93 & 11 & 12 & 7 & 4 & $* 100.9$ \\
\hline 93 & 11 & 16 & 7 & 3 & 140.8 \\
\hline & & & & & \\
\hline & & & & & \\
\hline
\end{tabular}

Data obtained from WHC Surveillance Analysis Computer System (SACS), November 16, 1993. 
Surface Level Data

Manual Tape

\begin{tabular}{|c|c|c|c|c|c|}
\hline Year & Month & Day & Hour & Minutes & Surface Level \\
\hline 91 & 1 & 2 & 15 & 25 & 108 \\
\hline 91 & 2 & 5 & 8 & 20 & 108 \\
\hline 91 & 3 & 4 & 14 & 56 & 108 \\
\hline 91 & 4 & 1 & 15 & 24 & 108 \\
\hline 91 & 5 & 7 & 13 & 24 & 151.75 \\
\hline 91 & 6 & 3 & 14 & 56 & 151 \\
\hline 91 & 7 & 1 & 14 & 56 & 152 \\
\hline 91 & 8 & 1 & 18 & 12 & 99.5 \\
\hline 91 & 8 & 5 & 18 & 12 & 99.75 \\
\hline 91 & 9 & 2 & 18 & 12 & 99.75 \\
\hline 91 & 10 & 1 & 18 & 12 & 99.75 \\
\hline 91 & 11 & 4 & $\overline{18}$ & 12 & 100 \\
\hline 91 & 12 & 2 & 18 & 12 & 100.25 \\
\hline 91 & 12 & 23 & 18 & 12 & 100.5 \\
\hline 92 & 1 & 1 & 10 & 0 & 100.75 \\
\hline 92 & 2 & 25 & 15 & 15 & 100 \\
\hline 92 & 3 & 4 & 8 & 37 & 100 \\
\hline 92 & 4 & 1 & 14 & 57 & 100 \\
\hline 92 & 5 & 6 & 7 & 52 & 98.5 \\
\hline 92 & 6 & 1 & 15 & 28 & 98.5 \\
\hline 92 & 7 & 2 & 15 & 33 & 97.75 \\
\hline 92 & 8 & 3 & 17 & 27 & 98.5 \\
\hline 92 & 9 & 1 & 15 & 25 & 98.25 \\
\hline 92 & 10 & 6 & 6 & 53 & 98.25 \\
\hline 92 & 11 & 2 & 15 & 20 & 98.5 \\
\hline 92 & 12 & 1 & 14 & 43 & 98.5 \\
\hline 93 & 2 & 3 & 2 & 28 & 98 \\
\hline 93 & 3 & 1 & 10 & 0 & 98.5 \\
\hline 93 & 3 & 1 & 17 & 22 & 98.5 \\
\hline 93 & 4 & 8 & 0 & 44 & 98.5 \\
\hline 93 & 6 & 1 & 14 & 48 & 98.5 \\
\hline 93 & 7 & 1 & 14 & 49 & 98.5 \\
\hline 93 & 8 & 2 & 14 & 39 & 148 \\
\hline 93 & 10 & 1 & 20 & 56 & 99 \\
\hline 93 & 11 & 1 & 15 & 44 & 99 \\
\hline & & & & & \\
\hline & & & & & \\
\hline & & & & & \\
\hline & & & & & \\
\hline & & & & & \\
\hline & & & & & \\
\hline & & & & & \\
\hline & & & & & \\
\hline & & & & & \\
\hline & & & & & \\
\hline & & & & & \\
\hline
\end{tabular}

Data obtained from WHC Surveillance Analysis Computer System (SACS), November 16, 1993. 


\begin{tabular}{|c|c|c|c|c|c|}
\hline \multicolumn{6}{|c|}{ Surface Level Data } \\
\hline \multicolumn{6}{|c|}{ Manual Tape } \\
\hline Year & Month & Day & Hour & Minutes & Surface Level \\
\hline 91 & 1 & 2 & 15 & 25 & 163.5 \\
\hline 91 & 2 & 5 & 8 & 20 & 163.5 \\
\hline 91 & 3 & 4 & 14 & 56 & 163.75 \\
\hline 91 & 4 & 1 & 15 & 24 & 163 \\
\hline 91 & 5 & 7 & 13 & 24 & 163 \\
\hline 91 & 6 & 3 & 14 & 56 & 163 \\
\hline 91 & 7 & 1 & 10 & 0 & 163 \\
\hline 91 & 8 & 5 & 18 & 12 & 163 \\
\hline 91 & 9 & 2 & 10 & 12 & 163 \\
\hline 91 & 10 & 1 & 10 & 12 & 163 \\
\hline 91 & 11 & 4 & 10 & 12 & 163.25 \\
\hline 91 & 12 & 2 & 10 & 12 & 163.25 \\
\hline 92 & 1 & 1 & 17 & 27 & 163.25 \\
\hline 92 & 2 & 25 & 15 & 15 & 163 \\
\hline 92 & 3 & 2 & 8 & 37 & 163 \\
\hline 92 & 3 & 30 & 8 & 37 & 163 \\
\hline 92 & 4 & 1 & 14 & 57 & 163 \\
\hline 92 & 5 & 6 & 7 & 52 & 163 \\
\hline 92 & 6 & 1 & 15 & 28 & 163 \\
\hline 92 & 7 & 2 & 15 & 33 & 163 \\
\hline 92 & 8 & 3 & 17 & 27 & 163.25 \\
\hline 92 & 9 & 1 & 15 & 25 & 163.5 \\
\hline 92 & 10 & 6 & 6 & 53 & 163 \\
\hline 92 & 11 & 2 & 15 & 20 & 163 \\
\hline 92 & 12 & 1 & 14 & 43 & 163 \\
\hline 93 & 2 & 3 & 2 & 28 & 163 \\
\hline 93 & 3 & 1 & 10 & 0 & 163 \\
\hline 93 & 3 & 1 & 17 & 22 & 163 \\
\hline 93 & 4 & 8 & 0 & 44 & 163.25 \\
\hline 93 & 6 & 1 & 14 & 48 & 163 \\
\hline 93 & 7 & 1 & 14 & 49 & 163 \\
\hline 93 & 8 & 2 & 14 & 39 & 162.75 \\
\hline 93 & 10 & 1 & 20 & 56 & 163 \\
\hline 93 & 11 & 1 & 15 & 44 & 163 \\
\hline & & & & & \\
\hline & & & & & \\
\hline & & & & & \\
\hline & & & & & \\
\hline & & & & & - \\
\hline & & & & & \\
\hline & & & & & \\
\hline & & & & & \\
\hline & & & & & \\
\hline & & & & & \\
\hline & & & & & \\
\hline & & & & & \\
\hline
\end{tabular}

Data obtained from WHC Surveillance Analysis Computer System (SACS), November 16, 1993. 


\begin{tabular}{|c|c|c|c|c|c|}
\hline \multicolumn{6}{|c|}{ Surface Level Data } \\
\hline \multicolumn{6}{|c|}{ Manual Tape } \\
\hline Year & Month & Day & Hour & Minutes & Surface Level \\
\hline 91 & 1 & 2 & 15 & 25 & 113 \\
\hline 91 & 1 & 19 & 15 & 25 & 113 \\
\hline 91 & 2 & 5 & 8 & 20 & 113 \\
\hline 91 & 3 & 4 & 14 & 56 & 113 \\
\hline 91 & 4 & 1 & 15 & 24 & 114 \\
\hline 91 & 4 & 2 & 10 & 0 & 112.75 \\
\hline 91 & 5 & 7 & 13 & 24 & 113.5 \\
\hline 91 & 6 & 3 & 14 & 56 & 113 \\
\hline 91 & 8 & 6 & 20 & 17 & 113 \\
\hline 91 & 9 & 2 & 10 & 0 & 113 \\
\hline 91 & 10 & 1 & 10 & 0 & 113 \\
\hline 91 & 11 & 4 & 10 & 0 & 113.25 \\
\hline 91 & 12 & 2 & 10 & 0 & 113.25 \\
\hline 91 & 12 & 23 & 10 & 0 & 113.25 \\
\hline 92 & 1 & 1 & 10 & 0 & 113.25 \\
\hline 92 & 2 & 25 & 15 & 15 & 113.25 \\
\hline 92 & 3 & 4 & 8 & 37 & 114.25 \\
\hline 92 & 3 & 30 & 10 & 0 & 113 \\
\hline 92 & 4 & 1 & 14 & 57 & 113 \\
\hline 92 & 5 & 6 & 7 & 52 & 112.75 \\
\hline 92 & 6 & 1 & 15 & 28 & 113 \\
\hline 92 & 7 & 2 & 15 & 33 & 113 \\
\hline 92 & 8 & 3 & 17 & 27 & 113.25 \\
\hline 92 & 9 & 1 & 15 & 25 & 113 \\
\hline 92 & 10 & 6 & 6 & 53 & 114.25 \\
\hline 92 & 11 & 2 & 15 & 20 & 113 \\
\hline 92 & 12 & 1 & 14 & 43 & 113 \\
\hline 93 & 2 & 3 & 2 & 28 & 113 \\
\hline 93 & 3 & 1 & 10 & 0 & 113.5 \\
\hline 93 & 3 & 1 & 17 & 22 & 113.5 \\
\hline 93 & 4 & 8 & 0 & 44 & 113.5 \\
\hline 93 & 6 & 1 & 14 & 48 & 113.25 \\
\hline 93 & 7 & 1 & 14 & 49 & 113.25 \\
\hline 93 & 8 & 2 & 14 & 39 & 113 \\
\hline 93 & 10 & 1 & 20 & 56 & 113 \\
\hline 93 & 10 & 22 & 20 & 42 & 113 \\
\hline 93 & 11 & 1 & 15 & 44 & 113.5 \\
\hline & & & & & \\
\hline & & & & & \\
\hline & & & & & \\
\hline & & & & & \\
\hline & & & & & \\
\hline & & & & & \\
\hline & & & & & \\
\hline & & & & & \\
\hline & & & & & \\
\hline
\end{tabular}

Data obtained from WHC Surveillance Analysis Computer System (SACS), November 16, 1993. 
WHC-SD-WM-ER-3.12, Rev. 0

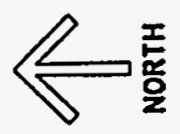
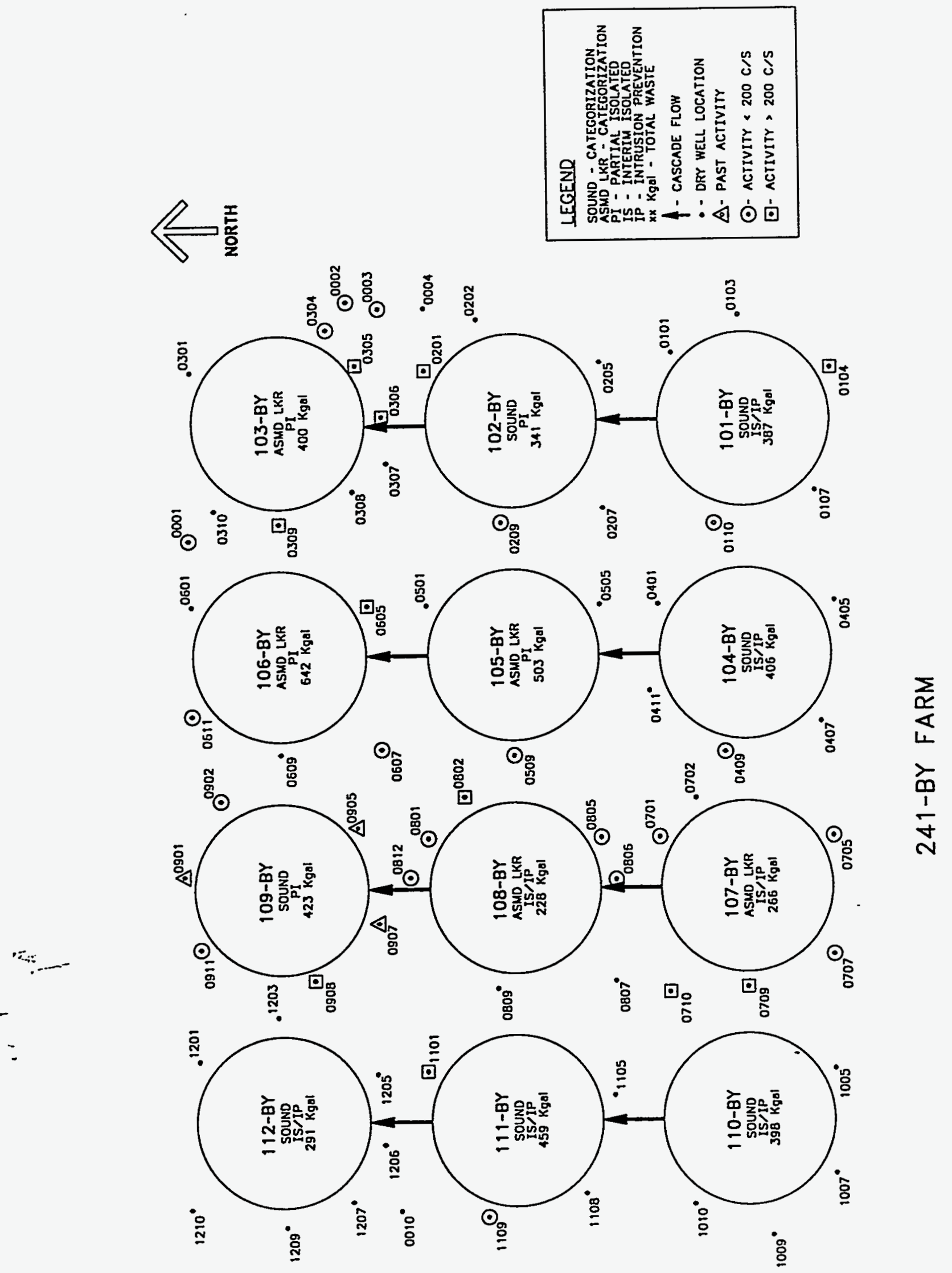


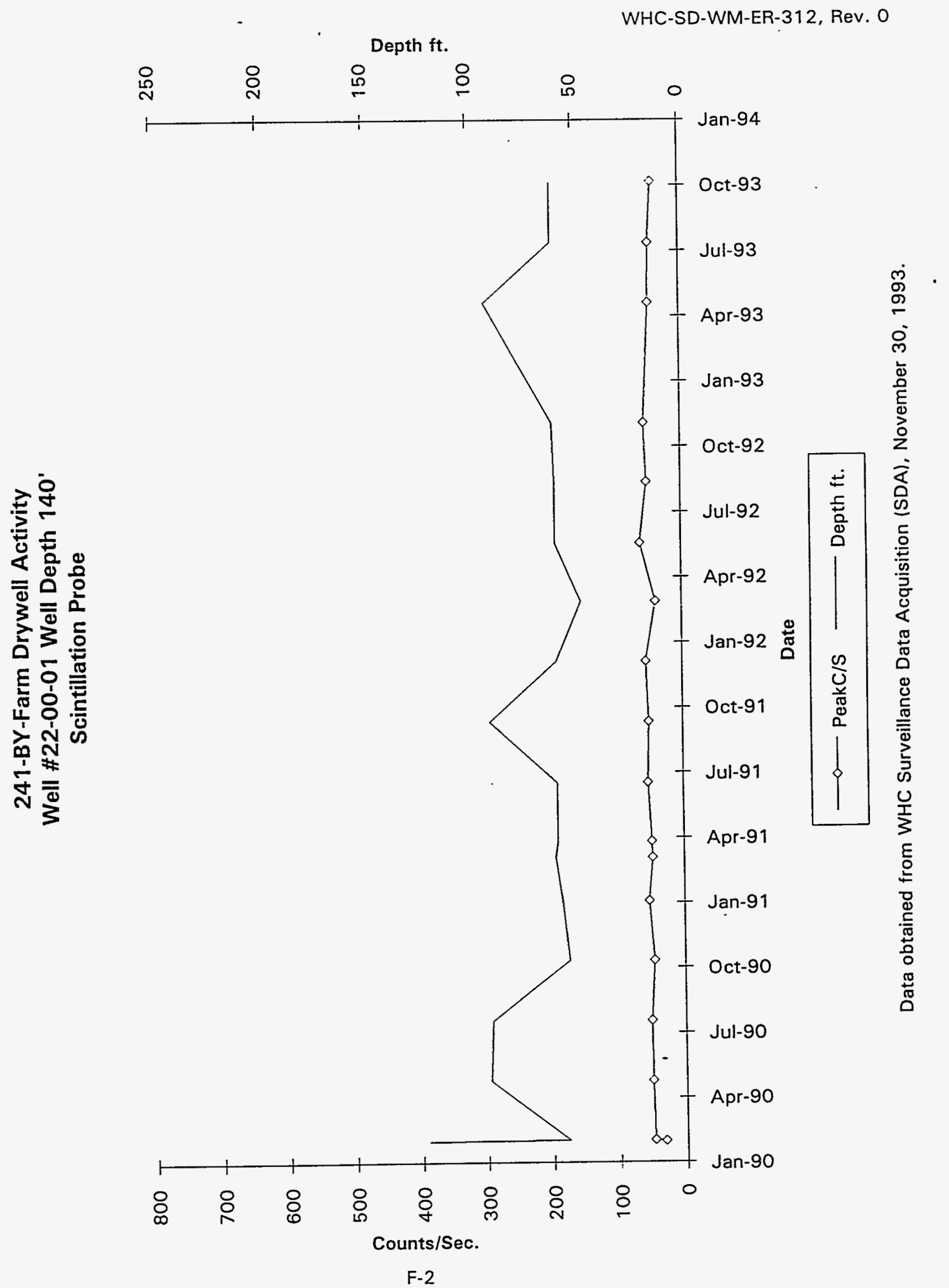




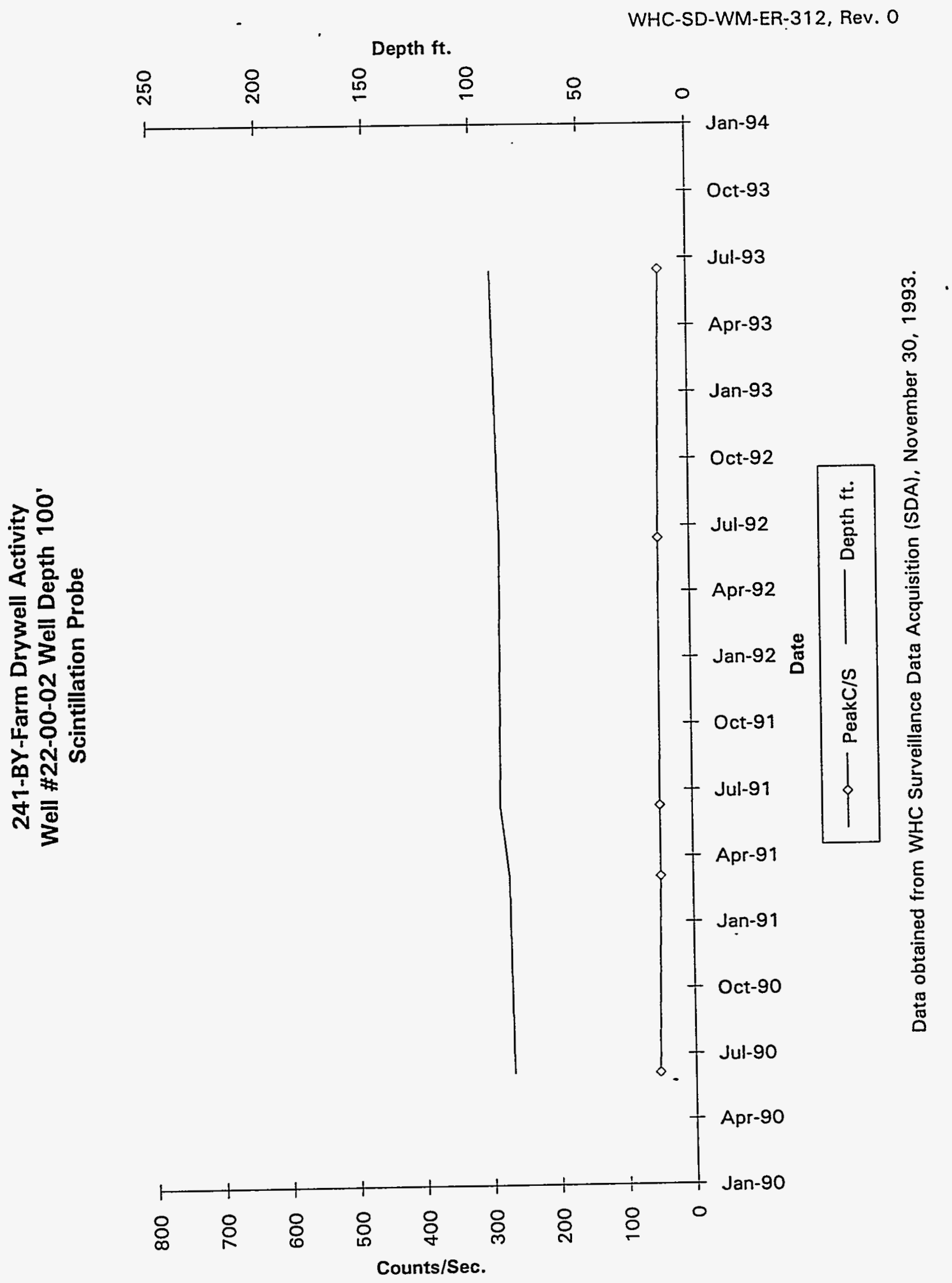

F-3 


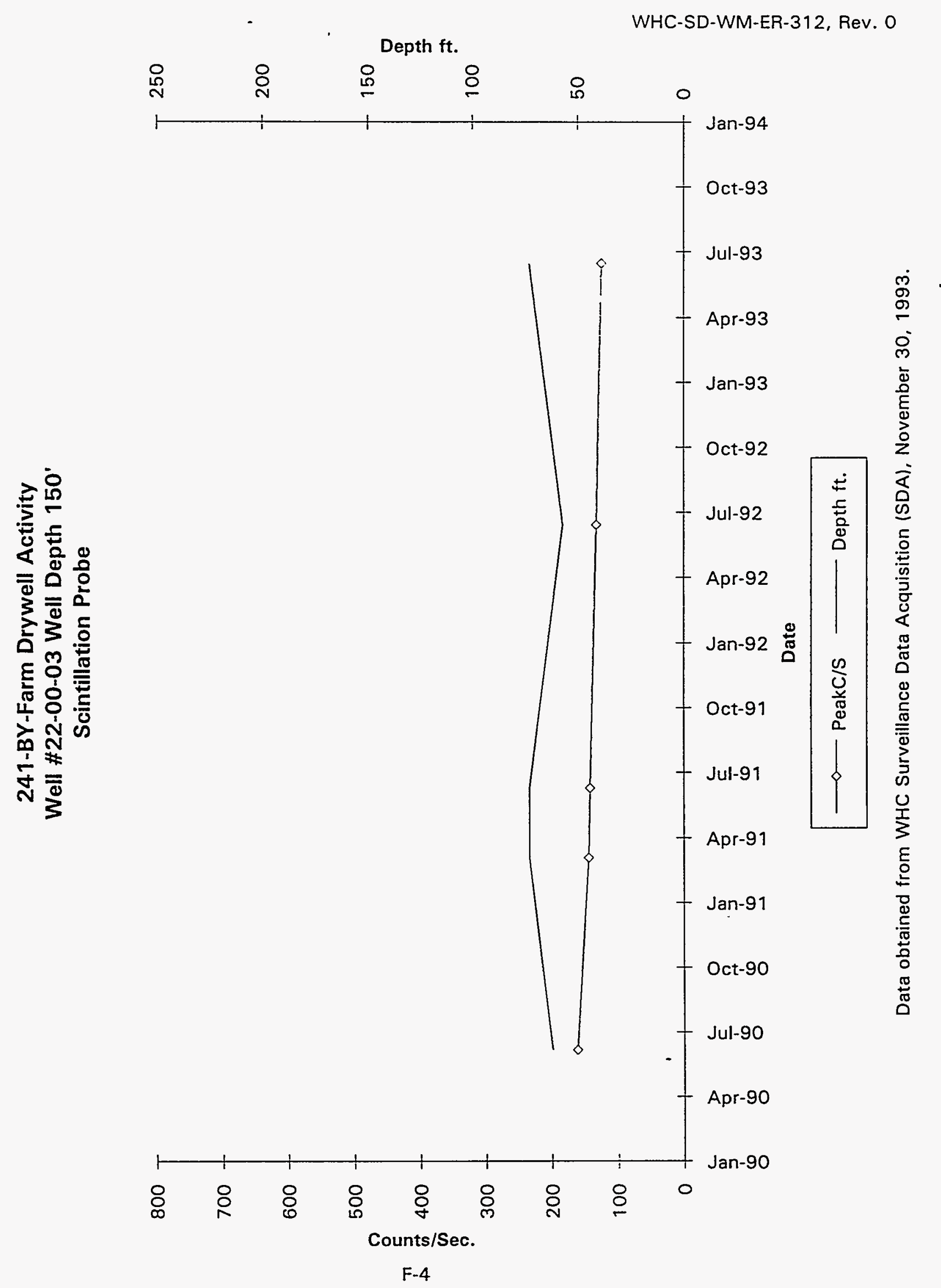


241-BY-101 Drywell Activity

Well \#22-01-10 Well Depth 100'

Scintillation Probe 1st Peak

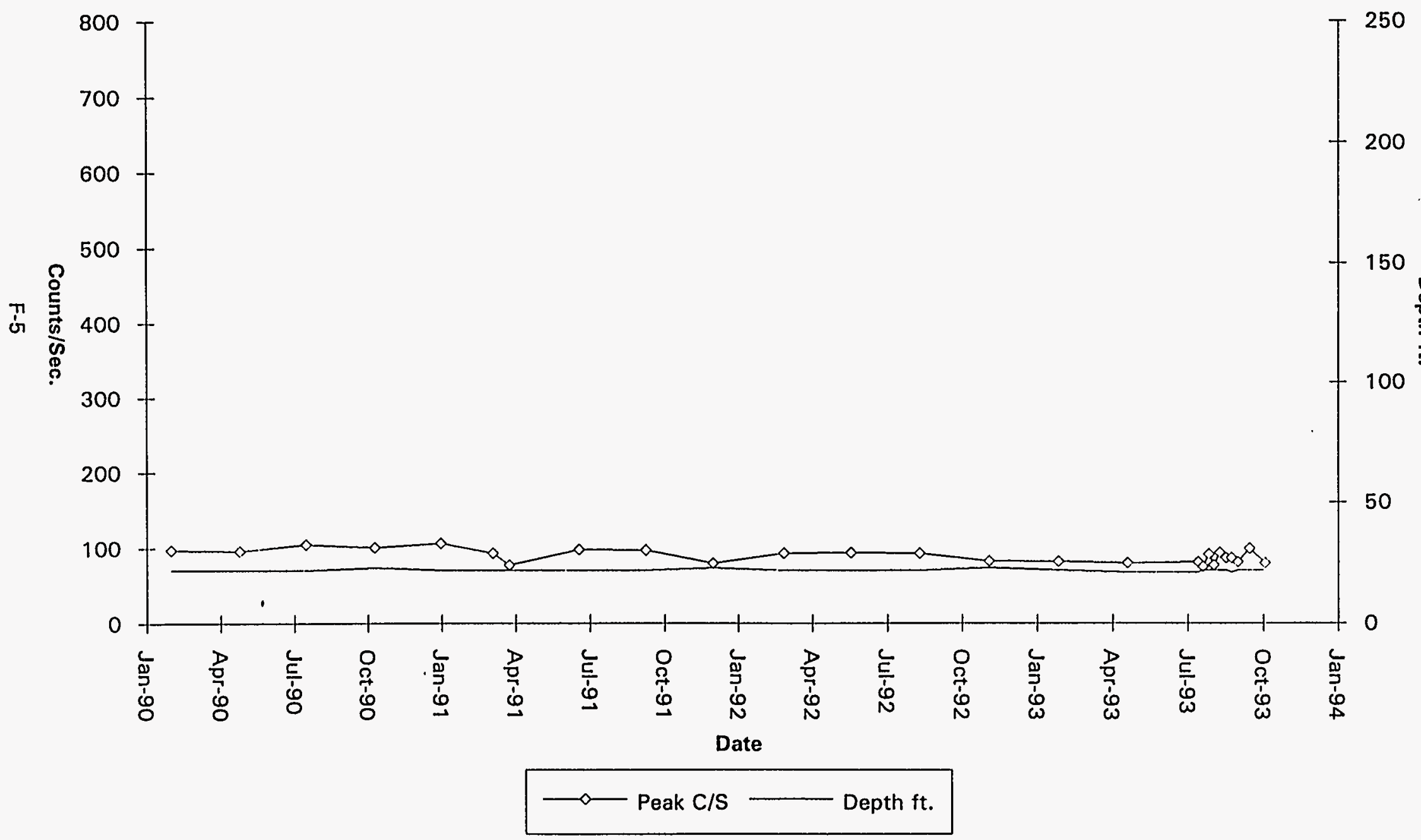

Data obtained from WHC Surveillance Data Acquisition (SDA), November 30, 1993. 


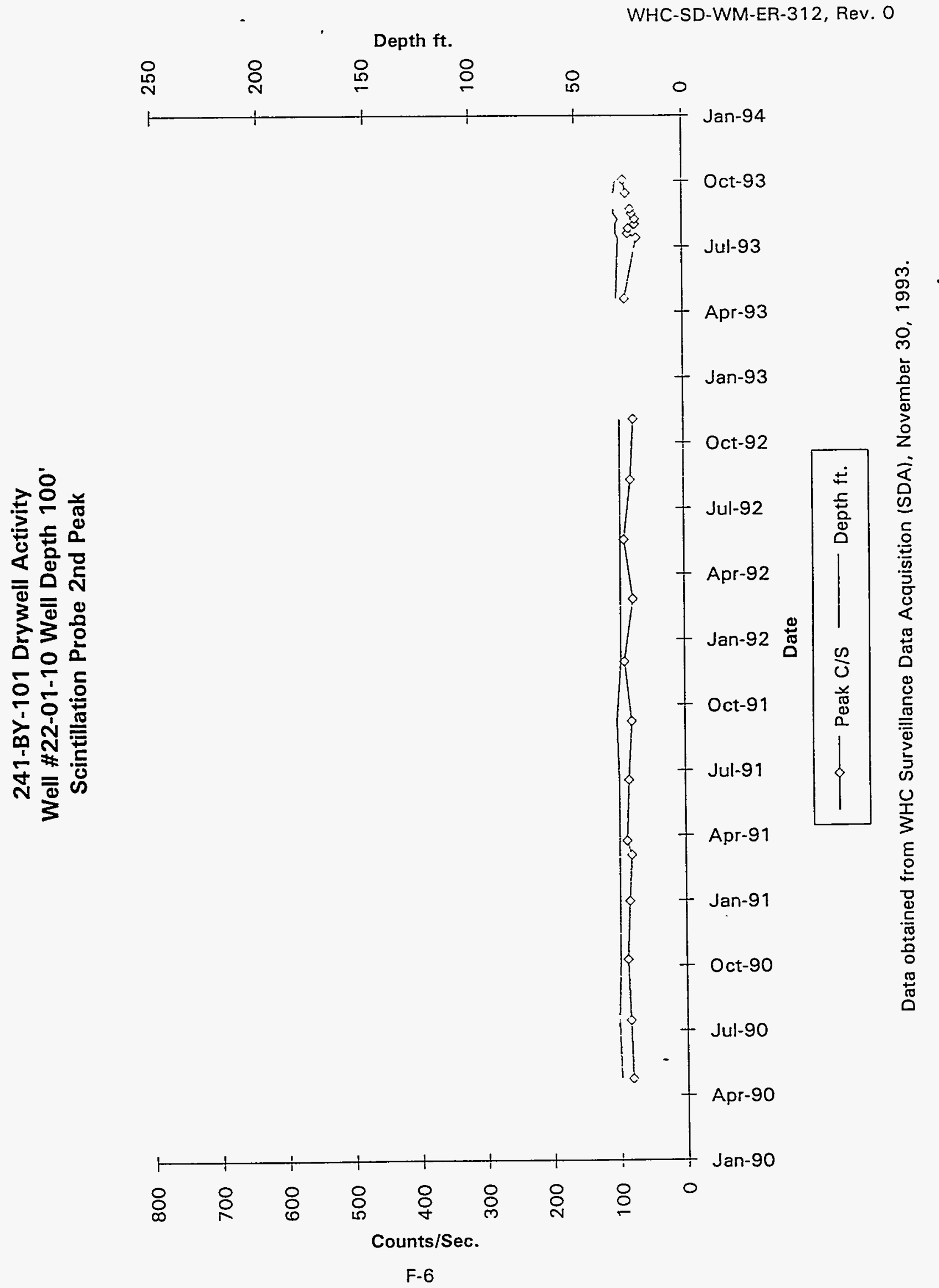


241-BY-101 Drywell Activity

Well \#22-01-10 Well Depth 100'

Scintillation Probe 3rd Peak

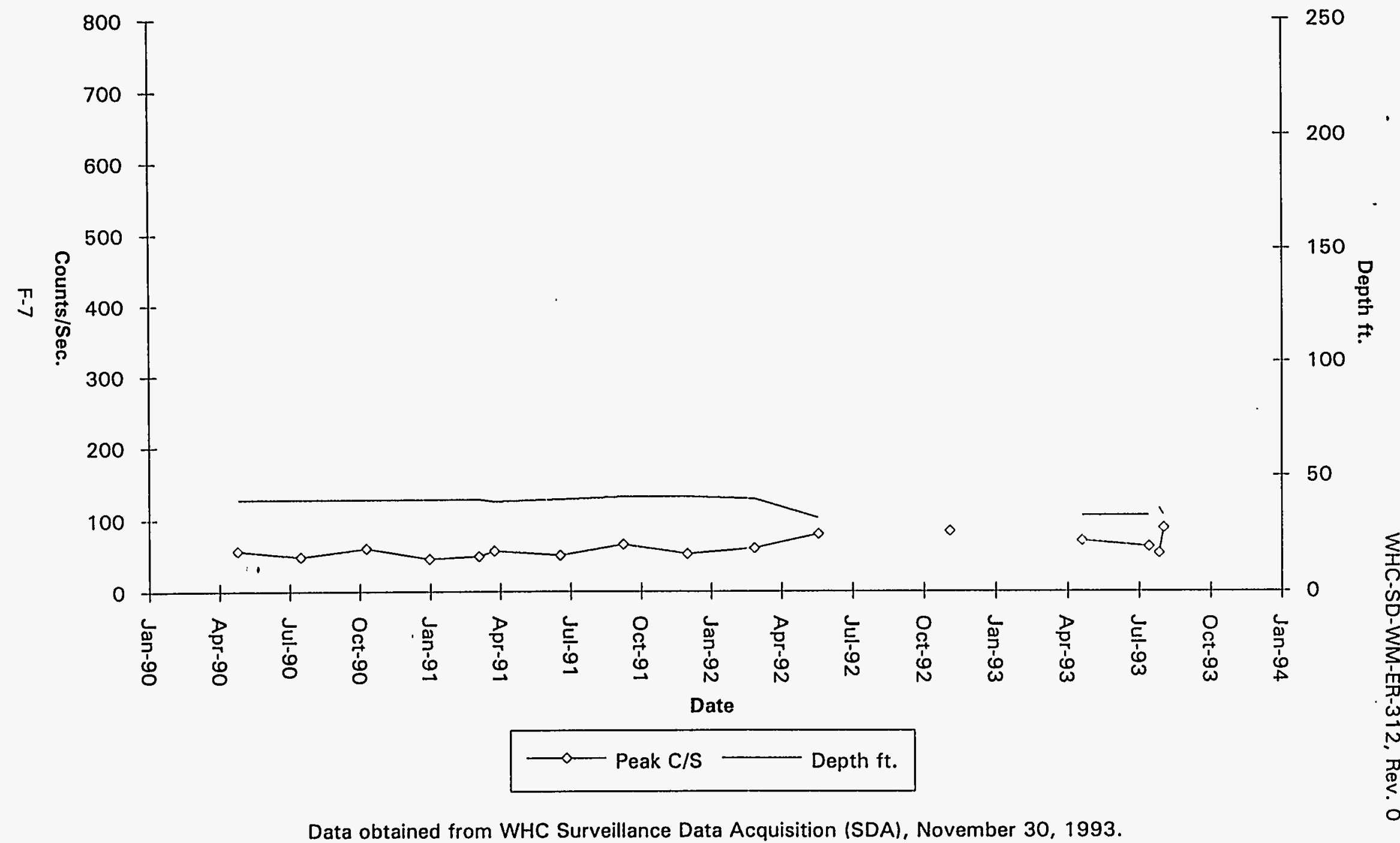

Data obtained from WHC Surveillance Data Acquisition (SDA), November 30, 1993. 

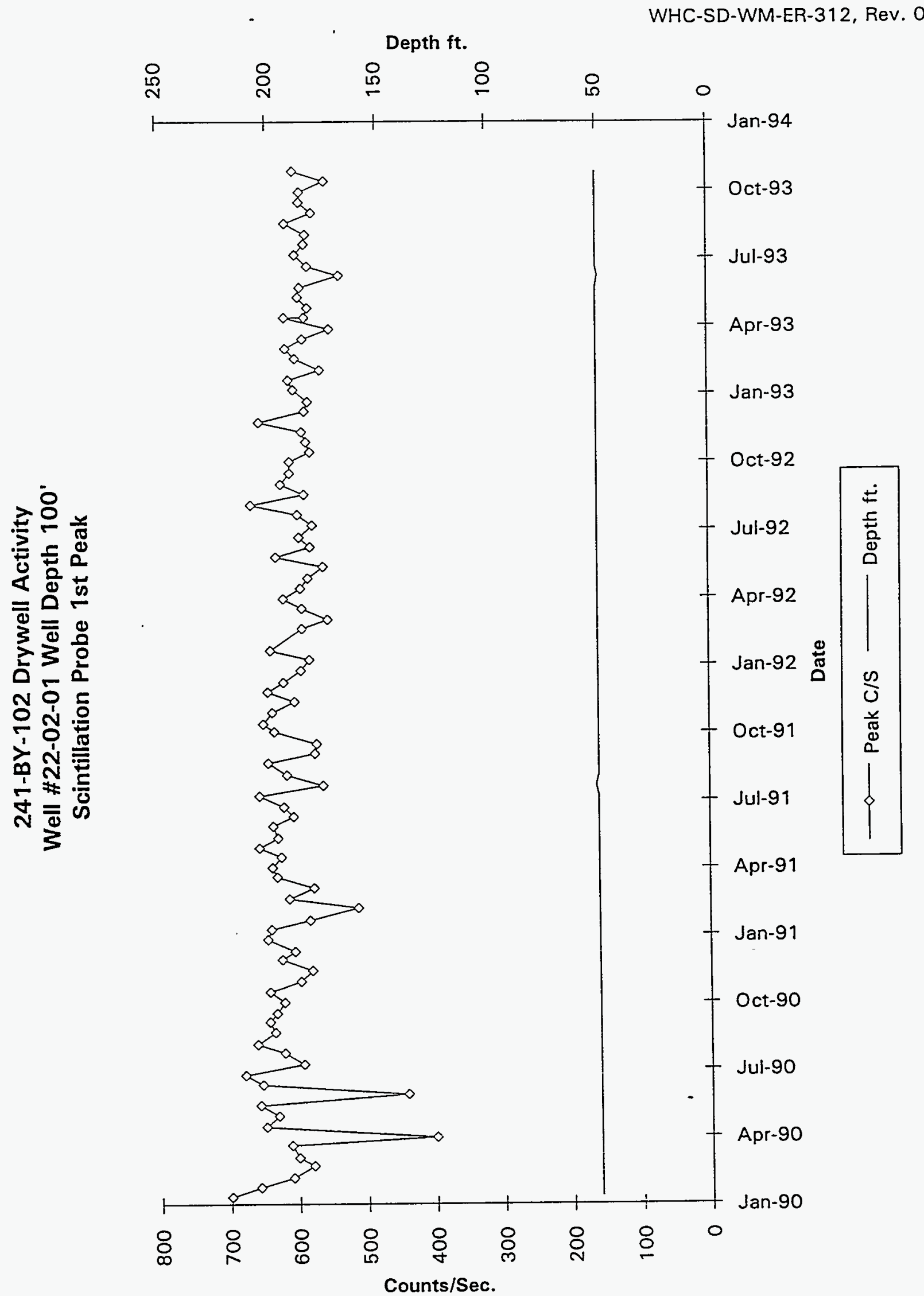


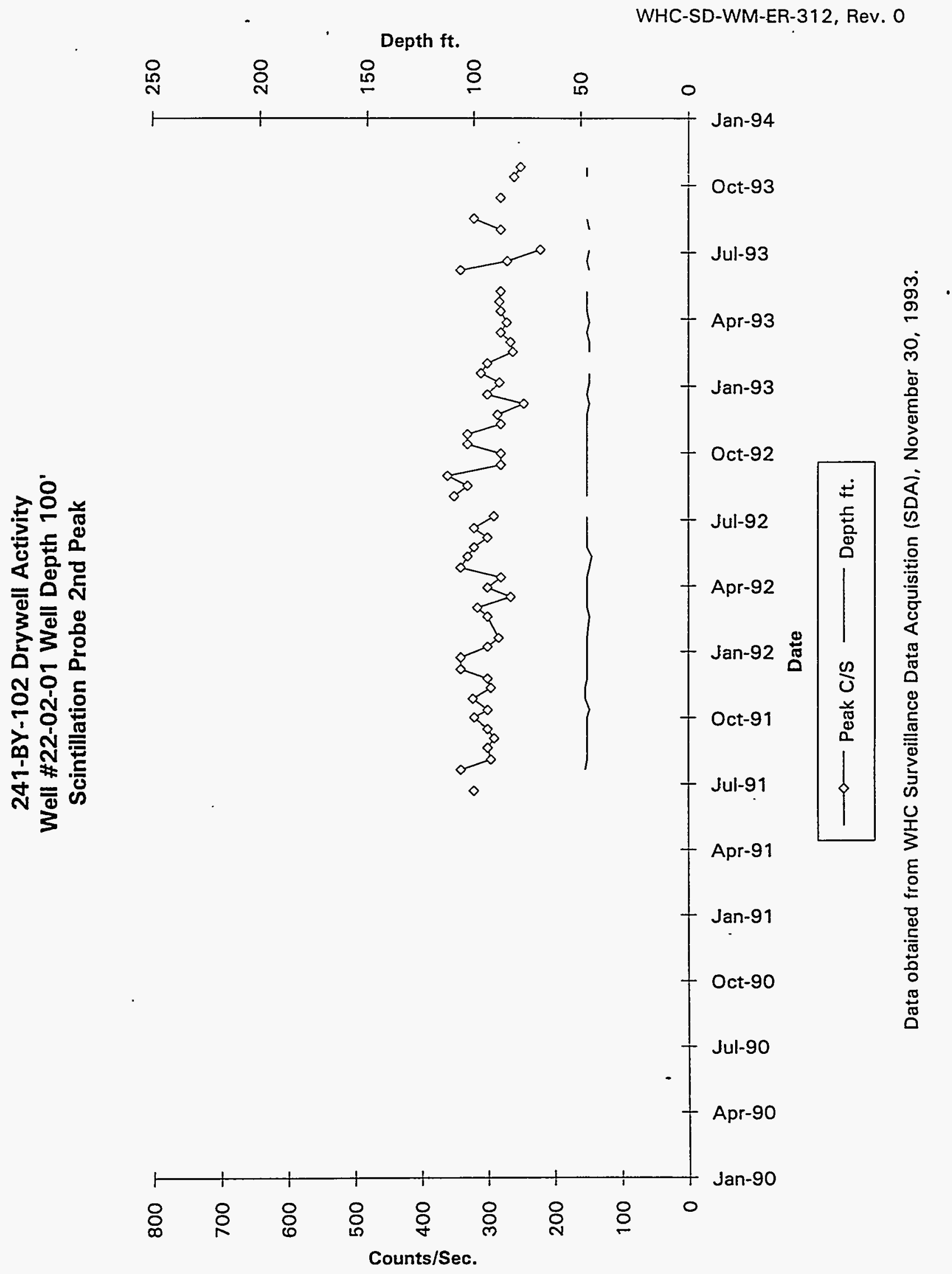

F-9 


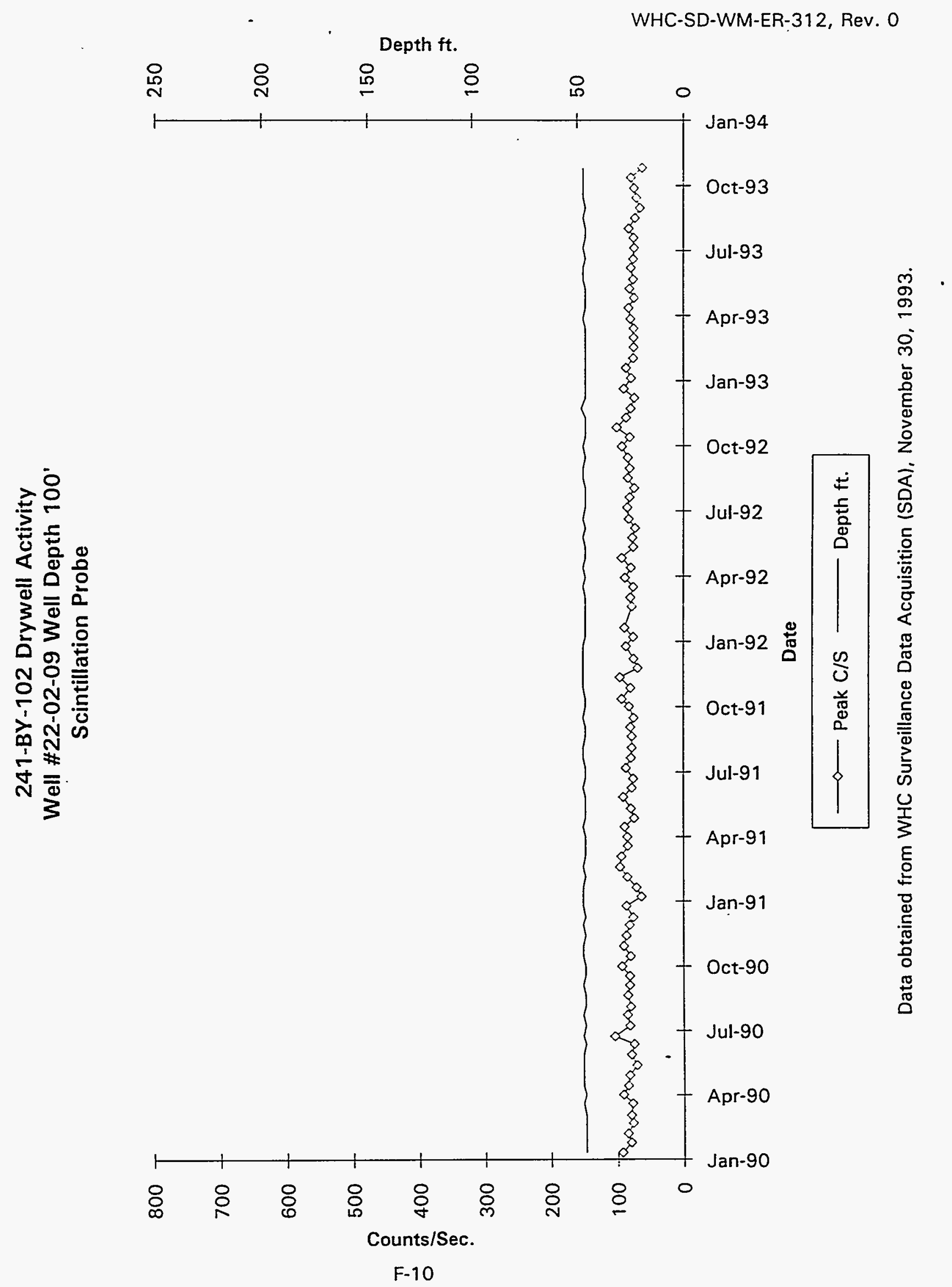



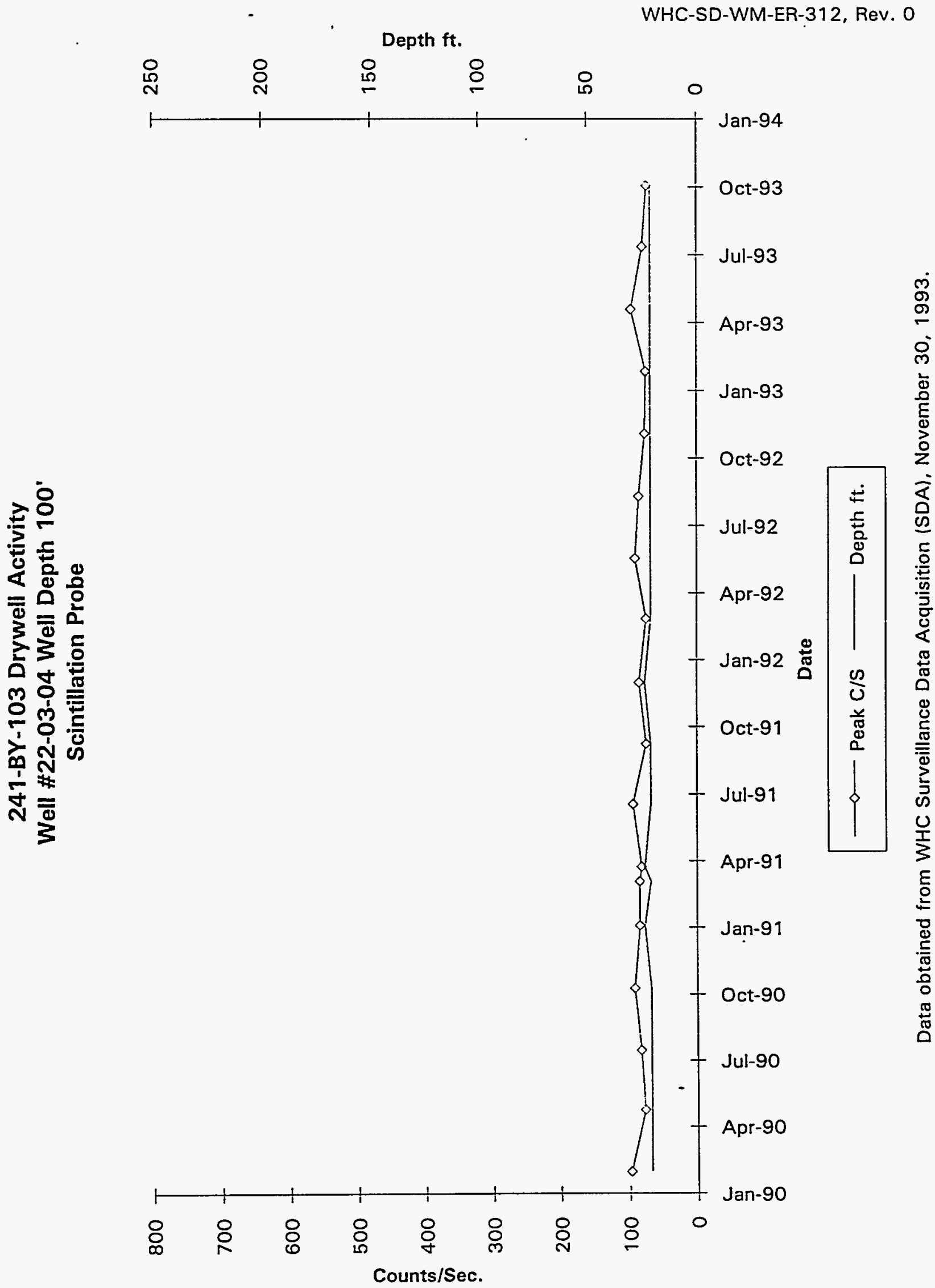

F-11 


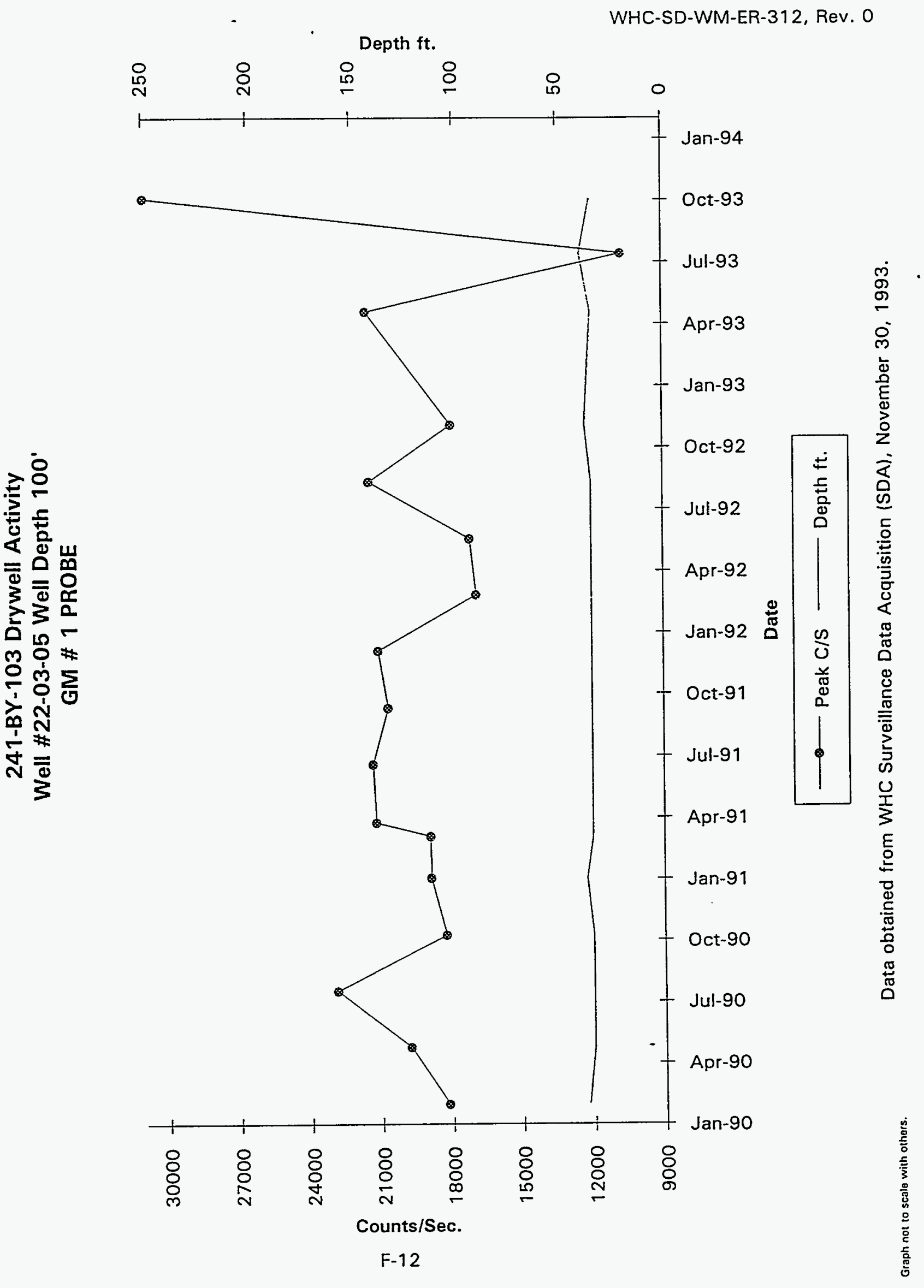




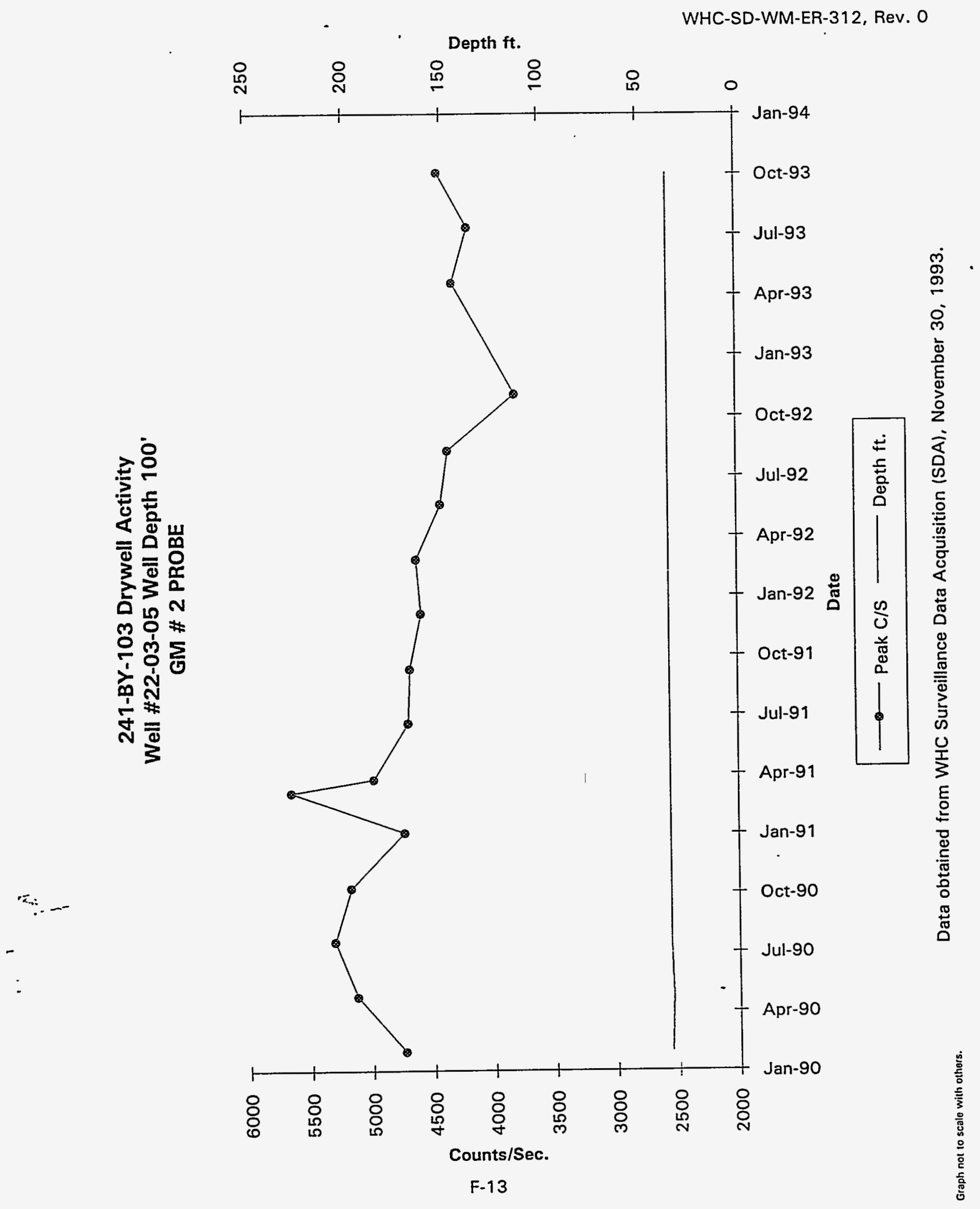


WHC-SD-WM-ER-312, Rev. 0

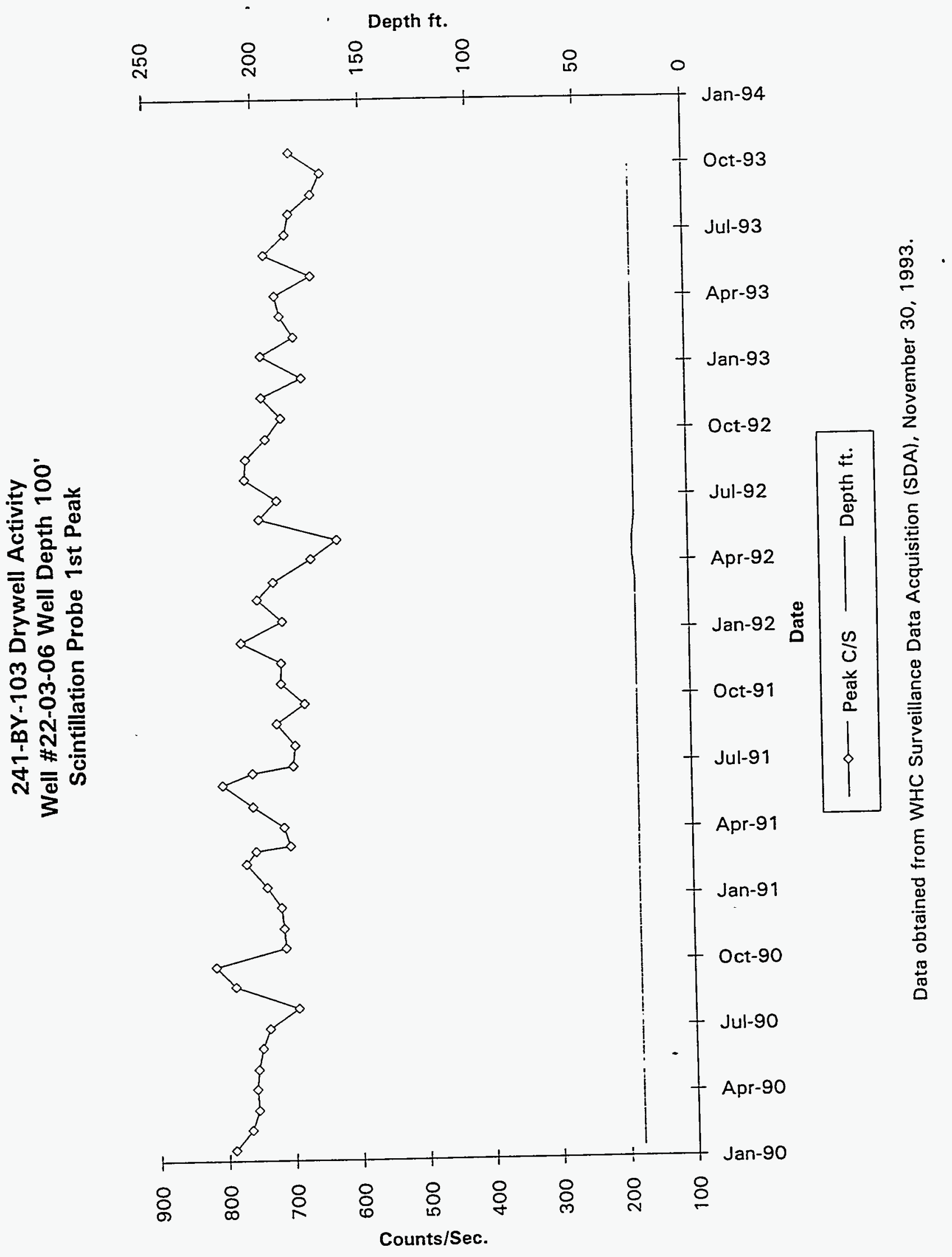

F-14 


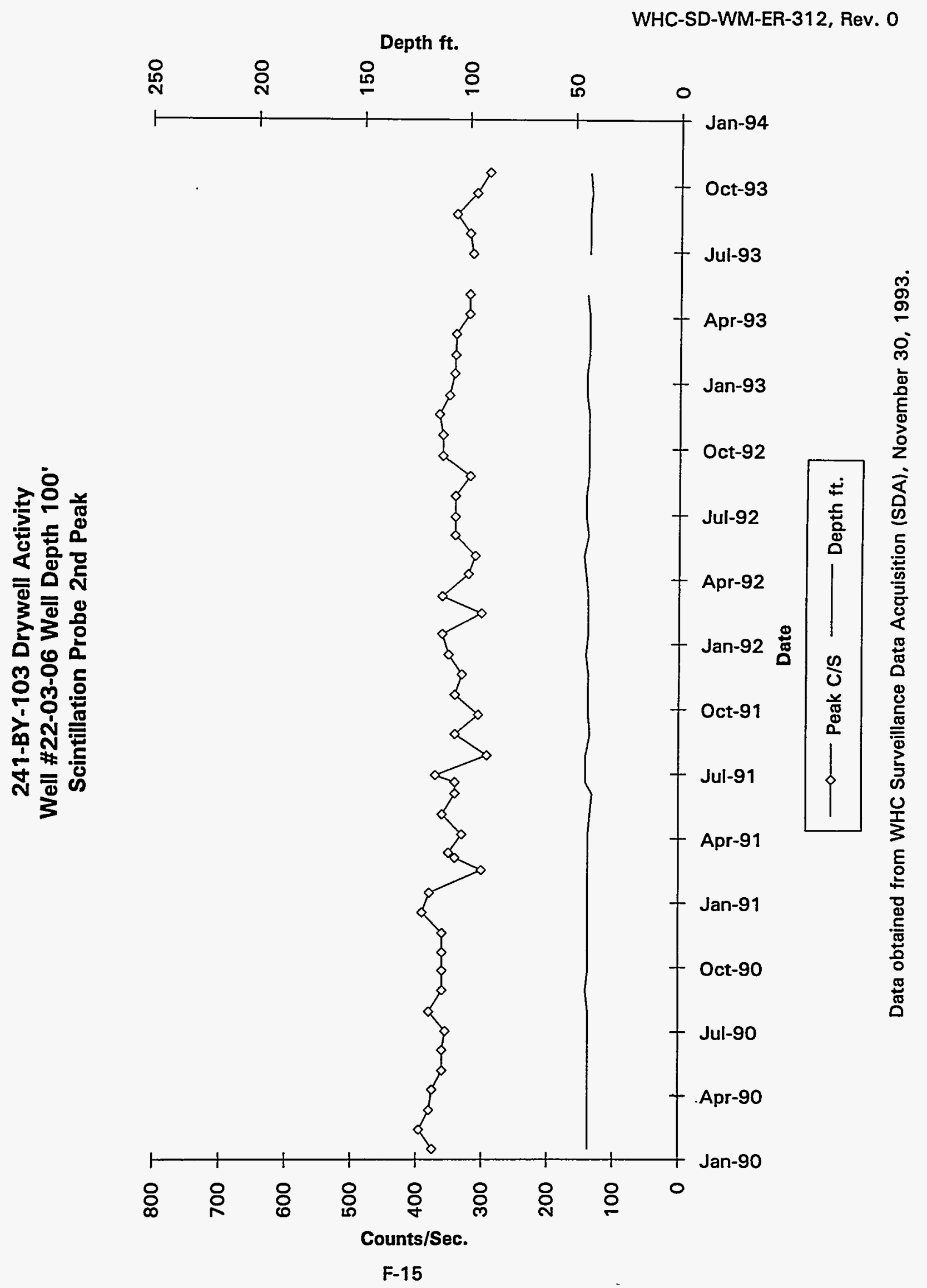


241-B-103 Drywell Activity

Well \#22-03-06 Well Depth 100'

Scintillation Probe 3rd Peak

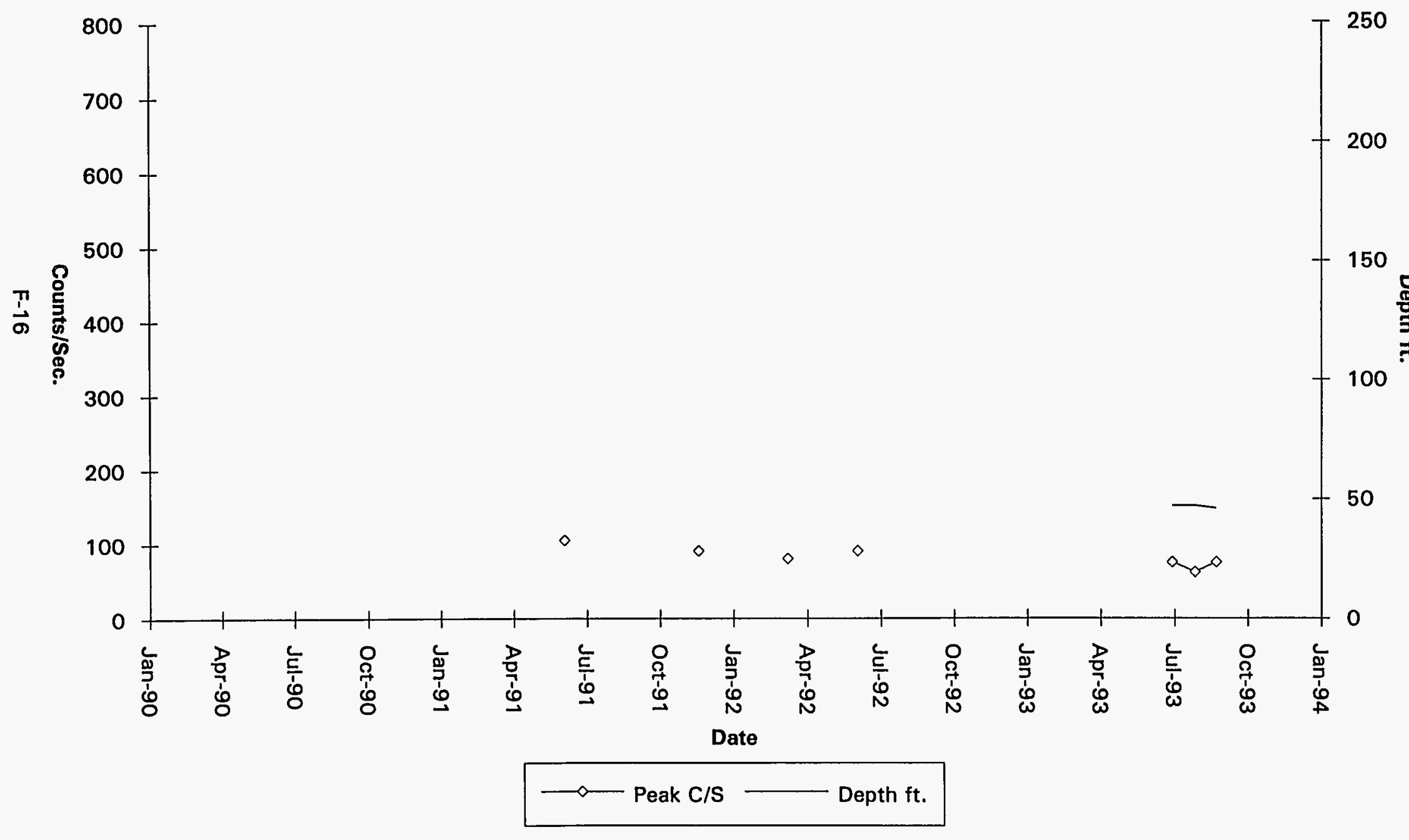

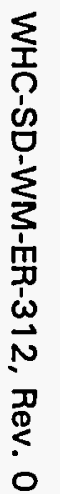

Data obtained from WHC Surveillance Data Acquisition (SDA), November 30, 1993. 


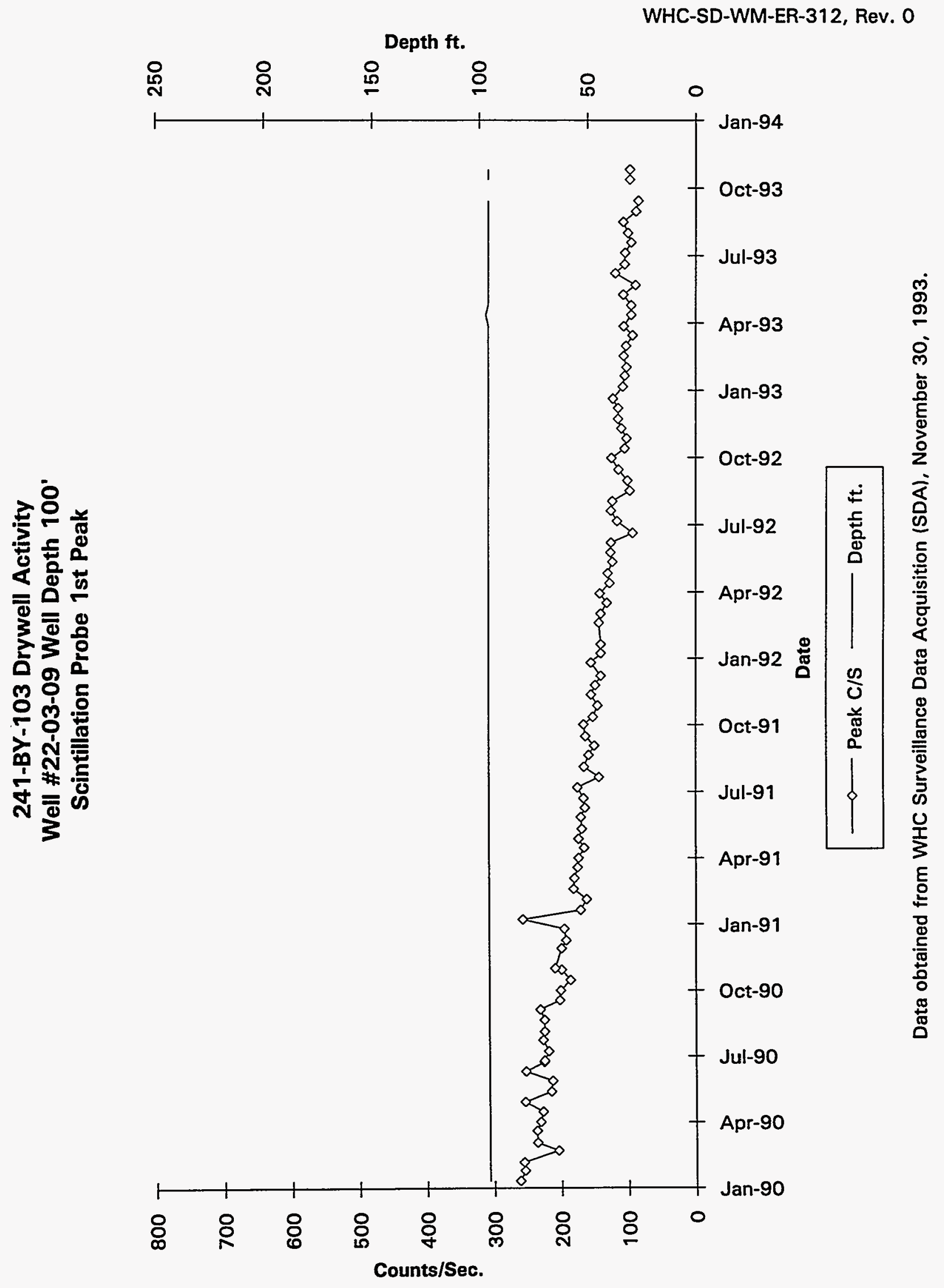

F-17 
241-BY-103 Drywell Activity

Well \#22-03-09 Well Depth 100'

Scintillation Probe 2nd Peak

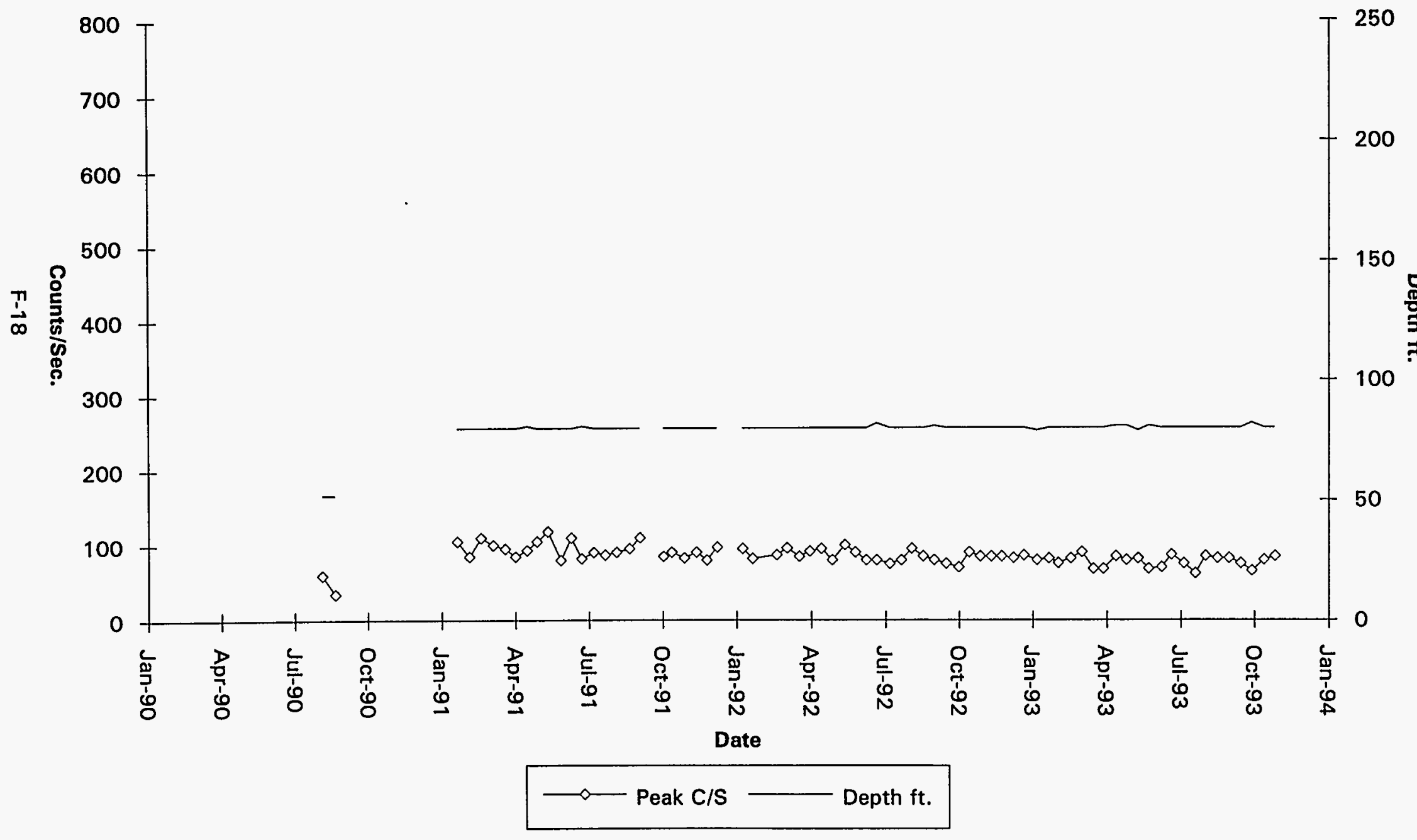

Data obtained from WHC Surveillance Data Acquisition (SDA), November 30, 1993. 


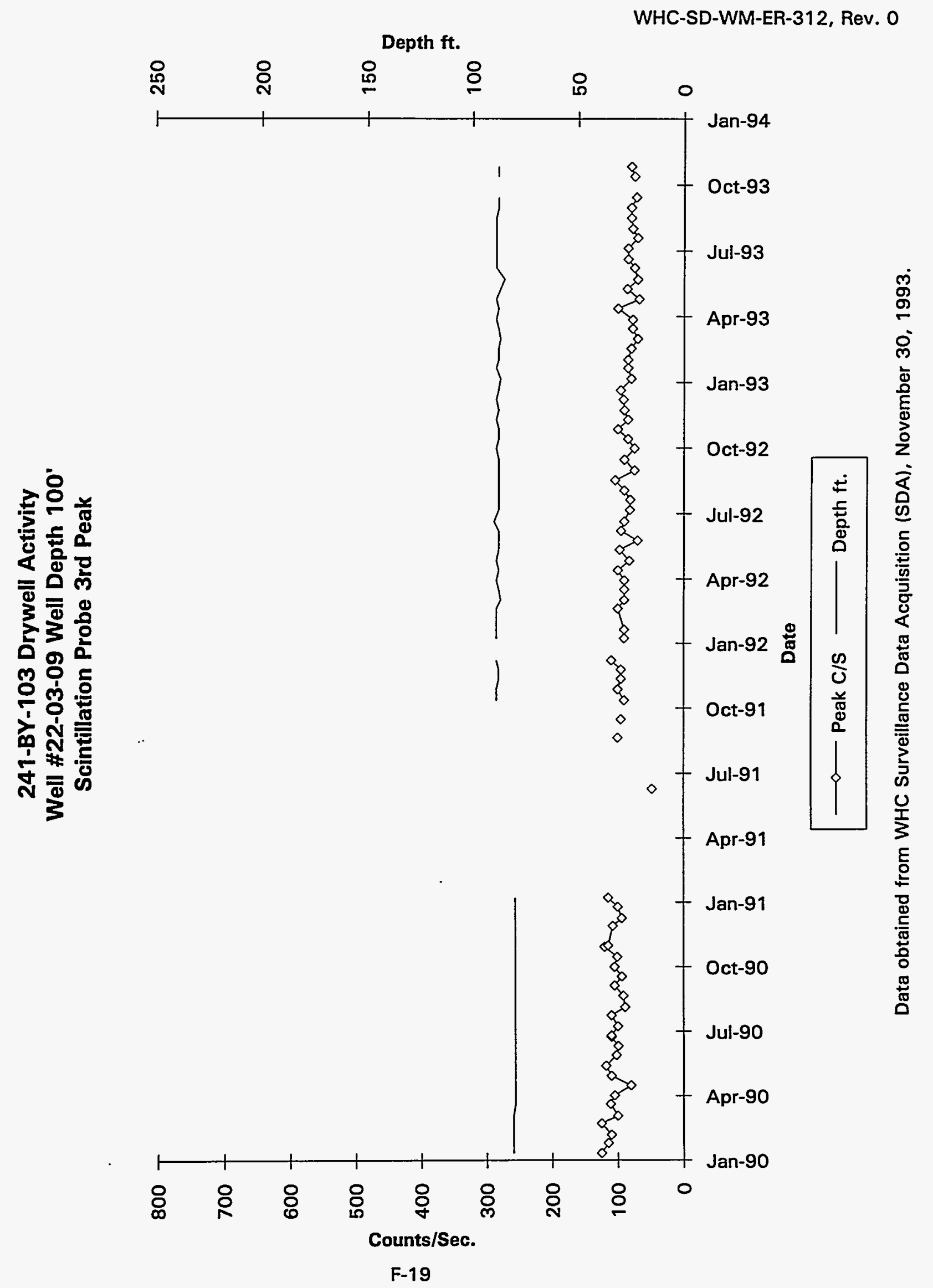




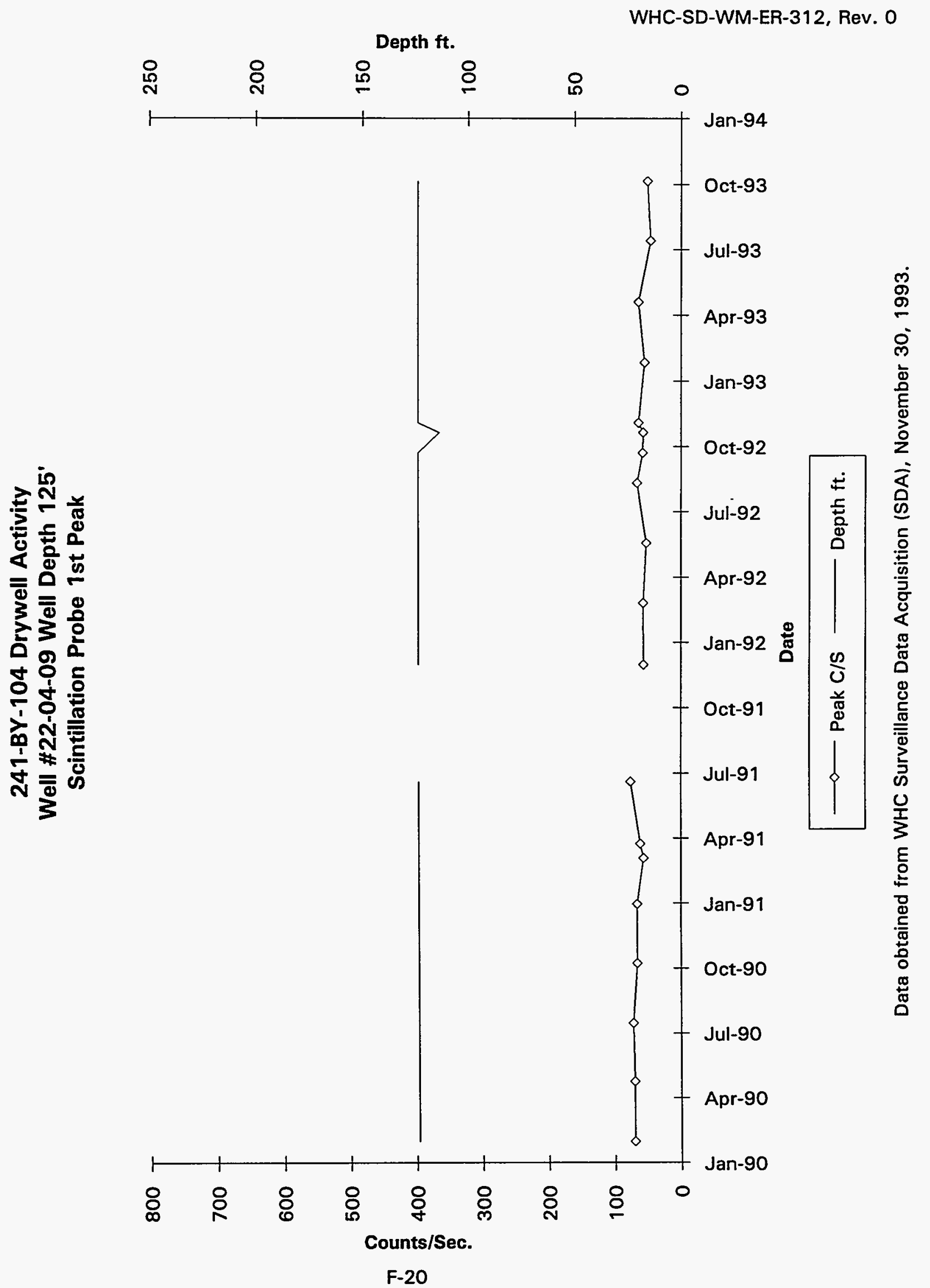



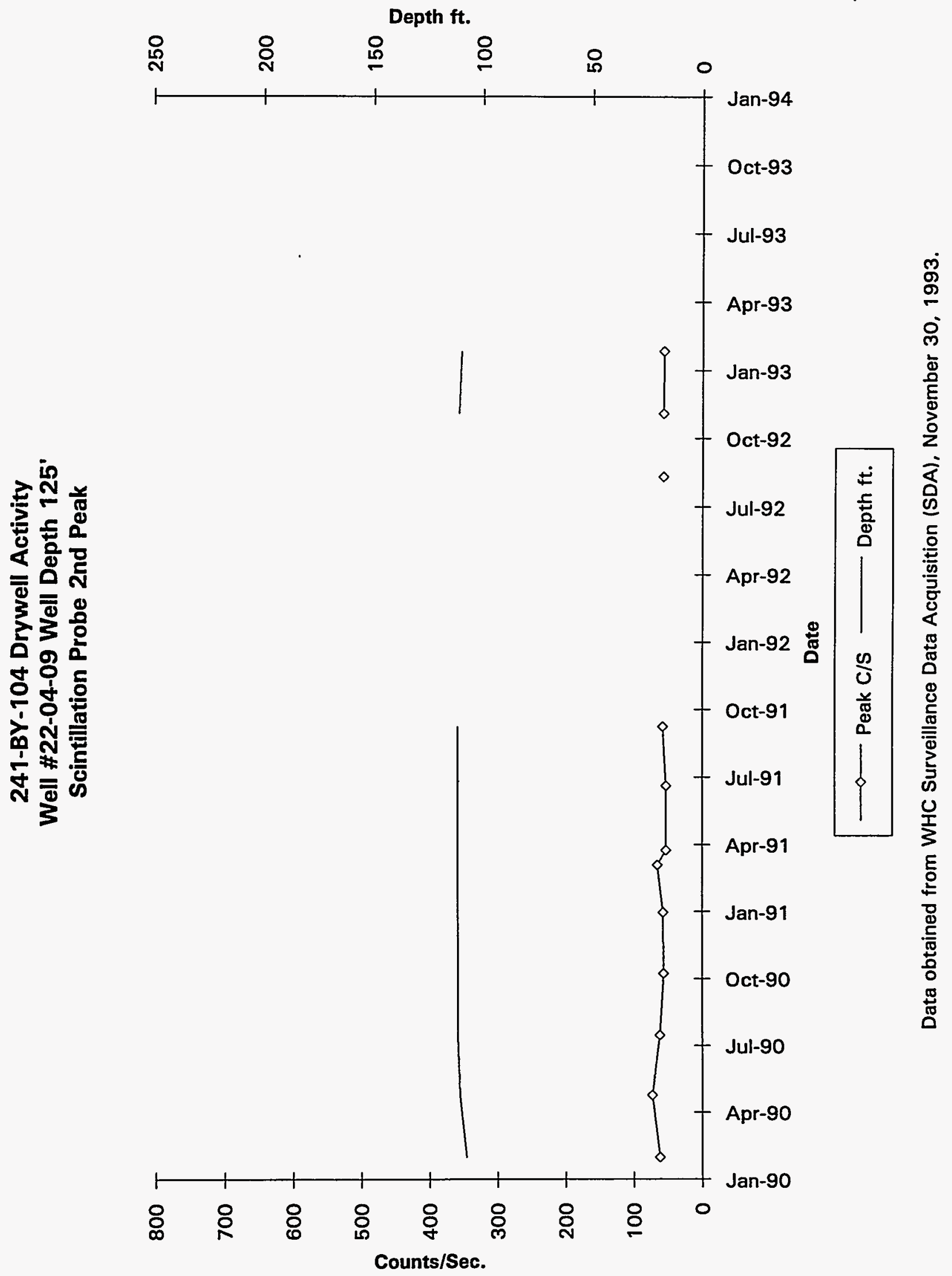
241-BY-104 Drywell Activity

Well \#22-04-09 Well Depth 125'

Scintillation Probe 3rd Peak

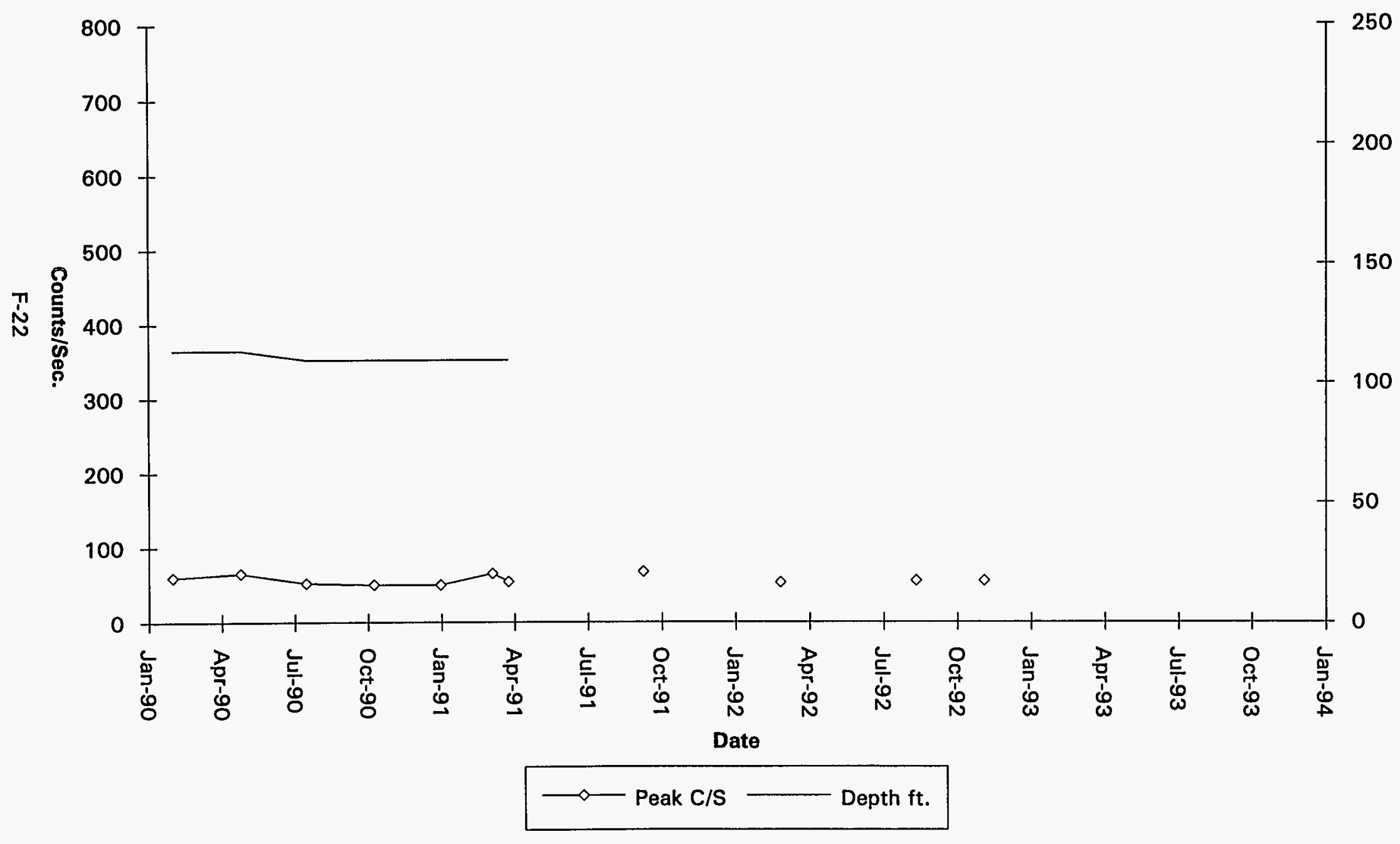

Data obtained from WHC Surveillance Data Acquisition (SDA), November 30, 1993. 

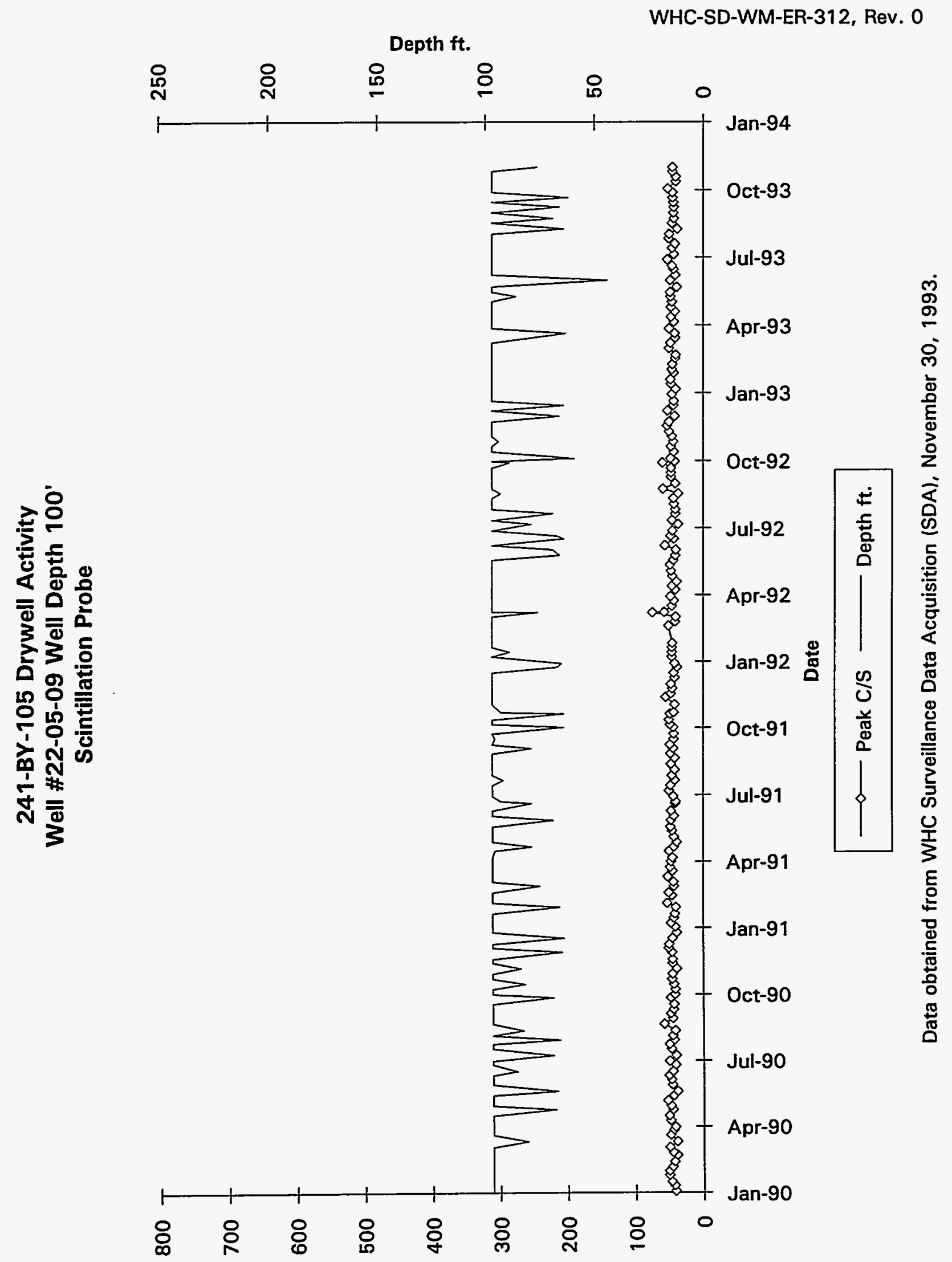

Counts/Sec.

F-23 
241-BY-106 Drywell Activity

Well \#22-06-05 Well Depth 100'

Scintillation Probe 1st Peak

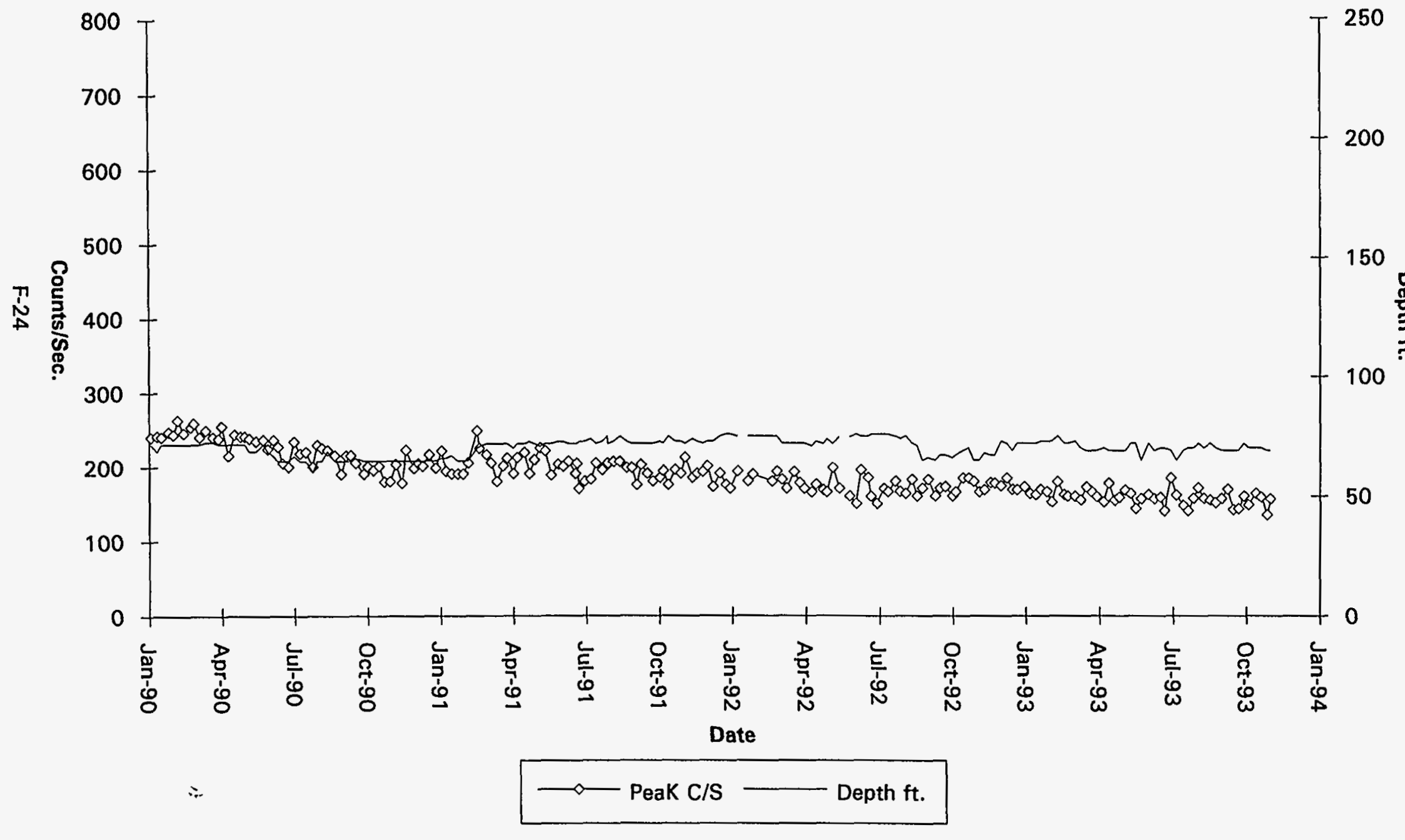

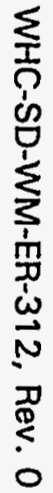

Data obtained from WHC Surveillance Data Acquisition (SDA), November 30, 1993. 

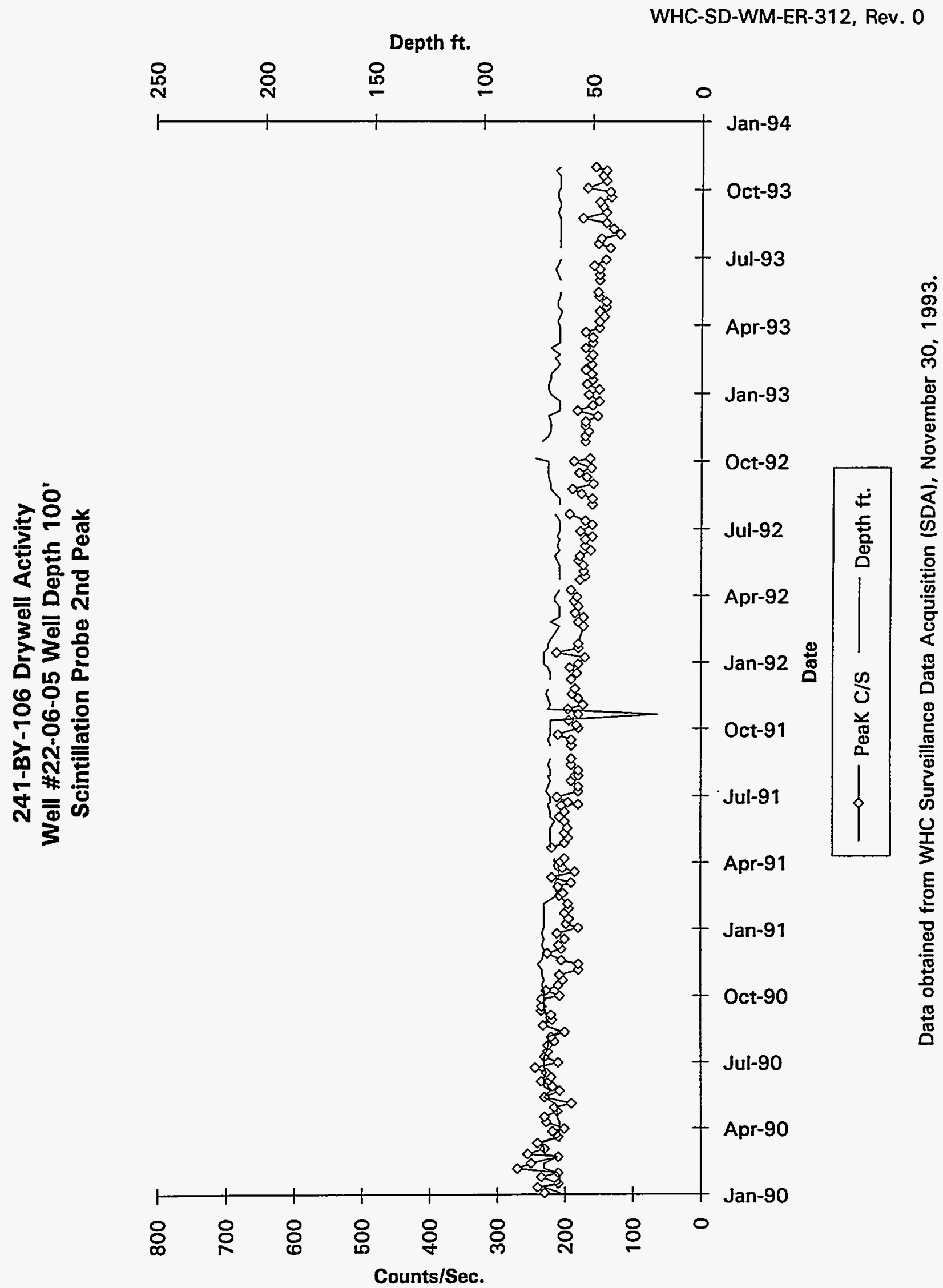

F-25 

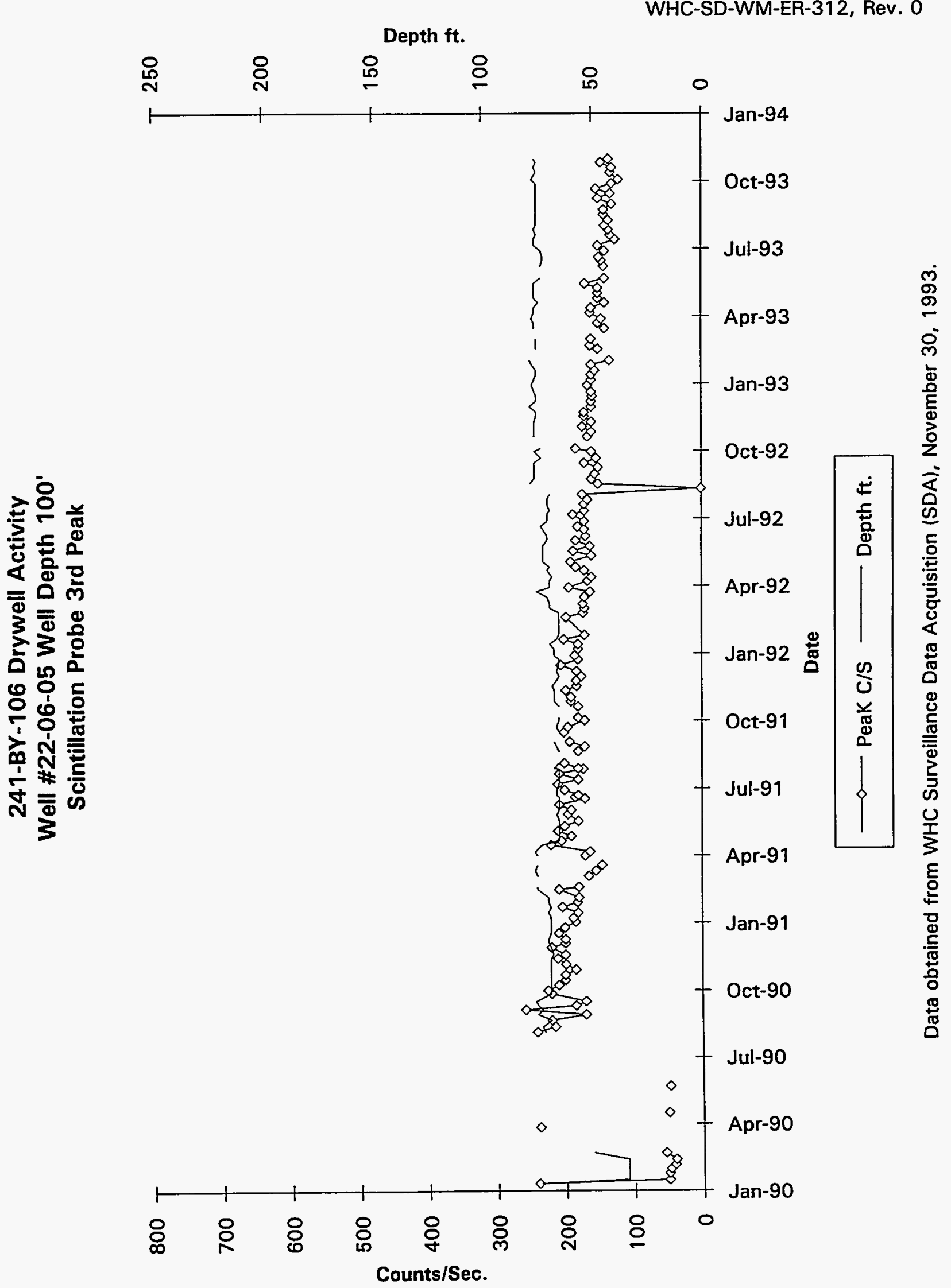

F-26 


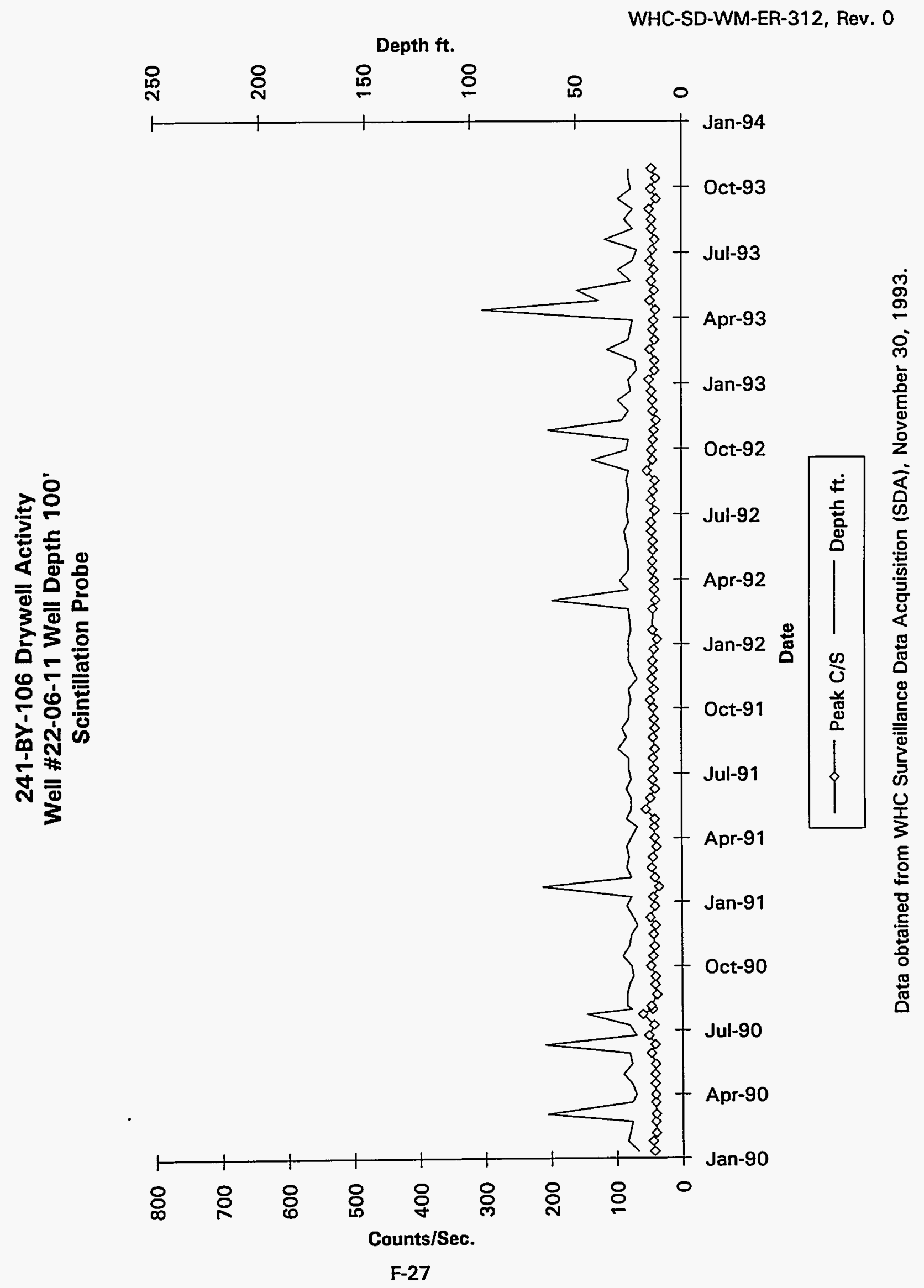


WHC-SD-WM-ER-312, Rev. 0

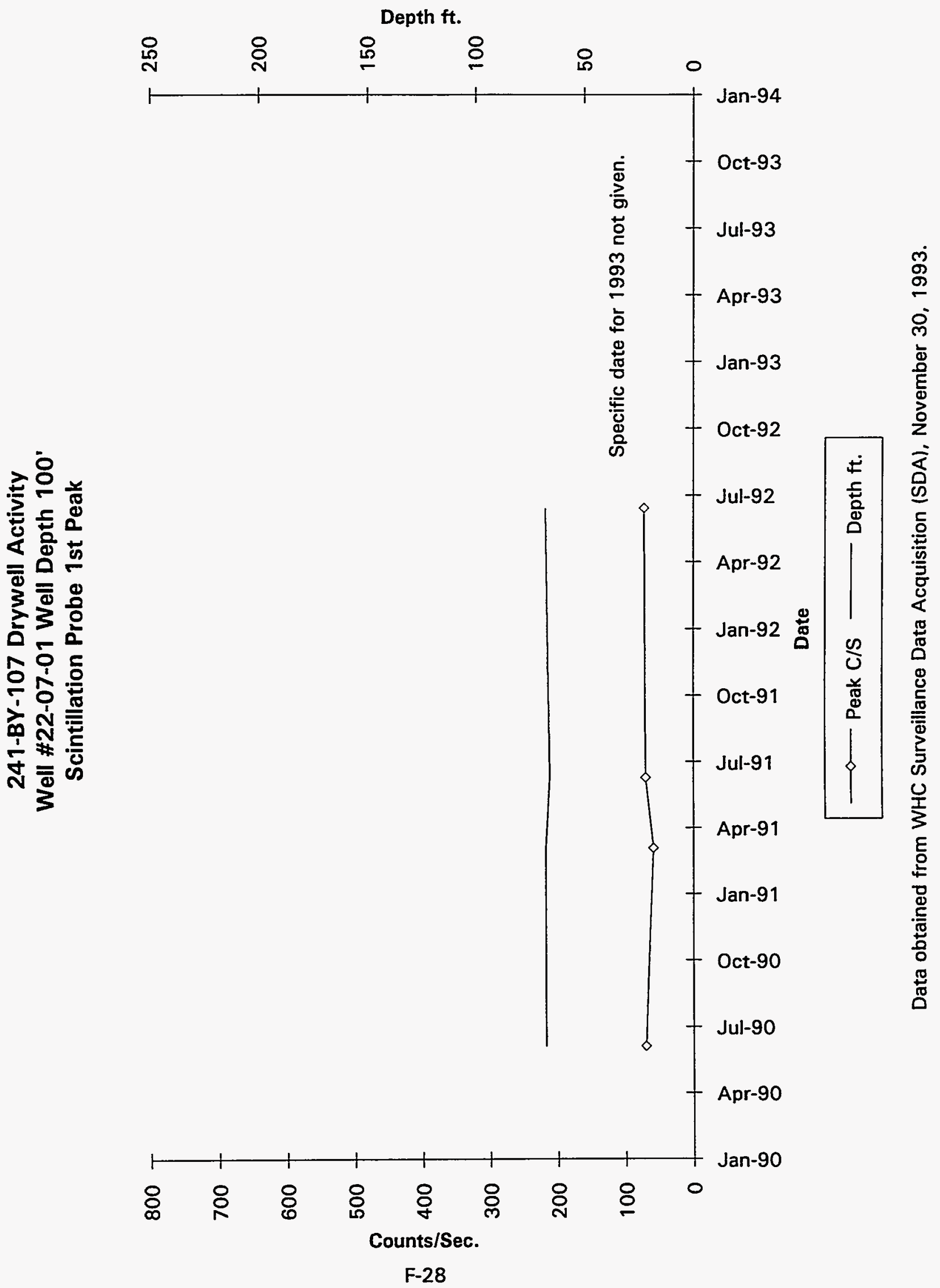


241-BY-107 Drywell Activity

Well \#22-07-01 Well Depth 100'

Scintillation Probe 2nd Peak

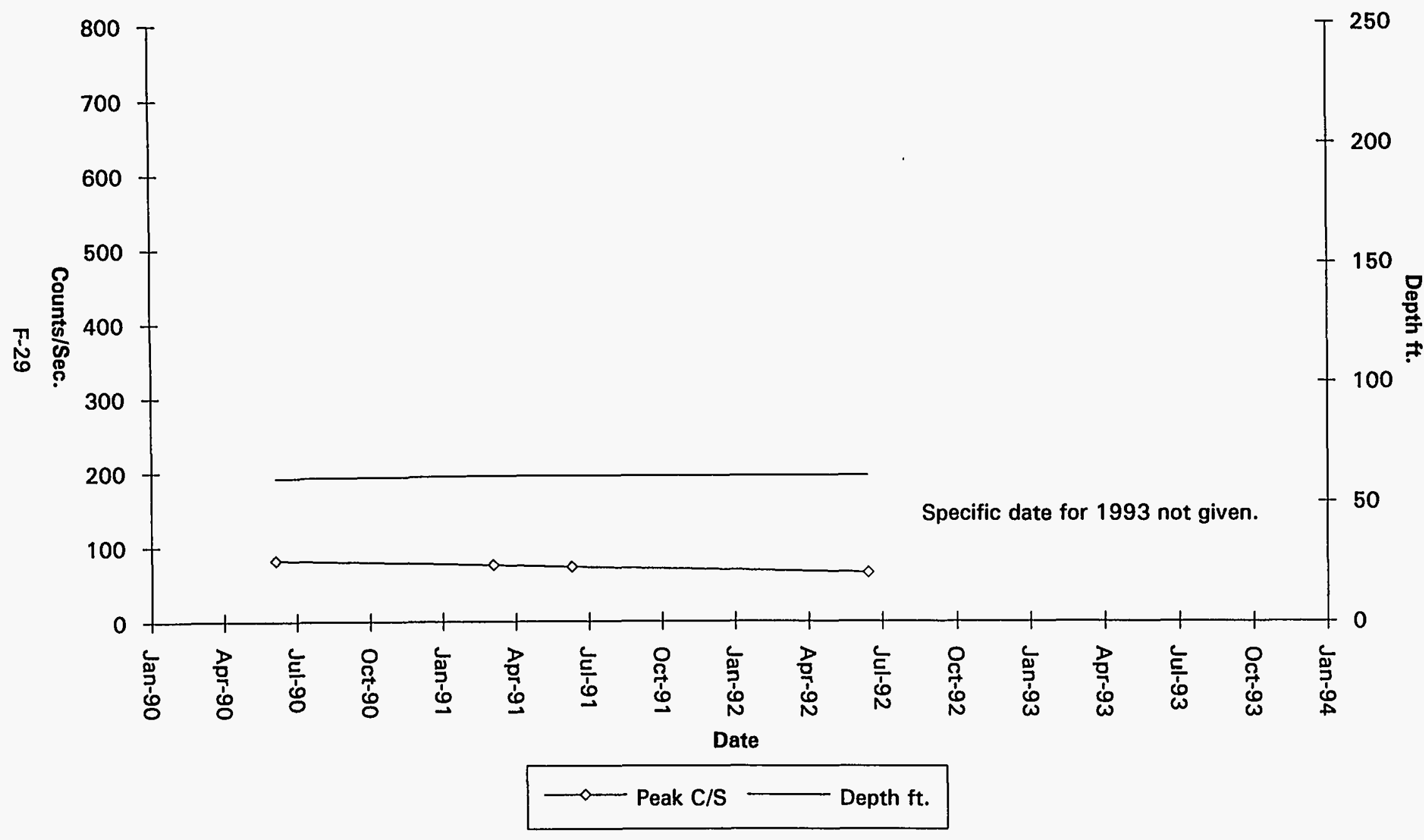

Data obtained from WHC Surveillance Data Acquisition (SDA), November 30, 1993. 
WHC-SD-WM-ER-312, Rev. 0

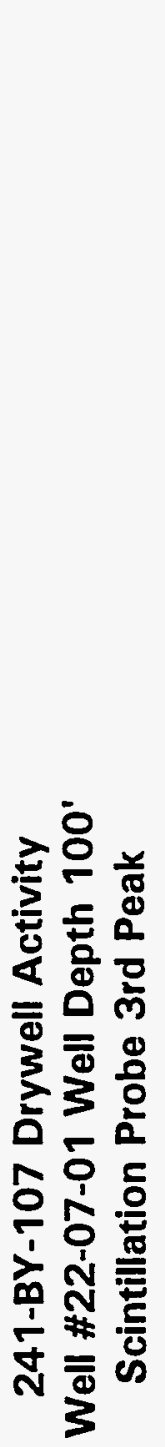

\section{Depth ft.}

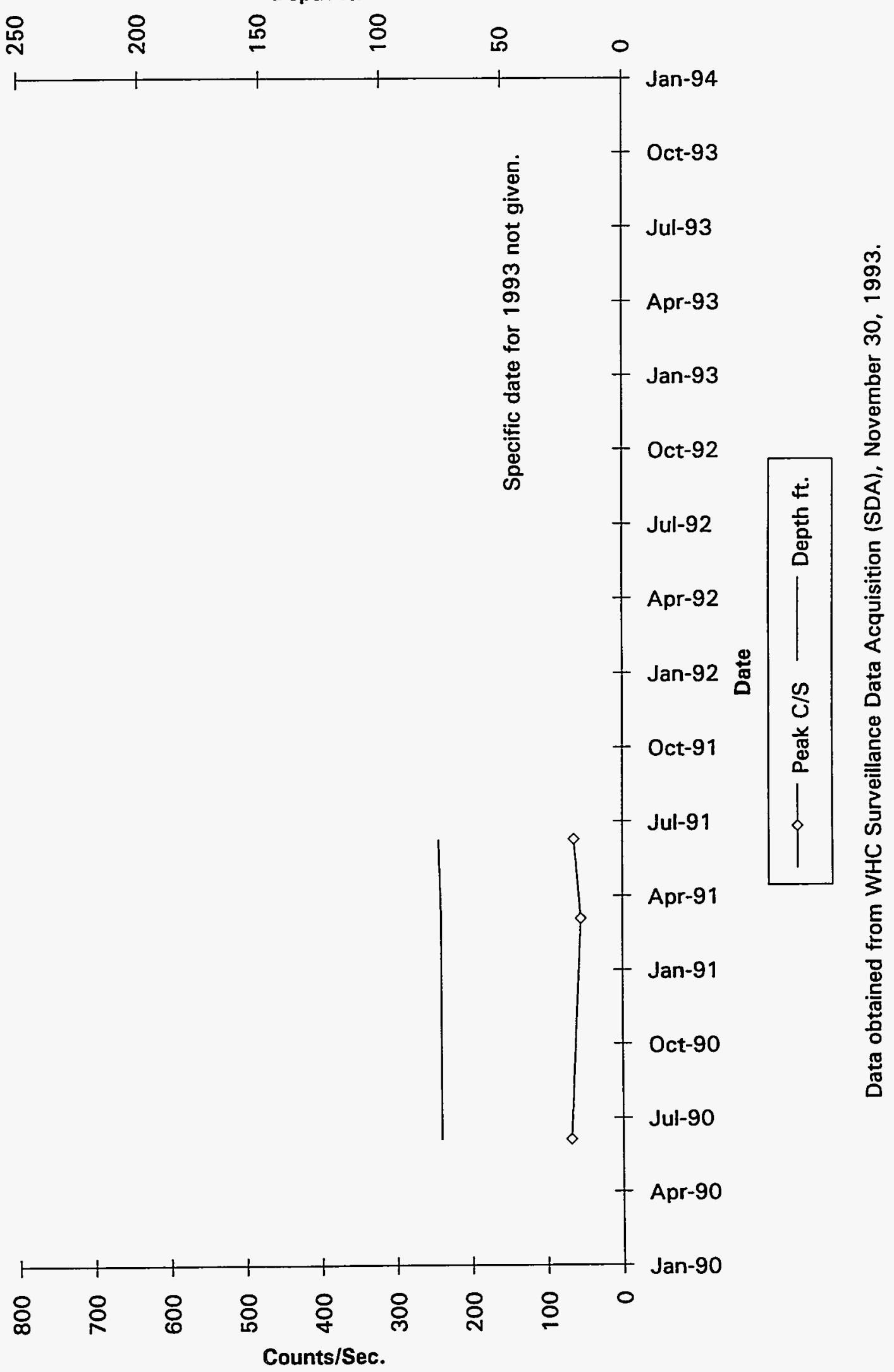

F-30 


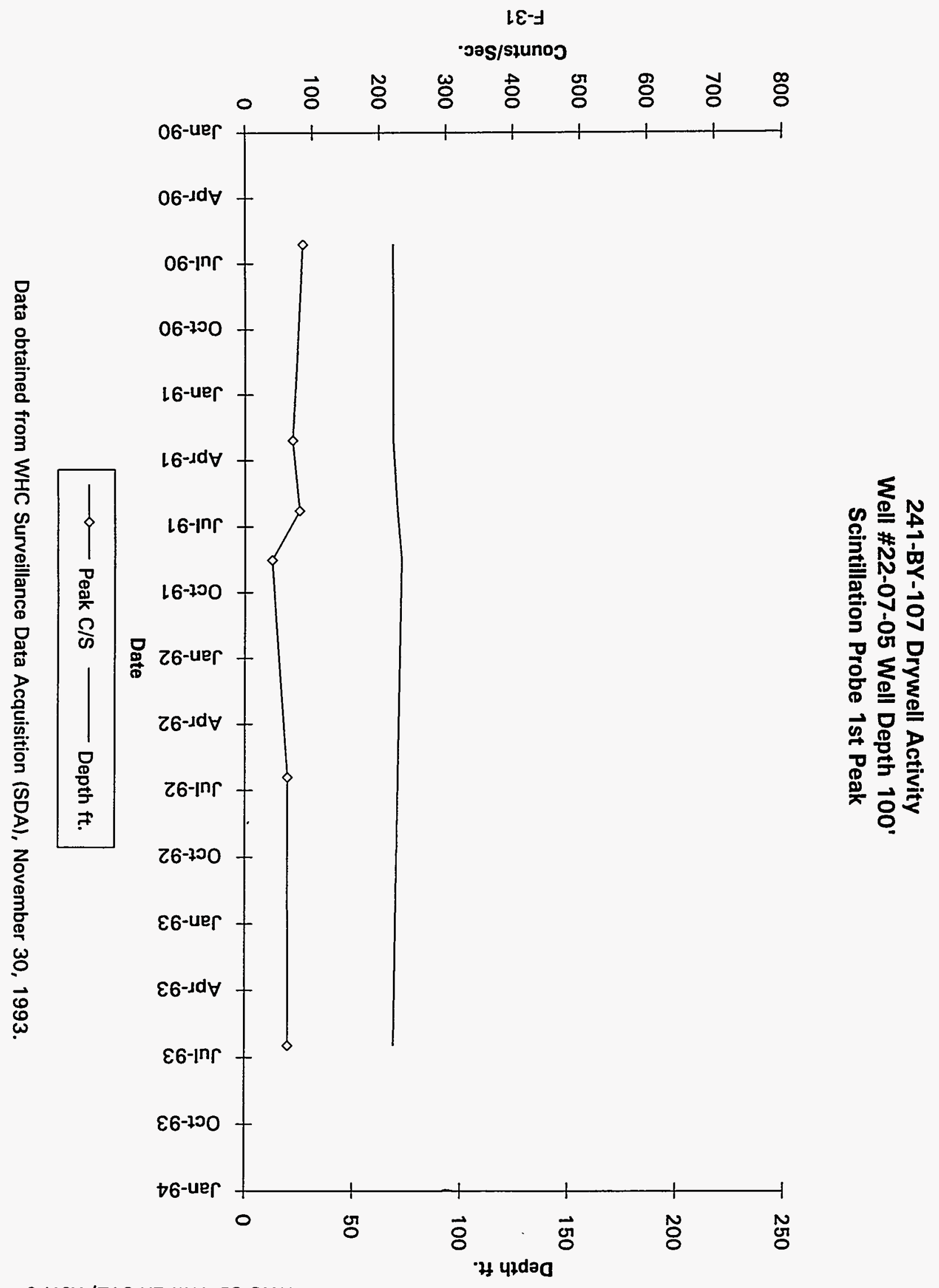

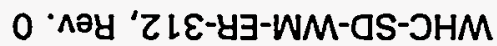


241-BY-107 Drywell Activity

Well \#22-07-05 Well Depth 100'

Scintillation Probe 2nd Peak

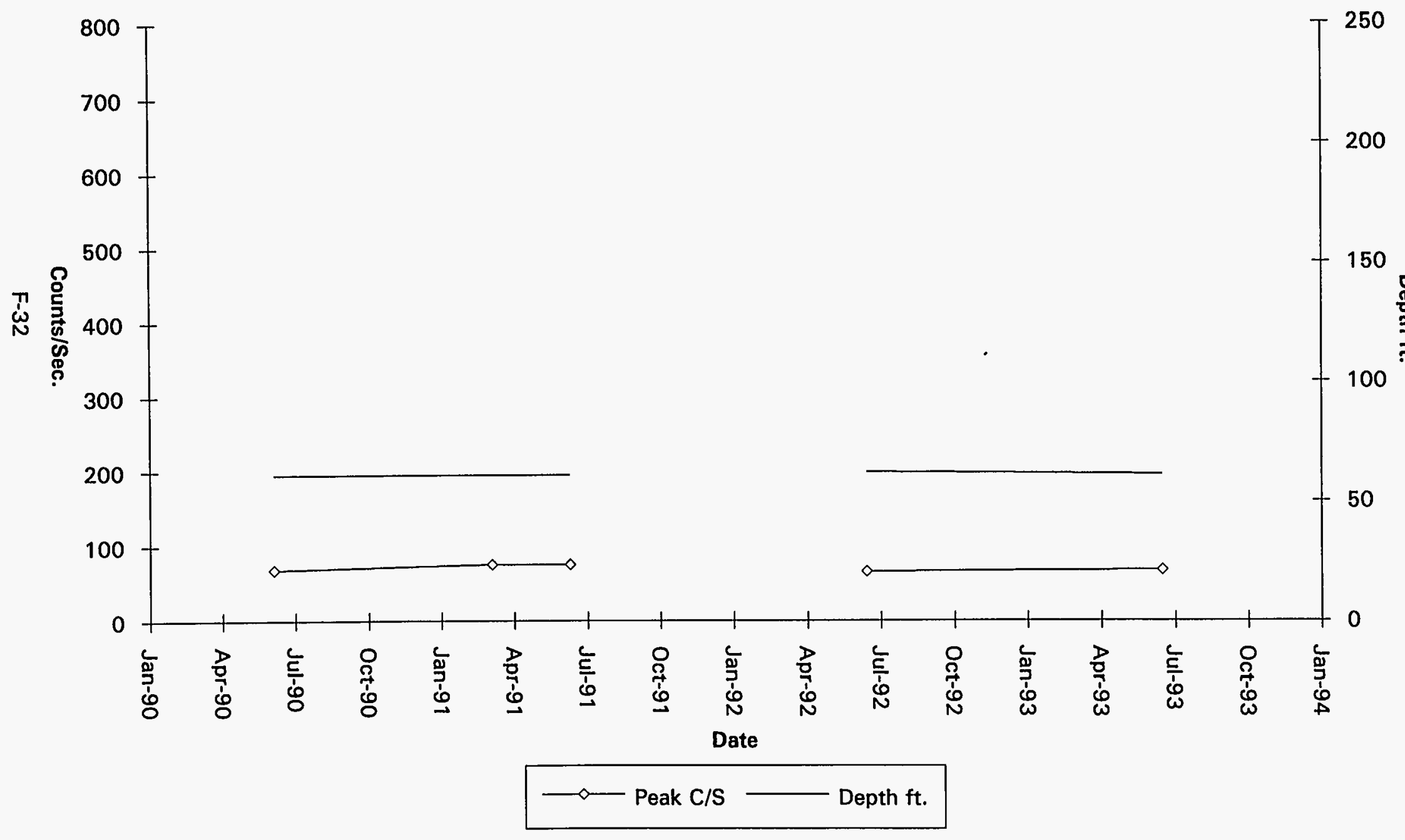

Data obtained from WHC Surveillance Data Acquisition (SDA), November 30, 1993. 


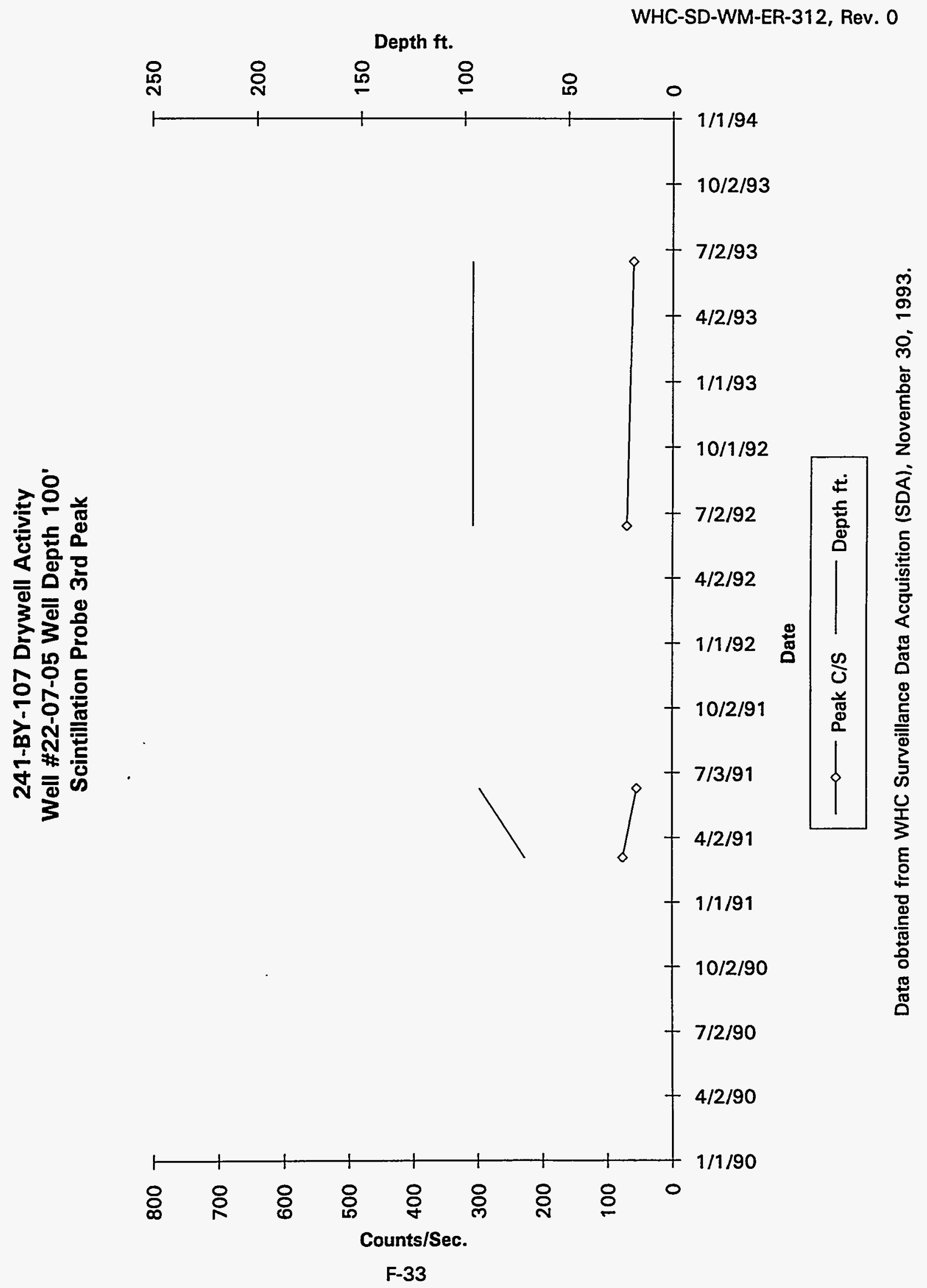




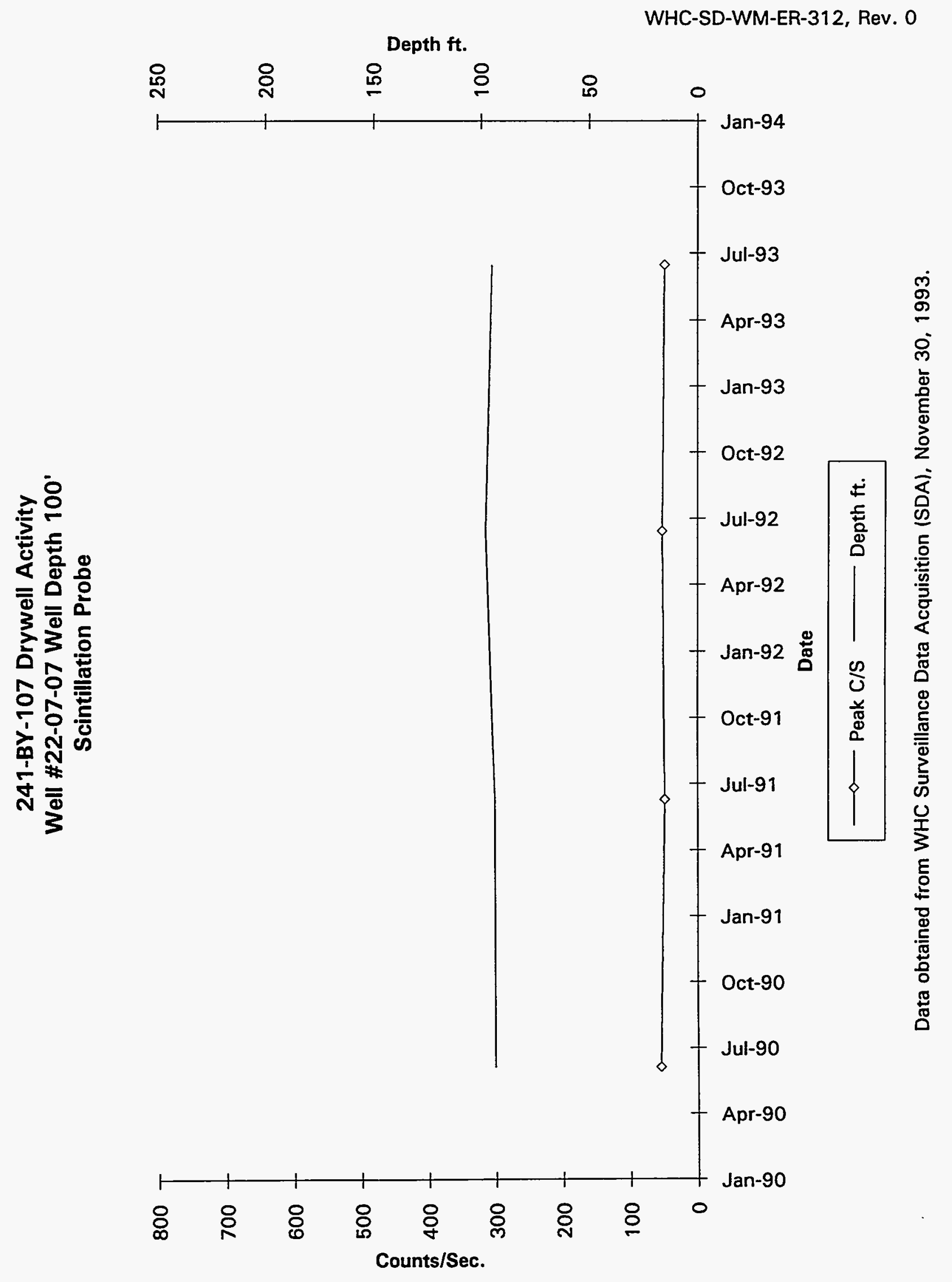




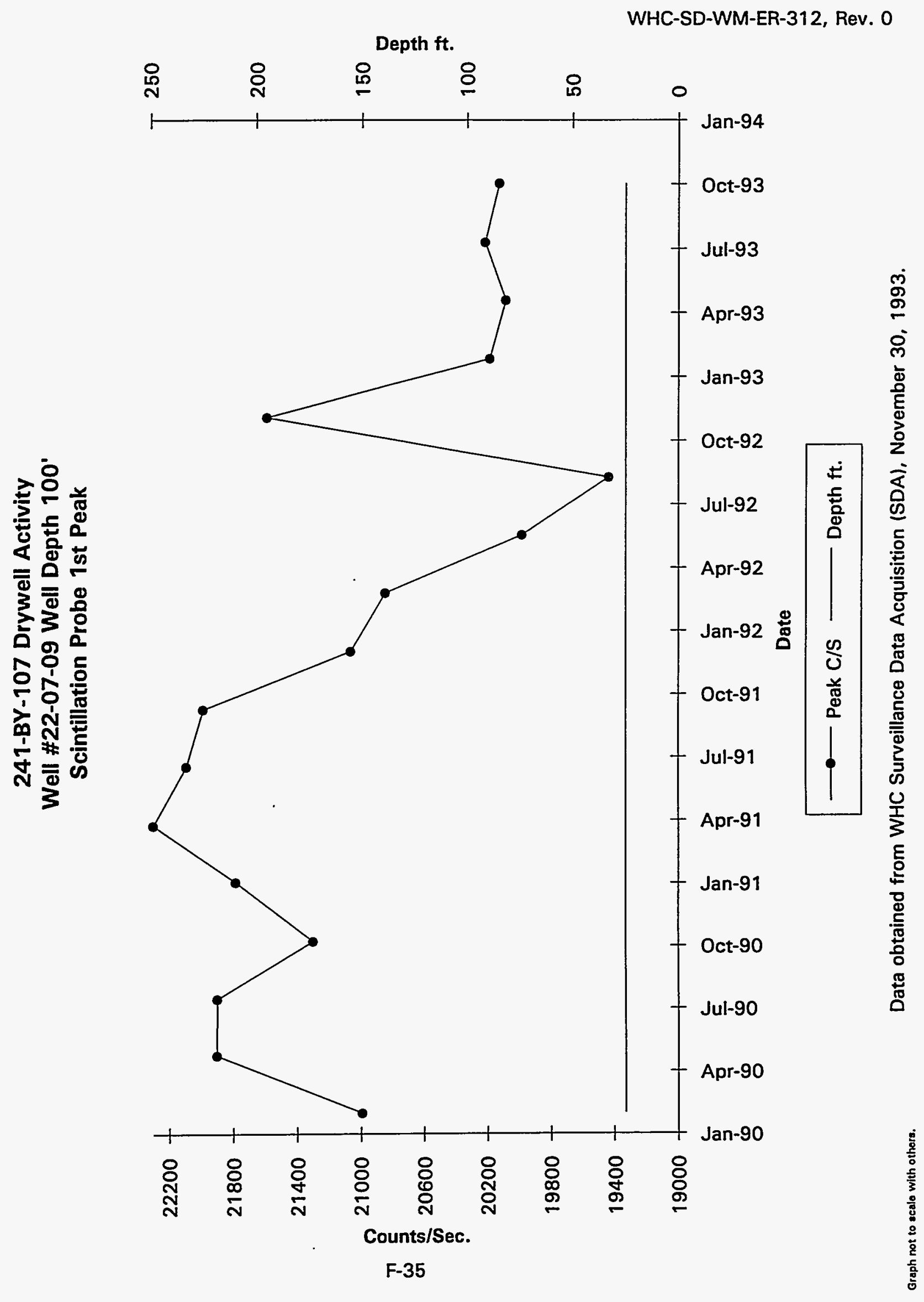


241-BY-107 Drywell Activity

Well \#22-07-09 Well Depth 100'

Scintillation Probe 2nd Peak

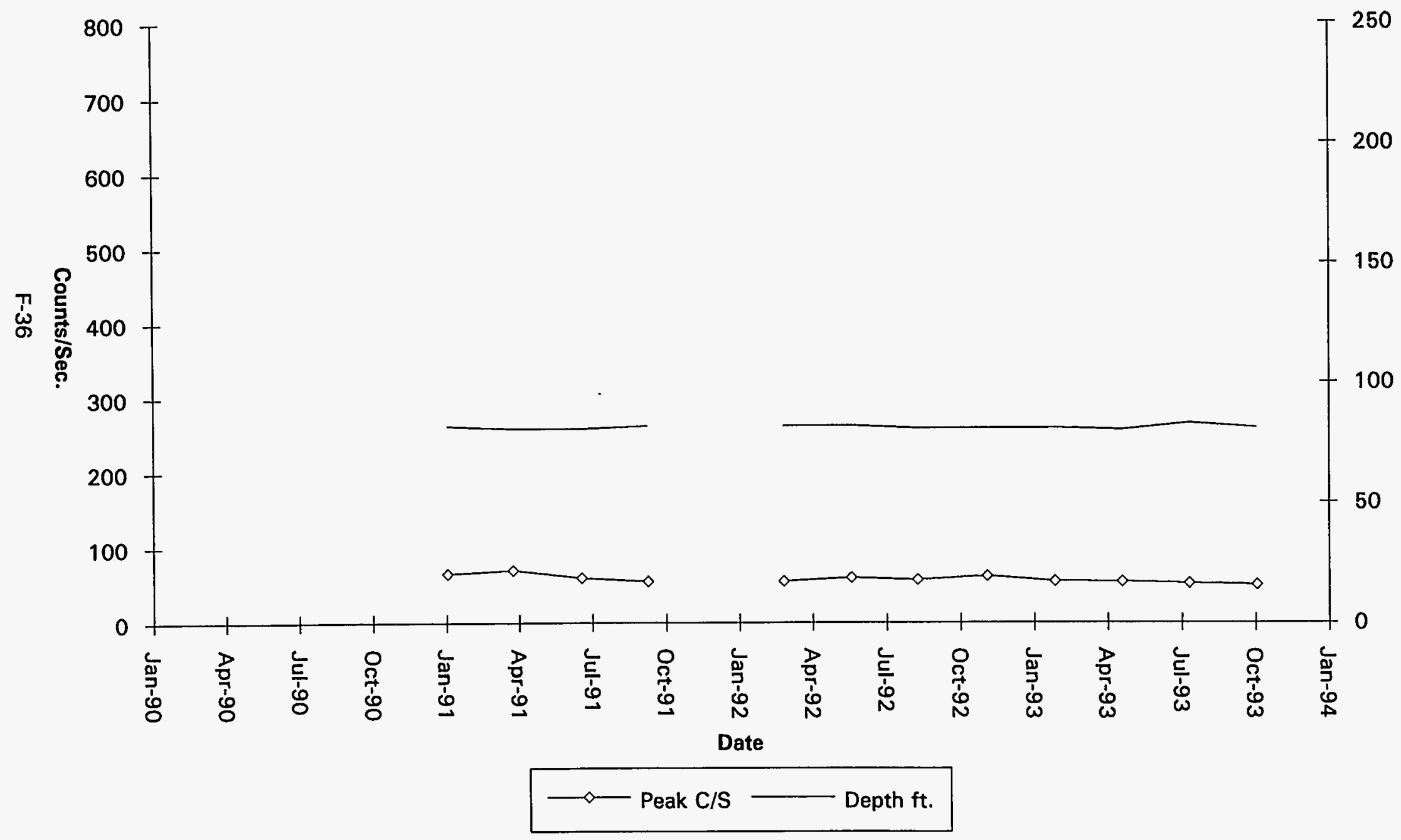

Data obtained from WHC Surveillance Data Acquisition (SDA), November 30, 1993. 


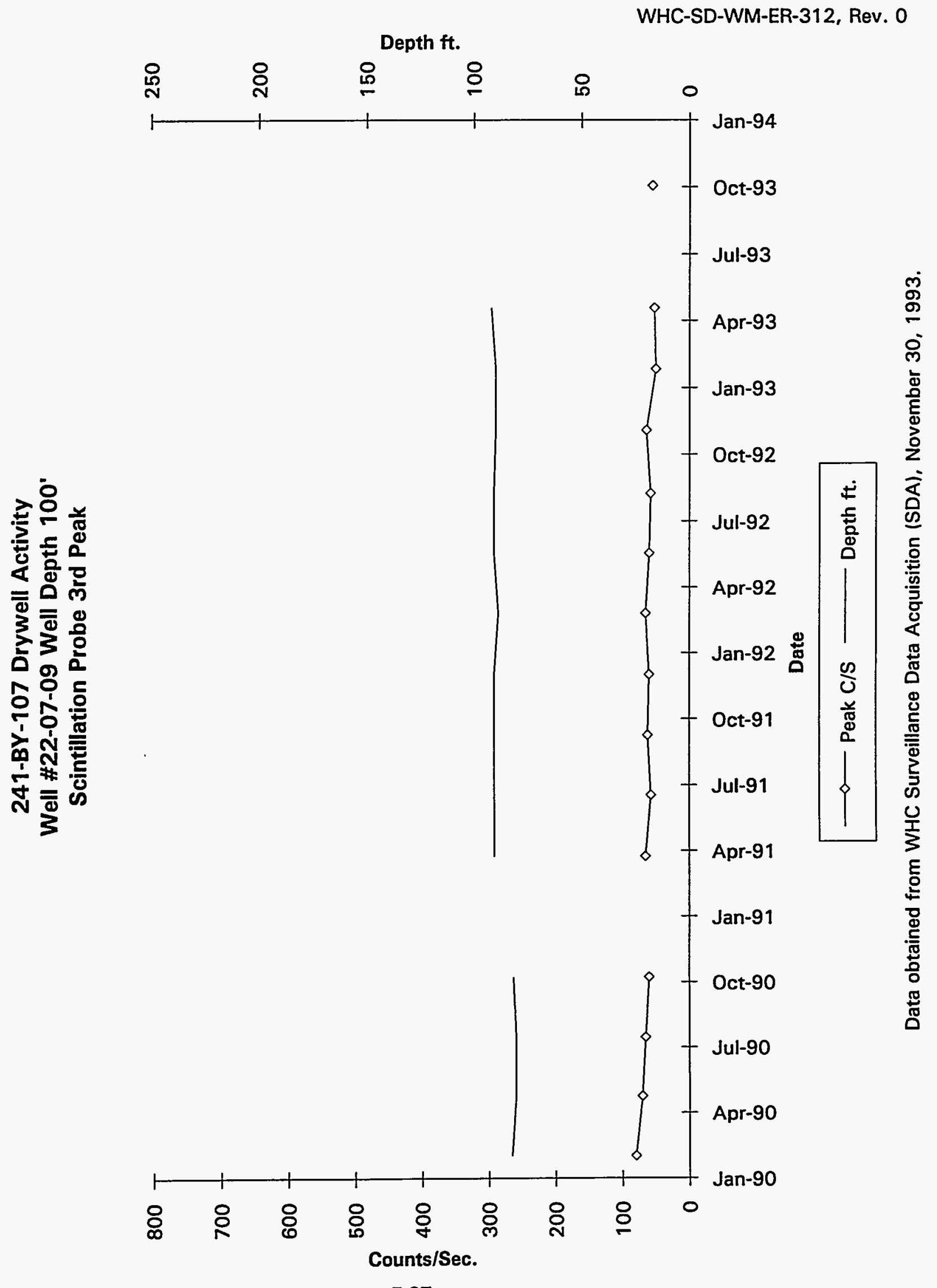

F-37 
241-BY-107 Drywell Activity

Well \#22-07-10 Well Depth 100'

Scintillation Probe 1st Peak

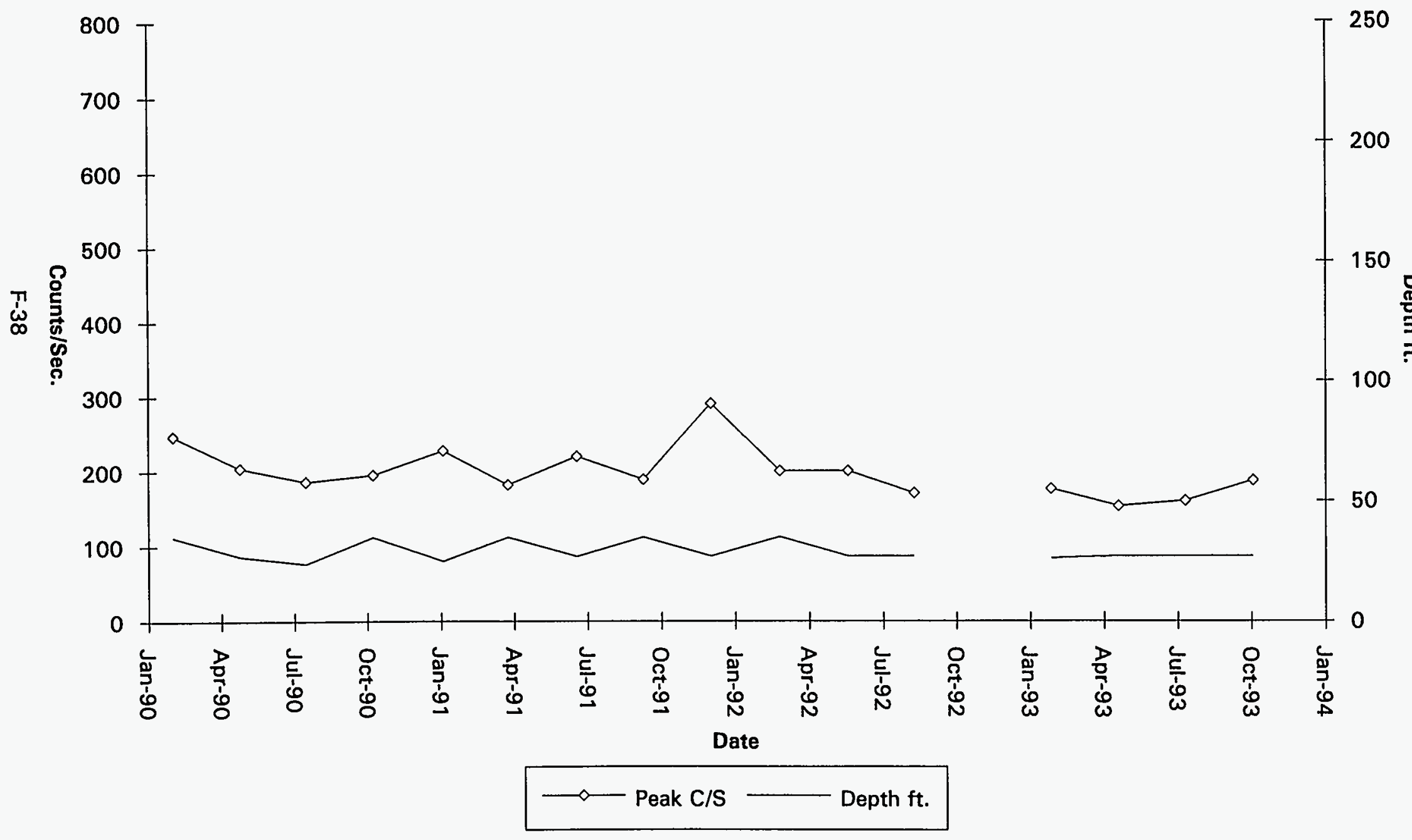


241-BY-107 Drywell Activity

Well \#22-07-10 Well Depth 100'

Scintillation Probe 2nd Peak

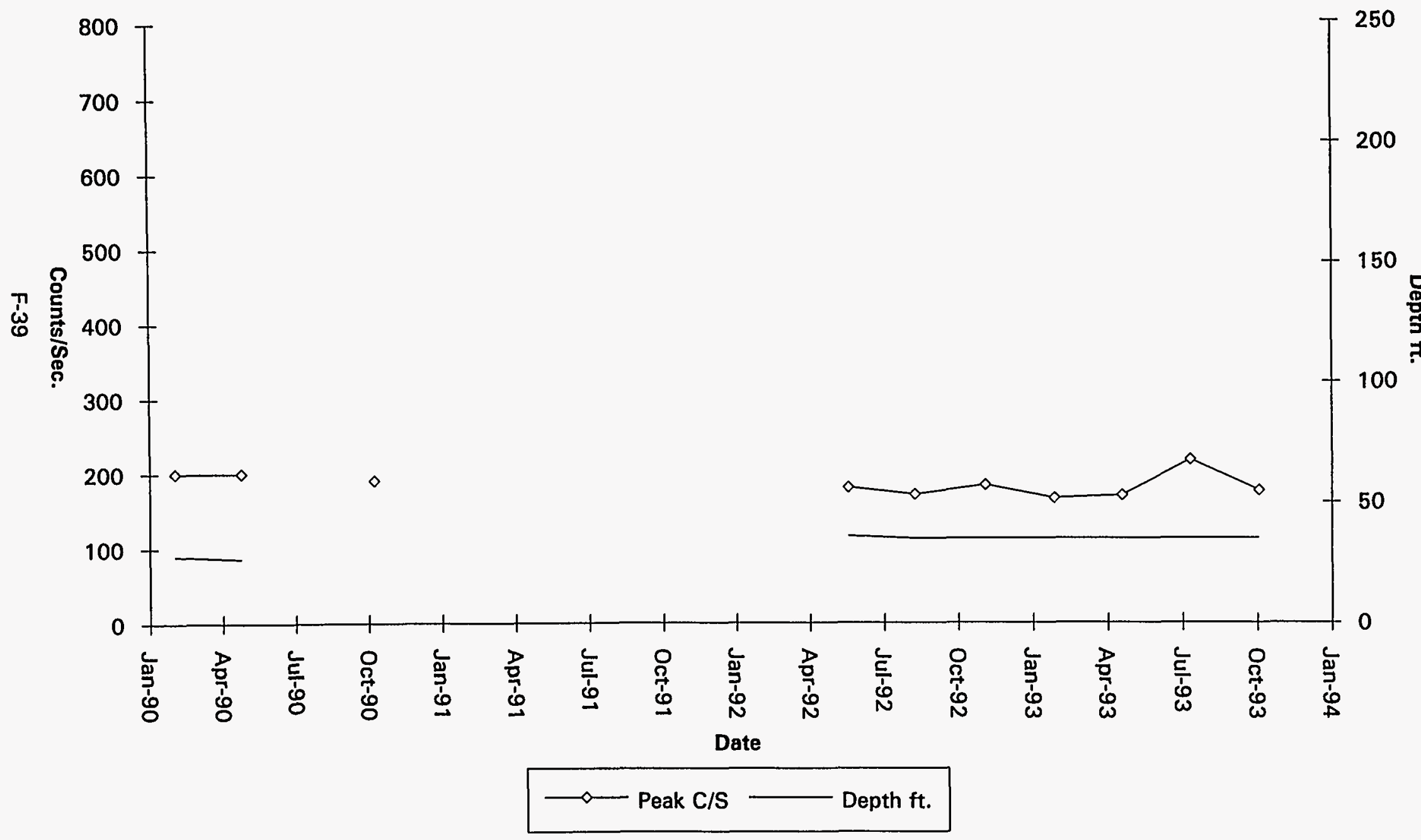

Data obtained from WHC Surveillance Data Acquisition (SDA), November 30, 1993. 


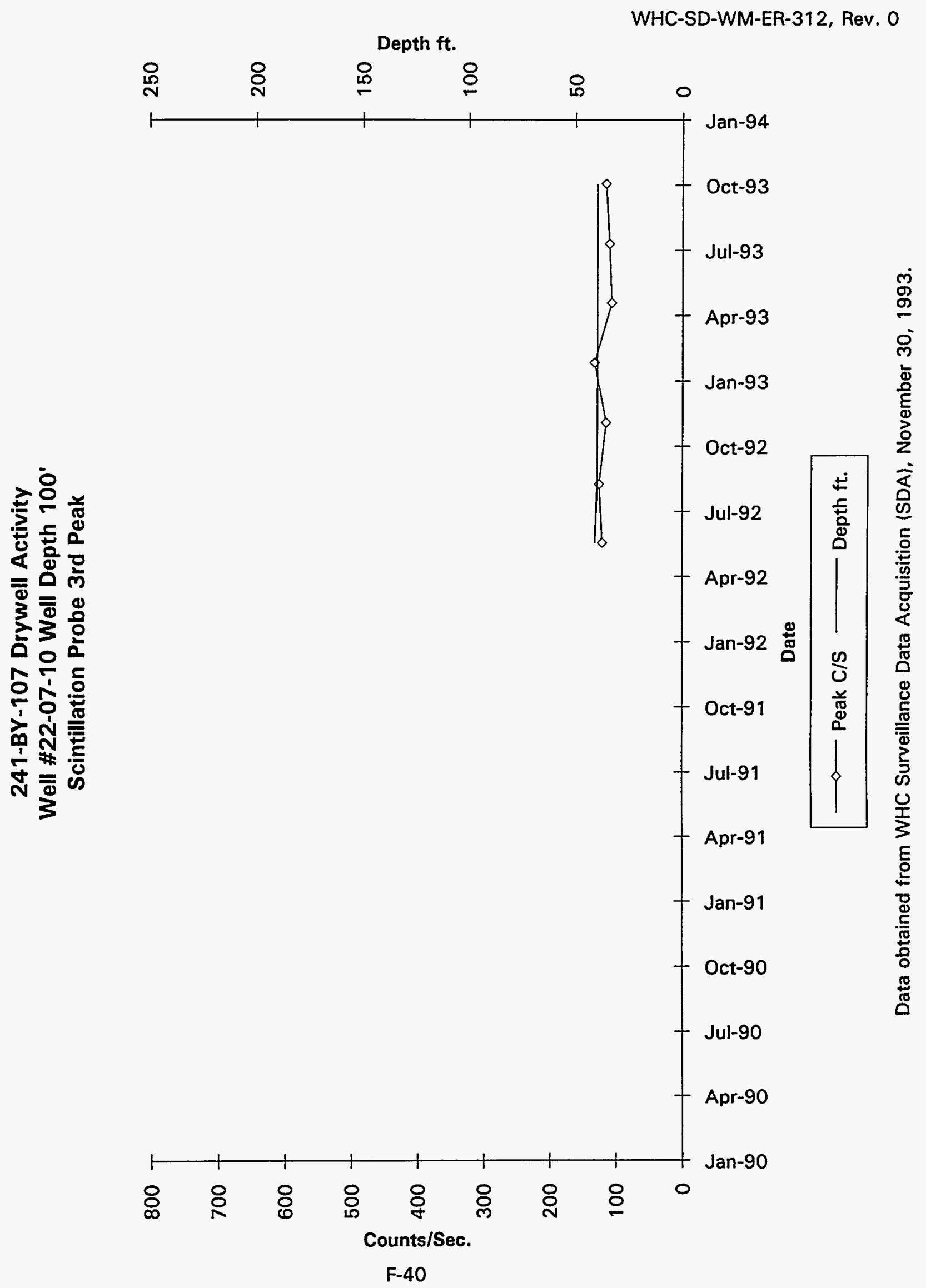




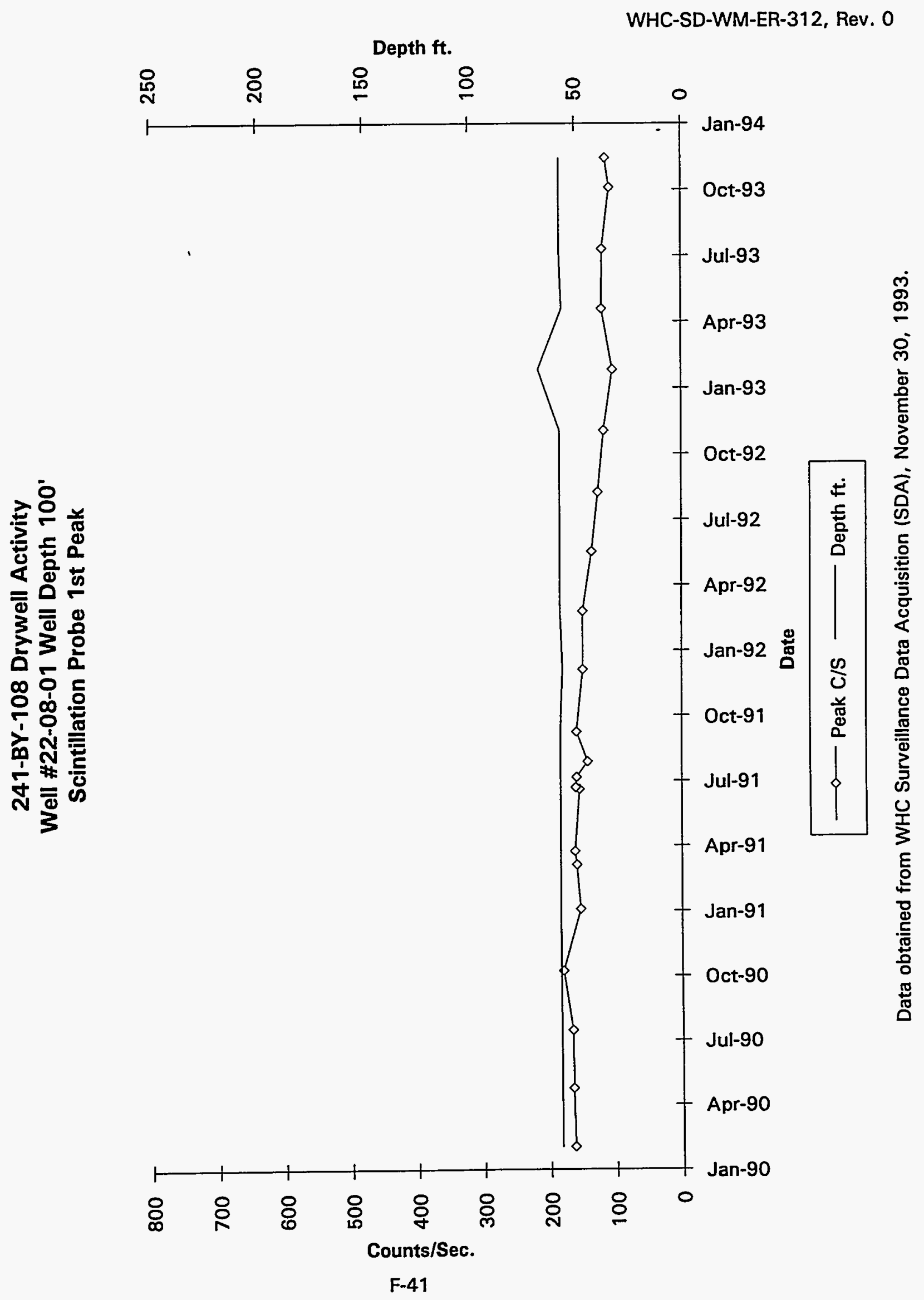




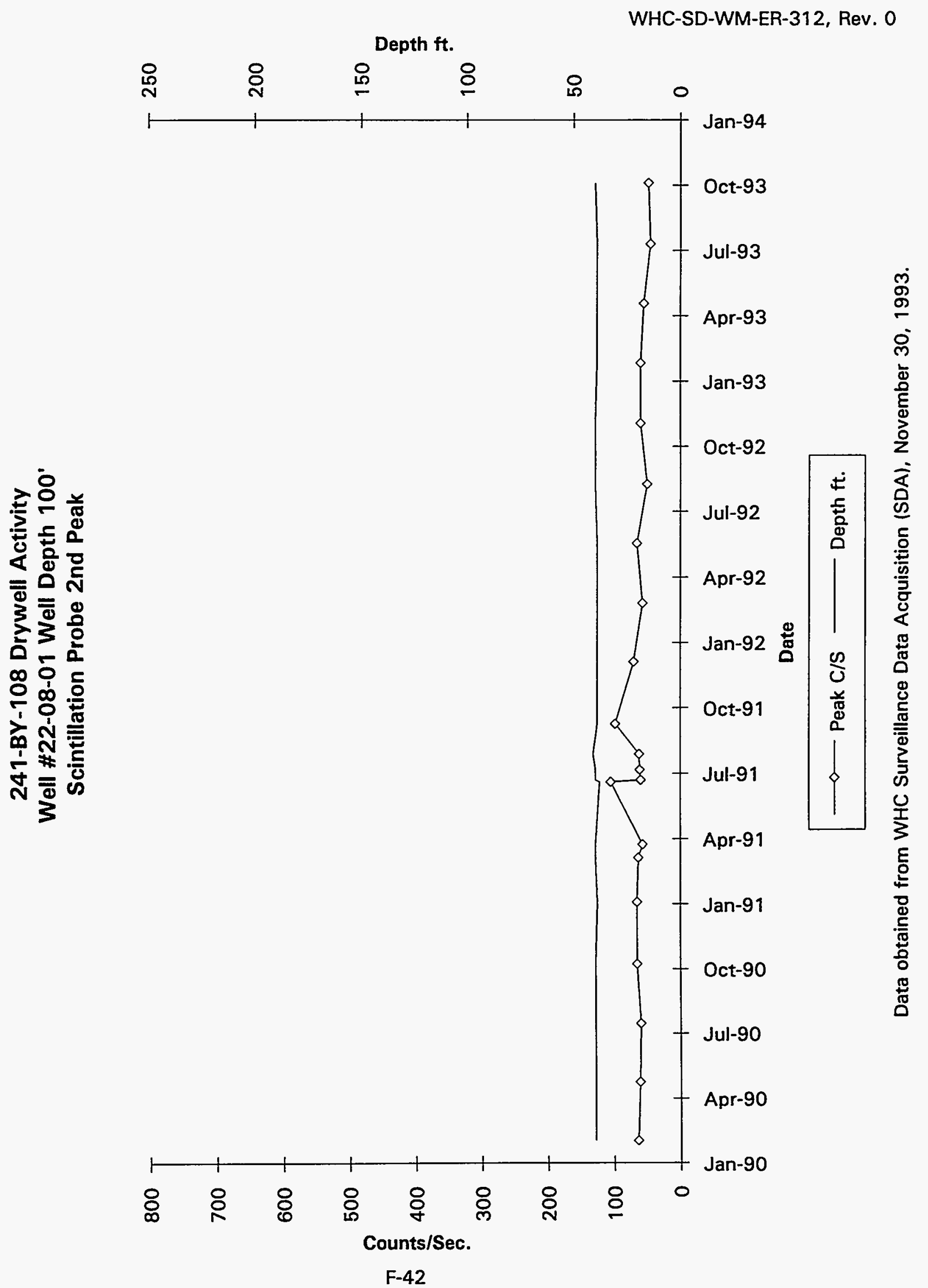




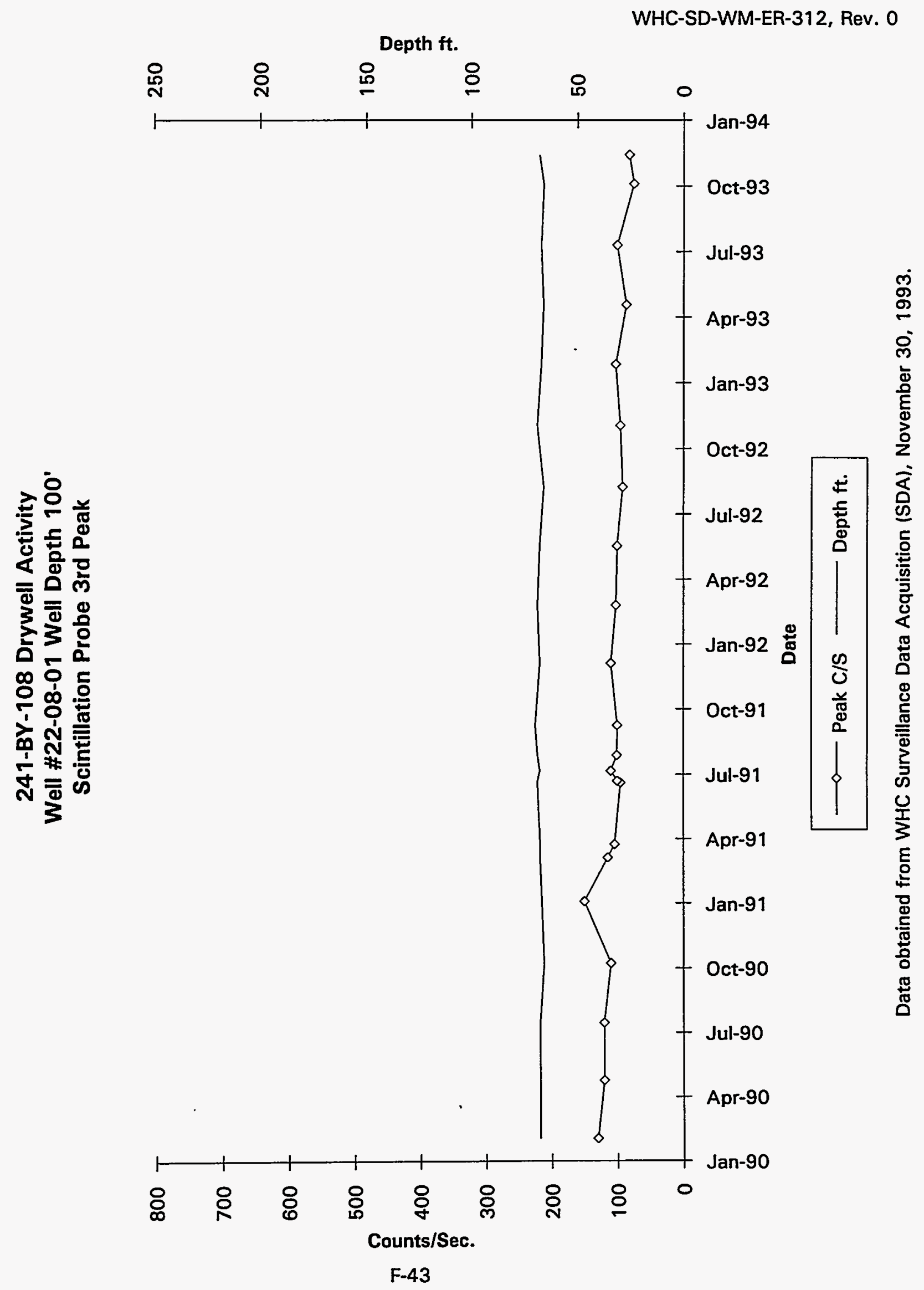




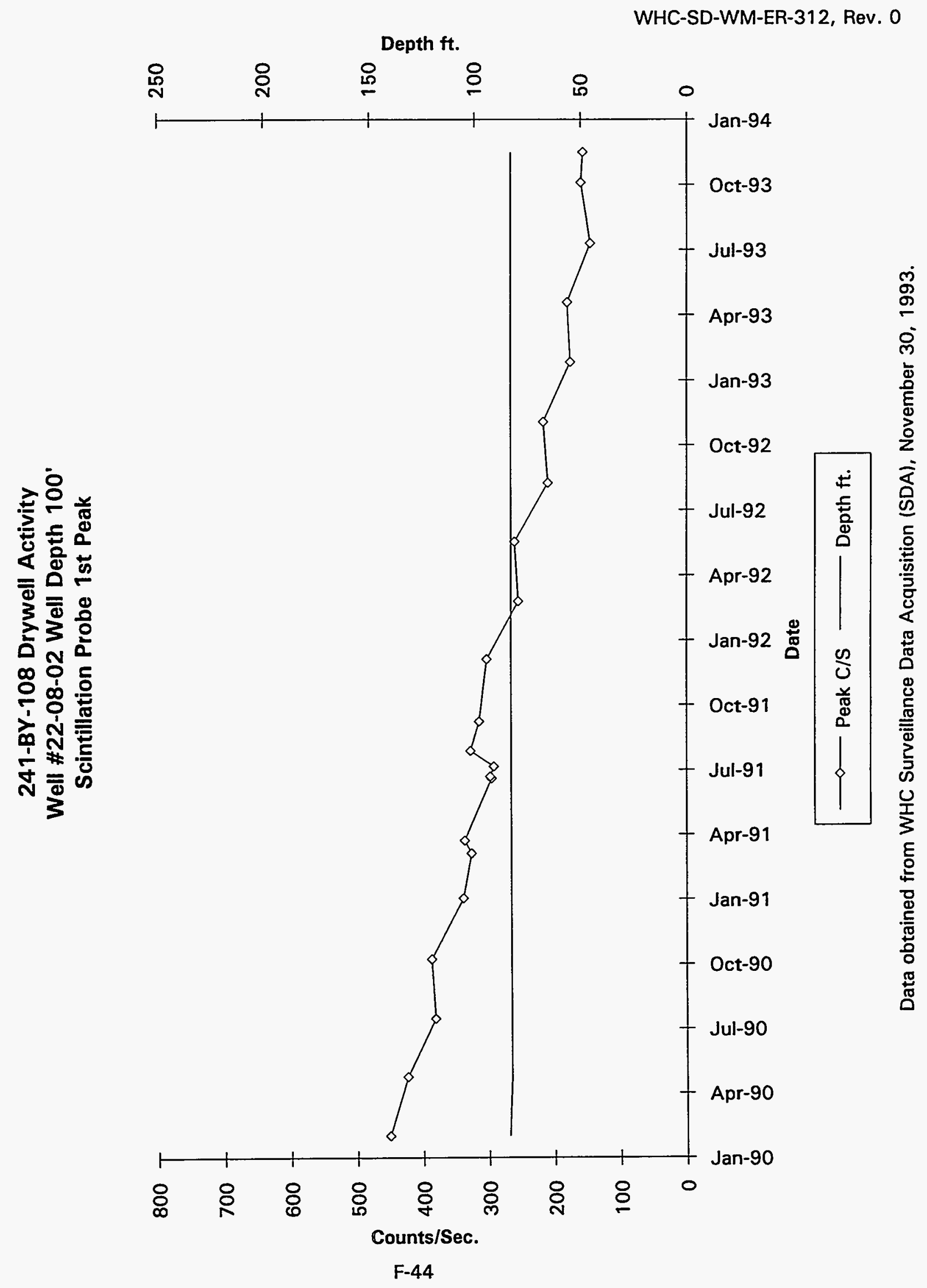




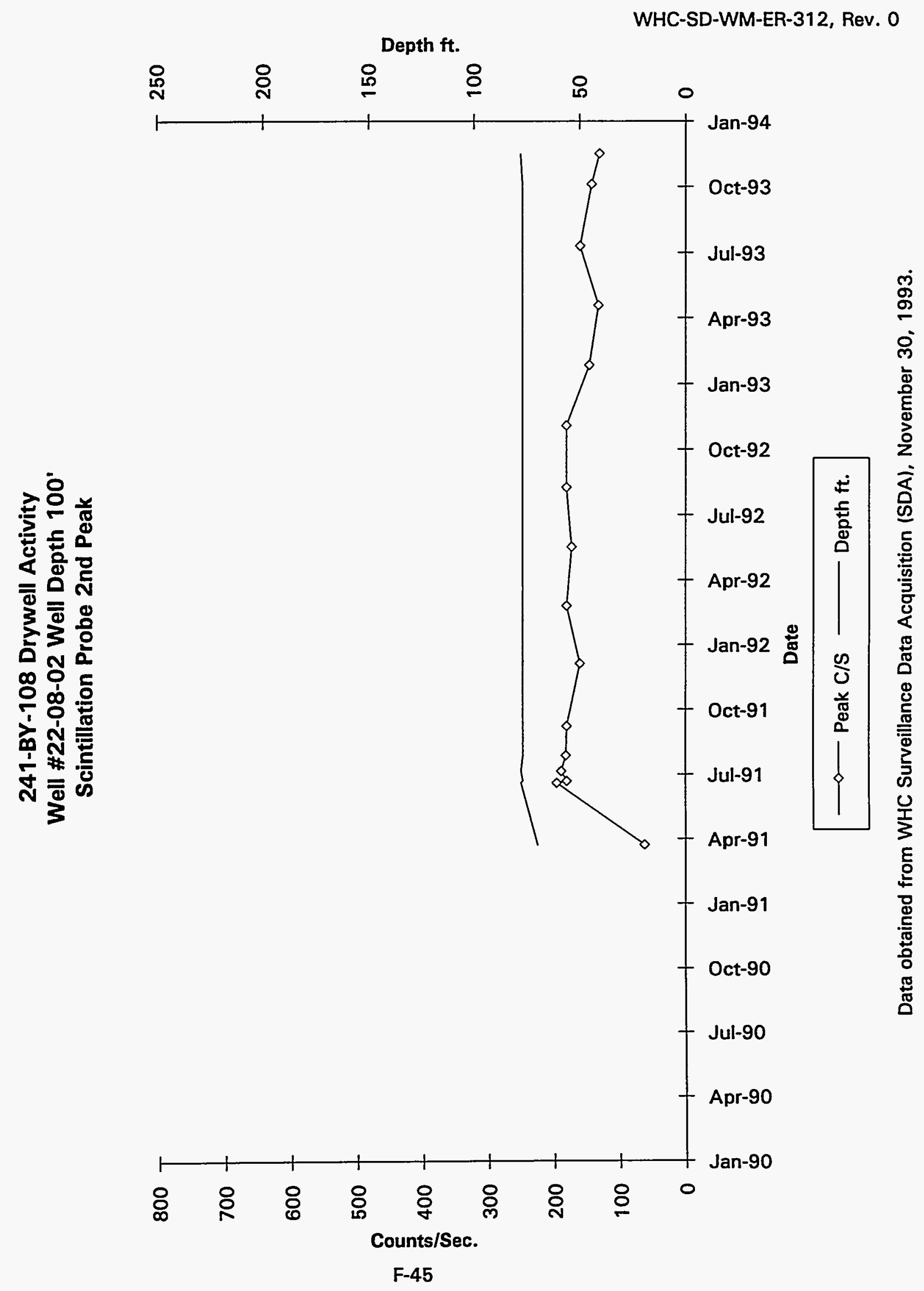




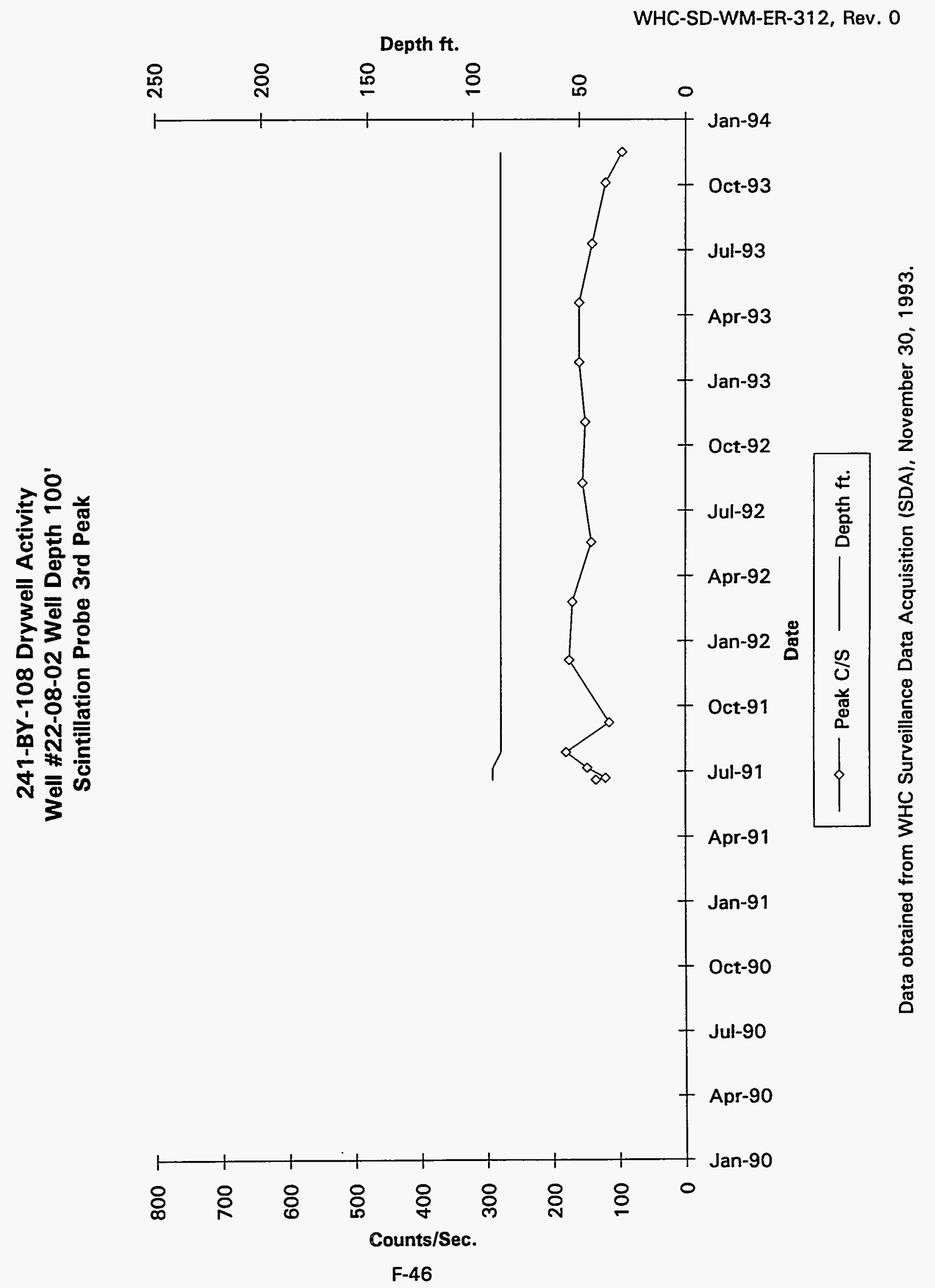




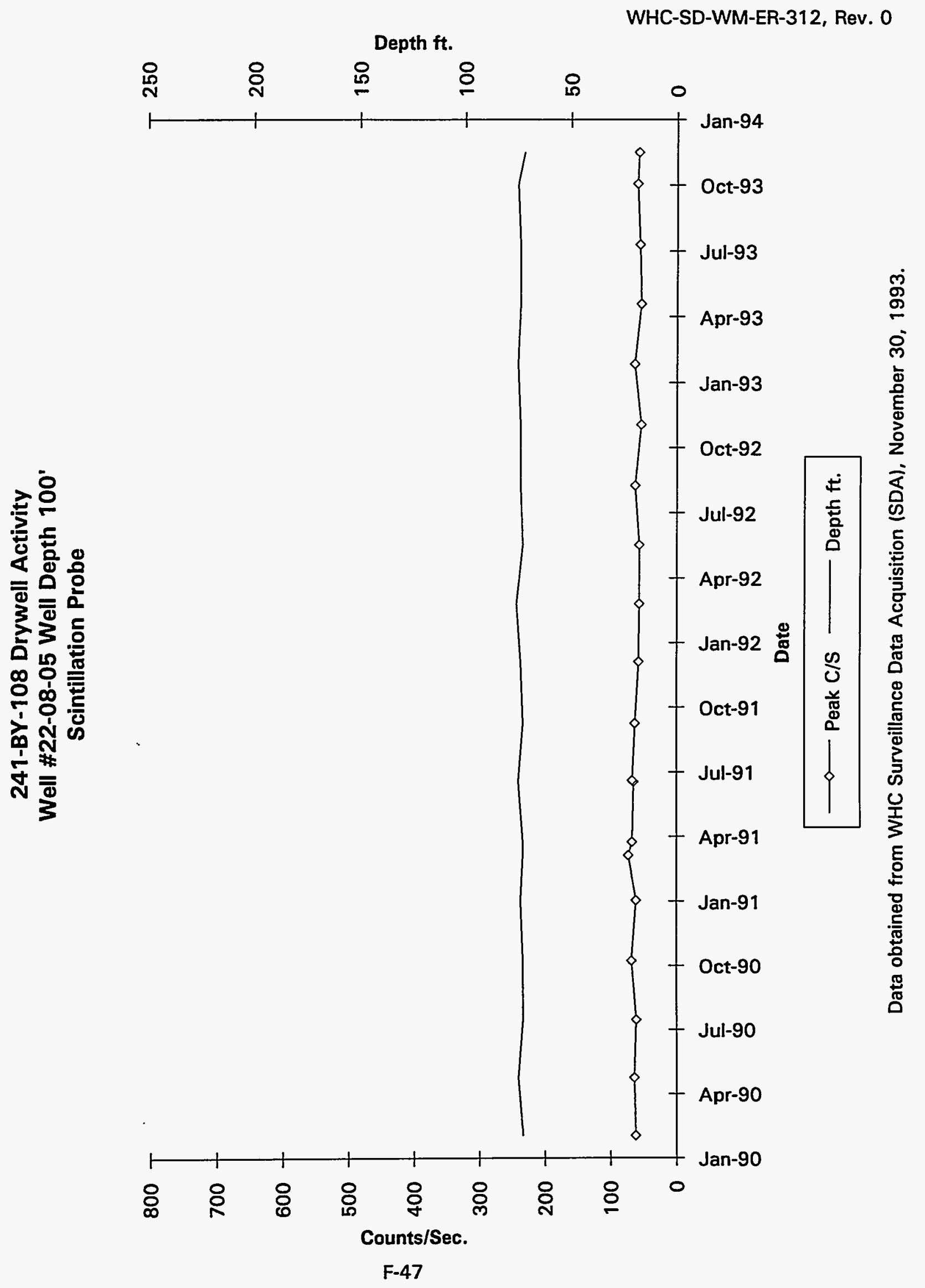




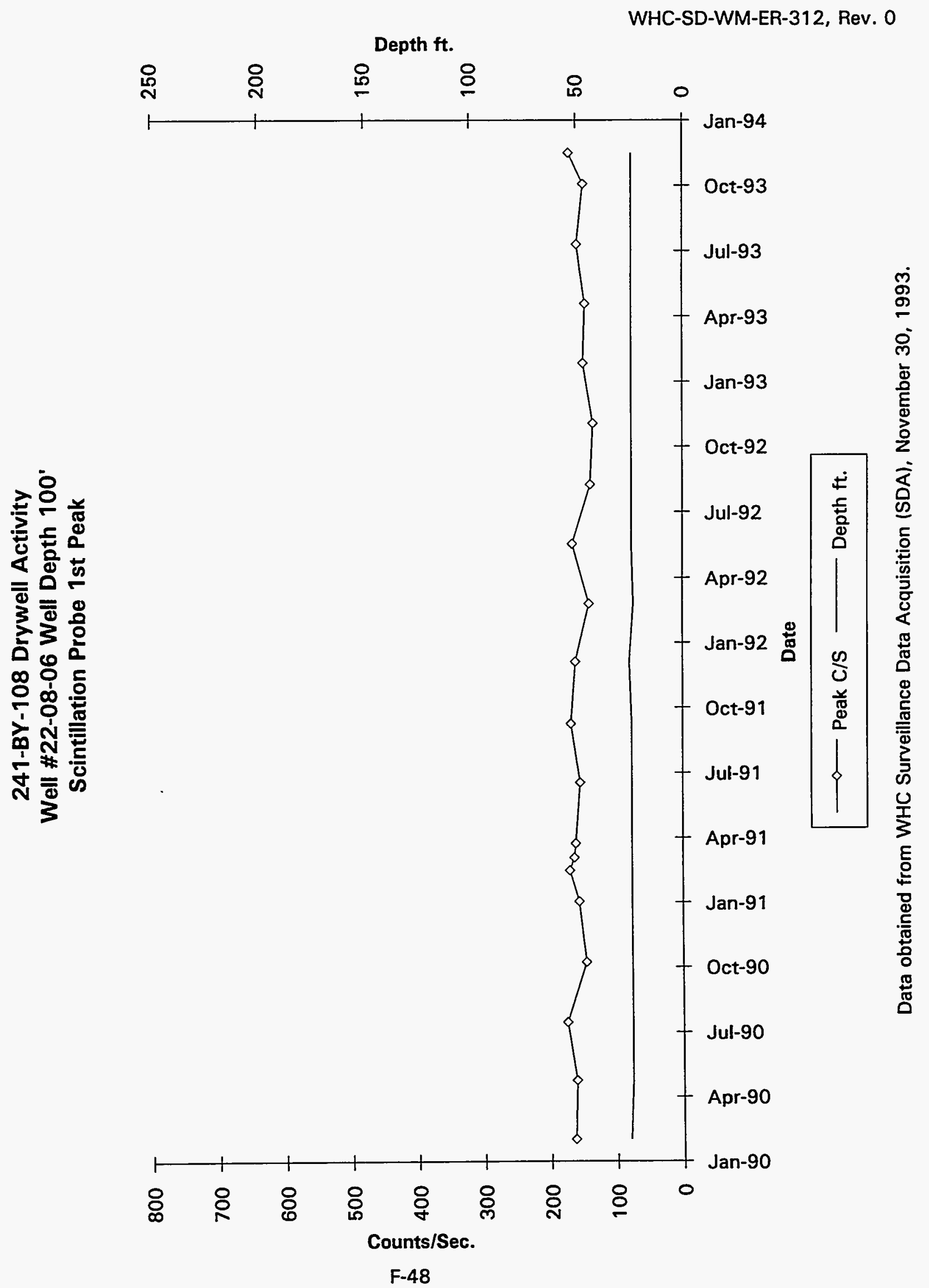




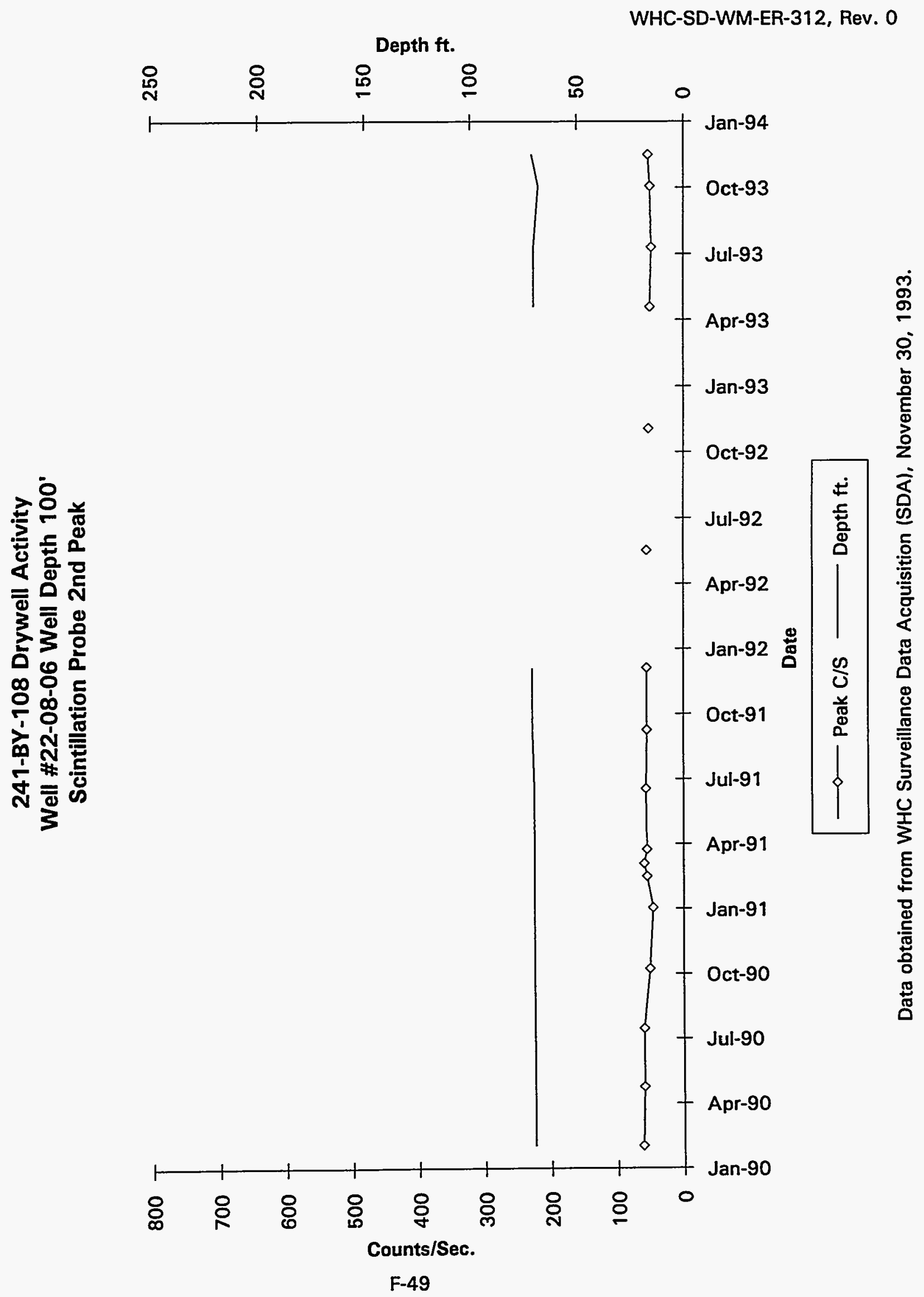


241-BY-108 Drywell Activity

Well \#22-08-12 Well Depth 100'

Scintillation Probe 1st Peak

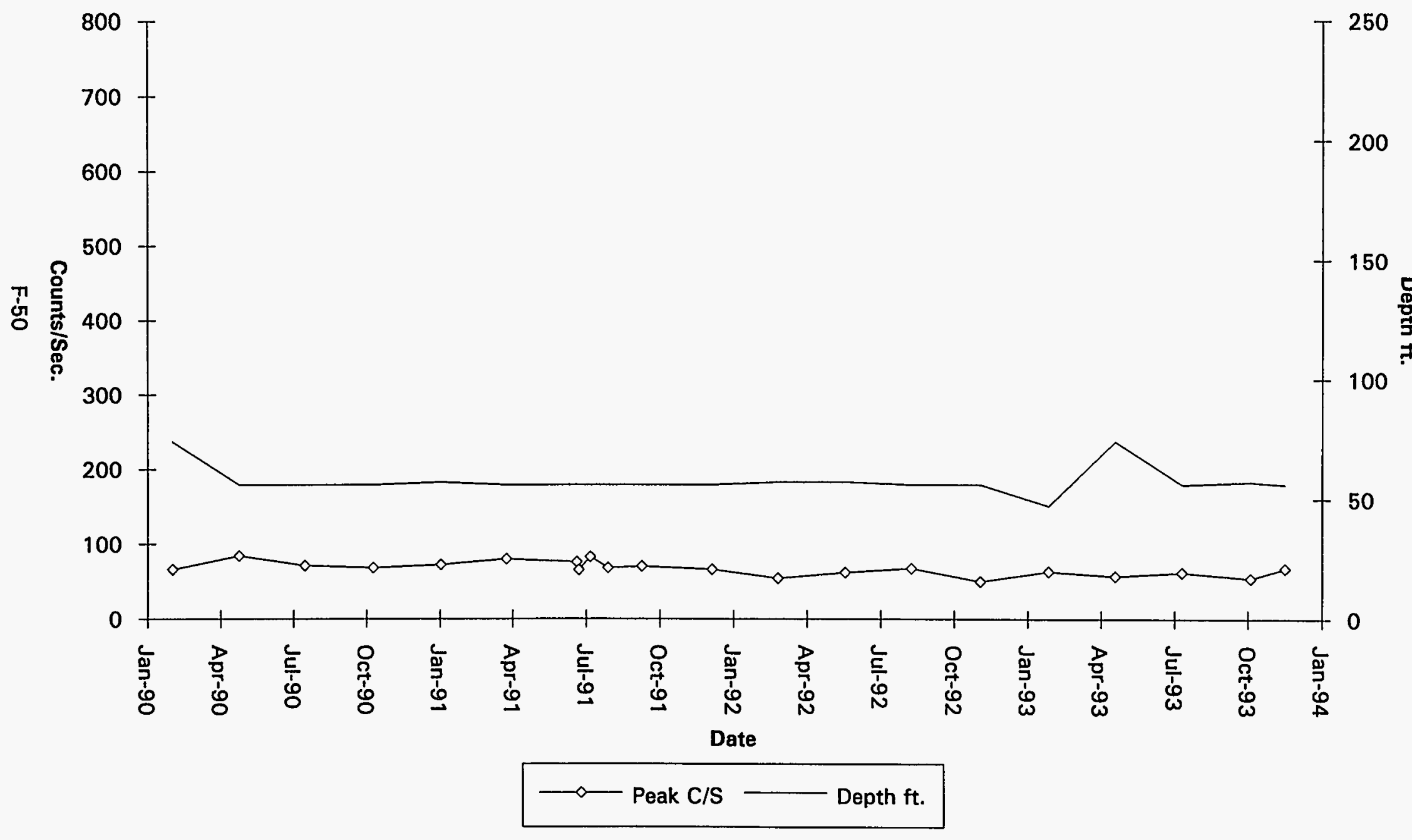

Data obtained from WHC Surveillance Data Acquisition (SDA), November 30, 1993. 


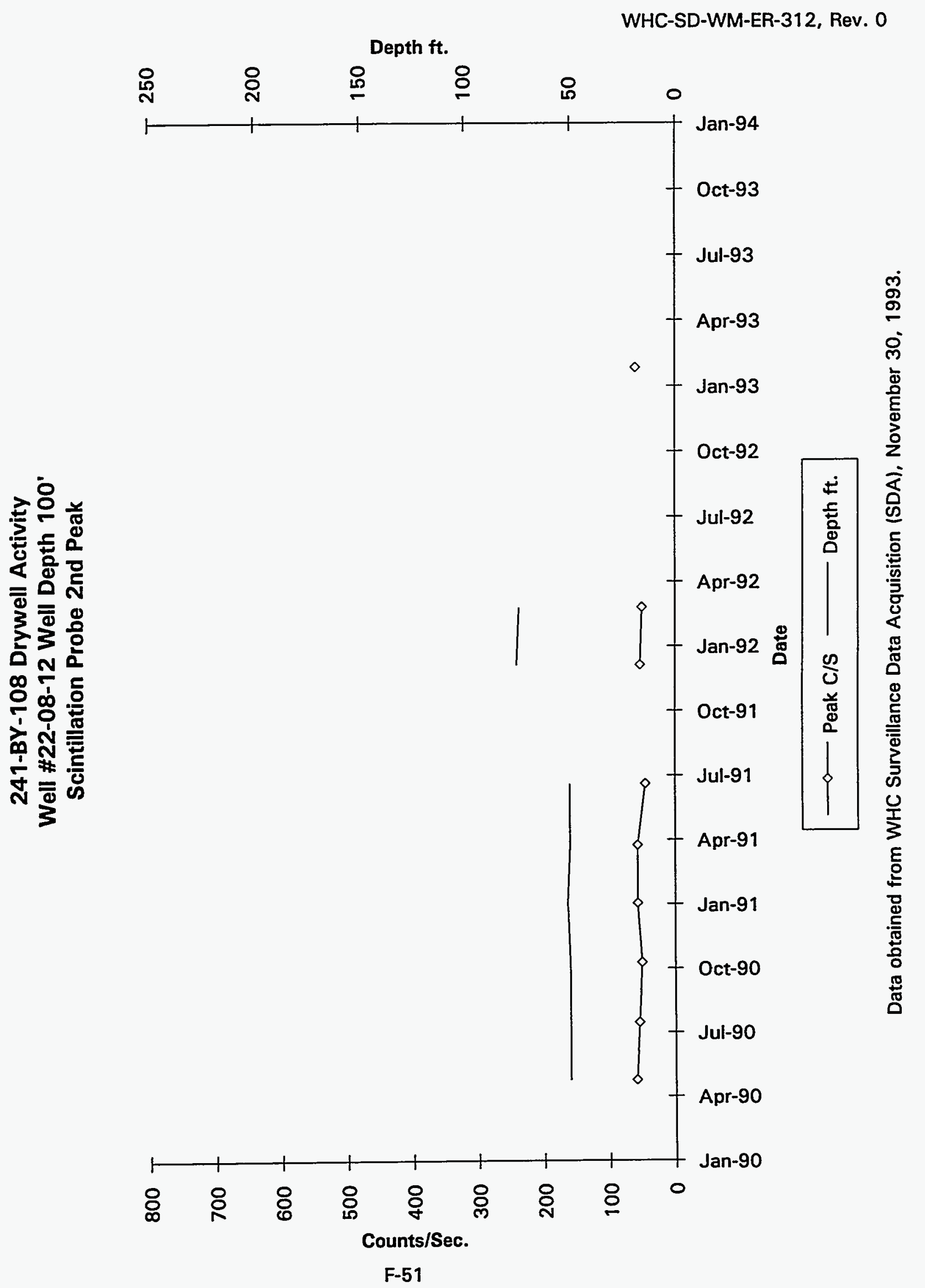


241-BY-109 Drywell Activity

Well \#22-09-02 Well Depth 100'

Scintillation Probe

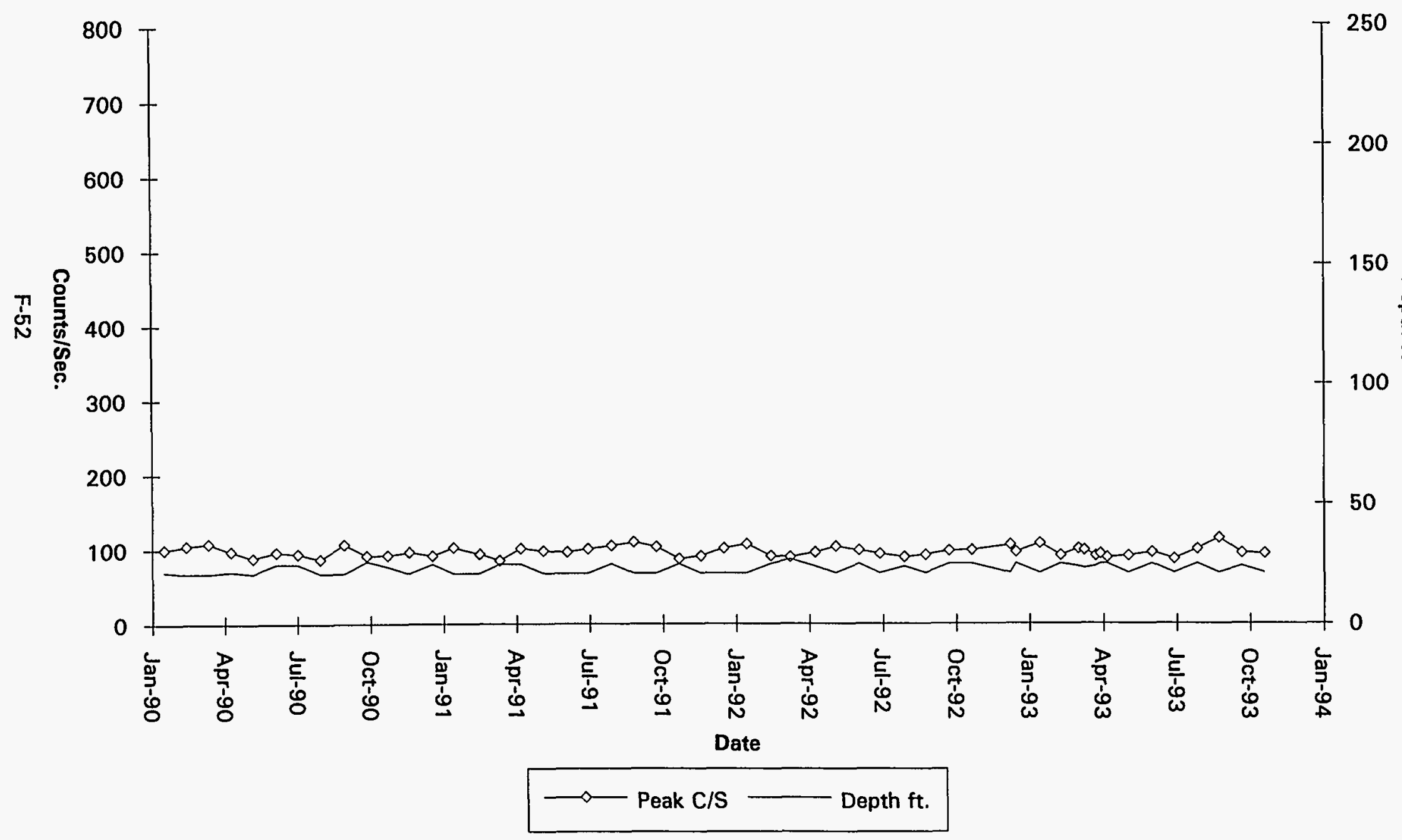

Data obtained from WHC Surveillance Data Acquisition (SDA), November 30, 1993. 


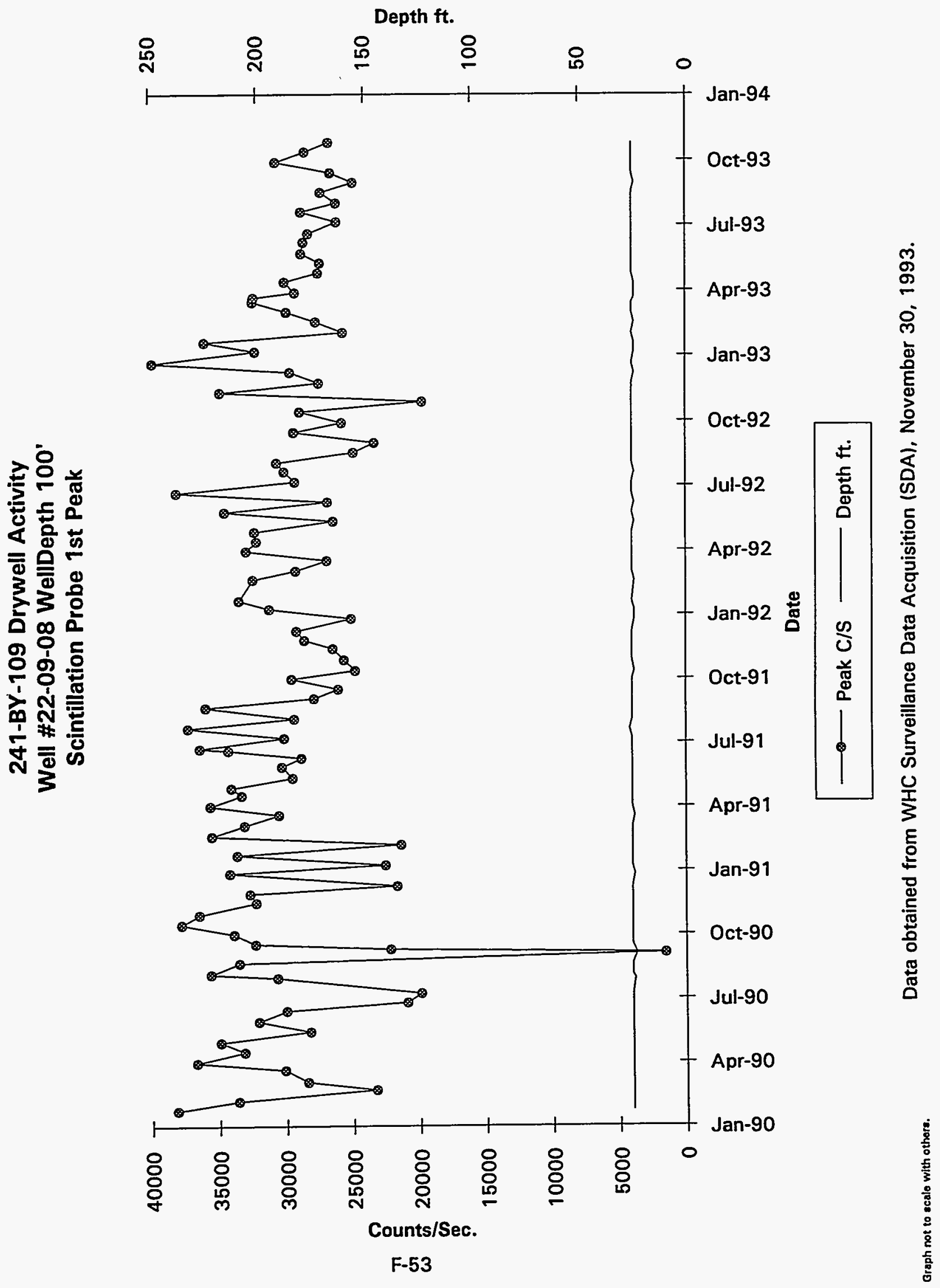




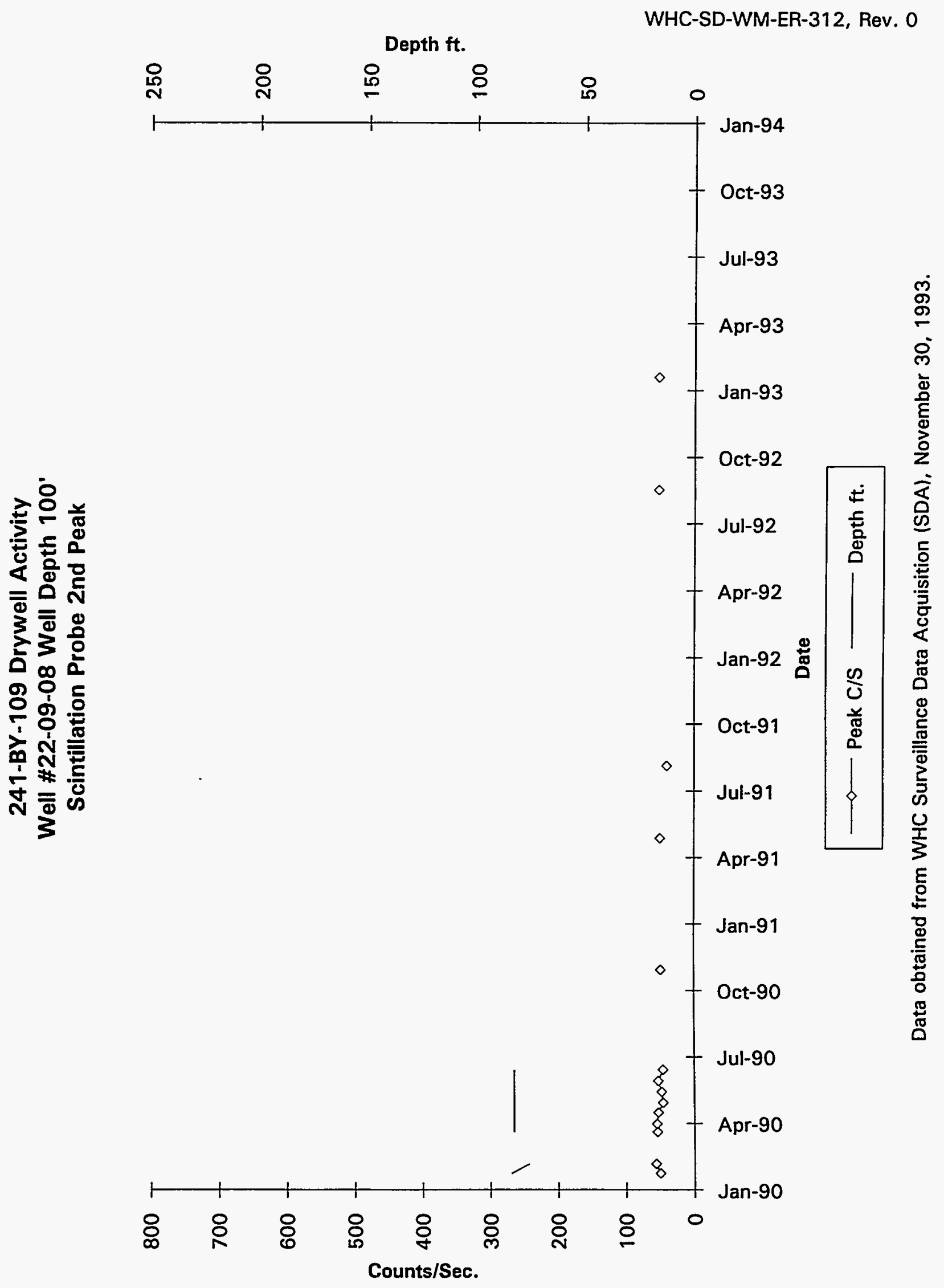




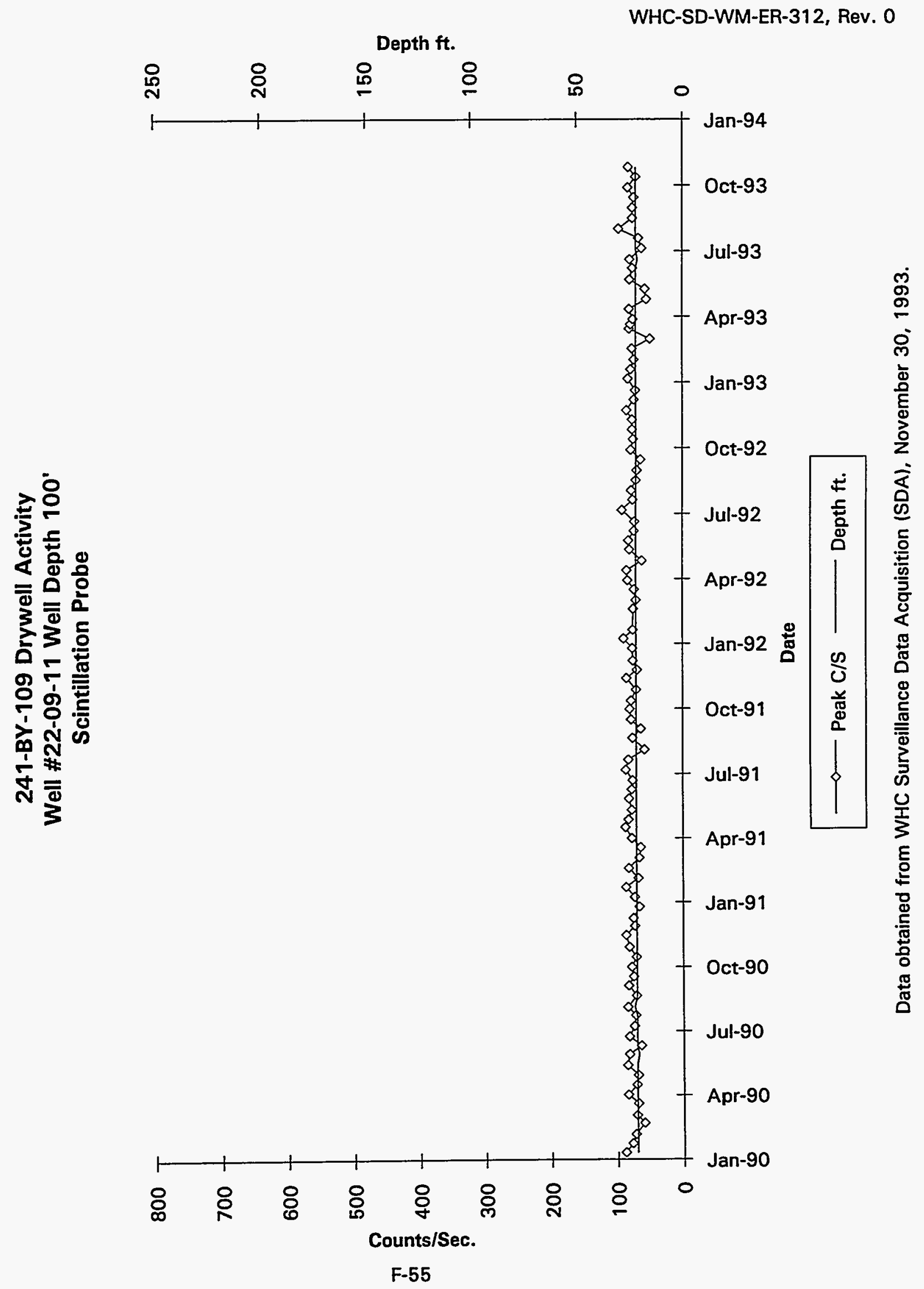




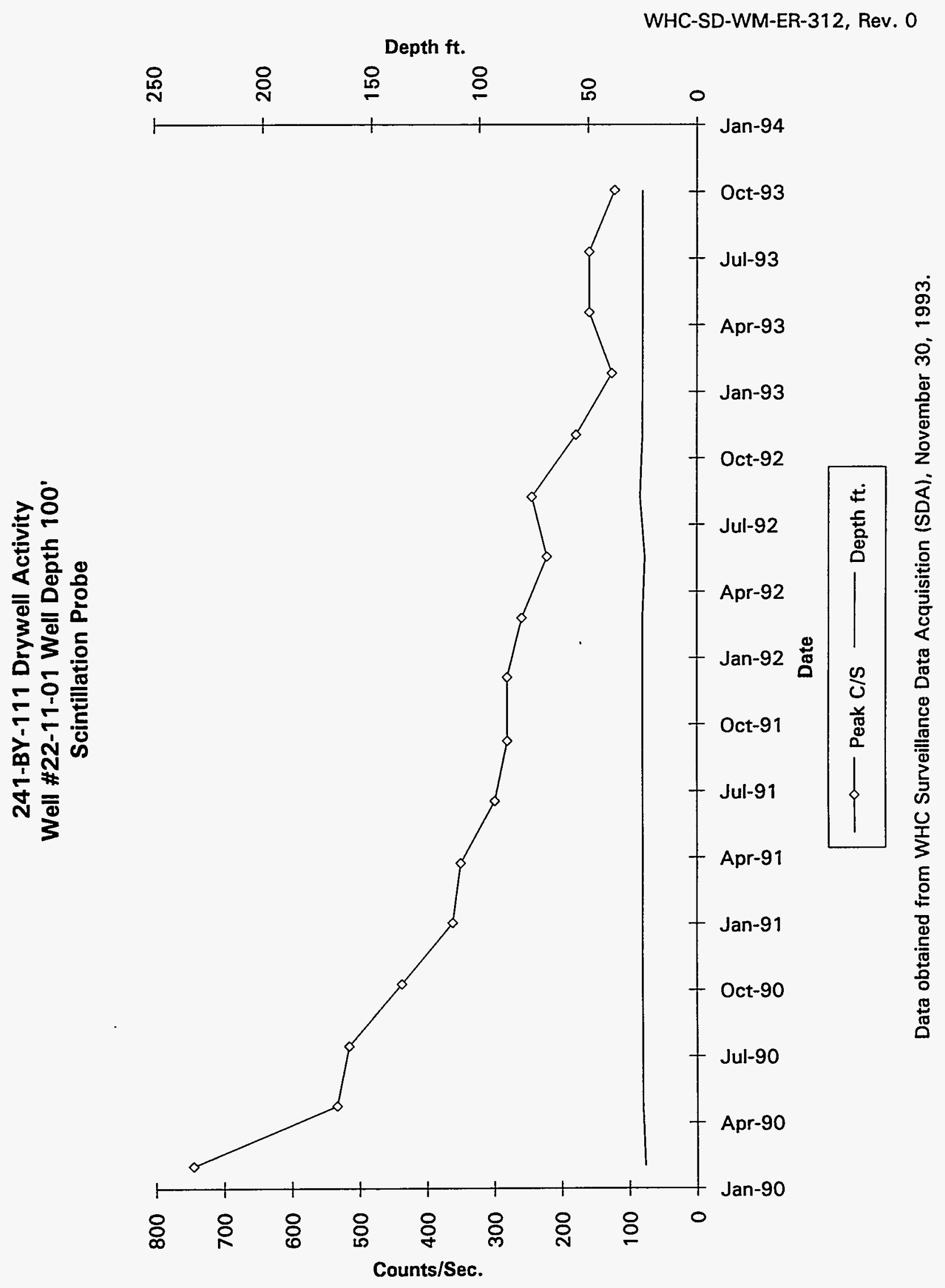

F-56 


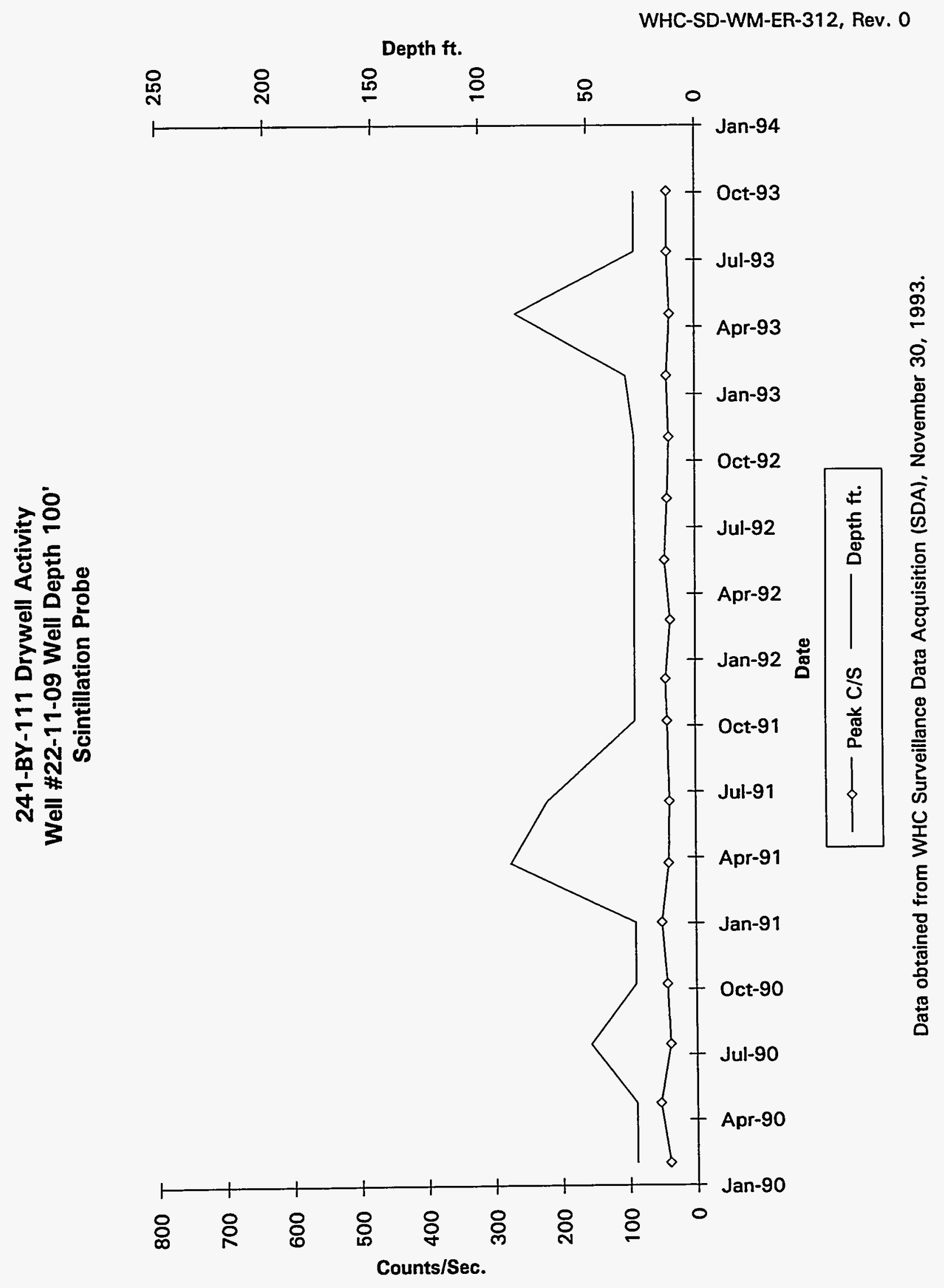

F-57 
WHC-SD-WM-ER-312, Rev. 0

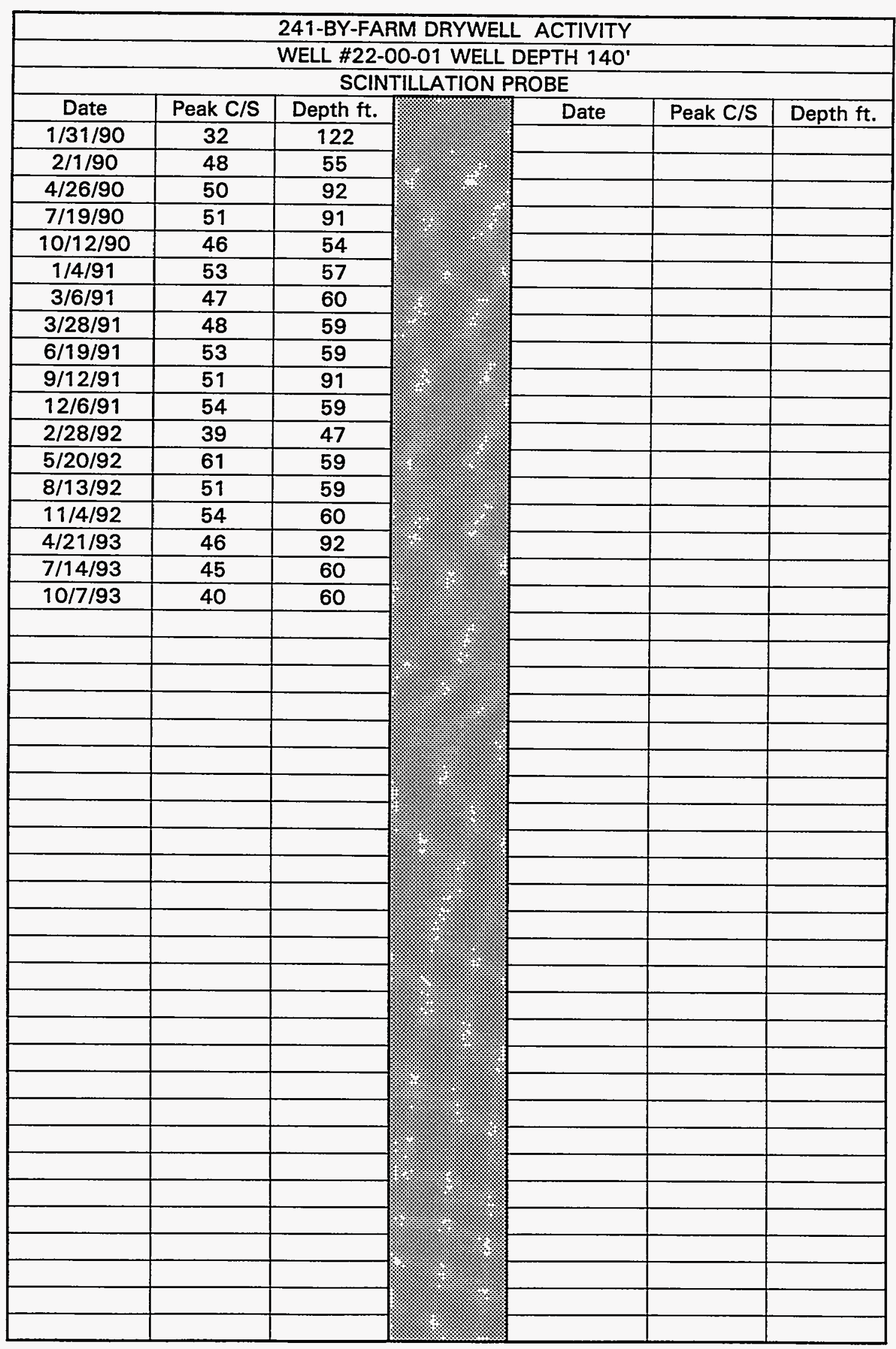

Data obtained from WHC Surveillance Data Acquisition (SDA), November 30, 1993. 


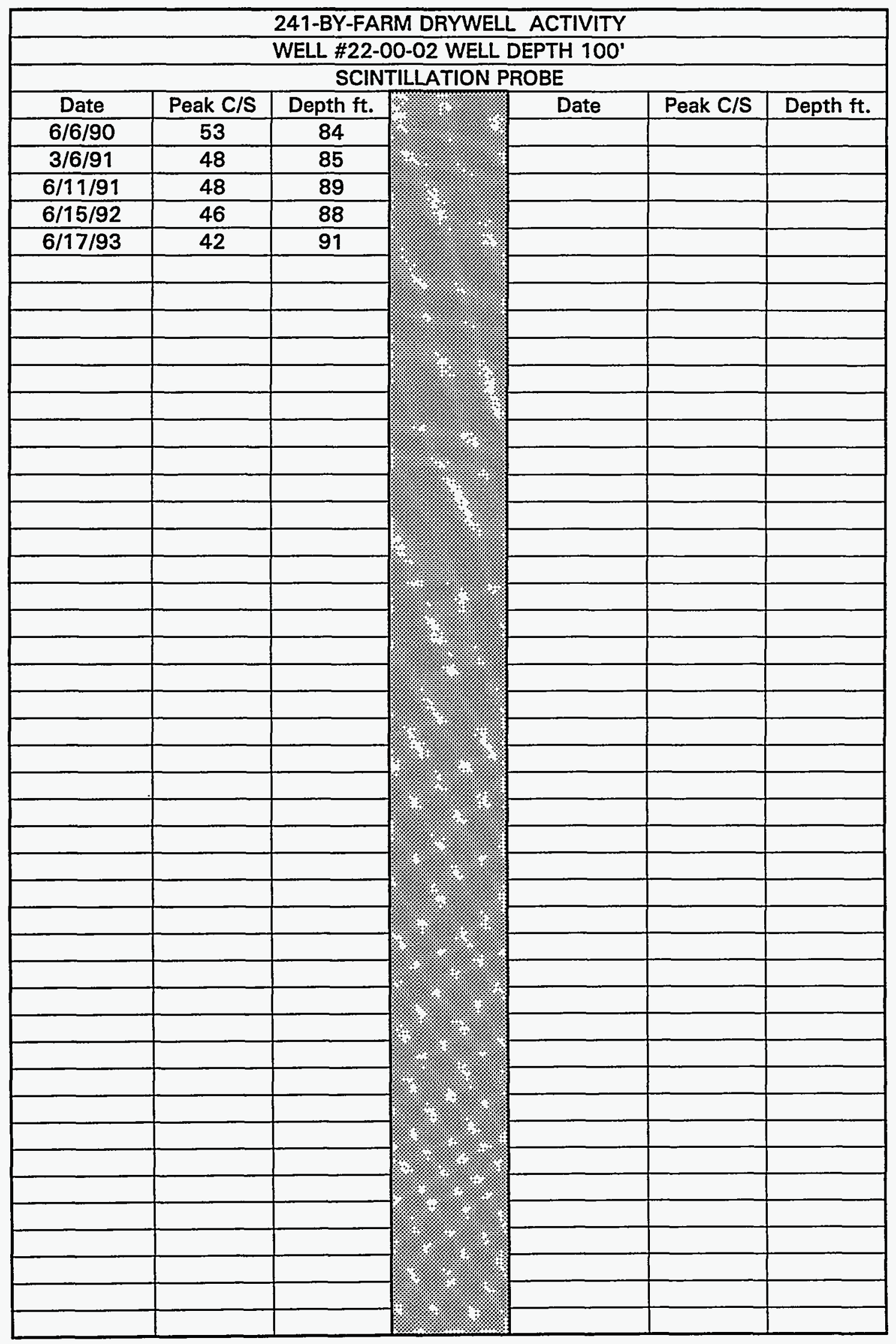

Data obtained from WHC Surveillance Data Acquisition (SDA), November 30, 1993. F-59 


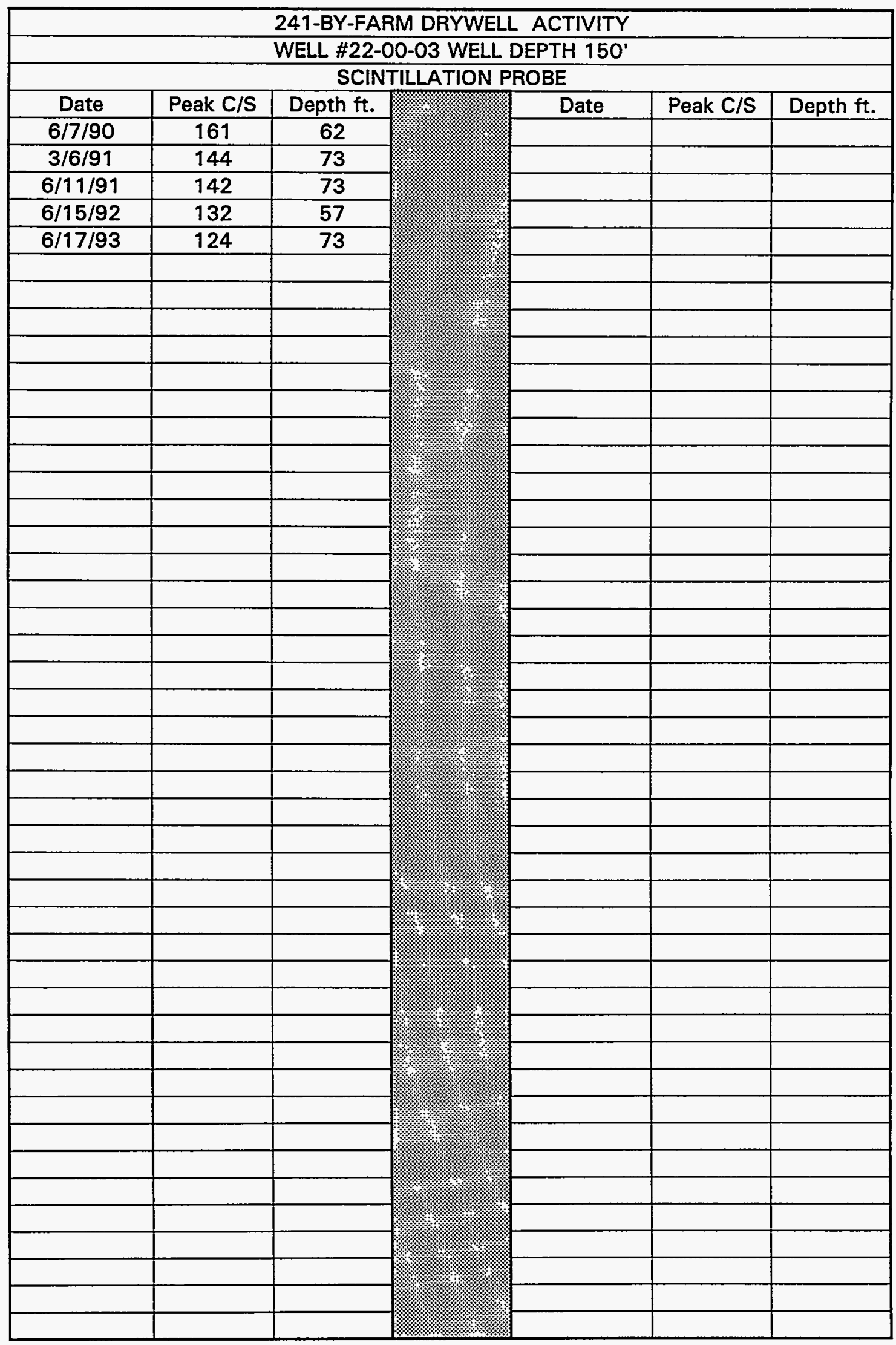

Data obtained from WHC Surveillance Data Acquisition (SDA), November 30, 1993. 


\begin{tabular}{|c|c|c|c|c|c|c|}
\hline & & $241-B Y-1 C$ & 1 DRYWELL & CTIVITY & & \\
\hline & & WELL \#22- & 1-10 WELL & PTH 100 & & \\
\hline & & SCINTILL & ATION PROBE & Ist Peak & & \\
\hline Date & Peak C/S & Depth ft. & & Date & Peak C/S & Depth ft. \\
\hline $1 / 31 / 90$ & 97 & 22 & & & & \\
\hline $4 / 26 / 90$ & 96 & 22 & & & & \\
\hline $7 / 17 / 90$ & 105 & 22 & & & & \\
\hline $10 / 10 / 90$ & 101 & 23 & & & & \\
\hline $12 / 31 / 90$ & 106 & 22 & & & & \\
\hline $3 / 6 / 91$ & 93 & 22 & ?. & & & \\
\hline $3 / 26 / 91$ & 77 & 22 & $\%$ & & & \\
\hline $6 / 19 / 91$ & 98 & 22 & & & & \\
\hline $9 / 9 / 91$ & 97 & 22 & & & & \\
\hline $12 / 2 / 91$ & 80 & 23 & & & & \\
\hline $2 / 27 / 92$ & 93 & 22 & & & & \\
\hline $5 / 20 / 92$ & 94 & 22 & & & & \\
\hline $8 / 11 / 92$ & 93 & 22 & 1 & & & \\
\hline $11 / 4 / 92$ & 83 & 23 & & & & \\
\hline $1 / 27 / 93$ & 82 & 22 & & & & \\
\hline $4 / 21 / 93$ & 80 & 21 & & & & \\
\hline $7 / 15 / 93$ & 81 & 21 & & & & \\
\hline $7 / 21 / 93$ & 75 & 22 & & & & \\
\hline $7 / 28 / 93$ & 92 & 22 & & & & \\
\hline $8 / 3 / 93$ & 77 & 22 & 8 & & & \\
\hline $8 / 10 / 93$ & 94 & 22 & & & & \\
\hline $8 / 18 / 93$ & 87 & 22 & & & & \\
\hline $8 / 24 / 93$ & 87 & 21 & & & & \\
\hline $9 / 1 / 93$ & 81 & 22 & \%. & & & \\
\hline $9 / 15 / 93$ & 99 & 22 & & & & \\
\hline $10 / 4 / 93$ & 80 & 22 & & & & \\
\hline & & & m & & & \\
\hline & & & 11: & & & \\
\hline & & & (ষ) & & & \\
\hline & & & & & & \\
\hline & & & & & & \\
\hline & & & 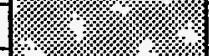 & & & \\
\hline & & & 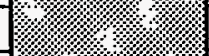 & & & \\
\hline & & & $f_{11}^{11}$ & & & \\
\hline & & & 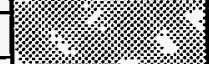 & & & \\
\hline & & & \% & & & \\
\hline & & & $\sqrt{11}$ & & & \\
\hline & & & $\sqrt{1}$ & & & \\
\hline & & & $\sqrt{19}$ & & & \\
\hline & & & & & & \\
\hline & & & ঋে. & & & \\
\hline & & & $f^{1}$ & & & \\
\hline & & & J & & & \\
\hline & & & 4 & & & \\
\hline
\end{tabular}

Data obtained from WHC Surveillance Data Acquisition (SDA), November 30, 1993. F-61 
WHC-SD-WM-ER-312, Rev. 0

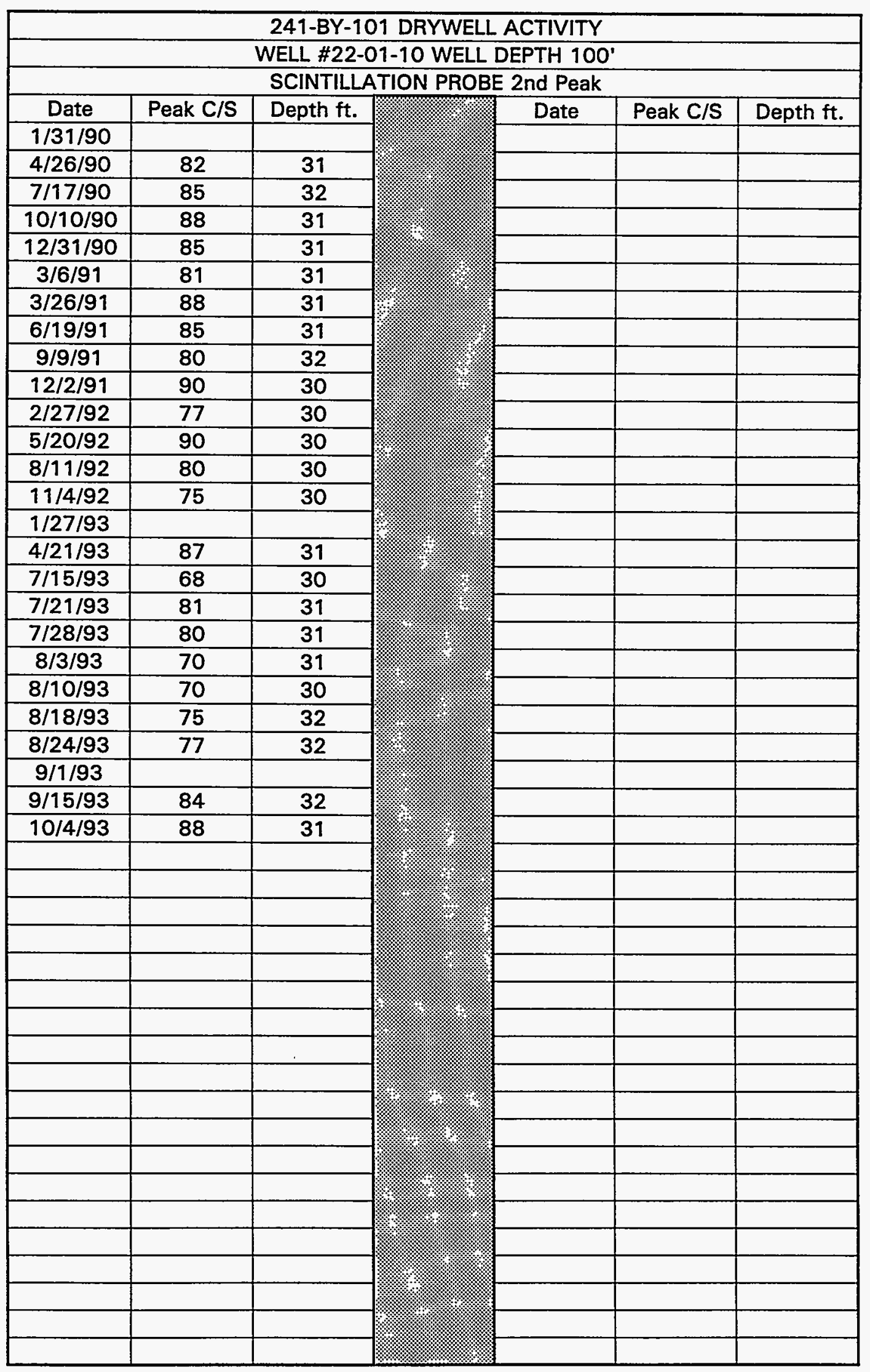

Data obtained from WHC Surveillance Data Acquisition (SDA), November 30, 1993. 


\begin{tabular}{|c|c|c|c|c|c|c|}
\hline \multicolumn{7}{|c|}{ 241-BY-101 DRYWELL ACTIVITY } \\
\hline \multicolumn{7}{|c|}{ WELL \#22-01-10 WELL DEPTH 100' } \\
\hline \multicolumn{7}{|c|}{ SCINTILLATION PROBE 3rd Peak } \\
\hline Date & Peak C/S & Depth ft. & & Date & Peak C/S & Depth ft. \\
\hline \multicolumn{7}{|l|}{$1 / 31 / 90$} \\
\hline $4 / 26 / 90$ & 56 & 40 & & & & \\
\hline $7 / 17 / 90$ & 48 & 40 & & & & \\
\hline $10 / 10 / 90$ & 60 & 40 & & & & \\
\hline $12 / 31 / 90$ & 45 & 40 & & & & \\
\hline $3 / 6 / 91$ & 49 & 40 & & & & \\
\hline $3 / 26 / 91$ & 56 & 39 & & & & \\
\hline $6 / 19 / 91$ & 50 & 40 & & & & \\
\hline $9 / 9 / 91$ & 65 & 41 & & & & \\
\hline $12 / 2 / 91$ & 52 & 41 & & & & \\
\hline $2 / 27 / 92$ & 60 & 40 & & & & \\
\hline $5 / 20 / 92$ & 80 & 32 & & & & \\
\hline \multicolumn{7}{|l|}{$8 / 11 / 92$} \\
\hline $11 / 4 / 92$ & 84 & 32 & & & & \\
\hline \multicolumn{7}{|l|}{$1 / 27 / 93$} \\
\hline $4 / 21 / 93$ & 70 & 33 & ঋ/: & & & \\
\hline $7 / 15 / 93$ & 62 & 33 & & & & \\
\hline \multicolumn{7}{|l|}{$7 / 21 / 93$} \\
\hline $7 / 28 / 93$ & 53 & 36 & & & & \\
\hline $8 / 3 / 93$ & 88 & $\overline{33}$ & & & & \\
\hline \multicolumn{7}{|l|}{$8 / 10 / 93$} \\
\hline \multicolumn{7}{|l|}{$8 / 18 / 93$} \\
\hline \multicolumn{7}{|l|}{$8 / 24 / 93$} \\
\hline \multicolumn{7}{|l|}{$9 / 1 / 93$} \\
\hline \multicolumn{7}{|l|}{$9 / 15 / 93$} \\
\hline \multicolumn{7}{|l|}{$10 / 4 / 93$} \\
\hline & & & & & & \\
\hline & & & & & & \\
\hline & & & & & & \\
\hline & & & $\sqrt{2}: 1$ & & & \\
\hline & & & "1. & & & \\
\hline & & & & & & \\
\hline & & & s: & & & \\
\hline & & & $1:$ & & & \\
\hline & & & & & & \\
\hline & & & & & & \\
\hline & & & & & & \\
\hline & & & স & & & \\
\hline & & & 1918.19 & & & \\
\hline & & & 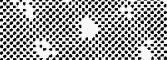 & & & \\
\hline & & & স4: & & & \\
\hline & & & "4. & & & \\
\hline & & & 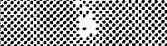 & & & \\
\hline & & & l' & & & \\
\hline & & & & & & \\
\hline
\end{tabular}

Data obtained from WHC Surveillance Data Acquisition (SDA), November 30, 1993. 


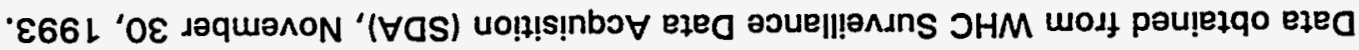

\begin{tabular}{|c|c|c|c|c|c|c|}
\hline 09 & $\varepsilon 19$ & $\varepsilon 6 / t / / t$ & \multirow[b]{5}{*}{4} & \multirow{2}{*}{$\frac{09}{09}$} & \multirow{2}{*}{$\frac{1+9}{t 19}$} & \multirow{2}{*}{$\begin{array}{c}16 / 2 Z / 8 \\
16 / 9 / 8\end{array}$} \\
\hline 09 & $\underline{G<G}$ & $\varepsilon 6 / \varepsilon L / t *$ & & & & \\
\hline 09 & $8+9$ & $\varepsilon 6 / 6 乙 / \varepsilon$ & & 19 & 199 & $L 6 / \varepsilon Z / L$ \\
\hline 09 & $\angle 89$ & $\varepsilon 6 / 9 L / \varepsilon$ & & $O G$ & 799 & $16 / 6 / L$ \\
\hline 09 & 219 & $\varepsilon 6 / \varepsilon / \varepsilon$ & & 09 & 619 & $16 / \triangleright Z / 9$ \\
\hline 09 & 869 & $\varepsilon 6 / \angle L / Z$ & & 09 & 909 & $L 6 / L L / 9$ \\
\hline OG & 299 & $\varepsilon 6 / 2 / 2$ & & 09 & g89 & $16 / 62 / 9$ \\
\hline OG & 809 & $\varepsilon 6 / 6 \mathrm{~L} / \mathrm{L}$ & & $O G$ & 829 & $\llcorner 6 / \varepsilon\llcorner/ G$ \\
\hline 09 & 109 & $\varepsilon 6 / 9 / \mathrm{l}$ & & 09 & 999 & 16/62/t \\
\hline$O S$ & 089 & $26 / L Z / Z L$ & & OS & $\varepsilon 29$ & $16 / \angle L / b$ \\
\hline 09 & 989 & $26 / 8 / 2 L$ & & 09 & $9 \varepsilon 9$ & $16 / \varepsilon / t$ \\
\hline 09 & L99 & $\overline{Z 6 / \varepsilon Z / \downarrow \downarrow ~}$ & & 09 & 629 & L6/LZ/E \\
\hline OG & 229 & Z6/E乙/LL* & & 09 & $9 \angle 9$ & $16 / 9 / \varepsilon$ \\
\hline OS & 689 & $26 / 0 \mathrm{~L} / \mathrm{LL}$ & & 09 & 229 & $16 / 9 / \varepsilon_{*}$ \\
\hline 09 & $\varepsilon 89$ & $26 / 82 / 0 \mathrm{~L}$ & & OG & 219 & $16 / 6 \mathrm{~L} / 2$ \\
\hline$\overline{O G}$ & $\angle L G$ & $26 / t L / 0 l$ & & OG & ZLG & $16 / L / 2$ \\
\hline 09 & $\angle 09$ & $26 / \mathrm{L} / 0 \mathrm{~L}$ & & 09 & 289 & $16 / 12 / L$ \\
\hline $\mathrm{OG}$ & $\angle 09$ & $26 / 9 \mathrm{l} / 6$ & & 09 & $8 \varepsilon 9$ & $L 6 / 6 / L$ \\
\hline OS & 029 & $26 / 1 / 6$ & & 09 & $t \nabla L$ & L6/8/L* \\
\hline 09 & 989 & $26 / 8\lfloor/ 8$ & & $\overline{O G}$ & $7 t 9$ & $06 / 9 Z / Z L$ \\
\hline 09 & 799 & $26 / t / 8$ & & 09 & 709 & $06 / 0 L / 2 L$ \\
\hline 09 & 969 & $26 / 22 ! L$ & & 09 & $\varepsilon 29$ & $06 / 62 / L L$ \\
\hline OG & $t \angle G$ & $26 / 8 / L$ & & 09 & $6 \angle 9$ & 06/†L/LL \\
\hline OS & 769 & $26 / 22 / 9$ & & 09 & 969 & $06 / 0 \varepsilon / 0 \mathrm{~L}$ \\
\hline 09 & $8 \angle 9$ & $26 / 6 / 9$ & & OG & 179 & $06 / 9 \mathrm{~L} / 0 \mathrm{~L}$ \\
\hline OG & 829 & Z6/9Z/9 & & $\overline{O G}$ & 029 & $06 / 2 / 0 \mathrm{~L}$ \\
\hline 09 & 699 & $26 / \varepsilon L / 9$ & & 09 & เع9 & $06 / \angle L / 6$ \\
\hline$O S$ & 189 & 乙6/8Z/† & & 09 & 279 & $06 / \mathcal{G} / 6$ \\
\hline 09 & 269 & $26 / t L / t$ & & 09 & $\succ \varepsilon 9$ & $06 / 22 / 8$ \\
\hline OG & $\angle L 9$ & $\tau 6 / L \varepsilon / \varepsilon$ & & OS & 099 & $06 / 9 / 8$ \\
\hline OS & 069 & $26 / 8 \mathrm{~L} / \varepsilon$ & & OS & 029 & $06 / 92 / L$ \\
\hline OS & $\varepsilon G G$ & $26 / \varepsilon / \varepsilon$ & & 09 & 269 & $06 / 0 \mathrm{~L} / \mathrm{L}$ \\
\hline 09 & 709 & $\tau 6 / \varepsilon / \varepsilon_{*}$ & & $O G$ & $8 \angle 9$ & $06 / 92 / 9$ \\
\hline OG & 069 & $26 / 02 / 2$ & & 09 & $\varepsilon 99$ & $06 / 2 \downarrow / 9$ \\
\hline OG & $\angle \varepsilon 9$ & $26 / L Z / L$ & $\%$ & 09 & $10 t$ & $06 / 0 \varepsilon / 9$ \\
\hline 09 & 089 & $26 / 6 / 1$ & & OS & 999 & $06 / 9 \mathrm{~L} / \mathrm{G}$ \\
\hline$\overline{09}$ & 269 & $16 / 9 Z / Z L$ & & 09 & $0 \varepsilon 9$ & $06 / \mathrm{b} / \mathrm{g}$ \\
\hline$\overline{O G}$ & 819 & $L 6 / 6 / Z L$ & & OG & 879 & $06 / 9 \mathrm{~L} / \mathrm{t}$ \\
\hline OG & $1+9$ & $16 / 9 Z / \downarrow L$ & & OS & $00 t$ & $06 / 2 / t$ \\
\hline 09 & 209 & $\llcorner 6 / \varepsilon \downarrow / L \downarrow$ & & 09 & 119 & 06/乙乙/घ \\
\hline 09 & ૬ع9 & $16 / 62 / 0 \mathrm{~L}$ & & 09 & 109 & $06 / 9 / \varepsilon$ \\
\hline 09 & $8+9$ & $16 / D L / 0 L$ & & OS & $6 \angle 9$ & $06 / 22 / 2$ \\
\hline OS & $2 \varepsilon 9$ & $\llcorner 6 / \varepsilon / O L$ & & 09 & 609 & $06 / 9 / 2$ \\
\hline 09 & $O \angle G$ & $16 / \angle L / 6$ & & $O G$ & $\angle 99$ & $06 / \hbar 2 / 1$ \\
\hline 09 & $\varepsilon \angle \mathcal{G}$ & $16 / t / 6$ & & OS & 669 & $06 / L L / L$ \\
\hline 'भ पदdag & S/J Yead & әfеव & & 'भ पदdəa & S/S Yeed & ә1еव \\
\hline & & yeod $151 \equiv$ & yd $N$ & $771 \perp N 135$ & & \\
\hline & & $.00 \perp H \perp d \exists C$ & $77 \exists M L$ & -22\# 7ר $\mathrm{N}$ & & \\
\hline & & $\wedge \perp|\wedge| \perp J \forall$ & & $2 L-\lambda g-l b 2$ & & \\
\hline
\end{tabular}




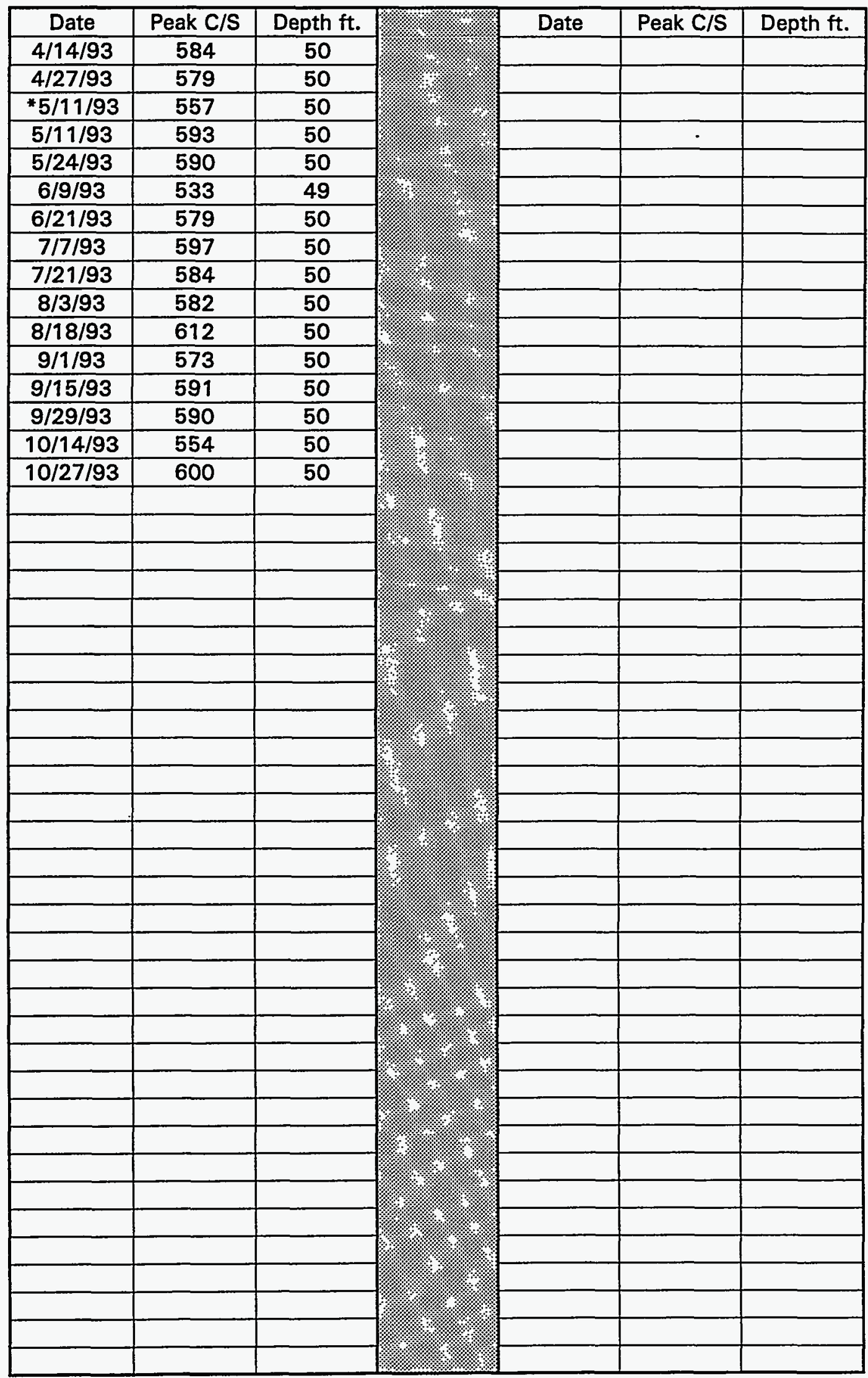

Data obtained from WHC Surveillance Data Acquisition (SDA), November 30, 1993. 
WHC-SD-WM-ER-312, Rev. 0

\begin{tabular}{|c|c|c|c|c|c|c|}
\hline & & $241-B Y-1$ & 2 DRYWELL & ACTIVITY & & \\
\hline & & WELL \#22- & 2-01 WELL & DEPTH 100' & & \\
\hline & & SCINTILL & TION PROB & 2nd Peak & & \\
\hline Date & Peak C/S & Depth ft. & & Date & Peak C/S & Depth ft. \\
\hline $1 / 11 / 90$ & & & & $9 / 4 / 91$ & 290 & 47 \\
\hline $1 / 24 / 90$ & & & & $9 / 17 / 91$ & 300 & 47 \\
\hline $2 / 6 / 90$ & & & & $10 / 3 / 91$ & 320 & 47 \\
\hline $2 / 22 / 90$ & & & & $10 / 14 / 91$ & 300 & 46 \\
\hline $3 / 5 / 90$ & & & & $10 / 29 / 91$ & 322 & 48 \\
\hline $3 / 22 / 90$ & & & & $11 / 13 / 91$ & 295 & 48 \\
\hline $4 / 2 / 90$ & & & & $11 / 26 / 91$ & 300 & 47 \\
\hline $4 / 16 / 90$ & & & & $12 / 9 / 91$ & 340 & 47 \\
\hline $5 / 1 / 90$ & & & & $12 / 25 / 91$ & 340 & 47 \\
\hline $5 / 15 / 90$ & & & & $1 / 9 / 92$ & 300 & 47 \\
\hline $5 / 30 / 90$ & & & & $1 / 21 / 92$ & 283 & 47 \\
\hline $6 / 12 / 90$ & & & & $2 / 20 / 92$ & 300 & 46 \\
\hline $6 / 25 / 90$ & & & & *3/3/92 & & \\
\hline $7 / 10 / 90$ & & & & $3 / 3 / 92$ & 315 & 47 \\
\hline $7 / 25 / 90$ & & & ? & $3 / 18 / 92$ & 265 & 47 \\
\hline $8 / 6 / 90$ & & & & $3 / 31 / 92$ & 300 & 47 \\
\hline $8 / 22 / 90$ & & & & $4 / 14 / 92$ & 280 & 47 \\
\hline $9 / 5 / 90$ & & & & $4 / 28 / 92$ & 340 & 46 \\
\hline $9 / 17 / 90$ & & & & $5 / 13 / 92$ & 330 & 45 \\
\hline $10 / 2 / 90$ & & & & $5 / 26 / 92$ & 320 & 47 \\
\hline $10 / 16 / 90$ & & & & $6 / 9 / 92$ & 300 & 47 \\
\hline $10 / 30 / 90$ & & & & $6 / 22 / 92$ & 320 & 47 \\
\hline $11 / 14 / 90$ & & & & $7 / 8 / 92$ & 290 & 47 \\
\hline $11 / 29 / 90$ & & & & $7 / 22 / 92$ & & \\
\hline $12 / 10 / 90$ & & & & $8 / 4 / 92$ & 350 & 47 \\
\hline $12 / 26 / 90$ & & & & $8 / 18 / 92$ & 330 & 47 \\
\hline$* 1 / 8 / 91$ & & & & $9 / 1 / 92$ & 360 & 47 \\
\hline $1 / 9 / 91$ & & & & $9 / 16 / 92$ & 280 & 47 \\
\hline $1 / 21 / 91$ & & & & $10 / 1 / 92$ & 280 & 47 \\
\hline $2 / 7 / 91$ & & & & $10 / 14 / 92$ & 330 & 47 \\
\hline $2 / 19 / 91$ & & & & $10 / 28 / 92$ & 330 & 47 \\
\hline${ }^{*} 3 / 5 / 91$ & & & & $11 / 10 / 92$ & 280 & 47 \\
\hline $3 / 6 / 91$ & & & & $* 11 / 23 / 92$ & 300 & 47 \\
\hline $3 / 21 / 91$ & & & & $11 / 23 / 92$ & 285 & 47 \\
\hline $4 / 3 / 91$ & & & & $12 / 8 / 92$ & 245 & 46 \\
\hline $4 / 17 / 91$ & & & & $12 / 21 / 92$ & 300 & 47 \\
\hline $4 / 29 / 91$ & & & & $1 / 6 / 93$ & 282 & 46 \\
\hline $5 / 13 / 91$ & & & & $1 / 19 / 93$ & 310 & 46 \\
\hline $5 / 29 / 91$ & & & & $2 / 2 / 93$ & 300 & \\
\hline $6 / 11 / 91$ & & & & $2 / 17 / 93$ & 262 & 46 \\
\hline $6 / 24 / 91$ & 321 & 47 & & $3 / 3 / 93$ & 265 & 46 \\
\hline $7 / 9 / 91$ & & & & $3 / 16 / 93$ & 280 & 47 \\
\hline $7 / 23 / 91$ & 340 & 48 & & $3 / 29 / 93$ & 271 & 46 \\
\hline $8 / 6 / 91$ & 295 & 47 & & * 4/13/93 & 290 & 47 \\
\hline $8 / 22 / 91$ & 300 & 47 & & $4 / 14 / 93$ & 280 & 47 \\
\hline
\end{tabular}

Data obtained from WHC Surveillance Data Acquisition (SDA), November 30, 1993. 


\begin{tabular}{|c|c|c|c|c|c|c|c|}
\hline Date & Peak C/S & Depth ft. & & & Date & Peak C/S & Depth ft. \\
\hline $4 / 14 / 93$ & 280 & 47 & & & & & \\
\hline $4 / 27 / 93$ & 282 & 47 & & & & & \\
\hline \multicolumn{8}{|l|}{ *5/11/93 } \\
\hline $5 / 11 / 93$ & 280 & 47 & & & & & \\
\hline \multicolumn{8}{|l|}{$5 / 24 / 93$} \\
\hline $6 / 9 / 93$ & 340 & 46 & & & & & \\
\hline $6 / 21 / 93$ & 270 & 47 & & & & & \\
\hline $7 / 7 / 93$ & 220 & 46 & & & & & \\
\hline \multicolumn{8}{|l|}{$7 / 21 / 93$} \\
\hline $8 / 3 / 93$ & 280 & 46 & & & & & \\
\hline $8 / 18 / 93$ & 320 & 47 & & & & & \\
\hline \multicolumn{8}{|l|}{$9 / 1 / 93$} \\
\hline $9 / 15 / 93$ & 280 & 48 & & & & & \\
\hline \multicolumn{8}{|l|}{$9 / 29 / 93$} \\
\hline $10 / 14 / 93$ & 260 & 47 & & & & & \\
\hline \multirow{2}{*}{\multicolumn{8}{|c|}{$10 / 27 / 93$}} \\
\hline & & & & & & & \\
\hline & & & & & & & \\
\hline & & & & & & & \\
\hline & & & & & & & \\
\hline & & & & & & & \\
\hline & & & & & & & \\
\hline & & & & 8 & & & \\
\hline & & & & & & & \\
\hline & & & & & & & \\
\hline & & & & & & & \\
\hline & & & & & & & \\
\hline & & & & & & & \\
\hline & & & & & & & \\
\hline & & & & 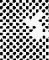 & & & \\
\hline & & & & & & & \\
\hline & & & & $:$ & & & \\
\hline & & & & & & & \\
\hline & & & & $\%$ & & & \\
\hline & & & & & & & \\
\hline & & & & : & & & \\
\hline & & & & & & & \\
\hline & & & & $\%$ & & & \\
\hline & & & & & & & \\
\hline & & & & \$. & & & \\
\hline & & & $4=$ & 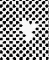 & & & \\
\hline & & & & 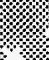 & & & \\
\hline & & & & & & & \\
\hline & & & 2 & : & & & \\
\hline & & & 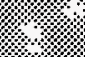 & 1: & & & \\
\hline & & & & & & & \\
\hline & & & & & & & \\
\hline & & & স স & & & & \\
\hline
\end{tabular}

Data obtained from WHC Surveillance Data Acquisition (SDA), November 30, 1993. 


\begin{tabular}{|c|c|c|c|c|c|}
\hline \multicolumn{6}{|c|}{ 241-BY-103 DRYWELL ACTIVITY } \\
\hline \multicolumn{6}{|c|}{ SCINTILLATION PROBE } \\
\hline Date & Peak C/S & Depth ft. & Date & Peak C/S & Depth $\mathrm{ft}$. \\
\hline $1 / 31 / 90$ & 98 & 21 & & & \\
\hline $4 / 26 / 90$ & 77 & 21 & & & \\
\hline $7 / 17 / 90$ & 83 & 21 & & & \\
\hline $10 / 10 / 90$ & 92 & 21 & & & \\
\hline$* 1 / 3 / 91$ & 467 & 83 & & & \\
\hline $1 / 4 / 91$ & 84 & 24 & & & \\
\hline $3 / 6 / 91$ & 84 & 21 & & & \\
\hline $3 / 26 / 91$ & 81 & 24 & & & \\
\hline $6 / 19 / 91$ & 94 & 21 & & & \\
\hline $9 / 9 / 91$ & 74 & 21 & & & \\
\hline $12 / 2 / 91$ & 85 & 24 & & & \\
\hline $2 / 27 / 92$ & 74 & 21 & & & \\
\hline $5 / 20 / 92$ & 90 & 21 & & & \\
\hline $8 / 11 / 92$ & 85 & 21 & & & \\
\hline $11 / 4 / 92$ & 76 & 21 & & & \\
\hline $1 / 27 / 93$ & 74 & 21 & & & \\
\hline $4 / 21 / 93$ & 96 & 21 & & & \\
\hline $7 / 14 / 93$ & 79 & 21 & & & \\
\hline $10 / 4 / 93$ & 73 & 21 & & & \\
\hline & & & & & \\
\hline & & & & & \\
\hline & & & & & \\
\hline & & & & & \\
\hline & & & & & \\
\hline & & & & & \\
\hline & & & & & \\
\hline & & & & & \\
\hline & & & & & \\
\hline & & & & & \\
\hline & & & & & \\
\hline & & & & & \\
\hline & & & & & \\
\hline & & & & & \\
\hline & & & & & \\
\hline & & & & & \\
\hline & & & & & \\
\hline & & & & & \\
\hline & & & & & \\
\hline & & & & & \\
\hline & & & & & \\
\hline
\end{tabular}

Data obtained from WHC Surveillance Data Acquisition (SDA), November 30, 1993. 


\begin{tabular}{|c|c|c|c|c|c|c|}
\hline & & $241-B Y-1$ & 3 DRYWELL & STIVIT & & \\
\hline & & NELL \#22- & 33-05 WELL L & PTH 1 & & \\
\hline & & & IM \# 1 PROB & & & \\
\hline Date & Peak C/S & Depth ft. & & Date & Peak C/S & Depth ft. \\
\hline $1 / 31 / 90$ & 18158 & 37 & & & & \\
\hline $4 / 26 / 90$ & 19786 & 34 & & & & \\
\hline $7 / 19 / 90$ & 22899 & 34 & & & & \\
\hline $10 / 10 / 90$ & 18232 & 34 & & & & \\
\hline $1 / 3 / 91$ & 18855 & 37 & & & & \\
\hline $3 / 6 / 91$ & 18874 & 34 & & & & \\
\hline $3 / 26 / 91$ & 21175 & $\overline{34}$ & & & & \\
\hline $6 / 21 / 91$ & 21319 & 34 & & & & \\
\hline $9 / 12 / 91$ & 20663 & 34 & & & & \\
\hline $12 / 6 / 91$ & 21074 & 34 & & & & \\
\hline $2 / 27 / 92$ & 16853 & 34 & & & & \\
\hline $5 / 20 / 92$ & 17140 & 34 & & & & \\
\hline *8/11/92 & 19348 & 33 & & & & \\
\hline $8 / 12 / 92$ & 21423 & 34 & & & & \\
\hline $11 / 4 / 92$ & 17924 & 37 & & & & \\
\hline $4 / 21 / 93$ & 21534 & 34 & & & & \\
\hline *7/14/93 & 0 & 98 & & & & \\
\hline $7 / 15 / 93$ & 10685 & 39 & \% & & & \\
\hline $10 / 5 / 93$ & 30924 & 34 & & & & \\
\hline & & & & & & \\
\hline & & & & & & \\
\hline & & & & & & \\
\hline & & & & & & \\
\hline & & & & & & \\
\hline & & & 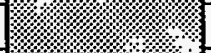 & & & \\
\hline & & & & & & \\
\hline & & & & & & \\
\hline & & & X & & & \\
\hline & & & & & & \\
\hline & & & & & & \\
\hline & & & & & & \\
\hline & & & : & & & \\
\hline & & & & & & \\
\hline & & & & & & \\
\hline & & & $\sqrt{1 / 2}=$ & & & \\
\hline & & & & & & \\
\hline & & & & & & \\
\hline & & & $\sqrt{17}$ & & & \\
\hline & & & & & & \\
\hline & & & & & & \\
\hline & & $\cdot$ & & & & \\
\hline & & & $11 \%$ & & & \\
\hline & & & $\sqrt{1 / 18}$ & & & \\
\hline & & & & & & \\
\hline
\end{tabular}

Data obtained from WHC Surveillance Data Acquisition (SDA), November 30, 1993. 


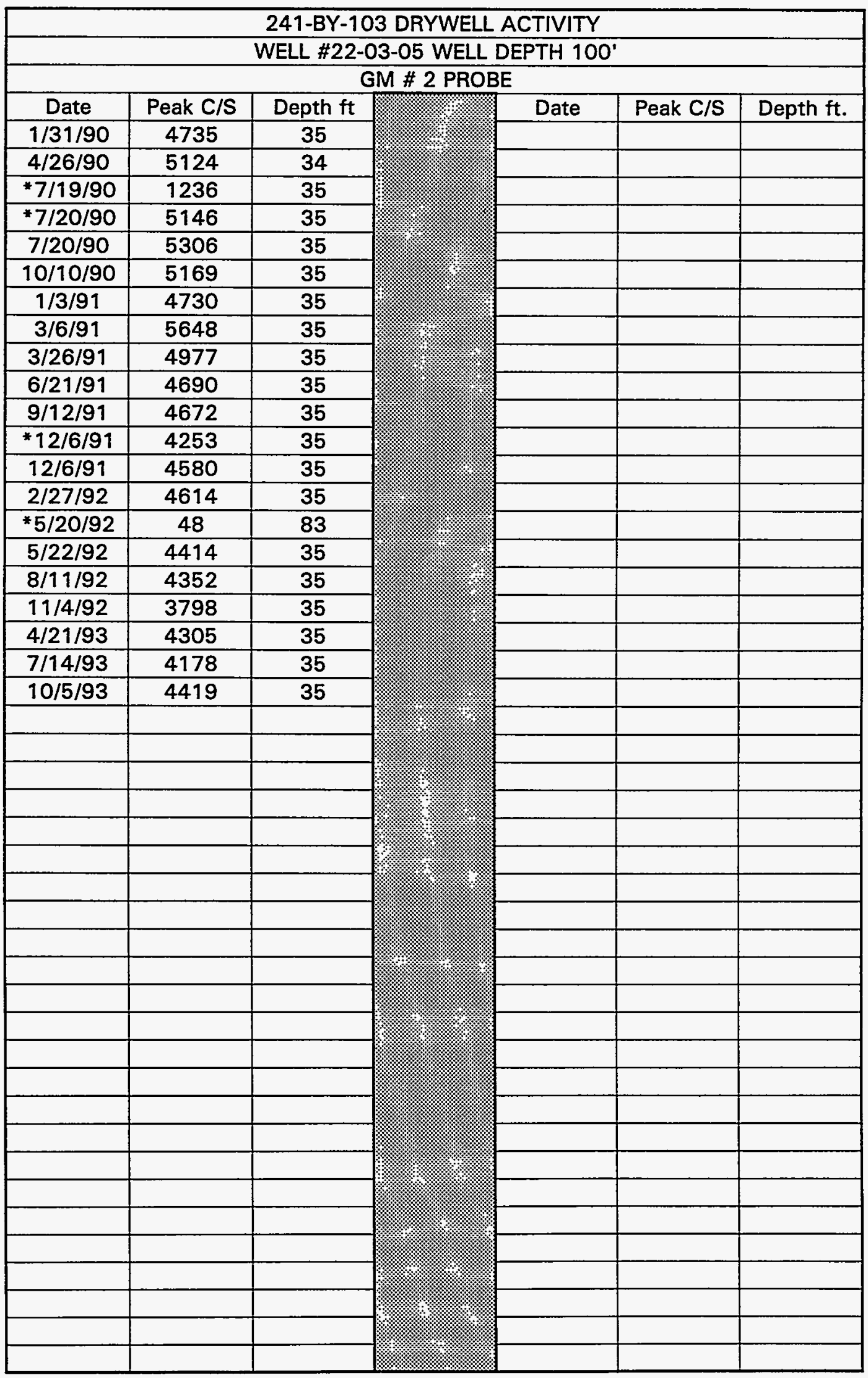

Data obtained from WHC Surveillance Data Acquisition (SDA), November 30, 1993. 


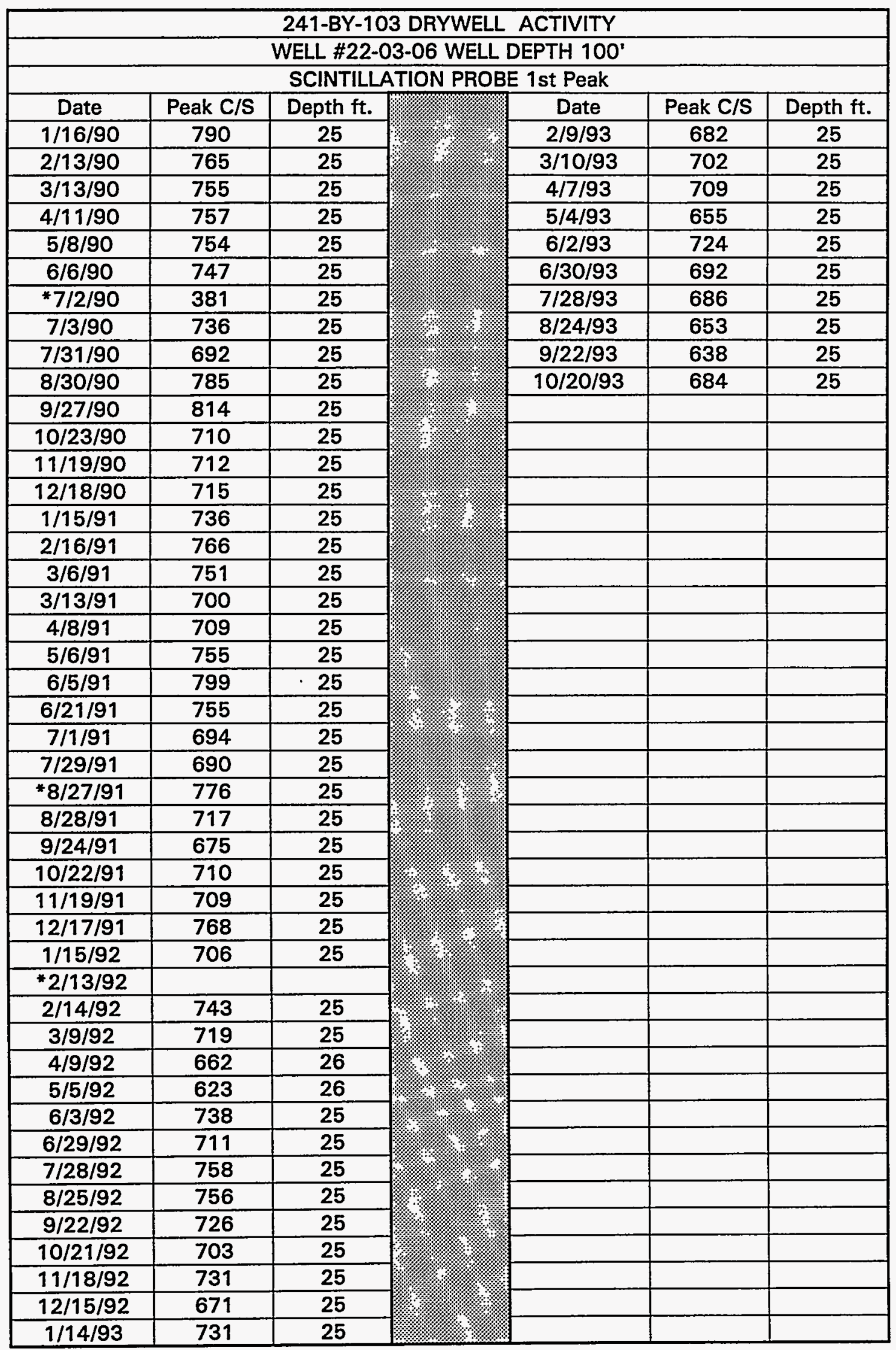

Data obtained from WHC Surveillance Data Acquisition (SDA), November 30, 1993. 
WHC-SD-EM-ER-308, Rev. 0

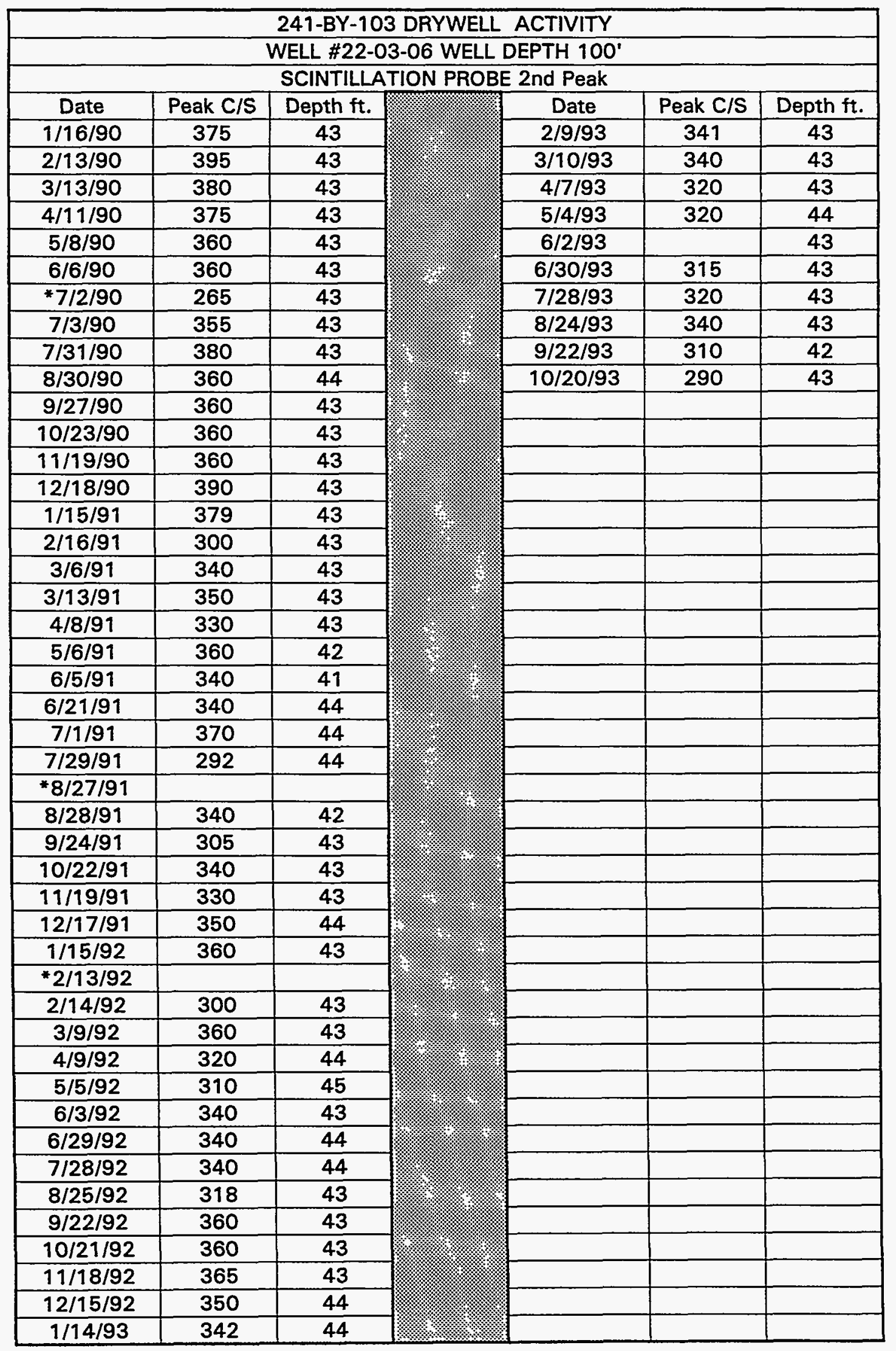

Data obtained from WHC Surveiliance Data Acquisition (SDA), November 30, 1993. 


\begin{tabular}{|c|c|c|c|c|c|c|}
\hline \multicolumn{7}{|c|}{ 241-BY-103 DRYWELL ACTIVITY } \\
\hline \multicolumn{7}{|c|}{ WELL \#22-03-06 WELL DEPTH 100' } \\
\hline \multicolumn{7}{|c|}{ SCINTILLATION PROBE 3rd Peak } \\
\hline Date & Peak C/S & Depth ft. & 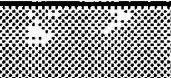 & Date & Peak C/S & Depth ft. \\
\hline $1 / 16 / 90$ & & & \multirow[b]{3}{*}{ 1/ } & $2 / 9 / 93$ & & \\
\hline $2 / 13 / 90$ & & & & $3 / 10 / 93$ & & \\
\hline $3 / 13 / 90$ & & & & $4 / 7 / 93$ & & \\
\hline $4 / 11 / 90$ & & & \multirow{3}{*}{ 1: } & $5 / 4 / 93$ & & \\
\hline $5 / 8 / 90$ & & & & $6 / 2 / 93$ & & \\
\hline $6 / 6 / 90$ & & & & $6 / 30 / 93$ & 75 & 47 \\
\hline * 7/2/90 & & & & $7 / 28 / 93$ & 62 & 47 \\
\hline $7 / 3 / 90$ & & & & $8 / 24 / 93$ & 75 & 46 \\
\hline $7 / 31 / 90$ & & & & $9 / 22 / 93$ & & \\
\hline $8 / 30 / 90$ & & & & $10 / 20 / 93$ & & \\
\hline \multicolumn{6}{|l|}{$9 / 27 / 90$} & \\
\hline \multicolumn{7}{|l|}{$10 / 23 / 90$} \\
\hline \multicolumn{7}{|l|}{$11 / 19 / 90$} \\
\hline \multicolumn{7}{|l|}{$12 / 18 / 90$} \\
\hline \multicolumn{7}{|l|}{$1 / 15 / 91$} \\
\hline \multicolumn{7}{|l|}{$2 / 16 / 91$} \\
\hline \multicolumn{7}{|l|}{$3 / 6 / 91$} \\
\hline \multicolumn{7}{|l|}{$3 / 13 / 91$} \\
\hline \multicolumn{7}{|l|}{$4 / 8 / 91$} \\
\hline $5 / 6 / 91$ & 105 & 47 & & & & \\
\hline \multicolumn{7}{|l|}{$6 / 5 / 91$} \\
\hline \multicolumn{7}{|l|}{$6 / 21 / 91$} \\
\hline \multicolumn{7}{|l|}{$7 / 1 / 91$} \\
\hline \multicolumn{7}{|l|}{$7 / 29 / 91$} \\
\hline \multicolumn{7}{|l|}{${ }^{*} 8 / 27 / 91$} \\
\hline $8 / 28 / 91$ & & & & & & \\
\hline $9 / 24 / 91$ & & & & & & \\
\hline $10 / 22 / 91$ & & & & & & \\
\hline $11 / 19 / 91$ & 90 & 47 & & & & \\
\hline $12 / 17 / 91$ & & & & & & \\
\hline $1 / 15 / 92$ & & & & & & \\
\hline *2/13/92 & 256 & 98 & & & & \\
\hline $2 / 14 / 92$ & & & : & & & \\
\hline $3 / 9 / 92$ & 80 & 47 & : & & & \\
\hline $4 / 9 / 92$ & & & & & & \\
\hline $5 / 5 / 92$ & & & & & & \\
\hline $6 / 3 / 92$ & 90 & 47 & 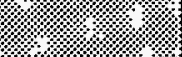 & & & \\
\hline $6 / 29 / 92$ & & & $=$ & & & \\
\hline $7 / 28 / 92$ & & & 1 & & & \\
\hline $8 / 25 / 92$ & & & & & & \\
\hline $9 / 22 / 92$ & & & & & & \\
\hline $10 / 21 / 92$ & & & \% & & & \\
\hline $11 / 18 / 92$ & & & 4 & & & \\
\hline $12 / 15 / 92$ & & & 4 & & & \\
\hline $1 / 14 / 93$ & & & & & & \\
\hline
\end{tabular}

Data obtained from WHC Surveillance Data Acquisition (SDA), November 30, 1993. 


\begin{tabular}{|c|c|c|c|c|c|c|}
\hline \multicolumn{7}{|c|}{ 241-BY-103 DRYWELL ACTIVITY } \\
\hline \multicolumn{7}{|c|}{ WELL \#22-03-09 WELL DEPTH 100' } \\
\hline \multicolumn{7}{|c|}{ SCINTILLATION PROBE 1st Peak } \\
\hline Date & Peak C/S & Depth ft. & & Date & Peak C/S & Depth ft. \\
\hline $1 / 11 / 90$ & 262 & 96 & & $8 / 6 / 91$ & 165 & 96 \\
\hline $1 / 25 / 90$ & 255 & 96 & & $* 8 / 21 / 91$ & 666 & 96 \\
\hline $2 / 6 / 90$ & 256 & 96 & & $8 / 22 / 91$ & 158 & 96 \\
\hline $2 / 22 / 90$ & 205 & 96 & & $9 / 4 / 91$ & 150 & 96 \\
\hline $3 / 5 / 90$ & 236 & 96 & & 9/17/91 & 163 & 96 \\
\hline $3 / 22 / 90$ & 237 & 96 & & $10 / 3 / 91$ & 166 & 96 \\
\hline $4 / 3 / 90$ & 231 & 96 & & $10 / 14 / 91$ & 152 & 96 \\
\hline$* 4 / 16 / 90$ & 229 & 96 & & $10 / 29 / 91$ & 145 & 96 \\
\hline $4 / 17 / 90$ & 228 & 96 & & $11 / 13 / 91$ & 155 & 96 \\
\hline $5 / 1 / 90$ & 254 & 96 & & $11 / 26 / 91$ & 149 & 96 \\
\hline $5 / 15 / 90$ & 215 & 96 & & $12 / 9 / 91$ & 140 & 96 \\
\hline $5 / 30 / 90$ & 213 & 96 & & *12/26/91 & 147 & 96 \\
\hline $6 / 12 / 90$ & 253 & 96 & & $12 / 27 / 91$ & 155 & 96 \\
\hline $6 / 25 / 90$ & 226 & 96 & & ${ }^{*} 1 / 8 / 92$ & 154 & 96 \\
\hline $6 / 27 / 90$ & 225 & 96 & & $1 / 9 / 92$ & 140 & 96 \\
\hline $7 / 10 / 90$ & 219 & 96 & & $1 / 21 / 92$ & 140 & 96 \\
\hline $7 / 25 / 90$ & 227 & 96 & & $2 / 20 / 92$ & 143 & 96 \\
\hline $8 / 6 / 90$ & 225 & 96 & & $3 / 3 / 92$ & 140 & 96 \\
\hline $8 / 22 / 90$ & 225 & 96 & & $3 / 18 / 92$ & 131 & 96 \\
\hline $9 / 5 / 90$ & 231 & 96 & & $3 / 31 / 92$ & 142 & 96 \\
\hline $9 / 18 / 90$ & 202 & 96 & & $4 / 14 / 92$ & 127 & 96 \\
\hline $10 / 2 / 90$ & 201 & 96 & & $4 / 28 / 92$ & 130 & 96 \\
\hline $10 / 16 / 90$ & 186 & 96 & & $5 / 13 / 92$ & 123 & 96 \\
\hline $10 / 30 / 90$ & 199 & 96 & & $5 / 26 / 92$ & 126 & 96 \\
\hline $11 / 1 / 90$ & 209 & 96 & & $6 / 9 / 92$ & 125 & 96 \\
\hline $11 / 29 / 90$ & 199 & 96 & & $6 / 22 / 92$ & 93 & 96 \\
\hline $12 / 10 / 90$ & 192 & 96 & & $7 / 8 / 92$ & 116 & 96 \\
\hline $12 / 26 / 90$ & 195 & 96 & & $7 / 22 / 92$ & 125 & 96 \\
\hline $1 / 8 / 91$ & 257 & 96 & & $8 / 4 / 92$ & 123 & 96 \\
\hline $1 / 21 / 91$ & 171 & 96 & \% & $8 / 18 / 92$ & 97 & 96 \\
\hline $2 / 5 / 91$ & 162 & 96 & & $9 / 1 / 92$ & 101 & 96 \\
\hline $2 / 19 / 91$ & 181 & 96 & & $9 / 16 / 92$ & 114 & 96 \\
\hline * $3 / 5 / 91$ & 192 & 96 & & * 9/29/92 & 2398 & 96 \\
\hline $3 / 6 / 91$ & 180 & 96 & & $10 / 1 / 92$ & 124 & 96 \\
\hline${ }^{*} 3 / 21 / 91$ & 172 & 96 & & $10 / 14 / 92$ & 105 & 96 \\
\hline $3 / 21 / 91$ & 175 & 96 & & $10 / 28 / 92$ & 102 & 96 \\
\hline $4 / 3 / 91$ & 174 & 96 & & $11 / 10 / 92$ & 110 & 96 \\
\hline $4 / 17 / 91$ & 165 & 96 & & $11 / 23 / 92$ & 115 & 96 \\
\hline $4 / 29 / 91$ & 174 & 96 & & $12 / 8 / 92$ & 115 & 96 \\
\hline $5 / 13 / 91$ & 169 & 96 & & $12 / 21 / 92$ & 122 & 96 \\
\hline $5 / 29 / 91$ & 170 & 96 & & $1 / 6 / 93$ & 108 & 96 \\
\hline $6 / 11 / 91$ & 164 & 96 & & $1 / 21 / 93$ & 105 & 96 \\
\hline $6 / 24 / 91$ & 166 & 96 & $\sqrt{1}$ & $2 / 2 / 93$ & 102 & 96 \\
\hline $7 / 9 / 91$ & 175 & 96 & & $2 / 17 / 93$ & 106 & 96 \\
\hline $7 / 23 / 91$ & 143 & 96 & & $3 / 3 / 93$ & 103 & 96 \\
\hline
\end{tabular}

Data obtained from WHC Surveillance Data Acquisition (SDA), November 30, 1993. 


\begin{tabular}{|c|c|c|c|c|c|}
\hline Date & Peak C/S & Depth ft. & Date & Peak C/S & Depth $\mathrm{ft}$. \\
\hline $3 / 17 / 93$ & 93 & 96 & & & \\
\hline $3 / 29 / 93$ & 106 & 96 & & & \\
\hline $4 / 14 / 93$ & 95 & 97 & & & \\
\hline $4 / 27 / 93$ & 95 & 96 & & & \\
\hline $5 / 11 / 93$ & 107 & 96 & & & \\
\hline $5 / 24 / 93$ & 89 & 96 & & & \\
\hline $6 / 9 / 93$ & 119 & 96 & & & \\
\hline $6 / 21 / 93$ & 105 & 96 & & & \\
\hline $7 / 7 / 93$ & 104 & 96 & & & \\
\hline $7 / 21 / 93$ & 95 & 96 & & & \\
\hline $8 / 3 / 93$ & 100 & 96 & & & \\
\hline $8 / 18 / 93$ & 107 & 96 & & & \\
\hline $9 / 1 / 93$ & 88 & 96 & & & \\
\hline $9 / 15 / 93$ & 85 & 96 & & & \\
\hline \multicolumn{6}{|l|}{$9 / 29 / 93$} \\
\hline $10 / 14 / 93$ & 97 & 96 & & & \\
\hline $10 / 27 / 93$ & 97 & 96 & & & \\
\hline & & & & - & \\
\hline & & & & & \\
\hline & & & & & \\
\hline & & & & & \\
\hline & & & & & \\
\hline & & & & & \\
\hline & & & & & \\
\hline & & & & & \\
\hline & & & & & \\
\hline & & & & & \\
\hline & & & & & \\
\hline & & & & & \\
\hline & & & & & \\
\hline & & & & & \\
\hline & & & & & \\
\hline & & & & & \\
\hline & & & & & \\
\hline & & & & & \\
\hline & & & & & \\
\hline & & & & & \\
\hline & & & & & \\
\hline & & & & & \\
\hline & & & & & \\
\hline & & & & & \\
\hline
\end{tabular}

Data obtained from WHC Surveillance Data Acquisition (SDA), November 30, 1993. 
WHC-SD-WM-ER-312, Rev. 0

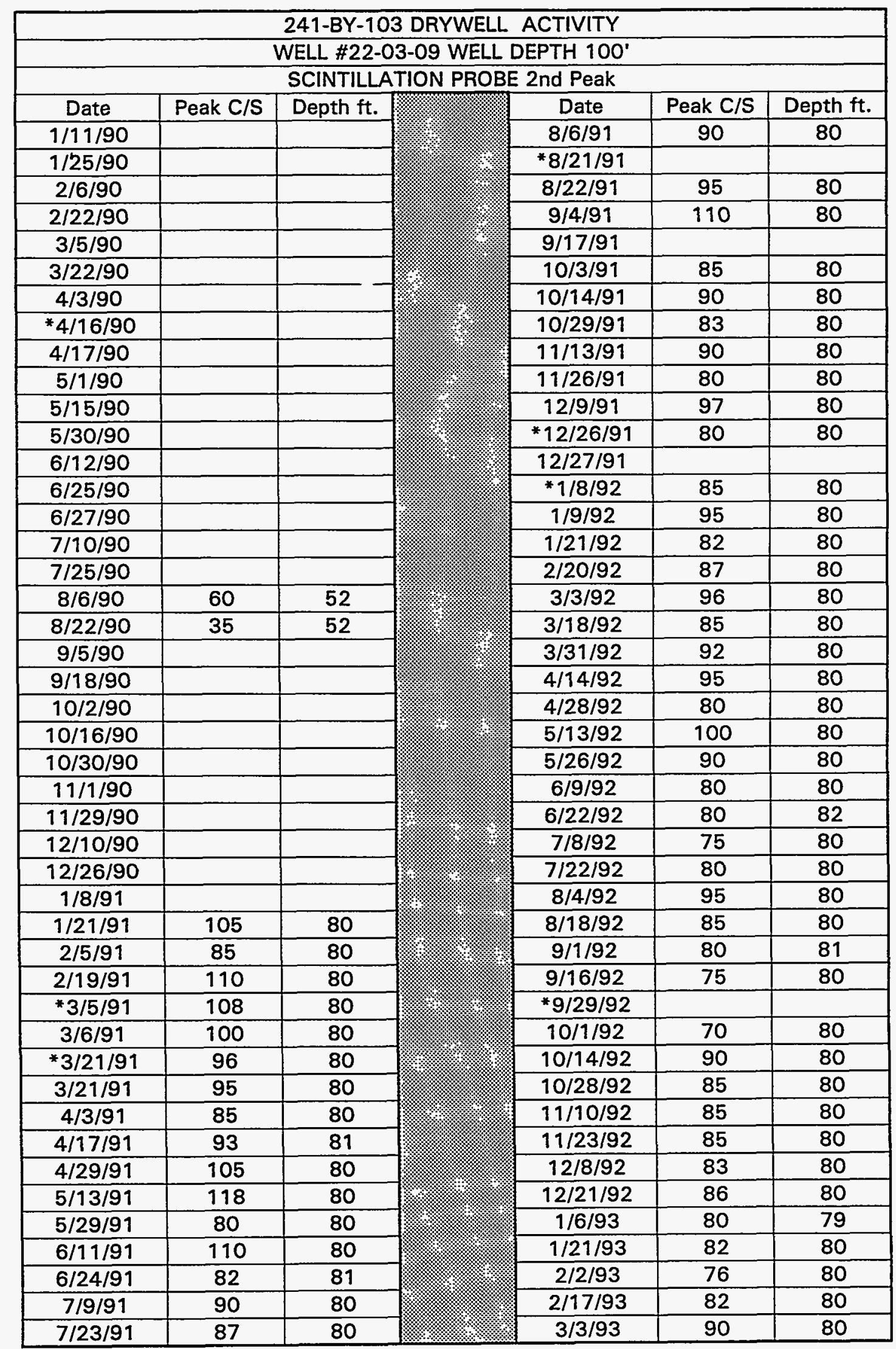

Data obtained from WHC Surveillance Data Acquisition (SDA), November 30, 1993. 


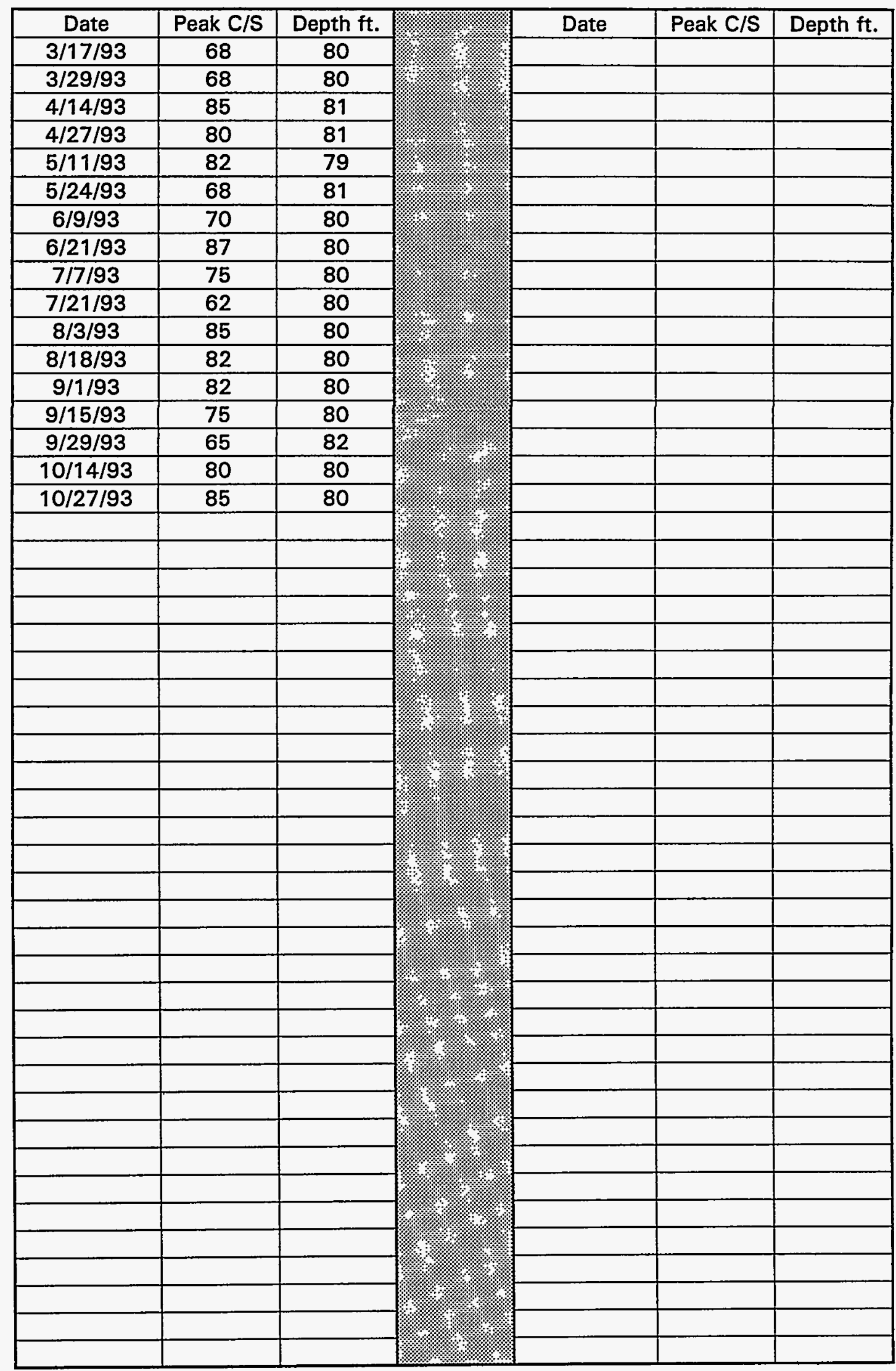

Data obtained from WHC Surveillance Data Acquisition (SDA), November 30, 1993. 
WHC-SD-WM-ER-312, Rev. 0

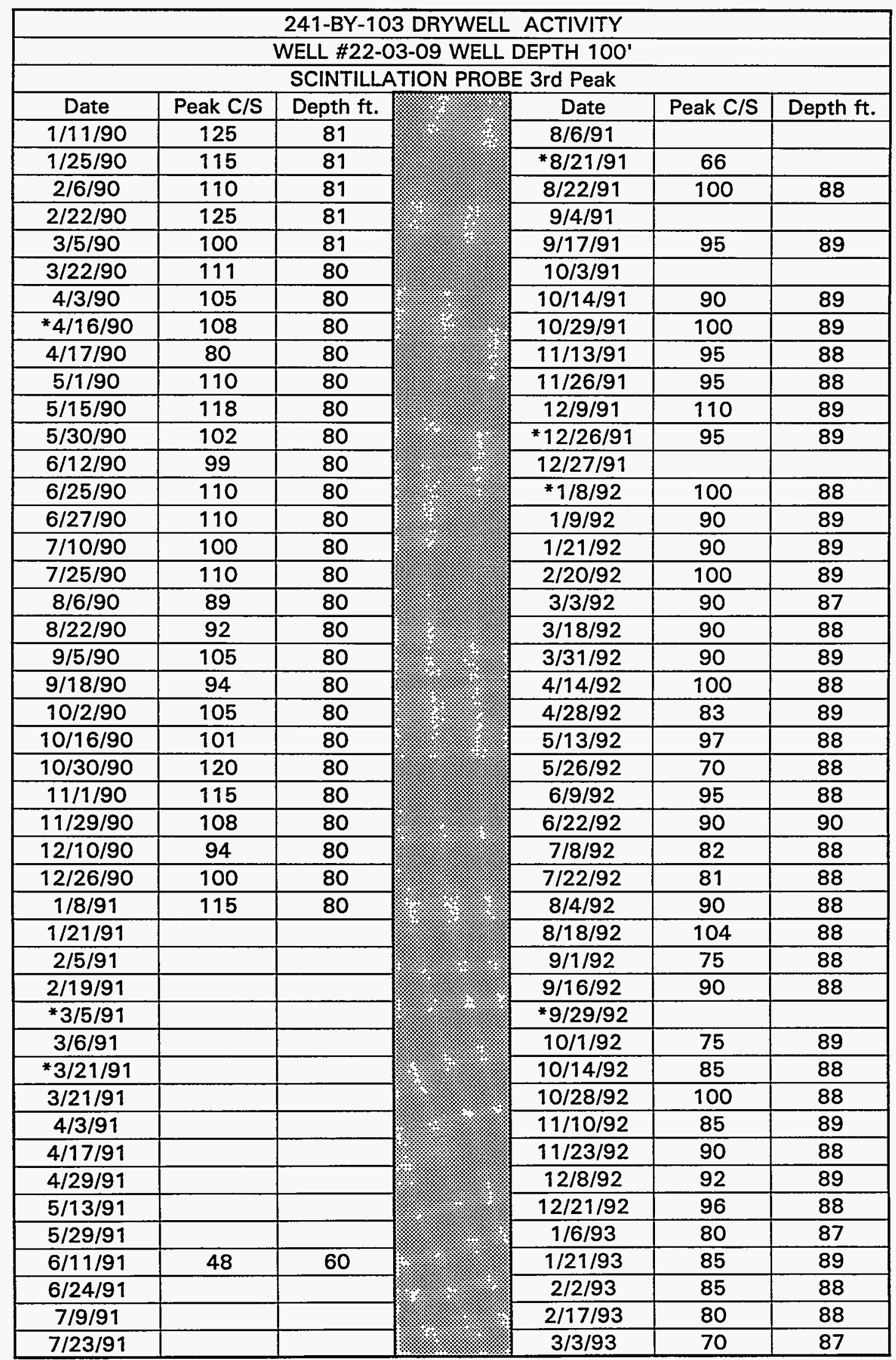

Data obtained from WHC Surveillance Data Acquisition (SDA), November 30, 1993. 
WHC-SD-WM-ER-312, Rev. 0

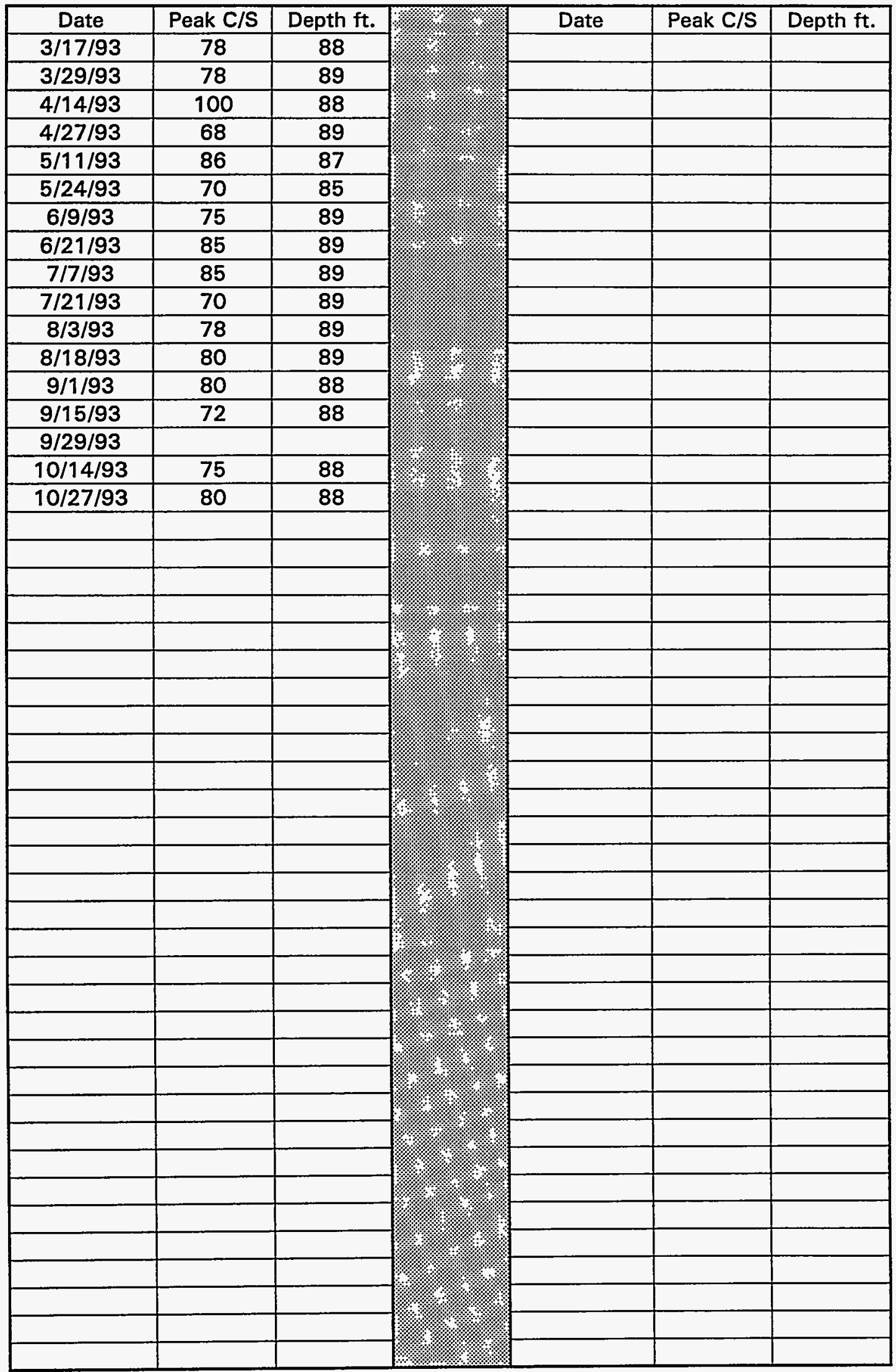

Data obtained from WHC Surveillance Data Acquisition (SDA), November 30, 1993. 
WHC-SD-WM-ER-312, Rev. 0

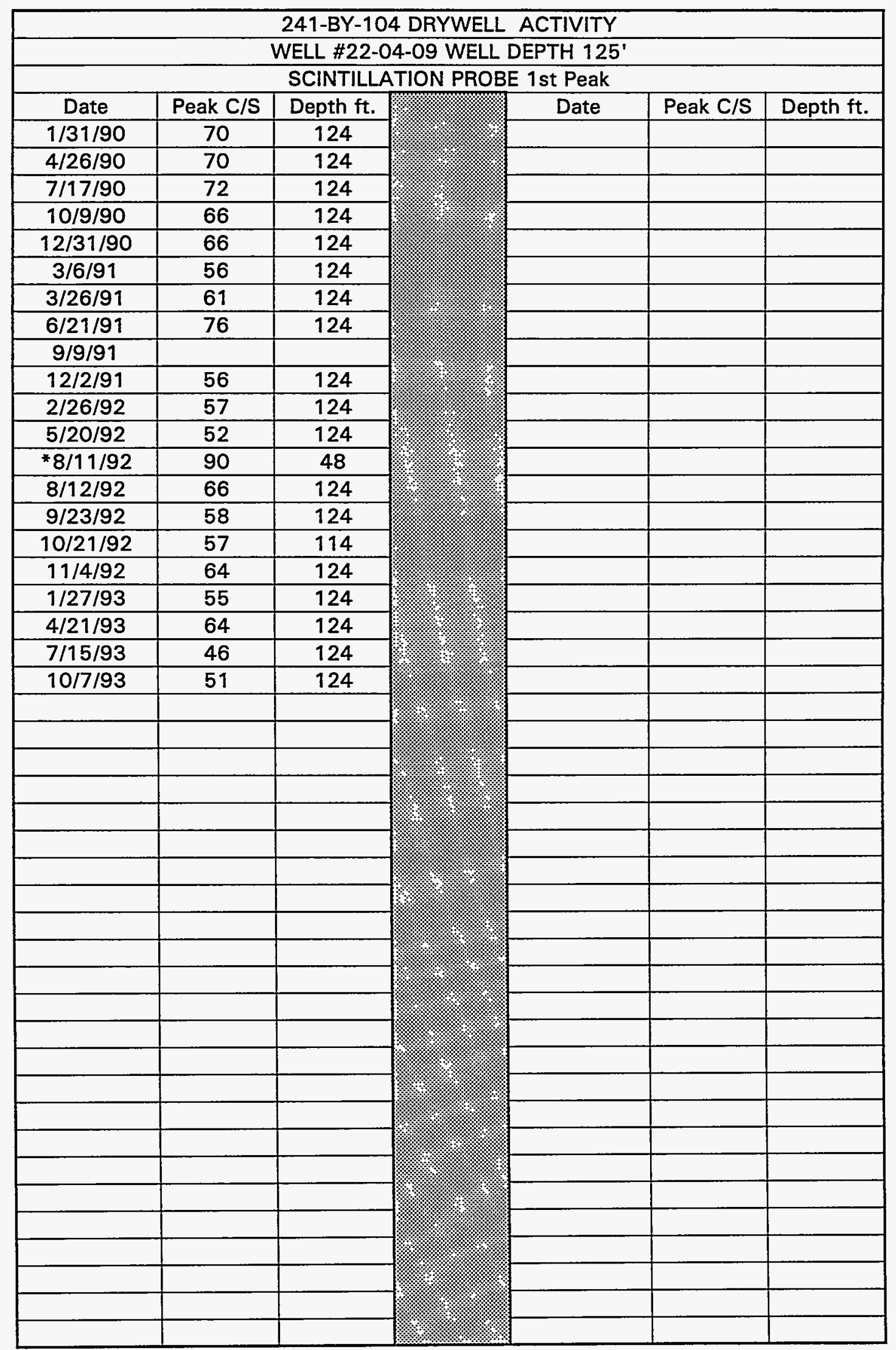

Data obtained from WHC Surveillance Data Acquisition (SDA), November 30, 1993. 


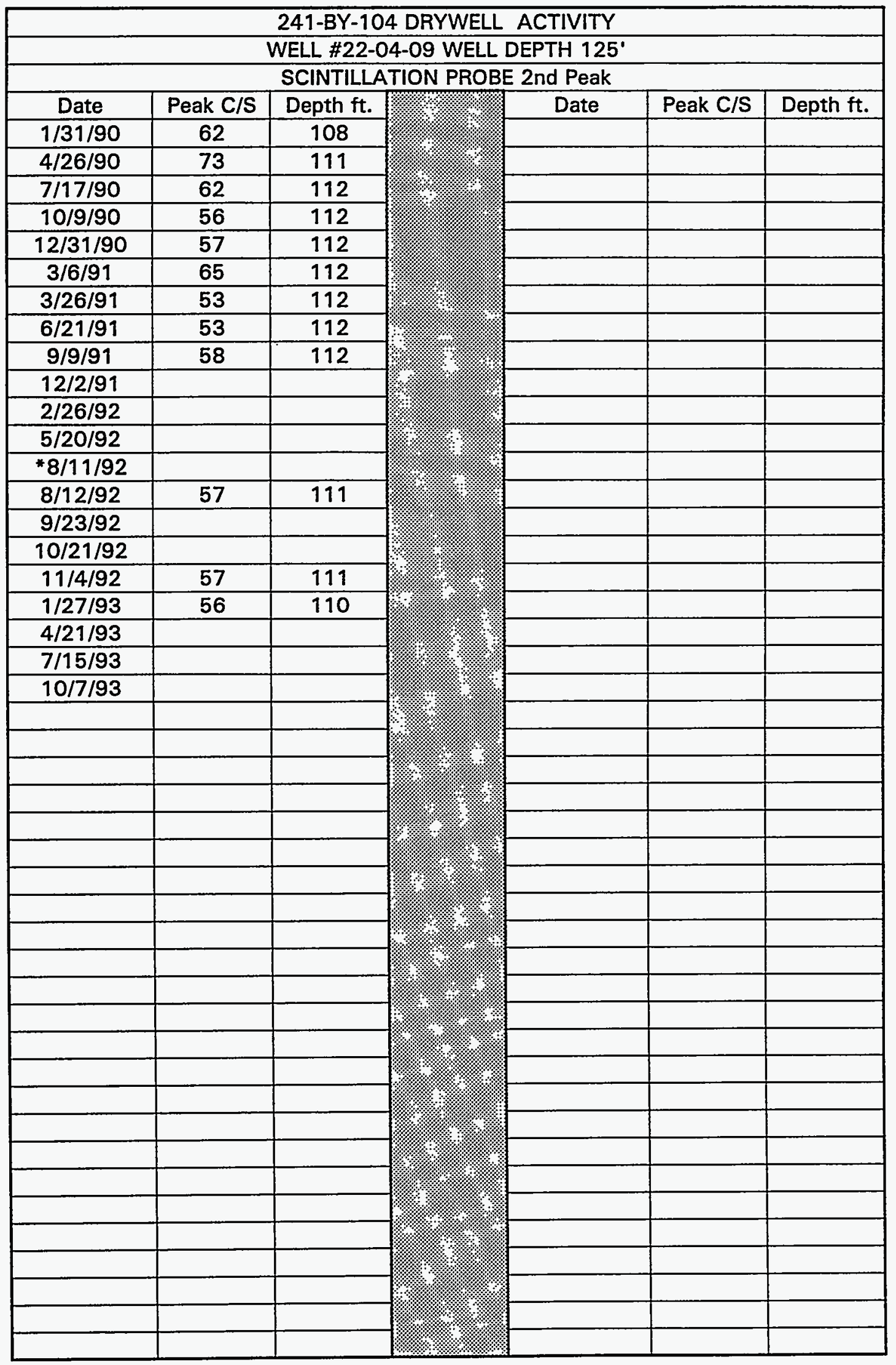

Data obtained from WHC Surveillance Data Acquisition (SDA), November 30, 1993. 


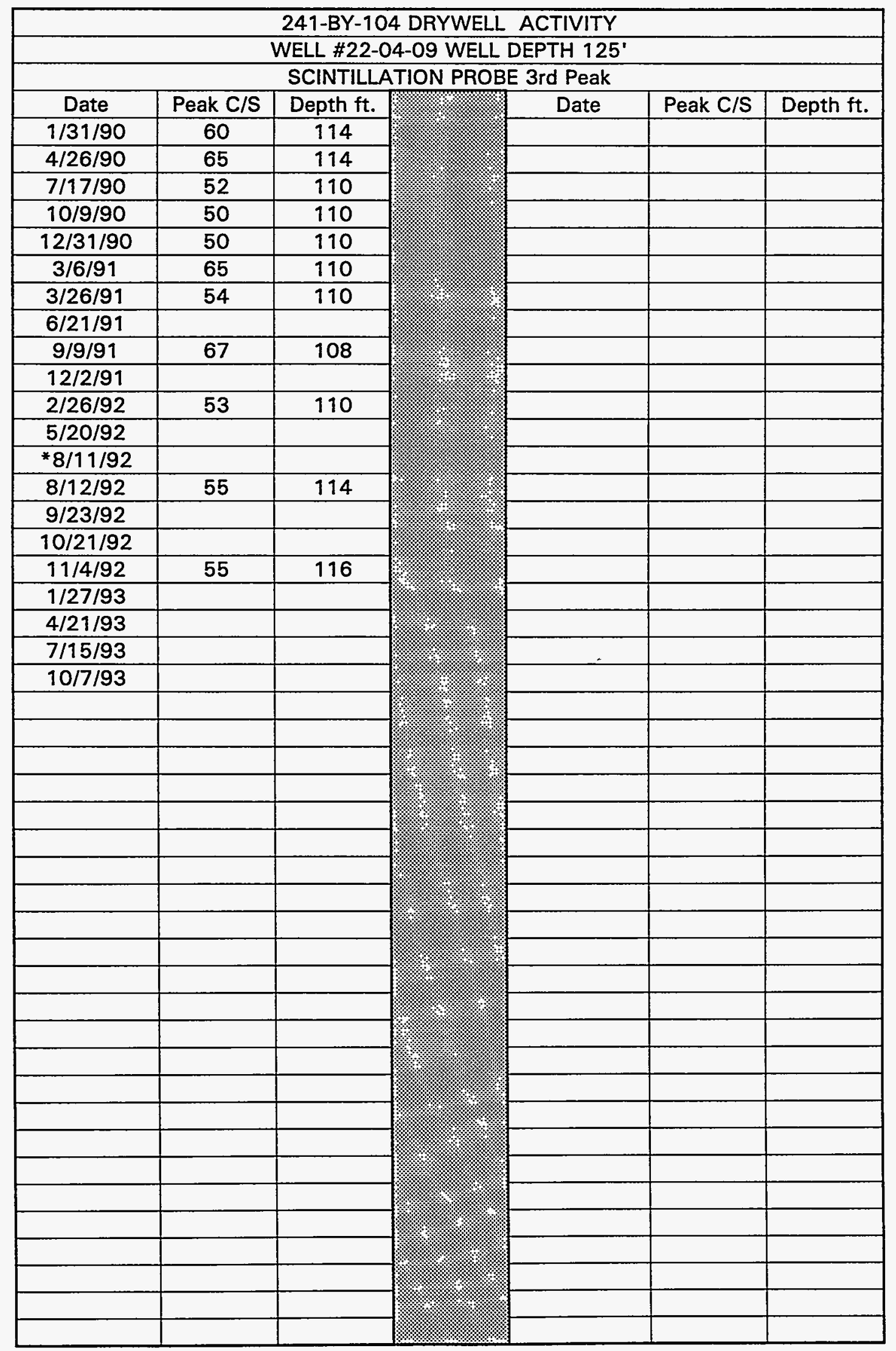

Data obtained from WHC Surveillance Data Acquisition (SDA), November 30, 1993. 


\begin{tabular}{|c|c|c|c|c|c|c|}
\hline \multicolumn{7}{|c|}{ 241-BY-105 DRYWELL ACTIVITY } \\
\hline \multicolumn{7}{|c|}{ WELL \#22-05-09 WELL DEPTH 100' } \\
\hline \multicolumn{7}{|c|}{ SCINTILLATION PROBE } \\
\hline Date & Peak C/S & Depth ft. & \multirow{10}{*}{ \%া. } & Date & Peak C/S & Depth $\mathrm{ft}$. \\
\hline $1 / 3 / 90$ & 42 & 97 & & $11 / 6 / 90$ & 39 & 84 \\
\hline $1 / 11 / 90$ & 43 & 97 & & $11 / 14 / 90$ & 45 & 97 \\
\hline $1 / 16 / 90$ & 47 & 97 & & $11 / 19 / 90$ & 45 & 97 \\
\hline $1 / 25 / 90$ & 51 & 97 & & $11 / 29 / 90$ & 46 & 65 \\
\hline $1 / 31 / 90$ & 51 & 97 & & $12 / 5 / 90$ & 51 & 97 \\
\hline $2 / 6 / 90$ & 46 & 97 & & $12 / 10 / 90$ & 50 & 97 \\
\hline $2 / 13 / 90$ & 43 & 97 & & $12 / 18 / 90$ & 45 & 64 \\
\hline $2 / 22 / 90$ & 39 & 97 & & $12 / 26 / 90$ & 39 & 97 \\
\hline $2 / 26 / 90$ & 45 & 97 & & $1 / 3 / 91$ & 41 & 97 \\
\hline $3 / 5 / 90$ & 50 & 97 & & $1 / 8 / 91$ & 47 & 97 \\
\hline $3 / 13 / 90$ & 39 & 81 & (1) & $1 / 15 / 91$ & 43 & 97 \\
\hline $3 / 22 / 90$ & 49 & 97 & & $1 / 21 / 91$ & 42 & 97 \\
\hline $3 / 29 / 90$ & 44 & 97 & & ${ }^{*} 1 / 29 / 91$ & 51 & 91 \\
\hline $4 / 2 / 90$ & 42 & 97 & & $1 / 30 / 91$ & 40 & 66 \\
\hline $4 / 11 / 90$ & 49 & 97 & & $2 / 5 / 91$ & 53 & 97 \\
\hline $4 / 17 / 90$ & 51 & 97 & & $2 / 15 / 91$ & 46 & 97 \\
\hline $4 / 26 / 90$ & 45 & 68 & & ${ }^{*} 2 / 19 / 91$ & 418 & 97 \\
\hline $5 / 1 / 90$ & 48 & 97 & & $2 / 19 / 91$ & 51 & 97 \\
\hline $5 / 8 / 90$ & 53 & 97 & & $2 / 28 / 91$ & 43 & 75 \\
\hline $5 / 15 / 90$ & 44 & 97 & & $3 / 6 / 91$ & 44 & 97 \\
\hline $5 / 21 / 90$ & 38 & 67 & & $3 / 13 / 91$ & 52 & 97 \\
\hline $5 / 30 / 90$ & 46 & 97 & & $3 / 21 / 91$ & 45 & 97 \\
\hline $6 / 6 / 90$ & 47 & 97 & & $3 / 26 / 91$ & 49 & 97 \\
\hline $6 / 12 / 90$ & 51 & 97 & & $4 / 3 / 91$ & 47 & 97 \\
\hline $6 / 18 / 90$ & 45 & 86 & & $4 / 8 / 91$ & 45 & 97 \\
\hline$* 6 / 25 / 90$ & 52 & 61 & & $4 / 17 / 91$ & 50 & 96 \\
\hline $6 / 27 / 90$ & 41 & 97 & & $4 / 23 / 91$ & 43 & 79 \\
\hline $7 / 2 / 90$ & 50 & 97 & & $4 / 29 / 91$ & 39 & 97 \\
\hline $7 / 10 / 90$ & 40 & 69 & & $5 / 6 / 91$ & 43 & 97 \\
\hline $7 / 19 / 90$ & 47 & 97 & & *5/13/91 & 51 & 97 \\
\hline $7 / 25 / 90$ & 50 & 97 & & $5 / 15 / 91$ & 46 & 97 \\
\hline $7 / 31 / 90$ & 43 & 66 & & $5 / 20 / 91$ & 48 & 97 \\
\hline $8 / 6 / 90$ & 45 & 97 & & $5 / 29 / 91$ & 47 & 69 \\
\hline $8 / 13 / 90$ & 42 & 83 & & $6 / 4 / 91$ & 43 & 97 \\
\hline $8 / 22 / 90$ & 58 & 97 & 1. & $6 / 11 / 91$ & 47 & 97 \\
\hline $8 / 30 / 90$ & 45 & 97 & . & $6 / 21 / 91$ & 41 & 79 \\
\hline $9 / 5 / 90$ & 48 & 97 & 4.4 & $6 / 24 / 91$ & 40 & 93 \\
\hline $9 / 11 / 90$ & 44 & 97 & & $7 / 1 / 91$ & 44 & 97 \\
\hline $9 / 18 / 90$ & 43 & 97 & & $7 / 9 / 91$ & 50 & 97 \\
\hline $9 / 27 / 90$ & 48 & 69 & 4: & $* 7 / 15 / 91$ & 53 & 97 \\
\hline $10 / 2 / 90$ & 42 & 97 & (1) & $7 / 16 / 91$ & 48 & 97 \\
\hline $10 / 9 / 90$ & 41 & 97 & প্ষে & $7 / 23 / 91$ & 42 & 92 \\
\hline $10 / 16 / 90$ & 43 & 82 & : & $7 / 29 / 91$ & 46 & 97 \\
\hline $10 / 23 / 90$ & 46 & 97 & (1) & $8 / 6 / 91$ & 42 & 97 \\
\hline $10 / 30 / 90$ & 45 & 97 & & $8 / 14 / 91$ & 47 & 97 \\
\hline
\end{tabular}

Data obtained from WHC Surveillance Data Acquisition (SDA), November 30, 1993. 


\begin{tabular}{|c|c|c|c|c|c|c|}
\hline Date & Peak C/S & Depth ft. & & Date & Peak C/S & Depth $\mathrm{ft}$. \\
\hline${ }^{*} 8 / 21 / 91$ & 1353 & 97 & & $7 / 8 / 92$ & 37 & 79 \\
\hline $8 / 22 / 91$ & 42 & 97 & & $7 / 13 / 92$ & 46 & 97 \\
\hline${ }^{*} 8 / 27 / 91$ & 61 & 97 & & $7 / 22 / 92$ & 41 & 69 \\
\hline $8 / 28 / 91$ & 48 & 97 & & $7 / 28 / 92$ & 41 & 97 \\
\hline $9 / 4 / 91$ & 44 & 79 & & $8 / 4 / 92$ & 43 & 97 \\
\hline 9/9/91 & 49 & 97 & & ${ }^{*} 8 / 11 / 92$ & 197 & 69 \\
\hline $9 / 17 / 91$ & 43 & 96 & & $8 / 12 / 92$ & 44 & 97 \\
\hline $9 / 24 / 91$ & 43 & 97 & & $8 / 18 / 92$ & 37 & 93 \\
\hline $10 / 3 / 91$ & 44 & 64 & & $8 / 25 / 92$ & 59 & 97 \\
\hline $10 / 7 / 91$ & 49 & 97 & & $9 / 1 / 92$ & 41 & 97 \\
\hline $10 / 14 / 91$ & 50 & 97 & & $9 / 10 / 92$ & 47 & 97 \\
\hline $10 / 22 / 91$ & 49 & 64 & 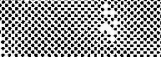 & $9 / 16 / 92$ & 47 & 97 \\
\hline $10 / 24 / 91$ & 43 & 93 & & $9 / 22 / 92$ & 47 & 97 \\
\hline $11 / 4 / 91$ & 42 & 97 & & $9 / 29 / 92$ & 60 & 89 \\
\hline $11 / 14 / 91$ & 55 & 97 & & $10 / 1 / 92$ & 42 & 97 \\
\hline $11 / 19 / 91$ & 47 & 97 & & $10 / 5 / 92$ & 48 & 59 \\
\hline $11 / 26 / 91$ & 45 & 97 & & $10 / 14 / 92$ & 43 & 97 \\
\hline $12 / 2 / 91$ & 47 & 97 & & $10 / 21 / 92$ & 47 & 97 \\
\hline $12 / 11 / 91$ & 42 & 97 & & $10 / 28 / 92$ & 44 & 94 \\
\hline $12 / 17 / 91$ & 43 & 97 & & $11 / 4 / 92$ & 46 & 97 \\
\hline $12 / 25 / 91$ & 38 & 67 & & $11 / 10 / 92$ & 50 & 97 \\
\hline $12 / 30 / 91$ & 42 & 65 & & $11 / 18 / 92$ & 53 & 97 \\
\hline $1 / 8 / 92$ & 46 & 97 & & $11 / 23 / 92$ & 50 & 97 \\
\hline $1 / 15 / 92$ & 46 & 89 & & $12 / 1 / 92$ & 42 & 66 \\
\hline $1 / 21 / 92$ & 46 & 97 & & $12 / 8 / 92$ & 52 & 97 \\
\hline $1 / 27 / 92$ & 45 & 97 & & $12 / 15 / 92$ & 44 & 64 \\
\hline $2 / 20 / 92$ & 51 & 97 & & $12 / 21 / 92$ & 43 & 97 \\
\hline $2 / 26 / 92$ & 41 & 97 & & $12 / 30 / 92$ & 46 & 97 \\
\hline $3 / 3 / 92$ & 40 & 97 & & $1 / 6 / 93$ & 40 & 97 \\
\hline $3 / 9 / 92$ & 74 & 76 & & $1 / 13 / 93$ & 47 & 97 \\
\hline $3 / 10 / 92$ & 57 & 97 & & $1 / 19 / 93$ & 48 & 97 \\
\hline $3 / 18 / 92$ & 46 & 97 & & $1 / 28 / 93$ & 43 & 97 \\
\hline $3 / 25 / 92$ & 43 & 97 & & $2 / 2 / 93$ & 46 & 97 \\
\hline $3 / 31 / 92$ & 48 & 97 & 4 & $2 / 9 / 93$ & 45 & 97 \\
\hline * 4/9/92 & 4100 & 25 & . & $2 / 17 / 93$ & 41 & 97 \\
\hline $4 / 9 / 92$ & 41 & 97 & & $2 / 22 / 93$ & 40 & 97 \\
\hline $4 / 14 / 92$ & 46 & 97 & & $3 / 3 / 93$ & 50 & 97 \\
\hline $4 / 20 / 92$ & 39 & 97 & 8 & $3 / 10 / 93$ & 48 & 97 \\
\hline $4 / 28 / 92$ & 45 & 97 & & $3 / 17 / 93$ & 41 & 78 \\
\hline $5 / 5 / 92$ & 47 & 97 & & $3 / 23 / 93$ & 42 & 63 \\
\hline $5 / 13 / 92$ & 49 & 97 & & $3 / 29 / 93$ & 50 & 97 \\
\hline $5 / 20 / 92$ & 44 & 97 & & $4 / 7 / 93$ & 43 & 97 \\
\hline $5 / 26 / 92$ & 41 & 66 & & $* 4 / 13 / 93$ & 450 & 92 \\
\hline $6 / 3 / 92$ & 40 & 69 & & $4 / 14 / 93$ & 47 & 97 \\
\hline $6 / 9 / 92$ & 56 & 97 & & $4 / 21 / 93$ & 42 & 97 \\
\hline $6 / 18 / 92$ & 43 & 64 & & ${ }^{*} 4 / 27 / 93$ & 485 & 62 \\
\hline $6 / 22 / 92$ & 48 & 67 & & $4 / 27 / 93$ & 47 & 97 \\
\hline $6 / 29 / 92$ & 45 & 97 & & $5 / 4 / 93$ & 46 & 97 \\
\hline
\end{tabular}

Data obtained from WHC Surveillance Data Acquisition (SDA), November 30, 1993. 


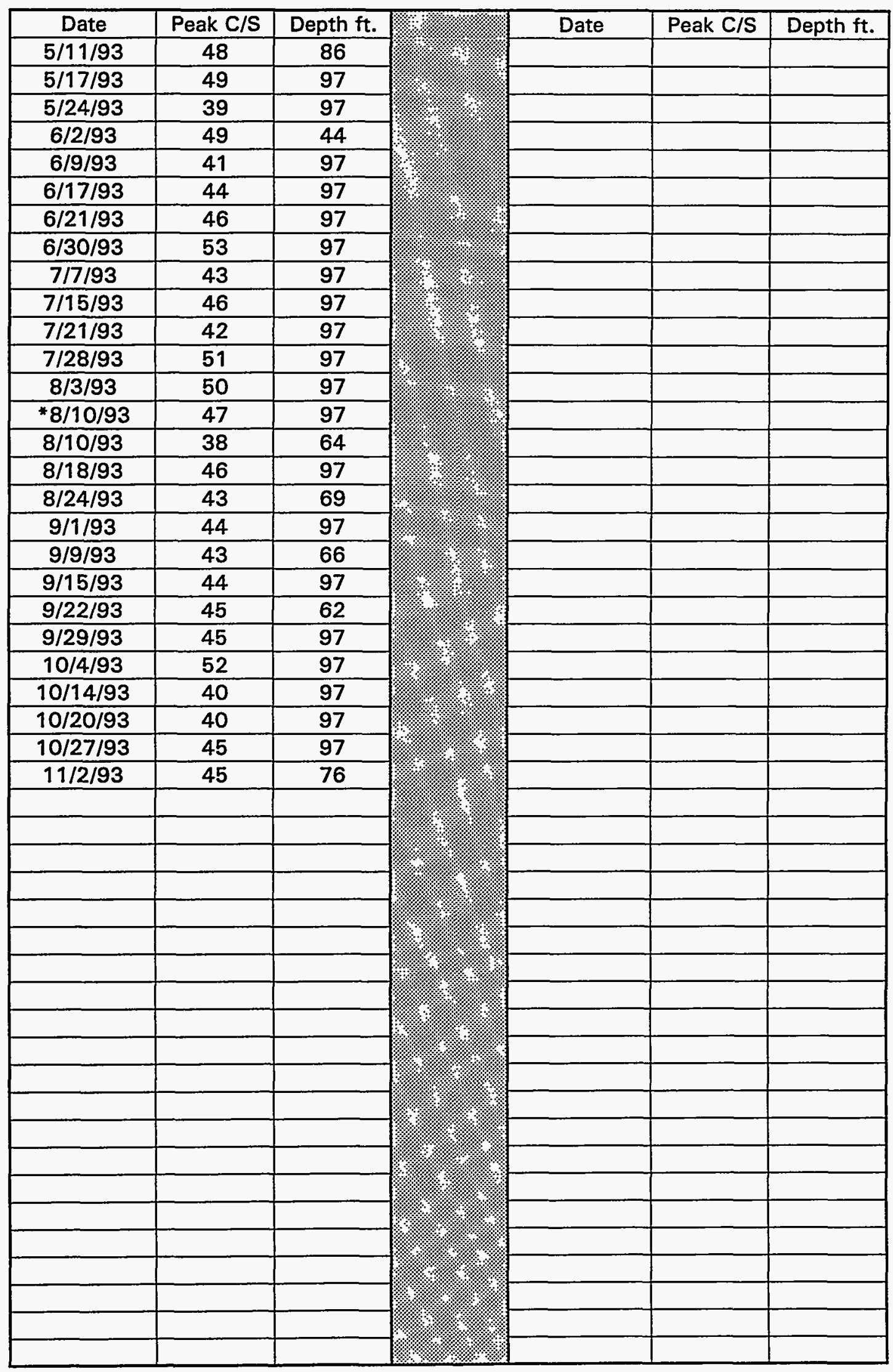

Data obtained from WHC Surveillance Data Acquisition (SDA), November 30, 1993. 


\begin{tabular}{|c|c|c|c|c|c|c|}
\hline \multicolumn{7}{|c|}{ 241-BY-106 DRYWELL ACTIVITY } \\
\hline \multicolumn{7}{|c|}{ WELL \#22-06-05 WELL DEPTH 100' } \\
\hline \multicolumn{7}{|c|}{ SCINTILLATION PROBE 1st Peak } \\
\hline Date & Peak C/S & Depth ft. & & Date & Peak C/S & Depth $\mathrm{ft}$. \\
\hline $1 / 3 / 90$ & 240 & 72 & & $11 / 6 / 90$ & 203 & 65 \\
\hline $1 / 11 / 90$ & 242 & 69 & & $11 / 14 / 90$ & 178 & 65 \\
\hline $1 / 16 / 90$ & 240 & 72 & & $11 / 19 / 90$ & 222 & 65 \\
\hline $1 / 25 / 90$ & 247 & 72 & & $11 / 29 / 90$ & 198 & 65 \\
\hline $1 / 31 / 90$ & 244 & 72 & & $12 / 5 / 90$ & 204 & 65 \\
\hline $2 / 6 / 90$ & 263 & 72 & & $12 / 10 / 90$ & 201 & 65 \\
\hline $2 / 13 / 90$ & 246 & 72 & & $12 / 18 / 90$ & 216 & 65 \\
\hline $2 / 22 / 90$ & 254 & 72 & & $12 / 26 / 90$ & 198 & 65 \\
\hline $2 / 26 / 90$ & 259 & 72 & & $1 / 3 / 91$ & 221 & 66 \\
\hline $3 / 5 / 90$ & 240 & 72 & & $1 / 8 / 91$ & 194 & 66 \\
\hline $3 / 13 / 90$ & 249 & 73 & & $1 / 15 / 91$ & 190 & 67 \\
\hline $3 / 22 / 90$ & 240 & 73 & & $1 / 23 / 91$ & 190 & 65 \\
\hline $3 / 29 / 90$ & 238 & 72 & & $1 / 29 / 91$ & 190 & 65 \\
\hline $4 / 2 / 90$ & 255 & 72 & & $2 / 5 / 91$ & 205 & 65 \\
\hline $4 / 11 / 90$ & 215 & 72 & & $2 / 16 / 91$ & 248 & 70 \\
\hline$* 4 / 17 / 90$ & 255 & 70 & & $2 / 19 / 91$ & 224 & 71 \\
\hline $4 / 18 / 90$ & 244 & 72 & & $2 / 28 / 91$ & 216 & 72 \\
\hline $4 / 26 / 90$ & 241 & 72 & & $* 3 / 5 / 91$ & 218 & 72 \\
\hline $5 / 1 / 90$ & 241 & 72 & & $3 / 6 / 91$ & 205 & 72 \\
\hline $5 / 7 / 90$ & 238 & 69 & & $3 / 13 / 91$ & 180 & 72 \\
\hline $5 / 15 / 90$ & 235 & 69 & & $3 / 21 / 91$ & 201 & 72 \\
\hline $5 / 24 / 90$ & 237 & 72 & & $3 / 26 / 91$ & 211 & 72 \\
\hline $5 / 30 / 90$ & 224 & 69 & & $4 / 3 / 91$ & 190 & 70 \\
\hline $6 / 6 / 90$ & 236 & 67 & & $4 / 8 / 91$ & 212 & 72 \\
\hline $6 / 12 / 90$ & 227 & 65 & & $4 / 17 / 91$ & 219 & 72 \\
\hline $6 / 18 / 90$ & 205 & 65 & & $4 / 23 / 91$ & 190 & 73 \\
\hline $6 / 25 / 90$ & 200 & 65 & & $4 / 29 / 91$ & 209 & 72 \\
\hline $7 / 2 / 90$ & 234 & 67 & & $5 / 6 / 91$ & 225 & 71 \\
\hline $7 / 10 / 90$ & 218 & 65 & & $5 / 13 / 91$ & 221 & 72 \\
\hline $7 / 17 / 90$ & 220 & 65 & & $5 / 20 / 91$ & 188 & 72 \\
\hline $7 / 25 / 90$ & 200 & 60 & & $5 / 29 / 91$ & 203 & 73 \\
\hline $7 / 31 / 90$ & 229 & 65 & & $6 / 4 / 91$ & 200 & 73 \\
\hline $8 / 6 / 90$ & 225 & 65 & & $6 / 11 / 91$ & 207 & 72 \\
\hline $8 / 13 / 90$ & 222 & 69 & & $6 / 19 / 91$ & 190 & 72 \\
\hline $8 / 22 / 90$ & 215 & 65 & & ${ }^{*} 6 / 21 / 91$ & 180 & 72 \\
\hline $8 / 30 / 90$ & 190 & 65 & & $6 / 21 / 91$ & 204 & 72 \\
\hline $9 / 5 / 90$ & 215 & 65 & & $6 / 24 / 91$ & 170 & 73 \\
\hline $9 / 11 / 90$ & 215 & 65 & & $7 / 1 / 91$ & 180 & 73 \\
\hline $9 / 17 / 90$ & 205 & 66 & & * $7 / 8 / 91$ & 203 & 72 \\
\hline $9 / 27 / 90$ & 190 & 65 & & $7 / 9 / 91$ & 183 & 74 \\
\hline $10 / 2 / 90$ & 200 & 65 & & $7 / 15 / 91$ & 204 & 72 \\
\hline $10 / 9 / 90$ & 195 & 65 & & $7 / 23 / 91$ & 195 & 73 \\
\hline $10 / 16 / 90$ & 201 & 65 & & $7 / 29 / 91$ & 202 & 75 \\
\hline $10 / 23 / 90$ & 180 & 65 & & $7 / 30 / 91$ & 204 & 72 \\
\hline $10 / 30 / 90$ & 180 & 65 & & $8 / 6 / 91$ & 206 & 73 \\
\hline
\end{tabular}

Data obtained from WHC Surveillance Data Acquisition (SDA), November 30, 1993. 


\begin{tabular}{|c|c|c|c|c|c|c|c|}
\hline Date & Peak C/S & Depth $\mathrm{ft}$. & \multirow{3}{*}{\multicolumn{2}{|c|}{ (1) }} & Date & Peak C/S & Depth $\mathrm{ft}$. \\
\hline $8 / 14 / 91$ & 206 & 75 & & & $7 / 8 / 92$ & 170 & 76 \\
\hline${ }^{*} 8 / 22 / 91$ & 248 & 73 & & & $7 / 13 / 92$ & 166 & 76 \\
\hline $8 / 22 / 91$ & 198 & 73 & \multirow{4}{*}{\multicolumn{2}{|c|}{ 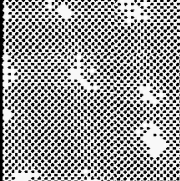 }} & $7 / 22 / 92$ & 180 & 75 \\
\hline $8 / 29 / 91$ & 198 & 72 & & & $7 / 28 / 92$ & 166 & 74 \\
\hline $9 / 4 / 91$ & 175 & 72 & & & $8 / 4 / 92$ & 163 & 75 \\
\hline $9 / 9 / 91$ & 202 & 72 & & & ${ }^{*} 8 / 11 / 92$ & 203 & 76 \\
\hline $9 / 17 / 91$ & 190 & 72 & \multirow{3}{*}{\multicolumn{2}{|c|}{ la }} & $8 / 12 / 92$ & 182 & 72 \\
\hline $9 / 24 / 91$ & 180 & 72 & & & $8 / 18 / 92$ & 160 & 71 \\
\hline $10 / 3 / 91$ & 185 & 73 & & & $8 / 25 / 92$ & 170 & 65 \\
\hline $10 / 7 / 91$ & 194 & 72 & \multirow{5}{*}{\multicolumn{2}{|c|}{ : }} & $9 / 1 / 92$ & 182 & 66 \\
\hline$* 10 / 14 / 91$ & & & & & $9 / 10 / 92$ & 160 & 65 \\
\hline $10 / 14 / 91$ & 175 & 75 & & & $9 / 16 / 92$ & 170 & 67 \\
\hline $10 / 22 / 91$ & 195 & 73 & & & $9 / 22 / 92$ & 172 & 67 \\
\hline $10 / 29 / 91$ & 190 & 73 & & & ${ }^{*} 9 / 29 / 92$ & 3973 & 31 \\
\hline $11 / 4 / 91$ & 212 & 72 & \multirow{9}{*}{\multicolumn{2}{|c|}{ : }} & $10 / 1 / 92$ & 160 & 66 \\
\hline $11 / 13 / 91$ & 185 & 74 & & & $10 / 5 / 92$ & 165 & 67 \\
\hline $11 / 19 / 91$ & 190 & 73 & & & $10 / 14 / 92$ & 184 & 69 \\
\hline $11 / 26 / 91$ & 192 & 72 & & & $10 / 21 / 92$ & 184 & 70 \\
\hline $12 / 2 / 91$ & 200 & 73 & & & $10 / 28 / 92$ & 179 & 65 \\
\hline $12 / 9 / 91$ & 172 & 73 & & & $11 / 4 / 92$ & 165 & 65 \\
\hline $12 / 17 / 91$ & 190 & 75 & & & $11 / 10 / 92$ & 168 & 68 \\
\hline $12 / 25 / 91$ & 175 & 76 & & & $11 / 18 / 92$ & 178 & 67 \\
\hline $12 / 30 / 91$ & 170 & 76 & & & $11 / 23 / 92$ & 177 & 67 \\
\hline $1 / 8 / 92$ & 193 & 75 & \multirow{2}{*}{\multicolumn{2}{|c|}{4}} & $12 / 1 / 92$ & 174 & 73 \\
\hline $1 / 15 / 92$ & & & & & $12 / 8 / 92$ & 184 & 72 \\
\hline $1 / 21 / 92$ & 180 & 75 & \multirow{4}{*}{\multicolumn{2}{|c|}{ ?la }} & $12 / 15 / 92$ & 169 & 69 \\
\hline $1 / 27 / 92$ & 189 & 75 & & & $12 / 21 / 92$ & 168 & 72 \\
\hline $2 / 20 / 92$ & 180 & 75 & & & $12 / 30 / 92$ & 172 & 72 \\
\hline $2 / 26 / 92$ & 193 & 75 & & & $1 / 6 / 93$ & 163 & 72 \\
\hline * $3 / 3 / 92$ & & & \multirow{4}{*}{\multicolumn{2}{|c|}{ 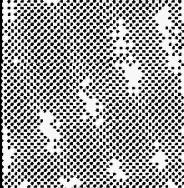 }} & $1 / 13 / 93$ & 162 & 72 \\
\hline $3 / 3 / 92$ & 182 & 72 & & & $1 / 19 / 93$ & 169 & 73 \\
\hline $3 / 9 / 92$ & 170 & 72 & & & $1 / 27 / 93$ & 166 & 73 \\
\hline $3 / 18 / 92$ & 192 & 72 & & & $2 / 2 / 93$ & 152 & 73 \\
\hline $3 / 25 / 92$ & 178 & 72 & & & $2 / 9 / 93$ & 179 & 75 \\
\hline $3 / 31 / 92$ & 170 & 72 & \multirow{2}{*}{\multicolumn{2}{|c|}{ 14: }} & $2 / 17 / 93$ & 162 & 72 \\
\hline $4 / 9 / 92$ & 165 & 71 & & & $2 / 22 / 93$ & 160 & 72 \\
\hline $4 / 14 / 92$ & 176 & 73 & \multirow{3}{*}{\multicolumn{2}{|c|}{$\frac{1}{6}$}} & $3 / 3 / 93$ & 160 & 73 \\
\hline $4 / 23 / 92$ & 168 & 72 & & & $3 / 10 / 93$ & 155 & 70 \\
\hline $4 / 28 / 92$ & 165 & 74 & & & $3 / 17 / 93$ & 172 & 69 \\
\hline $5 / 5 / 92$ & 198 & 72 & \multirow{3}{*}{\multicolumn{2}{|c|}{ 19:19: }} & ${ }^{*} 3 / 23 / 93$ & 150 & 70 \\
\hline $5 / 13 / 92$ & 170 & 75 & & & $3 / 24 / 93$ & 165 & 69 \\
\hline $5 / 20 / 92$ & & & & & $3 / 30 / 93$ & 159 & 69 \\
\hline $5 / 26 / 92$ & 160 & 75 & \multirow{6}{*}{ (3) } & & $4 / 7 / 93$ & 152 & 70 \\
\hline $6 / 3 / 92$ & 150 & 76 & & (1) & * 4/13/93 & & \\
\hline $6 / 9 / 92$ & 195 & 75 & & 1/. & $4 / 14 / 93$ & 177 & 69 \\
\hline $6 / 18 / 92$ & 185 & 75 & & 4 & $4 / 21 / 93$ & 154 & 69 \\
\hline $6 / 22 / 92$ & 160 & 76 & & & $4 / 27 / 93$ & 158 & 69 \\
\hline $6 / 29 / 92$ & 150 & 76 & & 18 & $5 / 4 / 93$ & 168 & 69 \\
\hline
\end{tabular}

Data obtained from WHC Surveillance Data Acquisition (SDA), November 30, 1993. 


\begin{tabular}{|c|c|c|c|c|c|c|}
\hline Date & Peak C/S & Depth ft. & 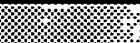 & Date & Peak C/S & Depth ft. \\
\hline $5 / 11 / 93$ & 163 & 72 & & & & \\
\hline $5 / 17 / 93$ & 143 & 72 & & & & \\
\hline $5 / 24 / 93$ & 156 & 65 & & & & \\
\hline $6 / 2 / 93$ & 162 & 72 & & & & \\
\hline $6 / 9 / 93$ & 156 & 69 & & & & \\
\hline $6 / 17 / 93$ & 158 & 70 & & & & \\
\hline $6 / 21 / 93$ & 140 & 70 & & & & \\
\hline $6 / 30 / 93$ & 184 & 69 & & & & \\
\hline $7 / 7 / 93$ & 161 & 65 & & & & \\
\hline${ }^{*} 7 / 14 / 93$ & 162 & 70 & & & & \\
\hline $7 / 15 / 93$ & 147 & 69 & & & & \\
\hline $7 / 21 / 93$ & 140 & 70 & & & & \\
\hline $7 / 28 / 93$ & 157 & 70 & & & & \\
\hline $8 / 3 / 93$ & 171 & 72 & & & & \\
\hline $8 / 10 / 93$ & 157 & 70 & & & & \\
\hline $8 / 18 / 93$ & 154 & 72 & & & & \\
\hline $8 / 24 / 93$ & 151 & 70 & & & & \\
\hline $9 / 1 / 93$ & 156 & 69 & & & & \\
\hline $9 / 9 / 93$ & 169 & 69 & & & & \\
\hline $9 / 15 / 93$ & 142 & 69 & & & & \\
\hline $9 / 22 / 93$ & 143 & 69 & & & & \\
\hline $9 / 29 / 93$ & 160 & 72 & & & & \\
\hline $10 / 4 / 93$ & 148 & 70 & & & & \\
\hline $10 / 14 / 93$ & 163 & 70 & & & & \\
\hline $10 / 20 / 93$ & 158 & 70 & & & & \\
\hline $10 / 27 / 93$ & 135 & 69 & & & & \\
\hline $11 / 1 / 93$ & 156 & 69 & & & & \\
\hline & & & & & & \\
\hline & & & & & & \\
\hline & & & & & & \\
\hline & & & & & & \\
\hline & & & & & & \\
\hline & & & & & & \\
\hline & & & & & & \\
\hline & & & & & & \\
\hline & & & & & & \\
\hline & & & & & & \\
\hline & & & & & & \\
\hline & & & & & & \\
\hline & & & & & & \\
\hline & & & & & & \\
\hline & & & & & & \\
\hline & & & & & & \\
\hline & & & & & & \\
\hline & & & & & & \\
\hline & & & & & & \\
\hline & & & $x$ & & & \\
\hline
\end{tabular}

Data obtained from WHC Surveillance Data Acquisition (SDA), November 30, 1993. 


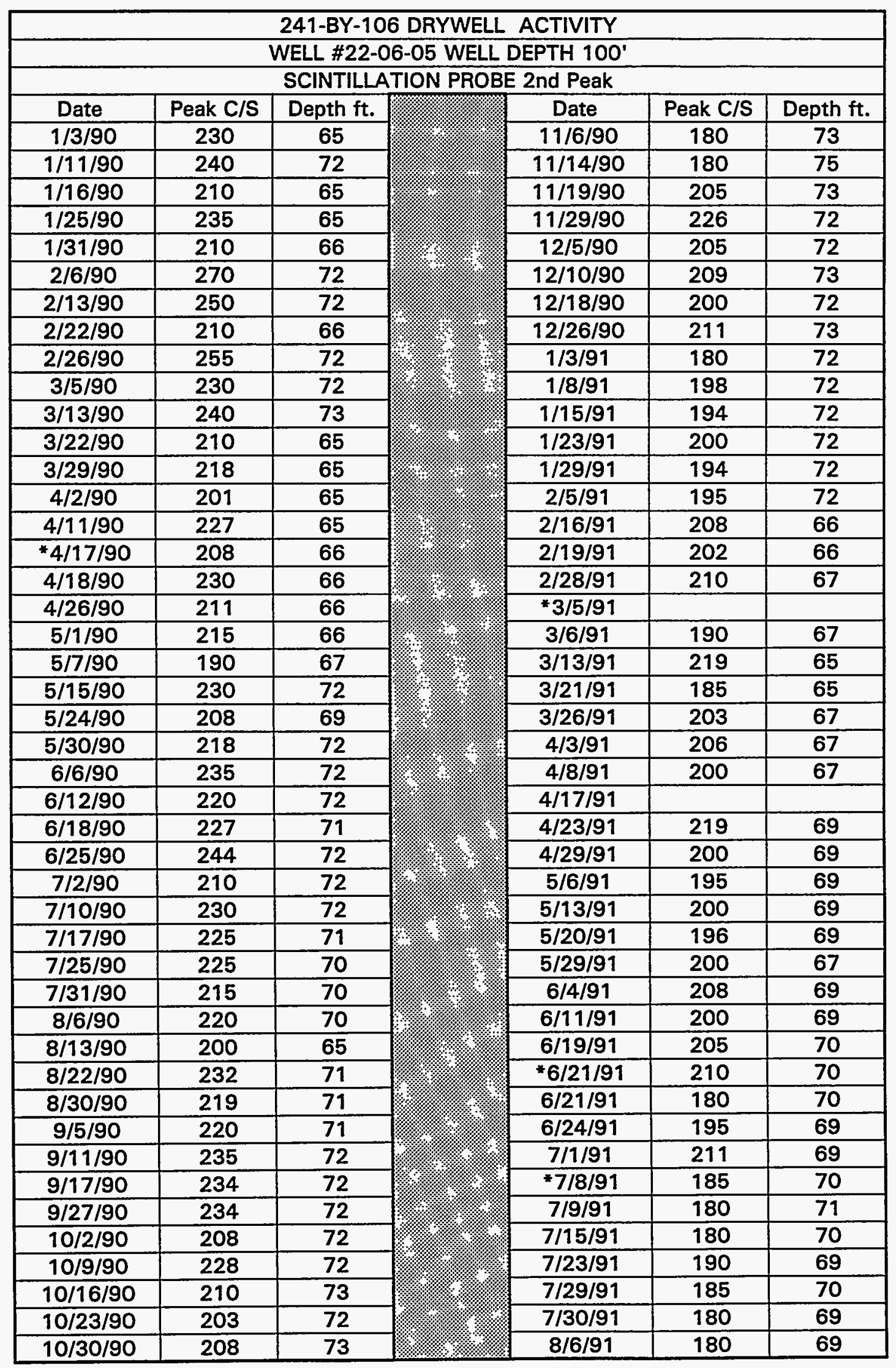

Data obtained from WHC Surveillance Data Acquisition (SDA), November 30, 1993. 
WHC-SD-WM-ER-312, Rev. 0

\begin{tabular}{|c|c|c|c|c|c|c|}
\hline Date & Peak C/S & Depth ft. & & Date & Peak C/S & Depth $\mathrm{ft}$. \\
\hline $8 / 14 / 91$ & 190 & 70 & & $7 / 8 / 92$ & 160 & 65 \\
\hline${ }^{*} 8 / 22 / 91$ & & & . & $7 / 13 / 92$ & 170 & 65 \\
\hline $8 / 22 / 91$ & 190 & 69 & & $7 / 22 / 92$ & 193 & 67 \\
\hline $8 / 29 / 91$ & & & & $7 / 28 / 92$ & & \\
\hline $9 / 4 / 91$ & & & & $8 / 4 / 92$ & 160 & 65 \\
\hline 9/9/91 & 190 & 69 & & $* 8 / 11 / 92$ & & 65 \\
\hline $9 / 17 / 91$ & 190 & 70 & & $8 / 12 / 92$ & 160 & 65 \\
\hline $9 / 24 / 91$ & 210 & 69 & & $8 / 18 / 92$ & 176 & 67 \\
\hline $10 / 3 / 91$ & 180 & 69 & & $8 / 25 / 92$ & 189 & 69 \\
\hline $10 / 7 / 91$ & 183 & 69 & & $9 / 1 / 92$ & 158 & 69 \\
\hline$* 10 / 14 / 91$ & & & & $9 / 10 / 92$ & 168 & 70 \\
\hline $10 / 14 / 91$ & 194 & 69 & & $9 / 16 / 92$ & 179 & 70 \\
\hline $10 / 22 / 91$ & 180 & 20 & & $9 / 22 / 92$ & 161 & 70 \\
\hline $10 / 29 / 91$ & 195 & 70 & & ${ }^{*} 9 / 29 / 92$ & & \\
\hline $11 / 4 / 91$ & 173 & 69 & & $10 / 1 / 92$ & 187 & 70 \\
\hline $11 / 13 / 91$ & 180 & 70 & & $10 / 5 / 92$ & 163 & 76 \\
\hline $11 / 19 / 91$ & 189 & 71 & & $10 / 14 / 92$ & & \\
\hline $11 / 26 / 91$ & 185 & 70 & & $10 / 21 / 92$ & & \\
\hline $12 / 2 / 91$ & & & & $10 / 28 / 92$ & 170 & 73 \\
\hline $12 / 9 / 91$ & 190 & 69 & & $11 / 4 / 92$ & 170 & 70 \\
\hline $12 / 17 / 91$ & 182 & 69 & & $11 / 10 / 92$ & 165 & 69 \\
\hline $12 / 25 / 91$ & 192 & 70 & & $11 / 18 / 92$ & 170 & 69 \\
\hline $12 / 30 / 91$ & 180 & 72 & & $11 / 23 / 92$ & 170 & 69 \\
\hline $1 / 8 / 92$ & 170 & 72 & & $12 / 1 / 92$ & 152 & 70 \\
\hline $1 / 15 / 92$ & 212 & 72 & & $12 / 8 / 92$ & 182 & 65 \\
\hline $1 / 21 / 92$ & 180 & 70 & & $12 / 15 / 92$ & 160 & 65 \\
\hline $1 / 27 / 92$ & 180 & 70 & & $12 / 21 / 92$ & 150 & 65 \\
\hline $2 / 20 / 92$ & 172 & 65 & & $12 / 30 / 92$ & 165 & 69 \\
\hline $2 / 26 / 92$ & 180 & 69 & & $1 / 6 / 93$ & 150 & 70 \\
\hline *3/3/92 & & & & $1 / 13 / 93$ & 168 & 70 \\
\hline $3 / 3 / 92$ & 172 & 65 & & $1 / 19 / 93$ & 160 & 69 \\
\hline $3 / 9 / 92$ & 185 & 65 & & $1 / 27 / 93$ & 161 & 69 \\
\hline $3 / 18 / 92$ & 180 & 65 & & $2 / 2 / 93$ & 170 & 67 \\
\hline $3 / 25 / 92$ & 186 & 67 & & $2 / 9 / 93$ & 161 & 65 \\
\hline $3 / 31 / 92$ & 182 & 67 & & $2 / 17 / 93$ & 163 & 67 \\
\hline $4 / 9 / 92$ & 191 & 65 & & $2 / 22 / 93$ & 160 & 65 \\
\hline $4 / 14 / 92$ & & & & $3 / 3 / 93$ & 170 & 69 \\
\hline $4 / 23 / 92$ & 178 & 65 & & $3 / 10 / 93$ & 160 & 65 \\
\hline $4 / 28 / 92$ & 170 & 65 & & $3 / 17 / 93$ & 160 & 65 \\
\hline $5 / 5 / 92$ & 172 & 65 & & $* 3 / 23 / 93$ & 160 & 67 \\
\hline $5 / 13 / 92$ & 173 & 65 & & $3 / 24 / 93$ & 170 & 65 \\
\hline $5 / 20 / 92$ & 180 & 66 & & $3 / 30 / 93$ & 150 & 65 \\
\hline $5 / 26 / 92$ & 178 & 67 & & $4 / 7 / 93$ & 150 & 66 \\
\hline $6 / 3 / 92$ & 162 & 65 & & $* 4 / 13 / 93$ & 155 & 67 \\
\hline $6 / 9 / 92$ & 170 & 66 & & $4 / 14 / 93$ & 143 & 65 \\
\hline $6 / 18 / 92$ & 170 & 65 & \% & $4 / 21 / 93$ & 150 & 64 \\
\hline $6 / 22 / 92$ & 160 & 66 & & $4 / 27 / 93$ & 140 & 66 \\
\hline $6 / 29 / 92$ & 177 & 65 & & $5 / 4 / 93$ & 140 & 66 \\
\hline
\end{tabular}

Data obtained from WHC Surveillance Data Acquisition (SDA), November 30, 1993. 
WHC-SD-WM-ER-312, Rev. 0

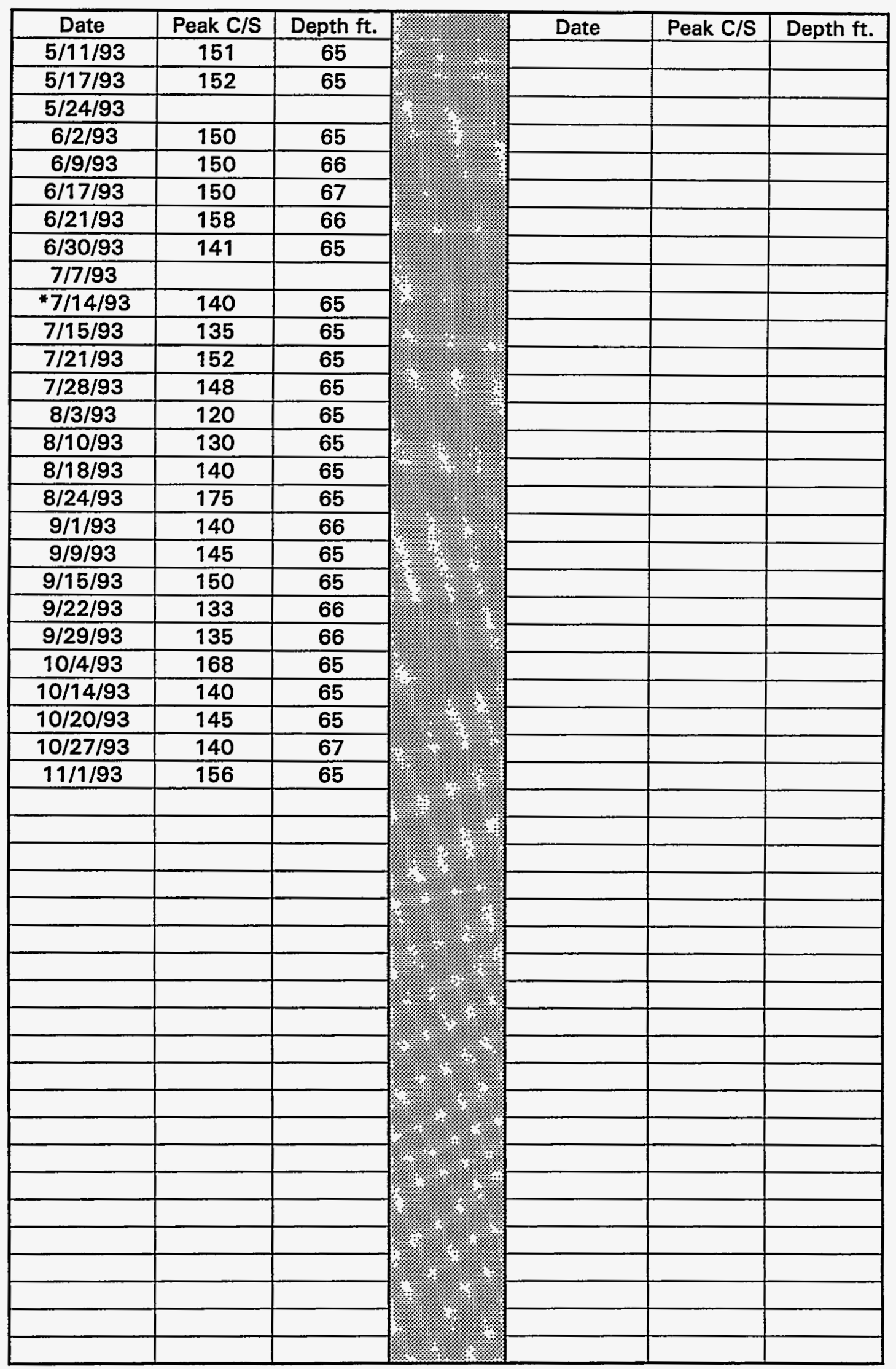

Data obtained from WHC Surveillance Data Acquisition (SDA), November 30, 1993. 
WHC-SD-WM-ER-312, Rev. 0

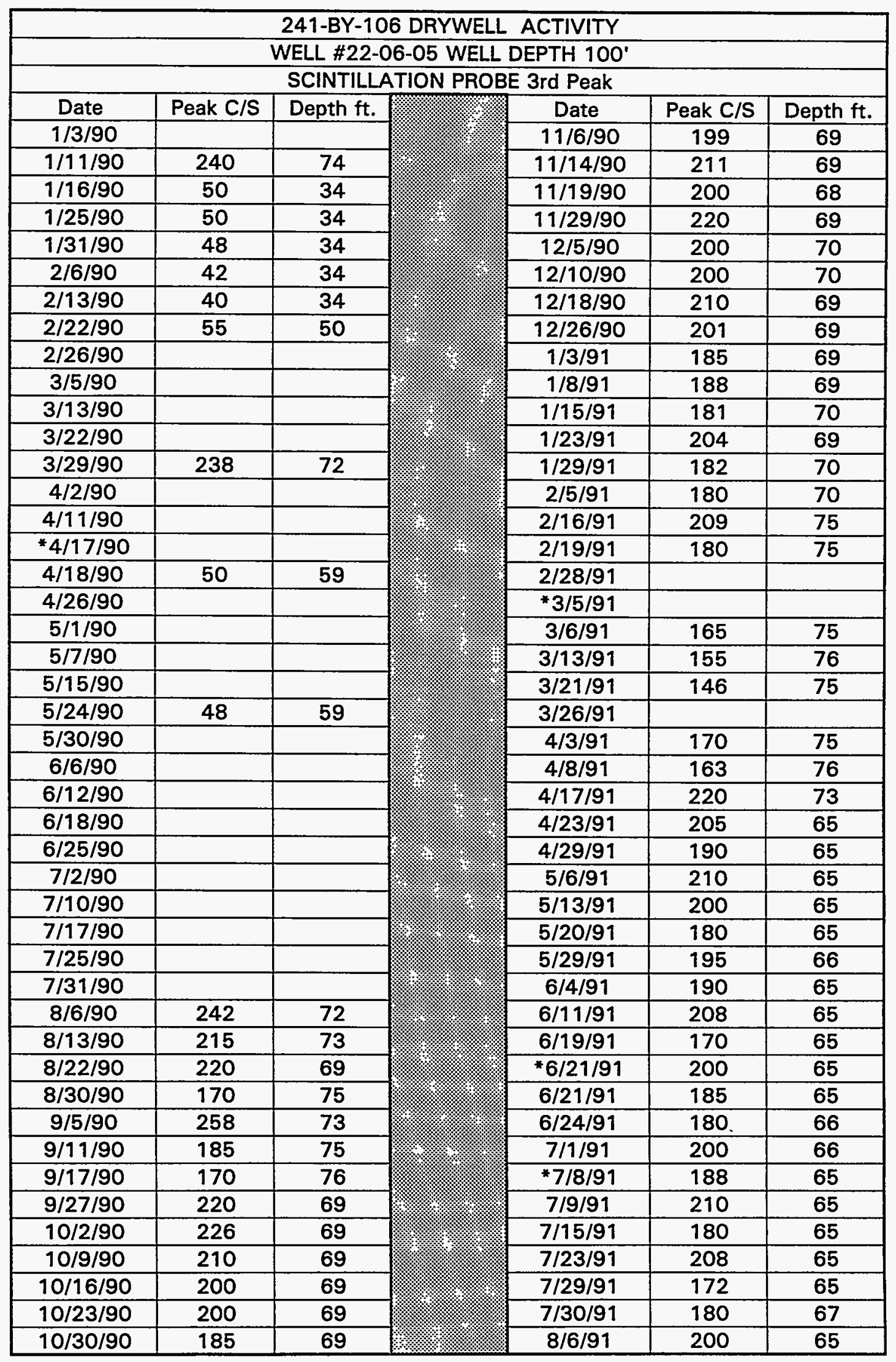

Data obtained from WHC Surveillance Data Acquisition (SDA), November 30, 1993. 
WHC-SD-WM-ER-312, Rev. 0

\begin{tabular}{|c|c|c|c|c|c|c|}
\hline Date & Peak C/S & Depth ft. & & Date & Peak C/S & Depth ft. \\
\hline $8 / 14 / 91$ & & & & $7 / 8 / 92$ & 187 & 70 \\
\hline${ }^{*} 8 / 22 / 91$ & & & \multirow{3}{*}{ 64: } & $7 / 13 / 92$ & 170 & 69 \\
\hline $8 / 22 / 91$ & 180 & 65 & & $7 / 22 / 92$ & 170 & 70 \\
\hline $8 / 29 / 91$ & 170 & 66 & & $7 / 28 / 92$ & 165 & 70 \\
\hline 9/4/91 & 192 & 67 & \multirow{3}{*}{ (1: } & $8 / 4 / 92$ & 173 & 69 \\
\hline 9/9/91 & & & & ${ }^{*} 8 / 11 / 92$ & 171 & 76 \\
\hline $9 / 17 / 91$ & 201 & 65 & & $8 / 12 / 92$ & 1 & \\
\hline $9 / 24 / 91$ & 195 & 66 & \multirow{9}{*}{ 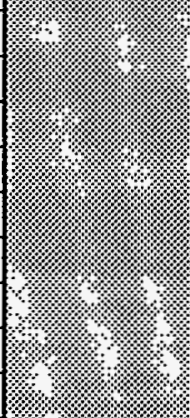 } & $8 / 18 / 92$ & 150 & 78 \\
\hline $10 / 3 / 91$ & 170 & 65 & & $8 / 25 / 92$ & 160 & 76 \\
\hline $10 / 7 / 91$ & 180 & 65 & & $9 / 1 / 92$ & 155 & 76 \\
\hline$* 10 / 14 / 91$ & 205 & 66 & & $9 / 10 / 92$ & 150 & 76 \\
\hline $10 / 14 / 91$ & & & & $9 / 16 / 92$ & 170 & 76 \\
\hline $10 / 22 / 91$ & 180 & 65 & & $9 / 22 / 92$ & 153 & 73 \\
\hline $10 / 29 / 91$ & 190 & 67 & & ${ }^{*} 9 / 29 / 92$ & & \\
\hline $11 / 4 / 91$ & 190 & 67 & & $10 / 1 / 92$ & 160 & 76 \\
\hline $11 / 13 / 91$ & 198 & 67 & & $10 / 5 / 92$ & 183 & 73 \\
\hline $11 / 19 / 91$ & 182 & 68 & \multirow[b]{3}{*}{ (1: } & $10 / 14 / 92$ & & \\
\hline $11 / 26 / 91$ & 183 & 66 & & $10 / 21 / 92$ & 165 & 76 \\
\hline $12 / 2 / 91$ & 175 & 65 & & $10 / 28 / 92$ & 160 & 76 \\
\hline $12 / 9 / 91$ & 182 & 66 & \multirow{4}{*}{ 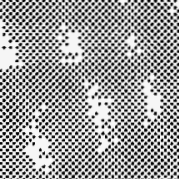 } & $11 / 4 / 92$ & 173 & 76 \\
\hline $12 / 17 / 91$ & 205 & 65 & & $11 / 10 / 92$ & 160 & 76 \\
\hline $12 / 25 / 91$ & 180 & 65 & & $11 / 18 / 92$ & 170 & 75 \\
\hline $12 / 30 / 91$ & 185 & 67 & & $11 / 23 / 92$ & 170 & 75 \\
\hline $1 / 8 / 92$ & 180 & 67 & \multirow{5}{*}{ 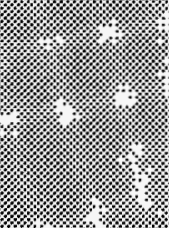 } & $12 / 1 / 92$ & 160 & 78 \\
\hline $1 / 15 / 92$ & 180 & 69 & & $12 / 8 / 92$ & 160 & 75 \\
\hline $1 / 21 / 92$ & 201 & 66 & & $12 / 15 / 92$ & 158 & 75 \\
\hline $1 / 27 / 92$ & 170 & 65 & & $12 / 21 / 92$ & 160 & 76 \\
\hline $2 / 20 / 92$ & 197 & 65 & & $12 / 30 / 92$ & 165 & 77 \\
\hline $2 / 26 / 92$ & 172 & 65 & \multirow{6}{*}{$\frac{16}{6}$} & $1 / 6 / 93$ & 160 & 76 \\
\hline$* 3 / 3 / 92$ & 261 & 66 & & $1 / 13 / 93$ & 160 & 75 \\
\hline $3 / 3 / 92$ & 170 & 69 & & $1 / 19 / 93$ & 155 & 75 \\
\hline $3 / 9 / 92$ & 172 & 69 & & $1 / 27 / 93$ & 160 & 77 \\
\hline $3 / 18 / 92$ & 170 & 70 & & $2 / 2 / 93$ & 133 & 78 \\
\hline $3 / 25 / 92$ & 162 & 75 & & $2 / 9 / 93$ & & \\
\hline $3 / 31 / 92$ & 193 & 69 & \multirow{3}{*}{ 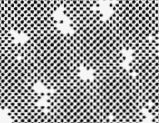 } & $2 / 17 / 93$ & 150 & 75 \\
\hline $4 / 9 / 92$ & 165 & 69 & & $2 / 22 / 93$ & 161 & 75 \\
\hline $4 / 14 / 92$ & 160 & 68 & & $3 / 3 / 93$ & 160 & 75 \\
\hline $4 / 23 / 92$ & 170 & 70 & \multirow{2}{*}{ (4) } & $3 / 10 / 93$ & & \\
\hline $4 / 28 / 92$ & 183 & 69 & & $3 / 17 / 93$ & 140 & 76 \\
\hline $5 / 5 / 92$ & 190 & 72 & \multirow{2}{*}{ 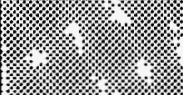 } & $3 / 23 / 93$ & & \\
\hline $5 / 13 / 92$ & 160 & 72 & & $3 / 24 / 93$ & 150 & 76 \\
\hline $5 / 20 / 92$ & 186 & 72 & \multirow{2}{*}{ 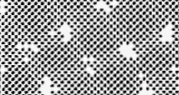 } & $3 / 30 / 93$ & 145 & 77 \\
\hline $5 / 26 / 92$ & 162 & 72 & & $4 / 7 / 93$ & 161 & 76 \\
\hline $6 / 3 / 92$ & 183 & 70 & \multirow{3}{*}{ (4) } & $* 4 / 13 / 93$ & & \\
\hline $6 / 9 / 92$ & 168 & 70 & & $4 / 14 / 93$ & 160 & 76 \\
\hline $6 / 18 / 92$ & 170 & 72 & & $4 / 21 / 93$ & 140 & 74 \\
\hline $6 / 22 / 92$ & 180 & 73 & \multirow{2}{*}{ 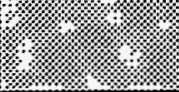 } & $4 / 27 / 93$ & 150 & 76 \\
\hline $6 / 29 / 92$ & 170 & 70 & & $5 / 4 / 93$ & 150 & 76 \\
\hline
\end{tabular}

Data obtained from WHC Surveillance Data Acquisition (SDA), November 30, 1993. 
WHC-SD-WM-ER-312, Rev. 0

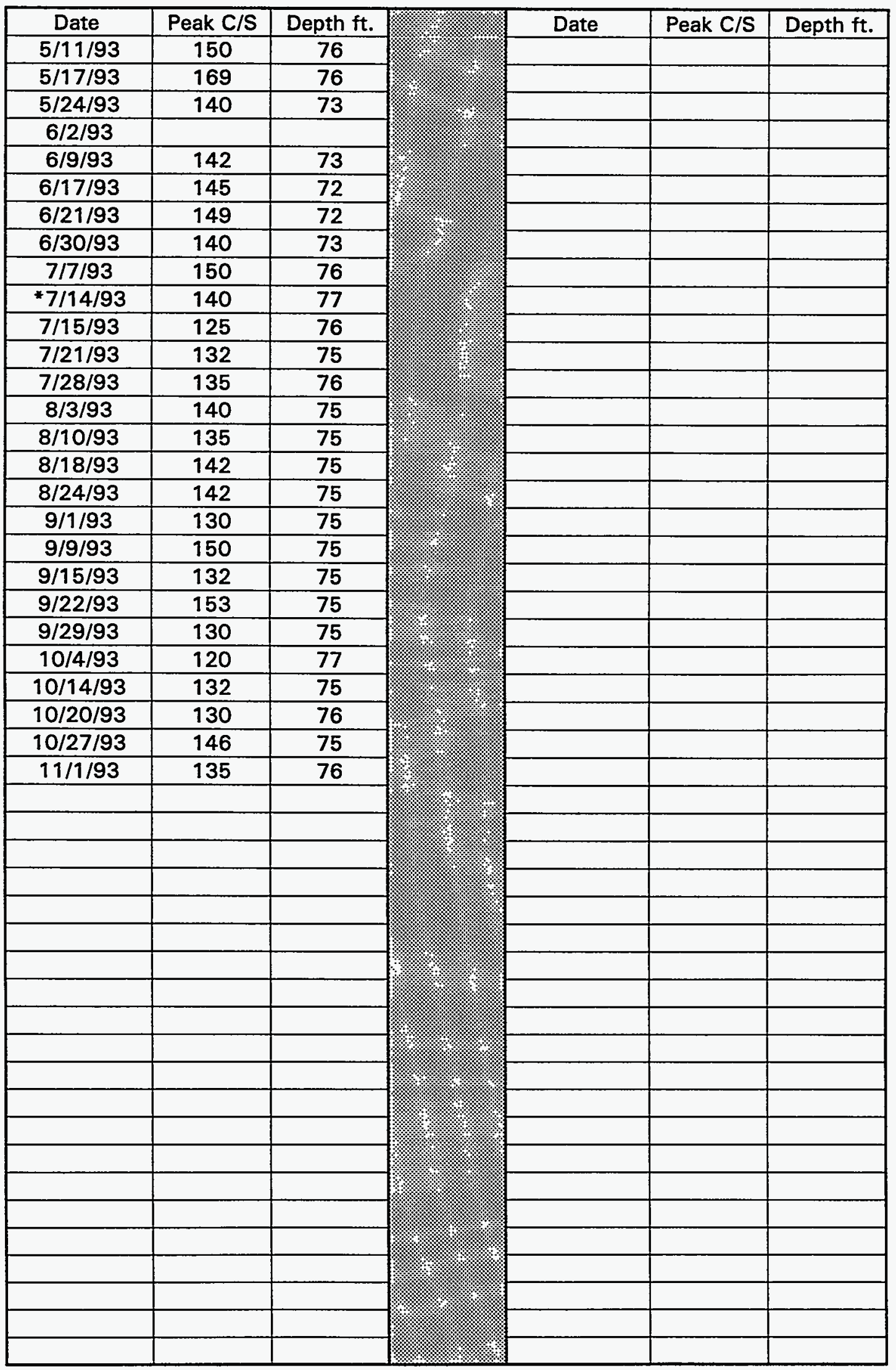

Data obtained from WHC Surveillance Data Acquisition (SDA), November 30, 1993. 


\begin{tabular}{|c|c|c|c|c|c|c|}
\hline \multicolumn{7}{|c|}{ 241-BY-106 DRYWELL ACTIVITY } \\
\hline \multicolumn{7}{|c|}{ WELL \#22-06-11 WELL DEPTH 100' } \\
\hline \multicolumn{7}{|c|}{ SCINTILLATION PROBE } \\
\hline Date & Peak C/S & Depth ft. & \multirow{8}{*}{ 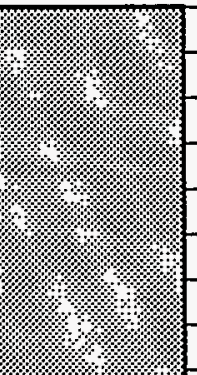 } & Date & Peak C/S & Depth ft. \\
\hline $1 / 11 / 90$ & 43 & 21 & & $7 / 24 / 91$ & 43 & 25 \\
\hline $1 / 25 / 90$ & 45 & 26 & & $8 / 6 / 91$ & 40 & 30 \\
\hline $2 / 6 / 90$ & 40 & 25 & & $8 / 22 / 91$ & 43 & 26 \\
\hline $2 / 22 / 90$ & 41 & 24 & & 9/4/91 & 40 & 28 \\
\hline $3 / 5 / 90$ & 40 & 64 & & $9 / 17 / 91$ & 42 & 25 \\
\hline $3 / 22 / 90$ & 41 & 24 & & $10 / 3 / 91$ & 43 & 25 \\
\hline $4 / 2 / 90$ & 41 & 22 & & $10 / 14 / 91$ & 47 & 24 \\
\hline $4 / 17 / 90$ & 42 & 24 & \multirow{4}{*}{ : } & $10 / 29 / 91$ & 42 & 25 \\
\hline $5 / 1 / 90$ & 42 & 28 & & $11 / 13 / 91$ & 45 & 21 \\
\hline $5 / 15 / 90$ & 41 & 24 & & $11 / 26 / 91$ & 43 & 23 \\
\hline $5 / 31 / 90$ & 47 & 25 & & $12 / 9 / 91$ & 44 & 25 \\
\hline $6 / 12 / 90$ & 42 & 65 & \multirow{9}{*}{ ?4 } & $12 / 25 / 91$ & 42 & 25 \\
\hline $6 / 25 / 90$ & 51 & 22 & & $1 / 8 / 92$ & 37 & 25 \\
\hline $7 / 10 / 90$ & 43 & 25 & & $1 / 21 / 92$ & 44 & 24 \\
\hline $7 / 25 / 90$ & 60 & 45 & & $2 / 20 / 92$ & 43 & 25 \\
\hline $8 / 1 / 90$ & 45 & 24 & & $3 / 3 / 92$ & 39 & 61 \\
\hline $8 / 6 / 90$ & 47 & 26 & & $3 / 18 / 92$ & 41 & 25 \\
\hline $8 / 22 / 90$ & 38 & 26 & & $3 / 31 / 92$ & 41 & 29 \\
\hline $9 / 5 / 90$ & 41 & 25 & & $4 / 14 / 92$ & 44 & 25 \\
\hline $9 / 17 / 90$ & 40 & 23 & & $4 / 28 / 92$ & 44 & 25 \\
\hline $10 / 2 / 90$ & 47 & 24 & \multirow{4}{*}{ 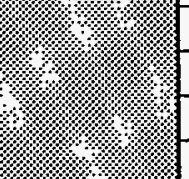 } & $5 / 13 / 92$ & 43 & 25 \\
\hline $10 / 16 / 90$ & 44 & 28 & & $5 / 26 / 92$ & 43 & 26 \\
\hline $10 / 30 / 90$ & 42 & 25 & & $6 / 9 / 92$ & 45 & 27 \\
\hline $11 / 16 / 90$ & 43 & 24 & & $6 / 22 / 92$ & 46 & 25 \\
\hline $11 / 29 / 90$ & 40 & 21 & \multirow{3}{*}{ r. } & $7 / 8 / 92$ & 40 & 26 \\
\hline $12 / 10 / 90$ & 48 & 23 & & $7 / 22 / 92$ & 46 & 25 \\
\hline $12 / 26 / 90$ & 41 & 26 & & $8 / 4 / 92$ & 43 & 25 \\
\hline $1 / 8 / 91$ & 44 & 24 & \multirow{2}{*}{ 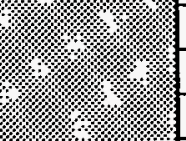 } & $8 / 18 / 92$ & 40 & 26 \\
\hline $1 / 23 / 91$ & 35 & 66 & & $9 / 1 / 92$ & 52 & 25 \\
\hline $2 / 5 / 91$ & 41 & 24 & \multirow[b]{2}{*}{ 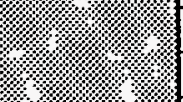 } & $9 / 16 / 92$ & 44 & 42 \\
\hline $2 / 19 / 91$ & 46 & 26 & & $9 / 29 / 92$ & 45 & 26 \\
\hline * $3 / 5 / 91$ & 139 & 43 & \multirow{4}{*}{$\begin{array}{l}4 \\
4\end{array}$} & $10 / 14 / 92$ & 43 & 25 \\
\hline $3 / 6 / 91$ & 44 & 25 & & $10 / 28 / 92$ & 42 & 63 \\
\hline $3 / 21 / 91$ & 38 & 26 & & $11 / 10 / 92$ & 38 & 28 \\
\hline $4 / 3 / 91$ & 41 & 24 & & $11 / 23 / 92$ & 43 & 25 \\
\hline * 4/18/91 & 52 & 24 & \multirow{2}{*}{ (4) } & $12 / 8 / 92$ & 44 & 30 \\
\hline $4 / 18 / 91$ & 42 & 21 & & $12 / 21 / 92$ & 45 & 24 \\
\hline $4 / 29 / 91$ & 41 & 26 & \multirow{2}{*}{ I } & $1 / 6 / 93$ & 49 & 25 \\
\hline $5 / 13 / 91$ & 54 & 24 & & $1 / 19 / 93$ & 40 & 21 \\
\hline $5 / 29 / 91$ & 47 & 24 & \multirow{2}{*}{ (1) } & $2 / 2 / 93$ & 40 & 22 \\
\hline $6 / 11 / 91$ & 40 & 26 & & $2 / 17 / 93$ & 47 & 35 \\
\hline $6 / 24 / 91$ & 44 & 24 & \multirow{2}{*}{ 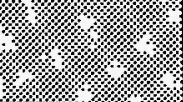 } & $3 / 3 / 93$ & 40 & 25 \\
\hline *7/9/91 & 50 & 25 & & $3 / 17 / 93$ & 43 & 24 \\
\hline $7 / 9 / 91$ & 42 & 25 & & $* 3 / 29 / 93$ & 43 & 23 \\
\hline * $7 / 23 / 91$ & 50 & 34 & 148.17 .18 & $3 / 30 / 93$ & 42 & 23 \\
\hline
\end{tabular}

Data obtained from WHC Surveillance Data Acquisition (SDA), November 30, 1993. 
WHC-SD-WM-ER-312, Rev. 0

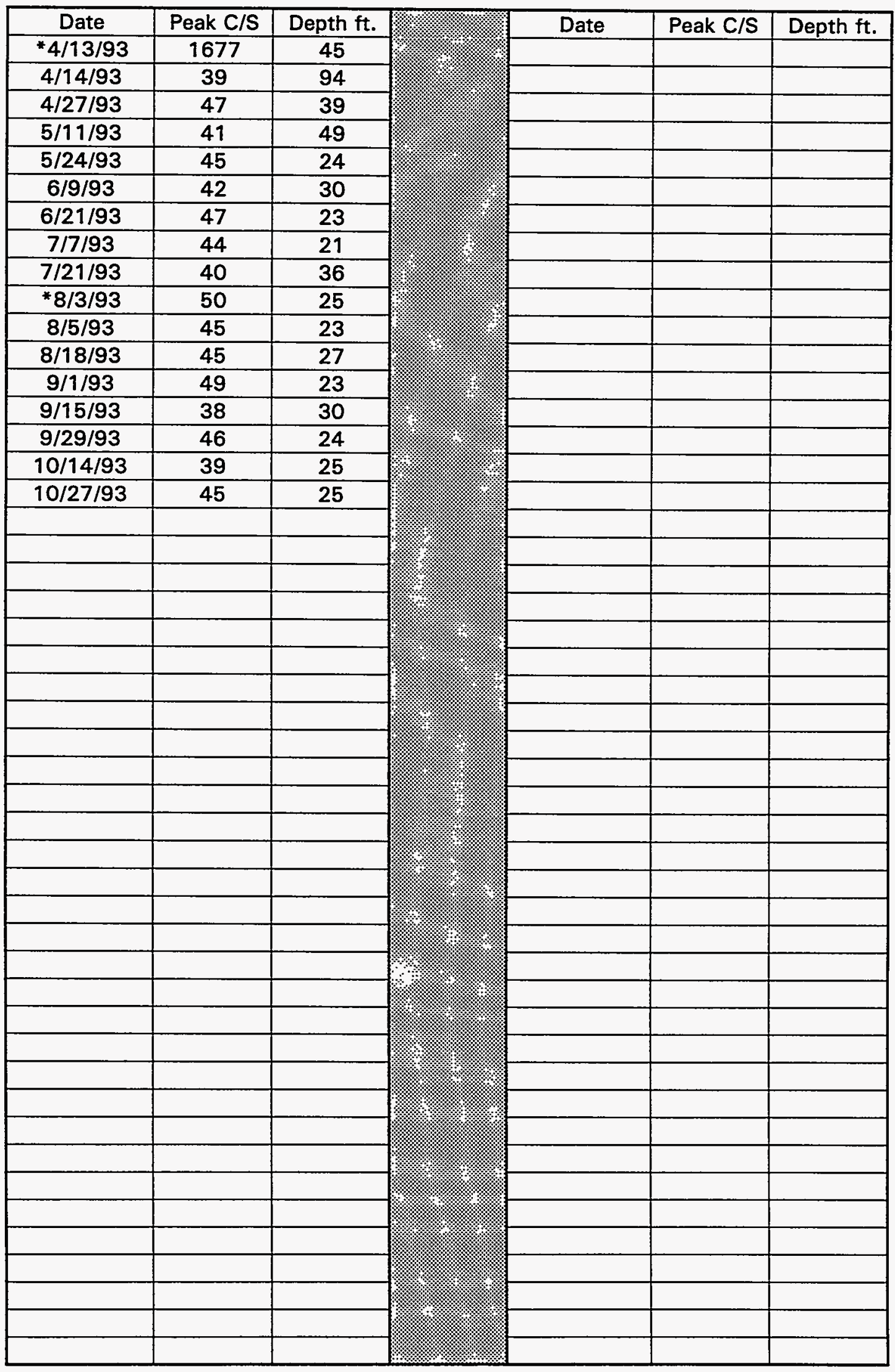

Data obtained from WHC Surveillance Data Acquisition (SDA), November 30, 1993. 


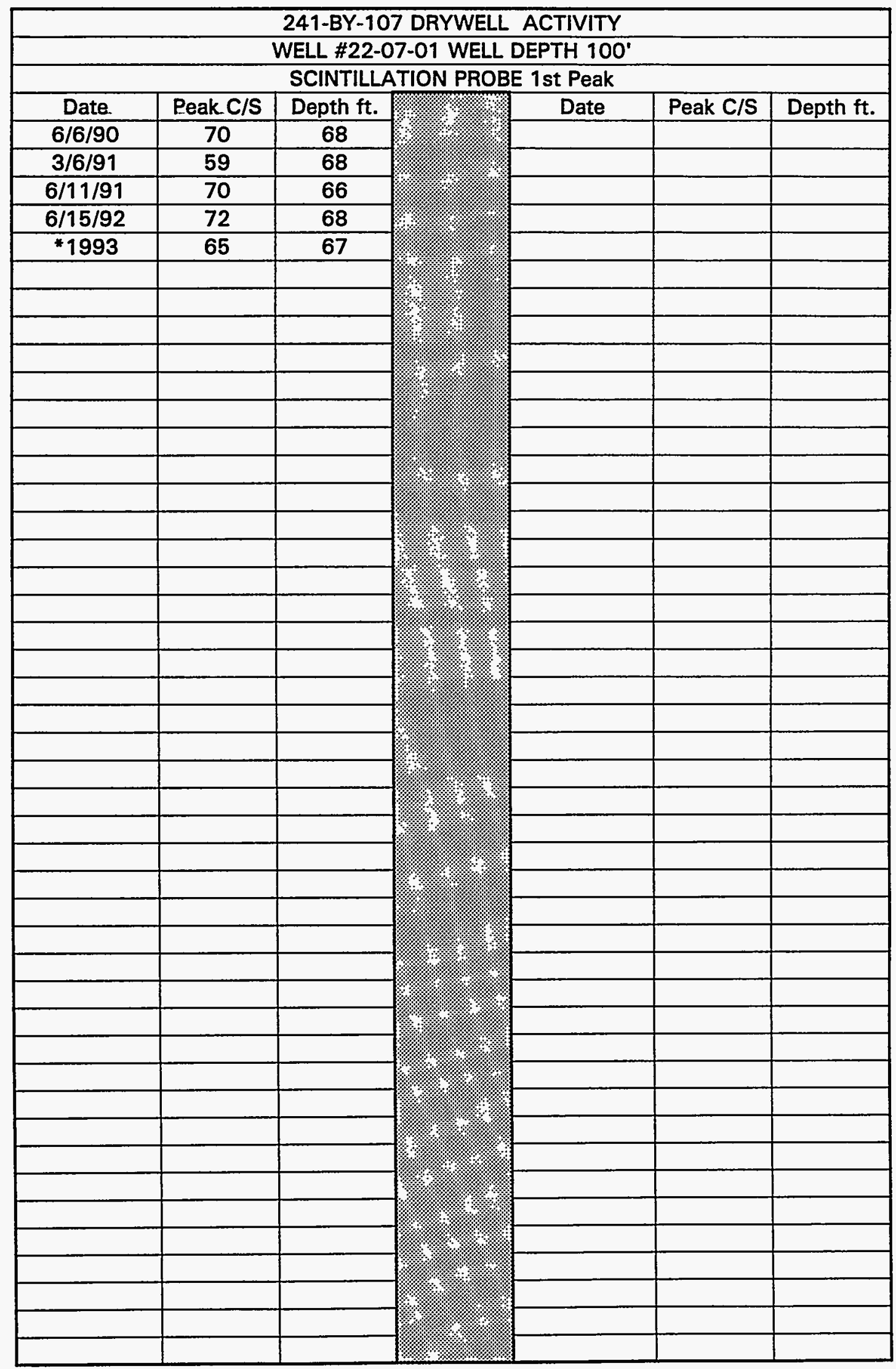

Data obtained from WHC Surveillance Data Acquisition (SDA), November 30, 1993. 
WHC-SD-WM-ER-312, Rev. 0

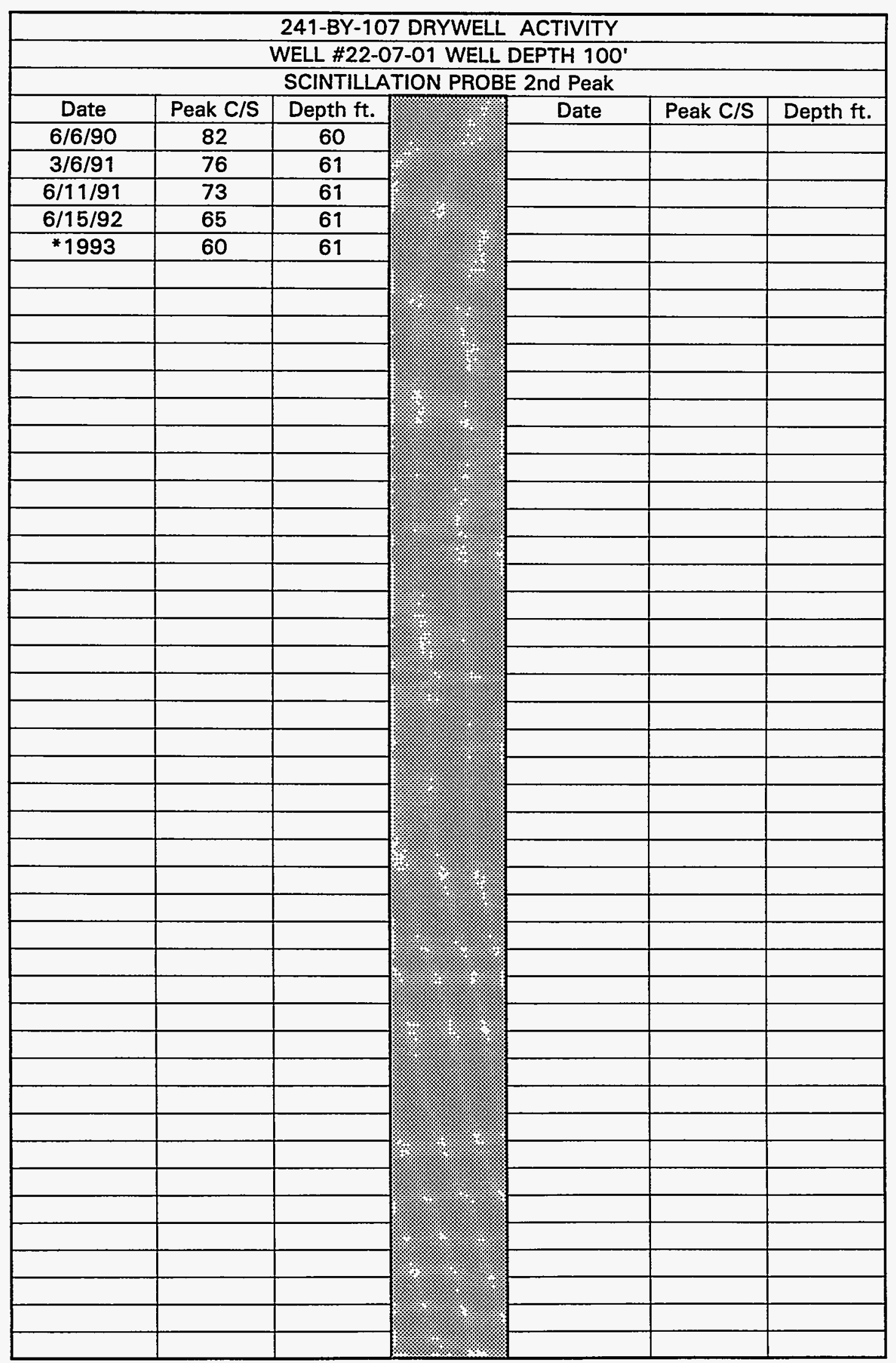

Data obtained from WHC Surveillance Data Acquisition (SDA), November 30, 1993. 


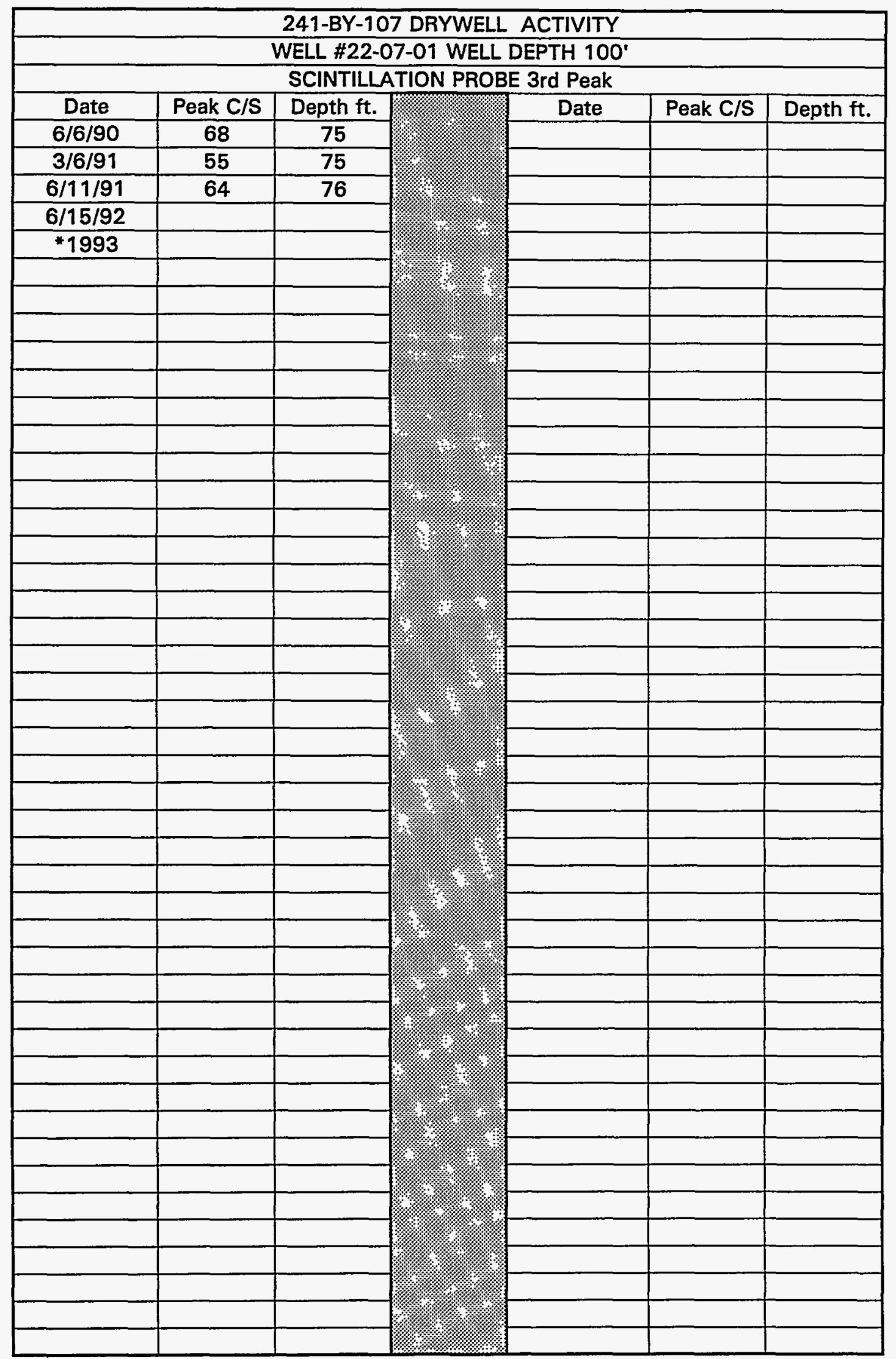

Data obtained from WHC Surveillance Data Acquisition (SDA), November 30, 1993. 
WHC-SD-WM-ER-312, Rev. 0

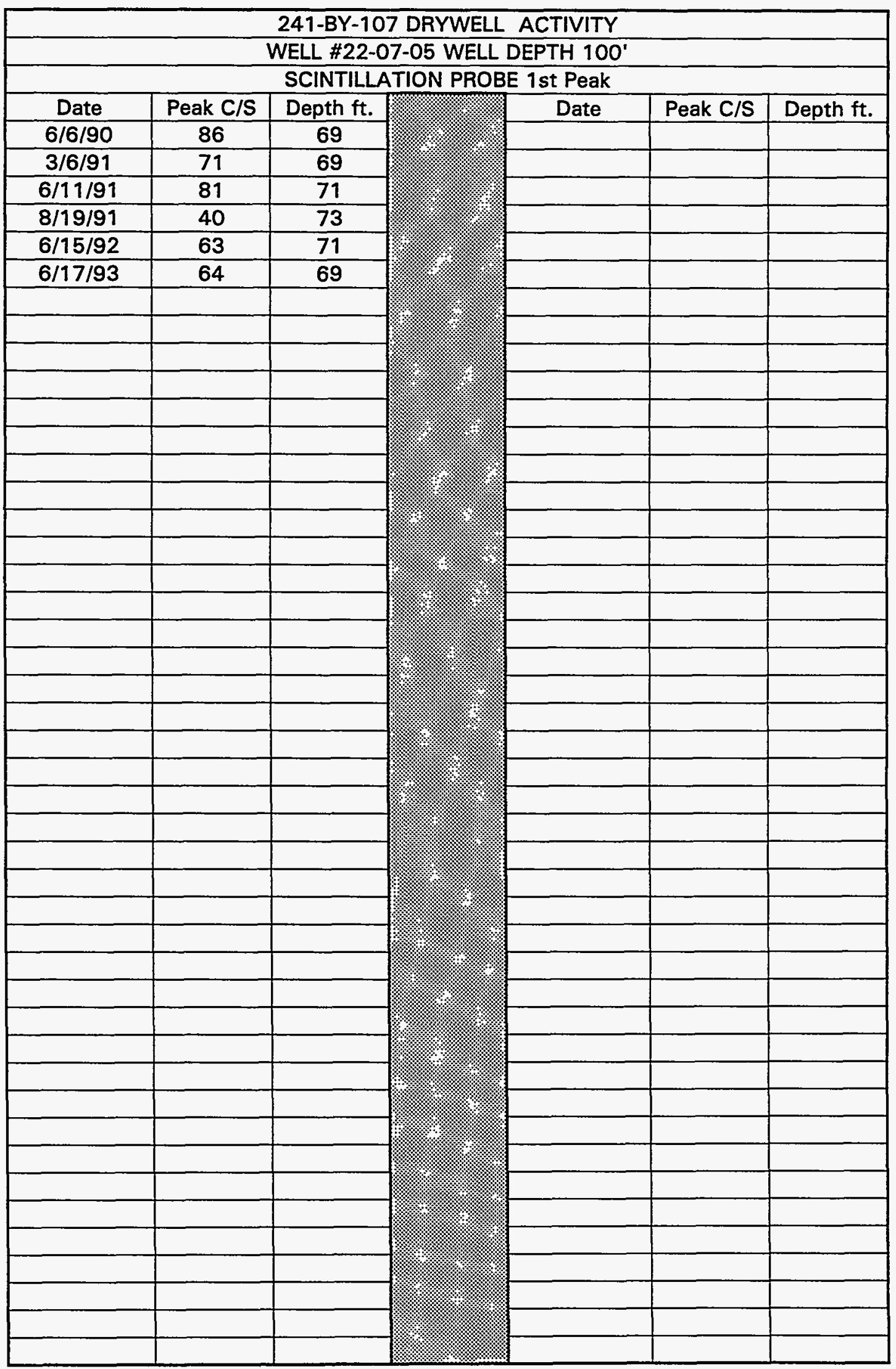

Data obtained from WHC Surveillance Data Acquisition (SDA), November 30, 1993. 


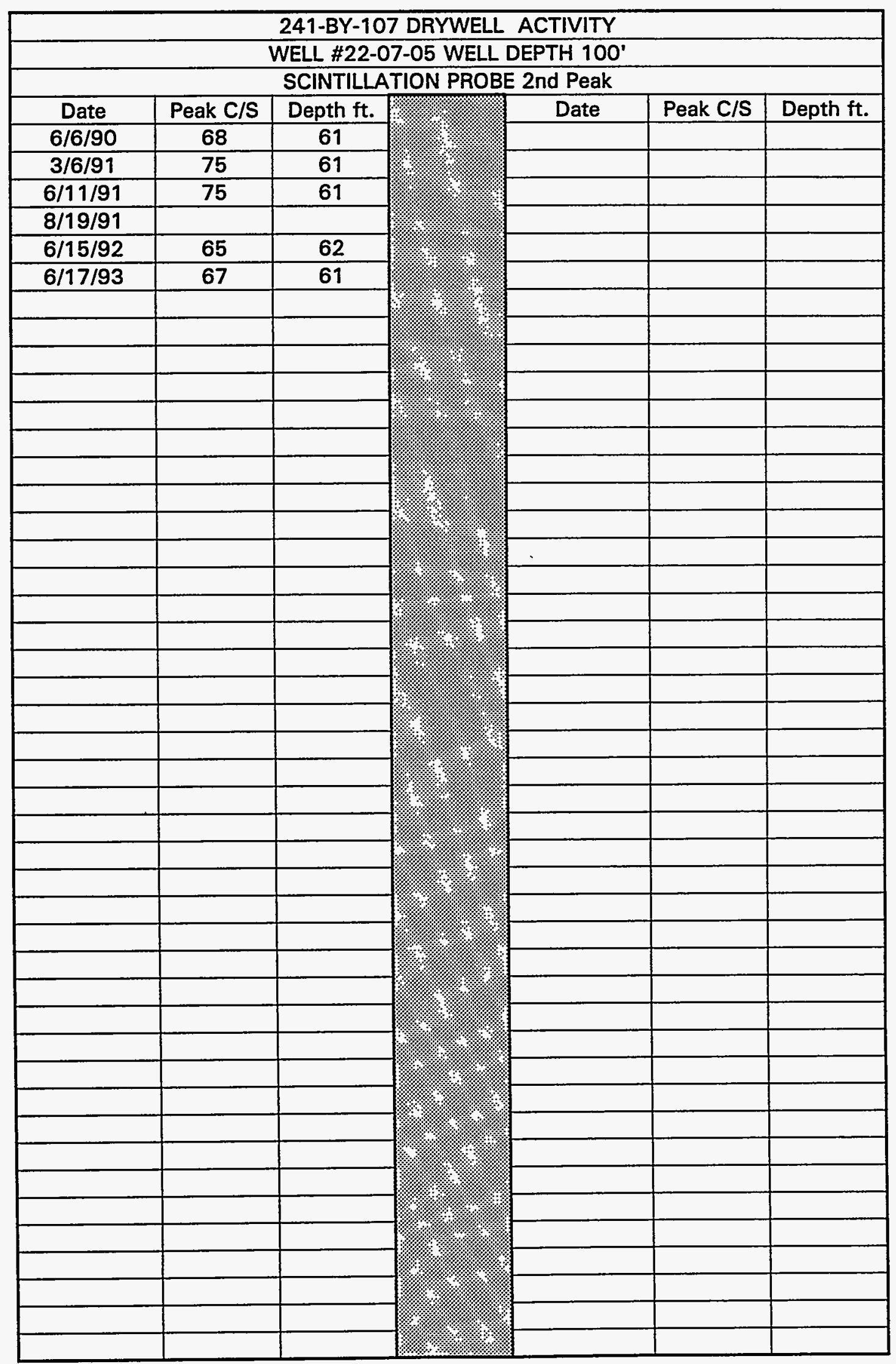

Data obtained from WHC Surveillance Data Acquisition (SDA), November 30, 1993. 


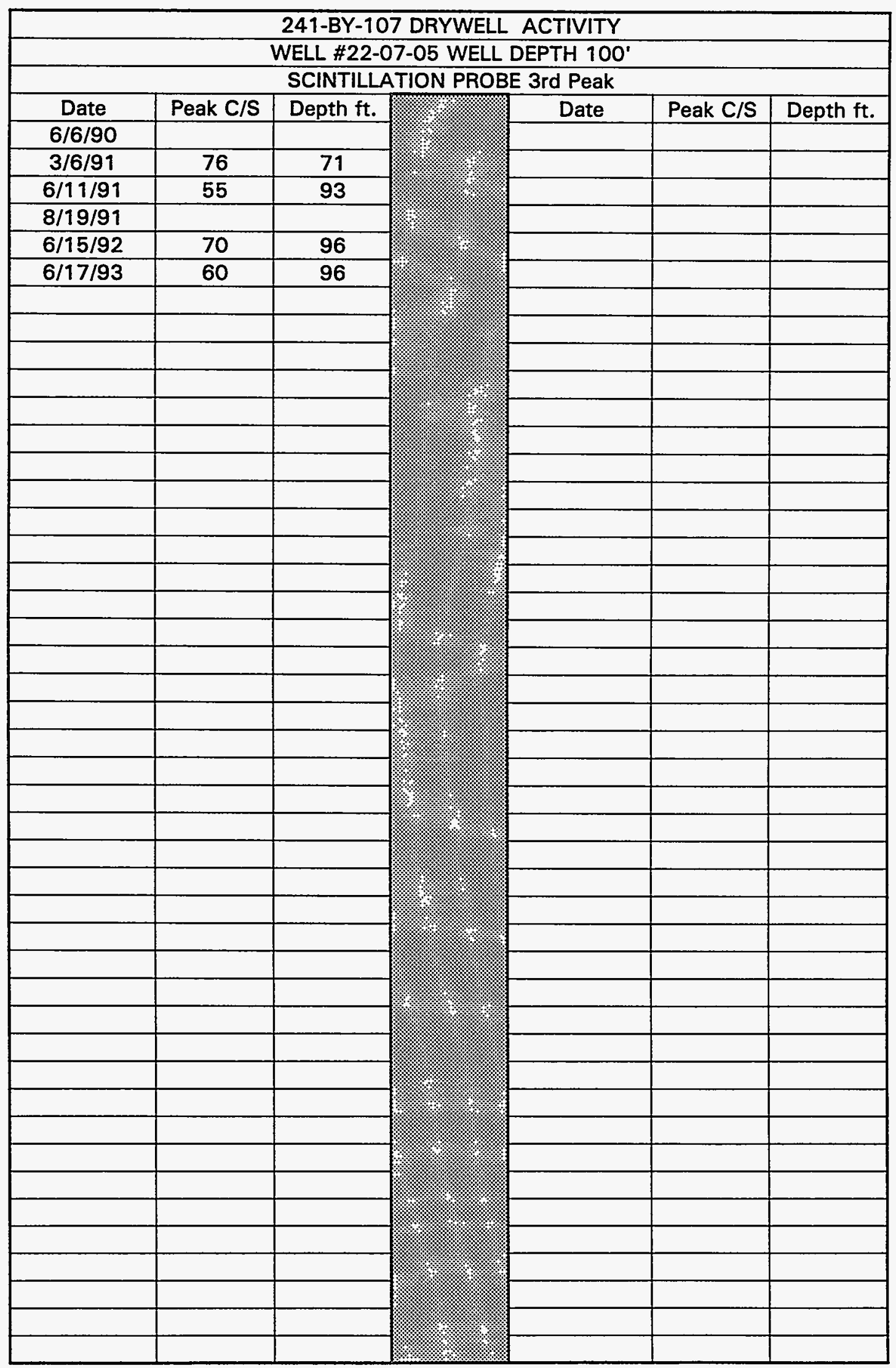

Data obtained from WHC Surveillance Data Acquisition (SDA), November 30, 1993. 


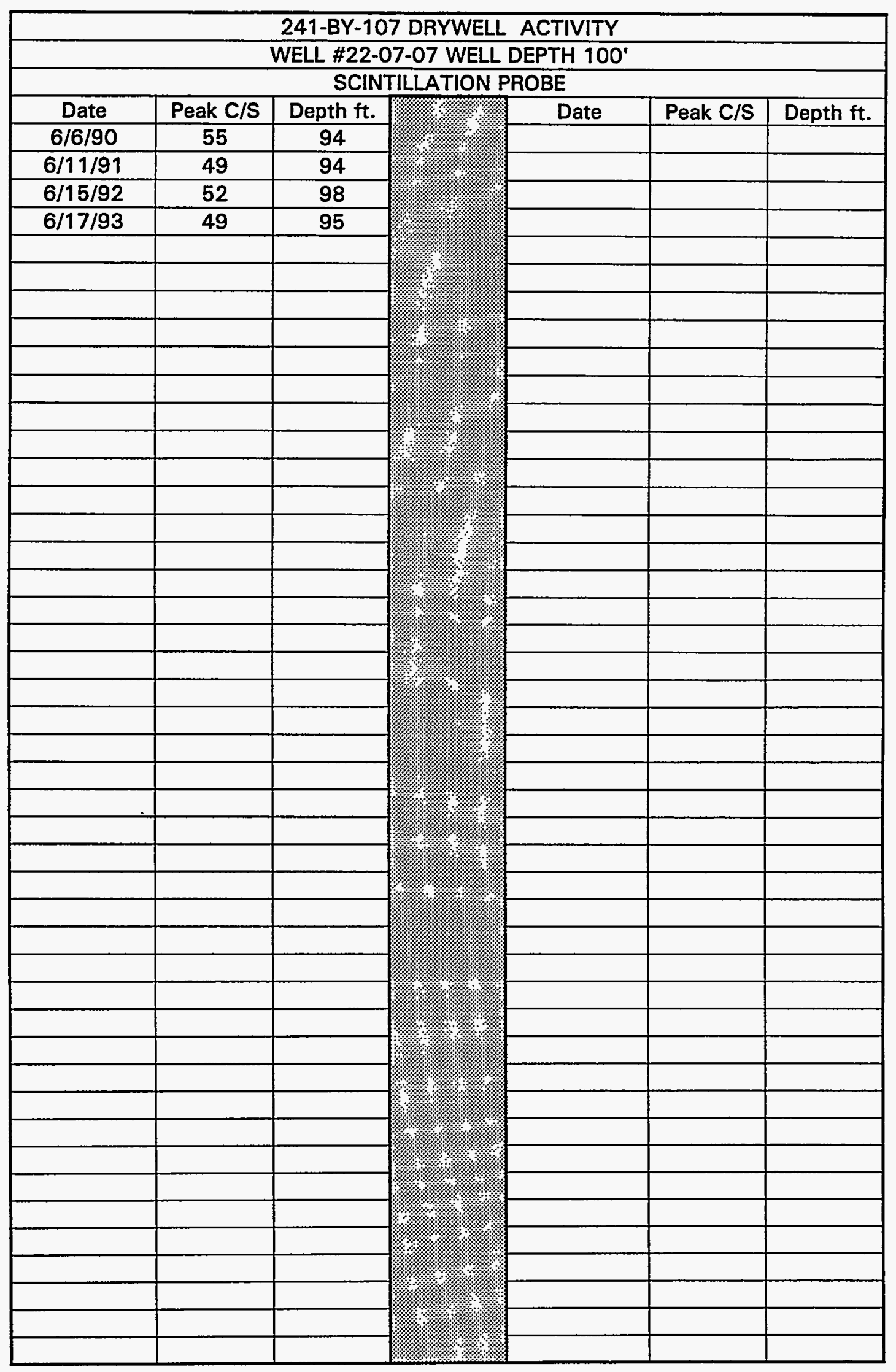

Data obtained from WHC Surveillance Data Acquisition (SDA), November 30, 1993. F-103 


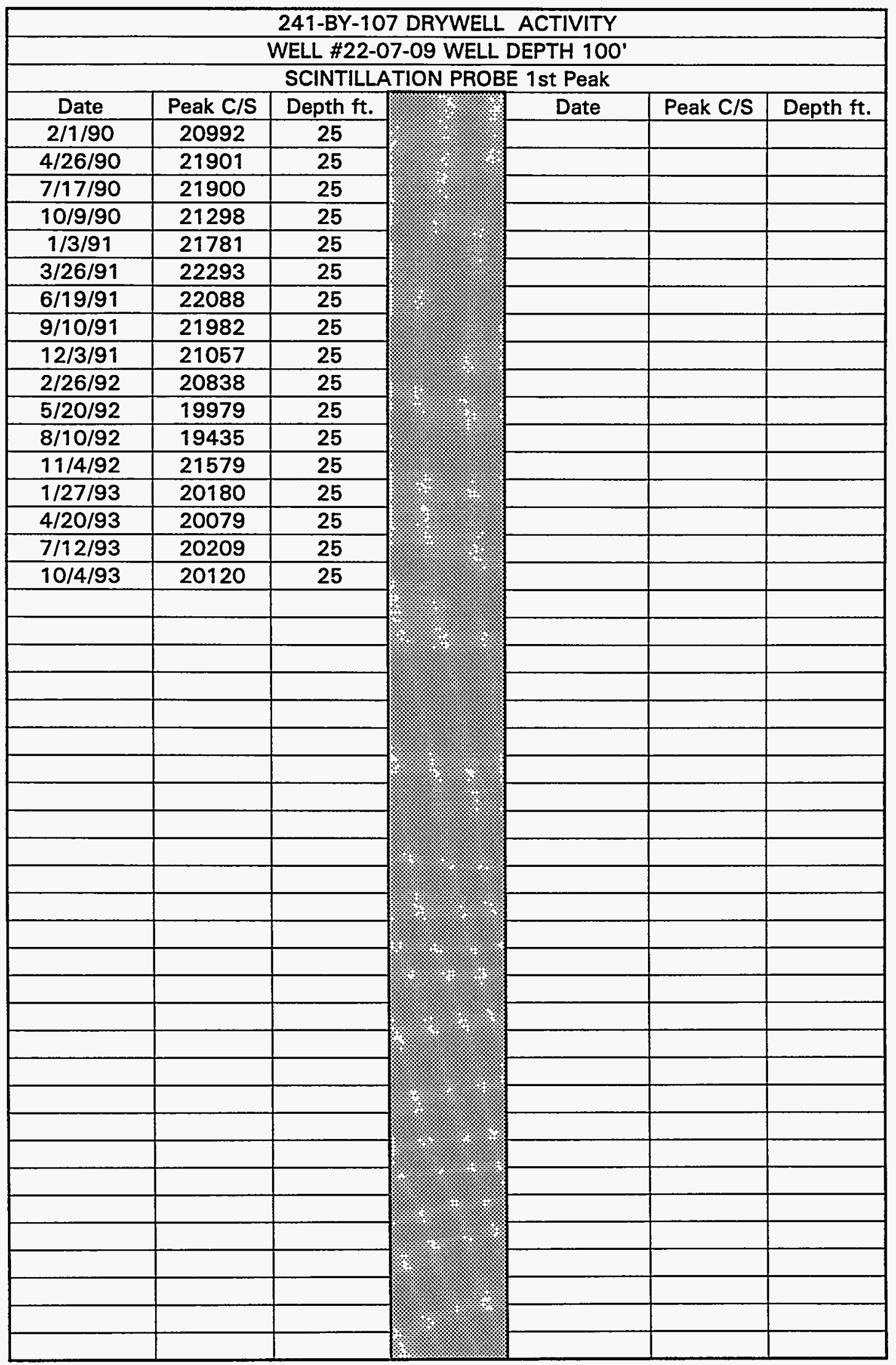

Data obtained from WHC Surveillance Data Acquisition (SDA), November 30, 1993. 


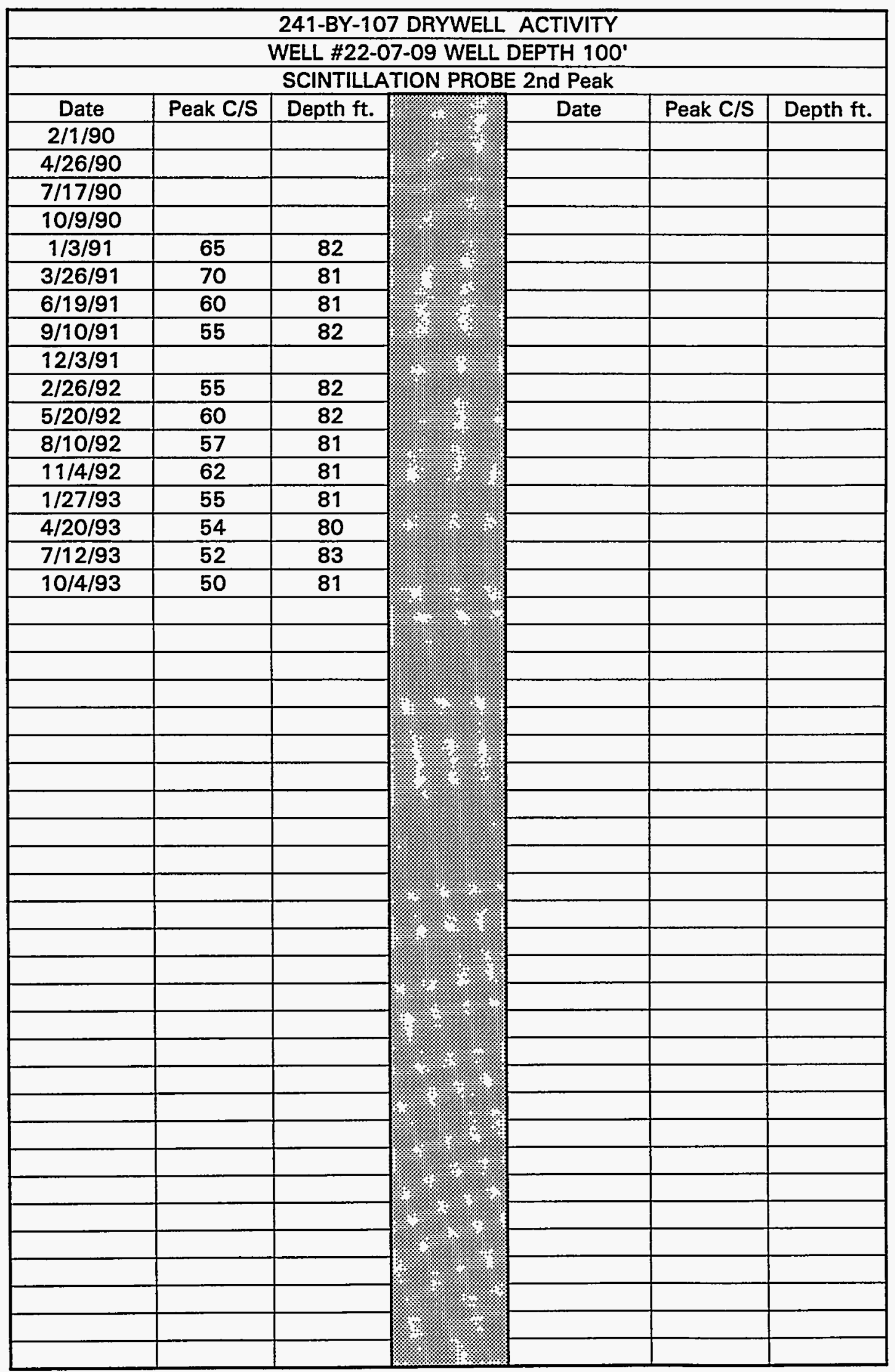

Data obtained from WHC Surveillance Data Acquisition (SDA), November 30, 1993. 
WHC-SD-WM-ER-312, Rev. 0

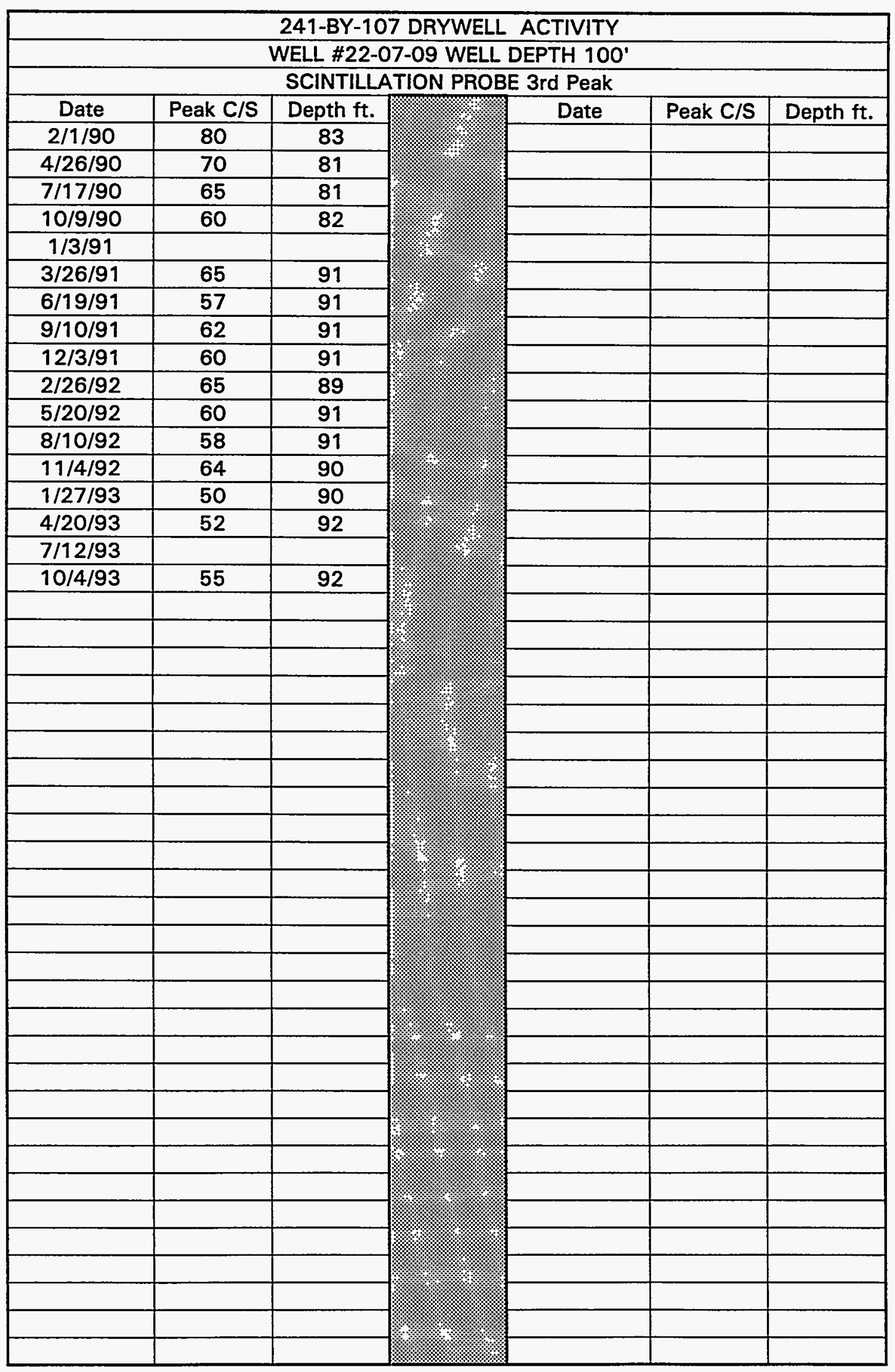

Data obtained from WHC Surveillance Data Acquisition (SDA), November 30, 1993. 


\begin{tabular}{|c|c|c|c|c|c|c|}
\hline \multicolumn{7}{|c|}{ 241-BY-107 DRYWELL ACTIVITY } \\
\hline \multicolumn{7}{|c|}{ WELL \#22-07-10 WELL DEPTH 100' } \\
\hline \multicolumn{7}{|c|}{ SCINTILLATION PROBE 1st Peak } \\
\hline Date & Peak C/S & Depth ft. & & Date & Peak C/S & Depth ft. \\
\hline $2 / 1 / 90$ & 247 & 35 & & & & \\
\hline $4 / 26 / 90$ & 204 & 27 & & & & \\
\hline $7 / 17 / 90$ & 186 & 24 & & & & \\
\hline $10 / 9 / 90$ & 195 & 35 & & & & \\
\hline$* 1 / 3 / 91$ & 246 & 35 & & & & \\
\hline $1 / 4 / 91$ & 228 & 25 & & & & \\
\hline $3 / 26 / 91$ & 182 & 35 & & & & \\
\hline $6 / 19 / 91$ & 220 & 27 & & & & \\
\hline $9 / 10 / 91$ & 189 & 35 & & & & \\
\hline $12 / 3 / 91$ & 290 & 27 & & & & \\
\hline *2/26/92 & 184 & 27 & & & & \\
\hline $2 / 26 / 92$ & 200 & 35 & & & & \\
\hline $5 / 20 / 92$ & 200 & 27 & & & & \\
\hline $8 / 10 / 92$ & 170 & 27 & & & & \\
\hline \multicolumn{7}{|l|}{$11 / 4 / 92$} \\
\hline $1 / 27 / 93$ & 176 & 26 & & & & \\
\hline $4 / 20 / 93$ & 153 & 27 & & & & \\
\hline $7 / 12 / 93$ & 160 & 27 & & & & \\
\hline $10 / 4 / 93$ & 187 & 27 & & & & \\
\hline & & & & & & \\
\hline & & & & & & \\
\hline & & & & & & \\
\hline & & & & & & \\
\hline & & & : & & & \\
\hline & & & & & & \\
\hline & & & & & & \\
\hline & & & & & & \\
\hline & & & & & & \\
\hline & & & & & & \\
\hline & & & & & & \\
\hline & & & & & & \\
\hline & & & (4) & & & \\
\hline & & & & & & \\
\hline & & & $\sqrt{1}$ & & & \\
\hline & & & 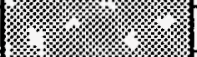 & & & \\
\hline & & & 18 & & & \\
\hline & & & \% & & & \\
\hline & & & 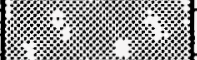 & & & \\
\hline & & & স4: & & & \\
\hline & & & & & & \\
\hline & & & & & & \\
\hline & & & 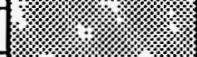 & & & \\
\hline & & & 19 & & & \\
\hline & & & & & & \\
\hline
\end{tabular}

Data obtained from WHC Surveillance Data Acquisition (SDA), November 30, 1993. 
WHC-SD-WM-ER-312, Rev. 0

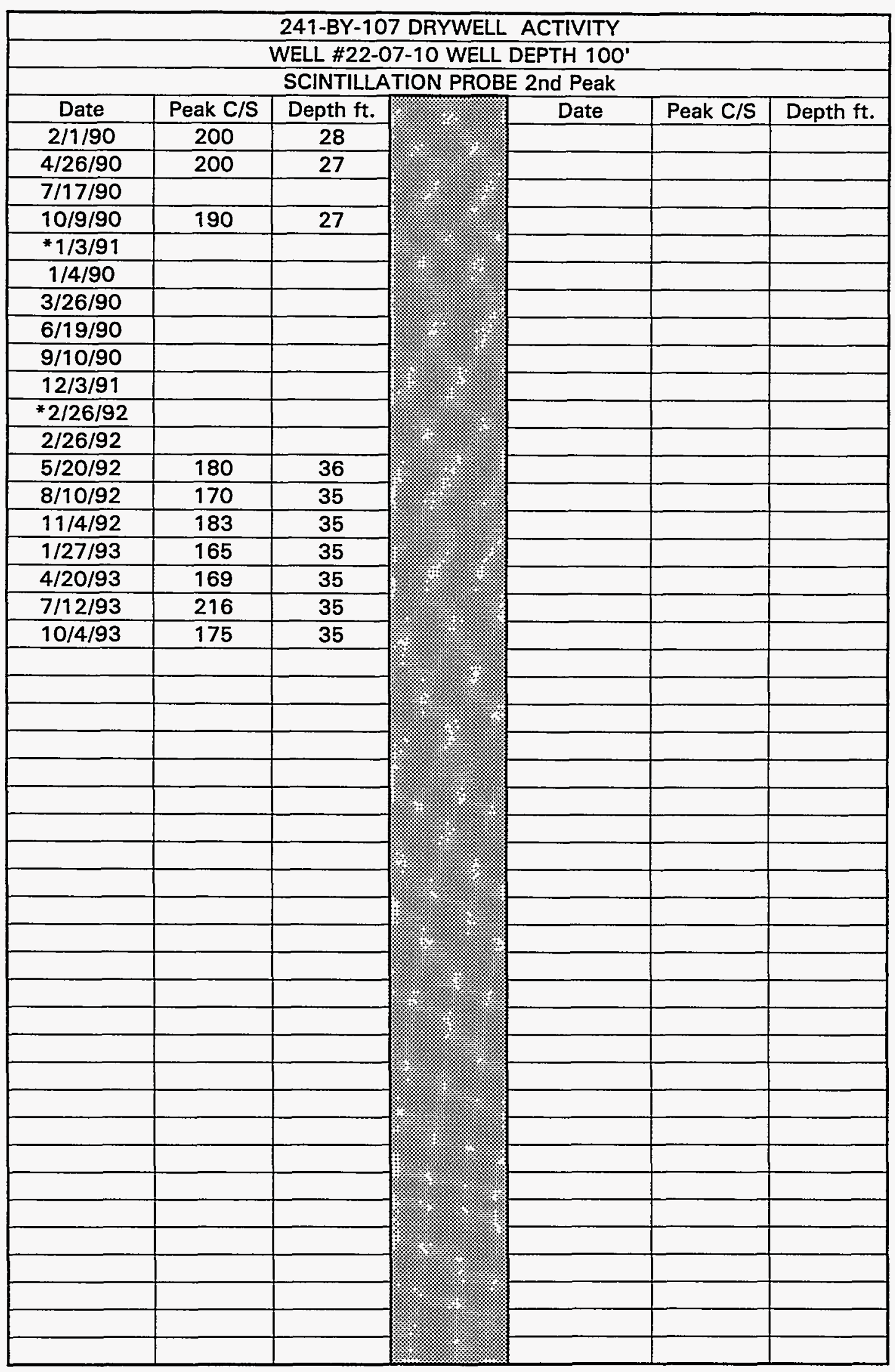

Data obtained from WHC Surveillance Data Acquisition (SDA), November 30, 1993. 


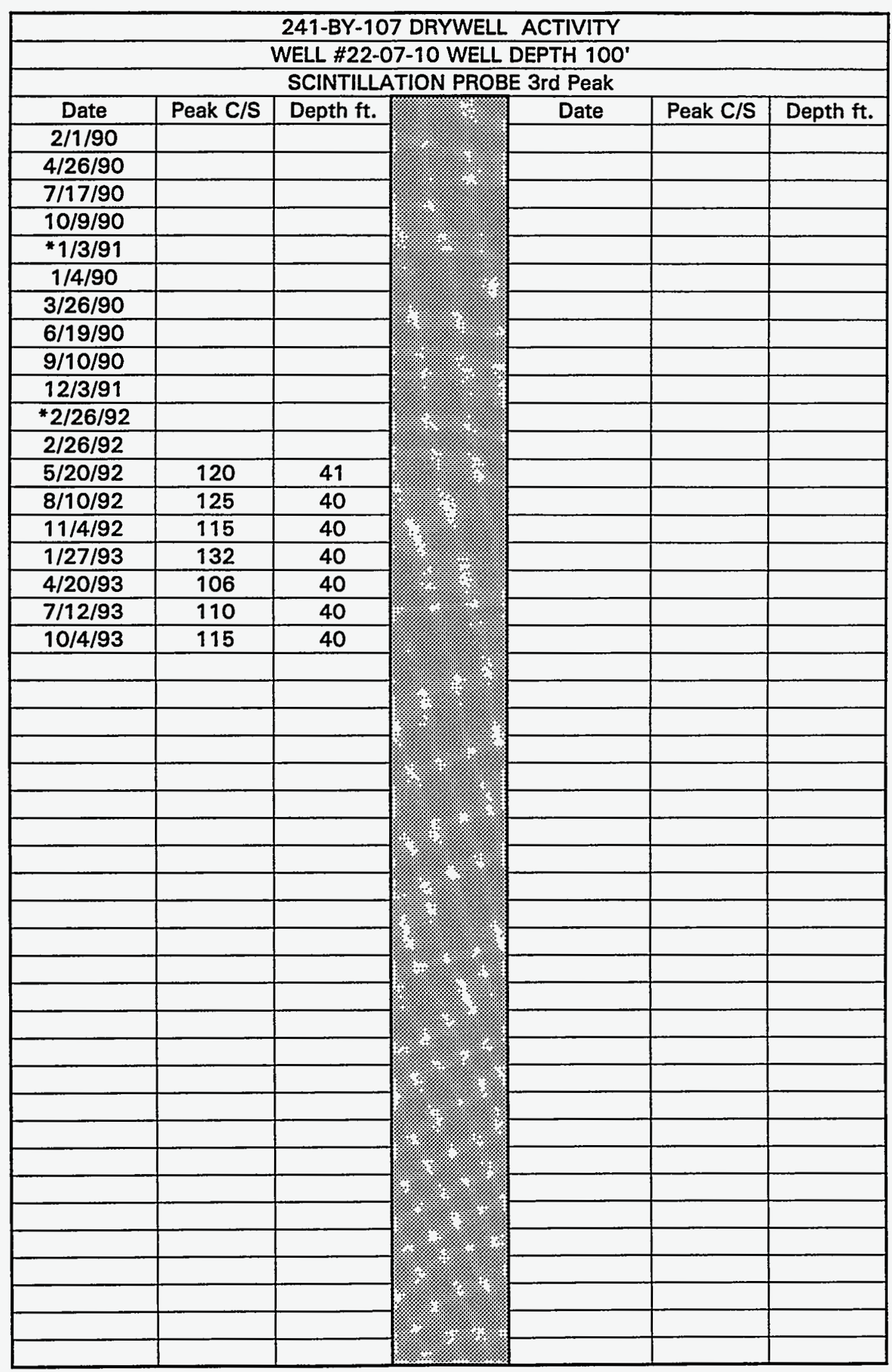

Data obtained from WHC Surveillance Data Acquisition (SDA), November 30, 1993. 
WHC-SD-WM-ER-312, Rev. 0

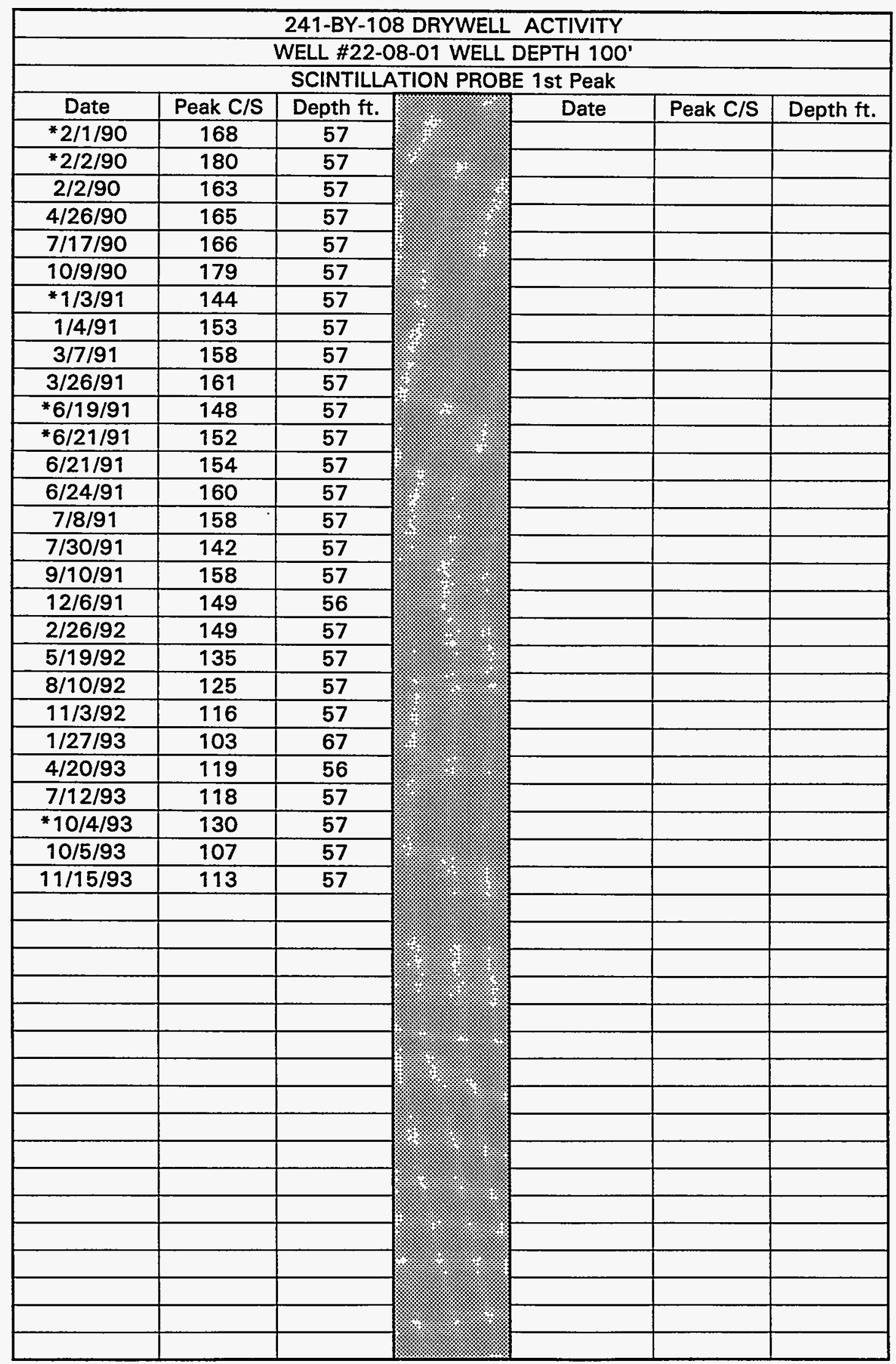

Data obtained from WHC Surveillance Data Acquisition (SDA), November 30, 1993. 
WHC-SD-WM-ER-312, Rev. 0

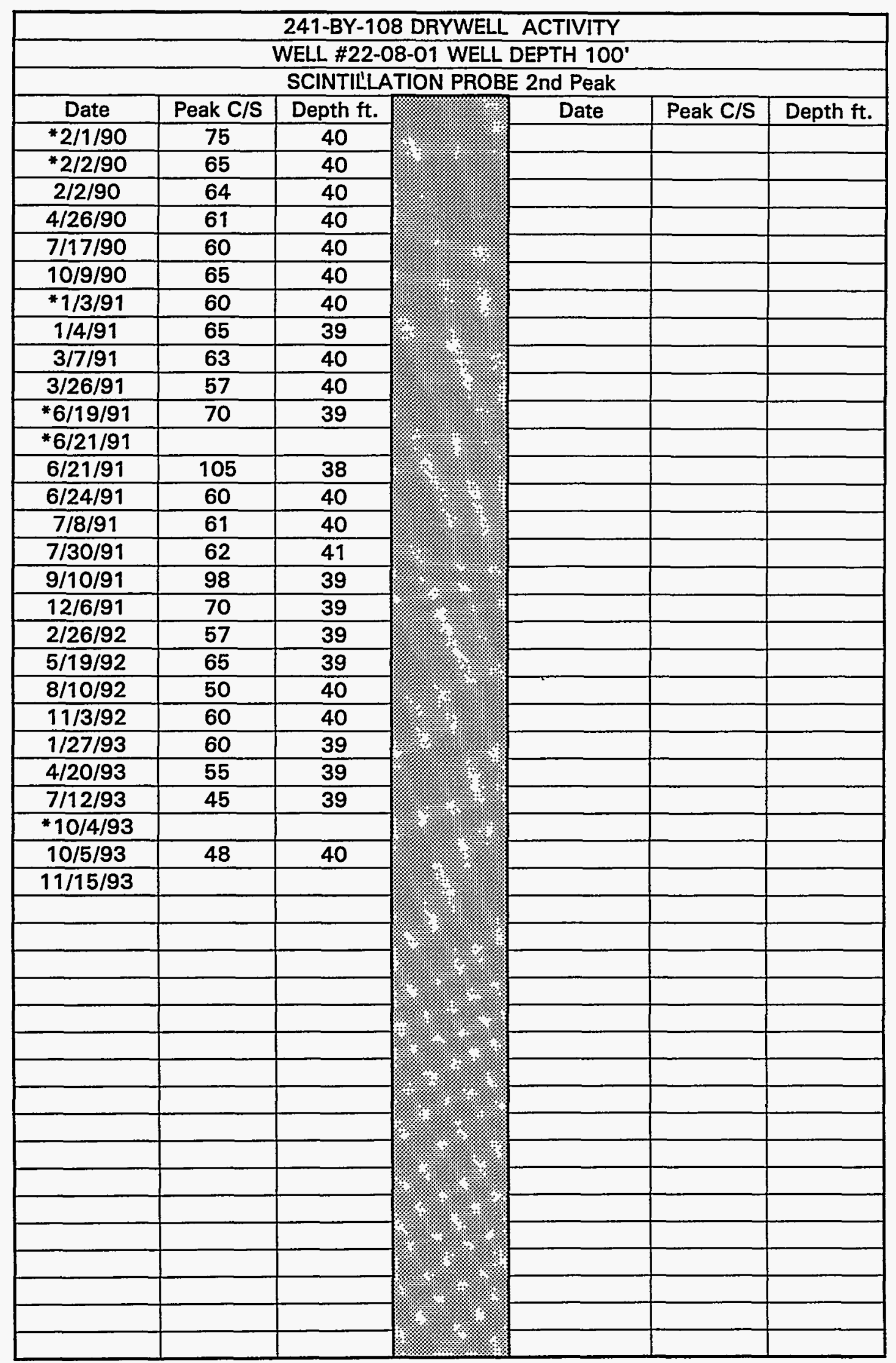

Data obtained from WHC Surveillance Data Acquisition (SDA), November 30, 1993. 
WHC-SD-WM-ER-312, Rev. 0

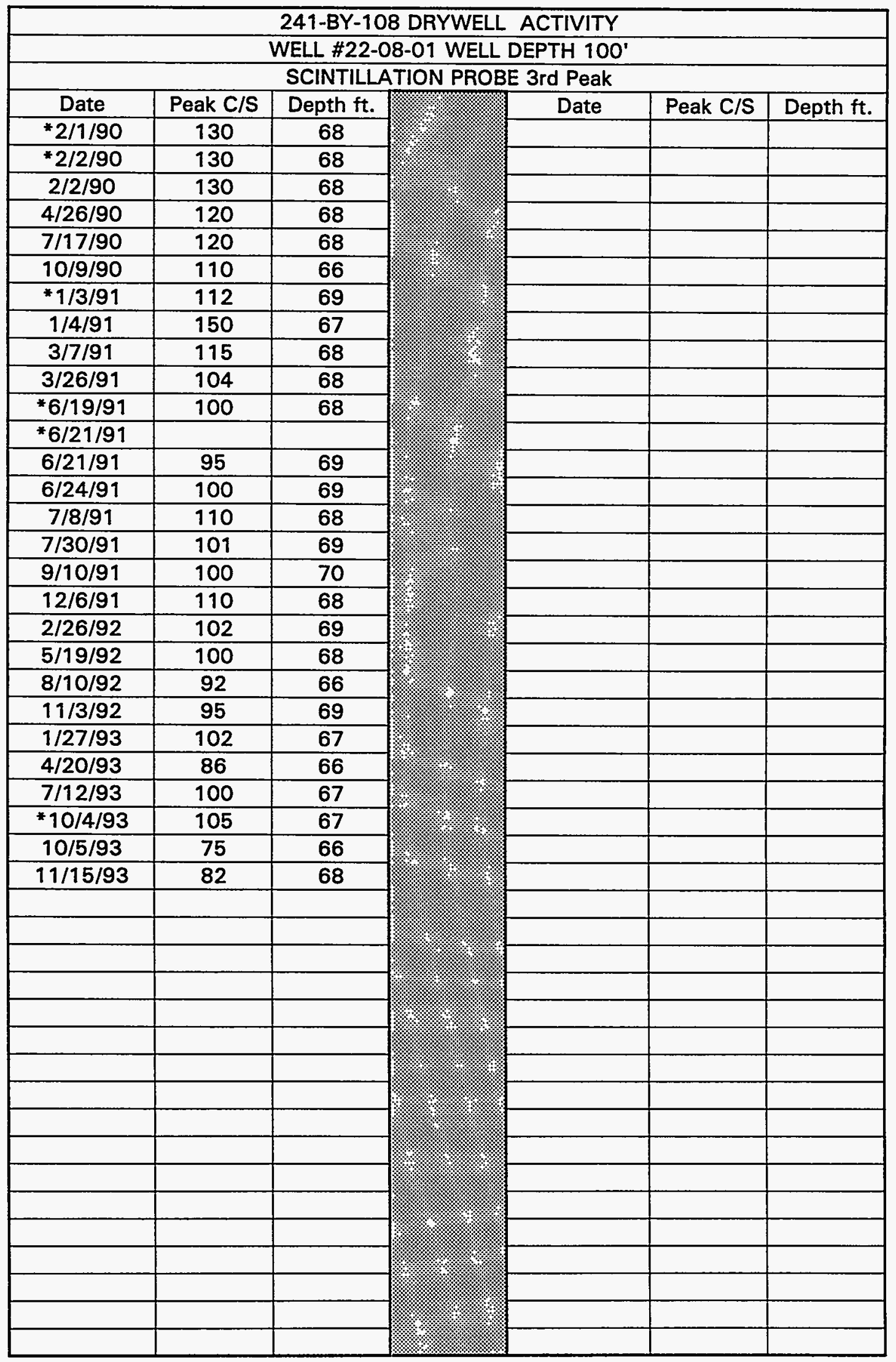

Data obtained from WHC Surveillance Data Acquisition (SDA), November 30, 1993. 


\begin{tabular}{|c|c|c|c|c|c|c|}
\hline \multicolumn{7}{|c|}{ 241-BY-108 DRYWELL ACTIVITY } \\
\hline \multicolumn{7}{|c|}{ WELL \#22-08-02 WELL DEPTH 100' } \\
\hline \multicolumn{7}{|c|}{ SCINTILLATION PROBE 1st Peak } \\
\hline Date & Peak C/S & Depth ft. & & Date & Peak C/S & Depth ft. \\
\hline $2 / 1 / 90$ & 451 & 84 & & & & \\
\hline $4 / 26 / 90$ & 424 & 83 & & & & \\
\hline $7 / 17 / 90$ & 381 & 83 & & & & \\
\hline $10 / 9 / 90$ & 387 & 83 & & & & \\
\hline $1 / 3 / 91$ & 338 & 83 & & & & \\
\hline $3 / 7 / 91$ & 326 & 83 & & & & \\
\hline $3 / 25 / 91$ & 336 & 83 & ঋ/: & & & \\
\hline${ }^{*} 6 / 19 / 91$ & 316 & 83 & & & & \\
\hline$* 6 / 21 / 91$ & 360 & 83 & & & & \\
\hline $6 / 21 / 91$ & 295 & 83 & & & & \\
\hline $6 / 24 / 91$ & 297 & 83 & & & & \\
\hline $7 / 8 / 91$ & 292 & 83 & & & & \\
\hline $7 / 30 / 91$ & 327 & 83 & 存 & & & \\
\hline $9 / 9 / 91$ & 314 & 83 & & & & \\
\hline $12 / 6 / 91$ & 302 & 83 & \% & & & \\
\hline $2 / 26 / 92$ & 254 & 83 & .8 & & & \\
\hline $5 / 19 / 92$ & 260 & 83 & & & & \\
\hline $8 / 10 / 92$ & 209 & 83 & & & & \\
\hline $11 / 4 / 92$ & 216 & 83 & $\%$ & & & \\
\hline $1 / 27 / 93$ & 175 & 83 & & & & \\
\hline $4 / 20 / 93$ & 180 & 83 & & & & \\
\hline $7 / 12 / 93$ & 145 & 83 & & & & \\
\hline * $10 / 4 / 93$ & 161 & 83 & & & & \\
\hline $10 / 5 / 93$ & 159 & 83 & & & & \\
\hline $11 / 17 / 93$ & 156 & 83 & & & & \\
\hline & & & & & & \\
\hline & & & & & & \\
\hline & & & & & & \\
\hline & & & & & & \\
\hline & & & 19 & & & \\
\hline & & & 1/ & & & \\
\hline & & & 9 & & & \\
\hline & & & $147=1918$ & & & \\
\hline & & & 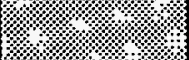 & & & \\
\hline & & & & & & \\
\hline & & & & & & \\
\hline & & & 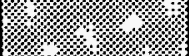 & & & \\
\hline & & & li & & & \\
\hline & & & & & & \\
\hline & & & & & & \\
\hline & & & lm. & & & \\
\hline & & & 1918 & & & \\
\hline & & & 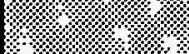 & & & \\
\hline & & & 4 & & & \\
\hline & & & 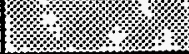 & & & \\
\hline
\end{tabular}

Data obtained from WHC Surveillance Data Acquisition (SDA), November 30, 1993. 


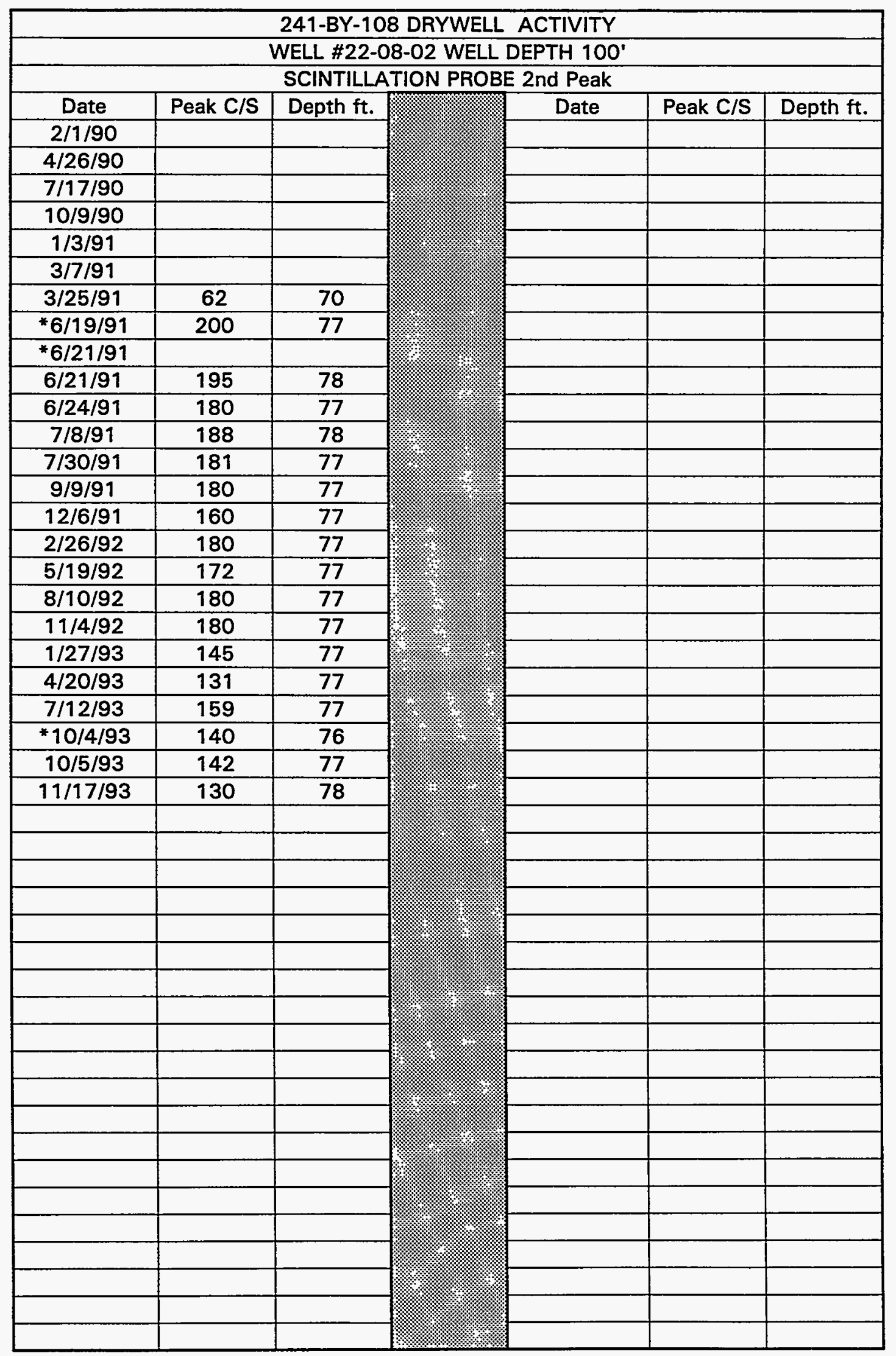

Data obtained from WHC Surveillance Data Acquisition (SDA), November 30, 1993. 


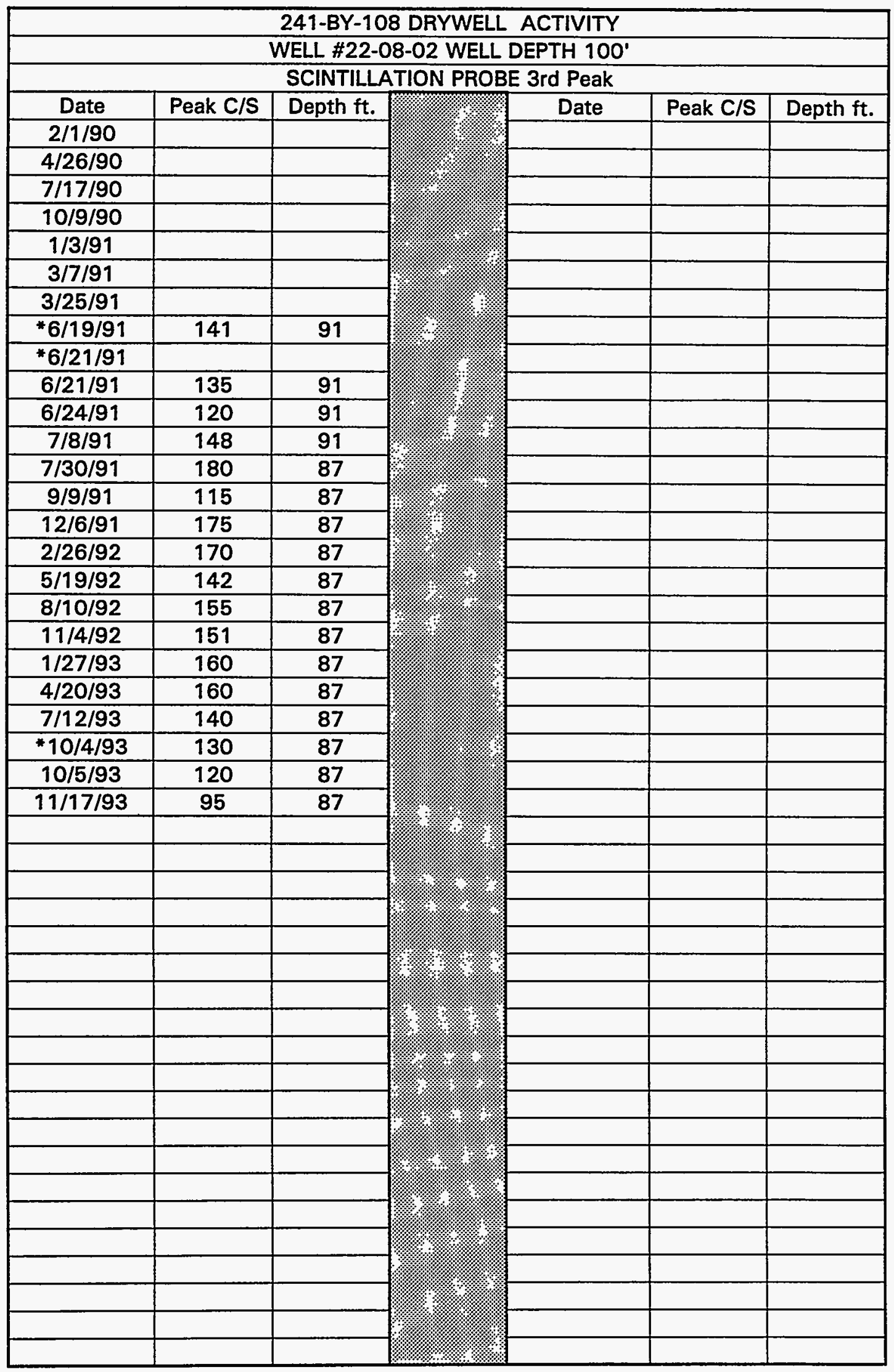

Data obtained from WHC Surveillance Data Acquisition (SDA), November 30, 1993. 


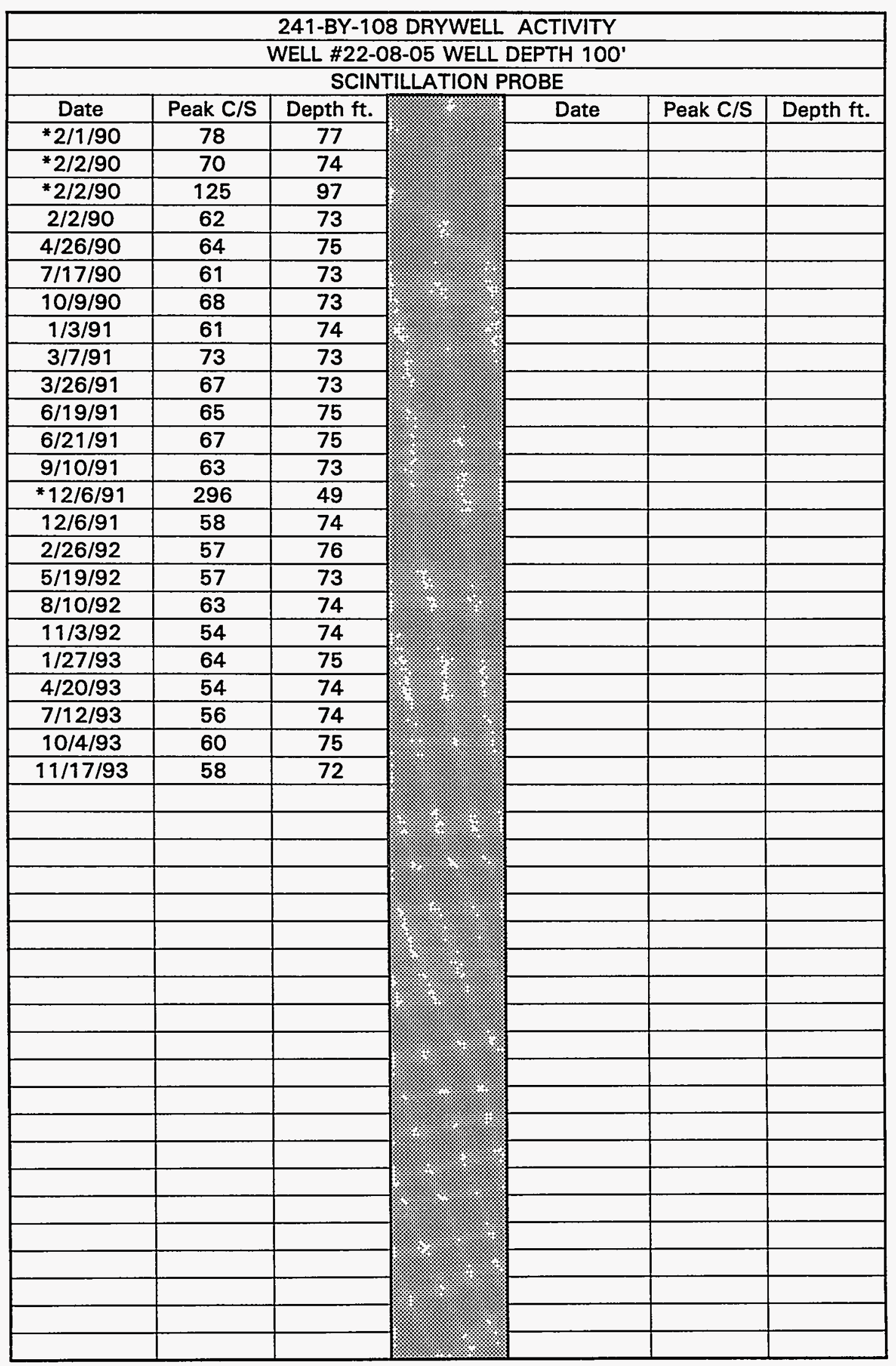

Data obtained from WHC Surveillance Data Acquisition (SDA), November 30, 1993. 


\begin{tabular}{|c|c|c|c|c|c|c|}
\hline \multicolumn{7}{|c|}{ 241-BY-108 DRYWELL ACTIVITY } \\
\hline \multicolumn{7}{|c|}{ WELL \#22-08-06 WELL DEPTH 100' } \\
\hline \multicolumn{7}{|c|}{ SCINTILLATION PROBE 1st Peak } \\
\hline Date & Peak C/S & Depth ft. & $=$ & Date & Peak C/S & Depth ft. \\
\hline $2 / 1 / 90$ & 163 & 25 & & & & \\
\hline $4 / 26 / 90$ & 161 & 24 & & & & \\
\hline $7 / 17 / 90$ & 175 & 24 & & & & \\
\hline $10 / 9 / 90$ & 146 & 24 & & & & \\
\hline $1 / 3 / 91$ & 156 & 24 & & & & \\
\hline$* 2 / 16 / 91$ & 218 & 24 & & & & \\
\hline $2 / 16 / 91$ & 170 & 24 & & & & \\
\hline $3 / 6 / 91$ & 163 & 24 & & & & \\
\hline $3 / 26 / 91$ & 161 & 24 & & & & \\
\hline $6 / 19 / 91$ & 154 & 24 & & & & \\
\hline $9 / 10 / 91$ & 168 & 24 & & & & \\
\hline$* 12 / 6 / 91$ & 195 & 25 & & & & \\
\hline $12 / 6 / 91$ & 161 & 25 & & & & \\
\hline $2 / 26 / 92$ & 140 & 23 & & & & \\
\hline $5 / 19 / 92$ & 165 & 24 & & & & \\
\hline $8 / 10 / 92$ & 138 & 24 & & & & \\
\hline $11 / 3 / 92$ & 134 & 24 & & & & \\
\hline $1 / 27 / 93$ & 149 & 24 & & & & \\
\hline $4 / 20 / 93$ & 146 & 24 & & & & \\
\hline $7 / 12 / 93$ & 158 & 24 & \%. & & & \\
\hline $10 / 4 / 93$ & 149 & 24 & & & & \\
\hline $11 / 17 / 93$ & 170 & 24 & & & & \\
\hline & & & 4 & & & \\
\hline & & & & & & \\
\hline & & & & & & \\
\hline & & & & & & \\
\hline & & & & & & \\
\hline & & & & & & \\
\hline & & & & & & \\
\hline & & & & & & \\
\hline & & & & & & \\
\hline & & & צ. & & & \\
\hline & & & 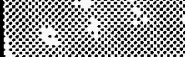 & & & \\
\hline & & & 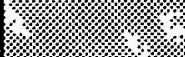 & & & \\
\hline & & & & & & \\
\hline & & & & & & \\
\hline & & & (1) & & & \\
\hline & & & 191819.19 & & & \\
\hline & & & $4=1.17 .14$ & & & \\
\hline & & & (1) & & & \\
\hline & & & $17=1.17$. & & & \\
\hline & & & 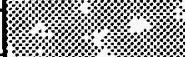 & & & \\
\hline & & & 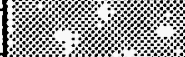 & & & \\
\hline & & & & & & \\
\hline
\end{tabular}

Data obtained from WHC Surveillance Data Acquisition (SDA), November 30, 1993. 
WHC-SD-WM-ER-312, Rev. 0

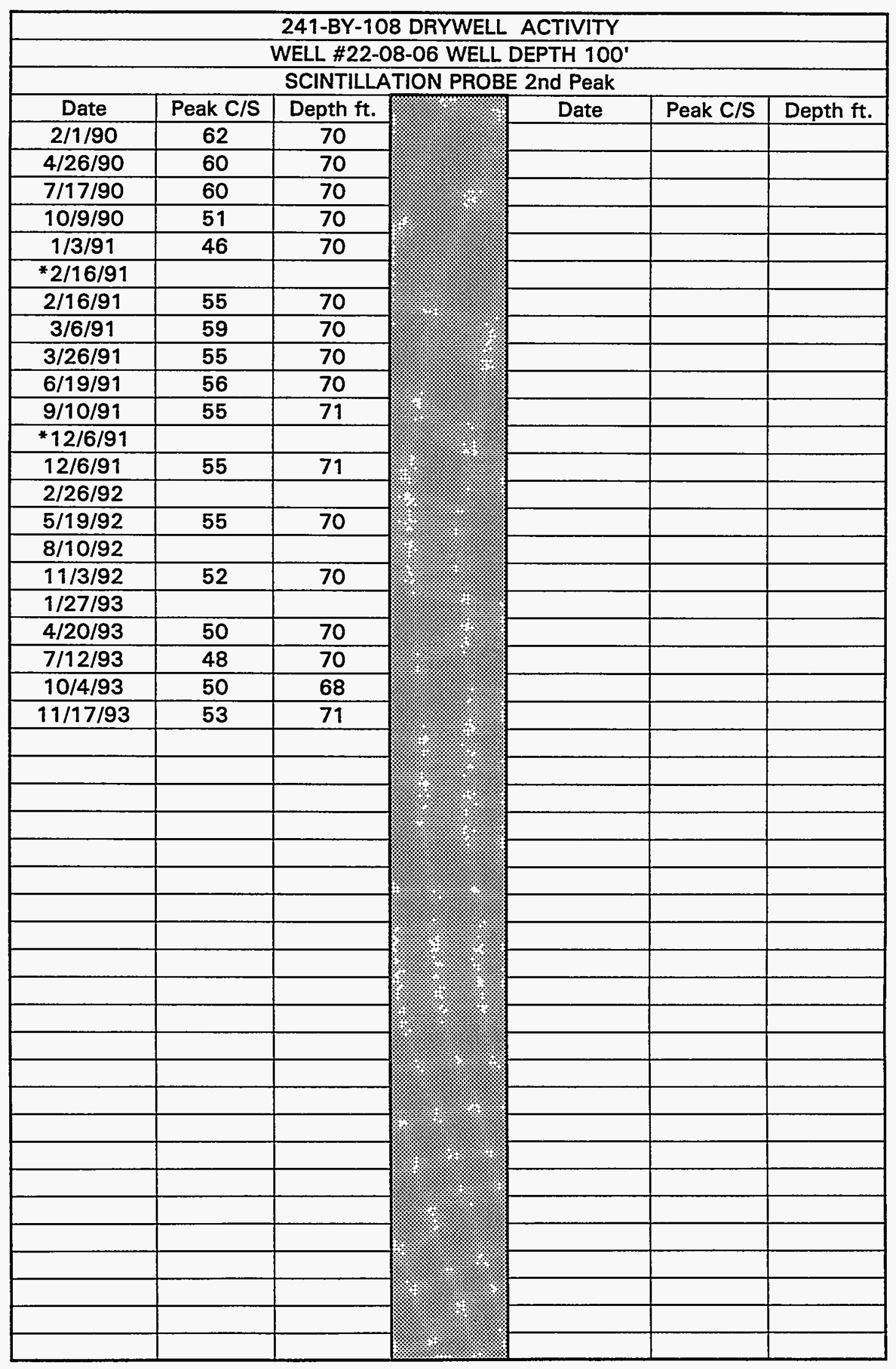

Data obtained from WHC Surveillance Data Acquisition (SDA), November 30, 1993. 
WHC-SD-WM-ER-312, Rev. 0

\begin{tabular}{|c|c|c|c|c|c|c|}
\hline \multicolumn{7}{|c|}{ 241-BY-108 DRYWELL ACTIVITY } \\
\hline \multicolumn{7}{|c|}{ WELL \#22-08-12 WELL DEPTH 100' } \\
\hline \multicolumn{7}{|c|}{ SCINTILLATION PROBE 1st Peak } \\
\hline Date & Peak C/S & Depth ft. & & Date & Peak C/S & Depth ft. \\
\hline $2 / 1 / 90$ & 66 & 74 & & & & \\
\hline $4 / 26 / 90$ & 84 & 56 & & & & \\
\hline $7 / 17 / 90$ & 71 & 56 & & & & \\
\hline $10 / 10 / 90$ & 68 & 56 & & & & \\
\hline $1 / 3 / 91$ & 72 & 57 & & & & \\
\hline $3 / 26 / 91$ & 80 & 56 & & & & \\
\hline *6/19/91 & 71 & 56 & & & & \\
\hline${ }^{*} 6 / 21 / 91$ & 78 & 56 & & & & \\
\hline $6 / 21 / 91$ & 76 & 56 & 3 & & & \\
\hline $6 / 24 / 91$ & 65 & 56 & \% & & & \\
\hline $7 / 8 / 91$ & 83 & 56 & & & & \\
\hline $7 / 30 / 91$ & 68 & 56 & & & & \\
\hline $9 / 10 / 91$ & 70 & 56 & & & & \\
\hline$* 12 / 6 / 91$ & 149 & 79 & & & & \\
\hline $12 / 6 / 91$ & 66 & 56 & & & & \\
\hline $2 / 26 / 92$ & 54 & 57 & & & & \\
\hline $5 / 20 / 92$ & 62 & 57 & & & & \\
\hline $8 / 10 / 92$ & 67 & 56 & & & & \\
\hline $11 / 3 / 92$ & 50 & 56 & & & & \\
\hline $1 / 27 / 93$ & 63 & 47 & & & & \\
\hline $4 / 20 / 93$ & 57 & 74 & & & & \\
\hline $7 / 12 / 93$ & 62 & 56 & & & & \\
\hline$* 10 / 4 / 93$ & 61 & 56 & & & & \\
\hline $10 / 5 / 93$ & 54 & 57 & & & & \\
\hline $11 / 17 / 93$ & 67 & 56 & & & & \\
\hline & & & & & & \\
\hline & & & 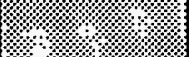 & & & \\
\hline & & & 41 & & & \\
\hline & & & $4:$ & & & \\
\hline & & & (3) & & & \\
\hline & & & & & & \\
\hline & & & & & & \\
\hline & & & $1=$ & & & \\
\hline & & & & & & \\
\hline & & & & & & \\
\hline & & & & & & \\
\hline & & & & & & \\
\hline & & & & & & \\
\hline & & & & & & \\
\hline & & & \% & & & \\
\hline & & & \% & & & \\
\hline & & & (1) & & & \\
\hline & & & 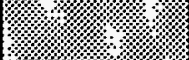 & & & \\
\hline & & & 18 & & & \\
\hline
\end{tabular}

Data obtained from WHC Surveillance Data Acquisition (SDA), November 30, 1993. 
WHC-SD-WM-ER-312, Rev. 0

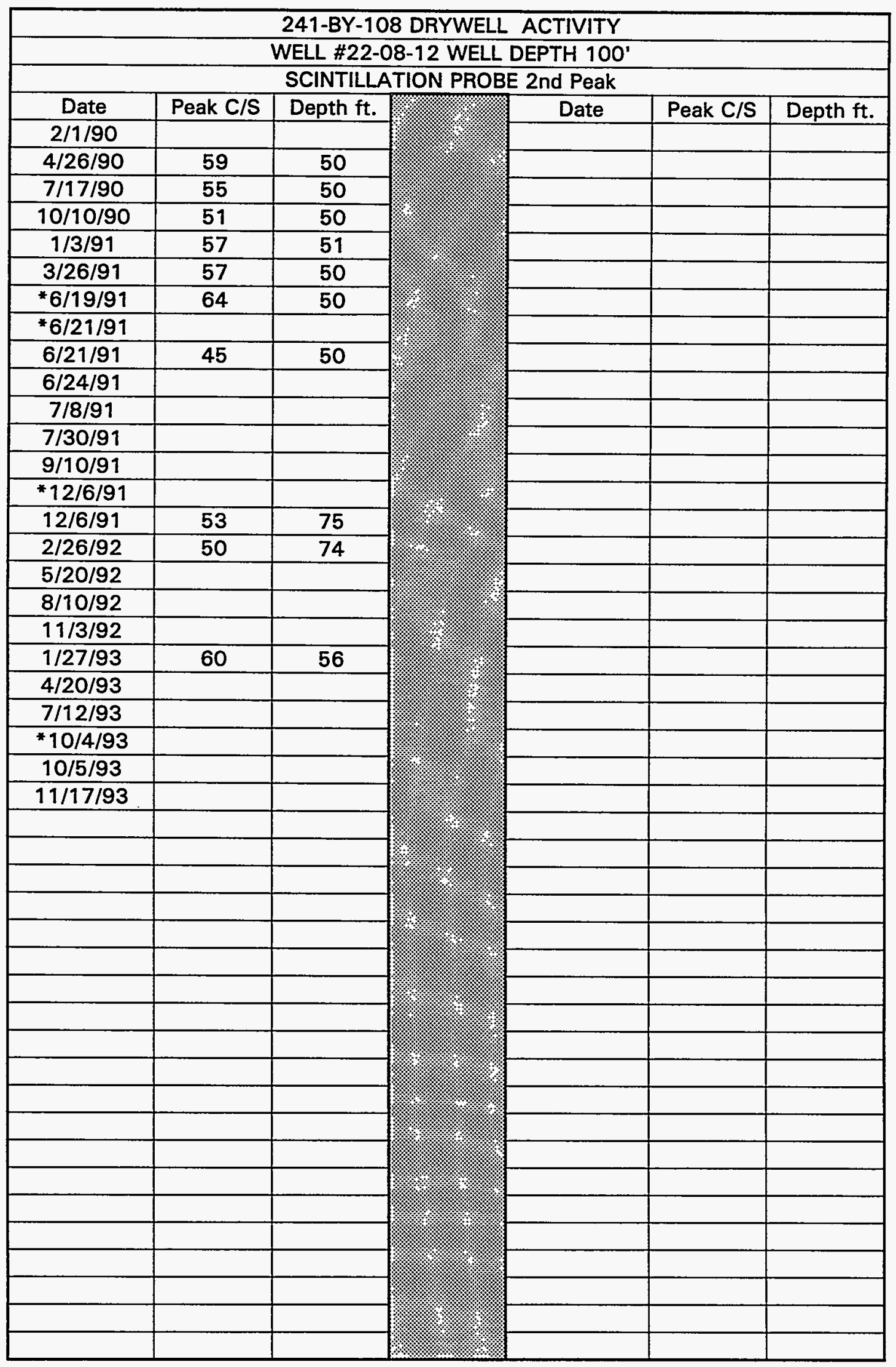

Data obtained from WHC Surveillance Data Acquisition (SDA), November 30, 1993. 


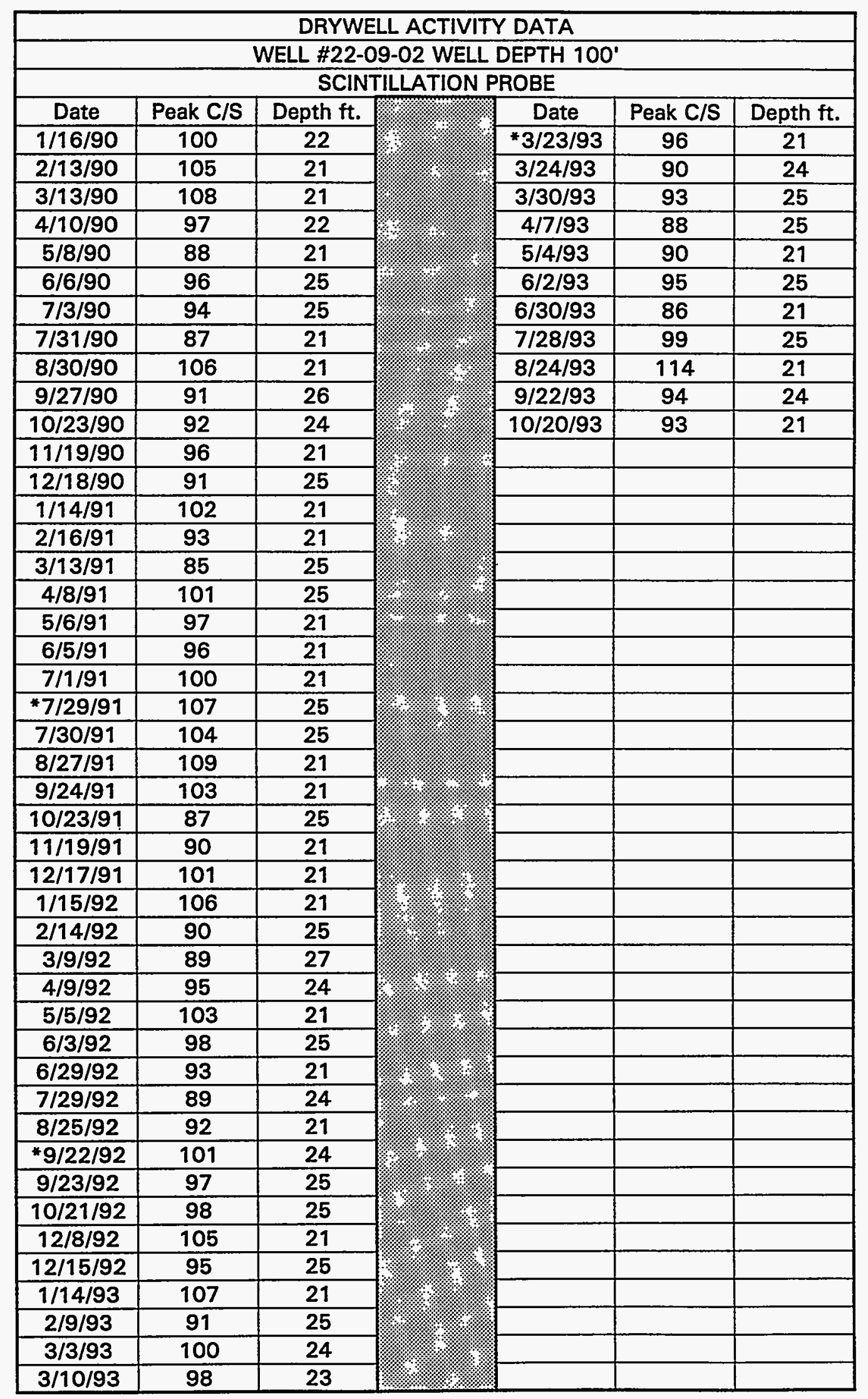

Data obtained from WHC Surveillance Data Acquisition (SDA), November 30, 1993. 


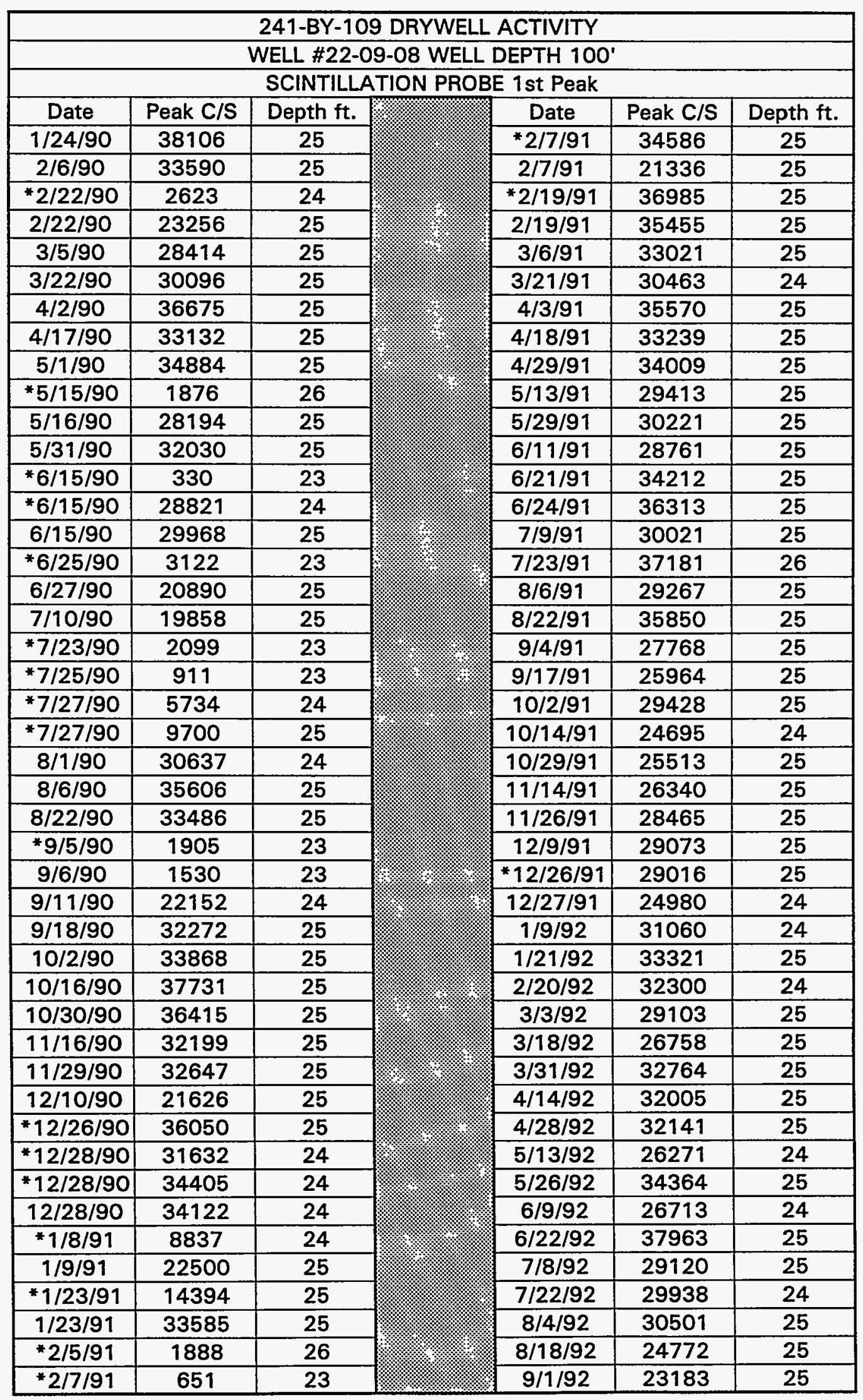

Data obtained from WHC Surveillance Data Acquisition (SDA), November 30, 1993. 


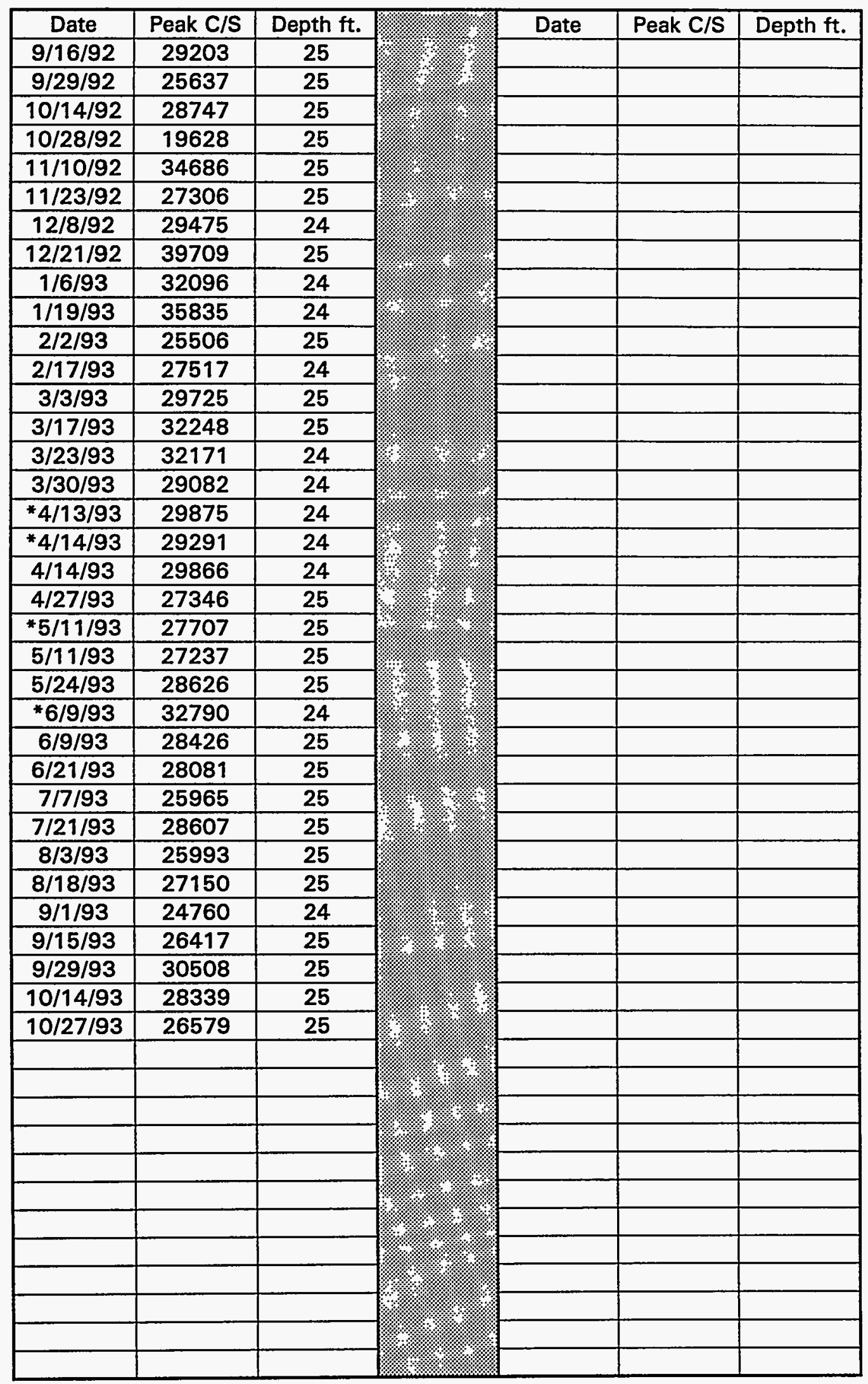

Data obtained from WHC Surveillance Data Acquisition (SDA), November 30, 1993. 


\begin{tabular}{|c|c|c|c|c|c|c|}
\hline & & 241-BY-10 & DRYWELL & ACTIVITY & & \\
\hline & & VELL \#22-C & 9-08 WELL & DEPTH $100^{\circ}$ & & \\
\hline & & SCINTILLA & FION PROBE & 2nd Peak & & \\
\hline Date & Peak C/S & Depth $\mathrm{ft}$ & & Date & Peak C/S & Depth $\mathrm{ft}$ \\
\hline $1 / 24 / 90$ & 50 & 84 & & *2/7/91 & & \\
\hline $2 / 6 / 90$ & 56 & 76 & & $2 / 7 / 91$ & & \\
\hline${ }^{*} 2 / 22 / 90$ & & & & ${ }^{*} 2 / 19 / 91$ & & \\
\hline $2 / 22 / 90$ & & & & $2 / 19 / 91$ & & \\
\hline $3 / 5 / 90$ & & & \% & $3 / 6 / 91$ & & \\
\hline $3 / 22 / 90$ & 54 & 83 & & $3 / 21 / 91$ & & \\
\hline $4 / 2 / 90$ & 55 & 83 & & $4 / 3 / 91$ & & \\
\hline $4 / 17 / 90$ & 53 & 83 & & $4 / 18 / 91$ & & \\
\hline $5 / 1 / 90$ & 46 & 83 & & $4 / 29 / 91$ & 50 & 83 \\
\hline *5/15/90 & & & & $5 / 13 / 91$ & & \\
\hline $5 / 16 / 90$ & 48 & 83 & & $5 / 29 / 91$ & & \\
\hline $5 / 31 / 90$ & 53 & 83 & & $6 / 11 / 91$ & & \\
\hline${ }^{*} 6 / 15 / 90$ & & & & $6 / 21 / 91$ & & \\
\hline${ }^{*} 6 / 15 / 90$ & 55 & 83 & & $6 / 24 / 91$ & & \\
\hline $6 / 15 / 90$ & 46 & 83 & & $7 / 9 / 91$ & & \\
\hline${ }^{*} 6 / 25 / 90$ & & & & $7 / 23 / 91$ & & \\
\hline $6 / 27 / 90$ & & & & $8 / 6 / 91$ & 40 & 82 \\
\hline $7 / 10 / 90$ & & & & $8 / 22 / 91$ & & \\
\hline$* 7 / 23 / 90$ & & & & $9 / 4 / 91$ & & \\
\hline${ }^{*} 7 / 25 / 90$ & & & & $9 / 17 / 91$ & & \\
\hline${ }^{*} 7 / 27 / 90$ & & & & $10 / 2 / 91$ & & \\
\hline$* 7 / 27 / 90$ & & & & $10 / 14 / 91$ & & \\
\hline $8 / 1 / 90$ & & & & $10 / 29 / 91$ & & \\
\hline $8 / 6 / 90$ & & & & $11 / 14 / 91$ & & \\
\hline $8 / 22 / 90$ & & & & $11 / 26 / 91$ & & \\
\hline * 9/5/90 & & & & $12 / 9 / 91$ & & \\
\hline $9 / 6 / 90$ & & & & *12/26/91 & 65 & 90 \\
\hline $9 / 11 / 90$ & & & & $12 / 27 / 91$ & & \\
\hline $9 / 18 / 90$ & & & & $1 / 9 / 92$ & & \\
\hline $10 / 2 / 90$ & & & 2 & $1 / 21 / 92$ & & \\
\hline $10 / 16 / 90$ & & & & $2 / 20 / 92$ & & \\
\hline $10 / 30 / 90$ & 49 & 83 & & $3 / 3 / 92$ & & \\
\hline $11 / 16 / 90$ & & & & $3 / 18 / 92$ & & \\
\hline $11 / 29 / 90$ & & & & $3 / 31 / 92$ & & \\
\hline $12 / 10 / 90$ & & & & $4 / 14 / 92$ & & \\
\hline * $12 / 26 / 90$ & 62 & 83 & & $4 / 28 / 92$ & & \\
\hline * $12 / 28 / 90$ & & & & $5 / 13 / 92$ & & \\
\hline *12/28/90 & & & & $5 / 26 / 92$ & & \\
\hline $12 / 28 / 90$ & & & & $6 / 9 / 92$ & & \\
\hline$* 1 / 8 / 91$ & & & & $6 / 22 / 92$ & & \\
\hline $1 / 9 / 91$ & & & & $7 / 8 / 92$ & & \\
\hline$* 1 / 23 / 91$ & & & & $7 / 22 / 92$ & & \\
\hline $1 / 23 / 91$ & & & & $8 / 4 / 92$ & & \\
\hline *2/5/91 & & & 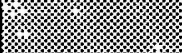 & $8 / 18 / 92$ & 53 & 83 \\
\hline$* 2 / 7 / 91$ & & & & $9 / 1 / 92$ & & \\
\hline
\end{tabular}

Data obtained from WHC Surveillance Data Acquisition (SDA), November 30, 1993. 


\begin{tabular}{|c|c|c|c|c|c|}
\hline \multirow{2}{*}{\multicolumn{6}{|c|}{\begin{tabular}{c|c} 
Date & Peak C/S \\
$9 / 16 / 92$ &
\end{tabular}}} \\
\hline & & & & & \\
\hline \multicolumn{6}{|l|}{$9 / 29 / 92$} \\
\hline \multicolumn{6}{|l|}{$10 / 14 / 92$} \\
\hline \multicolumn{6}{|l|}{$10 / 28 / 92$} \\
\hline \multicolumn{6}{|l|}{$11 / 10 / 92$} \\
\hline \multicolumn{6}{|l|}{$11 / 23 / 92$} \\
\hline \multicolumn{6}{|l|}{$12 / 8 / 92$} \\
\hline \multicolumn{6}{|l|}{$12 / 21 / 92$} \\
\hline \multicolumn{6}{|l|}{$1 / 6 / 93$} \\
\hline \multirow{2}{*}{\multicolumn{6}{|c|}{$\frac{1 / 79 / 93}{2 / 2 / 93}$}} \\
\hline & & & & & \\
\hline \multicolumn{6}{|l|}{$2 / 17 / 93$} \\
\hline \multicolumn{6}{|l|}{$3 / 3 / 93$} \\
\hline \multirow{2}{*}{\multicolumn{6}{|c|}{$3 / 17 / 93$}} \\
\hline \multicolumn{2}{|l|}{$3 / 23 / 93$} & & & & \\
\hline \multicolumn{6}{|l|}{$3 / 30 / 93$} \\
\hline \multirow{2}{*}{\multicolumn{6}{|c|}{ *4/13/93 }} \\
\hline \multicolumn{2}{|l|}{${ }^{* 4 / 14 / 93}$} & & & & \\
\hline \multirow{2}{*}{\multicolumn{6}{|c|}{$4 / 14 / 93$}} \\
\hline \multirow{2}{*}{\multicolumn{6}{|c|}{$\begin{array}{l}4 / 27 / 93 \\
* 5 / 11 / 93\end{array}$}} \\
\hline & & & & & \\
\hline \multicolumn{6}{|l|}{$5 / 11 / 93$} \\
\hline \multicolumn{6}{|l|}{$5 / 24 / 93$} \\
\hline \multirow{2}{*}{\multicolumn{6}{|c|}{$* 6 / 9 / 93$}} \\
\hline \multicolumn{2}{|l|}{$6 / 9 / 93$} & & & & \\
\hline $6 / 21 / 93$ & & & & & \\
\hline $7 / 7 / 93$ & & & & & \\
\hline $7 / 21 / 93$ & & & & & \\
\hline $8 / 3 / 93$ & & & & & \\
\hline $8 / 18 / 93$ & & & & & \\
\hline $9 / 1 / 93$ & & & & & \\
\hline $9 / 15 / 93$ & & & & & \\
\hline $9 / 29 / 93$ & & & & & \\
\hline $10 / 14 / 93$ & & & & & \\
\hline $10 / 27 / 93$ & & & & & \\
\hline & & & & & \\
\hline & & & & & \\
\hline & & & & & \\
\hline & & & & & \\
\hline & & & & & \\
\hline & & & & & \\
\hline & & & & & \\
\hline & & & & & \\
\hline & & & & & \\
\hline
\end{tabular}

Data obtained from WHC Surveillance Data Acquisition (SDA), November 30, 1993. 


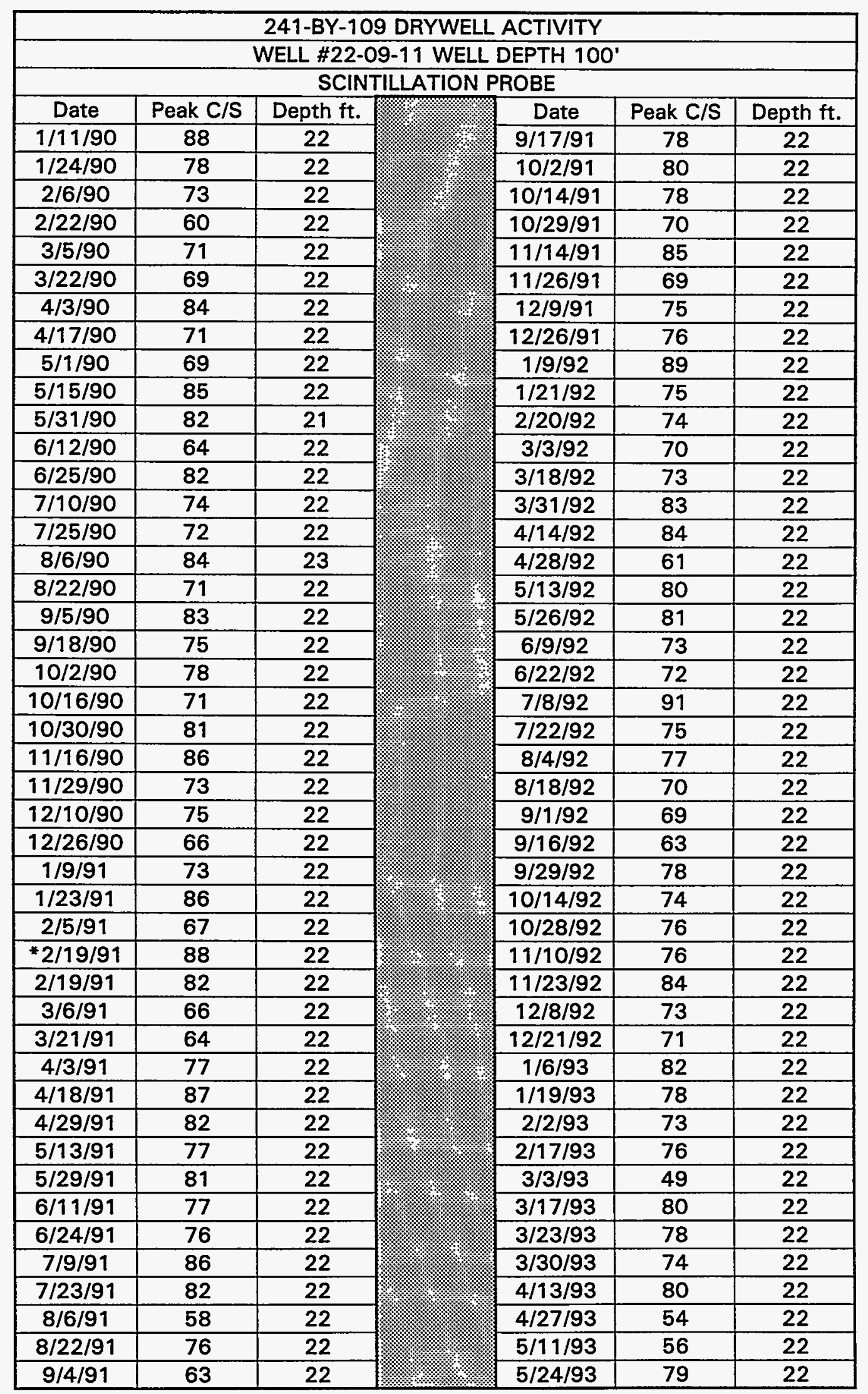

Data obtained from WHC Surveillance Data Acquisition (SDA), November 30, 1993. 
WHC-SD-WM-ER-312, Rev. 0

\begin{tabular}{|c|c|c|c|c|c|c|}
\hline Date & Peak C/S & Depth ft. & \multirow{4}{*}{ II) } & Date & Peak C/S & Depth ft. \\
\hline $6 / 9 / 93$ & 75 & 22 & & & & \\
\hline $6 / 21 / 93$ & 79 & 21 & & & & \\
\hline 7/7/93 & 61 & 22 & & & & \\
\hline $7 / 21 / 93$ & 66 & 22 & \multirow{4}{*}{$4 \sqrt{4}$} & & & \\
\hline $8 / 3 / 93$ & 96 & 22 & & & & \\
\hline $8 / 18 / 93$ & 75 & 22 & & & & \\
\hline $9 / 1 / 93$ & 75 & 22 & & & & \\
\hline $9 / 15 / 93$ & 73 & 22 & \multirow[b]{2}{*}{ 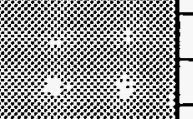 } & & & \\
\hline $9 / 29 / 93$ & 82 & 22 & & & & \\
\hline $10 / 14 / 93$ & 70 & 22 & \multirow{2}{*}{$4=18$} & & & \\
\hline $10 / 27 / 93$ & 81 & 22 & & & & \\
\hline & & & \multirow{5}{*}{ 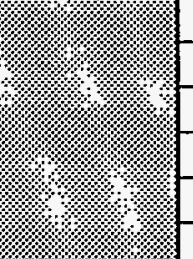 } & & & \\
\hline & & & & & & \\
\hline & & & & & & \\
\hline & & & & & & \\
\hline & & & & & & \\
\hline & & & \multirow[b]{2}{*}{ প্রে } & & & \\
\hline & & & & & & \\
\hline & & & \multirow[b]{2}{*}{47} & & & \\
\hline & & & & & & \\
\hline & & & & & & \\
\hline & & & \multirow[b]{2}{*}{ / } & & & \\
\hline & & & & & & \\
\hline & & & \multirow{2}{*}{$9=19=1: 19$} & & & \\
\hline & & & & & & \\
\hline & & & & & & \\
\hline & & & & & & \\
\hline & & & & & & \\
\hline & & & & & & \\
\hline & & & & & & \\
\hline & & & & & & \\
\hline & & & & & & \\
\hline & & & & & & \\
\hline & & & \multirow[b]{2}{*}{4} & & & \\
\hline & & & & & & \\
\hline & & & \multirow{5}{*}{ 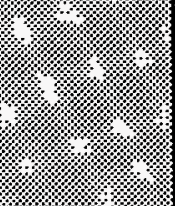 } & & & \\
\hline & & & & & & \\
\hline & & & & & & \\
\hline & & & & & & \\
\hline & & & & & & \\
\hline & & & \multirow{7}{*}{ 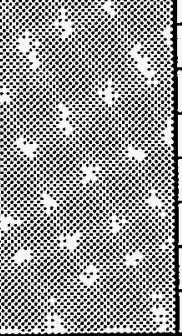 } & & & \\
\hline & & & & & & \\
\hline & & & & & & \\
\hline & & & & & & \\
\hline & & & & & & \\
\hline & & & & & & \\
\hline & & & & & & \\
\hline
\end{tabular}

Data obtained from WHC Surveillance Data Acquisition (SDA), November 30, 1993. 
WHC-SD-WM-ER-312, Rev. 0

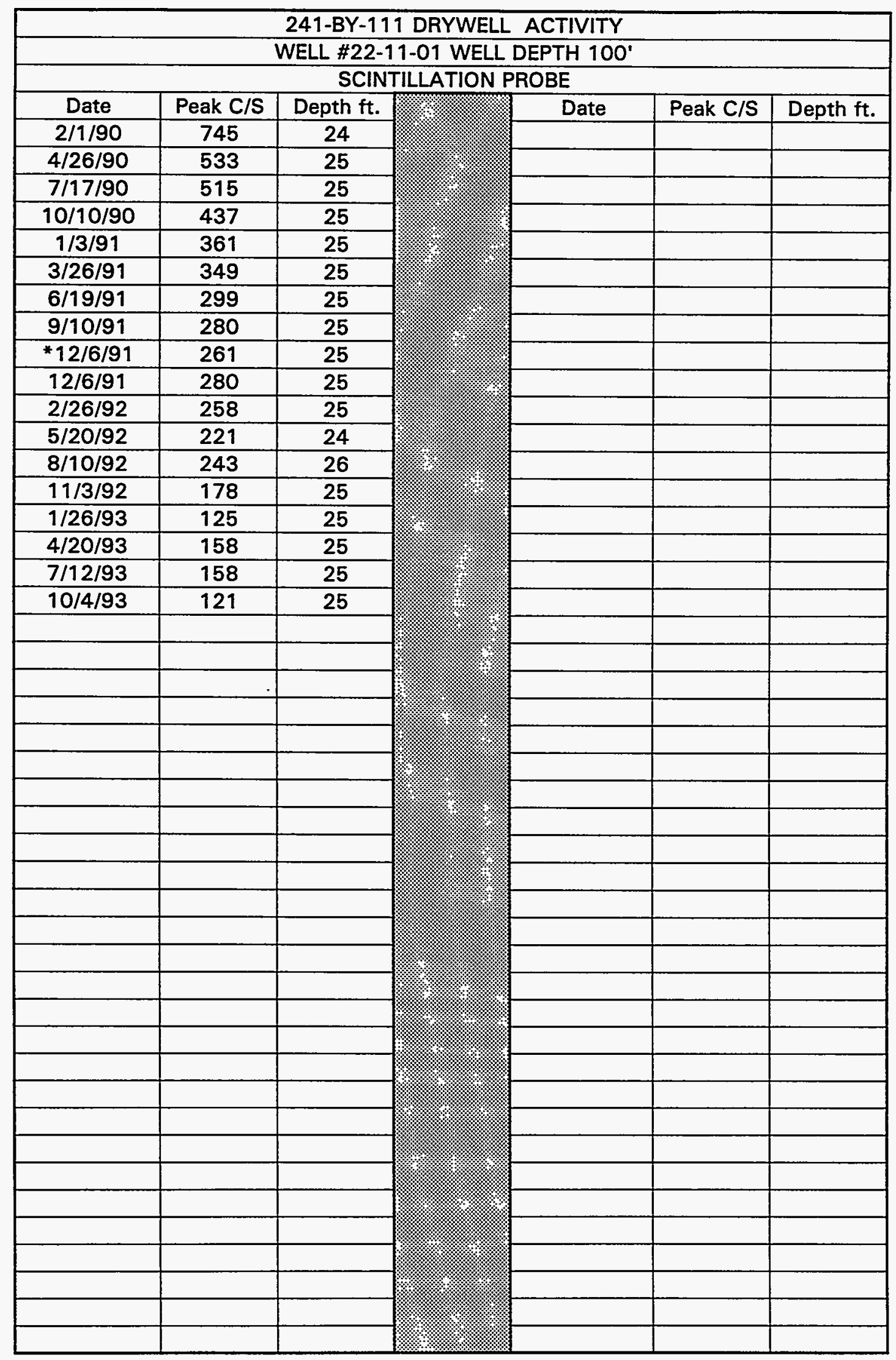

Data obtained from WHC Surveillance Data Acquisition (SDA), November 30, 1993. 


\begin{tabular}{|c|c|c|c|c|c|c|}
\hline \multicolumn{7}{|c|}{$\begin{array}{l}\text { 241-BY-111 DRYWELL ACTIVITY } \\
\text { WELL \#22-11-09 WELL DEPTH 100' }\end{array}$} \\
\hline \multicolumn{7}{|c|}{ SCINTILLATION PROBE } \\
\hline Date & Peak C/S & Depth ft. & & Date & Peak C/S & Depth ft. \\
\hline $2 / 1 / 90$ & 40 & 28 & & & & \\
\hline *4/25/90 & 48 & 28 & & & & \\
\hline $4 / 26 / 90$ & 54 & 28 & ] & & & \\
\hline $7 / 17 / 90$ & 39 & 49 & & & & \\
\hline $10 / 9 / 90$ & 43 & 28 & & & & \\
\hline $1 / 3 / 91$ & 51 & 28 & & & & \\
\hline $3 / 26 / 91$ & 40 & 86 & & & & \\
\hline $6 / 19 / 91$ & 39 & 69 & & & & \\
\hline $10 / 9 / 91$ & 42 & 28 & & & & \\
\hline $12 / 6 / 91$ & 44 & 28 & & & & \\
\hline $2 / 26 / 92$ & 37 & 28 & & & & \\
\hline $5 / 19 / 92$ & 45 & 28 & & & & \\
\hline * $8 / 10 / 92$ & 50 & 70 & & & & \\
\hline $8 / 11 / 92$ & 41 & 28 & 9 & & & \\
\hline $11 / 3 / 92$ & 38 & 28 & & & & \\
\hline $1 / 26 / 93$ & 42 & 32 & & & & \\
\hline $4 / 20 / 93$ & 37 & 83 & & & & \\
\hline $7 / 14 / 93$ & 41 & 28 & & & & \\
\hline $10 / 4 / 93$ & 41 & 28 & & & & \\
\hline & & & & & & \\
\hline & & & & & & \\
\hline & & & & & & \\
\hline & & & & & & \\
\hline & & & & & & \\
\hline & & & & & & \\
\hline & & & & & & \\
\hline & & & & & & \\
\hline & & & 9 & & & \\
\hline & & & & & & \\
\hline & & & & & & \\
\hline & & & $=4=1$ & & & \\
\hline & & & (19:-1: & & & \\
\hline & & & & & & \\
\hline & & & 19 & & & \\
\hline & & & & & & \\
\hline & & & $14: 1$ & & & \\
\hline & & & & & & \\
\hline & & & $12: 120$ & & & \\
\hline & & & ? & & & \\
\hline & & & & & & \\
\hline & & & & & & \\
\hline
\end{tabular}

Data obtained from WHC Surveillance Data Acquisition (SDA), November 30, 1993. 
WHC-SD-WM-ER-312, Rev . 0

THIS PAGE INTENTIONALLY LEFT BLANK

G-1 


\begin{tabular}{|c||c||l||}
\hline \multicolumn{2}{|c||}{ 241-BY-101 } \\
\hline \hline NO. & DIA. & \multicolumn{1}{|c||}{ DESCRIPTION AND COMMENTS } \\
\hline \hline 1 & $4^{\prime \prime}$ & TEMPERATURE PROBE, UNUSED \\
\hline 2 & $4^{\prime \prime}$ & BG \\
\hline 3 & $4^{\prime \prime}$ & PIT DRAIN \\
\hline 4 & $4^{\prime \prime}$ & BREATHER FILTER, G-1 HOUSING \\
\hline 5 & $4^{\prime \prime}$ & LIQUID LEVEL REEL , BM \\
\hline 6 & $12^{\prime \prime}$ & CONCRETE COVER \\
\hline 7 & $12^{\prime \prime}$ & SALTWELL SCREEN \& PUMP \\
\hline 8 & $12^{\prime \prime}$ & CONCRETE COVER \\
\hline 9 & $42^{\prime \prime}$ & PIT WC \\
\hline $9 \mathrm{~A}$ & $4^{\prime \prime}$ & FLANGE/B-436 LOW \\
\hline $9 \mathrm{~B}$ & $18^{\prime \prime}$ & FLANGE/45 AIR SWEEP \\
\hline 10 & $42^{\prime \prime}$ & BG \\
\hline $10 \mathrm{~A}$ & $12^{\prime \prime}$ & OBSV PORT \\
\hline 11 & $42^{\prime \prime}$ & PIT WC \\
\hline 12 & $42^{\prime \prime}$ & PIT WC \\
\hline $12 \mathrm{~A}$ & $12^{\prime \prime}$ & (WEIGHT FACTOR - SP GR) \\
\hline 13 & $42^{\prime \prime}$ & \\
\hline $13 \mathrm{~A}$ & $12^{\prime \prime}$ & (AIR LIFT CIRCULATOR, ITS UNIT) \\
\hline \hline
\end{tabular}




\section{$241-B Y-101$}

2,872,820 Liters

[758,000 Gallons]
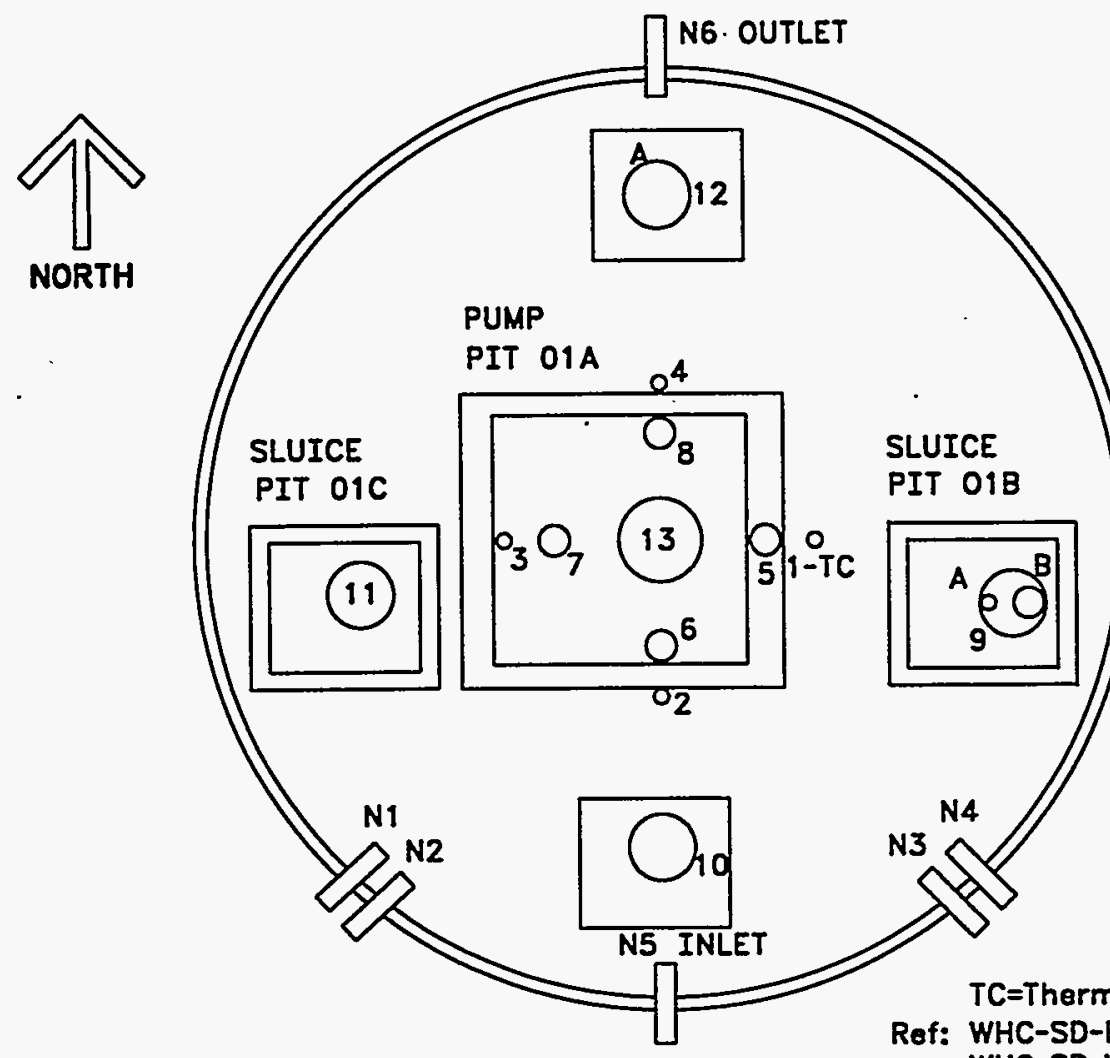

\section{TANK RISER LOCATION}

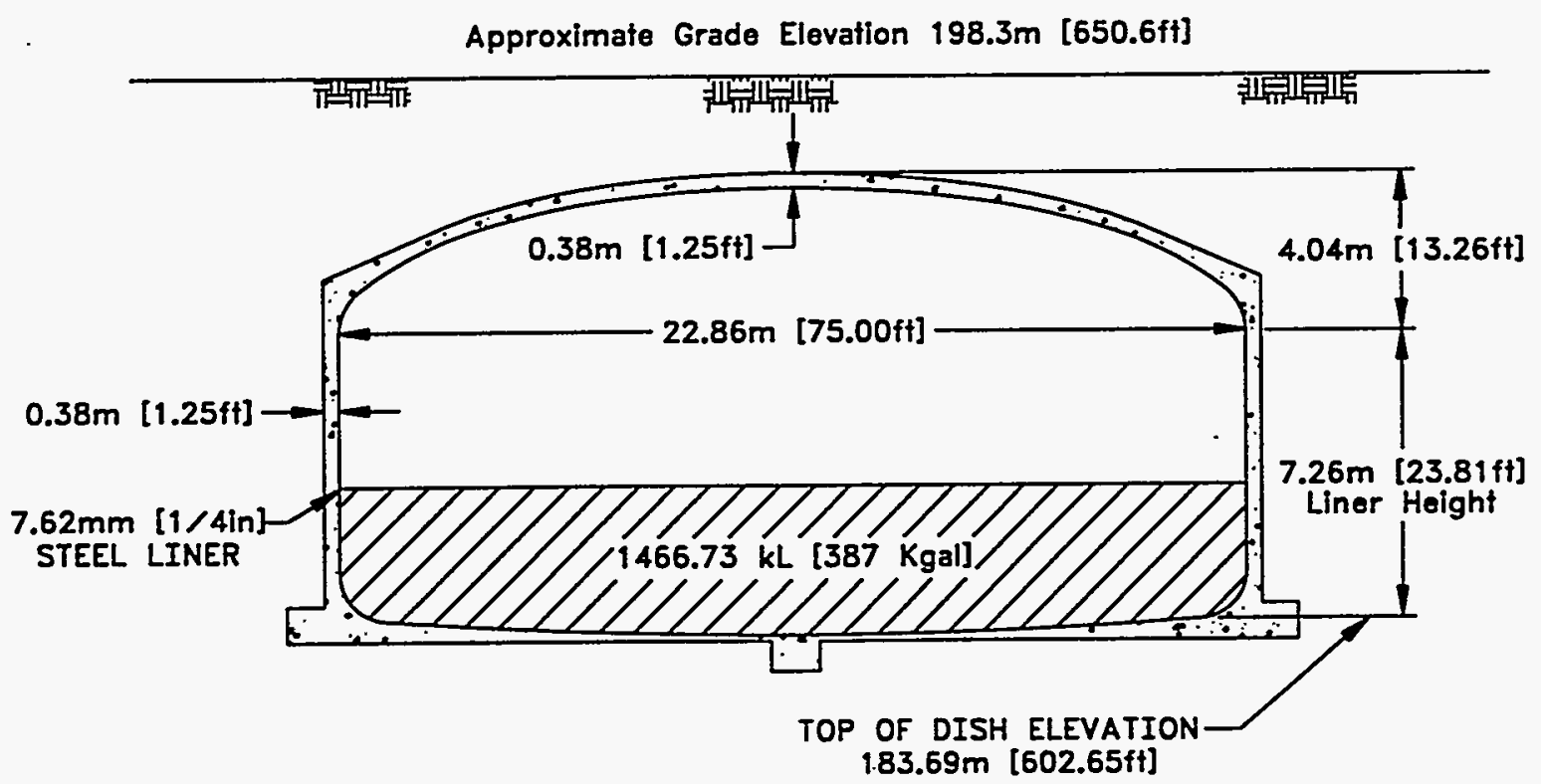

Ref: H-2-1312. Rev. 2 
WHC-SD-WM-ER-312, REV. 0

\begin{tabular}{|c|c|c|}
\hline \multicolumn{3}{|r|}{ 241-BY-102 } \\
\hline NO. & DIA. & DESCRIPTION AND COMMENTS \\
\hline 1 & 4" & $B-436$ LOW \\
\hline 2 & 4" & BG \\
\hline 3 & $4 "$ & \\
\hline 4 & 4" & SMP \\
\hline 5 & 4" & LIQUID LEVEL REEL , BM \\
\hline 6 & $12 "$ & CONCRETE PLUG \\
\hline$\overline{7}$ & $12 "$ & SALTWELL SCREEN AND PUMP \\
\hline 8 & $12 "$ & CONCRETE PLUG \\
\hline $9 A$ & $42 "$ & WC \\
\hline $9 \mathrm{~B}$ & $26 "$ & WC \\
\hline 10 & $42 "$ & WC, BG \\
\hline $10 \mathrm{~A}$ & $12 "$ & OBSV PORT \\
\hline 11 & $42 "$ & WC, BG \\
\hline 12 & $42 "$ & WC, $B G$ \\
\hline $12 \mathrm{~A}$ & 41 & $W C, \quad B G$ \\
\hline 13 & $42 "$ & (AIR LIFT CIRCULATOR, ITS UNIT) \\
\hline 14 & $4 "$ & BREATHER FILTER \\
\hline
\end{tabular}




\section{1-BY-102}

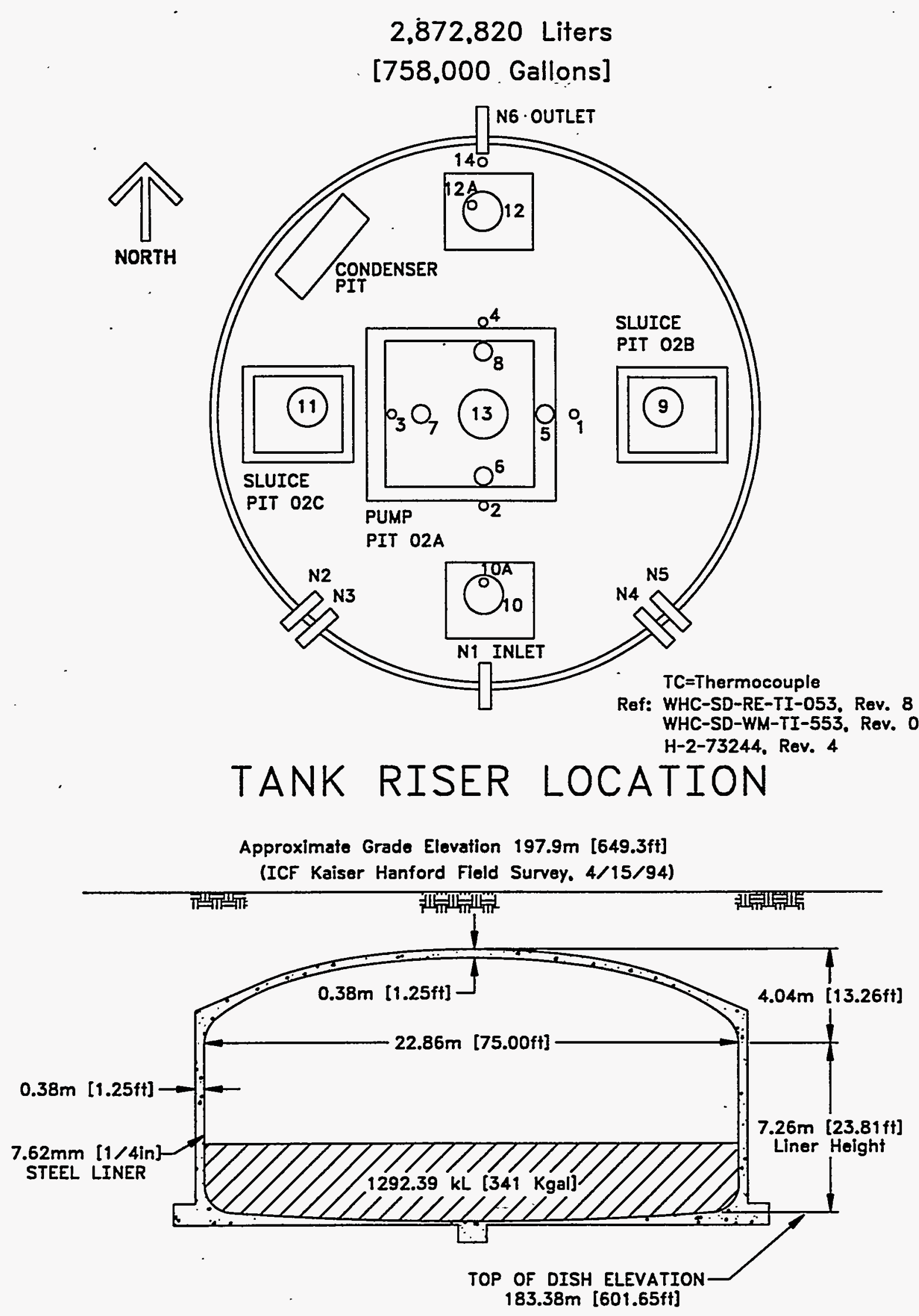

Ref: H-2-1312, Rev. 2 


\begin{tabular}{|c|c|c|}
\hline \multicolumn{3}{|r|}{ 241-BY-103 } \\
\hline NO. & DIA. & DESCRIPTION AND COMMENTS \\
\hline 1 & $4 "$ & TEMPERATURE PROBE, UNUSED \\
\hline 2 & $4 "$ & BG \\
\hline 3 & 4" & PIT DRAIN \\
\hline 4 & $4 "$ & FLANGE \\
\hline 5 & 4" & LIQUID LEVEL REEL \\
\hline 6 & $12 "$ & CONCRETE COVER \\
\hline 7 & $12 "$ & SALTWELL SCREEN AND PUMP \\
\hline 8 & $12 "$ & CONCRETE COVER \\
\hline $9 \mathrm{~A}$ & $4 "$ & WC \\
\hline $9 B$ & $14 "$ & WC \\
\hline $10 \mathrm{~A}$ & $4 "$ & B-436 LOW \\
\hline $10 \mathrm{~B}$ & $12^{\prime \prime}$ & OBSV PORT \\
\hline $10 \mathrm{C}$ & $14 "$ & BG, AIRLIFT CIRCULATOR (WELDED COVER) \\
\hline $10 D$ & $4 "$ & IIQUID LEVEL REEL \\
\hline 11 & $42 "$ & $B G, \quad W C$ \\
\hline 12 & $42 "$ & BG \\
\hline $12 \mathrm{~A}$ & 4" & BREATHER FILTER \\
\hline $12 \mathrm{~B}$ & $14 "$ & BG， AIR LIFT CIRCULATOR \\
\hline $12 \mathrm{C}$ & $18 "$ & BG \\
\hline 13 & $42 "$ & \\
\hline $13 \mathrm{~A}$ & $4 "$ & FLANGE \\
\hline $13 B$ & $14 "$ & AIRLIFT CIRCULATOR \\
\hline
\end{tabular}




\section{$241-B Y-103$}

2,872,820 Liters

[758,000 Gallons]
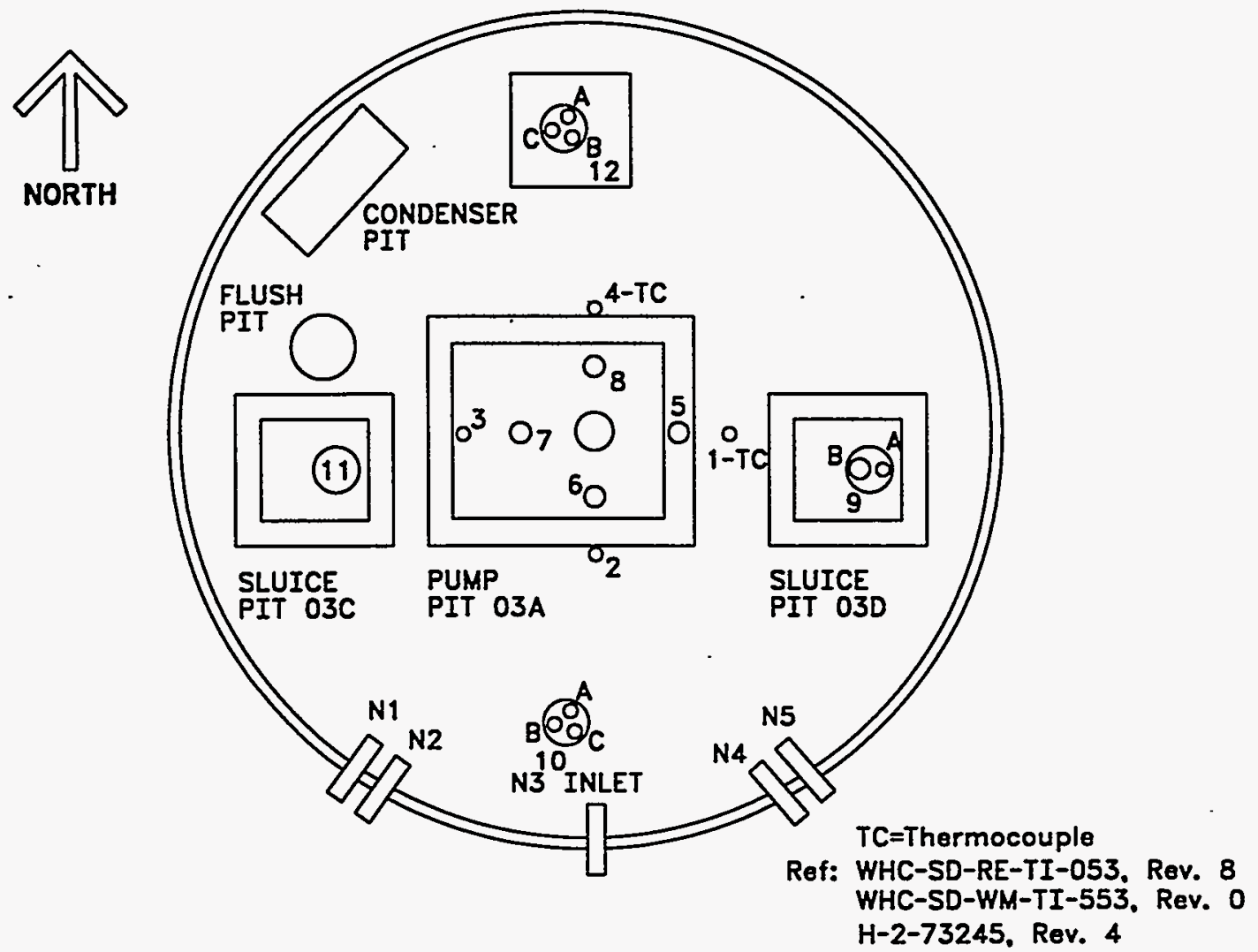

TANK RISER LOCATION

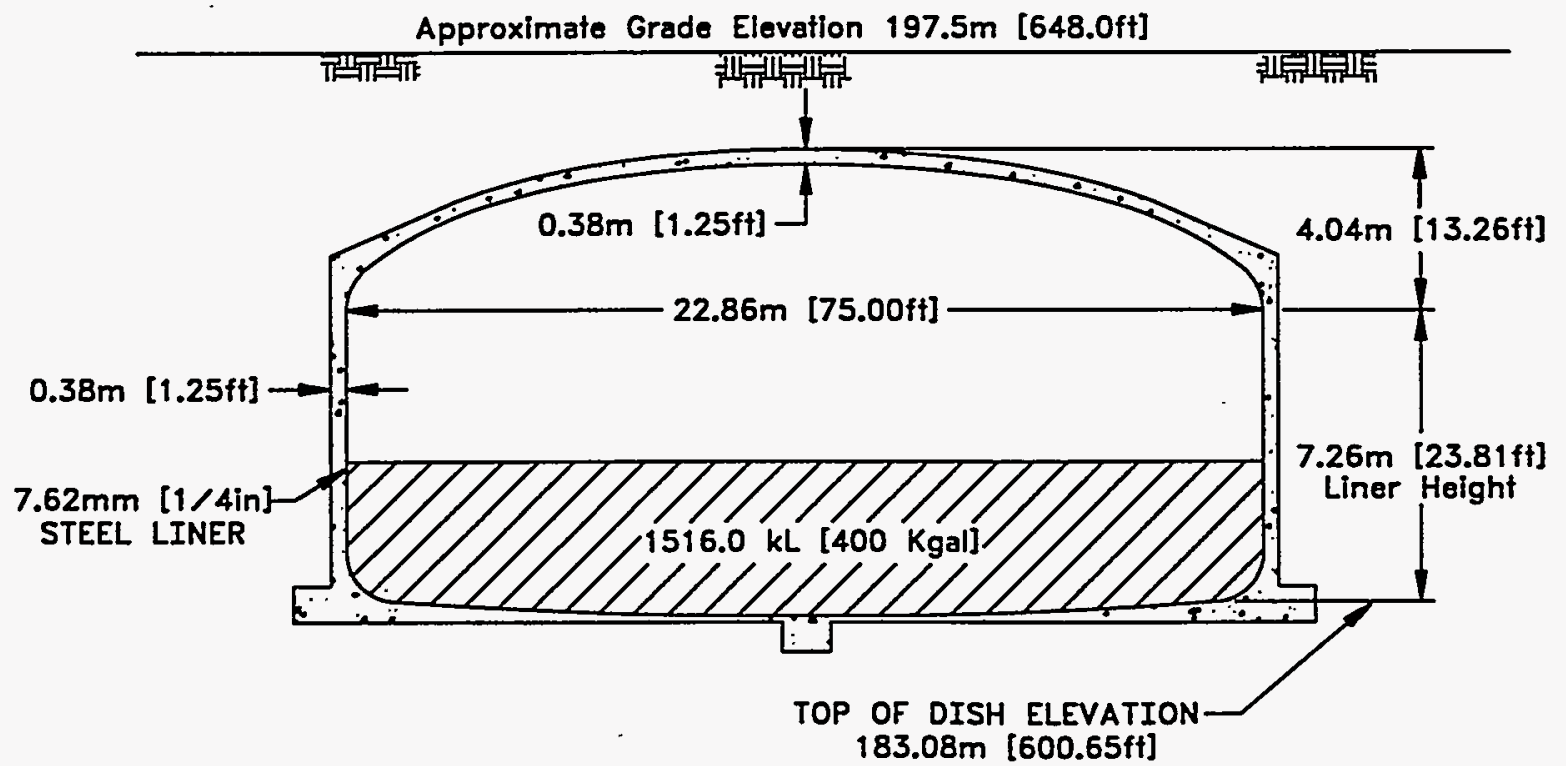

Ref: H-2-1312, Rev. 2 Merrick Maps of

200 Area's 89210406 


\begin{tabular}{|c||c||l||}
\hline \multicolumn{2}{|c||}{ 241-BY-104 } \\
\hline \hline NO. & DIA. & \multicolumn{1}{|c||}{ DESCRIPTION AND COMMENTS } \\
\hline \hline 1 & $4 "$ & TEMPERATURE PROBE, MANUAL B-221 \\
\hline 2 & $4 "$ & DOG LEG - BG \\
\hline 3 & $4 "$ & SPARE \\
\hline 4 & $4 "$ & BREATHER FILTER, G-1 HOUSING \\
\hline 5 & $12^{\prime \prime}$ & LIQUID LEVEL REEL, 4" SPOOL \\
\hline 6 & $12^{\prime \prime}$ & CONCRETE COVER \\
\hline 7 & $12^{\prime \prime}$ & (SALTWELL SCREEN \& PUMP) \\
\hline 8 & $12^{\prime \prime}$ & CONCRETE COVER \\
\hline 9 & $42^{\prime \prime}$ & WC \\
\hline $10 A$ & $18^{\prime \prime}$ & UNUSABLE OBSV PORT \\
\hline $10 B$ & $4 "$ & SPARE \\
\hline $10 C$ & $4^{\prime \prime}$ & B-436 LOW, BM \\
\hline 11 & $42^{\prime \prime}$ & WC \\
\hline 12 & $42^{\prime \prime}$ & WC AND SHIELDED \\
\hline 13 & $42^{\prime \prime}$ & \\
\hline
\end{tabular}




\section{1-BY-104}

2,872,820 Liters

- [758,000 Gallons]
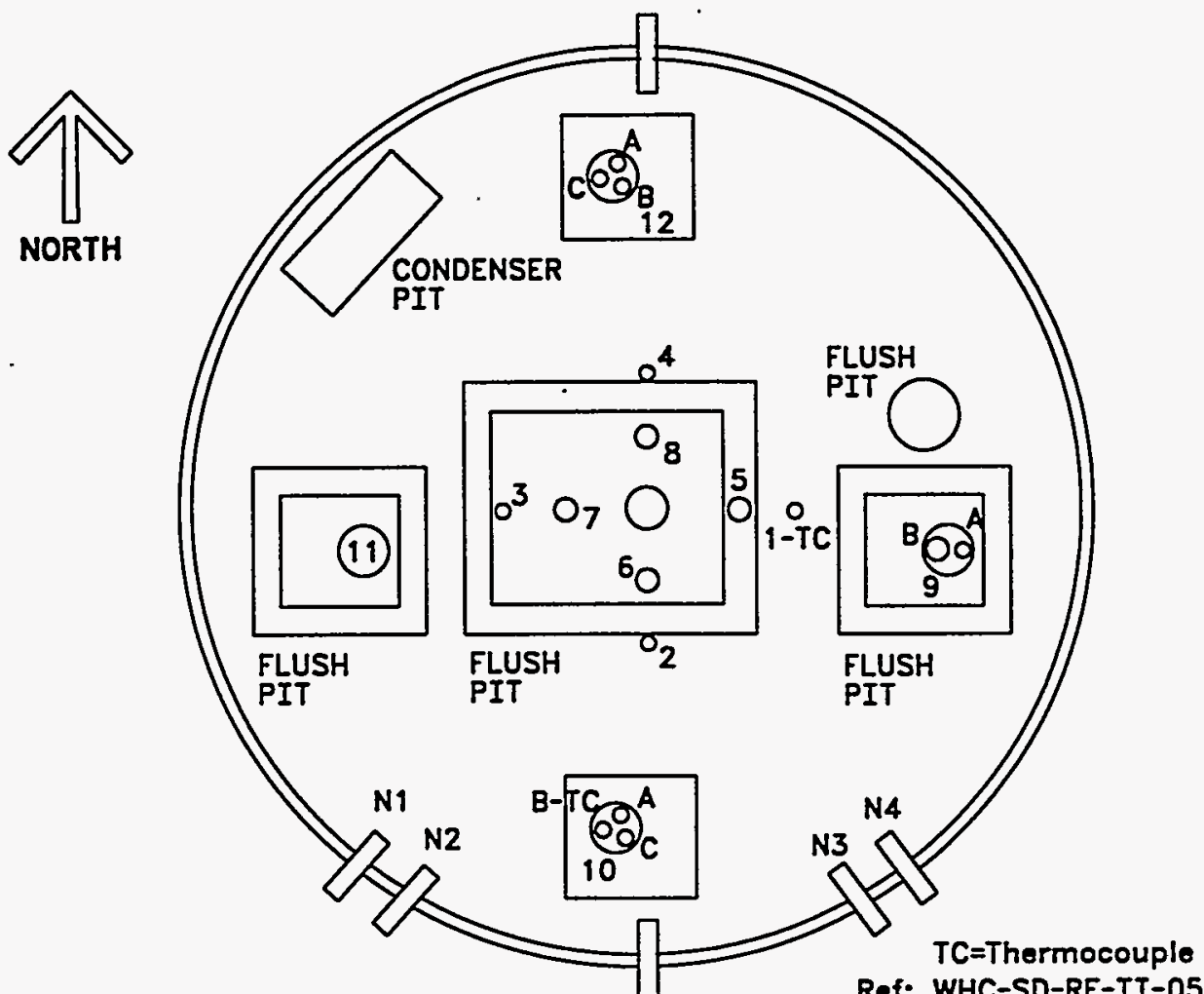

N5 INLET

Ref: WHC-SD-RE-TI-053, Rev. 8 WHC-SD-WM-TI-553, Rev. 0 H-2-73252, Rev. 2

TANK RISER LOCATION

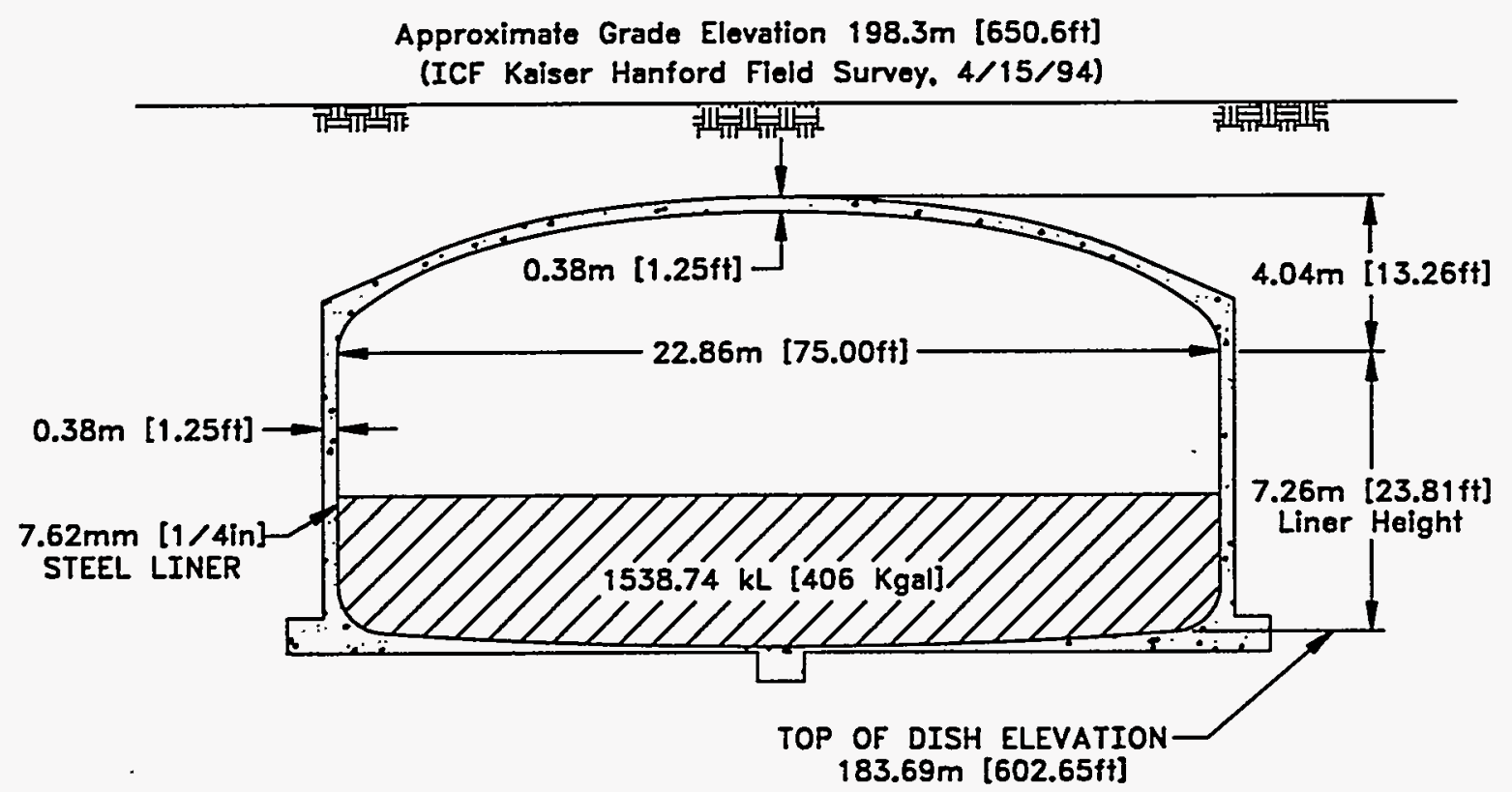

Ref: $H-2-1312$, Rev. 2 
WHC-SD-WM-ER-312, REV. 0

\begin{tabular}{|c|c|c|}
\hline NO. & DIA. & DESCRIPTION AND COMMENTS \\
\hline 1 & $4 "$ & TEMPERATURE PROBE - MANUAL B-221 \\
\hline 2 & $4 "$ & DOG LEG - BG \\
\hline 3 & $4 "$ & DRAIN \\
\hline 4 & 6" & FLANGE - DOG LEG \\
\hline 5 & $12 "$ & IIQUID LEVEI REEI, 4" SPOOI \\
\hline 6 & $12 "$ & CONCRETE COVER \\
\hline 7 & $12^{\prime \prime}$ & SPARE \\
\hline 8 & $12^{\prime \prime}$ & CONCRETE COVER \\
\hline 9 & $42 "$ & $\dot{-}$ \\
\hline $10 \mathrm{~A}$ & $18 "$ & DEENTRAINER AND AIR IIFT CIRCULATOR \\
\hline $10 \mathrm{~B}$ & $4 "$ & B-436 LOW \\
\hline $10 \mathrm{C}$ & $4 "$ & TEMPERATURE， BM \\
\hline 11 & $42^{\prime \prime}$ & WC \\
\hline $12 \mathrm{~A}$ & $4^{\prime \prime}$ & BREATHER FILTER \\
\hline $12 \mathrm{~B}$ & $18 "$ & AIR INLET \\
\hline $12 \mathrm{C}$ & $14 "$ & AIR IIFT CIRCULATOR, BG \\
\hline 13 & $42^{\prime \prime}$ & \\
\hline $13 \mathrm{~A}$ & $4 "$ & SPARE， COVERED \\
\hline $13 \mathrm{~B}$ & $12 "$ & SALTWELL SCREEN \\
\hline
\end{tabular}




\section{$241-B Y-105$}

2,872,820 Liters

[758,000 Gallons]
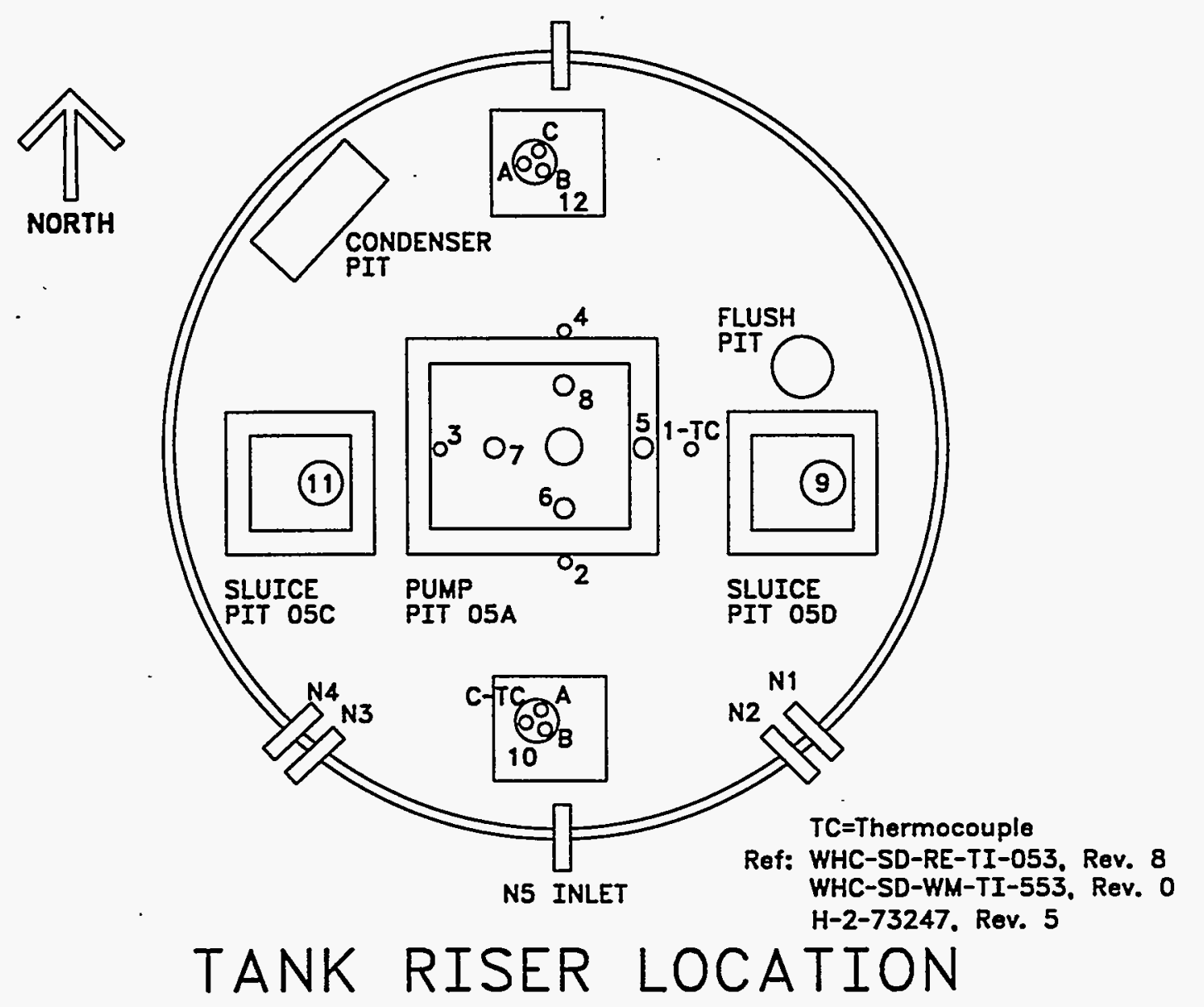

Approximate Grade Elevation 197.9m [649.3ft]

(ICF Kaiser Hanford Field Survey, 4/15/94)

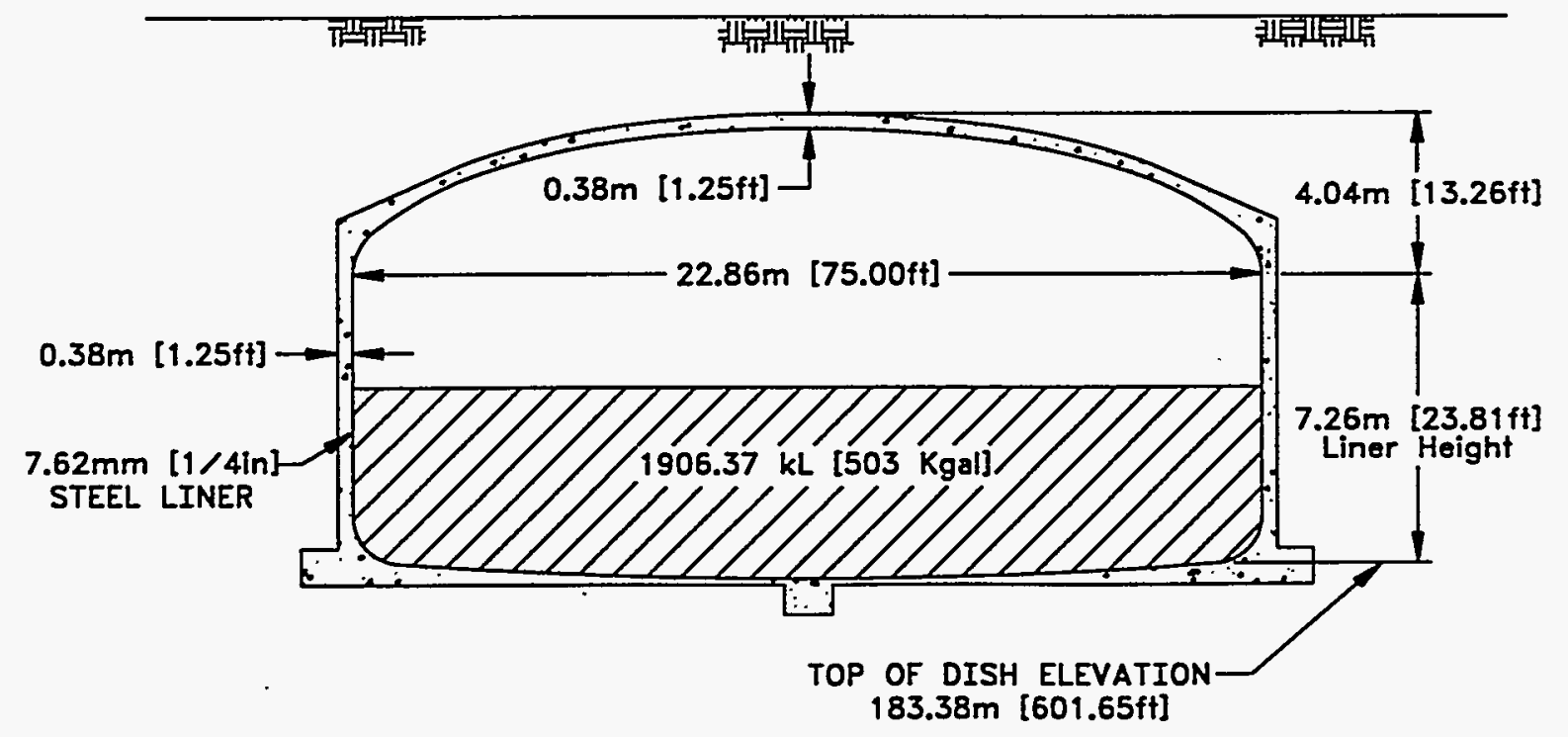

Ref: $\mathrm{H}-2-1312$. Rev. 2 


\begin{tabular}{|c|c|c|}
\hline NO. & DIA. & DESCRIPTION AND COMMENTS \\
\hline 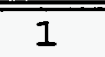 & 6" & TEMPERATURE PROBE - MANUAI B-221 \\
\hline 2 & $4 "$ & $\mathrm{BG}$ \\
\hline 3 & $4 "$ & PIT DRAIN \\
\hline 4 & $4 "$ & FIAGE \\
\hline 5 & $12 "$ & LIQUID LEVEL REEL, 4" SPOOL \\
\hline 6 & $4^{\prime \prime}$ & CONCRETE COVER \\
\hline 7 & $12^{\prime \prime}$ & SPARE \\
\hline 8 & $12^{\prime \prime}$ & CONCRETE COVER \\
\hline 9 & $42 "$ & SALTWELL SCREEN \\
\hline $10 A$ & $18 "$ & DEEDNTRAINER AND AIR LIFT CIRCULATOR \\
\hline $10 \mathrm{~B}$ & $4 "$ & BM, B-436 LOW \\
\hline $10 \mathrm{C}$ & $4^{\prime \prime}$ & BREATHER FILTER \\
\hline 11 & $42^{\prime \prime}$ & WC, SALTWELL SCREEN \\
\hline $12 \mathrm{~A}$ & $18 "$ & AIR INLET, BG \\
\hline $12 \mathrm{~B}$ & $12^{\prime \prime}$ & IIQUID LEVEI REEL, 4" SPOOL \\
\hline $12 \mathrm{C}$ & $14 "$ & AIRLIFT CIRCUI_ATOR， BG \\
\hline 13 & $42 "$ & \\
\hline $13 \mathrm{~A}$ & $12^{\prime \prime}$ & SALTWELI SCREEN \\
\hline
\end{tabular}




\section{1-BY-106}

2,872,820 Liters

[758,000, Gallons]
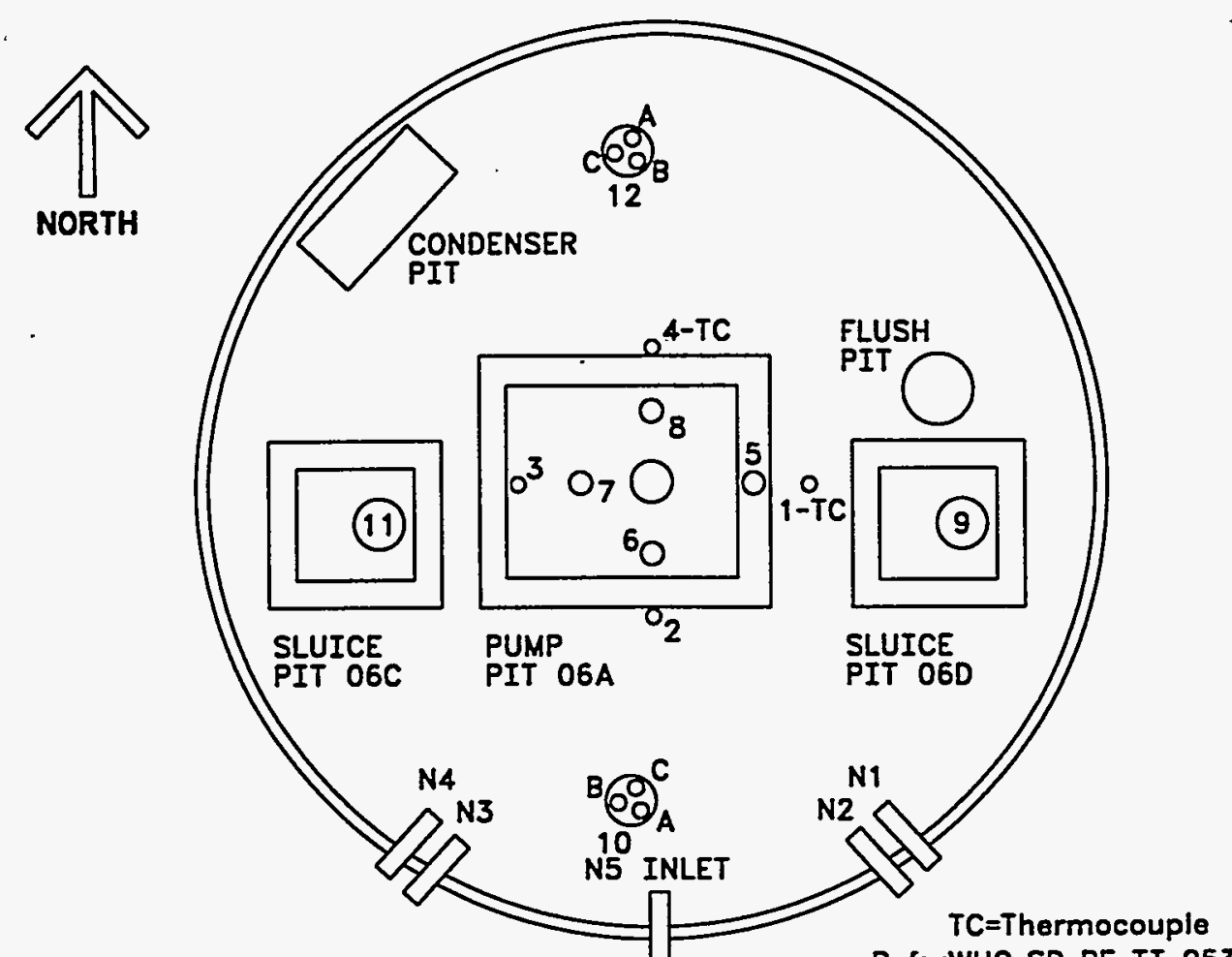

Ref: WHC-SD-RE-TI-053, Rev. 8 WHC-SD-WM-TI-553, Rev. 0 H-2-73248, Rev. 4

\section{TANK RISER LOCATION}

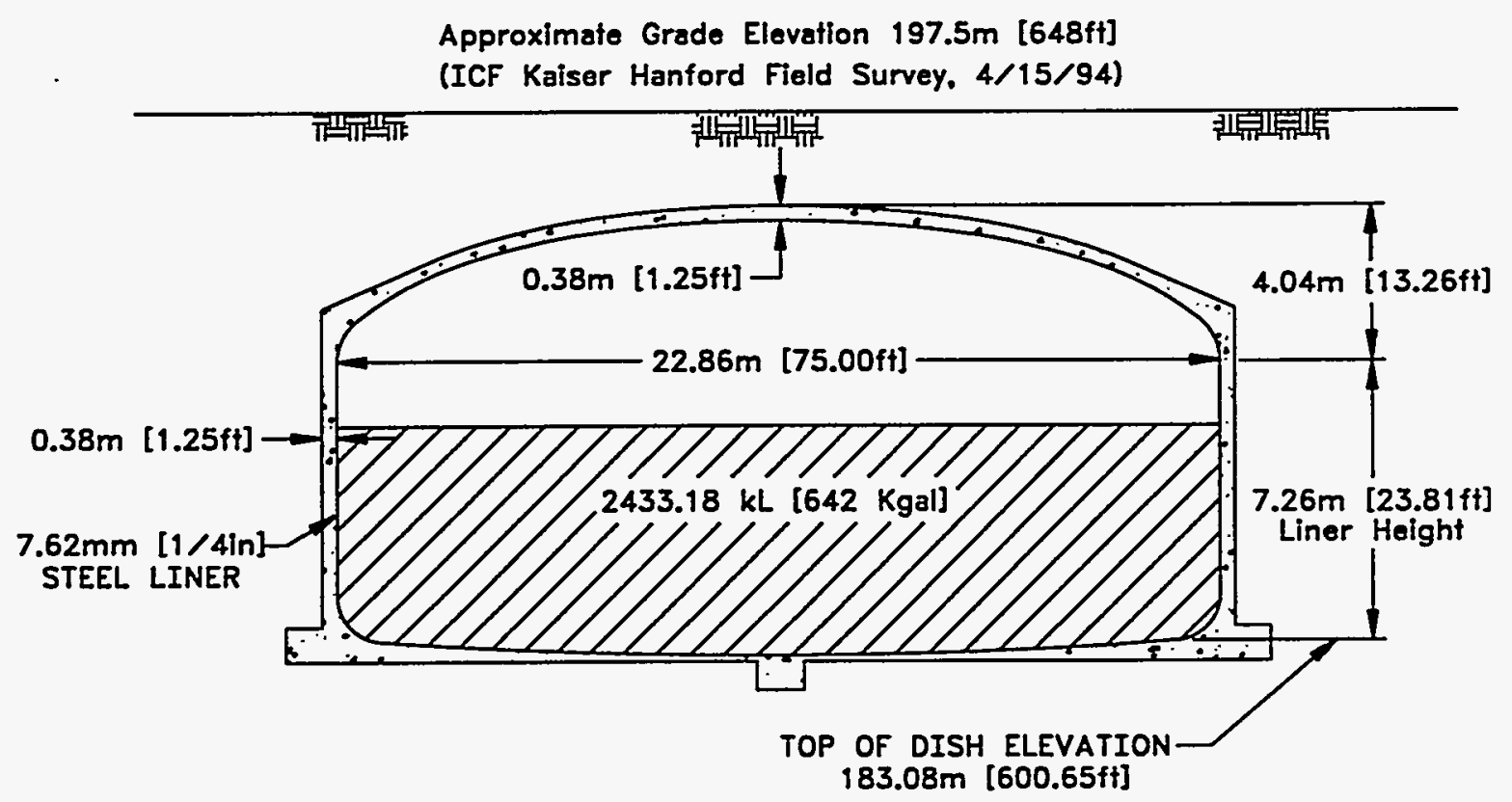

Ref: $\mathrm{H}-2-1312$. Rev. 2 


\begin{tabular}{|c|c|c|}
\hline NO. & DIA. & DESCRIPTION AND COMMENTS \\
\hline$\overline{1}$ & $4^{11}$ & TEMPERATURE PROBE \\
\hline 2 & $4 "$ & WC \\
\hline 3 & $4 "$ & BREATHER FIITER \\
\hline 4 & $4 "$ & LIQUID LEVEL REEL \\
\hline 5 & $4 "$ & FIANGE \\
\hline 6 & $12^{\prime \prime}$ & WC \\
\hline 7 & $12^{\prime \prime}$ & LOW \\
\hline 8 & $12^{\prime \prime}$ & FLANGE/B-222 OBSV PORT \\
\hline $9 A$ & $12 "$ & BG, AIRLIFT CIRCULATOR \\
\hline $9 B$ & $12 "$ & FLANGE WITH LEAD $3^{\prime}$ TALL \\
\hline $10 \mathrm{~A}$ & $18^{\prime \prime}$ & BG \\
\hline $10 \mathrm{~B}$ & $12 "$ & DIP TUBES \\
\hline $11 \mathrm{~A}$ & $12 "$ & BG, AIRLIFT CIRCULATOR \\
\hline $11 B$ & $12 "$ & FIAANGE WITH LEAD $3{ }^{\prime}$ TALL \\
\hline $12 \mathrm{~A}$ & $12 "$ & $\begin{array}{l}\text { FLANGE, LEAD CAP, BALE, BM, AIRLIFT } \\
\text { CIRCULATOR }\end{array}$ \\
\hline $12 \mathrm{~B}$ & & $\mathrm{BG}$ \\
\hline 13 & $42^{\prime \prime}$ & $B G$ \\
\hline
\end{tabular}




\section{$241-B Y-107$}

2,872,820 Liters

[758.000 Gallons]

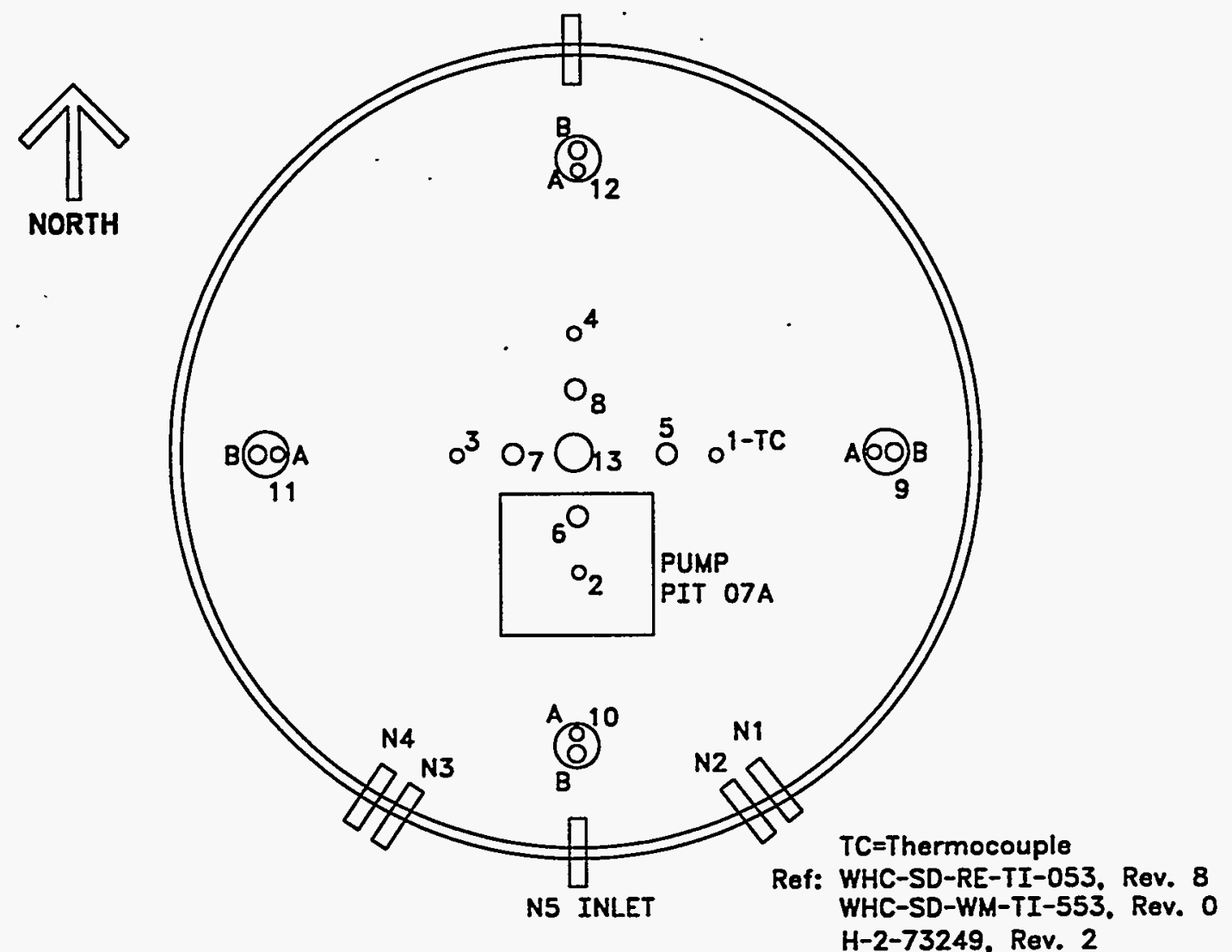

TANK RISER LOCATION

Approximate Grade Elevation $198.3 \mathrm{~m}$ [650.6ft]

(ICF Kaiser Hanford Field Survey, 4/15/94)

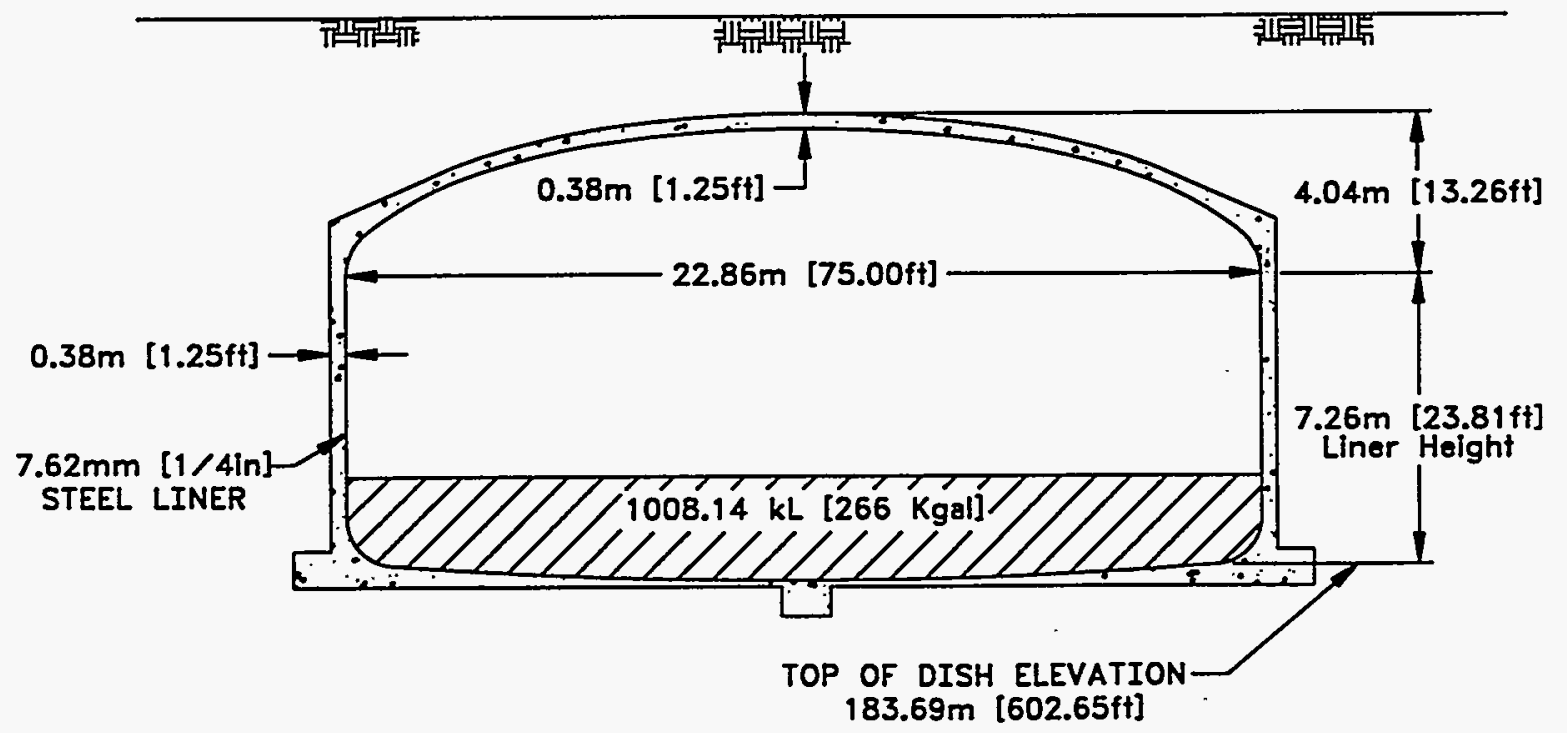

Ref: H-2-1312, Rev. 2 


\begin{tabular}{||c||c||l||}
\hline \multicolumn{2}{|c||}{ 241-BY-108 } \\
\hline \hline NO. & DIA. & \multicolumn{1}{|c||}{ DESCRIPTION AND COMMENTS } \\
\hline \hline 1 & $4 "$ & FLANGE, BALE \\
\hline 2 & $4 "$ & (FLANGE) \\
\hline 3 & $4 "$ & BREATHER FILTER \\
\hline 4 & $4 "$ & LIQUID LEVEL REEL, BM \\
\hline 5 & $4 "$ & FLANGE \\
\hline 6 & $12^{\prime \prime}$ & WC \\
\hline 7 & $12^{\prime \prime}$ & BG \\
\hline 8 & $12^{\prime \prime}$ & TEMPERATURE PROBE \\
\hline $9 \mathrm{~A}$ & & BG \\
\hline $9 \mathrm{~B}$ & $12^{\prime \prime}$ & FLANGE AND SPOOL \\
\hline $10 \mathrm{~A}$ & $12^{\prime \prime}$ & EXH PORT, BM \\
\hline 10 & $18^{\prime \prime}$ & EQUIPMENT G \\
\hline $11 \mathrm{~A}$ & $12^{\prime \prime}$ & BG, AIRLIFT CIRCULATOR \\
\hline $11 \mathrm{~B}$ & $4 "$ & CAPPED \\
\hline $12 \mathrm{~A}$ & $12^{\prime \prime}$ & OBSV PORT \\
\hline $12 \mathrm{~B}$ & $18^{\prime \prime}$ & BG, AIRLIFT CIRCULATOR \\
\hline 13 & $42^{\prime \prime}$ & BG \\
\hline \hline
\end{tabular}




\section{1-BY-108}

2,872,820 Liters

[758,000 Gallons]

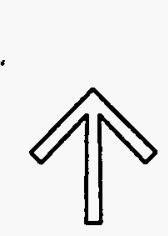
NORTH

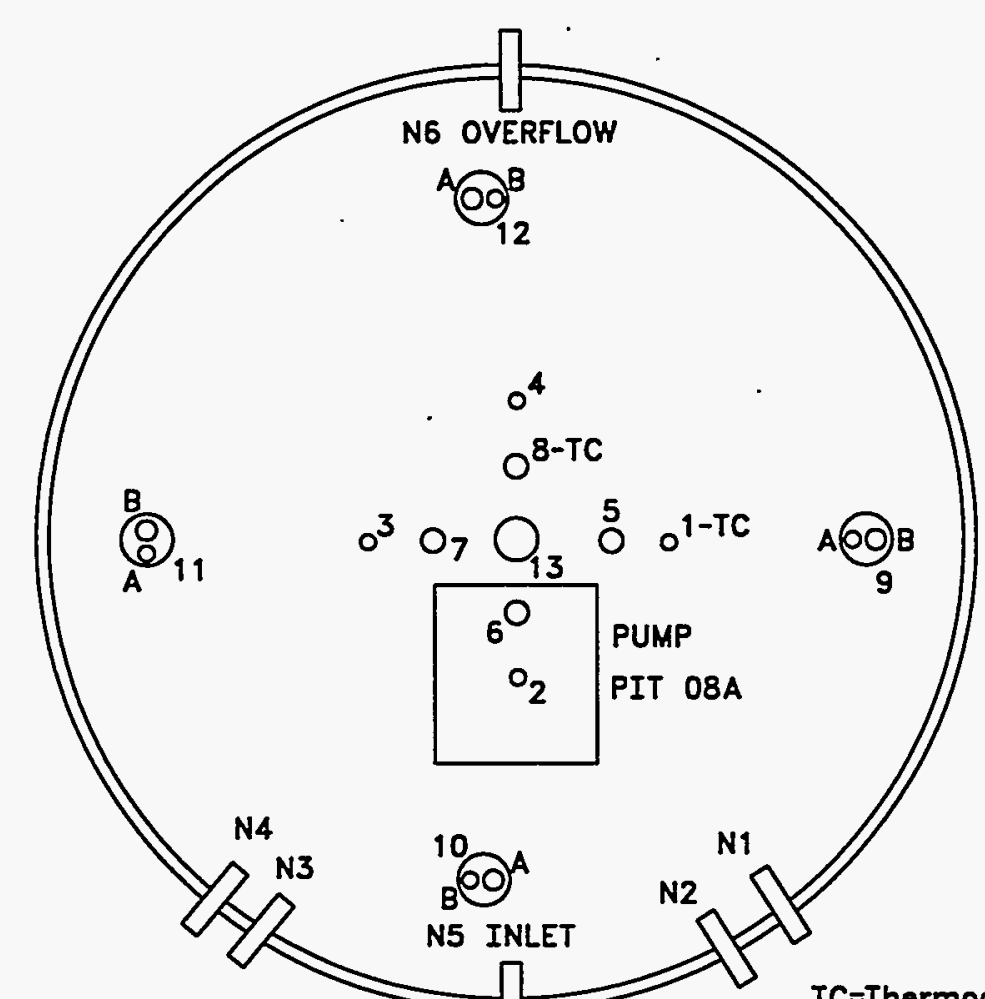

TC=Thermocouple

Ref: WHC-SD-RE-TI-053, Rev. 8 WHC-SD-WM-TI-553, Rev. 0 H-2-73250, Rov. 5

\section{TANK RISER LOCATION}

Approximate Grade Elevation 197.9m [649.3ff]

(ICF Kaiser Hanford Field Survey. 4/15/94)

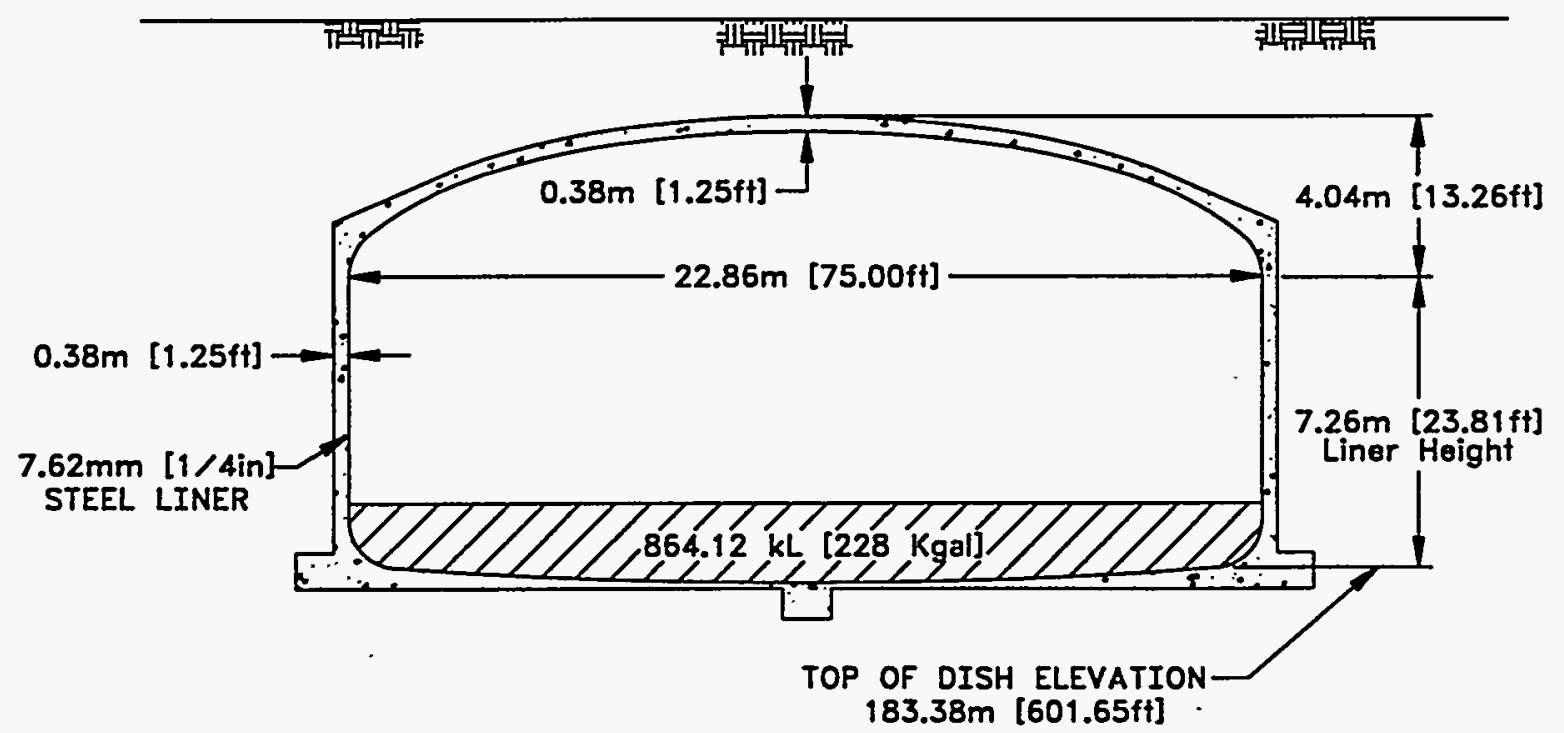

Ref: $H-2-1312$, Rev. 2 


\begin{tabular}{|c||c||l||}
\hline \multicolumn{2}{|c||}{ 241-BY-109 } \\
\hline \hline NO. & DIA. & \multicolumn{1}{|c||}{ DESCRIPTION AND COMMENTS } \\
\hline \hline 1 & $4 "$ & BG \\
\hline 2 & $4 "$ & (PIT DRAIN) \\
\hline 3 & $4 "$ & BG \\
\hline 4 & $4^{\prime \prime}$ & FIC, BM \\
\hline 5 & $4 "$ & \\
\hline 6 & $12^{\prime \prime}$ & SALTWELI SCREEN \\
\hline 7 & $12^{\prime \prime}$ & \\
\hline 8 & $12^{\prime \prime}$ & (COVERED WITH CONCRETE) \\
\hline $9 \mathrm{~A}$ & $12^{\prime \prime}$ & FLANGE \\
\hline $9 \mathrm{~B}$ & $12^{\prime \prime}$ & BG \\
\hline $10 \mathrm{~A}$ & $12^{\prime \prime}$ & BREATHER FILTER, G1 HOUSING \\
\hline $10 \mathrm{~B}$ & $12^{\prime \prime}$ & OBSV PORT \\
\hline $11 \mathrm{~A}$ & $4^{\prime \prime}$ & BLIND FLANGE WC \\
\hline $11 \mathrm{~B}$ & $42^{\prime \prime}$ & COVERED WITH PLYWOOD WC \\
\hline $12 \mathrm{~A}$ & $4^{\prime \prime}$ & 90 TURN ABOVE GRADE \\
\hline $12 \mathrm{~B}$ & $12^{\prime \prime}$ & B-436 LOW \\
\hline $12 \mathrm{C}$ & $12^{\prime \prime}$ & FLANGE, LEAD COVERED \\
\hline 13 & $42^{\prime \prime}$ & \\
\hline $13 \mathrm{C}$ & $12^{\prime \prime}$ & SALTWELL SCREEN AND PUMP \\
\hline
\end{tabular}




\section{1-BY-109}

$2,872,820$ Liters

[758,000 Gallons]
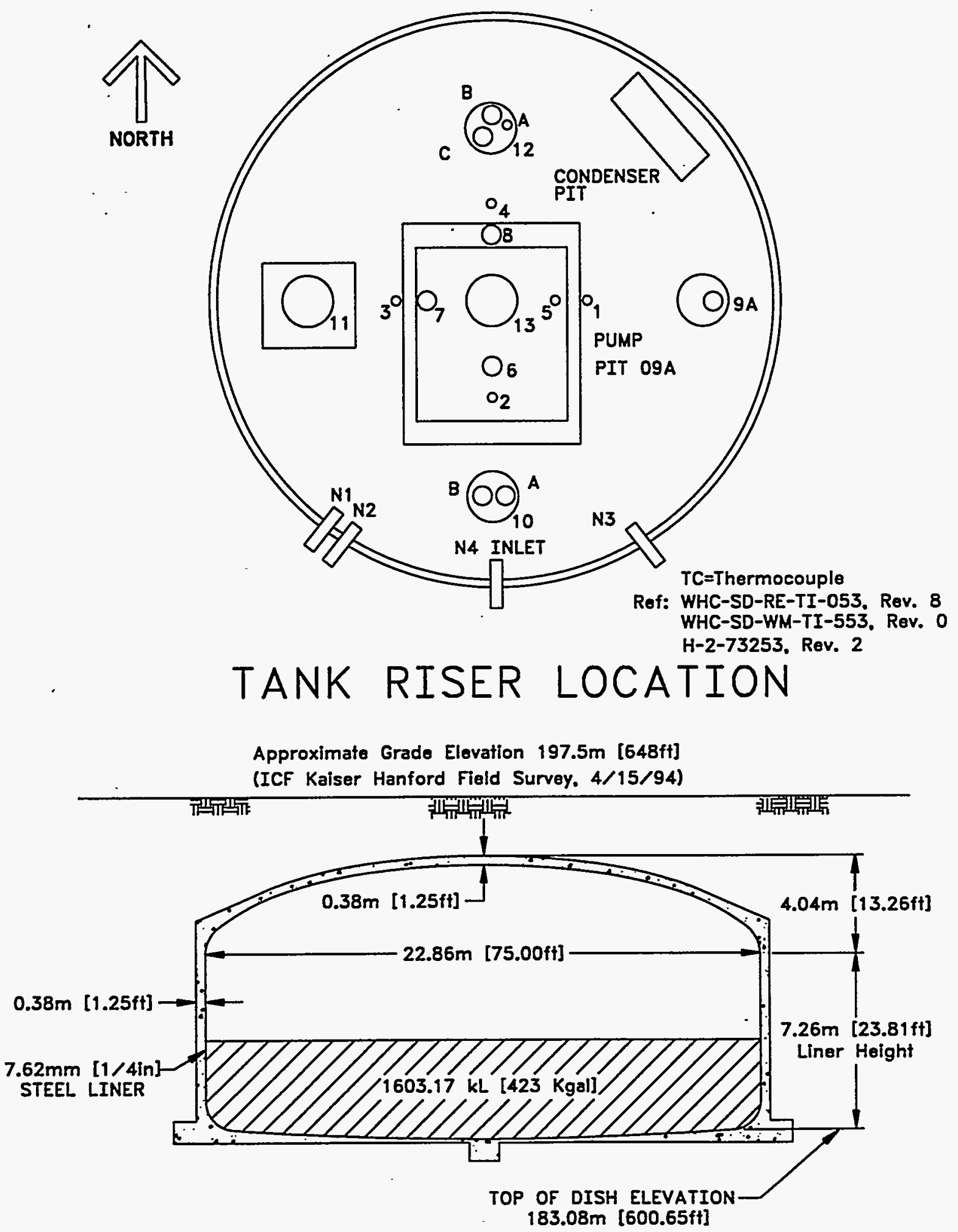

Ref: H-2-1312. Rev. 2 


\begin{tabular}{|c|c|c|}
\hline \multicolumn{3}{|r|}{ 241-BY-110 } \\
\hline NO. & DIA. & DESCRIPTION AND COMMENTS \\
\hline$\overline{1}$ & $4 "$ & TEMPERATURE PROBE \\
\hline 2 & $4 "$ & WC \\
\hline 3 & $4 "$ & $B-436$ LOW \\
\hline 4 & $4 "$ & LIQUID LEVEL REEL \\
\hline 5 & $12 "$ & TEMPERATURE PROBE BG \\
\hline 6 & $12^{\prime \prime}$ & SALTWELL SCREEN AND PUMP \\
\hline 7 & $12 "$ & FLANGE - PROCESS INLET BG \\
\hline 8 & $12 "$ & TEMPERATURE PROBE \\
\hline $9 A$ & $12 "$ & BG, AIRLIFT CIRCULATOR \\
\hline $9 \mathrm{~B}$ & & BG \\
\hline $10 \mathrm{~A}$ & $12 "$ & BREATHER FILTER, GI HOUSING \\
\hline $10 \mathrm{~B}$ & $18 "$ & OBSV PORT \\
\hline $11 \mathrm{~A}$ & $12 "$ & BG, AIRLIFT CIRCULATOR \\
\hline $11 B$ & & $B G$ \\
\hline $12 \mathrm{~A}$ & $12^{\prime \prime}$ & UNKNOWN EQUIPMENT \\
\hline 13 & $42 "$ & EQUIPMENT BG \\
\hline
\end{tabular}




\section{$241-B Y-110$}

\section{2,872,820 Liters}

[758,000 Gallons]
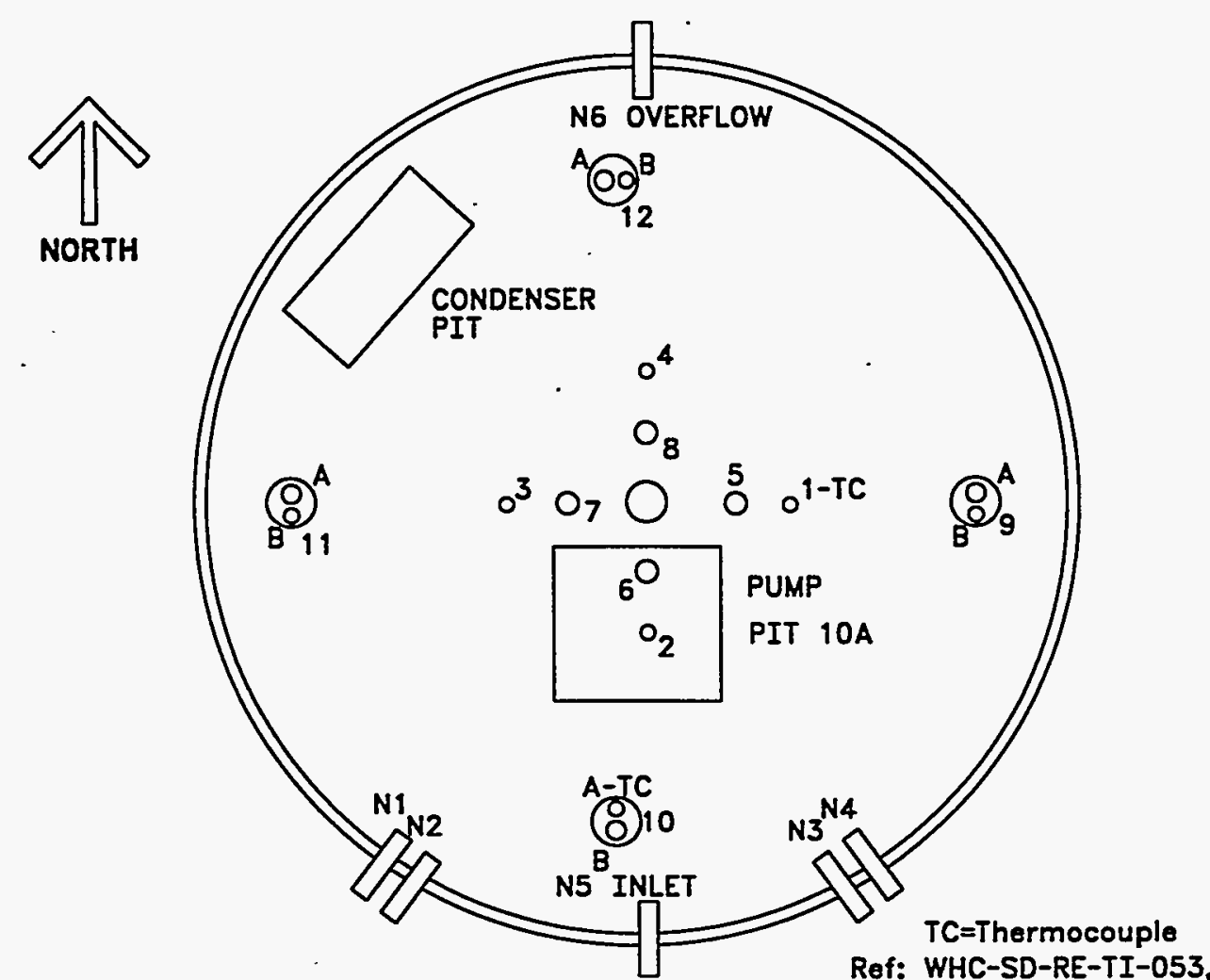

Ref: WHC-SD-RE-TI-053, Rev. 8 WHC-SD-WM-TI-553. Rev. 0 H-2-73254. Rev. 2

\section{TANK RISER LOCATION}

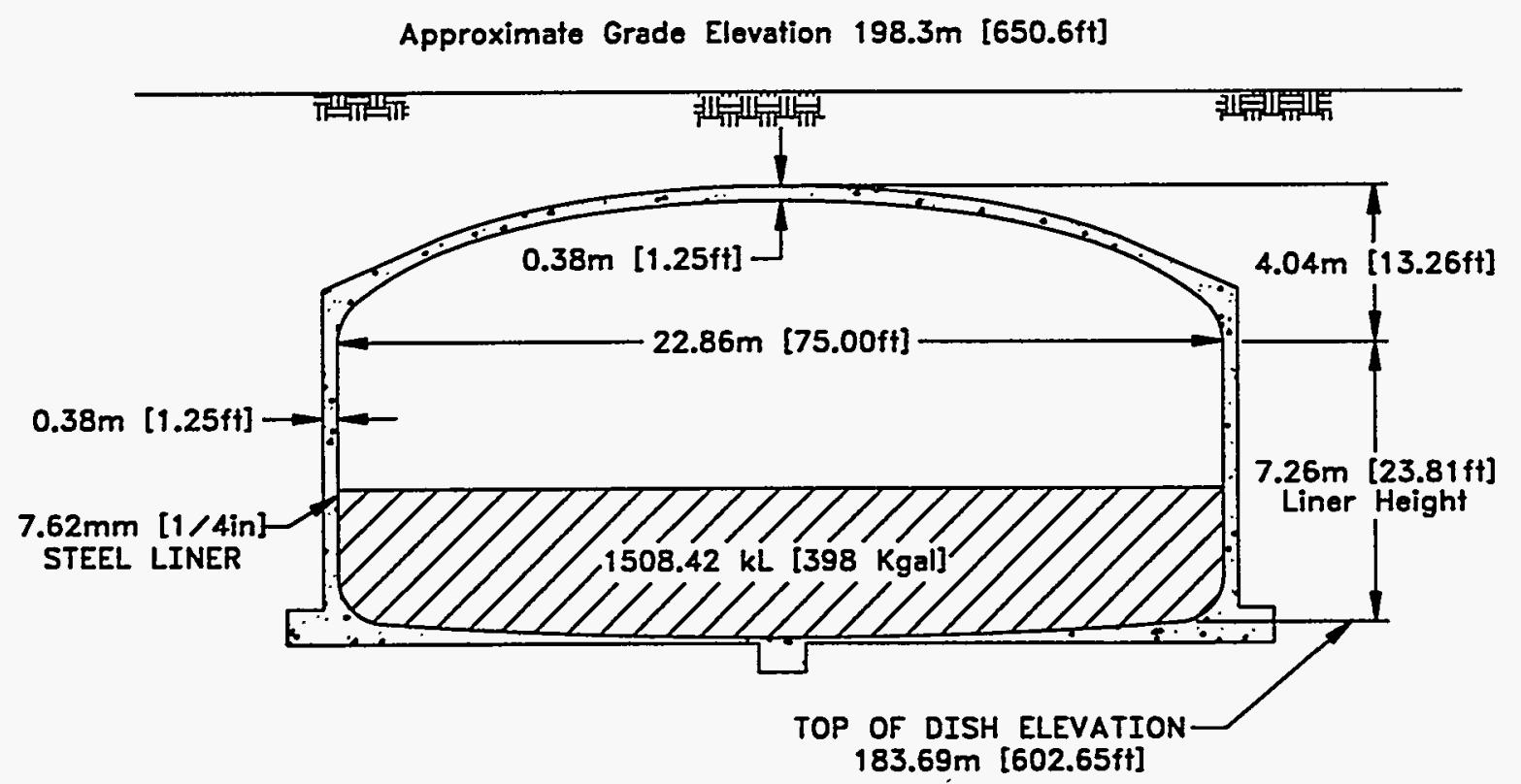

Ref: H-2-1312, Rev. 2 Merrick Maps of 200 Area's 89210406 


\begin{tabular}{|c||c||l||}
\hline \multicolumn{2}{|c||}{ 241-BY-111 } \\
\hline \hline NO. & DIA. & \multicolumn{1}{|c||}{ DESCRIPTION AND COMMENTS } \\
\hline \hline 1 & $4^{\prime \prime}$ & B-436 LOW \\
\hline 2 & $4^{\prime \prime}$ & EQUIPMENT INSTALLED BG \\
\hline 3 & $4 "$ & (PIT DRAIN) WC \\
\hline 4 & $4^{\prime \prime}$ & BREATHER FILTER, G1 HOUSING \\
\hline 5 & $4^{\prime \prime}$ & FLANGE \\
\hline 6 & $12^{\prime \prime}$ & WC \\
\hline 7 & $12^{\prime \prime}$ & (JET PUMP AND SALTWELL SCREEN) \\
\hline 8 & $12^{\prime \prime}$ & WC \\
\hline 9 & $42^{\prime \prime}$ & WC \\
\hline $10 A$ & $4^{\prime \prime}$ & FLANGED BG \\
\hline $10 B$ & $12^{\prime \prime}$ & BM, AIRLIFT CIRCULATOR \\
\hline 11 & $42^{\prime \prime}$ & WC \\
\hline $11 \mathrm{~A}$ & $12^{\prime \prime}$ & AIRLIFT CIRCULATOR \\
\hline 12 & $42^{\prime \prime}$ & FLANGE WC \\
\hline $12 \mathrm{~A}$ & $12^{\prime \prime}$ & OBSV PORT \\
\hline 13 & $42^{\prime \prime}$ & WC \\
\hline $13 \mathrm{~A}$ & $12^{\prime \prime}$ & AIRIIFT CIRCULATOR \\
\hline 14 & $6^{\prime \prime}$ & FLANGED \\
\hline 15 & $6^{\prime \prime}$ & LIQUID LEVEL REEL \\
\hline
\end{tabular}




\section{$241-B Y-111$}

2,872,820 Liters

[758,000 Gallons]

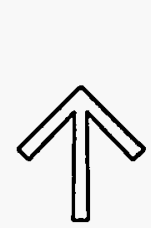
NORTH
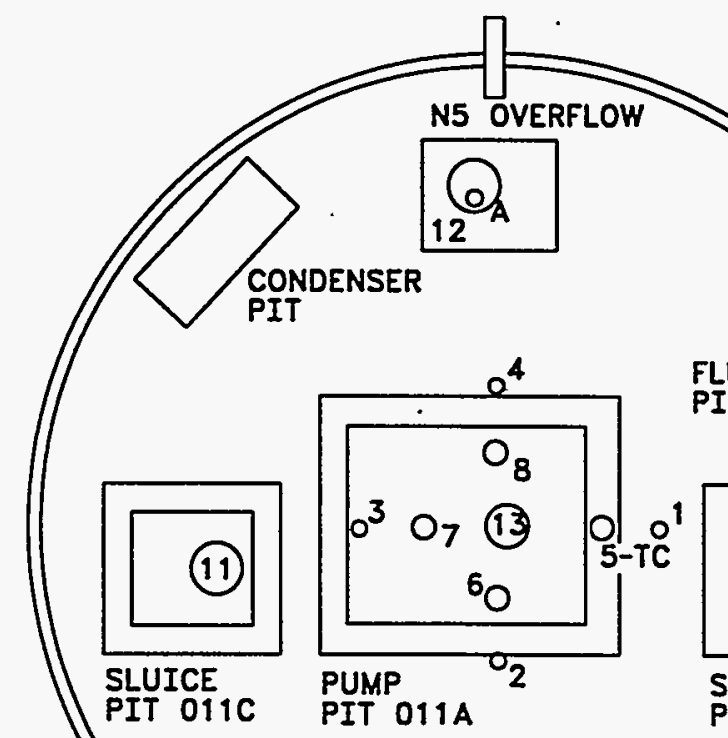


\begin{tabular}{||c||c||l||}
\hline \multicolumn{1}{|c||}{ 241-B Y-112 } \\
\hline \hline NO. & DIA. & \multicolumn{1}{|c||}{ DESCRIPTION AND COMMENTS } \\
\hline \hline 1 & $4^{\prime \prime}$ & BREATHER FILTER, GI HOUSING \\
\hline 2 & $4^{\prime \prime}$ & BLIND FLANGE \\
\hline 3 & $4^{\prime \prime}$ & WC \\
\hline 4 & $4^{\prime \prime}$ & BG \\
\hline 5 & $4^{\prime \prime}$ & FLANGE \\
\hline 6 & $12^{\prime \prime}$ & WC \\
\hline 7 & $12^{\prime \prime}$ & (SALTWELI SCREEN AND JET PUMP) WC \\
\hline 8 & $12^{\prime \prime}$ & WC \\
\hline 9 & $42^{\prime \prime}$ & WC \\
\hline $10 A$ & $12^{\prime \prime}$ & FLANGE - LEAD COVER/OBSV PORT \\
\hline $10 B$ & $12^{\prime \prime}$ & BLIND FLANGE \\
\hline 11 & $42^{\prime \prime}$ & WC \\
\hline 12 & $42^{\prime \prime}$ & WC \\
\hline 13 & $42^{\prime \prime}$ & WC \\
\hline 14 & $6^{\prime \prime}$ & WC \\
\hline 15 & $6^{\prime \prime}$ & FLANGE/B-436 LOW \\
\hline 16 & $6^{\prime \prime}$ & TEMPERATURE - NOT MOVABLE, (CAISSON) WC \\
\hline 18 & $6^{\prime \prime}$ & SPOOL AND FLANGE, BM \\
\hline 19 & $6^{\prime \prime}$ & LIQUID LEVEL \\
\hline 20 & $4^{\prime \prime}$ & BLIND FLANGE \\
\hline 21 & $4^{\prime \prime}$ & SPOOL AND FLANGE \\
\hline
\end{tabular}




\section{$241-B Y-112$}

2,872,820 Liters

[758,000 Gallons]
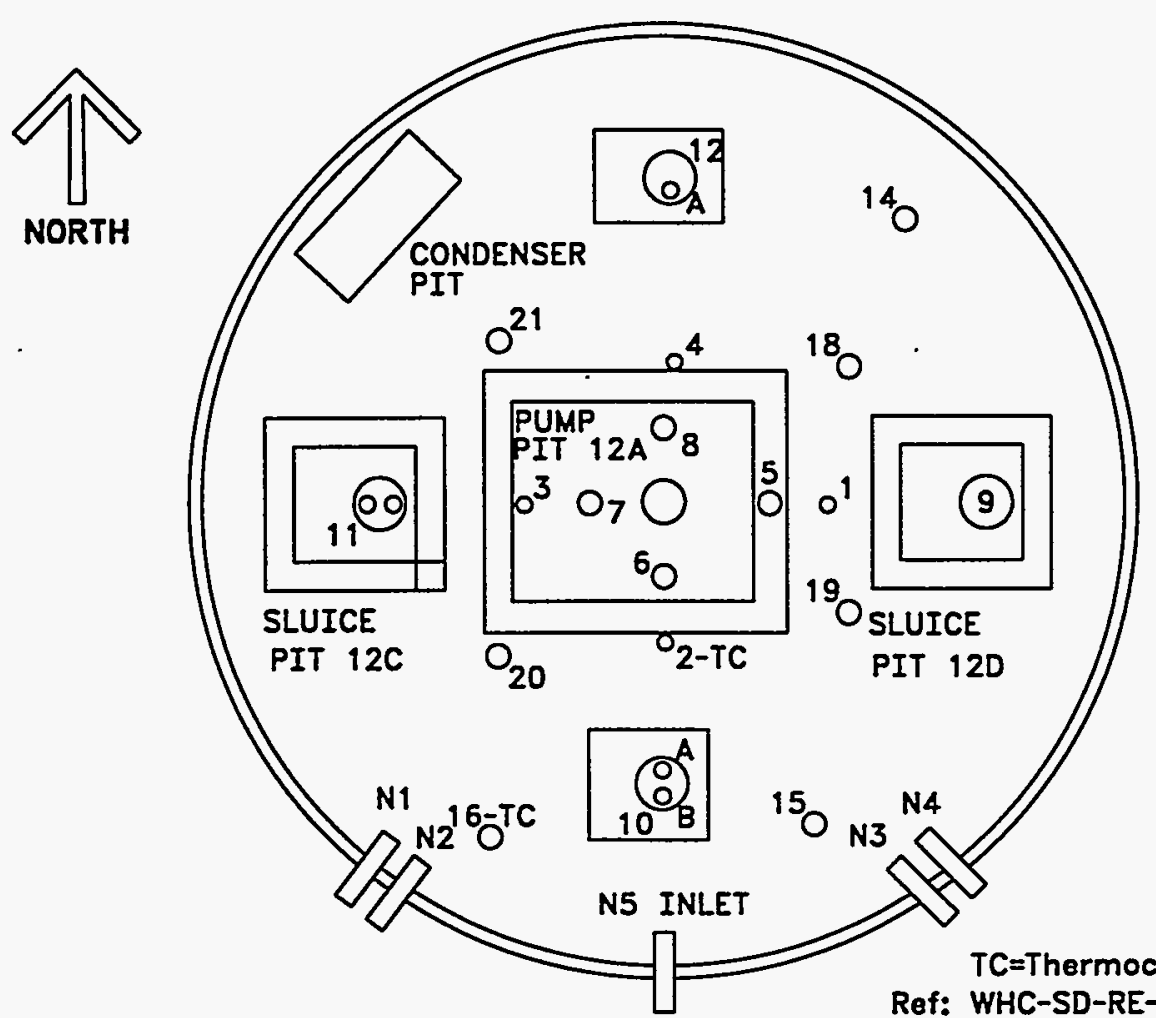

TC=Thermocouple

Ref: WHC-SD-RE-TI-053, Rev. 8 WHC-SD-WM-TI-553, Rev. 0 H-2-73256, Rev. 2

\section{TANK RISER LOCATION}

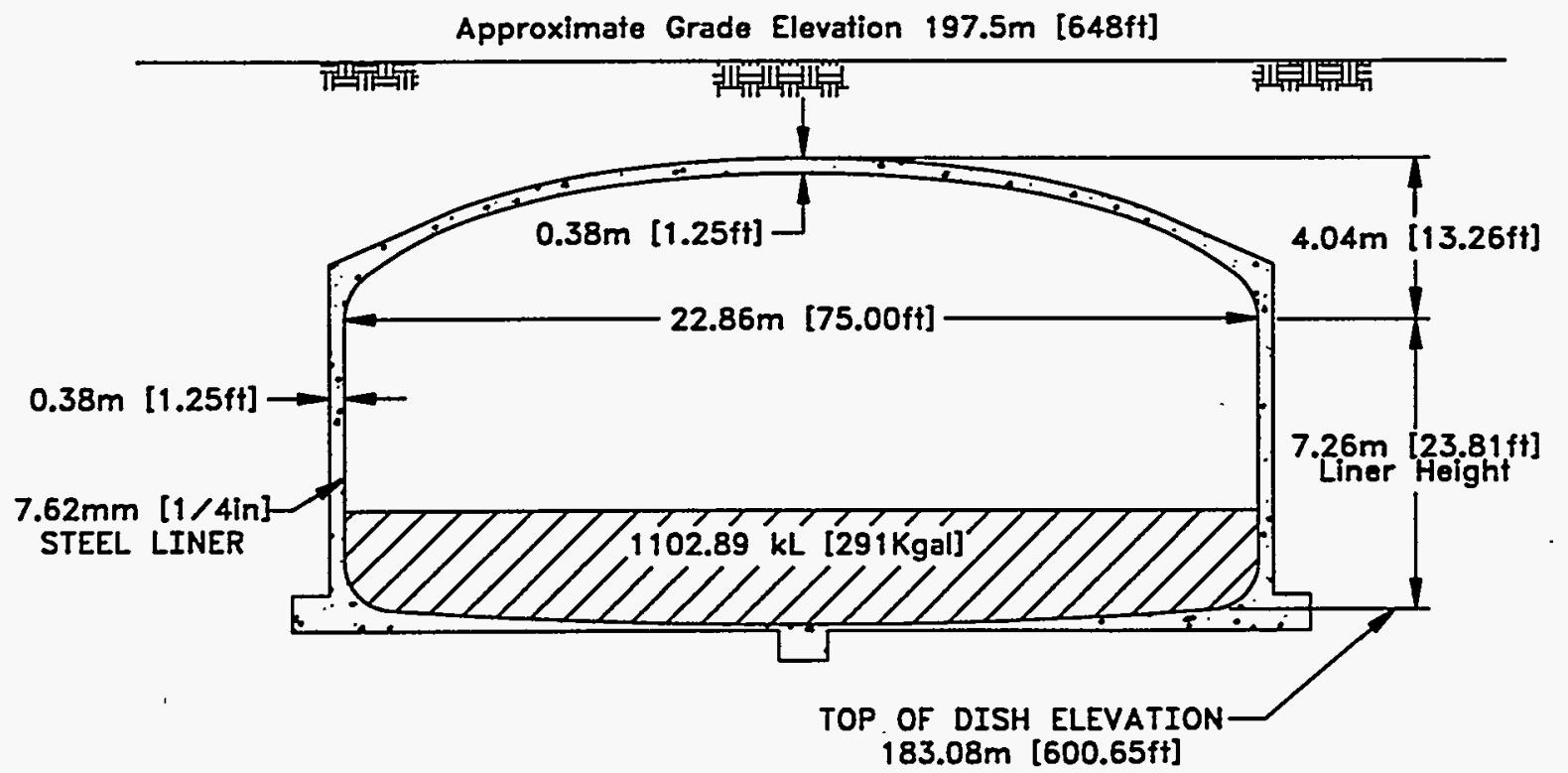

Ref: H-2-1312, Rev. 2

Merrick Maps of

200 Area's 89210406 


\begin{tabular}{|c|c|c|}
\hline \multicolumn{3}{|c|}{ Waste Tank 101-BY } \\
\hline \multicolumn{3}{|c|}{ STUDIES OF "IN FARM SCAVENGING" LABORATORY STUDIES } \\
\hline \multicolumn{3}{|c|}{ December 20,1955 } \\
\hline COMPONENT & LAB VALUE & LAB UNITS \\
\hline \multicolumn{3}{|c|}{ 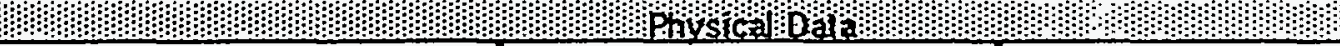 } \\
\hline $\mathrm{pH}$ & 9.5 & \\
\hline \multicolumn{3}{|c|}{ Gentichil Analysis } \\
\hline $\mathrm{PO} 4(3)$. & 0.14 & $M$ \\
\hline SO4 (2-) & 0.075 & $M$ \\
\hline $\mathrm{Nat}$ & 3.6 & $M$ \\
\hline $\mathrm{NiSO} 4$ & - & \\
\hline NaLFe(CN)6 & - & \\
\hline \multirow[t]{2}{*}{$\mathrm{Ce}(\mathrm{NO} 3) 2$} & 0.01 & M \\
\hline & 0.03 & $M$ \\
\hline \multicolumn{3}{|l|}{$\mathrm{Sr}(\mathrm{NO} 3) 2$} \\
\hline 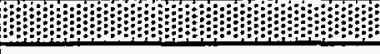 & lóógicallenan & \\
\hline \multicolumn{3}{|c|}{ Average Initial Composition } \\
\hline Cs 137 & 0.033 & $\mathrm{uCi} / \mathrm{ml}$ \\
\hline Sr 90 & 0.91 & uCi/ml \\
\hline \multicolumn{3}{|l|}{ Final Composition } \\
\hline Cs 137 & 0.018 & uci/ml \\
\hline Cs 137 & 0.027 & uCi/ml \\
\hline Sr 90 & 0.079 & uCi/ml \\
\hline Sr 90 & 0.022 & $\mathrm{uCi} / \mathrm{ml}$ \\
\hline
\end{tabular}


Wast Tank BY 101

\section{STUDIES OF"IN FARM SCAVENGING" LABORATORY STUDIES}

December 20, 1955

\begin{tabular}{|c|c|c|c|c|}
\hline COMPONENT & $\begin{array}{l}\text { AVERAGE INTIAL } \\
\text { COMPOSITION }\end{array}$ & $\begin{array}{c}\text { SCAVENGING } \\
\text { FLOWSHEET }\end{array}$ & $\begin{array}{c}\text { FINAL } \\
\text { CONCENTRATION }\end{array}$ & LAB UNITS \\
\hline \multicolumn{5}{|c|}{ popsica } \\
\hline $\mathrm{pH}$ & 9.5 & & & \\
\hline \multicolumn{5}{|c|}{ chencol Anjos } \\
\hline$P O 4$ (3-) & 0.14 & & & $\underline{M}$ \\
\hline $\mathrm{SO} 4\left(\mathrm{R}_{-}\right)$ & 0.075 & & & $\underline{M}$ \\
\hline $\mathrm{Nat}$ & 3.6 & & & $\underline{M}$ \\
\hline $\mathrm{Cs}(\mathrm{NO} 3) 2$ & & 0.01 & & $\underline{M}$ \\
\hline $\mathrm{Cs}(\mathrm{NO} 3) 2$ & & 0.03 & & $\underline{M}$ \\
\hline \multicolumn{5}{|c|}{ 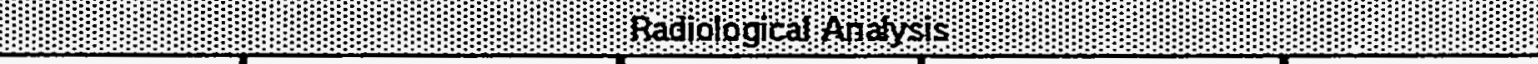 } \\
\hline Cs 137 & 0.033 & & 0.018 & $\mathrm{uCi} / \mathrm{ml}$ \\
\hline Cs 137 & & & 0.027 & $\mathrm{uCi} / \mathrm{ml}$ \\
\hline Sr90 & 0.91 & & 0.079 & $\mathrm{uCi} / \mathrm{ml}$ \\
\hline Sr9o & & & 0.022 & $\mathrm{uCi} / \mathrm{ml}$ \\
\hline
\end{tabular}




\begin{tabular}{|c|c|}
\hline \multicolumn{2}{|c|}{ Waste Tank 101-BY } \\
\hline \multicolumn{2}{|c|}{ ANALYSIS OF TRANK FARM SAMPLE } \\
\hline \multicolumn{2}{|c|}{ August 16,1972} \\
\hline \multicolumn{2}{|c|}{ Sample: T-5404 } \\
\hline COMPONENT & LAB VALUE \\
\hline \multicolumn{2}{|c|}{ p ayskad:Daba } \\
\hline \multicolumn{2}{|c|}{ Visible: Clear green $<10 \%$ solids, 20 RAD } \\
\hline$S p G$ & 1.423 \\
\hline Boildown st art ing volume & 50 \\
\hline Boildown $\%$ solids & 6 \\
\hline \multicolumn{2}{|c|}{ 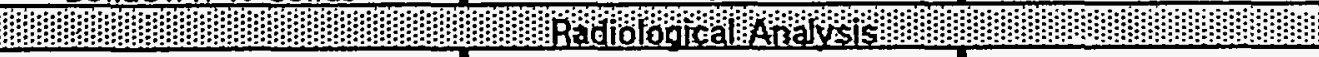 } \\
\hline GEA: $137 \mathrm{Cs}$ & 1.18E+06 \\
\hline GEA: $134 \mathrm{Cs}$ & $8.70 E+03$ \\
\hline SEP CS; CS 137 & $1.21 E+06$ \\
\hline SEP CS: CS 134 & $7.34 \mathrm{E}+03$ \\
\hline
\end{tabular}




\begin{tabular}{|c|c|c|c|c|}
\hline \multicolumn{5}{|c|}{ Waste Tank BY102 } \\
\hline \multicolumn{5}{|c|}{ SUMMARY OF"IN-FARM SCAVENGING" LABORATORY STUDIES } \\
\hline \multicolumn{5}{|c|}{ December 20.1955} \\
\hline \multirow[b]{2}{*}{ COMPONENT } & \multicolumn{3}{|c|}{ LAB VALUE } & \multirow[b]{2}{*}{ LAB UNIT } \\
\hline & $\begin{array}{l}\text { AVERAGE INTIAL } \\
\text { COMPOSITION }\end{array}$ & $\begin{array}{l}\text { SCAVENGING } \\
\text { FLOWSHEET }\end{array}$ & $\begin{array}{c}\text { FINAL CONCEN } \\
\text { TRATION }\end{array}$ & \\
\hline \multicolumn{5}{|c|}{ physedadata } \\
\hline $\mathrm{pH}$ & 9.40 & & & \\
\hline $\begin{array}{l}\mathrm{pH} \\
\mathrm{Adi}\end{array}$ & & & & \\
\hline \multicolumn{5}{|c|}{ 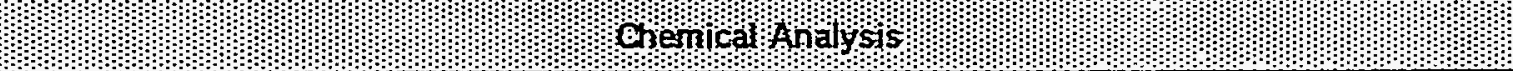 } \\
\hline$(\mathrm{PO} 4) 3$ & 0.13 & & & $\underline{M}$ \\
\hline$(\mathrm{SO} 4) 2$ & 0.21 & & & $\underline{M}$ \\
\hline $\mathrm{Na}+$ & 1.4 & & & $\underline{M}$ \\
\hline $\mathrm{Cs}(\mathrm{NO} 3) 2$ & & 0.01 & & M \\
\hline $\mathrm{Cs}(\mathrm{NO} 3) 2$ & & 0.03 & & $\underline{M}$ \\
\hline \multicolumn{5}{|c|}{ 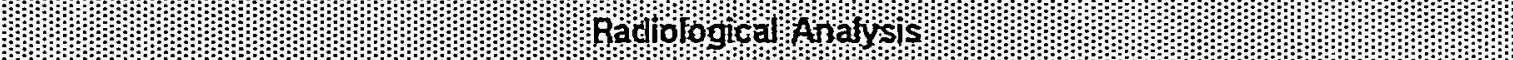 } \\
\hline Cs 137 & 0.044 & & 0.053 & $\mathrm{uCi} / \mathrm{ml}$. \\
\hline Cs 137 & & & 0.057 & $\mathrm{uCi} / \mathrm{ml}$ \\
\hline Sr 90 & 3.12 & & 0.99 & $\mathrm{uci} / \mathrm{ml}$ \\
\hline Sr 90 & & & 0.045 & uCi/ml \\
\hline
\end{tabular}




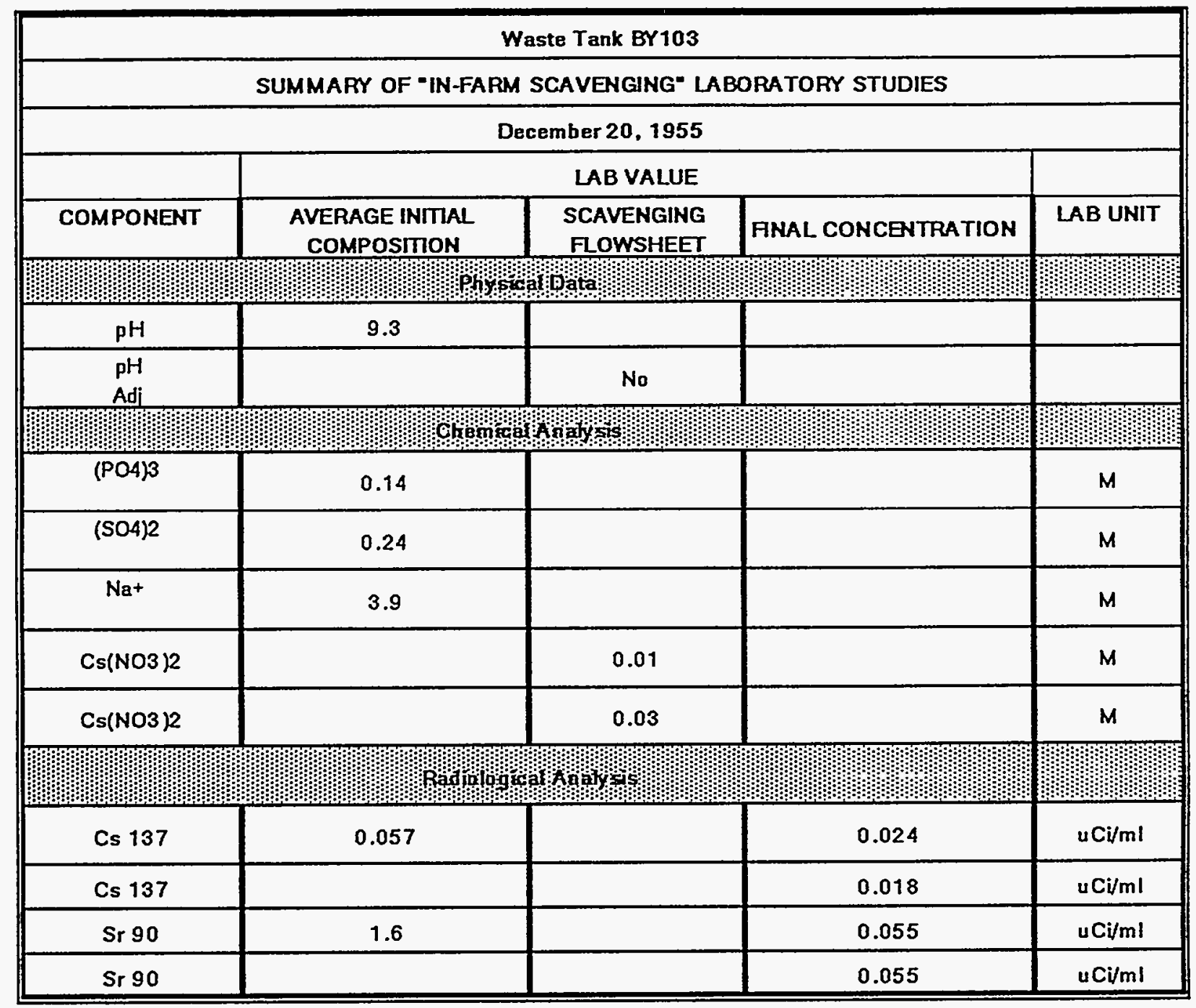




\begin{tabular}{|c|c|c|c|c|c|}
\hline \multicolumn{6}{|c|}{ WASTE TANK BY-103 } \\
\hline \multicolumn{6}{|c|}{ Analysis of 103-BY Supernatant Liquid } \\
\hline \multicolumn{6}{|c|}{ October 14, 1971} \\
\hline & \multicolumn{4}{|c|}{ LAB YALUE } & \multirow[b]{2}{*}{ LAB UNIT } \\
\hline COMPONENT & $30 \operatorname{deg} \mathrm{C}$ & 40 deg $C$ & 50 deg $C$ & $60 \mathrm{deg} C$ & \\
\hline \multicolumn{6}{|c|}{$p h s=10=$} \\
\hline Vpl. \% Solid & 22 & 14.8 & 8.9 & 0 & \\
\hline SpGr & 1.469 & 1.479 & 1.494 & 1.500 & \\
\hline \multicolumn{6}{|c|}{ 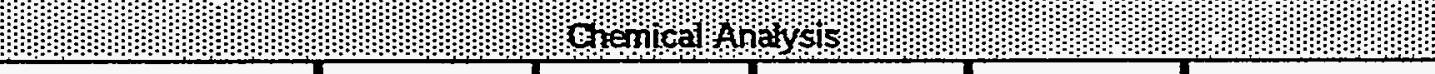 } \\
\hline $\mathrm{Na}$ & 11.9 & 11.6 & 11.7 & 12 & $M$ \\
\hline $\mathrm{Al}$ & 2.17 & 1.82 & 1.82 & 1.64 & M \\
\hline NO2 & 1.23 & 1.06 & 1.01 & 0.54 & M \\
\hline NO3 & 2.57 & 2.33 & 2.98 & 3.04 & M \\
\hline $\mathrm{CO} 3$ & 0.45 & 0.17 & 0.14 & $\cdots$ & $M$ \\
\hline SO4 & 0.015 & 0.022 & 0.017 & 0.017 & $M$ \\
\hline TAD & 7.87 & 8.07 & 7.34 & 6.99 & $M$ \\
\hline \multicolumn{6}{|c|}{ 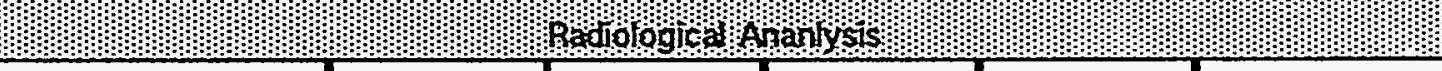 } \\
\hline $137 \mathrm{Cs}$ & $3.44 E+05$ & 3.37E+ 05 & $3.23 E+05$ & $4.21 E+05$ & uCin \\
\hline $134 \mathrm{Cs}$ & $3.83 E+03$ & $3.81 E+03$ & $3.56 E+03$ & 3.47E $\div 03$ & uCin \\
\hline
\end{tabular}




\begin{tabular}{|c|c|c|c|c|c|}
\hline \multicolumn{6}{|c|}{ Waste Tank 106-BY } \\
\hline \multicolumn{6}{|c|}{ Analysis of 106-BY Supernatant Liquid } \\
\hline \multicolumn{6}{|c|}{ October 14,1971} \\
\hline COMPONENT & \multicolumn{4}{|c|}{ LAB VALUE } & LAB UNIT \\
\hline \multicolumn{6}{|c|}{$\therefore$ and Physical Data } \\
\hline & $30 * \mathrm{C}$ & $40^{*} \mathrm{C}$ & $50 * \mathrm{C}$ & $60^{*} \mathrm{C}$ & \\
\hline Vol. \% Solid & 19.6 & 13.2 & 5.2 & 0 & \\
\hline $\mathrm{Sp} \mathrm{Gr}$ & 1.453 & 1.484 & 1.484 & 1.478 & \\
\hline \multicolumn{6}{|c|}{ Chemical Analysis } \\
\hline $\mathrm{Na}$ & 12.2 & 11.7 & 12.4 & 12.1 & $M$ \\
\hline Al & 2.00 & 2.00 & 2.22 & 1.79 & M \\
\hline NO2 & 1.00 & 1.08 & 1.00 & 1.00 & M \\
\hline NO3 & 2.79 & 2.86 & 3.73 & 4.17 & $M$ \\
\hline Co3, & 0.97 & 0.9 & 0.55 & 0.65 & M \\
\hline SO4 & 0.016 & 0.005 & 0.013 & 0.013 & $M$ \\
\hline TAD & 6.36 & 6.33 & 6.13 & 5.93 & $M$ \\
\hline \multicolumn{6}{|c|}{ Radiological Analysis } \\
\hline $137 \mathrm{Cs}$ & $3.30 E+05$ & $3.30 E+05$ & $3.20 \mathrm{E}+05$ & $3.23 E+05$ & Uci/l \\
\hline $134 \mathrm{Cs}$ & $3.44 E+03$ & $3.50 E+03$ & $3.26 \mathrm{E}+03$ & $3.28 E+03$ & Uci/l \\
\hline
\end{tabular}




\begin{tabular}{|c|c|c|c|c|c|}
\hline \multicolumn{6}{|c|}{ Waste Tank 106-BY } \\
\hline \multicolumn{6}{|c|}{ ANALYSES OF TANK 106-BY SAMPLES } \\
\hline \multicolumn{6}{|c|}{ April 13,1972} \\
\hline & \multicolumn{2}{|c|}{ No. 7395 , Surface } & \multicolumn{2}{|c|}{ No. $7394,33 \mathrm{ft} .10 \mathrm{in}}$. & \multirow[b]{2}{*}{ LAB UNIT } \\
\hline COMPONENT & LAB VALUE & WEIGHT \% & LAB VALUE & WEIGHT \% & \\
\hline \multicolumn{6}{|c|}{$\because$ Physical Data : } \\
\hline Density & 1.415 & & 1.414 & & g/cc \\
\hline \multicolumn{6}{|c|}{ Chemical Analyses. } \\
\hline $\mathrm{H} 2$ & & $53.20 \%$ & & $53.20 \%$ & \\
\hline $\mathrm{Na}$ & 9.85 & $16 \%$ & 10.32 & $16.80 \%$ & $M$ \\
\hline Al & 2.58 & $4.90 \%$ & 2.37 & $4.50 \%$ & $M$ \\
\hline NO2 & 1.11 & $3.60 \%$ & 1.13 & $3.70 \%$ & $M$ \\
\hline NO3 & 2.89 & $12.70 \%$ & 2.62 & $12 \%$ & $M$ \\
\hline$\overline{\mathrm{OH}}$ & 3.95 & $4.70 \%$ & 3.81 & $4.60 \%$ & $\bar{M}$ \\
\hline TAD & 6.76 & & 6.48 & & $M$ \\
\hline TOTAL & & 95.1 & & $94.80 \%$ & \\
\hline
\end{tabular}


WHC-SD-WM-ER-312, Rev. 0

\begin{tabular}{|c|c|c|c|}
\hline \multicolumn{4}{|c|}{ Waste Tank 109-BY } \\
\hline \multicolumn{4}{|c|}{ Analysis of Tank Farm Samples } \\
\hline \multicolumn{4}{|c|}{ August 3, 1972} \\
\hline \multicolumn{4}{|c|}{ Sample \#T-5953 } \\
\hline COMPONENT & \multicolumn{2}{|c|}{ LAB VALUE } & LAB UNIT \\
\hline & \multicolumn{2}{|c|}{ 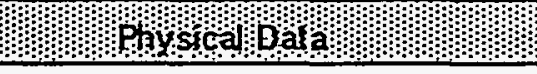 } & \\
\hline \multicolumn{4}{|c|}{ Visible - Clear Yellow; No organics; $25 \%$ solids } \\
\hline $\mathrm{SpG}$ & 1.381 & & \\
\hline Boildown starting volume & & 50 & $\mathrm{~mL}$ \\
\hline Boildown $\%$ solids & & $25 \%$ & \\
\hline \multicolumn{4}{|c|}{ 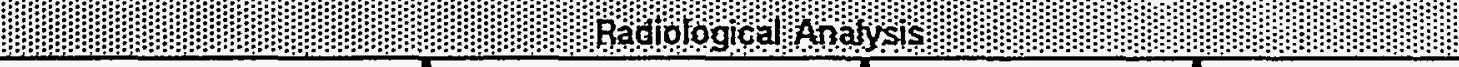 } \\
\hline GEA Cs-137 & $1.66 E+06$ & & uCilgal \\
\hline GEA Cs-134 & $2.41 E+04$ & & uCilgal \\
\hline
\end{tabular}




\begin{tabular}{|c|c|c|c|}
\hline \multicolumn{4}{|c|}{ Waste Tank 109-BY } \\
\hline \multicolumn{4}{|c|}{ Analysis of Tank Farm Samples } \\
\hline \multicolumn{4}{|c|}{ August 3,1972} \\
\hline \multicolumn{4}{|c|}{ Sample \#T-6968 } \\
\hline COMPONENT & \multicolumn{2}{|c|}{ LAB VALUE } & LAB UNIT \\
\hline \multicolumn{4}{|c|}{ fyspangat } \\
\hline \multicolumn{4}{|c|}{ Visible - Thick Yellow; $10 \%$ solids } \\
\hline SpG & 1.4425 & & \\
\hline \multicolumn{2}{|c|}{ Boildown Starting Volume } & 30 & $\mathrm{~mL}$ \\
\hline \multicolumn{2}{|c|}{ Boildown \% Solids } & $4 \%$ & \\
\hline \multicolumn{4}{|c|}{ padobggan Anass } \\
\hline GEA Cs-137 & $1.52 E+06$ & & uCilgal \\
\hline GEA Cs-134 & $2.26 \mathrm{E}+04$ & & uCi/gal \\
\hline
\end{tabular}




\begin{tabular}{|c|c|c|c|}
\hline \multicolumn{4}{|c|}{ Waste Tank 109-BY } \\
\hline \multicolumn{4}{|c|}{ Analysis of Tank Farm Samples } \\
\hline \multicolumn{4}{|c|}{ August 10,1972} \\
\hline \multicolumn{4}{|c|}{ Sample \#T-7993 } \\
\hline COMPONENT & \multicolumn{2}{|c|}{ LAB VALUE } & LAB UNIT \\
\hline & \multicolumn{2}{|c|}{ 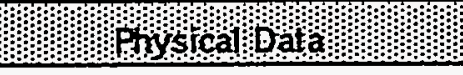 } & \\
\hline \multicolumn{4}{|c|}{ Visible - Clear Yellow: 15\% while solids } \\
\hline $\mathrm{SpG}$ & 1.463 & & \\
\hline \multicolumn{2}{|c|}{ Boildown Starting Volume } & 50 & $\mathrm{~mL}$ \\
\hline \multicolumn{2}{|c|}{ Boildown \% Solids } & $15 \%$ & \\
\hline \multicolumn{4}{|c|}{ 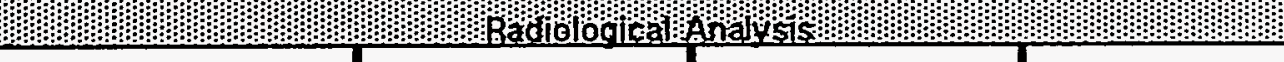 } \\
\hline GEA Cs-137 & 1.69E+06 & & uCi/gal \\
\hline GEA Cs-134 & $2.29 \mathrm{E}+04$ & & ucilaal \\
\hline
\end{tabular}




\begin{tabular}{|c|c|c|c|}
\hline \multicolumn{4}{|c|}{ Waste Tank 109-BY } \\
\hline \multicolumn{4}{|c|}{ Analysis of Tank Farm Samples } \\
\hline \multicolumn{4}{|c|}{ August 17,1972} \\
\hline \multicolumn{4}{|c|}{ Sample \#T-8966 } \\
\hline COMPONENT & \multicolumn{2}{|c|}{ LAB VALUE } & LAB UNIT \\
\hline $1 \boxminus$ & \multicolumn{2}{|c|}{ pyona Dob } & \\
\hline \multicolumn{4}{|c|}{ Visible - Clear, Yellow; $15 \%$ solids; $300 \mathrm{mRad}$} \\
\hline SpG & 1.455 & & \\
\hline \multicolumn{2}{|c|}{ Boildown Starting Volume } & 50 & $\mathrm{~mL}$ \\
\hline \multicolumn{2}{|c|}{ Boildown $\%$ Solids } & $10 \%$ & \\
\hline \multicolumn{4}{|c|}{ 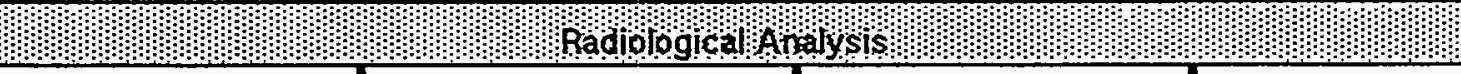 } \\
\hline GEA Cs-137 & $4.39 E+05$ & & uCi/gal \\
\hline GEA Cs-134 & $2.46 \mathrm{E}+04$ & & uciloal \\
\hline
\end{tabular}




\begin{tabular}{|c|c|c|c|}
\hline \multicolumn{4}{|c|}{ Analysis of Tank Farm Samples } \\
\hline \multicolumn{4}{|c|}{ August 25, 1972} \\
\hline \multicolumn{4}{|c|}{ Sample T-206 } \\
\hline COMPONENT & \multicolumn{2}{|c|}{ LAB VALUE } & LAB UNIT \\
\hline \multicolumn{4}{|c|}{ pgspangan } \\
\hline \multicolumn{4}{|c|}{ Visible - Clear, Yellow; - $5 \%$ white solids; $400 \mathrm{mRad}$} \\
\hline SpG & 1.4415 & & \\
\hline \multicolumn{2}{|c|}{ Boildown starting Volume } & 50 & $m L$ \\
\hline \multicolumn{2}{|c|}{ Boildown \%solids } & $0 \%$ & \\
\hline \multicolumn{4}{|c|}{ 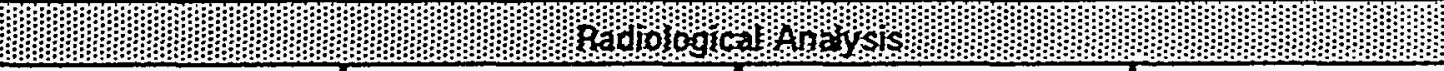 } \\
\hline GEA Cs-137 & $1.62 E+06$ & & ucigal \\
\hline GEA Cs-134 & $2.41 E+04$ & & uCi/gal \\
\hline
\end{tabular}




\begin{tabular}{|c|c|c|c|}
\hline \multicolumn{4}{|c|}{ Waste Tank 109-BY } \\
\hline \multicolumn{4}{|c|}{ Analysis of Tank Farm Samples } \\
\hline \multicolumn{4}{|c|}{ September 12,1972} \\
\hline \multicolumn{4}{|c|}{ Sample \#T-1832 } \\
\hline COMPONENT & \multicolumn{2}{|c|}{ LAB VALUE } & LAB UNIT \\
\hline 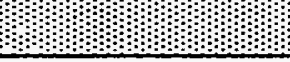 & \multicolumn{2}{|c|}{ 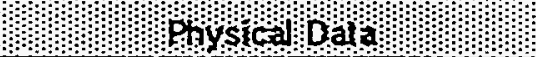 } & छ! \\
\hline \multicolumn{4}{|c|}{ Visible - Clear, Yellow; $10 \%$ while solids; $1 \mathrm{mRad}$} \\
\hline $\operatorname{SpG}$ & 1.420 & & \\
\hline \multicolumn{2}{|c|}{ Boildown starting Volume } & 50 & $\mathrm{~mL}$ \\
\hline \multicolumn{2}{|c|}{ Boildown \%solids } & $1 \%$ & \\
\hline \multicolumn{4}{|c|}{ 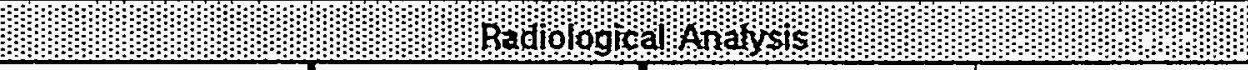 } \\
\hline GEA Cs-137 & $1.50 \mathrm{E}+06$ & & uCi/gal \\
\hline GEA Cs-134 & $2.69 \mathrm{E}+04$ & & uCi/gal \\
\hline
\end{tabular}




\begin{tabular}{|c|c|c|c|}
\hline \multicolumn{4}{|c|}{ Waste Tank 109-BY } \\
\hline \multicolumn{4}{|c|}{ Analysis of Tank Farm Samples } \\
\hline \multicolumn{4}{|c|}{ Septem ber 26, 1972} \\
\hline \multicolumn{4}{|c|}{ Sample \#T-2490 } \\
\hline COMPONENT & \multicolumn{2}{|c|}{ LAB VALUE } & LAB UNIT \\
\hline & \multicolumn{2}{|c|}{ 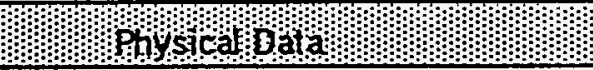 } & \\
\hline \multicolumn{4}{|c|}{ Visible - Yellow; $40 \%$ while solids; $400 \mathrm{mRad}$} \\
\hline SpG & 1.282 & & \\
\hline \multicolumn{2}{|c|}{ Boildown starting Volume } & $50 \mathrm{~mL}$ & $\mathrm{~mL}$ \\
\hline \multicolumn{2}{|c|}{ Boildown \%solids } & $1 \%$ & \\
\hline \multicolumn{4}{|c|}{ 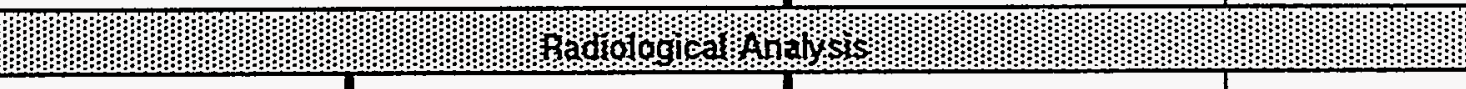 } \\
\hline GEA Cs-137 & $1.78 E+06$ & & uCilgal \\
\hline GEA Zrnb-95 & $-3.61 E+03$ & & uCilgal \\
\hline GEA Cs-134 & $3.66 \mathrm{E}+04$ & & uCilaal \\
\hline
\end{tabular}




\begin{tabular}{|c|c|c|c|}
\hline \multicolumn{4}{|c|}{ Waste Tank 109-BY } \\
\hline \multicolumn{4}{|c|}{ Analysis of Tank Farm Samples } \\
\hline \multicolumn{4}{|c|}{ October 6. 1972} \\
\hline \multicolumn{4}{|c|}{ Sample $T-2833$} \\
\hline COMPONENT & \multicolumn{2}{|c|}{ LAB VALUE } & LAB UNIT \\
\hline \multicolumn{4}{|c|}{ physúpanaba } \\
\hline \multicolumn{4}{|c|}{ Visible - Yellow: 3\% white solids; 1 Rad } \\
\hline SpG & 1.366 & & \\
\hline \multicolumn{2}{|c|}{ Boildown starting volume } & 50 & $m L$ \\
\hline \multicolumn{2}{|c|}{ Boildown \% Solids } & $4 \%$ & \\
\hline \multicolumn{4}{|c|}{ 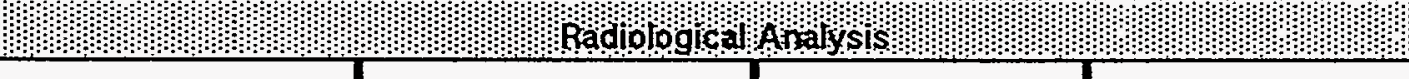 } \\
\hline GEA Cs-137 & $1.36 \mathrm{E}+06$ & & ucilgal \\
\hline GEA Cs-134 & $3.12 E+04$ & & uCilaal \\
\hline
\end{tabular}




\begin{tabular}{|c|c|c|}
\hline \multicolumn{3}{|c|}{ Waste Tank 109-BY } \\
\hline \multicolumn{3}{|c|}{ Analysis of Tank Farm Samples } \\
\hline \multicolumn{3}{|c|}{ November 16, 1972} \\
\hline \multicolumn{3}{|c|}{ Sample $T-3743$} \\
\hline COMPONENT & LAB VALUE & LAB UNIT \\
\hline \multicolumn{3}{|c|}{ 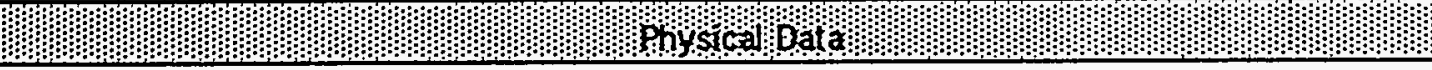 } \\
\hline \multicolumn{3}{|c|}{ Visible - Pea Green; $35 \%$ whit e solids; $200 \mathrm{mRad}$} \\
\hline$S p G$ & 1.402 & \\
\hline \multicolumn{3}{|c|}{ 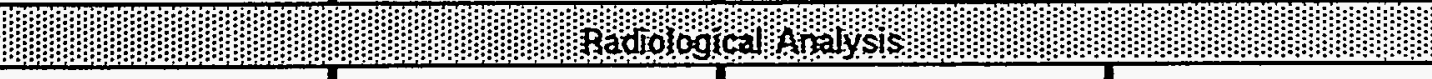 } \\
\hline GEA Cs-137 & $1.71 E+06$ & uCilgal \\
\hline GEA Cs-134 & $3.62 E+04$ & uCilgal \\
\hline
\end{tabular}




\begin{tabular}{|c|c|c|c|}
\hline \multicolumn{4}{|c|}{ Waste Tank 109-BY } \\
\hline \multicolumn{4}{|c|}{ Analysis of Tank Farm Samples } \\
\hline \multicolumn{4}{|c|}{ November 28, 1972} \\
\hline \multicolumn{4}{|c|}{ Sample \#T-4005 } \\
\hline COMPONENT & \multicolumn{2}{|c|}{ LAB VALUE } & LAB UNIT \\
\hline \multicolumn{4}{|c|}{ 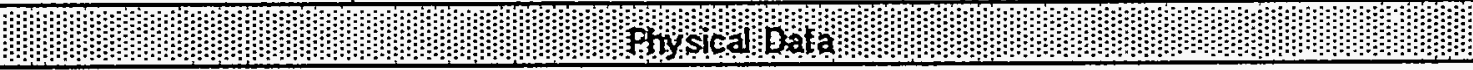 } \\
\hline \multicolumn{4}{|c|}{ Visible - Light green: $<1 \%$ white solids; 1.8 Rads } \\
\hline SpG & 1.3617 & & \\
\hline \multicolumn{2}{|c|}{ Boildown starting volume } & 50 & $\mathrm{~mL}$ \\
\hline \multicolumn{2}{|c|}{ Boildown \%solids } & $<1 \%$ & \\
\hline \multicolumn{4}{|c|}{ 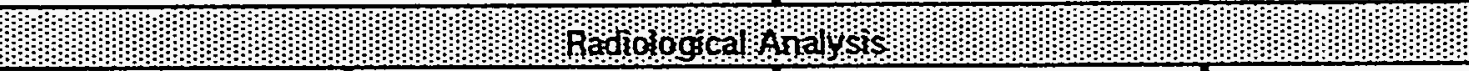 } \\
\hline GEA Cs -137 & $1.36 \mathrm{E}+06$ & & ua/gal \\
\hline GEA CS-134 & $3.36 E+04$ & & ualoal \\
\hline
\end{tabular}




\begin{tabular}{|c|c|c|c|}
\hline \multicolumn{4}{|c|}{ Waste Tank 109-BY } \\
\hline \multicolumn{4}{|c|}{ Analysis of Tank Farm Samples } \\
\hline \multicolumn{4}{|c|}{ December 18, 1972} \\
\hline \multicolumn{4}{|c|}{ Sample \#T-4316 } \\
\hline COMPONENT & \multicolumn{2}{|c|}{ LAB VALUE } & LAB UNIT \\
\hline \multicolumn{4}{|c|}{ Physical Data } \\
\hline \multicolumn{4}{|c|}{ Visible - Green with solids; $20 \%$ solids; 3.0 Rads } \\
\hline $\mathrm{SpG}$ & 1.422 & & \\
\hline \multicolumn{2}{|c|}{ Boildown starting volume } & 50 & $\mathrm{~mL}$ \\
\hline \multicolumn{2}{|c|}{ Boildown \%solids } & $20 \%$ & \\
\hline \multicolumn{4}{|c|}{ Radiological Analysis } \\
\hline GEA Cs-137 & $1.58 \mathrm{E}+06$ & & uCi/gal \\
\hline GEA Sb-125 & $5.53 E+03$ & & uCi/gal \\
\hline GEA Cs-134 & $3.44 E+04$ & & uCi/gal \\
\hline
\end{tabular}




\begin{tabular}{|c|c|c|c|}
\hline \multicolumn{4}{|c|}{ Waste Tank 109-BY } \\
\hline \multicolumn{4}{|c|}{ Analysis of Tank Farm Samples } \\
\hline \multicolumn{4}{|c|}{ January 8,1973} \\
\hline \multicolumn{4}{|c|}{ Sample \#T-4573 } \\
\hline COMPONENT & \multicolumn{2}{|c|}{ LAB VALUE } & LAB UNIT \\
\hline \multicolumn{4}{|c|}{ 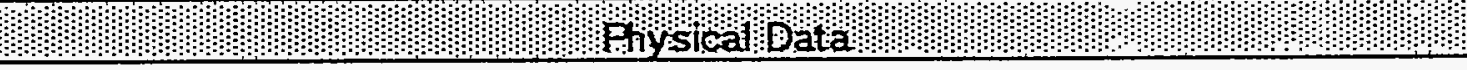 } \\
\hline \multicolumn{4}{|c|}{ Visible - Yellow: $5 \%$ white solids; 1.2 Rads } \\
\hline SpG & 1.225 & & \\
\hline \multicolumn{2}{|c|}{ Boildown starting volume } & 50 & $\mathrm{~mL}$ \\
\hline \multicolumn{2}{|c|}{ Boildown \% Solids } & $16 \%$ & \\
\hline \multicolumn{4}{|c|}{ 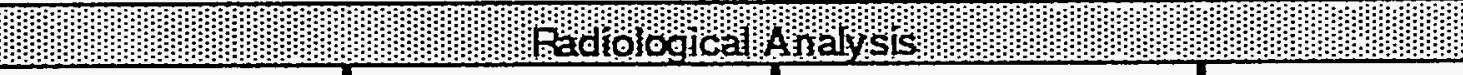 } \\
\hline GEA CS-137 & $7.34 E+05$ & & uCi/gal \\
\hline GEA Cs-134 & $1.85 E+04$ & & uCi/gal \\
\hline
\end{tabular}




\begin{tabular}{|c|c|c|c|}
\hline \multicolumn{4}{|c|}{ Waste Tank 109-BY } \\
\hline \multicolumn{4}{|c|}{ Analysis of Tank Farm Samples } \\
\hline \multicolumn{4}{|c|}{ January 16,1973} \\
\hline \multicolumn{4}{|c|}{ Sample \#T-4911 } \\
\hline COMPONENT & & & LAB UNIT \\
\hline \multicolumn{4}{|c|}{ 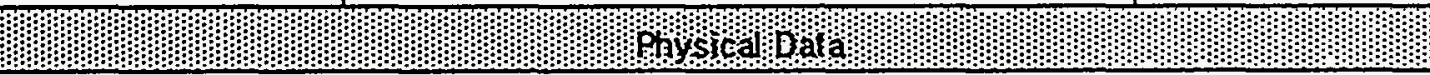 } \\
\hline \multicolumn{4}{|c|}{ Appearance-Yellow; - $5 \%$ while solids; 1.5 Rad } \\
\hline$S p G$ & 1.404 & & \\
\hline \multicolumn{2}{|c|}{ Boildown starting volume } & 50 & $\mathrm{~mL}$ \\
\hline \multicolumn{2}{|c|}{ Boildown \% Solids } & $-5 \%$ & \\
\hline \multicolumn{4}{|c|}{ 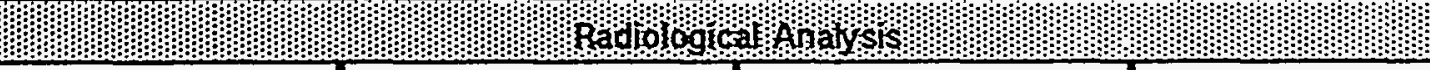 } \\
\hline GEA Cs-137 & $1.93 E+06$ & & uCilgal \\
\hline GEA Cs-134 & $3.62 E+04$ & & uCilaal \\
\hline
\end{tabular}




\begin{tabular}{|c|c|c|c|}
\hline \multicolumn{4}{|c|}{ Waste Tank 109-BY } \\
\hline \multicolumn{4}{|c|}{ Analysis of Tank Farm Samples } \\
\hline \multicolumn{4}{|c|}{ January 31,1973} \\
\hline \multicolumn{4}{|c|}{ Sample \#T-5124 } \\
\hline COMPONENT & \multicolumn{2}{|c|}{ LAB VALUE } & LAB UNIT \\
\hline & \multicolumn{2}{|c|}{ 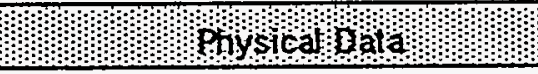 } & \\
\hline \multicolumn{4}{|c|}{ Visible - Yellow; $20 \%$ white solids; $1500 \mathrm{mRad}$} \\
\hline SpG & 1.396 & & \\
\hline \multicolumn{2}{|c|}{ Boildown starting volume } & 50 & $\mathrm{~mL}$ \\
\hline \multicolumn{2}{|c|}{ Boildown \% Solids } & $2 \%$ & \\
\hline \multicolumn{4}{|c|}{ 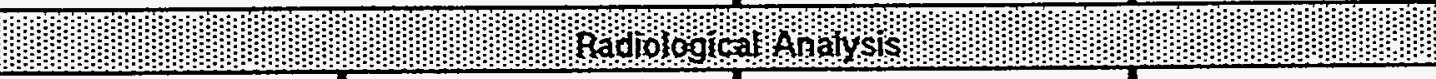 } \\
\hline GEA Cs-137 & $1.94 E+06$ & & uCi/gal \\
\hline GEA Cs-134 & $3.47 E+04$ & & uCiloal \\
\hline
\end{tabular}




\begin{tabular}{|c|c|c|c|}
\hline \multicolumn{4}{|c|}{ Waste Tank 109-BY } \\
\hline \multicolumn{4}{|c|}{ Analysis of Tank Farm Samples } \\
\hline \multicolumn{4}{|c|}{ February 7.1973} \\
\hline \multicolumn{4}{|c|}{ Sample $\quad$ T -5391} \\
\hline COMPONENT & \multicolumn{2}{|c|}{ LAB VALUE } & LAB UNIT \\
\hline & \multicolumn{2}{|c|}{ 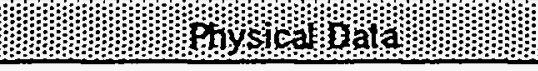 } & \\
\hline \multicolumn{4}{|c|}{ Visible - Yellow: $10 \%$ white solids: 1 Rad } \\
\hline$S p G$ & 1.324 & & \\
\hline \multicolumn{2}{|c|}{ Boildown starting volume } & 50 & $\mathrm{~mL}$ \\
\hline \multicolumn{2}{|c|}{ Boildown \%solids } & $10 \%$ & \\
\hline \multicolumn{4}{|c|}{ 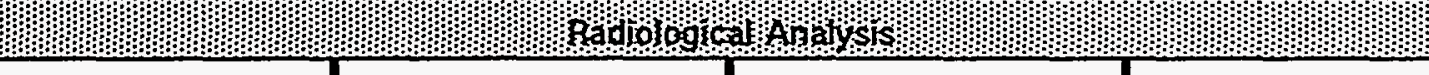 } \\
\hline GEA Cs-137 & $1.20 E+06$ & & uCigal \\
\hline GEA Cs-134 & $2.51 E+04$ & & uCilaal \\
\hline
\end{tabular}




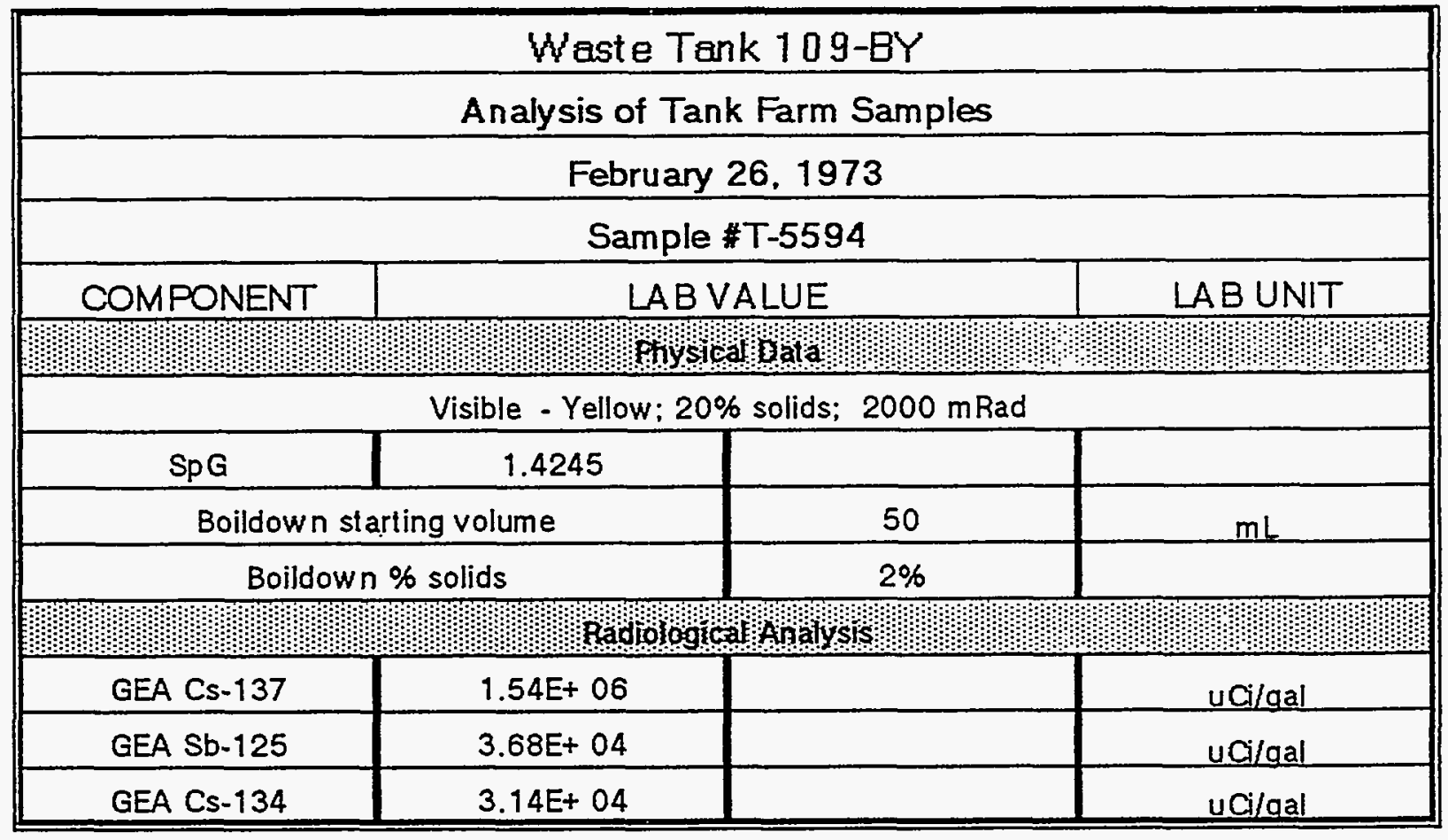




\begin{tabular}{|c|c|c|}
\hline \multicolumn{3}{|c|}{ Waste Tank 109-BY } \\
\hline \multicolumn{3}{|c|}{ Analysis of Tank Farm Samples } \\
\hline \multicolumn{3}{|c|}{ March 1, 1973} \\
\hline \multicolumn{3}{|c|}{ Sample \#T-5655 } \\
\hline COMPONENT & & LAB UNIT \\
\hline \multicolumn{3}{|c|}{ physedoat } \\
\hline \multicolumn{3}{|c|}{ Appearance - Yellow; $35 \%$ solids: 2 Rad } \\
\hline$S p G$ & 1.3895 & \\
\hline \multicolumn{3}{|c|}{ 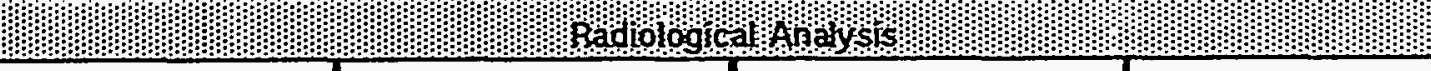 } \\
\hline GEA C $s-137$ & $1.64 \mathrm{E}+06$ & uCi/gal \\
\hline GEA Cs-134 & $2.87 E+04$ & uCi/gal \\
\hline
\end{tabular}




\begin{tabular}{|c|c|c|c|}
\hline \multicolumn{4}{|c|}{ Waste Tank 109-BY } \\
\hline \multicolumn{4}{|c|}{ Analysis of Tank Farm Samples } \\
\hline \multicolumn{4}{|c|}{ March 7. 1973} \\
\hline \multicolumn{4}{|c|}{ Sample \#T-5911 } \\
\hline COMPONENT & \multicolumn{2}{|c|}{ LAB VALUE } & LAB UNIT \\
\hline & \multicolumn{2}{|c|}{ 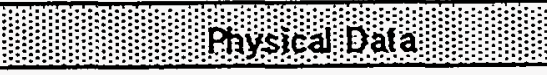 } & \\
\hline \multicolumn{4}{|c|}{ Visible - Yellow: 3\% solids; 2 Rad } \\
\hline $\mathrm{SpG}$ & 1.335 & & \\
\hline \multicolumn{2}{|c|}{ Boildown st arting volume } & 50 & $\mathrm{~mL}$ \\
\hline \multicolumn{2}{|c|}{ Boildown \% Solids } & $0 \%$ & \\
\hline \multicolumn{4}{|c|}{ 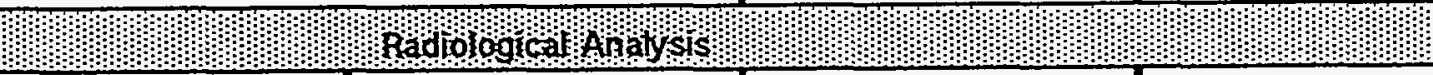 } \\
\hline GEA Cs-137 & $1.27 E+06$ & & uCiloal \\
\hline GEA Cs-134 & $2.21 E+04$ & & uCilgal \\
\hline
\end{tabular}




\begin{tabular}{|c|c|c|c|}
\hline \multicolumn{4}{|c|}{ Waste Tank 109-BY } \\
\hline \multicolumn{4}{|c|}{ Analysis of Tank Farm Samples } \\
\hline \multicolumn{4}{|c|}{ March 22, 1973} \\
\hline \multicolumn{4}{|c|}{ Sample } \\
\hline COMPONENT & \multicolumn{2}{|c|}{ LAB VALUE } & LAB UNIT \\
\hline \multicolumn{4}{|c|}{ phy } \\
\hline \multicolumn{4}{|c|}{ Visible - Yellow; $2 \%$ solids: $1.5 \mathrm{Rad}$} \\
\hline SpG & 1.316 & & \\
\hline \multicolumn{2}{|c|}{ Boildown starting volume } & 50 & $\mathrm{~mL}$ \\
\hline \multicolumn{2}{|c|}{ Boildown \%solids } & $0 \%$ & \\
\hline \multicolumn{4}{|c|}{ 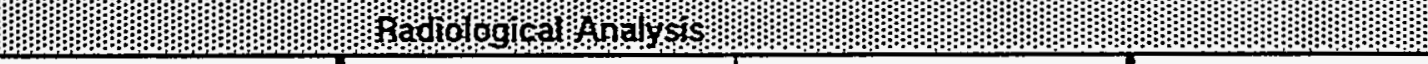 } \\
\hline GEA Cs-137 & $1.06 E+06$ & & uCilgal \\
\hline GEA Cs-134 & $1.60 E+04$ & & uCi/gal \\
\hline GEA RuRh-106 & $3.97 E+04$ & & uCi/gal \\
\hline GEA Eu-154 & $1.94 \mathrm{E}+03$ & & uci/gal \\
\hline
\end{tabular}




\begin{tabular}{|c|c|c|c|}
\hline \multicolumn{4}{|c|}{ Waste Tank 109-BY } \\
\hline \multicolumn{4}{|c|}{ Analysis of Tank Farm Samples } \\
\hline \multicolumn{4}{|c|}{ April 5, 1973} \\
\hline \multicolumn{4}{|c|}{ Sample \#T-6394 } \\
\hline COMPONENT & & & LAB UNIT \\
\hline \multicolumn{4}{|c|}{ 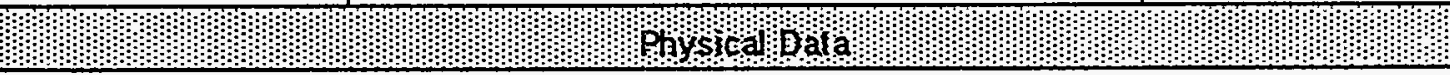 } \\
\hline \multicolumn{4}{|c|}{ Visible - Yellow; $3 \%$ solids; 1 Rad } \\
\hline $\mathrm{SpG}$ & 1.4432 & & \\
\hline \multicolumn{2}{|c|}{ Boildown starting volume } & 50 & $\mathrm{~mL}$ \\
\hline \multicolumn{2}{|c|}{ Boildown \%solids } & $8 \%$ & \\
\hline \multicolumn{4}{|c|}{ 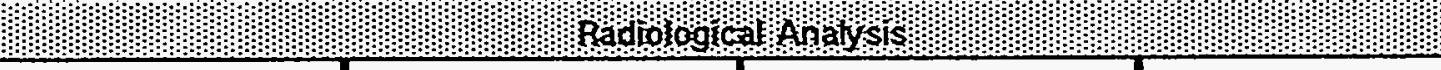 } \\
\hline GEA Cs-137 & $1.75 E+06$ & & uCi/gal \\
\hline GEA Cs-134 & $2.79 E+04$ & & uCi/gal \\
\hline
\end{tabular}


WHC-SD-WM-ER-312, Rev. 0

\begin{tabular}{|c|c|c|c|}
\hline \multicolumn{4}{|c|}{ Waste Tank 109-BY } \\
\hline \multicolumn{4}{|c|}{ April 19, 1973} \\
\hline \multicolumn{4}{|c|}{ Analysis of Tank Farm Samples } \\
\hline \multicolumn{4}{|c|}{ Sample \#T-6682 } \\
\hline COMPONENT & \multicolumn{2}{|c|}{ LAB VALUE } & LAB UNIT \\
\hline \multicolumn{4}{|c|}{ poysupda } \\
\hline \multicolumn{4}{|c|}{ Visible - Yellow; $20 \%$ solids: $1.3 \mathrm{Rad}$} \\
\hline Sp.G & 1.4028 & & \\
\hline \multicolumn{2}{|c|}{ Boildown starting volume } & 50 & $\mathrm{~mL}$ \\
\hline \multicolumn{2}{|c|}{ Boildown \%solids } & $0 \%$ & \\
\hline \multicolumn{4}{|c|}{ padolognan ans } \\
\hline GEA Cs-137 & $1.56 E+06$ & & uCi/gal \\
\hline GEA Cs-134 & $2.20 E+04$ & & uCi/gal \\
\hline
\end{tabular}




\begin{tabular}{|c|c|c|}
\hline \multicolumn{3}{|c|}{ Waste Tank 109-BY } \\
\hline \multicolumn{3}{|c|}{ Analysis of Tank Farm Samples } \\
\hline \multicolumn{3}{|c|}{ May 7,1973} \\
\hline \multicolumn{3}{|c|}{ Sample \#T-6877 } \\
\hline COMPONENT & & LAB UNIT \\
\hline 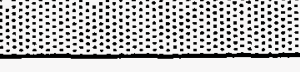 & \multicolumn{2}{|c|}{ nyston } \\
\hline \multicolumn{3}{|c|}{ Visible - Light green; Cruddy; 2.5 Rads } \\
\hline SpG & 1.439 & \\
\hline \multicolumn{3}{|c|}{ 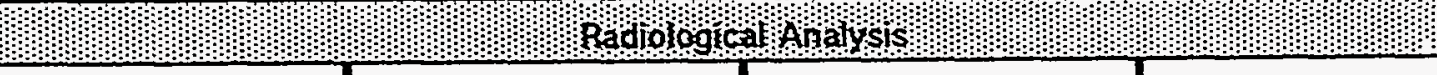 } \\
\hline GEA Cs-137 & 1.87E+ 06 & uCi/gal \\
\hline GEA Cs-134 & $2.58 \mathrm{E}+04$ & uCi/gal \\
\hline GEA Sb-125 & $2.57 E+03$ & uCi/gal \\
\hline
\end{tabular}




\begin{tabular}{|c|c|c|c|}
\hline \multicolumn{4}{|c|}{ Waste Tank 109-BY } \\
\hline \multicolumn{4}{|c|}{ Analysis of Tank Farm Samples } \\
\hline \multicolumn{4}{|c|}{ May 23, 1973} \\
\hline \multicolumn{4}{|c|}{ Sample } \\
\hline COMPONENT & \multicolumn{2}{|c|}{ LAB VALUE } & LAB UNIT \\
\hline & \multicolumn{2}{|c|}{ 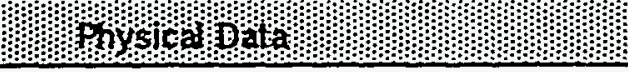 } & \\
\hline \multicolumn{4}{|c|}{ Visible - Cruddy; $50 \%$ solids; $200 \mathrm{mRad}$} \\
\hline Sp G & 1.3765 & & \\
\hline \multicolumn{2}{|c|}{ Boildown starting volume } & 50 & $\mathrm{~mL}$ \\
\hline \multicolumn{2}{|c|}{ Boildown \%solids } & $50 \%$ & \\
\hline \multicolumn{4}{|c|}{ 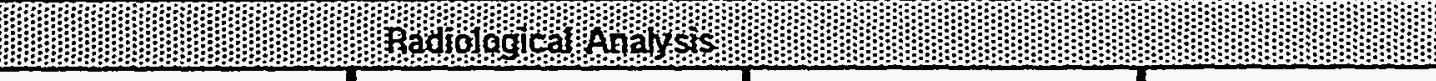 } \\
\hline GEA Cs -137 & $1.68 E+06$ & & uCi/gal \\
\hline GEA Cs-134 & $2.21 E+04$ & & uCi/gal \\
\hline
\end{tabular}




\begin{tabular}{|c|c|c|c|}
\hline \multicolumn{4}{|c|}{ Waste Tank 112-BY } \\
\hline \multicolumn{4}{|c|}{ Analysis of Supernatant, Sludge and Crust From Tank 112-BY } \\
\hline \multicolumn{4}{|c|}{ May 29.1971} \\
\hline Component & Supematant & Sludge & Crust \\
\hline \multicolumn{4}{|c|}{ Qfonum } \\
\hline $\mathrm{Na}$ & $11.16 \mathrm{M}$ & $16.9 \mathrm{M}$ & $20.7 \mathrm{M}$ \\
\hline $\mathrm{Fe}$ & $<1.81 \mathrm{E}-03$ & 0.42 & 0.06 \\
\hline Al & 2.05 & 1.71 & 0.58 \\
\hline $\mathrm{Si}$ & $9.08 \mathrm{E}-03$ & 0.22 & $<0.03$ \\
\hline NO2 & $1.4 \mathrm{M}$ & 1.54 & 0.012 \\
\hline NO3 & 2.1 & 6.17 & 4.32 \\
\hline $\cos$ & 0.018 & 0.67 & 3.05 \\
\hline SO4 & 0.05 & 0.12 & 0.46 \\
\hline PO4 & 0.023 & 0.26 & 0.12 \\
\hline TAD* & 7.20 & 8.11 & 9.84 \\
\hline \multicolumn{4}{|c|}{ 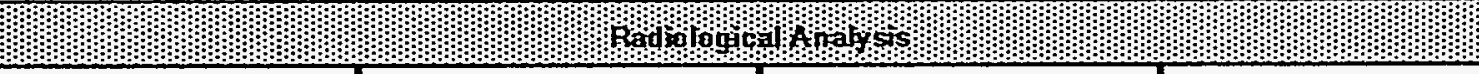 } \\
\hline $89+90 \mathrm{Sr}$ & $1.85 \mathrm{E}+03 \mathrm{uCi} / \mathrm{l}$ & $8.21 \mathrm{E}+04 \mathrm{uCi} / \mathrm{l}$ & $9.84 \mathrm{E}+04 \mathrm{uCi} / 1$ \\
\hline $137 \mathrm{Cs}$ & $4.30 E+05$ & $3.17 E+05$ & $2.51 E+05$ \\
\hline $134 \mathrm{Cs}$ & $4.48 E+03$ & $4.77 E+03$ & $3.11 E+03$ \\
\hline $106 \mathrm{Ru} 106 \mathrm{Rh}$ & & $3.91 E+04$ & $2.30 E+03$ \\
\hline $60 \mathrm{Co}$ & & 40 & $4.50 E+02$ \\
\hline $125 \mathrm{Sb}$ & & $3.77 E+02$ & $1.20 \mathrm{E}+03$ \\
\hline $144 \mathrm{Ce} 144 \mathrm{Pr}$ & & $3.29 E+04$ & $5.70 E+04$ \\
\hline $95 \mathrm{Zr} 95 \mathrm{Nb}$ & & & $5.30 E+02$ \\
\hline $154 \mathrm{Eu}$ & & & $8.30 E+02$ \\
\hline * Total acid deman & & & \\
\hline
\end{tabular}




\begin{tabular}{|c|c|c|c|}
\hline \multicolumn{4}{|c|}{ Waste Tank 112-BY } \\
\hline \multicolumn{4}{|c|}{ Analyses of 112-BY Samples } \\
\hline \multicolumn{4}{|c|}{ April 4.1972 } \\
\hline \multicolumn{4}{|c|}{ Sample Received: April 1971} \\
\hline Camponent & Top Solids & Liquid & Bottom Solids \\
\hline \multicolumn{4}{|c|}{ mententat } \\
\hline Density g/ce & 1.48 & 1.42 & 1.44 \\
\hline $\mathrm{H} 2 \mathrm{O} \%$ & - & - & 50.5 \\
\hline \multicolumn{4}{|c|}{ 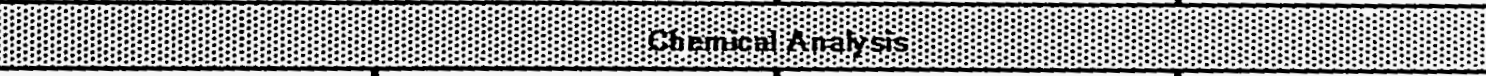 } \\
\hline $\mathrm{Na} \%$ & 24.1 & 19.1 & 20.6 \\
\hline Al \% & 2.8 & 4.1 & 0.6 \\
\hline $\mathrm{Fe} \%$ & $<0.3$ & - & $<0.5$ \\
\hline $\mathrm{Cr} \%$ & 0.15 & $\cdot$ & 4.8 \\
\hline $\mathrm{Ni} \%$ & 0.11 & - & 0.2 \\
\hline$M n \%$ & 0.02 & - & $<0.02$ \\
\hline Si \% & 0.7 & - & 0.6 \\
\hline NO2 $\%$ & 2.3 & 4.2 & 0.4 \\
\hline NO3 \% & 9.2 & 7.1 & 17.8 \\
\hline $\cos \%$ & 0.7 & 0.8 & 1.6 \\
\hline $\mathrm{OH} \%$ & 6.5 & 7.6 & 4.4 \\
\hline $\operatorname{TAD}(M)$ & 5.6 & 7.03 & 4.0 \\
\hline \multicolumn{4}{|c|}{ 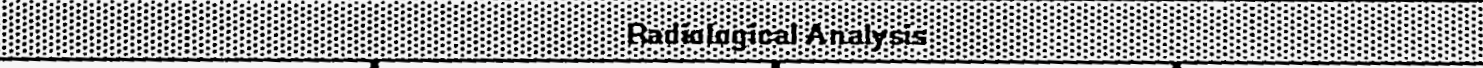 } \\
\hline $90 \mathrm{Sr}(\mathrm{uCi} / \mathrm{g})$ & 34.2 & 0.27 & 40.3 \\
\hline $137 \mathrm{Cs}(\mathrm{uCi} / \mathrm{g})$ & 151 & 483.0 & 20.6 \\
\hline $95 \mathrm{Zr} 95 \mathrm{Nb}(\mathrm{uCi} / \mathrm{g})$ & 25.2 & 8.6 & 23.6 \\
\hline $125 \mathrm{Sb}(\mathrm{uCi} / \mathrm{g})$ & - & . & 0.18 \\
\hline $106 \mathrm{Ru} 106 \mathrm{Rh}(\mathrm{uCi} / \mathrm{g})$ & - & $\therefore$ & 7.2 \\
\hline $144 \mathrm{Ce} 144 \operatorname{Pr}(\mathrm{Ci} / \mathrm{g})$ & - & - & 52.3 \\
\hline
\end{tabular}




\begin{tabular}{|c|c|c|c|}
\hline \multicolumn{4}{|c|}{ Waste Tank 112-BY } \\
\hline \multicolumn{4}{|c|}{ Compostion of 112-BY Samples } \\
\hline \multicolumn{4}{|c|}{ Apri 4, 1972} \\
\hline \multicolumn{4}{|c|}{ Sample Received: Apri 1971} \\
\hline & \multicolumn{3}{|c|}{ Weight Percent } \\
\hline Component & Top Solids & Liquid & Bottom Solids \\
\hline \multicolumn{4}{|c|}{ poy } \\
\hline $\mathrm{H} 2 \mathrm{O}$ & $50 . *$ & $50 . *$ & 50.5 \\
\hline \multicolumn{4}{|c|}{ 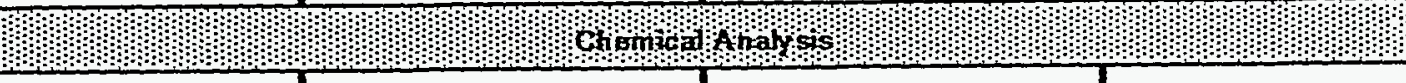 } \\
\hline $\mathrm{NaNO2}$ & 3.4 & 6.3 & 0.6 \\
\hline $\mathrm{NaNO} 3$ & 13.0 & 9.7 & 24.0 \\
\hline $\mathrm{NaAlO} 2$ & 8.50 & 12.0 & 1.8 \\
\hline $\mathrm{NaOH}$ & 15.00 & 18.0 & 10.0 \\
\hline $\mathrm{Na2} \mathrm{CO} 3$ & 1.20 & 1.4 & 2.8 \\
\hline $\mathrm{Cr} 2 \mathrm{O3}$ & 0.20 & - & 7.0 \\
\hline $\mathrm{NE2SiO3}$ & 3.0 & - & 2.6 \\
\hline Total & 94.3 & 97.4 & 99.3 \\
\hline * Estimated & & & \\
\hline
\end{tabular}




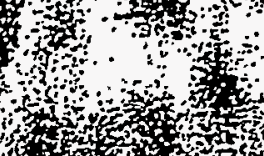
1.

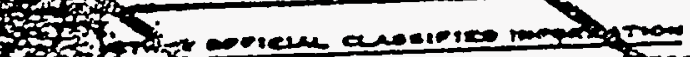

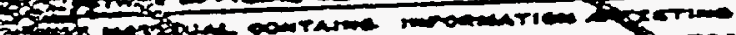

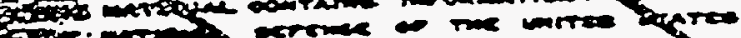

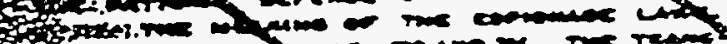

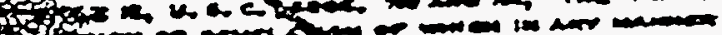

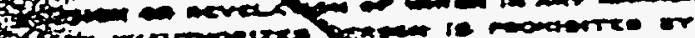
ind

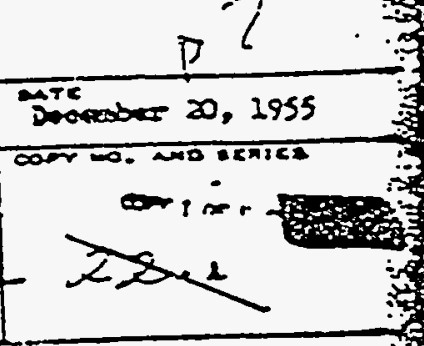

os

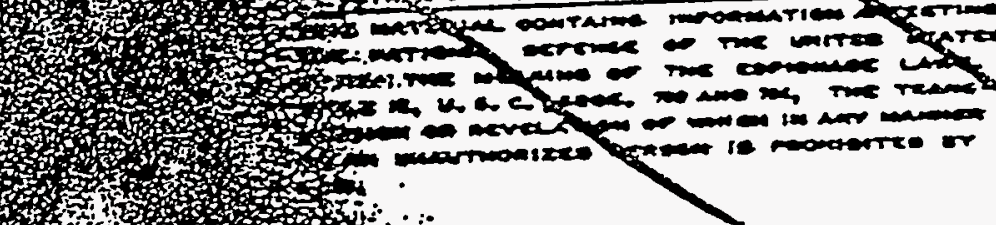

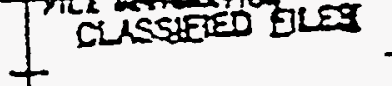

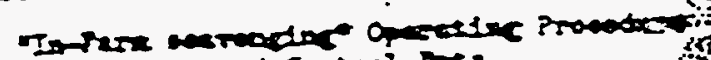
ind contel ins

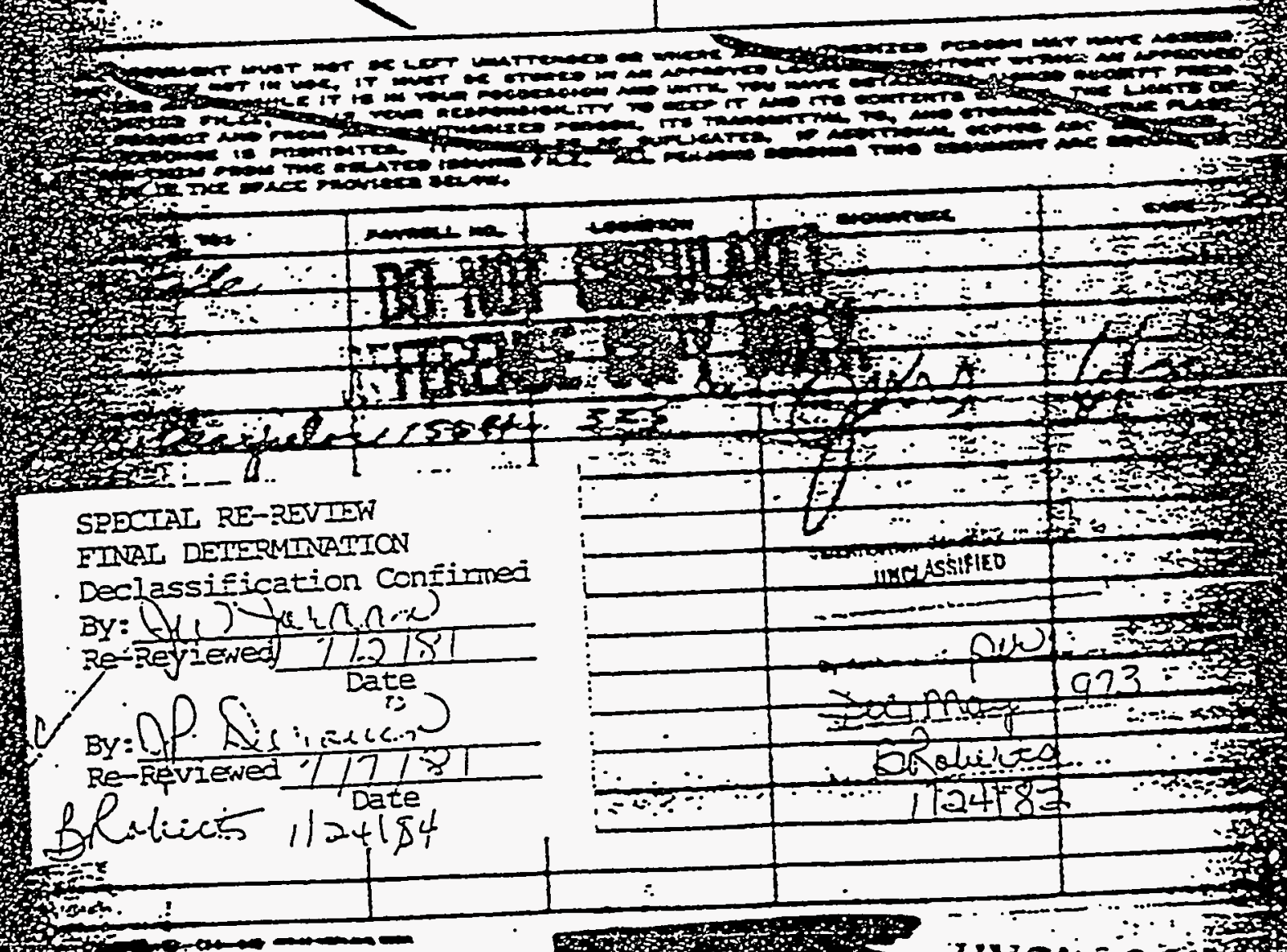

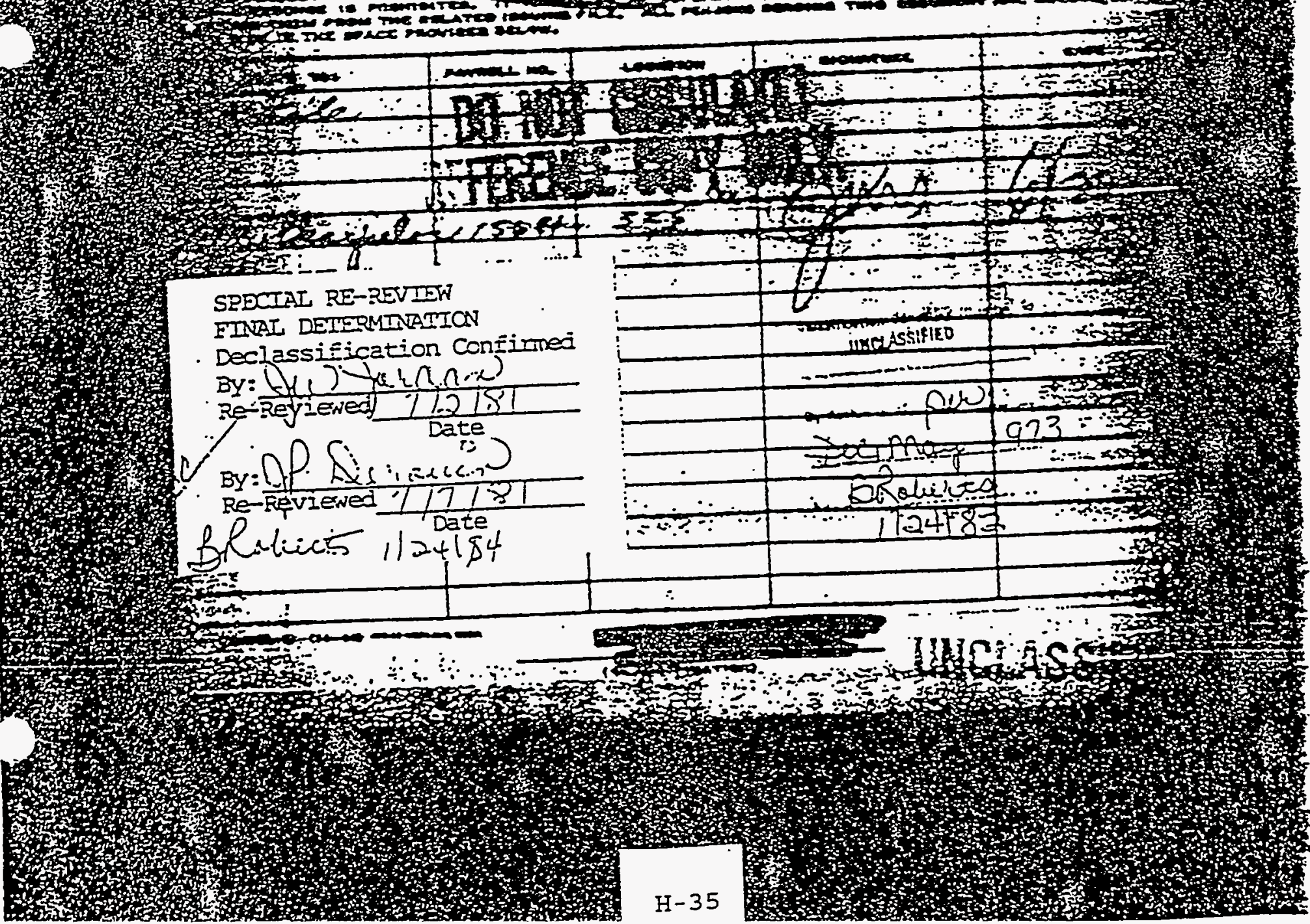




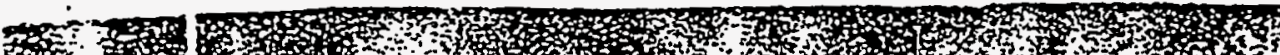

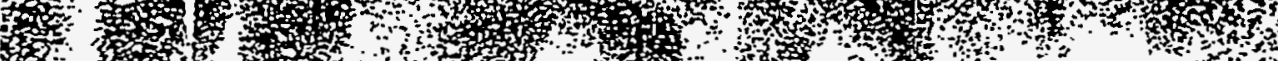

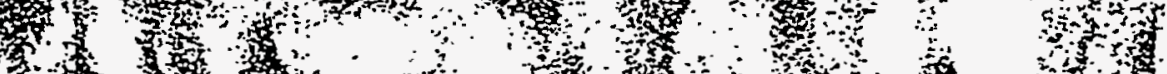

WHC-SD-WM-ER-312, Rev. 0

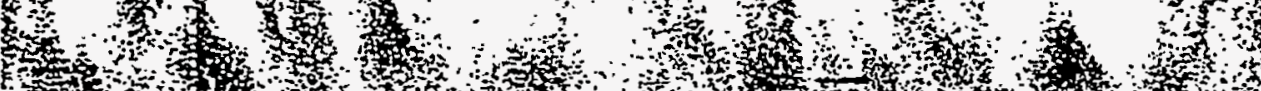

H.

H.

褯.

整

.

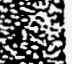

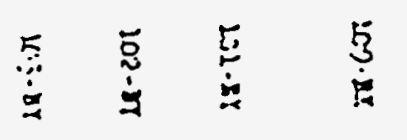

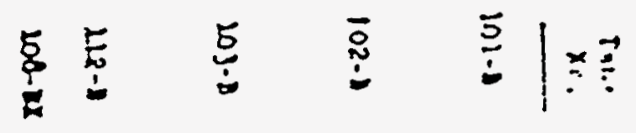

$$
\begin{aligned}
& \because \quad \vdots \quad \vdots \\
& i \vdots
\end{aligned}
$$

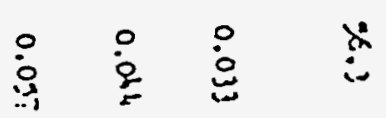

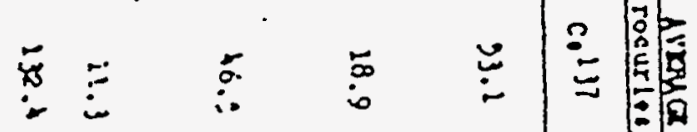

$$
\begin{aligned}
& \text { is } \quad \begin{array}{lll}
0 & 0 \\
\dot{0} & \vdots
\end{array} \\
& \text { 满 } \\
& \because \quad \vdots \quad \vdots \quad \because
\end{aligned}
$$

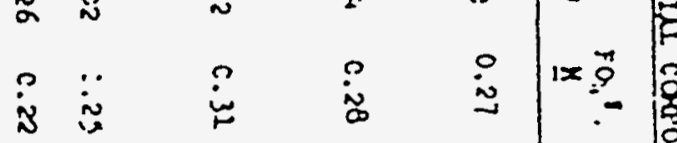

$$
\begin{aligned}
& \stackrel{i}{i} \stackrel{0}{i}
\end{aligned}
$$

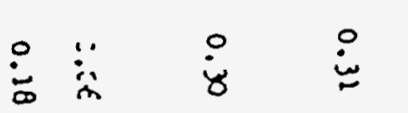

$$
\begin{aligned}
& \text { i. } \div \\
& \stackrel{9}{-1} \\
& \text { i } \\
& \vec{\infty} \quad \vec{i}
\end{aligned}
$$

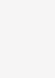

然

1. ' '

$\stackrel{8}{8}:$

然

$$
\text { (1) }
$$

०.: 0.: 0.: $1 \times \frac{2}{2}$

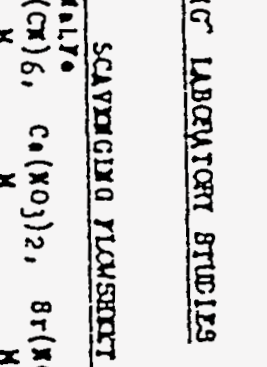

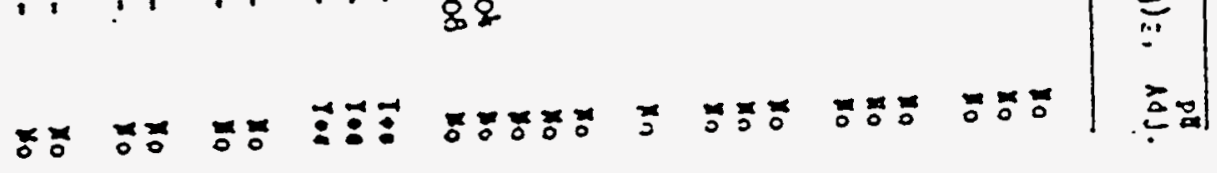

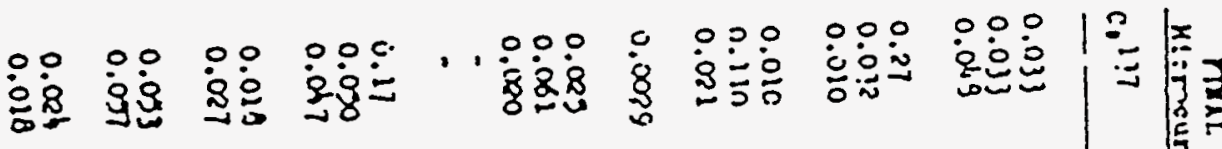
$\div 0$ 88.

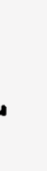

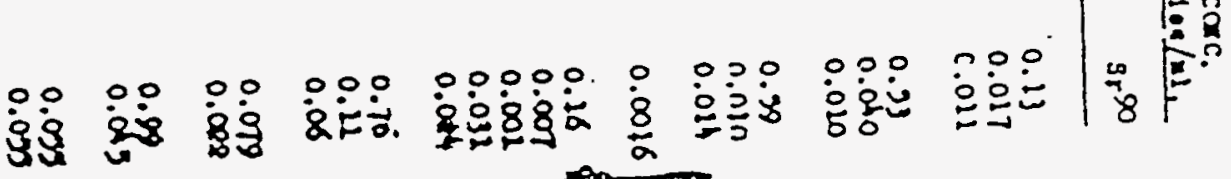
उU " $3 \times 3 \times$

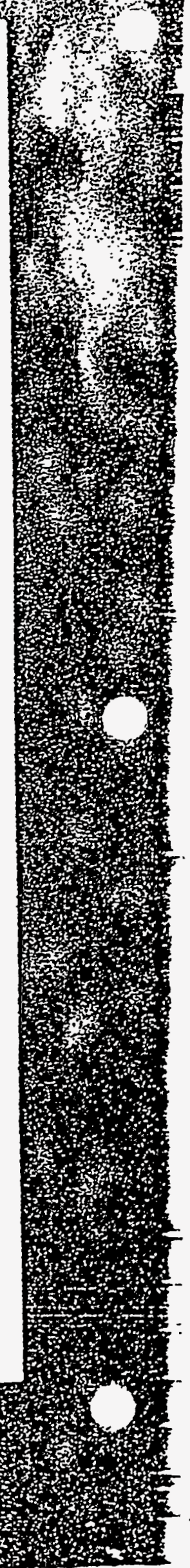


$T C R C-3$

Date: $\quad 8-16.72$

To: C. M. Walker

From: W. H. Sant

Subject: ANALYSIS OF TANK FARM SAMPLES

Sample: T $5404 \quad 601-B Y$

Appearance: Ceew, grum

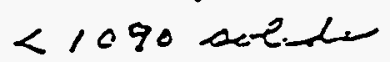

20 \&AD

Spg: 1.423

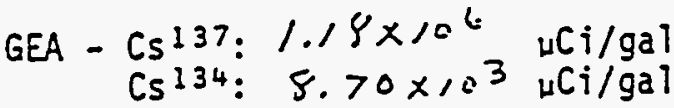

\section{BOILDOWN}

Starting Volume:50 $\mathrm{ml}$

Boiling Point: $1 / 7^{\circ} \mathrm{C}$

Volume at which solids formed: $42 \mathrm{ml}$

$M 1$ solids formed: $0.5 \mathrm{ml}$

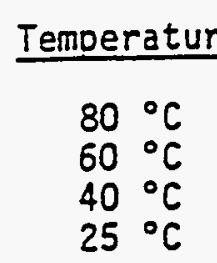

Volume

Volume of Solids

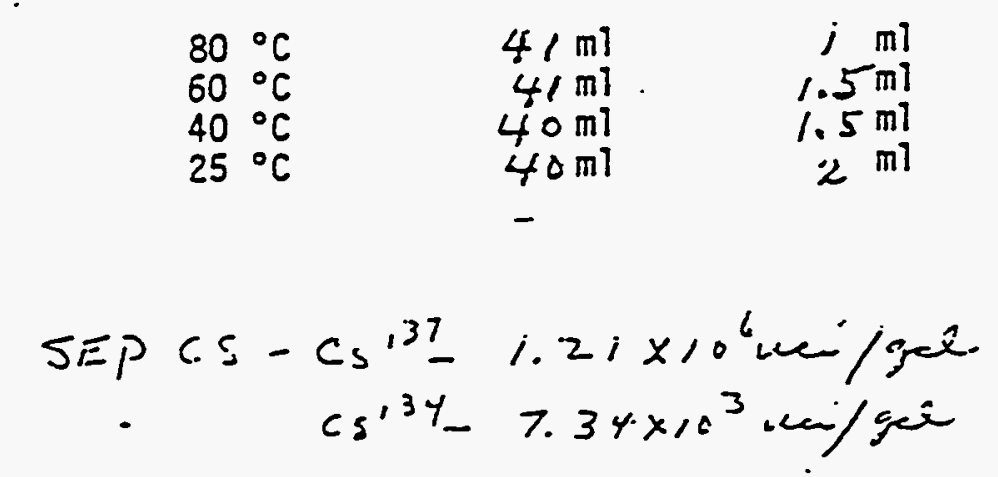




\section{$2 A-5=$}

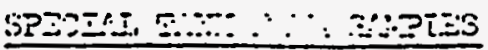

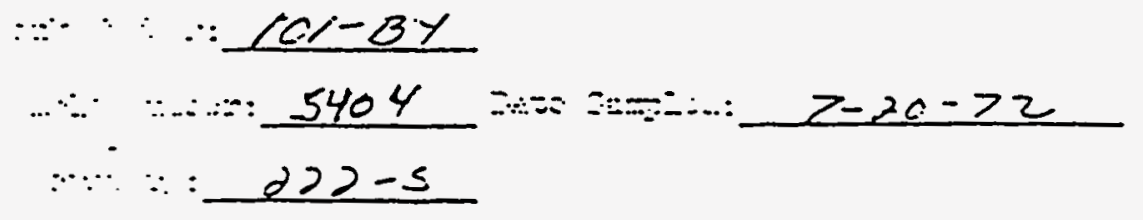

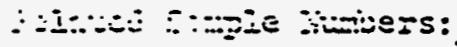

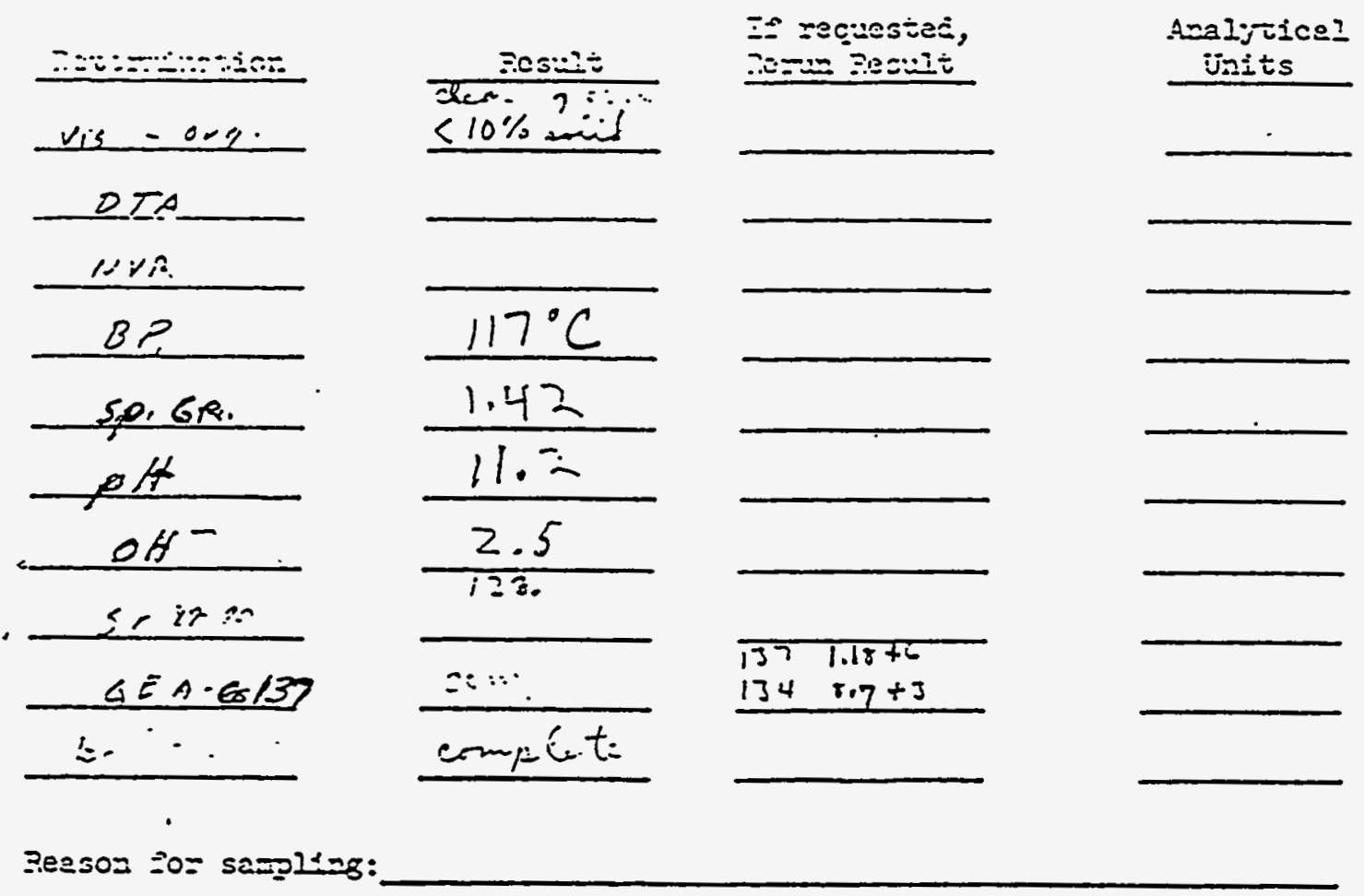

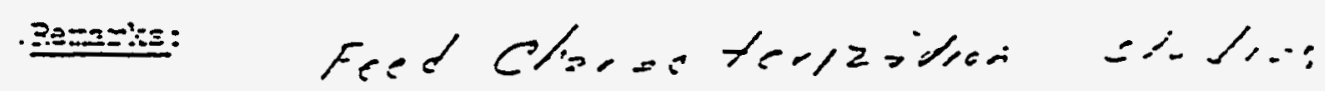

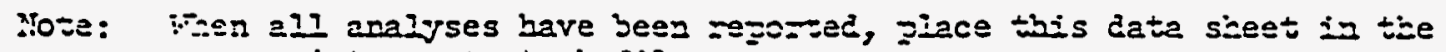
ezpopriate waste tark itle. 
and

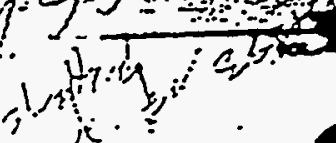

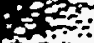

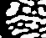

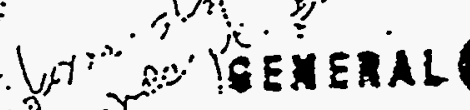

in

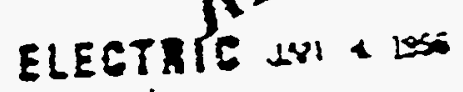

RECEIVE

$\frac{1}{01}$

(2)

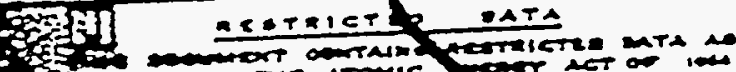

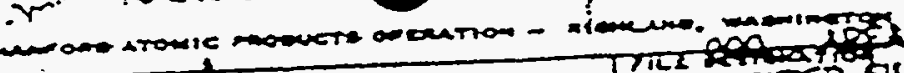

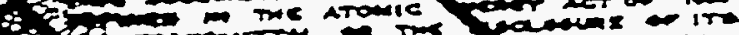
is 2.8?

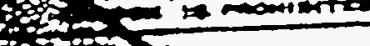

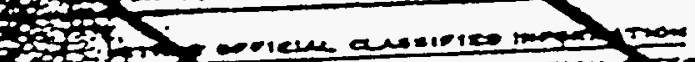

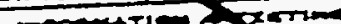

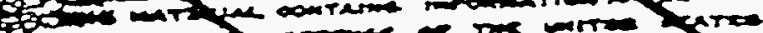

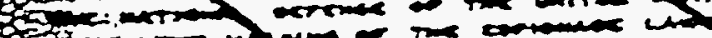

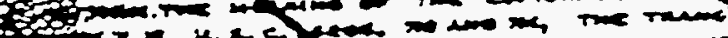

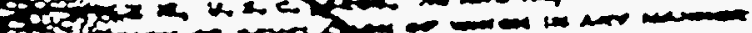

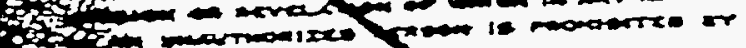
Cotam marmanises

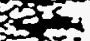

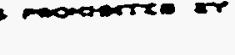
(x)

F

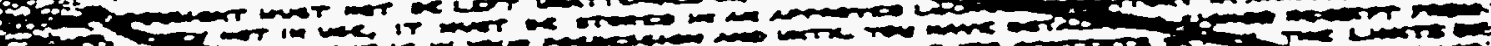
or it $\mathrm{h}-\mathrm{m}$ -

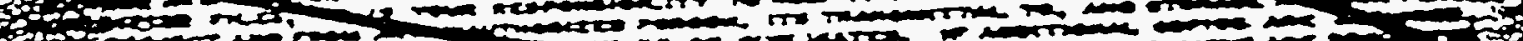
3.0

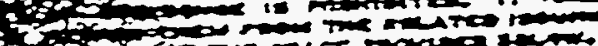

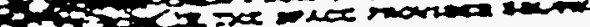

$-805020$

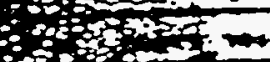

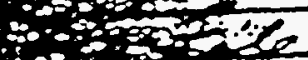

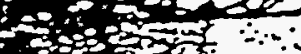

$30 \sin 2: 3$

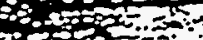

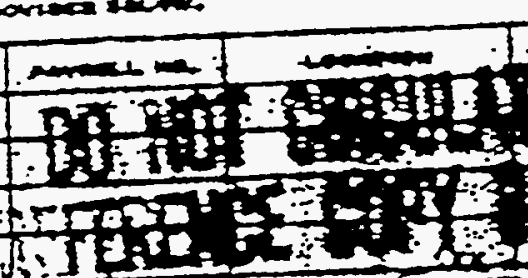

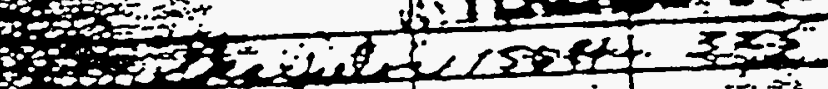

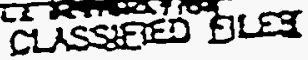

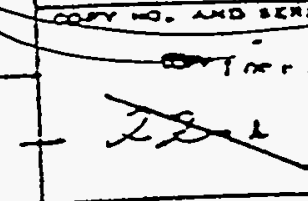

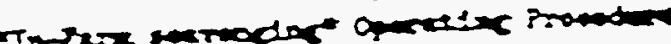
ind costin 3

$B y-101,02105$

S. Soet

\&

SEDCIRI RE-PDVIEN

EINTI DEIPEMINATION

Denlessification Confinmed

$\left.z y:()_{1}\right)-\frac{1}{x}+(n, n)$

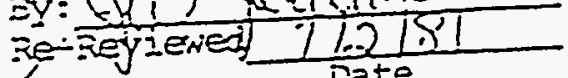

Date

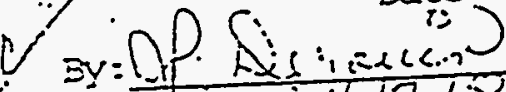

ze-Revienied! 710

BRulicis $112+184$

$\sum_{-=-x}$

स.

$=0=0-000$

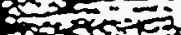

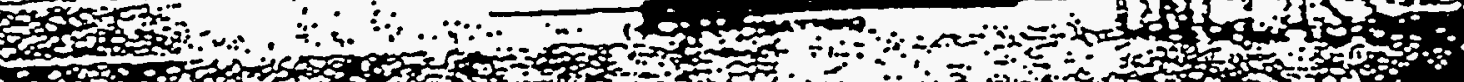

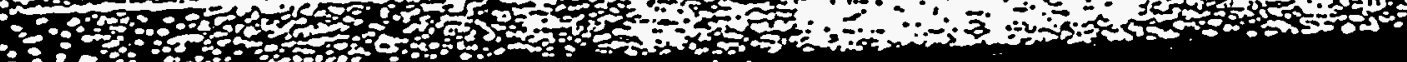

:

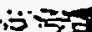

ix

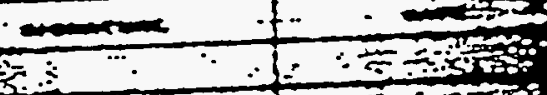

.

\section{$3 \div$}

1.

f.

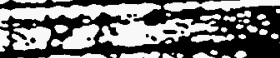

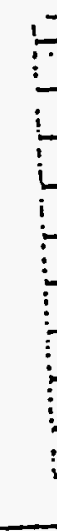




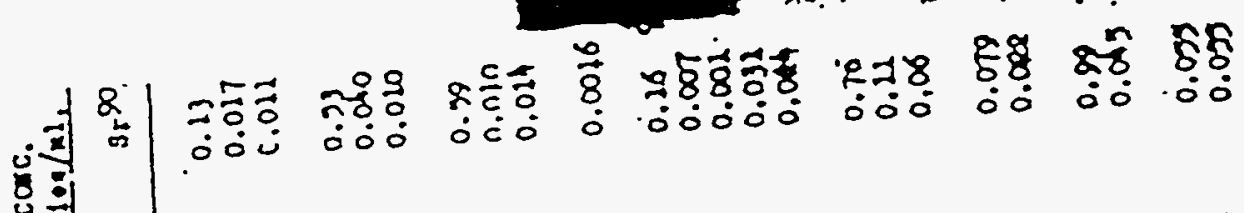

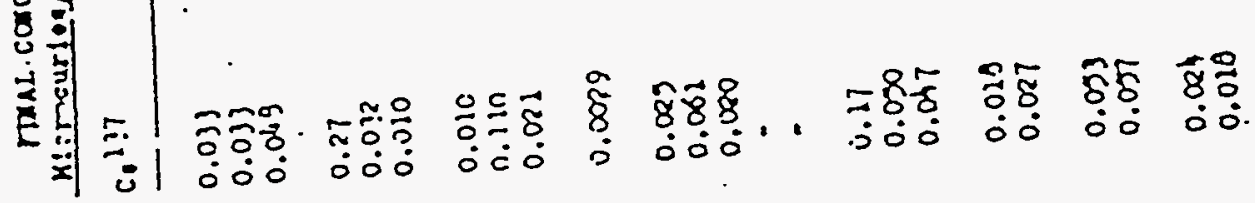

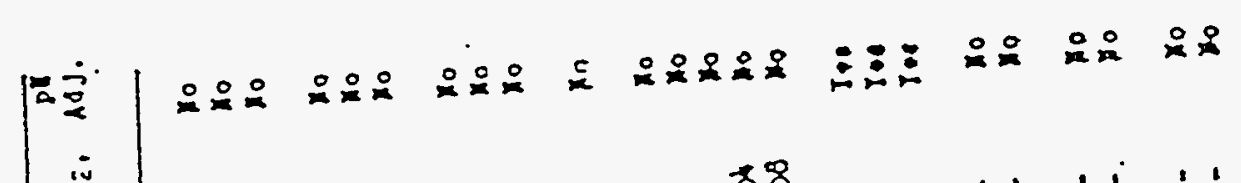




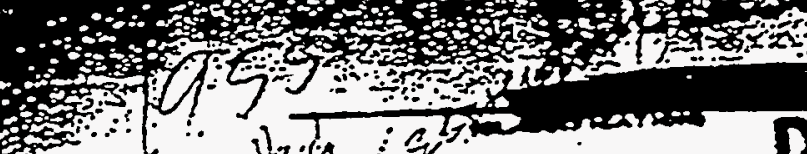

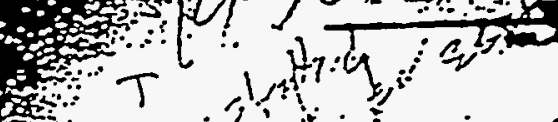
3.

X.t.

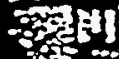

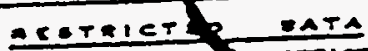

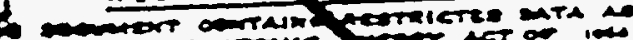
\%

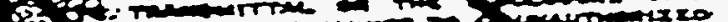
$\therefore$ Tim

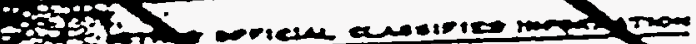

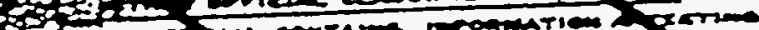

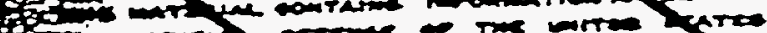

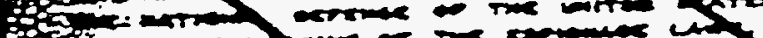

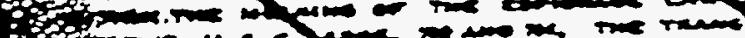

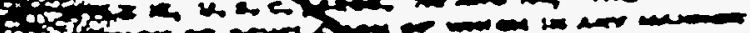
-5\%

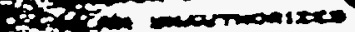
tos Pots

(1) S

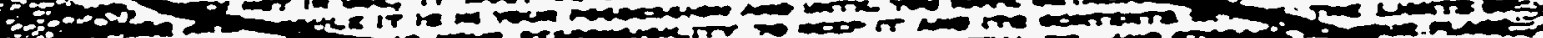

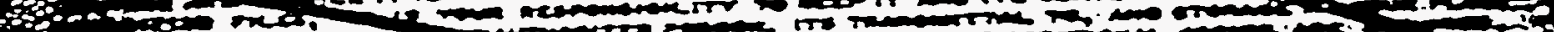
:

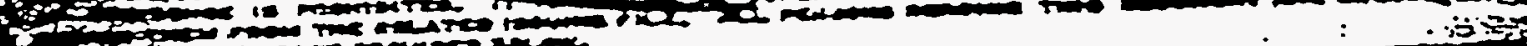

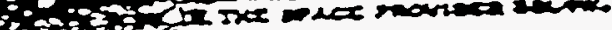

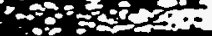

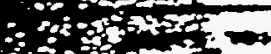

$50 \%$

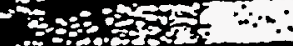

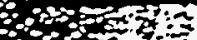

$\therefore$ :

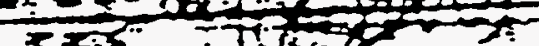
cusstept bus

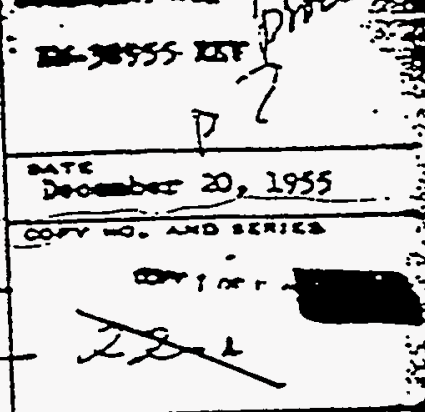

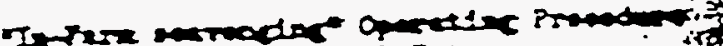
und copter bes

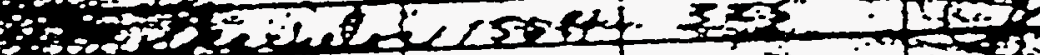
4 \%

SPXCIAI RE-REVIEW

FINAL DETERMINATION

Declassification Confirmed

By: $(5,1)-1(2,2,(n, k)$

ze-Rejiewed I 12 IS

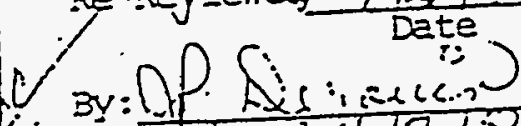

Re Keviened $171 \%$

BRilicis $112+184$

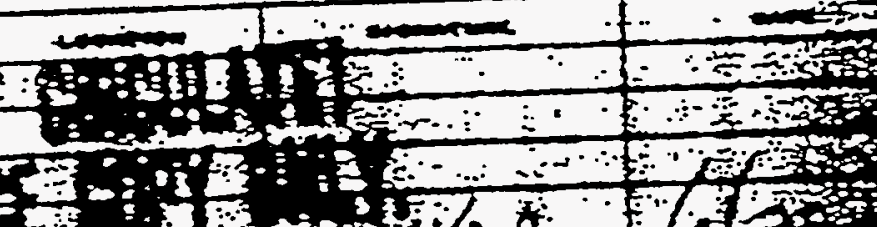

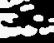

1. such

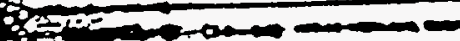

$30=0-1$

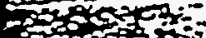

$\therefore: \vdots$

30

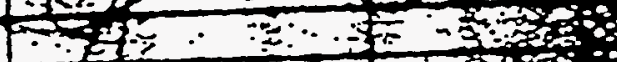

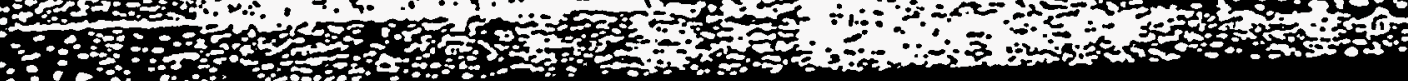




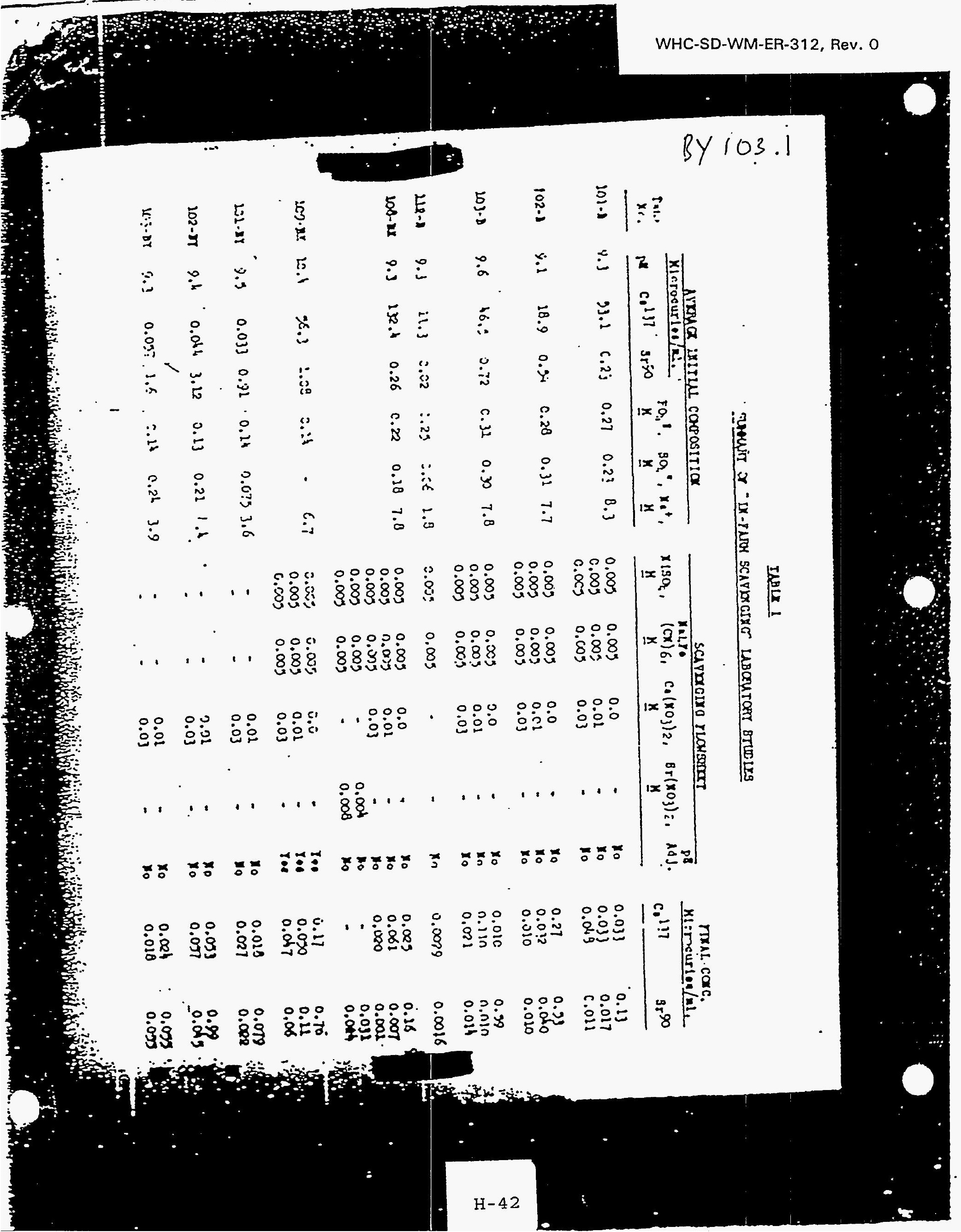


TCRC-4

Atlantic Richfield Hanford Company

in

Date: Octcber 14, 1971

TO: - D. J. Izzkin

From: J. S. 3uckinghim JSBuhlingham

Subject: COOLLRG CURVES AND ANAIYSIS OF SUPERITATANII

IIQUID FROH IOZBX AND 106 BY HASTX TAINKS

Volume percent sclias as a functicn or telloerature were determined on haste semples Jicm tent:s $103 \overline{B Y}$ and 1065Y; the supe:matant liquids $\varepsilon$ i $30,40,50$ and $60^{\circ} \mathrm{C}$ were anelyrec. The zesults are shown in tebles I ana 2 belcw and stapinically in the ettacina fiolures.

The "as received" samples were heated to between 80 and $90{ }^{\circ} \mathrm{C}$ to jissolve any solids thet had formed. Approximetely 30 milliliters of eech solution were transfezced to jacketed graciated certuifuze cones. The temperature of the solution iras adjusted by means of a thermostated circulating wate: beth. The sclutions were agitated itith a mecianical silirel as they care to the iesired temperatures. Aiter allowing solids to seitle, a one millilite= semple oi tive superatant was withoram and immediately jiluted into 10 milliliters $0:$ witer. The anelyses were run on ine diluted semole and the results multiplied by 17. Specific gravity was determired ficm the increase in weigit of the dilution when the ane milliliter of supe=netant was added.

\section{Table I}

\section{AITAIYSIS OF IO33Y SUPSRITATAIII IIQUDD}

Vol. I Solic

SDGr

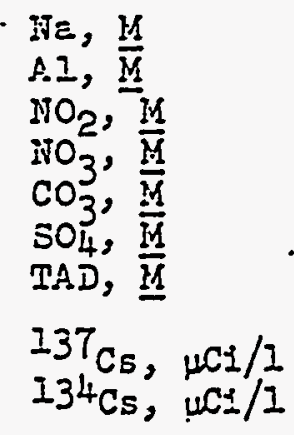

\begin{tabular}{|c|c|c|c|}
\hline $30^{\circ} \mathrm{C}$ & $40^{\circ} \mathrm{C}$ & $50^{\circ} \mathrm{C}$ & $60^{\circ} \mathrm{C}$ \\
\hline $\begin{array}{r}22 \\
1.469\end{array}$ & $\begin{array}{c}14.8 \\
1.479\end{array}$ & $\begin{array}{l}8.9 \\
1.494\end{array}$ & $\begin{array}{c}0 \\
1.500\end{array}$ \\
\hline $\begin{array}{l}11.9 \\
2.17 \\
1.23 \\
2.57 \\
0.45 \\
0.015 \\
7.87\end{array}$ & $\begin{array}{l}11.6 \\
1.22 \\
1.05 \\
2.33 \\
0.17 \\
0.022 \\
8.07\end{array}$ & $\begin{array}{l}11.7 \\
1.82 \\
1.01 \\
2.98 \\
0.14 \\
0.017 \\
7.34\end{array}$ & $\begin{array}{c}12.0 \\
1.64 \\
0.54 \\
3.04 \\
-. \\
0.017 \\
0.99\end{array}$ \\
\hline
\end{tabular}

$\begin{array}{llll}3.44 \times 10^{5} & 3.37 \times 10^{5} & 3.23 \times 10^{5} & 4.21 \times 10^{5} \\ 3.83 \times 103 & 3.81 \times 203 & 3.56 \times 10^{3} & 3.47 \times 10^{3}\end{array}$

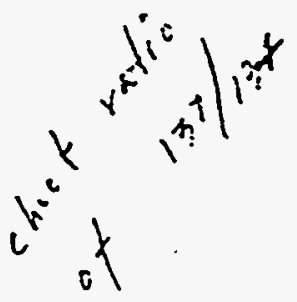


D. J. Is=bin

Pege 2

Ocicioer 1', 1971

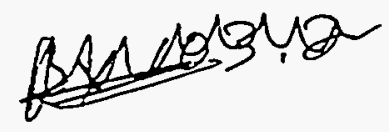

Table 2

, IXIYSIS OF IOS3Y SUPERTATAITP IIQUID

\begin{tabular}{|c|c|c|c|c|}
\hline & $30^{\circ} \mathrm{C}$ & $40^{\circ} \mathrm{C}$ & $50^{\circ} \mathrm{C}$ & $60^{\circ} \mathrm{C}$ \\
\hline $\begin{array}{l}\text { Vol. \& Solfa } \\
\text { SpGr }\end{array}$ & $\begin{array}{l}19.6 \\
1.453\end{array}$ & $\begin{array}{l}13.2 \\
1.484\end{array}$ & $\begin{array}{l}5.2 \\
1.484\end{array}$ & $\begin{array}{c}0 \\
1.1 \div 78\end{array}$ \\
\hline 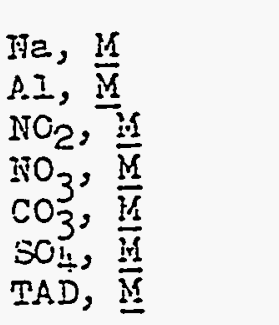 & $\begin{array}{l}12.2 \\
2.00 \\
1.00 \\
2.79 \\
0.97 \\
0.016 \\
6.36\end{array}$ & $\begin{array}{l}17.7 \\
2.00 \\
1.08 \\
2.06 \\
0.90 \\
0.005 \\
6.33\end{array}$ & $\begin{array}{l}12.4 \\
2.22 \\
1.00 \\
3.73 \\
0.55 \\
0.013 \\
0.13\end{array}$ & $\begin{array}{l}12.1 \\
1.79 \\
1.00 \\
4.17 \\
0.55 \\
0.013 \\
5.93\end{array}$ \\
\hline $\begin{array}{l}137 \mathrm{Cs}, \mu \mathrm{Ci} / 1 \\
13^{4} \mathrm{Cs}, \mu \mathrm{Ci} / I\end{array}$ & $\begin{array}{l}3.30 \times 105 \\
3.44 \times 10^{3}\end{array}$ & $\begin{array}{l}3.30 \times 10^{5} \\
3.50 \times 10^{3}\end{array}$ & $\begin{array}{l}3.20 \times 10^{5} \\
3.26 \times 10^{3}\end{array}$ & $\begin{array}{l}3.23 \times 10^{5} \\
3.28 \times 10^{3}\end{array}$ \\
\hline
\end{tabular}

Pleese ceil de if you have any questions resarcing tis rorts or if we can de of any ilutiner assistance.

JSB:فْ

cc: Dincerson

GI 3orsineis

NE Carobell

CJ Brancis

EC Pethron

RC Roe?

Jo Sticlnu

IH Sritin

File

I. 3

Process Aicis 
Date:

ADIII 33, IST2

To:

T. D. Arderson

In:

S. S. Bueks ashem. IS Bucking hain

Subject: ARAITSES OF TS IO6-II SAMFIDS

Tro dip samples were taken $1=0 m$ m IC6-Or to see if ery

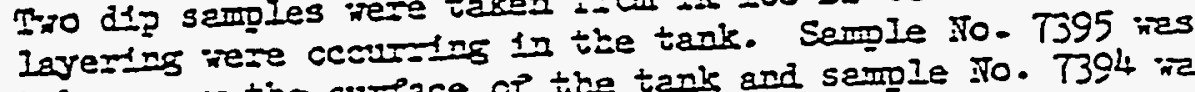
taiken rear ties surface or the tank and sample iTo. T304 ires taken at a cepth of 33 ieet 10 inches i=col s=cura level.

The araiyses, sicwi in Table I, Indecste that there is no s18pilicatt leyering in the tanis. The indiridual ensiyses ere ofthin expected aralytical precision and shor no ofontefcent difference betieen the tio samples.

\section{Table I}

Arairsts of $2 x$ I06-or

To. T395, Surpace To: T394, $33 \approx 0.10$ 1n.

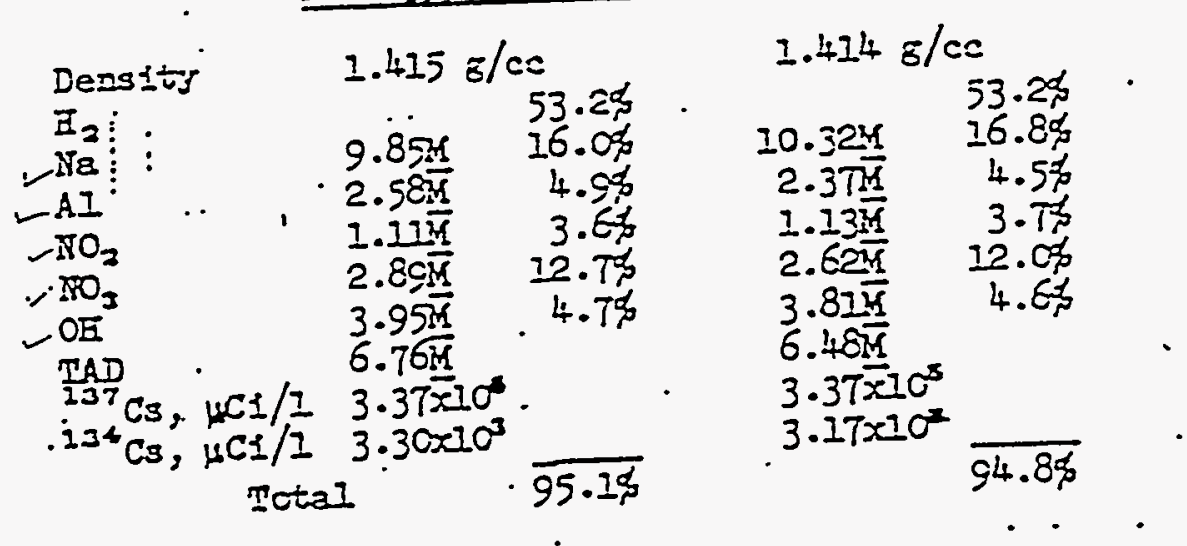

The andrses show that ire are accourting for arourd 95 percent of the materfal in the samples. In addition.the anfon anelyses are within 4 to $T$ percett of the cation analyses.

- Based on ari arerage of the tio anelyses, the ccmpcsition of the 106-aI is as shown in table II. 


\section{WHC-SD-WM-ER-312, Rev. 0}

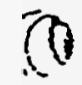

T. D: AndezS OD

Ease 2

ADII 13, I9T2

Table II

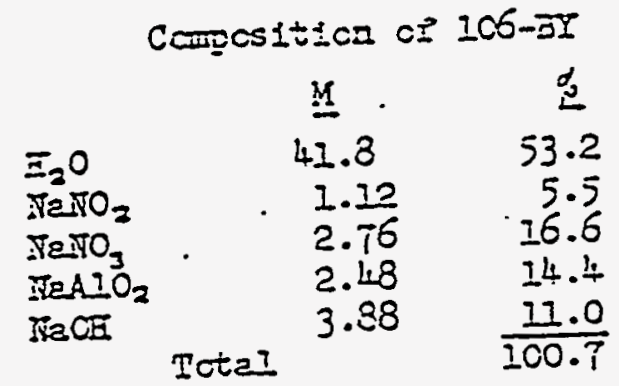

Please cell mo 12 you have any questicas regarifin this. roris.

$5 \sqrt{B}:$ ah

cs: Gr Zcrsineir

ine Carocein

CJ. İancis

DJ. Iaridn

퍼노

is

Process Afds

. 
Atlantic Rlchfleld Hanford Company

00359

Date: Octcoer 14, 1971

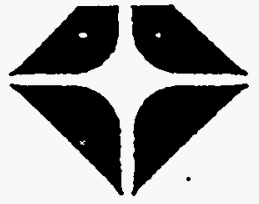

TO: - D. J. Ixxkin

Irom: 'J. 5. 3uckingsam JS Sulling ham

Subject: COOLIIG CURVES AND AINAIYSIS OF SUPERMATANI IIQUDD FROK IOZEY AND IO6 BY :IASTS IAITKS

Volume percenti solias as a tuncticn or tempezature rere determined on waste semples trom tents 103 BY and I065Y; the supe:natant liqutids at $30,40,50$ and $600^{\circ} \mathrm{C}$ jere axelyzec. Tre results are shown in tables 1 ana 2 belch and Jrapinically in the etircieo sigures.

The "as received" semples were heated to between 80 and" $90{ }^{\circ} \mathrm{C}$ to isissolve any solids that hed forded. Approxirately 30 milliliters of eech solution were trensfezred to jecketed graciuated centrifuge cones. The temperature $0:$ the solution iras adjusted by peans of a thermostated circulating wete= beti. The sclutions were agitated intin a Decinanical sitrrer as they cerle to the ciesized temoeratures. A Aver allowing solids to seitle, a one milifilite: sample on the supernetant ras winoraw ano innediately diluted into 10 milliliters ol witer. The anelyses were Iun on tive jiluted semple and the results multiplied by 11. Soecific sravity ies determiced ircm the increese in weigit of the diluticn when the cone riflililiter of supernetant was acied.

Teble I

AIATYSIS OF IO3FI SUPSRIVATANI IIQUDD

Vol. Ts Solica SDGI

고, $\underline{k}$

A.I, $\underline{M}$

$\mathrm{NO}_{2}, \mathrm{M}$

$\mathrm{iNO}_{3}, \overline{\mathrm{M}}$

$\mathrm{CO}_{3}^{3}, \bar{M}$

$\mathrm{SO}_{4}, \overline{\mathrm{H}}$

TAD, $\overline{\underline{H}}$

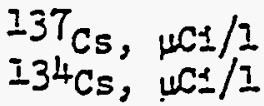

\begin{tabular}{|c|c|c|c|}
\hline $30^{\circ} \mathrm{C}$ & $40 \%$ & $50^{\circ} \mathrm{C}$ & $60^{\circ} \mathrm{C}$ \\
\hline$\frac{22}{1.469}$ & $\begin{array}{r}14.8 \\
1.479\end{array}$ & $\begin{array}{l}3.9 \\
1.494\end{array}$ & $\begin{array}{c}0 \\
1.500\end{array}$ \\
\hline $\begin{array}{l}11.9 \\
2.17 \\
1.23 \\
2.57 \\
0.45 \\
0.015 \\
7.87\end{array}$ & $\begin{array}{l}11.6 \\
1.82 \\
1.06 \\
2.33 \\
0.17 \\
0.022 \\
8.07\end{array}$ & $\begin{array}{l}11.7 \\
1.82 \\
1.01 \\
2.98 \\
0.14 \\
0.017 \\
1.34\end{array}$ & $\begin{array}{c}12.0 \\
1.64 \\
0.54 \\
3.04 \\
-0 \\
0.017 \\
0.99\end{array}$ \\
\hline
\end{tabular}

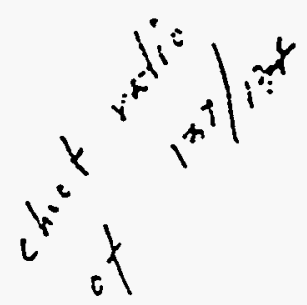


D. J. Is=kin

Pege 2

Octcier 1;, 1971

\section{Table 2}

A.NWIYSIS OF IOSSY SUPERIRTA ITI IIQUID

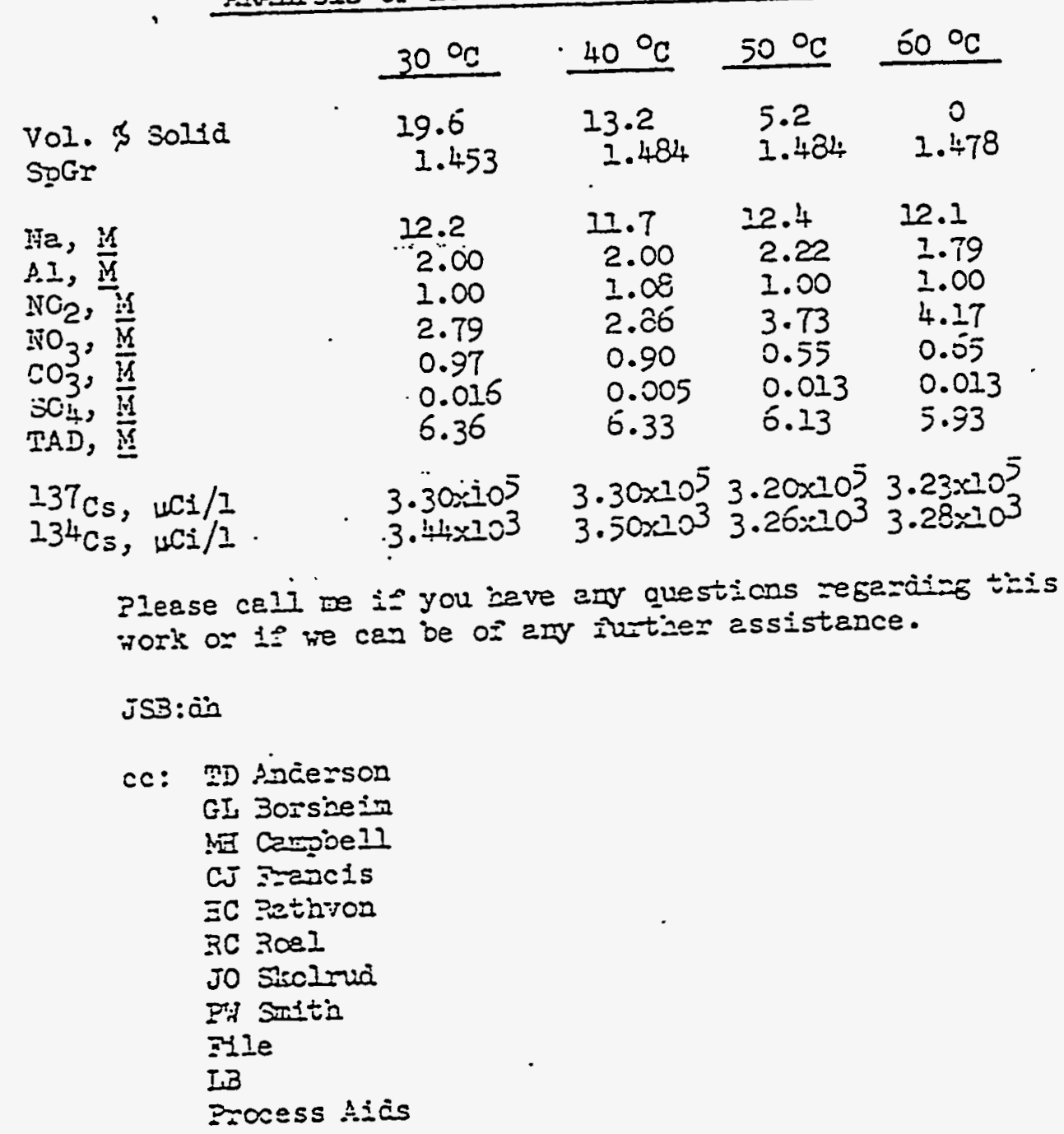


Atlantic Richfield Hanford Company

Date: $8 \cdot 3 \cdot 7^{2}$

To:

C. M. Walker

From:

W. H. Sant

Subject: ANALYSIS OF TANK FARM SAMPLES

Sample: $T-6968$

Appearance: Thick ifel/ow

$10 \% 0$ saciss

Spg: $-1.4-42.5^{-}$

$\begin{aligned} \text { GEA - } C^{2} 37: & 1.522 \times 10^{6} \mu \mathrm{Ci} / \mathrm{gal} \\ \mathrm{CS}^{134}: & 2.26 \times 10^{4} \mathrm{\mu Ci} / \mathrm{gal}\end{aligned}$

\section{BOILDOWN}

Starting Volume: $30 \mathrm{ml}$

$\%$ Solids: if \%

Boiling Point: $115^{\circ} \mathrm{C}$

Volume at which solids formed: $25 \mathrm{~m}$ ]

MI solids formed: I ml

\begin{tabular}{|c|c|c|}
\hline Temperature & Volume & Volume of Solids \\
\hline $\begin{array}{l}80^{\circ} \mathrm{C} \\
60^{\circ} \mathrm{C} \\
40^{\circ} \mathrm{C} \\
25^{\circ} \mathrm{C}\end{array}$ & $\begin{array}{l}25 \mathrm{ml} \\
25 \mathrm{ml} \\
25 \mathrm{ml} \\
25 \mathrm{ml}\end{array}$ & $\begin{array}{ll}5 & \mathrm{ml} \\
5 & \mathrm{ml} \\
5 & \mathrm{ml} \\
5 & \mathrm{ml}\end{array}$ \\
\hline
\end{tabular}


Date: $\quad 9-3-72$

To: C. M. Walker

From: W. H. Sant

Subject: ANALYSIS OF TANK FARM SAMPLES

Sample: $T-5953 \quad 1078 \mathrm{y}$

Appearance: Clew

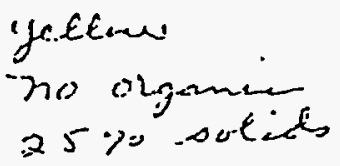

Spg:- 1.381

GEA - Cs137: i. o $6 \times 10^{6} \mu \mathrm{Ci} / \mathrm{gat}$ $\mathrm{Cs}^{134}: 0.41 \times 10 * 4 \mathrm{Ci} / \mathrm{gal}$

\section{BOILDOWN}

Starting Volume: $50 \mathrm{ml}$

$\%$ Solids: $25 \%$

Boiling Point: $115^{\circ} \mathrm{C}$

Volume at which solids formed: $38 \mathrm{ml}$

Ml solids formed:, $\mathrm{ml}$

\begin{tabular}{ccc} 
Temperature & volume & Volume of Solids \\
\hline $80^{\circ} \mathrm{C}$ & $38 \mathrm{ml}$ & $4 \mathrm{ml}$ \\
$60^{\circ} \mathrm{C}$ & $37 \mathrm{ml}$ & $7 \mathrm{ml}$ \\
$40^{\circ} \mathrm{C}$ & $37 \mathrm{ml}$ & if $\mathrm{ml}$ \\
$25^{\circ} \mathrm{C}$ & $36 \mathrm{ml}$ & $2 / \mathrm{ml}$
\end{tabular}


Date: $\quad 5-16-72$

To: C. M. Walker

From: W. H. Sant

Subject: ANALYSIS OF TANK FARM SAMPLES

Sample: T.7993 $109 \mathrm{BY}$

Appearance: Clecen

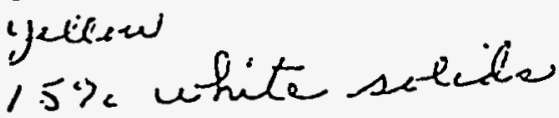

Spg: 1.463

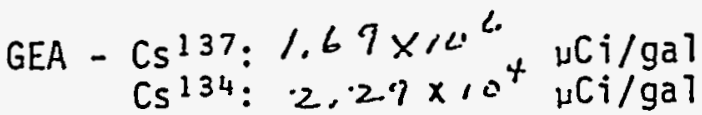

\section{BOILDOWN}

Starting Volume: $50 \mathrm{ml}$

Boiling Point: $114^{\circ} \mathrm{C}$

Volume at which solids formed: $43 \mathrm{ml}$

Ml solids formed: / $\mathrm{ml}$

\begin{tabular}{ccc} 
Temperature & Volume & Volume of Solids \\
\cline { 2 - 2 } & $4 / \mathrm{ml}$ & $2 \mathrm{ml}$ \\
$60{ }^{\circ} \mathrm{C}$ & $4 / \mathrm{ml}$ & $3 \mathrm{ml}$ \\
$40^{\circ} \mathrm{C}$ & $4 / \mathrm{ml}$ & $7 \mathrm{ml}$ \\
$25{ }^{\circ} \mathrm{C}$ & $40 \mathrm{ml}$ & $25 \mathrm{ml}$
\end{tabular}


Date: $8-17-72$

To: C. M. Walker

From: W. H. Sant

Subject: ANALYSIS OF TANK FARM SAMPLES

Sample: T $976.6 \quad 109 B \mathrm{Y}$

Appearance: Clisu, yelleres with 15\% seids 3 a

Spg: 1.455

GEA - Cs137: $4 i, 39 \times 10,5 \mu \mathrm{Ci} / \mathrm{ga} 1$ $\mathrm{Cs}^{134}: 2.46 \times 10^{4} \mu \mathrm{Ci} / \mathrm{gal}$

\section{BOILDOWN}

Starting Volume: $50 \mathrm{ml}$

Boiling Point: $/ 14^{\circ} \mathrm{C}$

Volume at which solids formed: $4 \mathrm{zml}$

M) solids formed: $5 \mathrm{ml}$

Temperature Volume Volume of Solids

$\begin{array}{llr}80^{\circ} \mathrm{C} & 42 \mathrm{ml} & 5 \mathrm{ml} \\ 60{ }^{\circ} \mathrm{C} & 41 \mathrm{ml} & 5 \mathrm{ml} \\ 40{ }^{\circ} \mathrm{C} & 40 \mathrm{ml} & -5 \mathrm{ml} \\ 25{ }^{\circ} \mathrm{C} & 40 \mathrm{ml} & 35 \mathrm{ml}\end{array}$


Date: $8-25-72$

To: C. M. Walker

From: W. H. Sant

Subject: ANALYSIS OF TANK FARM SAMPLES

Sample: TzOk j09 BY

Appearance: clean, yelerw

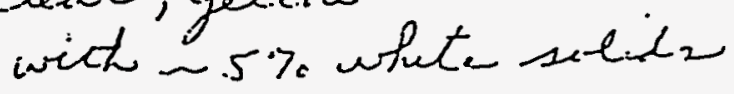

$$
\text { of OO MCAD }
$$

Spg: 1.4415

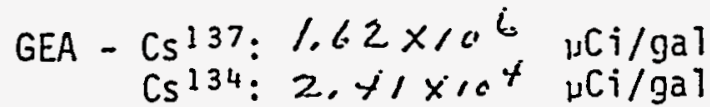

\section{BOILDOEN}

Starting Volume: $5^{\circ} \mathrm{ml}$

$\%$ Solids: 0 \&

Boiling Point: $112^{\circ} \mathrm{C}$

Volume at which solids formed: $41 \mathrm{ml}$

Ml solids formed: $1 \mathrm{ml}$

\begin{tabular}{ccc} 
Temperature & Volume & Volume of Solids \\
\cline { 3 - 3 } $80^{\circ} \mathrm{C}$ & $40 \mathrm{ml}$ & $2 \mathrm{ml}$ \\
$60^{\circ} \mathrm{C}$ & $40 \mathrm{ml}$ & $3 \mathrm{ml}$ \\
$40^{\circ} \mathrm{C}$ & $39 \mathrm{ml}$ & $15 \mathrm{ml}$ \\
$25^{\circ} \mathrm{C}$ & $39 \mathrm{ml}$ & $30 \mathrm{ml}$
\end{tabular}


Date: $\quad 7-12-72$

To: C. M. Walker

From: W. H. Sant

Subject: ANALYSIS OF TANK FARM SAMPLES

Sample: $7 / 832$ 1093Y

Appearance: (clean, yellete

- 1070 white silibs

IMRAD

Spg: $\quad 1.420$

GEA - $\begin{aligned} C_{5} 137: & 1.50 \times 10^{6}{ }^{13} \mathrm{Cj} / \mathrm{gal} \\ & \mathrm{Cs}^{134:} 2.69 \times 10^{\circ} \mathrm{HCi} / \mathrm{gal}\end{aligned}$

\section{BOILDO!N}

Starting Volume: $50 \mathrm{ml}$

\% Solids: $1 \%$

Boiling Point: $113^{\circ} \mathrm{C}$

Volume at which solids formed: $.57 \mathrm{ml}$

$M 1$ solids formed: $</ \mathrm{ml}$

Temperature

$80{ }^{\circ} \mathrm{C}$

$60{ }^{\circ} \mathrm{C}$

$40{ }^{\circ} \mathrm{C}$

$25{ }^{\circ} \mathrm{C}$
Volume

$37 \mathrm{ml}$

$36 \mathrm{ml}$

$\bar{s} .5 \mathrm{ml}$

$3+\mathrm{ml}$

Volume of Solids

$$
\begin{gathered}
<.1 \mathrm{ml} \\
5 \mathrm{ml} \\
30 \mathrm{ml} \\
3.2 \mathrm{ml}
\end{gathered}
$$


Date: $9-26-72$

To: C. M. Walker

From: W. H. Sant

Subject: ANALYSIS OF TANK FARM SAMPLES

Sample: T24-90 $109 B \mathrm{~B}$

Appearance: Yeférum
407\% aclide

$400 \mathrm{mRAD}$

Spg: 1.282

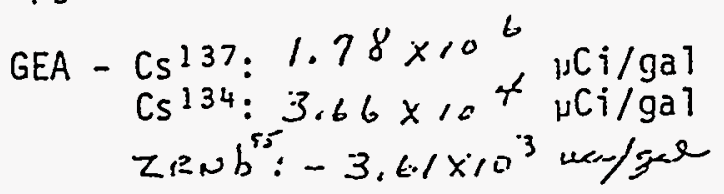

BOILDOHN

Starting Volume: $50 \mathrm{ml}$

$\%$ Solids: 1 \&

Boiling Point: $114^{\circ} \mathrm{C}$

Volume at which solids formed: $38 \mathrm{ml}$

MI solids formed: $</ \mathrm{ml}$

\begin{tabular}{ccc} 
Temperature & Volume & Volume of Solids \\
\cline { 1 - 2 } $80^{\circ} \mathrm{C}$ & $36.5 \mathrm{ml}$ & $1.5 \mathrm{ml}$ \\
$60^{\circ} \mathrm{C}$ & $36.0 \mathrm{ml}$ & $2.0 \mathrm{ml}$ \\
$40^{\circ} \mathrm{C}$ & $35.0 \mathrm{ml}$ & $5.0 \mathrm{ml}$ \\
$25{ }^{\circ} \mathrm{C}$ & $34.5 \mathrm{ml}$ & $33.0 \mathrm{ml}$
\end{tabular}


Date: $\quad 10-2 \ldots 72$

To:

C. M. Walker

From:

W. H. Sant

Subject: ANALYSIS OF TANK FARM SAMPLES

Sample: $125: 33 \quad 1098 y$

Appearance: Gasiér...

$$
\begin{aligned}
& 3 \% \text { cotide } \\
& 1 \text { CAD }
\end{aligned}
$$

Spg: 1.36 .6

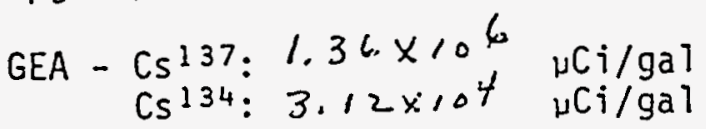

\section{BOILDOWN}

Starting Volume: $50 \mathrm{ml}$

$\%$ Solids: $4 \%$

Boiling Point: $/ 11^{\circ} \mathrm{C}$

Volume at which solids formed:37.5 51

\begin{tabular}{|c|c|c|}
\hline Temperature & Vol ume & Volume of Solids \\
\hline $\begin{array}{l}80{ }^{\circ} \mathrm{C} \\
60{ }^{\circ} \mathrm{C} \\
40{ }^{\circ} \mathrm{C} \\
25^{\circ} \mathrm{C}\end{array}$ & $\begin{array}{l}36.5 \mathrm{ml} \\
35.0 \mathrm{ml} \\
35.0 \mathrm{ml} \\
3, .0 \mathrm{ml}\end{array}$ & 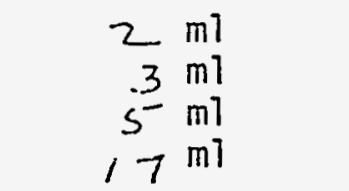 \\
\hline
\end{tabular}

$M 7$ solids formed: $1.5 \mathrm{ml}$ 


\section{น..}

Date: $\quad 11-16-7 Z$

To: C. M. Waiker

From: W. H. Sant

Subject: ANALYSIS OF TANK FARM SAMPLES

Sample: $T 3743 \quad 109 B \mathrm{~B}$

Appearance: PeA GReeN;

$$
35 \% \text { 50Lios }
$$

$20 \Leftrightarrow M \pi N D$

Spg: 1.402

GEA - Cs137: $1.71 \times 106,{ }_{13} \mathrm{Ci} / \mathrm{gal}$

\section{BOILDOHN}

Starting Volume: $\mathrm{m}$

\% Solids: $\quad$ \%

Boiling Point: $\quad{ }^{\circ} \mathrm{C}$

Volume at which solids formed: $\mathrm{ml}$

Ml solids formed: $\mathrm{ml}$

Temperature Volume Volume of Solids

$\begin{array}{lll}80{ }^{\circ} \mathrm{C} & \mathrm{ml} & \mathrm{ml} \\ 60{ }^{\circ} \mathrm{C} & \mathrm{ml} & \mathrm{ml} \\ 40{ }^{\circ} \mathrm{C} & \mathrm{ml} & \mathrm{ml} \\ 25{ }^{\circ} \mathrm{C} & \mathrm{ml} & \mathrm{ml}\end{array}$

Boiling Point - $109^{\circ} \mathrm{C}$ 
Date: $\quad 11-28-72$

To:

C. M. Walker

From:

W. H. Sant

Subject: ANALYSIS OF TANK FARM SAMPLES

Sample: T4005 107By

Appearance: Light ejsecent

$$
\begin{gathered}
<1 \% \text { sicios } \\
1,8 \text { Ratos }
\end{gathered}
$$

Spg: 1.3617

$$
\text { GEA - Cs } 137: 1.36 \times 10^{6}{ }^{13 C i / g a l}
$$

\section{BOILDOHN}

Starting Volume: $5 \circ \mathrm{ml}$

Boiling Point: $100^{\circ} \mathrm{C}$

Volume at which solids formed: if $2 \mathrm{ml}$

M] solids formed: $/ \mathrm{ml}$

\begin{tabular}{ccc} 
Temperature & Volume & Volume of Solids \\
\hline $80{ }^{\circ} \mathrm{C}$ & $42 \mathrm{ml}$ & $2 \mathrm{ml}$ \\
$60^{\circ} \mathrm{C}$ & $41 \mathrm{ml}$ & $2 \mathrm{ml}$ \\
$40{ }^{\circ} \mathrm{C}$ & $40 \mathrm{ml}$ & $2 \mathrm{ml}$ \\
$25{ }^{\circ} \mathrm{C}$ & $39 \mathrm{ml}$ & $2 \mathrm{ml}$
\end{tabular}




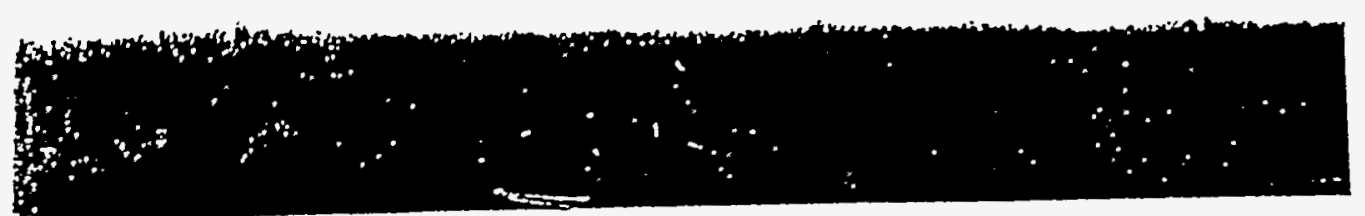

Atlantic Richfield Hanford Company

BY 109.11

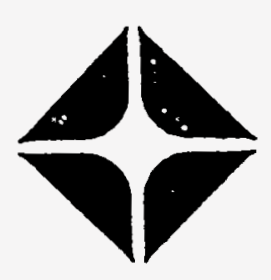

Date: $12 \cdot 18-72$

To: C. M. Walker

From: W. H. Sant

Subject: ANALYSIS OF TANK FARM SAMPLES

Sample: T-43i6 109-BY

Appearance: GReen with solide

3. 0 RAOS

Spg: 1.42 .2

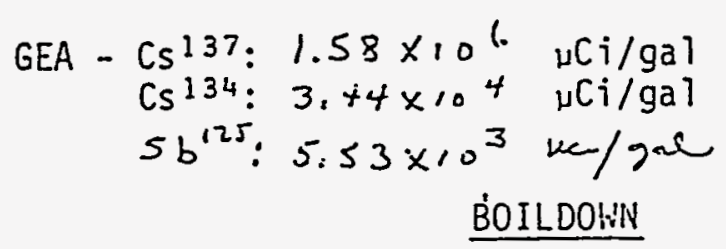

Starting Volume: $50 \mathrm{mi}$

$\%$ Solids: $20 \%$

Boiling Point: $189^{\circ} \mathrm{C}$

Volume at which solids formed: $45 \mathrm{ml}$

Ml solids formed: $1 \mathrm{ml}$

\begin{tabular}{|c|c|c|}
\hline Temperature & Voi ume & Volume of Solids \\
\hline $\begin{array}{l}80{ }^{\circ} \mathrm{C} \\
60{ }^{\circ} \mathrm{C} \\
40{ }^{\circ} \mathrm{C} \\
25^{\circ} \mathrm{C}\end{array}$ & $\begin{array}{l}45 \mathrm{ml} \\
44 \mathrm{ml} \\
43 \mathrm{ml} \\
43 \mathrm{ml}\end{array}$ & $\begin{array}{ll}1 & \mathrm{ml} \\
2 & \mathrm{ml} \\
3 & \mathrm{ml} \\
8 & \mathrm{ml}\end{array}$ \\
\hline
\end{tabular}


Date: $\quad 1-\ddot{8}-73$

To: C. M. Walker

From: W. H. Sant

Subject: ANALYSIS OF TANK FARM SAMPLES

Sample: T-4.57.3 $109 \mathrm{By}$

Appearance: $y$ ellow, $5 \%$ sic. os

$$
1.2 \text { RADS }
$$

Spg: $\quad 1.225$

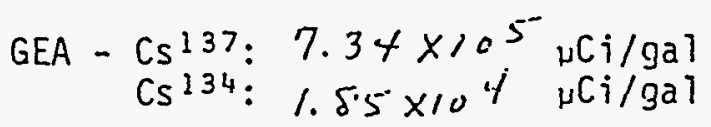

\section{BOILDOL:N}

Starting Volume: $50 \mathrm{ml}$ $\%$ Solids: $16 \%$

Boiling Point: $104^{\circ} \mathrm{C}$

Volume at which solids formed: $25 \mathrm{ml}$

Ml solids formed: $4 \mathrm{ml}$

\begin{tabular}{ccc} 
Temperature & Volume & Volume of Solids \\
\hline $80^{\circ} \mathrm{C}$ & $5 \mathrm{Cl} \mathrm{ml}$ & $2 \mathrm{ml}$ \\
$60^{\circ} \mathrm{C}$ & $50 \mathrm{ml}$ & $\circ \mathrm{ml}$ \\
$40^{\circ} \mathrm{C}$ & $50 \mathrm{ml}$ & $0 \mathrm{ml}$ \\
$25^{\circ} \mathrm{C}$ & $25 \mathrm{ml}$ & $4 \mathrm{ml}$
\end{tabular}


Date: $\quad 1-16-73$

To: C. M. Walker

From: H. H. Sant

Subject: ANALYSIS OF TANK FARM SAMPLES

Sample: T-4911 $1098 \%$

Appearance: $y e 110 \mathrm{w}, \sim 5 \%$ Silios

$$
1.5 \text { R.ti }
$$

Spg: $\quad 1.40 .1$

GEA - $\begin{aligned} \operatorname{Cs} 137: & 1.9 .3 \times 10^{6} \mu \mathrm{Ci} / \mathrm{gal} \\ \mathrm{Cs} 134: & 3.62 \times 10^{\circ} \mu \mathrm{Ci} / \mathrm{gal}\end{aligned}$

\section{BOILDOLIN}

Starting Volume:50 $\mathrm{ml}$

\% Solids:-5\%

Boiling Point: $110^{\circ} \mathrm{C}$

Volume at which solids formed: io ml

M] solids formed: $2 \mathrm{~m}$ ?

\begin{tabular}{|c|c|c|}
\hline Temperature & Volume & Volume of Solids \\
\hline & $40 \mathrm{ml}$ & $z \mathrm{mi}$ \\
\hline $60^{\circ} \mathrm{C}$ & fo ml & $-2.5 \mathrm{ml}$ \\
\hline $40^{\circ} \mathrm{C}$ & itc $\mathrm{ml}$ & $2.5 \mathrm{ml}$ \\
\hline $25^{\circ} \mathrm{C}$ & $a+c \mathrm{ml}$ & a. $5 \mathrm{ml}$ \\
\hline
\end{tabular}


Date: $\quad \mid-31-73$

To:

C. M. Walker

From:

W. H. Sant

Subject: ANALYSIS OF TANK FARM SAMPLES

Sample: $T-5 / 2 t \quad 109-B Y$

Appearance: yellew

$$
\begin{aligned}
& 20 \% \mathrm{solide} \\
& 1500 \mathrm{mr}
\end{aligned}
$$

Spg: 1.396

GEA - $\operatorname{Cs}^{137}: 1.94 \times 10^{6}{ }_{13 \mathrm{Ci} / \mathrm{gaT}}$

$$
\mathrm{Cs}^{134}: 3,47 \times 10^{+} \mathrm{Ci} / \mathrm{gal}
$$

\section{BOILDOUN}

Starting Volume: $507 \mathrm{ml}$

$\%$ Solids: $2 \%$

Boiling Point: $115^{\circ} \mathrm{C}$

Volume at which solids formed: to ml

M] solids formed: $</ \mathrm{ml}$

Temperature Volume Volume of Solids

$\begin{array}{llr}80{ }^{\circ} \mathrm{C} & 35 \mathrm{ml} & 6 \mathrm{ml} \\ 60^{\circ} \mathrm{C} & 3 \mathrm{y} \mathrm{ml} & 6 \mathrm{ml} \\ 40{ }^{\circ} \mathrm{C} & 3 \mathrm{f} \mathrm{ml}^{\circ} & 3 \mathrm{c} \mathrm{ml} \\ 25^{\circ} \mathrm{C} & 3 \mathrm{j} \mathrm{ml}^{\circ} & 34 \mathrm{ml}\end{array}$


Date: $\quad 2-7-73$

To: C. M. Walker

From: W. H. Sant

Subject: ANALYSIS OF TANK FARM SAMPLES

Sample: $T 5391 \quad 109-3 \mathrm{Y}$

Appearance: Yellow

$$
\begin{aligned}
& 10 \% \text { solios } \\
& 1 \text { KAD }
\end{aligned}
$$

Spg: 1.324

GEA - $\operatorname{Cs}^{137}: 1.20 \times 10^{6} \mathrm{HCi} / \mathrm{gal}$
$\left.\operatorname{Cs}^{134}: 2.51 \times 10^{4} \mathrm{HCi} / \mathrm{ga}\right]$

\section{BOILDOLIN}

Starting Volume: $50 \mathrm{ml}$

Boiling Point: $168 \mathrm{C}$

Volume at which solids formed: $49 \mathrm{ml}$

$M 7$ solids formed: $Q \mathrm{ml}$

\begin{tabular}{cccc} 
Temperature & & Volume & Volume of Solids \\
\cline { 1 - 2 } $80^{\circ} \mathrm{C}$ & & $36 \mathrm{ml}$ & $</ \mathrm{ml}$ \\
$60^{\circ} \mathrm{C}$ & $35 \mathrm{ml}$ & $</ \mathrm{ml}$ \\
$40^{\circ} \mathrm{C}$ & $33 \mathrm{ml}$ & $<i \mathrm{ml}$ \\
$25^{\circ} \mathrm{C}$ & $33 \mathrm{ml}$ & $3 \mathrm{ml}$
\end{tabular}


Date: $2-26-73$

To:

C. M. Walker

From:

W. H. Sant

Subject: ANALYSIS OF TANK FARM SAMPLES

Sample: T-5594 109BY

Appearance: yellow

$$
\begin{aligned}
& 20 \% \text { solios } \\
& 2000 \text { MAAD }
\end{aligned}
$$

Spg: 1.424 .5

$$
\begin{aligned}
\mathrm{GEA}-\mathrm{Cs}^{137}: 1.54 \times 10^{6} \mathrm{HCi} / \mathrm{gat} \\
\operatorname{cs}^{134}: 3.14 \times 10^{\circ} \mathrm{HCi} / \mathrm{gal} \\
\operatorname{sb}^{125}: 3.63 \times 10^{\circ} \mathrm{me} / \mathrm{ga}^{2}
\end{aligned}
$$

\section{BOILDOHIN}

Starting Volume: $50 \mathrm{ml}$

Boiling Point: $115^{\circ} \mathrm{C}$

Volume at which solids formed: $50 \mathrm{ml}$

$M 7$ solids formed: $/ \mathrm{ml}$

\begin{tabular}{ccc} 
Temperature & Volume & Volume of Solids \\
\hline $80{ }^{\circ} \mathrm{C}$ & $44 \mathrm{ml}$ & $/ \mathrm{ml}$ \\
$60^{\circ} \mathrm{C}$ & $44 \mathrm{ml}$ & $1 \mathrm{ml}$ \\
$40{ }^{\circ} \mathrm{C}$ & $44 \mathrm{ml}$ & $1 / \mathrm{ml}$ \\
$25{ }^{\circ} \mathrm{C}$ & $4.4 \mathrm{ml}$ & $1 \mathrm{ml}$
\end{tabular}


Date: $\quad 3-1-73$

To: C. M. Walker

From: - W. H. Sant

Subject: ANALYSIS OF TANK FARM SAMPLES

Sample: T5655 $107 \mathrm{BY}$

Appearance: Yelérw

$$
3.5 \% \text { SCLCDS }
$$

$2 R A D$

Spg: 1.3895

GEA - Cs137: $1.6 .4 \times 10^{6}, \mathrm{pCi} / \mathrm{gal}$

\section{BOILDOHIN}

Starting Volume: $\quad \mathrm{ml}$

\% Solids: $\quad$ \%

Boiling Point: ${ }^{\circ} \mathrm{C}$

Volume at which solids formed: $\mathrm{ml}$

M1 solids formed: $m$ T

Temperature volume volume of Solids

$\begin{array}{lll}80{ }^{\circ} \mathrm{C} & \mathrm{ml} & \mathrm{ml} \\ 60{ }^{\circ} \mathrm{C} & \mathrm{ml} & \mathrm{ml} \\ 40{ }^{\circ} \mathrm{C} & \mathrm{ml} & \mathrm{ml} \\ 25{ }^{\circ} \mathrm{C} & \mathrm{ml} & \mathrm{ml}\end{array}$

Insuf, Sample to RuV Baildowiv 
Date: $\quad 3-7-73$

BY 109.18

To: C. M. Walker

From:- W. H. Sant

Subject: ANALYSIS OF TANK FARM SAMPLES

Sample: $T 5911109 B Y$

Appearance: Yellow

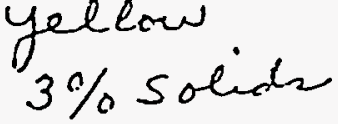
$2 R A D$

Spg: 1.335

GEA - Cs\$37: $1.27 \times 10^{6} \mathrm{uCi} / \mathrm{ga}$ $\mathrm{Cs}^{134}: 2.21 \times 10^{4} \mathrm{\mu Ci} / g a ?$

\section{BOILDOISN}

Starting Volume: $50 \mathrm{ml}$

Boiling Point: $/ 12 \circ \mathrm{C}$

Volume at which solids formed: $4.3 \mathrm{ml}$

MI solids formed: / $\mathrm{ml}$

\begin{tabular}{ccc} 
Temperature & Volume & Volume of Solids \\
\cline { 2 - 3 } $80^{\circ} \mathrm{C}$ & $4 \mathrm{zml}$ & $3 \mathrm{ml}$ \\
$60^{\circ} \mathrm{C}$ & $42 \mathrm{ml}$ & $3 \mathrm{ml}$ \\
$40^{\circ} \mathrm{C}$ & $40.5 \mathrm{ml}$ & $3 \mathrm{ml}$ \\
$25{ }^{\circ} \mathrm{C}$ & $40 \mathrm{ml}$ & $25 \mathrm{ml}$
\end{tabular}


Date: $3-22-73$

To: - C. M. Walker

From: W. H. Sant

Subject: ANALYSIS OF TANK FARM SAMPLES

Sample: $T 6.123$ is 9 i $3 y$

Appearance: yellow

$$
\begin{aligned}
& 2 \% \text { SOL.DS } \\
& 1.5 \text { S:2 }
\end{aligned}
$$

Spg: $1.3 / 6$

GEA - $\begin{array}{rll}\operatorname{Cs} 137: 1.06 \times 106 & \mu C i / g a 1 \\ \operatorname{Cs} 134: 1.60 \times 104 & \mu C i / g a 1\end{array}$

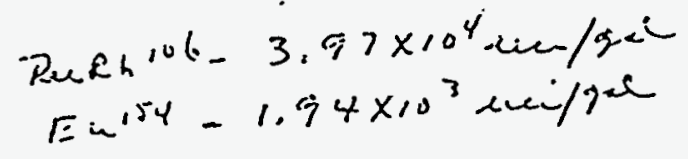

\section{BOILDOWN}

Starting Volume: $50 \mathrm{ml}$

\% Solids: 0 \%

Boiling Point: $106^{\circ} \mathrm{C}$

Volume at which solids formed: $3: \mathrm{ml}$

$M 1$ solids formed: $3 \mathrm{ml}$

Temperature

$80{ }^{\circ} \mathrm{C}$
$60{ }^{\circ} \mathrm{C}$
$40{ }^{\circ} \mathrm{C}$
$25{ }^{\circ} \mathrm{C}$

Volume

$27 \mathrm{ml}$

$27 \mathrm{ml}$

$25 \mathrm{ml}$

$25 \mathrm{ml}$
Volume of Solids

$\begin{array}{ll}5 & \mathrm{ml} \\ .5 & \mathrm{ml} \\ 6 & \mathrm{ml} \\ 6 & \mathrm{ml}\end{array}$


Date: $4-5-73$

To:

C. M. Walker

From:

W. H. Sant

Subject: ANALYSIS OF TANK FARM SAMPLES

Sample: $T$ k344 $109 B Y$

Appearance: yellow

$$
3 \% \text { SoL, OS }
$$

$$
\text { IRAD }
$$

Spg: 1.4432

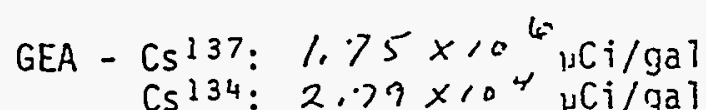

\section{BOILDOHN}

Starting Volume: $50 \mathrm{ml}$

$\%$ Solids: $\delta \%$

Boiling Point: $107^{\circ} \mathrm{C}$

Volume at which solids formed: $43 \mathrm{ml}$

$M 1$ solids formed: $2 \mathrm{~m}$ ?

\begin{tabular}{ccc} 
Temperature & Volume & Volume of Solids \\
\cline { 3 - 3 } $80^{\circ} \mathrm{C}$ & $4 / \mathrm{ml}$ & $4 \mathrm{ml}$ \\
$60^{\circ} \mathrm{C}$ & $40 \mathrm{ml}$ & $5 \mathrm{ml}$ \\
$40^{\circ} \mathrm{C}$ & $40 \mathrm{ml}$ & $5.5 \mathrm{ml}$ \\
$25^{\circ} \mathrm{C}$ & $40 \mathrm{ml}$ & $25 \mathrm{ml}$
\end{tabular}


Date: $4-19-73$

To: - C. M. Walker

From: H. H. Sant

Subject: ANALYSIS OF TANK FARM SAMPLES

Sample: T6682 109 BY

Appearance: Yeilow

$$
20 \% \text { sciids }
$$

1.3 R.AD

Spg: -1.4028

GEA - Cs137: $1.56 \times 10^{6} \mathrm{HCi} / \mathrm{gal}$ $\mathrm{Cs}^{134}: 2.20 \times 10^{4} \mathrm{HCi} / \mathrm{gal}$

\section{BOILDOWN}

Starting Volume: $5 \rho \mathrm{ml}$

$\%$ Solids: $\%$

Boiling Point: $11^{\circ \circ} \mathrm{C}$

Volume at which solids formed: $4 \notin \mathrm{ml}$

M1 solids formed: $5 \mathrm{ml}$

\begin{tabular}{cccc} 
Temperature & & Vol ume & Volume of Solids \\
\cline { 1 - 2 } $80{ }^{\circ} \mathrm{C}$ & & $40 \mathrm{ml}$ & $5 \mathrm{ml}$ \\
$600^{\circ} \mathrm{C}$ & $40 \mathrm{ml}$ & $5 \mathrm{ml}$ \\
$400^{\circ} \mathrm{C}$ & $40 \mathrm{ml}$ & $5 \mathrm{ml}$ \\
$25{ }^{\circ} \mathrm{C}$ & $35 \mathrm{ml}$ & $10 \mathrm{ml}$
\end{tabular}


Date: $5-7-73$

To:

C. M. Walker

From: W. H. Sant

subject: ANALYSIS OF TANK FARM SAMPLES

Sample: T 6877

$$
10 ; B Y
$$

Appearance: Light GiReen, c.eaddy

$$
\text { 2. } 5 \text { RAD }
$$

Spg: 1.439

$$
\begin{array}{r}
\text { GEA - } \operatorname{Cs}^{137}: 1.87 \times 10^{6} \mathrm{HCi} / \mathrm{gal} \\
\mathrm{Cs}^{134}: 2.55 \times 10^{124} \mathrm{HCi} / \mathrm{ga} \\
\mathrm{sb}^{125}: 2.57 \times 10^{3} \mathrm{we} / \mathrm{gel} \\
\text { BOILDOWN }
\end{array}
$$

Starting Volume: $\mathrm{ml}$

$\%$ Solids:

Boiling Point: $\quad{ }^{\circ} \mathrm{C}$

Volume at which solids formed: $\mathrm{ml}$

MI solids formed: $\mathrm{ml}$

\begin{tabular}{ccc} 
Temperature & Volume & Volume of Solids \\
\hline $80{ }^{\circ} \mathrm{C}$ & $\mathrm{ml}$ & $\mathrm{ml}$ \\
$60{ }^{\circ} \mathrm{C}$ & $\mathrm{ml}$ & $\mathrm{ml}$ \\
$40^{\circ} \mathrm{C}$ & $\mathrm{ml}$ & $\mathrm{ml}$ \\
$25{ }^{\circ} \mathrm{C}$ & $\mathrm{ml}$ & $\mathrm{ml}$
\end{tabular}

Sample Bilei oler
unable To Get BoilBown. 


\section{BY104.23}

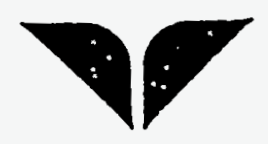

Date: $\quad 5-23 \cdot 7.3$

To: C. M. Walker

From: W. H. Sant

Subject: ANALYSIS OF TANK FARM SAMPLES

Sample: T-7,1T $1: T 13 y$

Appearance: ceudey $50 \%$ salios i2

Spg: 1.3 .765

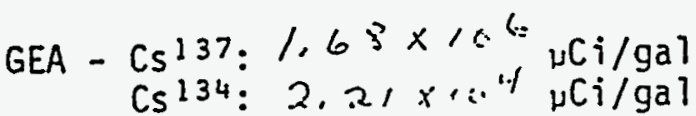

\section{BOILDOWN}

Starting Volume: $50 \mathrm{ml}$

$\%$ Solids: ses \&

Bojling Point: $11 \mathrm{~s}^{\circ} \mathrm{C}$

Volume at which solids formed: 5 is $\mathrm{ml}$

Ml solids formed: $5 \mathrm{ml}$

$\begin{array}{ccc}\text { Temperature } & \text { Volume } & \text { Volume of Solids } \\ 80^{\circ} \mathrm{C} & 50 \mathrm{ml} & 5 \mathrm{ml} \\ 60^{\circ} \mathrm{C} & 50 \mathrm{ml} & -5 \mathrm{ml} \\ 40^{\circ} \mathrm{C} & 50 \mathrm{ml} & 7.5 \mathrm{ml} \\ 25^{\circ} \mathrm{C} & 56 \mathrm{ml} & 10 \mathrm{ml}\end{array}$


Dete: Ney 29, 1971

To:

D. J. Jaried

G. C. Ooers

W. C. Schmat

In-crin:

J. S. Jucisingem J5 Suckinghaser

subject:

DISSOIJTIOX ATD ARATISIS OF 112-BI SIDDCE AND CRUST SALPIES

Semples.of sludge, crust, and the associsted nother liquor from tank 112-5I hare been tested for Bolubility and ansigzed in the laboratory.

About 70 percent of the sluage wes dissolved by $1.67 \mathrm{ml}$ act reter De $c c$ of sindige. About 9 percent of the sluage wes dissolired by not IIS feed ircon tenk 109-BY at a volume Ixtio of 0.6 Il ieed Der ce of sludige.

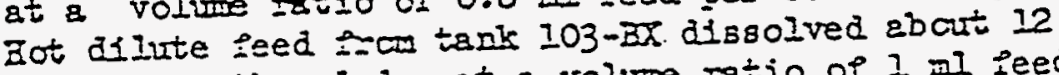
percent of the sladige at a volume ratio of 1 ill seed per cc of slucige.

The crust sppearea to be man less soluble then the sluage. At \& rolume ratio of $2 \mathrm{ml}$ of weter per $c c$ of crust, 31 percent of the solid dissolved. None of the crust dssolved in dilute feed from tank 103-BX. The Isboratory dete indicate that the dissolution of the sludge and crust in tenk 172-BI Fill be sIow end difictcult at best. Water agpeans to be the best solvent far dissolving the sluage.

Detrils of the dissolution studies are discussed belcw. Chemical arsiysis of the sluige, crust and nother $c r$ supernatant Ifquid are also shown.

\section{DISSOLUIION SIUDIES}

Sluoge

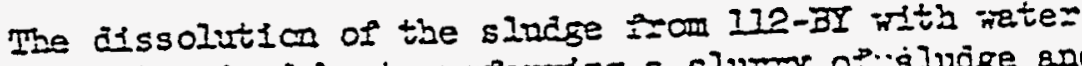
izs deternined by transferring a slurzy op"sluage anc superpatent Ifould to \& 15 ml greduated cent=1 huse cone. After 8 iloring the sludge to settle for six hours, the superastant liquid thes decanted leaving 
Distibution

Page 2

Kay 29, 2911

$4.8 \mathrm{cc}$ QI settled sludge. Tro mililiters of weter were pipetted on the surface of the siudge and allored to stand overnight at rocm tempersture and no egitaticn. In the moriting the sluage volume $\mathrm{kBs} 3.8 \mathrm{cc}$ Indicating that 21 percent of the sludge had alssolved. It should be polnted out thet part of the reduction in sludge volume could heve been due to furthe: setiling and compection of the sludge.

A second 2 fll increment of reter was added to the sludge. In 4 hours tice slucige rolume hed decressed to $3.6 \mathrm{cc}$. The temperature of the sludge and riter was rafsed to about $90^{\circ} \mathrm{C}$. Thinty minutes after raising the temperature the volume of sluase ind reauced to $3 \mathrm{cc}$; in 2.5 bours the rolume of sludige ics $2.7 \mathrm{cc}$. More reter was adced in 2 ml lnererents unt: I a total of 8 ml had been added. The volume of sludge iad cecreased to $1.55 \mathrm{cc}$. The izte= dissolution cets is sicwn in detail in Table I stischea.

As eny wate = tinet $\pm s$ added mist eventur $11 \mathrm{y}$ eveporate again, tine solubility of the sluige in 109-5I recjcis feed and 103-BX dilute leed ras tested. The results are shown in Table II attacined.

\section{Crust}

The crust irm the 112-5I tank wes solt and musty ind seemed to beve quite $a$ lot of ifquid assoclated Fith $1 t$. The eppezent density of the crust was 1.69. "The solubiIIties of tice crust of ieter and in 103-BX feed are shorn in Table III attached. Because the crust was quite dry conpared to the sludge, we could nct peasure the volme by ellowing a slurry of crust and supernatant to setile as was done with the sluage. For the caust the volume has deterraced by tapoing the materlal in 8 graduated $15 \mathrm{ml}$ centriliuge cone or by messuring the rolume of $\mathrm{CCI}_{4}$ a piece of the c-rist would atsplace.

In Table III, a particn of the zecuction in sludge volume Hith ieter migit be attulbutsile to the crust setiling and conpacting after the rate $=$ was added. When 103-5X feed was edied to the crust, the rolume of the arust 
PABLE I

DISSOLUTION OF 112-BY SLUDAR WTITI WATER

\begin{tabular}{|c|c|c|c|c|c|c|c|}
\hline Date & Ilours & $\begin{array}{l}\text { Incremontal } \\
\text { Tlime, hro. }\end{array}$ & $\begin{array}{l}\text { Sludgo } \\
\text { Volume, :cc } \\
\end{array}$ & $\begin{array}{l}\text { Total } \mathrm{M}_{\mathrm{g}} \mathrm{O} \\
\text { Added, } \mathrm{ml}\end{array}$ & Temp. & $\begin{array}{l}\text { Incremental } \\
\text { \& Digoolved. }\end{array}$ & $\begin{array}{l}\text { Total } \% \\
\text { D1saolved }\end{array}$ \\
\hline $4-28-71$ & 1500 & 0 & 4.8 & 2 & 25 & 0 & 0 \\
\hline \multirow[t]{8}{*}{$4-29-71$} & 0800 & 27 & 3.8 & 2 & 25 & 21 & 21 \\
\hline & 0800 & 0 & 3.8 & 4 & 25 & 0 & 21 \\
\hline & 1200 & 4 & 3.6 & 4 & 25 & 5 & 25 \\
\hline & 1230 & 0.5 & 3.0 & 4 & 90 & 17 & 38 \\
\hline & 1330 & 2 & 2.7 & 4 & 90 & 10 & 44 \\
\hline & $1 / 30$ & 1 & 2.7 & 4 & 90 & 0 & 4,4 \\
\hline & 1500 & 0.5 & 2.7 & 4 & 25 & 0 & 44 \\
\hline & 1500 & 0 & 2.7 & 6 & 25 & 0 & 44 \\
\hline \multirow[t]{4}{*}{$4-30-71$} & 0800 & 17. & 2.5 & 6 & 25 & 7 & 48 \\
\hline & 0800 & 0 & 2.5 & 6 & 90 & 0 & 48 \\
\hline & 1500 & 7 & 2.15 & 6 & 90 & 14 & 55 \\
\hline & 1500 & 0 & 2.15 & 8 & 25 & 0 & 55 \\
\hline \multirow[t]{2}{*}{$5-03-71$} & 0800 & 65 & 1.8 & 8 & 25 & 16 & 63 \\
\hline & 0800 & 0 & 1.8 & 8 & 90 & 0 & 63 \\
\hline $5-04-71$ & 0800 & 24 & 1.55 & 8 & 90 & 14 & 68 \\
\hline
\end{tabular}


DIstifibution

Pege 4

vay 29, 1971
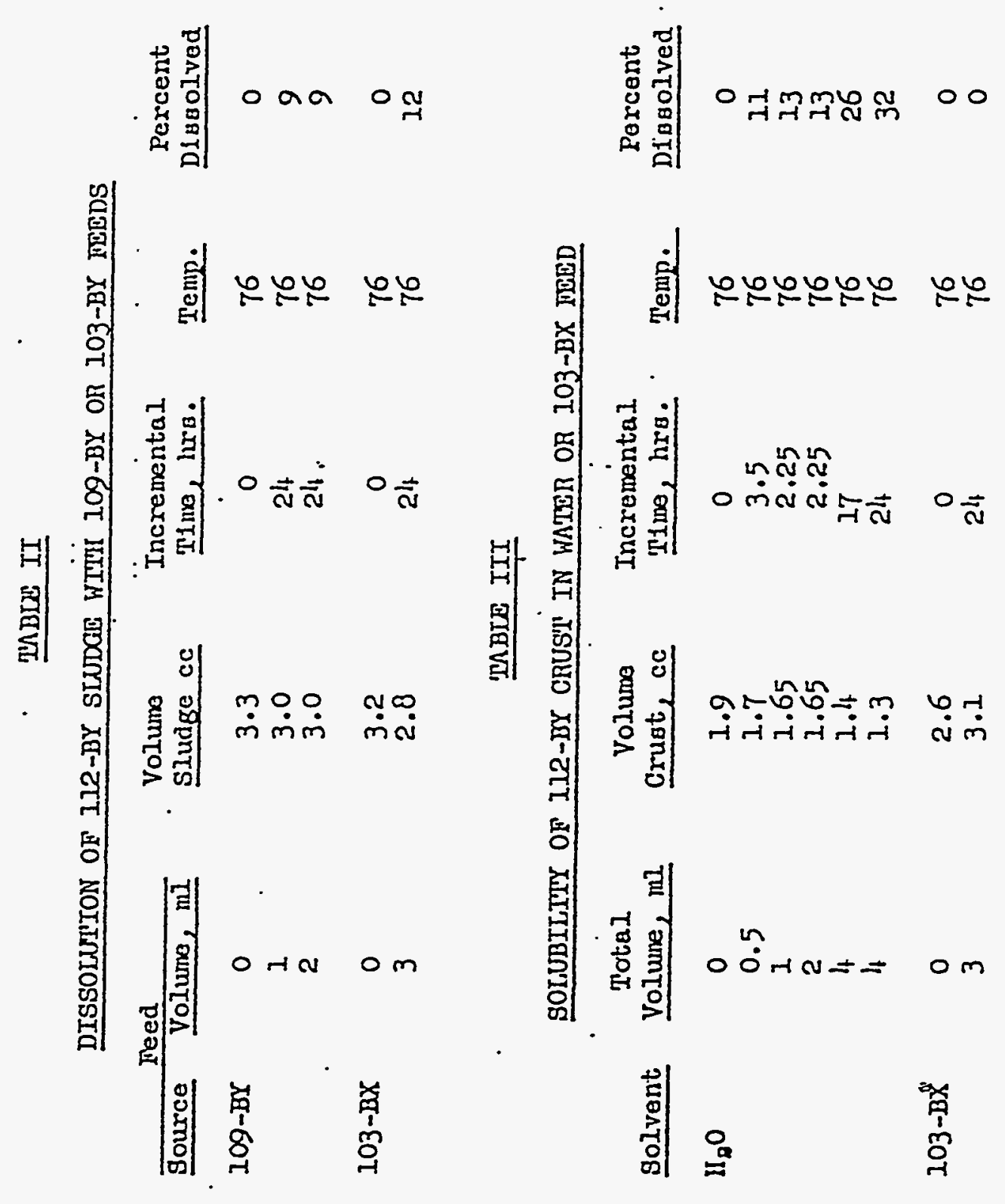
Distribution

Pege 5

key 29, 1971

incressed; however, most of the increase was caused by the crust expending and lind of sem-flceting in the Ifquid. A smell amount Irey have dissolved, but if it did it was quite smell.

Anslysis of Slucise, Superpetant Ifould and C.ust

The superzetant Ifquad associsted with the sludige and crist was ire lyzed directly. For the sludge, a slurry of sludge and superpetent pes transfezied to

a 15 inl centrifuge cone and"centrifuged to ccupsct the sludse. Atter determinting the rolume of the ccupected siucige, the superpetant liquid was decanted. Greate = ting 99 percent of the sludge wes dissclved by

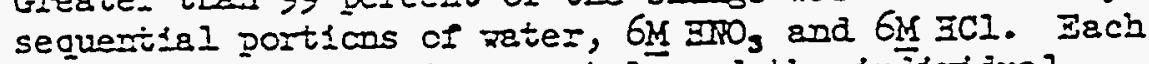
ifaction was anelyzed separately and the injit $\nabla$ icuel anslyses totaled to find the sincunt in the semple. AII results are based on a liter of conpacted sludge.

For the crast, the rolume of crust iras deteralined by CCI, aisplacement. Dissolution of the crust ins achieved by sequentisily relluding fith water, dilute and concentiated $\mathrm{BNO}_{3}$. The analyses are shown in Triole IV ettached. ToteI scid cemend, (TAD) includes all ions titrable by acid to the nejor an break between LE $T$ and 4. Icos tinst Fould be titrated by acid incluce $\mathrm{O}^{-}, \mathrm{AlO}_{2}^{-}$, and $\mathrm{CO}_{3}^{-2}$.

Please c8ll to if you have ang questions regaring this Fork.

JSB::abs

cc: in Cemplinel1

DA Dodd

CJ Francis

CA Iorengen

IN Roday

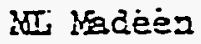

जW Schiniz:

JO Skólmä

PW Sorth

File

I. B 


\section{BYII2.1}

TABIE IV

ANALXSTS OF SUTBRNATANT, SLUDGE AND CRUST FROM TANK II2-IYY

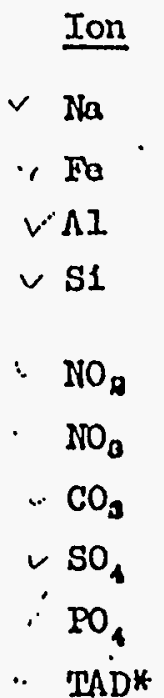

\section{Supernatant}

$11.16 \mathrm{M}$

$<1.81 \times 10^{-3}$

$\cdot 2.05$

$9.00 \times 10^{-3}$

Sludge

$16.9 \mathrm{M}$

0.12

1.71

0.22

Crust

出

ป

$20.7 \mathrm{M}$

0.06

0.58

$<0.03$

0.012

4.32

.05

0.16

$\begin{array}{lll}1.1 \mathrm{M} & 1.54 \mathrm{M} & 0.012 \\ 2.1 & 6.17 & 1.32 \\ 0.018 & 0.67 & 3.05 \\ 0.05 & 0.12 & 0.146 \\ 0.023 & 0.26 & 0.12 \\ 7.20 & 8.11 & 9.814\end{array}$

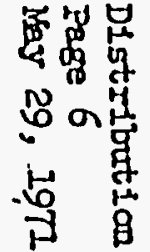

9.814

$\begin{array}{lll}1.85 \times 10^{3} \mu \mathrm{Cl} / 1 & 8.21 \times 10^{4} \mu \mathrm{Gl} / 1 & 9.84 \times 10^{4} \mu \mathrm{Cl} / 1 \\ 4.30 \times 10^{8} & 3.17 \times 10^{\circ} & 2.51 \times 10^{\circ} \\ 4.48 \times 10^{3} & 4.77 \times 10^{\circ} & 3.11 \times 10^{\circ} \\ & 3.91 \times 10^{4} & 2.30 \times 10^{3} \\ & 1.0 & 4.50 \times 10^{2} \\ 3.77 \times 10^{8} & 1.2 \times 10^{\circ} \\ 3.29 \times 10^{4} & 5.7 \times 10^{4} \\ & & 5.30 \times 10^{2} \\ & & 8.3 \times 10^{\circ}\end{array}$

* Total acid demand 
TERe-

Attantic Richfield Hanford Company

WHC-SD-WM-ER-312, Rev. O

2055

Date:

April 4, 1972

To:

T. D. Ande $=90 \pi$

Eェon:

J. S. 3uckingham is buckinghan

Subject:

ANAIYSIS OE TANK I12-3V SAMPIE

References:

(1) Lettez, Januazy 31, 1972,

T. D. Anderson to

J. S. Buckinghan, "Tank 112-3Y

Samples and Analyses"

(2) Ietter, May 29, 1971,

J. S. Buckingham to D. J. Ia $=k i n$

et al., "Dissolution and Analysis

OI $\overline{I I 2}-B Y$ siudge and Cxust

Samples"

Samples of too soiid, liquid, and bottom solid taken EIcm Tank II2-3Y near the end of Januazy have been analyzed as requested in Reference $I$. A sinilar set of analyses were run on samples taken in April 1971. The results were reported in Reference 2 . i

The analyses indicated that both the light and heavy solids were primazily NaNOz. Analyses showed there was not much difference between the two solids, so it is assumed that the light solid is an emulsion of very fine NaNOz crystals.

Differeatial thernal anaIysis.(DTA) of the liguid showed an exothermic reaction occurring between - 210 and 275 . C. Jowever, no unusual pressure or temperature excursions were observed when 100 milliliters of solution were evaporated to dzyress.

Aralyses of the samples are shown in Table $I$. Approximately 90 percent of the solia material was soluble in water, 4 percent soluble in aitzic acid, and 6 percent required a çaustic susion.

3ased on the analyses, the composition of the 112-BY samples aze assumed to be-as shown in Table II. 
T. D. Anderson

page 2

Agril 4, 1972

\section{5 \\ BY/10.2}

TABLE I

ANALYSES OF 112-BY SAMPLES

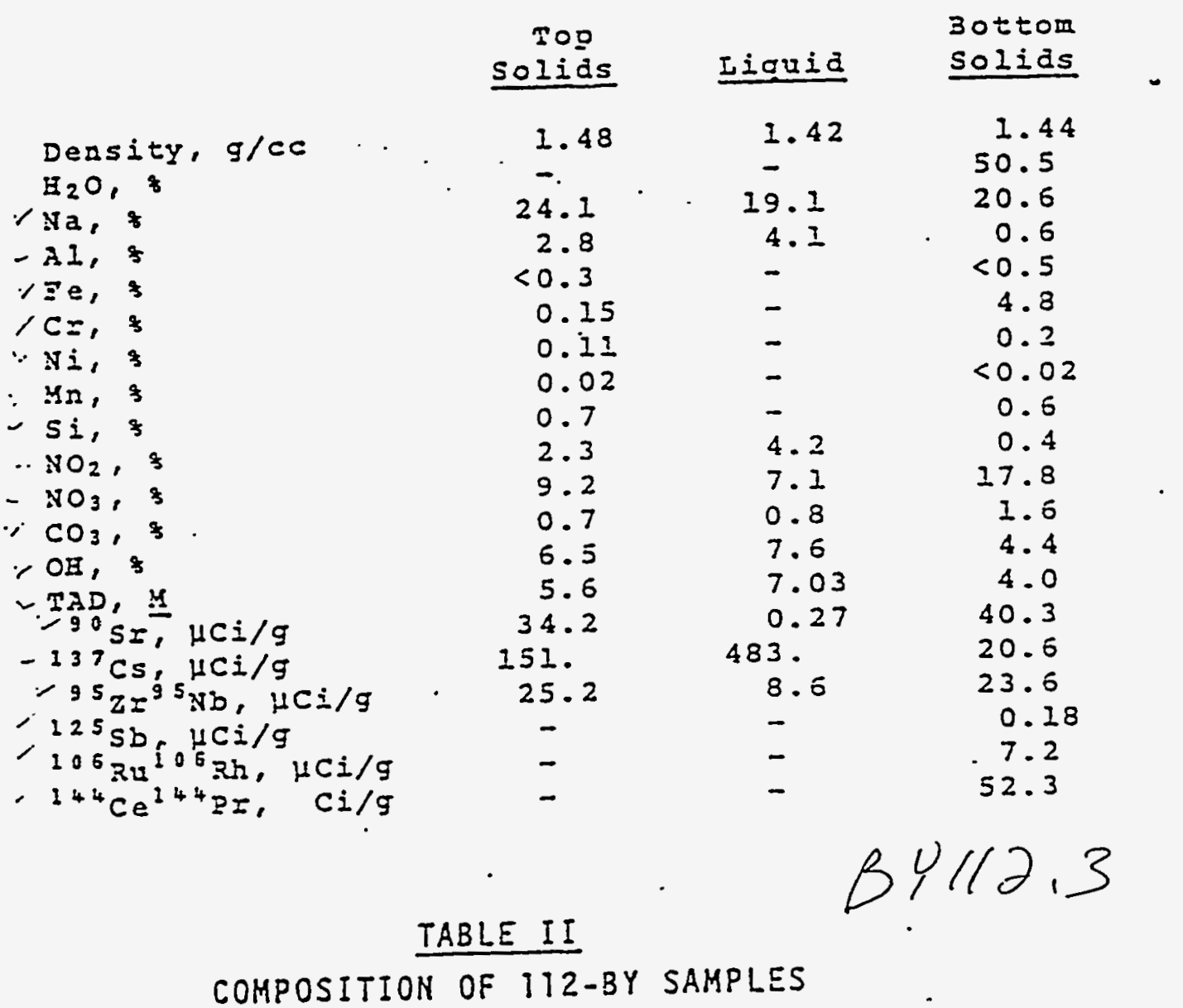

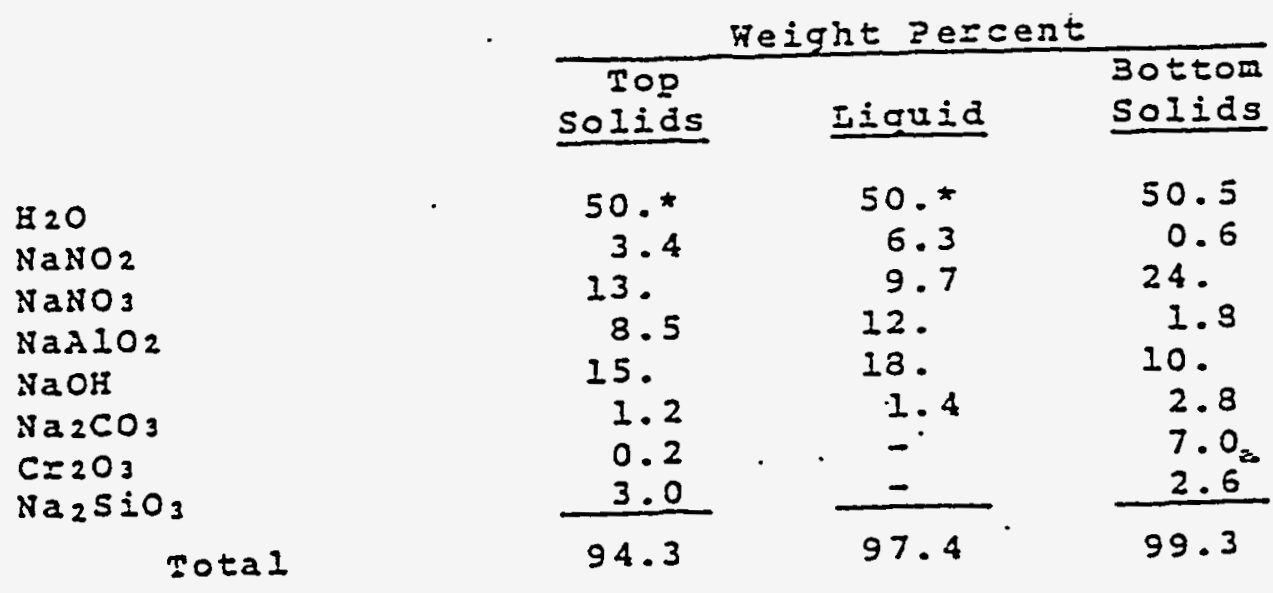

- Estimated. 
T. D. Andersor

Page 3

ApIi1 4, 1972

It should be pointed out that there aze other minor constituents in the waste, but theiz contribution to the weight percent is very smali. It is also interesting to note the unusually iigh percentage of chromiun in the bottom solids. The source of the chromium has not been identified.

Based on work done with synthetic wastes, the assumed value of 50 percent water in the top solid and liquid is probably fairly close. The ovezall material balances also indicate that our assumptions are reasonable.

Differential thernal analysis of the liguid showed an exotheraic reaction occurring between 210 and $275^{\circ} \mathrm{C}$. A second, saliler exothermic reaction occurred a=ound $360^{\circ} \mathrm{C}$.

No unusual temperature or pressure excursions were observed when 100 milliliters of liguid were evaporated to near dryness. Temperature of the concentrate rose to about $130^{\circ} \mathrm{C}$. Temperature of the heat transfer surface rose to $310^{\circ} \mathrm{C}$. Serere foaming was obserped during the boildown.

No exothermic reaction was observed on samples of the concentrated naterial. Difserential therzal analysis of the condensate showed.an exotheraic. reaction occurzing between 150 and $200^{\circ} \mathrm{C}$. The disappearance of an exotheric reaction in the concentrate and the appearance of an exothermic reaction in the concensate indicates that whatever was causing the exothermic reaction was distilling over. The. component causing the exothernic reaction has not been identified.

Please calI Ie li you have any questions regarding this work.

JS3:e

CE: GI Borshein
MA Campbell
DA Dodd
CJ Erancis
DJ Iarkin
HP Metz

\author{
DA Ruryear \\ RC Roal \\ Iit Roddy \\ WW SchuI = \\ Jo skolrud \\ Process Aids (5)
}




\section{BY Tank Farm}

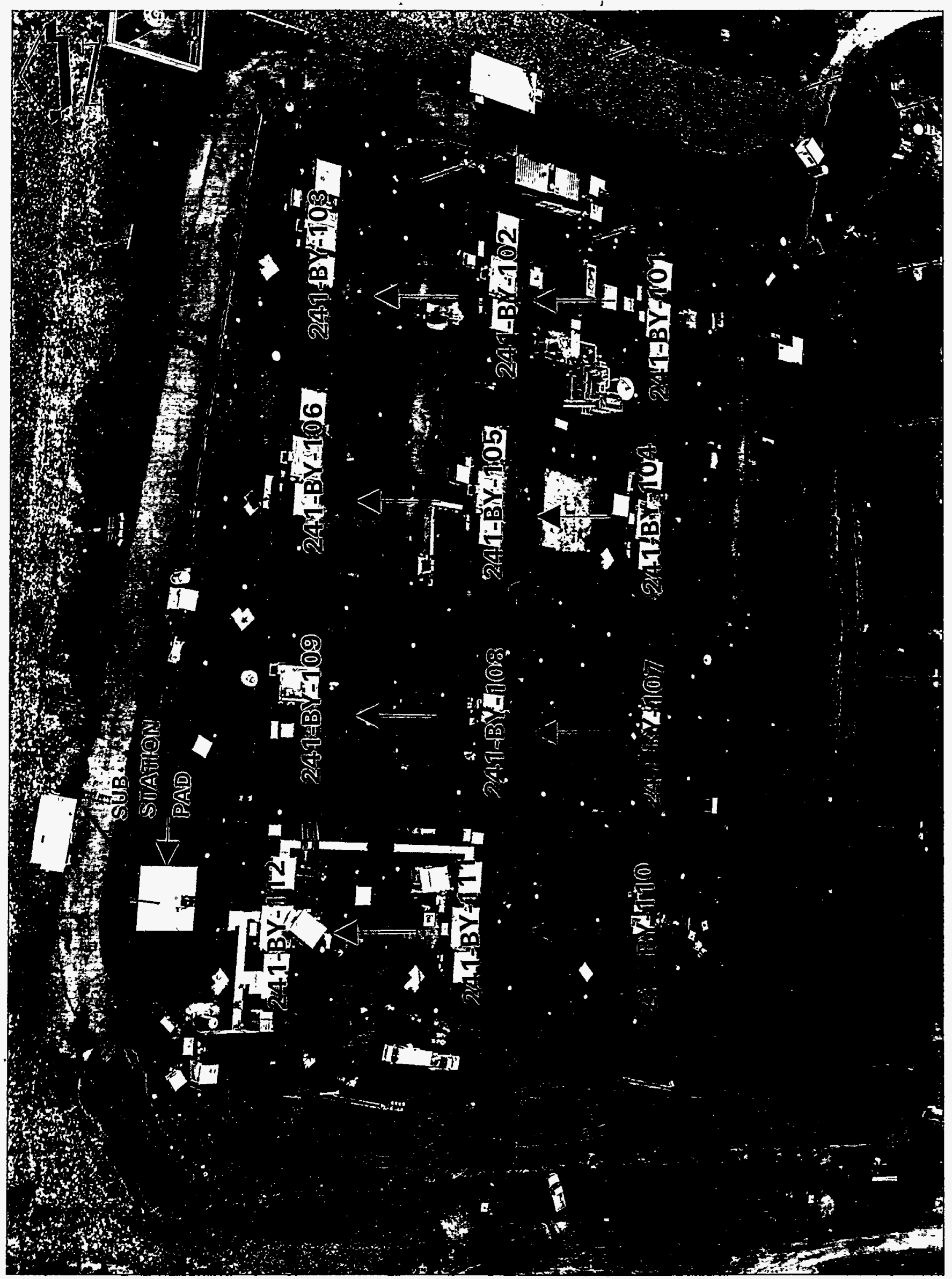




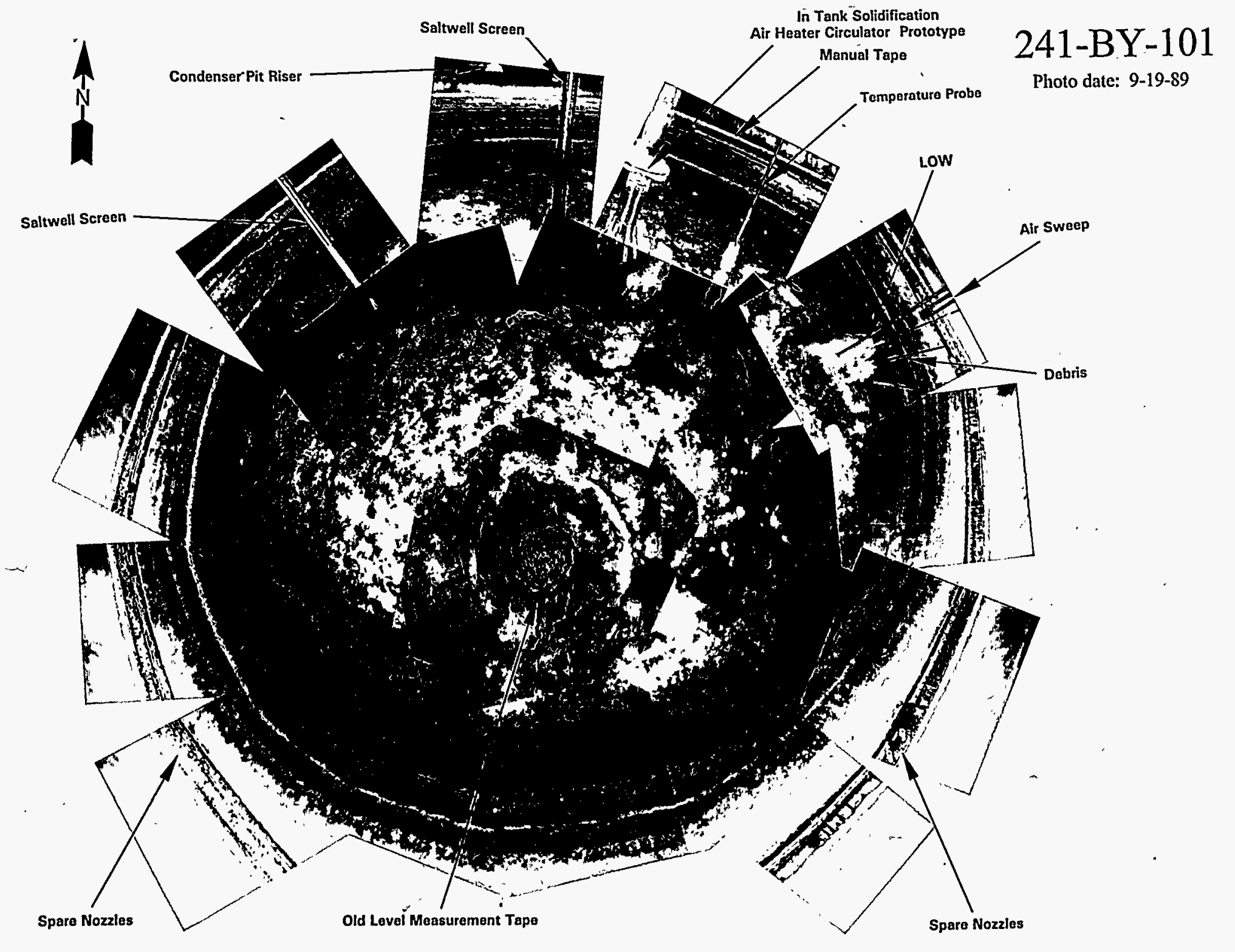


WHC-SD-WM-ER-312, Rev. 0

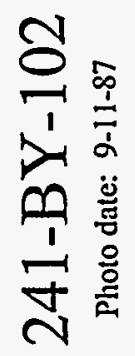


WHC-SD-WM-ER-312, Rev. 0

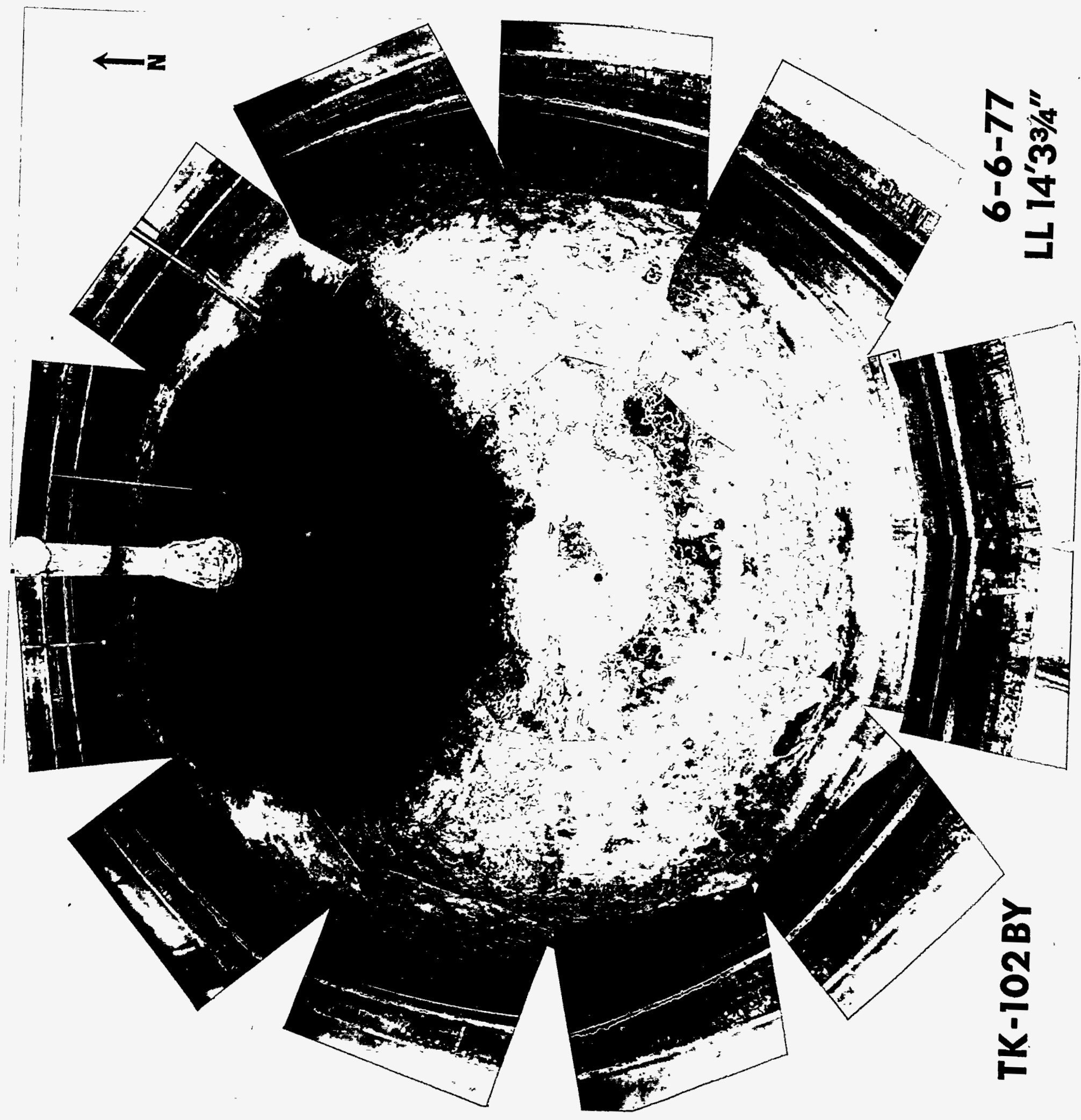


WHC-SD-WM-ER-312， Rev. 0
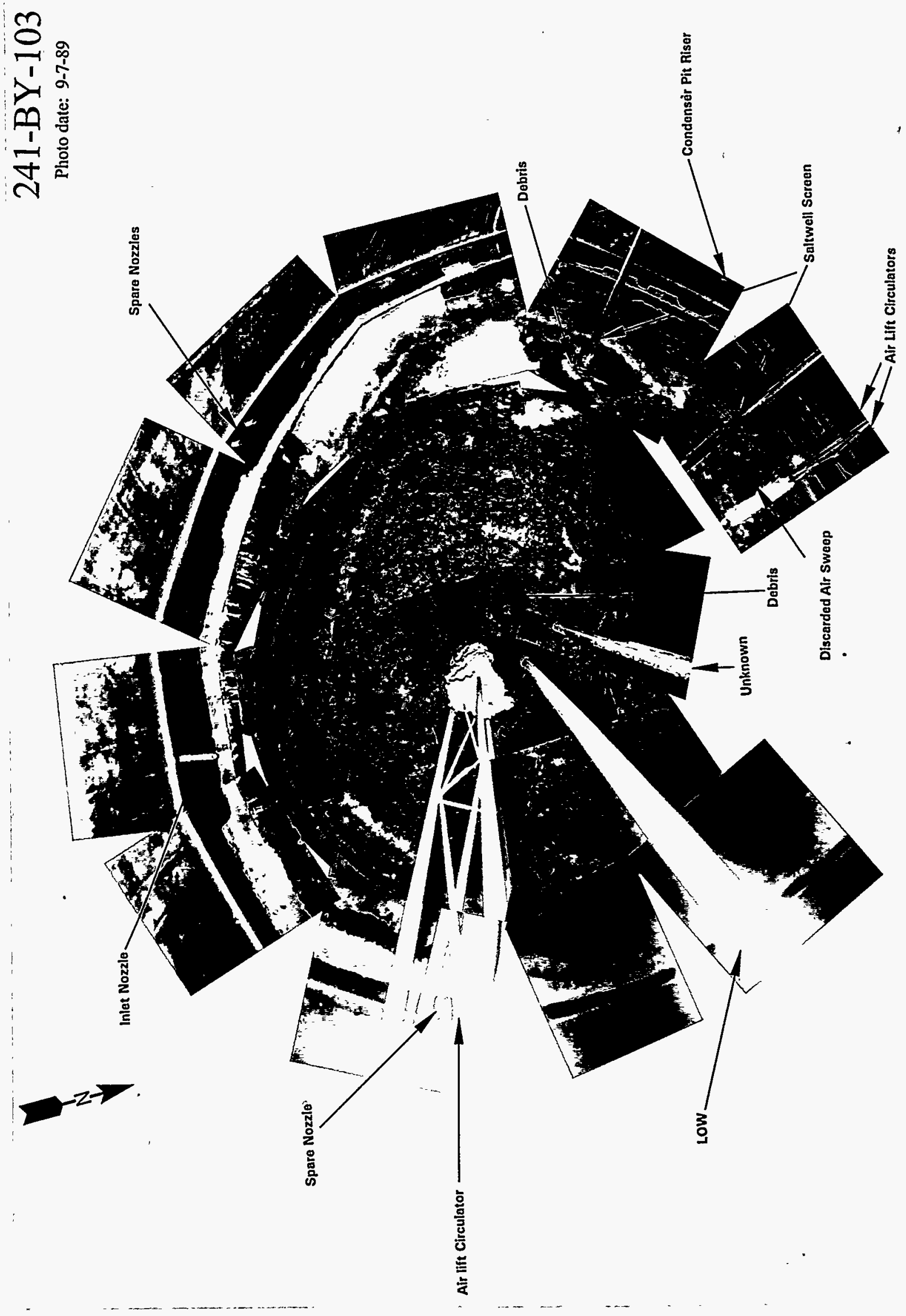


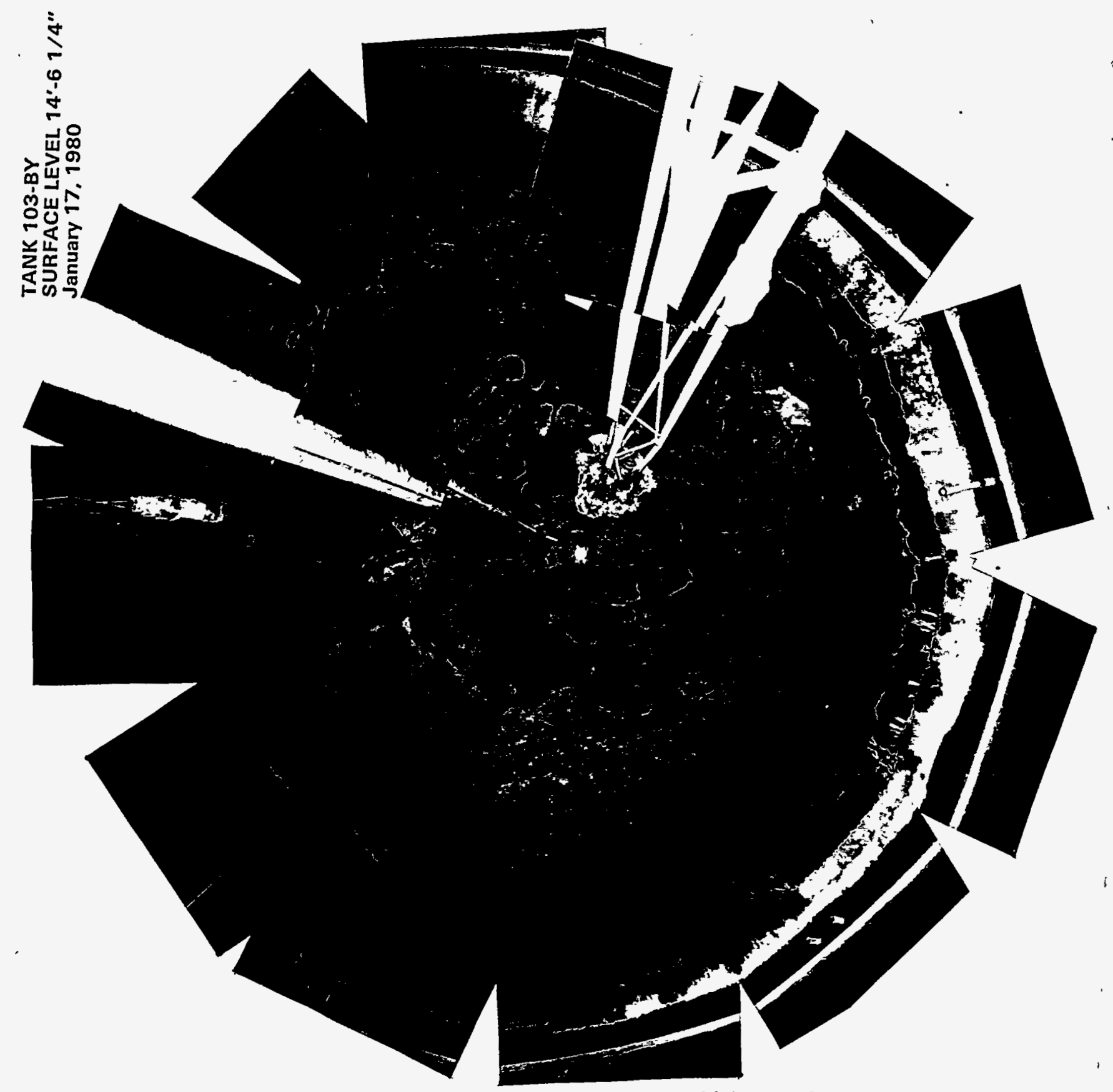


WHC-SD-WM-ER-312, Rev. 0

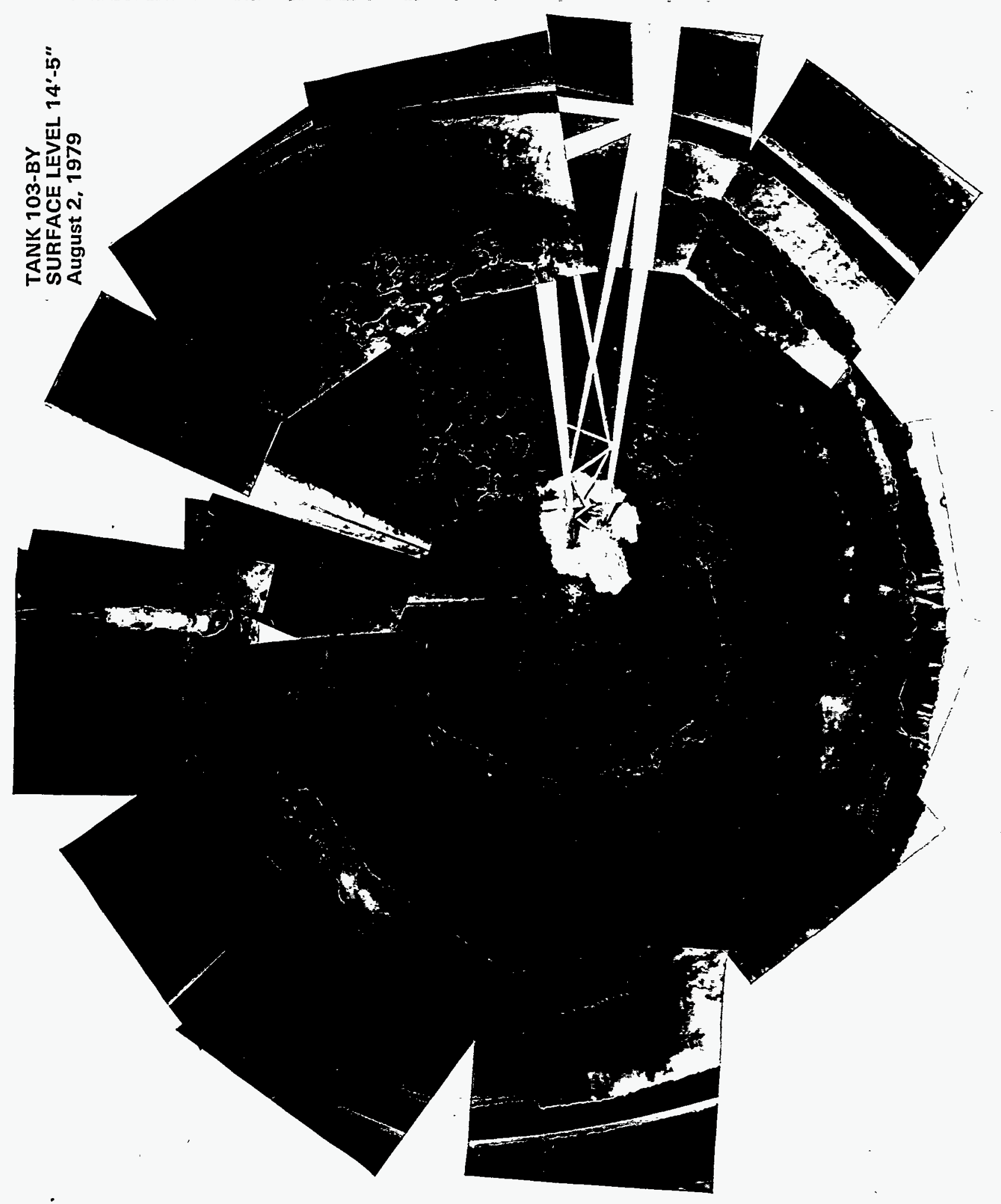




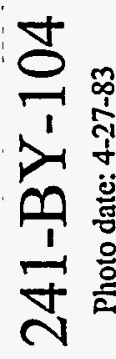
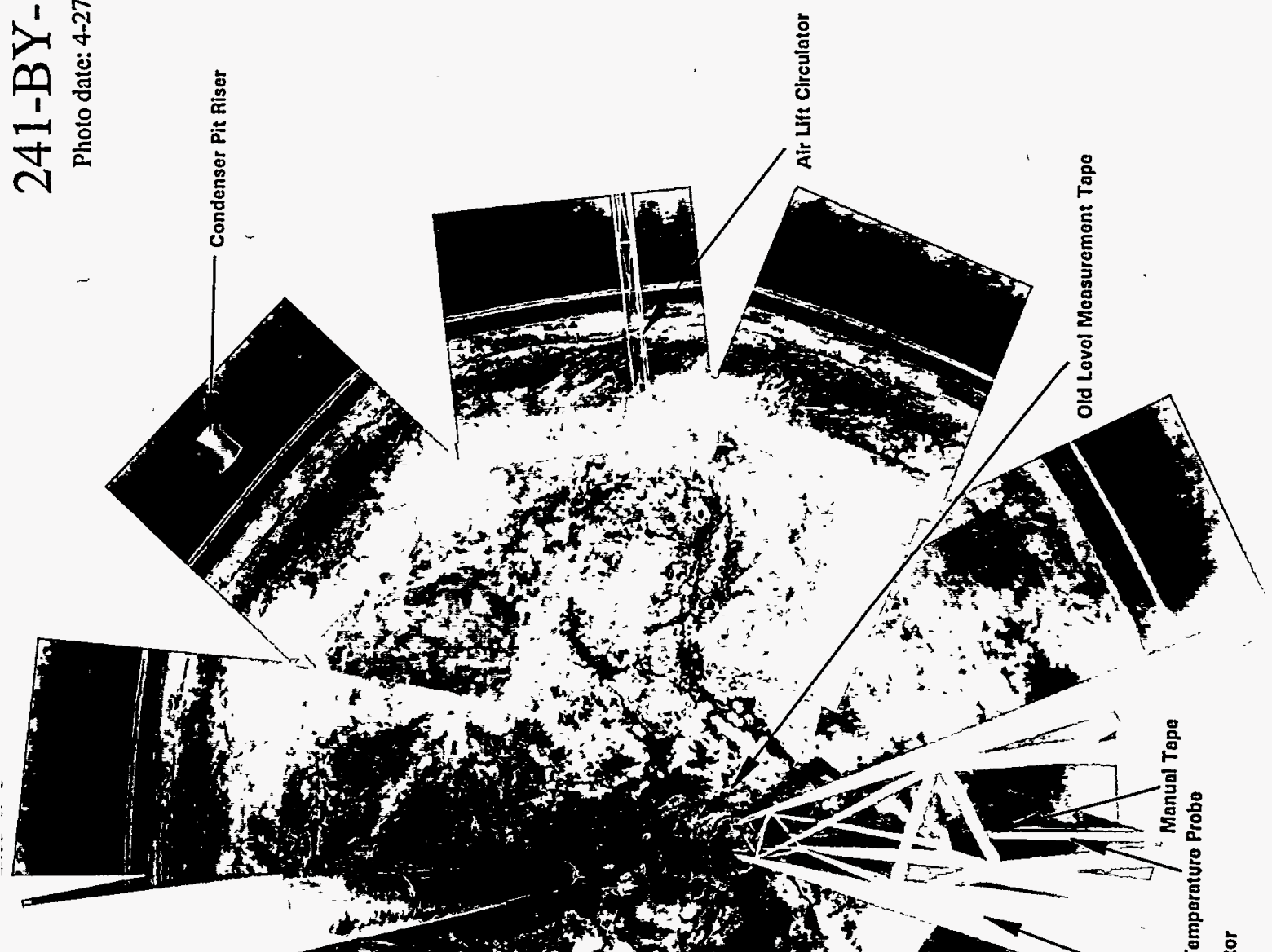
${ }^{6}$

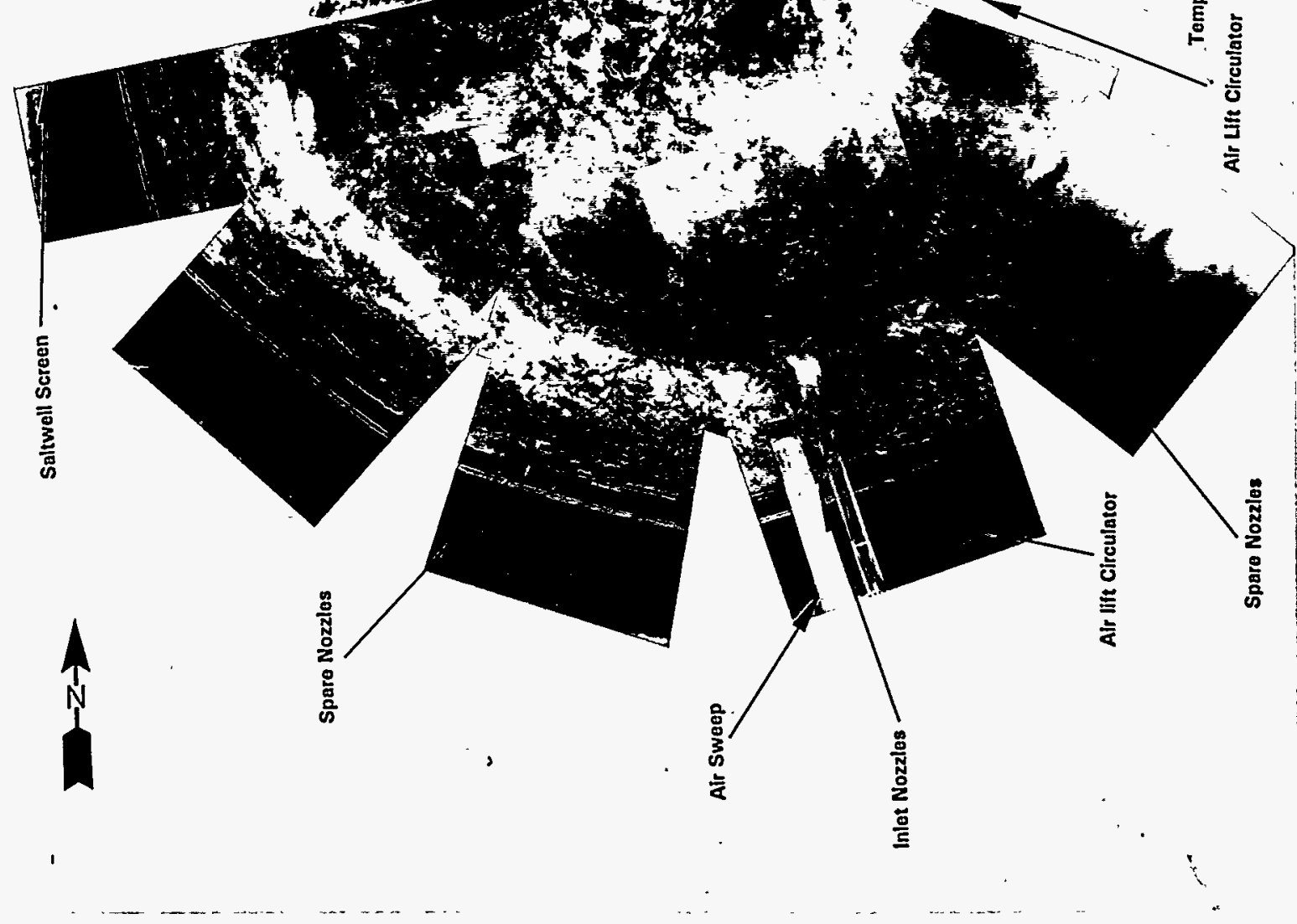


WHC-SD-WM-ER-312, Rev. 0

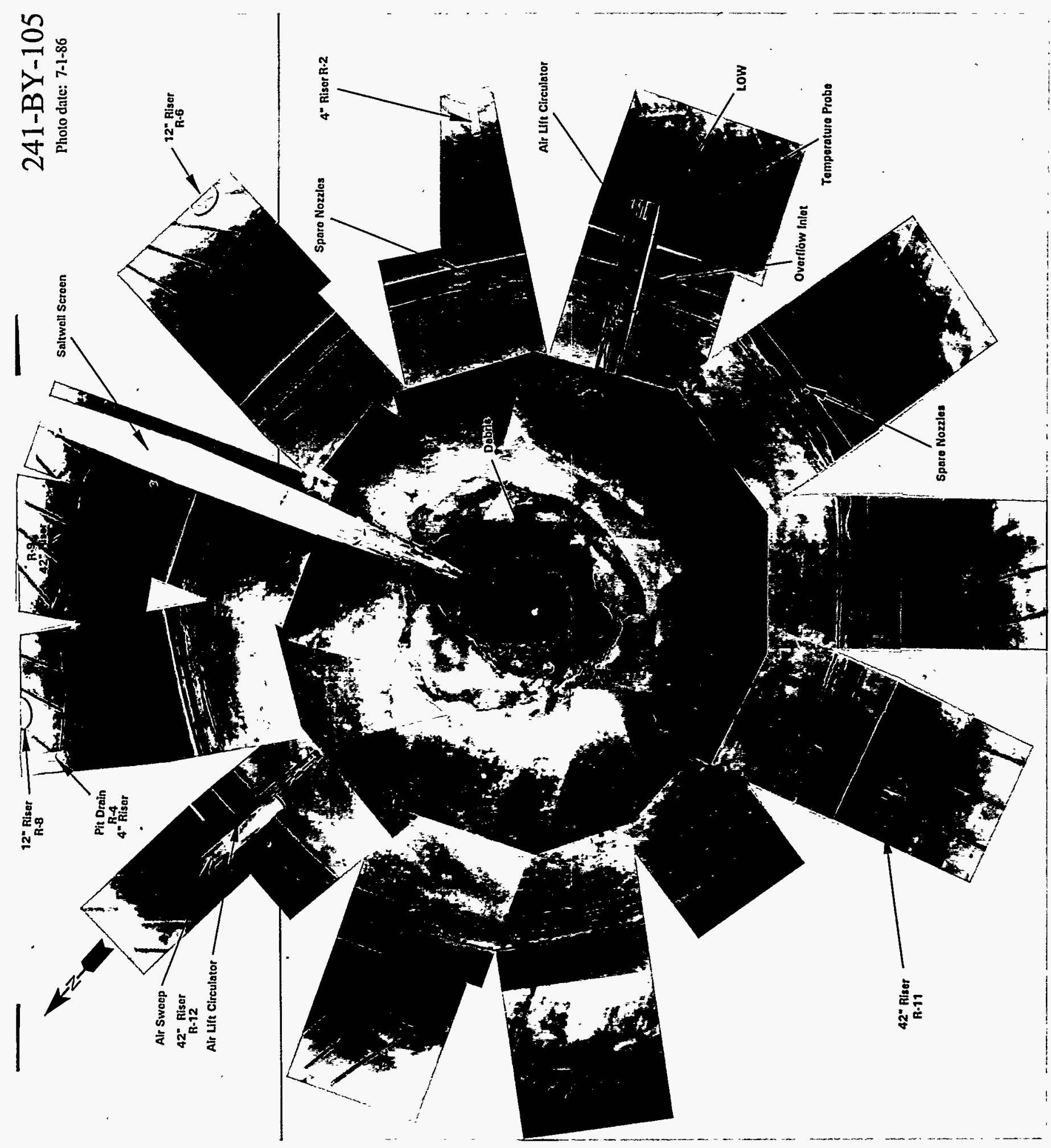


WHC-SD-WM-ER-312, Rev. 0
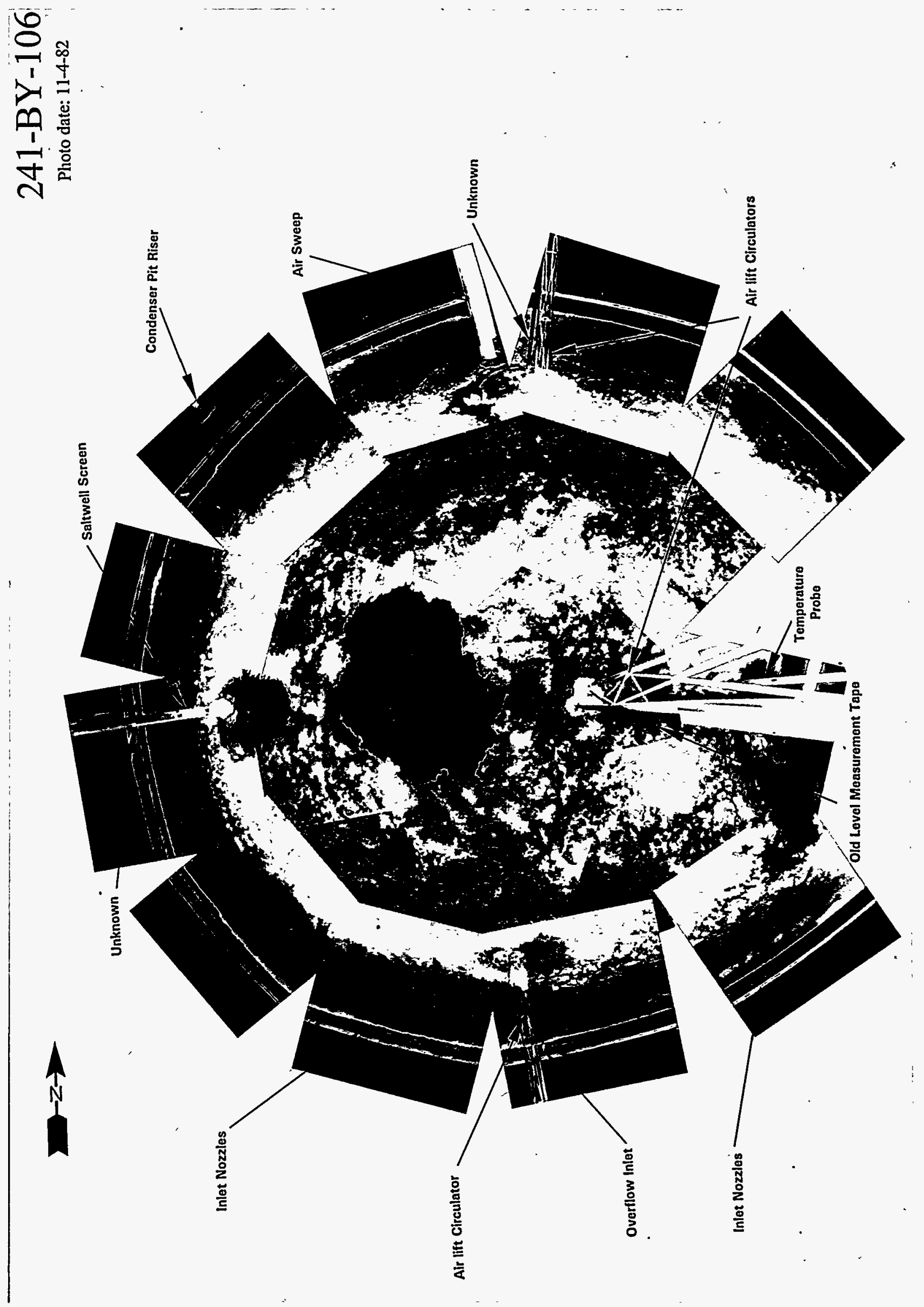


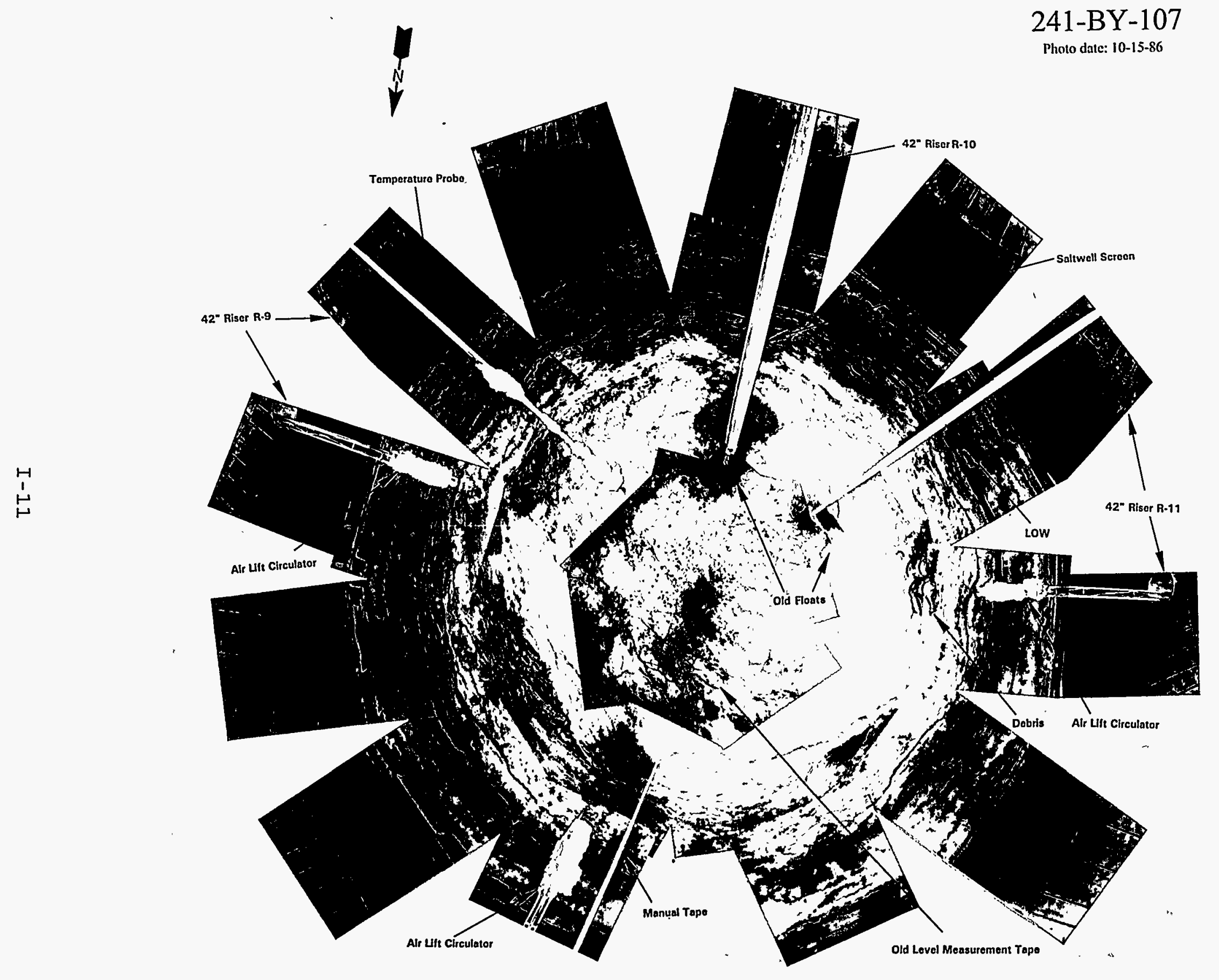




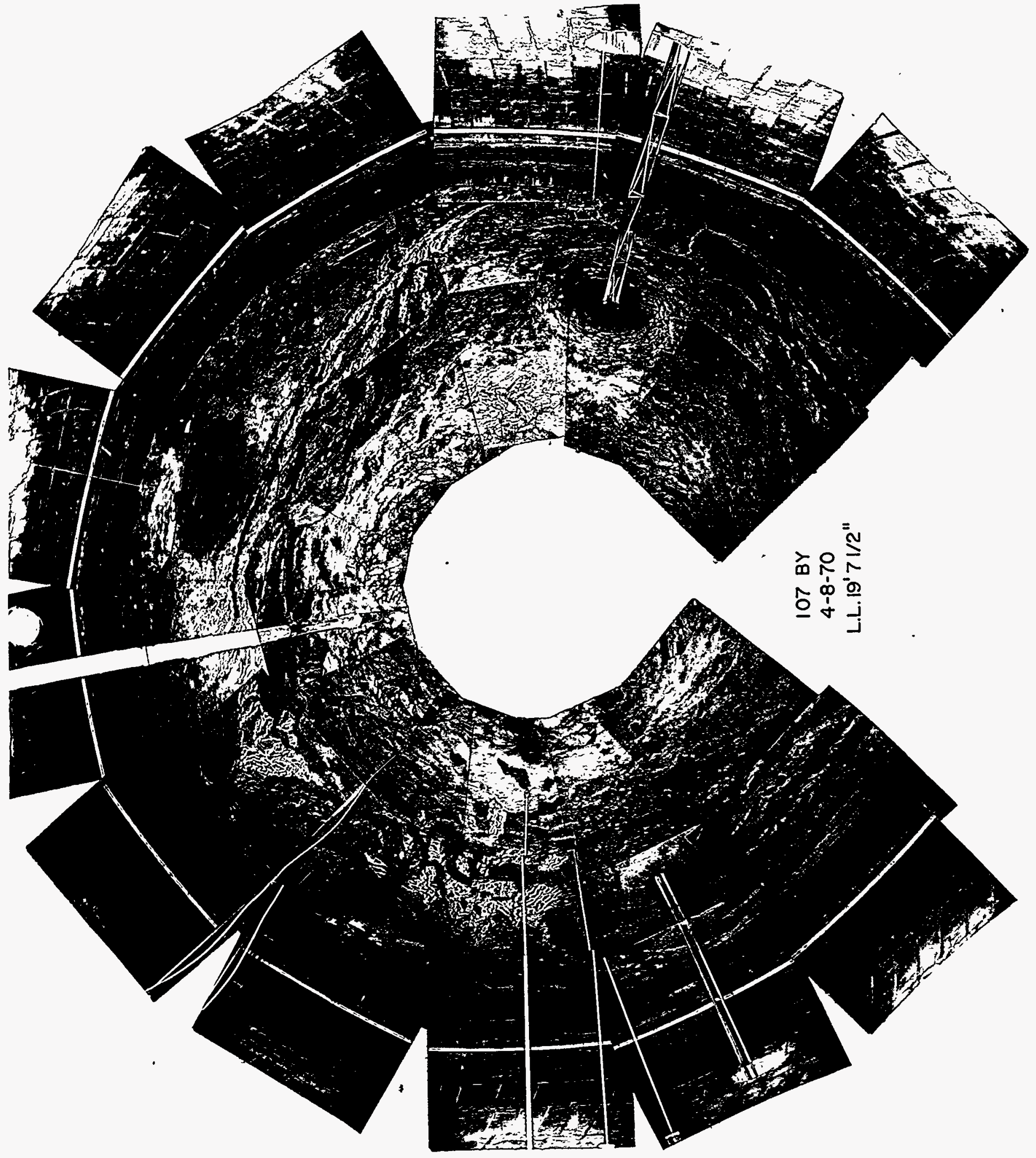




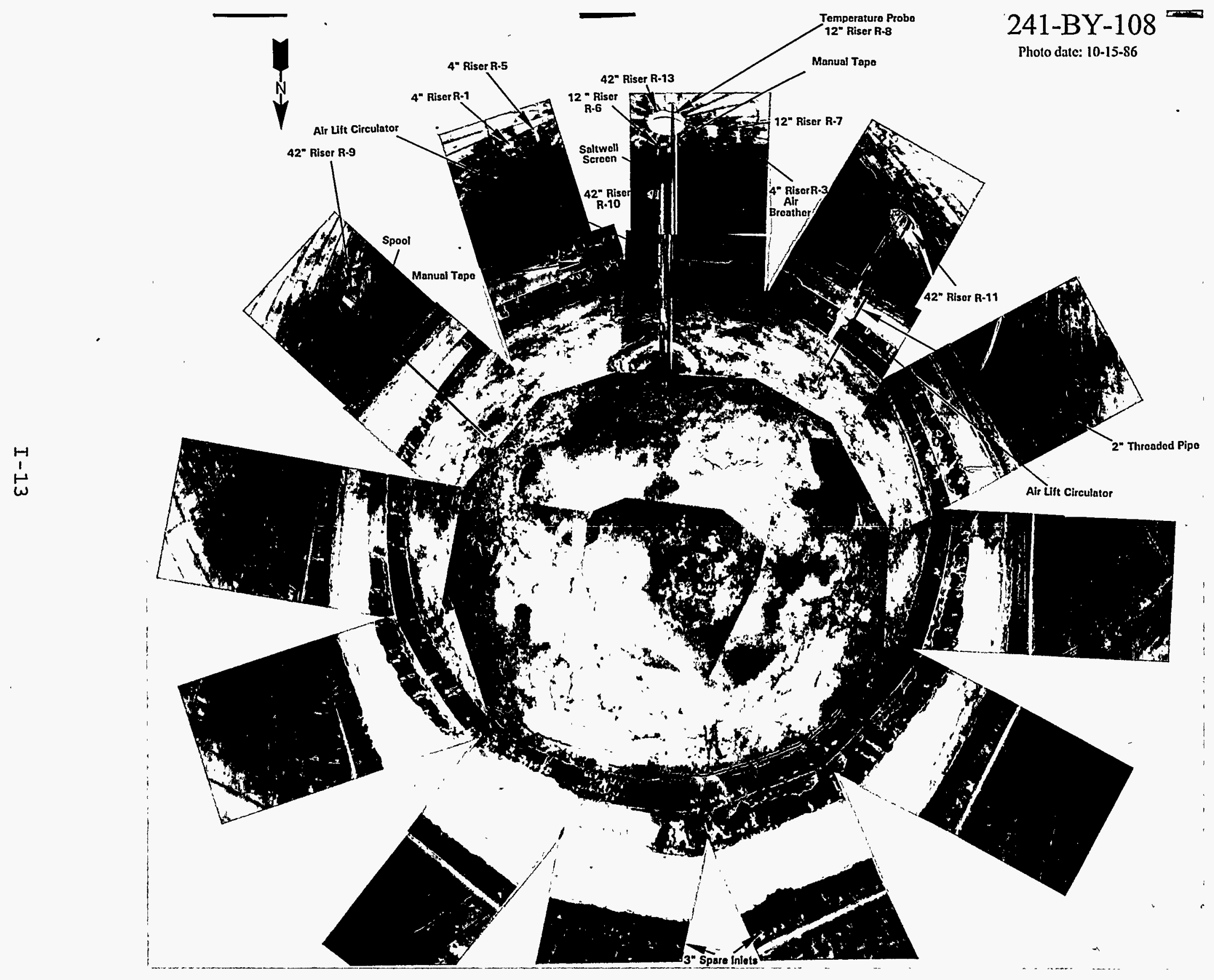


WHC-SD-WM-ER-312, Rev. 0

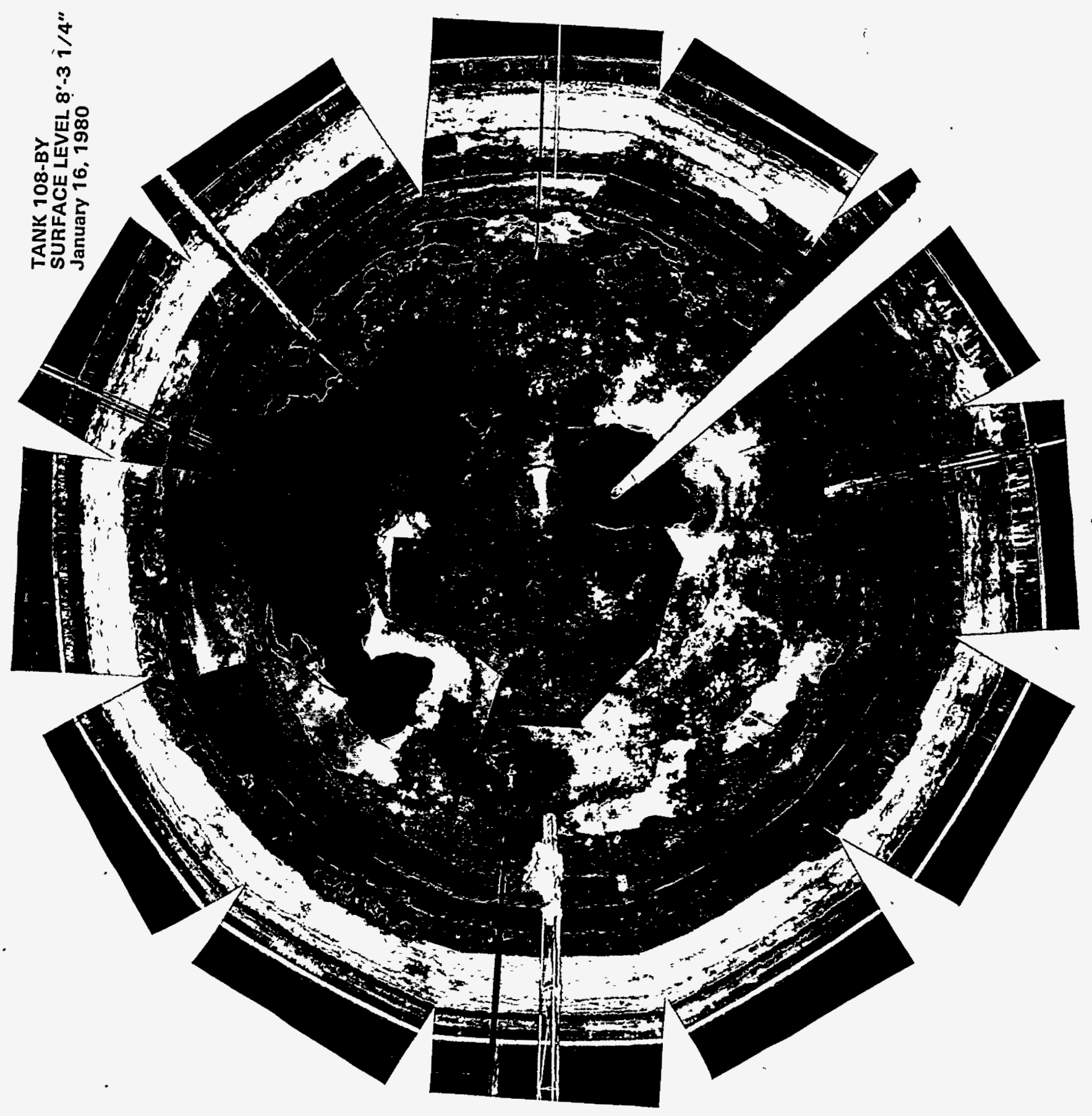




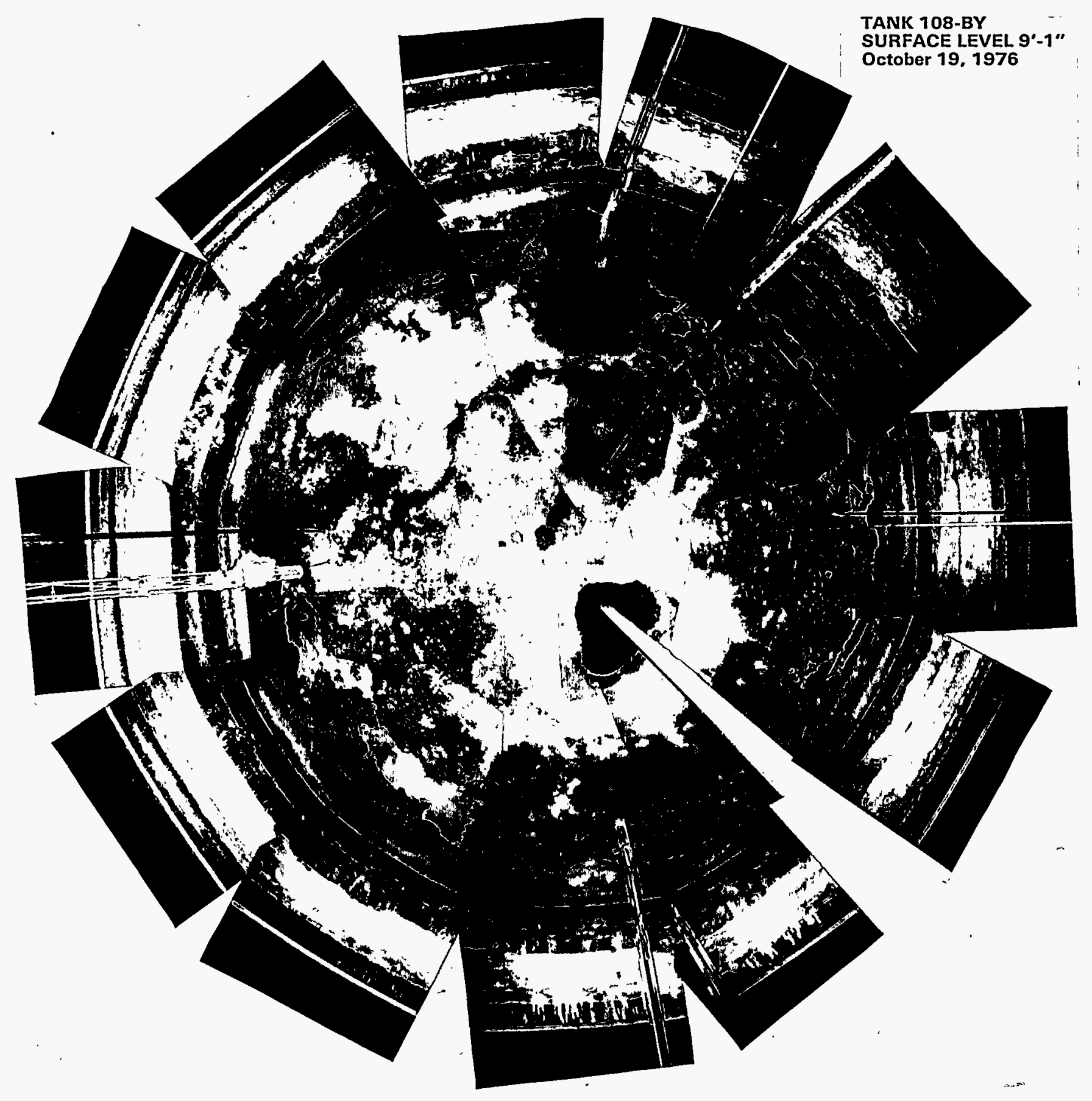




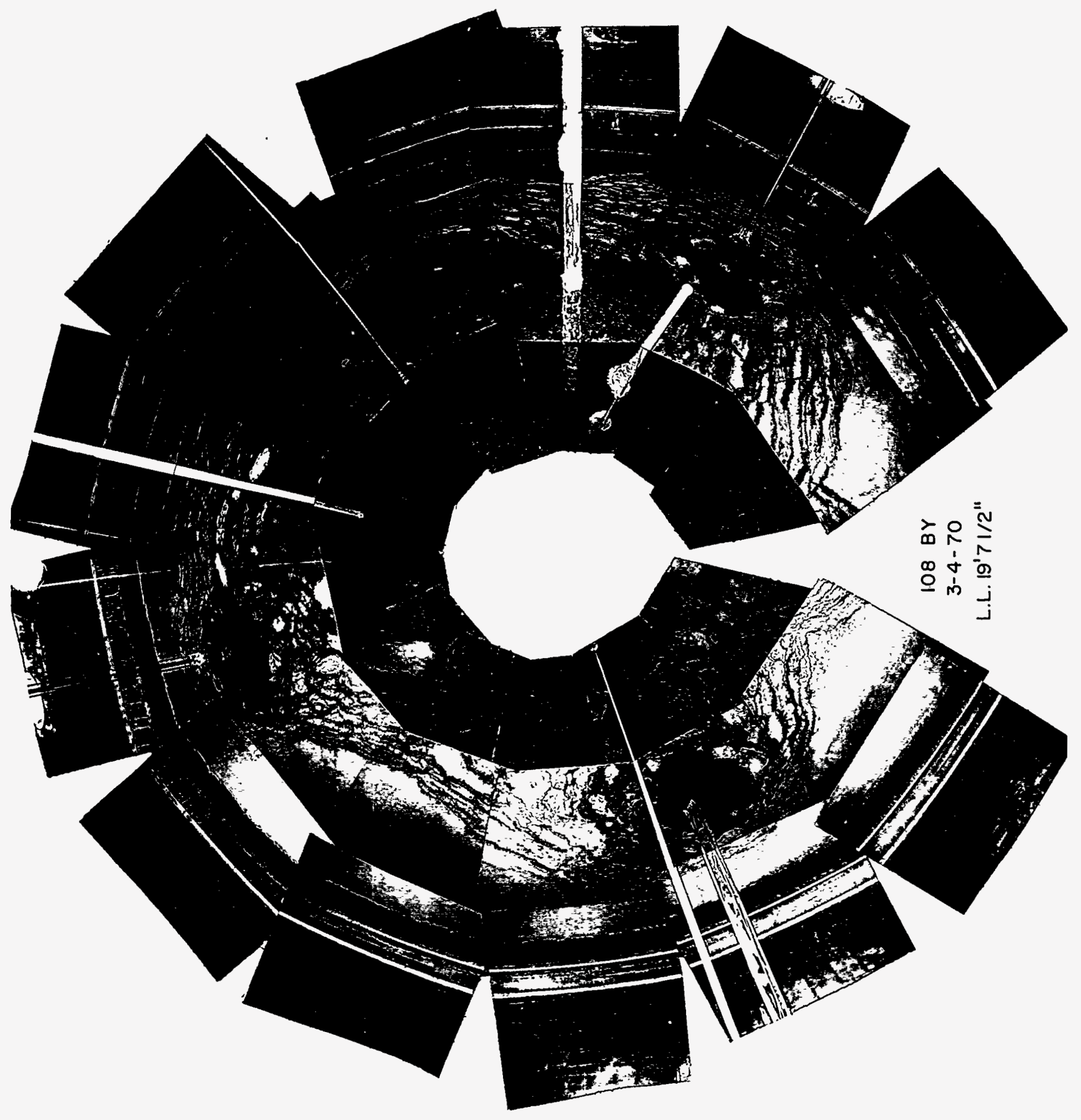




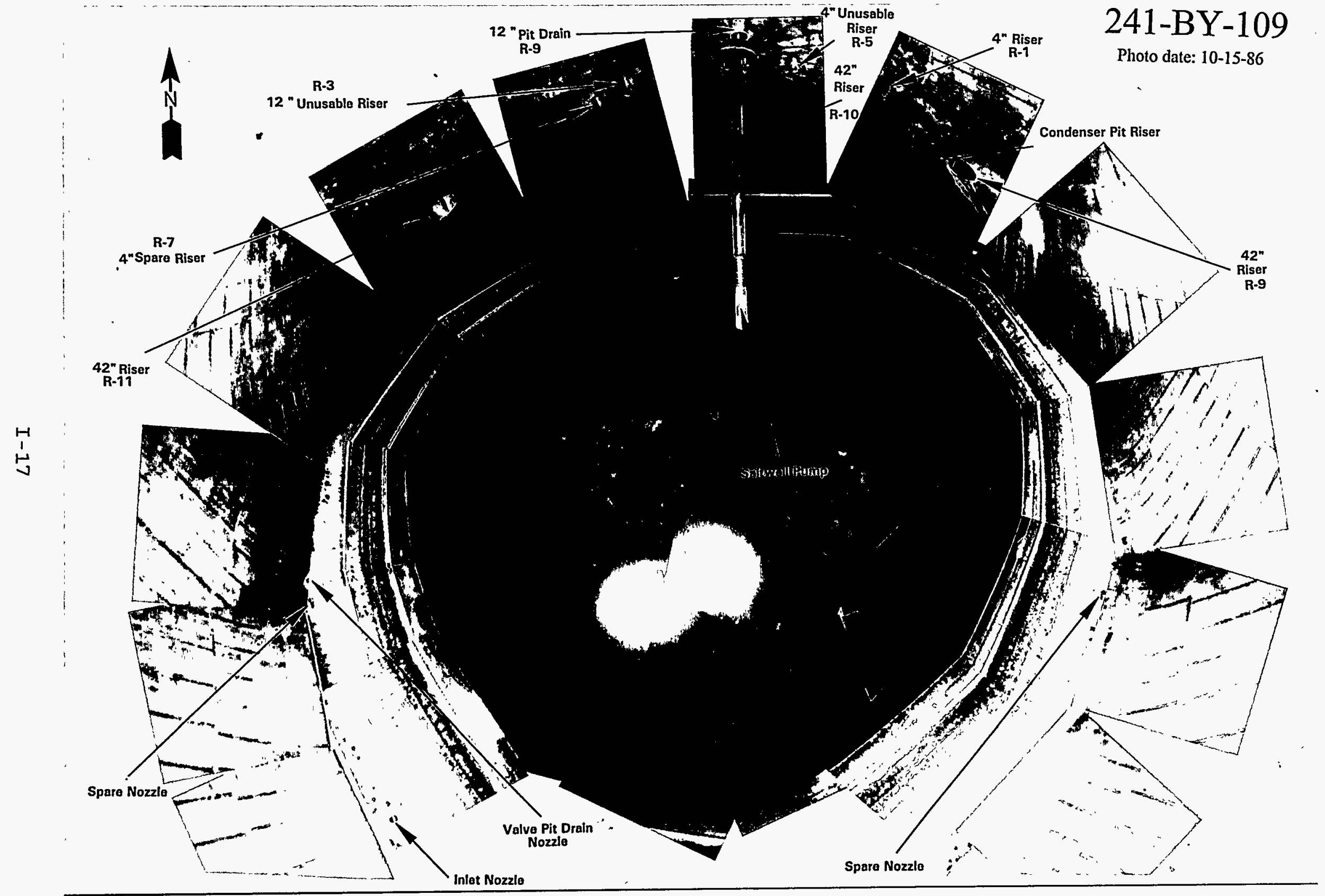


WHC-SD-WM-ER-312，Rev. 0

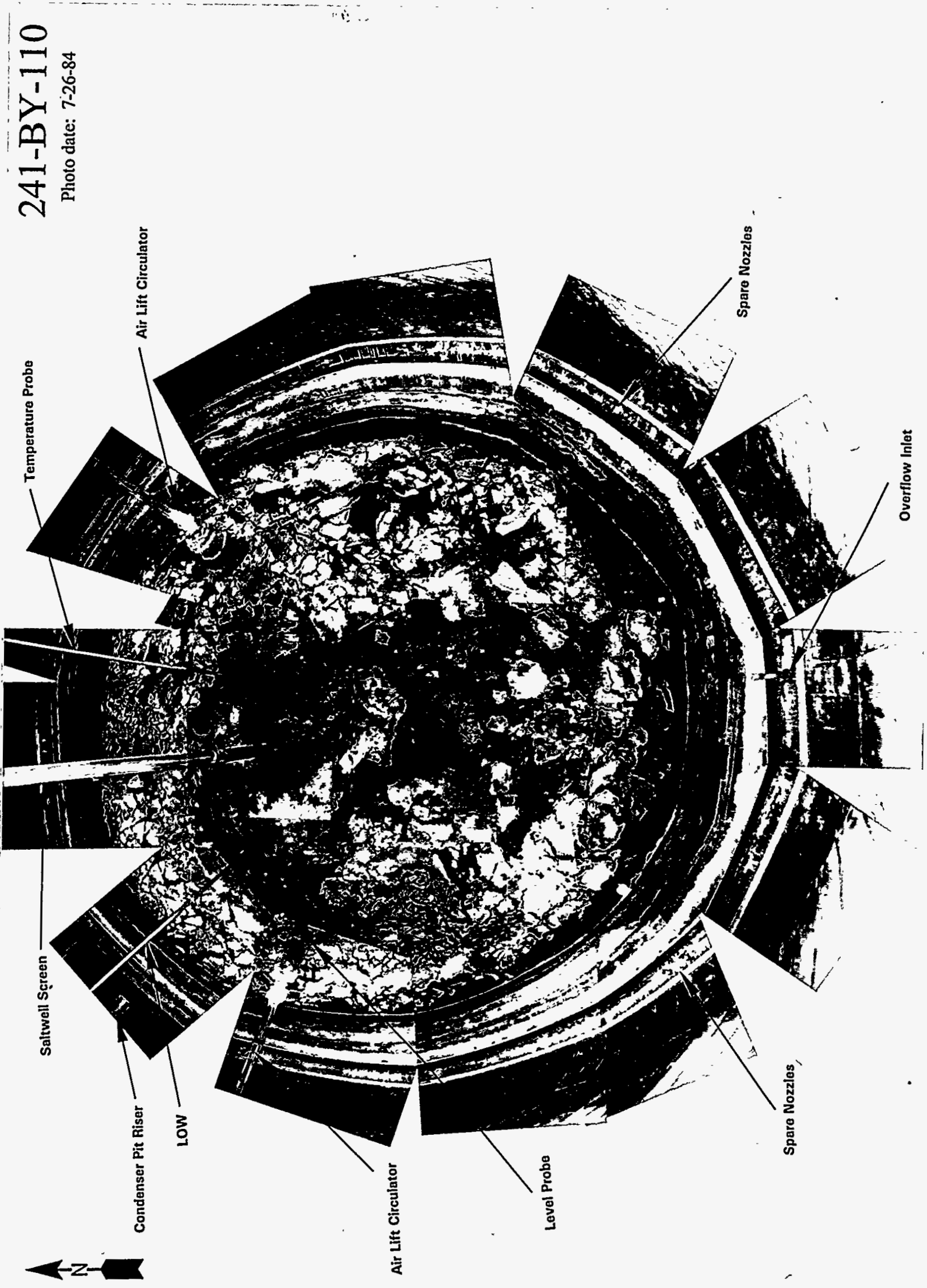


WHC-SD-WM-ER-312, Rev. 0

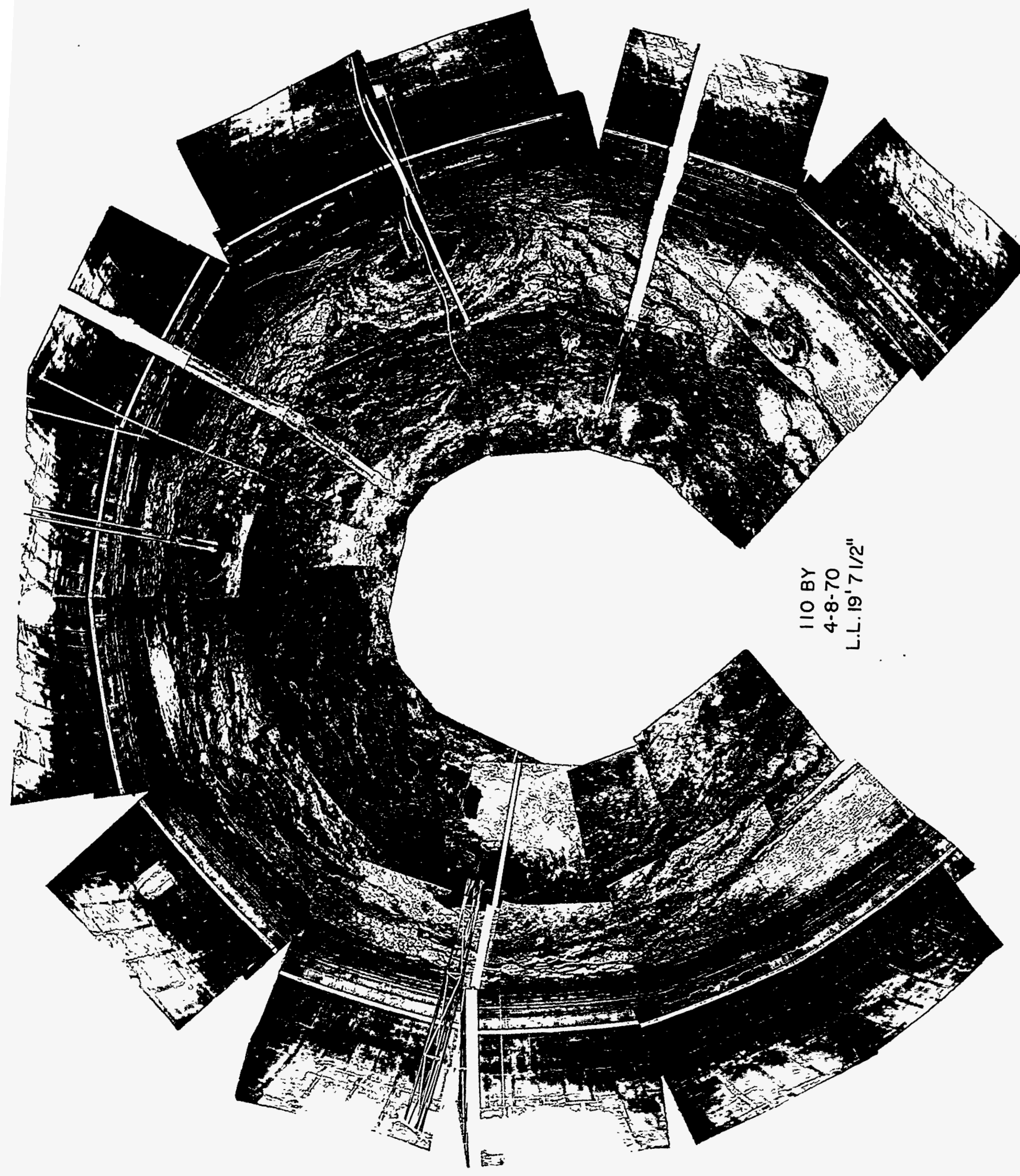




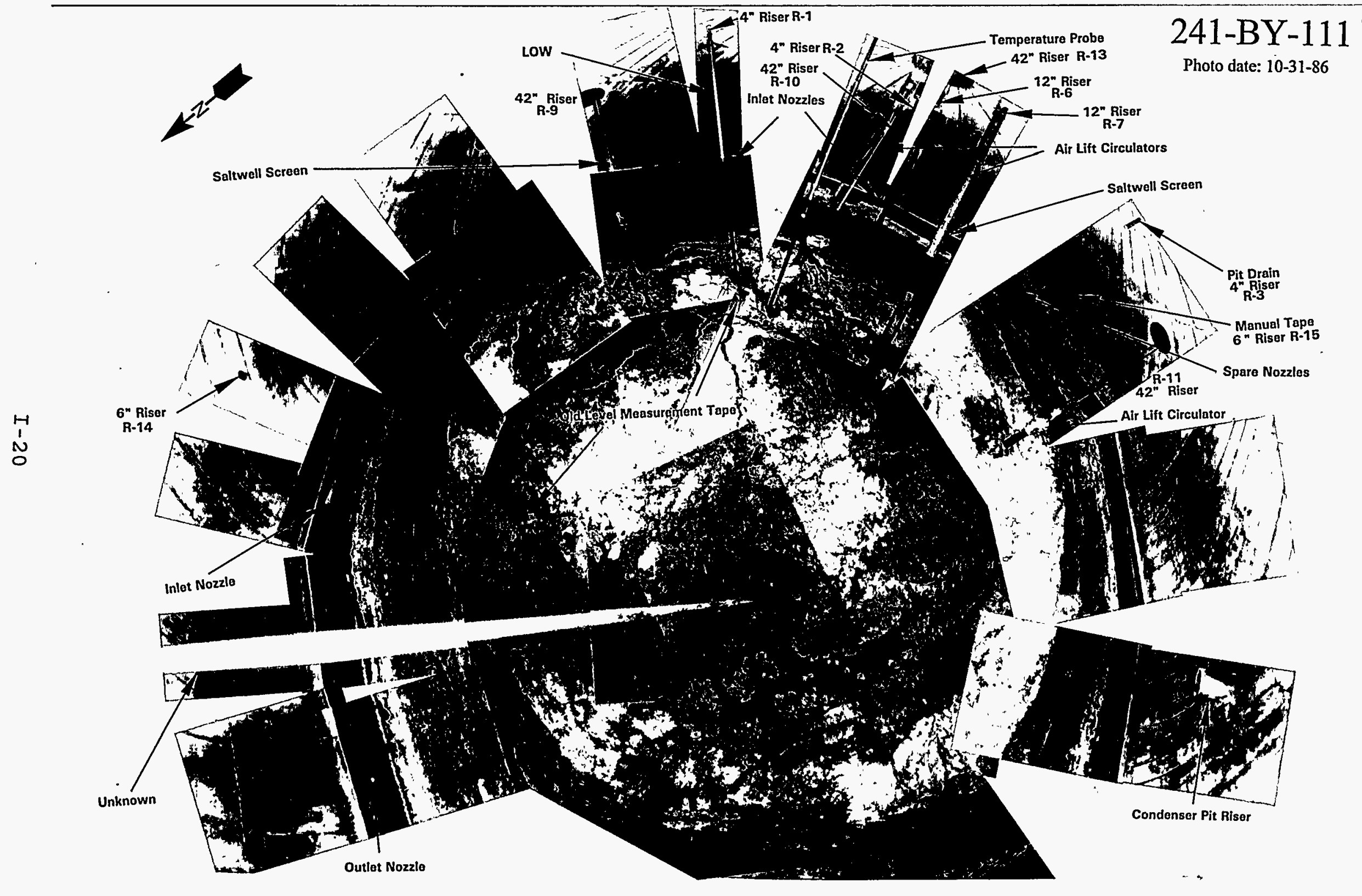




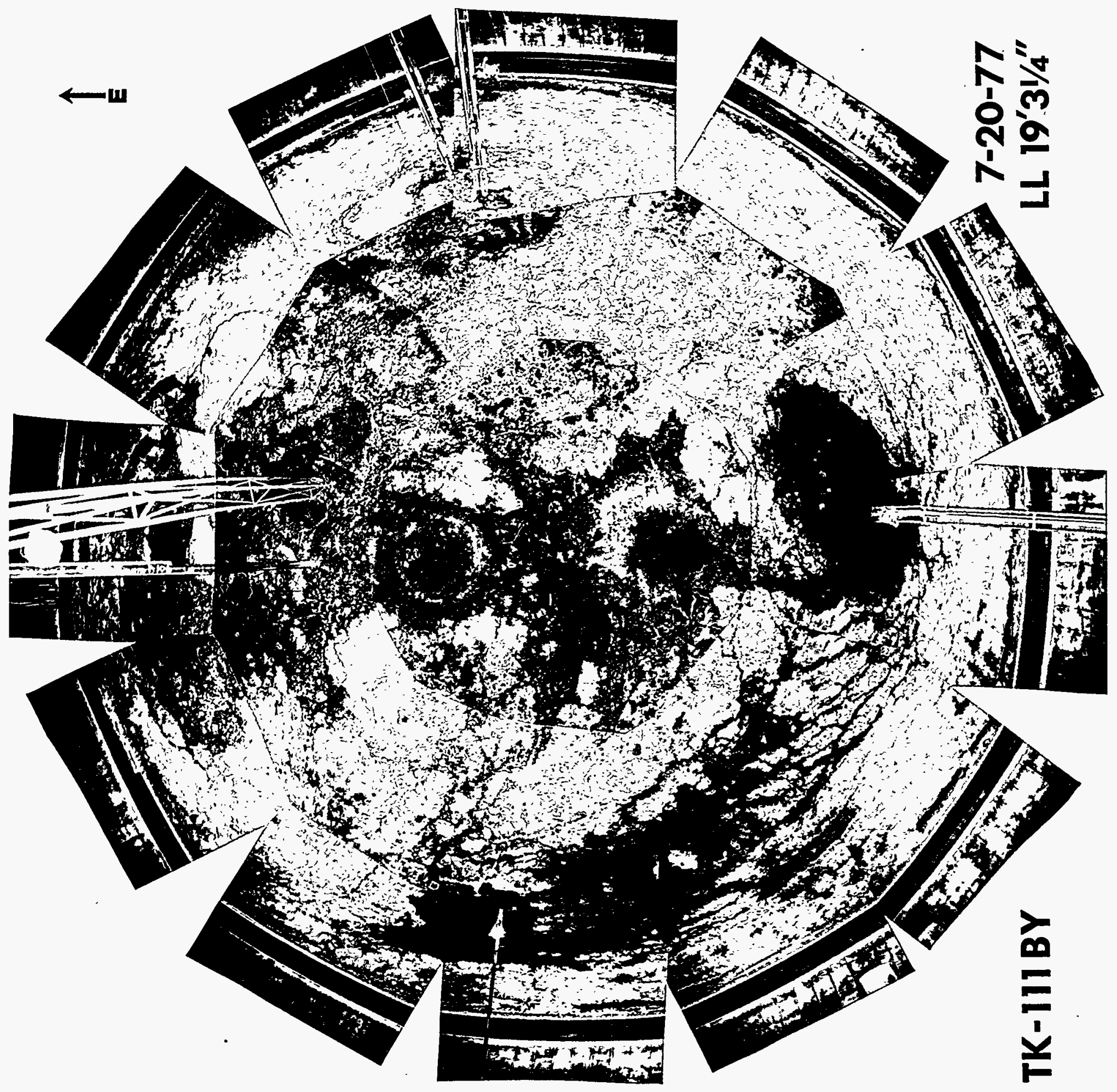




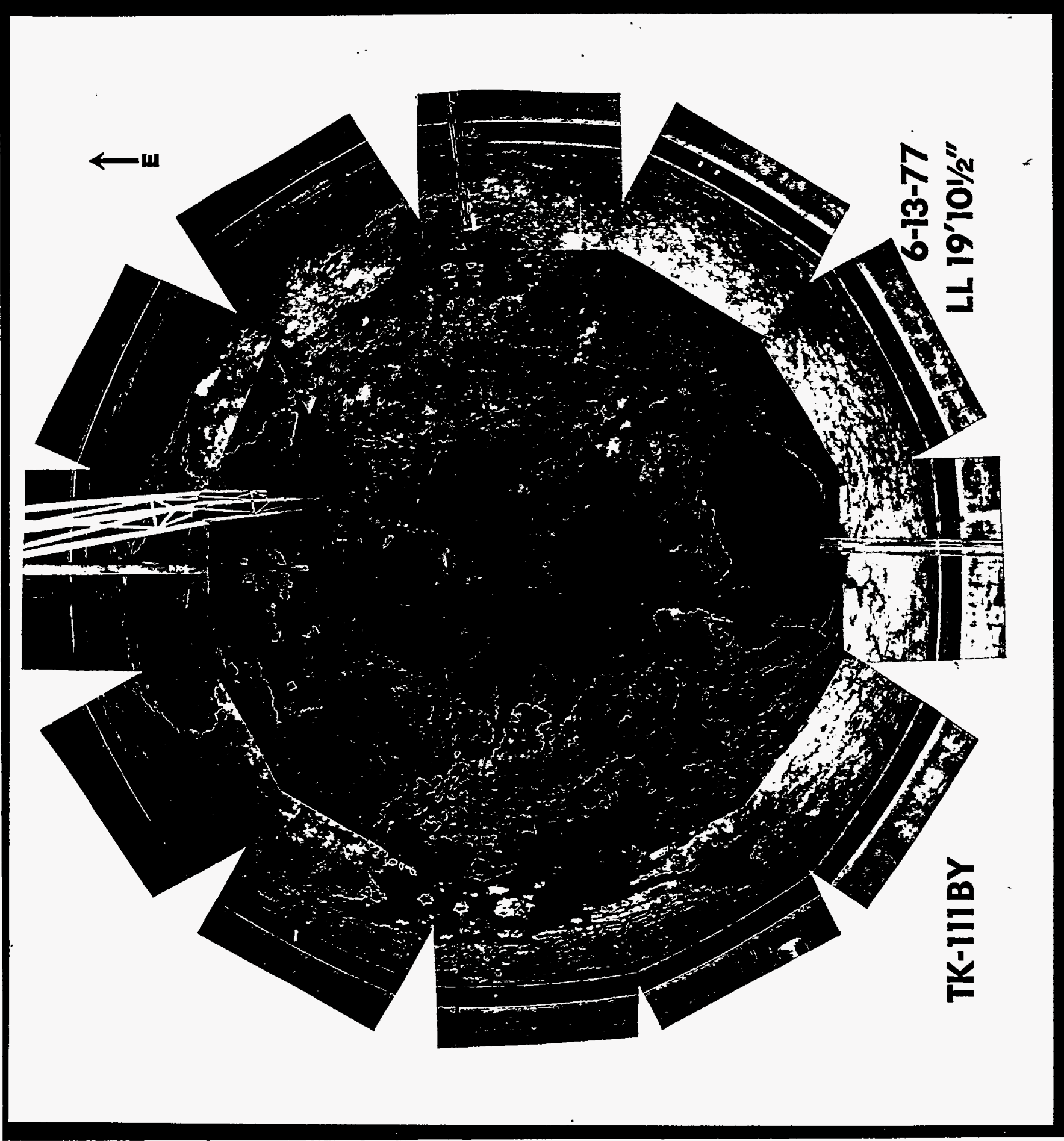


WHC-SD-WM-ER-312, Rev . 0

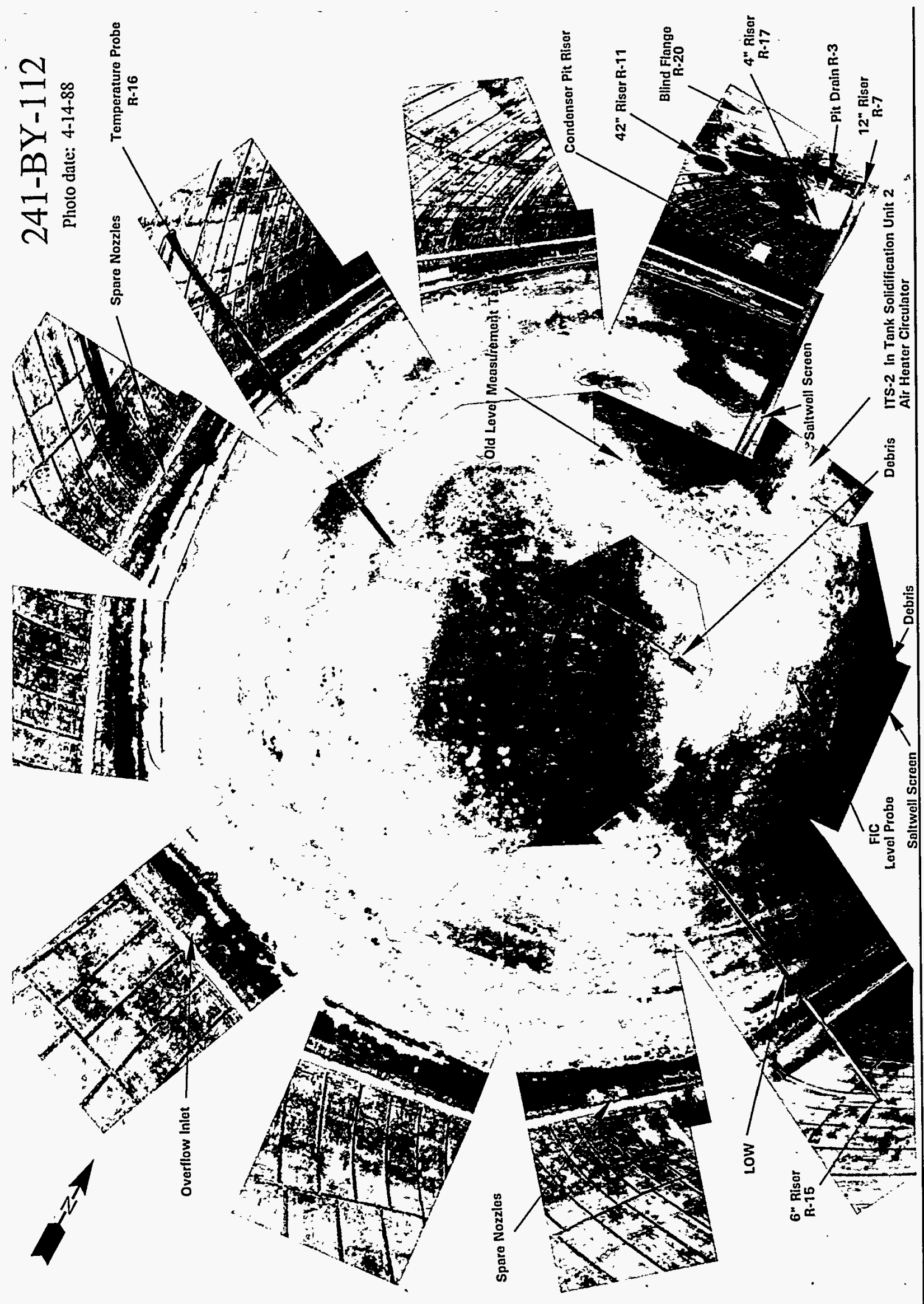




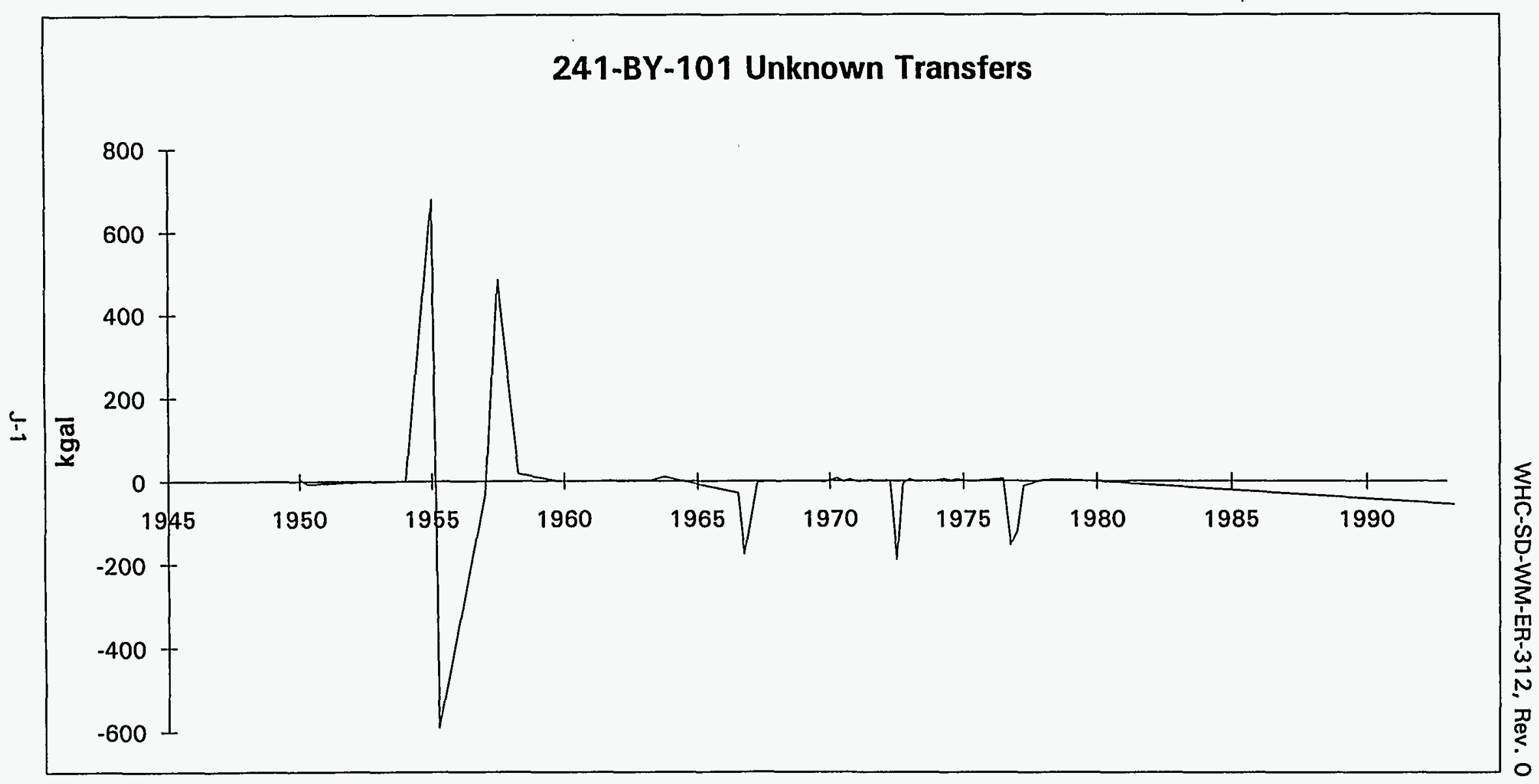

Derived from LANL Waste Status and Transaction Record Summary V2.0b (Agnew 1994) 


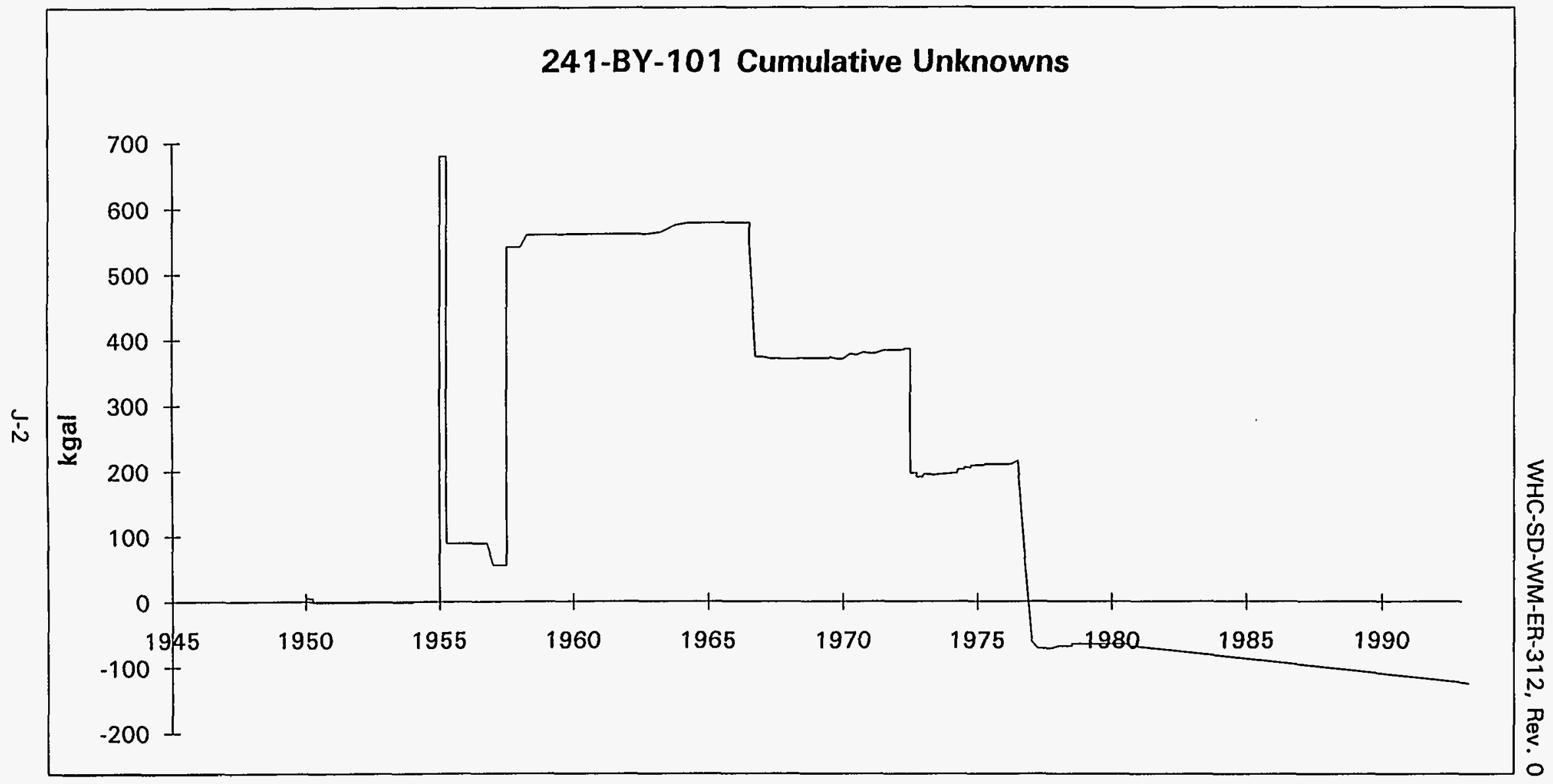

Derived from LANL Waste Status and Transaction Record Summary V2.0b (Agnew 1994) 
WHC-SD-WM-ER-312, Rev. 0

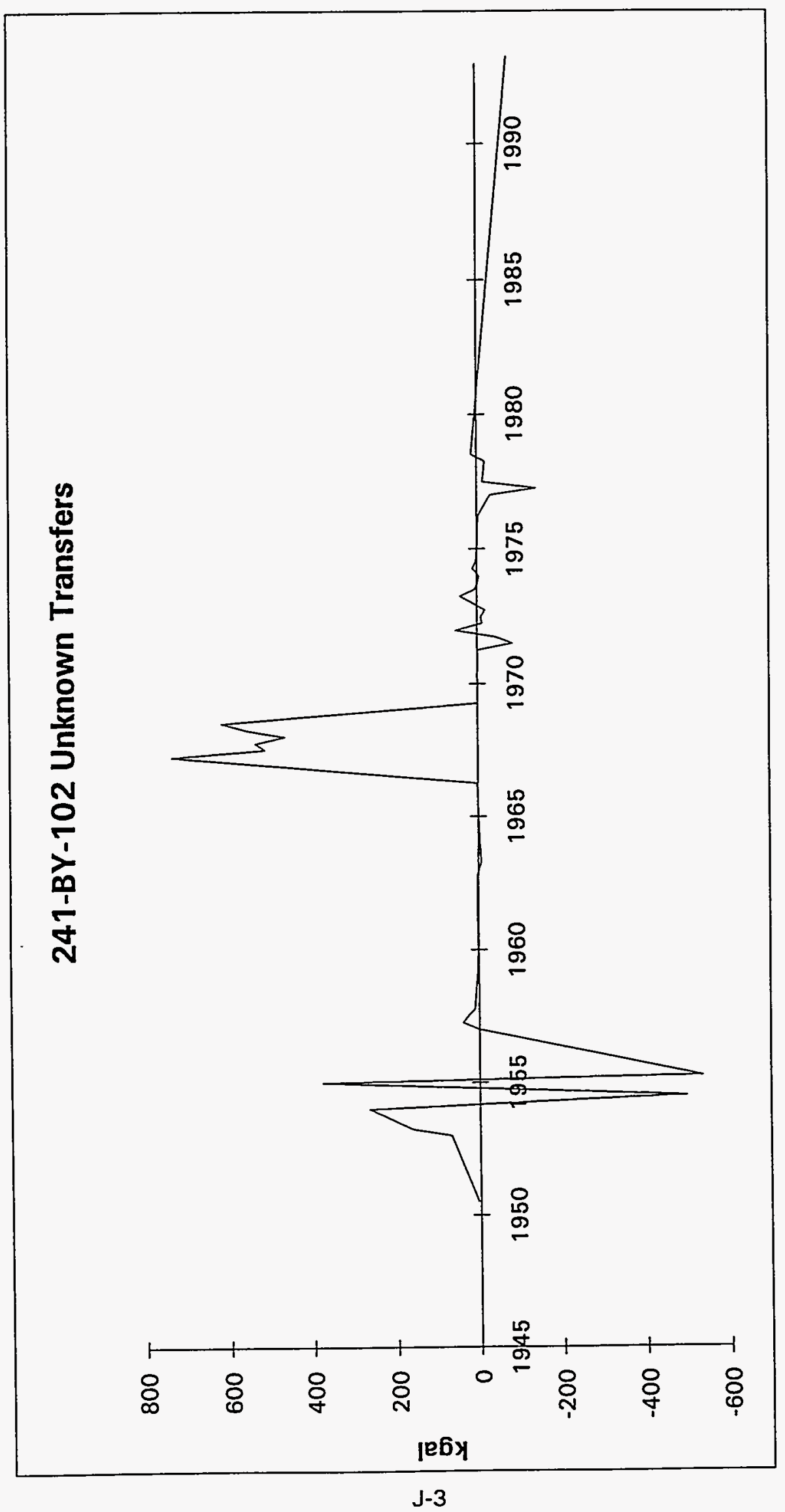

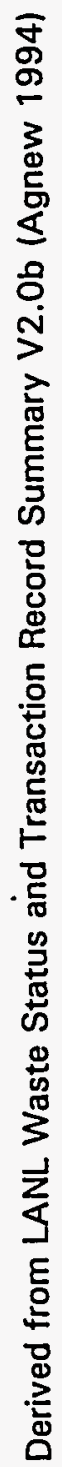




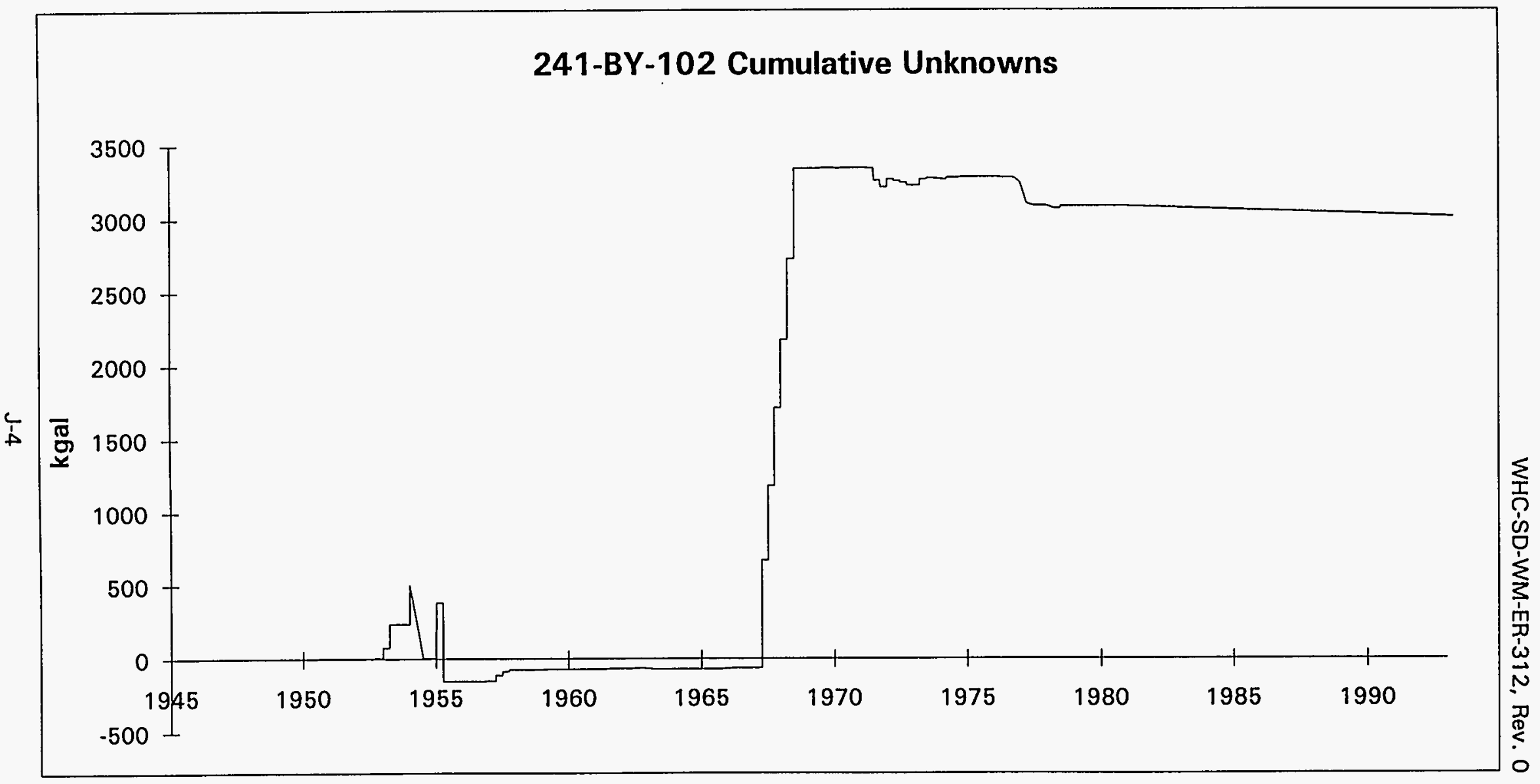


WHC-SD-WM-ER-312, Rev. O

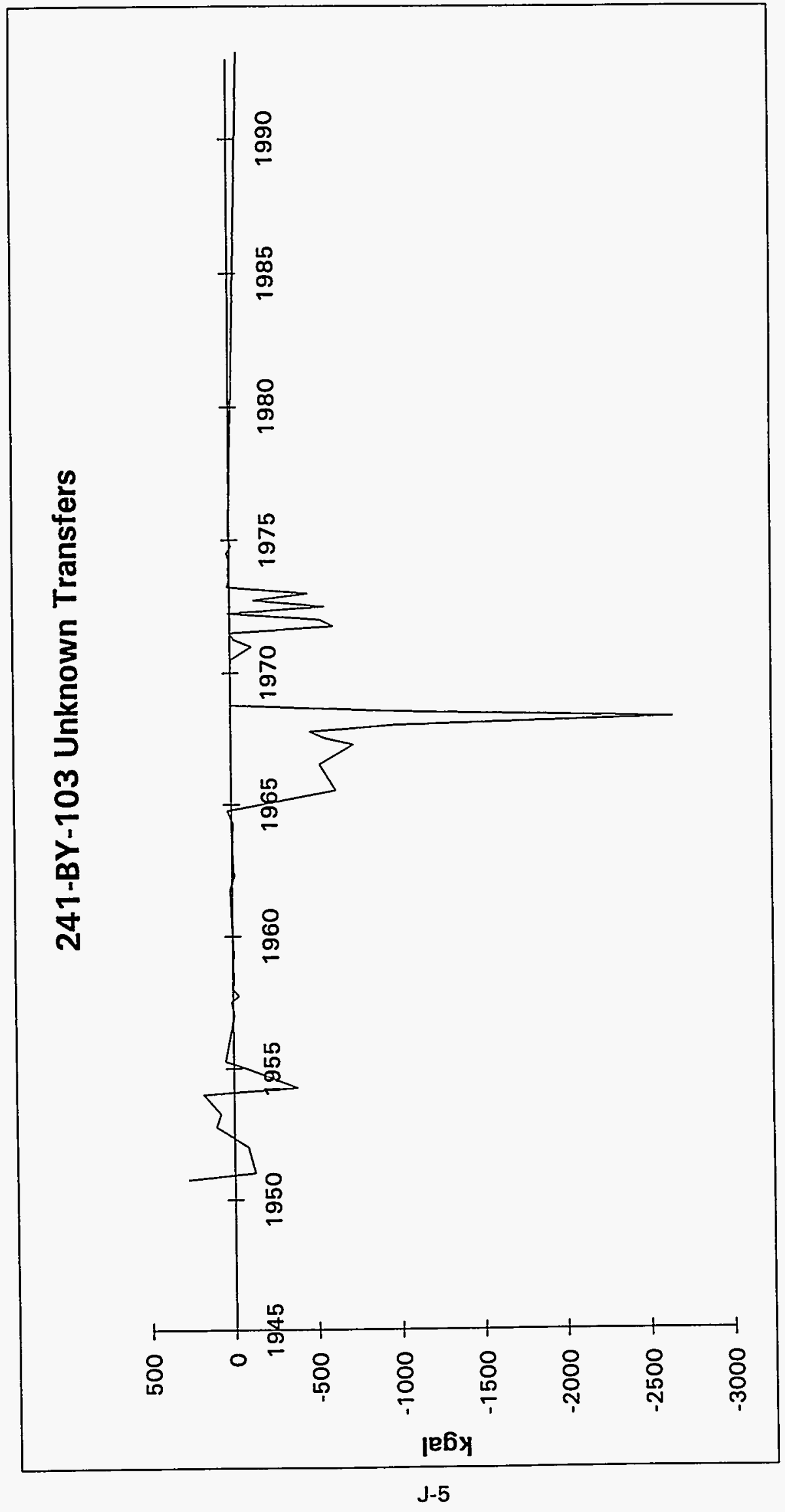

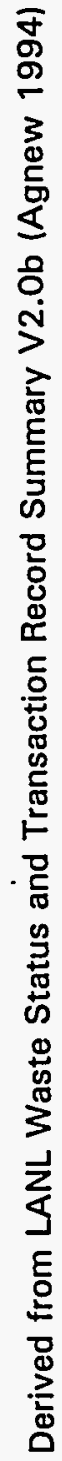




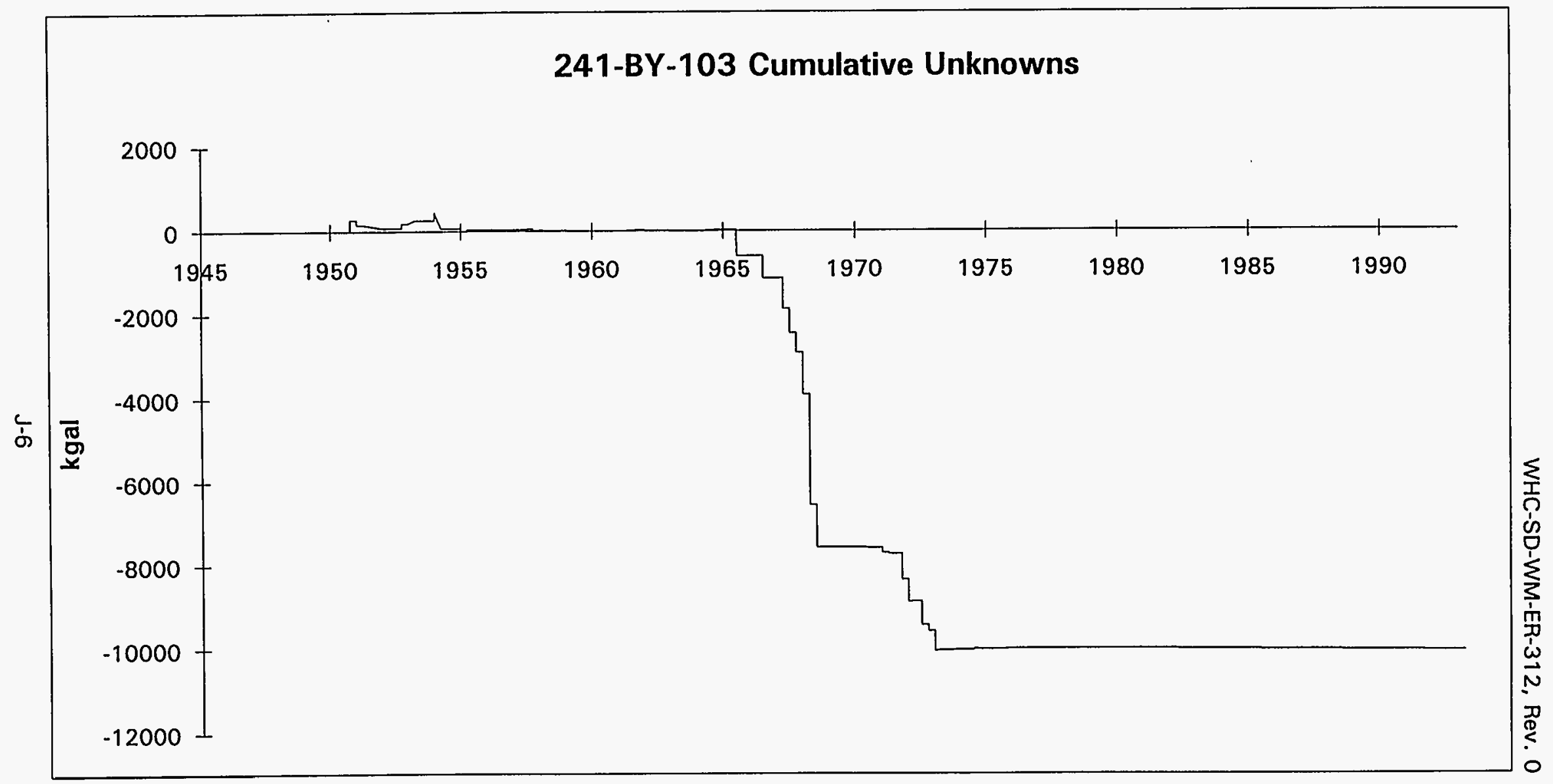

Derived from LANL Waste Status and Transaction Record Summary V2.0b (Agnew 1994) 
WHC-SD-WM-ER-312, Rev. 0

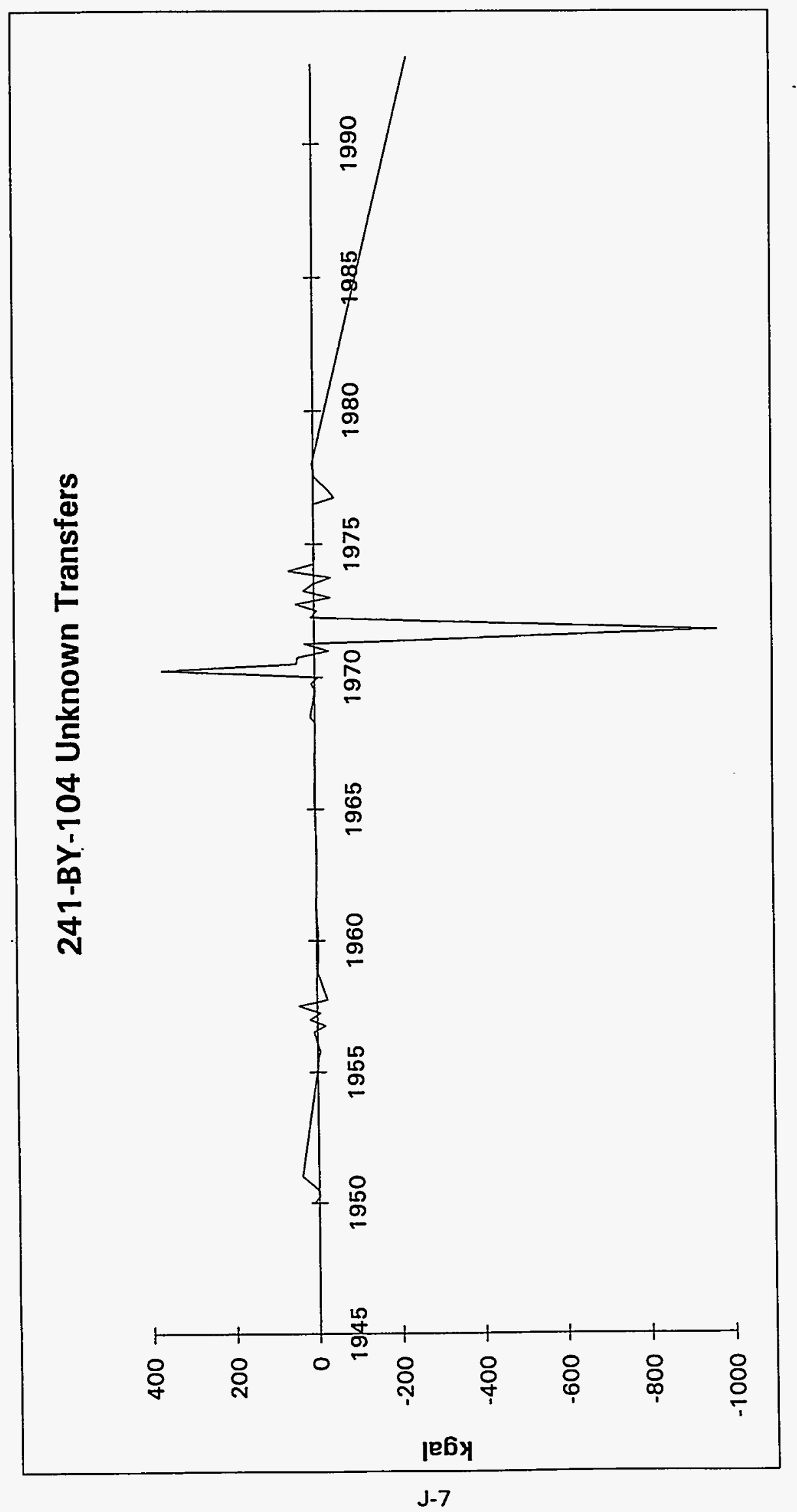

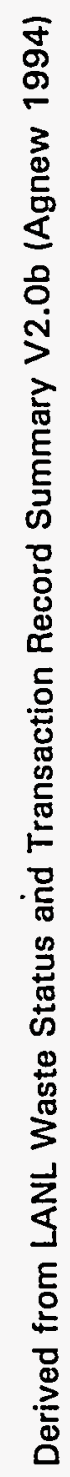


WHC-SD-WM-ER-312, Rev. 0

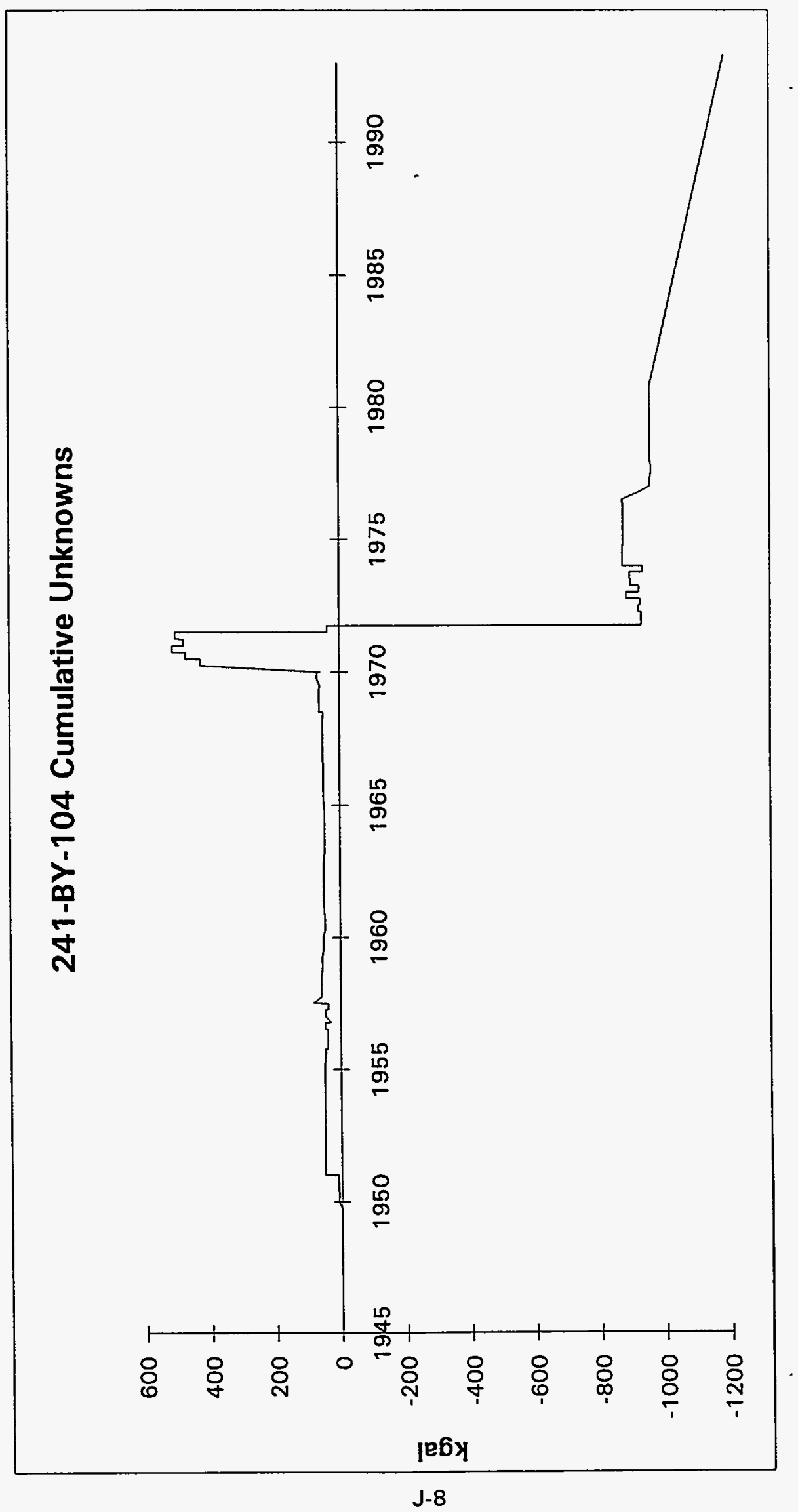

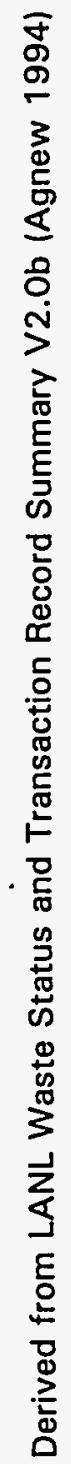


WHC-SD-WM-ER-312, Rev. 0

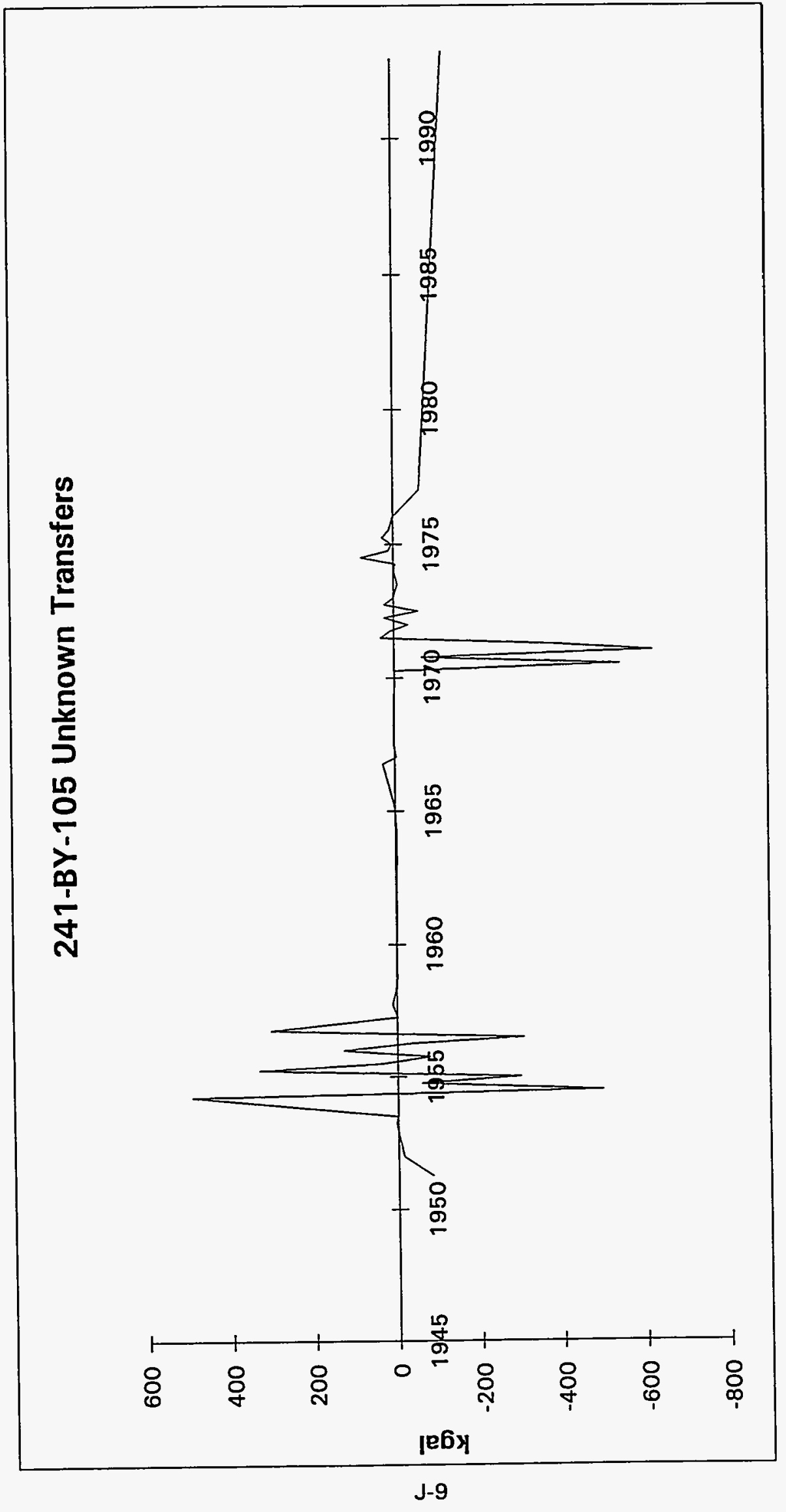


WHC-SD-WM-ER-312, Rev. 0

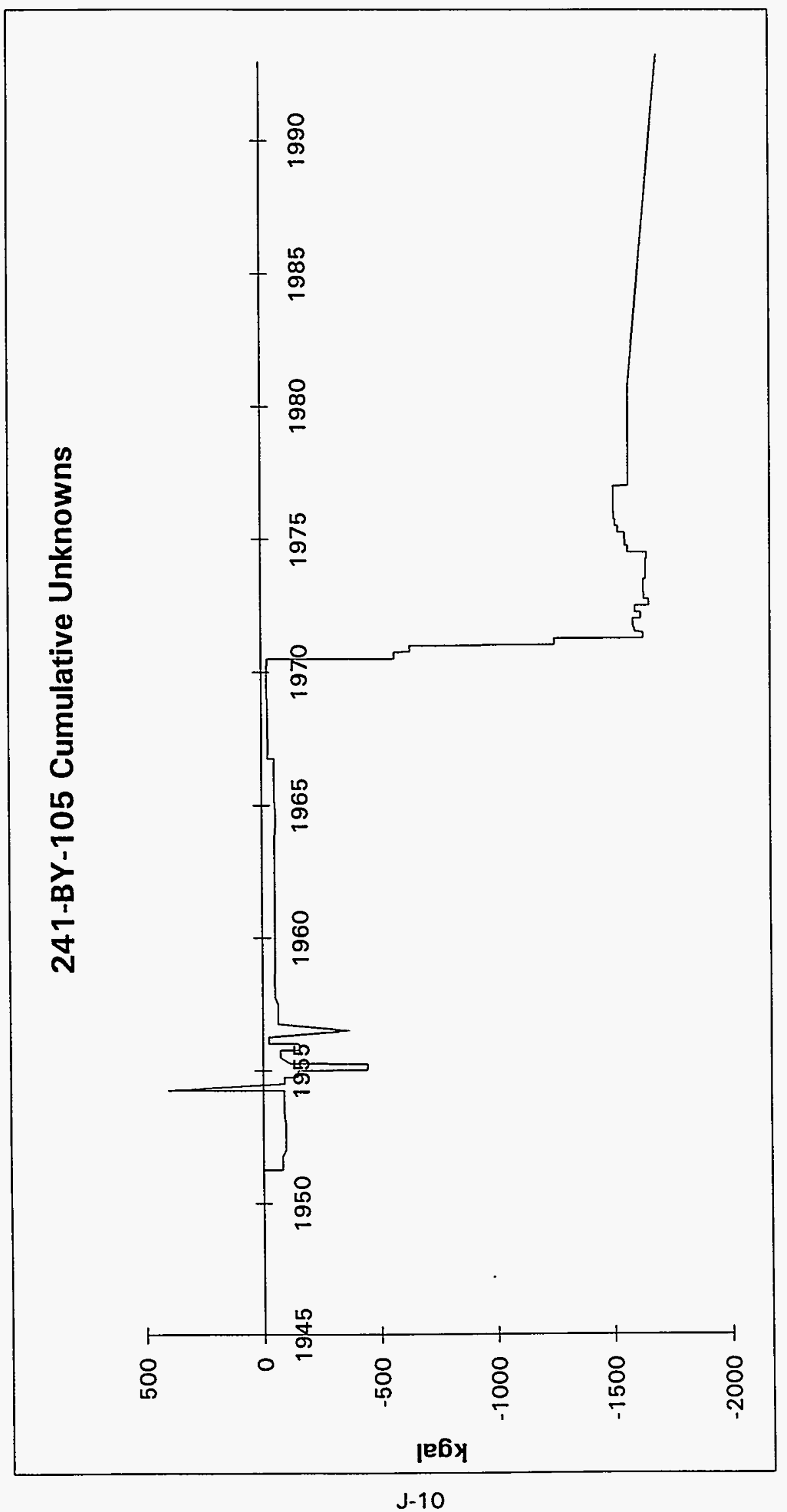

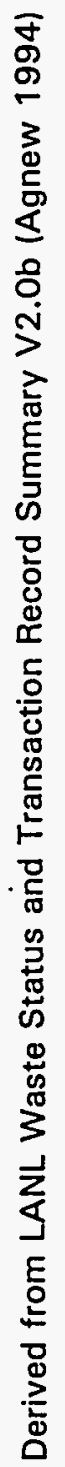


WHC-SD-WM-ER-312, Rev. O

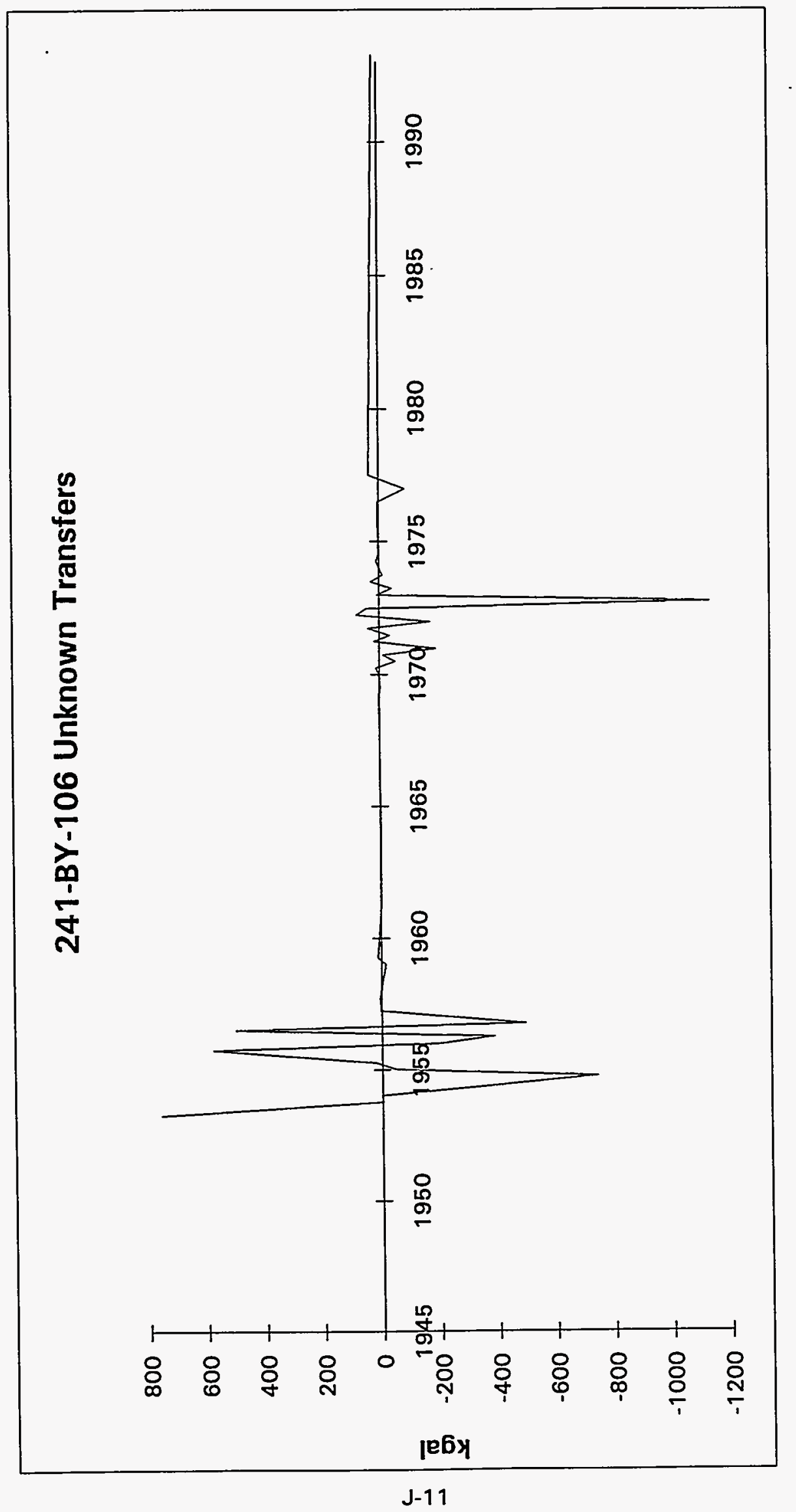

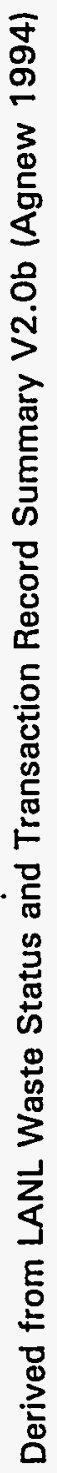




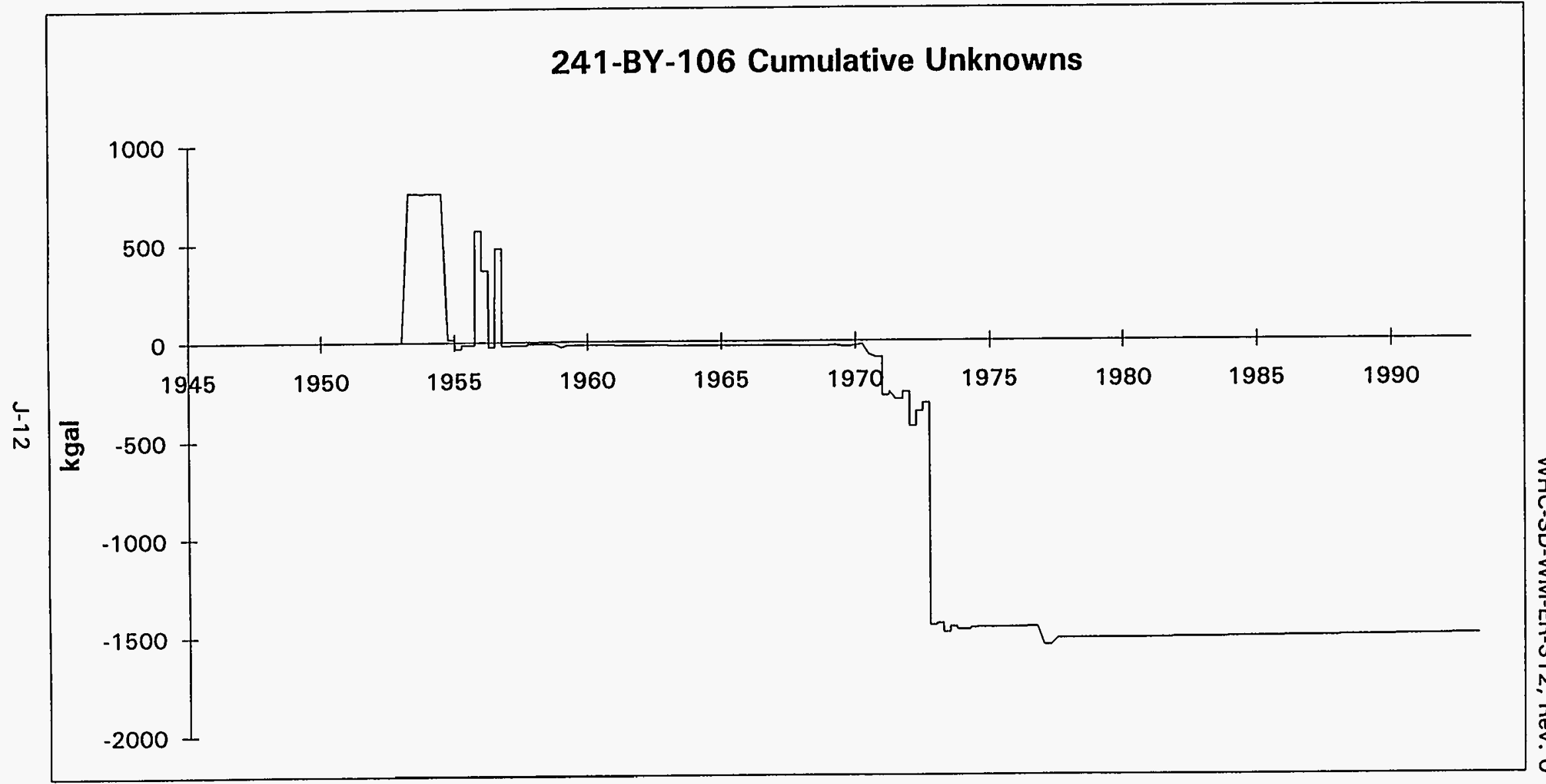

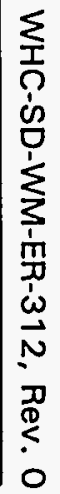


WHC-SD-WM-ER-312, Rev. 0

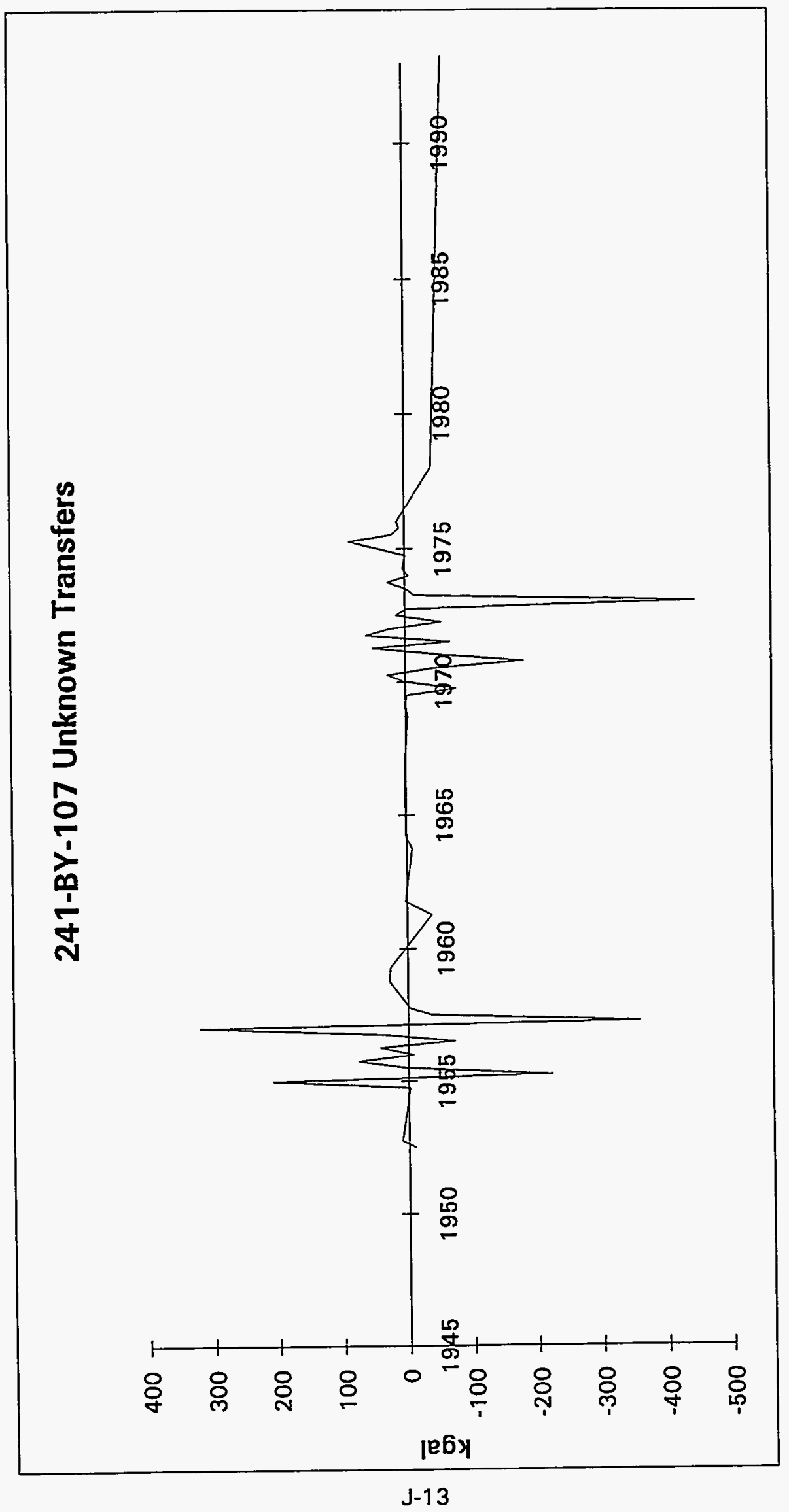

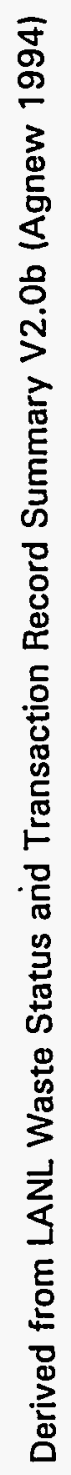




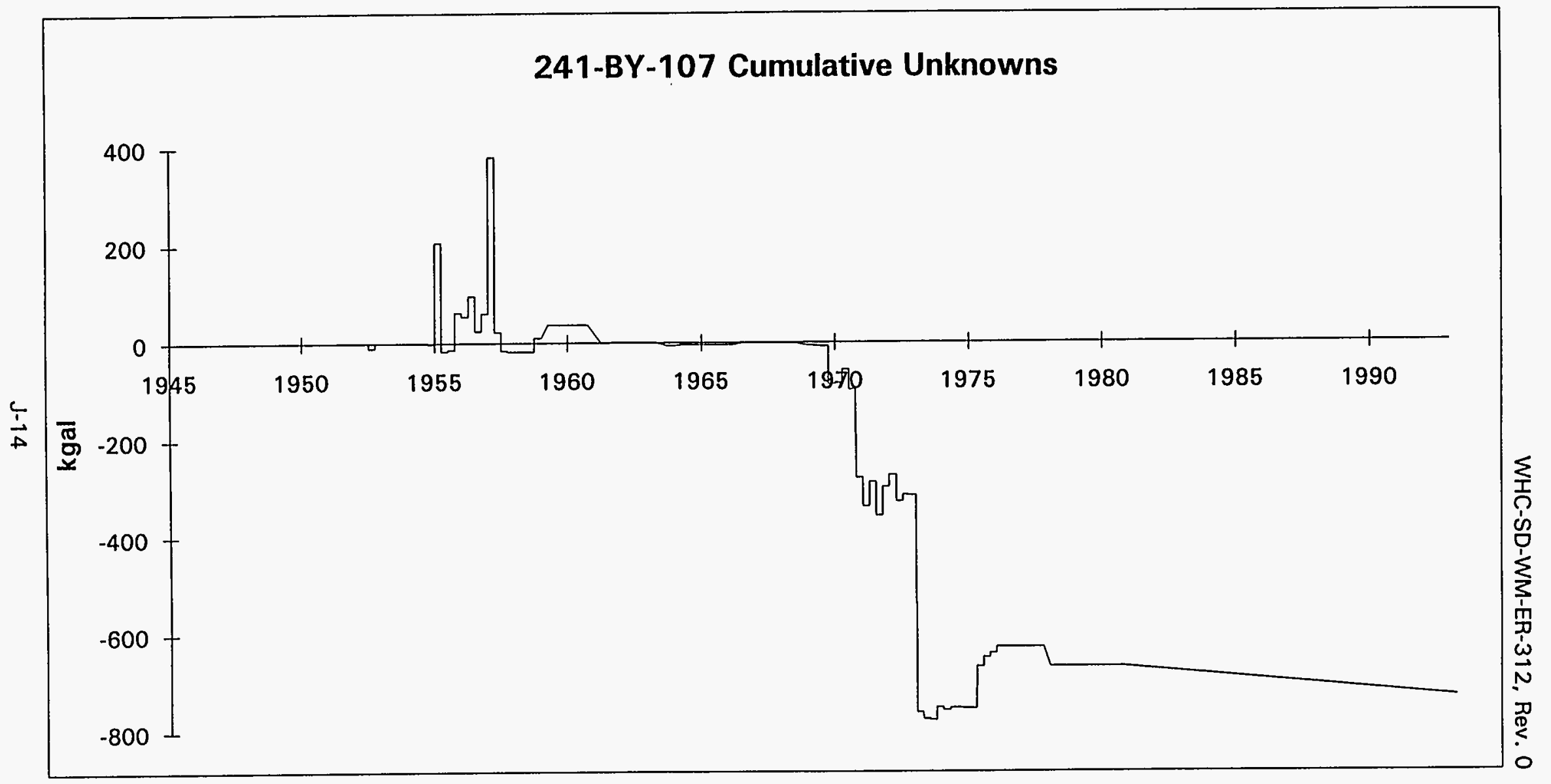

Derived from LANL Waste Status and Transaction Record Summary V2.0b (Agnew 1994) 


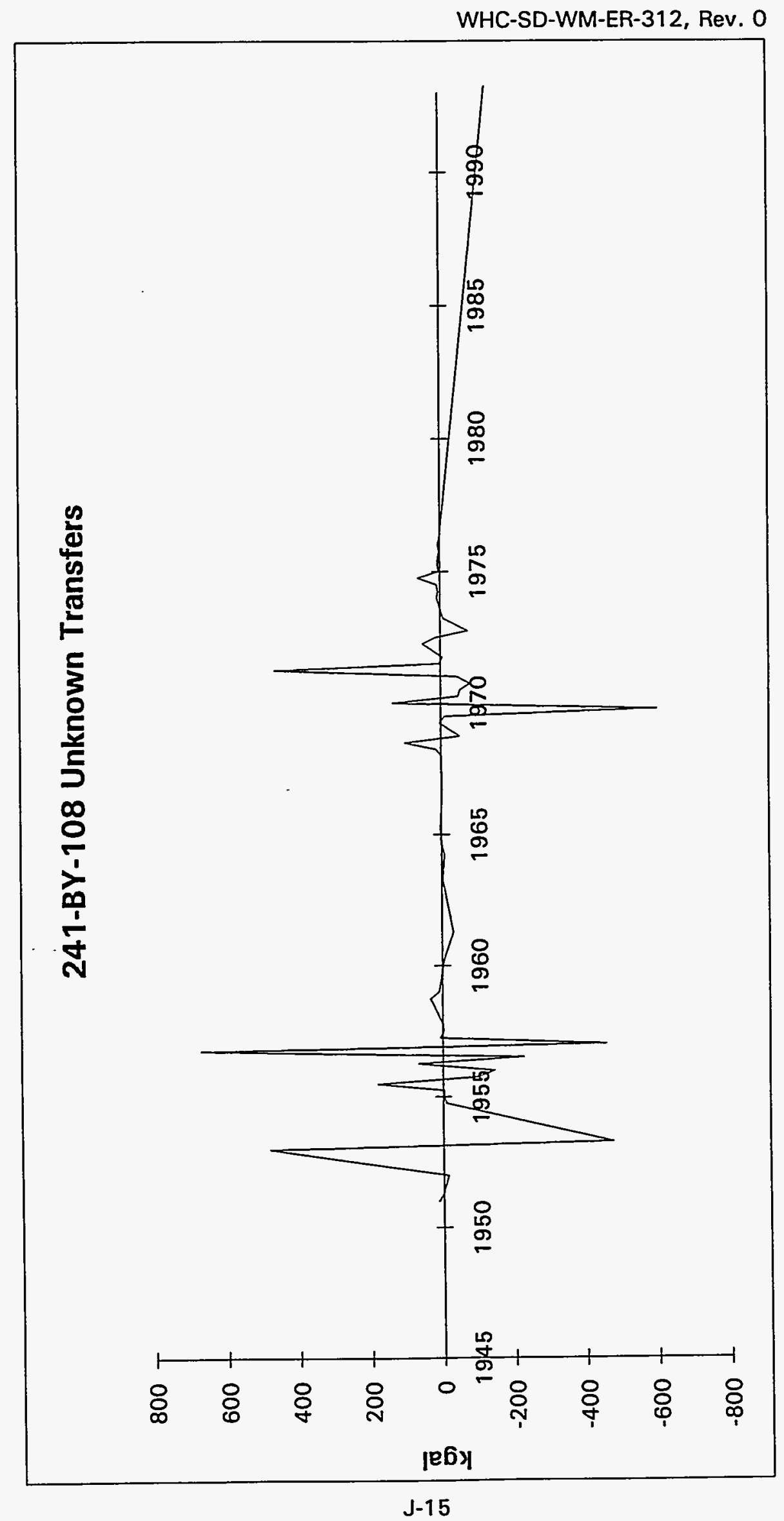

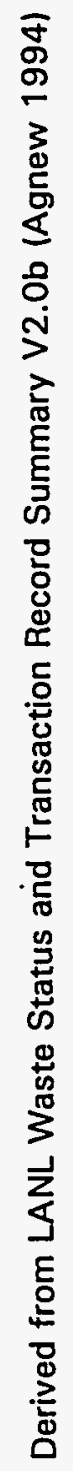




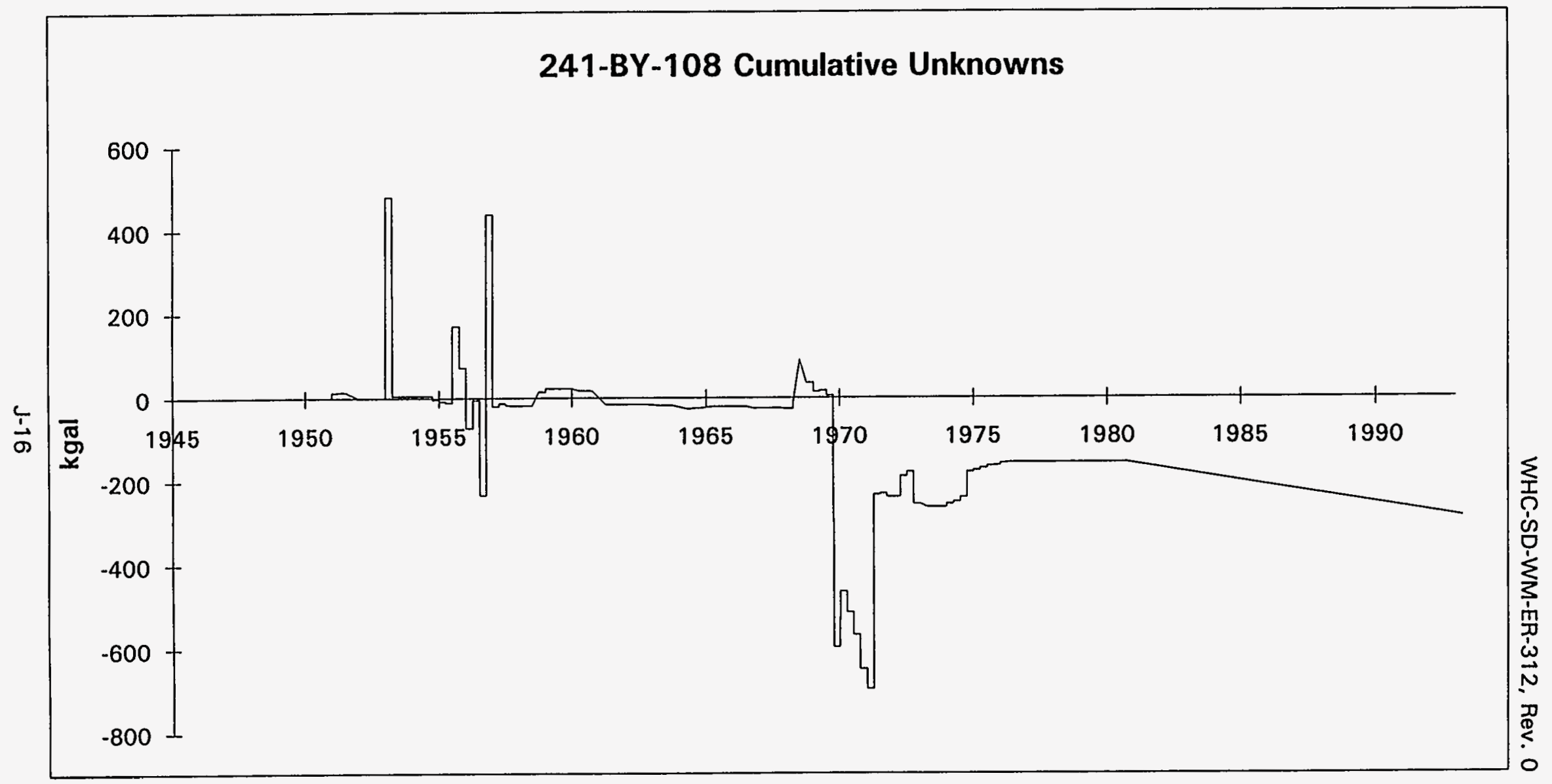

Derived from LANL Waste Status and Transaction Record Summary V2.0b (Agnew 1994) 
WHC-SD-WM-ER-312, Rev. 0

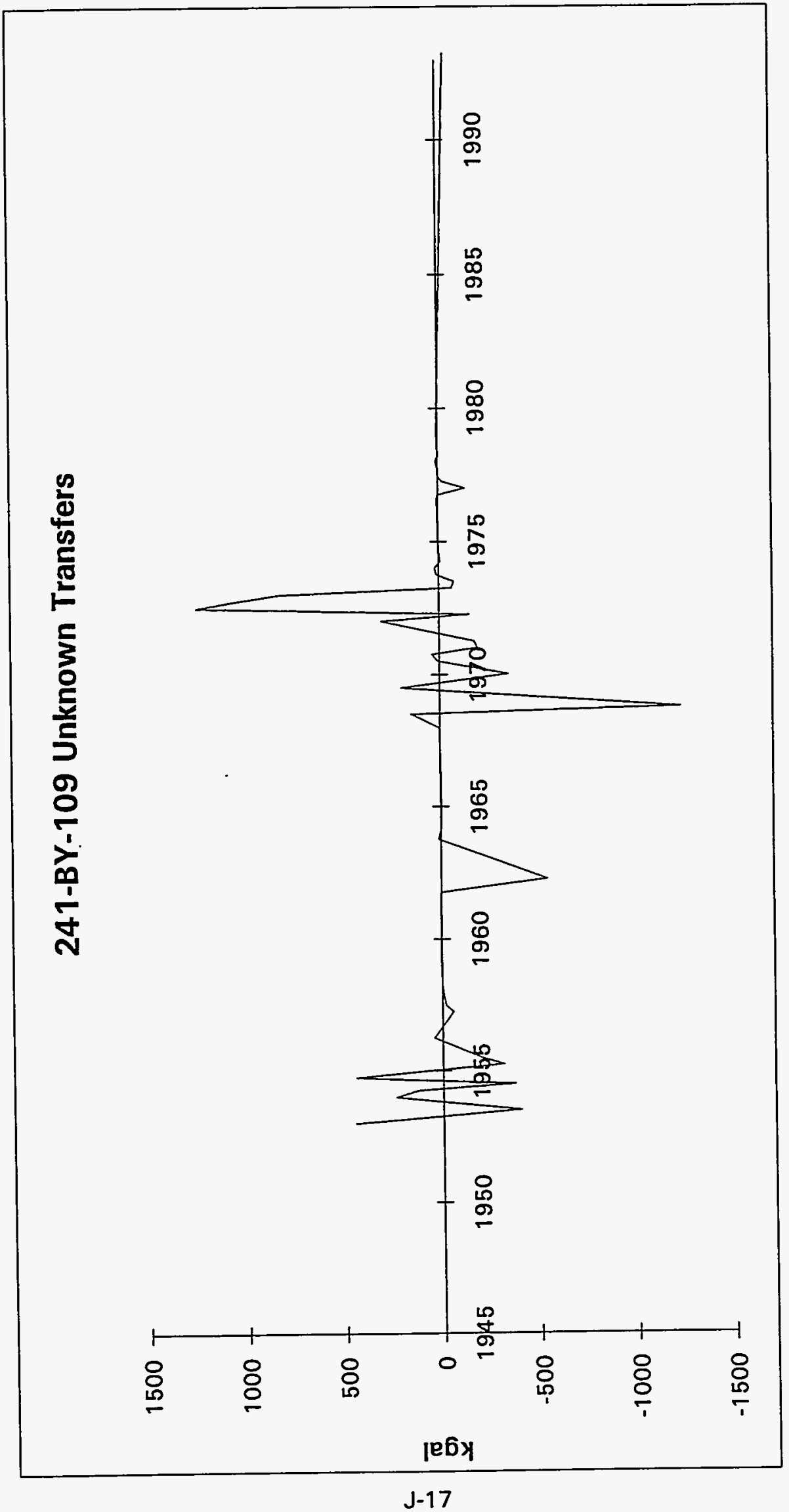

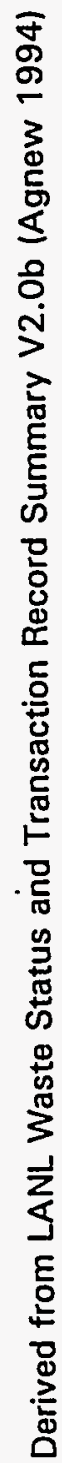


WHC-SD-WM-ER-312, Rev. 0

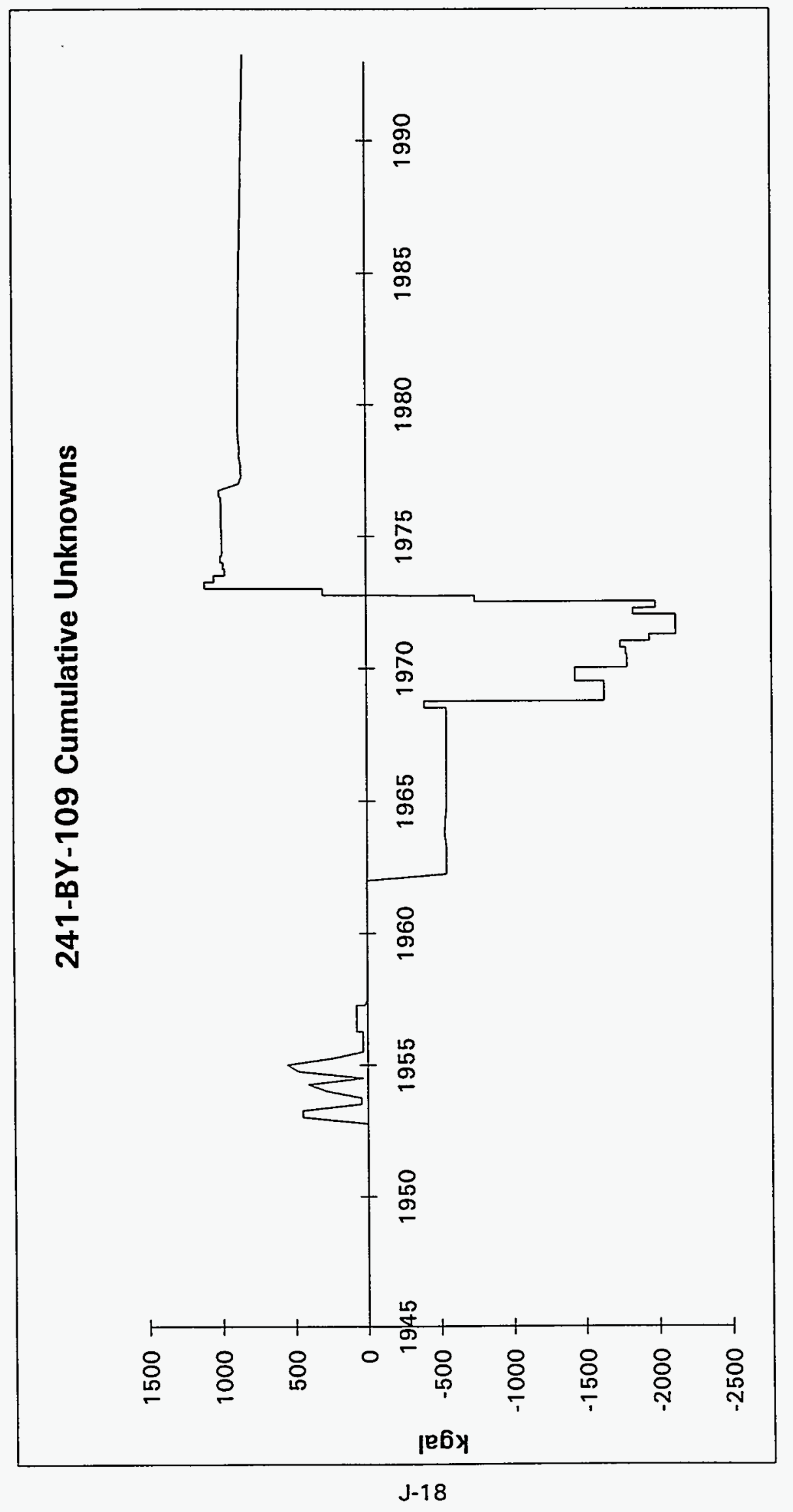

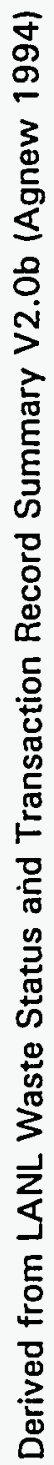


WHC-SD-WM-ER-312, Rev. 0

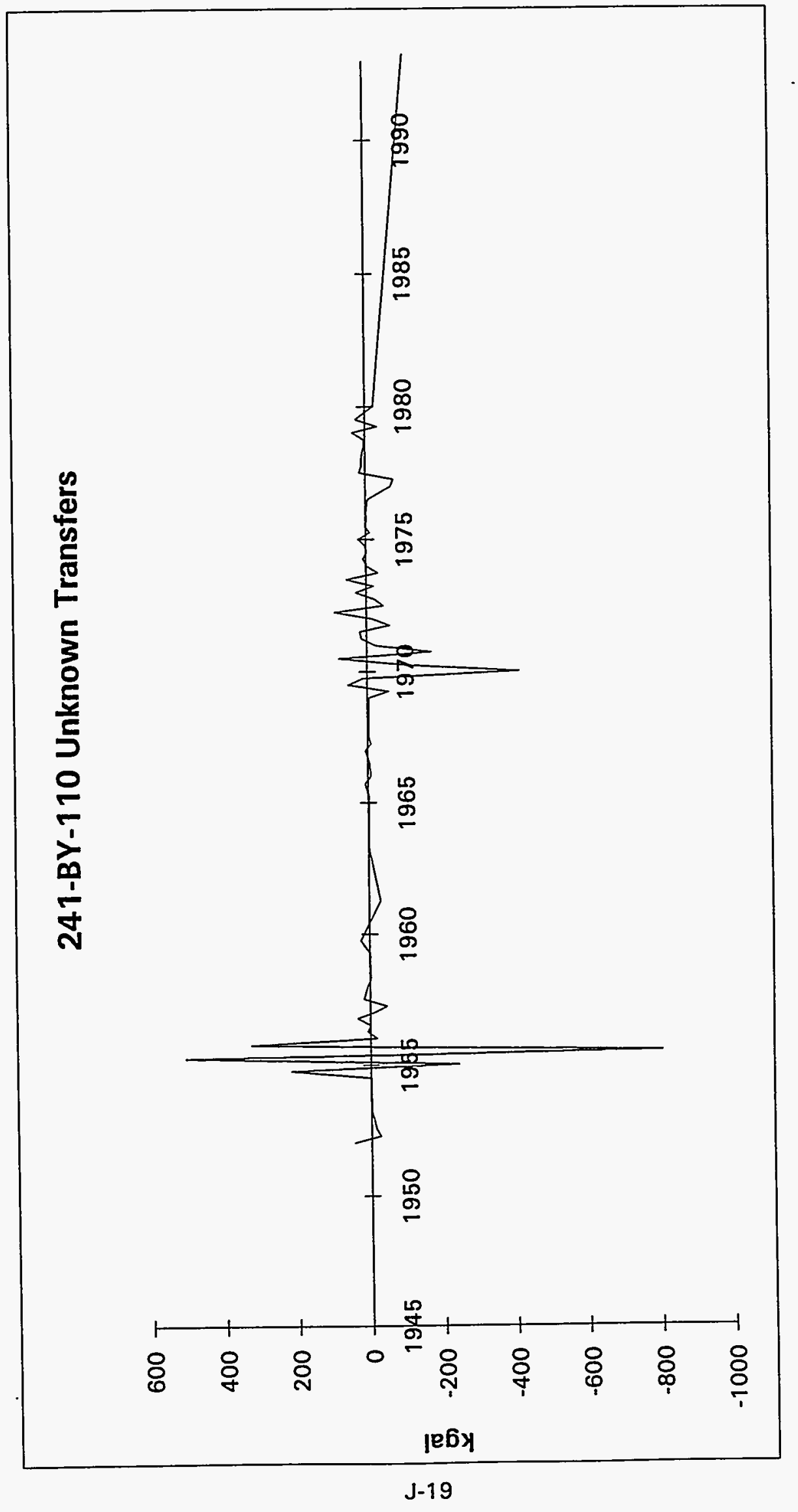




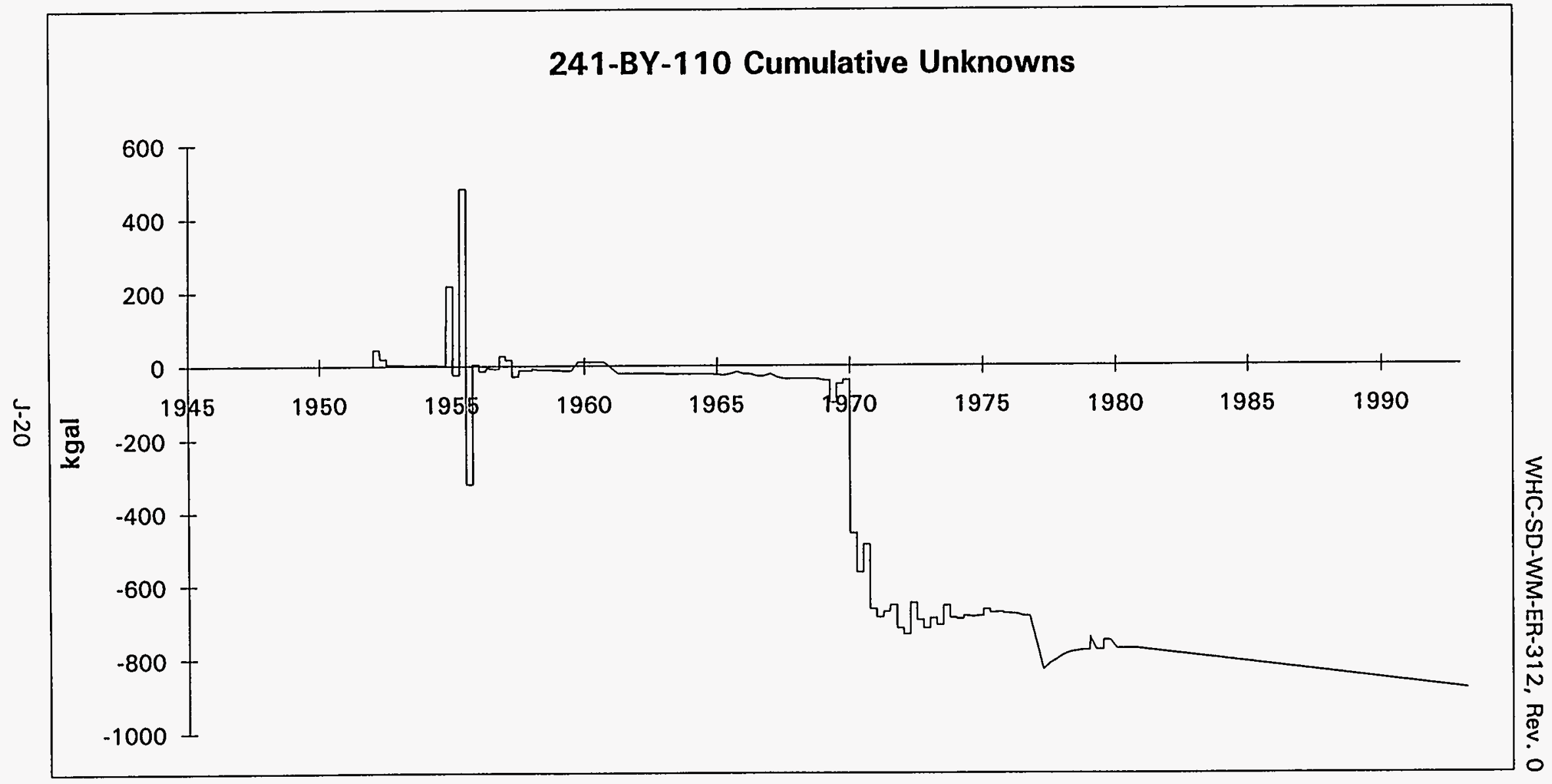

Derived from LANL Waste Status and Transaction Record Summary V2.0b (Agnew 1994) 
WHC-SD-WM-ER-312, Rev. 0

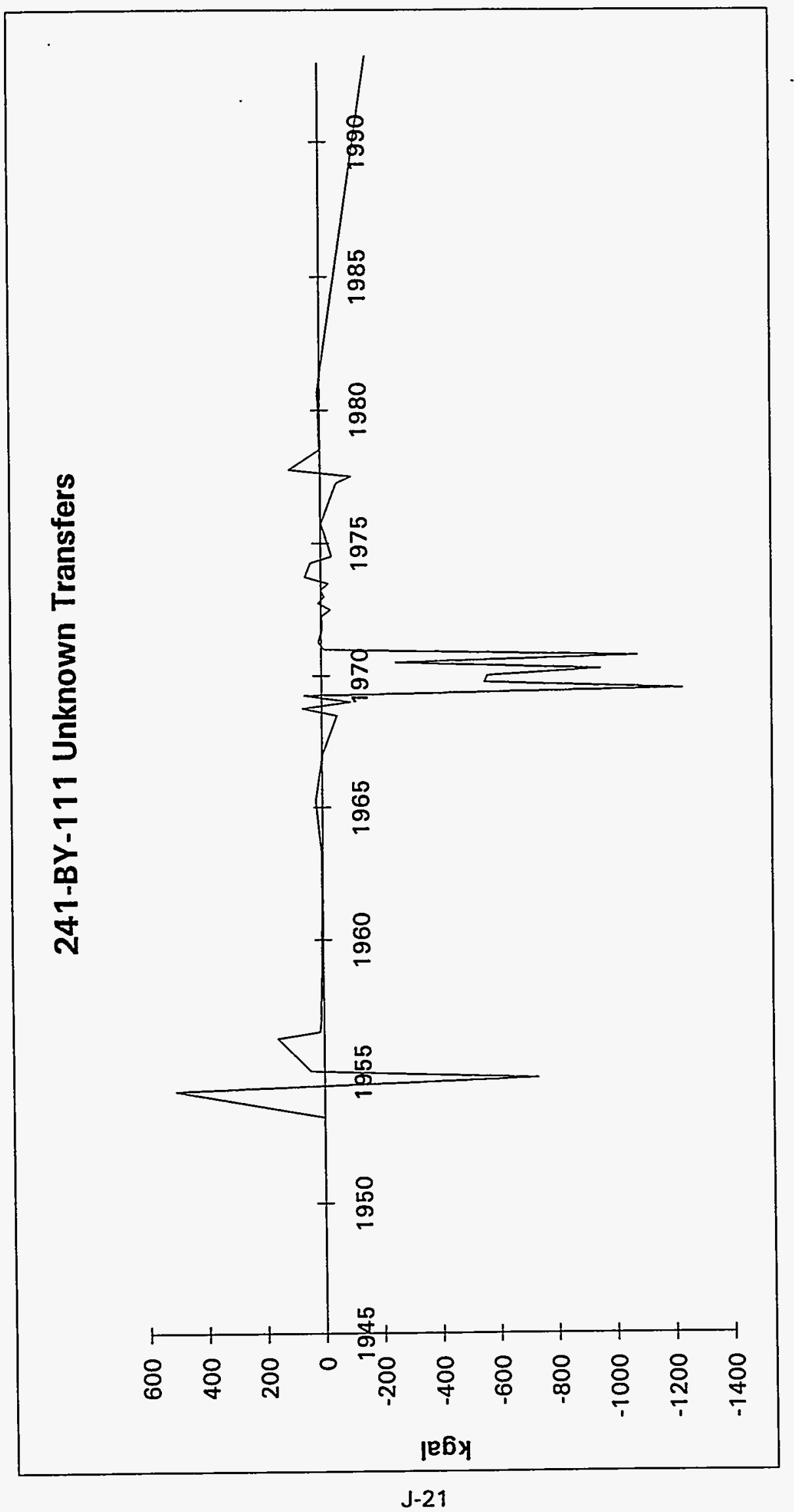

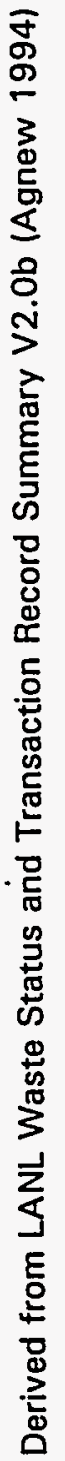


WHC-SD-WM-ER-312, Rev. 0

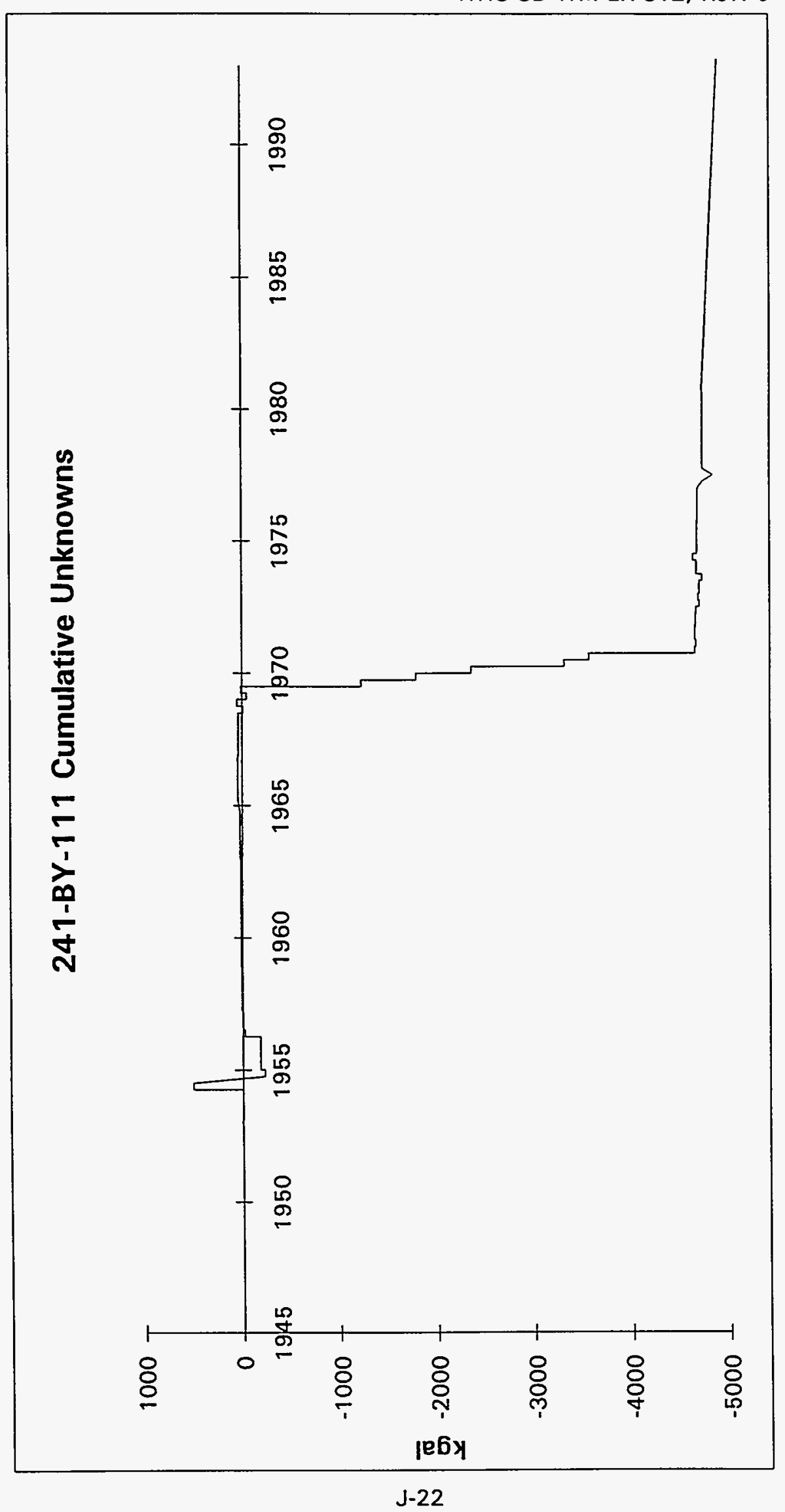

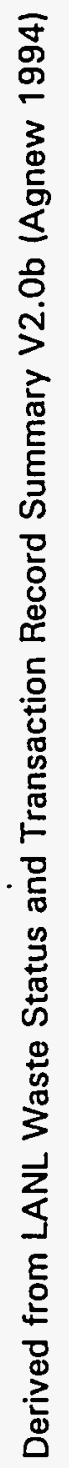


WHC-SD-WM-ER-312, Rev. 0

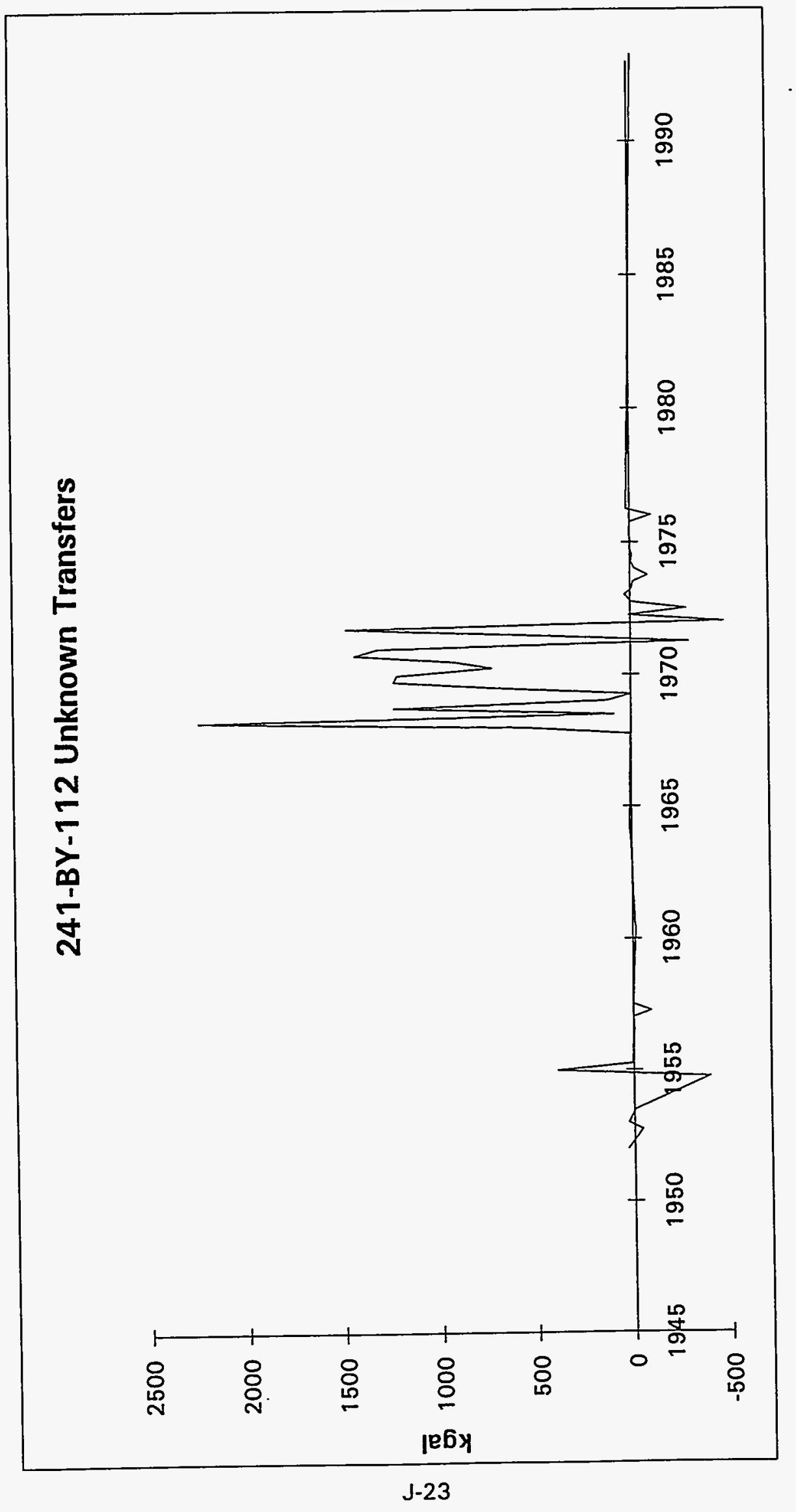


WHC-SD-WM-ER-312, Rev. 0

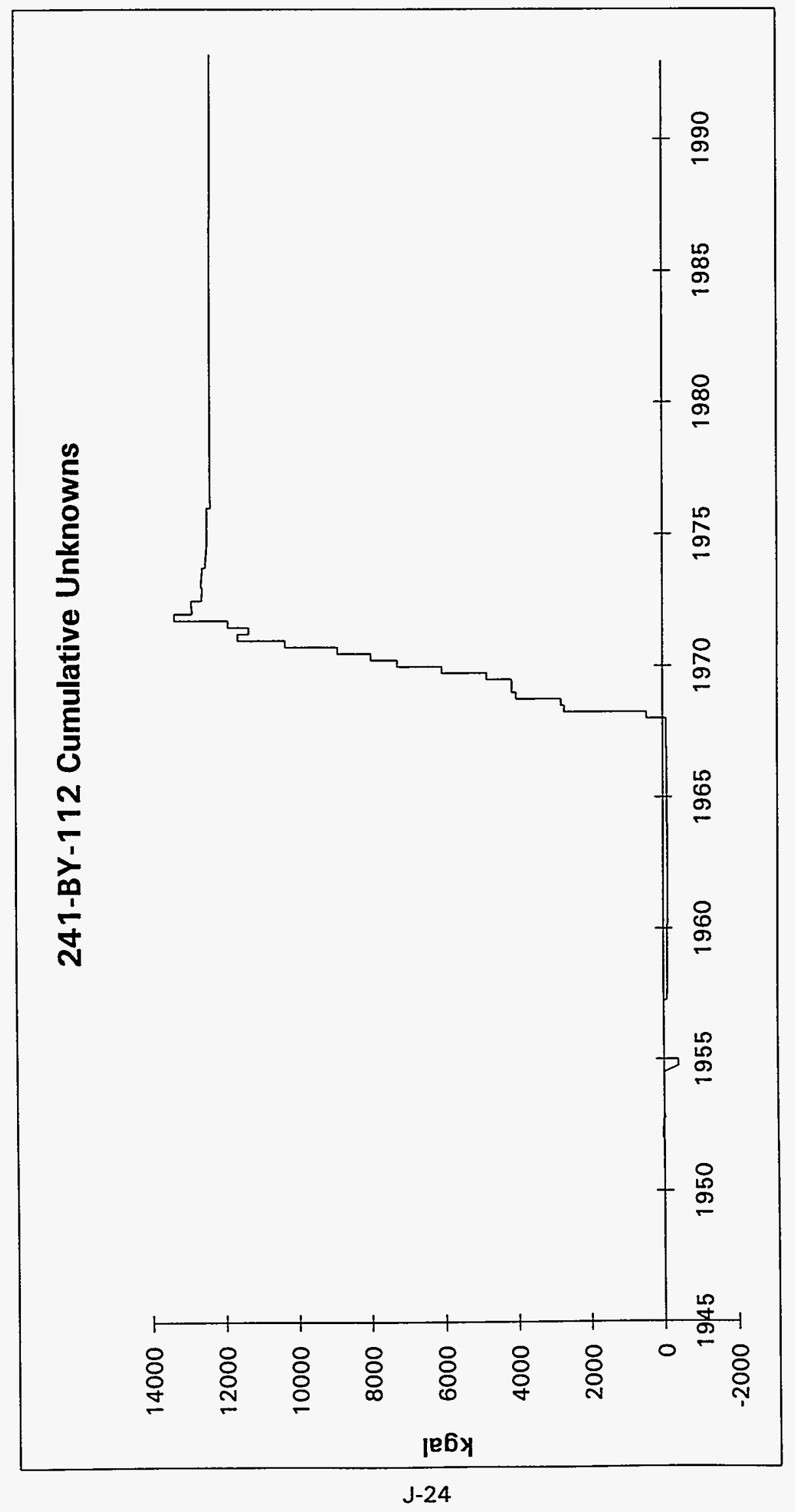

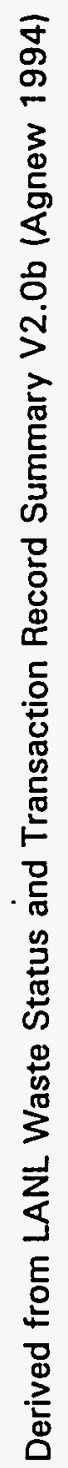




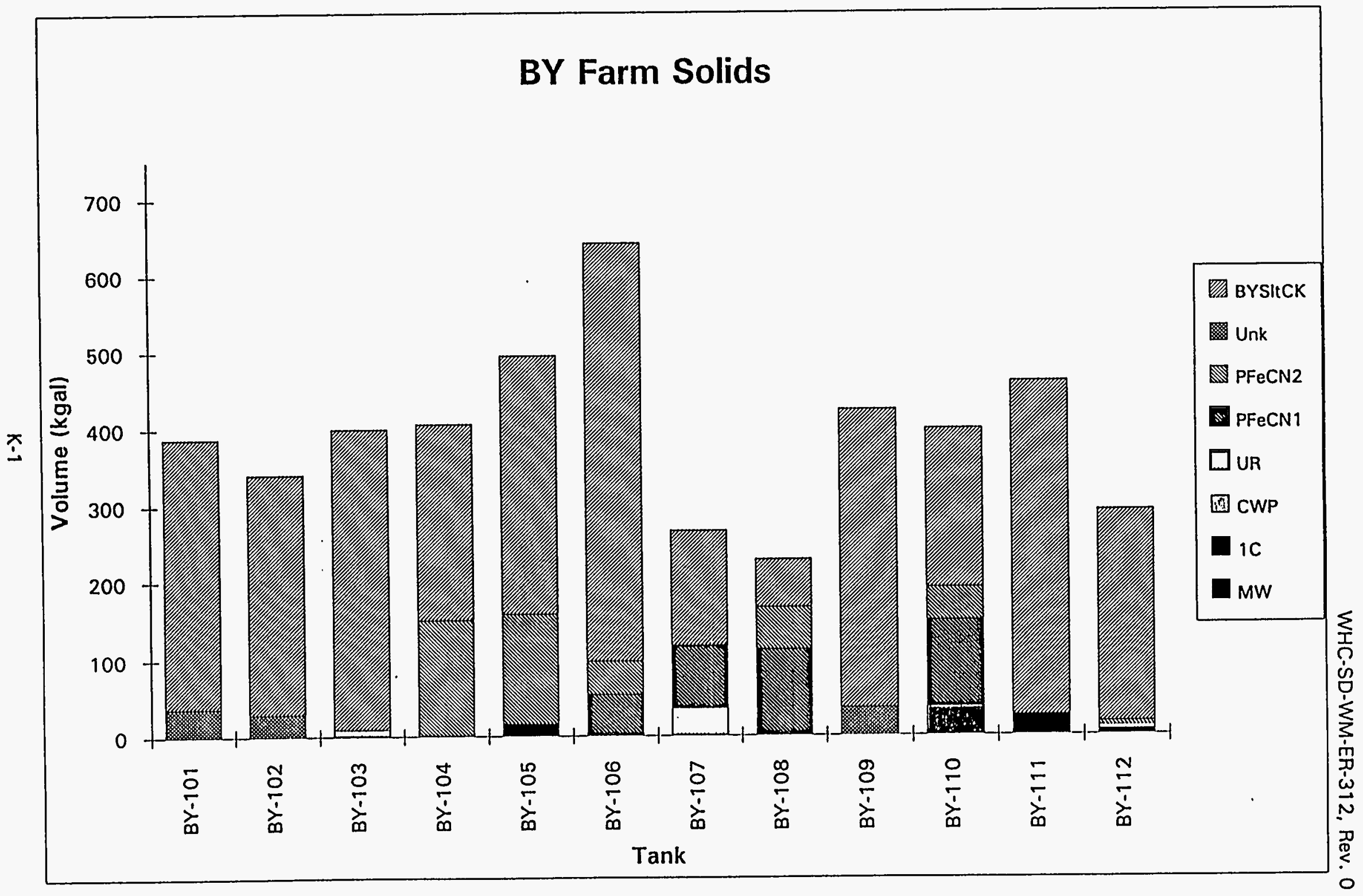

Derived from LANL Waste Status and Transaction Record Summary V2.0b (Agnew 1994) 


\begin{tabular}{|c|c|c|c|c|c|c|c|}
\hline Tank & Year & Qtr & $\begin{array}{l}\text { Meas. } \\
\text { Solids }\end{array}$ & $\begin{array}{c}\text { Solids } \\
\text { Change }\end{array}$ & $\begin{array}{c}\text { Predicted } \\
\text { Layer }\end{array}$ & $\begin{array}{l}\text { Layer } \\
\text { Type }\end{array}$ & $\begin{array}{l}\text { Waste } \\
\text { Volume } \\
\text { (K Gal) }\end{array}$ \\
\hline BY -101 & 1963 & 2 & 37 & 37 & \multicolumn{2}{|c|}{37 unk } & \\
\hline & 1967 & 3 & 109 & & & & \\
\hline & 1967 & 4 & 376 & & & & \\
\hline & 1968 & 1 & 378 & & & & \\
\hline & 1968 & 4 & 29 & & & & \\
\hline & 1969 & 1 & 281 & & & & \\
\hline & 1969 & 2 & 274 & & & & \\
\hline & 1969 & 3 & 310 & & & & \\
\hline & 1969 & 4 & 332 & & & & \\
\hline & 1970 & 1 & 337 & & & & \\
\hline & 1970 & 4 & 340 & & & & \\
\hline & 1971 & 2 & 386 & & & & \\
\hline & 1971 & 4 & 398 & & & & \\
\hline & 1972 & 3 & 418 & & & & \\
\hline & 1974 & 4 & 398 & & & & \\
\hline & 1977 & 2 & 439 & & & & \\
\hline & 1993 & 2 & 387 & 350 & 350 & BYSItCk & \\
\hline & & & & & & & \\
\hline$B Y-102$ & 1957 & 3 & 18 & 18 & 18 & Unk & \\
\hline & 1963 & 2 & 29 & 11 & 11 & Unk & \\
\hline & 1968 & 3 & 314 & & & & \\
\hline & 1968 & 4 & 250 & & & & \\
\hline & 1971 & 2 & 155 & & & & \\
\hline & 1972 & 1 & 197 & & & & \\
\hline & 1972 & 4 & 246 & & & & \\
\hline & 1973 & 1 & 194 & & & & \\
\hline & 1973 & 4 & 208 & & & & \\
\hline & 1974 & 4 & 200 & & & & \\
\hline & 1977 & 2 & 233 & & & & \\
\hline & 1977 & 3 & 436 & & & & \\
\hline & 1978 & 2 & 417 & & & & \\
\hline & 1993 & 2 & 341 & 312 & 312 & BYSItCk & \\
\hline & & & & & & & \\
\hline & & & & & & & \\
\hline & & & & & & & \\
\hline & & & & & & & \\
\hline & & & & & & & \\
\hline
\end{tabular}


WHC-SD-WM-ER-312, Rev. 0

\begin{tabular}{|c|c|c|c|c|c|c|c|}
\hline Tank & Year & Qtr & $\begin{array}{l}\text { Meas. } \\
\text { Solids }\end{array}$ & $\begin{array}{c}\text { Solids } \\
\text { Change }\end{array}$ & $\begin{array}{c}\text { Predicted } \\
\text { Layer }\end{array}$ & $\begin{array}{l}\text { Layer } \\
\text { Type }\end{array}$ & $\begin{array}{c}\text { Waste } \\
\text { Volume } \\
\text { (K Gal) } \\
\end{array}$ \\
\hline BY-103 & 1963 & 2 & 21 & & & & \\
\hline & 1966 & 3 & & 34 & & CWP & 1161 \\
\hline & 1969 & 1 & 8 & & & & \\
\hline & 1969 & 3 & 1 & & & & \\
\hline & 1969 & 4 & 8 & & & & \\
\hline & 1970 & 1 & 3 & & & & \\
\hline & 1970 & 2 & 4 & & & & \\
\hline & 1970 & 4. & 9 & -25 & 9 & CWP & \\
\hline & 1971 & 1 & 65 & & & & \\
\hline & 1971 & 2 & 128 & & & & \\
\hline & 1971 & 3 & 142 & & & & \\
\hline & 1971 & 4 & 202 & & & & \\
\hline & 1972 & 1 & 298 & & & & \\
\hline & 1972 & 2 & 351 & & & & \\
\hline & 1972 & 3 & 428 & & & & \\
\hline & 1973 & 1 & 469 & & & & \\
\hline & 1973 & 3 & 458 & & & & \\
\hline & 1974 & 3 & 469 & & & & \\
\hline & 1974 & 4 & 461 & & & & \\
\hline & 1980 & 4 & 458 & & & & \\
\hline & 1993 & 2 & 400 & 391 & 391 & BYSltCK & \\
\hline & & & & & & & \\
\hline & & & & & & & \\
\hline & & & & & & & \\
\hline & & & & & & & \\
\hline & & & & & & & \\
\hline & & & & & & & \\
\hline & & & & & & & \\
\hline & & & & & & & \\
\hline & & & & & & & \\
\hline & & & & & & & \\
\hline & & & & & & & \\
\hline & & & & & & & \\
\hline & & & & & & & \\
\hline & & & & & & & \\
\hline & & & & & & & \\
\hline & & & & & & & \\
\hline
\end{tabular}


WHC-SD-WM-ER-312, Rev, O

\begin{tabular}{|c|c|c|c|c|c|c|c|}
\hline Tank & Year & Qtr & $\begin{array}{l}\text { Meas. } \\
\text { Solids }\end{array}$ & $\begin{array}{c}\text { Solids } \\
\text { Change }\end{array}$ & $\begin{array}{c}\text { Predicted } \\
\text { Layer }\end{array}$ & $\begin{array}{l}\text { Layer } \\
\text { Type }\end{array}$ & $\begin{array}{l}\text { Waste } \\
\text { Volume } \\
\text { (K Gal) }\end{array}$ \\
\hline BY-104 & 1956 & 3 & 303 & & & $\mathrm{PFeCN} / 2$ & 317 \\
\hline & 1956 & 4 & 323 & & & & \\
\hline & 1957 & 2 & 277 & & & & \\
\hline & 1957 & 3 & 244 & & & & \\
\hline & 1963 & 2 & 263 & & & $\mathrm{PFeCN} / 2$ & 226 \\
\hline & 1965 & 2 & 227 & 227 & & $\mathrm{PFeCN} / 2$ & \\
\hline & 1967 & 2 & 205 & & & & \\
\hline & 1967 & 3 & 244 & & & & \\
\hline & 1968 & 2 & 212 & & & & \\
\hline & 1969 & 1 & 244 & & & & \\
\hline & 1969 & 2 & 233 & & & & \\
\hline & 1969 & 3 & 244 & & & & \\
\hline & 1969 & 4 & 240 & & & & \\
\hline & 1970 & 1 & 206 & & & & \\
\hline & 1970 & 2 & 44 & & & & \\
\hline & 1970 & 3 & 112 & & & & \\
\hline & 1970 & 4 & 131 & & & & \\
\hline & 1971 & 1 & 150 & -77 & 150 & $\mathrm{PFeCN} / 2$ & \\
\hline & 1971 & 3 & 425 & & & & \\
\hline & 1971 & 4 & 618 & & & & \\
\hline & 1972 & 3 & 310 & & & & \\
\hline & 1973 & 1 & 469 & & & & \\
\hline & 1973 & 4 & 628 & & & & \\
\hline & 1974 & 1 & 469 & & & & \\
\hline & 1977 & 3 & 623 & & & & \\
\hline & 1993 & 2 & 406 & 256 & 256 & BYSltCk & \\
\hline & & & & & & & \\
\hline & & & & & & & \\
\hline & & & & & & & \\
\hline & & & & & & & \\
\hline & & & & & & & \\
\hline & & & & & & & \\
\hline & & & & & & & \\
\hline & & & & & & & \\
\hline & & & & & & & \\
\hline & & & & & & & \\
\hline & & & & & & & \\
\hline
\end{tabular}

Derived from LANL Waste Status and Transaction Record Summary V2.Ob (Agnew 1994) K-4 
WHC-SD-WM-ER-312, Rev. 0

\begin{tabular}{|c|c|c|c|c|c|c|c|}
\hline Tank & Year & Qtr & $\begin{array}{l}\text { Meas. } \\
\text { Solids }\end{array}$ & $\begin{array}{c}\text { Solids } \\
\text { Change }\end{array}$ & $\begin{array}{c}\text { Predicted } \\
\text { Layer }\end{array}$ & $\begin{array}{l}\text { Layer } \\
\text { Type }\end{array}$ & $\begin{array}{l}\text { Waste } \\
\text { Volume } \\
\text { (K Gal) }\end{array}$ \\
\hline BY-105 & 1954 & 4 & 14 & & & & \\
\hline & 1955 & 1 & 16 & 16 & 16 & $\mathrm{MW}$ & \\
\hline & 1955 & 4 & & & & $\longdiv { \mathrm { PFeCN } 1 }$ & 91 \\
\hline & 1956 & 1 & 350 & & & & 51 \\
\hline & 1956 & 2 & & & & PFeCN2 & 245 \\
\hline & 1956 & 2 & 384 & & & & \\
\hline & 1957 & 3 & 213 & & & & \\
\hline & 1963 & 2 & 222 & 206 & & $\longdiv { \mathrm { PFeCN } 2 }$ & \\
\hline & 1965 & 2 & 186 & & & & \\
\hline & 1967 & 3 & 39 & & & & \\
\hline & 1967 & 4 & 88 & & & & \\
\hline & 1968 & 3 & 158 & -64 & 142 & $\mathrm{PFeCN} 2$ & \\
\hline & 1969 & 1 & 165 & & & & \\
\hline & 1969 & 2 & 153 & & & & \\
\hline & 1969 & 3 & 194 & & & & \\
\hline & 1969 & 4 & 189 & & & & \\
\hline & 1970 & 1 & 191 & & & & \\
\hline & 1970 & 2 & 216 & & & & \\
\hline & 1970 & 3 & 299 & & & & \\
\hline & 1971 & 1 & 340 & & & & \\
\hline & 1971 & 2 & 469 & & & & \\
\hline & 1971 & 4 & 653 & & & & \\
\hline & 1972 & 3 & 591 & & & & \\
\hline & 1973 & 1 & 628 & & & & \\
\hline & 1974 & 4 & 626 & & & & \\
\hline & 1977 & 1 & & & 337 & BYSltCk & \\
\hline & 1993 & 2 & 503 & -345 & 8 & Cem & 8 \\
\hline & & & & & & & \\
\hline & & & & & & & \\
\hline & & & & & & & \\
\hline & & & & & & & \\
\hline & & & & & & & \\
\hline & & & & & & & \\
\hline & & & & & & & \\
\hline & & & & & & & \\
\hline & & & & & & & \\
\hline & & & & & & & \\
\hline
\end{tabular}




\begin{tabular}{|c|c|c|c|c|c|c|c|}
\hline Tank & Year & Qtr & $\begin{array}{l}\text { Meas. } \\
\text { Solids }\end{array}$ & $\begin{array}{c}\text { Solids } \\
\text { Change } \\
\end{array}$ & $\begin{array}{c}\text { Predicted } \\
\text { Layer }\end{array}$ & $\begin{array}{l}\text { Layer } \\
\text { Type }\end{array}$ & $\begin{array}{c}\text { Waste } \\
\text { Volume } \\
\text { (K Gal) }\end{array}$ \\
\hline$B Y-106$ & 1955 & 1 & & 54 & & PFeCN1 & 1461 \\
\hline & 1955 & 1 & 40 & & & & \\
\hline & 1955 & 2 & 107 & & 54 & PFeCN1 & \\
\hline & 1955 & 4 & 120 & & & & \\
\hline & 1956 & 1 & 150 & & & & \\
\hline & 1956 & 2 & 180 & & & & \\
\hline & 1956 & 3 & 164 & & & & \\
\hline & 1956 & 4 & 168 & & & & -179 \\
\hline & 1957 & 1 & & 154 & & PFeCN2 & 4810 \\
\hline & 1957 & 1 & 111 & -97 & & PFeCN2 & \\
\hline & 1963 & 2 & 150 & & & & \\
\hline & 1965 & 2 & 103 & & & & \\
\hline & 1969 & 1 & 105 & & & & \\
\hline & 1969 & 2 & 93 & & & & \\
\hline & 1969 & 3 & 96 & & & & \\
\hline & 1969 & 4 & 95 & & & & \\
\hline & 1970 & 1 & 103 & & & & \\
\hline & 1970 & 2 & 96 & & & & \\
\hline & 1970 & 3 & 98 & -13 & 44 & PFeCN2 & \\
\hline & 1970 & 4 & 164 & & & & \\
\hline & 1971 & 1 & 249 & & & & \\
\hline & 1971 & 2 & 288 & & & & \\
\hline & 1972 & 1 & 296 & & & & \\
\hline & 1972 & 4 & 563 & & & & \\
\hline & 1973 & 1 & 447 & & & & \\
\hline & 1973 & 2 & 494 & & & & \\
\hline & 1974 & 4 & 420 & & & & \\
\hline & 1975 & 2 & 351 & & & & \\
\hline & 1977 & 1 & 593 & & & & \\
\hline & 1977 & 3 & 626 & & & & \\
\hline & 1993 & 2 & 642 & 544 & 544 & BYSItCK & \\
\hline & & & & & & & \\
\hline & & & & & & & \\
\hline & & & & & & & \\
\hline & & & & & & & \\
\hline & & & & & & & \\
\hline & & & & & & & \\
\hline
\end{tabular}

Derived from LANL Waste Status and Transaction Record Summary V2.Ob (Agnew 1994) 


\begin{tabular}{|c|c|c|c|c|c|c|c|}
\hline Tank & Year & Qtr & $\begin{array}{l}\text { Meas. } \\
\text { Solids }\end{array}$ & $\begin{array}{l}\text { Solids } \\
\text { Change }\end{array}$ & $\begin{array}{c}\text { Predicted } \\
\text { Layer }\end{array}$ & $\begin{array}{l}\text { Layer } \\
\text { Type }\end{array}$ & $\begin{array}{l}\text { Waste } \\
\text { Volume } \\
\text { (K Gal) }\end{array}$ \\
\hline BY-107 & 1951 & 3 & & 376 & & $1 \mathrm{C}$ & 1511 \\
\hline & 1953 & 1 & 1 & -375 & 1 & $1 \mathrm{C}$ & -757 \\
\hline & 1953 & 2 & & 35 & & IUR & 711 \\
\hline & 1954 & 3 & 44 & 8 & 34 & UR & \\
\hline & 1954 & 4 & 1 & -9 & & & \\
\hline & 1955 & 1 & 72 & & & & \\
\hline & 1955 & 2 & 45 & & & & \\
\hline & 1955. & 3 & & 145 & & PFeCN1 & 3916 \\
\hline & 1955 & 3 & 142 & & & & \\
\hline & 1955 & 4 & 147 & -33 & & PFeCN1 & \\
\hline & 1956 & 1 & 160 & & & & \\
\hline & 1956 & 2 & 168 & & & & \\
\hline & 1956 & 3 & 87 & & & & -191 \\
\hline & 1956 & 4 & 150 & & & & \\
\hline & 1957 & 2 & & 186 & & PFeCN2 & 5797 \\
\hline & 1957 & 3 & 178 & & & & \\
\hline & 1957 & 4 & 172 & & & & \\
\hline & 1958 & 4 & 150 & & & & \\
\hline & 1963 & 2 & 178 & & & & \\
\hline & 1965 & 2 & 150 & 115 & & PFeCN2 & \\
\hline & 1970 & 1 & 58 & & & & \\
\hline & 1970 & 2 & 61 & & & & \\
\hline & 1970 & 3 & 43 & & & & \\
\hline & 1970 & 4 & 106 & & & & \\
\hline & 1971 & 1 & 117 & -33 & 82 & $\mathrm{PFeCN1}$ & \\
\hline & 1973 & 1 & 175 & & & & \\
\hline & 1974 & 2 & 444 & & & & \\
\hline & 1974 & 4 & 183 & & & & \\
\hline & 1975 & 1 & 367 & & & & \\
\hline & 1978 & 1 & 326 & & & & \\
\hline & 1993 & 2 & 266 & 149 & 149 & BYSItCK & \\
\hline & & & & & & & \\
\hline & & & & & & & \\
\hline & & & & & & & \\
\hline & & & & & & & \\
\hline & & & & & & & \\
\hline & & & & & & & \\
\hline
\end{tabular}

Derived from LANL Waste Status and Transaction Record Summary V2.Ob (Agnew 1994) K-7 
WHC-SD-WM-ER-312, Rev. 0

\begin{tabular}{|c|c|c|c|c|c|c|c|}
\hline Tank & Year & Qtr & $\begin{array}{l}\text { Meas. } \\
\text { Solids }\end{array}$ & $\begin{array}{l}\text { Solids } \\
\text { Change }\end{array}$ & $\begin{array}{c}\text { Predicted } \\
\text { Layer }\end{array}$ & $\begin{array}{l}\text { Layer } \\
\text { Type }\end{array}$ & $\begin{array}{l}\text { Waste } \\
\text { Volume } \\
\text { (K Gal) }\end{array}$ \\
\hline \multirow[t]{2}{*}{ BY -108} & 1955 & 1 & 33 & & & & \\
\hline & 1955 & 2 & 98 & & & & \\
\hline & 1955 & 3 & 197 & & & & \\
\hline & 1955 & 4 & & 112 & 112 & PFeCN1 & 3018 \\
\hline & 1955 & 4 & & & & & -91 \\
\hline & 1955 & 4 & 200 & & & & \\
\hline & 1956 & 1 & 205 & & & & \\
\hline & 1956 & 2 & 179 & & & & \\
\hline & 1956 & 3 & 194 & & & & \\
\hline & 1956 & 4 & 201 & & & & -256 \\
\hline & 1957 & 1 & & 183 & & $\mathrm{PFeCN} 2$ & 5727 \\
\hline & 1957 & 2 & 249 & & & & \\
\hline & 1957 & 3 & 213 & & & & -64 \\
\hline & 1957 & 4 & 210 & & & & \\
\hline & 1963 & 2 & 202 & -93 & & & \\
\hline & 1965 & 2 & 178 & & & & \\
\hline & 1968 & 3 & 125 & & & & \\
\hline & 1969 & 1 & 165 & -37 & 53 & PFeCN2 & \\
\hline & 1969 & 2 & 222 & & & & \\
\hline & 1969 & 3 & 194 & & & & \\
\hline & 1969 & 4 & 233 & & & & \\
\hline & 1970 & 1 & 224 & & & & \\
\hline & 1970 & 2 & 176 & & & & \\
\hline & 1970 & 3 & 194 & & & & \\
\hline & 1970 & 4 & 65 & & & & \\
\hline & 1971 & 1 & 98 & & & & \\
\hline & 1971 & 2 & 385 & & & & \\
\hline & 1971 & 3 & 388 & & & & \\
\hline & 1971 & 4 & 381 & & & & \\
\hline & 1972 & 4 & 304 & & & & \\
\hline & 1974 & 4 & 359 & & & & \\
\hline & 1993 & 2 & 228 & 63 & 63 & BYSItCK & \\
\hline & & & & & & & \\
\hline & & & & & & & \\
\hline & & & & & & & \\
\hline & & & & & & & \\
\hline & & & & & & & \\
\hline
\end{tabular}

Derived from LANL Waste Status and Transaction Record Summary V2.Ob (Agnew 1994) 


\begin{tabular}{|c|c|c|c|c|c|c|c|}
\hline Tank & Year & Qtr & $\begin{array}{l}\text { Meas. } \\
\text { Solids }\end{array}$ & $\begin{array}{l}\text { Solids } \\
\text { Change }\end{array}$ & $\begin{array}{c}\text { Predicted } \\
\text { Layer }\end{array}$ & $\begin{array}{l}\text { Layer } \\
\text { Type }\end{array}$ & $\begin{array}{l}\text { Waste } \\
\text { Volume } \\
\text { (K Gal) }\end{array}$ \\
\hline BY-109 & 1956 & 3 & 36 & 36 & 36 & Unk & \\
\hline & 1956 & 4 & 66 & & & & \\
\hline & 1957 & 3 & 46 & & & & \\
\hline & 1963 & 2 & 76 & & & & \\
\hline & 1965 & 2 & 57 & & & & \\
\hline & 1968 & 3 & 37 & & & & \\
\hline & 1968 & 4 & 54 & & & & \\
\hline & 1969. & 1 & 72 & & & & \\
\hline & 1969 & 2 & 87 & & & & \\
\hline & 1969 & 3 & 79 & & & & \\
\hline & 1969 & 4 & 114 & & & & \\
\hline & 1970 & 1 & 190 & & & & \\
\hline & 1970 & 2 & 120 & & & & \\
\hline & 1970 & 3 & 128 & & & & \\
\hline & 1970 & 4. & 142 & & & & \\
\hline & 1971 & 1 & 150 & & & & \\
\hline & 1971 & 2 & 128 & & & & \\
\hline & 1971 & 4 & 153 & & & & \\
\hline & 1972 & 2 & 222 & & & & \\
\hline & 1972 & 3 & 260 & & & & \\
\hline & 1972 & 4 & 334 & & & & \\
\hline & 1973 & 1 & 381 & & & & \\
\hline & 1973 & 2 & 398 & & & & \\
\hline & 1973 & 4 & 411 & & & & \\
\hline & 1974 & 2 & 433 & & & & \\
\hline & 1974 & 4 & 425 & & & & \\
\hline & 1977 & 2 & 433 & & & & \\
\hline & 1993 & 2 & 423 & 387 & 387 & BYSitCk & \\
\hline & & & & & & & \\
\hline & & & & & & & \\
\hline & & & & & & & \\
\hline & & & & & & & \\
\hline & & & & & & & \\
\hline & & & & & & & \\
\hline & & & & & & & \\
\hline & & & & & & & \\
\hline & & & & & & & \\
\hline
\end{tabular}

Derived from LANL Waste Status and Transaction Record Summary V2.Ob (Agnew 1994) K-9 
WHC-SD-WM-ER-312, Rev. 0

\begin{tabular}{|c|c|c|c|c|c|c|c|}
\hline Tank & Year & Qtr & $\begin{array}{l}\text { Meas. } \\
\text { Solids }\end{array}$ & $\begin{array}{c}\text { Solids } \\
\text { Change }\end{array}$ & $\begin{array}{c}\text { Predicted } \\
\text { Layer }\end{array}$ & $\begin{array}{l}\text { Layer } \\
\text { Type }\end{array}$ & $\begin{array}{l}\text { Waste } \\
\text { Volume } \\
\text { (K Gal) }\end{array}$ \\
\hline BY-110 & 1952 & 3 & & 181 & & $1 \mathrm{C}$ & 728 \\
\hline & 1954 & 4 & 51 & -144 & 37 & $1 \mathrm{C}$ & \\
\hline & 1955 & 1 & 121 & & & & \\
\hline & 1955 & 2 & 85 & & & & \\
\hline & 1955 & 3 & & 114 & & PFeCN1 & 3094 \\
\hline & 1955 & 4 & 148 & -3 & 111 & PFeCN1 & \\
\hline & 1956 & 1 & 150 & & & & \\
\hline & 1956 & 3 & 211 & & & & \\
\hline & 1956 & 4 & 229 & & & & -189 \\
\hline & 1957 & 1 & 210 & & & & \\
\hline & 1957 & 2 & 205 & & & & \\
\hline & 1957 & 2 & & 185 & & $\mathrm{PFeCN} 2$ & 5777 \\
\hline & 1957 & 3 & 211 & & & & \\
\hline & 1957 & 4 & 210 & -123 & & & \\
\hline & 1963 & 2 & 246 & & & & \\
\hline & 1965 & 2 & 230 & & & & \\
\hline & 1969 & 2 & 189 & & & & \\
\hline " & 1969 & 3 & 123 & & & & \\
\hline & 1970 & 1 & 201 & & & & \\
\hline & 1970 & 2 & 190 & -20 & 42 & $\mathrm{PFeCN} 2$ & \\
\hline & 1970 & 3 & 211 & & & & \\
\hline & 1970 & 4 & 277 & & & & \\
\hline & 1974 & 4 & 296 & & & & \\
\hline & 1977 & 1 & 376 & & & & \\
\hline & 1977 & 3 & 469 & & & & \\
\hline & 1980 & 1 & 505 & & & & \\
\hline & 1993 & 2 & 398 & 208 & 208. & BYSItCk & \\
\hline & & & & & & & \\
\hline & & & & & & & \\
\hline & & & & & & & \\
\hline & & & & & & & \\
\hline & & & & & & & \\
\hline & & & & & & & \\
\hline & & & & & & & \\
\hline & & & & & & & \\
\hline & & & & & & & \\
\hline & & & & & & & \\
\hline
\end{tabular}


WHC-SD-WM-ER-312, Rev. 0

\begin{tabular}{|c|c|c|c|c|c|c|c|}
\hline Tank & Year & Qtr & $\begin{array}{l}\text { Meas. } \\
\text { Solids }\end{array}$ & $\begin{array}{c}\text { Solids } \\
\text { Change }\end{array}$ & $\begin{array}{c}\text { Predicted } \\
\text { Layer }\end{array}$ & $\begin{array}{l}\text { Layer } \\
\text { Type }\end{array}$ & $\begin{array}{c}\text { Waste } \\
\text { Volume } \\
\text { (K Gal) }\end{array}$ \\
\hline$B Y-111$ & 1952 & 1 & & 42 & & MW & 964 \\
\hline & 1952 & 4 & & 3 & & UR & 55 \\
\hline & 1955 & 1 & & & & & 390 \\
\hline & 1955 & 3 & & & & & -630 \\
\hline & 1957 & 1 & 26 & -19 & 26 & $\mathrm{MW}$ & \\
\hline & 1963 & 2 & 24 & & & & \\
\hline & 1965 & 2 & 26 & & & & \\
\hline & 1967. & 4 & & & & & 13 \\
\hline & 1968 & 3 & 145 & & & & \\
\hline & 1969 & 1 & 48 & & & & \\
\hline & 1969 & 2 & 52 & & & & \\
\hline & 1969 & 3 & 101 & & & & \\
\hline & 1969 & 4 & 142 & & & & \\
\hline & 1970 & 1 & 266 & & & & \\
\hline & 1970 & 2 & 290 & & & & \\
\hline & 1970 & 3 & 323 & & & & \\
\hline & 1970 & 4 & 673 & & & & \\
\hline & 1971 & 2 & 686 & & & & \\
\hline & 1972 & 1 & 194 & & & & \\
\hline & 1972 & 2 & 112 & & & & \\
\hline & 1972 & 3 & 142 & & & & \\
\hline & 1974 & 4 & 299 & & & & \\
\hline & 1977 & 3 & 510 & & & & \\
\hline & 1977 & 4 & 615 & & & & \\
\hline & 1980 & 4 & 622 & & & & \\
\hline & 1993 & 2 & 459 & 433 & 433 & BYSItCk & \\
\hline & & & & & & & \\
\hline & & & & & & & \\
\hline & & & & & & & \\
\hline & & & & & & & \\
\hline & & & & & & & \\
\hline & & & & & & & \\
\hline & & & & & & & \\
\hline & & & & & & & \\
\hline & & & & & & & \\
\hline & & & & & & & \\
\hline & & & & & & & \\
\hline
\end{tabular}

Derived from LANL Waste Status and Transaction Record Summary V2.0b (Agnew 1994) 


\begin{tabular}{|c|c|c|c|c|c|c|c|}
\hline Tank & Year & Otr & $\begin{array}{l}\text { Meas. } \\
\text { Solids }\end{array}$ & $\begin{array}{c}\text { Solids } \\
\text { Change }\end{array}$ & $\begin{array}{l}\text { Predicted } \\
\text { Layer }\end{array}$ & $\begin{array}{l}\text { Layer } \\
\text { Type }\end{array}$ & $\begin{array}{l}\text { Waste } \\
\text { Volume } \\
\text { (K Gal) }\end{array}$ \\
\hline \multirow{28}{*}{$B Y-112$} & 1952 & 3 & & 6 & 6 & MW & 126 \\
\hline & 1955 & 1 & & & & & -390 \\
\hline & 1957 & 2 & & 6 & 6 & PFeCN2 & 172 \\
\hline & & & & 5 & 5 & UR & 93 \\
\hline & 1963 & 2 & 29 & 12 & & & \\
\hline & 1965 & 2 & 24 & & & & \\
\hline & 1967 & 4 & & & & & 4 \\
\hline & 1968 & 2 & 17 & -12 & & & \\
\hline & 1968 & 3 & 2 & & & & \\
\hline & 1969 & 1 & 43 & & & & \\
\hline & 1969 & 2 & 59 & & & & \\
\hline & 1969 & 3 & 79 & & & & \\
\hline & 1970 & 1 & 85 & & & & \\
\hline & 1970 & 2 & 94 & & & & \\
\hline & 1970 & 3 & 145 & & & & \\
\hline & 1970 & 4 & 164 & & & & \\
\hline & 1971 & 1 & 255 & & & & \\
\hline & 1971 & 2 & 59 & & & & \\
\hline & 1971 & 3 & 166 & & & & \\
\hline & 1971 & 4 & 194 & & & & \\
\hline & 1972 & 1 & 263 & & & & \\
\hline & 1972 & 2 & 334 & & & & \\
\hline & 1972 & 4 & 315 & & & & \\
\hline & 1973 & 1 & 288 & & & & \\
\hline & 1973 & 3 & 311 & & & & \\
\hline & 1973 & 4 & 331 & & & & \\
\hline & 1974 & 4 & 310 & & & & \\
\hline & 1993 & 2 & 291 & 274 & 274 & BYSltCk & \\
\hline
\end{tabular}

